\title{
CERTS MICROGRID LABORATORY TEST BED
}

Prepared For:

California Energy Commission

Public Interest Energy Research Program

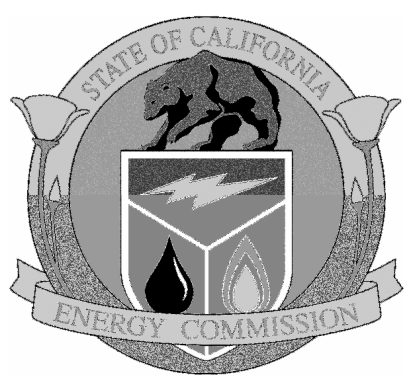

Arnold Schwarzenegger Governor

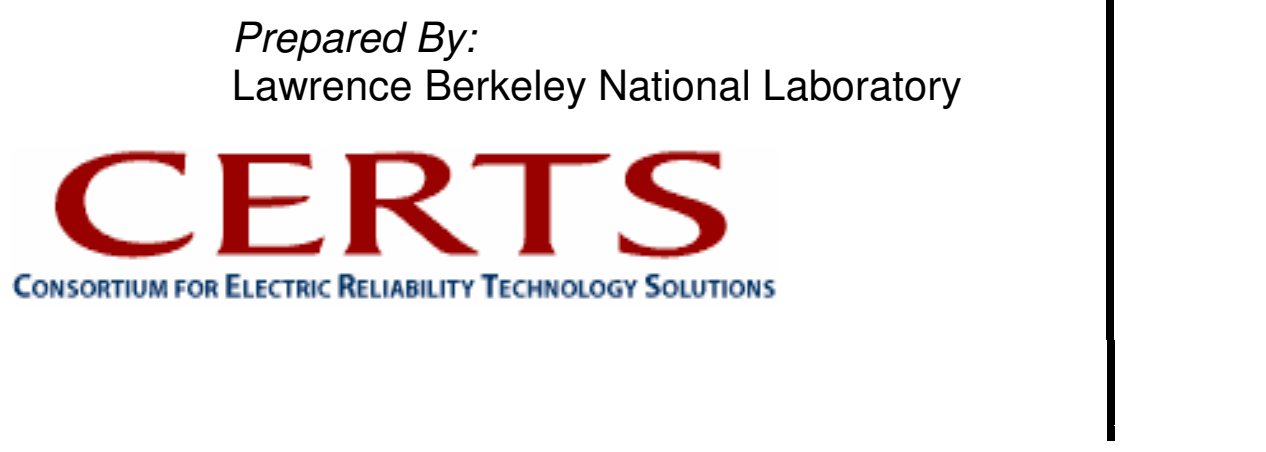





\section{Prepared By:}

Lawrence Berkeley National Laboratory

Joseph H. Eto, Principal Investigator

Berkeley, CA 94720

Robert Lasseter, University of Wisconsin

Ben Schenkman and John Stevens, Sandia National Laboratory

Harry Volkommer and Dave Klapp, American Electric Power

Ed Linton and Hector Hurtado, Northern Power Systems

Jean Roy, Tecogen, Inc.

Nancy Lewis, Lawrence Berkeley National Laboratory

Commission Contract No. 500-02-004

Commission Work Authorization No: MR-041

\section{Prepared For:}

Public Interest Energy Research (PIER)

\section{California Energy Commission}

Jamie Patterson

Contract Manager

Mike Gravely

Program Area Lead

ENERGY SYSTEMS INTEGRATION

Mike Gravely

Office Manager

ENERGY SYSTEMS RESEARCH

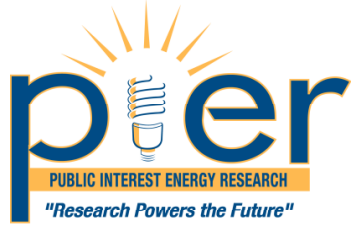

Martha Krebs, Ph.D.

PIER Director

Thom Kelly, Ph.D.

Deputy Director

ENERGY RESEARCH \& DEVELOPMENT DIVISION

Melissa Jones

Executive Director

\section{DISCLAIMER}

This report was prepared as the result of work sponsored by the California Energy Commission. It does not necessarily represent the views of the Energy Commission, its employees or the State of California. The Energy Commission, the State of California, its employees, contractors and subcontractors make no warrant, express or implied, and assume no legal liability for the information in this report; nor does any party represent that the uses of this information will not infringe upon privately owned rights. This report has not been approved or disapproved by the California Energy Commission nor has the California Energy Commission passed upon the accuracy or adequacy of the information in this report. 


\section{Disclaimer}

This document was prepared as an account of work sponsored by the United States

Government. While this document is believed to contain correct information, neither the United States Government nor any agency thereof, nor The Regents of the University of California, nor any of their employees, makes any warranty, express or implied, or assumes any legal responsibility for the accuracy, completeness, or usefulness of any information, apparatus, product, or process disclosed, or represents that its use would not infringe privately owned rights. Reference herein to any specific commercial product, process, or service by its trade name, trademark, manufacturer, or otherwise, does not necessarily constitute or imply its endorsement, recommendation, or favoring by the United States Government or any agency thereof, or The Regents of the University of California. The views and opinions of authors expressed herein do not necessarily state or reflect those of the United States Government or any agency thereof, or The Regents of the University of California.

Ernest Orlando Lawrence Berkeley National Laboratory is an equal opportunity employer. 


\section{Acknowledgments}

The authors are grateful for the technical direction provided by the Public Interest Energy Research (PIER), Energy Commission staff Bernard Treanton, and former staff Jose Palomo and Mark Rawson.

The authors also acknowledge the technical contributions of the members of the technical advisory committee:

Merrill Smith and, formerly, Poonum Agrawal, U.S. Department of Energy

Richard DeBlasio and Benjamin Kroposki, National Renewable Energy Laboratory

Mohammed Vaziri, Pacific Gas and Electric Company

Scott Lacy, Southern California Edison Company

Mark McGranaghan, EPRI Solutions

Narain Hingorani, independent consultant

This work was supported by the Office of Electricity Delivery and Energy Reliability, Office of Electric Transmission and Distribution, U. S. Department of Energy under contract No. DE-AC02-05CH11231.

\section{Citation}

Please cite this report as follows:

Eto, Joseph, Robert Lasseter, Ben Schenkman, John Stevens, Harry Volkommer, Dave Klapp, Ed Linton, Hector Hurtado, Jean Roy, Nancy Jo Lewis. Consortium for Electric Reliability Technology Solutions (CERTS). 2008. CERTS Microgrid Laboratory Test Bed. California Energy Commission, Public Interest Energy Research Program. CEC-500-2008-XXX. 


\section{Preface}

The Public Interest Energy Research (PIER) Program supports public interest energy research and development that will help improve the quality of life in California by bringing environmentally safe, affordable, and reliable energy services and products to the marketplace.

The PIER Program, managed by the California Energy Commission (Energy Commission), conducts public interest research, development, and demonstration (RD\&D) projects to benefit California.

The PIER Program strives to conduct the most promising public interest energy research by part nering with RD\&D entities, including individuals, businesses, utilities, and public or private res earch institutions.

PIER funding efforts are focused on the following RD\&D program areas:

Buildings End-Use Energy Efficiency

Energy Innovations Small Grants

Energy-Related Environmental Research

Energy Systems Integration

Environmentally Preferred Advanced Generation

Industrial/Agricultural/Water End-Use Energy Efficiency

Renewable Energy Technologies

Transportation

CERTS Microgrid Laboratory Test Bed is the final report for the CERTS Microgrid Laboratory Test Bed project (contract number 500-03-024) conducted by CERTS. The information from this project contributes to PIER's Energy Systems Integration Program.

For more information about the PIER Program, please visit the Energy Commission's website at www.energy.ca.gov/pier or contact the Energy Commission at 916-654-5164. 


\section{Table of Contents}

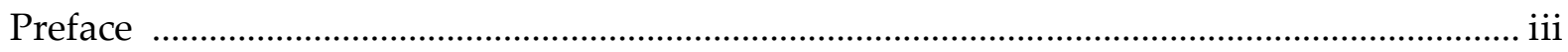

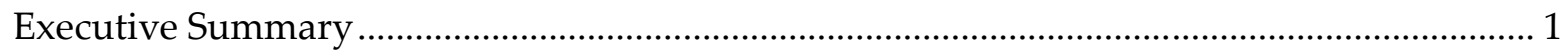

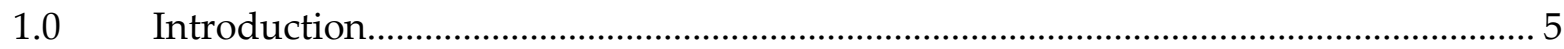

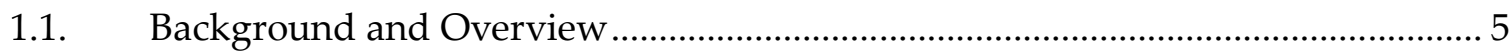

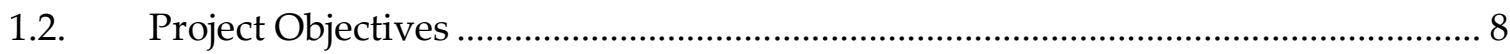

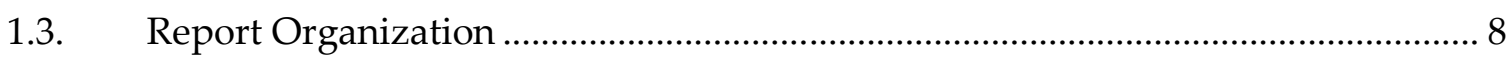

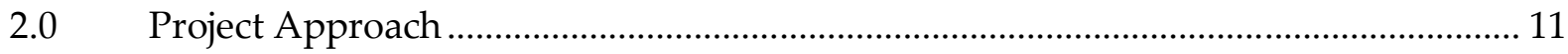

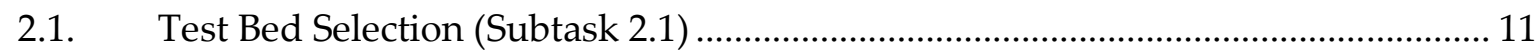

2.2. Equipment Preparation (Subtask 2.2) ................................................................. 11

2.3. Laboratory Tests (Subtask 2.3) ......................................................................... 12

2.4. Field Demonstration Planning (Subtask 2.4) ................................................... 17

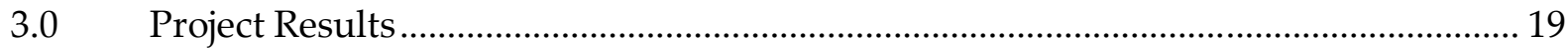

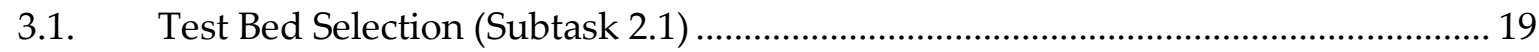

3.2. Equipment Preparation (Subtask 2.2) .................................................................. 21

3.3. CERTS Microgrid Laboratory Tests....................................................................... 24

3.4. Field Demonstration Planning (Task 2.4) ............................................................ 44

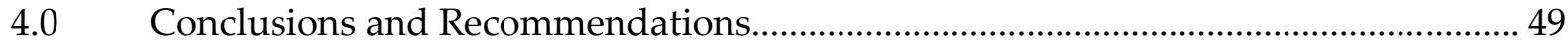

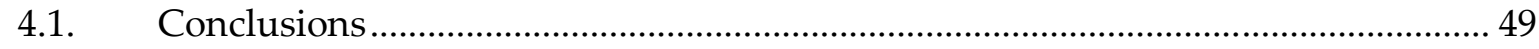

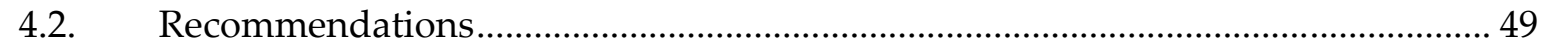

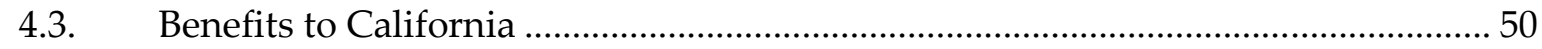

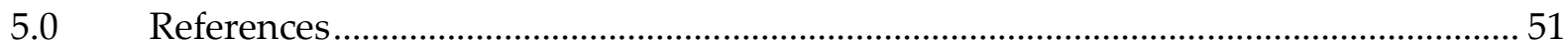

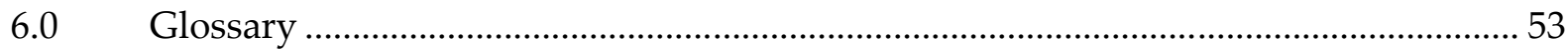

\section{Appendices}

Appendix A. Test Bed Design Schematics

Appendix B. CERTS Microgrid Test Plan,

Appendix C. Youtility Factory Test Plan Final Test Results

Appendix D. Tecogen 60kW Inverter-Based CHP Modules: Factory Testing

Appendix E. Tecogen CHP Modules Commissioning Report

Appendix F. CERTS Test Bed CERTEQUIP-V06-002, CERTS Switch, Low Power Factory Acceptance Test Report 
Appendix G. Summary of CERTS Microgrid Static Switch Power Quality Tests at AEP Dolan, CERTS Microgrid Static Switch Testing

Appendix H. CERTS Test Bed Design and Commissioning: Lessons Learned Summary Appendix I. Test Plan Section 6.0 Microgrid Test Bed System Checkout (Static Switch) Appendix J. Test Plan Section 7.0 Validate Protection Settings and Initial Fault Testing Appendix K. Test Plan Section 8.0 Reduced System Tests

Appendix L. Test Plan Section 9.0 Power Flow Control Tests

Appendix M. Test Plan Section 10.0 Difficult Loads

Appendix N. Test Log

Appendix O. Technical Advisory Committee Meeting Summary and Review Comments Appendix P. Microgrid Fault Protection Based on Symmetrical and Differential Current Components 


\section{List of Figures}

Figure 1. CERTS Microgrid Test Bed at American Electric Power .................................................

Figure 2. One-Line Diagram of CERTS Microgrid Test Bed ........................................................ 13

Figure 3. One-Line Diagram with Meter and Relay Locations CERTS Microgrid Test Bed .........14

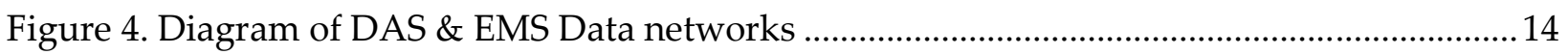

Figure 5. Tecogen Prime Mover with Inverter.......................................................................... 21

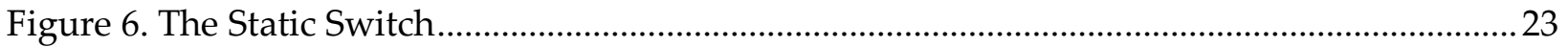

\section{List of Tables}

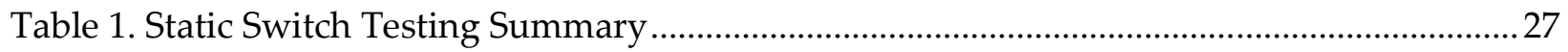

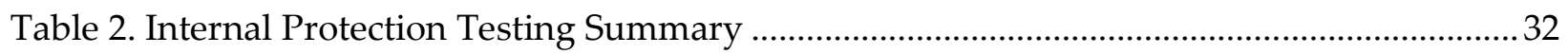

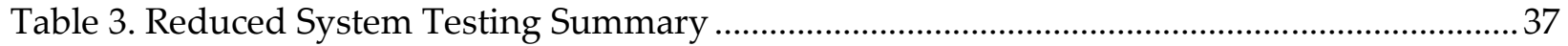

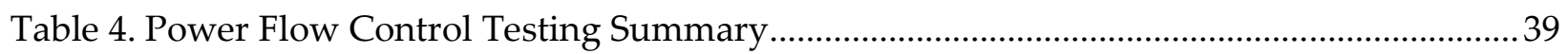

Table 5. Difficult Load Testing Summary …............................................................................... 41 


\begin{abstract}
The objective of the CERTS Microgrid Laboratory Test Bed project was to enhance the ease of integrating small energy sources into a microgrid. The project accomplished this objective by developing and demonstrating three advanced techniques, collectively referred to as the CERTS Microgrid concept, that significantly reduce the level of custom field engineering needed to operate microgrids consisting of small generating sources. The techniques comprising the CERTS Microgrid concept are: 1) a method for effecting automatic and seamless transitions between grid-connected and islanded modes of operation; 2) an approach to electrical protection within the microgrid that does not depend on high fault currents; and 3) a method for microgrid control that achieves voltage and frequency stability under islanded conditions without requiring high-speed communications.

The techniques were demonstrated at a full-scale test bed built near Columbus, Ohio and operated by American Electric Power. The testing fully confirmed earlier research that had been conducted initially through analytical simulations, then through laboratory emulations, and finally through factory acceptance testing of individual microgrid components. The islanding and resychronization method met all Institute of Electrical and Electronics Engineers 1547 and power quality requirements. The electrical protection system was able to distinguish between normal and faulted operation. The controls were found to be robust under all conditions, including difficult motor starts.

The results from these tests are expected to lead to additional testing of enhancements to the basic techniques at the test bed to improve the business case for microgrid technologies, as well to field demonstrations involving microgrids that involve one or more of the CERTS Microgrid concepts.
\end{abstract}

Keywords: CERTS, microgrid, control protection, electrical protection, voltage and frequency stability control, inverter, distributed energy resources, distributed generation, distributed resource, islanding. 


\section{Executive Summary}

Evolutionary changes in the regulatory and operational climate of traditional electric utilities and the emergence of smaller generating systems, including internal combustion engines, microturbines, photovoltaics, and fuel cells, have opened new opportunities for electricity users to generate power on site. In this context, distributed energy resources - small power generators typically located at sites where the energy (both electric and thermal) they generate is used - are a promising option to meet growing customer needs for economic and reliable electric power. In addition to generators, the distributed energy resources portfolio also includes energy storage, and load control. Organizing all of these resources into microgrids is a promising way to capture smaller distributed energy resources' significant potential to meet customers' and utilities' needs.

A key feature of a microgrid, is its ability, during a utility grid disturbance, to separate and isolate itself from the utility seamlessly with little or no disruption to the loads within the microgrid (e.g., in the CERTS Microgrid concept, no impacts on power quality). Then, when the utility grid returns to normal, the microgrid automatically resynchronizes and reconnects itself to the grid, in an equally seamless fashion.

The CERTS Microgrid concept seeks to provide this technically challenging functionality without extensive (i.e., expensive) custom engineering. In addition, the design of the CERTS Microgrid also provides high system reliability and great flexibility in the placement of distributed generation within the microgrid. The CERTS Microgrid is intended to offer these functionalities at much lower costs than traditional approaches by incorporating peer-to-peer and plug-and-play concepts for each component within the microgrid.

The peer-to-peer concept insures that no single component, such as a master controller or a central storage unit, is required for operation of the microgrid. Therefore, by its very design, the CERTS Microgrid can continue operating with loss of an individual component or generator. (With one additional source, $(\mathrm{N}+1)$ it can insure even higher levels of reliability.)

The plug-and-play concept means that a distributed energy resources unit can be placed at any point within the microgrid without re-engineering its controls. The plug-and-play functionality is similar to the flexibility one has with home appliances. That is, just as an appliance can be plugged in wherever there is an outlet, one can similarly locate distributed energy resources units at any location within a facility or building where they might be most needed. This is in sharp contrast to the traditional model, which clusters distributed generation at a single point in order to make the electrical integration tasks simpler. In combined heat and power applications, the plug-and-play model facilitates placing distributed energy resources immediately adjacent to heat loads, thereby allowing more effective use of waste heat without a complex heat distribution system, such as steam and chilled water pipes, and the energy losses associated with them.

A critical feature of the CERTS Microgrid is its presentation to the surrounding distribution grid as a single self-controlled entity. A CERTS Microgrid appears to the grid as indistinguishable from other customer sites that do not include DER. This presentation means that the microgrid 
avoids many of the current concerns associated with integrating DER, such as how many DER the system can tolerate before their collective electrical impact begins to create problems like excessive current flows into faults and voltage fluctuations.

These functionalities are provided through three advanced techniques comprising the CERTS Microgrid concept, including: 1) a method for effecting automatic and seamless transitions between grid-connected and islanded modes of operation; 2) an approach to electrical protection within the microgrid that does not depend on high fault currents; and 3) a method for microgrid control that achieves voltage and frequency stability under islanded conditions without requiring high-speed communications.

The techniques were demonstrated at a full-scale test bed built near Columbus, Ohio and operated by American Electric Power. See Figure 1. Tecogen and its inverter manufacturer, Youtility, modified and factory tested three combined heat and power units to incorporate the CERTS Microgrid control algorithms. Northern Power Systems designed, fabricated, and factory tested a static switch that implemented CERTS Microgrid islanding and resychronization procedures and also fabricated the other major hardware elements required, including load banks, power and control circuitry, protective relaying, and data-acquisition equipment, installed in metal-clad switchgear cabinets for protection in an outdoor location.

Five sets of tests were conducted by American Electric Power (AEP);

The first set of tests examined the operation of the static switch to determine that it and its digital signal processing control operated as designed. This included five tests of dead-bus and synchronized closing, reverse power and Institute of Electrical and Electronics Engineers (IEEE) 1547 protective relay functions. The goal was to confirm the correct operation (and thus the protection) of the static switch, which is located at the interface between the protected and unprotected portions of the microgrid.

The second set of tests examined a preliminary set of faults (i.e., overload simulating a high impedance fault) within the microgrid to ensure protection and safety of the test bed, prior to performing other planned tests. The fault condition tests were intended to cover the basic concepts of the protection design and to study its effectiveness (e.g., zero-sequence, negativesequence and residual currents for line-to-ground faults; negative-sequence or $\left(\mathrm{I}^{2} \mathrm{~T}\right)$ protection for phase-to-phase faults). For the majority of these test scenarios, the static switch was expected to open first, followed by the opening of the zone breaker of the faulted zone. The goal was to test and adjust protection settings to achieve the most ideal conditions and protection design within a limited fault current system. Sixteen separate tests were conducted. 


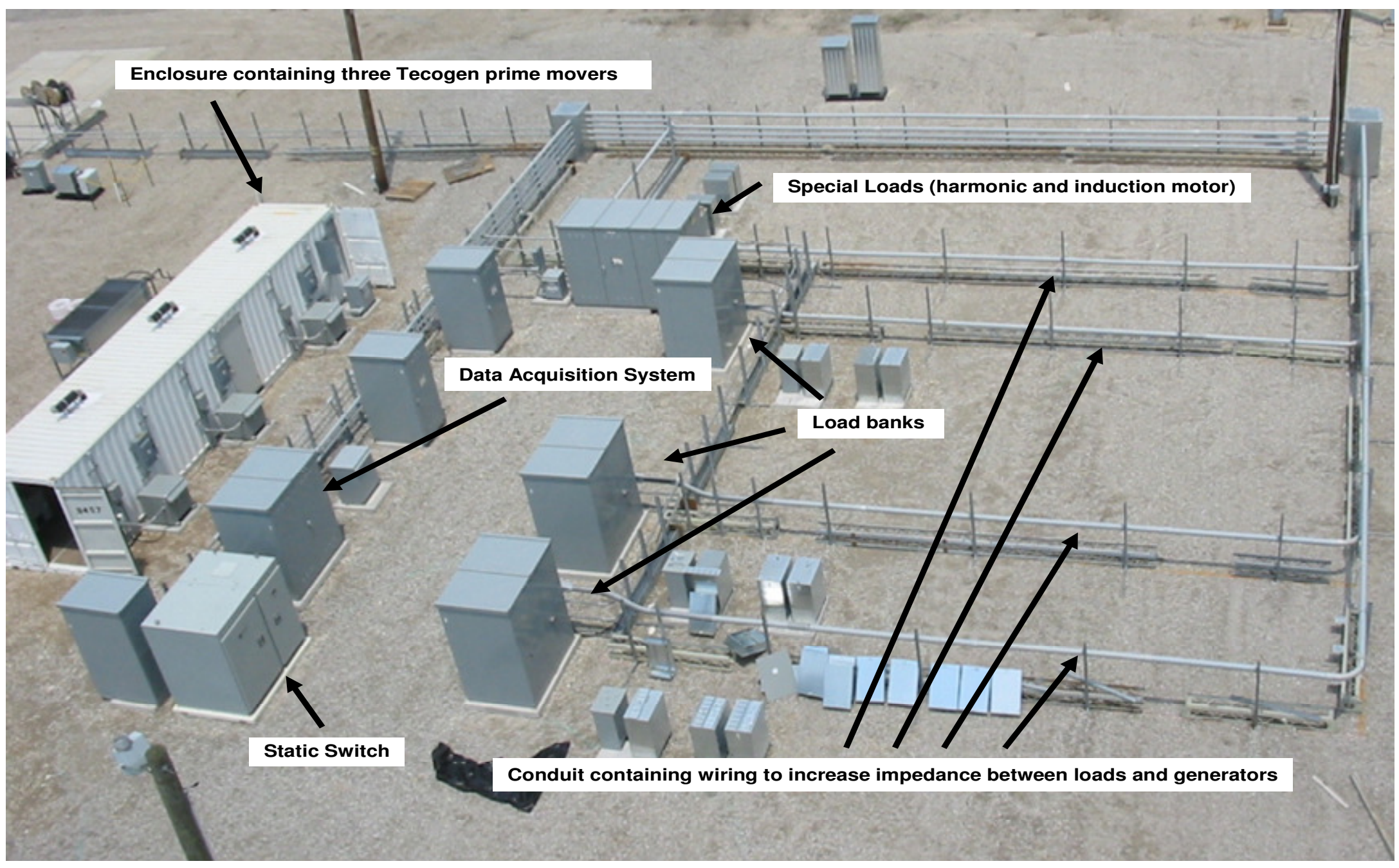

Figure 1. CERTS Microgrid Test Bed at American Electric Power

Photo Credit: American Electric Power 
The third set of tests was designed to ensure that the Gen-set inverter controls were working as designed during grid-connected and islanded modes of operation. This includes unit control, zone control, and mixed power controls, in conjunction with limiting controls and synchronized closing of the static switch. These tests were based on replicating tests that had previously been conducted by Tecogen and Youtility during the factory acceptance testing of the standalone inverters, and the engine-coupled inverters. The performance goal was to observe smooth transitions of the Gen-sets response to different step conditions. Thirteen separate tests were conducted.

The fourth set of tests demonstrated the flexibility of the microgrid while both grid connected and islanded, for different loads, power flows, and utility impact. These tests employed "weak grid" inductors in the circuit simulating the microgrid connection at the end of a lengthy feeder. Three sets of tests were conducted.

The fifth and final set of testing began to explore the operational limits of the microgrid with difficult induction motor starting loads. Two primary sets of tests were conducted under "weak grid" conditions.

Detailed, standalone reports (referenced as appendices to this report) were prepared containing narrative descriptions of the purpose and performance goal for each test, along with extensive graphical and tabular summaries of the results for each test.

The testing fully confirmed earlier research that had been conducted initially through analytical simulations, then through laboratory emulations, and finally through factory acceptance testing of individual microgrid components. The islanding and resychronization method met all IEEE 1547 and power quality requirements. The electrical protection system was able to distinguish between normal and faulted operation. The controls were found to be robust under all conditions, including difficult motor starts.

The results from these tests are expected to lead to additional testing of enhancements to the basic techniques at the test bed, to improve the business case for microgrid technologies, as well to field demonstrations involving microgrids that involve one or more of the CERTS Microgrid concepts. 


\subsection{Introduction}

This report describes the Consortium for Electric Reliability Solutions (CERTS) Microgrid Laboratory Test Bed project, which developed and tested innovative strategies for islanding and resychronizing microgrids with the grid, providing electrical protection within the microgrid, and controlling energy sources within the microgrid autonomously during islanded operation. Under earlier research contracts, the CERTS Microgrid team first developed these strategies using computer simulations, and then further refined them with laboratory-scale emulations. In this research contract, the team implemented them in commercial-grade hardware, with thorough testing at several stages of development, including at a full-scale test bed.

\subsection{Background and Overview}

Evolutionary changes in the regulatory and operational climate of traditional electric utilities and the emergence of smaller generating systems, including internal combustion engines, microturbines, photovoltaics, and fuel cells, have opened new opportunities for electricity users to generate power on site. In this context, distributed energy resources (DER) - small power resources typically located at sites where the energy (both electric and thermal) they provide is used locally - are a promising option to meet growing customer needs for economic and reliable electric power. In addition to generators, the DER portfolio also includes energy storage, and load control. Organizing all of these resources into microgrids is a promising way to capture smaller DER's significant potential to meet customers' and utilities' needs.

The CERTS Microgrid is an entirely new approach to integrating DER. Traditional approaches for integrating DER focus on the impacts on grid performance of a single or relatively small number of microsources. An example of the traditional approach to DER is the Institute of Electrical and Electronics Engineers (IEEE) Standard 1547 for Distributed Resources Interconnected with Electric Power Systems. This standard focuses on ensuring that interconnected systems will not affect the operation of the grid should problems arise on the grid. In compliance with this standard, the CERTS Microgrid is designed to seamlessly disconnect and operate as an island, separate from the grid, in case of problems and to reconnect to the grid once the problems are resolved. (Lasseter, et al. 2002. Lasseter, Piagi. 2005. Lasseter, Piagi. 2006.)

A critical feature of the CERTS Microgrid is its presentation to the surrounding distribution grid as a single, self-controlled entity. A CERTS Microgrid appears to the grid as indistinguishable from other customer sites that do not include DER. This presentation means that the microgrid avoids many of the current concerns associated with integrating DER, such as how many DER the system can tolerate before their collective electrical impact begins to create problems like excessive current flows into faults and voltage fluctuations. The CERTS Microgrid architecture insures that the microgrid will be a good citizen within the bulk power provider network, complying with grid rules and doing no harm beyond what would be acceptable from any existing customer. In return for appropriate compensation, the microgrid could provide interruptible load. Although technical barriers currently discourage it, the microgrid could also be a small source of power or ancillary services. The benefits it could offer to the distribution 
system include; congestion relief, postponement of new generation or delivery capacity, rapid response to load changes, and local voltage support.

A key feature of a microgrid is its ability, during a utility grid disturbance, to separate and isolate itself from the utility seamlessly with little or no disruption to the loads within the microgrid (e.g., in the CERTS Microgrid concept, with no impact on power quality). Then, when the utility grid returns to normal, the microgrid automatically resynchronizes and reconnects itself in an equally seamless fashion.

What is unique about the CERTS Microgrid is that it can provide this technically challenging functionality without extensive (i.e., expensive) custom engineering. In addition, the design of the CERTS Microgrid also provides high system reliability and great flexibility in the placement of distributed generation within the microgrid. The CERTS Microgrid is intended to offer these functionalities at much lower costs than traditional approaches by incorporating peer-to-peer and plug-and-play concepts for each component within the microgrid.

The peer-to-peer concept insures that no single component, such as a master controller or a central storage unit, is required for operation of the microgrid. Therefore, by its very design, the CERTS Microgrid can continue operating with loss of an individual component or generator. (With one additional source, $(\mathrm{N}+1)$ it can insure even higher levels of reliability.)

The plug-and-play concept means that a DER unit can be placed at any point within the microgrid without re-engineering its controls. The plug-and-play functionality is similar to the flexibility one has with home appliances. That is, just as an appliance can be plugged in wherever there is an outlet, one can similarly locate DER units at any location within a facility or building where they might be most needed. This is in sharp contrast to the traditional model, which clusters DG at a single point in order to make the electrical integration tasks simpler. In combined heat and power (CHP) applications, the plug-and-play model facilitates placing DER immediately adjacent to heat loads, thereby allowing more effective use of waste heat without a complex heat distribution system, such as steam and chilled water pipes, and the energy losses associated with them.

These functionalities are enabled by three innovations: First, the CERTS Microgrid concept relies on embedding intelligence in the interface between the microsources and the surrounding microgrid. ${ }^{1}$ This innovation enables autonomous operation of each microsource without highspeed communication or hierarchical control among microsources. This innovation is the key toward enabling a plug-and-play environment in which many microsources can operate harmoniously with one another with a minimum of expensive, custom site engineering.

\footnotetext{
${ }^{1}$ For pragmatic reasons of availability and controllability, this CERTS effort initially focused on small internal combustion (IC) engines. All DER technologies, especially those that rely on power electronic interfaces, including microturbines, fuel cells, photovoltaics, and energy storage, are candidates for inclusion in microgrids based on CERTS Microgrid concepts.
} 
Second, as a result of enabling an environment in which, in principle, any number of microsources might operate within a single microgrid, the CERTS Microgrid concept also incorporates an intelligent static switch for rapid disconnect and resynchronization of the entire microgrid to the larger, utility grid at the point of common coupling (PCC). A single, intelligent switch is innovative because it is a much more economic alternative to providing this functionality at each individual microsource.

Third, the CERTS Microgrid includes an innovative, additional level of protective relaying within the microgrid that complements traditional protection. The approach addresses that fact that power electronic interfaces can, by design, limit the fault current available to detect system faults, which is the traditional means used for detecting faults within an electrical network. Thus, while a microgrid can use bi-directional over-current protection devices when connected to the utility, it cannot rely on these approaches when the microgrid is islanded. The CERTS Microgrid protection scheme uses sequence components to detect low-fault currents in utilityconnected and islanded operation. This protection scheme allows for plug-and-play without changing the existing over-current devices on the electrical system.

The development and testing of the CERTS Microgrid concept is the culmination of over 7 years of research efforts supported by both the U.S. Department of Energy and (DOE) the California Energy Commission (Energy Commission). In 2001, the DOE provided research support to develop the initial CERTS Microgrid concept. In 2002, the Energy Commission began providing research support to demonstrate the CERTS Microgrid concept. Under prior Energy Commission Contract \# 150-99-003, Tasks 2.4 and 2.7, in the area of Distributed Energy Resources Integration, the CERTS team: 1) conducted analytical simulations of aspects of the concept (Lasseter, et. al. 2002); 2) prepared the design for test bed where a full-scale demonstration of the concepts could be tested (Appendix A. Test Bed Design Schematics); and 3) completed a review of potential sites and research partners where the test bed could be built (Akhil, et. al. 2002).

This document is the final report for the testing that has been conducted at the test bed.

The CERTS Microgrid Laboratory Test Bed project team initially included Lawrence Berkeley National Laboratory (LBNL); Sandia National Laboratory (SNL); the University of Wisconsin (UW); and Northern Power Systems (NPS), which designs, builds and installs electric power systems. Through this project, the CERTS team was expanded to include Tecogen, Inc., a manufacturer of engine-driven cooling and combined heat and power (CHP) systems, and Youtility, a supplier of power electronic systems for distributed power. In addition, American Electric Power (AEP), one of the largest electric utilities in the United States, delivering electricity to more than 5 million customers in 11 states, was selected by the team to host the test bed and conduct the tests.

The CERTS team was aided throughout the research process by a Technical Advisory Committee (TAC) consisting of representatives from 1) the DOE's Office of Electricity Delivery and Energy Reliability; 2) two major California electric utilities, Pacific Gas and Electric (PG\&E) and Southern California Edison (SCE) with expertise in interconnection of distributed 
generation; 3) the National Renewable Energy Laboratory (NREL) who manage aspects of the IEEE 1547 standards development process; 4) the Electric Power Research Institute (EPRI) with expertise in power quality; and 5) an independent consultant with expertise in power electronics.

\subsection{Project Objectives}

The objectives of this project were to:

- Demonstrate stable operation of the CERTS control algorithms during a variety of conditions including:

- Transitions between utility-interconnected and islanded operation

- Islanded operation that involved such traditionally difficult loads as motor starting

- Demonstrate the ability to detect faults within the microgrid under a variety of conditions when either interconnected to the utility or islanded

The CERTS Microgrid Laboratory Test Bed project meets the PIER Goal of Improving the Reliability/Quality of California's Electricity by creating technologies and control strategies needed to capture the full potential of DER to improve the reliability and quality of the California interconnected power system. This project also meets the secondary goal of Improving the Energy Cost/Value of California's Electricity by lowering the cost of power delivery.

\subsection{Report Organization}

The CERTS Microgrid Laboratory Test Bed project consists of one technical task, CERTS Microgrid Laboratory Test Bed, with four Subtasks and five Sub-subtasks, as follows:

Subtask 2.1: Select a laboratory test bed

Subtask 2.2: Prepare equipment for the test bed

Sub-subtask 2.2.1: Prepare three prime movers and inverters for the test bed, including factory acceptance testing

Sub-subtask 2.2.2: Design, fabricate and factory test other test bed equipment, including the static switch

Subtask 2.3: Conduct tests of the CERTS Microgrid concept at the test bed

Sub-subtask 2.3.1 Prepare the test bed for testing

Sub-subtask 2.3.2 Conduct tests of the CERTS Microgrid concepts

Sub-subtask 2.3.3 Prepare a microgrid protective relaying report

Subtask 2.4 Plan a field demonstration of the CERTS Microgrid concept

The next two major sections of this report, 2.0 Project Approach and 3.0 Project Outcomes, follow this Subtask and Sub-subtask structure. 
The report is supplemented by a number of stand-alone technical appendices that provide additional information and details on the results from all subtasks and sub-subtasks of this project. Several, separate appendices were required to organize voluminous tabular and graphical summaries of the data collected from each of the many tests conducted at the test bed. 


\subsection{Project Approach}

The CERTS Microgrid Laboratory Test Bed project implemented the CERTS Microgrid concept and test bed design initiated under Energy Commission Contact \#150-99-003, tasks 2.4 and 2.7, to develop tools and techniques for significant integration of distributed technologies in support of electricity reliability.

The subsections below describe the approach taken under each Subtask and Sub-subtask and in the project.

\subsection{Test Bed Selection (Subtask 2.1)}

The goal of this task was to select and execute a contract with a facility to conduct a full-scale test-bed demonstration of CERTS Microgrid concepts. The task included developing criteria for selecting a testing facility and, using information from past reviews conducted by the team to identify candidate sites, working with the Technical Advisory Committee (TAC) and Energy Commission Contract Manager to select the most appropriate site. Finally, following the Commission Contract Manager's approval, LBNL was to execute a subcontract with the Energy Commission-approved facility.

\subsection{Equipment Preparation (Subtask 2.2)}

The goal of this subtask was to enter into contracts with a microsource manufacturer, Tecogen/Youtility, to prepare three microsource prime movers with modified inverters and with a DER engineering firm, Northern Power Systems (NPS), to fabricate test bed hardware including a static switch, for use in the laboratory test bed demonstration (Subtask 2.3).

The first phase of work required Tecogen/Youtility to upgrade the current design of its inverter to incorporate CERTS Microgrid control algorithms and to conduct extensive factory tests of the modified microsource prime movers and inverters (Sub-subtask 2.2.1). The machines had to be as identical to one other as possible so that impacts from machines with differing characteristics did not mask important observations about performance, especially during transients, within the test bed. Because of the technical requirements for the test bed prime movers, the supplier had to be able to dedicate a small, technically skilled team to this project. The supplier also had to have the flexibility to revisit and rebuild aspects of the units because the team expected that initial testing would reveal the need for modifications.

In the original Energy Commission contract, Capstone Microturbines was expected to supply the modified microsource prime movers. However, Capstone Microturbines declined to execute a contract with LBNL due to a change in business priorities and withdrew from the project. Following an introduction provided by the Energy Commission contract manager, the team partnered with Tecogen/Youtility to supply the modified prime mover microsources and inverters. Aspects of the laboratory test bed design were modified by NPS to accommodate this change in prime movers.

The second phase of work required NPS to develop and conduct factory tests of a static switch that implements the CERTS Microgrid islanding and resynchronization procedures (Sub- 
subtask 2.2.2). As an additional element of the second phase of work, NPS also fabricated the major electrical components required by the test bed, such as load banks and emulators, switchgear, and the data acquisition system (DAS). NPS was well-qualified to conduct this work because they had, under a previous Energy Commission contract with CERTS, prepared the complete design of the laboratory test bed.

\subsection{Laboratory Tests (Subtask 2.3)}

The goal of this subtask was to conduct tests of the CERTS Microgrid concept. That is, the laboratory test facility selected in Subtask 2.1 was to build the test bed and conduct tests of the test bed equipment, prime movers, and static switch prepared under Subtask 2.2. This subtask was conducted through three sub-subtasks.

The goal of sub-subtask 2.3.1 was to prepare the test bed for conducting the tests. This involved preparation of the physical test bed site following the design of the test bed, which was prepared under an earlier contract. (See Figures 2, 3, and 4, which are based on Appendix A, Test Bed Design Schematics). The tasks included erection of enclosures to house the microsources and associated facilities to cool the engines in operation and the laying out of conduits and associated wiring to interconnect the microgrid elements. Finally, the task involved installing the cabinets assembled by NPS as well as the microsources prepared by Tecogen/Youtility. See Figure 1.

The goal of sub-subtask 2.3.2 was to conduct a large number of tests exercising all functions of the CERTS Microgrid concept. This goal was accomplished in three phases. First, a detailed test plan was developed. Second, the tests were executed following the test plan. Third, the test results were analyzed and assessed with respect to the test plan and overall project objectives.

The CERTS team, led by NPS, developed a comprehensive list of tests that would exercise every aspect of the CERTS Microgrid concept. The tests were presented to and refined through discussions with the TAC. After the list of tests was deemed complete, AEP developed "registered" test procedure, following AEP corporate rules that would meet all testing needs safely. This registered test procedure is presented in Appendix B. CERTS Microgrid Testbed Test Plan.

The registered test procedure contains ten sections. The first five are procedural. Sections six through ten refer to actual tests in which measurements are recorded. The tests were conducted by AEP in accordance with the registered test procedure. Testing was conducted sequentially, one section at a time.

Prior to each test day, the person in charge performed a job safety briefing including an inspection of the barricades and test setup for safety and compliance. A minimum of two people were on-site during each planned test 


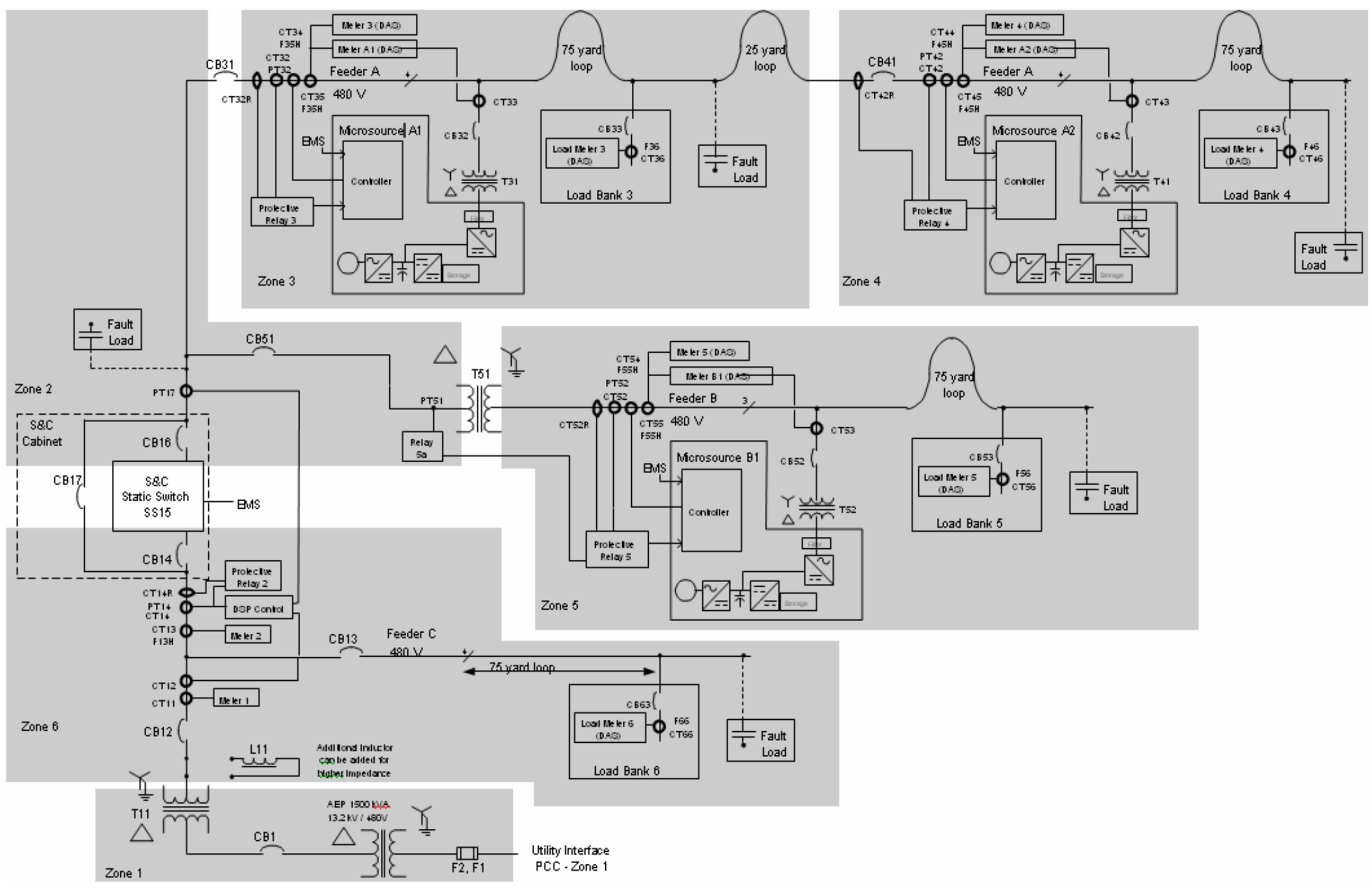

Figure 2. One-Line Diagram of CERTS Microgrid Test Bed 


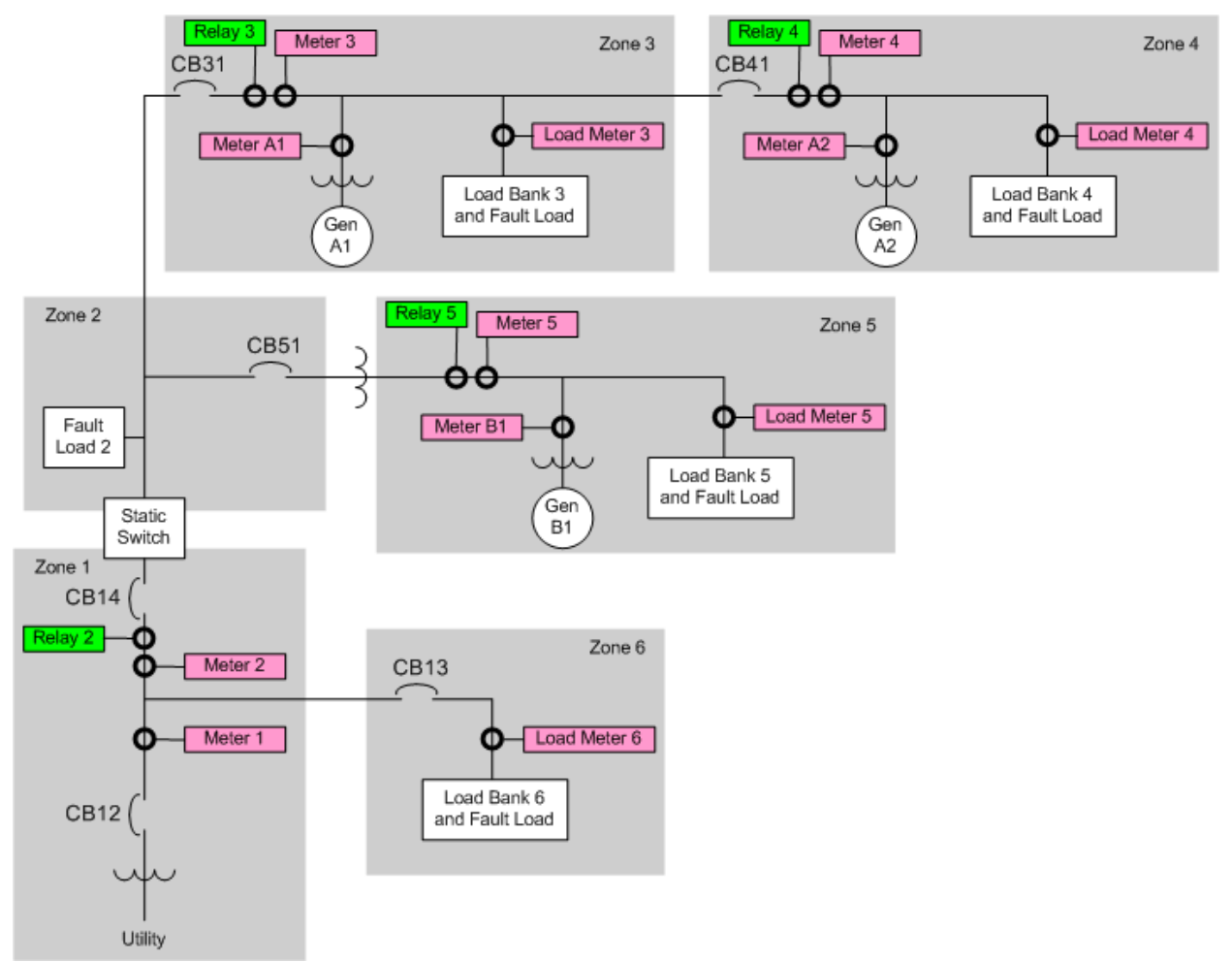

Figure 3. One-Line Diagram with Meter and Relay Locations CERTS Microgrid Test Bed

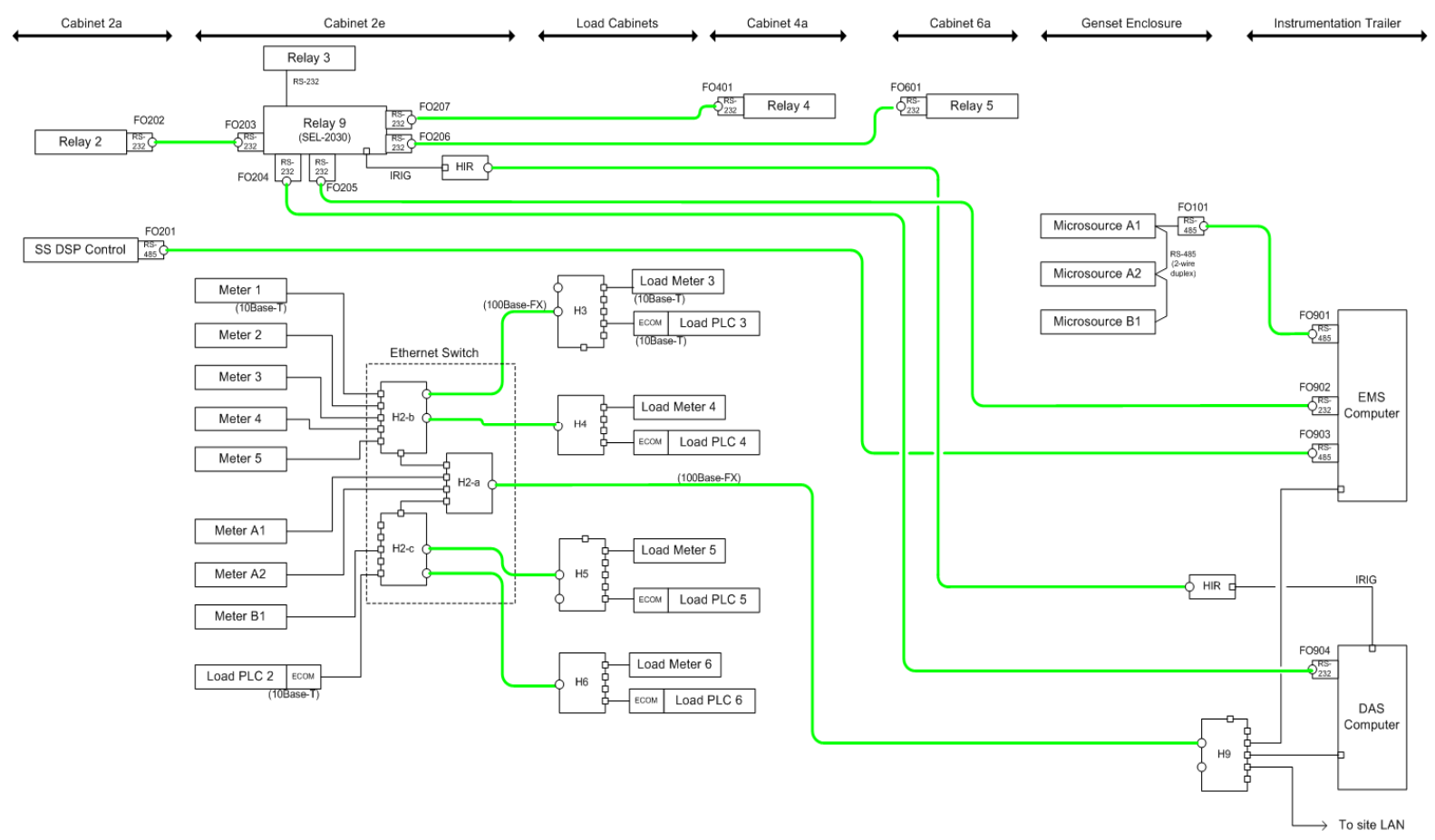

Figure 4. Diagram of DAS \& EMS Data networks 
Visual and audible alarms warned persons that energized testing was being performed in the Microgrid Test Bed area. The visual alarm consisted of a portable red flashing light, located between the Control Trailer and Gen-set Enclosure. An audible alarm, consisting of a portable wireless motion detector, was located at the front gate of the Walnut Test Site with the fence gate "Closed," not locked, and audible alarm in the trailer operational during test(s).

Barricades were set up around the Microgrid Test Bed area (i.e., saw-horse style barricades with a red plastic chain surrounded the test area containing the Gen-set Enclosure, Microgrid switching cabinets, plus load and fault bank cabinets).

Prior to performing tests, the Test Engineer or Technical Consultant verified that all personnel and visitors were properly protected and in assigned locations. Personnel were in or adjacent to the Control Trailer while tests were being performed. All nonessential personnel either left the main site or were sheltered in the Control Trailer.

The first set of tests (Section 6 of the test plan) examined the operation of the static switch to determine that it and its digital signal processing (DSP) control operated as designed. The goal was to confirm the correct operation (and thus the protection) of the static switch, which is located at the interface between the protected and unprotected portions of the microgrid. Successful completion of these tests was a prerequisite to performing subsequent tests in the test plan.

The tests were designed to check control and operation of the static switch, basic power and voltage control of the Gen-sets, and a preliminary check of the protection scheme. They included five tests of dead-bus and synchronized closing, reverse power and IEEE 1547 protective relay functions. The switch functions were tested with a single Gen-set A1 online. The measurements taken were unique to each test. Waveform and RMS data from Meter 2 were of prime interest.

The second set of tests (Section 7 of the test plan) examined a preliminary set of fault (i.e., overload simulating a fault) condition tests to ensure protection and safety of the test bed, prior to performing other planned tests. The goal was to confirm the new protection design developed in Task 2.3.3. This goal was to be accomplished by testing and adjusting protection settings to achieve the most ideal conditions and protection design. The tests included inductor L11 in the circuit, reflecting "weak grid" conditions. Sixteen separate tests were conducted.

The fault (i.e., overload simulating a fault) condition tests were intended to cover the basic concept of the protection design and to study its effectiveness (e.g., zero-sequence, negativesequence and residual currents for line-to-ground faults; negative-sequence or $\left(\mathrm{I}^{2} \mathrm{t}\right)$ protection for phase-to-phase faults). For the majority of these test scenarios, the static switch was expected to "OPEN" first, followed by the zone breaker of the faulted zone.

During each fault event waveforms of phase currents and line-to-neutral voltages at all Relay locations (i.e., Relays 2, 3, 4 \& 5) were recorded. The relay element that caused the "TRIP" with trip times for each relay relative to when the fault condition was applied was also recorded.

Two power control modes were defined for the third and fourth sets of tests: 
Unit Power Control Mode controls the amount of power (i.e., $\mathrm{kW}$ ) being injected into the Zone from the Gen-set being controlled.

Zone Power Control Mode controls the amount of power (i.e., $\mathrm{kW}$ ) entering/exiting the Zone using the Gen-set to offset the difference in that Zone.

The third set of tests (Section 8 of the test plan) was designed to ensure that the Gen-set inverter controls were working as designed. This includes unit control, zone control, and mixed power controls, in conjunction with limit controls and synchronized closing of the static switch. These tests were based on replicating tests that had previously been conducted by Tecogen and Youtility during the factory acceptance testing of the standalone inverters, and the engine coupled inverters. The performance goal was to observe smooth transitions of the Gen-sets response to different step conditions (i.e., static switch "OPEN"/ "CLOSE" and load steps). Thirteen separate tests were conducted.

Several measurements were made for each test:

- Sources -Injected power $(\mathrm{kW})$, reactive load (kVAr), frequency (freq), and voltage (V) for each Gen-set (i.e., A1p, A2p and B1p).

- Zone input power flow into Zones 3 (A1z), 4 (A2z), 5 (B1z) and 6.

- Loads - Voltage at Load Bank 3 (L3), 4 (L4), 5 (L5), and 6 (L6).

- Static Switch - Power (kW) and current (I) through the static switch.

- Static Switch Control Signal: Forced “OPEN” and release to allow self synchronization.

- Voltage (V) and frequency (Freq) difference across the static switch.

The fourth set of tests (Section 9 of the test plan) demonstrated the flexibility of the microgrid both grid connected and islanded for different loads, power flows and impact on the utility. The tests included the "weak grid" inductors (i.e., L11) in the circuit. Three sets of tests were conducted.

The measurements taken for these tests involved collecting RMS data for V, I, kW, kVAr, and Freq for each flow change at the following points:

- Meter $1-\mathrm{V}, \mathrm{I}, \mathrm{kW}, \mathrm{kVAr}$ for the utility connection

- Meter 2 - V, I, kW, kVAr and utility-side V and Freq

- Meter 3 - V, I, kW, and kVAr for Feeder A and Microgrid side V and Freq

- $\quad$ Meter A1p - V, I, kW, kVAr for Gen-set A1

- Load Meter $3-\mathrm{V}$, I, Zone 3 load kW and kVAr

- Meter 4-V, I, kW, kVAr for Zone 4

- $\quad$ Meter A2p - V, I, kW, kVAr for Gen-set A2

- Load Meter 4-V, I, Zone 4 load kW and kVAr

- Meter 5-V, I, kW, kVAr for Feeder B 
- $\quad$ Meter B1p - V, I, kW, kVAr for Gen-set B1

- Load Meter 5-V, I, Zone 5, Feeder B load kW and kVAr

- Load Meter 6- V, I, Zone 6, Feeder C load kW and kVAr

In addition $\mathrm{V}$ and $\mathrm{I}$ waveform data for unexpected events and for static switch transitions.

The fifth and final set of testing (Section 10 of the test plan) began to explore the operational limits of the microgrid (i.e., power quality, protection and inverter limits). Two primary sets of tests were conducted under "weak grid" conditions; the first involved induction motor starting loads under balanced and unbalanced load conditions; the second involved only unbalanced loads.

The measurements collected included the following:

- $\quad$ RMS data for $\mathrm{kW}, \mathrm{kVAr} \mathrm{V}$, and I injected by each Gen-set

- Freq at each connection point

- $\quad \mathrm{V}$ and I from Meters 1 - 5, and Load Meters 3 - 6

The analysis of the test results consisted of comparing test results to findings from earlier analyses of CERTS Microgrid concepts. As noted, successive analysis and testing of the CERTS Microgrid concepts have include analytical simulation, laboratory-scale emulations, and factory testing of the commercial-grade hardware installed at the test bed.

A number of software tools were used to process raw test data into a format suitable for review and presentation. These included: PQView, AcSELerator QuickSet Designer, Excel, and Word. Test results were posted by AEP onto a secure website where they could be reviewed and discussed by the entire CERTS team.

Test bed results were then presented and discussed with the TAC. TAC members were also invited to review and provide comments each of the extensive reports that have been prepared detailing the results of the testing.

The goal of sub-subtask 2.3.3 was to develop an innovative approach to provide protective relaying within the microgrid. The approach addresses that fact that power electronic interfaces, by design, limit the fault current available to detect system faults, which is the traditional means for detecting faults within an electrical network. Hence, in addition to traditional protection schemes that were already in place, the CERTS team, led by University of Wisconsin, needed to design an additional level of protection for faults that occur within the microgrid. These designs were then implemented and tested as part of the tests conducted in sub-subtask 2.3.2.

\subsection{Field Demonstration Planning (Subtask 2.4)}

The goal of subtask 2.4 was to identify and engage one or more partners for a field demonstration of the CERTS Microgrid concept. While a field demonstration was not to be conducted in this project, it was important to engage potential partners prior to and during the 
laboratory test-bed phase so that partner-specific technical or operational issues could be addressed in the test bed prior to conducting the field demonstration.

This task was a continuation of work initiated under a previous Energy Commission agreement (amendment \#150-99-003, task 2.7). That contract led to preparation of a field demonstration planning and partner recruitment strategy. The current task continued the implementation of that strategy.

The approach primarily involved conducting meetings with potential field demonstration partners to brief them on the CERTS Microgrid concept and test bed prior to and during the testing. Other activities that contributed to field demonstration planning by increasing the visibility of the CERTS Microgrid concept to a wide audience included presentations and publications of the CERTS Microgrid concept in a variety of fora, including professional meetings, domestic and international conferences, and academic and trade publications. These activities directly contributed to and supported follow-up discussions with potential field demonstration partners. 


\subsection{Project Results}

This section presents projects results from Subtasks 2.1-2.4 of the Microgrid Laboratory Test Bed project. As discussed earlier, the project produced a number of deliverables. Several of these are quite voluminous, as they contain extensive tabular and graphical summaries from each of the tests conducted at the test bed. For brevity, this report only summarizes and highlights selected findings from these deliverables. This report is supplemented by numerous, separately bound appendices, which contain these detail project findings; these appendices are referenced throughout the following discussions.

\subsection{Test Bed Selection (Subtask 2.1)}

The goal of Subtask 2.1 was accomplished through the selection of and execution of a contract with the American Electric Power Company to join the CERTS team to build the test bed and conduct the testing program. The steps including developing selection criteria, applying them to candidate facilities, and determining the most qualified facility.

The final test site selection criteria included the following:

1. Physical requirements

a) Adequate space for the test bed and generators (including safe working space around equipment)

b) Availability of a "stiff" distribution voltage (preferably $15 \mathrm{kV}$ class)

c) Availability of natural gas

d) Availability of "standard" test equipment

2. Personnel -Test engineers and technicians with appropriate experience

3. Project leader with appropriate experience

4. Corporate interest and involvement

5. Timing and availability to meet CERTS requirements

6. Utility-owned and operated site - Important for utility community acceptance of the credibility of test results

7. Accessibility of test site to interested researchers

Under an earlier Energy Commission contract 150-99-003, the CERTS team conducted a preliminary assessment of possible sites for the test bed. ${ }^{2}$ Based on this preliminary assessment, the team applied the above criteria and identified three candidate test sites:

1. Pacific Gas and Electric (PG\&E) Technology and Ecological Services, San Ramon, California;

2. Southern California Edison (SCE) Electric Vehicle Technology Center, Pomona, California

3. American Electric Power (AEP) Dolan Technology Center, Groveport, Ohio

2 "Review of Test Facilities for Distributed Energy Resources" by A. Akhil, C. Marnay, and T. Lipman, SAND2003-1602, May, 2003. 
Input from the TAC and discussions with the Energy Commission contract manager on the list of final candidate test sites and the test site selection criteria led the team to modify the scope of sub-subtask 2.2.2 to have NPS also fabricate the majority of the test bed hardware, rather than have it fabricated by the test facility. There were two reasons: First, the team determined that the test facility should be selected primarily based on its ability to conduct the tests, not based on its ability to fabricate test bed hardware. Second, NPS was uniquely qualified to fabricate the equipment because it had designed the equipment, as part of its preparation of the overall test bed design (conducted as part of an earlier Energy Commission contract 150-99-003.

With this revised scope for the test facility, the team then sent a questionnaire to each of the three final candidate sites requesting detailed written information on four topics:

1. Required steps to prepare the physical site;

2. Equipment availability, data acquisition hardware and approach, and energy management system (EMS);

3. Personnel; and

4. Project scheduling and coordination.

At this point, PG\&E indicated that would not able to respond because it would not be able to conduct the tests within the available time, largely due to prior commitments to other Energy Commission PIER DER Integration projects. This potential conflict was acknowledged by the Energy Commission contract manager and it was agreed that PG\&E should not be considered to host the tests.

The CERTS team and the Energy Commission contract manager then scheduled oral presentations from representatives from both SCE and AEP. On the basis of these presentations, the CERTS team and the Energy Commission contract manager further followed up with on-site visits to the proposed test sites at both SCE and AEP.

Following the site visits, the team recommended the selection of AEP, as the host for the test bed. The SCE site did not have a natural gas supply line and would have required extensive modifications to the electric service interconnection. The AEP site was well-suited physically, had natural gas service, and had an appropriate electric service interconnection.

In conjunction with the contract award, AEP and CERTS entered into a Memorandum of Understanding to work cooperatively in areas of mutual interest for research, development, and demonstration of distributed energy resource (DER) technology microgrids. Subsequent to the initiation of work on this Energy Commission project, CERTS and AEP have won a competitive solicitation from the U.S. Department of Energy to conduct additional tests using the microgrid equipment developed in this project. 


\subsection{Equipment Preparation (Subtask 2.2)}

The goal of subtask 2.2 was accomplished by work led by Tecogen and their subcontractor, Youtility, to modify three Tecogen prime movers to incorporate CERTS Microgrid control algorithms (Sub-subtask 2.2.1) and by work led by NPS to fabricate a static switch that implemented CERTS islanding and resychronization procedures and fabricate the other major hardware elements required by the CERTS laboratory test bed design. A critical element of these sub-subtasks involved detailed factory acceptance testing to confirm the performance of the equipment prior to shipment to AEP.

\subsubsection{The Prime Movers and Inverters (Sub-subtask 2.2.1)}

CERTS contracted with Tecogen to obtain three 100kW prime movers, each of which was modified to incorporate the CERTS Microgrid control algorithms. The complete systems, which were delivered and installed in the CERTS Microgrid test bed site at AEP, each included an engine/generator, a "surge module" and an inverter. See Figure 5.

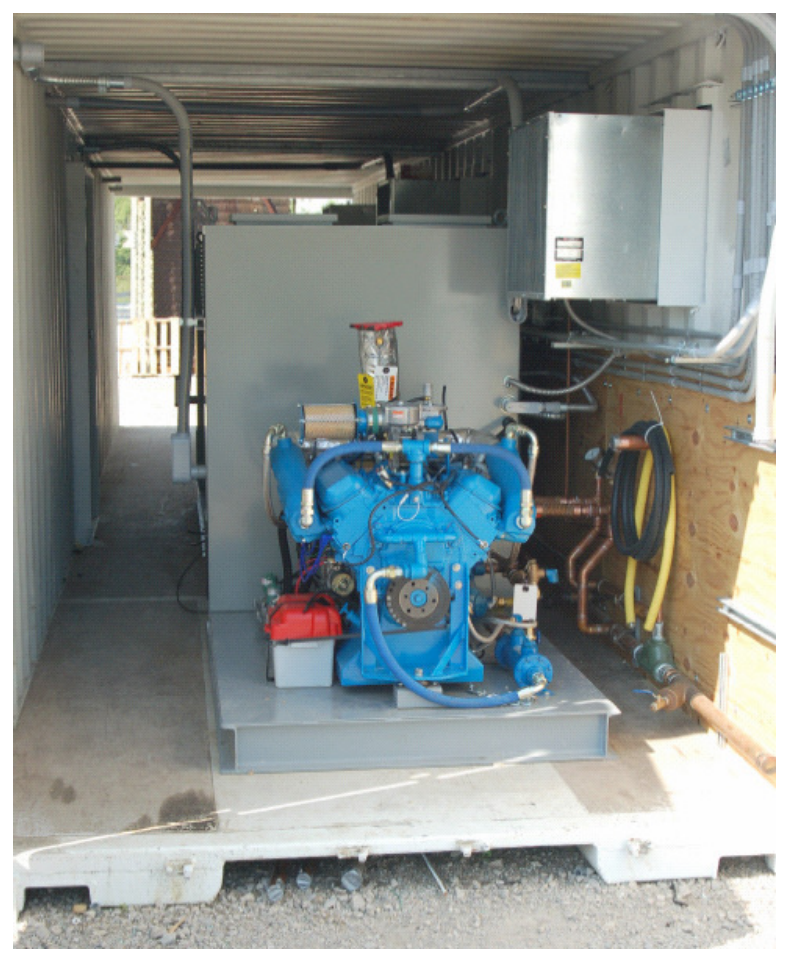

Figure 5. Tecogen Prime Mover with Inverter

A critical aspect of this project was the incorporation of the CERTS Microgrid control algorithms into the inverters provided by Youtility. This required not only providing the algorithms to Youtility, but also providing Youtility with an in-depth understanding of the function of these algorithms so that they could understand the expected response, and the significance of deviations from that response, when the completed inverters were subjected to test scenarios. Providing this understanding required considerable interaction between the Youtility designer and the project's technical director from the University of Wisconsin, Professor Robert Lasseter. 
Professor Lasseter also provided Youtility with a set of test scenarios that were used to confirm correct operation of the completed inverters. These test scenarios then comprised a Youtility factory acceptance test that was performed prior to sending the inverters to Tecogen. The test report for the Youtility factory acceptance tests is included as Appendix C. Youtility Factory Test Plan Final Test Results.

The prime mover for the engine/generator is a 454 cubic inch displacement natural gas-fired V8 reciprocating engine. This engine was designed to be operated at an engine speed selected to give the best combination of fuel efficiency and response to load changes. Thus, the engine speed will not necessarily be synchronous with a $60 \mathrm{~Hz}$ system. The electrical generator a variable AC output which is rectified to a direct current (DC) output. The inverter converts the DC to utility-synchronous AC. For this project, the inverter was modified to incorporate the CERTS Microgrid control algorithms. The surge module is an energy storage device, consisting of batteries, power electronics, and controls, which is tied to the DC bus in parallel with the electrical generator and the inverter. The purpose of the surge module is to provide instantaneous power when there is a load increase, while waiting for the engine/generator to adjust its speed to the new loading conditions. The inverters and surge modules were both supplied to Tecogen by Youtility, Inc. Tecogen integrated the entire prime mover system and performed a set of factory acceptance tests, as specified by the CERTS team, before Tecogen shipped the units to the test bed site. (See Appendix D. Tecogen 60kW Inverter-Based CHP Modules Factory Testing.)

Once at AEP's facilities, the Tecogen units were retested to ensure there had been no shipping damage. The tests confirmed that the performance of the units once installed at AEP was consistent with the factory tests. (Appendix E. Tecogen CHP Modules Commissioning Report.)

\subsubsection{The Static Switch and Other Test Bed Equipment}

CERTS contracted with NPS to design, fabricate, and test a static switch that implemented CERTS Microgrid islanding and resychronization procedures and to fabricate the other major hardware elements required by the CERTS laboratory test bed design, including load banks, power and control circuitry, protective relaying, and data-acquisition equipment, installed in metal-clad switchgear cabinets for protection in an outdoor location.

NPS designed and built the controls that would allow the static switch to implement the CERTS Microgrid islanding and resychronization procedures. The controls were implemented onto the static switch hardware associated with the Purewave uninterruptible power supply system manufactured by S\&C Electric. See Figure 6. The islanding procedures consisted of trip sensing (i.e., detect and trip on IEEE 1547-specified criteria such as voltage or frequency deviations and reverse power flow) followed by disconnection (islanding) of the microgrid from the utility. The voltage-trip function included trip-characteristics required by IEEE 1547 for distributed resource interconnection, those specified by the Information Technology Industry Council for power quality, as well as a custom-voltage-versus-time-trip characteristic that could be specified by the user. 
The resynchronizing function had to meet a more stringent requirement than the commonly accepted criteria of IEEE 1547. The reason for the more stringent requirement was to insure transient free transitions for power flow either into or out of the microgrid at the instant of switch closing. IEEE 1547 requires the phase difference across the switch is less than $20^{\circ}$ before the switch can close. This phase difference is too large for for our needs. We require closing at a zero phase difference. The high-speed static switch and digital signal processing (DSP), insures that the voltage phase difference a closing is zero.

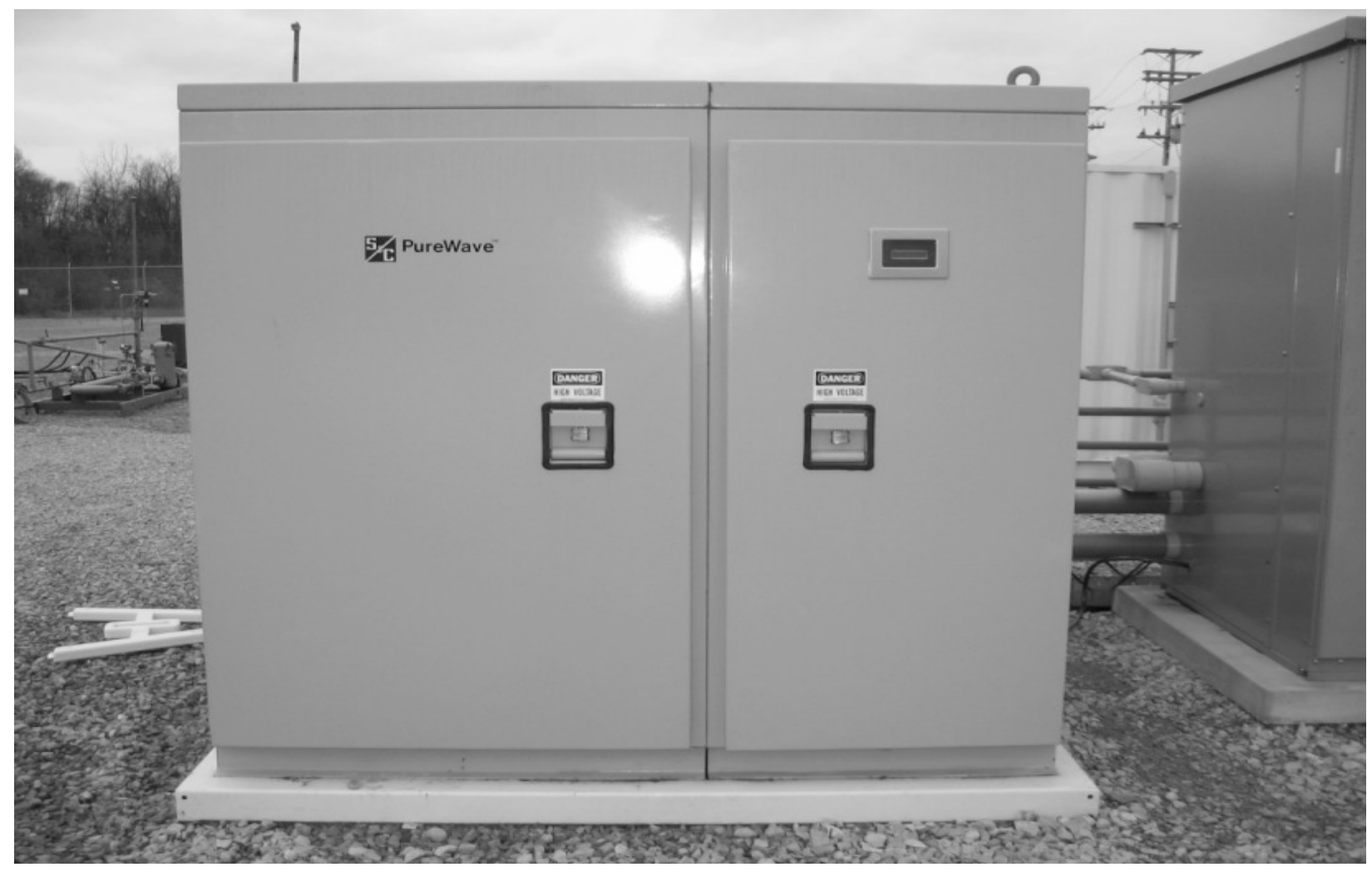

Figure 6. The Static Switch

NPS tested the static switch with low-level power (i.e., signal injection) to confirm proper operation before shipping the switch to AEP. TAC members from the National Renewable Energy Laboratory shipped additional testing equipment and lent expertise to NPS in support of this testing. (Appendix F. CERTS Test Bed CERTEQUIP-V06-002, CERTS Switch, Low Power Factory Acceptance Test Report.)

Once at AEP's facilities, the switch was retested with low power to ensure there had been no shipping damage. After confirming that the static switch was in good condition, AEP performed further testing at the Dolan Test Facility to examine selected power quality issues prior to installing equipment at the test bed. (Appendix G. Summary of CERTS Microgrid Static Switch Power Quality Tests at AEP Dolan, CERTS Microgrid Static Switch Testing.) 
NPS also fabricated cabinets containing all the sensing hardware for the protection and dataacquisition equipment; the circuit breakers, switches and fuses; and all the loads. The loads include a variety of resistive, inductive, capacitive and motor loads as well a six-pulse power rectifier to provide a non-linear load.

\subsection{CERTS Microgrid Laboratory Tests}

This section describes the preparation of the laboratory test bed for testing (Sub-subtask 2.3.1), the results of the CERTS microgrid laboratory tests (Sub-subtask 2.3.2), and the development of an approach for internal protection within the microgrid (Sub-subtask 2.3.3).

\subsubsection{Prepare Laboratory Test Bed for Testing}

After preparing the site, erecting supporting structures, cooling equipment, and wiring, and installing the equipment prepared by NPS and Tecogen/Youtility, AEP and Northern Power performed a field acceptance test. See Figure 1. The field acceptance testing also included exercising the complete data acquisition and control systems. After completing this testing and rectifying the minor issues that it uncovered, the team declared the test bed ready to conduct the full set of CERTS Microgrid tests, as outlined in the test plan. (Appendix H. CERTS Test Bed Design and Commissioning Lessons Learned Summary.)

\subsubsection{Test of CERTS Microgrid Concepts}

As described in section 2.3.2, five sets of tests were conducted. The results of these five sets of tests are contained in separate, stand-alone appendices to this report, as follows:

1. Static Switch (Section 6 of the test plan -6 tests - Appendix I)

2. Protection (Section 7 of the test plan -16 tests - Appendix J)

3. Reduced System (Section 8 of the test plan - 13 tests - Appendix K)

4. Power Flow Control (Section 9 of the test plan -3 banks of tests - Appendix L)

5. Difficult Loads (Section 10 of the test plan -1 set of motor starting tests - Appendix M) Each appendix contains a narrative description of the purpose and performance goal for each test, along with graphical and tabular summaries of the results for each test. In addition, a sixth appendix (Appendix N) contains the test log developed by AEP during the conduct of the tests.

For brevity, this report will summarize and highlight selected findings for each of the five sets of tests. This includes listing each test conducted and its performance objective. The interested reader is referred to the individual appendices to see detailed results from each test performed. 
The first set of tests (Section 6 of the test plan) examined the operation of the static switch to determine that it and its digital signal processing (DSP) control operated as designed. This included six tests of dead-bus and synchronized closing, reverse power and IEEE 1547 protective relay functions. These tests were designed to check control and operation of the static switch, basic power and voltage control of the Gen-sets, and a preliminary check of the protection scheme. The goal was to ensure that, by confirming the correct operation of the static switch, which is located at the interface (and thus serves as the protection) between the unprotected and protected portions of the microgrid, was ready to perform the remaining tests.

A synchronized closing test of the static switch was required to verify that when conditions were within synchronization limits set in the EMS, the static switch performed a synchronized close and thus provided a smooth connection transition. The dead-bus bus (de-energized bus) reclose test's goal was to verify that the static switch can close when de-energized bus conditions exist on the Gen-set side of the static switch; and that the dead-bus reclose algorithm requires user interaction (i.e., Operator needs to "Enable" the dead-bus reclose using pushbutton in the EMS).

The reverse power tests consisted of three tests: three-phase reverse power condition test, single-phase reverse power condition test, and anti-islanding Micro-grid settings reset test. Both three-phase and single-phase reverse power condition tests were required to verify the reverse power functionality of the static switch and confirm that the static switch islands the microgrid for a three-phase or single-phase reverse power conditions. The anti-islanding Micro-grid settings reset test was needed to verify that if a reverse power event occurred, due to a mismatch of Gen-set settings (i.e., total Gen-set power is greater than Microgrid load), the static switch will lockout and go to the "Fault" state, where user interaction is required.

The next goal was to verify the reconnection timers of the static switch (i.e., set by default at 300 seconds based on IEEE Standard 1547-2003). The length of time is programmed into the control system and designed to prevent reconnection until after the utility source voltage returns to nominal steady state conditions.

Table 1 summarizes the test performance goals and results from each of these tests. 


\begin{tabular}{|c|c|c|}
\hline Test Description & Performance Goal & Results \\
\hline $\begin{array}{l}\text { 6.2. Reverse power, } \\
\text { grid islanding (IEEE } \\
1547 \text {, loss of utility } \\
\text { service) }\end{array}$ & $\begin{array}{l}\text { Verify the reverse power functionality of the static } \\
\text { switch and confirm that the static switch islands the } \\
\text { Microgrid for a reverse power condition due to an } \\
\text { upstream utility operation at the PCC. Once the } \\
\text { utility voltage returns to the IEEE } 1547 \text { limits, verify } \\
\text { the proper operations of the reconnection timers } \\
\text { (set by default to } 300 \text { seconds based on IEEE } 1547 \\
\text { standard). }\end{array}$ & $\begin{array}{l}\text { The static switch islanded the microgrid from the utility grid due to an } \\
\text { undervoltage condition. This occurred after the utility feeder opened } \\
\text { separating the microgrid with } 500 \mathrm{~kW} \text { of feeder load which was } \\
\text { supplied by Gen-set A } 1 \text { until the static switch opened. Once the static } \\
\text { switch opened Gen-set A1 continued serving the protected load } \\
\text { within the microgrid. After } 300 \text { seconds, the static switch } \\
\text { synchronized and closed back into the utility grid with a smooth } \\
\text { transition. }\end{array}$ \\
\hline $\begin{array}{l}\text { 6.4. Reverse power, } \\
\text { anti-islanding } \\
\text { microgrid settings } \\
\text { reset }\end{array}$ & $\begin{array}{l}\text { Verify that if a reverse power event occurs, due to a } \\
\text { mismatch of Gen-set settings (Total Gen-set power } \\
>\text { Microgrid load), the static switch will lockout and } \\
\text { go to the "Fault" state, where user interaction is } \\
\text { required. }\end{array}$ & $\begin{array}{l}\text { Load was reduced within the microgrid which caused the static switch } \\
\text { to open on a reverse power condition. The Gen-sets picked up the } \\
\text { load within the microgrid. All loads were reset and the static switch } \\
\text { synchronized and closed back into the utility. }\end{array}$ \\
\hline
\end{tabular}




\begin{tabular}{|l|l|l|}
\hline $\begin{array}{l}\text { 6.5. De-energized } \\
\text { bus (dead bus) } \\
\text { reclose }\end{array}$ & $\begin{array}{l}\text { Verify that the static switch can close when de- } \\
\text { energized bus conditions }(<15 \mathrm{~V}) \text { on the DG side } \\
\text { are measured and that the Dead Bus Reclose } \\
\text { algorithm requires user interaction (i.e., Operator } \\
\text { needs to "Enable" the Dead Bus Reclose using } \\
\text { pushbutton in the EMS). }\end{array}$ & $\begin{array}{l}\text { The static switch synchronized and closed into the utility grid with } \\
\text { less than 15V on the DG side. }\end{array}$ \\
\hline
\end{tabular}

Table 1. Static Switch Testing Summary 
The second set of tests (Section 7 of the test plan) examined a preliminary set of fault (i.e., overload simulating a fault) condition tests to ensure protection and safety of the test bed, prior to performing other planned tests. The goal was to test and adjust protection settings to achieve the most ideal conditions and protection design. The tests included inductor L11 in the circuit, reflecting "weak grid" conditions. Sixteen separate tests were conducted. The fault (i.e., overload simulating a fault) condition tests were intended to cover the basic concept of the protection design and to study its effectiveness (e.g., zero-sequence, negative-sequence and residual currents for line-to-ground faults; negative-sequence or ( $\left.\mathrm{I}^{2} \mathrm{t}\right)$ protection for phase-tophase faults). For the majority of these test scenarios, the static switch was expected to "OPEN" first, followed by the zone breaker of the affected zone. The objective was to confirm the protective action detailed in the Protection document developed in Task 2.3.3.

Test 7.1 induced a three-phase-to-ground balanced overload fault condition in Zone 4 to verify the $I^{2} t$ protection and a single line-to-ground fault condition in Zone 4 to verify zero-sequence, negative-sequence or residual over-current protection. Zone 3 and Zone 5 were tested similar to Zone 4 by introducing a three-phase-to-ground balanced overload fault and a single line-toground fault conditions in the zones, verifying the protection scheme in Test 7.2 (Zone 3) and Test 7.3 (Zone 5). During the 7.1, 7.2 and 7.3 tests, all three Gen-sets were off-line and the CERTS Microgrid was connected to the utility. Test 7.4 tested a three-phase-to-ground balanced overload fault condition in Zone 4 with Gen-sets A1 and A2 operating in-parallel with the utility grid to verify $\mathrm{I}^{2} \mathrm{t}$ protection, plus to confirm a reverse power event after the zone breaker "opens".

Test 7.5 through Test 7.10 involved applying a single line-to-ground overload fault condition in each Zone, located beyond the static switch, while connected to the utility grid. Each one of the six tests differs from one another by which Gen-sets are on-line during the fault, which phase is faulted, and in which zone the fault is applied. These tests were designed to verify zerosequence, negative-sequence or residual over-current protection settings. Test 7.5 verified the protection in Zone 3 by applying a single line-to-ground overload fault condition with Gen-sets A1 operating in parallel with the utility grid. Test 7.6 tested a single line-to-ground overload fault condition in Zone 3 with Gen-sets A1 and A2 operating in parallel with the utility grid. Test 7.7 tested a single line-to-ground overload fault condition in Zone 5 with Gen-set B1 operating in parallel with the utility grid. Test 7.8 tested a single line-to-ground overload fault condition in Zone 5 with Gen-sets A1 and B1 operating in parallel with the utility grid. Test 7.9 tested a single line-to-ground overload fault condition in Zone 4 with Gen-sets A1 and A2 operating in parallel with the utility grid. Test 7.10 tested a single line-to-ground overload fault condition in Zone 2 with Gen-sets A1 and B1 operating in parallel with the utility grid.

Tests 7.11 and 7.12 both applied a single line-to-ground overload fault condition in Zone 6 while connected to the utility. Test 7.11 involved only Gen-set A1 operating in parallel with the grid; and Test 7.12 involved Gen-sets A1 and B1 operating in-parallel with the grid. Both tests were designed to verify the $I^{2} t$ protection of the breaker in Zone 6.

The four tests, 7.13 - 7.16, applied a line-to-line overload fault condition in one of the five zones beyond the static switch with a combination of two Gen-sets, operating in parallel with the 
utility grid. These tests were designed to test negative-sequence, $I^{2} t$ protection or residual overcurrent protection settings. Test 7.13 tested a phase-to-phase overload fault condition in Zone 3 with Gen-sets A1 and A2 operating in parallel with the utility grid. Test 7.14 tested a phase-tophase overload fault condition in Zone 4 with Gen-sets A1 and A2 operating in parallel with the utility grid. Test 7.15 tested a phase-to-phase overload fault condition in Zone 2 with Gen-sets A1 and B1 operating in-parallel with the utility grid. Test 7.16 tested a phase-to-phase overload fault condition in Zone 5 with Gen-sets A1 and B1 operating in parallel with the utility grid.

Table 2 summarizes the test performance goals and results from each of these tests. 


\begin{tabular}{|c|c|c|}
\hline Test Description & Performance Goal & Results \\
\hline $\begin{array}{l}\text { 7.1. Validate zone } 4 \\
\text { circuit breaker } \\
\text { settings, utility } \\
\text { connected }\end{array}$ & $\begin{array}{l}\text { Initially test a three-phase balanced fault } \\
\text { condition in Zone } 4 \text { to verify } I^{2} \text { t protection. Then } \\
\text { test a single line-to-ground fault condition in Zone } \\
4 \text { to verify zero-sequence, negative-sequence or } \\
\text { residual over-current protection. }\end{array}$ & $\begin{array}{l}\text { Relay } 4 \text { detected an } I^{2} \text { t protection event and opened circuit breaker } \\
\text { CB41 during the three-phase balanced fault. All other breakers } \\
\text { remained closed. Relay } 2 \text { detected a ground over-current during the } \\
\text { single line-to-ground fault and opened the static switch. All other } \\
\text { breakers remained closed. }\end{array}$ \\
\hline $\begin{array}{l}\text { 7.2. Validate zone } 3 \\
\text { circuit breaker } \\
\text { settings, utility } \\
\text { connected }\end{array}$ & $\begin{array}{l}\text { Initially test a three-phase balanced fault } \\
\text { condition in Zone } 3 \text { to verify } I^{2} \text { t protection. Then } \\
\text { test a single line-to-ground fault condition in Zone } \\
3 \text { to verify zero-sequence, negative-sequence or } \\
\text { residual over-current protection. }\end{array}$ & $\begin{array}{l}\text { Relay } 3 \text { detected an } I^{2} \text { t protection event and opened circuit breaker } \\
\text { CB31 during the three-phase balanced fault. All other breakers } \\
\text { remained closed. Relay } 2 \text { detected a ground over-current during the } \\
\text { single line-to-ground fault and opened the static switch. All other } \\
\text { breakers remained closed. }\end{array}$ \\
\hline $\begin{array}{l}\text { 7.3. Validate zone } 5 \\
\text { circuit breaker } \\
\text { settings, utility } \\
\text { connected }\end{array}$ & $\begin{array}{l}\text { Initially test a three-phase balanced fault } \\
\text { condition in Zone } 5 \text { to verify } \mathrm{I}^{2} \text { t protection. Then } \\
\text { test a single line-to-ground fault condition in Zone } \\
5 \text { to verify zero-sequence, negative-sequence or } \\
\text { residual over-current protection }\end{array}$ & $\begin{array}{l}\text { Relay } 5 \text { detected an } I^{2} t \text { protection event and opened circuit breaker } 5 \\
\text { during the three-phase balanced fault. All other breakers remained } \\
\text { closed. Relay } 5 \text { detected a negative-sequence over-current during } \\
\text { the single line-to-ground fault and opened circuit breaker CB51. All } \\
\text { other breakers remained closed. }\end{array}$ \\
\hline $\begin{array}{l}\text { 7.4. Zone } 4 \text { three- } \\
\text { phase un-grounded } \\
\text { fault, gen-sets } \\
\text { (A1+A2), utility } \\
\text { connected }\end{array}$ & $\begin{array}{l}\text { Test a three-phase balanced fault condition in } \\
\text { Zone } 4 \text { with Gen-sets A1 and A2 operating in- } \\
\text { parallel with the utility grid to verify } I^{2} \text { t protection, } \\
\text { plus confirm a reverse power event after the } \\
\text { Zone breaker "opens". }\end{array}$ & $\begin{array}{l}\text { Relay } 4 \text { detected an } I^{2} \text { t protection and opened circuit breaker CB } 41 . \\
\text { The static switch opened } 30 \text { seconds later due to a reverse power } \\
\text { event. }\end{array}$ \\
\hline $\begin{array}{l}\text { 7.5. Zone } 3 \text { A-phase } \\
\text { line-to-ground fault, } \\
\text { gen-set } A 1 \text {, utility } \\
\text { connected }\end{array}$ & $\begin{array}{l}\text { Test a single line-to-ground fault condition in } \\
\text { Zone } 3 \text { with Gen-sets A1 operating in-parallel } \\
\text { with the utility grid to verify zero-sequence, } \\
\text { negative-sequence or residual over-current } \\
\text { protection. }\end{array}$ & $\begin{array}{l}\text { Relay } 2 \text { detected a ground over-current and opened the static switch } \\
\text { within a cycle. Relay } 3 \text { opened circuit breaker CB31 } 0.15 \text { seconds } \\
\text { after the static switch when it detected a ground over-current }\end{array}$ \\
\hline $\begin{array}{l}\text { 7.6. Zone } 3 \text { A-phase } \\
\text { line-to-ground fault, }\end{array}$ & $\begin{array}{l}\text { Test a single line-to-ground fault condition in } \\
\text { Zone } 3 \text { with Gen-sets } A 1 \text { and } A 2 \text { operating in- }\end{array}$ & $\begin{array}{l}\text { Relay } 2 \text { detected a ground over-current and opened the static switch } \\
\text { in } 0.02 \text { seconds. Relay } 4 \text { opened circuit breaker CB41 } 0.07 \text { seconds }\end{array}$ \\
\hline
\end{tabular}




\begin{tabular}{|c|c|c|}
\hline $\begin{array}{l}\text { gen-sets }(A 1+A 2) \\
\text { utility connected }\end{array}$ & $\begin{array}{l}\text { parallel with the utility grid to verify zero- } \\
\text { sequence, negative-sequence or residual over- } \\
\text { current protection }\end{array}$ & $\begin{array}{l}\text { after the static switch when it detected a neutral over-current. Relay } \\
3 \text { opened circuit breaker CB31 } 0.04 \text { seconds after circuit breaker } \\
\text { CB41 when it detected a ground over-current. }\end{array}$ \\
\hline $\begin{array}{l}\text { 7.7. Zone } 5 \text { B-phase } \\
\text { line-to-ground fault, } \\
\text { gen-set B1, utility } \\
\text { connected }\end{array}$ & $\begin{array}{l}\text { Test a single line-to-ground fault condition in } \\
\text { Zone } 5 \text { with Gen-sets B1 operating in-parallel } \\
\text { with the utility grid to verify zero-sequence, } \\
\text { negative-sequence or residual over-current } \\
\text { protection }\end{array}$ & $\begin{array}{l}\text { Relay } 5 \text { opened circuit breaker CB51 in } 0.07 \text { seconds when it } \\
\text { detected a ground over-current. The static switch opened } 0.03 \\
\text { seconds after circuit breaker CB51 when it detected a negative } \\
\text { sequence over-current. }\end{array}$ \\
\hline $\begin{array}{l}\text { 7.8. Zone } 5 \text { B-phase } \\
\text { line-to-ground fault, } \\
\text { gen-sets }(A 1+B 1) \text {, } \\
\text { utility connected }\end{array}$ & $\begin{array}{l}\text { Test a single line-to-ground fault condition in } \\
\text { Zone } 5 \text { with Gen-sets A1 and B1 operating in- } \\
\text { parallel with the utility grid to verify zero- } \\
\text { sequence, negative-sequence or residual over- } \\
\text { current protection. This test is similar to the prior } \\
\text { test, but evaluates the resultant impact of two } \\
\text { Gen-sets operating during a fault condition }\end{array}$ & $\begin{array}{l}\text { Relay } 5 \text { opened circuit breaker CB51 in } 0.06 \text { seconds when it } \\
\text { detected a ground over-current. All other breakers remained closed. }\end{array}$ \\
\hline $\begin{array}{l}\text { 7.9. Zone } 4 \text { B-phase } \\
\text { line-to-ground fault, } \\
\text { gen-sets }(A 1+A 2) \\
\text { utility connected }\end{array}$ & $\begin{array}{l}\text { Test a single line-to-ground fault condition in } \\
\text { Zone } 4 \text { with Gen-sets A1 and A2 operating in- } \\
\text { parallel with the utility grid to verify zero- } \\
\text { sequence, negative-sequence or residual over- } \\
\text { current protection. }\end{array}$ & $\begin{array}{l}\text { Relay } 2 \text { detected a ground over-current and opened the static switch } \\
\text { within a cycle. Relay } 4 \text { opened circuit breaker } 40.058 \text { seconds after } \\
\text { the static switch when it detected a ground over-current. }\end{array}$ \\
\hline $\begin{array}{l}\text { 7.10. Zone } 2 \text { C-phase } \\
\text { line-to-ground fault, } \\
\text { gen-sets }(A 1+B 1) \\
\text { utility connected }\end{array}$ & $\begin{array}{l}\text { Test a single line-to-ground fault condition in } \\
\text { Zone } 2 \text { with Gen-sets A1 and B1 operating in- } \\
\text { parallel with the utility grid to verify zero- } \\
\text { sequence, negative-sequence or residual over- } \\
\text { current protection. }\end{array}$ & $\begin{array}{l}\text { Relay } 2 \text { detected a ground over-current and opened the static switch } \\
\text { within a cycle. Relay } 3 \text { opened circuit breaker CB31 } 0.17 \text { seconds } \\
\text { after the static switch when it detected a ground over-current. Relay } \\
5 \text { opened circuit breaker CB51 due to an under-voltage condition. }\end{array}$ \\
\hline $\begin{array}{l}\text { 7.11. Zone } 6 \text { C-phase } \\
\text { line-to-ground fault, } \\
\text { gen-set } A 1 \text {, utility } \\
\text { connected }\end{array}$ & $\begin{array}{l}\text { Test a single line-to-ground fault condition in } \\
\text { Zone } 6 \text { with Gen-set } A 1 \text { operating in-parallel with } \\
\text { the utility grid to verify } I^{2} t \text { protection. }\end{array}$ & $\begin{array}{l}\text { Relay } 2 \text { detected a neutral over-current and opened the static switch } \\
\text { in } 0.07 \text { seconds. Circuit breaker CB13 tripped on an } I^{2} \text { t protection } \\
\text { event. }\end{array}$ \\
\hline
\end{tabular}




\begin{tabular}{|c|c|c|}
\hline $\begin{array}{l}\text { 7.12. Zone } 6 \text { C-phase } \\
\text { line-to-ground fault, } \\
\text { gen-sets }(A 1+B 1) \\
\text { utility connected }\end{array}$ & $\begin{array}{l}\text { Test a single line-to-ground fault condition in } \\
\text { Zone } 6 \text { with Gen-sets } A 1 \text { and } B 1 \text { operating in- } \\
\text { parallel with the utility grid to verify } I^{2} t \text { protection. } \\
\text { This test is similar to the prior test, but evaluates } \\
\text { the resultant impact of two Gen-sets operating } \\
\text { during a fault condition. }\end{array}$ & $\begin{array}{l}\text { Relay } 2 \text { detected a neutral over-current and opened the static switch } \\
\text { in } 0.07 \text { seconds. Circuit breaker CB13 tripped on an I't protection } \\
\text { event. }\end{array}$ \\
\hline $\begin{array}{l}\text { 7.13. Zone } 3 \text { A-to-B } \\
\text { phase fault, gen-sets } \\
\text { (A1+A2), utility } \\
\text { connected }\end{array}$ & $\begin{array}{l}\text { Test a phase-to-phase fault condition in Zone } 3 \\
\text { with Gen-sets A1 and A2 operating in-parallel } \\
\text { with the utility grid to verify negative-sequence, } \\
I^{2} \text { t protection or residual over-current protection. }\end{array}$ & $\begin{array}{l}\text { Relay } 2 \text { detected a negative sequence over-current and opened the } \\
\text { static switch in } 0.028 \text { seconds. All other breakers remained online. }\end{array}$ \\
\hline $\begin{array}{l}\text { 7.14. Zone } 4 \text { A-to-B } \\
\text { phase fault, gen-sets } \\
(A 1+A 2) \text {, utility }\end{array}$ & $\begin{array}{l}\text { Test a phase-to-phase fault condition in Zone } 4 \\
\text { with Gen-sets A1 and A2 operating in-parallel } \\
\text { with the utility grid to verify negative-sequence, } \\
\mathrm{I}^{2} \text { t protection or residual over-current protection. }\end{array}$ & $\begin{array}{l}\text { Relay } 2 \text { detected a negative sequence over-current and opened the } \\
\text { static switch in } 0.078 \text { seconds. Relay } 4 \text { opened circuit breaker CB41 } \\
\text { after the static switch when it detected a negative sequence over- } \\
\text { current. }\end{array}$ \\
\hline $\begin{array}{l}\text { 7.15. Zone } 2 \text { A-to-B } \\
\text { phase fault, gen-sets } \\
\text { (A1+B1), utility } \\
\text { connected }\end{array}$ & $\begin{array}{l}\text { Test a phase-to-phase fault condition in Zone } 2 \\
\text { with Gen-sets A1 and B1 operating in-parallel } \\
\text { with the utility grid to verify negative-sequence, } \\
\mathrm{I}^{2} \text { t protection or residual over-current protection. }\end{array}$ & $\begin{array}{l}\text { There was not enough current produced by the fault, therefore, all } \\
\text { breakers remained closed during the test. }\end{array}$ \\
\hline $\begin{array}{l}\text { 7.16. Zone } 5 \text { A-to-B } \\
\text { phase fault, gen-sets } \\
\text { (A1+B1), utility } \\
\text { connected }\end{array}$ & $\begin{array}{l}\text { Test a phase-to-phase fault condition in Zone } 5 \\
\text { with Gen-sets } A 1 \text { and B1 operating in-parallel } \\
\text { with the utility grid to verify negative-sequence, } \\
I^{2} \text { t protection or residual over-current protection. }\end{array}$ & $\begin{array}{l}\text { Relay } 5 \text { detected a negative sequence over-current and opened } \\
\text { circuit breaker in } 0.075 \text { seconds. Relay } 2 \text { opened the static switch } \\
0.016 \text { seconds after circuit breaker CB51 when it detected a } \\
\text { negative sequence over-current. }\end{array}$ \\
\hline
\end{tabular}

\section{Table 2. Internal Protection Testing Summary}


The third set of tests (Section 8 of the test plan) was designed to ensure that the Gen-set inverter controls were working as designed. This includes unit control, zone control, and mixed power controls, in conjunction with limit controls and synchronized closing of the static switch. These tests were based on replicating tests that had previously been conducted by Tecogen and Youtility during the factory acceptance testing of the standalone inverters, and the engine coupled inverters. The performance goal was to observe smooth transitions of the Gen-sets response to different step conditions (i.e., static switch "OPEN"/ "CLOSE" and load steps). Thirteen separate tests were conducted.

Test 8.1 verified smooth transitions in Gen-set A1 when different step conditions of load are applied in Load Bank 3 with voltage set points ranging from $+5 \%$ to $-5 \%$. The same test was repeated for Gen-set A2 and Load Bank 4 and Gen-set B1 and Load Bank 5.

Test 8.2 verified smooth transitions of Gen-sets A1 and A2 response to different step conditions which drive Gen-set A1 to a lower limit of zero kW.

Test 8.3 verified smooth transitions of Gen-sets A1 and A2 response to different step conditions which drive Gen-set A2 to an upper limit of $60 \mathrm{~kW}$.

Test 8.4 verified smooth transitions of Gen-sets A1 and A2 response to different step conditions with an un-balanced load in Zone 3.

Test 8.5 verified smooth transitions of Gen-sets, A1 in Zone operation mode and A2 in Unit operation mode. During a load step change in Load Bank 3, Gen-set A1 was driven to an upper limit of $60 \mathrm{~kW}$.

Test 8.6 verified smooth transitions of Gen-sets, A1 in zone operation mode and Gen-set A2 in Unit operation mode. During a load step change in Load Bank 4, Gen-set A1 is driven to its maximum which causes an automatic reset of the zone set points.

Test 8.7 verified smooth transitions of Gen-sets, A1 in Zone operation mode and A2 in Unit operation mode, with a change of zone power in Feeder A.

Test 8.8 verified smooth transition of Gen-sets, A1 in Zone operation mode and A2 in Unit operation mode, with a static switch operation and a change of zone power in Zone 3 when islanded.

Test 8.9 verified smooth transition of Gen-sets, A1 in Zone operation mode and A2 in Unit operation mode, with a static switch operation and a change of zone power in Zone 3 when islanded. Gen-set A1 was driven to its maximum which caused an automatic reset of both Gensets set-points.

Test 8.10 verified smooth transitions of Gen-sets, A1 and B1 in Zone operation mode, with a static switch operation and a change of zone power in Zones 3 and 5 when islanded.

Test 8.11 verified smooth transitions of Gen-sets, A1 and B1 in Zone operation mode, with a static switch operation and a change of zone power in Zones 3 and 5 when islanded. When 
islanded, Gen-set A1 and B1 set-points were reset based on the remaining load in the islanded system.

Test 8.12 tested the manual procedure used to black-start the CERTS Micro-grid Test Bed in the event of a lengthy utility outage occurs with the Gen-sets off-line.

Test 8.13 was designed to determine the black-start capacity by increasing the amount of load on the CERTS Microgrid Test Bed from a black-out condition without generation or protection trips.

Table 3 summarizes the test performance goals and results from each of these tests 


\begin{tabular}{|c|c|c|}
\hline Test Description & Performance Goal & Results \\
\hline $\begin{array}{l}\text { 8.1. Initial voltage regulation test - } \\
\text { single zone, islanded with gen-set } A 1\end{array}$ & $\begin{array}{l}\text { Verify smooth transitions of Gen-set A1 response to } \\
\text { different step conditions of load in Load Bank } 3 \text { with } \\
\text { voltage set point changes ranging from }+5 \% \text { to }-5 \% \text {. } \\
\text { Repeat tests with Gen-set A2 in Load Bank } 4 \text {, and then } \\
\text { Gen-set B1 in Load Bank } 5\end{array}$ & $\begin{array}{l}\text { All Gen-sets had smooth transitions } \\
\text { through each load step and voltage } \\
\text { change. }\end{array}$ \\
\hline $\begin{array}{l}\text { 8.2. Open static switch test, check } P= \\
0 \text { limit, gen-set } A 1\end{array}$ & $\begin{array}{l}\text { Verify smooth transitions of Gen-sets A1 and A2 response } \\
\text { to different step conditions (i.e., static switch "OPEN"/ } \\
\text { "CLOSE") with the unit power limit of Gen-set A1 equal to } \\
\text { zero }\end{array}$ & $\begin{array}{l}\text { Gen-sets had smooth transition from } \\
\text { grid connected to island mode and vice } \\
\text { versa. All Gen-sets responded as } \\
\text { predicted. }\end{array}$ \\
\hline $\begin{array}{l}\text { 8.3. Open static switch test, check } P= \\
60 \mathrm{~kW} \text { limit, gen-set } A 2\end{array}$ & $\begin{array}{l}\text { Verify smooth transitions of Gen-sets A1 and A2 response } \\
\text { to different step conditions (i.e., static switch "OPEN"/ } \\
\text { "CLOSE") with the unit power limit of Gen-set A2 equal to } \\
60 \mathrm{~kW}\end{array}$ & $\begin{array}{l}\text { Gen-sets had smooth transition from } \\
\text { grid connected to island mode and vice } \\
\text { versa. All Gen-sets responded as } \\
\text { predicted. }\end{array}$ \\
\hline $\begin{array}{l}\text { 8.4. Test island operation, unbalanced } \\
\text { load }\end{array}$ & $\begin{array}{l}\text { Verify smooth transitions of Gen-sets A1 and A2 response } \\
\text { to different step conditions (i.e., static switch "OPEN"/ } \\
\text { "CLOSE") with an un-balanced load condition in Zone } 3\end{array}$ & $\begin{array}{l}\text { Gen-sets had smooth transition from } \\
\text { grid connected to island mode and vice } \\
\text { versa. All Gen-sets responded as } \\
\text { predicted. }\end{array}$ \\
\hline $\begin{array}{l}\text { 8.5. Mixed mode operation test - Zone } \\
3 \text { and } 4 \text {, gen-set } A 1 \text { to } 60 \mathrm{~kW} \text { maximum }\end{array}$ & $\begin{array}{l}\text { Verify smooth transitions of Gen-sets, A1 in Zone } \\
\text { operation mode with a } 60 \mathrm{~kW} \text { limit and A2 in Unit operation } \\
\text { mode, during a load step change in Load Bank } 3\end{array}$ & $\begin{array}{l}\text { Gen-set had smooth transition from grid } \\
\text { connected to island mode and vice } \\
\text { versa. Gen-sets responded as } \\
\text { predicted. }\end{array}$ \\
\hline $\begin{array}{l}\text { 8.6. Mixed mode operation test - } \\
\text { Zones } 3 \text { and } 4 \text {, automatic reset of zone } \\
\text { level set-point }\end{array}$ & $\begin{array}{l}\text { Verify smooth transitions of Gen-sets, A1 in Zone } \\
\text { operation mode with an automatic reset of the set point } \\
\text { and A2 in Unit operation mode, during a load step change } \\
\text { in Load Bank } 4\end{array}$ & $\begin{array}{l}\text { Gen-sets had smooth transition from } \\
\text { grid connected to island mode and vice } \\
\text { versa. All Gen-sets responded as } \\
\text { predicted. }\end{array}$ \\
\hline 8.7. Mixed mode operation test - & Verify smooth transitions of Gen-sets, A1 in Zone & Gen-sets had smooth transition from \\
\hline
\end{tabular}




\begin{tabular}{|c|c|c|}
\hline Zones 3 and 4 , zone power change & $\begin{array}{l}\text { operation mode and A2 in Unit operation mode, with a } \\
\text { change of zone power in Feeder A }\end{array}$ & $\begin{array}{l}\text { grid connected to island mode and vice } \\
\text { versa. All Gen-sets responded as } \\
\text { predicted. }\end{array}$ \\
\hline $\begin{array}{l}\text { 8.8. Mixed mode operation test - } \\
\text { Zones } 3 \text { and } 4 \text {, zone power change } \\
\text { when islanded }\end{array}$ & $\begin{array}{l}\text { Verify smooth transitions of Gen-sets, A1 in Zone } \\
\text { operation mode and A2 in Unit operation mode, with a } \\
\text { static switch operation and a change of zone power in } \\
\text { Zone } 3 \text { when islanded }\end{array}$ & $\begin{array}{l}\text { Gen-sets had smooth transition from } \\
\text { grid connected to island mode and vice } \\
\text { versa. All Gen-sets responded as } \\
\text { predicted. }\end{array}$ \\
\hline $\begin{array}{l}\text { 8.9. Mixed mode operation test - } \\
\text { Zones } 3 \text { and } 4 \text {, when islanded, } \\
\text { automatic reset of zone level set-point, } \\
\text { both gen-sets } P=60 \mathrm{~kW} \text { Maximum }\end{array}$ & $\begin{array}{l}\text { Verify smooth transitions of Gen-sets, A1 in Zone } \\
\text { operation mode with automatic reset of set point and A2 in } \\
\text { Unit operation mode, with a static switch operation and a } \\
\text { change of zone power in Zone } 3 \text { when islanded }\end{array}$ & $\begin{array}{l}\text { Gen-sets had smooth transition from } \\
\text { grid connected to island mode and vice } \\
\text { versa. All Gen-sets responded as } \\
\text { predicted. }\end{array}$ \\
\hline $\begin{array}{l}\text { 8.10. Two sources in zone control - } \\
\text { separate zones, when islanded, } \\
\text { automatic reset of zone level set-point, } \\
\text { new zones sum equal zero }\end{array}$ & $\begin{array}{l}\text { Verify smooth transitions of Gen-sets, A1 and B1 in Zone } \\
\text { operation mode, with a static switch operation and a } \\
\text { change of zone power in Zones } 3 \text { and } 5 \text { when islanded }\end{array}$ & $\begin{array}{l}\text { Gen-sets had smooth transition from } \\
\text { grid connected to island mode and vice } \\
\text { versa. All Gen-sets responded as } \\
\text { predicted. }\end{array}$ \\
\hline $\begin{array}{l}\text { 8.11. Two sources in zone control - } \\
\text { separate zones, when islanded, } \\
\text { automatic reset of zone level set-points }\end{array}$ & $\begin{array}{l}\text { Verify smooth transitions of Gen-sets, A1 and B1 in Zone } \\
\text { operation mode and both having automatic reset of set } \\
\text { points, with a static switch operation and a change of zone } \\
\text { power in Zones } 3 \text { and } 5 \text { when islanded }\end{array}$ & $\begin{array}{l}\text { Gen-sets had smooth transition from } \\
\text { grid connected to island mode and vice } \\
\text { versa. All Gen-sets responded as } \\
\text { predicted. }\end{array}$ \\
\hline 8.12. Test generator black-start & $\begin{array}{l}\text { Bring up the Microgrid Test Bed from a black-out condition } \\
\text { without generation or protection trips. } \\
\text { Measurement - Record the transition of the Gen-set } \\
\text { meters as they are started and brought on-line and as } \\
\text { load banks are switched on-line. Note, the transition time } \\
\text { between all events outage occurs with the Gen-sets off- } \\
\text { line } \\
\text { This test checks the manual procedure used to black-start } \\
\text { the Microgrid Test Bed in the event of a lengthy utility }\end{array}$ & $\begin{array}{l}\text { Gen-sets had smooth transitions and } \\
\text { met the load demand. }\end{array}$ \\
\hline
\end{tabular}


8.13. Test/establish generator black-

start capacity
Determine the Black-start capacity of the microgrid by

increasing the amount of load on the Microgrid Test Bed

from a black-out condition without generation or protection

trips

Total capacity was not determined because the Gen-set stayed online during each test supplying the load demand even for the $70 \mathrm{~kW}+\mathrm{j} 30 \mathrm{kVAr}$ load. This test proved that the Gen-sets are more robust for blackstart then previously predicted.

Table 3. Reduced System Testing Summary 
The fourth set of tests (Section 9 of the test plan) demonstrated the flexibility of the microgrid both grid connected and islanded for different loads, power flows and impact on the utility. The tests included the "weak grid" inductors (i.e., L11) in the circuit. Three sets of tests were conducted.

Tests 9.1 - 9.3 verified and documented power flow and microgrid frequency changes when transitioning from utility connected to an islanded mode of operation. In each test, 9.1-9.3, a series of tests were performed that vary in the amount of load that is applied to the microgrid in a weak grid scenario along with the power settings of each Gen-set. The difference between each test is the type of control mode that the Gen-sets are in. In Test 9.1, all the Gen-sets were set for unit control mode and then the next test, 9.2, all the Gen-sets were set for zone control mode. Test 9.3 mixed the unit and zone control modes of the Gen-sets during each test.

Table 4 summarizes the test performance goals and results from each of these tests 


\begin{tabular}{|l|l|l|}
\hline Test Description & Performance Goal & Results \\
\hline $\begin{array}{l}\text { 9.1. Unit control mode, weak } \\
\text { grid }\end{array}$ & $\begin{array}{l}\text { Verify and document power flow and Microgrid frequency } \\
\text { changes when transitioning from utility connected to an } \\
\text { islanded mode of operation. During each sequence of } \\
\text { tests, maintain a weak grid connection with L11 in the } \\
\text { circuit and the static switch "Closed"; all zone and load } \\
\text { bank circuit breakers "Closed"; Gen-sets A1, A2 and B1 } \\
\text { set for Unit (injection power) control mode; and all load } \\
\text { banks initially set at zero }\end{array}$ & $\begin{array}{l}\text { Voltage and frequency remained stable during grid } \\
\text { connected to island mode and vice versa for all four } \\
\text { tests. Control of Power was as expected from Gen-sets } \\
\text { to load demand. }\end{array}$ \\
\hline $\begin{array}{l}\text { 9.2. Zone control mode, } \\
\text { weak grid }\end{array}$ & $\begin{array}{l}\text { Verify and document power flow and Microgrid frequency } \\
\text { changes when transitioning from utility connected to an } \\
\text { islanded mode of operation. }\end{array}$ & $\begin{array}{l}\text { Voltage and frequency remained stable during grid } \\
\text { connected to island mode and vice versa for all four } \\
\text { tests. Control of Power was as expected from Gen-sets } \\
\text { to load demand. }\end{array}$ \\
\hline $\begin{array}{l}\text { 9.3. Mixed zone and unit } \\
\text { control modes, weak grid }\end{array}$ & $\begin{array}{l}\text { Verify and document power flow and Microgrid frequency } \\
\text { changes when transitioning from utility connected to an } \\
\text { islanded mode of operation. }\end{array}$ & $\begin{array}{l}\text { Voltage and frequency remained stable during grid } \\
\text { connected to island mode and vice versa for all four } \\
\text { tests. Control of Power was as expected from Gen-sets } \\
\text { to load demand. }\end{array}$ \\
\hline
\end{tabular}

Table 4. Power Flow Control Testing Summary 
The fifth and final set of testing (Section 10 of the test plan) began to explore the operation limits of the microgrid (i.e., power quality, protection and inverter limits). Two primary sets of tests were conducted under weak grid conditions; the first involved induction motor starting loads under balanced and unbalanced load conditions; the second involved only unbalanced loads.

Motor start tests conducted in Test 10.2 had balanced loads at a 0.9 power factor under weak grid conditions. These tests verified and documented power flow, microgrid frequency changes, and protection design with different Gen-set settings during motor starts when utility connected and then again during islanded mode of operation. Test 10.3 was similar to Test 10.2, except that the loads were unbalanced with a 0.9 power factor.

In Test 10.4 verified and documented power flow, microgrid frequency changes, and protection design with different Gen-set settings during changes of unbalanced load in load banks.

Table 5 summarizes the test performance goals and results from each of these tests. 


\begin{tabular}{|c|c|c|}
\hline Test Description & Performance Goal & Results \\
\hline $\begin{array}{l}\text { 10.2. Motor Start Tests, } \\
\text { Weak Grid, Balanced } \\
\text { Load }\end{array}$ & $\begin{array}{l}\text { Verify and document power flow, Micro-grid } \\
\text { frequency changes and protection design with } \\
\text { different Gen-set settings during motor starts when } \\
\text { utility connected and island mode of operation. }\end{array}$ & $\begin{array}{l}\text { CERTS Microgrid remained electrically stable } \\
\text { during the } 10 \mathrm{Hp} \text { induction motor start in Zone } 3 \\
\text { while utility connected and islanded mode for all } \\
\text { six test. }\end{array}$ \\
\hline $\begin{array}{l}\text { 10.3. Motor Start Tests, } \\
\text { Weak Grid, Unbalanced } \\
\text { Load }\end{array}$ & $\begin{array}{l}\text { Verify and document power flow, Micro-grid } \\
\text { frequency changes and protection design with } \\
\text { different Gen-set settings during motor starts when } \\
\text { utility connected and island mode of operation. }\end{array}$ & $\begin{array}{l}\text { CERTS Microgrid remained electrically stable } \\
\text { during the } 10 \mathrm{Hp} \text { induction motor start in Zone } 3 \\
\text { while utility connected and islanded mode for all } \\
\text { six test. }\end{array}$ \\
\hline $\begin{array}{l}\text { 10.4. Unbalanced Load } \\
\text { Tests, Weak Grid }\end{array}$ & $\begin{array}{l}\text { Verify and document power flow, Micro-grid } \\
\text { frequency changes and protection design with } \\
\text { different Gen-set settings during changes of } \\
\text { unbalanced load in Load Banks while utility } \\
\text { connected and in islanded mode of operation. }\end{array}$ & $\begin{array}{l}\text { Static switch opened on a reverse power trip } \\
\text { while utility connected in Tests } 10.4 .12 \text { and } 10.4 .14 \\
\text { when Load Banks } 3 \text { and } 6 \text { A-phase was reduced } \\
\text { by } 50 \% \text {. CERTS Microgrid remained electrically } \\
\text { stable when the A-phase in the load demands } \\
\text { equaled 0kW and in island mode of operation. } \\
\text { The static switched opened on a reverse power } \\
\text { trip for all three tests when the microgrid was } \\
\text { reconnected to the utility grid. Once the static } \\
\text { switch opened, the CERTS microgrid remained } \\
\text { electrically stable. All test results performed as } \\
\text { predicted. }\end{array}$ \\
\hline
\end{tabular}

Table 5. Difficult Load Testing Summary 
The testing fully confirmed earlier research that had been conducted initially through analytical simulations, then through laboratory emulations, and finally through factory acceptance testing of individual microgrid components. The islanding and resychronization method met all IEEE 1547 and power quality requirements. The electrical protection system was able to distinguish between normal and faulted operation. The controls were found to be robust under all conditions, including difficult motor starts.

Test bed results were then presented and discussed jointly with and then reviewed individually by members of the TAC. The reviews were uniformly positive:

"Concepts are elegant, yet simple. Simple translates to more affordable."

“Demonstration that static switch can comply with 1547, with non-compliant generation behind it, is a major accomplishment. The team has confirmed that a static switch is very fast. It is recognized that ground fault testing on the utility side of the static switch is still missing from this aspect of 1547 compliance."

“Inverter controls are impressive."

“Test plan was well laid-out. A wide range of concerns that were identified have now been successfully addressed. In particular, motor starting was a challenging test and the results are very encouraging. Should be a major contribution to the literature."

"The test bed platform should be used for future research."

A summary of the TAC meeting at which test bed results were reviewed as well as the additional written comments received from the TAC are contained in Appendix O. Technical Advisory Committee Meeting Summary and Review Comments.

In addition, AEP has prepared a standalone technical assessment and recommendations:

“The major objective of plug and play gen-sets has been proven to work very well and is seen as a major stepping stone toward increasing the commercial adoption of microgrids. The keystone of this plug and play approach is the CERTS frequency versus power and voltage versus VAR algorithms which have proven to be robust in the control of multiple voltage source inverters on a common bus. The specific advantages offered by this approach are expected to include: improved operation, more manageable equipment requirements, all at lower total system costs compared to a traditional custom-designed distributed generation power system involving multiple prime movers.

In addition, the concept of placing generation, which is non-compliant with IEEE Standard 1547, beyond a static switch, which is compliant, was tested and proven to be successful. This is expected to be another effective tool in reducing the cost of a 
traditional custom-designed, distributed generation power system involving multiple prime movers, while still maintaining safe interconnection with a utility system.

It is recognized that the tests conducted by AEP represent the first full-scale tests of the CERTS Microgrid Concept. AEP looks forward to participation in future research and testing to advance this Concept. Toward this end, we recommend focus on the following areas:

1. Demonstrate the ability of power electronic inverters to adjust fault contribution from the gen-sets.

2. Re-examine and potentially re-tune the anti-islanding philosophy governing operation of the static switch, in light of expected unbalanced voltages presented by utility distribution systems to the microgrid.

3. Develop and test means for minimizing VAR flows by using the Energy Manager to direct voltage set-point changes to the gen-sets based on actual voltages on the utility distribution system.

4. Continue testing to explore performance of the CERTS Microgrid in supporting difficult loads.

5. Confirm the adequacy or revise the management of the static switch when the utility closes into a dead bus.

6. Examine enhanced functionalities for the Energy Manager to address customer and utility needs, such as power factor correction, fault and error handling, energy efficiency, and price- or reliability-driven demand management.

7. Examine system impacts for scenarios involving significant deployment of microgrids."

\subsubsection{Microgrid Protective Relaying Design}

Traditionally, power flow in the existing electrical infrastructure flows from the power plant through transmission and possibly distribution lines to the load that is demanding power. Protection schemes were based on this concept, which allowed for uni-directional over-current protection devices to be installed. Uni-directional over-current protection devices sense when there is a fault on the electrical system by opening up when the current exceeds a certain value in a certain direction.

Uni-directional over-current protection schemes are not feasible within microgrids, because of microgrids' reverse power-flow capability; if current is flowing in the opposite direction (i.e., from the microsources toward the point of common coupling (PCC)), it will not trip the overcurrent device when a fault occurs. A microgrid can use bi-directional over-current protection devices when the microgrid is connected to the utility but not while the microgrid is islanded. During islanded operation, the microgrid fault current magnitudes are less significant than 
when the microgrid is connected to the utility, so uni-directional and bi-directional detection may not detect the fault current preventing the protection device from operating.

The team developed a protection scheme that uses sequence components to detect low-fault currents in utility-connected and islanded operation. Sequence components take a balanced or unbalanced voltage or current and break it up into three separate components: positive, negative, and zero sequence. Adding these three components together gives the magnitude and phase angle of balanced and unbalanced currents and voltages. A single line-to-ground fault produces a zero sequence current, providing a trip signal for the zone with the fault that does not depend on the direction of the current and magnitude as is the case with traditional overcurrent protection devices. A line-to-line fault produces a negative sequence current that signals the zone with the fault to trip. This protection scheme allows for plug-and-play without changing the existing over-current devices on the electrical system.

The technical report on the microgrid protective relaying design is contained in Appendix O. Microgrid Fault Protection Based on Symmetrical and Differential Current Components.

\subsection{Field Demonstration Planning (Task 2.4)}

The goal of task 2.4 was to identify and engage one or more partners for a field demonstration of the CERTS Microgrid concept. While a field demonstration was not included in the project plan for this project, it was important to engage potential partners prior to and during the laboratory test bed phase so that partner-specific technical or operational issues could be addressed in the laboratory test bed prior to conducting a field demonstration.

The identification of potential field demonstration partners took place through a variety of means. Central to most of them was outreach conducted through a variety of presentations and publications.

\section{Presentations}

R.H. Lasseter, “Advanced Distribution using DER," Rethinking T\&D Architecture for DER, 24 April 2008, T\&D Panel, Chicago

R.H. Lasseter, “DER and Microgrids,” IEEE Distinguish Lecturer, IEEE PES St. Louis chapter, 6 November 2007

R.H. Lasseter, “Microgrids and Distributed Generation,” ASCE Journal Energy Engineering, Volume 133, Number 3, September 2007.

H. Nikkhajoei and R.H. Lasseter, "Microgrid Protection," IEEE Panel: Microgrid Research and Field Testing, IEEE PES General Meeting, 24-28 June 2007, Tampa, FL

R.H. Lasseter, "Extended Microgrid Using (DER) Distributed Energy Resources," IEEE Panel: Sustainable Energy, IEEE PES General Meeting, 24-28 June 2007, Tampa, FL

R.H. Lasseter, “CERTS Microgrid," Panel on Microgrids Systems, International Conference on System of Systems Engineering, April 16-18, 2007 San Antonio 
R.H. Lasseter, “CERTS Microgrid,” ERDC/CERL_RDCOM, Army Engineers, 18 January 2007, Champaign, Ill.

R.H. Lasseter, "Microgrids on Distribution Scale," Panel, Army Installation Energy Security \& Independence Conference, Greensboro, N.C., 12-13 December 2006

R.H. Lasseter. "Methods for Controlling Multiple Independent Generators in an Intentional Island (Microgrids)," Minnesota Power 11/8/06

R.H. Lasseter. “Distributed Energy Resources and Microgrids,” IEEE Distinguish Lecturer, IEEE Cuernavaca, Mexico, 2 July 2006.

R.H. Lasseter. “Directions In Microgrid research”, PSerc Lecture, 6 June 2006, Madison, Wi

R.H. Lasseter." Autonomous Control", 2006 PES General Meeting, 22 June 2006, Montréal, Québec Canada

R.H. Lasseter. "Microgrids and Protection”, CERTS IAB Meeting, 27 June 2006, Cleveland, Ohio

R.H. Lasseter. “Enhanced Microgrid”, Microgrid Road Map. 18 May 2006, Office of Electric Energy, Washington, DC

R.H. Lasseter. “Dynamics Distribution”, IEEE T\&D Meeting, Panel on Future of Distribution, Dallas, 20 May 2006 "Enhanced Business Case for CERTS Microgrid”, Peer Review Meeting for the Department of Energy's , Electric Distribution R\&D Program, 26 May 2006, San Ramon Ca

R.H. Lasseter. "The Role of Distributed Energy Resources in Future Electric Power Systems", Energy System Seminar, 27 March 2006, Madison, Wi

R.H. Lasseter. “DER based Distribution”, DOE Energy Workshop, Panel, January 31 to February 1 2006, Tallahassee, Fl

R.H. Lasseter. “Microgrid Test Plan”, CERTS IAB Meeting, 6 October 2005, Cleveland, Ohio

R.H. Lasseter. "CERTS Microgrid”, International Microgrid Workshop, Panel Speaker, 17 June 2005, University of California-Berkeley, Berkeley, California

R.H. Lasseter. "CERTS Microgrid", California Energy Commission R\&D Forum, 4 May 2005, Sacramento, $\mathrm{Ca}$

R.H. Lasseter. "Microgrid: A Conceptual Solution," Wisconsin Distributed Resources Collaborative, September 16, 2004

R.H. Lasseter. “CERTS Microgrid,” CEC Technical Advisory Committee Meeting, July 19, 2004

R.H. Lasseter. "Where are Microgrids Going?,“ Distributed Resources Workshop, CEA, May 9-11, 2004 Calgrary

R.H. Lasseter. "Microgrids: What's Next," PSerc Industrial Advisory Board, May 20-21, 2004

\section{Publications}


Klapp, D., H.T. Vollkommer. Application of an Intelligent Static Switch to the Point of Common Coupling to Satisfy IEEE 1547 Compliance. Panel: Microgrid Research and Field Testing IEEE PES General Meeting, 24-28 June 2007, Tampa, FL. 4 pages.

Lasseter, R.H., P. Piagi. 2006. Control and Design of Microgrid Components. January. 257 pages. http://www.pserc.org/ecow/getpublicatio/reports/2006report

Lasseter, R. Dynamic Distribution using (DER) Distributed Energy Resources. 2006. IEEE PES T \& D Meeting, Dallas, May. 3 pages.

Lasseter, R.H. 2007. “Microgrids and Distributed Generation.” Journal of Energy Engineering, Volume 133, Issue3, ASCE, Sept. 7 pages.

Lasseter, R.H. 2007. “CERTS Microgrid.” International Conference on System of Systems Engineering , April 16-18. 6 pages.

Nichols, D.K., J. Stevens, R.H. Lasseter, J.H. Eto, H.T. Vollkommer. 2006 Validation of the CERTS Microgrid Concept: The CEC/CERTS Microgrid Testbed. Power Engineering Society General Meeting, IEEE. 3 pages.

Nikkhajoei, H., R. H. Lasseter. 2007. Microgrid Protection. IEEE PES General Meeting, 24-28, Tampa, FL. June. 6 pages.

Nikkhajoei, H., R. H. Lasseter. 2006. Microgrid Fault Protection Based on Symmetrical and Differential Current Components. December. 72 pages. (Power Systems Engineering Research Center (PSERC) website)

Panora, R.A., J.B. Gehret Jr., P. Piagi. 2007. Design and Testing of an Inverter-Based Combined Heat and Power Module for Special Application in a Microgrid. Panel: Microgrid Research and Field Testing IEEE PES General Meeting, June 24-28, Tampa FL. 7 pages.

Stevens, J., H. Vollkommer, D.Klapp. CERTS Microgrid System Tests. Power Engineering Society General Meeting, June 24-28. 4 pages.

In addition, the CERTS Microgrid concepts have appeared in Distributed Energy Journal, May/June 2008, which can be found at http://www.distributedenergy.com/de 0805 micro.html.

Through this outreach, members of the CERTS team met with a wide variety of potential field demonstrations partners during the course of the project. A separate Energy Commission contract to Navigant Consulting further augmented efforts to develop field demonstrations involving the CERTS Microgrid concept.

At the time of the preparation of this report, the status of discussions with potential partners for field demonstrations involving the CERTS Microgrid Concept is as follows:

Tecogen, a partner in the CERTS Microgrid Test Bed project, is currently conducting a research project for the New York State Energy Research and Development Authority to demonstrate aspects of the CERTS Microgrid Concept at a school in upstate New York. 
The Energy Commission PIER program is considering funding for two demonstrations involving aspects of the CERTS Microgrid Concept. Chevron Energy Solutions is proposed to lead a demonstration, which would involve battery storage, renewable energy, and engine generators at a correctional facility in Northern California. Sacramento Municipal Utilities District (SMUD) is proposed to lead a demonstration that would involve engine generators at SMUD corporate headquarters.

Two additional demonstrations involving aspects of the CERTS Microgrid Concept have been submitted in response to a competitive solicitation from the U.S. Department of Energy (DOE). The identities of the firms leading these proposals are being withheld pending the announcement of solicitation awardees by DOE.

In addition, active discussions are also under way for additional field demonstrations, involving the U.S. Army Corp of Engineers/Construction Research Laboratory and with Cummins Engines. 


\subsection{Conclusions and Recommendations}

This section describes the conclusions drawn from the successful demonstration of the CERTS Microgrid concept at a full-scale test bed operated by AEP and lists recommendations for future work.

\subsection{Conclusions}

The objective of the CERTS Microgrid Laboratory Test Bed Demonstration project was to demonstrate three advanced techniques comprising the CERTS Microgrid concept at a full-scale test bed operated by AEP.

The advance techniques, which included: 1) a method for effecting automatic and seamless transitions between grid-connected and islanded modes of operation; 2) an approach to electrical protection within the microgrid that does not depend on high fault currents; and 3) a new method for microgrid control that achieves voltage and frequency stability under islanded conditions without requiring high-speed communications, were demonstrated through a carefully orchestrated sequence of five sets of tests by AEP.

The first set of tests examined the operation of the static switch to determine that it and its digital signal processing (DSP) control operated as designed. The second set of tests examined a preliminary set of fault (i.e., overload simulating a fault) condition tests to ensure protection and safety of the test bed, prior to performing other planned tests. The third set of tests was designed to ensure that the Gen-set inverter controls were working as designed. The fourth set of tests demonstrated the flexibility of the microgrid both grid connected and islanded for different loads, power flows and impact on the utility. The fifth and final set of testing began to explore the operation limits of the microgrid (i.e., power quality, protection and inverter limits) with motor starting loads.

The testing fully confirmed earlier research that had been conducted initially through analytical simulations, then through laboratory emulations, and finally through factory acceptance testing of individual microgrid components. The islanding and resychronization method met all IEEE 1547 and power quality requirements. The electrical protection system was able to distinguish between normal and faulted operation. The controls were found to be robust under all conditions.

\subsection{Recommendations}

The next logical phase for RD\&D on the CERTS Microgrid Concept is to build from the base established by the currently operational CERTS Microgrid to prioritize, develop, and then demonstrate needed additional technology enhancements required to optimize the microgrid from the explicit perspective of enhancing the business case for microgrids. That is, having demonstrated the technical feasibility of microgrid functions, RD\&D optimization efforts are now needed to accelerate commercial deployment. The should pay special attention to the economic drivers, such as economic dispatch responsive to pricing signals and demand management programs, customer willingness to pay premiums for increased power reliability and quality, etc. 
In order to enhance the business case for microgrids, the cost and functionalities of the CERTS Microgrid need to be compared directly against traditional solutions for a) CHP including the heat distribution systems and system reliability, and b) power quality, including multiple feeders and/or UPS systems. That is, enhancing the business case for microgrids involves, first, identifying the cost and performance targets of the traditional approaches for providing the values offered by a microgrid and then, second, prioritizing development and testing of CERTS Microgrid technology enhancements to beat these targets. Some promising areas to consider include: 1) lowering the costs of providing protection among microsources and loads; 2) determining the optimal amount of storage required on the DC bus; 3 ) the best size, technology, and control strategy (or strategies) for integration of AC storage; and 4) inclusion of noninverter-based prime-movers along side of inverter-based prime-movers within a microgrid.

\subsection{Benefits to California}

DER - small power generators typically located at sites where the energy (both electric and thermal) they generate is used - are a promising option to meet growing customer needs for economic and reliable electric power (the DER portfolio also includes energy storage, and load control). Organizing all of these resources into microgrids is a promising way to capture smaller DER's significant potential to meet customers' and utilities' needs.

A key feature of a microgrid is its ability, during a utility grid disturbance, to separate and isolate itself from the utility seamlessly with no disruption to the loads within the microgrid (including no reduction in power quality). Then, when the utility grid returns to normal, the microgrid automatically resynchronizes and reconnects itself to the grid, in an equally seamless fashion.

The CERTS Microgrid concept seeks to provide this technically challenging functionality without extensive (i.e., expensive) custom engineering. In addition, the design of the CERTS Microgrid also provides high system reliability and great flexibility in the placement of distributed generation within the microgrid. The CERTS Microgrid is intended to offer these functionalities at much lower costs than traditional approaches by incorporating peer-to-peer and plug-and-play concepts for each component within the microgrid.

In so doing, the CERTS Microgrid concept will help improve the quality of life in California by bringing environmentally safe, affordable, and reliable energy services and products to the marketplace. 


\subsection{References}

Akhil, A., Marnay, C. Lipman, T. 2002. Review of Test Facilities for Distributed Energy Resources. SAND2003-1602. October 2002. 43 pages.

Klapp, D., H.T. Vollkommer. Application of an Intelligent Static Switch to the Point of Common Coupling to Satisfy IEEE 1547 Compliance. Panel: Microgrid Research and Field Testing IEEE PES General Meeting, 24-28 June 2007, Tampa, FL. 4 pages.

Lasseter, R.H., A. Akhil, C. Marnay, J. Stephens, J. Dagle, R. Guttromson, A.S. Meliopoulous, R. Yinger, and J. Eto. 2002. Consortium for Electric Reliability Technology Solutions White Paper on Integration of Distributed Energy Resources: The MicroGrid Concept. LBNL-50829. April. 29 pages.

Lasseter, R.H., P. Piagi. Microgrid: A Conceptual Solution. PESC'04 Aachen, Germany 20-25 June 2004. 7 pages.

Lasseter, R.H., P. Piagi. 2005. Control and Design of CERTS Microgrid. July.

Lasseter, R.H., P. Piagi. 2006. Control and Design of Microgrid Components. January. 257 pages. http://www.pserc.org/ecow/getpublicatio/reports/2006report

Lasseter, R. Dynamic Distribution using (DER) Distributed Energy Resources. 2006. IEEE PES T \& D Meeting, Dallas, May. 3 pages.

Lasseter, R.H. 2007. “Microgrids and Distributed Generation.” Journal of Energy Engineering, Volume 133, Issue3, ASCE, Sept. 7 pages.

Lasseter, R.H. 2007. “CERTS Microgrid.” International Conference on System of Systems Engineering, April 16-18. 6 pages.

Nichols, D.K., J. Stevens, R.H. Lasseter, J.H. Eto, H.T. Vollkommer. 2006 Validation of the CERTS Microgrid Concept: The CEC/CERTS Microgrid Testbed. Power Engineering Society General Meeting, IEEE. 3 pages.

Nikkhajoei, H., R. H. Lasseter. 2007. Microgrid Protection. IEEE PES General Meeting, 24-28, Tampa, FL. June. 6 pages.

Nikkhajoei, H., R. H. Lasseter. 2006. Microgrid Fault Protection Based on Symmetrical and Differential Current Components. December. 72 pages. (Power Systems Engineering Research Center (PSERC) website)

Panora, R.A., J.B. Gehret Jr., P. Piagi. 2007. Design and Testing of an Inverter-Based Combined Heat and Power Module for Special Application in a Microgrid. Panel: Microgrid Research and Field Testing IEEE PES General Meeting, June 24-28, Tampa FL. 7 pages.

Piagi, P., H.R. Lasseter. 2006. Autonomous Control of Microgrids. IEEE PES Meeting, Montreal, June. 8 pages.

Stevens, J., H. Vollkommer, D.Klapp. CERTS Microgrid System Tests. Power Engineering Society General Meeting, June 24-28. 4 pages. 


\subsection{Glossary}

\begin{tabular}{|c|c|}
\hline Acronym & Definition \\
\hline $\mathrm{AC}$ & alternating current \\
\hline AEP & American Electric Power \\
\hline CERTS & Consortium for Electric Reliability Technology Solutions \\
\hline $\mathrm{CHP}$ & combined heat and power \\
\hline DC & direct current \\
\hline DER & distributed energy resources \\
\hline DOE & Department of Energy \\
\hline EMS & $\begin{array}{l}\text { energy management system (a control system that optimizes operation of } \\
\text { the Microgrid to economically meet electricity and heat loads) }\end{array}$ \\
\hline EPRI & Electric Power Research Institute \\
\hline $\begin{array}{l}\text { Energy } \\
\text { Commission }\end{array}$ & California Energy Commission \\
\hline IEEE & Institute of Electrical and Electronics Engineers \\
\hline LBNL & Lawrence Berkeley National Laboratory \\
\hline NPS & Northern Power Systems \\
\hline NREL & National Renewable Energy Laboratory \\
\hline PCC & point of common coupling \\
\hline PIER & Public Interest Energy Research \\
\hline PSERC & Power Systems Engineering Research Center \\
\hline RD\&D & research, development, and demonstration \\
\hline SCE & Southern California Edison \\
\hline SMUD & Sacramento Municipal Utilities District \\
\hline SNL & Sandia National Laboratories \\
\hline Static Switch & Separation device used to interconnect microgrid with distribution system \\
\hline TAC & Technical Advisory Committee \\
\hline UW & University of Wisconsin \\
\hline
\end{tabular}




\section{Appendices}

\section{Appendices are available as separate documents}

Appendix A. Test Bed Design Schematics

Appendix B. CERTS Microgrid Test Plan

Appendix C. Youtility Factory Test Plan Final Test Results

Appendix D. Tecogen 60kW Inverter-Based CHP Modules: Factory Testing

Appendix E. Tecogen CHP Modules Commissioning Report

Appendix F. CERTS Test Bed CERTEQUIP-V06-002, CERTS Switch, Low Power Factory Acceptance Test Report

Appendix G. Summary of CERTS Microgrid Static Switch Power Quality Tests at AEP Dolan, CERTS Microgrid Static Switch Testing

Appendix H. CERTS Test Bed Design and Commissioning: Lessons Learned Summary

Appendix I. Test Plan Section 6.0 Microgrid Test Bed System Checkout (Static Switch)

Appendix J. Test Plan Section 7.0 Validate Protection Settings and Initial Fault Testing

Appendix K. Test Plan Section 8.0 Reduced System Tests

Appendix L. Test Plan Section 9.0 Power Flow Control Tests

Appendix M. Test Plan Section 10.0 Difficult Loads

Appendix N. Test Log

Appendix O. Technical Advisory Committee Meeting Summary and Review Comments

Appendix P. Microgrid Fault Protection Based on Symmetrical and Differential Current Components 


\section{Preface}

The Public Interest Energy Research (PIER) Program supports public interest energy research and development that will help improve the quality of life in California by bringing environmentally safe, affordable, and reliable energy services and products to the marketplace.

The PIER Program, managed by the California Energy Commission (Energy Commission), conducts public interest research, development, and demonstration (RD\&D) projects to benefit California.

The PIER Program strives to conduct the most promising public interest energy research by part nering with RD\&D entities, including individuals, businesses, utilities, and public or private res earch institutions.

PIER funding efforts are focused on the following RD\&D program areas:

Buildings End-Use Energy Efficiency

Energy Innovations Small Grants

Energy-Related Environmental Research

Energy Systems Integration

Environmentally Preferred Advanced Generation

Industrial/Agricultural/Water End-Use Energy Efficiency

Renewable Energy Technologies

Transportation

CERTS Microgrid Laboratory Test Bed is the final report for the CERTS Microgrid Laboratory Test Bed project (contract number 500-03-024) conducted by CERTS. The information from this project contributes to PIER's Energy Systems Integration Program.

For more information about the PIER Program, please visit the Energy Commission's website at www.energy.ca.gov/pier or contact the Energy Commission at 916-654-5164. 


\section{Table of Contents}

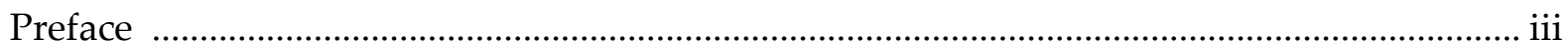

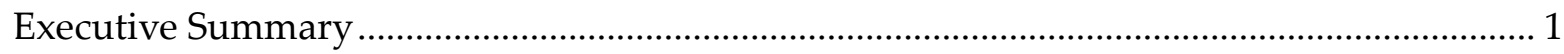

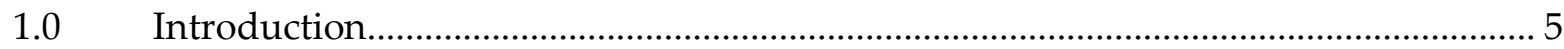

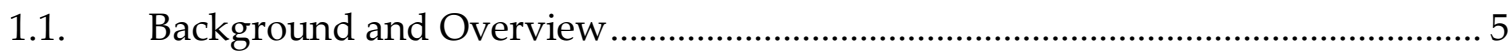

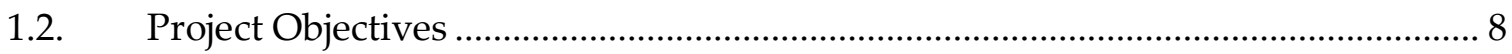

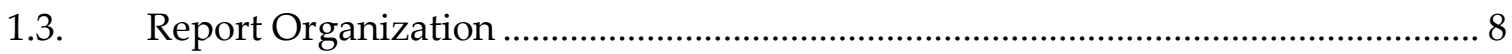

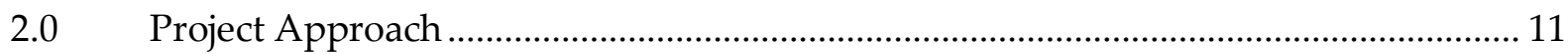

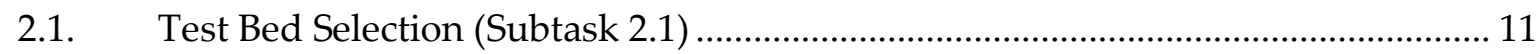

2.2. Equipment Preparation (Subtask 2.2) ................................................................. 11

2.3. Laboratory Tests (Subtask 2.3) ........................................................................ 12

2.4. Field Demonstration Planning (Subtask 2.4) ................................................... 17

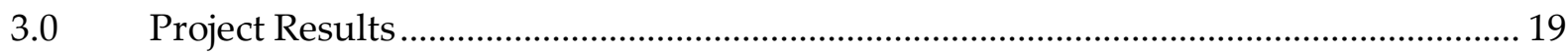

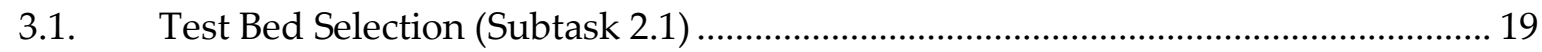

3.2. Equipment Preparation (Subtask 2.2) .................................................................. 21

3.3. CERTS Microgrid Laboratory Tests....................................................................... 24

3.4. Field Demonstration Planning (Task 2.4) ............................................................ 44

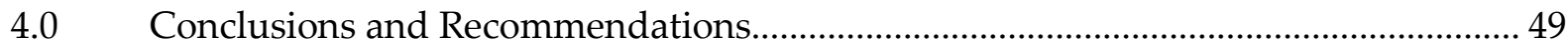

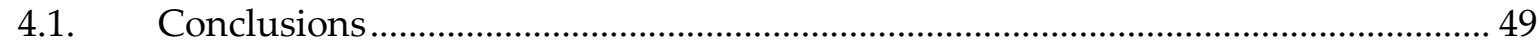

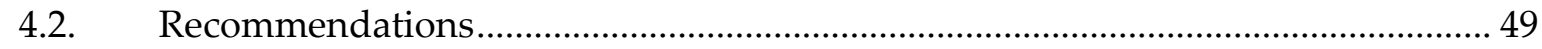

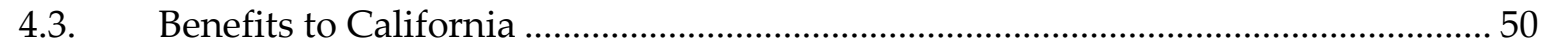

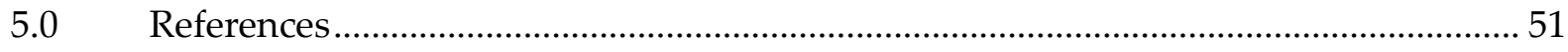

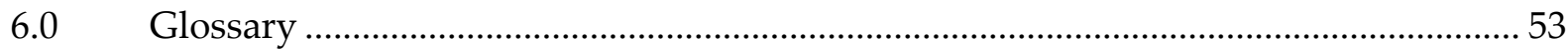

\section{Appendices}

Appendix A. Test Bed Design Schematics

Appendix B. CERTS Microgrid Test Plan,

Appendix C. Youtility Factory Test Plan Final Test Results

Appendix D. Tecogen 60kW Inverter-Based CHP Modules: Factory Testing

Appendix E. Tecogen CHP Modules Commissioning Report

Appendix F. CERTS Test Bed CERTEQUIP-V06-002, CERTS Switch, Low Power Factory Acceptance Test Report 
Appendix G. Summary of CERTS Microgrid Static Switch Power Quality Tests at AEP Dolan, CERTS Microgrid Static Switch Testing

Appendix H. CERTS Test Bed Design and Commissioning: Lessons Learned Summary

Appendix I. Test Plan Section 6.0 Microgrid Test Bed System Checkout (Static Switch)

Appendix J. Test Plan Section 7.0 Validate Protection Settings and Initial Fault Testing

Appendix K. Test Plan Section 8.0 Reduced System Tests

Appendix L. Test Plan Section 9.0 Power Flow Control Tests

Appendix M. Test Plan Section 10.0 Difficult Loads

Appendix N. Test Log

Appendix O. Technical Advisory Committee Meeting Summary and Review Comments

Appendix P. Microgrid Fault Protection Based on Symmetrical and Differential Current Components 


\section{List of Figures}

Figure 1. CERTS Microgrid Test Bed at American Electric Power .................................................

Figure 2. One-Line Diagram of CERTS Microgrid Test Bed …........................................................ 13

Figure 3. One-Line Diagram with Meter and Relay Locations CERTS Microgrid Test Bed ......... 14

Figure 4. Diagram of DAS \& EMS Data networks ..................................................................... 14

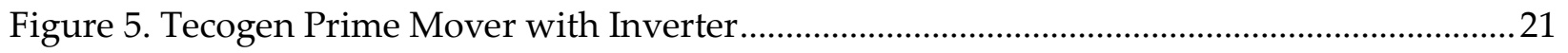

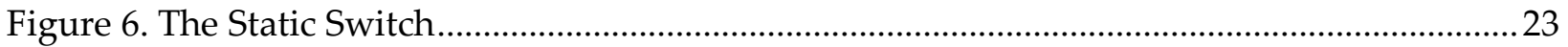

\section{List of Tables}

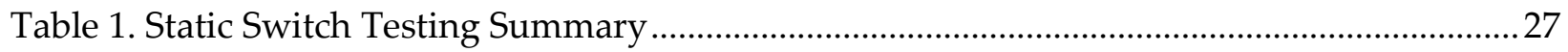

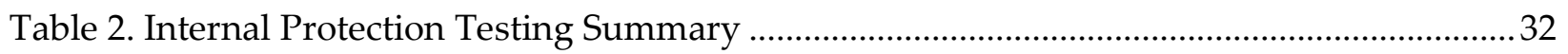

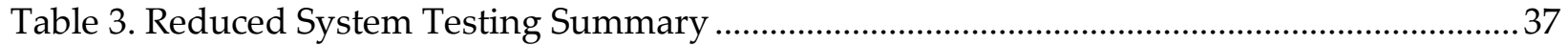

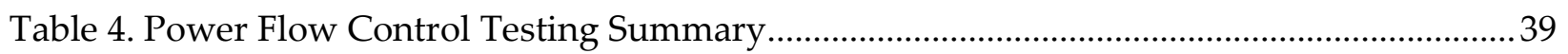

Table 5. Difficult Load Testing Summary ................................................................................... 41 


\begin{abstract}
The objective of the CERTS Microgrid Laboratory Test Bed project was to enhance the ease of integrating small energy sources into a microgrid. The project accomplished this objective by developing and demonstrating three advanced techniques, collectively referred to as the CERTS Microgrid concept, that significantly reduce the level of custom field engineering needed to operate microgrids consisting of small generating sources. The techniques comprising the CERTS Microgrid concept are: 1) a method for effecting automatic and seamless transitions between grid-connected and islanded modes of operation; 2) an approach to electrical protection within the microgrid that does not depend on high fault currents; and 3) a method for microgrid control that achieves voltage and frequency stability under islanded conditions without requiring high-speed communications.

The techniques were demonstrated at a full-scale test bed built near Columbus, Ohio and operated by American Electric Power. The testing fully confirmed earlier research that had been conducted initially through analytical simulations, then through laboratory emulations, and finally through factory acceptance testing of individual microgrid components. The islanding and resychronization method met all Institute of Electrical and Electronics Engineers 1547 and power quality requirements. The electrical protection system was able to distinguish between normal and faulted operation. The controls were found to be robust under all conditions, including difficult motor starts.

The results from these tests are expected to lead to additional testing of enhancements to the basic techniques at the test bed to improve the business case for microgrid technologies, as well to field demonstrations involving microgrids that involve one or more of the CERTS Microgrid concepts.
\end{abstract}

Keywords: CERTS, microgrid, control protection, electrical protection, voltage and frequency stability control, inverter, distributed energy resources, distributed generation, distributed resource, islanding. 


\section{Executive Summary}

Evolutionary changes in the regulatory and operational climate of traditional electric utilities and the emergence of smaller generating systems, including internal combustion engines, microturbines, photovoltaics, and fuel cells, have opened new opportunities for electricity users to generate power on site. In this context, distributed energy resources - small power generators typically located at sites where the energy (both electric and thermal) they generate is used - are a promising option to meet growing customer needs for economic and reliable electric power. In addition to generators, the distributed energy resources portfolio also includes energy storage, and load control. Organizing all of these resources into microgrids is a promising way to capture smaller distributed energy resources' significant potential to meet customers' and utilities' needs.

A key feature of a microgrid, is its ability, during a utility grid disturbance, to separate and isolate itself from the utility seamlessly with little or no disruption to the loads within the microgrid (e.g., in the CERTS Microgrid concept, no impacts on power quality). Then, when the utility grid returns to normal, the microgrid automatically resynchronizes and reconnects itself to the grid, in an equally seamless fashion.

The CERTS Microgrid concept seeks to provide this technically challenging functionality without extensive (i.e., expensive) custom engineering. In addition, the design of the CERTS Microgrid also provides high system reliability and great flexibility in the placement of distributed generation within the microgrid. The CERTS Microgrid is intended to offer these functionalities at much lower costs than traditional approaches by incorporating peer-to-peer and plug-and-play concepts for each component within the microgrid.

The peer-to-peer concept insures that no single component, such as a master controller or a central storage unit, is required for operation of the microgrid. Therefore, by its very design, the CERTS Microgrid can continue operating with loss of an individual component or generator. (With one additional source, $(\mathrm{N}+1)$ it can insure even higher levels of reliability.)

The plug-and-play concept means that a distributed energy resources unit can be placed at any point within the microgrid without re-engineering its controls. The plug-and-play functionality is similar to the flexibility one has with home appliances. That is, just as an appliance can be plugged in wherever there is an outlet, one can similarly locate distributed energy resources units at any location within a facility or building where they might be most needed. This is in sharp contrast to the traditional model, which clusters distributed generation at a single point in order to make the electrical integration tasks simpler. In combined heat and power applications, the plug-and-play model facilitates placing distributed energy resources immediately adjacent to heat loads, thereby allowing more effective use of waste heat without a complex heat distribution system, such as steam and chilled water pipes, and the energy losses associated with them.

A critical feature of the CERTS Microgrid is its presentation to the surrounding distribution grid as a single self-controlled entity. A CERTS Microgrid appears to the grid as indistinguishable from other customer sites that do not include DER. This presentation means that the microgrid 
avoids many of the current concerns associated with integrating DER, such as how many DER the system can tolerate before their collective electrical impact begins to create problems like excessive current flows into faults and voltage fluctuations.

These functionalities are provided through three advanced techniques comprising the CERTS Microgrid concept, including: 1) a method for effecting automatic and seamless transitions between grid-connected and islanded modes of operation; 2) an approach to electrical protection within the microgrid that does not depend on high fault currents; and 3) a method for microgrid control that achieves voltage and frequency stability under islanded conditions without requiring high-speed communications.

The techniques were demonstrated at a full-scale test bed built near Columbus, Ohio and operated by American Electric Power. See Figure 1. Tecogen and its inverter manufacturer, Youtility, modified and factory tested three combined heat and power units to incorporate the CERTS Microgrid control algorithms. Northern Power Systems designed, fabricated, and factory tested a static switch that implemented CERTS Microgrid islanding and resychronization procedures and also fabricated the other major hardware elements required, including load banks, power and control circuitry, protective relaying, and data-acquisition equipment, installed in metal-clad switchgear cabinets for protection in an outdoor location.

Five sets of tests were conducted by American Electric Power (AEP);

The first set of tests examined the operation of the static switch to determine that it and its digital signal processing control operated as designed. This included five tests of dead-bus and synchronized closing, reverse power and Institute of Electrical and Electronics Engineers (IEEE) 1547 protective relay functions. The goal was to confirm the correct operation (and thus the protection) of the static switch, which is located at the interface between the protected and unprotected portions of the microgrid.

The second set of tests examined a preliminary set of faults (i.e., overload simulating a high impedance fault) within the microgrid to ensure protection and safety of the test bed, prior to performing other planned tests. The fault condition tests were intended to cover the basic concepts of the protection design and to study its effectiveness (e.g., zero-sequence, negativesequence and residual currents for line-to-ground faults; negative-sequence or $\left(\mathrm{I}^{2} \mathrm{~T}\right)$ protection for phase-to-phase faults). For the majority of these test scenarios, the static switch was expected to open first, followed by the opening of the zone breaker of the faulted zone. The goal was to test and adjust protection settings to achieve the most ideal conditions and protection design within a limited fault current system. Sixteen separate tests were conducted. 


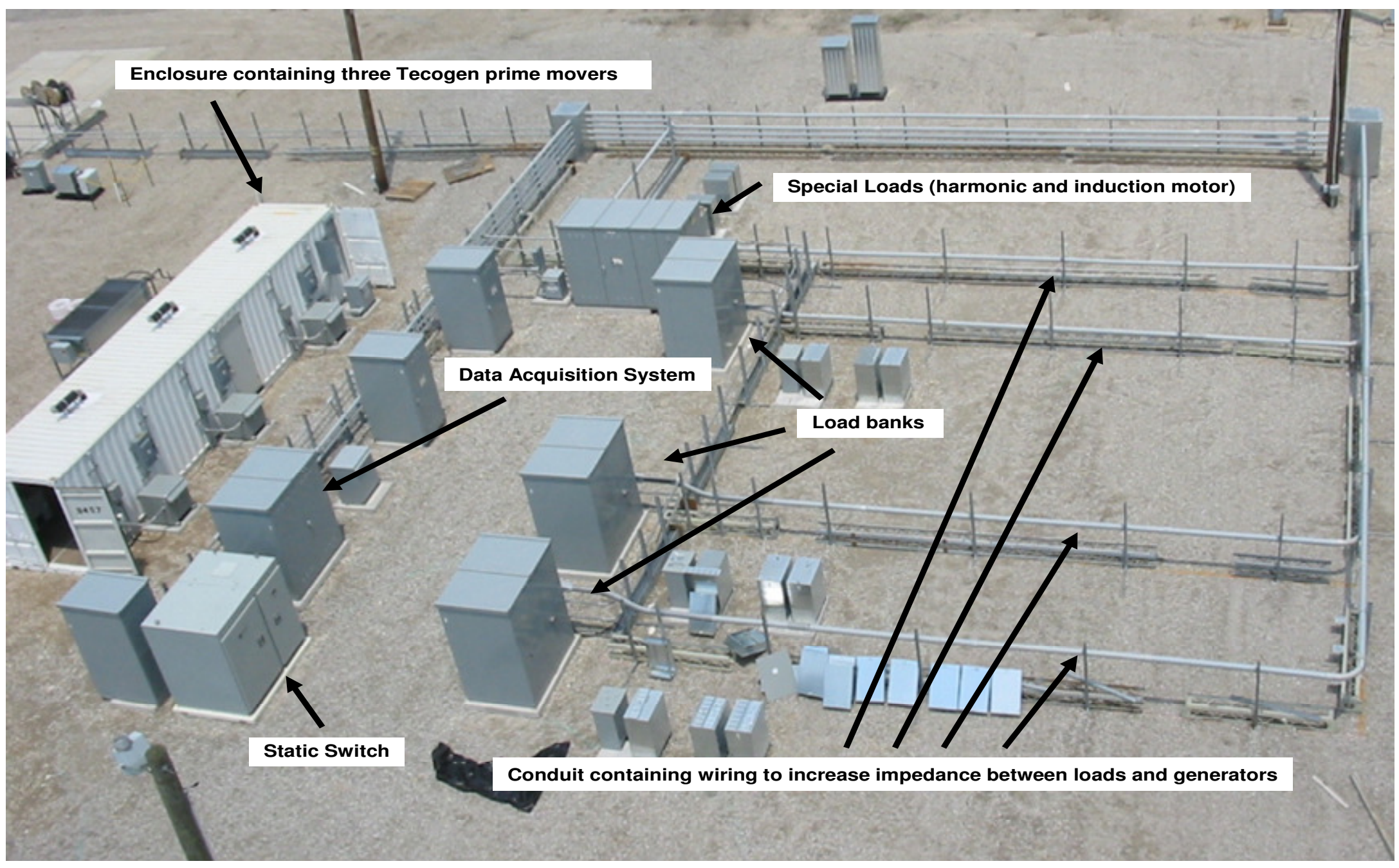

Figure 1. CERTS Microgrid Test Bed at American Electric Power

Photo Credit: American Electric Power 
The third set of tests was designed to ensure that the Gen-set inverter controls were working as designed during grid-connected and islanded modes of operation. This includes unit control, zone control, and mixed power controls, in conjunction with limiting controls and synchronized closing of the static switch. These tests were based on replicating tests that had previously been conducted by Tecogen and Youtility during the factory acceptance testing of the standalone inverters, and the engine-coupled inverters. The performance goal was to observe smooth transitions of the Gen-sets response to different step conditions. Thirteen separate tests were conducted.

The fourth set of tests demonstrated the flexibility of the microgrid while both grid connected and islanded, for different loads, power flows, and utility impact. These tests employed "weak grid" inductors in the circuit simulating the microgrid connection at the end of a lengthy feeder. Three sets of tests were conducted.

The fifth and final set of testing began to explore the operational limits of the microgrid with difficult induction motor starting loads. Two primary sets of tests were conducted under "weak grid" conditions.

Detailed, standalone reports (referenced as appendices to this report) were prepared containing narrative descriptions of the purpose and performance goal for each test, along with extensive graphical and tabular summaries of the results for each test.

The testing fully confirmed earlier research that had been conducted initially through analytical simulations, then through laboratory emulations, and finally through factory acceptance testing of individual microgrid components. The islanding and resychronization method met all IEEE 1547 and power quality requirements. The electrical protection system was able to distinguish between normal and faulted operation. The controls were found to be robust under all conditions, including difficult motor starts.

The results from these tests are expected to lead to additional testing of enhancements to the basic techniques at the test bed, to improve the business case for microgrid technologies, as well to field demonstrations involving microgrids that involve one or more of the CERTS Microgrid concepts. 


\subsection{Introduction}

This report describes the Consortium for Electric Reliability Solutions (CERTS) Microgrid Laboratory Test Bed project, which developed and tested innovative strategies for islanding and resychronizing microgrids with the grid, providing electrical protection within the microgrid, and controlling energy sources within the microgrid autonomously during islanded operation. Under earlier research contracts, the CERTS Microgrid team first developed these strategies using computer simulations, and then further refined them with laboratory-scale emulations. In this research contract, the team implemented them in commercial-grade hardware, with thorough testing at several stages of development, including at a full-scale test bed.

\subsection{Background and Overview}

Evolutionary changes in the regulatory and operational climate of traditional electric utilities and the emergence of smaller generating systems, including internal combustion engines, microturbines, photovoltaics, and fuel cells, have opened new opportunities for electricity users to generate power on site. In this context, distributed energy resources (DER) - small power resources typically located at sites where the energy (both electric and thermal) they provide is used locally - are a promising option to meet growing customer needs for economic and reliable electric power. In addition to generators, the DER portfolio also includes energy storage, and load control. Organizing all of these resources into microgrids is a promising way to capture smaller DER's significant potential to meet customers' and utilities' needs.

The CERTS Microgrid is an entirely new approach to integrating DER. Traditional approaches for integrating DER focus on the impacts on grid performance of a single or relatively small number of microsources. An example of the traditional approach to DER is the Institute of Electrical and Electronics Engineers (IEEE) Standard 1547 for Distributed Resources Interconnected with Electric Power Systems. This standard focuses on ensuring that interconnected systems will not affect the operation of the grid should problems arise on the grid. In compliance with this standard, the CERTS Microgrid is designed to seamlessly disconnect and operate as an island, separate from the grid, in case of problems and to reconnect to the grid once the problems are resolved. (Lasseter, et al. 2002. Lasseter, Piagi. 2005. Lasseter, Piagi. 2006.)

A critical feature of the CERTS Microgrid is its presentation to the surrounding distribution grid as a single, self-controlled entity. A CERTS Microgrid appears to the grid as indistinguishable from other customer sites that do not include DER. This presentation means that the microgrid avoids many of the current concerns associated with integrating DER, such as how many DER the system can tolerate before their collective electrical impact begins to create problems like excessive current flows into faults and voltage fluctuations. The CERTS Microgrid architecture insures that the microgrid will be a good citizen within the bulk power provider network, complying with grid rules and doing no harm beyond what would be acceptable from any existing customer. In return for appropriate compensation, the microgrid could provide interruptible load. Although technical barriers currently discourage it, the microgrid could also be a small source of power or ancillary services. The benefits it could offer to the distribution 
system include; congestion relief, postponement of new generation or delivery capacity, rapid response to load changes, and local voltage support.

A key feature of a microgrid is its ability, during a utility grid disturbance, to separate and isolate itself from the utility seamlessly with little or no disruption to the loads within the microgrid (e.g., in the CERTS Microgrid concept, with no impact on power quality). Then, when the utility grid returns to normal, the microgrid automatically resynchronizes and reconnects itself in an equally seamless fashion.

What is unique about the CERTS Microgrid is that it can provide this technically challenging functionality without extensive (i.e., expensive) custom engineering. In addition, the design of the CERTS Microgrid also provides high system reliability and great flexibility in the placement of distributed generation within the microgrid. The CERTS Microgrid is intended to offer these functionalities at much lower costs than traditional approaches by incorporating peer-to-peer and plug-and-play concepts for each component within the microgrid.

The peer-to-peer concept insures that no single component, such as a master controller or a central storage unit, is required for operation of the microgrid. Therefore, by its very design, the CERTS Microgrid can continue operating with loss of an individual component or generator. (With one additional source, $(\mathrm{N}+1)$ it can insure even higher levels of reliability.)

The plug-and-play concept means that a DER unit can be placed at any point within the microgrid without re-engineering its controls. The plug-and-play functionality is similar to the flexibility one has with home appliances. That is, just as an appliance can be plugged in wherever there is an outlet, one can similarly locate DER units at any location within a facility or building where they might be most needed. This is in sharp contrast to the traditional model, which clusters DG at a single point in order to make the electrical integration tasks simpler. In combined heat and power (CHP) applications, the plug-and-play model facilitates placing DER immediately adjacent to heat loads, thereby allowing more effective use of waste heat without a complex heat distribution system, such as steam and chilled water pipes, and the energy losses associated with them.

These functionalities are enabled by three innovations: First, the CERTS Microgrid concept relies on embedding intelligence in the interface between the microsources and the surrounding microgrid. ${ }^{1}$ This innovation enables autonomous operation of each microsource without highspeed communication or hierarchical control among microsources. This innovation is the key toward enabling a plug-and-play environment in which many microsources can operate harmoniously with one another with a minimum of expensive, custom site engineering.

\footnotetext{
${ }^{1}$ For pragmatic reasons of availability and controllability, this CERTS effort initially focused on small internal combustion (IC) engines. All DER technologies, especially those that rely on power electronic interfaces, including microturbines, fuel cells, photovoltaics, and energy storage, are candidates for inclusion in microgrids based on CERTS Microgrid concepts.
} 
Second, as a result of enabling an environment in which, in principle, any number of microsources might operate within a single microgrid, the CERTS Microgrid concept also incorporates an intelligent static switch for rapid disconnect and resynchronization of the entire microgrid to the larger, utility grid at the point of common coupling (PCC). A single, intelligent switch is innovative because it is a much more economic alternative to providing this functionality at each individual microsource.

Third, the CERTS Microgrid includes an innovative, additional level of protective relaying within the microgrid that complements traditional protection. The approach addresses that fact that power electronic interfaces can, by design, limit the fault current available to detect system faults, which is the traditional means used for detecting faults within an electrical network. Thus, while a microgrid can use bi-directional over-current protection devices when connected to the utility, it cannot rely on these approaches when the microgrid is islanded. The CERTS Microgrid protection scheme uses sequence components to detect low-fault currents in utilityconnected and islanded operation. This protection scheme allows for plug-and-play without changing the existing over-current devices on the electrical system.

The development and testing of the CERTS Microgrid concept is the culmination of over 7 years of research efforts supported by both the U.S. Department of Energy and (DOE) the California Energy Commission (Energy Commission). In 2001, the DOE provided research support to develop the initial CERTS Microgrid concept. In 2002, the Energy Commission began providing research support to demonstrate the CERTS Microgrid concept. Under prior Energy Commission Contract \# 150-99-003, Tasks 2.4 and 2.7, in the area of Distributed Energy Resources Integration, the CERTS team: 1) conducted analytical simulations of aspects of the concept (Lasseter, et. al. 2002); 2) prepared the design for test bed where a full-scale demonstration of the concepts could be tested (Appendix A. Test Bed Design Schematics); and 3) completed a review of potential sites and research partners where the test bed could be built (Akhil, et. al. 2002).

This document is the final report for the testing that has been conducted at the test bed.

The CERTS Microgrid Laboratory Test Bed project team initially included Lawrence Berkeley National Laboratory (LBNL); Sandia National Laboratory (SNL); the University of Wisconsin (UW); and Northern Power Systems (NPS), which designs, builds and installs electric power systems. Through this project, the CERTS team was expanded to include Tecogen, Inc., a manufacturer of engine-driven cooling and combined heat and power (CHP) systems, and Youtility, a supplier of power electronic systems for distributed power. In addition, American Electric Power (AEP), one of the largest electric utilities in the United States, delivering electricity to more than 5 million customers in 11 states, was selected by the team to host the test bed and conduct the tests.

The CERTS team was aided throughout the research process by a Technical Advisory Committee (TAC) consisting of representatives from 1) the DOE's Office of Electricity Delivery and Energy Reliability; 2) two major California electric utilities, Pacific Gas and Electric (PG\&E) and Southern California Edison (SCE) with expertise in interconnection of distributed 
generation; 3) the National Renewable Energy Laboratory (NREL) who manage aspects of the IEEE 1547 standards development process; 4) the Electric Power Research Institute (EPRI) with expertise in power quality; and 5) an independent consultant with expertise in power electronics.

\subsection{Project Objectives}

The objectives of this project were to:

- Demonstrate stable operation of the CERTS control algorithms during a variety of conditions including:

- Transitions between utility-interconnected and islanded operation

- Islanded operation that involved such traditionally difficult loads as motor starting

- Demonstrate the ability to detect faults within the microgrid under a variety of conditions when either interconnected to the utility or islanded

The CERTS Microgrid Laboratory Test Bed project meets the PIER Goal of Improving the Reliability/Quality of California's Electricity by creating technologies and control strategies needed to capture the full potential of DER to improve the reliability and quality of the California interconnected power system. This project also meets the secondary goal of Improving the Energy Cost/Value of California's Electricity by lowering the cost of power delivery.

\subsection{Report Organization}

The CERTS Microgrid Laboratory Test Bed project consists of one technical task, CERTS Microgrid Laboratory Test Bed, with four Subtasks and five Sub-subtasks, as follows:

Subtask 2.1: Select a laboratory test bed

Subtask 2.2: Prepare equipment for the test bed

Sub-subtask 2.2.1: Prepare three prime movers and inverters for the test bed, including factory acceptance testing

Sub-subtask 2.2.2: Design, fabricate and factory test other test bed equipment, including the static switch

Subtask 2.3: Conduct tests of the CERTS Microgrid concept at the test bed

Sub-subtask 2.3.1 Prepare the test bed for testing

Sub-subtask 2.3.2 Conduct tests of the CERTS Microgrid concepts

Sub-subtask 2.3.3 Prepare a microgrid protective relaying report

Subtask 2.4 Plan a field demonstration of the CERTS Microgrid concept

The next two major sections of this report, 2.0 Project Approach and 3.0 Project Outcomes, follow this Subtask and Sub-subtask structure. 
The report is supplemented by a number of stand-alone technical appendices that provide additional information and details on the results from all subtasks and sub-subtasks of this project. Several, separate appendices were required to organize voluminous tabular and graphical summaries of the data collected from each of the many tests conducted at the test bed. 


\subsection{Project Approach}

The CERTS Microgrid Laboratory Test Bed project implemented the CERTS Microgrid concept and test bed design initiated under Energy Commission Contact \#150-99-003, tasks 2.4 and 2.7, to develop tools and techniques for significant integration of distributed technologies in support of electricity reliability.

The subsections below describe the approach taken under each Subtask and Sub-subtask and in the project.

\subsection{Test Bed Selection (Subtask 2.1)}

The goal of this task was to select and execute a contract with a facility to conduct a full-scale test-bed demonstration of CERTS Microgrid concepts. The task included developing criteria for selecting a testing facility and, using information from past reviews conducted by the team to identify candidate sites, working with the Technical Advisory Committee (TAC) and Energy Commission Contract Manager to select the most appropriate site. Finally, following the Commission Contract Manager's approval, LBNL was to execute a subcontract with the Energy Commission-approved facility.

\subsection{Equipment Preparation (Subtask 2.2)}

The goal of this subtask was to enter into contracts with a microsource manufacturer, Tecogen/Youtility, to prepare three microsource prime movers with modified inverters and with a DER engineering firm, Northern Power Systems (NPS), to fabricate test bed hardware including a static switch, for use in the laboratory test bed demonstration (Subtask 2.3).

The first phase of work required Tecogen/Youtility to upgrade the current design of its inverter to incorporate CERTS Microgrid control algorithms and to conduct extensive factory tests of the modified microsource prime movers and inverters (Sub-subtask 2.2.1). The machines had to be as identical to one other as possible so that impacts from machines with differing characteristics did not mask important observations about performance, especially during transients, within the test bed. Because of the technical requirements for the test bed prime movers, the supplier had to be able to dedicate a small, technically skilled team to this project. The supplier also had to have the flexibility to revisit and rebuild aspects of the units because the team expected that initial testing would reveal the need for modifications.

In the original Energy Commission contract, Capstone Microturbines was expected to supply the modified microsource prime movers. However, Capstone Microturbines declined to execute a contract with LBNL due to a change in business priorities and withdrew from the project. Following an introduction provided by the Energy Commission contract manager, the team partnered with Tecogen/Youtility to supply the modified prime mover microsources and inverters. Aspects of the laboratory test bed design were modified by NPS to accommodate this change in prime movers.

The second phase of work required NPS to develop and conduct factory tests of a static switch that implements the CERTS Microgrid islanding and resynchronization procedures (Sub- 
subtask 2.2.2). As an additional element of the second phase of work, NPS also fabricated the major electrical components required by the test bed, such as load banks and emulators, switchgear, and the data acquisition system (DAS). NPS was well-qualified to conduct this work because they had, under a previous Energy Commission contract with CERTS, prepared the complete design of the laboratory test bed.

\subsection{Laboratory Tests (Subtask 2.3)}

The goal of this subtask was to conduct tests of the CERTS Microgrid concept. That is, the laboratory test facility selected in Subtask 2.1 was to build the test bed and conduct tests of the test bed equipment, prime movers, and static switch prepared under Subtask 2.2. This subtask was conducted through three sub-subtasks.

The goal of sub-subtask 2.3.1 was to prepare the test bed for conducting the tests. This involved preparation of the physical test bed site following the design of the test bed, which was prepared under an earlier contract. (See Figures 2, 3, and 4, which are based on Appendix A, Test Bed Design Schematics). The tasks included erection of enclosures to house the microsources and associated facilities to cool the engines in operation and the laying out of conduits and associated wiring to interconnect the microgrid elements. Finally, the task involved installing the cabinets assembled by NPS as well as the microsources prepared by Tecogen/Youtility. See Figure 1.

The goal of sub-subtask 2.3.2 was to conduct a large number of tests exercising all functions of the CERTS Microgrid concept. This goal was accomplished in three phases. First, a detailed test plan was developed. Second, the tests were executed following the test plan. Third, the test results were analyzed and assessed with respect to the test plan and overall project objectives.

The CERTS team, led by NPS, developed a comprehensive list of tests that would exercise every aspect of the CERTS Microgrid concept. The tests were presented to and refined through discussions with the TAC. After the list of tests was deemed complete, AEP developed "registered" test procedure, following AEP corporate rules that would meet all testing needs safely. This registered test procedure is presented in Appendix B. CERTS Microgrid Testbed Test Plan.

The registered test procedure contains ten sections. The first five are procedural. Sections six through ten refer to actual tests in which measurements are recorded. The tests were conducted by AEP in accordance with the registered test procedure. Testing was conducted sequentially, one section at a time.

Prior to each test day, the person in charge performed a job safety briefing including an inspection of the barricades and test setup for safety and compliance. A minimum of two people were on-site during each planned test 


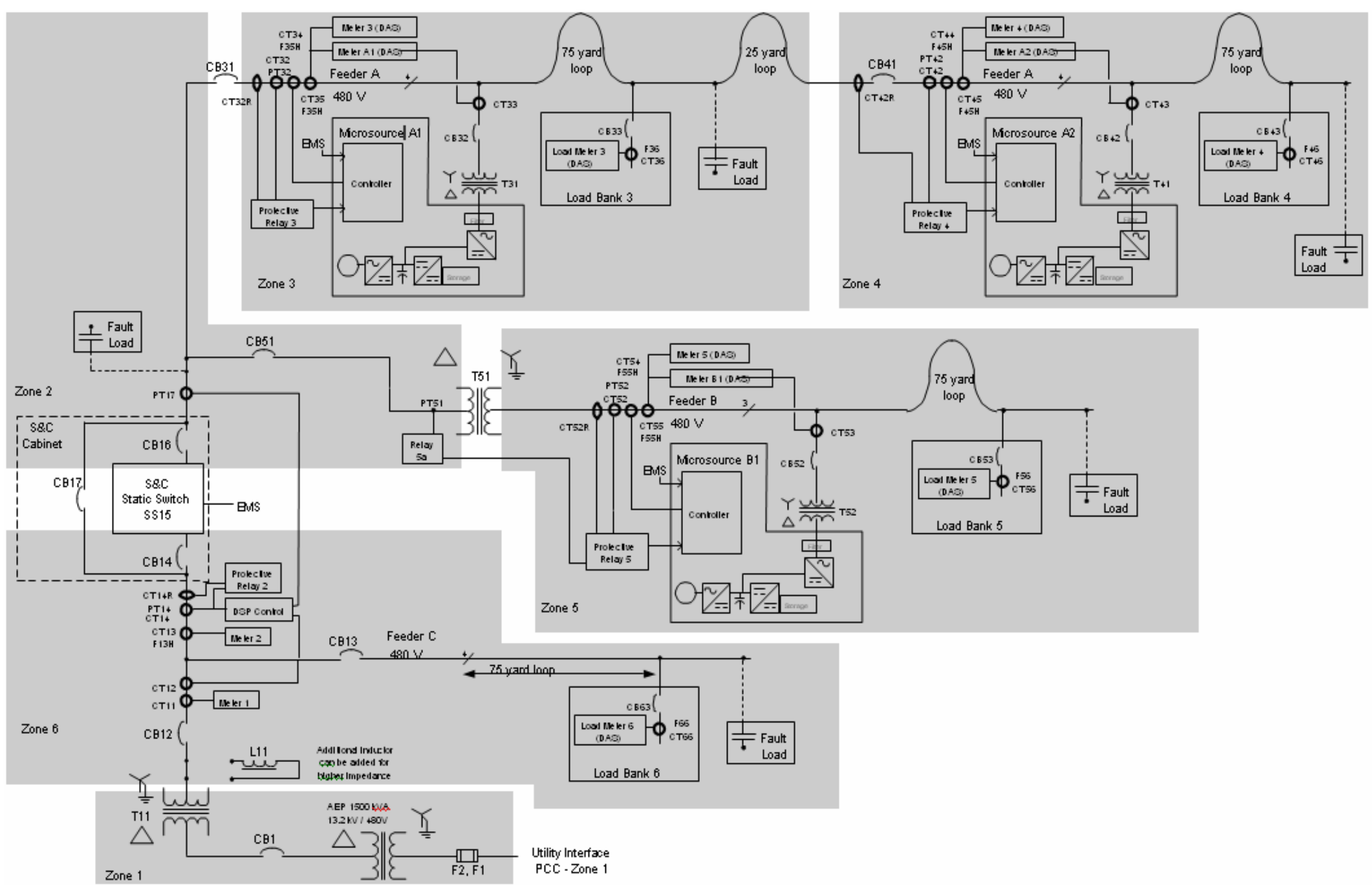

Figure 2. One-Line Diagram of CERTS Microgrid Test Bed 


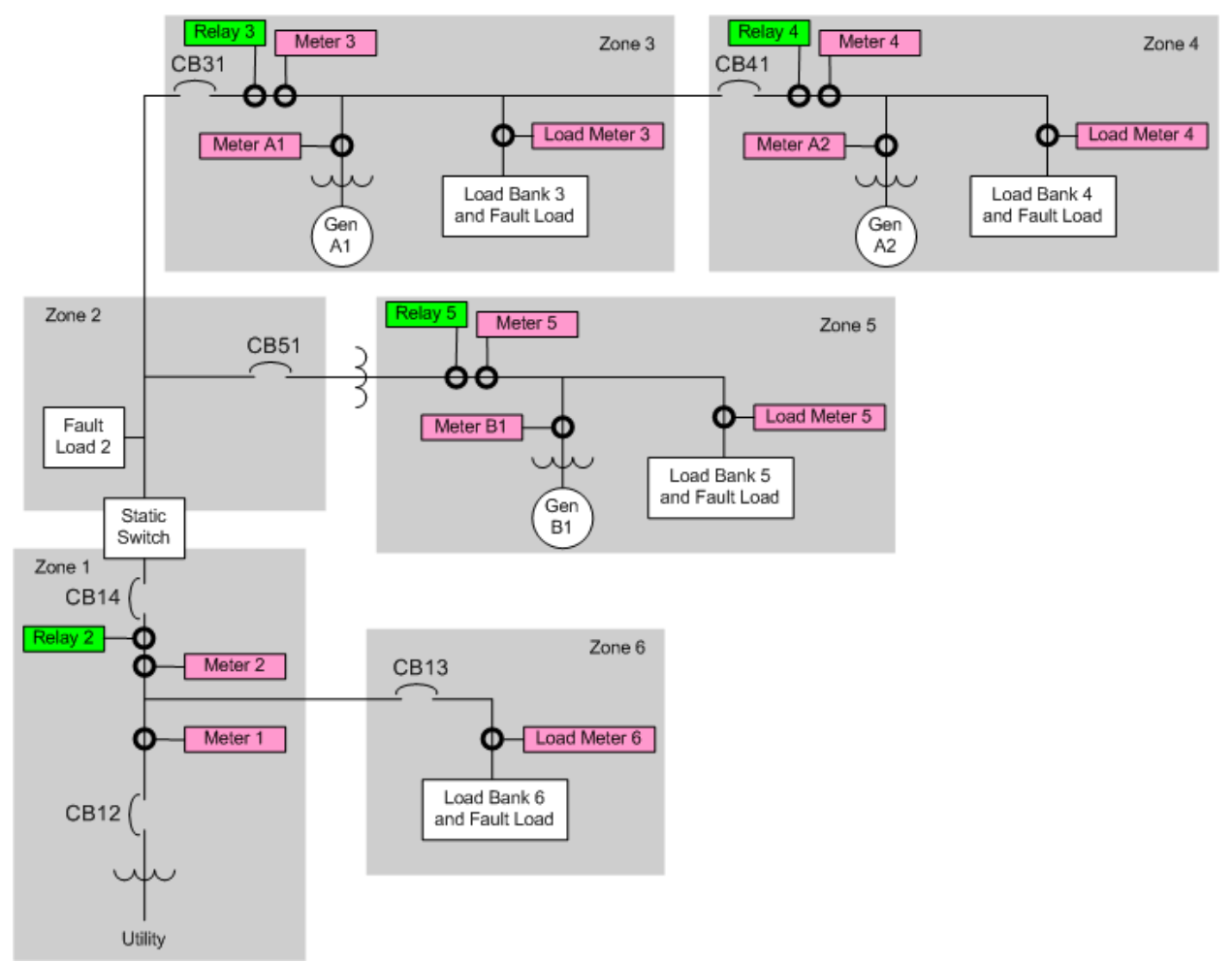

Figure 3. One-Line Diagram with Meter and Relay Locations CERTS Microgrid Test Bed

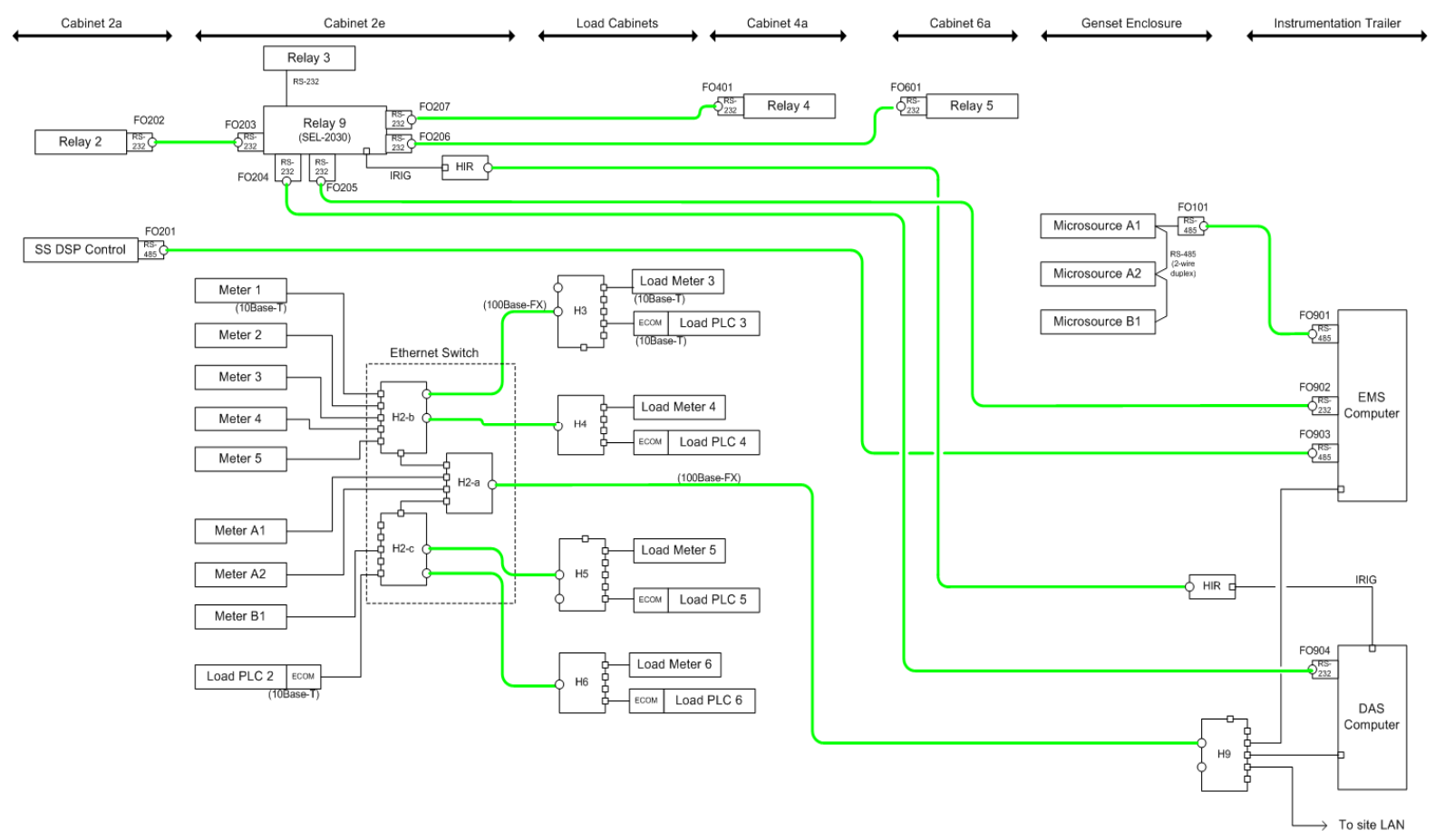

Figure 4. Diagram of DAS \& EMS Data networks 
Visual and audible alarms warned persons that energized testing was being performed in the Microgrid Test Bed area. The visual alarm consisted of a portable red flashing light, located between the Control Trailer and Gen-set Enclosure. An audible alarm, consisting of a portable wireless motion detector, was located at the front gate of the Walnut Test Site with the fence gate "Closed," not locked, and audible alarm in the trailer operational during test(s).

Barricades were set up around the Microgrid Test Bed area (i.e., saw-horse style barricades with a red plastic chain surrounded the test area containing the Gen-set Enclosure, Microgrid switching cabinets, plus load and fault bank cabinets).

Prior to performing tests, the Test Engineer or Technical Consultant verified that all personnel and visitors were properly protected and in assigned locations. Personnel were in or adjacent to the Control Trailer while tests were being performed. All nonessential personnel either left the main site or were sheltered in the Control Trailer.

The first set of tests (Section 6 of the test plan) examined the operation of the static switch to determine that it and its digital signal processing (DSP) control operated as designed. The goal was to confirm the correct operation (and thus the protection) of the static switch, which is located at the interface between the protected and unprotected portions of the microgrid. Successful completion of these tests was a prerequisite to performing subsequent tests in the test plan.

The tests were designed to check control and operation of the static switch, basic power and voltage control of the Gen-sets, and a preliminary check of the protection scheme. They included five tests of dead-bus and synchronized closing, reverse power and IEEE 1547 protective relay functions. The switch functions were tested with a single Gen-set A1 online. The measurements taken were unique to each test. Waveform and RMS data from Meter 2 were of prime interest.

The second set of tests (Section 7 of the test plan) examined a preliminary set of fault (i.e., overload simulating a fault) condition tests to ensure protection and safety of the test bed, prior to performing other planned tests. The goal was to confirm the new protection design developed in Task 2.3.3. This goal was to be accomplished by testing and adjusting protection settings to achieve the most ideal conditions and protection design. The tests included inductor L11 in the circuit, reflecting "weak grid" conditions. Sixteen separate tests were conducted.

The fault (i.e., overload simulating a fault) condition tests were intended to cover the basic concept of the protection design and to study its effectiveness (e.g., zero-sequence, negativesequence and residual currents for line-to-ground faults; negative-sequence or $\left(\mathrm{I}^{2} \mathrm{t}\right)$ protection for phase-to-phase faults). For the majority of these test scenarios, the static switch was expected to "OPEN" first, followed by the zone breaker of the faulted zone.

During each fault event waveforms of phase currents and line-to-neutral voltages at all Relay locations (i.e., Relays 2, 3, 4 \& 5) were recorded. The relay element that caused the "TRIP" with trip times for each relay relative to when the fault condition was applied was also recorded.

Two power control modes were defined for the third and fourth sets of tests: 
Unit Power Control Mode controls the amount of power (i.e., $\mathrm{kW}$ ) being injected into the Zone from the Gen-set being controlled.

Zone Power Control Mode controls the amount of power (i.e., $\mathrm{kW}$ ) entering/exiting the Zone using the Gen-set to offset the difference in that Zone.

The third set of tests (Section 8 of the test plan) was designed to ensure that the Gen-set inverter controls were working as designed. This includes unit control, zone control, and mixed power controls, in conjunction with limit controls and synchronized closing of the static switch. These tests were based on replicating tests that had previously been conducted by Tecogen and Youtility during the factory acceptance testing of the standalone inverters, and the engine coupled inverters. The performance goal was to observe smooth transitions of the Gen-sets response to different step conditions (i.e., static switch "OPEN"/ "CLOSE" and load steps). Thirteen separate tests were conducted.

Several measurements were made for each test:

- Sources -Injected power $(\mathrm{kW})$, reactive load (kVAr), frequency (freq), and voltage (V) for each Gen-set (i.e., A1p, A2p and B1p).

- Zone input power flow into Zones 3 (A1z), 4 (A2z), 5 (B1z) and 6.

- Loads - Voltage at Load Bank 3 (L3), 4 (L4), 5 (L5), and 6 (L6).

- Static Switch - Power (kW) and current (I) through the static switch.

- Static Switch Control Signal: Forced “OPEN” and release to allow self synchronization.

- Voltage (V) and frequency (Freq) difference across the static switch.

The fourth set of tests (Section 9 of the test plan) demonstrated the flexibility of the microgrid both grid connected and islanded for different loads, power flows and impact on the utility. The tests included the "weak grid" inductors (i.e., L11) in the circuit. Three sets of tests were conducted.

The measurements taken for these tests involved collecting RMS data for V, I, kW, kVAr, and Freq for each flow change at the following points:

- $\quad$ Meter $1-\mathrm{V}, \mathrm{I}, \mathrm{kW}, \mathrm{kVAr}$ for the utility connection

- Meter 2 - V, I, kW, kVAr and utility-side V and Freq

- Meter 3 - V, I, kW, and kVAr for Feeder A and Microgrid side V and Freq

- $\quad$ Meter A1p - V, I, kW, kVAr for Gen-set A1

- Load Meter 3 - V, I, Zone 3 load kW and kVAr

- Meter $4-\mathrm{V}, \mathrm{I}, \mathrm{kW}, \mathrm{kVAr}$ for Zone 4

- $\quad$ Meter A2p - V, I, kW, kVAr for Gen-set A2

- Load Meter 4 - V, I, Zone 4 load kW and kVAr

- Meter $5-\mathrm{V}, \mathrm{I}, \mathrm{kW}, \mathrm{kVAr}$ for Feeder B 
- $\quad$ Meter B1p - V, I, kW, kVAr for Gen-set B1

- Load Meter 5 - V, I, Zone 5, Feeder B load kW and kVAr

- Load Meter 6 - V, I, Zone 6, Feeder C load kW and kVAr

In addition $\mathrm{V}$ and $\mathrm{I}$ waveform data for unexpected events and for static switch transitions.

The fifth and final set of testing (Section 10 of the test plan) began to explore the operational limits of the microgrid (i.e., power quality, protection and inverter limits). Two primary sets of tests were conducted under "weak grid" conditions; the first involved induction motor starting loads under balanced and unbalanced load conditions; the second involved only unbalanced loads.

The measurements collected included the following:

- $\quad$ RMS data for $\mathrm{kW}, \mathrm{kVAr} \mathrm{V}$, and I injected by each Gen-set

- Freq at each connection point

- $\quad \mathrm{V}$ and I from Meters 1 - 5, and Load Meters 3 - 6

The analysis of the test results consisted of comparing test results to findings from earlier analyses of CERTS Microgrid concepts. As noted, successive analysis and testing of the CERTS Microgrid concepts have include analytical simulation, laboratory-scale emulations, and factory testing of the commercial-grade hardware installed at the test bed.

A number of software tools were used to process raw test data into a format suitable for review and presentation. These included: PQView, AcSELerator QuickSet Designer, Excel, and Word. Test results were posted by AEP onto a secure website where they could be reviewed and discussed by the entire CERTS team.

Test bed results were then presented and discussed with the TAC. TAC members were also invited to review and provide comments each of the extensive reports that have been prepared detailing the results of the testing.

The goal of sub-subtask 2.3.3 was to develop an innovative approach to provide protective relaying within the microgrid. The approach addresses that fact that power electronic interfaces, by design, limit the fault current available to detect system faults, which is the traditional means for detecting faults within an electrical network. Hence, in addition to traditional protection schemes that were already in place, the CERTS team, led by University of Wisconsin, needed to design an additional level of protection for faults that occur within the microgrid. These designs were then implemented and tested as part of the tests conducted in sub-subtask 2.3.2.

\subsection{Field Demonstration Planning (Subtask 2.4)}

The goal of subtask 2.4 was to identify and engage one or more partners for a field demonstration of the CERTS Microgrid concept. While a field demonstration was not to be conducted in this project, it was important to engage potential partners prior to and during the 
laboratory test-bed phase so that partner-specific technical or operational issues could be addressed in the test bed prior to conducting the field demonstration.

This task was a continuation of work initiated under a previous Energy Commission agreement (amendment \#150-99-003, task 2.7). That contract led to preparation of a field demonstration planning and partner recruitment strategy. The current task continued the implementation of that strategy.

The approach primarily involved conducting meetings with potential field demonstration partners to brief them on the CERTS Microgrid concept and test bed prior to and during the testing. Other activities that contributed to field demonstration planning by increasing the visibility of the CERTS Microgrid concept to a wide audience included presentations and publications of the CERTS Microgrid concept in a variety of fora, including professional meetings, domestic and international conferences, and academic and trade publications. These activities directly contributed to and supported follow-up discussions with potential field demonstration partners. 


\subsection{Project Results}

This section presents projects results from Subtasks 2.1-2.4 of the Microgrid Laboratory Test Bed project. As discussed earlier, the project produced a number of deliverables. Several of these are quite voluminous, as they contain extensive tabular and graphical summaries from each of the tests conducted at the test bed. For brevity, this report only summarizes and highlights selected findings from these deliverables. This report is supplemented by numerous, separately bound appendices, which contain these detail project findings; these appendices are referenced throughout the following discussions.

\subsection{Test Bed Selection (Subtask 2.1)}

The goal of Subtask 2.1 was accomplished through the selection of and execution of a contract with the American Electric Power Company to join the CERTS team to build the test bed and conduct the testing program. The steps including developing selection criteria, applying them to candidate facilities, and determining the most qualified facility.

The final test site selection criteria included the following:

1. Physical requirements

a) Adequate space for the test bed and generators (including safe working space around equipment)

b) Availability of a "stiff" distribution voltage (preferably $15 \mathrm{kV}$ class)

c) Availability of natural gas

d) Availability of "standard" test equipment

2. Personnel -Test engineers and technicians with appropriate experience

3. Project leader with appropriate experience

4. Corporate interest and involvement

5. Timing and availability to meet CERTS requirements

6. Utility-owned and operated site - Important for utility community acceptance of the credibility of test results

7. Accessibility of test site to interested researchers

Under an earlier Energy Commission contract 150-99-003, the CERTS team conducted a preliminary assessment of possible sites for the test bed.2 Based on this preliminary assessment, the team applied the above criteria and identified three candidate test sites:

1. Pacific Gas and Electric (PG\&E) Technology and Ecological Services, San Ramon, California;

2. Southern California Edison (SCE) Electric Vehicle Technology Center, Pomona, California

3. American Electric Power (AEP) Dolan Technology Center, Groveport, Ohio

2 "Review of Test Facilities for Distributed Energy Resources" by A. Akhil, C. Marnay, and T. Lipman, SAND2003-1602, May, 2003. 
Input from the TAC and discussions with the Energy Commission contract manager on the list of final candidate test sites and the test site selection criteria led the team to modify the scope of sub-subtask 2.2.2 to have NPS also fabricate the majority of the test bed hardware, rather than have it fabricated by the test facility. There were two reasons: First, the team determined that the test facility should be selected primarily based on its ability to conduct the tests, not based on its ability to fabricate test bed hardware. Second, NPS was uniquely qualified to fabricate the equipment because it had designed the equipment, as part of its preparation of the overall test bed design (conducted as part of an earlier Energy Commission contract 150-99-003.

With this revised scope for the test facility, the team then sent a questionnaire to each of the three final candidate sites requesting detailed written information on four topics:

1. Required steps to prepare the physical site;

2. Equipment availability, data acquisition hardware and approach, and energy management system (EMS);

3. Personnel; and

4. Project scheduling and coordination.

At this point, PG\&E indicated that would not able to respond because it would not be able to conduct the tests within the available time, largely due to prior commitments to other Energy Commission PIER DER Integration projects. This potential conflict was acknowledged by the Energy Commission contract manager and it was agreed that PG\&E should not be considered to host the tests.

The CERTS team and the Energy Commission contract manager then scheduled oral presentations from representatives from both SCE and AEP. On the basis of these presentations, the CERTS team and the Energy Commission contract manager further followed up with on-site visits to the proposed test sites at both SCE and AEP.

Following the site visits, the team recommended the selection of AEP, as the host for the test bed. The SCE site did not have a natural gas supply line and would have required extensive modifications to the electric service interconnection. The AEP site was well-suited physically, had natural gas service, and had an appropriate electric service interconnection.

In conjunction with the contract award, AEP and CERTS entered into a Memorandum of Understanding to work cooperatively in areas of mutual interest for research, development, and demonstration of distributed energy resource (DER) technology microgrids. Subsequent to the initiation of work on this Energy Commission project, CERTS and AEP have won a competitive solicitation from the U.S. Department of Energy to conduct additional tests using the microgrid equipment developed in this project. 


\subsection{Equipment Preparation (Subtask 2.2)}

The goal of subtask 2.2 was accomplished by work led by Tecogen and their subcontractor, Youtility, to modify three Tecogen prime movers to incorporate CERTS Microgrid control algorithms (Sub-subtask 2.2.1) and by work led by NPS to fabricate a static switch that implemented CERTS islanding and resychronization procedures and fabricate the other major hardware elements required by the CERTS laboratory test bed design. A critical element of these sub-subtasks involved detailed factory acceptance testing to confirm the performance of the equipment prior to shipment to AEP.

\subsubsection{The Prime Movers and Inverters (Sub-subtask 2.2.1)}

CERTS contracted with Tecogen to obtain three 100kW prime movers, each of which was modified to incorporate the CERTS Microgrid control algorithms. The complete systems, which were delivered and installed in the CERTS Microgrid test bed site at AEP, each included an engine/generator, a "surge module" and an inverter. See Figure 5.

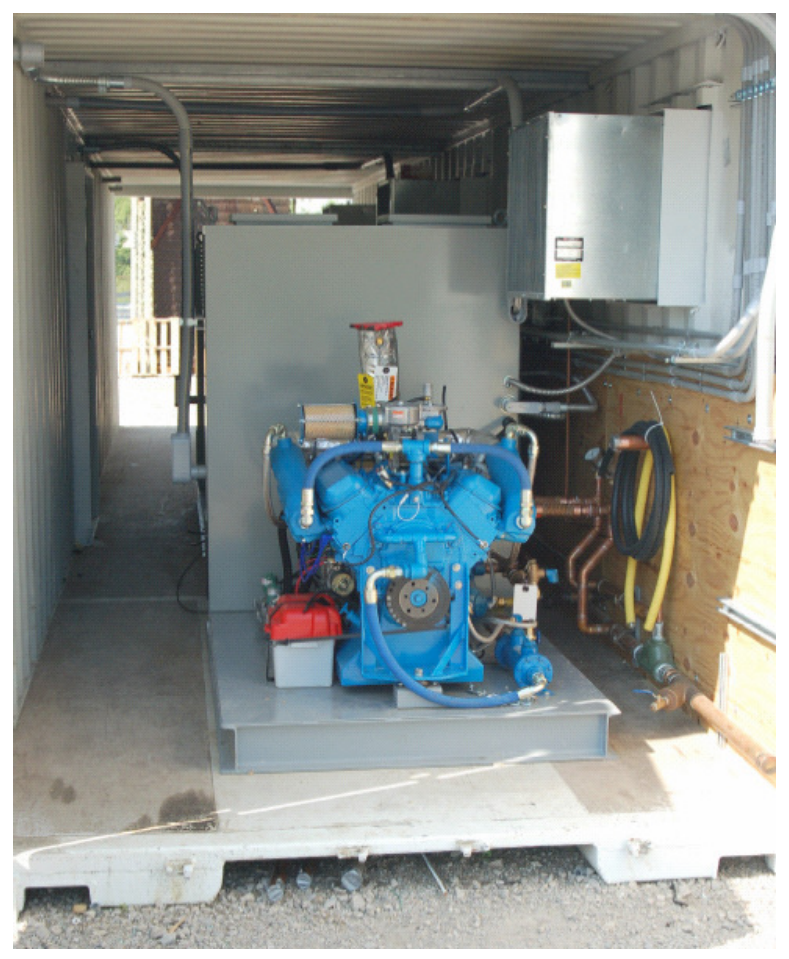

Figure 5. Tecogen Prime Mover with Inverter

A critical aspect of this project was the incorporation of the CERTS Microgrid control algorithms into the inverters provided by Youtility. This required not only providing the algorithms to Youtility, but also providing Youtility with an in-depth understanding of the function of these algorithms so that they could understand the expected response, and the significance of deviations from that response, when the completed inverters were subjected to test scenarios. Providing this understanding required considerable interaction between the Youtility designer and the project's technical director from the University of Wisconsin, Professor Robert Lasseter. 
Professor Lasseter also provided Youtility with a set of test scenarios that were used to confirm correct operation of the completed inverters. These test scenarios then comprised a Youtility factory acceptance test that was performed prior to sending the inverters to Tecogen. The test report for the Youtility factory acceptance tests is included as Appendix C. Youtility Factory Test Plan Final Test Results.

The prime mover for the engine/generator is a 454 cubic inch displacement natural gas-fired V8 reciprocating engine. This engine was designed to be operated at an engine speed selected to give the best combination of fuel efficiency and response to load changes. Thus, the engine speed will not necessarily be synchronous with a $60 \mathrm{~Hz}$ system. The electrical generator a variable AC output which is rectified to a direct current (DC) output. The inverter converts the DC to utility-synchronous AC. For this project, the inverter was modified to incorporate the CERTS Microgrid control algorithms. The surge module is an energy storage device, consisting of batteries, power electronics, and controls, which is tied to the DC bus in parallel with the electrical generator and the inverter. The purpose of the surge module is to provide instantaneous power when there is a load increase, while waiting for the engine/generator to adjust its speed to the new loading conditions. The inverters and surge modules were both supplied to Tecogen by Youtility, Inc. Tecogen integrated the entire prime mover system and performed a set of factory acceptance tests, as specified by the CERTS team, before Tecogen shipped the units to the test bed site. (See Appendix D. Tecogen 60kW Inverter-Based CHP Modules Factory Testing.)

Once at AEP's facilities, the Tecogen units were retested to ensure there had been no shipping damage. The tests confirmed that the performance of the units once installed at AEP was consistent with the factory tests. (Appendix E. Tecogen CHP Modules Commissioning Report.)

\subsubsection{The Static Switch and Other Test Bed Equipment}

CERTS contracted with NPS to design, fabricate, and test a static switch that implemented CERTS Microgrid islanding and resychronization procedures and to fabricate the other major hardware elements required by the CERTS laboratory test bed design, including load banks, power and control circuitry, protective relaying, and data-acquisition equipment, installed in metal-clad switchgear cabinets for protection in an outdoor location.

NPS designed and built the controls that would allow the static switch to implement the CERTS Microgrid islanding and resychronization procedures. The controls were implemented onto the static switch hardware associated with the Purewave uninterruptible power supply system manufactured by S\&C Electric. See Figure 6. The islanding procedures consisted of trip sensing (i.e., detect and trip on IEEE 1547-specified criteria such as voltage or frequency deviations and reverse power flow) followed by disconnection (islanding) of the microgrid from the utility. The voltage-trip function included trip-characteristics required by IEEE 1547 for distributed resource interconnection, those specified by the Information Technology Industry Council for power quality, as well as a custom-voltage-versus-time-trip characteristic that could be specified by the user. 
The resynchronizing function had to meet a more stringent requirement than the commonly accepted criteria of IEEE 1547. The reason for the more stringent requirement was to insure transient free transitions for power flow either into or out of the microgrid at the instant of switch closing. IEEE 1547 requires the phase difference across the switch is less than $20^{\circ}$ before the switch can close. This phase difference is too large for for our needs. We require closing at a zero phase difference. The high-speed static switch and digital signal processing (DSP), insures that the voltage phase difference a closing is zero.

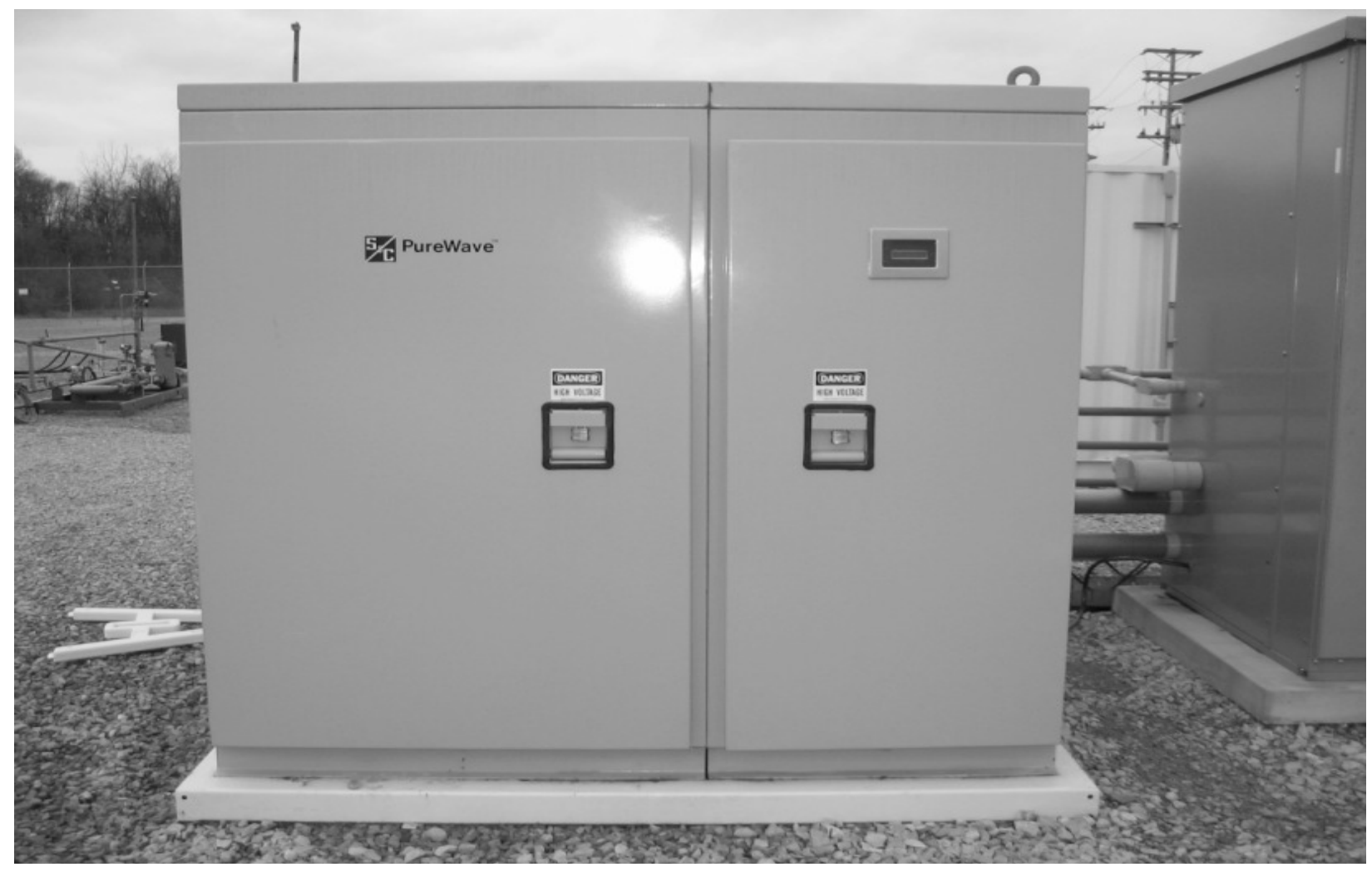

Figure 6. The Static Switch

NPS tested the static switch with low-level power (i.e., signal injection) to confirm proper operation before shipping the switch to AEP. TAC members from the National Renewable Energy Laboratory shipped additional testing equipment and lent expertise to NPS in support of this testing. (Appendix F. CERTS Test Bed CERTEQUIP-V06-002, CERTS Switch, Low Power Factory Acceptance Test Report.)

Once at AEP's facilities, the switch was retested with low power to ensure there had been no shipping damage. After confirming that the static switch was in good condition, AEP performed further testing at the Dolan Test Facility to examine selected power quality issues prior to installing equipment at the test bed. (Appendix G. Summary of CERTS Microgrid Static Switch Power Quality Tests at AEP Dolan, CERTS Microgrid Static Switch Testing.) 
NPS also fabricated cabinets containing all the sensing hardware for the protection and dataacquisition equipment; the circuit breakers, switches and fuses; and all the loads. The loads include a variety of resistive, inductive, capacitive and motor loads as well a six-pulse power rectifier to provide a non-linear load.

\subsection{CERTS Microgrid Laboratory Tests}

This section describes the preparation of the laboratory test bed for testing (Sub-subtask 2.3.1), the results of the CERTS microgrid laboratory tests (Sub-subtask 2.3.2), and the development of an approach for internal protection within the microgrid (Sub-subtask 2.3.3).

\subsubsection{Prepare Laboratory Test Bed for Testing}

After preparing the site, erecting supporting structures, cooling equipment, and wiring, and installing the equipment prepared by NPS and Tecogen/Youtility, AEP and Northern Power performed a field acceptance test. See Figure 1. The field acceptance testing also included exercising the complete data acquisition and control systems. After completing this testing and rectifying the minor issues that it uncovered, the team declared the test bed ready to conduct the full set of CERTS Microgrid tests, as outlined in the test plan. (Appendix H. CERTS Test Bed Design and Commissioning Lessons Learned Summary.)

\subsubsection{Test of CERTS Microgrid Concepts}

As described in section 2.3.2, five sets of tests were conducted. The results of these five sets of tests are contained in separate, stand-alone appendices to this report, as follows:

1. Static Switch (Section 6 of the test plan -6 tests - Appendix I)

2. Protection (Section 7 of the test plan -16 tests - Appendix J)

3. Reduced System (Section 8 of the test plan - 13 tests - Appendix K)

4. Power Flow Control (Section 9 of the test plan - 3 banks of tests - Appendix L)

5. Difficult Loads (Section 10 of the test plan -1 set of motor starting tests - Appendix M) Each appendix contains a narrative description of the purpose and performance goal for each test, along with graphical and tabular summaries of the results for each test. In addition, a sixth appendix (Appendix N) contains the test log developed by AEP during the conduct of the tests.

For brevity, this report will summarize and highlight selected findings for each of the five sets of tests. This includes listing each test conducted and its performance objective. The interested reader is referred to the individual appendices to see detailed results from each test performed. 
The first set of tests (Section 6 of the test plan) examined the operation of the static switch to determine that it and its digital signal processing (DSP) control operated as designed. This included six tests of dead-bus and synchronized closing, reverse power and IEEE 1547 protective relay functions. These tests were designed to check control and operation of the static switch, basic power and voltage control of the Gen-sets, and a preliminary check of the protection scheme. The goal was to ensure that, by confirming the correct operation of the static switch, which is located at the interface (and thus serves as the protection) between the unprotected and protected portions of the microgrid, was ready to perform the remaining tests.

A synchronized closing test of the static switch was required to verify that when conditions were within synchronization limits set in the EMS, the static switch performed a synchronized close and thus provided a smooth connection transition. The dead-bus bus (de-energized bus) reclose test's goal was to verify that the static switch can close when de-energized bus conditions exist on the Gen-set side of the static switch; and that the dead-bus reclose algorithm requires user interaction (i.e., Operator needs to "Enable" the dead-bus reclose using pushbutton in the EMS).

The reverse power tests consisted of three tests: three-phase reverse power condition test, single-phase reverse power condition test, and anti-islanding Micro-grid settings reset test. Both three-phase and single-phase reverse power condition tests were required to verify the reverse power functionality of the static switch and confirm that the static switch islands the microgrid for a three-phase or single-phase reverse power conditions. The anti-islanding Micro-grid settings reset test was needed to verify that if a reverse power event occurred, due to a mismatch of Gen-set settings (i.e., total Gen-set power is greater than Microgrid load), the static switch will lockout and go to the "Fault" state, where user interaction is required.

The next goal was to verify the reconnection timers of the static switch (i.e., set by default at 300 seconds based on IEEE Standard 1547-2003). The length of time is programmed into the control system and designed to prevent reconnection until after the utility source voltage returns to nominal steady state conditions.

Table 1 summarizes the test performance goals and results from each of these tests. 


\begin{tabular}{|c|c|c|}
\hline Test Description & Performance Goal & Results \\
\hline $\begin{array}{l}\text { 6.2. Reverse power, } \\
\text { grid islanding (IEEE } \\
1547 \text {, loss of utility } \\
\text { service) }\end{array}$ & $\begin{array}{l}\text { Verify the reverse power functionality of the static } \\
\text { switch and confirm that the static switch islands the } \\
\text { Microgrid for a reverse power condition due to an } \\
\text { upstream utility operation at the PCC. Once the } \\
\text { utility voltage returns to the IEEE } 1547 \text { limits, verify } \\
\text { the proper operations of the reconnection timers } \\
\text { (set by default to } 300 \text { seconds based on IEEE } 1547 \\
\text { standard). }\end{array}$ & $\begin{array}{l}\text { The static switch islanded the microgrid from the utility grid due to an } \\
\text { undervoltage condition. This occurred after the utility feeder opened } \\
\text { separating the microgrid with } 500 \mathrm{~kW} \text { of feeder load which was } \\
\text { supplied by Gen-set A } 1 \text { until the static switch opened. Once the static } \\
\text { switch opened Gen-set A } 1 \text { continued serving the protected load } \\
\text { within the microgrid. After } 300 \text { seconds, the static switch } \\
\text { synchronized and closed back into the utility grid with a smooth } \\
\text { transition. }\end{array}$ \\
\hline $\begin{array}{l}\text { 6.4. Reverse power, } \\
\text { anti-islanding } \\
\text { microgrid settings } \\
\text { reset }\end{array}$ & $\begin{array}{l}\text { Verify that if a reverse power event occurs, due to a } \\
\text { mismatch of Gen-set settings (Total Gen-set power } \\
>\text { Microgrid load), the static switch will lockout and } \\
\text { go to the "Fault" state, where user interaction is } \\
\text { required. }\end{array}$ & $\begin{array}{l}\text { Load was reduced within the microgrid which caused the static switch } \\
\text { to open on a reverse power condition. The Gen-sets picked up the } \\
\text { load within the microgrid. All loads were reset and the static switch } \\
\text { synchronized and closed back into the utility. }\end{array}$ \\
\hline
\end{tabular}




\begin{tabular}{|l|l|l|}
\hline $\begin{array}{l}\text { 6.5. De-energized } \\
\text { bus (dead bus) } \\
\text { reclose }\end{array}$ & $\begin{array}{l}\text { Verify that the static switch can close when de- } \\
\text { energized bus conditions }(<15 \mathrm{~V}) \text { on the DG side } \\
\text { are measured and that the Dead Bus Reclose } \\
\text { algorithm requires user interaction (i.e., Operator } \\
\text { needs to "Enable" the Dead Bus Reclose using } \\
\text { pushbutton in the EMS). }\end{array}$ & $\begin{array}{l}\text { The static switch synchronized and closed into the utility grid with } \\
\text { less than 15V on the DG side. }\end{array}$ \\
\hline
\end{tabular}

Table 1. Static Switch Testing Summary 
The second set of tests (Section 7 of the test plan) examined a preliminary set of fault (i.e., overload simulating a fault) condition tests to ensure protection and safety of the test bed, prior to performing other planned tests. The goal was to test and adjust protection settings to achieve the most ideal conditions and protection design. The tests included inductor L11 in the circuit, reflecting "weak grid" conditions. Sixteen separate tests were conducted. The fault (i.e., overload simulating a fault) condition tests were intended to cover the basic concept of the protection design and to study its effectiveness (e.g., zero-sequence, negative-sequence and residual currents for line-to-ground faults; negative-sequence or ( $\left.\mathrm{I}^{2} \mathrm{t}\right)$ protection for phase-tophase faults). For the majority of these test scenarios, the static switch was expected to "OPEN" first, followed by the zone breaker of the affected zone. The objective was to confirm the protective action detailed in the Protection document developed in Task 2.3.3.

Test 7.1 induced a three-phase-to-ground balanced overload fault condition in Zone 4 to verify the $I^{2} t$ protection and a single line-to-ground fault condition in Zone 4 to verify zero-sequence, negative-sequence or residual over-current protection. Zone 3 and Zone 5 were tested similar to Zone 4 by introducing a three-phase-to-ground balanced overload fault and a single line-toground fault conditions in the zones, verifying the protection scheme in Test 7.2 (Zone 3) and Test 7.3 (Zone 5). During the 7.1, 7.2 and 7.3 tests, all three Gen-sets were off-line and the CERTS Microgrid was connected to the utility. Test 7.4 tested a three-phase-to-ground balanced overload fault condition in Zone 4 with Gen-sets A1 and A2 operating in-parallel with the utility grid to verify $\mathrm{I}^{2} \mathrm{t}$ protection, plus to confirm a reverse power event after the zone breaker "opens".

Test 7.5 through Test 7.10 involved applying a single line-to-ground overload fault condition in each Zone, located beyond the static switch, while connected to the utility grid. Each one of the six tests differs from one another by which Gen-sets are on-line during the fault, which phase is faulted, and in which zone the fault is applied. These tests were designed to verify zerosequence, negative-sequence or residual over-current protection settings. Test 7.5 verified the protection in Zone 3 by applying a single line-to-ground overload fault condition with Gen-sets A1 operating in parallel with the utility grid. Test 7.6 tested a single line-to-ground overload fault condition in Zone 3 with Gen-sets A1 and A2 operating in parallel with the utility grid. Test 7.7 tested a single line-to-ground overload fault condition in Zone 5 with Gen-set B1 operating in parallel with the utility grid. Test 7.8 tested a single line-to-ground overload fault condition in Zone 5 with Gen-sets A1 and B1 operating in parallel with the utility grid. Test 7.9 tested a single line-to-ground overload fault condition in Zone 4 with Gen-sets A1 and A2 operating in parallel with the utility grid. Test 7.10 tested a single line-to-ground overload fault condition in Zone 2 with Gen-sets A1 and B1 operating in parallel with the utility grid.

Tests 7.11 and 7.12 both applied a single line-to-ground overload fault condition in Zone 6 while connected to the utility. Test 7.11 involved only Gen-set A1 operating in parallel with the grid; and Test 7.12 involved Gen-sets A1 and B1 operating in-parallel with the grid. Both tests were designed to verify the $I^{2} t$ protection of the breaker in Zone 6.

The four tests, 7.13 - 7.16, applied a line-to-line overload fault condition in one of the five zones beyond the static switch with a combination of two Gen-sets, operating in parallel with the 
utility grid. These tests were designed to test negative-sequence, $I^{2} t$ protection or residual overcurrent protection settings. Test 7.13 tested a phase-to-phase overload fault condition in Zone 3 with Gen-sets A1 and A2 operating in parallel with the utility grid. Test 7.14 tested a phase-tophase overload fault condition in Zone 4 with Gen-sets A1 and A2 operating in parallel with the utility grid. Test 7.15 tested a phase-to-phase overload fault condition in Zone 2 with Gen-sets A1 and B1 operating in-parallel with the utility grid. Test 7.16 tested a phase-to-phase overload fault condition in Zone 5 with Gen-sets A1 and B1 operating in parallel with the utility grid.

Table 2 summarizes the test performance goals and results from each of these tests. 


\begin{tabular}{|c|c|c|}
\hline Test Description & Performance Goal & Results \\
\hline $\begin{array}{l}\text { 7.1. Validate zone } 4 \\
\text { circuit breaker } \\
\text { settings, utility } \\
\text { connected }\end{array}$ & $\begin{array}{l}\text { Initially test a three-phase balanced fault } \\
\text { condition in Zone } 4 \text { to verify } I^{2} \text { t protection. Then } \\
\text { test a single line-to-ground fault condition in Zone } \\
4 \text { to verify zero-sequence, negative-sequence or } \\
\text { residual over-current protection. }\end{array}$ & $\begin{array}{l}\text { Relay } 4 \text { detected an } I^{2} \text { t protection event and opened circuit breaker } \\
\text { CB41 during the three-phase balanced fault. All other breakers } \\
\text { remained closed. Relay } 2 \text { detected a ground over-current during the } \\
\text { single line-to-ground fault and opened the static switch. All other } \\
\text { breakers remained closed. }\end{array}$ \\
\hline $\begin{array}{l}\text { 7.2. Validate zone } 3 \\
\text { circuit breaker } \\
\text { settings, utility } \\
\text { connected }\end{array}$ & $\begin{array}{l}\text { Initially test a three-phase balanced fault } \\
\text { condition in Zone } 3 \text { to verify } I^{2} \text { t protection. Then } \\
\text { test a single line-to-ground fault condition in Zone } \\
3 \text { to verify zero-sequence, negative-sequence or } \\
\text { residual over-current protection. }\end{array}$ & $\begin{array}{l}\text { Relay } 3 \text { detected an } I^{2} \text { t protection event and opened circuit breaker } \\
\text { CB31 during the three-phase balanced fault. All other breakers } \\
\text { remained closed. Relay } 2 \text { detected a ground over-current during the } \\
\text { single line-to-ground fault and opened the static switch. All other } \\
\text { breakers remained closed. }\end{array}$ \\
\hline $\begin{array}{l}\text { 7.3. Validate zone } 5 \\
\text { circuit breaker } \\
\text { settings, utility } \\
\text { connected }\end{array}$ & $\begin{array}{l}\text { Initially test a three-phase balanced fault } \\
\text { condition in Zone } 5 \text { to verify } 1^{2} \text { t protection. Then } \\
\text { test a single line-to-ground fault condition in Zone } \\
5 \text { to verify zero-sequence, negative-sequence or } \\
\text { residual over-current protection }\end{array}$ & $\begin{array}{l}\text { Relay } 5 \text { detected an } I^{2} t \text { protection event and opened circuit breaker } 5 \\
\text { during the three-phase balanced fault. All other breakers remained } \\
\text { closed. Relay } 5 \text { detected a negative-sequence over-current during } \\
\text { the single line-to-ground fault and opened circuit breaker CB51. All } \\
\text { other breakers remained closed. }\end{array}$ \\
\hline $\begin{array}{l}\text { 7.4. Zone } 4 \text { three- } \\
\text { phase un-grounded } \\
\text { fault, gen-sets } \\
\text { (A1+A2), utility } \\
\text { connected }\end{array}$ & $\begin{array}{l}\text { Test a three-phase balanced fault condition in } \\
\text { Zone } 4 \text { with Gen-sets A1 and A2 operating in- } \\
\text { parallel with the utility grid to verify } I^{2} \text { t protection, } \\
\text { plus confirm a reverse power event after the } \\
\text { Zone breaker "opens". }\end{array}$ & $\begin{array}{l}\text { Relay } 4 \text { detected an } I^{2} \text { t protection and opened circuit breaker CB41. } \\
\text { The static switch opened } 30 \text { seconds later due to a reverse power } \\
\text { event. }\end{array}$ \\
\hline $\begin{array}{l}\text { 7.5. Zone } 3 \text { A-phase } \\
\text { line-to-ground fault, } \\
\text { gen-set } A 1 \text {, utility } \\
\text { connected }\end{array}$ & $\begin{array}{l}\text { Test a single line-to-ground fault condition in } \\
\text { Zone } 3 \text { with Gen-sets A1 operating in-parallel } \\
\text { with the utility grid to verify zero-sequence, } \\
\text { negative-sequence or residual over-current } \\
\text { protection. }\end{array}$ & $\begin{array}{l}\text { Relay } 2 \text { detected a ground over-current and opened the static switch } \\
\text { within a cycle. Relay } 3 \text { opened circuit breaker CB31 } 0.15 \text { seconds } \\
\text { after the static switch when it detected a ground over-current }\end{array}$ \\
\hline $\begin{array}{l}\text { 7.6. Zone } 3 \text { A-phase } \\
\text { line-to-ground fault, }\end{array}$ & $\begin{array}{l}\text { Test a single line-to-ground fault condition in } \\
\text { Zone } 3 \text { with Gen-sets A1 and A2 operating in- }\end{array}$ & $\begin{array}{l}\text { Relay } 2 \text { detected a ground over-current and opened the static switch } \\
\text { in } 0.02 \text { seconds. Relay } 4 \text { opened circuit breaker CB41 } 0.07 \text { seconds }\end{array}$ \\
\hline
\end{tabular}




\begin{tabular}{|c|c|c|}
\hline $\begin{array}{l}\text { gen-sets }(A 1+A 2) \text {, } \\
\text { utility connected }\end{array}$ & $\begin{array}{l}\text { parallel with the utility grid to verify zero- } \\
\text { sequence, negative-sequence or residual over- } \\
\text { current protection }\end{array}$ & $\begin{array}{l}\text { after the static switch when it detected a neutral over-current. Relay } \\
3 \text { opened circuit breaker CB31 } 0.04 \text { seconds after circuit breaker } \\
\text { CB41 when it detected a ground over-current. }\end{array}$ \\
\hline $\begin{array}{l}\text { 7.7. Zone } 5 \text { B-phase } \\
\text { line-to-ground fault, } \\
\text { gen-set B1, utility } \\
\text { connected }\end{array}$ & $\begin{array}{l}\text { Test a single line-to-ground fault condition in } \\
\text { Zone } 5 \text { with Gen-sets B1 operating in-parallel } \\
\text { with the utility grid to verify zero-sequence, } \\
\text { negative-sequence or residual over-current } \\
\text { protection }\end{array}$ & $\begin{array}{l}\text { Relay } 5 \text { opened circuit breaker CB51 in } 0.07 \text { seconds when it } \\
\text { detected a ground over-current. The static switch opened } 0.03 \\
\text { seconds after circuit breaker CB51 when it detected a negative } \\
\text { sequence over-current. }\end{array}$ \\
\hline $\begin{array}{l}\text { 7.8. Zone } 5 \text { B-phase } \\
\text { line-to-ground fault, } \\
\text { gen-sets }(A 1+B 1) \\
\text { utility connected }\end{array}$ & $\begin{array}{l}\text { Test a single line-to-ground fault condition in } \\
\text { Zone } 5 \text { with Gen-sets A1 and B1 operating in- } \\
\text { parallel with the utility grid to verify zero- } \\
\text { sequence, negative-sequence or residual over- } \\
\text { current protection. This test is similar to the prior } \\
\text { test, but evaluates the resultant impact of two } \\
\text { Gen-sets operating during a fault condition }\end{array}$ & $\begin{array}{l}\text { Relay } 5 \text { opened circuit breaker CB51 in } 0.06 \text { seconds when it } \\
\text { detected a ground over-current. All other breakers remained closed. }\end{array}$ \\
\hline $\begin{array}{l}\text { 7.9. Zone } 4 \text { B-phase } \\
\text { line-to-ground fault, } \\
\text { gen-sets }(A 1+A 2) \\
\text { utility connected }\end{array}$ & $\begin{array}{l}\text { Test a single line-to-ground fault condition in } \\
\text { Zone } 4 \text { with Gen-sets A1 and A2 operating in- } \\
\text { parallel with the utility grid to verify zero- } \\
\text { sequence, negative-sequence or residual over- } \\
\text { current protection. }\end{array}$ & $\begin{array}{l}\text { Relay } 2 \text { detected a ground over-current and opened the static switch } \\
\text { within a cycle. Relay } 4 \text { opened circuit breaker } 40.058 \text { seconds after } \\
\text { the static switch when it detected a ground over-current. }\end{array}$ \\
\hline $\begin{array}{l}\text { 7.10. Zone } 2 \text { C-phase } \\
\text { line-to-ground fault, } \\
\text { gen-sets }(A 1+B 1) \\
\text { utility connected }\end{array}$ & $\begin{array}{l}\text { Test a single line-to-ground fault condition in } \\
\text { Zone } 2 \text { with Gen-sets } A 1 \text { and B1 operating in- } \\
\text { parallel with the utility grid to verify zero- } \\
\text { sequence, negative-sequence or residual over- } \\
\text { current protection. }\end{array}$ & $\begin{array}{l}\text { Relay } 2 \text { detected a ground over-current and opened the static switch } \\
\text { within a cycle. Relay } 3 \text { opened circuit breaker CB31 } 0.17 \text { seconds } \\
\text { after the static switch when it detected a ground over-current. Relay } \\
5 \text { opened circuit breaker CB51 due to an under-voltage condition. }\end{array}$ \\
\hline $\begin{array}{l}\text { 7.11. Zone } 6 \text { C-phase } \\
\text { line-to-ground fault, } \\
\text { gen-set } A 1 \text {, utility } \\
\text { connected }\end{array}$ & $\begin{array}{l}\text { Test a single line-to-ground fault condition in } \\
\text { Zone } 6 \text { with Gen-set } A 1 \text { operating in-parallel with } \\
\text { the utility grid to verify } I^{2} t \text { protection. }\end{array}$ & $\begin{array}{l}\text { Relay } 2 \text { detected a neutral over-current and opened the static switch } \\
\text { in } 0.07 \text { seconds. Circuit breaker CB13 tripped on an } I^{2} \text { t protection } \\
\text { event. }\end{array}$ \\
\hline
\end{tabular}




\begin{tabular}{|c|c|c|}
\hline $\begin{array}{l}\text { 7.12. Zone } 6 \text { C-phase } \\
\text { line-to-ground fault, } \\
\text { gen-sets }(A 1+B 1) \\
\text { utility connected }\end{array}$ & $\begin{array}{l}\text { Test a single line-to-ground fault condition in } \\
\text { Zone } 6 \text { with Gen-sets } A 1 \text { and } B 1 \text { operating in- } \\
\text { parallel with the utility grid to verify } I^{2} t \text { protection. } \\
\text { This test is similar to the prior test, but evaluates } \\
\text { the resultant impact of two Gen-sets operating } \\
\text { during a fault condition. }\end{array}$ & $\begin{array}{l}\text { Relay } 2 \text { detected a neutral over-current and opened the static switch } \\
\text { in } 0.07 \text { seconds. Circuit breaker CB13 tripped on an I't protection } \\
\text { event. }\end{array}$ \\
\hline $\begin{array}{l}\text { 7.13. Zone } 3 \text { A-to-B } \\
\text { phase fault, gen-sets } \\
\text { (A1+A2), utility } \\
\text { connected }\end{array}$ & $\begin{array}{l}\text { Test a phase-to-phase fault condition in Zone } 3 \\
\text { with Gen-sets } A 1 \text { and A2 operating in-parallel } \\
\text { with the utility grid to verify negative-sequence, } \\
I^{2} \text { t protection or residual over-current protection. }\end{array}$ & $\begin{array}{l}\text { Relay } 2 \text { detected a negative sequence over-current and opened the } \\
\text { static switch in } 0.028 \text { seconds. All other breakers remained online. }\end{array}$ \\
\hline $\begin{array}{l}\text { 7.14. Zone } 4 \text { A-to-B } \\
\text { phase fault, gen-sets } \\
(A 1+A 2) \text {, utility }\end{array}$ & $\begin{array}{l}\text { Test a phase-to-phase fault condition in Zone } 4 \\
\text { with Gen-sets A1 and A2 operating in-parallel } \\
\text { with the utility grid to verify negative-sequence, } \\
\mathrm{I}^{2} \text { t protection or residual over-current protection. }\end{array}$ & $\begin{array}{l}\text { Relay } 2 \text { detected a negative sequence over-current and opened the } \\
\text { static switch in } 0.078 \text { seconds. Relay } 4 \text { opened circuit breaker CB41 } \\
\text { after the static switch when it detected a negative sequence over- } \\
\text { current. }\end{array}$ \\
\hline $\begin{array}{l}\text { 7.15. Zone } 2 \text { A-to-B } \\
\text { phase fault, gen-sets } \\
\text { (A1+B1), utility } \\
\text { connected }\end{array}$ & $\begin{array}{l}\text { Test a phase-to-phase fault condition in Zone } 2 \\
\text { with Gen-sets } A 1 \text { and } B 1 \text { operating in-parallel } \\
\text { with the utility grid to verify negative-sequence, } \\
I^{2} \text { t protection or residual over-current protection. }\end{array}$ & $\begin{array}{l}\text { There was not enough current produced by the fault, therefore, all } \\
\text { breakers remained closed during the test. }\end{array}$ \\
\hline $\begin{array}{l}\text { 7.16. Zone } 5 \text { A-to-B } \\
\text { phase fault, gen-sets } \\
\text { (A1+B1), utility } \\
\text { connected }\end{array}$ & $\begin{array}{l}\text { Test a phase-to-phase fault condition in Zone } 5 \\
\text { with Gen-sets } A 1 \text { and B1 operating in-parallel } \\
\text { with the utility grid to verify negative-sequence, } \\
I^{2} \text { t protection or residual over-current protection. }\end{array}$ & $\begin{array}{l}\text { Relay } 5 \text { detected a negative sequence over-current and opened } \\
\text { circuit breaker in } 0.075 \text { seconds. Relay } 2 \text { opened the static switch } \\
0.016 \text { seconds after circuit breaker CB51 when it detected a } \\
\text { negative sequence over-current. }\end{array}$ \\
\hline
\end{tabular}

Table 2. Internal Protection Testing Summary 
The third set of tests (Section 8 of the test plan) was designed to ensure that the Gen-set inverter controls were working as designed. This includes unit control, zone control, and mixed power controls, in conjunction with limit controls and synchronized closing of the static switch. These tests were based on replicating tests that had previously been conducted by Tecogen and Youtility during the factory acceptance testing of the standalone inverters, and the engine coupled inverters. The performance goal was to observe smooth transitions of the Gen-sets response to different step conditions (i.e., static switch "OPEN"/ "CLOSE" and load steps). Thirteen separate tests were conducted.

Test 8.1 verified smooth transitions in Gen-set A1 when different step conditions of load are applied in Load Bank 3 with voltage set points ranging from $+5 \%$ to $-5 \%$. The same test was repeated for Gen-set A2 and Load Bank 4 and Gen-set B1 and Load Bank 5.

Test 8.2 verified smooth transitions of Gen-sets A1 and A2 response to different step conditions which drive Gen-set A1 to a lower limit of zero kW.

Test 8.3 verified smooth transitions of Gen-sets A1 and A2 response to different step conditions which drive Gen-set A2 to an upper limit of $60 \mathrm{~kW}$.

Test 8.4 verified smooth transitions of Gen-sets A1 and A2 response to different step conditions with an un-balanced load in Zone 3.

Test 8.5 verified smooth transitions of Gen-sets, A1 in Zone operation mode and A2 in Unit operation mode. During a load step change in Load Bank 3, Gen-set A1 was driven to an upper limit of $60 \mathrm{~kW}$.

Test 8.6 verified smooth transitions of Gen-sets, A1 in zone operation mode and Gen-set A2 in Unit operation mode. During a load step change in Load Bank 4, Gen-set A1 is driven to its maximum which causes an automatic reset of the zone set points.

Test 8.7 verified smooth transitions of Gen-sets, A1 in Zone operation mode and A2 in Unit operation mode, with a change of zone power in Feeder A.

Test 8.8 verified smooth transition of Gen-sets, A1 in Zone operation mode and A2 in Unit operation mode, with a static switch operation and a change of zone power in Zone 3 when islanded.

Test 8.9 verified smooth transition of Gen-sets, A1 in Zone operation mode and A2 in Unit operation mode, with a static switch operation and a change of zone power in Zone 3 when islanded. Gen-set A1 was driven to its maximum which caused an automatic reset of both Gensets set-points.

Test 8.10 verified smooth transitions of Gen-sets, A1 and B1 in Zone operation mode, with a static switch operation and a change of zone power in Zones 3 and 5 when islanded.

Test 8.11 verified smooth transitions of Gen-sets, A1 and B1 in Zone operation mode, with a static switch operation and a change of zone power in Zones 3 and 5 when islanded. When 
islanded, Gen-set A1 and B1 set-points were reset based on the remaining load in the islanded system.

Test 8.12 tested the manual procedure used to black-start the CERTS Micro-grid Test Bed in the event of a lengthy utility outage occurs with the Gen-sets off-line.

Test 8.13 was designed to determine the black-start capacity by increasing the amount of load on the CERTS Microgrid Test Bed from a black-out condition without generation or protection trips.

Table 3 summarizes the test performance goals and results from each of these tests 


\begin{tabular}{|c|c|c|}
\hline Test Description & Performance Goal & Results \\
\hline $\begin{array}{l}\text { 8.1. Initial voltage regulation test - } \\
\text { single zone, islanded with gen-set } A 1\end{array}$ & $\begin{array}{l}\text { Verify smooth transitions of Gen-set A1 response to } \\
\text { different step conditions of load in Load Bank } 3 \text { with } \\
\text { voltage set point changes ranging from }+5 \% \text { to }-5 \% \text {. } \\
\text { Repeat tests with Gen-set A2 in Load Bank } 4 \text {, and then } \\
\text { Gen-set B1 in Load Bank } 5\end{array}$ & $\begin{array}{l}\text { All Gen-sets had smooth transitions } \\
\text { through each load step and voltage } \\
\text { change. }\end{array}$ \\
\hline $\begin{array}{l}\text { 8.2. Open static switch test, check } P= \\
0 \text { limit, gen-set } A 1\end{array}$ & $\begin{array}{l}\text { Verify smooth transitions of Gen-sets A1 and A2 response } \\
\text { to different step conditions (i.e., static switch "OPEN"/ } \\
\text { "CLOSE") with the unit power limit of Gen-set A1 equal to } \\
\text { zero }\end{array}$ & $\begin{array}{l}\text { Gen-sets had smooth transition from } \\
\text { grid connected to island mode and vice } \\
\text { versa. All Gen-sets responded as } \\
\text { predicted. }\end{array}$ \\
\hline $\begin{array}{l}\text { 8.3. Open static switch test, check } P= \\
60 \mathrm{~kW} \text { limit, gen-set } A 2\end{array}$ & $\begin{array}{l}\text { Verify smooth transitions of Gen-sets A1 and A2 response } \\
\text { to different step conditions (i.e., static switch "OPEN"/ } \\
\text { "CLOSE") with the unit power limit of Gen-set A2 equal to } \\
60 \mathrm{~kW}\end{array}$ & $\begin{array}{l}\text { Gen-sets had smooth transition from } \\
\text { grid connected to island mode and vice } \\
\text { versa. All Gen-sets responded as } \\
\text { predicted. }\end{array}$ \\
\hline $\begin{array}{l}\text { 8.4. Test island operation, unbalanced } \\
\text { load }\end{array}$ & $\begin{array}{l}\text { Verify smooth transitions of Gen-sets A1 and A2 response } \\
\text { to different step conditions (i.e., static switch "OPEN"/ } \\
\text { "CLOSE") with an un-balanced load condition in Zone } 3\end{array}$ & $\begin{array}{l}\text { Gen-sets had smooth transition from } \\
\text { grid connected to island mode and vice } \\
\text { versa. All Gen-sets responded as } \\
\text { predicted. }\end{array}$ \\
\hline $\begin{array}{l}\text { 8.5. Mixed mode operation test - Zone } \\
3 \text { and } 4 \text {, gen-set } A 1 \text { to } 60 \mathrm{~kW} \text { maximum }\end{array}$ & $\begin{array}{l}\text { Verify smooth transitions of Gen-sets, A1 in Zone } \\
\text { operation mode with a } 60 \mathrm{~kW} \text { limit and A2 in Unit operation } \\
\text { mode, during a load step change in Load Bank } 3\end{array}$ & $\begin{array}{l}\text { Gen-set had smooth transition from grid } \\
\text { connected to island mode and vice } \\
\text { versa. Gen-sets responded as } \\
\text { predicted. }\end{array}$ \\
\hline $\begin{array}{l}\text { 8.6. Mixed mode operation test - } \\
\text { Zones } 3 \text { and } 4 \text {, automatic reset of zone } \\
\text { level set-point }\end{array}$ & $\begin{array}{l}\text { Verify smooth transitions of Gen-sets, A1 in Zone } \\
\text { operation mode with an automatic reset of the set point } \\
\text { and A2 in Unit operation mode, during a load step change } \\
\text { in Load Bank } 4\end{array}$ & $\begin{array}{l}\text { Gen-sets had smooth transition from } \\
\text { grid connected to island mode and vice } \\
\text { versa. All Gen-sets responded as } \\
\text { predicted. }\end{array}$ \\
\hline 8.7. Mixed mode operation test - & Verify smooth transitions of Gen-sets, A1 in Zone & Gen-sets had smooth transition from \\
\hline
\end{tabular}




\begin{tabular}{|c|c|c|}
\hline Zones 3 and 4 , zone power change & $\begin{array}{l}\text { operation mode and A2 in Unit operation mode, with a } \\
\text { change of zone power in Feeder A }\end{array}$ & $\begin{array}{l}\text { grid connected to island mode and vice } \\
\text { versa. All Gen-sets responded as } \\
\text { predicted. }\end{array}$ \\
\hline $\begin{array}{l}\text { 8.8. Mixed mode operation test - } \\
\text { Zones } 3 \text { and } 4 \text {, zone power change } \\
\text { when islanded }\end{array}$ & $\begin{array}{l}\text { Verify smooth transitions of Gen-sets, A1 in Zone } \\
\text { operation mode and A2 in Unit operation mode, with a } \\
\text { static switch operation and a change of zone power in } \\
\text { Zone } 3 \text { when islanded }\end{array}$ & $\begin{array}{l}\text { Gen-sets had smooth transition from } \\
\text { grid connected to island mode and vice } \\
\text { versa. All Gen-sets responded as } \\
\text { predicted. }\end{array}$ \\
\hline $\begin{array}{l}\text { 8.9. Mixed mode operation test - } \\
\text { Zones } 3 \text { and } 4 \text {, when islanded, } \\
\text { automatic reset of zone level set-point, } \\
\text { both gen-sets } P=60 \mathrm{~kW} \text { Maximum }\end{array}$ & $\begin{array}{l}\text { Verify smooth transitions of Gen-sets, A1 in Zone } \\
\text { operation mode with automatic reset of set point and A2 in } \\
\text { Unit operation mode, with a static switch operation and a } \\
\text { change of zone power in Zone } 3 \text { when islanded }\end{array}$ & $\begin{array}{l}\text { Gen-sets had smooth transition from } \\
\text { grid connected to island mode and vice } \\
\text { versa. All Gen-sets responded as } \\
\text { predicted. }\end{array}$ \\
\hline $\begin{array}{l}\text { 8.10. Two sources in zone control - } \\
\text { separate zones, when islanded, } \\
\text { automatic reset of zone level set-point, } \\
\text { new zones sum equal zero }\end{array}$ & $\begin{array}{l}\text { Verify smooth transitions of Gen-sets, A1 and B1 in Zone } \\
\text { operation mode, with a static switch operation and a } \\
\text { change of zone power in Zones } 3 \text { and } 5 \text { when islanded }\end{array}$ & $\begin{array}{l}\text { Gen-sets had smooth transition from } \\
\text { grid connected to island mode and vice } \\
\text { versa. All Gen-sets responded as } \\
\text { predicted. }\end{array}$ \\
\hline $\begin{array}{l}\text { 8.11. Two sources in zone control - } \\
\text { separate zones, when islanded, } \\
\text { automatic reset of zone level set-points }\end{array}$ & $\begin{array}{l}\text { Verify smooth transitions of Gen-sets, A1 and B1 in Zone } \\
\text { operation mode and both having automatic reset of set } \\
\text { points, with a static switch operation and a change of zone } \\
\text { power in Zones } 3 \text { and } 5 \text { when islanded }\end{array}$ & $\begin{array}{l}\text { Gen-sets had smooth transition from } \\
\text { grid connected to island mode and vice } \\
\text { versa. All Gen-sets responded as } \\
\text { predicted. }\end{array}$ \\
\hline 8.12. Test generator black-start & $\begin{array}{l}\text { Bring up the Microgrid Test Bed from a black-out condition } \\
\text { without generation or protection trips. } \\
\text { Measurement - Record the transition of the Gen-set } \\
\text { meters as they are started and brought on-line and as } \\
\text { load banks are switched on-line. Note, the transition time } \\
\text { between all events outage occurs with the Gen-sets off- } \\
\text { line } \\
\text { This test checks the manual procedure used to black-start } \\
\text { the Microgrid Test Bed in the event of a lengthy utility }\end{array}$ & $\begin{array}{l}\text { Gen-sets had smooth transitions and } \\
\text { met the load demand. }\end{array}$ \\
\hline
\end{tabular}


8.13. Test/establish generator black-

start capacity

Determine the Black-start capacity of the microgrid by

increasing the amount of load on the Microgrid Test Bed

from a black-out condition without generation or protection

trips

Table 3. Reduced System Testing Summary 
The fourth set of tests (Section 9 of the test plan) demonstrated the flexibility of the microgrid both grid connected and islanded for different loads, power flows and impact on the utility. The tests included the "weak grid" inductors (i.e., L11) in the circuit. Three sets of tests were conducted.

Tests 9.1 - 9.3 verified and documented power flow and microgrid frequency changes when transitioning from utility connected to an islanded mode of operation. In each test, 9.1-9.3, a series of tests were performed that vary in the amount of load that is applied to the microgrid in a weak grid scenario along with the power settings of each Gen-set. The difference between each test is the type of control mode that the Gen-sets are in. In Test 9.1, all the Gen-sets were set for unit control mode and then the next test, 9.2, all the Gen-sets were set for zone control mode. Test 9.3 mixed the unit and zone control modes of the Gen-sets during each test.

Table 4 summarizes the test performance goals and results from each of these tests 


\begin{tabular}{|l|l|l|}
\hline Test Description & Performance Goal & Results \\
\hline $\begin{array}{l}\text { 9.1. Unit control mode, weak } \\
\text { grid }\end{array}$ & $\begin{array}{l}\text { Verify and document power flow and Microgrid frequency } \\
\text { changes when transitioning from utility connected to an } \\
\text { islanded mode of operation. During each sequence of } \\
\text { tests, maintain a weak grid connection with L11 in the } \\
\text { circuit and the static switch "Closed"; all zone and load } \\
\text { bank circuit breakers "Closed"; Gen-sets A1, A2 and B1 } \\
\text { set for Unit (injection power) control mode; and all load } \\
\text { banks initially set at zero }\end{array}$ & $\begin{array}{l}\text { Voltage and frequency remained stable during grid } \\
\text { connected to island mode and vice versa for all four } \\
\text { tests. Control of Power was as expected from Gen-sets } \\
\text { to load demand. }\end{array}$ \\
\hline $\begin{array}{l}\text { 9.2. Zone control mode, } \\
\text { weak grid }\end{array}$ & $\begin{array}{l}\text { Verify and document power flow and Microgrid frequency } \\
\text { changes when transitioning from utility connected to an } \\
\text { islanded mode of operation. }\end{array}$ & $\begin{array}{l}\text { Voltage and frequency remained stable during grid } \\
\text { connected to island mode and vice versa for all four } \\
\text { tests. Control of Power was as expected from Gen-sets } \\
\text { to load demand. }\end{array}$ \\
\hline $\begin{array}{l}\text { 9.3. Mixed zone and unit } \\
\text { control modes, weak grid }\end{array}$ & $\begin{array}{l}\text { Verify and document power flow and Microgrid frequency } \\
\text { changes when transitioning from utility connected to an } \\
\text { islanded mode of operation. }\end{array}$ & $\begin{array}{l}\text { Voltage and frequency remained stable during grid } \\
\text { connected to island mode and vice versa for all four } \\
\text { tests. Control of Power was as expected from Gen-sets } \\
\text { to load demand. }\end{array}$ \\
\hline
\end{tabular}

\section{Table 4. Power Flow Control Testing Summary}


The fifth and final set of testing (Section 10 of the test plan) began to explore the operation limits of the microgrid (i.e., power quality, protection and inverter limits). Two primary sets of tests were conducted under weak grid conditions; the first involved induction motor starting loads under balanced and unbalanced load conditions; the second involved only unbalanced loads.

Motor start tests conducted in Test 10.2 had balanced loads at a 0.9 power factor under weak grid conditions. These tests verified and documented power flow, microgrid frequency changes, and protection design with different Gen-set settings during motor starts when utility connected and then again during islanded mode of operation. Test 10.3 was similar to Test 10.2, except that the loads were unbalanced with a 0.9 power factor.

In Test 10.4 verified and documented power flow, microgrid frequency changes, and protection design with different Gen-set settings during changes of unbalanced load in load banks.

Table 5 summarizes the test performance goals and results from each of these tests. 


\begin{tabular}{|c|c|c|}
\hline Test Description & Performance Goal & Results \\
\hline $\begin{array}{l}\text { 10.2. Motor Start Tests, } \\
\text { Weak Grid, Balanced } \\
\text { Load }\end{array}$ & $\begin{array}{l}\text { Verify and document power flow, Micro-grid } \\
\text { frequency changes and protection design with } \\
\text { different Gen-set settings during motor starts when } \\
\text { utility connected and island mode of operation. }\end{array}$ & $\begin{array}{l}\text { CERTS Microgrid remained electrically stable } \\
\text { during the } 10 \mathrm{Hp} \text { induction motor start in Zone } 3 \\
\text { while utility connected and islanded mode for all } \\
\text { six test. }\end{array}$ \\
\hline $\begin{array}{l}\text { 10.3. Motor Start Tests, } \\
\text { Weak Grid, Unbalanced } \\
\text { Load }\end{array}$ & $\begin{array}{l}\text { Verify and document power flow, Micro-grid } \\
\text { frequency changes and protection design with } \\
\text { different Gen-set settings during motor starts when } \\
\text { utility connected and island mode of operation. }\end{array}$ & $\begin{array}{l}\text { CERTS Microgrid remained electrically stable } \\
\text { during the } 10 \mathrm{Hp} \text { induction motor start in Zone } 3 \\
\text { while utility connected and islanded mode for all } \\
\text { six test. }\end{array}$ \\
\hline $\begin{array}{l}\text { 10.4. Unbalanced Load } \\
\text { Tests, Weak Grid }\end{array}$ & $\begin{array}{l}\text { Verify and document power flow, Micro-grid } \\
\text { frequency changes and protection design with } \\
\text { different Gen-set settings during changes of } \\
\text { unbalanced load in Load Banks while utility } \\
\text { connected and in islanded mode of operation. }\end{array}$ & $\begin{array}{l}\text { Static switch opened on a reverse power trip } \\
\text { while utility connected in Tests } 10.4 .12 \text { and } 10.4 .14 \\
\text { when Load Banks } 3 \text { and } 6 \text { A-phase was reduced } \\
\text { by } 50 \% \text {. CERTS Microgrid remained electrically } \\
\text { stable when the A-phase in the load demands } \\
\text { equaled 0kW and in island mode of operation. } \\
\text { The static switched opened on a reverse power } \\
\text { trip for all three tests when the microgrid was } \\
\text { reconnected to the utility grid. Once the static } \\
\text { switch opened, the CERTS microgrid remained } \\
\text { electrically stable. All test results performed as } \\
\text { predicted. }\end{array}$ \\
\hline
\end{tabular}

Table 5. Difficult Load Testing Summary 
The testing fully confirmed earlier research that had been conducted initially through analytical simulations, then through laboratory emulations, and finally through factory acceptance testing of individual microgrid components. The islanding and resychronization method met all IEEE 1547 and power quality requirements. The electrical protection system was able to distinguish between normal and faulted operation. The controls were found to be robust under all conditions, including difficult motor starts.

Test bed results were then presented and discussed jointly with and then reviewed individually by members of the TAC. The reviews were uniformly positive:

"Concepts are elegant, yet simple. Simple translates to more affordable."

“Demonstration that static switch can comply with 1547, with non-compliant generation behind it, is a major accomplishment. The team has confirmed that a static switch is very fast. It is recognized that ground fault testing on the utility side of the static switch is still missing from this aspect of 1547 compliance."

"Inverter controls are impressive."

“Test plan was well laid-out. A wide range of concerns that were identified have now been successfully addressed. In particular, motor starting was a challenging test and the results are very encouraging. Should be a major contribution to the literature."

“The test bed platform should be used for future research."

A summary of the TAC meeting at which test bed results were reviewed as well as the additional written comments received from the TAC are contained in Appendix O. Technical Advisory Committee Meeting Summary and Review Comments.

In addition, AEP has prepared a standalone technical assessment and recommendations:

“The major objective of plug and play gen-sets has been proven to work very well and is seen as a major stepping stone toward increasing the commercial adoption of microgrids. The keystone of this plug and play approach is the CERTS frequency versus power and voltage versus VAR algorithms which have proven to be robust in the control of multiple voltage source inverters on a common bus. The specific advantages offered by this approach are expected to include: improved operation, more manageable equipment requirements, all at lower total system costs compared to a traditional custom-designed distributed generation power system involving multiple prime movers.

In addition, the concept of placing generation, which is non-compliant with IEEE Standard 1547, beyond a static switch, which is compliant, was tested and proven to be successful. This is expected to be another effective tool in reducing the cost of a 
traditional custom-designed, distributed generation power system involving multiple prime movers, while still maintaining safe interconnection with a utility system.

It is recognized that the tests conducted by AEP represent the first full-scale tests of the CERTS Microgrid Concept. AEP looks forward to participation in future research and testing to advance this Concept. Toward this end, we recommend focus on the following areas:

1. Demonstrate the ability of power electronic inverters to adjust fault contribution from the gen-sets.

2. Re-examine and potentially re-tune the anti-islanding philosophy governing operation of the static switch, in light of expected unbalanced voltages presented by utility distribution systems to the microgrid.

3. Develop and test means for minimizing VAR flows by using the Energy Manager to direct voltage set-point changes to the gen-sets based on actual voltages on the utility distribution system.

4. Continue testing to explore performance of the CERTS Microgrid in supporting difficult loads.

5. Confirm the adequacy or revise the management of the static switch when the utility closes into a dead bus.

6. Examine enhanced functionalities for the Energy Manager to address customer and utility needs, such as power factor correction, fault and error handling, energy efficiency, and price- or reliability-driven demand management.

7. Examine system impacts for scenarios involving significant deployment of microgrids."

\subsubsection{Microgrid Protective Relaying Design}

Traditionally, power flow in the existing electrical infrastructure flows from the power plant through transmission and possibly distribution lines to the load that is demanding power. Protection schemes were based on this concept, which allowed for uni-directional over-current protection devices to be installed. Uni-directional over-current protection devices sense when there is a fault on the electrical system by opening up when the current exceeds a certain value in a certain direction.

Uni-directional over-current protection schemes are not feasible within microgrids, because of microgrids' reverse power-flow capability; if current is flowing in the opposite direction (i.e., from the microsources toward the point of common coupling (PCC)), it will not trip the overcurrent device when a fault occurs. A microgrid can use bi-directional over-current protection devices when the microgrid is connected to the utility but not while the microgrid is islanded. During islanded operation, the microgrid fault current magnitudes are less significant than 
when the microgrid is connected to the utility, so uni-directional and bi-directional detection may not detect the fault current preventing the protection device from operating.

The team developed a protection scheme that uses sequence components to detect low-fault currents in utility-connected and islanded operation. Sequence components take a balanced or unbalanced voltage or current and break it up into three separate components: positive, negative, and zero sequence. Adding these three components together gives the magnitude and phase angle of balanced and unbalanced currents and voltages. A single line-to-ground fault produces a zero sequence current, providing a trip signal for the zone with the fault that does not depend on the direction of the current and magnitude as is the case with traditional overcurrent protection devices. A line-to-line fault produces a negative sequence current that signals the zone with the fault to trip. This protection scheme allows for plug-and-play without changing the existing over-current devices on the electrical system.

The technical report on the microgrid protective relaying design is contained in Appendix O. Microgrid Fault Protection Based on Symmetrical and Differential Current Components.

\subsection{Field Demonstration Planning (Task 2.4)}

The goal of task 2.4 was to identify and engage one or more partners for a field demonstration of the CERTS Microgrid concept. While a field demonstration was not included in the project plan for this project, it was important to engage potential partners prior to and during the laboratory test bed phase so that partner-specific technical or operational issues could be addressed in the laboratory test bed prior to conducting a field demonstration.

The identification of potential field demonstration partners took place through a variety of means. Central to most of them was outreach conducted through a variety of presentations and publications.

\section{Presentations}

R.H. Lasseter, “Advanced Distribution using DER," Rethinking T\&D Architecture for DER, 24 April 2008, T\&D Panel, Chicago

R.H. Lasseter, “DER and Microgrids,” IEEE Distinguish Lecturer, IEEE PES St. Louis chapter, 6 November 2007

R.H. Lasseter, “Microgrids and Distributed Generation,” ASCE Journal Energy Engineering, Volume 133, Number 3, September 2007.

H. Nikkhajoei and R.H. Lasseter, “Microgrid Protection," IEEE Panel: Microgrid Research and Field Testing, IEEE PES General Meeting, 24-28 June 2007, Tampa, FL

R.H. Lasseter, "Extended Microgrid Using (DER) Distributed Energy Resources," IEEE Panel: Sustainable Energy, IEEE PES General Meeting, 24-28 June 2007, Tampa, FL

R.H. Lasseter, "CERTS Microgrid," Panel on Microgrids Systems, International Conference on System of Systems Engineering, April 16-18, 2007 San Antonio 
R.H. Lasseter, “CERTS Microgrid,” ERDC/CERL_RDCOM, Army Engineers, 18 January 2007, Champaign, Ill.

R.H. Lasseter, "Microgrids on Distribution Scale," Panel, Army Installation Energy Security \& Independence Conference, Greensboro, N.C., 12-13 December 2006

R.H. Lasseter. "Methods for Controlling Multiple Independent Generators in an Intentional Island (Microgrids)," Minnesota Power 11/8/06

R.H. Lasseter. “Distributed Energy Resources and Microgrids,” IEEE Distinguish Lecturer, IEEE Cuernavaca, Mexico, 2 July 2006.

R.H. Lasseter. “Directions In Microgrid research”, PSerc Lecture, 6 June 2006, Madison, Wi

R.H. Lasseter." Autonomous Control", 2006 PES General Meeting, 22 June 2006, Montréal, Québec Canada

R.H. Lasseter. “Microgrids and Protection”, CERTS IAB Meeting, 27 June 2006, Cleveland, Ohio

R.H. Lasseter. “Enhanced Microgrid”, Microgrid Road Map. 18 May 2006, Office of Electric Energy, Washington, DC

R.H. Lasseter. “Dynamics Distribution”, IEEE T\&D Meeting, Panel on Future of Distribution, Dallas, 20 May 2006 "Enhanced Business Case for CERTS Microgrid”, Peer Review Meeting for the Department of Energy's , Electric Distribution R\&D Program, 26 May 2006, San Ramon Ca

R.H. Lasseter. "The Role of Distributed Energy Resources in Future Electric Power Systems", Energy System Seminar, 27 March 2006, Madison, Wi

R.H. Lasseter. “DER based Distribution”, DOE Energy Workshop, Panel, January 31 to February 1 2006, Tallahassee, Fl

R.H. Lasseter. “Microgrid Test Plan”, CERTS IAB Meeting, 6 October 2005, Cleveland, Ohio

R.H. Lasseter. "CERTS Microgrid”, International Microgrid Workshop, Panel Speaker, 17 June 2005, University of California-Berkeley, Berkeley, California

R.H. Lasseter. "CERTS Microgrid", California Energy Commission R\&D Forum, 4 May 2005, Sacramento, $\mathrm{Ca}$

R.H. Lasseter. "Microgrid: A Conceptual Solution," Wisconsin Distributed Resources Collaborative, September 16, 2004

R.H. Lasseter. “CERTS Microgrid,” CEC Technical Advisory Committee Meeting, July 19, 2004

R.H. Lasseter. "Where are Microgrids Going?,“ Distributed Resources Workshop, CEA, May 9-11, 2004 Calgrary

R.H. Lasseter. "Microgrids: What's Next," PSerc Industrial Advisory Board, May 20-21, 2004

\section{Publications}


Klapp, D., H.T. Vollkommer. Application of an Intelligent Static Switch to the Point of Common Coupling to Satisfy IEEE 1547 Compliance. Panel: Microgrid Research and Field Testing IEEE PES General Meeting, 24-28 June 2007, Tampa, FL. 4 pages.

Lasseter, R.H., P. Piagi. 2006. Control and Design of Microgrid Components. January. 257 pages. http://www.pserc.org/ecow/getpublicatio/reports/2006report

Lasseter, R. Dynamic Distribution using (DER) Distributed Energy Resources. 2006. IEEE PES T \& D Meeting, Dallas, May. 3 pages.

Lasseter, R.H. 2007. “Microgrids and Distributed Generation.” Journal of Energy Engineering, Volume 133, Issue3, ASCE, Sept. 7 pages.

Lasseter, R.H. 2007. “CERTS Microgrid.” International Conference on System of Systems Engineering , April 16-18. 6 pages.

Nichols, D.K., J. Stevens, R.H. Lasseter, J.H. Eto, H.T. Vollkommer. 2006 Validation of the CERTS Microgrid Concept: The CEC/CERTS Microgrid Testbed. Power Engineering Society General Meeting, IEEE. 3 pages.

Nikkhajoei, H., R. H. Lasseter. 2007. Microgrid Protection. IEEE PES General Meeting, 24-28, Tampa, FL. June. 6 pages.

Nikkhajoei, H., R. H. Lasseter. 2006. Microgrid Fault Protection Based on Symmetrical and Differential Current Components. December. 72 pages. (Power Systems Engineering Research Center (PSERC) website)

Panora, R.A., J.B. Gehret Jr., P. Piagi. 2007. Design and Testing of an Inverter-Based Combined Heat and Power Module for Special Application in a Microgrid. Panel: Microgrid Research and Field Testing IEEE PES General Meeting, June 24-28, Tampa FL. 7 pages.

Stevens, J., H. Vollkommer, D.Klapp. CERTS Microgrid System Tests. Power Engineering Society General Meeting, June 24-28. 4 pages.

In addition, the CERTS Microgrid concepts have appeared in Distributed Energy Journal, May/June 2008, which can be found at http://www.distributedenergy.com/de 0805 micro.html.

Through this outreach, members of the CERTS team met with a wide variety of potential field demonstrations partners during the course of the project. A separate Energy Commission contract to Navigant Consulting further augmented efforts to develop field demonstrations involving the CERTS Microgrid concept.

At the time of the preparation of this report, the status of discussions with potential partners for field demonstrations involving the CERTS Microgrid Concept is as follows:

Tecogen, a partner in the CERTS Microgrid Test Bed project, is currently conducting a research project for the New York State Energy Research and Development Authority to demonstrate aspects of the CERTS Microgrid Concept at a school in upstate New York. 
The Energy Commission PIER program is considering funding for two demonstrations involving aspects of the CERTS Microgrid Concept. Chevron Energy Solutions is proposed to lead a demonstration, which would involve battery storage, renewable energy, and engine generators at a correctional facility in Northern California. Sacramento Municipal Utilities District (SMUD) is proposed to lead a demonstration that would involve engine generators at SMUD corporate headquarters.

Two additional demonstrations involving aspects of the CERTS Microgrid Concept have been submitted in response to a competitive solicitation from the U.S. Department of Energy (DOE). The identities of the firms leading these proposals are being withheld pending the announcement of solicitation awardees by DOE.

In addition, active discussions are also under way for additional field demonstrations, involving the U.S. Army Corp of Engineers/Construction Research Laboratory and with Cummins Engines. 


\subsection{Conclusions and Recommendations}

This section describes the conclusions drawn from the successful demonstration of the CERTS Microgrid concept at a full-scale test bed operated by AEP and lists recommendations for future work.

\subsection{Conclusions}

The objective of the CERTS Microgrid Laboratory Test Bed Demonstration project was to demonstrate three advanced techniques comprising the CERTS Microgrid concept at a full-scale test bed operated by AEP.

The advance techniques, which included: 1) a method for effecting automatic and seamless transitions between grid-connected and islanded modes of operation; 2) an approach to electrical protection within the microgrid that does not depend on high fault currents; and 3) a new method for microgrid control that achieves voltage and frequency stability under islanded conditions without requiring high-speed communications, were demonstrated through a carefully orchestrated sequence of five sets of tests by AEP.

The first set of tests examined the operation of the static switch to determine that it and its digital signal processing (DSP) control operated as designed. The second set of tests examined a preliminary set of fault (i.e., overload simulating a fault) condition tests to ensure protection and safety of the test bed, prior to performing other planned tests. The third set of tests was designed to ensure that the Gen-set inverter controls were working as designed. The fourth set of tests demonstrated the flexibility of the microgrid both grid connected and islanded for different loads, power flows and impact on the utility. The fifth and final set of testing began to explore the operation limits of the microgrid (i.e., power quality, protection and inverter limits) with motor starting loads.

The testing fully confirmed earlier research that had been conducted initially through analytical simulations, then through laboratory emulations, and finally through factory acceptance testing of individual microgrid components. The islanding and resychronization method met all IEEE 1547 and power quality requirements. The electrical protection system was able to distinguish between normal and faulted operation. The controls were found to be robust under all conditions.

\subsection{Recommendations}

The next logical phase for RD\&D on the CERTS Microgrid Concept is to build from the base established by the currently operational CERTS Microgrid to prioritize, develop, and then demonstrate needed additional technology enhancements required to optimize the microgrid from the explicit perspective of enhancing the business case for microgrids. That is, having demonstrated the technical feasibility of microgrid functions, RD\&D optimization efforts are now needed to accelerate commercial deployment. The should pay special attention to the economic drivers, such as economic dispatch responsive to pricing signals and demand management programs, customer willingness to pay premiums for increased power reliability and quality, etc. 
In order to enhance the business case for microgrids, the cost and functionalities of the CERTS Microgrid need to be compared directly against traditional solutions for a) CHP including the heat distribution systems and system reliability, and b) power quality, including multiple feeders and/or UPS systems. That is, enhancing the business case for microgrids involves, first, identifying the cost and performance targets of the traditional approaches for providing the values offered by a microgrid and then, second, prioritizing development and testing of CERTS Microgrid technology enhancements to beat these targets. Some promising areas to consider include: 1) lowering the costs of providing protection among microsources and loads; 2) determining the optimal amount of storage required on the DC bus; 3 ) the best size, technology, and control strategy (or strategies) for integration of AC storage; and 4) inclusion of noninverter-based prime-movers along side of inverter-based prime-movers within a microgrid.

\subsection{Benefits to California}

DER - small power generators typically located at sites where the energy (both electric and thermal) they generate is used - are a promising option to meet growing customer needs for economic and reliable electric power (the DER portfolio also includes energy storage, and load control). Organizing all of these resources into microgrids is a promising way to capture smaller DER's significant potential to meet customers' and utilities' needs.

A key feature of a microgrid is its ability, during a utility grid disturbance, to separate and isolate itself from the utility seamlessly with no disruption to the loads within the microgrid (including no reduction in power quality). Then, when the utility grid returns to normal, the microgrid automatically resynchronizes and reconnects itself to the grid, in an equally seamless fashion.

The CERTS Microgrid concept seeks to provide this technically challenging functionality without extensive (i.e., expensive) custom engineering. In addition, the design of the CERTS Microgrid also provides high system reliability and great flexibility in the placement of distributed generation within the microgrid. The CERTS Microgrid is intended to offer these functionalities at much lower costs than traditional approaches by incorporating peer-to-peer and plug-and-play concepts for each component within the microgrid.

In so doing, the CERTS Microgrid concept will help improve the quality of life in California by bringing environmentally safe, affordable, and reliable energy services and products to the marketplace. 


\subsection{References}

Akhil, A., Marnay, C. Lipman, T. 2002. Review of Test Facilities for Distributed Energy Resources. SAND2003-1602. October 2002. 43 pages.

Klapp, D., H.T. Vollkommer. Application of an Intelligent Static Switch to the Point of Common Coupling to Satisfy IEEE 1547 Compliance. Panel: Microgrid Research and Field Testing IEEE PES General Meeting, 24-28 June 2007, Tampa, FL. 4 pages.

Lasseter, R.H., A. Akhil, C. Marnay, J. Stephens, J. Dagle, R. Guttromson, A.S. Meliopoulous, R. Yinger, and J. Eto. 2002. Consortium for Electric Reliability Technology Solutions White Paper on Integration of Distributed Energy Resources: The MicroGrid Concept. LBNL-50829. April. 29 pages.

Lasseter, R.H., P. Piagi. Microgrid: A Conceptual Solution. PESC'04 Aachen, Germany 20-25 June 2004. 7 pages.

Lasseter, R.H., P. Piagi. 2005. Control and Design of CERTS Microgrid. July.

Lasseter, R.H., P. Piagi. 2006. Control and Design of Microgrid Components. January. 257 pages. http://www.pserc.org/ecow/getpublicatio/reports/2006report

Lasseter, R. Dynamic Distribution using (DER) Distributed Energy Resources. 2006. IEEE PES T \& D Meeting, Dallas, May. 3 pages.

Lasseter, R.H. 2007. “Microgrids and Distributed Generation.” Journal of Energy Engineering, Volume 133, Issue3, ASCE, Sept. 7 pages.

Lasseter, R.H. 2007. “CERTS Microgrid.” International Conference on System of Systems Engineering , April 16-18. 6 pages.

Nichols, D.K., J. Stevens, R.H. Lasseter, J.H. Eto, H.T. Vollkommer. 2006 Validation of the CERTS Microgrid Concept: The CEC/CERTS Microgrid Testbed. Power Engineering Society General Meeting, IEEE. 3 pages.

Nikkhajoei, H., R. H. Lasseter. 2007. Microgrid Protection. IEEE PES General Meeting, 24-28, Tampa, FL. June. 6 pages.

Nikkhajoei, H., R. H. Lasseter. 2006. Microgrid Fault Protection Based on Symmetrical and Differential Current Components. December. 72 pages. (Power Systems Engineering Research Center (PSERC) website)

Panora, R.A., J.B. Gehret Jr., P. Piagi. 2007. Design and Testing of an Inverter-Based Combined Heat and Power Module for Special Application in a Microgrid. Panel: Microgrid Research and Field Testing IEEE PES General Meeting, June 24-28, Tampa FL. 7 pages.

Piagi, P., H.R. Lasseter. 2006. Autonomous Control of Microgrids. IEEE PES Meeting, Montreal, June. 8 pages.

Stevens, J., H. Vollkommer, D.Klapp. CERTS Microgrid System Tests. Power Engineering Society General Meeting, June 24-28. 4 pages. 


\subsection{Glossary}

\begin{tabular}{|c|c|}
\hline Acronym & Definition \\
\hline $\mathrm{AC}$ & alternating current \\
\hline AEP & American Electric Power \\
\hline CERTS & Consortium for Electric Reliability Technology Solutions \\
\hline CHP & combined heat and power \\
\hline DC & direct current \\
\hline DER & distributed energy resources \\
\hline DOE & Department of Energy \\
\hline EMS & $\begin{array}{l}\text { energy management system (a control system that optimizes operation of } \\
\text { the Microgrid to economically meet electricity and heat loads) }\end{array}$ \\
\hline EPRI & Electric Power Research Institute \\
\hline $\begin{array}{l}\text { Energy } \\
\text { Commission }\end{array}$ & California Energy Commission \\
\hline IEEE & Institute of Electrical and Electronics Engineers \\
\hline LBNL & Lawrence Berkeley National Laboratory \\
\hline NPS & Northern Power Systems \\
\hline NREL & National Renewable Energy Laboratory \\
\hline PCC & point of common coupling \\
\hline PIER & Public Interest Energy Research \\
\hline PSERC & Power Systems Engineering Research Center \\
\hline RD\&D & research, development, and demonstration \\
\hline SCE & Southern California Edison \\
\hline SMUD & Sacramento Municipal Utilities District \\
\hline SNL & Sandia National Laboratories \\
\hline Static Switch & Separation device used to interconnect microgrid with distribution system \\
\hline TAC & Technical Advisory Committee \\
\hline UW & University of Wisconsin \\
\hline
\end{tabular}




\section{Appendices}

\section{Appendices are available as separate documents}

Appendix A. Test Bed Design Schematics

Appendix B. CERTS Microgrid Test Plan

Appendix C. Youtility Factory Test Plan Final Test Results

Appendix D. Tecogen 60kW Inverter-Based CHP Modules: Factory Testing

Appendix E. Tecogen CHP Modules Commissioning Report

Appendix F. CERTS Test Bed CERTEQUIP-V06-002, CERTS Switch, Low Power Factory Acceptance Test Report

Appendix G. Summary of CERTS Microgrid Static Switch Power Quality Tests at AEP Dolan, CERTS Microgrid Static Switch Testing

Appendix H. CERTS Test Bed Design and Commissioning: Lessons Learned Summary

Appendix I. Test Plan Section 6.0 Microgrid Test Bed System Checkout (Static Switch)

Appendix J. Test Plan Section 7.0 Validate Protection Settings and Initial Fault Testing

Appendix K. Test Plan Section 8.0 Reduced System Tests

Appendix L. Test Plan Section 9.0 Power Flow Control Tests

Appendix M. Test Plan Section 10.0 Difficult Loads

Appendix N. Test Log

Appendix O. Technical Advisory Committee Meeting Summary and Review Comments

Appendix P. Microgrid Fault Protection Based on Symmetrical and Differential Current Components 


\title{
CERTS MICROGRID LABORATORY TEST BED
}

\author{
Test Bed Design Schematics
}

\section{Prepared For: \\ California Energy Commission \\ Public Interest Energy Research Program}

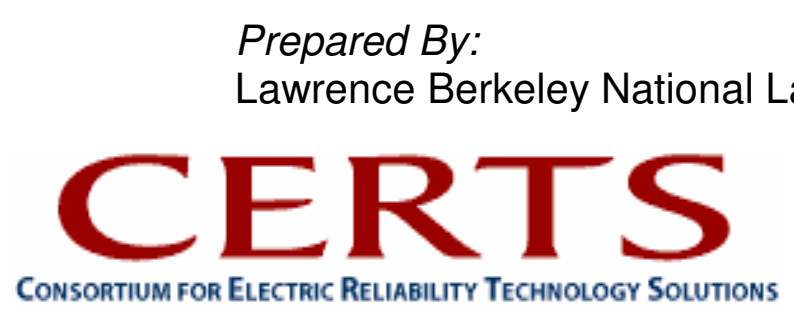

Arnold Schwarzenegger Governor

$\frac{x}{2}$ 

Prepared By:

Lawrence Berkeley National Laboratory

Joseph H. Eto, Principal Investigator

Berkeley, CA 94720

Ed Linton, Northern Power Systems

Commission Contract No. 500-03-024

Prepared For:

Public Interest Energy Research (PIER) California Energy Commission

Bernard Treanton

Contract Manager

Mike Gravely

Program Area Lead

ENERGY SYSTEMS INTEGRATION

Mike Gravely

Office Manager

ENERGY SYSTEMS RESEARCH

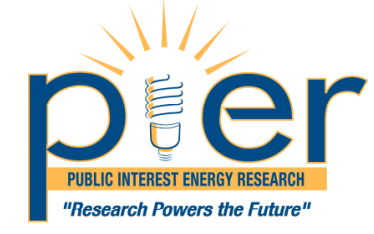

Martha Krebs, Ph.D.

PIER Director

Thom Kelly, Ph.D.

Deputy Director

ENERGY RESEARCH \& DEVELOPMENT DIVISION

Melissa Jones

Executive Director

\section{DISCLAIMER}

This report was prepared as the result of work sponsored by the California Energy Commission. It does not necessarily represent the views of the Energy Commission, its employees or the State of California. The Energy Commission, the State of California, its employees, contractors and subcontractors make no warrant, express or implied, and assume no legal liability for the information in this report; nor does any party represent that the uses of this information will not infringe upon privately owned rights. This report has not been approved or disapproved by the California Energy Commission nor has the California Energy Commission passed upon the accuracy or adequacy of the information in this report. 



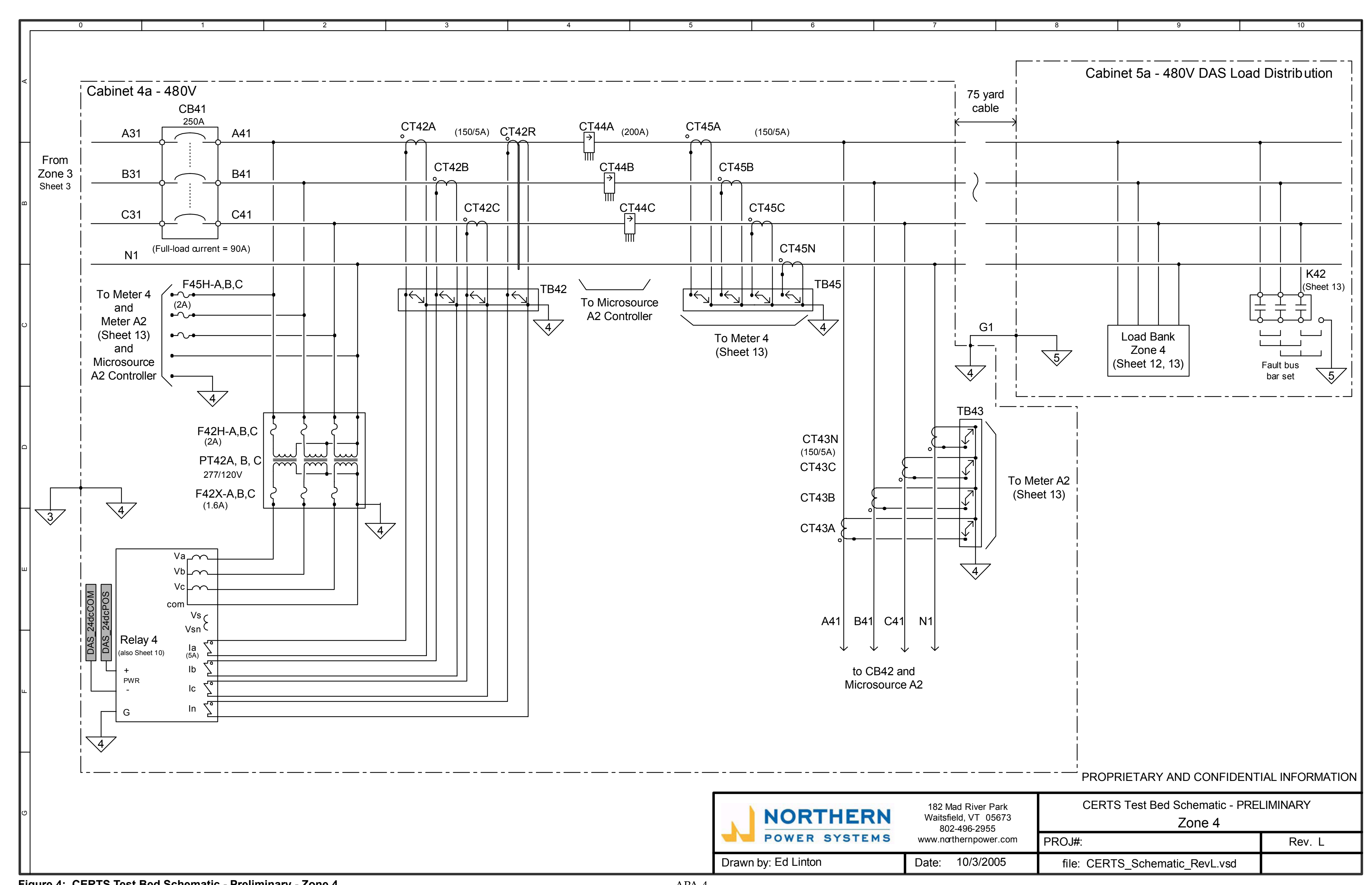




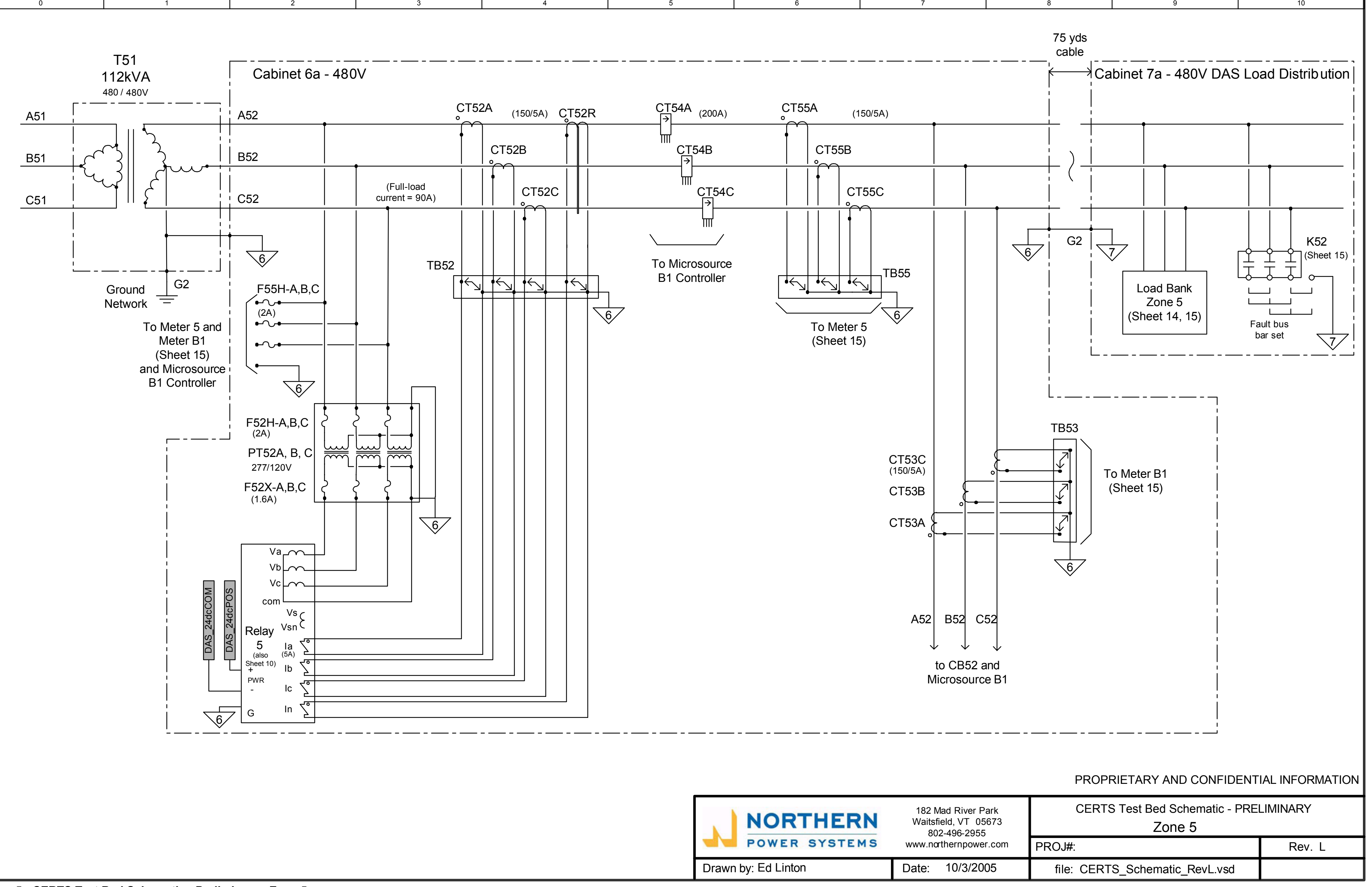


Cabinet 2a -

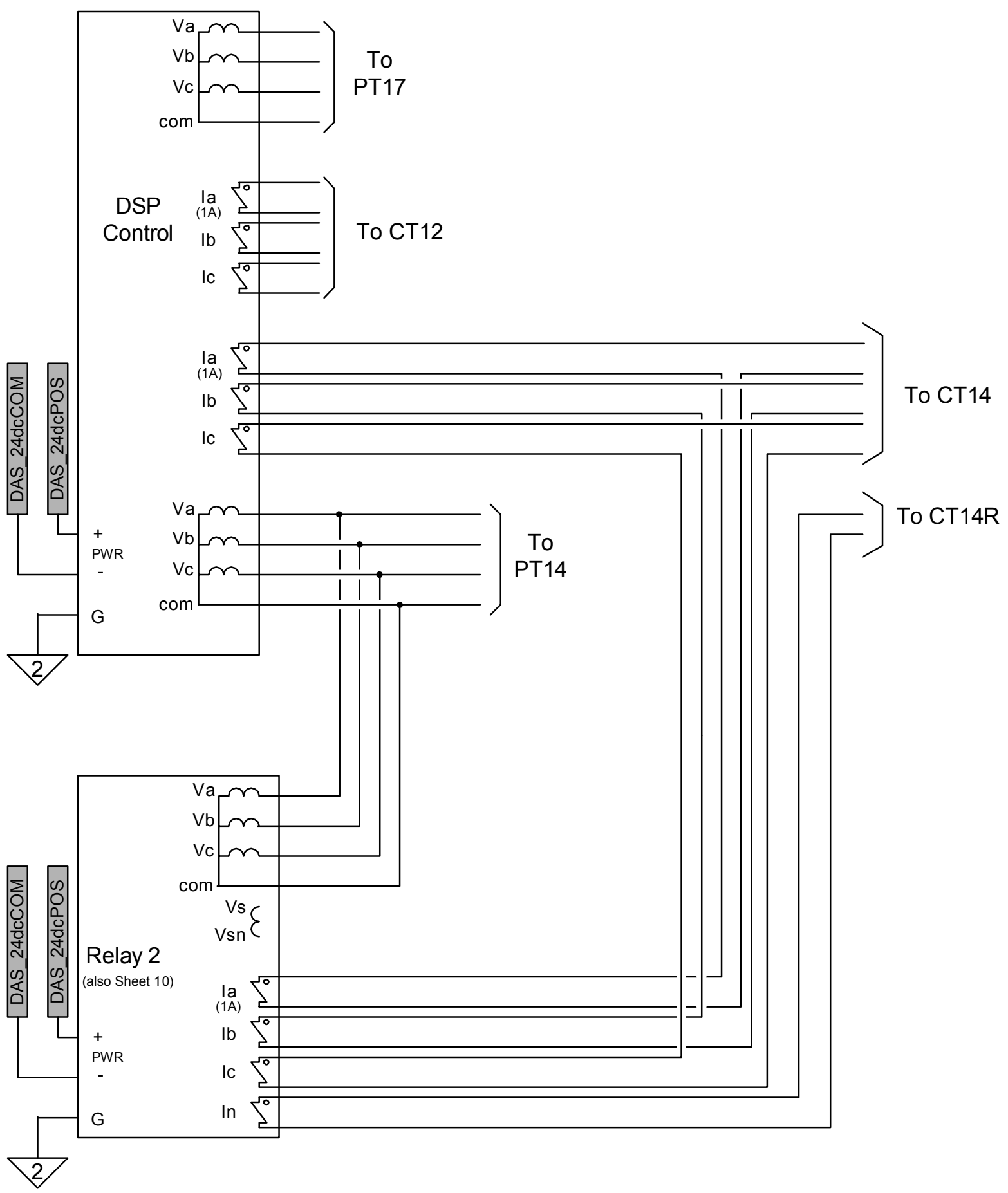

Cabinet $2 \mathrm{e}$ -

LV SS Control

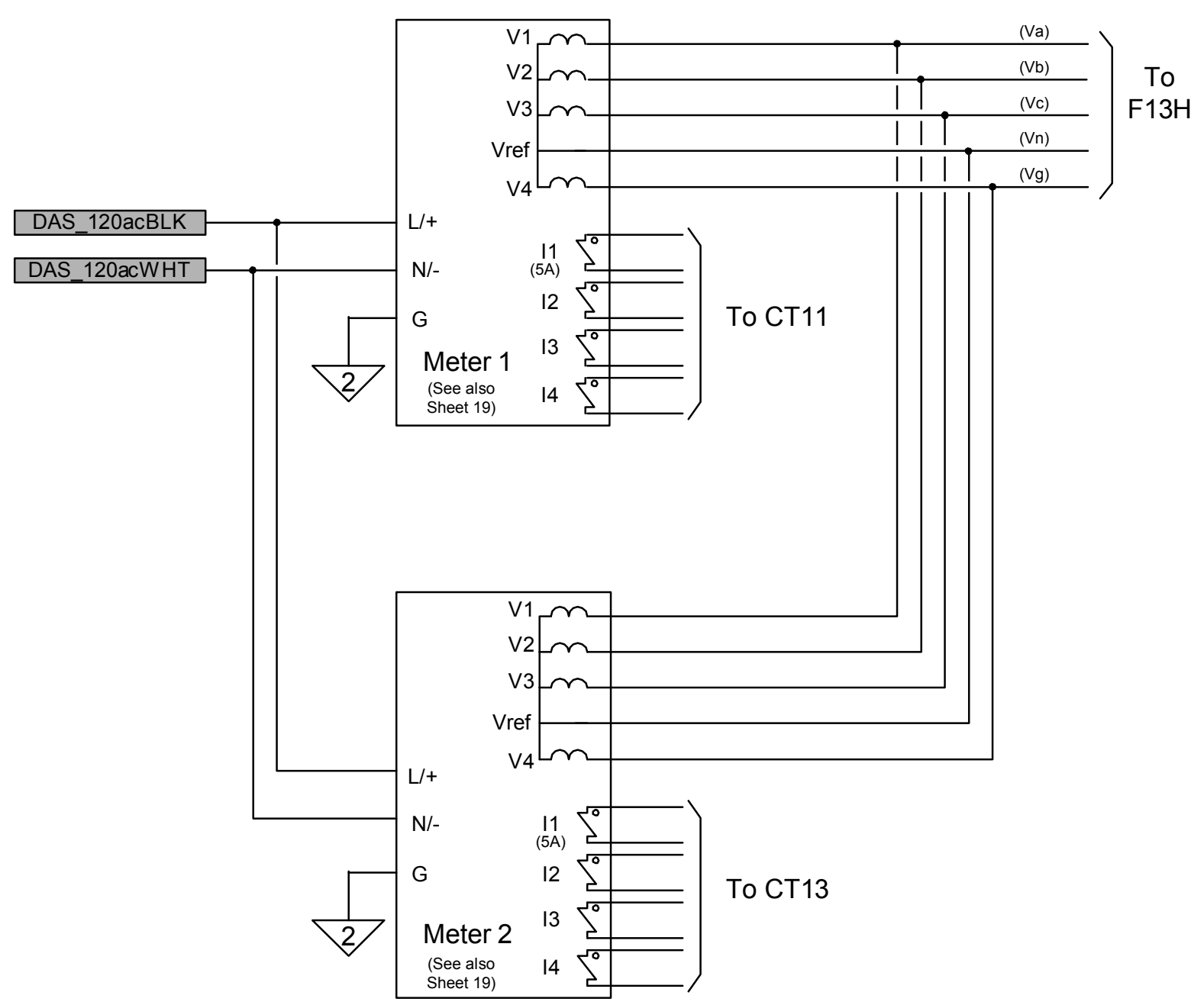

PROPRIETARY AND CONFIDENTIAL INFORMATION CERTS Test Bed Schematic - PRELIMINARY Zone 2 Controls www.northernpower.com

Date: 10/3/2005

PROJA: 


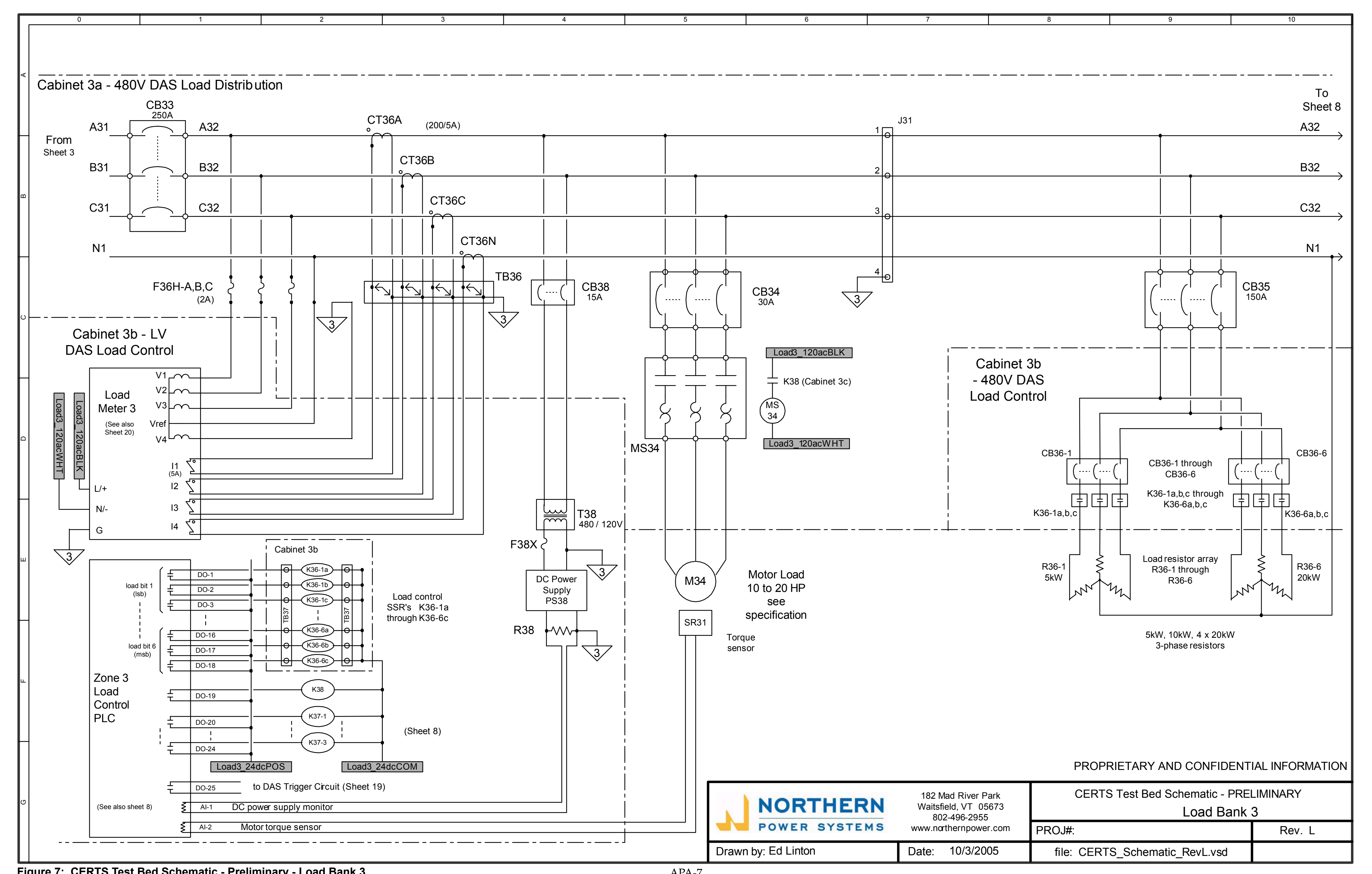




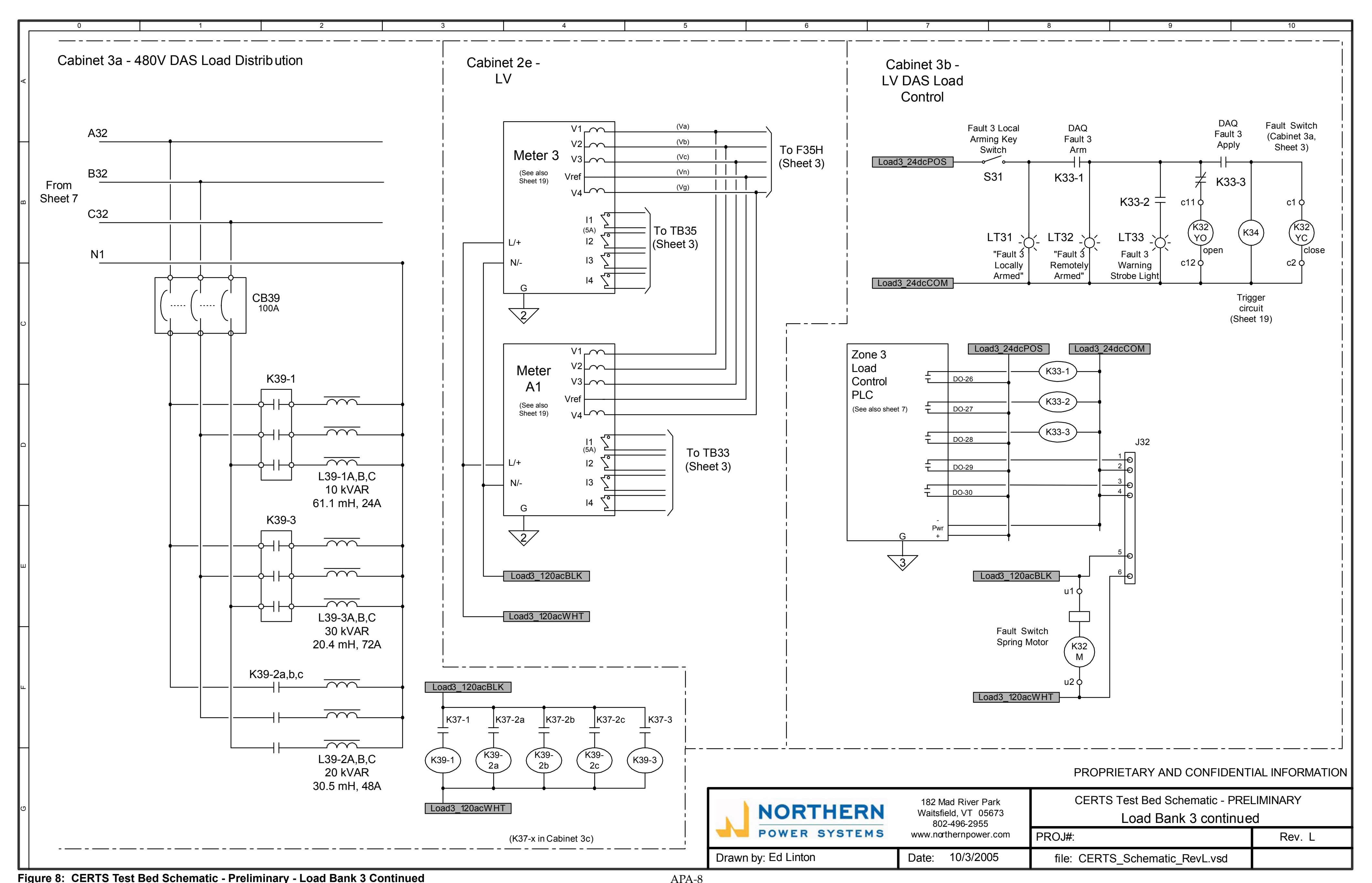




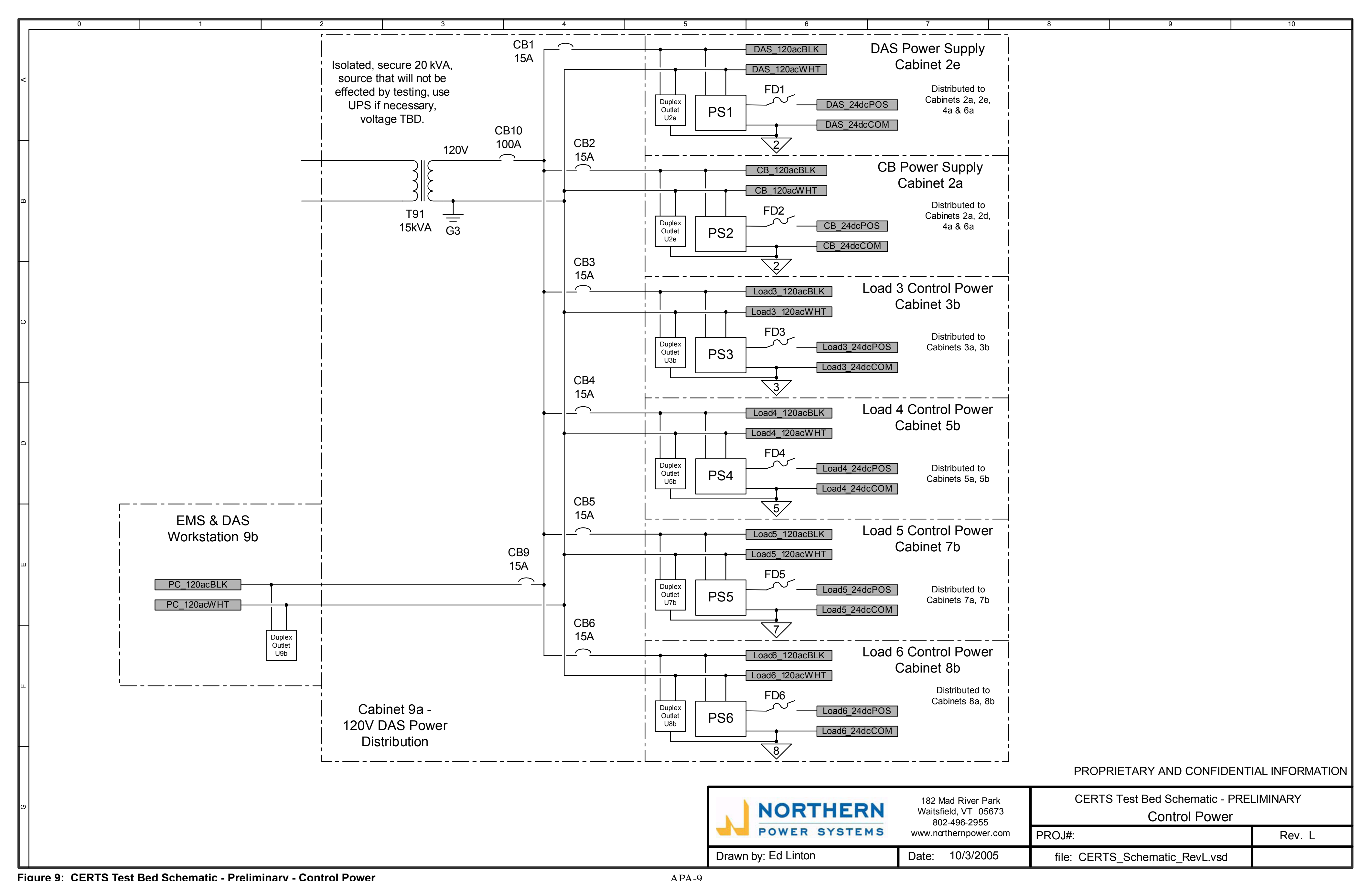


Cabinet 5a - 480V DAS Load Distribution

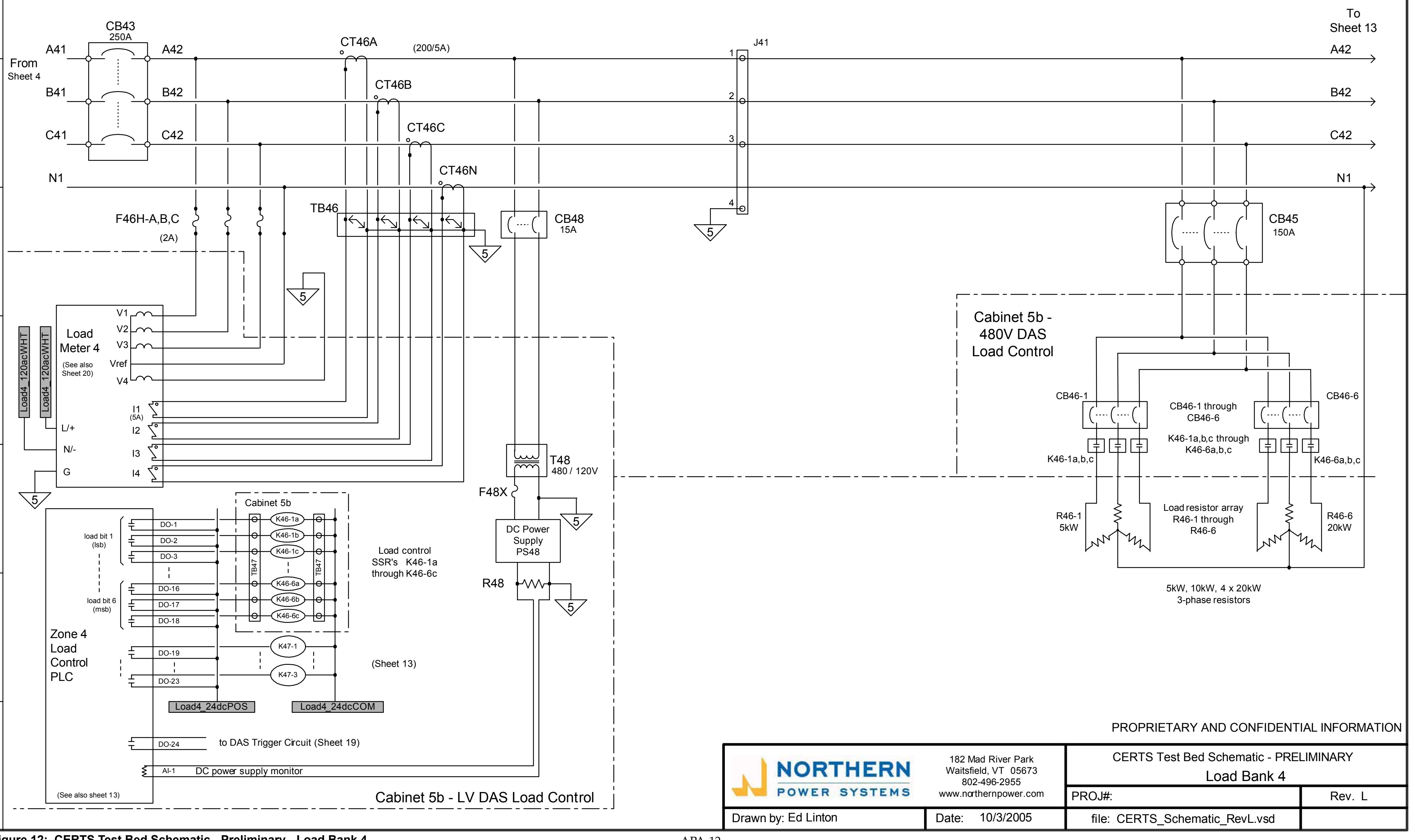




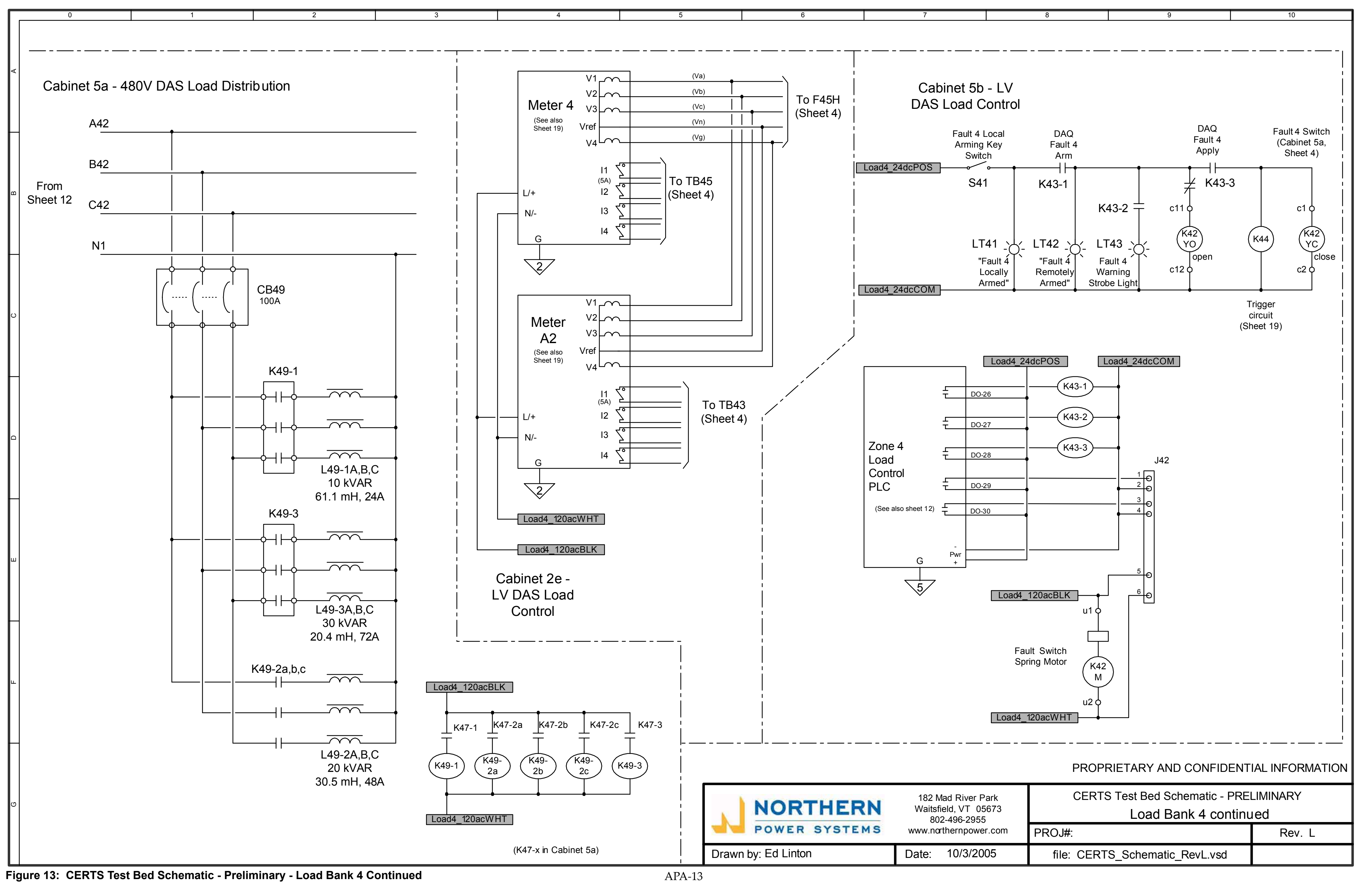


Cabinet 7a - 480V DAS Load Distribution

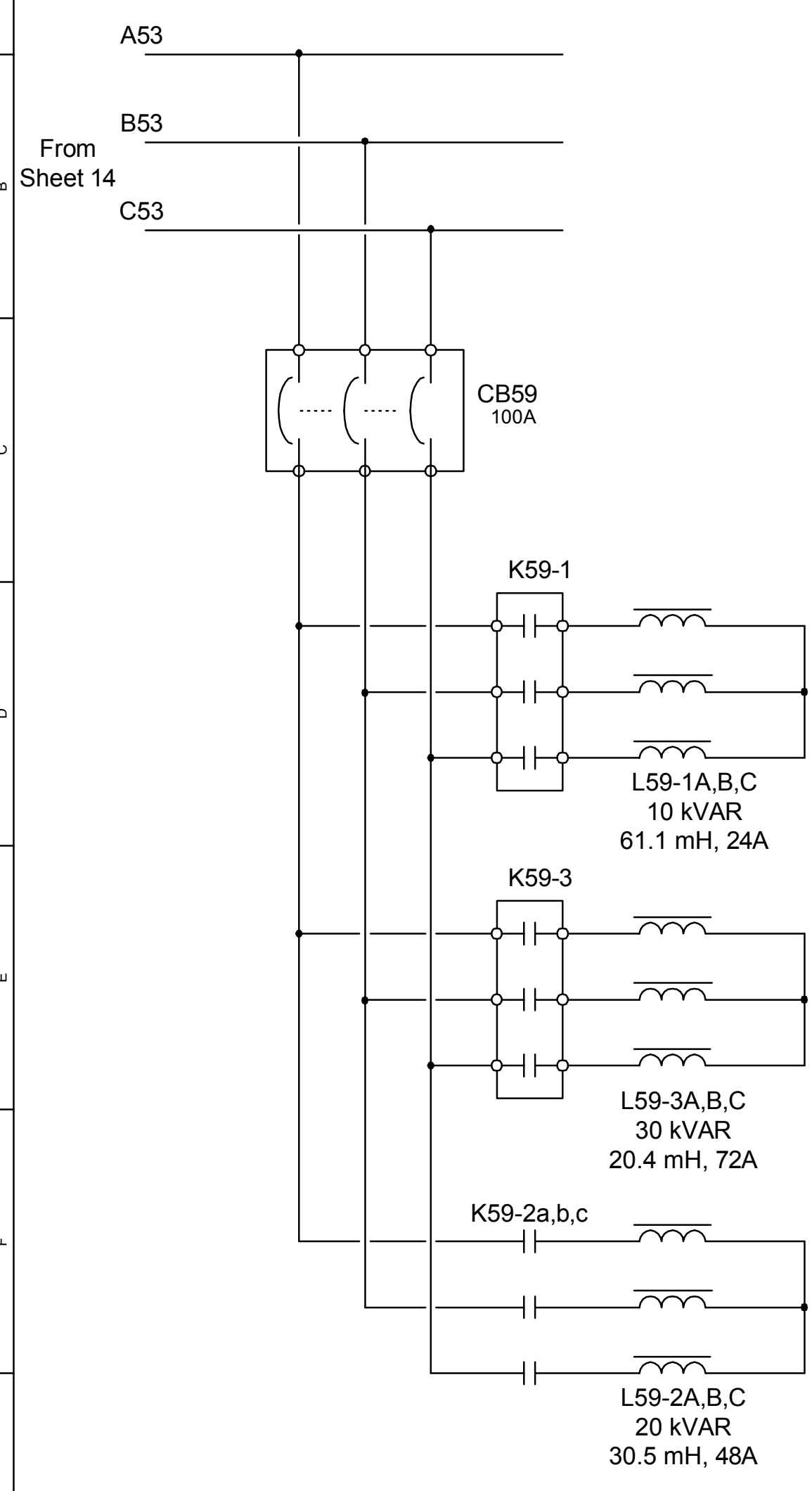

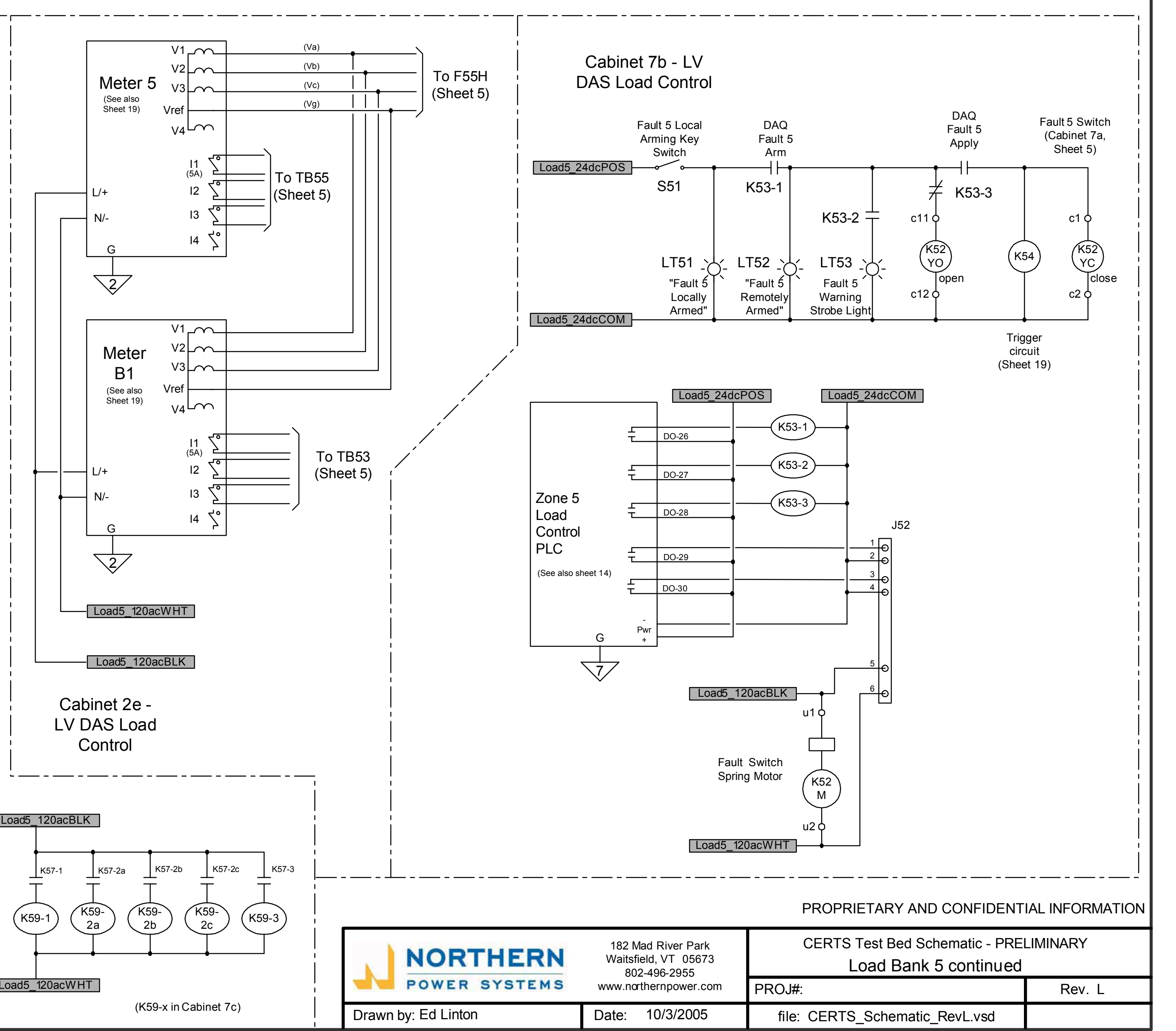

APA-15 
Cabinet $10-480 \mathrm{~V}$

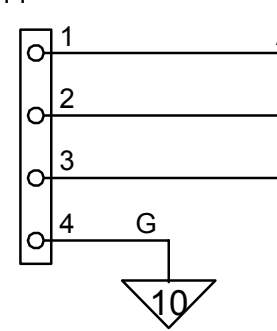

To Load Bank

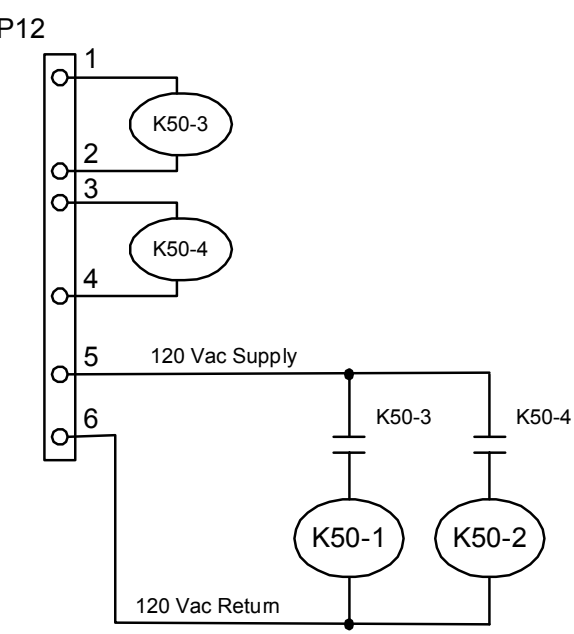

Typical Fault Bus Bar \& Load Set
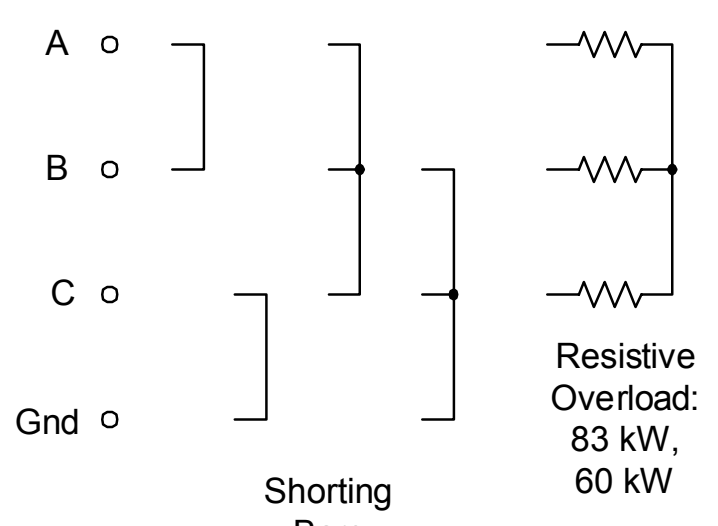
Lmened Rotor

$70 \mathrm{kVAR}$
$8.7 \mathrm{mH}$

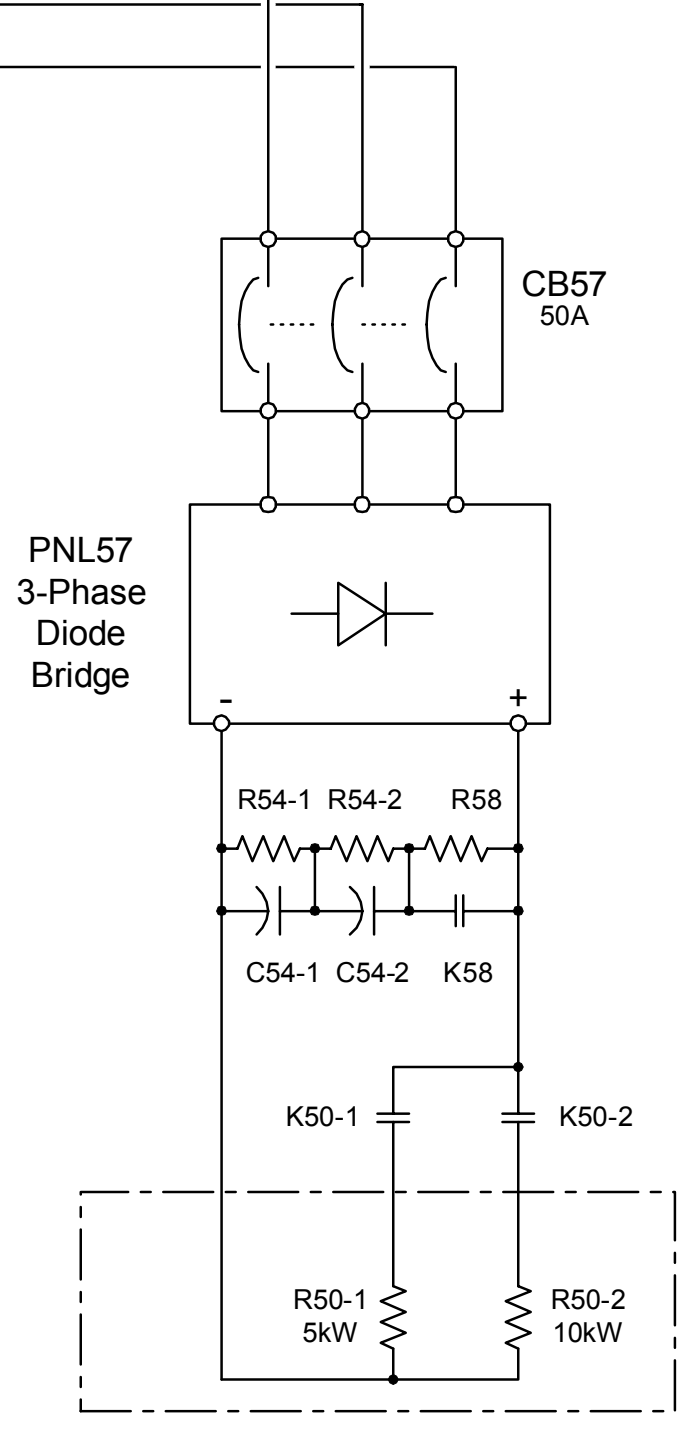

Harmonic Load
Cabinet 2e - LV DAS

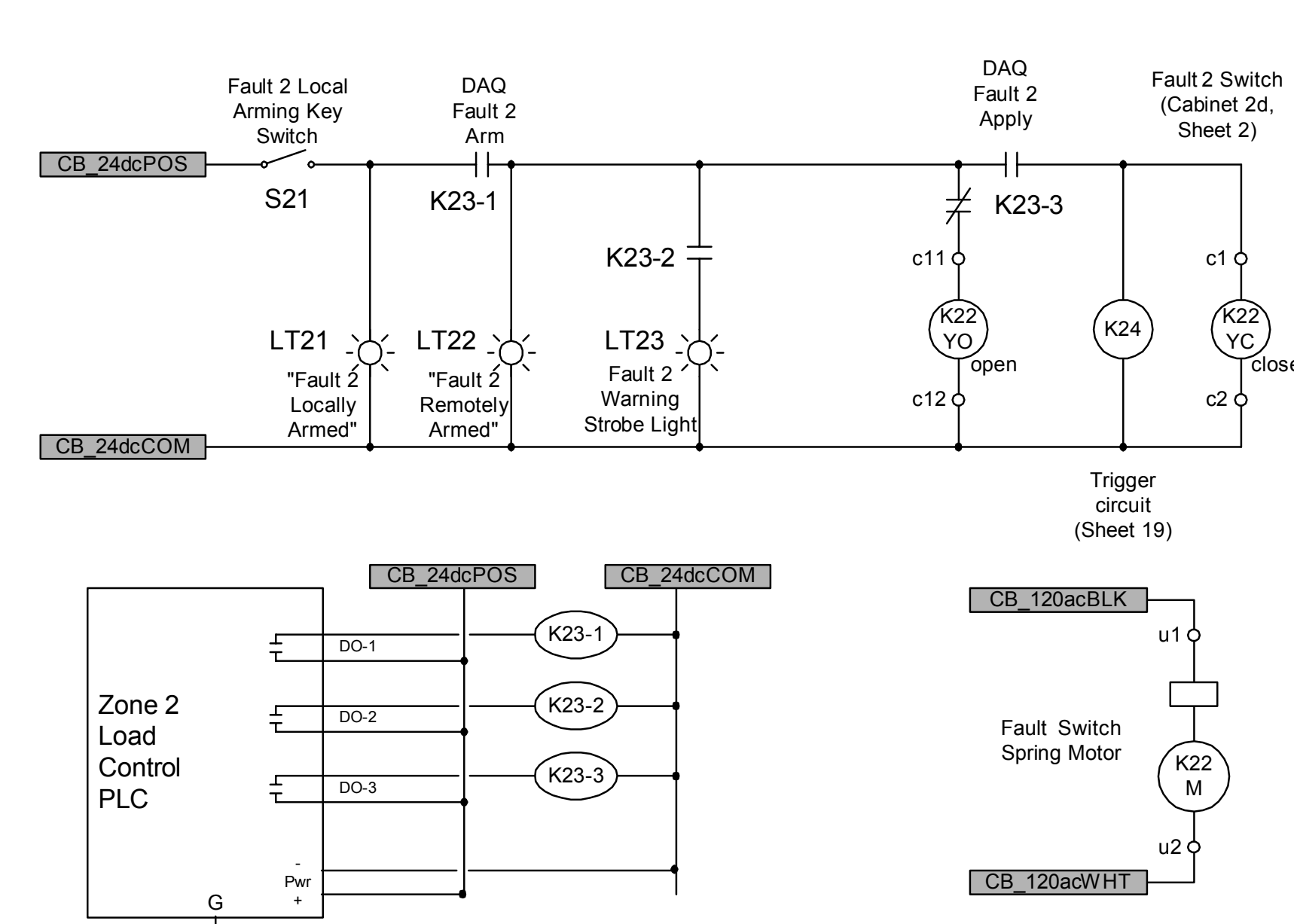

PROPRIETARY AND CONFIDENTIAL INFORMATION CERTS Test Bed Schematic - PRELIMINARY Harmonic Load and Zone 2 Fault Control

182 Mad River Park 802-496-2955 unw.nothernernower.com

\begin{tabular}{|l|l|}
\hline Drawn by: Ed Linton & Date: 10/3/2005
\end{tabular}

PROJ\#: 


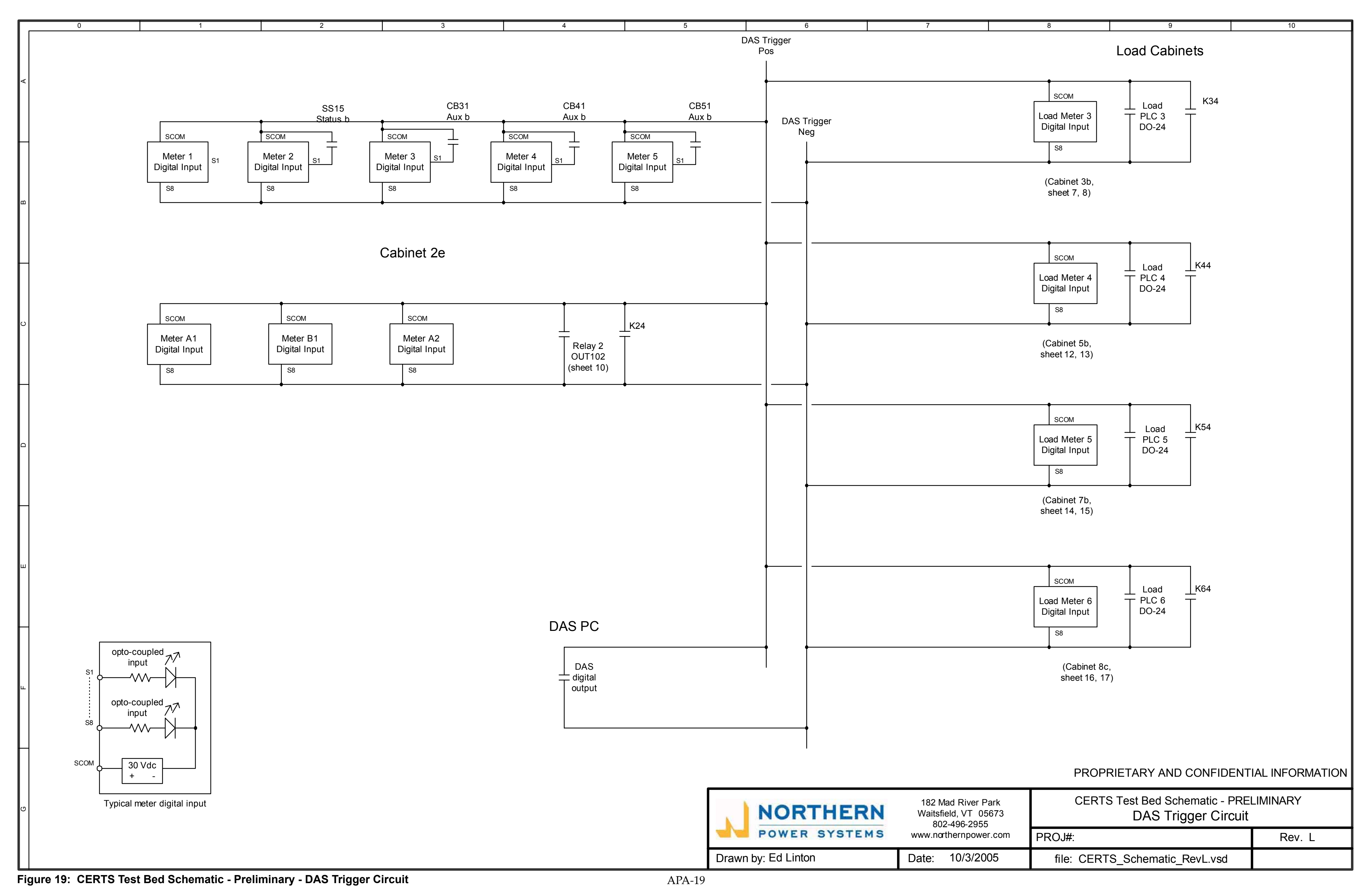




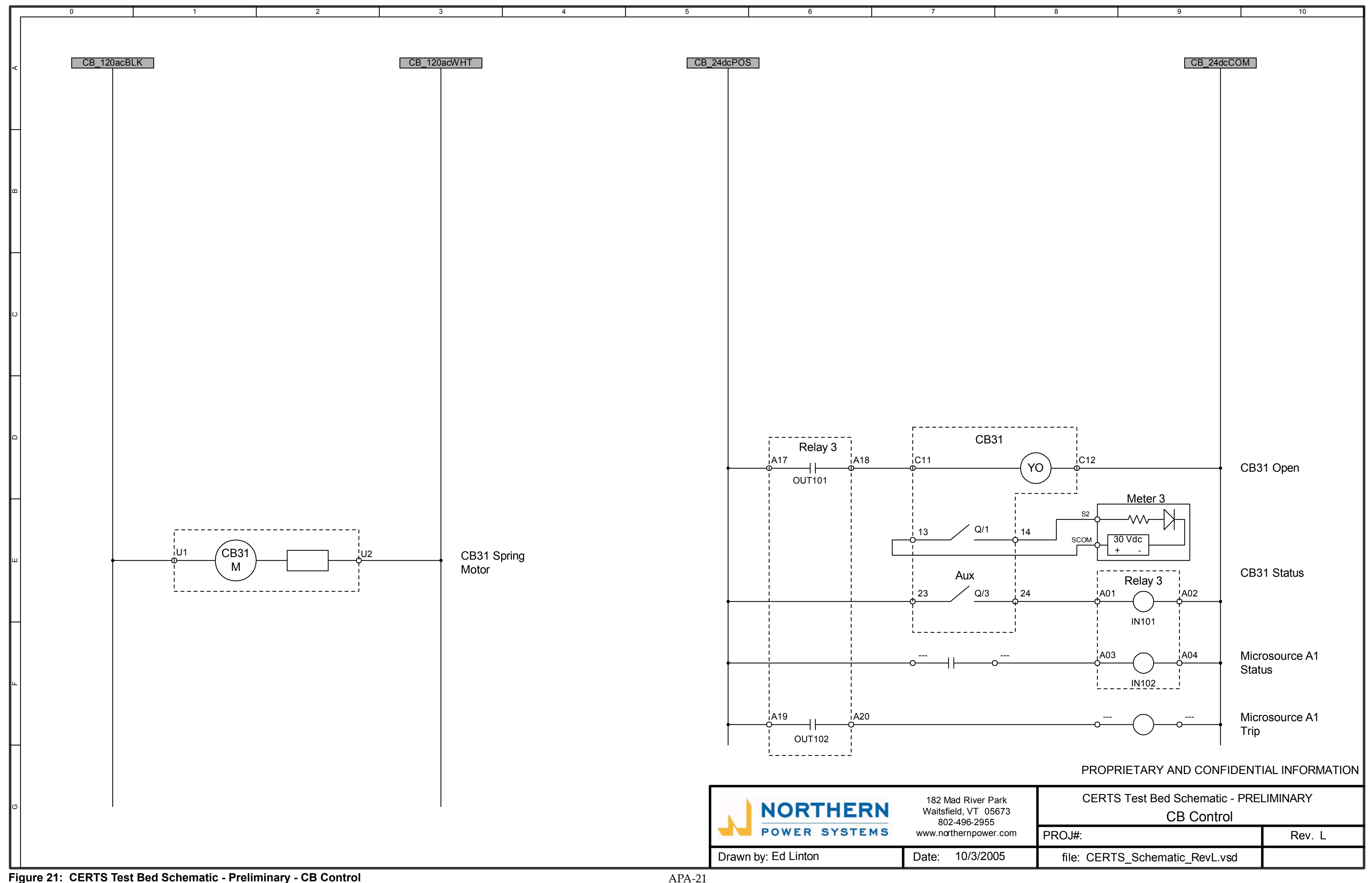




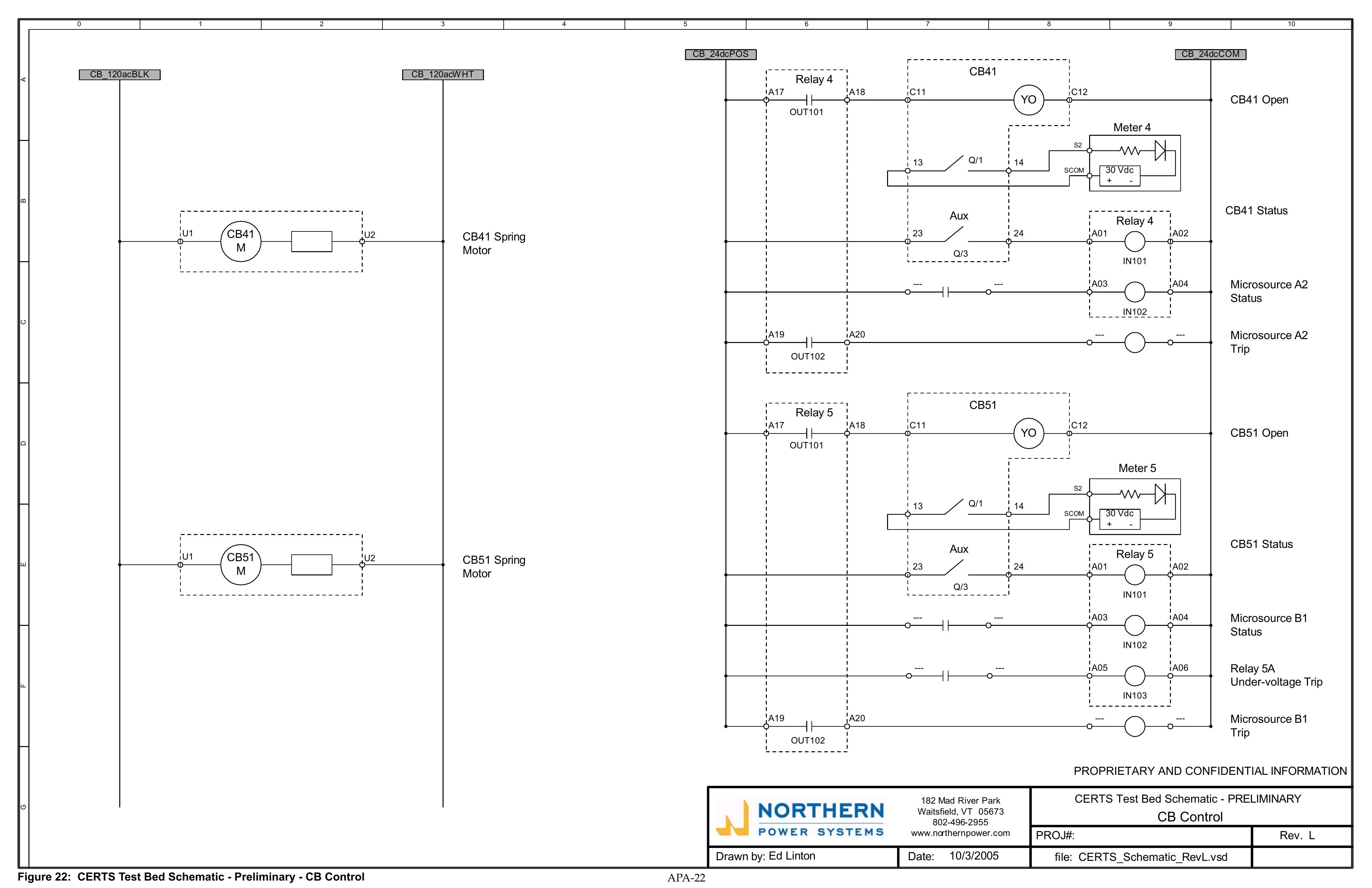




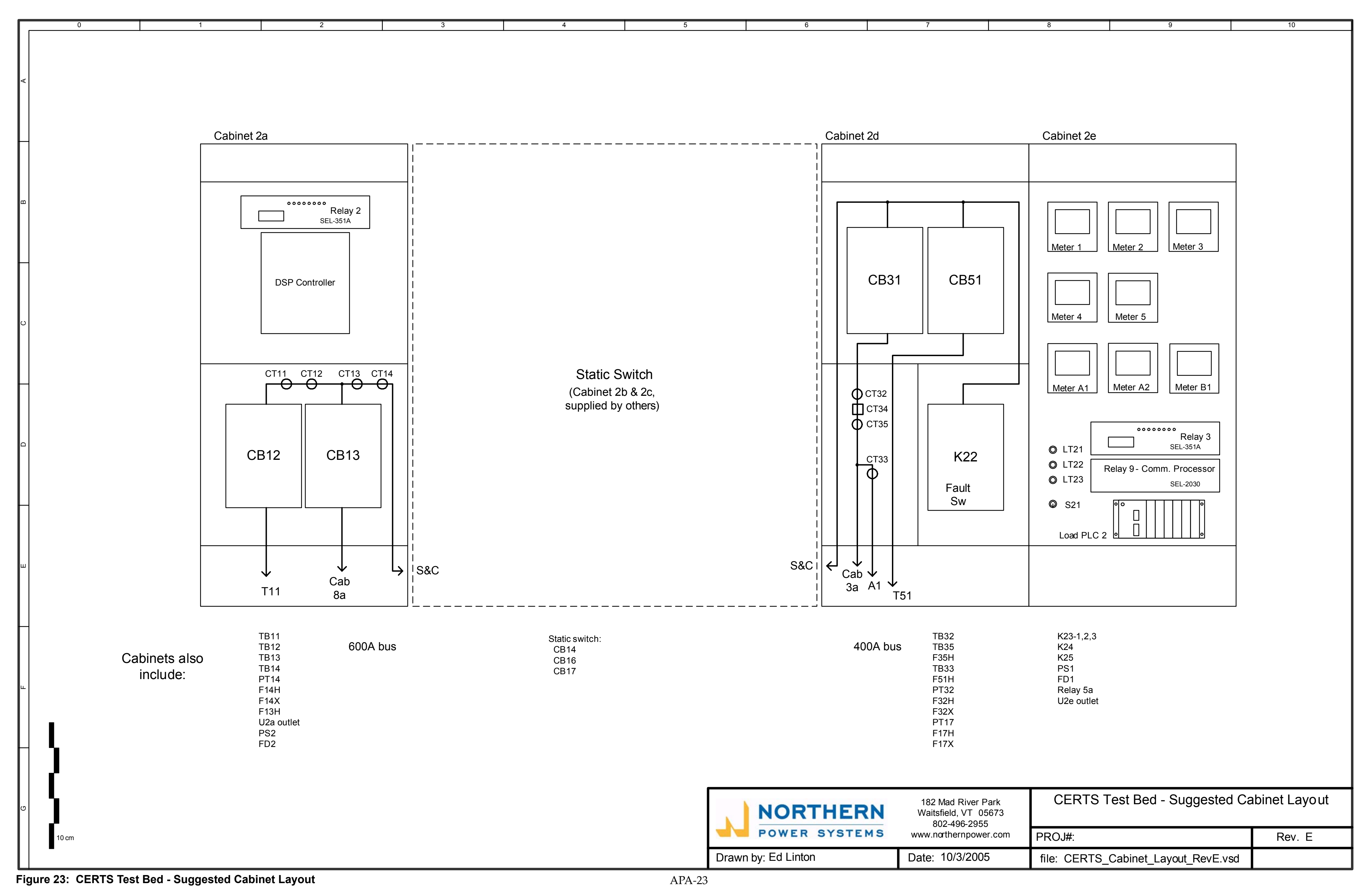




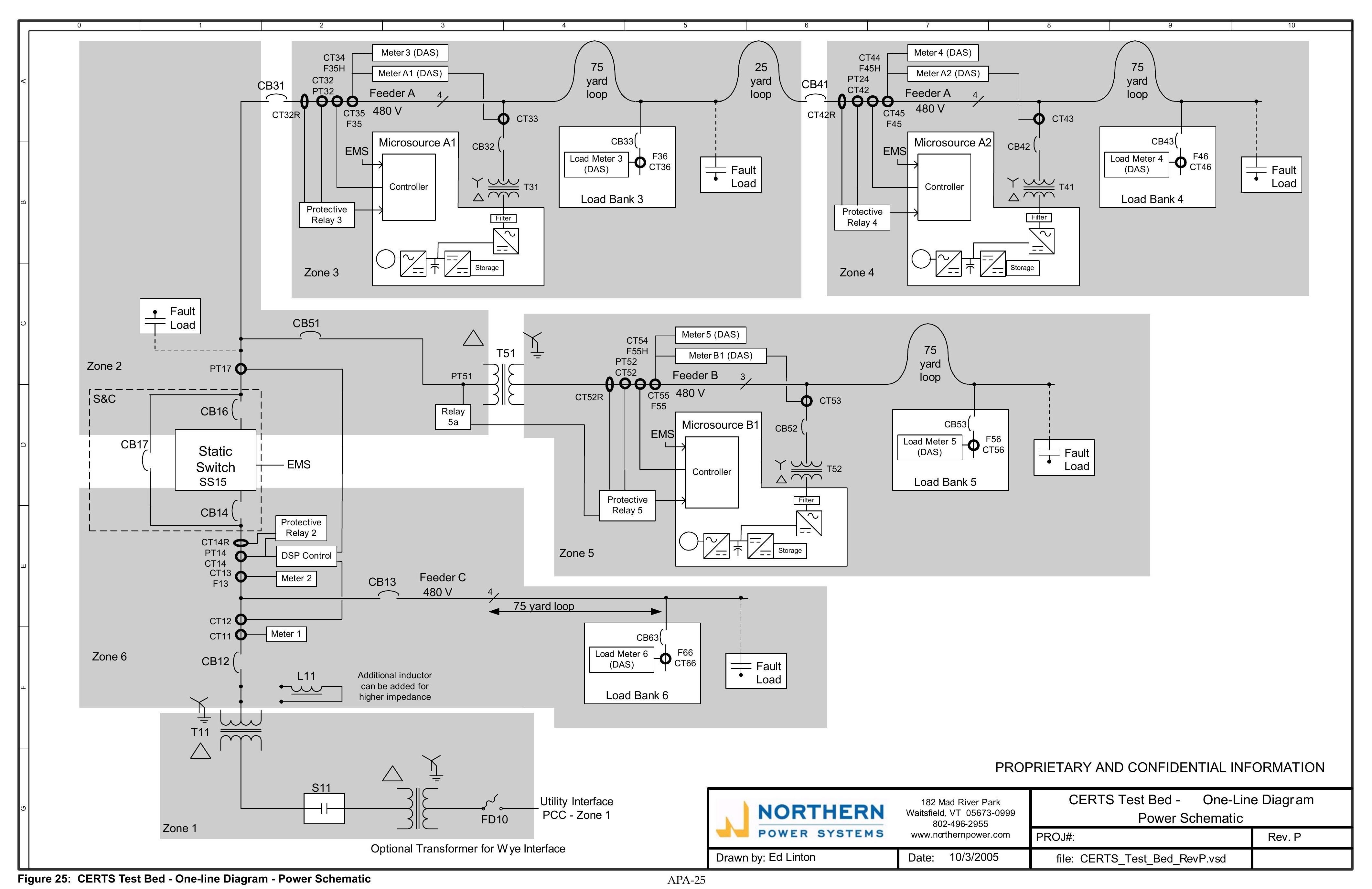




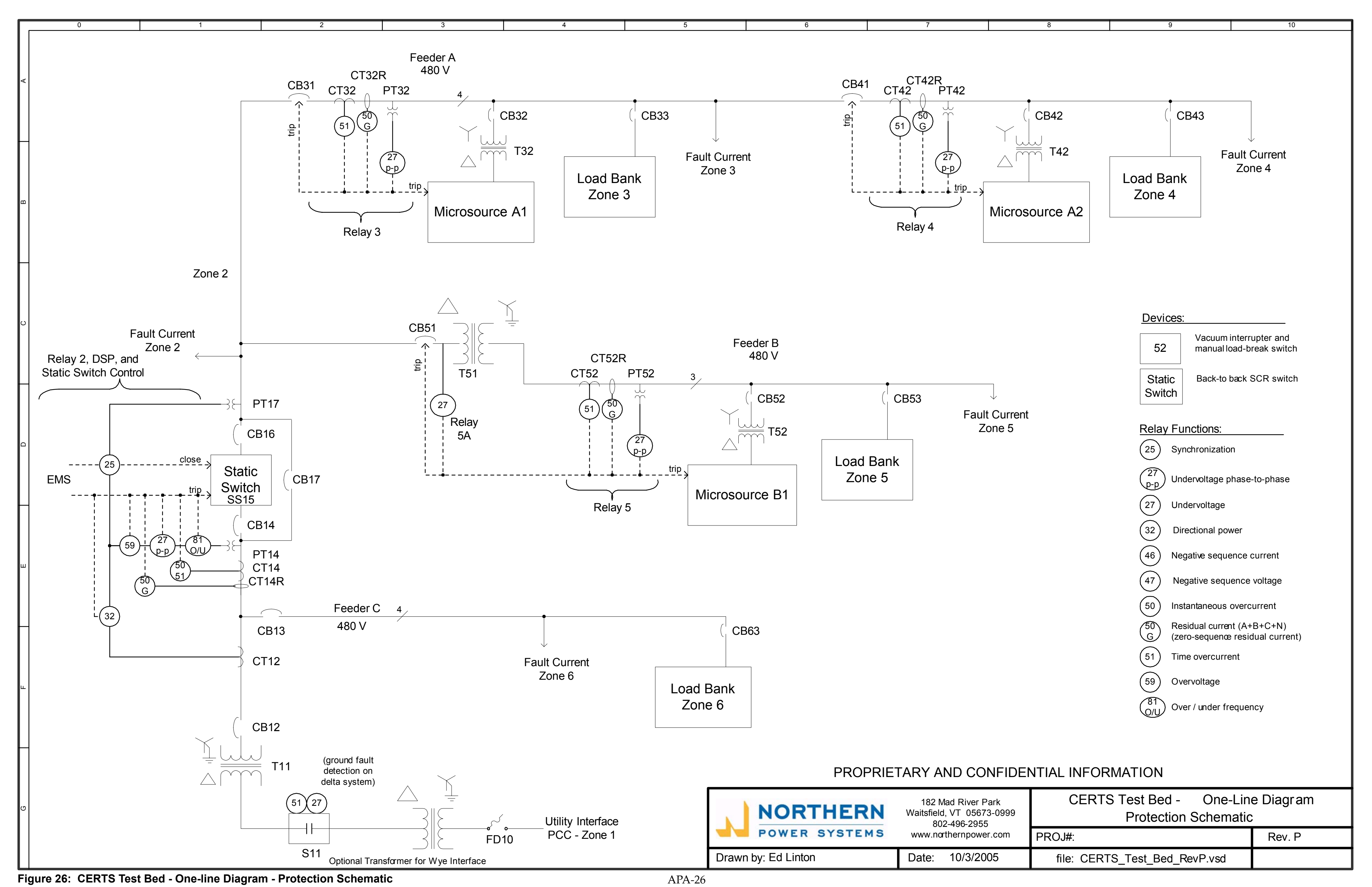




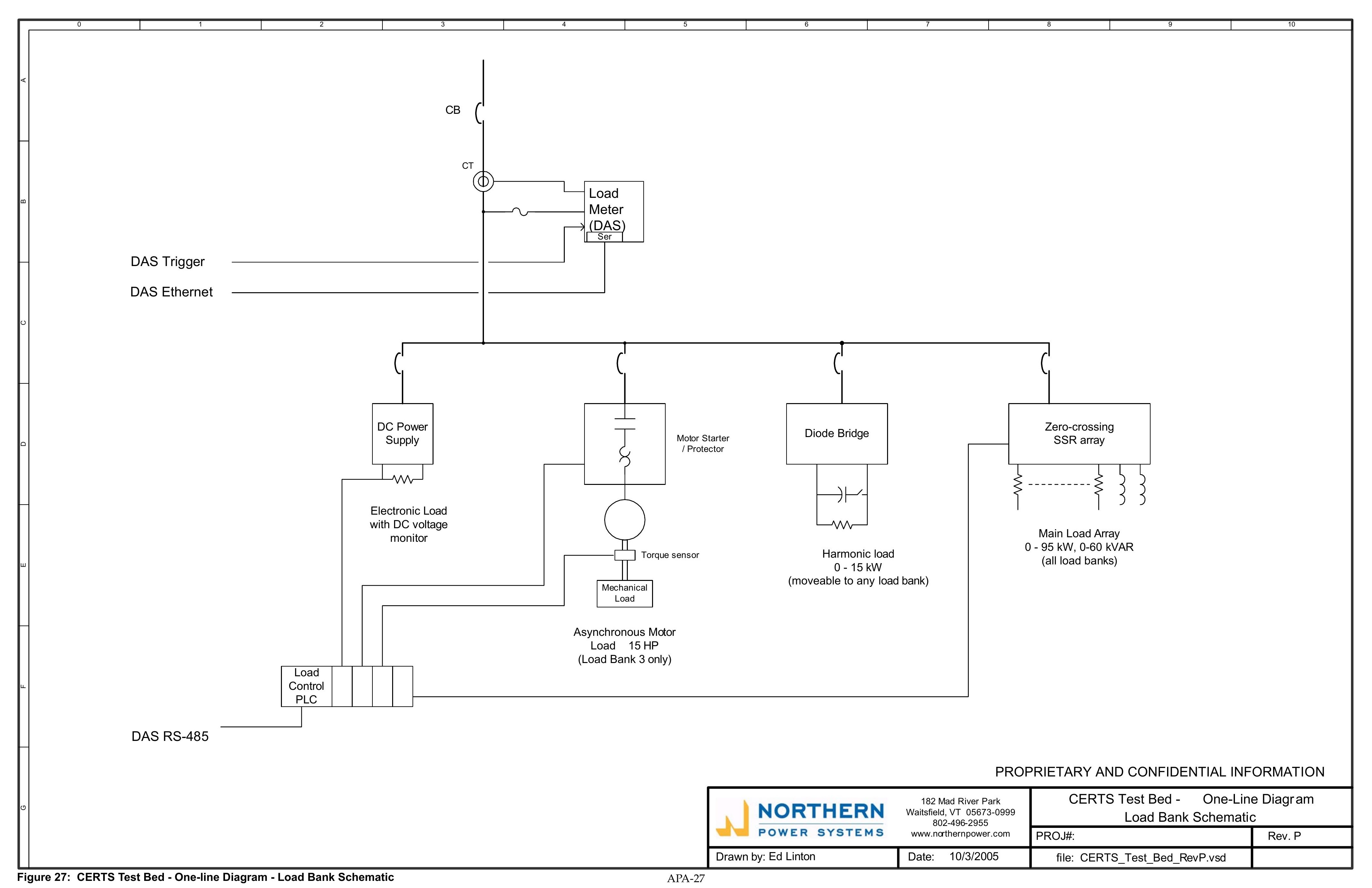




\title{
CERTS MICROGRID LABORATORY TEST BED
}

\author{
CERTS Microgrid Test Plan
}

Prepared For:

California Energy Commission

Public Interest Energy Research Program

$\frac{n}{x}$

Prepared By:

Lawrence Berkeley National Laboratory

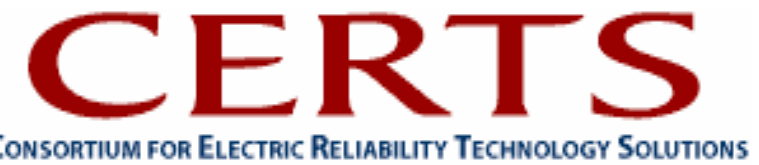



Prepared By:

Lawrence Berkeley National Laboratory

Joseph H. Eto, Principal Investigator

Berkeley, CA 94720

Harry Volkommer and Dave Klapp, American Electric Power

Commission Contract No. 500-03-024

Prepared For:

Public Interest Energy Research (PIER)

California Energy Commission

Bernard Treanton

Contract Manager

Mike Gravely

Program Area Lead

ENERGY SYSTEMS INTEGRATION

Mike Gravely

Office Manager

ENERGY SYSTEMS RESEARCH

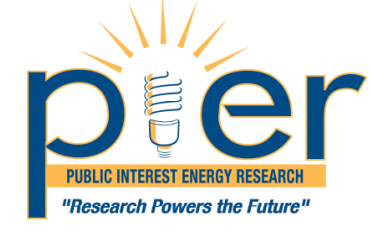

Martha Krebs, Ph.D.

PIER Director

Thom Kelly, Ph.D.

Deputy Director

ENERGY RESEARCH \& DEVELOPMENT DIVISION

Melissa Jones

Executive Director

\section{DISCLAIMER}

This report was prepared as the result of work sponsored by the California Energy Commission. It does not necessarily represent the views of the Energy Commission, its employees or the State of California. The Energy Commission, the State of California, its employees, contractors and subcontractors make no warrant, express or implied, and assume no legal liability for the information in this report; nor does any party represent that the uses of this information will not infringe upon privately owned rights. This report has not been approved or disapproved by the California Energy Commission nor has the California Energy Commission passed upon the accuracy or adequacy of the information in this report. 



\begin{tabular}{|l|l|}
\hline DTC Registered Procedure \\
CERTS Microgrid Test Plan \\
\begin{tabular}{|l|l|l|}
\hline Dolan Technology Center & Document No: DTC212372.000.01A \\
\hline Written by: AEP & Effective Date: 24 Jan. 2007 & Target Group: Assigned \\
\hline Approved by: K. P. Loving & Procedure Review Date: 24 Jan. 2008 & \\
\hline
\end{tabular}
\end{tabular}

\section{TABLE OF CONTENTS \\ DTC Register Procedure \\ CERTS Microgrid Test Plan \\ Date: January 24, 2007}

1.0 Purpose, References, Definitions

1.1 Purpose ...

1.2 Procedures ...

1.3 Pre-energizing tests ...

1.4 Site Commissioning ...

1.5 Reference Documents

1.6 Definitions

2.0 Responsibilities

2.1 Team Members

2.2 Walnut Test Site Issuing Authority Designee

3.0 Training - Team Members

3.1 Skills ...

3.2 Basic Electrical Safety ...

3.3 Protective equipment ...

3.4 Barricades, barriers ...

3.5 Test equipment operation ...

4.0 Procedures - CERTS Microgrid Test Bed Lockout/Tagout

5.0 Procedures - General

5.1 Job Safety Briefing ...

5.2 Alarms - Visual \& Audible

5.3 Barricades, Barriers or Guarding of Test Area

5.4 Other Precautions

5.5 Items to Verify Prior to Energizing Microgrid Test Bed

5.6 Verify Operation of DAS and EMS, Prior to Performing Test Plan

6.0 Procedures - Microgrid Test Bed System Checkout

6.1 Function of Static Switch

6.1.1 Startup System, Synchronized Closing

6.1.2 Reverse Power, Grid Islanding

6.1.3 Reverse Power, Single-Phase (IEEE 1547 Voltage Event)

6.1.4 Reverse Power, Anti-islanding, Microgrid Settings Reset

6.1.5 De-energized Bus (Dead Bus) Reclose

7.0 Validate Protection Settings \& Initial Fault Testing

7.1 Validate Zone 4 Circuit Breaker Settings, Utility Connected

This document contains information confidential and proprietary to AEPSC. It shall not be reproduced in whole or in part or released to any third party without expressed written consent of AEPSC. 


\begin{tabular}{|l|l|}
\hline DTC Registered Procedure \\
CERTS Microgrid Test Plan \\
\begin{tabular}{|l|l|l|}
\hline Dolan Technology Center & Document No: DTC212372.000.01A \\
\hline Written by: AEP & Effective Date: 24 Jan. 2007 & Target Group: Assigned \\
\hline Approved by: K. P. Loving & Procedure Review Date: 24 Jan. 2008 & \\
\hline
\end{tabular}
\end{tabular}

7.2 Validate Zone 3 Circuit Breaker Settings, Utility Connected

7.3 Validate Zone 5 Circuit Breaker Settings, Utility Connected

7.4 Zone 4 Three-Phase Un-grounded Fault, Gen-set (A1+A2) \& Utility Connected

7.5 Zone 3 A-Phase Line-to-Ground Fault, Gen-set A1 \& Utility Connected

7.6 Zone 3 A-Phase Line-to-Ground Fault, Gen-set (A1+A2) \& Utility Connected

7.7 Zone 5 B-Phase Line-to-Ground Fault, Gen-set B1 \& Utility Connected

7.8 Zone 5 B-Phase Line-to-Ground Fault, Gen-set (A1+B1) \& Utility Connected

7.9 Zone 4 B-Phase Line-to-Ground Fault, Gen-set (A1+A2) \& Utility Connected

7.10 Zone 2 C-Phase Line-to-Ground Fault, Gen-set (A1+B1) \& Utility Connected

7.11 Zone 6 C-Phase Line-to-Ground Fault, Gen-set A1 \& Utility Connected

7.12 Zone 6 C-Phase Line-to-Ground Fault, Gen-set (A1+B1) \& Utility Connected

7.13 Zone 3 A-to-B Phase Fault, Gen-set (A1+A2) \& Utility Connected

7.14 Zone 4 A-to-B Phase Fault, Gen-set (A1+A2) \& Utility Connected

7.15 Zone 2 A-to-B Phase Fault, Gen-set $(\mathrm{A} 1+\mathrm{B} 1)$ \& Utility Connected

7.16 Zone 5 A-to-B Phase Fault, Gen-set $(\mathrm{A} 1+\mathrm{B} 1)$ \& Utility Connected

8.0 Procedure - Reduced System Tests

8.1 Initial Voltage Regulation Test - Single Zone, Islanded, Gen-set A1

8.2 Open Static Switch Test, Check P=0 Limit, Gen-set A1

8.3 Open Static Switch Test, Check $\mathrm{P}=60 \mathrm{~kW}$ Limit, Gen-set A2

8.4 Test Island Operation, Unbalanced Load

8.5 Mixed Mode Operation Test - Feeder A, Gen-set A1 to 60kW Maximum

8.6 Mixed Mode Operation Test - Feeder A, Automatic Reset of Zone Level SetPoint

8.7 Mixed Mode Operation Test - Feeder A, Zone Power Change

8.8 Mixed Mode Operation Test - Feeder A, Zone Power Change When Islanded

8.9 Mixed Mode Operation Test - Feeder A, When Islanded Automatic Reset of Zone Level Set-point, Both Gen-sets P=60kW Maximum

8.10 Two Sources (A1 \& A2) Zone Control - Separate Feeders, When Islanded Automatic Reset of Zone Level Set-point, New Zone Sum Equal Zero

8.11 Two Sources (A1 \& B1) Zone Control - Separate Feeders, When Islanded Automatic Reset of Zone Level Set-points

8.12 Test Generator Black-Start Procedure

8.13 Test/Establish Generator Black-Start Capacity

9.0 Procedure - Demonstration Tests of Control Power Flow

9.1 Unit Control Mode, Weak Grid

9.2 Zone Control Mode, Weak Grid

9.3 Mixed Zone \& Unit Control Modes, Weak Grid

9.4 Mixed Zone \& Unit Control Modes, Strong Grid

10.0 Procedure - Test Difficult Loads

This document contains information confidential and proprietary to AEPSC. It shall not be reproduced in whole or in part or released to any third party without expressed written consent of AEPSC. 


\section{DTC Registered Procedure CERTS Microgrid Test Plan}

\begin{tabular}{l|l}
\hline Dolan Technology Center & Document No: DTC212372.000.01A
\end{tabular}

\begin{tabular}{|l|l|l|}
\hline Written by: AEP & Effective Date: 24 Jan. 2007 & Target Group: Assigned \\
\hline
\end{tabular}

\begin{tabular}{l|l}
\hline Approved by: K. P. Loving & Procedure Review Date: 24 Jan. 2008 \\
\hline
\end{tabular}

10.1 Motor Start Tests, Strong Grid, Balanced Load, 0.9 Power Factor

10.2 Motor Start Tests, Weak Grid, Balanced Load, 0.9 Power Factor

10.3 Motor Start Tests, Weak Grid, Unbalanced Load, 0.9 Power Factor

10.4 Unbalanced Load Test, Weak Grid, 0.9 Power Factor

10.5 Power Factor Tests, Balanced Load, Weak Grid

10.6 Power Factor Tests, Unbalanced Load, Weak Grid

10.7 Zone 3, 5kW Harmonic Injection Tests, Motor Start with Balanced Load, Weak Grid

10.8 Zone 3, 10kW Harmonic Injection Tests, Motor Start with Balanced Load, Weak Grid

10.9 Zone 3, 15kW Harmonic Injection Tests, Motor Start with Balanced Load, Weak Grid

10.10 Zone 5, 5kW Harmonic Injection Tests, Motor Start with Balanced Load, Weak Grid

10.11 Zone 5, 10kW Harmonic Injection Tests, Motor Start with Balanced Load, Weak Grid

10.12 Zone 5, 15kW Harmonic Injection Tests, Motor Start with Balanced Load, Weak Grid

10.13 Zone 6, 5kW Harmonic Injection Tests, Motor Start with Balanced Load, Weak Grid

10.14 Zone 6, 10kW Harmonic Injection Tests, Motor Start with Balanced Load, Weak Grid

10.15 Zone 6, 15kW Harmonic Injection Tests, Motor Start with Balanced Load, Weak Grid

11.0 Hazards \& Mitigation

12.0 Quality Assurance

Appendix A "CERTS Microgrid Test Bed Single Line Diagram" Appendix B "CERTS Microgrid Test Bed - Lockout/Tagout Log" Appendix C "CERTS Microgrid Test Bed - Test Log"

\begin{tabular}{|c|l|l|}
\hline Revision \# & Date & Revision Description \\
\hline 0 & $01 / 24 / 07$ & Original \\
\hline & & \\
\hline
\end{tabular}

This document contains information confidential and proprietary to AEPSC. It shall not be reproduced in whole or in part or released to any third party without expressed written consent of AEPSC. 


\section{DTC Registered Procedure CERTS Microgrid Test Plan}

Dolan Technology Center

Document No: DTC212812.207.01A

\begin{tabular}{|l|l|l|}
\hline Written by: AEP & Effective Date: 23 Feb 2007 & Target Group: Assigned \\
\hline Approved by: K. P. Loving & Procedure Review Date: 23 Feb. 2008 & \\
\hline
\end{tabular}

\begin{tabular}{|c|l|l|}
\hline Revision \# & Date & Revision Description \\
\hline 0 & $02 / 23 / 07$ & Original \\
\hline & & \\
\hline
\end{tabular}

Team Members and Roles

\begin{tabular}{|lll|}
\hline Name & Group & Primary Roles \\
\hline Kevin P. Loving & DTC Operations & Test Site Coordinator \\
\hline Dave A. Klapp & DTC Operations & Project \& Test Engineer \#1 \\
\hline Galen W. Perry & DTC Operations-Contractor & Test Operator/Technician \#1 \\
\hline H. T. Vollkommer & Consultant-Contractor & Technical Support Consultant \\
\hline John Stevens & Sandia National Lab. & CERTS Microgrid Test Team \\
\hline Robert Lasseter & University of Wisconsin & CERTS Microgrid Test Team \\
\hline Ed Linton & Northern Power Systems & CERTS Microgrid Test Team \\
\hline Jean Roy & TECOGEN, Inc. & CERTS Microgrid Test Team \\
\hline
\end{tabular}

\subsection{Purpose, References, Definitions}

1.1 The purpose of this document is to establish procedures for testing of the CERTS Microgrid Test Bed, located at the Walnut Test Site near Columbus, Ohio. This Test Site is part of AEP's Dolan Technology Center (DTC) campus. The CERTS Microgrid Test Bed (referred to as "Microgrid") is connected on the load side of the 2000 ampere safety breaker of Test Cell \#1, identified as CB1 in the single line diagram, CERTEQUIP-J01-001, dated March 24, 2006, and located in Appendix A.

1.2 Procedures necessary to perform tests on the Microgrid require:

1.2.1 Walnut Test Site specifically adapted "DTC Clearance Permit System" procedures to facilitate on site testing of CERTS Microgrid Test Bed.

1.2.2 Verification that the equipment is properly installed and wired in accordance with Northern Power System (NPS) and TECOGEN wiring prints, the controls operate as designed, protective equipment is installed and each piece of equipment is ready to be placed in service.

1.2.3 On-line verification of voltages, phase rotations, control operations, and communications.

1.3 Pre-energizing tests of electrical components shall be performed to verify that no short-circuits exist or possible faulty equipment will be energized.

This document contains information confidential and proprietary to AEPSC. It shall not be reproduced in whole or in part or released to any third party without expressed written consent of AEPSC. 


\begin{tabular}{|l|l|}
\hline DTC Registered Procedure \\
CERTS Microgrid Test Plan \\
\begin{tabular}{|l|l|l|}
\hline Dolan Technology Center & Document No: DTC212812.207.01A \\
\hline Written by: AEP & Effective Date: 23 Feb 2007 & Target Group: Assigned \\
\hline Approved by: K. P. Loving & Procedure Review Date: 23 Feb. 2008 & \\
\hline
\end{tabular}
\end{tabular}

1.4 The Microgrid shall be considered commissioned and available for use only after all the requirements identified in the NPS document, titled CERTS Test Bed, CERTEQUIP-T06-001-X9, Site Commissioning Plan, have been met.

1.5 References documents:

1.5.1 DTC Standard Operating Procedures

1.5.1.1 SOP - DTC Clearance Permit System, 204-DTC-05

1.5.1.2 SOP - Barricades, Barriers and Guards, 204-DTC-11

1.5.1.3 SOP - Personal Protective Equipment, 204-DTC-32

1.5.1.4 SOP - DTC Parent SOP for Primary Distribution Switching, 204-DTC-50.0

1.5.1.5 SOP - Sub-Procedure Primary Distribution Switching Walnut Test Site, 204-DTC-50.08

1.5.1.6 SOP - Job Safety Briefing, 204-DTC-70

1.5.2 $\quad$ AEP Safety Manual

1.5.2.1 Section 1.00 "General Safety"

1.5.2.2 Section 6.00 "Basic Electrical Safety, clauses 6.01, 6.02, 6.04, 6.05, and 6.16.

1.5.3 NFPA 70: "National Electric Code 2005"

1.5.4 OSHA 1910.269 Electric Power Generation, Transmission and Distribution: Electrical Protective Equipment

1.5.4.1 Subpart $\mathrm{o}-$ Testing and Test Facilities

1.5.4.2 Subpart v - Power Generation

1.5.5 Northern Power Systems (NPS) Documents - CERTS Test Bed

1.5.5.1 CERTEQUIP-J01-001, Single Line Diagram

1.5.5.2 CERTEQUIP-J06-001, Top Level Schematic

1.5.5.3 CERTEQUIP-B02-001, General Arrangement

1.5.5.4 CERTEQUIP-J06-003, Load Bank Schematic

1.5.5.5 CERTEQUIP-J14-001, Grounding and Neutral Schematic

1.5.5.6 CERTEQUIP-T06-001, Site Commissioning Plan

1.5.5.7 CERTEQUIP-Y01-001, DAS Manual - LabView

1.5.5.8 CERTEQUIP-Y01-002, EMS Manual

1.5.5.9 CERTEQUIP-Y01-003, DAS Manual - Ion Meter

1.5.5.10 CERTEQUIP-Y01-004, DAS \& EMS System Overview

This document contains information confidential and proprietary to AEPSC. It shall not be reproduced in whole or in part or released to any third party without expressed written consent of AEPSC. 


\begin{tabular}{|l|l|}
\hline DTC Registered Procedure \\
CERTS Microgrid Test Plan \\
\begin{tabular}{|l|l|l|}
\hline Dolan Technology Center & Document No: DTC212812.207.01A \\
\hline Written by: AEP & Effective Date: 23 Feb 2007 & Target Group: Assigned \\
\hline Approved by: K. P. Loving & Procedure Review Date: 23 Feb. 2008 & \\
\hline
\end{tabular}
\end{tabular}

\subsubsection{CERTEQUIP-C16-001, Protection Document}

\section{$1.6 \quad$ Definitions}

1.6.1 Walnut Test Site Issuing Authority Designee

1.6.1.1 A Designee assigned by the "DTC Issuing Authority" who will temporarily serve as the Walnut Test Site Issuing Authority Designee for this registered procedure.

1.6.1.2 Shall not be the Permit Holder for the CERTS Microgrid Test Bed or any part of this procedure or related equipment.

1.6.1.3 Is restricted to the CERTS Microgrid Test Bed and the switching of the Walnut Test Site 2000 ampere Station Safety Breaker, CB1.

1.6.2 CERTS Microgrid Test Bed - Test bed consisting of the Northern Power System (NPS) and TECOGEN equipment located at the Walnut Test Site. The boundary between the CERTS Microgrid Test Bed and AEP Grid is at the delta-wye transformer (T11) connected to the terminal box of the Walnut Test Site Station Safety Breaker CB1. Note, between T11 transformer and the CERTS Test Bed Safety Breaker (CB12) is a selectable High/Low in-series inductive impedance cabinet (L11).

1.6.3 Lockout/Tagout $\log -\mathrm{A} \log$ listing the date and time of the Lock / Tag "in" and "out" operation of a designated disconnect device or circuit breaker that shall be tagged and locked out for a particular test sequence and test change.

1.6.4 Electrical One-Line Status Board - A cork bulletin board with the CERTS Microgrid Test Bed electrical one-line overlaid on the corkboard.

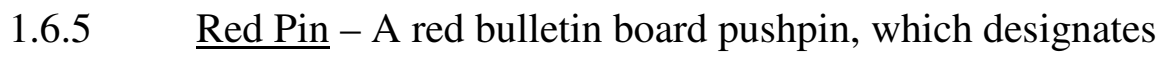
location and a visual Red Clearance Permit Tag presence.

1.6.6 Lockout Lock Board - A board with five (5) green locks to be used for each lockout/tagout procedure. Each lock shall have a designated breaker or disconnect device and the board shall reflect labeling to this effect.

This document contains information confidential and proprietary to AEPSC. It shall not be reproduced in whole or in part or released to any third party without expressed written consent of AEPSC. 


\section{DTC Registered Procedure CERTS Microgrid Test Plan}

Dolan Technology Center

\begin{tabular}{|l|l|l}
\hline Written by: AEP & Effective Date: 23 Feb 2007 & Target Group: Assigned
\end{tabular}

Approved by: K. P. Loving $\quad$ Procedure Review Date: 23 Feb. 2008

1.6.7 Walnut Circuit Breaker Program - AEP created software to control the Walnut Test Site Recloser and Station Safety Breakers. This software is found on the Walnut Test Site Computer in the test trailer.

\subsection{Responsibilities}

Scheduling:

Test Site Coordinator

Parameter Specifications:

Project Engineer, Technical Support Consultant, CERTS Microgrid Test Team

Setup:

Test Engineer \#1 \& Test Operator/Technician \#1

Execution:

(Alternate: Technical Support Consultant)

Report:

Test Engineer \#1 \& Test Operator/Technician \#1 (Alternate: Technical Support Consultant)

Report:

Project Engineer, Technical Support Consultant,

Analysis: and CERTS Microgrid Test Team

Approval:

Project Engineer, Technical Support Consultant, CERTS Microgrid Test Team

2.1 Team Members

2.1.1 Team members shall comply with DTC operating procedures, as required by this registered procedure.

2.1.2 At least two people shall be on-site during test(s) and either Test Engineer \#1 or Technical Support Consultant shall be onsite and in charge during the test(s). Test Engineer \#1, Test Operator/Technician \#1 and Technical Support Consultant, must be trained and qualified in the operation of both the DAS (Data Acquisition System) and EMS (Energy Management System) equipment, familiar with the test plan and emergency shutdown procedures, and has the most immediate control of the hazard-producing devices that is practical.

2.1.3 During a test event, the test shall be initiated and performed from the Control Trailer with "NO" humans physically located in the Microgrid Test Bed area or on the East, South or West sides of the Gen-set Enclosure during the test.

2.1.4 In preparation of and/or prior to performing a test event, one person may enter the Microgrid Test Bed with power enclosure doors in the Test Bed "latched" with no energized equipment

This document contains information confidential and proprietary to AEPSC. It shall not be reproduced in whole or in part or released to any third party without expressed written consent of AEPSC. 


\section{DTC Registered Procedure CERTS Microgrid Test Plan}

Dolan Technology Center

\begin{tabular}{|l|l|l|}
\hline Written by: AEP & Effective Date: 23 Feb 2007 & Target Group: Assigned
\end{tabular}

Approved by: K. P. Loving Procedure Review Date: 23 Feb. 2008

terminals exposed or in contact with people. The second person on-site will be in the Control Trailer and situated such that he/she can view the test area and available to perform an immediate Emergency Shutdown Procedure.

2.1.5 The Test Engineer \#1 or Technical Support Consultant shall be in the Control Trailer and situated such that he/she can view the test area, while the Test Operator/Technician \#1 is performing the test(s). If an unsafe condition arises while the test area is energized, the Test Operator/Technician \#1 shall be directed to perform an immediate Emergency Shutdown Procedure.

2.1.6 The Test Site Coordinator shall ensure that the required qualifications of all participants are met, including training cited in Section 3.0 of this Procedure.

2.1.7 The Test Site Coordinator shall be responsible for approving this procedure and any revisions.

2.2 Walnut Test Site Issuing Authority Designee

2.2.1 Shall comply with the DTC Clearance Permit System or the "CERTS Microgrid Test Bed Lockout/Tagout" procedure, cited in Section 4.0.

2.2.2 Shall place all tags, lockout devices and/or grounds associated with the CERTS Microgrid Test Bed.

2.2.3 Shall use AEP System safety procedures, PPE, and approved tools when isolating equipment and/or placing grounds for the CERTS Microgrid Test Bed.

2.2.4 Shall be knowledgeable about the CERTS Microgrid Test Bed and other systems distributing hazardous energy.

2.2.5 Shall not be a Clearance Permit holder for the CERTS Microgrid Test Bed. This ensures that at least two independent judgments are involved in deciding that the Clearance Permit provides a safe arrangement of devices and that the tags are properly placed.

2.2.6 Is responsible for addressing any observed non-compliance issues.

\subsection{Training - Team Members}

This document contains information confidential and proprietary to AEPSC. It shall not be reproduced in whole or in part or released to any third party without expressed written consent of AEPSC. 


\section{DTC Registered Procedure CERTS Microgrid Test Plan}

\begin{tabular}{l|l}
\hline Dolan Technology Center & Document No: DTC212812.207.01A
\end{tabular} \begin{tabular}{|l|l|l|}
\hline Written by: AEP & Effective Date: 23 Feb 2007 & Target Group: Assigned \\
\hline
\end{tabular}

\begin{tabular}{l|l}
\hline Approved by: K. P. Loving & Procedure Review Date: 23 Feb. 2008 \\
\hline
\end{tabular}

3.1 The skills necessary to distinguish exposed high voltage/energy parts of the test equipment.

3.2 Basic Electrical Safety practices of the AEP Safety Manual shall be a followed with Section 6.02 being the guiding principle and minimum approach distances specified in Section 6.03.

3.3 The proper use of personal protective equipment and insulated tools when working on or near exposed energized equipment.

3.4 The proper use of barricades, barriers or guards.

3.5 The proper operation of associated test equipment, based on the manufacturer's operator's manual, and of the DAS and EMS computerbased operating systems.

\subsection{Procedure - CERTS Microgrid Test Bed Lockout/Tagout}

4.1 The CERTS Microgrid Test Bed Lockout/Tagout procedure is designed to provide the same level of safety afforded by the DTC Clearance Permit system with less implementation overhead. Because of this, it can ONLY be applied under a limited set of applications, described as follows:

4.1.1 To make adjustments of Fault/Overload breaker connections;
or

4.1.2 To make adjustments to the weakening grid inductor in Cabinet L11.

4.2 These are the ONLY two reasons that the CERTS Microgrid Test Bed Lockout/Tagout procedure may be used. Any other application requiring the lockout/tagout of hazardous energy equipment will default back to the DTC Clearance Permit Procedure.

4.3 The Walnut Test Site Issuing Authority Designee shall:

4.3.1 Issue a "Microgrid Clearance Permit" for the CERTS

Microgrid Test Bed prior to testing and advance the permit to a "Permission to Test Operate" status.

4.3.2 Assign this Clearance Permit to the Walnut Test Site Station Safety Breaker CB1, the Microgrid Main Breaker (CB12), the three Fused Disconnects on Gen-sets A1, B1 and A2.

4.3.3 Be responsible for Lockout/Tagout of Walnut Test Site Station Safety Breaker (CB1), the Microgrid Main Breaker (CB12), the three Fused Disconnects on Gen-sets A1, B1 and A2, as required in these procedures.

This document contains information confidential and proprietary to AEPSC. It shall not be reproduced in whole or in part or released to any third party without expressed written consent of AEPSC. 


\begin{tabular}{|l|l|}
\hline DTC Registered Procedure \\
CERTS Microgrid Test Plan \\
\begin{tabular}{|l|l|l|}
\hline Dolan Technology Center & Document No: DTC212812.207.01A \\
\hline Written by: AEP & Effective Date: 23 Feb 2007 & Target Group: Assigned \\
\hline Approved by: K. P. Loving & Procedure Review Date: 23 Feb. 2008 & \\
\hline
\end{tabular}
\end{tabular}

4.3.4 Verify that all tags and lockout devices are properly installed at the designated locations prior to a test.

4.3.5 Assure a "Job Safety Briefing" (JSB) has taken place prior to each test.

4.4 Walnut Test Site Issuing Authority Designee and Team Members shall independently confirm that the test configuration provides the protection required for the procedures to be undertaken.

4.5 A re-useable Microgrid Clearance Permit Tag shall be assigned to each circuit breaker and disconnect device that requires lockout/tagout for a particular test sequence.

4.6 Lockout Devices

4.6.1 In addition to the tag types allowed by "AEP Fossil and Hydro Generation Clearance Permit System", keyed padlocks will be placed in combination with each red tag placed on a device that is capable of being locked out, in accordance with the AEP Control of Hazardous Energy Policy.

4.6.2 A green keyed-alike padlock shall be used as the default lockout device for the CERTS Microgrid Test Bed.

4.6.3 Keys for the green keyed-alike padlocks shall be located:

4.6.3.1 One key provided to the Issuing Authority.

4.6.3.2 One key provided to the Walnut Test Site Issuing Authority Designee.

4.6.3.3 Spare keys will be secured in the DTC key safe

4.7 Each time a CERTS Microgrid Test Bed Lockout/Tagout is required, the Walnut Test Site Issuing Authority Designee shall isolate the CERTS Microgrid Test Bed from the utility as follows:

4.7.1 Remotely "Shutdown" all Gen-sets (i.e., A1, B1 \& A2).

4.7.2 Remotely “Trip” the Station Safety Breaker CB1, using the Walnut Circuit Breaker Program found on the Walnut Test Site Computer in the Control Trailer.

4.7.3 Verify that no voltage exists on the Microgrid Test Bed, using the DAS meter readouts.

This document contains information confidential and proprietary to AEPSC. It shall not be reproduced in whole or in part or released to any third party without expressed written consent of AEPSC. 


\begin{tabular}{|l|l|}
\hline DTC Registered Procedure \\
CERTS Microgrid Test Plan \\
\begin{tabular}{|l|l|l|}
\hline Dolan Technology Center & Document No: DTC212812.207.01A \\
\hline Written by: AEP & Effective Date: 23 Feb 2007 & Target Group: Assigned \\
\hline Approved by: K. P. Loving & Procedure Review Date: 23 Feb. 2008 & \\
\hline
\end{tabular}
\end{tabular}

4.7.4 Lockout/Tagout the Station Safety Breaker CB1, using the designated green lock.

4.7.5 Manually "Trip" circuit breaker CB12 to the CERTS Microgrid Test Bed.

4.7.6 Lockout/Tagout the CERTS Microgrid Test Bed circuit breaker $\mathrm{CB} 12$, using the designated green lock.

4.7.7 Open the Fused Disconnects on Gen-sets A1, B1 and A2.

4.7.8 Lockout/Tagout the Fused Disconnects to Gen-sets A1, B1 and A2, using the designated green locks.

4.7.9 The area (i.e., within/near cabinets) where work is to be performed shall then be checked with a voltage detector to confirm that hazardous potential has been removed.

4.7.10 Using the proper PPE, an approximately sized grounding conductor shall be connected between the electrical bus and ground, as close to the work area as reasonably feasible.

4.7.11 The Lockout/Tagout Log is in Appendix B and shall be completed, reflecting completion of the Lockout/Tagout procedure. The horizontal axis includes the breakers and disconnects, while the vertical axis includes the date, time and specific test described in this procedure document.

4.7.12 The work requiring this Logout/Tagout procedure may now be completed with the Red Clearance Permit Tag pins positioned on the Electrical One-Line Status Board to inform others of site status.

4.7.13 Perform these steps in reverse order. Then:

4.7.13.1 Check to verify all grounds have been removed before energizing;

4.7.13.2 Re-energize the CERTS Microgrid Test Bed; and

4.7.13.3 Verify the proper nominal voltages in Meter 1.

\subsection{CERTS Microgrid Test Bed-Test Log}

4.8.1 A list of test events shall be organized in a logical sequence to complete all tests involving energizing work progressing from the utility source and Gen-sets to the last piece of equipment.

This document contains information confidential and proprietary to AEPSC. It shall not be reproduced in whole or in part or released to any third party without expressed written consent of AEPSC. 


\begin{tabular}{|c|c|c|}
\hline \multirow{2}{*}{$\begin{array}{l}\text { DTC Registered Procedure } \\
\text { CERTS Microgrid Test Plan } \\
\text { Dolan Technology Center }\end{array}$} & $\begin{array}{l}\text { cedure } \\
\text { est Plan }\end{array}$ & \\
\hline & \begin{tabular}{l|l} 
& Document No:
\end{tabular} & DTC212812.207.01A \\
\hline Written by: AEP & Effective Date: 23 Feb 2007 & Target Group: Assigned \\
\hline Approved by: K. P. Loving & Procedure Review Date: $23 \mathrm{~F}$ & eb. 2008 \\
\hline
\end{tabular}

4.8.2 Appendix C lists the "CERTS Microgrid Test Bed-Test Log" to be completed.

4.8.3 A single copy of Appendix C shall be printed out, initialed by the Test Engineer \#1 performing the test or Technical Support Consultant, and dated.

4.8.4 Once Appendix $\mathrm{C}$ is completed, the appendix shall be attached to this document and stored in an electronic file.

4.8.5 All Team Members involved with the actual test shall acknowledge to the Test Engineer \#1 or designated person that they are "Ready to go".

4.8.6 Test Engineer \#1 or designated person shall verify that the front gate to the Walnut Test Site is "Closed", not locked, with an audible alarm in operation.

4.8.7 Test Engineer \#1 or designated person shall then start the visual alarms.

4.8.8 Test Engineer \#1 or designated person shall notify the Team Members "The test is about to begin".

\subsection{Procedure - General}

5.1 Prior to testing each day, the person in charge during the planned test $(\mathrm{s})$ or his designee shall perform a JSB, and the barricades and test setup shall be inspected for safety and compliance.

5.2 Alarms - Visual \& Audible

5.2.1 These alarms shall warn persons that energized testing is being performed in the Microgrid Test Bed area.

5.2.2 Visual alarm, consisting of a portable red flashing light, shall be located between the Control Trailer and Gen-set Enclosure (i.e., North side of Enclosure between the coolant system structure and natural gas lines into the Enclosure).

5.2.3 Audible alarm, consisting of a portable buzzer, shall be located at the front gate of the Walnut Test Site with the fence gate "Closed", not locked, and an audible alarm in operation during test(s).

5.3 Barricades, Barriers, or Guarding of Test Area

12

This document contains information confidential and proprietary to AEPSC. It shall not be reproduced in whole or in part or released to any third party without expressed written consent of AEPSC. 


\begin{tabular}{|l|l|}
\hline DTC Registered Procedure \\
CERTS Microgrid Test Plan \\
\begin{tabular}{|l|l|l|}
\hline Dolan Technology Center & Document No: DTC212812.207.01A \\
\hline Written by: AEP & Effective Date: 23 Feb 2007 & Target Group: Assigned \\
\hline Approved by: K. P. Loving & Procedure Review Date: 23 Feb. 2008 & \\
\hline
\end{tabular}
\end{tabular}

5.3.1 Barricades shall be set up around major equipment of the Microgrid Test Bed (i.e., saw-horse style barricades with plastic chain shall surround the test area containing the Gen-set Enclosure, Microgrid switching cabinets, plus load and fault bank cabinets).

5.3.2 Barricades set up at the entrance area to the Gen-set Enclosure and Microgrid Test Bed switching cabinets shall include "Danger - Test In Progress" signs.

5.4 Other Precautions

5.4.1 During initial energizing tests all personnel shall remain within or adjacent to the Control Trailer while tests are being performed.

5.4.2 All nonessential personnel shall either leave the main site or shall shelter in the Control Trailer.

5.5 Items to Verify Prior to Energizing Microgrid Test Bed Equipment.

5.5.1 Test Engineer \#1 or Technical Support Consultant shall verify that all personnel and visitors are properly protected and in assigned locations prior to performing each test.

5.5.2 The test area is properly reviewed and secured.

5.5.3 Auxiliary power sources fed from circuit breakers in electrical cabinets $9 \mathrm{a}$ and $9 \mathrm{~b}$ are switched "On", energizing down-line equipment, located in the Test Bed and Gen-set Enclosure.

5.5.4 Gen-set Enclosure doors (i.e., located on East, South and West sides) must remain "Open" for proper airflow during Gen-set operation.

5.5.5 Gen-set Enclosure exhaust fans and the coolant system/pump shall be "On" whenever one or more Gen-sets are operating.

5.6 Verify Operation of DAS and EMS, Prior to Performing Test Plan.

5.6.1 All recording instrumentation shall be checked and readied for operation.

5.6.2 Twelve PML Ion 7650 meters are distributed within the Microgrid Test Bed with locations shown in Figure 1. These meters are prime data collection elements in the system for DAS and EMS. They monitor electrical system conditions, plus acquire phase current and voltage waveforms; and

13

This document contains information confidential and proprietary to AEPSC. It shall not be reproduced in whole or in part or released to any third party without expressed written consent of AEPSC. 


\section{DTC Registered Procedure CERTS Microgrid Test Plan}

Dolan Technology Center

Document No: DTC212812.207.01A

\begin{tabular}{l|l|l} 
Written by: AEP & Effective Date: 23 Feb 2007 & Target Group: Assigned \\
\hline
\end{tabular}

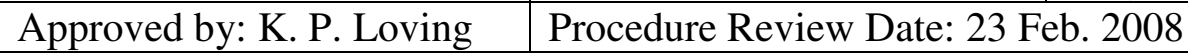

calculate RMS values of voltage, current, active power, reactive power, and frequency.

5.6.3 An Ethernet network is provided, as shown in Figure 2, for communications between all meters, load control PLCs, and the DAS computer, using fiber-optic links and switches. The DAS and EMS computers are also networked into the local Dolan LAN. Additional serial links connect all SEL-351 relays, the static switch DSP control, and the Tecogen Gen-set controls to the EMS computer, using fiber optic converters.

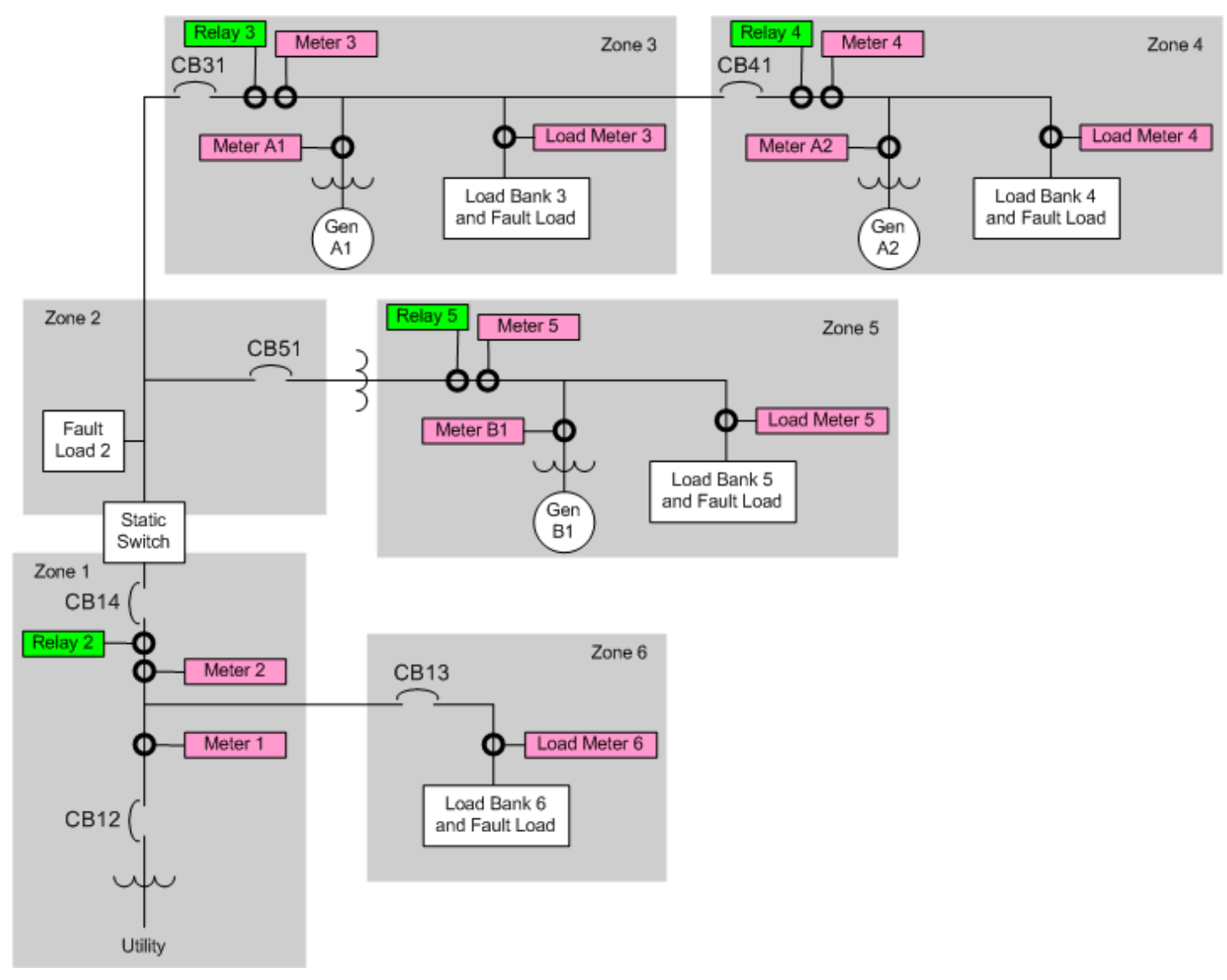

Figure 1 - Test Bed 1-Line with Meter and Relay Locations 


\section{DTC Registered Procedure CERTS Microgrid Test Plan}

Dolan Technology Center

Document No: DTC212812.207.01A

\begin{tabular}{|l|l|l}
\hline Written by: AEP & Effective Date: 23 Feb 2007 & Target Group: Assigned
\end{tabular}

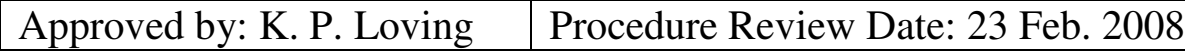

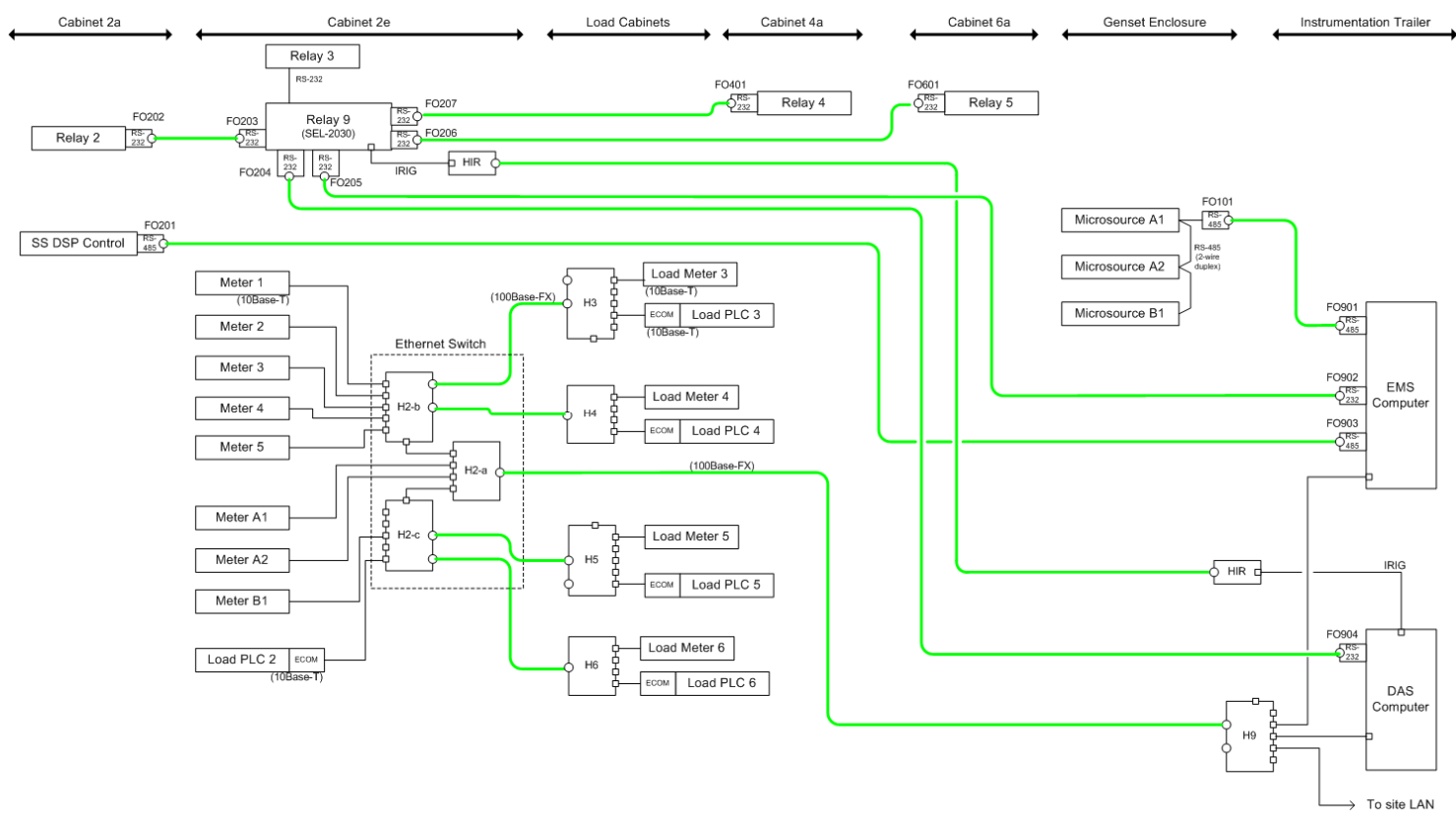

Figure 2 - DAS \& EMS Data Network

\subsection{Procedure - Microgrid Test Bed System Checkout}

Objective: The tests described in Section 6 are designed to check control and operation of the static switch, basic power and voltage control of the Gen-sets, and a preliminary check of the protection scheme. The goal is to assure that the test bed is operating and ready to perform the tests described in the remaining sections of this test document. During these system checkout tests, the series inductor L11 shall be connected in the circuit to minimize any impact to the Test Bed.

\subsection{Function of Static Switch}

Objective: Check operation of the static switch, to assure it and its DSP control is operating as designed. This will include tests of dead-bus and synchronized closing, reverse power and IEEE 1547 protective relay functions. Waveform and RMS data from Meter 2 will be of prime interest. At this stage, the switch functions will be tested with a single Gen-set A1 online.

\subsubsection{Start-up System, Synchronized Closing}

Measure - Voltage waveforms for phases A, B \& C on both sides of the static switch, current waveforms for phase A at the switch. To obtain the voltage across each phase of the switch, using Meter 2 and Meter A1 to measure the voltage on both sides of the switch. 


\begin{tabular}{|l|l|}
\hline DTC Registered Procedure \\
CERTS Microgrid Test Plan \\
\begin{tabular}{|l|l|l|}
\hline Dolan Technology Center & Document No: DTC212812.207.01A \\
\hline Written by: AEP & Effective Date: 23 Feb 2007 & Target Group: Assigned \\
\hline Approved by: K. P. Loving & Procedure Review Date: 23 Feb. 2008 & \\
\hline
\end{tabular}
\end{tabular}

Performance Goal - Verification that when conditions are appropriate (within synchronization limits set in the EMS), the switch can perform a synchronized closing and thus give smooth closing transitions.

6.1.1.1 Verify the following items:

6.1.1.1.1 All alarms are reset on both the S\&C PES and DSP, and that system is ready to start.

6.1.1.1.2 Circuit breakers in Cabinet 3 and Cabinet 8 to Load Banks are "Closed".

6.1.1.1.3 CB 32 is "Closed".

6.1.1.1.4 Set Gen-set A1 to the following set-points:

Gen-set A1 = Unit Power Control

Output Power Command $=20 \mathrm{~kW}$

MG Power/Frequency Droop $=-0.0833 \mathrm{~Hz} / \mathrm{kW}$

MG Voltage Command $=277 \mathrm{~V}$

6.1.1.1.5 Set loads on Load Bank 3 and Load Bank 6 to $40 \mathrm{~kW}$.

6.1.1.2 Start the system with the "Start" command from the EMS. The system should enter the "Disconnect" State on the DSP, and the Gen-set side voltage at Meter A1 should indicate a frequency of approximately $59.65 \mathrm{~Hz}$.

6.1.1.3 When conditions are appropriate, the static switch should do a synchronized "Close". A smooth transition should occur from island to utility-connected mode in Gen-set A1. This will happen relatively quickly, after the "Start" command is issued.

6.1.1.4 When the static switch closes, "CONNECT" is displayed in the Control State block of the EMS, and "PES OK" is displayed in the PES Status block. Verify that the readings of power flow measurements are accurate.

6.1.1.5 Once steady state is reached, power flow should be $\sim 60$ $\mathrm{kW}$ at the remote location (Meter 1) and $20 \mathrm{~kW}$ at the static switch (Meter 2); the frequency should stabilize to the grid frequency $(\sim 60 \mathrm{~Hz})$.

\subsubsection{Reverse Power, Grid Islanding (IEEE 1547, Loss of Utility Source).}

This document contains information confidential and proprietary to AEPSC. It shall not be reproduced in whole or in part or released to any third party without expressed written consent of AEPSC. 


\begin{tabular}{|l|l|}
\hline DTC Registered Procedure \\
CERTS Microgrid Test Plan \\
\begin{tabular}{|l|l|l|}
\hline Dolan Technology Center & Document No: DTC212812.207.01A \\
\hline Written by: AEP & Effective Date: 23 Feb 2007 & Target Group: Assigned \\
\hline Approved by: K. P. Loving & Procedure Review Date: 23 Feb. 2008 & \\
\hline
\end{tabular}
\end{tabular}

Measure - RMS voltage and active power at static switch, current waveforms at the static switch and timing of event sequence.

Performance Goal - Verify the reverse power functionality of the static switch and confirm that the static switch islands the Microgrid for a reverse power condition due to an upstream utility operation at the PCC. Once the utility voltage returns to the IEEE 1547 limits, verify the proper operations of the reconnection timers (set by default to 300 seconds based on IEEE 1547 standard).

6.1.2.1 Set Gen-set A1 to the following set-points:

Gen-set A1 = Unit Power Control

Output Power Command $=20 \mathrm{~kW}$

MG Power/Frequency Droop $=-0.0833 \mathrm{~Hz} / \mathrm{kW}$

MG Voltage Command $=277 \mathrm{~V}$

6.1.2.2 Set load on Load Bank 3 to $40 \mathrm{~kW}$ and on Load Bank 6 to 20kW. Also, set load on the AEP Load Bank to 500kW.

6.1.2.3 Perform a synchronized "Close" of the static switch and verify closing after one minute.

6.1.2.4 Next disconnect the utility grid from the Microgrid via "Opening" CB1 (three-pole breaker). Note, the static switch should "Open" within 2 seconds of opening CB1. Once the static switch opens, no voltage is present on the grid side of the switch, which creates an "IEEE 1547 voltage test" event. Verify in the EMS screen that the indicator of this event is turned "ON".

6.1.2.5 Verify in the "Event" Logger file that the above "Reverse Power" event was recorded, that the data acquisition was triggered and data was recorded, via a review of the DAS database.

6.1.2.6 Wait for approximately 20 seconds, and verify that the "IEEE 1547 voltage test" event remains high.

6.1.2.7 Reduce load on the AEP Load Bank to zero, than reconnect the grid by remote manual "Close" of CB1. The "IEEE 1547 voltage test" indicator should turn "Off".

6.1.2.8 The static switch should not attempt to reconnect immediately, since IEEE 1547 reconnection timer is set to 300 seconds. When synchronization conditions are

This document contains information confidential and proprietary to AEPSC. It shall not be reproduced in whole or in part or released to any third party without expressed written consent of AEPSC. 


\begin{tabular}{|l|l|}
\hline DTC Registered Procedure \\
CERTS Microgrid Test Plan \\
\begin{tabular}{|l|l|l|}
\hline Dolan Technology Center & Document No: DTC212812.207.01A \\
\hline Written by: AEP & Effective Date: 23 Feb 2007 & Target Group: Assigned \\
\hline Approved by: K. P. Loving & Procedure Review Date: 23 Feb. 2008 & \\
\hline
\end{tabular}
\end{tabular}

appropriate, the switch should attempt to do a synchronized "Close".

6.1.2.9 Verify a synchronized "Close" of the static switch, after the IEEE 1547 reconnection timer has elapsed.

6.1.2.10 Verify in the DAS database that the synchronized "Close" of the static switch was triggered and data was recorded.

6.1.3 Reverse Power, Single -Phase (IEEE 1547 Voltage Event). Measure - RMS voltage and active power at static switch, current waveforms for phase A at static switch, and timing of event sequence.

Performance Goal - Verification that the static switch islands the Microgrid when a reverse power condition, due an open-phase occurs. Note there are no single-phase breakers to disconnect the utility; reducing the load on one phase of the Microgrid will simulate the "open phase" condition. This condition does not properly test the reconnection logic, after an open phase, since the utility voltage will still be present on all three phases (i.e., test didn't really disconnected a phase).

6.1.3.1 Set Gen-set A1 to the following set-points:

Gen-set A1 = Unit Power Control

Output Power Command $=20 \mathrm{~kW}$

MG Power/Frequency Droop $=-0.0833 \mathrm{~Hz} / \mathrm{kW}$

MG Voltage Command $=277 \mathrm{~V}$

6.1.3.2 Select load on Load Bank 3 and Load Bank 6 to 40kW.

6.1.3.3 Start by removing the entire load on phase A of Load Bank 6. After this load change, the power measurement at the remote location (Meter 1) in phase A should be approximately $6.6 \mathrm{~kW}$, and in phases B and C should each be approximately $20 \mathrm{~kW}$.

6.1.3.4 Maintain zero load on phase A of Load Bank 6 and repeat the above load step change for Load Bank 3. Note, since the power measurement at remote location (Meter 1) in phase A is below the set threshold of $3.3 \mathrm{~kW}$, this should create a reverse power condition on phase $\mathrm{A}$ and open the

18

This document contains information confidential and proprietary to AEPSC. It shall not be reproduced in whole or in part or released to any third party without expressed written consent of AEPSC. 


\begin{tabular}{|l|l|}
\hline DTC Registered Procedure \\
CERTS Microgrid Test Plan \\
\begin{tabular}{|l|l|l|}
\hline Dolan Technology Center & Document No: DTC212812.207.01A \\
\hline Written by: AEP & Effective Date: 23 Feb 2007 & Target Group: Assigned \\
\hline Approved by: K. P. Loving & Procedure Review Date: 23 Feb. 2008 & \\
\hline
\end{tabular}
\end{tabular}

static switch within 2 seconds. Once the switch opens, voltage should still be present on all phases of the utility grid side of the switch, the static switch will go to lockout by the DSP stopping the S\&C PES switch (i.e., opening CB14 and going to the "Fault" state in the EMS).

6.1.3.5 Verify in the EMS that the system entered the "Fault" state, and check that the "Reverse power Anti-islanding, Microgrid Settings Reset" Alarm indicator has turned "On".

6.1.3.6 Verify in "Event" Logger file that at the time when this test was performed, a "Reverse Power" event was recorded, DAS was triggered and data was recorded.

6.1.3.7 Remove the reverse power single phase condition by bringing all phase A loads at Zone 6 and at Zone 3 back to default conditions (i.e., $40 \mathrm{~kW}$ each). The "Reverse power Anti-islanding, Microgrid Settings Reset Alarm" indicator should turn "Off". Note, the static switch will need to be "Reset" to return it to service, as it will think this is a misdispatch of the generation within the Microgrid. Also, the static switch will not wait for the 300 seconds to reconnect to the utility grid.

6.1.3.8 Use the EMS to give a "Reset" command to clear all faults and events.

6.1.3.9 Once all events and alarms are cleared, "Start" the system and observe a synchronized closing of the static switch.

\subsubsection{Reverse Power, Anti-islanding Microgrid Settings Reset}

Measure - RMS voltage and active power at static switch, current waveforms for phase $\mathrm{A}$ at switch and timing of event sequence.

Performance Goal - Verify that if a reverse power event occurs, due to a mismatch of Gen-set settings (Total Gen-set power > Microgrid load), the static switch will lockout and go to the "Fault" state, where user intervention is required.
6.1.4.1 Set Gen-set A1 to the following set-points:
Gen-set A1 = Unit Power Control
Output Power Command $=20 \mathrm{~kW}$

This document contains information confidential and proprietary to AEPSC. It shall not be reproduced in whole or in part or released to any third party without expressed written consent of AEPSC. 


\section{DTC Registered Procedure CERTS Microgrid Test Plan}

Dolan Technology Center \begin{tabular}{l|l|l} 
Written by: AEP & Effective Date: 23 Feb 2007 & Target Group: Assigned \\
\hline
\end{tabular}

Approved by: K. P. Loving $\quad$ Procedure Review Date: 23 Feb. 2008

MG Power/Frequency Droop= $-0.0833 \mathrm{~Hz} / \mathrm{kW}$

MG Voltage Command $=277 \mathrm{~V}$

6.1.4.2 Select load on Load Bank 3 and Load Bank 6 to 40kW.

6.1.4.3 Once the static switch "Closes", the power flow at Meter 1 is approximately $60 \mathrm{~kW}$.

6.1.4.4 Reduce the load to $0 \mathrm{~kW}$ at Load Bank 6 and verify the power measurement at Meter 1 drops to roughly $20 \mathrm{~kW}$. Wait 5 seconds and note that no reverse power trip should occur (i.e., above minimum $10 \mathrm{~kW}$ threshold value).

6.1.4.5 Reduce the load at Load Bank 3 by $5 \mathrm{~kW}$, wait about 5 seconds and verify a reverse power trip occurs. If not, repeat the step 6.1.4.4, reducing load at Load Bank 3 by an additional $5 \mathrm{~kW}$. Continue until the reverse power condition occurs and the static switch "Opens".

6.1.4.6 Once the static switch opens, since there is still voltage present in all phases of the grid side of the switch, the system will lockout by the DSP stopping the S\&C PES switch (i.e., opening CB14 and going to the "Fault" state in the EMS).

6.1.4.7 Verify in the EMS that the system entered the "Fault" state, and check that the "Reverse Power Anti-islanding, Microgrid Settings Reset" alarm indicator has turned "On".

6.1.4.8 Verify in the "Event" Logger file that at the time when this test was performed, a "Reverse Power" event was recorded, DAS was triggered and data was recorded.

6.1.4.9 Return load in Load Banks 3 and 6 back to 40kW.

6.1.4.10 Using the EMS, give a "Reset" command to clear all faults and events.

6.1.4.11 Once all events and alarms are cleared, "Start" the system and verify a synchronized closing of the static switch.

\subsubsection{De-energized Bus (Dead Bus) Reclose}

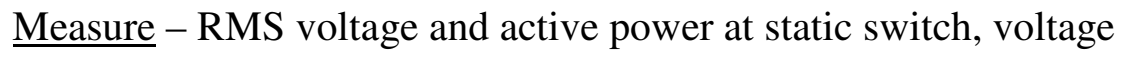
on both sides of the switch, and timing of event sequence. 


\begin{tabular}{|l|l|}
\hline DTC Registered Procedure \\
CERTS Microgrid Test Plan \\
\begin{tabular}{|l|l|l|}
\hline Dolan Technology Center & Document No: DTC212812.207.01A \\
\hline Written by: AEP & Effective Date: 23 Feb 2007 & Target Group: Assigned \\
\hline Approved by: K. P. Loving & Procedure Review Date: 23 Feb. 2008 & \\
\hline
\end{tabular}
\end{tabular}

Performance Goal - Verify that the static switch can close when de-energized bus conditions $(<15 \mathrm{~V})$ on the DG side are measured and that the Dead Bus Reclose algorithm requires user intervention (i.e., Operator needs to "Enable" the Dead Bus Reclose using pushbutton in the EMS).

6.1.5.1 Set Gen-set A1 to the following set-points:

Gen-set A1 = Unit Power Control

Output Power Command $=20 \mathrm{~kW}$

MG Power/Frequency Droop $=-0.0833 \mathrm{~Hz} / \mathrm{kW}$

MG Voltage Command $=277 \mathrm{~V}$

6.1.5.2 Select load on Load Bank 3, and Load Bank 6 to 40kW.

6.1.5.3 Open the static switch using "Manual Open" command in the EMS.

6.1.5.4 Perform a controlled "Shutdown" of Gen-set A1.

6.1.5.5 Verify in the EMS display that "DG side Dead Bus" event indicator turns "On". This confirms that the DSP detects a de-energized bus; the static switch doesn't "CLOSE" because "Manual Open" is enabled and "Dead Bus Reclose" is disabled.

6.1.5.6 Remove "Manual Open" and verify that the switch still remains "Open".

6.1.5.7 Enable "Dead Bus Reclose" by pressing the pushbutton in the EMS and verify the static switch "Close" event.

\subsubsection{Power Quality Trip, Grid Islanding (IEEE 1547, Loss of Utility Source).}

Measure - RMS voltage and active power at static switch, current waveforms at the static switch and timing of event sequence.

Performance Goal - Verify the reverse power functionality of the static switch and confirm that the static switch islands the Microgrid for a reverse power condition due to an upstream utility operation at the PCC. Once the utility voltage returns to the IEEE 1547 limits, verify the proper operations of the reconnection timers (set by default to 300 seconds based on IEEE 1547 standard).

This document contains information confidential and proprietary to AEPSC. It shall not be reproduced in whole or in part or released to any third party without expressed written consent of AEPSC. 


\section{DTC Registered Procedure CERTS Microgrid Test Plan}

Dolan Technology Center

\begin{tabular}{l|l|l} 
Written by: AEP & Effective Date: 23 Feb 2007 & Target Group: Assigned \\
\hline
\end{tabular}

Approved by: K. P. Loving $\quad$ Procedure Review Date: 23 Feb. 2008

6.1.6.1 Set Gen-set A1 to the following set-points:

Gen-set A1 = Unit Power Control

Output Power Command $=20 \mathrm{~kW}$

MG Power/Frequency Droop= $-0.0833 \mathrm{~Hz} / \mathrm{kW}$

MG Voltage Command $=277 \mathrm{~V}$

6.1.6.2 Set load on Load Bank 3 to $40 \mathrm{~kW}$ and on Load Bank 6 to 20kW. Also, set load on the AEP Load Bank to 500kW.

6.1.6.3 Perform a synchronized "Close" of the static switch and verify closing after one minute.

6.1.6.4 Next disconnect the utility grid from the Microgrid via "Opening" CB1 (three-pole breaker). Note, the static switch should "Open" within the CBEMA requirement after the opening of CB1. Once the static switch opens, no voltage is present on the grid side of the switch, which creates an "IEEE 1547 voltage test" event. Verify in the EMS screen that the indicator of this event is turned "ON".

6.1.6.5 Verify in the "Event" Logger file that the above "Reverse Power" event was recorded, that the data acquisition was triggered and data was recorded, via a review of the DAS database.

6.1.6.6 Wait for approximately 20 seconds, and verify that the "IEEE 1547 voltage test" event remains high.

6.1.6.7 Reduce load on the AEP Load Bank to zero, than reconnect the grid by remote manual "Close" of CB1. The "IEEE 1547 voltage test" indicator should turn "Off".

6.1.6.8 The static switch should not attempt to reconnect immediately, since IEEE 1547 reconnection timer is set to 300 seconds. When synchronization conditions are appropriate, the switch should attempt to do a synchronized "Close".

6.1.6.9 Verify a synchronized "Close" of the static switch, after the IEEE 1547 reconnection timer has elapsed.

6.1.6.10 Verify in the DAS database that the synchronized "Close" of the static switch was triggered and data was recorded. 


\section{DTC Registered Procedure CERTS Microgrid Test Plan}

Dolan Technology Center

Document No: DTC212812.207.01A

Approved by: K. P. Loving

Effective Date: 23 Feb 2007 Target Group: Assigned

Procedure Review Date: 23 Feb. 2008

\subsection{Procedure - Validate Protection Settings \& Initial Fault Testing}

Objective: The tests described in Section 7 are designed to examine a

preliminary set of fault (i.e., overload simulating a fault) condition tests to ensure protection and safety of the Microgrid Test Bed, while performing other planned tests. The goal is to test and adjust protection settings to achieve the most ideal conditions and protection design. Tests performed in this section will include inductor L11 in the circuit, reflecting weak grid conditions.

Definitions - To maintain a common understanding of Gen-set control modes during tests proposed in the following paragraphs:

- Unit Power Control Mode involves the amount of power (i.e., $\mathrm{kW}$ ) being injected into the Zone from the Gen-set being controlled.

- Zone Power Control Mode involves the amount of power (i.e., kW) entering/exiting the Zone which controls the output of the Gen-set in that Zone.

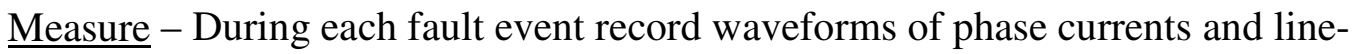
to-neutral voltages at all Relay locations (i.e., Relays 2, 3, 4 \& 5). Record the relay element that caused the "TRIP" with trip times for each relay relative to when the fault condition was applied.

Performance Goal - The fault (i.e., overload simulating a fault) condition tests are intended to cover the basic concept of the protection design and to study its effectiveness (e.g., zero-sequence, negative-sequence and residual currents for line-to-ground faults; negative-sequence or $\left(\mathrm{I}^{2} \mathrm{t}\right)$ protection for phase-to-phase faults). For the majority of these test scenarios, the static switch should "OPEN" first, followed by a "TRIP" at the specified relay. Confirm the protective action detailed in the Protection document.

\subsection{Validate Zone 4 Circuit Breaker Settings, Utility Connected}

Performance Goal - Initially test a three-phase balanced fault condition in Zone 4 to verify $\mathrm{I}^{2} \mathrm{t}$ protection. Then test a single line-to-ground fault condition in Zone 4 to verify zero-sequence, negative-sequence or residual over-current protection.

7.1.1 Perform the Microgrid Test Bed Lockout/Tagout Procedure, described in Section 4.7.

7.1.2 To simulate a high impedance three-phase ungrounded fault, setup the Overload Load Bank for $85 \mathrm{~kW}$ and connect portable cable/plug into the exterior receptacle of Cabinet $5 \mathrm{a}$. 


\section{DTC Registered Procedure CERTS Microgrid Test Plan}

Dolan Technology Center

\begin{tabular}{|l|l|l|}
\hline Written by: AEP & Effective Date: 23 Feb 2007 & Target Group: Assigned \\
\hline
\end{tabular}

\begin{tabular}{l|l}
\hline Approved by: K. P. Loving & Procedure Review Date: 23 Feb. 2008 \\
\hline
\end{tabular}

7.1.3 Install/physically connect portable overload cabling inside the cabinet at the outlet receptacle to phases A, B, and C (i.e., connecting and tightening the bolted connection).

7.1.4 Reverse the Microgrid Test Bed Lockout/Tagout Procedure.

7.1.5 Verify CB43 is Closed along with the circuit breakers to the Overload and Load Banks, manually "ARM" the key switch in the cabinet and close/lock the entry doors to Cabinet 5 .

7.1.6 Verify that the Microgrid Test Bed is energized with nominal voltages indicated on all three phases at Meter 1.

7.1.7 Using the Load Control program, set the load in Load Bank 4 to $60 \mathrm{~kW}$.

7.1.8 Using the EMS, give a "Reset" command to clear all faults and events in the system.

7.1.9 After the dead-bus closing of the static switch from the EMS, the Microgrid Test Bed is connected to the utility grid with $60 \mathrm{~kW}$ at Meter 2 and Meter 1.

7.1.10 Using the DAS Load Control program set the duration of the simulated fault to 100 seconds, set the software to "ARM" and initiate a three-phase, ungrounded fault (overload) in Zone 4, which adds $85 \mathrm{~kW}$ to the $60 \mathrm{~kW}$ load.

7.1.11 Verify that CB41 "TRIPS" first on over-current clearing the fault. Breaker K42 should Open under PLC control, removing the fault of the Overload Load Bank.

7.1.12 Verify in the DAS Database that:

7.1.12.1 The fault event waveforms of phase currents and voltages at all Relay locations (i.e., Relays 2, 3, 4 \& 5) were recorded, as well as the relay element that caused the "Trip" with trip times for each relay relative to when the fault was applied.

7.1.12.2 The waveform and RMS Meter data were recorded. Note the above collection of all data and waveforms will take approximately 30 minutes to arrive.

7.1.13 Remove load from Load Bank 4 to complete this simulated fault test event. Turn the key switch in Cabinet 5 to the "Off" position. 


\section{DTC Registered Procedure CERTS Microgrid Test Plan}

Dolan Technology Center

Written by: AEP

Approved by: K. P. Loving

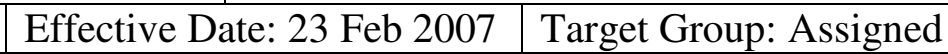

Procedure Review Date: 23 Feb. 2008

7.1.14 Perform the Microgrid Test Bed lockout/Tagout Procedure in Section 4.7 to prepare for a single-phase-to-ground fault (i.e., overload) test in Zone 4.

7.1.15 Within Cabinet 5a, physically remove the overload cabling inside the cabinet from phases B and C.

7.1.16 Change the wiring in Cabinet 5 between the external connector and the fault contactor $\mathrm{K} 42$ for a single phase fault of $28 \mathrm{~kW}$ between Phase A and ground (i.e., connecting and tightening the bolted connections).

7.1.17 Reverse the Microgrid Test Bed Lockout/Tagout Procedure.

7.1.18 Verify CB43 is "Closed" along with the circuit breakers to the Load Bank, manually "ARM" the key switch in the cabinet and close entry doors to Cabinet 5 .

7.1.19 Using the Load Control program, set the load in Load Bank 4 to 60 $\mathrm{kW}$.

7.1.20 Using the EMS, give a "Reset" command to clear all faults and events in the system.

7.1.21 After performing a dead-bus closing of the static switch, the Microgrid Test Bed is connected to the utility grid with $60 \mathrm{~kW}$ at Meter 2 and Meter 1.

7.1.22 Using the DAS Load Control program, set the duration of the simulated fault to 10 seconds, set the software to "ARM" and initiate a single-phase to ground fault (overload) in Zone 4, which adds $28 \mathrm{~kW}$ to the $20 \mathrm{~kW}$ load on A-phase.

7.1.23 Verify that the static switch "TRIPS" first on Ground over-current, which clears the fault (overload), followed by breaker CB41. Breaker K42 should Open under PLC control, removing the fault of the Overload Load Bank.

7.1.24 Verify in the DAS Database that:

7.1.24.1 The fault event waveforms of phase currents and voltages at all Relay locations (i.e., Relays 2, 3, 4 \& 5) were recorded, as well as the relay element that caused the "TRIP" with trip times for each relay relative to when the fault was applied.

This document contains information confidential and proprietary to AEPSC. It shall not be reproduced in whole or in part or released to any third party without expressed written consent of AEPSC. 


\section{DTC Registered Procedure \\ CERTS Microgrid Test Plan}

\begin{tabular}{l|l}
\hline Dolan Technology Center & Document No: DTC212812.207.01A
\end{tabular}

\begin{tabular}{|l|l|l|l|}
\hline Written by: AEP & Effective Date: 23 Feb 2007 & Target Group: Assigned \\
\hline Approved by: K. P. Loving & Procedure Review Date: 23 Feb. 2008 & \\
\hline
\end{tabular}

7.1.24.2 The waveform and RMS Meter data were recorded.

Note the above collection of all data and waveforms will take approximately 30 minutes to arrive.

7.1.25 Remove load from Load Bank 4 and "STOP" the static switch.

7.1.26 Perform the Microgrid Test Bed Lockout/Tagout Procedure, described in Section 4.7.

7.1.27 Remove the Overload Load Bank portable cable/plug from the exterior receptacle of Cabinet 5 a.

7.1.28 Physically remove the portable overload cabling inside Cabinet 5 between A-phase to ground.

7.1.29 Reverse the Microgrid Test Bed Lockout/Tagout Procedure.

7.1.30 Verify CB41 and the breaker to the Load Bank is closed. Close the entry doors to Cabinet 5 to complete this fault test event.

7.2 Validate Zone 3 Circuit Breaker Settings, Utility Connected Performance Goal - Initially test a three-phase balanced fault condition in Zone 3 to verify $I^{2}$ t protection. Then test a single line-to-ground fault condition in Zone 3 to verify zero-sequence, negative-sequence or residual over-current protection.

7.2.1 Repeat steps 7.1.1 through 7.1.30 to simulate a three-phase ungrounded fault (i.e., overload), and then a single-phase to ground fault (i.e., overload) on B-phase in Zone 3, Cabinet 3 and circuit breaker CB31. Note, for this test $60 \mathrm{~kW}$ of additional load is required in Zone 4 on Load Bank 4.

\subsection{Validate Zone 5 Circuit Breaker Settings, Utility Connected}

Performance Goal - Initially test a three-phase balanced fault condition in Zone 5 to verify $I^{2} t$ protection. Then test a single line-to-ground fault condition in Zone 5 to verify zero-sequence, negative-sequence or residual over-current protection.

7.3.1 Repeat steps 7.1.1 through 7.1.30 to simulate a three-phase ungrounded fault (i.e., overload), and then a single-phase to ground fault (i.e., overload) on C-phase in Zone 5, Cabinet 7b and circuit breaker CB51. 


\begin{tabular}{|l|l|}
\hline DTC Registered Procedure \\
CERTS Microgrid Test Plan \\
\begin{tabular}{|l|l|l|}
\hline Dolan Technology Center & Document No: DTC212812.207.01A \\
\hline Written by: AEP & Effective Date: 23 Feb 2007 & Target Group: Assigned \\
\hline Approved by: K. P. Loving & Procedure Review Date: 23 Feb. 2008 & \\
\hline
\end{tabular}
\end{tabular}

7.4 Zone 4 Three-Phase Un-grounded Fault, Gen-sets (A1+A2) \& Utility Connected

Performance Goal - Test a three-phase balanced fault condition in Zone 4 with Gen-sets A1 and A2 operating in-parallel with the utility grid to verify $I^{2} t$ protection, plus confirm a reverse power event after the Zone breaker "opens".

7.4.1 Perform the Microgrid Test Bed Lockout/Tagout Procedure, described in Section 4.7.

7.4.2 Setup the Overload Load Bank for a three-phase un-grounded fault of $85 \mathrm{~kW}$ and connect portable cable/plug into the exterior receptacle of Cabinet 5a.

7.4.3 Install/physically connect portable overload cabling inside the cabinet from the outlet receptacle to phases A, B, and C (i.e., connecting and tightening the bolted connections).

7.4.4 Reverse the Microgrid Test Bed Lockout/Tagout Procedure.

7.4.5 Verify CB43 is "Closed" along with the circuit breakers to the Load Banks, and manually "ARM" the key switch in the cabinet and close the entry doors to Cabinet 5.

7.4.6 Verify that the static switch is "Open".

7.4.7 Set Gen-sets $\mathrm{A} 1$ and $\mathrm{A} 2$ to the following set-points:

Gen-set A1 and A2 = Unit Power Control Mode

Output Power Command of $\mathrm{A} 1=50 \mathrm{~kW}$

Output Power Command of A2 $=20 \mathrm{~kW}$

MG Power/Frequency Droop $=-0.0833 \mathrm{~Hz} / \mathrm{kW}$

MG Voltage Command $=277 \mathrm{~V}$.

7.4.8 Select load on Load Bank 3 to $10 \mathrm{~kW}$ and Load Bank 6 to $40 \mathrm{~kW}$, and load on Load Bank 4 to $50 \mathrm{~kW}$.

7.4.9 Using EMS, give a "Reset" command to clear all faults and events.

7.4.10 Once all events and alarms are cleared, "Start" the Gen-sets. Note, there is $60 \mathrm{~kW}$ of load in the microgrid when the first Gen-set starts, causing it to go to full (i.e., $60 \mathrm{~kW}$ ) power as soon as it connects and simulating a Black Start into $60 \mathrm{~kW}$.

7.4.11 Once the Gen-sets are running and on-line, which will take a few minutes to occur, use the EMS to put the static switch in "Start" mode and verify a synchronized closing of the static switch. Note, if the static switch does not "Close", reduce output of Gen-set A1 27

This document contains information confidential and proprietary to AEPSC. It shall not be reproduced in whole or in part or released to any third party without expressed written consent of AEPSC. 


\begin{tabular}{|l|l|}
\hline DTC Registered Procedure \\
CERTS Microgrid Test Plan \\
\begin{tabular}{|l|l|l|}
\hline Dolan Technology Center & Document No: DTC212812.207.01A \\
\hline Written by: AEP & Effective Date: 23 Feb 2007 & Target Group: Assigned \\
\hline Approved by: K. P. Loving & Procedure Review Date: 23 Feb. 2008 & \\
\hline
\end{tabular}
\end{tabular}

to $30 \mathrm{~kW}$ (this will reduce frequency as A1 and A2 change their frequency to balance generation loss of $10 \mathrm{~kW}$ ).

7.4.12 After synchronized closing of the static switch, the Microgrid Test Bed is connected to the utility grid with $-10 \mathrm{~kW}$ at Meter 2 and $30 \mathrm{~kW}$ at Meter 1.

7.4.13 Using the DAS Load Control program, set the duration of the fault (overload) to 100 seconds, set the software to "ARM" and initiate a three-phase, ungrounded fault (overload) in Zone 4, which adds $85 \mathrm{~kW}$ to the $50 \mathrm{~kW}$ load.

7.4.14 Verify that CB41 "TRIPS" first on over-current, which clears the fault and shuts down Gen-set A2. Breaker K42 should Open under PLC control, removing the fault of the Overload Load Bank.

7.4.15 Verify that the static switch "Opens", due to reverse power and Gen-set A1 continues to operate, but reduces its output power to $10 \mathrm{~kW}$.

7.4.16 Verify in the DAS Database that:

7.4.16.1 The fault event waveforms of phase currents and voltages at all Relay locations (i.e., Relays $2,3,4$ \& 5) were recorded, as well as the relay element that caused the "TRIP" with trip times for each relay relative to when the fault was applied.

7.4.16.2 The waveform and RMS Meter data were recorded. Note the above collection of all data and waveforms will take approximately 30 minutes to arrive.

7.4.17 Shutdown Gen-set A1, remove remaining load, "STOP" the static switch, and re-close CB41 to complete this fault (overload) test event.

\subsection{Zone 3 A-Phase Line-to-Ground Fault, Gen-set A1 \& Utility Connected} Performance Goal - Test a single line-to-ground fault condition in Zone 3 with Gen-sets A1 operating in-parallel with the utility grid to verify zerosequence, negative-sequence or residual over-current protection.

\subsubsection{Perform the Microgrid Test Bed Lockout/Tagout Procedure,} described in Section 4.7. whole or in part or released to any third party without expressed written consent of AEPSC. 


\section{DTC Registered Procedure CERTS Microgrid Test Plan}

Dolan Technology Center

Document No: DTC212812.207.01A

\begin{tabular}{|l|l|l|}
\hline Written by: AEP & Effective Date: 23 Feb 2007 & Target Group: Assigned \\
\hline
\end{tabular}

Approved by: K. P. Loving Procedure Review Date: 23 Feb. 2008

7.5.2 Remove the Overload Load Bank portable cable/plug from the exterior receptacle and physically remove the portable overload cabling inside Cabinet 5a.

7.5.3 Setup the Overload Load Bank for a single-phase fault (overload) on A-phase of $28 \mathrm{~kW}$ and connect the portable cable/plug into the exterior receptacle of Cabinet 3a

7.5.4 Install/physically connect the portable overload cabling inside Cabinet 3 from the outlet receptacle to A-phase and Ground (i.e., connecting and tightening the bolted connections).

7.5.5 Reverse the Microgrid Test Bed Lockout/Tagout Procedure.

7.5.6 Verify CB33 is "Closed" along with the circuit breakers to the Load Banks, manually "ARM" key switch in the cabinet and close the entry door to Cabinet 3 .

7.5.7 Set Gen-set A1 to the following set-points:

Gen-set A1 = Unit Power Control Mode

Output Power Command $=20 \mathrm{~kW}$

MG Power/Frequency Droop $=-0.0833 \mathrm{~Hz} / \mathrm{kW}$

MG Voltage Command $=277 \mathrm{~V}$.

7.5.8 Select loads on Load Bank 3 and Load Bank 6 to 40kW.

7.5.9 Using EMS, give a "Reset" command to clear all faults and events

7.5.10 Once all events and alarms are cleared, "Start" Gen-set A1.

7.5.11 Once the Gen-set is running and on-line, use EMS to put the static switch in "Start" mode. Verify synchronized closing of the static switch.

7.5.12 After synchronized closing of the static switch, the Microgrid Test Bed is connected to the utility grid with $20 \mathrm{~kW}$ at Meter 2 and $60 \mathrm{~kW}$ at Meter 1.

7.5.13 Using the DAS Load Control program, set the duration of the fault (overload) to 10 seconds, set the software to "ARM" and initiate a Phase A-to ground fault in Zone 3.

7.5.14 Verify that the static switch "TRIPS" first followed by breaker CB31, which clears the fault and shuts down Gen-set A1. Breaker K32 should then Open under PLC control, removing the fault of the Overload Load Bank.

This document contains information confidential and proprietary to AEPSC. It shall not be reproduced in whole or in part or released to any third party without expressed written consent of AEPSC. 


\begin{tabular}{|l|l|}
\hline DTC Registered Procedure \\
CERTS Microgrid Test Plan \\
\begin{tabular}{|l|l|l|}
\hline Dolan Technology Center & Document No: DTC212812.207.01A \\
\hline Written by: AEP & Effective Date: 23 Feb 2007 & Target Group: Assigned \\
\hline Approved by: K. P. Loving & Procedure Review Date: 23 Feb. 2008 & \\
\hline
\end{tabular}
\end{tabular}

7.5.15 Verify power flow change of $0 \mathrm{~kW}$ at Meter 2 and $40 \mathrm{~kW}$ at Meter 1, serving the $40 \mathrm{~kW}$ in Load Bank 6.

7.5.16 Verify in the DAS Database that:

7.5.16.1 The fault event waveforms of phase currents and voltages at all Relay locations (i.e., Relays 2, 3, 4 \& 5) were recorded, as well as the relay element that caused the "TRIP" with trip times for each relay relative to when the fault was applied.

7.5.16.2 The waveform and RMS Meter data were recorded. Note the above collection of all data and waveforms will take approximately 30 minutes to arrive.

7.5.17 Remove remaining load from the Microgrid Test Bed, "STOP" the static switch, and re-close breaker CB31 to complete this fault test event.

\subsection{Zone 3 A-Phase Line-to-Ground Fault, Gen-sets (A1+A2) \& Utility Connected}

Performance Goal - Test a single line-to-ground fault condition in Zone 3 with Gen-sets A1 and A2 operating in-parallel with the utility grid to verify zero-sequence, negative-sequence or residual over-current protection.

7.6.1 Verify CB33 is "Closed" along with the circuit breakers to the Load Banks, manually "ARM" key switch in the cabinet and close the entry doors to Cabinet 3 .

7.6.2 Set Gen-sets A1 \& A2 to the following set-points:

Gen-set A1 \& A2 = Unit Power Control Mode

Output Power Command $=20 \mathrm{~kW}$ each

MG Power/Frequency Droop $=-0.0833 \mathrm{~Hz} / \mathrm{kW}$

MG Voltage Command $=277 \mathrm{~V}$.

7.6.3 Using EMS, give a "Reset" command to clear all faults and events.

7.6.4 Once all events and alarms are cleared, "Start" the Gen-sets.

7.6.5 Once the Gen-sets are running and on-line, select loads on Load Bank 3, Load Bank 4 and Load Bank 6 to $40 \mathrm{~kW}$.

7.6.6 Use EMS to put the static switch in "Start" mode. Verify synchronized closing of the static switch.

This document contains information confidential and proprietary to AEPSC. It shall not be reproduced in whole or in part or released to any third party without expressed written consent of AEPSC. 


\begin{tabular}{|l|l|}
\hline DTC Registered Procedure \\
CERTS Microgrid Test Plan \\
\begin{tabular}{|l|l|l|}
\hline Dolan Technology Center & Document No: DTC212812.207.01A \\
\hline Written by: AEP & Effective Date: 23 Feb 2007 & Target Group: Assigned \\
\hline Approved by: K. P. Loving & Procedure Review Date: 23 Feb. 2008 & \\
\hline
\end{tabular}
\end{tabular}

7.6.7 After the synchronized closing of the static switch, the Microgrid Test Bed is connected to the utility grid with $40 \mathrm{~kW}$ at Meter 2 and $80 \mathrm{~kW}$ at Meter 1.

7.6.8 Using the DAS Load Control program, set the duration of the fault (overload) to 10 seconds, set the software to "ARM" and initiate a A-phase to ground fault in Zone 3.

7.6.9 Verify that the static switch "Trips/Opens" first followed by breaker CB41 opening on zero-sequence current and Gen-set A2 will shut down. About 7 cycles after this protection sequence, CB31 will Trip/Open and Gen-set A1 will shut down. Breaker K32 should "Open" under PLC control, removing the fault on the Overload Load Bank.

7.6.10 Verify power flow change of $0 \mathrm{~kW}$ at Meter 2 and $40 \mathrm{~kW}$ at Meter 1 , serving the $40 \mathrm{~kW}$ in Load Bank 6.

7.6.11 Verify in the DAS Database that:

7.6.11.1 The fault event waveforms of phase currents and voltages at all Relay locations (i.e., Relays 2, 3, 4 \& 5) were recorded, as well as the relay element that caused the "TRIP" with trip times for each relay relative to when the fault was applied.

7.6.11.2 The waveform and RMS Meter data were recorded. Note the above collection of all data and waveforms will take approximately 30 minutes to arrive.

7.6.12 Remove remaining load from the Microgrid Test Bed, "STOP" the static switch, and re-close breakers CB41 and CB31 to complete this fault test.

\subsection{Zone 5 B-Phase Line-to-Ground Fault, Gen-set B1 \& Utility \\ Connected}

Performance Goal - Test a single line-to-ground fault condition in Zone 5 with Gen-sets B1 operating in-parallel with the utility grid to verify zerosequence, negative-sequence or residual over-current protection.

7.7.1 Perform the Microgrid Test Bed Lockout/Tagout Procedure, described in Section 4.7.

7.7.2 Remove the Overload Load Bank portable cable/plug from the exterior receptacle and physically remove the portable overload cabling inside Cabinet 3a.

This document contains information confidential and proprietary to AEPSC. It shall not be reproduced in whole or in part or released to any third party without expressed written consent of AEPSC. 


\begin{tabular}{|l|l|}
\hline DTC Registered Procedure \\
CERTS Microgrid Test Plan \\
\begin{tabular}{|l|l|l|}
\hline Dolan Technology Center & Document No: DTC212812.207.01A \\
\hline Written by: AEP & Effective Date: 23 Feb 2007 & Target Group: Assigned \\
\hline Approved by: K. P. Loving & Procedure Review Date: 23 Feb. 2008 & \\
\hline
\end{tabular}
\end{tabular}

7.7.3 Setup the Overload Load Bank for a single-phase fault (overload) on B-phase of $28 \mathrm{~kW}$ and connect the portable cable/plug into the exterior receptacle of Cabinet $7 \mathrm{a}$.

7.7.4 Install/physically connect the portable overload cabling inside Cabinet 7 from the outlet receptacle to B-phase and Ground (i.e., connecting and tightening the bolted connections).

7.7.5 Reverse the Microgrid Test Bed Lockout/Tagout Procedure.

7.7.6 Verify breaker CB53 is "Closed" along with the circuit breakers to the Load Banks, manually "ARM" the key switch in the cabinet, and close the entry doors to Cabinet 7.

7.7.7 Set Gen-sets B1 to the following set-points:

Gen-set B1 = Unit Power Control Mode

Output Power Command $=20 \mathrm{~kW}$

MG Power/Frequency Droop $=-0.0833 \mathrm{~Hz} / \mathrm{kW}$

MG Voltage Command= $277 \mathrm{~V}$.

7.7.8 Select loads on Load Bank 3 and Load Bank 6 to 40kW.

7.7.9 Using EMS, give a "Reset" command to clear all faults and events

7.7.10 Once all events and alarms are cleared, "Start" Gen-set B1.

7.7.11 Once the Gen-set is running and on-line, use EMS to put the static switch in "Start" mode. Verify synchronized closing of the static switch.

7.7.12 After synchronized closing of the static switch, the Microgrid Test Bed is connected to the utility grid with $20 \mathrm{~kW}$ at Meter 2 and $60 \mathrm{~kW}$ at Meter 1.

7.7.13 Using the DAS Load Control program, set the duration of the fault (overload) to 10 seconds, set the software to "ARM" and initiate a B-phase to ground fault in Zone 5.

7.7.14 Verify that CB51 "Opens" first on differential or zero-sequence current which clears the fault and shuts down Gen-set B1. Breaker K52 should then Open under PLC control, removing the fault on the Overload Load Bank. Note, the static switch is not expected to "Trip" during this test event.

7.7.15 Verify power flow change of $40 \mathrm{~kW}$ at Meter 2 and $80 \mathrm{~kW}$ at Meter 1 , serving the $40 \mathrm{~kW}$ in Load Bank 3 and Load Bank 6.

7.7.16 Verify in the DAS Database that:

32

This document contains information confidential and proprietary to AEPSC. It shall not be reproduced in whole or in part or released to any third party without expressed written consent of AEPSC. 


\section{DTC Registered Procedure CERTS Microgrid Test Plan}

Dolan Technology Center

\begin{tabular}{l|l|l} 
Written by: AEP & Effective Date: 23 Feb 2007 & Target Group: Assigned \\
\hline
\end{tabular}

Approved by: K. P. Loving $\quad$ Procedure Review Date: 23 Feb. 2008

7.7.16.1 The fault event waveforms of phase currents and voltages at all Relay locations (i.e., Relays 2, 3, 4 \& 5) were recorded, as well as the relay element that caused the "TRIP" with trip times for each relay relative to when the fault was applied.

7.7.16.2 The waveform and RMS Meter data were recorded. Note the above collection of all data and waveforms will take approximately 30 minutes to arrive.

7.7.17 Remove remaining load from the Microgrid Test Bed, "STOP" the static switch, and re-close breaker CB51 to complete this fault test event.

\subsection{Zone 5 B-Phase Line-to-Ground Fault, Gen-sets (A1+B1) \& Utility} Connected Performance Goal - Test a single line-to-ground fault condition in Zone 5 with Gen-sets A1 and B1 operating in-parallel with the utility grid to verify zero-sequence, negative-sequence or residual over-current protection. This test is similar to the prior test, but evaluates the resultant impact of two Gen-sets operating during a fault condition.

7.8.1 Verify CB31 and CB51 are "Closed" along with the circuit breakers to the Load Banks, manually "ARM" key switch in the cabinet and close the entry doors to Cabinet 7.

7.8.2 Set Gen-sets A1 \& B1 to the following set-points:

Gen-set A1 \& B1 = Unit Power Control Mode

Output Power Command $=20 \mathrm{~kW}$ each

MG Power/Frequency Droop $=-0.0833 \mathrm{~Hz} / \mathrm{kW}$

MG Voltage Command $=277 \mathrm{~V}$.

7.8.3 Using EMS, give a "Reset" command to clear all faults and events.

7.8.4 Once all events and alarms are cleared, "Start" the Gen-sets.

7.8.5 Once the Gen-sets are running and on-line, select loads on Load Bank 3, Load Bank 4 and Load Bank 6 to 40kW.

7.8.6 Use EMS to put the static switch in "Start" mode. Verify synchronized closing of the static switch.

7.8.7 After the synchronized closing of the static switch, the Microgrid Test Bed is connected to the utility grid with $40 \mathrm{~kW}$ at Meter 2 and $80 \mathrm{~kW}$ at Meter 1. 


\begin{tabular}{|l|l|}
\hline DTC Registered Procedure \\
CERTS Microgrid Test Plan \\
\begin{tabular}{|l|l|l|}
\hline Dolan Technology Center & Document No: DTC212812.207.01A \\
\hline Written by: AEP & Effective Date: 23 Feb 2007 & Target Group: Assigned \\
\hline Approved by: K. P. Loving & Procedure Review Date: 23 Feb. 2008 & \\
\hline
\end{tabular}
\end{tabular}

7.8.8 Using the DAS Load Control program, set the duration of the fault (overload) to 10 seconds, set the software to "ARM" and initiate a B-phase to ground fault in Zone 3.

7.8.9 Verify that CB51 "Opens" first on zero-sequence current which clears the fault and shuts down Gen-set B1. Breaker K52 should then Open under PLC control, removing the fault of the Overload Load Bank. Note, the static switch is not expected to "Trip" during this test event.

7.8.10 Verify the static switch remains "Closed", Gen-set A1 continues to operate, and the power flow change of $60 \mathrm{~kW}$ at Meter 2 and $100 \mathrm{~kW}$ at Meter 1.

7.8.11 Verify in the DAS Database that:

7.8.11.1 The fault event waveforms of phase currents and voltages at all Relay locations (i.e., Relays 2, 3, 4 \& 5) were recorded, as well as the relay element that caused the "TRIP" with trip times for each relay relative to when the fault was applied.

7.8.11.2 The waveform and RMS Meter data were recorded. Note the above collection of all data and waveforms will take approximately 30 minutes to arrive.

7.8.12 Remove remaining load from the Microgrid Test Bed, shut down Gen-set A1, "STOP" the static switch, and re-close breaker CB51 to complete this fault test.

\subsection{Zone 4 B-Phase Line-to-Ground Fault, Gen-sets (A1+A2) \& Utility Connected}

Performance Goal - Test a single line-to-ground fault condition in Zone 4 with Gen-sets A1 and A2 operating in-parallel with the utility grid to verify zero-sequence, negative-sequence or residual over-current protection.

7.9.1 Perform the Microgrid Test Bed Lockout/Tagout Procedure, described in Section 4.7.

7.9.2 Remove the Overload Load Bank portable cable/plug from the exterior receptacle and physically remove the portable overload cabling inside Cabinet 7a. whole or in part or released to any third party without expressed written consent of AEPSC. 


\begin{tabular}{|l|l|}
\hline DTC Registered Procedure \\
CERTS Microgrid Test Plan \\
\begin{tabular}{|l|l|l|}
\hline Dolan Technology Center & Document No: DTC212812.207.01A \\
\hline Written by: AEP & Effective Date: 23 Feb 2007 & Target Group: Assigned \\
\hline Approved by: K. P. Loving & Procedure Review Date: 23 Feb. 2008 & \\
\hline
\end{tabular}
\end{tabular}

7.9.3 Setup the Overload Load Bank for a single-phase fault (overload) on B-phase of $28 \mathrm{~kW}$ and connect the portable cable/plug into the exterior receptacle of Cabinet $5 \mathrm{a}$

7.9.4 Install/physically connect the portable overload cabling inside Cabinet 5 from the outlet receptacle to B-phase and Ground (i.e., connecting and tightening the bolted connections).

7.9.5 Reverse the Microgrid Test Bed Lockout/Tagout Procedure.

7.9.6 Verify CB43 is "Closed" along with the circuit breakers to the Load Banks, manually "ARM" key switch in the cabinet and close the entry doors to Cabinet 5 .

7.9.7 Set Gen-sets A1 \& A2 to the following set-points:

Gen-set A1 \& A2 = Unit Power Control Mode

Output Power Command $=20 \mathrm{~kW}$ each

MG Power/Frequency Droop $=-0.0833 \mathrm{~Hz} / \mathrm{kW}$

MG Voltage Command= $277 \mathrm{~V}$.

7.9.8 Using EMS, give a "Reset" command to clear all faults and events

7.9.9 Once all events and alarms are cleared, "Start" the Gen-sets.

7.9.10 Once the Gen-sets are running and on-line, select loads on Load Bank 3, Load Bank 4 and Load Bank 6 to 40kW.

7.9.11 Use EMS to put the static switch in "Start" mode. Verify synchronized closing of the static switch.

7.9.12 After the synchronized closing of the static switch, the Microgrid Test Bed is connected to the utility grid with $40 \mathrm{~kW}$ at Meter 2 and $80 \mathrm{~kW}$ at Meter 1.

7.9.13 Using the DAS Load Control program, set the duration of the fault (overload) to 10 seconds, set the software to "ARM" and initiate a B-phase to ground fault in Zone 4.

7.9.14 Verify that the static switch "Trips/Opens" first followed by breaker CB41 opening zero-sequence current, which clears the fault and shuts down Gen-set A2. Breaker K42 should "Open" under PLC control, removing the fault of the Overload Load Bank. Gen-set A1 should increase to $40 \mathrm{~kW}$ to serve the load in Zone 3.

7.9.15 Verify power flow change of $0 \mathrm{~kW}$ at Meter 2 and $40 \mathrm{~kW}$ at Meter 1, serving the 40kW in Load Bank 6.

7.9.16 Verify in the DAS Database that:

This document contains information confidential and proprietary to AEPSC. It shall not be reproduced in whole or in part or released to any third party without expressed written consent of AEPSC. 


\section{DTC Registered Procedure CERTS Microgrid Test Plan}

Dolan Technology Center

\begin{tabular}{l|l|l} 
Written by: AEP & Effective Date: 23 Feb 2007 & Target Group: Assigned \\
\hline
\end{tabular}

Approved by: K. P. Loving $\quad$ Procedure Review Date: 23 Feb. 2008

7.9.16.1 The fault event waveforms of phase currents and voltages at all Relay locations (i.e., Relays 2, 3, 4 \& 5) were recorded, as well as the relay element that caused the "TRIP" with trip times for each relay relative to when the fault was applied.

7.9.16.2 The waveform and RMS Meter data were recorded. Note the above collection of all data and waveforms will take approximately 30 minutes to arrive.

7.9.17 Remove remaining load from the Microgrid Test Bed, shut down Gen-set A1, "STOP" the static switch, and re-close breaker CB41 to complete this fault test.

\subsection{Zone 2 C-Phase Line-to-Ground Fault, Gen-sets (A1+B1) \& Utility Connected} Performance Goal - Test a single line-to-ground fault condition in Zone 2 with Gen-sets A1 and B1 operating in-parallel with the utility grid to verify zero-sequence, negative-sequence or residual over-current protection.

7.10.1 Perform the Microgrid Test Bed Lockout/Tagout Procedure, described in Section 4.7.

7.10.2 Remove the Overload Load Bank portable cable/plug from the exterior receptacle and physically remove the portable overload cabling inside Cabinet 5a.

7.10.3 Setup the Overload Load Bank for a single-phase fault (overload) on C-phase of $28 \mathrm{~kW}$ and connect the portable cable/plug into the exterior receptacle of Cabinet $2 \mathrm{~d}$

7.10.4 Install/physically connect the portable overload cabling inside Cabinet 2 from the outlet receptacle to $\mathrm{C}$-phase and Ground (i.e., connecting and tightening the bolted connections).

7.10.5 Reverse the Microgrid Test Bed Lockout/Tagout Procedure.

7.10.6 Verify CB31 and CB51 are "Closed" along with the circuit breakers to the Load Banks, manually "ARM" key switch in the cabinet and close the entry doors to Cabinet $2 \mathrm{~d}$.

7.10.7 Set Gen-sets A1 \& B1 to the following set-points: Gen-set A1 \& B1 = Unit Power Control Mode Output Power Command $=20 \mathrm{~kW}$ each 


\section{DTC Registered Procedure CERTS Microgrid Test Plan}

\begin{tabular}{l|l}
\hline Dolan Technology Center & Document No: DTC212812.207.01A
\end{tabular}

\begin{tabular}{|l|l|l|}
\hline Written by: AEP & Effective Date: 23 Feb 2007 & Target Group: Assigned \\
\hline
\end{tabular}

\begin{tabular}{l|l} 
Approved by: K. P. Loving & Procedure Review Date: 23 Feb. 2008 \\
\hline
\end{tabular}

MG Power/Frequency Droop $=-0.0833 \mathrm{~Hz} / \mathrm{kW}$

MG Voltage Command $=277 \mathrm{~V}$.

7.10.8 Using EMS, give a "Reset" command to clear all faults and events

7.10.9 Once all events and alarms are cleared, "Start" the Gen-sets.

7.10.10 Once the Gen-sets are running and on-line, select loads on Load Bank 3, Load Bank 4 and Load Bank 6 to $40 \mathrm{~kW}$

7.10.11 Use EMS to put the static switch in "Start" mode. Verify synchronized closing of the static switch.

7.10.12 After the synchronized closing of the static switch, the Microgrid Test Bed is connected to the utility grid with $40 \mathrm{~kW}$ at Meter 2 and $80 \mathrm{~kW}$ at Meter 1.

7.10.13 Using the DAS Load Control program, set the duration of the fault (overload) to 10 seconds, set the software to "ARM" and initiate a C-phase to ground fault in Zone 2.

7.10.14 Verify that the static switch "Trips/Opens" first followed by breaker CB51 opening on line-to-neutral under-voltage and shuts down Gen-set B1. About 7 cycles after this protection sequence, CB31 will Trip/Open on differential current and Gen-set A1 will shut down. Breaker K22 should "Open" under PLC control, removing the fault of the Overload Load Bank.

7.10.15 Verify power flow change of $0 \mathrm{~kW}$ at Meter 2 and $40 \mathrm{~kW}$ at Meter 1 , serving the $40 \mathrm{~kW}$ in Load Bank 6.

7.10.16 Verify in the DAS Database that:

7.10.16.1 The fault event waveforms of phase currents and voltages at all Relay locations (i.e., Relays 2, 3, 4 \& 5) were recorded, as well as the relay element that caused the "TRIP" with trip times for each relay relative to when the fault was applied.

7.10.16.2 The waveform and RMS Meter data were recorded. Note the above collection of all data and waveforms will take approximately 30 minutes to arrive.

7.10.17 Remove remaining load from the Microgrid Test Bed, "STOP" the static switch, and re-close breakers CB51 and CB31 to complete this fault test.

This document contains information confidential and proprietary to AEPSC. It shall not be reproduced in whole or in part or released to any third party without expressed written consent of AEPSC. 


\begin{tabular}{|l|l|}
\hline DTC Registered Procedure \\
CERTS Microgrid Test Plan \\
\begin{tabular}{|l|l|l|}
\hline Dolan Technology Center & Document No: DTC212812.207.01A \\
\hline Written by: AEP & Effective Date: 23 Feb 2007 & Target Group: Assigned \\
\hline Approved by: K. P. Loving & Procedure Review Date: 23 Feb. 2008 & \\
\hline
\end{tabular}
\end{tabular}

\subsection{Zone 6 C-Phase Line-to-Ground Fault, Gen-set A1 \& Utility Connected}

Performance Goal - Test a single line-to-ground fault condition in Zone 6 with Gen-set A1 operating in-parallel with the utility grid to verify $\mathrm{I}^{2} \mathrm{t}$ protection.

7.11.1 Perform the Microgrid Test Bed Lockout/Tagout Procedure, described in Section 4.7.

7.11.2 Remove the Overload Load Bank portable cable/plug from the exterior receptacle and physically remove the portable overload cabling inside Cabinet 2d.

7.11.3 Setup the Overload Load Bank for a single-phase fault (overload) on C-phase of $28 \mathrm{~kW}$ and connect the portable cable/plug into the exterior receptacle of Cabinet $8 \mathrm{a}$

7.11.4 Install/physically connect the portable overload cabling inside Cabinet 8 from the outlet receptacle to C-phase and Ground (i.e., connecting and tightening the bolted connections).

7.11.5 Reverse the Microgrid Test Bed Lockout/Tagout Procedure.

7.11.6 Verify CB63 is "Closed" along with the circuit breakers to the Load Banks, manually "ARM" the key switch in the cabinet and close the entry doors to Cabinet 8 .

7.11.7 Set Gen-set A1 to the following set-points:

Gen-set A1 = Unit Power Control Mode

Output Power Command $=20 \mathrm{~kW}$

MG Power/Frequency Droop $=-0.0833 \mathrm{~Hz} / \mathrm{kW}$

MG Voltage Command= $277 \mathrm{~V}$.

7.11.8 Select loads on Load Bank 3 and Load Bank 6 to 40kW.

7.11.9 Using EMS, give a "Reset" command to clear all faults and events.

7.11.10 Once all events and alarms are cleared, "Start" the Gen-sets.

7.11.11 Once the Gen-sets are running and on-line, use EMS to put the static switch in "Start" mode. Verify synchronized closing of the static switch.

7.11.12 After the synchronized closing of the static switch, the Microgrid Test Bed is connected to the utility grid with $20 \mathrm{~kW}$ at Meter 2 and $60 \mathrm{~kW}$ at Meter 1 .

This document contains information confidential and proprietary to AEPSC. It shall not be reproduced in whole or in part or released to any third party without expressed written consent of AEPSC. 


\begin{tabular}{|l|l|}
\hline DTC Registered Procedure \\
CERTS Microgrid Test Plan \\
\begin{tabular}{|l|l|l|}
\hline Dolan Technology Center & Document No: DTC212812.207.01A \\
\hline Written by: AEP & Effective Date: 23 Feb 2007 & Target Group: Assigned \\
\hline Approved by: K. P. Loving & Procedure Review Date: 23 Feb. 2008 & \\
\hline
\end{tabular}
\end{tabular}

7.11.13 Using the DAS Load Control program, set the duration of the fault (overload) to 100 seconds, set the software to "ARM" and initiate a Phase C-to-ground fault in Zone 6.

7.11.14 Verify that the static switch "TRIPS" first, followed by breaker CB13 opening, which clears the fault. Breaker K62 should "Open" under PLC control, removing the fault of the Overload Load Bank.

7.11.15 Verify that Gen-set A1 power output increases from $20 \mathrm{~kW}$ to $40 \mathrm{~kW}$ to serve the $40 \mathrm{~kW}$ load in Load Bank 3.

7.11.16 Verify in the DAS Database that:

7.11.16.1 The fault event waveforms of phase currents and voltages at all Relay locations (i.e., Relays 2, 3, 4 \& 5) were recorded, as well as the relay element that caused the "TRIP" with trip times for each relay relative to when the fault was applied.

7.11.16.2 The waveform and RMS Meter data were recorded. Note the above collection of all data and waveforms will take approximately 30 minutes to arrive.

7.11.17 Shutdown Gen-set A1, "STOP" the static switch, and remove load from the Microgrid Test Bed to complete this fault test event.

\subsection{Zone 6 C-Phase Line-to-Ground Fault, Gen-set (A1+B1) \& Utility Connected} Performance Goal - Test a single line-to-ground fault condition in Zone 6 with Gen-sets A1 and B1 operating in-parallel with the utility grid to verify $\mathrm{I}^{2} \mathrm{t}$ protection. This test is similar to the prior test, but evaluates the resultant impact of two Gen-sets operating during a fault condition.

7.12.1 Verify CB63 is "Closed" along with the circuit breakers to the Load Banks. Manually "ARM" the key switch in the cabinet and close the entry doors to Cabinet 8 .

7.12.2 Set Gen-set A1 \& B1 to the following set-points: Gen-set A1 \& B1 = Unit Power Control Mode Output Power Command $=20 \mathrm{~kW}$ each MG Power/Frequency Droop $=-0.0833 \mathrm{~Hz} / \mathrm{kW}$ MG Voltage Command $=277 \mathrm{~V}$. whole or in part or released to any third party without expressed written consent of AEPSC. 


\begin{tabular}{|l|l|}
\hline DTC Registered Procedure \\
CERTS Microgrid Test Plan \\
\begin{tabular}{|l|l|l|}
\hline Dolan Technology Center & Document No: DTC212812.207.01A \\
\hline Written by: AEP & Effective Date: 23 Feb 2007 & Target Group: Assigned \\
\hline Approved by: K. P. Loving & Procedure Review Date: 23 Feb. 2008 & \\
\hline
\end{tabular}
\end{tabular}

7.12.3 Select loads on Load Bank 3, Load Bank 5 and Load Bank 6 to $40 \mathrm{~kW}$.

7.12.4 Using EMS, give a "Reset" command to clear all faults and events.

7.12.5 Once all events and alarms are cleared, "Start" the Gen-sets.

7.12.6 Once the Gen-sets are running and on-line, use EMS to put the static switch in "Start" mode. Verify synchronized closing of the static switch.

7.12.7 After the synchronized closing of the static switch, the Microgrid Test Bed is connected to the utility grid with $40 \mathrm{~kW}$ at Meter 2 and $80 \mathrm{~kW}$ at Meter 1.

7.12.8 Using the DAS Load Control program, set the duration of the fault (overload) to 100 seconds, set the software to "ARM" and initiate a Phase C-to-ground fault in Zone 6.

7.12.9 Verify that the static switch "TRIPS" first, followed by breaker CB13 opening, which clears the fault. Breaker K62 should "Open" under PLC control, removing the fault of the Overload Load Bank.

7.12.10 Verify that Gen-set A1 and B1 power output increases from $20 \mathrm{~kW}$ to $40 \mathrm{~kW}$ to serve the $40 \mathrm{~kW}$ load in Load Bank 3 and Load Bank 5.

7.12.11 Verify in the DAS Database that:

7.12.11.1 The fault event waveforms of phase currents and voltages at all Relay locations (i.e., Relays 2, 3, 4 \& 5) were recorded, as well as the relay element that caused the "TRIP" with trip times for each relay relative to when the fault was applied.

7.12.11.2 The waveform and RMS Meter data were recorded. Note the above collection of all data and waveforms will take approximately 30 minutes to arrive.

7.12.12 Shutdown Gen-set A1 \& A2, "STOP" the static switch, and remove load from the Microgrid Test Bed to complete this fault test event.

7.12.13 Verify CB63 is "Closed" along with the circuit breakers to the Load Bank; and close the entry doors to Cabinet 8.

\subsection{Zone 3 A-to-B Phase Fault, Gen-set (A1+A2) \& Utility Connected}

Performance Goal - Test a phase-to-phase fault condition in Zone 3 with

40

This document contains information confidential and proprietary to AEPSC. It shall not be reproduced in whole or in part or released to any third party without expressed written consent of AEPSC. 


\begin{tabular}{|l|l|}
\hline DTC Registered Procedure \\
CERTS Microgrid Test Plan \\
\begin{tabular}{|l|l|l|}
\hline Dolan Technology Center & Document No: DTC212812.207.01A \\
\hline Written by: AEP & Effective Date: 23 Feb 2007 & Target Group: Assigned \\
\hline Approved by: K. P. Loving & Procedure Review Date: 23 Feb. 2008 & \\
\hline
\end{tabular}
\end{tabular}

Gen-sets A1 and A2 operating in-parallel with the utility grid to verify negative-sequence, $\mathrm{I}^{2} \mathrm{t}$ protection or residual over-current protection.

7.13.1 Perform the Microgrid Test Bed Lockout/Tagout Procedure, described in Section 4.7.

7.13.2 Remove the Overload Load Bank portable cable/plug from the exterior receptacle and physically remove the portable overload cabling inside Cabinet 8a.

7.13.3 Setup the Overload Load Bank for a phase-to-phase fault (overload) between A and B phases of $84 \mathrm{~kW}$ and connect the portable cable/plug into the exterior receptacle of Cabinet $3 \mathrm{a}$

7.13.4 Install/physically connect the portable overload cabling inside Cabinet 3 from the outlet receptacle to A and B phases (i.e., connecting and tightening the bolted connections).

7.13.5 Reverse the Microgrid Test Bed Lockout/Tagout Procedure.

7.13.6 Verify CB33 is "Closed" along with the circuit breakers to the Load Banks, manually "ARM" the key switch in the cabinet and close the entry doors to Cabinet 3 .

7.13.7 Set Gen-set A1 \& A2 to the following set-points: Gen-set A1 \& A2 = Unit Power Control Mode Output Power Command $=20 \mathrm{~kW}$ each MG Power/Frequency Droop $=-0.0833 \mathrm{~Hz} / \mathrm{kW}$ MG Voltage Command $=277 \mathrm{~V}$.

7.13.8 Using EMS, give a "Reset" command to clear all faults and events.

7.13.9 Once all events and alarms are cleared, "Start" the Gen-sets.

7.13.10 Once the Gen-sets are running and on-line, select loads on Load Bank 3, Load Bank 4 and Load Bank 6 to 40kW.

7.13.11 Use EMS to put the static switch in "Start" mode. Verify synchronized closing of the static switch.

7.13.12 After the synchronized closing of the static switch, the Microgrid Test Bed is connected to the utility grid with $40 \mathrm{~kW}$ at Meter 2 and $80 \mathrm{~kW}$ at Meter 1 .

7.13.13 Using the DAS Load Control program, set the duration of the fault (overload) to 10 seconds, set the software to "ARM" and initiate a Phase A-to-Phase B fault in Zone 3.

This document contains information confidential and proprietary to AEPSC. It shall not be reproduced in whole or in part or released to any third party without expressed written consent of AEPSC. 


\section{DTC Registered Procedure CERTS Microgrid Test Plan}

Dolan Technology Center

Document No: DTC212812.207.01A

\begin{tabular}{|l|l|l} 
Written by: AEP & Effective Date: 23 Feb 2007 & Target Group: Assigned
\end{tabular}

Approved by: K. P. Loving $\quad$ Procedure Review Date: 23 Feb. 2008

7.13.14 Verify that the static switch "Trips/Opens" first, followed by breakers CB31, CB41 and CB51 clearing the fault. Gen-set A1 \& A2 will shut down and breaker K32 should "Open" under PLC control, removing the fault of the Overload Load Bank.

7.13.15 Verify power flow change of $0 \mathrm{~kW}$ at Meter 2 and $40 \mathrm{~kW}$ at Meter 1 to Load Bank 6.

7.13.16 Verify in the DAS Database that:

7.13.16.1 The fault event waveforms of phase currents and voltages at all Relay locations (i.e., Relays 2, 3, 4 \& 5) were recorded, as well as the relay element that caused the "TRIP" with trip times for each relay relative to when the fault was applied.

7.13.16.2 The waveform and RMS Meter data were recorded. Note the above collection of all data and waveforms will take approximately 30 minutes to arrive.

7.13.17 Remove remaining load from the Microgrid Test Bed, "STOP" the static switch, and re-close breakers CB31, CB41 and CB51 to complete this fault test event.

\subsection{Zone 4 A-to-B Phase Fault, Gen-set (A1+A2) \& Utility Connected}

Performance Goal - Test a phase-to-phase fault condition in Zone 4 with Gen-sets A1 and A2 operating in-parallel with the utility grid to verify negative-sequence, $\mathrm{I}^{2} \mathrm{t}$ protection or residual over-current protection.

7.14.1 Perform the Microgrid Test Bed Lockout/Tagout Procedure, described in Section 4.7.

7.14.2 Remove the Overload Load Bank portable cable/plug from the exterior receptacle and physically remove the portable overload cabling inside Cabinet 3a.

7.14.3 Setup the Overload Load Bank for a phase-to-phase fault (overload) between A and B phases of $84 \mathrm{~kW}$ and connect the portable cable/plug into the exterior receptacle of Cabinet 5a

7.14.4 Install/physically connect the portable overload cabling inside Cabinet 5 from the outlet receptacle to A and B phases (i.e., connecting and tightening the bolted connections).

7.14.5 Reverse the Microgrid Test Bed Lockout/Tagout Procedure. 


\section{DTC Registered Procedure CERTS Microgrid Test Plan}

Dolan Technology Center

Document No: DTC212812.207.01A

\begin{tabular}{|l|l|l|}
\hline Written by: AEP & Effective Date: 23 Feb 2007 & Target Group: Assigned \\
\hline
\end{tabular}

\begin{tabular}{l|l}
\hline Approved by: K. P. Loving & Procedure Review Date: 23 Feb. 2008 \\
\hline
\end{tabular}

7.14.6 Verify CB43 is "Closed" along with the circuit breakers to the Load Banks, manually "ARM" the key switch in the cabinet and close the entry doors to Cabinet 5 .

7.14.7 Set Gen-set A1 \& A2 to the following set-points:

Gen-set A1 \& A2 = Unit Power Control Mode

Output Power Command $=20 \mathrm{~kW}$ each

MG Power/Frequency Droop $=-0.0833 \mathrm{~Hz} / \mathrm{kW}$

MG Voltage Command $=277 \mathrm{~V}$.

7.14.8 Using EMS, give a "Reset" command to clear all faults and events.

7.14.9 Once all events and alarms are cleared, "Start" the Gen-sets.

7.14.10 Once the Gen-sets are running and on-line, select loads on Load Bank 3, Load Bank 4 and Load Bank 6 to 40kW.

7.14.11 Use EMS to put the static switch in "Start" mode. Verify synchronized closing of the static switch.

7.14.12 After the synchronized closing of the static switch, the Microgrid Test Bed is connected to the utility grid with $40 \mathrm{~kW}$ at Meter 2 and $80 \mathrm{~kW}$ at Meter 1.

7.14.13 Using the DAS Load Control program, set the duration of the fault (overload) to 10 seconds, set the software to "ARM" and initiate a Phase A-to-Phase B fault in Zone 4.

7.14.14 Verify that the static switch "Trips/Opens" first, followed by breakers CB31, CB41 and CB51 clearing the fault. Gen-set A1 \& A2 will shut down and breaker K42 should "Open" under PLC control, removing the fault of the Overload Load Bank.

7.14.15 Verify power flow change of $0 \mathrm{~kW}$ at Meter 2 and $40 \mathrm{~kW}$ at Meter 1 to Load Bank 6.

7.14.16 Verify in the DAS Database that:

7.14.16.1 The fault event waveforms of phase currents and voltages at all Relay locations (i.e., Relays 2, 3, 4 \& 5) were recorded, as well as the relay element that caused the "TRIP" with trip times for each relay relative to when the fault was applied.

7.14.16.2 The waveform and RMS Meter data were recorded. Note the above collection of all data and waveforms will take approximately 30 minutes to arrive.

This document contains information confidential and proprietary to AEPSC. It shall not be reproduced in whole or in part or released to any third party without expressed written consent of AEPSC. 


\begin{tabular}{|l|l|}
\hline DTC Registered Procedure \\
CERTS Microgrid Test Plan \\
\begin{tabular}{|l|l|l|}
\hline Dolan Technology Center & Document No: DTC212812.207.01A \\
\hline Written by: AEP & Effective Date: 23 Feb 2007 & Target Group: Assigned \\
\hline Approved by: K. P. Loving & Procedure Review Date: 23 Feb. 2008 & \\
\hline
\end{tabular}
\end{tabular}

7.14.17 Remove remaining load from the Microgrid Test Bed, "STOP" the static switch, and re-close breakers CB31, CB41 and CB51 to complete this fault test event.

\subsection{Zone 2 A-to-B Phase Fault, Gen-set (A1+B1) \& Utility Connected}

Performance Goal - Test a phase-to-phase fault condition in Zone 2 with Gen-sets A1 and B1 operating in-parallel with the utility grid to verify negative-sequence, $I^{2} t$ protection or residual over-current protection

7.15.1 Perform the Microgrid Test Bed Lockout/Tagout Procedure, described in Section 4.7.

7.15.2 Remove the Overload Load Bank portable cable/plug from the exterior receptacle and physically remove the portable overload cabling inside Cabinet 5a.

7.15.3 Setup the Overload Load Bank for a phase-to-phase fault (overload) between A and B phases of $84 \mathrm{~kW}$ and connect the portable cable/plug into the exterior receptacle of Cabinet $2 \mathrm{~d}$.

7.15.4 Install/physically connect the portable overload cabling inside Cabinet $2 \mathrm{~d}$ from the outlet receptacle to A and B phases (i.e., connecting and tightening the bolted connections).

7.15.5 Reverse the Microgrid Test Bed Lockout/Tagout Procedure.

7.15.6 Verify CB31, CB41, CB51, and K22 are "Closed" along with the circuit breakers to the Load Banks, manually "ARM" the key switch in the cabinet and close the entry doors to Cabinet $2 \mathrm{~d}$.

7.15.7 Set Gen-set A1 \& B1 to the following set-points: Gen-set A1 \& A2 = Unit Power Control Mode Output Power Command $=20 \mathrm{~kW}$ each MG Power/Frequency Droop $=-0.0833 \mathrm{~Hz} / \mathrm{kW}$ MG Voltage Command= $277 \mathrm{~V}$.

7.15.8 Using EMS, give a "Reset" command to clear all faults and events.

7.15.9 Once all events and alarms are cleared, "Start" the Gen-sets.

7.15.10 Once the Gen-sets are running and on-line, select loads on Load Bank 3, Load Bank 5 and Load Bank 6 to 40kW.

7.15.11 Use EMS to put the static switch in "Start" mode. Verify synchronized closing of the static switch. whole or in part or released to any third party without expressed written consent of AEPSC. 


\begin{tabular}{|l|l|}
\hline DTC Registered Procedure \\
CERTS Microgrid Test Plan \\
\begin{tabular}{|l|l|l|}
\hline Dolan Technology Center & Document No: DTC212812.207.01A \\
\hline Written by: AEP & Effective Date: 23 Feb 2007 & Target Group: Assigned \\
\hline Approved by: K. P. Loving & Procedure Review Date: 23 Feb. 2008 & \\
\hline
\end{tabular}
\end{tabular}

7.15.12 After the synchronized closing of the static switch, the Microgrid Test Bed is connected to the utility grid with $40 \mathrm{~kW}$ at Meter 2 and $80 \mathrm{~kW}$ at Meter 1.

7.15.13 Using the DAS Load Control program, set the duration of the fault (overload) to 10 seconds, set the software to "ARM" and initiate a Phase A-to-Phase B fault in Zone 2.

7.15.14 Verify that the static switch "Trips/Opens" first, followed by breakers CB31, CB41 and CB51 clearing the fault. Gen-set A1 \& B1 will shut down and breaker K22 should "Open" under PLC control, removing the fault of the Overload Load Bank.

7.15.15 Verify power flow change of $0 \mathrm{~kW}$ at Meter 2 and $40 \mathrm{~kW}$ at Meter 1 to Load Bank 6.

7.15.16 Verify in the DAS Database that:

7.15.16.1 The fault event waveforms of phase currents and voltages at all Relay locations (i.e., Relays 2, 3, 4 \& 5) were recorded, as well as the relay element that caused the "TRIP" with trip times for each relay relative to when the fault was applied.

7.15.16.2 The waveform and RMS Meter data were recorded. Note the above collection of all data and waveforms will take approximately 30 minutes to arrive.

7.15.17 Remove remaining load from the Microgrid Test Bed, "STOP" the static switch, and re-close breakers CB31, CB41 and CB51 to complete this fault test event.

\subsection{Zone 5 A-to-B Phase Fault, Gen-set (A1+B1) \& Utility Connected} Performance Goal - Test a phase-to-phase fault condition in Zone 5 with Gen-sets A1 and B1 operating in-parallel with the utility grid to verify negative-sequence, $I^{2} t$ protection or residual over-current protection.

7.16.1 Perform the Microgrid Test Bed Lockout/Tagout Procedure, described in Section 4.7.

7.16.2 Remove the Overload Load Bank portable cable/plug from the exterior receptacle and physically remove the portable overload cabling inside Cabinet 2d.

7.16.3 Setup the Overload Load Bank for a phase-to-phase fault (overload) between A and B phases of $84 \mathrm{~kW}$ and connect the portable cable/plug into the exterior receptacle of Cabinet $7 \mathrm{a}$. 


\section{DTC Registered Procedure CERTS Microgrid Test Plan}

Dolan Technology Center

Document No: DTC212812.207.01A

\begin{tabular}{|l|l|l|}
\hline Written by: AEP & Effective Date: 23 Feb 2007 & Target Group: Assigned
\end{tabular}

\begin{tabular}{l|l}
\hline Approved by: K. P. Loving & Procedure Review Date: 23 Feb. 2008 \\
\hline
\end{tabular}

7.16.4 Install/physically connect the portable overload cabling inside Cabinet 7 from the outlet receptacle to A and B phases (i.e., connecting and tightening the bolted connections).

7.16.5 Reverse the Microgrid Test Bed Lockout/Tagout Procedure.

7.16.6 Verify CB53 is "Closed" along with the circuit breakers to the Load Banks, manually "ARM" the key switch in the cabinet and close the entry doors to Cabinet 7 .

7.16.7 Set Gen-set A1 \& B1 to the following set-points:

Gen-set A1 \& A2 = Unit Power Control Mode

Output Power Command $=20 \mathrm{~kW}$ each

MG Power/Frequency Droop $=-0.0833 \mathrm{~Hz} / \mathrm{kW}$

MG Voltage Command $=277 \mathrm{~V}$.

7.16.8 Using EMS, give a "Reset" command to clear all faults and events.

7.16.9 Once all events and alarms are cleared, "Start" the Gen-sets.

7.16.10 Once the Gen-sets are running and on-line, select loads on Load Bank 3, Load Bank 5 and Load Bank 6 to 40kW.

7.16.11 Use EMS to put the static switch in "Start" mode. Verify synchronized closing of the static switch.

7.16.12 After the synchronized closing of the static switch, the Microgrid Test Bed is connected to the utility grid with $40 \mathrm{~kW}$ at Meter 2 and $80 \mathrm{~kW}$ at Meter 1.

7.16.13 Using the DAS Load Control program, set the duration of the fault (overload) to 10 seconds, set the software to "ARM" and initiate a Phase A-to-Phase B fault in Zone 5.

7.16.14 Verify that the static switch "Trips/Opens" first, followed by breakers CB31, CB41 and CB51 clearing the fault. Gen-set A1 \& B1 will shut down and breaker K52 should "Open" under PLC control, removing the fault of the Overload Load Bank. (Note, due to the delta-wye transformer (T51) the zero-sequence and negative-sequence detection is less sensitive to faults in Zone 5.)

7.16.15 Verify power flow change of $0 \mathrm{~kW}$ at Meter 2 and $40 \mathrm{~kW}$ at Meter 1 to Load Bank 6.

7.16.16 Verify in the DAS Database that:

This document contains information confidential and proprietary to AEPSC. It shall not be reproduced in whole or in part or released to any third party without expressed written consent of AEPSC. 


\section{DTC Registered Procedure \\ CERTS Microgrid Test Plan}

Dolan Technology Center

\begin{tabular}{|l|l|l|}
\hline Written by: AEP & Effective Date: 23 Feb 2007 & Target Group: Assigned
\end{tabular}

\begin{tabular}{l|l}
\hline Approved by: K. P. Loving & Procedure Review Date: 23 Feb. 2008 \\
\hline
\end{tabular}

7.16.16.1 The fault event waveforms of phase currents and voltages at all Relay locations (i.e., Relays 2, 3, 4 \& 5) were recorded, as well as the relay element that caused the "TRIP" with trip times for each relay relative to when the fault was applied.

7.16.16.2 The waveform and RMS Meter data were recorded. Note the above collection of all data and waveforms will take approximately 30 minutes to arrive.

7.16.17 Remove remaining load from the Microgrid Test Bed, "STOP" the static switch, and re-close breakers CB31, CB41 and CB51 to complete this fault test event.

\subsection{Procedure - Reduced System Tests}

Objective: The tests described in Section 8 are designed to ensure that the Genset inverter controls are working correctly. This includes unit control, feeder control, and mixed power controls, in conjunction with limit controls and synchronized closing of the static switch. These tests are based on the Tecogen / Youtility factory acceptance testing.

Measurement

- Sources - Injected power $(\mathrm{kW})$, reactive load (kVAr), frequency (freq), and voltage (V) for each Gen-set (i.e., A1p, A2p and B1p).

- Zone input power flow into Zones 3 (A1z), 4 (A2z), 5 (B1z) and 6.

- Loads - Voltage at Load Bank 3 (L3), 4 (L4), 5 (L5), and 6 (L6).

- Static Switch (SS) - Power (kW) and current (I) through the static switch.

- Static Switch Control Signal: Forced "OPEN" and release to allow self synchronization.

- Voltage (V) and frequency (Freq) difference across the static switch.

Performance Goal - Expect to observe smooth transitions of the Gen-sets response to different step conditions (i.e., static switch "OPEN"/ "CLOSE" and load steps).

8.1 Initial Voltage Regulation Test - Single Zone, Islanded With Gen-set A1

Performance Goal - Verify smooth transitions of Gen-set A1 response to different step conditions of load in Load Bank 3 with voltage set point

This document contains information confidential and proprietary to AEPSC. It shall not be reproduced in whole or in part or released to any third party without expressed written consent of AEPSC. 


\begin{tabular}{|l|l|}
\hline DTC Registered Procedure \\
CERTS Microgrid Test Plan \\
\begin{tabular}{|l|l|l|}
\hline Dolan Technology Center & Document No: DTC212812.207.01A \\
\hline Written by: AEP & Effective Date: 23 Feb 2007 & Target Group: Assigned \\
\hline Approved by: K. P. Loving & Procedure Review Date: 23 Feb. 2008 & \\
\hline
\end{tabular}
\end{tabular}

changes ranging from $+5 \%$ to $-5 \%$. Repeat tests with Gen-set A2 in Load Bank 4, and then Gen-set B1 in Load Bank 5.

8.1.1 Verify the utility's CB1 breaker is "Closed" and the Microgrid Test Bed is energized with nominal voltages indicated on all three phases at Meter 1.

8.1.2 During the next sequence of tests, verify in the "DAS Database" file that when the test was performed, the event waveforms at all relay locations (i.e., Relays 2, 3, 4 \& 5) were recorded, along with the test event times when the event was initiated.

8.1.3 Maintain the weak grid connection at L11 during this sequence of tests with the static switch "Open", all zone and load bank circuit breakers are "Closed", Gen-sets A1, A2 and B1 set for injection power control, and all load banks initially set at zero load.

8.1.4 Start Gen-set A1 in Zone 3 and then bring Load Bank 3 up to $20 \mathrm{~kW}$, balanced load, verify during this event that the voltage magnitude remains unchanged with minimum transients.

8.1.5 Verify in the DAS Database that the event waveforms of phase currents and voltages, plus RMS Meter data were recorded at all locations when the event was applied.

8.1.6 Apply step change of load event in Load Bank 3 from 20kW to $40 \mathrm{~kW}$, balanced load and observe the voltage magnitude remains unchanged with minimum transients.

8.1.7 Verify in the DAS Database that the event waveforms of phase currents and voltages, plus RMS Meter data were recorded at all locations when the event was applied.

8.1.8 Apply another step change of load event in Load Bank 3 from $40 \mathrm{~kW}$ to $60 \mathrm{~kW}$, balanced load, and observe the voltage magnitude remains unchanged with minimum transients.

8.1.9 Verify in the DAS Database that the event waveforms of phase currents and voltages, plus RMS Meter data were recorded at all locations when the event was applied.

This document contains information confidential and proprietary to AEPSC. It shall not be reproduced in whole or in part or released to any third party without expressed written consent of AEPSC. 


\section{DTC Registered Procedure CERTS Microgrid Test Plan}

\begin{tabular}{l|l} 
Dolan Technology Center & Document No: DTC212812.207.01A
\end{tabular}

\begin{tabular}{|l|l|l} 
Written by: AEP & Effective Date: 23 Feb 2007 & Target Group: Assigned
\end{tabular}

Approved by: K. P. Loving $\quad$ Procedure Review Date: 23 Feb. 2008

8.1.10 Change the voltage set point from nominal to $+5 \%$. Then, repeat steps 8.1.4 through 8.1.9, and observe stable voltage control. Verify that the event waveforms of phase currents and voltages, plus RMS Meter data were recorded.

8.1.11 Change the voltage set point from $+5 \%$ to $-5 \%$. Then, repeat steps 8.1.4 through 8.1.9, and observe stable voltage control. Verify that the event waveforms of phase currents and voltages, plus RMS Meter data were recorded

8.1.12 Reduce Load Bank 3 to zero and shutdown Gen-set A1.

8.1.13 Repeat steps 8.1.4, through 8.1.11 for Zone 4, using Load Bank 4 and Gen-set A2.

8.1.14 Reduce Load Bank 4 to zero and shutdown Gen-set A2.

8.1.15 Repeat steps 8.1.4, through 8.1.11 for Zone 5, using Load Bank 5 and Gen-set B1.

8.1.16 Reduce Load Bank 5 to zero and shutdown Gen-set B1.

\subsection{Open Static Switch Test, Check P = 0 Limit, Gen-set A1}

Performance Goal - Verify smooth transitions of Gen-sets A1 and A2 response to different step conditions (i.e., static switch "OPEN"/

"CLOSE") with the unit power limit of Gen-set A1 equal to zero.

8.2.1 Maintain the weak grid connection at L11 during this sequence of tests with the static switch "Closed", all zone and load bank circuit breakers are "Closed", and Gen-sets A1, A2 and B1 set for injection power control.

8.2.2 Set balanced load in Load Banks 3, 4 \& 6 to the $\mathrm{kW}$ values in the Start column. Then, start Gen-sets A1 and A2 in unit control mode with the settings indicated in Start column. 


\section{DTC Registered Procedure CERTS Microgrid Test Plan}

Dolan Technology Center

Document No: DTC212812.207.01A

\begin{tabular}{|l|l|l} 
Written by: AEP & Effective Date: 23 Feb 2007 & Target Group: Assigned
\end{tabular}

\begin{tabular}{l|l} 
Approved by: K. P. Loving & Procedure Review Date: 23 Feb. 2008 \\
\hline
\end{tabular}

\begin{tabular}{|c|c|c|c|c|}
\hline \multicolumn{4}{|c|}{ Test Event - Open Static Switch } & \multirow[b]{2}{*}{ End } \\
\hline & Mode & Start & Event & \\
\hline $\mathrm{A} 1_{\mathrm{P}}$ & Unit & $5 \mathrm{~kW}$ & & $0.0 \mathrm{~kW}$ \\
\hline $\mathrm{A} 2_{\mathrm{P}}$ & Unit & $55 \mathrm{~kW}$ & & $40 \mathrm{~kW}$ \\
\hline $\mathrm{B} 1_{\mathrm{P}}$ & & Off & & Off \\
\hline Freq. & & $\sim 60 \mathrm{~Hz}$ & & $\sim 60.12$ \\
\hline L3 & & $20 \mathrm{~kW}$ & & $20 \mathrm{~kW}$ \\
\hline L4 & & $20 \mathrm{~kW}$ & & $20 \mathrm{~kW}$ \\
\hline L5 & & 0 & & \\
\hline L6 & & $20 \mathrm{~kW}$ & & $20 \mathrm{~kW}$ \\
\hline SS & CLOSED & $-20 \mathrm{~kW}$ & OPEN & $0.0 \mathrm{~kW}$ \\
\hline Grid & & 0 & & $20 \mathrm{~kW}$ \\
\hline Let $S$ & lo & & & \\
\hline
\end{tabular}

8.2.3 Allow a few minutes for the Gen-sets to warm up. When the system is in steady state condition, force "Open" static switch control signal.

8.2.4 Verify that the Gen-set and load conditions are similar to the values in the End column.

8.2.5 Verify in the "DAS Database" file that at the time when this test was performed, that the test event data and waveforms of phase currents and voltages at affected locations were recorded in the DAS, along with the test event date/time when the event was initiated.

8.2.6 Release the static switch "Open" condition and allow self synchronization of the static switch to "Re-close".

8.2.7 Verify in the "DAS Database" file that at the time when this synchronization event was performed, that the event data and waveforms of phase currents and voltages at affected locations were recorded in the DAS, along with the test event date/time when the event was initiated.

8.2.8 Verify that system conditions returned to the values listed in the Start column.

8.2.9 Shutdown Gen-sets A1 \& A2 and reduce load in Load Banks 3, 4, \& 6 to zero.

This document contains information confidential and proprietary to AEPSC. It shall not be reproduced in whole or in part or released to any third party without expressed written consent of AEPSC. 


\section{DTC Registered Procedure CERTS Microgrid Test Plan}

Dolan Technology Center

Document No: DTC212812.207.01A

\begin{tabular}{|l|l|l} 
Written by: AEP & Effective Date: 23 Feb 2007 & Target Group: Assigned
\end{tabular}

Approved by: K. P. Loving $\quad$ Procedure Review Date: 23 Feb. 2008

8.3 Open Static Switch Test, Check $P=60 \mathrm{~kW}$ Limit, Gen-set A2

Performance Goal - Verify smooth transitions of Gen-sets A1 and A2 response to different step conditions (i.e., static switch "OPEN"/

"CLOSE") with the unit power limit of Gen-set A2 equal to 60kW.

8.3.1 Maintain the weak grid connection at L11 during this sequence of tests with the static switch "Closed", all zone and load bank circuit breakers are "Closed" and Gen-sets A1, A2 and B1 set for injection power control.

8.3.2 Set balanced load in Load Banks $3 \& 4$ to the $\mathrm{kW}$ values in the Start column. Then, start Gen-sets A1 and A2 in unit control mode with the settings indicated in Start column.

\begin{tabular}{|l|l|l|l|l|}
\hline \multicolumn{5}{|c|}{ Event Open SS } \\
\hline & Mode & Start & Event & End \\
\hline A1 $\mathrm{P}$ & Unit & $5 \mathrm{~kW}$ & & $40 \mathrm{~kW}$ \\
\hline A2 & Unit & $55 \mathrm{~kW}$ & & $60 \mathrm{~kW}$ \\
\hline B1 & & Off & & Off \\
\hline Freq & & $\sim 60 \mathrm{~Hz}$ & & $\sim 59.72$ \\
\hline L3 & & $60 \mathrm{~kW}$ & & $60 \mathrm{~kW}$ \\
\hline L4 & & $40 \mathrm{~kW}$ & & $40 \mathrm{~kW}$ \\
\hline L5 & & 0 & & 0 \\
\hline L6 & & 0 & & 0 \\
\hline SS & CLOSED & $40 \mathrm{~kW}$ & OPEN & $0 \mathrm{~kW}$ \\
\hline Grid & & $40 \mathrm{~kW}$ & & $0 \mathrm{~kW}$ \\
\hline Let SS re-close & & \\
\hline
\end{tabular}

8.3.3 Allow a few minutes for the Gen-sets to warm up. When the system is in steady state condition, force "Open" static switch control signal.

8.3.4 Verify that the Gen-set and load conditions are similar to the values in the End column (i.e., Gen-set A1 power increases from $5 \mathrm{~kW}$ to $40 \mathrm{~kW}$ and Gen-set A2 power increases from $55 \mathrm{~kW}$ to $60 \mathrm{~kW})$.

8.3.5 Verify in the "DAS Database" file that at the time when this test was performed, that the test event data and waveforms of phase currents and voltages at affected locations were recorded in the DAS, along with the test event date/time when the event was

This document contains information confidential and proprietary to AEPSC. It shall not be reproduced in whole or in part or released to any third party without expressed written consent of AEPSC. 


\section{DTC Registered Procedure CERTS Microgrid Test Plan}

Dolan Technology Center

Document No: DTC212812.207.01A

\begin{tabular}{|l|l|l|}
\hline Written by: AEP & Effective Date: 23 Feb 2007 & Target Group: Assigned
\end{tabular}

Approved by: K. P. Loving $\quad$ Procedure Review Date: 23 Feb. 2008

initiated.

8.3.6 Release the static switch "Open" condition and allow self synchronization of the static switch to "Re-close".

8.3.7 Verify in the "DAS Database" file that at the time when this synchronization event was performed, that the event data and waveforms of phase currents and voltages at affected locations were recorded in the DAS, along with the test event date/time when the event was initiated.

8.3.8 Verify that system conditions return to the values listed in the Start column.

8.3.9 Shutdown Gen-sets A1 \& A2 and reduce load in Load Banks 3 \& 4 to zero.

\subsection{Test Island Operation, Unbalanced Load}

Performance Goal -Verify smooth transitions of Gen-sets A1 and A2 response to different step conditions (i.e., static switch "OPEN"/

"CLOSE") with an un-balanced load condition in Zone 3.

8.4.1 During this sequence of tests, maintain the static switch "Open", all zone and load bank circuit breakers are "Closed", and Gen-sets A1, A2 and B1 set for injection power control.

8.4.2 Start Gen-sets A1 and A2 in unit control mode with the settings indicated in Start column. Then, set balanced load in Load Bank 3 to the $\mathrm{kW}$ value in the Start column.

\begin{tabular}{|c|c|c|c|c|}
\hline \multicolumn{4}{|c|}{ Reduce A-Phase Load in Load Bank 3} & \multirow[b]{2}{*}{ End } \\
\hline & Mode & Start & Event & \\
\hline $\mathrm{A} 1_{\mathrm{P}}$ & Unit & $15 \mathrm{~kW}$ & & $\sim 2.5 \mathrm{~kW}$ \\
\hline $\mathrm{A} 22_{\mathrm{P}}$ & Unit & $45 \mathrm{~kW}$ & & $\sim 37.5 \mathrm{~kW}$ \\
\hline $\mathrm{B} 1_{\mathrm{P}}$ & & Off & & Off \\
\hline Freq & & $\sim 60 \mathrm{~Hz}$ & & $\sim 60.09$ \\
\hline L3 & & $60 \mathrm{~kW}$ & A-phase $=0 \mathrm{~kW}$ & $40 \mathrm{~kW}$ \\
\hline L4 & & $0 \mathrm{~kW}$ & & $0 \mathrm{~kW}$ \\
\hline L5 & & OkW & & $0 \mathrm{~kW}$ \\
\hline L6 & & $0 \mathrm{~kW}$ & & $0 \mathrm{~kW}$ \\
\hline SS & OPEN & $0 \mathrm{~kW}$ & & $0 \mathrm{~kW}$ \\
\hline Grid & & $0 \mathrm{~kW}$ & & $0 \mathrm{~kW}$ \\
\hline Shou & no cl & it in & e a & \\
\hline
\end{tabular}

This document contains information confidential and proprietary to AEPSC. It shall not be reproduced in whole or in part or released to any third party without expressed written consent of AEPSC. 


\begin{tabular}{|l|l|}
\hline DTC Registered Procedure \\
CERTS Microgrid Test Plan \\
\begin{tabular}{|l|l|l|}
\hline Dolan Technology Center & Document No: DTC212812.207.01A \\
\hline Written by: AEP & Effective Date: 23 Feb 2007 & Target Group: Assigned \\
\hline Approved by: K. P. Loving & Procedure Review Date: 23 Feb. 2008 & \\
\hline
\end{tabular}
\end{tabular}

8.4.3 Allow a few minutes for the Gen-sets to warm up. When the system is in steady state condition, reduce A-phase load in Load Bank 3 from $20 \mathrm{~kW}$ to $0 \mathrm{~kW}$.

8.4.4 Verify that there is zero $\mathrm{kW}$ (approximate) in Phase A, and that Gen-set and load conditions are similar to the values in the End column.

8.4.5 Verify in the "DAS Database" file that at the time when this test was performed, that the test event data and waveforms of phase currents and voltages at affected locations were recorded in the DAS, along with the test event date/time when the event was initiated.

8.4.6 Shutdown Gen-sets A1 \& A2 and reduce load in Load Banks 3 to zero.

\subsection{Mixed Mode Operation Test - Feeder A, Gen-set A1 to $60 \mathrm{~kW}$} Maximum

Performance Goal -Verify smooth transitions of Gen-sets, A1 in Zone operation mode with a $60 \mathrm{~kW}$ limit and A2 in Unit operation mode, during a load step change in Load Bank 3.

8.5.1 Maintain the weak grid connection at L11 during this sequence of tests with the static switch "Closed", all zone and load bank circuit breakers are "Closed", and Gen-sets A1, A2 and B1 set for injection power control.

8.5.2 Start Gen-sets A1 in zone control mode and A2 in unit control mode with the settings indicated in Start column. Then, set balanced load in Load Banks 3 \& 4 to the $\mathrm{kW}$ values in the Start column. whole or in part or released to any third party without expressed written consent of AEPSC. 


\section{DTC Registered Procedure CERTS Microgrid Test Plan}

Dolan Technology Center

Document No: DTC212812.207.01A

\begin{tabular}{|l|l|l} 
Written by: AEP & Effective Date: 23 Feb 2007 & Target Group: Assigned
\end{tabular}

Approved by: K. P. Loving $\quad$ Procedure Review Date: 23 Feb. 2008

\begin{tabular}{|c|c|c|c|c|}
\hline \multicolumn{4}{|c|}{ Step load } & \multirow[b]{2}{*}{ End } \\
\hline & Mode & Start & Event & \\
\hline $\mathrm{A} 1 \mathrm{z}$ & Zone & $54 \mathrm{~kW}$ & & $54 \mathrm{~kW}$ \\
\hline $\mathrm{A} 1_{\mathrm{P}}$ & $\begin{array}{l}\text { Injected } \\
\text { Power }\end{array}$ & $10 \mathrm{~kW}$ & & $\begin{array}{l}60 \mathrm{~kW} \\
\mathrm{Max}\end{array}$ \\
\hline $\mathrm{A} 2_{\mathrm{P}}$ & Unit & $6 \mathrm{~kW}$ & & $6 \mathrm{~kW}$ \\
\hline $\mathrm{B} 1_{\mathrm{P}}$ & & Off & & Off \\
\hline Freq & & $\sim 60 \mathrm{~Hz}$ & & $\sim 60 \mathrm{~Hz}$ \\
\hline L3 & & $35 \mathrm{~kW}$ & Add $50 \mathrm{~kW}$ & $85 \mathrm{~kW}$ \\
\hline L4 & & $35 \mathrm{~kW}$ & & $35 \mathrm{~kW}$ \\
\hline L5 & & 0 & & 0 \\
\hline L6 & & 0 & & 0 \\
\hline SS & CLOSED & $54 \mathrm{~kW}$ & & $54 \mathrm{~kW}$ \\
\hline Grid & & $54 \mathrm{~kW}$ & & $54 \mathrm{~kW}$ \\
\hline
\end{tabular}

8.5.3 Allow a few minutes for the Gen-sets to warm up. When the system is in steady state condition, increase balanced three phase load in Load Bank 3 from $35 \mathrm{~kW}$ to $85 \mathrm{~kW}$.

8.5.4 Verify that Gen-set and load conditions are similar to the values in the End column (i.e., injected power from Gen-set A1 increases from $10 \mathrm{~kW}$ to $60 \mathrm{~kW}$ with the Zone power in Feeder A maintained at $54 \mathrm{~kW})$.

8.5.5 Verify in the "DAS Database" file that at the time when this test was performed, that the test event data and waveforms of phase currents and voltages at affected locations were recorded in the DAS, along with the test event date/time when the event was initiated.

8.5.6 Shutdown Gen-sets A1 \& A2 and reduce load in Load Banks 3 \& 4 to zero.

\subsection{Mixed Mode Operation Test - Feeder A, Automatic Reset of Zone Level Set-Point}

Performance Goal -Verify smooth transitions of Gen-sets, A1 in Zone operation mode with an automatic reset of the set point and A2 in Unit operation mode, during a load step change in Load Bank 4. 


\section{DTC Registered Procedure CERTS Microgrid Test Plan}

Dolan Technology Center

Document No: DTC212812.207.01A

\begin{tabular}{|l|l|l|}
\hline Written by: AEP & Effective Date: 23 Feb 2007 & Target Group: Assigned
\end{tabular}

Approved by: K. P. Loving $\quad$ Procedure Review Date: 23 Feb. 2008

8.6.1 Maintain the weak grid connection at L11 during this sequence of tests with the static switch "Closed", all zone and load bank circuit breakers are "Closed", and Gen-sets A1, A2 and B1 set for injection power control.

8.6.2 Start Gen-sets A1 in zone control mode and A2 in unit control mode with the settings indicated in Start column. Then, set balanced load in Load Banks 3 \& 4 to the $\mathrm{kW}$ values in the Start column.

\begin{tabular}{|c|c|c|c|c|}
\hline \multicolumn{4}{|c|}{ Step load } & \multirow[b]{2}{*}{ End } \\
\hline & Mode & Start & Event & \\
\hline $\mathrm{A} 1 \mathrm{z}$ & Zone & $55 \mathrm{~kW}$ & & $65 \mathrm{~kW}$ \\
\hline A1p & $\begin{array}{l}\text { Injected } \\
\text { Power }\end{array}$ & $50 \mathrm{~kW}$ & & $\begin{array}{l}60 \mathrm{~kW}, \mathrm{~A} 1 \\
\text { at Max }\end{array}$ \\
\hline $\mathrm{A} 2_{\mathrm{P}}$ & Unit & $5 \mathrm{~kW}$ & & $5 \mathrm{~kW}$ \\
\hline $\mathrm{B} 1_{\mathrm{P}}$ & & Off & & Off \\
\hline Freq & & $\sim 60 \mathrm{~Hz}$ & & $\sim 60 \mathrm{~Hz}$ \\
\hline L3 & & $75 \mathrm{~kW}$ & & $75 \mathrm{~kW}$ \\
\hline $\mathrm{L} 4$ & & $35 \mathrm{~kW}$ & Add $20 \mathrm{~kW}$ & $55 \mathrm{~kW}$ \\
\hline L5 & & 0 & & 0 \\
\hline L6 & & 0 & & 0 \\
\hline SS & CLOSED & $55 \mathrm{~kW}$ & & $65 \mathrm{~kW}$ \\
\hline Grid & & $55 \mathrm{~kW}$ & & $65 \mathrm{~kW}$ \\
\hline SS st & Closed & & & \\
\hline
\end{tabular}

8.6.3 Allow a few minutes for the Gen-sets to warm up. When the system is in steady state condition, increase balanced three phase load in Load Bank 4 from $35 \mathrm{~kW}$ to $55 \mathrm{~kW}$.

8.6.4 Verify that Gen-set and load conditions are similar to the values in the End column (i.e., load change forces the injected power of Gen-set A1 to exceed its maximum and the system should automatically reset its Zone power level set-point from $55 \mathrm{~kW}$ to $65 \mathrm{~kW}$ to hold Gen-set A1 to $60 \mathrm{~kW}$ with power from the grid increased from $55 \mathrm{~kW}$ to $65 \mathrm{~kW}$ ).

8.6.5 Verify in the "DAS Database" file that at the time when this test was performed, that the test event data and waveforms of phase currents and voltages at affected locations were recorded in the 


\section{DTC Registered Procedure CERTS Microgrid Test Plan}

Dolan Technology Center

Document No: DTC212812.207.01A

\begin{tabular}{|l|l|l|}
\hline Written by: AEP & Effective Date: 23 Feb 2007 & Target Group: Assigned \\
\hline
\end{tabular}

Approved by: K. P. Loving $\quad$ Procedure Review Date: 23 Feb. 2008

DAS, along with the test event date/time when the event was initiated.

8.6.6 Shutdown Gen-sets A1 \& A2 and reduce load in Load Banks 3 \& 4 to zero.

\subsection{Mixed Mode Operation Test - Feeder A, Zone Power Change} Performance Goal -Verify smooth transitions of Gen-sets, A1 in Zone operation mode and A2 in Unit operation mode, with a change of zone power in Feeder A.

8.7.1 Maintain the weak grid connection at L11 during this sequence of tests with the static switch "Closed", all zone and load bank circuit breakers are "Closed", and Gen-sets A1, A2 and B1 set for injection power control.

8.7.2 Start Gen-sets A1 in zone control mode and A2 in unit control mode with the settings indicated in Start column. Then, set a balanced load in Load Banks 3 \& 4 to the $\mathrm{kW}$ values in the Start column.

\begin{tabular}{|c|c|c|c|c|}
\hline \multicolumn{4}{|c|}{ Large Zone Set to Pickup System } & \multirow[b]{2}{*}{ End } \\
\hline & Mode & Start & Event & \\
\hline $\mathrm{A} 1 \mathrm{z}$ & Zone & $54 \mathrm{~kW}$ & Add $56 \mathrm{~kW}$ & $110 \mathrm{~kW}$ \\
\hline $\mathrm{A} 1_{\mathrm{P}}$ & $\begin{array}{l}\text { Injected } \\
\text { Power }\end{array}$ & $\begin{array}{l}60 \mathrm{~kW} \\
\text { A1 at max }\end{array}$ & & $4 \mathrm{~kW}$ \\
\hline $\mathrm{A} 2_{\mathrm{P}}$ & Unit & $6 \mathrm{~kW}$ & & $6 \mathrm{~kW}$ \\
\hline $\mathrm{B} 1_{\mathrm{P}}$ & & Off & & Off \\
\hline Freq & & $\sim 60 \mathrm{~Hz}$ & & $\sim 60 \mathrm{~Hz}$ \\
\hline L3 & & $85 \mathrm{~kW}$ & & $85 \mathrm{~kW}$ \\
\hline L4 & & $35 \mathrm{~kW}$ & & $35 \mathrm{~kW}$ \\
\hline L5 & & 0 & & 0 \\
\hline L6 & & 0 & & 0 \\
\hline SS & CLOSED & $54 \mathrm{~kW}$ & & $110 \mathrm{~kW}$ \\
\hline Grid & & $54 \mathrm{~kW}$ & & $110 \mathrm{~kW}$ \\
\hline EMS & Lone A1 & $\mathrm{m} 54 \mathrm{~kW}$ & $10 \mathrm{~kW}$ & \\
\hline
\end{tabular}

8.7.3 Allow a few minutes for the Gen-sets to warm up. When the system is in steady state condition, use the EMS to change/increase the Zone power level in Feeder A from $54 \mathrm{~kW}$ to $110 \mathrm{~kW}$. 


\begin{tabular}{|c|c|c|}
\hline \multirow{2}{*}{$\begin{array}{l}\text { DTC Registered Procedure } \\
\text { CERTS Microgrid Test Plan } \\
\text { Dolan Technology Center }\end{array}$} & $\begin{array}{l}\text { cedure } \\
\text { est Plan }\end{array}$ & \\
\hline & \begin{tabular}{l|l} 
& Document No:
\end{tabular} & DTC212812.207.01A \\
\hline Written by: AEP & Effective Date: 23 Feb 2007 & Target Group: Assigned \\
\hline Approved by: K. P. Loving & Procedure Review Date: $23 \mathrm{~F}$ & eb. 2008 \\
\hline
\end{tabular}

8.7.4 Verify that Gen-set and load conditions are similar to the values in the End column (i.e., the Zone power change in Feeder A forces Gen-set A1 to reduce its injected power from $60 \mathrm{~kW}$ to $4 \mathrm{~kW}$ and power from the grid increased from $54 \mathrm{~kW}$ to $110 \mathrm{~kW}$ ).

8.7.5 Verify in the "DAS Database" file that at the time when this test was performed, that the test event data and waveforms of phase currents and voltages at affected locations were recorded in the DAS, along with the test event date/time when the event was initiated.

8.7.6 Shutdown Gen-sets A1 \& A2 and reduce load in Load Banks $3 \& 4$ to zero.

\subsection{Mixed Mode Operation Test - Feeder A, Zone Power Change When}

\section{Islanded}

Performance Goal -Verify smooth transitions of Gen-sets, A1 in Zone operation mode and A2 in Unit operation mode, with a static switch operation and a change of zone power in Feeder A when islanded.

8.8.1 Maintain the weak grid connection at L11 during this sequence of tests with the static switch "Closed", all zone and load bank circuit breakers are "Closed", and Gen-sets A1, A2 and B1 set for injection power control.

8.8.2 Start Gen-sets A1 in zone control mode and A2 in unit control mode with the settings indicated in Start column. Then, set a balanced load in Load Banks 3 \& 4 to the $\mathrm{kW}$ values in the Start column. whole or in part or released to any third party without expressed written consent of AEPSC. 


\section{DTC Registered Procedure CERTS Microgrid Test Plan}

Dolan Technology Center

Document No: DTC212812.207.01A

Written by: AEP

Approved by: K. P. Loving Effective Date: 23 Feb 2007 Target Group: Assigned Procedure Review Date: 23 Feb. 2008

\begin{tabular}{|c|c|c|c|c|}
\hline \multicolumn{4}{|c|}{ Open SS } & \multirow[b]{2}{*}{ End } \\
\hline & Mode & Start & Event & \\
\hline $\mathrm{A} 1 \mathrm{z}$ & Zone & $50 \mathrm{~kW}$ & & $0 \mathrm{~kW}$ \\
\hline $\mathrm{A} 1_{\mathrm{P}}$ & $\begin{array}{l}\text { Injected } \\
\text { Power }\end{array}$ & $14 \mathrm{~kW}$ & & $\sim 18 \mathrm{~kW}$ \\
\hline $\mathrm{A} 2_{\mathrm{P}}$ & Unit & $6 \mathrm{~kW}$ & & $\sim 52 \mathrm{~kW}$ \\
\hline $\mathrm{B} 1_{\mathrm{P}}$ & & Off & & Off \\
\hline Freq & & $\sim 60 \mathrm{~Hz}$ & & $\sim 59.6 \mathrm{~Hz}$ \\
\hline L3 & & $35 \mathrm{~kW}$ & & $35 \mathrm{~kW}$ \\
\hline L4 & & $35 \mathrm{~kW}$ & & $35 \mathrm{~kW}$ \\
\hline L5 & & 0 & & 0 \\
\hline L6 & & 0 & & 0 \\
\hline SS & CLOSED & $50 \mathrm{~kW}$ & OPEN & $0 \mathrm{~kW}$ \\
\hline Grid & & $50 \mathrm{~kW}$ & & 0kW \\
\hline \multicolumn{4}{|c|}{ Allow SS to Re-close } & \\
\hline
\end{tabular}

8.8.3 Allow a few minutes for the Gen-sets to warm up. When the system is in steady state condition, force "Open" static switch control signal.

8.8.4 Verify that the Gen-set and load conditions are similar to the values in the End column (i.e., Zone power in Feeder A decreases from $50 \mathrm{~kW}$ to $0 \mathrm{~kW}$, Gen-set A1 power increases from $14 \mathrm{~kW}$ to about $18 \mathrm{~kW}$, Gen-set A2 power increases from $6 \mathrm{~kW}$ to about $52 \mathrm{~kW}$, frequency decreases by about $0.4 \mathrm{~Hz}$, and power from the grid decreases from $50 \mathrm{~kW}$ to zero).

8.8.5 Verify in the "DAS Database" file that at the time when this test was performed, that the test event data and waveforms of phase currents and voltages at affected locations were recorded in the DAS, along with the test event date/time when the event was initiated.

8.8.6 Release the static switch "Open" condition and allow self synchronization of the static switch to "Re-close".

8.8.7 Verify in the "DAS Database" file that at the time when this synchronization event was performed, that the event data and waveforms of phase currents and voltages at affected locations 


\section{DTC Registered Procedure CERTS Microgrid Test Plan}

Dolan Technology Center

Document No: DTC212812.207.01A

\begin{tabular}{|l|l|l|}
\hline Written by: AEP & Effective Date: 23 Feb 2007 & Target Group: Assigned
\end{tabular}

Approved by: K. P. Loving $\quad$ Procedure Review Date: 23 Feb. 2008

were recorded in the DAS, along with the test event date/time when the event was initiated.

8.8.8 Verify that system conditions returned to the values listed in the Start column.

8.8.9 Shutdown Gen-sets A1 \& A2 and reduce load in Load Banks 3 \& 4 to zero.

8.9 Mixed Mode Operation Test - Feeder A, When Islanded Automatic Reset of Zone Level Set-point, Both Gen-sets $P=60 \mathrm{~kW}$ Maximum Performance Goal -Verify smooth transitions of Gen-sets, A1 in Zone operation mode with automatic reset of set point and A2 in Unit operation mode, with a static switch operation and a change of zone power in Feeder A when islanded.

8.9.1 Maintain the weak grid connection at L11 during this sequence of tests with the static switch "Closed", all zone and load bank circuit breakers are "Closed", and Gen-sets A1, A2 and B1 set for injection power control.

8.9.2 Start Gen-sets A1 in zone control mode and A2 in unit control mode with the settings indicated in Start column. Then, set a balanced load in Load Banks 3 \& 4 to the $\mathrm{kW}$ values in the Start column.

\begin{tabular}{|l|l|l|l|l|}
\hline \multicolumn{4}{|c|}{ Open SS, Test Gen-set $\mathrm{P}_{\text {max. }}$} & \\
\hline & Mode & Start & Event & End \\
\hline $\mathrm{A} 1 \mathrm{z}$ & Zone & $54 \mathrm{~kW}$ & & $0 \mathrm{~kW}$ \\
\hline $\mathrm{A} 1_{\mathrm{P}}$ & $\begin{array}{l}\text { Injected } \\
\text { Power }\end{array}$ & $60 \mathrm{~kW}$ & & $\begin{array}{l}60 \mathrm{~kW} \\
\text { A1 at Max. }\end{array}$ \\
\hline $\mathrm{A} 2_{\mathrm{P}}$ & Unit & $6 \mathrm{~kW}$ & & $\begin{array}{l}60 \mathrm{~kW} \\
\text { A2 at Max. }\end{array}$ \\
\hline B1 $1_{\mathrm{P}}$ & & Off & & Off \\
\hline Freq & & $\sim 60 \mathrm{~Hz}$ & & $\sim 59.5 \mathrm{~Hz}$ \\
\hline L3 & & $60 \mathrm{~kW}$ & & $60 \mathrm{~kW}$ \\
\hline L4 & & $60 \mathrm{~kW}$ & & $60 \mathrm{~kW}$ \\
\hline L5 & & 0 & & 0 \\
\hline L6 & & 0 & & 0 \\
\hline SS & CLOSED & $54 \mathrm{~kW}$ & OPEN & $0 \mathrm{~kW}$ \\
\hline Grid & $54 \mathrm{~kW}$ & & $0 \mathrm{~kW}$ \\
\hline Allow SS to Re-close & \\
\hline
\end{tabular}

This document contains information confidential and proprietary to AEPSC. It shall not be reproduced in whole or in part or released to any third party without expressed written consent of AEPSC. 


\begin{tabular}{|l|l|}
\hline DTC Registered Procedure \\
CERTS Microgrid Test Plan \\
\begin{tabular}{|l|l|l|}
\hline Dolan Technology Center & Document No: DTC212812.207.01A \\
\hline Written by: AEP & Effective Date: 23 Feb 2007 & Target Group: Assigned \\
\hline Approved by: K. P. Loving & Procedure Review Date: 23 Feb. 2008 & \\
\hline
\end{tabular}
\end{tabular}

8.9.3 Allow a few minutes for the Gen-sets to warm up. When the system is in steady state condition, force "Open" static switch control signal.

8.9.4 Verify that the Gen-set and load conditions are similar to the values in the End column (i.e., Zone power in Feeder A decreases from $50 \mathrm{~kW}$ to $0 \mathrm{~kW}$, Gen-set A1 power remains at $60 \mathrm{~kW}$, Gen-set A2 power increases from $6 \mathrm{~kW}$ to about $60 \mathrm{~kW}$, frequency decreases by about $0.5 \mathrm{~Hz}$, and power from the grid decreases from $50 \mathrm{~kW}$ to zero).

8.9.5 Verify in the "DAS Database" file that at the time when this test was performed, that the test event data and waveforms of phase currents and voltages at affected locations were recorded in the DAS, along with the test event date/time when the event was initiated.

8.9.6 Release the static switch "Open" condition and allow self synchronization of the static switch to "Re-close".

8.9.7 Verify in the "DAS Database" file that at the time when this synchronization event was performed, that the event data and waveforms of phase currents and voltages at affected locations were recorded in the DAS, along with the test event date/time when the event was initiated.

8.9.8 Verify that system conditions returned to the values listed in the Start column.

8.9.9 Shutdown Gen-sets A1 \& A2 and reduce load in Load Banks 3 \& 4 to zero.

8.10 Two Sources in Zone Control - Separate Feeders, When Islanded Automatic Reset of Zone Level Set-point, New Zones Sum Equal Zero Performance Goal-Verify smooth transitions of Gen-sets, A1 and B1 in Zone operation mode, with a static switch operation and a change of zone power in Feeder A and Feeder B when islanded.

8.10.1 Maintain the weak grid connection at L11 during this sequence of tests with the static switch "Closed", all zone and load bank circuit breakers are "Closed", and Gen-sets A1, A2 and B1 set for injection power control.

This document contains information confidential and proprietary to AEPSC. It shall not be reproduced in whole or in part or released to any third party without expressed written consent of AEPSC. 


\section{DTC Registered Procedure CERTS Microgrid Test Plan}

Dolan Technology Center

Document No: DTC212812.207.01A

Written by: AEP

Approved by: K. P. Loving Effective Date: 23 Feb 2007 Target Group: Assigned Procedure Review Date: 23 Feb. 2008

8.10.2 Start Gen-sets A1 and B1 in zone control mode with the settings indicated in Start column. Then, set a balanced load in Load Banks $3 \& 5$ to the $\mathrm{kW}$ values in the Start column.

\begin{tabular}{|l|l|l|l|l|}
\hline \multicolumn{2}{|c|}{ Two Zone Control Test: Separate Event } & \\
\hline & \multicolumn{1}{|c|}{ Opode } & \multicolumn{1}{c|}{ Start } & Event & \multicolumn{1}{|c|}{ End } \\
\hline $\mathrm{A} 1 \mathrm{z}$ & Zone & $40 \mathrm{~kW}$ & & $10 \mathrm{~kW}$ \\
\hline $\mathrm{A} 1_{\mathrm{P}}$ & $\begin{array}{l}\text { Injected } \\
\text { Power }\end{array}$ & $0 \mathrm{~kW}$ & & $30 \mathrm{~kW}$ \\
\hline $\mathrm{A} 2_{\mathrm{P}}$ & & Off & & Off \\
\hline B1z & Zone & $10 \mathrm{~kW}$ & & $-10 \mathrm{KW}$ \\
\hline $\mathrm{B} 1_{\mathrm{P}}$ & $\begin{array}{l}\text { Injected } \\
\text { Power }\end{array}$ & $40 \mathrm{~kW}$ & & $60 \mathrm{~kW}$ \\
\hline Freq & & $\sim 60 \mathrm{~Hz}$ & & B 1 at Max. \\
\hline L3 & & $40 \mathrm{~kW}$ & & $40 \mathrm{~kW}$ \\
\hline L4 & & 0 & & 0 \\
\hline L5 & & $50 \mathrm{~kW}$ & & $50 \mathrm{~kW}$ \\
\hline L6 & & 0 & & 0 \\
\hline SS & CLOSED & $50 \mathrm{~kW}$ & OPEN & $0.0 \mathrm{~kW}$ \\
\hline Grid & & $50 \mathrm{~kW}$ & & $0.0 \mathrm{~kW}$ \\
\hline Let SS Re-close & & \\
\hline
\end{tabular}

8.10.3 Allow a few minutes for the Gen-sets to warm up. When the system is in steady state condition, force "Open" static switch control signal.

8.10.4 Verify that the Gen-set and load conditions are similar to the values in the End column (i.e., Zone power in Feeder A decreases from $40 \mathrm{~kW}$ to $10 \mathrm{~kW}$ and Gen-set A1 injected power increases from $0 \mathrm{~kW}$ to $30 \mathrm{~kW}$; Zone power in Feeder B decreases from $+10 \mathrm{~kW}$ to $-10 \mathrm{~kW}$ and Gen-set B 1 injected power increases from $40 \mathrm{~W}$ to about $60 \mathrm{~kW}$, frequency decreases by about $0.25 \mathrm{~Hz}$, and power from the grid decreases from $50 \mathrm{~kW}$ to zero). Also, verify when the system islands, the sum of the new zone settings equal zero (i.e., $-10 \mathrm{~kW}+10 \mathrm{~kW}$ ).

8.10.5 Verify in the "DAS Database" file that at the time when this test was performed, that the test event data and waveforms of phase currents and voltages at affected locations were recorded in the

This document contains information confidential and proprietary to AEPSC. It shall not be reproduced in whole or in part or released to any third party without expressed written consent of AEPSC. 


\begin{tabular}{|c|c|c|}
\hline \multirow{2}{*}{$\begin{array}{l}\text { DTC Registered Procedure } \\
\text { CERTS Microgrid Test Plan } \\
\text { Dolan Technology Center }\end{array}$} & $\begin{array}{l}\text { cedure } \\
\text { est Plan }\end{array}$ & \\
\hline & \begin{tabular}{l|l} 
& Document No:
\end{tabular} & DTC212812.207.01A \\
\hline Written by: AEP & Effective Date: 23 Feb 2007 & Target Group: Assigned \\
\hline Approved by: K. P. Loving & Procedure Review Date: $23 \mathrm{~F}$ & eb. 2008 \\
\hline
\end{tabular}

DAS, along with test event date/time when the event was initiated.

8.10.6 Release the static switch "Open" condition and allow self synchronization of the static switch to "Re-close".

8.10.7 Verify in the "DAS Database" file that at the time when this synchronization event was performed, that the event data and waveforms of phase currents and voltages at affected locations were recorded in the DAS, along with the test event date/time when the event was initiated.

8.10.8 Verify that system conditions returned to the values listed in the Start column.

8.10.9 Shutdown Gen-sets A1 \& A2 and reduce load in Load Banks 3 \& 4 to zero

\subsection{Two Sources in Zone Control - Separate Feeders, When Islanded} Automatic Reset of Zone Level Set-points

Performance Goal -Verify smooth transitions of Gen-sets, A1 and B1 in Zone operation mode and both having automatic reset of set points, with a static switch operation and a change of zone power in Feeder A and Feeder B when islanded.

8.11.1 Maintain the weak grid connection at L11 during this sequence of tests with the static switch "Closed", all zone and load bank circuit breakers are "Closed", and Gen-sets A1, A2 and B1 set for injection power control.

8.11.2 Start Gen-sets A1 and B1 in zone control mode with the settings indicated in Start column. Then, set a balanced load in Load Banks 3, 5, \& 6 to the $\mathrm{kW}$ values in the Start column. whole or in part or released to any third party without expressed written consent of AEPSC. 


\section{DTC Registered Procedure CERTS Microgrid Test Plan}

Dolan Technology Center

Document No: DTC212812.207.01A

Written by: AEP

Approved by: K. P. Loving Effective Date: 23 Feb 2007 Target Group: Assigned Procedure Review Date: 23 Feb. 2008

\begin{tabular}{|c|c|c|c|c|}
\hline \multicolumn{4}{|c|}{ Two-Zones: Event Open SS } & \multirow[b]{2}{*}{ End } \\
\hline & Mode & Start & Event & \\
\hline $\mathrm{A} 1 \mathrm{z}$ & Zone & $-30 \mathrm{~kW}$ & & $-25 \mathrm{~kW}$ \\
\hline $\mathrm{A} 1_{\mathrm{P}}$ & $\begin{array}{l}\text { Injected } \\
\text { Power }\end{array}$ & $50 \mathrm{~kW}$ & & $45 \mathrm{~kW}$ \\
\hline $\mathrm{A} 2_{\mathrm{P}}$ & & Off & & Off \\
\hline $\mathrm{B} 1 \mathrm{z}$ & Zone & $20 \mathrm{~kW}$ & & $25 \mathrm{Kw}$ \\
\hline $\mathrm{B} 1_{\mathrm{P}}$ & $\begin{array}{l}\text { Injected } \\
\text { Power }\end{array}$ & $20 \mathrm{~kW}$ & & $15 \mathrm{~kW}$ \\
\hline Freq & & $\sim 60 \mathrm{~Hz}$ & & $\sim 60.04 \mathrm{~Hz}$ \\
\hline L3 & & $20 \mathrm{~kW}$ & & $20 \mathrm{~kW}$ \\
\hline L4 & & 0 & & 0 \\
\hline L5 & & $40 \mathrm{~kW}$ & & $40 \mathrm{~kW}$ \\
\hline L6 & & $20 \mathrm{~kW}$ & & $20 \mathrm{~kW}$ \\
\hline SS & CLOSED & $-10 \mathrm{~kW}$ & OPEN & $0.0 \mathrm{~kW}$ \\
\hline Grid & & $10 \mathrm{~kW}$ & & $20 \mathrm{~kW}$ \\
\hline \multicolumn{4}{|c|}{ Let SS Re-close } & \\
\hline
\end{tabular}

8.11.3 Allow a few minutes for the Gen-sets to warm up. When the system is in steady state condition, force "Open" static switch control signal.

8.11.4 Verify that the Gen-set and load conditions are similar to the values in the End column (i.e., Zone power in Feeder A changes from $-30 \mathrm{~kW}$ to $-25 \mathrm{~kW}$ and Gen-set A1 injected power decreases from $50 \mathrm{~kW}$ to $45 \mathrm{~kW}$; Zone power in Feeder B increases from $+20 \mathrm{~kW}$ to $+25 \mathrm{~kW}$; and Gen-set B1 injected power decreases from $20 \mathrm{~W}$ to $15 \mathrm{~kW}$, frequency increases by about $0.04 \mathrm{~Hz}$, and power from the grid increases from $10 \mathrm{~kW}$ to $20 \mathrm{~kW}$ ). Also, verify when the system islands, the automatic reset of the Zones with one Zone exporting power and the other Zone importing power. Note, at the start of this test $10 \mathrm{~kW}$ of power is being exported through the Static Switch to Feeder C

8.11.5 Verify in the "DAS Database" file that at the time when this test was performed, that the test event data and waveforms of phase currents and voltages at affected locations were recorded in the 


\section{DTC Registered Procedure CERTS Microgrid Test Plan}

\begin{tabular}{l|l} 
Dolan Technology Center & Document No: DTC212812.207.01A
\end{tabular}

\begin{tabular}{l|l|l} 
Written by: AEP & Effective Date: 23 Feb 2007 & Target Group: Assigned \\
\hline
\end{tabular}

Approved by: K. P. Loving $\quad$ Procedure Review Date: 23 Feb. 2008

DAS, along with test event date/time when the event was initiated.

8.11.6 Release the static switch "Open" condition and allow self synchronization of the static switch to "Re-close".

8.11.7 Verify in the "DAS Database" file that at the time when this synchronization event was performed, that the event data and waveforms of phase currents and voltages at affected locations were recorded in the DAS, along with the test event date/time when the event was initiated.

8.11.8 Verify that system conditions returned to the values listed in the Start column.

8.11.9 Shutdown Gen-sets A1 \& B1 and reduce load in Load Banks 3, 5, \& 6 to zero.

\subsection{Test Generator Black-Start Procedure}

Objective: This test will check the manual procedure used to black-start the Microgrid Test Bed in the event of a lengthy utility outage occurs with the Gen-sets off-line.

Measurement - Record the transition of the Gen-set meters as they are started and brought on-line and as load banks are switched on-line. Note, the transition time between all events.

Performance Goal - Bring up the Microgrid Test Bed from a black-out condition without generation or protection trips.

8.12.1 Maintain the weak grid connection at L11 during this sequence of tests with the static switch "Closed", all zone and load bank circuit breakers are "Closed" and all load banks initially set at zero load.

8.12.2 Begin with Gen-sets A1, A2 and B1 set for injection power control, off-line and shut down. Using the Walnut Breaker Control Program, "Open" CB1 to simulate a utility outage with the Microgrid de-energized, referred to as a black-out.

8.12.3 Using the EMS and DAS programs:

8.12.3.1 Set a manual "Open" on the static switch. 


\begin{tabular}{|l|l|}
\hline DTC Registered Procedure \\
CERTS Microgrid Test Plan \\
\begin{tabular}{|l|l|l|}
\hline Dolan Technology Center & Document No: DTC212812.207.01A \\
\hline Written by: AEP & Effective Date: 23 Feb 2007 & Target Group: Assigned \\
\hline Approved by: K. P. Loving & Procedure Review Date: 23 Feb. 2008 & \\
\hline
\end{tabular}
\end{tabular}

8.12.3.2 Using the DAS Load Control program, reduce load on Load Banks 3, 4 and 5 to zero.

8.12.3.3 Individually start Gen-set A1, Gen-set A2 and Gen-set B1; wait a few minutes (e.g., 3-4 minutes) to allow them to warm up and come on-line.

8.12.3.4 Using the DAS Load Control program, select the load in each Load Bank (i.e., 3, 4 \& 5) to $27 \mathrm{~kW}+\mathrm{j} 13 \mathrm{kVAr}$, and then switch the load on-line. The Microgrid is now manually islanded and supporting 90kVA of loads.

\subsubsection{Remove the manual "Open" on the static switch.}

8.12.4 Using the Walnut Breaker Control Program, "Close" circuit breaker CB1 to restore utility voltage and observe automatic reconnection (i.e., synchronization) after the IEEE 1547 restoration timer elapses, which is 300 seconds (recall utility outage creates an IEEE 1547 voltage event).

8.12.5 Verify in the DAS Database file that at the time when this synchronization event was performed, that the event data and waveforms of phase currents and voltages at affected locations were recorded in the DAS, along with test event date/time when the event was initiated.

8.12.6 Shutdown Gen-sets A1, A2, \& B1 and reduce load in Load Banks $3,4 \& 5$ to zero.

\subsection{Test/Establish Generator Black-Start Capacity}

Performance Goal - Determine the Black-start capacity of the microgrid by increasing the amount of load on the Microgrid Test Bed from a blackout condition without generation or protection trips.

8.13.1 Maintain the weak grid connection at L11 during this sequence of tests with the static switch "Closed", all zone and load bank circuit breakers are "Closed" and all load banks initially set at zero load.

8.13.2 Begin with Gen-sets A1, A2 and B1 set for injection power control, off-line and shut down. Using the Walnut Breaker Control Program, "Open" CB1 to simulate a utility outage with the Microgrid de-energized, referred to as a black-out.

$$
65
$$

This document contains information confidential and proprietary to AEPSC. It shall not be reproduced in whole or in part or released to any third party without expressed written consent of AEPSC. 


\begin{tabular}{|l|l|}
\hline DTC Registered Procedure \\
CERTS Microgrid Test Plan \\
\begin{tabular}{|l|l|l|}
\hline Dolan Technology Center & Document No: DTC212812.207.01A \\
\hline Written by: AEP & Effective Date: 23 Feb 2007 & Target Group: Assigned \\
\hline Approved by: K. P. Loving & Procedure Review Date: 23 Feb. 2008 & \\
\hline
\end{tabular}
\end{tabular}

8.13.3 Using the EMS and DAS programs:

8.13.3.1 Set a manual "Open" on the static switch.

8.13.3.2 Set the load in Load Bank 3 at $27 \mathrm{~kW}+\mathrm{j} 13 \mathrm{kVAr}$, and verify that load bank circuit breaker CB33 is "Closed".

8.13.3.3 Start Gen-set A1 with Load Bank 3 load connected when starting the generator wait a few minutes (e.g., 35 minutes) for the engine to warm up, than the inverter contactor will "Close" to serve load on-line.

8.13.3.4 Verify in the DAS Database file that at the time when the inverter contactor Closed, the test event data and waveforms of phase currents and voltages were recorded in the DAS, along with test event date/time when the event was initiated.

8.13.3.5 Shutdown Gen-set A1. Note, Gen-set A1 was manually islanded and supporting $30 \mathrm{kVA}$ of load.

8.13.3.6 Increase load in Load Bank 3 to $36 \mathrm{~kW}+\mathrm{j}$ 19.5VAr, and verify that load bank circuit breaker CB33 is "Closed".

8.13.3.7 Start Gen-set A1 with Load Bank 3 load connected when starting the generator wait a few minutes (e.g., 35 minutes) for the engine to warm up, than the inverter contactor will "Close" to serve load on-line

8.13.3.8 Verify in the DAS Database file that at the time when the inverter contactor Closed, the test event data and waveforms of phase currents and voltages were recorded in the DAS, along with test event date/time when the event was initiated.

8.13.3.9 Shutdown Gen-set A1. Note, Gen-set A1 was manually islanded and supporting 40kVA of load.

8.13.3.10 Increase load in Load Bank 3 to $54 \mathrm{~kW}+\mathrm{j} 26 \mathrm{VAr}$, and verify that load bank circuit breaker CB33 is "Closed".

This document contains information confidential and proprietary to AEPSC. It shall not be reproduced in whole or in part or released to any third party without expressed written consent of AEPSC. 


\section{DTC Registered Procedure CERTS Microgrid Test Plan}

Dolan Technology Center

Document No: DTC212812.207.01A

\begin{tabular}{|l|l|l|l|}
\hline Written by: AEP & Effective Date: 23 Feb 2007 & Target Group: Assigned \\
\hline Approved by: K. P. Loving & Procedure Review Date: 23 Feb. 2008 & \\
\hline
\end{tabular}

8.13.3.11 Start Gen-set A1 with Load Bank 3 load connected when starting the generator wait a few minutes (e.g., 34 minutes) for the engine to warm up, than the inverter contactor will "Close" to serve load on-line.

8.13.3.12 Verify in the DAS Database file that at the time when the inverter contactor Closed, the test event data and waveforms of phase currents and voltages were recorded in the DAS, along with test event date/time when the event was initiated.

8.13.3.13 Shutdown Gen-set A1. Note, Gen-set A1 was manually islanded and supporting 60kVA of load.

8.13.3.14 If Gen-set A1 successfully islanded and supported $60 \mathrm{kVA}$ of load, increase load in Load Bank 3 to $63 \mathrm{~kW}+\mathrm{j} 30.5 \mathrm{kVAr}$, and verify that load bank circuit breaker CB33 is "Closed".

8.13.3.15 Start Gen-set A1 with Load Bank 3 load connected when starting the generator wait a few minutes (e.g., 34 minutes) for the engine to warm up, than the inverter contactor will "Close" to serve load on-line.

8.13.3.16 Verify in the DAS Database file that at the time when the inverter contactor Closed, the test event data and waveforms of phase currents and voltages were recorded in the DAS, along with test event date/time when the event was initiated.

8.13.3.17 Shutdown Gen-set A1. Note, if successful, then Genset A1 manually islanded and supported 70kVA of load.

\subsection{Procedure - Demonstration Tests of Control Power Flow}

Objective - To demonstrate the flexibility of the Microgrid both grid connected and islanded for different loads, power flows and impact on the utility. First sequence of tests will include the weak grid inductors (i.e., L11) in the circuit. 


\begin{tabular}{|l|l|}
\hline DTC Registered Procedure \\
CERTS Microgrid Test Plan \\
\begin{tabular}{|l|l|l|}
\hline Dolan Technology Center & Document No: DTC212812.207.01A \\
\hline Written by: AEP & Effective Date: 23 Feb 2007 & Target Group: Assigned \\
\hline Approved by: K. P. Loving & Procedure Review Date: 23 Feb. 2008 & \\
\hline
\end{tabular}
\end{tabular}

Second sequence of tests will repeat a select number of the most interesting results under strong grid conditions.

Measurements: Collect RMS data for V, I, kW, kVAr, and Freq for each flow change at the following points:

- $\quad$ Meter $1-\mathrm{I}, \mathrm{kW}, \mathrm{kVAr}$ for the utility connection

- $\quad$ Meter 2 - I, kW, kVAr and utility-side V and Freq

- $\quad$ Meter 3 - I, kW, and kVAr for Feeder A and Microgrid side V and Freq

- $\quad$ Meter A1p - I, kW, kVAr for Gen-set A1

- $\quad$ Load Meter 3 - Zone 3 load kW and kVAr

- $\quad$ Meter 4 - I, kW, kVAr for Zone 4

- $\quad$ Meter A2p-I, kW, kVAr for Gen-set A2

- $\quad$ Load Meter 4 - Zone 4 load $\mathrm{kW}$ and $\mathrm{kVAr}$

- $\quad$ Meter 5 - I, kW, kVAr for Feeder B

- $\quad$ Meter B1p - I, kW, kVAr for Gen-set B1

- $\quad$ Load Meter 5-Zone 5, Feeder B load kW and kVAr

- $\quad$ Load Meter 6 - Zone 6, Feeder C load kW and kVAr

In addition collect:

- V and I waveform data for unexpected events and for static switch transitions.

- Motor torque waveforms for all switch transitions.

Loading: Load on Feeder A \& Feeder B must be less than $170 \mathrm{~kW}$ and Feeder C should be such that there is no power into the utility grid. All loads should include an approximate 0.9 power factor (pf), if possible. The motor load will be included as part of Zone 3.

\subsection{Unit Control Mode, Weak Grid}

Performance Goal - Verify and document power flow and Microgrid frequency changes when transitioning from utility connected to an islanded mode of operation. During each sequence of tests in Table 1, maintain a weak grid connection with L11 in the circuit and the static switch "Closed"; all zone and load bank circuit breakers "Closed"; Gensets A1, A2 and B1 set for Unit (injection power) control mode; and all load banks initially set at zero.

9.1.1 Each test event procedure begins with setting the load (i.e., balanced load) conditions as indicated in the Table, using the EMS with reactive load set at each Load Bank to achieve an whole or in part or released to any third party without expressed written consent of AEPSC. 


\title{
CERTS MICROGRID LABORATORY TEST BED
}

\author{
Youtility Factory Test Plan
} Final Test Results

Prepared For:

California Energy Commission

Public Interest Energy Research Program

Arnold Schwarzenegger Governor

Prepared By:

Lawrence Berkeley National Laboratory

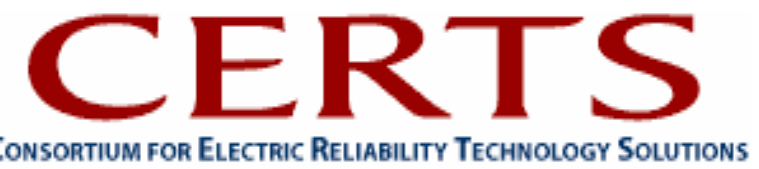



Prepared By:

Lawrence Berkeley National Laboratory

Joseph H. Eto, Principal Investigator

Berkeley, CA 94720

Jian Wan, Youtility

Commission Contract No. 500-03-024

Prepared For:

Public Interest Energy Research (PIER)

California Energy Commission

Bernard Treanton

Contract Manager

Mike Gravely

Program Area Lead

ENERGY SYSTEMS INTEGRATION

Mike Gravely

Office Manager

ENERGY SYSTEMS RESEARCH

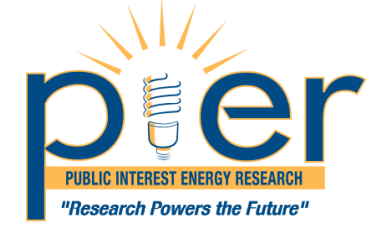

Martha Krebs, Ph.D.

PIER Director

Thom Kelly, Ph.D.

Deputy Director

ENERGY RESEARCH \& DEVELOPMENT DIVISION

Melissa Jones

Executive Director

\section{DISCLAIMER}

This report was prepared as the result of work sponsored by the California Energy Commission. It does not necessarily represent the views of the Energy Commission, its employees or the State of California. The Energy Commission, the State of California, its employees, contractors and subcontractors make no warrant, express or implied, and assume no legal liability for the information in this report; nor does any party represent that the uses of this information will not infringe upon privately owned rights. This report has not been approved or disapproved by the California Energy Commission nor has the California Energy Commission passed upon the accuracy or adequacy of the information in this report. 



\title{
Youtility Factory Test Plan Final Test Results
}

\author{
For Inverter Functions
}




\section{Test Results:}

The data provided in this paper are the final results to demonstrate the capabilities of the microgrid algorithm implemented in the Youtility inverter microgrid system. Also, the data provided in this paper only depicts two-unit series operation. In each plot the data is formatted in the following fashion:

Ch1 = Unit RMS Power Output;

$0.0 \mathrm{~V}=-15 \mathrm{KW}$

$0.5 \mathrm{~V}=0 \mathrm{KW}$

$1.5 \mathrm{~V}=30 \mathrm{KW}$

$2.5 \mathrm{~V}=60 \mathrm{KW}$

$\mathrm{Ch} 2=$ Unit Operating Frequency;

$60 \mathrm{~Hz}-->2.3 \mathrm{~V}$

$59.5 \mathrm{~Hz}-->1.66 \mathrm{~V}$

$60.5 \mathrm{~Hz}-->2.94 \mathrm{~V}$

Ch 3 = Micro Grid Voltage; $1 \mathrm{~V}=1 \mathrm{~V}$

Ch4 = Unit Output Current; 1V = 100Amps

Also, the result of each test are organized in a table, shown below in Table 1, which define the set points of the load and the individual micro gird inverters (abbreviated S.P) and the actual load of the load bank and the actual power output from the unit. The table also contains the microgrid operating frequency before and after the transient event occurs. Data contained in column A defines the operating condition and the state of the microgrid before the transient condition being tested occurs. Data contained in column B defines the operating condition and the state of the microgrid after the transient condition being tested has occurred.

\begin{tabular}{|c|c|c|}
\hline & A (Grid or Island) & B (Grid or Island) \\
\hline Load Bank \#1(S.P/Actual) KW & XX KW & XX KW \\
\hline Load Bank \#2(S.P/Actual) KW & XX KW & XX KW \\
\hline Unit \#1 (S.P/Pout) KW & XX KW Feeder or Unit & XX KW Feeder or Unit \\
\hline Unit \#2 (S.P/Pout) KW & XX KW Feeder or Unit & XX KW Feeder or Unit \\
\hline Frequency Hz & XX.X Hz & XX.X Hz \\
\hline
\end{tabular}

Table 1 Sample Test Data Table 
Phase One Testing:

Phase one testing consists of data taken while operating individual inverters in an islanded condition. The data provided in this section depicts step loads of various magnitudes applied on the inverter. In each test, the inverter is operated in unit power mode with a command of $20 \mathrm{KW}$. In this section, the plots for the individual units and the information contained in each table are grouped together for ease of comparison. The units ARE NOT operating synchronized together as a microgrid.

Test I_a_i (0KW-30KW step load)

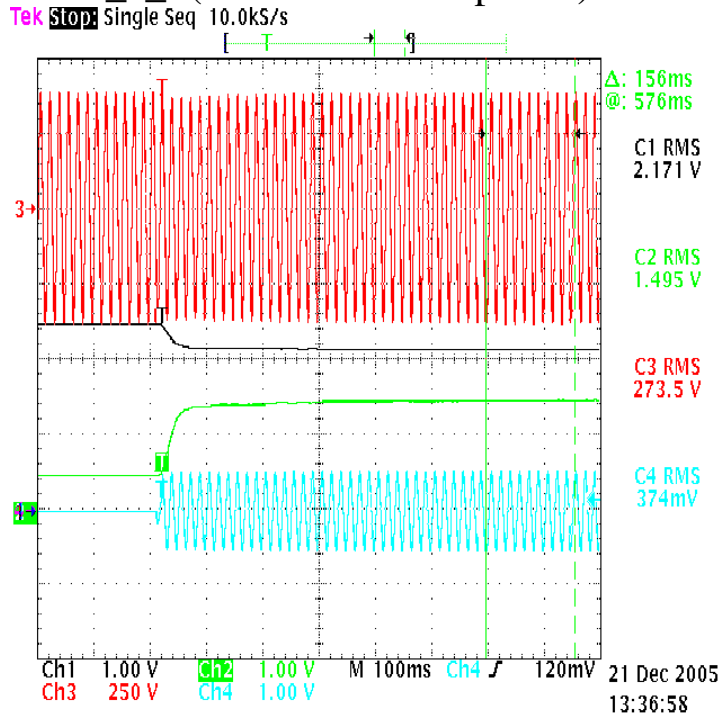

Unit \#1 Transient Response

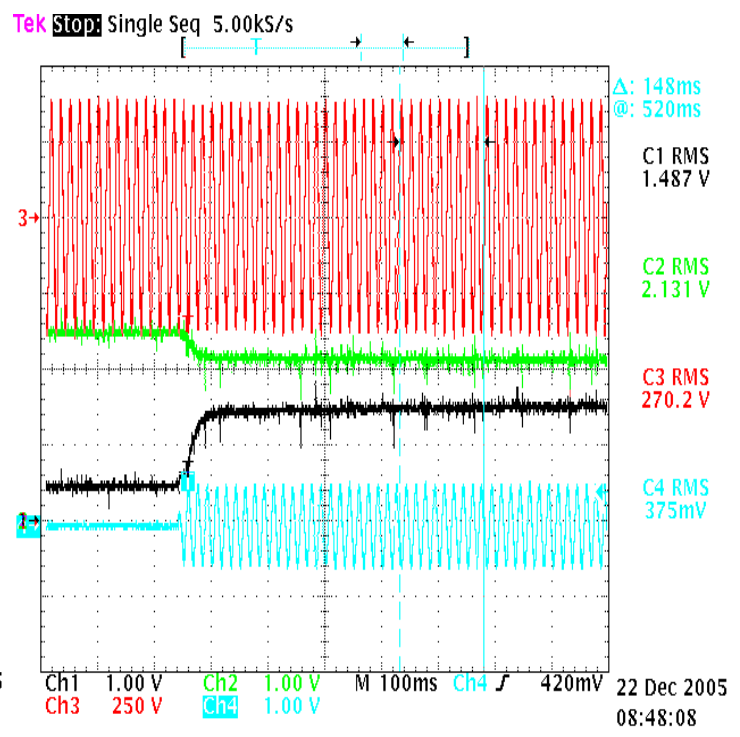

Unit \#2 Transient Response

Figure 1 Individual Inverter Transient Response 0 - 30 kW

\begin{tabular}{|c|c|c|}
\hline & A (Island) & B (Island) \\
\hline Load Bank \#1(S.P/Actual) KW & $0.0 / 0.0 \mathrm{KW}$ & $30 / 31.4 \mathrm{KW}$ \\
\hline Load Bank \#2(S.P/Actual) KW & $0.0 / 0.0 \mathrm{KW}$ & $0.0 / 0.0 \mathrm{KW}$ \\
\hline Unit \#1 (S.P/Actual) KW & $20.0 / 0.0 \mathrm{KW}$ Unit & $20.0 / 30.7 \mathrm{KW}$ Unit \\
\hline Unit \#2 (S.P/Actual) KW & $20.0 / 0.0 \mathrm{KW} \mathrm{Unit}$ & $20.0 / 30.3 \mathrm{KW}$ Unit \\
\hline Frequency Hz & $60.12 \mathrm{~Hz}$ & $59.91 \mathrm{~Hz}$ \\
\hline
\end{tabular}

Table 2 Individual Inverter Transient Response 0 - 30 kW 
Test I_a_ii (0KW-60KW step load)
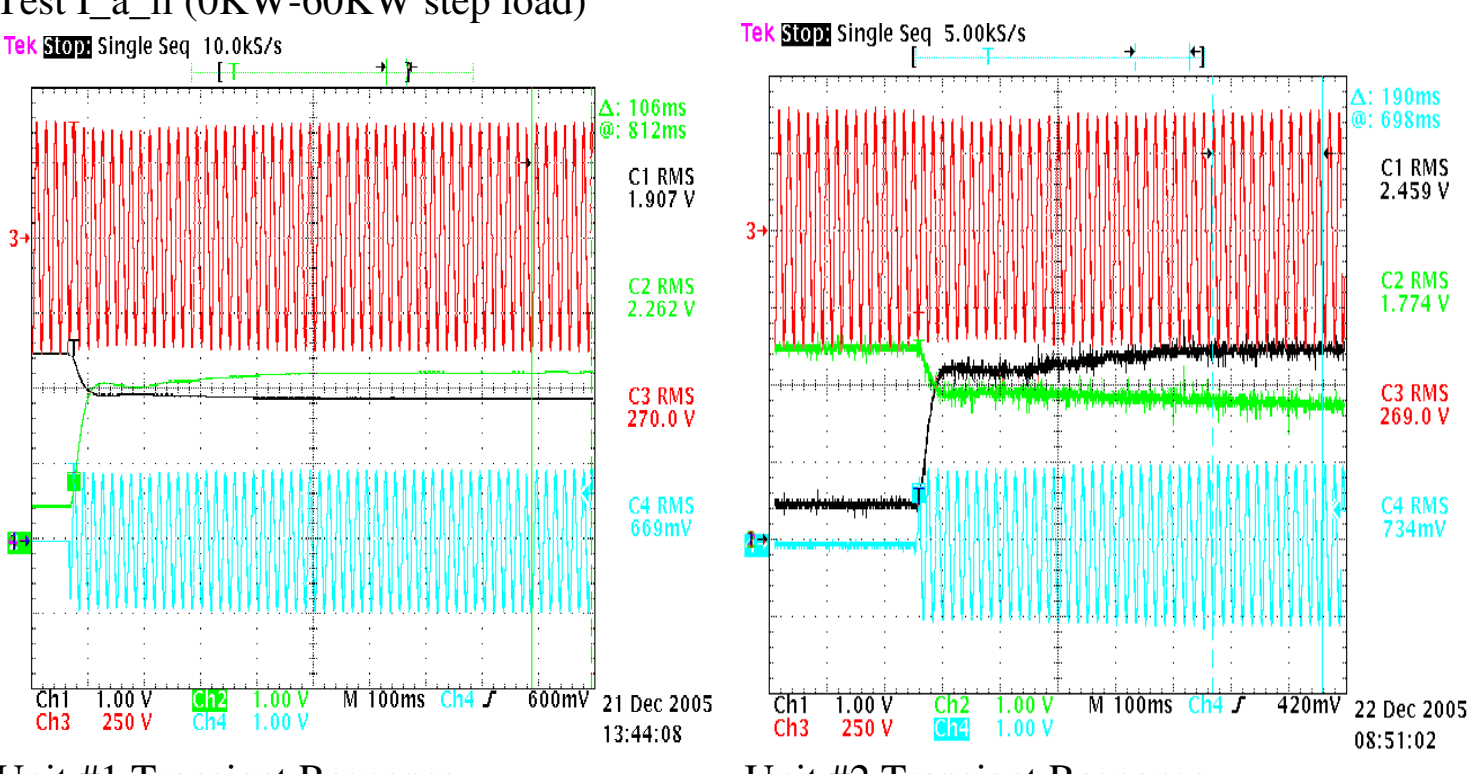

Unit \#1 Transient Response

Unit \#2 Transient Response

Figure 2 Individual Inverter Transient Response 0 - 60 kW

\begin{tabular}{|c|c|c|}
\hline & A (Island) & B (Island) \\
\hline Load Bank \#1(S.P/Actual) KW & $0.0 / 0.0 \mathrm{KW}$ & $60 / 62.8 \mathrm{KW}$ \\
\hline Load Bank \#2(S.P/Actual) KW & $0.0 / 0.0 \mathrm{KW}$ & $0.0 / 0.0 \mathrm{KW}$ \\
\hline Unit \#1 (S.P/Pout) KW & $20.0 / 0.0 \mathrm{KW}$ Unit & $20.0 / 54.1 \mathrm{KW}$ Unit \\
\hline Unit \#2 (S.P/Pout) KW & $20.0 / 0.0 \mathrm{KW}$ Unit & $20.0 / 59.2 \mathrm{KW}$ Unit \\
\hline Frequency Hz & $60.12 \mathrm{~Hz}$ & $59.61 \mathrm{~Hz}$ \\
\hline
\end{tabular}

Table 3 Individual Inverter Transient Response 0 - 60 kW 
Test I_a_iii (15KW-45KW step load)

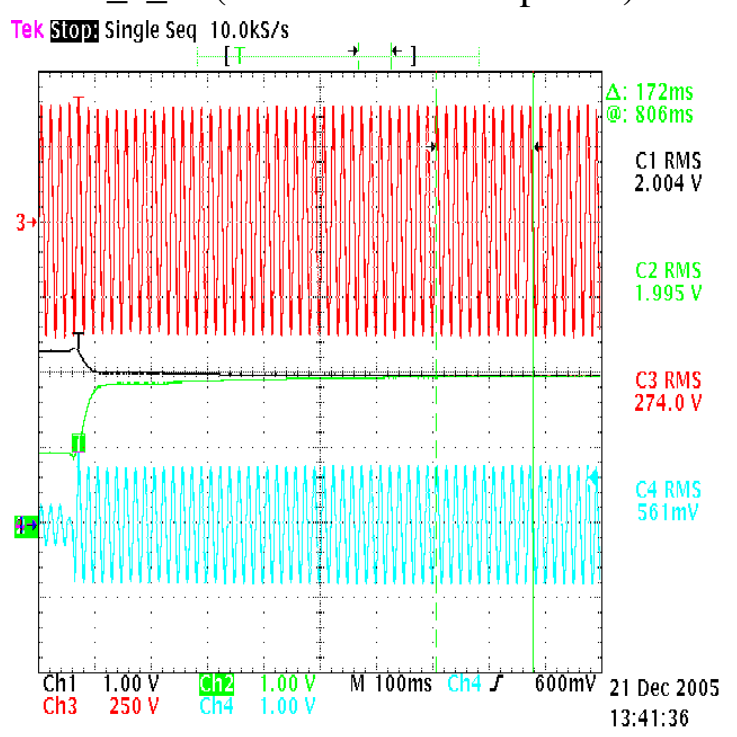

Unit \#1 Transient Response

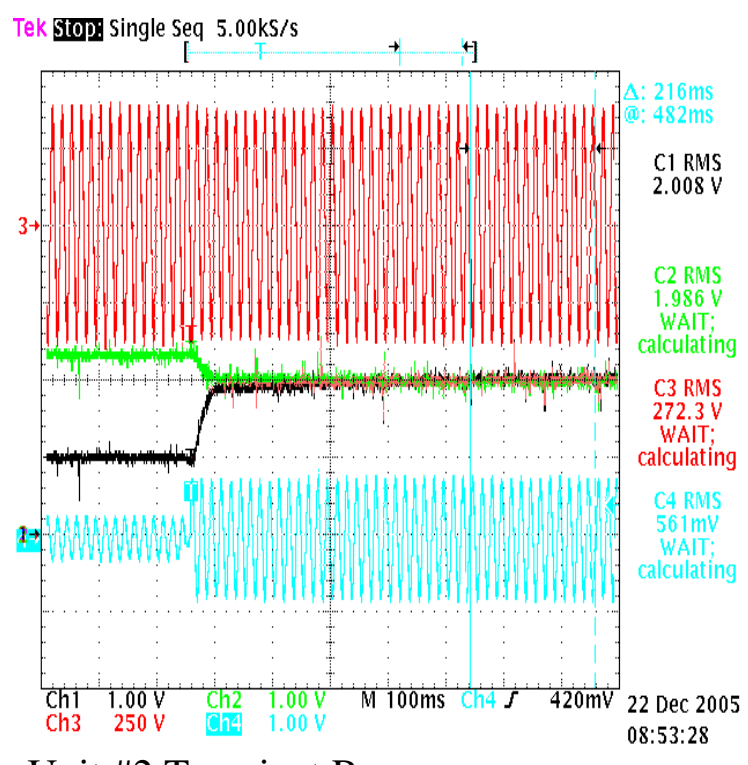

Unit \#2 Transient Response

Figure 3 Individual Inverter Transient Response 15 - 45 kW

\begin{tabular}{|c|c|c|}
\hline & A (Island) & B (Island) \\
\hline Load Bank \#1(S.P/Actual) KW & $15.0 / 15.5 \mathrm{KW}$ & $45.0 / 47.1 \mathrm{KW}$ \\
\hline Load Bank \#2(S.P/Actual) KW & $0.0 / 0.0 \mathrm{KW}$ & $0.0 / 0.0 \mathrm{KW}$ \\
\hline Unit \#1 (S.P/Pout) KW & $20.0 / 15.5 \mathrm{KW}$ Unit & $20.0 / 46.1 \mathrm{KW}$ Unit \\
\hline Unit \#2 (S.P/Pout) KW & $20.0 / 15.3 \mathrm{KW}$ Unit & $20.0 / 45.8 \mathrm{KW}$ Unit \\
\hline Frequency Hz & $59.99 \mathrm{~Hz}$ & $59.77 \mathrm{~Hz}$ \\
\hline
\end{tabular}

Table 4 Individual Inverter Transient Response 15 - 45 kW 
Test I_a_iv (30KW-60KW step load)

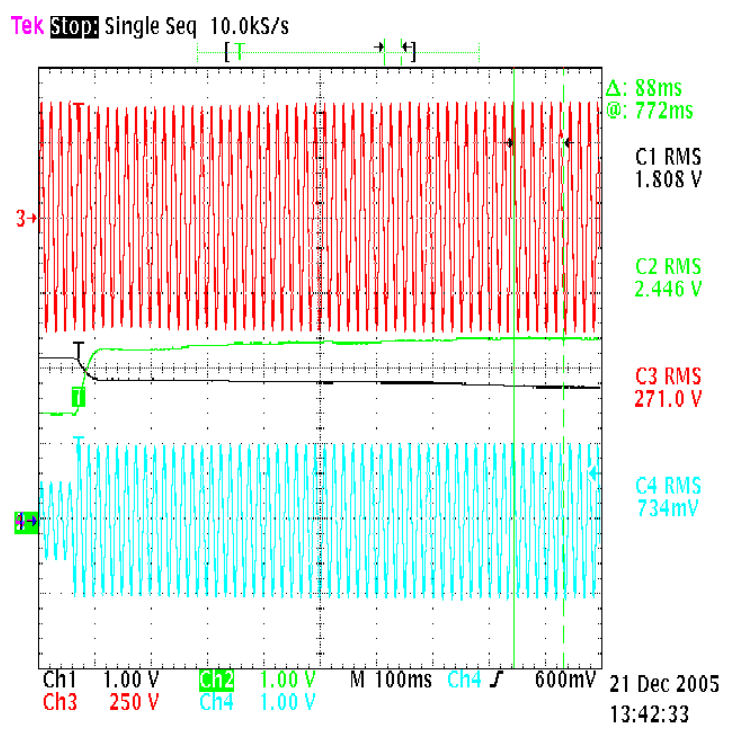

Unit \#1 Transient Response

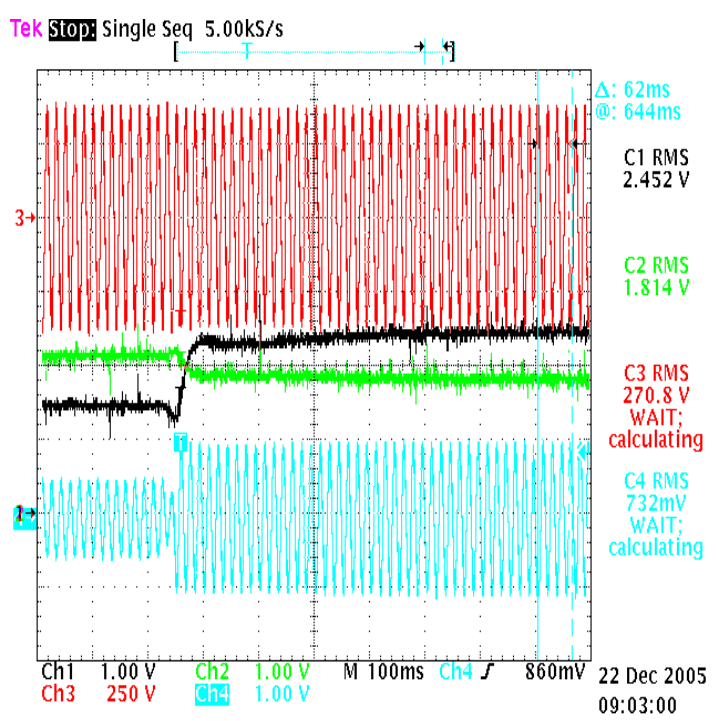

Unit \#2 Transient Response

Figure 4 Individual Inverter Transient Response 30 - 60 kW

\begin{tabular}{|c|c|c|}
\hline & A (Island) & B (Island) \\
\hline Load Bank \#1(S.P/Actual) KW & $30.0 / 31.4 \mathrm{KW}$ & $60.0 / 62.8 \mathrm{KW}$ \\
\hline Load Bank \#2(S.P/Actual) KW & $0.0 / 0.0 \mathrm{KW}$ & $0.0 / 0.0 \mathrm{KW}$ \\
\hline Unit \#1 (S.P/Pout) KW & $20.0 / 30.7 \mathrm{KW}$ Unit & $20.0 / 59.6 \mathrm{KW}$ Unit \\
\hline Unit \#2 (S.P/Pout) KW & $20.0 / 30.3 \mathrm{KW} \mathrm{Unit}$ & $20.0 / 59.4 \mathrm{KW}$ Unit \\
\hline Frequency Hz & $59.92 \mathrm{~Hz}$ & $59.57 \mathrm{~Hz}$ \\
\hline
\end{tabular}

Table 5 Individual Inverter Transient Response 30 - 60 kW 
Test I_a_vi (60KW-0KW step load)
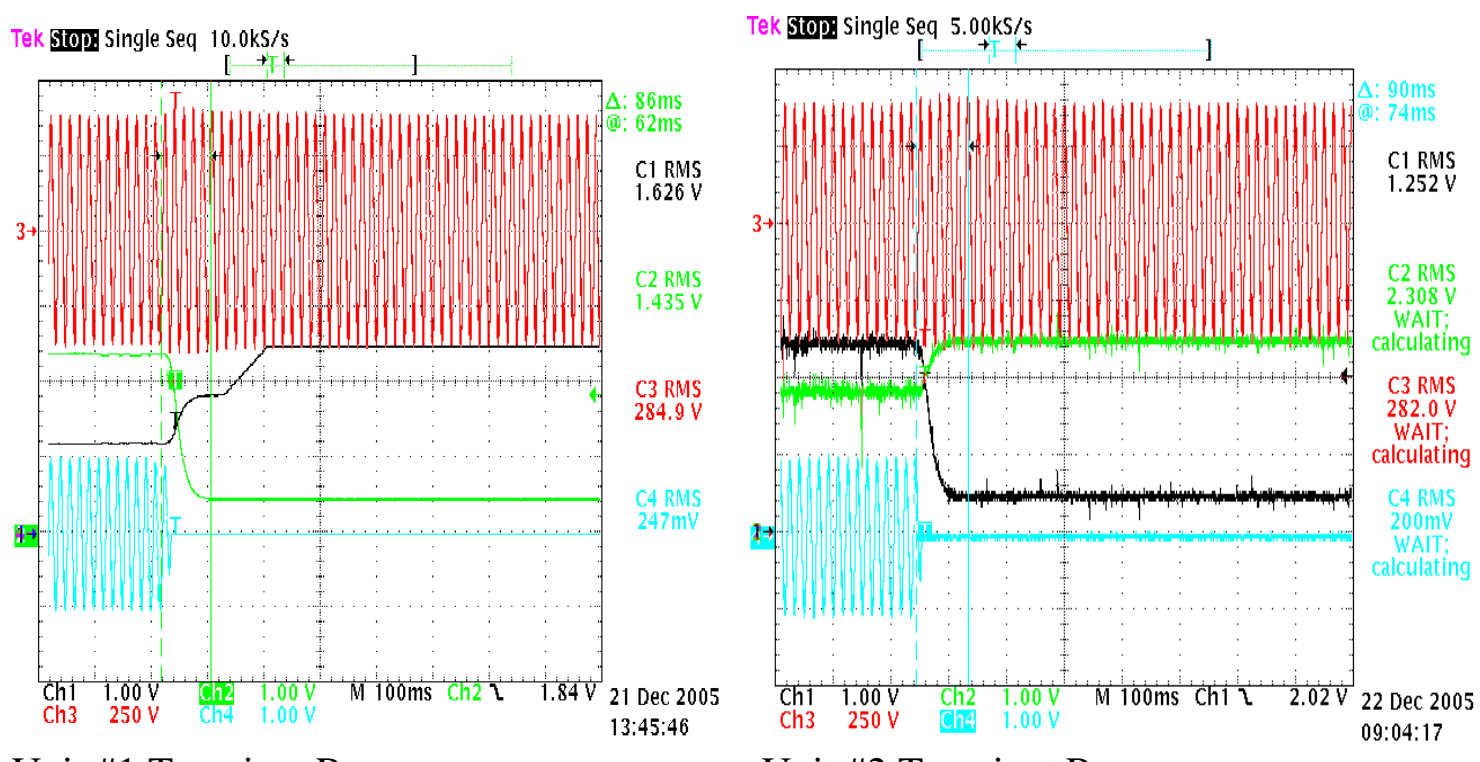

Unit \#1 Transient Response

Unit \#2 Transient Response

Figure 5 Individual Inverter Transient Response 60 - 0 kW

\begin{tabular}{|c|c|c|}
\hline & A (Island) & B (Island) \\
\hline Load Bank \#1(S.P/Actual) KW & $60.0 / 62.8 \mathrm{KW}$ & $0.0 / 0.0 \mathrm{KW}$ \\
\hline Load Bank \#2(S.P/Actual) KW & $0.0 / 0.0 \mathrm{KW}$ & $0.0 / 0.0 \mathrm{KW}$ \\
\hline Unit \#1 (S.P/Pout) KW & $20.0 / 59.6 \mathrm{KW}$ Unit & $20.0 / 0.0 \mathrm{KW}$ Unit \\
\hline Unit \#2 (S.P/Pout) KW & $20.0 / 59.4 \mathrm{KW}$ Unit & $20.0 / 0.0 \mathrm{KW}$ Unit \\
\hline Frequency Hz & $59.62 \mathrm{~Hz}$ & $60.12 \mathrm{~Hz}$ \\
\hline
\end{tabular}

Table 6 Individual Inverter Transient Response 60 - 0 kW 
Test I_a_vii (30KW-0KW step load)

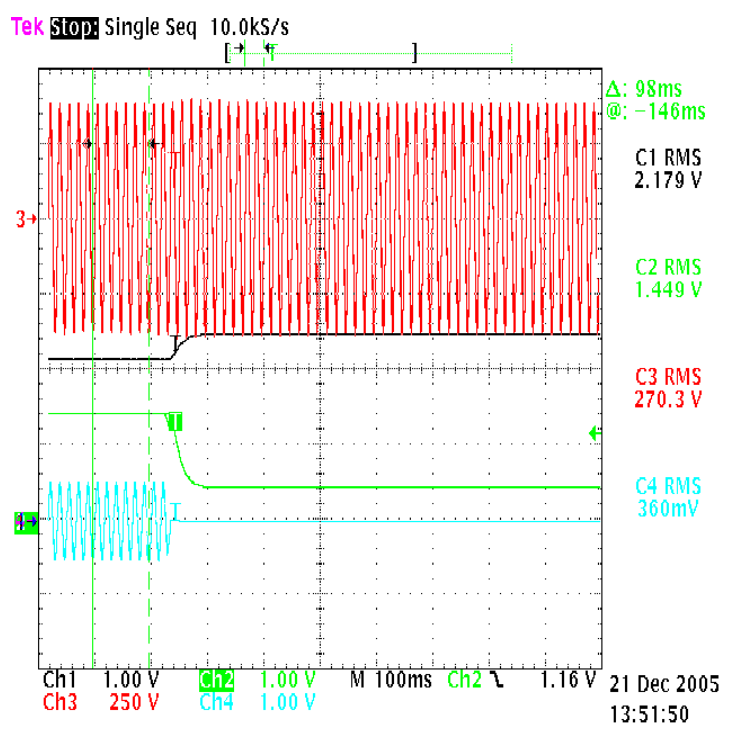

Unit \#1 Transient Response

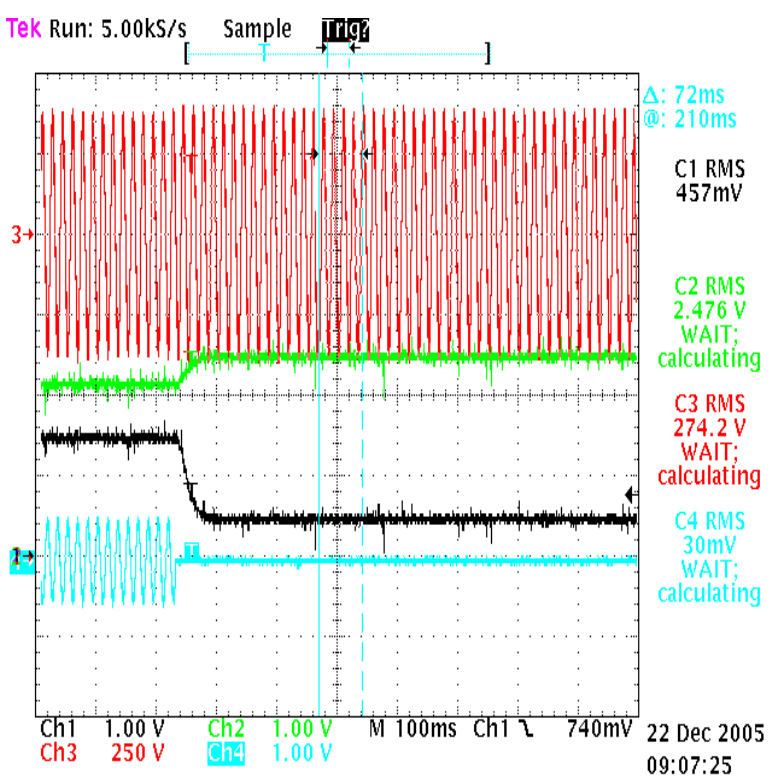

Unit \#2 Transient Response

Figure 6 Individual Inverter Transient Response 30 - 0 kW

\begin{tabular}{|c|c|c|}
\hline & A (Island) & B (Island) \\
\hline Load Bank \#1(S.P/Actual) KW & $30.0 / 31.4 \mathrm{KW}$ & $30.0 / 0.0 \mathrm{KW}$ \\
\hline Load Bank \#2(S.P/Actual) KW & $0.0 / 0.0 \mathrm{KW}$ & $0.0 / 0.0 \mathrm{KW}$ \\
\hline Unit \#1 (S.P/Pout) KW & XX KW Feeder or Unit & XX KW Feeder or Unit \\
\hline Unit \#2 (S.P/Pout) KW & XX KW Feeder or Unit & XX KW Feeder or Unit \\
\hline Frequency Hz & $59.91 \mathrm{~Hz}$ & $60.13 \mathrm{~Hz}$ \\
\hline
\end{tabular}

Table 7 Individual Inverter Transient Response 30 - 0 kW 
Phase Two Testing:

Phase two testing consists of two inverters connected in parallel operating in unit power control mode while connected to the grid, transitioning from a grid connection to an island case and while operating in an islanded case. The data provided in this section depicts various changes in the micro grid system including step loads, changes in commanded output power and changes in the state of the gird. For each test case, the initial conditions and the final conditions after the transient event of the micro grid occurred are given.

a.Unit Power Control Mode Grid Connected Evaluation

i. Step Load Evaluation 1

1. Load Bank \#1 Start Load $=10 \mathrm{KW}$

2. Load Bank \#2 Start Load $=20 \mathrm{KW}$

3. Unit $\mathrm{A} C \mathrm{CMD}=20 \mathrm{KW}$

4. Unit $\mathrm{B} C \mathrm{CMD}=40 \mathrm{KW}$

5. Load Bank \#1 End Load $=70 \mathrm{KW}$

6. Load Bank \#2 End Load $=20 \mathrm{KW}$

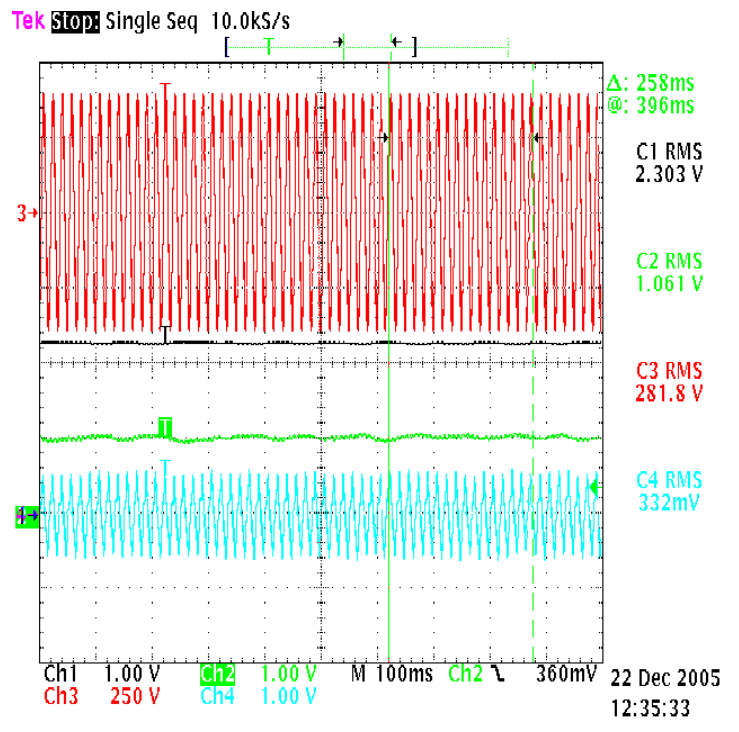

Unit \#1 Transient Response

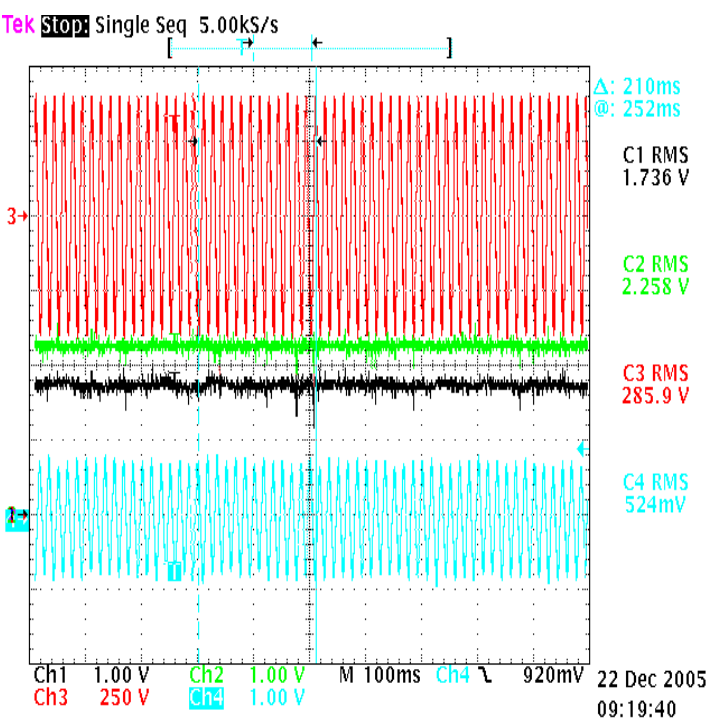

Unit \#2 Transient Response

Figure 7 Parallel Inverters -Unit Power Control, Grid Connect-Step Load Evaluation 1

\begin{tabular}{|c|c|c|}
\hline & A (Grid) & B (Grid) \\
\hline Load Bank \#1(S.P/Actual) KW & $10.0 / 10.5 \mathrm{KW}$ & $70.0 / 73.5 \mathrm{KW}$ \\
\hline Load Bank \#2(S.P/Actual) KW & $20.0 / 21.5 \mathrm{KW}$ & $20.0 / 21.5 \mathrm{KW}$ \\
\hline Unit \#1 (S.P/Pout) KW & $20.0 / 16.8 \mathrm{KW}$ Unit & $20.0 / 16.8 \mathrm{KW}$ Unit \\
\hline Unit \#2 (S.P/Pout) KW & $40.0 / 37.8 \mathrm{KW}$ Unit & $40.0 / 37.8 \mathrm{KW}$ Unit \\
\hline Frequency Hz & $59.97 \mathrm{~Hz}$ & $59.97 \mathrm{~Hz}$ \\
\hline
\end{tabular}

Table 8 Parallel Inverters -Unit Power Control, Grid Connect-Step Load Evaluation 1 
ii. Step Load Evaluation 2

1.Load Bank \#1 Start Load $=70 \mathrm{KW}$

2.Load Bank \#2 Start Load $=20 \mathrm{KW}$

3.Unit $\mathrm{A} \mathrm{CMD}=20 \mathrm{KW}$

4.Unit $\mathrm{B} \mathrm{CMD}=40 \mathrm{KW}$

5.Load Bank \#1 End Load $=25 \mathrm{KW}$

6.Load Bank \#2 End Load = 20KW

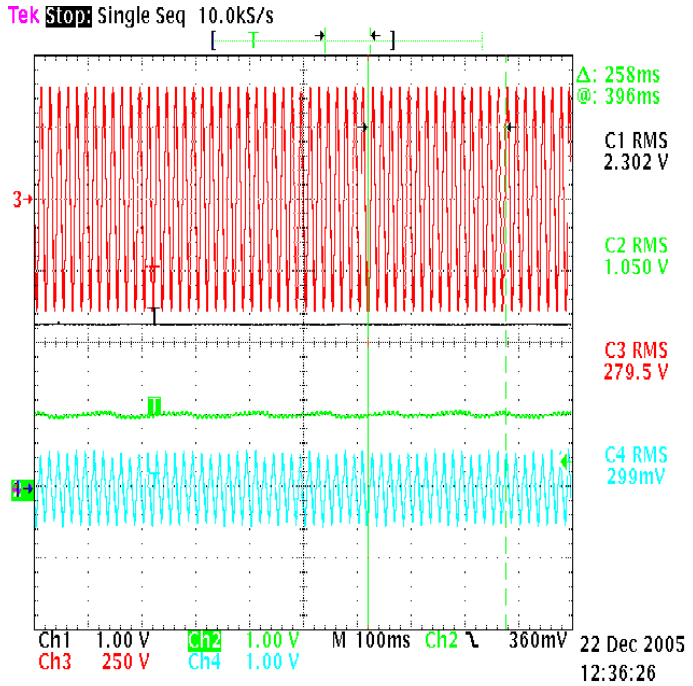

Unit \#1 Transient Response

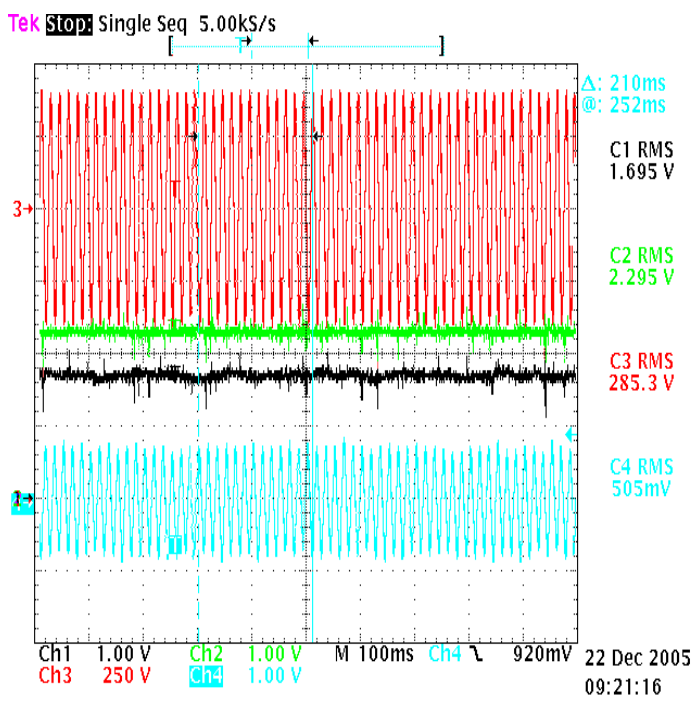

Unit \#2 Transient Response

Figure 8 Parallel Inverters -Unit Power Control, Grid Connect-Step Load Evaluation 2

\begin{tabular}{|c|c|c|}
\hline & A (Grid) & B (Grid) \\
\hline Load Bank \#1(S.P/Actual) KW & $70.0 / 73.5 \mathrm{KW}$ & $25.0 / 26.1 \mathrm{KW}$ \\
\hline Load Bank \#2(S.P/Actual) KW & $20.0 / 21.5 \mathrm{KW}$ & $20.0 / 21.5 \mathrm{KW}$ \\
\hline Unit \#1 (S.P/Pout) KW & $20.0 / 16.5 \mathrm{KW}$ Unit & $20.0 / 16.5 \mathrm{KW}$ Unit \\
\hline Unit \#2 (S.P/Pout) KW & $40.0 / 36.8 \mathrm{KW} \mathrm{Unit}$ & $40.0 / 36.8 \mathrm{KW}$ Unit \\
\hline Frequency Hz & $60.01 \mathrm{~Hz}$ & $60.01 \mathrm{~Hz}$ \\
\hline
\end{tabular}

Table 9 Parallel Inverters -Unit Power Control, Grid Connect-Step Load Evaluation 2 
b.) Unit Power Control Mode Grid Tie to Island Transition Evaluation

i.)Unit Power Limit Evaluation

7. Load Bank \#1 Start Load $=20 \mathrm{KW}$

8. Load Bank \#2 Start Load $=20 \mathrm{KW}$

9. Unit A Power CMD $=6 \mathrm{KW}$

10. Unit B Power CMD $=54 \mathrm{KW}$

11. Transition to Island

12. Load Bank \#1 End Load = 20KW

13. Load Bank \#2 End Load = 20KW

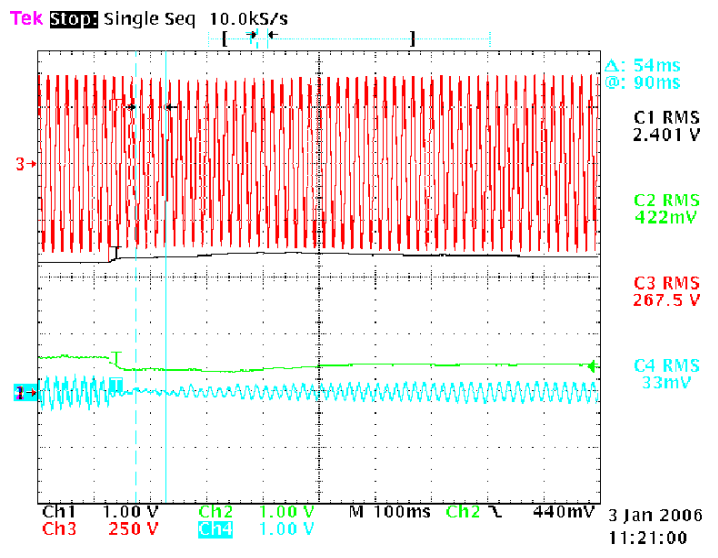

Unit \#1 Transient Response

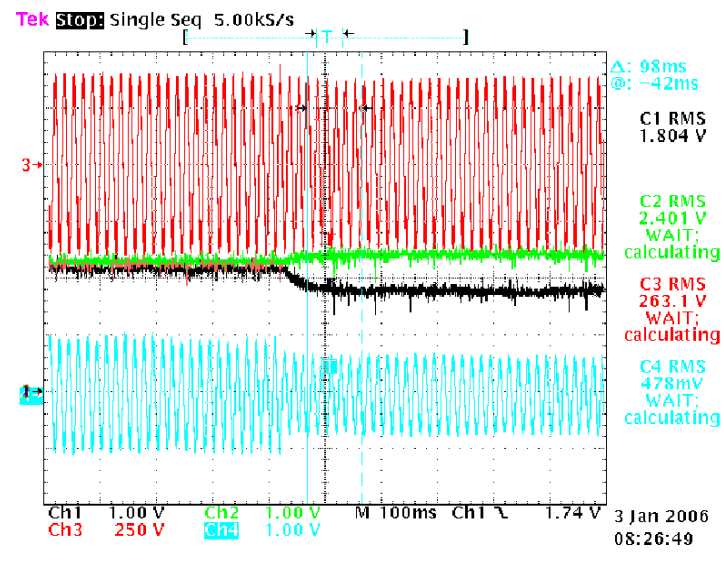

Unit \#2 Transient Response

Figure 9 Parallel Inverters - Unit Power Control - Grid to Island Transition

\begin{tabular}{|c|c|c|}
\hline & A (Grid) & B (Island) \\
\hline Load Bank \#1(S.P/Actual) KW & $20.0 / 21.5 \mathrm{KW}$ & $20.0 / 21.5 \mathrm{KW}$ \\
\hline Load Bank \#2(S.P/Actual) KW & $20.0 / 21.3 \mathrm{KW}$ & $20.0 / 21.3 \mathrm{KW}$ \\
\hline Unit \#1 (S.P/Pout) KW & $6.0 / 3.0 \mathrm{KW} \mathrm{Unit}$ & $6.0 / \sim 0.0 \mathrm{KW}$ Unit \\
\hline Unit \#2 (S.P/Pout) KW & $54.0 / 51.0 \mathrm{KW}$ Unit & $54.0 / 39.1 \mathrm{KW}$ Unit \\
\hline Frequency Hz & $59.92 \mathrm{~Hz}$ & $60.08 \mathrm{~Hz}$ \\
\hline
\end{tabular}

Table 10 Parallel Inverters - Unit Power Control - Grid to Island Transition 
ii. Unit Power Limit Evaluation

14. Load Bank \#1 Start Load $=60 \mathrm{KW}$

15. Load Bank \#2 Start Load $=40 \mathrm{KW}$

16. Unit A Power CMD $=54 \mathrm{KW}$

17. Unit B Power CMD $=6 \mathrm{KW}$

18. Transition to Island

19. Load Bank \#1 End Load $=60 \mathrm{KW}$

20. Load Bank \#2 End Load 40KW
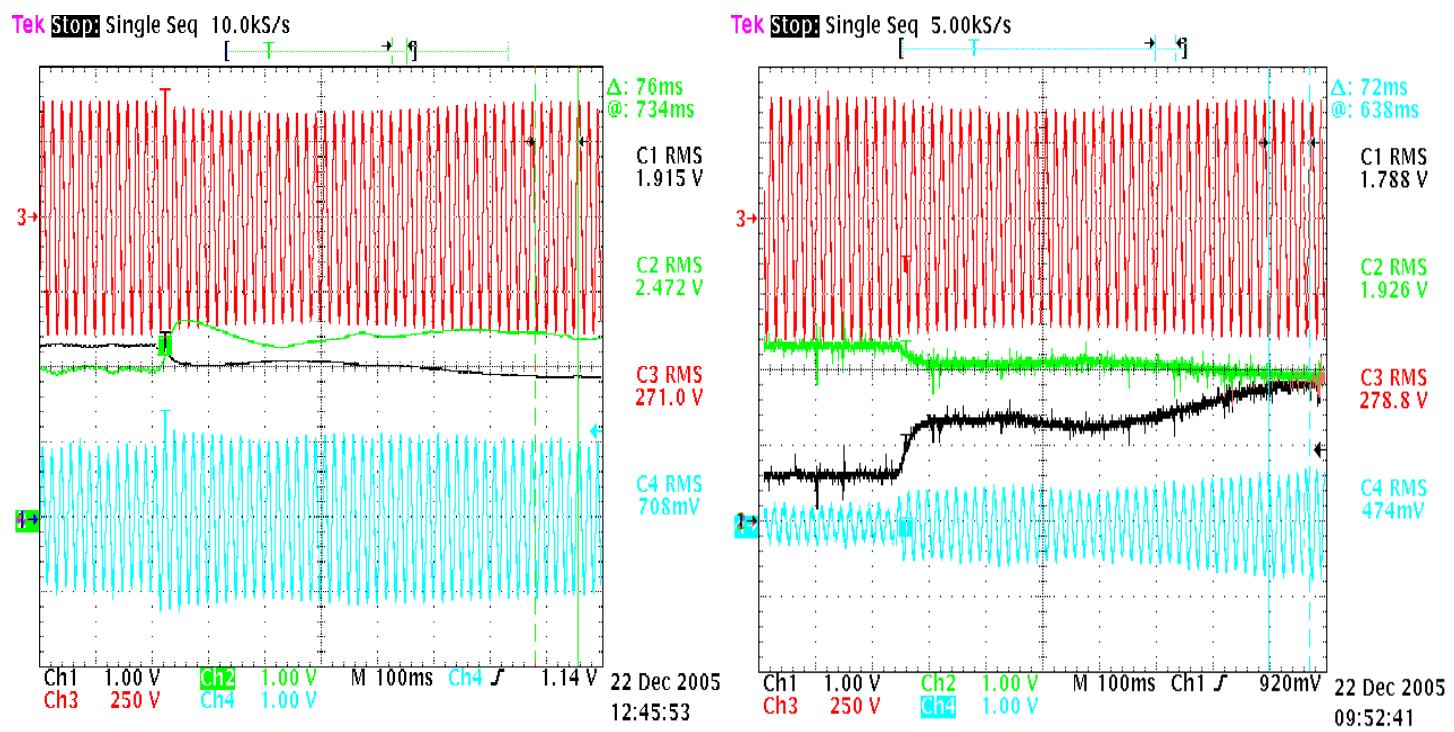

Unit \#1 Transient Response

Unit \#2 Transient Response

Figure 10 Parallel Inverters -Unit Power Control - Unit Power Limit Evaluation

\begin{tabular}{|c|c|c|}
\hline & A (Grid) & B (Island) \\
\hline Load Bank \#1(S.P/Actual) KW & $60.0 / 62.8 \mathrm{KW}$ & $60.0 / 62.8 \mathrm{KW}$ \\
\hline Load Bank \#2(S.P/Actual) KW & $40.0 / 42.3 \mathrm{KW}$ & $40.0 / 42.3 \mathrm{KW}$ \\
\hline Unit \#1 (S.P/Pout) KW & $54.0 / 50.0 \mathrm{KW}$ Unit & $54.0 / 60.1 \mathrm{KW}$ Unit \\
\hline Unit \#2 (S.P/Pout) KW & $6.0 / 3.0 \mathrm{KW} \mathrm{Unit}$ & $6.0 / 39.0 \mathrm{KW}$ Unit \\
\hline Frequency Hz & $59.99 \mathrm{~Hz}$ & $59.69 \mathrm{~Hz}$ \\
\hline
\end{tabular}

Table 11 Parallel Inverters -Unit Power Control - Unit Power Limit Evaluation 
c.) Unit Power Control Mode Island Evaluation

i.)Unit Power Limit Evaluation

1.) Load Bank \#1 Start Load $=0 \mathrm{KW}$

2.) Load Bank \#2 Start Load $=50 \mathrm{KW}$

3.) Unit A Power $\mathrm{CMD}=0 \mathrm{KW}$ (will reduce frequency)

4.) Unit $\mathrm{B}$ Power $\mathrm{CMD}=6 \mathrm{KW}$ (into energized line)

5.) Load Bank \#1 End Load = 0KW

6.) Load Bank \#2 End Load = $120 \mathrm{KW}$

Unit 1 closes into an islanded 50KW load, Unit 2 is off
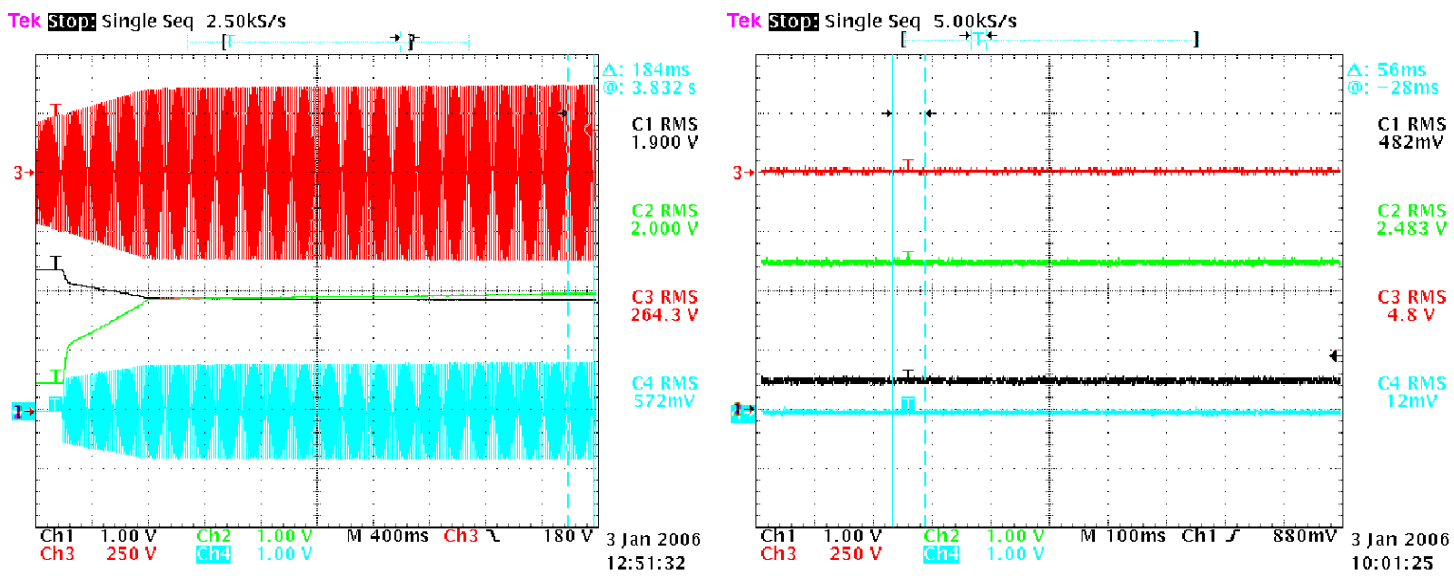

Figure 11 Parallel Inverters -Power Control, Island - Unit Power Limit Evaluation 1 (Step 1)

\begin{tabular}{|c|c|c|}
\hline & A (Island) & B (Island) \\
\hline Load Bank \#1(S.P/Actual) KW & $0.0 / 0.0 \mathrm{KW}$ & $0.0 / 0.0 \mathrm{KW}$ \\
\hline Load Bank \#2(S.P/Actual) KW & $50.0 / 51.6 \mathrm{KW}$ & $50.0 / 51.6 \mathrm{KW}$ \\
\hline Unit \#1 (S.P/Pout) KW & $6.0 / 0.0 \mathrm{KW} \mathrm{Unit}$ & $6.0 / 49.5 \mathrm{KW}$ Unit \\
\hline Unit \#2 (S.P/Pout) KW & NA & NA \\
\hline Frequency Hz & $60.12 \mathrm{~Hz}$ & $59.68 \mathrm{~Hz}$ \\
\hline
\end{tabular}

Table 12 Parallel Inverters - Power Control, Island - Unit Power Limit Evaluation 1 (Step 1) 
$50 \mathrm{KW}$ load on Unit 1 and then Unit 2 energizes into the islanded micro grid

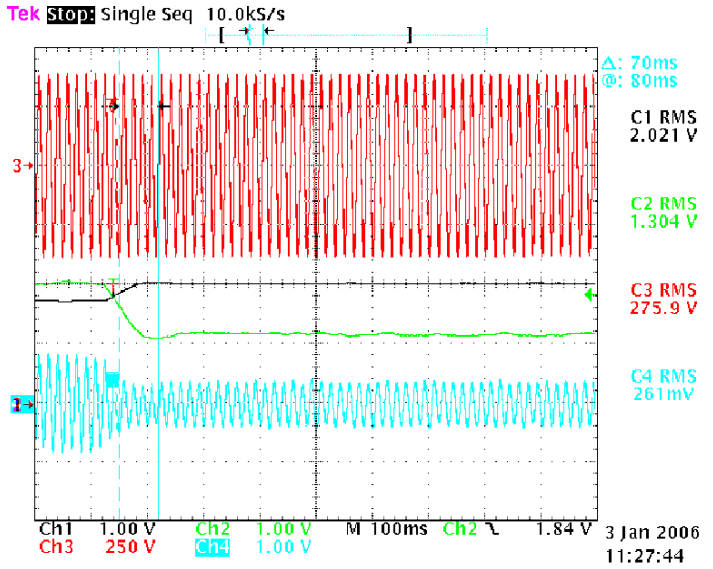

Unit \#1 Transient Response

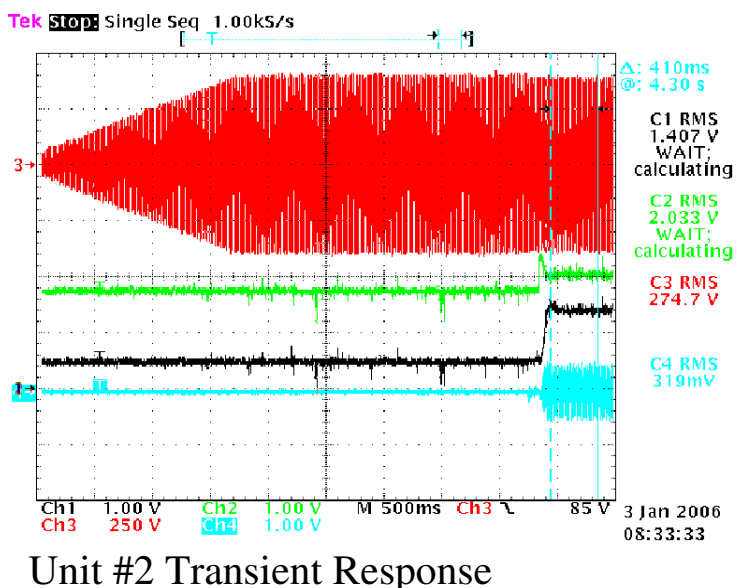

Figure 12 Parallel Inverters - Power Control, Island- Unit Power Limit Evaluation 1 (Step 2)

\begin{tabular}{|c|c|c|}
\hline & A (Island) & B (Island) \\
\hline Load Bank \#1(S.P/Actual) KW & $0.0 / 0.0 \mathrm{KW}$ & $0.0 / 0.0 \mathrm{KW}$ \\
\hline Load Bank \#2(S.P/Actual) KW & $50.0 / 51.6 \mathrm{KW}$ & $50.0 / 51.6 \mathrm{KW}$ \\
\hline Unit \#1 (S.P/Pout) KW & $0.0 / 49.1 \mathrm{KW}$ Unit & $0.0 / 21.8 \mathrm{KW}$ Unit \\
\hline Unit \#2 (S.P/Pout) KW & $6.0 / 0.0 \mathrm{KW} \mathrm{Unit}$ & $6.0 / 26.2 \mathrm{KW}$ Unit \\
\hline Frequency Hz & $59.61 \mathrm{~Hz}$ & $59.79 \mathrm{~Hz}$ \\
\hline
\end{tabular}

Table 13 Parallel Inverters - Power Control, Island - Unit Power Limit Evaluation 1 (Step 2) 


\section{Load change from $50 \mathrm{Kw}$ to $120 \mathrm{KW}$}

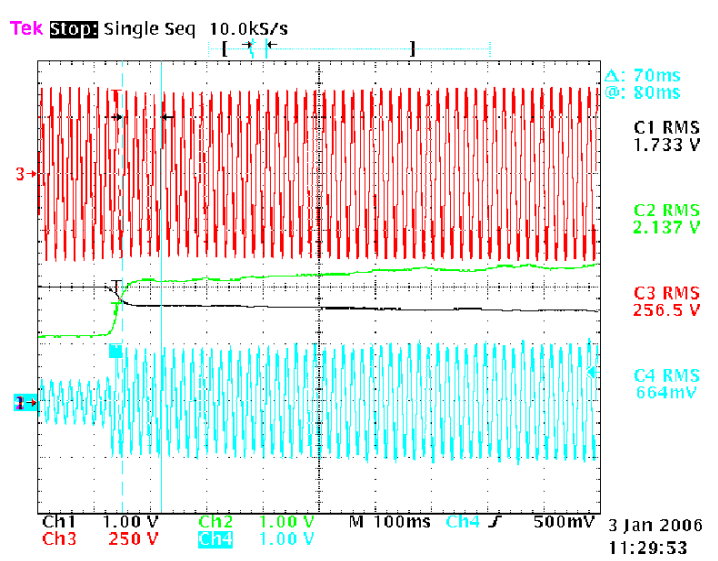

Unit \#1 Transient Response

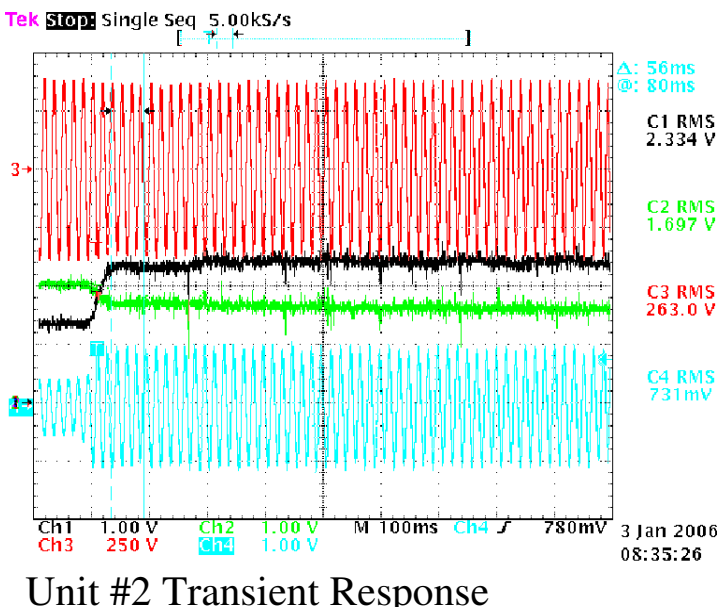

Figure 13 Parallel Inverters -Power Control, Island - Unit Power Limit Evaluation 1 (Step 3)

\begin{tabular}{|c|c|c|}
\hline & A (Island) & B (Island) \\
\hline Load Bank \#1(S.P/Actual) KW & $0.0 / 0.0 \mathrm{KW}$ & $0.0 / 0.0 \mathrm{KW}$ \\
\hline Load Bank \#2(S.P/Actual) KW & $50.0 / 51.6 \mathrm{KW}$ & $50.0 / 125.6 \mathrm{KW}$ \\
\hline Unit \#1 (S.P/Pout) KW & $0.0 / 21.8 \mathrm{KW}$ Unit & $0.0 / 58.6 \mathrm{KW}$ Unit \\
\hline Unit \#2 (S.P/Pout) KW & $6.0 / 26.2 \mathrm{KW} \mathrm{Unit}$ & $6.0 / 57.9 \mathrm{KW}$ Unit \\
\hline Frequency Hz & $59.79 \mathrm{~Hz}$ & $59.45 \mathrm{~Hz}$ \\
\hline
\end{tabular}

Table 14 Parallel Inverters - Power Control, Island - Unit Power Limit Evaluation 1 (Step 3) 
ii.) Unit Power Limit Evaluation

1.) Load Bank \#1 Start Load $=120 \mathrm{KW}$

2.) Load Bank \#2 Start Load $=0 \mathrm{KW}$

3.) Unit $\mathrm{A}$ Power $\mathrm{CMD}=54 \mathrm{KW}$

4.) Unit $B$ Power $C M D=6 \mathrm{KW}$

5.) Load Bank \#1 End Load = $0 \mathrm{KW}$

6.) Load Bank \#2 End Load = 0KW

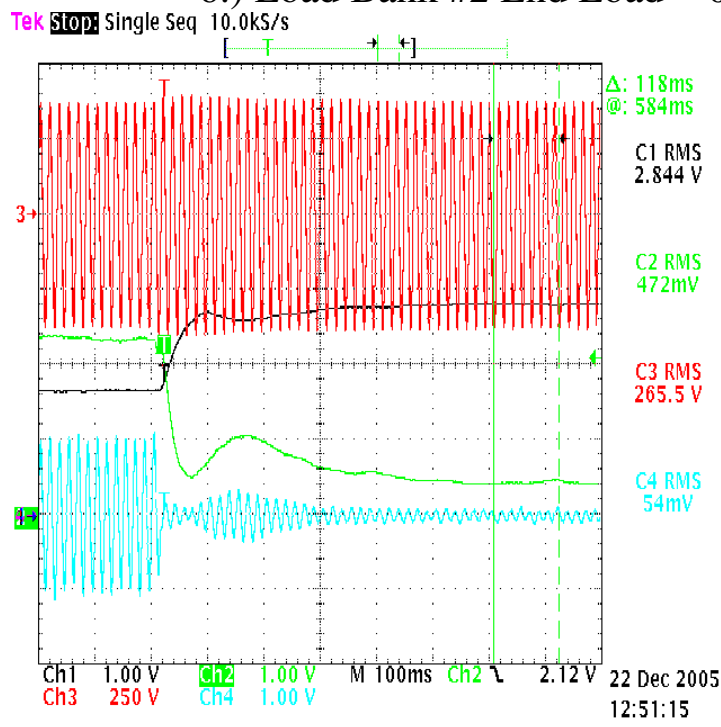

Unit \#1 Transient Response

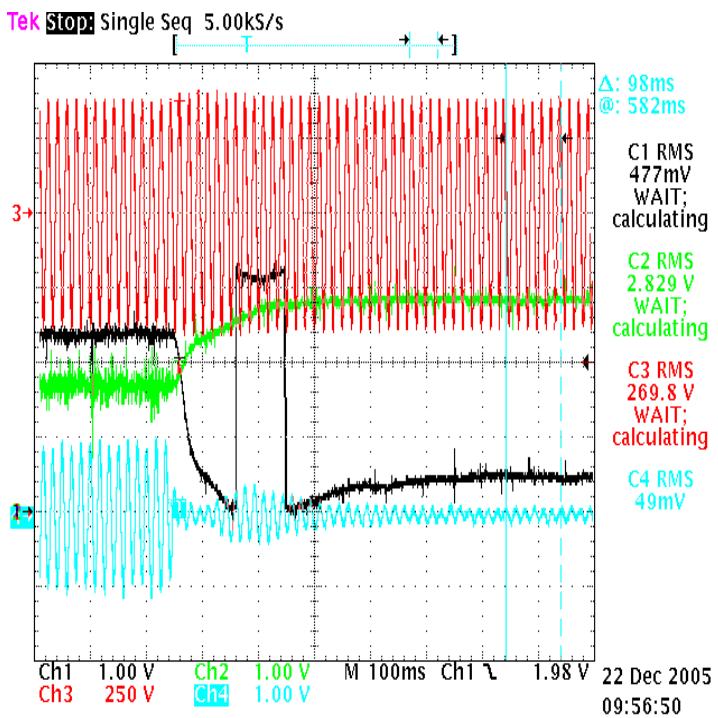

Unit \#2 Transient Response

Figure 14 Parallel Inverters - Power Control, Island - Unit Power Limit Evaluation 2

\begin{tabular}{|c|c|c|}
\hline & A (Island) & B (Island) \\
\hline Load Bank \#1(S.P/Actual) KW & $120.0 / 124.6 \mathrm{KW}$ & $0.0 / 0.0 \mathrm{KW}$ \\
\hline Load Bank \#2(S.P/Actual) KW & $0.0 / 0.0 \mathrm{KW}$ & $0.0 / 0.0 \mathrm{KW}$ \\
\hline Unit \#1 (S.P/Pout) KW & $54.0 / 58.2 \mathrm{KW}$ Unit & $54.0 / 0.0 \mathrm{KW}$ Unit \\
\hline Unit \#2 (S.P/Pout) KW & $6.0 / 57.4 \mathrm{KW} \mathrm{Unit}$ & $6.0 / 0.0 \mathrm{KW}$ Unit \\
\hline Frequency Hz & $59.45 \mathrm{~Hz}$ & $60.42 \mathrm{~Hz}$ \\
\hline
\end{tabular}

Table 15 Parallel Inverters - Power Control, Island - Unit Power Limit Evaluation 2 
iii.Unbalanced Load

1.Load Bank \#1 Start Load = 0KW

2.Load Bank \#2 Start Load $=50 \mathrm{KW}$

3.Unit A Power CMD $=54 \mathrm{KW}$

4.Unit B Power CMD $=6 \mathrm{KW}$

5.Load Bank \#1 End Load = 0KW

6.Load Bank \#2 End Load = $25 \mathrm{KW}$ (Inverter phase A opened to delta load bank)

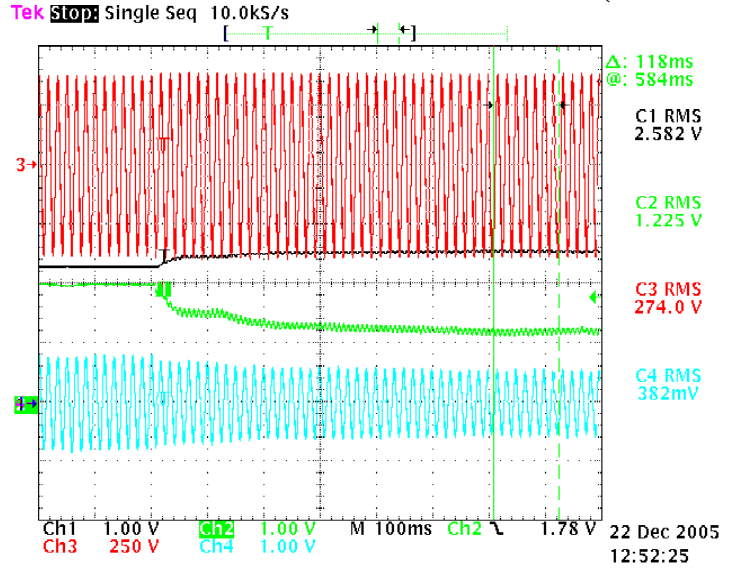

Unit \#1 Transient Response

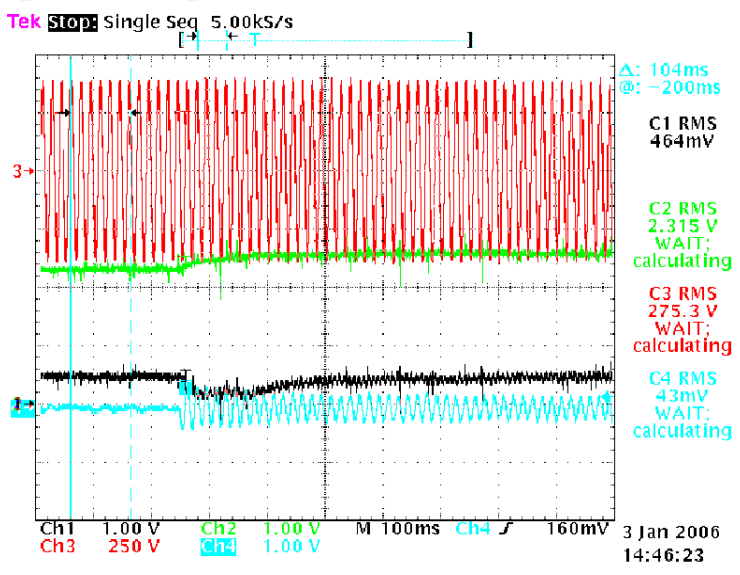

Unit \#2 Transient Response

Figure 15 Parallel Inverters - Power Control, Island - Unbalanced Load

\begin{tabular}{|c|c|c|}
\hline & A (Island) & B (Island) \\
\hline Load Bank \#1(S.P/Actual) KW & $0.0 / 0.0 \mathrm{KW}$ & $0.0 / 0.0 \mathrm{KW}$ \\
\hline Load Bank \#2(S.P/Actual) KW & $50.0 / 51.9 \mathrm{KW}$ & $33.3 / 34.6 \mathrm{KW}$ \\
\hline Unit \#1 (S.P/Pout) KW & $54.0 / 45.6 \mathrm{KW}$ Unit & $54.0 / 23.0 \mathrm{KW}$ Unit \\
\hline Unit \#2 (S.P/Pout) KW & $6.0 / 0.0 \mathrm{KW} \mathrm{Unit}$ & $6.0 / 3.0 \mathrm{KW}$ Unit \\
\hline Frequency Hz & $60.01 \mathrm{~Hz}$ & $60.22 \mathrm{~Hz}$ \\
\hline
\end{tabular}

Table 16 Parallel Inverters - Power Control, Island - Unbalanced Load 
Phase Three Testing:

Phase three testing consists of two inverters connected in parallel operating with unit one operating in power control mode and unit two operating in unit flow control while connected to the grid, transitioning from a grid connection to an island case and while operating in an islanded case. The data provided in this section depicts various changes in the micro grid system including step loads, changes in commanded output power and changes in the state of the gird. For each test case, the initial conditions and the final conditions after the transient event of the micro grid occurred are given.

a. Grid Tie Feeder Control Mode Evaluation

i. Step Load Evaluation 1

1. Load Bank \#1 Start Load = 35KW

2. Load Bank \#2 Start Load $=35 \mathrm{KW}$

3. Unit A Feeder CMD $=54 \mathrm{KW}$

4. Unit B Power CMD $=6 \mathrm{KW}$

5. Load Bank \#1 End Load $=85 \mathrm{KW}$

6. Load Bank \#2 End Load $=35 \mathrm{KW}$

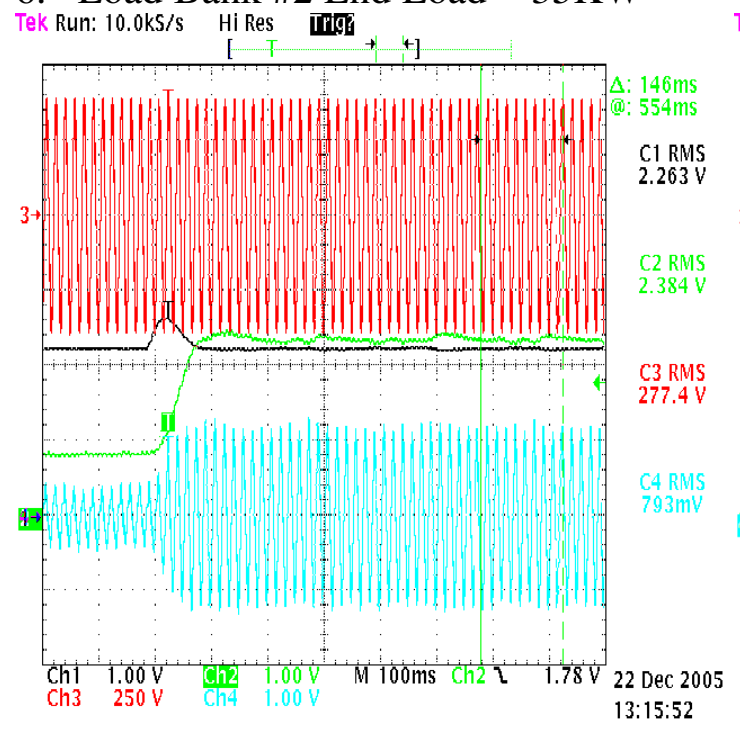

Unit \#1 Transient Response

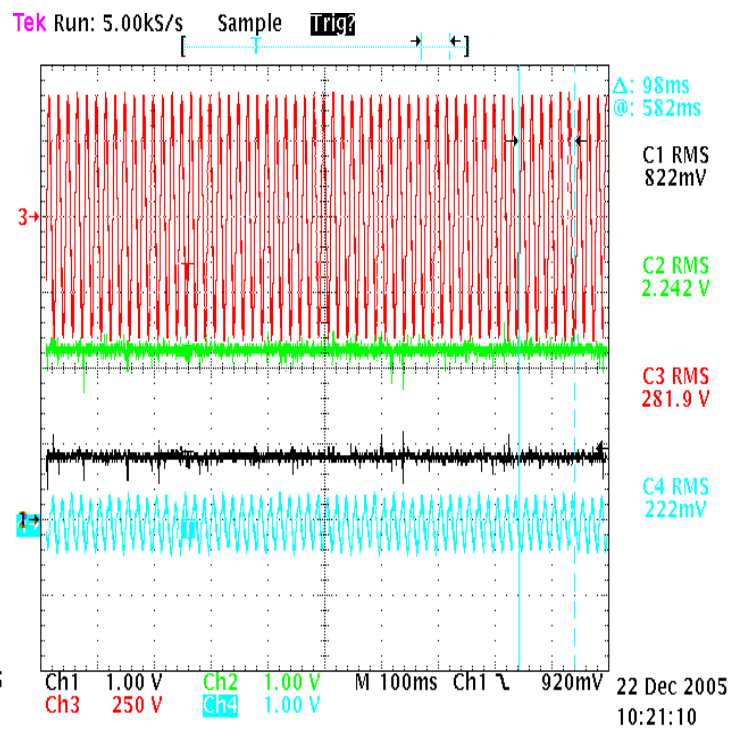

Unit \#2 Transient Response

Figure 16 Parallel Inverters - Feeder Control, Grid Tie - Step Load Evaluation 1

\begin{tabular}{|c|c|c|}
\hline & A (Grid) & B (Grid) \\
\hline Load Bank \#1(S.P/Actual) KW & $35.0 / 37.2 \mathrm{KW}$ & $85.0 / 88.1 \mathrm{KW}$ \\
\hline Load Bank \#2(S.P/Actual) KW & $35.0 / 36.9 \mathrm{KW}$ & $35.0 / 36.9 \mathrm{KW}$ \\
\hline Unit \#1 (S.P/Pout) KW & $54.0 / 8.2 \mathrm{KW}$ Feeder & $54.0 / 56.8 \mathrm{KW}$ Feeder \\
\hline Unit \#2 (S.P/Pout) KW & $6.0 / 7.2 \mathrm{KW} \mathrm{Unit}$ & $6.0 / 7.2 \mathrm{KW}$ Unit \\
\hline Frequency Hz & $59.97 \mathrm{~Hz}$ & $59.96 \mathrm{~Hz}$ \\
\hline
\end{tabular}

Table 17 Parallel Inverters - Feeder Control, Grid Tie - Step Load Evaluation 1 
ii.) Step Load Evaluation 2

1.) Load Bank \#1 Start Load $=85 \mathrm{KW}$

2.) Load Bank \#2 Start Load $=35 \mathrm{KW}$

3.) Unit A Feeder $\mathrm{CMD}=40 \mathrm{KW}$

4.) Unit $B$ Power $C M D=6 \mathrm{KW}$

5.) Load Bank \#1 End Load $=25 \mathrm{KW}$

6.) Load Bank \#2 End Load = 35KW
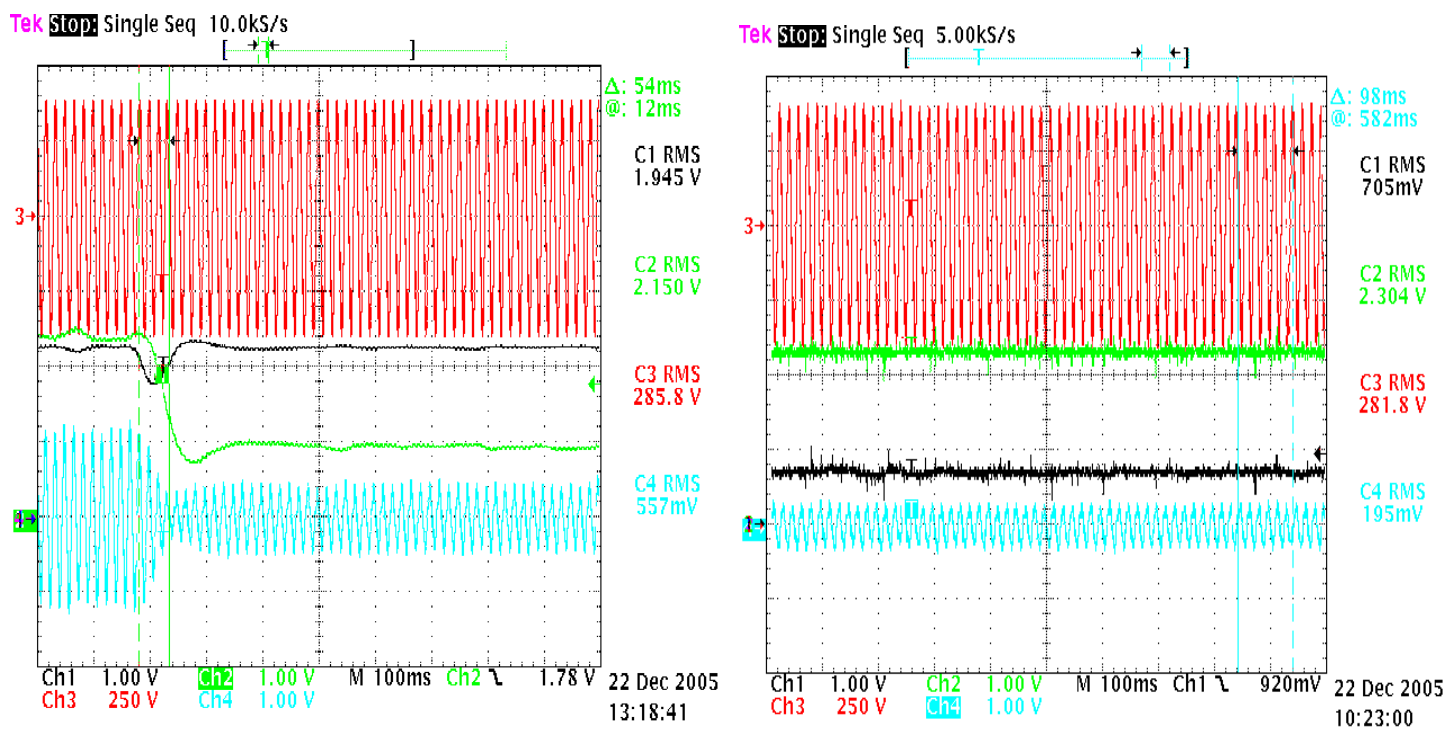

Unit \#1 Transient Response

Unit \#2 Transient Response

Figure 17 Parallel Inverters - Feeder Control, Grid Tie - Step Load Evaluation 2

\begin{tabular}{|c|c|c|}
\hline & A (Grid) & B (Grid) \\
\hline Load Bank \#1(S.P/Actual) KW & $85.0 / 88.1 \mathrm{KW}$ & $35.0 / 37.2 \mathrm{KW}$ \\
\hline Load Bank \#2(S.P/Actual) KW & $35.0 / 36.9 \mathrm{KW}$ & $25.0 / 38.1 \mathrm{KW}$ \\
\hline Unit \#1 (S.P/Pout) KW & $40.0 / 61.1 \mathrm{KW}$ Feeder & $40.0 / 14.0 \mathrm{KW}$ Feeder \\
\hline Unit \#2 (S.P/Pout) KW & $6.0 / \mathrm{KW} \mathrm{Unit}$ & $6.0 / 6.5 \mathrm{KW}$ Unit \\
\hline Frequency Hz & $59.7 \mathrm{~Hz}$ & $59.8 \mathrm{~Hz}$ \\
\hline
\end{tabular}

Table 18 Parallel Inverters - Feeder Control, Grid Tie - Step Load Evaluation 2 
b. Feeder Control Mode to Island Transition Evaluation

i. Unit Power Limit Evaluation

1. Load Bank \#1 Start Load $=35 \mathrm{KW}$

2. Load Bank \#2 Start Load $=35 \mathrm{KW}$

3. Unit A Feeder $\mathrm{CMD}=50 \mathrm{KW}$

4. Unit B Power CMD $=6 \mathrm{KW}$

5. Transition to island

6. Load Bank \#1 End Load $=35 \mathrm{KW}$

7. Load Bank \#2 End Load $=35 \mathrm{KW}$

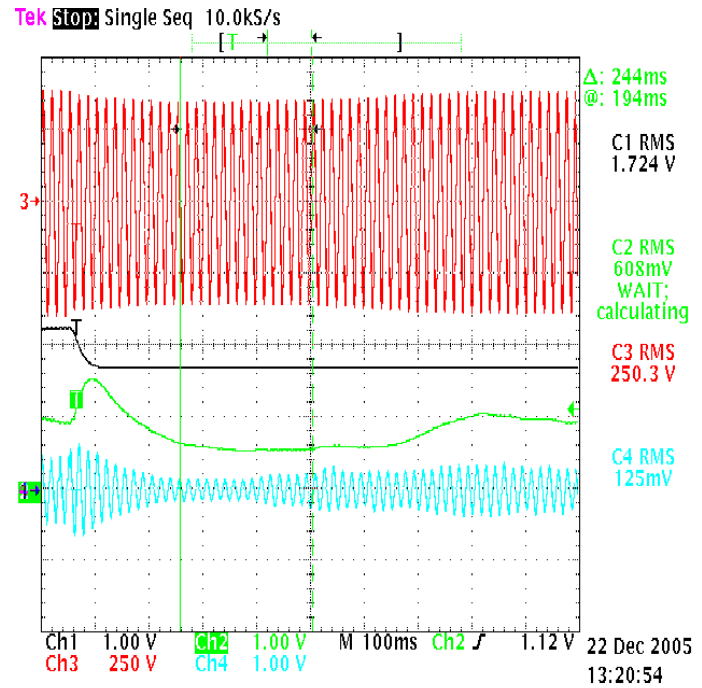

Unit \#1 Transient Response

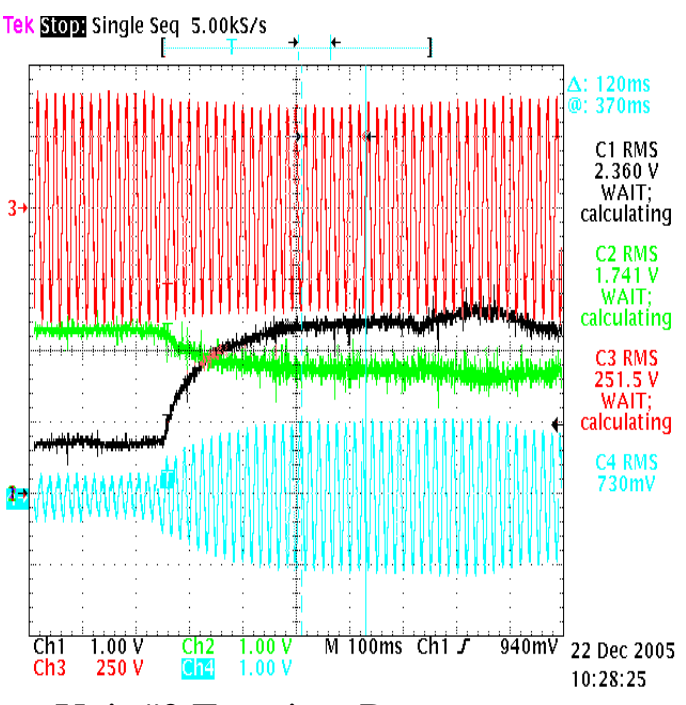

Unit \#2 Transient Response

Figure 18 Parallel Inverters - Feeder Control, Island Transition - Unit Power Limit Evaluation 1

\begin{tabular}{|c|c|c|}
\hline & A (Grid) & B (Island) \\
\hline Load Bank \#1(S.P/Actual) KW & $35.0 / 37.2 \mathrm{KW}$ & $35.0 / 37.2 \mathrm{KW}$ \\
\hline Load Bank \#2(S.P/Actual) KW & $35.0 / 36.9 \mathrm{KW}$ & $35.0 / 36.9 \mathrm{KW}$ \\
\hline Unit \#1 (S.P/Pout) KW & $50.0 / 9.0 \mathrm{KW} \mathrm{Feeder}$ & $50.0 / 10.0 \mathrm{KW}$ Feeder \\
\hline Unit \#2 (S.P/Pout) KW & $6.0 / \mathrm{KW} \mathrm{Unit}$ & $6.0 / 51.0 \mathrm{KW}$ Unit \\
\hline Frequency Hz & $59.92 \mathrm{~Hz}$ & $59.54 \mathrm{~Hz}$ \\
\hline
\end{tabular}

Table 19 Parallel Inverters - Feeder Control, Island Transition - Unit Power Limit Evaluation 1 
ii. Unit Power Limit Evaluation

1. Load Bank \#1 Start Load $=70 \mathrm{KW}$

2. Load Bank \#2 Start Load $=50 \mathrm{KW}$

3. Unit A Feeder $\mathrm{CMD}=54 \mathrm{KW}$

4. Unit B Power $\mathrm{CMD}=6 \mathrm{KW}$

5. Transition to island

6. Load Bank \#1 End Load $=70 \mathrm{KW}$

7. Load Bank \#2 End Load $=50 \mathrm{KW}$

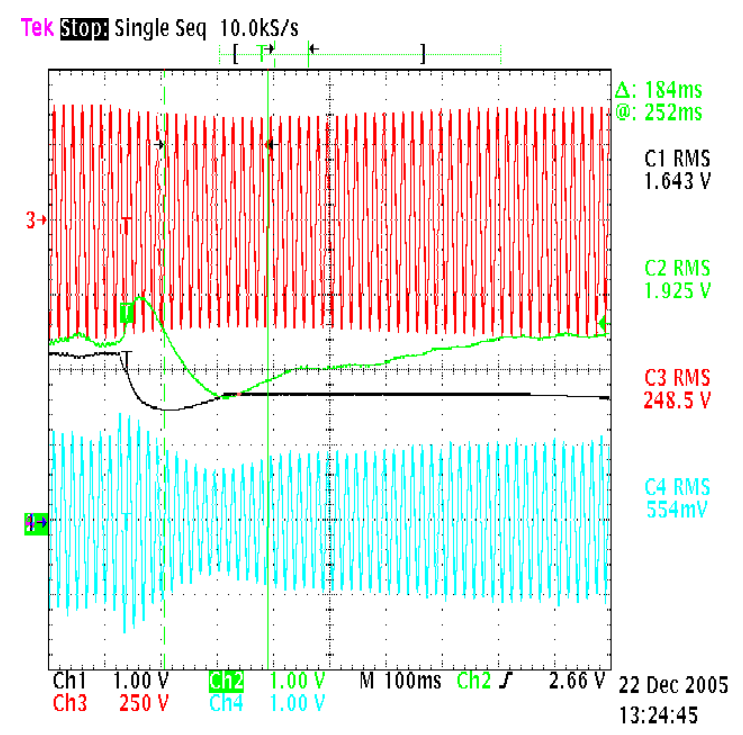

Unit \#1 Transient Response

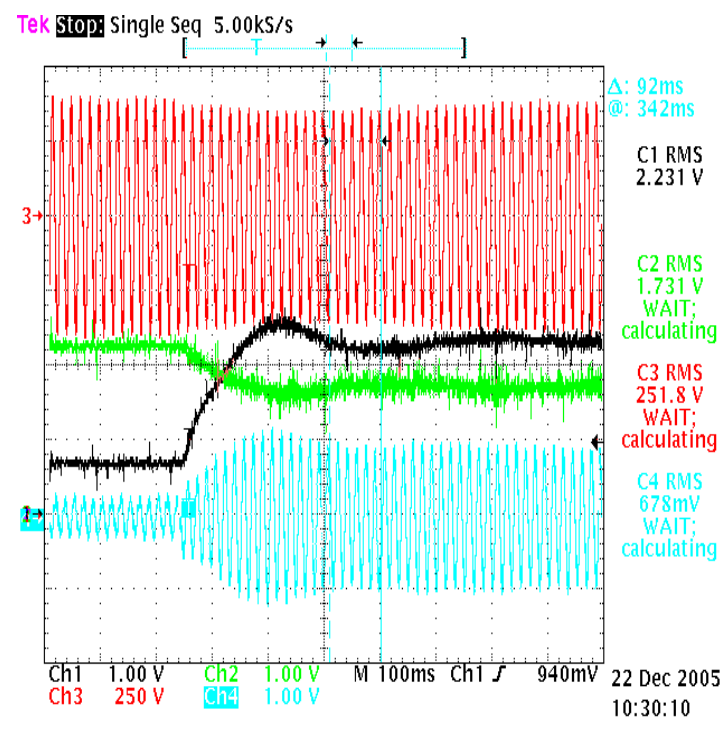

Unit \#2 Transient Response

Figure 19 Parallel Inverters - Feeder Control, Island Transition - Unit Power Limit Evaluation 2

\begin{tabular}{|c|c|c|}
\hline & A (Grid) & B (Island) \\
\hline Load Bank \#1(S.P/Actual) KW & $70.0 / 73.5 \mathrm{KW}$ & $70.0 / 73.5 \mathrm{KW}$ \\
\hline Load Bank \#2(S.P/Actual) KW & $50.0 / 51.9 \mathrm{KW}$ & $50.0 / 51.9 \mathrm{KW}$ \\
\hline Unit \#1 (S.P/Pout) KW & $54.0 / 61.2 \mathrm{KW} \mathrm{Feeder}$ & $54.0 / 61.2 \mathrm{KW}$ Feeder \\
\hline Unit \#2 (S.P/Pout) KW & $6.0 / 5.5 \mathrm{KW} \mathrm{Unit}$ & $6.0 / 60.9 \mathrm{KW}$ Unit \\
\hline Frequency Hz & $59.92 \mathrm{~Hz}$ & $59.53 \mathrm{~Hz}$ \\
\hline
\end{tabular}

Table 20 Parallel Inverters - Feeder Control, Island Transition - Unit Power Limit Evaluation 2 
c. Feeder Control; grid connected

i. Feeder control to check digital round-off.

1. Load Bank \#1 Start Load $=80 \mathrm{KW}$

2. Load Bank \#2 Start Load $=80 \mathrm{KW}$

3. Unit A Feeder CMD $=60 \mathrm{KW}$

4. Unit B Power CMD $=40 \mathrm{KW}$

5. Change unit $A$ Feeder $C M D=120 K W$

6. Change unit B Power CMD $=10 \mathrm{KW}$

7. Change unit A Feeder $\mathrm{CMD}=150 \mathrm{KW}$

8. Load Bank \#1 End Load $=80 \mathrm{KW}$

9. Load Bank \#2 End Load $=80 \mathrm{KW}$

Changed unit A Feeder CMD $=120 \mathrm{KW}$
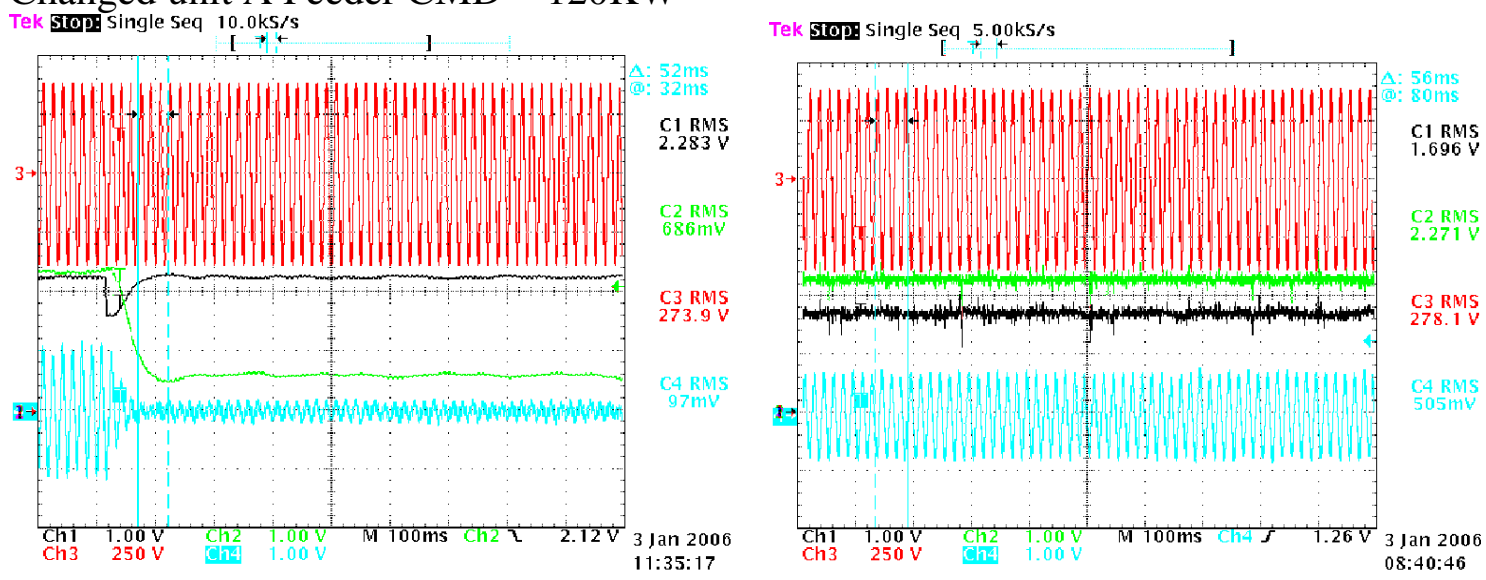

Unit \#1 Transient Response

Unit \#2 Transient Response

Figure 20 Parallel Inverters - Feeder Control, Grid Connected - Digital Round-off Check 1

\begin{tabular}{|c|c|c|}
\hline & A (Grid) & B (Grid) \\
\hline Load Bank \#1(S.P/Actual) KW & $80.0 / 83.9 \mathrm{KW}$ & $80.0 / 83.9 \mathrm{KW}$ \\
\hline Load Bank \#2(S.P/Actual) KW & $80.0 / 84.3 \mathrm{KW}$ & $80.0 / 84.3 \mathrm{KW}$ \\
\hline Unit \#1 (S.P/Pout) KW & $60 / 57.5 \mathrm{KW}$ Feeder & $120.0 / 2.2 \mathrm{KW}$ Feeder \\
\hline Unit \#2 (S.P/Pout) KW & $40 / 37.5 \mathrm{KW}$ Unit & $40 / 37.5 \mathrm{KW}$ Unit \\
\hline Frequency Hz & $59.97 \mathrm{~Hz}$ & $59.97 \mathrm{~Hz}$ \\
\hline
\end{tabular}

Table 21 Parallel Inverters - Feeder Control, Grid Connected - Digital Round-off Check 1 
Changed unit B Power CMD $=10 \mathrm{KW}$

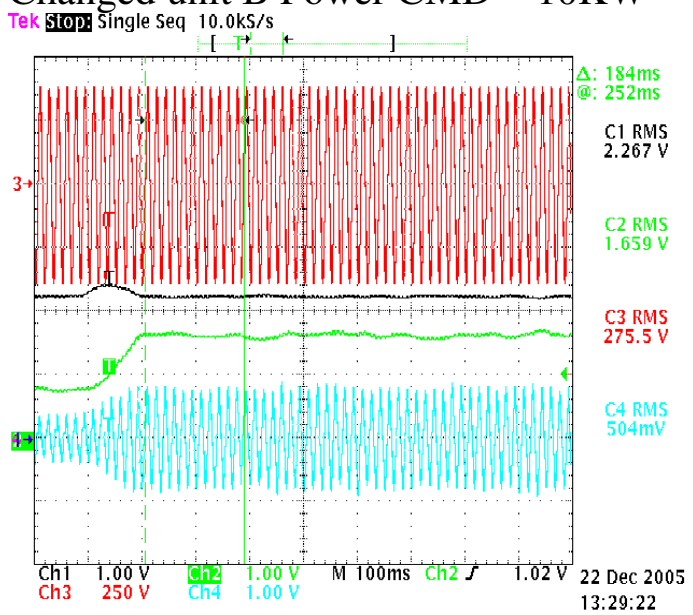

Unit \#1 Transient Response

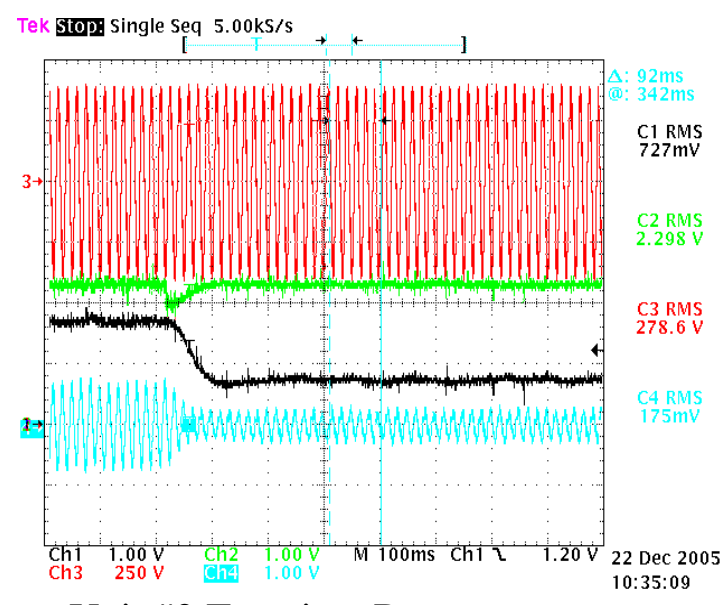

Unit \#2 Transient Response

Figure 21 Parallel Inverters - Feeder Control, Grid Connected - Digital Round-off Check 2

\begin{tabular}{|c|c|c|}
\hline & A (Grid) & B (Grid) \\
\hline Load Bank \#1(S.P/Actual) KW & $80.0 / 83.9 \mathrm{KW}$ & $80.0 / 83.9 \mathrm{KW}$ \\
\hline Load Bank \#2(S.P/Actual) KW & $80.0 / 84.3 \mathrm{KW}$ & $80.0 / 84.3 \mathrm{KW}$ \\
\hline Unit \#1 (S.P/Pout) KW & $120 / 2.2 \mathrm{KW} \mathrm{Feeder}$ & $120.0 / 34.6 \mathrm{KW}$ Feeder \\
\hline Unit \#2 (S.P/Pout) KW & $40 / 37.5 \mathrm{KW}$ Unit & $10 / 8.3 \mathrm{KW}$ Unit \\
\hline Frequency Hz & $59.97 \mathrm{~Hz}$ & $59.99 \mathrm{~Hz}$ \\
\hline
\end{tabular}

Table 22 Parallel Inverters - Feeder Control, Grid Connected - Digital Round-off Check 2 
Changed unit A Feeder CMD $=150 \mathrm{KW}$

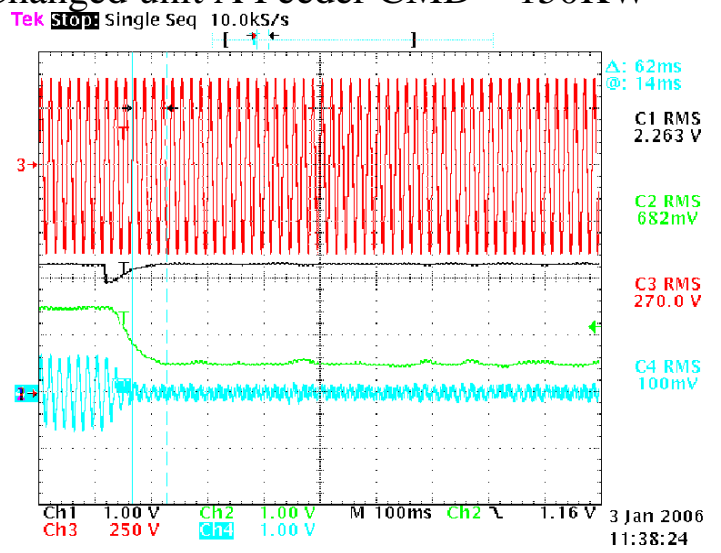

Unit \#1.) Transient Response

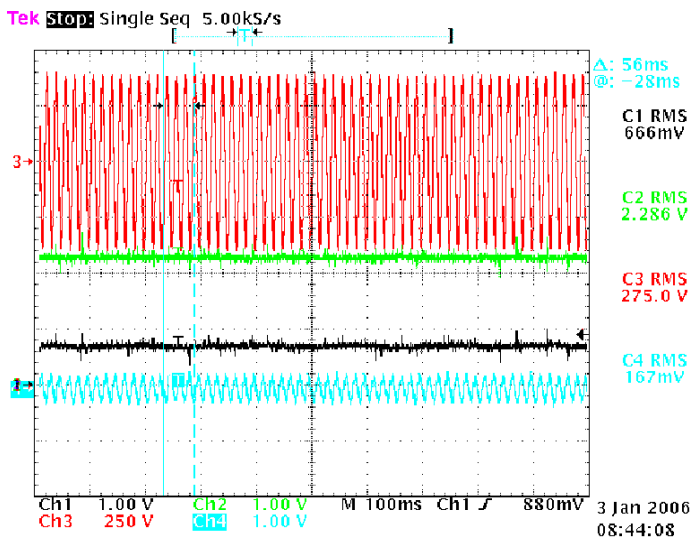

Unit \#2.) Transient Response

Figure 22 Parallel Inverters - Feeder Control, Grid Connected - Digital Round-off Check 3

\begin{tabular}{|c|c|c|}
\hline & A (Grid) & B (Grid) \\
\hline Load Bank \#1(S.P/Actual) KW & $80.0 / 83.9 \mathrm{KW}$ & $80.0 / 83.9 \mathrm{KW}$ \\
\hline Load Bank \#2(S.P/Actual) KW & $80.0 / 84.3 \mathrm{KW}$ & $80.0 / 84.3 \mathrm{KW}$ \\
\hline Unit \#1 (S.P/Pout) KW & $120 / 34.6 \mathrm{KW}$ Feeder & $150.0 / 0.5 \mathrm{KW}$ Feeder \\
\hline Unit \#2 (S.P/Pout) KW & $10.0 / 5.5 \mathrm{KW} \mathrm{Unit}$ & $10.0 / 5.5 \mathrm{KW}$ Unit \\
\hline Frequency Hz & $59.98 \mathrm{~Hz}$ & $59.99 \mathrm{~Hz}$ \\
\hline
\end{tabular}

Table 23 Parallel Inverters - Feeder Control, Grid Connected - Digital Round-off Check 3 


\section{CERTS MICROGRID LABORATORY TEST BED}

Tecogen 60kW Inverter-Based CHP Modules Factory Testing

Prepared For:

California Energy Commission

Public Interest Energy Research Program

Prepared By:

Lawrence Berkeley National Laboratory

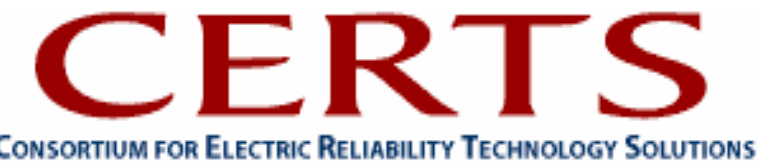



Prepared By:

Lawrence Berkeley National Laboratory

Joseph H. Eto, Principal Investigator

Berkeley, CA 94720

Jean Roy, Tecogen, Inc.

Commission Contract No. 500-03-024

Prepared For:

Public Interest Energy Research (PIER)

California Energy Commission

Bernard Treanton

Contract Manager

Mike Gravely

Program Area Lead

ENERGY SYSTEMS INTEGRATION

Mike Gravely

Office Manager

ENERGY SYSTEMS RESEARCH

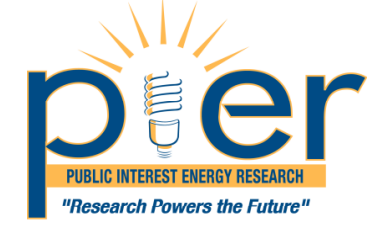

Martha Krebs, Ph.D.

PIER Director

Thom Kelly, Ph.D.

Deputy Director

ENERGY RESEARCH \& DEVELOPMENT DIVISION

Melissa Jones

Executive Director

\section{DISCLAIMER}

This report was prepared as the result of work sponsored by the California Energy Commission. It does not necessarily represent the views of the Energy Commission, its employees or the State of California. The Energy Commission, the State of California, its employees, contractors and subcontractors make no warrant, express or implied, and assume no legal liability for the information in this report; nor does any party represent that the uses of this information will not infringe upon privately owned rights. This report has not been approved or disapproved by the California Energy Commission nor has the California Energy Commission passed upon the accuracy or adequacy of the information in this report. 



\section{TECOGEN}

Natural Gas Engine-Driven Products

\section{TECOGEN $60 \mathrm{~kW}$ Inverter-Based CHP Modules}

\section{CERTS Microgrid Test Bed Project}

\section{Tecogen Factory Testing}

Unit Serial \#200835

3/15/06 


\section{Introduction}

This report presents the factory test data of the first Tecogen CHP unit (Serial \# 200835), equipped with a customized inverter (by Youtility) that is configured with the CERTS microgrid control algorithm. Figure 1 presents a one-line diagram of the test set-up. The unit generates power in a stand-alone mode (no grid-tie) and is connected to a load bank. The output of the inverter is connected to the Delta side of a transformer in a 3-wire configuration. The output of the transformer is wired to the load bank in a 4-wire configuration with a 25-yard loop.

The inverter is also equipped with a Surge Module wired directly to the DC Bus. The Surge Module is a battery powered energy storage device with a rating of $20 \mathrm{~kW}$ for 3 seconds. Its purpose is to assist the engine/inverter's response to step loads. It is housed separately in an adjacent cabinet (DC power wiring length:12.5 feet).

All testing was done with the inverters set to Power Control Mode.

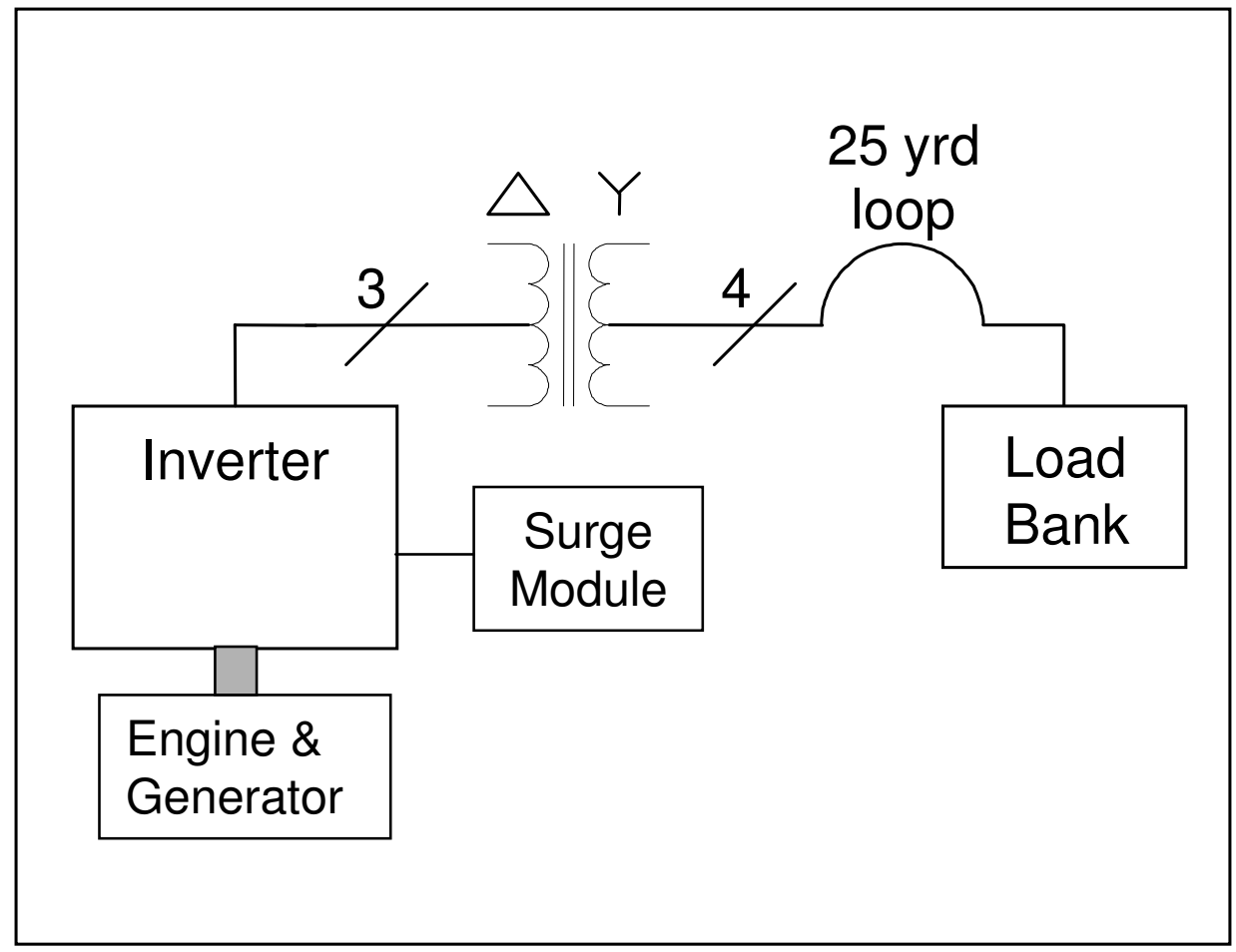

Figure 1 Factory Test One-line Diagram 


\section{B.2. Step Load Evaluations with Surge Module - Variable Speed Operation}

The following step loads were tested:

\begin{tabular}{|c|c|c|l|}
\hline & Test Plan & $\begin{array}{c}\text { Actual Test } \\
\text { Point }\end{array}$ & \multicolumn{1}{c|}{ Comments } \\
\hline i. & $0-30 \mathrm{~kW}$ & $0-30 \mathrm{~kW}$ & \multicolumn{1}{c|}{} \\
\hline ii. & $0-60 \mathrm{~kW}$ & $0-50 \mathrm{~kW}$ & $\begin{array}{l}\text { Engine stalls at a step load of 0 - 55 kW in } \\
\text { this variable speed mode. A 0 - 60 kW step } \\
\text { load can be achieved at fixed speed (see } \\
\text { section B.3). Minimum engine speed will be } \\
\text { raised in testing of Unit \#2 to 1560 rpm (52 } \\
\text { Hz) in order to achieve 0 - 60 kW in variable } \\
\text { speed mode with the surge module. }\end{array}$ \\
\hline iii. & $15-45 \mathrm{~kW}$ & $15-50 \mathrm{~kW}$ & $\begin{array}{l}\text { A 15 - 50 increment was easier to implement } \\
\text { with the load bank. }\end{array}$ \\
\hline iv. & $30-60 \mathrm{~kW}$ & $30-55 \mathrm{~kW}$ & $\begin{array}{l}\text { As was done in Phase 1 at Youtility, 55 kW } \\
\text { was done to ensure the frequency did not go } \\
\text { out of range if the load slightly exceeded 60 } \\
\text { kW from the load bank. }\end{array}$ \\
\hline v. & $60-0 \mathrm{~kW}$ & $60-0 \mathrm{~kW}$ & \\
\hline vi. & $30-0 \mathrm{~kW}$ & $30-0 \mathrm{~kW}$ & \\
\hline
\end{tabular}

Table 1: Test Point Summary - Step Loads with Surge Module/Variable Speed

For each step load point, an oscilloscope trace was captured that measured the power output, frequency, voltage, and current. These measurements were taken on the microgrid side (Y side) of the transformer. In each plot the data is formatted in the following way:

Ch1 = Unit RMS Power Output;

$0.0 \mathrm{~V}=-15 \mathrm{KW}$

$0.5 \mathrm{~V}=0 \mathrm{KW}$

$1.5 \mathrm{~V}=30 \mathrm{KW}$

$2.5 \mathrm{~V}=60 \mathrm{KW}$

$\mathrm{Ch} 2=$ Unit Operating Frequency;

$60 \mathrm{~Hz}-->2.3 \mathrm{~V}$

$59.5 \mathrm{~Hz}-->1.66 \mathrm{~V}$

$60.5 \mathrm{~Hz}-->2.94 \mathrm{~V}$

Ch 3 - The graphical trace is Grid Voltage L-N, nominal 277 Volts. The displayed value on right hand side is the measured frequency after the step load.

Channel 4

Ch4 = Unit Output Current; 1V = 100Amps 
Also, the Step "Up" load points (i-iv.) were run two separate times so that additional data on the system dynamics, with the surge module, could be captured. This oscillocope data is formatted as follows:

Channel 1 = Engine RPM;

$120 \mathrm{~Hz}(3600 \mathrm{rpm})$ per 3.3 volts

1 volt per division

Zero point is at black " 1 " marker on left-hand side of Figure

Nominal Minimum Speed $=50 \mathrm{~Hz}(1500 \mathrm{rpm})$

Channel 2 = Bus Voltage

Zero point is actually 500 volts and measures from black "l" marker on left-hand side of Figure

100 volts per division

Nominal Voltage; 860 volts

Channel $3=$ AC Current ; $1 \mathrm{~V}=100$ amps

Channel $4=$ Surge Module Current

$10 \mathrm{mV}=20 \mathrm{amps}$

$10 \mathrm{mV}$ per division

Nominal Current; 26 amps

The results of each test is organized in a table that defines the load setpoint (abbreviated S.P) and the actual power output from the unit. The table also contains the microgrid operating frequency before and after the transient event occurs (These frequency values are obtained from the units software and not the oscilloscope measurement). Data contained in column A defines the operating condition and the state of the microgrid before the transient condition being tested occurs. Data contained in column B defines the operating condition and the state of the microgrid after the transient condition being tested has occurred.

i. $\quad$ Step Load Evaluation 1

1.Load Bank Start Load $=0 \mathrm{~kW}$

2.Unit A CMD $=20 \mathrm{~kW}$

3.Load Bank End Load $=30 \mathrm{~kW}$

\begin{tabular}{|c|c|c|}
\hline & A & B \\
\hline Unit \#1 (S.P/Actual) kW & $20.0 / 0.0 \mathrm{~kW}$ & $20.0 / 30.45 \mathrm{~kW}$ \\
\hline Frequency Hz & $60.16 \mathrm{~Hz}$ & $59.91 \mathrm{~Hz}$ \\
\hline
\end{tabular}

Table 2 Step Load Evaluation 1: 0 - 30 kW (w/Surge Module) 


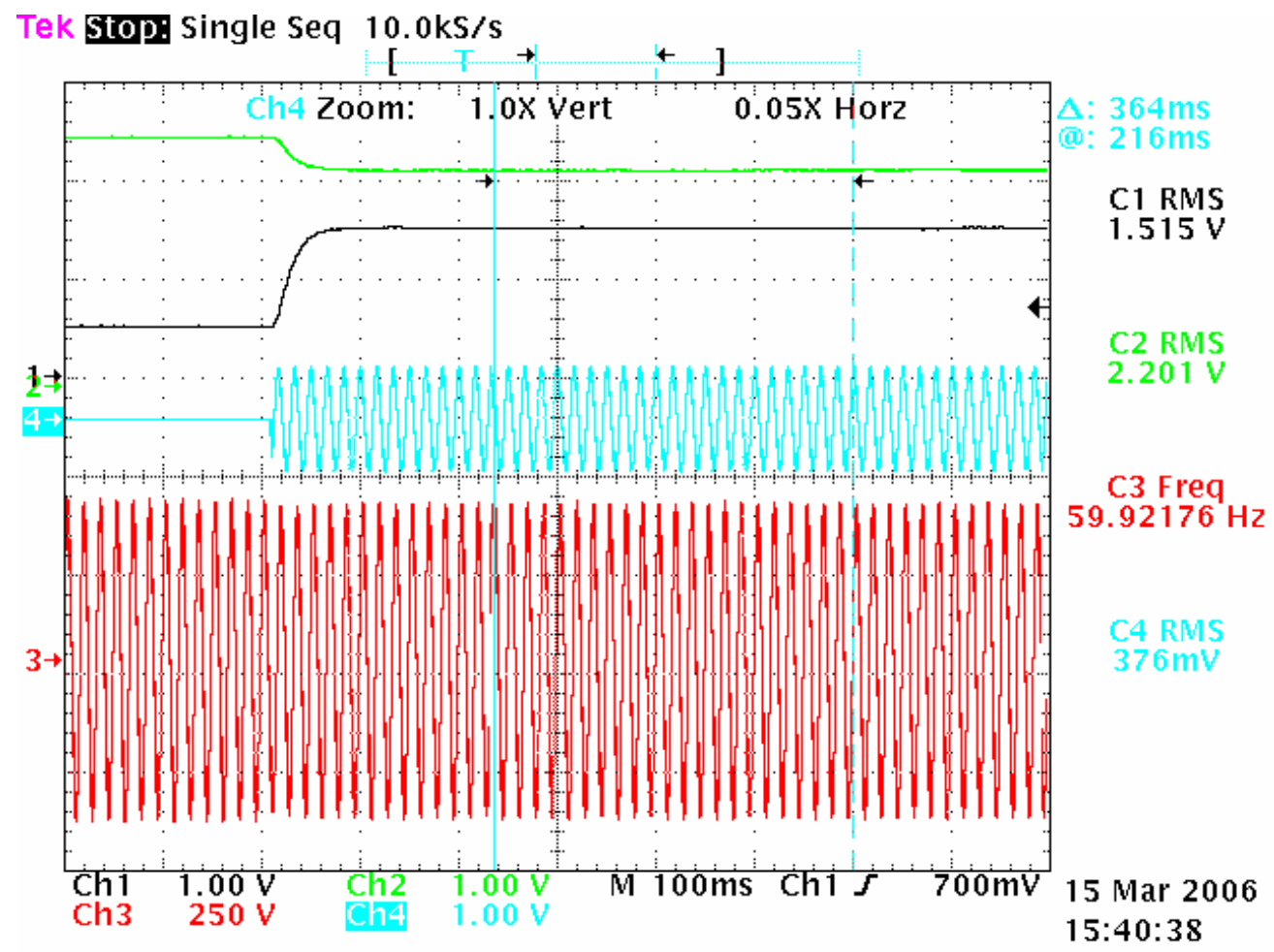

Figure 2 0-30 kW: Power, Frequency, Voltage, Current

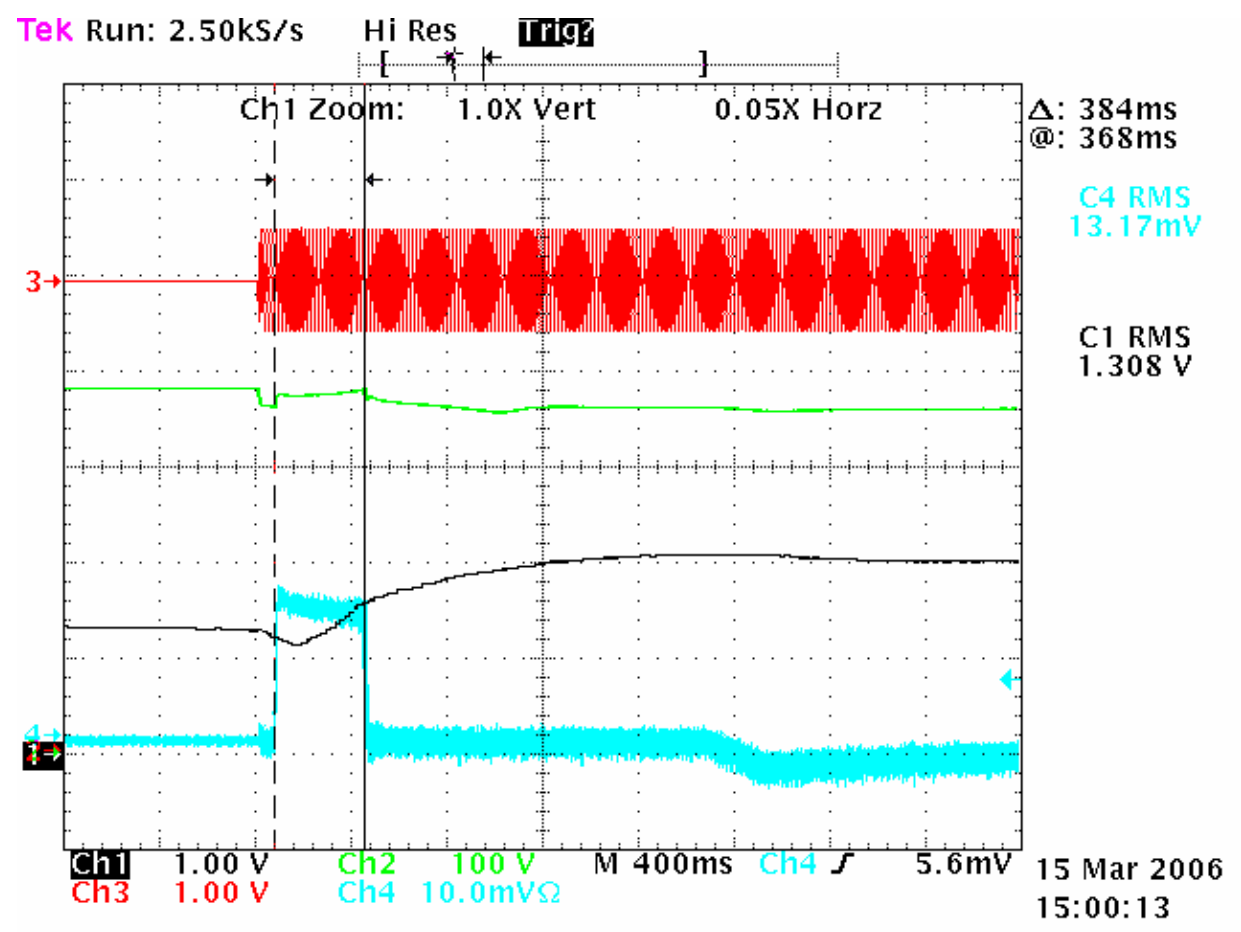

Figure 3 0-30 kW: Engine RPM, Bus Voltage, AC Current, Surge Module Current 
ii. Step Load Evaluation 2

1. Load Bank Start Load $=0 \mathrm{KW}$

2. Unit $\mathrm{A} C \mathrm{CMD}=20 \mathrm{KW}$

3. Load Bank End Load $=60 \mathrm{KW}$ (Engine Stalled)

Load Bank End Load $=55 \mathrm{~kW}$ (Engine Stalled)

Load Bank End Load $=50 \mathrm{~kW}$ (Okay)

\begin{tabular}{|c|c|c|}
\hline & A & B \\
\hline Unit \#1 (S.P/Actual) kW & $20.0 / 0.0 \mathrm{~kW}$ & $20.0 / 50.97 \mathrm{~kW}$ \\
\hline Frequency Hz & $60.16 \mathrm{~Hz}$ & $59.75 \mathrm{~Hz}$ \\
\hline
\end{tabular}

Table 3 Step Load Evaluation 2: 0 - 60 kW (w/Surge Module)

O - 50 kW Step Load -Surge Module- Variable Speed

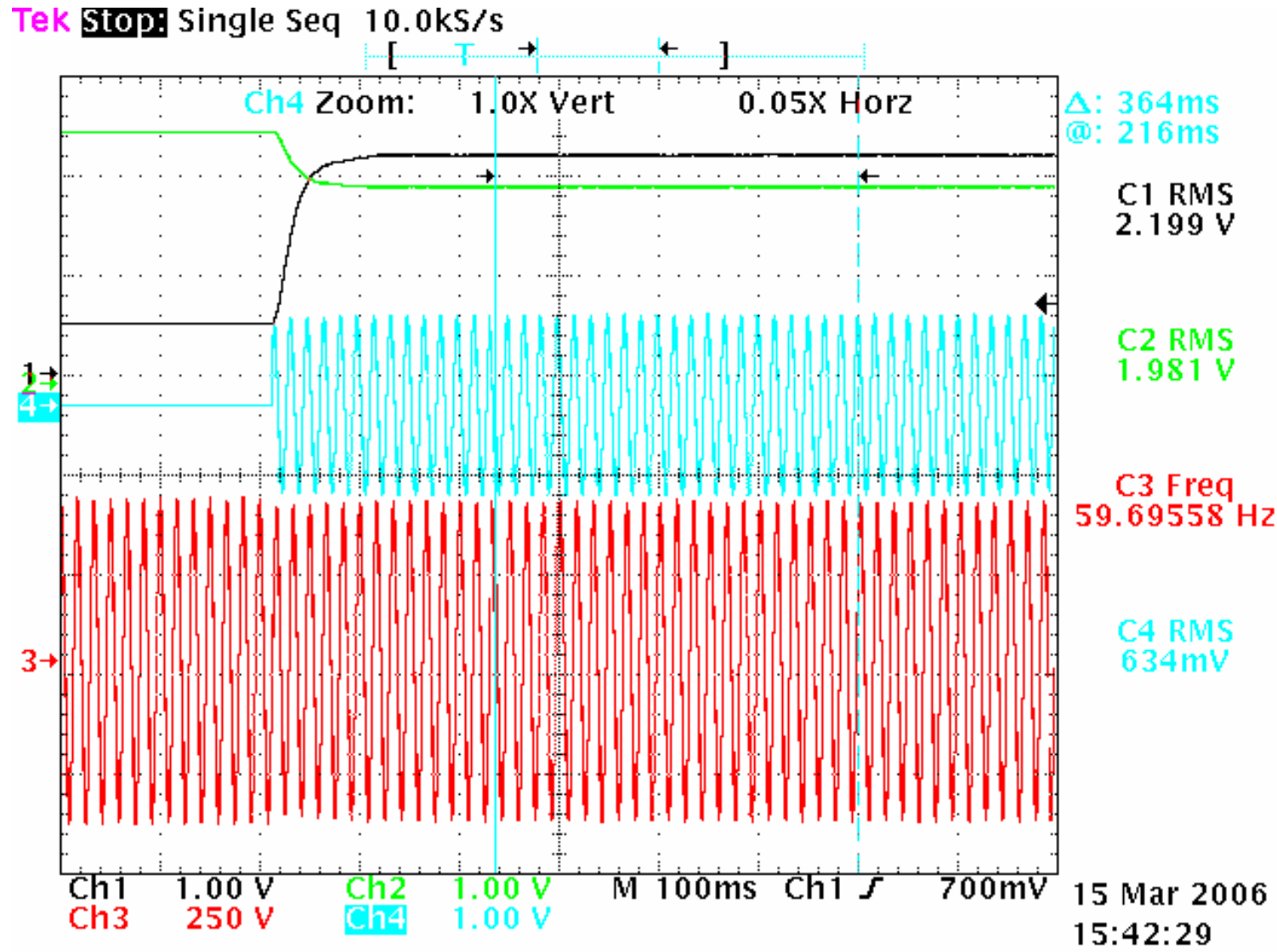

Figure 4 0-50 kW: Power, Frequency, Voltage, Current 


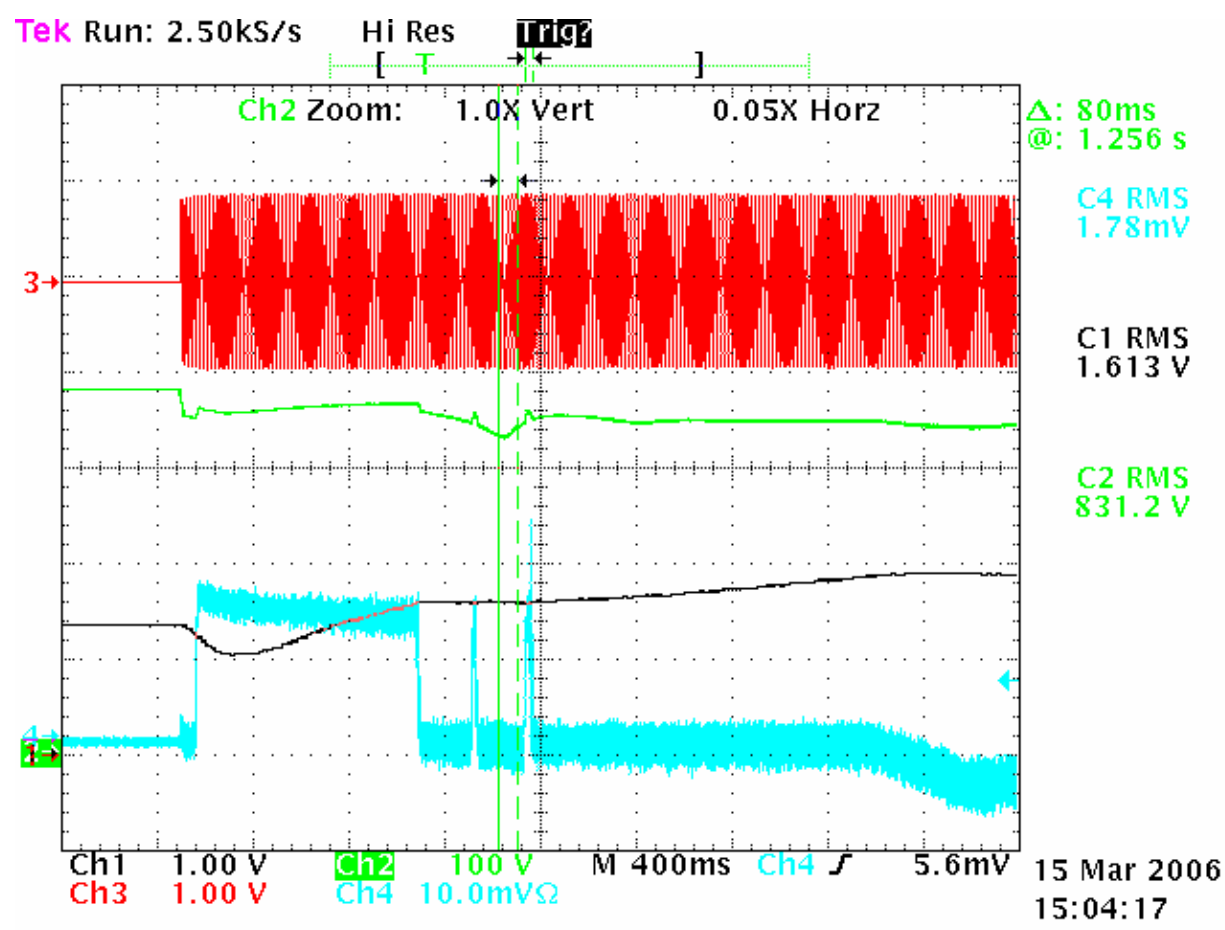

Figure 5 0-50 kW: Engine RPM, Bus Voltage, AC Current, Surge Module Current

0 - 55 KW Step Load -Surge Module- Variable Speed Tek Stop: Single Seq $2.50 \mathrm{kS} / \mathrm{s}$

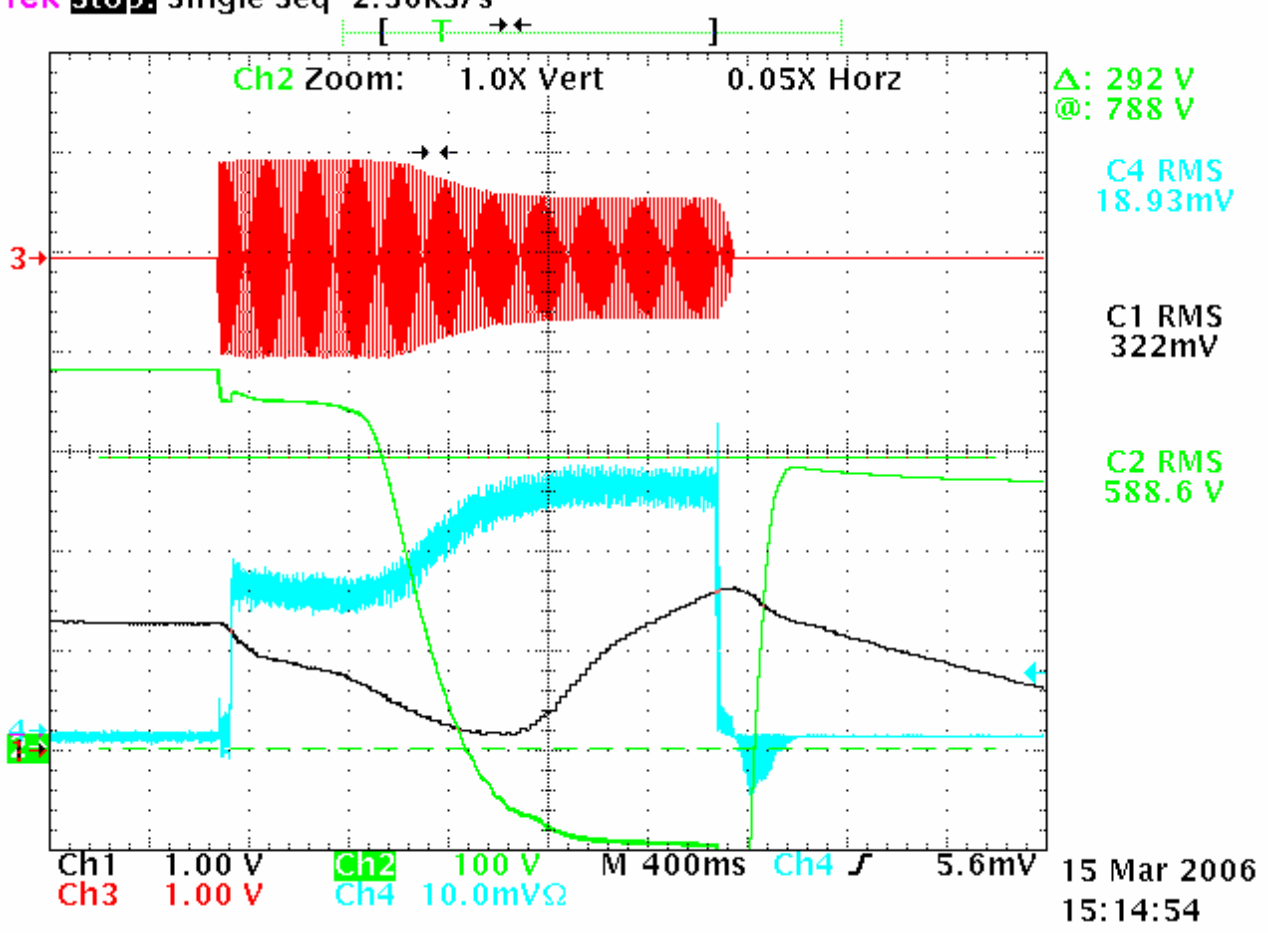

Engine Stalled

Figure 6 0-55 kW: Engine RPM, Bus Voltage, AC Current, Surge Module Current 
iii. $\quad$ Step Load Evaluation 3

1.Load Bank Start Load $=15 \mathrm{~kW}$

2.Unit $\mathrm{A} C \mathrm{CMD}=20 \mathrm{~kW}$

3.Load Bank End Load $=50 \mathrm{~kW}$ (Changed from $45 \mathrm{~kW}$ because easier to implement on Load Bank)

\begin{tabular}{|c|c|c|}
\hline & A & B \\
\hline Unit \#1 (S.P/Actual) $\mathrm{kW}$ & $20.0 / 0.0 \mathrm{~kW}$ & $20.0 / 49.86 \mathrm{~kW}$ \\
\hline Frequency Hz & $60.16 \mathrm{~Hz}$ & $59.75 \mathrm{~Hz}$ \\
\hline
\end{tabular}

Table 4 Step Load Evaluation 3: 15 - 50 kW (w/Surge Module)

15 - 50 KW Step Load -Surge Module- Variable Speed

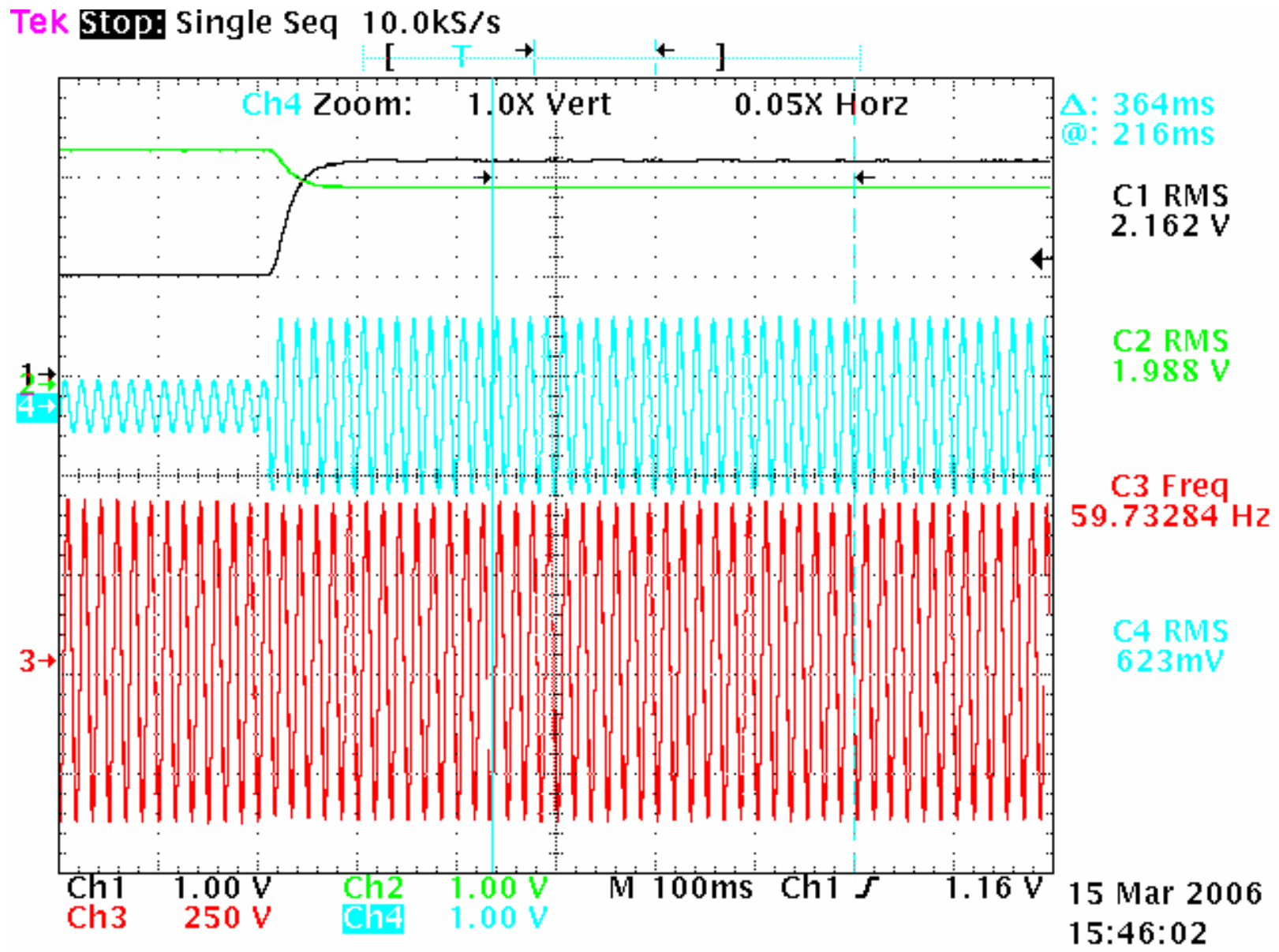

Figure 7 15-50 kW: Power, Frequency, Voltage, Current 


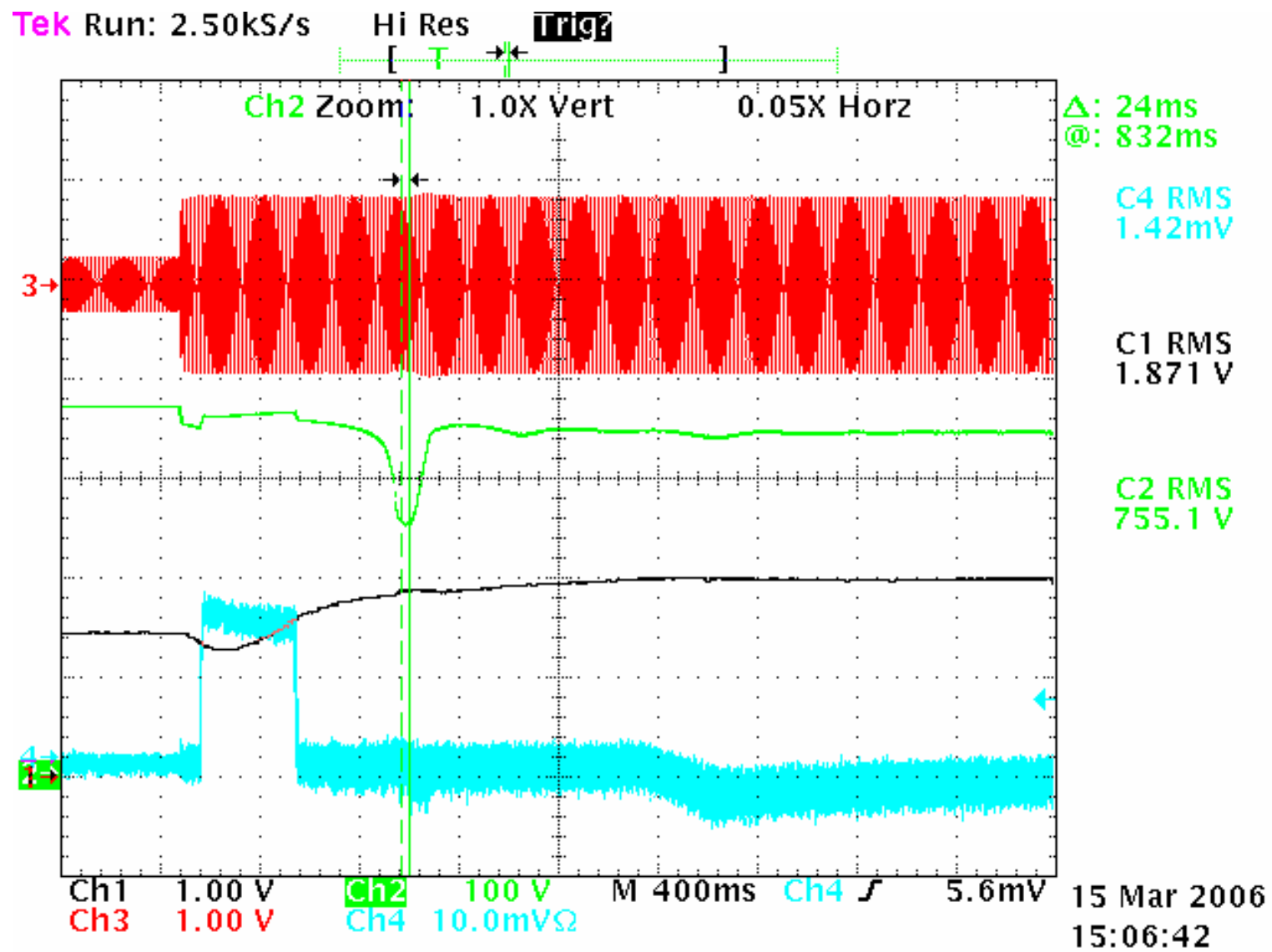

Figure 815 - 50 kW: Engine RPM, Bus Voltage, AC Current, Surge Module Current

iv. $\quad$ Step Load Evaluation 4

1.Load Bank Start Load $=30 \mathrm{~kW}$

2.Unit A CMD $=20 \mathrm{~kW}$

3.Load Bank End Load $=55 \mathrm{~kW}$

\begin{tabular}{|c|c|c|}
\hline & A & B \\
\hline Unit \#1 (S.P/Actual) KW & $20.0 / 0.0 \mathrm{~kW}$ & $20.0 / 55.5 \mathrm{~kW}$ \\
\hline Frequency Hz & $60.16 \mathrm{~Hz}$ & $59.70 \mathrm{~Hz}$ \\
\hline
\end{tabular}

Table 5 Step Load Evaluation 4: (30 - 55 kW) (w/Surge Module) 


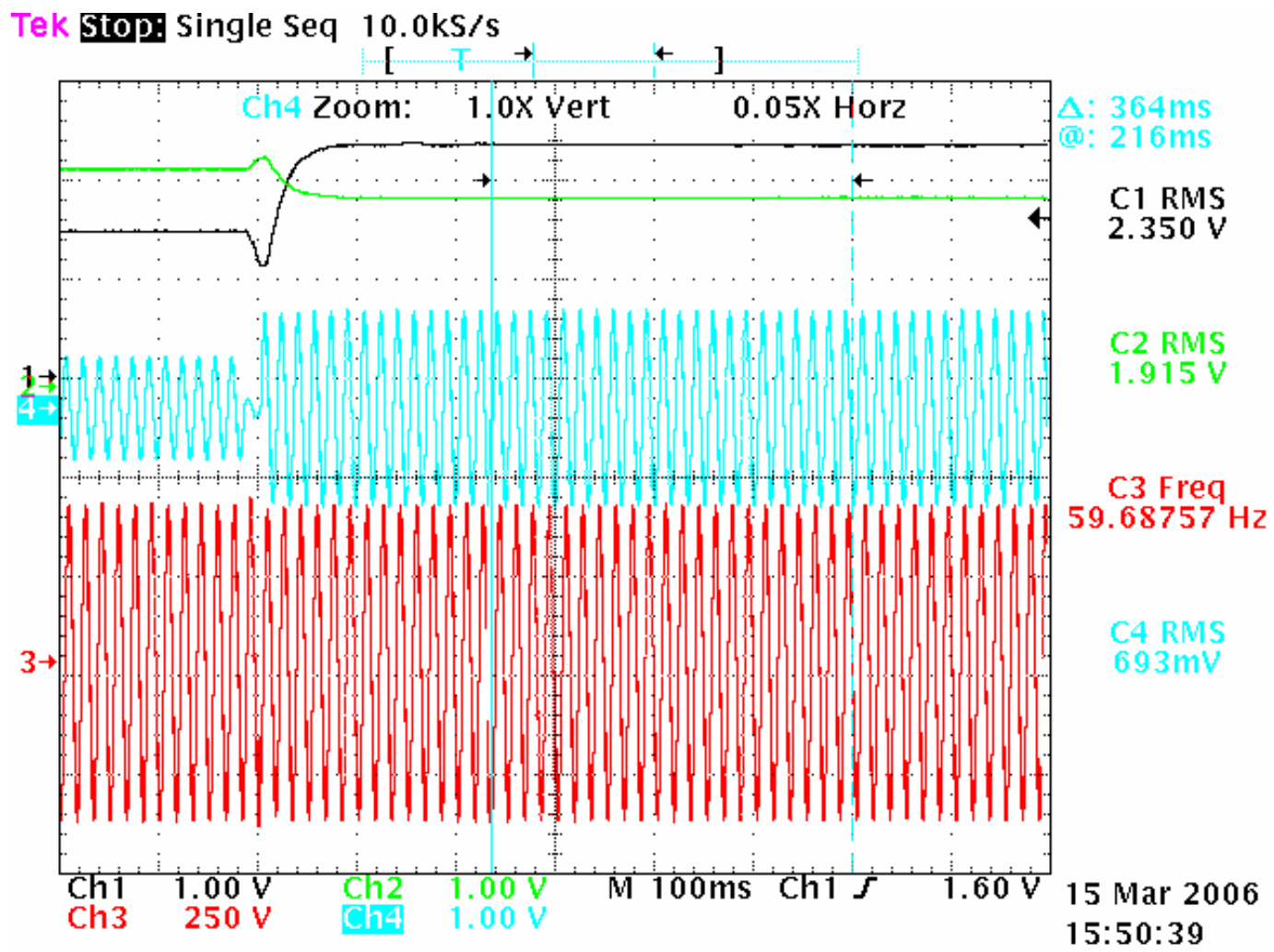

Figure $930-55$ kW Power, Frequency, Voltage, Current

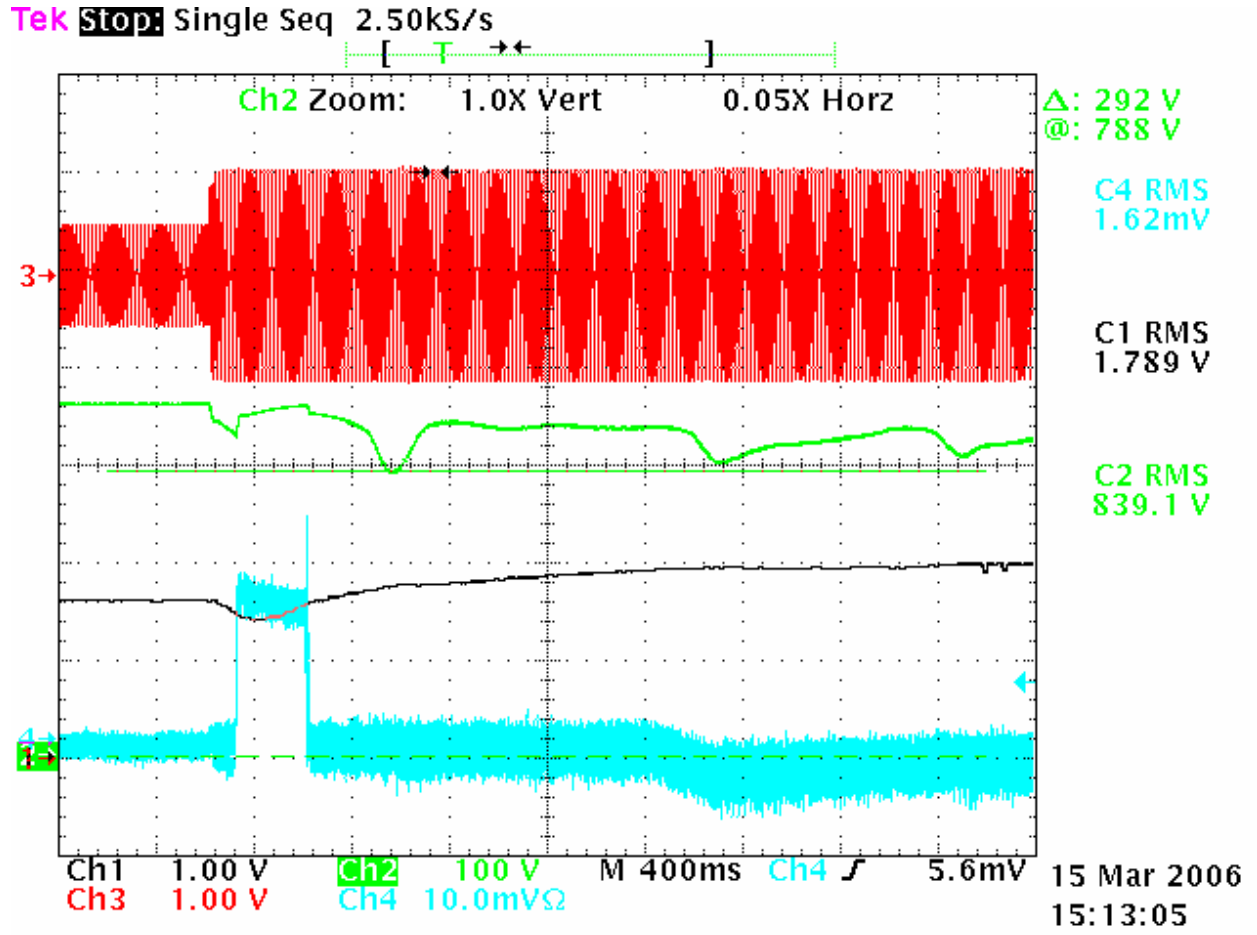

Figure 1030 - 55 kW: Engine RPM, Bus Voltage, AC Current, Surge Module Current 
v. Step Load Evaluation 5

1.Load Bank Start Load $=60 \mathrm{~kW}$

2.Unit $\mathrm{A} C \mathrm{CMD}=20 \mathrm{~kW}$

3. Load Bank End Load $=0 \mathrm{~kW}$

\begin{tabular}{|c|c|c|}
\hline & A & B \\
\hline Unit \#1 (S.P/Actual) $\mathrm{kW}$ & $20.0 / 60 \mathrm{~kW}$ & $20.0 / 0 \mathrm{~kW}$ \\
\hline Frequency Hz & $59.67 \mathrm{~Hz}$ & $60.6 \mathrm{~Hz}$ \\
\hline
\end{tabular}

Table 6 Step Load Evaluation 5: 60 - 0 kW (w/Surge Module)

\section{0- 0 kW Step Load -Surge Module- Variable Speed}

Tek Stop: Single Seq $10.0 \mathrm{kS} / \mathrm{s}$

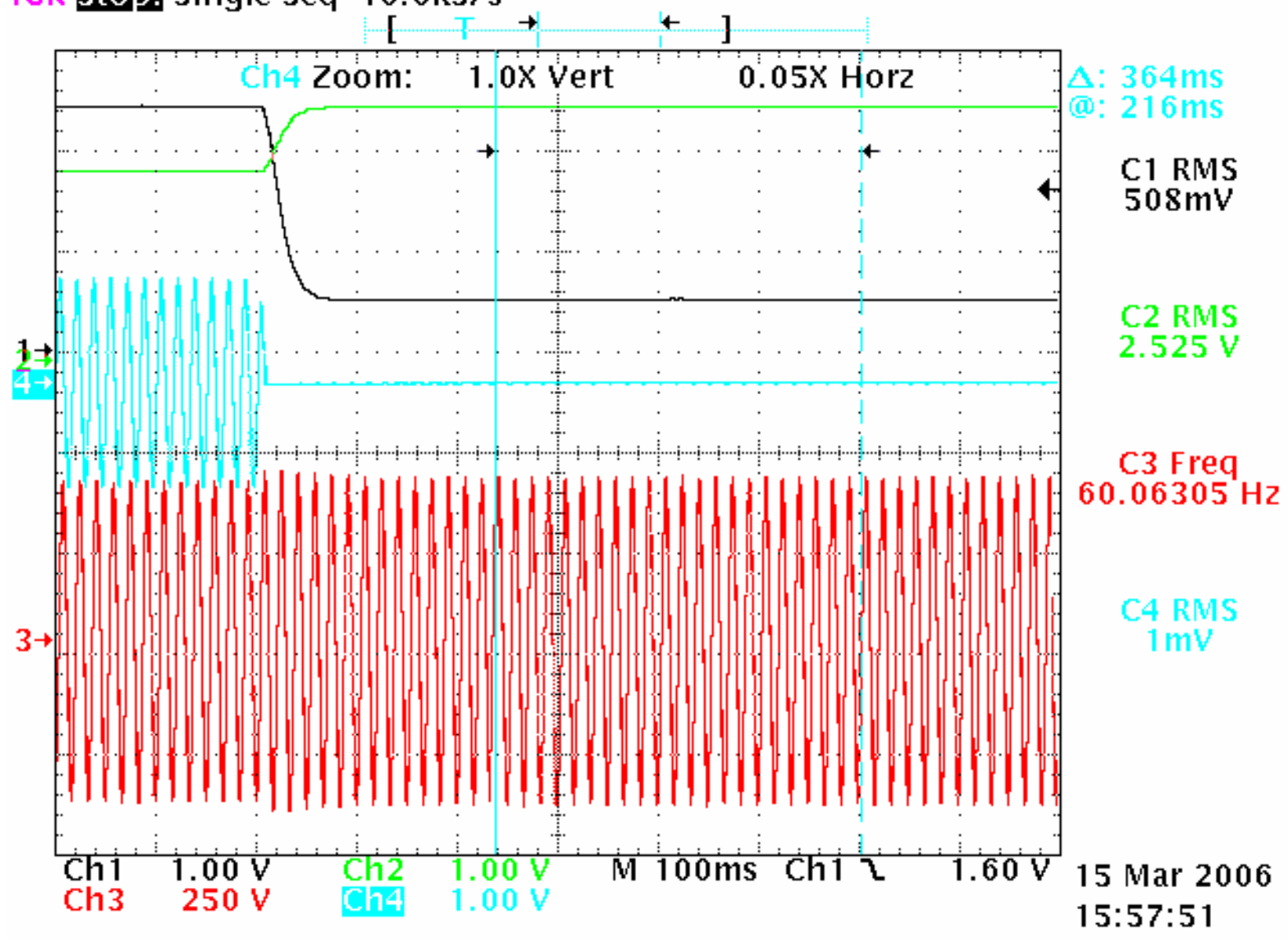

Figure 11 60-0 kW: Power, Frequency, Voltage, Current 
vi. Step Load Evaluation 6

1. Load Bank Start Load $=30 \mathrm{~kW}$

2. Unit $\mathrm{A} \mathrm{CMD}=20 \mathrm{~kW}$

3. Load Bank End Load $=0 \mathrm{~kW}$

\begin{tabular}{|c|c|c|}
\hline & A & B \\
\hline Unit \#1 (S.P/Actual) kW & $20.0 / 30 \mathrm{~kW}$ Unit & $20.0 / 0 \mathrm{~kW}$ \\
\hline Frequency Hz & $59.91 \mathrm{~Hz}$ & $60.16 \mathrm{~Hz}$ \\
\hline
\end{tabular}

Table 7 Step Load Evaluation 6: 30 - 0 kW (w/Surge Module)

$$
\text { 30- } 0 \text { kW Step Load -Surge Module- Variable Speed }
$$

Tek Stop: Single Seq $10.0 \mathrm{kS} / \mathrm{s}$

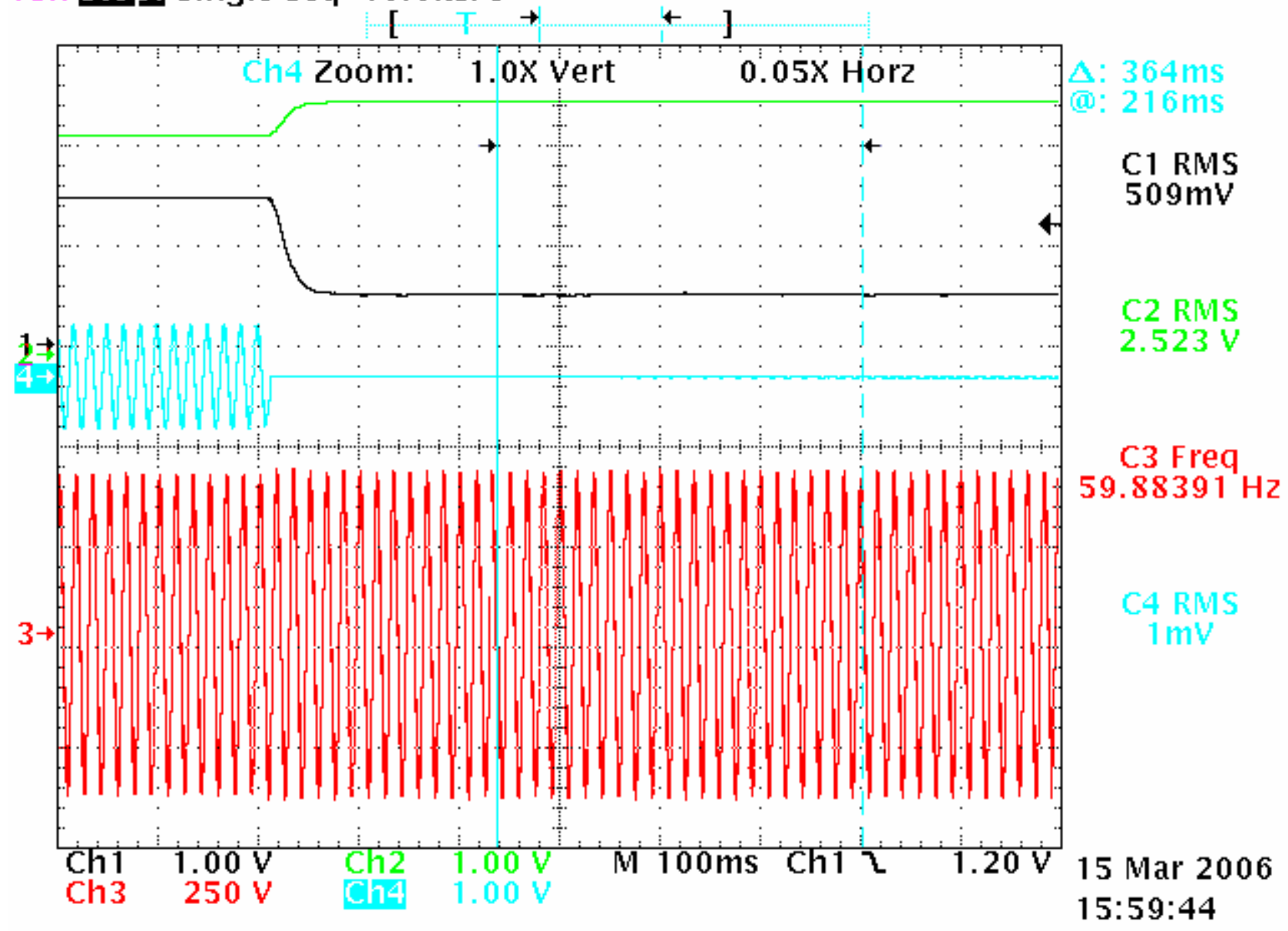

Figure 1230 - 0 kW Power, Frequency, Voltage, Current 


\section{B.3. Step Load Evaluations without Surge Module - Fixed Speed Operation}

The CHP unit was tested with the surge module disabled. The results showed that the dynamics of the engine/inverter could successfully handle a step load without the surge module. However, the engine must operate at a fixed speed and there is a minimum engine operating speed for each load step to prevent the engine from stalling. This minimum speed was determined for each "step-up" load.

The following table summarizes the test results. The minimum operational speed required for the given step load is highlighted with a bold box.

\begin{tabular}{|c|c|c|c|}
\hline \multicolumn{4}{|c|}{ Fixed Engine Speed - No Surge Module } \\
\hline $\begin{array}{l}\text { Step } \\
\text { Loads }\end{array}$ & $\begin{array}{c}\text { Engine } \\
\text { Speed [Hz] }\end{array}$ & $\begin{array}{c}\text { Engine } \\
\text { Speed [RPM] }\end{array}$ & Pass/Fail \\
\hline \multirow[t]{2}{*}{$0-30$} & 65 & 1950 & Pass \\
\hline & 60 & 1800 & Fail \\
\hline \multirow[t]{3}{*}{$0-60$} & 72 & 2160 & Pass \\
\hline & 70 & 2100 & Pass \\
\hline & 68 & 2040 & Fail \\
\hline \multirow[t]{8}{*}{$15-50$} & 68 & 2040 & Pass \\
\hline & 66 & 1980 & Pass \\
\hline & 65 & 1950 & Pass \\
\hline & 64 & 1920 & Pass \\
\hline & 63 & 1890 & Pass \\
\hline & 62 & 1860 & Pass \\
\hline & 61 & 1830 & Pass \\
\hline & 60 & 1800 & Fail \\
\hline \multirow[t]{7}{*}{$30-60$} & 70 & 2100 & Pass \\
\hline & 68 & 2040 & Pass \\
\hline & 67 & 2010 & Pass \\
\hline & 66 & 1980 & Pass \\
\hline & 65 & 1950 & Pass \\
\hline & 64 & 1920 & Pass \\
\hline & 63 & 1890 & Fail \\
\hline
\end{tabular}

Table 8 Test Point Summary -Step Loads without Surge Module/Fixed Speed 
i.) Step Load Evaluation 1

1.Load Bank Start Load $=0 \mathrm{~kW}$

2.Unit A CMD $=20 \mathrm{~kW}$

3.Load Bank End Load $=30 \mathrm{~kW}$

\section{Engine Speed: $1950 \mathrm{rpm}(65 \mathrm{~Hz})$}

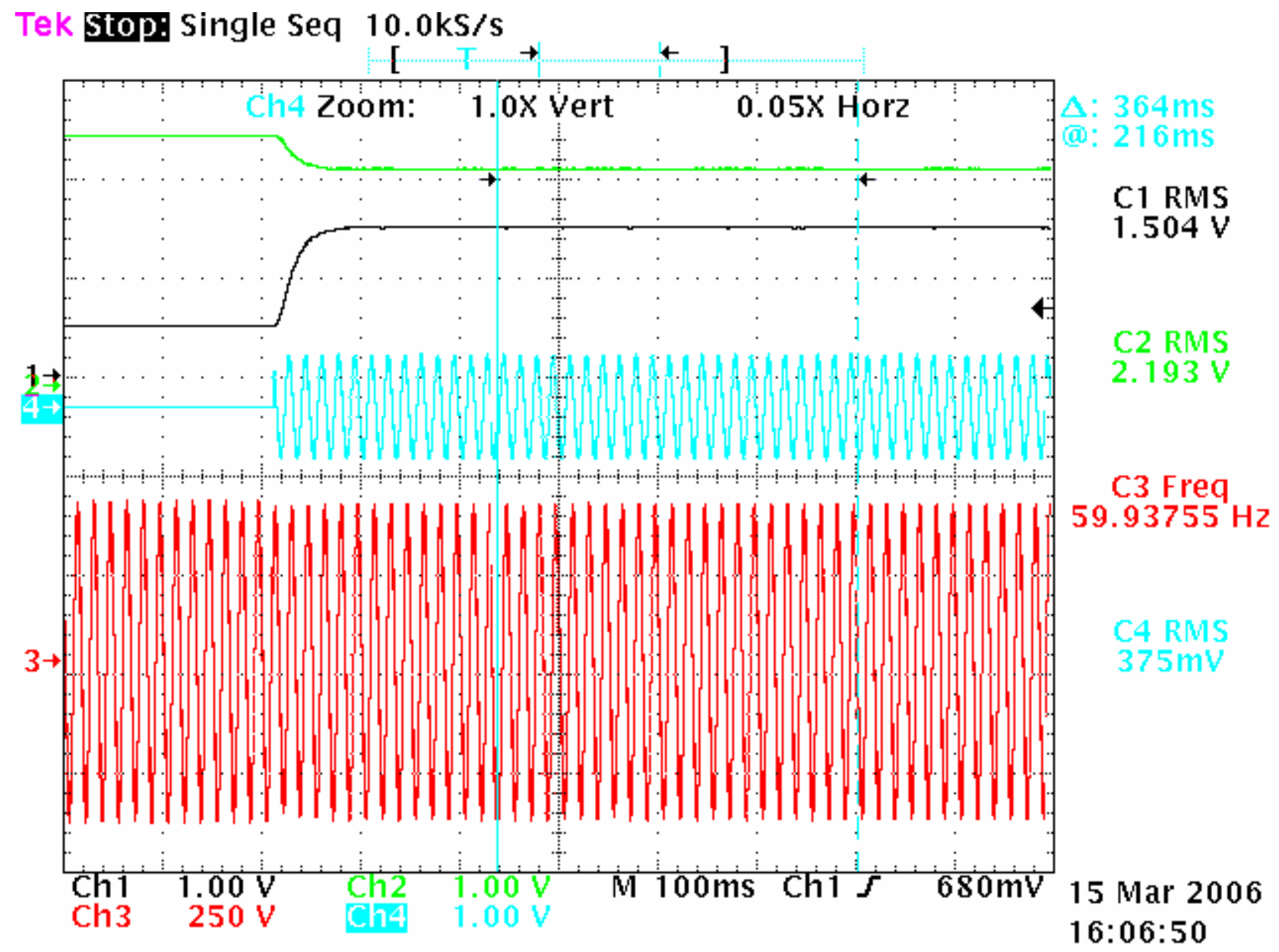

Figure 13 0-30 kW (Fixed Speed): Power, Frequency, Voltage, Current 
ii.) Step Load Evaluation 2

4. Load Bank Start Load $=0 \mathrm{~kW}$

5. Unit $\mathrm{A} \mathrm{CMD}=20 \mathrm{~kW}$

6. Load Bank End Load $=60 \mathrm{~kW}$

\section{Engine Speed: $2160 \mathrm{rpm}(72 \mathrm{~Hz})$}

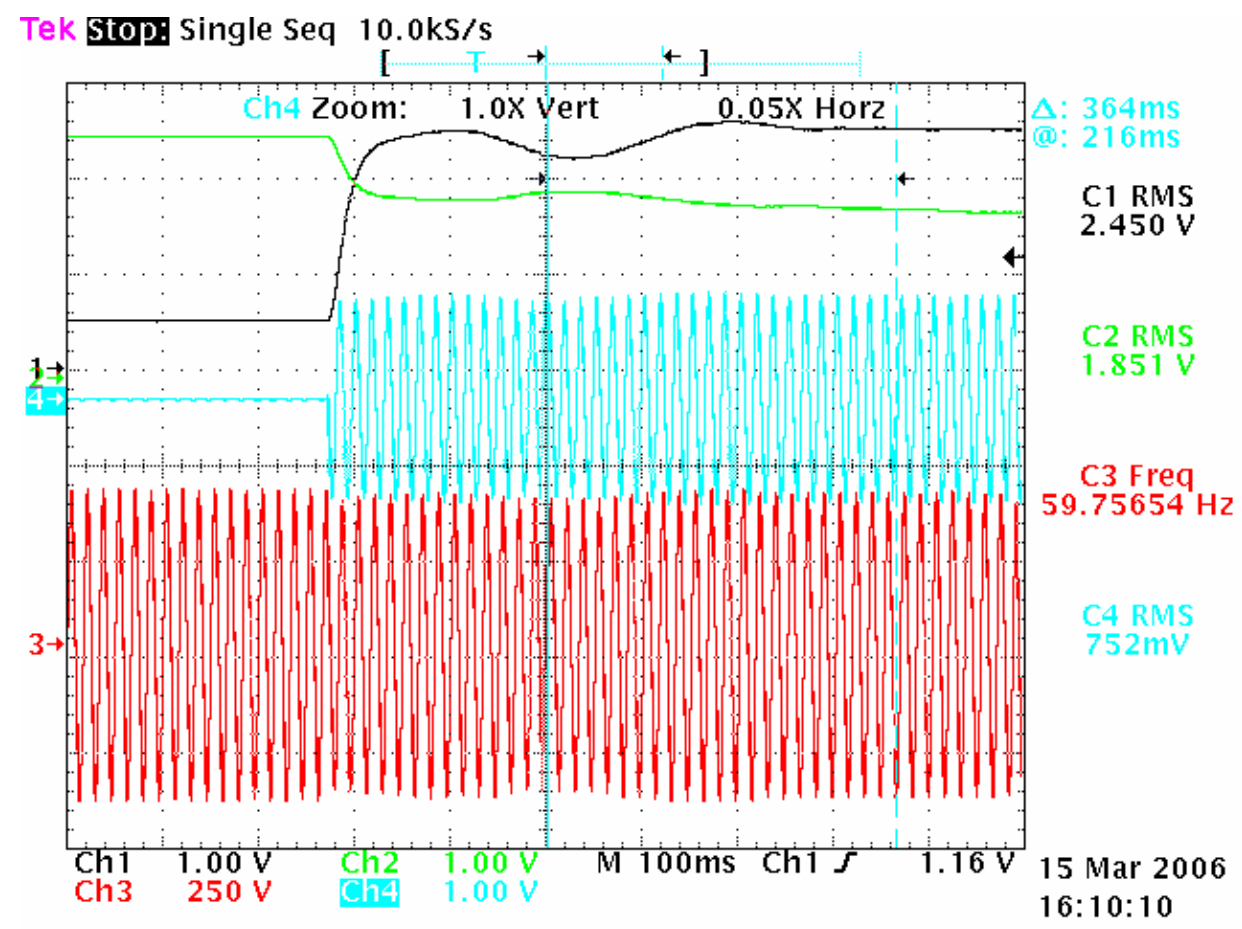

Figure 14 0-60 kW (Fixed Speed - 2160 rpm) Power, Frequency, Voltage, Current 
Engine Speed: $2100 \mathrm{rpm}(70 \mathrm{~Hz})$

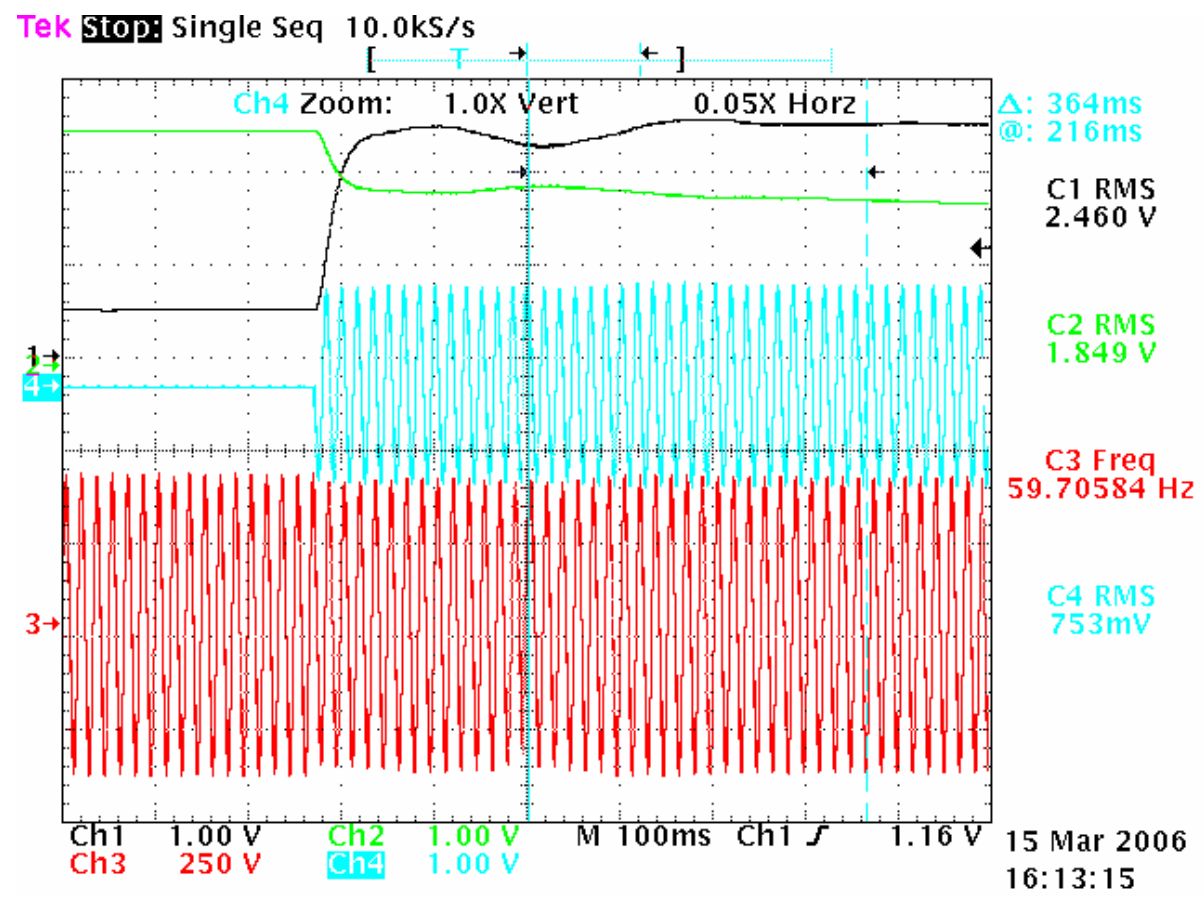

Figure 15 0-60 kW (Fixed Speed - 2100 rpm) Power, Frequency, Voltage, Current 
iii.) Step Load Evaluation 3

1.Load Bank Start Load $=15 \mathrm{~kW}$

2.Unit $\mathrm{A} \mathrm{CMD}=20 \mathrm{~kW}$

3.Load Bank End Load $=50 \mathrm{~kW}$ (Changed from $45 \mathrm{~kW}$ because easier to implement on Load Bank)

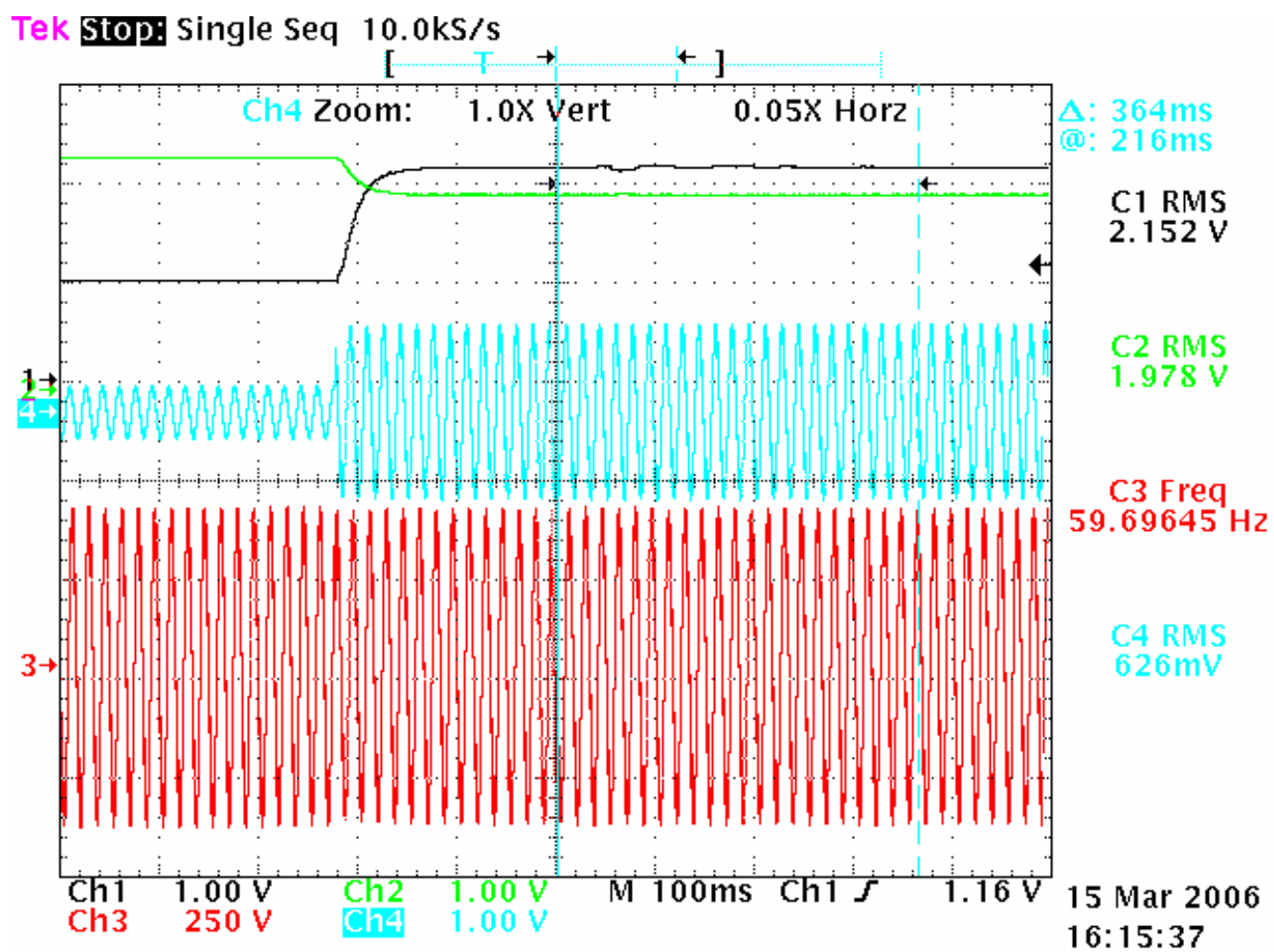

Figure 1615 - 45 kW (Fixed Speed) Power, Frequency, Voltage, Current 
iv.) Step Load Evaluation 4

1.Load Bank Start Load $=30 \mathrm{~kW}$

2.Unit A CMD $=20 \mathrm{~kW}$

3.Load Bank End Load $=60 \mathrm{~kW}$

\section{Engine Speed: $2100 \mathrm{rpm}(70 \mathrm{~Hz})$}

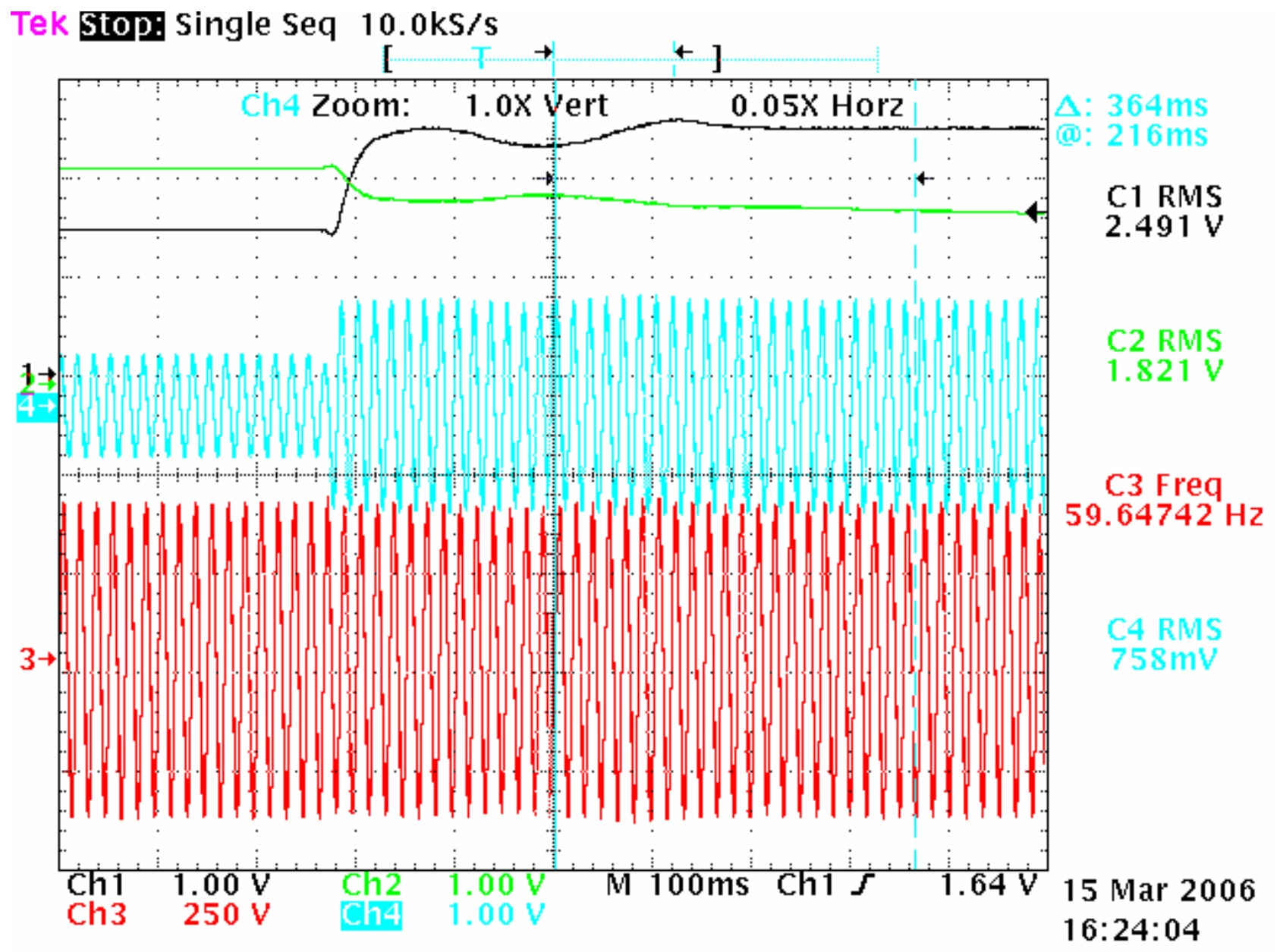

Figure 1730 - 60 kW (Fixed Speed) Power, Frequency, Voltage, Current 


\section{TECOGEN $60 \mathrm{~kW}$ Inverter-Based CHP Modules}

\section{CERTS Microgrid Test Bed Project \\ Tecogen Factory Testing \\ Unit Serial \#200836 \\ $4 / 13 / 06$}




\section{Introduction}

This report presents the factory test data of the first Tecogen CHP unit (Serial \# 200836), equipped with a customized inverter (by Youtility) that is configured with the CERTS microgrid control algorithm. Figure 1 presents a one-line diagram of the test set-up. The unit generates power in a stand-alone mode (no grid-tie) and is connected to a load bank. The output of the inverter is connected to the Delta side of a transformer in a 3-wire configuration. The output of the transformer is wired to the load bank in a 4-wire configuration with a 25-yard loop.

The inverter is also equipped with a Surge Module wired directly to the DC Bus. The Surge Module is a battery powered energy storage device with a rating of $20 \mathrm{~kW}$ for 3 seconds. Its purpose is to assist the engine/inverter's response to step loads. It is housed separately in an adjacent cabinet (DC power wiring length:12.5 feet).

All testing was done with the inverters set to Power Control Mode.

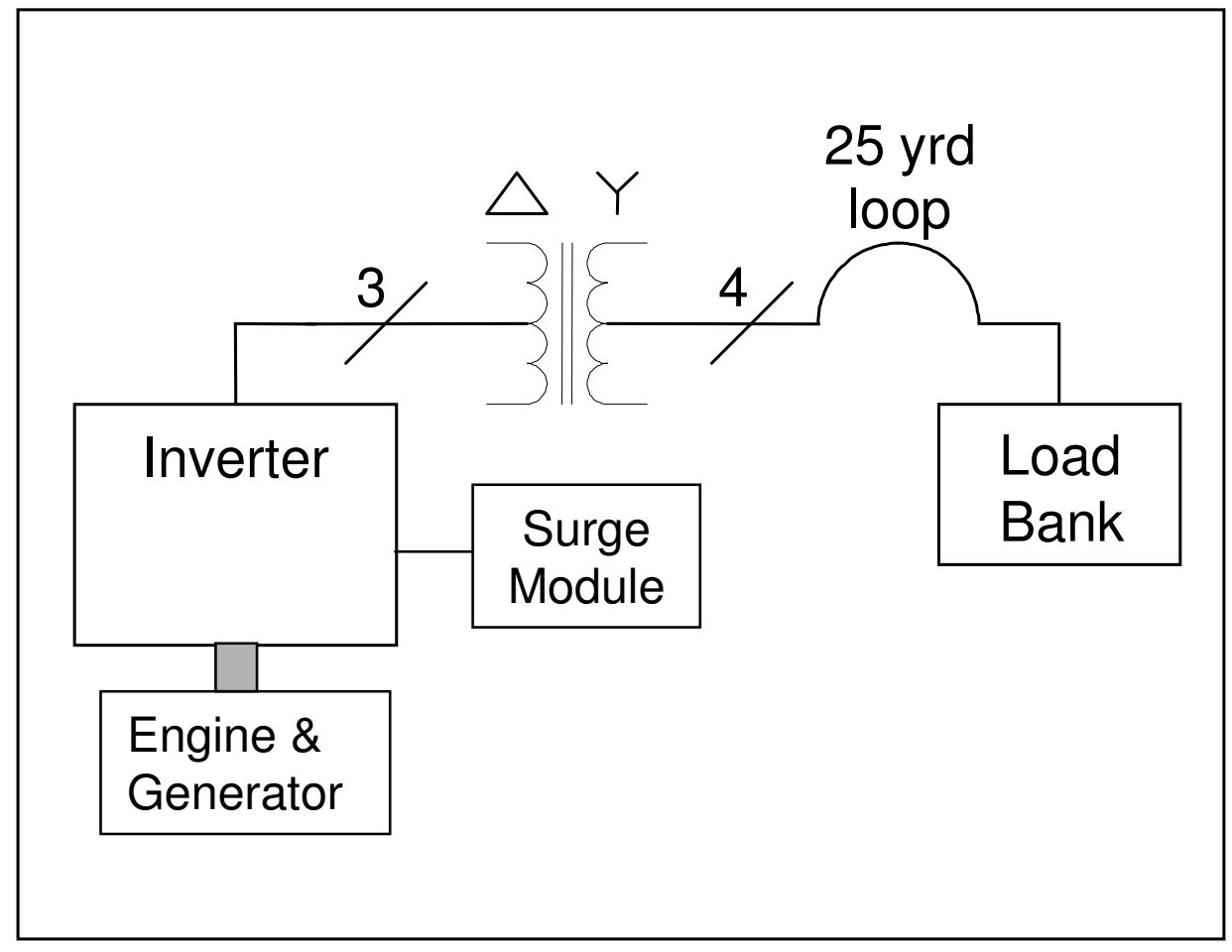

Figure 18 Factory Test One-line Diagram 


\section{B.2. Step Load Evaluations with Surge Module - Variable Speed Operation}

In prior testing on Unit\# 200835, it was determined that with a minimum speed setting of $1500 \mathrm{rpm}(50 \mathrm{~Hz})$, the system could not achieve the $0-60 \mathrm{~kW}$ step load. The objective for testing this second unit was to raise the minimum engine speed in Variable Speed mode to a level that would successfully accomplish the $0-60 \mathrm{~kW}$ step load. This would then establish the lower speed limit for Variable Speed operation. This speed was determined to be $1800 \mathrm{rpm}(60 \mathrm{~Hz})$. Therefore, all data was taken with the following settings in the software:

Minimum Engine Speed: $60 \mathrm{~Hz}$ (1800 rpm)

Maximum Engine Speed: $72 \mathrm{~Hz}$ (2160 rpm)

The following step loads were tested :

\begin{tabular}{|c|c|c|l|}
\hline & Test Plan & $\begin{array}{c}\text { Actual Test } \\
\text { Point }\end{array}$ & \multicolumn{1}{c|}{ Comments } \\
\hline i. & $0-30 \mathrm{~kW}$ & $0-30 \mathrm{~kW}$ & \\
\hline ii. a & & $0-50 \mathrm{~kW}$ & $\begin{array}{l}\text { Done to ensure the frequency did not go out } \\
\text { of range if the load slightly exceeded } 60 \mathrm{~kW} \\
\text { from the load bank. }\end{array}$ \\
\hline ii. b & $0-60 \mathrm{~kW}$ & $0-60 \mathrm{~kW}$ & \\
\hline iii. & $15-45 \mathrm{~kW}$ & $15-50 \mathrm{~kW}$ & $\begin{array}{l}\text { Changed to } 50 \mathrm{~kW} \text { because easier to } \\
\text { implement on load bank }\end{array}$ \\
\hline iv. a & & $30-55 \mathrm{~kW}$ & $\begin{array}{l}\text { Done to ensure the frequency did not go out } \\
\text { of range if the load slightly exceeded } 60 \mathrm{~kW} \\
\text { from the load bank. }\end{array}$ \\
\hline iv. b & $30-60 \mathrm{~kW}$ & $30-60 \mathrm{~kW}$ & \\
\hline v. a & $60-0 \mathrm{~kW}$ & $60-0 \mathrm{~kW}$ & \\
\hline v. b & & $55-0 \mathrm{~kW}$ & $\begin{array}{l}\text { Done to ensure the frequency did not go out } \\
\text { of range if the load slightly exceeded } 60 \mathrm{~kW} \\
\text { from the load bank. }\end{array}$ \\
\hline vi. & $30-0 \mathrm{~kW}$ & $30-0 \mathrm{~kW}$ & \\
\hline
\end{tabular}

Table 9 Test Point Summary - Step Loads with Surge Module/Variable Speed

For each step load point, an oscilloscope trace was captured that measured the power output, frequency, current, and voltage. These measurements were taken on the microgrid side (Y side) of the transformer. In each plot the data is formatted in the following way:

Ch1 = Unit RMS Power Output;

$0.0 \mathrm{~V}=-15 \mathrm{KW}$

$0.5 \mathrm{~V}=0 \mathrm{KW}$

$1.5 \mathrm{~V}=30 \mathrm{KW}$

$2.5 \mathrm{~V}=60 \mathrm{KW}$ 
$\mathrm{Ch} 2=$ Unit Operating Frequency;

$60 \mathrm{~Hz}-->2.3 \mathrm{~V}$

$59.5 \mathrm{~Hz}-->1.66 \mathrm{~V}$

$60.5 \mathrm{~Hz}-->2.94 \mathrm{~V}$

\section{Ch 3 -Grid Voltage L-N}

Channel 4

Ch4 = Unit Output Current; $1 \mathrm{~V}=100 \mathrm{Amps}$

Also, the Step "Up" load points (i-iv.) were run two separate times so that additional data on the system dynamics, with the surge module, could be captured. This oscillocope data is formatted as follows:

Channel 1 = Engine RPM;

Not Recorded

Channel $2=$ Bus Voltage

Zero point is actually 500 volts and measures from black "l" marker on left-hand side of Figure

100 volts per division

Nominal Voltage; 860 volts

Channel $3=$ AC Current $; 1 \mathrm{~V}=100 \mathrm{amps}$

Channel $4=$ Surge Module Current

$10 \mathrm{mV}=10 \mathrm{amps}$ (Need to verify with $\mathrm{J}$. Wen of Youtility)

$10 \mathrm{mV}$ per division

Nominal Current; 26 amps

The results of each test is organized in a table that defines the load setpoint (abbreviated S.P) and the actual power output from the unit. The table also contains the microgrid operating frequency before and after the transient event occurs (These frequency values are obtained from the units software and not the oscilloscope measurement). Also recorded in the table is the engine speed before and after the transient. Data contained in column A defines the operating condition and the state of the microgrid before the transient condition being tested occurs. Data contained in column B defines the operating condition and the state of the microgrid after the transient condition being tested has occurred. 
i. Step Load Evaluation 1

4.Load Bank Start Load $=0 \mathrm{~kW}$

5.Unit A CMD $=20 \mathrm{~kW}$

6.Load Bank End Load $=30 \mathrm{~kW}$

\begin{tabular}{|c|c|c|}
\hline & A & B \\
\hline Unit \#1 (S.P/Actual) kW & $20.0 / 0.0 \mathrm{~kW}$ & $20.0 / 30.5 \mathrm{~kW}$ \\
\hline Frequency Hz & $60.16 \mathrm{~Hz}$ & $59.91 \mathrm{~Hz}$ \\
\hline Engine Speed (Hz/rpm) & $60 / 1800$ & $60 / 1800$ \\
\hline
\end{tabular}

Table 10 Step Load Evaluation 1: 0 - 30 kW (w/Surge Module)

\section{i. 0 - 30 KW Step Load -Surge Module- Variable Speed}

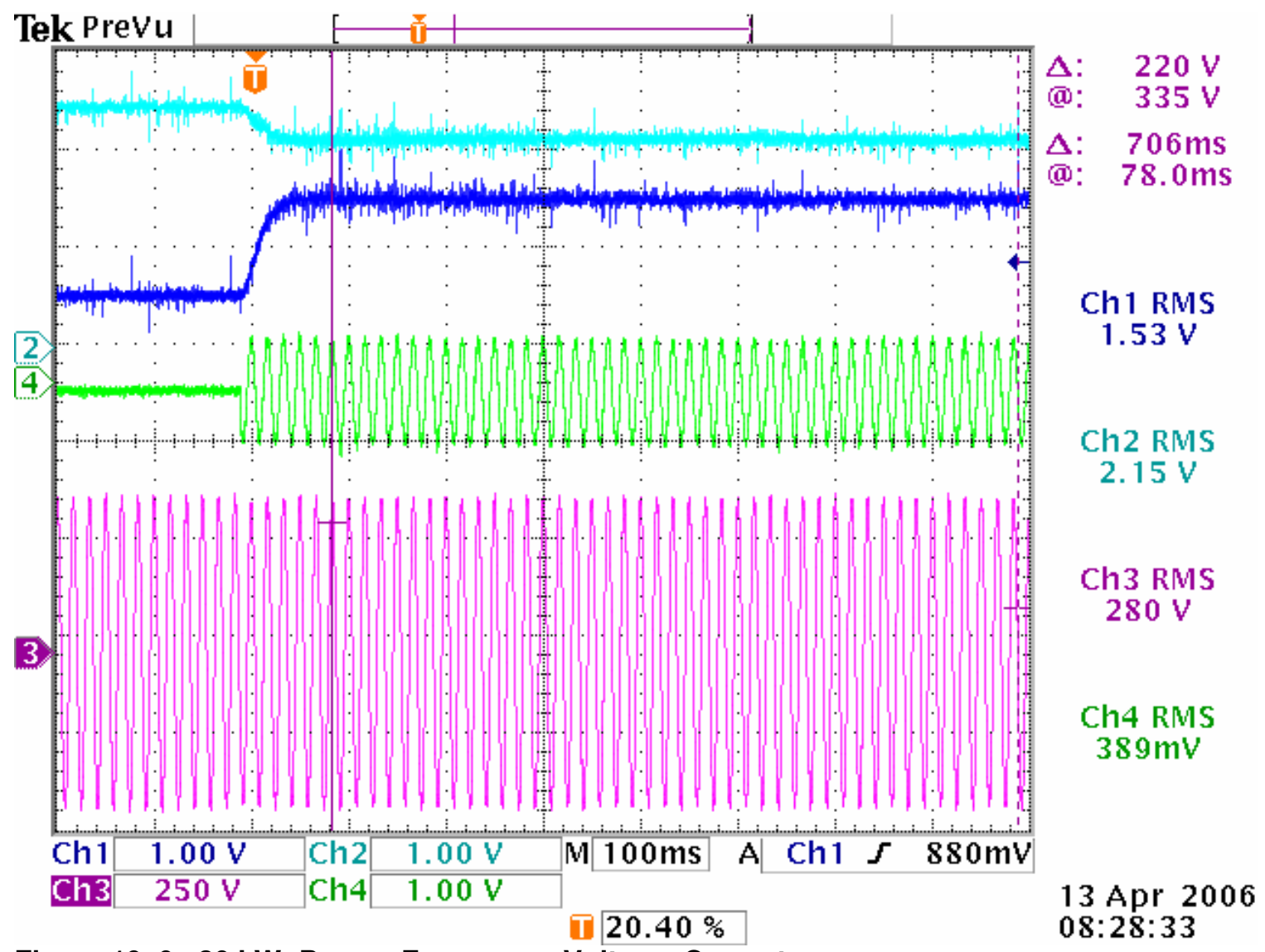

Figure 19 0-30 kW: Power, Frequency, Voltage, Current 


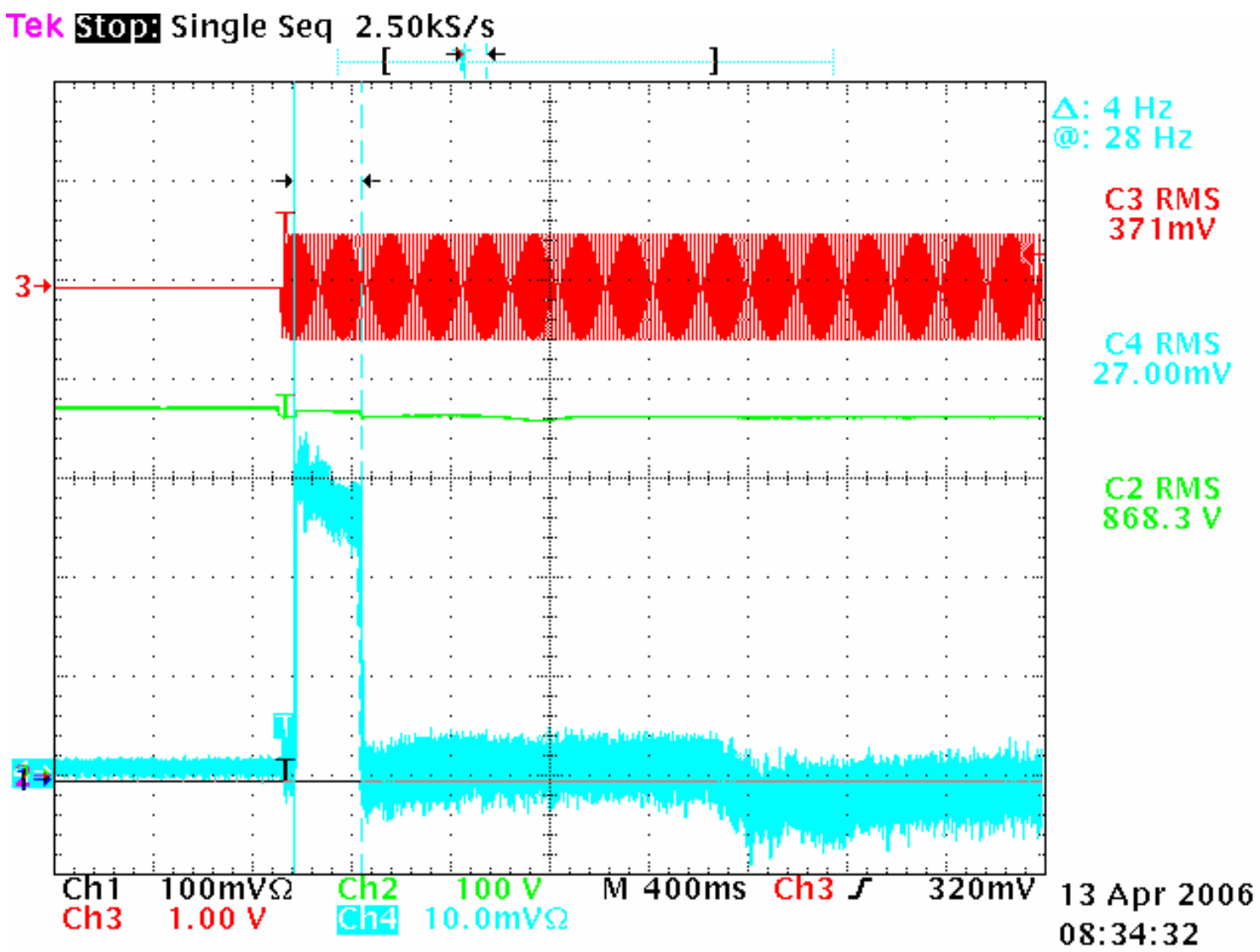

Figure 200 - 30 kW: Engine RPM, Bus Voltage, AC Current, Surge Module Current

vi. $\quad$ Step Load Evaluation 2

7. Load Bank Start Load $=0 \mathrm{KW}$

8. Unit $\mathrm{A} C \mathrm{CMD}=20 \mathrm{KW}$

9. Load Bank End Load $=60 \mathrm{~kW}$

ii a. $0-50 \mathrm{~kW}$ (Additional test point done to ensure the frequency did not go out of range if the load slightly exceeded $60 \mathrm{~kW}$ from the load bank)

\begin{tabular}{|c|c|c|}
\hline & A & B \\
\hline Unit \#1 (S.P/Actual) kW & $20.0 / 0.0 \mathrm{~kW}$ & $20.0 / 50.9 \mathrm{~kW}$ \\
\hline Frequency Hz & $60.16 \mathrm{~Hz}$ & $59.74 \mathrm{~Hz}$ \\
\hline Engine Speed (Hz/rpm) & $60 / 1800$ & $67.7 / 2031$ \\
\hline
\end{tabular}

Table 11 Step Load Evaluation 2a: 0-50 kW (w/Surge Module) 


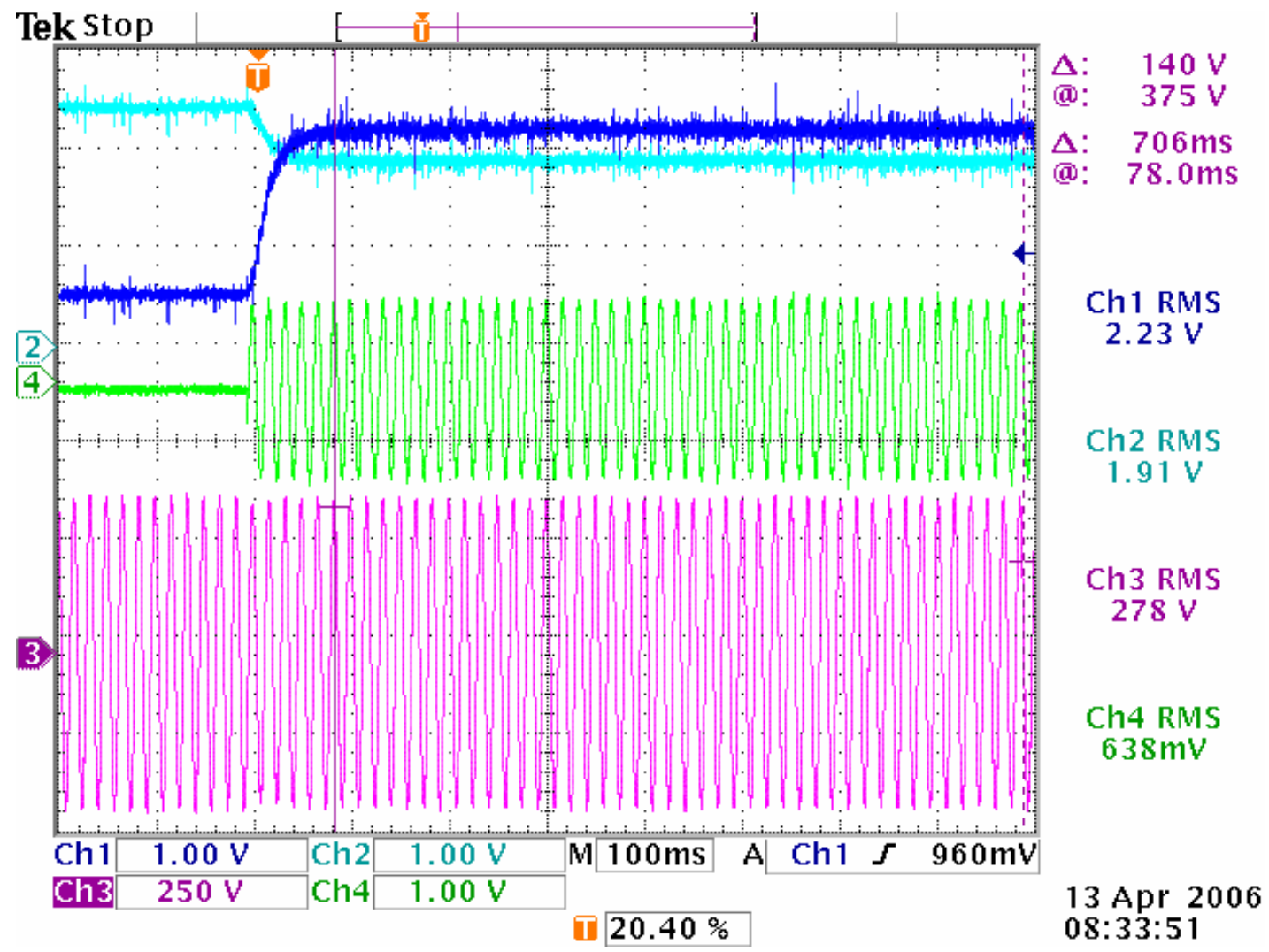

Figure 210 -50 kW: Power, Frequency, Voltage, Current

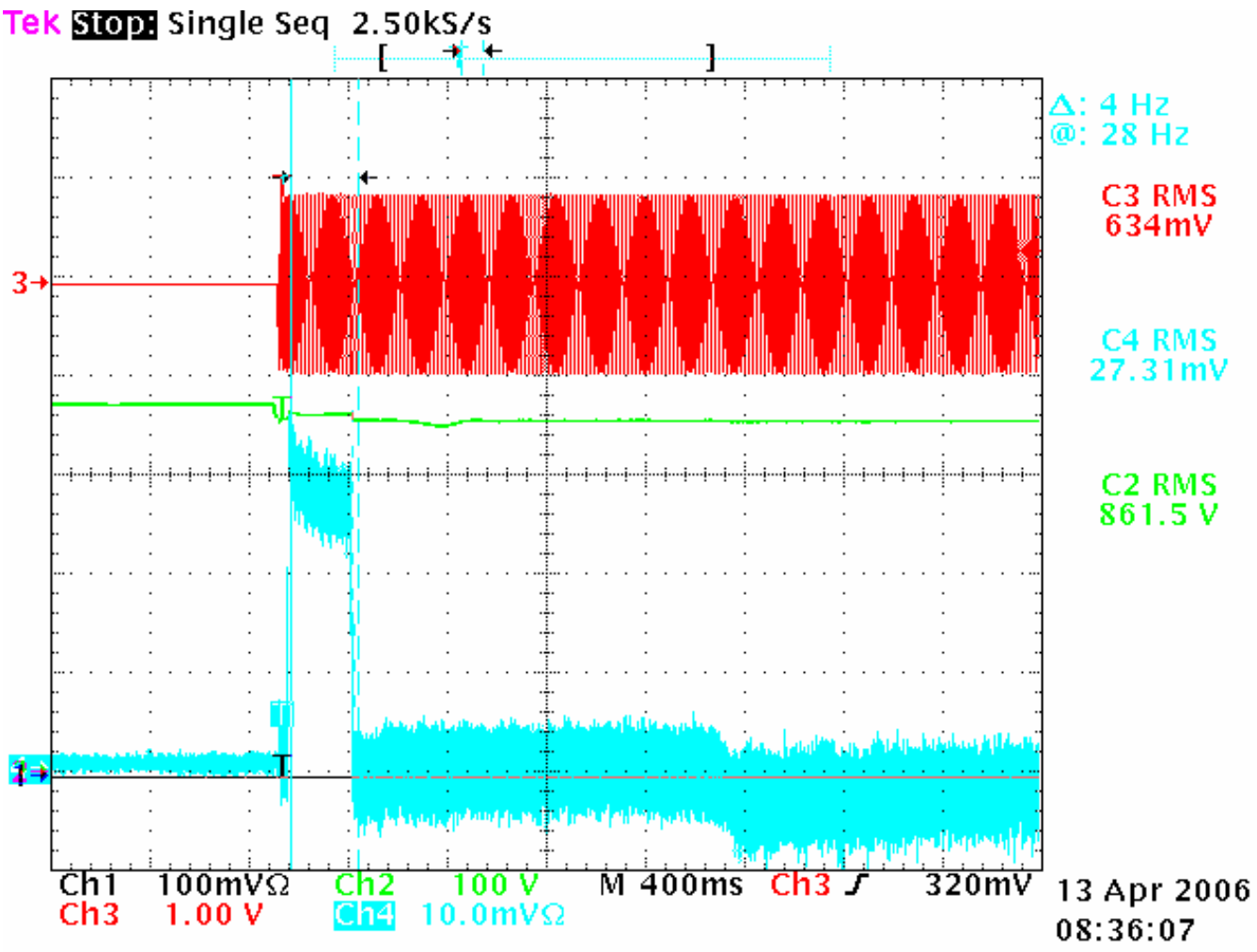

Figure 220 -50 kW: Engine RPM, Bus Voltage, AC Current, Surge Module Current 
ii b. $0-60 \mathrm{~kW}$

\begin{tabular}{|c|c|c|}
\hline & A & B \\
\hline Unit \#1 (S.P/Actual) kW & $20.0 / 0.0 \mathrm{~kW}$ & $20.0 / 60.7 \mathrm{~kW}$ \\
\hline Frequency Hz & $60.16 \mathrm{~Hz}$ & $59.18 \mathrm{~Hz}$ \\
\hline Engine Speed $(\mathrm{Hz} / \mathrm{rpm})$ & $60 / 1800$ & $72.7 / 2181$ \\
\hline
\end{tabular}

Table 12 Step Load Evaluation 2b: 0-60 kW (w/Surge Module)

ii b. $0-60 \mathrm{KW}$ Step Load -Surge Module- Variable Speed

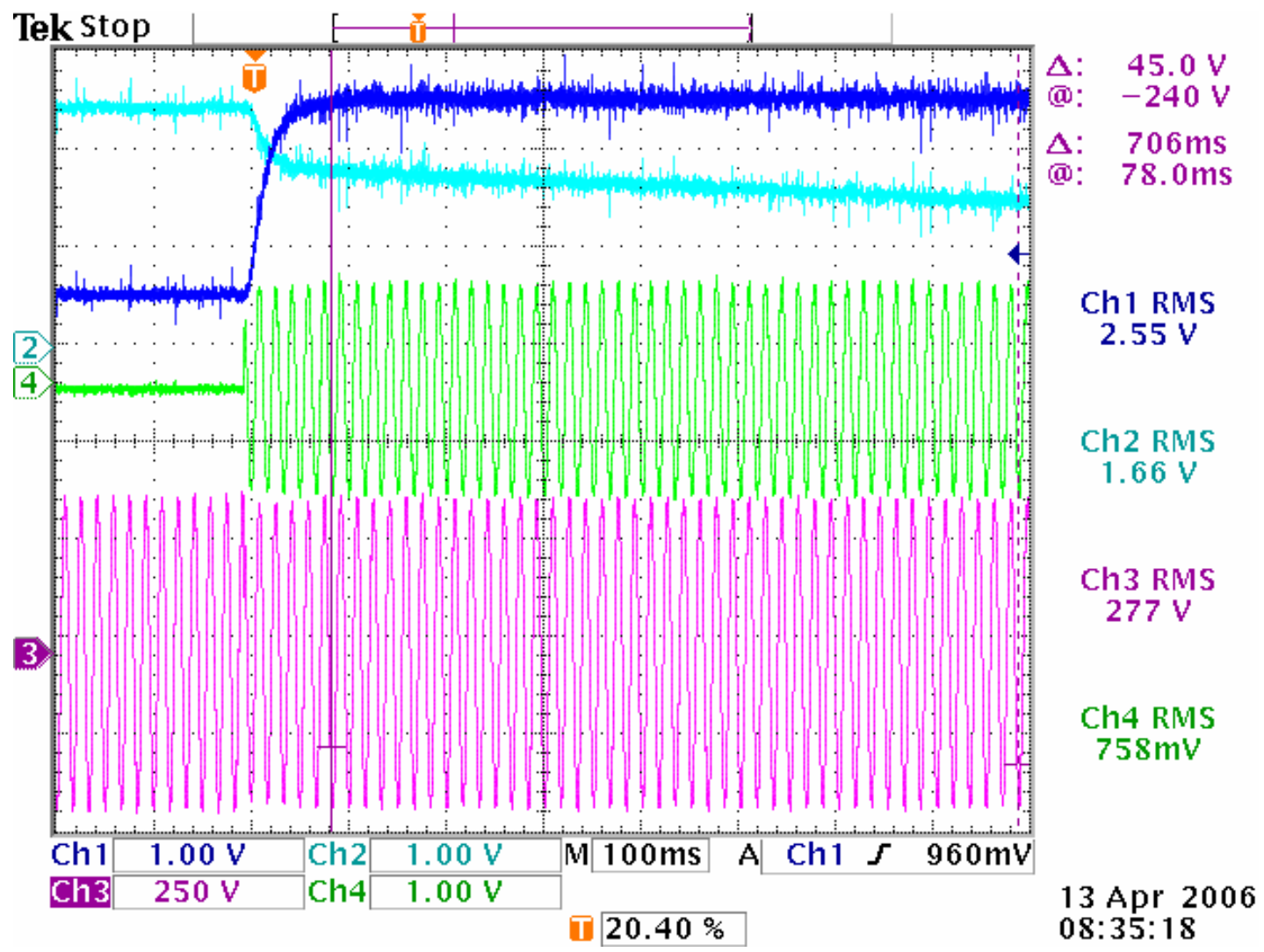

Figure $230-60$ kW: Power, Frequency, Voltage, Current 


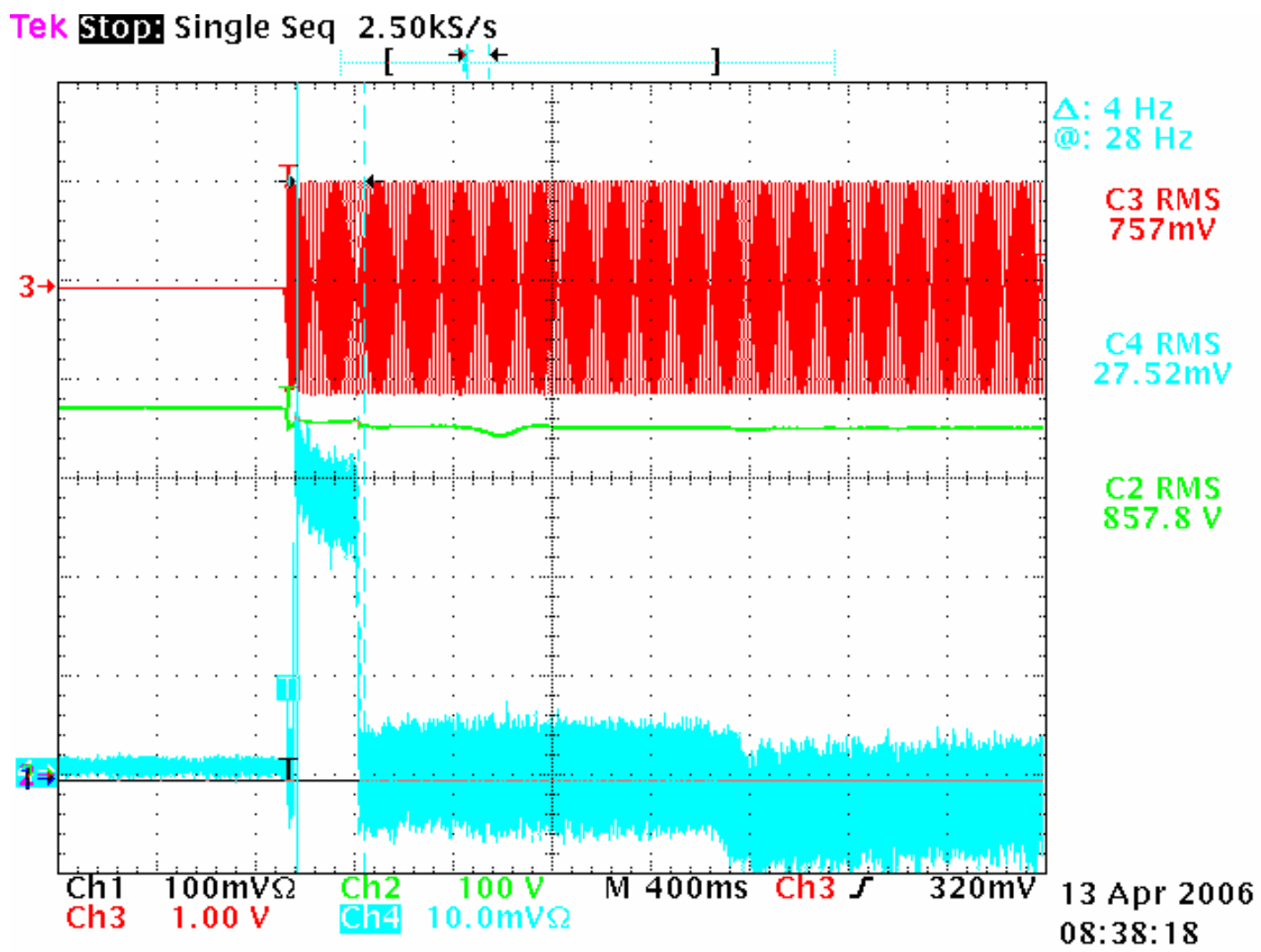

Figure 240 - 60 kW: Engine RPM, Bus Voltage, AC Current, Surge Module Current

vii. $\quad$ Step Load Evaluation 3

1.Load Bank Start Load $=15 \mathrm{~kW}$

2.Unit A CMD $=20 \mathrm{~kW}$

3.Load Bank End Load $=50 \mathrm{~kW}$ (Changed from $45 \mathrm{~kW}$ because easier to implement on Load Bank)

\begin{tabular}{|c|c|c|}
\hline & A & B \\
\hline Unit \#1 (S.P/Actual) kW & $20.0 / 15.5 \mathrm{~kW}$ & $20.0 / 50.9 \mathrm{~kW}$ \\
\hline Frequency Hz & $60.03 \mathrm{~Hz}$ & $59.74 \mathrm{~Hz}$ \\
\hline Engine Speed (Hz/rpm) & $60 / 1800$ & $67.7 / 2031$ \\
\hline
\end{tabular}

Table 13 Step Load Evaluation 3: 15 - 50 kW (w/Surge Module) 
iii. 15 - 50 KW Step Load -Surge Module- Variable Speed

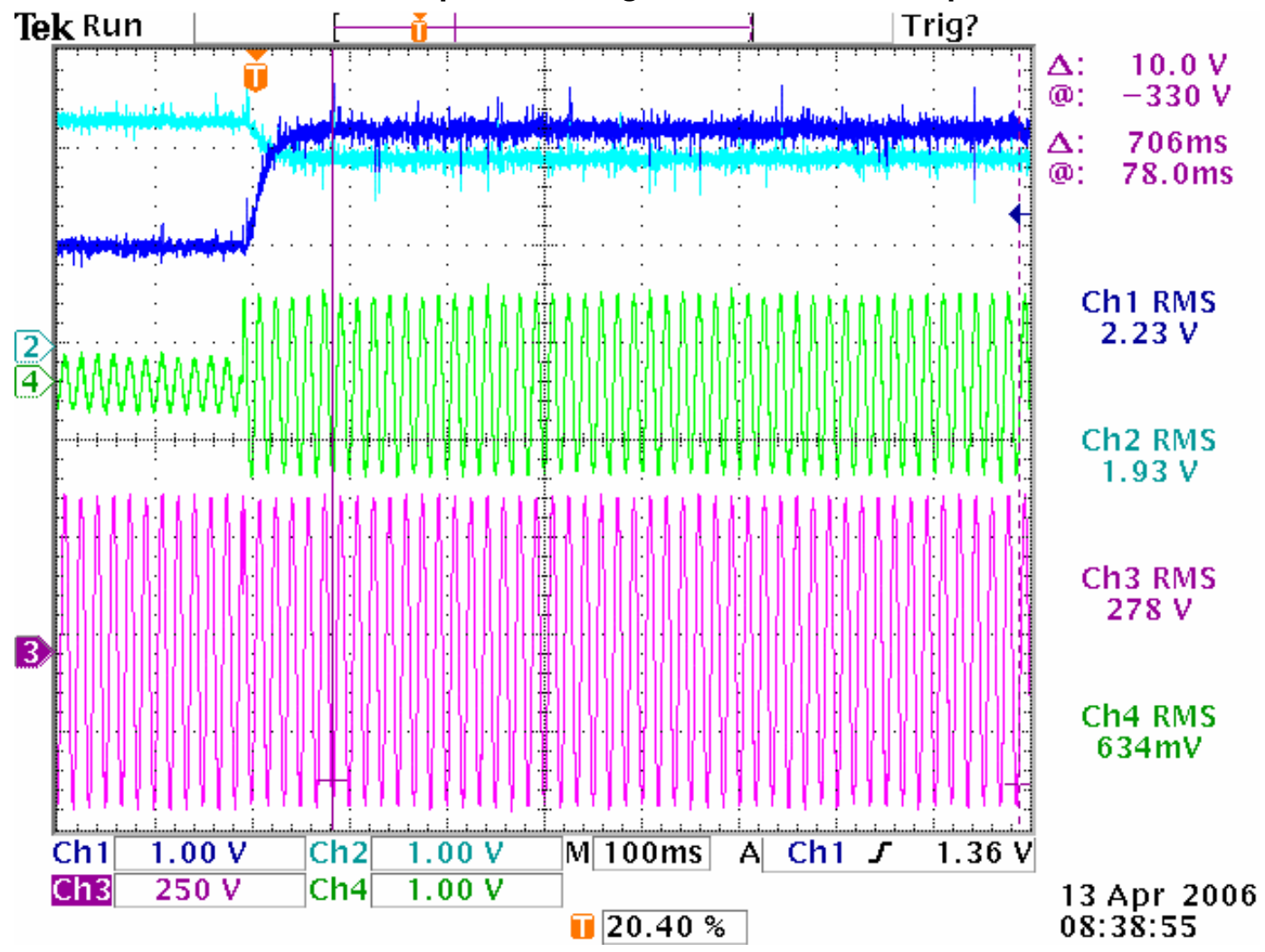

Figure 2515 - 50 kW: Power, Frequency, Voltage, Current

Tek Stop: Single Seq $2.50 \mathrm{kS} / \mathrm{s}$

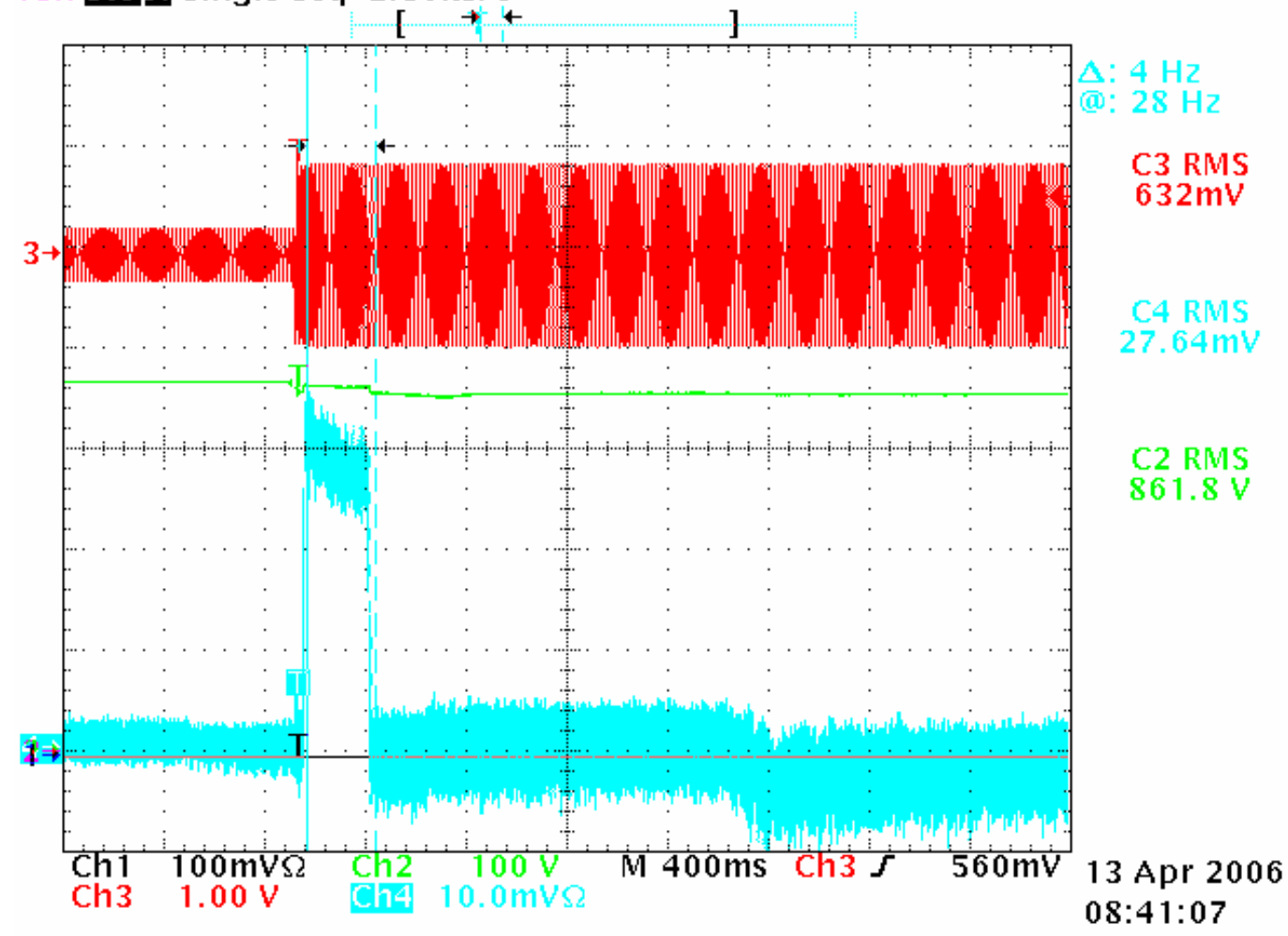

Figure 2615 - 50 kW: Engine RPM, Bus Voltage, AC Current, Surge Module Current 
viii. $\quad$ Step Load Evaluation 4

1.Load Bank Start Load $=30 \mathrm{~kW}$

2.Unit A CMD $=20 \mathrm{~kW}$

3.Load Bank End Load $=60 \mathrm{~kW}$

iv a. 30 - $55 \mathrm{~kW}$ (Additional test point done to ensure the frequency did not go out of range if the load slightly exceeded $60 \mathrm{~kW}$ from the load bank)

\begin{tabular}{|c|c|c|}
\hline & A & B \\
\hline Unit \#1 (S.P/Actual) KW & $20.0 / 30.4 \mathrm{~kW}$ & $20.0 / 55.8 \mathrm{~kW}$ \\
\hline Frequency Hz & $59.91 \mathrm{~Hz}$ & $59.70 \mathrm{~Hz}$ \\
\hline Engine Speed (Hz/rpm) & $60 / 1800$ & $70.1 / 2103$ \\
\hline
\end{tabular}

Table 14 Step Load Evaluation 4a: 30 - 55 kW (w/Surge Module)

iv.a 30-55 KW Step Load -Surge Module- Variable Speed

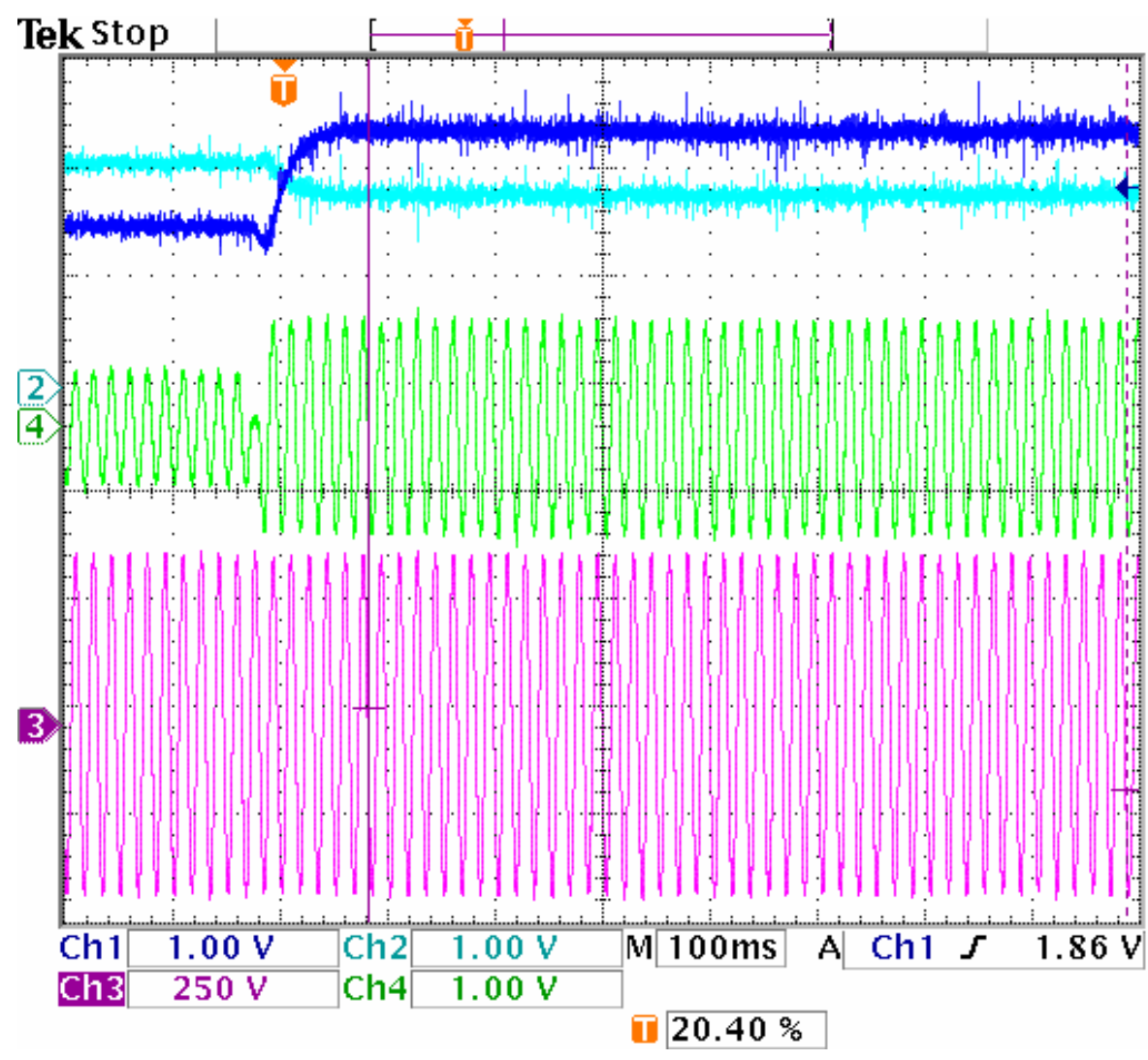

$\triangle: \quad 190 \mathrm{~V}$

(a): $40.0 \mathrm{~V}$

$\Delta: \quad 706 \mathrm{~ms}$

(a): $78.0 \mathrm{~ms}$

Ch1 RMS

$2.38 \mathrm{~V}$

Ch2 RVIS

$1.79 \mathrm{~V}$

Ch3 RMS

$277 \mathrm{~V}$

Ch4 RMS

$692 \mathrm{mV}$

Figure 2730 -55 kW: Power, Frequency, Voltage, Current 


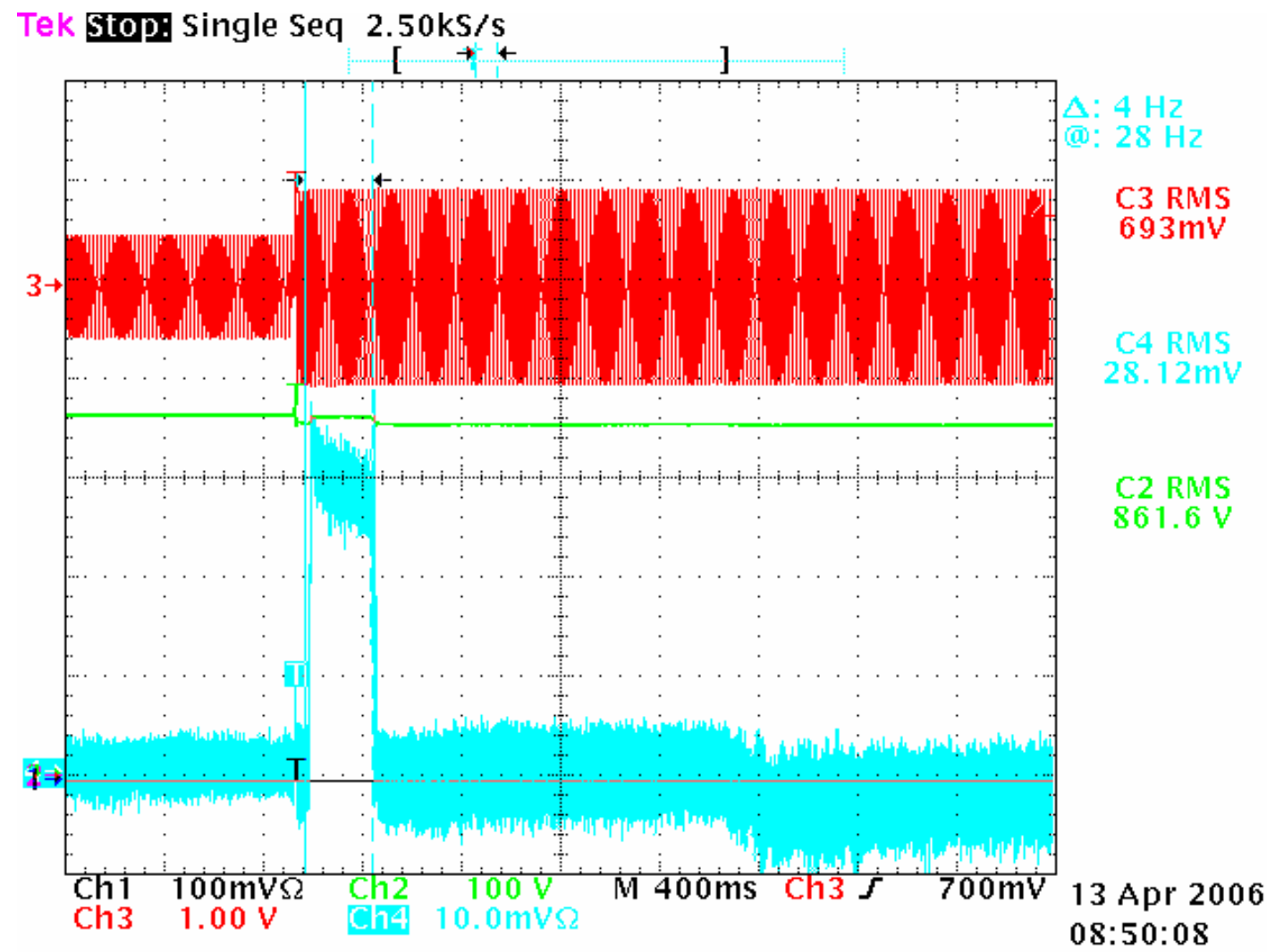

Figure 2830 - 55 kW: Engine RPM, Bus Voltage, AC Current, Surge Module Current iv b. $30-60 \mathrm{~kW}$

\begin{tabular}{|c|c|c|}
\hline & A & B \\
\hline Unit \#1 (S.P/Actual) KW & $20.0 / 30.4 \mathrm{~kW}$ & $20.0 / 60.7 \mathrm{~kW}$ \\
\hline Frequency Hz & $59.91 \mathrm{~Hz}$ & $59.16 \mathrm{~Hz}$ \\
\hline Engine Speed (Hz/rpm) & $60 / 1800$ & $72.5 / 2175$ \\
\hline
\end{tabular}

Table 15 Step Load Evaluation 4b: $30-60$ kW (w/Surge Module) 
iv.b 30-60 KW Step Load -Surge Module- Variable Speed

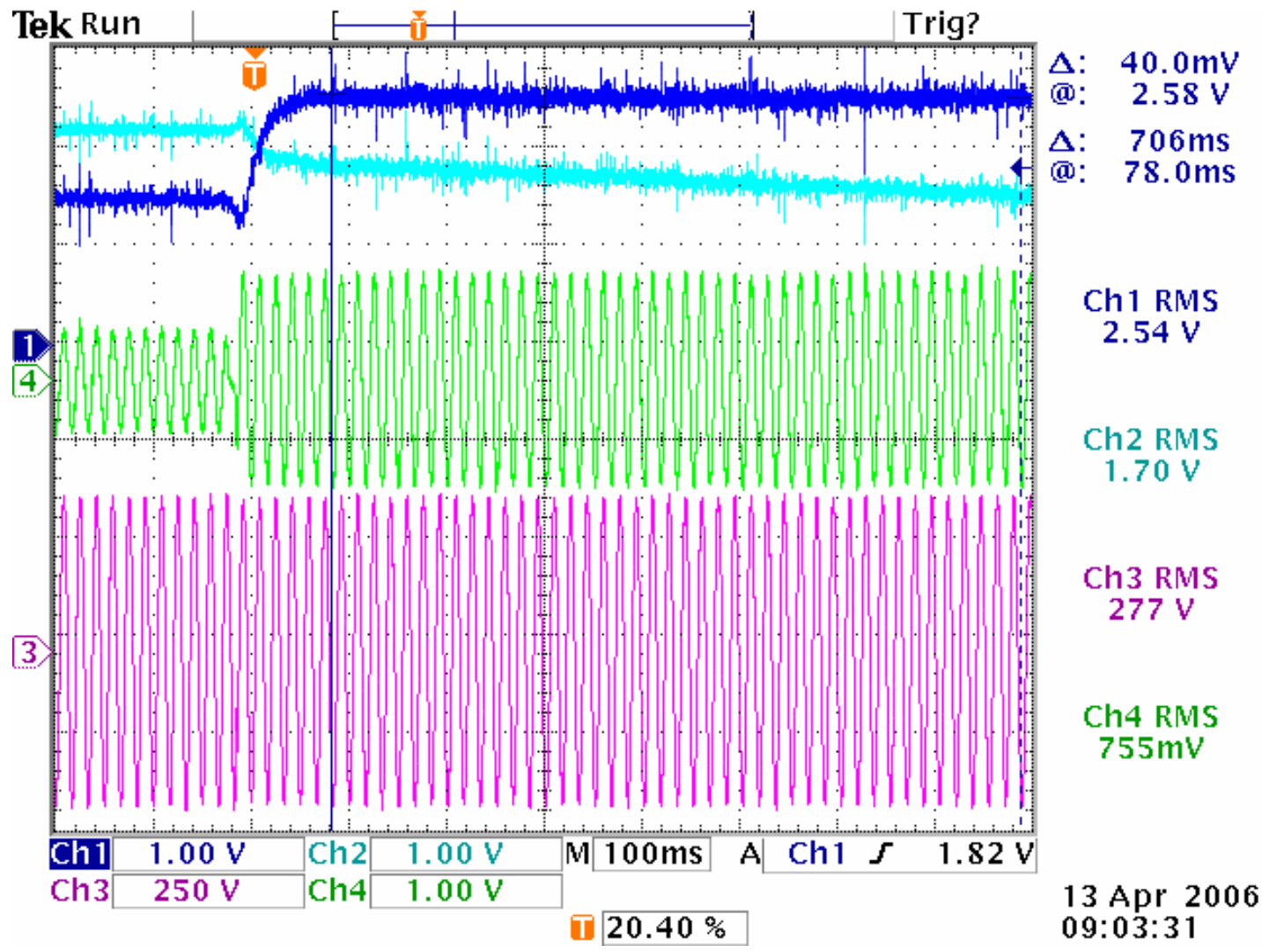

Figure 2930 -60 kW: Power, Frequency, Voltage, Current

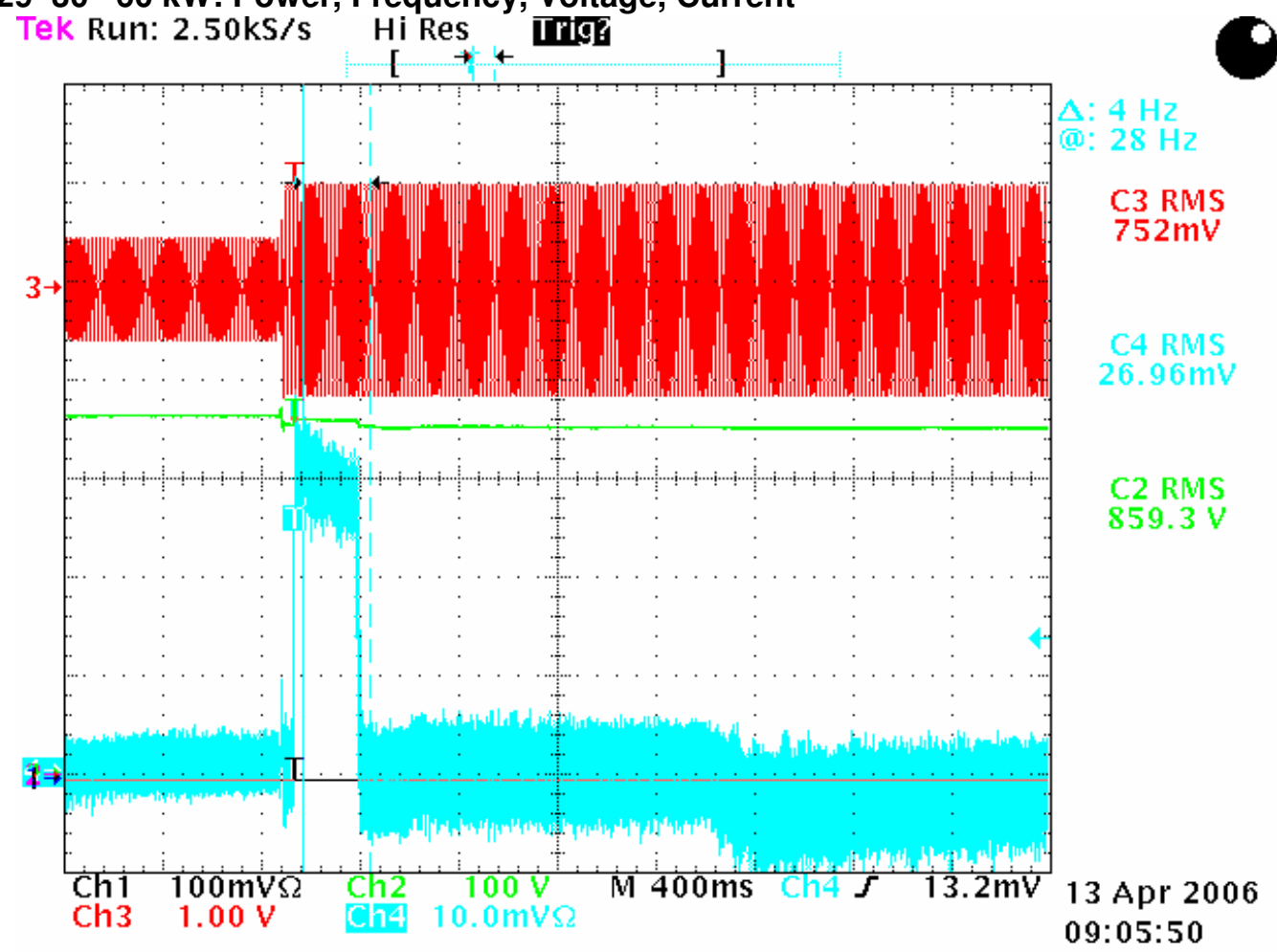

Figure 3030 - 60 kW: Engine RPM, Bus Voltage, AC Current, Surge Module Current 
ix. $\quad$ Step Load Evaluation 5

1.Load Bank Start Load $=60 \mathrm{~kW}$

2.Unit A CMD $=20 \mathrm{~kW}$

3.Load Bank End Load $=0 \mathrm{~kW}$

v a. $60-0 \mathrm{~kW}$

\begin{tabular}{|c|c|c|}
\hline & A & B \\
\hline Unit \#1 (S.P/Actual) kW & $20.0 / 60.7 \mathrm{~kW}$ & $20.0 / 0 \mathrm{~kW}$ \\
\hline Frequency Hz & $59.16 \mathrm{~Hz}$ & $60.16 \mathrm{~Hz}$ \\
\hline Engine Speed (Hz/rpm) & $72.5 / 2175$ & $60 / 1800$ \\
\hline
\end{tabular}

Table 16 Step Load Evaluation 5a: 60 - 0 kW (w/Surge Module)

\section{0- 0 kW Step Load -Surge Module- Variable Speed}

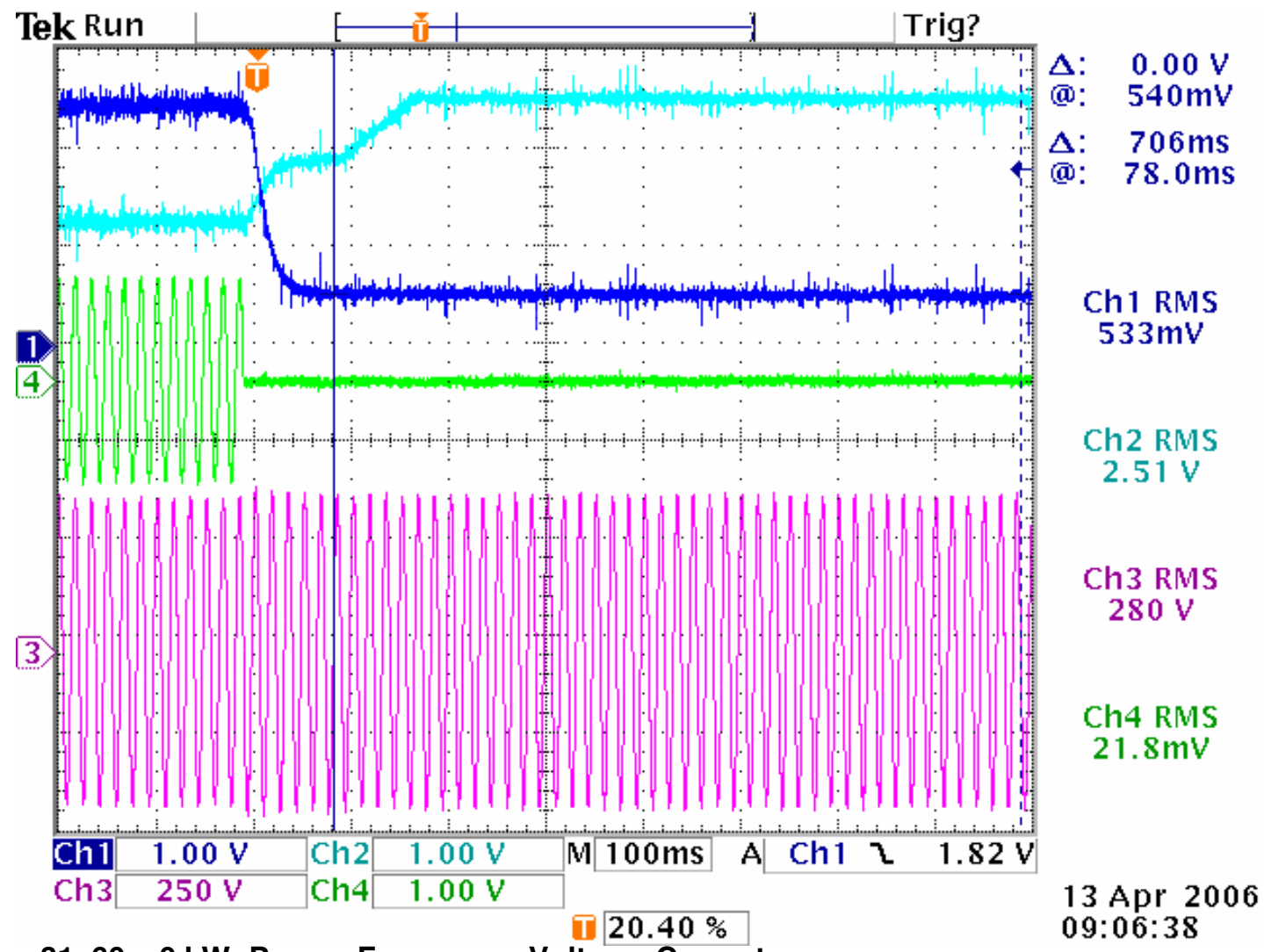

Figure 31 60-0 kW: Power, Frequency, Voltage, Current 
v b. 55 - $0 \mathrm{~kW}$ (Additional test point done to ensure the frequency did not go out of range if the load slightly exceeded $60 \mathrm{~kW}$ from the load bank)

\begin{tabular}{|c|c|c|}
\hline & A & B \\
\hline Unit \#1 (S.P/Actual) kW & $20.0 / 55.8 \mathrm{~kW}$ & $20.0 / 0 \mathrm{~kW}$ \\
\hline Frequency Hz & $59.70 \mathrm{~Hz}$ & $60.16 \mathrm{~Hz}$ \\
\hline Engine Speed (Hz/rpm) & $70.1 / 2103$ & $60 / 1800$ \\
\hline
\end{tabular}

Table 17 Step Load Evaluation 5b: 55 - 0 kW (w/Surge Module)

\section{5- 0 kW Step Load -Surge Module- Variable Speed}

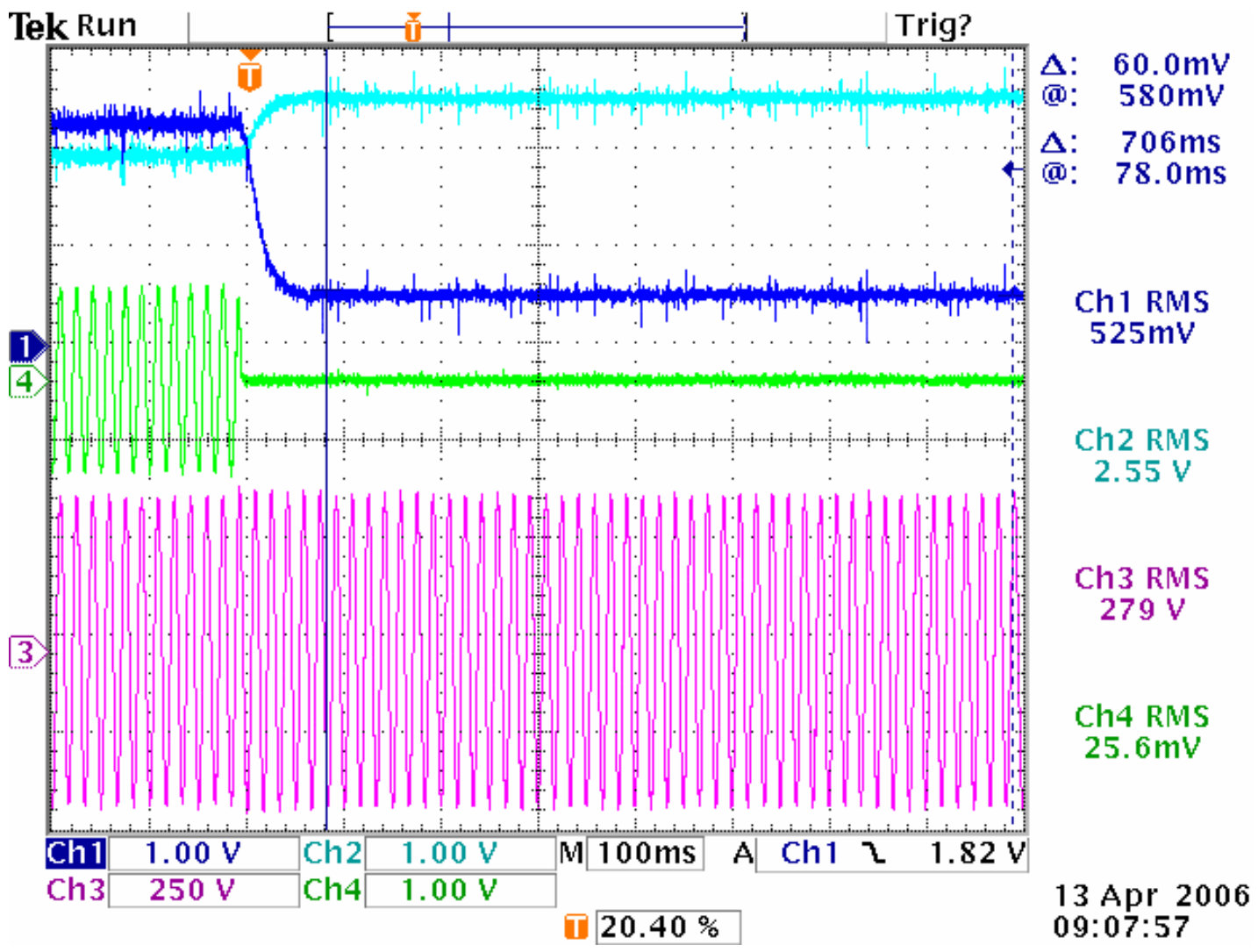

Figure 32 55 - 0 kW: Power, Frequency, Voltage, Current 
vi. Step Load Evaluation 6

4. Load Bank Start Load $=30 \mathrm{~kW}$

5. Unit A CMD $=20 \mathrm{~kW}$

6. Load Bank End Load $=0 \mathrm{~kW}$

\begin{tabular}{|c|c|c|}
\hline & A & B \\
\hline Unit \#1 (S.P/Actual) kW & $20.0 / 30.4 \mathrm{~kW}$ Unit & $20.0 / 0 \mathrm{~kW}$ \\
\hline Frequency Hz & $59.91 \mathrm{~Hz}$ & $60.16 \mathrm{~Hz}$ \\
\hline Engine Speed (Hz/rpm) & $60 / 1800$ & $60 / 1800$ \\
\hline
\end{tabular}

Table 18 Step Load Evaluation 6: $30-0$ kW (w/Surge Module)

\section{0- 0 kW Step Load -Surge Module- Variable Speed}

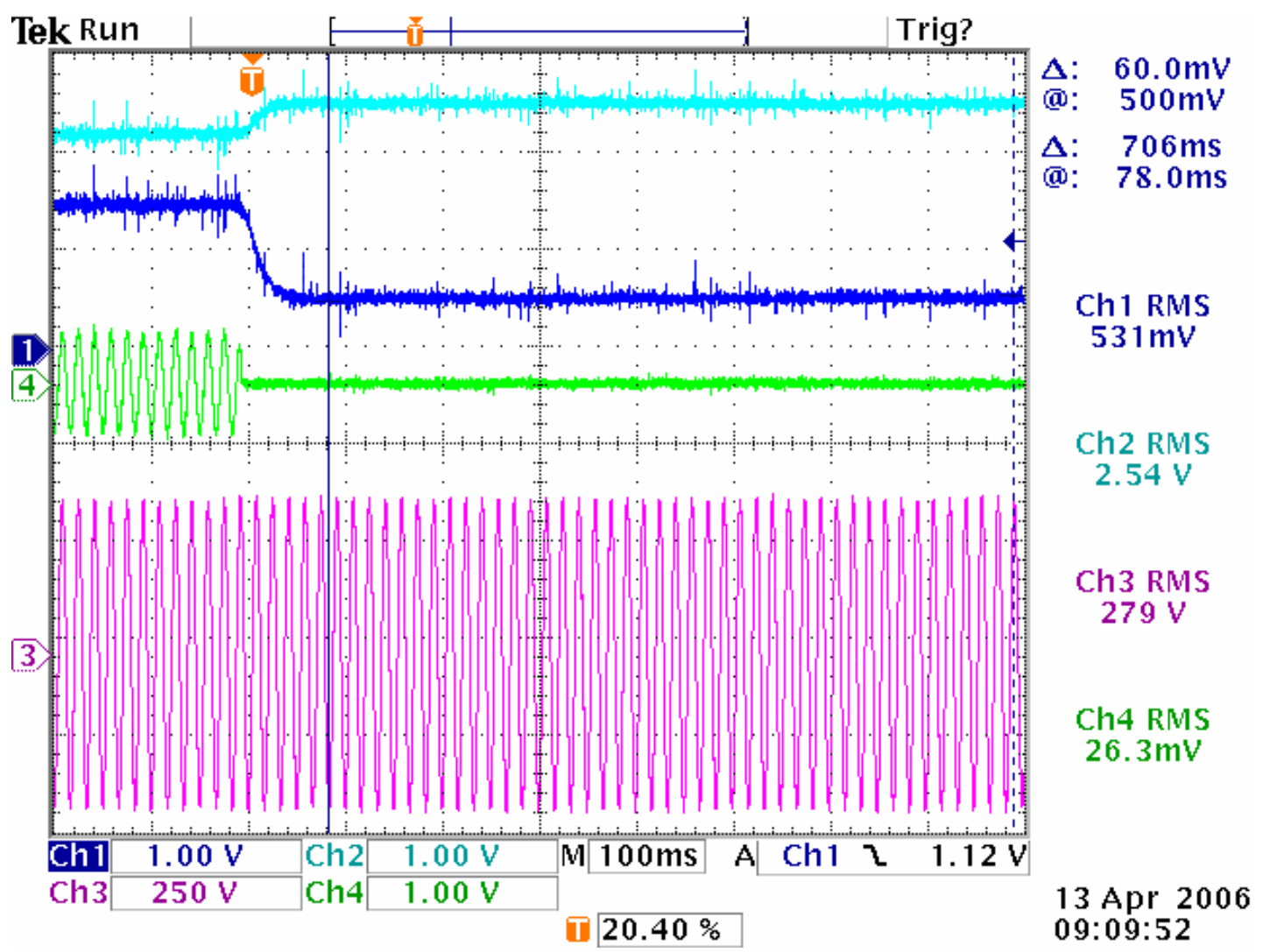

Figure 33 30-0 kW: Power, Frequency, Voltage, Current 


\section{B.3. Step Load Evaluations without Surge Module - Fixed Speed Operation}

The CHP unit was tested with the surge module disabled. As with the first unit (Serial \# 200835), the results confirmed that the dynamics of the engine/inverter allows the unit to successfully handle a step load without the surge module. The engine was set at the fixed speed required for the maximum load step of $0-60 \mathrm{~kW}(2160 \mathrm{rpm})$. Each step load point was run to confirm Pass/Fail. Oscilloscope data was not recorded since, as demonstrated in the testing of Unit \#200835, if the unit "Passes", the electrical output signatures (Figure 1) are the same as in Variable Speed operation.

The following table summarizes the test results.

\begin{tabular}{|c|c|c|}
\hline \multicolumn{3}{|c|}{$\begin{array}{c}\text { Fixed Engine Speed } 2160 \mathrm{rpm} \\
(72 \mathrm{~Hz}) \\
\text { No Surge Module }\end{array}$} \\
\hline & Step Load & Pass/Fail \\
\hline i. & $0-30 \mathrm{~kW}$ & Pass \\
\hline ii. $\mathrm{a}$ & $0-50 \mathrm{~kW}$ & Pass \\
\hline ii. $b$ & $0-60 \mathrm{~kW}$ & Pass \\
\hline iii. & $15-50 \mathrm{~kW}$ & Pass \\
\hline iv. a & $30-55 \mathrm{~kW}$ & Pass \\
\hline iv. b & $30-60 \mathrm{~kW}$ & Pass \\
\hline v. a & $60-0 \mathrm{~kW}$ & Pass \\
\hline v. b & $55-0 \mathrm{~kW}$ & Pass \\
\hline vi. & $30-0 \mathrm{~kW}$ & Pass \\
\hline
\end{tabular}

Table 19 Test Point Summary - Step Loads without Surge Module/Fixed Speed 


\section{TECOGEN $60 \mathrm{~kW}$ Inverter-Based CHP Modules}

\section{CERTS Microgrid Test Bed Project \\ Tecogen Factory Testing \\ Unit Serial \#200837}

$5 / 09 / 06$ 


\section{Introduction}

This report presents the factory test data of the third Tecogen CHP unit (Serial \# 200837), equipped with a customized inverter (by Youtility) that is configured with the CERTS microgrid control algorithm. Figure 1 presents a one-line diagram of the test set-up. The unit generates power in a stand-alone mode (no grid-tie) and is connected to a load bank. The output of the inverter is connected to the Delta side of a transformer in a 3-wire configuration. The output of the transformer is wired to the load bank in a 4-wire configuration with a 25-yard loop.

The inverter is also equipped with a Surge Module wired directly to the DC Bus. The Surge Module is a battery powered energy storage device with a rating of $20 \mathrm{~kW}$ for 3 seconds. Its purpose is to assist the engine/inverter's response to step loads. It is housed separately in an adjacent cabinet (DC power wiring length:12.5 feet).

All testing was done with the inverters set to Power Control Mode.

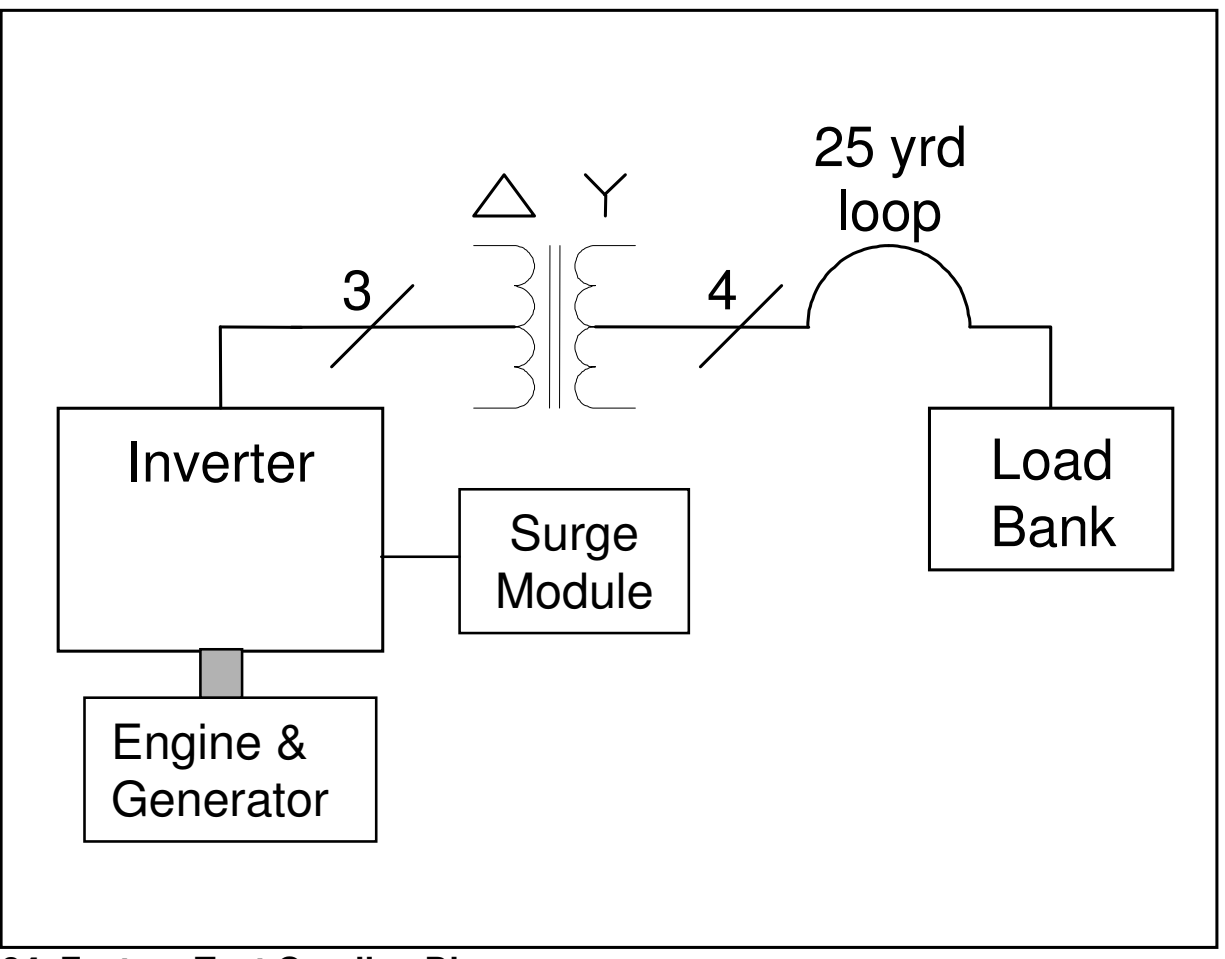

Figure 34 Factory Test One-line Diagram 


\section{B.2. Step Load Evaluations with Surge Module - Variable Speed Operation}

All data was taken with the following settings in the software:

Minimum Engine Speed: $60 \mathrm{~Hz}$ (1800 rpm)

Maximum Engine Speed: $72 \mathrm{~Hz}$ (2160 rpm)

The following step loads were tested :

\begin{tabular}{|c|c|c|l|}
\hline & Test Plan & $\begin{array}{c}\text { Actual Test } \\
\text { Point }\end{array}$ & \multicolumn{1}{|c|}{ Comments } \\
\hline i. & $0-30 \mathrm{~kW}$ & $0-30 \mathrm{~kW}$ & \\
\hline ii. a & & $0-50 \mathrm{~kW}$ & $\begin{array}{l}\text { Done to ensure the frequency did not go out } \\
\text { of range if the load slightly exceeded } 60 \mathrm{~kW} \\
\text { from the load bank. }\end{array}$ \\
\hline ii. b & $0-60 \mathrm{~kW}$ & $0-60 \mathrm{~kW}$ & \\
\hline iii. & $15-45 \mathrm{~kW}$ & $15-50 \mathrm{~kW}$ & $\begin{array}{l}\text { Changed to } 50 \mathrm{~kW} \text { because easier to } \\
\text { implement on load bank }\end{array}$ \\
\hline iv. a & & $30-55 \mathrm{~kW}$ & $\begin{array}{l}\text { Done to ensure the frequency did not go out } \\
\text { of range if the load slightly exceeded } 60 \mathrm{~kW} \\
\text { from the load bank. }\end{array}$ \\
\hline iv. b & $30-60 \mathrm{~kW}$ & $30-60 \mathrm{~kW}$ & \\
\hline v. a & $60-0 \mathrm{~kW}$ & $60-0 \mathrm{~kW}$ & \\
\hline v. b & & $55-0 \mathrm{~kW}$ & $\begin{array}{l}\text { Done to ensure the frequency did not go out } \\
\text { of range if the load slightly exceeded } 60 \mathrm{~kW} \\
\text { from the load bank. }\end{array}$ \\
\hline vi. & $30-0 \mathrm{~kW}$ & $30-0 \mathrm{~kW}$ & \\
\hline
\end{tabular}

Table 20 Test Point Summary - Step Loads with Surge Module/Variable Speed

For each step load point, an oscilloscope trace was captured that measured the power output, frequency, current, and voltage. These measurements were taken on the microgrid side (Y side) of the transformer. In each plot the data is formatted in the following way:

Ch1 = Unit RMS Power Output;

$0.0 \mathrm{~V}=-15 \mathrm{KW}$

$0.5 \mathrm{~V}=0 \mathrm{KW}$

$1.5 \mathrm{~V}=30 \mathrm{KW}$

$2.5 \mathrm{~V}=60 \mathrm{KW}$

$\mathrm{Ch} 2=$ Unit Operating Frequency;

$60 \mathrm{~Hz}-->2.3 \mathrm{~V}$

$59.5 \mathrm{~Hz}-->1.66 \mathrm{~V}$

$60.5 \mathrm{~Hz}-->2.94 \mathrm{~V}$ 


\title{
Ch 3 -Grid Voltage L-N
}

Channel 4

Ch4 = Unit Output Current; 1V = 100Amps

\begin{abstract}
Also, the Step "Up" load points (i-iv.) were run two separate times so that additional data on the system dynamics, with the surge module, could be captured. This oscillocope data is formatted as follows:
\end{abstract}

Channel 1 = Engine RPM;

Not Recorded

Channel 2 = Bus Voltage

Zero point is actually 500 volts and measures from black "l" marker on left-hand side of figure

100 volts per division

Nominal Voltage; 860 volts

Channel $3=$ AC Current ; $1 \mathrm{~V}=100$ amps

Channel $4=$ Surge Module Current

$10 \mathrm{mV}=10 \mathrm{amps}$

$10 \mathrm{mV}$ per division

Nominal Current; 26 amps

The results of each test is organized in a table that defines the load setpoint (abbreviated S.P) and the actual power output from the unit. The table also contains the microgrid operating frequency before and after the transient event occurs (These frequency values are obtained from the units software and not the oscilloscope measurement). Also recorded in the table is the engine speed before and after the transient. Data contained in column A defines the operating condition and the state of the microgrid before the transient condition being tested occurs. Data contained in column B defines the operating condition and the state of the microgrid after the transient condition being tested has occurred. 
i. Step Load Evaluation 1

4.Load Bank Start Load $=0 \mathrm{~kW}$

5.Unit A CMD $=20 \mathrm{~kW}$

6.Load Bank End Load $=30 \mathrm{~kW}$

\begin{tabular}{|c|c|c|}
\hline & A & B \\
\hline Unit \#1 (S.P/Actual) kW & $20.0 / 0.0 \mathrm{~kW}$ & $20.0 / 30.5 \mathrm{~kW}$ \\
\hline Frequency Hz & $60.16 \mathrm{~Hz}$ & $59.91 \mathrm{~Hz}$ \\
\hline Engine Speed (Hz/rpm) & $60 / 1800$ & $60 / 1800$ \\
\hline
\end{tabular}

Table 21 Step Load Evaluation 1: 0 - 30 kW (w/Surge Module)

\section{i. 0 - 30 KW Step Load -Surge Module- Variable Speed}

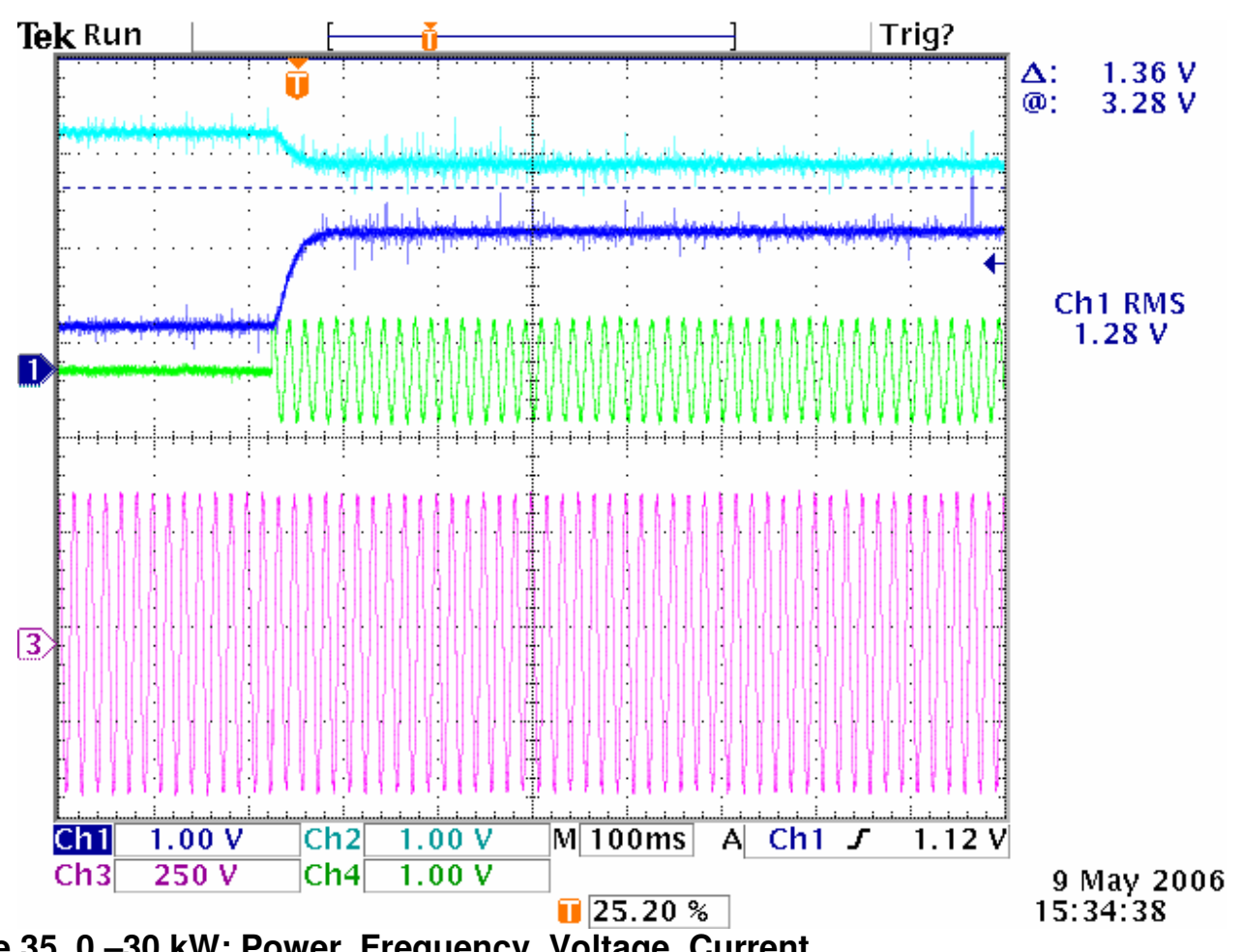

Figure 350 -30 kW: Power, Frequency, Voltage, Current 
x. $\quad$ Step Load Evaluation 2

10. Load Bank Start Load $=0 \mathrm{KW}$

11. Unit A CMD $=20 \mathrm{KW}$

12. Load Bank End Load $=60 \mathrm{~kW}$

ii a. 0 - $50 \mathrm{~kW}$ (Additional test point done to ensure the frequency did not go out of range if the load slightly exceeded $60 \mathrm{~kW}$ from the load bank)

\begin{tabular}{|c|c|c|}
\hline & A & B \\
\hline Unit \#1 (S.P/Actual) kW & $20.0 / 0.0 \mathrm{~kW}$ & $20.0 / 51.3 \mathrm{~kW}$ \\
\hline Frequency Hz & $60.16 \mathrm{~Hz}$ & $59.73 \mathrm{~Hz}$ \\
\hline Engine Speed (Hz/rpm) & $60 / 1800$ & $65.9 / 1977$ \\
\hline
\end{tabular}

Table 22 Step Load Evaluation 2a: 0 - 50 kW (w/Surge Module)

iia. 0 - 50 kW Step Load -Surge Module- Variable Speed

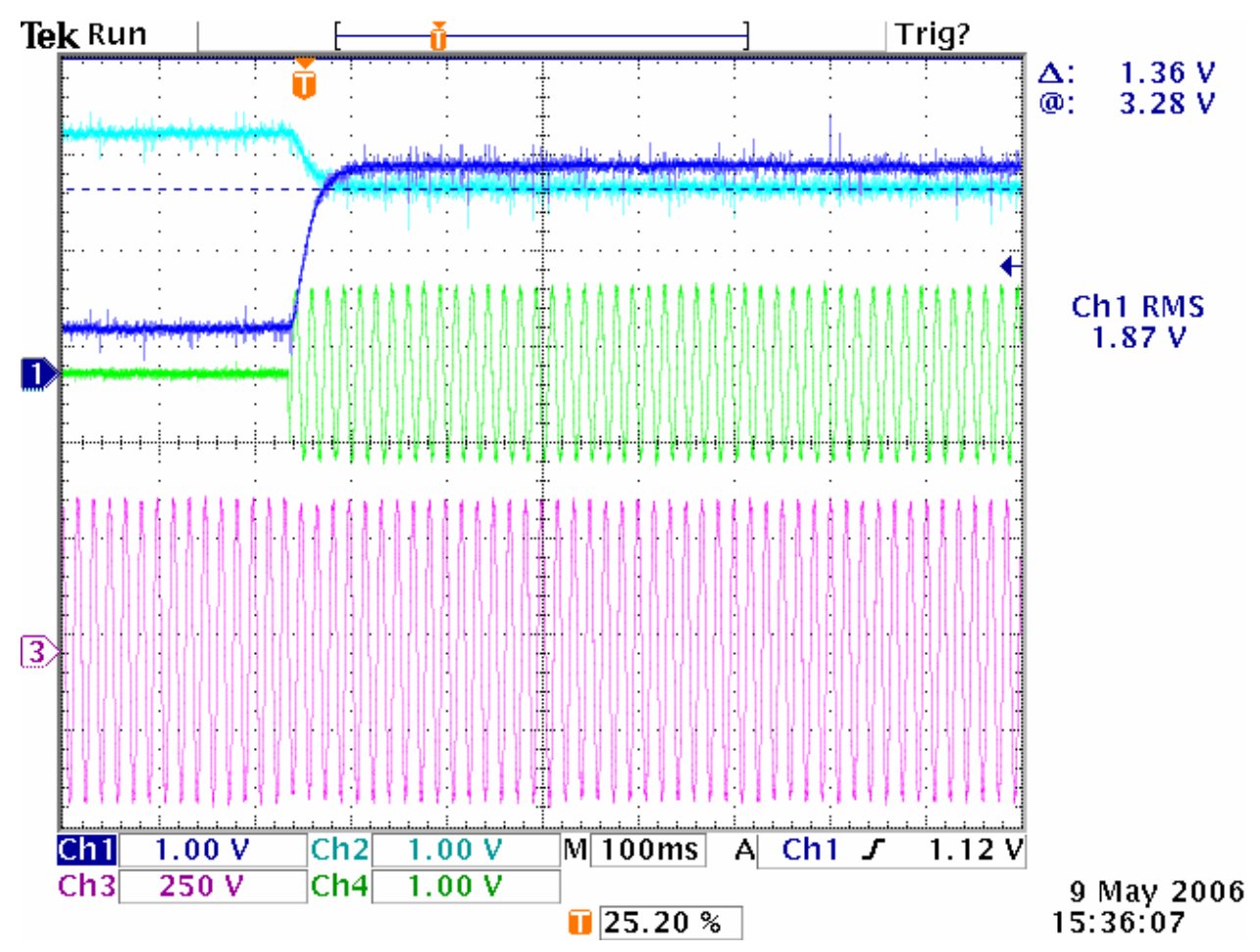

Figure 36 0-50 kW: Power, Frequency, Voltage, Current 


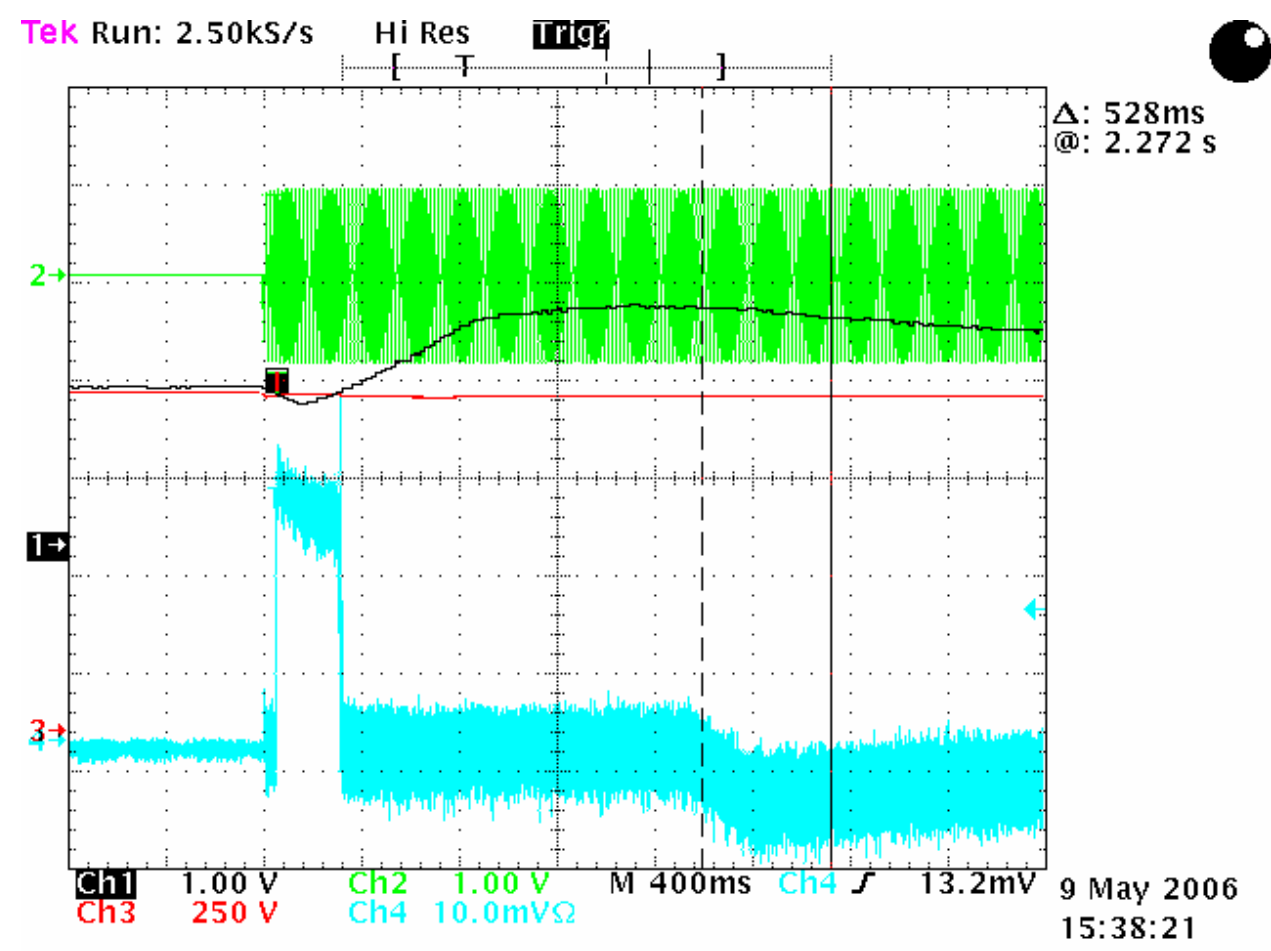

Figure 37 0-50 kW: Engine RPM, Bus Voltage, AC Current, Surge Module Current

ii b. $0-60 \mathrm{~kW}$

\begin{tabular}{|c|c|c|}
\hline & A & B \\
\hline Unit \#1 (S.P/Actual) kW & $20.0 / 0.0 \mathrm{~kW}$ & $20.0 / 61.0 \mathrm{~kW}$ \\
\hline Frequency Hz & $60.16 \mathrm{~Hz}$ & $59.16 \mathrm{~Hz}$ \\
\hline Engine Speed $(\mathrm{Hz} / \mathrm{rpm})$ & $60 / 1800$ & $67.8 / 2034$ \\
\hline
\end{tabular}

Table 23 Step Load Evaluation 2b: 0 - 60 kW (w/Surge Module) 
ii b. 0-60 KW Step Load-Surge Module- Variable Speed

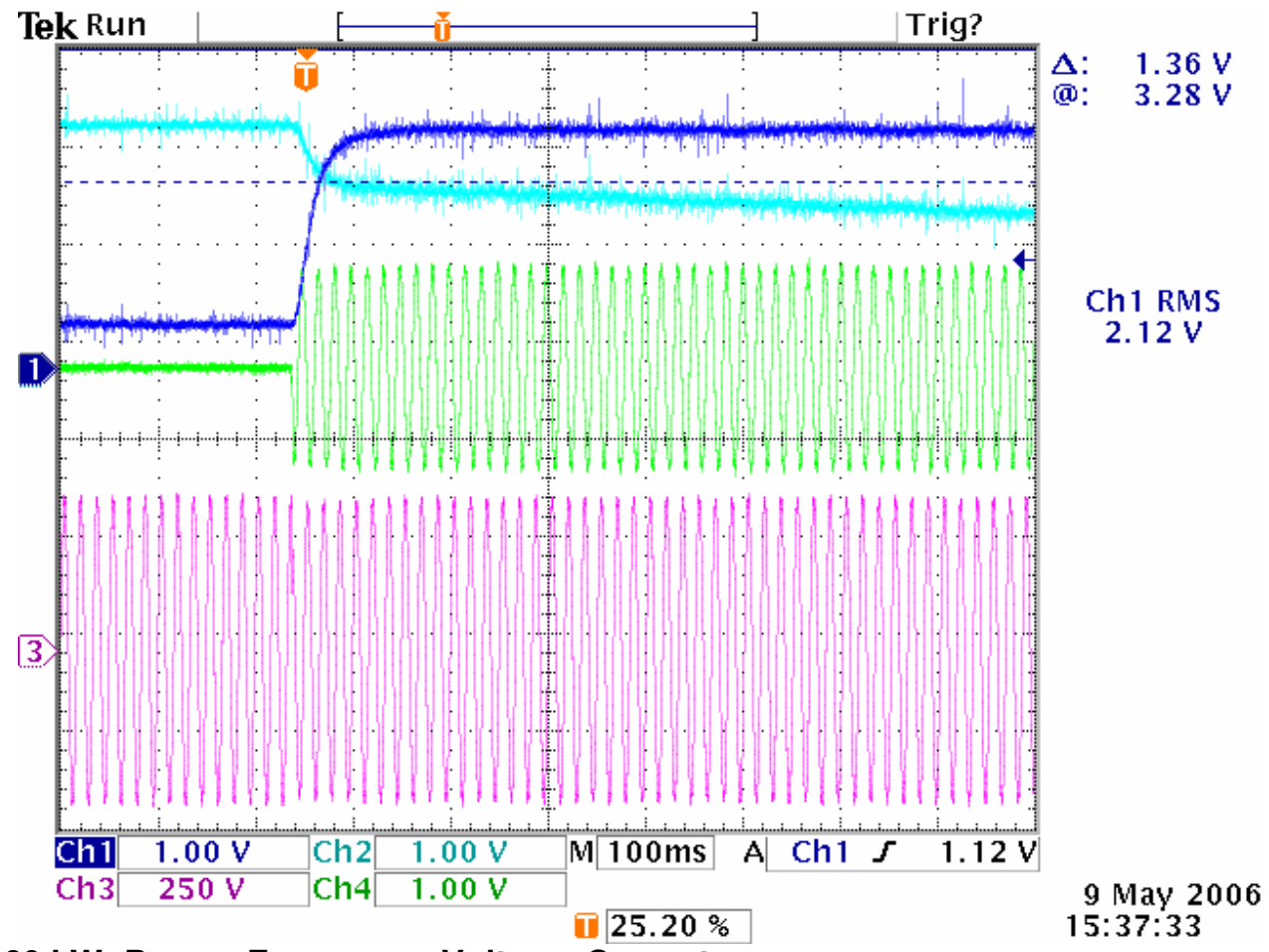

Figure 38 0 -60 kW: Power, Frequency, Voltage, Current

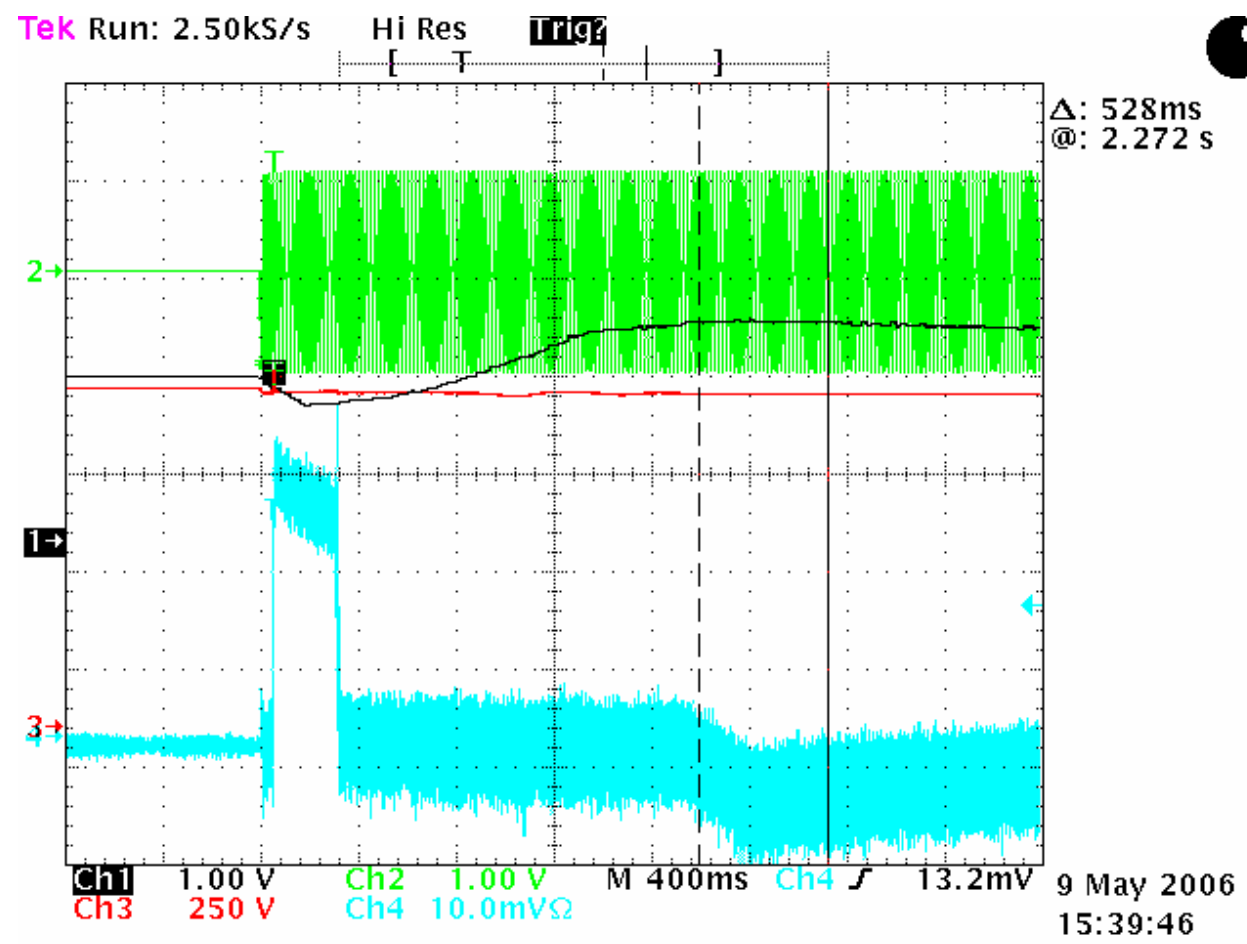

Figure 39 0-60 kW: Engine RPM, Bus Voltage, AC Current, Surge Module Current 
xi. $\quad$ Step Load Evaluation 3

1.Load Bank Start Load $=15 \mathrm{~kW}$

2.Unit A CMD $=20 \mathrm{~kW}$

3.Load Bank End Load $=50 \mathrm{~kW}$ (Changed from $45 \mathrm{~kW}$ because easier to implement on Load Bank)

\begin{tabular}{|c|c|c|}
\hline & A & B \\
\hline Unit \#1 (S.P/Actual) kW & $20.0 / 15.5 \mathrm{~kW}$ & $20.0 / 50.9 \mathrm{~kW}$ \\
\hline Frequency Hz & $60.03 \mathrm{~Hz}$ & $59.74 \mathrm{~Hz}$ \\
\hline Engine Speed (Hz/rpm) & $60 / 1800$ & $67.7 / 2031$ \\
\hline
\end{tabular}

Table 24 Step Load Evaluation 3: 15 - 50 kW (w/Surge Module)

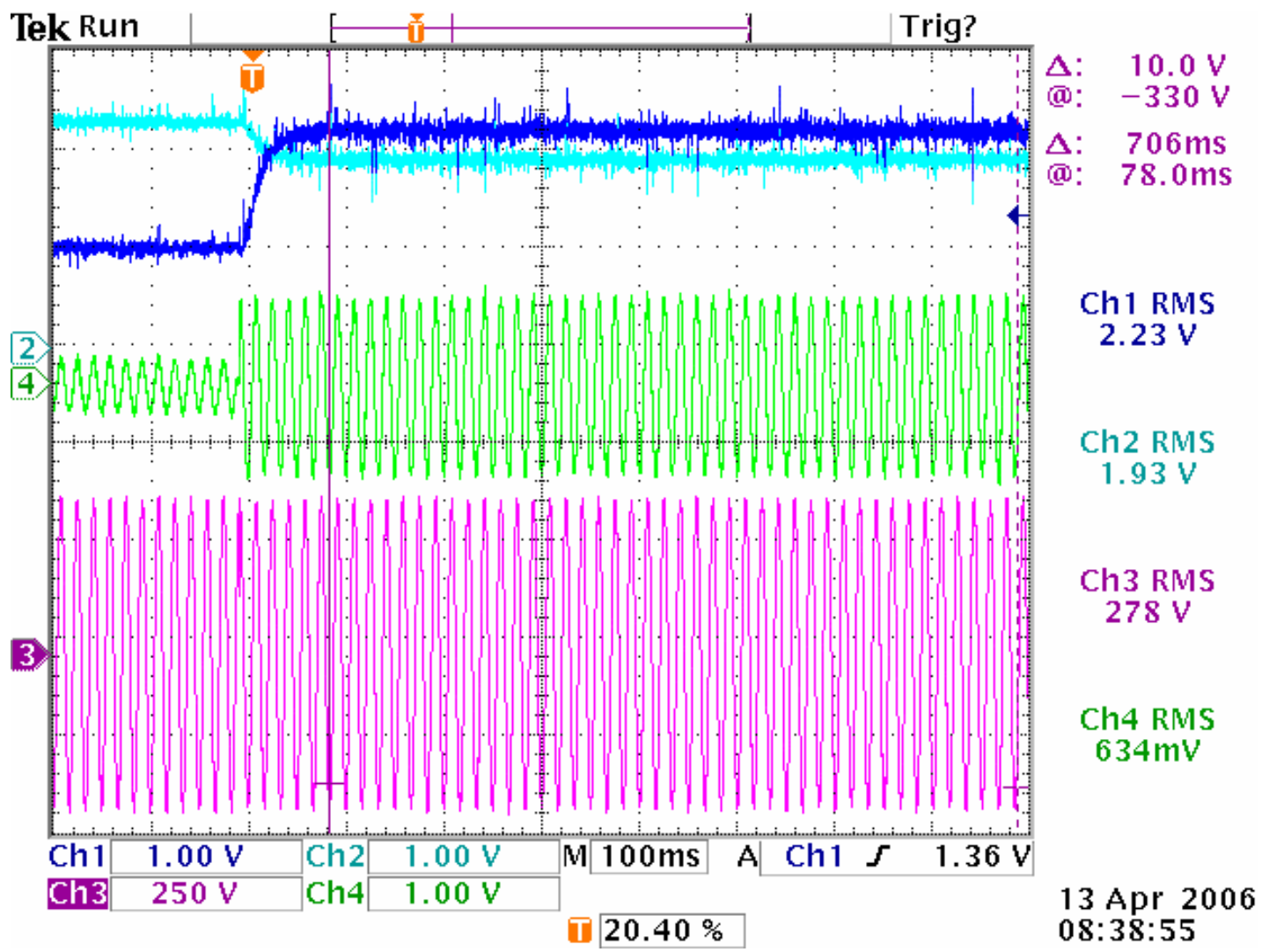

Figure 4015 - 50 kW: Power, Frequency, Voltage, Current 


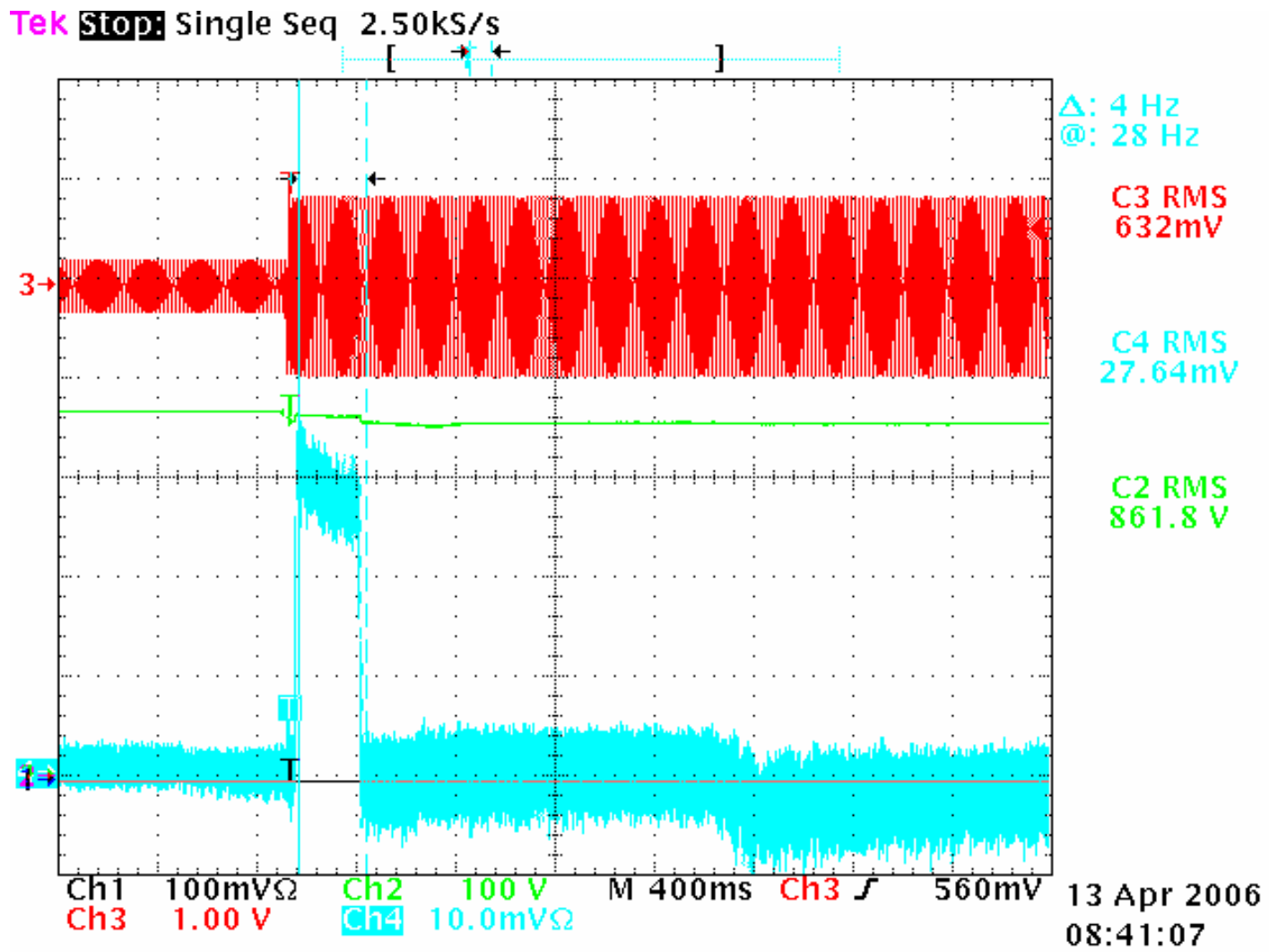

Figure 4115 - 50 kW: Engine RPM, Bus Voltage, AC Current, Surge Module Current

xii. $\quad$ Step Load Evaluation 4

1.Load Bank Start Load $=30 \mathrm{~kW}$

2.Unit A CMD $=20 \mathrm{~kW}$

3.Load Bank End Load $=60 \mathrm{~kW}$

iv a. $30-55 \mathrm{~kW}$ (Additional test point done to ensure the frequency did not go out of range if the load slightly exceeded $60 \mathrm{~kW}$ from the load bank)

\begin{tabular}{|c|c|c|}
\hline & A & B \\
\hline Unit \#1 (S.P/Actual) KW & $20.0 / 30.4 \mathrm{~kW}$ & $20.0 / 55.8 \mathrm{~kW}$ \\
\hline Frequency Hz & $59.91 \mathrm{~Hz}$ & $59.70 \mathrm{~Hz}$ \\
\hline Engine Speed (Hz/rpm) & $60 / 1800$ & $70.1 / 2103$ \\
\hline
\end{tabular}

Table 25 Step Load Evaluation 4a: $30-55$ kW (w/Surge Module) 
iv.a 30-55 KW Step Load -Surge Module- Variable Speed

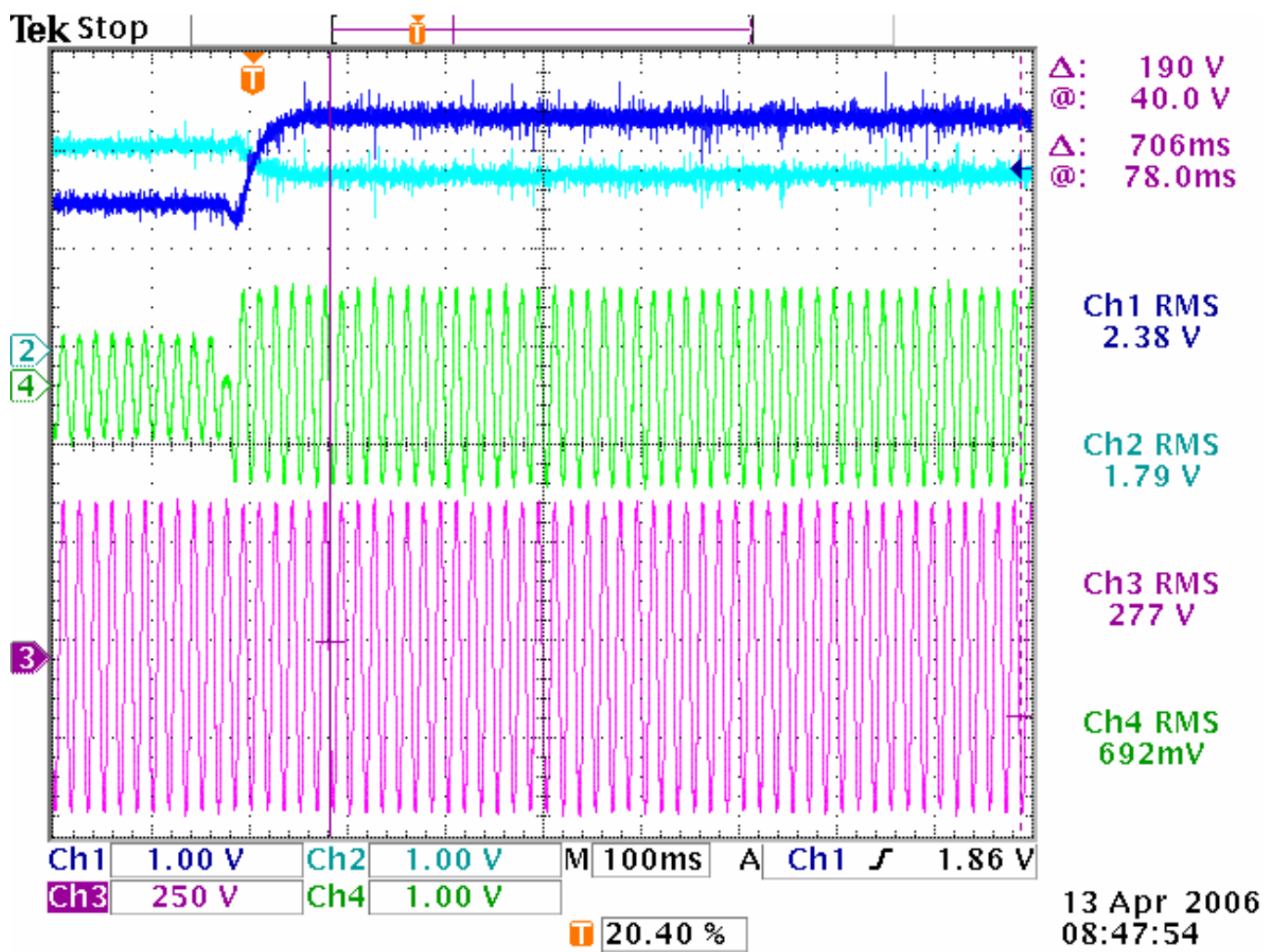

Figure $4230-55$ kW: Power, Frequency, Voltage, Current

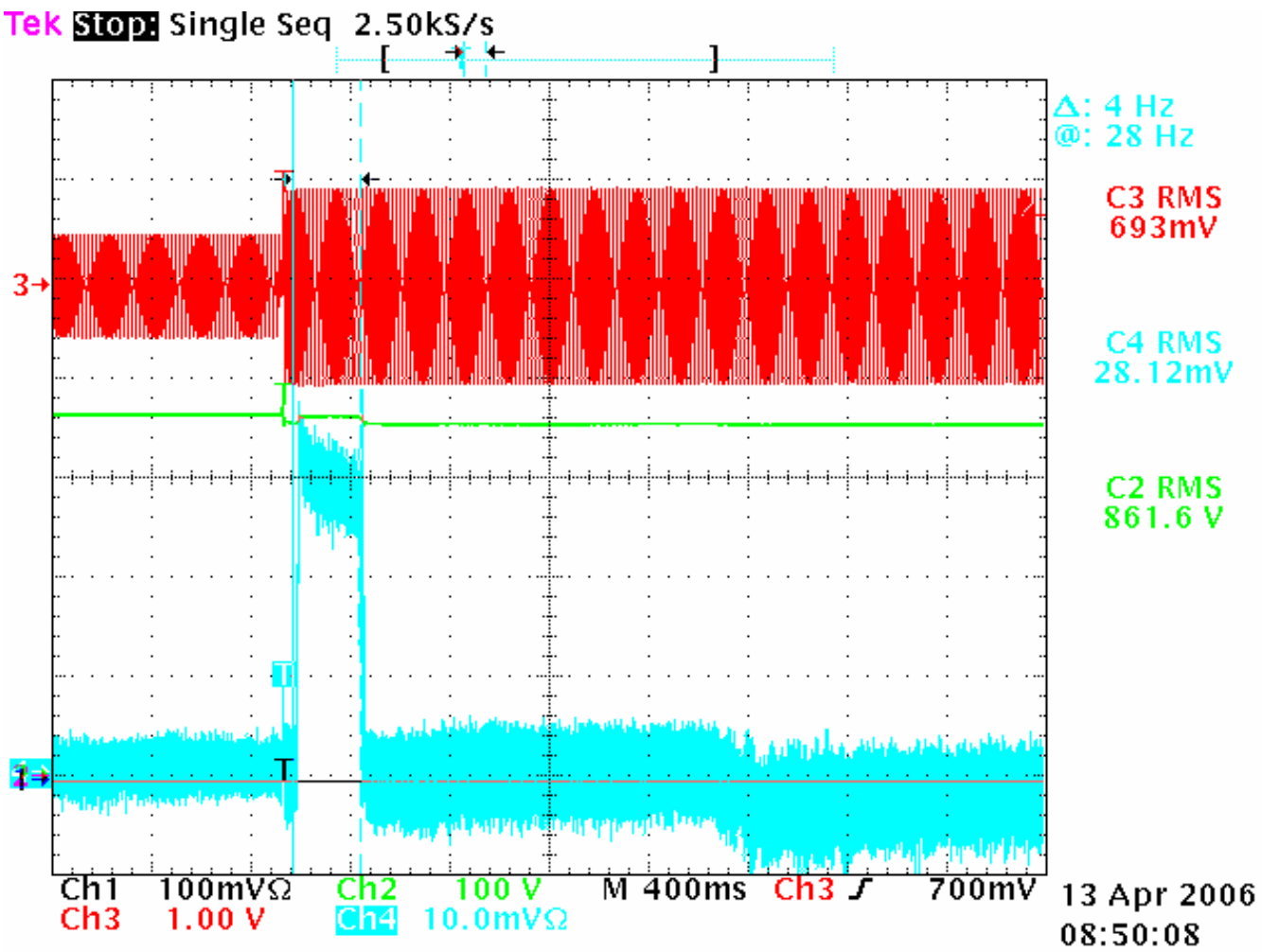

Figure 4330 - 55 kW: Engine RPM, Bus Voltage, AC Current, Surge Module Current 
iv b. $30-60 \mathrm{~kW}$

\begin{tabular}{|c|c|c|}
\hline & A & B \\
\hline Unit \#1 (S.P/Actual) KW & $20.0 / 30.4 \mathrm{~kW}$ & $20.0 / 60.7 \mathrm{~kW}$ \\
\hline Frequency Hz & $59.91 \mathrm{~Hz}$ & $59.16 \mathrm{~Hz}$ \\
\hline Engine Speed (Hz/rpm) & $60 / 1800$ & $72.5 / 2175$ \\
\hline
\end{tabular}

Table 26 Step Load Evaluation 4b: $30-60$ kW (w/Surge Module)

iv.b 30-60 KW Step Load -Surge Module- Variable Speed

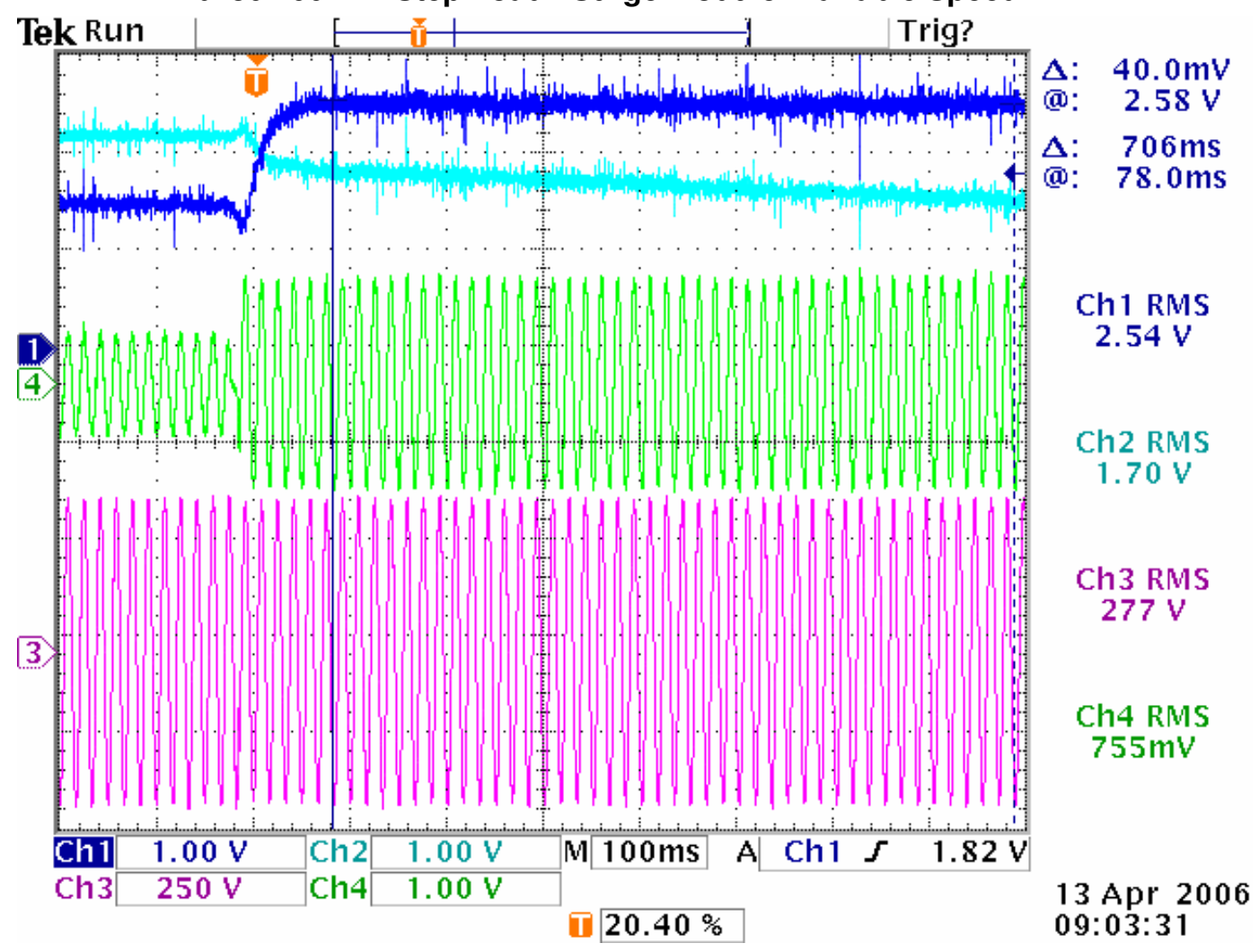

Figure $4430-60$ kW: Power, Frequency, Voltage, Current 


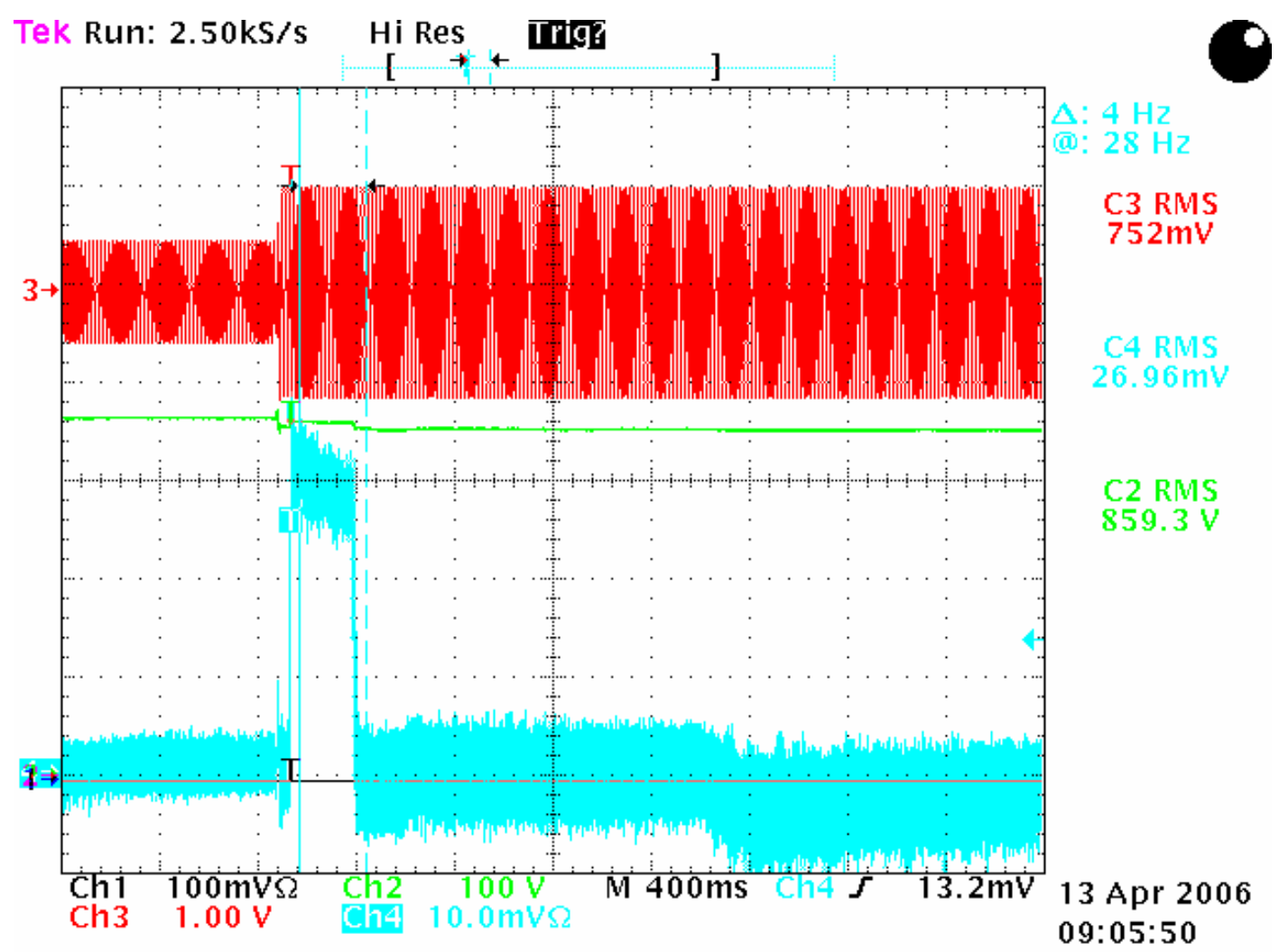

Figure 4530 - 60 kW: Engine RPM, Bus Voltage, AC Current, Surge Module Current 
xiii. $\quad$ Step Load Evaluation 5

1.Load Bank Start Load $=60 \mathrm{~kW}$

2.Unit A CMD $=20 \mathrm{~kW}$

3.Load Bank End Load $=0 \mathrm{~kW}$

v a. $60-0 \mathrm{~kW}$

\begin{tabular}{|c|c|c|}
\hline & A & B \\
\hline Unit \#1 (S.P/Actual) kW & $20.0 / 60.7 \mathrm{~kW}$ & $20.0 / 0 \mathrm{~kW}$ \\
\hline Frequency Hz & $59.16 \mathrm{~Hz}$ & $60.16 \mathrm{~Hz}$ \\
\hline Engine Speed (Hz/rpm) & $72.5 / 2175$ & $60 / 1800$ \\
\hline
\end{tabular}

Table 27 Step Load Evaluation 5a: 60 - 0 kW (w/Surge Module)

\section{0- 0 kW Step Load -Surge Module- Variable Speed}

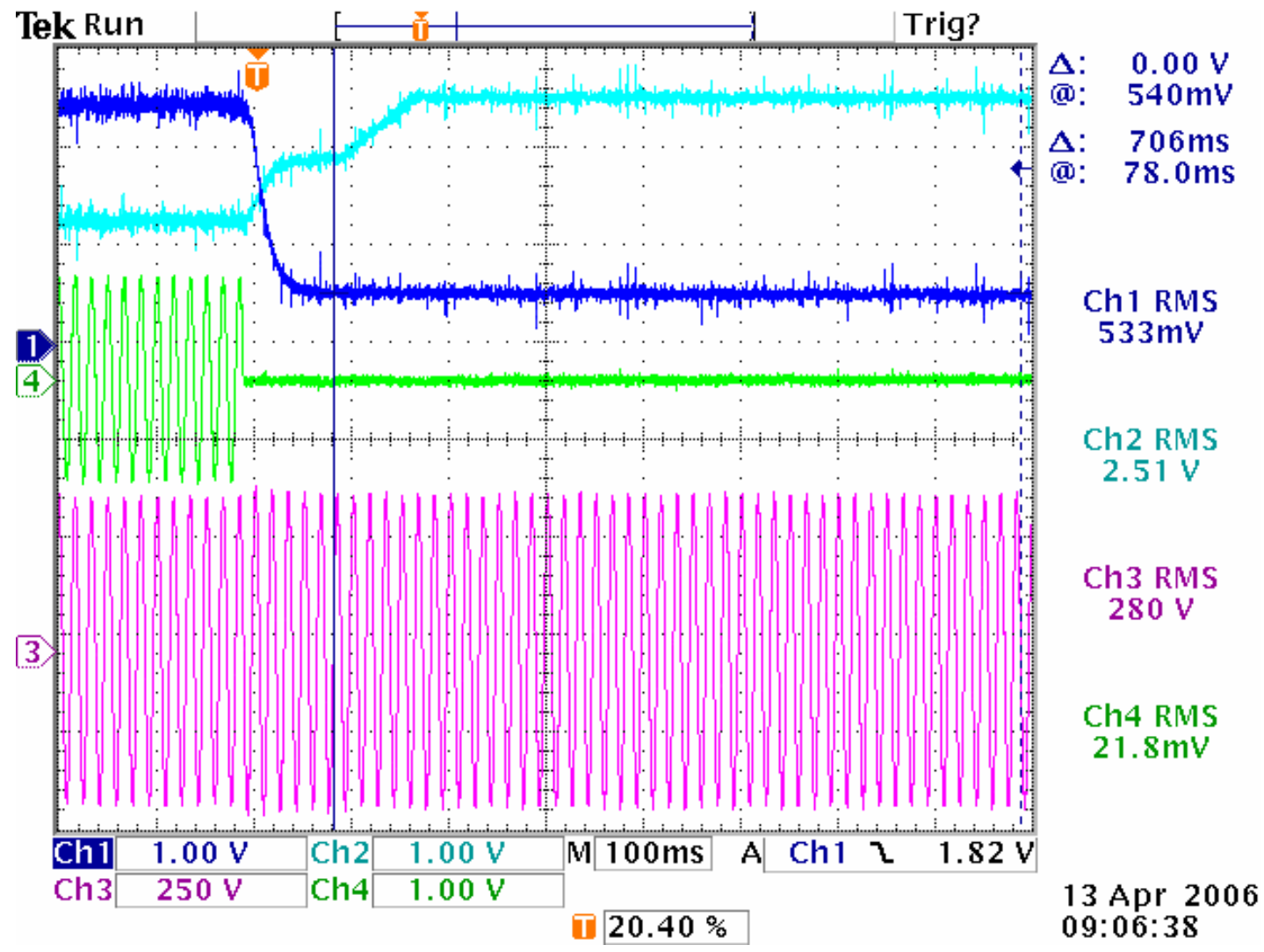

Figure 46 60-0 kW: Power, Frequency, Voltage, Current 
v b. $55-0 \mathrm{~kW}$ (Additional test point done to ensure the frequency did not go out of range if the load slightly exceeded $60 \mathrm{~kW}$ from the load bank)

\begin{tabular}{|c|c|c|}
\hline & A & B \\
\hline Unit \#1 (S.P/Actual) kW & $20.0 / 55.8 \mathrm{~kW}$ & $20.0 / 0 \mathrm{~kW}$ \\
\hline Frequency Hz & $59.70 \mathrm{~Hz}$ & $60.16 \mathrm{~Hz}$ \\
\hline Engine Speed (Hz/rpm) & $70.1 / 2103$ & $60 / 1800$ \\
\hline
\end{tabular}

Table 28 Step Load Evaluation 5b: 55 - 0 kW (w/Surge Module)

\section{5- 0 kW Step Load -Surge Module- Variable Speed}

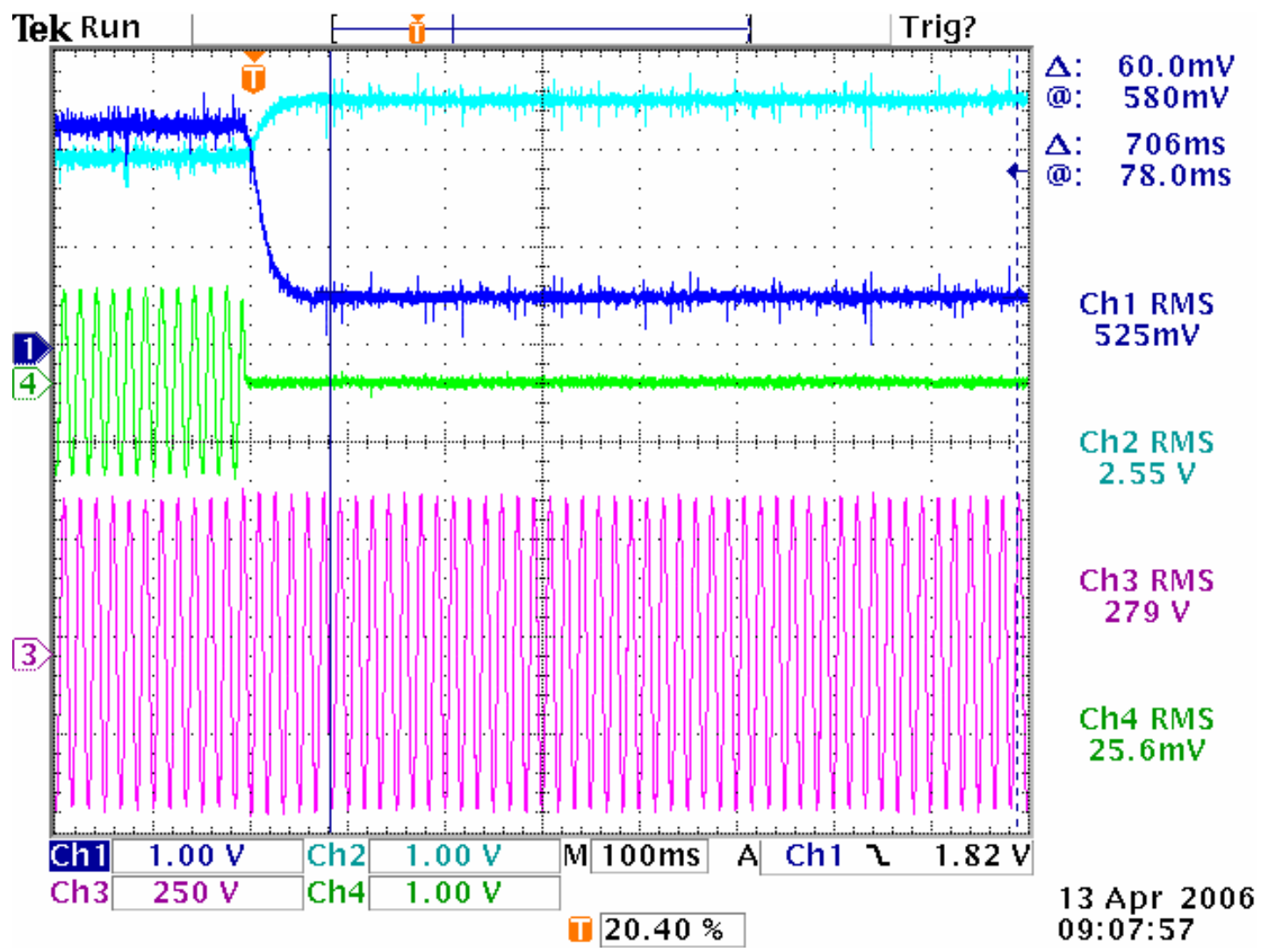

Figure 47 55-0 kW: Power, Frequency, Voltage, Current 
vi. Step Load Evaluation 6

7. Load Bank Start Load $=30 \mathrm{~kW}$

8. Unit $\mathrm{A} C \mathrm{CMD}=20 \mathrm{~kW}$

9. Load Bank End Load $=0 \mathrm{~kW}$

\begin{tabular}{|c|c|c|}
\hline & A & B \\
\hline Unit \#1 (S.P/Actual) kW & $20.0 / 30.4 \mathrm{~kW}$ Unit & $20.0 / 0 \mathrm{~kW}$ \\
\hline Frequency Hz & $59.91 \mathrm{~Hz}$ & $60.16 \mathrm{~Hz}$ \\
\hline Engine Speed (Hz/rpm) & $60 / 1800$ & $60 / 1800$ \\
\hline
\end{tabular}

Table 29 Step Load Evaluation 6: 30 - 0 kW (w/Surge Module)

\section{0- 0 kW Step Load -Surge Module- Variable Speed}

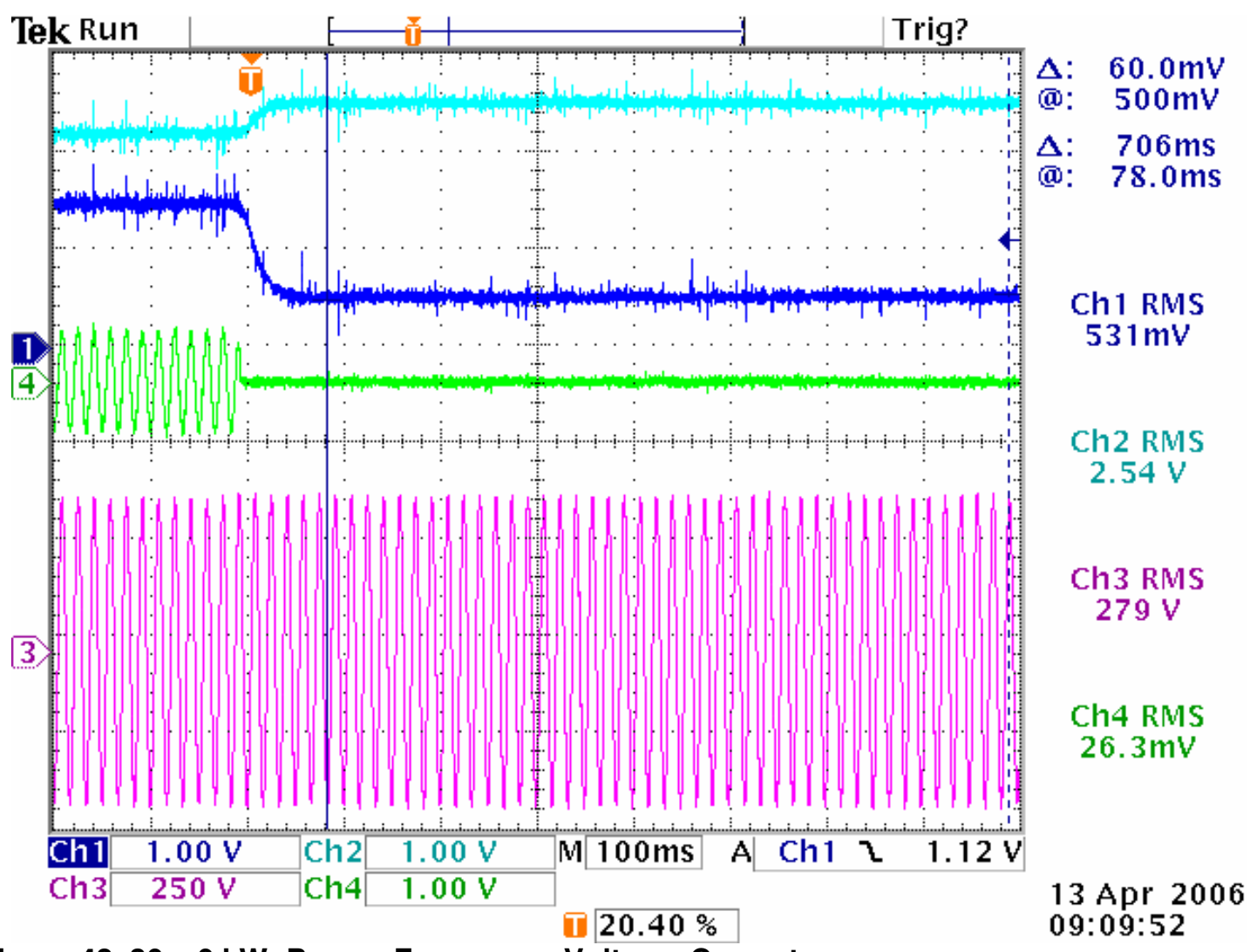

Figure 4830 - 0 kW: Power, Frequency, Voltage, Current 


\section{B.3. Step Load Evaluations without Surge Module - Fixed Speed Operation}

The CHP unit was tested with the surge module disabled. As with the first unit (Serial \# 200835), the results confirmed that the dynamics of the engine/inverter allows the unit to successfully handle a step load without the surge module. The engine was set at the fixed speed required for the maximum load step of $0-60 \mathrm{~kW}(2160 \mathrm{rpm})$. Each step load point was run to confirm Pass/Fail. Oscilloscope data was not recorded since, as demonstrated in the testing of Unit \#200835, if the unit "Passes", the electrical output signatures (Figure 1) are the same as in Variable Speed operation.

The following table summarizes the test results.

\begin{tabular}{|c|c|c|}
\hline \multicolumn{3}{|c|}{$\begin{array}{c}\text { Fixed Engine Speed } 2160 \mathrm{rpm} \\
(72 \mathrm{~Hz}) \\
\text { No Surge Module }\end{array}$} \\
\hline & Step Load & Pass/Fail \\
\hline i. & $0-30 \mathrm{~kW}$ & Pass \\
\hline ii. a & $0-50 \mathrm{~kW}$ & Pass \\
\hline ii. $b$ & $0-60 \mathrm{~kW}$ & Pass \\
\hline iii. & $15-50 \mathrm{~kW}$ & Pass \\
\hline iv. $a$ & $30-55 \mathrm{~kW}$ & Pass \\
\hline iv. $b$ & $30-60 \mathrm{~kW}$ & Pass \\
\hline v. $a$ & $60-0 \mathrm{~kW}$ & Pass \\
\hline v. b & $55-0 \mathrm{~kW}$ & Pass \\
\hline vi. & $30-0 \mathrm{~kW}$ & Pass \\
\hline
\end{tabular}

Table 30 Test Point Summary - Step Loads without Surge Module/Fixed Speed 
Changed unit A Feeder CMD $=150 \mathrm{KW}$

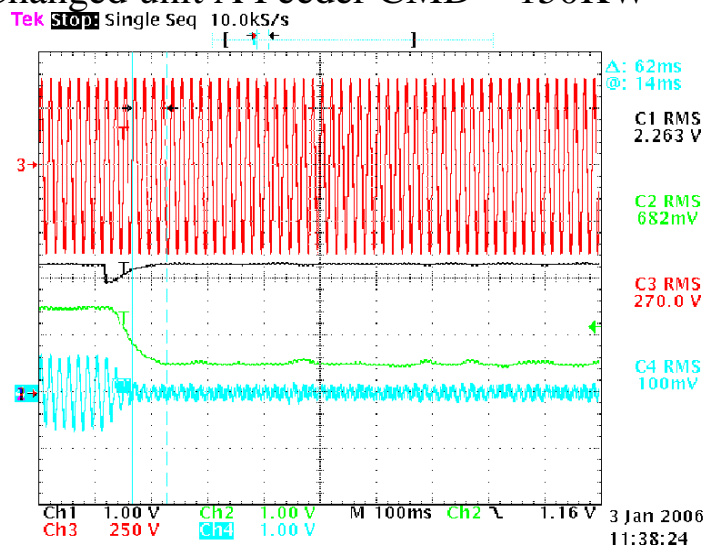

Unit \#1.) Transient Response

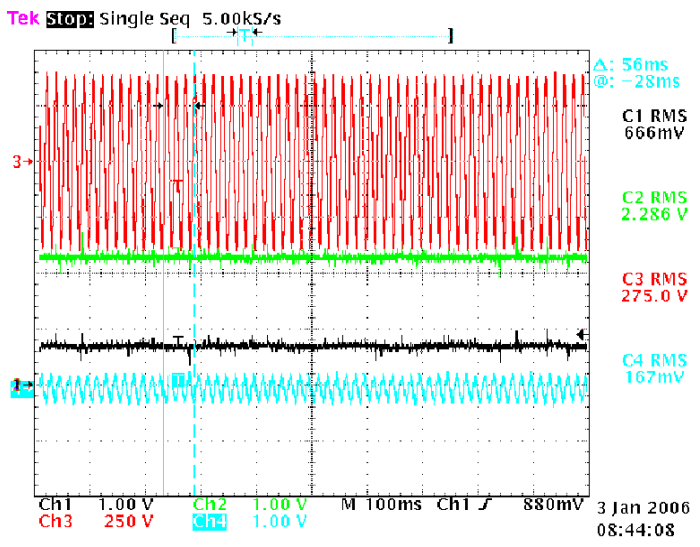

Unit \#2.) Transient Response

Figure 22 Parallel Inverters - Feeder Control, Grid Connected - Digital Round-off Check 3

\begin{tabular}{|c|c|c|}
\hline & A (Grid) & B (Grid) \\
\hline Load Bank \#1(S.P/Actual) KW & $80.0 / 83.9 \mathrm{KW}$ & $80.0 / 83.9 \mathrm{KW}$ \\
\hline Load Bank \#2(S.P/Actual) KW & $80.0 / 84.3 \mathrm{KW}$ & $80.0 / 84.3 \mathrm{KW}$ \\
\hline Unit \#1 (S.P/Pout) KW & $120 / 34.6 \mathrm{KW}$ Feeder & $150.0 / 0.5 \mathrm{KW}$ Feeder \\
\hline Unit \#2 (S.P/Pout) KW & $10.0 / 5.5 \mathrm{KW} \mathrm{Unit}$ & $10.0 / 5.5 \mathrm{KW}$ Unit \\
\hline Frequency Hz & $59.98 \mathrm{~Hz}$ & $59.99 \mathrm{~Hz}$ \\
\hline
\end{tabular}

Table 23 Parallel Inverters - Feeder Control, Grid Connected - Digital Round-off Check 3 


\section{CERTS MICROGRID LABORATORY TEST BED}

Tecogen CHP Modules Commissioning Report

Prepared For:

California Energy Commission

Public Interest Energy Research Program

늘

Prepared By:

Lawrence Berkeley National Laboratory

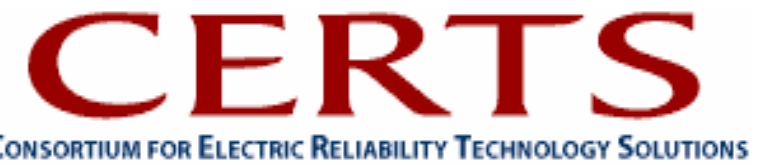



Prepared By:

Lawrence Berkeley National Laboratory

Joseph H. Eto, Principal Investigator

Berkeley, CA 94720

Jean Roy, Tecogen, Inc.

Commission Contract No. 500-03-024

Prepared For:

Public Interest Energy Research (PIER)

California Energy Commission

Bernard Treanton

Contract Manager

Mike Gravely

Program Area Lead

ENERGY SYSTEMS INTEGRATION

Mike Gravely

Office Manager

ENERGY SYSTEMS RESEARCH

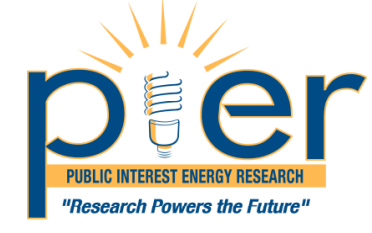

Martha Krebs, Ph.D.

PIER Director

Thom Kelly, Ph.D.

Deputy Director

ENERGY RESEARCH \& DEVELOPMENT DIVISION

Melissa Jones

Executive Director

\section{DISCLAIMER}

This report was prepared as the result of work sponsored by the California Energy Commission. It does not necessarily represent the views of the Energy Commission, its employees or the State of California. The Energy Commission, the State of California, its employees, contractors and subcontractors make no warrant, express or implied, and assume no legal liability for the information in this report; nor does any party represent that the uses of this information will not infringe upon privately owned rights. This report has not been approved or disapproved by the California Energy Commission nor has the California Energy Commission passed upon the accuracy or adequacy of the information in this report. 



\section{TECOGEN}

Natural Gas Engine-Driven Products

\section{Tecogen CHP Modules Commissioning Report}




\section{Introduction}

Tecogen, Inc. supplied (3) $60 \mathrm{~kW}$, inverter-based, natural gas engine driven generators to the CERTS research program for the AEP Microgrid Test Bed. Tecogen subcontracted with Youtility Inc. (Hudson, NH) to supply the inverter cabinet. Each unit was packaged on a skid that includes the engine and its subsystems, synchronous generator, Youtility inverter, and the master control system. The master control system was equipped with a communications link to the Northern Power Energy Management System. The master control system also communicates internally with the inverter via a CAN Bus.

Each unit was also equipped with a separate energy storage device, or Surge Module that was also supplied by Youtility. It was packaged in a cabinet for wall mount at the test site. The Surge Module provides $20 \mathrm{~kW}$ of battery power for 250 milliseconds to assist the engine/ inverter's response to a step load when the unit is islanded.

All three units were extensively tested prior to shipment to the AEP test site. First, each inverter cabinet was bench-tested at Youtility both individually, and in pairs as a simulated microgrid. Rather than the engine/generator providing the input power, the grid was used as the power source. The purpose of this testing was to verify the implementation of the various microgrid software control algorithms.

Once the Youtility bench testing was complete, the three inverter cabinets were sent to Tecogen for final packaging with the engine/generator. Each module was then tested to verify the integration of the inverter with the engine/generator. The three Surge Modules were also included in the scope of the factory testing at Tecogen to verify their performance. Both phases of the factory testing were successfully completed with each unit meeting the requirements established by the CERTS technical team.

Commissioning of the units commenced in July of 2006. In general, there were some normal shakedown issues encountered in getting the units on line. The most significant problem discovered during the commissioning was the speed control of the engine. The inverter was designed to directly control the engine throttle, via an electronic actuator, to adjust the engine 
speed in reaction to the load. It was able to sufficiently accomplish this during normal grid-tie operation where load changes were gradual. However, the units had problems with step loads, even with the assistance of the surge module.

Although this feature was extensively tested at the Tecogen factory, only specific step loads were checked. The control software was tuned to be able to handle these, but it was not possible to check the wide range of possible step-load scenarios at the Tecogen factory. Although additional software tuning by Youtility at the AEP test site may have possibly resolved the problem, Tecogen and Youtility felt a more prudent resolution was to remove the inverter control of the engine speed and use a conventional engine governor. This modification was successfully implemented and the governor was set to control the engine at fixed speed. The engine was then able to reliably handle the various step loads.

However, the change in the method of speed control caused a problem with the surge modules. The inverter software was using the speed signal to trigger the on/off control of the surge modules. When the inverter speed control was disabled, it corrupted the surge module control sequence such that the batteries were allowed to discharge, but not recharge. As a result, all the batteries discharged to such a low level that most of them were permanently damaged. Once this problem was identified, the inverter software was modified and the batteries replaced. The surge modules then successfully operated with the independent governor speed control. Subsequently, the Commissioning Test Matrix was then successfully completed by AEP.

In the following section, the details of the commissioning are summarized in a tabular format. Several documents are referenced in the table and attached to this document, including the test matrix, commissioning checklists, and the test data collected throughout the process. 


\section{Tecogen Commissioning Summary}

\begin{tabular}{|c|c|c|c|c|}
\hline Date & Activity & Attendees & Events & Outstanding Issues \\
\hline $\begin{array}{l}7 / 10 / 06- \\
7 / 12 / 06\end{array}$ & Engine Commissioning & $\begin{array}{l}\text { John Freeman } \\
\text { (Tecogen) }\end{array}$ & $\begin{array}{l}\text { - Got all three engines } \\
\text { operating } \\
\text { - See Attachments 2, 3, and } 4\end{array}$ & \\
\hline $\begin{array}{l}7 / 26 / 06- \\
7 / 28 / 06\end{array}$ & $\begin{array}{l}\text { Commissioning of } \\
\text { inverters and surge } \\
\text { modules }\end{array}$ & $\begin{array}{l}\text { John Freeman } \\
\text { (Tecogen) } \\
\text { Jian Wen (Youtility) }\end{array}$ & $\begin{array}{l}\text { - } \text { Corrected Phase Rotation } \\
\text { - Ran each unit through } \\
\text { individual step loads - } \\
\text { islanded. } \\
\text { - Paralleled all } 3 \text { units } \\
\text { together - islanded. } \\
\text { - Paralleled all } 3 \text { units to the } \\
\text { grid and transferred from } \\
\text { grid-tie mode to micro-grid } \\
\text { mode. } \\
\text { - Transferred generator } \\
\text { from micro-grid mode to } \\
\text { grid-tie mode. } \\
\text { - Refer to Attachment } 5\end{array}$ & $\begin{array}{l}\text { - Only could run engines in fixed } \\
\text { speed mode - engine would } \\
\text { stall with large step loads in } \\
\text { variable speed mode } \\
\text { - Even in fixed speed mode, } \\
\text { engine would stall with } 0-60 \\
\text { kW } \\
\text { - Full Matrix testing not done } \\
\text { - Required waveform data not } \\
\text { collected } \\
\text { - Load banks on generator } \\
\text { feeders overheated }\end{array}$ \\
\hline $8 / 23 / 06$ & $\begin{array}{l}\text { Execute Test Matrix in } \\
\text { Fixed Speed Mode }\end{array}$ & $\begin{array}{l}\text { John Freeman } \\
\text { (Tecogen) }\end{array}$ & $\begin{array}{l}\text { - Ran single unit step load } \\
\text { tests on all three units } \\
\text { - Frequency droop did not } \\
\text { respond properly on all units } \\
\text { for the } 0 \text { - } 60 \mathrm{~kW} \text { tests. The } \\
\text { reason was because the load } \\
\text { banks actually produced } \\
\text { slightly more the } 60 \mathrm{~kW} \\
\text { putting the software out of its } \\
\text { control range. } \\
\text { - Unit A2 had a software } \\
\text { problem where the frequency } \\
\text { droop was not functioning } \\
\text { properly at all. It was later } \\
\text { determined some of the } \\
\text { software constants were } \\
\text { inadvertently reset by AEP } \\
\text { when testing the EMS } \\
\text { system. } \\
\text { Refer to Attachment } 6 \text { and } 7\end{array}$ & $\begin{array}{l}\text { - The single unit testing was } \\
\text { redone on } 8 / 28 / 06 \text { by Dave } \\
\text { Klapp of AEP. } \\
\text { - Repeat testing of Units } \mathrm{A} 1 \text { and } \\
\text { B1 at slightly less than } 60 \mathrm{~kW} \\
\text { showed correct frequency } \\
\text { droop response. } \\
\text { - Refer to Attachments } 8,9,10\end{array}$ \\
\hline $\begin{array}{l}9 / 19 / 06- \\
9 / 21 / 06\end{array}$ & $\begin{array}{l}\text { Install commercial } \\
\text { governor to maintain } \\
\text { engine speed at a fixed } \\
\text { value independent of } \\
\text { Youtility's inverter } \\
\text { software. }\end{array}$ & $\begin{array}{l}\text { John Freeman } \\
\text { (Tecogen) }\end{array}$ & $\begin{array}{l}\text { - Governor control was } \\
\text { successful } \\
\text { - Individual unit island tests } \\
\text { were completed on units A1, } \\
\text { A2 and B1. Surge modules } \\
\text { were energized. } \\
\text { - Problem arose with units A1 } \\
\text { and A2 unable to phase lock. } \\
\text { - See Attachments } 11 \text { and } 12\end{array}$ & $\begin{array}{l}\text { The Microgrid interface board } \\
\text { was removed from one of the } \\
\text { units and brought back to be } \\
\text { inspected by Youtility. }\end{array}$ \\
\hline
\end{tabular}




\begin{tabular}{|c|c|c|c|c|}
\hline Date & Activity & Attendees & Events & Outstanding Issues \\
\hline $\begin{array}{l}10 / 11 / 06- \\
10 / 14 / 06\end{array}$ & $\begin{array}{l}\text { Correct phase lock } \\
\text { problem of } A 1 \text { and } A 2 \\
\text { Capture oscilloscope } \\
\text { data on surge modules } \\
\text { Complete test matrix for } \\
\text { multiple units }\end{array}$ & $\begin{array}{l}\text { Jian Wen (Youtility) } \\
\text { Paolo Piagi (Youtility) } \\
\text { John Freeman } \\
\text { (Tecogen) }\end{array}$ & $\begin{array}{l}\text { - Two CT's, installed on the } \\
\text { feeders by AEP were found } \\
\text { to be reversed causing the } \\
\text { phase lock problem. This } \\
\text { was corrected. } \\
\text { - It was discovered that the } \\
\text { testing that occurred after the } \\
\text { governors had been installed } \\
\text { damaged the surge modules. } \\
\text { By disabling the Youtility } \\
\text { engine speed control } \\
\text { software, the surge module } \\
\text { control was adversely } \\
\text { affected. The batteries } \\
\text { discharged to such a low } \\
\text { value that they were } \\
\text { permanently damaged. } \\
\text { The software was updated } \\
\text { on all three units. New } \\
\text { batteries were obtained to } \\
\text { repair the A2 surge module. } \\
\text { The governor speed setting } \\
\text { was reduced from } 2000 \text { rpm } \\
\text { to } 1750 \text { rpm on A2 to allow } \\
\text { the surge module to trigger. } \\
\text { The single unit step-load } \\
\text { tests were run on unit A2 and } \\
\text { oscilloscope waveforms were } \\
\text { obtained. The surge module } \\
\text { function on unit A2 was } \\
\text { found to be acceptable. } \\
\text { The Commissioning Test } \\
\text { Matrix was completed on all } \\
\text { three units. Units A1 and B1 } \\
\text { had the surge modules off } \\
\text { with the engine speed fixed } \\
\text { at } 2200 \text { rpm. Unit A2 had the } \\
\text { surge module functioning. All } \\
\text { tests passed. } \\
\text { See Attachments } 13 \text { and } 14\end{array}$ & $\begin{array}{l}\text { - New batteries for surge } \\
\text { modules A1 and B1 were } \\
\text { going to be obtained and } \\
\text { installed by AEP. John } \\
\text { Stevens was going to evaluate } \\
\text { the surge module design to } \\
\text { determine if any design } \\
\text { changes or additional sensors, } \\
\text { for diagnostics or fault } \\
\text { detection, would be required } \\
\text { before commencing with } \\
\text { further testing. } \\
\text { - Once the surge modules got } \\
\text { back on line, AEP would redo } \\
\text { the matrix testing for all three } \\
\text { units. }\end{array}$ \\
\hline $1 / 07$ & $\begin{array}{l}\text { Replace cylinder head } \\
\text { on engine A2 }\end{array}$ & Bob Buday (Tecogen) & $\begin{array}{l}\text { The cylinder head had a } \\
\text { factory defect causing water } \\
\text { to leak into the exhaust. }\end{array}$ & - None \\
\hline
\end{tabular}




\section{ATTACHMENTS}

\section{Commissioning Test Matrix}

\begin{tabular}{|c|c|c|c|}
\hline & \multicolumn{3}{|c|}{ Microsource Generators } \\
\hline Test Point & A1 & A2 & B1 \\
\hline \multicolumn{4}{|c|}{ Island Tests } \\
\hline \multicolumn{4}{|c|}{$\begin{array}{l}\text { Individual Unit - Power Control Mode } \\
\text { Scheduled } 7 / 11-7 / 12 \text { by Tecogen }\end{array}$} \\
\hline I.a.i & $\mathrm{X}$ & $x$ & $x$ \\
\hline I.a.ii & $\mathrm{X}$ & $X$ & $\mathrm{X}$ \\
\hline I.a.iii & $\mathrm{X}$ & $X$ & $X$ \\
\hline I.a.iv & $\mathrm{X}$ & $\mathrm{X}$ & $\mathrm{X}$ \\
\hline I.a.v & $\mathrm{X}$ & $X$ & $\mathrm{X}$ \\
\hline I.a.vi & $\mathrm{X}$ & $\mathrm{X}$ & $\mathrm{X}$ \\
\hline \multicolumn{4}{|c|}{$\begin{array}{c}\text { Two Unit-Power Control Mode } \\
\text { Scheduled } 7 / 26-7 / 28 \text { by Tecogen \& Youtility }\end{array}$} \\
\hline & & & \\
\hline II.c.i & $X$ & $X$ & \\
\hline II.c.ii & $\mathrm{X}$ & $X$ & \\
\hline II.c.i & $\mathrm{X}$ & & $\mathrm{X}$ \\
\hline II.c.ii & $\mathrm{X}$ & & $\mathrm{X}$ \\
\hline \multicolumn{4}{|c|}{ Grid Tie Tests } \\
\hline \multicolumn{4}{|c|}{$\begin{array}{c}\text { Two Unit -Power Control Mode } \\
\text { Scheduled } 7 / 26-7 / 28 \text { by Tecogen \& Youtility }\end{array}$} \\
\hline II.a.i & $X$ & $\mathrm{X}$ & \\
\hline II.a.i & & $\mathrm{X}$ & $\mathrm{X}$ \\
\hline \multicolumn{4}{|c|}{$\begin{array}{c}\text { Two Unit - Mixed Mode } \\
\text { Scheduled } 7 / 26-7 / 28 \text { by Tecogen \& Youtility }\end{array}$} \\
\hline III.a.i & $\mathrm{X}$ & $\mathrm{X}$ & \\
\hline III.a.i & & $X$ & $X$ \\
\hline \multicolumn{4}{|c|}{ Grid Tie to Island Tests } \\
\hline \multicolumn{4}{|c|}{$\begin{array}{c}\text { Two Unit -Power Control Mode } \\
\text { Scheduled } 7 / 26-7 / 28 \text { by Tecogen \& Youtility }\end{array}$} \\
\hline II.b.i & $\mathrm{X}$ & $X$ & \\
\hline Il.b.i & & $\mathrm{X}$ & $\mathrm{X}$ \\
\hline \multicolumn{4}{|c|}{$\begin{array}{c}\text { Two Unit }- \text { Mixed Mode } \\
\text { Scheduled } 7 / 26-7 / 28 \text { by Tecogen \& Youtility }\end{array}$} \\
\hline III.b.i & $\mathrm{X}$ & $\mathrm{X}$ & \\
\hline III.b.i & & $\mathrm{X}$ & $\mathrm{X}$ \\
\hline
\end{tabular}


Attachment 2

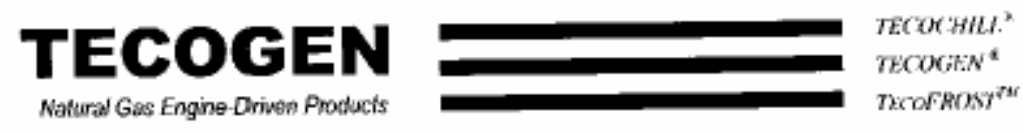

\section{Tecogen Generator Commissioning Plan \\ Revision 1, 06/09/06}

Unit Serial Number: zoo837 unit A 1

Date: $z-11-06$

By: Iokn Freeman 
Each t'ecogen unit will go through the procedure presented in this document.

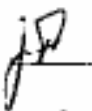

1. Inspection

1. Inspect Exhaust System

a. Self-supporting - no significant force applied to exhaust connection

b. Insulated and with fire - resistant roof penctration

$\checkmark \star$. Proper termination that prevents rainwater from entering piping system

2. Inspect Natural Gas System

a. Proper pipe size

b. Dead-end Lock-up type regulator

$\checkmark$ c. Gas supply pressure: $10-28$ "wc

3. Inspect Engine Coolant and Oil systems for leaks.

\section{Energize Control Power}

1. Ensure breaker on cabinet door is open.

1.2. Reconncet battery nogative cable.

$\checkmark 3$. Energize 208 Volt power to coolant pump.

$\checkmark 4$. Close microprocessor switch to boot up control system.

$\checkmark 5$. Check all Input switch positions

\begin{tabular}{|c|c|c|}
\hline Safety Switch & Position & Check \\
\hline Low Engine Oil Level & On & $V$ \\
\hline Low Coolant Pressure & Off & $r$ \\
\hline Low Engine Ol Pressure & Off & $\checkmark$ \\
\hline Hgh Coolant Pressure & On & $\checkmark$ \\
\hline
\end{tabular}

6. Chcck all Temperatures

\begin{tabular}{|l|c|}
\hline \multicolumn{1}{|c|}{ Thermistors } & Record \\
\hline Engine Cootant Temperature & 27.6 \\
\hline Engine Oil Temperature & 75.3 \\
\hline Ambient Temperature & 74.9 \\
\hline tigh Geetant Pressure & \\
\hline Water Temperature in & 72.4 \\
\hline Water Temperature Out & 72.3 \\
\hline
\end{tabular}

2 of 4 


\section{Encrgize Outputs in Calibrate Modc}

a. Coolant System Pump

i. Open shut-off valves to unit

ii. Record System Static Pressure:

iii. Energize Main System Cïrculating Pump

iv. Record Inlet and Outlet Pressure of Main Pump Inlet Pressure:

Outlet Pressure:

v. Check low Coolant Pressurc Switch is "On"

b. Natural Gas System

i. Open shut-off valve to unit

ii. Energize Gas Solenoid

iii. Bleed Gas Line

iv. Record Gas Pressure: $16^{*}$ ialet $4^{-}$outlet

c. Energize Inverter Control Power

i. Record MAP Pressure: 2\%.9

d. Encrgize Alarm Output

\section{Start Engine - No Electric Power Output}

$\sim 1$. Ensure breaker on cabinet door is still open.

$\sim_{2}$. Ensure system is set to Variable Speed mode.

13. Disconnect Ficld Control (J5).

$\sqrt{4}$. Start engine and verify proper specd loop operation. It should shut down after approximatcly 10 seconds.

v5. Reconnect Field Control.

6. Restart cngine.

7. Check and record the following:
a. Engine Timing: $30^{\circ}$
b. Engine Oil Pressure: 50
c. DC Bus Voltage:

8. Engine will shut down once PCS is energized. 


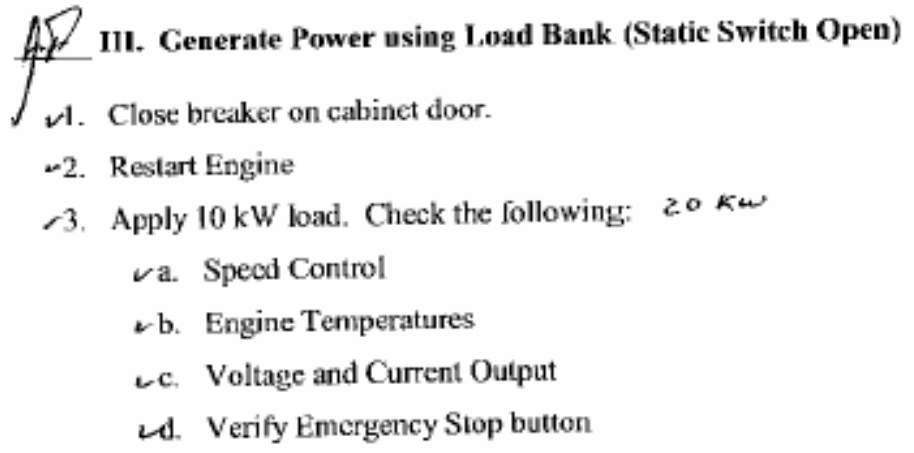

4. Restart and slowly bring load up to $60 \mathrm{~kW}$. Continuously check the following:

ra. Specd Control

$\sim$ b. Engine Temperatures

vc. Voltage and Current Output

5. Download $60 \mathrm{~kW}$ data with RMCS

IV. Perform Step Load Tests - Refer to Northern Power's Commissioning Plan Section 10

1. $0-30 \mathrm{~kW}$
2. $0-60 \mathrm{~kW}, \mathrm{c}$
3. $15-50 \mathrm{~kW}$
4. $30-60 \mathrm{~kW}$
3. $60-0 \mathrm{~kW}$
$\checkmark 6.30-0 \mathrm{~kW}$


Attachment 3

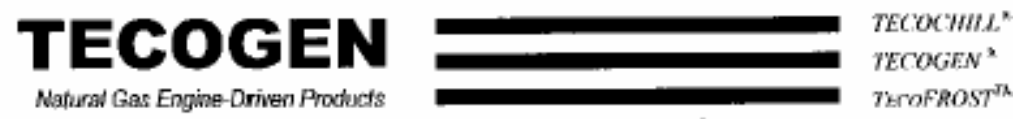

\title{
Tecogen Generator Commissioning Plan \\ Revision 1, 06/09/06
}

\author{
Unit Serial Number: 200836 unit $A 2$ \\ Date: $2-11-06$ \\ By: Ioln Freatman
}


Each Tccogen unit will go through the procedurc prescnted in this document.

\section{$\frac{27}{1}$ I. Inspection}

1. Inspect Exhaust System

$\checkmark$ a. Self-supporting - no significant force applied to exhaust connection

b. Insulated and with fire - resistant roof penctration NoT $\varphi_{t} t$

$\checkmark$ c. Proper termination that prevents rainwater from entering piping system

2. Inspect Natural Gas System

2a. Proper pipe size

$\checkmark$ b. Dead-cnd Lock-up type rcgulator

rc. Gas supply pressure: 10- 28 "wc $16^{\prime \prime}$ jtatic

3. Inspect Engine Coolant and Oil systems for leaks.

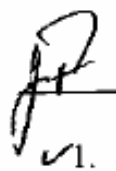

\section{Energize Control Power}

$\checkmark 1$. Ensure breaker on cabinct door is open.

2. Reconnect battery negative cable.

3. Energize 208 Voll power to coolant pump.

$\checkmark$. Close microprocessor switch to boot up control system.

$\checkmark 5$. Check all Input switch positions

\begin{tabular}{|l|c|c|}
\hline \multicolumn{1}{|c|}{ Safety Switch } & $\frac{\text { Position }}{\text { On }}$ & Check \\
\hline Low Engine Oil Level & - & $\checkmark$ \\
\hline Low Coolant Pressure & Off & Off \\
\hline Low Engine Oil Pressure & On & \\
\hline High Coolant Pressure & On \\
\hline
\end{tabular}

6. Check all Temperatures

\begin{tabular}{|l|c|}
\hline \multicolumn{1}{|c|}{ Thermistors } & Record \\
\cline { 2 - 2 } \cline { 2 - 2 } Engine Coolant Temperature & 72.2 \\
\hline Engine Oil Temperature & 73.5 \\
\hline Ambient Temperature & 78.7 \\
\hline Hight Cootant Presaufe & \\
\hline Water Temperature In & 72.2 \\
\hline Water Temperature Out & 74.4 \\
\hline
\end{tabular}


7. Energize Outputs in Calibrate Mode

a. Coolant System Pump

i. Open shut-off valves to unit

ii. Record System Static Pressure: 15 pst

iii. Energize Main System Circulating Pump

iv. Record Inlet and Outlet Pressure of Main Pump

Inlet Pressure:

Outlet Pressure:

v. Check Low Coolant Pressure Switch is "On"

b. Natural Gas System

i. Open shut-off valve to unit

ii. Energizc Gas Solenoid

iii. Bleed Gas Line

iv. Record Gas Pressure: $16^{\prime \prime}$

c. Energize Inverter Control Power

i. Record MAP Pressure: 29.5

d. Encrgize Alarm Output

fit

III. Start Engine - No Ělectric Power Output

1. Ensurc breaker on cabinct door is still open.

$\checkmark 2$. Ensure system is set to Variable Speed mode.

$\checkmark 3$. Disconnect Field Control (J5).

i- 4. Start engine and verify proper speed loop operation. It should shut down after approximately 10 seconds.

$\checkmark 5$. Reconnect Ficld Control.

V6. Restart enginc.

$\sim 7$. Check and record the following:
a. Engine Timing: $30^{\circ}$
b. Engine Oil Pressure: 4$\}$
c. DC Bus Voltage: $\quad 4.32$

8. Engine will shut down once PCS is energized.

3 of 4 


\section{(2) III. Generate Power using Load Bank (Static Switch Open)}

$\int / 1$. Close brcaker on cabinct door.

$\sqrt{2}$. Restart Engine

3. Apply $10 \mathrm{~kW}$ load. Chock the following:

a. Speed Control

b. Engine Temperaturcs

c. Voltage and Current Output

d. Verify Emergency Stop bution

4. Restart and slowly bring load up to $60 \mathrm{~kW}$. Continuously check the following:
a. Speed Control
b. Engine Tcmperatures
c. Voltage and Current Output

5. Download $60 \mathrm{~kW}$ data with RMCS

1. $0-30 \mathrm{~kW}$
2. $0-60 \mathrm{~kW}$
2ection 10
3. $15-50 \mathrm{~kW}$
$\because 4.30 .60 \mathrm{~kW}$
4- 5. $60.0 \mathrm{~kW}$
6. $30-0 \mathrm{~kW}$


Attachment 4

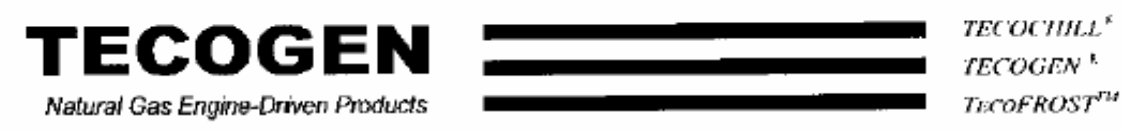

\title{
Tecogen Generator Commissioning Plan \\ Revision 1, 06/09/06
}

\author{
Unit Serial Number: $\quad 200835$ unst $B 1$ \\ Date: $>-11-06$ \\ By: Jokn Freemen
}


Each Tecogen unit will go through the procedure presented in this document.

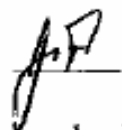

\section{Inspection}

1. Inspect Exhaust System

-á. Self-supporting no significant force applied to exhaust connection

b. Insulated and with fire - resistant roof penetration stack not yet in sulu ted.

c. Proper termination that prevents rainwater from entering piping system

2. Inspect Natural Gas System

ح. Proper pipe size

6. Dead-cnd Lock-up type regulator

$\checkmark$ c. Gas supply pressure: $10-28^{\prime \prime}$ we $/ 6$ "

3. Inspect Engine Coolant and Oil systems for leaks.

fo

\section{Energize Control Power}

1. Ensure brcaker on cabinet door is open.

$\checkmark 2$. Reconnect battery negativc cable.

$\checkmark 3$. Energize 208 Volt power to coolant pump.

$\checkmark 4$. Closc microprocessor switch to boot up control system.

$\sqrt{5}$. Check all Input switch positions

\begin{tabular}{|l|c|c|}
\hline \multicolumn{1}{|c|}{ Safety Switch } & Position & Check \\
\hline Low Engine Oil Level & On & $\checkmark$ \\
\hline Low Coolant Pressure & Off & $\checkmark$ \\
\hline Low Engine Oil Pressure & Off & $\checkmark$ \\
\hline High Coolant Pressure & On & $\checkmark$ \\
\hline
\end{tabular}

6. Check all Temperatures

\begin{tabular}{|l|c|}
\hline \multicolumn{1}{c|}{ Thermistors } & Record \\
\hline Engine Coolant Temperature & 74.4 \\
\hline Engine Oil Temperature & 74.3 \\
\hline Ambient Temperature & 74.4 \\
\hline Htigit Cootant Prestroce. & \\
\hline Water Temperature In & 73.9 \\
\hline Water Temperature Out & 73.4 \\
\hline
\end{tabular}


7. Energizc Outputs in Calibrate Mode

a. Coolant System Pump

i. Open shut-off valves to unit

ii. Rocord System Static Pressure: 15 psi

$\checkmark$ iii. Energize Main System Circulating Pump

iv. Record Inlet and Outlet Pressurc of Main Pump

Inlet Pressure:

Outlet Pressure:

v. Check Low Coolant Pressure Switch is "On"

b. Natural Gas System

i. Open shut-off valve to urit

ii. Encrgize Gas Solenoid

iii. Bleed Gas Line

iv. Record Gas Pressure: it "

c. Fncrgize Inverter Control Power

i. Record MAP Pressure: . 29.4

d. Energize Alarm Output

\section{Start Engine - No Electric Power Output}

$\checkmark 1$. Ensure brcaker on cabinct door is still open.

$\checkmark 2$. Ensure system is set to Variable Specd mode.

$\checkmark$ 3. Disconnect Field Control (J5).

4. Start engine and verify proper speed loop operation. It should shut down after approximately 10 scconds.

5. Reconnect Field Control.

6. Restart engine.

7. Chack and record the following:
a. Engine Timing: .30 .
b. Engine Oil Pressurc: y9
c. DC Bus Voltage: 432

8. Engine will shut down once PCS is energized. 


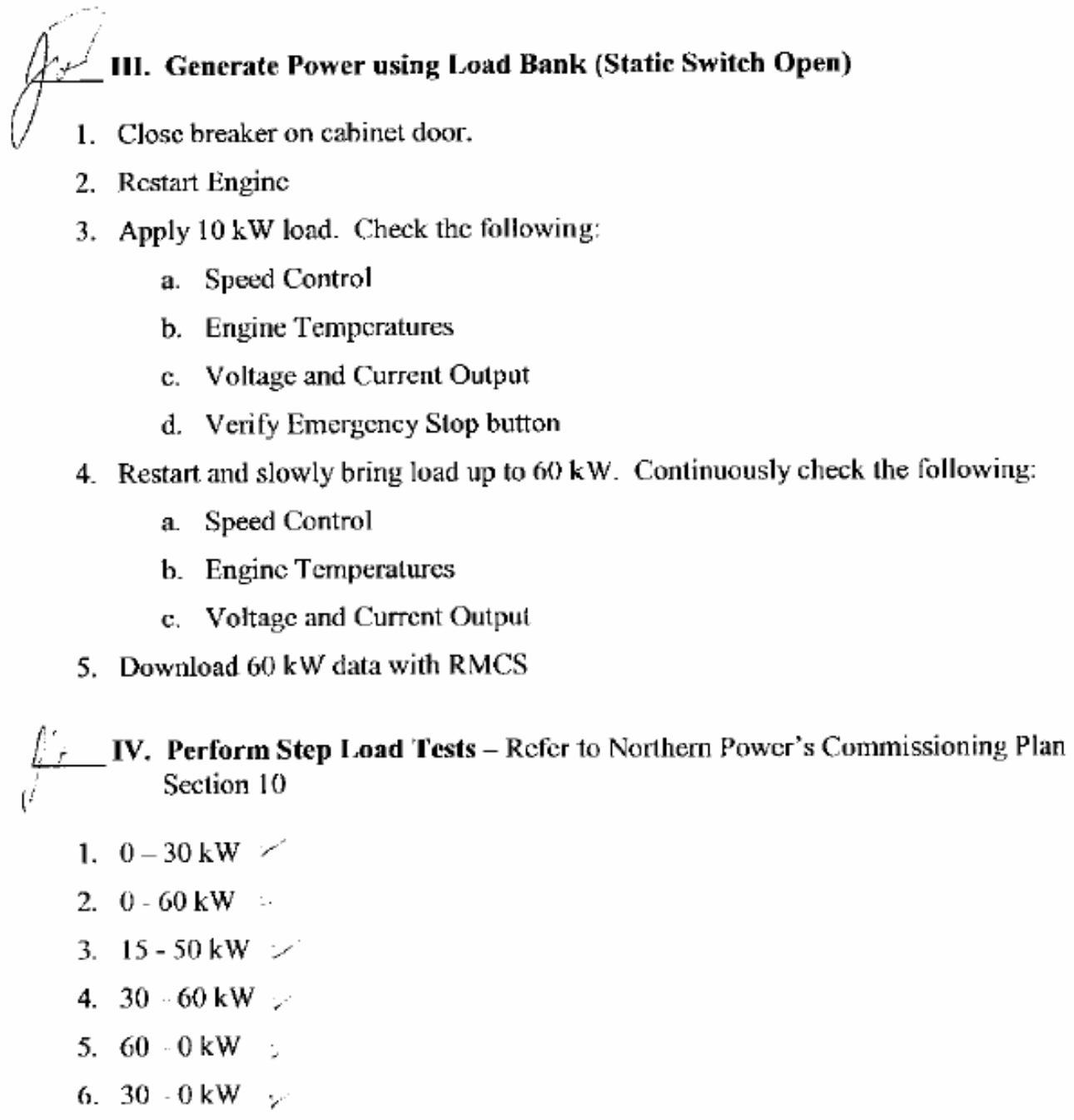

4 of 4 


\section{CERTS Microgrid Initial Test at AEP (7/26/06 - 7/28/06)}

- Fixed unit A1 speed control problem.

- Updated firmware for all 3 units.

- $\quad$ Tested each unit in variable speed mode using Zone 4 load bank.

- Step up 30KW

- Step down $30 \mathrm{KW}$

- Step up 60KW

- Step down $60 \mathrm{KW}$

- Paralleled A1 and A2 units. Set variable speed mode as initial test, however, engine control failed to handle $60 \mathrm{KW}$ step load.

Changed to fix speed mode, and ran engine at 2070 RPW. Tested A1 and A2 using Zone 4 load bank.

- Step up 60KW

- Step down $60 \mathrm{KW}$

- Step up $90 \mathrm{KW}$

- Step down $90 \mathrm{KW}$

After certain run time, Zone 4 load bank was over heat and failed.

- Paralleled all 3 A1, A2 and B1 units together.

Set engine control as fix speed mode, and engine speed at 2070 RPW. Used the load near the grid input site, and tested

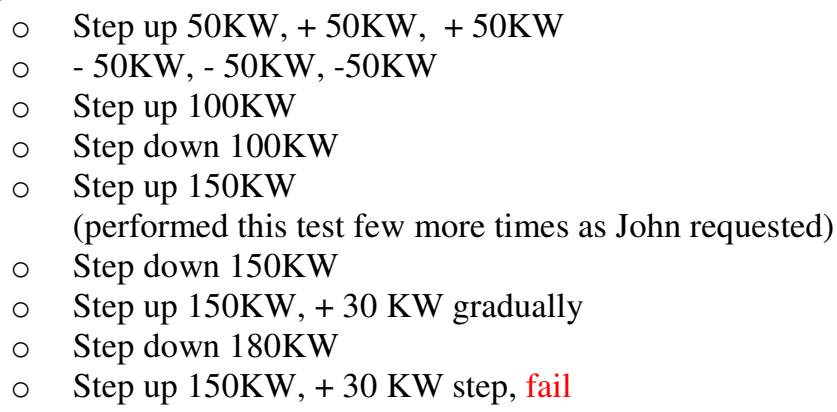

- Turned on grid, turned on static switch.

Set load bank to $150 \mathrm{KW}$.

Set all 3 units as power control mode and set power command to $10 \mathrm{KW}$.

Tied all 3 units to the grid in the sequence of A1, A2, and B1.

Each unit exported about 10KW.

Opened the grid switch, all 3 unit ran at micro-grid mode and power $150 \mathrm{KW}$.

- Turned on grid, turned on static switch.

Set load bank to $150 \mathrm{KW}$.

Set all 3 units as power control mode and set power command to $50 \mathrm{KW}$.

Tied all 3 units to the grid in the sequence of $\mathrm{A} 1, \mathrm{~A} 2$, and $\mathrm{B} 1$.

Each unit exported about 50KW.

Opened the grid switch, all 3 unit ran at micro-grid mode and power $150 \mathrm{KW}$.

- Turned on B1, and step 50KW load.

Ran 30 min.

Tuned on A1 and A2.

Set load bank to $150 \mathrm{KW}$.

Ran 90 min.

Shuted down all 3 units. 
- $\quad$ Turned on B1.

Set $\mathrm{B} 1$ as power control mode, and power command $=10 \mathrm{KW}$.

Set Zone 5 load bank $=15 \mathrm{KW}$.

Turned on gird.

Set load bank near the grid to 50KW.

Enable the static switch, few minuets later, static switch automatically closed, and B1 tied to the grid successfully.

- Turned on B1.

Set $\mathrm{B} 1$ as power control mode, and power command $=10 \mathrm{KW}$.

Set Zone 5 load bank $=30 \mathrm{KW}$.

Turned on gird.

Set load bank near the grid to $50 \mathrm{KW}$.

Enable the static switch, few minuets later, static switch automatically closed, and B1 tied to the grid successfully. 


\section{Test Log for 8-23-06}

\begin{tabular}{|c|c|c|c|c|}
\hline$\underline{\text { Time }}$ & $\underline{\text { Test }}$ & $\underline{\text { Unit }}$ & Load Bank & $\underline{\text { Load }(\mathrm{kW})}$ \\
\hline $10: 06 \mathrm{am}$ & \multicolumn{4}{|l|}{ Trigger Test } \\
\hline 10:49 am & I.a.i & A1 & \multicolumn{2}{|r|}{$0-30$} \\
\hline $10: 51 \mathrm{am}$ & I.a.vi & A1 & 3 & $30-0$ \\
\hline $10: 56 \mathrm{am}$ & I.a.ii & A1 & 3 & $0-60$ \\
\hline $11: 00 \mathrm{am}$ & I.a.v & A1 & 3 & $60-0$ \\
\hline 11:04 am & I.a.iii & A1 & 3 & $15-50$ \\
\hline$\underline{\text { Time }}$ & $\underline{\text { Test }}$ & $\underline{\text { Unit }}$ & $\underline{\text { Load Bank }}$ & $\underline{\text { Load }(\mathrm{kW})}$ \\
\hline $11: 12 \mathrm{am}$ & I.a.i & A2 & \multirow[t]{2}{*}{4} & $0-30$ \\
\hline $11: 16 \mathrm{am}$ & Ignore data & & & \\
\hline $11: 20 \mathrm{am}$ & I.a.vi & A2 & 4 & $30-0$ \\
\hline $11: 24$ am & I.a.ii & A2 & 4 & $0-60$ \\
\hline $11: 28 \mathrm{am}$ & I.a.v & A2 & 4 & $60-0$ \\
\hline $11: 32 \mathrm{am}$ & I.a.iii & $\mathrm{A} 2$ & 4 & $15-50$ \\
\hline$\underline{\text { Time }}$ & $\underline{\text { Test }}$ & $\underline{\text { Unit }}$ & Load Bank & $\underline{\text { Load }(\mathrm{kW})}$ \\
\hline 11:46 am & \multicolumn{3}{|c|}{ Ignore Data (Engine Overspeed) } & \\
\hline $11: 56$ am & I.a.i & B1 & 5 & $0-30$ \\
\hline $12: 00 \mathrm{pm}$ & I.a.vi & B1 & 5 & $30-0$ \\
\hline $12: 04 \mathrm{pm}$ & I.a.ii & B1 & 5 & $0-60$ \\
\hline $12: 08 \mathrm{pm}$ & I.a.v & B1 & 5 & $60-0$ \\
\hline $12: 12 \mathrm{pm}$ & I.a.iii & $\mathrm{B} 1$ & 5 & $15-50$ \\
\hline$\underline{\text { Time }}$ & $\underline{\text { Test }}$ & $\underline{\text { Unit }}$ & $\underline{\text { Load Bank }}$ & Load $(\mathrm{kW})$ \\
\hline 01:44 pm & \multicolumn{4}{|c|}{ Closed CB41 A1 was running with 50kW on Load Bank 3, No load on Load Bank 4} \\
\hline 02:15 pm & \multicolumn{4}{|c|}{$\begin{array}{l}\text { Generator A1 and A2 were load sharing } 50 \mathrm{~kW} \text {, Generators carrying } 50 \mathrm{~kW} \text { of load at } 60 \mathrm{~Hz} \\
\text { frequency, A2 performing as if it were Utility connected. Altering power output command would } \\
\text { result in corresponding change in actual power output. We believe event was captured with a } \\
\text { manual trigger. }\end{array}$} \\
\hline \multicolumn{5}{|c|}{ Note: "cpt" means cycles pre-trigger } \\
\hline 03:10 pm & I.I.c.i (part2)(5 cpt) & $\mathrm{A} 1 * \mathrm{~A} 2$ & 50 & \\
\hline $03: 16 \mathrm{pm}$ & I.I.c.i (part2)(13 cpt) & $\mathrm{A} 1 * \mathrm{~A} 2$ & 50 & \\
\hline $03: 26 \mathrm{pm}$ & I.I.c.i (part3) & $\mathrm{A} 1 * \mathrm{~A} 2$ & \#3/50 \#4/70 & $50-120$ \\
\hline
\end{tabular}


7. Test Results for $\mathbf{8 - 2 3 - 0 6}$

I.a.i A1

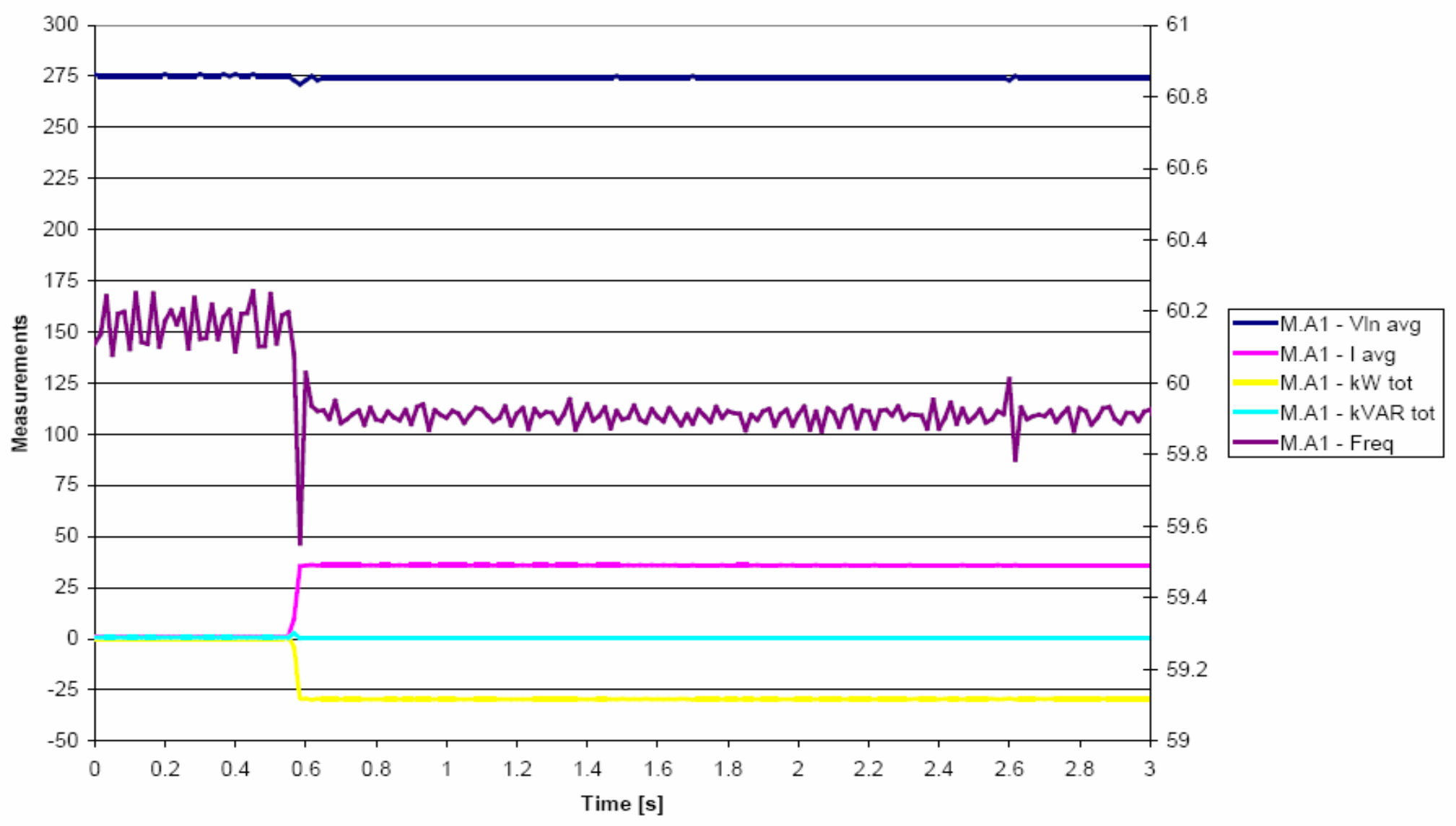




\section{Test Log for 8-28-06}

Time

Generator A1

$12: 44 \mathrm{pm}$

$12: 58 \mathrm{pm}$

1:03 pm I.a.ii

1:06 pm I.a.iii

1:09 pm I.a.iv

1:12 pm I.a.v

1:15 pm I.a.vi

1:28 pm I.a.i

1:31 pm I.a.ii

1:34 pm I.a.iii

1:37 pm I.a.iv

1:42 pm I.a.iv

1:46 pm I.a.v

1:49 pm I.a.vi

5:04 pm I.a.i

5:08 pm I.a.i

5:13 pm I.a.ii

5:16 pm I.a.iii

5:22 pm I.a.iii

5:25 pm I.a.iv

5:29 pm I.a.iv

5:37 pm I.a.v

5:40 pm I.a.vi $\underline{\text { Test }}$

Trigger Test

I.a.i

A1

A1

A1

A1

A1

A2

A2

A2

A2

A2

A2

A2

B1

B1

B1

B1

B1

B1

B1

B1

B1 $\underline{\text { Load Bank }}$

$\underline{\operatorname{Load}(\mathrm{kW})}$

A1

3

$0-30$

$0-55$

$15-45$

$30-55$

55-0

30-0

$0-30$

$0-55$

$15-45$

Genset No Field Sig 30-55

Genset No Field Sig 30-55

55-0

30-0

Genset Overspeed 0-30

Genset Overspeed 0-30

$0-55$

Genset No Field Sig 15-45

$15-45$

Genset No Field Sig 30-55

Genset No Field Sig 30-55

$55-0$

$30-0$ 


\section{Test Log for 8-31-06}

$\underline{\text { Time }} \underline{\text { Test }} \underline{\text { Unit }} \quad \underline{\text { Load Bank }} \quad \underline{\text { Load }(\mathrm{kW})}$

Generator A1 and B1 w/out surge module

8:22 am II.c.i (Part 2) A1+B1

3

55

A1 initially loaded to $50 \mathrm{~kW}, \mathrm{~B} 1$ was then connected.

Power Command: $\mathrm{A} 1=6, \mathrm{~B} 1=0$

8:27 am II.c.i (Part 3) A1+B1

$3+5 \quad$ Gensets Stalled 55-120

$\mathrm{A} 1$ and B1 load step together. Power Command: $\mathrm{A} 1=6, \mathrm{~B} 1=0$

A1 = No Field Sig, B1 = Boost Fault

8:38 am II.c.i (Part 2) A1+B1 3

A1 initially loaded to $50 \mathrm{~kW}, \mathrm{~B} 1$ was then connected.

Power commanded: $\mathrm{A} 1=0, \mathrm{~B} 1=6$

Repeated due to setpoint mismatch

8:44 am II.c.i (Part 3) A1+B1

3+5 Gensets Stalled 55-120

A1 and B1 load step together. Power Command: A1 = 0, B1 = 6

A1 = No Field Sig, B1 = Boost Fault

$\begin{array}{ccc}\text { 8:54 am Custom Test } & \text { A1+B1 } & \text { Gensets S } \\ \text { A1 and B1 load step together. Power Command: A1 }=0, B 1=6\end{array}$

9:01 am Custom Test $\quad$ A1+B1 $\quad 3+5 \quad$ Gensets Stalled 50-120

A1 and B1 load step together. Power Command: A1 = 0, B1 = 6

A1 = No Field Sig, B1 = Boost Fault

9:15 am II c.ii

$\mathrm{A} 1+\mathrm{B} 1$

$95-0$

A1 and B1 load step together. Power Command: A1 = 0, B1 = 6

Altered test because a single load step of $95 \mathrm{~kW}$ is the maximum possible.

9:49 am Ignore Data Trigger Test of CB12 Opening

$\begin{array}{llll}10: 16 & \text { II.a.i } & \text { A1+A2 3+4 }\end{array}$

$30-90$

A1 and A2 load step together. Power Command: A1 = 20, A2 = 40

10:23 am II.b.i A1+A2 3+4

40

$\mathrm{A} 1$ and $\mathrm{A} 2$ Island Power Command: $\mathrm{A} 1=6, \mathrm{~A} 2=54$

$\begin{array}{ccc}11: 12 \mathrm{am} \text { II.a.i } & \text { A1+B1 } & 3+5 \\ \text { A1 and B1 load step together Power Command: } \mathrm{A} 1=40, \mathrm{~B} 1=20\end{array}$

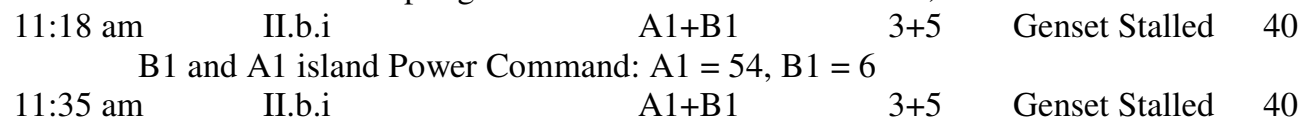

$\mathrm{B} 1$ and $\mathrm{A} 1$ island Power Command: $\mathrm{A} 1=54, \mathrm{~B} 1=6$ 


\section{Test Results for 8-31-06}

Test I.a.i A1 $=0-29.5 \mathrm{~kW}$ load step

I.a.i A1

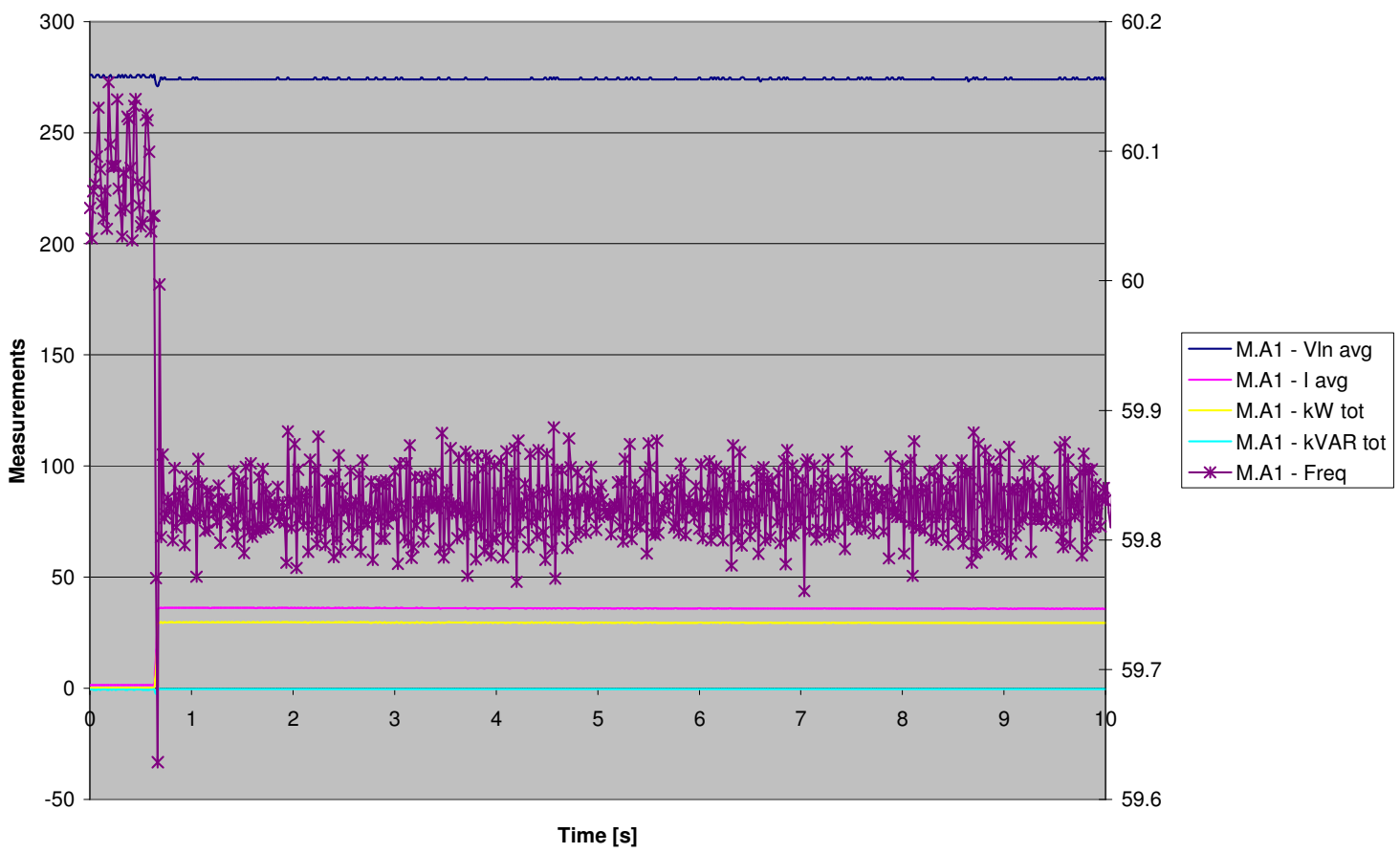

Test I.a.ii A1 $=0-53 \mathrm{~kW}$ load step

I.a.ii A1

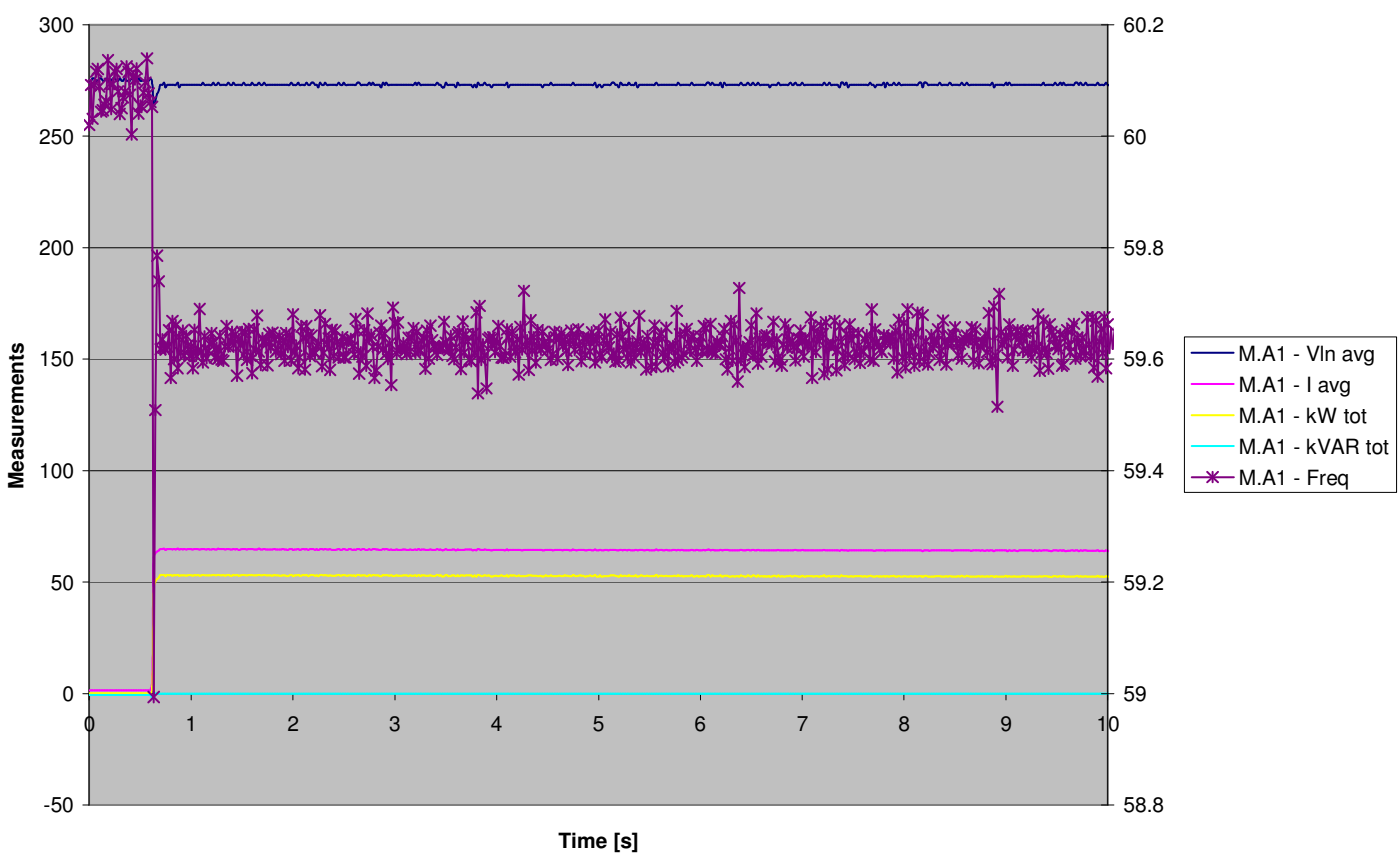


Test I.a.iii A1 $=17.6-40.5 \mathrm{~kW}$ load step

I.a.iii A1

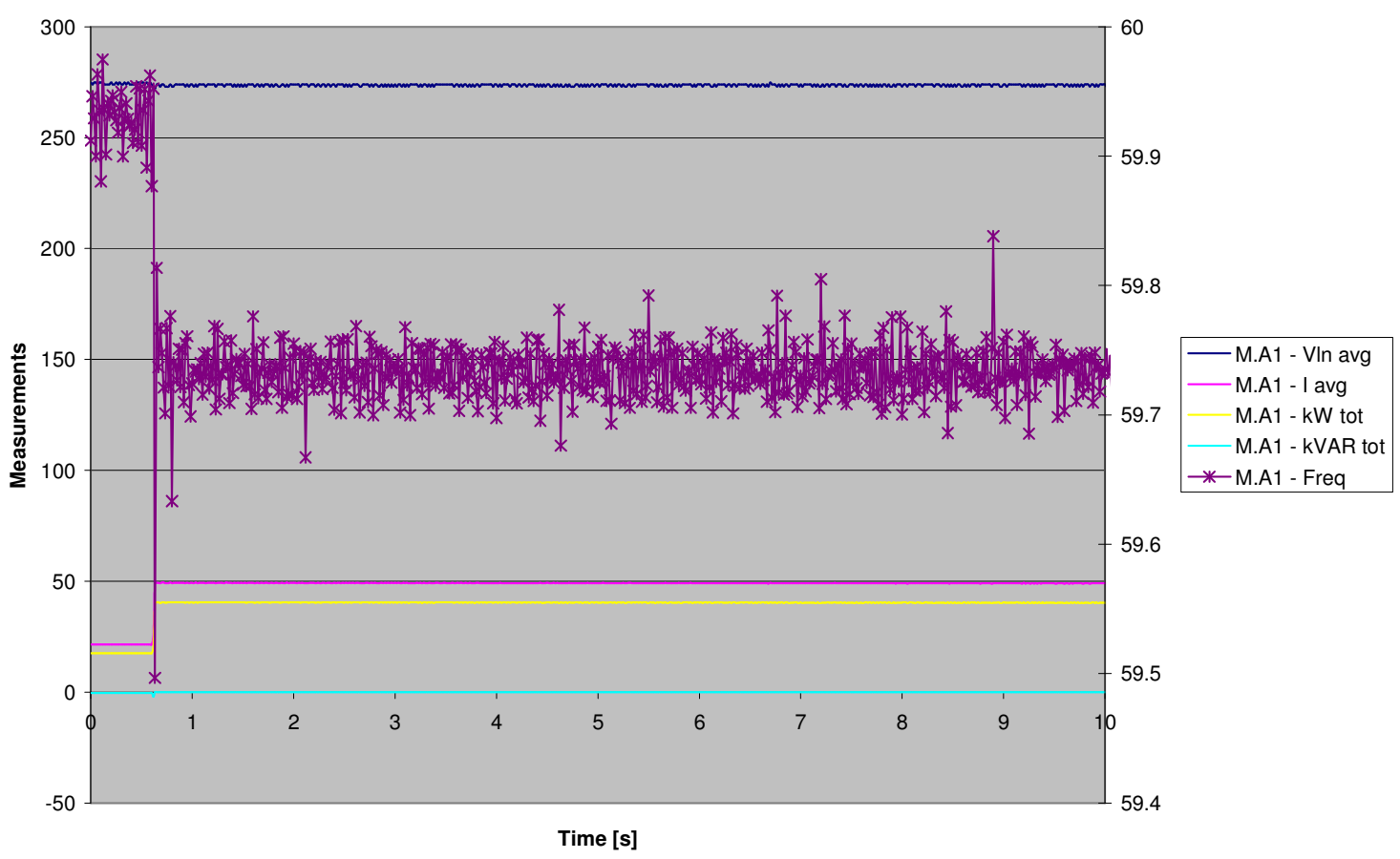

Test I.a.iv A1 $=32.6-50.8 \mathrm{~kW}$ load step

I.a.iv A1

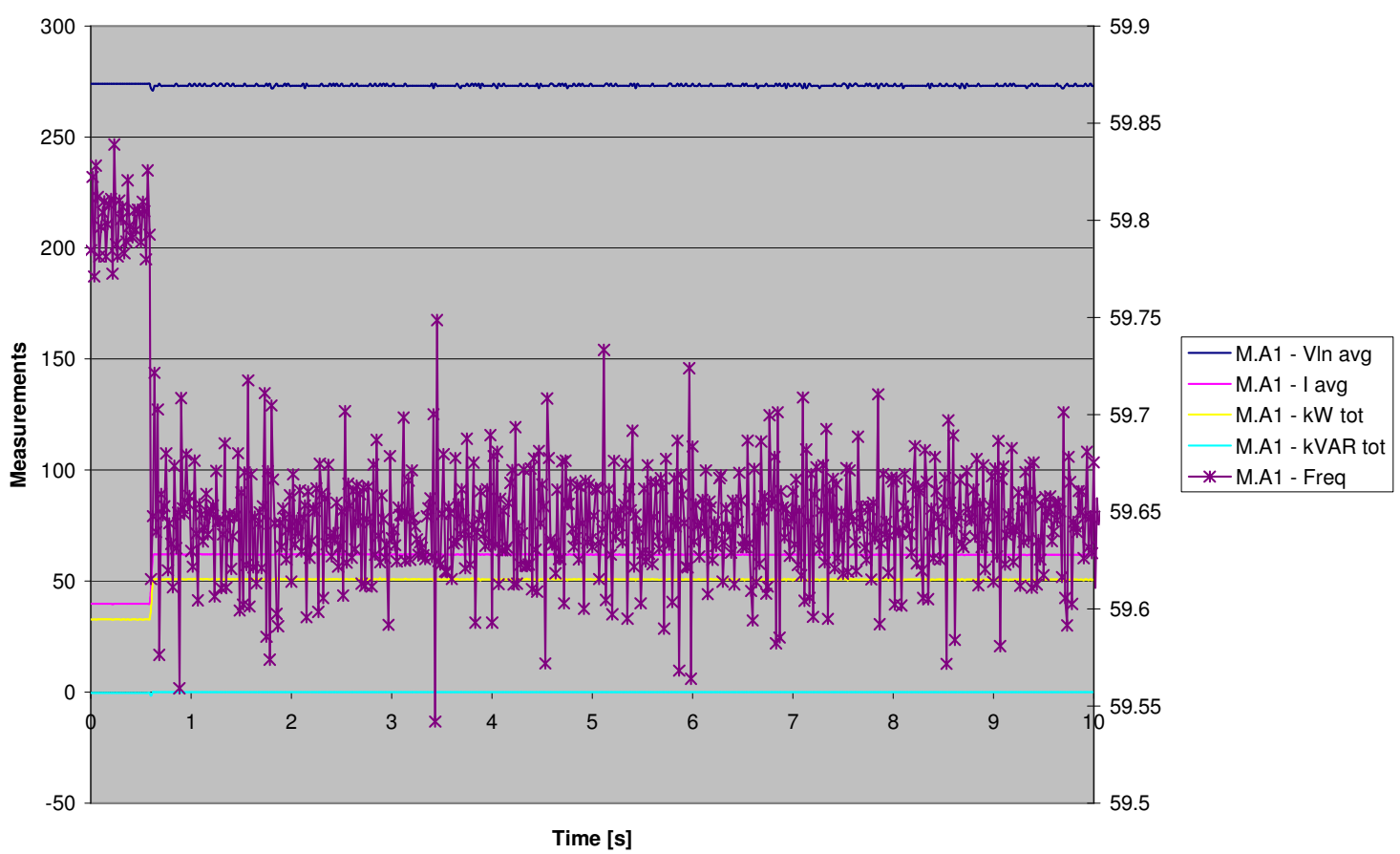


Test I.a.v A1 $=49.5-0 \mathrm{~kW}$ load step

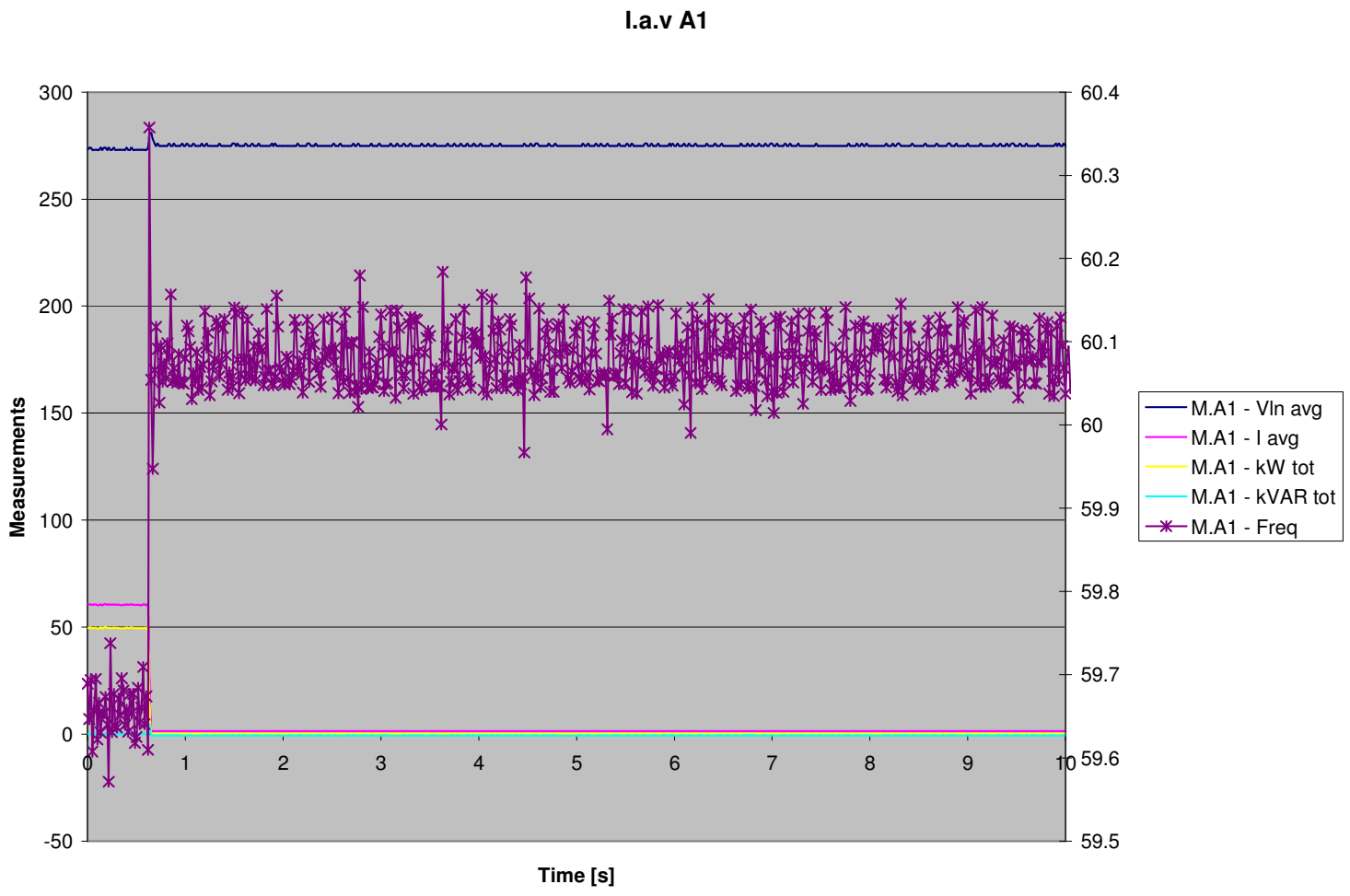

Test I.a.vi A1 $=32.5-0 \mathrm{~kW}$ load step

I.a.vi A1

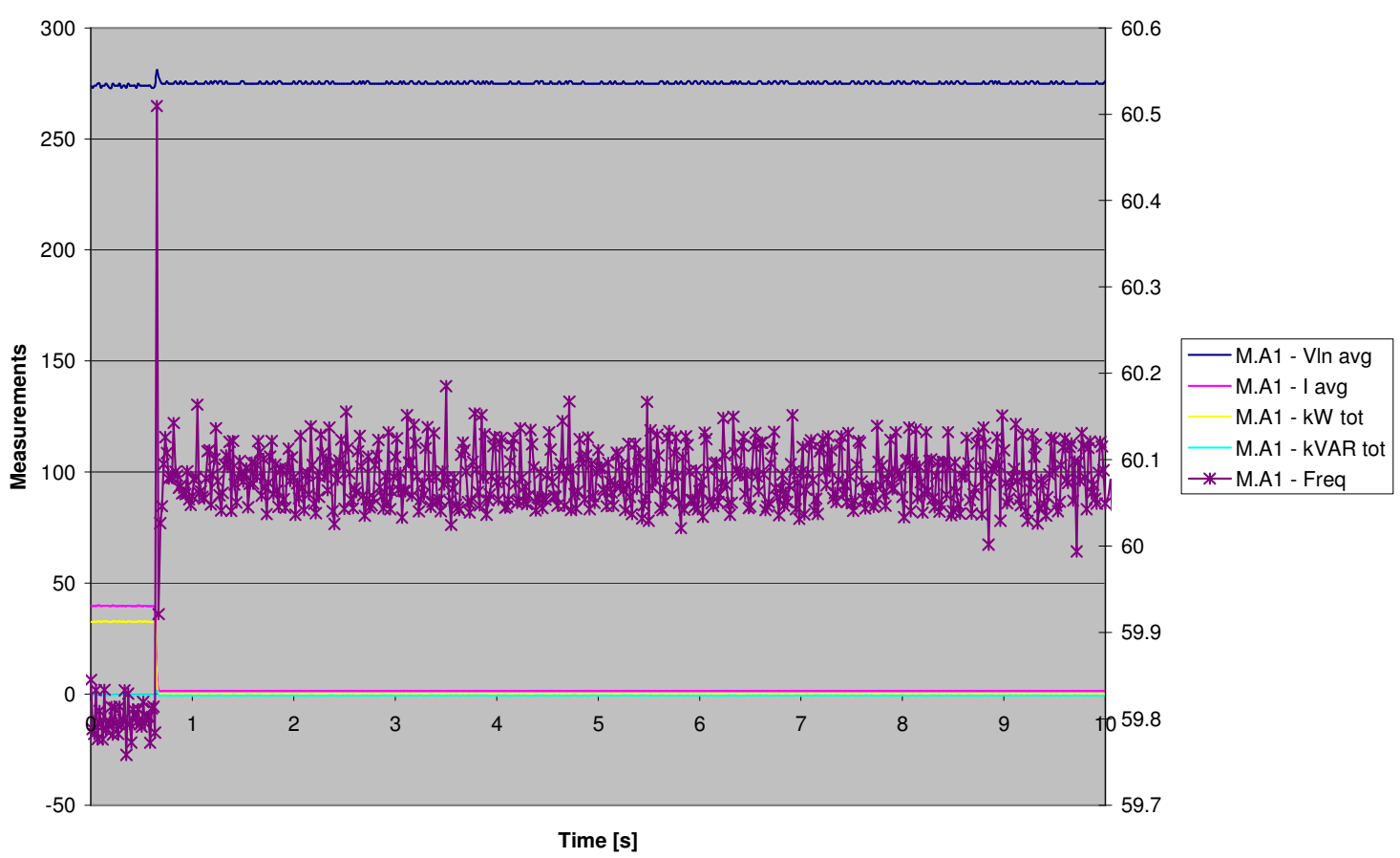


Test I.a.i A2 $=0-29.9 \mathrm{~kW}$ load step

I.a.i A2

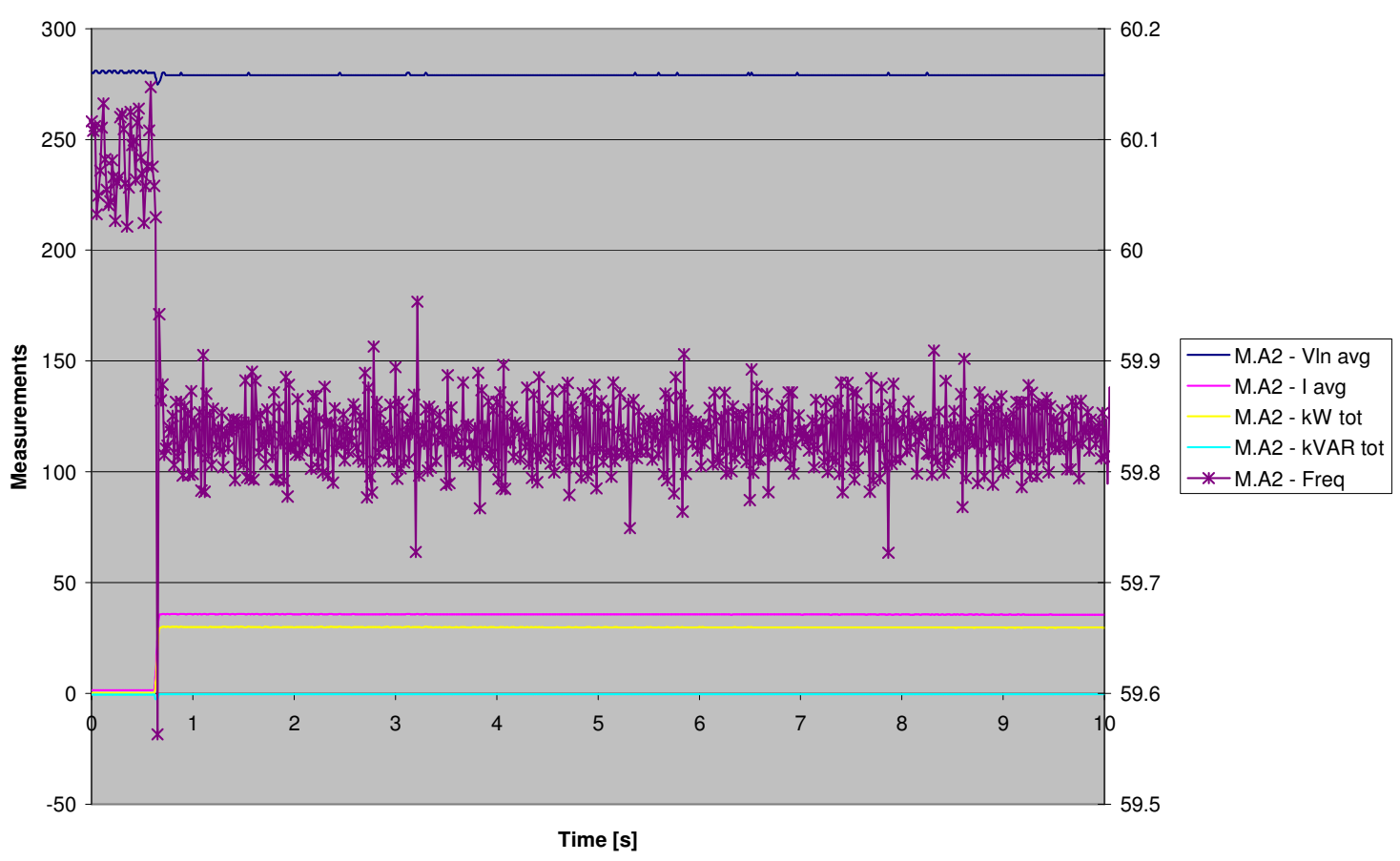

Test I.a.ii A2 $=0-53.9 \mathrm{~kW}$ load step

I.a.ii A2

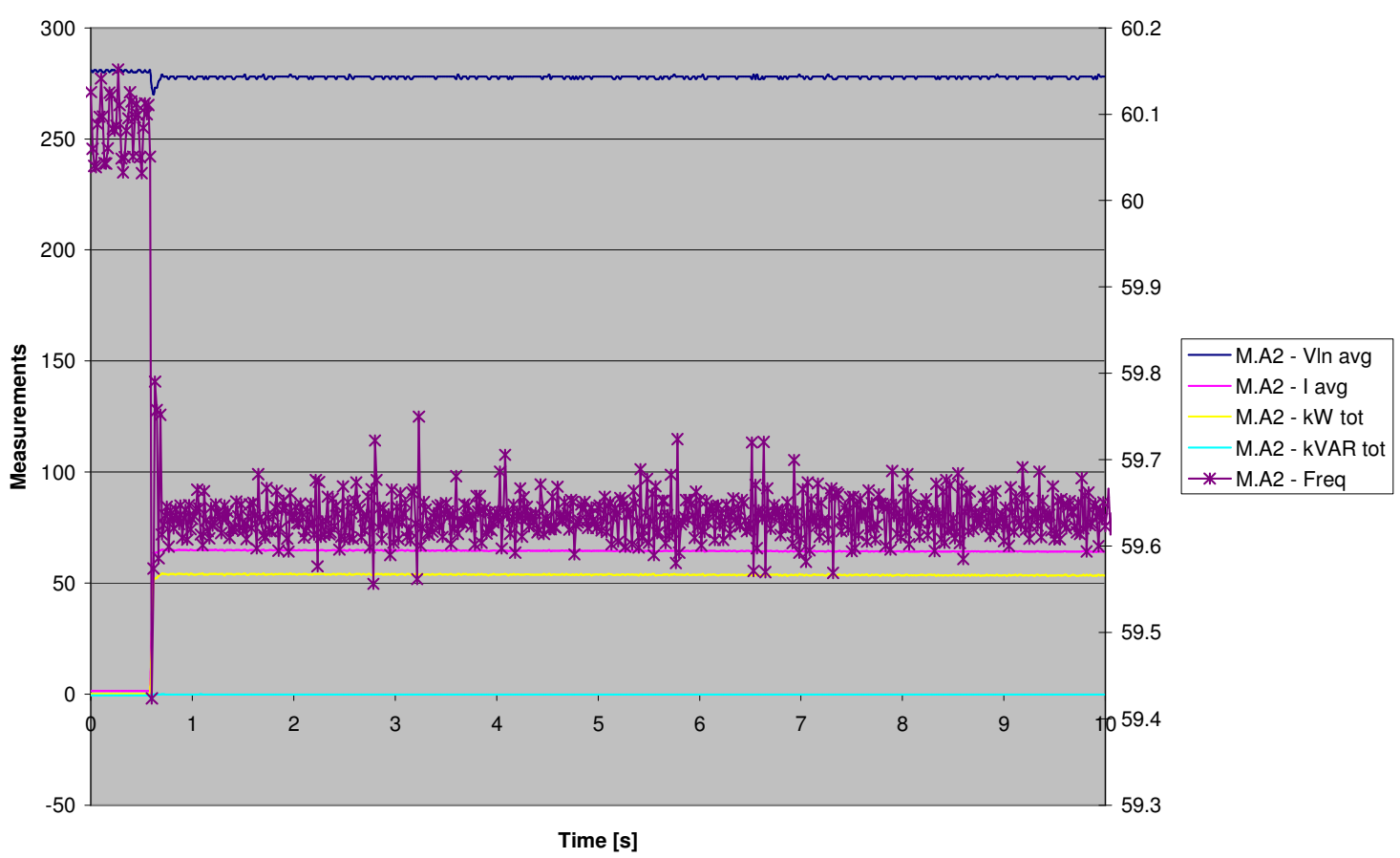


Test I.a.iii A2 $=18-42.2 \mathrm{~kW}$ load step

I.a.iii A2

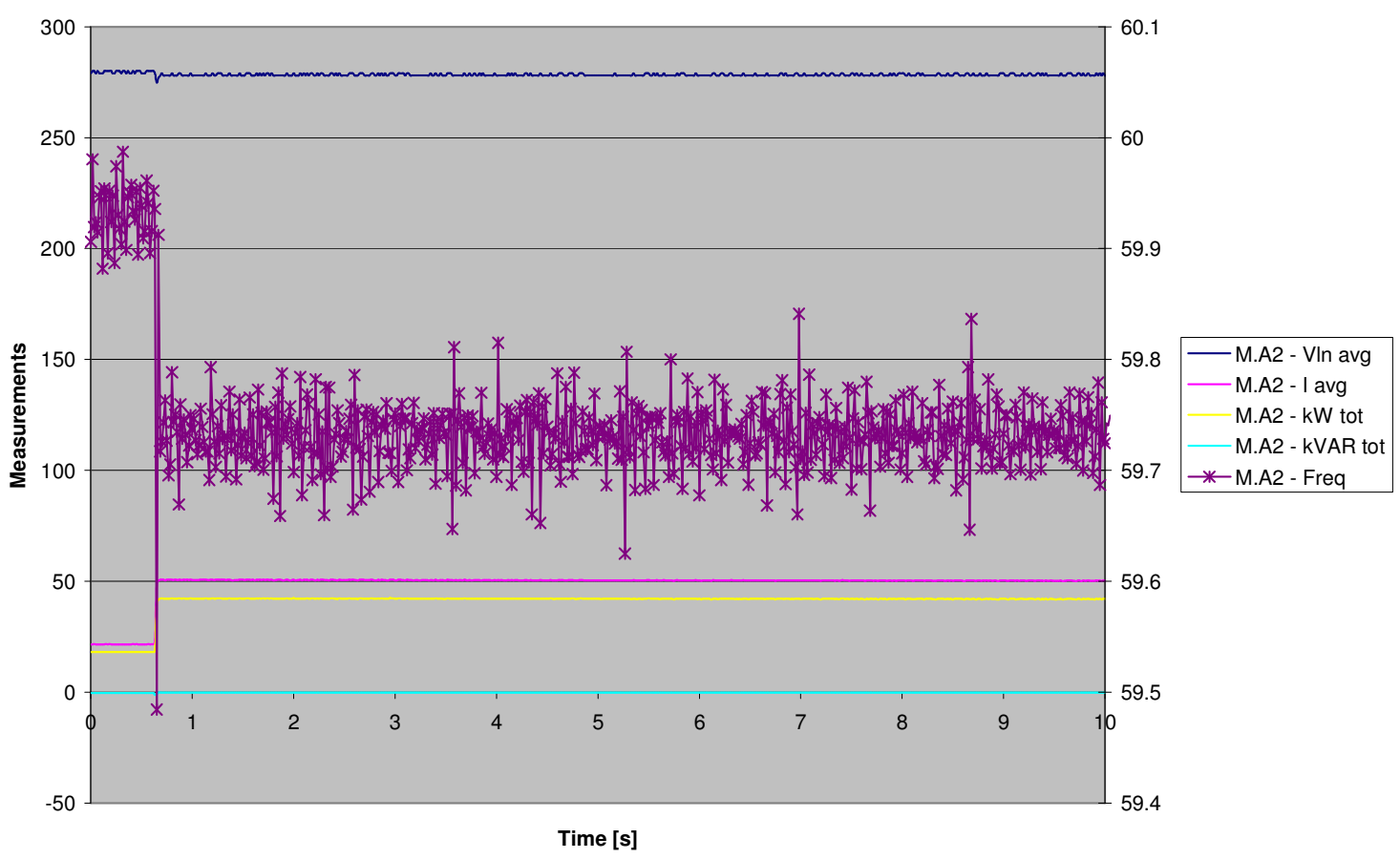

Test I.a.iv A2 $=28.5-52.4 \mathrm{~kW}$ load step, Genset Stalled with No Field Sig

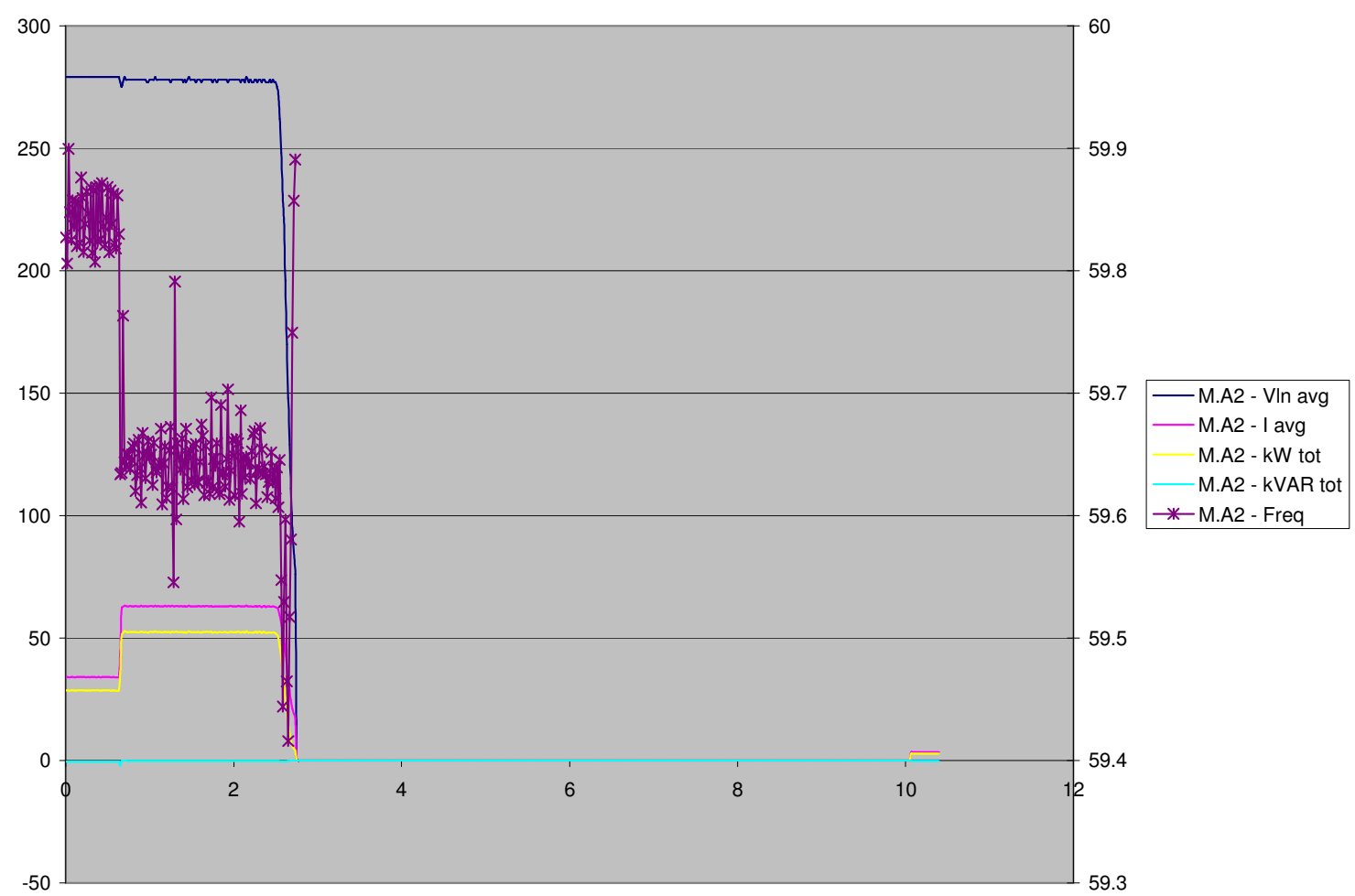


Test I.a.iv A2 $=29-51 \mathrm{~kW}$ load step, Genset Stalled with No Field Sig

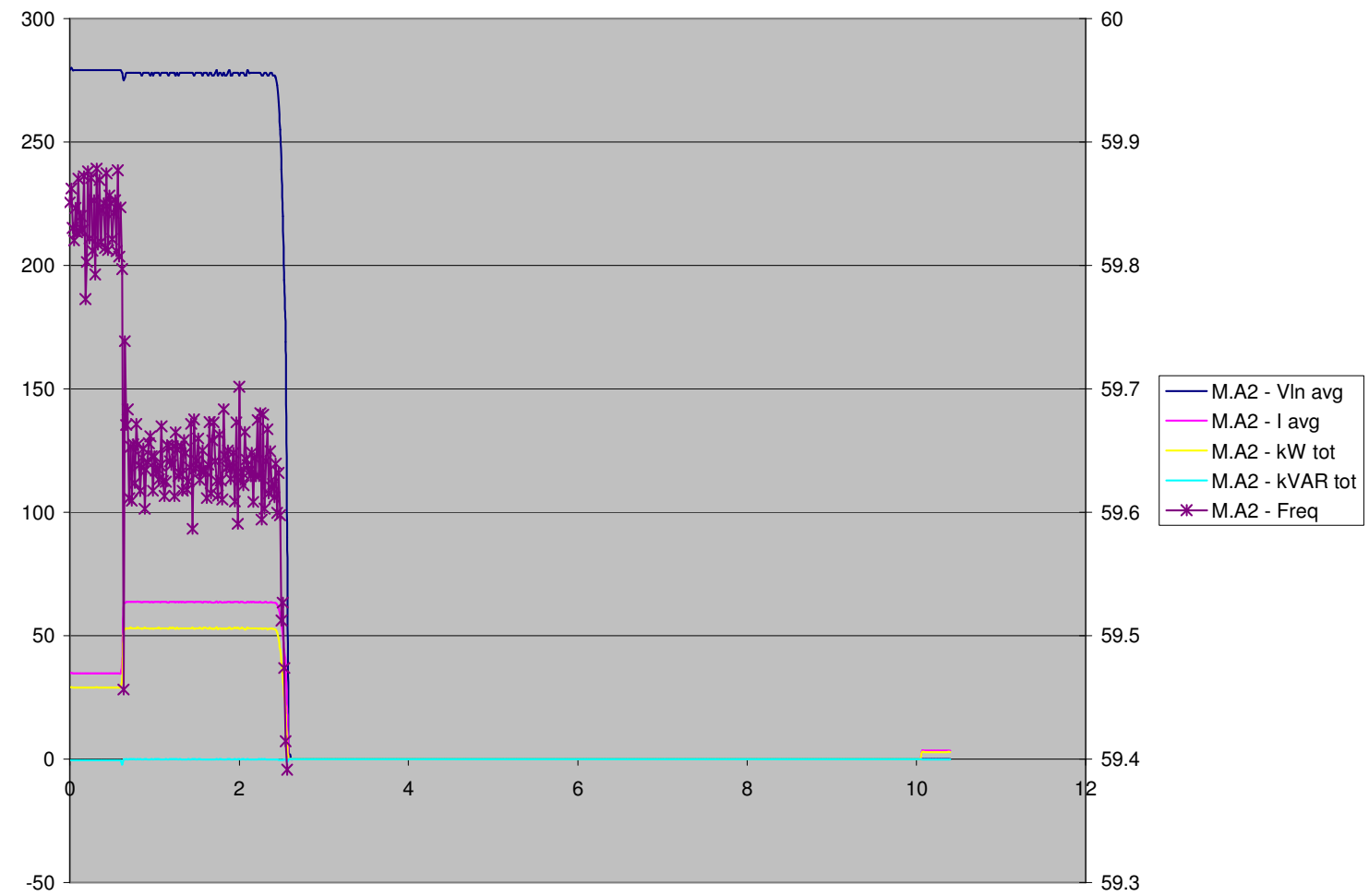

Test I.a.v A2 $=53.5-0 \mathrm{~kW}$ load step

I.a.v A2

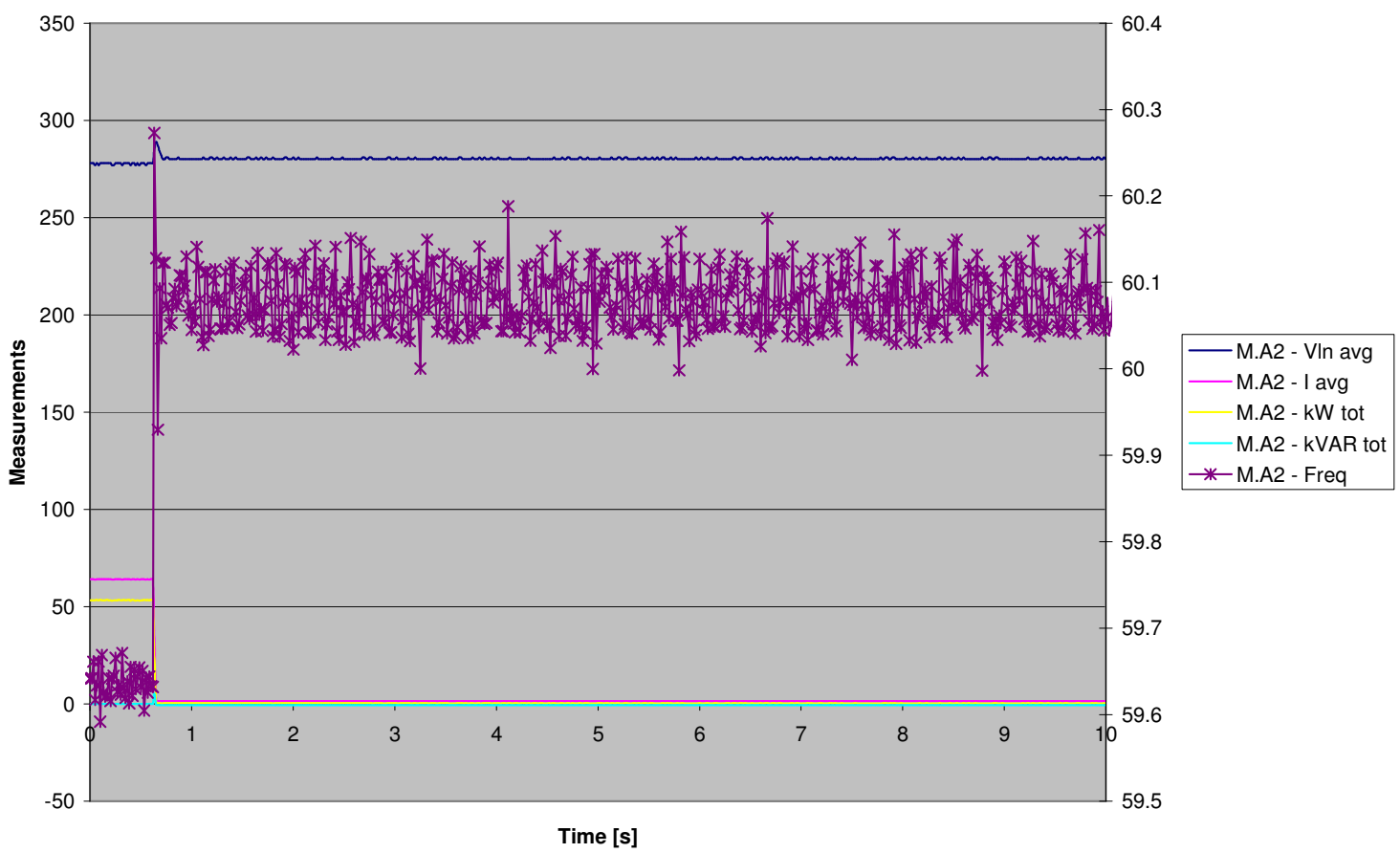


Test I.a.vi A2 $=33.9-0 \mathrm{~kW}$ load step

\section{l.a.vi A2}

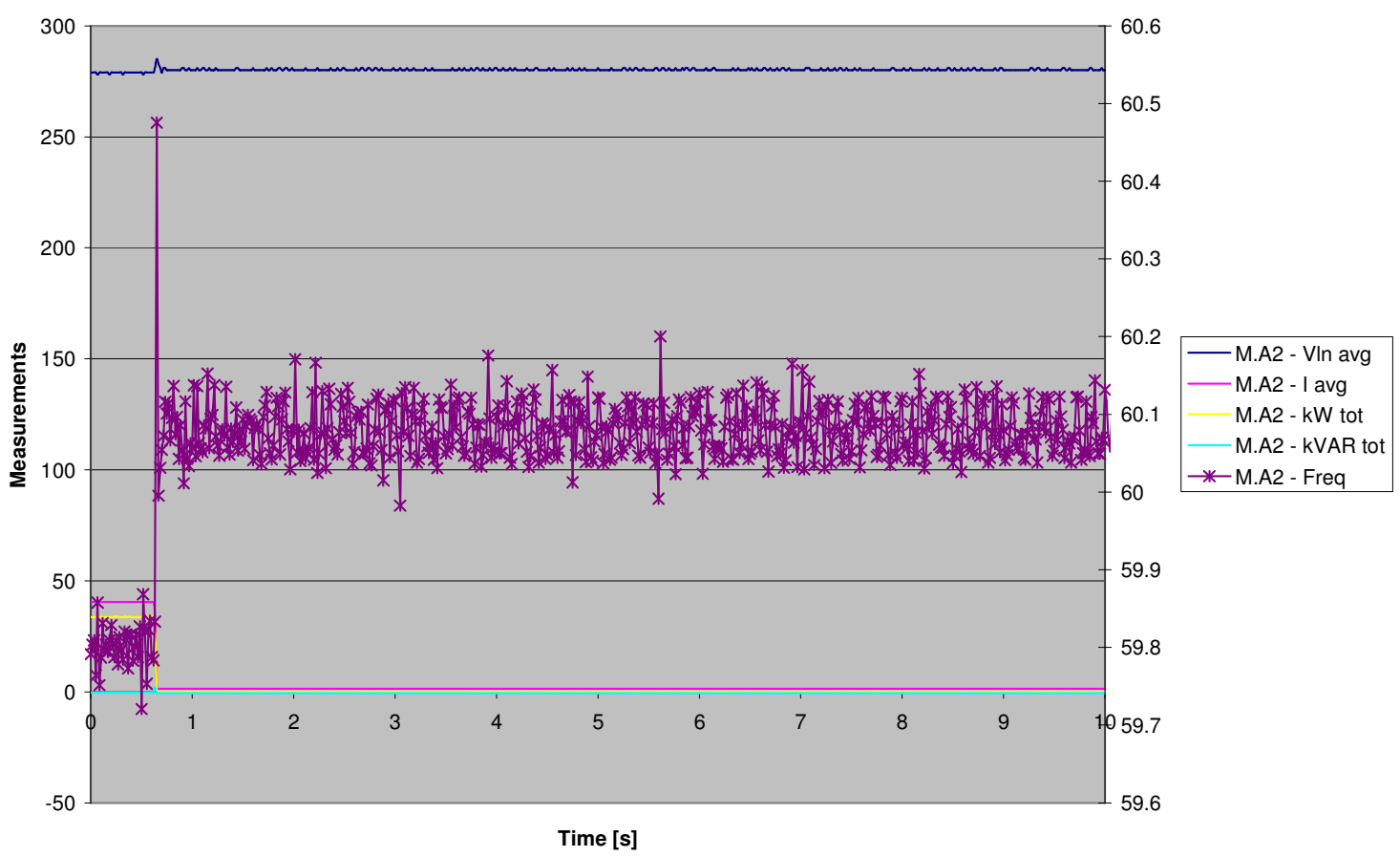

Test I.a.i B1 $=0-31 \mathrm{~kW}$ load step Genset Stalled Overspeed

\section{I.a.i B1 1st Stall Overspeed}

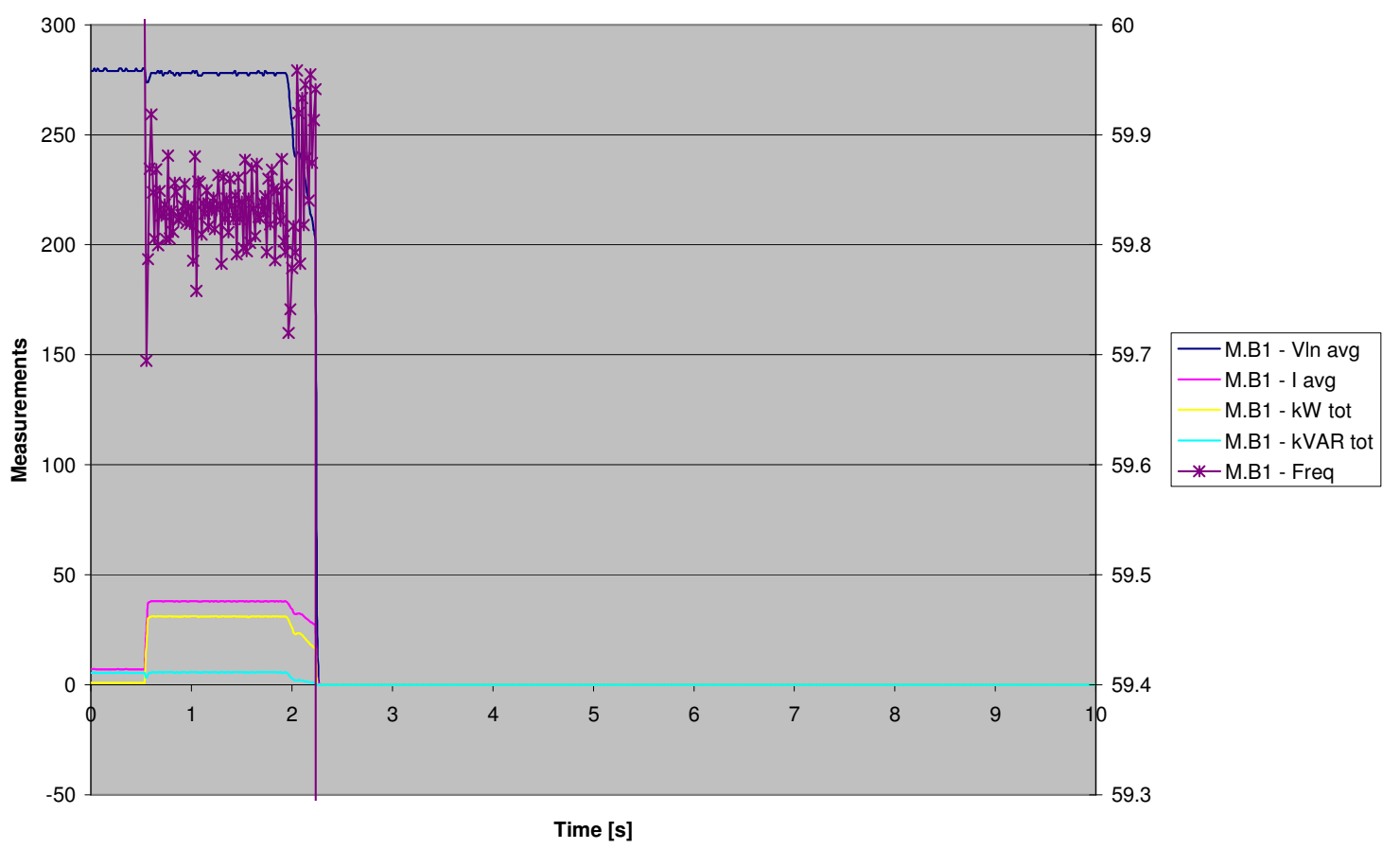


Test I.a.i B1 $=0-31 \mathrm{~kW}$ load step Genset Stalled Overspeed

\section{I.a.i B1 2nd Stall Overspeed}

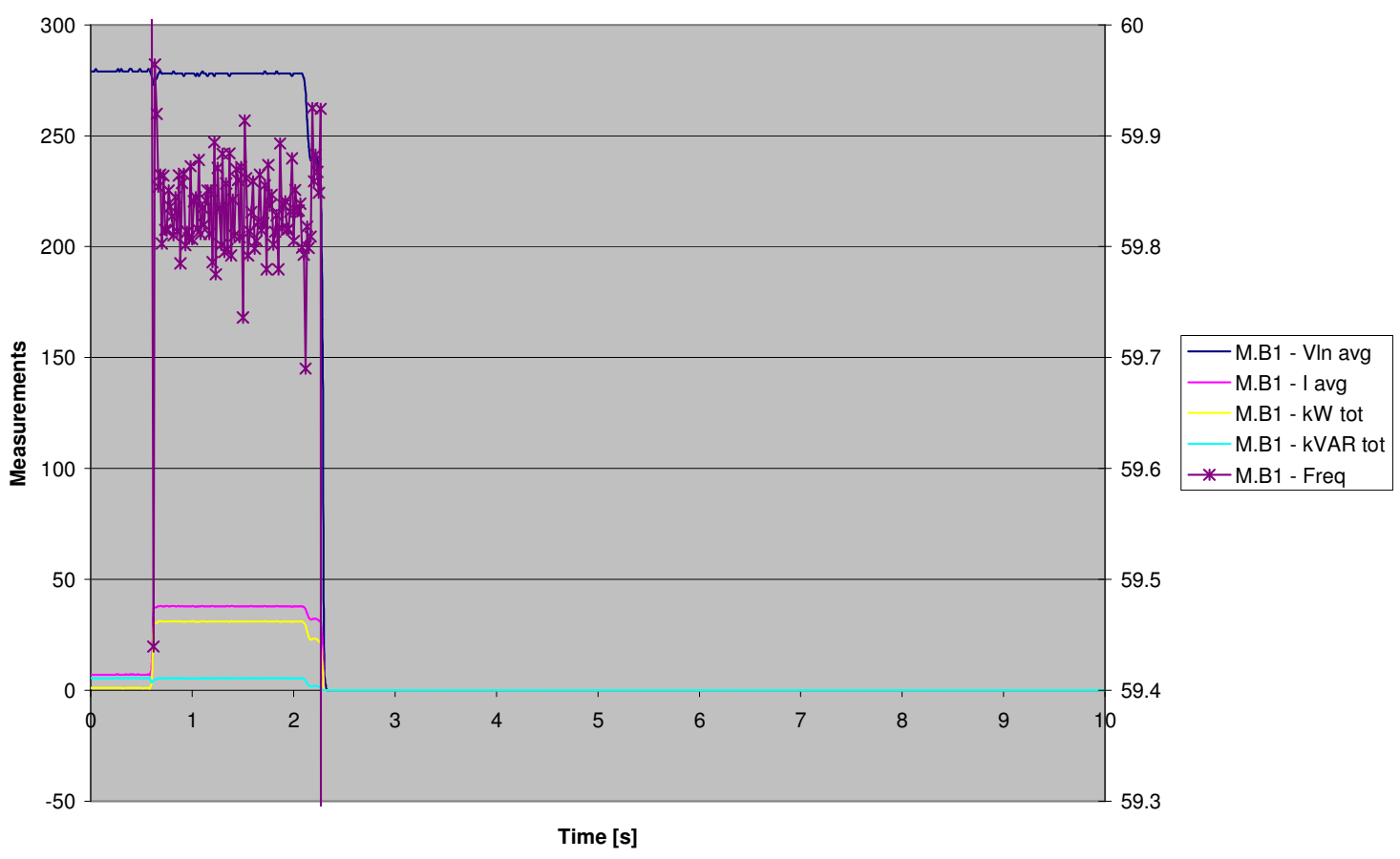

Test I.a.ii B1 $=0-54.4 \mathrm{~kW}$ load step

I.a.ii B1

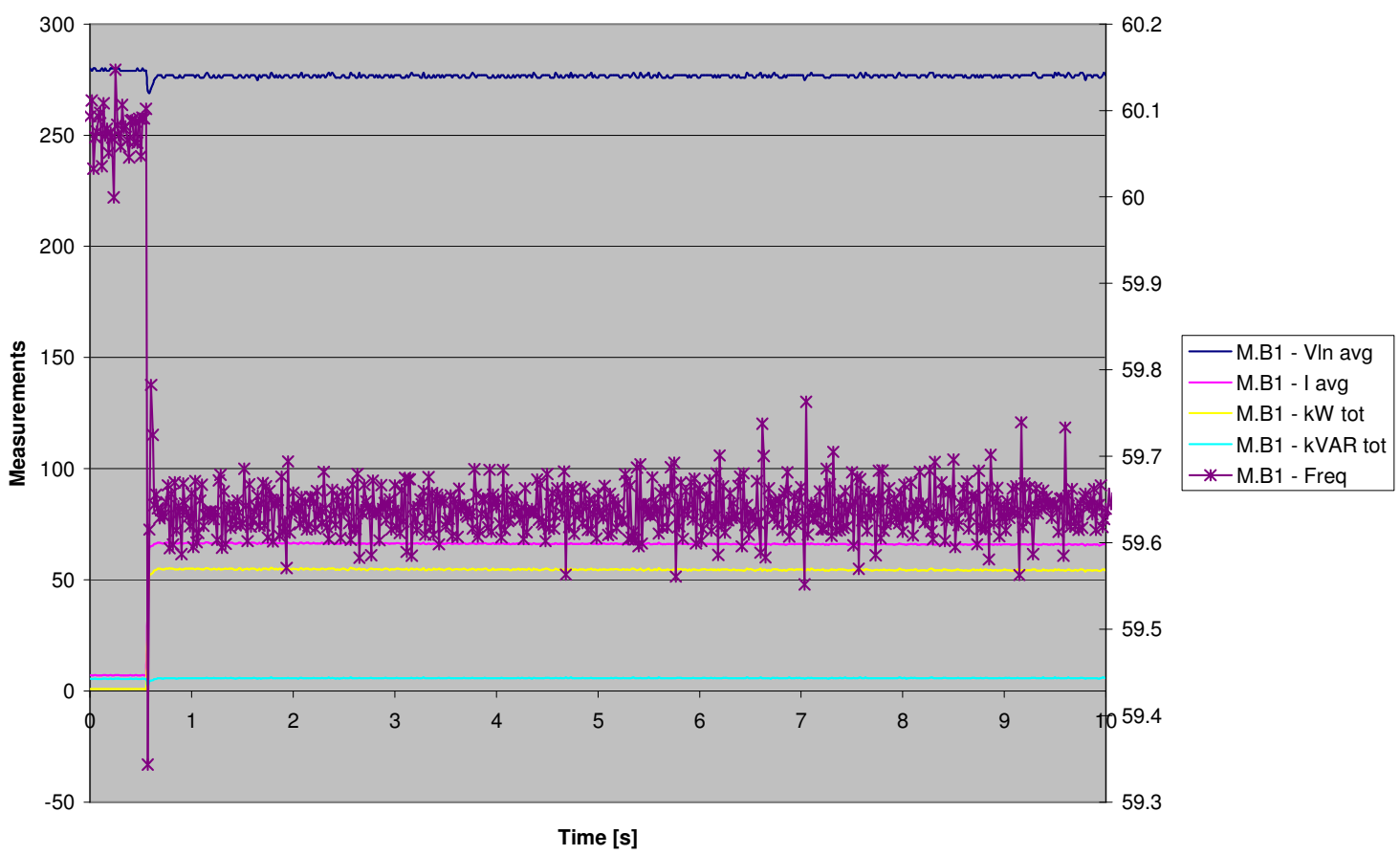


Test I.a.iii B1 $=16.7-43.5 \mathrm{~kW}$ load step Genset Stalled No Field Signal

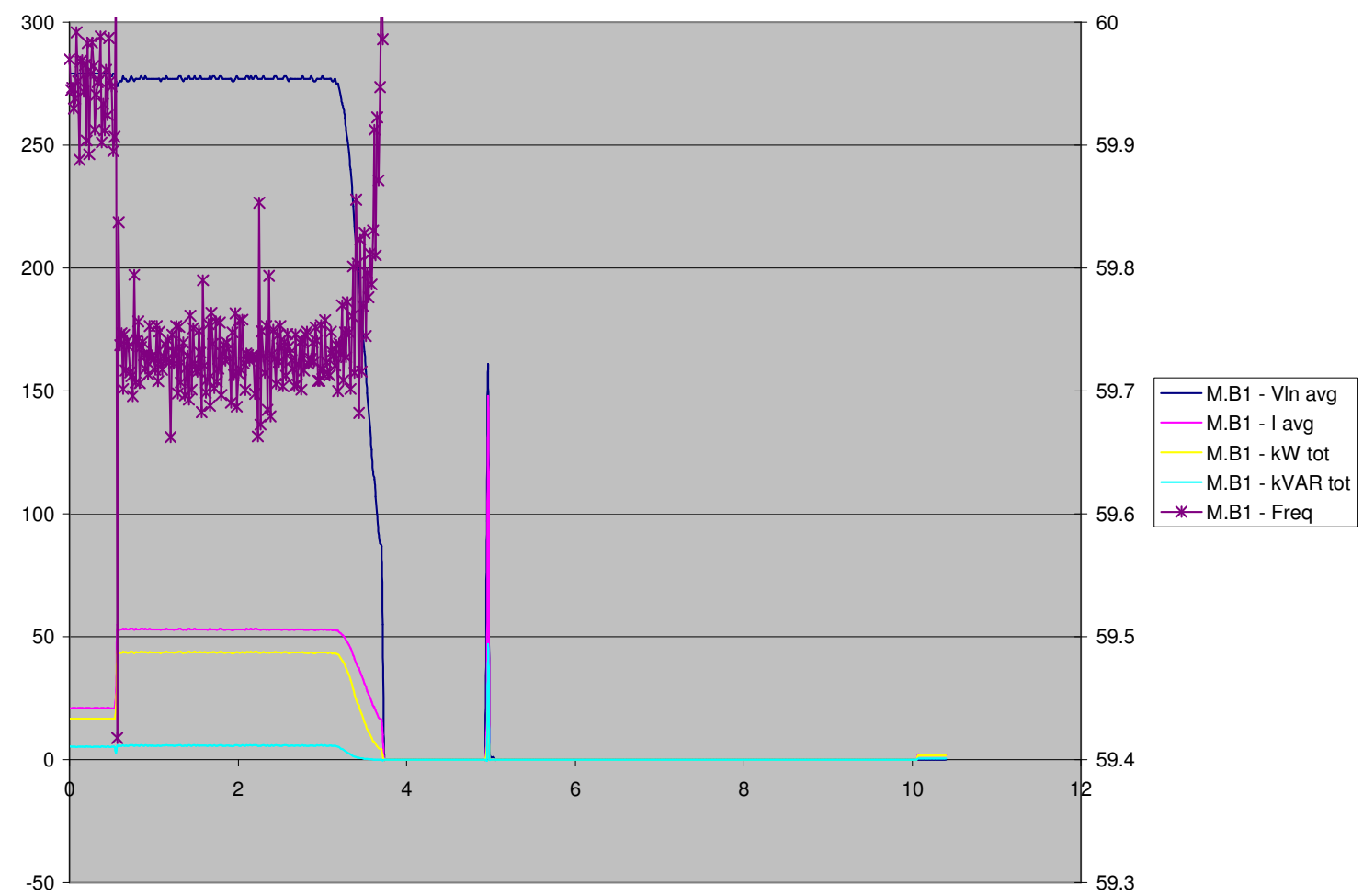

Test I.a.iii B1 $=16.7-44.4 \mathrm{~kW}$ load step

I.a.iii B1

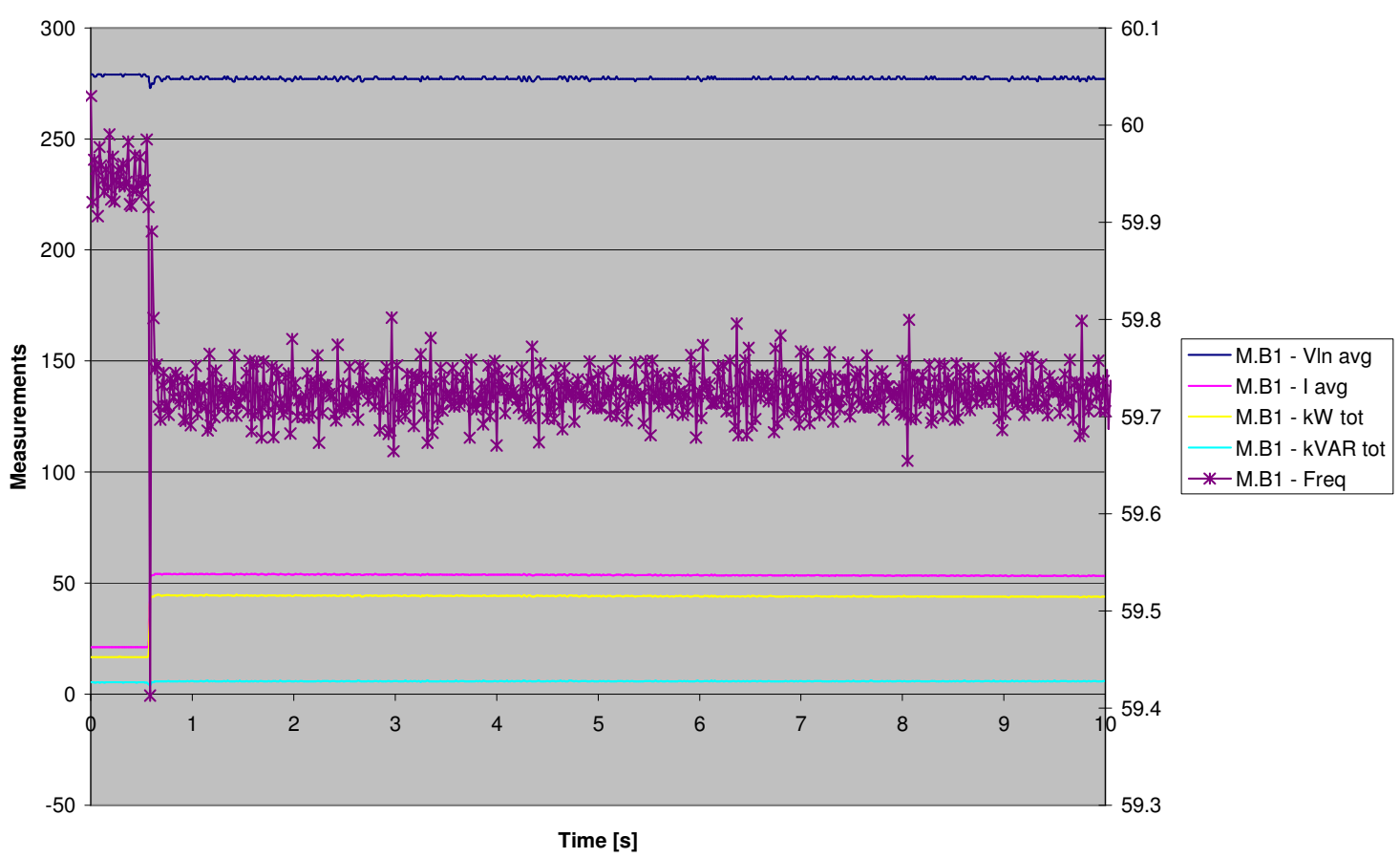


Test I.a.iv B1 $=29.2-52.9 \mathrm{~kW}$ load step No Field Sig

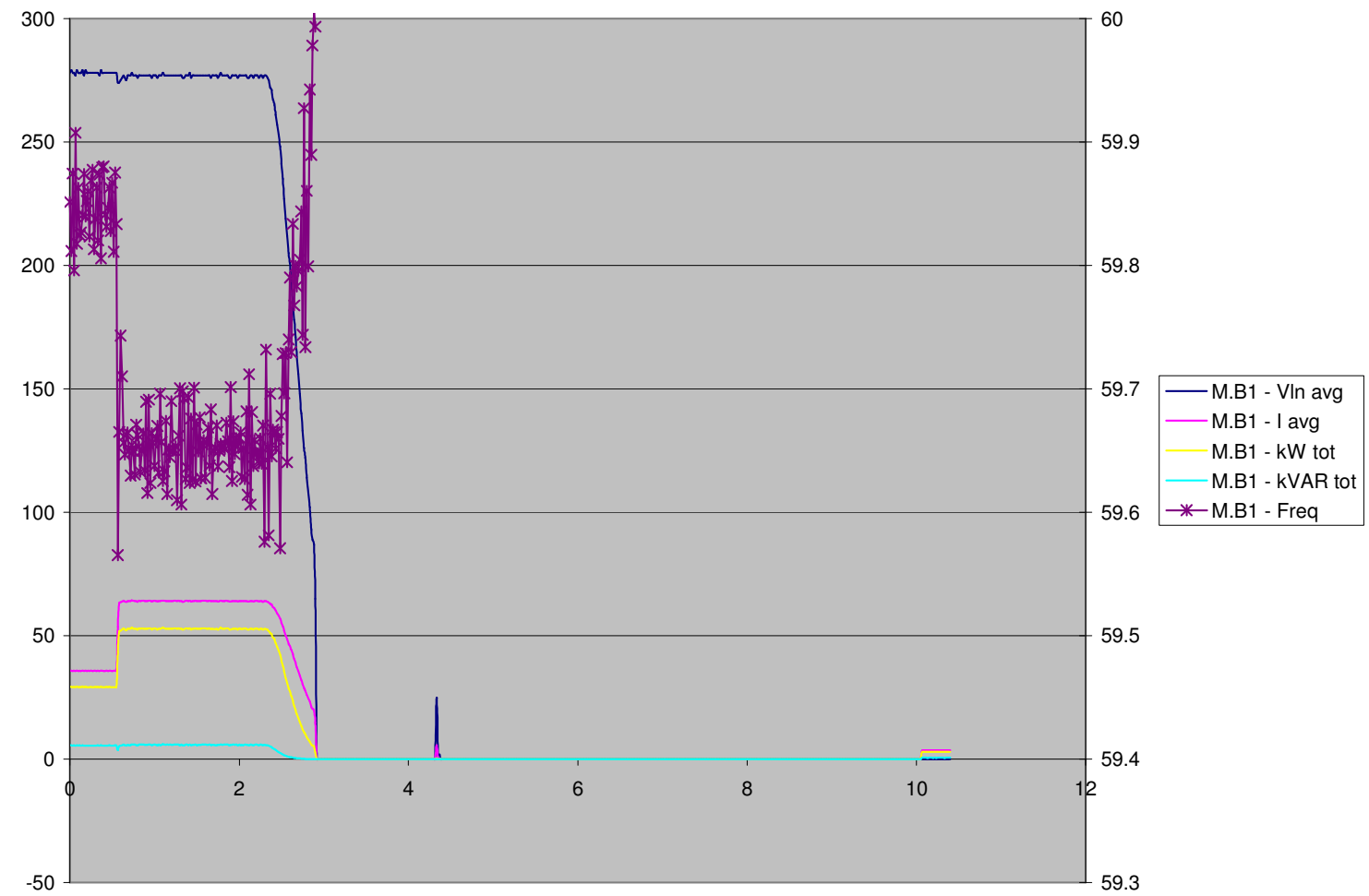

Test I.a.iv B1 $=29.5-53.1 \mathrm{~kW}$ load step No Field Sig

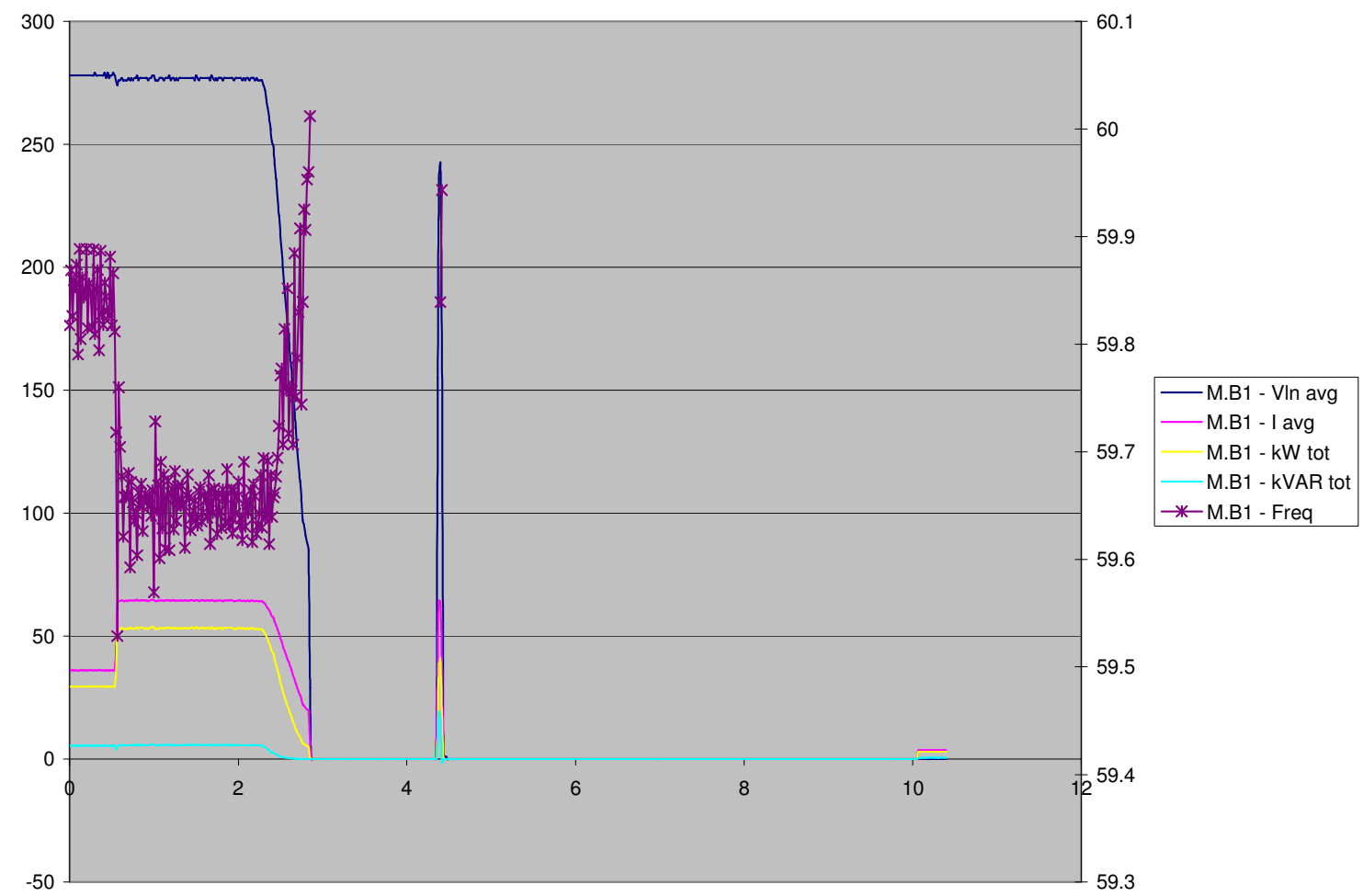


Test I.a.v B1 $=52.2-0 \mathrm{~kW}$ load step

I.a.v B1

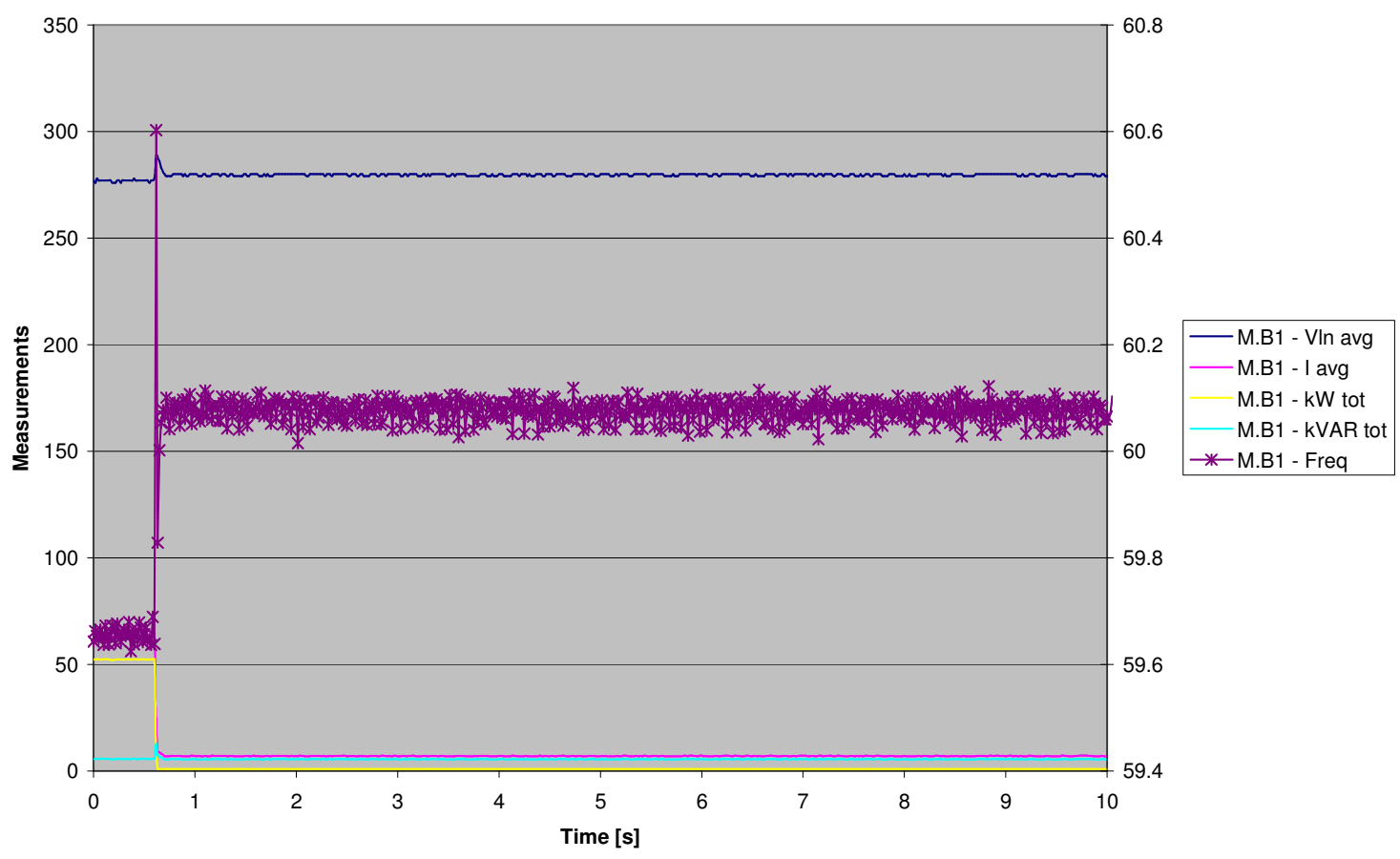

Test I.a.vi B1 $=29.2-0 \mathrm{~kW}$ load step

\section{I.a.vi B1}

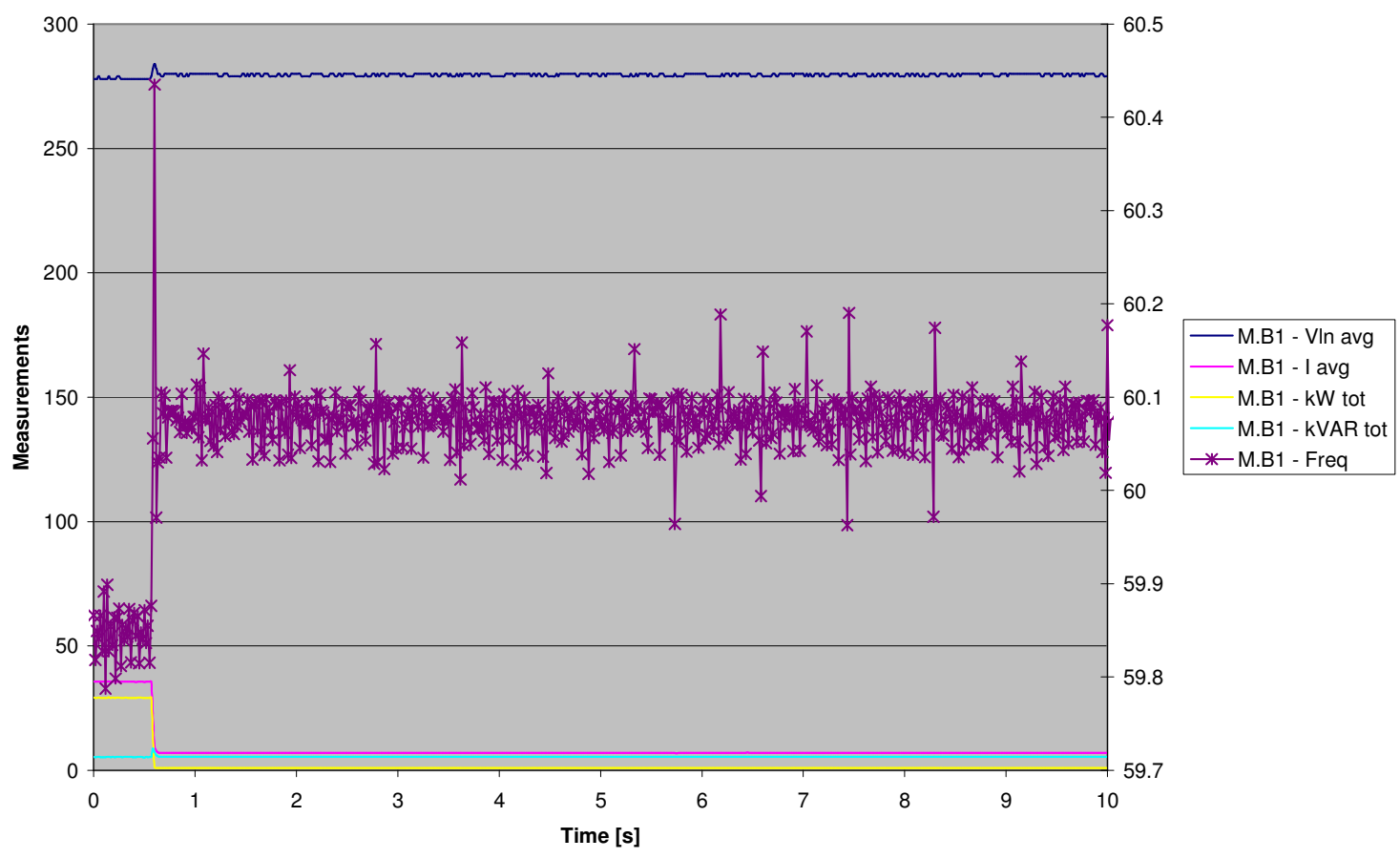




\section{Test Log for 9-20-06}

\begin{tabular}{|c|c|c|c|}
\hline \multirow{2}{*}{\multicolumn{4}{|c|}{$\frac{\text { Time }}{\text { Generator B1 with surge module }}$}} \\
\hline & & & \\
\hline 1:30 pm I.a.i & B1 & 5 & $0-30$ \\
\hline 1:33 pm I.a.ii & B1 & 5 & $0-60$ \\
\hline 1:36 pm I.a.iii & B1 & 5 & $15-50$ \\
\hline 1:40 pm I.a.iv & B1 & 5 & $30-60$ \\
\hline 1:43 pm I.a.v & B1 & 5 & $60-0$ \\
\hline 1:46 pm I.a.vi & B1 & 5 & $30-0$ \\
\hline \multicolumn{4}{|c|}{ Generator A 1 w/out surge module } \\
\hline 1:55 pm II.c.i (Part 1) & A1 & 4 & 50 \\
\hline 1:57 pm A1 Shutdown & A1 & 4 & 50 \\
\hline \multicolumn{4}{|c|}{ Generator A1 and A2 with surge module } \\
\hline 2:01 pm II.c.i (Part 1) & A1 & 4 & 50 \\
\hline
\end{tabular}




\section{Test Log for 9-21-06}

\begin{tabular}{|c|c|c|c|c|}
\hline \multirow{2}{*}{\multicolumn{5}{|c|}{$\frac{\text { Time }}{\text { Generator A1 with surge module }}$}} \\
\hline & & & & \\
\hline 7:49 am & I.a.i & A1 & 3 & $0-30$ \\
\hline 8:00 am & I.a.ii & A1 & 3 & $0-60$ \\
\hline 8:03 am & I.a.iii & A1 & 3 & $15-50$ \\
\hline $8: 19 \mathrm{am}$ & I.a.iv & A1 & 3 & $30-60$ \\
\hline $8: 22 \mathrm{am}$ & I.a.v & A1 & 3 & $60-0$ \\
\hline $8: 25 \mathrm{am}$ & I.a.vi & A1 & 3 & $30-0$ \\
\hline \multicolumn{5}{|c|}{ Generator A2 with surge module } \\
\hline $10: 18 \mathrm{am}$ & Trigge & & & \\
\hline $10: 21 \mathrm{am}$ & I.a.i & A2 & 4 & $0-30$ \\
\hline $10: 24 \mathrm{am}$ & I.a.ii & A2 & 4 & $0-60$ \\
\hline $10: 28 \mathrm{am}$ & I.a.iii & A2 & 4 & $15-50$ \\
\hline 10:31 am & I.a.iv & A2 & 4 & $30-60$ \\
\hline $10: 34 \mathrm{am}$ & I.a.v & A2 & 4 & $60-0$ \\
\hline $10: 37 \mathrm{am}$ & I.a.vi & A2 & 4 & $30-0$ \\
\hline
\end{tabular}


Attachment 13

\section{Test Results for 10-11-06}

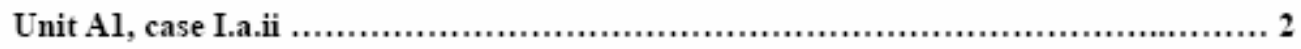

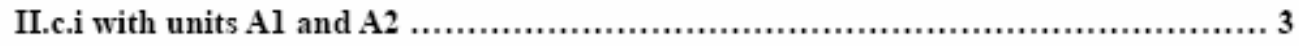

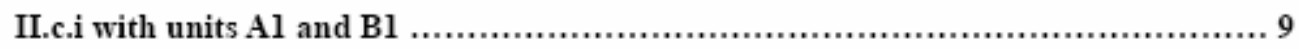

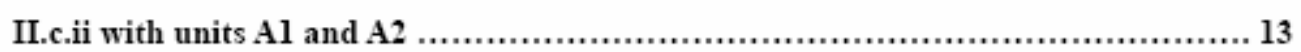

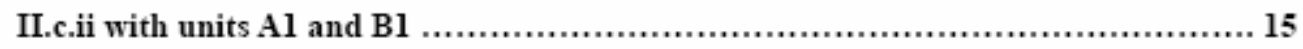

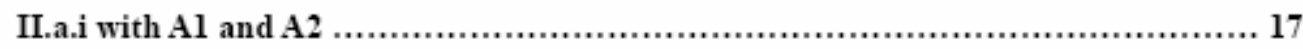

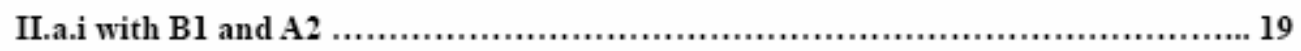

Event to reach III.a.l with unit $A 1$ and $A 2$ (connection of $A 1$ in feeder mode) .........21

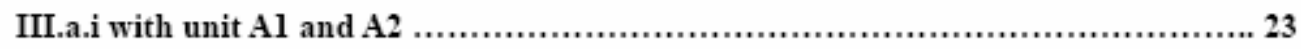

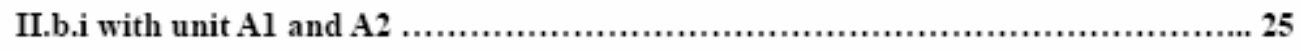

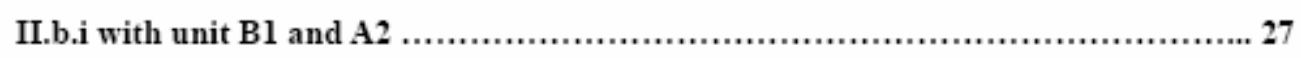

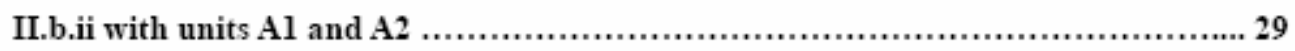

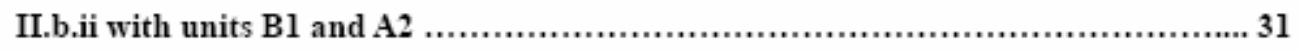

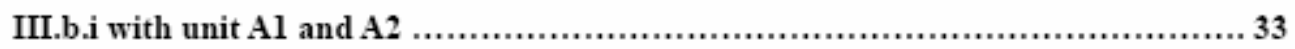




\section{Unit Al, case I.a.ii}

Single Unit A1 $0-60 \mathrm{kH}$

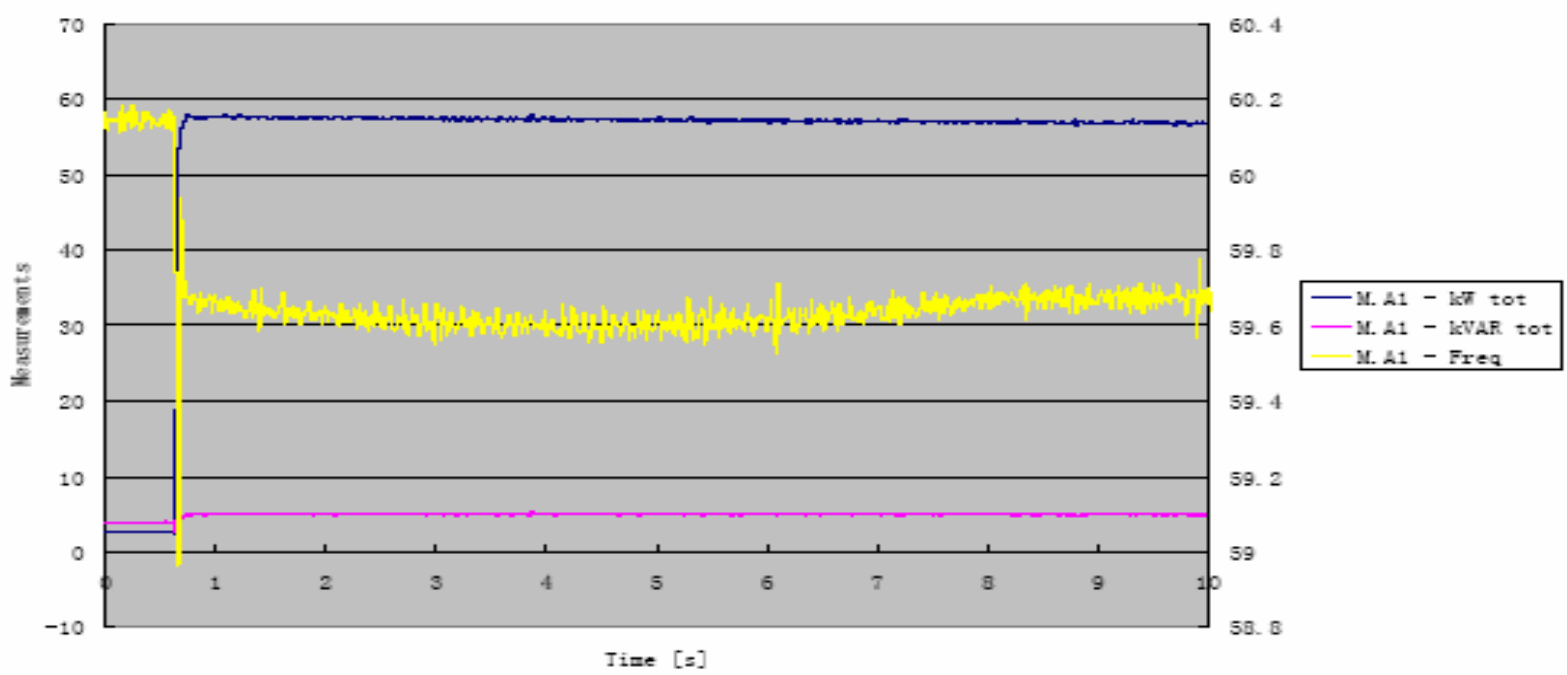

Sing1e Unit A1 $0-60 \mathrm{~kJ}$

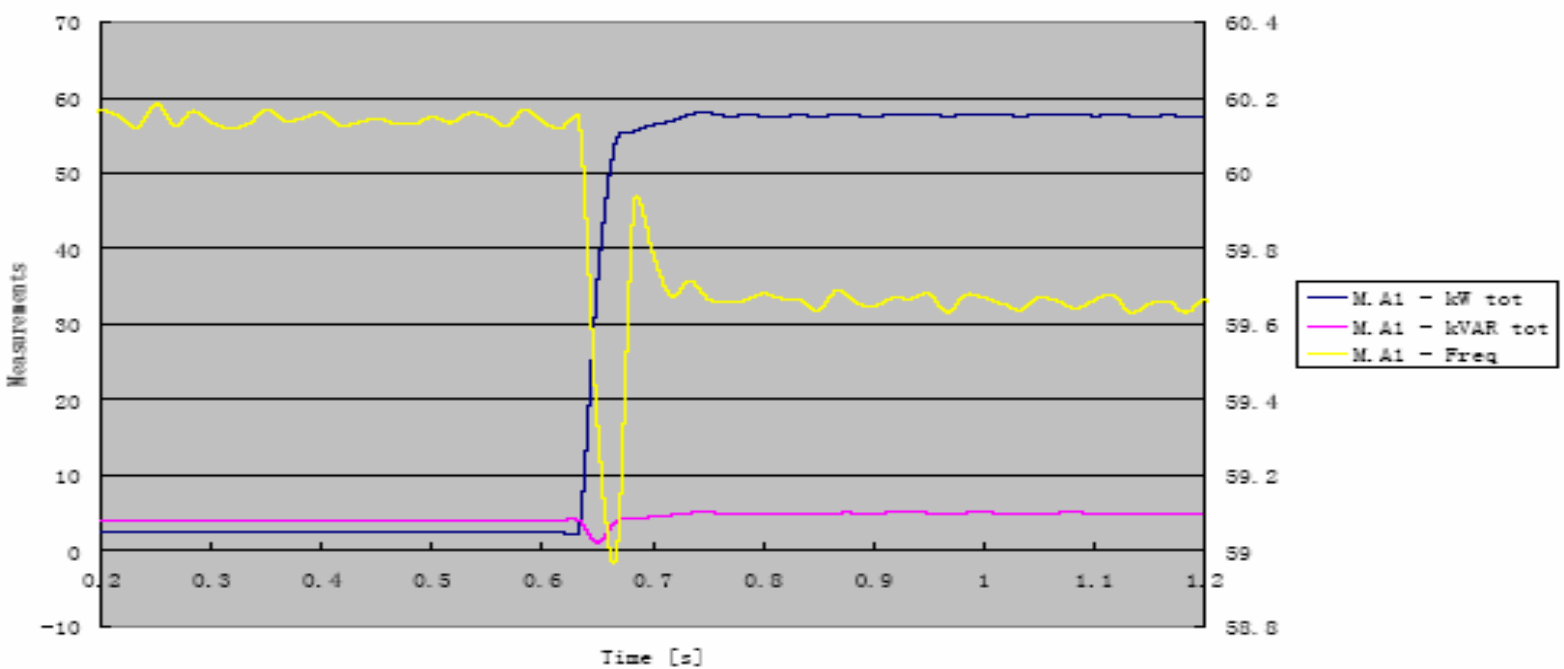




\section{II.c.i with units A1 and A2}

II. C. i (Part 1) A1, A2

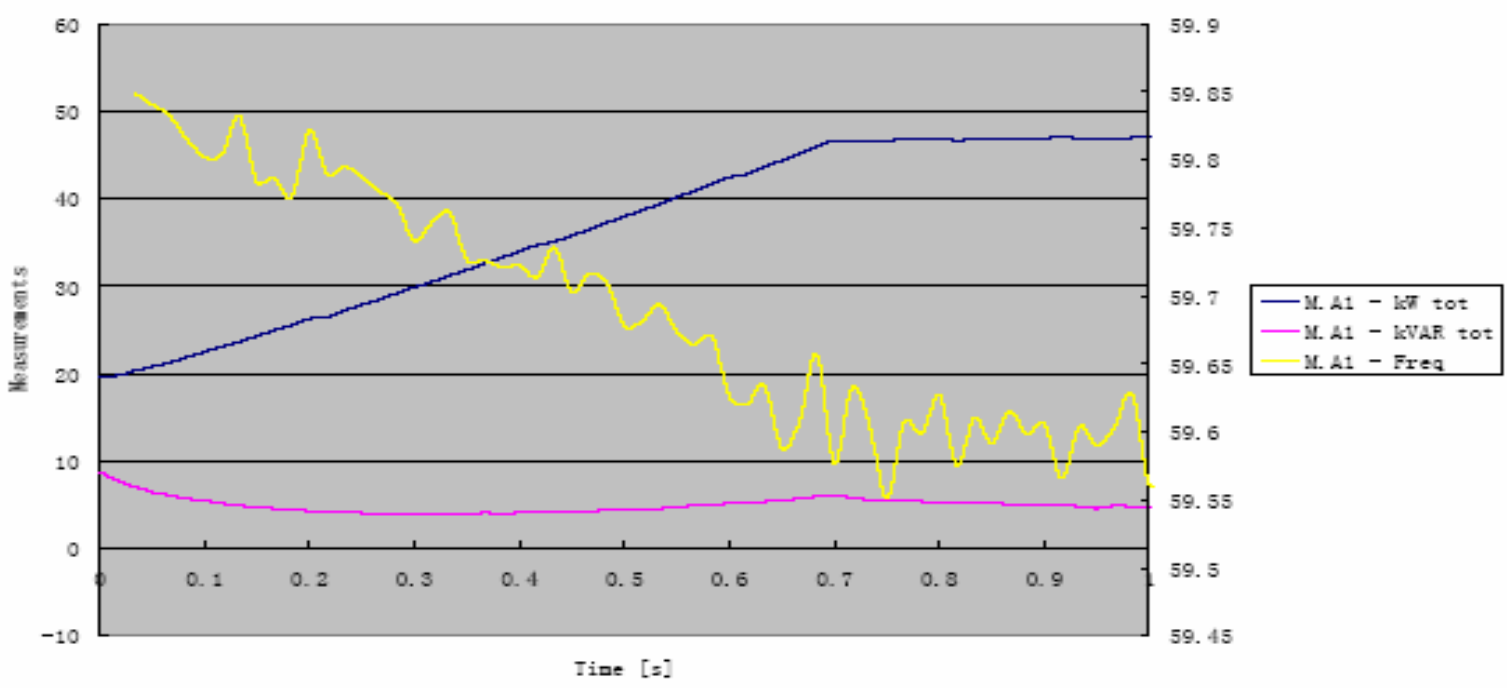

II. c. i (Part 1) A1, A2

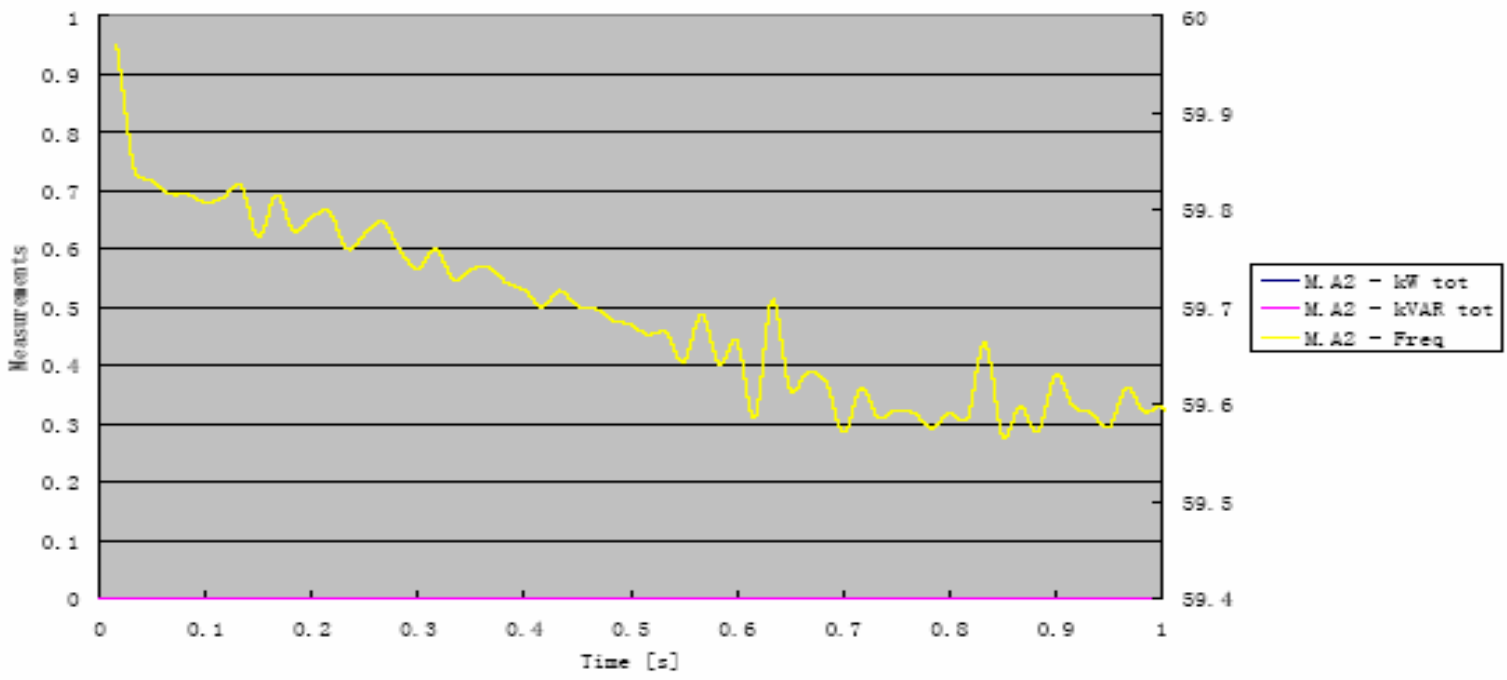


II. c. i (Part 1) A1, A2

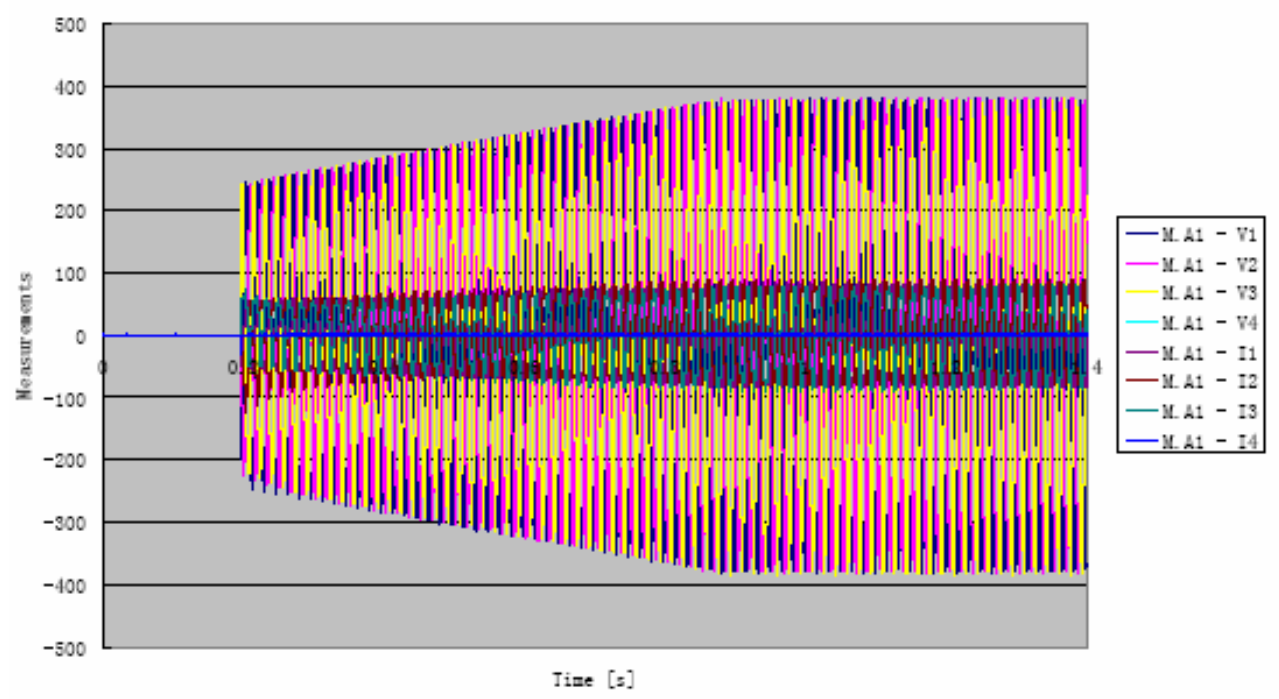

II. c. i (Part 1) A1, A2

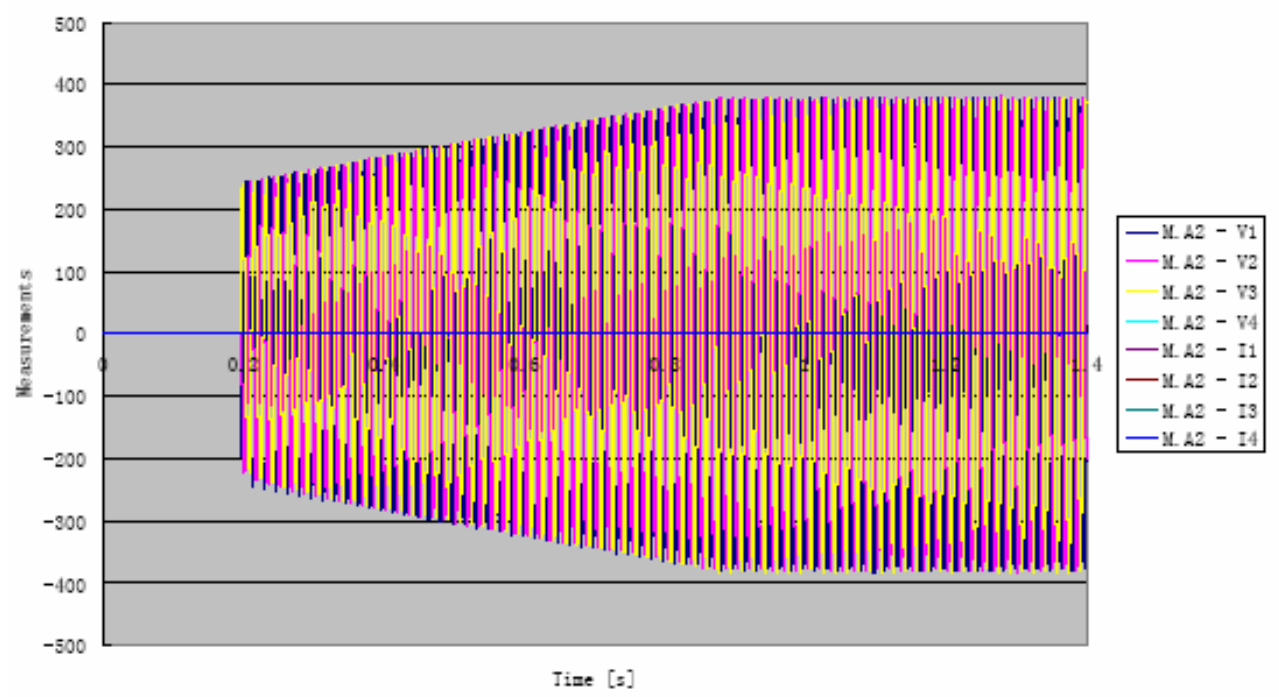


II. c. i (Part 2) A1, A2

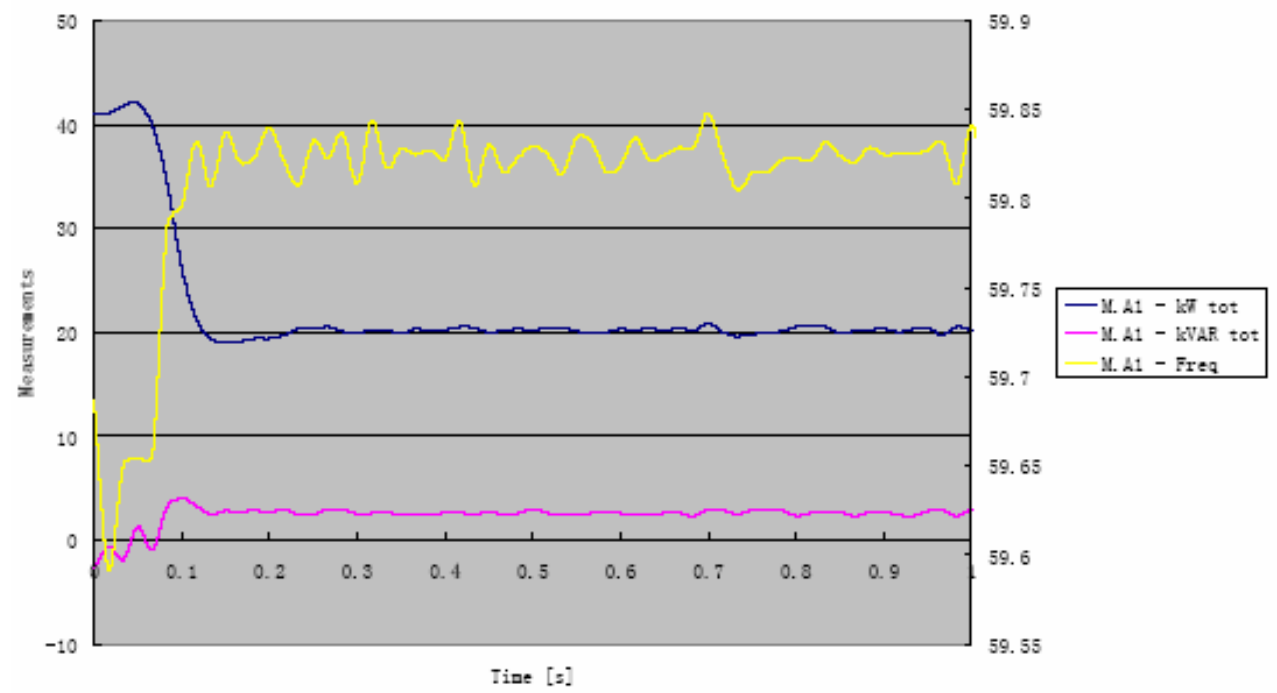

II. C.i (Part 2) A1, A2

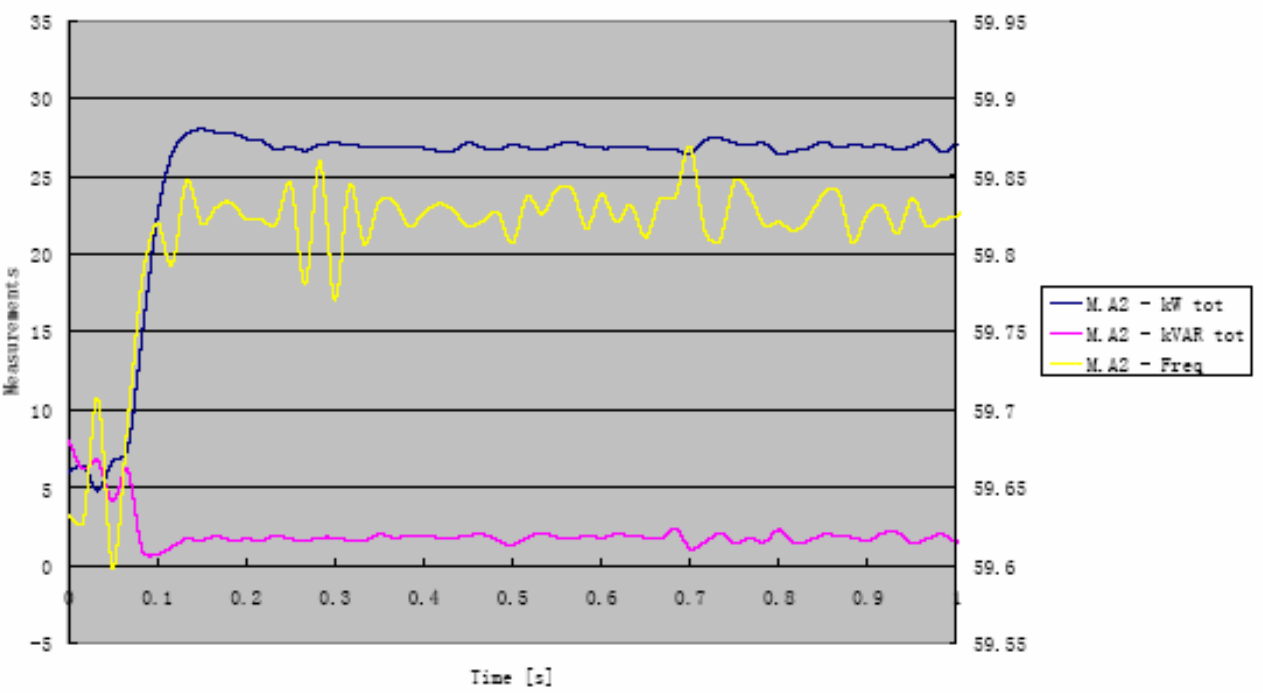


II. c.i (Part 2) A1, A2

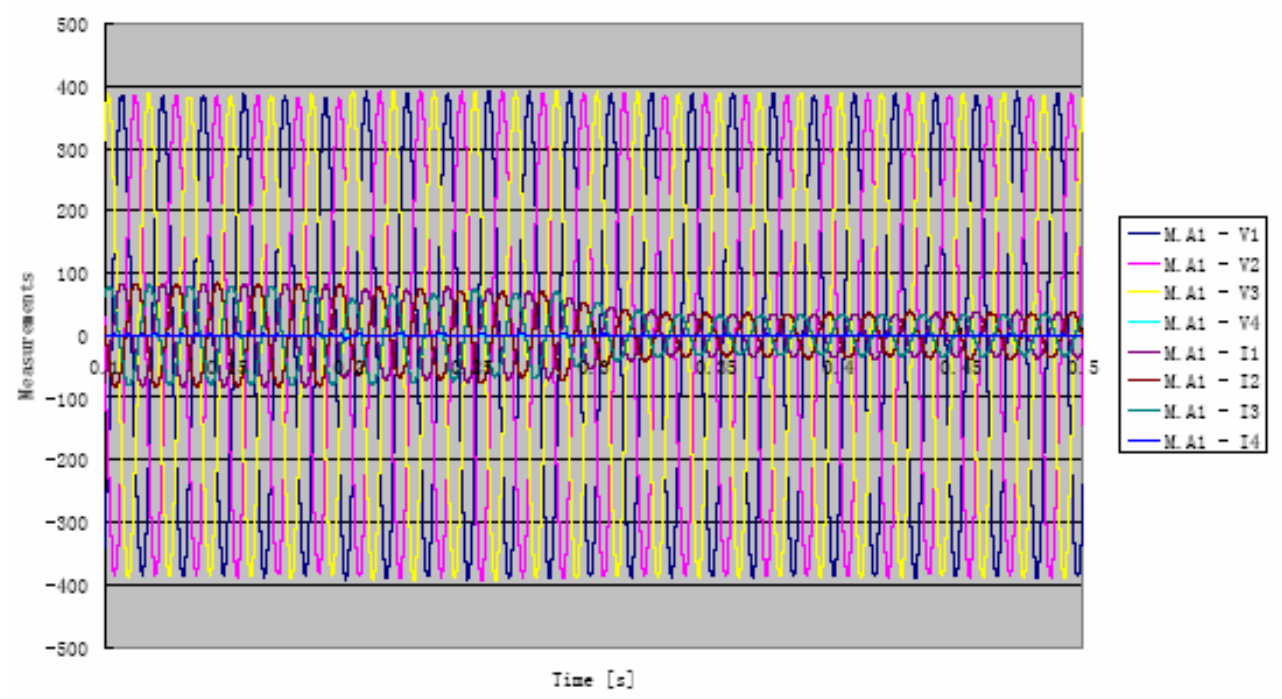

II.c.i (Part 2) A1, A2

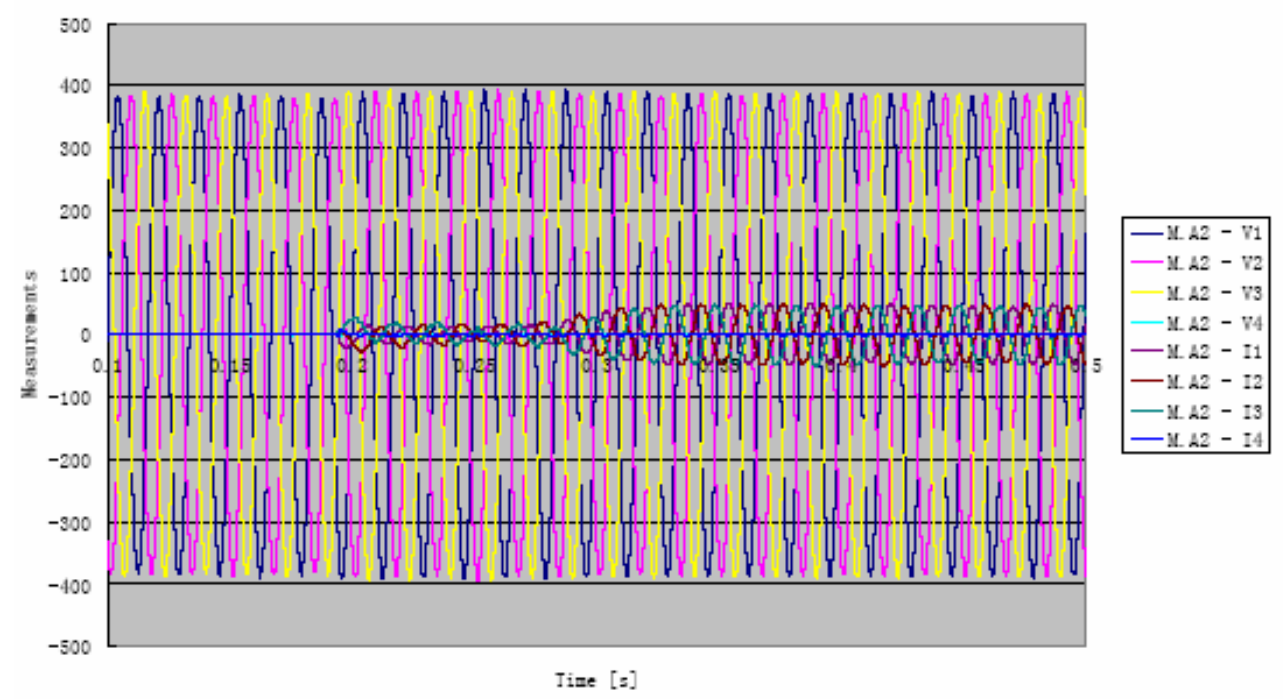


II.c. i (Part 3) A1, A2

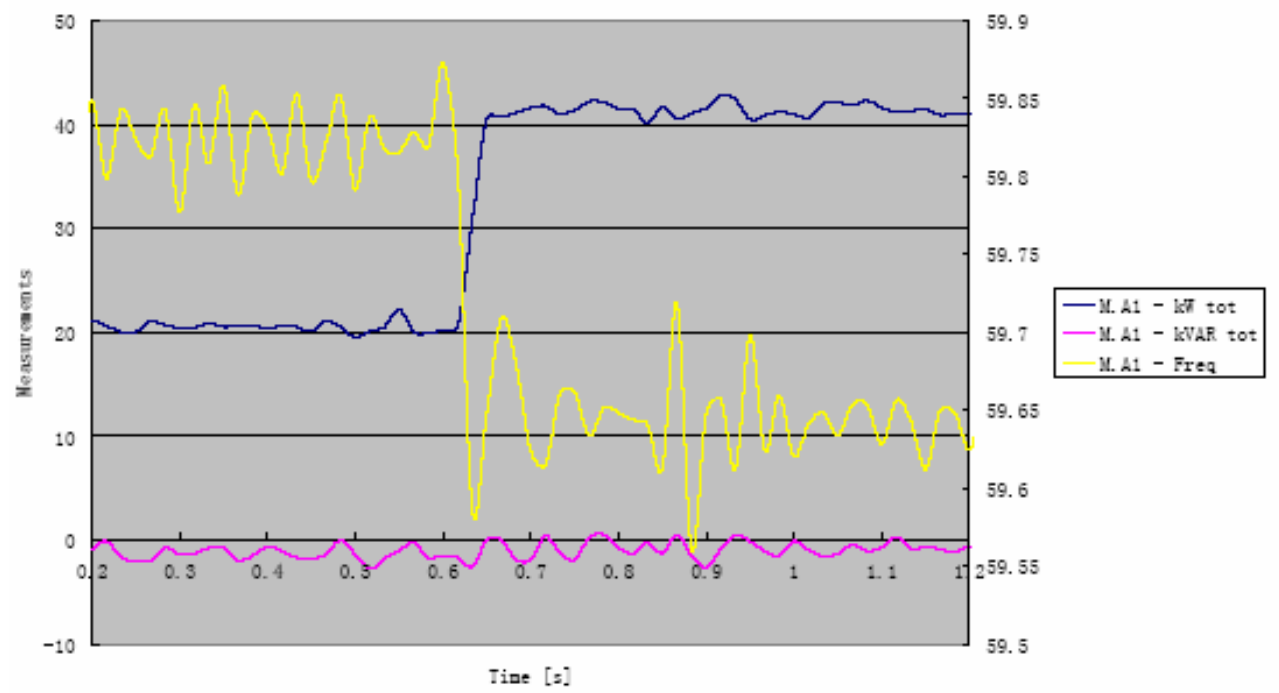

II. c. i (Part 3) A1, A2

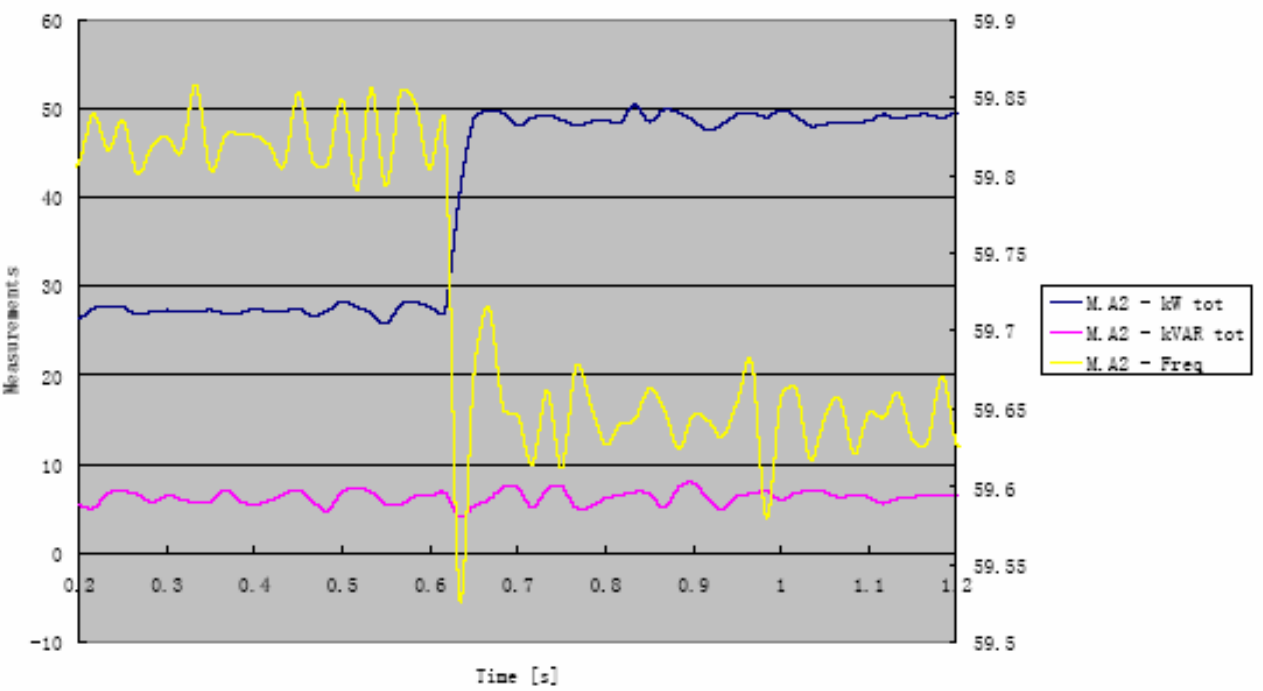


II. C. i (Part 3) A1, A2

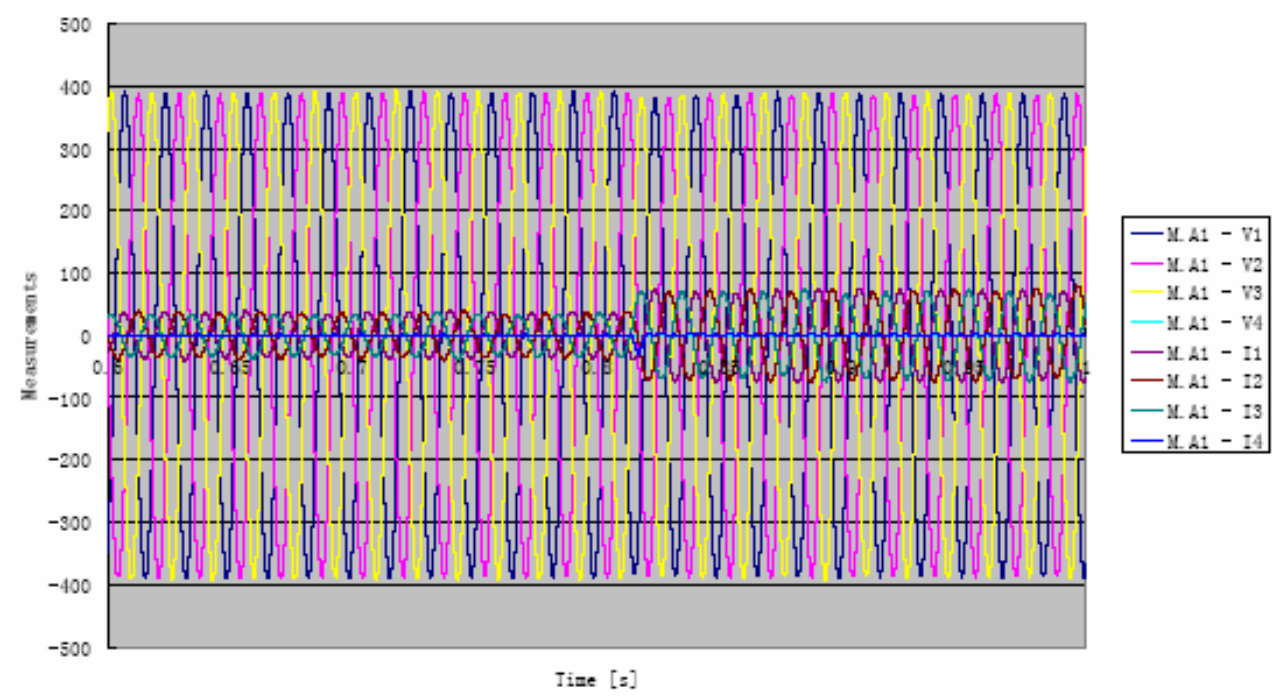

II.c.i (Part 3) A1, A2

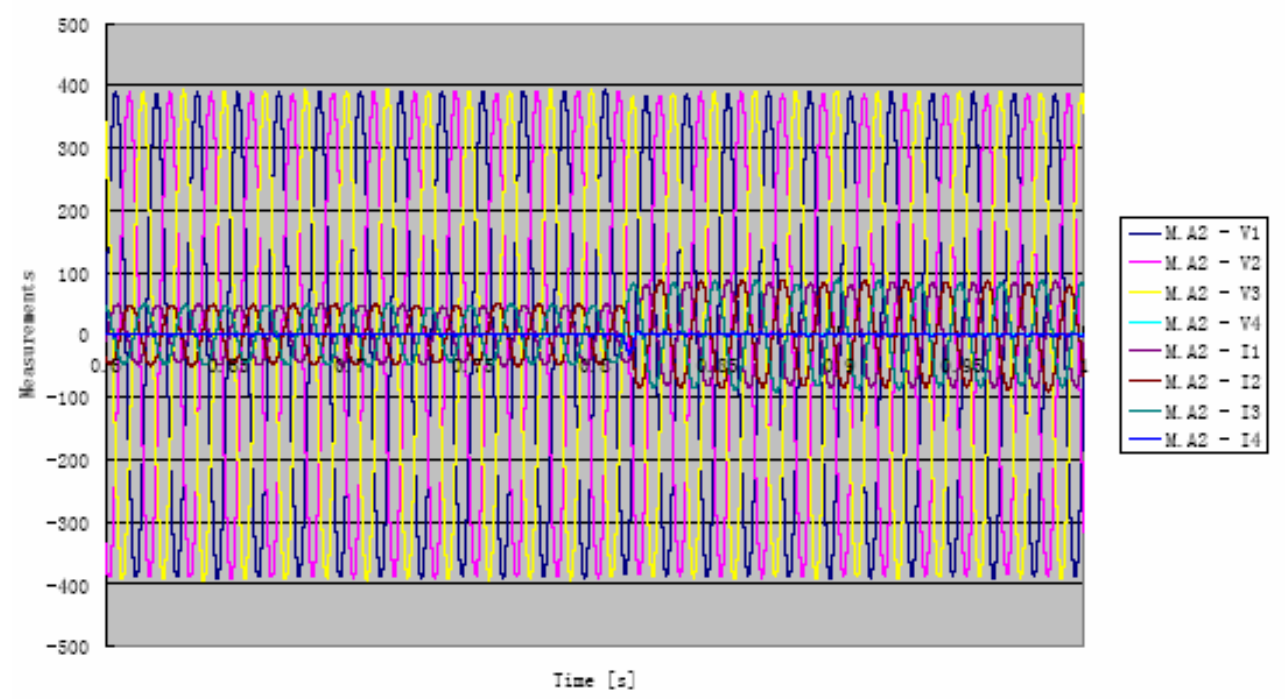




\section{II.c.i with units Al and Bl}

II. C. i (Part 2) A1, B:

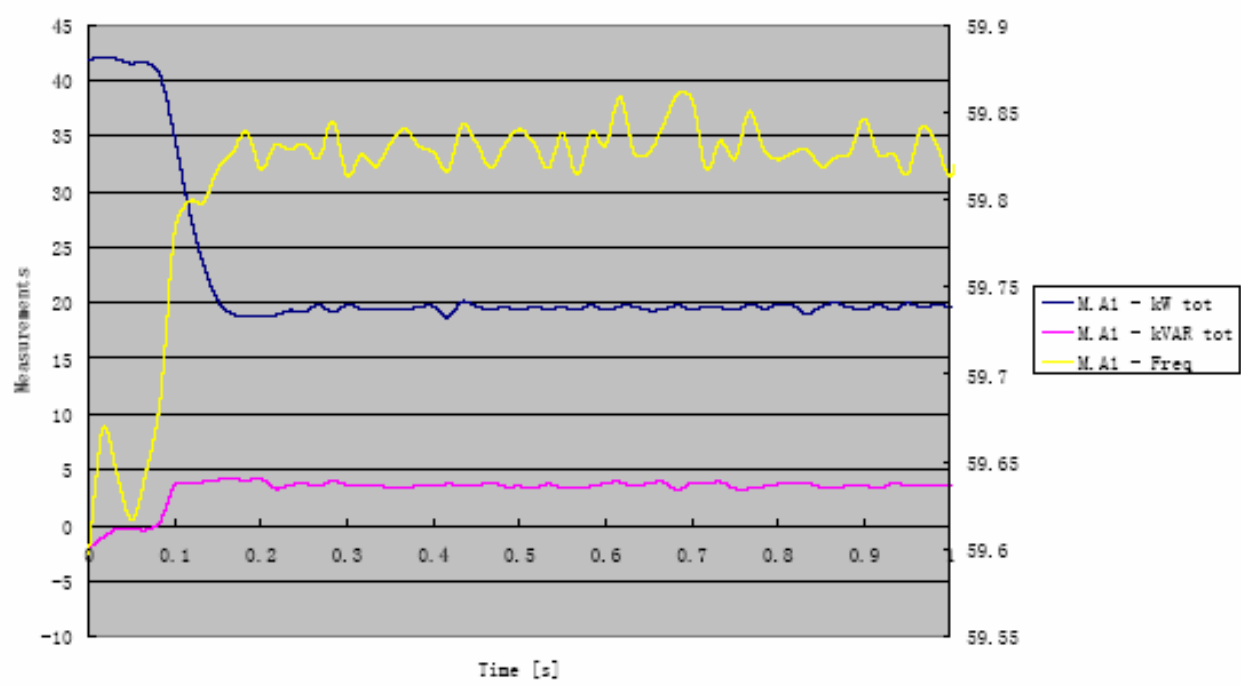

II. C. i (Part 2) A1, B:

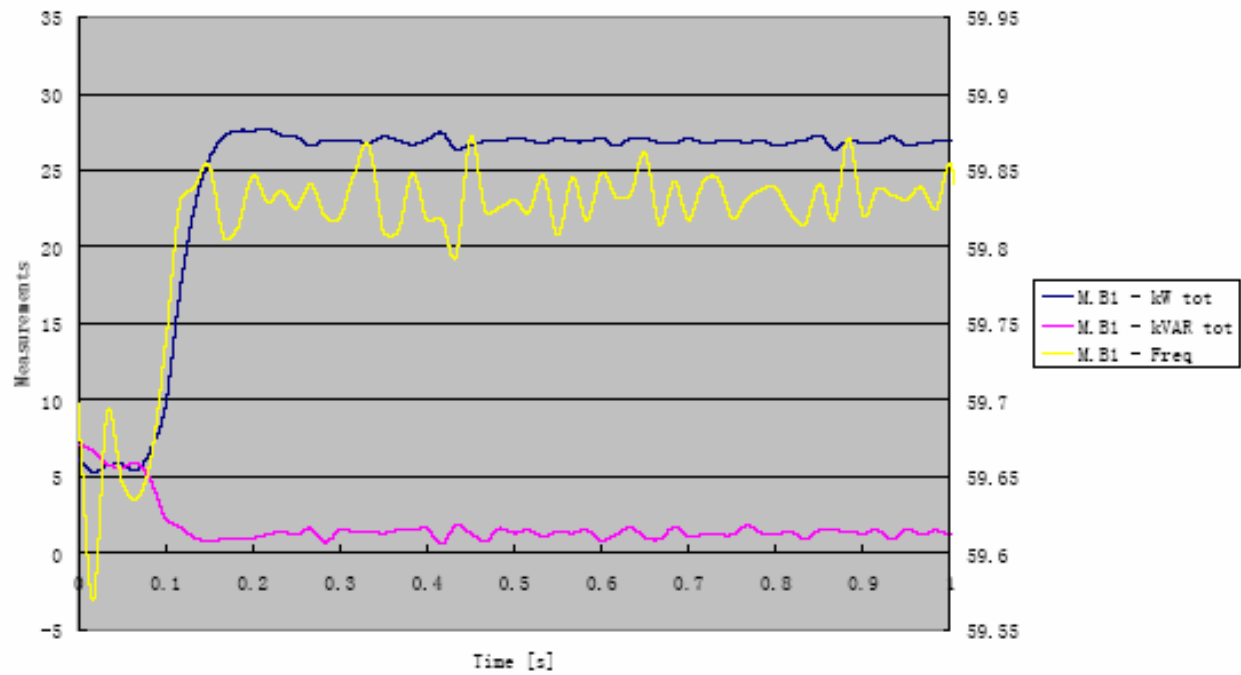


II. C. i (Part 2) A1, B1

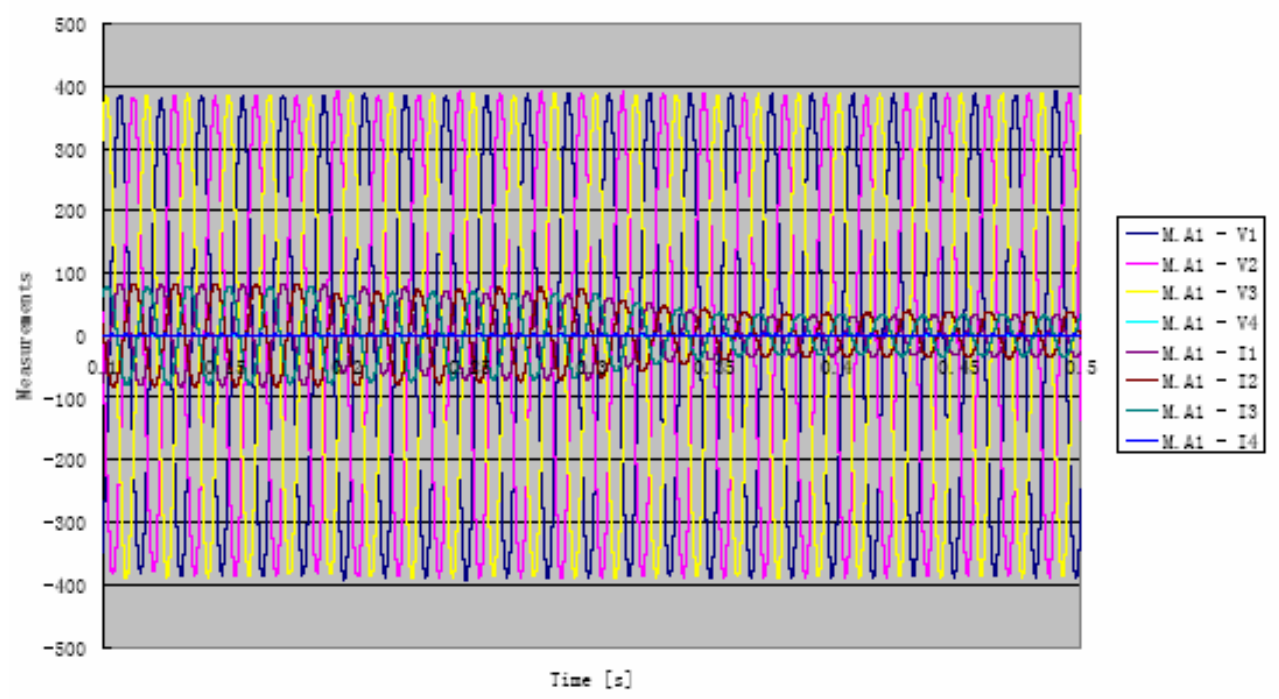

II. C. i (Part 2) A1, B1

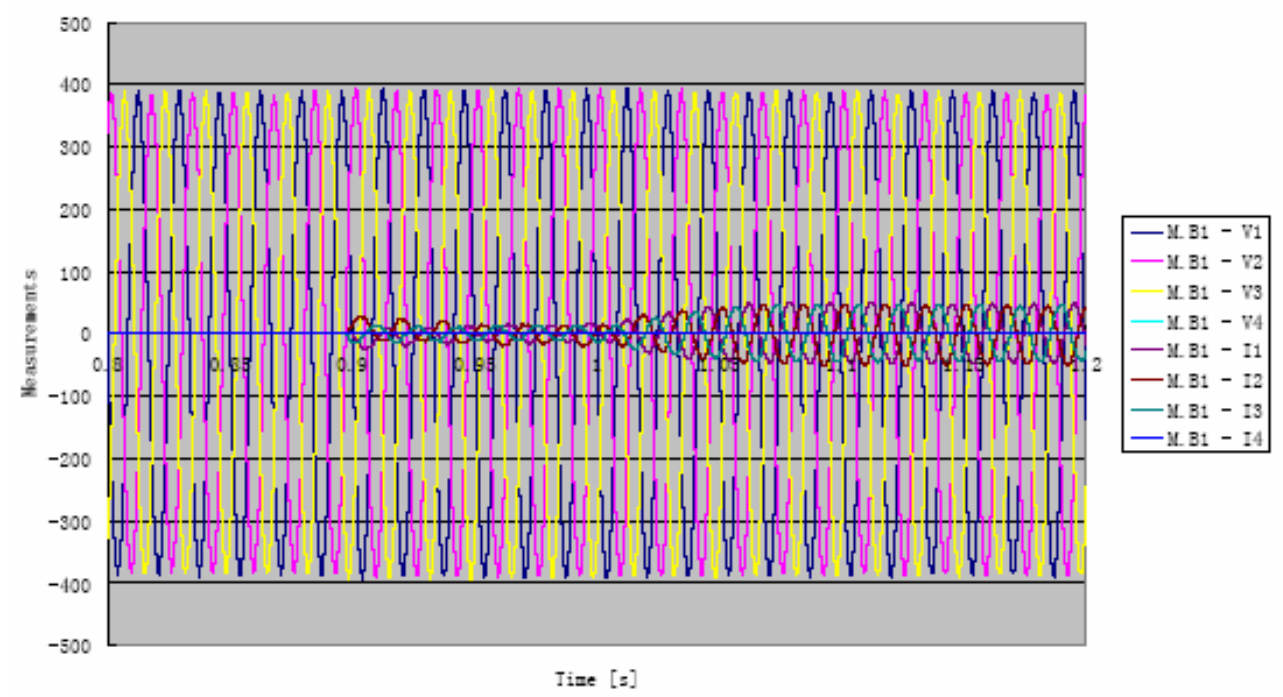


II. C. i (Part 3) A1, B1

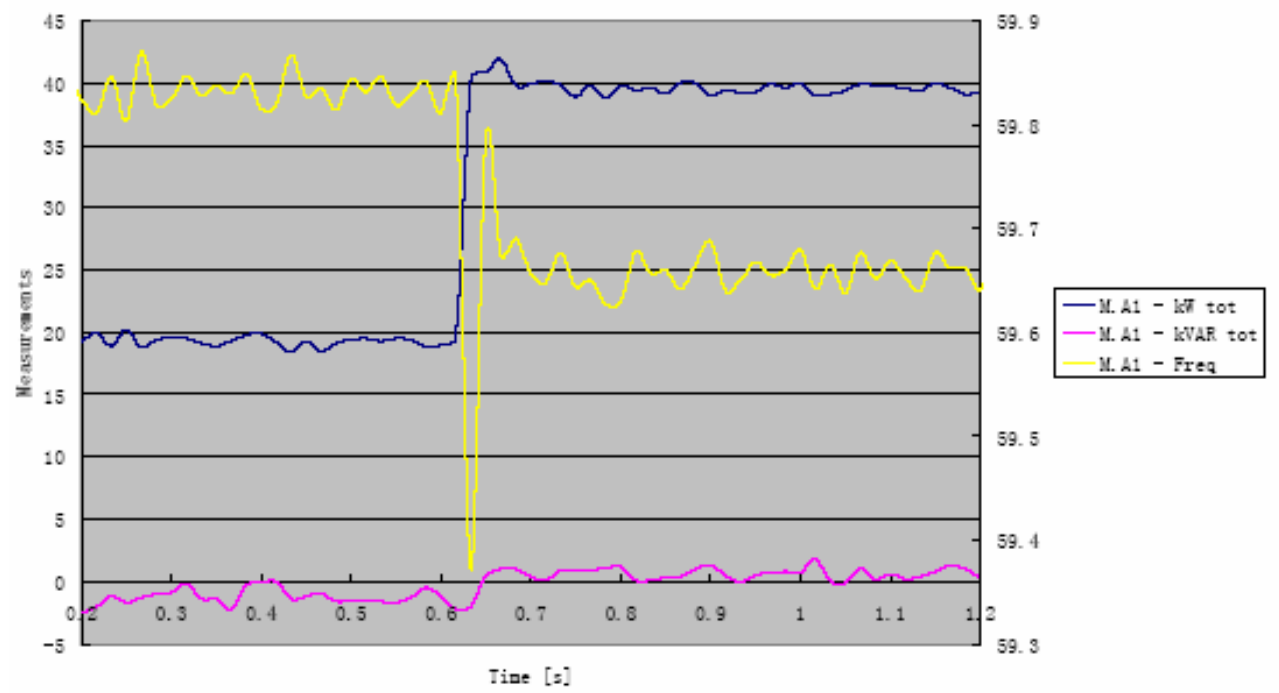

II. C. i (Part 3) A1, B1

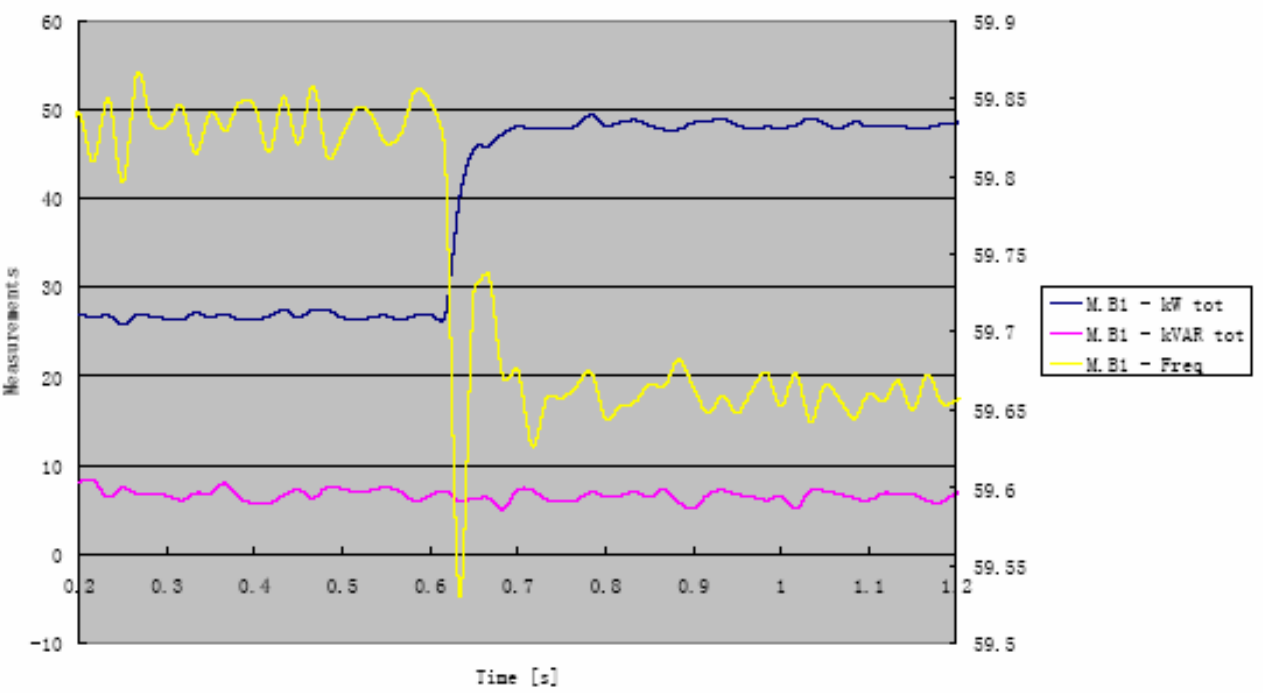


II. c. i (Part 3) A1, B1

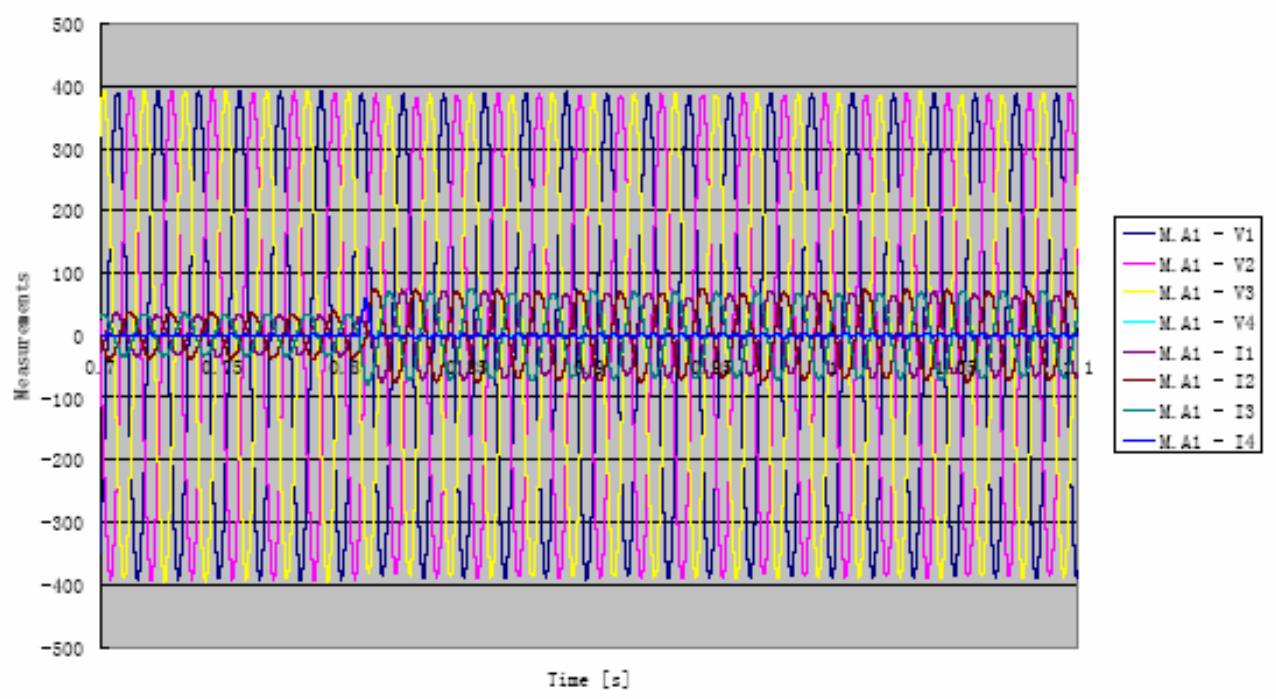

Event non captured 


\section{II.c.ii with units $\mathrm{Al}$ and $\mathrm{A} 2$}

II. c. ii A1, $A 2$

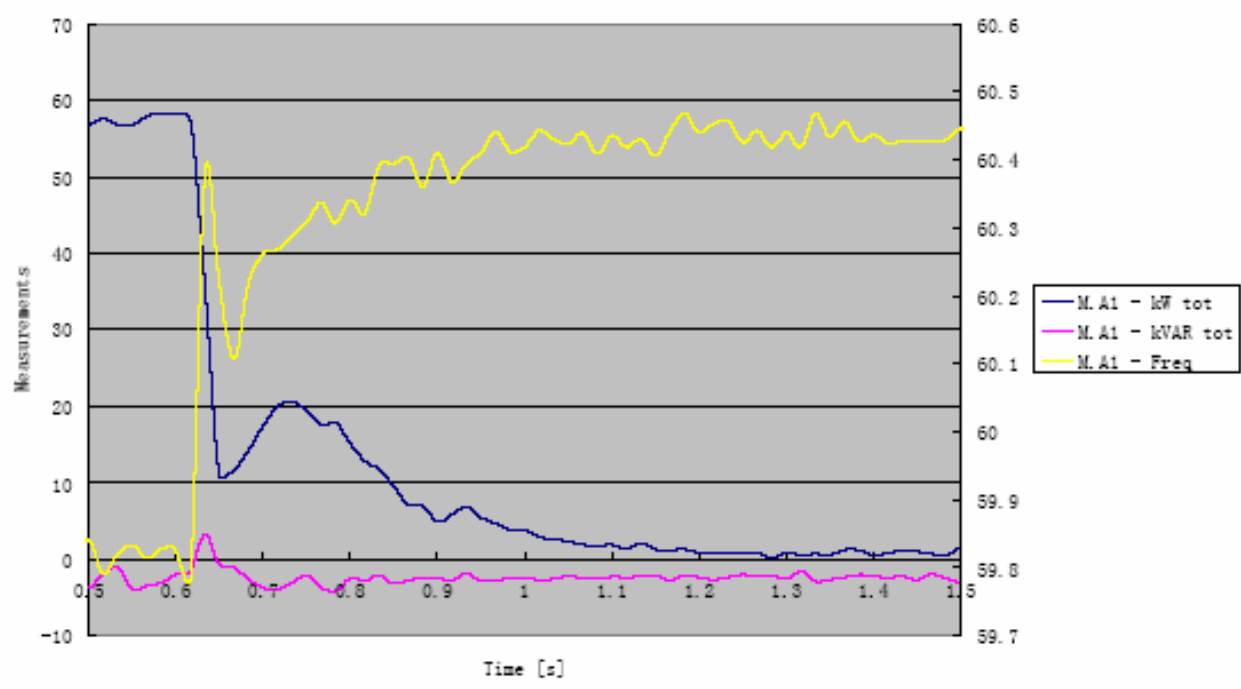

II. C. ii A1, A2

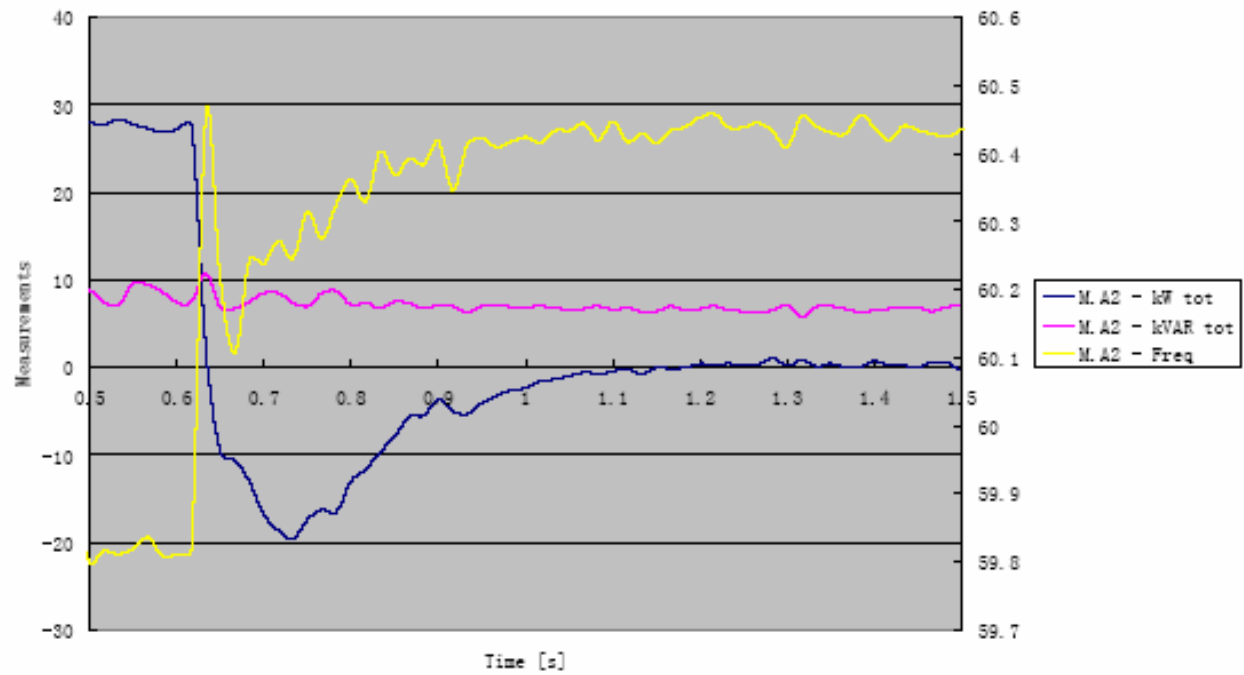


II. c. ii A1, A2 Part 1

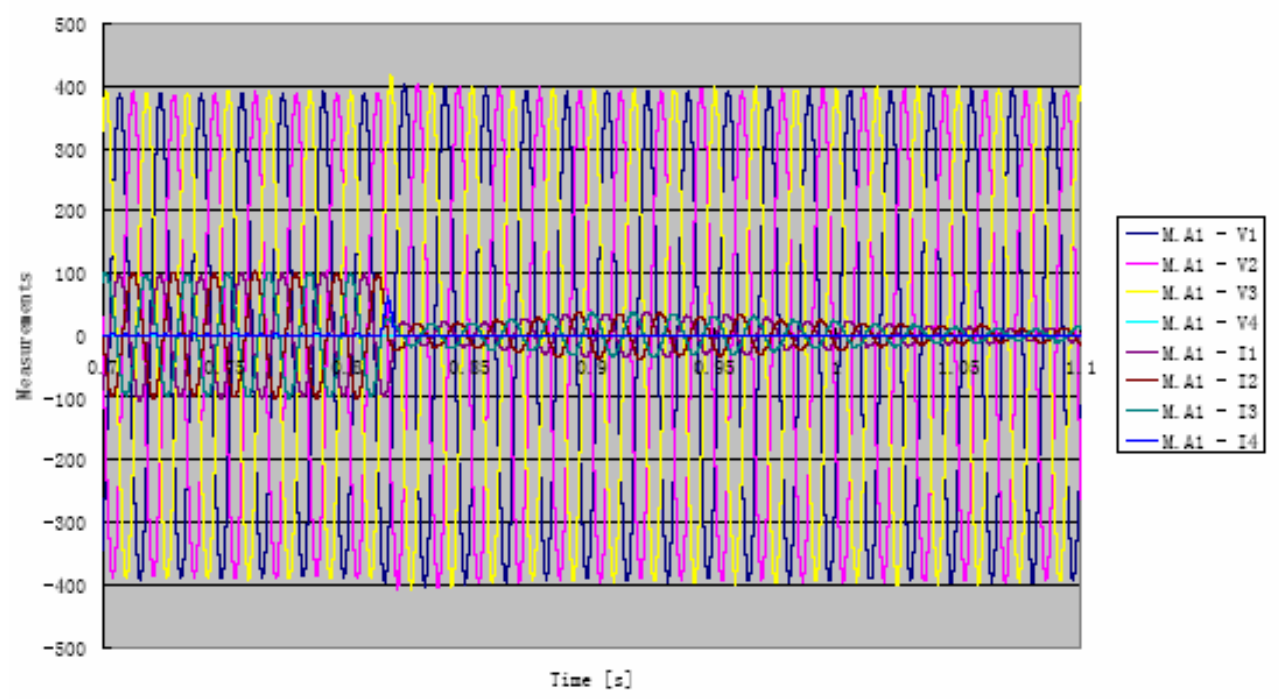

II. c. ii A1, A2 Part 1

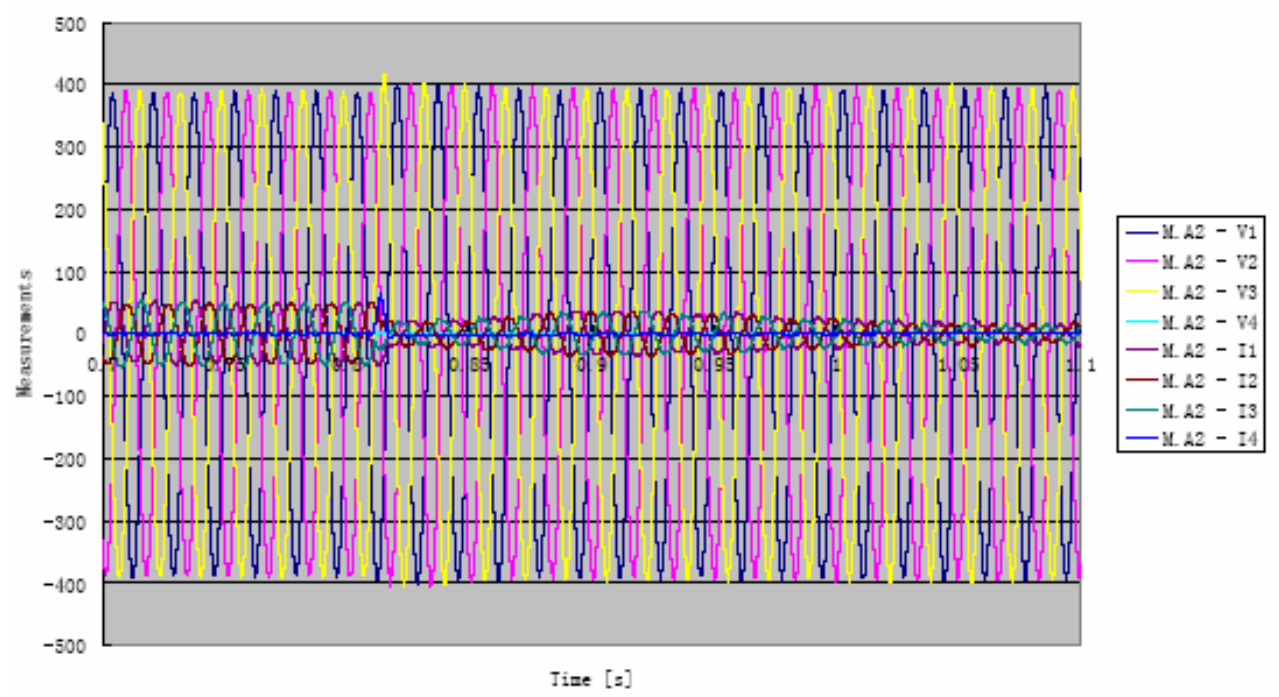




\section{II.c.ii with units Al and Bl}

II. c. ii A1, B1

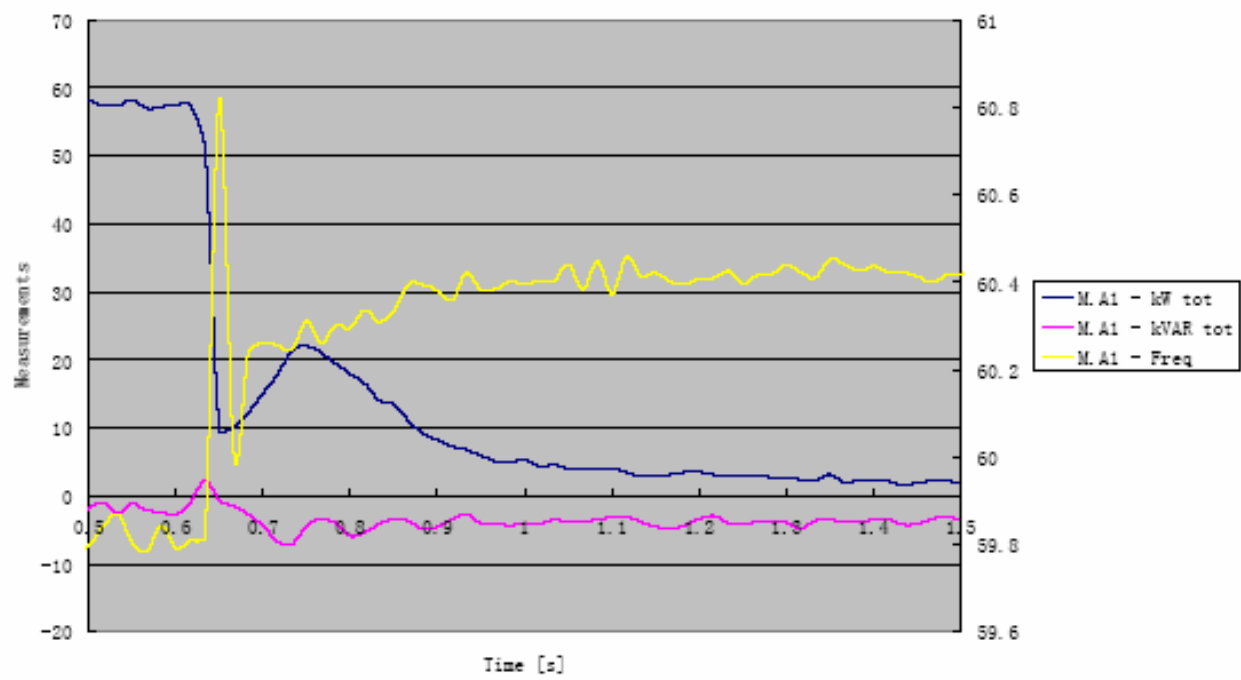

II. c. ii A1, B1

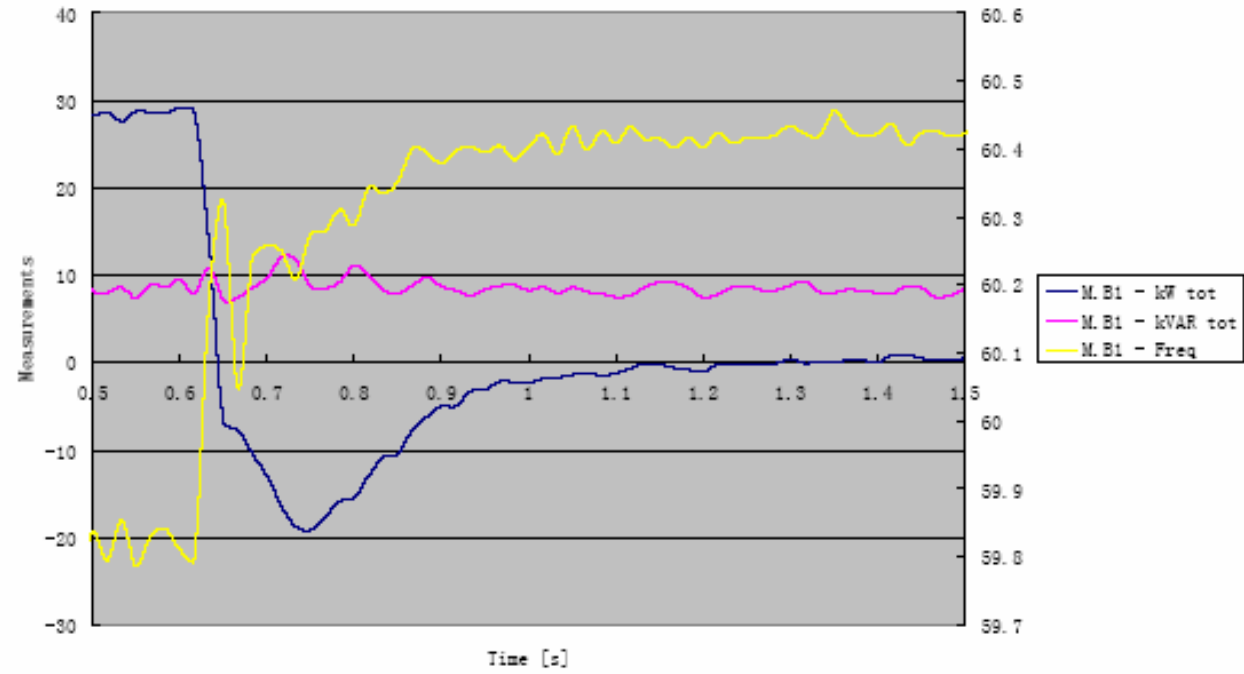


IICH As, :

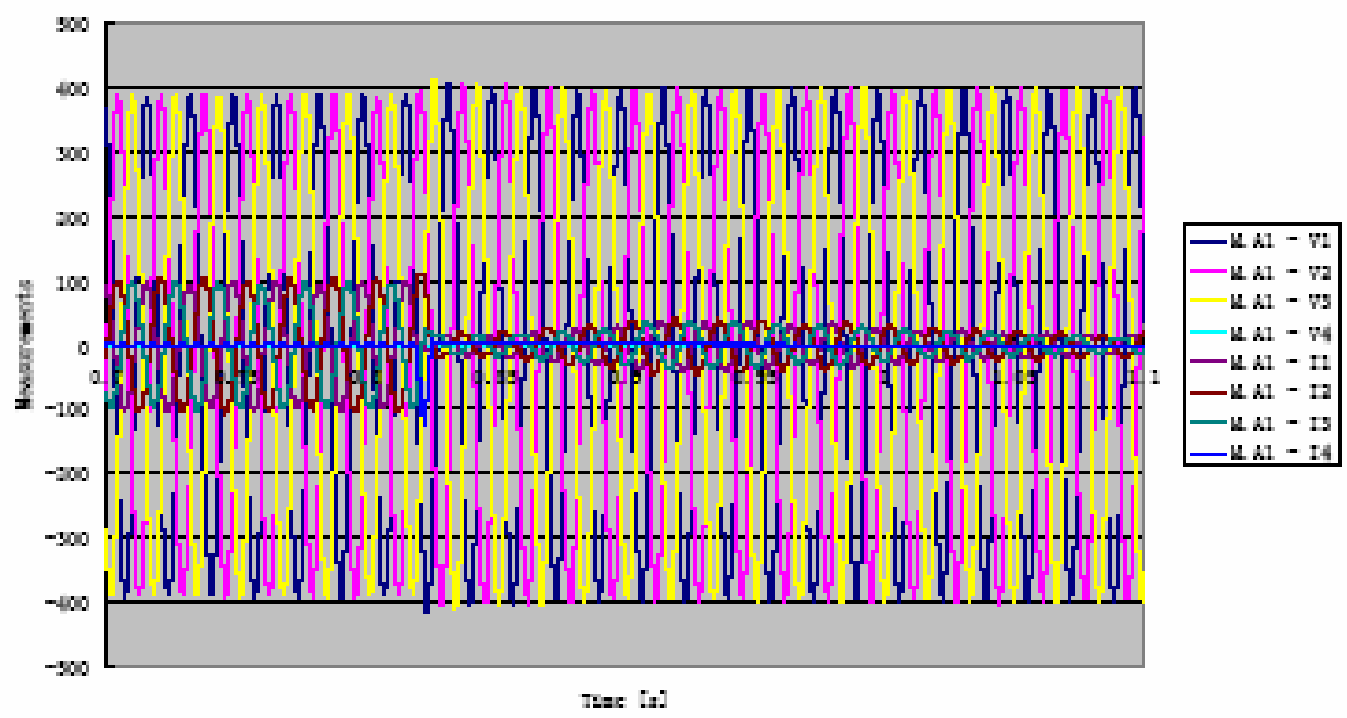

Event not captured 


\section{II.a.i with $\mathrm{Al}$ and $\mathrm{A2}$}

11. 2.1 2.1, a2

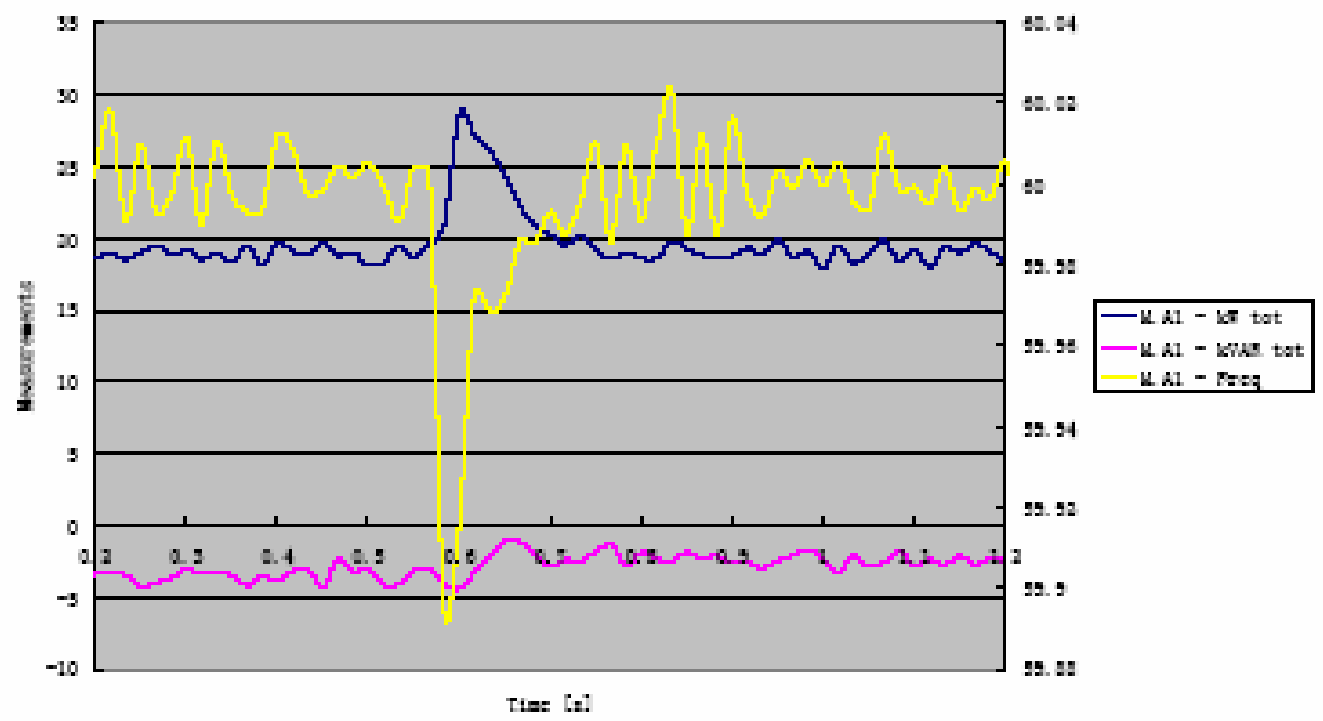

11. 2.1 2.1, a2

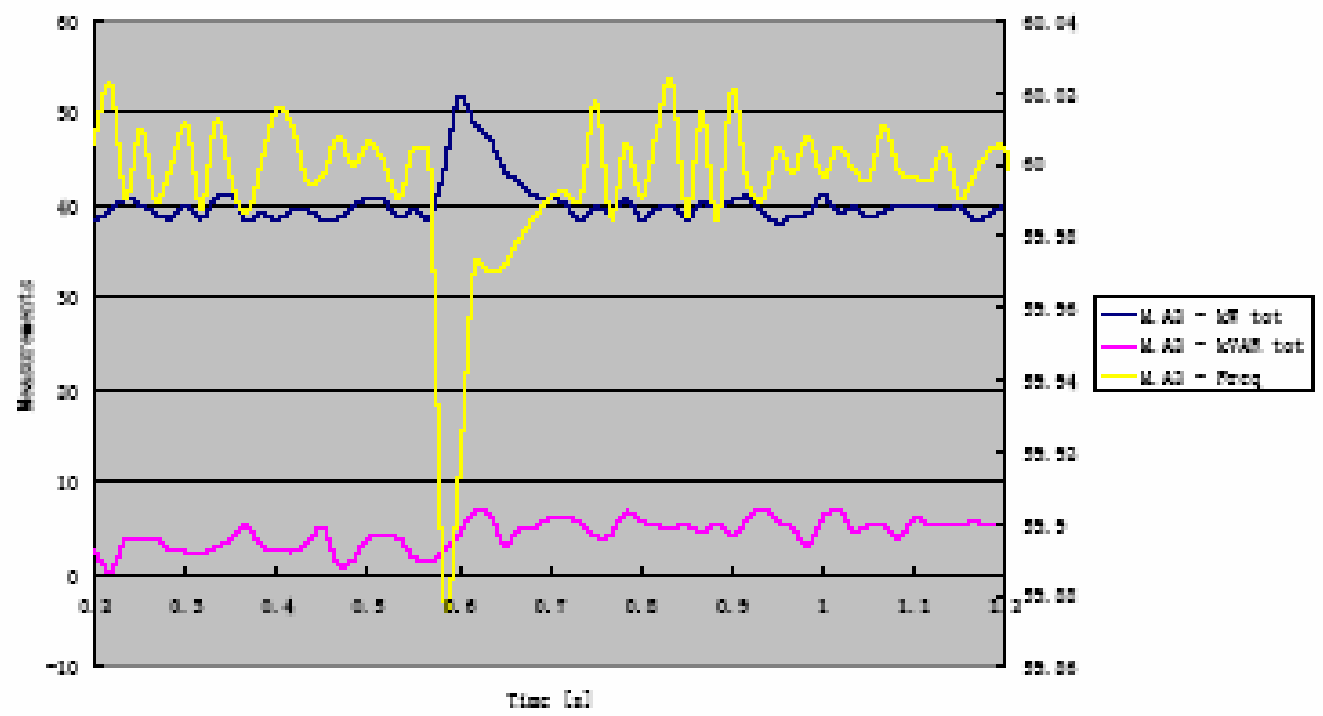


11. $2.121,22$

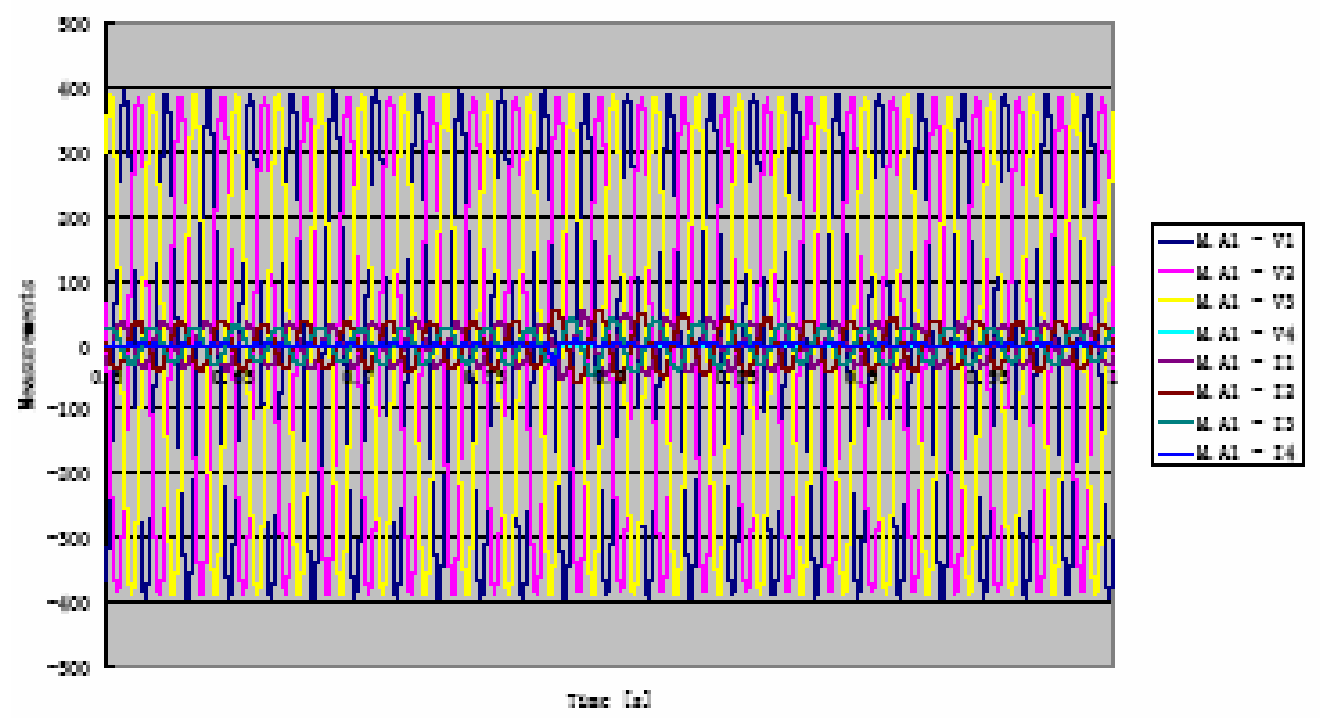

11. $2.421,22$

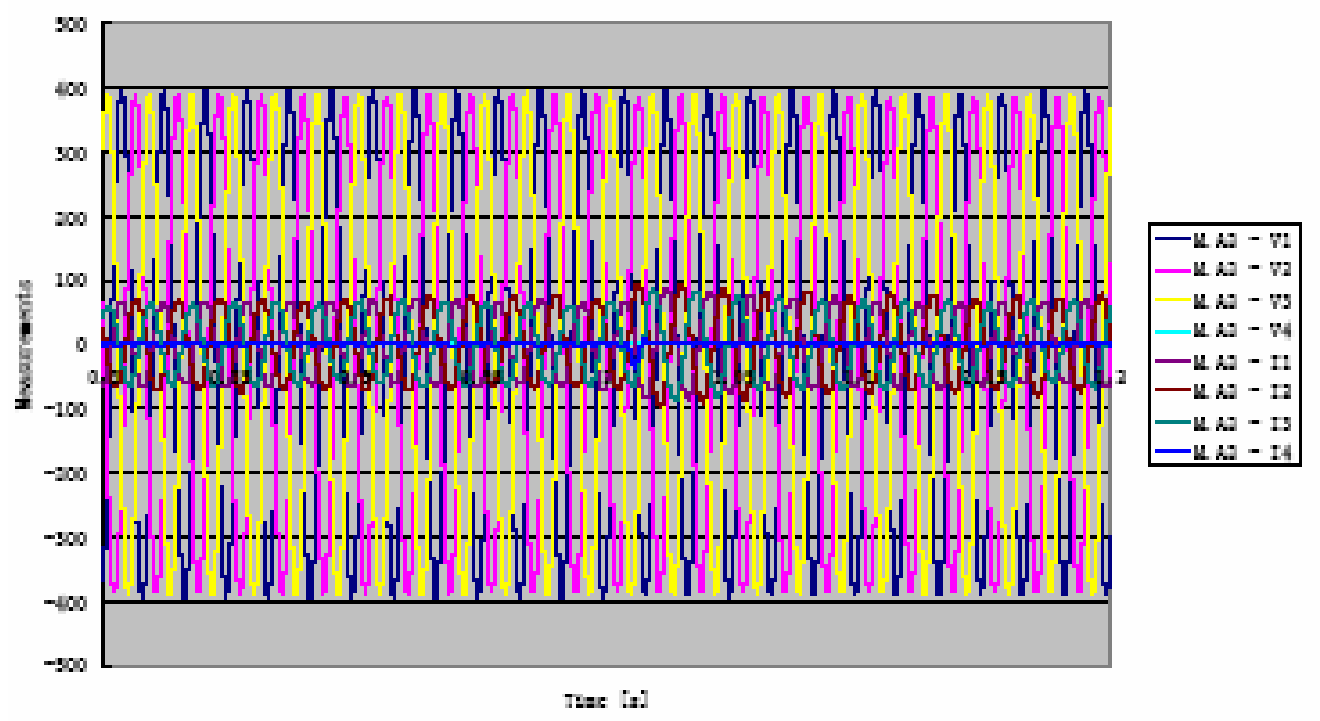




\section{II.a.i with $\mathrm{Bl}$ and $\mathrm{A2}$}

11. 2.4 21, 81

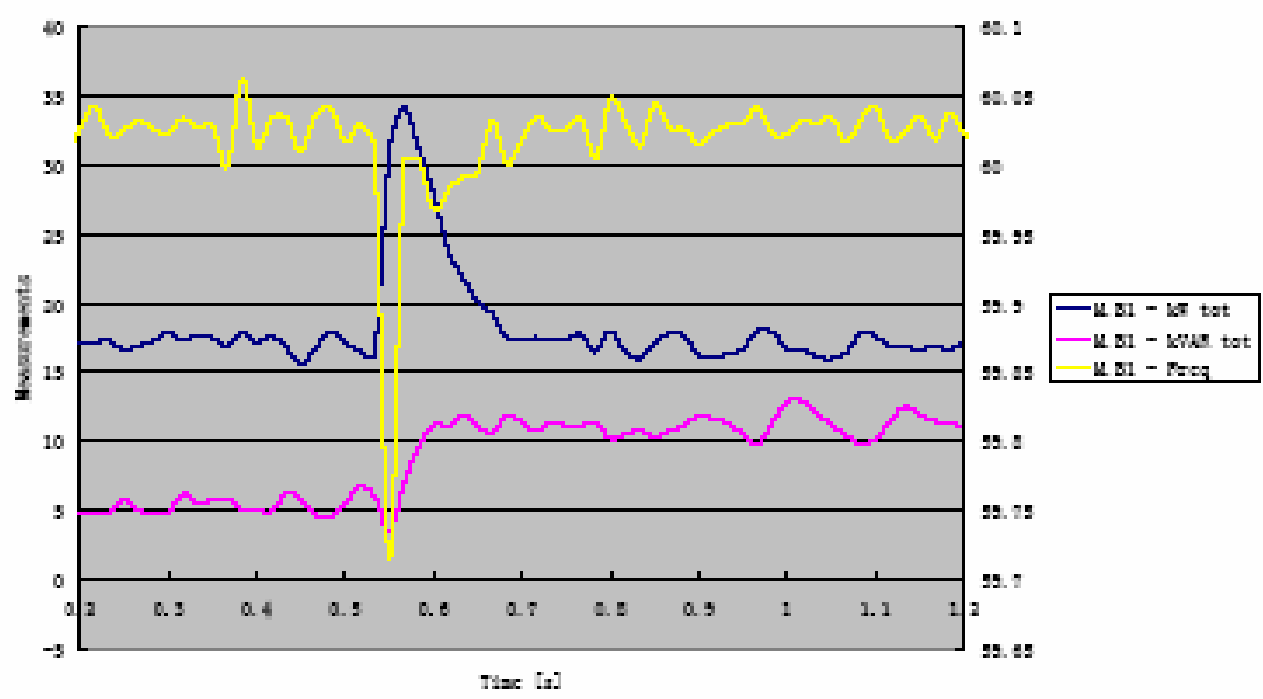

11. 8.4 . 21, 81

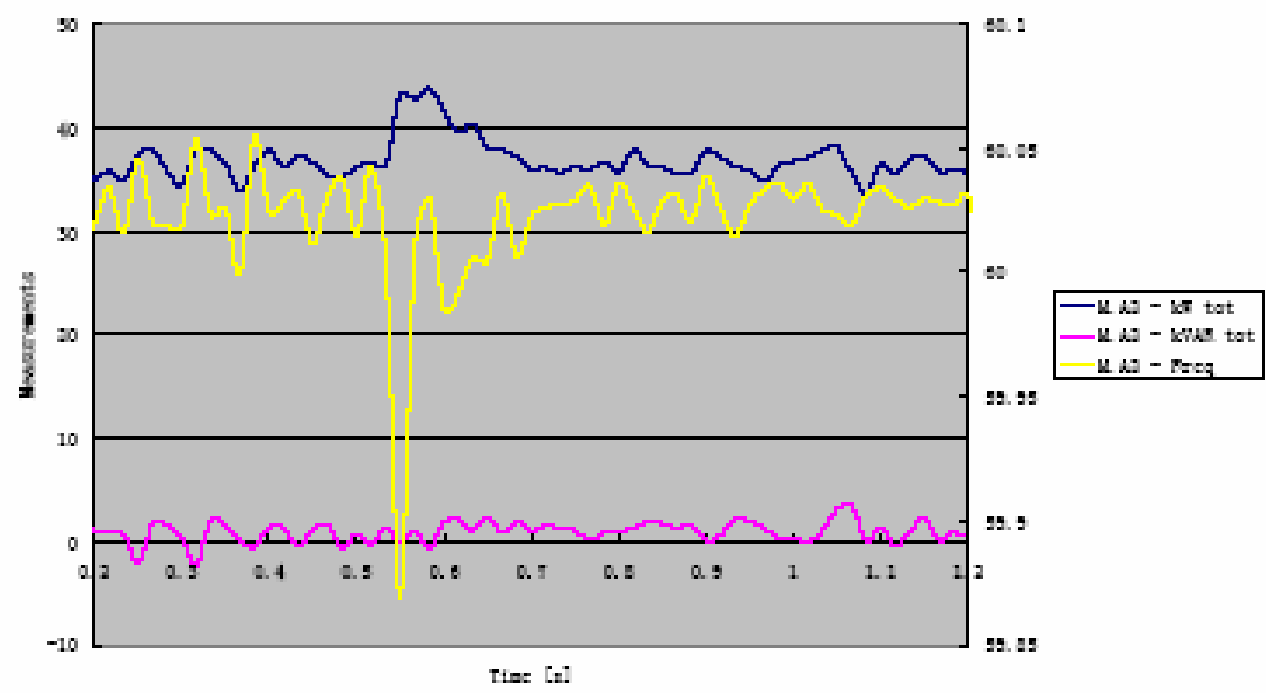


Event not captured

11. $2.4 \mathrm{B1}$, a2

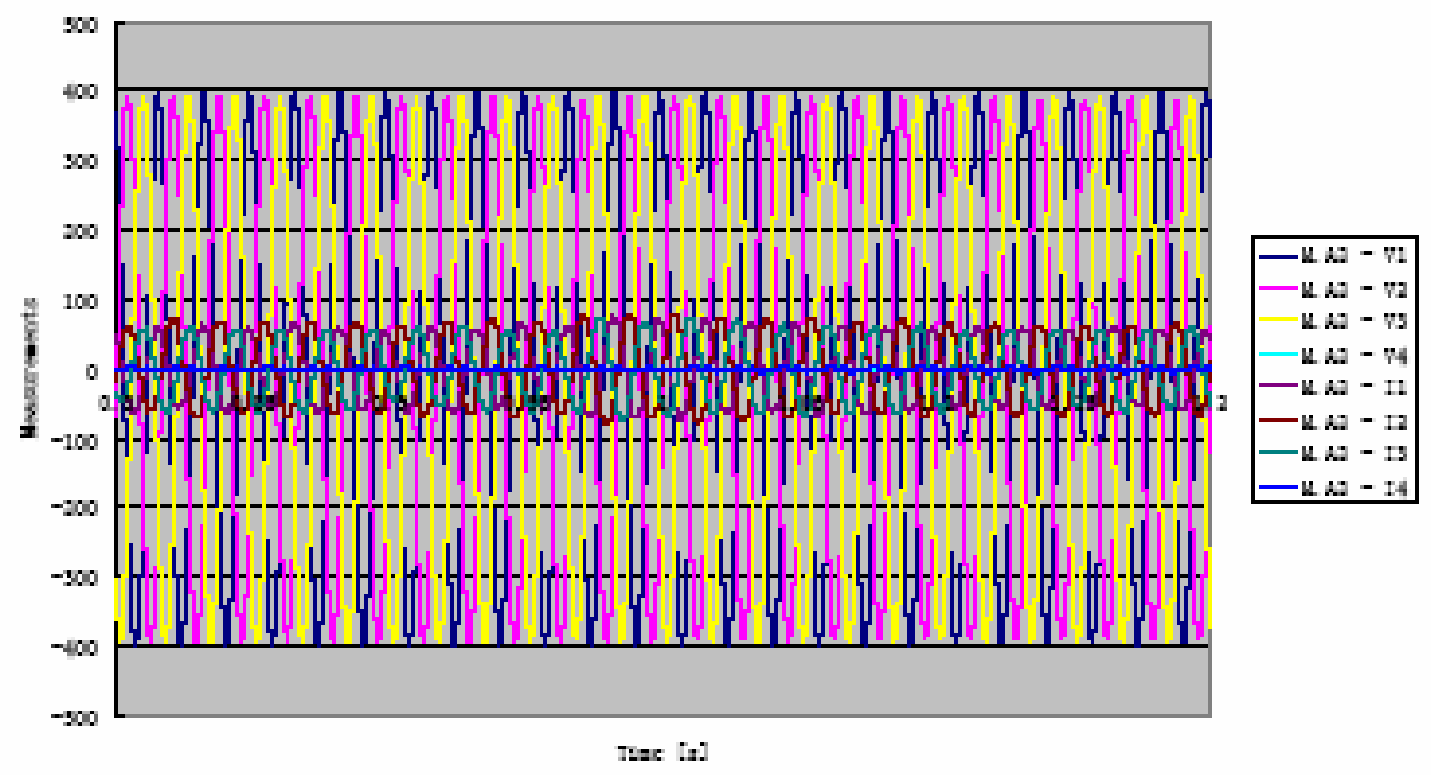




\section{Event to reach III.a.l with unit $\mathrm{Al}$ and $\mathrm{A} 2$ (connection of $\mathrm{Al}$ in feeder mode)}

A1 Convettien in Fetter wede

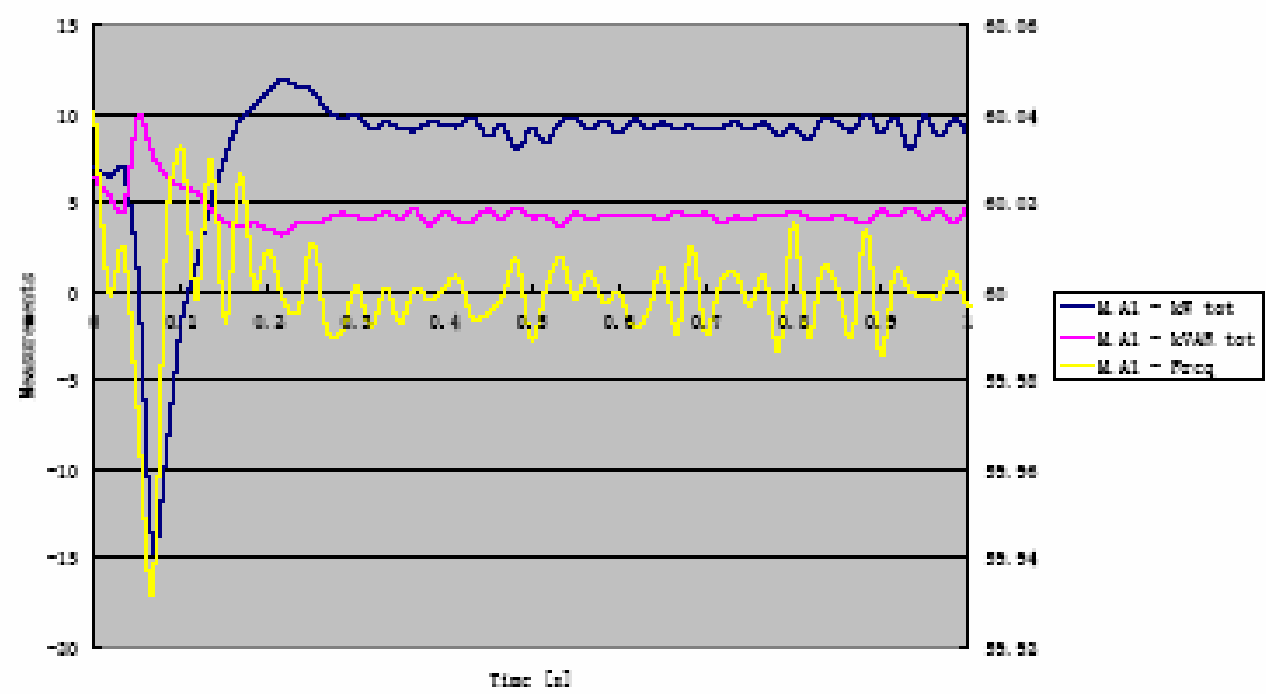

A1 Consettion in Fester weds

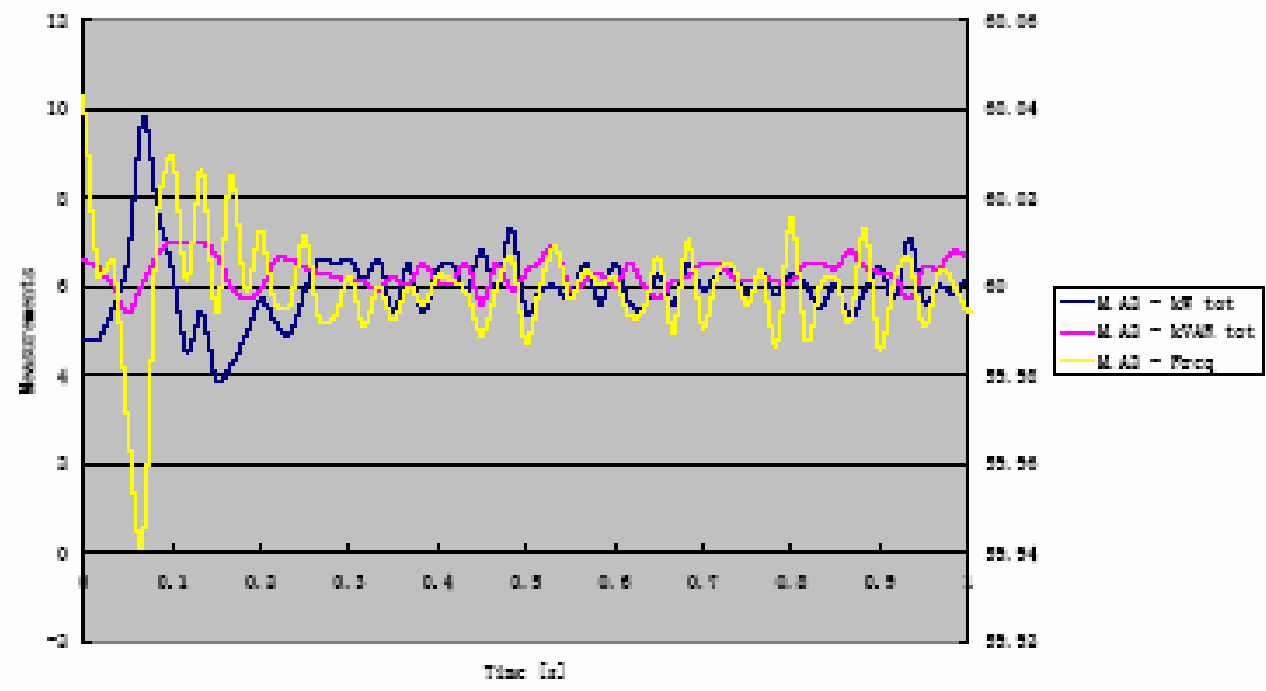



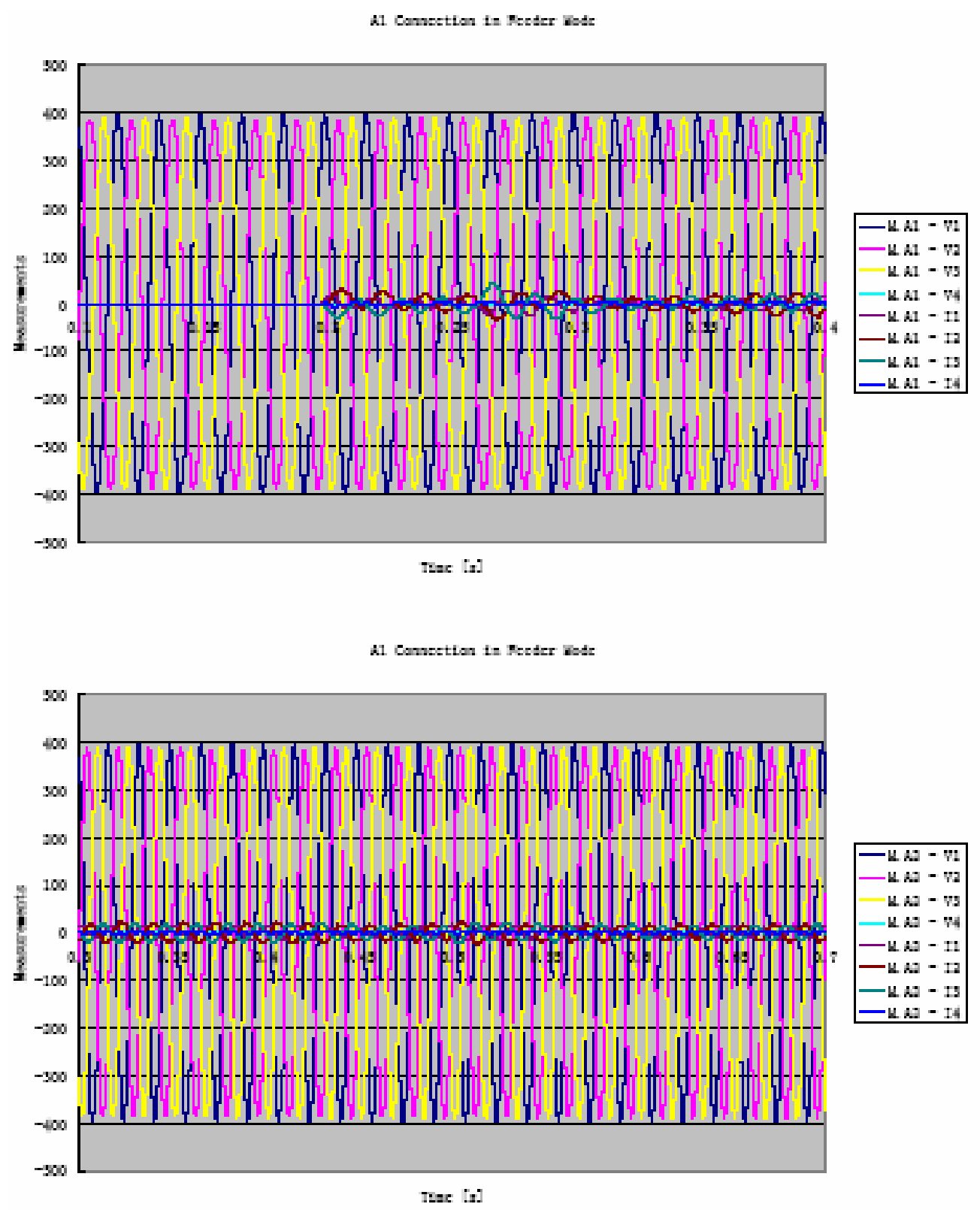


\section{III.a.i with unit $\mathrm{Al}$ and A2}

tII. Q. A A2, A2

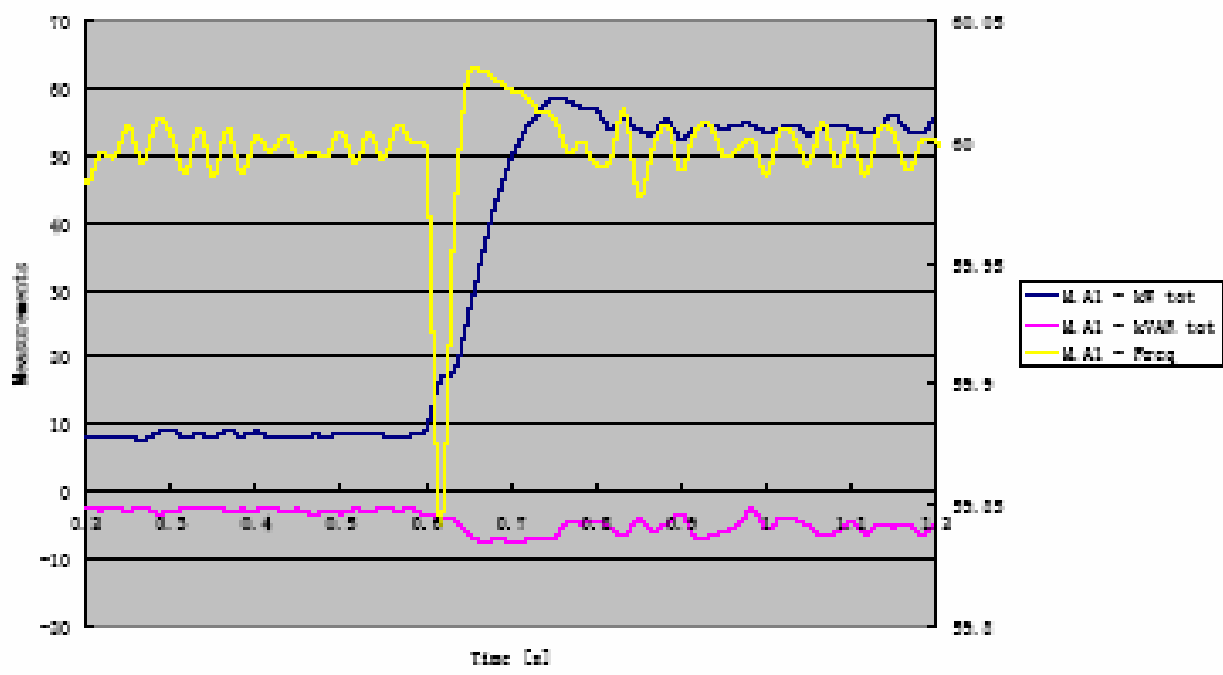

trtat A2, a2

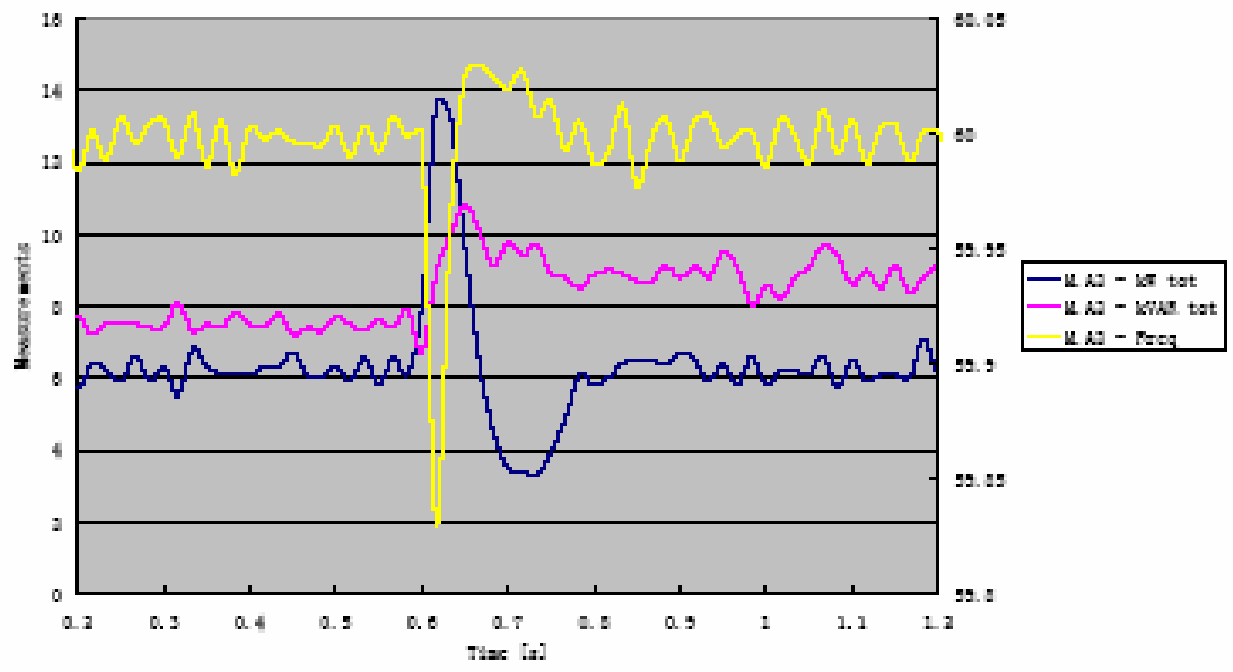


IIteA A2, A2

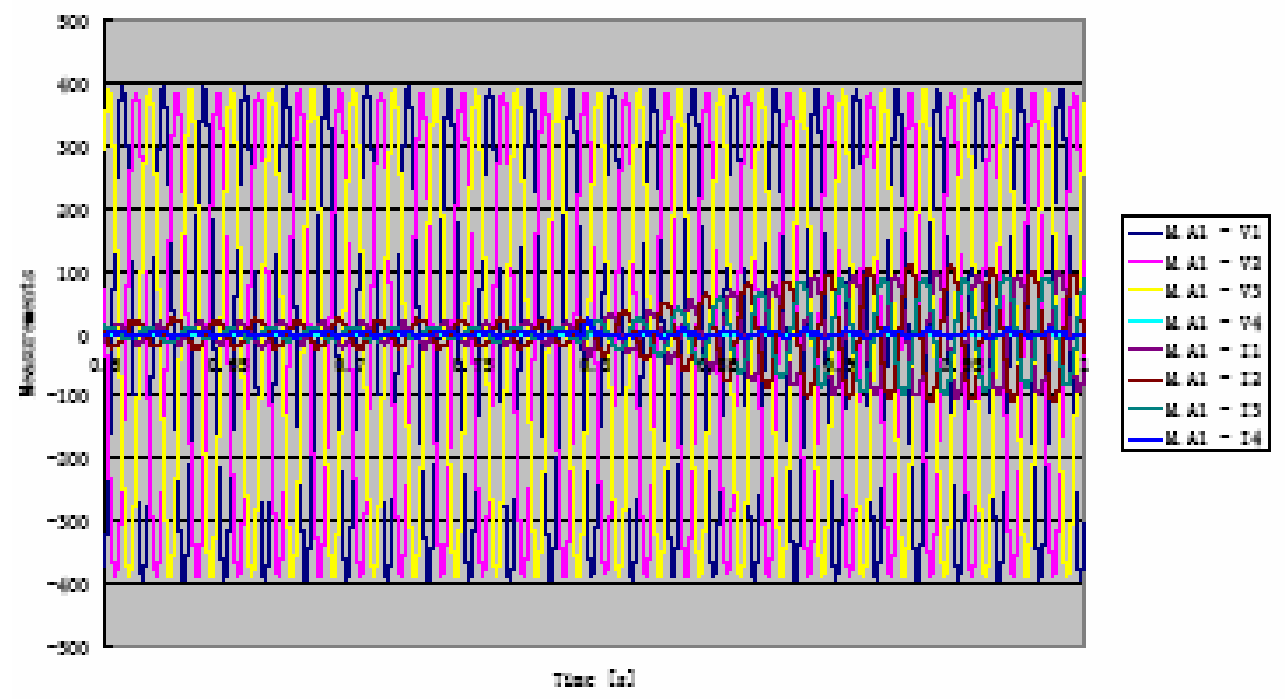

III Q A A2, A2

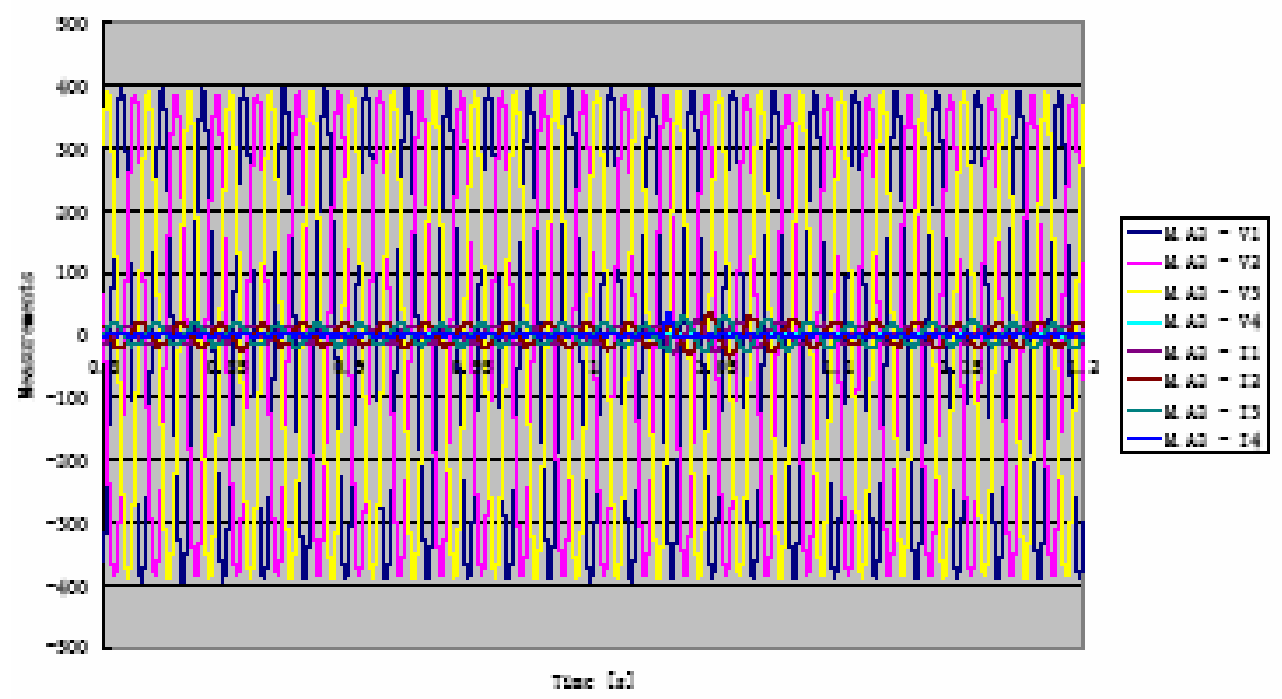




\section{II.b.i with unit $\mathrm{Al}$ and $\mathrm{A} 2$}

11. t. 1 21, a2

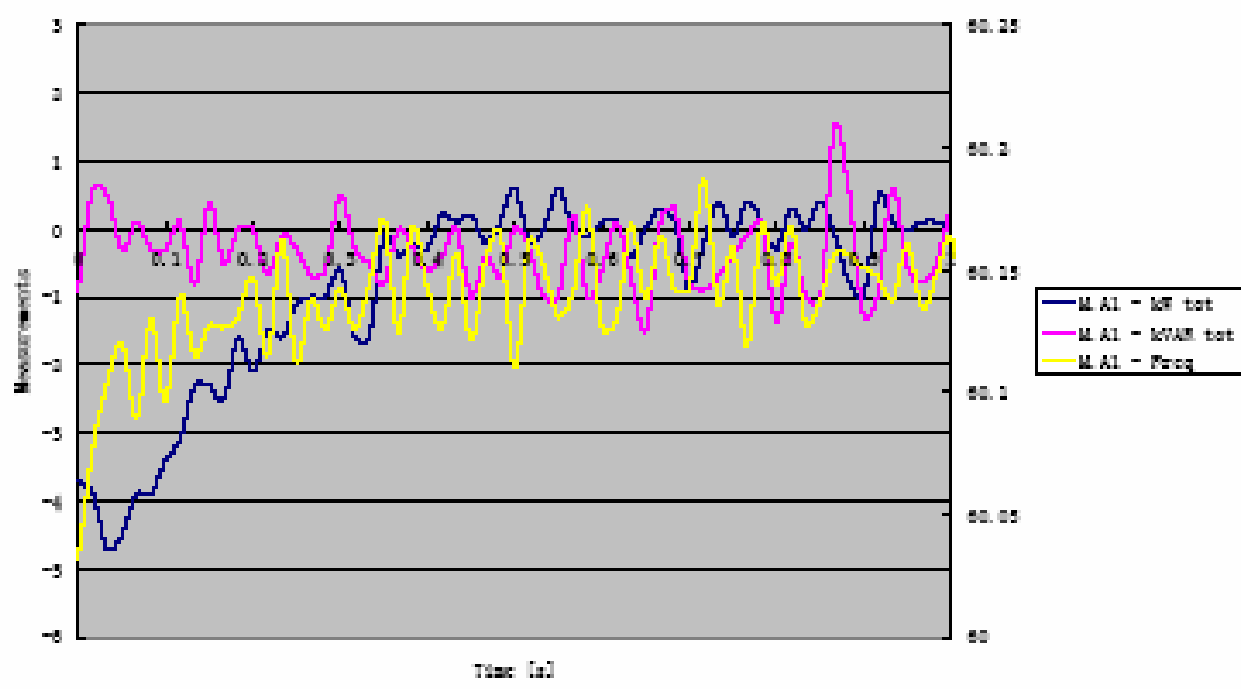

11. k. 4 21, a.

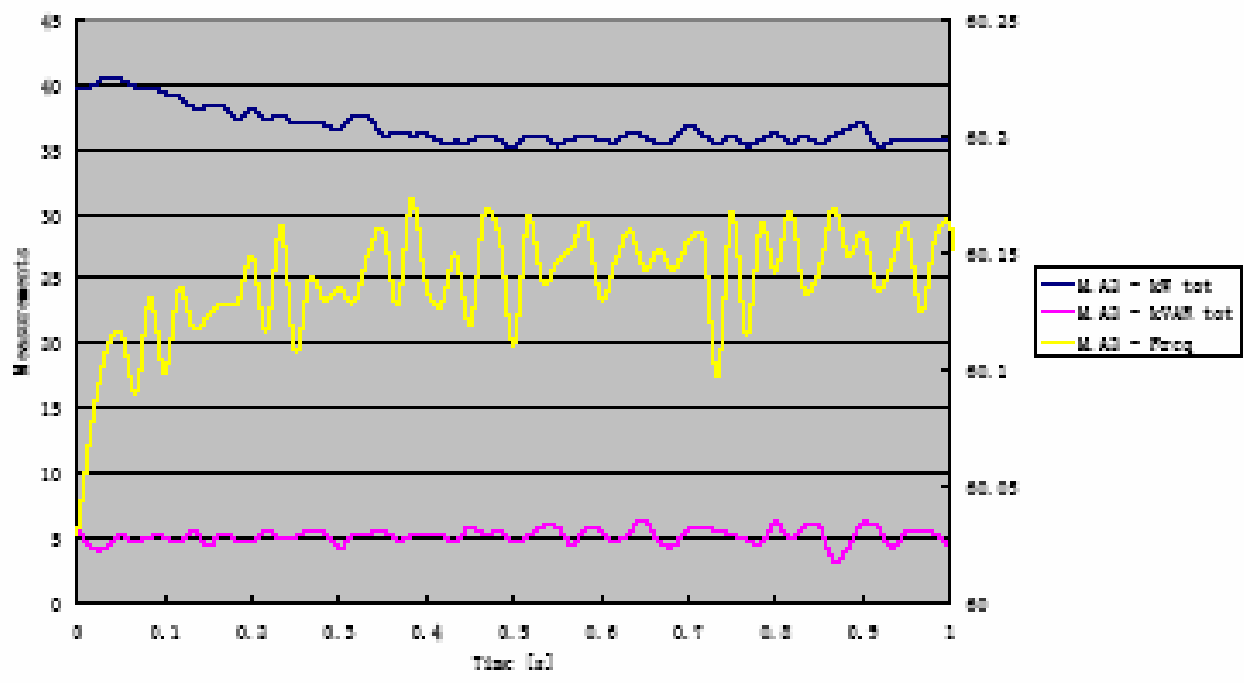


11.t. 4 21, a:

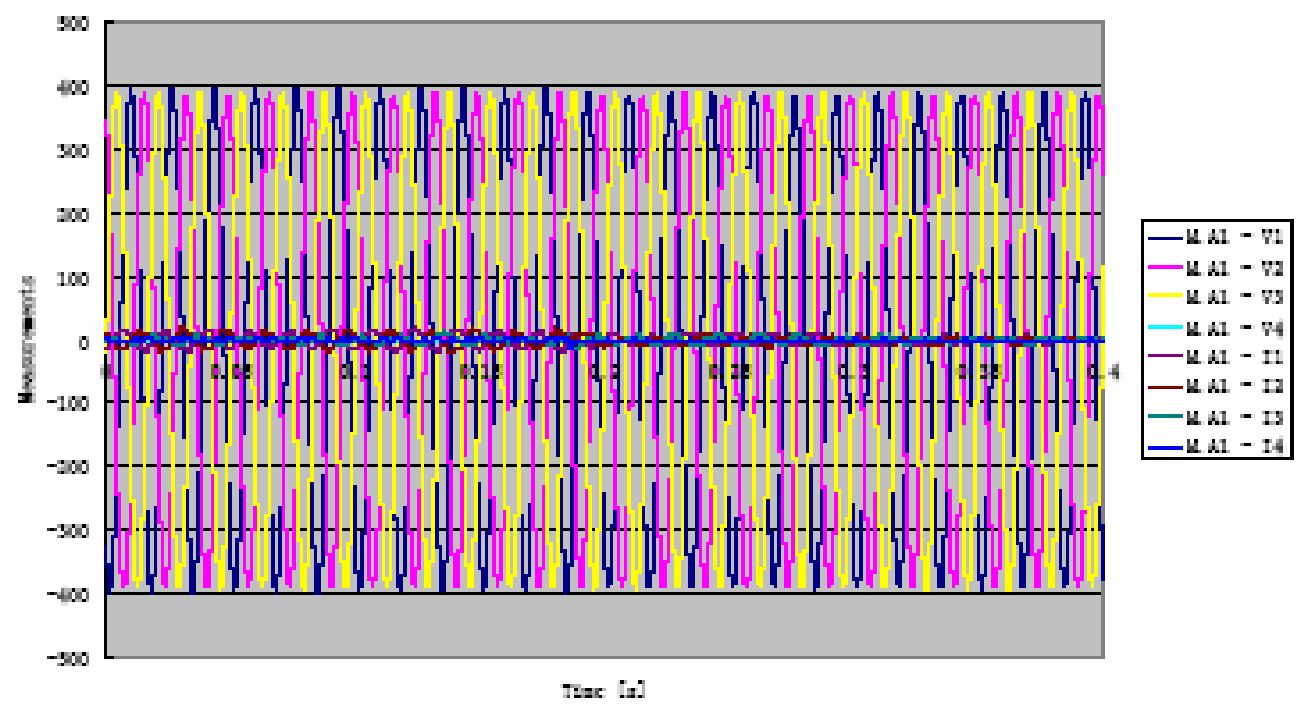

11.t. 4 21, a:

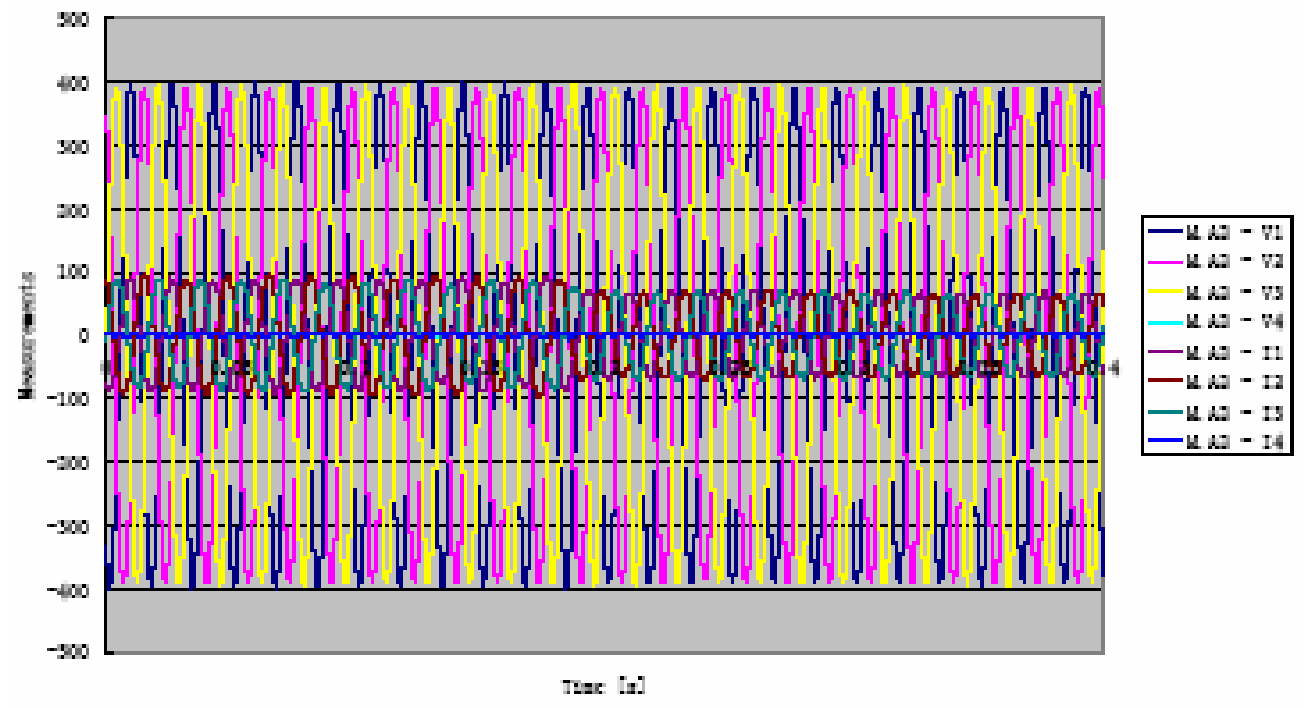




\section{II.b.i with unit $\mathrm{Bl}$ and $\mathrm{A} 2$}

11. t. 1 B1, a2

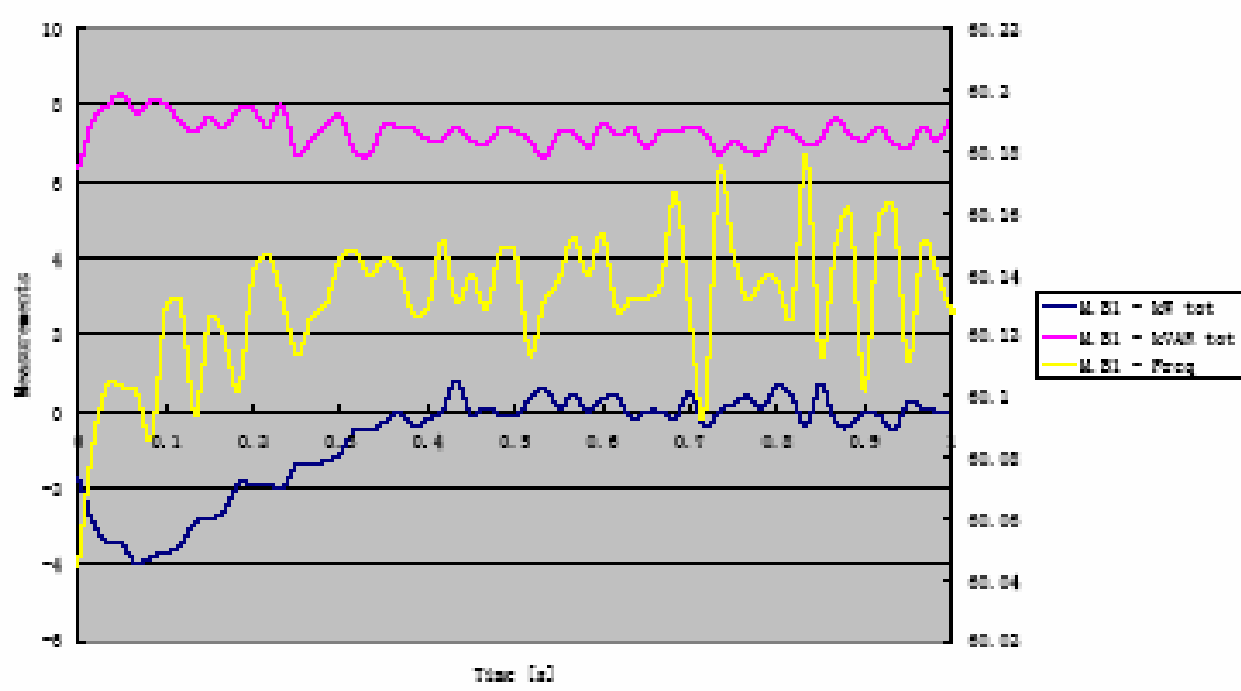

11. b. 1 81, a2

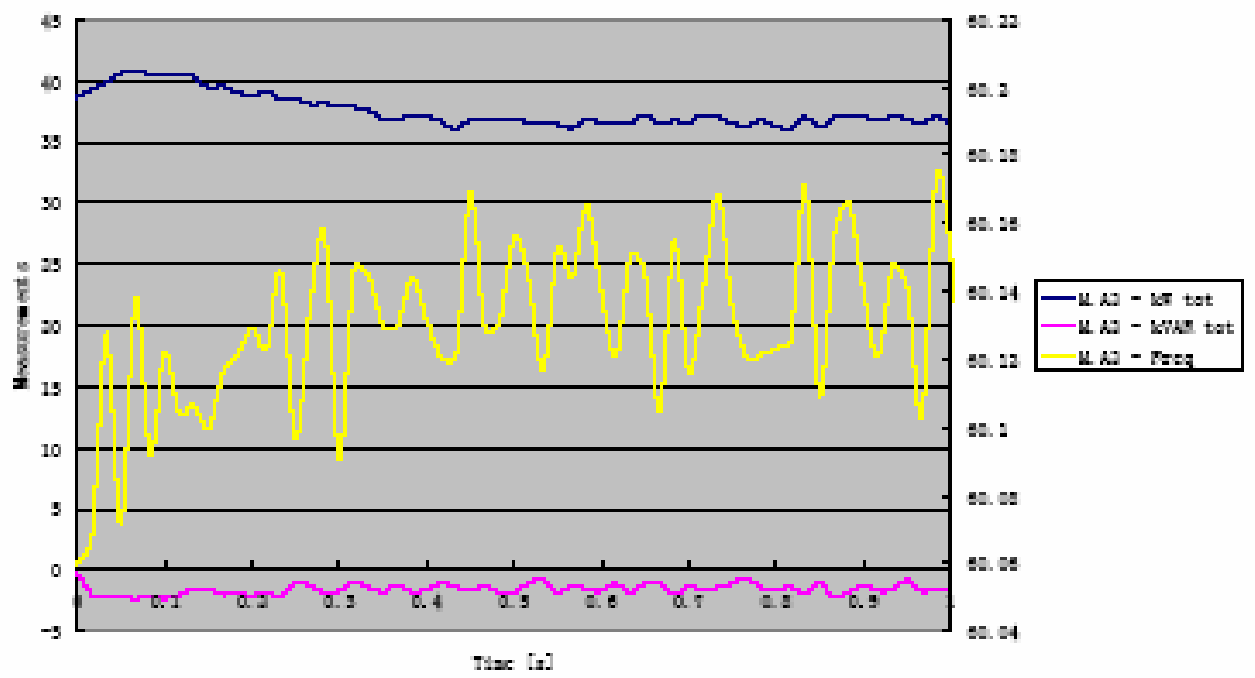


11. t. 1 81, a

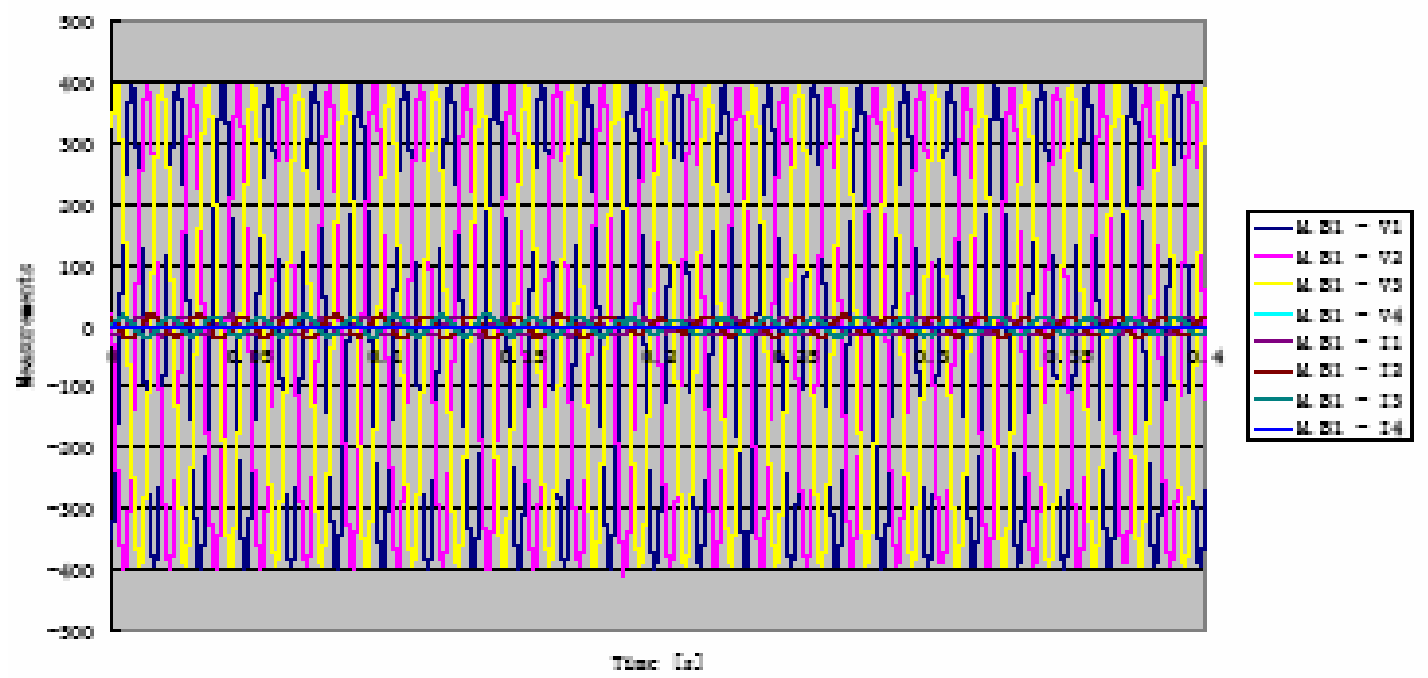

11. b. 1 81, 2a

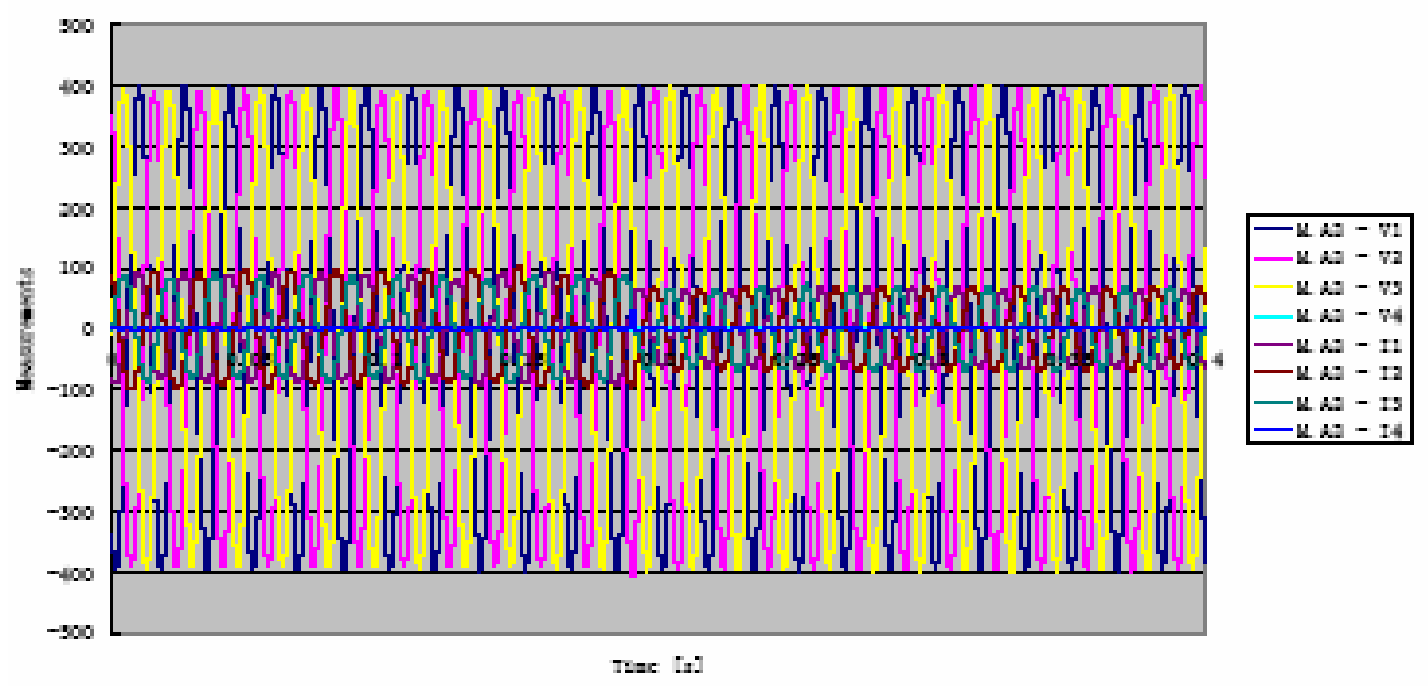




\section{II.b.ii with units $\mathrm{Al}$ and $\mathrm{A2}$}

II. 211 a2, a2

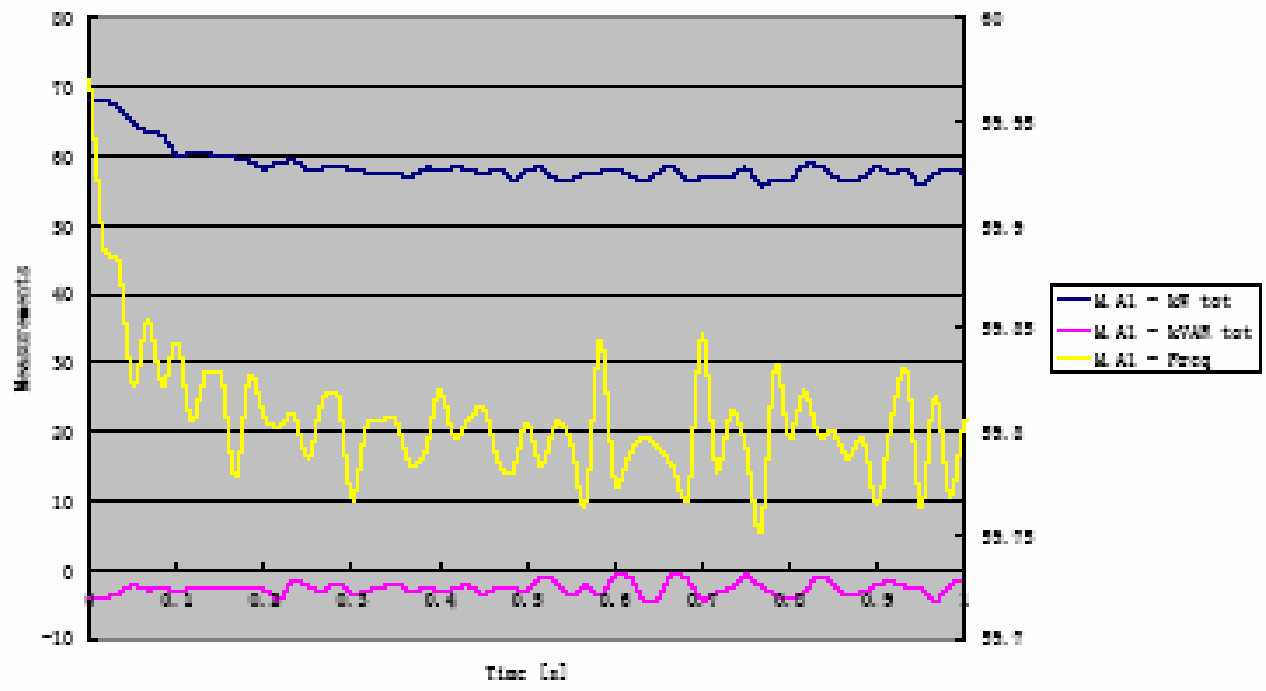

II. 2.11 A2, A2

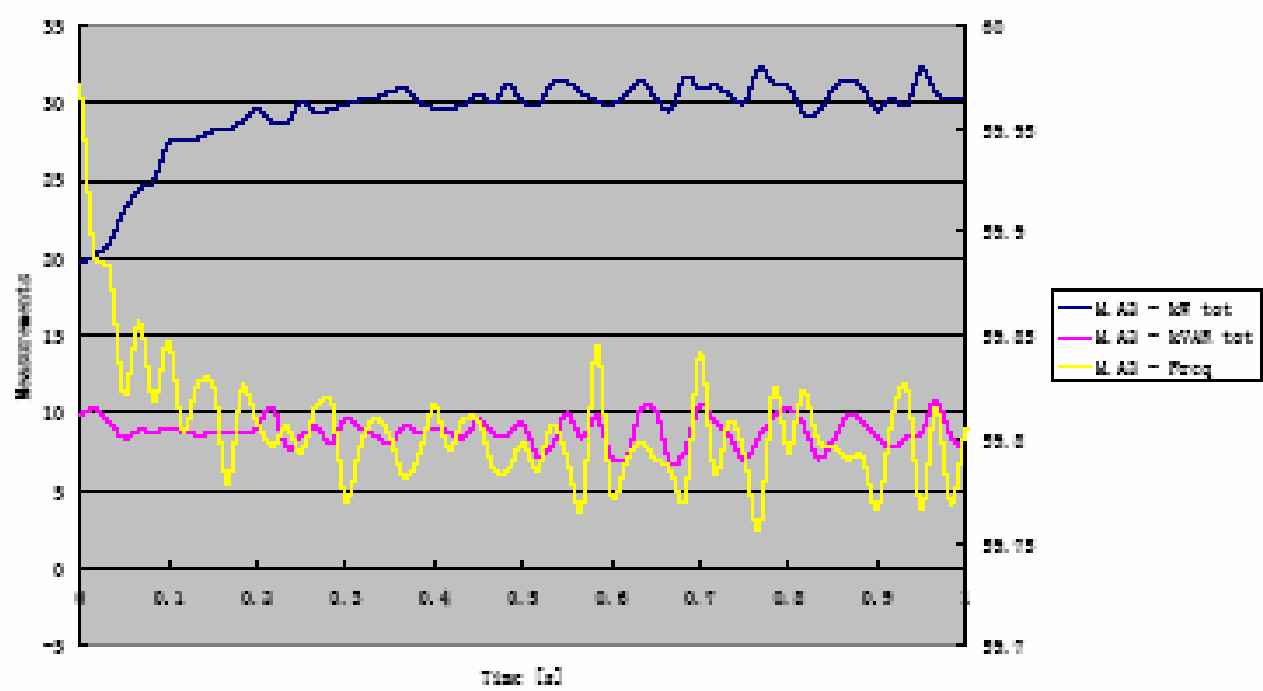




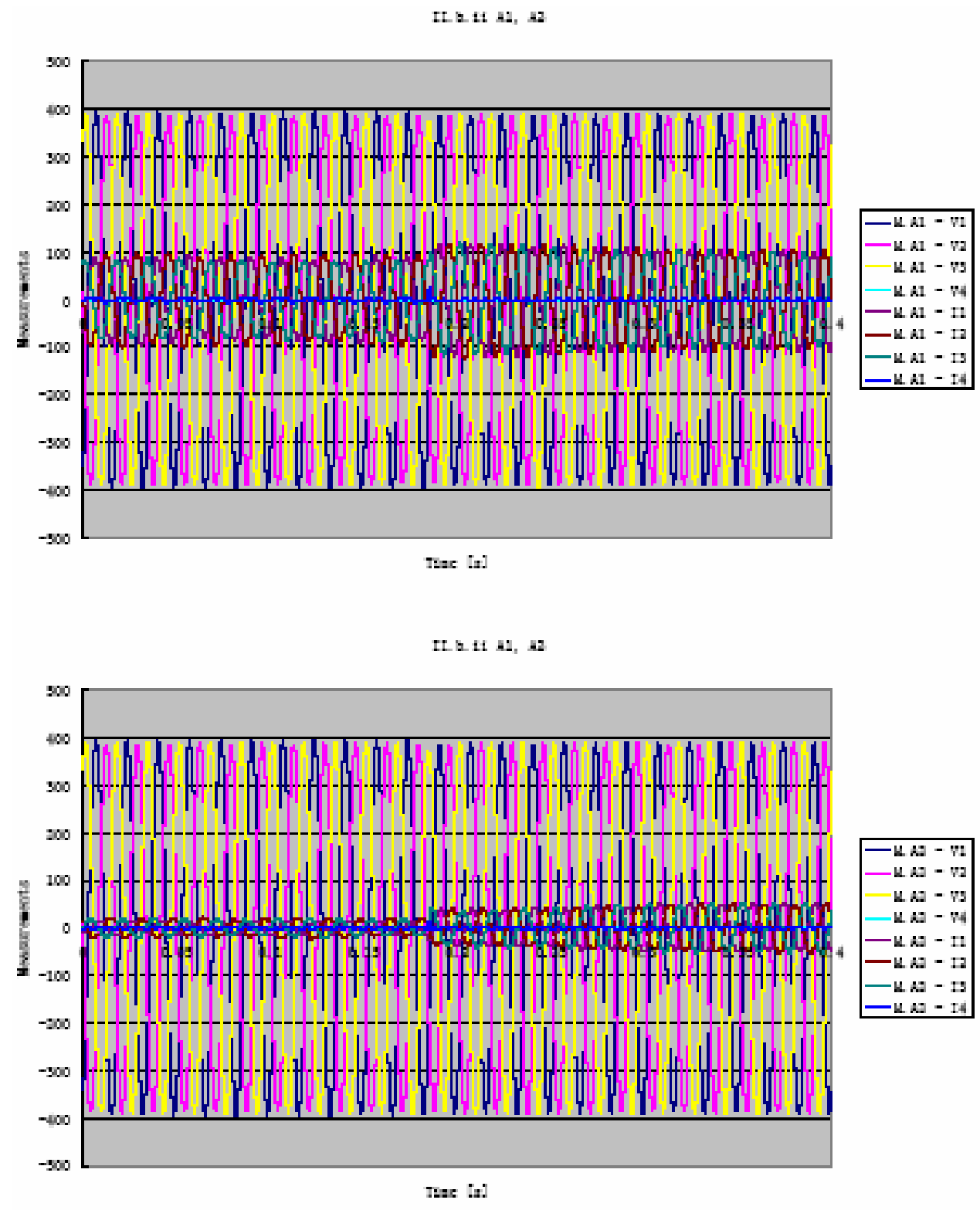




\section{II.b.ii with units $\mathrm{Bl}$ and $\mathrm{A} 2$}

IE. 2.11 :2, 12

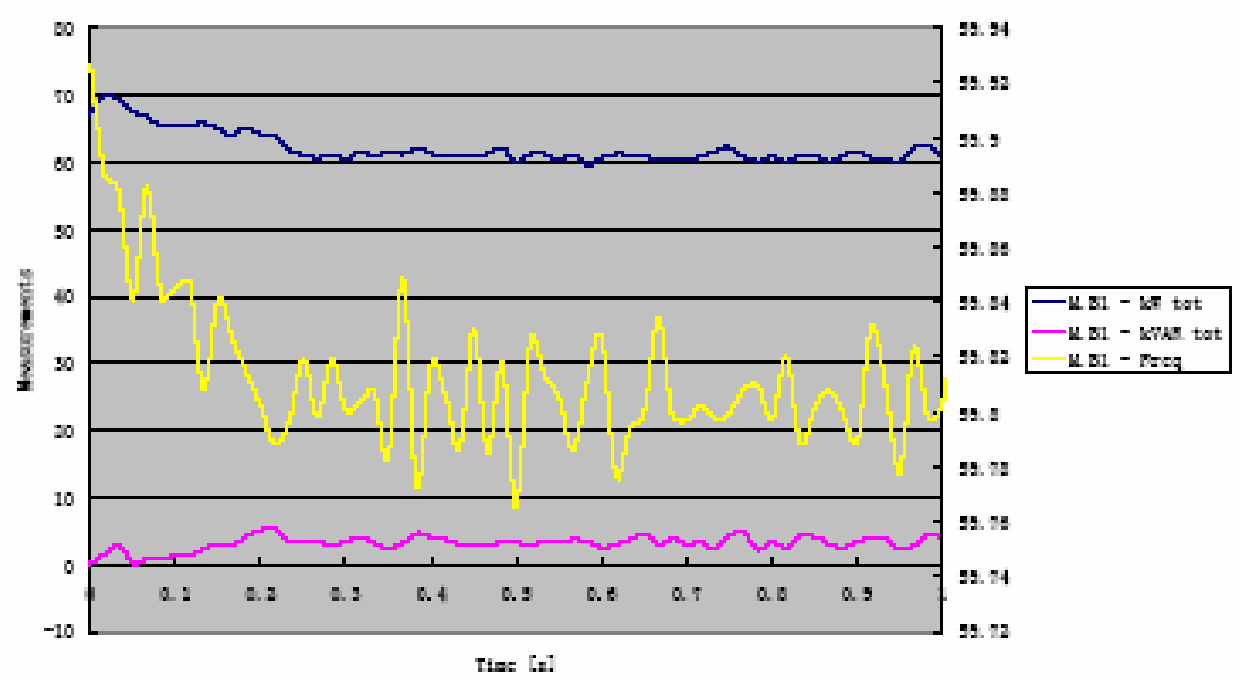

II. $2.11 \mathrm{28,} .2$

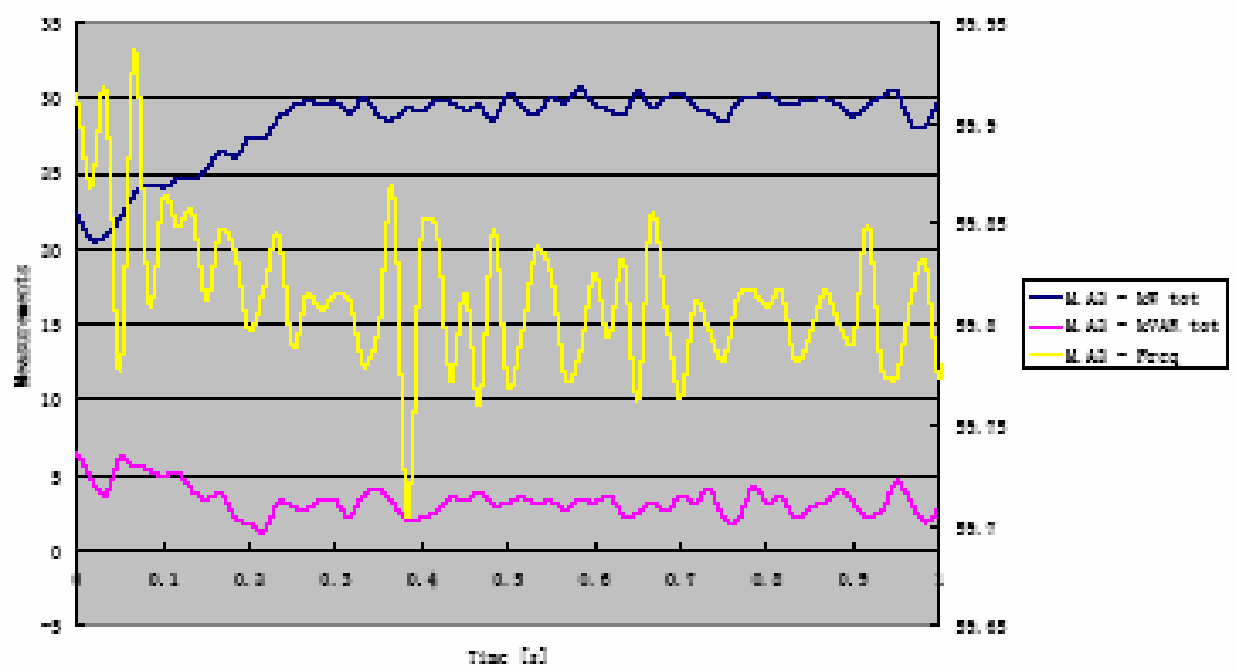




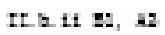

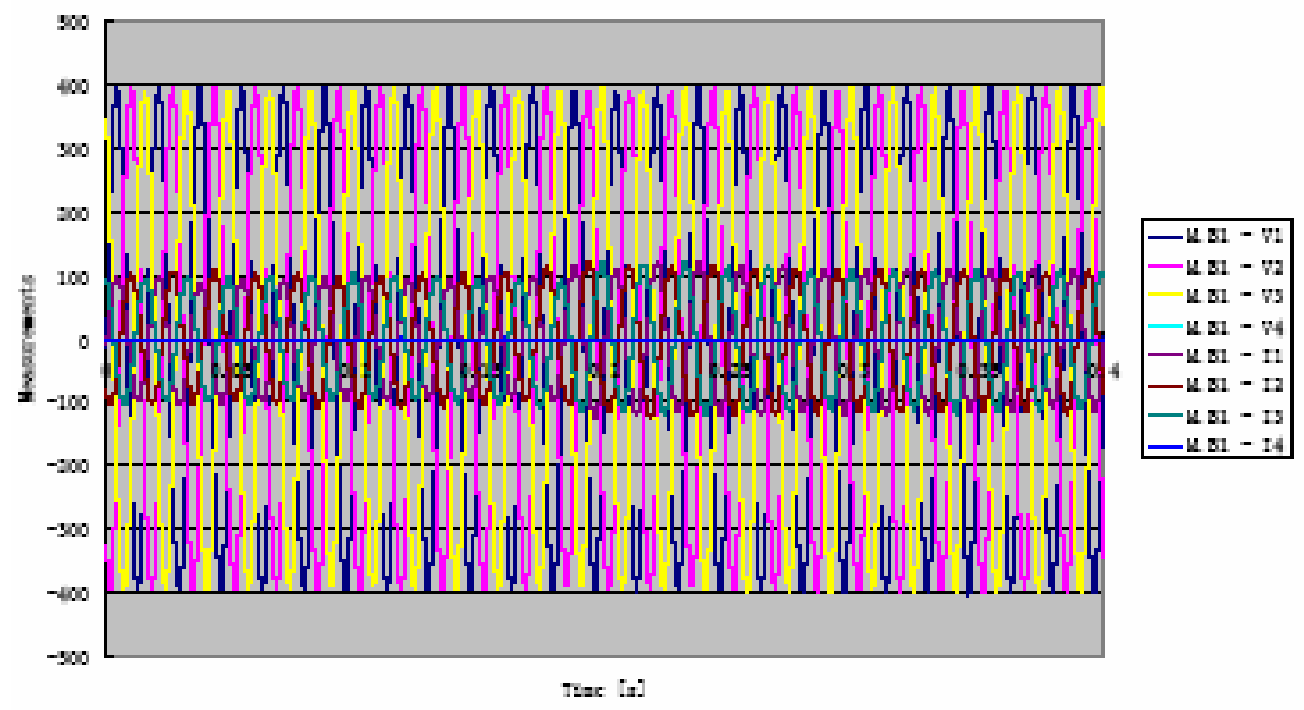

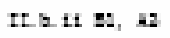

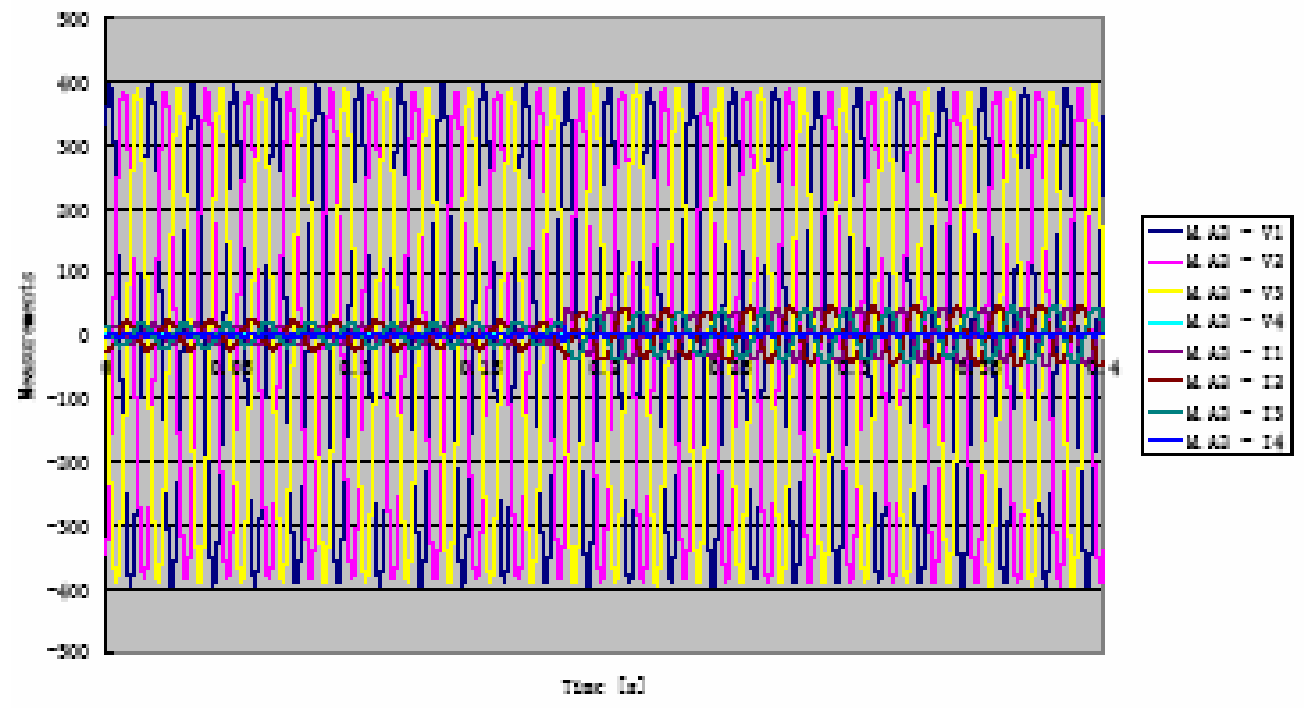




\section{III.b.i with unit $\mathrm{Al}$ and $\mathrm{A} 2$}

$\operatorname{trt} 21 \mathrm{aA}, \mathrm{az}$

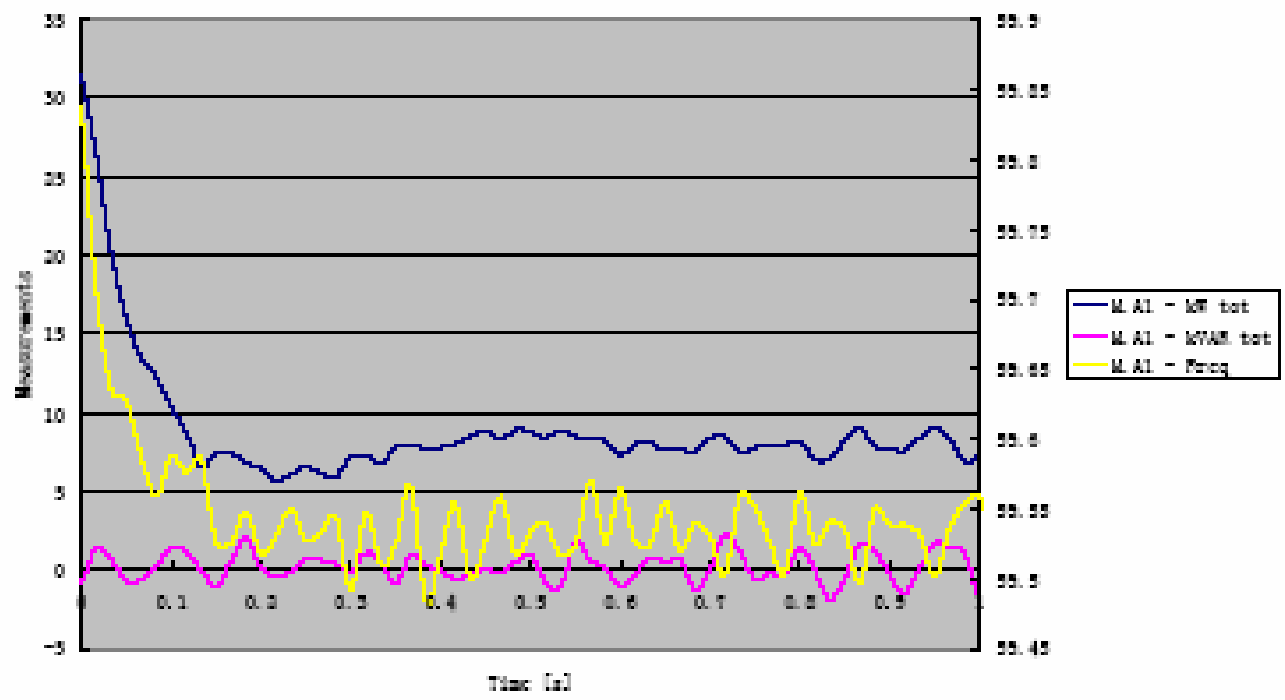

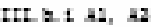

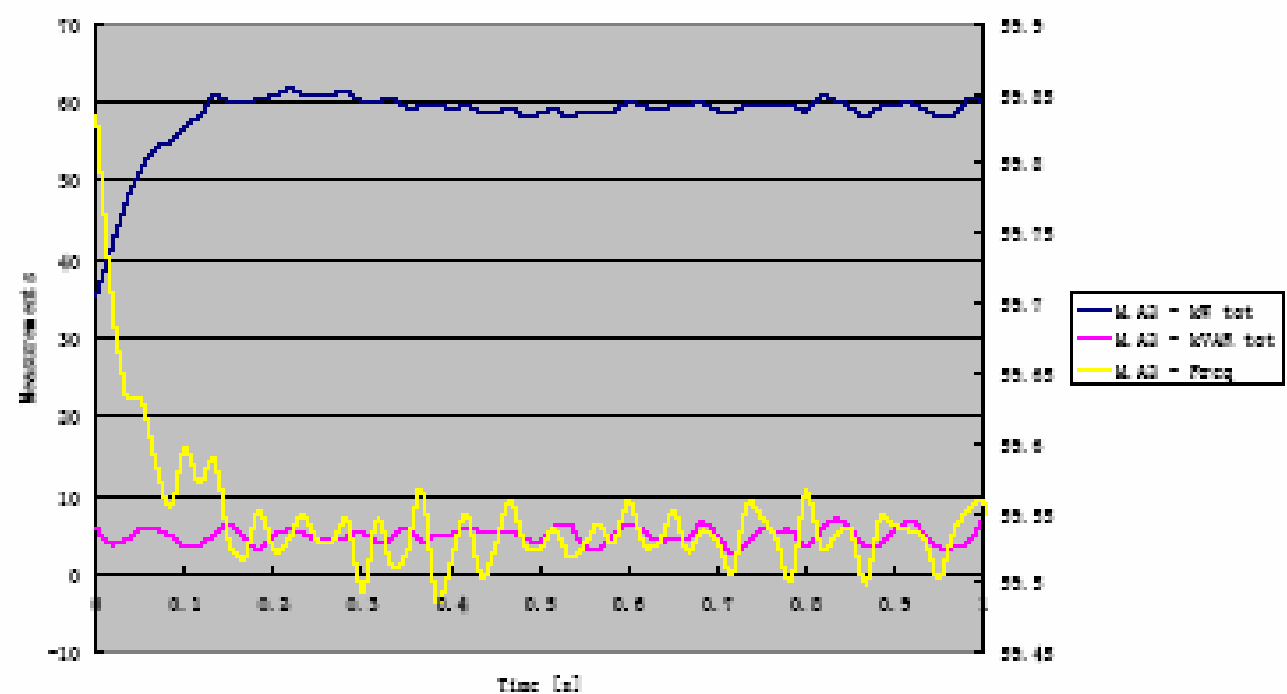


tte.2. A2, a2

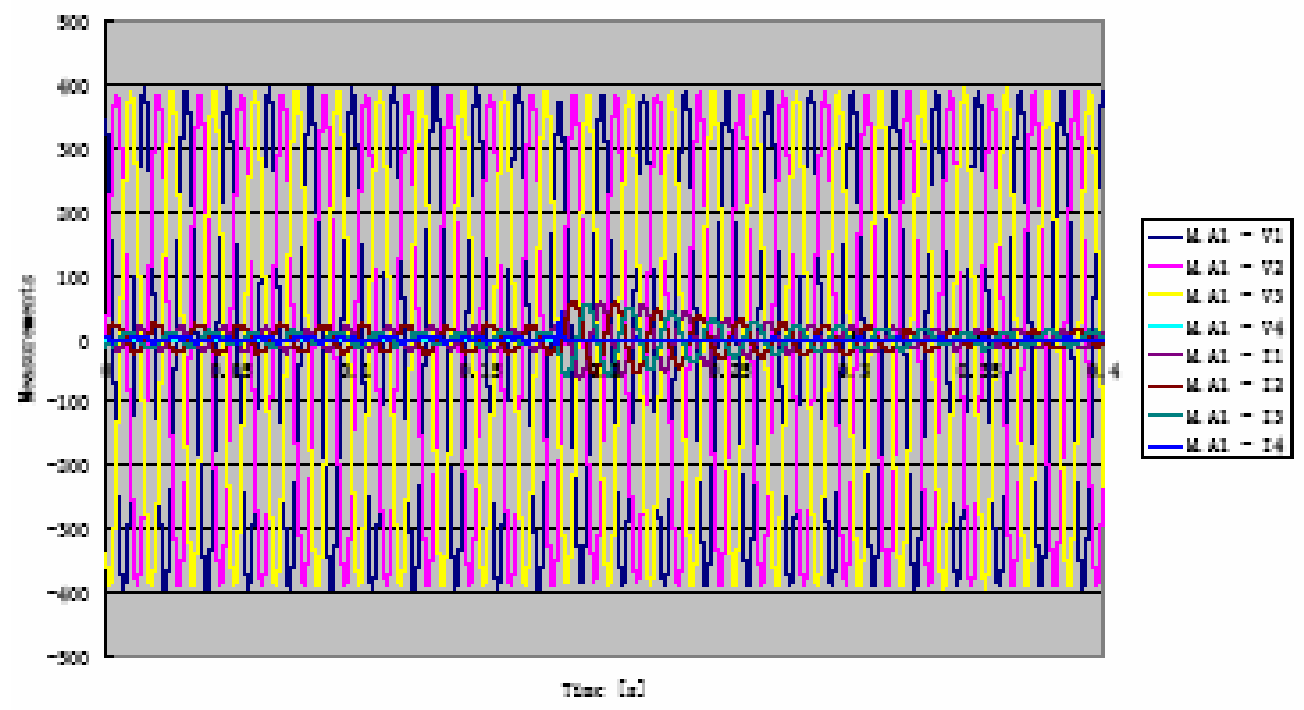

$\operatorname{trI} 21 \mathrm{AB}, \mathrm{Az}$

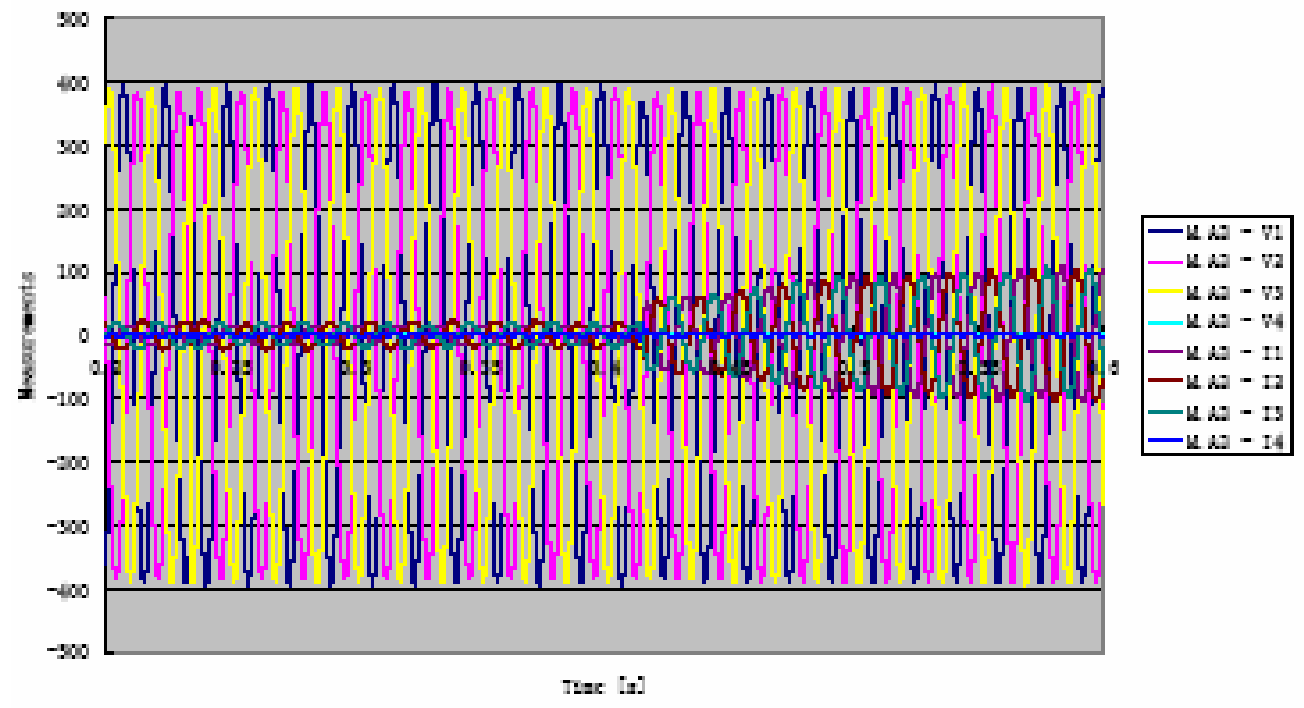





\section{Oscilloscope Waveforms, Unit A2, 10-11-06}

\section{Oscilloscope waveforms with and without weakened genset}

This report focuses in identifying the contributions of the surge module to the operation of a single genset during several loading and unloading conditions. It was shown that the new stiff engine control would survive all the load changes without the assistance of the surge module. For that, see data in "10_11_06_test_results.pdf", obtained with surge module off and engine running at 2000rpm.

This document shows the oscilloscope traces obtained with the stiff control at unit A1 in two basic series of tests: A) with the engine setpoint 2000rpm and the surge module on at all times; B) with a weakened engine, operating at a 1850rpm setpoint and with the surge module on in some tests and off in others.

The traces show a matrix of plots with DC bus voltage, surge module current, inverter current and voltage, synchronous machine current and engine rpm. The speed was calculated from zero crossing of the synch. mach. current.

The other time (i.e. "Oscilloscope_8_31_06.pdf") the DC bus voltage was obtained from subtracting low rail voltage from the high rail voltage. At this time, only the high rail voltage was captured: the full rail to rail voltage was calculated multiplying by two this available voltage.

The two series of tests can be found:

A) Engine set to run at 2000rpm pg. 2

B) Engine set to run at $1850 \mathrm{rpm}$ 8

Notes:

i) the current from the surge module (when it is activated) seems to be larger than previously measured in August. I am not sure if before there was a filter in the probe and this time it was without the filtering. Or the issue could be related to the sampling rate. The average current should be equal to $20 \mathrm{~A}$.

ii) in the case A) with load from $60 \mathrm{~kW}=>0$, (Figure 6) the DC bus exceeds $900 \mathrm{~V}$. It could be that the mid point voltage experienced a sag and the high rail was larger than the low rail. Because this plot was obtained by multiplying by two the high rail voltage, it is possible that the actual overall DC bus never exceeded $900 \mathrm{~V}$. iii) I agree with Dave that when the surge module is injecting current, the sychronous machine current has a strong non zero DC offset. This behaviour was identically present also in the August waveforms. 


\section{A) Engine at 2000 RPM}

OKW to 25kW Load Step with Surge Module On shows No Surge Assist.
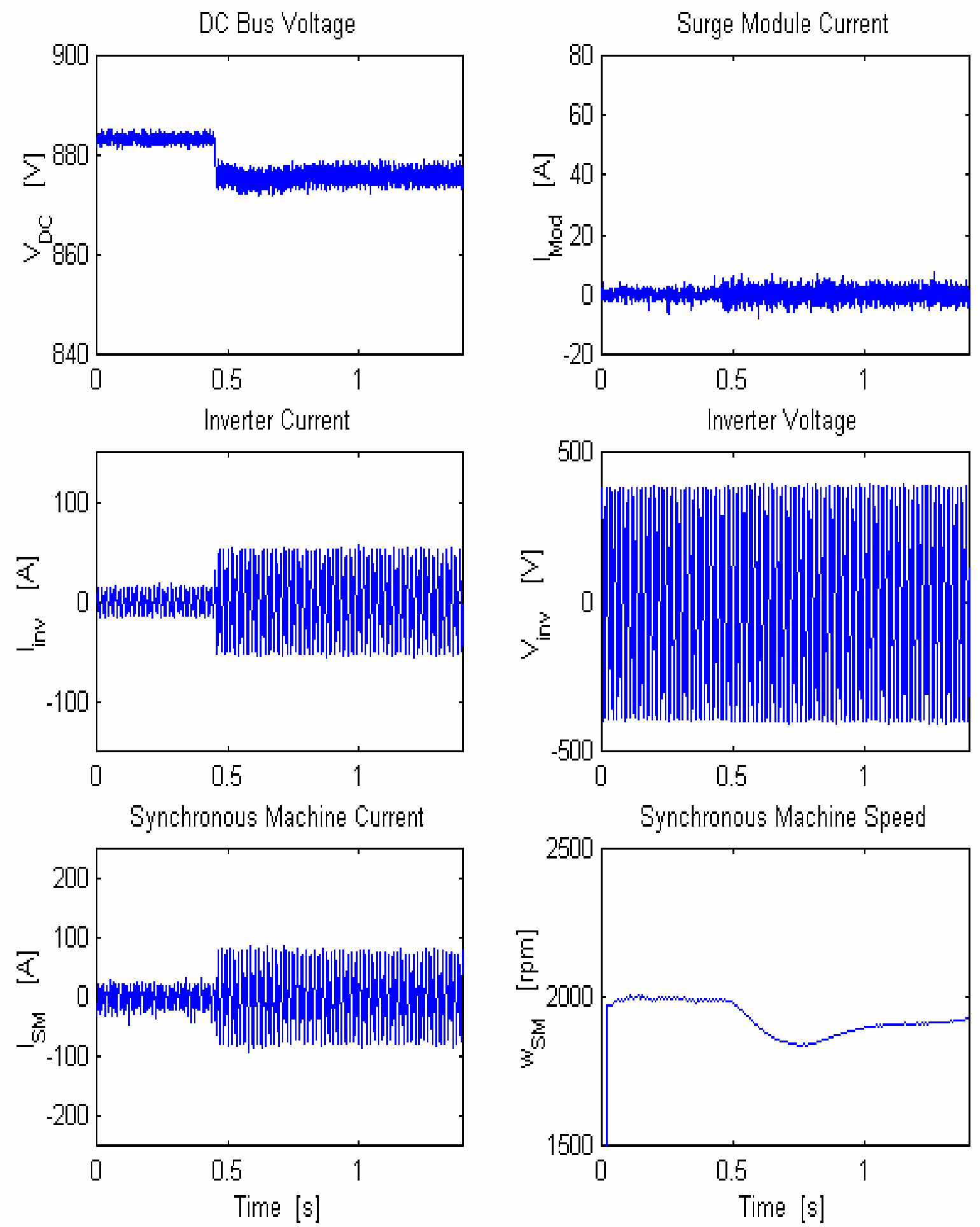

Figure 1. 
OKW to 30kW Load Step with Surge Module On shows Surge Assist.
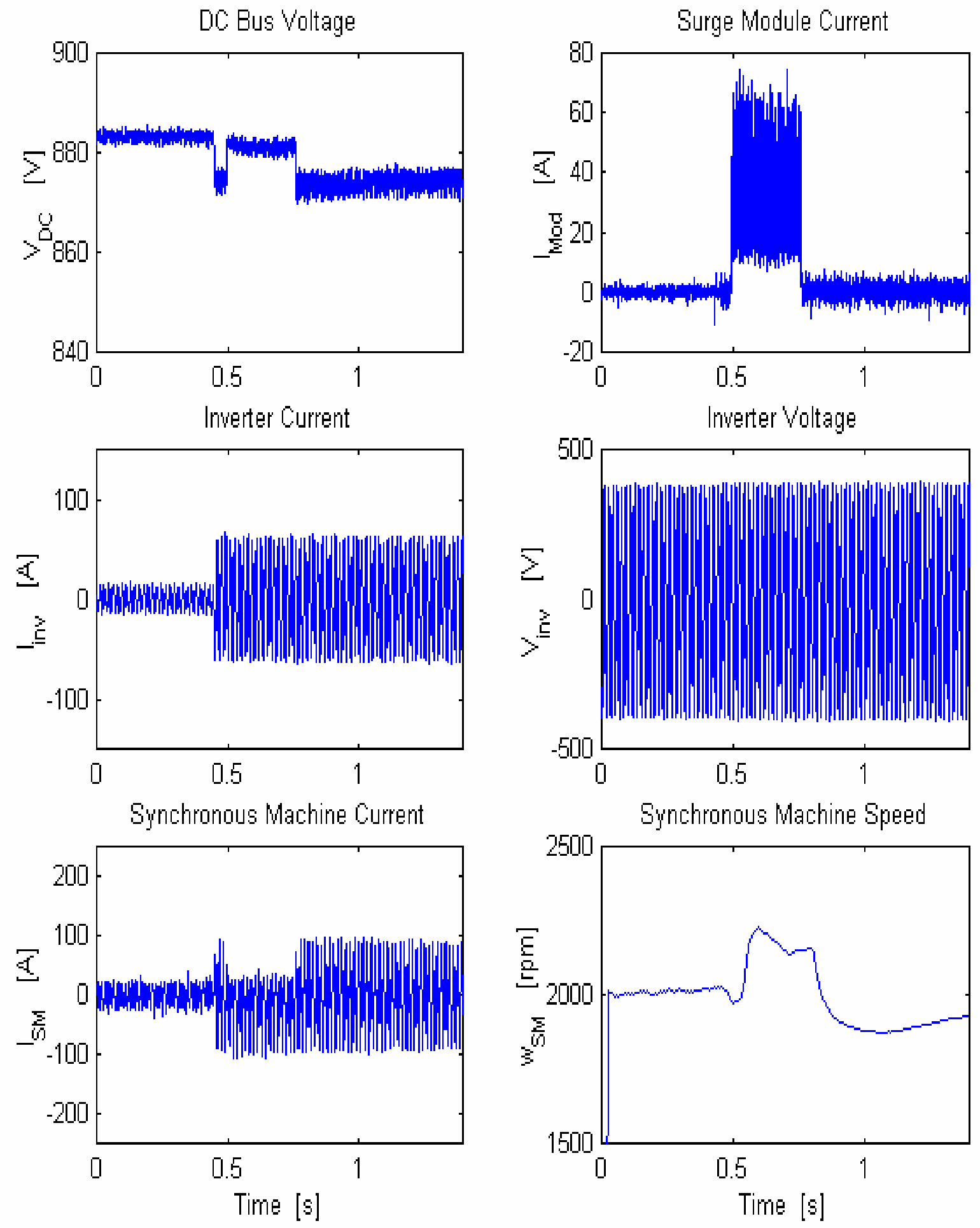

Figure 2. 
OKW to 60kW Load Step with Surge Module On shows Surge Assist.
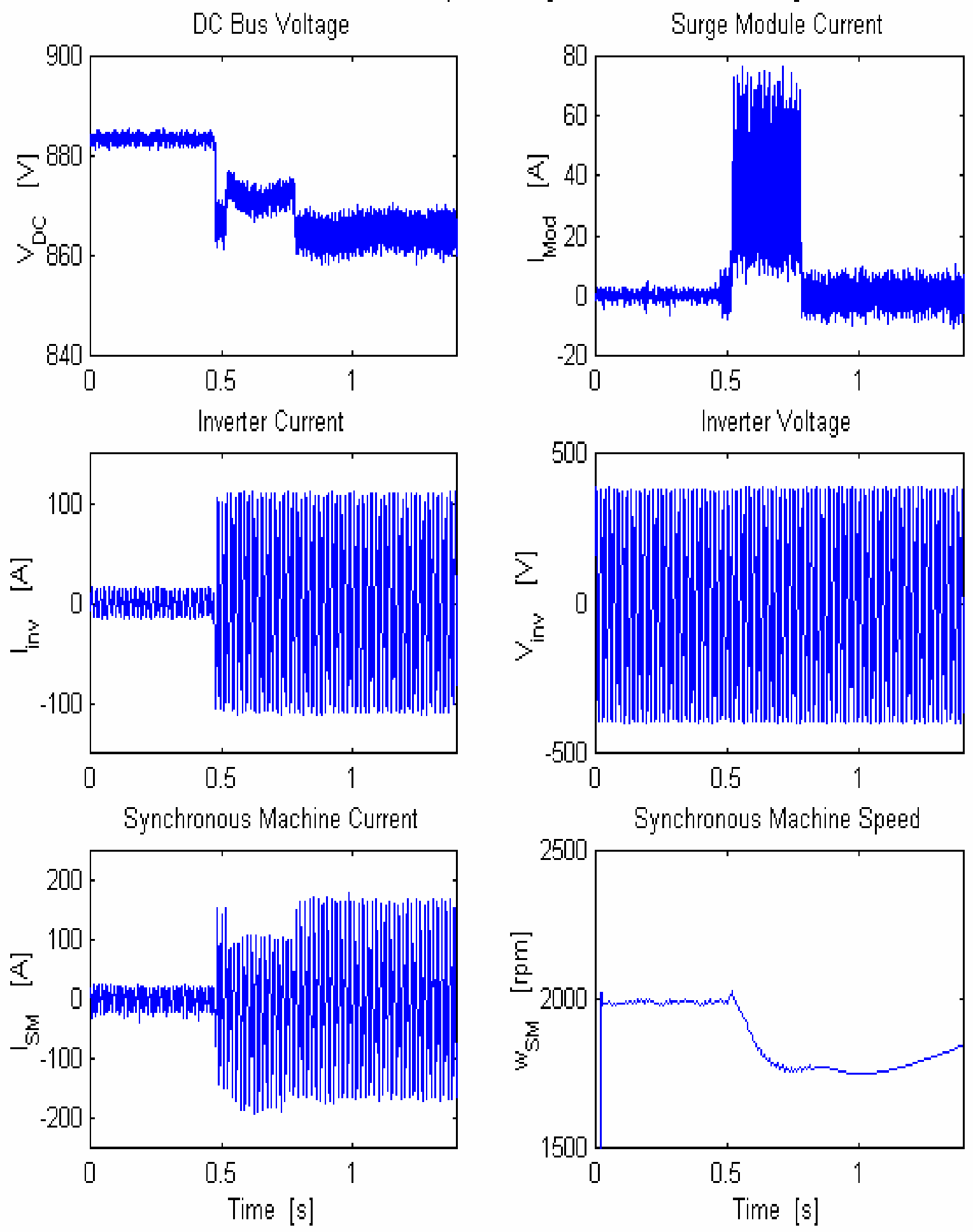

Figure 3. 
$25 \mathrm{KW}$ to OKW Load Step with Surge Module On.
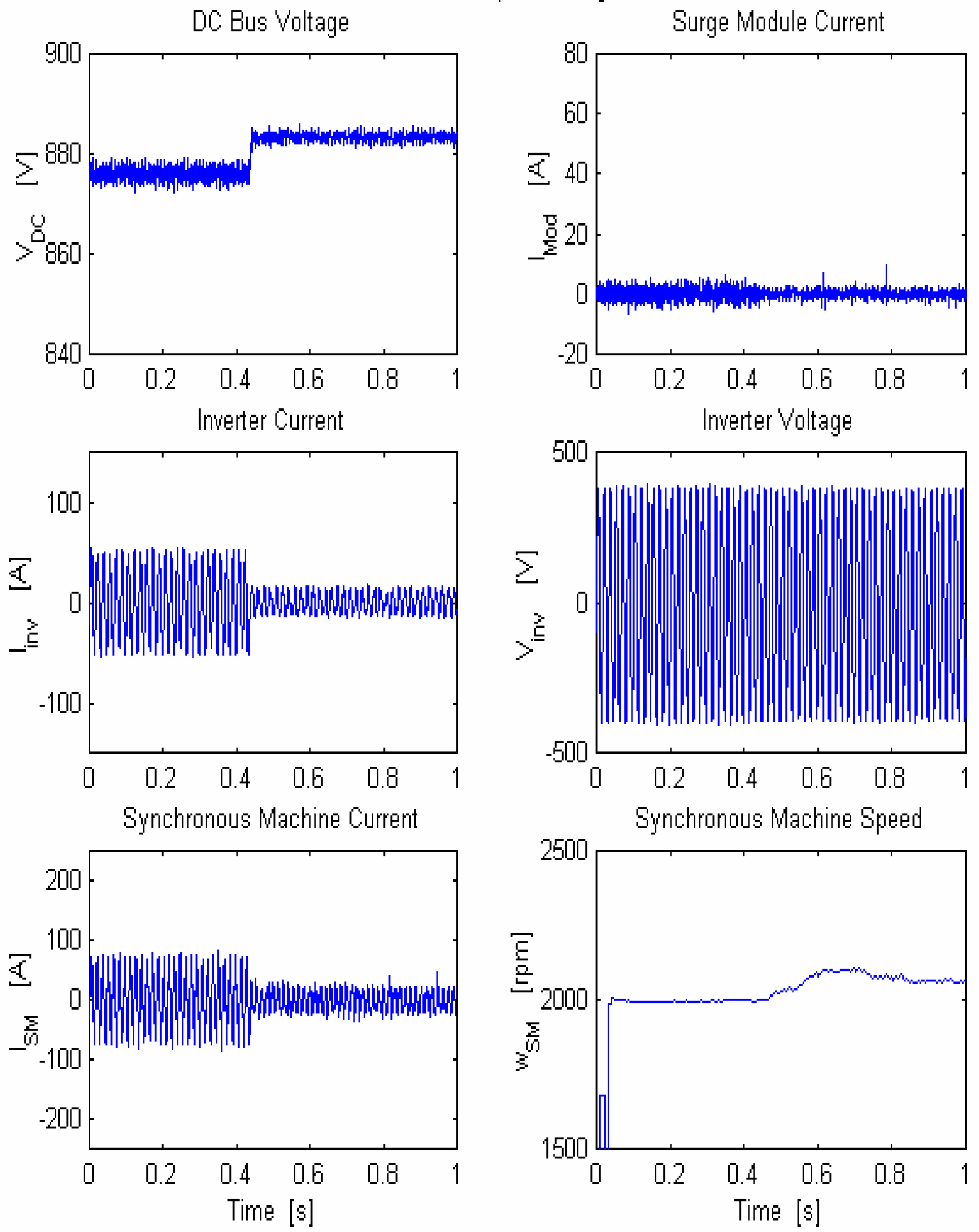

Figure 4. 
$30 \mathrm{~kW}$ to OKW Load Step with Surge Module On.
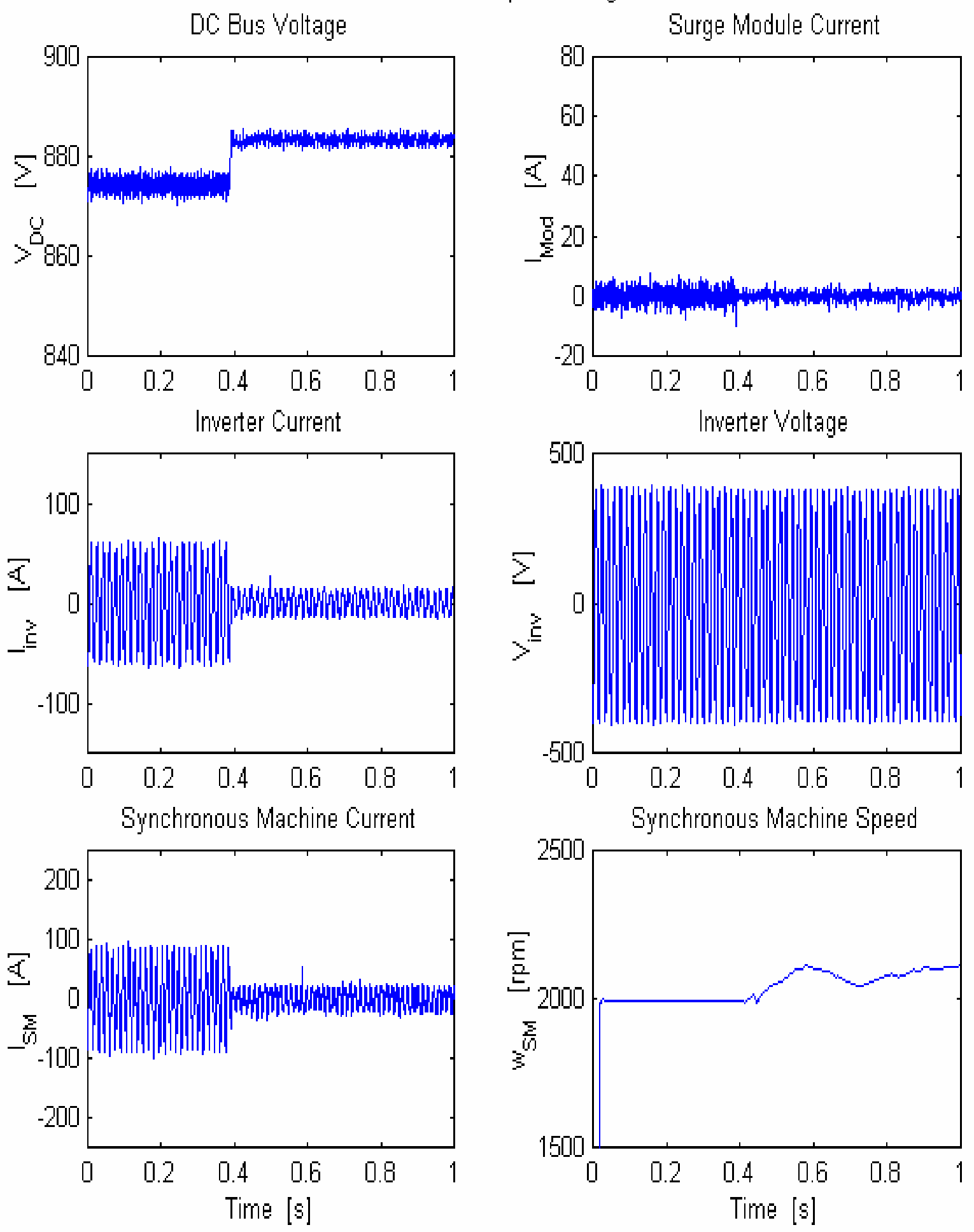

Figure 5. 
G0KW to OKW Load Step with Surge Module On.
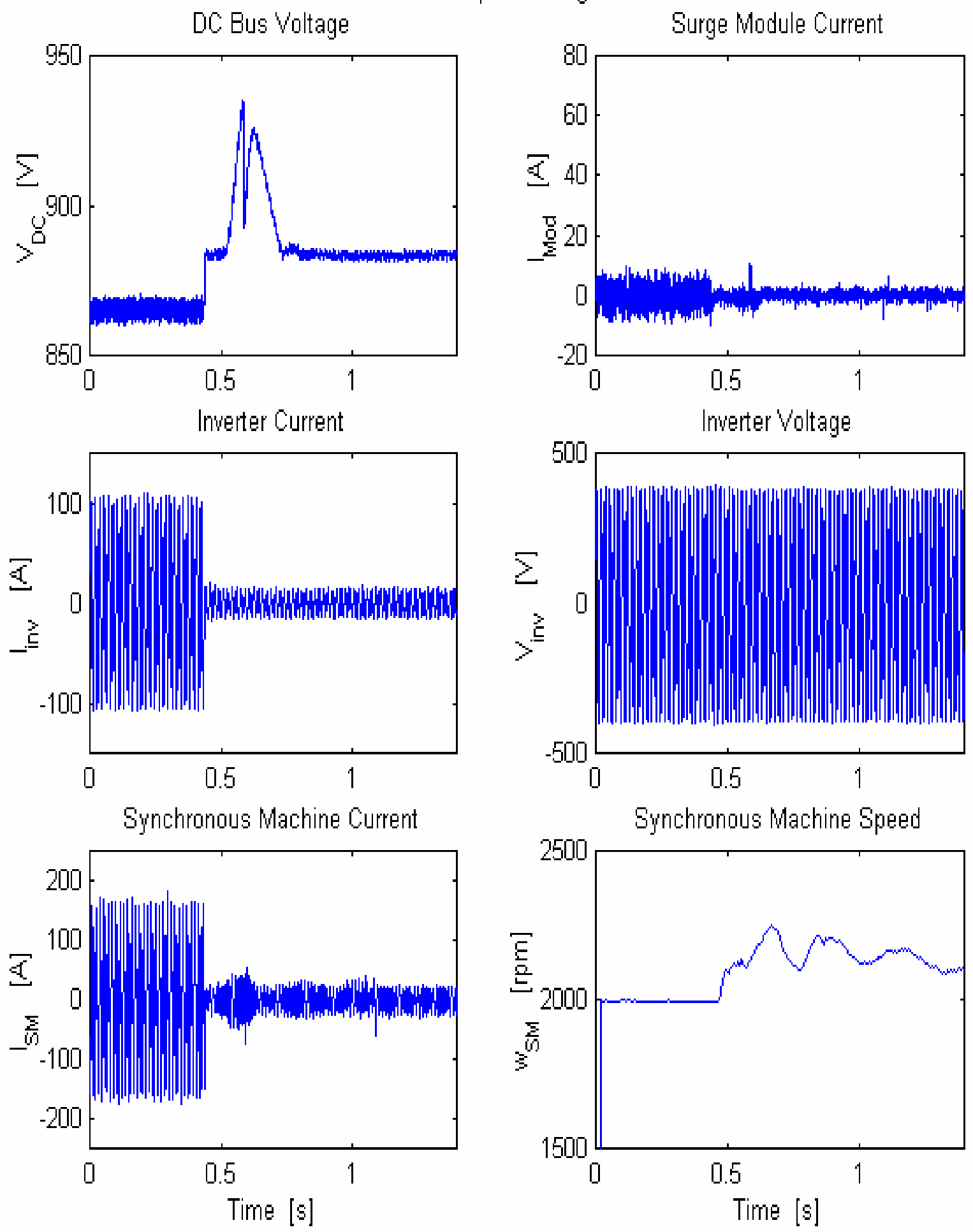

Figure 6. 


\section{B) Engine at 1850 RPM}

OKW to 25kW Load Step with Surge Module Off.
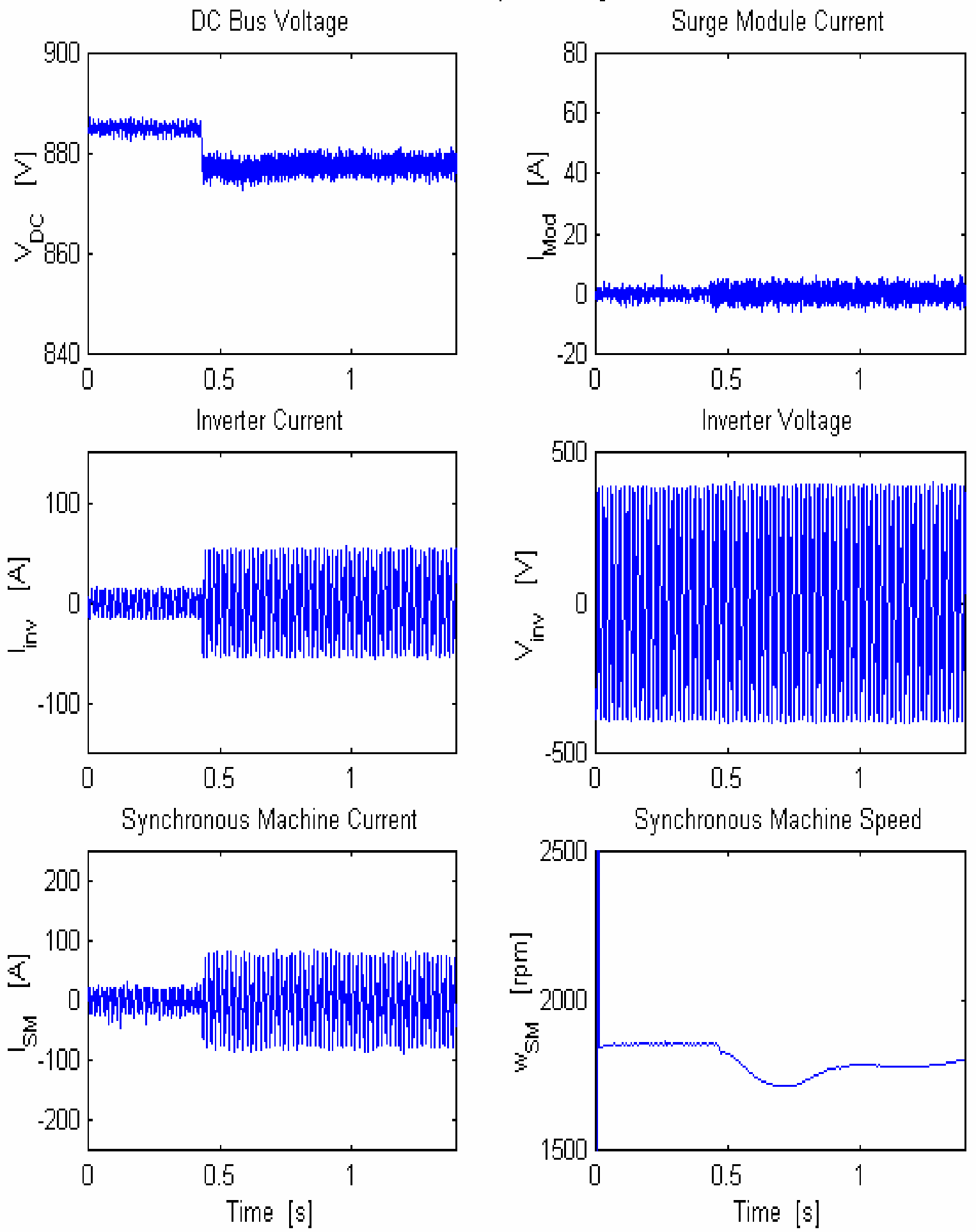

Figure 7. 
OKW to 25KW Load Step with Surge Module On.
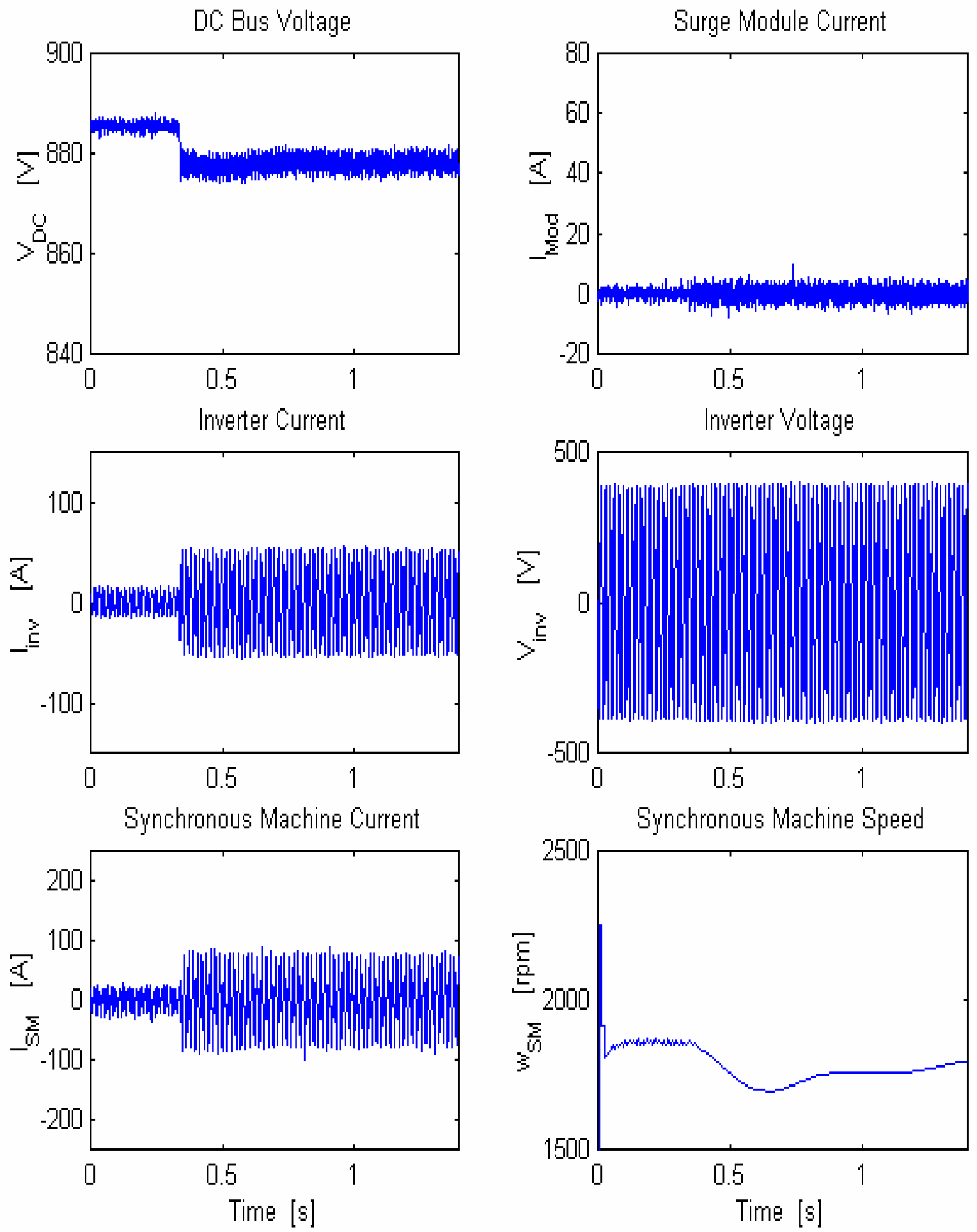

Figure 8. 

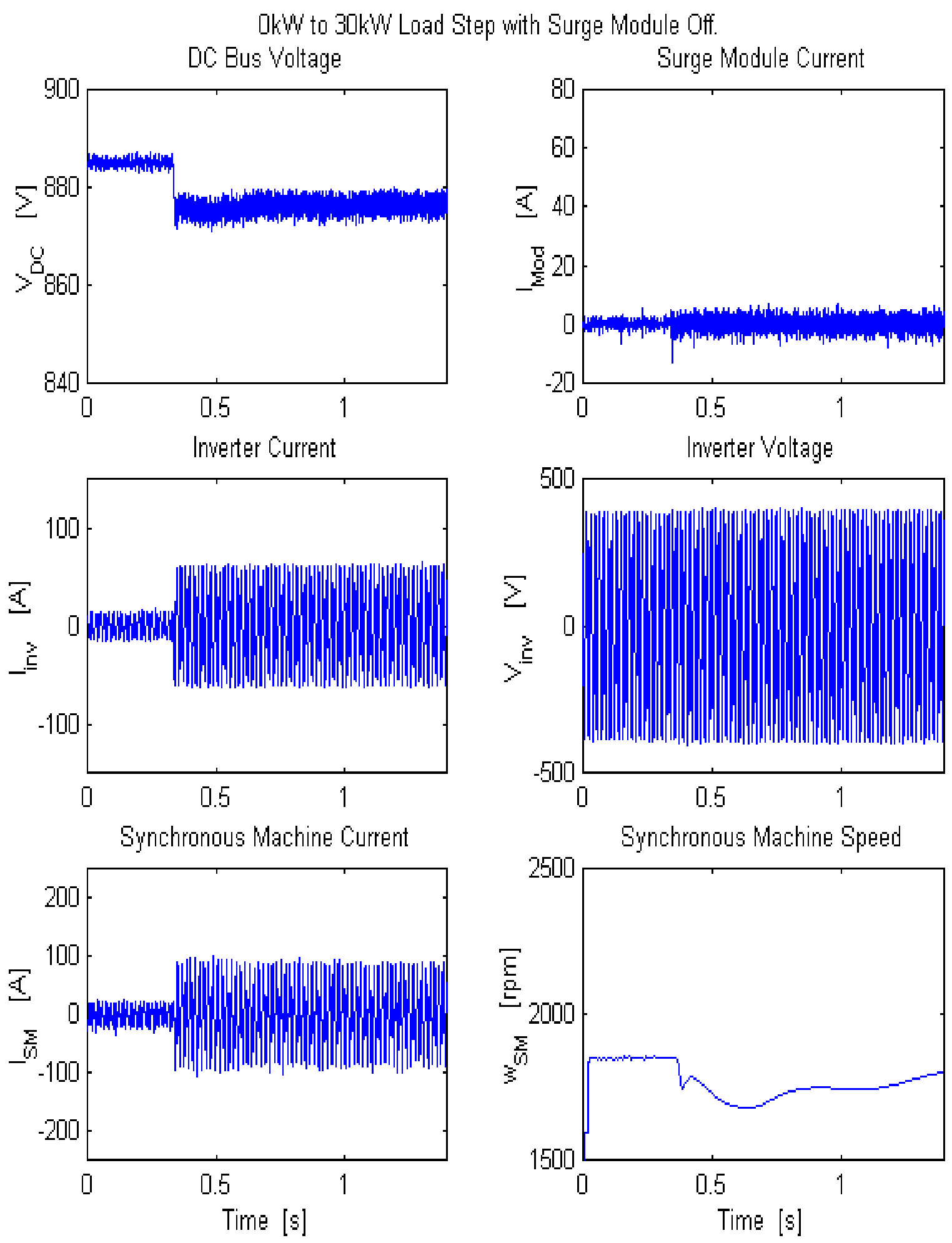

Figure 9. 
OKW to 30kW Load Step with Surge Module On shows Surge Assist.
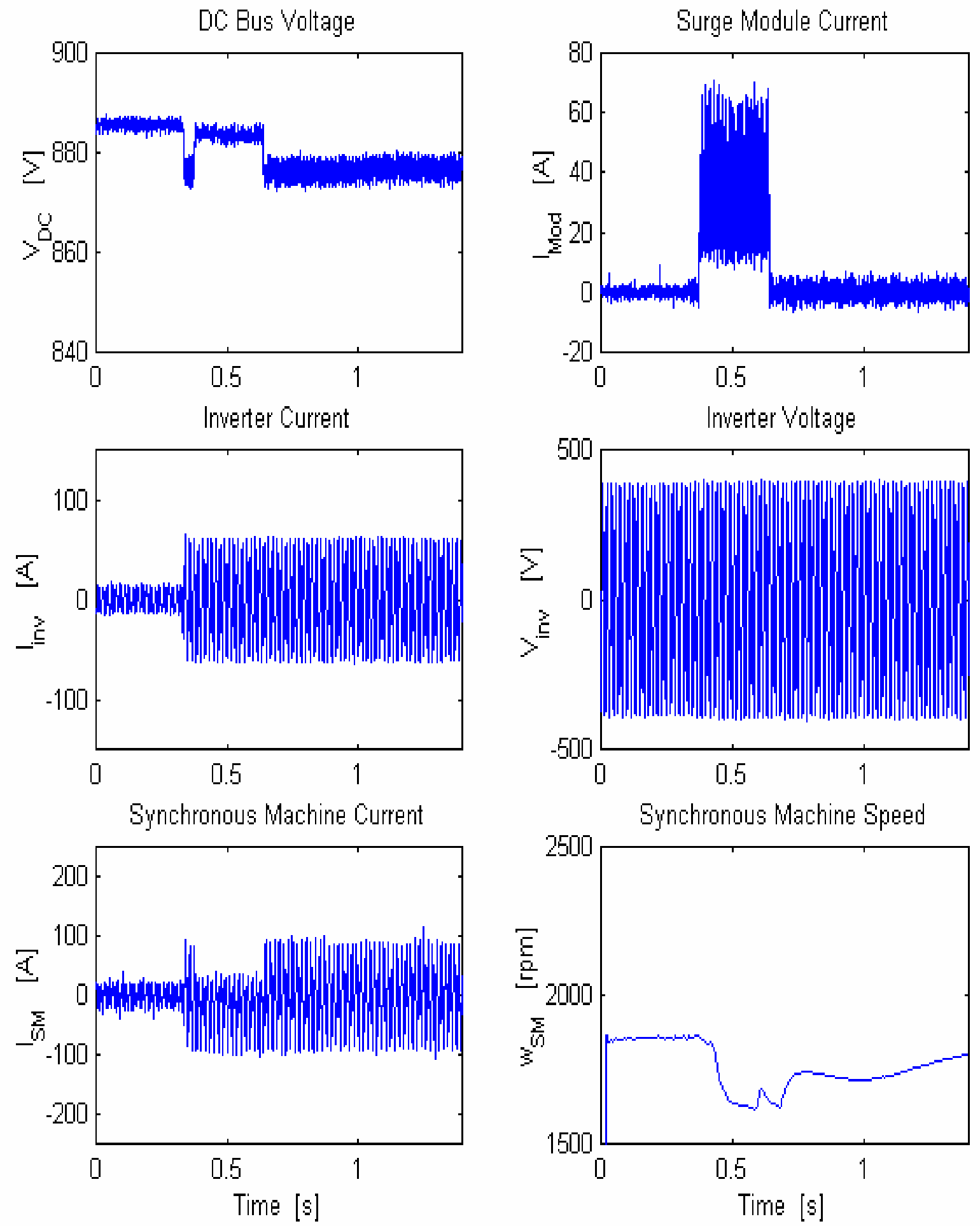

Figure 10. 

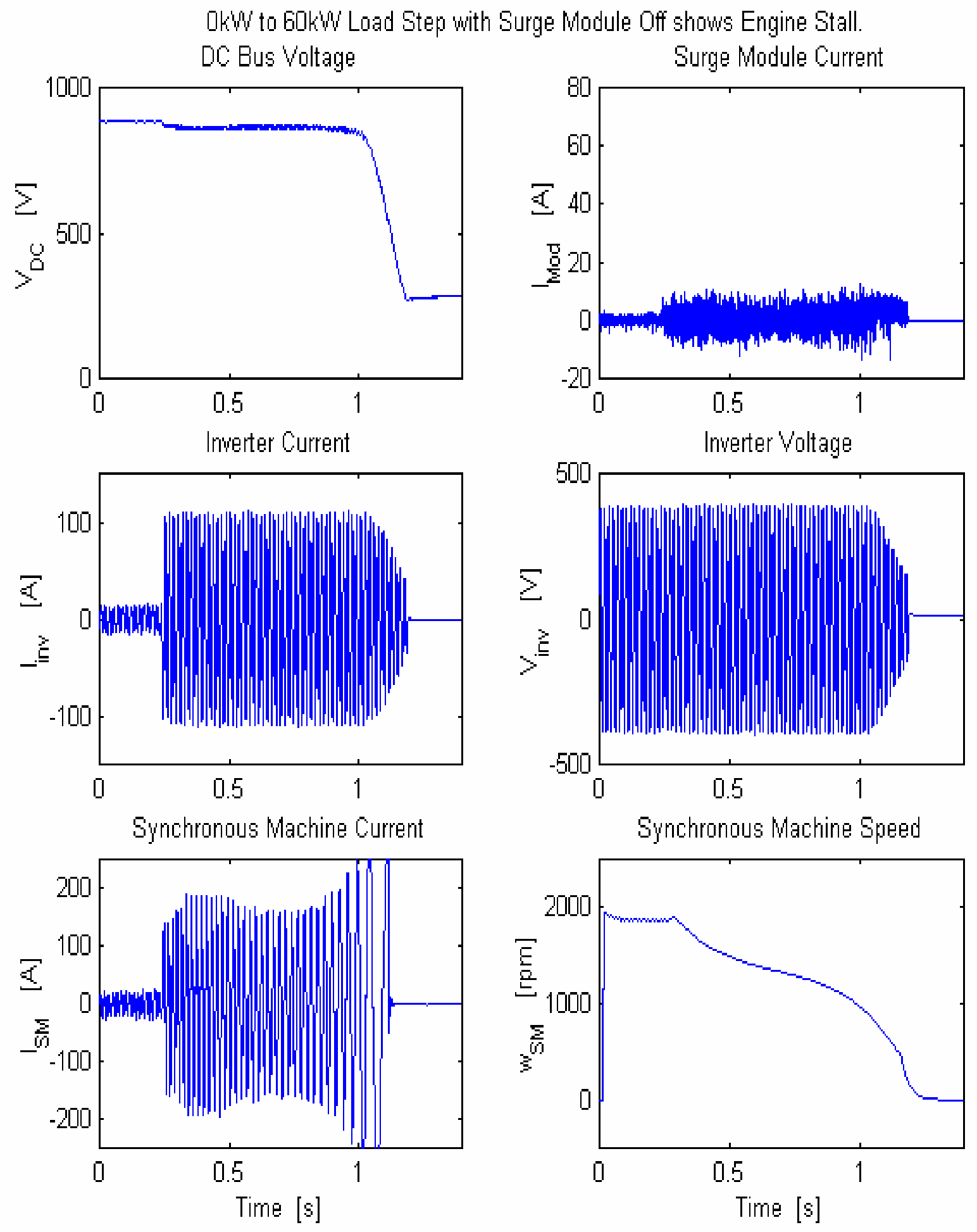

Figure 11. 

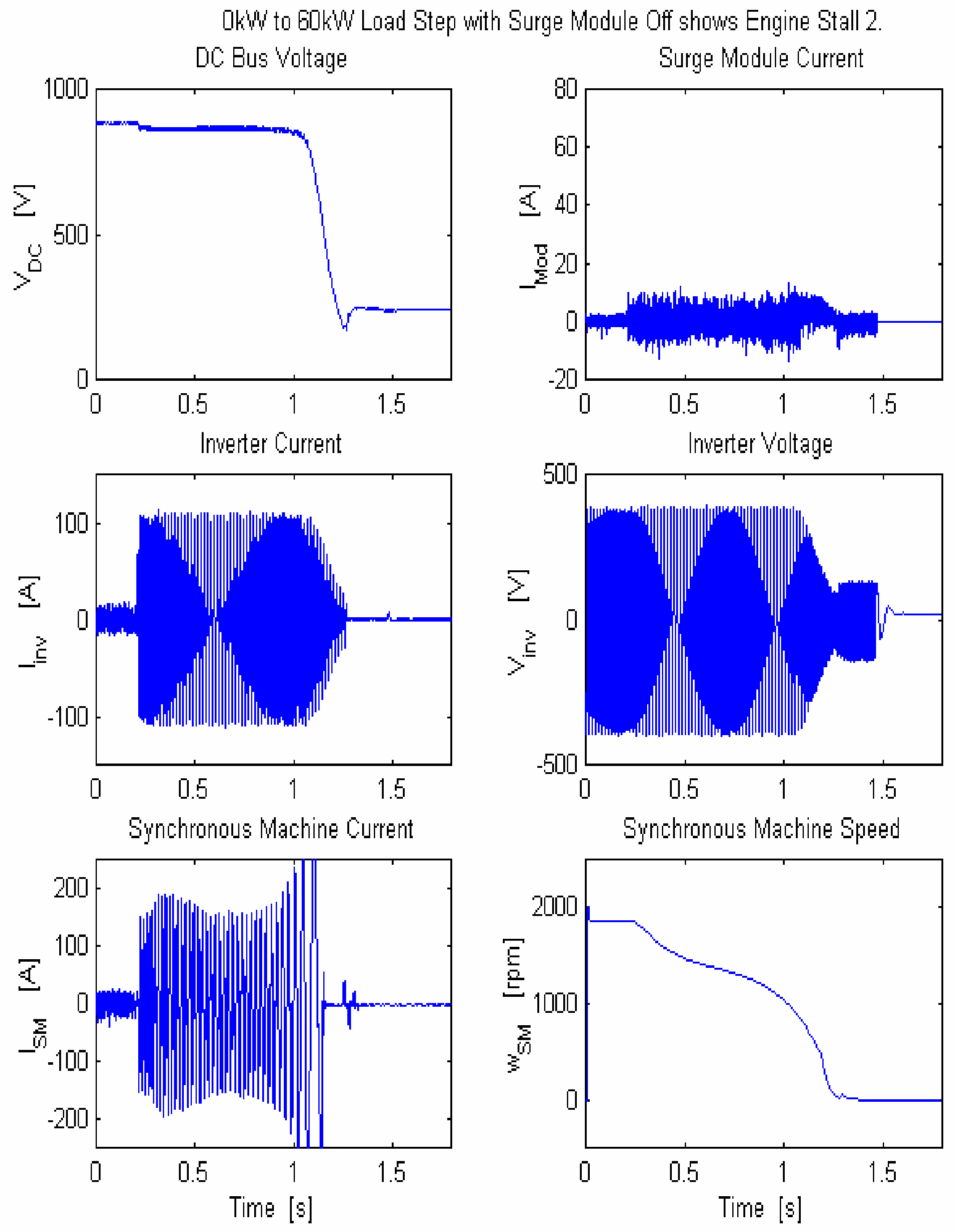

Figure 12. 
OKW to 60kW Load Step with Surge Module On shows Surge Assist.
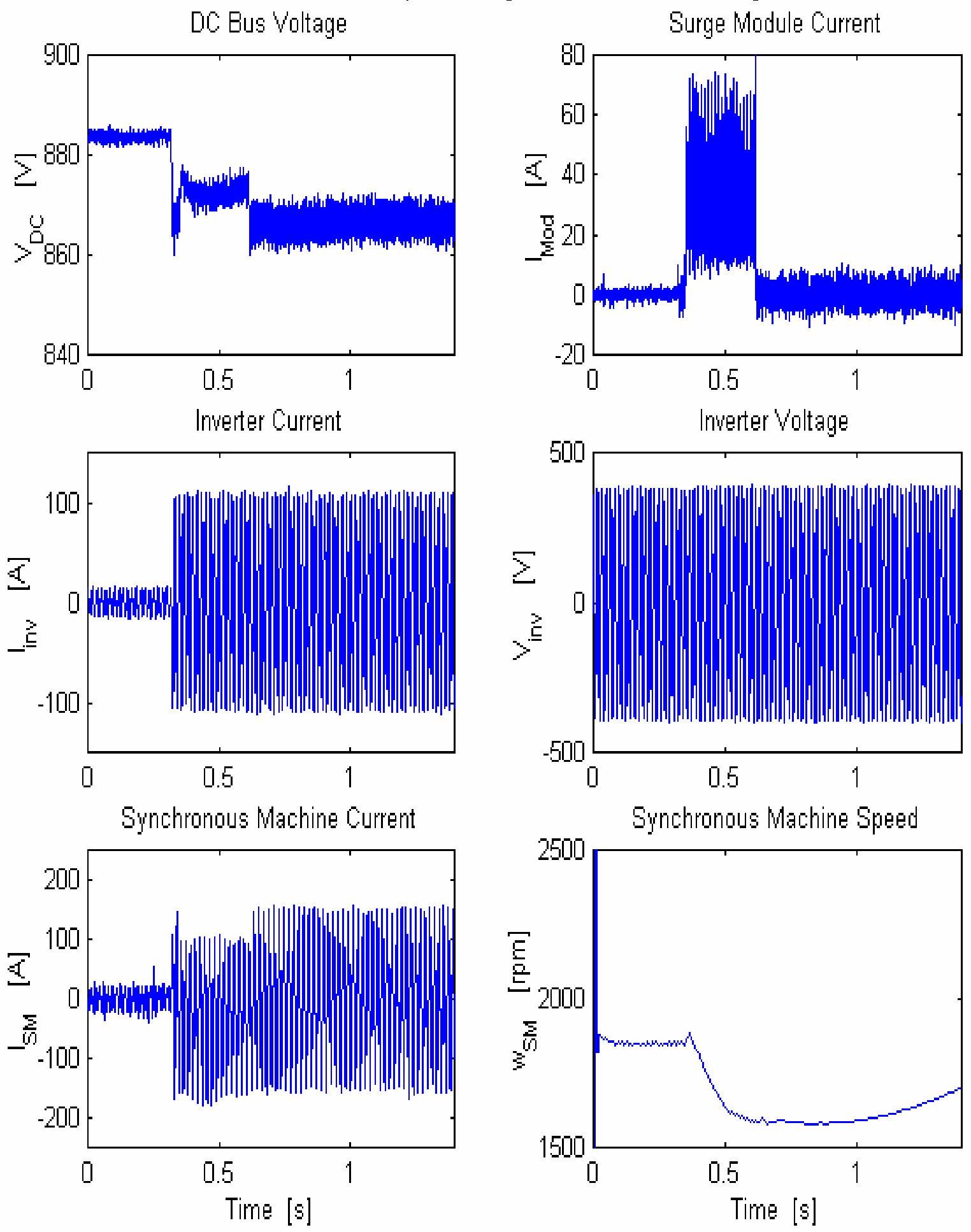

Figure 13. 
$25 \mathrm{KW}$ to OKW Load Step with Surge Module Off.
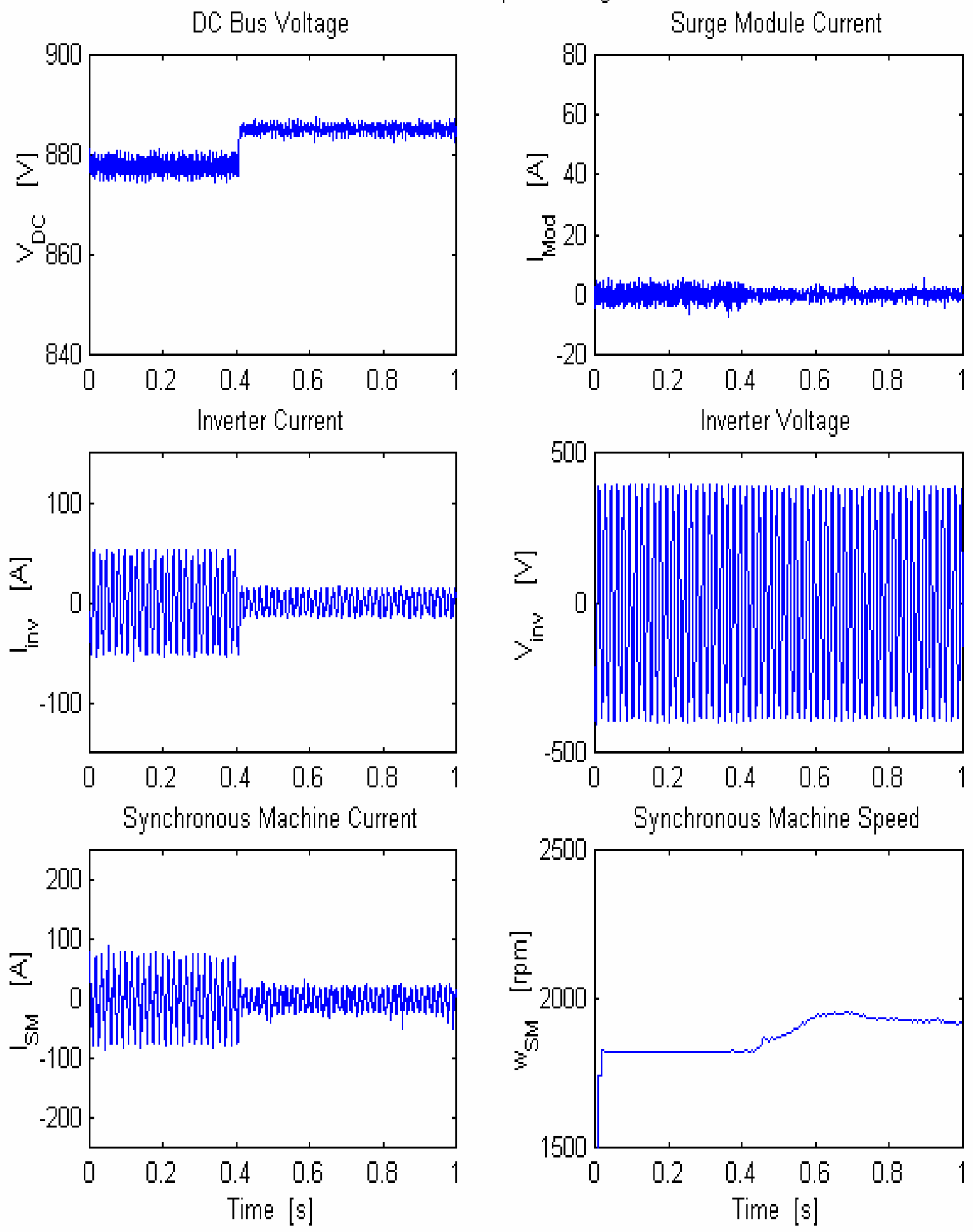

Figure 14. 

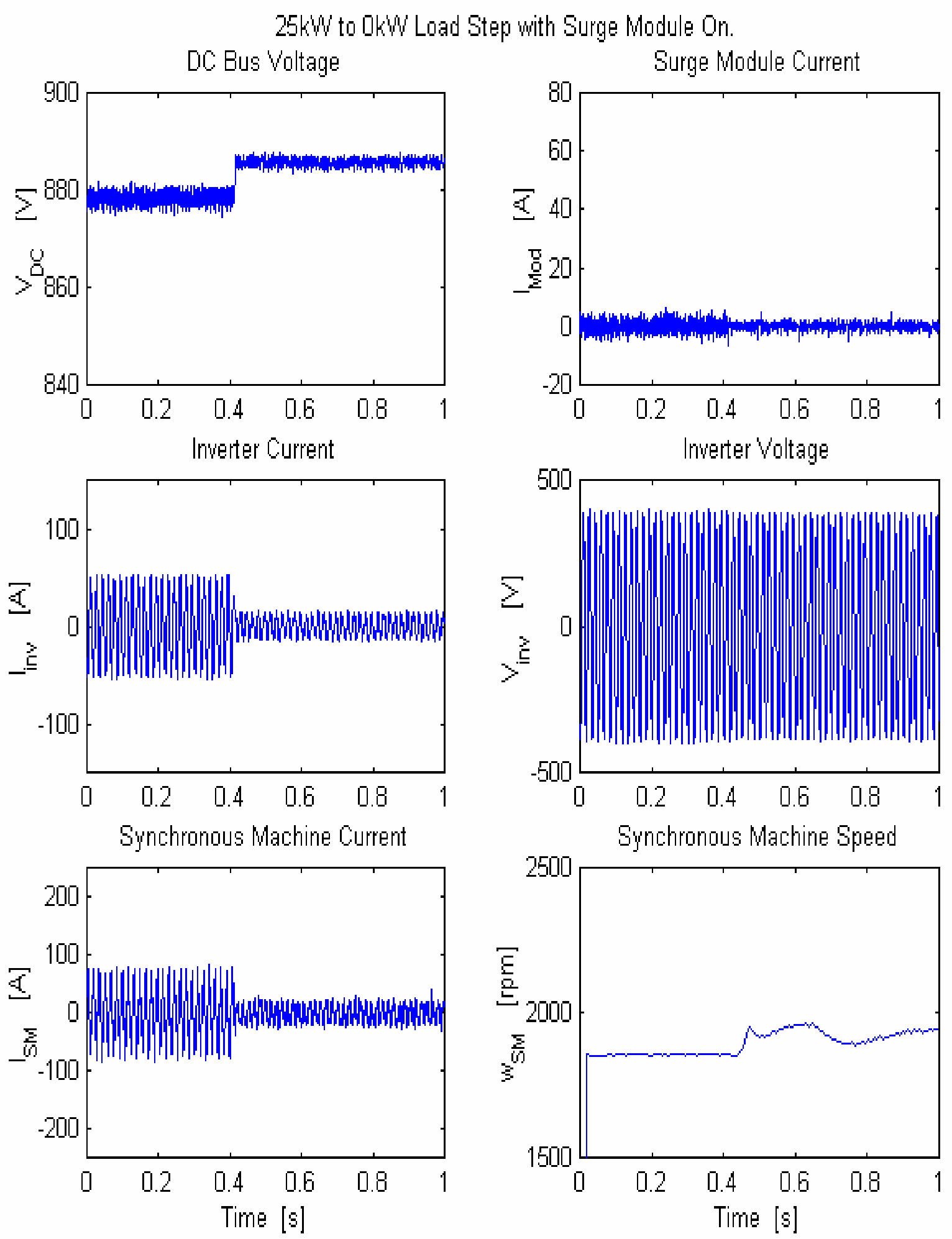

Figure 15. 
$30 \mathrm{~kW}$ to OKW Load Step with Surge Module Off.
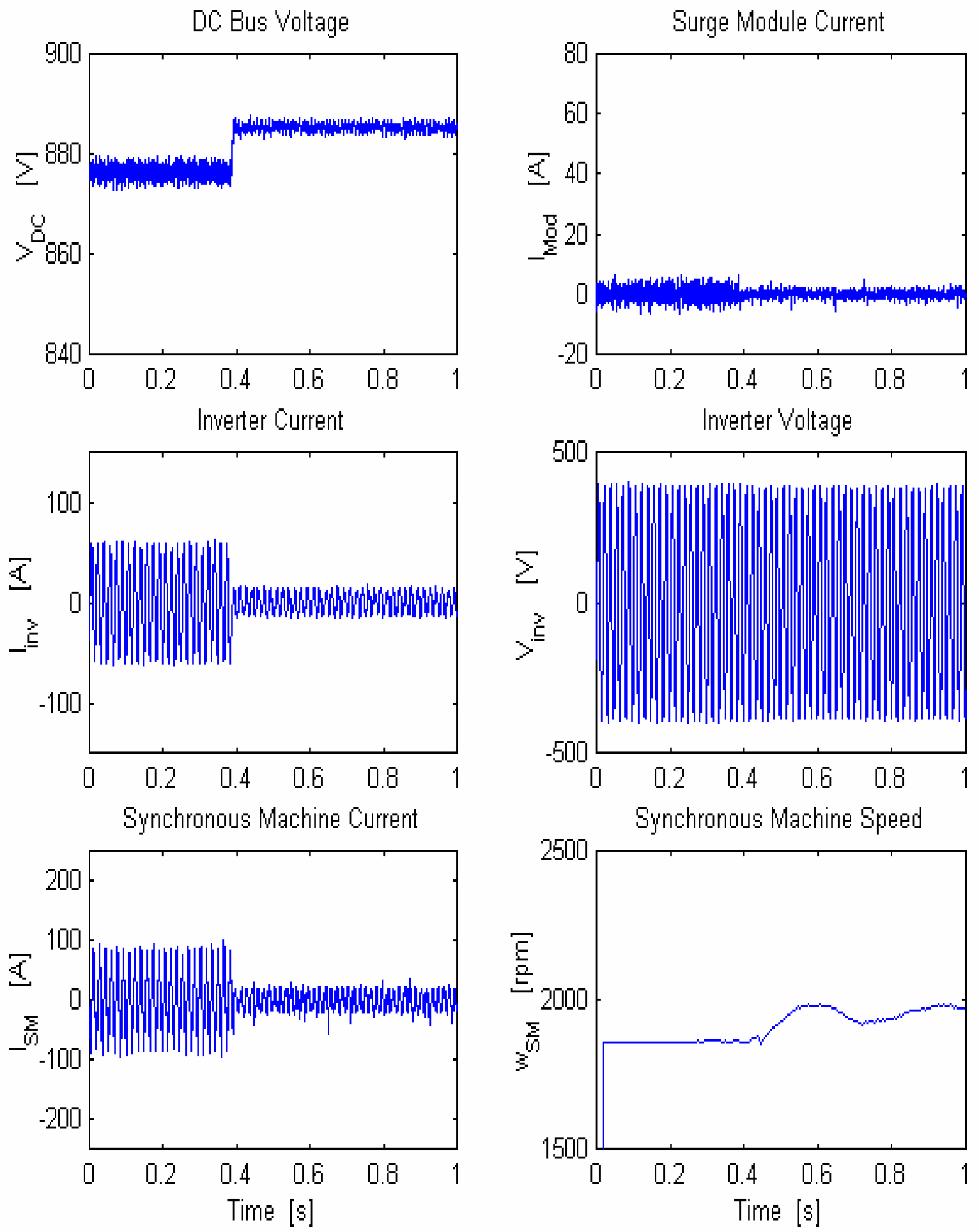

Figure 16. 
$30 \mathrm{~W}$ to OKW Load Step with Surge Module On.
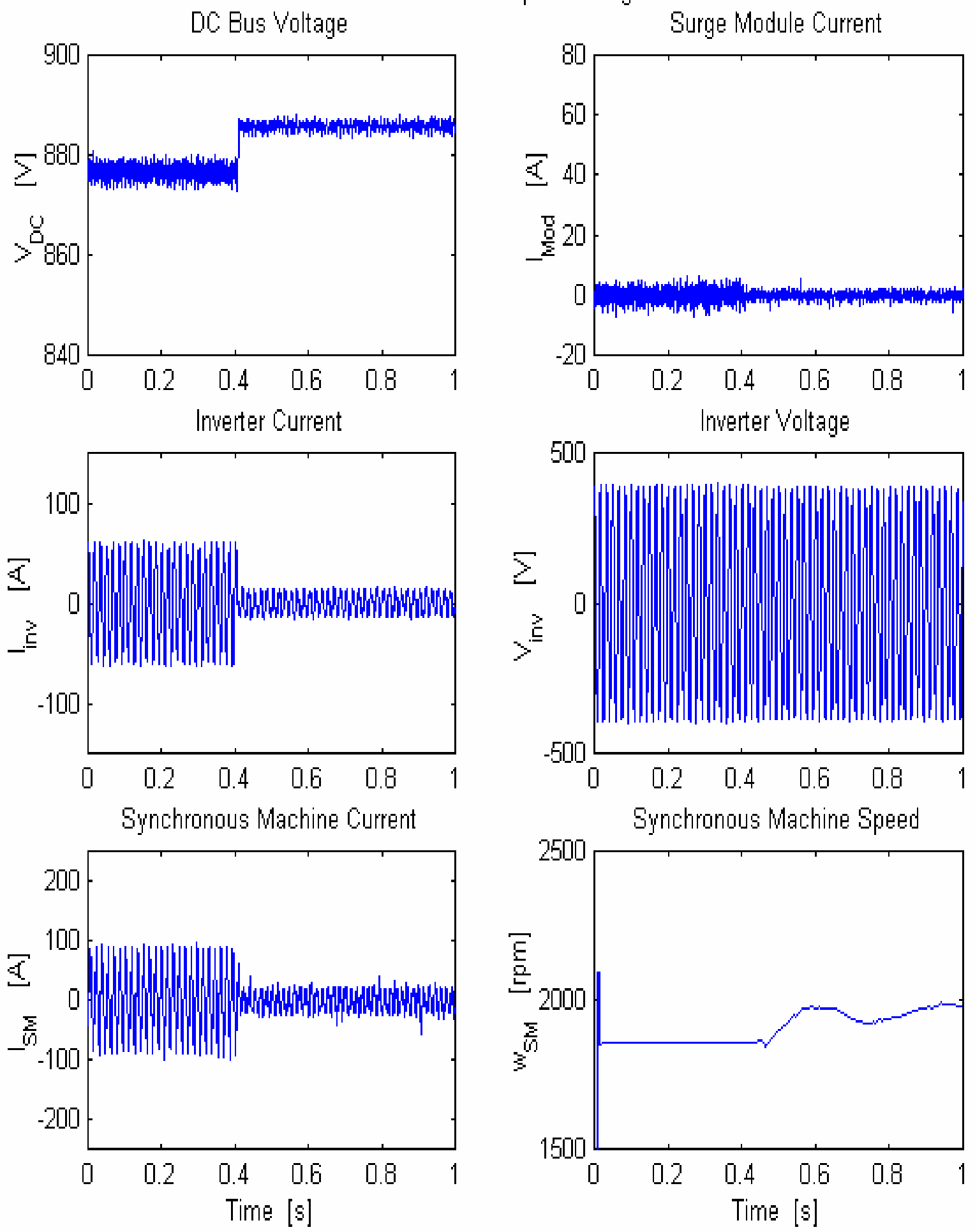

Figure 17. 
60KW to OKW Load Step with Surge Module On.
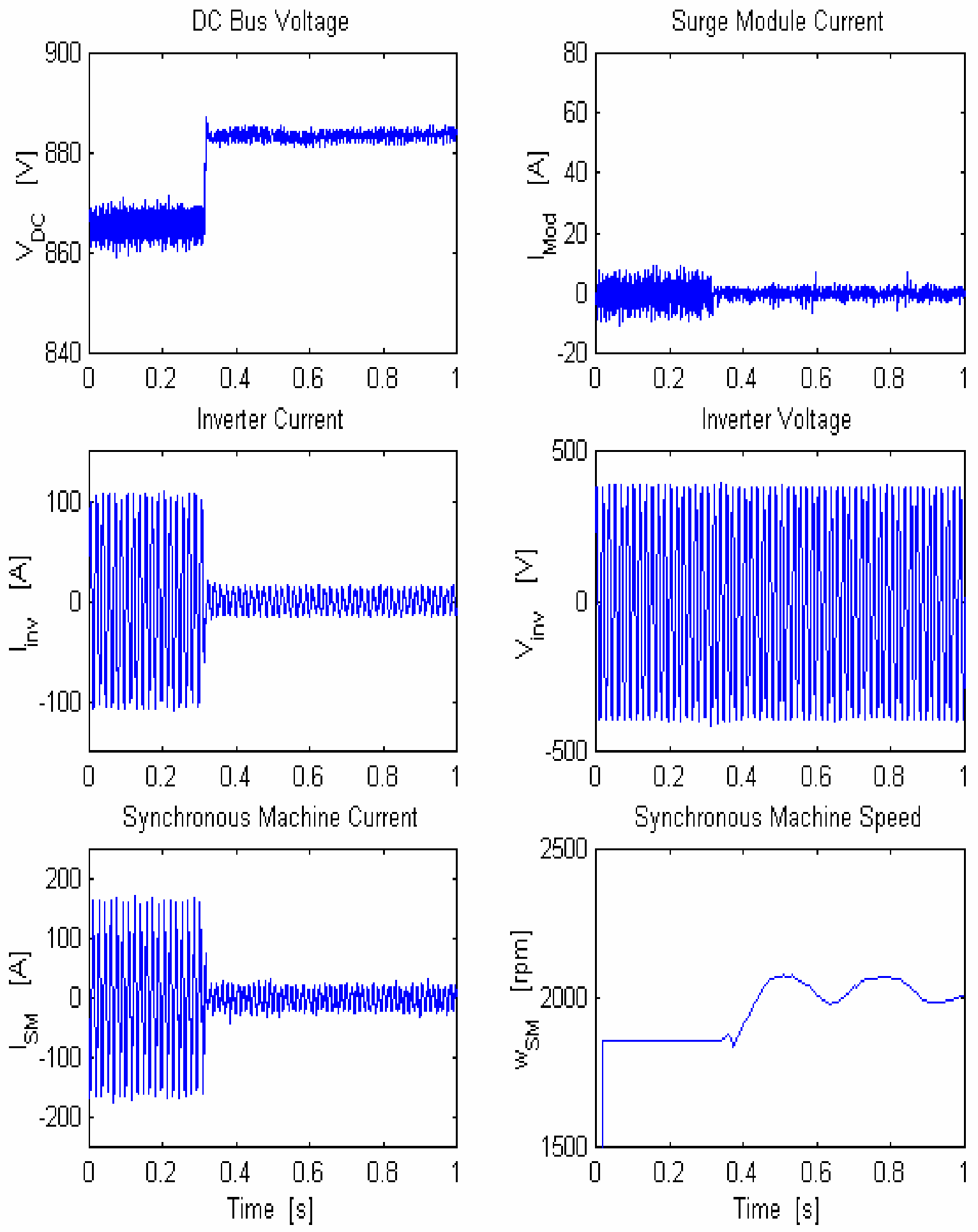

Figure 18. 


\section{CERTS MICROGRID LABORATORY TEST BED}

\section{CERTS Test Bed CERTEQUIP-V06-002, CERTS Static Switch, Low Power Factory Acceptance Test Report}

Prepared For:

California Energy Commission

Public Interest Energy Research Program

Arnold Schwarzenegger Governor

Prepared By:

Lawrence Berkeley National Laboratory

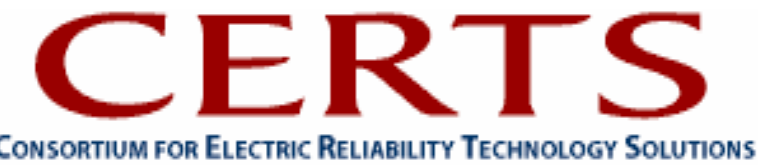



Prepared By:

Lawrence Berkeley National Laboratory

Joseph H. Eto, Principal Investigator

Berkeley, CA 94720

Ed Linton and Hector Hurtado, Northern Power Systems

Commission Contract No. 500-03-024

Prepared For:

Public Interest Energy Research (PIER)

California Energy Commission

Bernard Treanton

Contract Manager

Mike Gravely

Program Area Lead

ENERGY SYSTEMS INTEGRATION

Mike Gravely

Office Manager

ENERGY SYSTEMS RESEARCH

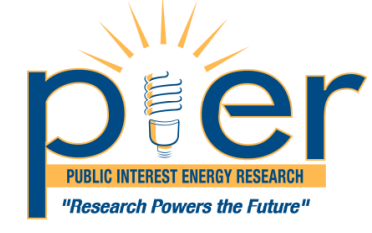

Martha Krebs, Ph.D.

PIER Director

Thom Kelly, Ph.D.

Deputy Director

ENERGY RESEARCH \& DEVELOPMENT DIVISION

Melissa Jones

Executive Director

\section{DISCLAIMER}

This report was prepared as the result of work sponsored by the California Energy Commission. It does not necessarily represent the views of the Energy Commission, its employees or the State of California. The Energy Commission, the State of California, its employees, contractors and subcontractors make no warrant, express or implied, and assume no legal liability for the information in this report; nor does any party represent that the uses of this information will not infringe upon privately owned rights. This report has not been approved or disapproved by the California Energy Commission nor has the California Energy Commission passed upon the accuracy or adequacy of the information in this report. 



\section{CERTS Test Bed CERTEQUIP-V06-002 CERTS Switch Low Power Factory Acceptance Test Report}




\section{Contents}

1 Introduction

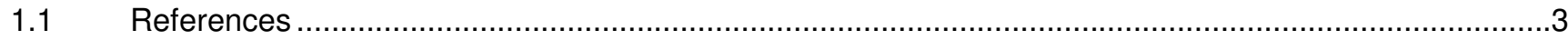

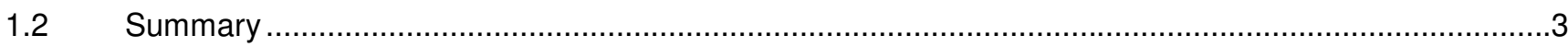

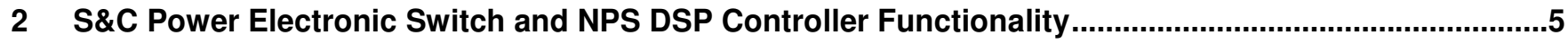

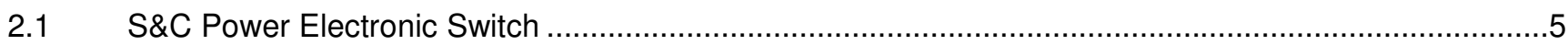

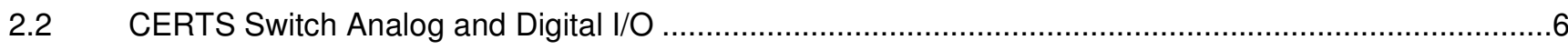

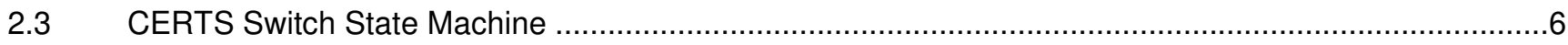

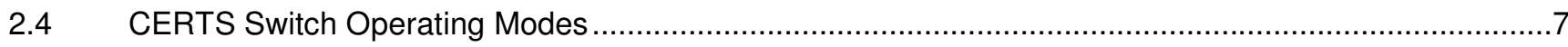

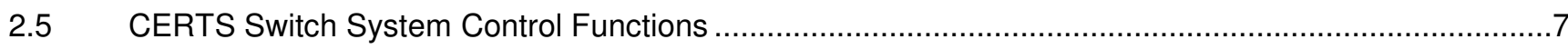

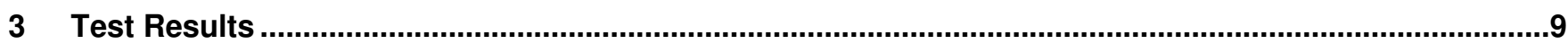

3.1 S\&C-DSP Interface Commissioning Test [Test Plan Section 2.1] …..............................................10

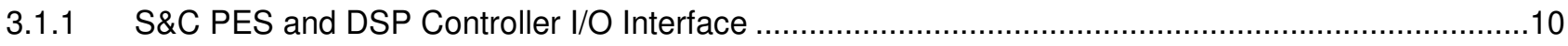

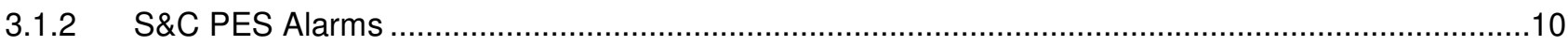

3.2 Synchronization [Test Plan Section 3.1] ….......................................................................12

3.2.1 Limits Accuracy (using Omicron CMA256-6 secondary injection test set from NREL) ....................12

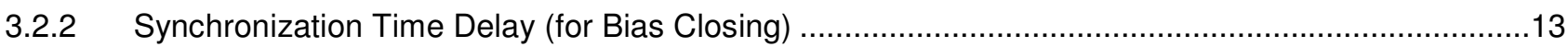

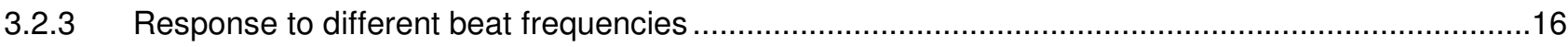

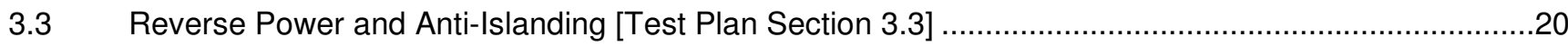

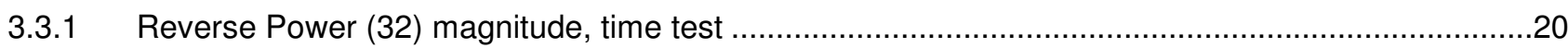

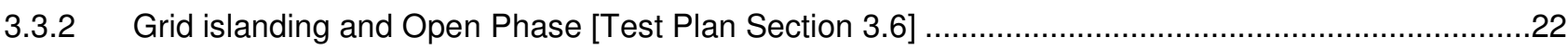

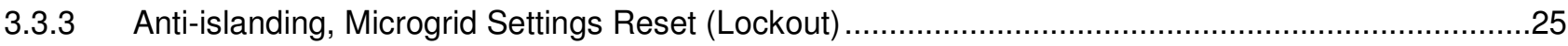

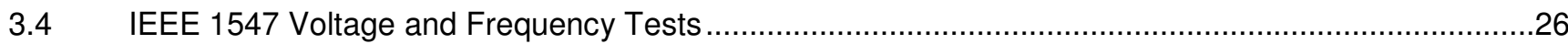

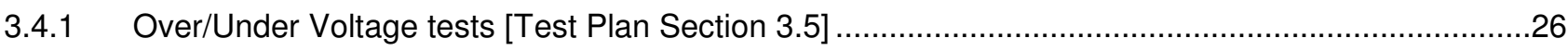

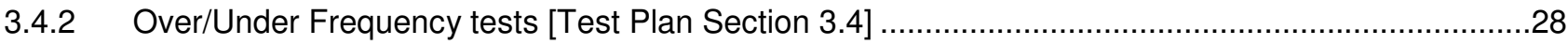

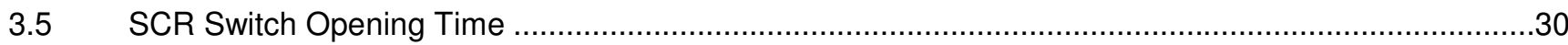

3.6 SEL-351 Relay - DSP Controller Functionality [Test Plan Section 4] ...............................................31

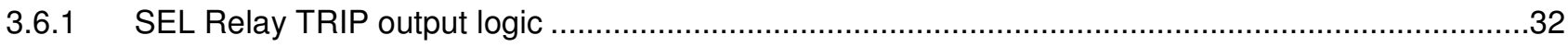

3.6.2 SEL Relay minimum opening time and associated DSP reclosing ...........................................33

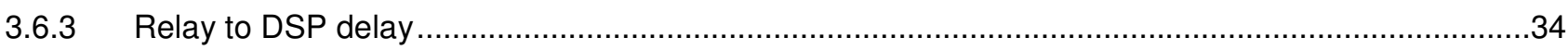

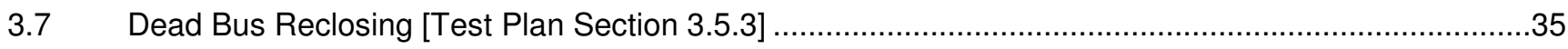

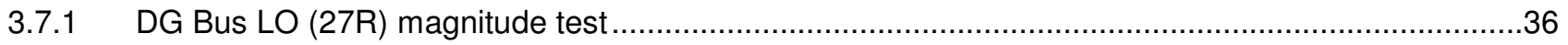

3.7.2 DG Bus LO (27R) time test; "Dead Bus Reclose" operating mode ..............................................36

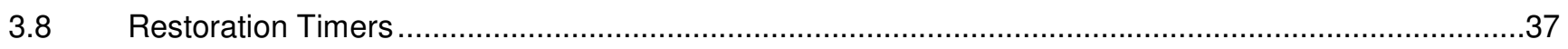

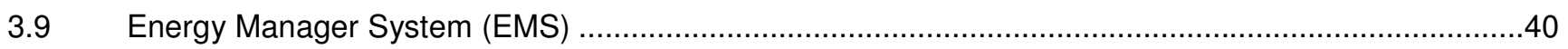

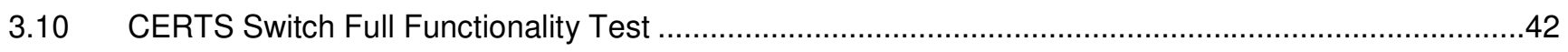

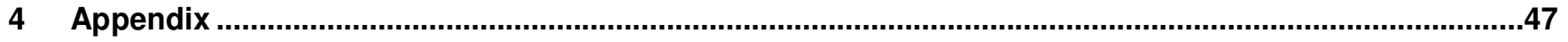

4.1 Appendix 1: IEEE1547 Tests Results with NREL's Omicron Equipment (Section 3.3) .......................47

$4.2 \quad$ Appendix 2: List of DSP Controller settings in EMS (Section 3.9) ................................................54 
\lrcorner Northern

A Distributed Energy

Systems Company

4.3 Appendix 3: Synchronization Tests (Section 3.2) plots................................................................56 


\subsection{References}

* Reference 1: CERTEQUIP-T05-004, "CERTS Test Bed, Factory Acceptance Test, Static Switch Low Power Test Plan"

* Reference 2: CERTEQUIP-H03-002, "CERTS Static Switch Operation \& Control"

* Reference 3: "Control and Design of CERTS Microgrid", R. H. Lasseter and Paolo Piagi, Spetember 28,2005

* Reference 4: "Static Switch Test V2" (2/06), R.H. Lasseter

\subsection{Summary}

The CERTS Test Bed is designed to test advanced microgrid and distributed generation control concepts developed by Professor Robert Lasseter at the University of Wisconsin. It consists of inverter-coupled generators, loads, low-voltage distribution and data gathering equipment and a static switch to separate the microgrid from the utility. Separation allows the microgrid to operate as an island, protecting its loads when there is a problem with the utility supply. The switch implements IEEE-1547 DG interface requirements, protecting the utility from reverse power and the microgrid from utility power quality issues. Disconnection and reconnection are accomplished smoothly with reduced transients presented to either side.

The switch chosen for the Test Bed was supplied by S\&C Electric. It is a 480V, 400A thyristor (SCR) switch with controller, a modified Pure-Wave UPS product without the inverter and battery. To implement the required CERTS function we have added Northern Power's DSP controller and a programmable SEL-351 protective relay. This assembly was tested at reduced power in our lab. A one-line schematic of the test set up is given in Figure 1 below.

We used an existing inverter development test bed to provide controllable Microgrid and utility sources. The utility side inverter was operated as a voltage source with the ability to control voltage and frequency. The microgrid inverter used the University of Wisconsin control method with power / frequency droop. Control code for this method had been developed under an earlier phase of this CERTS contract. Each inverter was capable of $15 \mathrm{kVA}$ output and included controllable load banks.

The test plan is given in Reference1, test results are detailed in this report. Some revisions were made during testing to meet the capacities of the source inverters and to follow up on discovered issues. S\&C provided an applications engineer, Rodger Troyer, for three days on site to rectifying problems found during the initial test runs. Also during testing it was decided to add measurements made by NREL using their Omicron test equipment, applying secondary signal injection to test the IEEE1547 voltage and frequency functions of the DSP Controller. Those measurements, made by Chris Pink of NREL, are included in this report. A final round of static switch testing (Power Quality CBEMA and SEMIF47 events) is planned for the Dolan Labs at AEP before the switch is installed at AEP in the CERTS Test Bed. 
A Distributed Energ

Systems Company

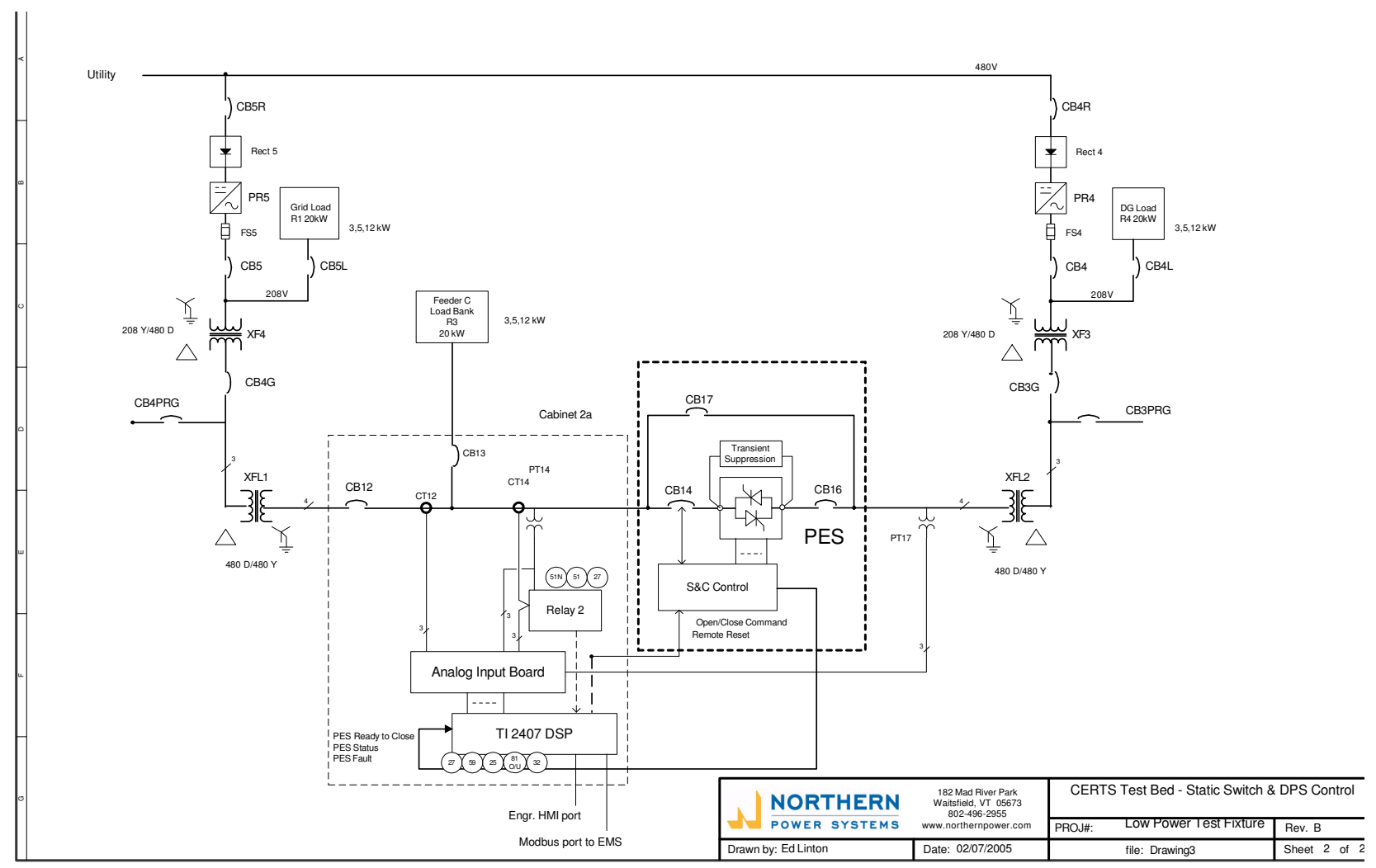

Figure 1. CERTS Switch Low Power test setup 1-line diagram 


\section{S\&C Power Electronic Switch and NPS DSP Controller Functionality}

This section is intended to provide a brief description of the control functions that the Northern Power DSP Controller will perform, as well as a description of the interface between the S\&C SCR switch control and the DSP Controller. For a complete description of this functionality and Interface, refer to Reference [2].

\subsection{S\&C Power Electronic Switch}

S\&C Electric Company was selected as the provider of the SCR-based switch. They provided their 480V, 400A PureWave ${ }^{\circledR}$ Power Electronic Switch (PES), which consists of and SCR-based switch (using Semicron SKKT 250/16E Dual SCR modules) with input, output and bypass breakers, and fuses.

For operation, the PES requires a turn on/off input signal, a Remote reset input for clearing latched alarms and a Remote Disabled (Start/Stop command); these signals are provided by the DSP. It also contains a "Reset" pushbutton for local reset, an "Enable" Selector switch to enable/disable the PES.

Below, is a brief description of the Digital I/O interface of the PES with the Northern Power DSP controller; as well as alarms and operating states that will be available on the LCD display of the PES:

PES Pushbutton/Selector

- $\quad$ Reset (Pushbutton)

- Disable/Enable (Selector)

PES Digital Inputs (24 V, opto-isolated)

- PES Command

- Remote Reset (This reset will come from HMI)

- DSP Fault

- Remote Disable (works as the Start/Stop Command in DSP state machine)

PES Digital Outputs (5-60 V, opto-isolated)

- PES Ready to Close

- PES Over-temperature (Alarm and Pre-alarm)

- PES SCR Failure

- PES Alarm (includes all alarms)

- PES is Closed (PES State)

- PES Disabled (Disable/Enable status)

- Enable/Disable Selector state

- PES Reset Pushbutton state

- PES Warning

PES Alarms (LCD Display in PES)

- $\quad$ SCR failure (PES Open or PES shorted, latching)

- Over-temperature

- Any Alarm (including DSP faults)

- Disable PES

- Warnings 


\subsection{CERTS Switch Analog and Digital I/O}

The external analog inputs for current measurement assume the use of $1 \mathrm{~A}$ rms secondary CT. Analog inputs for grid and DG AC voltage measurements are made using $120 \mathrm{Vrms}$ nominal at the PT secondary. Sixteen DSP board analog channels that are directly available for high speed sampling are utilized in the controller.

The analog inputs are:

1. Switch Current CTs - (CT14) phase A, B, C

2. Remote Current CTs - (CT12) phase A, B, C

3. $\quad$ Grid side sensing - $(\mathrm{Vg})$ phase $A, B, C$

4. $\quad$ DG side sensing - (Vdg) phase $A, B, C$ Digital inputs to the DSP are opto-isolated and debounced. The $10 \mathrm{~mA}$ digital outputs of the DSP are opto-isolated. For a full description of the DSP Controller Digital I/O refer to Reference [2].

\subsection{CERTS Switch State Machine}

The state machine controls the operation of the CERTS Switch. The primary operating states of the CERTS Switch are to connect or disconnect the grid and Dg sides by closing or opening the switch. A number of the other control states are used for the startup sequence, faults and disable. The behavior of the CERTS Switch when operating in the controller states is described below.

OFF State: The CERTS Switch following power-up and reset of the controls is considered to be the OFF state. The DSP controller conditions for the OFF state are: no faults are latched and all alarms are enabled. To leave the OFF state, a "Start" command is required to be given to the S\&C PES; the "Start" command controls the motor operated input breaker.

[STARTUP] Test State: A start command in the Off state initiates the transition to the Test state. Once the Start command is given, the motor operated input breaker as well as the Output Breaker should be close. In the Test State, the DSP Controller checks the "Ready to Close" signal from the PES; if the signal is HIGH, then the system will enter the ON States; if not, it will go back to the OFF state.

[ON] Disconnect State: In the Disconnect state the CERTS Switch checks to see if DG is present in the system using the dead bus relay (27R) function. If synchronization function check evaluates to True or if the Dead Bus Relay check is True (and the operator has enable the "Dead Bus Reclose" Operating Mode) then the switch transitions to the Connect State.

[ON]Connect State: In the Connect state the CERTS Switch checks for power quality, reverse power anti-islanding, SEL Relay and other IEEE1547 functions and fault events. If any of these events are true, the DER Switch transitions to the Disconnect state

Fault State: If there are faults the CERTS Switch can shut down rapidly into a safe state. In this state, the switch is commanded to open, and also the S\&C PES is commanded to "Stop"; which opens the motor operated input breaker. The types of faults range from S\&C PES internal Alarms ("Fail to Open", "SCR Failure", etc) to conditions that require lockout of the system within the DSP Controller (Such as Reverse Power Anti-islanding event). To leave the Fault State, all faults should be reset and cleared.

PES Disable State: The S\&C PES has a two-position switch that within its state machine switches the system from Enable to Disable (when Disable, both the motor operated input breaker and the shunt-trip operated output breaker are open). If the DSP Controller receives this signal, it will enter this State, in which an Open command and a "Stop" Command are passed to the PES. 


\subsection{CERTS Switch Operating Modes}

Auto mode: In this mode, when conditions are appropriate synchronized closing of the switch is attempted; also all events are checked.

Manual Connection Mode: In this mode, the switch can be manually controlled to open or close. Also, during this mode, events are not checked (Except for the "External Relay Trip" event, which in any mode will always open the SCR switch).

Sync Test Bypass: If manual connection mode is enabled, it will try to attempt to close when synchronization conditions are appropriate. However, if the Sync Test Bypass option is also enabled at the same time, then it will skip the synchronization test and force a switch close regardless of the grid and DG conditions.

EFVST (Enable Fast Voltage Sag Trip) Mode: If this operating mode is enable, during "Auto" Mode, Power Quality (CBEMA,SEMIF47) events and trips will be enabled. If disabled, the switch will not be commanded to open is these events occur. This mode can be disabled manually through the EMS; also the EMS may disabled it automatically based on wether there is enough microsources available to cover the microgrid load.

Dead Bus Reclose: If this operating mode is enable, when running in "Auto" Mode, reclosing to a DG side dead bus is allowed in order to perform system black starts. Notice that the operating mode simply allows or blocks the dead bus reclosing; the detection of the dead bus is perform by the relay function "Dead Bus Reclose Relay" described in section 2.5

\subsection{CERTS Switch System Control Functions}

Several protection, IEE1547, Power Quality and control functions are performed by the CERTS switch; they are listed below:

Synchronism Check: The calculation of this algorithm is performed in the Disconnect state. This function checks to verify that the voltage amplitude (for all three phases), frequency and phase angle are within an acceptable window to enable the closing of the DER Switch. Note that other CERTS Switch control functions may also need to be true to enable the closing.

The voltage magnitude and phase comparison is performed at the high speed ISR rate of the DSP. This ensures that any sudden jump in voltage or magnitude on either side of the CERTS Switch does not cause any false synchronization.

IEEE 1547 Voltage and Frequency Events: The recently approved IEEE 1547 standard contains requirements under certain voltage and frequency events. The levels on these events are fully described in section 3.3.3. If any of these events occur when operating in the "Auto" Mode, the switch will transition from the Connect to the Disconnect State, and if it is already in the Disconnect State, it will prevent the switch from closing.

Relay Trip Signal Event: The SEL-351 Relay (Relay 2) associated with the CERTS Switch will perform protection functions that include phase overcurrent, zero sequence overcurrent and line to line undervoltage. When the Relay detects these conditions, it will provide a trip signal to the CERTS controller so it can command the switch to open.

Dead Bus Reclose Relay: This relay function is provided in the CERTS Switch, so that black start of loads is allowed when there are no microsources present on the DG side.

Phase Sequence Error: This function checks for phase rotation direction, missing phase information or lack of signal on the phase voltage. In addition this function checks if the controller's internal data is 
synchronized with the grid and DG operating frequencies. This event in the Connect state will make the CERTS Switch to transition to Disconnect state. An event in the Disconnect state will prevent the operation of synchronization function.

Remote Location RMS Reverse Power (32) and Anti-islanding: The CERTS controller evaluates single and three phase power flow at the remote Current transformer (CT12) location, which is located at the PCC (so its measurement includes protected and unprotected loads of the microgrid). The power measurement is compared with an adjustable threshold and time delay for coordination. If the power flow crosses the threshold then an event is set. An event in the Connect state will cause the CERTS Switch to transition to the Disconnect state. Depending on the state of the grid voltage after transitioning to the Disconnect state (which allows the Controller to decide wether the reverse power event was caused due to grid islanding, open phase or Erroneous Microgrid settings), the Switch may be allowed to reclose when conditions are appropriate (grid islanding, open phase) or the system will enter the fault state and require user intervention (Anti-islanding, Microgrid Settings Reset required).

Controller Digital Event: In addition to the above, functions it is possible to set a bit in the CERTS Switch controller to simulate an event. Also it is possible to use the external digital input to simulate an event occurrence in the controller.

Restoration Timers: IEEE 1547 and Power Quality events will have restoration timers, meaning that once the measured parameters (voltage and frequency) return to normal levels, they should stayed within acceptable limits for at least the time specified in these timers. Two separate timers will exist: one for IEEE 1547 events, which will be a longer time (default 5 minutes) while Power Quality Events will have a faster separate timer ( 2 seconds). Both timers will be adjustable. 


\section{$3 \quad$ Test Results}

This section presents the results of all the tests performed at the Northern Power test facility Below are given some aspects of the general procedure taken in order to perform the low-power test for the CERTS switch. Specific settings for the testing of each kind of event and algorithm are specified in each subsection; notice that some settings used may differ from the original settings in the test plan due to limitations of the source inverters used. The changes are not significant, the intent of the test plan has been maintained.

Figure 1 shows a 1-line diagram of the test setup used for the low-power testing.

Notes about the general test procedure:

1. The CERTS Switch unit was connected to the source inverters and loads, with one inverter and load pair emulating the grid and another pair emulating the DG source. The Grid source operates in open loop mode (Constant voltage magnitude and frequency) while the DG source operates using CERTS microgrid controls (Reference [3]).

2. During the testing of the different types of algorithms, only the event functions under test were enabled, while all other event functions were disabled. The event settings were adjusted to the appropriate values related to the test objective.

3. During the testing of most algorithms and events, the Northern Power Engineering HMI was used as the interface between the user and the DSP. Through this display the operator was able to observe the status of all alarms and events as well as the status of the switch (information from the S\&C PES); also the operator was able to adjust any settings on the fly. In section ("EMS testing") most of these tests are repeated but using the Energy Manager System that will be available at $A E P$ in order to verify required communications. 


\subsection{S\&C-DSP Interface Commissioning Test [Test Plan Section 2.1]}

\subsubsection{S\&C PES and DSP Controller I/O Interface}

The first sets of tests performed are to verify that basic functionality, wiring and interface between the S\&C switch and the DSP Controller were working properly. The following steps were performed, and all of them passed successfully.

- Wiring Check: All wiring was verified for proper connection according to schematics, as well as check for mechanical and electrical integrity (DSP Controller only)

- Power Supply: 24V power supply for DSP Controller and Relay2 was checked successfully.

- Digital Inputs and Outputs: Correct operation and logic polarity of all inputs and outputs was verified by simulating several kind of events.

- State Machine: We loop through the state machine as detailed in section 2 of this report several times, and both the S\&C and DSP operate as expected. All desired status are communicated between the S\&C and the controller

- $\quad$ Alarms: Some of the S\&C Alarms were tested; results are detailed in section 3.1.2

- Analog Inputs: All analog inputs (voltage, current) were checked for correct scaling and response. Some calibration was performed to achieve the following accuracies:

$$
\begin{aligned}
& \text { Voltage: }<1 \% \text { error }(2.7 \text { Vrms, measured at rated voltage } 277 \mathrm{Vrms} \text { ) } \\
& \text { Current }<2 \text { Arms error (maximum current simulated }=20 \text { Arms, switch rating }=400 \mathrm{~A} \text { ) } \\
& \text { Power error }<1 \mathrm{~kW} \text { (maximum power simulated }=15 \mathrm{~kW} \text {, switch rating is } 330 \mathrm{kVA} \text { ) }
\end{aligned}
$$

\subsubsection{S\&C PES Alarms}

Some additional simple tests were attempted in order to verify the functionality of the S\&C Internal Alarms. A detail description of each of these alarms can be seen in Reference [2]. The status of these alarms is communicated to the DSP controller by the following digital outputs:

PES Alarm: This output includes toggles HIGH if any of the S\&C alarms in enabled (Over temperature, Open PES, Shorted PES, PES Fail to Open)

SCR Failure: This output toggles HIGH if either the Open PES or Shorted PES alarms is enabled

\subsubsection{Fail to Open Alarm}

A strategy was devised to test the operation of this alarm. The grid side inverter was set to operate in open loop mode (constant voltage, frequency) while the DG side had only a passive load (5 kW). Also, a single phase contactor across phase A of the SCR switch was connected, but initially left open.

After performing a Manual Connect of the SCR switch with the DSP controller, the single phase contactor was closed. Then, the Manual Connect command was removed; even though the SCR switch opens, the S\&C controls will not measure any voltage buildup across the SCR phase A (since the single phase contactor is shorting the connection); thus assuming that the SCR switch has failed to open. When this occurs, the "PES Alarm" digital output goes $\mathrm{HIGH}$, and after the DSP receives this signal, it transfers the CERTS switch to the Fault State and gives a "Stop" command to the S\&C PES. 
This response can be observed in Figure 2; this figure shows the voltage across phase A of the SCR switch (channel 1); phase A switch current (channel 2, 20A/division) and the DSP switch command (channel 3). When the DSP command goes Low (to open the switch); the current across the SCR goes to zero (proving that the SCR opened) but the voltage across the SCR terminals is still zero. After approximately 7 cycles, we see the voltage across the switch build up; this is because it takes 2 cycles for the "Fail to Open" detection to operate, and after the Stop command is given, it takes 4-5 cycles for the motor operated input breaker to open. This verifies the correct functionality of the alarm.

This test was repeated, but with the Alarms disabled in the DSP controller; the reason to do this is to verify that the S\&C PES will internally open the input breaker if this alarm occurs, independently of wether it receives the Stop Command from the DSP. After repeating the test, the input breaker was open so the S\&C PES operates appropriately. Notice that for the other alarms (Open PES, Shorted PES) the DSP needs to provide the Stop command to the S\&C PES; it will not open the breaker on its own for these alarms).

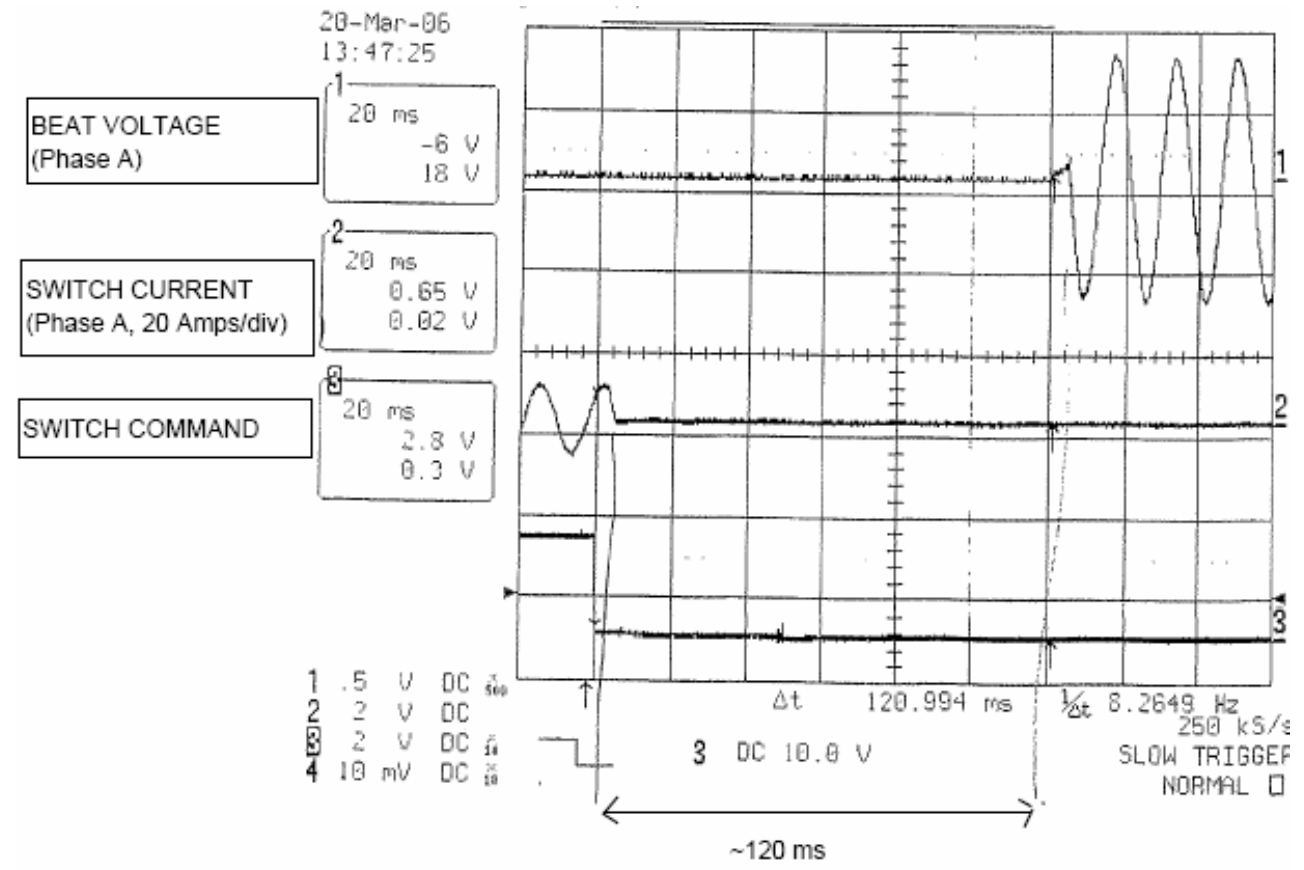

Figure 2: S\&C "Fail to Open” Alarm Tests

\subsubsection{SCR Failure Alarms}

The conditions to test these alarms were not possible to be recreated at Northern Power test site.

The "PES Shorted" alarm only operates when the switch current is above $5 \%$ SCR rating (400 A); this corresponds to 20A, which can't be output by the inverters at Northern Power's site (max $15 \mathrm{kVA}$ ).

The "Open PES" alarm is hard to recreate, although an issue was observed during the whole testing process. After performing several srynchronized closings and manual Opening of the switch, sometimes this alarm will show up when the switch is commanded to open. During Roger Troyer (S\&C) visit to the NPS site to resolve some of these issues, the possibility of this issue being a noise problem arose; Northern Power continued the testing since these alarms will not interfere with the test procedure for the control functions; S\&C will be visiting the AEP site to resolve the issue of the "Open PES" Alarm. 


\subsection{Synchronization [Test Plan Section 3.1]}

The synchronization tests were intended to demonstrate that the CERTS Switch would permit connection between the grid and DG terminals when both systems are synchronized within an allowable window for voltage, frequency and phase.

The following tests were performed to verify the synchronization functionality:

1) Limits Accuracy (using Omicron CMA256-6 secondary injection test set from NREL)

2) Synchronization Time Delay (for Bias Closing)

3) Response to different beat frequencies

\subsubsection{Limits Accuracy (using Omicron CMA256-6 secondary injection test set from NREL)}

During the time NREL's Omicron Equipment was available at NPS site, calibration tests were performed with secondary level (120V) signals input into the grid and DG PTs (PT14 and PT17 in figure 1). During this test, the CERTS switch was set to "Auto" mode in order to allow synchronized closing when the conditions are appropriate. The DSP limit settings used during testing were as follows:

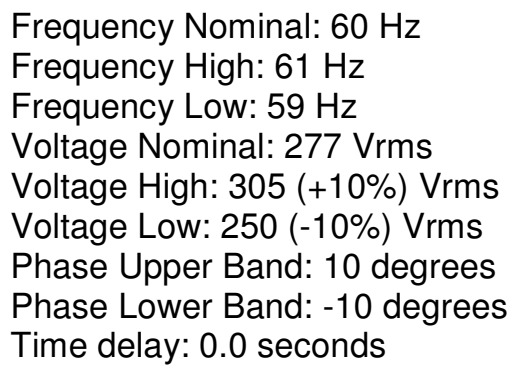

It is important to make a few notes about the synchronization settings. First, the reason to allow the larger range for frequency $(+/-1 \mathrm{~Hz})$ is so that the DG side can droop that much if necessary while open and still allow to reclose (the grid side will be limited to the tighter IEEE1547 range 60.5-50.3 Hz since the IEEE1547 events are monitored on the grid side PT only). Also, the phase band settings are simply outer limits to ensure that the switch will not close if the beat voltage is off the zero crossing by this amount; however the DSP control is still trying to reclose it at precisely the zero crossing.

The Omicron equipment performed the calibration test by simulating the grid side voltage to nominal parameters $(277 \mathrm{Vrms}, 60 \mathrm{~Hz}$ ) and then setting the DG side to a wide range of voltage and frequency deviations. Figure 3 shows the results of this test; the Omicron equipment simulated each of the test conditions for a 1-minute duration and recorded whether the switch closed or not within a 10 degree band of the zero crossing. Figure 3 presents the results in the following format:

- "Failed" test corresponds to a closing outside limits or a failure to close if all conditions are within limits

- "Passed" test corresponds to closing within limits or preventing closing when outside limits

Most of the tests performed gave desired results with the synchronization passing the tests; however, a common "failed test" condition was observed when the DG voltage was just below the upper limit (lower 
by $2.7 \mathrm{Vrms}(1 \%))$. Is possible that there is a calibration issue with the DG voltage measurements at this level; however, $1 \%$ is a relatively acceptable accuracy.

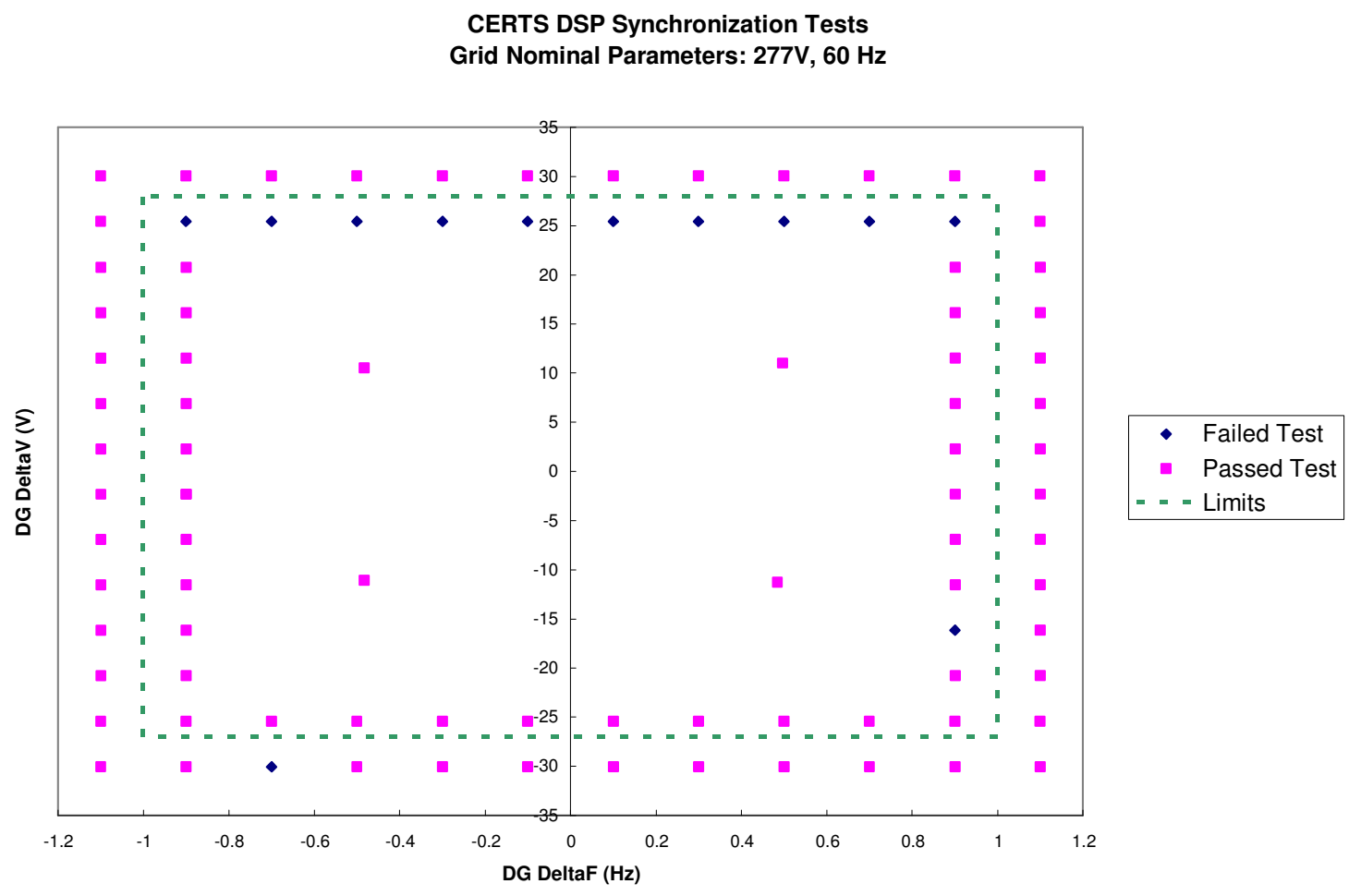

Figure 3: DSP Synchronization Limits Accuracy Test using NREL's Omicron Equipment

\subsubsection{Synchronization Time Delay (for Bias Closing)}

One of the settings available in Northern Power's DSP Controller is a "time delay" for synchronization closing. The initial purpose of this time delay is to compensate for opening time of devices such as circuit breakers (which typically can take up to 5 cycles to open). However, for the CERTS switch project this time delay can also be used to test the concept of biasing the closing such that switch closes when the fastest rotating voltage must be leading the voltage that rotates slower (check Reference [3]). Without the bias, the controller will try to close when both voltages are on phase (meaning the average phase of the three beat voltages $\mathrm{A}, \mathrm{B}$, $\mathrm{C}$ is zero).

The bias closing can be achieved by using a positive time delay; a negative time delay will perform the opposite effect (switch closes when the fastest rotating voltage is lagging the voltage that rotates slower). In this situation, the initial direction of power flow will be opposite to the expected steady state power flow direction, thus creating a significant transient response. Also notice that the setting in the Engineering $\mathrm{HMI}$ is a time value (in seconds); the equivalent phase bias is obtained by multiplying the beat frequency of the voltage across the switch and this time delay value.

To examine the effect of the bias closing, the following setup was used:

\section{Grid Side:}


A Distributed Energy

Systems Company

Voltage: $277 \mathrm{Vrms}$

Frequency: $60 \mathrm{~Hz}$

Grid Load (R1), Feeder C Load (R3) $=0 \mathrm{~kW}$

DG side:

Psetpoint $=6 \mathrm{~kW}$

Dg Load (R4) $=12 \mathrm{~kW}$

Droop: $0.025 \mathrm{~Hz} / \mathrm{kW}$

Frequency Measured (before closing): $59.7 \mathrm{~Hz}$

This setup allows us to test an scenario with a beat frequency of $0.3 \mathrm{~Hz}$. When the switch closes, a power flow from the grid side to the DG side of approximately $6 \mathrm{~kW}$ results. The following waveforms were recorded:

- Figure 4: Beat Voltage

- Figure 5: Synchronized closing delay= $0 \mathrm{sec}$ (right at zero crossing of beat voltage)

- Figure 6: Synchronized closing delay $=+0.25 \mathrm{sec}(\sim 27$ degrees after zero crossing)

- Figure 7: Synchronized closing delay= $-0.25 \mathrm{sec}(\sim 27$ degrees after zero crossing)

Figures 5-7 show the following measurements:

- Channel 1: Voltage across the switch phase A (Beat Voltage)

- Channel 2: Phase A switch current (20A/division)

- Channel 3: Phase B switch current (20A/division)

- Channel 4: Phase C switch current (20A/division)

Also, the time axis shows an arrow (trigger) which is where the Switch command (open/close) transition from open to close.

These figures show that with a zero delay, the closing transition was very smooth and the current is slowly growing to its steady state value. Also, with the positive time delay, the current is biased in the correct power flow direction, so the transition is also relatively smooth. However, with the negative time delay, we can see that the current in all the phases takes initially the wrong direction, so an unwanted transient is observed. This shows that it is desired to avoid closing of the switch when the fastest voltage is lagging the slower voltage.

Since is shown that the DSP can measure phase very accurately, from here on it is expected that synchronized closing will be performed without any bias (time delay). This setting will be available if desired to test on the field for certain conditions where the phase measurement by the DSP may not be as accurate (harmonic content, significant phase unbalance) and thus the bias could smooth out the transitions.

An important observation to note during this test is that with no delay, we see the switch current is slowly growing to its steady state and taking 8-10 cycles to reach that point. The reason for this behavior is that what we call the Grid side is not really a stiff voltage source because of the impedance of the two transformers (total $X \sim 10 \%$ at $15 \mathrm{kVA}, 480 \mathrm{~V}$ ). If this was a stiff source, the current should take $\sim 1$ cycle to reach its steady state when closing at exactly the zero crossing; this kind of response will be observed in future synchronization tests, and as explained here it is very probable it occurs due to impedance of the grid connection in the test setup. 


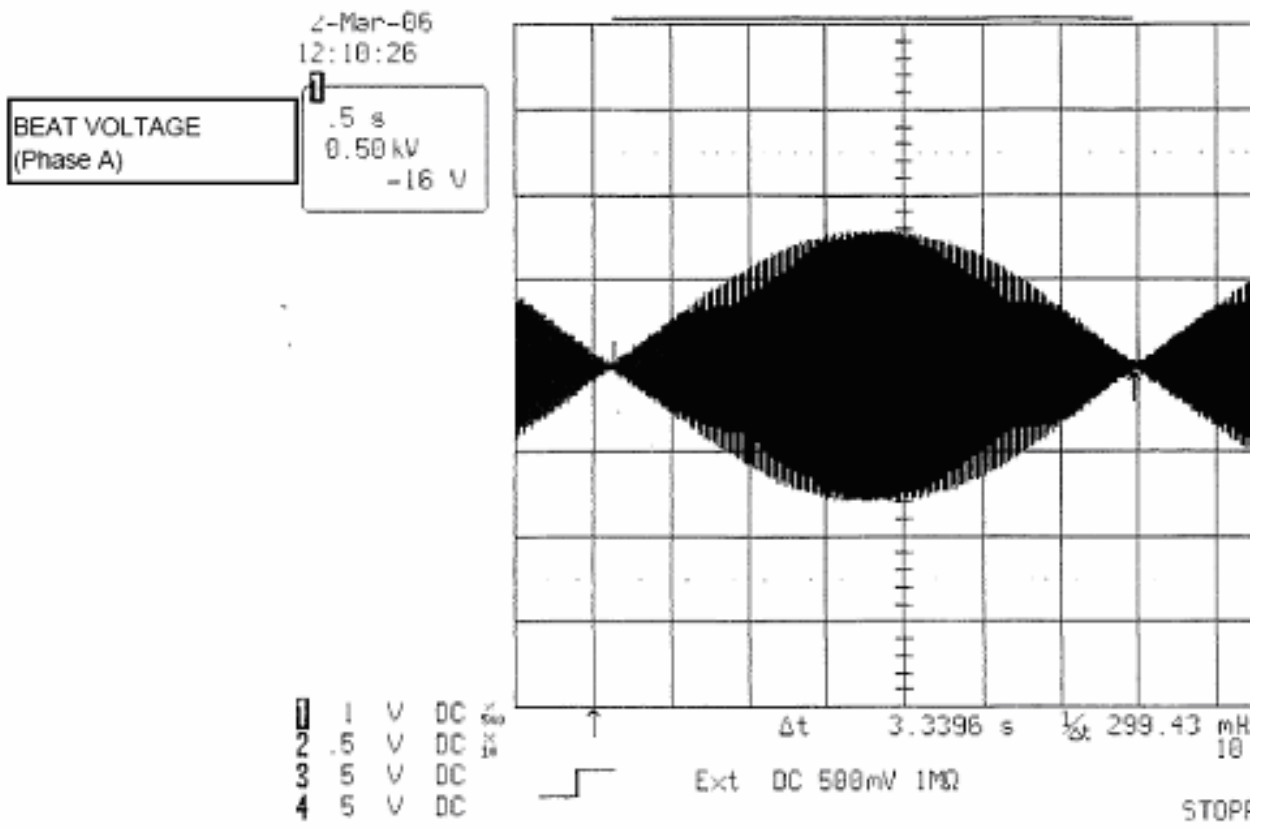

Figure 4: Synchronization Time delay test, Beat Voltage phase A

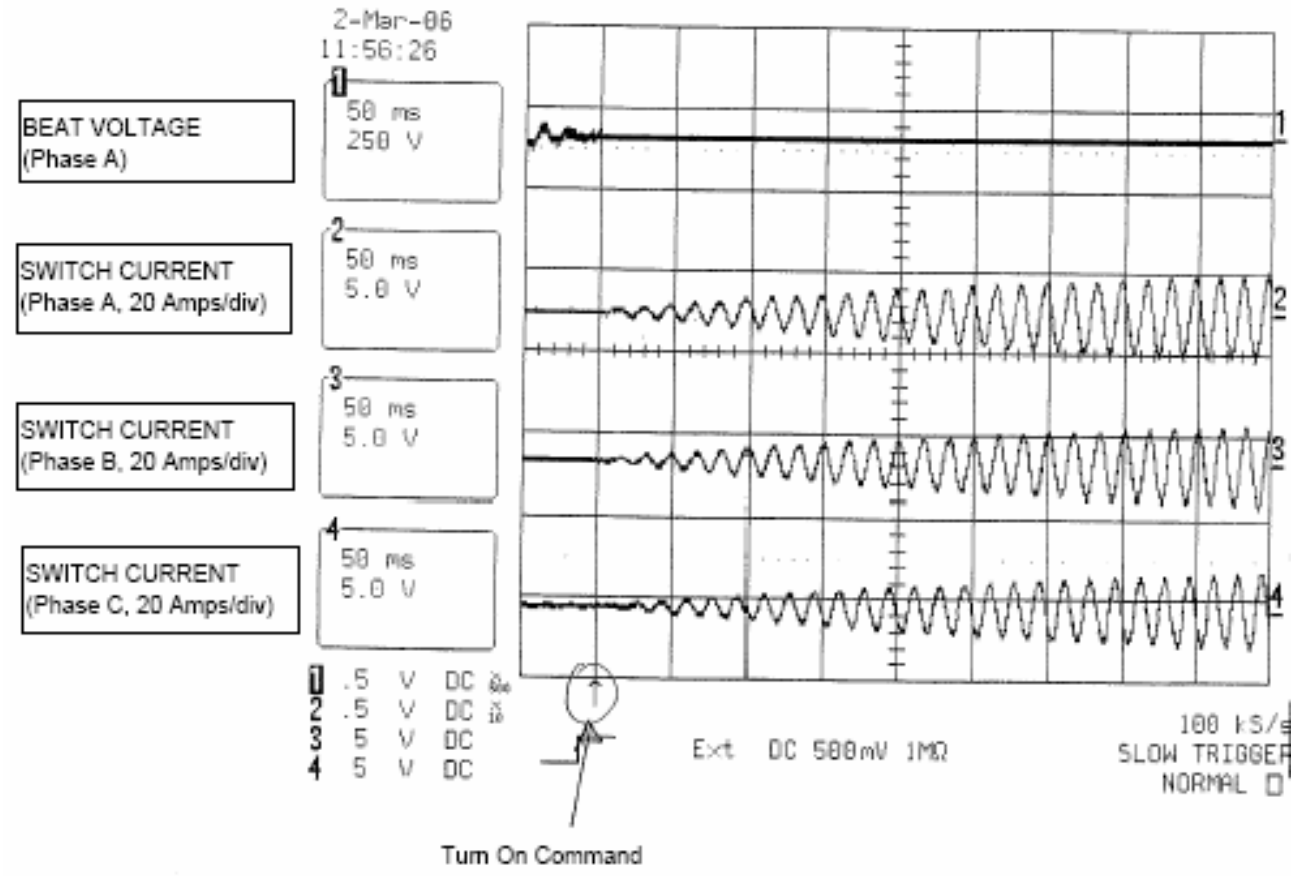

Figure 5: Synchronized closing delay= 0 sec (right at zero crossing of beat voltage) 


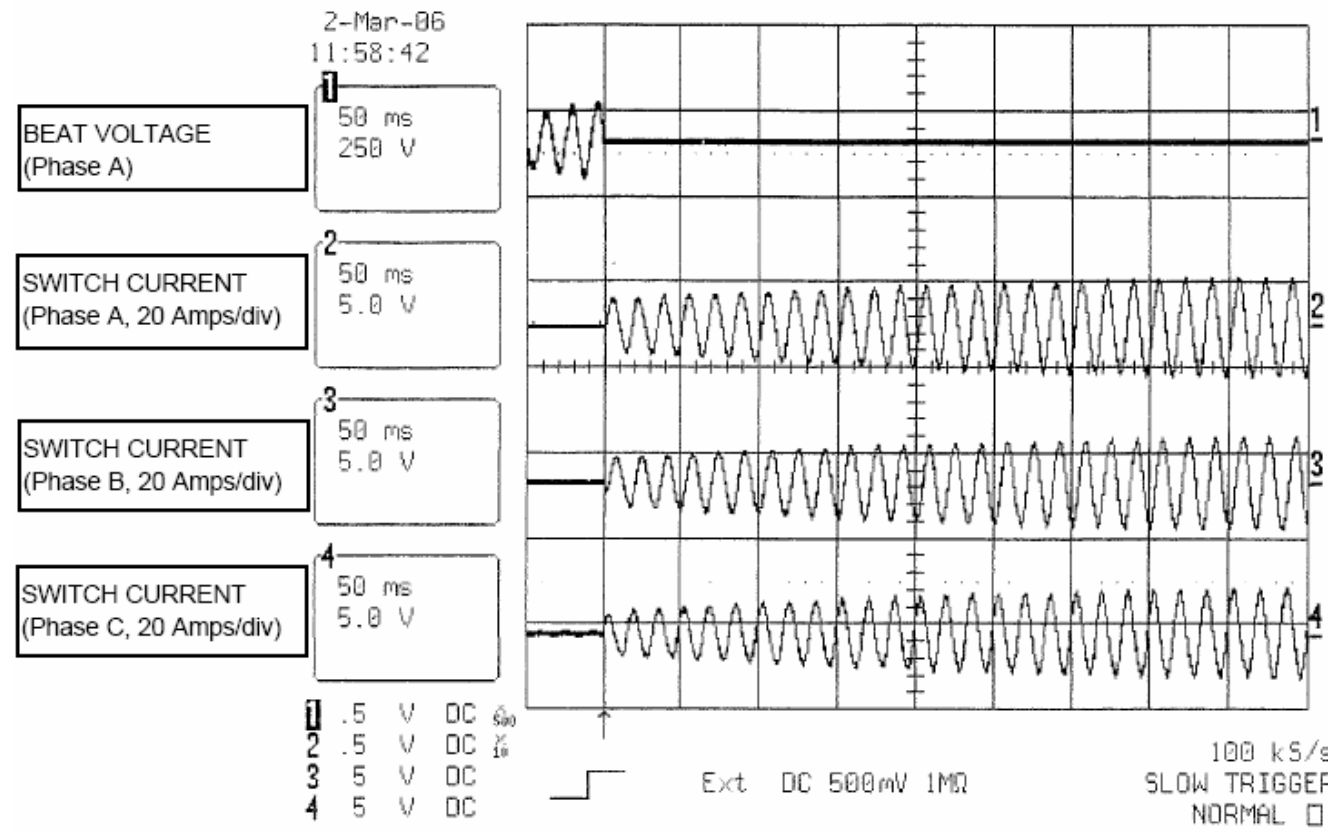

Figure 6: Synchronized closing delay $=+0.25 \mathrm{sec}$ ( 27 degrees after zero crossing)

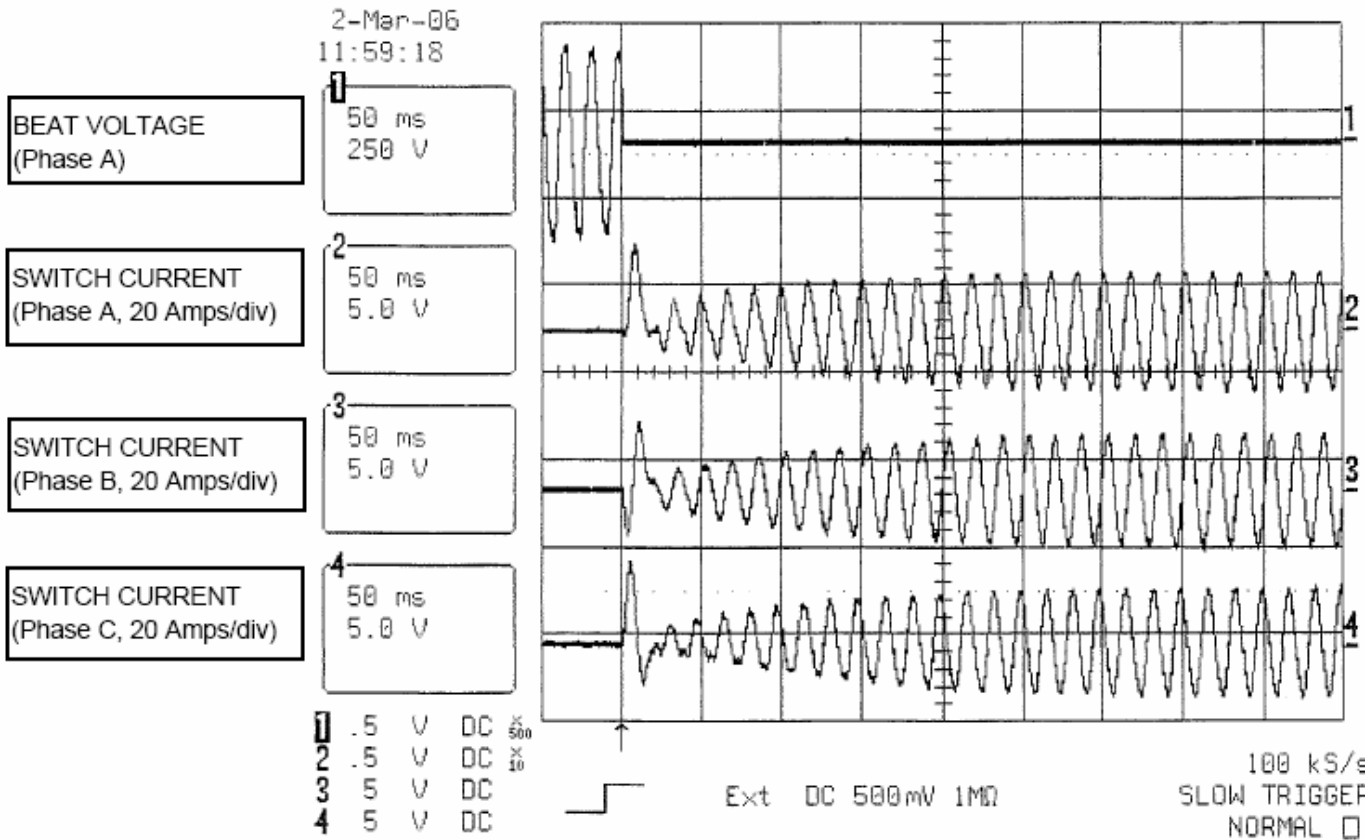

Figure 7: Synchronized closing delay= $-0.25 \sec (\sim 27$ degrees before zero crossing)

\subsubsection{Response to different beat frequencies}


The final task of synchronization testing is to evaluate the ability of Northern Power DSP controller to perform the synchronized closing at a wide range of beat frequencies. Table 2 below shows all the different cases that were evaluated. Some important notes about these tests:

1. Table 1 explains the setup used (set points, DG droop, loads, measurements before closing, etc).

2. For all these tests, the grid inverter setpoints were rated values $(277 \mathrm{Vrms}, 60 \mathrm{~Hz})$

3. The DG voltage droop used for all cases was 2.7 Volts/kVAR. The reasoning behind using this setting is based on the reactive power droop control explained in Reference [3].

Average Voltage Measured at No Load $=277$ Vrms

Average Voltage at DG full load $(15 \mathrm{~kW})=250 \mathrm{Vrms}$

Max kVAR output $=10 \mathrm{kVAR}$

Reactive power droop $=(277-250) /(10)=2.7 \mathrm{~V} / \mathrm{kVAR}$

4. Tests 1-9 show cases where the power flow when the switch closes will be from the DG side to the grid side of the switch. Tests 10-12 present cases with the power flow outside of the microgrid side.

5. For Tests 1-6 the real power droop $(\mathrm{m})$ was not selected precisely according to Reference[3]; rather, a fixed droop $(0.025 \mathrm{~kW} / \mathrm{Hz})$ was used. The purpose during these runs was to artificially create a wide range of beat frequencies without major adjustments in the setup (from $0.1 \mathrm{~Hz}$ to 0.5 $\mathrm{Hz})$

6. Tests 7-12 have the droop selected accordingly to the "Control and Design of CERTS Microgrid" document (Reference [3]). The assumption in selecting the droop were:

Max Power $=15 \mathrm{~kW}$

Minimum frequency $=59,25 \mathrm{~Hz}$

$M$ droop $=(60 \mathrm{~Hz}-$ Minimum Frequency $) /($ Max Power - Pset $)$

The DG power setpoints selected for tests 7-12 were chosen so the beat frequencies obtained will approximately cover the range of frequencies in which the DSP is expected to perform the synchronized closing $(0.05 \mathrm{~Hz}-0.5 \mathrm{~Hz})$

\begin{tabular}{|c|c|c|c|c|c|c|c|c|c|}
\hline $\begin{array}{c}\text { Test } \\
\#\end{array}$ & $\begin{array}{c}\text { Feeder } \\
\text { C }\end{array}$ & $\begin{array}{c}\text { DG } \\
(\mathrm{kW})\end{array}$ & $\begin{array}{c}\text { DG } \\
(\mathrm{kW})\end{array}$ & $\begin{array}{c}\text { DG } \\
\text { Pset }\end{array}$ & $\begin{array}{c}\text { Vgrid } \\
\text { droop }\end{array}$ & $\begin{array}{c}\text { VDG } \\
\text { measured } \\
\text { measured }\end{array}$ & $\begin{array}{c}\text { Fgrid } \\
\text { measured } \\
(\mathrm{Hz} / \mathrm{kW})\end{array}$ & $\begin{array}{c}\text { F_DG } \\
\text { measured } \\
(\mathrm{Vrms})\end{array}$ & $\begin{array}{c}\text { DeltaF } \\
(\mathrm{Hz})\end{array}$ \\
\hline
\end{tabular}




\begin{tabular}{|c|c|c|c|c|c|c|c|c|c|}
1 & 0 & 12 & 2.7 & 0.025 & 272 & 275 & 60 & 59.5 & +0.5 \\
\hline 2 & 0 & 12 & 4.2 & 0.025 & 272 & 274 & 60 & 59.6 & +0.4 \\
\hline 3 & 0 & 12 & 6 & 0.025 & 272 & 269 & 60 & 59.7 & +0.3 \\
\hline 4 & 0 & 12 & 7.5 & 0.025 & 272 & 268 & 60 & 59.8 & +0.2 \\
\hline 5 & 0 & 12 & 9.5 & 0.025 & 272 & 265 & 60 & 59.9 & +0.1 \\
\hline 6 & 0 & 12 & 10.5 & 0.025 & 272 & 264 & 60 & 59.95 & +0.05 \\
\hline 7 & 0 & 12 & 6 & 0.083 & 272 & 273 & 60 & 59.5 & +0.5 \\
\hline 8 & 0 & 8 & 4 & 0.068 & 272 & 262 & 60 & 59.73 & +0.27 \\
\hline 9 & 0 & 3 & 1 & 0.053 & 272 & 272 & 60 & 59.86 & +0.14 \\
\hline 10 & 12 & 3 & 8 & 0.107 & 272 & 282 & 60 & 60.49 & -0.49 \\
\hline 11 & 12 & 3 & 6 & 0.083 & 273 & 277 & 60 & 60.23 & -0.23 \\
\hline 12 & 12 & 3 & 4.5 & 0.071 & 273 & 275 & 60 & 60.08 & -0.08 \\
\hline
\end{tabular}

Table 1: Synchronization Test Runs for range of beat frequencies and power flow direction across switch

For analysis, in this section the results from tests \#7 (beat frequency $=0.5 \mathrm{~Hz}$ ) and \#6 (beat frequency $=0.05 \mathrm{~Hz}$ ) are presented since there are the extreme cases of the beat frequencies evaluated. Figures 8 and 9 shows the synchronized closing for both of these tests. Channel 1 shows the voltage across phase A of the SCR switch (refer as "beat voltage" in later figures); Channels 2, 3,and 4 show the SCR switch currents $A, B$, and $C$ respectively (20 A/division).

It can be seen that both transitions are smooth without any unwanted transients, and that the initial direction of the switch current in all phases is in the same direction as the steady state power flow after closing. These results show the capability of NPS the controller, combined with the DG settings and controls to provide seamless transitions. Notice that, as explained in section 3.2.1, the current slow growth to steady state can be explained by the significant grid impedance in the test setup.

In the appendix section of this report, waveforms are shown for all of these cases. The waveforms for each test include phase $A$ beat voltage, as well as the switch currents $A, B, C$ during synchronized closing and also after a manual opening of the switch. 


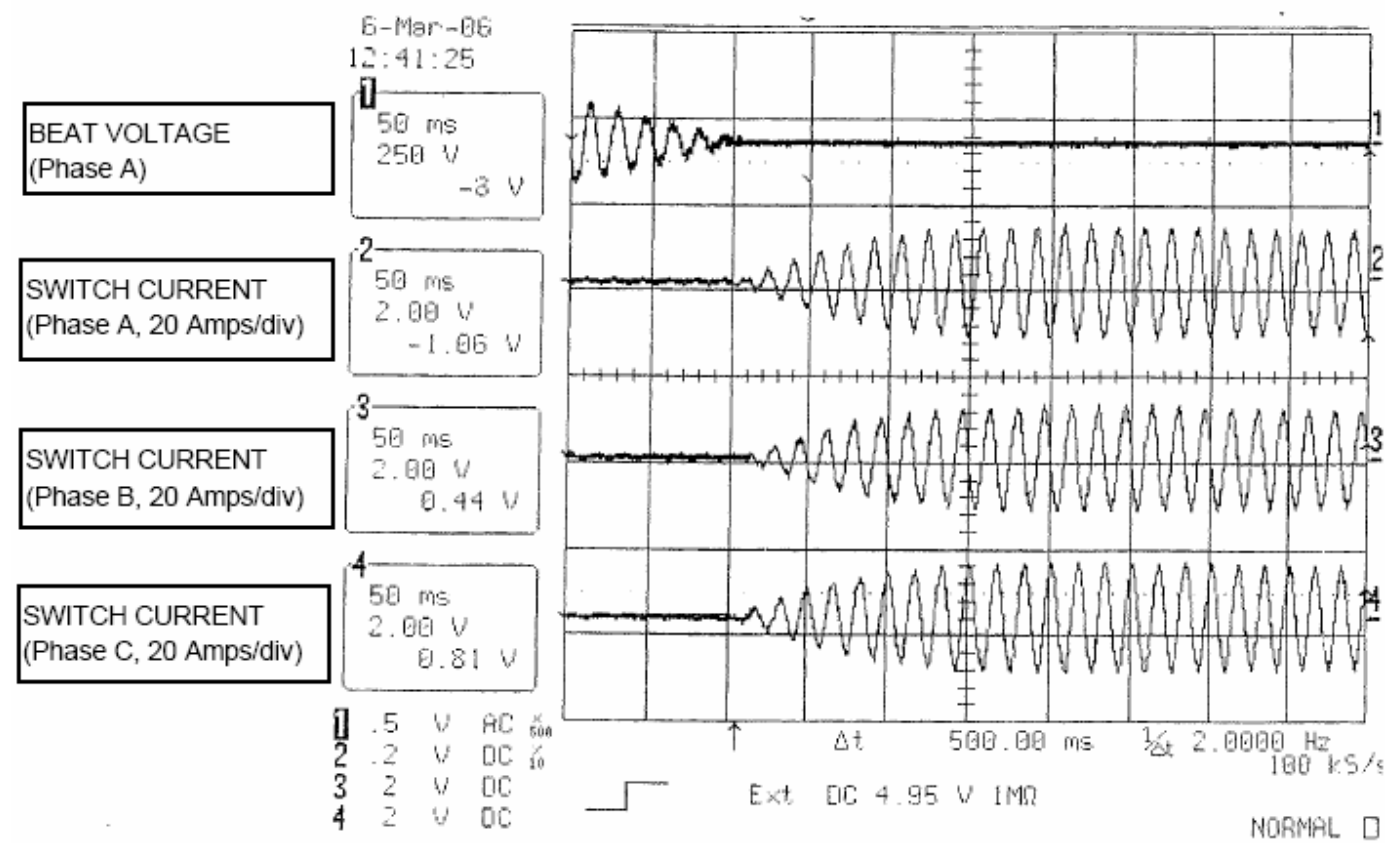

Figure 8: Synchronized Closing for Test \#7 (beat frequency= $0.5 \mathrm{~Hz}$ )

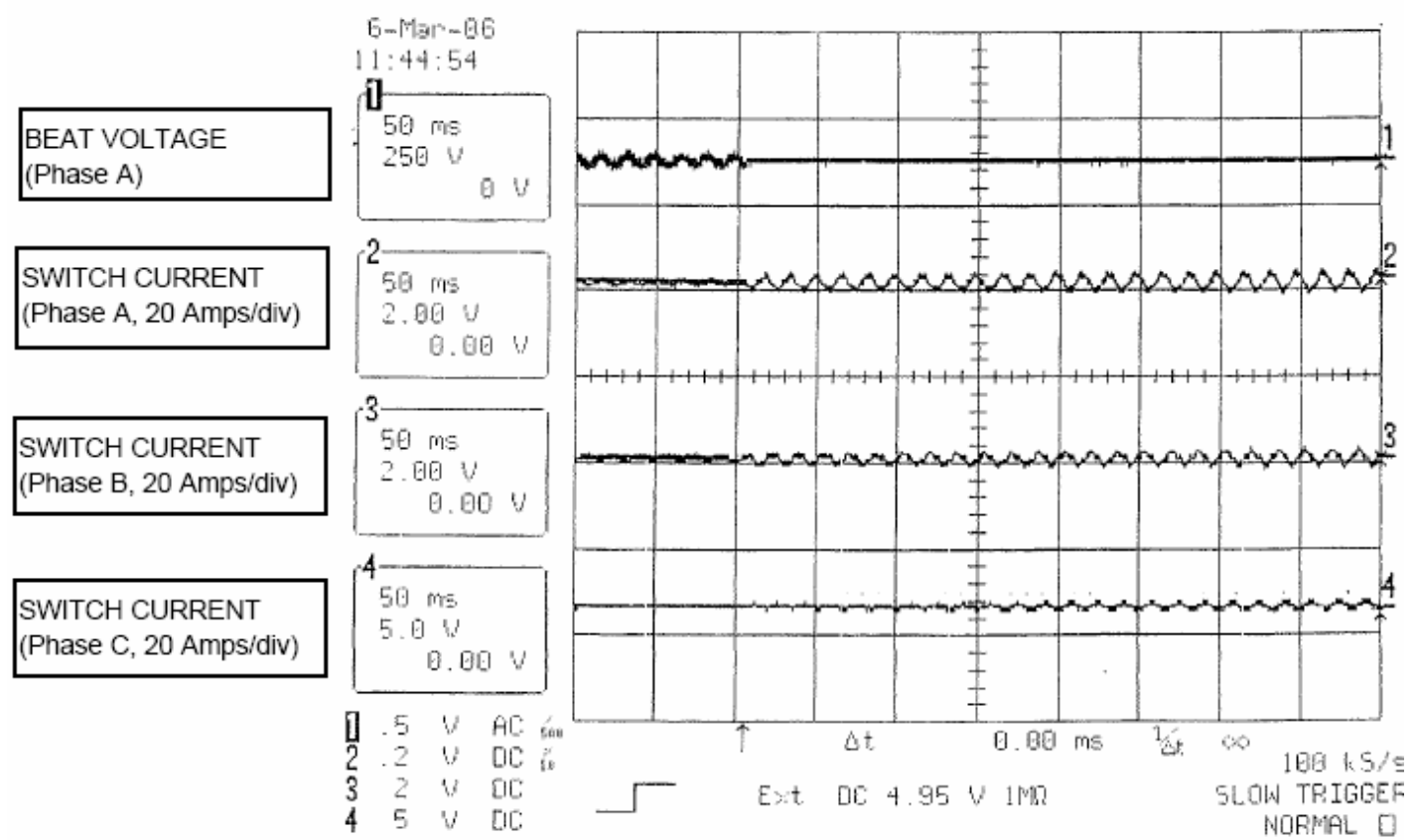

Figure 9: Synchronized Closing for Test \#6 (beat frequency= $0.05 \mathrm{~Hz}$ ) 


\subsection{Reverse Power and Anti-Islanding [Test Plan Section 3.3]}

In this section, the reverse power algorithm as well as the functionalities that it is part of will be tested. The reverse power algorithm event will be running when operating in the "Auto" Mode, and if the conditions of the power flow make this event true, then the DSP will command the switch to open.

Additionally, based on the kind of condition that causes the reverse power event, the DSP will take a decision wether to reclose the switch when conditions are appropriate, or simply lockout the system. This decision will be based on what the grid side voltage is after the switch has opened after a reverse power event:

- If after the switch opens on a reverse power event, any of phases of the grid voltage is low (due to a grid upstream islanding or due to an open phase) then, once conditions are normal for synchronization and all events are cleared, the DSP will try to reclose the switch.

- If after the switch opens on a reverse power event, all phases of the grid voltage is normal, then it means that the reverse power condition was created due to incorrect power setpoints in the DG sources (DG Pset > microgrid load); in this situation, then the DSP should create a fault that will be displayed to the user though the Engineering HMI or EMS as "Reverse Power Anti-islanding = Microgrid Settings Reset" and the switch will be lockout (enter the "OFF" state which opens the SCR switch and the input breaker, thus requiring alarms reset and restarting of the system).

\subsubsection{Reverse Power (32) magnitude, time test}

The first step in testing the reverse power functionality is to test the accuracy the magnitude and time delay of the relay. The settings associated with the reverse power event (which is monitored at the remote location current transformers CT12) are:

- $\quad$ Remote Reverse Power (per phase): In kW; if the reverse power measured at the remote location in any phase is less than this threshold, the "Remote Power connect delay" timer will be triggered. The sign convention is set so that a positive number corresponds to power flow into the microgrid (minimum import convention). The threshold can be set negative or positive (negative settings will allow power export into the grid). The power is measured in every phase so all conditions (including open phase) can be detected.

- Remote Power connect delay timer: In seconds; once the reverse power measurement in any phase is below the threshold, a timer will start to run; if this timer reaches the time delay mentioned here, a "Reverse Power" event will be enabled, which in turn will give an Open command to the SCR switch. This time delay will typically be set to 2 seconds as recommended by utilities on their interconnection agreements.

Two sets of magnitude and time tests were performed: a minimum import case (positive setting) and a maximum export case (negative settings). Recall that during all these tests, all other events (Power Quality, IEEE 1547, etc. were disabled; only Remote reverse power (32) was enabled)

\section{Minimum Import Test Setup}


Initial DG Pset: $1 \mathrm{~kW}$

DG load $(R 4)=12 \mathrm{~kW}$

Grid Load (R1) $=0 \mathrm{~kW}$

Feeder C Load $(\mathrm{R} 3)=0 \mathrm{~kW}$

RMS Remote Reverse Power Setting per phase: $1.66 \mathrm{~kW}$ (5 kW total)

Remote Power connect delay timer: 2 seconds

To run this test, the DSP controller was set in the Auto Mode and when the conditions were appropriate, the DSP performed a synchronized closing. Once switch closes, initial power flow is approximately $\sim 8 \mathrm{~kW}$ into the microgrid, which does not trigger the reverse power event. To create the reverse power condition, the DG source Power Setpoint was increased in $1 \mathrm{~kW}$ steps (for approximately 10 seconds) until a reverse power trip occurs (this is observed by an open command to the switch and also the Reverse Power event bit is enabled in the Engineering $\mathrm{HMI}$ ). This test was repeated three times, and the power magnitude and time delay during the trip were recorded; these results are shown in Table 2.

\author{
Maximum Export Test Setup \\ Initial DG Pset: $2 \mathrm{~kW}$ \\ DG load $(R 4)=0 \mathrm{~kW}$ \\ Grid Load (R1) $=8 \mathrm{~kW}$ \\ Feeder C Load (R3) $=0 \mathrm{~kW}$ \\ RMS Remote Reverse Power Setting per phase: $-1.66 \mathrm{~kW}$ (-5 kW total) \\ Remote Power connect delay timer: 2 seconds
}

This test was performed in a similar way as the Minimum Import Test. The DG source Power Setpoint was increased in $1 \mathrm{~kW}$ steps (for approximately 10 seconds) until a reverse power trip occurs. This test was repeated three times, and the power magnitude and time delay during the trip were recorded; these results are also shown in Table 2.

\begin{tabular}{|r|c|c|c|c|}
\cline { 2 - 5 } \multicolumn{1}{c|}{} & \multicolumn{2}{c|}{ Minimum Import (+5kW, 2 seconds) } & \multicolumn{2}{c|}{ Maximum Export (- 5kW, 2 seconds) } \\
\hline Try \# & $\begin{array}{c}\text { Magnitude } \\
\mathbf{( k W )}\end{array}$ & Time (s) & $\begin{array}{c}\text { Magnitude } \\
\mathbf{( k W )}\end{array}$ & Time (s) \\
\hline 1st & 4.2 & 2.16 & -4.32 & 2.13 \\
\hline 2nd & 4.1 & 2.11 & -4.2 & 2.23 \\
\hline 3rd & 4.1 & 2.07 & -4.4 & 2.2 \\
\hline
\end{tabular}

Table 2: Reverse Power Magnitude and Time tests for Minimum Import and Maximum Export conditions

The results above show a good accuracy for the reverse power detection as well as the time delay; it also shows the capability of the algorithm to work for both power directions (import and export to the grid). Notice that the additional time delay ( 0.2 seconds) could be due to the filtering of the RMS power measurement. The power measurement accuracy in our DSP controller is approximately within $1 \mathrm{~kW}$ which might explain the trips not occurring at exactly the setpoint $(5 \mathrm{~kW})$; also recall that the steps performed during this tests were +1 $\mathrm{kW}$, so the resolution of the test wasn't more precise than that. 


\title{
3.3.2 Grid islanding and Open Phase [Test Plan Section 3.6]
}

Using the reverse power algorithm tested in 3.3.1, an upstream grid islanding as well as an open phase condition can be detected. If this conditions occurs, it is expected that at least one phase of the grid voltage will be dead after the switch opens on the reverse power event; if the DSP controller observes this condition, then it will decide not to lockout the system, and thus allow to reclose when conditions are appropriate.

For the tests described above, and during normal operation, an additional setting is required:

- Voltage test delay timer: In seconds; once the reverse power event is enable, this new timer starts to run, and after it expires it looks at the voltage on the grid side (the switch should still be open from the reverse power event); if all the three phases voltages are normal, then it will create a fault and lockout the system, requiring user intervention; but if any of the phases voltage is low (grid islanding upstream or open-phase) then it will simply keep the switch open until conditions are appropriate for reclosing. For testing purposes, this timer will be set to 1 second.

\author{
Grid islanding and Open Phase Test Setup \\ Initial DG Pset: $1 \mathrm{~kW}$ \\ $\mathrm{DG}$ load $(\mathrm{R} 4)=8 \mathrm{~kW}$ \\ Grid Load (R1) $=0 \mathrm{~kW}$ \\ Feeder C Load $=0 \mathrm{~kW}$ \\ RMS Remote Reverse Power Setting per phase: $1.66 \mathrm{~kW}$ (5 kW total) \\ Remote Power connect delay timer: 2 seconds
}

The test setup described above was used for both the grid-islanding and the Open phase test. The grid islanding test condition was created by disconnecting the grid side inverter, thus no power flow exist across the switch which enables the reverse power event. The open-phase condition was created by disconnecting one of the phases on the grid side.

Figures 10 and 11 show the response of the controller and the switch to the grid upstream islanding test. These figures show the following:

Channel 1: beat voltage across phase A of the SCR

Channel 2: phase A switch current (20A/division)

Channel 3: DSP switch command

In Figure 10 it can be observed when the grid islanding condition occurs by disconnecting the grid inverter; the switch current is reduced to almost zero (the small current is due to the S\&C control load), and after approximately 2 seconds, the switch is commanded to open (this can be seen by the switch command going $\mathrm{HIGH}$, and also by the beat voltage going from zero when the switch is still closed to rated voltage once the switch opens). More than 1 second elapses, and the system is still running in the "Disconnect" mode, waiting for the right synchronization conditions to close.

In figure 11, the grid side is reconnected at approximately 0.25 seconds in the oscilloscope plot; after the grid is reconnected, the voltage across the switch now changes from rated voltage to a beat voltage waveform, and once synchronization conditions are appropriate (phase of beat voltage at zero) the DSP commands the switch to reclose. This is the desired behavior. 


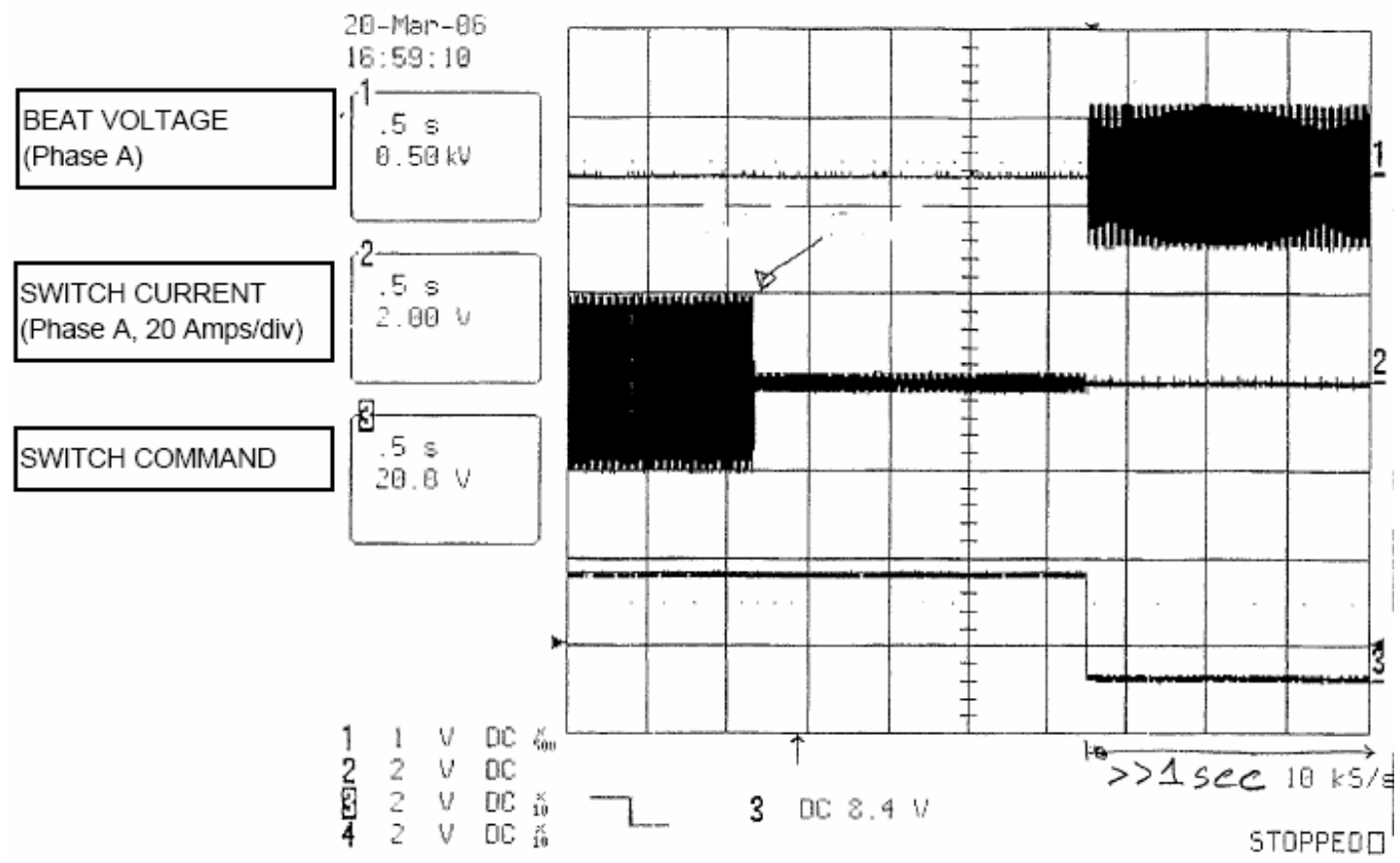

Figure 10: Grid Islanding Test, Disconnection after reverse power event

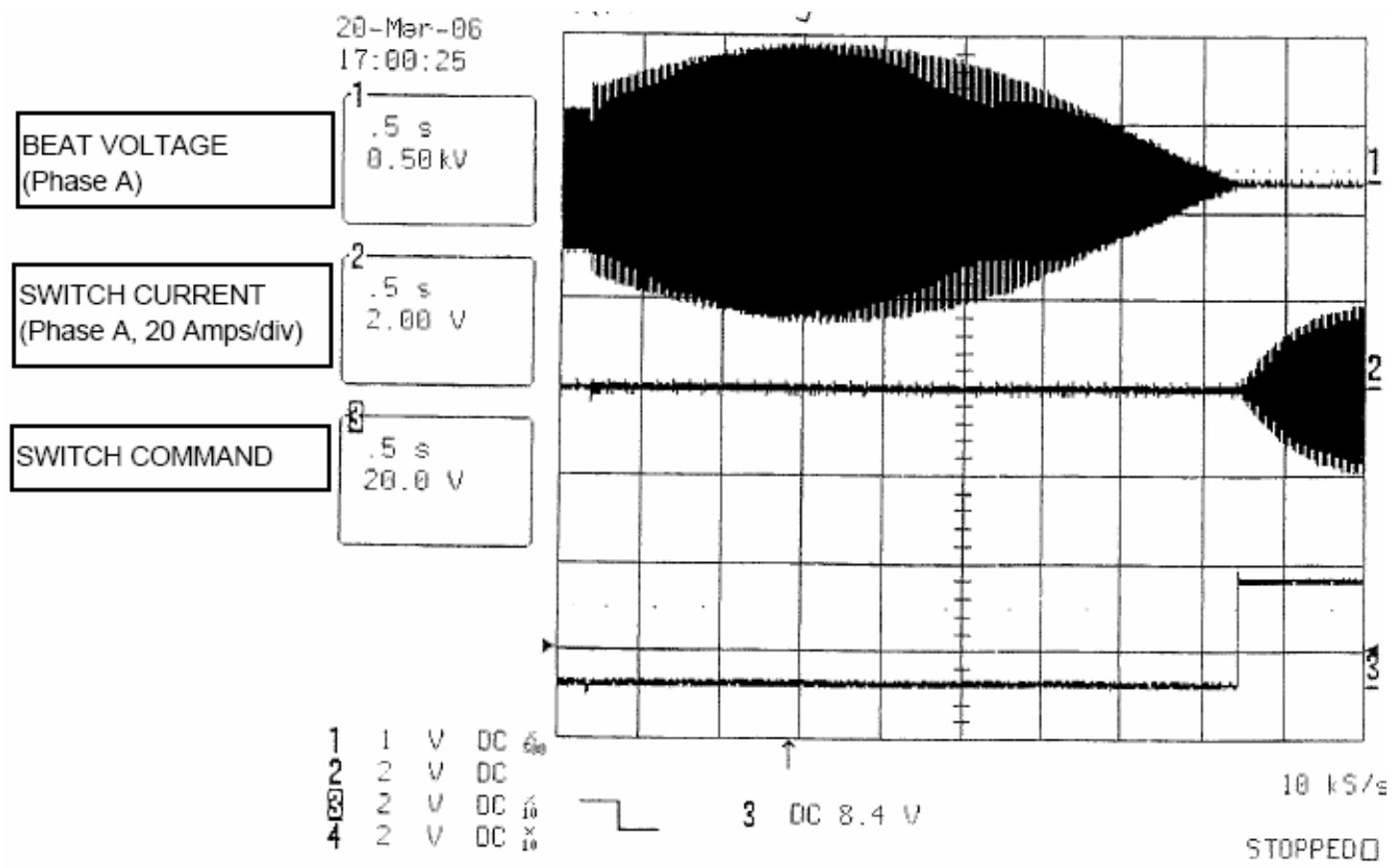

Figure 11: Grid Islanding Test, Reconnection 
A Distributed Energy

Systems Company

In figure 12, the response to an open phase condition is observed. This figure shows the following:

Channel 1: beat voltage across phase A of the SCR

Channel 2: phase A switch current (20A/division)

Channel 3: phase B switch current (20A/division)

Channel 4: DSP switch command

Phase C switch current isn't shown due to oscilloscope channel limitations. This figure verifies that an open-phase condition was created by showing the phase A switch current going to zero while phase B (and phase C) still supply the load. After $\sim 2$ seconds, the reverse power event requires the DSP to open the switch without locking out the system. Just like the previous test, when synchronization conditions are appropriate, the DSP will attempt to reclose the switch.

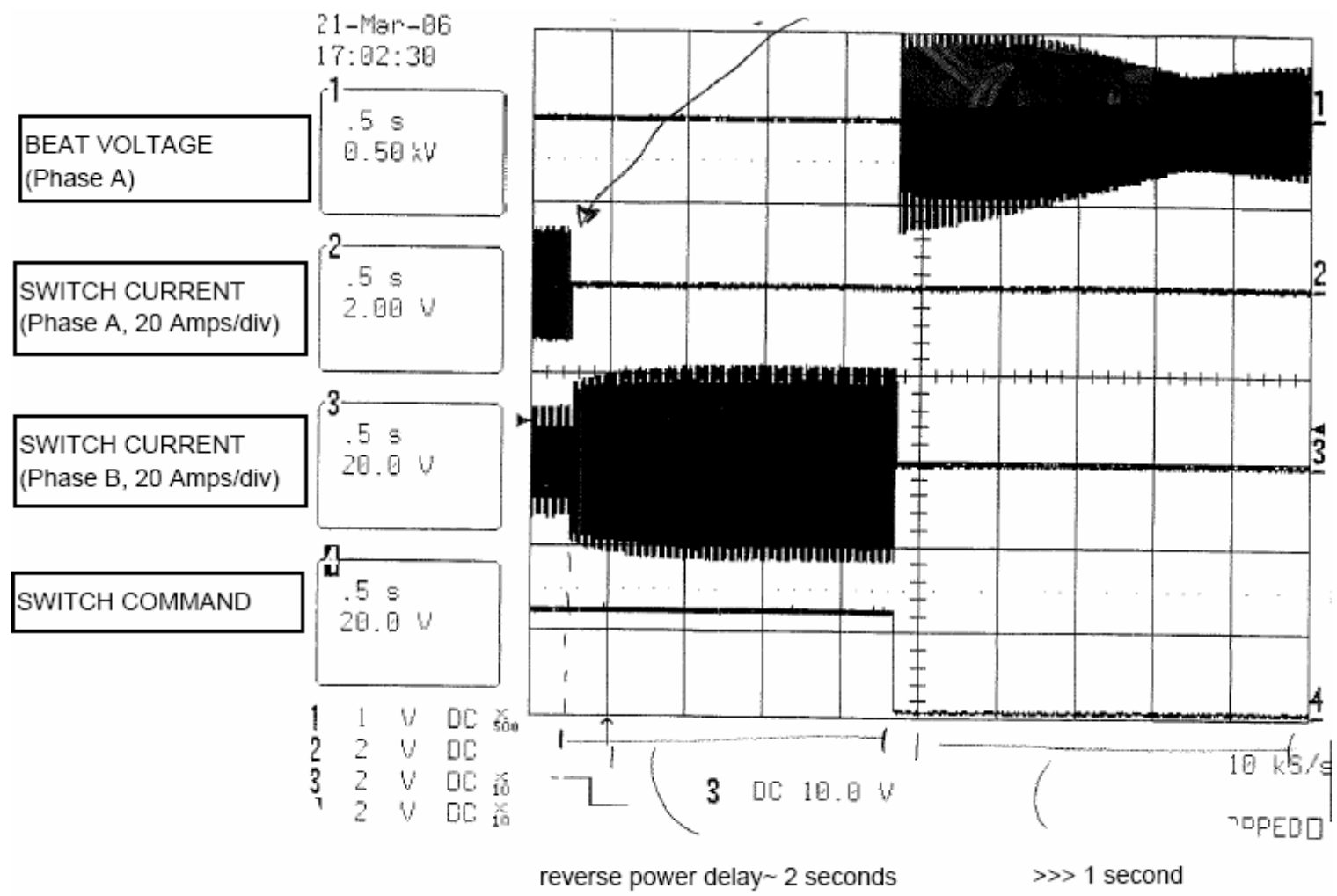

Figure 12: Open Phase Test 


\subsubsection{Anti-islanding, Microgrid Settings Reset (Lockout)}

The final reverse power test in this section involves the possible islanding condition of the microgrid due to a reverse power event created by erroneous microgrid settings (power setpoints larger than microgrid load). If this occurs, once the switch opens, the voltages on the grid and DG sides will all be normal, and it is expected that by detecting this condition, the DSP controller will lockout the system. The lockout occurs by creating a fault, transferring switch to the "Fault State" which opens the input breaker and requires user interface to restart the system.

The setup used for this test is the same setup as the grid islanding and open phase tests in section 3.3.2. Once the switch has done the synchronized closing and reach steady state, the power flow into the microgrid is approximately $7 \mathrm{~kW}$ (over the reverse power threshold). Then, the DG load bank (R4) is reduced from $8 \mathrm{~kW}$ to $3 \mathrm{~kW}$, reducing the power flow into the microgrid side to $2 \mathrm{~kW}$, which will enable the reverse power event. Figure 13 shows the system response to this test:

Channel 2: phase A switch current (20A/division)

Channel 3: DSP Switch command

\section{Channel 4: "Start/Stop" Command}

After approximately 2 seconds after the load reduction, the DSP switch command opens the SCR switch as expected, but 1 second after that, the "Start/Stop" command goes low, which stops the S\&C PES by opening the input breaker.

In the Engineering HMI, it was observed that the system entered the "Fault" state, which will require user interface. The alarm displayed in the HMI is "Reverse Power Anti-islanding, Microgrid Settings Reset" which should inform the operator that adjustments in the power setpoints of the microsources is required.

For future consideration it is possible that rather than locking out the system when this condition occurs, the EMS will automatically update the microsource settings to eliminate the reverse power condition, but this was not considered for this project.

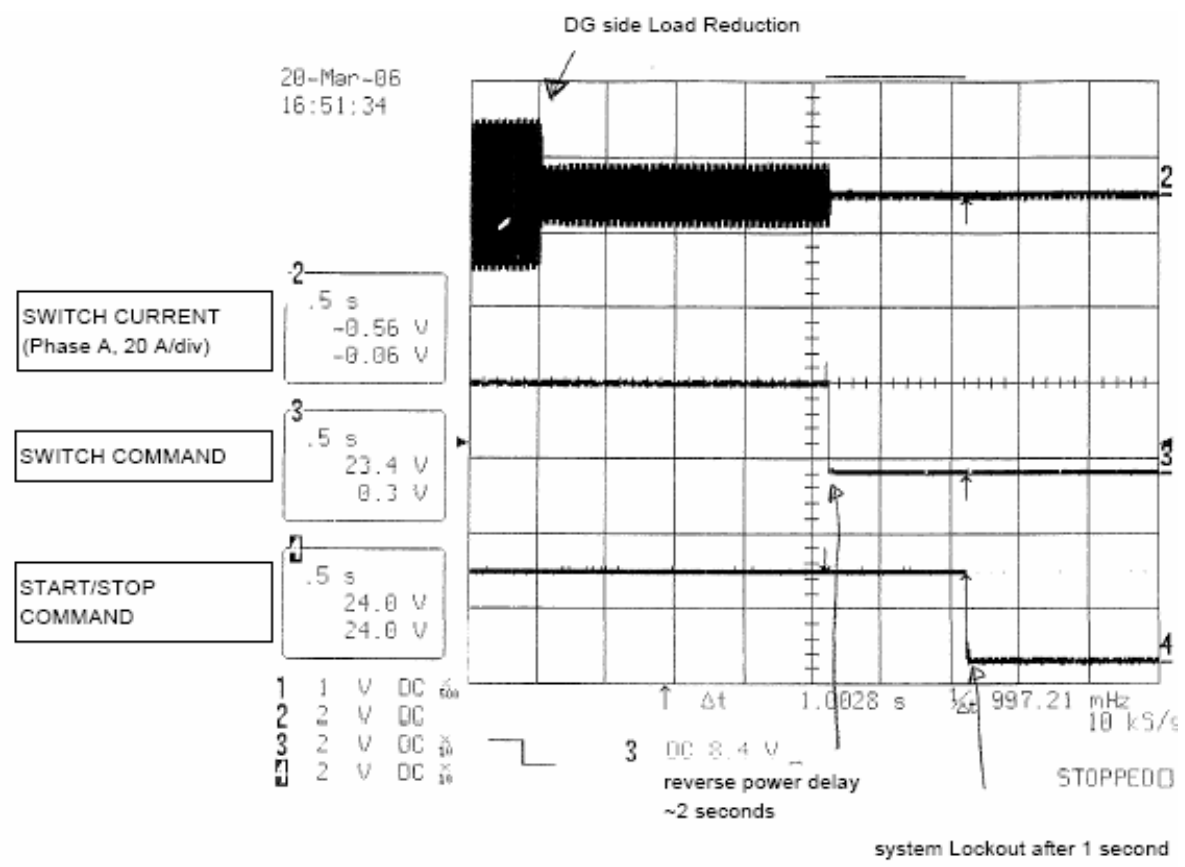

Figure 13: Anti-islanding, Microgrid Settings Reset Test 


\subsection{IEEE 1547 Voltage and Frequency Tests}

The IEEE 1547 standard specifies the voltage ranges and clearing times for over-voltage, under-voltage, over-frequency and under-frequency events. The values specified in the standard are repeated in each section for reference.

NREL's Omicron CMA256-6 secondary injection test set was used to perform the IEEE 1547 voltage and frequency tests. These tests closely reflect the method required by the IEEE 1547.1 standard, where tests are repeated 5 times to get more accurate results. Two types of tests are performed:

- $\quad$ Magnitude Test: The Omicron equipment ramps the voltage or frequency up or down (depending on the setpoint tested) at a relatively slow rate, and stays at each level long enough to allow the DSP to give the trip command if necessary. The magnitude at which the trip occurs is recorded.

- Time test: The Omicron equipment ramp the voltage or frequency from rated level to just above or below the magnitude of the setpoint that is tested, and records the time delay of the switch command to trip.

As explained before, all these tests are run in the "Auto" model the Omicron Equipment simulates appropriate synchronization conditions on both the DG and Grid side PT secondary signals. After the DSP switch command goes high (close), it performs the tests mentioned above.

Also, it is important to note that these tests are performed at low power, so the S\&C switch is off during this test; the time delays recorded relate to the DSP switch command, and does not include the opening time of the SCR (which is expected to be up to $1 / 2$ cycles). The next subsection shows some tests that were done to verify the SCR opening time.

\subsubsection{Over/Under Voltage tests [Test Plan Section 3.5]}

In this subsection, a summary of the results of the voltage tests (magnitude and time) is presented. Tables 3 and 4 show the specified responses in the IEEE1547.1 standard to over and under-voltage event.

Table 3. IEEE 1547 specification for interconnection response to over-voltages

\begin{tabular}{ll}
\hline $\begin{array}{l}\text { Voltage range } \\
\text { (\% of base voltage) }\end{array}$ & $\begin{array}{l}\text { Clearing time } \\
(\mathbf{s})\end{array}$ \\
\hline $110<\mathrm{V}<120$ & 1 \\
$\mathrm{~V} \geq 120$ & 0.16 \\
\hline
\end{tabular}

Table 4. IEEE 1547 specification for interconnection response to under-voltages

\begin{tabular}{ll}
\hline $\begin{array}{l}\text { Voltage range (\% of } \\
\text { base voltage) }\end{array}$ & Clearing time (s) \\
\hline $\mathrm{V}<50$ & 0.16 \\
$50 \leq \mathrm{V}<88$ & 2 \\
\hline
\end{tabular}


Tables 5 and 6 below shows a summary of the tests that were performed; it presents average results of the 5 tries that were run for each voltage setpoint compared to the actual setting that was set in the DSP controller while running the test. Notice that for all setpoints, three phase tests were performed. However, for single phase tests different phases were alternated for different setpoints; this procedure was done this way because due to limited availability of the Omicron Equipment at Northern Power's facility, there would not have been time to perform all single-phase tests for all the different setpoints. However, by alternating the phases with different setpoints we were able to obtain a good picture of the overall accuracy of the controller.

For the voltage magnitude tests, the magnitude was ramped with $0.1 \mathrm{~V}$ steps. Also, for the time tests, the steps were done from rated voltage $(277 \mathrm{~V})$ to $5 \mathrm{~V}$ above or below the setpoint.

\begin{tabular}{|l|c|c|c|}
\hline \multicolumn{4}{|c|}{ IEEE 1547 VOLTAGE MAGNITUDE TESTS (AVERAGES FROM 5 TRIES) } \\
\hline Test & Setting (V) & $\begin{array}{c}\text { Average Trip } \\
\text { Magnitude (V) }\end{array}$ & $\begin{array}{c}\text { Average } \\
\text { Deviation (V) }\end{array}$ \\
\hline Under-voltage 50\%, Three Phase & 138.5 & 139.00 & 0.50 \\
Under-voltage 50\%, Phase A & 138.5 & 138.98 & 0.48 \\
Under-voltage 88\%, Three Phase & 243.8 & 244.02 & 0.22 \\
Under-voltage 88\%, Phase C & 243.8 & 243.78 & -0.02 \\
Over-voltage 110\%, Three Phase & 304.7 & 305.08 & 0.38 \\
Over-voltage 110\%, Phase A & 304.7 & 306.24 & 1.54 \\
Over-voltage 110\%, Phase B & 304.7 & 305.08 & 0.38 \\
Over-voltage 110\%, Phase C & 304.7 & 305.66 & 0.96 \\
Over-voltage 120\%, Three Phase & 332.4 & 332.50 & 0.10 \\
Over-voltage 120\%, Phase B & 332.4 & 332.50 & 0.10 \\
\hline
\end{tabular}

Table 5. Summary of IEEE 1547 voltage magnitude tests

\begin{tabular}{|l|c|c|c|}
\hline \multicolumn{4}{|c|}{ IEEE 1547 VOLTAGE TIME TESTS (AVERAGES FROM 5 TRIES) } \\
\hline Test & Setting (ms) & $\begin{array}{c}\text { Actual Trip } \\
\text { Time (ms) }\end{array}$ & $\begin{array}{c}\text { Average } \\
\text { Deviation (ms) }\end{array}$ \\
\hline Under-voltage 50\%, Three Phase & 167 & 181.94 & 14.94 \\
Under-voltage 50\%, Phase A & 167 & 181.68 & 14.68 \\
Under-voltage 50\%, Phase B & 167 & 181.84 & 14.84 \\
Under-voltage 88\%, Three Phase & 2000 & 2014.98 & 14.98 \\
Under-voltage 88\%, Phase C & 2000 & 2015.16 & 15.16 \\
Over-voltage 110\%, Three Phase & 1000 & 1008.48 & 8.48 \\
Over-voltage 110\%, Phase A & 1000 & 1015.34 & 15.34 \\
Over-voltage 120\%, Three Phase & 167 & 181.70 & 14.7 \\
Over-voltage 120\%, Phase B & 167 & 178.68 & 11.68 \\
\hline
\end{tabular}

Table 6. Summary of IEEE 1547 voltage time tests

The results in Table 5 show a very good accuracy for the voltage magnitude tests. The maximum deviation occurred at the $110 \%$ over-voltage setting for a single phase $(A)$ test; the deviation for this test was $1.54 \mathrm{~V}$, which corresponds to $0.55 \%$ of rated voltage. 
The results in Table 6 also show good accuracies for the voltage time delays; the maximum deviation for the faster trips (167 ms) was less than 1 cycle (14.98 ms).

The IEEE 1547 under and over-voltage function tests results confirmed that the controller achieved its software programmed functionality.

\subsubsection{Over/Under Frequency tests [Test Plan Section 3.4]}

In this subsection, a summary of the results of the frequency tests (magnitude and time) is presented. Tables 7 and 8 show the specified responses in the IEEE1547.1 standard to over and under-frequency events.

Table 7. IEEE 1547 specification for interconnection response to over-frequency

\begin{tabular}{ll}
\hline Frequency range $(\mathbf{H z})$ & Clearing time $(\mathbf{s})$ \\
\hline$>60.5$ & 0.16
\end{tabular}

Table 8. IEEE 1547 specification for interconnection response to under-frequency

\begin{tabular}{ll}
\hline Frequency range $\mathbf{( H z )}$ & Clearing time $\mathbf{( s )}$ \\
\hline$<57.0$ & 0.16 \\
$59.8>$ freq $>57$ & Adjustable $0.16-300$ seconds $^{*}$ \\
\hline
\end{tabular}

* The IEEE 1547.1 standard specifies this time setting to be adjustable, and does not provide an actual value. For this testing it will be set to 1 second, and if necessary it can be adjusted in the microgrid test site using the EMS.

Tables 9 and 10 show the summary results for the frequency magnitude and time tests. The frequency magnitude tests were ramped at a rate of 10 milliHertz per step; for the time tests, Table 10 describes precisely to what frequency was the step done for each of the tests.

\begin{tabular}{|l|c|c|c|}
\hline \multicolumn{4}{|c|}{ IEEE 1547 FREQUENCY MAGNITUDE TESTS (AVERAGES FROM 5 TRIES) } \\
\hline & Setting (V) & $\begin{array}{c}\text { Average Trip } \\
\text { Magnitude (Hz) }\end{array}$ & $\begin{array}{c}\text { Average } \\
\text { Deviation (Hz) }\end{array}$ \\
\hline Ovest & 60.50 & 60.51 & 0.01 \\
Under-frequency $60.5 \mathrm{~Hz}$ & 59.30 & 59.30 & 0.00 \\
Under-frequency $59.3 \mathrm{~Hz}$ & 57.00 & 57.00 & 0.00 \\
\hline
\end{tabular}

Table 9. Summary of IEEE 1547 frequency magnitude tests 


\begin{tabular}{|ll|c|c|c|}
\hline \multicolumn{5}{|c|}{ IEEE 1547 FREQUENCY TIME TESTS (AVERAGES FROM 5 TRIES) } \\
\hline Test & & Setting (ms) & $\begin{array}{c}\text { Actual Trip } \\
\text { Time (ms) }\end{array}$ & $\begin{array}{c}\text { Average } \\
\text { Deviation } \\
\text { (ms) }\end{array}$ \\
\hline Over-frequency $60.5 \mathrm{~Hz}$ & (step:60.0 to $60.55 \mathrm{~Hz})$ & 167 & 266.06 & 99.06 \\
Over-frequency $60.5 \mathrm{~Hz}$ & (step:60.45 to $60.55 \mathrm{~Hz})$ & 167 & 202.66 & 35.66 \\
Over-frequency $60.5 \mathrm{~Hz}$ & (step:60.40 to $60.70 \mathrm{~Hz})$ & 167 & 183.04 & 16.04 \\
Over-frequency $60.5 \mathrm{~Hz}$ & (step:60.0 to $60.55 \mathrm{~Hz})$ & 100 & 186.56 & 86.56 \\
Under-frequency $59.3 \mathrm{~Hz}$ (step:60.0 to $59 \mathrm{~Hz}$ ) & 1000 & 1069.54 & 69.54 \\
Under-frequency $57 \mathrm{~Hz}$ & (step:60.0 to $56 \mathrm{~Hz}$ ) & 167 & 251.08 & 84.08 \\
\hline
\end{tabular}

Table 10. Summary of IEEE 1547 frequency time tests

The magnitude tests in Table 9 show that the maximum deviation was $0.01 \mathrm{~Hz}$, therefore the frequency magnitude trips are extremely accurate.

However, some issues were observed for the frequency time tests in Table 10; longer time delays than expected were observed. Notice that for the frequency time tests, rather than simply doing a large step from $60 \mathrm{~Hz}$ to just above or below the thresholds, also smaller steps were taken.

Similar results were obtained from the testing of Northern Power Controller for the DER Switch project (another NREL subcontract) The investigation of this issue shows that when a large frequency step change was applied, it can also cause a large PLL transient response, possibly large enough to briefly break the lock detector. In reality, a power system would not be subjected to instant frequency swings so drastic. In order to obtain the expected time response with a more realistic test condition, the frequency tests were repeated for smaller step changes centered at the threshold (see Table 10); these tests provides much better time responses. 


\subsection{SCR Switch Opening Time}

In the IEEE 1547 section, the trip time measurement only monitored the opening command from the DSP Controller, and does not include the opening time of the switch (since the S\&C PES was not powered). It was considered important to perform some runs and verify that unless an asymmetrical component is present in any of the switch currents, the SCR switch will open on approximately $1 / 2$ cycles $(8 \mathrm{~ms})$.

To perform this test, a very simple test setup was used. The inverter side of the switch was setup as an open-loop source (constant voltage and frequency; $277 \mathrm{~V}, 60 \mathrm{~Hz}$ ) while the other side of the switch was set to an $8 \mathrm{~kW}$ resistive load.

The test was operated in the "Manual" mode and "Sync Test Bypass", so the switch could be manually opened and close. With this setup, the switch was cycled 20 times, and the opening time was recorded (from when the DSP command to open is given until all the phase currents go to zero).

The results are presented in Table 11. Figure 14 shows the oscilloscope waveforms (switch currents $A, B, C$ and DSP switch command) for try\#4 in table 11. The average opening time from these results was $8.13 \mathrm{~ms}$, which verifies the expected performance of the SCR switch.

\begin{tabular}{|c|c|}
\hline Try\# & $\begin{array}{c}\text { Opening Time } \\
\text { (ms) }\end{array}$ \\
1 & 7.96 \\
2 & 7.67 \\
3 & 8.96 \\
4 & 7.62 \\
5 & 8.25 \\
6 & 7.31 \\
7 & 7.38 \\
8 & 8.07 \\
9 & 7.92 \\
10 & 9.18 \\
11 & 7.59 \\
12 & 9.00 \\
13 & 8.39 \\
14 & 8.73 \\
15 & 8.57 \\
16 & 8.56 \\
17 & 7.95 \\
18 & 8.85 \\
19 & 7.03 \\
20 & 7.68 \\
\hline Average (ms) & 8.13 \\
\hline
\end{tabular}

Table 11. SCR Opening Time Test Results 


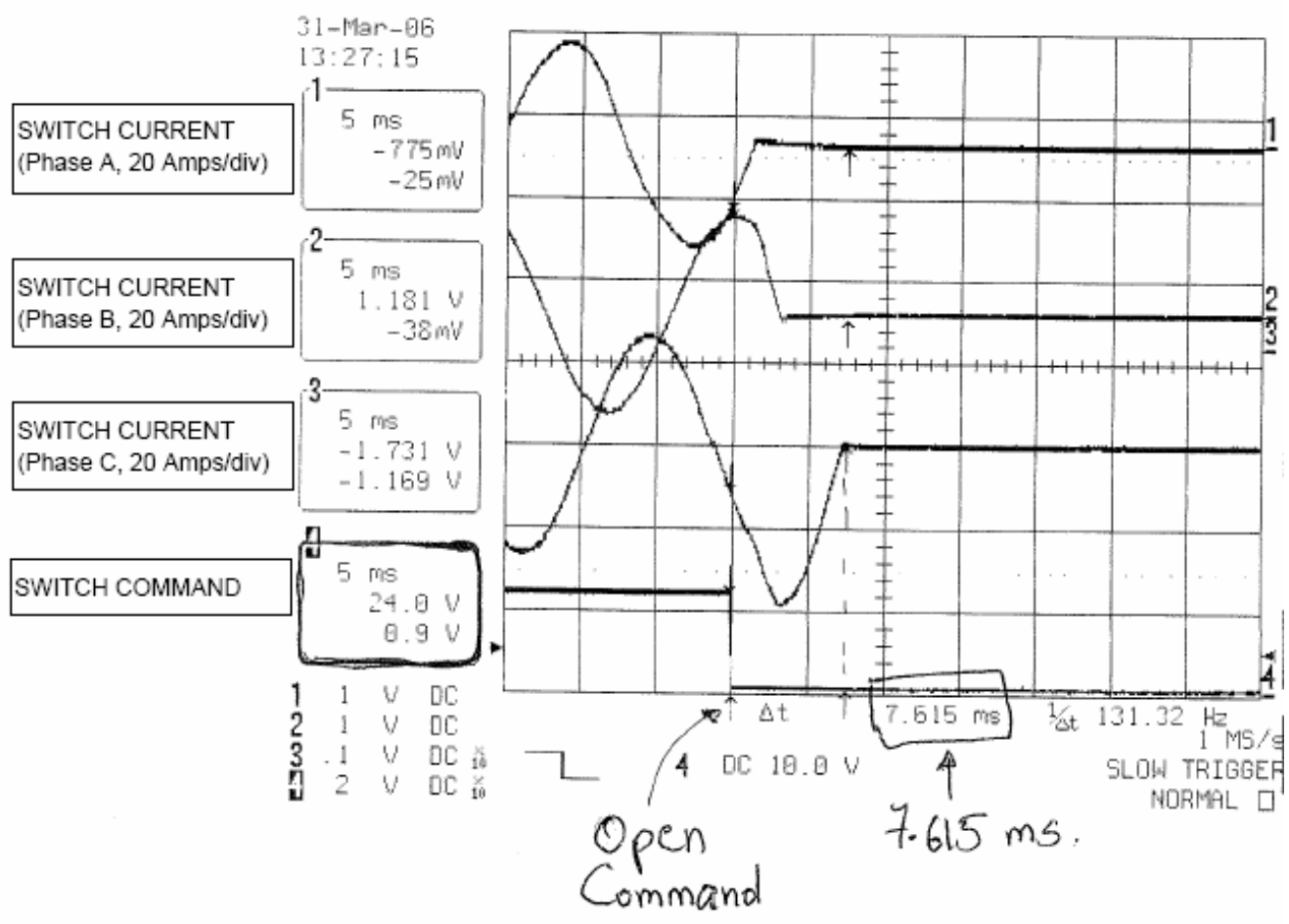

Figure 14. SCR Opening Time Test; Try \#4

3.6 SEL-351 Relay - DSP Controller Functionality [Test Plan Section 4] 
Several tests were performed to verify the basic functionality of the SEL-351 relay and its interaction with the Static Switch DSP control. The Relay input is considered an "Event" in the DSP controller; anytime the Relay communicates a trip to the DSP Controller, the DSP will give an opening command to the switch, no matter what the control mode is (recall that for the other events, such IEEE1547, Power Quality and Reverse Power, the DSP Controller opens the switch only in the "Auto" Mode)

As explained before, the Static Switch SEL 351 Relay (Relay2) is expected to perform the following protection functions:

- Phase overcurrent protection (instantaneous and time-overcurrent)

- $\quad$ Line to Line undervoltage protection (on the grid side of the switch PT14)

- Zero Sequence Current (using the neutral current input)

However, notice that for this set of tests, the programming of the SEL-351 relay was set to very simple trip levels and timers so that the trip conditions could be created based on the capabilities of the NPS test setup. The purpose is to test the interaction of Relay-DSP and not the trip accuracies of the Relay. The accuracies of the SEL-351 Relays can be trusted due to their wide use in the industry.

The tests performed in this section attempted to verify the following:

1) SEL Relay TRIP output logic

2) SEL Relay minimum opening time and associated DSP reconnection timer and reclosing

3) Relay to DSP delay

\section{Settings}

To perform the testing of this simple functionality, the Relay was programmed to the following settings (given in primary values):

- $50 \mathrm{P} 1$ (Instantaneous overcurrent): 15A

- $\quad$ 27PP (Line-Line undervoltage): $50 \%$

- $\quad$ SV1 = 27PP (SV1 logic associated with line-line voltage)

- SV1 Pickup time: 0 cycles

- SV1 Dropout time: 5 seconds

- Relay minimum opening time: 20 seconds

- $\quad$ TRIP $=50 \mathrm{P} 1+\mathrm{SV} 1$

- $\quad$ OUT101 =! TRIP (OUT101 is the output that will give the command to DSP)

\subsubsection{SEL Relay TRIP output logic}

The purpose of this test is to verify the output logic of the SEL-351. The DSP is expected to open the switch whenever the relay gives a trip command to it. There is no reconnection timers in the DSP associated with the Relay input (the final programming of the Relay will have its minimum opening time $~ 20$ seconds in order to allow utility protection equipment to reclose first under fault conditions).

OUT101 has been programmed with an inverse logic to the trip condition (!TRIP); also the DSP was programmed to the following logic:

- If input from Relay is HIGH (24V); keep switch closed

- If input from Relay is LOW (0V); open switch 
A Distributed Energy

Systems Company

The reason for this is to have a fail-safe mode; if for some reason communication is lost between the Relay and the DSP, the input to the DSP from the Relay will go low, thus providing the trip and making the operator aware that something wrong has occurred.

To verify this functionality, a test was done creating and under-voltage condition. The grid side inverter was disconnected, dropping the voltage below $50 \%$ and thus creating an under-voltage trip condition (27PP). The inverter was kept disconnected for about 1 minute (to allow minimum opening time to run off); then, the inverter is turned on again.

Figure 15 shows the response to the test described above:

Channel 1: Grid Voltage Phase A

Channel 4: Relay output signal to the DSP

We can see that the relay signal is initially low as expected; once the grid voltage has recovered, approximately 5 seconds after (dropout time) it can be seen that the Relay signal goes high $(+24 \mathrm{~V})$, which means the relay trip conditions are cleared and now the DSP can turn on the switch when synchronization conditions allow it.

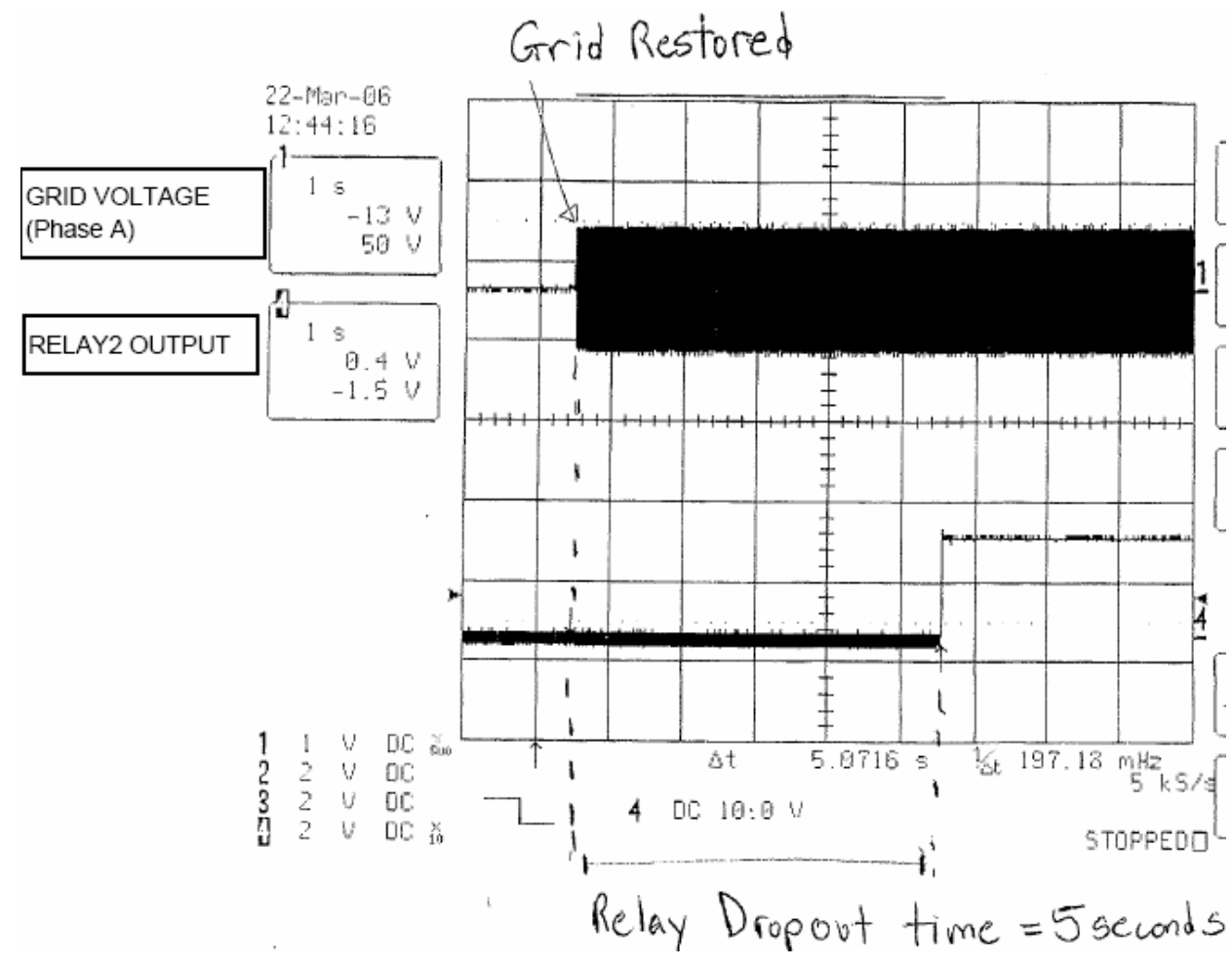

Figure 15. SEL Relay Trip Output Logic Test

\subsubsection{SEL Relay minimum opening time and associated DSP reclosing}

The second test performed verifies that the DSP opens the static switch on the Relay input signal going Low, and that once the command goes High again; the DSP closes the switch once synchronization conditions are appropriate. 
A Distributed Energy

Systems Company

For this test, both the grid and DG power routers are on, and their initial conditions and loads were set such that the beat frequency of the voltage across the switch before closing is approximately $0.08 \mathrm{~Hz}$. Once the switch performs synchronized closing, the grid supplies $12 \mathrm{~kW}$ of the DG load ( $14 \mathrm{Arms}$ ).

To test the interaction of DSP and Relay, an over-current condition was created. On the DG side, the load was increased from $12 \mathrm{~kW}$ to $15 \mathrm{~kW}$. This will increase the current measured by the Relay from $14 \mathrm{Arms}$ to approximately $18 \mathrm{Arms}$, thus requiring an instantaneous current trip (recall threshold was set to 15A).

Figure 16 shows the response to this test; it shows the following waveforms:

Channel 1: switch phase A beat voltage

Channel 2: phase A switch current (20 A/division),

Channel 3: Switch Command

Channel 4: Relay output signal to the DSP

The switch current curve shows when the over-current condition is created, and it can be seen that, instantaneously, the Relay signal goes Low thus requiring the DSP command to open the switch. The switch opens, which can be observed by the formation of the beat voltage across the switch phase A.

The load is immediately reduced back to $12 \mathrm{~kW}$ (to avoid any overcurrent condition when reconnecting). After 20 seconds (minimum opening time), the Relay signal goes back High $(+24 \mathrm{~V})$, but the DSP does not attempt to reclose the switch right away because synchronization conditions are not appropriate. Once synchronization conditions are appropriate, the DSP switch command closes the switch again for normal operation.

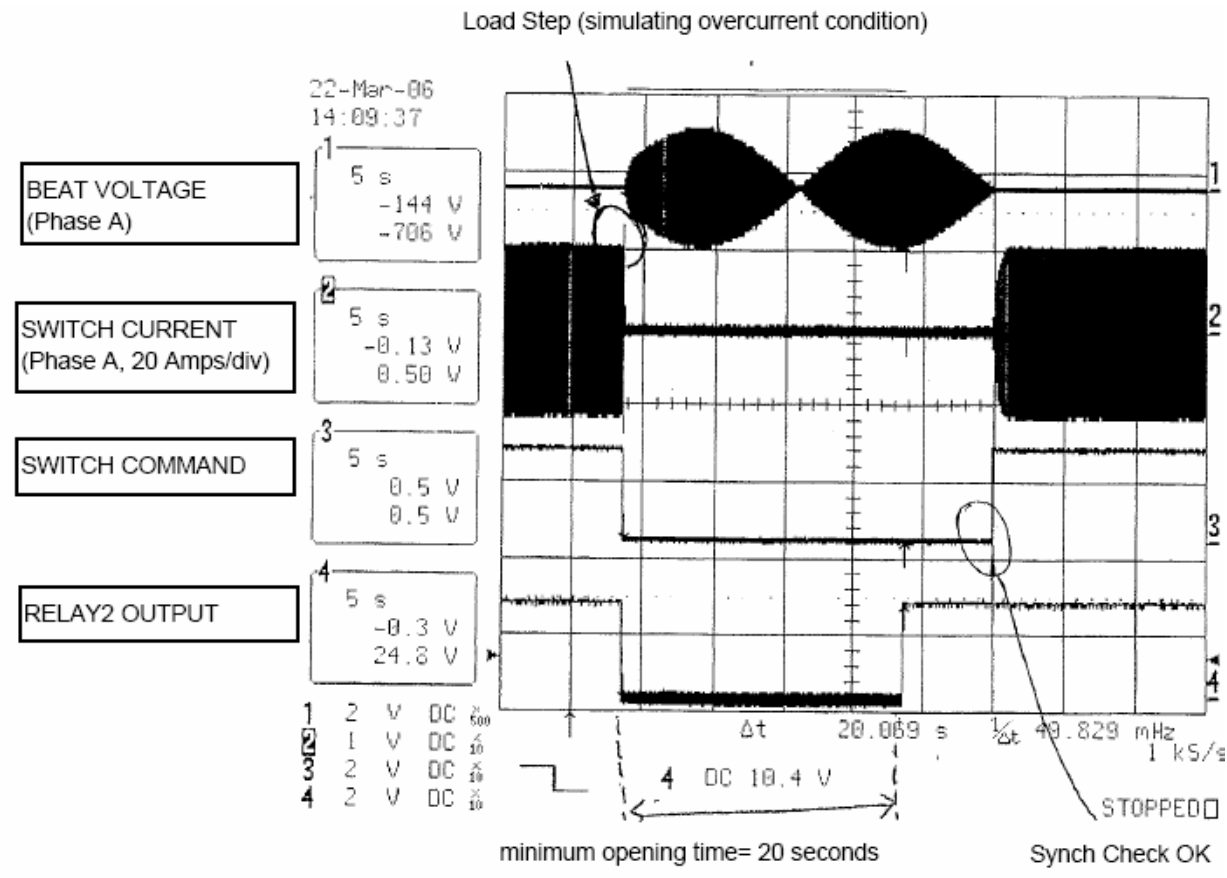

Figure 16. SEL Relay minimum opening time and associated DSP reclosing Test

\subsubsection{Relay to DSP delay}

The final Relay functionality test was performed in order to have an estimation of time delays between the Relay trip signal and the DSP switch command when required to open the switch. Five tries were run, creating the same over-current condition as in section 3.6.2, recording the time delay between the two signals. Table 
12 below shows the data collected. On average, the time delay was $1.1 \mathrm{~ms}$, which is not significant and does not provide any concerns.

\begin{tabular}{|c|c|}
\hline \multicolumn{2}{|c|}{ Relay to DSP Delay } \\
\hline Try\# & Time $\mathbf{( m s )}$ \\
\hline 1 & 1.22 \\
\hline 2 & 0.975 \\
\hline 3 & 1.52 \\
\hline 4 & 1.19 \\
\hline 5 & 0.81 \\
\hline Average & $\mathbf{1 . 1 4 3}$ \\
\hline
\end{tabular}

Table 12. Relay to DSP time delay Test data

\subsection{Dead Bus Reclosing [Test Plan Section 3.5.3]}

This section presents results of testing the accuracy and operation of the Dead Bus Reclosing functionality of the DSP. The purpose of this algorithm is to allow for reclosing to a dead bus on the DG side to allow for black starts of loads. The test will be divided in two sections:

1) DG Bus LO (27R) magnitude test 
2) DG Bus LO (27R) time test; "Dead Bus Reclose" operating mode

The settings for the algorithm during this test are:

Dead Bus 27R Magnitude: 15Vrms

Dead Bus 27R Time constant:: 1 second

\subsubsection{DG Bus LO (27R) magnitude test}

The first step is to test the accuracy of the DG Bus LO (27R) relay function, which toggles HIGH if ALL phases of the DG side voltage are below the threshold. This test was performed with voltage on the DG side only; no attempt to reclose the switch was done, only the 27R relay event was checked to see when it detects the Dead Bus.

The test was performed in each test by setting the voltage in two of the phases (B,C for the first test) close to zero volts, and setting the remaining phase (A for the first test) just above the threshold (set it to $25 \mathrm{Vrms}$ ). At this point, the 27R event should be Low since one phase is above the threshold. Then, in $0.5 \mathrm{~V}$ steps, the phase A voltage was reduced and settle at that level for 5 seconds until the 27R relay event toggles High. Results for the test performed on each phase were recorded and shown in table 13:

\begin{tabular}{|c|c|}
\hline \multicolumn{2}{|c|}{$\begin{array}{c}\text { Dead Bus Reclose Magnitude Test } \\
\text { Threshold= 15 Vrms }\end{array}$} \\
\hline Phase Tested & Voltage at Trip (Vrms) \\
\hline A & 14.33 \\
\hline B & 14.23 \\
\hline C & 14.34 \\
\hline
\end{tabular}

\subsubsection{DG Bus LO (27R) time test; "Dead Bus Reclose" operating mode}

This test will verify the Dead Bus reclosing Logic and the time delay in the 27R LO relay. The setup used for this test was:

Operating Mode: Auto

Grid Side Voltage: 277 Vrms

DG Side Voltage: 25 Vrms

Dead Bus Reclose Mode: Enabled

Figure 17 shows the response for this test. This waveform shows:

Channel 1: DG side voltage

Channel 2: Grid side Voltage

Channel 3: Switch command 
A Distributed Energy

Systems Company

Once the system is started, the system remains in the Disconnect state because synchronization conditions are not appropriate since the DG side voltage is well below the limits, and also it doesn't attempt to reclose since the voltage is not below the Dead Bus Relay threshold. To achieve the dead bus reclose, the DG side inverter was disconnected, dropping the DG voltage to zero. After a 0.944 seconds time delay (very close to the 1 second setting) the Switch Command goes high, closing the SCR switch; the fact that the switch closes can be observed since the grid and DG voltages are now equal.

The test was repeated, but disabling the operating mode "Dead Bus Reclose" in the HMI. With this change, even after disconnecting the DG inverter, closing of the switch did not occur; this verifies that the operator can control through the HMI (or EMS) whether Dead Bus Reclosing is allowed or not.

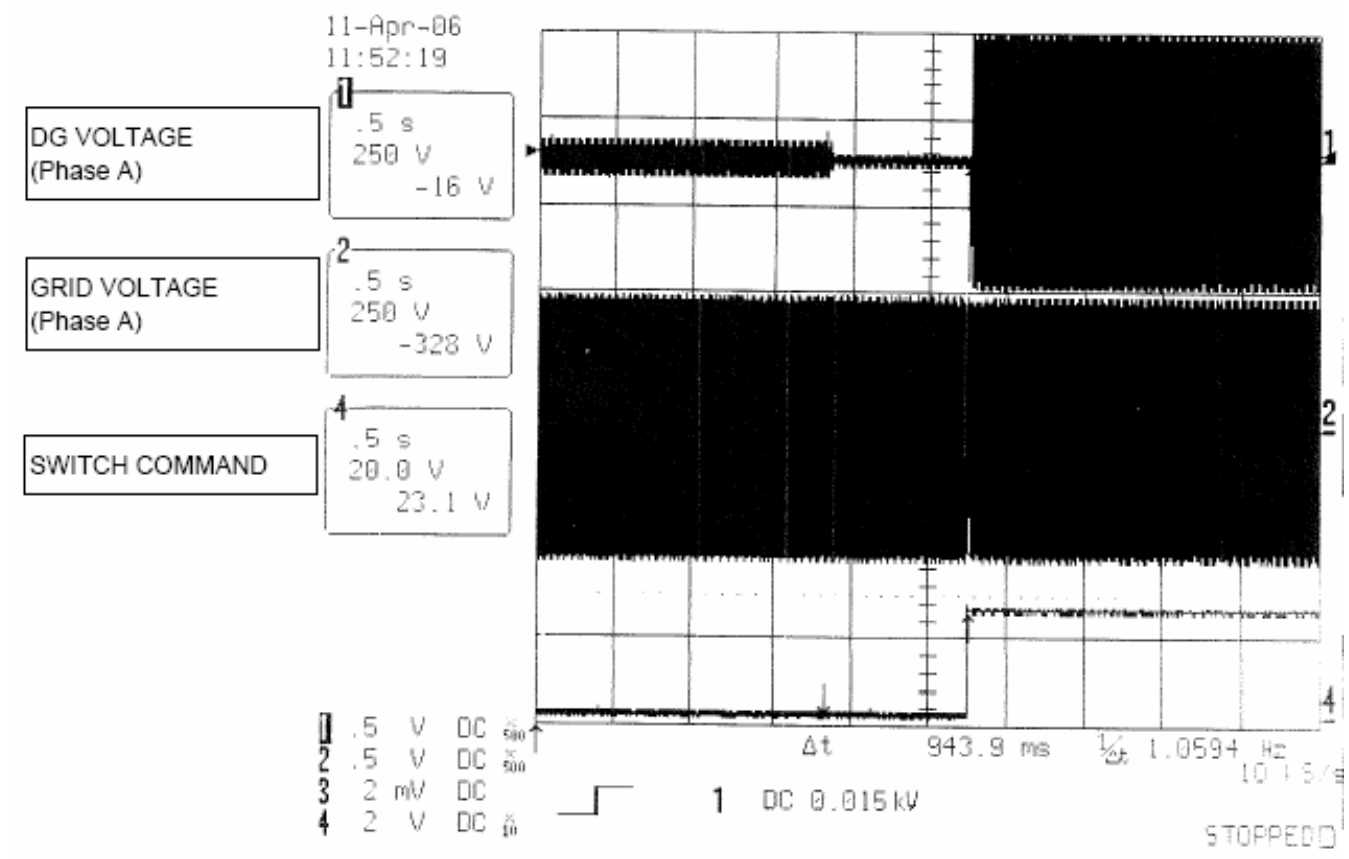

Figure 17. Dead Bus Reclosing Test

\subsection{Restoration Timers}

In this section, the accuracy of the restoration timers was tested. There are two separate restoration timers:

IEEE 1547 restoration timer: This restoration timer is used whenever a IEEE 1547 voltage or frequency event occurs. The timer is adjustable, and it will be set by default to 300 seconds ( 5 minutes) as recommended in the IEEE1547.1 standard.

$P Q$ restoration timer: This restoration timer is used whenever a Power Quality event occurs. The timer is adjustable, and is set by default to 2 second. 
Additionally, it is important to mention that a "Disconnect" timer also exist in the DSP controller, this timer is not related to restoration conditions but rather to a minimum opening time for the switch. This timer is set by default to 1 second.

To test the IEEE1547 timer, a reverse power event due to a grid upstream was created (similar to section 3.3.2). Once the switch opens, since there is no voltage in the grid side, an IEEE1547 and a Power Quality event occur (this was verified by observing the alarms displayed in the Engineering $\mathrm{HMI}$ ).

Figure 18 shows the response to this event. This figure shows the following waveforms:

\section{Channel 1: grid voltage phase A}

Channel 2: Switch command

After a couple of minutes, the grid was reconnected (as seen in figure 18, channel 1 the grid voltage recovers). However, the switch is not commanded to reconnect after the voltage has returned within limits for approximately 300 seconds, verifying the functionality of the IEEE1547 restoration timer. This test was repeated, but after approximately 2 minutes of restoring the grid, the grid was disconnected again; then the grid was brought back to normal conditions again, and it took and additional 300 seconds to reconnect (as expected).

To test the PQ restoration timer, the $90 \%$ under-voltage point in the CBEMA curve was simulated (this is the only event that can be simulated with the test setup without triggering other events). Initially, the grid is supplying $8 \mathrm{~kW}$ load to the DG side.

Figure 19 shows the response to this event. This figure shows the following waveforms:

Channel 1: grid voltage phase $A$

Channel 2: phase A beat voltage

Channel 3: Switch command

Approximately 4 seconds into the time axis of the plot, a voltage drop can be observed occurring in the grid voltage; this drop is an $11 \%$ drop (from $277 \mathrm{~V}$ to $247 \mathrm{~V}$ ) which after 10 seconds (time delay setting for $90 \%$ undervoltage), enables a Power Quality event. After the 10 seconds, the switch is commanded to open. Once the switch opens, due to the load reduction, the voltage grows up above the $90 \%$ threshold, but it takes at least 2 seconds (PQ restoration timer) before it attempts to close. It actually closed at precisely 3.7 seconds. The reason why it didn't close at 2 seconds is because at that time, synchronization conditions were not optimum for reclosing (see channel 2). 


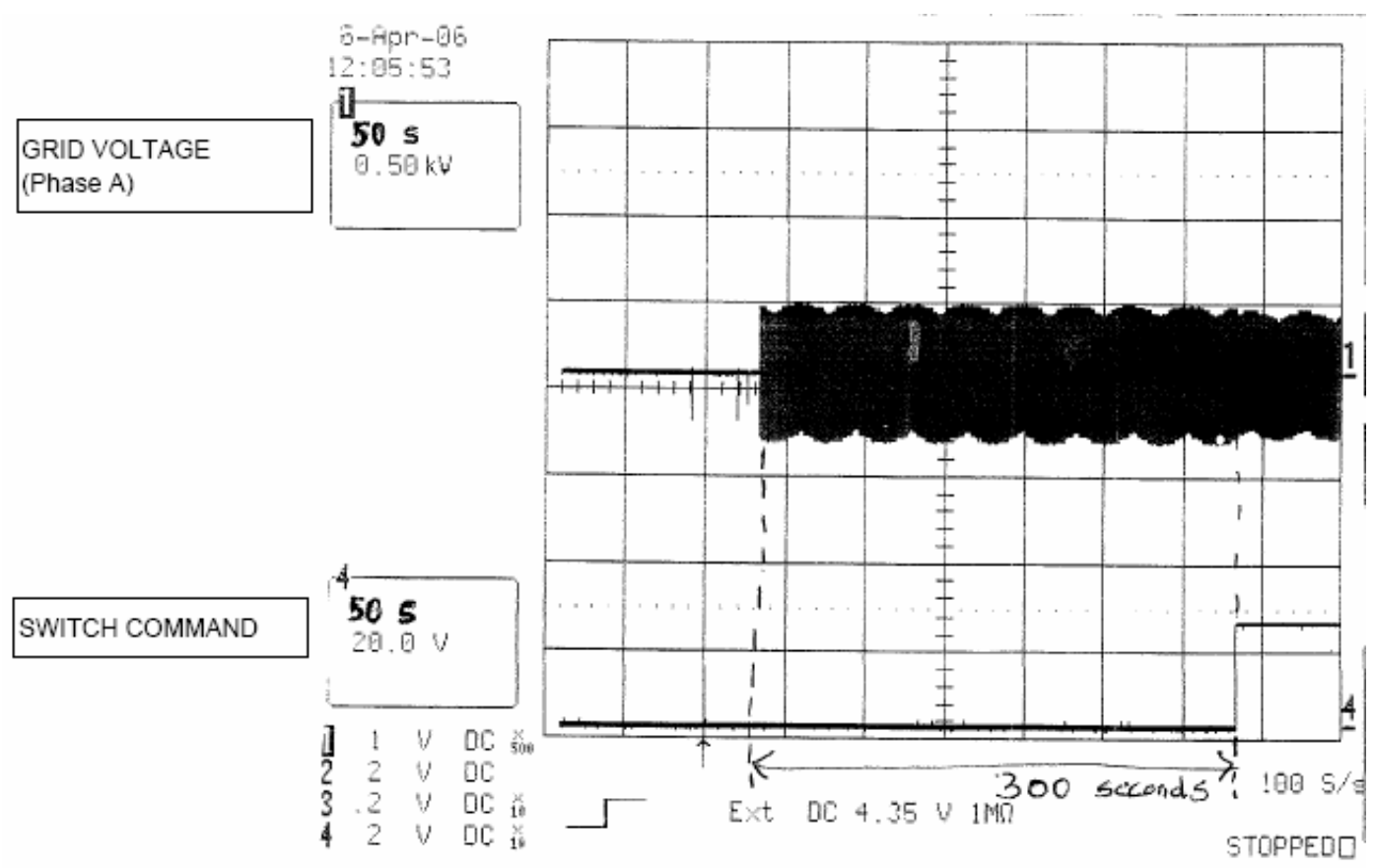

Figure 18. IEEE 1547 Restoration Timer test

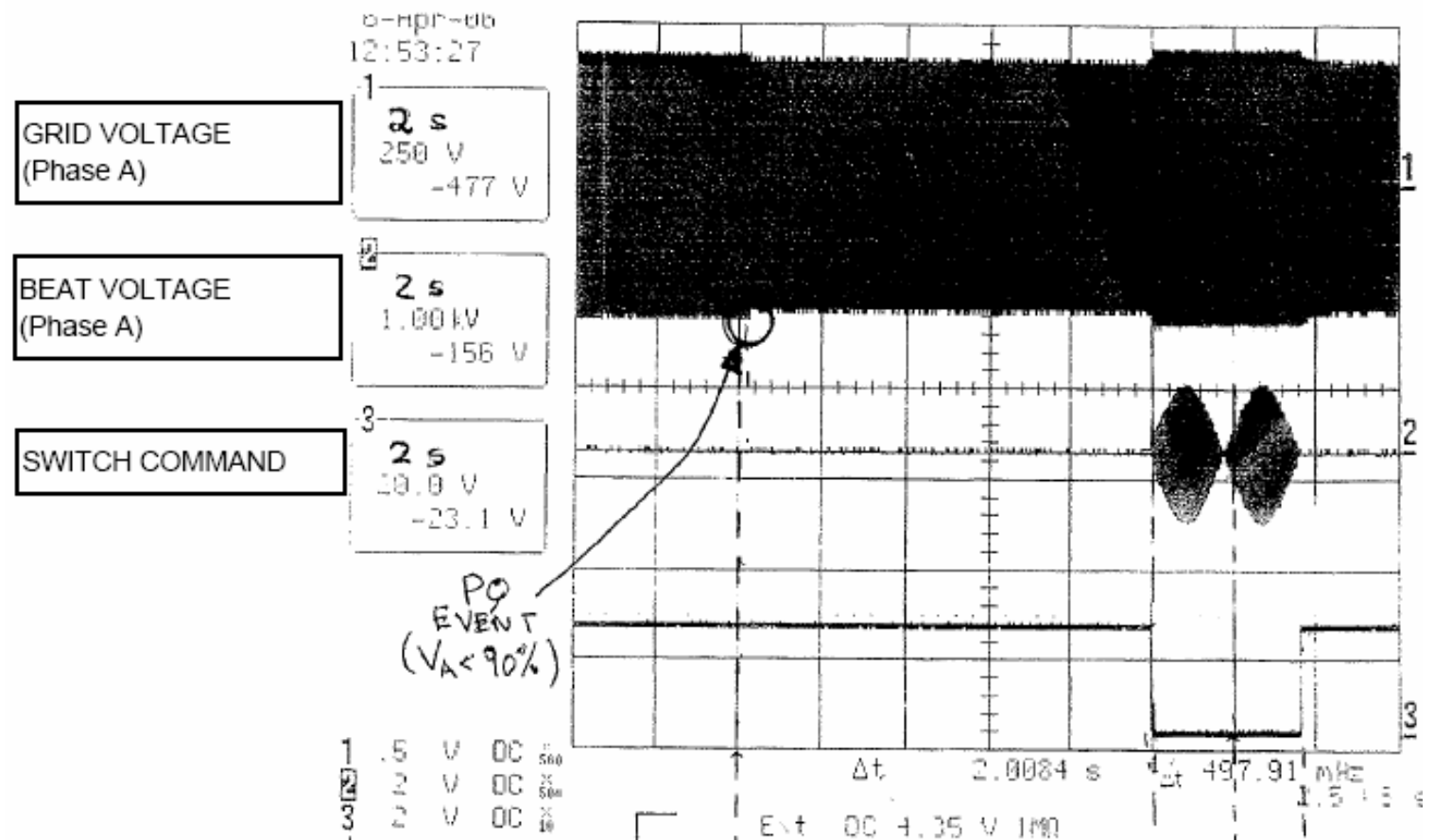

Figure 19. Power Quality Restoration Timer Test 
A Distributed Energy

Systems Company

\subsection{Energy Manager System (EMS)}

All the functionality tests so far have been performed using Northern Power Engineering HMI. However, at the AEP site, control and monitoring of the Static Switch (as well as the operation of the rest of the zones in the testbed) is performed using an Energy Manager System (EMS) developed with LABVIEWTM.

For control and monitoring of the Static Switch, the EMS will have two windows associated to this feature:

Static Switch Control: This window includes the information of the operating status of the switch, status and enabling control of all alarms and events, interface with the switch control (Start, Stop, Reset commands) as well as overview of measurements (Remote location and Switch RMS power, DG and Grid voltages). Figure 20 shows a snapshot of this display in the EMS.

Static Switch Parameters: This window includes all the necessary setpoints that can be adjusted by the user for the different kind of events:

- $\quad$ IEEE 1547 events (magnitude and time delay)

- $\quad$ Power Quality events (magnitude and time delay)

- $\quad$ Reverse Power

- $\quad$ Synchronization Limits

- Dead Bus Reclose

- Reconnection Timers

The complete list of settings can be observed in Appendix.

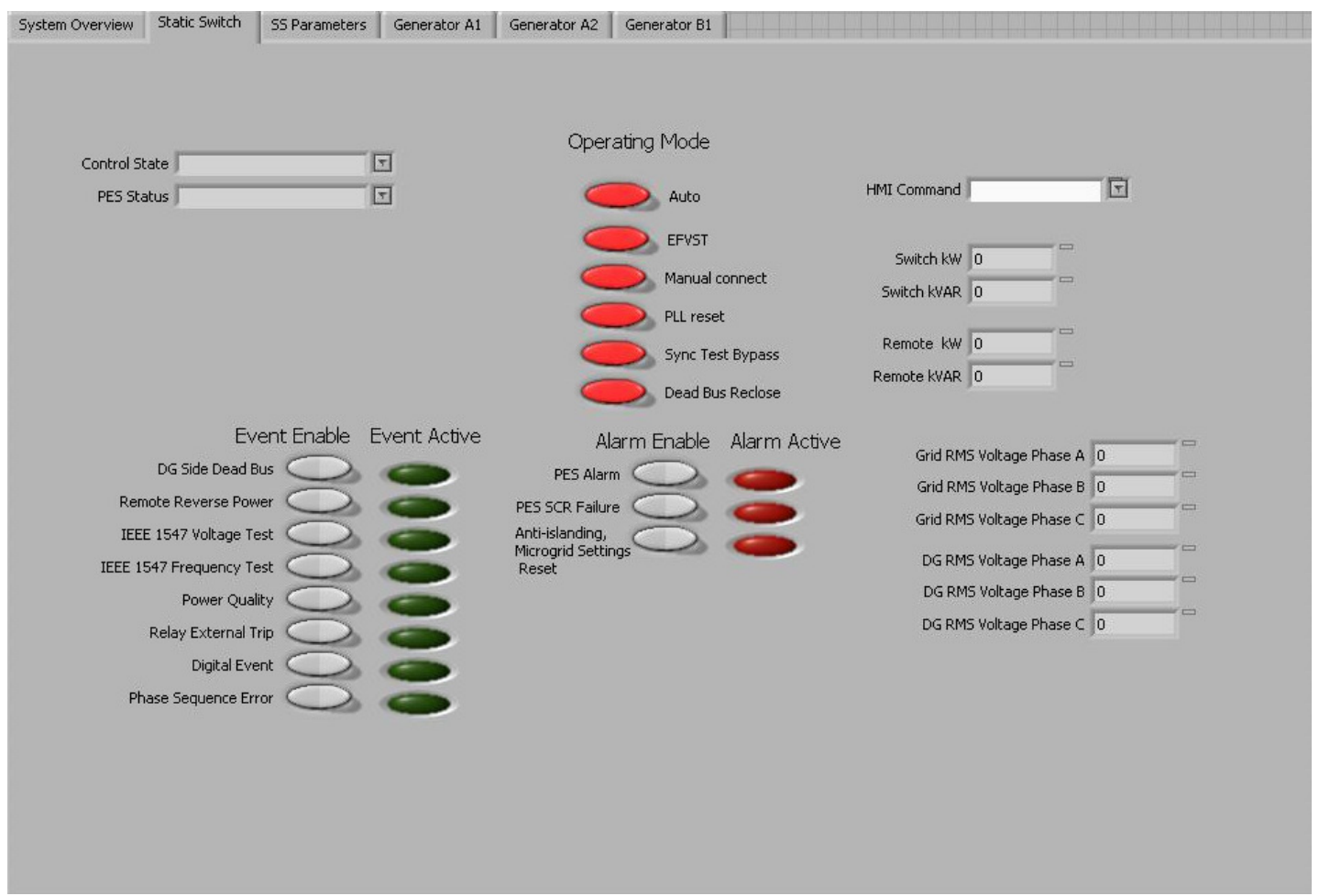

Figure 20. EMS "Static Switch Control” Window Display

As part of this Factory Acceptance Test procedure, a set of simplified tests were preformed: 
HMI Command: The HMI Command includes the "Start", "Stop" and "Reset" command. These commands were cycled around about 5 times, and the expected response of switch was observed (by opening and closing the input breaker, or by Resetting any alarms that appear).

Control State: The Control State window display in which state of the DSP Controller State Machine the system is at the moment. These states include:

$$
\begin{array}{ll}
\text { - } & \text { OFF } \\
\text { - } & \text { FAULT } \\
\text { - } & \text { DISABLE } \\
\text { - } & \text { TEST } \\
\text { - } & \text { CONNECT, DISCONNECT }
\end{array}
$$

To test this feature, some simple functionality tests from the previous sections were performed, and it was observed that this display will show the correct state in which the switch was at the moment (which was also the case when using the Engineering $\mathrm{HMI}$ ).

PES Status: The Control State window display in which state of the S\&C PES is at the moment. These states include:

- PES OK (running, no alarms, but the switch open)

- PES Closed

- PES Alarm

- $\quad$ PES SCR failure

- PES Warning

To test this feature, several open/close of the switch as well as alarm conditions were created, and the expected display was observed. To understand in full detail the meaning of each of this alarm, check Reference [2].

Events and Alarms: All the events and alarms that the DSP Controller will be monitoring have to bit associated with them:

- Enable: When this event is on, the switch will be checking this event, if not, the event is disabled

- Active: Indicator that tells whether conditions that create this event or alarm are present at the moment.

To test the "Enable" bit, overcurrent condition was created (same as section 3.6.2) which requires and External Relay trip; it was observed that, with the "External Relay Trip" event enabled, the Active indicator for this event was on, and the switch goes to disconnect state on this event. Then, the test was repeated but the "Relay trip event" was disable, and in this condition no opening of the switch occurs as expected.

To test the "Active" bit, a reverse power condition requiring anti-islanding lockout (section 3.3.3); once the switch command the system to "Stop" the "Anti-islanding Microgrid Settings Reset" indicator turns on as expected, communicating the operator that adjustments of power setpoints in the DG sources is required. 


\subsection{CERTS Switch Full Functionality Test}

The purpose of these section is to verify the complete functionality of CERTS switch Controller by creating events that will simulate all the conditions tested previously, but while having all the functions Enabled (with all the default functions and settings when the Switch goes to the AEP site). Also, during all these tests, the interface between the operator the DSP controller will be the Energy Manager System (EMS) only.

Below, a description of all the tests performed is included. All the tests were run continuously without stopping the system (unless required by the control logic); during all these tests, all signals and alarms are checked in the EMS to see if it operates as expected. In each of the tests below, a brief summary with observations of the CERTS switch response is presented.

\section{CERTS Switch Full Functionality Test}

\section{Initial System Setup}

Grid side

- Grid inverter voltage setpoint: $277 \mathrm{~V}$

- Grid inverter Frequency: $60 \mathrm{~Hz}$

- $\quad$ Grid Load (R1) $=0$ kW

DG side

- DG inverter Power Setpoint $=1 \mathrm{~kW}$

- $\quad D G$ inverter droop= $0.05 \mathrm{~Hz} / \mathrm{kW}$ (assuming Fmin=59.3Hz, Pmax=15 kW)

- $\quad$ DG Load (R4) = 12kW

- $\quad$ Frequency (before closing) $~ 59.45 \mathrm{~Hz}$

\section{DSP Default Setpoints}

- Synchronization frequency limits: (Nominal $=60 \mathrm{~Hz}$, Freq $\min =59 \mathrm{~Hz}$, Freq $\max =61 \mathrm{~Hz}$ )

- Synchronization voltage limits: (Nominal $=277 \mathrm{~V}$,Voltage $\mathrm{min}=305 \mathrm{~V}(110 \%)$, Voltage $\mathrm{max}=250 \mathrm{~V}(90 \%)$

- Synchronizations phase outer limits: 10, -10 degrees

- $\quad$ Reverse power per phase $=3.33 \mathrm{~kW}(10 \mathrm{~kW}$ total)

- $\quad$ Reverse power time delay $=2$ seconds

- $\quad$ Reverse power voltage test delay (for lockout decision) $=0.5$ seconds

- IEEE 1547 voltage, frequency points: based on IEEE 1547.1 standard (same settings tested in section 3.4 ; tables $4,5,8,9)$

- $\quad$ Power Quality: Based on CBEMA, SEMIF47 standard (4 undervoltages and 3 overvoltages; check Reference [2]). The testpoint that will be tested in this process is:

○ Voltage \#7 (sag): magnitude=90\% (249.3 Vrms), time delay= 10 seconds

- Dead Bus reclose test voltage: $15 \mathrm{~V}$

- $\quad$ Dead bus reclose test time delay: 1 second 
- Reconnection timer for PQ events: 2 seconds

- Reconnection timer for IEEE1547 events: 300 seconds

- Disconnect timer: 1 second

\section{SEL 351 Relay2 Settings}

- $50 \mathrm{P} 1$ (Instantaneous overcurrent): 15A

- Relay minimum opening time: 20 seconds

- $\quad$ TRIP $=50 P 1+$ SV1

- $\quad$ OUT101 =! TRIP (OUT101 is the output that will give the command to DSP)

Note: under-voltage protection was not included in the Relay programming for this test so it wouldn't interfere with the IEE1547 and Power Quality voltage tests.

\section{Test \#1: Start up system, Synchronized Closing}

The initial setup of the DG and grid side will provide a beat frequency of about $0.55 \mathrm{~Hz}$.. Also all voltages should be within the default synchronization limits so system should be ready to automatically performed a synchronized closing.

- Measurements were setup to observe:

- Voltage across phase A of the switch

- Phase A switch current.

The following steps were performed:

1) Make sure all alarms are reset on both the S\&C PES and DSP, and that system is ready to start. (S\&C PES should be in "Output disconnect" mode)

2) Start the system with the "Start" command. The system should enter the "Disconnect" State on the DSP, and we should observe the beat voltage with a frequency of approximately $\sim 0.2 \mathrm{~Hz}$.

3) After passing through a zero crossing in the beat voltage, the DSP should provide a "Close" command to the PES and the switch will close. Should see the beat voltage drop to approximately zero Volts, and the switch current should increase to approximately 14Arms (the Grid needs to supply 12 of the $15 \mathrm{~kW}$ in the DG side)

Observations: The response of the CERTS switch during this test was as expected; once the system was started, synchronization closing occurred with a very smooth transition. After waiting a few minutes, the switch remaining closed without any alarms or events, meaning the system is in normal operation.

When the switch closes, "CONNECT" is displayed in the Control State block of the EMS, and "PES OK" is displayed in the PES Status block.

\section{Test \#2: Reverse power due to grid islanding (Check for IEEE1547 voltage event)}

For this test, monitor:

- Switch command

- Grid side voltage phase A

- Voltage across phase A of the switch

- Switch current phase A

1) Disconnect grid side inverter from system. The switch current should drop from 14Arms to zero since the grid is gone and won't be providing any power (in any of the phases) to the DG side of the switch. 
2) After two seconds, the DSP should provide an open command to the S\&C PES due to a reverse power event. The beat voltage in Phase A should change from zero to approximately rated voltage (since DG side: rated voltage and Grid side: $0 \mathrm{~V}$ ). Switch current remains zero since switch is opened.

Observe the "Events Active" list in the EMS. Once the switch opens, verify that the "IEEE1547 voltage type test" turn on, due to the fact that once the switch opens, the voltage on the grid side PT drops to zero.(Notice that the "Power Quality Function" event also should turn on)

3) Wait for $\sim 20-30$ seconds, and observe that the "IEEE1547 voltage type test" event remain high.

4) Reconnect the grid inverter to bring the grid voltage back. The "IEEE1547 voltage type test" event should toggle back low since the grid voltage is back to normal, but switch shouldn't try to reclose due to IEEE1547 reconnection timer being 300 seconds.

5) Observe the beat voltage of the switch phase A (which should again be a beat waveform with $\sim 0.2 \mathrm{~Hz}$ frequency). After approximately 5 minutes, once synchronization conditions are appropriate, it should reclose. Switch current should go back to 14 Arms.

Observations: The response of the CERTS switch during this test was as expected; the switch is disconnected 2.05 seconds after the reverse power event occurs.

When the switch opens, "DISCONNECT" is displayed in the Control State block of the EMS, and "PES OPEN" is displayed in the PES Status block. In the Events list, the "IEEE1547 voltage type test" and "Power Quality Function" events are on.

Once the grid was reconnected, it took over 300 seconds ( 305 seconds) for the switch to reclose. The Control State and PES Status block returned to "CONNECT" and "PES CLOSED" respectively.

\section{Test \#3: Reverse power due to open phase (Also check for IEEE1547 voltage event)}

For this test, monitor:

- Switch command

- Grid side voltage phase A

- Voltage across phase A of the switch

- Switch current phasse A and B

1) Disconnect grid side phase $A$ from system by using a single phase contactor. Phase $A$ current should drop from 14Arms to zero while phase B (and C) currents increase a bit. Also, grid Phase A to neutral voltage should drop to zero, but phase $B$ (and C) will remain approximately at rated voltage.

2) After two seconds, the DSP should provide an open command to the PES due to a reverse power event (setting is at $3.3 \mathrm{~kW}$ per phase). The beat voltage in Phase A should change from zero to approximately rated voltage (since $D G=$ rated voltage and $G$ rid $=0 \mathrm{~V}$ ). Now both phase $A$ and $B$ switch current drop to zero since switch is opened.

3) Observe the "Alarms" menu in the HMI display. Once the switch opens, we should see the "IEEE1547 voltage type test" event go high, due to the fact that one of the phases (phase A) the voltage on the grid side PT drops to zero.

4) Wait for $~ 20-30$ seconds, and observe that the "IEEE1547 voltage type test" event remains high

5) Close the single-phase contactor to bring phase A grid voltage back. The "IEEE1547 voltage type test" event should toggle back low since the grid voltage is back to normal, but switch shouldn't try to reclose due to IEEE1547 reconnection timer being 300 seconds.

6) Observe the beat voltage $A$ (which should again be a waveform with $\sim 0.2 \mathrm{~Hz}$ frequency). After approximately 5 minutes, once synchronization conditions are appropriate, it should reclose. Switch current should go back to $\sim 14$ Arms. 


\section{Test \#4: Reverse power Anti-islanding, Microgrid Settings Reset (Check Anti- islanding lockout logic)}

For this test, monitor:

- Switch command

- Start/Stop Command

- Grid side Voltage phase A

- Switch Current phase A

1) Currently, the grid is supplying $\sim 11 \mathrm{~kW}$ to the $\mathrm{DG}$ side (monitored by the remote CT12 current transformers).

2) Change the DG setpoint from $1 \mathrm{~kW}$ to $4 \mathrm{~kW}$. This will require the grid to supply a per phase power of $\sim 2.66 \mathrm{~kW}$, just below the threshold (3.3 kW per phase).

3) After 2 seconds, a reverse power event occurs, so the Switch command should open the S\&C PES.

4) Approximately 0.5 seconds after the trip, the reverse power voltage test algorithm will see that all the voltages on the grid side are normal. This will create a fault condition that requires the switch to lockout. The DSP will provide a "Stop" command to the PES, opening the input breaker and keeping the DSP state machine in the "Fault" state.

5) Restart the system. The switch should performed automatically a synchronized closing, but two seconds fter that it will see the reverse power condition again, and lockout the system

6) Change the microgrid setpoint back to $3 \mathrm{~kW}$.

7) Restart the system. The system should perform the synchronized close. Wait for 1 minute, and we should see no alarms or events come on.

Observations: The CERTS Switch controller responded as expected; the reverse power event transition the switch to the "DISCONNECT" State, but after 0.5 seconds, the switch was commanded to Stop and the input breaker was Open. The Control State Block display "Fault- Anti-islanding Microgrid Settings Reset".

Faults were cleared and the switch Started. After attempting the first switch reclosing (without adjusting the DG setpoint back to $1 \mathrm{~kW}$ ) the switch closed by opened and Stop again. Once the DG setpoint was adjusted back to $1 \mathrm{~kW}$, the switch closed and remained in the "CONNECT" State.

\section{Test \#5: Power Quality Event}

- For this test, monitor:

- Switch command

- Grid side Voltage phase A

- Voltage across switch phase A

1) Drop all phases of the grid side voltage from rated voltage to $91 \%$ (252Vrms)

2) Drop phase A by $0.5 \%$ (1.38V). Wait for 30 seconds; no events should occur.

3) Repeat step 2 until a PQ event (and no IEEE1547) occurs. The event should occur approximately 10 seconds after the voltage step is performed.

4) Once the event occurs, the Switch Command should open the switch. 
5) Observe the "Power Quality" event go high. No other events should be enabled at this point.

6) Wait for $\sim 1$ minute.

7) Step the phase A voltage back to $100 \%$ (277Vrms). Approximately two seconds (PQ reconnection timer) after the step, the switch should attempt to reclose (once synchronization conditions are acceptable)

8) System goes back to normal, no alarms present.

Observations: CERTS Switch responded appropriately; in the events list on the EMS, the "Power Quality Function" event turn on, and after 9.7 seconds of the voltage step, the switch was commanded to open and transitioned to the "DISCONNECT" state. The voltage came back within the Power Quality thresholds (>90\%) almost immediately (since there is no voltage drop across the grid inverter transformers). The switch reconnected after 3.7 seconds rather than 2 seconds ( $P Q$ restoration timer) because synchronization conditions were not appropriate yet.

\section{Test \# 6: SEL351 Relay2 Event}

For this test, monitor:

- Switch command

- Relay Output Signal

- $\quad$ Switch Current Phase A

- Voltage across switch phase A.

1) After the switch recloses in the final step of test \#5, the switch current is approximately $\sim 14 \mathrm{~A} \mathrm{rms} \mathrm{(12 \textrm {kW }}$ supplied by grid).

2) Check the Relay current readings (in the display panel); the three phases should be approximately 14 Arms.

3) Increase the DG side load to $15 \mathrm{~kW}$.. The current now will increase to 18 Arms. Since the overcurrent level in the relay was set to $15 \mathrm{Arms}$, then the relay should provide a trip command to the DSP, and the DSP should command the switch to open instantaneously. Reduce the DG load back to $12 \mathrm{~kW}$.

4) Check that the "Relay Trip event" alarm goes high.

5) After $\sim 20$ seconds (relay minimum opening time) the relay trip signal will go off, and the DSP will immediately try to reclose the switch.

Observations: The CERTS Switch controller responded as expected; when the DG side load was increased, the Relay Output signal went Low, and the Switch command immediately opened the switch. At this time, the "Relay External Trip" event turned on, and all other alarms and events remained off. After 20 seconds, the Relay Output signal toggle HIGH again, and after an additional $\sim 1.8$ seconds, the DSP reclosed the switch (once synchronization conditions were appropriate). 


\subsection{Appendix 1: IEEE1547 Tests Results with NREL's Omicron Equipment (Section 3.3)}

Table 1 - Under-voltage Magnitude Test Results

SUMMARY OF IEEE 1547 UNDERVOLTAGE MAGNITUDE TESTS

UNDERVOLTAGE 50\%, Three Phase

$\mathrm{dV} / \mathrm{dT}=$

dT per step
0.10

$1.00 \mathrm{~s}$
UNDERVOLTAGE 50\%, Phase A

$\mathrm{dV} / \mathrm{dT}=\quad 0.10$

$\begin{array}{lll}\text { dT per step } & 1.00 \mathrm{~S}\end{array}$

\begin{tabular}{|r|r|r|}
\hline \multicolumn{1}{|l|}{$\begin{array}{l}\text { Nominal } \\
\text { (V) }\end{array}$} & \multicolumn{1}{|l|}{$\begin{array}{l}\text { Actual } \\
\text { (V) }\end{array}$} & \multicolumn{1}{|l|}{$\begin{array}{l}\text { Deviation } \\
\text { (mV) }\end{array}$} \\
\hline 138.50 & 139.00 & 500.00 \\
138.50 & 139.00 & 500.00 \\
138.50 & 139.00 & 500.00 \\
138.50 & 139.00 & 500.00 \\
138.50 & 139.00 & 500.00 \\
\hline Avg: & $\mathbf{1 3 9 . 0 0}$ & V \\
\hline
\end{tabular}

\begin{tabular}{|c|r|r|}
\hline $\begin{array}{c}\text { Nominal } \\
\text { (V) }\end{array}$ & $\begin{array}{c}\text { Actual } \\
\text { (V) }\end{array}$ & \multicolumn{1}{c|}{$\begin{array}{l}\text { Deviation } \\
\text { (mV) }\end{array}$} \\
\hline 138.50 & 139.00 & 500.00 \\
138.50 & 139.00 & 500.00 \\
138.50 & 138.90 & 400.00 \\
138.50 & 139.00 & 500.00 \\
138.50 & 139.00 & 500.00 \\
\hline Avg: & $\mathbf{1 3 8 . 9 8}$ & $\mathbf{V}$ \\
\hline
\end{tabular}

\begin{tabular}{|c|c|c|}
\hline \multicolumn{3}{|c|}{ UNDERVOLTAGE $88 \%$, Three Pha } \\
\hline \multirow{2}{*}{\multicolumn{2}{|c|}{$\begin{array}{l}\mathrm{dV} / \mathrm{dT}= \\
\mathrm{dT} \text { per step }\end{array}$}} & \\
\hline & & 1.00 \\
\hline $\begin{array}{l}\text { Nominal } \\
\text { (V) }\end{array}$ & $\begin{array}{l}\text { Actual } \\
\text { (V) }\end{array}$ & $\begin{array}{l}\text { Deviation } \\
(\mathrm{mV})\end{array}$ \\
\hline 243.80 & 244.00 & 200.00 \\
\hline 243.80 & 244.00 & 200.00 \\
\hline 243.80 & 244.10 & 300.00 \\
\hline 243.80 & 244.00 & 200.00 \\
\hline 243.80 & 244.00 & 200.00 \\
\hline Avg: & 244.02 & V \\
\hline
\end{tabular}

UNDERVOLTAGE 88\%, Phase C dV/dT= $0.10 \mathrm{~V} / \mathrm{s}$ dT per step

$1.00 \mathrm{~s}$


Table 2 - Over-voltage Magnitude Test Results

SUMMARY OF IEEE 1547 OVERVOLTAGE MAGNITUDE TESTS

OVERVOLTAGE 110\%, Three Phase

$\mathrm{dV} / \mathrm{dT}=$

dT per step

$0.10 \mathrm{~V} / \mathrm{s}$

$1.00 \mathrm{~s}$
OVERVOLTAGE $110 \%$, Phase A

$\mathrm{dV} / \mathrm{dT}=$

$0.10 \mathrm{~V} / \mathrm{s}$

dT per step

\begin{tabular}{|c|c|r|}
\hline $\begin{array}{c}\text { Nominal } \\
\text { (V) }\end{array}$ & $\begin{array}{c}\text { Actual } \\
\text { (V) }\end{array}$ & \multicolumn{1}{c|}{$\begin{array}{l}\text { Deviation } \\
\text { (mV) }\end{array}$} \\
\hline 304.70 & 305.10 & 400.00 \\
304.70 & 305.10 & 400.00 \\
304.70 & 305.10 & 400.00 \\
304.70 & 305.10 & 400.00 \\
304.70 & 305.00 & 300.00 \\
\hline Avg: & \multicolumn{3}{|c}{$\mathbf{3 0 5 . 0 8}$} & $\mathbf{V}$ \\
\hline
\end{tabular}

\begin{tabular}{|c|r|r|}
\hline $\begin{array}{c}\text { Nominal } \\
\text { (V) }\end{array}$ & $\begin{array}{c}\text { Actual } \\
(\mathbf{V})\end{array}$ & $\begin{array}{c}\text { Deviation } \\
(\mathbf{m V})\end{array}$ \\
\hline 304.70 & 306.20 & 1500.00 \\
304.70 & 306.20 & 1500.00 \\
304.70 & 306.20 & 1500.00 \\
304.70 & 306.30 & 1600.00 \\
304.70 & 306.30 & 1600.00 \\
\hline Avg: & $\mathbf{3 0 6 . 2 4}$ & $\mathbf{V}$ \\
\hline
\end{tabular}

\begin{tabular}{|c|c|c|}
\hline \multicolumn{3}{|c|}{ OVERVOLTAGE $110 \%$, Phase B } \\
\hline \multicolumn{2}{|c|}{$\begin{array}{l}\mathrm{dV} / \mathrm{dT}= \\
\mathrm{dT} \text { per step }\end{array}$} & $\begin{array}{l}0.10 \\
1.00\end{array}$ \\
\hline $\begin{array}{c}\text { Nominal } \\
\text { (V) }\end{array}$ & $\begin{array}{l}\text { Actual } \\
\text { (V) }\end{array}$ & $\begin{array}{l}\text { Deviation } \\
(\mathrm{mV})\end{array}$ \\
\hline 304.70 & 305.00 & 300.00 \\
\hline 304.70 & 305.10 & 400.0 \\
\hline 304.70 & 305.10 & 400.00 \\
\hline 304.70 & 305.10 & 400.00 \\
\hline 304.70 & 305.10 & 400.00 \\
\hline Avg & 305.08 & V \\
\hline
\end{tabular}

OVERVOLTAGE $110 \%$, Phase C

$d V / d T=$

$0.10 \mathrm{~V} / \mathrm{s}$

dT per step

$1.00 \mathrm{~s}$

$\mathrm{V}$

\begin{tabular}{|c|c|c|}
\hline \multicolumn{3}{|c|}{ OOVERVOLTAGE $120 \%$, Three Ph } \\
\hline \multirow{2}{*}{\multicolumn{2}{|c|}{$\begin{array}{l}\mathrm{dV} / \mathrm{dT}= \\
\mathrm{dT} \text { per step }\end{array}$}} & 0.10 \\
\hline & & 1.00 \\
\hline $\begin{array}{c}\text { Nominal } \\
\text { (V) }\end{array}$ & $\begin{array}{c}\text { Actual } \\
\text { (V) }\end{array}$ & $\begin{array}{l}\text { Deviation } \\
(\mathrm{mV})\end{array}$ \\
\hline 332.40 & 332.50 & 100.00 \\
\hline 332.40 & 332.40 & 0.00 \\
\hline 332.40 & 332.60 & 200.00 \\
\hline 332.40 & 332.50 & 100.00 \\
\hline 332.40 & 332.50 & 100.00 \\
\hline Avg: & 332.50 & $\mathbf{V}$ \\
\hline
\end{tabular}

\begin{tabular}{|c|r|r|}
\hline $\begin{array}{c}\text { Nominal } \\
(\mathbf{V})\end{array}$ & $\begin{array}{c}\text { Actual } \\
(\mathbf{V})\end{array}$ & \multicolumn{1}{c|}{$\begin{array}{c}\text { Deviation } \\
(\mathbf{m V})\end{array}$} \\
\hline 304.70 & 305.60 & 900.00 \\
304.70 & 305.70 & 1000.00 \\
304.70 & 305.60 & 900.00 \\
304.70 & 305.70 & 1000.00 \\
304.70 & 305.70 & 1000.00 \\
\hline Avg: & $\mathbf{3 0 5 . 6 6}$ & $\mathbf{V}$ \\
\hline
\end{tabular}


Table 3 - Under-voltage Time Test Results

SUMMARY OF IEEE 1547 UNDERVOLTAGE TIME TESTS

\begin{tabular}{|c|c|c|}
\hline \multicolumn{3}{|c|}{ UNDERVOLTAGE 50\%, Three P } \\
\hline \multicolumn{2}{|c|}{ Ramp from } & 277.00 \\
\hline \multicolumn{2}{|l|}{ Ramp to } & 130.00 \\
\hline $\begin{array}{c}\text { Tnominal } \\
(\mathrm{ms})\end{array}$ & $\begin{array}{c}\text { Tactual } \\
\text { (ms) }\end{array}$ & $\begin{array}{l}\text { Tdev } \\
\text { (ms) }\end{array}$ \\
\hline 167 & 176.6 & 9.60 \\
\hline 167 & 181.6 & 14.60 \\
\hline 167 & 183.8 & 16.80 \\
\hline 167 & 183.8 & 16.80 \\
\hline 167 & 183.9 & 16.90 \\
\hline
\end{tabular}

Avg:

$181.94 \quad \mathrm{msec}$

\begin{tabular}{lll}
\multicolumn{2}{l}{ UNDERVOLTAGE } & $\mathbf{5 0 \%}$, Phase A \\
Ramp from & 277.00 & V \\
Ramp to & 130.00 & V
\end{tabular}

\begin{tabular}{|c|c|c|}
\hline \multicolumn{3}{|c|}{$\begin{array}{l}\text { UNDERVOLTAGE 50\%, Phase B } \\
\end{array}$} \\
\hline \multirow{2}{*}{\multicolumn{2}{|c|}{$\begin{array}{c}\text { Ramp from } \\
\text { Ramp to }\end{array}$}} & 277.00 \\
\hline & & 130.00 \\
\hline$\underset{(\mathrm{ms})}{\text { Tnominal }}$ & $\begin{array}{c}\text { Tactual } \\
(\mathrm{ms})\end{array}$ & $\begin{array}{l}\text { Tdev } \\
\text { (ms) }\end{array}$ \\
\hline 167 & 176.1 & 9.10 \\
\hline 167 & 183.7 & 16.70 \\
\hline 167 & 181.8 & 14.80 \\
\hline 167 & 183.8 & 16.80 \\
\hline 167 & 183.8 & 16.80 \\
\hline
\end{tabular}

Avg:

181.84

msec

\begin{tabular}{|c|c|c|}
\hline $\begin{array}{c}\text { Tnominal } \\
\text { (ms) }\end{array}$ & $\begin{array}{c}\text { Tactual } \\
\text { (ms) }\end{array}$ & $\begin{array}{c}\text { Tdev } \\
\text { (ms) }\end{array}$ \\
\hline 167 & 175.4 & 8.40 \\
167 & 183.6 & 16.60 \\
167 & 183.9 & 16.90 \\
167 & 183.7 & 16.70 \\
167 & 181.8 & 14.80 \\
\hline
\end{tabular}

Avg: $\quad 181.68 \quad$ msec

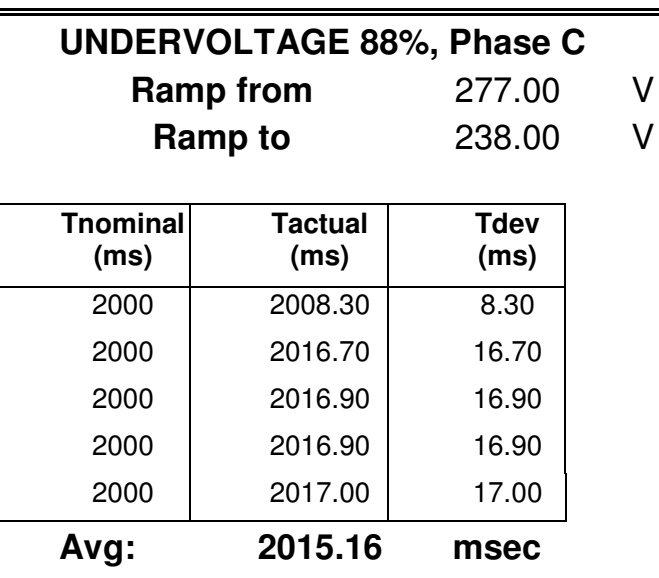


Table 4 - Over-voltage Time Test Results

SUMMARY OF IEEE 15470VERVOLTAGE TIME TESTS

\begin{tabular}{|c|c|c|}
\hline \multicolumn{3}{|c|}{ OVERVOLTAGE $110 \%$, Three } \\
\hline Ramp frc & & 277.00 \\
\hline Ramp to & & 310.00 \\
\hline $\begin{array}{l}\text { Tnominal } \\
\text { (ms) }\end{array}$ & $\begin{array}{l}\text { Tactual } \\
\text { (ms) }\end{array}$ & $\begin{array}{l}\text { Tdev } \\
\text { (ms) }\end{array}$ \\
\hline 1000 & 1009.7 & 9.70 \\
\hline 1000 & 1017.0 & 17.00 \\
\hline 1000 & 1015.0 & 15.00 \\
\hline 1000 & 1000.3 & 0.30 \\
\hline 1000 & 1000.4 & 0.40 \\
\hline
\end{tabular}

Avg: $\quad 1008.48 \quad$ msec

\begin{tabular}{|c|c|c|}
\hline $\begin{array}{l}\text { Tnominal } \\
\text { (ms) }\end{array}$ & $\begin{array}{c}\text { Tactual } \\
\text { (ms) }\end{array}$ & $\begin{array}{c}\text { Tdev } \\
\text { (ms) }\end{array}$ \\
\hline 1000 & 1008.8 & 8.80 \\
1000 & 1016.8 & 16.80 \\
1000 & 1017.1 & 17.10 \\
1000 & 1017.1 & 17.10 \\
1000 & 1016.9 & 16.90 \\
\hline
\end{tabular}

Avg: $\quad 1015.34 \quad \mathrm{msec}$

\begin{tabular}{|c|c|c|}
\hline \multicolumn{3}{|c|}{ OVERVOLTAGE $120 \%$, Three } \\
\hline Ramp frc & & 277.00 \\
\hline Ramp to & & 340.00 \\
\hline $\begin{array}{l}\text { Tnominal } \\
\text { (ms) }\end{array}$ & $\begin{array}{l}\text { Tactual } \\
\text { (ms) }\end{array}$ & $\begin{array}{l}\text { Tdev } \\
\text { (ms) }\end{array}$ \\
\hline 167 & 175.4 & 8.40 \\
\hline 167 & 183.6 & 16.60 \\
\hline 167 & 183.9 & 16.90 \\
\hline 167 & 183.8 & 16.80 \\
\hline 167 & 181.8 & 14.80 \\
\hline
\end{tabular}

Avg: $\quad 181.70 \quad \mathrm{msec}$

\begin{tabular}{|l|c|c|}
\hline $\begin{array}{l}\text { Tnominal } \\
(\mathbf{m s})\end{array}$ & $\begin{array}{c}\text { Tactual } \\
(\mathbf{m s})\end{array}$ & $\begin{array}{c}\text { Tdev } \\
(\mathbf{m s})\end{array}$ \\
\hline 167 & 175.0 & 8.00 \\
167 & 166.9 & -0.10 \\
167 & 183.9 & 16.90 \\
167 & 183.8 & 16.80 \\
167 & 183.8 & 16.80 \\
\hline
\end{tabular}

$\begin{array}{lc}\text { OVERVOLTAGE } & \text { 120\%, Phase B } \\ \text { Ramp from } & 277.00 \\ \text { Ramp to } & 340.00\end{array}$

$\begin{array}{ll}\text { Ramp from } & 277.00 \\ \text { Ramp to } & 310.00\end{array}$

Avg:

178.68 msec 
Table 5 - Over-frequency Magnitude Test Results

SUMMARY OF IEEE 1547 OVERFREQUENCY MAGNITUDE TESTS

OVERFREQUENCY $60.5 \mathrm{~Hz}$

$\begin{array}{lrl}\mathbf{d V} / \mathbf{d T}= & 10.00 \mathrm{mHz} / \mathrm{s} \\ \text { dT per step } & 1.00 & \end{array}$

dT per step $\quad 1.00 \mathrm{~s}$

\begin{tabular}{|c|c|c|}
\hline $\begin{array}{c}\text { Nominal } \\
\mathbf{( H z )}\end{array}$ & $\begin{array}{c}\text { Actual } \\
(\mathbf{H z})\end{array}$ & $\begin{array}{c}\text { Deviation } \\
(\mathbf{H z})\end{array}$ \\
\hline 60.50 & 60.51 & 0.01 \\
60.50 & 60.51 & 0.01 \\
60.50 & 60.51 & 0.01 \\
60.50 & 60.51 & 0.01 \\
60.50 & 60.51 & 0.01 \\
\hline
\end{tabular}

Avg:

$60.51 \quad \mathrm{~Hz}$

Table 6 - Under-frequency Magnitude Test Results

SUMMARY OF IEEE 1547 UNDERFREQUENCY MAGNITUDE

TESTS

\begin{tabular}{|c|c|c|c|c|c|c|c|}
\hline \multicolumn{4}{|c|}{ UNDERFREQUENCY 57 Hz } & \multicolumn{4}{|c|}{ UNDERFREQUENCY $59.3 \mathrm{~Hz}$} \\
\hline \multirow{2}{*}{\multicolumn{2}{|c|}{$\begin{array}{l}d V / d T= \\
d T \text { per step }\end{array}$}} & \multirow{2}{*}{$\begin{array}{r}10.00 \\
1.00\end{array}$} & \multirow{2}{*}{$\begin{array}{l}\mathrm{mHz} / \mathrm{s} \\
\mathrm{s}\end{array}$} & \multirow{2}{*}{\multicolumn{2}{|c|}{$\begin{array}{l}d V / d T= \\
d T \text { per step }\end{array}$}} & \multirow{2}{*}{$\begin{array}{l}2.00 \\
1.00\end{array}$} & \multirow{2}{*}{$\begin{array}{l}\mathrm{mHz} / \mathrm{s} \\
\mathrm{s}\end{array}$} \\
\hline & & & & & & & \\
\hline$\underset{(\mathrm{Hz})}{\text { Nominal }}$ & $\begin{array}{c}\text { Actual } \\
(\mathrm{Hz})\end{array}$ & $\begin{array}{c}\text { Deviation } \\
(\mathrm{Hz})\end{array}$ & & $\underset{(\mathrm{Hz})}{\text { Nominal }}$ & $\begin{array}{c}\text { Actual } \\
(\mathrm{Hz})\end{array}$ & $\begin{array}{c}\text { Deviation } \\
(\mathrm{Hz})\end{array}$ & \\
\hline 57.00 & 57.00 & 0.00 & & 59.30 & 59.30 & 0.00 & \\
\hline 57.00 & 57.00 & 0.00 & & 59.30 & 59.30 & 0.00 & \\
\hline 57.00 & 57.00 & 0.00 & & 59.30 & 59.30 & 0.00 & \\
\hline 57.00 & 57.00 & 0.00 & & 59.30 & 59.30 & 0.00 & \\
\hline 57.00 & 57.00 & 0.00 & & 59.30 & 59.30 & 0.00 & \\
\hline Avg: & 57.00 & $\mathrm{~Hz}$ & & Avg: & 59.30 & $\mathrm{~Hz}$ & \\
\hline
\end{tabular}


Table 7 - Over-frequency Time Test Results

SUMMARY OF IEEE 1547 OVERFREQUENCY TIME

TESTS

\begin{tabular}{l}
\hline \hline \multicolumn{2}{|l|}{ OVERFREQUENCY } \\
Ramp from \\
$\begin{array}{c}\text { Ra_TEST } \\
\text { Ramp to }\end{array}$ \\
\begin{tabular}{|c|c|c|}
\hline $\begin{array}{c}\text { Nominal } \\
\text { Time } \\
\text { (ms) }\end{array}$ & $\begin{array}{c}\text { Actual } \\
\text { Time } \\
\text { (ms) }\end{array}$ & $\begin{array}{c}\text { Deviation } \\
\text { (ms) }\end{array}$ \\
\hline 167.00 & 274.20 & 107.20 \\
167.00 & 263.00 & 96.00 \\
167.00 & 265.00 & 98.00 \\
167.00 & 265.00 & 98.00 \\
167.00 & 263.10 & 96.10 \\
\hline
\end{tabular}
\end{tabular}

Avg:

$266.06 \quad \mathrm{msec}$

OVERFREQUENCY $60.5 \mathrm{~Hz}$ TEST \# 2

Ramp from $\quad 60.45 \mathrm{~Hz}$

Ramp to $\quad 60.55 \mathrm{~Hz}$

\section{OVERFREQUENCY $60.5 \mathrm{~Hz}$ _TEST \# 3}

Ramp from $\quad 60.40 \mathrm{~Hz}$

Ramp to $\quad 60.70 \mathrm{~Hz}$

\begin{tabular}{|c|c|c|}
\hline $\begin{array}{c}\text { Nominal } \\
\text { Time } \\
\text { (ms) }\end{array}$ & $\begin{array}{c}\text { Actual } \\
\text { Time } \\
\text { (ms) }\end{array}$ & $\begin{array}{c}\text { Deviation } \\
\text { (ms) }\end{array}$ \\
\hline 167.00 & 203.00 & 36.00 \\
167.00 & 190.10 & 23.10 \\
167.00 & 206.70 & 39.70 \\
167.00 & 206.80 & 39.80 \\
167.00 & 206.70 & 39.70 \\
\hline
\end{tabular}

Avg: 202.66 msec

\begin{tabular}{|c|c|c|}
\hline $\begin{array}{c}\text { Nominal } \\
\text { Time } \\
(\mathbf{m s})\end{array}$ & $\begin{array}{c}\text { Actual } \\
\text { Time } \\
(\mathbf{m s})\end{array}$ & $\begin{array}{c}\text { Deviation } \\
\text { (ms) }\end{array}$ \\
\hline 167.00 & 190.40 & 23.40 \\
167.00 & 181.70 & 14.70 \\
167.00 & 181.80 & 14.80 \\
167.00 & 179.50 & 12.50 \\
167.00 & 181.80 & 14.80 \\
\hline
\end{tabular}

Avg: $\quad \mathbf{1 8 3 . 0 4}$ msec

\begin{tabular}{|c|c|c|}
\hline $\begin{array}{c}\text { Nominal } \\
\text { Time } \\
\text { (ms) }\end{array}$ & $\begin{array}{c}\text { Actual } \\
\text { Time } \\
\mathbf{( m s )}\end{array}$ & $\begin{array}{c}\text { Deviation } \\
\text { (ms) }\end{array}$ \\
\hline 100.00 & 207.80 & 107.80 \\
100.00 & 198.40 & 98.40 \\
100.00 & 165.10 & 65.10 \\
100.00 & 165.10 & 65.10 \\
100.00 & 196.40 & 96.40 \\
\hline Avg: & $\mathbf{1 8 6 . 5 6}$ & msec \\
\hline
\end{tabular}


Table 8 - Under-frequency Time Test Results

SUMMARY OF IEEE 1547 UNDERFREQUENCY TIME TESTS

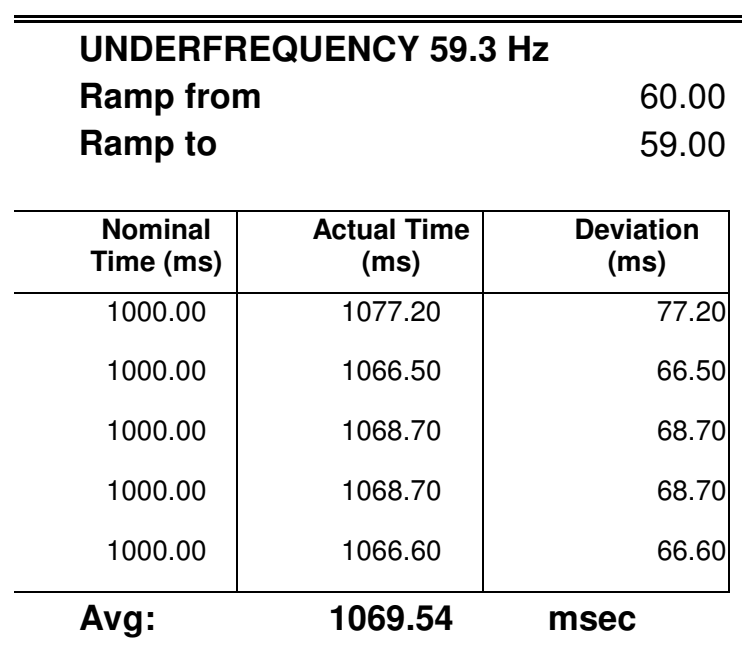

\begin{tabular}{|c|c|c|}
\hline $\begin{array}{l}\text { UNDERFR } \\
\text { Ramp fror } \\
\text { Ramp to }\end{array}$ & UENCY 57 & $\begin{array}{l}60.45 \\
60.55\end{array}$ \\
\hline $\begin{array}{c}\text { Nominal } \\
\text { Time }(\mathrm{ms})\end{array}$ & $\begin{array}{c}\text { Actual Time } \\
(\mathrm{ms})\end{array}$ & $\begin{array}{c}\text { Deviation } \\
(\mathrm{ms})\end{array}$ \\
\hline 167.00 & 255.60 & 88.60 \\
\hline 167.00 & 250.40 & 83.40 \\
\hline 167.00 & 248.40 & 81.40 \\
\hline 167.00 & 250.40 & 83.40 \\
\hline 167.00 & 250.60 & 83.60 \\
\hline Avg: & 251.08 & msec \\
\hline
\end{tabular}




\subsection{Appendix 2: List of DSP Controller settings in EMS (Section 3.9)}

Table 9 - Static Switch DSP Controller Settings

\section{Control Constants}

\begin{tabular}{|l|l|c|c|}
\hline Type & Event & Value & unit \\
\hline Filter time constant & 27R (Dead Bus Relay) & 1.00 & $\mathrm{sec}$ \\
\hline Timeout & 1547 voltage deep sag & 0.17 & $\mathrm{sec}$ \\
\hline Timeout & 1547 voltage shallow sag & 2.00 & $\mathrm{sec}$ \\
\hline Timeout & 1547 voltage swell & 1.00 & $\mathrm{sec}$ \\
\hline Timeout & 1547 voltage over & 0.17 & $\mathrm{sec}$ \\
\hline Timeout & 1547 frequency high swing & 0.17 & $\mathrm{sec}$ \\
\hline Timeout & 1547 frequency low droop & 1.00 & $\mathrm{sec}$ \\
\hline Timeout & 1547 frequency critically low & 0.17 & $\mathrm{sec}$ \\
\hline Time delay & Reverse power test delay & 2.00 & $\mathrm{sec}$ \\
\hline Time delay & Reverse power voltage fault time delay & 0.50 & $\mathrm{sec}$ \\
\hline CBEMA time constant & $\# 1$, instantaneous voltage & 0.02 & $\mathrm{sec}$ \\
\hline CBEMA time constant & $\# 2$, instantaneous voltage & 0.00 & $\mathrm{sec}$ \\
\hline CBEMA time constant & $\# 3$, filtered med speed voltage & 0.20 & $\mathrm{sec}$ \\
\hline CBEMA time constant & $\# 4$, filtered med speed voltage & 0.50 & $\mathrm{sec}$ \\
\hline CBEMA time constant & $\# 5$, filtered int speed voltage & 0.50 & $\mathrm{sec}$ \\
\hline CBEMA time constant & $\# 6$, filtered int speed voltage & 0.50 & $\mathrm{sec}$ \\
\hline CBEMA time constant & $\# 7$, filtered low speed voltage & 10.00 & $\mathrm{sec}$ \\
\hline
\end{tabular}

\section{Setpoints}

\begin{tabular}{|l|l|r|c|}
\hline Type & Event & Value & unit \\
\hline Event 25 & Sync voltage HH & 305.00 & $\mathrm{~V}$ \\
\hline Event 25 & Sync Voltage LL & 250.00 & $\mathrm{~V}$ \\
\hline Event 25 & Sync voltage nominal & 277.00 & $\mathrm{~V}$ \\
\hline Event 25 & Sync frequency HH & 61.00 & $\mathrm{~Hz}$ \\
\hline Event 25 & Sync Frequency LL & 59.00 & $\mathrm{~Hz}$ \\
\hline Event 25 & Sync frequency nominal & 60.00 & $\mathrm{~Hz}$ \\
\hline Event 25 & Sync Phase HH & 10.00 & degrees \\
\hline Event 25 & Sync Phase LL & -10.00 & degrees \\
\hline Event 25 & Time advance & 0.00 & sec \\
\hline
\end{tabular}

\section{State Controller}

\begin{tabular}{|l|c|c|}
\hline Timer & Value & unit \\
\hline Time disconnect & 1 & $\mathrm{sec}$ \\
\hline Restoration 1547 & 2 & $\mathrm{sec}$ \\
\hline Restoration $\mathrm{PQ}$ & 300 & $\mathrm{sec}$ \\
\hline
\end{tabular}


Table 10 - Static Switch DSP Controller Parameters

\section{DSP Controller - Parameters}

\section{Event Levels}

\section{Filtered}

\begin{tabular}{|l|c|c|}
\hline \multicolumn{1}{|c|}{ Event } & Value & unit \\
\hline 27R (rms dead DG bus) & 15 & $\mathrm{~V}$ \\
\hline AC Remote Power per Phase & 3.333 & $\mathrm{~kW}$ \\
\hline 1547 voltage deep sag & 50 & $\%$ of rated \\
\hline 1547 voltage shallow sag & 88 & $\%$ of rated \\
\hline 1547 voltage swell & 110 & $\%$ of rated \\
\hline 1547 voltage over & 120 & $\%$ of rated \\
\hline 1547 frequency high swing & 60.50 & $\mathrm{~Hz}$ \\
\hline 1547 frequency low droop & 59.30 & $\mathrm{~Hz}$ \\
\hline 1547 frequency critically low & 57.00 & $\mathrm{~Hz}$ \\
\hline RMS CBEMA voltage \#3 & 194 & $\mathrm{~V}$ \\
\hline RMS CBEMA voltage \#4 & 332 & $\mathrm{~V}$ \\
\hline RMS CBEMA voltage \#5 & 222 & $\mathrm{~V}$ \\
\hline RMS CBEMA voltage \#6 & 332 & $\mathrm{~V}$ \\
\hline RMS CBEMA voltage \#7 & 249 & $\mathrm{~V}$ \\
\hline
\end{tabular}

Instantaneous

\begin{tabular}{|c|c|c|}
\hline Event & Value & unit \\
\hline Instantaneous CBEMA voltage \#1 & 196 & $\mathrm{~V}$ \\
\hline Instantaneous CBEMA voltage \#2 & 548 & $\mathrm{~V}$ \\
\hline
\end{tabular}




\subsection{Appendix 3: Synchronization Tests (Section 3.2) plots}

In this appendix, a complete set of waveforms for the synchronization tests that were performed in Section 3.2 is presented. Results in this appendix include all the cases that are described in Table 1 (Section 3.2.3).

The results for each of the 12 tests include three figures:

1) Phase A Voltage Across Switch (Beat Voltage) before closing

2) Response to a "Manual Opening" of the switch

3) Response to a "Synchronized Closing" of the switch

For figures 2) and 3), the following channels represent:

Channel 1: Phase A Switch Current (20 Amps per division)

Channel 2: Phase B Switch Current (20 Amps per division)

Channel 3: Phase C Switch Current (20 Amps per division)

For figures 2) and 3), the vertical arrow in the time axis represents the transition of the switch command from open-to-close or close-to-open (the switch command was used to trigger the oscilloscope plots). 


\section{CERTS MICROGRID LABORATORY TEST BED}

Summary of CERTS Microgrid Static Switch Power Quality Tests at AEP Dolan, CERTS Microgrid Static Switch Testing

Prepared For:

California Energy Commission

Public Interest Energy Research Program

Arnold Schwarzenegger

Governor

0
$\frac{2}{2}$
$\frac{2}{\alpha}$

Prepared By:

Lawrence Berkeley National Laboratory

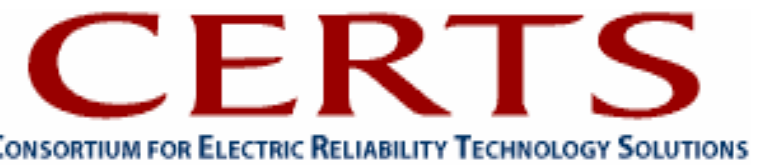



Prepared By:

Lawrence Berkeley National Laboratory

Joseph H. Eto, Principal Investigator

Berkeley, CA 94720

Harry Volkommer and Dave Klapp, American Electric Power

Commission Contract No. 500-03-024

Prepared For:

Public Interest Energy Research (PIER)

California Energy Commission

Bernard Treanton

Contract Manager

Mike Gravely

Program Area Lead

ENERGY SYSTEMS INTEGRATION

Mike Gravely

Office Manager

ENERGY SYSTEMS RESEARCH

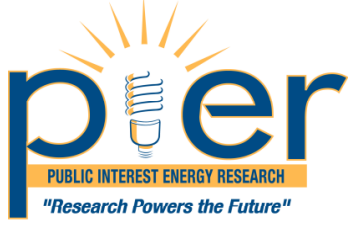

Martha Krebs, Ph.D.

PIER Director

Thom Kelly, Ph.D.

Deputy Director

ENERGY RESEARCH \& DEVELOPMENT DIVISION

Melissa Jones

Executive Director

\section{DISCLAIMER}

This report was prepared as the result of work sponsored by the California Energy Commission. It does not necessarily represent the views of the Energy Commission, its employees or the State of California. The Energy Commission, the State of California, its employees, contractors and subcontractors make no warrant, express or implied, and assume no legal liability for the information in this report; nor does any party represent that the uses of this information will not infringe upon privately owned rights. This report has not been approved or disapproved by the California Energy Commission nor has the California Energy Commission passed upon the accuracy or adequacy of the information in this report. 



\section{Background}

The Static Switch (SS) will be the power gateway between the protected and unprotected portions of the Microgrid Test Bed. This gateway is required to incorporate many different functions in order that the Microgrid will seamlessly transition from grid connection to grid disconnection for certain reasons. These reasons include, but aren't limited to, Power Quality Events and Power Export to the utility grid. This gateway must also handle the seamless reconnection of the Microgrid following any of these events. Originally, it was discussed that the SS would meet IEEE Standard 1547 for interconnecting DR with electric power systems. Since these tests are not trying to qualify the SS at this time, some portions of testing will be left for a later date (i.e. Thermal, EMI, Harmonics, etc). Therefore, DTC testing will focus on how the SS disconnects and reconnects to the utility grid. Although the SS function may not completely fit into IEEE Standard 1547, it does not cause any problems for the intended testing of the Microgrid, however testing of the SS needs to incorporate this knowledge. A brief example of the differences and a suggested test plan at AEP's DTC follows.

From a protection point of view the SS can be setup to meet the IEEE 1547 criteria or more stringent criteria, such as ITIC, CBEMA, etc. The deviation from standard deals primarily with reconnection to the utility grid. Briefly, IEEE 1547 has windows for frequency difference, voltage difference, and phase angle difference between the two electrical buses. It is understood that there are possible operating points under which the Microgrid would not reconnect to the utility grid, if the SS strictly followed IEEE Standard 1547. Therefore, the SS will have somewhat looser criteria under which it is acceptable to reconnect to the utility grid and will be tested in the Microgrid Test Bed to see what the results show.

\subsection{Proposed DTC Test Plan}

The proposed DTC tests are designed to test the functionality of the protection portion of the SS. For example, does the switch open when out of voltage, current, and frequency criteria, etc. From discussions with Ed, Northern Power Systems (NPS) has put together a good basic test sequence for SS functionality. This is appropriate, as any system debugging will be easiest to perform at the NPS site. Thus, more advanced testing of the SS will be performed at the DTC, primarily dealing with power quality events. Examples would be sags, swells, switching transient events, etc. It is our intention to treat this SS as a protection relay and test it similarly. To achieve this, we will inject various stimuli and capture the SS's response. This stimulus is best kept low power in nature as larger power may cause damage to the SS. It is also appropriate for the team to agree on what the stimulus should be prior to testing. Currently, AEP budgeted/scheduled 10 days to setup and test the SS at the DTC site and we must make the most of it without delaying the project schedule. 
Originally, we discussed testing the SS with high power stimuli, such as fault currents. Because the SS is likely to be the most critical path device during Microgrid installation, it would cause large delays in the project schedule if damage to the SS occurred during DTC tests. Also, there is adequate secondary protection within the Walnut Test Site, so that this high power testing may take place on site. We believe power fault tests would be more appropriately tested once in the Microgrid. However, we would like to simulate faults at the DTC site both in the utility system and faults within the Microgrid to determine if the SS reacts properly. This testing would inject voltage and current signals into the sensing portion of the SS and capture its response.

Based on the above information, we recommend the following tests take place at the DTC site.

- Ramp testing of Over/Under Voltage, and Over/Under Frequency protection. This protection is part of IEEE Standard 1547, which may be somewhat difficult for NPS to complete. Currently, NPS is performing the step technique portion of this test. The ramp test technique will complete the test requirements.

- Synchronization to the utility grid. As mentioned, above, the criterion for synchronization is likely to be different to IEEE Standard 1547; however, it should be tested to the CERTS Microgrid criteria set-points, i.e., Frequency, Voltage, and Phase Angle. Currently, NPS is testing the SS with an inverter source, mimicking the Tecogen generator devices. This testing needs further verification to demonstrate that the SS alone meets its design criteria.

- Power Quality Events.

- Sags/Swells in Voltage, reflecting ITIC, CBEMA, and/or SEMI

- Sags/Swells in Frequency

- Switching/Line Transients

- Simulated Utility/Microgrid Faults

We also recommend the following tests take place at NPS.

- Open phase combinations

We recommend this test be done as this can be a common occurrence in the utility system. This testing can be satisfied, if the minimum import relay is tested successfully.

- SCR failure to "Open"

We recommend this test be done to satisfy concerns that the SS may be unable to "Open" under asymmetrical faults. This testing can take place at either NPS or DTC; however, AEP will need more detail of this functionality if done here. 
Most, if not all, of this testing can be accomplished with the capabilities of a single set of test equipment. This includes a 480volts 3-phase variac, an Elgar SW 5250A Smart Wave, an Elgar SS-66 3-phase power supply, a Manta MTS 1710 relay test set, and a Wavetek Datron Model 195 Universal Waveform Generator.

\subsection{DTC Test Details}

For all of the following tests the SCR switches will be disconnected from the circuit. This is to prevent any stress on the SCR switches and damage to any test equipment. The stimuli will be injected into the sensing PTs and CTs with the resultant response measured on the signal that "opens" and "closes" the SCR switches.

1) Ramp testing of Over/Under Voltage and Over/Under Frequency protection. This testing is used to determine if the SS meets the design criteria for voltage and frequency protection. Specifically, ramping the voltage and frequency will allow accurate measurement of where the trip points are set. This test setup will require only the Manta 1710 Relay Test Set (Manta) and shown in Figure 1. The measurement PTs of the SS will be disconnected and the Manta connected in their place. The Manta will be configured to deliver the same stimuli to both sides of the switch. These stimuli will be the ramping functions as laid out in IEEE Standard 1547.1. Individual tests for voltage and frequency will be performed. The SS will be energized to nominal conditions and then ramped from them. The magnitude of the ramp at the command to "open" the SS will be recorded for 5 test runs. For voltage each phase will be tested, individually, as well as all three phases together. For frequency all three phases will be tested together. 


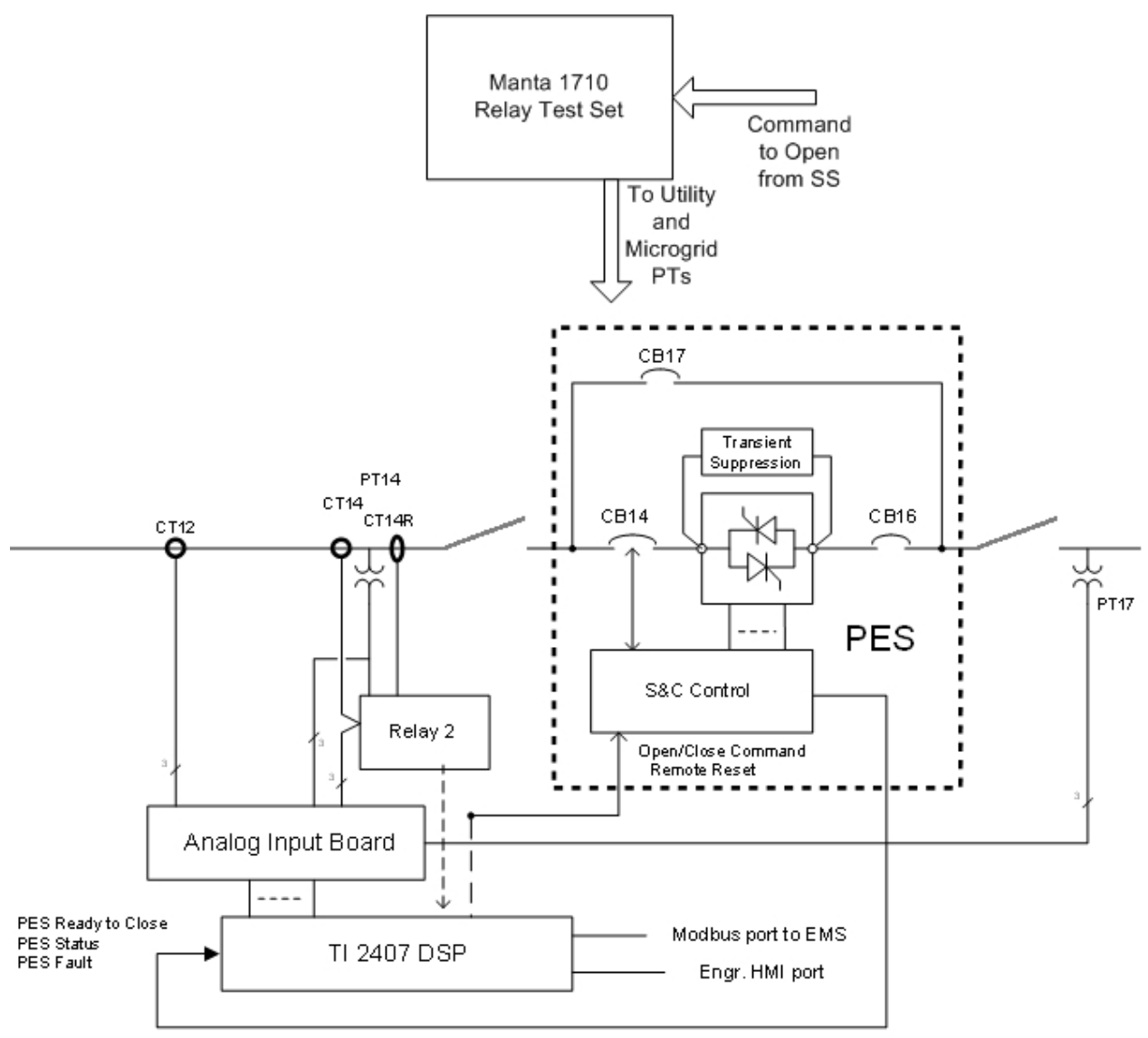

Figure 1: Ramp Test Setup for Over/Under Voltage \& Over/Under Frequency.

2) Synchronization to the utility grid. This testing is used to determine if the SS meets the CERTS Microgrid criteria for synchronizing to the utility grid. Specifically, this test will allow us to test the each condition for acceptable synchronization, individually, instead of as a combined process. This test setup will require both the Manta 1710 Relay Test Set and a 3phase variac, as shown in Figure 2. The Manta will provide stimulus to the Microgrid side of the SS. The 3-phase variac will connect to the Utility side of the SS and will primarily correct the utility voltage, if other than nominal.

a. For testing the synchronization of voltage, the SS will be energized on the utility side through the variac to nominal voltage. The Manta will energize the Microgrid side of the SS with a frequency and phase locked voltage. This voltage magnitude will be ramped from out of tolerance to in to tolerance. The magnitude of the ramp at the command to "close" the SS will be recorded for 5 test runs. Each phase will be tested, individually, as well as all three phases together.

b. For testing the synchronization of frequency and phase angle, the SS will be energized on the utility side through the variac to nominal voltage. The Manta will energize the Microgrid side of the SS with the nominal voltage and a frequency that is outside the synchronization criteria. The frequency will then be ramped from out of tolerance and 
AEP DTC Proposed Test Plan - CERTS Micro-grid Static Switch

Project Engineer: David A. Klapp

Date: March 3, 2006

in to tolerance. The magnitude of the ramp and the phase angle at the command to "close" the SS will be recorded for 5 test runs. All three phases will be tested together.

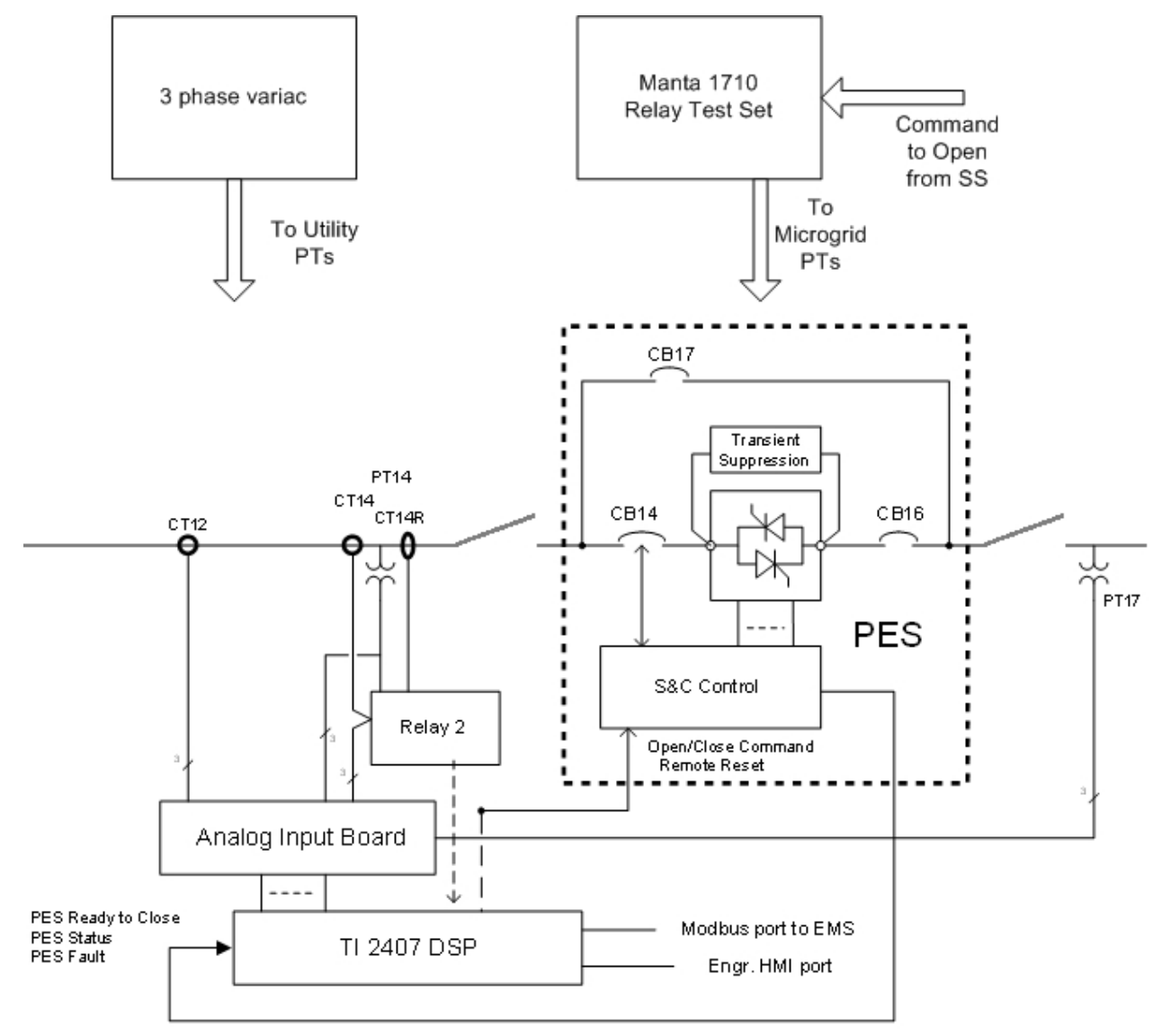

Figure 2: Test Setup for Synchronization to the utility grid.

3) Power Quality Events. This testing is used to determine how the SS will respond to power quality events within the utility system. The test setup for both sags and swells in voltage and frequency, as well as switching and line transients will be similar to the voltage and frequency ramp testing, described above. This will require only the Manta 1710 Relay Test Set. The measurement PTs of the SS will be disconnected and the Manta connected in their place. The Manta will be configured to deliver the same stimuli to both sides of the SS. These stimuli will need to be decided, prior to testing. A recommendation follows:

a. Voltage sags and swells for at least 7 points on the ITIC or CBEMA curve

b. If desired, voltage sags at 3 points for the SEMIF47 curve.

c. Frequency sags and swells for at least 3 points to meet IEEE Standard 1547. 
AEP DTC Proposed Test Plan - CERTS Micro-grid Static Switch

Project Engineer: David A. Klapp

Date: March 3, 2006

d. At least 6 switching and line transients to be decided upon.

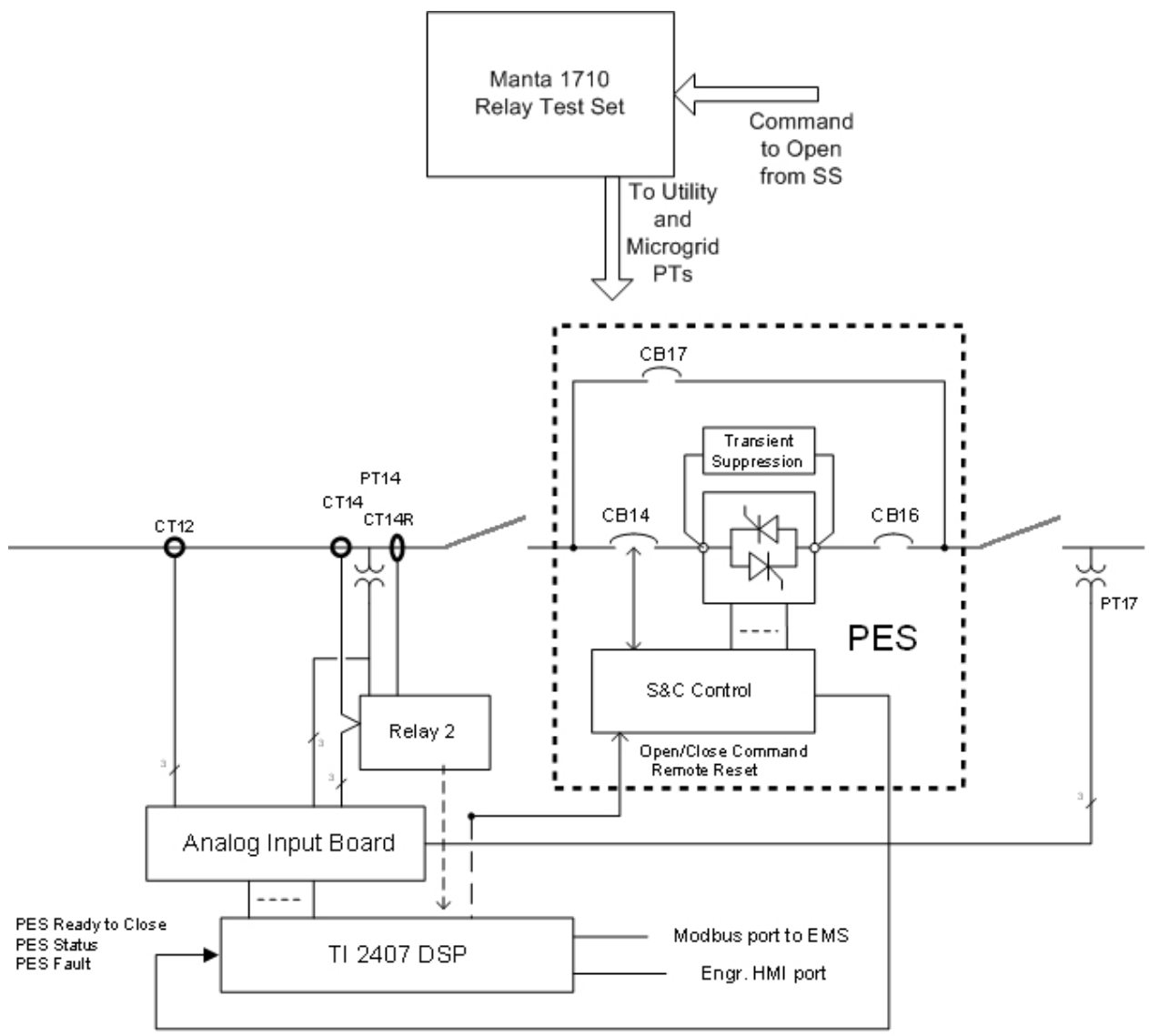

Figure 3: Test Setup for Power Quality Events.

4) Utility and Microgrid Faults. Test setup for simulated Utility and Microgrid Faults will be similar to the sag and swell testing above, except the measurement CTs of the SS will also be disconnected and the Manta connected in their place. This test setup is shown in Figure 4. The Manta will be configured to deliver the same voltage stimuli to both sides of the SS and current stimuli to the utility side. The stimulus will match what the anticipated faults will look like within the Microgrid. Testing of each type of fault, as well as three different levels of fault currents will be performed. For simulating the utility faults, data from past test and 
the simulation of the Microgrid Test bed will be used to design the stimuli. This test will take into account the effect of the Reverse power relay.

The SS will be energized to nominal conditions and then the power quality event will be triggered. The event details (type, magnitude, duration, etc.) and the response of the SS will be recorded for each of the 5 test runs.

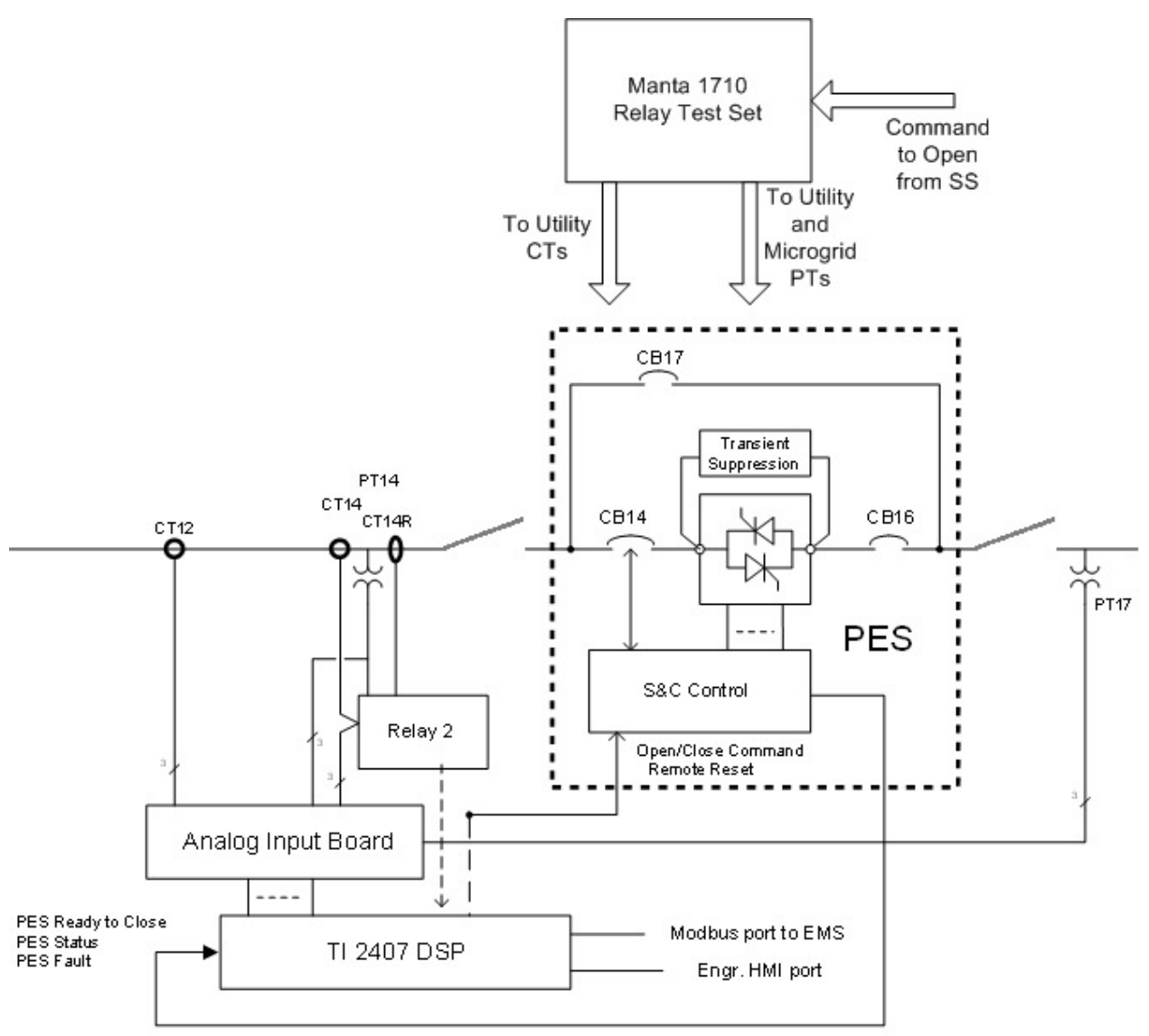

Figure 4: Test Setup for Utility and Microgrid Faults. 
SUMMARY OF CERTS MICROGRID STATIC SWITCH POWER QUALITY TESTS @ AEP DOLAN

Notes: AEP EMS is setup with only PQ Events Enableded, The SS Parameters for CBEMA\#7 Time was changed to 3.0223 and CBEMA\#2 Voltage was changed to 470 Volts

The Manta has been setup to deliver secondary voltages and currents to the DSP and Relay 2

CBEMA/ITIC/SEMI VOLTAGE SAG/SWELL TESTS (AVERAGES FROM 5 TRIES)

\begin{tabular}{|c|c|c|c|c|c|}
\hline \multicolumn{6}{|c|}{ CBEMA/ITIC/SEMI VOLTAGE SAG/SWELL TESTS (AVERAGES FROM 5 TRIES) } \\
\hline Test & Voltage & Cycles & $\begin{array}{c}\text { Average } \\
\text { Trip Time } \\
\text { (ms) }\end{array}$ & Standard Deviation (ms) & Pass / Fail \\
\hline Undervoltage $0 \%$, Three Phase & 0.0 Peak & 0.5 & $\mathrm{~N} / \mathrm{A}$ & $\mathrm{N} / \mathrm{A}$ & Pass \\
\hline Undervoltage $0 \%$, Three Phase & 0.0 Peak & 2 & $\mathrm{~N} / \mathrm{A}$ & N/A & Fail \\
\hline Undervoltage $30 \%$, Three Phase & 117.5 Peak & 5 & $\mathrm{~N} / \mathrm{A}$ & $\mathrm{N} / \mathrm{A}$ & Fail \\
\hline Undervoltage $67 \%$, Three Phase & 262.5 Peak & 15 & 0.2084 & 0.0004 & Pass \\
\hline Undervoltage $73 \%$, Three Phase & 286.0 Peak & 3 & $\mathrm{~N} / \mathrm{A}$ & $\mathrm{N} / \mathrm{A}$ & Pass \\
\hline Undervoltage $77 \%$, Three Phase & 213.3 RMS & 35 & 0.4582 & 0.0008 & Pass \\
\hline Undervoltage 83\%, Three Phase & 229.9 RMS & 45 & $\mathrm{~N} / \mathrm{A}$ & $\mathrm{N} / \mathrm{A}$ & Pass \\
\hline Overvoltage $107 \%$, Three Phase & 296.4 RMS & 60 & $\mathrm{~N} / \mathrm{A}$ & $\mathrm{N} / \mathrm{A}$ & Pass \\
\hline Overvoltage $113 \%$, Three Phase & 313.0 RMS & 45 & $\mathrm{~N} / \mathrm{A}$ & $\mathrm{N} / \mathrm{A}$ & Fail \\
\hline Overvoltage $117 \%$, Three Phase & 458.3 Peak & 20 & $\mathrm{~N} / \mathrm{A}$ & $\mathrm{N} / \mathrm{A}$ & Pass \\
\hline Overvoltage $123 \%$, Three Phase & 481.8 Peak & 10 & $\mathrm{~N} / \mathrm{A}$ & $\mathrm{N} / \mathrm{A}$ & Fail \\
\hline Undervoltage $0 \%$, Single Phase A & 0.0 Peak & 0.5 & $\mathrm{~N} / \mathrm{A}$ & $\mathrm{N} / \mathrm{A}$ & Pass \\
\hline Undervoltage $45 \%$, Single Phase B & 176.3 Peak & 20 & 0.1415 & 0.0006 & Pass \\
\hline Undervoltage $67 \%$, Single Phase C & 185.6 RMS & 40 & 0.1848 & 0.0004 & Pass \\
\hline Undervoltage $77 \%$, Single Phase A & 213.3 RMS & 45 & 0.4376 & 0.0004 & Pass \\
\hline Undervoltage $83 \%$, Single Phase B & $229.9 \mathrm{RMS}$ & 70 & $\mathrm{~N} / \mathrm{A}$ & $\mathrm{N} / \mathrm{A}$ & Pass \\
\hline Undervoltage $0 \%$, Single Phase A & 0.0 Peak & 2 & $\mathrm{~N} / \mathrm{A}$ & $\mathrm{N} / \mathrm{A}$ & Fail \\
\hline Undervoltage $0 \%$, Single Phase A & 0.0 Peak & 3 & $\mathrm{~N} / \mathrm{A}$ & $\mathrm{N} / \mathrm{A}$ & Fail \\
\hline Undervoltage $0 \%$, Single Phase A & 0.0 Peak & 4 & $\mathrm{~N} / \mathrm{A}$ & $\mathrm{N} / \mathrm{A}$ & Fail \\
\hline Undervoltage $0 \%$, Single Phase A & 0.0 Peak & 5 & $\mathrm{~N} / \mathrm{A}$ & $\mathrm{N} / \mathrm{A}$ & Fail \\
\hline Undervoltage $0 \%$, Single Phase A & 0.0 Peak & 6 & $\mathrm{~N} / \mathrm{A}$ & $\mathrm{N} / \mathrm{A}$ & Fail \\
\hline Undervoltage $0 \%$, Single Phase A & 0.0 Peak & 7 & 0.1077 & 0.0006 & Pass \\
\hline Undervoltage $0 \%$, Single Phase A & 0.0 Peak & 8 & 0.1081 & 0.0010 & Pass \\
\hline Undervoltage $0 \%$, Single Phase A & 0.0 Peak & 9 & 0.1082 & 0.0004 & Pass \\
\hline Undervoltage $0 \%$, Single Phase A & 0.0 Peak & 10 & 0.1079 & 0.0008 & Pass \\
\hline Overvoltage $120 \%$, Three Phase & 470.1 Peak & 20 & $\mathrm{~N} / \mathrm{A}$ & N/A & Fail \\
\hline
\end{tabular}


SUMMARY OF CERTS MICROGRID STATIC SWITCH POWER QUALITY TESTS @ AEP DOLAN

Notes: AEP EMS is setup with only PQ Events Enableded, The SS Parameters for CBEMA\#7 Time was changed to 3.0223 and CBEMA\#2 Voltage was changed to 470 Volts

The Manta has been setup to deliver secondary voltages and currents to the DSP and Relay 2

Overvoltage $121 \%$, Three Phase

Overvoltage $122 \%$, Three Phase

Overvoltage $123 \%$, Three Phase

Overvoltage 124\%, Three Phase

Overvoltage 125\%, Three Phase

Overvoltage $126 \%$, Three Phase

Overvoltage $127 \%$, Three Phase

Overvoltage $128 \%$, Three Phase

Overvoltage $129 \%$, Three Phase

Overvoltage $130 \%$, Three Phase

Table 1: Voltage Sag/Swell Test Results

\begin{tabular}{|l|l|l|l|l|} 
474.0 Peak & 20 & N/A & N/A & Fail \\
477.9 Peak & 20 & N/A & N/A & Fail \\
481.8 Peak & 20 & N/A & N/A & Fail \\
485.8 Peak & 20 & N/A & N/A & Fail \\
489.7 Peak & 20 & N/A & N/A & Fail \\
493.6 Peak & 20 & N/A & N/A & Fail \\
497.5 Peak & 20 & N/A & 0.0008 & Pass \\
501.4 Peak & 20 & 0.3248 & 0.0004 & Pass \\
505.3 Peak & 20 & 0.3253 & 0.0008 & Pass \\
509.3 Peak & 20 & 0.3083 &
\end{tabular}

SIMULATED FAULT TESTS (AVERAGES FROM 5 TRIES)

\begin{tabular}{|l|c|c|c|c|}
\hline \multicolumn{4}{|c|}{ SIMULATED FAULT TESTS (AVERAGES FROM 5 TRIES) } \\
\hline Test & Current & Cycles & $\begin{array}{c}\text { Average } \\
\text { Trip Time } \\
\text { (ms) }\end{array}$ & Standard Deviation (ms) \\
\hline Phase A to B, Bolted Fault & & 300 & 0.0231 & 0.0002 \\
Phase A to Ground, Bolted Fault & & 300 & 0.0214 & 0.0005 \\
Phase A to B to Ground, Bolted Fault & & 300 & 0.0224 & 0.0012 \\
Phase A to B to C to Ground, Bolted Fault & & 300 & 0.0212 & 0.0010 \\
Phase A to B to Ground, High Impedance Fault & & 300 & 0.0235 & 0.0006 \\
Phase A to B to C to Ground, High Impedance Fault & & 300 & 0.0214 & 0.0007 \\
\hline
\end{tabular}

Table 2: Simulated Fault Test Results

\begin{tabular}{|l|c|c|c|c|}
\hline \multicolumn{4}{|c|}{ OPEN PHASE TESTS (AVERAGES FROM 5 TRIES) } \\
\hline Test & & $\begin{array}{c}\text { Average } \\
\text { Trip Time } \\
\text { (ms) }\end{array}$ & Standard Deviation (ms) & Pass / Fail \\
\hline Open Phase A in Delta-Delta Feed & & 300 & 0.1582 & 0.0006 \\
Open Phase A in Wye Secondary Feed & 300 & 0.1074 & 0.0005 \\
Open Phase A and B in Wye Secondary Feed & & 300 & 0.1075 & 0.0008 \\
\hline
\end{tabular}

Table 3: Open Phase Test Results 
SUMMARY OF CERTS MICROGRID STATIC SWITCH POWER QUALITY TESTS @ AEP DOLAN

Notes: AEP EMS is setup with only PQ Events Enableded, The SS Parameters for CBEMA\#7 Time was changed to 3.0223 and CBEMA\#2 Voltage was changed to 470 Volts

The Manta has been setup to deliver secondary voltages and currents to the DSP and Relay 2

\begin{tabular}{|l|c|c|c|c|c|}
\hline \multicolumn{4}{|c|}{ VOLTAGE TRANSIENT/DISTORTION TESTS (AVERAGES FROM 5 TRIES) } \\
\hline & Cycles & Trip & $\begin{array}{c}\text { Average } \\
\text { Trip Time } \\
\text { (ms) }\end{array}$ & Standard Deviation (ms) & Pass / Fail \\
Test & $<1$ & No & N/A & N/A & N/A \\
\hline Lightning Surge 1.2-50us & $<1$ & No & N/A & N/A \\
900 MVar Capacitor Switch & 40 & Yes & 0.4749 & 0.0007 & N/A \\
Motor Start Non-CBEMA/ITIC/SEMI Violation & 40 & Yes & 0.1918 & 0.0010 \\
Motor Start CBEMA/ITIC/SEMI Violation & 300 & No & N/A & N/A \\
Six Pulse Bridge Load & 240 & No & N/A & N/A & N/A \\
Harmonic Distortion & & & N/A \\
\hline
\end{tabular}

Table 4: Voltage Transient/Distortion Test Reults 


\section{SUMMARY OF CBEMA/ITIC/SEMI VOLTAGE SAG/SURGE TESTS}

UNDERVOLTAGE SAG 0\%, Three

Phase, 0.5 Cycle

\begin{tabular}{|c|c|c|c|c|}
\hline Name & $\begin{array}{l}\text { Anticipated } \\
\text { Reaction }\end{array}$ & $\begin{array}{l}\text { Actual } \\
\text { Reaction }\end{array}$ & $\begin{array}{l}\text { Reaction } \\
\text { Time }\end{array}$ & $\begin{array}{l}\text { Pass / } \\
\text { Fail }\end{array}$ \\
\hline Test 1 & $\begin{array}{l}\text { Remain } \\
\text { Online }\end{array}$ & $\begin{array}{l}\text { Remain } \\
\text { Online }\end{array}$ & $\mathrm{N} / \mathrm{A}$ & Pass \\
\hline Test 2 & $\begin{array}{l}\text { Remain } \\
\text { Online }\end{array}$ & $\begin{array}{l}\text { Remain } \\
\text { Online }\end{array}$ & $\mathrm{N} / \mathrm{A}$ & Pass \\
\hline Test 3 & $\begin{array}{l}\text { Remain } \\
\text { Online }\end{array}$ & $\begin{array}{l}\text { Remain } \\
\text { Online }\end{array}$ & $\mathrm{N} / \mathrm{A}$ & Pass \\
\hline Test 4 & $\begin{array}{l}\text { Remain } \\
\text { Online }\end{array}$ & $\begin{array}{l}\text { Remain } \\
\text { Online }\end{array}$ & $\mathrm{N} / \mathrm{A}$ & Pass \\
\hline Test 5 & $\begin{array}{l}\text { Remain } \\
\text { Online }\end{array}$ & $\begin{array}{l}\text { Remain } \\
\text { Online }\end{array}$ & $\mathrm{N} / \mathrm{A}$ & Pass \\
\hline
\end{tabular}

AVERAGE TRIP TIME

$N / A$

ST DEV N/A

\begin{tabular}{|c|c|c|c|c|}
\hline \multicolumn{5}{|c|}{$\begin{array}{l}\text { UNDERVOLTAGE SAG 30\%, Three } \\
\text { Phase, } 5 \text { Cycles }\end{array}$} \\
\hline Name & $\begin{array}{l}\text { Anticipated } \\
\text { Reaction }\end{array}$ & $\begin{array}{l}\text { Actual } \\
\text { Reaction }\end{array}$ & $\begin{array}{l}\text { Reaction } \\
\text { Time }\end{array}$ & $\begin{array}{l}\text { Pass / } \\
\text { Fail }\end{array}$ \\
\hline Test 1 & Trip Open & $\begin{array}{l}\text { Remain } \\
\text { Online }\end{array}$ & $\mathrm{N} / \mathrm{A}$ & Fail \\
\hline Test 2 & Trip Open & $\begin{array}{l}\text { Remain } \\
\text { Online }\end{array}$ & $\mathrm{N} / \mathrm{A}$ & Fail \\
\hline Test 3 & Trip Open & $\begin{array}{l}\text { Remain } \\
\text { Online }\end{array}$ & $\mathrm{N} / \mathrm{A}$ & Fail \\
\hline Test 4 & Trip Open & $\begin{array}{l}\text { Remain } \\
\text { Online }\end{array}$ & $\mathrm{N} / \mathrm{A}$ & Fail \\
\hline Test 5 & Trip Open & $\begin{array}{l}\text { Remain } \\
\text { Online }\end{array}$ & $\mathrm{N} / \mathrm{A}$ & Fail \\
\hline
\end{tabular}

AVERAGE TRIP TIME N/A ST DEV N/A

UNDERVOLTAGE SAG 73\%, Three

Phase, 3 Cycles

\begin{tabular}{|l|l|l|l|l|}
\hline Name & $\begin{array}{l}\text { Anticipated } \\
\text { Reaction }\end{array}$ & $\begin{array}{l}\text { Actual } \\
\text { Reaction }\end{array}$ & $\begin{array}{l}\text { Reaction } \\
\text { Time }\end{array}$ & $\begin{array}{l}\text { Pass / } \\
\text { Fail }\end{array}$ \\
\hline Test 1 & Remain & Remain & N/A & Pass
\end{tabular}

UNDERVOLTAGE SAG 0\%, Three

Phase, 2 Cycles

\begin{tabular}{|c|c|c|c|c|}
\hline Name & $\begin{array}{l}\text { Anticipated } \\
\text { Reaction }\end{array}$ & $\begin{array}{l}\text { Actual } \\
\text { Reaction }\end{array}$ & $\begin{array}{l}\text { Reaction } \\
\text { Time }\end{array}$ & $\begin{array}{l}\text { Pass / } \\
\text { Fail }\end{array}$ \\
\hline Test 1 & Trip Open & $\begin{array}{l}\text { Remain } \\
\text { Online }\end{array}$ & $\mathrm{N} / \mathrm{A}$ & Fail \\
\hline Test 2 & Trip Open & $\begin{array}{l}\text { Remain } \\
\text { Online }\end{array}$ & $\mathrm{N} / \mathrm{A}$ & Fail \\
\hline Test 3 & Trip Open & $\begin{array}{l}\text { Remain } \\
\text { Online }\end{array}$ & $\mathrm{N} / \mathrm{A}$ & Fail \\
\hline Test 4 & Trip Open & $\begin{array}{l}\text { Remain } \\
\text { Online }\end{array}$ & $\mathrm{N} / \mathrm{A}$ & Fail \\
\hline Test 5 & Trip Open & $\begin{array}{l}\text { Remain } \\
\text { Online }\end{array}$ & $\mathrm{N} / \mathrm{A}$ & Fail \\
\hline
\end{tabular}

AVERAGE TRIP TIME N/A ST DEV N/A

UNDERVOLTAGE SAG $67 \%$, Three

Phase, 15 Cycles

\begin{tabular}{|c|c|c|c|c|}
\hline Name & $\begin{array}{l}\text { Anticipated } \\
\text { Reaction }\end{array}$ & $\begin{array}{l}\text { Actual } \\
\text { Reaction }\end{array}$ & $\begin{array}{l}\text { Reaction } \\
\text { Time }\end{array}$ & $\begin{array}{l}\text { Pass / } \\
\text { Fail }\end{array}$ \\
\hline Test 1 & Trip Open & Trip Open & 0.2085 & Pass \\
\hline Test 2 & Trip Open & Trip Open & 0.208 & Pass \\
\hline Test 3 & Trip Open & Trip Open & 0.2085 & Pass \\
\hline Test 4 & Trip Open & Trip Open & 0.208 & Pass \\
\hline Test 5 & Trip Open & Trip Open & 0.209 & Pass \\
\hline
\end{tabular}

AVERAGE TRIP TIME $\quad 0.2084 \quad$ ST DEV $\quad 0.0004$

UNDERVOLTAGE SAG 77\%, Three

Phase, 35 Cycles

\begin{tabular}{|l|l|l|l|l|}
\hline Name & $\begin{array}{l}\text { Anticipated } \\
\text { Reaction }\end{array}$ & $\begin{array}{l}\text { Actual } \\
\text { Reaction }\end{array}$ & $\begin{array}{l}\text { Reaction } \\
\text { Time }\end{array}$ & $\begin{array}{l}\text { Pass / } \\
\text { Fail }\end{array}$ \\
\hline Test 1 & Trip Open & Trip Open & 0.4585 & Pass \\
\hline
\end{tabular}


SUMMARY OF CBEMA/ITIC/SEMI VOLTAGE SAG/SURGE TESTS

\begin{tabular}{|l|l|l|l|l|} 
& Online & Online & & \\
Test 2 & $\begin{array}{l}\text { Remain } \\
\text { Online }\end{array}$ & $\begin{array}{l}\text { Remain } \\
\text { Online }\end{array}$ & N/A & Pass \\
Test 3 & $\begin{array}{l}\text { Remain } \\
\text { Online }\end{array}$ & $\begin{array}{l}\text { Remain } \\
\text { Online }\end{array}$ & N/A & Pass \\
Test 4 & $\begin{array}{l}\text { Remain } \\
\text { Online }\end{array}$ & $\begin{array}{l}\text { Remain } \\
\text { Online }\end{array}$ & N/A & Pass \\
Test 5 & $\begin{array}{l}\text { Remain } \\
\text { Online }\end{array}$ & Ondine & N/A & Pass \\
& Onlin & & \\
\hline
\end{tabular}

\begin{tabular}{|l|l|l|r|l|} 
Test 2 & Trip Open & Trip Open & 0.4575 & Pass \\
Test 3 & Trip Open & Trip Open & 0.4575 & Pass \\
Test 4 & Trip Open & Trip Open & 0.4595 & Pass \\
Test 5 & Trip Open & Trip Open & 0.458 & Pass \\
\hline
\end{tabular}

$N / A$

ST DEV N/A

AVERAGE TRIP TIME

0.4582

ST DEV

0.0008

\begin{tabular}{|c|c|c|c|c|}
\hline \multicolumn{5}{|c|}{$\begin{array}{l}\text { UNDERVOLTAGE SAG 83\%, Three } \\
\text { Phase, } 45 \text { Cycles }\end{array}$} \\
\hline Name & $\begin{array}{l}\text { Anticipated } \\
\text { Reaction }\end{array}$ & $\begin{array}{l}\text { Actual } \\
\text { Reaction }\end{array}$ & $\begin{array}{l}\text { Reaction } \\
\text { Time }\end{array}$ & $\begin{array}{l}\text { Pass / } \\
\text { Fail }\end{array}$ \\
\hline Test 1 & $\begin{array}{l}\text { Remain } \\
\text { Online }\end{array}$ & $\begin{array}{l}\text { Remain } \\
\text { Online }\end{array}$ & $\mathrm{N} / \mathrm{A}$ & Pass \\
\hline Test 2 & $\begin{array}{l}\text { Remain } \\
\text { Online }\end{array}$ & $\begin{array}{l}\text { Remain } \\
\text { Online }\end{array}$ & $\mathrm{N} / \mathrm{A}$ & Pass \\
\hline Test 3 & $\begin{array}{l}\text { Remain } \\
\text { Online }\end{array}$ & $\begin{array}{l}\text { Remain } \\
\text { Online }\end{array}$ & $\mathrm{N} / \mathrm{A}$ & Pass \\
\hline Test 4 & $\begin{array}{l}\text { Remain } \\
\text { Online }\end{array}$ & $\begin{array}{l}\text { Remain } \\
\text { Online }\end{array}$ & $\mathrm{N} / \mathrm{A}$ & Pass \\
\hline Test 5 & $\begin{array}{l}\text { Remain } \\
\text { Online }\end{array}$ & $\begin{array}{l}\text { Remain } \\
\text { Online }\end{array}$ & $\mathrm{N} / \mathrm{A}$ & Pass \\
\hline
\end{tabular}

OVERVOLTAGE SURGE 107\%, Three

Phase, 60 Cycles

\begin{tabular}{|c|c|c|c|c|}
\hline Name & $\begin{array}{l}\text { Anticipated } \\
\text { Reaction }\end{array}$ & $\begin{array}{l}\text { Actual } \\
\text { Reaction }\end{array}$ & $\begin{array}{l}\text { Reaction } \\
\text { Time }\end{array}$ & $\begin{array}{l}\text { Pass / } \\
\text { Fail }\end{array}$ \\
\hline Test 1 & $\begin{array}{l}\text { Remain } \\
\text { Online }\end{array}$ & $\begin{array}{l}\text { Remain } \\
\text { Online }\end{array}$ & $\mathrm{N} / \mathrm{A}$ & Pass \\
\hline Test 2 & $\begin{array}{l}\text { Remain } \\
\text { Online }\end{array}$ & $\begin{array}{l}\text { Remain } \\
\text { Online }\end{array}$ & $\mathrm{N} / \mathrm{A}$ & Pass \\
\hline Test 3 & $\begin{array}{l}\text { Remain } \\
\text { Online }\end{array}$ & $\begin{array}{l}\text { Remain } \\
\text { Online }\end{array}$ & $\mathrm{N} / \mathrm{A}$ & Pass \\
\hline Test 4 & $\begin{array}{l}\text { Remain } \\
\text { Online }\end{array}$ & $\begin{array}{l}\text { Remain } \\
\text { Online }\end{array}$ & $\mathrm{N} / \mathrm{A}$ & Pass \\
\hline Test 5 & $\begin{array}{l}\text { Remain } \\
\text { Online }\end{array}$ & $\begin{array}{l}\text { Remain } \\
\text { Online }\end{array}$ & $\mathrm{N} / \mathrm{A}$ & Pass \\
\hline
\end{tabular}

AVERAGE TRIP TIME

N/A

ST DEV N/A

AVERAGE TRIP TIME N/A

ST DEV N/A

\begin{tabular}{|c|c|c|c|c|}
\hline \multicolumn{5}{|c|}{$\begin{array}{l}\text { OVERVOLTAGE SURGE } 113 \% \text {, Three } \\
\text { Phase, } 45 \text { Cycles }\end{array}$} \\
\hline Name & $\begin{array}{l}\text { Anticipated } \\
\text { Reaction }\end{array}$ & $\begin{array}{l}\text { Actual } \\
\text { Reaction }\end{array}$ & $\begin{array}{l}\text { Reaction } \\
\text { Time }\end{array}$ & $\begin{array}{l}\text { Pass / } \\
\text { Fail }\end{array}$ \\
\hline Test 1 & Trip Open & $\begin{array}{l}\text { Remain } \\
\text { Online }\end{array}$ & $\mathrm{N} / \mathrm{A}$ & Fail \\
\hline Test 2 & Trip Open & $\begin{array}{l}\text { Remain } \\
\text { Online }\end{array}$ & $\mathrm{N} / \mathrm{A}$ & Fail \\
\hline Test 3 & Trip Open & $\begin{array}{l}\text { Remain } \\
\text { Online }\end{array}$ & $\mathrm{N} / \mathrm{A}$ & Fail \\
\hline
\end{tabular}

OVERVOLTAGE SAG 117\%, Three
Phase, 20 Cycles
\begin{tabular}{|l|l|l|l|l|}
\hline Name & $\begin{array}{l}\text { Anticipated } \\
\text { Reaction }\end{array}$ & $\begin{array}{l}\text { Actual } \\
\text { Reaction }\end{array}$ & $\begin{array}{l}\text { Reaction } \\
\text { Time }\end{array}$ & $\begin{array}{l}\text { Pass / } \\
\text { Fail }\end{array}$ \\
\hline Test 1 & $\begin{array}{l}\text { Remain } \\
\text { Online }\end{array}$ & $\begin{array}{l}\text { Remain } \\
\text { Online }\end{array}$ & N/A & Pass \\
Test 2 & $\begin{array}{l}\text { Remain } \\
\text { Remain }\end{array}$ & N/A & Pass \\
Test 3 & $\begin{array}{l}\text { Online } \\
\text { Remain } \\
\text { Online }\end{array}$ & $\begin{array}{l}\text { Online } \\
\text { Remain } \\
\text { Online }\end{array}$ & N/A & Pass \\
\end{tabular}




\section{SUMMARY OF CBEMA/ITIC/SEMI VOLTAGE SAG/SURGE TESTS}

\begin{tabular}{|c|c|c|c|c|}
\hline Test 4 & Trip Open & Remain & $\mathrm{N} / \mathrm{A}$ & Fail \\
\hline & Trip Open & $\begin{array}{l}\text { Remain } \\
\text { Online }\end{array}$ & $\mathrm{N} / \mathrm{A}$ & Fail \\
\hline
\end{tabular}

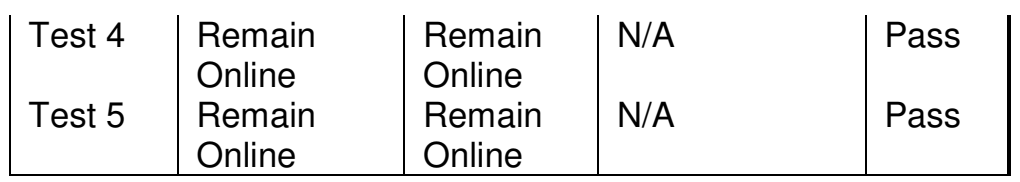

N/A ST DEV N/A

AVERAGE TRIP TIME

$N / A$

ST DEV

N/A

OVERVOLTAGE SAG 123\%, Three
Phase, 10 Cycles
\begin{tabular}{|l|l|l|l|l|}
\hline Name & $\begin{array}{l}\text { Anticipated } \\
\text { Reaction }\end{array}$ & $\begin{array}{l}\text { Actual } \\
\text { Reaction }\end{array}$ & $\begin{array}{l}\text { Reaction } \\
\text { Time }\end{array}$ & $\begin{array}{l}\text { Pass / } \\
\text { Fail }\end{array}$ \\
\hline Test 1 & Trip Open & $\begin{array}{l}\text { Remain } \\
\text { Online } \\
\text { Test } 2\end{array}$ & N/A & Fail \\
Test 3 & Trip Open & $\begin{array}{l}\text { Online } \\
\text { Temain } \\
\text { Online }\end{array}$ & N/A & Fail \\
Test 4 & Trip Open & $\begin{array}{l}\text { Remain } \\
\text { Online } \\
\text { Remain } \\
\text { Online }\end{array}$ & N/A & Fail \\
Test 5 & Trip Open & Fail \\
\hline
\end{tabular}

UNDERVOLTAGE SAG 0\%, Single

Phase A, 0.5 Cycle

\begin{tabular}{|c|c|c|c|c|}
\hline Name & $\begin{array}{l}\text { Anticipated } \\
\text { Reaction }\end{array}$ & $\begin{array}{l}\text { Actual } \\
\text { Reaction }\end{array}$ & $\begin{array}{l}\text { Reaction } \\
\text { Time }\end{array}$ & $\begin{array}{l}\text { Pass / } \\
\text { Fail }\end{array}$ \\
\hline Test 1 & $\begin{array}{l}\text { Remain } \\
\text { Online }\end{array}$ & $\begin{array}{l}\text { Remain } \\
\text { Online }\end{array}$ & $\mathrm{N} / \mathrm{A}$ & Pass \\
\hline Test 2 & $\begin{array}{l}\text { Remain } \\
\text { Online }\end{array}$ & $\begin{array}{l}\text { Remain } \\
\text { Online }\end{array}$ & $\mathrm{N} / \mathrm{A}$ & Pass \\
\hline Test 3 & $\begin{array}{l}\text { Remain } \\
\text { Online }\end{array}$ & $\begin{array}{l}\text { Remain } \\
\text { Online }\end{array}$ & $\mathrm{N} / \mathrm{A}$ & Pass \\
\hline Test 4 & $\begin{array}{l}\text { Remain } \\
\text { Online }\end{array}$ & $\begin{array}{l}\text { Remain } \\
\text { Online }\end{array}$ & $\mathrm{N} / \mathrm{A}$ & Pass \\
\hline Test 5 & $\begin{array}{l}\text { Remain } \\
\text { Online }\end{array}$ & $\begin{array}{l}\text { Remain } \\
\text { Online }\end{array}$ & $\mathrm{N} / \mathrm{A}$ & Pass \\
\hline
\end{tabular}

ST DEV N/A

AVERAGE TRIP TIME N/A

ST DEV N/A

UNDERVOLTAGE SAG 45\%, Single
Phase B, 20 Cycles
\begin{tabular}{|l|l|l|l|l|}
\hline Name & $\begin{array}{l}\text { Anticipated } \\
\text { Reaction }\end{array}$ & $\begin{array}{l}\text { Actual } \\
\text { Reaction }\end{array}$ & $\begin{array}{l}\text { Reaction } \\
\text { Time }\end{array}$ & $\begin{array}{l}\text { Pass / } \\
\text { Fail }\end{array}$ \\
\hline Test 1 & Trip Open & $\begin{array}{l}\text { Trip } \\
\text { Open } \\
\text { Trip } \\
\text { Open } 2\end{array}$ & 0.142 & Pass \\
Test 3 & Trip Open & 0.142 & Pass \\
Test 4 & Trip Open & $\begin{array}{l}\text { Trip } \\
\text { Open } \\
\text { Trip } \\
\text { Open } \\
\text { Trip } \\
\text { Open }\end{array}$ & 0.1405 & Pass \\
Test 5 & Trip Open & 0.1415 & Pass \\
\hline
\end{tabular}

UNDERVOLTAGE SAG $67 \%$, Single

Phase C, 40 Cycles

\begin{tabular}{|c|c|c|c|c|}
\hline Name & $\begin{array}{l}\text { Anticipated } \\
\text { Reaction }\end{array}$ & $\begin{array}{l}\text { Actual } \\
\text { Reaction }\end{array}$ & $\begin{array}{l}\text { Reaction } \\
\text { Time }\end{array}$ & $\begin{array}{l}\text { Pass / } \\
\text { Fail }\end{array}$ \\
\hline Test 1 & Trip Open & Trip Open & 0.18425 & Pass \\
\hline Test 2 & Trip Open & Trip Open & 0.185 & Pass \\
\hline Test 3 & Trip Open & Trip Open & 0.185 & Pass \\
\hline Test 4 & Trip Open & Trip Open & 0.1845 & Pass \\
\hline Test 5 & Trip Open & Trip Open & 0.185 & Pass \\
\hline
\end{tabular}




\section{SUMMARY OF CBEMA/ITIC/SEMI VOLTAGE SAG/SURGE TESTS}

AVERAGE TRIP TIME

0.1415 ST DEV $\quad 0.0006$

AVERAGE TRIP TIME

$0.1848 \quad$ ST DEV

0.0004

\begin{tabular}{|c|c|c|c|c|}
\hline \multicolumn{5}{|c|}{$\begin{array}{l}\text { UNDERVOLTAGE SAG } 77 \% \text {, Single } \\
\text { Phase A, } 45 \text { Cycles }\end{array}$} \\
\hline Name & $\begin{array}{l}\text { Anticipated } \\
\text { Reaction }\end{array}$ & $\begin{array}{l}\text { Actual } \\
\text { Reaction }\end{array}$ & $\begin{array}{l}\text { Reaction } \\
\text { Time }\end{array}$ & $\begin{array}{l}\text { Pass / } \\
\text { Fail }\end{array}$ \\
\hline Test 1 & Trip Open & $\begin{array}{l}\text { Trip } \\
\text { Open }\end{array}$ & 0.4375 & Pass \\
\hline Test 2 & Trip Open & $\begin{array}{l}\text { Trip } \\
\text { Open }\end{array}$ & 0.438 & Pass \\
\hline Test 3 & Trip Open & $\begin{array}{l}\text { Trip } \\
\text { Open }\end{array}$ & 0.437 & Pass \\
\hline Test 4 & Trip Open & $\begin{array}{l}\text { Trip } \\
\text { Open }\end{array}$ & 0.4375 & Pass \\
\hline Test 5 & Trip Open & $\begin{array}{l}\text { Trip } \\
\text { Open }\end{array}$ & 0.438 & Pass \\
\hline
\end{tabular}

\section{UNDERVOLTAGE SAG 83\%, Single}

Phase B, 70 Cycles

\begin{tabular}{|c|c|c|c|c|}
\hline Name & $\begin{array}{l}\text { Anticipated } \\
\text { Reaction }\end{array}$ & $\begin{array}{l}\text { Actual } \\
\text { Reaction }\end{array}$ & $\begin{array}{l}\text { Reaction } \\
\text { Time }\end{array}$ & $\begin{array}{l}\text { Pass / } \\
\text { Fail }\end{array}$ \\
\hline Test 1 & Remain & Remain & $\mathrm{N} / \mathrm{A}$ & Pass \\
\hline Test 2 & $\begin{array}{l}\text { Remain } \\
\text { Online }\end{array}$ & $\begin{array}{l}\text { Remain } \\
\text { Online }\end{array}$ & $\mathrm{N} / \mathrm{A}$ & Pass \\
\hline Test 3 & $\begin{array}{l}\text { Remain } \\
\text { Online }\end{array}$ & $\begin{array}{l}\text { Remain } \\
\text { Online }\end{array}$ & $\mathrm{N} / \mathrm{A}$ & Pass \\
\hline Test 4 & $\begin{array}{l}\text { Remain } \\
\text { Online }\end{array}$ & $\begin{array}{l}\text { Remain } \\
\text { Online }\end{array}$ & $\mathrm{N} / \mathrm{A}$ & Pass \\
\hline Test 5 & $\begin{array}{l}\text { Remain } \\
\text { Online }\end{array}$ & $\begin{array}{l}\text { Remain } \\
\text { Online }\end{array}$ & $\mathrm{N} / \mathrm{A}$ & Pass \\
\hline
\end{tabular}

\section{$\begin{array}{llll}\text { AVERAGE TRIP TIME } & 0.4376 & \text { ST DEV } & 0.0004\end{array}$}

\section{AVERAGE TRIP TIME N/A}

ST DEV N/A

UNDERVOLTAGE SAG 0\%, Single
Phase A, 2 Cycles
\begin{tabular}{|l|l|l|l|l|}
\hline Name & $\begin{array}{l}\text { Anticipated } \\
\text { Reaction }\end{array}$ & $\begin{array}{l}\text { Actual } \\
\text { Reaction }\end{array}$ & $\begin{array}{l}\text { Reaction } \\
\text { Time }\end{array}$ & $\begin{array}{l}\text { Pass / } \\
\text { Fail }\end{array}$ \\
\hline Test 1 & Trip Open & $\begin{array}{l}\text { Remain } \\
\text { Online } \\
\text { Test } 2\end{array}$ & N/A & Fail \\
Test 3 & Trip Open & $\begin{array}{l}\text { Remain } \\
\text { Online } \\
\text { Temain } \\
\text { Online }\end{array}$ & N/A & Fail \\
Test 4 & Trip Open & $\begin{array}{l}\text { Remain } \\
\text { Online } \\
\text { Remain } \\
\text { Online }\end{array}$ & N/A & Fail \\
Test 5 & Trip Open & Fail \\
\hline
\end{tabular}

\section{UNDERVOLTAGE SAG $0 \%$, Single}

Phase A, 3 Cycles

\begin{tabular}{|c|c|c|c|c|}
\hline Name & $\begin{array}{l}\text { Anticipated } \\
\text { Reaction }\end{array}$ & $\begin{array}{l}\text { Actual } \\
\text { Reaction }\end{array}$ & $\begin{array}{l}\text { Reaction } \\
\text { Time }\end{array}$ & $\begin{array}{l}\text { Pass / } \\
\text { Fail }\end{array}$ \\
\hline Test 1 & Trip Open & $\begin{array}{l}\text { Remain } \\
\text { Online }\end{array}$ & $\mathrm{N} / \mathrm{A}$ & Fail \\
\hline Test 2 & Trip Open & $\begin{array}{l}\text { Remain } \\
\text { Online }\end{array}$ & $N / A$ & Fail \\
\hline Test 3 & Trip Open & $\begin{array}{l}\text { Remain } \\
\text { Online }\end{array}$ & $N / A$ & Fail \\
\hline Test 4 & Trip Open & $\begin{array}{l}\text { Remain } \\
\text { Online }\end{array}$ & $N / A$ & Fail \\
\hline Test 5 & Trip Open & $\begin{array}{l}\text { Remain } \\
\text { Online }\end{array}$ & $N / A$ & Fail \\
\hline
\end{tabular}

AVERAGE TRIP TIME N/A NT DEV N/A

AVERAGE TRIP TIME N/A

ST DEV

$\mathrm{N} / \mathrm{A}$

UNDERVOLTAGE SAG 0\%, Single

Phase A, 4 Cycles

\section{UNDERVOLTAGE SAG 0\%, Single}

Phase A, 5 Cycles 


\section{SUMMARY OF CBEMA/ITIC/SEMI VOLTAGE SAG/SURGE TESTS}

\begin{tabular}{|c|c|c|c|c|}
\hline Name & $\begin{array}{l}\text { Anticipated } \\
\text { Reaction }\end{array}$ & $\begin{array}{l}\text { Actual } \\
\text { Reaction }\end{array}$ & $\begin{array}{l}\text { Reaction } \\
\text { Time }\end{array}$ & $\begin{array}{l}\text { Pass / } \\
\text { Fail }\end{array}$ \\
\hline Test 1 & Trip Open & $\begin{array}{l}\text { Remain } \\
\text { Online }\end{array}$ & $\mathrm{N} / \mathrm{A}$ & Fail \\
\hline Test 2 & Trip Open & $\begin{array}{l}\text { Remain } \\
\text { Online }\end{array}$ & $\mathrm{N} / \mathrm{A}$ & Fail \\
\hline Test 3 & Trip Open & $\begin{array}{l}\text { Remain } \\
\text { Online }\end{array}$ & $\mathrm{N} / \mathrm{A}$ & Fail \\
\hline Test 4 & Trip Open & $\begin{array}{l}\text { Remain } \\
\text { Online }\end{array}$ & $\mathrm{N} / \mathrm{A}$ & Fail \\
\hline Test 5 & Trip Open & $\begin{array}{l}\text { Remain } \\
\text { Online }\end{array}$ & $\mathrm{N} / \mathrm{A}$ & Fail \\
\hline
\end{tabular}

\begin{tabular}{|c|c|c|c|c|}
\hline Name & $\begin{array}{l}\text { Anticipated } \\
\text { Reaction }\end{array}$ & $\begin{array}{l}\text { Actual } \\
\text { Reaction }\end{array}$ & $\begin{array}{l}\text { Reaction } \\
\text { Time }\end{array}$ & $\begin{array}{l}\text { Pass / } \\
\text { Fail }\end{array}$ \\
\hline Test 1 & Trip Open & $\begin{array}{l}\text { Remain } \\
\text { Online }\end{array}$ & $\mathrm{N} / \mathrm{A}$ & Fail \\
\hline Test 2 & Trip Open & $\begin{array}{l}\text { Remain } \\
\text { Online }\end{array}$ & $\mathrm{N} / \mathrm{A}$ & Fail \\
\hline Test 3 & Trip Open & $\begin{array}{l}\text { Remain } \\
\text { Online }\end{array}$ & $\mathrm{N} / \mathrm{A}$ & Fail \\
\hline Test 4 & Trip Open & $\begin{array}{l}\text { Remain } \\
\text { Online }\end{array}$ & $\mathrm{N} / \mathrm{A}$ & Fail \\
\hline Test 5 & Trip Open & $\begin{array}{l}\text { Remain } \\
\text { Online }\end{array}$ & $\mathrm{N} / \mathrm{A}$ & Fail \\
\hline
\end{tabular}

AVERAGE TRIP TIME N/A ST DEV N/A

$\begin{array}{lll}\text { AVERAGE TRIP TIME N/A NT DEV N/A } & \text { S }\end{array}$

UNDERVOLTAGE SAG 0\%, Single

\section{Phase A, 6 Cycles}

\begin{tabular}{|l|l|l|l|l|}
\hline Name & $\begin{array}{l}\text { Anticipated } \\
\text { Reaction }\end{array}$ & $\begin{array}{l}\text { Actual } \\
\text { Reaction }\end{array}$ & $\begin{array}{l}\text { Reaction } \\
\text { Time }\end{array}$ & $\begin{array}{l}\text { Pass / } \\
\text { Fail }\end{array}$ \\
\hline Test 1 & Trip Open & $\begin{array}{l}\text { Remain } \\
\text { Online }\end{array}$ & N/A & Fail \\
Test 3 & Trip Open & $\begin{array}{l}\text { Remain } \\
\text { Online } \\
\text { Temain } \\
\text { Online }\end{array}$ & N/A & Fail \\
Test 4 & Trip Open & $\begin{array}{l}\text { Remain } \\
\text { Online } \\
\text { Remain } \\
\text { Online }\end{array}$ & N/A & Fail \\
Test 5 & Trip Open & Fail \\
\hline
\end{tabular}

AVERAGE TRIP TIME N/A ST DEV N/A

UNDERVOLTAGE SAG $0 \%$, Single
Phase A, 8 Cycles
\begin{tabular}{|l|l|l|l|l|}
\hline Name & $\begin{array}{l}\text { Anticipated } \\
\text { Reaction }\end{array}$ & $\begin{array}{l}\text { Actual } \\
\text { Reaction }\end{array}$ & $\begin{array}{l}\text { Reaction } \\
\text { Time }\end{array}$ & $\begin{array}{l}\text { Pass / } \\
\text { Fail }\end{array}$ \\
\hline Test 1 & Trip Open & $\begin{array}{l}\text { Trip } \\
\text { Open }\end{array}$ & 0.108 & Pass \\
\hline
\end{tabular}

UNDERVOLTAGE SAG 0\%, Single

Phase A, 7 Cycles

\begin{tabular}{|c|c|c|c|c|}
\hline Name & $\begin{array}{l}\text { Anticipated } \\
\text { Reaction }\end{array}$ & $\begin{array}{l}\text { Actual } \\
\text { Reaction }\end{array}$ & $\begin{array}{l}\text { Reaction } \\
\text { Time }\end{array}$ & $\begin{array}{l}\text { Pass / } \\
\text { Fail }\end{array}$ \\
\hline Test 1 & Trip Open & Trip Open & 0.108 & Pass \\
\hline Test 2 & Trip Open & Trip Open & 0.1075 & Pass \\
\hline Test 3 & Trip Open & Trip Open & 0.107 & Pass \\
\hline Test 4 & Trip Open & Trip Open & 0.1085 & Pass \\
\hline Test 5 & Trip Open & Trip Open & 0.1075 & Pass \\
\hline
\end{tabular}

\section{$\begin{array}{llll}\text { AVERAGE TRIP TIME } & 0.1077 & \text { ST DEV } & 0.0006\end{array}$}

UNDERVOLTAGE SAG 0\%, Single

Phase A, 9 Cycles

\begin{tabular}{|l|l|l|l|l|}
\hline Name & $\begin{array}{l}\text { Anticipated } \\
\text { Reaction }\end{array}$ & $\begin{array}{l}\text { Actual } \\
\text { Reaction }\end{array}$ & $\begin{array}{l}\text { Reaction } \\
\text { Time }\end{array}$ & $\begin{array}{l}\text { Pass / } \\
\text { Fail }\end{array}$ \\
\hline Test 1 & Trip Open & Trip Open & 0.108 & Pass
\end{tabular}


SUMMARY OF CBEMA/ITIC/SEMI VOLTAGE SAG/SURGE TESTS

\begin{tabular}{|l|l|l|r|l|} 
Test 2 & Trip Open & $\begin{array}{l}\text { Trip } \\
\text { Open }\end{array}$ & 0.107 & Pass \\
Test 3 & Trip Open & $\begin{array}{l}\text { Trip } \\
\text { Open } \\
\text { Trip }\end{array}$ & 0.1085 & Pass \\
Test 4 & Trip Open & $\begin{array}{l}\text { Open } \\
\text { Trip }\end{array}$ & 0.1075 & Pass \\
Open & Trip Open & $\begin{array}{l}\text { Pon } \\
\text { Test }\end{array}$ & Pass \\
\hline
\end{tabular}

\begin{tabular}{|l|l|l|l|l|} 
Test 2 & Trip Open & Trip Open & 0.108 & Pass \\
Test 3 & Trip Open & Trip Open & 0.108 & Pass \\
Test 4 & Trip Open & Trip Open & 0.108 & Pass \\
Test 5 & Trip Open & Trip Open & 0.109 & Pass \\
\hline
\end{tabular}

AVERAGE TRIP TIME $\quad 0.1081 \quad$ ST DEV 0.0010

$\begin{array}{llll}\text { AVERAGE TRIP TIME } & 0.1082 & \text { ST DEV } & 0.0004\end{array}$

\begin{tabular}{|c|c|c|c|c|}
\hline \multicolumn{5}{|c|}{$\begin{array}{l}\text { UNDERVOLTAGE SAG 0\%, Single } \\
\text { Phase A, } 10 \text { Cycles }\end{array}$} \\
\hline Name & $\begin{array}{l}\text { Anticipated } \\
\text { Reaction }\end{array}$ & $\begin{array}{l}\text { Actual } \\
\text { Reaction }\end{array}$ & $\begin{array}{l}\text { Reaction } \\
\text { Time }\end{array}$ & $\begin{array}{l}\text { Pass / } \\
\text { Fail }\end{array}$ \\
\hline Test 1 & Trip Open & $\begin{array}{l}\text { Trip } \\
\text { Open }\end{array}$ & 0.1085 & Pass \\
\hline Test 2 & Trip Open & $\begin{array}{l}\text { Trip } \\
\text { Open }\end{array}$ & 0.109 & Pass \\
\hline Test 3 & Trip Open & $\begin{array}{l}\text { Trip } \\
\text { Open }\end{array}$ & 0.1075 & Pass \\
\hline Test 4 & Trip Open & $\begin{array}{l}\text { Trip } \\
\text { Open }\end{array}$ & 0.107 & Pass \\
\hline Test 5 & Trip Open & $\begin{array}{l}\text { Trip } \\
\text { Open }\end{array}$ & 0.1075 & Pass \\
\hline
\end{tabular}

OVERVOLTAGE SAG 120\%, Three

Phase, 20 Cycles

\begin{tabular}{|c|c|c|c|c|}
\hline Name & $\begin{array}{l}\text { Anticipated } \\
\text { Reaction }\end{array}$ & $\begin{array}{l}\text { Actual } \\
\text { Reaction }\end{array}$ & $\begin{array}{l}\text { Reaction } \\
\text { Time }\end{array}$ & $\begin{array}{l}\text { Pass / } \\
\text { Fail }\end{array}$ \\
\hline Test 1 & Trip Open & $\begin{array}{l}\text { Remain } \\
\text { Online }\end{array}$ & $\mathrm{N} / \mathrm{A}$ & Fail \\
\hline Test 2 & Trip Open & $\begin{array}{l}\text { Remain } \\
\text { Online }\end{array}$ & $\mathrm{N} / \mathrm{A}$ & Fail \\
\hline Test 3 & Trip Open & $\begin{array}{l}\text { Remain } \\
\text { Online }\end{array}$ & $\mathrm{N} / \mathrm{A}$ & Fail \\
\hline Test 4 & Trip Open & $\begin{array}{l}\text { Remain } \\
\text { Online }\end{array}$ & $\mathrm{N} / \mathrm{A}$ & Fail \\
\hline Test 5 & Trip Open & $\begin{array}{l}\text { Remain } \\
\text { Online }\end{array}$ & $\mathrm{N} / \mathrm{A}$ & Fail \\
\hline
\end{tabular}

AVERAGE TRIP TIME

$0.1079 \quad$ ST DEV $\quad 0.0008$

AVERAGE TRIP TIME N/A

ST DEV N/A

OVERVOLTAGE SAG 121\%, Three
Phase, 20 Cycles
\begin{tabular}{|l|l|l|l|l|}
\hline Name & $\begin{array}{l}\text { Anticipated } \\
\text { Reaction }\end{array}$ & $\begin{array}{l}\text { Actual } \\
\text { Reaction }\end{array}$ & $\begin{array}{l}\text { Reaction } \\
\text { Time }\end{array}$ & $\begin{array}{l}\text { Pass / } \\
\text { Fail }\end{array}$ \\
\hline Test 1 & Trip Open & $\begin{array}{l}\text { Remain } \\
\text { Online } \\
\text { Remain }\end{array}$ & N/A & Fail \\
Test 2 & Trip Open & $\begin{array}{l}\text { Online } \\
\text { Remain } \\
\text { Online } \\
\text { Remain }\end{array}$ & N/A & Fail \\
Test 3 & Nrip Open & Fail \\
Test 4 & Trip Open & Fail
\end{tabular}

OVERVOLTAGE SAG 122\%, Three

Phase, 20 Cycles

\begin{tabular}{|c|c|c|c|c|}
\hline Name & $\begin{array}{l}\text { Anticipated } \\
\text { Reaction }\end{array}$ & $\begin{array}{l}\text { Actual } \\
\text { Reaction }\end{array}$ & $\begin{array}{l}\text { Reaction } \\
\text { Time }\end{array}$ & $\begin{array}{l}\text { Pass / } \\
\text { Fail }\end{array}$ \\
\hline Test 1 & Trip Open & $\begin{array}{l}\text { Remain } \\
\text { Online }\end{array}$ & $\mathrm{N} / \mathrm{A}$ & Fail \\
\hline Test 2 & Trip Open & $\begin{array}{l}\text { Remain } \\
\text { Online }\end{array}$ & $\mathrm{N} / \mathrm{A}$ & Fail \\
\hline Test 3 & Trip Open & $\begin{array}{l}\text { Remain } \\
\text { Online }\end{array}$ & $\mathrm{N} / \mathrm{A}$ & Fail \\
\hline Test 4 & Trip Open & Remain & $N / A$ & Fail \\
\hline
\end{tabular}


SUMMARY OF CBEMA/ITIC/SEMI VOLTAGE SAG/SURGE TESTS

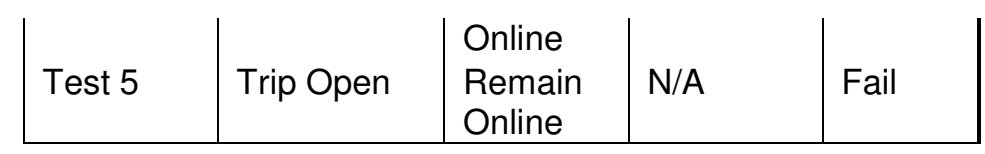

AVERAGE TRIP TIME N/A ST DEV N/A

OVERVOLTAGE SAG 123\%, Three

Phase, 20 Cycles

\begin{tabular}{|l|l|l|l|l|}
\hline Name & $\begin{array}{l}\text { Anticipated } \\
\text { Reaction }\end{array}$ & $\begin{array}{l}\text { Actual } \\
\text { Reaction }\end{array}$ & $\begin{array}{l}\text { Reaction } \\
\text { Time }\end{array}$ & $\begin{array}{l}\text { Pass / } \\
\text { Fail }\end{array}$ \\
\hline Test 1 & Trip Open & $\begin{array}{l}\text { Remain } \\
\text { Online } \\
\text { Rest } 2\end{array}$ & N/A & Fail \\
Trip Open & $\begin{array}{l}\text { Online } \\
\text { Online } \\
\text { Remain }\end{array}$ & N/A & Fail \\
Test 4 & Trip Open & $\begin{array}{l}\text { Online } \\
\text { Remain } \\
\text { Online } \\
\text { Trip Openain } \\
\text { Online }\end{array}$ & N/A & Fail \\
Test 5 & Trip Open & Fail \\
\hline
\end{tabular}

AVERAGE TRIP TIME

N/A

ST DEV

$\mathrm{N} / \mathrm{A}$

\begin{tabular}{|l|l|l|l|l|} 
Test 5 & Trip Open & $\begin{array}{l}\text { Online } \\
\text { Remain } \\
\text { Online }\end{array}$ & N/A & Fail \\
\hline
\end{tabular}

AVERAGE TRIP TIME N/A ST DEV N/A

\begin{tabular}{|c|c|c|c|c|}
\hline \multicolumn{5}{|c|}{$\begin{array}{l}\text { OVERVOLTAGE SAG 124\%, Three } \\
\text { Phase, } 20 \text { Cycles }\end{array}$} \\
\hline Name & $\begin{array}{l}\text { Anticipated } \\
\text { Reaction }\end{array}$ & $\begin{array}{l}\text { Actual } \\
\text { Reaction }\end{array}$ & $\begin{array}{l}\text { Reaction } \\
\text { Time }\end{array}$ & $\begin{array}{l}\text { Pass / } \\
\text { Fail }\end{array}$ \\
\hline Test 1 & Trip Open & $\begin{array}{l}\text { Remain } \\
\text { Online }\end{array}$ & $\mathrm{N} / \mathrm{A}$ & Fail \\
\hline Test 2 & Trip Open & $\begin{array}{l}\text { Remain } \\
\text { Online }\end{array}$ & $\mathrm{N} / \mathrm{A}$ & Fail \\
\hline Test 3 & Trip Open & $\begin{array}{l}\text { Remain } \\
\text { Online }\end{array}$ & $\mathrm{N} / \mathrm{A}$ & Fail \\
\hline Test 4 & Trip Open & $\begin{array}{l}\text { Remain } \\
\text { Online }\end{array}$ & $\mathrm{N} / \mathrm{A}$ & Fail \\
\hline Test 5 & Trip Open & $\begin{array}{l}\text { Remain } \\
\text { Online }\end{array}$ & $\mathrm{N} / \mathrm{A}$ & Fail \\
\hline
\end{tabular}

AVERAGE TRIP TIME N/A ST DEV N/A

\begin{tabular}{|c|c|c|c|c|}
\hline \multicolumn{5}{|c|}{$\begin{array}{l}\text { OVERVOLTAGE SAG 125\%, Three } \\
\text { Phase, } 20 \text { Cycles }\end{array}$} \\
\hline Name & $\begin{array}{l}\text { Anticipated } \\
\text { Reaction }\end{array}$ & $\begin{array}{l}\text { Actual } \\
\text { Reaction }\end{array}$ & $\begin{array}{l}\text { Reaction } \\
\text { Time }\end{array}$ & $\begin{array}{l}\text { Pass / } \\
\text { Fail }\end{array}$ \\
\hline Test 1 & Trip Open & $\begin{array}{l}\text { Remain } \\
\text { Online }\end{array}$ & $\mathrm{N} / \mathrm{A}$ & Fail \\
\hline Test 2 & Trip Open & $\begin{array}{l}\text { Remain } \\
\text { Online }\end{array}$ & $\mathrm{N} / \mathrm{A}$ & Fail \\
\hline Test 3 & Trip Open & $\begin{array}{l}\text { Remain } \\
\text { Online }\end{array}$ & $\mathrm{N} / \mathrm{A}$ & Fail \\
\hline Test 4 & Trip Open & $\begin{array}{l}\text { Remain } \\
\text { Online }\end{array}$ & $\mathrm{N} / \mathrm{A}$ & Fail \\
\hline Test 5 & Trip Open & $\begin{array}{l}\text { Remain } \\
\text { Online }\end{array}$ & $\mathrm{N} / \mathrm{A}$ & Fail \\
\hline
\end{tabular}

OVERVOLTAGE SAG 126\%, Three

Phase, 20 Cycles

\begin{tabular}{|c|c|c|c|c|}
\hline Name & $\begin{array}{l}\text { Anticipated } \\
\text { Reaction }\end{array}$ & $\begin{array}{l}\text { Actual } \\
\text { Reaction }\end{array}$ & $\begin{array}{l}\text { Reaction } \\
\text { Time }\end{array}$ & $\begin{array}{l}\text { Pass / } \\
\text { Fail }\end{array}$ \\
\hline Test 1 & Trip Open & $\begin{array}{l}\text { Remain } \\
\text { Online }\end{array}$ & $\mathrm{N} / \mathrm{A}$ & Fail \\
\hline Test 2 & Trip Open & $\begin{array}{l}\text { Remain } \\
\text { Online }\end{array}$ & $\mathrm{N} / \mathrm{A}$ & Fail \\
\hline Test 3 & Trip Open & $\begin{array}{l}\text { Remain } \\
\text { Online }\end{array}$ & $\mathrm{N} / \mathrm{A}$ & Fail \\
\hline Test 4 & Trip Open & $\begin{array}{l}\text { Remain } \\
\text { Online }\end{array}$ & $\mathrm{N} / \mathrm{A}$ & Fail \\
\hline Test 5 & Trip Open & $\begin{array}{l}\text { Remain } \\
\text { Online }\end{array}$ & $\mathrm{N} / \mathrm{A}$ & Fail \\
\hline
\end{tabular}




\section{SUMMARY OF CBEMA/ITIC/SEMI VOLTAGE SAG/SURGE TESTS}

$\begin{array}{lllllll}\text { AVERAGE TRIP TIME } & \text { N/A } & \text { ST DEV N/A } & \text { AVERAGE TRIP TIME } & \text { N/A } & \text { ST DEV N/A }\end{array}$

OVERVOLTAGE SAG 127\%, Three
Phase, 20 Cycles
\begin{tabular}{|l|l|l|l|l|}
\hline Name & $\begin{array}{l}\text { Anticipated } \\
\text { Reaction }\end{array}$ & $\begin{array}{l}\text { Actual } \\
\text { Reaction }\end{array}$ & $\begin{array}{l}\text { Reaction } \\
\text { Time }\end{array}$ & $\begin{array}{l}\text { Pass / } \\
\text { Fail }\end{array}$ \\
\hline Test 1 & Trip Open & $\begin{array}{l}\text { Remain } \\
\text { Online } \\
\text { Nemain }\end{array}$ & N/A & Fail \\
Test 2 & Trip Open & $\begin{array}{l}\text { Remain } \\
\text { Online } \\
\text { Remain } \\
\text { Online } \\
\text { Test } 3\end{array}$ & Trip Open & Fail \\
Test 4 & Trip Open & N/A & Fail \\
Test 5 & Trip Open & $\begin{array}{l}\text { Remain } \\
\text { Online }\end{array}$ & N/A & Fail \\
\hline
\end{tabular}

OVERVOLTAGE SAG 128\%, Three

Phase, 20 Cycles

\begin{tabular}{|c|c|c|c|c|}
\hline Name & $\begin{array}{l}\text { Anticipated } \\
\text { Reaction }\end{array}$ & $\begin{array}{l}\text { Actual } \\
\text { Reaction }\end{array}$ & $\begin{array}{l}\text { Reaction } \\
\text { Time }\end{array}$ & $\begin{array}{l}\text { Pass / } \\
\text { Fail }\end{array}$ \\
\hline Test 1 & Trip Open & Trip Open & 0.325 & Pass \\
\hline Test 2 & Trip Open & Trip Open & 0.326 & Pass \\
\hline Test 3 & Trip Open & Trip Open & 0.3245 & Pass \\
\hline Test 4 & Trip Open & Trip Open & 0.3245 & Pass \\
\hline Test 5 & Trip Open & Trip Open & 0.324 & Pass \\
\hline
\end{tabular}

AVERAGE TRIP TIME N/A ST DEV N/A

AVERAGE TRIP TIME $\quad 0.3248 \quad$ ST DEV $\quad 0.0008$

\begin{tabular}{|c|c|c|c|c|}
\hline \multicolumn{5}{|c|}{$\begin{array}{l}\text { OVERVOLTAGE SAG 129\%, Three } \\
\text { Phase, } 20 \text { Cycles }\end{array}$} \\
\hline Name & $\begin{array}{l}\text { Anticipated } \\
\text { Reaction }\end{array}$ & $\begin{array}{l}\text { Actual } \\
\text { Reaction }\end{array}$ & $\begin{array}{l}\text { Reaction } \\
\text { Time }\end{array}$ & $\begin{array}{l}\text { Pass / } \\
\text { Fail }\end{array}$ \\
\hline Test 1 & Trip Open & $\begin{array}{l}\text { Trip } \\
\text { Open }\end{array}$ & 0.3255 & Pass \\
\hline Test 2 & Trip Open & $\begin{array}{l}\text { Trip } \\
\text { Open }\end{array}$ & 0.3255 & Pass \\
\hline Test 3 & Trip Open & $\begin{array}{l}\text { Trip } \\
\text { Open }\end{array}$ & 0.3245 & Pass \\
\hline Test 4 & Trip Open & $\begin{array}{l}\text { Trip } \\
\text { Open }\end{array}$ & 0.3255 & Pass \\
\hline Test 5 & Trip Open & $\begin{array}{l}\text { Trip } \\
\text { Open }\end{array}$ & 0.3255 & Pass \\
\hline
\end{tabular}

OVERVOLTAGE SAG 130\%, Three

Phase, 20 Cycles

\begin{tabular}{|c|c|c|c|c|}
\hline Name & $\begin{array}{l}\text { Anticipated } \\
\text { Reaction }\end{array}$ & $\begin{array}{l}\text { Actual } \\
\text { Reaction }\end{array}$ & $\begin{array}{l}\text { Reaction } \\
\text { Time }\end{array}$ & $\begin{array}{l}\text { Pass / } \\
\text { Fail }\end{array}$ \\
\hline Test 1 & Trip Open & Trip Open & 0.3075 & Pass \\
\hline Test 2 & Trip Open & Trip Open & 0.3075 & Pass \\
\hline Test 3 & Trip Open & Trip Open & 0.309 & Pass \\
\hline Test 4 & Trip Open & Trip Open & 0.309 & Pass \\
\hline Test 5 & Trip Open & Trip Open & 0.3085 & Pass \\
\hline
\end{tabular}

AVERAGE TRIP TIME $\quad 0.3253 \quad$ ST DEV 0.0004

Table 5: Detailed Voltage Sag/Swell Test Results

$\begin{array}{llll}\text { AVERAGE TRIP TIME } & 0.3083 & \text { ST DEV } & 0.0008\end{array}$ 
PHASE A TO B, BOLTED, 300 Cycles

\begin{tabular}{|l|l|l|r|l|}
\hline Name & $\begin{array}{l}\text { Anticipated } \\
\text { Reaction }\end{array}$ & $\begin{array}{l}\text { Actual } \\
\text { Reaction }\end{array}$ & \multicolumn{1}{l|}{$\begin{array}{l}\text { Reaction } \\
\text { Time }\end{array}$} & $\begin{array}{l}\text { Pass } / \\
\text { Fail }\end{array}$ \\
\hline Test 1 & Trip Open & Trip Open & 0.0235 & Pass \\
Test 2 & Trip Open & Trip Open & 0.023 & Pass \\
Test 3 & Trip Open & Trip Open & 0.023 & Pass \\
Test 4 & Trip Open & Trip Open & 0.023 & Pass \\
Test 5 & Trip Open & Trip Open & 0.023 & Pass \\
\hline
\end{tabular}

AVERAGE TRIP TIME
PHASE A TO GROUND, BOLTED, 300 Cycles

\begin{tabular}{|l|l|l|r|l|}
\hline Name & $\begin{array}{l}\text { Anticipated } \\
\text { Reaction }\end{array}$ & $\begin{array}{l}\text { Actual } \\
\text { Reaction }\end{array}$ & \multicolumn{1}{l|}{ Reaction } & $\begin{array}{l}\text { Pass / } \\
\text { Time }\end{array}$ \\
\hline Test 1 & Trip Open & Trip Open & 0.022 & Pass \\
Test 2 & Trip Open & Trip Open & 0.021 & Pass \\
Test 3 & Trip Open & Trip Open & 0.021 & Pass \\
Test 4 & Trip Open & Trip Open & 0.022 & Pass \\
Test 5 & Trip Open & Trip Open & 0.021 & Pass \\
\hline
\end{tabular}

\section{AVERAGE TRIP TIME $\quad 0.0214 \quad$ ST DEV}

0.0005

\section{PHASE A TO B TO GROUND, BOLTED, 300 Cycles}

\begin{tabular}{|l|l|l|r|l|}
\hline Name & $\begin{array}{l}\text { Anticipated } \\
\text { Reaction }\end{array}$ & $\begin{array}{l}\text { Actual } \\
\text { Reaction }\end{array}$ & \multicolumn{1}{l|}{$\begin{array}{l}\text { Reaction } \\
\text { Time }\end{array}$} & $\begin{array}{l}\text { Pass / } \\
\text { Fail }\end{array}$ \\
\hline Test 1 & Trip Open & Trip Open & 0.021 & Pass \\
Test 2 & Trip Open & Trip Open & 0.023 & Pass \\
Test 3 & Trip Open & Trip Open & 0.024 & Pass \\
Test 4 & Trip Open & Trip Open & 0.0215 & Pass \\
Test 5 & Trip Open & Trip Open & 0.0225 & Pass \\
\hline
\end{tabular}

AVERAGE TRIP TIME
PHASE A TO B TO C TO GROUND, BOLTED, 300

Cycles

\begin{tabular}{|l|l|l|r|l|}
\hline Name & $\begin{array}{l}\text { Anticipated } \\
\text { Reaction }\end{array}$ & $\begin{array}{l}\text { Actual } \\
\text { Reaction }\end{array}$ & $\begin{array}{l}\text { Reaction } \\
\text { Time }\end{array}$ & $\begin{array}{l}\text { Pass / } \\
\text { Fail }\end{array}$ \\
\hline Test 1 & Trip Open & Trip Open & 0.0205 & Pass \\
Test 2 & Trip Open & Trip Open & 0.02 & Pass \\
Test 3 & Trip Open & Trip Open & 0.0225 & Pass \\
Test 4 & Trip Open & Trip Open & 0.022 & Pass \\
Test 5 & Trip Open & Trip Open & 0.021 & Pass \\
\hline
\end{tabular}

AVERAGE TRIP TIME

0.0212 ST DEV

0.0010
PHASE A TO B TO GROUND, HIGH IMPEDANCE, 300 Cycles

\begin{tabular}{|l|l|l|r|l|}
\hline Name & $\begin{array}{l}\text { Anticipated } \\
\text { Reaction }\end{array}$ & $\begin{array}{l}\text { Actual } \\
\text { Reaction }\end{array}$ & \multicolumn{1}{l|}{$\begin{array}{l}\text { Reaction } \\
\text { Time }\end{array}$} & $\begin{array}{l}\text { Pass / } \\
\text { Fail }\end{array}$ \\
\hline Test 1 & Trip Open & Trip Open & 0.0235 & Pass \\
Test 2 & Trip Open & Trip Open & 0.024 & Pass \\
Test 3 & Trip Open & Trip Open & 0.024 & Pass \\
Test 4 & Trip Open & Trip Open & 0.0235 & Pass \\
Test 5 & Trip Open & Trip Open & 0.0225 & Pass \\
\hline
\end{tabular}

$\begin{array}{llll}\text { AVERAGE TRIP TIME } & 0.0235 & \text { ST DEV } & 0.0006\end{array}$
PHASE A TO B TO C TO GROUND, HIGH IMPEDANCE, 300 Cycles

\begin{tabular}{|l|l|l|r|l|}
\hline Name & $\begin{array}{l}\text { Anticipated } \\
\text { Reaction }\end{array}$ & $\begin{array}{l}\text { Actual } \\
\text { Reaction }\end{array}$ & \multicolumn{1}{l|}{$\begin{array}{l}\text { Reaction } \\
\text { Time }\end{array}$} & $\begin{array}{l}\text { Pass / } \\
\text { Fail }\end{array}$ \\
\hline Test 1 & Trip Open & Trip Open & 0.0205 & Pass \\
Test 2 & Trip Open & Trip Open & 0.022 & Pass \\
Test 3 & Trip Open & Trip Open & 0.021 & Pass \\
Test 4 & Trip Open & Trip Open & 0.0215 & Pass \\
Test 5 & Trip Open & Trip Open & 0.022 & Pass \\
\hline
\end{tabular}

$\begin{array}{llll}\text { AVERAGE TRIP TIME } & 0.0214 & \text { ST DEV } & 0.0007\end{array}$ 
SUMMARY OF OPEN PHASE TESTS

OPEN PHASE A IN DELTA-DELTA FEED, 300

\begin{tabular}{|c|c|c|c|c|}
\hline Name & $\begin{array}{l}\text { Anticipated } \\
\text { Reaction }\end{array}$ & $\begin{array}{l}\text { Actual } \\
\text { Reaction }\end{array}$ & $\begin{array}{l}\text { Reaction } \\
\text { Time }\end{array}$ & $\begin{array}{l}\text { Pass / } \\
\text { Fail }\end{array}$ \\
\hline Test 1 & Trip Open & $\begin{array}{l}\text { Trip } \\
\text { Open }\end{array}$ & 0.158 & Pass \\
\hline Test 2 & Trip Open & $\begin{array}{l}\text { Trip } \\
\text { Open }\end{array}$ & 0.1585 & Pass \\
\hline Test 3 & Trip Open & $\begin{array}{l}\text { Trip } \\
\text { Open }\end{array}$ & 0.159 & Pass \\
\hline Test 4 & Trip Open & $\begin{array}{l}\text { Trip } \\
\text { Open }\end{array}$ & 0.1575 & Pass \\
\hline Test 5 & Trip Open & $\begin{array}{l}\text { Trip } \\
\text { Open }\end{array}$ & 0.158 & Pass \\
\hline
\end{tabular}

AVERAGE TRIP TIME $\quad 0.1582 \quad$ ST DEV 0.0006

\begin{tabular}{|l|l|l|r|l|}
\hline PHASE A AND B IN WYE SECONDARY FEED, 300 Cycles \\
\hline Name & $\begin{array}{l}\text { Anticipated } \\
\text { Reaction }\end{array}$ & $\begin{array}{l}\text { Actual } \\
\text { Reaction }\end{array}$ & $\begin{array}{l}\text { Reaction } \\
\text { Time }\end{array}$ & $\begin{array}{l}\text { Pass / } \\
\text { Fail }\end{array}$ \\
\hline Test 1 & Trip Open & $\begin{array}{l}\text { Trip } \\
\text { Open }\end{array}$ & 0.107 & Pass \\
Test 2 & Trip Open & $\begin{array}{l}\text { Trip } \\
\text { Open } \\
\text { Trip }\end{array}$ & 0.1085 & Pass \\
Test 4 & Trip Open & $\begin{array}{l}\text { Open } \\
\text { Trip } \\
\text { Open } \\
\text { Trip } \\
\text { Open }\end{array}$ & 0.108 & Pass \\
Test 5 & Trip Open & 0.1065 & Pass \\
\hline
\end{tabular}

AVERAGE TRIP TIME $\quad 0.1075 \quad$ ST DEV 0.0008

Table 6: Detailed Simulated Fault Test Results
OPEN PHASE A IN WYE SECONDARY FEED, 300

\begin{tabular}{|c|c|c|c|c|}
\hline Name & $\begin{array}{l}\text { Anticipated } \\
\text { Reaction }\end{array}$ & $\begin{array}{l}\text { Actual } \\
\text { Reaction }\end{array}$ & $\begin{array}{l}\text { Reaction } \\
\text { Time }\end{array}$ & $\begin{array}{l}\text { Pass / } \\
\text { Fail }\end{array}$ \\
\hline Test 1 & Trip Open & Trip Open & 0.107 & Pass \\
\hline Test 2 & Trip Open & Trip Open & 0.108 & Pass \\
\hline Test 3 & Trip Open & Trip Open & 0.108 & Pass \\
\hline Test 4 & Trip Open & Trip Open & 0.107 & Pass \\
\hline Test 5 & Trip Open & Trip Open & 0.107 & Pass \\
\hline
\end{tabular}

$\begin{array}{llll}\text { AVERAGE TRIP TIME } & 0.1074 & \text { ST DEV } & 0.0005\end{array}$ 
SUMMARY OF SIMULATED FAULT TESTS

LIGHTNING SURGE 1.2-50us

\begin{tabular}{|c|c|c|c|c|}
\hline Name & $\begin{array}{l}\text { Anticipated } \\
\text { Reaction }\end{array}$ & $\begin{array}{l}\text { Actual } \\
\text { Reaction }\end{array}$ & $\begin{array}{l}\text { Reaction } \\
\text { Time }\end{array}$ & $\begin{array}{l}\text { Pass / } \\
\text { Fail }\end{array}$ \\
\hline Test 1 & ??? & $\begin{array}{l}\text { Remain } \\
\text { Online }\end{array}$ & $\mathrm{N} / \mathrm{A}$ & $\mathrm{N} / \mathrm{A}$ \\
\hline Test 2 & ??? & $\begin{array}{l}\text { Remain } \\
\text { Online }\end{array}$ & $\mathrm{N} / \mathrm{A}$ & $\mathrm{N} / \mathrm{A}$ \\
\hline Test 3 & ??? & $\begin{array}{l}\text { Remain } \\
\text { Online }\end{array}$ & $\mathrm{N} / \mathrm{A}$ & $\mathrm{N} / \mathrm{A}$ \\
\hline Test 4 & ??? & $\begin{array}{l}\text { Remain } \\
\text { Online }\end{array}$ & $\mathrm{N} / \mathrm{A}$ & $\mathrm{N} / \mathrm{A}$ \\
\hline Test 5 & ??? & $\begin{array}{l}\text { Remain } \\
\text { Online }\end{array}$ & $\mathrm{N} / \mathrm{A}$ & $\mathrm{N} / \mathrm{A}$ \\
\hline
\end{tabular}

900 MVAR CAPACITOR SWITCH

\begin{tabular}{|l|l|l|l|l|} 
Name & $\begin{array}{l}\text { Anticipated } \\
\text { Reaction }\end{array}$ & $\begin{array}{l}\text { Actual } \\
\text { Reaction }\end{array}$ & $\begin{array}{l}\text { Reaction } \\
\text { Time }\end{array}$ & $\begin{array}{l}\text { Pass / } \\
\text { Fail }\end{array}$ \\
\hline Test 1 & ??? & Remain Online & N/A & N/A \\
Test 2 & ??? & Remain Online & N/A & N/A \\
Test 3 & ??? & Remain Online & N/A & N/A \\
Test 4 & ??? & Remain Online & N/A & N/A \\
Test 5 & ??? & Remain Online & N/A & N/A \\
\hline
\end{tabular}

AVERAGE TRIP TIME

$\mathrm{N} / \mathrm{A}$

ST DEV N/A

AVERAGE TRIP TIME

$\mathrm{N} / \mathrm{A}$

ST DEV N/A

\section{MOTOR START NON-CBEMA/ITIC/SEMI VIOLATION}

\begin{tabular}{|l|l|l|r|l|}
\hline Name & $\begin{array}{l}\text { Anticipated } \\
\text { Reaction }\end{array}$ & $\begin{array}{l}\text { Actual } \\
\text { Reaction }\end{array}$ & $\begin{array}{l}\text { Reaction } \\
\text { Time }\end{array}$ & $\begin{array}{l}\text { Pass / } \\
\text { Fail }\end{array}$ \\
\hline Test 1 & $? ? ?$ & Trip Open & 0.476 & Pass \\
Test 2 & ??? & Trip Open & 0.475 & Pass \\
Test 3 & ??? & Trip Open & 0.4745 & Pass \\
Test 4 & $? ? ?$ & Trip Open & 0.474 & Pass \\
Test 5 & $? ? ?$ & Trip Open & 0.475 & Pass \\
\hline
\end{tabular}

MOTOR START CBEMA/ITIC/SEMI VIOLATION
\begin{tabular}{|l|l|l|r|l|}
\hline Name & $\begin{array}{l}\text { Anticipated } \\
\text { Reaction }\end{array}$ & $\begin{array}{l}\text { Actual } \\
\text { Reaction }\end{array}$ & $\begin{array}{l}\text { Reaction } \\
\text { Time }\end{array}$ & $\begin{array}{l}\text { Pass } / \\
\text { Fail }\end{array}$ \\
\hline Test 1 & Trip Open & Trip Open & 0.1925 & Pass \\
Test 2 & Trip Open & Trip Open & 0.1925 & Pass \\
Test 3 & Trip Open & Trip Open & 0.191 & Pass \\
Test 4 & Trip Open & Trip Open & 0.1925 & Pass \\
Test 5 & Trip Open & Trip Open & 0.1905 & Pass \\
\hline
\end{tabular}

\section{AVERAGE TRIP TIME}

$0.4749 \quad$ ST DEV $\quad 0.0007$

AVERAGE TRIP TIME

0.1918 ST DEV 0.0010

SIX PULSE BRIDGE
LOAD
\begin{tabular}{|l|l|l|l|l|}
\hline Name & $\begin{array}{l}\text { Anticipated } \\
\text { Reaction }\end{array}$ & $\begin{array}{l}\text { Actual } \\
\text { Reaction }\end{array}$ & $\begin{array}{l}\text { Reaction } \\
\text { Time }\end{array}$ & $\begin{array}{l}\text { Pass / } \\
\text { Fail }\end{array}$ \\
\hline Test 1 & $? ? ?$ & $\begin{array}{l}\text { Remain } \\
\text { Online }\end{array}$ & N/A & N/A \\
Test 2 & $? ? ?$ & $\begin{array}{l}\text { Remain } \\
\text { Online } \\
\text { Remain } \\
\text { Online }\end{array}$ & N/A & N/A \\
Test 3 & $? ? ?$ & N/A & N/A \\
\hline
\end{tabular}

HARMONIC DISTORTION

\begin{tabular}{|l|l|l|l|l|}
\hline Name & $\begin{array}{l}\text { Anticipated } \\
\text { Reaction }\end{array}$ & $\begin{array}{l}\text { Actual } \\
\text { Reaction }\end{array}$ & $\begin{array}{l}\text { Reaction } \\
\text { Time }\end{array}$ & $\begin{array}{l}\text { Pass / } \\
\text { Fail }\end{array}$ \\
\hline Test 1 & ??? & Remain Online & N/A & N/A \\
Test 2 & ??? & Remain Online & N/A & N/A \\
Test 3 & ??? & Remain Online & N/A & N/A
\end{tabular}


SUMMARY OF SIMULATED FAULT TESTS

\begin{tabular}{|l|l|l|l|l|} 
Test 4 & $? ? ?$ & $\begin{array}{l}\text { Remain } \\
\text { Online } \\
\text { Remain } \\
\text { Online }\end{array}$ & N/A & N/A \\
Test 5 & $? ? ?$ & N/A \\
\hline
\end{tabular}

\begin{tabular}{|l|l|l|l|l|} 
Test 4 & ??? & Remain Online & N/A & N/A \\
Test 5 & ??? & Remain Online & N/A & N/A \\
\hline
\end{tabular}

\section{AVERAGE TRIP TIME N/A \\ ST DEV N/A}

AVERAGE TRIP TIME

$\mathrm{N} / \mathrm{A}$

ST DEV N/A

\section{Table 7: Detailed Miscellaneous Test Results}

Notes

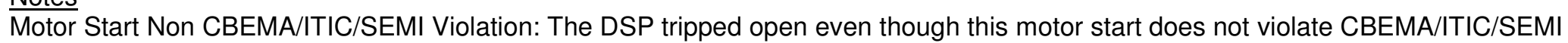
Curves

Motor Start CBEMA/ITIC/SEMI Violation: The DSP properly tripped open and reclosed after the event, but then tripped open again for unknown reasons 


\begin{tabular}{|l|l|l|l|}
\hline \multicolumn{3}{|c|}{ DTC Registered Test Procedure } \\
CERTS Microgrid Static Switch Testing \\
\hline Dolan Technology Center & Document No: DTC212812.206 \\
\hline Written by: David A. Klapp & Effective Date: April 04, 2006 & Target Group: Assigned \\
\hline Approved by: K. P. Loving, P.E. & Procedure Review Date: As Needed & \\
\hline
\end{tabular}

\begin{tabular}{|l|c|lc|}
\hline Revision \# & Date & \multicolumn{1}{c|}{ Revision Description } \\
\hline 0 & $04 / 04 / 06$ & Original \\
\hline & & & \\
\hline
\end{tabular}

Team Members and Roles

\begin{tabular}{|c|c|c|c|c|c|}
\hline 3.0 & Name & 4.0 & Group & 5.0 & Primary Roles \\
\hline David Klapp & & Operations & & \multicolumn{2}{|c|}{ Test Engineer } \\
\hline Kevin Loving & & Operations & & \multicolumn{2}{|c|}{ Test Coordinator } \\
\hline CERTS & & CERTS & & \multicolumn{2}{|l|}{ Sponsor } \\
\hline
\end{tabular}

\subsection{Purpose, References, Definitions}

Purpose: The purpose of this document is to define the procedure for testing the CERTS Microgrid Static Switch DSP and SEL-351 Relay for detection and reaction to various power quality events and simulated faults (see Figure 5).

This procedure defines the functional requirements for testing and documenting the results.

Criterion: The CERTS Microgrid Static Switch DSP or SEL-351 Relay should correctly identify and respond to predetermined power quality events and simulated faults, demonstrating its ability to island the Microgrid and properly protect the load.

Materials to be used include, but are not limited to; an Manta MTS 1710 relay test set, an Elgar SW 5250A Smart Wave, an Elgar SS-66 3-phase power supply, a 480 volt 3phase variac, and a Wavetek Datron 195 Universal Waveform Generator.

Definition: The CERTS Microgrid Static Switch DSP and SEL-351 Relay located in Cabinet 2a.

This document contains information confidential and proprietary to AEPSC. It shall not be reproduced in whole or in part or released to any third party without expressed written consent of AEPSC 
Reference documents:

DTC Standard Operating Procedures

SOP - Barricades, Barriers and Guards, 204-DTC-011

SOP - Personal Protective Equipment, 204-DTC-032

SOP - Job Safety Analysis, 204-DTC-070

\subsection{Responsibilities}

Scheduling:

Test Coordinator

Parameter Specification: Sponsor, Test Engineer

Setup: $\quad$ Test Engineer

Execution: Test Engineer

Report: $\quad$ Test Engineer

2.1 The Test Engineer shall comply with all the appropriate DTC operating procedures that are listed above in Clause 1.6.

2.2 The Test Engineer in charge during a test must be familiar with the test plan.

2.3 All persons who are directly involved during the execution of the tests shall wear the appropriate personal protective equipment.

2.4 The Test Coordinator shall ensure the required qualifications of all participants are met.

2.5 The Test Coordinator shall be responsible for approving this procedure and any revisions to it.

\subsection{Procedure}

General Safety Requirements

Testing in Test Cell A shall be in accordance with:

SOP 204-DTC-011, Barricades, Barriers and Guards.

SOP 204-DTC-032, Personal Protective Equipment.

The proper operation of any associated test equipment based on the manufacturer's operator's manual.

A Job Safety Briefing shall be performed prior to testing.

\section{Test Setup}

This document contains information confidential and proprietary to AEPSC. It shall not be reproduced in whole or in part or released to any third party without expressed written consent of AEPSC 


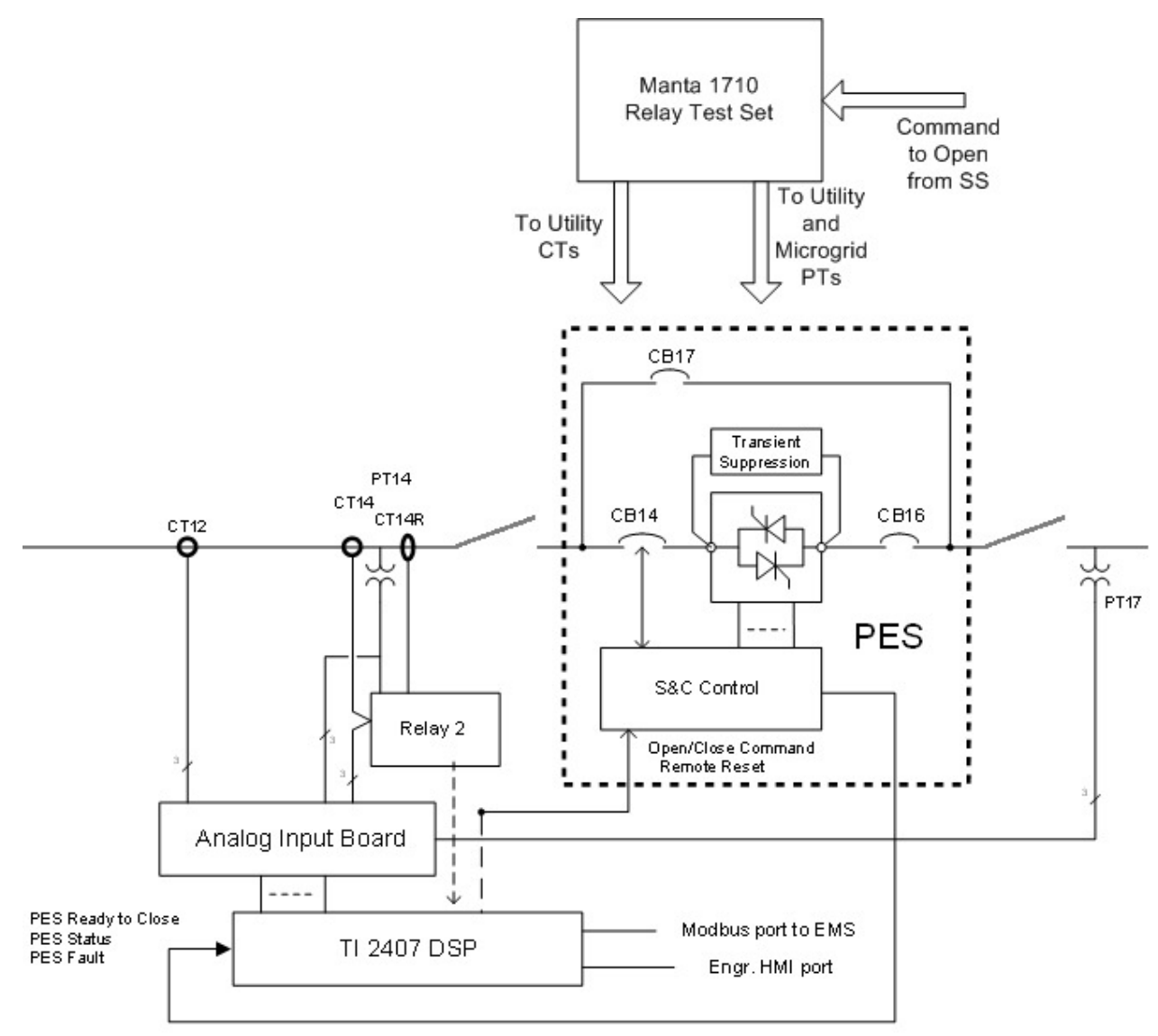

Figure 5: Test setup for Power Quality and Simulated Fault Testing.

All testing will be preformed at low power to reduce the possibility of damage to the SS and test equipment.

The SS should be assembled and connected to all of the appropriate control cabinets as needed for functionality.

The measurement PTs and CTs of the SS will be disconnected and the Manta relay test set will be connected in their place.

The reverse power relay will either be disabled or will be connected to a source to simulate continuous power import.

Test Procedure

The Manta will be configured to deliver the same stimulus to both sides of the SS.

Each individual test will consist of three parts, a pre-fault, a fault, and a postfault. Note: the word "fault" as referred to above means the condition of abnormality under test. This is not solely limited to electrical faults but also includes the power quality events of interest.

This document contains information confidential and proprietary to AEPSC. It shall not be reproduced in whole or in part or released to any third party without expressed written consent of AEPSC 
The pre-fault configuration which will lie within the normal operation conditions where the Microgrid can be connected to the utility.

The fault configuration will consist of the desired power quality or simulated fault event

The post-fault configuration will return to the normal operation conditions where the Microgrid is connected to the utility.

The Manta will be set to the pre-fault configuration and the SS will be commanded closed.

The Manta will then be triggered into the fault configuration.

Once the SS responds to the fault configuration the Manta move to the post-fault configuration.

The SS response to the fault and the time for the response will be recorded in the results form, CERTS Static Switch PQ Results.xls.

This testing will not be concerned with whether or not the SS resynchronizes with the utility source once the Manta is in post-fault configuration.

The power quality and simulated fault tests in CERTS Static Switch PQ Tests.doc will be performed.

All results shall be recorded in the results form, CERTS Static Switch PQ

Results.xls.

\subsection{Hazards \& Mitigation}

Hazards that exist during testing of the "Purple Power Cleaner" include dangerous voltage levels and electrical flashover. These hazards are mitigated by the following measures:

Compliance with all applicable SOP's, including

204-DTC-11 Barricades, Barriers, and Guards

204-DTC-32 Personal Protective Equipment

204-DTC-70 Job Safety Analysis

\subsection{Quality Assurance}

Quality assurance is maintained by ensuring that:

The measurement equipment used is within the specified calibration interval.

Measurement uncertainty is appropriate to the needs of the test.

All appropriate information on the test results form is filled in.

All setup parameters, calculations and assumptions are fully documented.

This document contains information confidential and proprietary to AEPSC. It shall not be reproduced in whole or in part or released to any third party without expressed written consent of AEPSC 


\section{CERTS MICROGRID LABORATORY TEST BED}

\section{CERTS Test Bed Design and Commissioning Lessons Learned Summary}

Prepared For:

California Energy Commission

Public Interest Energy Research Program

Arnold Schwarzenegger

Governor

$\frac{I}{x}$

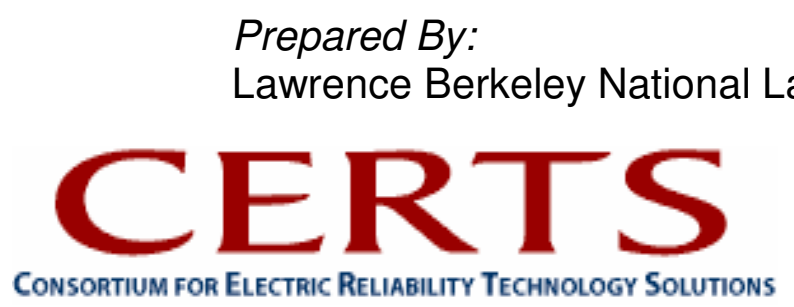



Prepared By:

Lawrence Berkeley National Laboratory

Joseph H. Eto, Principal Investigator

Berkeley, CA 94720

Ed Linton and Hector Hurtado, Northern Power Systems

Harry Volkommer and Dave Klapp, American Electric Power

Commission Contract No. 500-03-024

Prepared For:

Public Interest Energy Research (PIER)

California Energy Commission

Bernard Treanton

Contract Manager

Mike Gravely

Program Area Lead

ENERGY SYSTEMS INTEGRATION

Mike Gravely

Office Manager

ENERGY SYSTEMS RESEARCH

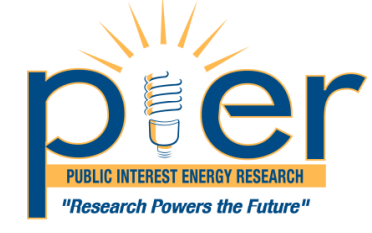

Martha Krebs, Ph.D.

PIER Director

Thom Kelly, Ph.D.

Deputy Director

ENERGY RESEARCH \& DEVELOPMENT DIVISION

Melissa Jones

Executive Director

\section{DISCLAIMER}

This report was prepared as the result of work sponsored by the California Energy Commission. It does not necessarily represent the views of the Energy Commission, its employees or the State of California. The Energy Commission, the State of California, its employees, contractors and subcontractors make no warrant, express or implied, and assume no legal liability for the information in this report; nor does any party represent that the uses of this information will not infringe upon privately owned rights. This report has not been approved or disapproved by the California Energy Commission nor has the California Energy Commission passed upon the accuracy or adequacy of the information in this report. 



\section{CERTS Test Bed \\ Design and Commissioning Lessons Learned Summary}

\section{Date: December 11, 2006}

This document is intended to summarize some of the most important issues that were found during the design stage of the Testbed as well as during commissioning at the AEP site. The document includes a range of issues that go from safety, control, protection and data acquisition; the correspondent solutions implemented are also described. Safety Review recommendations were obtained via outside safety review of the design by Jim Daley, P.E., DGCP

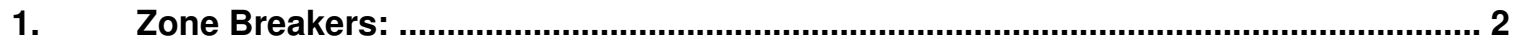

2. Other Safety Review Issues: .................................................................................. 3

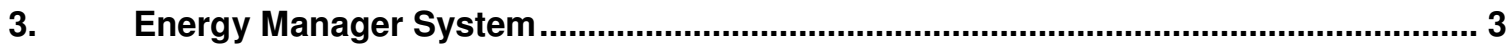

4. Load Control Software and Zone/Fault Loads............................................................ 4

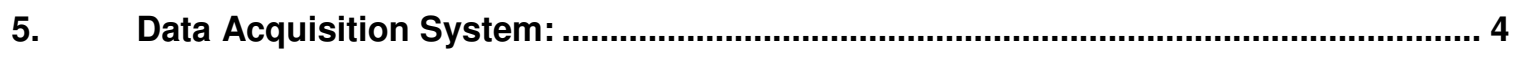

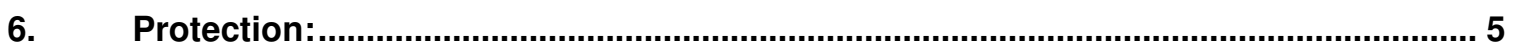

7. S\&C Power Electronic Switch (PES) …............................................................. 5

8. TECOGEN Engine Control-Communications ..................................................... 6 


\section{Zone Breakers:}

Initially, Molded-Case circuit breakers (ABB Isomax) were selected based on the CERTS Testbed criteria. However, due to the multiple fault testing that is expected for this project, these breakers were replaced in the design with Insulated-Case breakers (ABB Emax). Molded-Case breakers are not adequate for the following reasons:

a. They are not built to withstand repeated fault currents. Expected life would be 2 or 3 faults and they would have to be replaced. The molded-case design is not conducive to inspection and maintenance between test runs. Subsequent failures could be explosive and could damage other equipment.

b. Nuisance trips can be expected at $\sim 10$ times the continuous current rating due to the magnetic release mechanism in spite of the rapid static switch opening. This may interfere with testing the intended protection scheme. For example, in the current CERTS Tesbed design Strong Grid Case, the fault current in Zone 2 (just downstream of the Static Switch) was calculated to be $\sim 8000$ A sym. Zone Breaker CB31 would require a continuous rating larger than $800 \mathrm{~A}$. ABB Isomax $S 7$ would have meet that requirement ( $\mathrm{In}=1200 \mathrm{~A}$; S6 would have been borderline $\mathrm{In}=800 \mathrm{~A}$ ); however, the $\mathrm{CT}$ ratings and microprocessor unit available in $\mathrm{S} 7$ would only allow for a minimum overload setting of $400 \mathrm{~A}$, which is much larger than the rated load in Zone $3(\sim 225 \mathrm{~A})$ so proper time-overcurrent protection wouldn't be possible. For CERTS Testbed testing this issue could be avoided by using the external protection CTs and SEL-351 Relays available at each zone, but for a commercial design or the next phase of CERTS testing (where these relays and CTs will not be present) careful evaluation of the rated load and fault currents of each zone breaker would be required to verify is Molded-Case breakers are appropriate.

Insulated-Case breakers were recommended for the following reasons:

a. They can handle much higher fault currents without damage

b. They can be more easily inspected and rebuilt.

c. They can stand much higher short time (6 to 18 cycles) currents without tripping, allowing the static switch time to act.

Other recommendations that were provided in the Safety Review include: 


\section{Other Safety Review Issues:}

Fault contactors (K22, K32, K42, K52, K62): should be insulated-case circuit breakers with latching spring driven actuators. A common contactor may not be able to stay closed under fault currents due to high magnetic fields. This recommendation has been implemented in the CERTS Testbed.

Cable trays are preferred over conduit. The insulation on cable in conduit may be worn and may fail due to conductor whip under fault conditions and resulting magnetic forces. Conductors should be tied down on every rung of the cable trays. On the longer power cables, outbound conductor runs should be separated from returns to mitigate magnetic forces at fault current levels. Phase and neutral conductors should be tightly bundled.

AEP reviewed this safety comment and decided that significant forces are not expected, so in the present CERTS Testbed, conduit was used.

Power conductor termination lugs should be crimp type and not mechanical type. This recommendation has been implemented in the CERTS Testbed.

\section{Energy Manager System}

AEP made several recommendations that were implemented to the Energy Manager System (EMS) program that includes the following:

a. In the System Overview Display, status (Open/Closed) of all zone breakers was added to the already present information (Static Switch Status, power flows, etc).

b. Remote Operation of Breaker CB12 was added to the Energy Manager System; this breaker is used to backup the rest of the system and also its disconnection provides a testing condition for reverse power alarms. This option was added to avoid the operator from having to open CB12 locally.

c. Emergency Stop was added to the EMS. Every tab (window) in the EMS includes and E-Stop button that remotely shuts down every microsource and also opens CB12 to disconnect the microgrid from the utility. 


\section{Load Control Software and Zone/Fault Loads}

a. The Load Control Labview Software initialization was modified so that upon initialization (Powering up computer/ Starting Program) all initial settings that are displayed in the screen are uploaded from the PLCs or DSP boards (this fix was also implemented in the EMS software). Before this correction, if the computers were restarted, the programs will be showing initial values that may not match the current controls, thus providing a confusing picture to the operator.

b. Remote Operation of Breaker CB12 was added to the Energy Manager System; this breaker is used to backup the rest of the system and also its disconnection provides a testing condition for reverse power alarms. This option was added to avoid the operator from having to open CB12 locally.

c. AEP recommended that an Emergency Stop be added to the EMS. Presently, every tab (window) in the EMS includes and E-Stop button that remotely shuts down every microsource and also opens CB12 to disconnect the microgrid from the utility.

d. A Load Bank as originally designed had temperature issues when running at full load. Post-Glover recommended the addition of ventilation on top of the load bank to improve the airflow.

\section{Data Acquisition System:}

a. PML ION 7650 Meters are used as the data acquisition equipment at every important location of the Testbed (Zones, Loads, Static Switch). These meters have fast sampling (up to 3 cycles 1024 samples/cycle; 96 cycles at 16 samples/cycles). It also includes a 1cycle-RMS calculation for the variables that are required to be monitored in CERTS (Real Power, Reactive Power, Voltage, Current, Frequency). Initially during commissioning, several data collection tests were performed with waveform resolution of 128 samples/cycle and 10second duration RMS calculations at 1cycle sampling. The waveform collection was optimum; however, the RMS collection had issues because the ION 7650 does not include a pre-trigger option for this type of calculation (which the waveform collection does). This is an important requirement since it is useful to know the initial state of the system before step loads or switch transitions occur in order to understand the response of the inverter-gensets. Several solutions were attempted, such as implementing pre-triggering signals directly from the EMS-DAS Labview programs, however, this provided 
mismatching between the starting times of the waveform and RMS data, which added confusion in interpreting the data. Also, with this strategy, a Software "Manual Trigger" from the operator would have been required, thus adding further complication to the system.

b. To eliminate this problem, the approach taken was to use the meters only as a front-end data acquisition system (waveform data collection) and allow external software to download the data and process it and perform the RMS calculation. By taking this approach, any pretriggering mismatches are eliminated (every meter gets the same trigger signal, and each meter has a \#cycles pre-trigger setting). PQView, software developed by Electrotek Concepts, will be used as the processing tool and also as a Database Management System to share data in the internet (PQWeb). Setup of PQ View is currently under way.

\section{Protection:}

a. In the original design, neutral point the Y-side of every microsource transformer was connected to a ground-conductor that will run from the neutral point of the microgrid main transformer (T11) through the length of all feeders. However, due to a safety recommendation from AEP, the microsource transformers $\mathrm{Y}$-side neutral is now tied to a neutral conductor that runs back to the neutral point of $T 11$, where it is solidly grounded. These change affected the original intent of the differential current (CT around phase and neutral conductors) scheme; now, this scheme will only detect ground faults that are downstream of the microsource (rather than independent on location). Professor Lasseter investigated and provided recommendations to implement additional zero-sequence and negative sequence current elements to the protection scheme. The addition of these elements is possible due to the flexibility provided by the SEL-351 Relays.

\section{S\&C Power Electronic Switch (PES)}

During Low-Power Testing of the Static Switch (at NPS Lab), some issues were found with nuisance alarms from the S\&C PES (power electronic switch) local controls:

a. When the Switch was commanded to Open, with a source only on the "Input" (Grid) side, the system will "Stop", opening the input CB. The 
reasoning behind was that control power is usually taken from the "Output" (DG) side, thus when the switch opens, control power is lost temporarily, which sent the system "Stop". This issue was verified by performing a similar test but with the source connected to the "output" side, so control power was always available; during this test, the problem disappear. Roger Troyer from S\&C visited the AEP Dolan Lab and performed modifications to the control power circuit of the $S \& C$ to eliminate this problem.

b. The PES also includes a "Fail to Open" Alarm, which will Stop the system (open the input breaker) in the switch if the switch is still closed (measured $>15 \mathrm{Vpk}$ across any phase of the SCR switch) after 2-cycles of being commanded open. This issue was found sporadically during low power testing at NPS, and was replicated during the first visit of Roger Troyer to the AEP site. This issue occurs because this method of detection is not design to account for having sources of approximately the same frequency on both sides of the switch (if this is the case, then voltage will not develop, or will take time to develop, across the switch when it has opened). Based on the CERTS microsource control, this situation will occur when the power across the switch is zero or very low (there is usually at least some real power taken by the PES controls). To fix this issue, Roger Troyer provided a modification in the PES code so the "Fail to Open" detection will only occur if the current across the switch has fallen below $\sim 20 \mathrm{~A}$ after 2 cycles.

\section{TECOGEN Engine Control-Communications}

During the commissioning process, it was noticed that communications between the EMS computer and the TECOGEN inverter controls was very slow; commands for frequency-voltage-power were modified from the EMS computer, and it will take approximately 20 seconds until values were updated on the Engine Control side.

Modifications to the communications between gensets and EMS computer were required. Initially, the gensets were connected together (2-wire duplex) using RS485 connection to the EMS.

This setup was replaced with an Ethernet connection from the EMS to the gensets; on the genset side, an Ethernet serial switch is used to split the Ethernet link into $4 \mathrm{COM}$ ports; three of these are used to communicate with each genset, using RS-232. With this setup, each generator will be polled individually, thus speeding up communications. 


\title{
CERTS MICROGRID LABORATORY TEST BED
}

\author{
Test Plan Section 6.0 \\ Microgrid Test Bed System Checkout \\ (Static Switch)
}

Prepared For:

California Energy Commission

Public Interest Energy Research Program

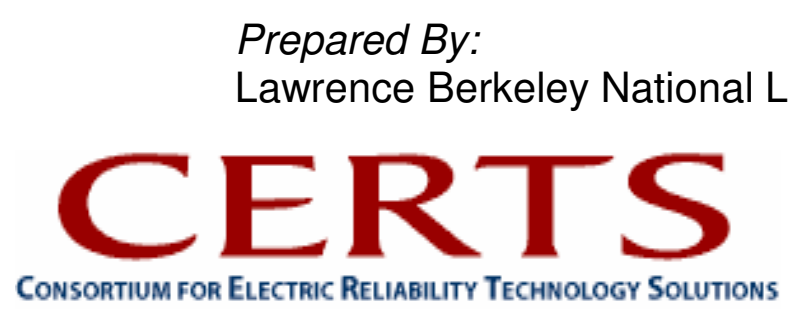

Arnold Schwarzenegger Governor 

Prepared By:

Lawrence Berkeley National Laboratory

Joseph H. Eto, Principal Investigator

Berkeley, CA 94720

Ben Schenkman, Sandia National Laboratory

Harry Volkommer and Dave Klapp, American Electric Power

Commission Contract No. 500-03-024

\section{Prepared For:}

Public Interest Energy Research (PIER)

California Energy Commission

Bernard Treanton

Contract Manager

Mike Gravely

Program Area Lead

ENERGY SYSTEMS INTEGRATION

Mike Gravely

Office Manager

ENERGY SYSTEMS RESEARCH

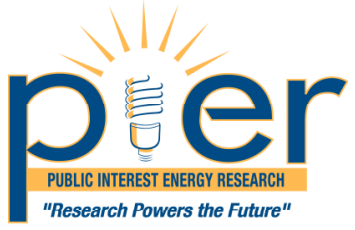

Martha Krebs, Ph.D.

PIER Director

Thom Kelly, Ph.D.

Deputy Director

ENERGY RESEARCH \& DEVELOPMENT DIVISION

Melissa Jones

Executive Director

\section{DISCLAIMER}

This report was prepared as the result of work sponsored by the California Energy Commission. It does not necessarily represent the views of the Energy Commission, its employees or the State of California. The Energy Commission, the State of California, its employees, contractors and subcontractors make no warrant, express or implied, and assume no legal liability for the information in this report; nor does any party represent that the uses of this information will not infringe upon privately owned rights. This report has not been approved or disapproved by the California Energy Commission nor has the California Energy Commission passed upon the accuracy or adequacy of the information in this report. 



\title{
CERTS MICROGRID TEST REPORT
}

\author{
SECTION 6.0 \\ "Microgrid Test Bed System Checkout \\ (Static Switch)"
}




\section{Table of Contents}

1.0

2.0

3.0

4.0

5.0

6.0

6.1

\subsection{1}

6.12

INTRODUCTION

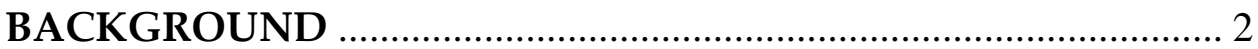

MICROGRID TESTBED SETUP .................................................. 3

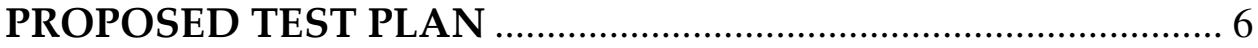

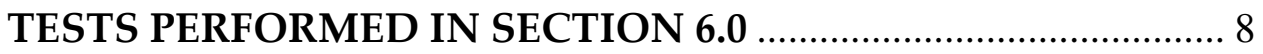

ANALYSES OF TEST RESULTS ........................................... 10

SECTION 6 - MICROGRID TEST BED SYSTEM CHECKOUT 10

6.1.3 Single-Phase Reverse Power Test (Simulate Loss of Phase) ........ 22

6.1.4 Reverse Power, Anti-Islanding Microgrid Setting Reset Test .... 26

6.1.5 De-energized Bus (Dead Bus) Reclose Test ................................... 30

7.0

CONCLUSION 


\section{List Of Figures}

Figure 1 - CERTS Microgrid Aerial Photo.................................................................... 4

Figure 2 - One Line Diagram of CERTS Microgrid Test Bed...................................... 5

Figure 3 - Simplified diagram of Test Bed showing Meter and Relay locations .... 5

Figure 4 - Diagram of DAS \& EMS Data networks................................................... 6

Figure 5a - A phase voltage waveforms and current at Meter 2 on the Microgrid during a synchronized "Close" of the static switch................................. 11

Figure $5 b$ - Change in frequency during a synchronized close of the static switch, during the transition from island to utility-connected mode................ 12

Figure 5c - Single and three phase $\mathrm{kW}$ load in Zone 3 at Load Meter 3 ............... 13

Figure 5d - RMS voltage at Load Bank3, during the transition from island to utility-connected mode. .......................................................................... 14

Figure 5e - RMS voltage on grounded-wye, 480/277 volts, side of T11 transformer during the transition from island to utility-connected mode................. 14

Figure $5 \mathrm{f}$ - The $\mathrm{kW}$ change at Meter 2 when served from the utility grid ............. 15

Figure $5 \mathrm{~g}$ - The $\mathrm{kW}$ change at Meter A1 on the output of Gen-set A1 ................... 15

Figure $5 \mathrm{~h}$ - The kVAr change at Meter A1 on the output of Gen-set A1 ............... 16

Figure $6 \mathrm{a}$ - Meter 3 voltage waveform during breaker CB1 opening and the microgrid islanded within 2 cycles........................................................... 18

Figure $6 \mathrm{~b}$ - Current waveforms at Meter A1 during breaker CB1 opening .......... 18

Figure $6 \mathrm{c}$ - Meter 2 load during synchronized close of the static switch............... 19

Figure $6 \mathrm{~d}$ - Meter A1 load when microgrid transitioned from island mode to utility-connected mode ............................................................................ 20

Figure 6e - Zone 3 load when microgrid transitioned from island mode to utilityconnected mode

Figure $6 \mathrm{f}$ - kVAr changes in Zone 3 when Microgrid transitioned from island mode to utility-connected mode.

Figure $6 \mathrm{~g}$ - Single-phase $\mathrm{kW}$ at PCC when microgrid load was below reverse

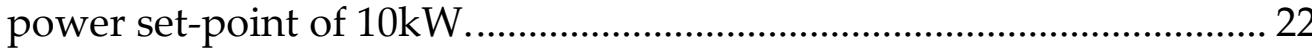

Figure 7a - Load Bank 6 real power before and after the static switch opened.... 23 Figure $7 \mathrm{~b}$ - Load change at Meter 1 when static switch opened on reverse power

Figure 7c - Gen-set A1 kW load change when Microgrid was islanded................. 25

Figure 7d - Frequency change on the Microgrid after being islanded .................... 26

Figure 8a - Meter 1 load decrease after the static switch opened........................... 28

Figure $8 \mathrm{~b}$ - Load increase at Meter 1 during synchronized close of static switch.

Figure 8c - Gen-set A1 output during synchronized close of static switch ........... 30 
Figure 8d - Frequency on the Microgrid during synchronized close of static switch.

Figure 9a - Load increase at Meter 1 (PCC) during a Dead-Bus Reclose of the static switch............................................................................................. 32

Figure 9b - Load Bank 3 connected in the Microgrid during a Dead-Bus Reclose

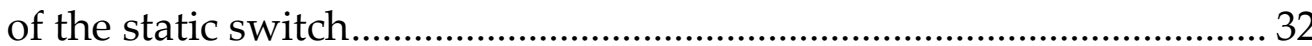




\section{List Of Tables}

Table 1 - Microgrid kW loading before/after the static switch opened................. 25

Table 2 - Microgrid kW loading before/after the static switch opened.................. 28 


\subsection{INTRODUCTION}

A series of tests were performed on the CERTS Microgrid by American Electric Power at the Walnut test site in Groveport, Ohio with support from Lawrence Berkeley National laboratory, Sandia National Laboratory, TECOGEN, The Switch (originally Youtility), Distributed-Energy (originally Northern Power) and University of Wisconsin. These tests were designed to demonstrate the CERTS Microgrid concepts of control and protection while connected to the utility electrical system and isolated (i.e., referred to as "islanded" from it. This paper describes the tests that were performed in Section 6.0 "Procedure - Microgrid Test Bed System Checkout" of the CERTS Micro-grid Test Plan.

\subsection{BACKGROUND}

The CERTS Microgrid Concept is an advanced approach for enabling integration of, in principle, an unlimited quantity of DER (e.g., distributed generation (DG), energy storage, etc.) into the electric utility grid. A key feature of a microgrid is its ability to separate and island itself from the utility system, during a utility grid disturbance. This is accomplished via intelligent power electronic interfaces and a single, high-speed, switch which is used for disconnection from the grid and synchronization to the grid. During a disturbance, the DER and corresponding loads can autonomously be separated from the utility's distribution system, isolating the microgrid's load from the disturbance (and thereby maintaining high level of service) without harming the integrity of the utility's electrical system. Thus, when the utility grid returns to normal, the microgrid automatically synchronizes and reconnects itself to the grid, in an equally seamless fashion. Intentional islanding of DER and loads has the potential to provide a higher level of reliability than that provided by the distribution system as a whole.

What is unique about the CERTS Microgrid is that it can provide this technically challenging functionality without extensive (i.e., expensive) custom engineering. In addition, the design of the CERTS Microgrid provides a high level of system reliability and great flexibility in the placement of DER within the microgrid. The CERTS Microgrid offers these functionalities at much lower costs than traditional approaches by incorporating peer-to-peer and plug-and-play concepts for each component within the microgrid.

The original concept was driven by two fundamental principles: 1.) A systems perspective was necessary for customers, utilities, and society to capture the full benefits of integrating DER into an energy system; and 2.) The business case for accelerating adoption of these advanced concepts will be driven, primarily, by lowering the up-front cost and enhancing the value offered by microgrids. 
Each innovation was created specifically to lower the cost and improve the reliability of small-scale DG systems (i.e., installed systems with capacities ranging from less than $100 \mathrm{~kW}$ to $1000 \mathrm{~kW}$ ). The goal was to increase and accelerate realization of the many benefits offered by small-scale DG, such as their ability to supply waste heat at the point of need or to provide a higher level of reliability to some but not all loads within a facility. From an electric utility perspective, the CERTS Microgrid Concept is attractive because it recognizes that the nation's distribution system is extensive, aging, and will change over time which impacts power quality. The CERTS Microgrid Concept enables high penetration of DG systems without requiring re-design or re-engineering of the utility's distribution system.

Prospective applications of the CERTS Microgrid include industrial parks, commercial and institutional campuses, situations that require uninterrupted power supplies and high power quality, CHP systems, Greenfield communities, and remote applications. In short, wherever economic and DG location considerations indicate the need for multiple DG units within a (or among) site, the CERTS Microgrid offers the potential for a much more reliable, flexible, and lower cost solution compared to traditional engineering approaches for integrating DG.

\subsection{MICROGRID TESTBED SETUP}

The CERTS Microgrid Test Bed is operated at 480/277 volts (i.e., three-phase, four-wire) and consists of three TECOGEN Generators at 480 volts capable of producing $60 \mathrm{~kW}$ plus 60kVAr (Gen-set A1, Gen-set A2 and Gen-set B1) and four load banks (Load Bank 3, Load Bank 4, Load Bank 5 and Load Bank 6) capable of consuming 100kW plus 20kVAr each, as shown in Figure 2. Each of the generators are connected to an 112kVA isolation transformer and interfaced to the CERTS Microgrid through an inverter, developed by The Switch, where the algorithms for the CERTS Microgrid controls are embedded. . A semiconductor switch made by S\&C Electric Company, known as the static switch, connects the CERTS Microgrid to the utility grid. Load Banks $3-5$ are the local loads in zones located beyond the static switch; and Load Bank 6 is a customer load in Zone 6 located on the utility side of the static switch. 


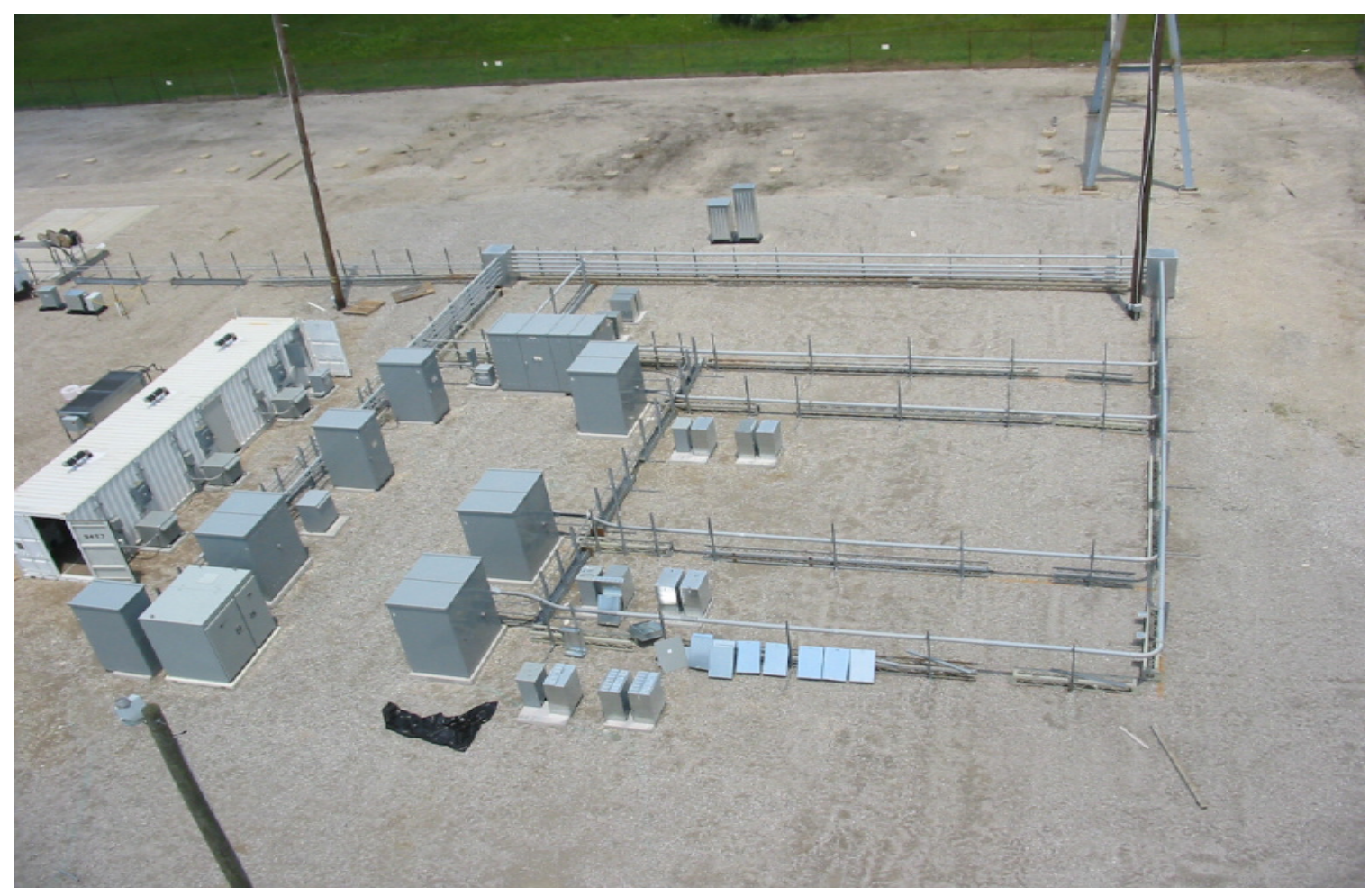

Figure 1 - CERTS Microgrid Aerial Photo

There are 6 zones in the Test Bed with Zones 2 - 6 contained within the CERTS Microgrid design and Zone 1 being the utility interface and referred to as the point-of-common coupling (PCC) to the grid. Each zone is protected by a Schweitzer SEL-351 relay. Faults of varying magnitude can be applied to each zone through an additional breaker which allows fault application and removal. 


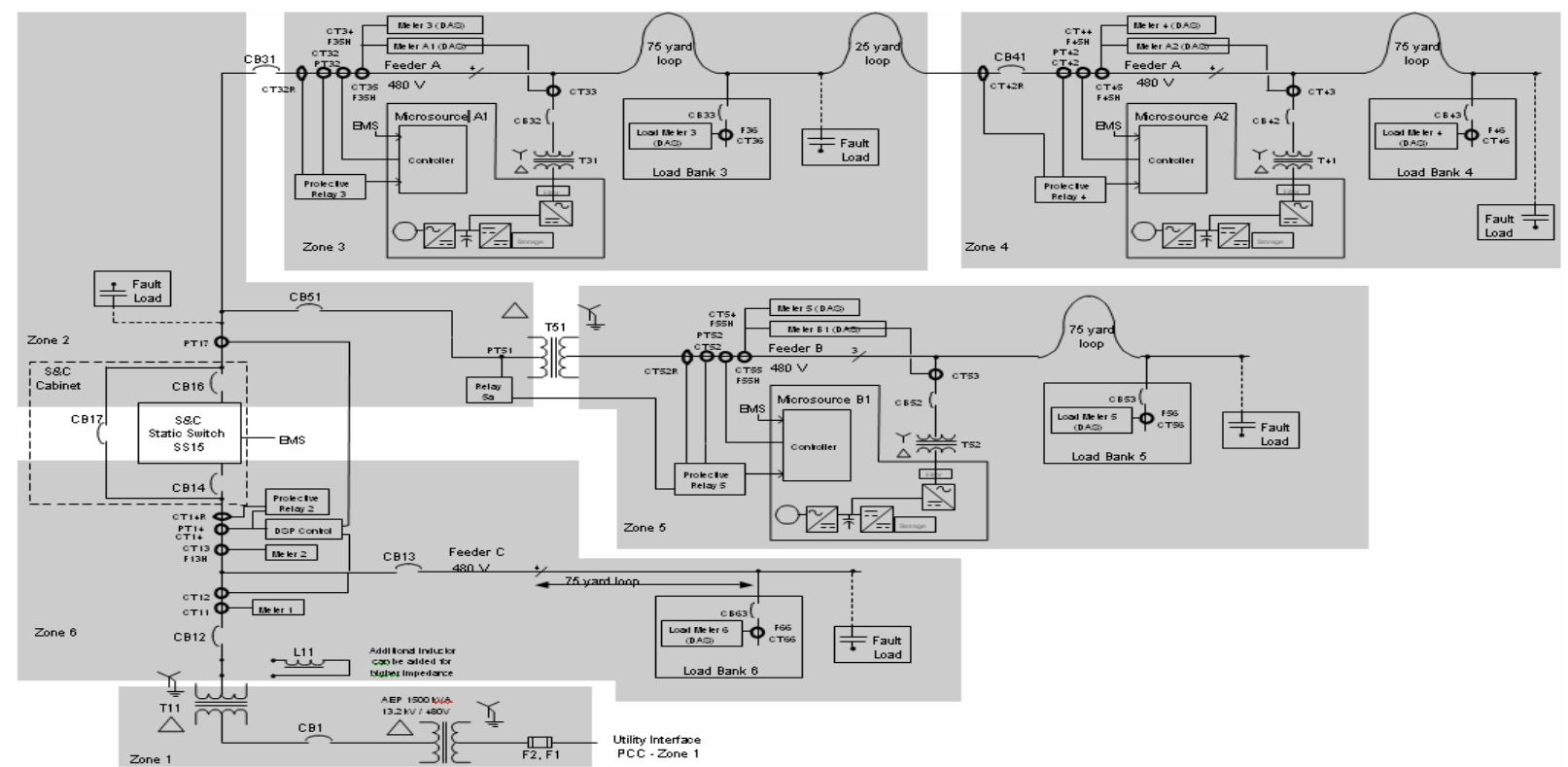

Figure 2 - One Line Diagram of CERTS Microgrid Test Bed

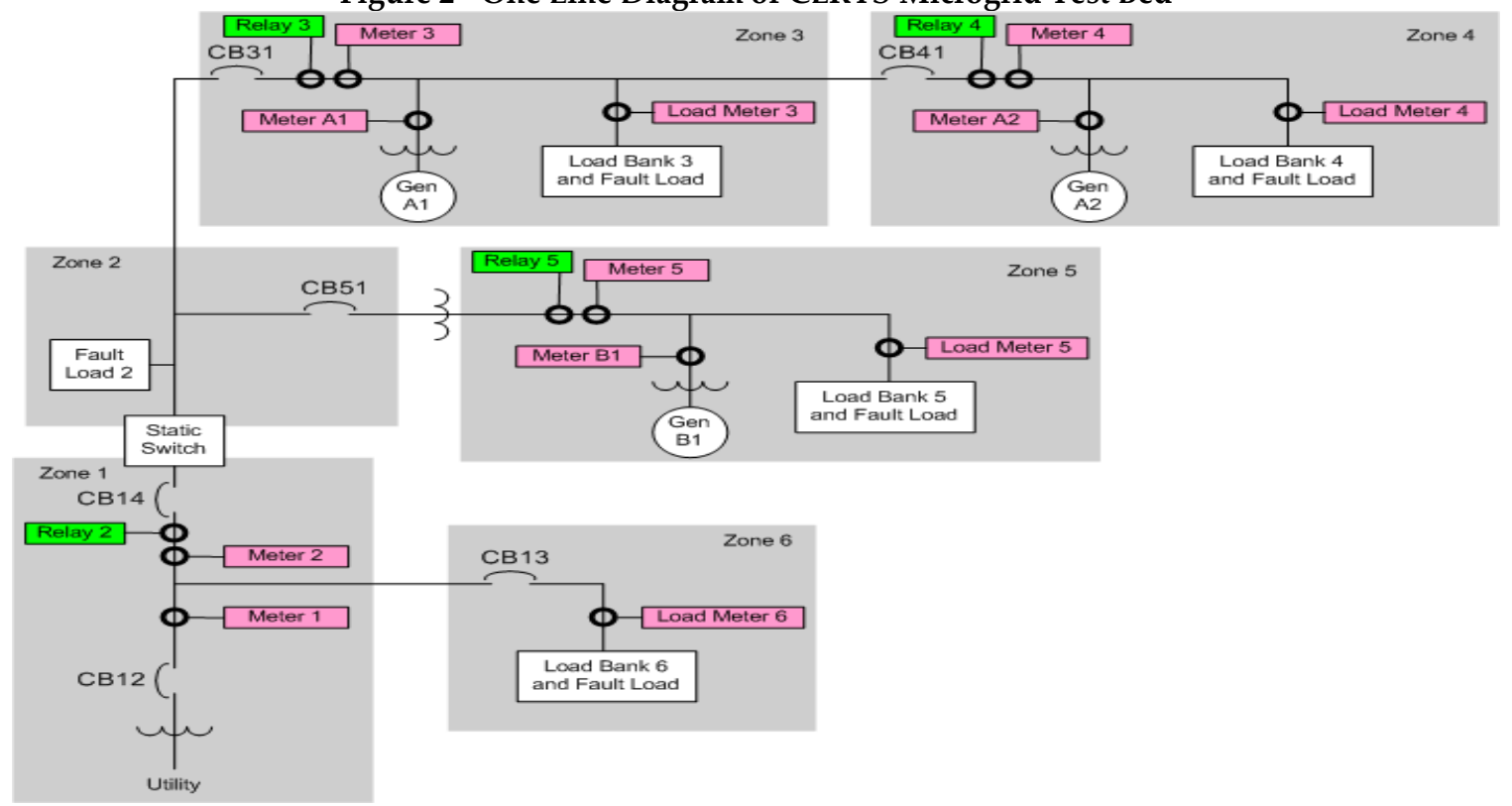

Figure 3 - Simplified diagram of Test Bed showing Meter and Relay locations

There are twelve PML ION 7650 meters placed through out the microgrid and shown in Figure 3, which monitor electrical system conditions, plus acquire phase current and voltage waveforms; and calculate RMS values of voltage, current, active power, reactive power, and frequency. 


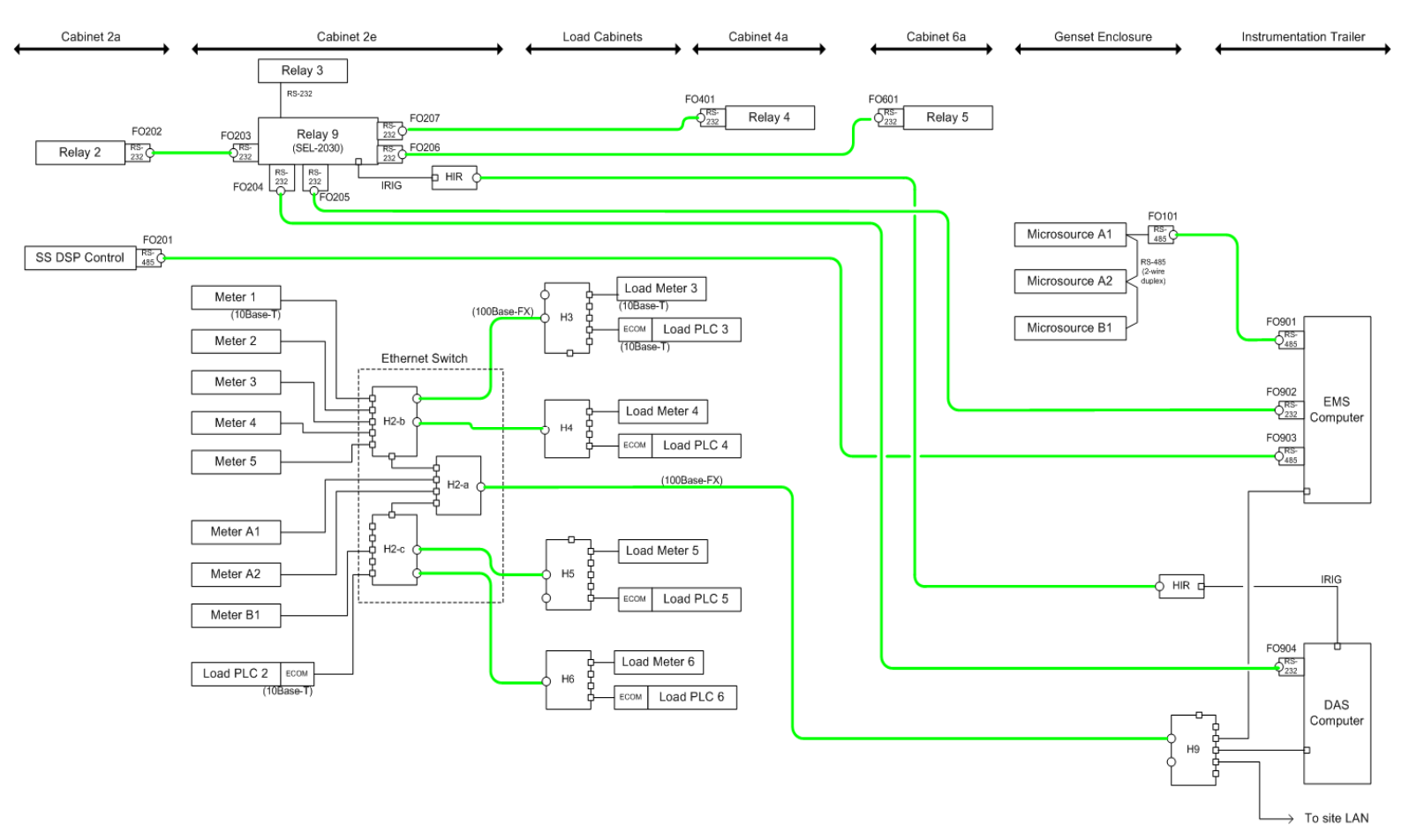

Figure 4 - Diagram of DAS \& EMS Data networks

An Ethernet network was provided as shown in Figure 4, for communications between all meters, load control PLCs, and the Data Acquisition System (DAS) computer, using fiber-optic links and switches. The DAS and Energy Management System (EMS) computers were also networked into the local Dolan Local Area Network (LAN) and to a secure Website with user ID and password protection. Additional serial links, using fiber optic converters, connect all relays, static switch Digital Signal Processor (DSP) controller, and TECOGEN Gen-set controls to the EMS computer.

\subsection{PROPOSED TEST PLAN}

The CERTS Microgrid Test Plan was developed by the CERTS Microgrid Team to demonstrate the unique concepts of control and protection of the CERTS Microgrid. This test plan was reviewed by a Technical Advisory Committee outside the CERTS Microgrid Team and then implemented by American Electric Power. CERTS Microgrid Test Plan consists of 12 sections with 5 of them detailing desired tests, starting at section 6.0, to demonstrate the controls and concepts of the CERTS Microgrid. The other 7 sections pertain to safety procedures, equipment calibration, and documentation. Each section of the test plan is described below.

- Section 1 - "Purpose, References, and Definitions" describes the purpose of the test plan, helpful references for further explanation of how the test bed was created, and definitions used through out the test plan. 
- Section 2 - "Responsibilities" informs personnel of their responsibilities while working on or near the CERTS Microgrid test site.

- Section 3 - “Training - Team Members" lists the mandatory training needed by personnel before they can work on or near the CERTS Microgrid test site.

- Section 4 - "Procedure - CERTS Microgrid Test Bed Lockout/Tagout" entails how to safely shut down the equipment and lockout/tagout the closest upstream disconnect to work on or near equipment.

- Section 5 - "Procedure - General" is the daily procedures performed at the CERTS Microgrid Test Site, prior to performing a test from Section 6 through Section 10.

- Section 6 - "Procedure - Microgrid Test Bed System Checkout" was designed to check control and operation of the static switch, basic power and voltage control of the Gen-sets, and a preliminary check of the protection scheme. The goal is to assure that the test bed is operating and ready to perform the tests described in the remaining sections of the test plan document.

- Section 7 - "Validate Protection Settings \& Initial Fault Testing" is designed to examine a preliminary set of fault (i.e. overload simulating a fault) condition tests to ensure protection and safety of the Micro-grid test Bed, while performing other planned tests. The goal is to test and adjust protection settings to achieve the most ideal conditions and protection design.

- Section 8 - "Procedure - Reduced System Tests" is a limited set of tests to build confidence that the Gen-set inverter controls are working correctly. This includes unit control, zone control, and mixed power controls, in conjunction with limit controls and synchronized closing of the static switch. These tests are based on the TECOGEN/THE SWITCH factory acceptance testing.

- Section 9 - "Procedure - Demonstration Tests of Control Power Flow" demonstrates the flexibility of the Micro-grid both grid connected and islanded for different loads, power flows and impact on the utility. 
- Section 10 - "Procedure - Test Difficult Loads" determines operation limits of the Micro-grid (i.e. power quality, protection and inverter limits) with low pf loads, motor loads, harmonic loads and unbalance loads.

- Section 11 - "Hazards \& Mitigation" informs the personnel of hazards that may exist while working on or near the CERTS Micro-grid test site and how to mitigate them.

- Section 12 - “Quality Assurance” ensures quality for the acquiring data results by providing a checklist reminder for personnel.

\subsection{TESTS PERFORMED IN SECTION 6.0}

Prior to each test day, the person in charge performed a job safety briefing (JSB) with barricades and test setup inspected for safety and compliance. A minimum of two people were on-site during each planned test.

Visual and audible alarms were used to warn persons that energized testing was being performed in the Microgrid Test Bed area. The visual alarm consisted of a portable red flashing light, located between the Control Trailer and Gen-set Enclosure. An audible alarm, consisting of a portable wireless motion detector, was located at the front gate of the Walnut Test Site with the fence gate "Closed", not locked, and audible alarm in the trailer operational during test(s).

Barricades were set up around the Micro-grid Test Bed area (i.e., saw-horse style barricades with a "Red" plastic chain surrounded the test area containing the Gen-set Enclosure, Micro-grid switching cabinets, plus load and fault bank cabinets).

Prior to performing tests, the Test Engineer or Technical Consultant verified that all personnel and visitors were properly protected and in assigned locations. Personnel were in or adjacent to the Control Trailer while tests were being performed. All nonessential personnel either left the main site or were sheltered in the Control Trailer.

For all tests the following waveforms were captured and recorded in the DAS for voltage $(\mathrm{V})$ and current $(\mathrm{I})$. From these waveforms real power $(\mathrm{kW})$, reactive power $(\mathrm{kVAr})$, and frequency (freq) were post calculated by the PQView software. Frequency measurements in this report should be used for steady state information and not used for transient analysis, due to the calculation and filtering methods employed. Below is a list of the meters capturing this data. 
- Meters 1, 2, 3, 4 \& 5

- Load Meters 3, 4, 5, \& 6

- Meters A1, A2 \& B1

- Meter 2 also measures the voltage across the static switch on phase A

Schweitzer event reports were also captured for each event, along with breaker and static switch status, such as Open or Close.

The first sets of tests came from section 6.0 where the static switch's controls and operation were checked, all three Gen-sets basic power and voltage controls were verified for correct operation, and a preliminary check of the protection scheme that was later tested in section 7.0. The static switch function tests checked the operation of the static switch, to assure it and its DSP control were operating as designed. This section included tests of dead-bus and synchronized closing, reverse power, and IEEE 1547 protective relay functions.

A synchronized closing test of the static switch was required to verify that when conditions were within synchronization limits set in the EMS, the static switch performed a synchronized close and thus provided a smooth connection transition. The de-energized bus (dead-bus) reclose test's goal was to verify that the static switch can close when de-energized bus conditions exist on the Gen-set side of the static switch; and that the dead-bus reclose algorithm requires user intervention (i.e., Operator needs to "Enable" the dead-bus reclose using pushbutton in the EMS).

The reverse power tests consisted of three tests: three-phase reverse power condition test, single-phase reverse power condition test, and anti-islanding Micro-grid settings reset test. Both three-phase and single-phase reverse power condition tests were required to verify the reverse power functionality of the static switch and confirm that the static switch islands the microgrid for a three-phase or single-phase reverse power condition The anti-islanding Micro-grid settings reset test was needed to verify that if a reverse power event occurred, due to a mismatch of Gen-set settings (i.e., total Gen-set power is greater than Microgrid load), the static switch will lockout and go to the "Fault" state, where user intervention is required.

The next goal was to verify the reconnection timers of the static switch (i.e., set by default at 300 seconds based on IEEE Standard 1547-2003). The length of time is programmed into the control system and designed to prevent reconnection from island to utility-connected mode until after the utility source voltage returns to nominal steady state conditions. 


\subsection{ANALYSES OF TEST RESULTS}

\subsection{SECTION 6 - MICROGRID TEST BED SYSTEM CHECKOUT}

\subsubsection{System Start-Up and Synchronized Closing of Static Switch}

Performance Goal:

Verify that when conditions are appropriate (within synchronization limits set in the EMS), the switch can perform a synchronized closing and thus give smooth closing transitions.

Initial Setup:

Gen-set A1 = Unit Power Control

Output Power Command $=20 \mathrm{~kW}$

MG Power/Frequency Droop $=-0.0833 \mathrm{~Hz} / \mathrm{kW}$

MG Voltage Command $=277 \mathrm{~V}$

Load Bank $3=40 \mathrm{~kW}(13.3 \mathrm{~kW}$ per phase $)$

Load Bank 6=40kW (13.3kW per phase)

After Gen-set A1 was running for a few minutes and supplied power to Load Bank 3, the test was started with a "Start" command from the EMS. 


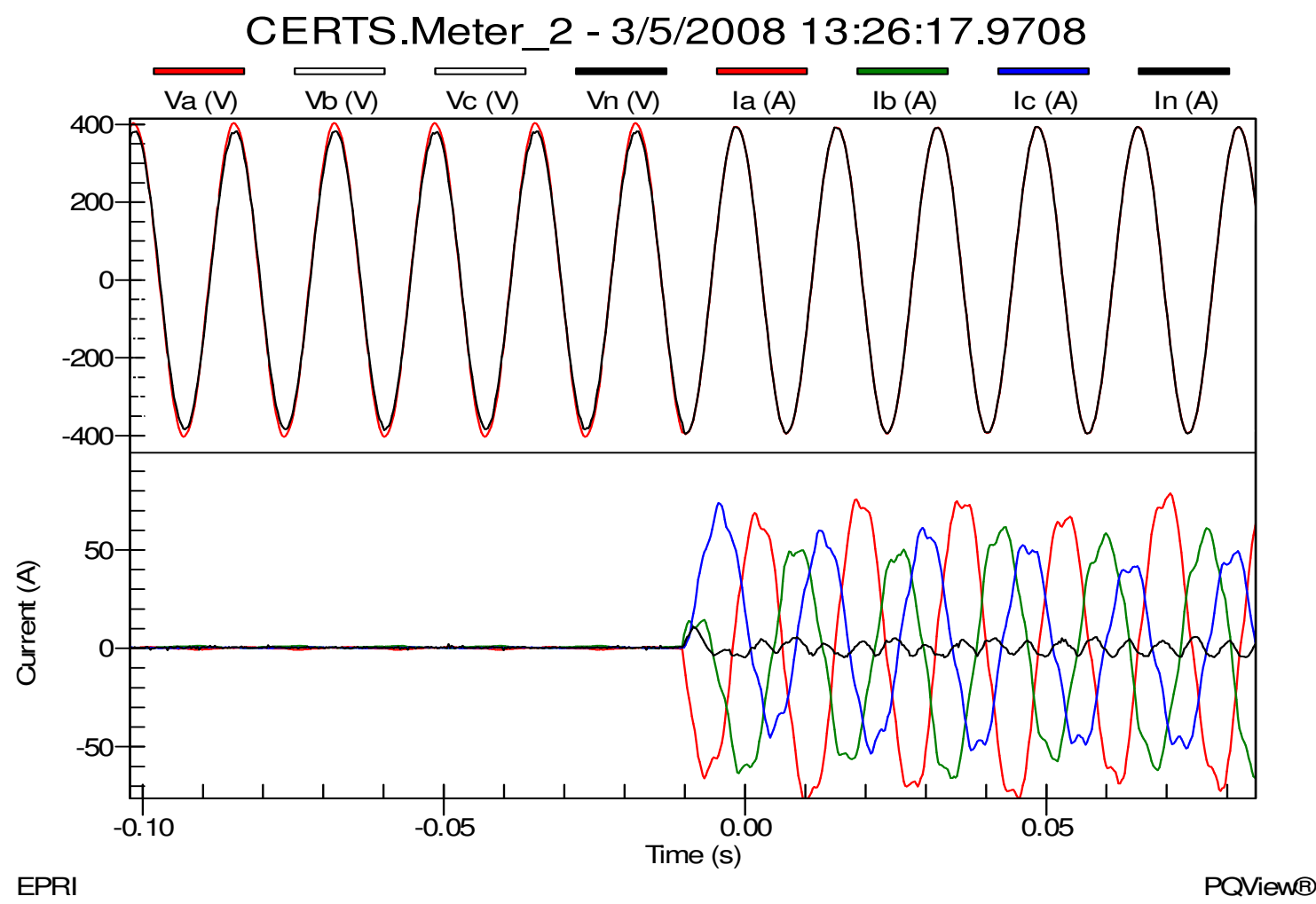

Figure 5a - A phase voltage waveforms and current at Meter 2 on the Microgrid during a synchronized "Close" of the static switch

When electrical system conditions met established criteria, the static switch did a synchronized "Close". A smooth voltage transition occurred from island to utilityconnected mode. This transition occurred relatively quickly, after the "Start" command with the A-phase voltage waveforms at Meter 2 shown in Figure 5a. It can be seen that the utility A-phase voltage (red) closely matches the microgrid A-phase voltage (black) prior to synchronization. The transition occurred at approximately minus 0.01 seconds.

After the static switch closed and steady state conditions were established, the power flow at Meter 1, Meter 2, and Meter A1 was approximately 52kW, 16kW, and 20kW, respectively and the frequency stabilized, as shown in Figure $5 \mathrm{~b}$ from $59.86 \mathrm{~Hz}$ to the grid frequency of $60.01 \mathrm{~Hz}$.

The resistive load banks in each test zone were nominally rated with deviations from nominal expected due to changes in ambient temperature, per phase voltages, heat rise based on duration of test, etc. Thus, during this test the load banks were set at $13.3 \mathrm{~kW}$ 
per phase (i.e., approximately $40 \mathrm{~kW}$ ), but in reality the actual $\mathrm{kW}$ value recorded at Load Meter 3 and at load Meter 6 was approximately 36kW.

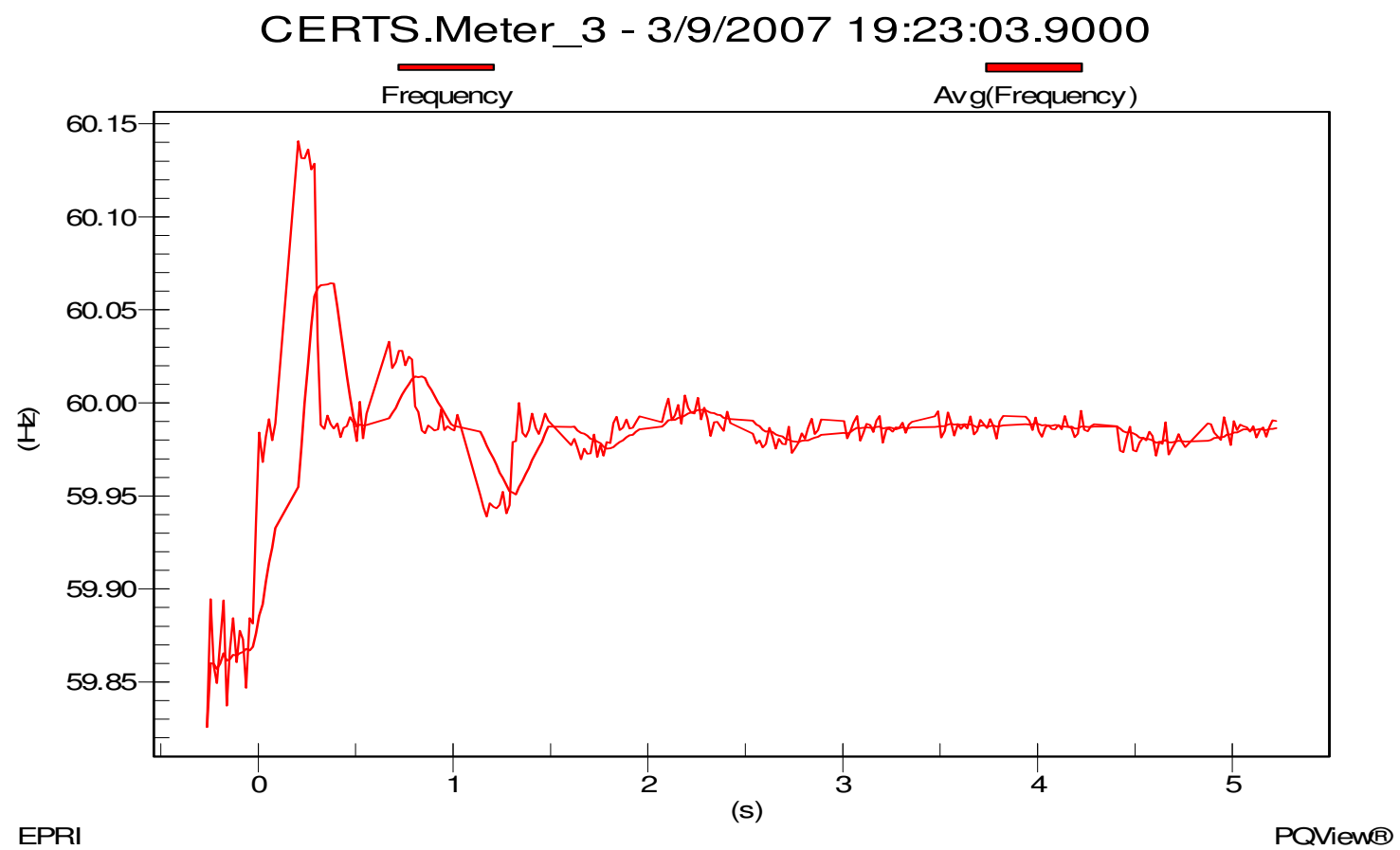

Figure $5 \mathrm{~b}$ - Change in frequency during a synchronized close of the static switch, during the transition from island to utility-connected mode.

The first full scale test on the Microgrid Test Bed and synchronizing an islanded microgrid to the utility required a thorough review of the electrical conditions and affects of the corresponding algorithms designed into the CERTS Microgrid Concept. Thus, the following paragraphs provide a more extensive review and focus-in on the $\mathrm{kW}$ and $\mathrm{kVAr}$ load flows in Test Zone 3 which occurred before and after the transition from island to utility-connected mode.

Figure 5c includes the single-phase and three-phase load being served in Zone 3 of the microgrid. When in island mode and prior to synchronization, Gen-set A1 at Load Meter 3 was serving $33.75 \mathrm{~kW}$ with A, B and C phases being $11.15 \mathrm{~kW}, 11.35 \mathrm{~kW}$ and $11.25 \mathrm{~kW}$, respectively. After synchronizing to the utility grid, the load at Load Meter 3 increased to $35.4 \mathrm{~kW}$ with $\mathrm{A}, \mathrm{B}$ and $\mathrm{C}$ phases being $11.95 \mathrm{~kW}, 11.75 \mathrm{~kW}$ and $11.70 \mathrm{~kW}$, respectively. 


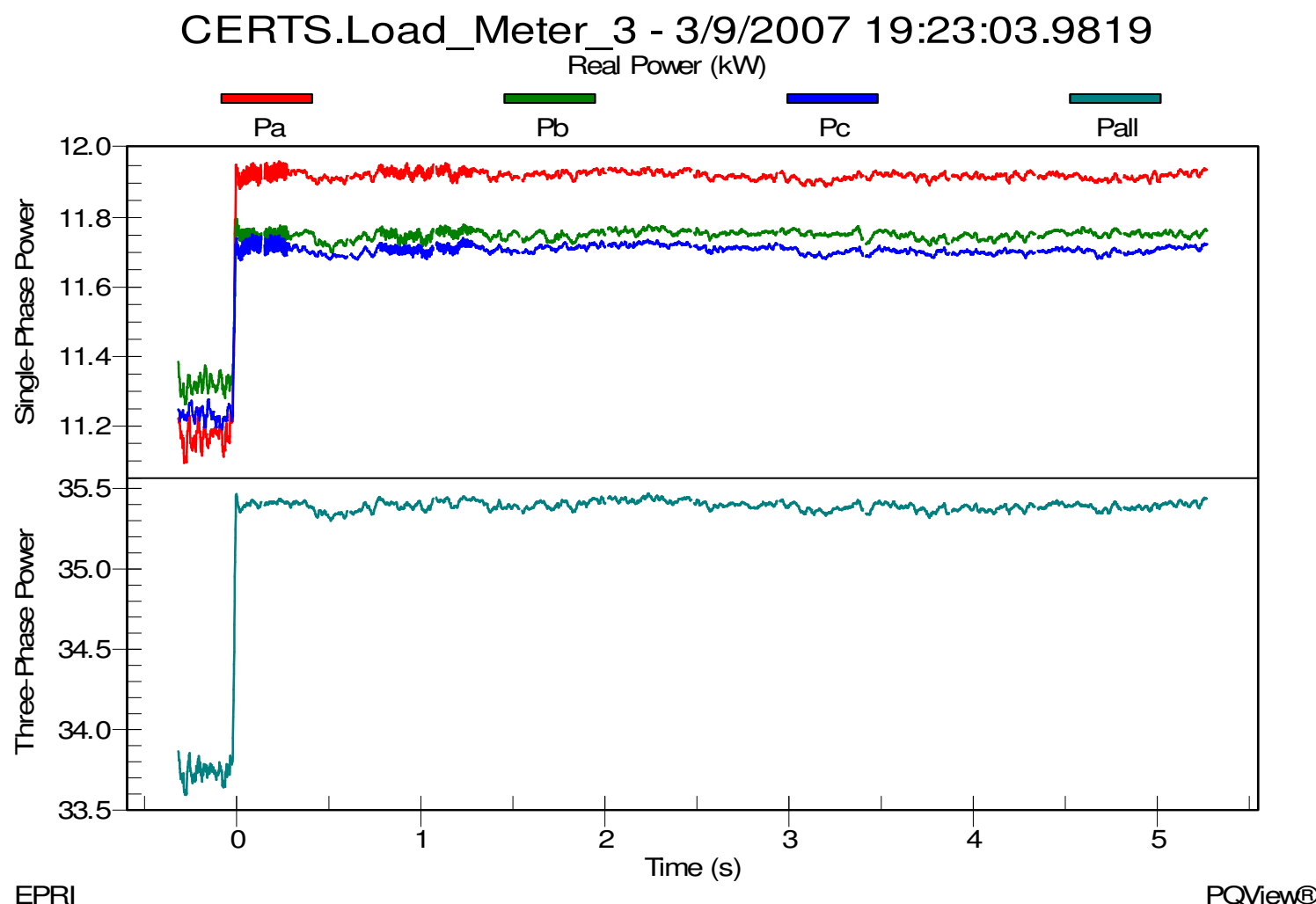

Figure 5c - Single and three phase kW load in Zone 3 at Load Meter 3

The increase in $\mathrm{kW}$ load at Load Bank 3 can be attributed to the slight change in the per phase voltages when interconnected to the utility grid. As illustrated in Figure 5d, Aphase RMS voltage was below B and C phases when the microgrid was in island mode, but is above $\mathrm{B}$ and $\mathrm{C}$ phase voltages when connected to the utility grid.

Prior to synchronization, Figure 5e show A-phase voltage at the point of common coupling (PCC) being higher at $283 \mathrm{~V}$ with B and C phase voltages at $280 \mathrm{~V}$. After the microgrid synchronized to the utility grid, each of the phase voltages dropped at the PCC with A-phase reducing to approximately $280 \mathrm{~V}$, and B and C phases reducing to approximately $277 \mathrm{~V}$. These voltages are similar to the voltages at Meter 2 and closely match the voltage change at Load Bank 3.

With the slight change in $\mathrm{kW}$ load explained at Load Bank 3, the question remains as to how much of the microgrid's protected load is being served from the Gen-set A1 and how much is being served from the utility grid. 


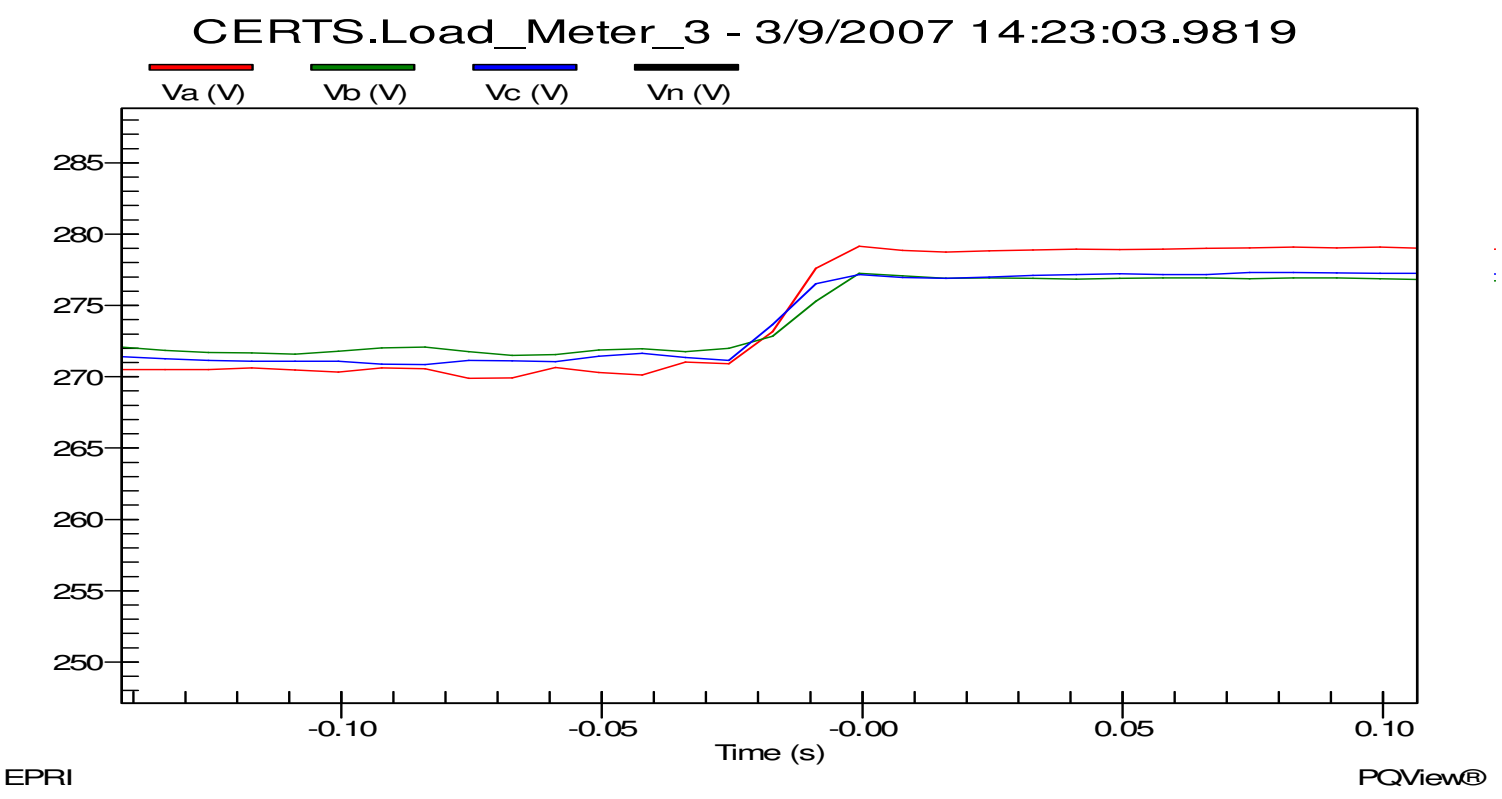

Figure 5d - RMS voltage at Load Bank3, during the transition from island to utility-connected mode.

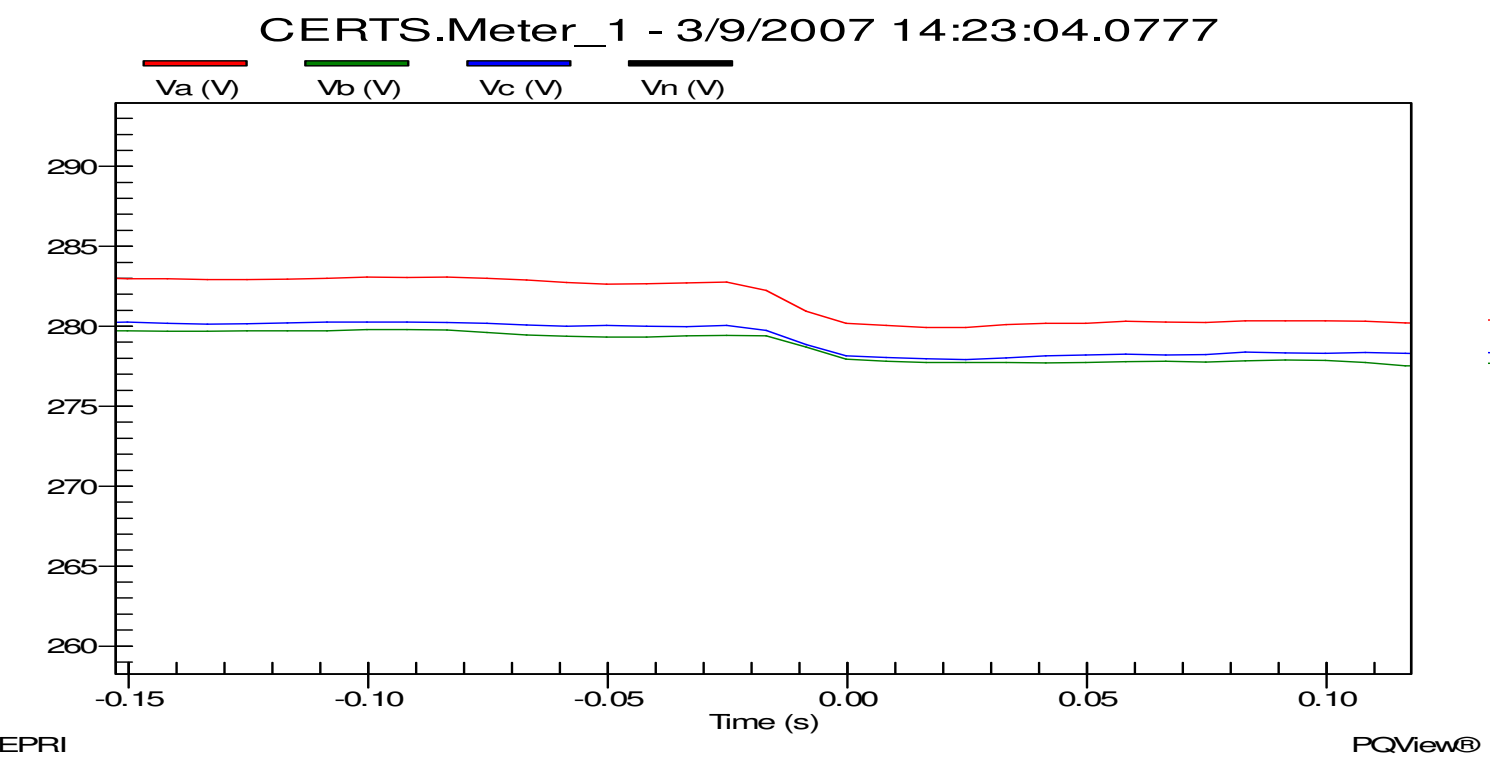

Figure 5e - RMS voltage on grounded-wye, 480/277 volts, side of T11 transformer during the transition from island to utility-connected mode

Figure $5 \mathrm{f}$ shows the change in $\mathrm{kW}$ load being served from the utility at Meter 2 when the microgrid is interconnected to the utility grid. As indicated, after the static switch closed, the total three phase load increased from $0 \mathrm{~kW}$ to $16 \mathrm{~kW}$ with $\mathrm{A}, \mathrm{B}$ and $\mathrm{C}$ phase loads increasing from 0 to approximately $5.5 \mathrm{~kW}, 4.0 \mathrm{~kW}$ and $6.5 \mathrm{~kW}$, respectively. 


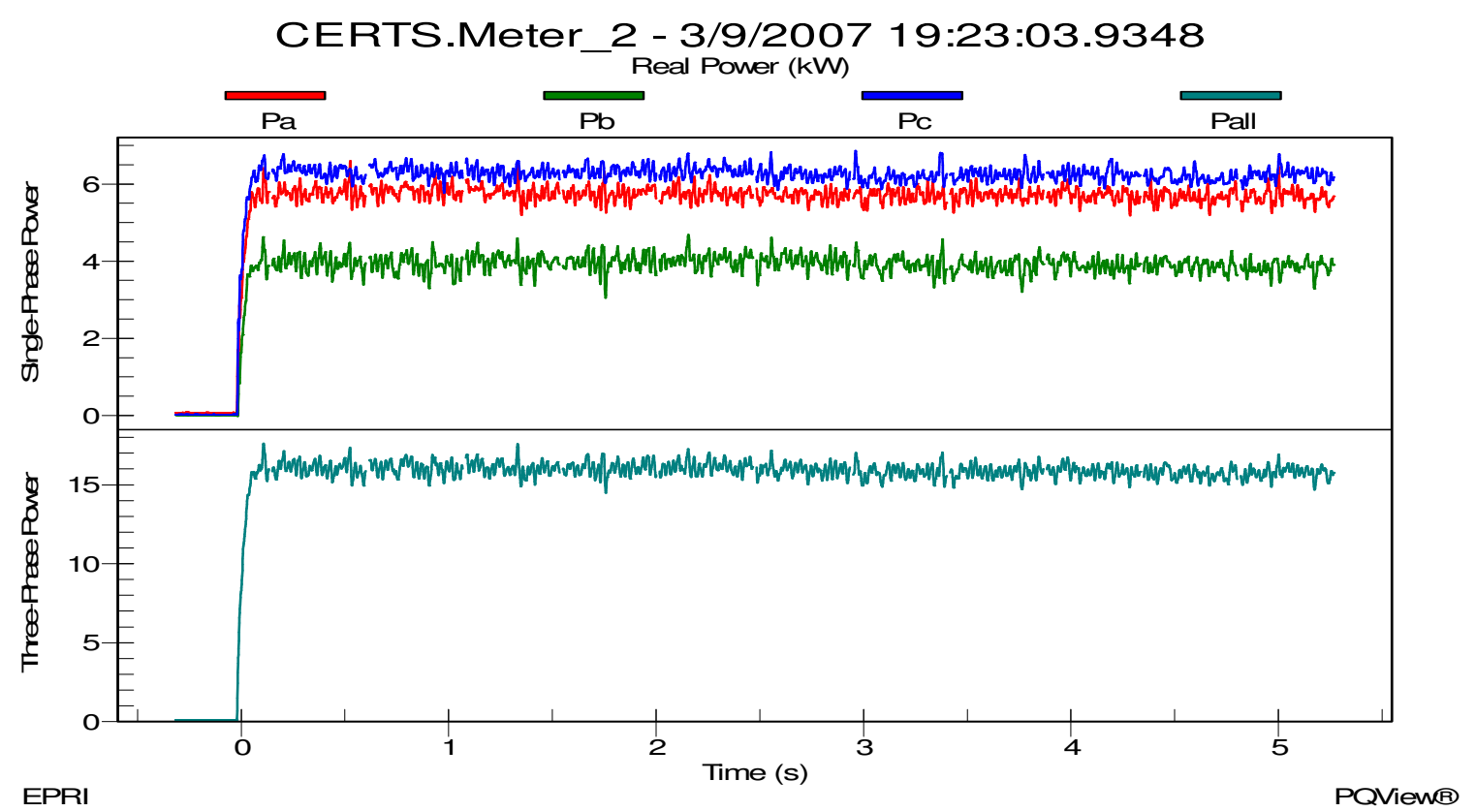

Figure 5f - The kW change at Meter 2 when served from the utility grid

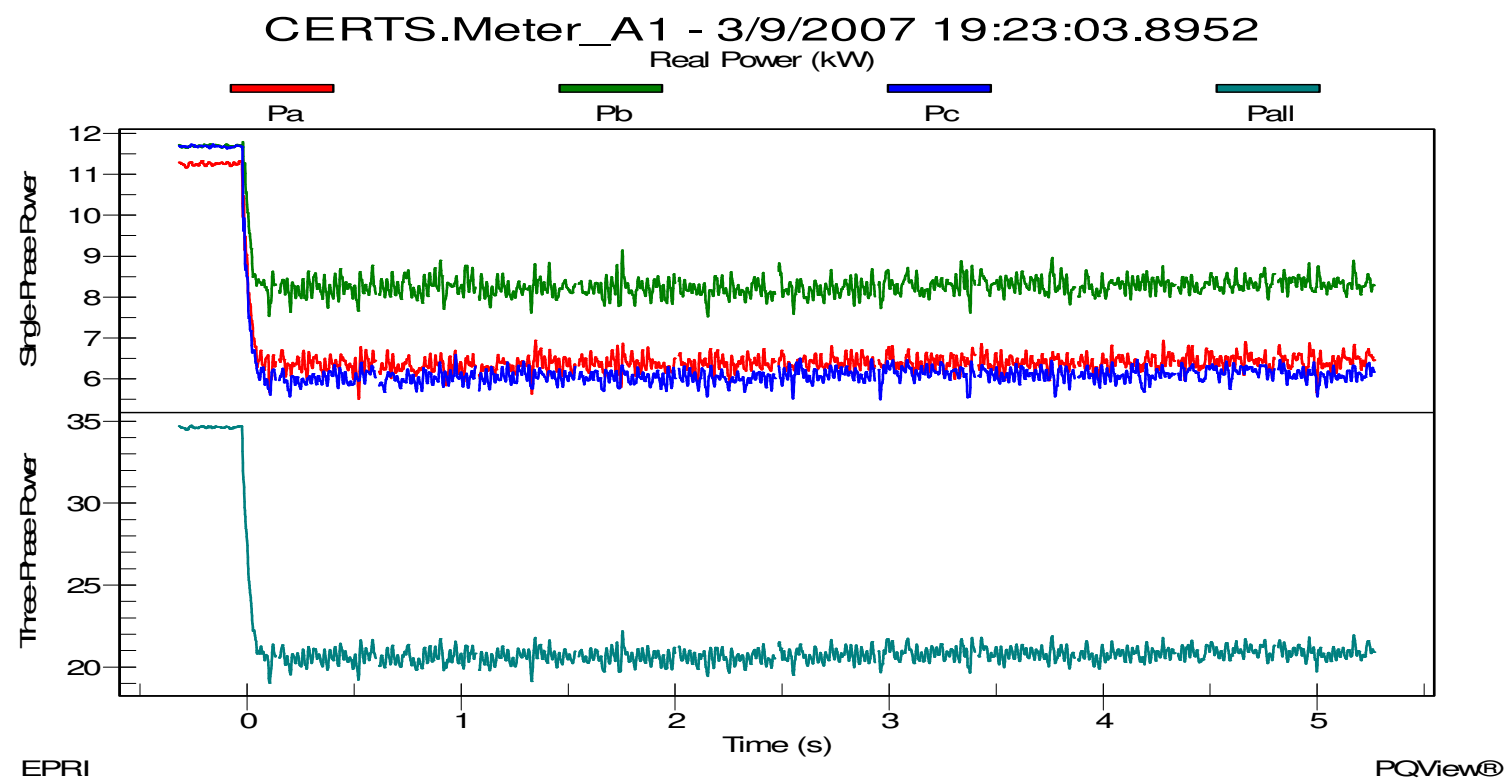

Figure $5 \mathrm{~g}$ - The $\mathrm{kW}$ change at Meter A1 on the output of Gen-set A1

Figure $5 \mathrm{~g}$ shows the corresponding change in $\mathrm{kW}$ output of Gen-set A1 at Meter A1, after the transition from island mode to utility-connected mode. As illustrated, the three-phase $\mathrm{kW}$ output of Gen-set A1 changed from approximately $34.6 \mathrm{~kW}$ to $20 \mathrm{~kW}$ with A-phase changing from approximately $11.2 \mathrm{~kW}$ to $6 \mathrm{~kW}$; B-phase changing from $11.7 \mathrm{~kW}$ to $8 \mathrm{~kW}$, and C-phase changing from $11.7 \mathrm{~kW}$ to $6 \mathrm{~kW}$. 
Note the $20 \mathrm{~kW}$ value was the original Unit Power output set point in the controller of Gen-set A1. When in island mode, Gen-set A1 had sufficient capacity to serve the $35 \mathrm{~kW}$ of load in Zone 3; and when the static switch synchronized with the utility grid, Gen-set A1 reduced its $\mathrm{kW}$ output to the selected set-point value.

Once the synchronization criteria were within limits, the static switch closed, connecting the microgrid to the utility. After this connection Gen-set A1 adjusted its frequency to match that of the utility grid, as shown in Figure $5 \mathrm{~b}$. This increase in frequency caused Gen-set A1 to reduce its real power output to the unit power mode set-point of $20 \mathrm{~kW}$, as shown in Figure 5g. Finally, Gen-set A1 reactive power output transitioned from producing to absorbing power in response to the increased voltage of the utility in an attempt to lower the microgrid nominal voltage of 277 volts, as shown in Figure 5h.

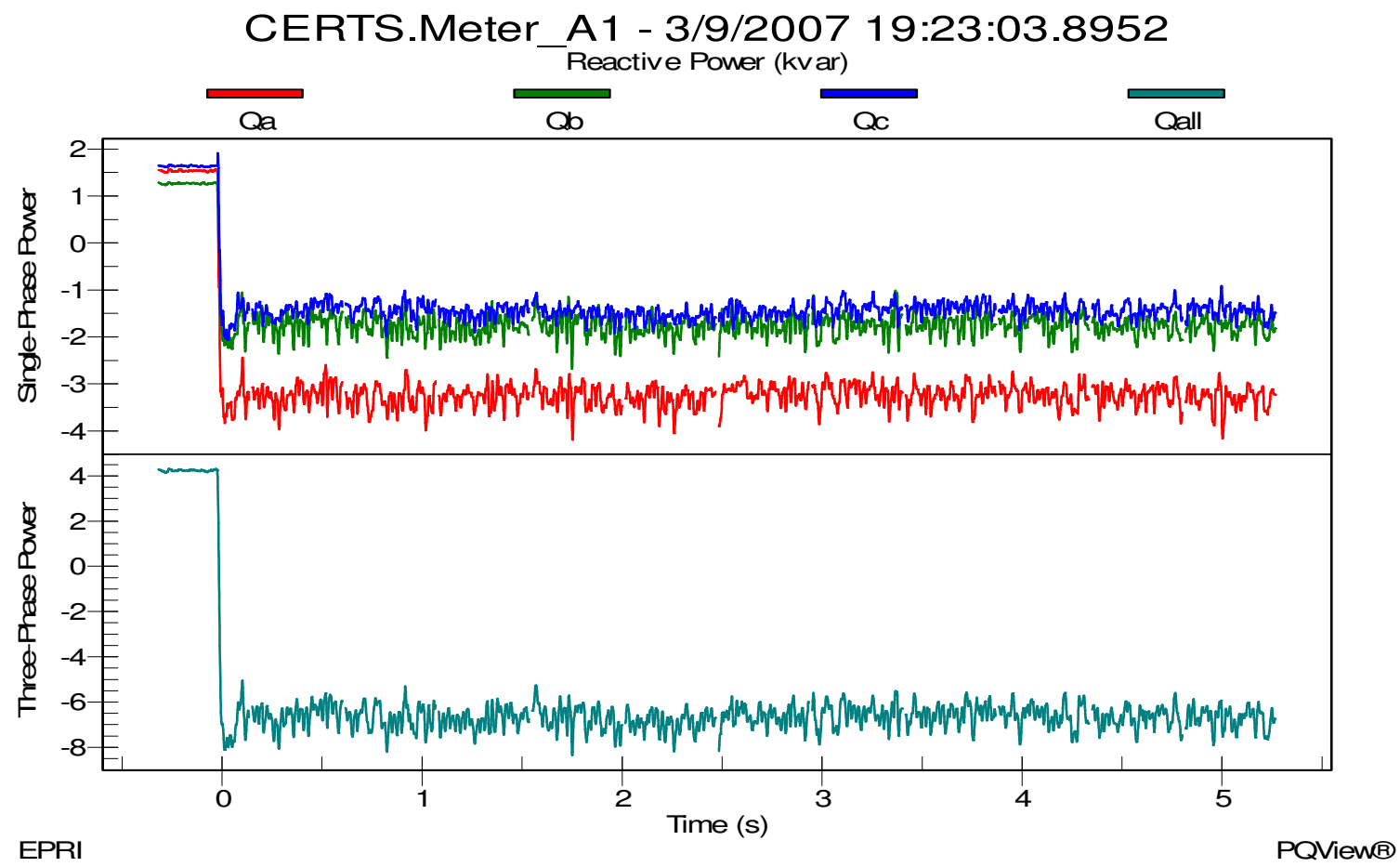

Figure 5h - The kVAr change at Meter A1 on the output of Gen-set A1

Figure 5h shows the inverter adjusting the total $\mathrm{kVAr}$ value at Gen-set A1 from $+4 \mathrm{kVAr}$ when in island mode to approximately -7kVAr when utility-connected. For the static switch to synchronize with the utility grid, A-phase voltage needed to be raised high on the Microgrid than the voltages on phases B and C. This is evident in Figure 5h, where the $\mathrm{kVAr}$ change in A-phase was much greater (i.e., almost twice the kVAr change) than in phases $B$ and $C$. Thus, the voltages raised and frequency adjusted on the Microgrid, 
as designed in the algorithm (i.e., MG Power/Frequency Droop setting), for the static switch to synchronize with the utility grid.

\subsubsection{Loss of Utility Source Test \\ Performance Goal:}

Verify that the microgrid detects and islands itself upon the loss of the utility source upstream of the PCC. This is detected in two ways: first, for a large load loss on the utility system an under-voltage event will occur; and second, for a small load loss a reverse power event will occur. Once the utility voltage returns to the IEEE 1547 limits, verify the proper operations of the reconnection timers (set by default to 300 seconds based on IEEE 1547 standards).

Initial Setup for large loss on utility system:

Gen-set A1 = Unit Power Control

Output Power Command $=20 \mathrm{~kW}$

MG Power/Frequency Droop $=-0.0833 \mathrm{~Hz} / \mathrm{kW}$

MG Voltage Command $=277 \mathrm{~V}$

Load Bank $3=40 \mathrm{~kW}$

Load Bank $6=20 \mathrm{~kW}$

AEP Load Bank $=500 \mathrm{~kW}$

After Gen-set A1 was running for a few minutes and supplying power to Load Bank 3, a synchronized "Close" of the static switch was initiated. Once synchronized, the microgrid was connected to the utility grid with a separate AEP Load Bank connected to the electrical system between transformer T11 and circuit breaker CB1 with $500 \mathrm{~kW}$ of additional load being served from the utility through breaker CB1.

Figure 6a shows the voltage waveform results when breaker CB1 to the utility grid was "Opened/Tripped". It can be seen that the voltage collapses quickly causing the static switch to open and the microgrid load remains served by the Gen-set A1. Likewise, Figure $6 \mathrm{~b}$ shows the current waveforms at Meter A1 when breaker CB1 was opened, the microgrid islanded in approximately 2 cycles. Note, Gen-set A1 current waveform increased to approximately 500 amperes, peak, which is well beyond its design rating. The EMS indicated that an under-voltage PQ event did occur and that a static switch 
opened, successfully. During this event the under-voltage relay of CB-51 operated, deenergizing Zone 5 in the test bed.

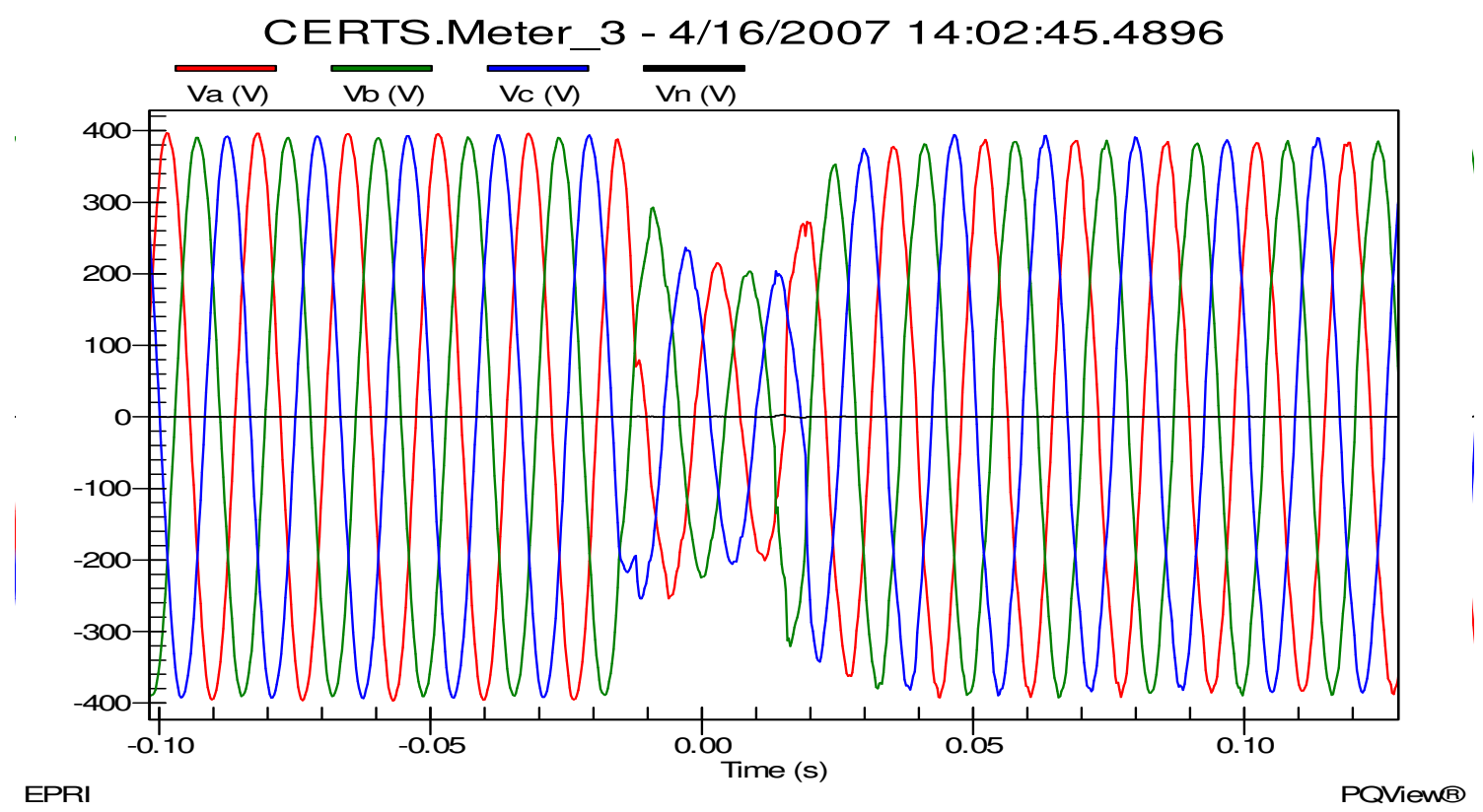

Figure 6a - Meter 3 voltage waveform during breaker CB1 opening and the microgrid islanded within 2 cycles

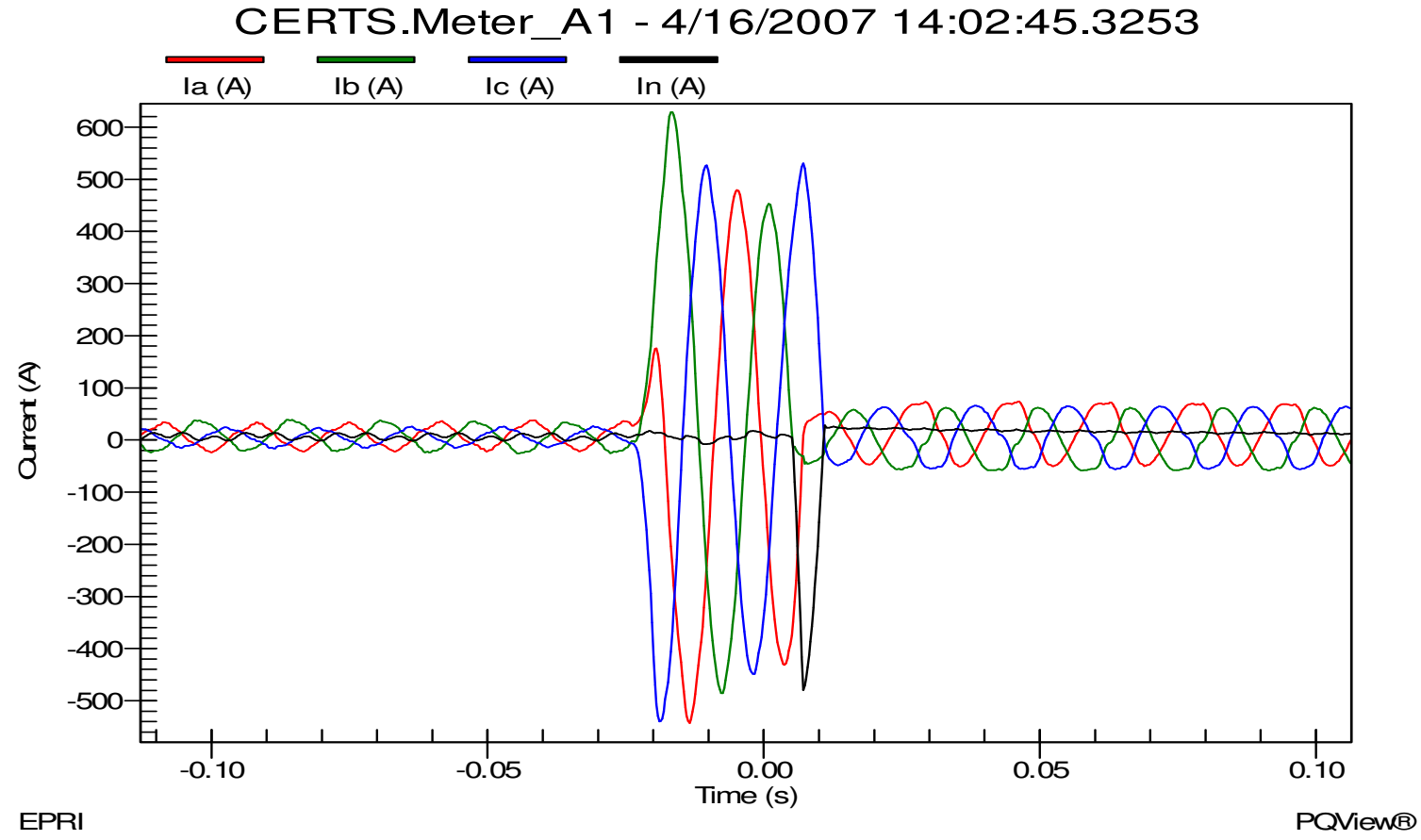

Figure $6 \mathrm{~b}$ - Current waveforms at Meter A1 during breaker CB1 opening 
Load on the AEP Load Bank was reduced to $0 \mathrm{~kW}$ and the utility grid was reconnected by a manual "Close" of breaker CB1. After CB1 was closed, the IEEE 1547 voltage test did shut-off and the static switch did not immediately attempt to reconnect, since the reconnection timer was set to 300 seconds. After 300 seconds, the static switch did a synchronized "Close" and the microgrid transitioned from island mode to utilityconnected mode of operation. Figure $6 \mathrm{c}$ shows $\mathrm{kW}$ load at Meter 2 increasing from $0 \mathrm{~kW}$ to approximately $28 \mathrm{~kW}$ from the utility grid. Coincidently, $\mathrm{kW}$ load at Meter A1 decreased from $34.5 \mathrm{~kW}$ to $10 \mathrm{~kW}$, as shown in Figure $6 \mathrm{~d}$.

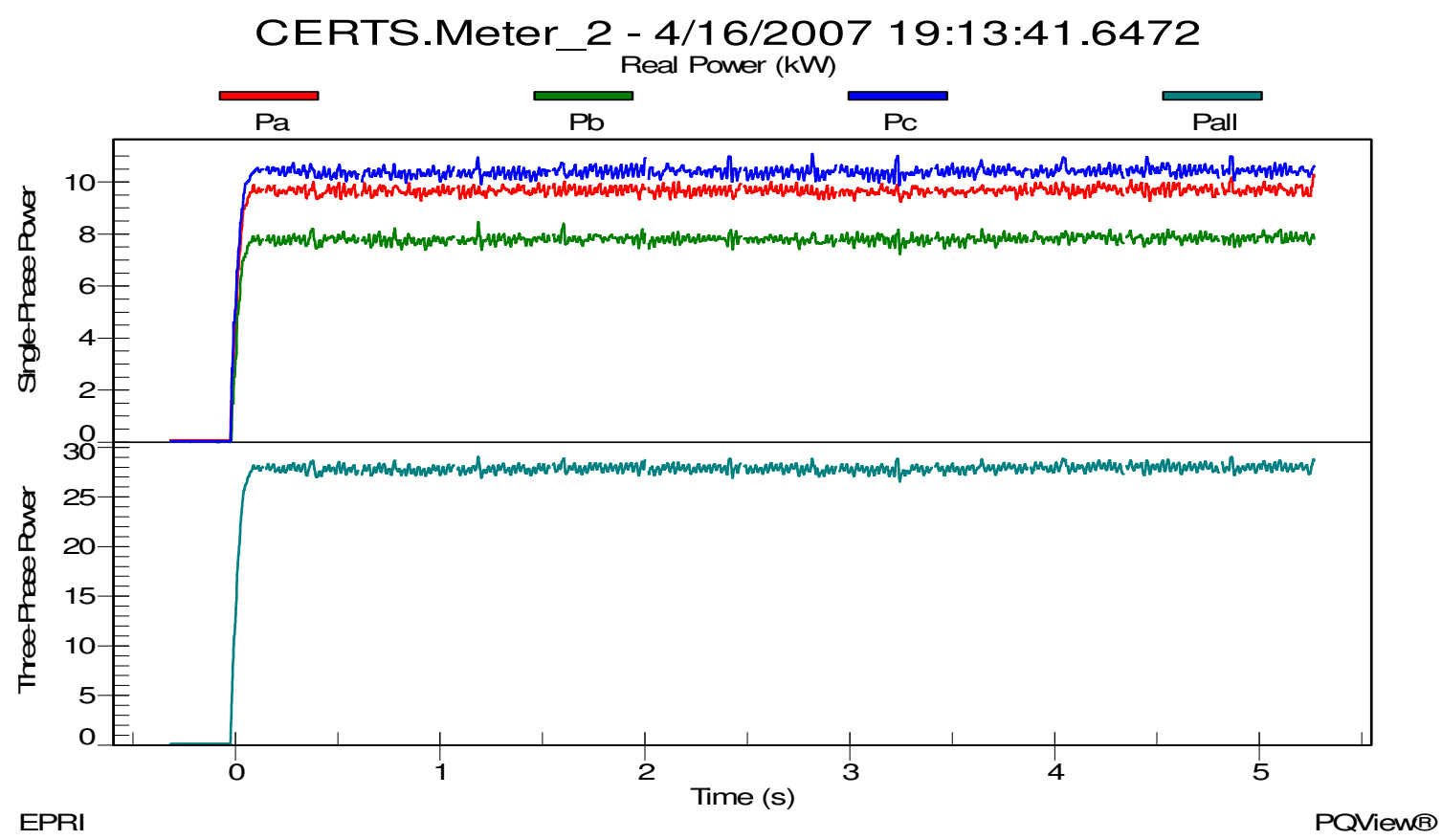

Figure 6c - Meter 2 load during synchronized close of the static switch 


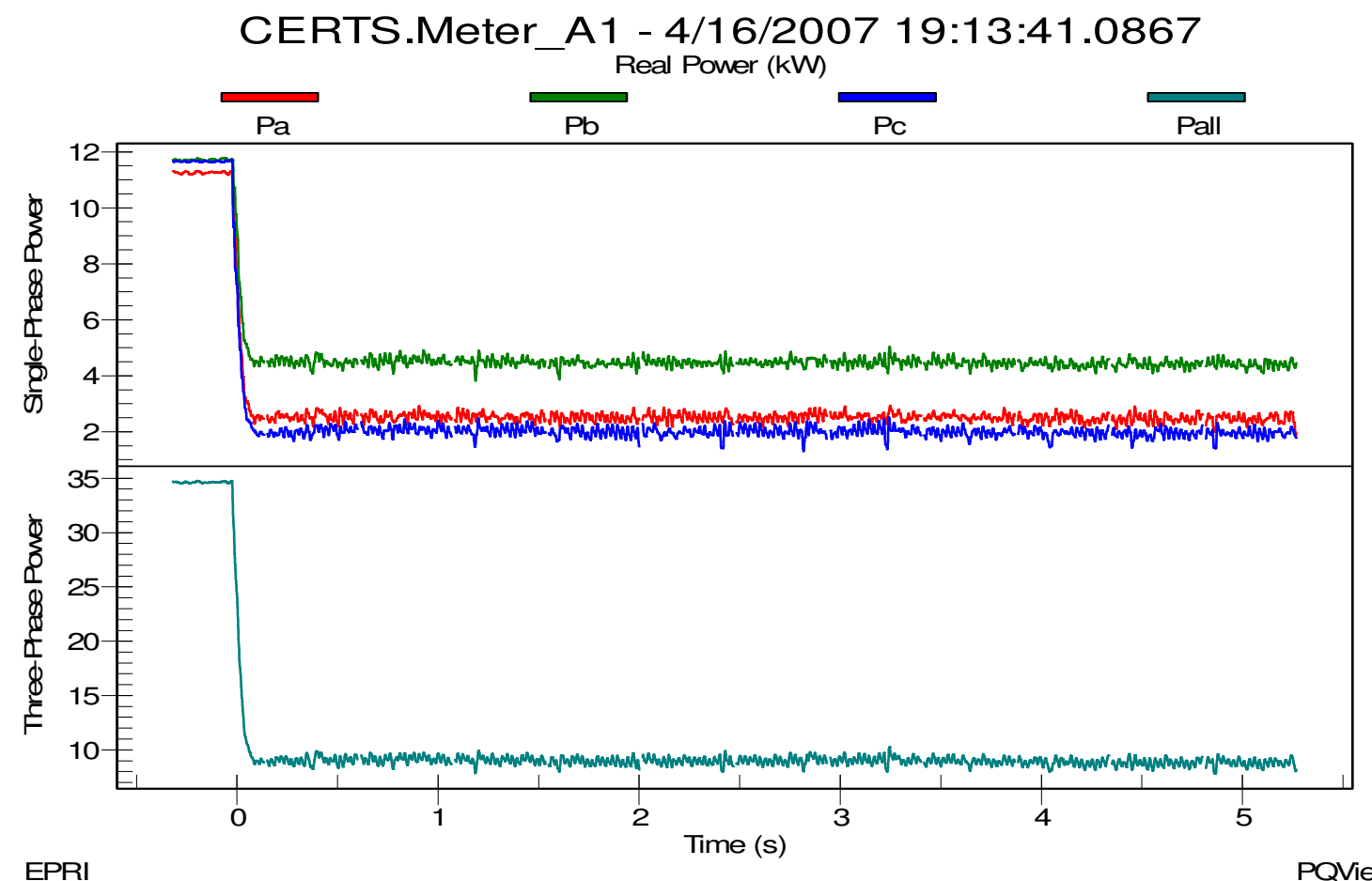

EPRI

PQVien®

Figure 6d - Meter A1 load when microgrid transitioned from island mode to utility-connected mode Figures 6e and 6f show the $\mathrm{kW}$ load changes in Zone 3 at Load Meter 3 and $\mathrm{kVAr}$ changes at Meter A1, respectively, when the microgrid transitioned from island mode to utility-connected mode.

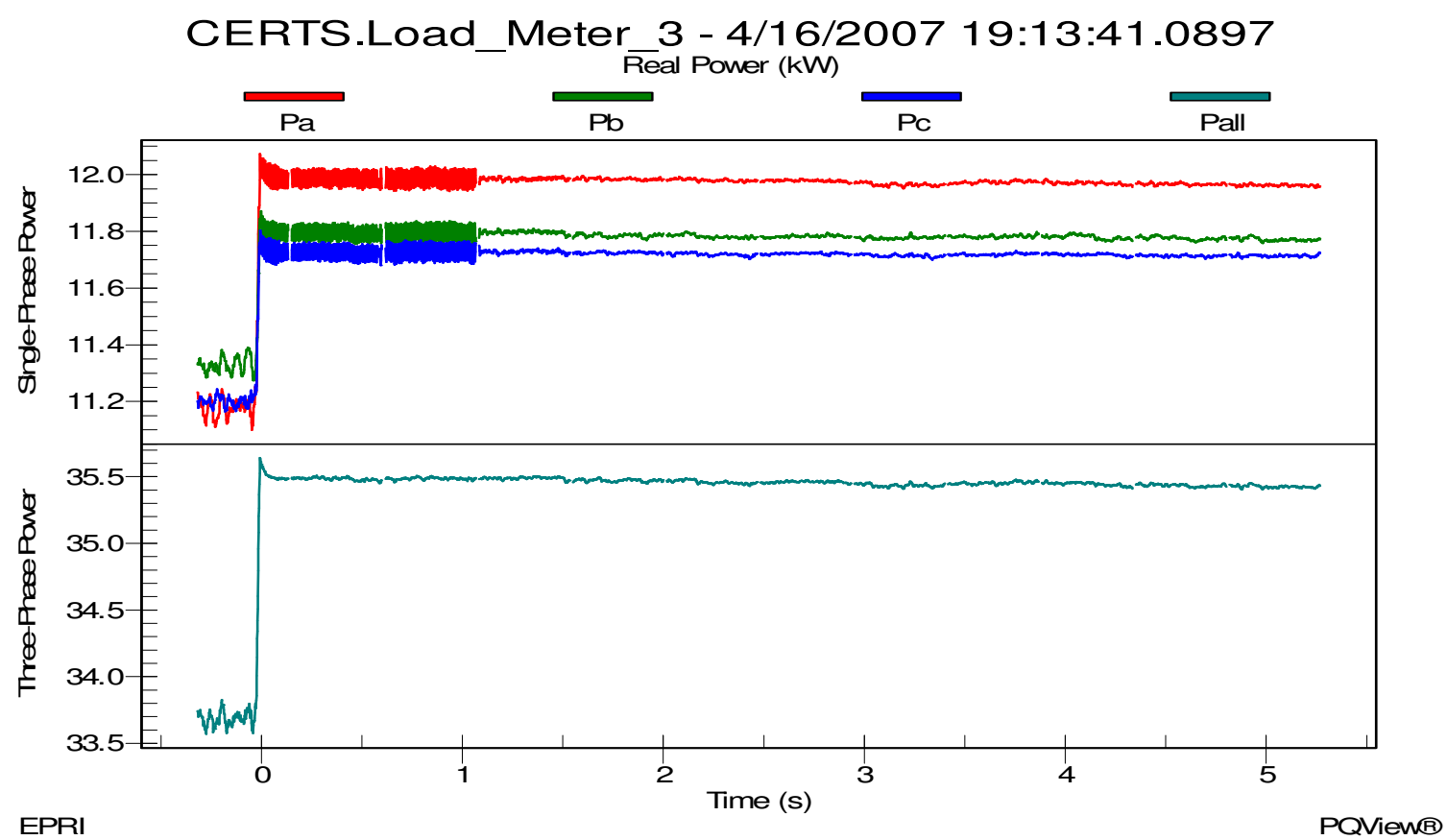

Figure 6e - Zone 3 load when microgrid transitioned from island mode to utility-connected mode 


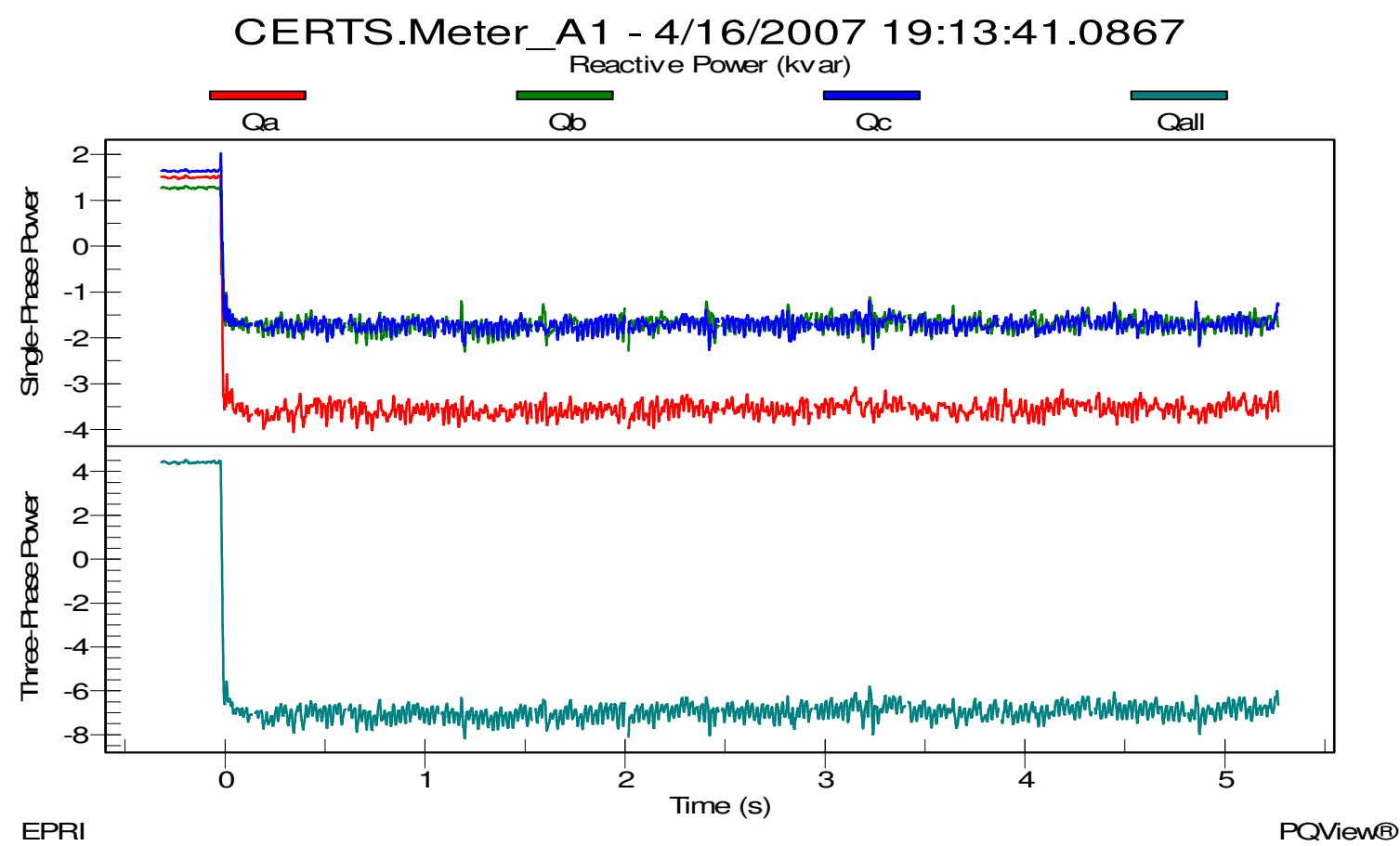

Figure $6 \mathrm{f}-\mathrm{kVAr}$ changes in Zone 3 when Microgrid transitioned from island mode to utility-connected mode.

Figure $6 \mathrm{f}$ shows the inverter adjusting the total $\mathrm{kVAr}$ value at Gen-set $\mathrm{A} 1 \mathrm{from}+4.5 \mathrm{kVAr}$ when in island mode to $-7 \mathrm{kVAr}$ when utility-connected. For the static switch to synchronize with the utility grid, A-phase voltage on the microgrid needed to be raised higher than the voltages on phases B and C. In Figure 6f the kVAr change in A-phase was much greater than in phases B and C. Thus, the voltages raised and the frequency adjusted on the microgrid, as designed in the algorithm, for the static switch to synchronize with the utility grid.

The test below verifies that the microgrid detected and islanded itself upon the loss of the utility source upstream of the PCC for a small load loss with a reverse power event occurring.

Initial Setup for small loss on utility system:

Overload-Load Bank $=85 \mathrm{~kW}$

Load Bank $4=60 \mathrm{~kW}$

Load Bank $6=10 \mathrm{~kW}$ 
Reverse Power Set-point $=10 \mathrm{~kW}$ (i.e., import 3.33kW per phase)

In Figure $6 \mathrm{~g}$ a large load of approximately $135 \mathrm{~kW}$ is removed from the microgrid. Only a small amount of load remains beyond the PCC (i.e., actually less than $10 \mathrm{~kW}$ ) in Load Bank 6. After two seconds a reverse power event occurs and the static switch opens. This demonstrates that any load below the reverse power set-point for more than two seconds will initiate an anti-islanding event.

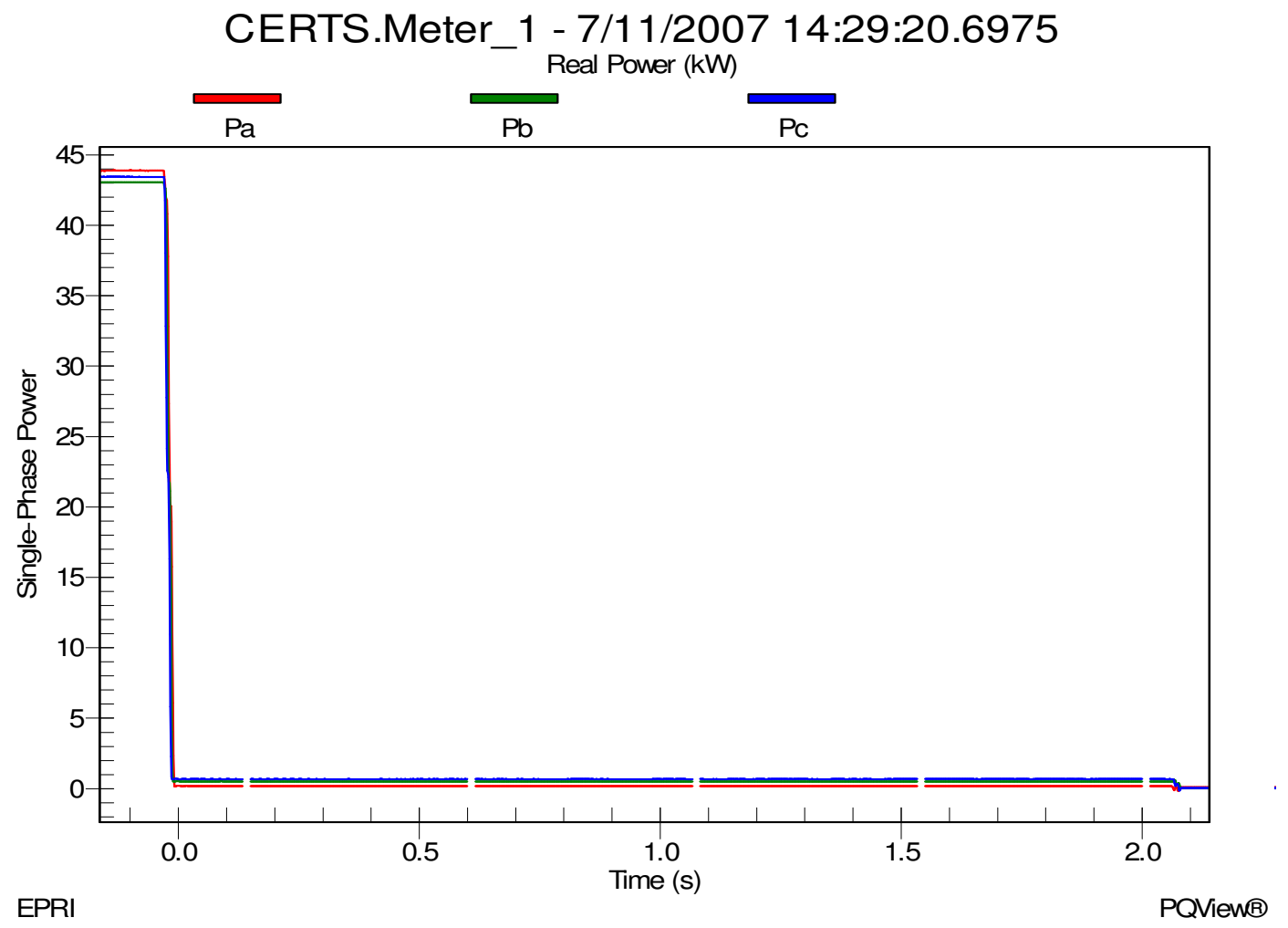

Figure $6 \mathrm{~g}$ - Single-phase $\mathrm{kW}$ at PCC when microgrid load was below reverse power set-point of $10 \mathrm{~kW}$.

\subsubsection{Single-Phase Reverse Power Test (Simulate Loss of Phase)}

Performance Goal:

Verify that the static switch islands the microgrid when a reverse power condition, due to an open-phase occurs.

Initial Setup:

Gen-set A1 = Unit Power Control 
Output Power Command $=20 \mathrm{~kW}$

MG Power/Frequency Droop $=-0.0833 \mathrm{~Hz} / \mathrm{kW}$

MG Voltage Command $=277 \mathrm{~V}$

Reverse Power Setting $=10 \mathrm{~kW}$

Load Bank $3=40 \mathrm{~kW}$

Load Bank $6=40 \mathrm{~kW}$

After the Gen-set A1 was running for a few minutes and supplying power to Load Bank 3, a synchronized "Close" of the static switch was initiated. After the static switch closed and steady-state conditions established, the microgrid and Load Bank 6 were connected to the utility grid.

This test began by removing the load on A-phase of Load Bank 6, shown in Figure 7a, with approximately $23.7 \mathrm{~kW}$ remaining (i.e., $11.85 \mathrm{~kW}$ in each of the two phases). While maintaining $0 \mathrm{~kW}$ on A-phase of Load Bank 6, the $\mathrm{kW}$ load on A-phase of Load Bank 3 was reduced from approximately $11.8 \mathrm{~kW}$ to $4 \mathrm{~kW}$, which in turn reduced the $\mathrm{kW}$ load on the A-phase at Meter 1 at the PCC to below the set threshold of $3.3 \mathrm{~kW}$, shown in Figure $7 \mathrm{~b}$. This created a reverse power condition on A-phase and opened the static switch.

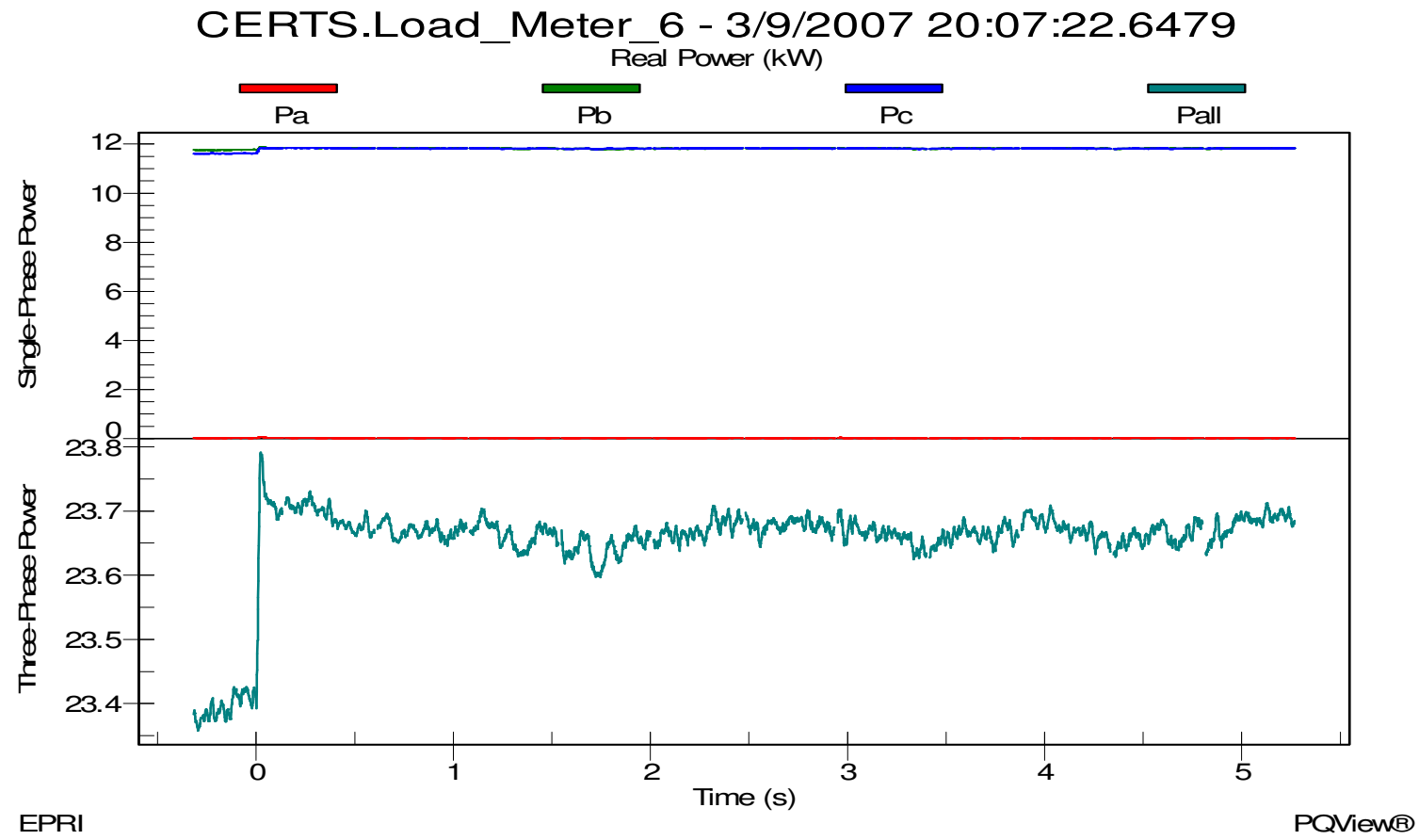

Figure 7a - Load Bank 6 real power before and after the static switch opened. 


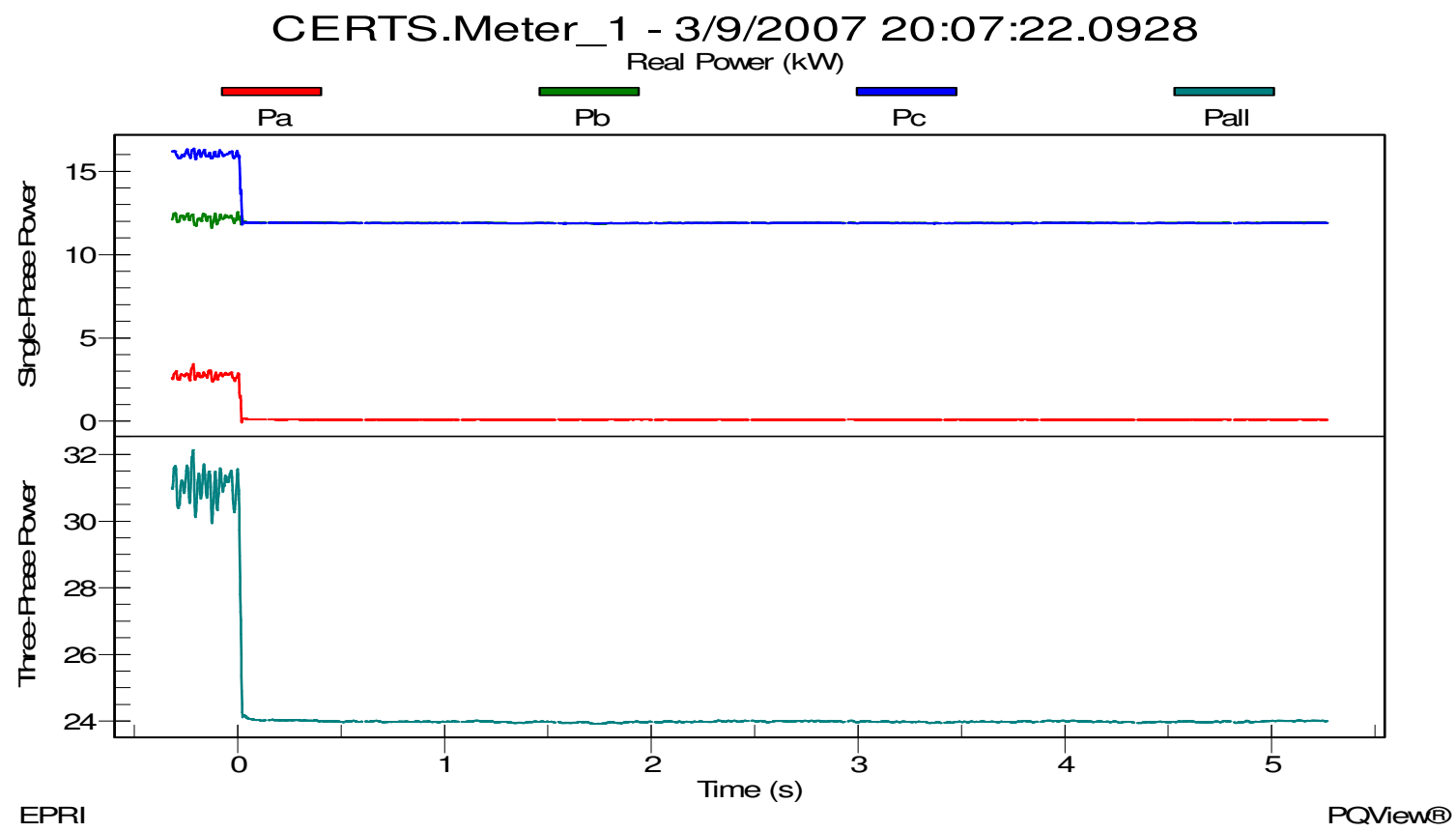

Figure $7 \mathrm{~b}$ - Load change at Meter 1 when static switch opened on reverse power

Once the static switch opened and voltage remained on the utility side of the switch, the load at Meter 1 decreased from approximately $31 \mathrm{~kW}$ to $24 \mathrm{~kW}$, serving only Load Bank 6 . A review of the sequence of actions which took place revealed that the static switch went to a lockout condition and fault condition in EMS (i.e., the DSP stopping the S\&C PES switch and opening CB14). The EMS did indicate a "Fault" condition and the "Reverse power Anti-islanding Alarm did turn "On". 


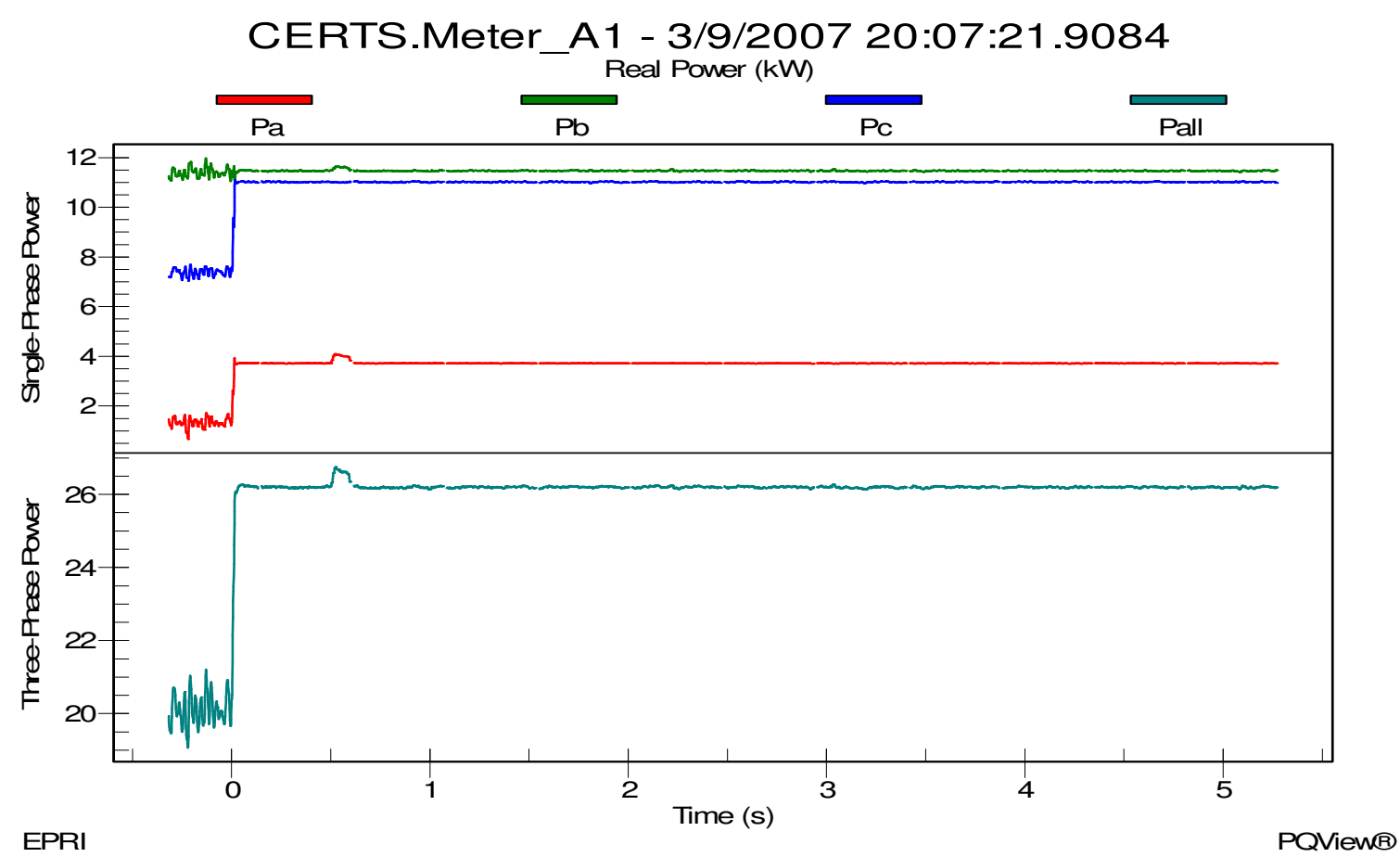

Figure 7c - Gen-set A1 kW load change when Microgrid was islanded

After the static switch opened, Gen-set A1 successfully picked up the Microgrid load remaining in Load bank 3. Figure 7c shows the change in $\mathrm{kW}$ load at Meter A1 when the static switch opened. Note, even though the Gen-set setting was $20 \mathrm{~kW}$, generator output was unbalanced on a per phase basis. This could lead to complication when operating the microgrid near the reverse power set-point at the PCC.

$\begin{array}{llll}\text { Monitoring Location } & \begin{array}{l}\text { Phase-A }(\mathbf{k W}) \\ \text { Before/After }\end{array} & \begin{array}{l}\text { Phase-B }(\mathbf{k W}) \\ \text { Before/After }\end{array} & \begin{array}{l}\text { Phase-C }(\mathbf{k W}) \\ \text { Before/After }\end{array} \\ \text { Meter 1 } & 3.0 / 0 & 12.0 / 12.0 & 16.0 / 12.0 \\ \text { Load Meter 6 } & 0 / 0 & 11.8 / 12.0 & 11.6 / 11.8 \\ \text { Meter 3 } & 2.5 / 0 & 0.5 / 0 & 4.2 / 0 \\ \text { Meter A1 } & 1.5 / 3.8 & 11.5 / 11.7 & 7.5 / 11.2 \\ \text { Load Meter 3 } & 3.8 / 3.5 & 11.8 / 11.5 & 11.6 / 11.0\end{array}$

Table 1 - Microgrid kW loading before/after the static switch opened

Although the output power set-point of Gen-set A1 was set at 20kW, the remaining load on the microgrid was approximately $26 \mathrm{~kW}$. Table 1 shows the relative change in $\mathrm{kW}$ load beyond the static switch before and after the static switch opened. Note the $\mathrm{kW}$ difference between Meter A1 and Load Meter 3 can be attributed to the voltage drop and electrical loss in the 225 feet of conductor between Load Bank 3 and A1 source, as well as 
transformer T51. In addition to $\mathrm{kW}$ load and voltage changes, the change in frequency when the microgrid was islanded is shown in Figure $7 \mathrm{~d}$. This figure shows the frequency changed from approximately $59.99 \mathrm{~Hz}$ to $59.95 \mathrm{~Hz}$ after the microgrid was islanded.

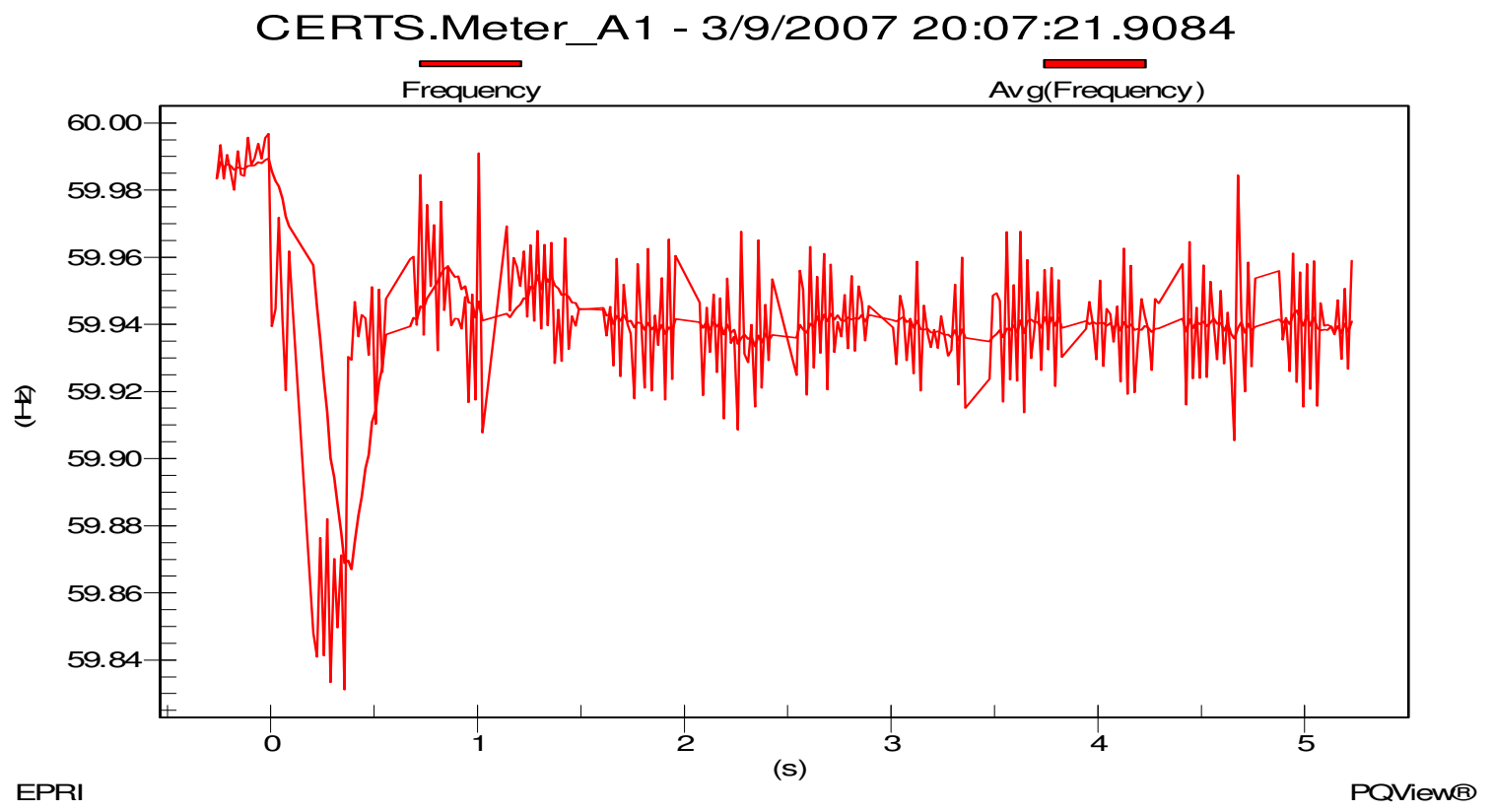

Figure 7d - Frequency change on the Microgrid after being islanded

The reverse power single phase condition was removed by bringing all A-phase loads at Zone 6 and at Zone 3 back to pretest conditions (i.e., 40kW each). The reverse power anti-islanding Alarm was turned "Off". Note the static switch needed to be "Reset" to return it to service, because to the EMS this was a mis-dispatch of the generation within the microgrid. Also, the static switch did not wait for the 300 seconds to reconnect to the utility grid, since voltage remained on the utility side of the static switch. Once all event and alarms were cleared, a "Start" command was initiated on the EMS and a synchronized closing of the static switch was observed. (Note the 300 seconds reconnect time was proven during later tests on the microgrid, satisfying this requirement.)

\subsubsection{Reverse Power, Anti-Islanding Microgrid Setting Reset Test Performance Goal:}

Verify that if a reverse power event occurs, due to a mismatch of Gen-set settings (Total Gen-set power > microgrid load), the static switch will lockout and go to the "Fault" state, where user intervention is required. 


\author{
Initial Setup: \\ Gen-set A1 = Unit Power Control \\ Output Power Command $=20 \mathrm{~kW}$ \\ MG Power/Frequency Droop $=-0.0833 \mathrm{~Hz} / \mathrm{kW}$ \\ MG Voltage Command $=277 \mathrm{~V}$ \\ Reverse Power Setting $=10 \mathrm{~kW}$ \\ Load Bank $3=40 \mathrm{~kW}$
}

After Gen-set A1 was running for a few minutes and supplying power to Load Bank 3, a synchronized "Close" of the static switch was initiated. As soon as the static switch closed and steady-state conditions established, the microgrid was connected to the utility grid with the total power flow at Meter 1 approximately $18 \mathrm{~kW}$.

When the test began the power measurement at Meter 1 was $18 \mathrm{~kW}$. Waited 5 seconds with no reverse power trip occurring (i.e., value was above the minimum $10 \mathrm{~kW}$ threshold value). Then, reduced load at Load Bank 3 by $5 \mathrm{~kW}$, waited about 5 seconds and no reverse power trip occurred. Reduced load at Load Bank 3 by an additional 5kW and the static switch opened, after the second $5 \mathrm{~kW}$ load reduction. Once the static switch opened, shown in Figure 8a, the Event Logger recorded a reverse power event, DAS was triggered and data was recorded. The EMS entered a "Fault" state, opening CB14, and the "Reverse Power Anti-islanding Microgrid Settings Reset" alarm turned "On".

Although the output power set-point of Gen-set A1 was set at 20kW, the remaining load on the microgrid was approximately $28.5 \mathrm{~kW}$, which was successfully served by Gen-set A1. Table 2 shows the relative change in $\mathrm{kW}$ load per phase in the microgrid before and after the static switch opened. As mentioned earlier, the $\mathrm{kW}$ differences can be attributed to the voltage drop and electrical loss in the microgrid. In addition to load and voltage changes, the frequency changed from approximately $59.99 \mathrm{~Hz}$ to $59.95 \mathrm{~Hz}$ after the Microgrid was islanded.

$\begin{array}{llll}\text { Monitoring Location } & \begin{array}{l}\text { Phase-A }(\mathbf{k W}) \\ \text { Before/After }\end{array} & \begin{array}{l}\text { Phase-B }(\mathbf{k W}) \\ \text { Before/After }\end{array} & \begin{array}{l}\text { Phase-C }(\mathbf{k W}) \\ \text { Before/After }\end{array} \\ \text { Meter } 1 & 3.2 / 0 & 2.0 / 0 & 4.0 / 0\end{array}$




$\begin{array}{llll}\text { Meter } 3 & 3.0 /-0.2 & 1.3 /-0.5 & 3.4 /-0.5 \\ \text { Meter A1 } & 6.7 / 9.3 & 8.2 / 9.6 & 6.2 / 9.6 \\ \text { Load Meter 3 } & 9.6 / 9.0 & 9.4 / 9.1 & 9.4 / 9.1\end{array}$

Table 2 - Microgrid kW loading before/after the static switch opened

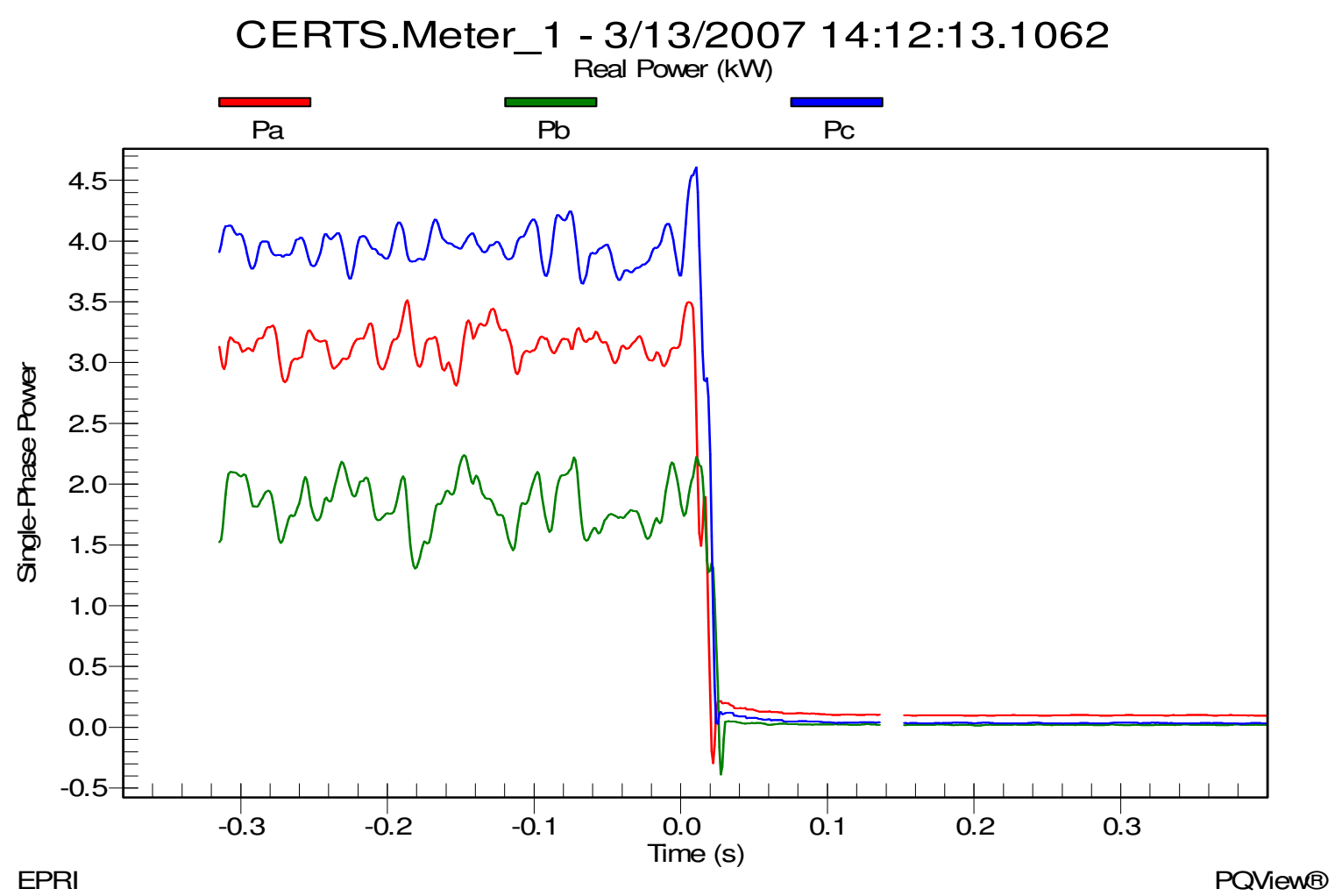

Figure 8a-Meter 1 load decrease after the static switch opened. 


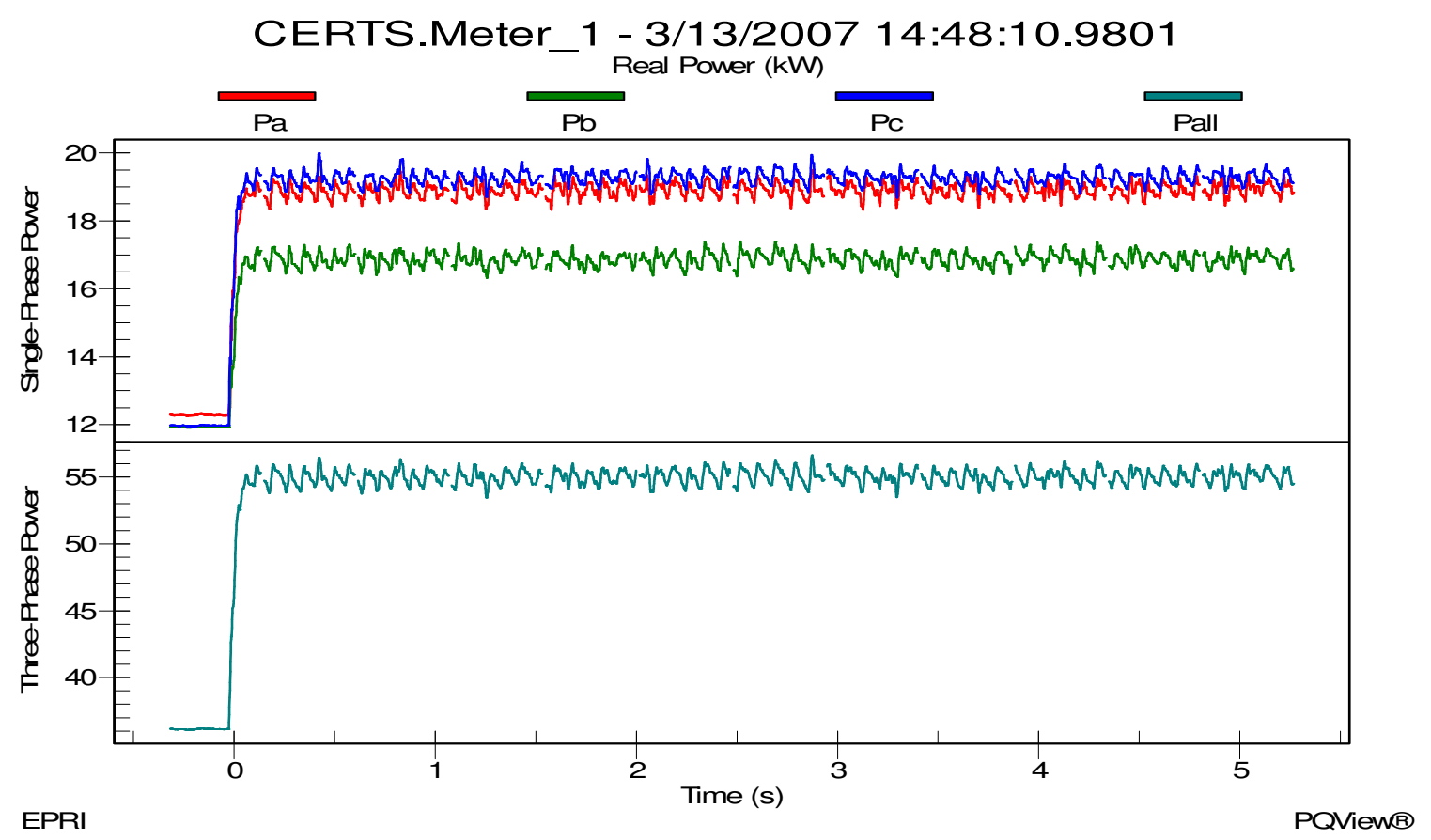

Figure $8 b$ - Load increase at Meter 1 during synchronized close of static switch.

Load Bank 3 and 6 were each set at 40kW. All events and alarms were cleared. The static switch successfully synchronized and reconnected after the "Start" command was initiated with load at Meter 1 increasing from approximately $36 \mathrm{~kW}$ to $55 \mathrm{~kW}$, shown in Figure $8 \mathrm{~b}$. During this test the first trigger was noted on reset of the static switch, and the second trigger was caused after the static switch was issued the start command and successfully closed. Likewise, during this transition from island to grid-connected mode, Gen-set A1 decreased its output from $35 \mathrm{~kW}$ to $18 \mathrm{~kW}$, shown in Figure $8 \mathrm{c}$, and frequency increased from $59.86 \mathrm{~Hz}$ to $60.01 \mathrm{~Hz}$ shown in Figure 8d. 


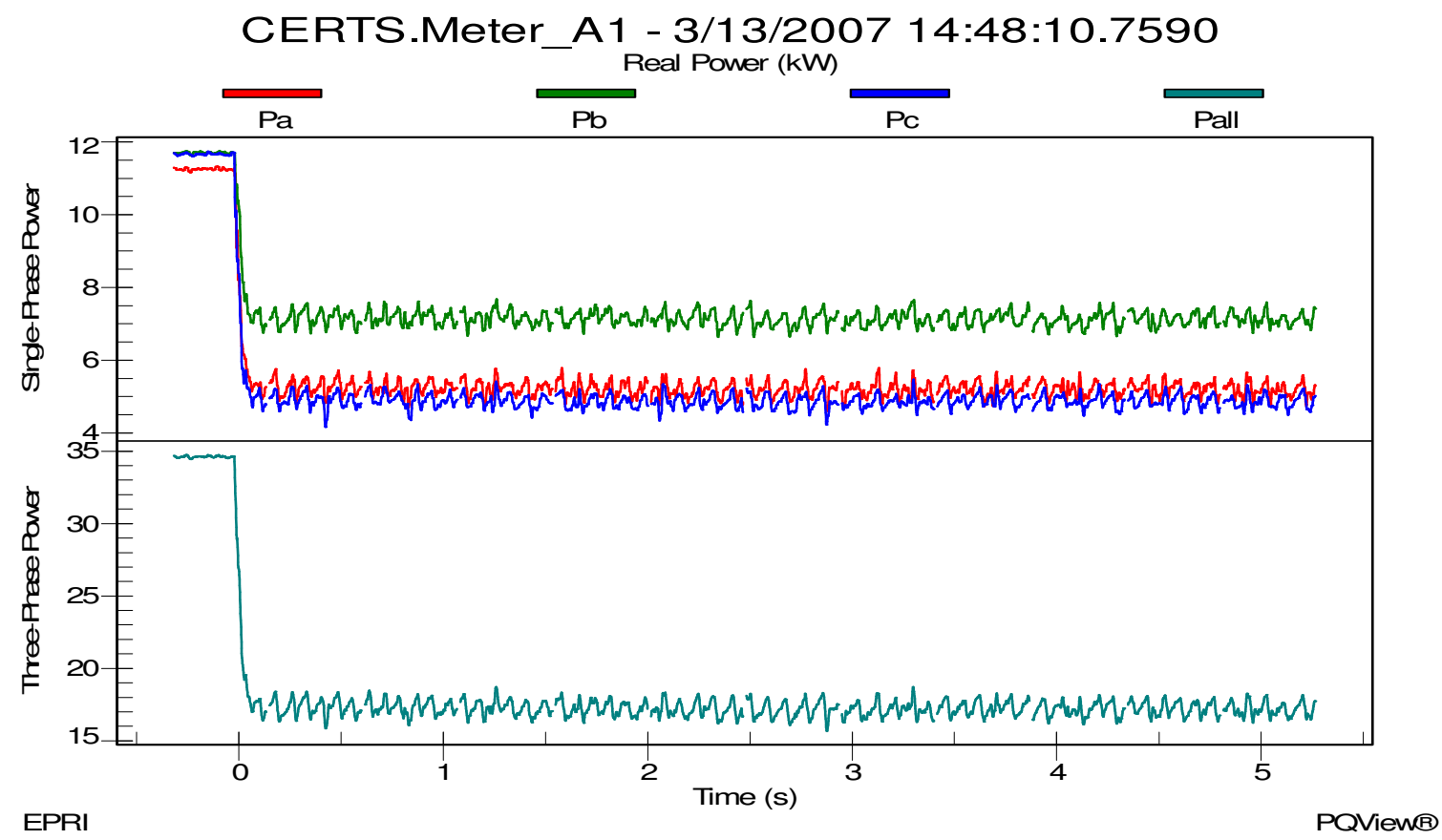

Figure 8c - Gen-set A1 output during synchronized close of static switch

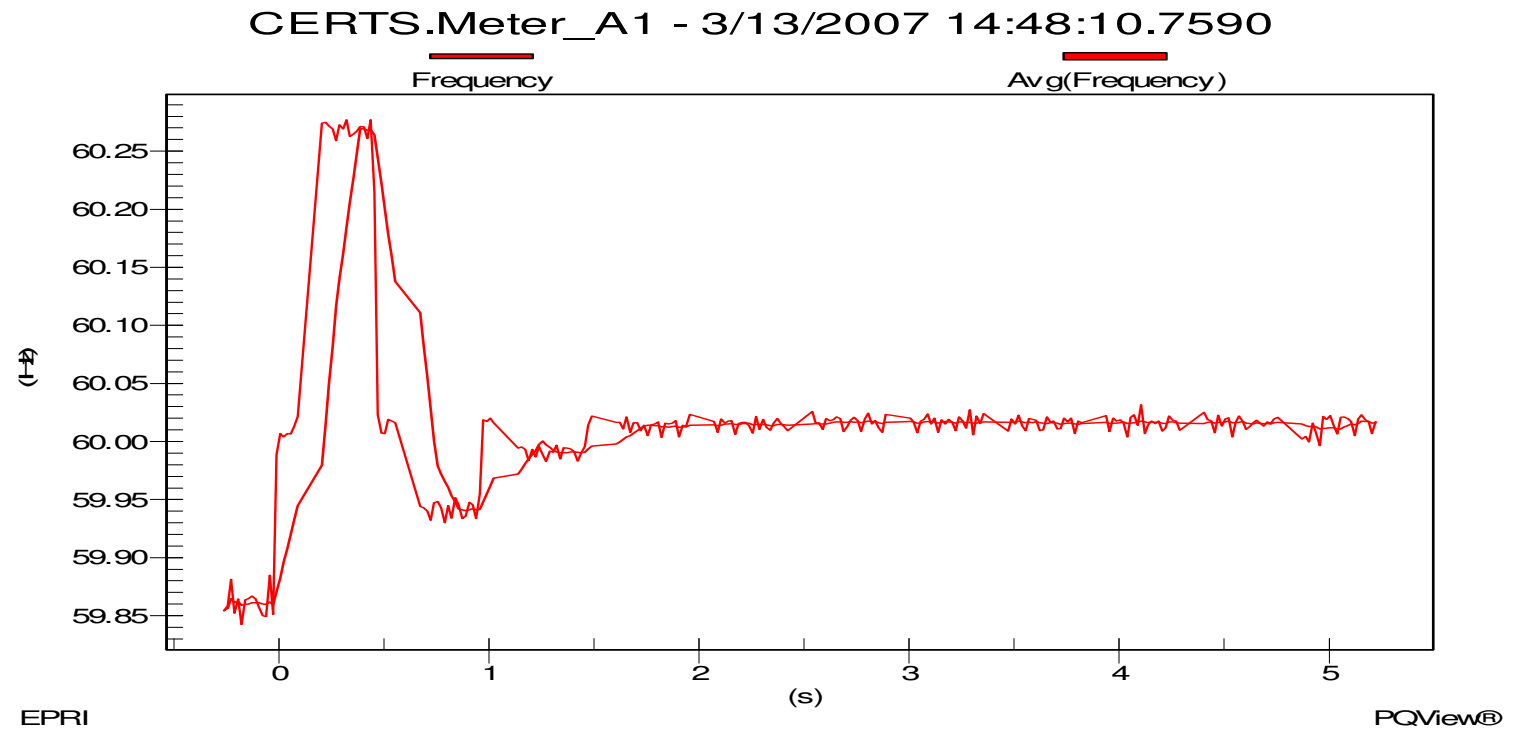

Figure 8d - Frequency on the Microgrid during synchronized close of static switch

\subsubsection{De-energized Bus (Dead Bus) Reclose Test}

Performance Goal:

Verify that the static switch can close when de-energized bus conditions $(<15 \mathrm{~V})$ on the DG side are measured and that the Dead Bus Reclose algorithm requires user intervention (i.e. Operator needs to "Enable: the Dead Bus Reclose using pushbutton in the EMS). 
Initial Setup:

Gen-set A1 = Unit Power Control

Output Power Command $=20 \mathrm{~kW}$

MG Power $/$ Frequency Droop $=-0.0833 \mathrm{~Hz} / \mathrm{kW}$

MG Voltage Command $=277 \mathrm{~V}$

Relay Settings $=$ A $($ Delayed $)$

Load Bank $3=40 \mathrm{~kW}$

Load Bank $6=40 \mathrm{~kW}$

After a few minutes, the "Manual Open" command was removed in the EMS and the static switch remained in the "Open" position. Then a "Dead-Bus Reclose". This DeadBus Reclose event was captured in the Event Logger with the change in load at Meter 1 increasing from $36 \mathrm{~kW}$ to $75 \mathrm{~kW}$, shown in Figure 9a. Note, prior to the static switch closing, Meter 1 is serving Load Bank 6 in Zone 6.

During the Dead-Bus Reclose of the microgrid, Figure 9a shows the expected kW inrush, due to transformer T51 (i.e., 112.5kVA rated) which is connected within the microgrid. 


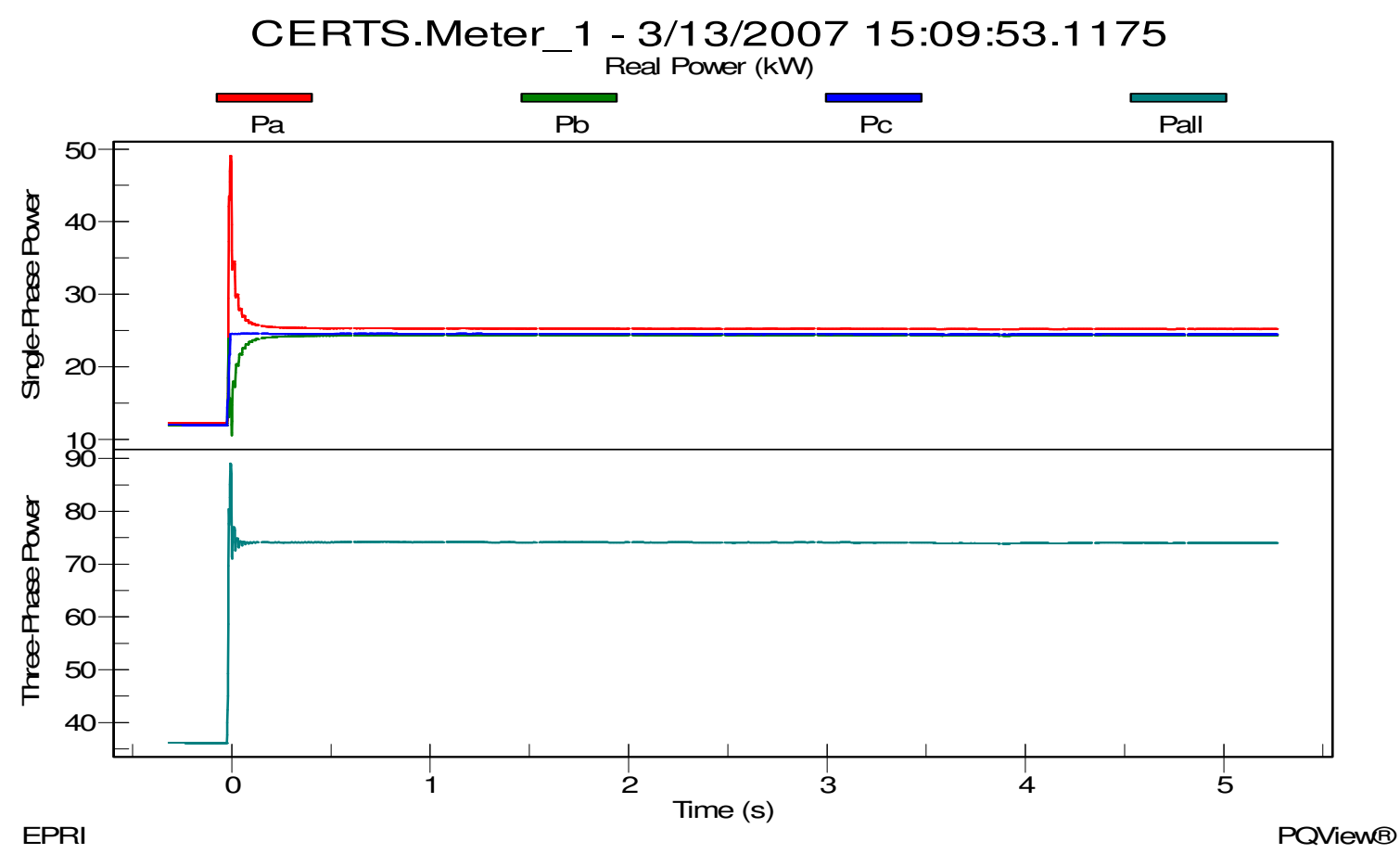

Figure 9a - Load increase at Meter 1 (PCC) during a Dead-Bus Reclose of the static switch

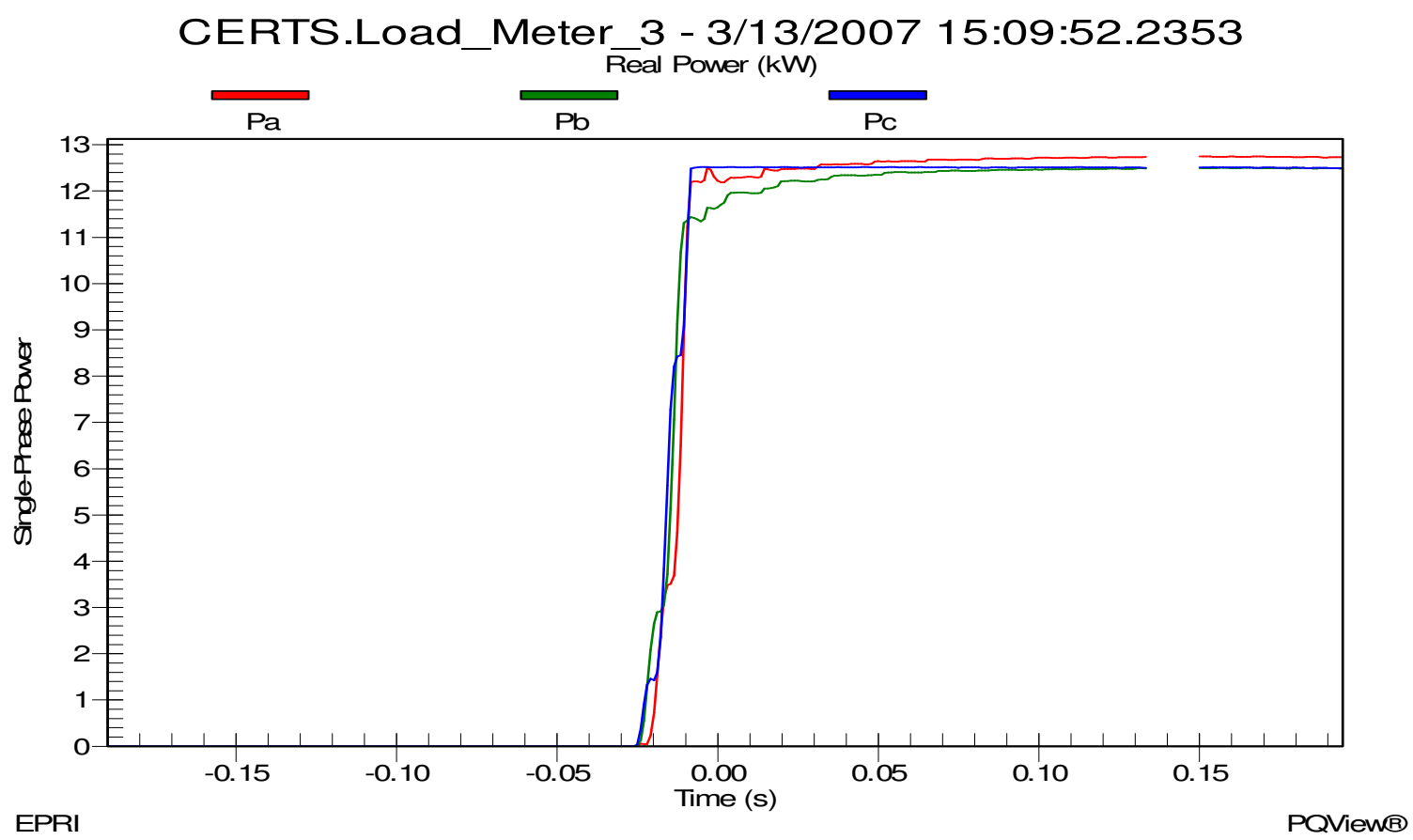

Figure 9b - Load Bank 3 connected in the Microgrid during a Dead-Bus Reclose of the static switch 
Load Bank 3 was also connected in the microgrid and set at $40 \mathrm{~kW}$ (i.e., $13.3 \mathrm{~kW}$ per phase), but the actual load was approximately $38 \mathrm{~kW}$ with minimal inrush, shown in Figure $9 b$, when energized and connected to the utility grid.

After this testing was performed the protection settings were revised, increasing their sensitivity. The updated protection settings no longer allow for a Dead-Bus close to take place successfully, due to the transformer inrush currents.

\subsection{CONCLUSION}

The series of tests performed in section 6.0 validated the proper control and operation of the static switch, basic power and voltage control of the Gen-sets, and a preliminary check of the protection scheme. These tests provided confidence that the static switch would provide protection and fast disconnect from the utility for the CERTS microgrid during a disturbance for future tests.

One interesting test came during the large load condition in the "Loss of Utility Source Test" in section 6.1.2 where 6 p.u. current was recorded before the Gen-set turned off which seems high but was expected. The inverters on the Gen-sets are 125kVA rated which is twice the rating of the Gen-sets but were artificially limited to half. Also, the inverters were set up to deliver 2.5 p.u. fault current which is approximately 550A peak. This is the close to the 6 p.u. current recorded during the test. 


\title{
CERTS MICROGRID LABORATORY TEST BED
}

\author{
Test Plan Section 7.0 \\ Validate Protection Settings and \\ Initial Fault Testing
}

Prepared For:

California Energy Commission

Public Interest Energy Research Program

Arnold Schwarzenegger Governor

줄

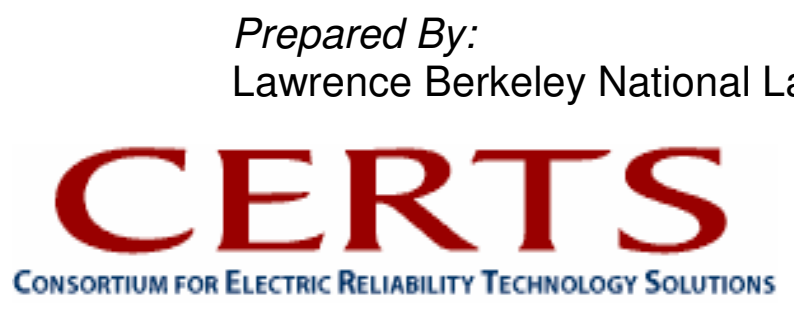



Prepared By:

Lawrence Berkeley National Laboratory

Joseph H. Eto, Principal Investigator

Berkeley, CA 94720

Ben Schenkman, Sandia National Laboratory

Harry Volkommer and Dave Klapp, American Electric Power

Commission Contract No. 500-03-024

Prepared For:

Public Interest Energy Research (PIER)

California Energy Commission

Bernard Treanton

Contract Manager

Mike Gravely

Program Area Lead

ENERGY SYSTEMS INTEGRATION

Mike Gravely

Office Manager

ENERGY SYSTEMS RESEARCH

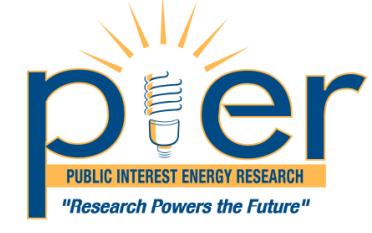

Martha Krebs, Ph.D.

PIER Director

Thom Kelly, Ph.D.

Deputy Director

ENERGY RESEARCH \& DEVELOPMENT DIVISION

Melissa Jones

Executive Director

\section{DISCLAIMER}

This report was prepared as the result of work sponsored by the California Energy Commission. It does not necessarily represent the views of the Energy Commission, its employees or the State of California. The Energy Commission, the State of California, its employees, contractors and subcontractors make no warrant, express or implied, and assume no legal liability for the information in this report; nor does any party represent that the uses of this information will not infringe upon privately owned rights. This report has not been approved or disapproved by the California Energy Commission nor has the California Energy Commission passed upon the accuracy or adequacy of the information in this report. 



\section{CERTS MICROGRID TEST REPORT}

\section{SECTION 7.0}

"Validate Protection Settings and Initial Fault Testing" 


\section{Table of Contents}

1.0

2.0

3.0

4.0

5.0

6.0

6.1

INTRODUCTION 1

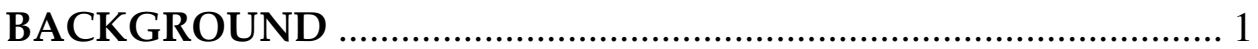

MICROGRID TESTBED SETUP ............................................... 2

PROPOSED TEST PLAN ...................................................... 5

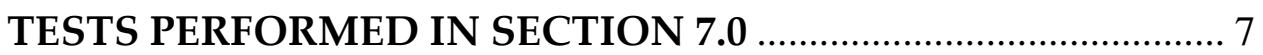

ANALYSES OF TEST RESULTS …........................................... 9

SECTION 7 - VALIDATE PROTECTION SETTINGS \& INITIAL

FAULT TESTING.

6.1.1 Zone 4 Circuit Breaker Settings and Utility Connected ................. 9

6.1.2 Zone 3 Circuit Breaker Settings and Utility Connected ............... 13

6.1.3 Zone 5 Circuit Breaker Settings and Utility Connected ............... 18

6.1.4 Zone 4 Three-Phase Grounded Fault, Gen-sets (A1+A2) and Utility Connected

6.1.5 Zone 3 A-Phase Line-to-Ground Fault, Gen-set A1 and Utility

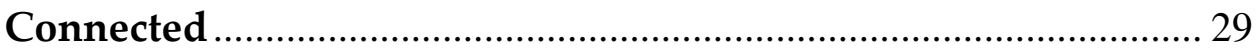

6.1.6 Zone 3 A-Phase Line-to-Ground Fault, Gen-sets (A1+A2) and Utility Connected ..................................................................... 33

6.1.7 Zone 5 B-Phase Line-to-Ground Fault, Gen-set B1 and Utility Connected.

6.1.8 Zone 5 B-Phase Line-to-Ground Fault, Gen-sets (A1+B1) and Utility Connected

6.1.9 Zone 4 B-Phase Line-to-Ground Fault, Gen-sets (A1+A2) and Utility Connected

6.1.10 Zone 2 C-Phase Line-to-Ground Fault, Gen-sets (A1+B1) and Utility Connected

6.1.11 Zone 6 C-Phase Line-to-Ground Fault, Gen-set A1 and Utility Connected.....

6.1.12 Zone 6 C-Phase Line-to-Ground fault, Gen-sets (A1+B1) and Utility Connected

6.1.13 Zone 3 A-to-B Phase Fault, Gen-sets (A1+A2) and Utility

Connected.

6.1.14 Zone 4 A-to-B Phase Fault, Gen-sets (A1+A2) and Utility Connected.

6.1.15 Zone 2 A-to-B Phase Fault, Gen-sets (A1+B1) and Utility Connected. 
6.1.16 Zone 5 A-to-B Phase Fault, Gen-sets (A1+B1) and Utility Connected.... CONCLUSION 


\section{List Of Figures}

Figure 1 - CERTS Microgrid Aerial Photo.................................................................... 3

Figure 2 - One Line Diagram of CERTS Microgrid Test Bed...................................... 4

Figure 3 - Simplified diagram of Test Bed showing Meter and Relay locations .... 4

Figure 4 - Diagram of DAS \& EMS Data networks..................................................... 5

Figure 10a - Load in Zone 4 when the overload fault is initiated ........................... 10

Figure 10b - Load in Zone 4 after circuit breaker CB41 "Opens" to isolate and

clear the fault ........................................................................................... 11

Figure 10d - Load in Zone 4 before/after the single phase-to-ground fault is

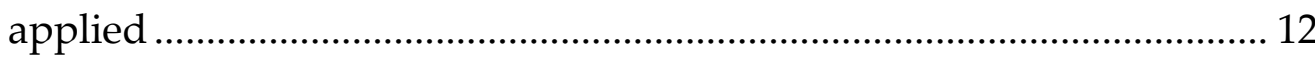

Figure 10e - Voltages, Currents, and Ground Current (IN) at Relay 2 when the

fault is detected and cleared by the static switch................................... 13

Figure 11a - Load change due to Zone 3 when the overload fault was initiated. 15

Figure 11b - Load change in Zone 3 after CB31 opened" to isolate and clear the

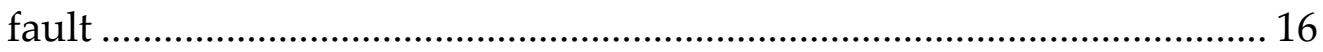

Figure 11c - Load change before/after the single phase-to-ground fault was

applied in Zone 3. .............................................................................. 17

Figure 11d - Voltages, Currents, and Ground current (IN) at Relay 2 when the

fault is detected and cleared by the static switch .................................... 18

Figure 12a - Load change when the overload fault was initiated in Zone 5......... 19

Figure 12b - Load change before/after CB51 opened to isolate and clear the fault

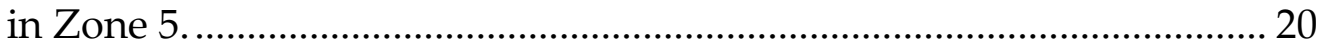

Figure 12c - Load in Zone 5 before/after the single phase-to-ground fault was

applied...................................................................................................... 21

Figure $12 \mathrm{~d}$ - Load change before/after the single phase-to-ground fault was

applied in Zone 5. ..................................................................................... 22

Figure 12e - RMS Voltage in Zone 3 before/after the single phase-to-ground fault

was applied ........................................................................................... 22

Figure 12f - Voltages, Currents, and Sequence Currents at Relay 2 when the fault

was detected and cleared by the static switch........................................ 23

Figure 13a - Power flow at Meter 1 before/after the overload fault was applied. 25

Figure 13b - Power flow at Meter 2 before/after CB41 opened............................... 26

Figure 13c - Gen-set A2 shutting down after CB41 opens ...................................... 27

Figure 13d - Gen-set A1 power flow before/after CB41 opened.............................. 28

Figure 13e - Gen-set A1 power flow reducing its output power to approximately

$12 \mathrm{~kW}$ after the static switch opens from a reverse power condition. .. 29

Figure 14a - Power flow in Meter 1 before/after the fault was cleared in Zone 3. 30

Figure 14b - Power flow in Meter 2 before/after the fault was cleared in Zone 3.31 
Figure 14c - Relay 2 (static switch) tripped due to ground over-current (67N1T) in 1 cycle after the single phase-to-ground fault was applied in Zone 3. 32 Figure 14d - Relay 3 (CB31) tripped on a ground over-current (67N1T) after the single phase-to-ground fault was applied in Zone 3.

Figure 14e - Gen-set A1 output power before/after the static switch opens and then when Zones 3 and 4 are isolated from the rest of the Microgrid. 33

Figure 15a - Meter 1 power flow before/after the static switch opens, islanding the microgrid from the utility grid after a single phase-to-ground fault was applied to Zone 3.

Figure 15b - Meter 2 power flow before/after the static switch opened, isolating the microgrid from the utility grid after a single phase-to-ground fault was applied to Zone 3. 35

Figure 15c - Relay 2 (static switch) tripped after the single phase-to-ground fault was applied in Zone 3. 36

Figure 15d - Relay 4 (CB41) tripped after the single phase-to-ground fault was applied in Zone 3.

Figure 15e - Meter 4 (CB41) voltage and current for a single phase-to-ground fault applied in Zone 3. 38

Figure $15 \mathrm{f}$ - Meter A2 voltage and current for a single phase-to-ground fault applied in Zone 3. 38

Figure 15g - Meter A1 voltage and current for a single phase-to-ground fault applied in Zone 3. 39

Figure 15h - Gen-set A2 output power before/after the static switch "Opens" and when Zone 4 is separated from the rest of the microgrid. 40

Figure 15i - Relay 3 (CB31) tripped due to a ground over-current (67N1T) when the single phase-to-ground fault was initiated in Zone 3 41

Figure $15 \mathrm{j}$ - Gen-set A1 output power before/after the static switch opened, when CB41 opens isolating Zone 4, and when Zone 3 is isolated from the rest of the microgrid. 41

Figure 16a - Meter 1 power flow before/after the static switch opened, islanding the microgrid from the utility grid when a single phase-to-ground fault was applied in Zone 5. 43

Figure 16b - Meter 2 power flow before/after the static switch opened, islanding the microgrid from the utility grid when a single phase-to-ground fault was applied in Zone 5. 44

Figure 16c - Relay 5 (CB51) tripped due to ground over-current (67N1T) after the single phase-to-ground fault was initiated in Zone 5. 45

Figure 16d - Meter 5 power flow for a single phase-to-ground fault applied in

Zone 5. 46 
Figure 16e - Relay 2 (static switch) tripped due to negative sequence (67Q1T) after the single phase-to-ground fault was applied in Zone 5.

Figure 17a-Meter 1 power flow for a single phase-to-ground fault applied in Zone 5.

Figure 17b Meter 2 power flow for a single phase-to-ground fault applied in Zone 5.

Figure 17c - Power produced by Gen-set A1 before/after a B-phase phase-toground fault in Zone 5.

Figure 17d - Power produced by Gen-set A2 before/after a B-phase phase-toground fault in Zone 5.

Figure17e - Relay 5 (CB51) tripped due to a ground over-current (67N1) after the single phase-to-ground fault was initiated in Zone 5. 50

Figure17f - Meter B1 voltage and current for a single phase-to-ground fault applied in Zone 5.

Figure17g- Meter 5 (CB51) voltage and current for a single phase-to-ground fault applied in Zone 5 52

Figure 18a Meter 1 power flow before/after the static switch opened, islanding the microgrid from the utility grid when a single phase-to-ground fault was applied in Zone 4.

Figure 18b Meter 2 power flow before/after the static switch opened, islanding the microgrid from the utility grid when a single phase-to-ground fault was applied in Zone 4.

Figure 18c - Power produced by Gen-set A1 before/after the static switch and CB41 opened when a B-phase-to-ground fault was applied in Zone 4.

Figure 18d - Power produced by Gen-set A2 before/after the static switch and CB41 opened when a B-phase-to-ground fault was applied in Zone 4.

Figure 18e - Relay 2 (static switch) tripped due to a ground over-current (67N1T) when the single phase-to-ground fault is applied in Zone 4................. 56 Figure 18f - Relay 4 (CB41) tripped due to a ground over-current (67N1T) when the single phase-to-ground fault was initiated in Zone 4 ........................ 57

Figure 19a Meter 1 power flow before/after the static switch opened, islanding the microgrid from the utility grid when a single phase-to-ground fault was applied in Zone 2. 58

Figure 19b - Meter 2 power flow before/after the static switch opened, islanding the microgrid from the utility grid when a single phase-to-ground fault was applied in Zone 2. 
Figure 19c - Power produced by Gen-set A1 before/after the static switch opened from a C-phase-to-ground fault in Zone 2.

Figure 19d - Power produced by Gen-set B1 before/after the static switch opened from a C-phase-to-ground fault in Zone 2.

Figure 19e - Relay 2 (static switch) tripped due to a gournd over-current (67N1T)

when the single phase-to-ground fault was initiated in Zone 2.

Figure 19f - Meter 5 voltage and current during the applied single-phase-toground fault in Zone 2

Figure 19g - Meter 2 RMS voltage before/after CB31 opened.

Figure 19h - Relay 5 (CB51) tripped due to an undervoltage at T51 when the single phase-to-ground fault was initiated in Zone 2.

Figure 20a - Meter 1 power flow before/after the static switch opened, islanding the microgrid from the utility grid when a single phase-to-ground fault was applied in Zone 6.

Figure 20b - Meter 2 power flow before/after the static switch opened, islanding the microgrid from the utility grid when a single phase-to-ground fault was applied in Zone 6.

Figure 20c - Power produced by Gen-set A1 before/after the static switch opened from a C-phase phase-to-ground fault in Zone 6. 66

Figure 20d - Relay 2 (static switch) tripping due to a ground over-current (50GR) when the single phase-to-ground fault was initiated in Zone 6.

Figure 21a Meter 1 power flow before/after the static switch opened, islanding the microgrid from the utility grid when a single phase-to-ground fault was applied in Zone 6.

Figure 21b Meter 1 power flow before/after the static switch opened, islanding the microgrid from the utility grid when a single phase-to-ground fault was applied in Zone 6.

Figure 21c - Power produced by Gen-set A1 before/after the static switch opened from a C-phase-to-ground fault in Zone 6. 70

Figure 21d - Power produced by Gen-set B1 before/after the static switch opened from a C-phase-to-ground fault in Zone 6. 71

Figure 21e - Relay 2 (static switch) tripped due to a neutral over-current (67G1T) when the single phase-to-ground fault was initiated in Zone 6.......... 72

Figure 21f - Gen-set A1 shutting down due to a "No Field" fault......................... 73

Figure 21g - Gen-set B1 shutting down due to a "Boost" fault ................................ 73

Figure 21h - Gen-set A1 shutting down due to a "No Field" fault........................ 74

Figure 22a - Meter 1 power flow before/after the static switch opened, islanding the microgrid from the utility grid when the phase-to-phase fault was applied in Zone 3. 
Figure $22 \mathrm{~b}$ - Meter 2 power flow before/after the static switch opened, islanding the microgrid from the utility grid when a phase-to-phase fault was applied in Zone 3.

Figure 225c - Power produced by Gen-set A1 before/after the static switch opened when an A-to-B phase fault was applied in Zone 3.................. 78

Figure 22d - Power produced by Gen-set A2 before/after the static switch opened when an A-to-B phase fault was applied in Zone 3............................... 79

Figure 22e - Relay 2 (static switch) tripped due to a negative-sequence overcurrent (67Q1T) when the phase-to-phase fault was initiated in Zone 3.

Figure 23a - Meter 1 power flow before/after the static switch opened, islanding the microgrid from the utility grid when a phase-to-phase fault was applied in Zone 4.

Figure $23 \mathrm{~b}$ - Meter 2 power flow before/after the static switch opened, islanding the microgrid from the utility grid when a phase-to-phase fault was applied in Zone 4 .

Figure 23c - Power produced by Gen-set A1 before/after the static switch opened when an A-phase to B-phase fault was applied in Zone 4 84

Figure 23d - Power produced by Gen-set A2 before/after the static switch opened when an A-phase toB-phase fault was applied in Zone 4. 85

Figure 23e - Relay 2 (static switch) tripped due to a negative-sequence overcurrent (67Q1T) when the phase-to-phase fault was initiated in Zone 4.

Figure 23f - Relay 4 (CB41) tripped due to a negative-sequence over-current (67Q1T) when the phase-to-phase fault was initiated in Zone 4. 86

Figure 24a - Meter 1 power flow before/after the phase-to-phase fault was applied in Zone 2.

Figure $24 b$ - Meter 2 power flow before/after the phase-to-phase fault was applied in Zone 2.

Figure 24c - Power produced by Gen-set A1 before/after an A-phase to B-phase fault was applied in Zone 2.

Figure 24d - Power produced by Gen-set B1 before/after an A-to-B phase-tophase fault in Zone 2 is applied

Figure 25a - Meter 1 power flow before/after the static switch opened, islanding the microgrid from the utility grid when a phase-to-phase fault was applied in Zone 5.

Figure $25 \mathrm{~b}$ - Meter 2 power flow before/after the static switch opened, islanding the microgrid from the utility grid when a phase-to-phase fault was applied in Zone 5. 
Figure 25c - Power produced by Gen-set A1 before/after the static switch opened from an A-phase to B-phase fault in Zone 5.

Figure 25d - Power produced by Gen-set B1 before/after the static switch opened

from an A-phase to B-phase fault in Zone 5. ........................................... 96

Figure 25e - Relay 2 (static switch) tripped due to a negative-sequence overcurrent (67N1T) when the phase-to-phase fault was initiated in Zone 5.

Figure $25 f$ - Relay 5 (CB51) tripped due to a negative sequence over-current

(67N1T) when the phase-to-phase fault was initiated in Zone 5.

Figure 25g - Gen-set B1 current before/after a phase-to-phase fault was initiated

in Zone 5 98

Figure $25 \mathrm{~h}$ - Gen-set A1 current before/after a phase-to-phase fault was initiated

in Zone 5. 99 


\subsection{INTRODUCTION}

A series of tests were performed on the CERTS Microgrid by American Electric Power at the Walnut test site in Groveport, Ohio with support from Lawrence Berkeley National laboratory, Sandia National Laboratory, TECOGEN, The Switch (originally Youtility), Distributed-Energy (originally Northern Power) and University of Wisconsin. These tests were designed to demonstrate the CERTS Microgrid concepts of control and protection while connected to the utility electrical system and isolated (i.e., referred to as "islanded" from it. This paper describes the tests that were performed in Section 7.0 "Validate Protection Settings \& Initial Fault Testing" of the CERTS Micro-grid Test Plan.

\subsection{BACKGROUND}

The CERTS Microgrid Concept is an advanced approach for enabling integration of, in principle, an unlimited quantity of DER (e.g., distributed generation (DG), energy storage, etc.) into the electric utility grid. A key feature of a microgrid is its ability to separate and island itself from the utility system, during a utility grid disturbance. This is accomplished via intelligent power electronic interfaces and a single, high-speed, switch which is used for disconnection from the grid and synchronization to the grid. During a disturbance, the DER and corresponding loads can autonomously be separated from the utility's distribution system, isolating the microgrid's load from the disturbance (and thereby maintaining high level of service) without harming the integrity of the utility's electrical system. Thus, when the utility grid returns to normal, the microgrid automatically synchronizes and reconnects itself to the grid, in an equally seamless fashion. Intentional islanding of DER and loads has the potential to provide a higher level of reliability than that provided by the distribution system as a whole.

What is unique about the CERTS Microgrid is that it can provide this technically challenging functionality without extensive (i.e., expensive) custom engineering. In addition, the design of the CERTS Microgrid provides a high level of system reliability and great flexibility in the placement of DER within the microgrid. The CERTS Microgrid offers these functionalities at much lower costs than traditional approaches by incorporating peer-to-peer and plug-and-play concepts for each component within the microgrid.

The original concept was driven by two fundamental principles: 1.) A systems perspective was necessary for customers, utilities, and society to capture the full benefits of integrating DER into an energy system; and 2.) The business case for accelerating 
adoption of these advanced concepts will be driven, primarily, by lowering the up-front cost and enhancing the value offered by microgrids.

Each innovation was created specifically to lower the cost and improve the reliability of small-scale DG systems (i.e., installed systems with capacities ranging from less than $100 \mathrm{~kW}$ to $1000 \mathrm{~kW}$ ). The goal was to increase and accelerate realization of the many benefits offered by small-scale DG, such as their ability to supply waste heat at the point of need or to provide a higher level of reliability to some but not all loads within a facility. From an electric utility perspective, the CERTS Microgrid Concept is attractive because it recognizes that the nation's distribution system is extensive, aging, and will change over time which impacts power quality. The CERTS Microgrid Concept enables high penetration of DG systems without requiring re-design or re-engineering of the utility's distribution system.

Prospective applications of the CERTS Microgrid include industrial parks, commercial and institutional campuses, situations that require uninterrupted power supplies and high power quality, CHP systems, Greenfield communities, and remote applications. In short, wherever economic and DG location considerations indicate the need for multiple DG units within a (or among) site, the CERTS Microgrid offers the potential for a much more reliable, flexible, and lower cost solution compared to traditional engineering approaches for integrating DG.

\subsection{MICROGRID TESTBED SETUP}

The CERTS Microgrid Test Bed is operated at 480/277 volts (i.e., three-phase, four-wire) and consists of three TECOGEN Generators at 480 volts capable of producing $60 \mathrm{~kW}$ plus 60kVAr (Gen-set A1, Gen-set A2 and Gen-set B1) and four load banks (Load Bank 3, Load Bank 4, Load Bank 5 and Load Bank 6) capable of consuming 100kW plus 20kVAr each, as shown in Figure 2. Each of the generators are connected to a 112kVA isolation transformer and interfaced to the CERTS Microgrid through an inverter, developed by The Switch, where the algorithms for the CERTS Microgrid controls are embedded. . A semiconductor switch made by S\&C Electric Company, known as the static switch, connects the CERTS Microgrid to the utility grid. Load Banks 3 - 5 are the local loads in zones located beyond the static switch; and Load Bank 6 is a customer load in Zone 6 located on the utility side of the static switch. 


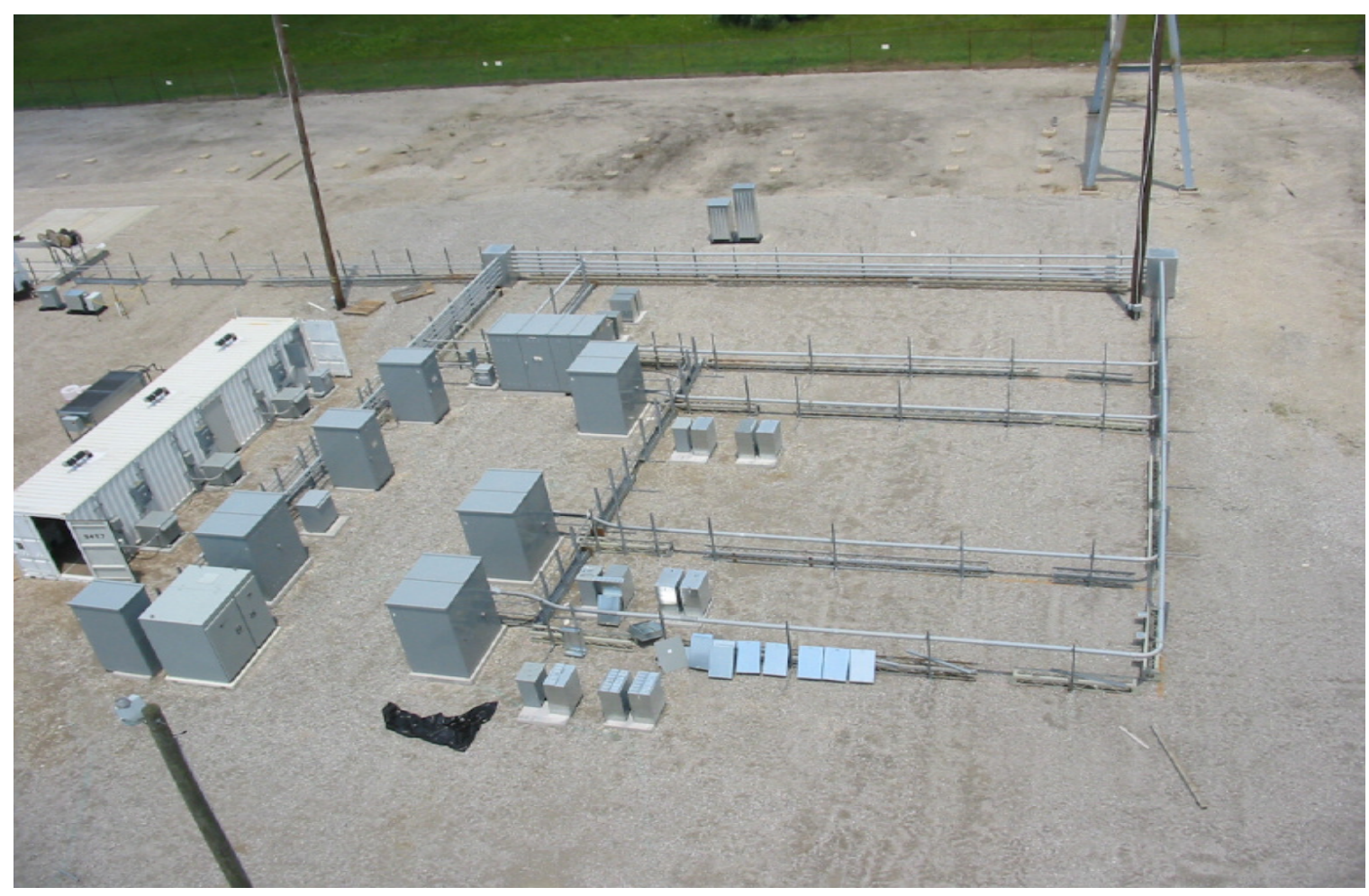

Figure 1 - CERTS Microgrid Aerial Photo

There are 6 zones in the Test Bed with Zones 2 - 6 contained within the CERTS Microgrid design and Zone 1 being the utility interface and referred to as the point-of-common coupling (PCC) to the grid. Each zone is protected by a Schweitzer SEL-351 relay. Faults of varying magnitude can be applied to each zone through an additional breaker which allows fault application and removal. 


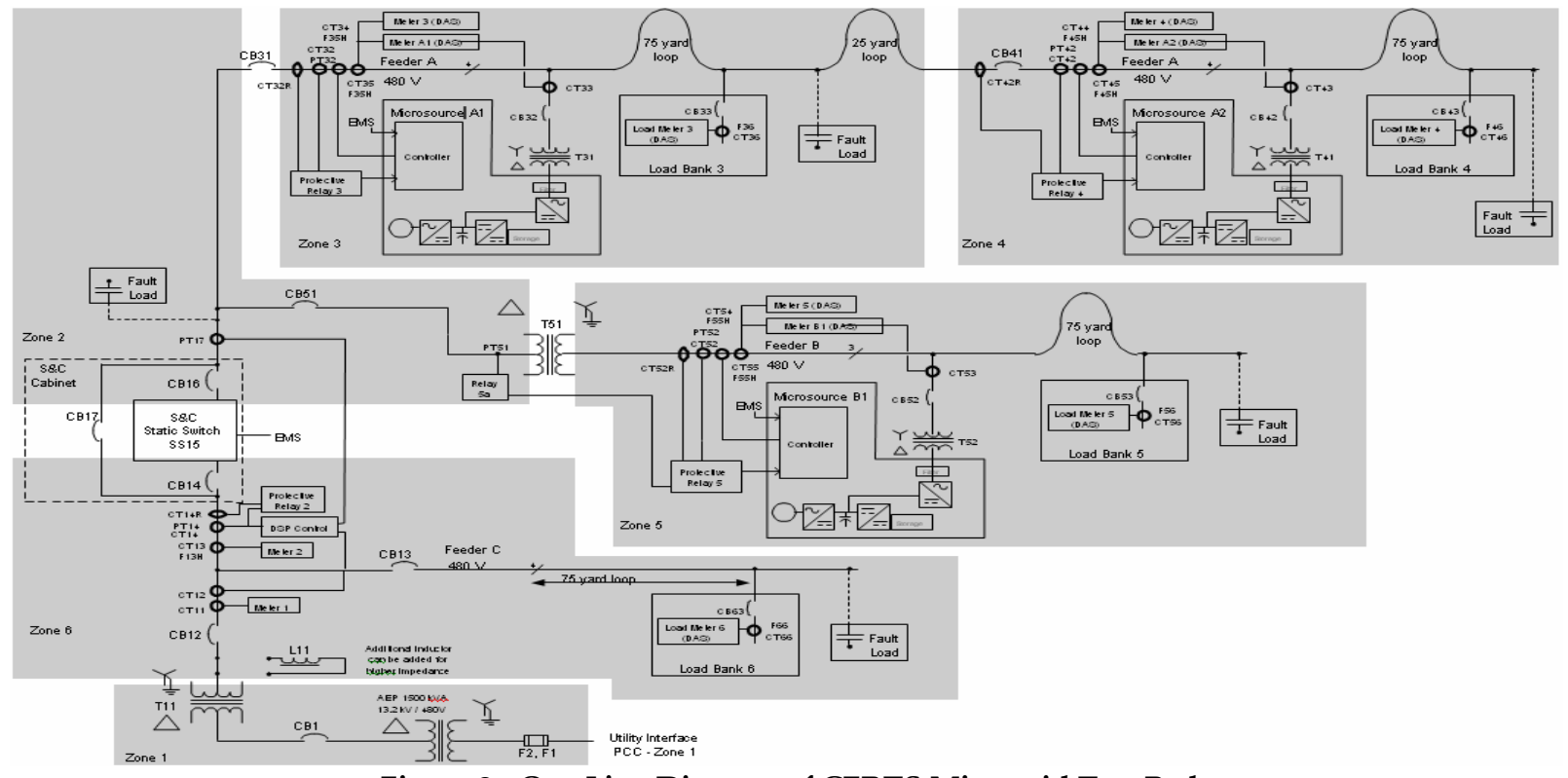

Figure 2 - One Line Diagram of CERTS Microgrid Test Bed

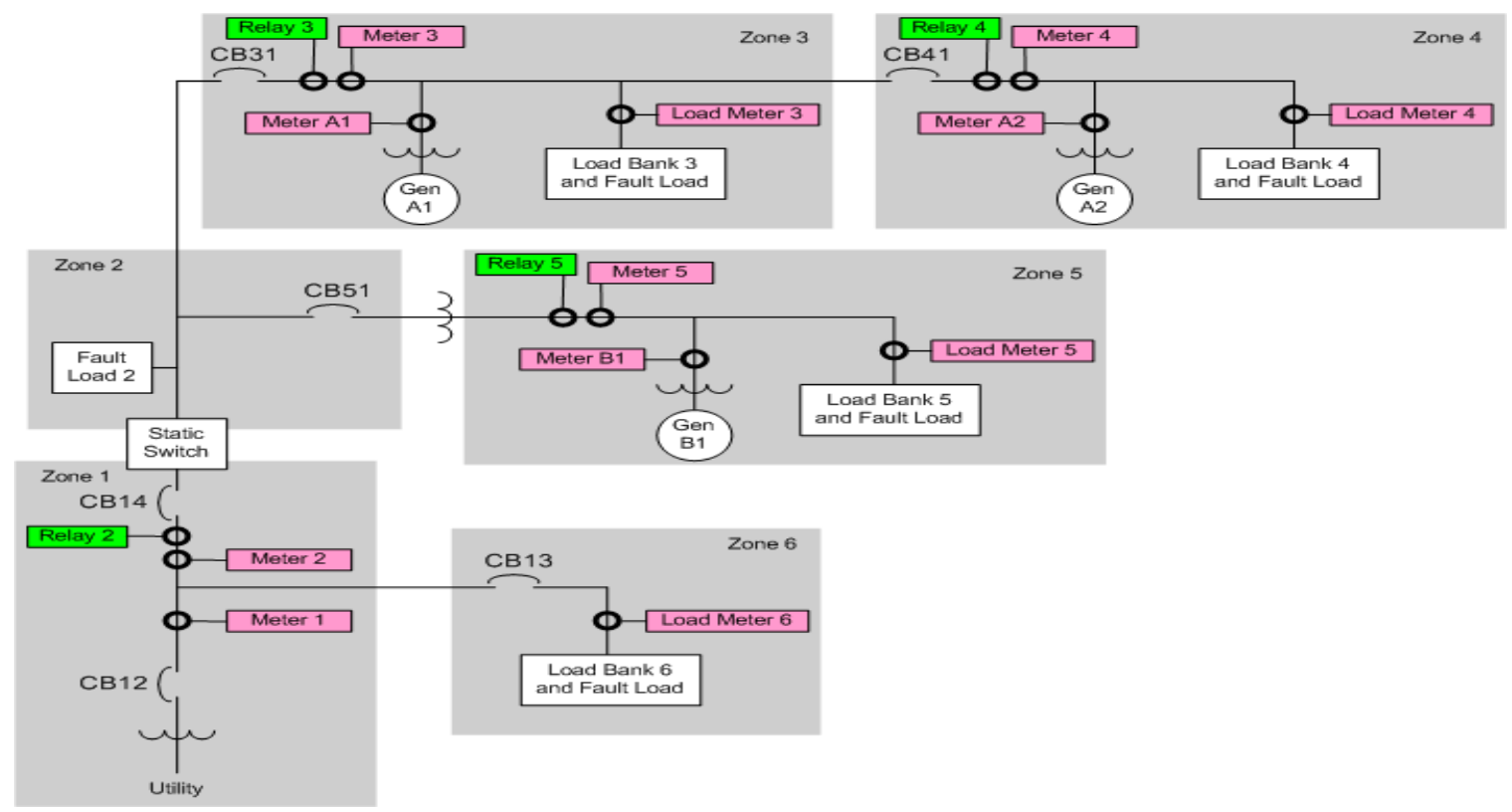

Figure 3 - Simplified diagram of Test Bed showing Meter and Relay locations

There are twelve PML ION 7650 meters placed through out the microgrid and shown in Figure 3, which monitor electrical system conditions, plus acquire phase current and voltage waveforms; and calculate RMS values of voltage, current, active power, reactive power, and frequency. 


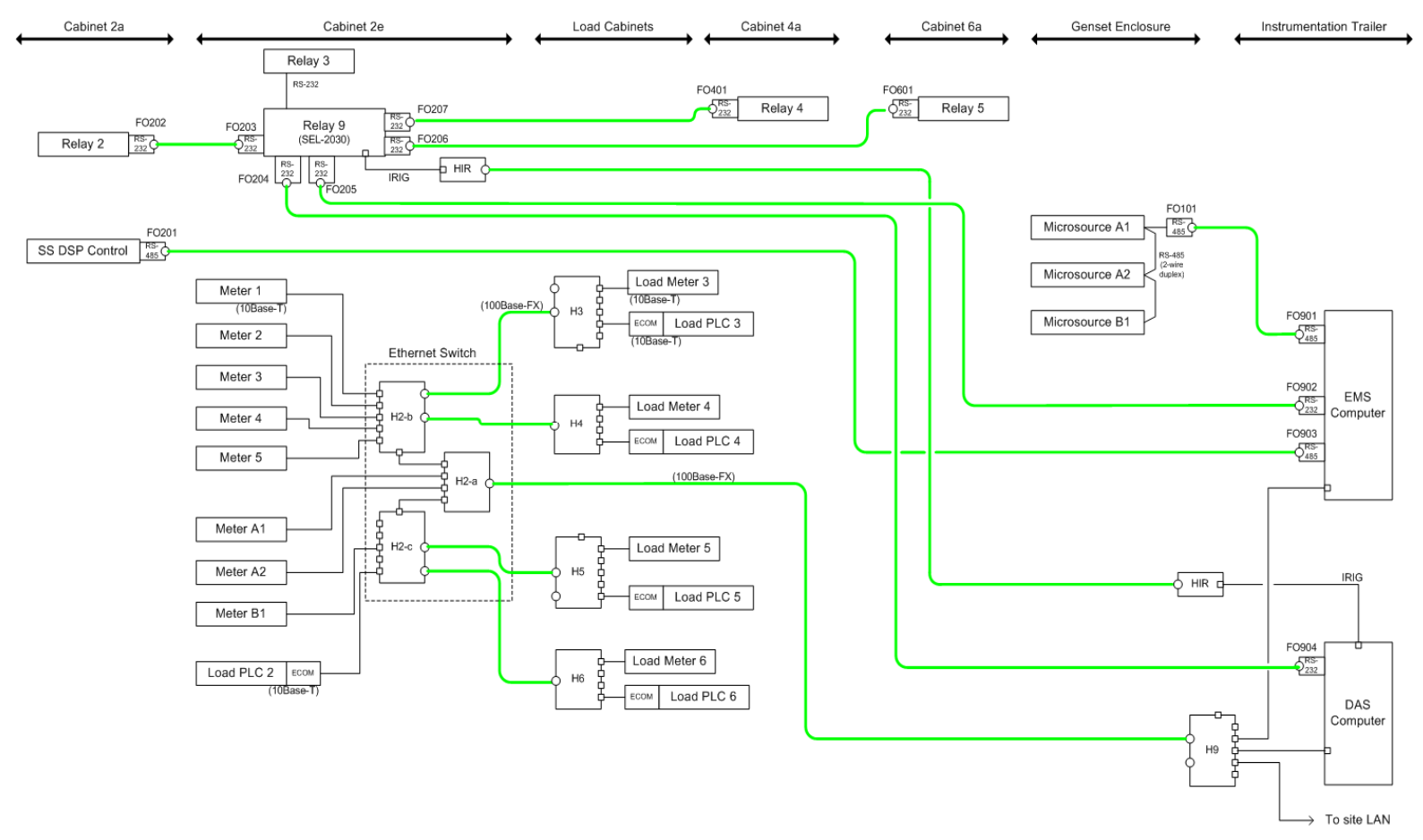

Figure 4 - Diagram of DAS \& EMS Data networks

An Ethernet network was provided as shown in Figure 4, for communications between all meters, load control PLCs, and the Data Acquisition System (DAS) computer, using fiber-optic links and switches. The DAS and Energy Management System (EMS) computers were also networked into the local Dolan Local Area Network (LAN) and to a secure Website with user ID and password protection. Additional serial links, using fiber optic converters, connect all relays, static switch Digital Signal Processor (DSP) controller, and TECOGEN Gen-set controls to the EMS computer.

\subsection{PROPOSED TEST PLAN}

The CERTS Microgrid Test Plan was developed by the CERTS Microgrid Team to demonstrate the unique concepts of control and protection of the CERTS Microgrid. This test plan was reviewed by a Technical Advisory Committee outside the CERTS Microgrid Team and then implemented by American Electric Power. CERTS Microgrid Test Plan consists of 12 sections with 5 of them detailing desired tests, starting at section 6.0, to demonstrate the controls and concepts of the CERTS Microgrid. The other 7 sections pertain to safety procedures, equipment calibration, and documentation. Each section of the test plan is described below. 
- Section 1 - "Purpose, References, and Definitions" describes the purpose of the test plan, helpful references for further explanation of how the test bed was created, and definitions used through out the test plan.

- Section 2 - "Responsibilities" informs personnel of their responsibilities while working on or near the CERTS Microgrid test site.

- Section 3 - "Training - Team Members" lists the mandatory training needed by personnel before they can work on or near the CERTS Microgrid test site.

- Section 4 - "Procedure - CERTS Microgrid Test Bed Lockout/Tagout” entails how to safely shut down the equipment and lockout/tagout the closest upstream disconnect to work on or near equipment.

- Section 5 - "Procedure - General" is the daily procedures performed at the CERTS Microgrid Test Site, prior to performing a test from Section 6 through Section 10.

- Section 6 - "Procedure - Microgrid Test Bed System Checkout" was designed to check control and operation of the static switch, basic power and voltage control of the Gen-sets, and a preliminary check of the protection scheme. The goal is to assure that the test bed is operating and ready to perform the tests described in the remaining sections of the test plan document.

- Section 7 - "Validate Protection Settings \& Initial Fault Testing" is designed to examine a preliminary set of fault (i.e. overload simulating a fault) condition tests to ensure protection and safety of the Micro-grid test Bed, while performing other planned tests. The goal is to test and adjust protection settings to achieve the most ideal conditions and protection design.

- Section 8 - "Procedure - Reduced System Tests" is a limited set of tests to build confidence that the Gen-set inverter controls are working correctly. This includes unit control, zone control, and mixed power controls, in conjunction with limit controls and synchronized closing of the static switch. These tests are based on the TECOGEN/THE SWITCH factory acceptance testing.

- Section 9 - "Procedure - Demonstration Tests of Control Power Flow" demonstrates the flexibility of the Micro-grid both grid connected and islanded for different loads, power flows and impact on the utility. 
- Section 10 - "Procedure - Test Difficult Loads" determines operation limits of the Micro-grid (i.e. power quality, protection and inverter limits) with low pf loads, motor loads, harmonic loads and unbalance loads.

- Section 11 - "Hazards \& Mitigation" informs the personnel of hazards that may exist while working on or near the CERTS Micro-grid test site and how to mitigate them.

- Section 12 - “Quality Assurance” ensures quality for the acquiring data results by providing a checklist reminder for personnel.

\subsection{TESTS PERFORMED IN SECTION 7.0}

Prior to each test day, the person in charge performed a job safety briefing (JSB) with barricades and test setup inspected for safety and compliance. A minimum of two people were on-site during each planned test.

Visual and audible alarms were used to warn persons that energized testing was being performed in the Microgrid Test Bed area. The visual alarm consisted of a portable red flashing light, located between the Control Trailer and Gen-set Enclosure. An audible alarm, consisting of a portable wireless motion detector, was located at the front gate of the Walnut Test Site with the fence gate "Closed", not locked, and audible alarm in the trailer operational during test(s).

Barricades were set up around the Micro-grid Test Bed area (i.e., saw-horse style barricades with a "Red" plastic chain surrounded the test area containing the Gen-set Enclosure, Micro-grid switching cabinets, plus load and fault bank cabinets).

Prior to performing tests, the Test Engineer or Technical Consultant verified that all personnel and visitors were properly protected and in assigned locations. Personnel were in or adjacent to the Control Trailer while tests were being performed. All nonessential personnel either left the main site or were sheltered in the Control Trailer.

For all tests the following waveforms were captured and recorded in the DAS for voltage $(\mathrm{V})$ and current $(\mathrm{I})$. From these waveforms real power $(\mathrm{kW})$, reactive power $(\mathrm{kVAr})$, and frequency (freq) were post calculated by the PQView software. Frequency measurements in this report should be used for steady state information and not used for transient analysis, due to the calculation and filtering methods employed. Below is a list of the meters capturing this data. 
- Meters 1, 2, 3, 4 \& 5

- Load Meters 3, 4, 5, \& 6

- Meters A1, A2 \& B1

- Meter 2 also measures the voltage across the static switch on phase A

Schweitzer event reports were also captured for each event, along with breaker and static switch status, such as Open or Close.

Section 7 was the next set of tests that were developed to demonstrate the CERTS Microgrid protection scheme while connected to the grid and islanded under different overload fault conditions. During each overload fault test event, waveforms of phase currents and line-to-neutral voltages were recorded in DAS at all meter locations and at all relay locations (i.e., Relays 2, 3, 4, \& 5). In addition, the relay element that caused the "TRIP" was recorded with trip times for each relay relative to when the fault condition was applied. Tests performed in this section included inductor L11 in the circuit, reflecting weak utility grid conditions.

Test 7.1 induced a three-phase-to-ground balanced overload fault condition in Zone 4 to verify the $\mathrm{I}^{2} \mathrm{t}$ protection and a single line-to-ground fault condition in Zone 4 to verify zero-sequence, negative-sequence or residual over-current protection. Zone 3 and Zone 5 were tested similar to Zone 4 by introducing a three-phase-to-ground balanced overload fault and a single line-to-ground fault conditions in the zones, verifying the protection scheme in Test 7.2 (Zone 3) and Test 7.3 (Zone 5). During the 7.1, 7.2 and 7.3 tests, all three Gen-sets were off-line and the CERTS Microgrid was connected to the utility. Test 7.4 tested a three-phase-to-ground balanced overload fault condition in Zone 4 with Gen-sets A1 and A2 operating in-parallel with the utility grid to verify I2t protection, plus to confirm a reverse power event after the zone breaker "opens".

Test 7.5 through Test 7.10 involved applying a single line-to-ground overload fault condition in each Zone, located beyond the static switch, while connected to the utility grid. Each one of the six tests differs from one another by which Gen-sets are on-line during the fault, which phase is faulted, and in which zone the fault is applied. These tests were designed to verify zero-sequence, negative-sequence or residual over-current protection settings. Test 7.5 verified the protection in Zone 3 by applying a single lineto-ground overload fault condition with Gen-sets A1 operating in parallel with the utility grid. Test 7.6 tested a single line-to-ground overload fault condition in Zone 3 with Gen-sets A1 and A2 operating in parallel with the utility grid. Test 7.7 tested a single line-to-ground overload fault condition in Zone 5 with Gen-set B1 operating in parallel with the utility grid. Test 7.8 tested a single line-to-ground overload fault condition in Zone 5 with Gen-sets A1 and B1 operating in parallel with the utility grid. Test 7.9 tested a single line-to-ground overload fault condition in Zone 4 with Gen-sets 
A1 and A2 operating in parallel with the utility grid. Test 7.10 tested a single line-toground overload fault condition in Zone 2 with Gen-sets A1 and B1 operating in parallel with the utility grid.

Tests 7.11 and 7.12 both applied a single line-to-ground overload fault condition in Zone 6 while connected to the utility. Test 7.11 involved only Gen-set A1 operating in parallel with the grid; and Test 7.12 involved Gen-sets A1 and B1 operating in-parallel with the grid. Both tests were designed to verify the $\mathrm{I}^{2} \mathrm{t}$ protection of the breaker in Zone 6 .

The four tests, 7.13 - 7.16, applied a line-to-line overload fault condition in one of the five zones beyond the static switch with a combination of two Gen-sets, operating in parallel with the utility grid. These tests were designed to test negative-sequence, $I^{2} t$ protection or residual over-current protection settings. Test 7.13 tested a phase-to-phase overload fault condition in Zone 3 with Gen-sets A1 and A2 operating in parallel with the utility grid. Test 7.14 tested a phase-to-phase overload fault condition in Zone 4 with Gen-sets A1 and A2 operating in parallel with the utility grid. Test 7.15 tested a phaseto-phase overload fault condition in Zone 2 with Gen-sets A1 and B1 operating inparallel with the utility grid. Test 7.16 tested a phase-to-phase overload fault condition in Zone 5 with Gen-sets A1 and B1 operating in parallel with the utility grid.

\subsection{ANALYSES OF TEST RESULTS}

\subsection{SECTION 7 - VALIDATE PROTECTION SETTINGS \& INITIAL FAULT TESTING}

\subsubsection{Zone 4 Circuit Breaker Settings and Utility Connected} Performance Goal:

Initially test a three-phase-to-ground balanced overload fault in Zone 4 to verify $\mathrm{I}^{2} \mathrm{t}$ protection. Then test a single line-to-ground fault condition in Zone 4 to verify zerosequence, negative-sequence or residual over-current protection.

\footnotetext{
Initial Setup:

Relay Settings $=$ A $($ Delayed $)$

Load Bank $4=60 \mathrm{~kW}$

Load Bank $6=10 \mathrm{~kW}$
} 
To simulate a high impedance three-phase-to-ground fault in Zone 4, the

Overload Load Bank of $85 \mathrm{~kW}$ was connected by cable/plug into the exterior receptacle of Cabinet 5a. This would add an additional $85 \mathrm{~kW}$ to Zone 4 when initiated, causing an overload because the load is currently set at $60 \mathrm{~kW}$ from Load Bank 4 .

After the dead-bus closing of the static switch from the EMS, the Microgrid Test

Bed was connected to the utility grid with approximately $55 \mathrm{~kW}$ at Meter 2 . A simulated fault (i.e., Overload Load Bank of 85kW) with a duration set for 99 seconds was initiated to Zone 4 from the DAS Load Control Program. Figure 10a shows when the overload bank was initiated and the load in Zone 4 increased from approximately $55 \mathrm{~kW}$ to $130 \mathrm{~kW}$.

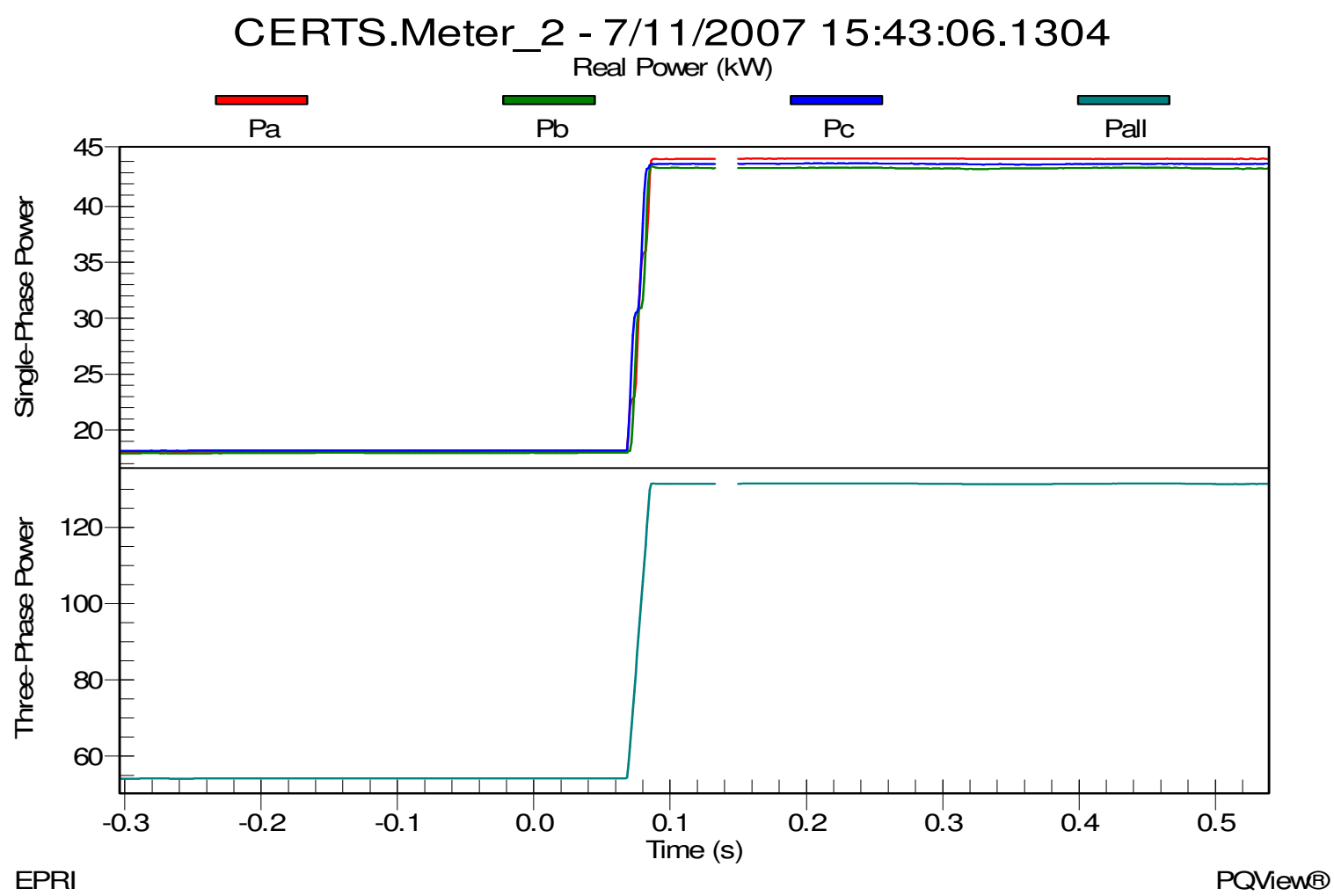

Figure 10a - Load in Zone 4 when the overload fault is initiated 
When the load increased in Zone 4, CB41 internally detected an I²t protection event and opened after 71.3 seconds, clearing the fault from Zone 4, shown in Figure 10b.

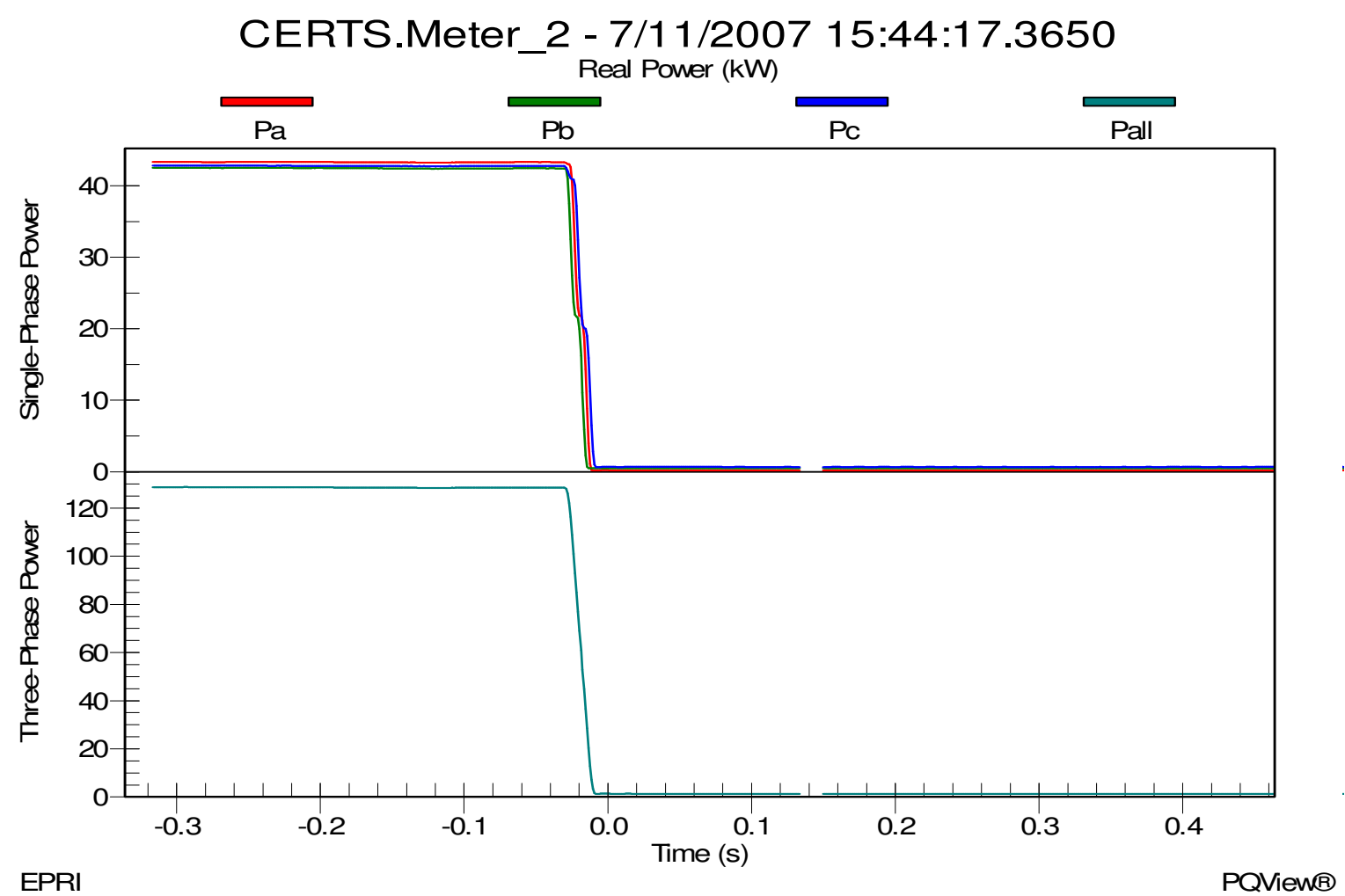

Figure 10b - Load in Zone 4 after circuit breaker CB41 "Opens" to isolate and clear the fault

The static switch, nor any of the other breakers, opened up at the time the fault was initiated. The main purpose of this test was designed for CB41 to isolate the fault and then clear it.

Once the fault was cleared and all the alarms and circuit breakers were reset and brought back to the initial setup, the Overload Load Bank in Cabinet 5A was reconfigured from a three-phase-to-ground fault into a single phase-to-ground fault. This was accomplished by removing phases B and C from the Overload Load Bank cabling which leaves approximately $28.3 \mathrm{~kW}$ on A-phase when the fault is initiated. 
After the dead-bus closing of the static switch from the EMS, the Microgrid Test

Bed was connected to the utility grid with approximately $51 \mathrm{~kW}$ at Meter 2 . A simulated overload fault (i.e., Overload Load Bank of $28.3 \mathrm{~kW}$ on A-phase) with a duration set for 10 seconds was initiated to Zone 4 from the DAS Load Control Program. Figure 10d shows when the overload bank was initiated and load in Zone 4 increased from approximately $52 \mathrm{~kW}$ to $75 \mathrm{~kW}$ three-phase power and approximately $17.5 \mathrm{~kW}$ to $41 \mathrm{~kW}$ on A-phase. Also in Figure 10d, you can see where the fault is cleared and the load is reduced to $0 \mathrm{~kW}$.

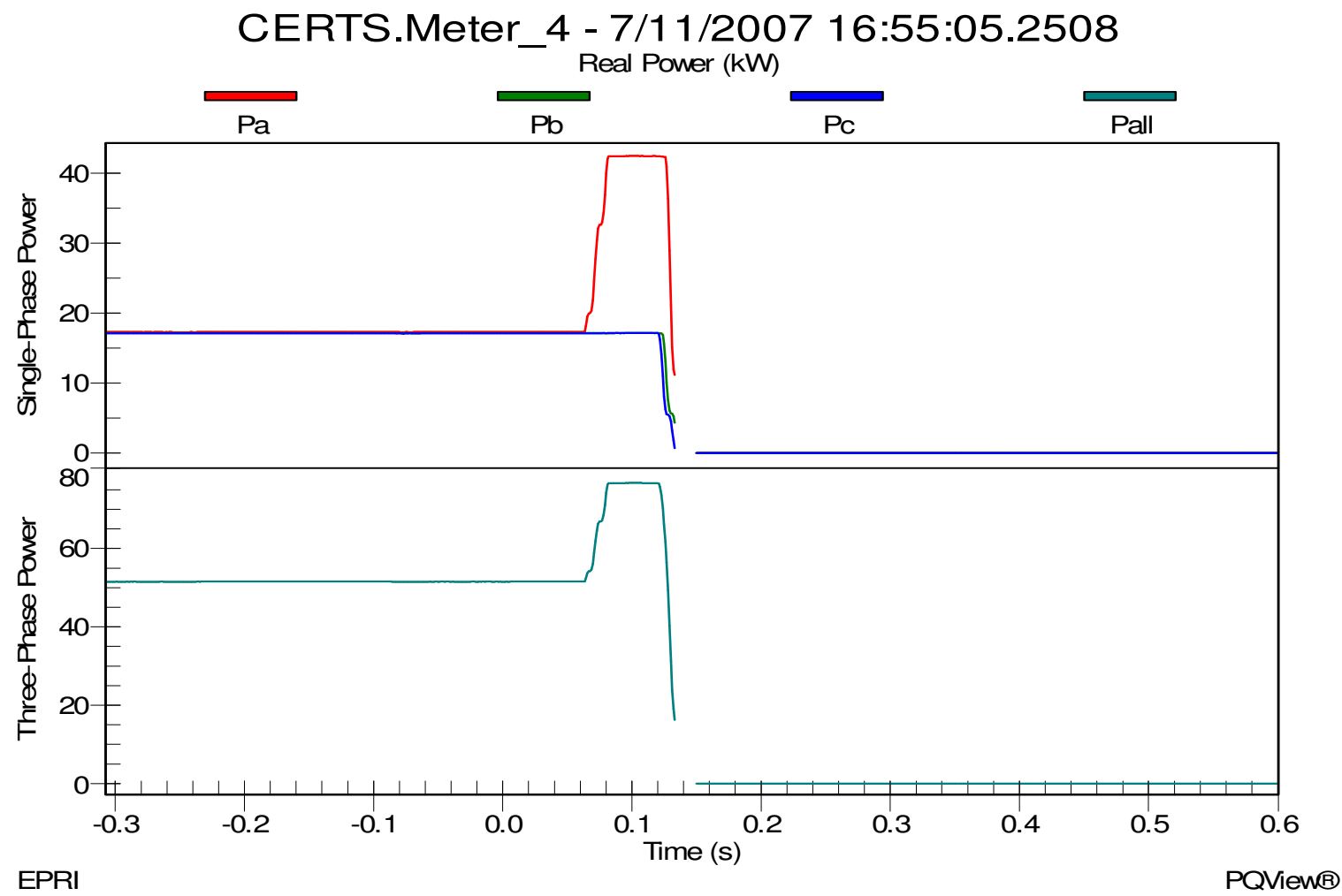

Figure 10d - Load in Zone 4 before/after the single phase-to-ground fault is applied

It's important to note that all four protection relays (i.e., relays 2 through 5) have residual CT's wired to the neutral input of the relay. Thus, any neutral current measurements are actually measurements of ground current. 
When the load increased in Zone 4, the static switch (i.e., Relay 2) detected a ground over-current (67N1T), shown in Figure 10e. The static switch opened after 0.023 seconds (1.5 cycles) clearing the fault from Zone 4, shown in Figures 10d and 10e.

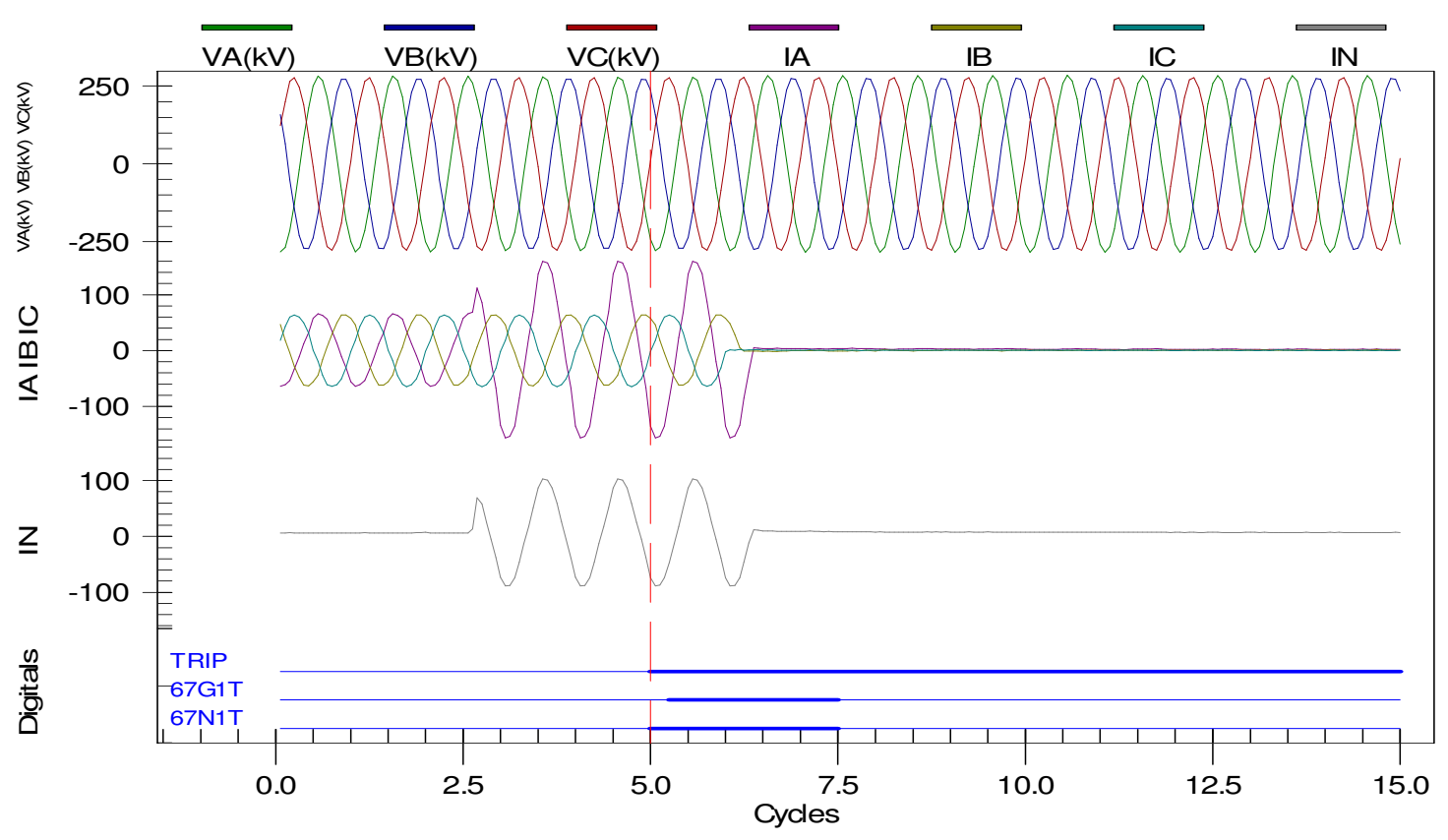

Figure 10e - Voltages, Currents, and Ground Current (IN) at Relay 2 when the fault is detected and cleared by the static switch.

After the static switch opened, the breakers in protection zones (i.e., CB31 in Zone 3, CB41 in Zone 4 and CB51 in Zone 5) remained closed. This was expected since the fault was isolated and cleared by the static switch.

The electrical system was stable in both the microgrid and utility grid, after CB41 opened for the three-phase-to-ground fault. In addition the static switch opened for a single phase-to-ground fault condition in Zone 4, separating the microgrid from the utility system. All loads and alarms were reset and equipment was prepared for the next test.

\subsubsection{Zone 3 Circuit Breaker Settings and Utility Connected} Performance Goal: 
Initially test a three-phase-to-ground balanced overload fault condition in Zone 3 to verify $\mathrm{I}^{2} \mathrm{t}$ protection. Then test a single line-to-ground fault condition in Zone 3 to verify zero-sequence, negative-sequence or residual over-current protection.

\author{
Initial Setup: \\ Relay Settings $=$ A $($ Delayed $)$ \\ Load Bank $3=75 \mathrm{~kW}$ \\ Load Bank $4=75 \mathrm{~kW}$ \\ Load Bank $6=10 \mathrm{~kW}$
}

To simulate a high impedance three-phase-to-ground fault in Zone 3, the

Overload load Bank of $85 \mathrm{~kW}$ was connected by cable/plug into the exterior receptacle of Cabinet 3a. This additional $85 \mathrm{~kW}$ of load in Zone 3 when initiated would cause an overload in Zone 3, because load is currently set at 75kW in Load Bank 3 and in Load Bank 4.

After the dead-bus closing of the static switch from the EMS, the Microgrid Test

Bed was connected to the utility grid with approximately $140 \mathrm{~kW}$ at Meter 2. A simulated fault (i.e., Overload Load Bank of $85 \mathrm{~kW}$ ) with a duration set for 99 seconds was initiated to Zone 3 from the DAS Load Control Program. Figure 11a shows when the overload bank was initiated and load in Zone 3 increased from approximately $140 \mathrm{~kW}$ to $220 \mathrm{~kW}$. 


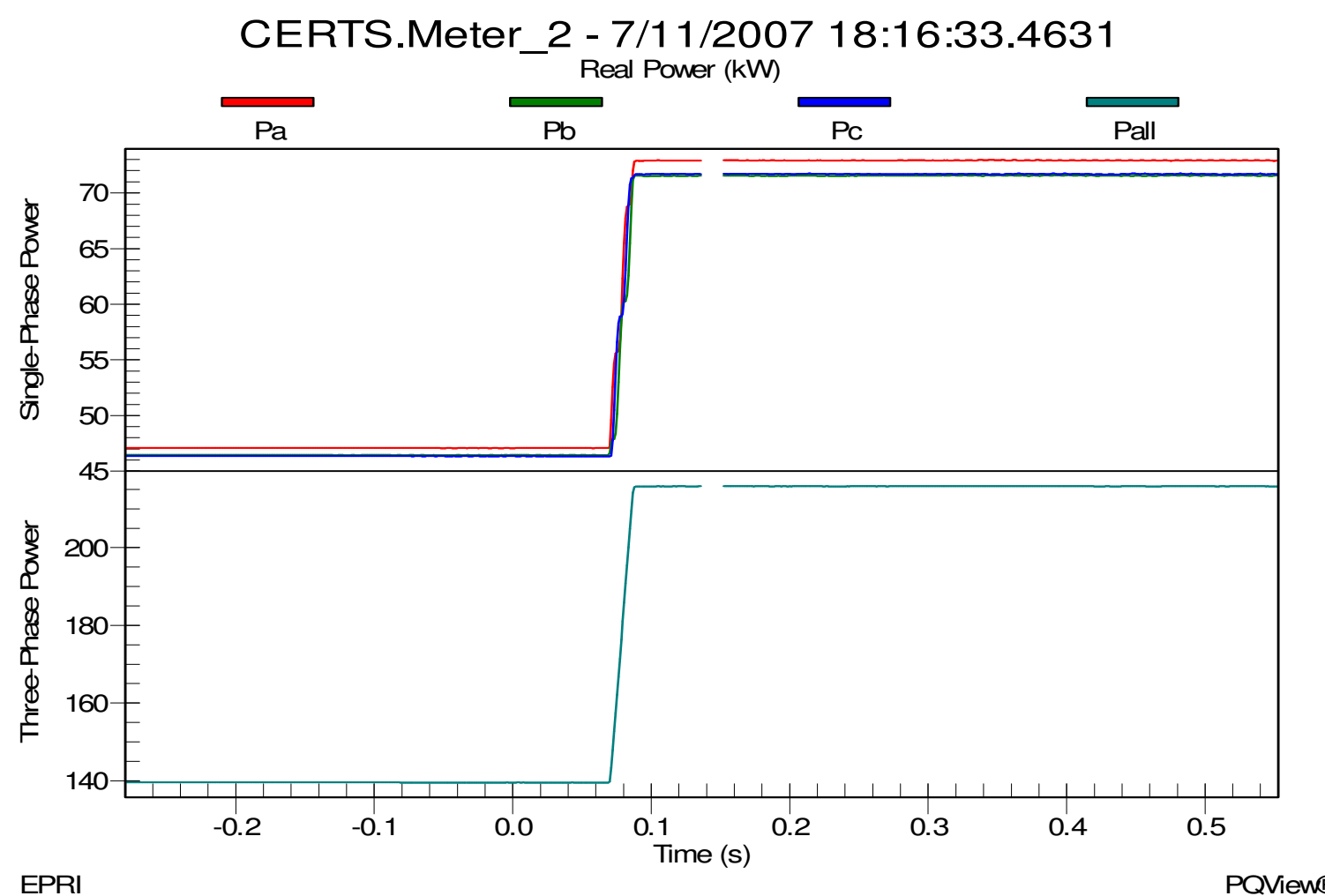

Figure 11a - Load change due to Zone 3 when the overload fault was initiated

When the load increased in Zone 3, CB31 internally detected an I²t protection event and opened after 84 seconds, clearing the fault from Zone 3, as shown in Figure 11b. 


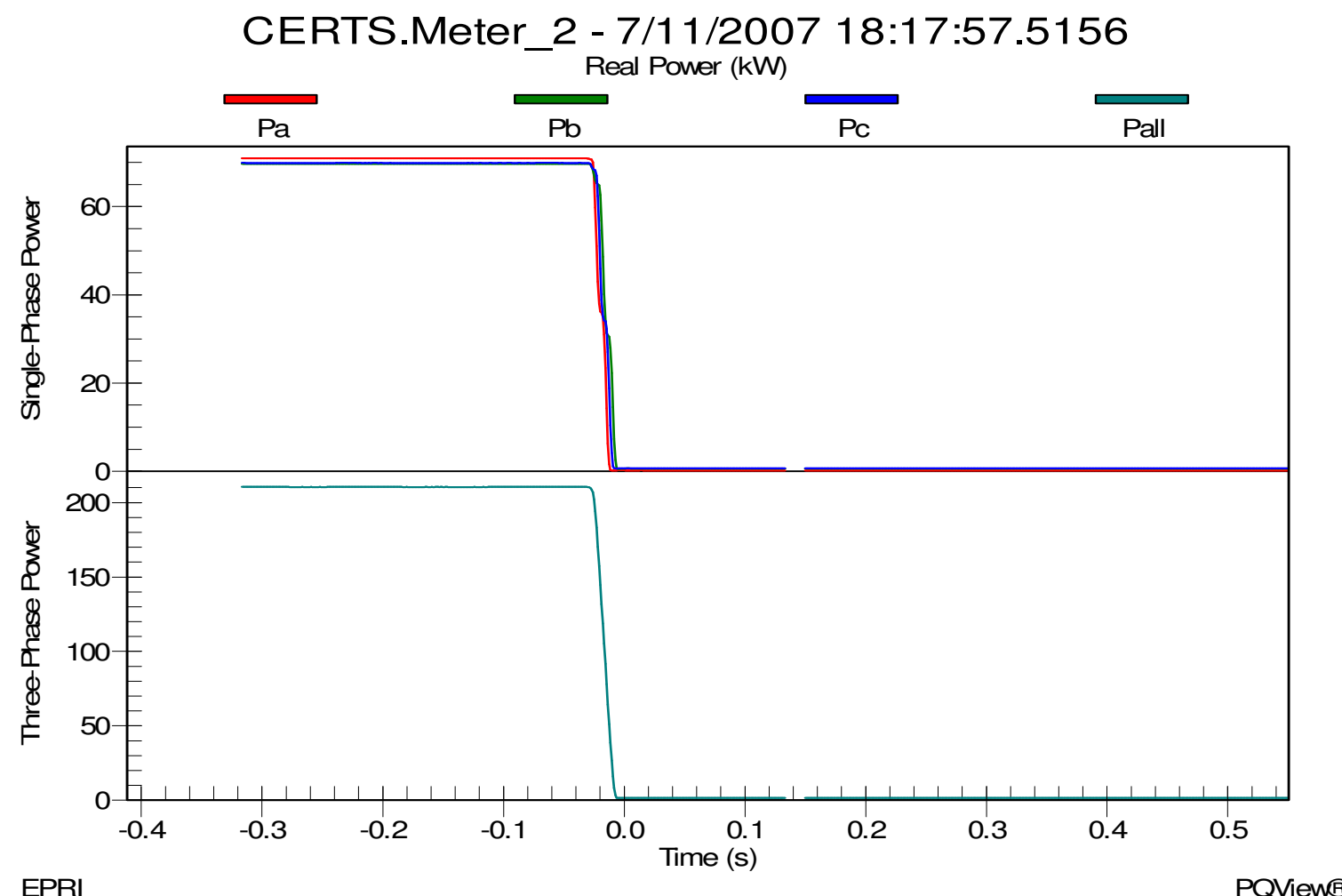

Figure 11b - Load change in Zone 3 after CB31 opened" to isolate and clear the fault

The static switch, nor any of the other breakers, opened at the time when the fault was initiated. The main purpose of this test was designed for CB31 to isolate the fault in Zone 3 and then clear it.

Once the fault was cleared, all alarms and circuit breakers were reset and brought back to the initial setup. In addition, the load in Load Bank 3 and in Load Bank 4 was reset to $60 \mathrm{~kW}$. The Overload Load Bank in Cabinet 3 was reconfigured from a three-phase-toground fault into a single phase-to-ground fault. This was accomplished by removing phases $\mathrm{A}$ and $\mathrm{C}$ from the Overload Load Bank cabling which leaves approximately $28.3 \mathrm{~kW}$ on B-phase when the fault is initiated.

After the dead-bus closing of the static switch from the EMS, the Microgrid Test

Bed was connected to the utility grid with approximately $111 \mathrm{~kW}$ at Meter 2 . A simulated fault (i.e., Overload Load Bank of $28.3 \mathrm{~kW}$ on B-phase) with a duration set for 
10 seconds was initiated to Zone 3 from the DAS Load Control Program. Figure 11c shows when the overload bank was initiated and load in Zone 3 increased from approximately $111 \mathrm{~kW}$ to $133 \mathrm{~kW}$ three-phase power and approximately $35 \mathrm{~kW}$ to $60 \mathrm{~kW}$ on B-phase. Also, Figure 11c, shows where the fault is cleared and load is reduced to $0 \mathrm{~kW}$.

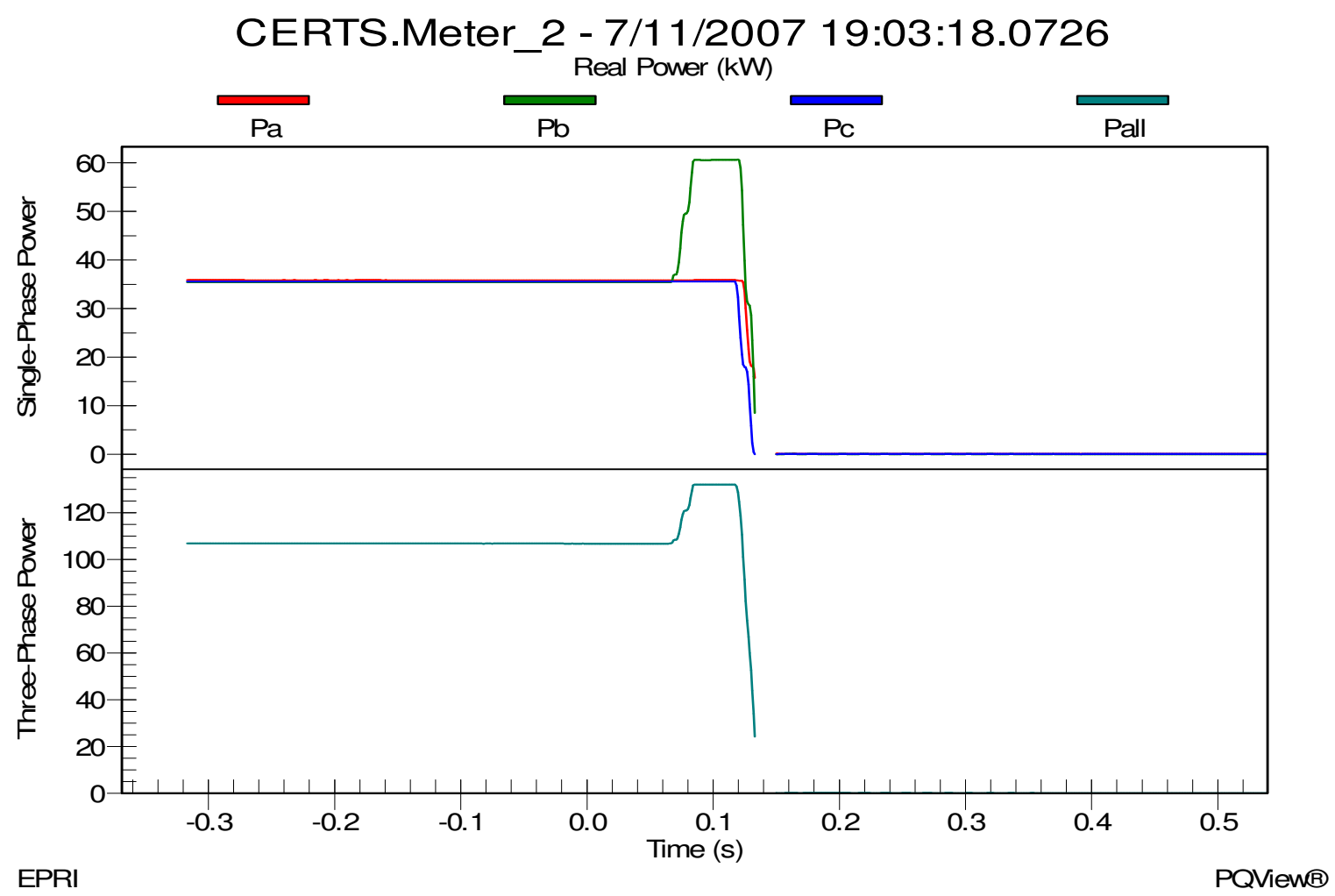

Figure 11c - Load change before/after the single phase-to-ground fault was applied in Zone 3.

When load increased in Zone 3, the static switch (Relay 2) detected a ground overcurrent (67N1T) shown in Figure 11d. The static switch then "Opens" after 0.016 seconds (1 cycle) clearing the fault from Zone 3 shown in Figures 11c and 11d. 


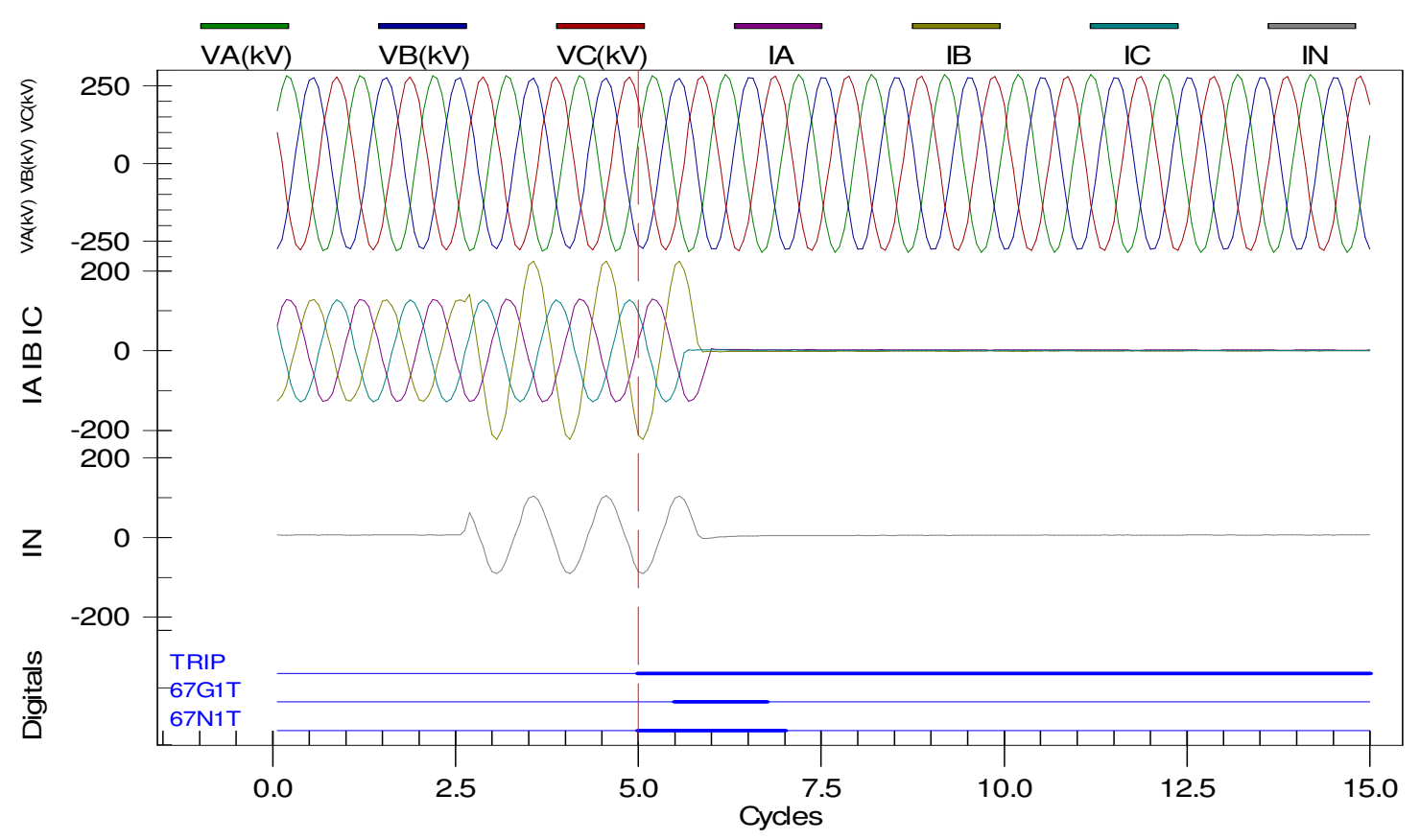

Figure 11d - Voltages, Currents, and Ground current (IN) at Relay 2 when the fault is detected and cleared by the static switch

After the static switch opened, the breakers in the protection zones (i.e., CB31 in Zone 3, CB41 in Zone 4 and CB51 in Zone 5) remained closed. This was expected, since the fault was isolated and cleared by the static switch.

The electrical system was stable in both the microgrid and utility grid, after circuit breaker CB31 opened for the three-phase-to-ground overload fault. In addition, the static switch opened for a single phase-to-ground fault condition in Zone 3, separating the microgrid from the utility system. All loads and alarms were reset and equipment was prepared for the next test.

\subsubsection{Zone 5 Circuit Breaker Settings and Utility Connected} Performance Goal:

Initially test a three-phase-to-ground balanced overload fault condition in Zone 5 to verify $\mathrm{I}^{2} \mathrm{t}$ protection. Then test a single line-to-ground fault condition in Zone 5 to verify zero-sequence, negative-sequence or residual over-current protection. 


\section{Initial Setup:}

Relay Settings $=$ A (Delayed)

Load Bank $5=60 \mathrm{~kW}$

To simulate a high impedance three-phase-to-ground fault in Zone 5, the

Overload Load Bank of $85 \mathrm{~kW}$ was connected by cable/plug into the exterior receptacle of Cabinet 7a. This additional $85 \mathrm{~kW}$ of load in Zone 5 when combined with the $60 \mathrm{~kW}$ in Load Bank 5 causes an overload condition.

After the dead-bus closing of the static switch from the EMS, the Microgrid Test

Bed was connected to the utility grid with approximately $55 \mathrm{~kW}$ at Meter 2. A simulated fault (i.e., Overload Load Bank of $85 \mathrm{~kW}$ ) with the duration set for 99 seconds was initiated in Zone 5 from the DAS Load Control Program. Figure 12a shows when the overload bank was initiated and load in Zone 5 increased from approximately $55 \mathrm{~kW}$ to $130 \mathrm{~kW}$.

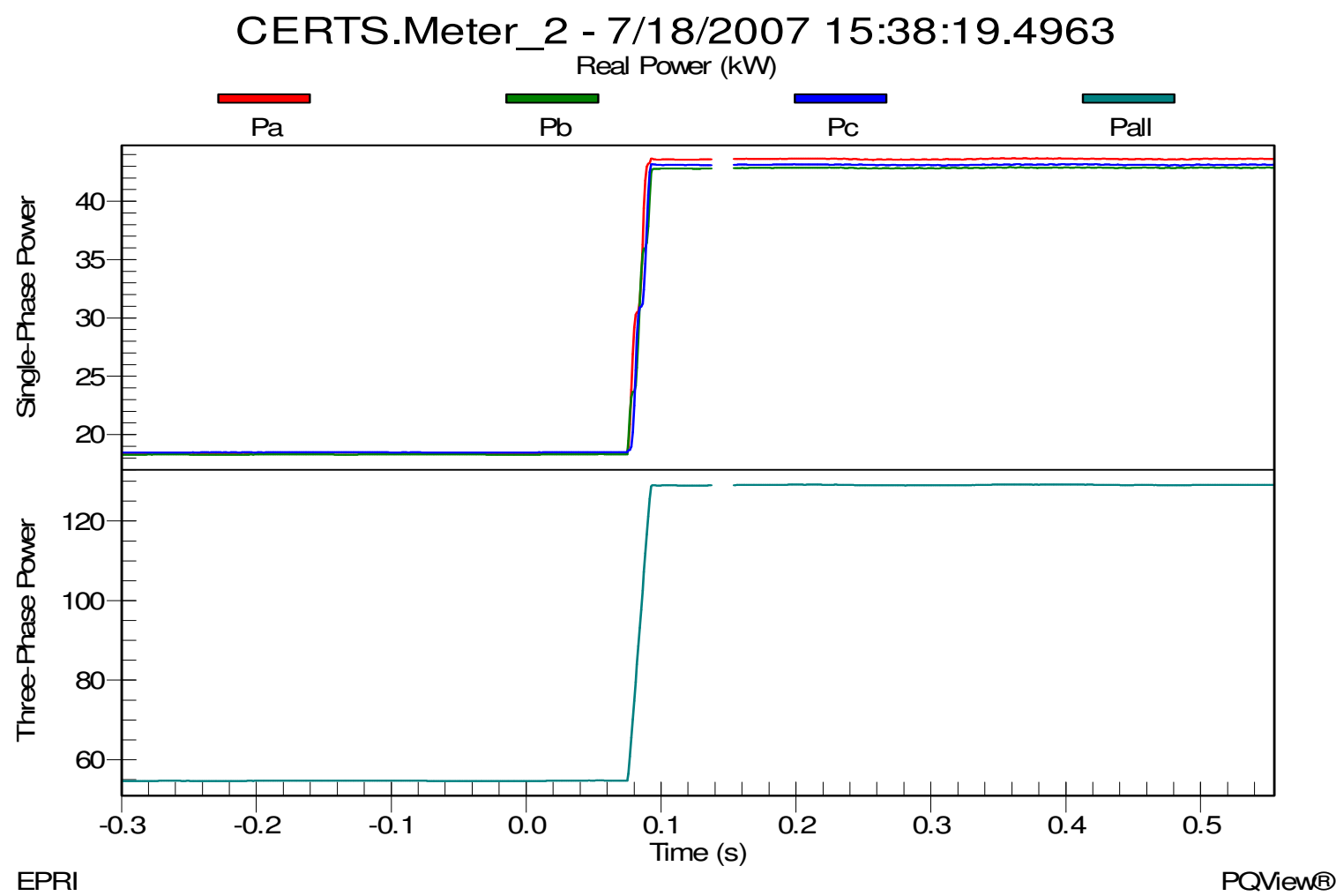

Figure 12a - Load change when the overload fault was initiated in Zone 5. 
When the load increased in Zone 5, CB51 internally detected an $\mathrm{I}^{2} \mathrm{t}$ protection event and opened after 72.8 seconds, clearing the fault from Zone 5, as shown in Figure 12b.

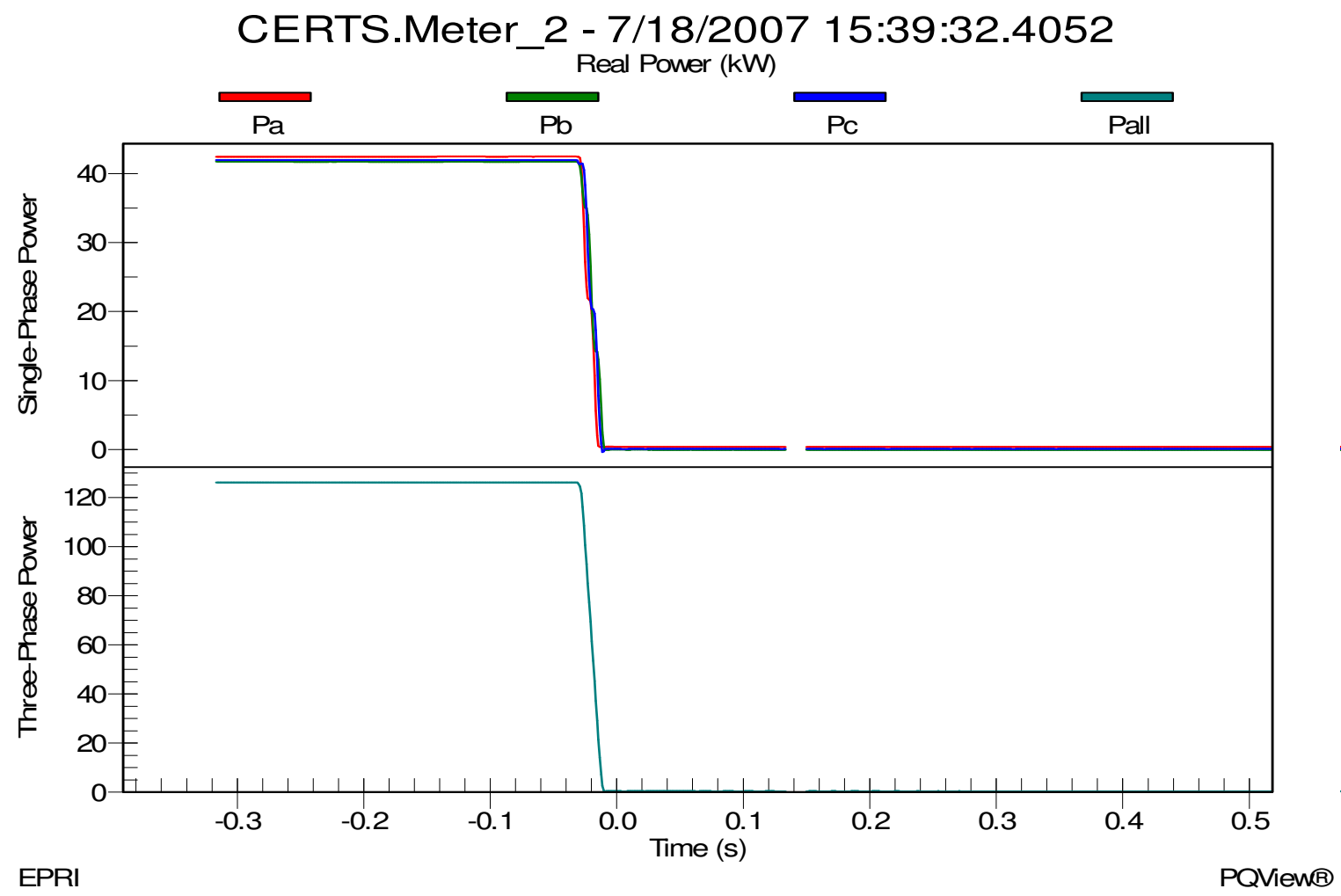

Figure 12b - Load change before/after CB51 opened to isolate and clear the fault in Zone 5.

The static switch, nor any of the other breakers, opened at the time the fault was initiated. The main purpose of this test was designed for CB51 to isolate the fault in Zone 5 and then clear it.

Once the fault was cleared and all the alarms and circuit breakers were reset and brought back to the initial setup, the Overload Load Bank in Cabinet 7a was reconfigured from a three-phase-to-ground fault into a single phase-to-ground fault. This was accomplished by removing phases A and B from the Overload Load Bank cabling which leaves approximately $28.3 \mathrm{~kW}$ on the C-phase when the fault is initiated. 
After the dead-bus closing of the static switch from the EMS, the Microgrid Test

Bed was connected to the utility grid with approximately $54 \mathrm{~kW}$ at Meter 2. A simulated fault (i.e., Overload Load Bank of $28.3 \mathrm{~kW}$ on C-phase) with a duration set for 10 seconds was initiated to Zone 5 from the DAS Load Control Program. Figure 12c shows when the overload bank was initiated and the load in Zone 5 increased from approximately $51 \mathrm{~kW}$ to $74 \mathrm{~kW}$ three-phase power and approximately $16.5 \mathrm{~kW}$ to $40 \mathrm{~kW}$ on C-phase. Also, Figure 12ce, shows where the fault is cleared and the load is reduced to $0 \mathrm{~kW}$.

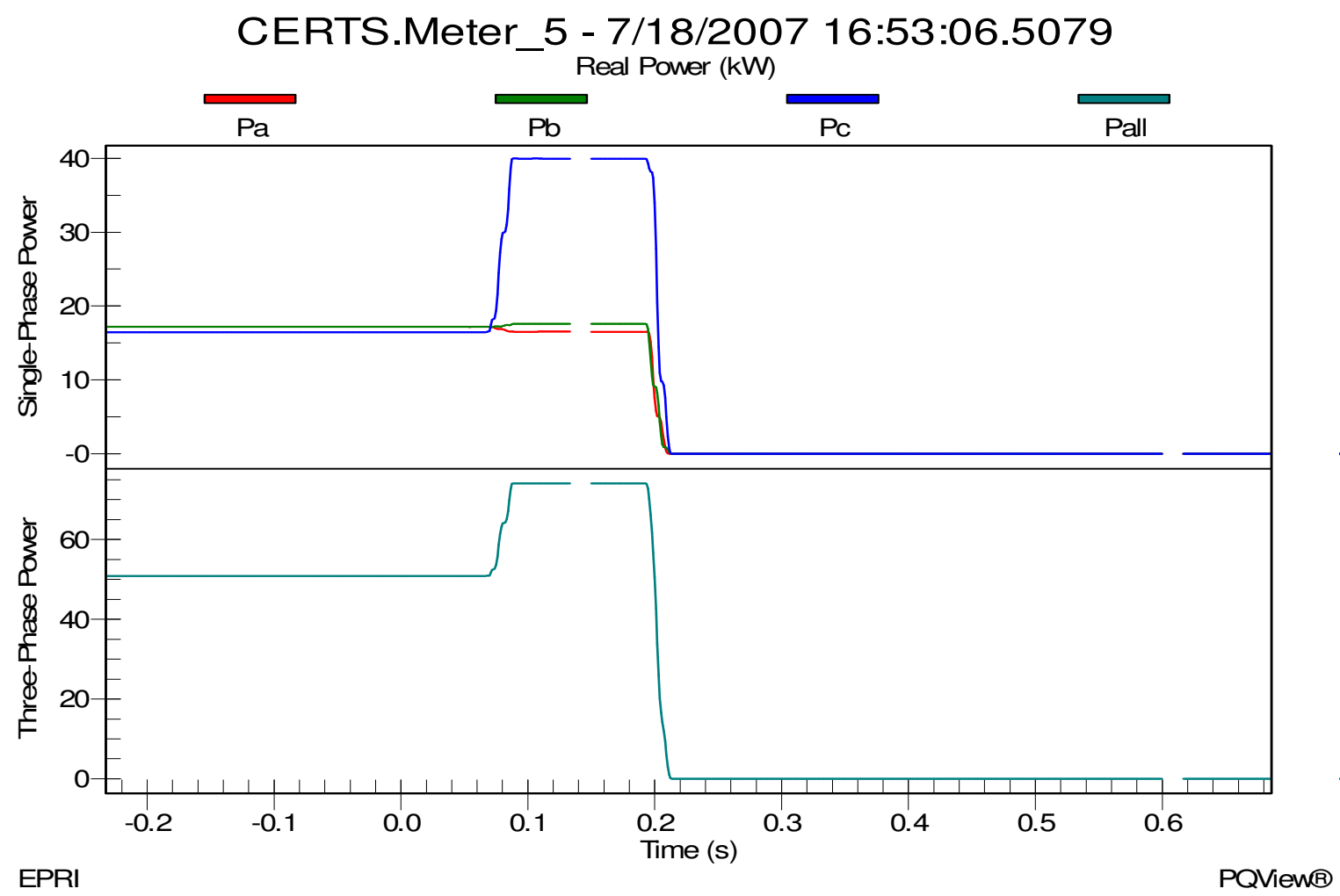

Figure 12c - Load in Zone 5 before/after the single phase-to-ground fault was applied. 


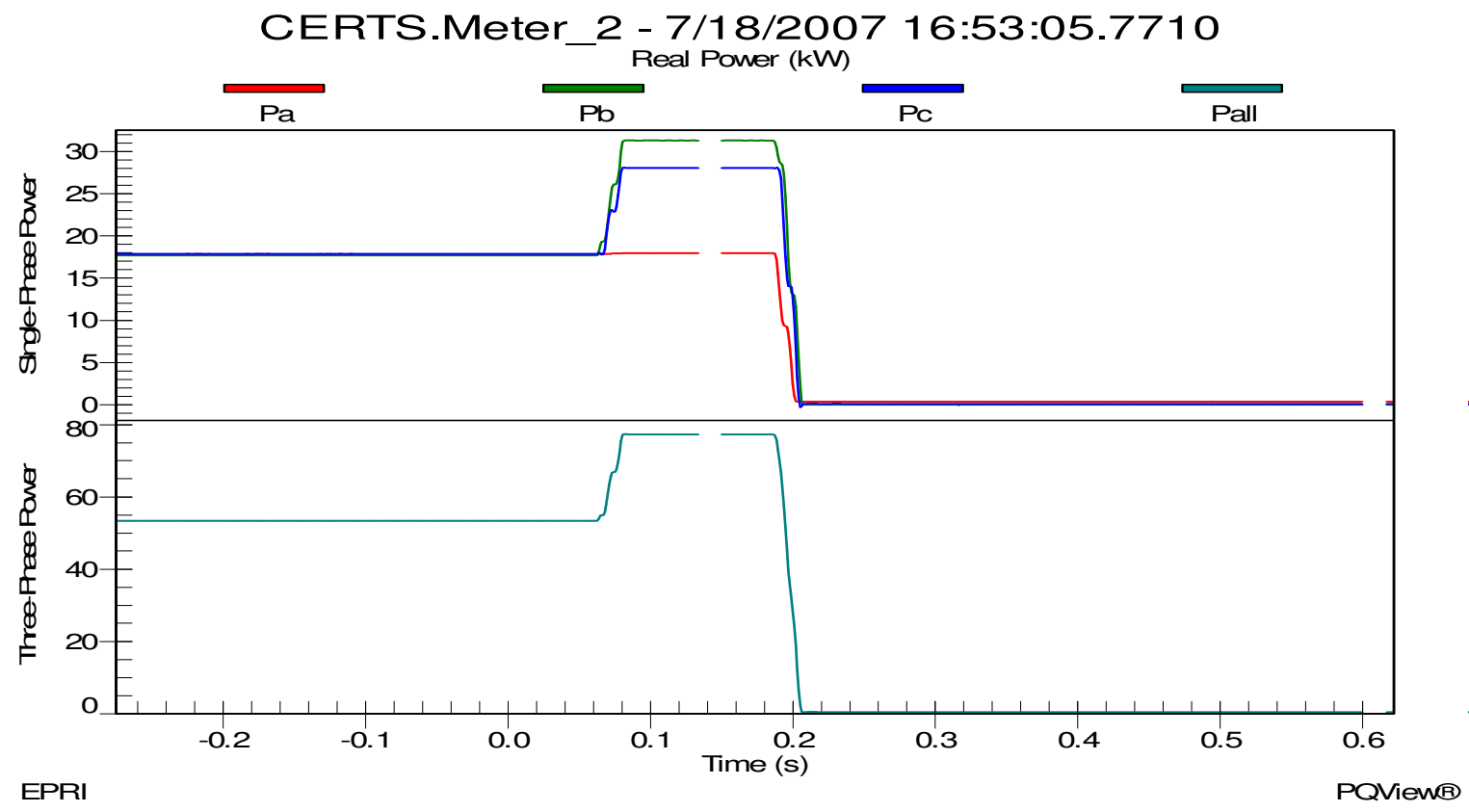

Figure 12d - Load change before/after the single phase-to-ground fault was applied in Zone 5.

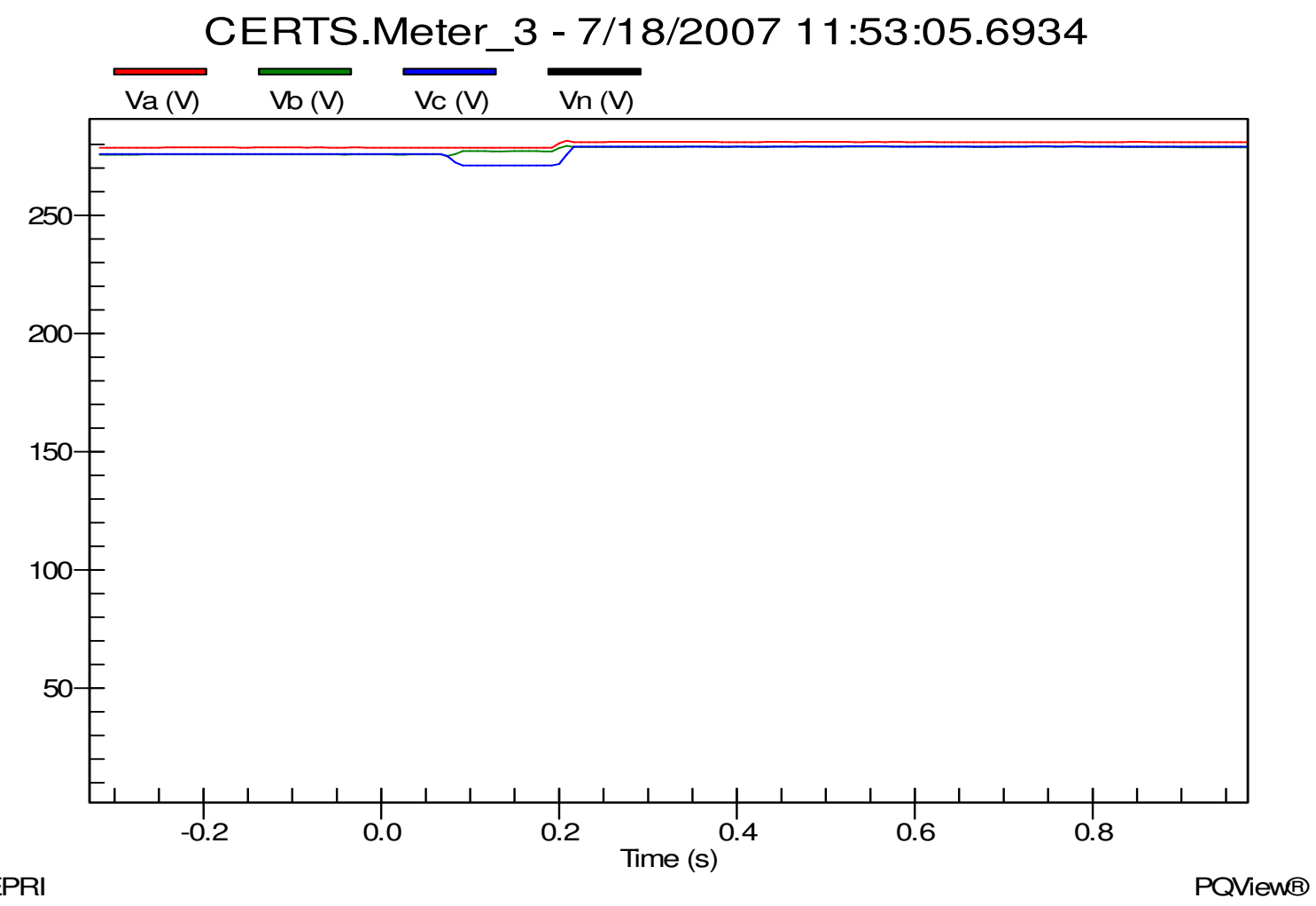

Figure 12e - RMS Voltage in Zone 3 before/after the single phase-to-ground fault was applied 
From Figure $12 \mathrm{~d}$ it can be seen at the static switch that the phase $\mathrm{C}$ real power appears reduced in magnitude and distributed over both phases B and C. This reduces the sensitivity of the static switch to this type of fault in Zone 5. From Figure 12e it is apparent that the static switch did not operate, since the RMS voltage never collapsed.

When the load increased in Zone 5, Relay 5 detected a negative-sequence over-current (67Q1T) event, shown in Figure 12f. CB51 opened and cleared the fault from Zone 5 shown in Figure 12f. The opening of CB51 was past the end of the recorded relay waveform, but it can be seen that the real power at Meter 5 reduces to zero, shown in Figure 12c.

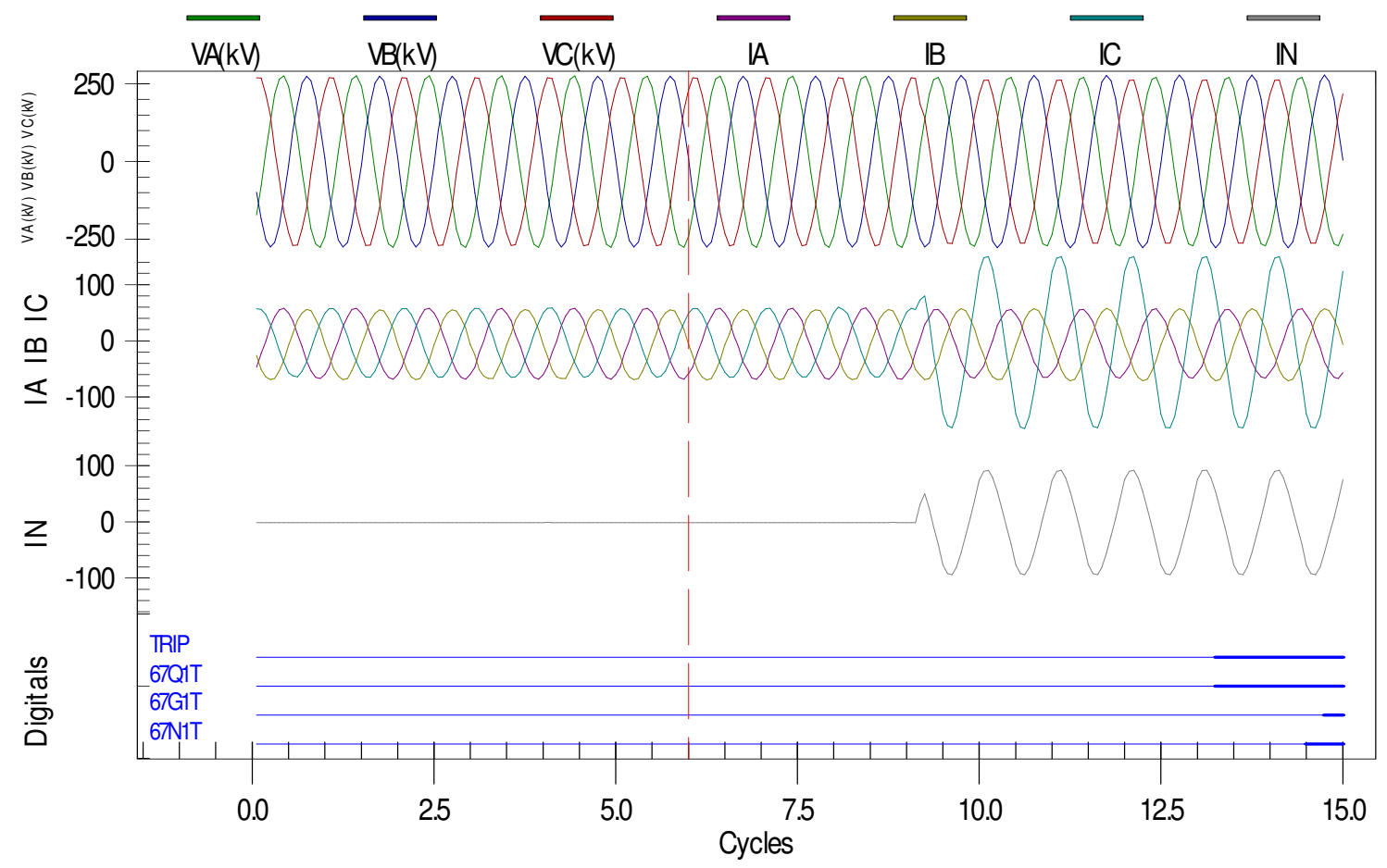

Figure 12f - Voltages, Currents, and Sequence Currents at Relay 2 when the fault was detected and cleared by the static switch.

After circuit breaker CB51 opened, the breakers in other protection zones, (i.e., CB31 in Zone 3 and CB41 in Zone 4) and the static switch remained closed. This was expected since the fault was isolated and cleared by CB51 in Zone 5. 
The electrical system was stable in both the microgrid and utility grid after CB51 opened for both single phase-to-ground and three-phase-to-ground fault conditions in Zone 5. All loads and alarms were reset and equipment was prepared for the next test.

\title{
6.1.4 Zone 4 Three-Phase Grounded Fault, Gen-sets (A1+A2) and Utility Connected Performance Goal:
}

Test a three-phase-to-ground balanced overload fault condition in Zone 4 with Gen-sets $\mathrm{A} 1$ and A2 operating in-parallel with the utility grid to verify $\mathrm{I}^{2} \mathrm{t}$ protection, plus confirm a reverse power event after the Zone breaker "Opens".

\author{
Initial Setup: \\ Gen-set A1 = Unit Power Control \\ Gen-set A1 Output Power Command $=50 \mathrm{~kW}$ \\ Gen-set A2 Output Power Command $=20 \mathrm{~kW}$ \\ MG Power/Frequency Droop $=-0.0833 \mathrm{~Hz} / \mathrm{kW}$ \\ MG Voltage Command $=277 \mathrm{~V}$ \\ Reverse Power Set-point $=0 \mathrm{~kW}$ \\ Load Bank $3=10 \mathrm{~kW}$ \\ Load Bank $6=40 \mathrm{~kW}$ \\ Load Bank $4=65 \mathrm{~kW}$
}

To simulate a high impedance three-phase-to-ground fault in Zone 4, the

Overload Load Bank of $85 \mathrm{~kW}$ was connected by cable/plug into the exterior receptacle of Cabinet 5a. This additional $85 \mathrm{~kW}$ of load in Zone 4 when initiated and combined with the $65 \mathrm{~kW}$ in Load Bank 4 would cause an overload condition.

After both Gen-sets A1 and A2 were running for a few minutes and supplied power to Load Bank 3 and Load Bank 4, the test was started with a "Start" command from the EMS. As soon as the static switch closed and steady-state conditions established, the microgrid was connected to the utility grid with total power flow at Meter 1 equaling approximately $45 \mathrm{~kW}$ and approximately $5 \mathrm{~kW}$ at Meter 2. Figure $13 \mathrm{a}$ shows when the 
overload fault was applied in Zone 4, the load increased from 45kW to approximately $125 \mathrm{~kW}$ at Meter 1.

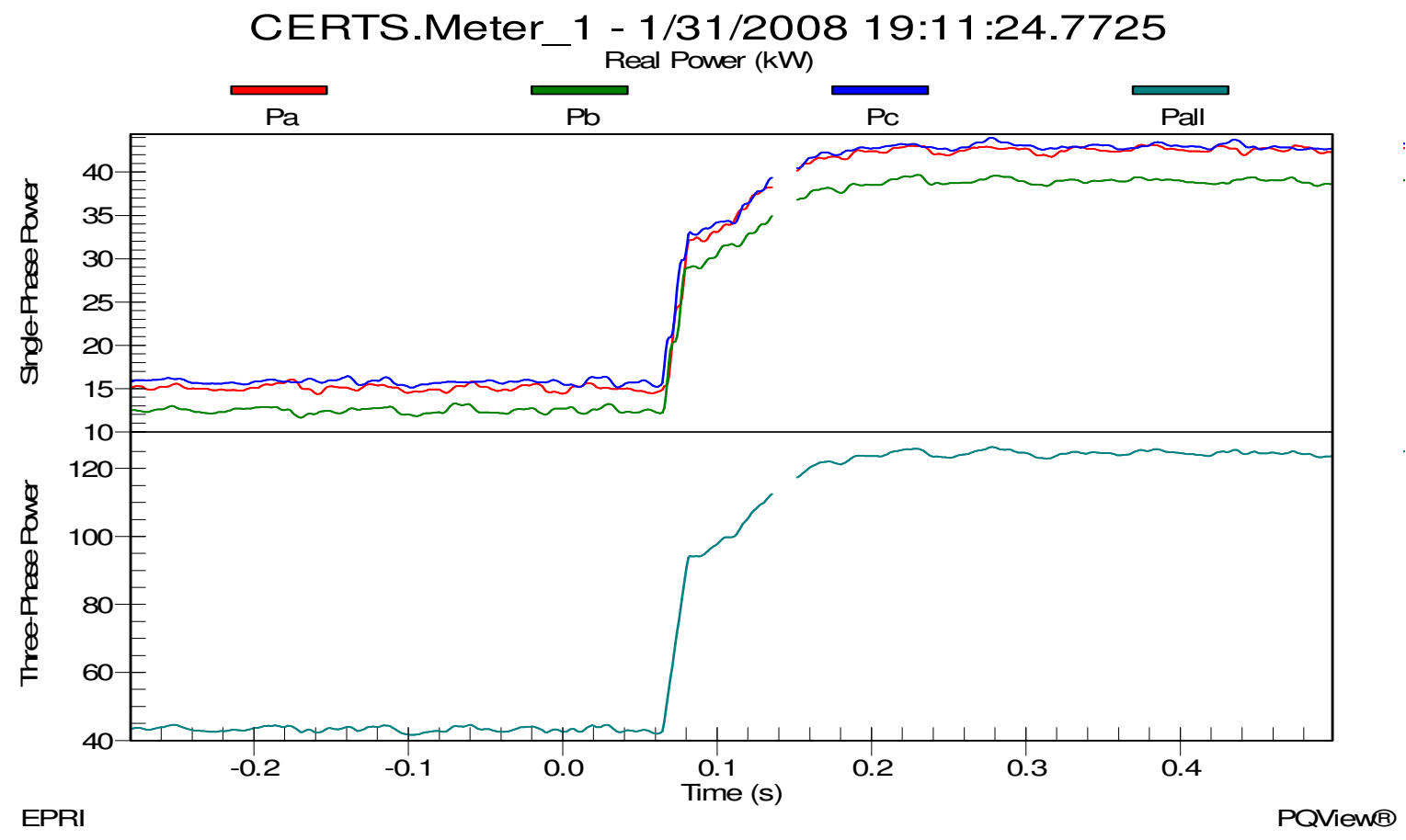

Figure 13a - Power flow at Meter 1 before/after the overload fault was applied. 
Using the DAS Load Control program, the three-phase ground fault was set for a duration of 99 seconds and then initiated. CB41 tripped on an $\mathrm{I}^{2} \mathrm{t}$ protection event in 86 seconds, shown in Figures 13b, which isolated and cleared the fault as expected in Zone 4. Zone 3 and Gen-set A1 remained connected, displacing the load in Zone 6, as shown in Figure $13 \mathrm{~b}$ with minus $37 \mathrm{~kW}$ through the static switch. This results in approximately zero load at the PCC, which in-turn caused the static switch to open after 30 seconds due to a reverse power event.

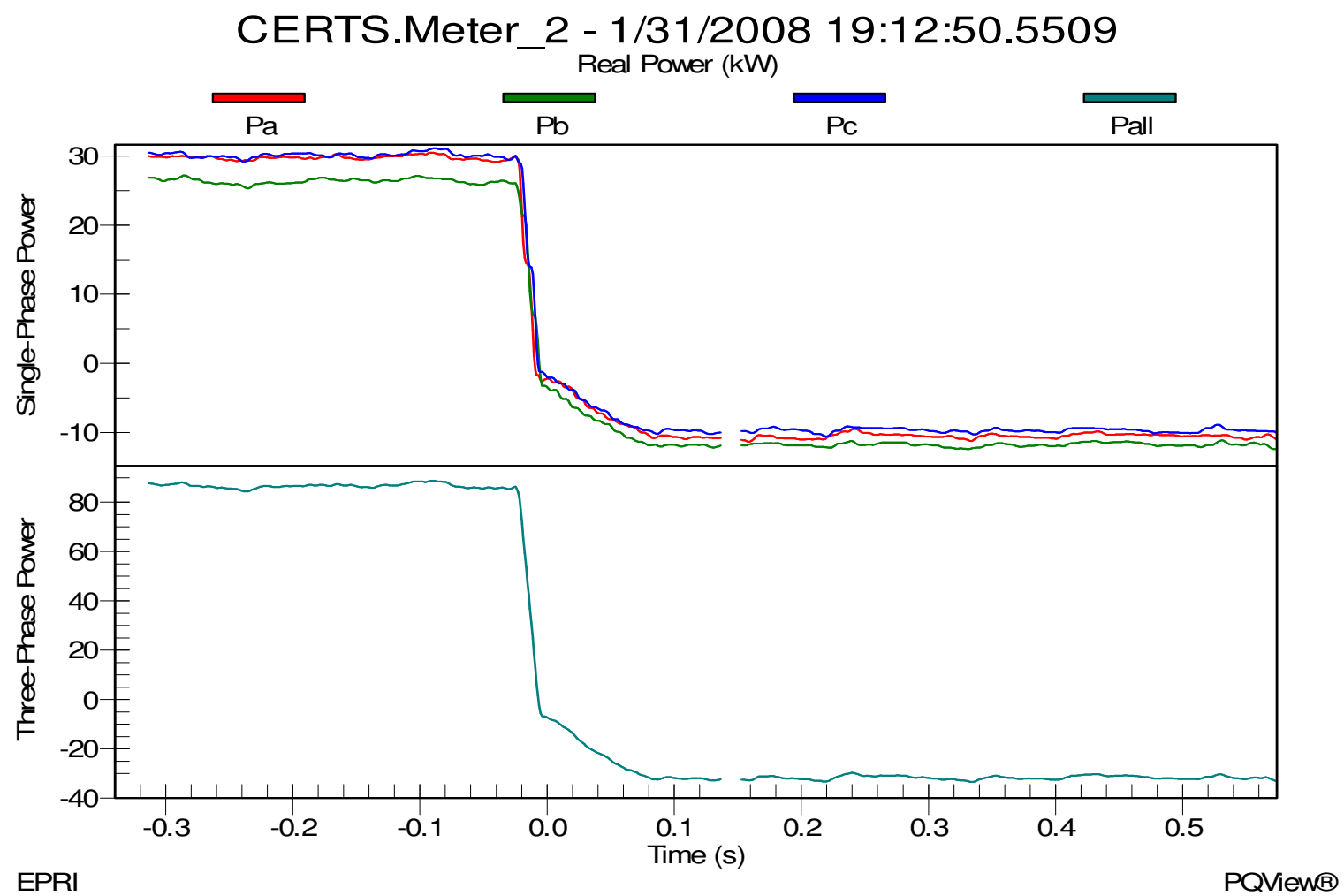

Figure 13b - Power flow at Meter 2 before/after CB41 opened.

Gen-set A2 shut down 0.2619 seconds ( 16 cycles) after CB41 opened to clear the fault, shown in Figure 13c. Gen-set A1 remained online after Gen-set A2 shutdown producing approximately $47 \mathrm{~kW}$. Note, in Figure $13 \mathrm{~d}$ there is an instance where Gen-set A1 drops from approximately $47 \mathrm{~kW}$ to $22 \mathrm{~kW}$, which is due to Load Bank 4 being disconnected 
from the microgrid. Since Gen-set A1 was set for a Unit Power Control of 50kW and there was only $10 \mathrm{~kW}$ worth of load from Load Bank 3 within the microgrid a reverse power flow occurred opening the static switch islanding it from the utility grid. The static switched opened approximately 56.2 seconds after CB41 opened and Gen-set A1 remained online, serving the microgrid load. Figure 13e shows Gen-set A1 picking up the load from Load Bank 3, approximately 12kW after the static switch opened.

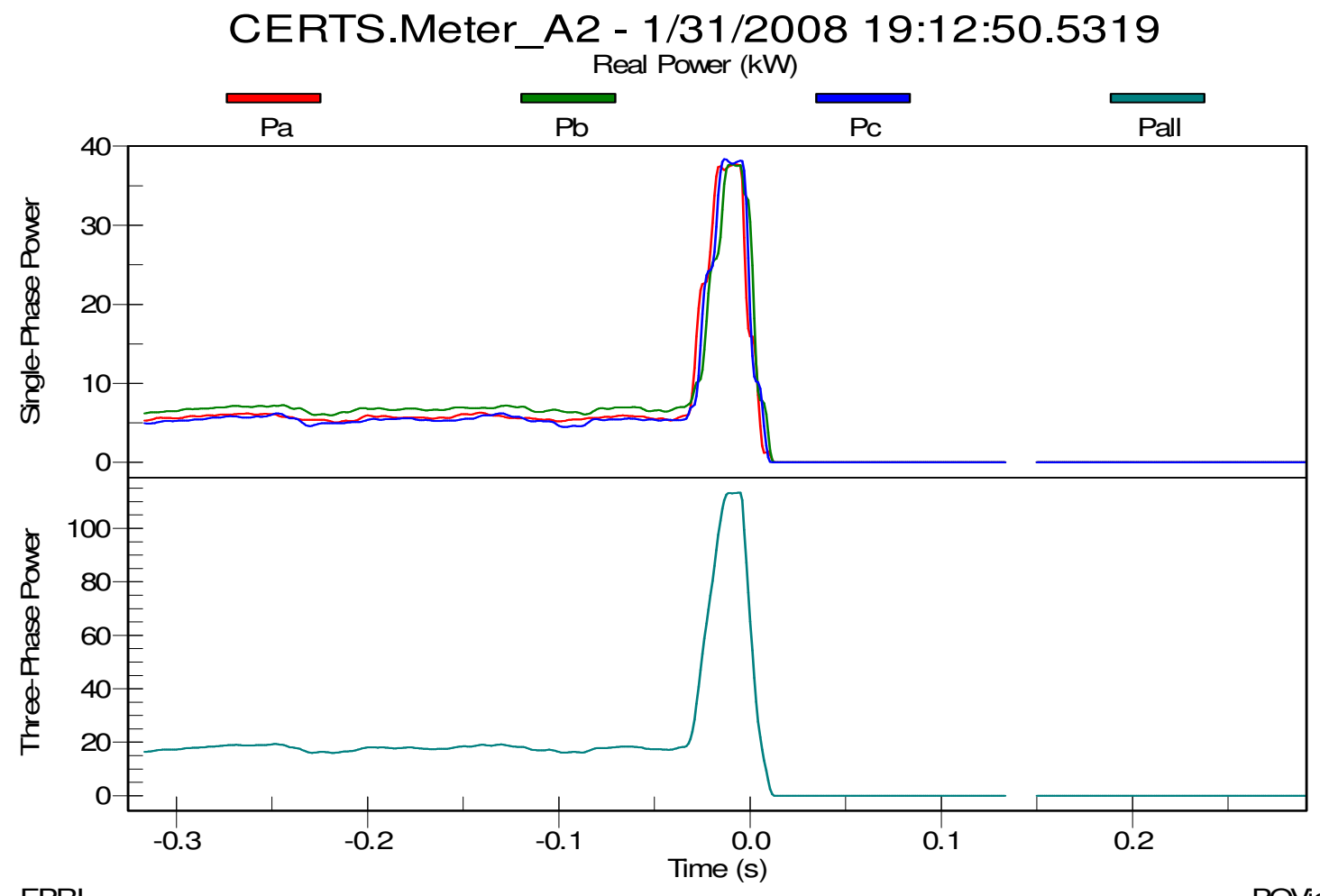

EPRI

Figure 13c - Gen-set A2 shutting down after CB41 opens 


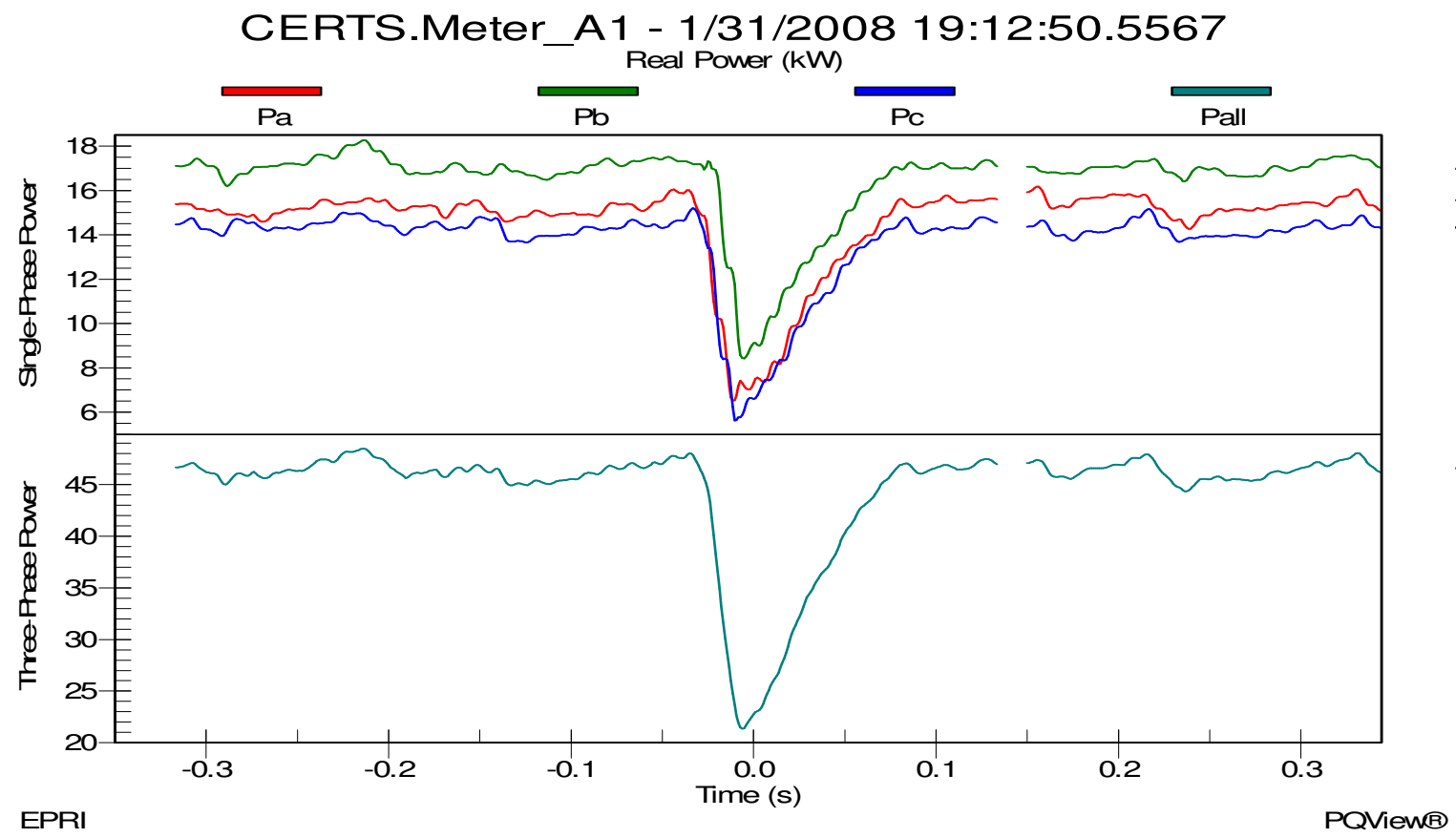

Figure 13d - Gen-set A1 power flow before/after CB41 opened.

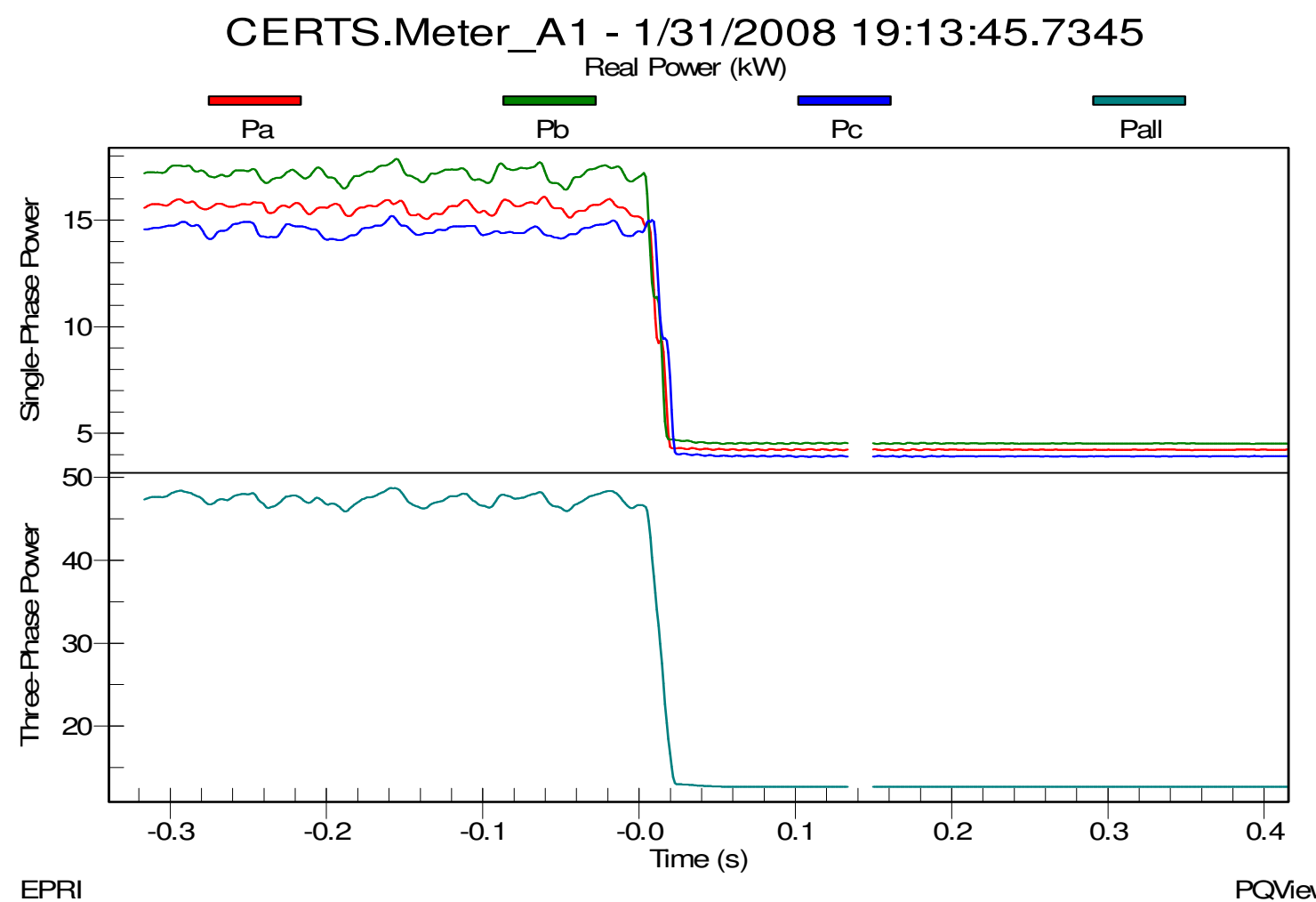


Figure 13e - Gen-set A1 power flow reducing its output power to approximately 12kW after the static switch opens from a reverse power condition.

Gen-set A1 dropped its output power from $50 \mathrm{~kW}$ to $12 \mathrm{~kW}$ to satisfy the load requirement. The electrical system was stable in both the microgrid and utility grid after CB31 opened and Gen-set A2 shutdown. Gen-set A1 was manually shutdown, all loads and alarms were reset, and equipment was prepared for the next test.

\subsubsection{Zone 3 A-Phase Line-to-Ground Fault, Gen-set A1 and Utility Connected}

Performance Goal:

Test a single line-to-ground overload fault condition in Zone 3 with Gen-sets A1 operating in-parallel with the utility grid to verify zero-sequence, negative-sequence or residual over-current protection.

Initial Setup:

Gen-set A1 = Unit Power Control Mode

Output Power Command $=20 \mathrm{~kW}$

MG Power /Frequency Droop $=-0.0833 \mathrm{~Hz} / \mathrm{kW}$

MG Voltage Command $=277 \mathrm{~V}$

Relay Settings $=$ B(Inst)

Reverse Power Set-point $=0 \mathrm{~kW}$

Load Bank $3=40 \mathrm{~kW}$

Load Bank $6=40 \mathrm{~kW}$

To simulate a high impedance single phase-to-ground fault in Zone 3 on the A-phase the Overload Load Bank of $85 \mathrm{~kW}$ (approximately $28.3 \mathrm{~kW}$ per phase) was connected by cable/plug into the exterior receptacle of Cabinet $3 \mathrm{a}$ with phases B and C disconnected. This, when combined with the load in Load Bank 3 of $40 \mathrm{~kW}$ (approximately $13.3 \mathrm{~kW}$ per phase), added $28.3 \mathrm{~kW}$ to A-phase of Zone 3 causing an overload condition.

After Gen-set A1 was running for a few minutes and supplying power to Load Bank 3, the test was started with a "Start" command from the EMS. As soon as the static switch 
closed and steady-state conditions established, the microgrid and Load Bank 6 were connected to the utility grid with the total power flow at Meter 1 equaling approximately $57.5 \mathrm{~kW}$, shown in Figure $14 \mathrm{a}$, and $21 \mathrm{~kW}$ at Meter 2 shown in Figure 14b before the fault was applied.

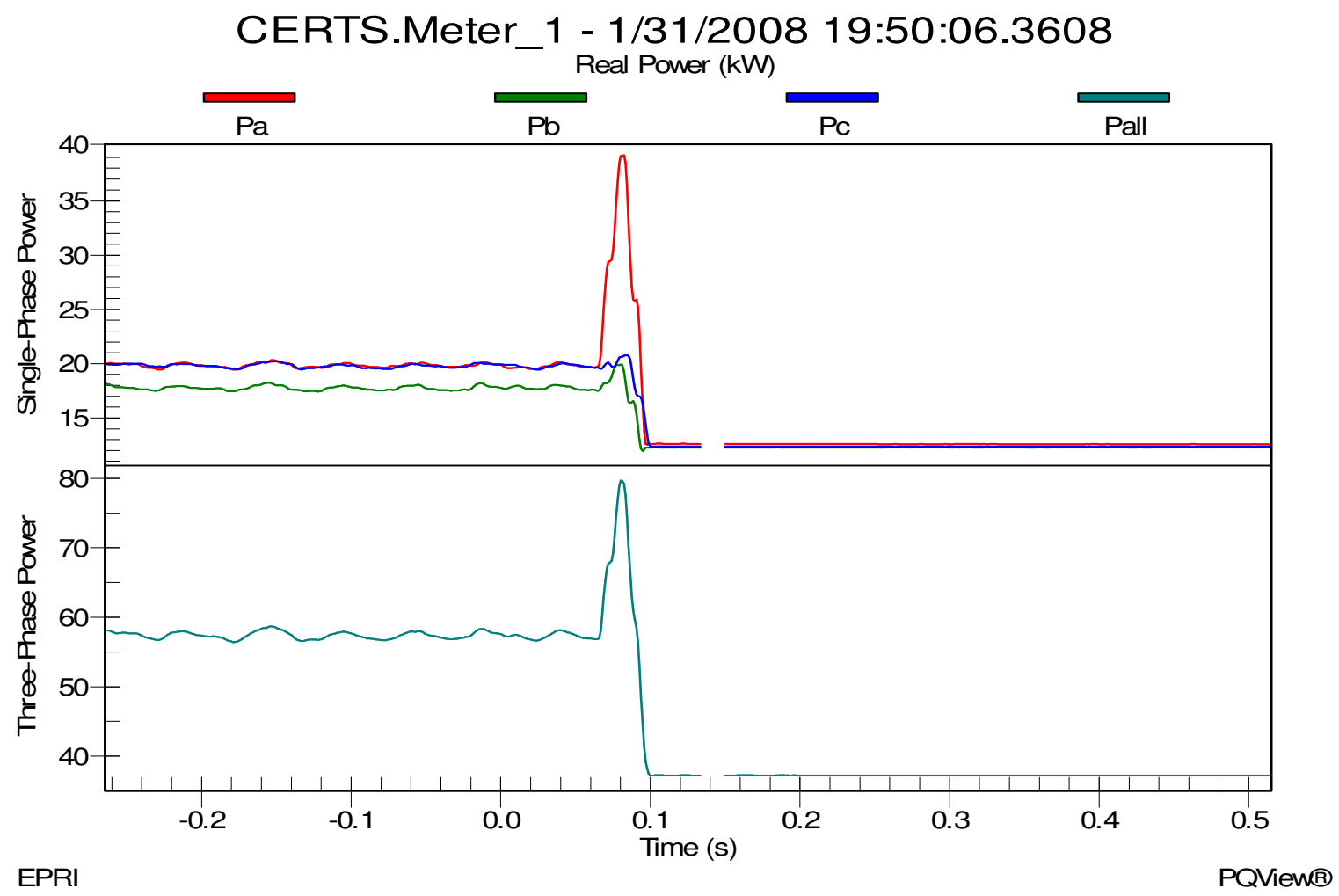

Figure 14a - Power flow in Meter 1 before/after the fault was cleared in Zone 3. 


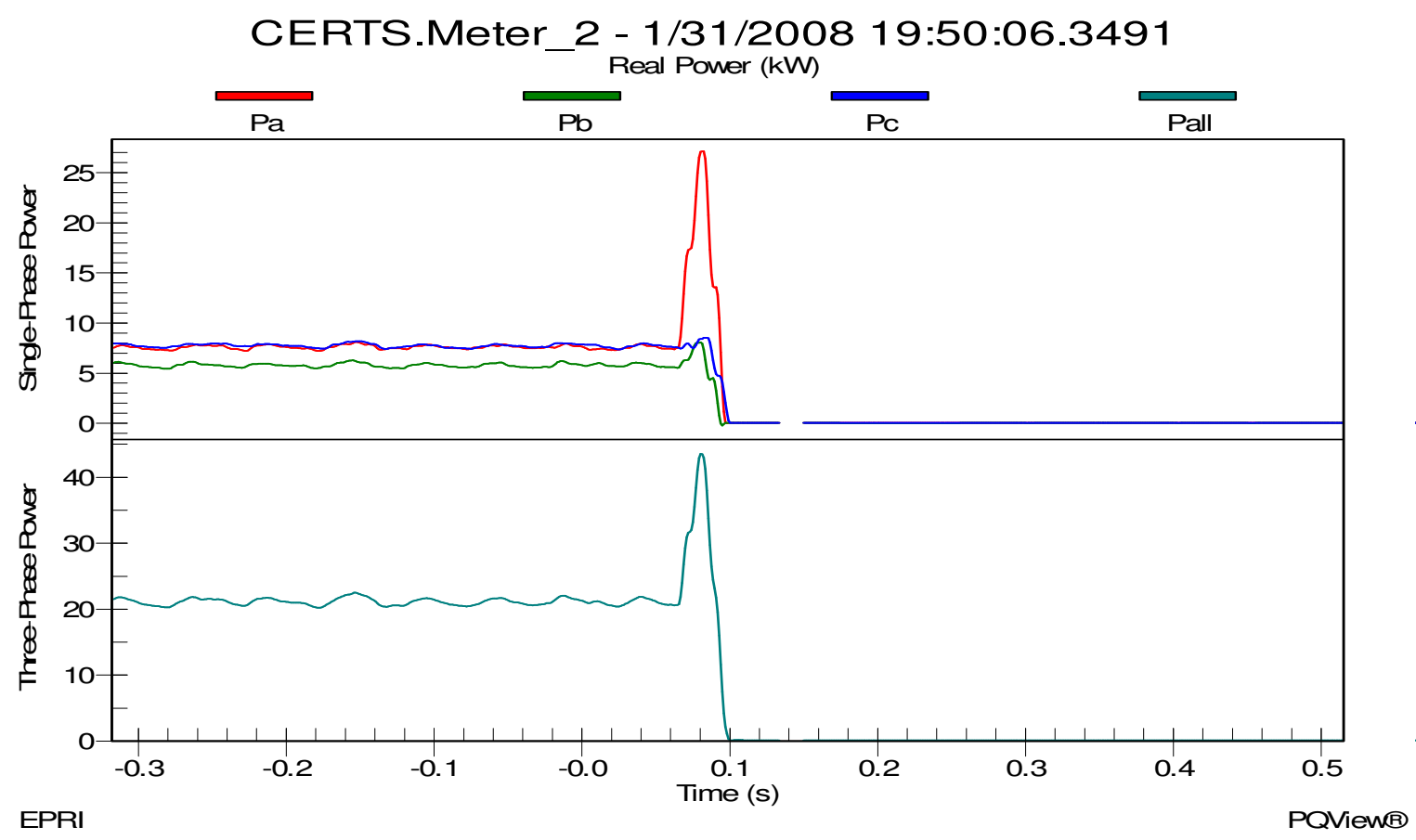

Figure 14b - Power flow in Meter 2 before/after the fault was cleared in Zone 3.

Using the DAS Load Control program, the single phase-to-ground fault on A-phase was set for 10 seconds and then initiated. The static switch tripped first in approximately 0.016 seconds ( 1 cycle) from a ground over-current $(67 \mathrm{~N} 1 \mathrm{~T})$, shown in Figure 14c, isolating the microgrid from the utility grid. Relay 3 tripped CB31, opening in 0.15 seconds ( $\sim 9$ cycles) after the static switch opened due to a ground over-current (67N1T), shown in Figure 14d, isolating Zones 3 and 4 from the rest of the microgrid and shutting down Gen-set A1. It is also worth noting that once the static switch opens the phase currents measured by the relay reduce to zero. However, because of the grounding layout, ground current from the fault, fed by Gen-set A1, is still measured and tripped. Once CB31 opens Zones 3 and 4 are isolated, no longer being satisfied by the grid or Gen-set A1 as shown by the collapsed voltage in Figure $14 \mathrm{~d}$. The approximate $39 \mathrm{~kW}$ load in Load Bank 6 is still being satisfied by the grid as shown in Figure 14a. 


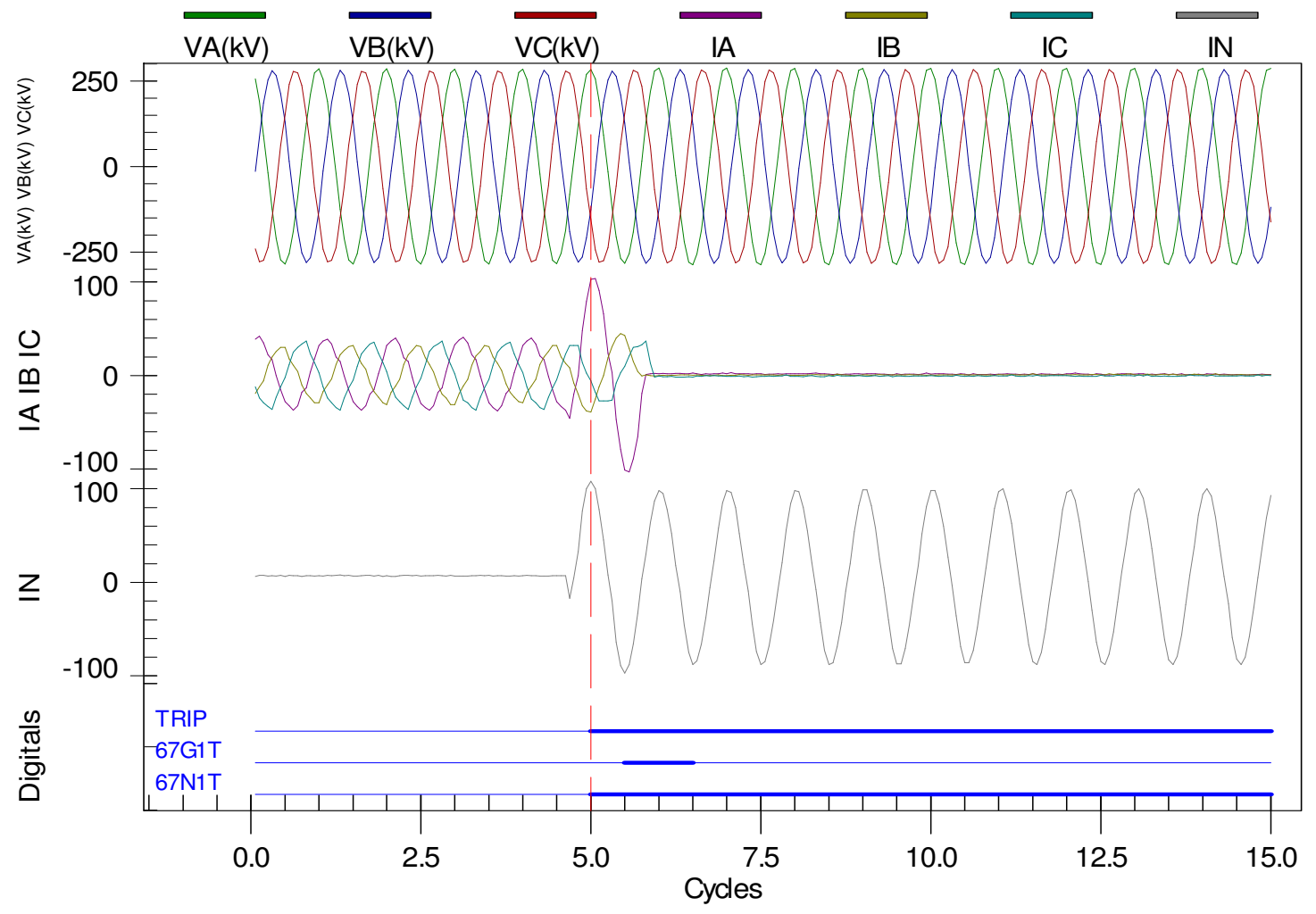

Figure 14c - Relay 2 (static switch) tripped due to ground over-current (67N1T) in 1 cycle after the single phase-to-ground fault was applied in Zone 3.

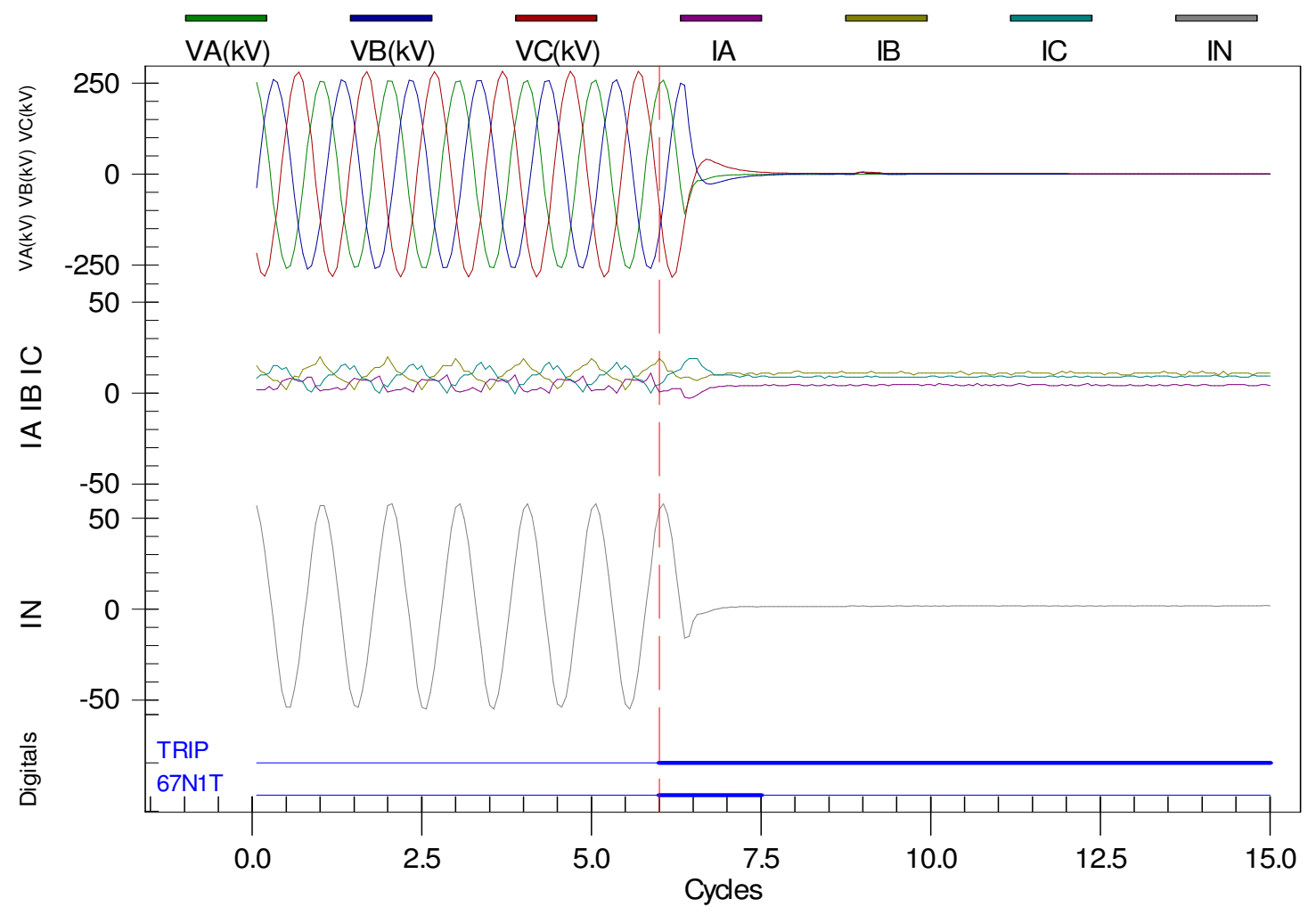


Figure 14d - Relay 3 (CB31) tripped on a ground over-current (67N1T) after the single phase-to-ground fault was applied in Zone 3.

Gen-set A1 shutdown 0.166 seconds ( 10 cycles) after Zones 3 and 4 were separated when CB31 opened. Figure 14e shows Gen-set A1 produced approximately $19 \mathrm{~kW}$ before the single phase-to-ground fault was initiated, increased to approximately $57 \mathrm{~kW}$ to satisfy Load Bank 3 when the static switch opened and shutdown when CB31 separated Zones 3 and 4 .

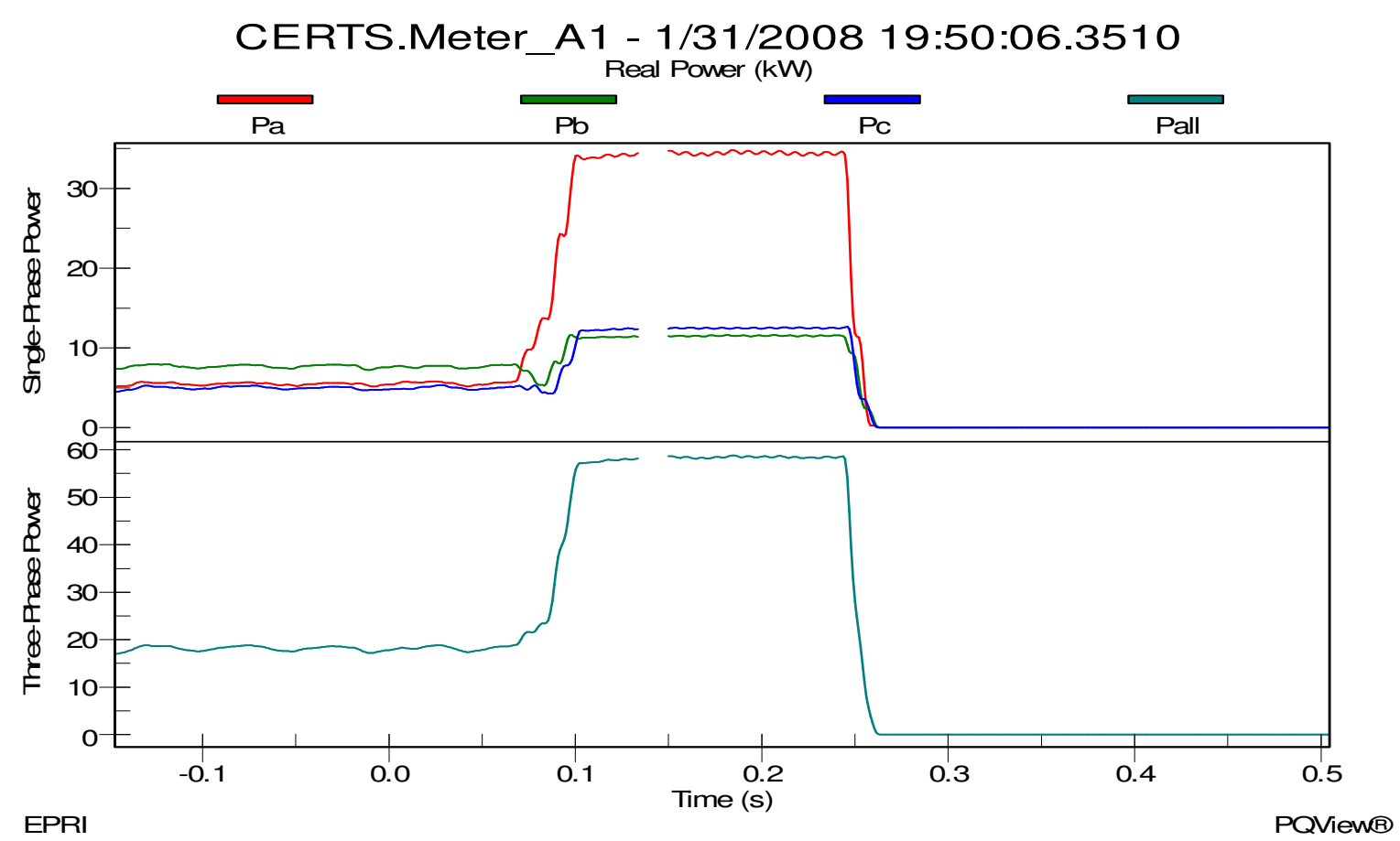

Figure 14e - Gen-set A1 output power before/after the static switch opens and then when Zones 3 and 4 are isolated from the rest of the Microgrid.

The electrical system was stable in the microgrid and utility grid after the static switch opened, CB31 opened and Gen-set A1 shutdown. All loads and alarms were reset and equipment was prepared for the next test.

\subsubsection{Zone 3 A-Phase Line-to-Ground Fault, Gen-sets (A1+A2) and Utility Connected} Performance Goal:

Test a single line-to-ground overload fault condition in Zone 3 with Gen-sets A1 and A2, operating in-parallel with the utility grid, to verify zero-sequence, negative-sequence or residual over-current protection. 


\author{
Initial Setup: \\ Gen-set A1 = Unit Power Control \\ Gen-set A2 = Unit Power Control \\ Gen-set A1 Output Power Command $=20 \mathrm{~kW}$ \\ Gen-set A2 Output Power Command $=20 \mathrm{~kW}$ \\ MG Power/Frequency Droop $=-0.0833 \mathrm{~Hz} / \mathrm{kW}$ \\ MG Voltage Command $=277 \mathrm{~V}$ \\ Relay Settings = B (Inst) \\ Reverse Power Set-point $=0 \mathrm{~kW}$ \\ Load Bank $3=40 \mathrm{~kW}$ \\ Load Bank $4=40 \mathrm{~kW}$ \\ Load Bank $6=40 \mathrm{~kW}$
}

To simulate a high impedance single phase-to-ground fault in Zone 3 on A-phase, the Overload Load Bank of $85 \mathrm{~kW}$ (approximately $28.3 \mathrm{~kW}$ per phase) was connected by cable/plug into the exterior receptacle of Cabinet 3 a with phases B and C disconnected. This, when combined with the load in Load Bank 3 of $40 \mathrm{~kW}$ (approximately $13.3 \mathrm{~kW}$ per phase), added $28.3 \mathrm{~kW}$ to A-phase when initiated and caused an overload condition in Zone 3.

After both Gen-sets A1 and A2 were running for a few minutes and supplying power to Load Banks 3 and 4, the test was started with a "Start" command from the EMS. As soon as the static switch closed and steady-state conditions established, the microgrid and Load Bank 6 were connected to the utility grid. Before the fault was applied the total power flow at Meter 1 was approximately $72.5 \mathrm{~kW}$ shown in Figure 15a and approximately 35kW at Meter 2 shown in Figure 15b.

Using the DAS Load Control program, the single phase-to-ground overload fault on Aphase was set for 10 seconds and then initiated. The static switch tripped first in approximately 0.02 seconds ( 1.3 cycles) from a ground over-current $(67 \mathrm{~N} 1 \mathrm{~T})$, shown in Figure 15c, islanding the microgrid from the utility grid. 


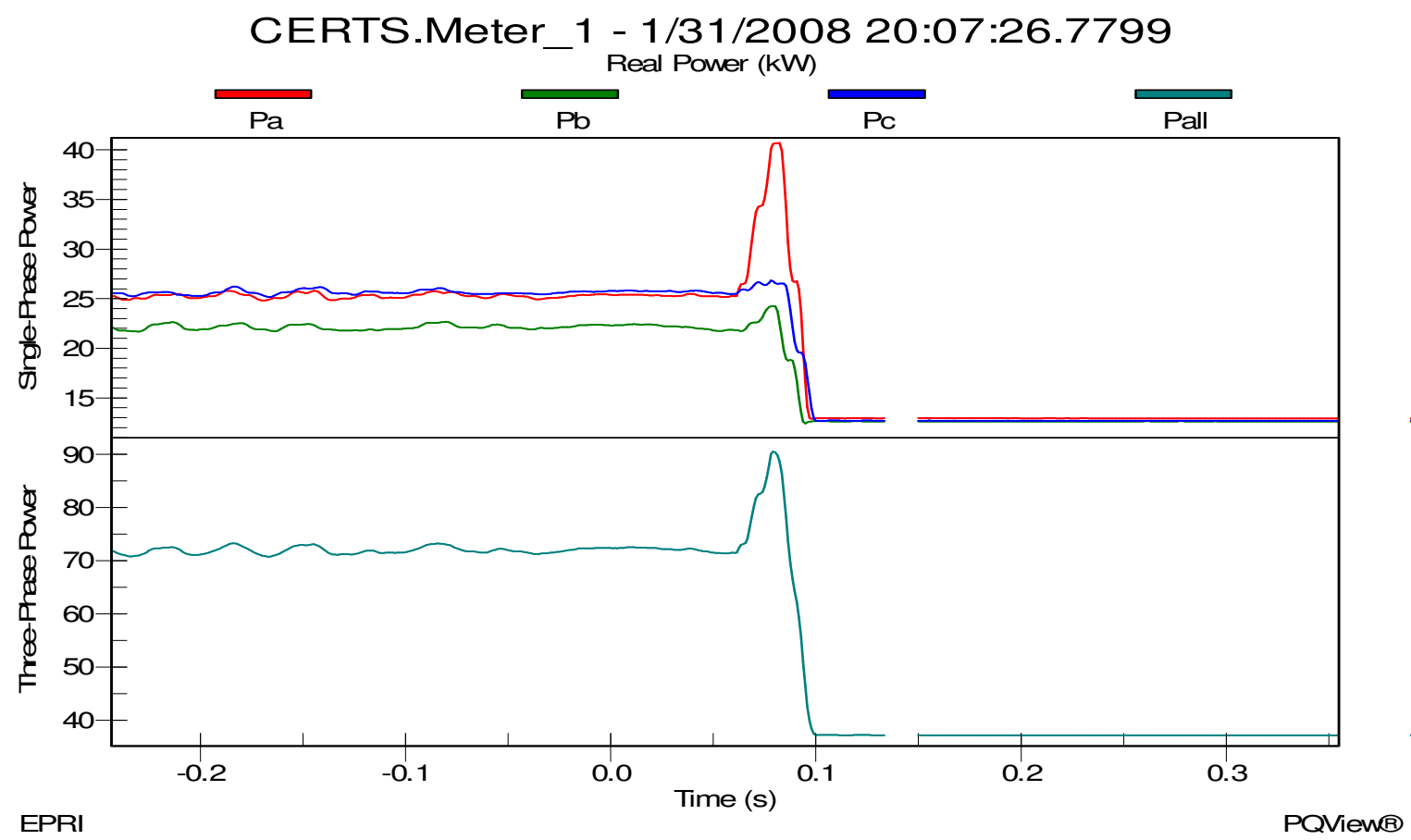

Figure 15a - Meter 1 power flow before/after the static switch opens, islanding the microgrid from the utility grid after a single phase-to-ground fault was applied to Zone 3.

CERTS.Meter_2 - 1/31/2008 20:07:26.7687 Real Power (kW)

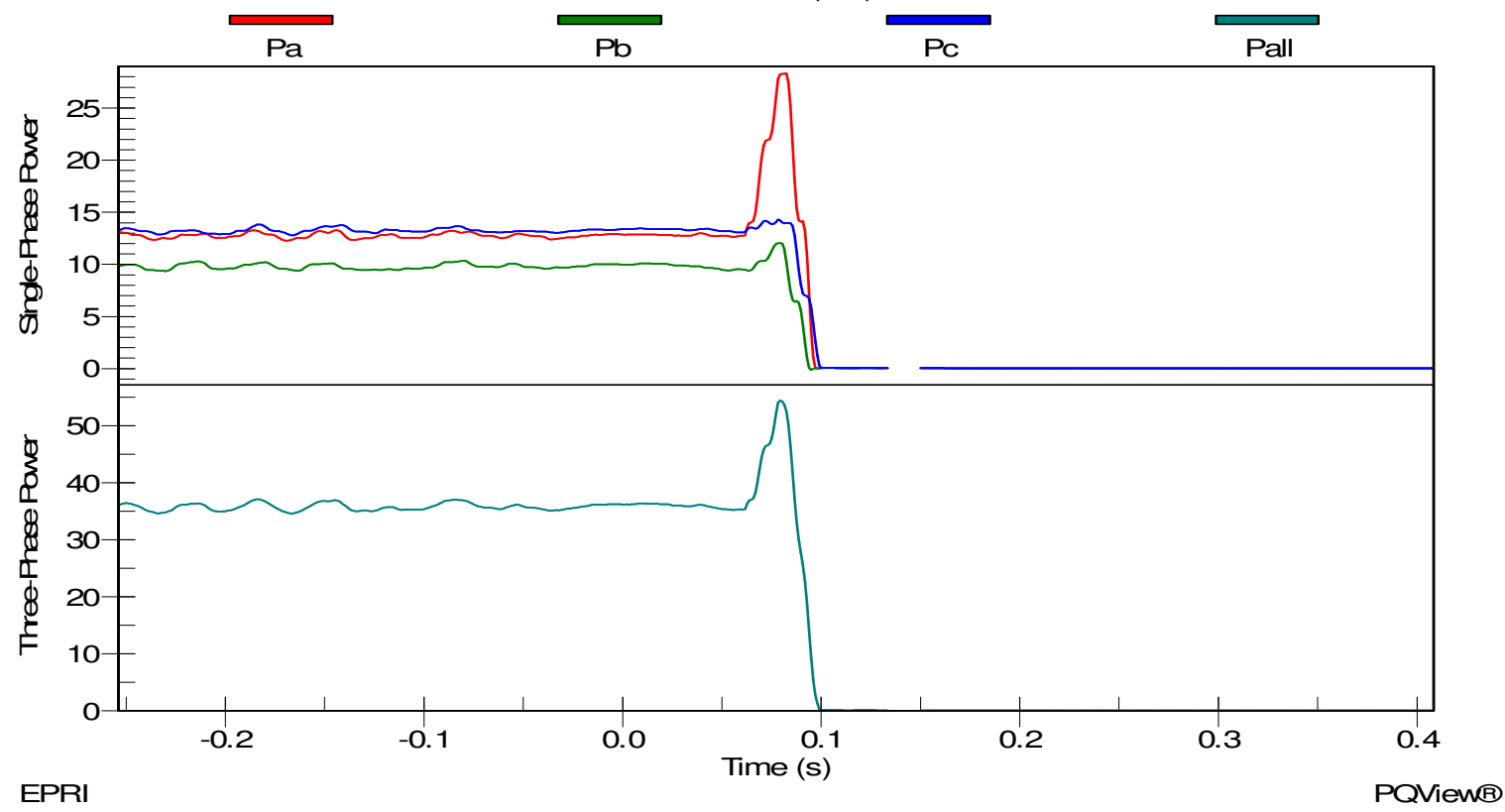

Figure 15b - Meter 2 power flow before/after the static switch opened, isolating the microgrid from the utility grid after a single phase-to-ground fault was applied to Zone 3 . 


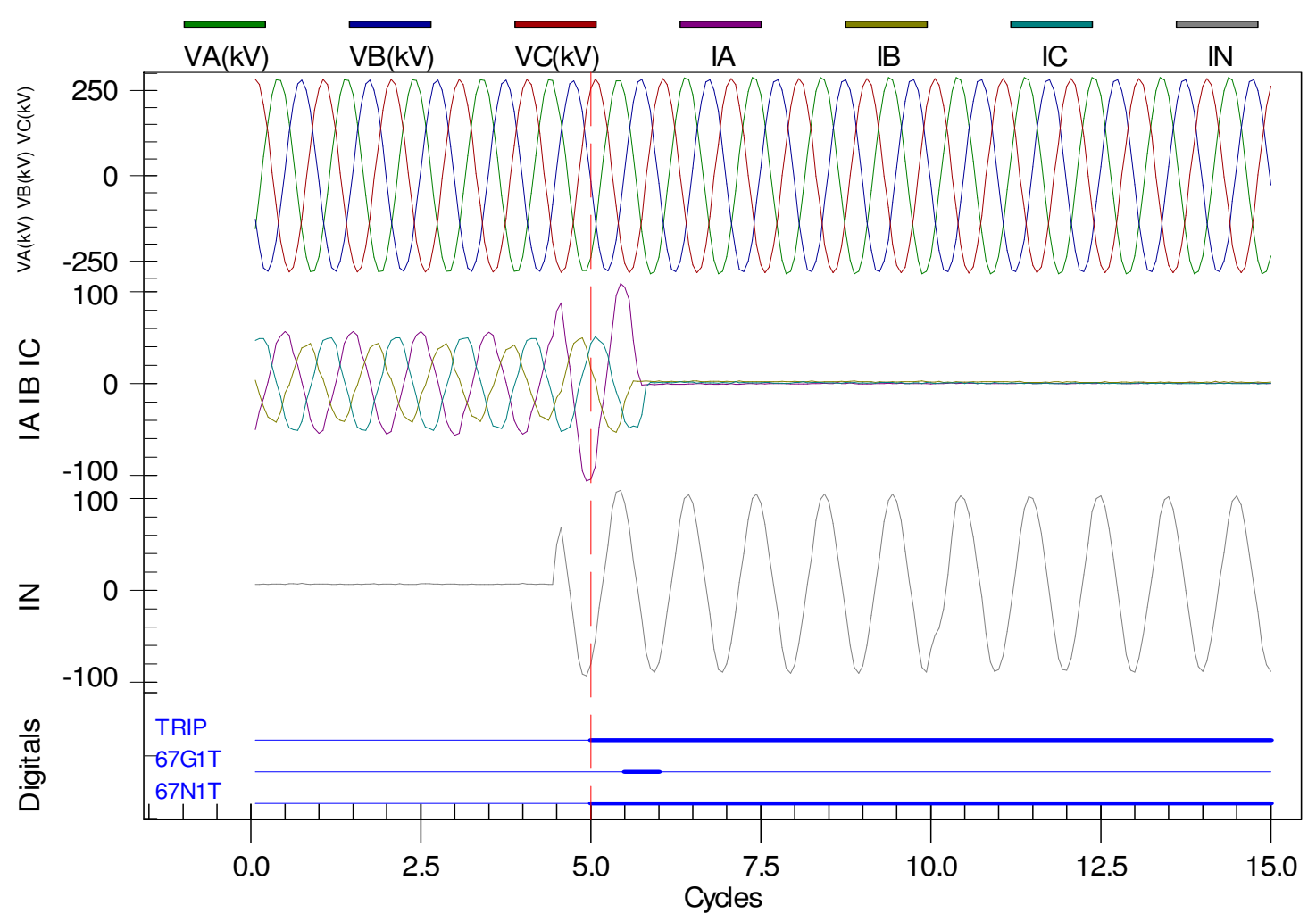

Figure 15c - Relay 2 (static switch) tripped after the single phase-to-ground fault was applied in Zone 3.

Relay 4 trips 0.07 seconds ( 4.3 cycles) after the static switch opened due to a neutral over-current (67G1T), shown in Figure 15d, separating Zone 4 from the rest of the microgrid. 


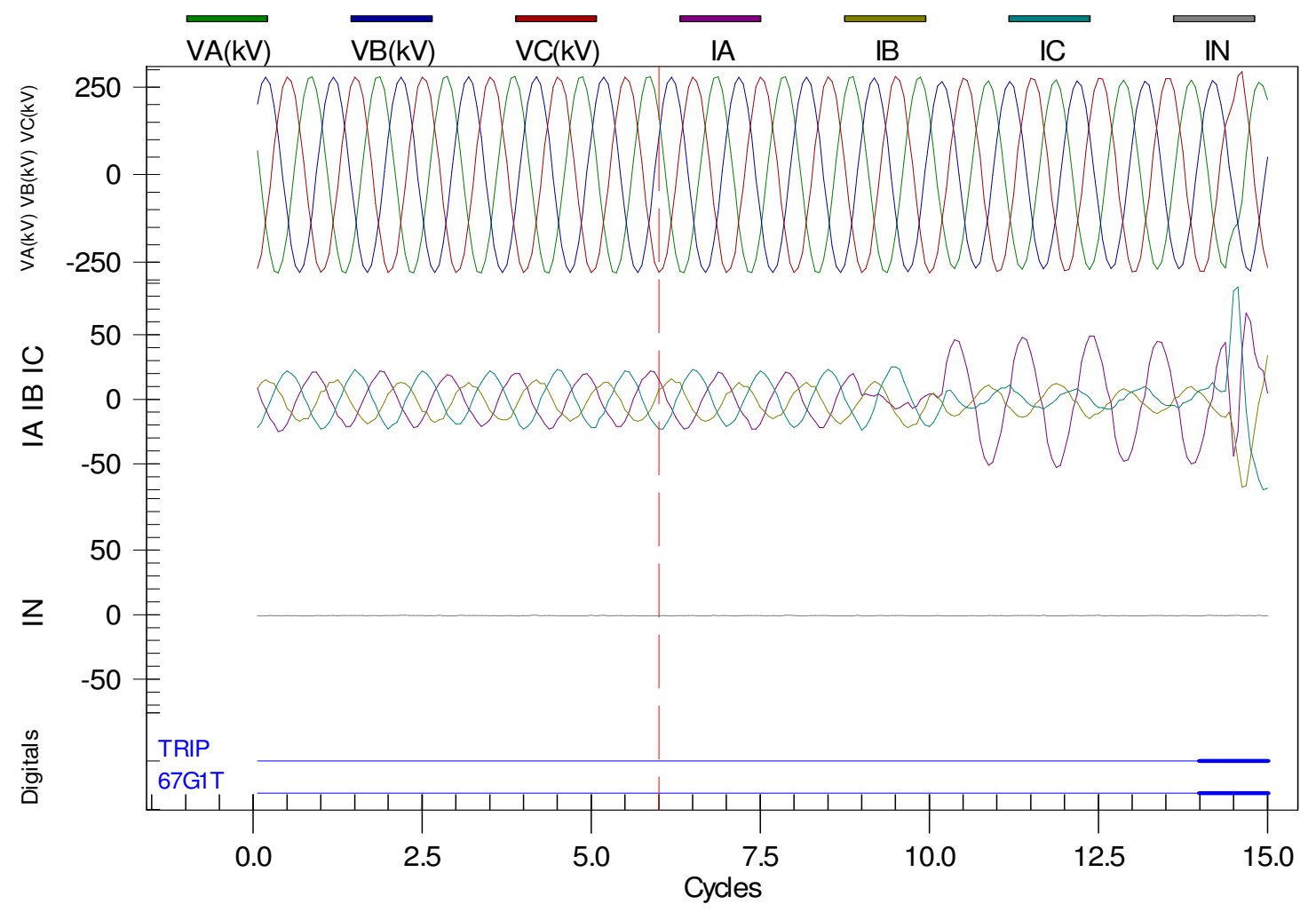

Figure 15d - Relay 4 (CB41) tripped after the single phase-to-ground fault was applied in Zone 3.

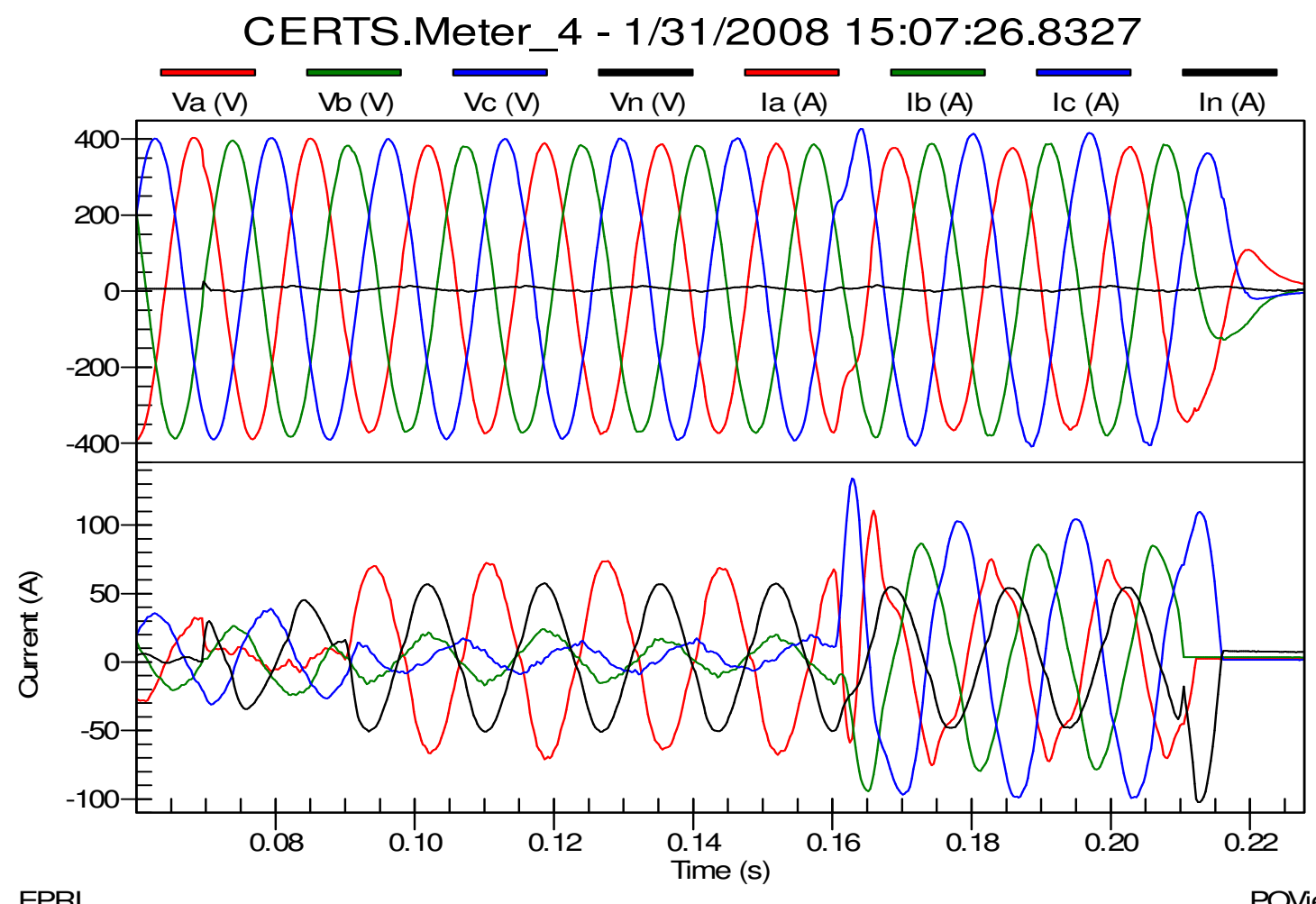


Figure 15e - Meter 4 (CB41) voltage and current for a single phase-to-ground fault applied in Zone 3. A complex situation took place in Figures $15 \mathrm{e}, 15 \mathrm{f}$ and $15 \mathrm{~g}$. The fault was applied at 0.07 seconds which caused the static switch to open at 0.09 seconds. This was followed by a short period where both Gen-sets A1 and A2 were supporting the microgrid load, as well as the fault overload, shown in Figures $15 \mathrm{f}$ and $15 \mathrm{~g}$ between 0.09 and 0.16 seconds. At 0.16 seconds Relay 4 commanded Gen-set A2 to shutdown and CB41 to open. Genset A2 first stopped delivering power to the microgrid, as seen by the sudden increase of current at Meter 4, Figure 15e, and Meter A1, Figure 15g. Even though Gen-set A2 stopped switching the inverter output, its interface transformer remained connected to the microgrid. This is evident by the continued current flow though Meter A2 after 0.16 seconds, Figure 15f, due to the Wye/Delta transformation, and also evident in the real power transferred from B and C phases to A phase at Meter A2 in Figure 15h. At 0.215 seconds CB41 opened reducing the current through CB41 to zero and denergizing Zone 4 of the microgrid.

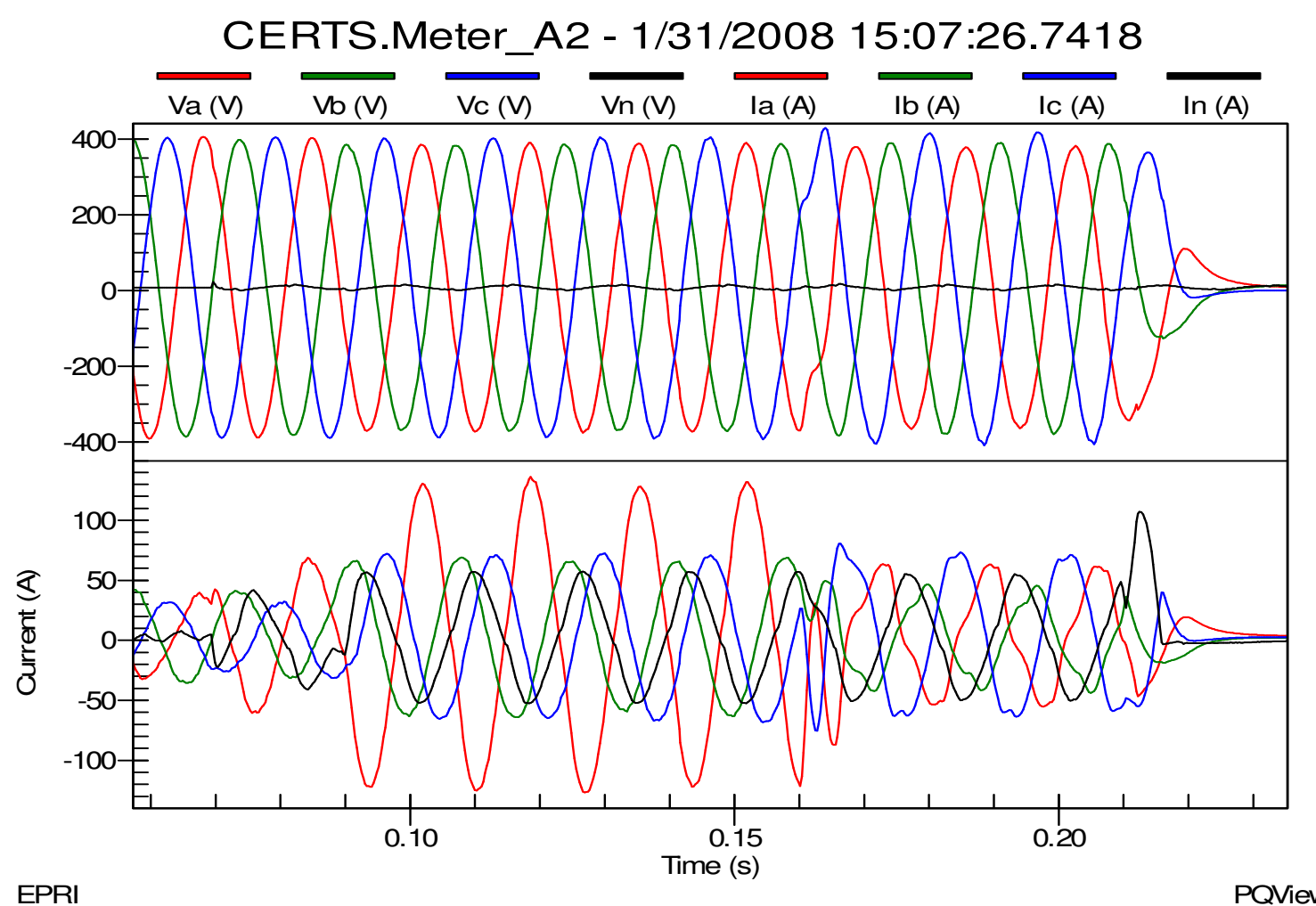

Figure 15f - Meter A2 voltage and current for a single phase-to-ground fault applied in Zone 3. 


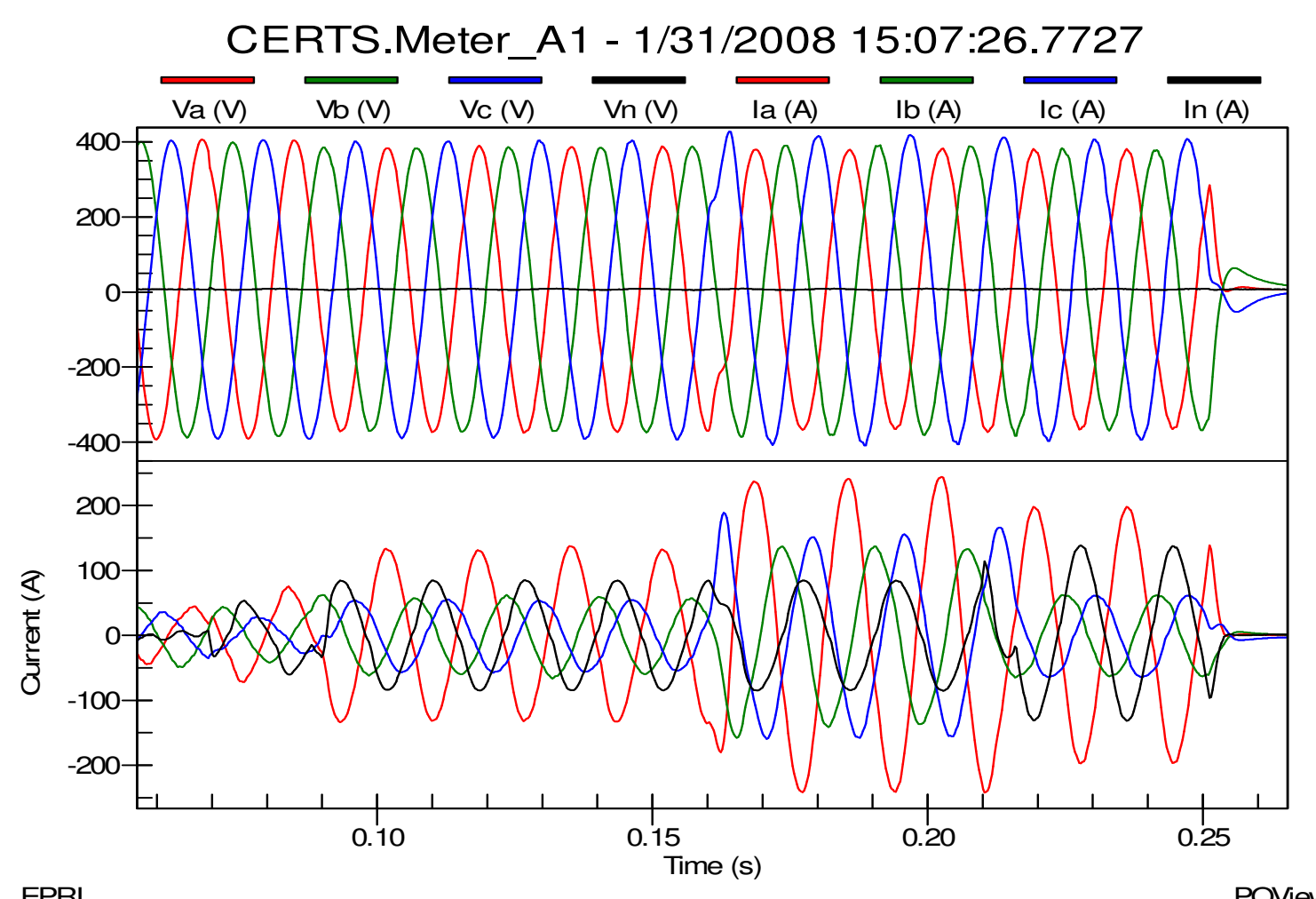

EPRI

Figure 15g - Meter A1 voltage and current for a single phase-to-ground fault applied in Zone 3.

Relay 3 tripped Gen-setA1 and opened CB31 0.04 seconds ( 2.3 cycles) after CB41 opened, due to ground over-current (67N1T), shown in Figure 15i, separating Zone 3 from the rest of the microgrid. In Figure 15j Gen-set A1 produced approximately $20 \mathrm{~kW}$ before the single phase-to-ground fault was initiated and increased to approximately $48.5 \mathrm{~kW}$ to satisfy Load Banks 3 and 4 along with generation from Gen-set A2 once the static switch opened. Output power for Gen-set A1 also increased to approximately $100 \mathrm{~kW}$ for a brief period of time due to Gen-set A2 shutting down while the $40 \mathrm{~kW}$ of Load Bank 3 and 4 remained connected. 


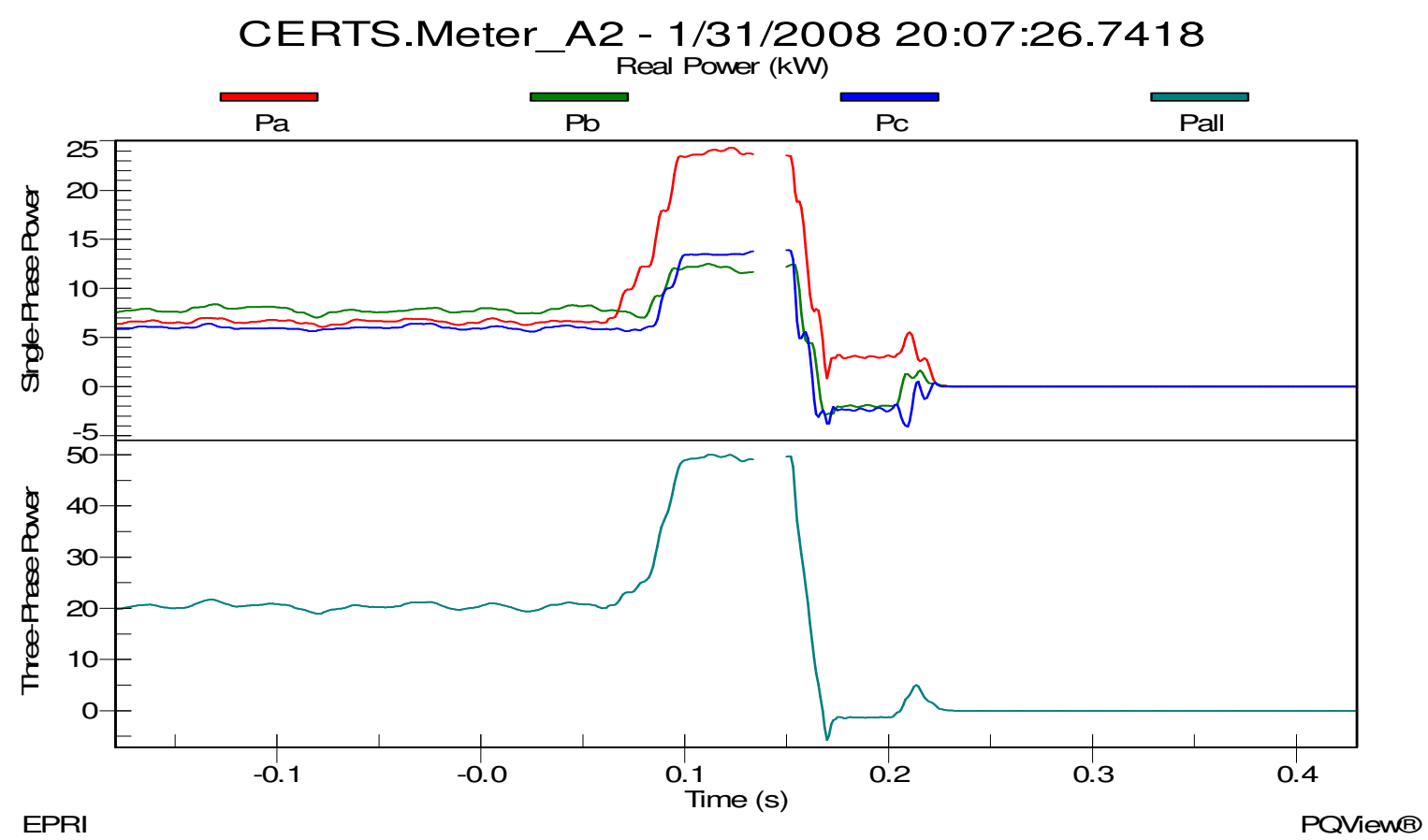

Figure 15h - Gen-set A2 output power before/after the static switch "Opens" and when Zone 4 is separated from the rest of the microgrid.

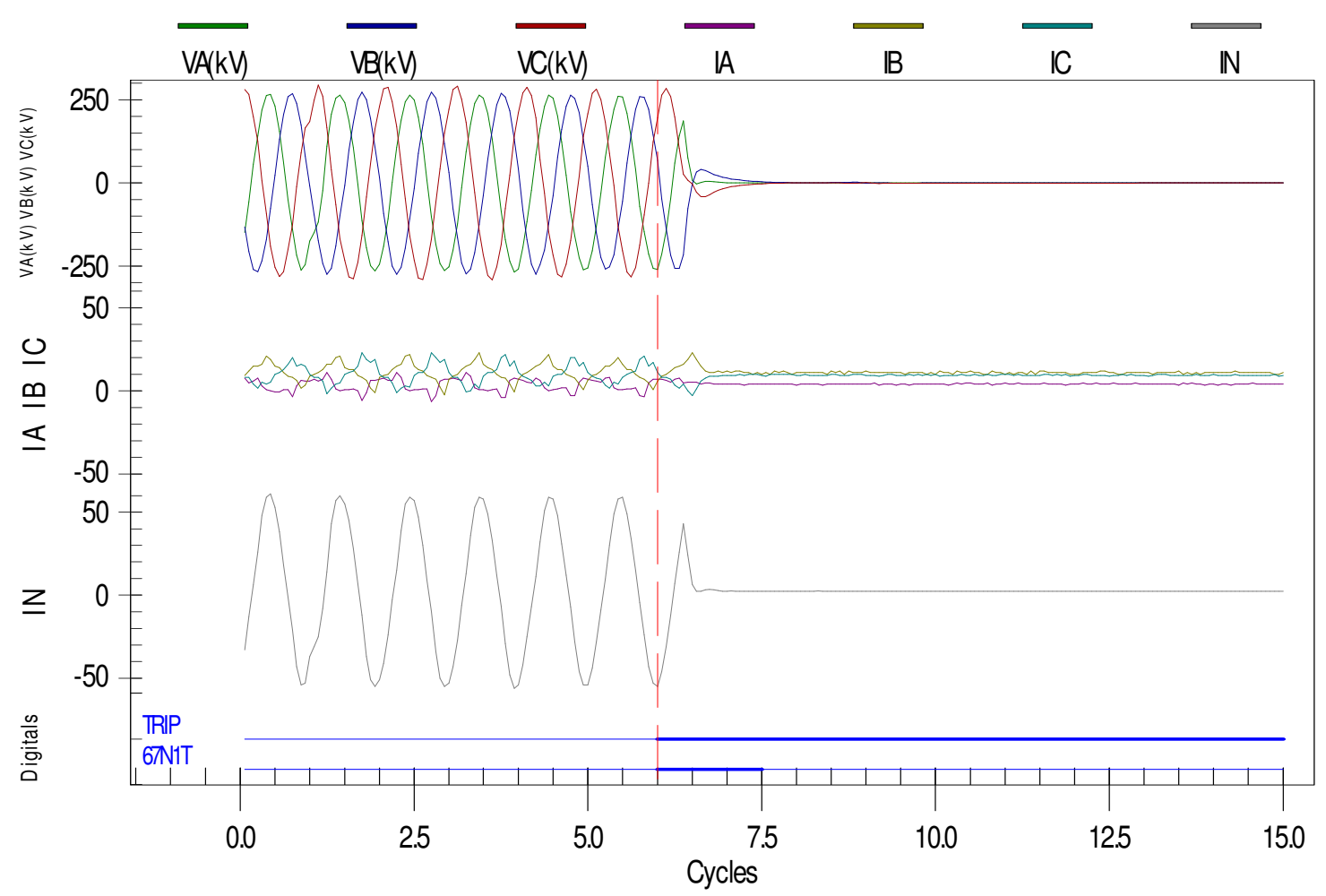


Figure 15i - Relay 3 (CB31) tripped due to a ground over-current (67N1T) when the single phase-toground fault was initiated in Zone 3

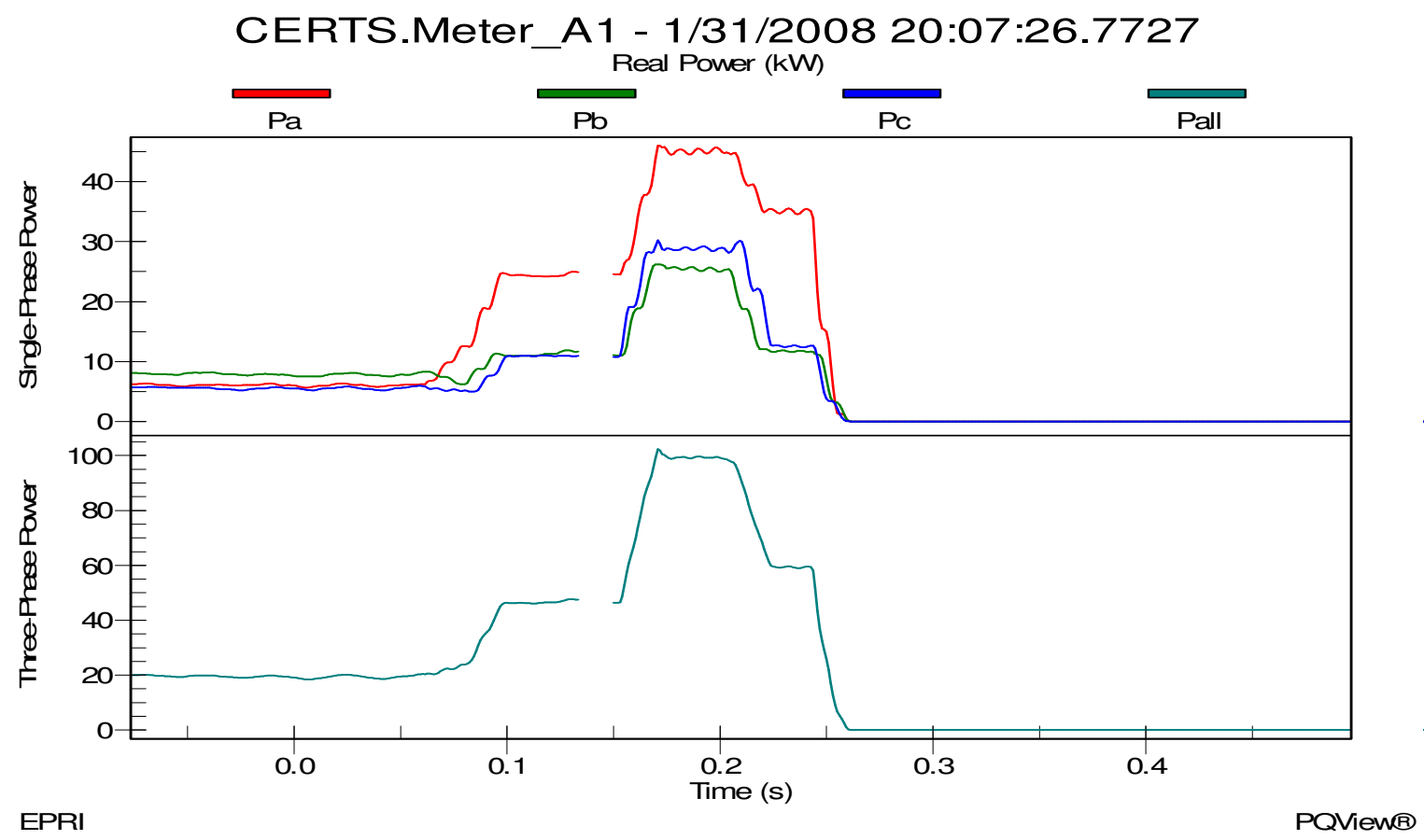

Figure 15j - Gen-set A1 output power before/after the static switch opened, when CB41 opens isolating Zone 4, and when Zone 3 is isolated from the rest of the microgrid.

The electrical system was stable in both the microgrid and utility grid after the static switch had opened, circuit breakers (i.e., CB31, CB41 andCB51) had opened, and Gensets A1 and A2 shut down. All loads and alarms were reset and equipment was prepared for the next test.

\subsubsection{Zone 5 B-Phase Line-to-Ground Fault, Gen-set B1 and Utility Connected} Performance Goal:

Test a single line-to-ground overload fault condition in Zone 5 with Gen-sets B1 operating in-parallel with the utility grid to verify zero-sequence, negative-sequence or residual over-current protection.

Initial Setup:

Gen-set B1 = Unit Power Control 
Output Power Command $=20 \mathrm{~kW}$

MG Power/Frequency Droop $=-0.0833 \mathrm{~Hz} / \mathrm{kW}$

MG Voltage Command $=277 \mathrm{~V}$

Relay Settings $=$ B (Inst)

Reverse Power Set-point $=0 \mathrm{~kW}$

Load Bank $3=40 \mathrm{~kW}$

Load Bank $6=40 \mathrm{~kW}$

To simulate a high impedance single phase-to-ground fault in Zone 5 on the B-phase the Overload Load Bank of $85 \mathrm{~kW}$ (approximately $28.3 \mathrm{~kW}$ per phase) was connected by cable/plug into the exterior receptacle of Cabinet 7a with phases A and C disconnected. When initiated, this $28.3 \mathrm{~kW}$ on B-phase, with the $40 \mathrm{~kW}$ in Load Bank 3 of Zone 3, causes an overload.

After Gen-set B1 was running for a few minutes and supplying power to Load Bank 3, the test was started with a "Start" command from the EMS. As soon as the static switch closed and steady-state conditions established, the microgrid and Load Bank 6 were connected to the utility grid with total power flow at Meter 1 being approximately $54 \mathrm{~kW}$, shown in Figure 16a, and approximately 17.5kW at Meter 2 shown in Figure 16b before the fault was applied. 


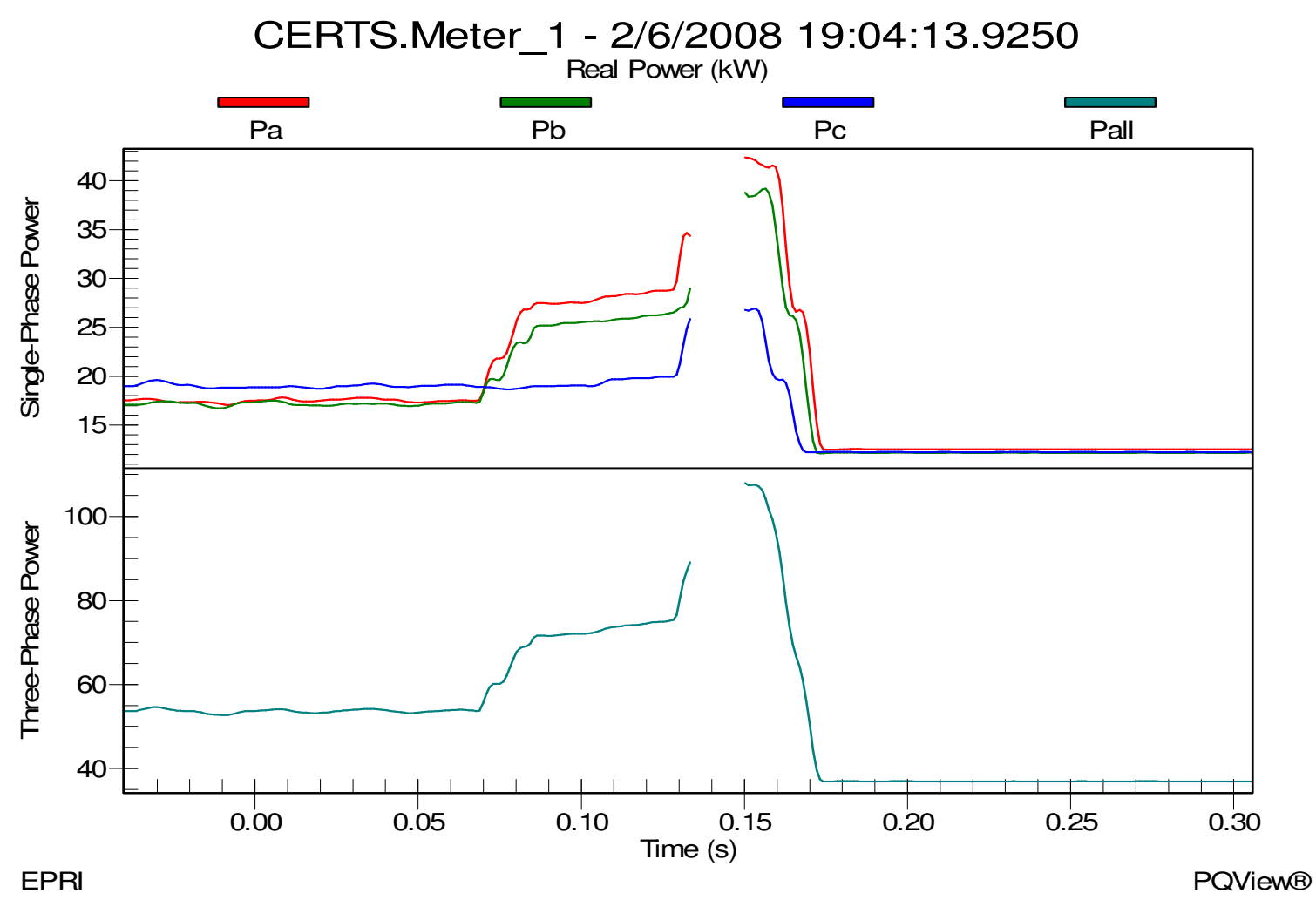

Figure 16a - Meter 1 power flow before/after the static switch opened, islanding the microgrid from the utility grid when a single phase-to-ground fault was applied in Zone 5. 


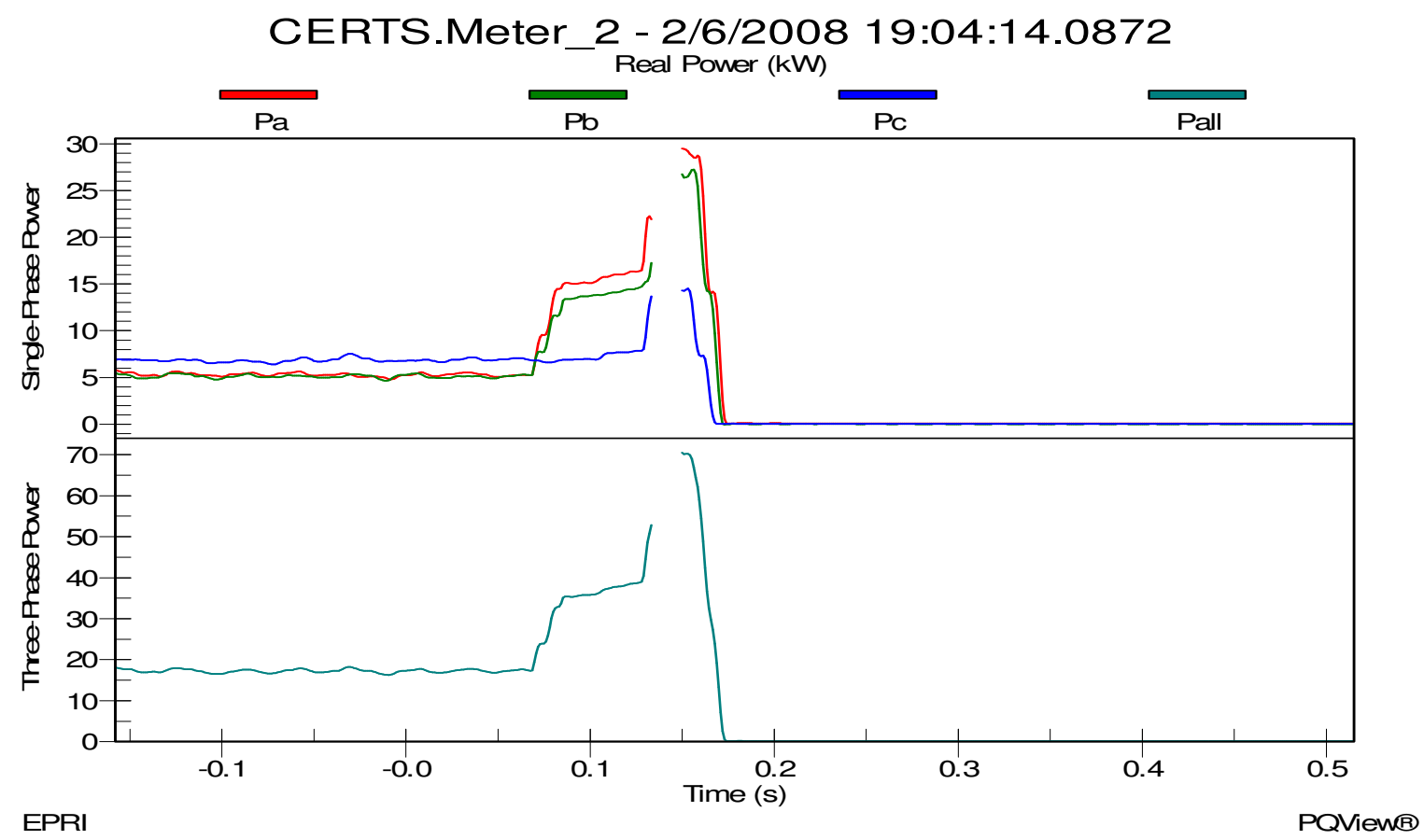

Figure 16b - Meter 2 power flow before/after the static switch opened, islanding the microgrid from the utility grid when a single phase-to-ground fault was applied in Zone 5.

Using the DAS Load Control program, a single phase-to-ground overload fault on Bphase was set for 10 seconds and then initiated. The protection coordination for this event appears to be different than expected. After the fault is initiated Relay 5 first trips shutting down Gen-set B1 and opening CB51, Figure 16c. The Gen-set stops switching the inverter output, however its transformer contactor and CB51 remains closed for a brief period of time, Figure 16d. After the Gen-set stops switching the fault current at the static switch becomes large enough to cause Relay 2 to trip on negative sequence (67Q1T), Figure 16e. Relay 5 tripped first approximately 0.07 seconds ( 4.2 cycles) separating Zone 5 from the rest of the microgrid. The static switch opened 0.03 seconds ( $\sim 75$ cycles) after Relay 5 tripped, islanding the microgrid from the utility. 


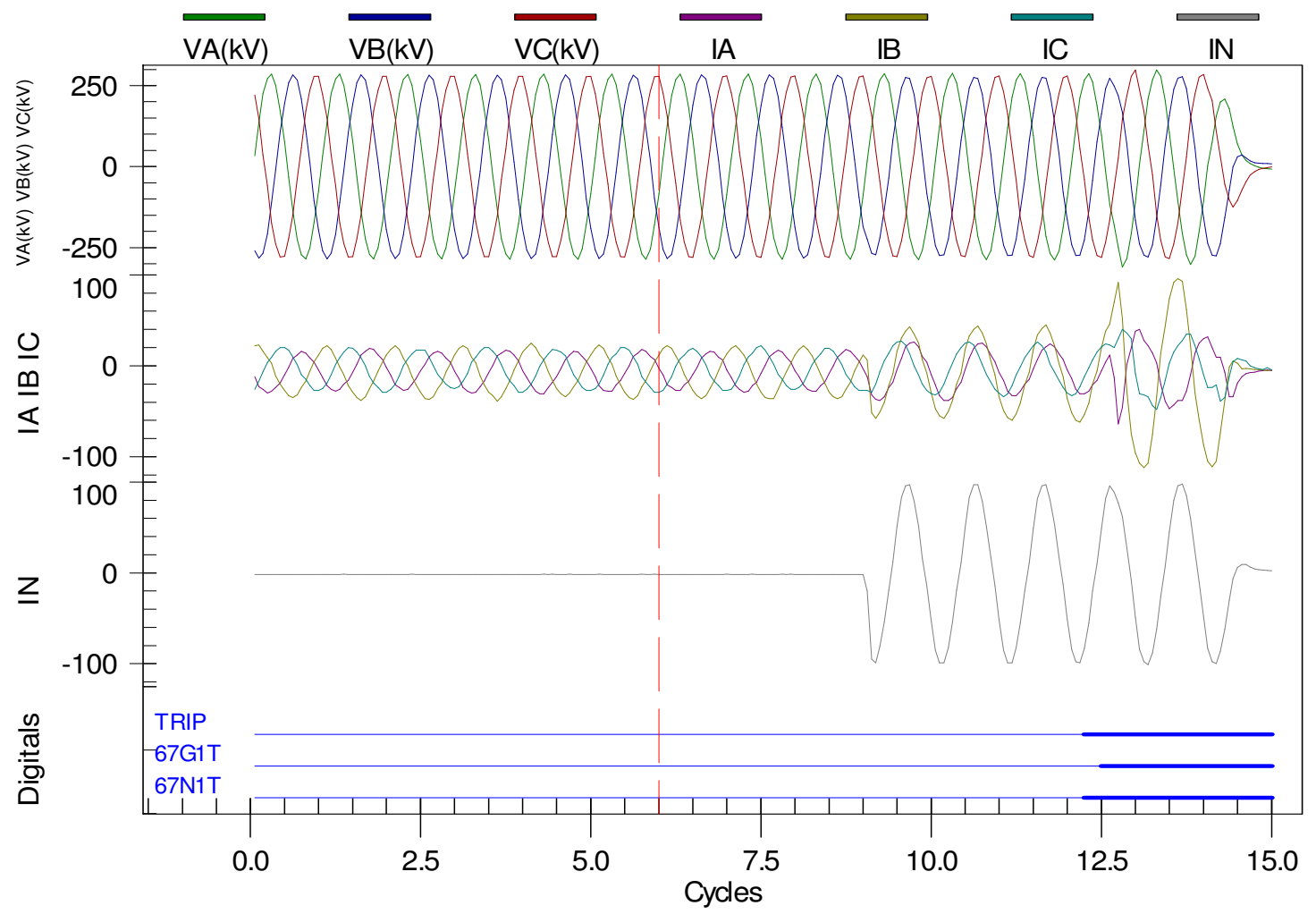

Figure 16c - Relay 5 (CB51) tripped due to ground over-current (67N1T) after the single phase-toground fault was initiated in Zone 5.

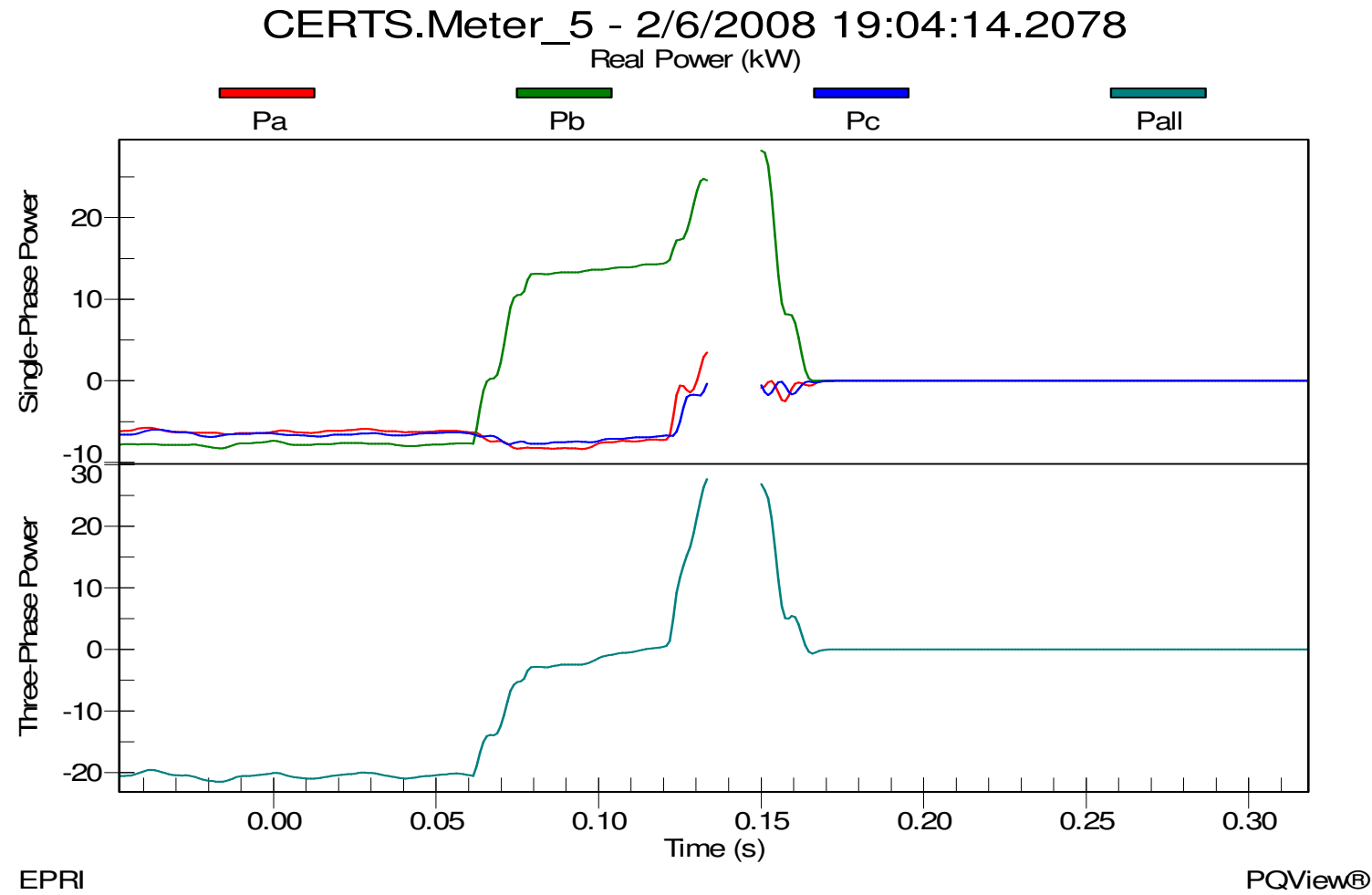


Figure 16d - Meter 5 power flow for a single phase-to-ground fault applied in Zone 5.

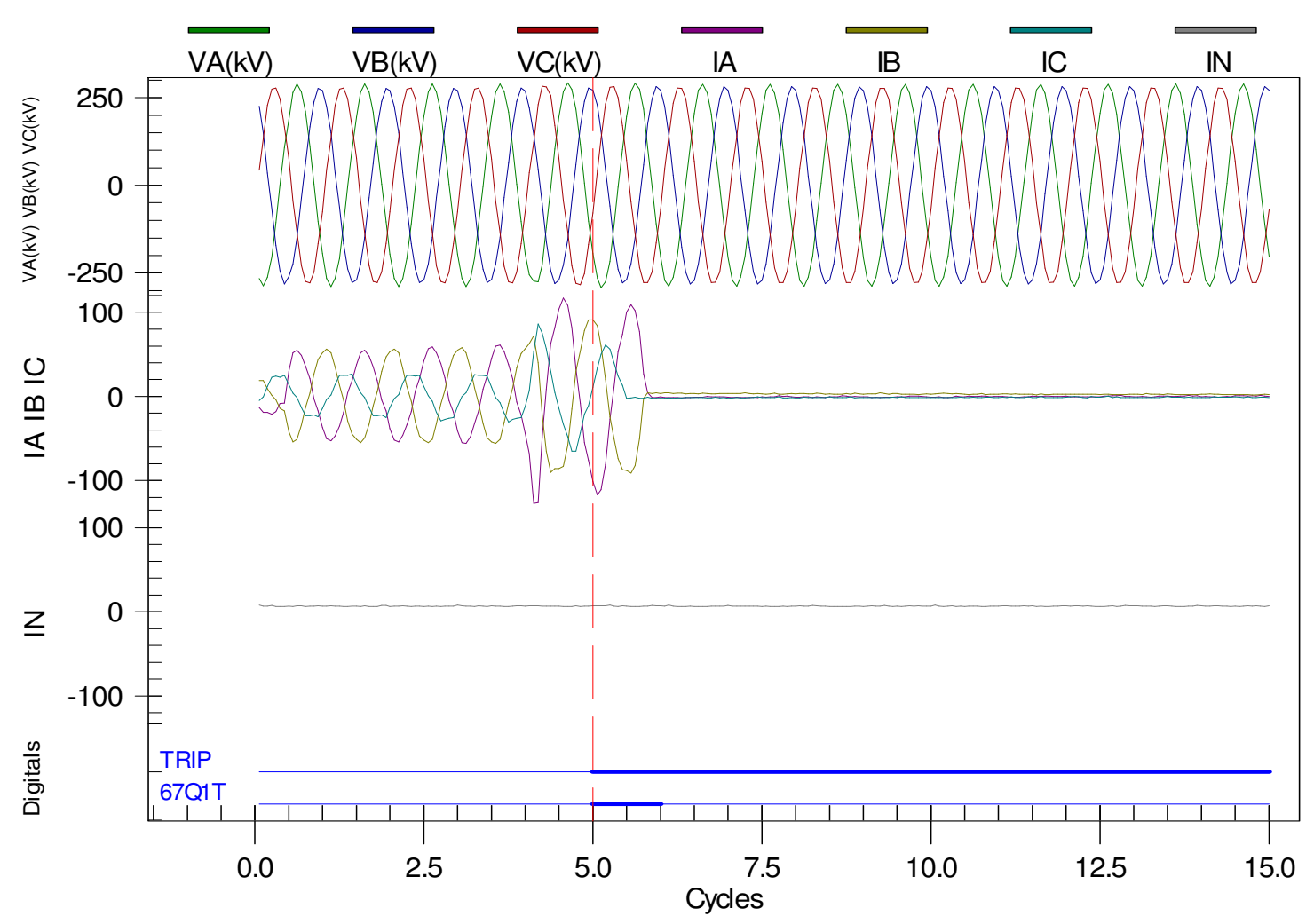

Figure 16e - Relay 2 (static switch) tripped due to negative sequence (67Q1T) after the single phase-toground fault was applied in Zone 5.

The electrical system was stable in the microgrid and utility grid after the static switch opened, CB51 opened, and Gen-set B1 shut down. All loads and alarms were reset and equipment was prepared for the next test.

\subsubsection{Zone 5 B-Phase Line-to-Ground Fault, Gen-sets (A1+B1) and Utility Connected Performance Goal:}

Test a single line-to-ground overload fault condition in Zone 5 with Gen-sets A1 and B1 operating in-parallel with the utility grid to verify zero-sequence, negative-sequence or residual over-current protection.

\section{Initial Setup:}

Gen-set A1 = Unit Power Control

Gen-set B1 = Unit Power Control 
Gen-set A1 Output Power Command $=20 \mathrm{~kW}$

Gen-set B1 Output Power Command $=20 \mathrm{~kW}$

MG Power/Frequency Droop $=-0.0833 \mathrm{~Hz} / \mathrm{kW}$

MG Voltage Command $=277 \mathrm{~V}$

Relay Settings = B (Inst)

Reverse Power Set-point $=0 \mathrm{~kW}$

Load Bank $3=40 \mathrm{~kW}$

Load Bank $4=40 \mathrm{~kW}$

Load Bank $6=40 \mathrm{~kW}$

To simulate a high impedance single phase-to-ground fault in Zone 5 on B-phase the Overload Load Bank of $85 \mathrm{~kW}$ (approximately $28.3 \mathrm{~kW}$ per phase) was connected by cable/plug into the exterior receptacle of Cabinet $7 \mathrm{a}$ with phases $\mathrm{A}$ and $\mathrm{C}$ disconnected. When initiated, this $28.3 \mathrm{~kW}$ on B-phase, with the $40 \mathrm{~kW}$ in Load Banks 3 and 4 , causes an overload.

After both Gen-sets A1 and B1 were running for a few minutes and supplying power to Load Banks 3 and 4, the test was started with a "Start" command from the EMS. As soon as the static switch closed and steady-state conditions established, the microgrid and Load Bank 6 were connected to the utility grid with the total power flow at Meter 1 being approximately $75 \mathrm{~kW}$, shown in Figure $17 \mathrm{a}$, and approximately $40 \mathrm{~kW}$ at Meter 2, shown in Figure 17b. Gen-sets A1 and B1 were producing between 17 to $18 \mathrm{~kW}$ each, shown in Figures 17c and 17d, before the overload fault was applied. 


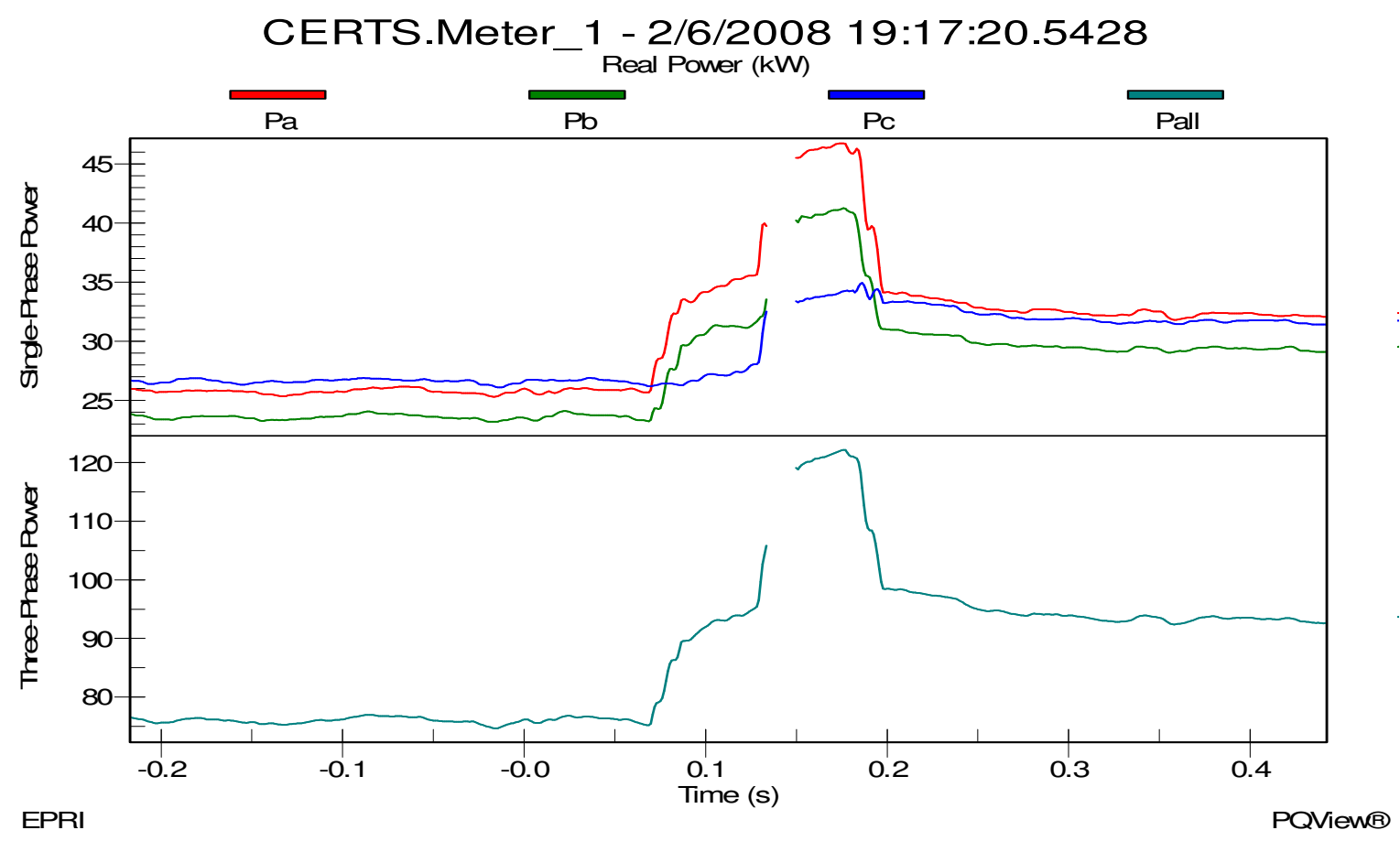

Figure 17a - Meter 1 power flow for a single phase-to-ground fault applied in Zone 5.

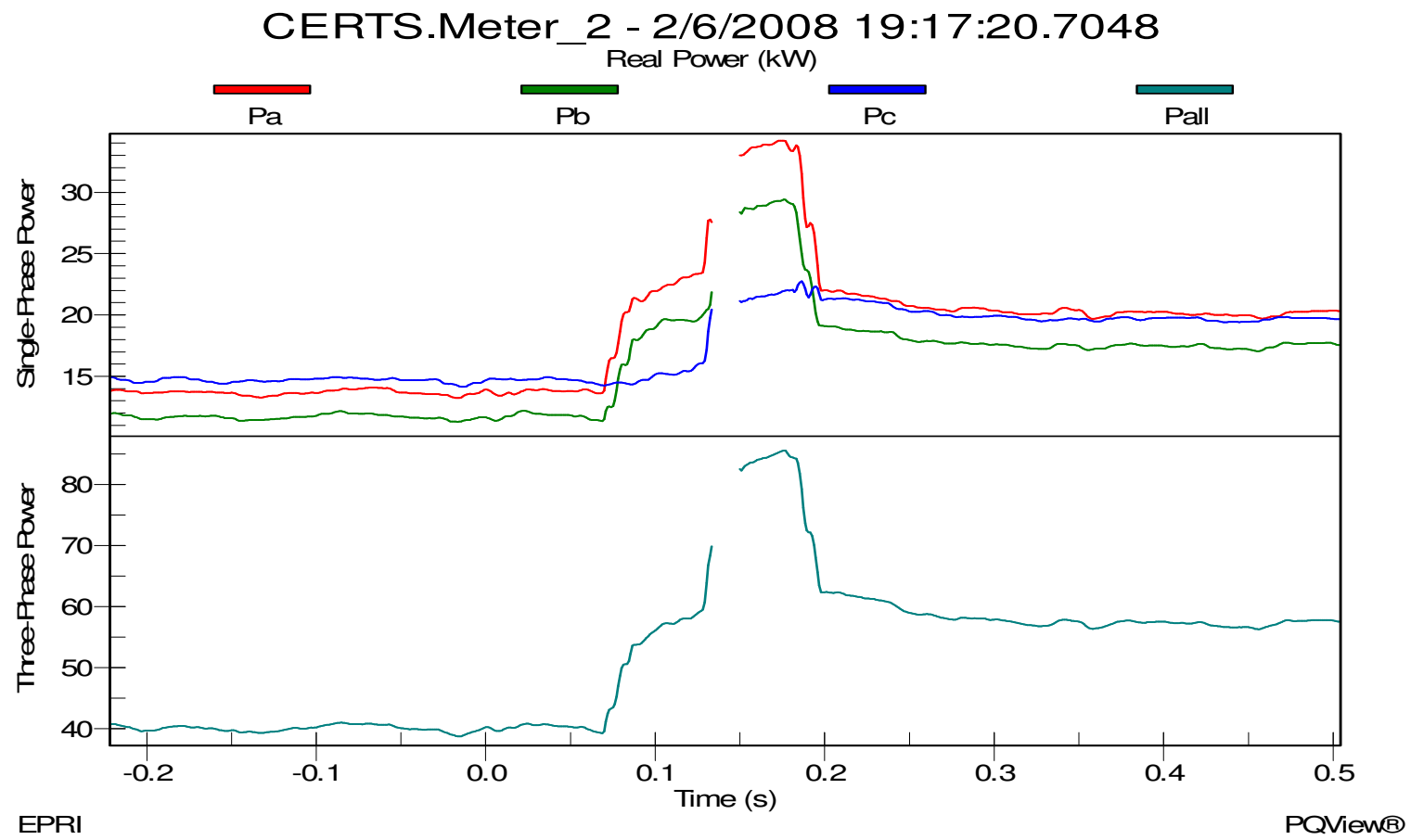

Figure 17b Meter 2 power flow for a single phase-to-ground fault applied in Zone 5. 


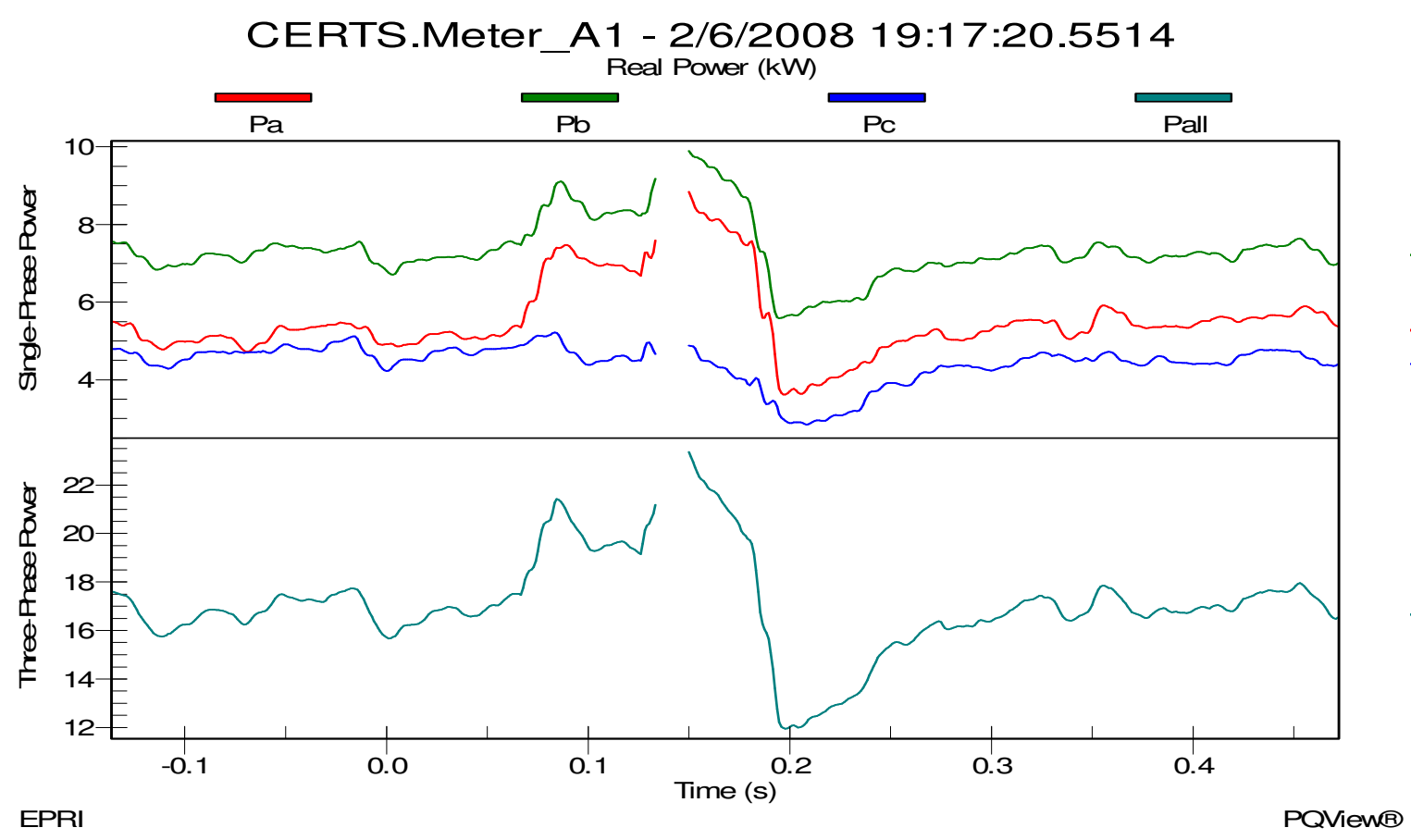

Figure 17c - Power produced by Gen-set A1 before/after a B-phase phase-to-ground fault in Zone 5.

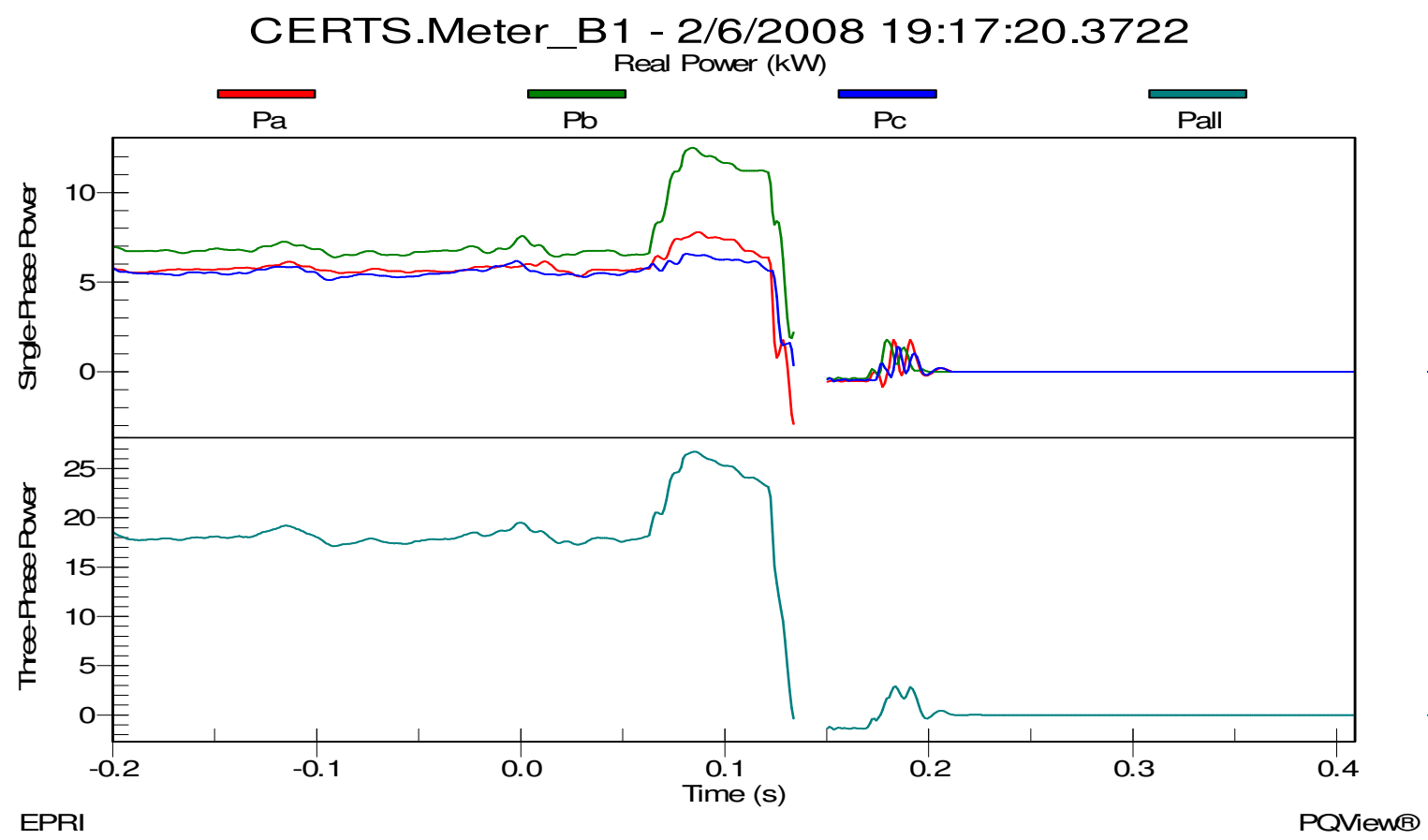

Figure 17d - Power produced by Gen-set A2 before/after a B-phase phase-to-ground fault in Zone 5 
Using the DAS Load Control program, the single phase-to-ground fault on B-phase was set for 10 seconds and then initiated.

The protection coordination for this event appears to be different than expected but similar to the previous test. After the fault was initiated, Relay 5 tripped on ground over-current (67N1T), shutting down Gen-set B1 and opening CB51, Figures 17d and 17e. The Gen-set stopped switching the inverter output; however, its transformer contactor and CB51 remained closed for a brief period of time, Figure 17f. Once CB51 cleared 0.06 seconds ( 3.8 cycles) later the fault was removed and Zone 5 was deenergized, Figure $17 \mathrm{~g}$. The static switch and remaining zones of the microgrid remained online and connected.

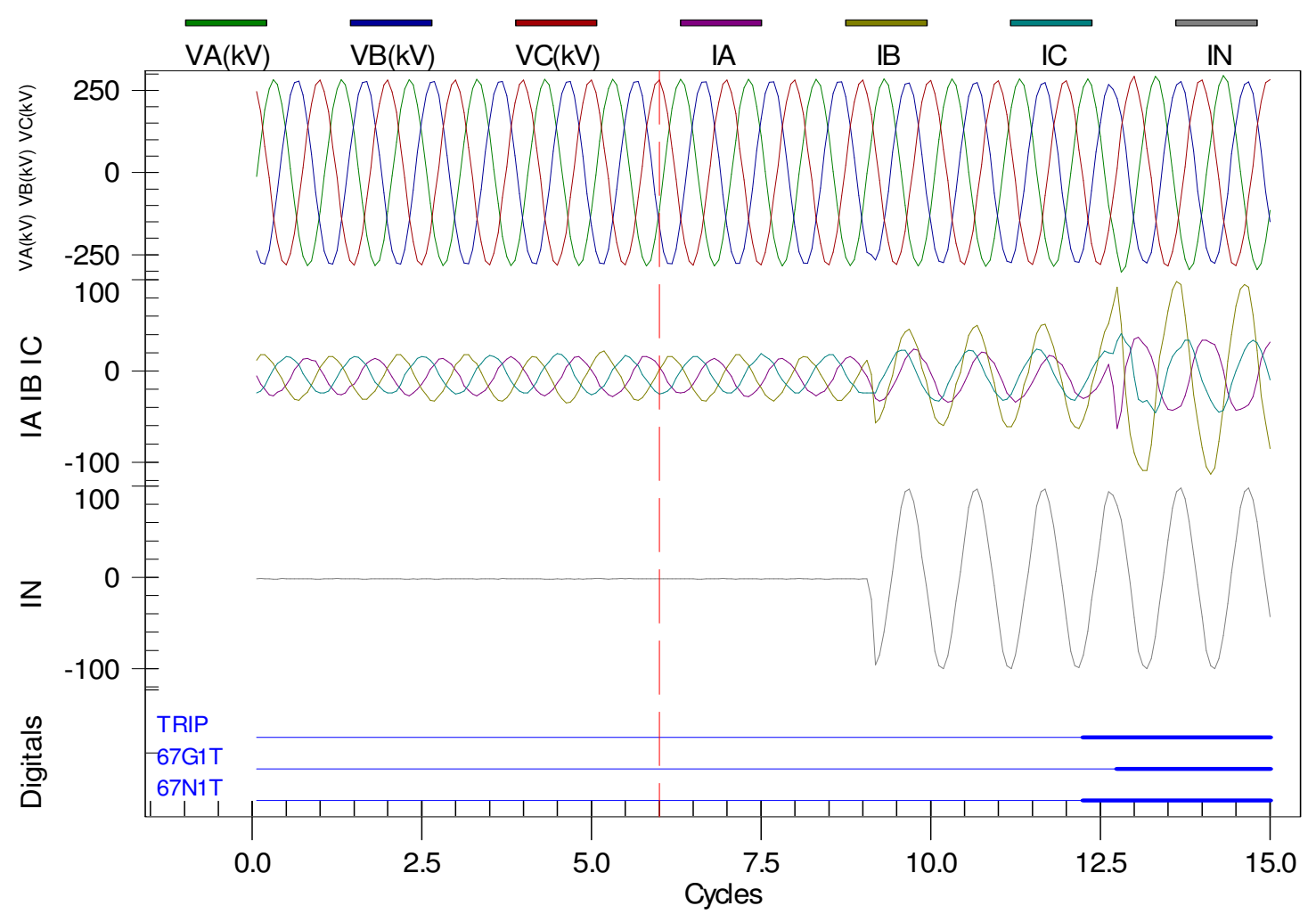

Figure17e - Relay 5 (CB51) tripped due to a ground over-current (67N1) after the single phase-to-ground fault was initiated in Zone 5. 


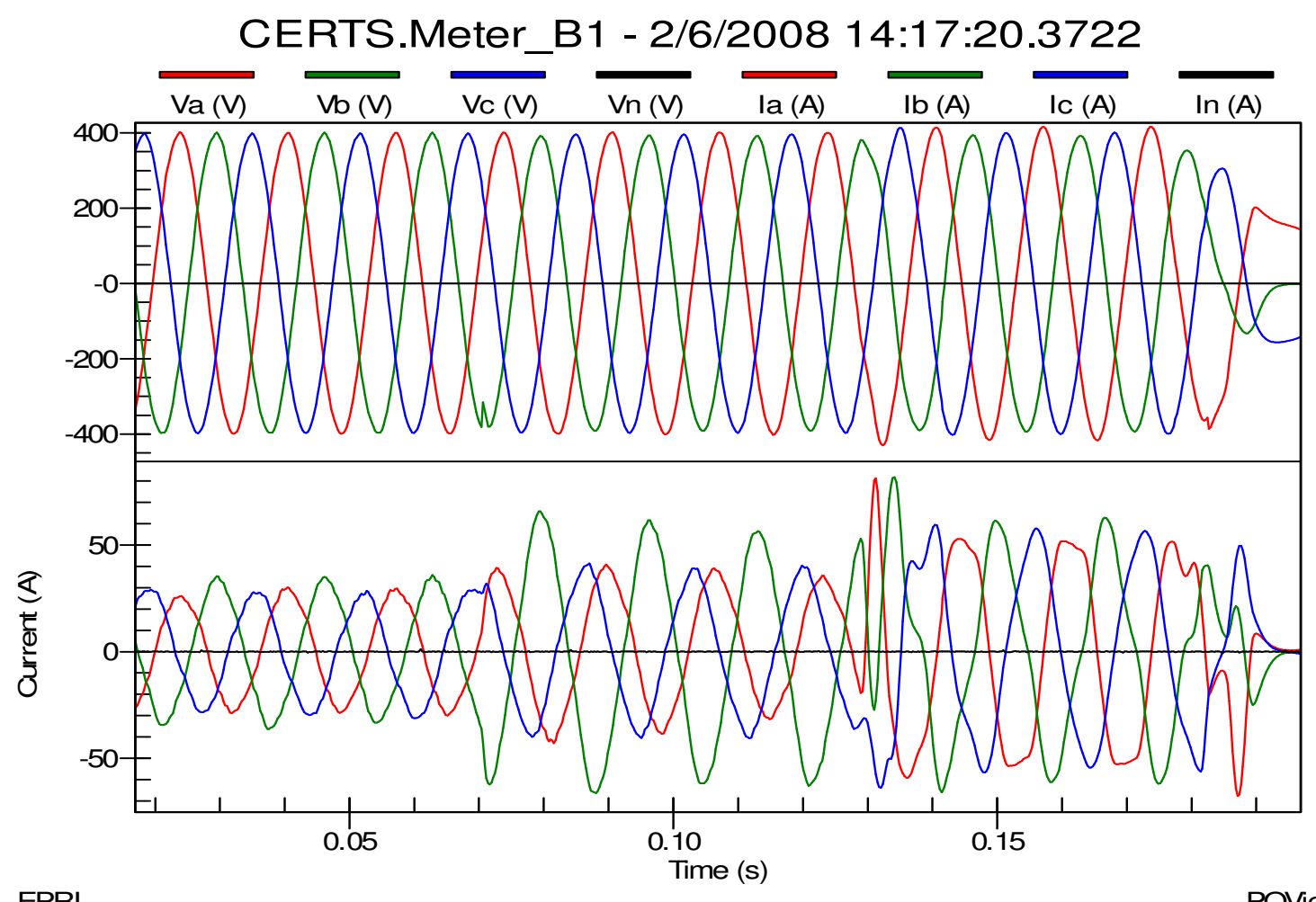

EPRI

Figure17f - Meter B1 voltage and current for a single phase-to-ground fault applied in Zone 5.

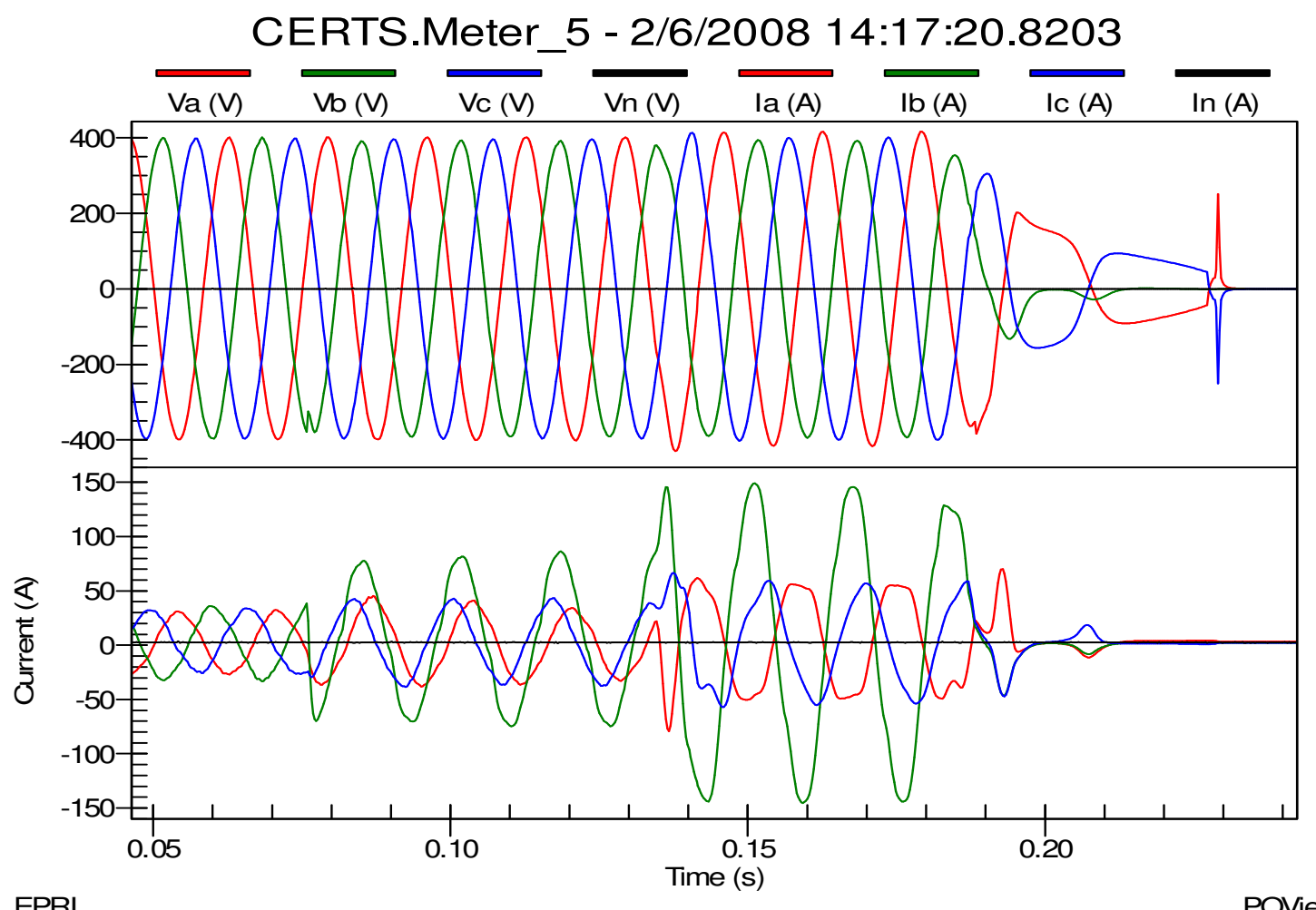

EPRI

PQViewA 
Figure17g- Meter 5 (CB51) voltage and current for a single phase-to-ground fault applied in Zone 5

The electrical system was stable in both the microgrid and utility grid after CB51 opened and Gen-set B1 shut down. Gen-set A1 was shut down manually, all loads and alarms were reset, and equipment was prepared for the next test.

\subsubsection{Zone 4 B-Phase Line-to-Ground Fault, Gen-sets (A1+A2) and Utility Connected} Performance Goal:

Test a single line-to-ground overload fault condition in Zone 4 with Gen-sets A1 and A2 operating in-parallel with the utility grid to verify zero-sequence, negative-sequence or residual over-current protection.

Initial Setup:

Gen-set A1 = Unit Power Control

Gen-set A2 = Unit Power Control

Gen-set A1 Output Power Command $=20 \mathrm{~kW}$

Gen-set A2 Output Power Command $=20 \mathrm{~kW}$

MG Power/Frequency Droop $=-0.0833 \mathrm{~Hz} / \mathrm{kW}$

MG Voltage Command $=277 \mathrm{~V}$

Relay Settings = B (Inst)

Reverse Power Set-point $=0 \mathrm{~kW}$

Load Bank $3=40 \mathrm{~kW}$

Load Bank $4=40 \mathrm{~kW}$

Load Bank $6=40 \mathrm{~kW}$

To simulate a high impedance single phase-to-ground fault in Zone 4 on B-phase the Overload Load Bank of $85 \mathrm{~kW}$ (approximately $28.3 \mathrm{~kW}$ per phase) was connected by cable/plug into the exterior receptacle of Cabinet $5 \mathrm{a}$ with phases $\mathrm{A}$ and $\mathrm{C}$ disconnected. This, when combined with the load in Load Bank 4 of $40 \mathrm{~kW}$ (approximately $13.3 \mathrm{~kW}$ per phase), added $28.3 \mathrm{~kW}$ to B-phase of Zone 4, causing an overload condition in Zone 4.

After both Gen-sets A1 and A2 were running for a few minutes and supplying power to Load Banks 3 and 4, the test was started with a "Start" command from the EMS. As 
soon as the static switch closed and steady-state conditions established, the microgrid and Load Bank 6 were connected to the utility grid with the total power flow at Meter 1 being approximately $74 \mathrm{~kW}$, shown in Figure 18a, and approximately $36 \mathrm{~kW}$ at Meter 2, shown in Figure 18b. Gen-sets A1 and A2 were producing about $20 \mathrm{~kW}$ each, shown in Figures $18 \mathrm{c}$ and $18 \mathrm{~d}$ before the fault was applied after 0 seconds.

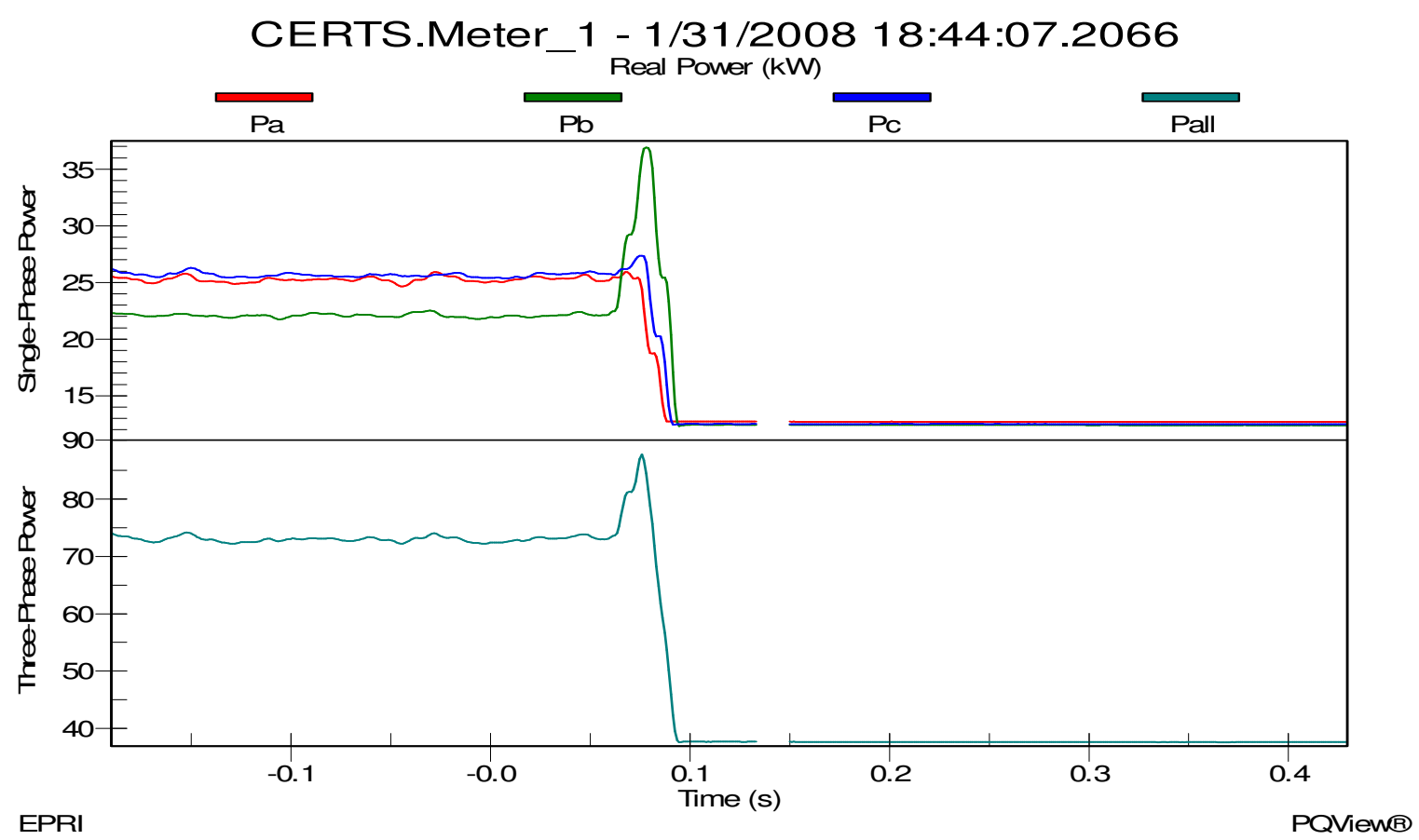

Figure 18a Meter 1 power flow before/after the static switch opened, islanding the microgrid from the utility grid when a single phase-to-ground fault was applied in Zone 4. 


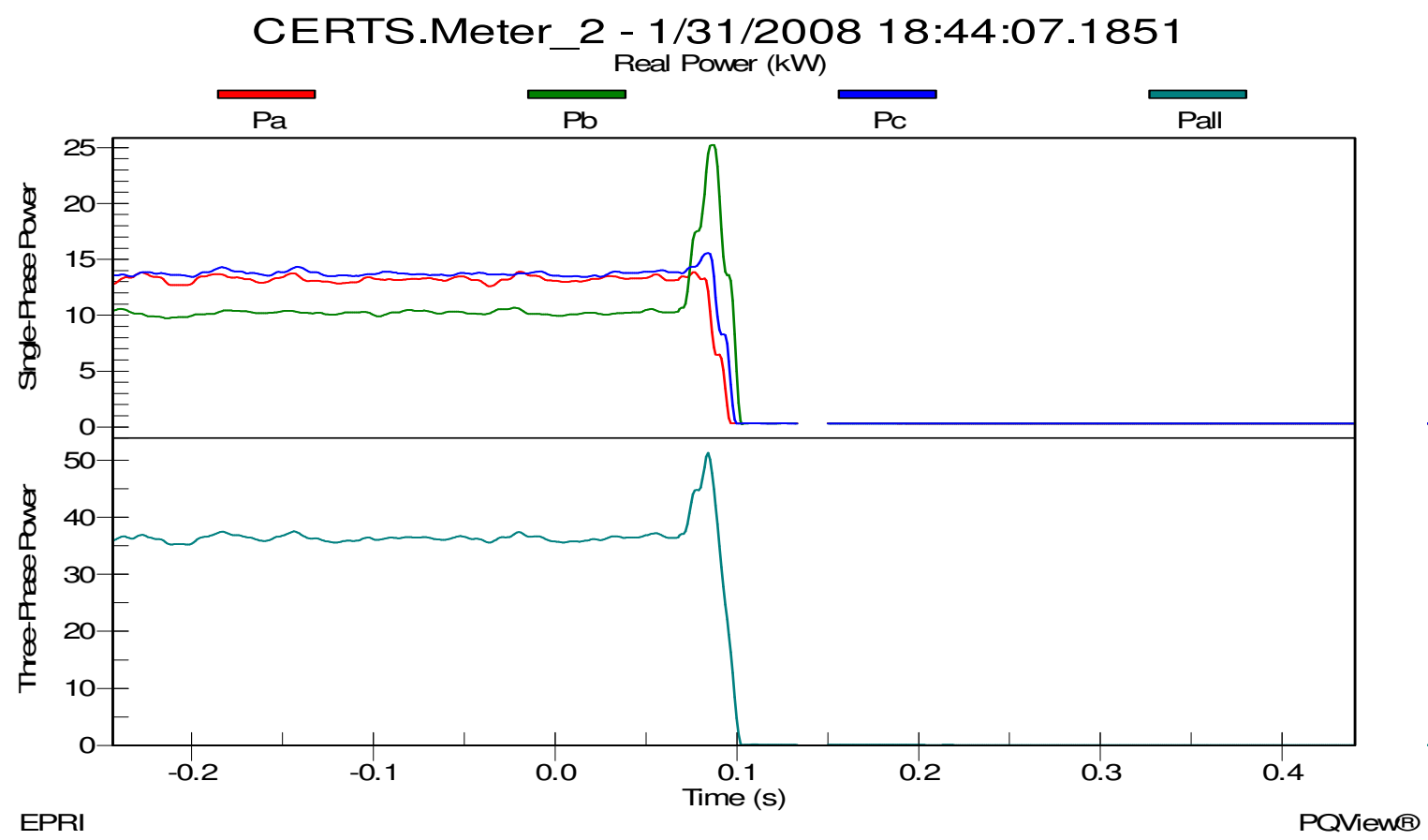

Figure 18b Meter 2 power flow before/after the static switch opened, islanding the microgrid from the utility grid when a single phase-to-ground fault was applied in Zone 4.

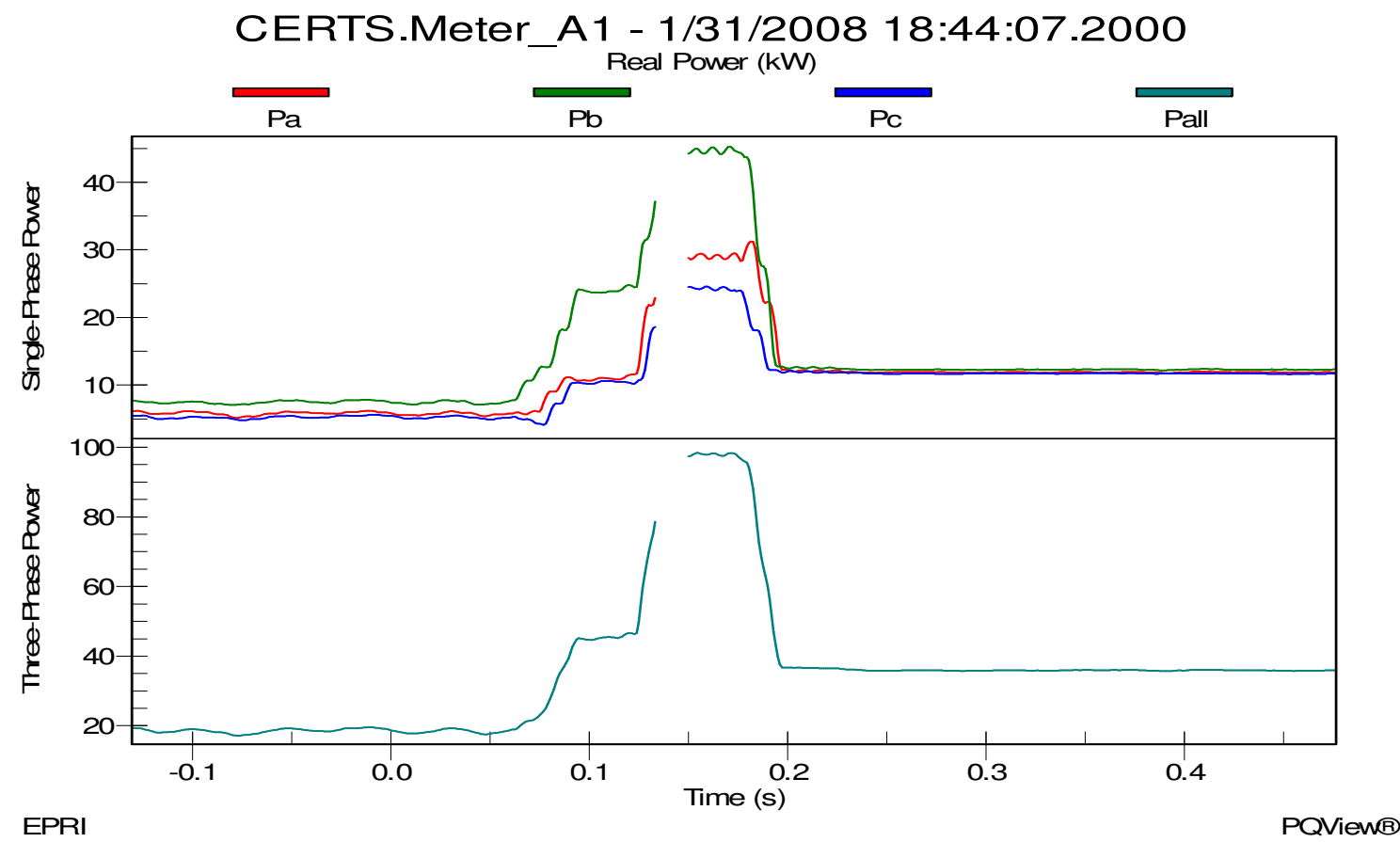

Figure 18c - Power produced by Gen-set A1 before/after the static switch and CB41 opened when a Bphase-to-ground fault was applied in Zone 4. 


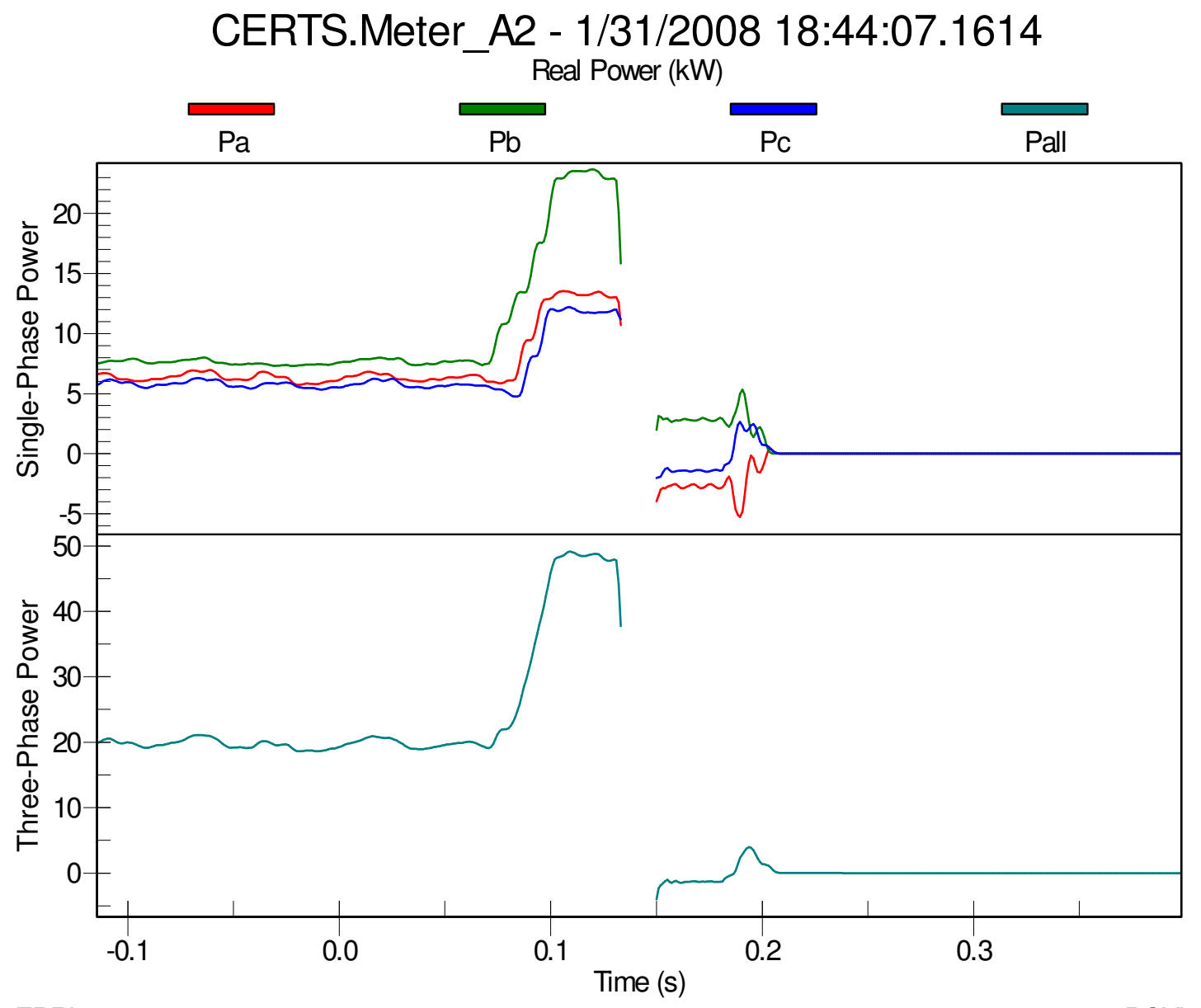

EPRI

PQView

Figure 18d - Power produced by Gen-set A2 before/after the static switch and CB41 opened when a Bphase-to-ground fault was applied in Zone 4.

Using the DAS Load Control program, the single phase-to-ground fault on B-phase was set for 10 seconds and then initiated. The static switch tripped in approximately 0.007 seconds ( 0.4 cycles) from a ground over-current (67N1T), shown in Figure 18e, islanding the microgrid from the rest of the utility grid. Relay 4 tripped after approximately 0.058 seconds ( 3.5 cycles) on a ground over-current $(67 \mathrm{~N} 1 \mathrm{~T})$ signal, isolating Zone 4 from the rest of the microgrid, shown in Figure 18f. 


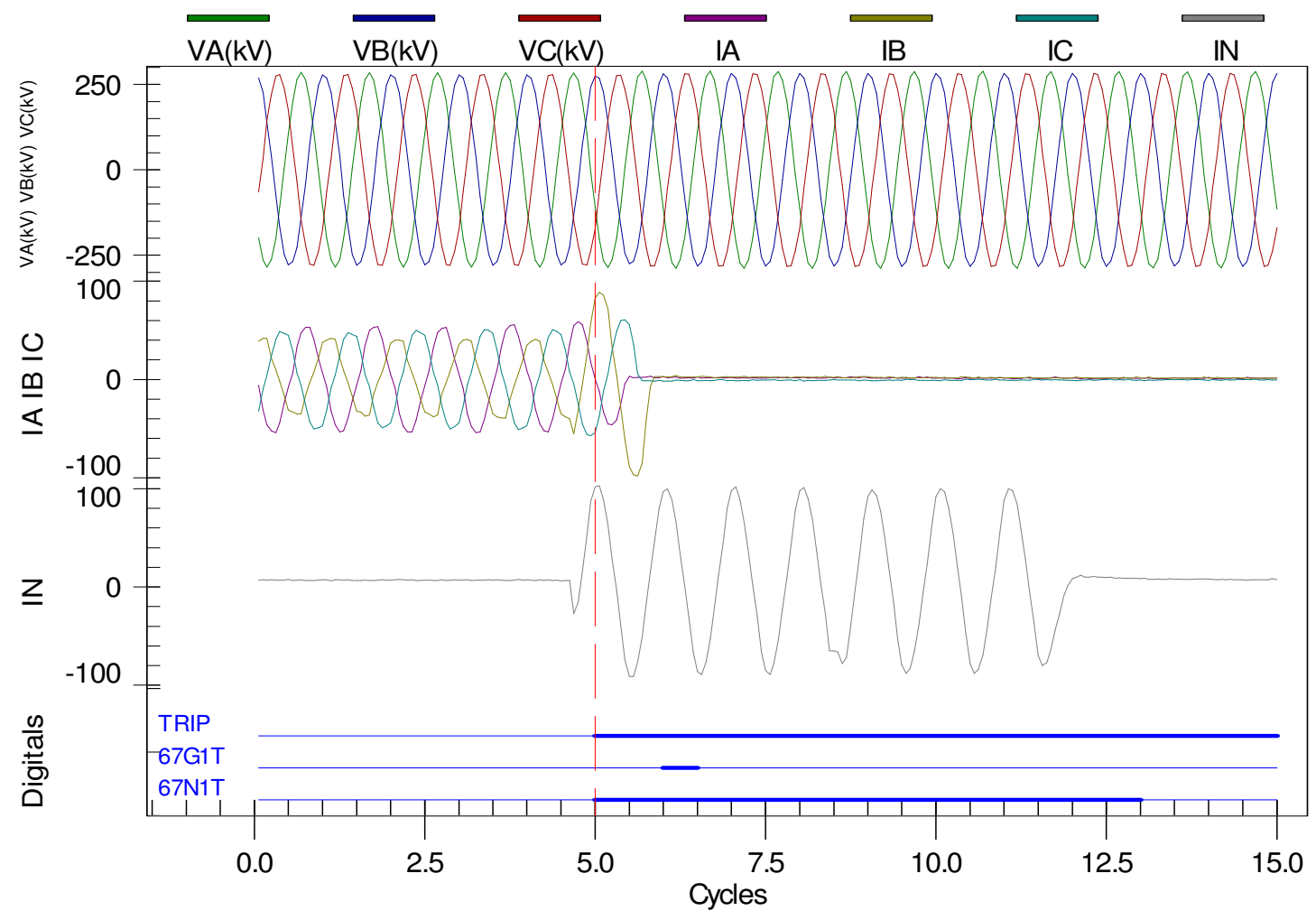

Figure 18e - Relay 2 (static switch) tripped due to a ground over-current (67N1T) when the single phaseto-ground fault is applied in Zone 4.

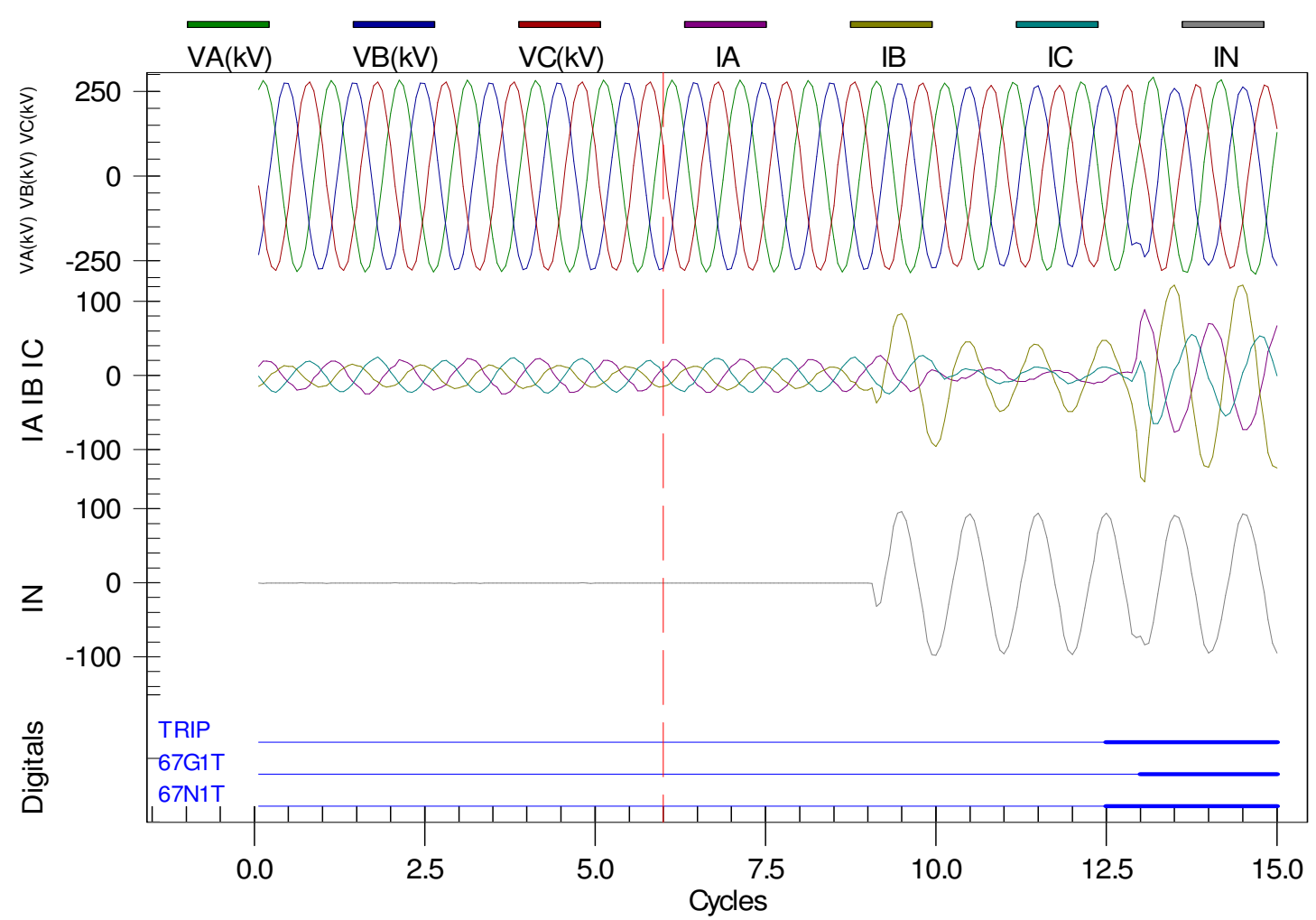


Figure 18f - Relay 4 (CB41) tripped due to a ground over-current (67N1T) when the single phase-toground fault was initiated in Zone 4

Gen-set A2 stopped switching approximately 0.008 seconds ( 0.5 cycles) after Relay 4 tripped; however, its transformer contactor and CB41 remained closed for a short period. In Figures 18a and 18b power flow through Meter 1 was approximately $37.5 \mathrm{~kW}$, supplying only the load in Load Bank 6 from the utility. Load Bank 3 was supplied power from Gen-set A1, within the islanded microgrid, with output power raised from approximately $20 \mathrm{~kW}$ to about $36 \mathrm{~kW}$ to meet the load demand, Figure 18c. CB31 and CB51 remained closed throughout the entire test.

The electrical system was stable in both the microgrid and utility grid after the static switch opened, CB41 opened and Gen-set A2 shut down. A short duration later the static switch performed a synchronized close and reconnected the microgrid to the utility grid. Gen-set A1 was shutdown manually, all loads and alarms were reset, and equipment was prepared for the next test.

\title{
6.1.10 Zone 2 C-Phase Line-to-Ground Fault, Gen-sets (A1+B1) and Utility Connected
} Performance Goal:

Test a single line-to-ground overload fault condition in Zone 2 with Gen-sets A1 and B1 operating in-parallel with the utility grid to verify zero-sequence, negative-sequence or residual over-current protection.

\author{
Initial Setup: \\ Gen-set A1 = Unit Power Control \\ Gen-set B1 = Unit Power Control \\ Gen-set A1 Output Power Command $=20 \mathrm{~kW}$ \\ Gen-set B1 Output Power Command $=20 \mathrm{~kW}$ \\ MG Power/Frequency Droop $=-0.0833 \mathrm{~Hz} / \mathrm{kW}$ \\ MG Voltage Command $=277 \mathrm{~V}$ \\ Relay Settings $=$ B (Inst) \\ Reverse Power Set-point $=0 \mathrm{~kW}$ \\ Load Bank $3=40 \mathrm{~kW}$ \\ Load Bank $4=40 \mathrm{~kW}$
}


Load Bank $6=40 \mathrm{~kW}$

To simulate a high impedance single phase-to-ground fault in Zone 2 on C-phase the Overload Load Bank of $85 \mathrm{~kW}$ (approximately $28.3 \mathrm{~kW}$ per phase) was connected by cable/plug into the exterior receptacle of Cabinet $2 \mathrm{~d}$ with phases $\mathrm{A}$ and $\mathrm{B}$ disconnected. This, when combined with the load in Load Banks 3 and 4 of $40 \mathrm{~kW}$ each (each at approximately $13.3 \mathrm{~kW}$ per phase), added $28.3 \mathrm{~kW}$ to C-phase in Zone 2 and caused an overload condition in Zone 2.

After both Gen-sets A1 and B1 were running for a few minutes and supplied power to Load Banks 3 and 4, the test was started with a "Start" command from the EMS. As soon as the static switch closed and steady-state conditions established, the microgrid and Load Bank 6 were connected to the utility grid with the total power flow at Meter 1 being approximately $68 \mathrm{~kW}$, shown in Figure $19 \mathrm{a}$, and approximately $32 \mathrm{~kW}$ at Meter 2, shown in Figure 19b. Gen-sets A1 and B1 were producing about 20kW each, shown in Figures $19 \mathrm{c}$ and $19 \mathrm{~d}$ before the fault was applied after 0 seconds.

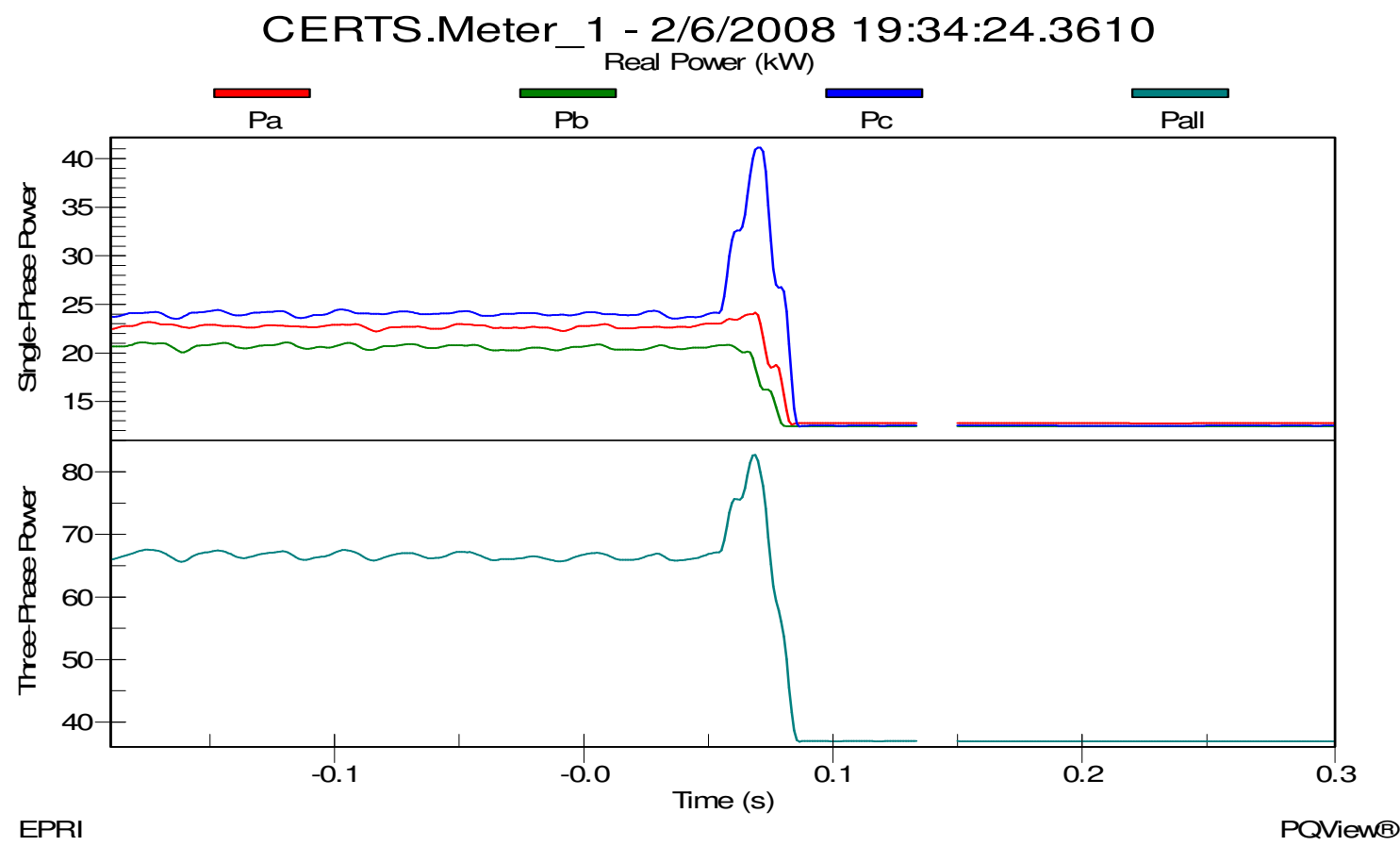

Figure 19a Meter 1 power flow before/after the static switch opened, islanding the microgrid from the utility grid when a single phase-to-ground fault was applied in Zone 2. 


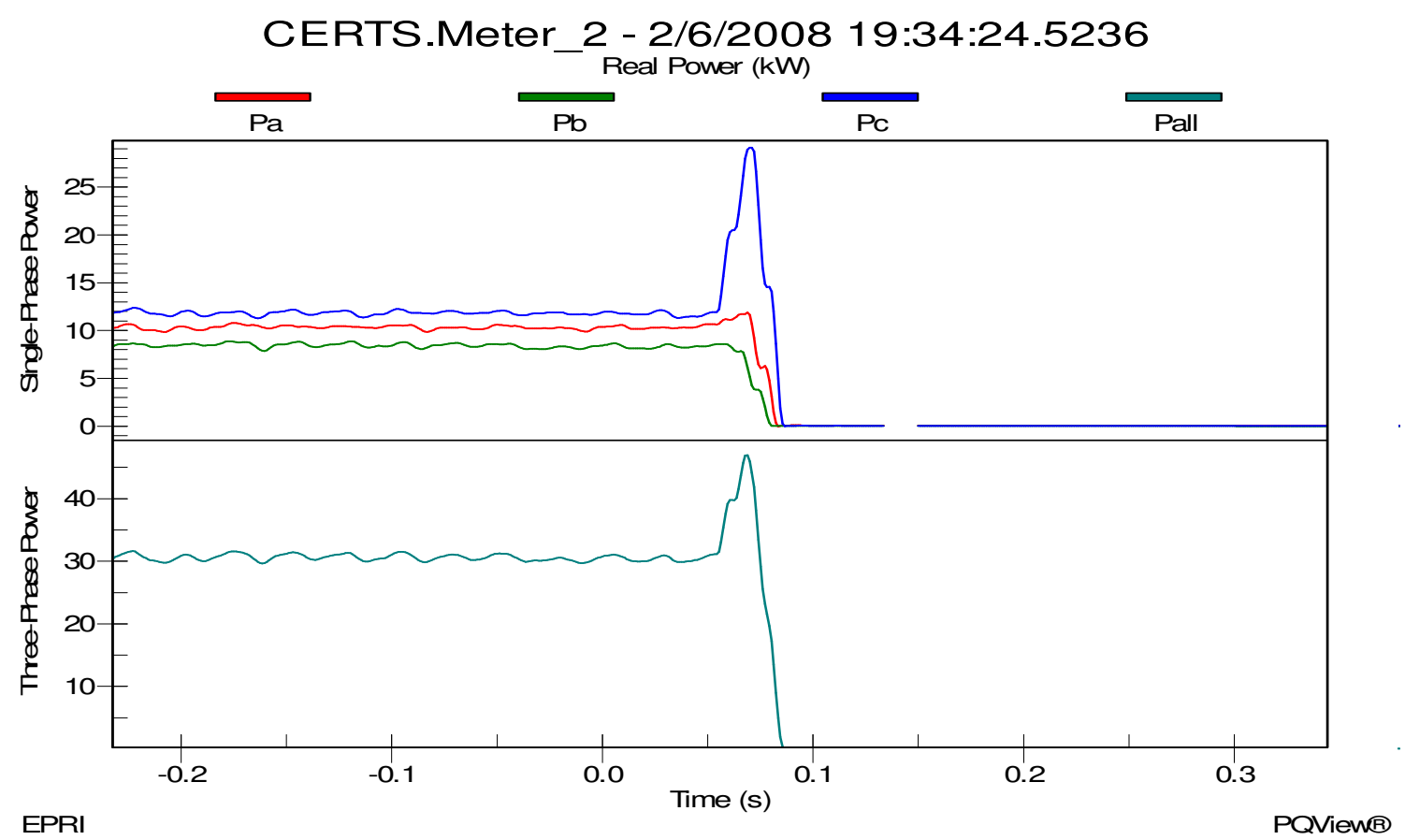

Figure 19b - Meter 2 power flow before/after the static switch opened, islanding the microgrid from the utility grid when a single phase-to-ground fault was applied in Zone 2. 


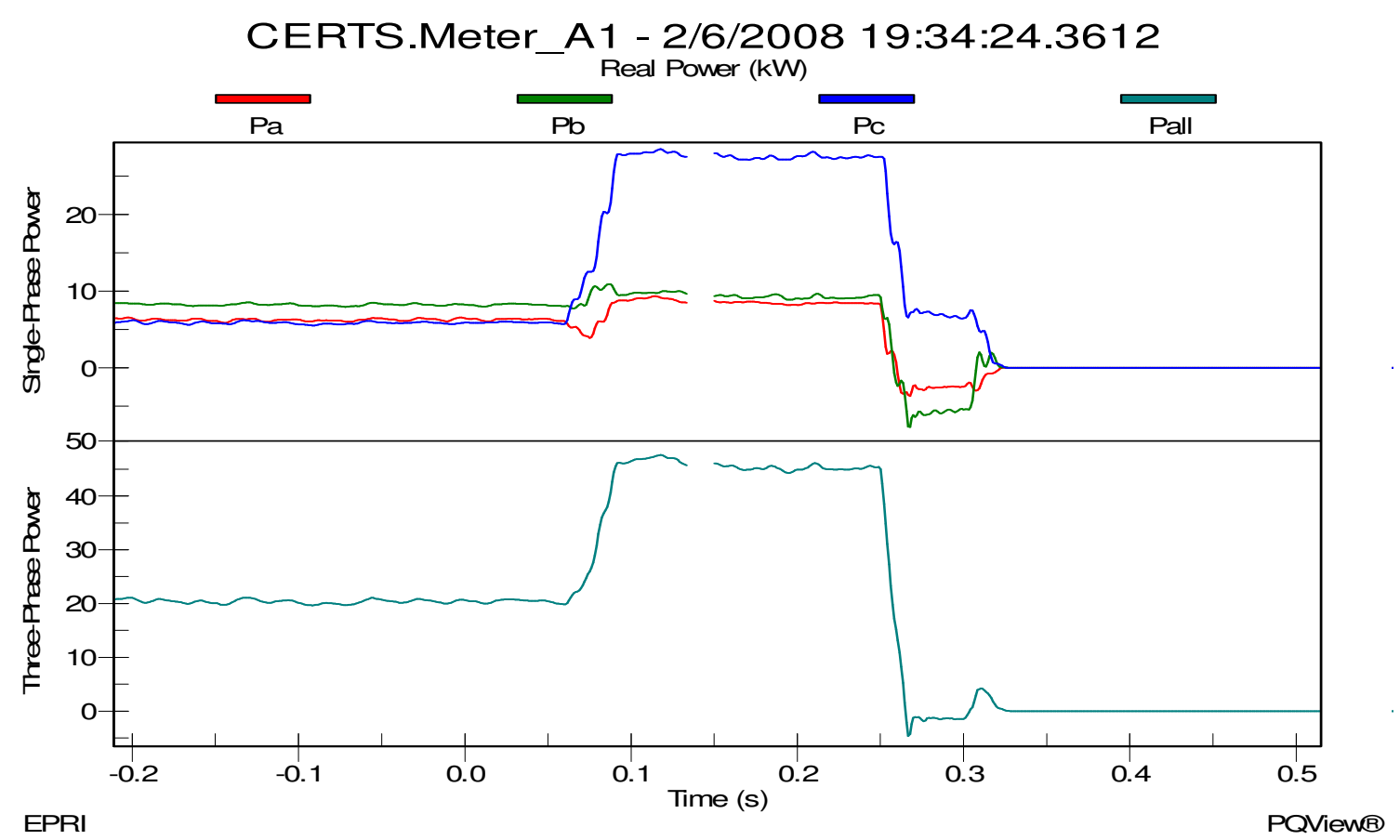

Figure 19c - Power produced by Gen-set A1 before/after the static switch opened from a C-phase-toground fault in Zone 2.

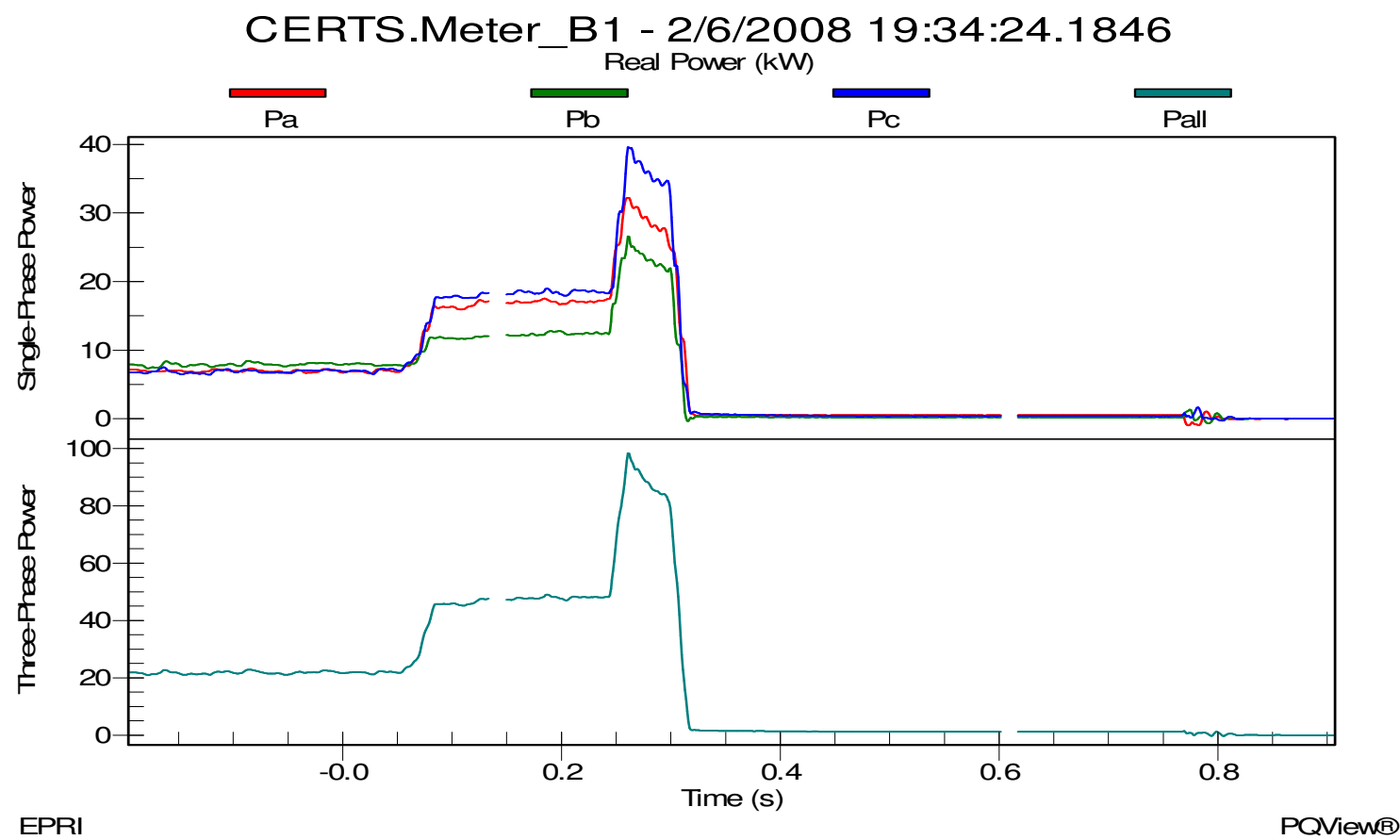

Figure 19d - Power produced by Gen-set B1 before/after the static switch opened from a C-phase-toground fault in Zone 2. 
Using the DAS Load Control program, the single phase-to-ground fault on C-phase was set for 10 seconds and then initiated. Relay 2 tripped the static switch first on ground over-current $(67 \mathrm{~N} 1 \mathrm{~T})$ in approximately 0.02 seconds $(1$ cycle) after the fault was applied, Figure 19e. Both Gen-sets A1 and B1 ramped up carrying Load Banks 3 and 4, as well as the C phase overload fault. Relay 3 tripped approximately 0.17 seconds ( 10.4 cycles) after the static switch opened, causing Gen-set A1 to stop switching its output, although its transformer contactor and CB31 remained closed for a few cycles longer. This can be seen by the reverse power on $A$ and $B$ phases supplying $C$ phase even though the net three phase power remains zero, Figure 19c. CB31 opened 0.046 seconds (2.75 cycles) after receiving the trip signal from Relay 3, separating Zones 3 and 4 from the microgrid.

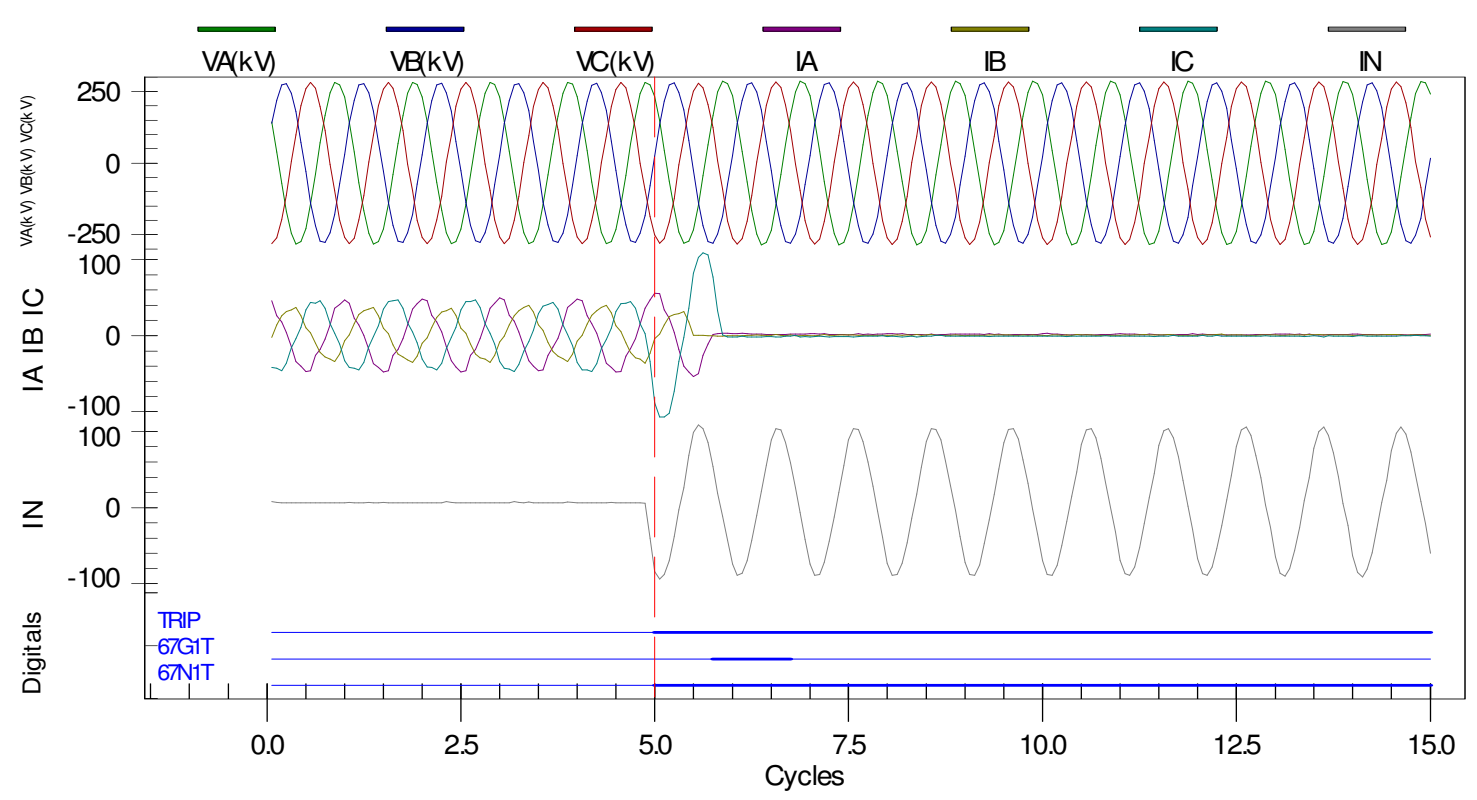

Figure 19e - Relay 2 (static switch) tripped due to a gournd over-current (67N1T) when the single phaseto-ground fault was initiated in Zone 2.

Gen-set B1 remained on-line supporting the fault load bank in Zone 2, Figure 19f. Because the static switch and CB31 were now open, Zone 2 was dominated by the Delta winding of T51 and effectively turned the entire zone into a 3-wire system. However, the measurement transformers which feed the DSP controller and the under-voltage relay 5a were connected wye-wye and likely experienced a full phase to phase voltage $(480 \mathrm{~V})$ across their primary windings $(277 \mathrm{~V})$, Figure $19 \mathrm{~g}$. Because only high impedance measurement equipment was connected to the secondaries of these transformers, negligible fault current flowed through them. Since the fault was applied from $C$ phaseto-ground there remained no path for current flow and the fault was effectively removed. 


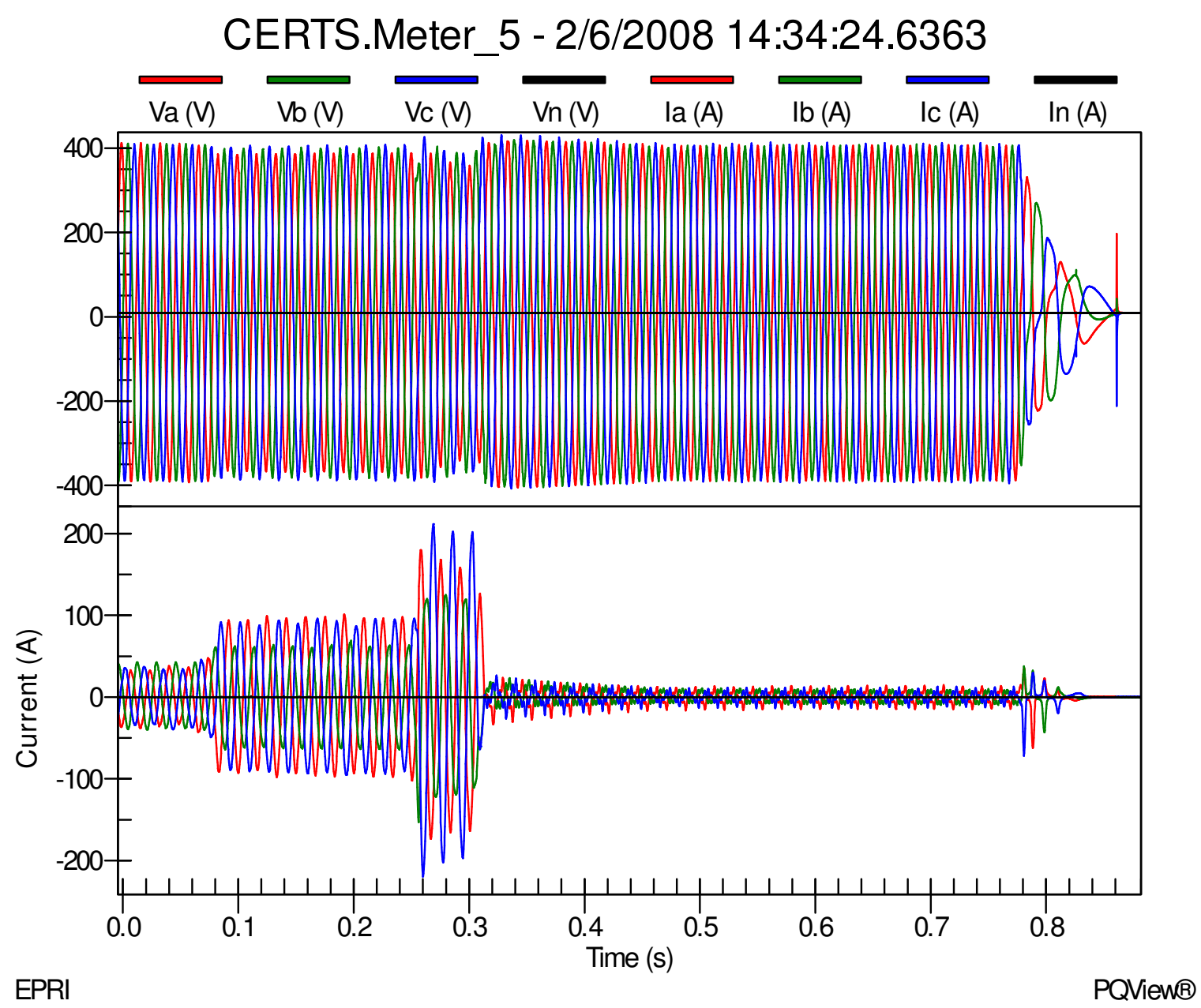

Figure 19f - Meter 5 voltage and current during the applied single-phase-to-ground fault in Zone 2. 


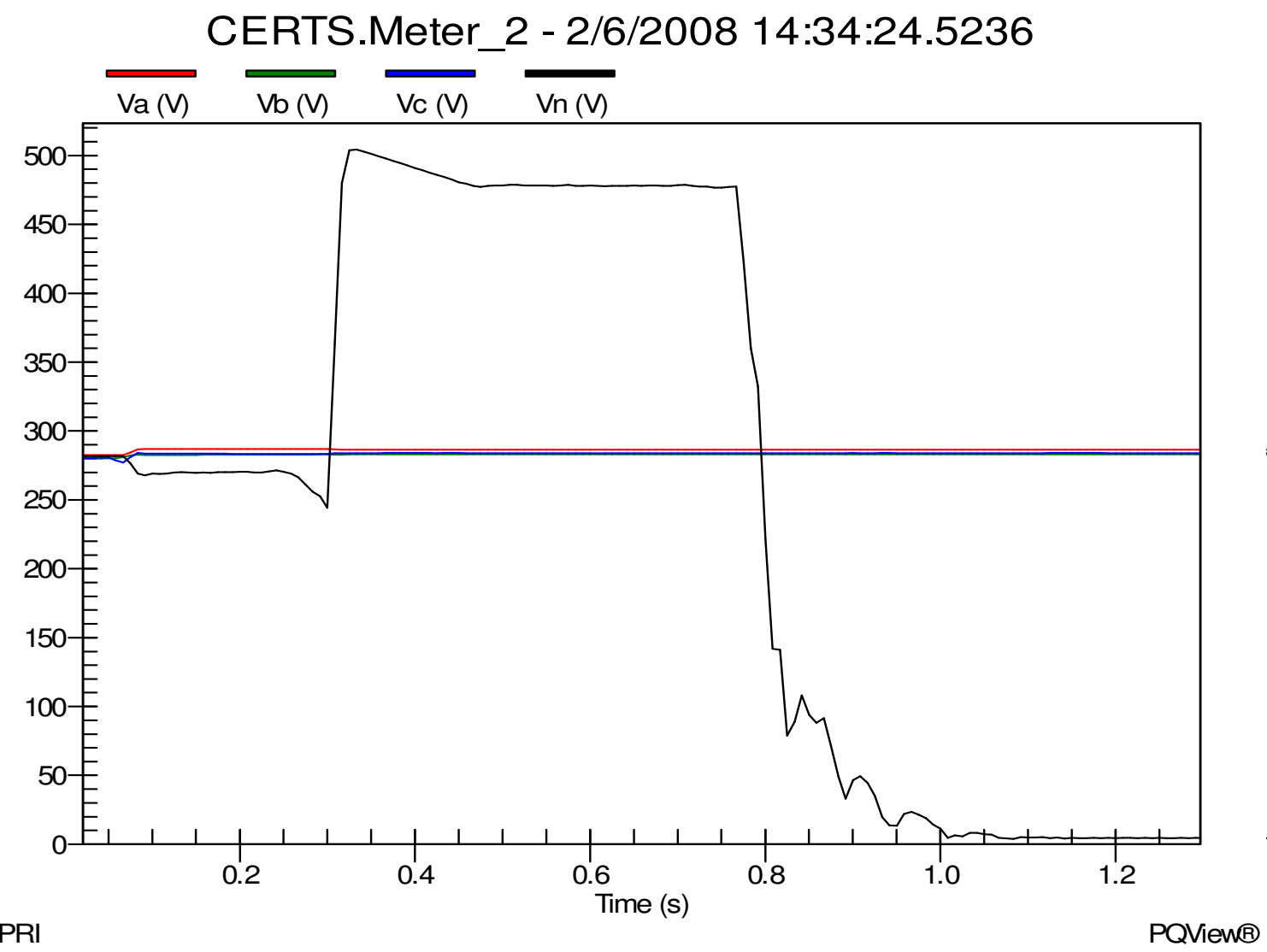

Figure 19g - Meter 2 RMS voltage before/after CB31 opened.

Finally Gen-set B1 was tripped off-line by under-voltage Relay 5a (SV4T) as can be seen in Relay 5, Figure 19h. As mentioned above, voltage remained within Zone 2 after the static switch and CB31 opened, therefore it must be assumed that either the fusing on Relay 5 a operated or that it was damaged by excessive voltage that occurred during the fault. This was interpreted as an under-voltage and caused Relay 5 to trip incorrectly, shutting down Gen-set B1 and opening CB51. Relay 5 tripped approximately 0.5 seconds (30 cycles) after CB31 opened. 


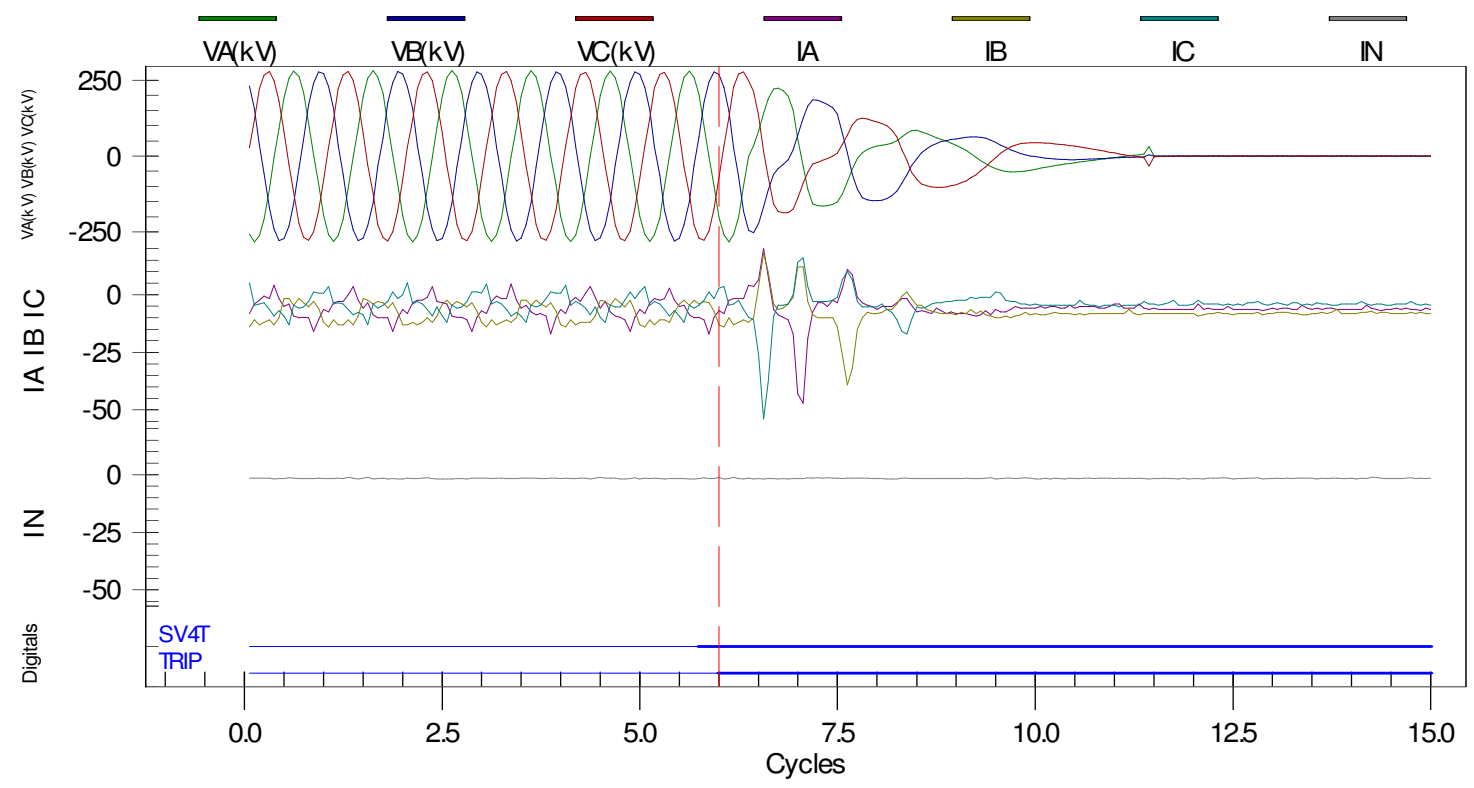

Figure 19h - Relay 5 (CB51) tripped due to an undervoltage at T51 when the single phase-to-ground fault was initiated in Zone 2.

The electrical system was stable in both the microgrid and utility grid after the static switch opened, CB31 and CB51 opened, and Gen-sets A1 and B1 shut down. All loads and alarms were reset and equipment was prepared for the next test.

\subsubsection{Zone 6 C-Phase Line-to-Ground Fault, Gen-set A1 and Utility Connected} Performance Goal:

Test a single line-to-ground overload fault condition in Zone 6 with Gen-set A1 operating in-parallel with the utility grid to verify $\mathrm{I}^{2} \mathrm{t}$ protection.

\section{Initial Setup:}

Gen-set A1 = Unit Power Control

Output Power Command $=20 \mathrm{~kW}$

MG Power/Frequency Droop $=-0.0833 \mathrm{~Hz} / \mathrm{kW}$

MG Voltage Command $=277 \mathrm{~V}$

Relay Settings $=$ A $($ Delayed $)$

Load Bank $3=40 \mathrm{~kW}$

Load Bank $6=60 \mathrm{~kW}$ 
To simulate a high impedance single phase-to-ground fault in Zone 6 on C-phase the Overload Load Bank of $85 \mathrm{~kW}$ (approximately $28.3 \mathrm{~kW}$ per phase) was connected by cable/plug into the exterior receptacle of Cabinet $8 \mathrm{a}$ with phases A and B disconnected. This, when combined with the load in Load Bank 6 of $60 \mathrm{~kW}(20 \mathrm{~kW}$ per phase), added $28.3 \mathrm{~kW}$ to $\mathrm{C}$-phase in Zone 6 and caused an overload condition.

After Gen-set A1 was running for a few minutes and supplied power to Load Bank 3, the test was started with a "Start" command from the EMS. As soon as the static switch closed and steady-state conditions established, the microgrid and Load Bank 6 were connected to the utility grid with the total power flow at Meter 1 being approximately $68.5 \mathrm{~kW}$, shown in Figure 20a, and approximately 16kW at Meter 2, shown in Figure 20b. Gen-set A1 was producing about 20.5kW, shown in Figures 20c before the fault was applied after 0 seconds.

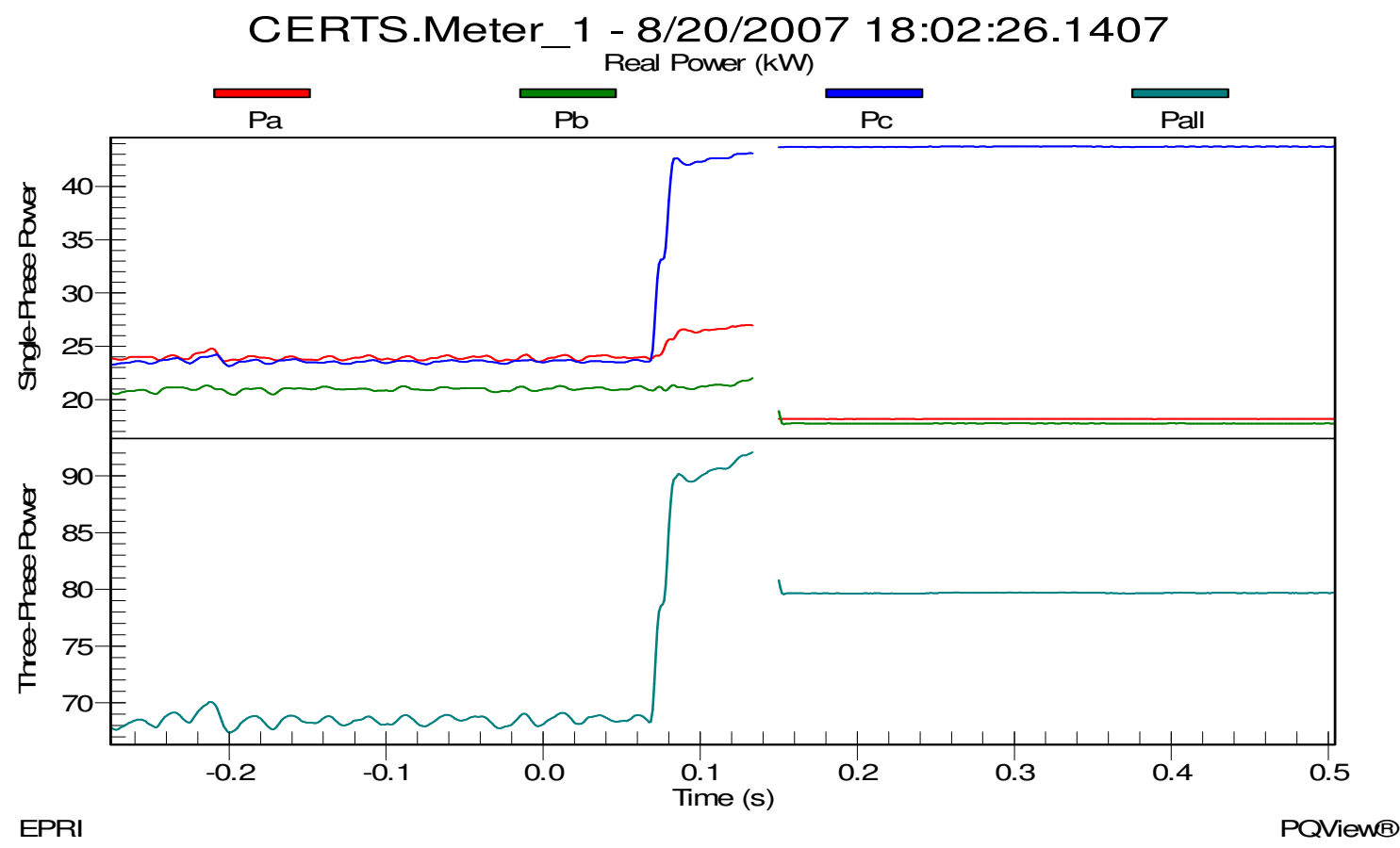

Figure 20a - Meter 1 power flow before/after the static switch opened, islanding the microgrid from the utility grid when a single phase-to-ground fault was applied in Zone 6. 


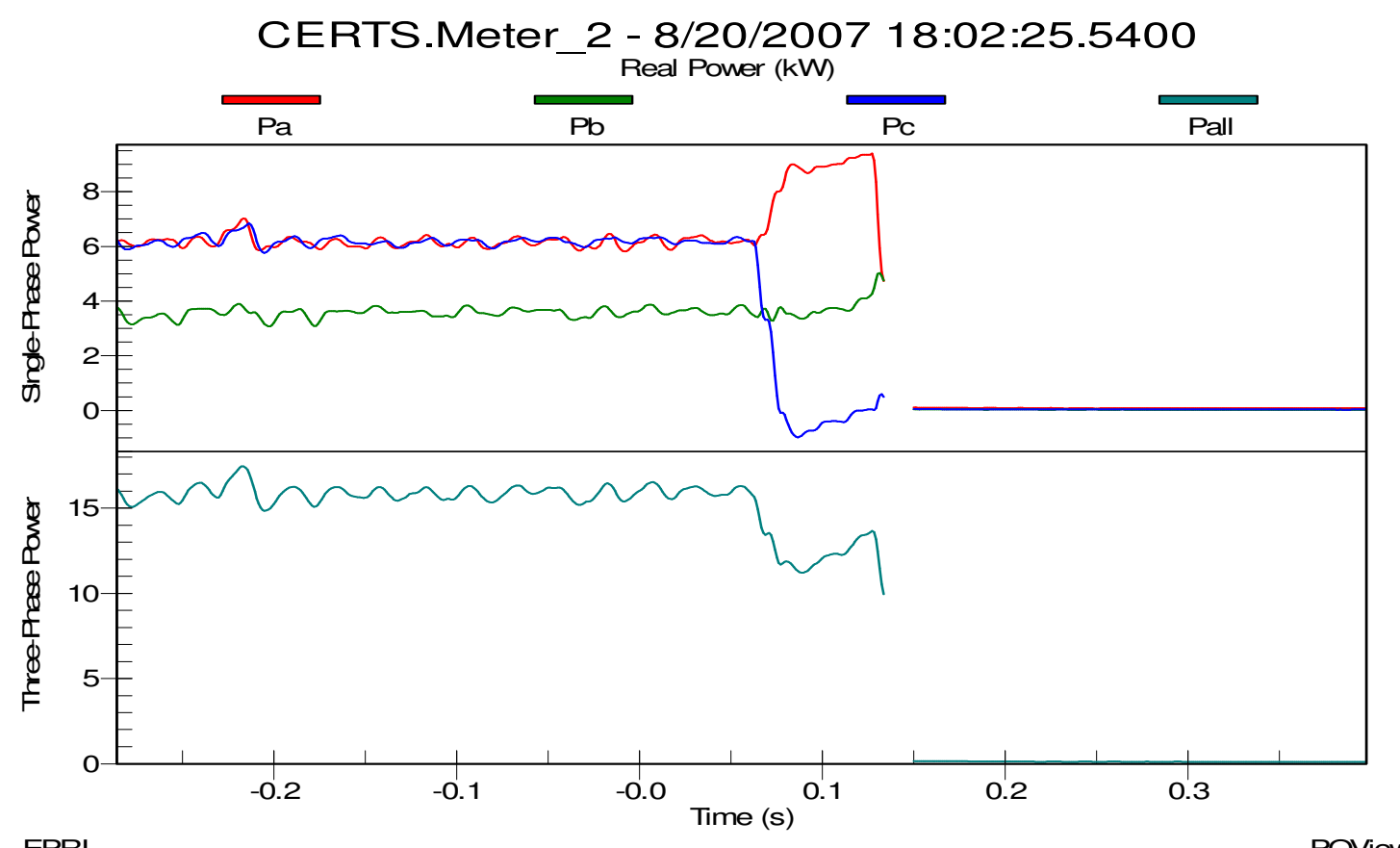

EPRI

PQVienA

Figure 20b - Meter 2 power flow before/after the static switch opened, islanding the microgrid from the utility grid when a single phase-to-ground fault was applied in Zone 6.

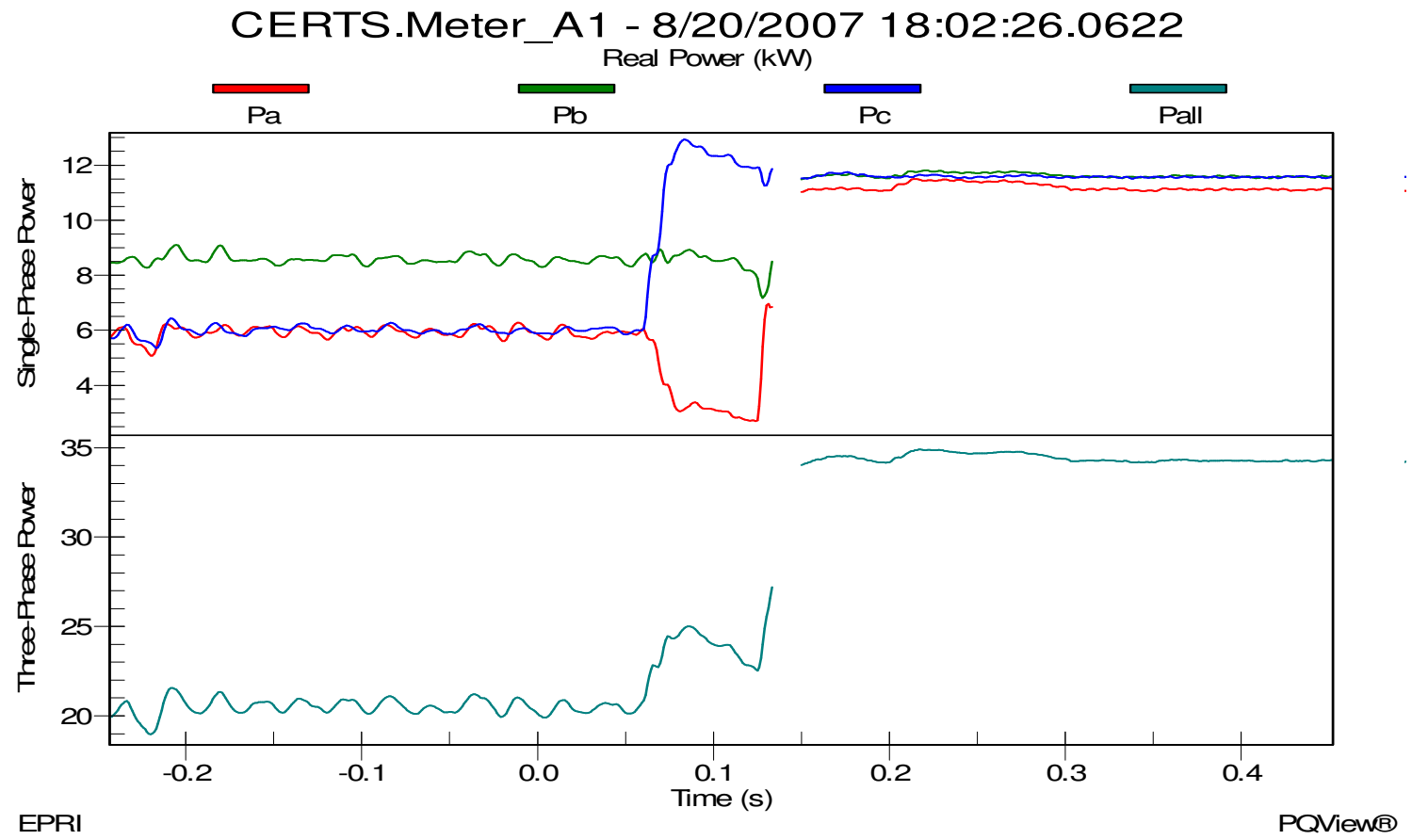

Figure 20c - Power produced by Gen-set A1 before/after the static switch opened from a C-phase phaseto-ground fault in Zone 6. 
Using the DAS Load Control program, the single phase-to-ground fault on C-phase was set for 10 seconds and then initiated. The static switch tripped in approximately 0.07 seconds ( 4.3 cycles) from a neutral over-current (67G1T), shown in Figure 20d, islanding the microgrid from the utility grid. Following the static switch operation, CB13, which provides protection to Zone 6, tripped on $I^{2}$ t protection with CB31, CB41 and CB51 remaining closed, since the overload fault was not part of the microgrid, located beyond the static switch.

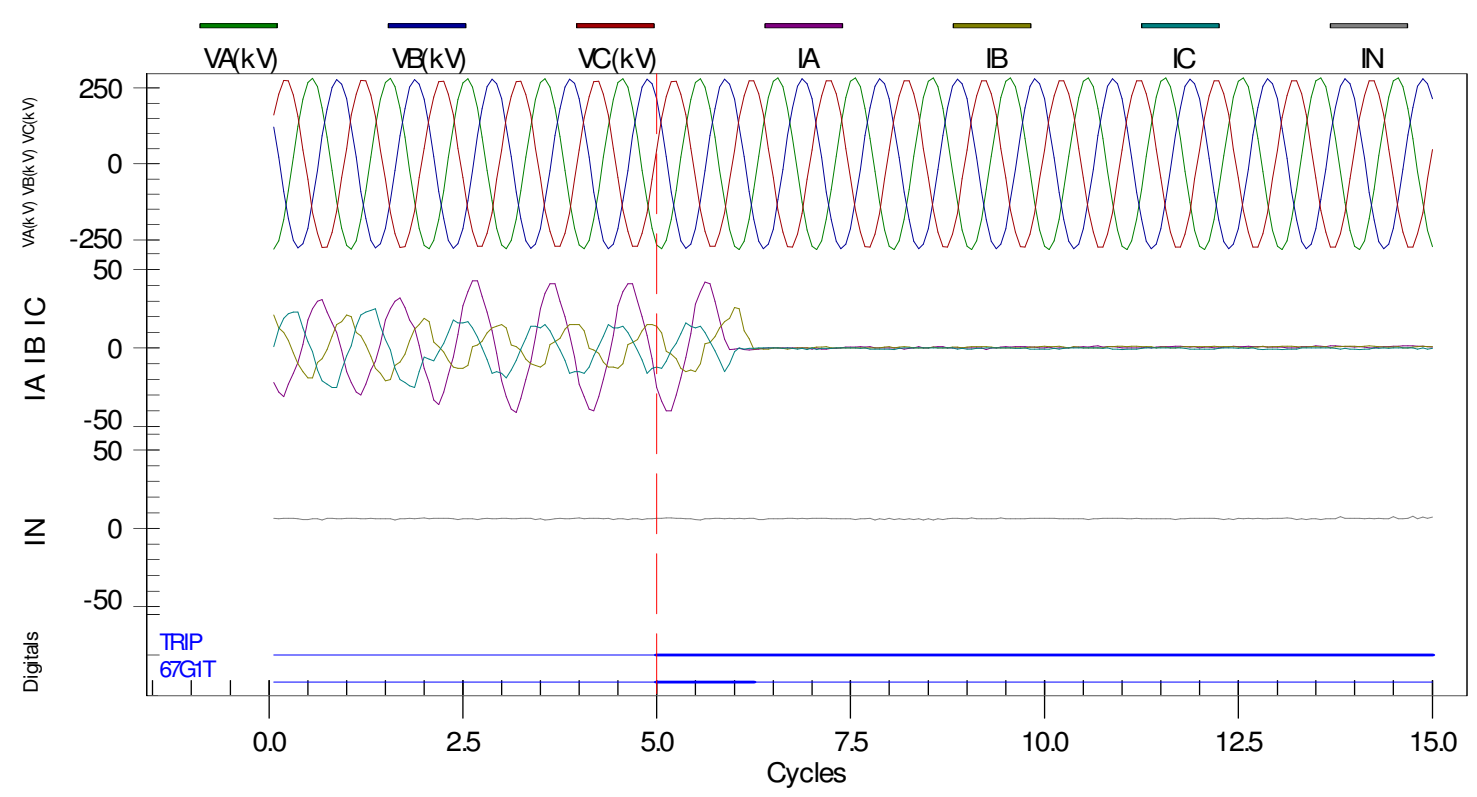

Figure 20d - Relay 2 (static switch) tripping due to a ground over-current (50GR) when the single phaseto-ground fault was initiated in Zone 6.

The electrical system was stable in both the microgrid and utility grid after the static switch opened and CB13 opened. Gen-set A1 was manually shut down with all loads and alarms reset and equipment was prepared for the next test.

A few points of interest for this test include the Gen-set contribution to the fault and the sensitivity of the static switch to an unbalanced load within the unprotected portion (i.e., Zone 6) of the microgrid. In Figure 20c it can be seen that at Meter A1, just after the fault was applied, three phase power increased slightly from $20 \mathrm{~kW}$ to $25 \mathrm{~kW}$, with C-phase output increasing and A-phase output decreasing. Because the three phase power increased by approximately $5 \mathrm{~kW}$, it must be concluded that Gen-set A1 did increase its power output. The reduction in A-phase power which contributed to the C-phase 
increase in power is likely due to the delta winding of the inverter transformer, which cannot be confirmed, since there are no meter provisions on that side.

The second point of interest for this test is that the static switch is sensitive to load unbalance in Zone 6 of the microgrid. In this test, had the unbalance not tripped CB13, removing it from the system, the static switch after a short period of time would have reconnected to the utility briefly before tripping off again. This process would have continued indefinitely or until some other system parameter was altered. In future installations, consideration must be given to this to prevent unattended operation in this manner.

\subsubsection{Zone 6 C-Phase Line-to-Ground fault, Gen-sets (A1+B1) and Utility Connected} Performance Goal:

Test a single line-to-ground overload fault condition in Zone 6 with Gen-sets A1 and B1 operating in-parallel with the utility grid to verify $\mathrm{I}^{2} \mathrm{t}$ protection.

\section{Initial Setup:}

Gen-set A1 = Unit Power Control

Gen-set B1 = Unit Power Control

Gen-set A1 Output Power Command $=20 \mathrm{~kW}$

Gen-set B1 Output Power Command $=20 \mathrm{~kW}$

MG Power/Frequency Droop $=-0.0833 \mathrm{~Hz} / \mathrm{kW}$

MG Voltage Command $=277 \mathrm{~V}$

Relay Settings $=$ A $($ Delayed $)$

Reverse Power Set-point $=0 \mathrm{~kW}$

Load Bank $3=40 \mathrm{~kW}$

Load Bank $5=40 \mathrm{~kW}$

Load Bank $6=60 \mathrm{~kW}$

To simulate a high impedance single phase-to-ground fault in Zone 6 on C-phase the Overload Load Bank of $85 \mathrm{~kW}$ (approximately $28.3 \mathrm{~kW}$ per phase) was connected by cable/plug into the exterior receptacle of Cabinet $8 \mathrm{a}$ with phases $\mathrm{A}$ and $\mathrm{B}$ disconnected. 
This, when combined with the load in Load Bank 6 of $60 \mathrm{~kW}$ (approximately 20kW per phase), added $28.3 \mathrm{~kW}$ to C-phase in Zone 6 and caused an overload condition in Zone 6.

After both Gen-sets A1 and B1 were running for a few minutes and supplied power to Load Banks 3 and 5, the test started with a "Start" command from the EMS. As soon as the static switch closed and steady-state conditions established, the microgrid and Load Bank 6 were connected to the utility grid with the total power flow at Meter 1 being approximately $89 \mathrm{~kW}$, shown in Figure 21a, and approximately $35 \mathrm{~kW}$ at Meter 2, shown in Figure 21b. Before the overload fault was applied, Gen-set A1 was producing approximately $17 \mathrm{~kW}$ and Gen-set B1 was producing approximately $19 \mathrm{~kW}$, shown in Figures 21c and 21d, respectively.

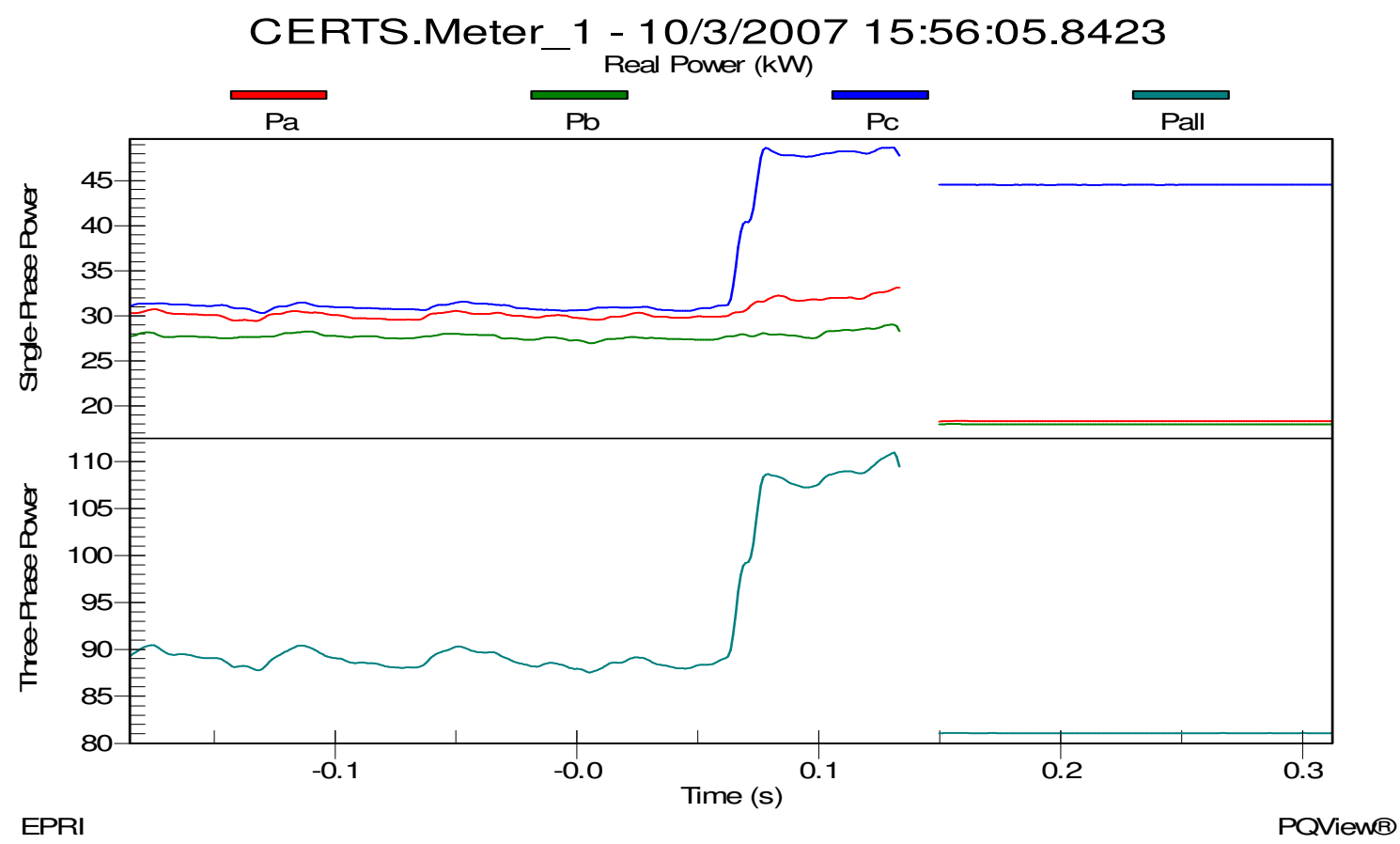

Figure 21a Meter 1 power flow before/after the static switch opened, islanding the microgrid from the utility grid when a single phase-to-ground fault was applied in Zone 6. 


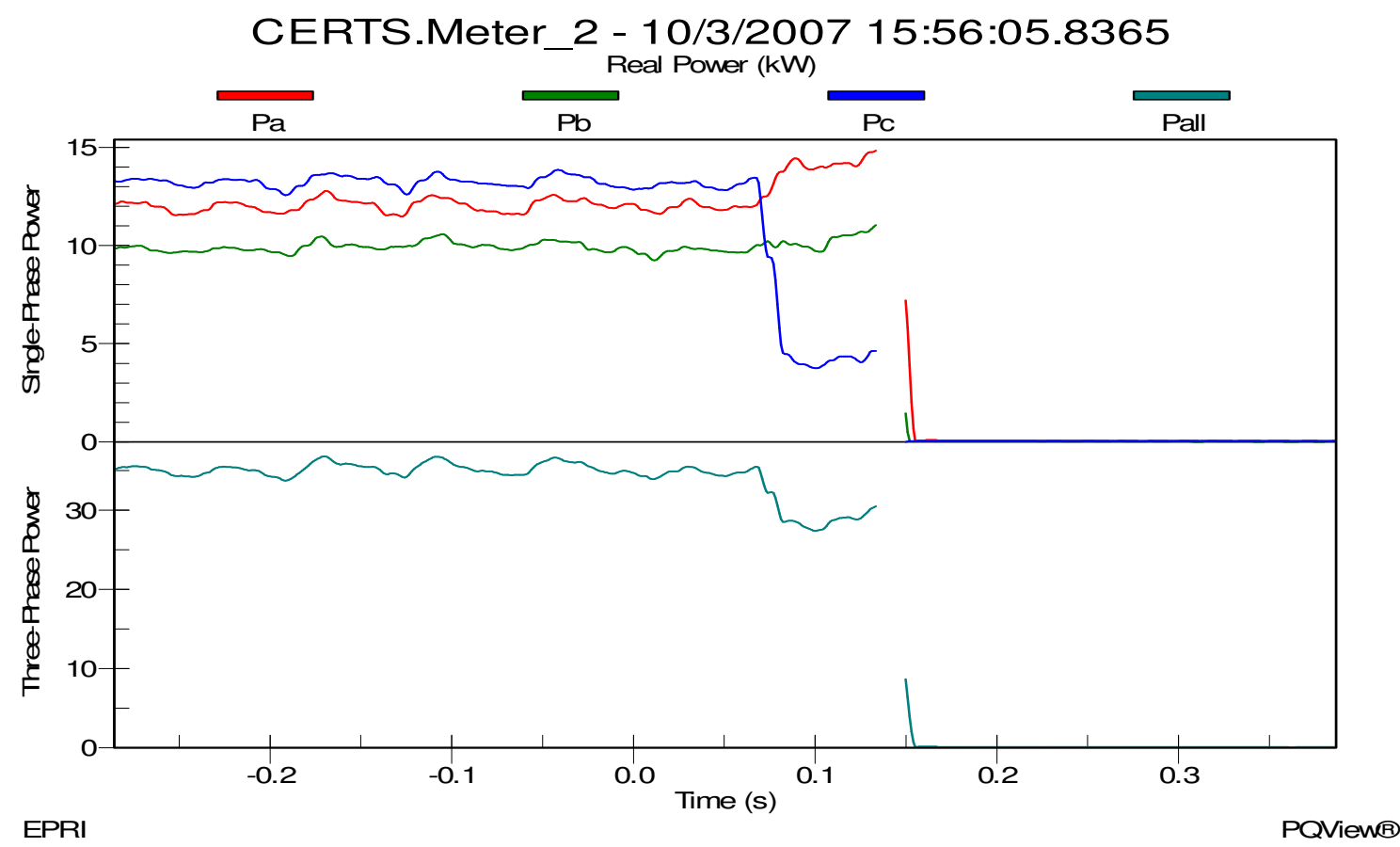

Figure 21b Meter 1 power flow before/after the static switch opened, islanding the microgrid from the utility grid when a single phase-to-ground fault was applied in Zone 6.

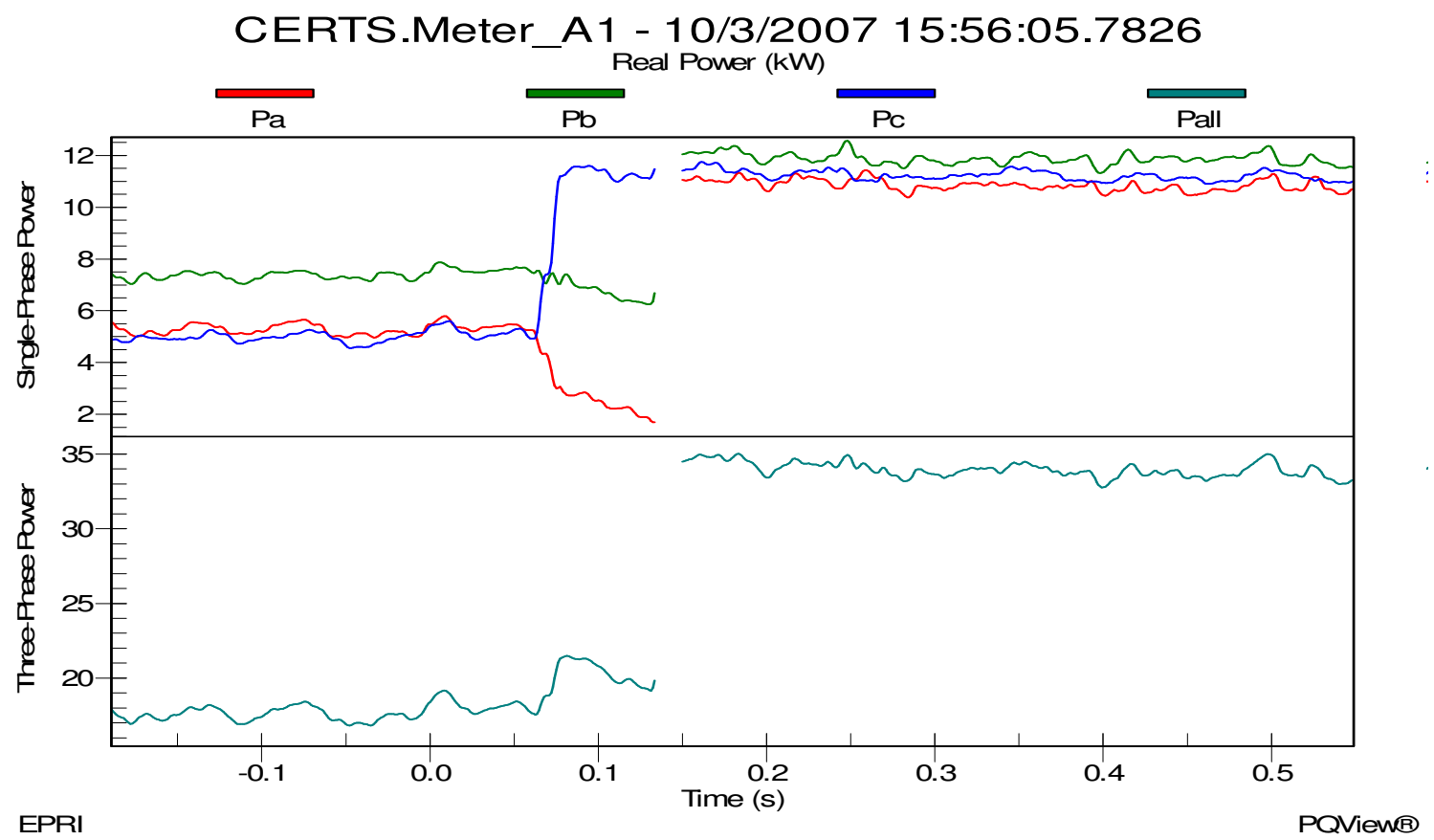

Figure 21c - Power produced by Gen-set A1 before/after the static switch opened from a C-phase-toground fault in Zone 6. 


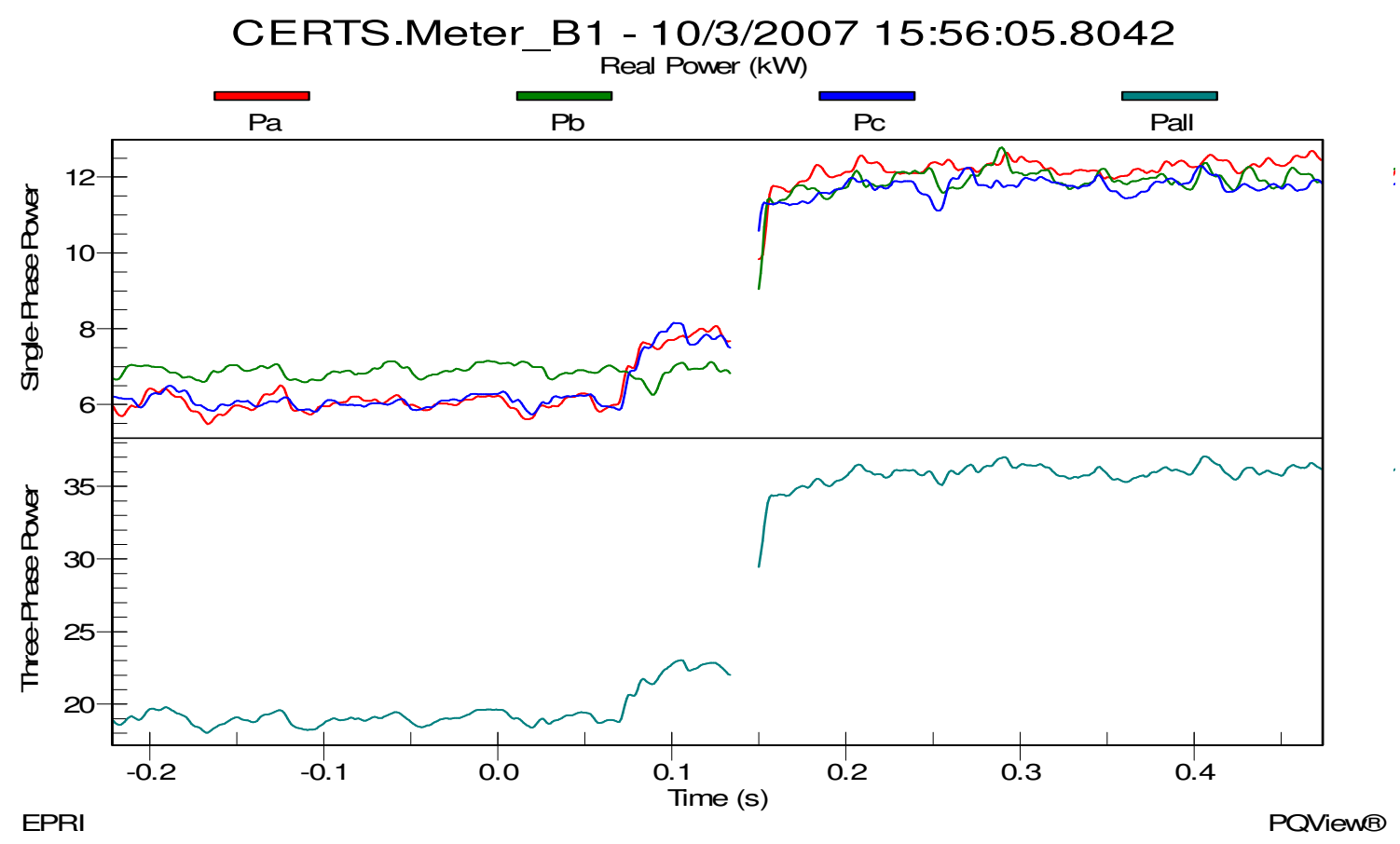

Figure 21d - Power produced by Gen-set B1 before/after the static switch opened from a C-phase-toground fault in Zone 6.

Using the DAS Load Control program, the single phase-to-ground fault on C-phase was set for 10 seconds and then initiated. The static switch tripped in approximately 0.07 seconds ( 4.3 cycles) from a neutral over-current (67G1T), shown in Figure 21e, islanding the microgrid from the utility grid. Following the static switch operation, CB13, which provides protection to Zone 6, tripped on $I^{2} t$ protection with CB31, CB41 and CB51 remaining closed, since the overload fault was not part of the microgrid, located beyond the static switch.

Within the islanded microgrid, Gen-sets A1 and B1 increased their output power to Load Banks 3 and 5. Gen-set A1 increased its output power from approximately $17 \mathrm{~kW}$ to $34 \mathrm{~kW}$, and Gen-set B1 increased its output power from approximately $19 \mathrm{~kW}$ to $36 \mathrm{~kW}$, shown in Figures 21c and 21d, respectively. Meter 2 (i.e., Figure 21b) power flow decreased to $0 \mathrm{~kW}$ after the fault was initiated and the static switch opened. After the overload fault was cleared from the electrical system, Meter 1 (i.e., Figure 21a) power flow decreased to $80 \mathrm{~kW}$ from the utility grid. The utility grid and microgrid remained in this stable state until the fault was removed. 


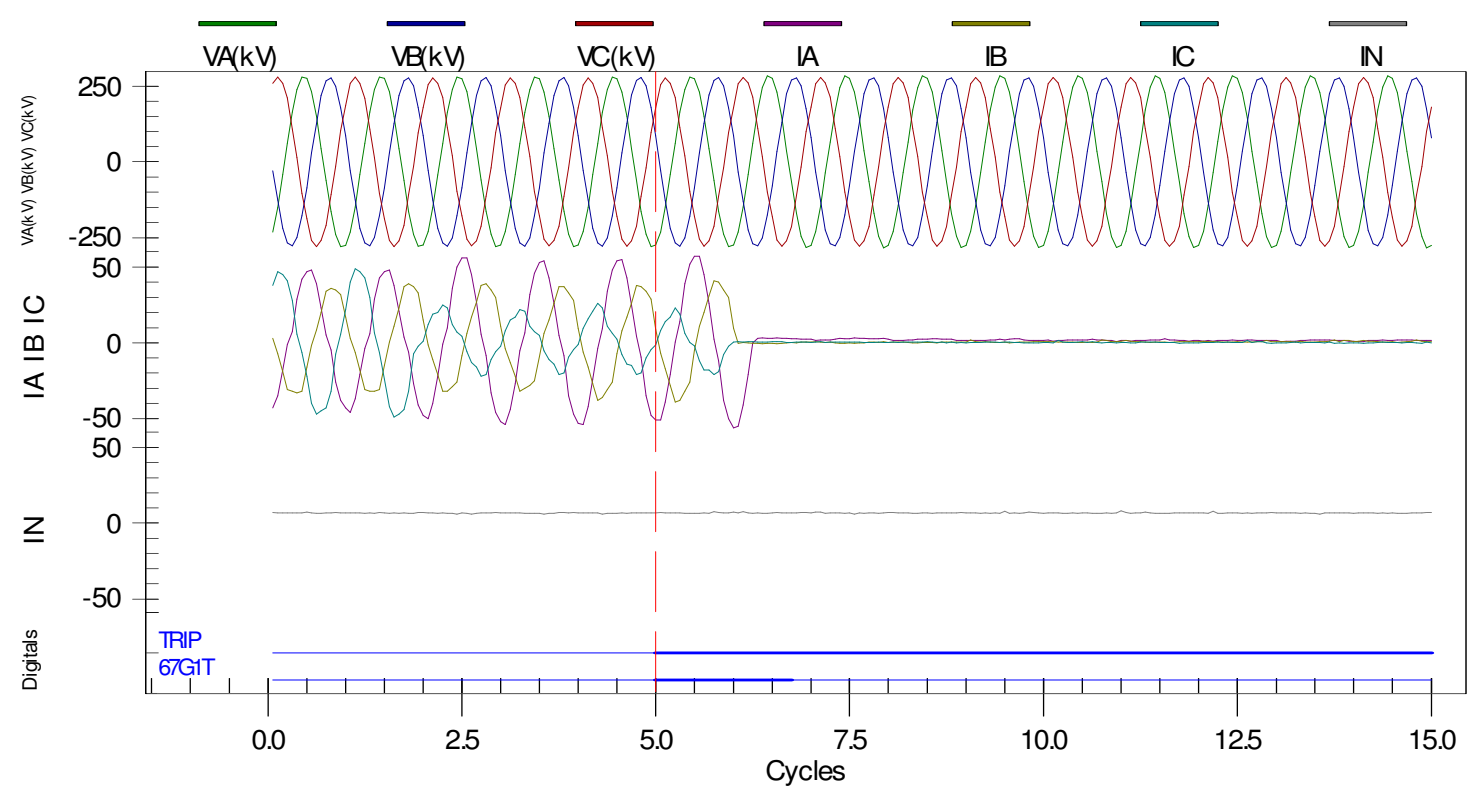

Figure 21e - Relay 2 (static switch) tripped due to a neutral over-current (67G1T) when the single phaseto-ground fault was initiated in Zone 6.

This test also produced the two points of interest mentioned in the previous test. These tests were similar with the exception that Gen-set B1 was less affected by the phase-toground fault due to transformer T51. Gen-set B1 did increase its power output by approximately $2 \mathrm{~kW}$; however, it was spread more evenly across A- and C-phases. Also, both A- and C-phases increased in power output instead of A-phase reducing as in the last test. The static switch still remains sensitive to unbalanced load within Zone 6 of the microgrid.

A third point of interest developed in this test, while waiting for the data to finish being collected from the metering equipment. Both Gen-set A1 and B1 shut down prematurely after the test was completed. This shutdown was captured by the metering equipment and can be used as insight into what caused the shutdown. Gen-set A1 reported a "No Field Fault" and B1 reported a "Boost Fault". Figure 21f indicated Genset A1 produced a $150 \mathrm{~kW}$ increase of power for a short period of time. This matched up with Figure $21 \mathrm{~g}$ indicating Gen-set B1 draws-in a large amount (i.e., 95kW) of power for a short period of time. These Gen-sets were not designed to absorb power, thus, it can be assume that the cause for this premature shutdown was attributed to Gen-set B1. 


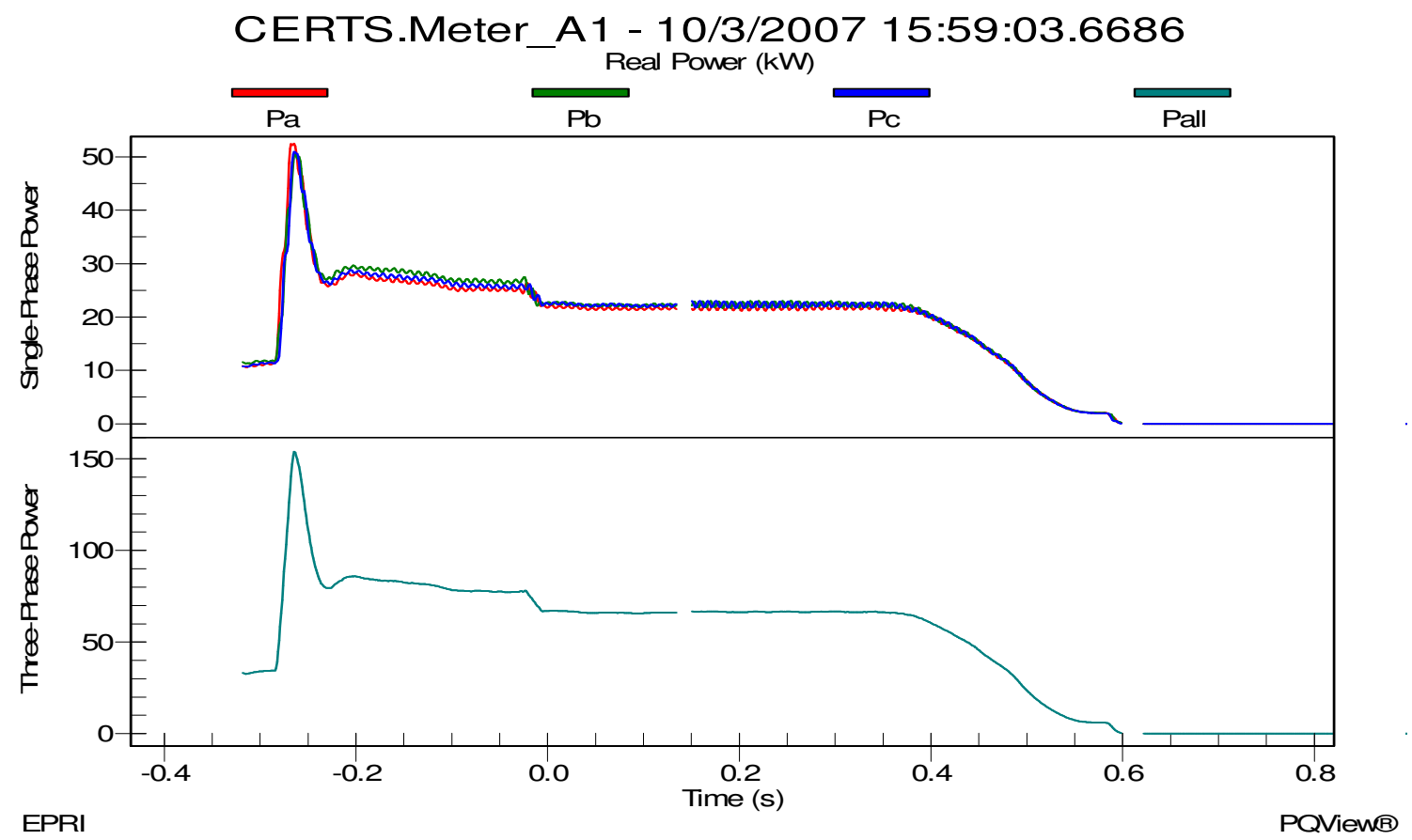

Figure 21f - Gen-set A1 shutting down due to a "No Field" fault.

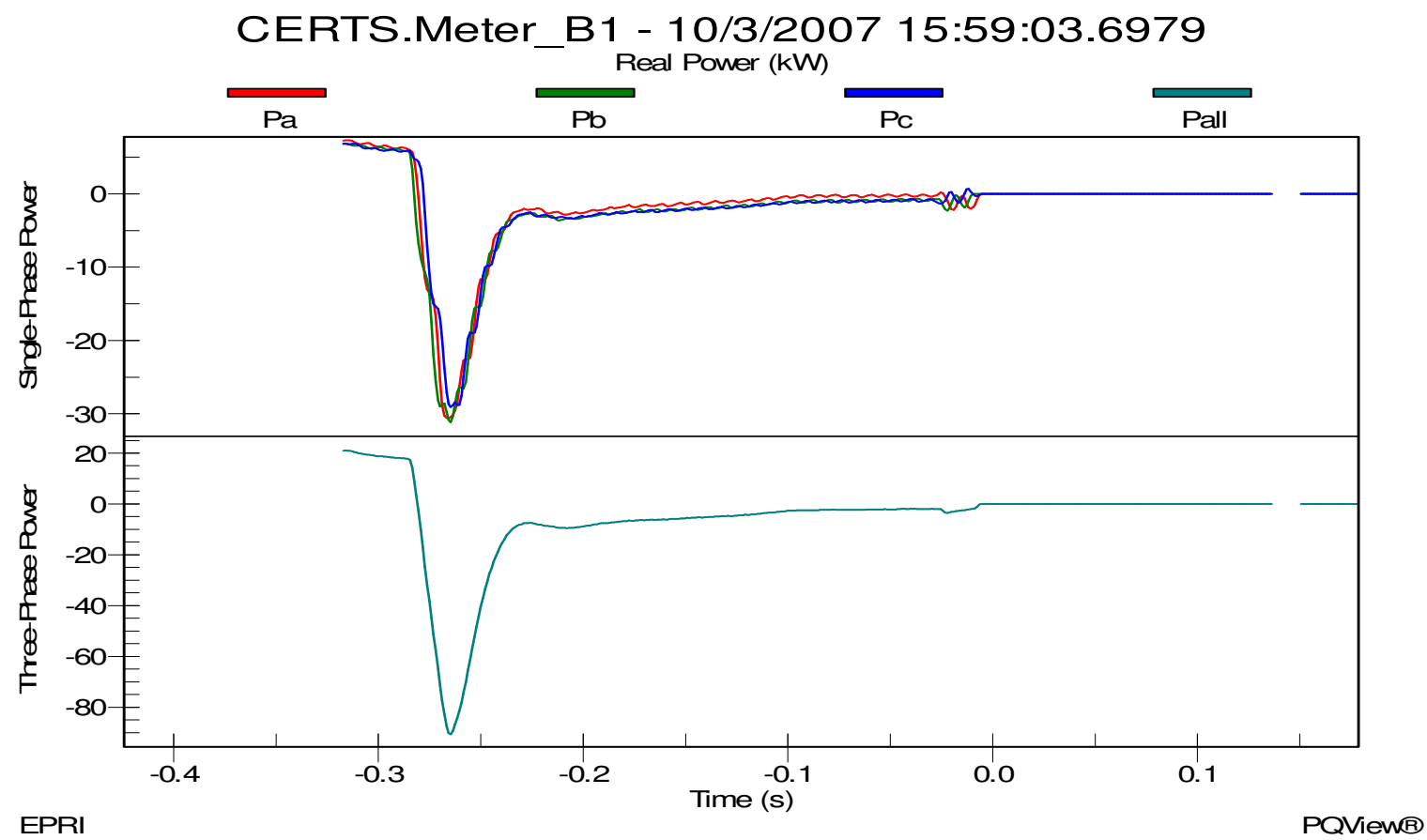

Figure 21g - Gen-set B1 shutting down due to a "Boost" fault 
In Figure 21h, voltage at Gen-set A1 suddenly dropped to $245 \mathrm{~V}$ and current increased to approximately 200A. After a short period of time, 0.28 seconds, Gen-set B1 was removed from the system and Gen-set A1 remained on-line, now serving both Load Banks 3 and 5, measured at approximately 65kW. Gen-set A1 continued to serve this load for approximately 0.38 seconds and then shut down, unexpectedly. Two conditions need be examined; the sudden import of power into Gen-set B1 which initiated the problem, and the short duration of Gen-set A1 operation afterwards which should have been within its overload envelope capability. These problems need to be corrected, as they unintentionally lead to a decrease in reliability.

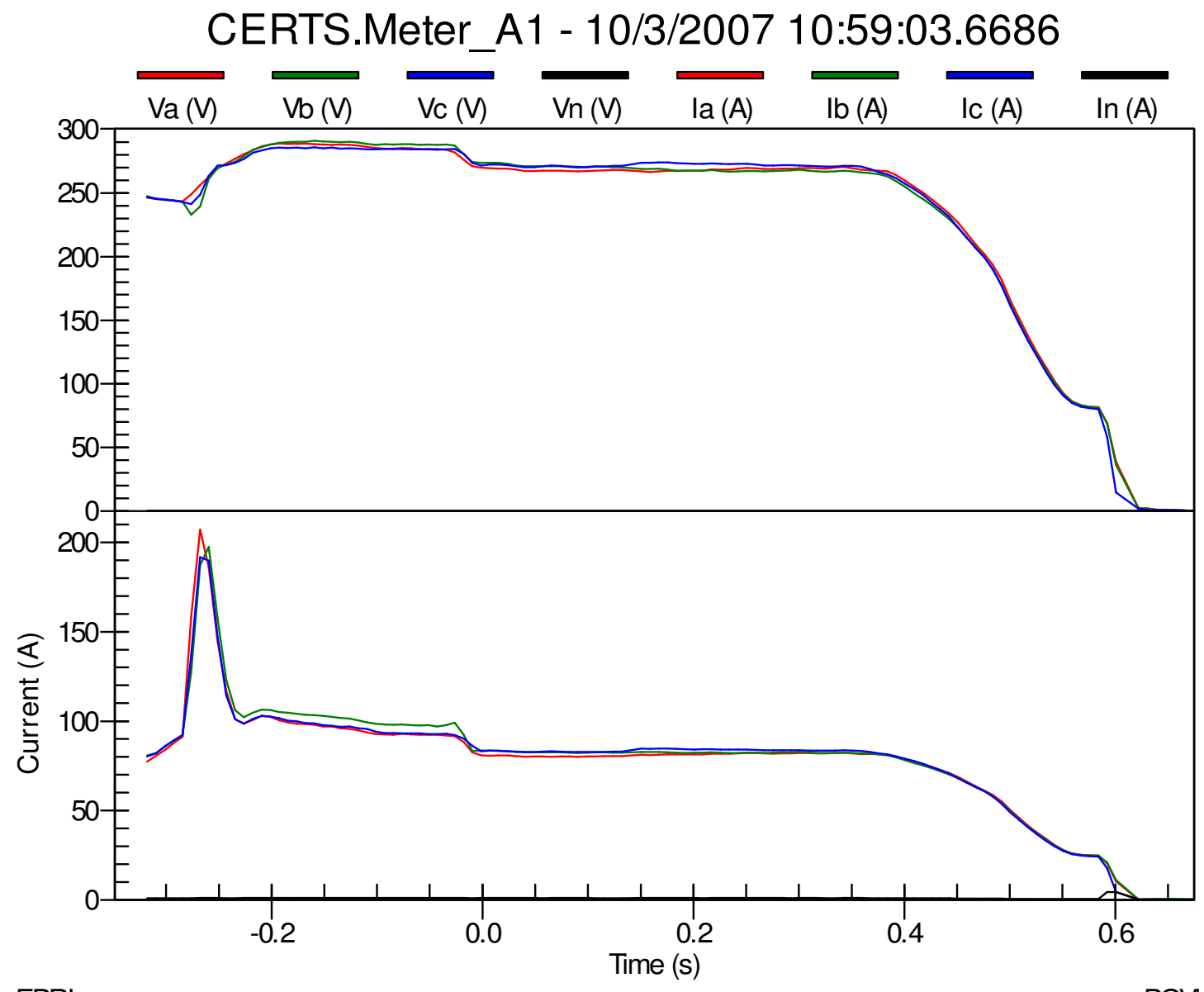

EPRI

PQView尺

Figure 21h - Gen-set A1 shutting down due to a "No Field" fault.

\subsubsection{Zone 3 A-to-B Phase Fault, Gen-sets (A1+A2) and Utility Connected Performance Goal:}

Test a phase-to-phase overload fault condition in Zone 3 on A- and B-phases with Gensets A1 and A2 operating in-parallel with the utility grid to verify negative-sequence, $I^{2} t$ protection or residual over-current protection. 
Initial Setup:

Gen-set A1 = Unit Power Control

Gen-set A2 = Unit Power Control

Gen-set A1 Output Power Command $=20 \mathrm{~kW}$

Gen-set A2 Output Power Command $=20 \mathrm{~kW}$

MG Power/Frequency Droop $=-0.0833 \mathrm{~Hz} / \mathrm{kW}$

MG Voltage Command $=277 \mathrm{~V}$

Relay Settings $=$ B (Inst)

Reverse Power Set-point $=0 \mathrm{~kW}$

Load Bank $3=40 \mathrm{~kW}$

Load Bank $4=40 \mathrm{~kW}$

Load Bank $6=40 \mathrm{~kW}$

To simulate a high impedance phase-to-phase fault in Zone 3 on A-B phases the Overload Load Bank of $85 \mathrm{~kW}$ (i.e., approximately $42.5 \mathrm{~kW}$ phase-to-phase) was connected by cable/plug into the exterior receptacle of Cabinet 3 with C-phase disconnected. This, combined with load in Load Banks 3 and 4, added an additional $21.2 \mathrm{~kW}$ on A- and B phases of Zone 3 when initiated causing an overload.

After Gen-sets A1 and A2 were running for a few minutes and supplied power to Load Banks 3 and 4, the test started with a "Start" command from the EMS. As soon as the static switch closed and steady-state conditions established, the microgrid and Load Bank 6 were connected to the utility grid with power flow at Meter 1 being approximately $74 \mathrm{~kW}$, shown in Figure $22 \mathrm{a}$, and approximately $38 \mathrm{~kW}$ at Meter 2 , shown in Figure 22b. Before the overload fault was applied, Gen-sets A1 and A2 were producing about 19kW, shown in Figures 22c and 22d. 


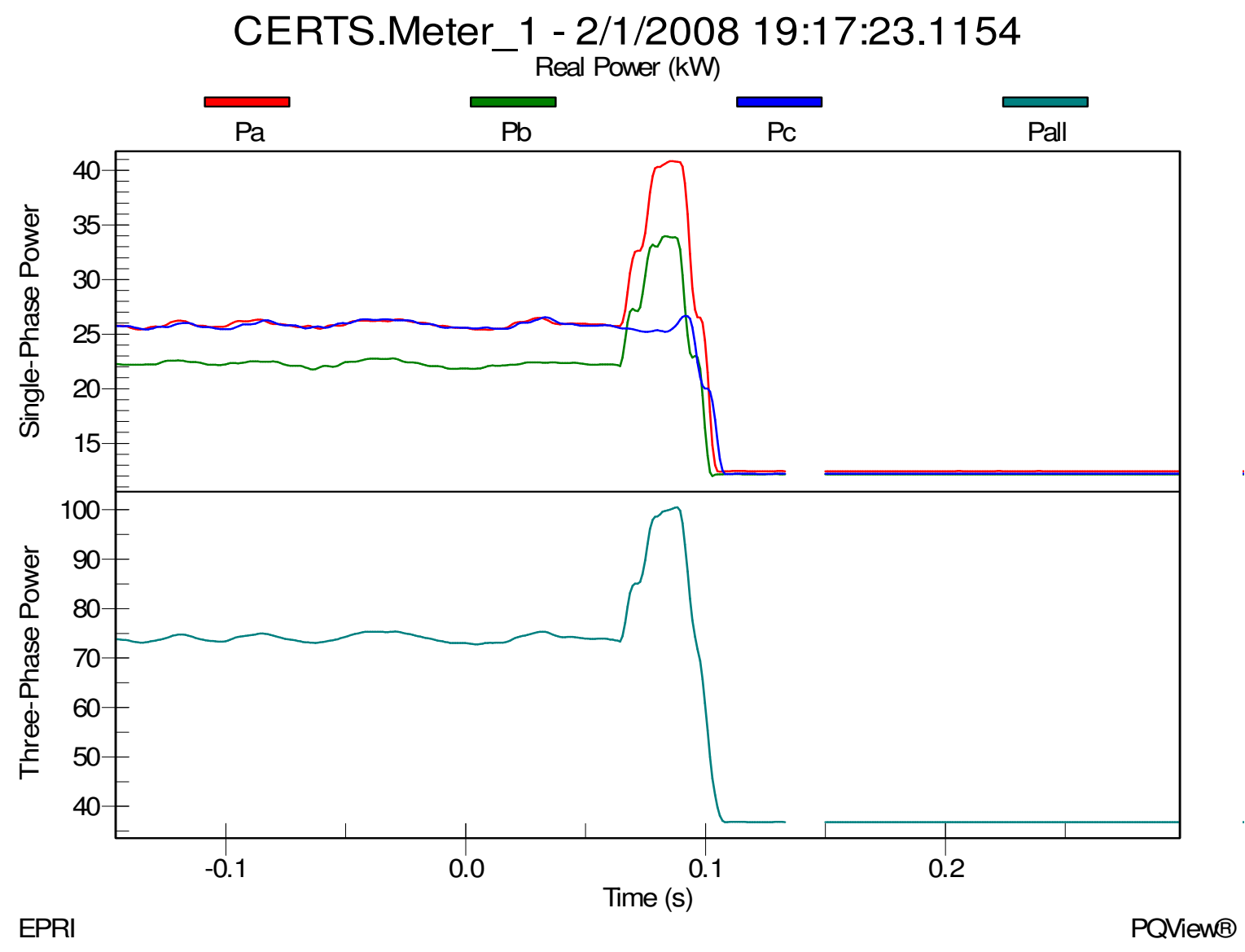

Figure 22a - Meter 1 power flow before/after the static switch opened, islanding the microgrid from the utility grid when the phase-to-phase fault was applied in Zone 3. 


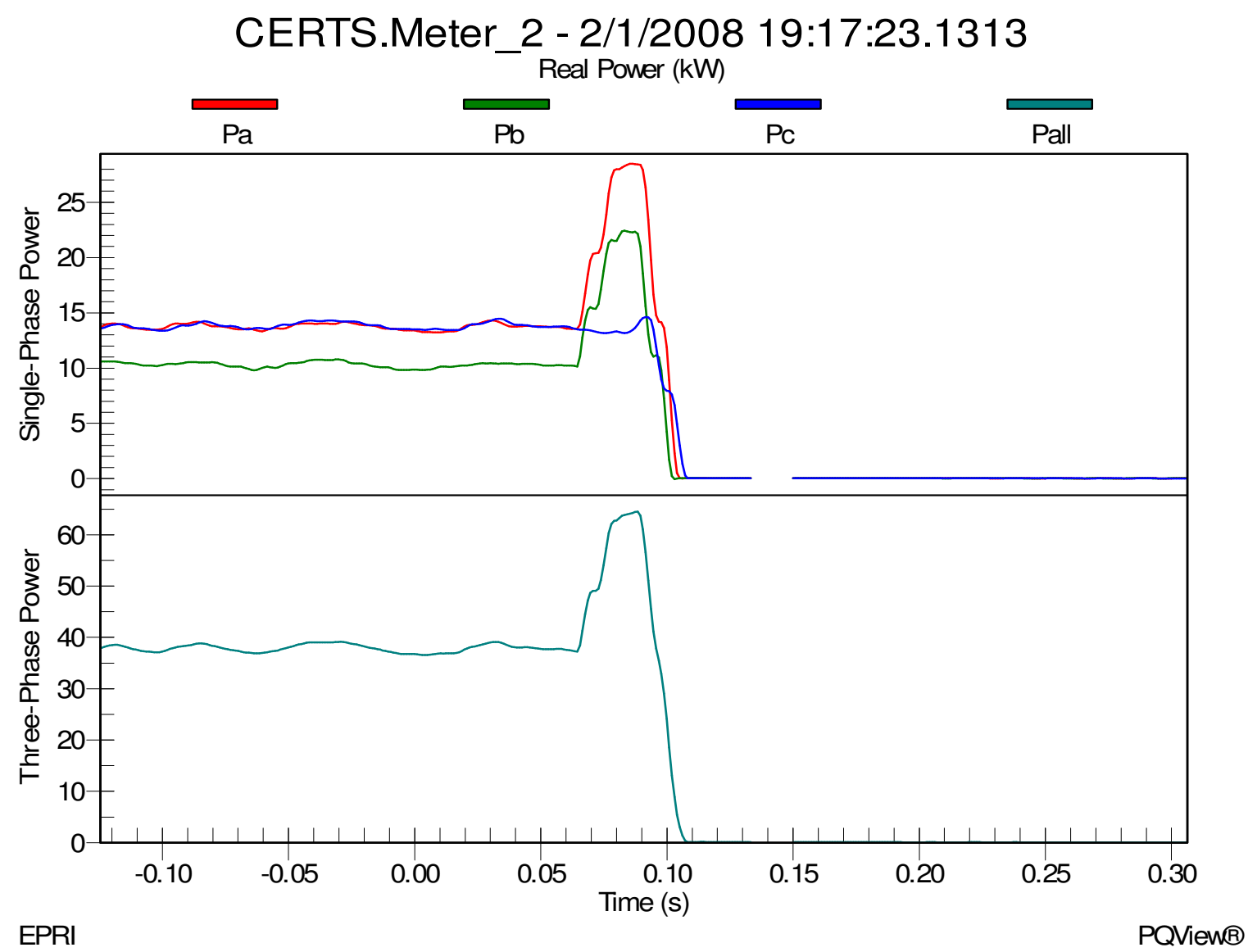

Figure 22b - Meter 2 power flow before/after the static switch opened, islanding the microgrid from the utility grid when a phase-to-phase fault was applied in Zone 3. 


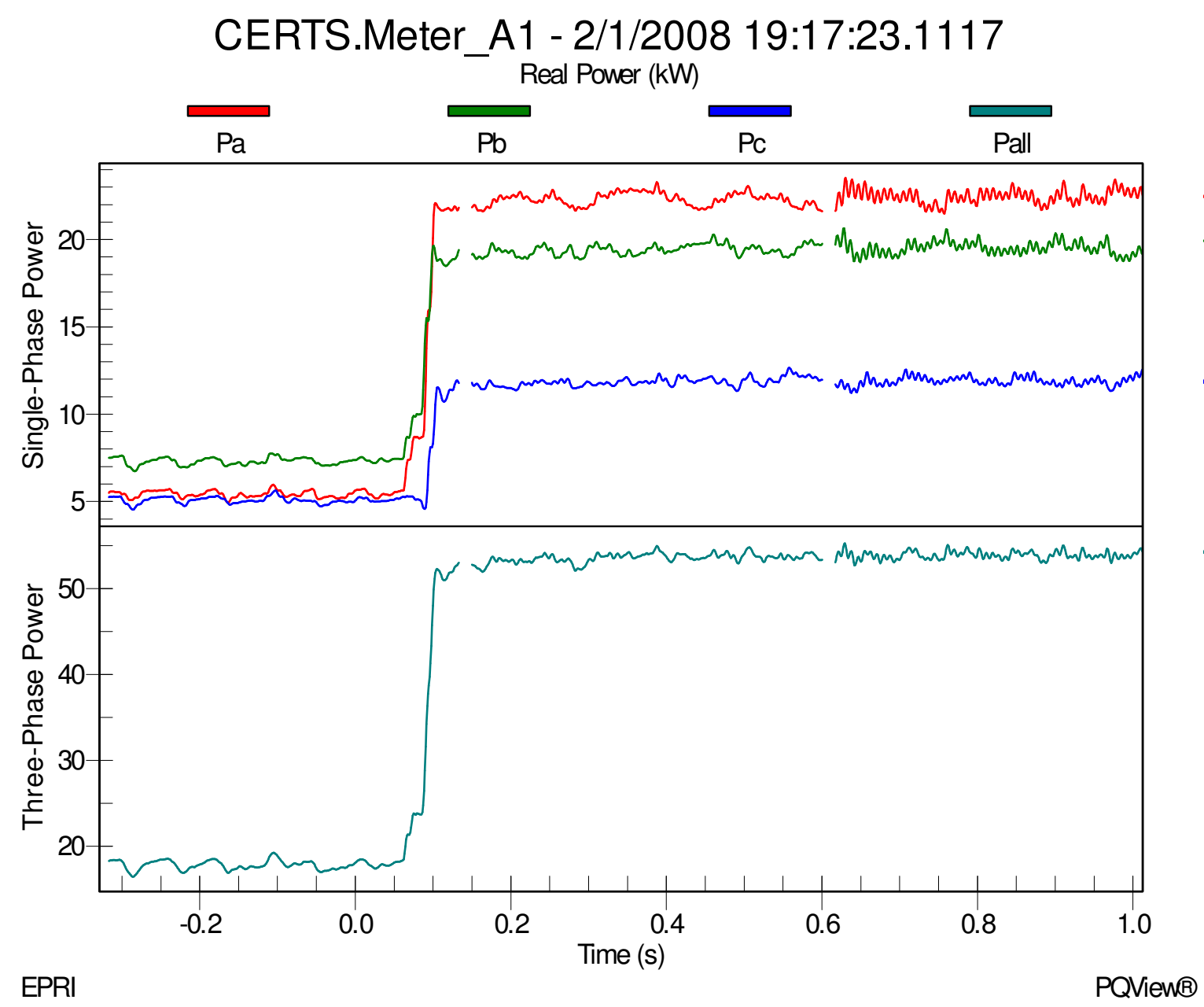

Figure 225c - Power produced by Gen-set A1 before/after the static switch opened when an A-to-B phase fault was applied in Zone 3. 


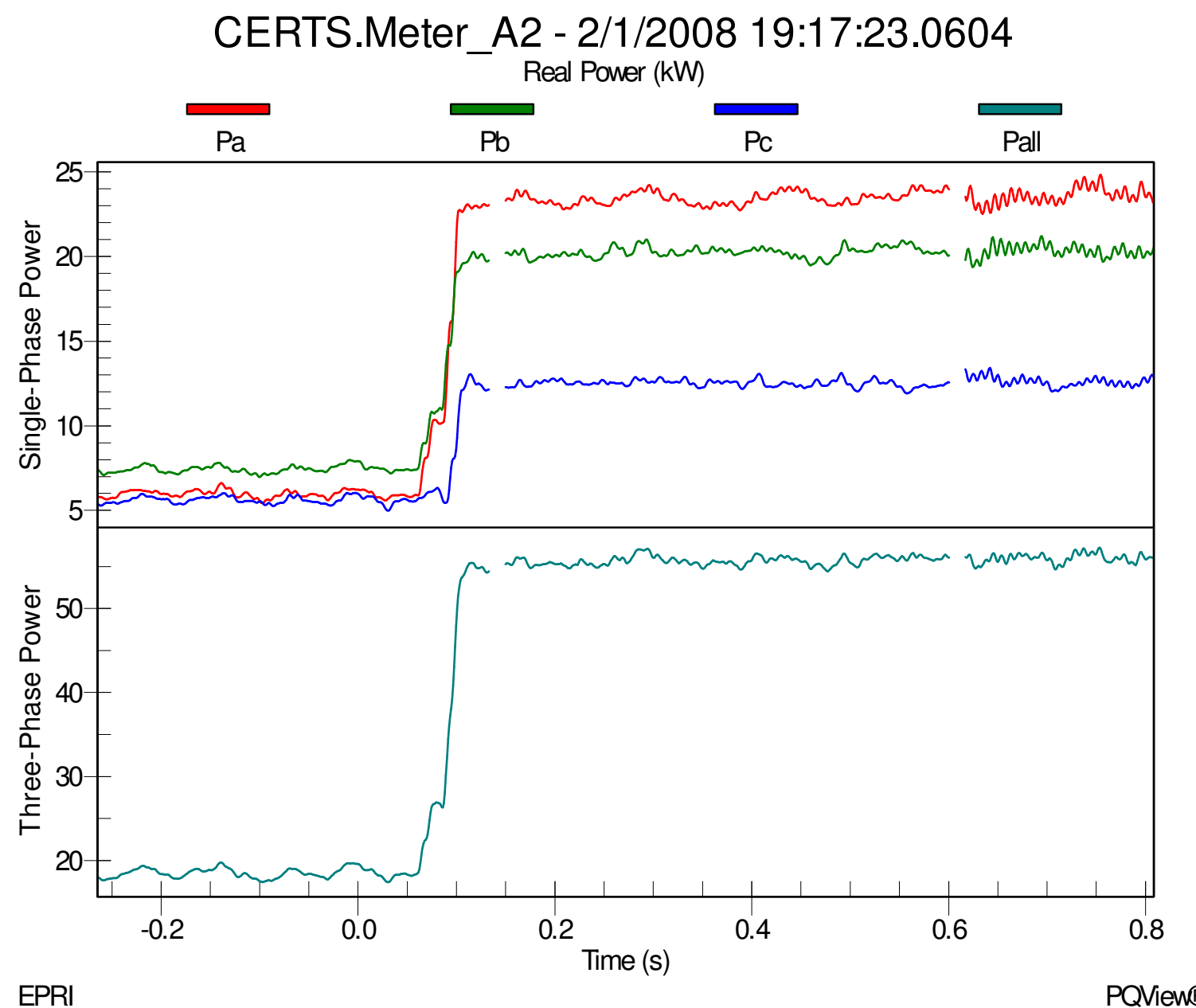

Figure 22d - Power produced by Gen-set A2 before/after the static switch opened when an A-to-B phase fault was applied in Zone 3.

Using the DAS Load Control program, the phase-to-phase fault on A and B phases was set for 10 seconds and then initiated. The static switch tripped in approximately 0.028 seconds ( 1.7 cycles) from a negative-sequence over-current (67Q1T), shown in Figure 22e, islanding the microgrid from the utility grid. CB31, CB41 and CB51 remained closed throughout the 10 second phase-to-phase fault. Gen-sets A1 and A2 increased their output power from approximately $19 \mathrm{~kW}$ to $54 \mathrm{~kW}$, each shown in Figures 22c and 22d supplying power to Load Banks 3 and 4 . Load Bank 6 was supplied power from the utility grid, shown in Figure 22a. 


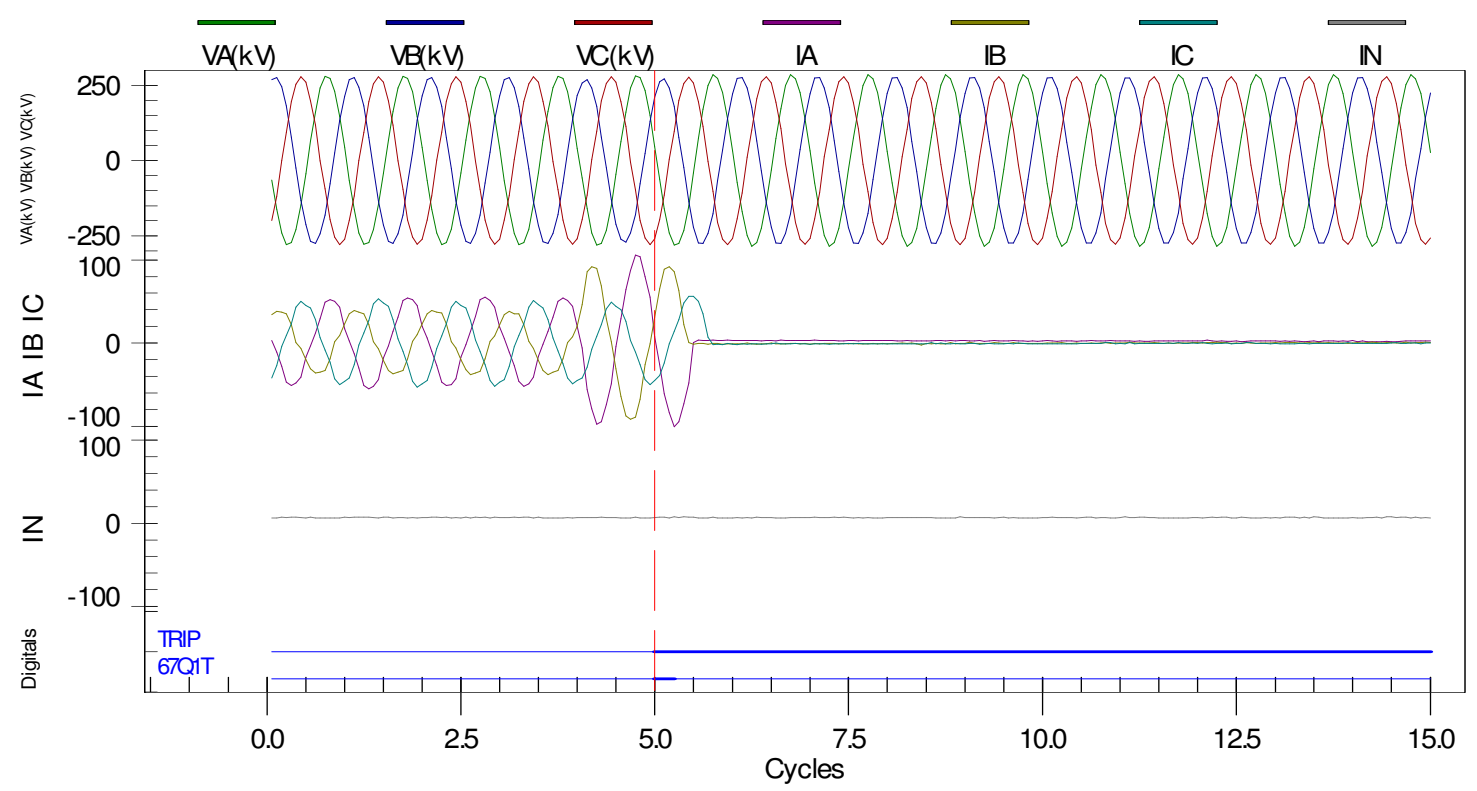

Figure 22e - Relay 2 (static switch) tripped due to a negative-sequence over-current (67Q1T) when the phase-to-phase fault was initiated in Zone 3.

After the fault was removed from Zone 3, the static switch was synchronized and shortly closed back into the utility grid. The electrical system was stable in both the microgrid and utility grid after the static switch opened and then when it closed. Gensets A1 and A2 were shut down manually, all loads and alarms were reset, and equipment was prepared for the next test.

\subsubsection{Zone 4 A-to-B Phase Fault, Gen-sets (A1+A2) and Utility Connected} Performance Goal:

Test a phase-to-phase overload fault condition in Zone 4 on A- and B-phases with Gensets A1 and A2 operating in-parallel with the utility grid to verify negative-sequence, $I^{2} t$ protection or residual over-current protection.

Initial Setup:

Gen-set A1 = Unit Power Control

Gen-set A2 = Unit Power Control

Gen-set A1 Output Power Command $=20 \mathrm{~kW}$ 
Gen-set A2 Output Power Command $=20 \mathrm{~kW}$

MG Power/Frequency Droop $=-0.0833 \mathrm{~Hz} / \mathrm{kW}$

MG Voltage Command $=277 \mathrm{~V}$

Relay Settings $=$ B (Inst)

Reverse Power Set-point $=0 \mathrm{~kW}$

Load Bank $3=40 \mathrm{~kW}$

Load Bank $4=40 \mathrm{~kW}$

Load Bank $6=40 \mathrm{~kW}$

To simulate a high impedance phase-to-phase fault in Zone 4 on A-and B-phases, the Overload Load Bank of $85 \mathrm{~kW}$ (i.e., approximately $42.5 \mathrm{~kW}$ phase-phase) was connected by cable/plug into the exterior receptacle of Cabinet $5 \mathrm{a}$ with $\mathrm{C}$-phase disconnected. This, combined with Load Bank 4 load, added $21.2 \mathrm{~kW}$ on both A and B phases in Zone 4, when initiated, causing an overload.

After Gen-sets A1 and A2 were running for a few minutes and supplied power to Load Banks 3 and 4, the test started with a "Start" command from the EMS. As soon as the static switch closed and steady-state conditions established, the microgrid and Load Bank 6 were connected to the utility grid with power flow at Meter 1 being approximately $67.5 \mathrm{~kW}$, shown in Figure 23a, and approximately $36 \mathrm{~kW}$ at Meter 2, shown in Figure 23b, Before the overload fault was applied, Gen-sets A1 and A2 were producing about $20 \mathrm{~kW}$, each shown in Figures $23 \mathrm{c}$ and $23 \mathrm{~d}$. 


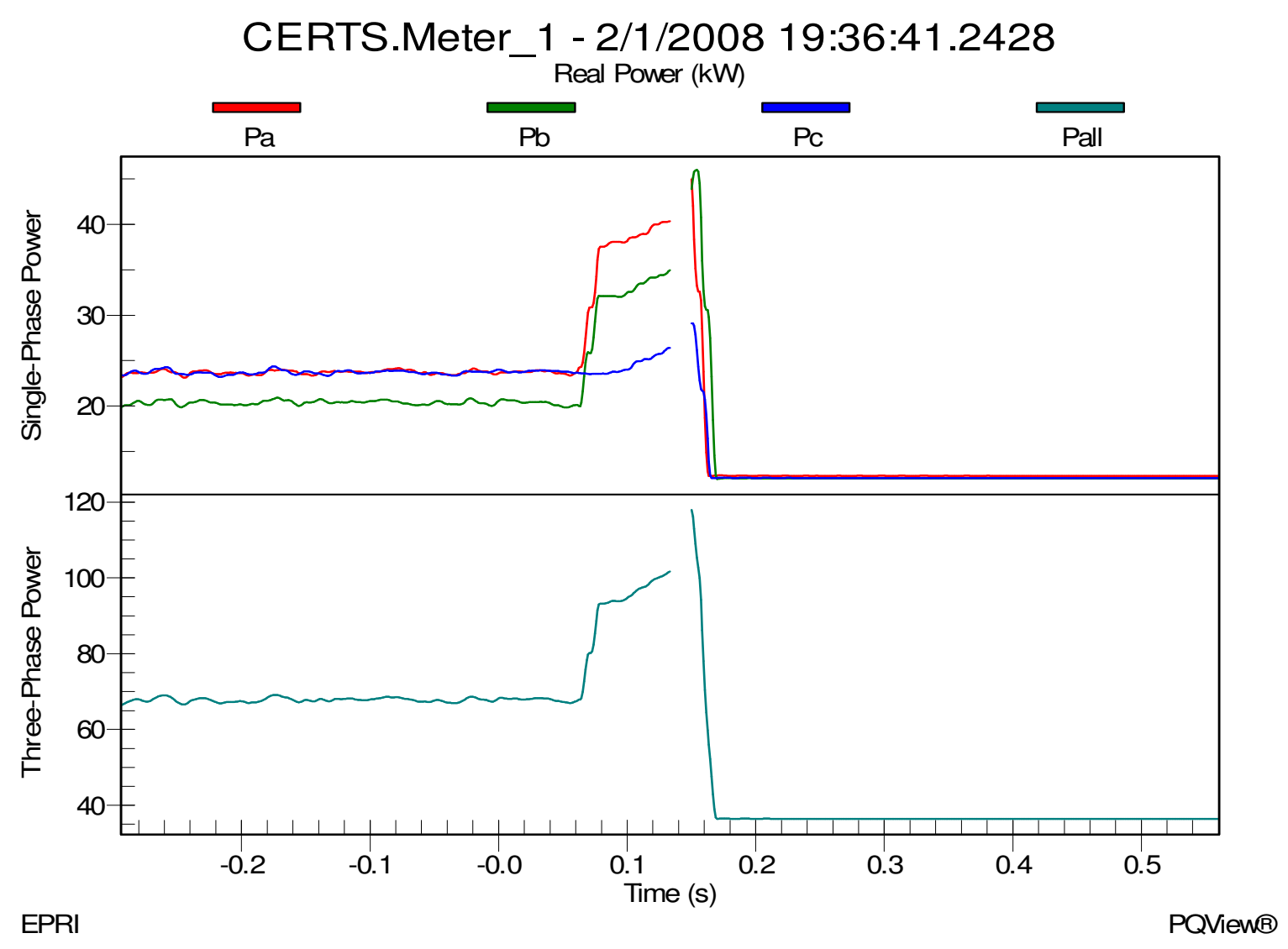

Figure 23a - Meter 1 power flow before/after the static switch opened, islanding the microgrid from the utility grid when a phase-to-phase fault was applied in Zone 4. 


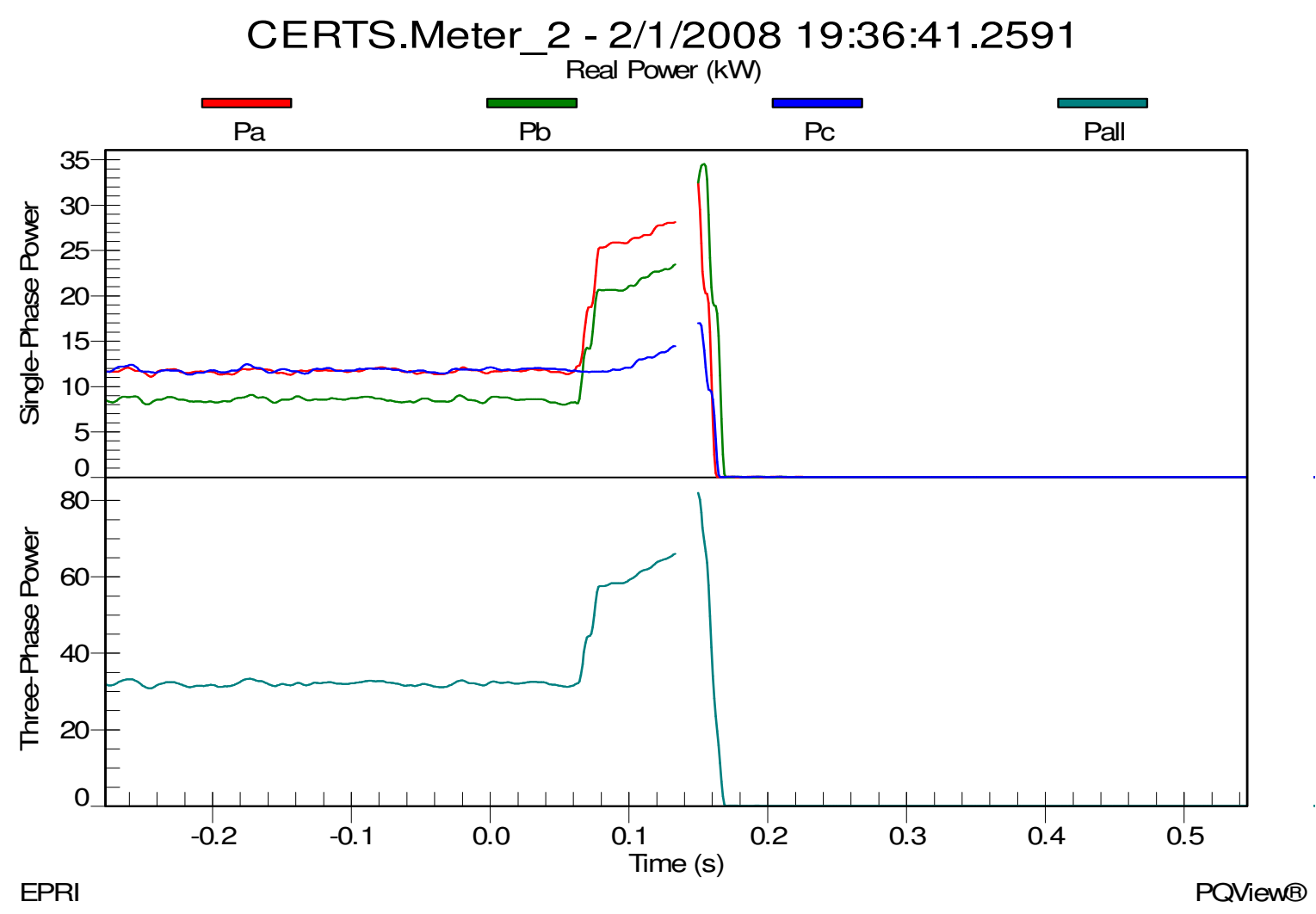

Figure $23 \mathrm{~b}$ - Meter 2 power flow before/after the static switch opened, islanding the microgrid from the utility grid when a phase-to-phase fault was applied in Zone 4. 


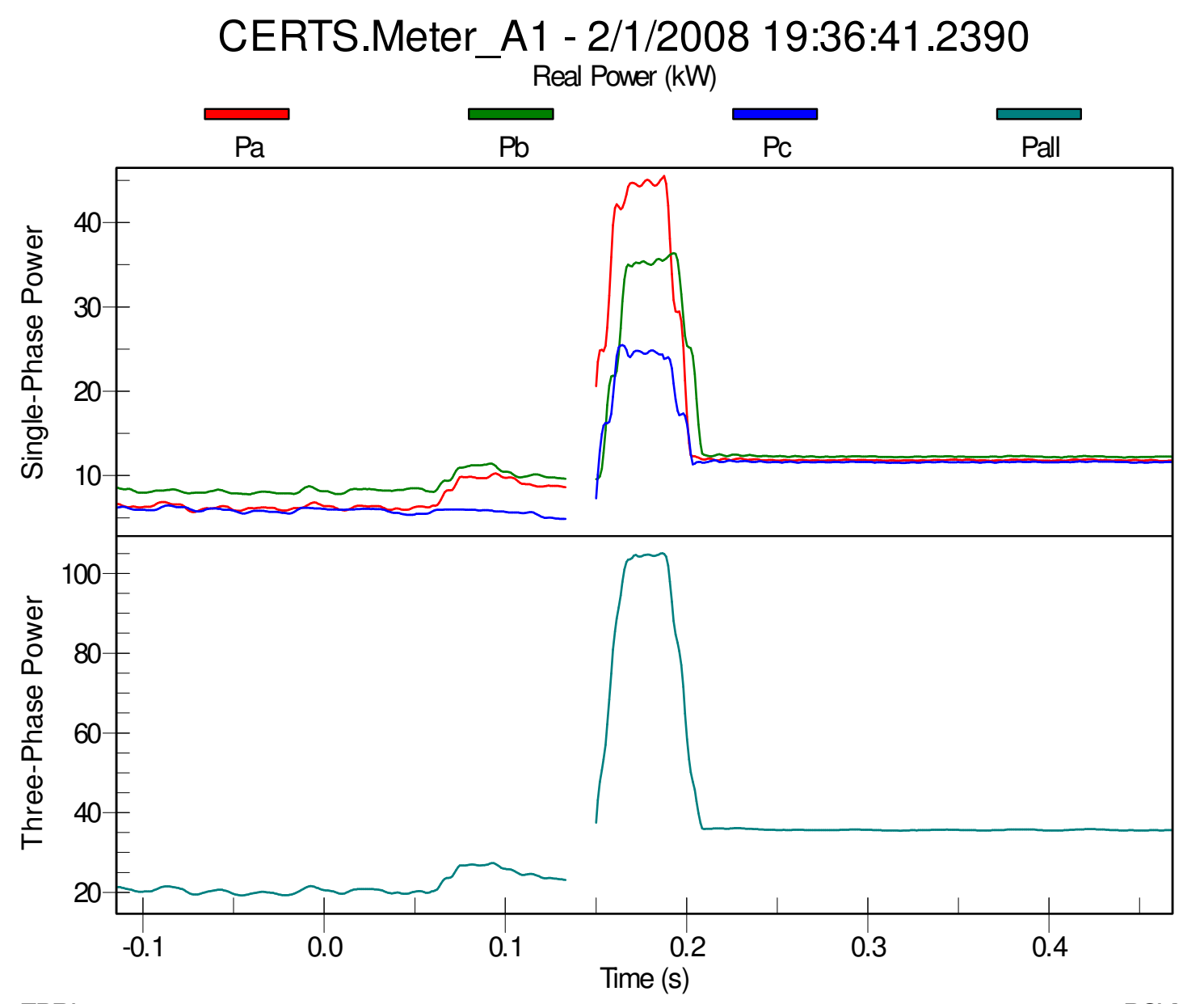

EPRI

PQView ${ }^{\circledR}$

Figure 23c - Power produced by Gen-set A1 before/after the static switch opened when an A-phase to Bphase fault was applied in Zone 4. 


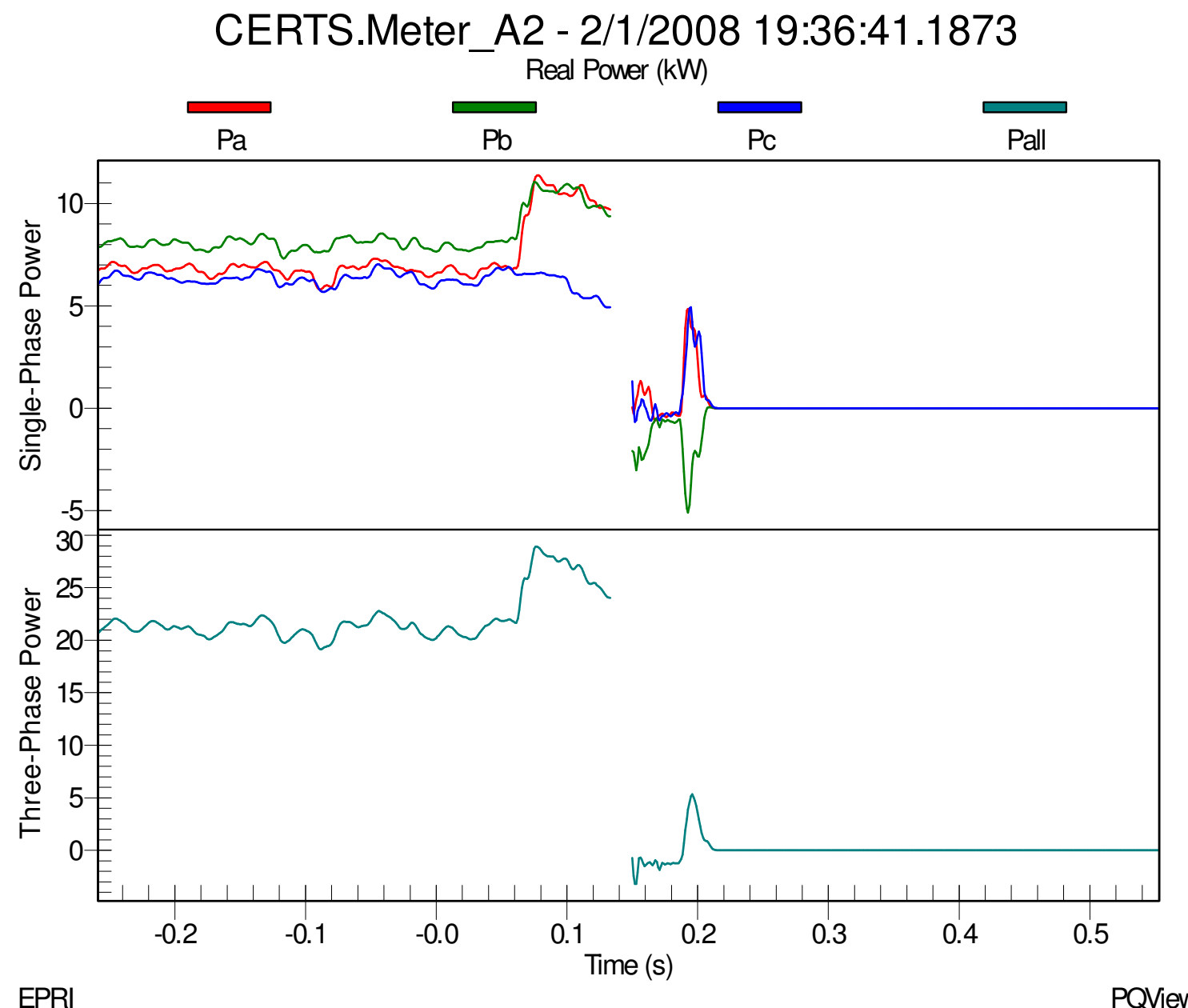

Figure 23d - Power produced by Gen-set A2 before/after the static switch opened when an A-phase toBphase fault was applied in Zone 4.

Using the DAS Load Control program, the phase-to-phase fault on A and B phases was set for 10 seconds and then initiated. The static switch tripped in approximately 0.078 seconds ( 4.7 cycles) from a negative-sequence over-current (67Q1T), shown in Figure $23 \mathrm{e}$, islanding the microgrid from the utility grid. CB41 tripped, after the static switch opened, due to a negative-sequence over-current (67Q1T) isolating Zone 4 from the rest of the microgrid, and shutting down Gen-set A2, shown in Figures 23d and 23f. CB31 and CB51 remained closed throughout the 10 second phase-to-phase fault. Gen-set A1 increased its output power from approximately $20 \mathrm{~kW}$ to $35 \mathrm{~kW}$, shown in Figure $23 \mathrm{c}$, supplying power to Load Bank 3. Load Bank 6 was supplied power from the utility grid, shown in Figure 23a. 


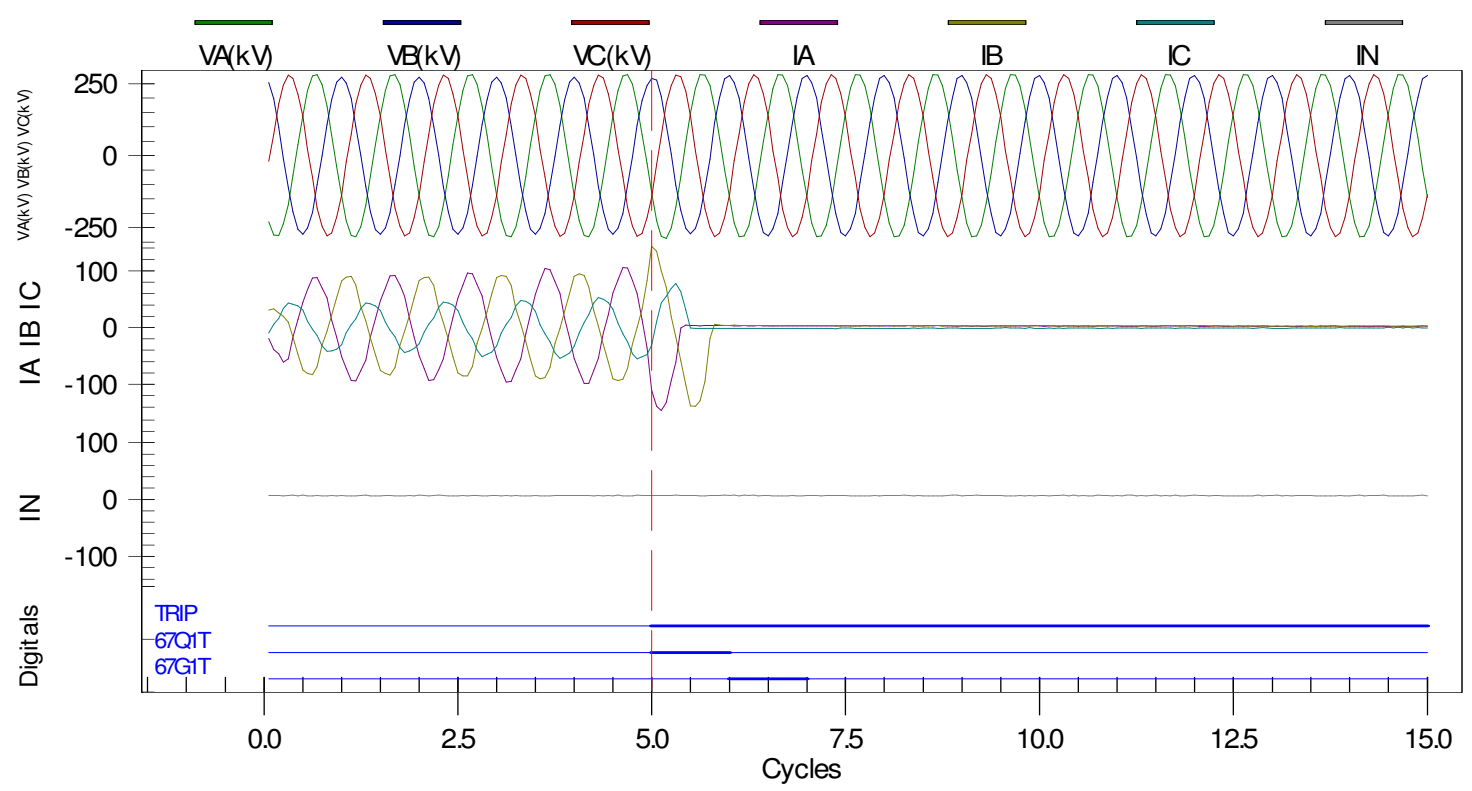

Figure 23e - Relay 2 (static switch) tripped due to a negative-sequence over-current (67Q1T) when the phase-to-phase fault was initiated in Zone 4.

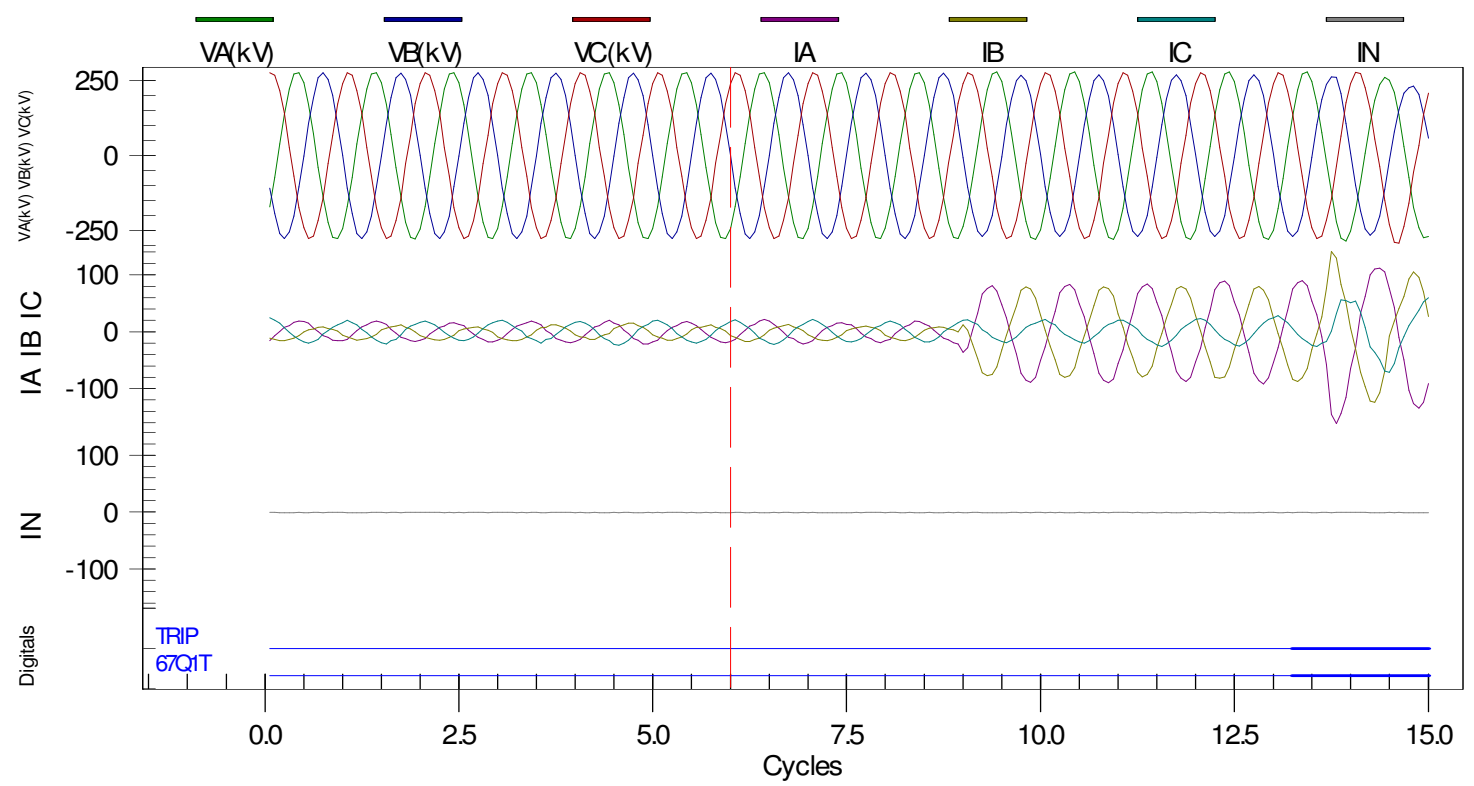

Figure 23f - Relay 4 (CB41) tripped due to a negative-sequence over-current (67Q1T) when the phase-tophase fault was initiated in Zone 4.

After the fault was removed in Zone 4, the static switch was synchronized and shortly closed back into the utility grid. The electrical system was stable in the microgrid and utility grid after the static switch opened, CB41 opened, and Gen-set A2 shutdown. Gen-set A1 was shut down manually, all loads and alarms were reset, and equipment was prepared for the next test. 


\title{
6.1.15 Zone 2 A-to-B Phase Fault, Gen-sets (A1+B1) and Utility Connected Performance Goal:
}

Test a phase-to-phase overload fault condition in Zone 2 on A- and B-phases with Gensets $\mathrm{A} 1$ and $\mathrm{B} 1$ operating in-parallel with the utility grid to verify negative-sequence, $\mathrm{I}^{2} \mathrm{t}$ protection or residual over-current protection.

\author{
Initial Setup: \\ Gen-set A1 = Unit Power Control \\ Gen-set B1 = Unit Power Control \\ Gen-set A1 Output Power Command $=20 \mathrm{~kW}$ \\ Gen-set B1 Output Power Command $=20 \mathrm{~kW}$ \\ MG Power/Frequency Droop $=-0.0833 \mathrm{~Hz} / \mathrm{kW}$ \\ MG Voltage Command $=277 \mathrm{~V}$ \\ Relay Settings $=$ B (Inst) \\ Reverse Power Set-point $=0 \mathrm{~kW}$ \\ Load Bank $3=40 \mathrm{~kW}$ \\ Load Bank $5=40 \mathrm{~kW}$ \\ Load Bank $6=40 \mathrm{~kW}$
}

To simulate a high impedance phase-to-phase fault in Zone 2 on A- and B- phases, the Overload Load Bank of $85 \mathrm{~kW}$ (i.e., approximately $42.5 \mathrm{~kW}$ phase-phase) was connected by cable/plug into the exterior receptacle of Cabinet $2 \mathrm{~d}$ with C-phase disconnected. This, when combined with load of Load Banks 3 and 5, added $21.2 \mathrm{~kW}$ on A and B phases of Zone 2, when initiated, causing an overload.

After Gen-sets A1 and B1 were running for a few minutes and supplied power to Load Banks 3 and 5, the test started with a "Start" command from the EMS. As soon as the static switch closed and steady-state conditions established, the microgrid and Load Bank 6 were connected to the utility grid with the total power flow at Meter 1 being approximately $70 \mathrm{~kW}$, shown in Figure 24a, and approximately $34 \mathrm{~kW}$ at Meter 2, shown in Figure 24b. Before the overload fault was applied, Gen-sets A1 and B1 were producing about 20kW each, shown in Figures 24c and 24d. 


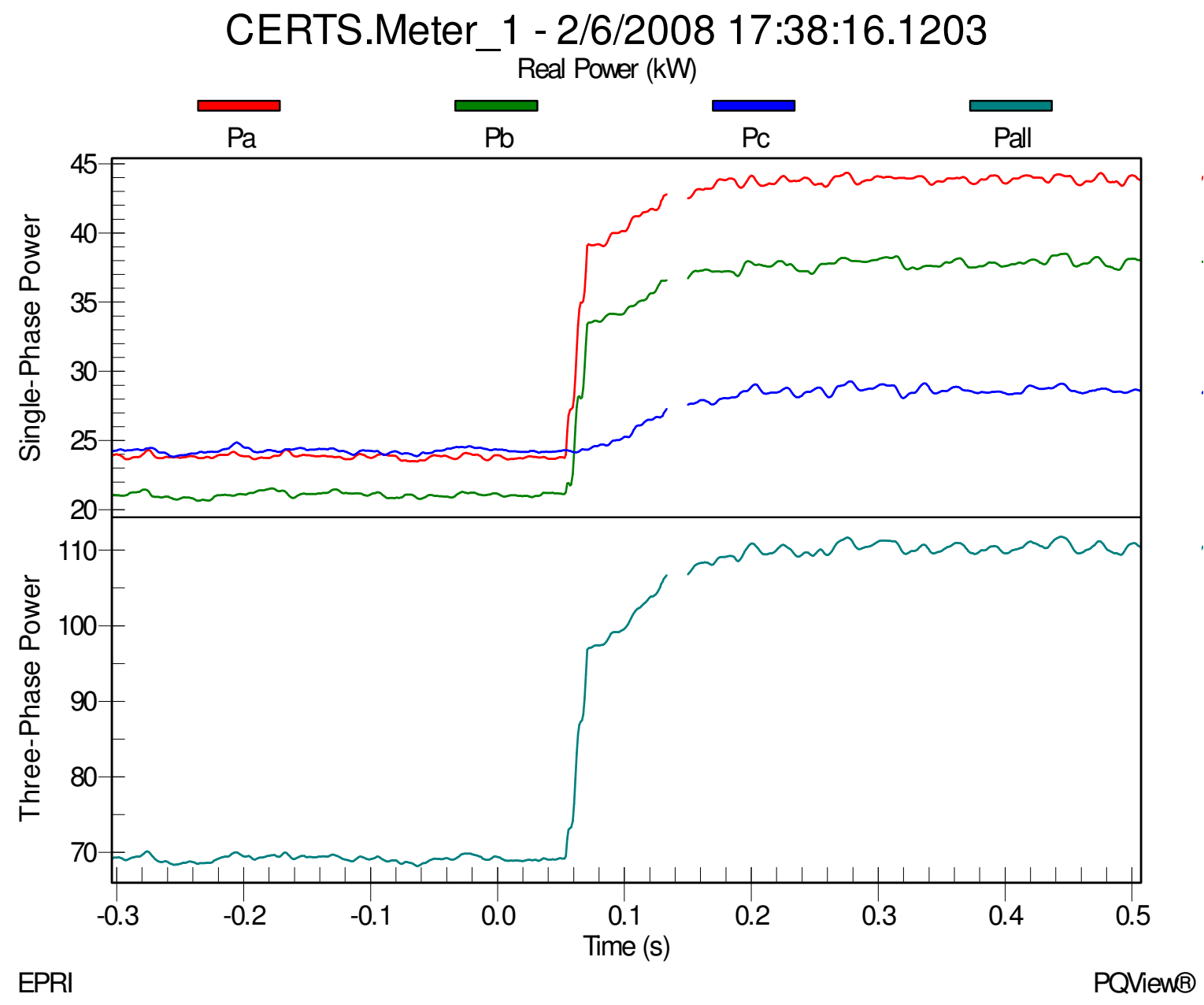

Figure 24a - Meter 1 power flow before/after the phase-to-phase fault was applied in Zone 2. 


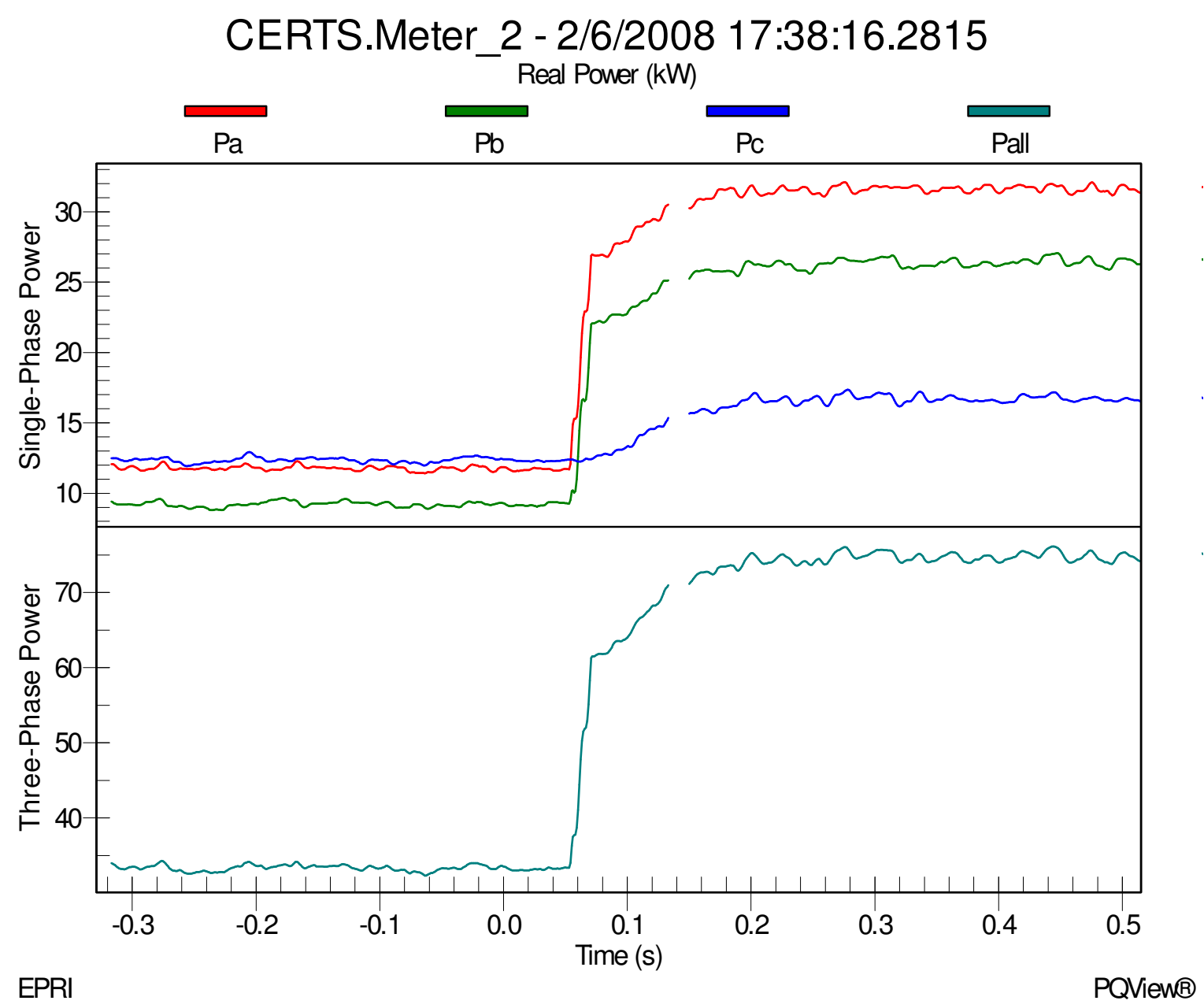

Figure 24b - Meter 2 power flow before/after the phase-to-phase fault was applied in Zone 2. 


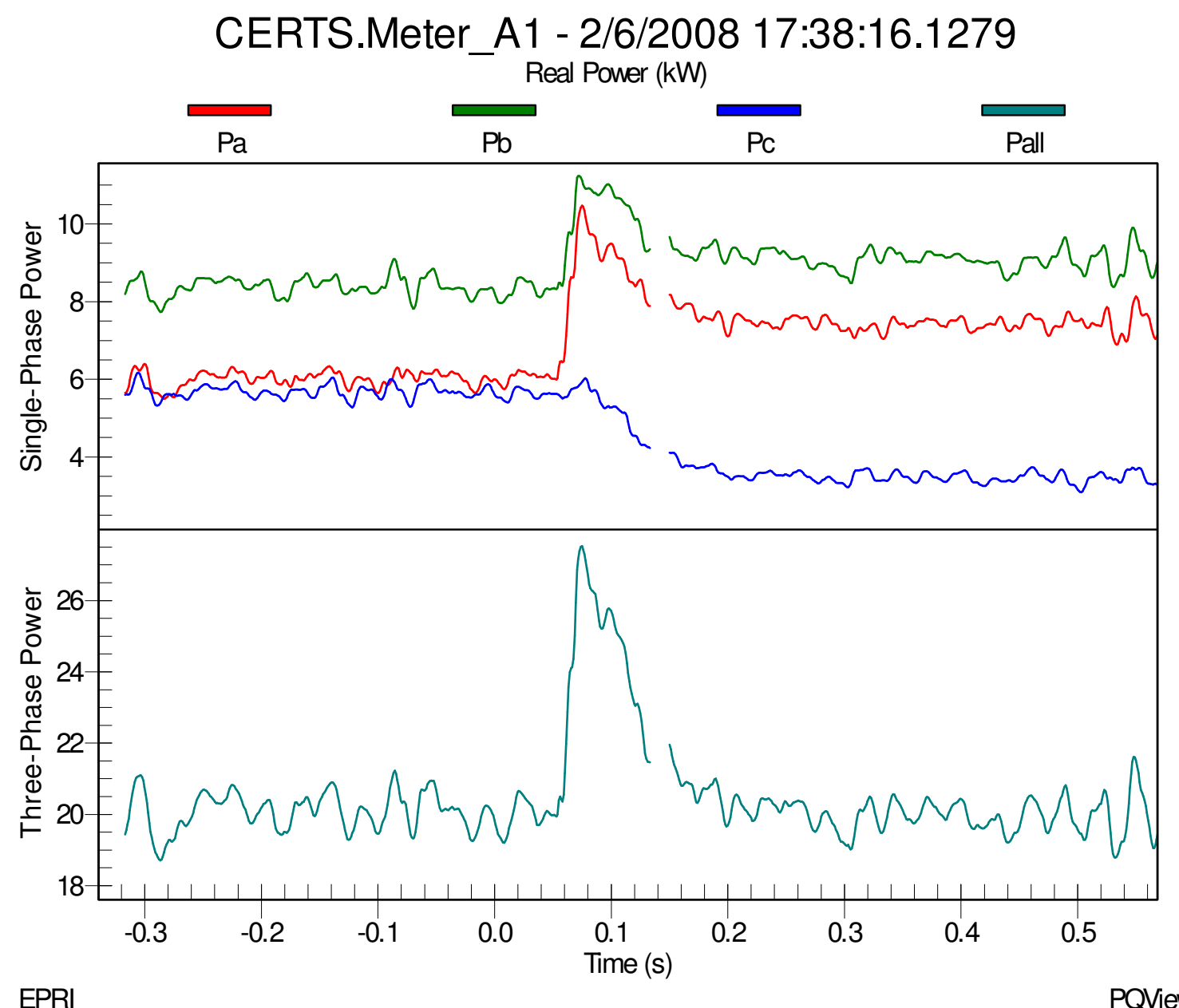

EPRI

Figure 24c - Power produced by Gen-set A1 before/after an A-phase to B-phase fault was applied in Zone 2. 


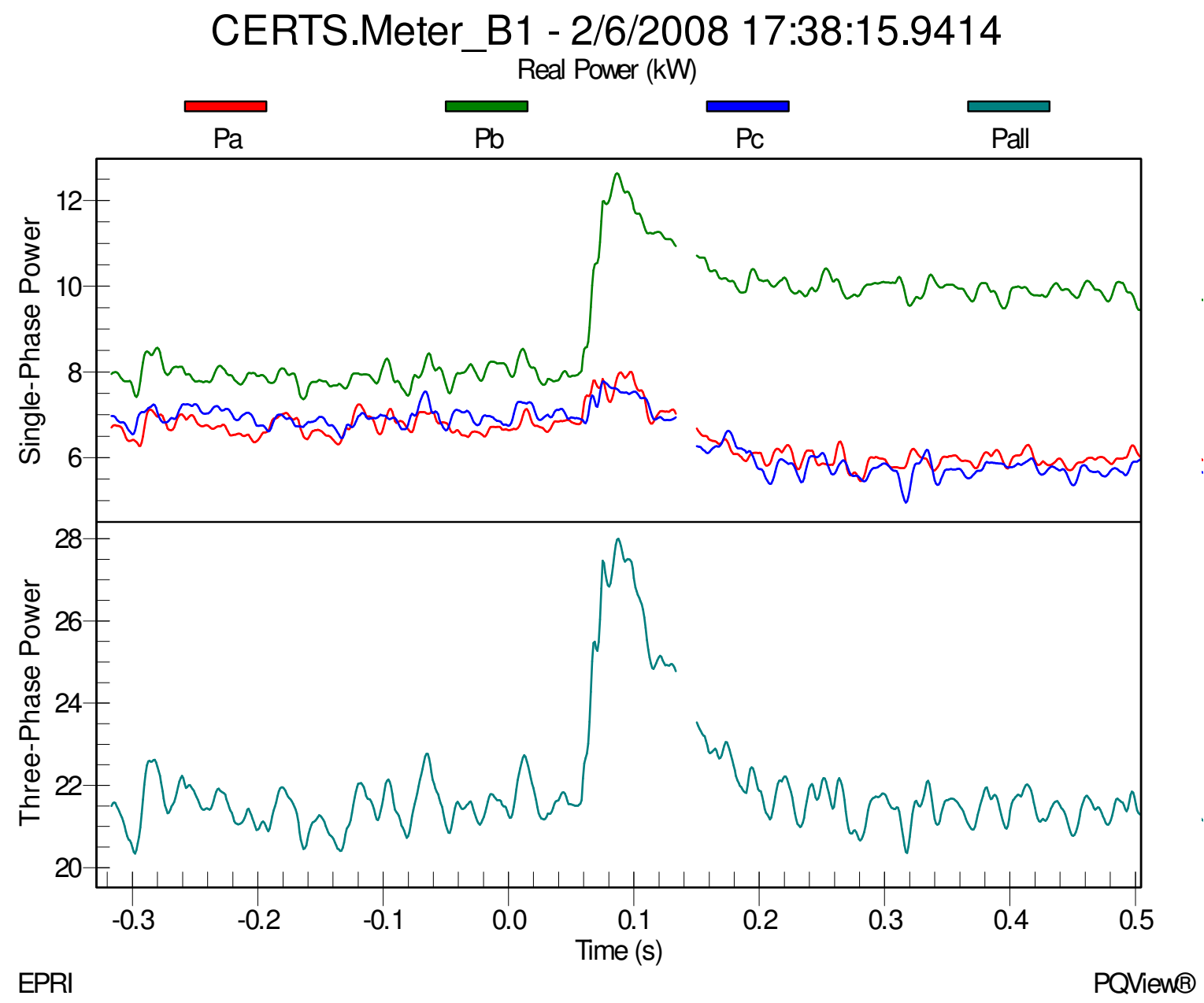

Figure 24d - Power produced by Gen-set B1 before/after an A-to-B phase-to-phase fault in Zone 2 is applied

Using the DAS Load Control program, the phase-to-phase fault on A- and B- phases was set for 10 seconds and then initiated. The static switch, CB31, CB41 and CB51 did not detect enough current from Zone 2 to trip. Power flow in Meter 1, Figure 24a, increased from approximately $70 \mathrm{~kW}$ (i.e., A-phase $=24 \mathrm{~kW}$, B-phase $=22 \mathrm{~kW}$ and C-phase $=24 \mathrm{~kW}$ ) to $110 \mathrm{~kW}$ (i.e., A-phase $=44 \mathrm{~kW}$, B-phase $=38 \mathrm{~kW}$ and C-phase $=28 \mathrm{~kW}$ ) when the fault was initiated in Zone 2. A-phase increased by approximately $20 \mathrm{~kW}$ and B-phase by $26 \mathrm{~kW}$, due to the overload bank connected in Zone 2. The microgrid treated this as an unbalanced load and supplied it power by the utility grid, since both Gen-sets were set for unit power control.

The Overload Load Bank was on-line for the allotted time of 10 seconds and then was removed. The electrical system was stable in both the microgrid and utility grid through the entire duration of the fault with no tripping of the static switch or circuit breakers. 
Gen-sets A1 and B1 were shut down manually, all loads and alarms were reset, and equipment was prepared for the next test.

\subsubsection{Zone 5 A-to-B Phase Fault, Gen-sets (A1+B1) and Utility Connected} Performance Goal:

Test a phase-to-phase overload fault condition in Zone 5 on A- and B-phases with Gensets $\mathrm{A} 1$ and $\mathrm{B} 1$ operating in-parallel with the utility grid to verify negative-sequence, $\mathrm{I}^{2} \mathrm{t}$ protection or residual over-current protection.

Initial Setup:

Gen-set A1 = Unit Power Control

Gen-set B1 = Unit Power Control

Gen-set A1 Output Power Command $=20 \mathrm{~kW}$

Gen-set B1 Output Power Command $=20 \mathrm{~kW}$

MG Power/Frequency Droop $=-0.0833 \mathrm{~Hz} / \mathrm{kW}$

MG Voltage Command $=277 \mathrm{~V}$

Relay Settings $=$ B (Inst)

Reverse Power Set-point $=0 \mathrm{~kW}$

Load Bank $3=40 \mathrm{~kW}$

Load Bank $5=40 \mathrm{~kW}$

Load Bank $6=40 \mathrm{~kW}$

To simulate a high impedance phase-to-phase fault in Zone 5 on A and B phases, the Overload Load Bank of $85 \mathrm{~kW}$ (i.e., approximately $42.5 \mathrm{~kW}$ phase-phase) was connected by cable/plug into the exterior receptacle of Cabinet 7a with C-phase disconnected. This, combined with Load Bank 5 load, added $21.2 \mathrm{~kW}$ on A and B phases of Zone 5 when initiated causing an overload.

After Gen-sets A1 and B1 were running for a few minutes and supplied power to Load Banks 3 and 5, the test started with a "Start" command from the EMS. As soon as the static switch closed and steady-state conditions established, the microgrid and Load 
Bank 6 were connected to the utility grid with the total power flow at Meter 1 being approximately $69 \mathrm{~kW}$, shown in Figure 25a, and approximately $34 \mathrm{~kW}$ at Meter 2, shown in Figure 25b. Before the overload fault was applied, Gen-sets A1 and B1 were producing about $20 \mathrm{~kW}$ and $22 \mathrm{~kW}$, respectively, with each shown in Figures $25 \mathrm{c}$ and $25 \mathrm{~d}$.

CERTS.Meter_1 - 2/6/2008 17:52:26.4620

Real Power (kW)

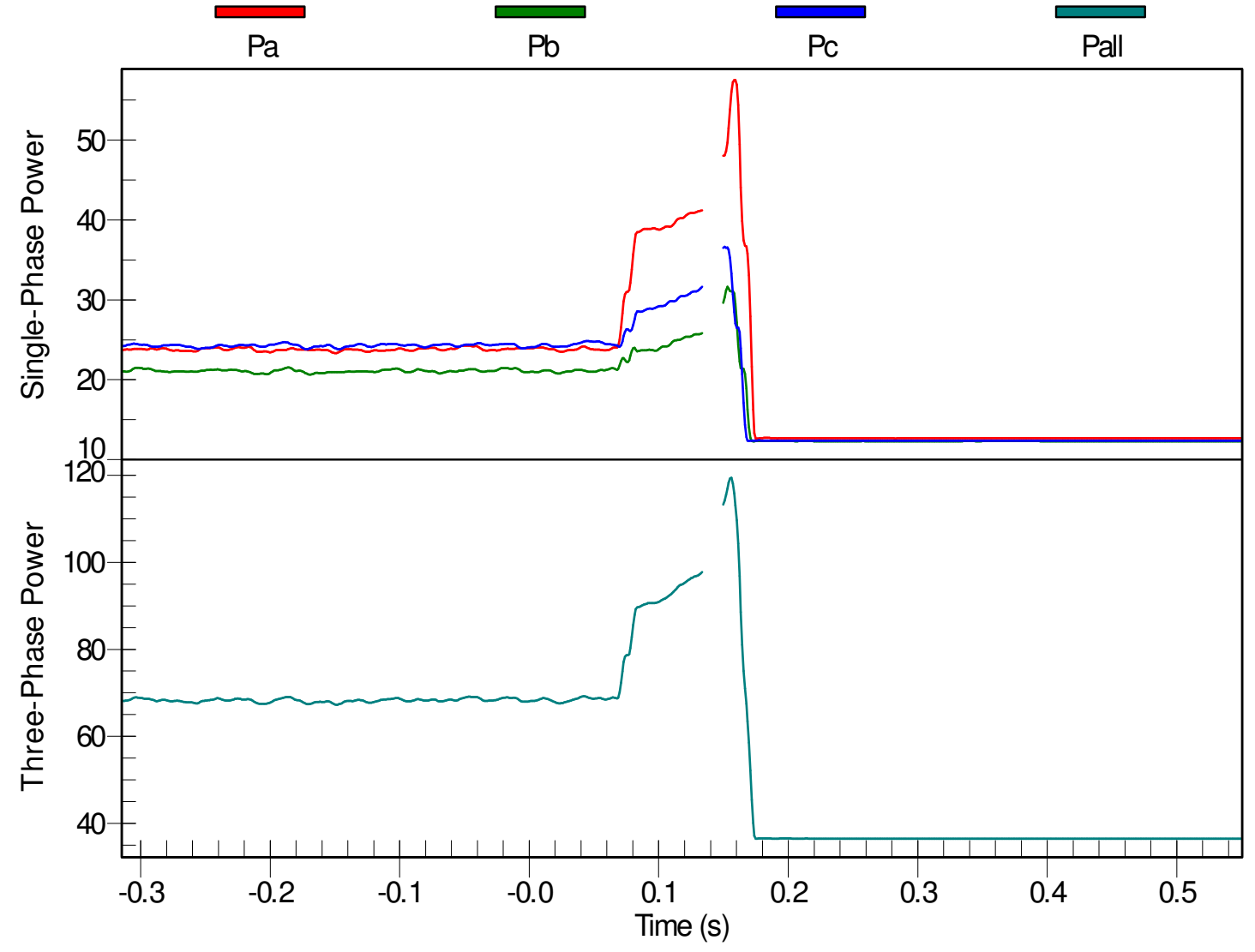

EPRI

PQView®

Figure 25a - Meter 1 power flow before/after the static switch opened, islanding the microgrid from the utility grid when a phase-to-phase fault was applied in Zone 5. 


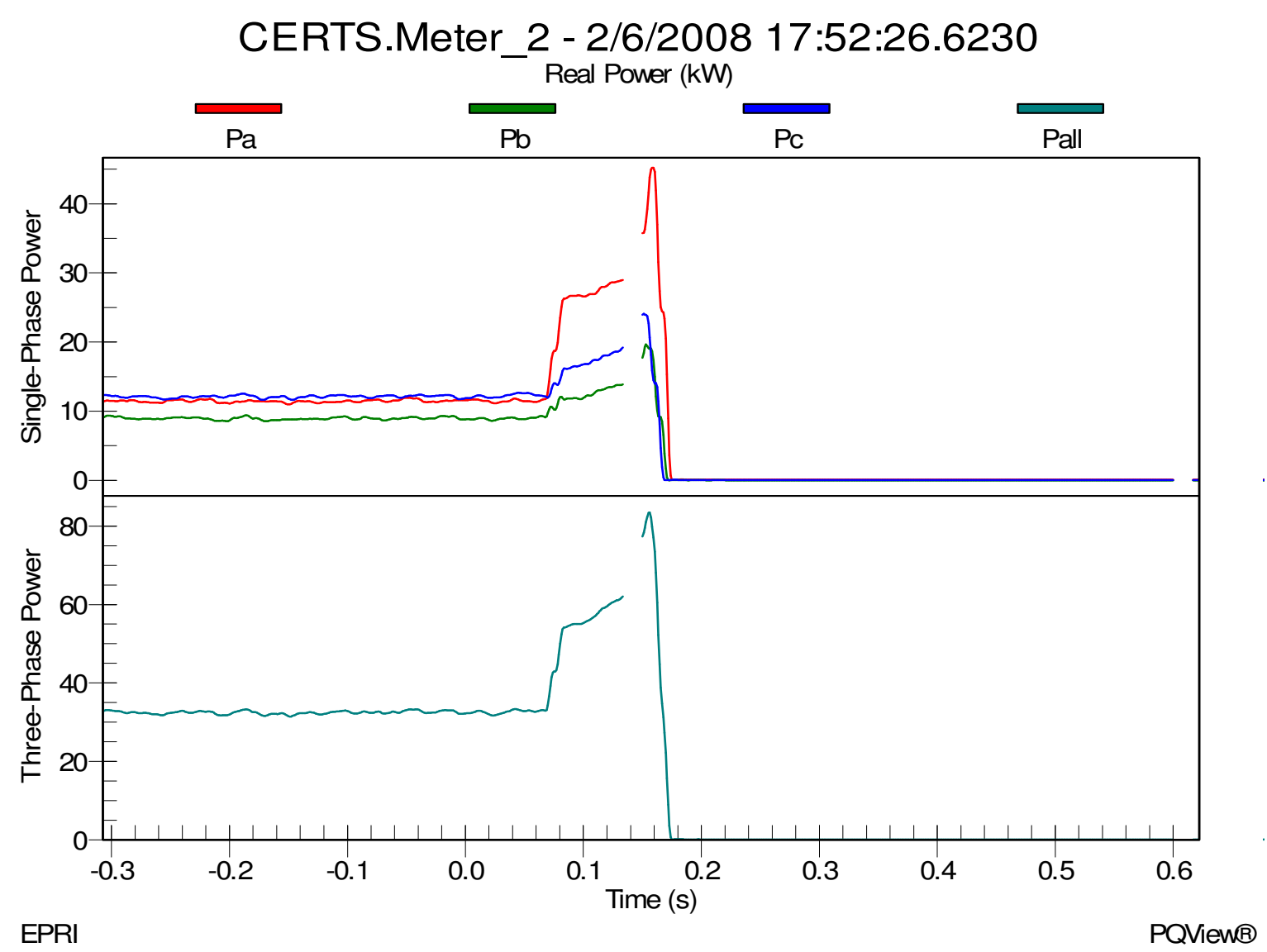

Figure 25b - Meter 2 power flow before/after the static switch opened, islanding the microgrid from the utility grid when a phase-to-phase fault was applied in Zone 5. 


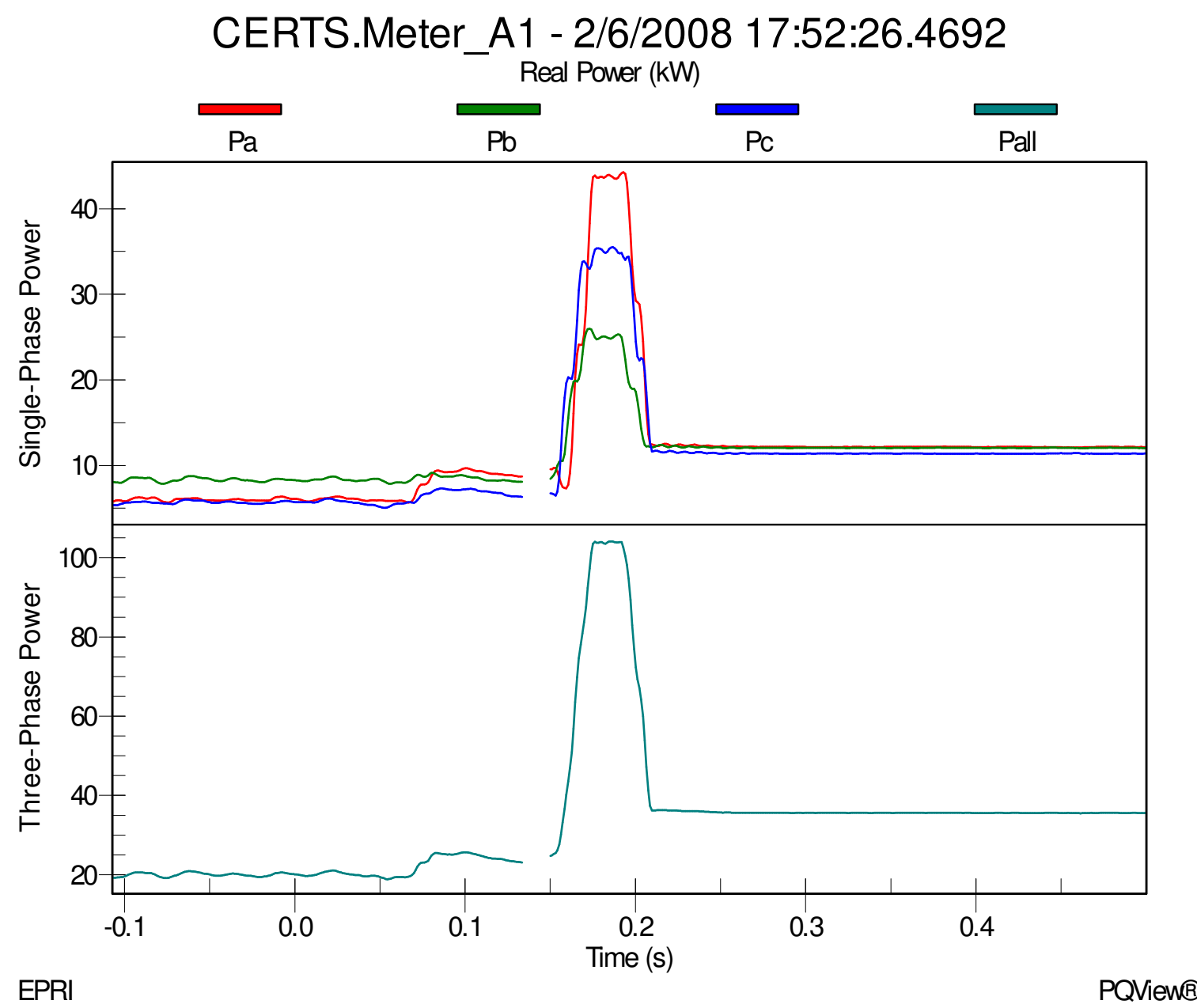

Figure 25c - Power produced by Gen-set A1 before/after the static switch opened from an A-phase to Bphase fault in Zone 5. 


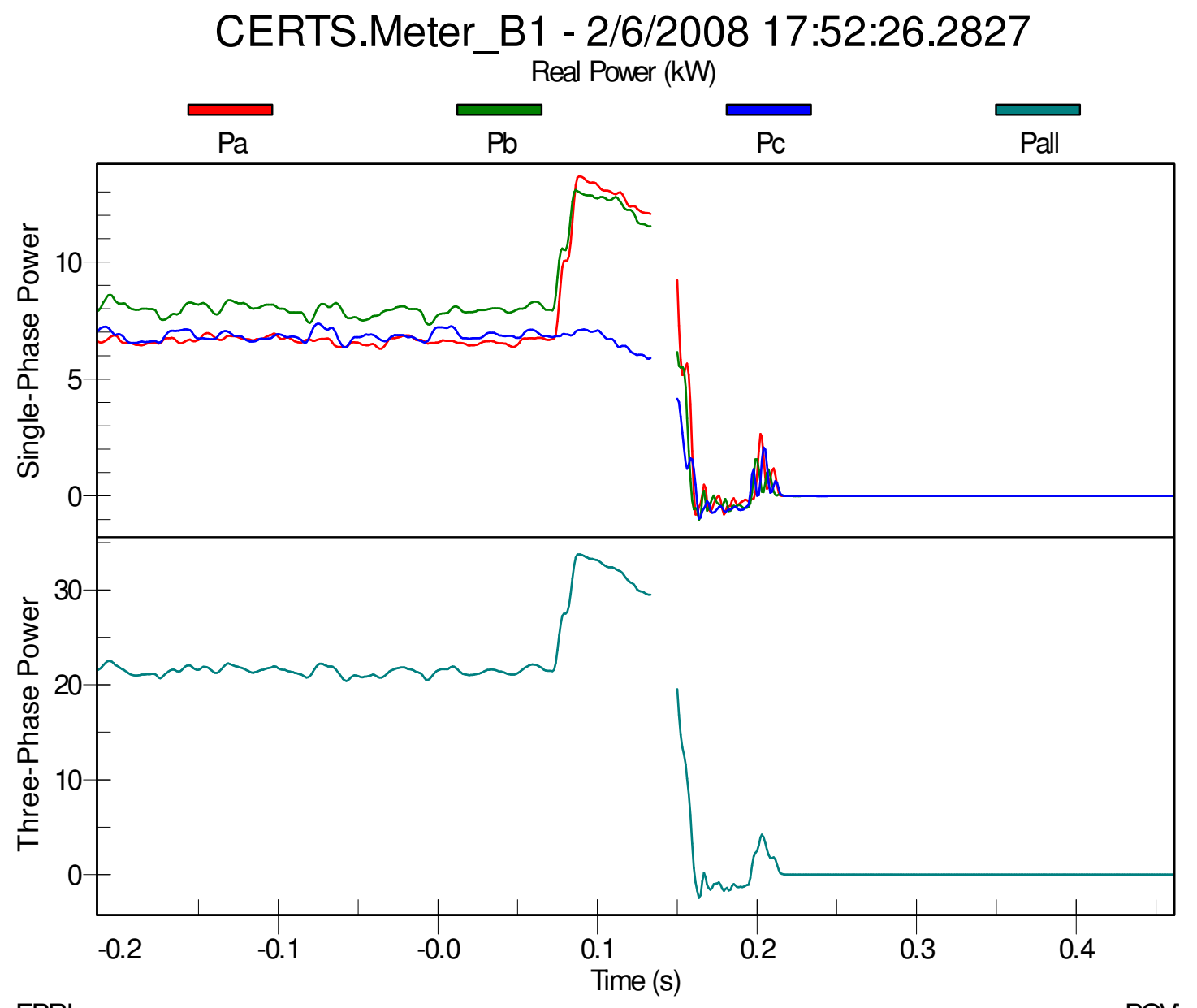

EPRI

PQViewß

Figure 25d - Power produced by Gen-set B1 before/after the static switch opened from an A-phase to Bphase fault in Zone 5.

Using the DAS Load Control program, the phase-to-phase fault on A- and B-phases was set for 10 seconds and then initiated. Relay 5 tripped in approximately 0.075 seconds ( 4.5 cycles) from a negative-sequence over-current (67N1T), shown in Figure 25f. Genset B1 stopped switching its output even though its transformer contactor and CB51 remained closed, shown in Figure 25g. This loss of generation increased the negative sequence current seen by the static switch enough to cause it to trip within approximately 0.016 seconds ( 1 cycle), shown in Figure 25e. Gen-set A1 increased its output briefly to support the load in Load Banks 3 and 5, as well as the overload fault. After approximately 0.043 seconds ( 2.5 cycles) CB51 cleared and Gen-set A1 remained on-line, supplying power to loads in zones 2,3 , and 4 .

CB31 and CB41 remained closed throughout the 10 second phase-to-phase fault. Genset A1 increased its output power from approximately $21 \mathrm{~kW}$ to $36 \mathrm{~kW}$, shown in Figure 
25c, supplying power to Load Bank 3. Load Bank 6 was supplied by the utility grid, shown in Figure 25a.

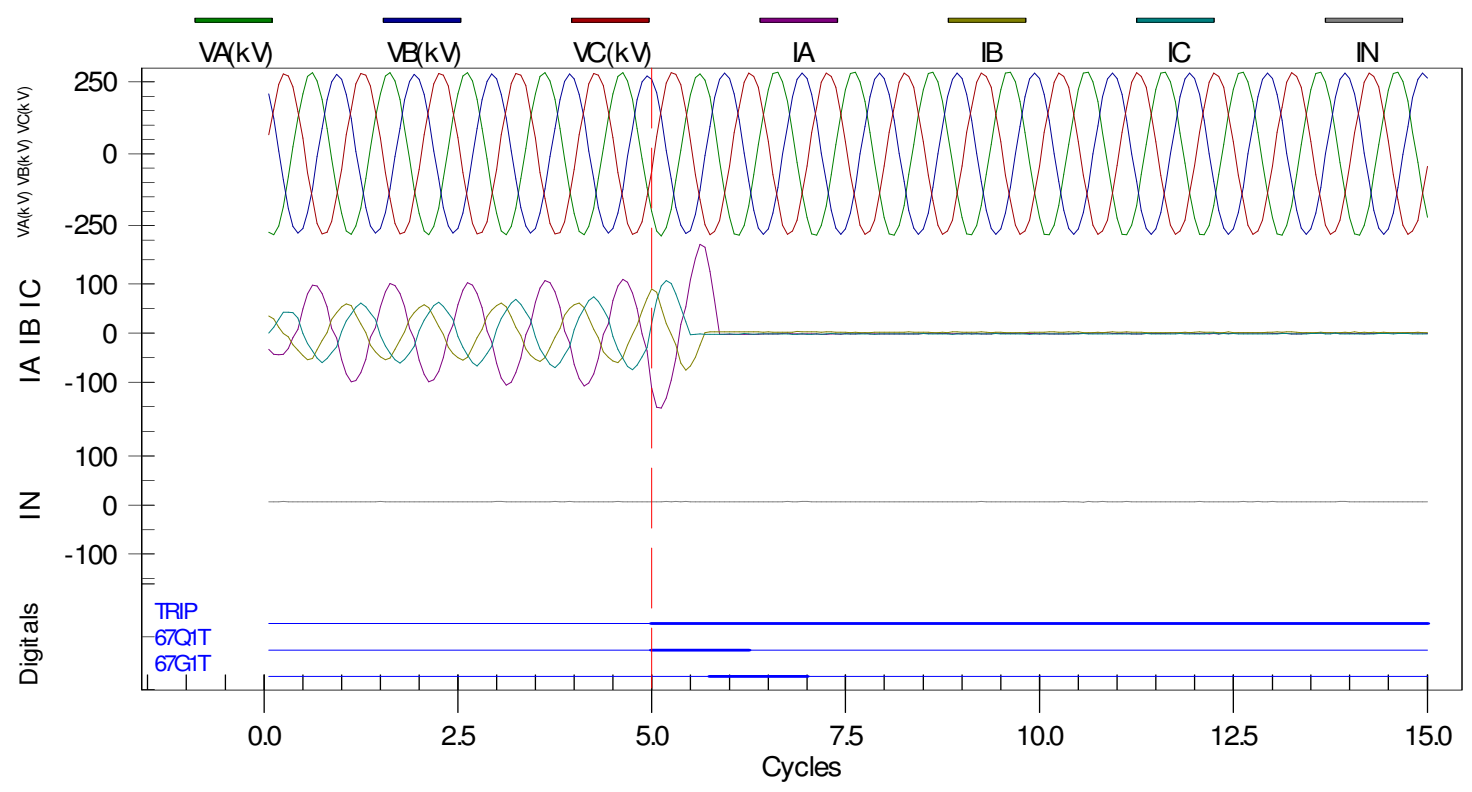

Figure 25e - Relay 2 (static switch) tripped due to a negative-sequence over-current (67N1T) when the phase-to-phase fault was initiated in Zone 5.

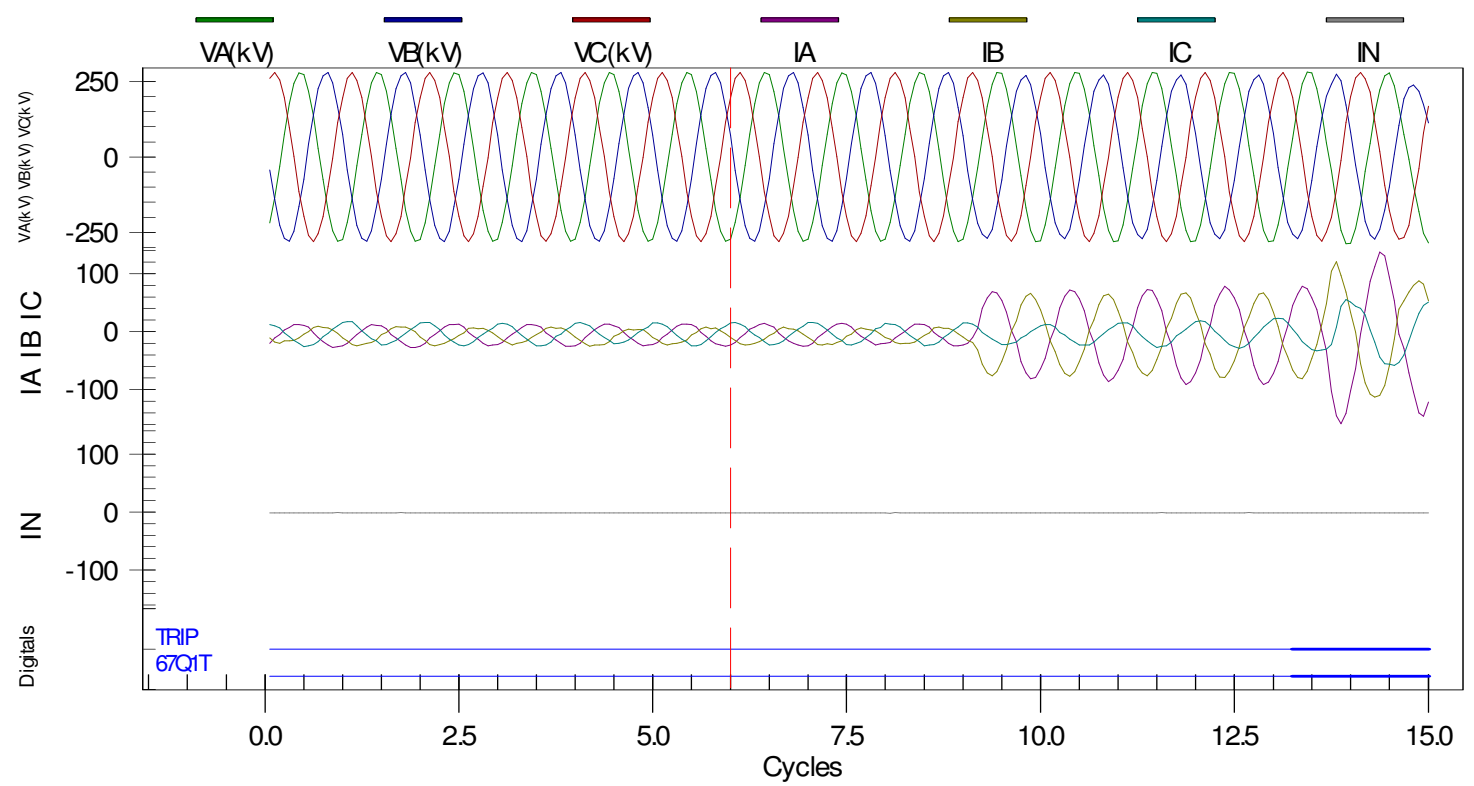

Figure 25f - Relay 5 (CB51) tripped due to a negative sequence over-current (67N1T) when the phase-tophase fault was initiated in Zone 5. 


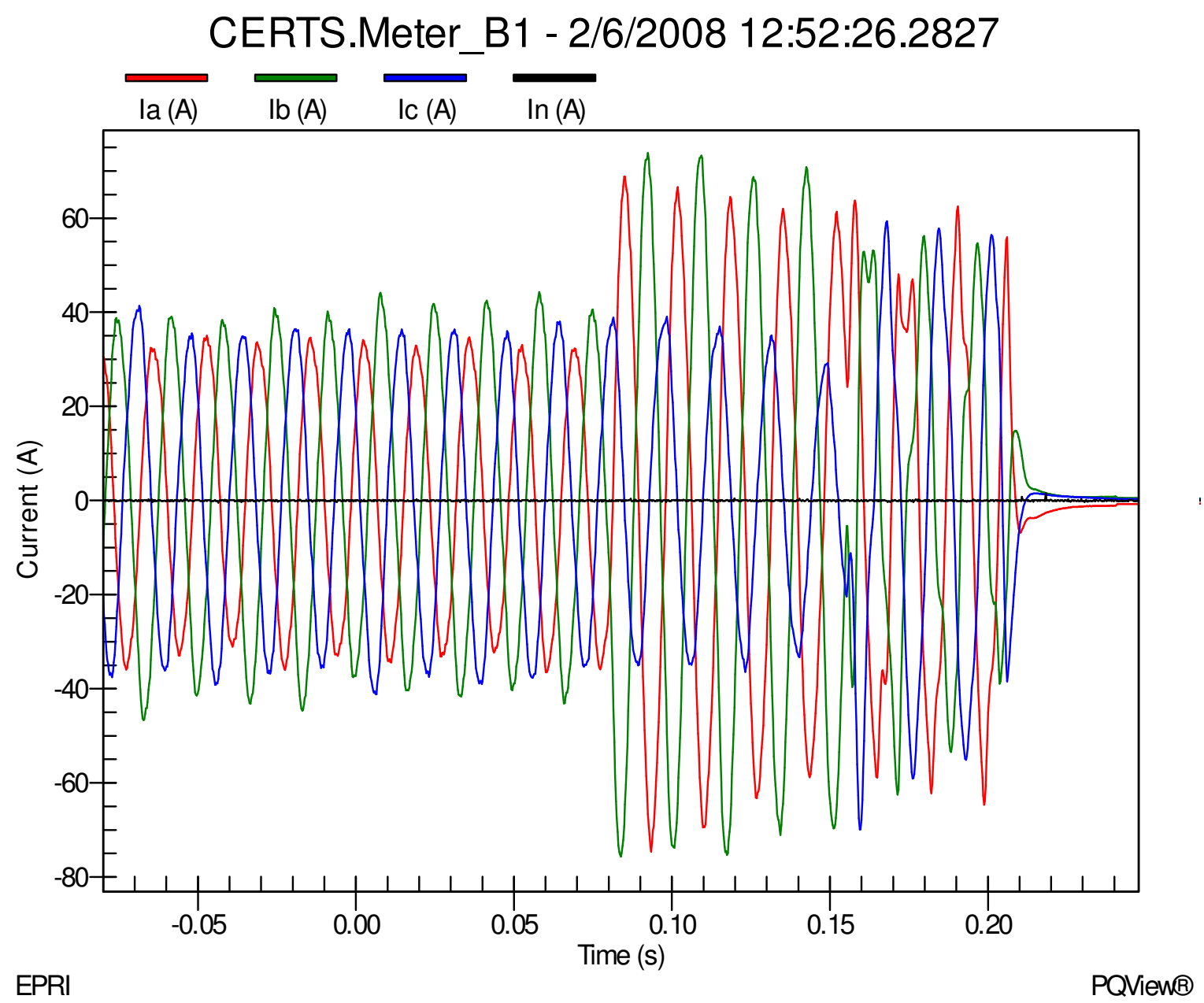

Figure 25g - Gen-set B1 current before/after a phase-to-phase fault was initiated in Zone 5. 


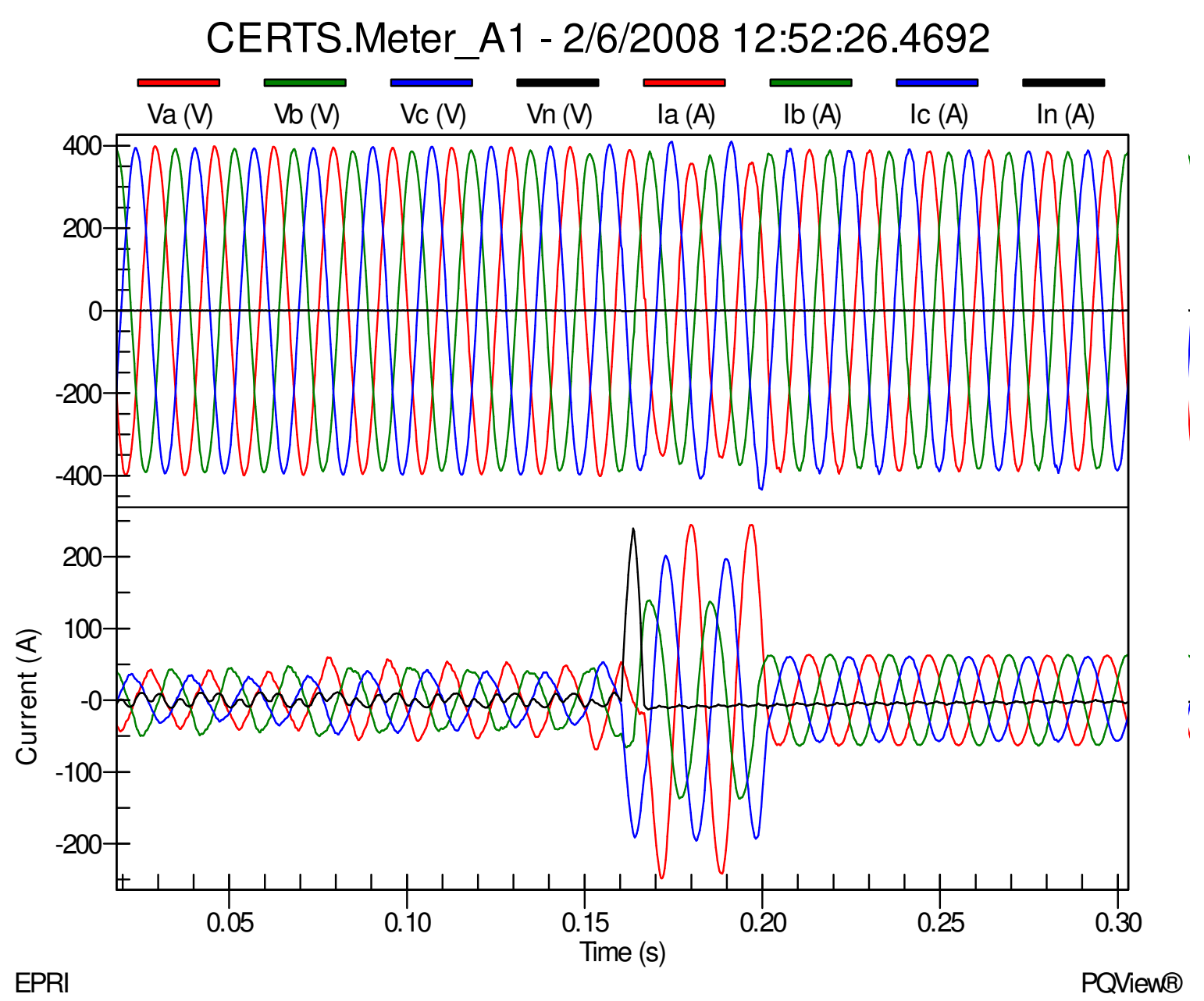

Figure 25h - Gen-set A1 current before/after a phase-to-phase fault was initiated in Zone 5.

The electrical system was stable in both the microgrid and utility grid, after the static switch opened, CB51 opened, and Gen-set B1 shut down. All loads and alarms were reset and equipment was prepared for the next test.

\subsection{CONCLUSION}

The tests performed in section 7.0 were performed to validate the protection settings for various combinations of type of faults, placement of faults, and amount of real power provided by the Gen-sets. In the first three sub-sections of section 7.0 (7.1, 7.2 and 7.3) the delays were increased from the initial values to avoid the transformer inrush current which was causing nuisance tripping of the relays. In the remaining tests of section 7.0, the Gen-sets were allowed to warm up before the faults were applied to the CERTS microgrid which allowed the delay settings in the relays to be reset to the initial values. The protection settings in the relays proved to be sufficient for protecting the microgrid 
during single line-to-ground, three phase-to-ground and line-to-line faults downstream of the PCC.

One test that was not performed during this section was a fault on the utility side of the PCC. This test was not performed due to the utility not allowing intentional faults to be applied to their system, but will be performed in future tests. 


\title{
CERTS MICROGRID LABORATORY TEST BED
}

\author{
Test Plan Section 8.0 \\ Reduced System Tests
}

Prepared For:

California Energy Commission

Public Interest Energy Research Program

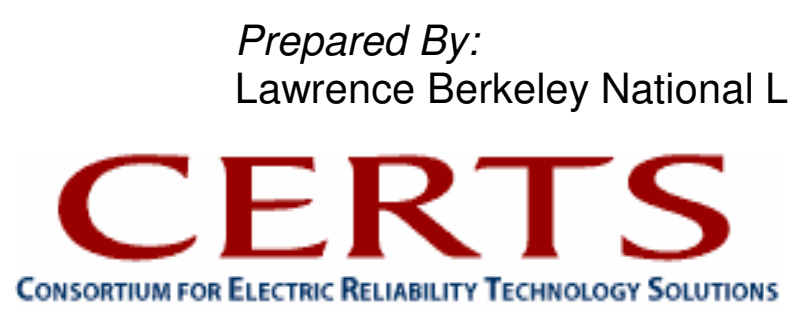

Arnold Schwarzenegger Governor 

Prepared By:

Lawrence Berkeley National Laboratory

Joseph H. Eto, Principal Investigator

Berkeley, CA 94720

Ben Schenkman, Sandia National Laboratory

Harry Volkommer and Dave Klapp, American Electric Power

Commission Contract No. 500-03-024

Prepared For:

Public Interest Energy Research (PIER)

California Energy Commission

Bernard Treanton

Contract Manager

Mike Gravely

Program Area Lead

ENERGY SYSTEMS INTEGRATION

Mike Gravely

Office Manager

ENERGY SYSTEMS RESEARCH

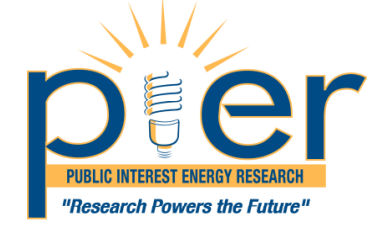

Martha Krebs, Ph.D.

PIER Director

Thom Kelly, Ph.D.

Deputy Director

ENERGY RESEARCH \& DEVELOPMENT DIVISION

Melissa Jones

Executive Director

\section{DISCLAIMER}

This report was prepared as the result of work sponsored by the California Energy Commission. It does not necessarily represent the views of the Energy Commission, its employees or the State of California. The Energy Commission, the State of California, its employees, contractors and subcontractors make no warrant, express or implied, and assume no legal liability for the information in this report; nor does any party represent that the uses of this information will not infringe upon privately owned rights. This report has not been approved or disapproved by the California Energy Commission nor has the California Energy Commission passed upon the accuracy or adequacy of the information in this report. 



\section{CERTS MICROGRID TEST REPORT}

\section{SECTION 8.0}

"Reduced System Tests" 


\section{Table of Contents}

1.0

2.0

3.0

4.0

5.0

6.0

6.1

\subsection{1}

6.1 .2

6.1 .3

6.1 .4

6.1 .5

\subsection{6}

6.1.7

6.1.8 When Islanded.

INTRODUCTION

BACKGROUND .

MICROGRID TESTBED SETUP.

PROPOSED TEST PLAN

TESTS PERFORMED IN SECTION 8.0

ANALYSES OF TEST RESULTS

SECTION 8 - REDUCED SYSTEM TESTS .

Initial Voltage Regulation Test - Single Zone, Islanded

Open Static Switch Test, Gen-set A1

Open Static Switch Test, Gen-set A2

Test Island Operation, Unbalanced Load

Mixed Mode Operation Test - Feeder A, Gen-set A1 Upper Limit, $60 \mathrm{~kW}$.

6.1.9 Mixed Mode Operation Test - Feeder A, When Islanded Automatic Reset of Zone Level Set-point

6.1.10 Two Sources in Zone Control - Separate Feeders, When Islanded Automatic Reset of Zone Set Points, Zones Sum Equal 0kW ..... 26

6.1.11 Two Sources in Zone Control - Separate Feeders, When Islanded Automatic Reset of Zone Set Point............................................... 28

6.1.12 Test Generator Black-Start Procedure ............................................. 31

6.1.13 Test/Establish Generator Black-Start Capacity ............................. 32 


\section{$\underline{\text { List Of Figures }}$}

Figure 1 - CERTS Microgrid Aerial Photo ..................................................................... 3

Figure 2 - One Line Diagram of CERTS Microgrid Test Bed...................................... 4

Figure 3 - Simplified diagram of Test Bed showing Meter and Relay locations .... 4

Figure 4 - Diagram of DAS \& EMS Data networks....................................................... 5 


\section{List Of Tables}

Table 3 - Voltage regulation test results of Gen-set A1, A2 and B1 in Zone 3, 4 and 5, respectively............................................................................. 12

Table 4 - Open Static Switch (SS) Test Results, Gen-set A1.................................... 14

Table 5 - Open Static Switch (SS) Test Results, Gen-set A2 ..................................... 16

Table 6 - Test Results, Island Operation, Unbalanced Load ..................................... 18

Table 7 - Mixed Mode Operation Test Results, Feeder A, Gen-set A1 Upper Limit,

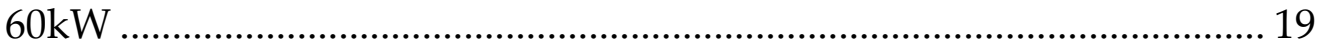

Table 8 - Mixed Mode Operation Test Results, Feeder A, Automatic Reset of Zone Level Set-point................................................................................. 21

Table 9 - Mixed Mode Operation Test Results, Feeder A Zone Power Change ... 22

Table 10 - Mixed Mode Operation Test Results, Feeder A, Zone Power Change When Islanded.......................................................................................... 24

Table 11 - Mixed Mode Operation Test Results, Feeder A When Islanded Automatic Reset of Zone Level Set-point ................................................. 26

Table 12 - Two Sources in Zone Control Test Results, Separate Feeders When Islanded, Automatic Reset of Zone Set-points, Zone Sums Equal Zero $\mathrm{kW}$

Table 13 - Two Sources in Zone Control Test Results, Separate Feeders When Islanded, Automatic Reset of Zone Set-point 30

Table 14 - Generator Black Start Procedure Test Results .......................................... 32

Table 15a - Black Start Test Results, 20kW + j20kVAr ............................................. 33

Table 15b - Black Start Test Results, 35kW + j20kVAr .............................................. 34

Table 15c - Black Start Test Results, 55kW + j20kVAr................................................ 34

Table 15d - Black Start Test Results, 60kW + j30kVAr .............................................. 35

Table 15e - Black Start Test Results, 70kW + j30kVAr ............................................... 36 


\subsection{INTRODUCTION}

A series of tests were performed on the CERTS Microgrid by American Electric Power at the Walnut test site in Groveport, Ohio with support from Lawrence Berkeley National laboratory, Sandia National Laboratory, TECOGEN, The Switch (originally Youtility), Distributed-Energy (originally Northern Power) and University of Wisconsin. These tests were designed to demonstrate the CERTS Microgrid concepts of control and protection while connected to the utility electrical system and isolated (i.e., referred to as "islanded" from it. This paper describes the tests that were performed in Section 8.0 "Reduced System Tests" of the CERTS Micro-grid Test Plan.

\subsection{BACKGROUND}

The CERTS Microgrid Concept is an advanced approach for enabling integration of, in principle, an unlimited quantity of DER (e.g., distributed generation (DG), energy storage, etc.) into the electric utility grid. A key feature of a microgrid is its ability to separate and island itself from the utility system, during a utility grid disturbance. This is accomplished via intelligent power electronic interfaces and a single, high-speed, switch which is used for disconnection from the grid and synchronization to the grid. During a disturbance, the DER and corresponding loads can autonomously be separated from the utility's distribution system, isolating the microgrid's load from the disturbance (and thereby maintaining high level of service) without harming the integrity of the utility's electrical system. Thus, when the utility grid returns to normal, the microgrid automatically synchronizes and reconnects itself to the grid, in an equally seamless fashion. Intentional islanding of DER and loads has the potential to provide a higher level of reliability than that provided by the distribution system as a whole.

What is unique about the CERTS Microgrid is that it can provide this technically challenging functionality without extensive (i.e., expensive) custom engineering. In addition, the design of the CERTS Microgrid provides a high level of system reliability and great flexibility in the placement of DER within the microgrid. The CERTS Microgrid offers these functionalities at much lower costs than traditional approaches by incorporating peer-to-peer and plug-and-play concepts for each component within the microgrid.

The original concept was driven by two fundamental principles: 1.) A systems perspective was necessary for customers, utilities, and society to capture the full benefits of integrating DER into an energy system; and 2.) The business case for accelerating adoption of these advanced concepts will be driven, primarily, by lowering the up-front cost and enhancing the value offered by microgrids. 
Each innovation was created specifically to lower the cost and improve the reliability of small-scale DG systems (i.e., installed systems with capacities ranging from less than $100 \mathrm{~kW}$ to $1000 \mathrm{~kW}$ ). The goal was to increase and accelerate realization of the many benefits offered by small-scale DG, such as their ability to supply waste heat at the point of need or to provide a higher level of reliability to some but not all loads within a facility. From an electric utility perspective, the CERTS Microgrid Concept is attractive because it recognizes that the nation's distribution system is extensive, aging, and will change over time which impacts power quality. The CERTS Microgrid Concept enables high penetration of DG systems without requiring re-design or re-engineering of the utility's distribution system.

Prospective applications of the CERTS Microgrid include industrial parks, commercial and institutional campuses, situations that require uninterrupted power supplies and high power quality, CHP systems, Greenfield communities, and remote applications. In short, wherever economic and DG location considerations indicate the need for multiple DG units within a (or among) site, the CERTS Microgrid offers the potential for a much more reliable, flexible, and lower cost solution compared to traditional engineering approaches for integrating DG.

\subsection{MICROGRID TESTBED SETUP}

The CERTS Microgrid Test Bed is operated at 480/277 volts (i.e., three-phase, four-wire) and consists of three TECOGEN Generators at 480 volts capable of producing $60 \mathrm{~kW}$ plus 60kVAr (Gen-set A1, Gen-set A2 and Gen-set B1) and four load banks (Load Bank 3, Load Bank 4, Load Bank 5 and Load Bank 6) capable of consuming 100kW plus 20kVAr each, as shown in Figure 2. Each of the generators are connected to a 112kVA isolation transformer and interfaced to the CERTS Microgrid through an inverter, developed by The Switch, where the algorithms for the CERTS Microgrid controls are embedded. . A semiconductor switch made by S\&C Electric Company, known as the static switch, connects the CERTS Microgrid to the utility grid. Load Banks $3-5$ are the local loads in zones located beyond the static switch; and Load Bank 6 is a customer load in Zone 6 located on the utility side of the static switch. 


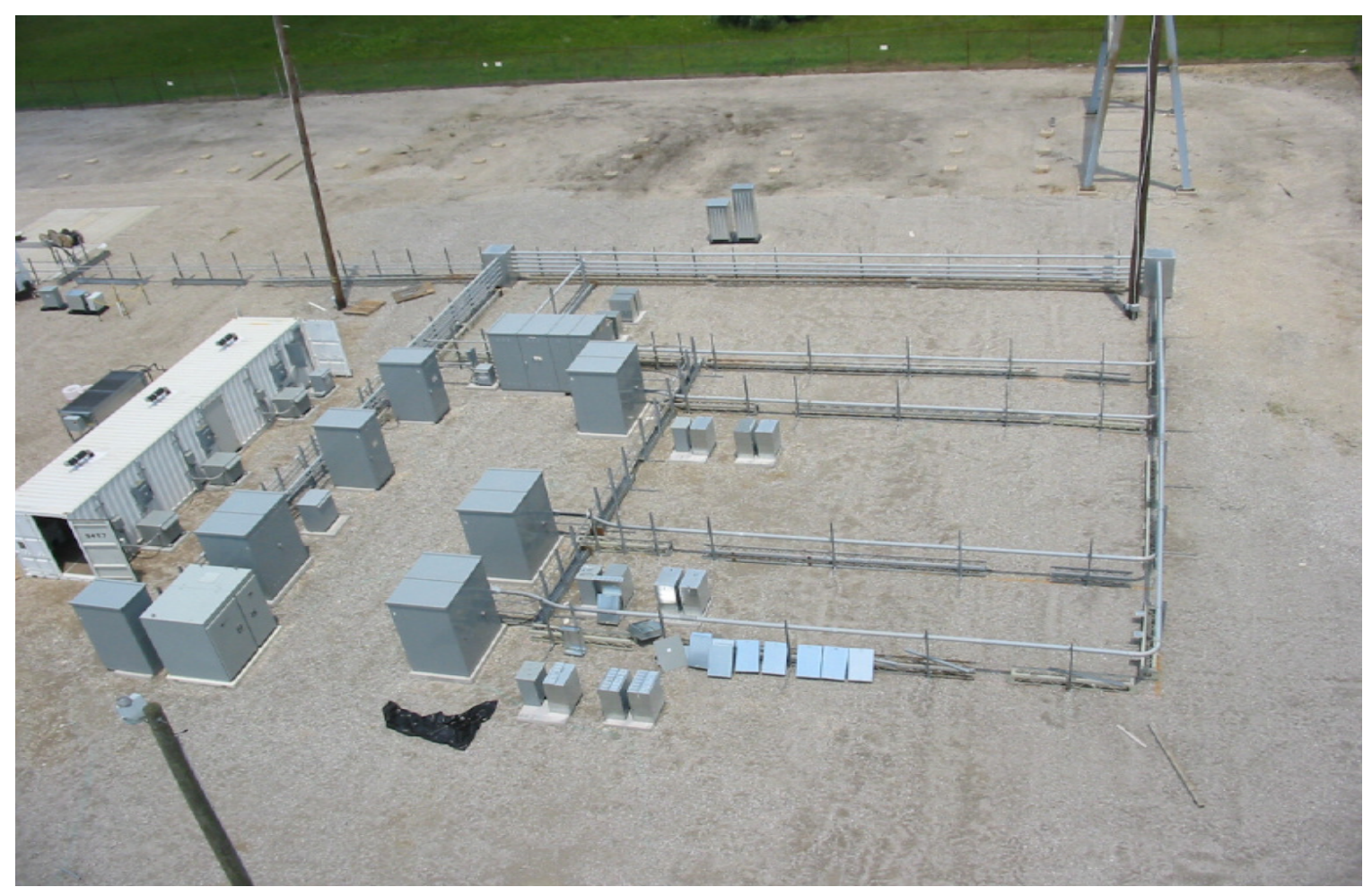

Figure 1 - CERTS Microgrid Aerial Photo

There are 6 zones in the Test Bed with Zones 2 - 6 contained within the CERTS Microgrid design and Zone 1 being the utility interface and referred to as the point-of-common coupling (PCC) to the grid. Each zone is protected by a Schweitzer SEL-351 relay. Faults of varying magnitude can be applied to each zone through an additional breaker which allows fault application and removal.

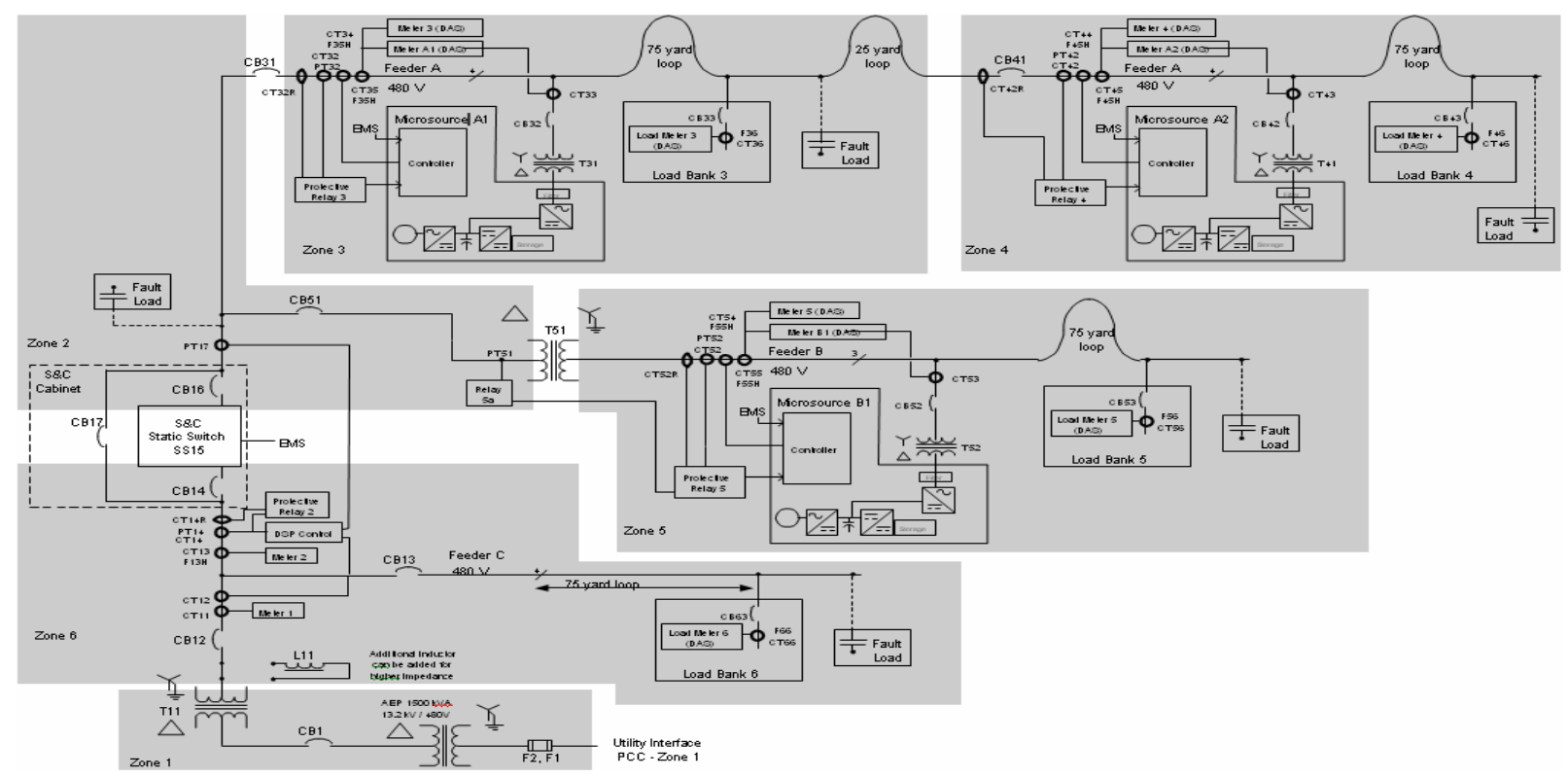




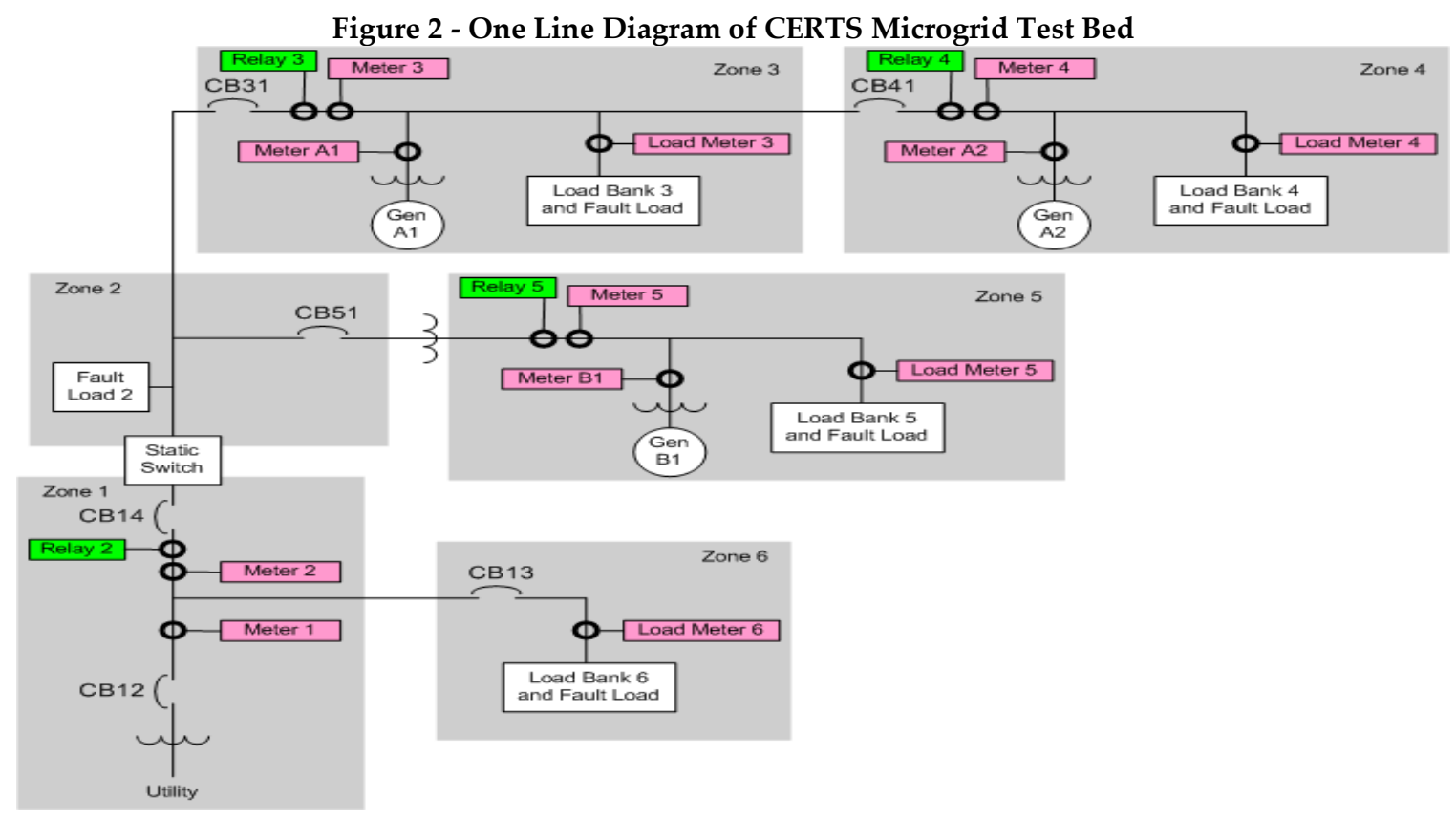

Figure 3 - Simplified diagram of Test Bed showing Meter and Relay locations

There are twelve PML ION 7650 meters placed through out the microgrid and shown in Figure 3, which monitor electrical system conditions, plus acquire phase current and voltage waveforms; and calculate RMS values of voltage, current, active power, reactive power, and frequency. 


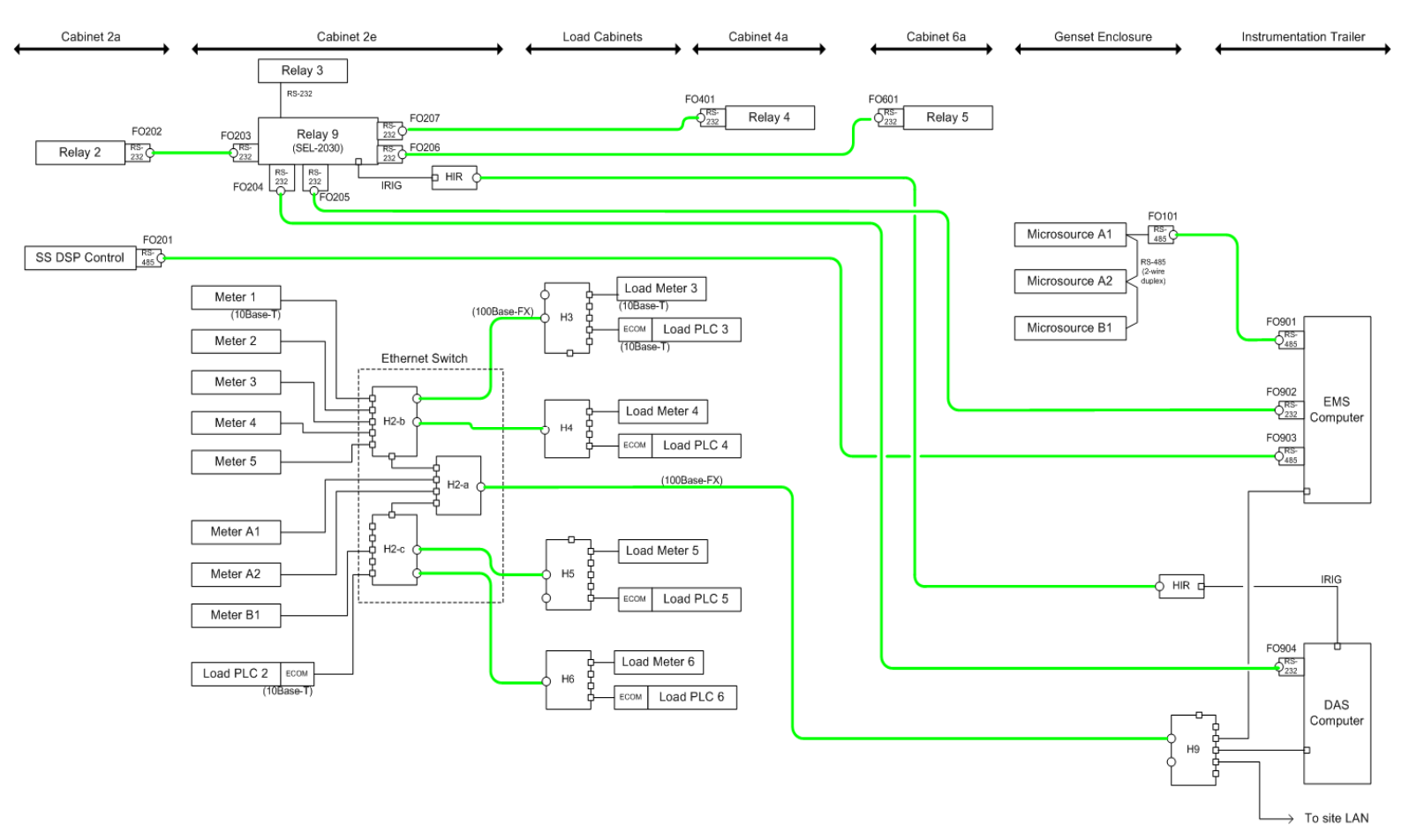

Figure 4 - Diagram of DAS \& EMS Data networks

An Ethernet network was provided as shown in Figure 4, for communications between all meters, load control PLCs, and the Data Acquisition System (DAS) computer, using fiber-optic links and switches. The DAS and Energy Management System (EMS) computers were also networked into the local Dolan Local Area Network (LAN) and to a secure Website with user ID and password protection. Additional serial links, using fiber optic converters, connect all relays, static switch Digital Signal Processor (DSP) controller, and TECOGEN Gen-set controls to the EMS computer.

\subsection{PROPOSED TEST PLAN}

The CERTS Microgrid Test Plan was developed by the CERTS Microgrid Team to demonstrate the unique concepts of control and protection of the CERTS Microgrid. This test plan was reviewed by a Technical Advisory Committee outside the CERTS Microgrid Team and then implemented by American Electric Power. CERTS Microgrid Test Plan consists of 12 sections with 5 of them detailing desired tests, starting at section 6.0, to demonstrate the controls and concepts of the CERTS Microgrid. The other 7 sections pertain to safety procedures, equipment calibration, and documentation. Each section of the test plan is described below.

- Section 1 - "Purpose, References, and Definitions" describes the purpose of the test plan, helpful references for further explanation of how the test bed was created, and definitions used through out the test plan. 
- Section 2 - "Responsibilities" informs personnel of their responsibilities while working on or near the CERTS Microgrid test site.

- Section 3 - “Training - Team Members" lists the mandatory training needed by personnel before they can work on or near the CERTS Microgrid test site.

- Section 4 - "Procedure - CERTS Microgrid Test Bed Lockout/Tagout" entails how to safely shut down the equipment and lockout/tagout the closest upstream disconnect to work on or near equipment.

- Section 5 - "Procedure - General" is the daily procedures performed at the CERTS Microgrid Test Site, prior to performing a test from Section 6 through Section 10.

- Section 6 - "Procedure - Microgrid Test Bed System Checkout" was designed to check control and operation of the static switch, basic power and voltage control of the Gen-sets, and a preliminary check of the protection scheme. The goal is to assure that the test bed is operating and ready to perform the tests described in the remaining sections of the test plan document.

- Section 7 - "Validate Protection Settings \& Initial Fault Testing" is designed to examine a preliminary set of fault (i.e. overload simulating a fault) condition tests to ensure protection and safety of the Micro-grid test Bed, while performing other planned tests. The goal is to test and adjust protection settings to achieve the most ideal conditions and protection design.

- Section 8 - "Procedure - Reduced System Tests" is a limited set of tests to build confidence that the Gen-set inverter controls are working correctly. This includes unit control, zone control, and mixed power controls, in conjunction with limit controls and synchronized closing of the static switch. These tests are based on the TECOGEN/THE SWITCH factory acceptance testing.

- Section 9 - "Procedure - Demonstration Tests of Control Power Flow" demonstrates the flexibility of the Micro-grid both grid connected and islanded for different loads, power flows and impact on the utility. 
- Section 10 - "Procedure - Test Difficult Loads" determines operation limits of the Micro-grid (i.e. power quality, protection and inverter limits) with low pf loads, motor loads, harmonic loads and unbalance loads.

- Section 11 - "Hazards \& Mitigation" informs the personnel of hazards that may exist while working on or near the CERTS Micro-grid test site and how to mitigate them.

- Section 12 - “Quality Assurance” ensures quality for the acquiring data results by providing a checklist reminder for personnel.

\subsection{TESTS PERFORMED IN SECTION 8.0}

Prior to each test day, the person in charge performed a job safety briefing (JSB) with barricades and test setup inspected for safety and compliance. A minimum of two people were on-site during each planned test.

Visual and audible alarms were used to warn persons that energized testing was being performed in the Microgrid Test Bed area. The visual alarm consisted of a portable red flashing light, located between the Control Trailer and Gen-set Enclosure. An audible alarm, consisting of a portable wireless motion detector, was located at the front gate of the Walnut Test Site with the fence gate "Closed", not locked, and audible alarm in the trailer operational during test(s).

Barricades were set up around the Micro-grid Test Bed area (i.e., saw-horse style barricades with a "Red" plastic chain surrounded the test area containing the Gen-set Enclosure, Micro-grid switching cabinets, plus load and fault bank cabinets).

Prior to performing tests, the Test Engineer or Technical Consultant verified that all personnel and visitors were properly protected and in assigned locations. Personnel were in or adjacent to the Control Trailer while tests were being performed. All nonessential personnel either left the main site or were sheltered in the Control Trailer.

For all tests the following waveforms were captured and recorded in the DAS for voltage $(\mathrm{V})$ and current $(\mathrm{I})$. From these waveforms real power $(\mathrm{kW})$, reactive power $(\mathrm{kVAr})$, and frequency (freq) were post calculated by the PQView software. Frequency measurements in this report should be used for steady state information and not used for transient analysis, due to the calculation and filtering methods employed. Below is a list of the meters capturing this data. 
- Meters 1, 2, 3, 4 \& 5

- Load Meters 3, 4, 5, \& 6

- Meters A1, A2 \& B1

- Meter 2 also measures the voltage across the static switch on phase A

Schweitzer event reports were also captured for each event, along with breaker and static switch status, such as Open or Close.

To maintain a common understanding of Gen-set control modes during tests described in the following paragraphs, it is important to know the difference between unit power control mode and zone control mode. Unit power control mode involves the amount of power (i.e., $\mathrm{kW}$ ) injected into the Zone from the Gen-set being controlled. Zone power control mode involves the amount of power (i.e., $\mathrm{kW}$ ) entering/exiting the Zone which controls the output of the Gen-set in that zone.

Section 8 consists of 13 tests designed to validate the inverter controls tested by TECONGEN/The Switch in a laboratory setting with field demonstration. In each test waveforms were captured and recorded in the DAS, as described below.

Test 8.1 was designed to verify smooth transitions in Gen-set A1 when different step conditions of load are applied in Load Bank 3 with voltage set points ranging from $+5 \%$ to $-5 \%$. The same test was repeated for Gen-set A2 and Load Bank 4 and Gen-set B1 and Load Bank 5.

Test 8.2 was designed to verify smooth transitions of Gen-sets A1 and A2 response to different step conditions which drive Gen-set A1 to a lower limit of zero kW.

Test 8.3 verified smooth transitions of Gen-sets A1 and A2 response to different step conditions which drive Gen-set A2 to an upper limit of $60 \mathrm{~kW}$.

Test 8.4 verified smooth transitions of Gen-sets A1 and A2 response to different step conditions with an un-balanced load in Zone 3.

Test 8.5 verified smooth transitions of Gen-sets, A1 in Zone operation mode and A2 in Unit operation mode. During a load step change in Load Bank 3, Gen-set A1 was driven to an upper limit of $60 \mathrm{~kW}$.

Test 8.6 verified smooth transitions of Gen-sets, A1 in zone operation mode and Gen-set A2 in Unit operation mode. During a load step change in Load Bank 4, Gen-set A1 is driven to its maximum which causes an automatic reset of the zone set points.

Test 8.7 verified smooth transitions of Gen-sets, A1 in Zone operation mode and A2 in Unit operation mode, with a change of zone power in Feeder A. 
Test 8.8 verified smooth transition of Gen-sets, A1 in zone operation mode and A2 in unit operation mode, with a static switch operation and a change of zone power in Feeder A when islanded.

Test 8.9 verified smooth transition of Gen-sets, A1 in Zone operation mode and A2 in Unit operation mode, with a static switch operation and a change of zone power in Feeder A when islanded. Gen-sets A1 and A2 were driven to their maximum which caused an automatic reset of their set-points.

Test 8.10 verified smooth transitions of Gen-sets, A1 and B1 in Zone operation mode, with a static switch operation and a change of zone power in Feeder A and Feeder B when islanded.

Test 8.11 verified smooth transitions of Gen-sets, A1 and B1 in Zone operation mode, with a static switch operation and a change of zone power in Feeder A and Feeder B when islanded. When islanded, Gen-set A1 and B1 set-points were reset based on the remaining load in the islanded system.

Test 8.12 tested the manual procedure used to black-start the CERTS Micro-grid Test Bed in the event of a lengthy utility outage occurs with the Gen-sets off-line.

Test 8.13 was designed to determine the black-start capacity by increasing the amount of load on the CERTS Microgrid Test Bed from a black-out condition without generation or protection trips.

\subsection{ANALYSES OF TEST RESULTS}

\subsection{SECTION 8 - REDUCED SYSTEM TESTS}

\subsubsection{Initial Voltage Regulation Test - Single Zone, Islanded} Performance Goal:

Verify smooth transitions of Gen-sets A1 response to different step conditions of load in Load Bank 3 with voltage set point changes ranging from $+5 \%$ to $-5 \%$. Repeat tests with Gen-set A2 and Load Bank 4, then with Gen-set B1 and Load Bank 5.

During all tests in this voltage regulation test section, the static switch was open isolating the microgrid from the utility grid. All data graphs from each voltage regulation test are available in Appendix A. Table 3 provides a brief summary of test 
results obtained during these twenty-seven voltage step changes in Zone 3, Zone 4 and Zone 5 of the microgrid.

Initially, Gen-set A1 in Zone 3 was running for a few minutes to warm-up with the voltage set-point at $277 \mathrm{~V}$, with zero load at Load Bank 3. Load Bank 3 was set at $20 \mathrm{~kW}$ which was measured as approximately $19 \mathrm{~kW}$. Real and reactive power output response to this load increase was approximately $21 \mathrm{~kW}$ and $4 \mathrm{kVAr}$, respectively. The electrical impedance in the 75 yards of distribution conductors between Gen-set A1 and Load Bank 3 was the primary cause of the power difference. The voltage at Gen-set A1 was steady at approximately $273 \mathrm{~V}$ and the frequency was approximately $59.99 \mathrm{~Hz}$. Once all the data was recorded and validated, Load Bank 3 was increased from $20 \mathrm{~kW}$ to $40 \mathrm{~kW}$. A smooth transition occurred when the load increased by $\sim 20 \mathrm{~kW}$ with the resultant load being $36 \mathrm{~kW}$, as measured by Load Meter 3. At the time of this load change, Gen-set A1 increased its output power from approximately $21 \mathrm{~kW}$ and $4 \mathrm{kVAr}$ to $38 \mathrm{~kW}$ and $4.4 \mathrm{kVAr}$. The voltage remained the same at the Gen-set (i.e., 273V) from the previous reading and the frequency dropped to $59.84 \mathrm{~Hz}$. Once all the data was recorded and validated, Load Bank 3 was increased by an additional $20 \mathrm{~kW}$ to a total of $60 \mathrm{~kW}$. Power flow at Load Bank 3 was approximately $52.5 \mathrm{~kW}$ and the Gen-set responded to this load step with a smooth transition producing an output power of $55 \mathrm{~kW}$ and $4.8 \mathrm{kVAr}$. The voltage at Gen-set A1 measured the same as the previous load change, at 273V. During this load change, frequency was reduced from approximately $59.84 \mathrm{~Hz}$ to $59.70 \mathrm{~Hz}$. The drop in frequency was caused by the CERTS algorithm for Real Power versus Frequency droop control. Gen-set A1 and the microgrid remained stable through all the load step changes of $20 \mathrm{~kW}$ at $277 \mathrm{~V}$. All data was recorded in DAS and the next test was implemented.

The voltage set-point (i.e., MG Voltage) of Gen-set A1 was increased by $5 \%$ to $291 \mathrm{~V}$ and Load Bank3 was reset to 0kW. Then, Load Bank 3 was brought on-line with a $20 \mathrm{~kW}$ setting. In response to this load step change, output power at Gen-set A1 was $22.5 \mathrm{~kW}$ and $6.5 \mathrm{kVAr}$. The voltage was measured at approximately $287 \mathrm{~V}$, which remained the same at Gen-set A1. The rise in reactive power from the previous test (i.e., load step of $20 \mathrm{~kW}$ at $277 \mathrm{~V}$ ) was adjusted by the Gen-set to increase its voltage from $273 \mathrm{~V}$ to $287 \mathrm{~V}$. Frequency at Gen-set A1 was $59.98 \mathrm{~Hz}$. Once all data was recorded and validated, Load Bank 3 was increased from $20 \mathrm{~kW}$ to $40 \mathrm{~kW}$. Load Meter 3 measured a power flow of approximately $40 \mathrm{~kW}$ at Load Bank 3 with voltage at Gen-set A1 of 287V. The voltage held constant, allowing for a smooth transition between the load step changes. Gen-set A1 increased its output power from approximately $22.5 \mathrm{~kW}$ and $6.5 \mathrm{kVAr}$ to $41.5 \mathrm{~kW}$ and $6.8 \mathrm{kVAr}$, supplying power to Load Bank 3 and the frequency within the microgrid decreased from $59.98 \mathrm{~Hz}$ to $59.82 \mathrm{~Hz}$. Once all data was recorded and validated, Load Bank 3 was increased from $40 \mathrm{~kW}$ to $60 \mathrm{~kW}$. The voltage at Gen-set A1 remained the same at $287 \mathrm{~V}$, resulting in a smooth transition. Load Meter 3 measured a power flow of approximately 57kW at Load Bank 3 and the resultant output power of Gen-set A1 
increased to $59.5 \mathrm{~kW}$ and $7.7 \mathrm{kVAr}$. The microgrid frequency during this load step change decreased from $59.82 \mathrm{~Hz}$ to $59.15 \mathrm{~Hz}$. All data was recorded in the DAS and the next test was implemented.

The voltage set-point (i.e., MG Voltage) of Gen-set A1 was decreased from 291V to 263V (i.e., minus $5 \%$ of nominal $277 \mathrm{~V}$ ) and Load Bank 3 was reset to $0 \mathrm{~kW}$. Load Bank 3 was then brought on-line with a $20 \mathrm{~kW}$ setting and a resultant measurement of approximately $17 \mathrm{~kW}$. The voltage was measured at approximately $260 \mathrm{~V}$ and remained the same at Gen-set A1. In response to the load step change, the output power at Gen-set A1 was $19 \mathrm{~kW}$ and 2.55kVAr. A decrease in the reactive power from the previous test (i.e., load step of $20 \mathrm{~kW}$ at $277 \mathrm{~V}$ ) was adjusted by the Gen-set to decrease its voltage to approximately $260 \mathrm{~V}$. Frequency in the microgrid from Gen-set A1 was $60.00 \mathrm{~Hz}$. Once all data was recorded and validated, Load Bank 3 was increased from $20 \mathrm{~kW}$ to $40 \mathrm{~kW}$. Load Meter 3 measured a power flow of approximately $32 \mathrm{~kW}$ with voltage at Load Bank 3 of $260 \mathrm{~V}$. The voltage held constant, allowing for a smooth transition between load step changes. Gen-set A1 increased its output power from approximately $19 \mathrm{~kW}$ and $2.55 \mathrm{kVAr}$ to $34.5 \mathrm{~kW}$ and $2.9 \mathrm{kVAr}$, supplying power to Load Bank 3, and frequency within the microgrid decreased from $60.00 \mathrm{~Hz}$ to $59.87 \mathrm{~Hz}$. Once all data was recorded and validated, Load Bank 3 was increased from $40 \mathrm{~kW}$ to $60 \mathrm{~kW}$. Voltage at Gen-set A1 remained the same from the $40 \mathrm{~kW}$ load step test at $260 \mathrm{~V}$, resulting in a smooth transition. Load Meter 3 measured a power flow of approximately $47.5 \mathrm{~kW}$ at Load Bank 3 and the output power of Gen-set A1 increased to $50 \mathrm{~kW}$ and $3.3 \mathrm{kVAr}$. The microgrid frequency during this load step change decreased from $59.87 \mathrm{~Hz}$ to $59.74 \mathrm{~Hz}$. Once all data was recorded and validated, Gen-set A1 was shut down, manually, and all loads and alarms were reset in preparation for the next set of tests.

The above tests were repeated with adding 3 load steps of $20 \mathrm{~kW}$ and varying the voltage by $+5 \%$ and $-5 \%$ of the nominal $277 \mathrm{~V}$ for Gen-set A2 with Load Bank 4; and repeated again for Gen-set B1 with Load Bank 5. In each test, the Gen-sets transitioned smoothly through each load step change and voltage change.

\begin{tabular}{|c|c|c|c|c|c|c|c|}
\hline & \multicolumn{2}{|c|}{ Set-point Values } & \multicolumn{5}{|c|}{ Test Results } \\
\hline \multirow{4}{*}{$\begin{array}{l}\bar{\sigma} \\
\frac{c}{d} \\
0\end{array}$} & $\begin{array}{c}\text { Gen- } \\
\text { set } \\
\text { Voltage }\end{array}$ & $\begin{array}{c}\text { Load } \\
\text { Bank } \\
\text { kW }\end{array}$ & $\begin{array}{c}\text { Load Bank } \\
\quad(k W)\end{array}$ & $\begin{array}{c}\text { Gen-set Output } \\
\text { Real } \\
\text { Power (kW) }\end{array}$ & $\begin{array}{c}\text { Gen-set Output } \\
\text { Reactive Power } \\
\text { (kVAr) }\end{array}$ & $\begin{array}{l}\text { Freq. } \\
(\mathrm{Hz})\end{array}$ & Voltage \\
\hline & 277 & 20 & 19 & 21 & 4 & 59.99 & 273 \\
\hline & 277 & 40 & 36 & 38 & 4.4 & 59.84 & 273 \\
\hline & 277 & 60 & 52.5 & 55 & 4.8 & 59.70 & 273 \\
\hline
\end{tabular}




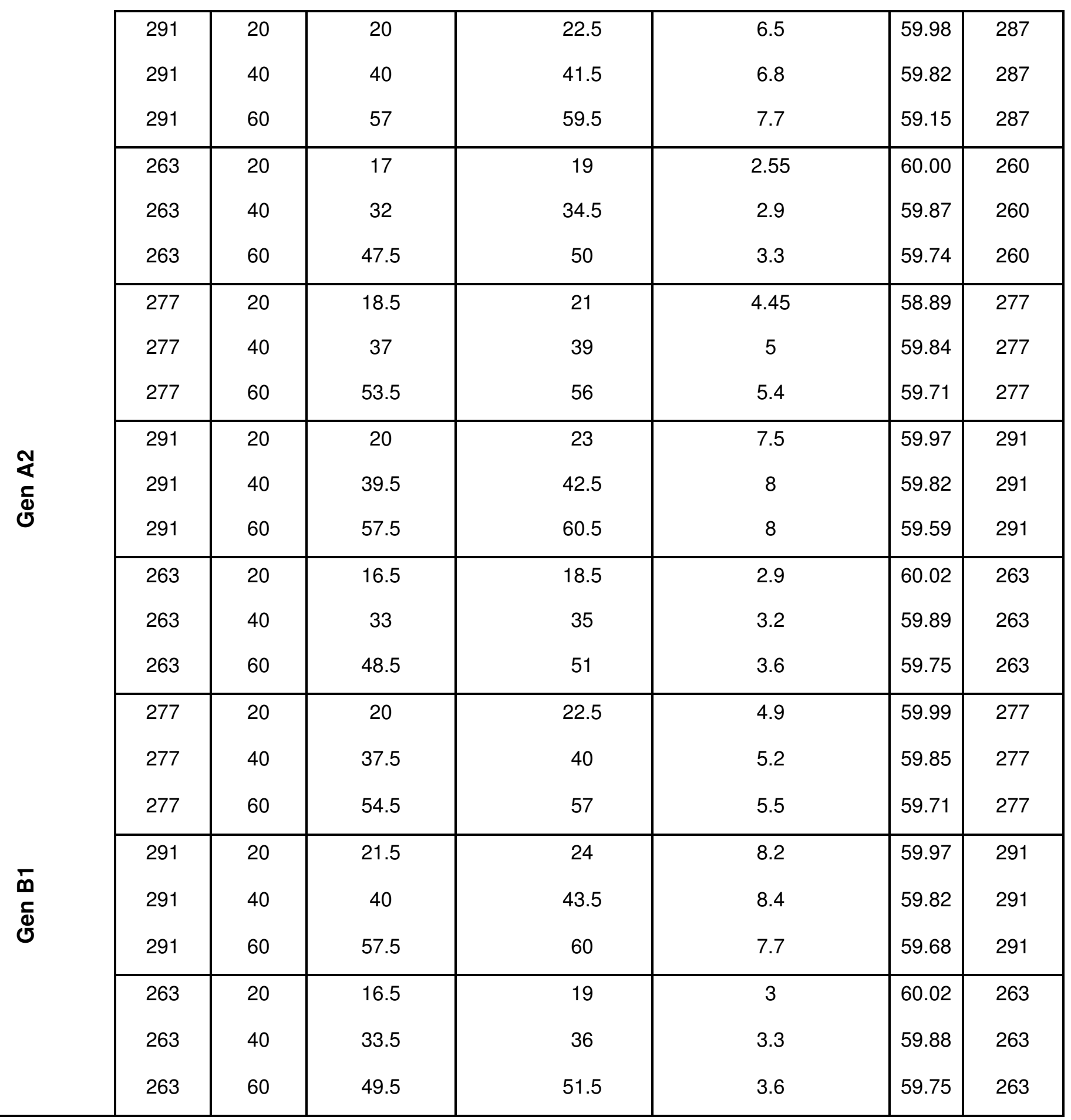

Table 1 - Voltage regulation test results of Gen-set A1, A2 and B1 in Zone 3, 4 and 5, respectively.

\subsubsection{Open Static Switch Test, Gen-set A1}

Performance Goal: 
Verify smooth transitions in Unit power control mode of Gen-sets A1 and A2 response to different step conditions (i.e., static switch "OPEN" / "CLOSE") when the lower power control limit, $0 \mathrm{~kW}$, of Gen-set A1 is reached.

Table 4 shows the values of Gen-sets, loads, power flows and frequency with the static switch closed and the final values after the static switch was opened, islanding the microgrid. The static switch was also allowed to reclose in this test and resultant values were expected to return to the initial values before the static switch was opened. During this test, the series inductor L11 was connected to the utility grid to simulate a weak grid condition at the PCC to the microgrid.

After Gen-sets A1 and A2 were running for a few minutes and in a steady state condition, Load Banks 3, 4 and 6 were connected with approximately $18.2 \mathrm{~kW}, 18.1 \mathrm{~kW}$ and $28.7 \mathrm{~kW}$, respectively. Gen-set A1 output power was approximately $5 \mathrm{~kW}$ and Genset A2 output power was approximately 55kW. Power flow through the static switch, before it was open, was approximately $-21.5 \mathrm{~kW}$. Power flow from the utility grid was approximately $7 \mathrm{~kW}$ which combined with the power flowing from the microgrid supplying power for Load Bank 6.

As soon as the manual open was asserted to the static switch, Load Bank 6 was no longer powered by the Gen-sets but by the utility grid and was approximately $29.35 \mathrm{~kW}$. Load Banks 3 and 4 were picked up by Gen-set A2, because of the CERTS power versus frequency droop control, and Gen-set A1 output power was reduced to 0kW. Gen-set A2 output power was approximately $37 \mathrm{~kW}$ and Load Banks 3 and 4 were $17.5 \mathrm{~kW}$ and $17.3 \mathrm{~kW}$, respectively. The frequency increased when the microgrid islanded from $60.00 \mathrm{~Hz}$ to $60.14 \mathrm{~Hz}$, because the load demand decreased within the microgrid and because of the CERTS algorithm. Power flow at the static switch was $0 \mathrm{~kW}$ and approximately $29.5 \mathrm{~kW}$ at Meter 1 on the utility side of the switch. The islanded microgrid operated this way for a few minutes before the static switch manual open was removed.

After the manual open was removed, the static switch reconnected the microgrid to the utility grid in a smooth transition and Load Bank 6 was supplied power by the microgrid and utility grid. Gen-set A1 increased its output power to approximately $6 \mathrm{~kW}$ and Gen-set A2 increased output power to approximately 56.5kW. Load Banks 3, 4 and 6 measured approximately $18.2 \mathrm{~kW}, 18.1 \mathrm{~kW}$ and $28.7 \mathrm{~kW}$, respectively. Power flow at the static switch was approximately $-22.5 \mathrm{~kW}$ with $7 \mathrm{~kW}$ at the utility side. Frequency within the microgrid decreased from $60.15 \mathrm{~Hz}$ to approximately $60.00 \mathrm{~Hz}$. The resultant 
values, after the static switch reconnected the microgrid to the utility grid, were very similar to the values at the beginning of the test.

Gen-sets A1 and A2 were shut down manually and all loads and alarms were reset and equipment was prepared for the next test.

\begin{tabular}{|l|l|l|l|l|l|l|l|}
\hline \multicolumn{2}{|c|}{ Test Results } \\
\hline \multicolumn{3}{|c|}{ Event Open SS } & \multicolumn{2}{c|}{$\begin{array}{l}\text { Event Results } \\
\text { Open SS }\end{array}$} & \multicolumn{2}{c|}{ Event Results } \\
Close SS
\end{tabular}

Table 4-Open Static Switch (SS) Test Results, Gen-set A1

\subsubsection{Open Static Switch Test, Gen-set A2}

Performance Goal: 
Verify smooth transitions in Unit power control mode of Gen-sets A1 and A2 response to different step conditions (i.e., static switch "OPEN" / "CLOSE") when the upper power control limit, $60 \mathrm{~kW}$, of Gen-set A2 is reached.

Table 5 shows the values of Gen-sets, loads, power flows and frequency with the static switch closed and the final values after the static switch was opened, islanding the microgrid. The static switch was also allowed to reclose in this test and resultant values were expected to return to the initial values before the static switch opened. During this test, the series inductor L11 was connected to the utility grid to simulate a weak grid condition at the PCC to the microgrid.

After Gen-sets A1 and A2 were running for a few minutes and in a steady state condition, Load Banks 3 and 4 were connected with approximately $53.9 \mathrm{~kW}$ and $35.6 \mathrm{~kW}$, respectively. Gen-set A1 output power was approximately $5 \mathrm{~kW}$ and Gen-set A2 output power was approximately 55kW. Power flow from the utility grid through the static switch was approximately $32 \mathrm{~kW}$, indicating that part of the loads within the microgrid were being supported by the utility grid.

As soon as the static switch opened, the utility grid was no longer providing the additional power needed to satisfy loads in the microgrid. Therefore, Gen-set A1 and A2 had to increase their output power, accordingly. Gen-set A1 output power increased from approximately $5 \mathrm{~kW}$ to $29 \mathrm{~kW}$ and Gen-set A2 increased from $55 \mathrm{~kW}$ to $60 \mathrm{~kW}$. The frequency decreased when the microgrid islanded from $59.94 \mathrm{~Hz}$ to $59.80 \mathrm{~Hz}$, because load demand increased within the microgrid and because of the CERTS algorithm. Likewise, voltage in the system dropped from approximately $280 \mathrm{~V}$ to $277 \mathrm{~V}$, decreasing load demands in Load Banks 3 and 4 to approximately $51.75 \mathrm{~kW}$ and $34.2 \mathrm{~kW}$, respectively. Power flow from the utility and through the static switch was $0 \mathrm{~kW}$. The islanded microgrid operated for a few minutes before the static switch manual open was removed.

The static switch reconnected the microgrid to the utility in a smooth transition with Load Banks 3 and 4 being supplied by the microgrid and utility grid. Gen-set A1 decreased its output power to approximately $2 \mathrm{~kW}$ and Gen-set A2 had an output power of approximately 53kW. Load Banks 3 and 4 measured approximately $53.7 \mathrm{~kW}$ and $35.5 \mathrm{~kW}$, respectively. The power flow at the static switch was approximately $37 \mathrm{~kW}$ at the utility side. Voltage within the microgrid increased from approximately $277 \mathrm{~V}$ to $280 \mathrm{~V}$ and the frequency increased from $59.80 \mathrm{~Hz}$ to approximately $60.02 \mathrm{~Hz}$. The values after the static switch reconnected the microgrid to the utility grid are very similar to the values at the beginning of the test which was expected. 
Gen-sets A1 and A2 were shut down manually and all loads and alarms were reset, and equipment was prepared for the next test.

\begin{tabular}{|c|c|c|c|c|c|c|c|}
\hline \multicolumn{8}{|c|}{ Test Results } \\
\hline \multicolumn{4}{|c|}{ Event Open SS } & \multicolumn{2}{|c|}{$\begin{array}{c}\text { Event Results } \\
\text { Open SS }\end{array}$} & \multicolumn{2}{|c|}{$\begin{array}{c}\text { Event Results } \\
\text { Close SS }\end{array}$} \\
\hline & Mode & $\begin{array}{l}\text { Set- } \\
\text { point }\end{array}$ & $\begin{array}{l}\text { Expected } \\
\text { Results }\end{array}$ & Start & End & Start & End \\
\hline $\mathrm{A} 1$ & Unit & $5 \mathrm{~kW}$ & $40 \mathrm{~kW}$ & $5 \mathrm{~kW}$ & $29 \mathrm{~kW}$ & $29 \mathrm{~kW}$ & $2 \mathrm{~kW}$ \\
\hline A2 & Unit & $55 \mathrm{~kW}$ & $60 \mathrm{~kW}$ & $55 \mathrm{~kW}$ & $60 \mathrm{~kW}$ & $60 \mathrm{~kW}$ & $53 \mathrm{~kW}$ \\
\hline B1 & & Off & Off & Off & Off & Off & Off \\
\hline $\mathrm{Fq}$ & & $\sim 60 \mathrm{~Hz}$ & $\sim 59.72 \mathrm{~Hz}$ & $59.94 \mathrm{~Hz}$ & $59.8 \mathrm{~Hz}$ & $59.80 \mathrm{~Hz}$ & $60.02 \mathrm{~Hz}$ \\
\hline L3 & & $60 \mathrm{~kW}$ & $60 \mathrm{~kW}$ & $53.9 \mathrm{~kW}$ & $51.75 \mathrm{~kW}$ & $51.7 \mathrm{~kW}$ & $53.7 \mathrm{~kW}$ \\
\hline L4 & & $40 \mathrm{~kW}$ & $40 \mathrm{~kW}$ & $35.6 \mathrm{~kW}$ & $34.2 \mathrm{~kW}$ & $34.25 \mathrm{~kW}$ & $35.5 \mathrm{~kW}$ \\
\hline L5 & & $0 \mathrm{~kW}$ & OkW & OkW & $0 \mathrm{~kW}$ & $0 \mathrm{~kW}$ & $0 \mathrm{~kW}$ \\
\hline L6 & & OkW & OkW & OkW & OkW & OkW & OkW \\
\hline SS & & $40 \mathrm{~kW}$ & OkW & $32 \mathrm{~kW}$ & $0 \mathrm{~kW}$ & $0 \mathrm{~kW}$ & $37 \mathrm{~kW}$ \\
\hline Grid & & $40 \mathrm{~kW}$ & OkW & $32 \mathrm{~kW}$ & OkW & $0 \mathrm{~kW}$ & $37 \mathrm{~kW}$ \\
\hline & & S re-clo & & & & & \\
\hline Exp & ct the $\mathrm{s}$ & $\begin{array}{l}\text { em to } r \\
\text { alues }\end{array}$ & n to Start & & & & \\
\hline
\end{tabular}

Table 5 - Open Static Switch (SS) Test Results, Gen-set A2

\subsubsection{Test Island Operation, Unbalanced Load}

Performance Goal:

Verify smooth transitions in Unit power control mode of Gen-sets A1 and A2 response to different step conditions (i.e., static switch "OPEN" / "CLOSE") with an un-balanced load in Zone 3. 
Table 6 shows the values of Gen-sets, loads, power flows and frequency before and after the load on A-phase of Load Bank 3 was reduced. During this test, the static switch remained open.

After Gen-sets A1 and A2 were running for a few minutes and in a steady state condition, Load Bank 3 was connected with approximately 54kW. Gen-set A1 output power was approximately $13 \mathrm{~kW}$ and Gen-set A2 output power was approximately $43 \mathrm{~kW}$.

As soon as A-phase load in Load Bank 3 was reduced, as verified by 0A flowing at Load Meter 3 on A-phase, Gen-sets A1 and A2 output power decreased to meet the new load demand. Gen-set A1 output power decreased from approximately $13 \mathrm{~kW}$ to $4 \mathrm{~kW}$ and Gen-set A2 decreased from approximately $43 \mathrm{~kW}$ to $34 \mathrm{~kW}$. The frequency increased when the microgrid was islanded from approximately $60.00 \mathrm{~Hz}$ to $60.09 \mathrm{~Hz}$, because load demand decreased within the microgrid and because of the CERTS algorithm. Likewise, voltage in the system increased from approximately $275 \mathrm{~V}$ to $277 \mathrm{~V}$ on phases A and B and decreased on C-phase from approximately $275 \mathrm{~V}$ to $270 \mathrm{~V}$. After reducing A-phase load to zero, Load Bank 3 decreased to approximately 35kW. Gen-sets A1 and A2 were shut down manually and all loads and alarms were reset, and equipment was prepared for the next test.

\begin{tabular}{|l|l|l|l|l|l|}
\hline \multicolumn{5}{|c|}{ Test Results } \\
\hline \multicolumn{2}{|c|}{ Event Open Phase-A of Load Bank 3 } & \multicolumn{2}{c|}{ Event Results } \\
\hline & Mode & $\begin{array}{l}\text { Set- } \\
\text { point }\end{array}$ & $\begin{array}{l}\text { Expected } \\
\text { Results }\end{array}$ & Start & End \\
\hline A1 & Unit & $15 \mathrm{~kW}$ & $\sim 2.5 \mathrm{~kW}$ & $13 \mathrm{~kW}$ & $4 \mathrm{~kW}$ \\
B1 & Unit & $45 \mathrm{~kW}$ & $\sim 37.5 \mathrm{~kW}$ & $43 \mathrm{~kW}$ & $34 \mathrm{~kW}$ \\
Freq & & Off & Off & Off & Off \\
L3 & & $\sim 60 \mathrm{~Hz}$ & $\sim 60.09 \mathrm{~Hz}$ & $60 \mathrm{~Hz}$ & $60.09 \mathrm{~Hz}$ \\
L4 & & $60 \mathrm{~kW}$ & $40 \mathrm{~kW}$ & $54 \mathrm{~kW}$ & $35 \mathrm{~kW}$ \\
L5 & & $0 \mathrm{~kW}$ & $0 \mathrm{~kW}$ & $0 \mathrm{~kW}$ & $0 \mathrm{~kW}$ \\
L6 & & $0 \mathrm{~kW}$ & $0 \mathrm{~kW}$ & $0 \mathrm{~kW}$ & $0 \mathrm{~kW}$ \\
S5 & Open & 0kW & $0 \mathrm{~kW}$ & $0 \mathrm{~kW}$ & $0 \mathrm{~kW}$ \\
& & $0 \mathrm{~kW}$ & $0 \mathrm{~kW}$ & $0 \mathrm{~kW}$ \\
\end{tabular}




\begin{tabular}{|c|l|l|l|} 
Grid $\mid$\begin{tabular}{ll|}
$0 \mathrm{~kW} \mid$ \\
Should be no current in Phase A
\end{tabular} & $0 \mathrm{~kW}$ & $0 \mathrm{~kW}$ \\
\hline \multicolumn{3}{|c|}{ Note: SS Open } & \\
\hline
\end{tabular}

Table 6 - Test Results, Island Operation, Unbalanced Load

\subsubsection{Mixed Mode Operation Test - Feeder A, Gen-set A1 Upper Limit, 60kW Performance Goal:}

Verify smooth transitions of Gen-set, A1 in Zone power control mode with a $60 \mathrm{~kW}$ upper limit and Gen-set A2 in Unit power control mode, during a load step change in Load Bank 3.

Table 7 shows the values of Gen-sets, loads, power flows and frequency before load in Zone 3 was expected to increase by $50 \mathrm{~kW}$ and the final values after load in Zone 3 was increased. During this test, the series inductor L11 was connected to the utility grid to simulate a weak grid condition at the PCC to the microgrid.

Gen-set A1 was set for zone power control mode and Gen-set A2 was set for unit power control mode. After Gen-sets A1 and A2 were running for a few minutes and in a steady state condition with the static switch closed, Load Banks 3 and 4 were connected and measured approximately $35 \mathrm{~kW}$ and $33.5 \mathrm{~kW}$, respectively. Gen-set A1 output power was approximately $10 \mathrm{~kW}$ and Gen-set A2 output power was approximately $5.5 \mathrm{~kW}$. Power flow through the static switch before additional load was added to Zone 3 was approximately 55kW. Likewise, power flow from the utility grid was approximately $55 \mathrm{~kW}$. Frequency in the microgrid before Zone 3 load was increased was approximately $60.005 \mathrm{~Hz}$ and voltage was approximately $277 \mathrm{~V}$.

As soon as load was added in Zone 3 by Load Bank 3, increasing its load from approximately $35 \mathrm{~kW}$ to $80 \mathrm{~kW}$, Gen-set A1 increased its output power from approximately $10 \mathrm{~kW}$ to $55 \mathrm{~kW}$ due to its Zone control set-point, which satisfied the increased load demand. Because frequency remained at approximately $60.005 \mathrm{~Hz}$ no additional power was supplied from Gen-set A2. The resultant voltage in the system dropped from approximately $277 \mathrm{~V}$ to $276 \mathrm{~V}$, decreasing load power demand in Load Bank 4 to approximately $33.25 \mathrm{~kW}$. Power flow from the utility through the static switch was $57 \mathrm{~kW}$. Although it was desired to put Gen-set A1 at its upper power control limit, differences in actual power delivered by the load banks prevented it from being reached. Gen-set A1 did correctly meet its zone control set point of $54 \mathrm{~kW}$; however, it only needed to provide $55 \mathrm{~kW}$ instead of the anticipated $60 \mathrm{~kW}$. The electrical system was 
allowed to operate for a couple of minutes to see if an event would occur, which did not happen.

Gen-sets A1 and A2 were shut down manually, all loads and alarms were reset, and equipment was prepared for the next test.

\begin{tabular}{|l|l|l|l|l|l|}
\hline \multicolumn{5}{|c|}{ Test Results } \\
\hline & Mode & $\begin{array}{l}\text { Set- } \\
\text { point }\end{array}$ & $\begin{array}{l}\text { Expected } \\
\text { Results }\end{array}$ & Start & End \\
A1 & Zone & $54 \mathrm{~kW}$ & $54 \mathrm{~kW}$ & $52 \mathrm{~kW}$ & $54 \mathrm{~kW}$ \\
A1 & Injected P & $10 \mathrm{~kW}$ & $60 \mathrm{~kW}$ & $10 \mathrm{~kW}$ & $55 \mathrm{~kW}$ \\
A2 & Unit & $6 \mathrm{~kW}$ & $6 \mathrm{~kW}$ & $5.5 \mathrm{~kW}$ & $5.5 \mathrm{~kW}$ \\
B1 & & Off & Off & Off & Off \\
Freq & & $\sim 60 \mathrm{~Hz}$ & $\sim 60 \mathrm{~Hz}$ & $60.005 \mathrm{~Hz}$ & $60.005 \mathrm{~Hz}$ \\
L3 & & $35 \mathrm{~kW}$ & $85 \mathrm{~kW}$ & $35 \mathrm{~kW}$ & $80 \mathrm{~kW}$ \\
L4 & & $35 \mathrm{~kW}$ & $35 \mathrm{~kW}$ & $33.45 \mathrm{~kW}$ & $33.25 \mathrm{~kW}$ \\
L5 & & $0 \mathrm{~kW}$ & $0 \mathrm{~kW}$ & $0 \mathrm{~kW}$ & $0 \mathrm{~kW}$ \\
L6 & & $0 \mathrm{~kW}$ & $0 \mathrm{~kW}$ & $0 \mathrm{~kW}$ & $0 \mathrm{~kW}$ \\
S5 & Closed & $54 \mathrm{~kW}$ & $54 \mathrm{~kW}$ & $55 \mathrm{~kW}$ & $57 \mathrm{~kW}$ \\
Grid & & $54 \mathrm{~kW}$ & $54 \mathrm{~kW}$ & $55 \mathrm{~kW}$ & $57 \mathrm{~kW}$ \\
\hline
\end{tabular}

Table 7 - Mixed Mode Operation Test Results, Feeder A, Gen-set A1 Upper Limit, 60kW

\subsubsection{Mixed Mode Operation Test - Feeder A, Automatic Reset of Zone Level Set- Point}

Performance Goal:

Verify smooth transitions of Gen-set A1 in Zone power control mode with an automatic reset of the set-point and Gen-set A2 in Unit power control mode, during a load step change in Load Bank 4.

Table 8 shows the values of Gen-sets, loads, power flows and frequency before load in Zone 4 was increased by $20 \mathrm{~kW}$ and the resultant values after load in Zone 4 was 
increased. During this test, the series inductor L11 was connected to the utility grid to simulate a weak grid condition at the PCC to the microgrid.

Gen-set A1 was set for zone power control mode and Gen-set A2 was set for unit power control mode. After Gen-sets A1 and A2 were running for a few minutes and in a steady state condition with the static switch closed, Load Banks 3 and 4 were connected and measured approximately $68.95 \mathrm{~kW}$ and $33.5 \mathrm{~kW}$, respectively. Gen-set A1 output power was approximately $44 \mathrm{~kW}$ and Gen-set A2 output power was approximately $6.5 \mathrm{~kW}$. Power flow through the static switch before additional load was added to Zone 4 was approximately 55kW. Likewise, power flow from the utility grid was approximately $55 \mathrm{~kW}$ which means that about half of the load in Load Banks 3 and 4 was supplied power by the utility grid. Frequency in the microgrid, before Zone 4 load was increased, was approximately $59.95 \mathrm{~Hz}$ and voltage was approximately $277 \mathrm{~V}$.

As soon as load was added in Zone 4 by Load Bank 4, increasing its load from approximately $33.5 \mathrm{~kW}$ to $52.5 \mathrm{~kW}$, Gen-set A1 increased its output power from approximately $44 \mathrm{~kW}$ to $58 \mathrm{~kW}$ due to its Zone control set-point, which satisfied the increased load demand. No additional power was supplied from Gen-set A2, which remained the same at approximately $6.5 \mathrm{~kW}$, and load demand in Load Bank 3 stayed the same at approximately $68.95 \mathrm{~kW}$. The resultant voltage in the system dropped from approximately $277 \mathrm{~V}$ to $276 \mathrm{~V}$ and frequency increased from approximately59.95Hz to $59.98 \mathrm{~Hz}$. Power flow from the utility through the static switch was $61 \mathrm{~kW}$. Gen-set A1 did correctly meet its zone control set-point of $55 \mathrm{~kW}$; however, differences in actual power delivered by the load banks prevented it from reaching the anticipated $60 \mathrm{~kW}$. The electrical system was allowed to operate for a couple of minutes to see if an event would occur, which did not happen.

Gen-sets A1 and A2 were shut down manually, all loads and alarms were reset and equipment was prepared for the next test.

\begin{tabular}{|l|l|l|l|l|l|}
\hline \multicolumn{2}{|c|}{ Test Results } \\
\hline \multirow{2}{|c|}{ Event Load step in Zone 4 } & \multicolumn{3}{c|}{ Event Results } \\
\hline & Mode & Set-point & $\begin{array}{l}\text { Expected } \\
\text { Results }\end{array}$ & Start & End \\
\hline A1 & Zone & $55 \mathrm{~kW}$ & $65 \mathrm{~kW}$ & $52 \mathrm{~kW}$ & $58 \mathrm{~kW}$ \\
A1 & Injected P & $50 \mathrm{~kW}$ & $60 \mathrm{~kW}$ at Max & $44 \mathrm{~kW}$ & $58 \mathrm{~kW}$
\end{tabular}




\begin{tabular}{|l|l|l|l|l|l|} 
A2 & Unit & $5 \mathrm{~kW}$ & $5 \mathrm{~kW}$ & $6.5 \mathrm{~kW}$ & $6.5 \mathrm{~kW}$ \\
Freq & & Off & Off & Off & Off \\
L3 & $\sim 60 \mathrm{~Hz}$ & $\sim 60 \mathrm{~Hz}$ & $59.95 \mathrm{~Hz}$ & $59.98 \mathrm{~Hz}$ \\
L4 & & $75 \mathrm{~kW}$ & $75 \mathrm{~kW}$ & $68.95 \mathrm{~kW}$ & $68.95 \mathrm{~kW}$ \\
L5 & $35 \mathrm{~kW}$ & $55 \mathrm{~kW}$ & $33.5 \mathrm{~kW}$ & $52.5 \mathrm{~kW}$ \\
L6 & OkW & $0 \mathrm{~kW}$ & $0 \mathrm{~kW}$ & $0 \mathrm{~kW}$ \\
S5 & Closed & $55 \mathrm{~kW}$ & $65 \mathrm{~kW}$ & $0 \mathrm{~kW}$ & $0 \mathrm{~kW}$ \\
Grid & $55 \mathrm{~kW}$ & $65 \mathrm{~kW}$ & $55 \mathrm{~kW}$ & $61 \mathrm{~kW}$ \\
\hline
\end{tabular}

Table 8 - Mixed Mode Operation Test Results, Feeder A, Automatic Reset of Zone Level Set-point.

\subsubsection{Mixed Mode Operation Test-Feeder A, Zone Power Change Performance Goal:}

Verify smooth transitions of Gen-set A1 in Zone power control mode and Gen-set A2 in Unit power control mode, with a change of zone power in Feeder A. Table 9 shows the values of Gen-sets, loads, power flows and frequency before the EMS requested Zone 3 power be increased by $56 \mathrm{~kW}$ and the resultant values after Zone 3 power was increased. During this test, the series inductor L11 was connected to the utility grid to simulate a weak grid condition at the PCC to the microgrid.

Gen-set A1 was set for zone power control mode and Gen-set A2 was set for unit power control mode. After Gen-sets A1 and A2 were running for a few minutes and in a steady state condition with the static switch closed, Load Banks 3 and 4 were connected and measured approximately $75.7 \mathrm{~kW}$ and $33.3 \mathrm{~kW}$, respectively. Gen-set A1 output power was approximately $50.5 \mathrm{~kW}$ and Gen-set A2 output power was approximately $5.25 \mathrm{~kW}$. Power flow through the static switch was approximately $57 \mathrm{~kW}$ before the EMS increased power in Zone 3 and was approximately $54 \mathrm{~kW}$ in Zone 3. Frequency in the microgrid before the EMS changed the power in Zone 3 was approximately $60.008 \mathrm{~Hz}$ and voltage was approximately $277 \mathrm{~V}$.

As soon as the EMS increased the power set-point setting of Gen-set A1 in Zone 3, Genset A1 decreased its output power from approximately $50.5 \mathrm{~kW}$ to $0 \mathrm{~kW}$, due to its zone control set-point which increased load demand to Zone 3. Gen-set A2 output power increased slightly from $5.25 \mathrm{~kW}$ to approximately $5.6 \mathrm{~kW}$. The resultant voltage in the 
system dropped from approximately $277 \mathrm{~V}$ to $276 \mathrm{~V}$ and frequency decreased slightly (i.e., from $60.008 \mathrm{~Hz}$ to $60.003 \mathrm{~Hz}$ ), reducing load at Load Banks 3 and 4 to approximately $75.55 \mathrm{~kW}$ and $33.24 \mathrm{~kW}$, respectively. Power flow at the static switch increased from $57 \mathrm{~kW}$ to $107.5 \mathrm{~kW}$. This $50.5 \mathrm{~kW}$ increase by the utility grid was required to meet the increased set-point setting in Zone 3, and Gen-set A1 reduced to 0kW. The electrical system was allowed to operate for a couple of minutes to see if an event would occur, which did not happen.

Gen-sets A1 and A2 was shut down manually, all loads and alarms were reset and equipment was prepared for the next test.

\begin{tabular}{|c|c|c|c|c|c|}
\hline \multicolumn{6}{|c|}{ Test Results } \\
\hline \multicolumn{4}{|c|}{ Event Increase Zone 3 set-point } & \multicolumn{2}{|c|}{ Event Results } \\
\hline & Mode & $\begin{array}{l}\text { Set- } \\
\text { point }\end{array}$ & $\begin{array}{l}\text { Expected } \\
\text { Results }\end{array}$ & Start & End \\
\hline A1 & Zone & $54 \mathrm{~kW}$ & $110 \mathrm{~kW}$ & $54 \mathrm{~kW}$ & $104 \mathrm{~kW}$ \\
\hline A1 & Injected P & $\begin{array}{l}60 \mathrm{~kW} \\
\text { at Max }\end{array}$ & $4 \mathrm{~kW}$ & $50.5 \mathrm{~kW}$ & $0 \mathrm{~kW}$ \\
\hline $\mathrm{A} 2$ & Unit & $6 \mathrm{~kW}$ & $6 \mathrm{~kW}$ & $5.25 \mathrm{~kW}$ & $5.6 \mathrm{~kW}$ \\
\hline B1 & & Off & Off & Off & Off \\
\hline Freq & & $\sim 60 \mathrm{~Hz}$ & $\sim 60 \mathrm{~Hz}$ & $60.008 \mathrm{~Hz}$ & $60.003 \mathrm{~Hz}$ \\
\hline L3 & & $85 \mathrm{~kW}$ & $85 \mathrm{~kW}$ & $75.7 \mathrm{~kW}$ & $75.55 \mathrm{~kW}$ \\
\hline L4 & & $35 \mathrm{~kW}$ & $35 \mathrm{~kW}$ & $33.3 \mathrm{~kW}$ & $33.24 \mathrm{~kW}$ \\
\hline L5 & & OkW & OkW & OkW & OkW \\
\hline L6 & & OkW & $0 \mathrm{~kW}$ & OkW & OkW \\
\hline SS & Closed & $54 \mathrm{~kW}$ & $110 \mathrm{~kW}$ & $57 \mathrm{~kW}$ & $107.5 \mathrm{~kW}$ \\
\hline Grid & & $54 \mathrm{~kW}$ & $110 \mathrm{~kW}$ & $57 \mathrm{~kW}$ & $107.5 \mathrm{~kW}$ \\
\hline Rese & Zone Powe & or Gen-s & $\mathrm{A} 1$ to $\sim 11$ & & \\
\hline
\end{tabular}

Table 9 - Mixed Mode Operation Test Results, Feeder A Zone Power Change

\subsubsection{Mixed Mode Operation Test - Feeder A, Zone Power Change When Islanded} Performance Goal: 
Verify smooth transition of Gen-sets, A1 in Zone power control mode and A2 in Unit power control mode, with a static switch operation to island the microgrid and a change of zone power control in Feeder A.

Table 10 shows the values of Gen-sets, loads, power flows and frequency before the static switch was opened and the resultant values after the static switch islanded the microgrid. The static switch was also allowed to reclose in this test to compare values indicated in the table before the static switch was opened. During this test, the series inductor L11 was connected to the utility grid to simulate a weak grid condition at the PCC to the microgrid.

Gen-set A1 was set for zone power control mode and Gen-set A2 was set for unit power control mode. After Gen-sets A1 and A2 were running for a few minutes and in a steady state condition with the static switch closed, Load Banks 3 and 4 were connected and measured approximately $33.75 \mathrm{~kW}$ and $33.4 \mathrm{~kW}$, respectively. Gen-set A1 output power was approximately $14 \mathrm{~kW}$ and Gen-set A2 output power was approximately $5 \mathrm{~kW}$. Power flow through the static switch, from the utility grid, was approximately $52.5 \mathrm{~kW}$ with approximately $75 \%$ of the loads within the microgrid being supplied by the utility grid.

As soon as the static switch was manually opened, the utility grid was no longer providing power to the microgrid with Gen-sets A1 and A2 adjusting their output power, accordingly. Gen-set A1 output power decreased from approximately $14 \mathrm{~kW}$ to $9 \mathrm{~kW}$ because Zone 3 power set-point was decreased from $50 \mathrm{~kW}$; and Gen-set A2 output power increased from $5 \mathrm{~kW}$ to $59 \mathrm{~kW}$. Gen-set A1 continued producing power in Zone 3, because Gen-set A2 was at its maximum power limit and could not satisfy load demand from Load Banks 3 and 4. The resultant frequency decreased when the microgrid islanded from $60.02 \mathrm{~Hz}$ to $59.58 \mathrm{~Hz}$, because the load increased within the microgrid and because of the CERTS algorithm. In addition, voltage in the microgrid dropped from approximately $280 \mathrm{~V}$ to $277 \mathrm{~V}$, and decreased load power demands in Load Banks 3 and 4 to approximately $32.8 \mathrm{~kW}$ and $32.6 \mathrm{~kW}$, respectively. Power flow at the static switch decreased from $52.5 \mathrm{~kW}$ to $0 \mathrm{~kW}$. The islanded microgrid operated this way for a few minutes before the static switch manual open was removed.

The static switch reconnected the microgrid to the utility grid in a smooth transition with Load Banks 3 and 4 supplied power by the microgrid and utility grid. Gen-set A1 increased its output power to approximately $14 \mathrm{~kW}$ and Gen-set A2 decreased its output power to approximately 5kW. Load Banks 3 and 4 were measured at approximately $33.65 \mathrm{~kW}$ and $33.4 \mathrm{~kW}$, respectively. Power flow through the static switch was 
approximately $52.5 \mathrm{~kW}$ from the utility. Voltage within the microgrid increased from approximately $277 \mathrm{~V}$ to $280 \mathrm{~V}$ and frequency increased from approximately $59.55 \mathrm{~Hz}$ to $60.00 \mathrm{~Hz}$. The resultant values after the static switch reconnected the microgrid to the utility grid were very similar to the values at the beginning of the test.

Gen-sets A1 and A2 were shut down manually, all loads and alarms were reset, and equipment was prepared for the next test.

\begin{tabular}{|c|c|c|c|c|c|c|c|}
\hline \multicolumn{8}{|c|}{ Test Results } \\
\hline \multicolumn{4}{|c|}{ Test Event Open SS } & \multicolumn{2}{|c|}{$\begin{array}{c}\text { Event Results } \\
\text { Open SS }\end{array}$} & \multicolumn{2}{|c|}{$\begin{array}{c}\text { Event Results } \\
\text { Close SS }\end{array}$} \\
\hline & Mode & Start & End & Start & End & Start & End \\
\hline $\mathrm{A} 1$ & Zone & $50 \mathrm{~kW}$ & $0 \mathrm{~kW}$ & $50 \mathrm{~kW}$ & $0 \mathrm{~kW}$ & $0 \mathrm{~kW}$ & $50 \mathrm{~kW}$ \\
\hline A1 & Injected P & $14 \mathrm{~kW}$ & $\sim 18 \mathrm{~kW}$ & $14 \mathrm{~kW}$ & $9 \mathrm{~kW}$ & $9 \mathrm{~kW}$ & $14 \mathrm{~kW}$ \\
\hline $\mathrm{A} 2$ & Unit & $6 \mathrm{~kW}$ & $\sim 52 \mathrm{~kW}$ & $5 \mathrm{~kW}$ & $59 \mathrm{~kW}$ & $59 \mathrm{~kW}$ & $5 \mathrm{~kW}$ \\
\hline B1 & & Off & Off & Off & Off & Off & Off \\
\hline Freq & & $\sim 60 \mathrm{~Hz}$ & $\sim 59.6 \mathrm{~Hz}$ & $60.02 \mathrm{~Hz}$ & $59.58 \mathrm{~Hz}$ & $59.55 \mathrm{~Hz}$ & $60 \mathrm{~Hz}$ \\
\hline L3 & & $35 \mathrm{~kW}$ & $35 \mathrm{~kW}$ & $33.75 \mathrm{~kW}$ & $32.8 \mathrm{~kW}$ & $32.7 \mathrm{~kW}$ & $33.65 \mathrm{~kW}$ \\
\hline L4 & & $35 \mathrm{~kW}$ & $35 \mathrm{~kW}$ & $33.4 \mathrm{~kW}$ & $32.6 \mathrm{~kW}$ & $32.6 \mathrm{~kW}$ & $33.4 \mathrm{~kW}$ \\
\hline L5 & & $0 \mathrm{~kW}$ & $0 \mathrm{~kW}$ & OkW & $0 \mathrm{~kW}$ & $0 \mathrm{~kW}$ & $0 \mathrm{~kW}$ \\
\hline L6 & & $0 \mathrm{~kW}$ & $0 \mathrm{~kW}$ & OkW & OkW & $0 \mathrm{~kW}$ & $0 \mathrm{~kW}$ \\
\hline SS & Closed & $50 \mathrm{~kW}$ & OkW & $52.5 \mathrm{~kW}$ & $0 \mathrm{~kW}$ & $0 \mathrm{~kW}$ & $52.5 \mathrm{~kW}$ \\
\hline Grid & & $50 \mathrm{~kW}$ & OkW & $52.5 \mathrm{~kW}$ & OkW & OkW & $52.5 \mathrm{~kW}$ \\
\hline & Allow $S$ & to re-clo & & & & & \\
\hline & pect the sys & $\begin{array}{l}\text { to retu } \\
\text { es. }\end{array}$ & to Start & & & & \\
\hline
\end{tabular}

Table 10 - Mixed Mode Operation Test Results, Feeder A, Zone Power Change When Islanded

\subsubsection{Mixed Mode Operation Test - Feeder A, When Islanded Automatic Reset of Zone Level Set-point \\ Performance Goal:}


Verify smooth transitions of Gen-sets A1 in Zone power control mode with automatic reset of set point and Gen-set A2 in Unit power control mode, with a static switch operation and a change of zone power control set-point in Feeder A, when islanded.

Table 11 shows the values of Gen-sets, loads, power flows and frequency before the static switch opened and the resultant values after the static switch islanded the microgrid. The static switch was also allowed to reclose in this test to compare values indicated in the table before the static switch was opened. During this test, the series inductor L11 is connected to the utility grid to simulate a weak grid condition at the PCC to the microgrid.

Gen-set A1 was set for zone power control mode and Gen-set A2 was set for unit power control mode. After Gen-sets A1 and A2 were running for a few minutes and in a steady state condition, Load Banks 3 and 4 were connected and measured approximately $54 \mathrm{~kW}$ and $54 \mathrm{~kW}$, respectively. Gen-set A1 output power was approximately 50kW and Genset A2 output power was approximately $10 \mathrm{~kW}$. Power flow from the utility through the static switch, before it was opened, was approximately $53 \mathrm{~kW}$, indicating that about half of the load within the microgrid was being supplied by the utility grid.

As soon as the static switch was manually opened, the utility grid was no longer providing additional power needed to satisfy the loads in the microgrid; therefore, Genset A1 and A2 had to increase or decrease their output powers, accordingly. Gen-set A1 output power decreased from approximately $50 \mathrm{~kW}$ to $47 \mathrm{~kW}$ and Gen-set A2 increased from $10 \mathrm{~kW}$ to $60 \mathrm{~kW}$. Gen-set A1 continued producing power because Gen-set A2 was at its maximum power point and could not satisfy load demand from Load Banks 3 and 4. The frequency decreased when the microgrid islanded from $59.965 \mathrm{~Hz}$ to $59.78 \mathrm{~Hz}$, because the load demand increased within the microgrid and because of the CERTS algorithm. The resultant voltage in the system dropped from approximately $280 \mathrm{~V}$ to $275 \mathrm{~V}$, decreasing load power demands in Load Banks 3 and 4 to approximately 52kW and $52.25 \mathrm{~kW}$, respectively. Power flow from the utility through the static switch was $0 \mathrm{~kW}$. The islanded microgrid operated this way for a few minutes before the static switch manual open was removed.

The static switch reconnected the microgrid to the utility grid in a smooth transition with Load Banks 3 and 4 supplied power from the microgrid and utility grid. Gen-set A1 increased its output power to approximately $49 \mathrm{~kW}$ and Gen-set A2 decreased its output power to approximately $4 \mathrm{~kW}$. Load Banks 3 and 4 measured approximately 
$53.5 \mathrm{~kW}$ and $52.8 \mathrm{~kW}$, respectively. Power flow at the static switch was approximately $57.5 \mathrm{~kW}$ from the utility side. Voltage within the microgrid increased from approximately $275 \mathrm{~V}$ to $280 \mathrm{~V}$ and frequency increased approximately to $60.02 \mathrm{~Hz}$. The values after the static switch reconnected the microgrid to the utility grid were very similar to the values at the beginning of the test, which was expected.

Gen-sets A1 and A2 were shut down manually, all loads and alarms were reset, and equipment was prepared for the next test.

\begin{tabular}{|c|c|c|c|c|c|c|c|}
\hline \multicolumn{8}{|c|}{ Test Results } \\
\hline \multicolumn{4}{|c|}{ Event Open SS } & \multicolumn{2}{|c|}{$\begin{array}{c}\text { Event Results } \\
\text { Open SS }\end{array}$} & \multicolumn{2}{|c|}{$\begin{array}{c}\text { Event Results } \\
\text { Close SS }\end{array}$} \\
\hline & Mode & $\begin{array}{l}\text { Set- } \\
\text { point }\end{array}$ & $\begin{array}{l}\text { Expected } \\
\text { Results }\end{array}$ & Start & End & Start & End \\
\hline A1 & Zone & $54 \mathrm{~kW}$ & $0 \mathrm{~kW}$ & $50 \mathrm{~kW}$ & $0 \mathrm{~kW}$ & $0 \mathrm{~kW}$ & $55 \mathrm{~kW}$ \\
\hline $\mathrm{A} 1$ & Injected P & $60 \mathrm{~kW}$ & $60 \mathrm{~kW}$ at Max & $50 \mathrm{~kW}$ & $47 \mathrm{~kW}$ & $45 \mathrm{~kW}$ & $49 \mathrm{~kW}$ \\
\hline A2 & Unit & $6 \mathrm{~kW}$ & $60 \mathrm{~kW}$ & $10 \mathrm{~kW}$ & $60 \mathrm{~kW}$ & $60 \mathrm{~kW}$ & $4 \mathrm{~kW}$ \\
\hline B1 & & Off & Off & Off & Off & Off & Off \\
\hline Freq & & $\sim 60 \mathrm{~Hz}$ & $\sim 59.5 \mathrm{~Hz}$ & $59.965 \mathrm{~Hz}$ & $59.54 \mathrm{~Hz}$ & $59.79 \mathrm{~Hz}$ & $60.02 \mathrm{~Hz}$ \\
\hline L3 & & $60 \mathrm{~kW}$ & $60 \mathrm{~kW}$ & $54 \mathrm{~kW}$ & $52 \mathrm{~kW}$ & $51.7 \mathrm{~kW}$ & $53.5 \mathrm{~kW}$ \\
\hline L4 & & $60 \mathrm{~kW}$ & $60 \mathrm{~kW}$ & $54 \mathrm{~kW}$ & $52.25 \mathrm{~kW}$ & $51.3 \mathrm{~kW}$ & $52.8 \mathrm{~kW}$ \\
\hline L5 & & $0 \mathrm{~kW}$ & $0 \mathrm{~kW}$ & $0 \mathrm{~kW}$ & $0 \mathrm{~kW}$ & $0 \mathrm{~kW}$ & $0 \mathrm{~kW}$ \\
\hline L6 & & $0 \mathrm{~kW}$ & $0 \mathrm{~kW}$ & $0 \mathrm{~kW}$ & OkW & $0 \mathrm{~kW}$ & $0 \mathrm{~kW}$ \\
\hline SS & Closed & $54 \mathrm{~kW}$ & $0 \mathrm{~kW}$ & $53 \mathrm{~kW}$ & OkW & $0 \mathrm{~kW}$ & $57.5 \mathrm{~kW}$ \\
\hline Grid & & $54 \mathrm{~kW}$ & $0 \mathrm{~kW}$ & $53 \mathrm{~kW}$ & 0kW & $0 \mathrm{~kW}$ & $57.5 \mathrm{~kW}$ \\
\hline Ex & $\begin{array}{l}\text { Allow } \\
\text { he systen }\end{array}$ & $\begin{array}{l}\text { o Re-c } \\
\text { return }\end{array}$ & Start values. & & & & \\
\hline
\end{tabular}

Table 11 - Mixed Mode Operation Test Results, Feeder A When Islanded Automatic Reset of Zone Level Set-point

\subsubsection{Two Sources in Zone Control - Separate Feeders, When Islanded Automatic Reset of Zone Set Points, Zones Sum Equal 0kW}




\section{Performance Goal:}

Verify smooth transitions of Gen-sets, A1 and B1 in Zone power control mode, with a static switch operation and a change of zone power control settings in Feeder $\mathrm{A}$ and Feeder B, when islanded.

Table 12 shows the values of Gen-sets, loads, power flows and frequency before the static switch opened and resultant values after the static switch opened, islanding the microgrid. The static switch was also allowed to reclose in this test to compare values with initial values of the test before the static switch was opened. During this test, the series inductor L11 was connected to the utility grid to simulate weak grid conditions at the PCC to the microgrid.

Gen-sets A1 and B1 are both set for zone power control mode. After Gen-sets A1 and B1 were running for a few minutes and in a steady state condition, Load Banks 3 and 5 were connected, measuring approximately $35.5 \mathrm{~kW}$ and $46.4 \mathrm{~kW}$, respectively. Gen-set A1 output power was approximately $0 \mathrm{~kW}$ and Gen-set B1 output power was approximately $34 \mathrm{~kW}$. Power flow from the utility through the static switch, before it opened, was approximately $50 \mathrm{~kW}$, with a little more than half of the load within the microgrid being supplied by the utility grid.

As soon as the static switch was manually opened, the utility grid was no longer providing additional power needed to satisfy the loads in the microgrid; therefore, Genset A1 and B1 increased their output power, accordingly. Gen-set A1 output power increased from approximately $0 \mathrm{~kW}$ to $22 \mathrm{~kW}$ and Gen-set B1 increased from $34 \mathrm{~kW}$ to $61 \mathrm{~kW}$. Zone 3 received $15 \mathrm{~kW}$ from Zone 5 to cover the load demand of Load Bank 3. Frequency decreased when the microgrid islanded from $60.03 \mathrm{~Hz}$ to $59.78 \mathrm{~Hz}$, because load demand increased within the microgrid and because of the CERTS algorithm. The resultant voltage in the system dropped from approximately $277 \mathrm{~V}$ to $272 \mathrm{~V}$, decreasing load power demands in Load Banks 3 and 5 to approximately $34.2 \mathrm{~kW}$ and $45.4 \mathrm{~kW}$, respectively. Power flow from the utility through the static switch was $0 \mathrm{~kW}$. The islanded microgrid operated this way for a few minutes before the static switch manual open was removed.

The static switch reconnected the microgrid to the utility grid in a smooth transition with Load Banks 3 and 5 being supplied power by the microgrid and utility grid. Genset A1 decreased its output power to approximately $0 \mathrm{~kW}$ and Gen-set B1 decreased its output power to approximately $34 \mathrm{~kW}$. Load Banks 3 and 5 measured approximately $35.7 \mathrm{~kW}$ and $46.3 \mathrm{~kW}$, respectively. Power flow at the static switch was approximately 
$50 \mathrm{~kW}$ from the utility grid. Voltage within the microgrid increased from approximately $272 \mathrm{~V}$ to $277 \mathrm{~V}$ and frequency increased to approximately $60.015 \mathrm{~Hz}$. The resultant values after the static switch reconnected the microgrid to the utility grid were very similar to the values at the beginning of the test, which were expected.

Gen-sets A1 and B1 were manually shut down, all loads and alarms were reset, and equipment was prepared for the next test.

\begin{tabular}{|c|c|c|c|c|c|c|c|}
\hline \multicolumn{8}{|c|}{ Test Results } \\
\hline \multicolumn{4}{|c|}{ Event Open SS } & \multicolumn{2}{|c|}{$\begin{array}{c}\text { Event Results } \\
\text { Open SS }\end{array}$} & \multicolumn{2}{|c|}{$\begin{array}{c}\text { Event Results } \\
\text { Close SS }\end{array}$} \\
\hline & Mode & Start & End & Start & End & Start & End \\
\hline A1 & Zone & $40 \mathrm{~kW}$ & $10 \mathrm{~kW}$ & $35 \mathrm{~kW}$ & $13 \mathrm{~kW}$ & $13 \mathrm{~kW}$ & $36 \mathrm{~kW}$ \\
\hline A1 & Injected P & $0 \mathrm{~kW}$ & $30 \mathrm{~kW}$ & OkW & $22 \mathrm{~kW}$ & $21.5 \mathrm{~kW}$ & OkW \\
\hline A2 & & Off & Off & Off & Off & Off & Off \\
\hline B1 & Zone & $10 \mathrm{~kW}$ & $-10 \mathrm{~kW}$ & $13 \mathrm{~kW}$ & $-15 k W$ & $-15.5 \mathrm{~kW}$ & $12 \mathrm{~kW}$ \\
\hline B1 & Injected P & $40 \mathrm{~kW}$ & $60 \mathrm{~kW}$ & $34 \mathrm{~kW}$ & $61 \mathrm{~kW}$ & $61 \mathrm{~kW}$ & $34 \mathrm{~kW}$ \\
\hline $\mathrm{Fq}$ & & $\sim 60 \mathrm{~Hz}$ & $\sim 59.76 \mathrm{~Hz}$ & $60.03 \mathrm{~Hz}$ & $59.78 \mathrm{~Hz}$ & $59.87 \mathrm{~Hz}$ & $60.015 \mathrm{~Hz}$ \\
\hline L3 & & $40 \mathrm{~kW}$ & $40 \mathrm{~kW}$ & $35.5 \mathrm{~kW}$ & $34.2 \mathrm{~kW}$ & $34.4 \mathrm{~kW}$ & $35.7 \mathrm{~kW}$ \\
\hline L4 & & $0 \mathrm{~kW}$ & $0 \mathrm{~kW}$ & OkW & OkW & OkW & $0 \mathrm{~kW}$ \\
\hline L5 & & $50 \mathrm{~kW}$ & $50 \mathrm{~kW}$ & $46.4 \mathrm{~kW}$ & $45.4 \mathrm{~kW}$ & $45.3 \mathrm{~kW}$ & $46.3 \mathrm{~kW}$ \\
\hline L6 & & $0 \mathrm{~kW}$ & $0 \mathrm{~kW}$ & OkW & OkW & OkW & $0 \mathrm{~kW}$ \\
\hline SS & Closed & $50 \mathrm{~kW}$ & $0 \mathrm{~kW}$ & $50 \mathrm{~kW}$ & OkW & OkW & $50 \mathrm{~kW}$ \\
\hline Grid & & $50 \mathrm{~kW}$ & OkW & $50 \mathrm{~kW}$ & OkW & OkW & $50 \mathrm{~kW}$ \\
\hline & $\begin{array}{r}\text { Allow S } \\
\text { eect the syst } \\
\mathrm{V}\end{array}$ & $\begin{array}{l}\text { re-clos } \\
\text { to retur } \\
\text { es }\end{array}$ & o Start & & & & \\
\hline
\end{tabular}

Table 12 - Two Sources in Zone Control Test Results, Separate Feeders When Islanded, Automatic Reset of Zone Set-points, Zone Sums Equal Zero kW

\subsubsection{Two Sources in Zone Control - Separate Feeders, When Islanded Automatic $\underline{\text { Reset of Zone Set Point }}$}




\section{Performance Goal:}

Verify smooth transitions of Gen-sets A1 and B1 in Zone power control mode, both having automatic reset of set points, with a static switch operation and a change of zone control power in Feeder A and Feeder B, when islanded.

Table 13 shows the values of Gen-sets, loads, power flows and frequency before the static switch opened and resultant final values after the static switch opened, islanding the microgrid. The static switch was also allowed to reclose in this test to compare values indicated in the table with initial values of the test before the static switch opened. During this test, the series inductor L11 was connected to the utility grid to simulate a weak grid condition at the PCC to the microgrid.

Gen-sets A1 and B1 were both set for zone power control mode. After Gen-sets A1 and B1 were running for a few minutes and in a steady state condition, Load Banks 3, 5 and 6 were connected, measuring approximately $18.1 \mathrm{~kW}, 35.8 \mathrm{~kW}$, and $18.15 \mathrm{~kW}$ respectively. Gen-set A1 output power was approximately $43.6 \mathrm{~kW}$ and Gen-set B1 output power was approximately $14 \mathrm{~kW}$. Power flow through the static switch before it opened was approximately $-0.5 \mathrm{~kW}$, meaning that power was flowing beyond the static switch. Power flow from the utility grid was approximately $17.7 \mathrm{~kW}$ which means that Load Bank 6 was being supported mainly by the utility grid. Gen-set A1 was supporting Load Bank 3 and sending approximately $25.1 \mathrm{~kW}$ to Zone 5 and Load Bank 6 .

As soon as the static switch was manually opened, the utility grid was no longer providing additional power needed to satisfy loads in the microgrid, therefore, Gen-set A1 and B1 had to increase and decrease their output powers, accordingly. Because nearly $0 \mathrm{~kW}$ was being exported from the microgrid very little changed once the static switch opened. The frequency increased from $60.014 \mathrm{~Hz}$ to $60.024 \mathrm{~Hz}$ because load demand decreased within the microgrid and because of the CERTS algorithm. The resultant voltage in the system dropped from approximately $280 \mathrm{~V}$ to $272 \mathrm{~V}$ decreasing the load power demands in Load Banks 3 and 5 to approximately $17.45 \mathrm{~kW}$ and $34.2 \mathrm{~kW}$, respectively. Power flow at the static switch was $0 \mathrm{~kW}$ with approximately $18.6 \mathrm{~kW}$ from the utility feeding Load Bank 6. The islanded microgrid operated this way for a few minutes before the static switch manual open was removed.

The static switch reconnected the microgrid to the utility grid in a smooth transition with Load Banks 3, 5 and 6 now being supplied power by the microgrid and utility grid. Gen-set A1 increased its output power to approximately $45 \mathrm{~kW}$ and Gen-set B1 increased its output power to approximately $15.25 \mathrm{~kW}$. Load Banks 3, 5 and 6 measured 
approximately $18.15 \mathrm{~kW}, 35.7 \mathrm{~kW}$ and $18.1 \mathrm{~kW}$, respectively. Power flow at the static switch was approximately $-3.5 \mathrm{~kW}$ and $14.75 \mathrm{~kW}$ from the utility side. Gen-set A1 increased its power output which was distributed to Zone 5 and Load Bank 6. Voltage within the microgrid increased from approximately $272 \mathrm{~V}$ to $280 \mathrm{~V}$ and frequency decreased to approximately $60.005 \mathrm{~Hz}$. The resultant values after the static switch reconnected the microgrid to the utility grid were very similar to the values at the beginning of the test, which was expected.

Gen-sets A1 and B1 were manually shut down, all loads and alarms were reset, and equipment was prepared for the next test.

\begin{tabular}{|c|c|c|c|c|c|c|c|}
\hline \multicolumn{8}{|c|}{ Test Results } \\
\hline \multicolumn{4}{|c|}{ Event Open SS } & \multicolumn{2}{|c|}{$\begin{array}{c}\text { Event Results } \\
\text { Open SS }\end{array}$} & \multicolumn{2}{|c|}{$\begin{array}{c}\text { Event Results } \\
\text { Close SS }\end{array}$} \\
\hline & Mode & $\begin{array}{l}\text { Set- } \\
\text { point }\end{array}$ & $\begin{array}{l}\text { Expected } \\
\text { Results }\end{array}$ & Start & End & Start & End \\
\hline $\mathrm{A} 1$ & Zone & $-30 \mathrm{~kW}$ & $-25 \mathrm{~kW}$ & $-25.1 \mathrm{~kW}$ & $-25.4 \mathrm{~kW}$ & $-25.5 \mathrm{~kW}$ & $-26.75 \mathrm{~kW}$ \\
\hline $\mathrm{A} 1$ & Injected P & $50 \mathrm{~kW}$ & $45 \mathrm{~kW}$ & $43.6 \mathrm{~kW}$ & $42.9 \mathrm{~kW}$ & $43 \mathrm{~kW}$ & $45 \mathrm{~kW}$ \\
\hline $\mathrm{A} 2$ & & Off & Off & Off & Off & Off & Off \\
\hline B1 & Zone & $20 \mathrm{~kW}$ & $25 \mathrm{~kW}$ & $21.7 \mathrm{~kW}$ & $22.7 \mathrm{~kW}$ & $22.5 \mathrm{~kW}$ & $20.5 \mathrm{~kW}$ \\
\hline B1 & Injected P & $20 \mathrm{~kW}$ & $15 \mathrm{~kW}$ & $14 \mathrm{~kW}$ & $12 \mathrm{~kW}$ & $12.25 \mathrm{~kW}$ & $15.25 \mathrm{~kW}$ \\
\hline Freq & & $\sim 60 \mathrm{~Hz}$ & $\sim 60.04 \mathrm{~Hz}$ & $60.014 \mathrm{~Hz}$ & $60.024 \mathrm{~Hz}$ & $60.01 \mathrm{~Hz}$ & $60.005 \mathrm{~Hz}$ \\
\hline L3 & & $20 \mathrm{~kW}$ & $20 \mathrm{~kW}$ & $18.1 \mathrm{~kW}$ & $17.45 \mathrm{~kW}$ & $17.55 \mathrm{~kW}$ & $18.15 \mathrm{~kW}$ \\
\hline L4 & & OkW & $0 \mathrm{~kW}$ & OkW & OkW & OkW & OkW \\
\hline L5 & & $40 \mathrm{~kW}$ & $40 \mathrm{~kW}$ & $35.8 \mathrm{~kW}$ & $34.2 \mathrm{~kW}$ & $34.4 \mathrm{~kW}$ & $35.7 \mathrm{~kW}$ \\
\hline L6 & & $20 \mathrm{~kW}$ & $20 \mathrm{~kW}$ & $18.15 \mathrm{~kW}$ & $18.475 \mathrm{~kW}$ & $18.425 \mathrm{~kW}$ & $18.1 \mathrm{~kW}$ \\
\hline SS & Closed & $-10 \mathrm{~kW}$ & $0 \mathrm{~kW}$ & $-0.5 \mathrm{~kW}$ & $0.1 \mathrm{~kW}$ & $0 \mathrm{~kW}$ & $-3.5 \mathrm{~kW}$ \\
\hline Grid & & $10 \mathrm{~kW}$ & $20 \mathrm{~kW}$ & $17.7 \mathrm{~kW}$ & $18.6 \mathrm{~kW}$ & $18.5 \mathrm{~kW}$ & $14.75 \mathrm{~kW}$ \\
\hline Expe & $\begin{array}{r}\text { Allow S } \\
\text { the system }\end{array}$ & $\begin{array}{l}\text { to re-clos } \\
\text { return tc }\end{array}$ & Start values & & & & \\
\hline
\end{tabular}

Table 13 - Two Sources in Zone Control Test Results, Separate Feeders When Islanded, Automatic Reset of Zone Set-point 


\subsubsection{Test Generator Black-Start Procedure}

Performance Goal:

Bring up the Micro-grid Test Bed from a black-out condition without generation or protection trips. During this test, the series inductor L11 was connected to the utility grid to simulate a weak grid condition at the PCC to the microgrid.

Gen-set A1, A2 and B1 were set for injection power control, but were left off-line. Breaker CB1 was then opened where the microgrid was isolated from the utility with Load Bank 6 still part of the microgrid loads. A manual open was sent to the static switch to isolate the microgrid, islanding it from the utility grid. Gen-sets A1, A2 and B1 were started and allowed to run for a few minutes until steady state conditions were established. At this time Load Banks 3, 4, and 5 were applied to the microgrid at approximately $22.55 \mathrm{~kW}+\mathrm{j} 10.35 \mathrm{kVAr}, 22.3 \mathrm{~kW}+\mathrm{j} 9.95 \mathrm{kVAr}$ and $22.625 \mathrm{~kW}+\mathrm{j} 10.45 \mathrm{kVAr}$, respectively. Gen-set A1, A2 and B1 output powers were approximately $23 \mathrm{~kW}, 23 \mathrm{~kW}$ and $24 \mathrm{~kW}$, respectively. Frequency on the microgrid was $59.978 \mathrm{~Hz}$ at a voltage level of approximately $271 \mathrm{~V}$. The grid supplied approximately $6 \mathrm{~kW}$ to Load bank 6 which measured approximately $5.9 \mathrm{~kW}+\mathrm{j} 10.95 \mathrm{kVAr}$.

The manual "Open" was removed from the static switch and CB1 was closed. The static switch waited 300 seconds before closing into the utility grid, because of IEEE Standard 1547 requirements. A smooth transition occurred when the static switch closed into the utility grid.

The voltage and frequency increased in the microgrid to approximately $277 \mathrm{~V}$ and $60.006 \mathrm{~Hz}$ which in turn increased the load demands in Load Banks 3, 4 and 5 to approximately $23.35 \mathrm{~kW}+\mathrm{j} 10.75 \mathrm{kVAr}, 23.075 \mathrm{~kW}+\mathrm{j} 10.34 \mathrm{kVAr}$ and $23.275 \mathrm{~kW}+$ j10.775kVAr, respectively. Load Bank 6 reduced to approximately $5.715 \mathrm{~kW}+\mathrm{j} 10.61 \mathrm{kVAr}$. Gen-sets A1, A2 and B1 decreased their output power to approximately $18.5 \mathrm{~kW}, 19.5 \mathrm{~kW}$ and $19.5 \mathrm{~kW}$, respectively. The additional power needed to support all loads in the microgrid and Load Bank 6 was supplied by the utility grid, which increased from approximately $6 \mathrm{~kW}$ to $21 \mathrm{~kW}$. Zones 3,4 and 5 increased to approximately $9 \mathrm{~kW}, 4 \mathrm{~kW}$ and $3.75 \mathrm{~kW}$. The microgrid was operated like this for a couple of minutes to monitor performance and fault events, which did not occur.

Gen-sets A1, A2 and B1 were manually shut down, all loads and alarms were reset, and equipment was prepared for the next test. 


\begin{tabular}{|l|c|c|c|}
\hline \multicolumn{3}{|c|}{ Test Results } \\
\hline & Mode & $\begin{array}{c}\text { Event Results } \\
\text { Start }\end{array}$ & $\begin{array}{c}\text { Event Results } \\
\text { End }\end{array}$ \\
\hline A1 & Unit & $23 \mathrm{~kW}$ & $18.5 \mathrm{~kW}$ \\
A2 & Zone & $23 \mathrm{~kW}$ & $19.5 \mathrm{~kW}$ \\
B1 & Zone & $24 \mathrm{~kW}$ & $19.5 \mathrm{~kW}$ \\
Freq & & $59.978 \mathrm{~Hz}$ & $60.006 \mathrm{~Hz}$ \\
L3 & & $22.55 \mathrm{~kW}$ & $23.35 \mathrm{~kW}$ \\
L4 & & $22.3 \mathrm{~kW}$ & $23.075 \mathrm{~kW}$ \\
L5 & & $22.625 \mathrm{~kW}$ & $23.275 \mathrm{~kW}$ \\
L6 & & $5.9 \mathrm{~kW}$ & $5.715 \mathrm{~kW}$ \\
SS & & $0 \mathrm{~kW}$ & $15.5 \mathrm{~kW}$ \\
Grid & Open & $6 \mathrm{~kW}$ & $21 \mathrm{~kW}$ \\
\hline
\end{tabular}

Table 14 - Generator Black Start Procedure Test Results

\subsubsection{Test/Establish Generator Black-Start Capacity Performance Goal:}

Determine the Black-start capacity of the microgrid by increasing the amount of load on the Micro-grid Test Bed from a black-out condition without generation or protection trips. During this test the series inductor L11 was connected to the utility grid to simulate a weak grid condition at the PCC to the microgrid.

Gen-set A1 was set for unit power control mode, but was left off-line. CB1 was opened isolating the microgrid with Load Bank 6 still part of the microgrid loads. A manual open was sent to the static switch to isolate the microgrid, islanding it from the utility grid. At this time Load Bank 3 was connected to the microgrid at approximately $18 \mathrm{~kW}+$ j17.5kVAr. Gen-set A1 was started and closed into the load in Load Bank 3. Gen-set A1 output power was approximately $20 \mathrm{~kW}+\mathrm{j} 20.5 \mathrm{kVAr}$. Zone 3 was approximately $-2.3 \mathrm{~kW}$ + j2.75kVAr supporting transformer T51 and the load of the static switch controls. Frequency on the microgrid was $60.00 \mathrm{~Hz}$ at a voltage level of approximately 270V. Genset A1 was shut down manually after if ran for a few minutes. Load Bank 3 was then increased to approximately $31 \mathrm{~kW}+\mathrm{j} 17.5 \mathrm{kVAr}$ and then Gen-set A1 was restarted. 
The process above continued for three more load increases in Load Bank 3 (i.e., $47 \mathrm{~kW}+$ $\mathrm{j} 17.8 \mathrm{kVAr}, 50 \mathrm{~kW}+\mathrm{j} 26.5 \mathrm{kVAr}$ and $59 \mathrm{~kW}+\mathrm{j} 26.5 \mathrm{kVAr}$ ). The results for all the Black-start load step tests are shown in Tables $15 \mathrm{a}-15 \mathrm{e}$. Gen-sets A1 was manually shut down, all loads and alarms were reset, and equipment was prepared for the next test.

\section{Event Results}

Mode $\quad$ Open SS and Gen-set A1 Start

Load

LOAD 20kW+j20kVar

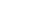

A1

A1

A2

B1

Freq

L3

L4

L5

L6

SS

Grid

Start End

$\begin{array}{lll}\text { Unit } & 20 \mathrm{~kW} & -2.3 \mathrm{~kW}-\mathrm{j} 2.75 \mathrm{kVar} \\ \text { Injected P } & \text { OkW } & 20 \mathrm{~kW}+\mathrm{j} 20.5 \mathrm{kVar} \\ & \text { Off } & \text { Off } \\ \text { Off } & \text { Off } \\ \text { OHz } & 60 \mathrm{~Hz} \\ \text { OkW } & 18 \mathrm{~kW}+\mathrm{j} 17.5 \mathrm{kVar} \\ \text { Off } & \text { Off } \\ \text { Off } & \text { Off } \\ \text { Off } & \text { Off } \\ \text { OkW+j0kVar } & \text { OkW +j0kVar } \\ \text { OkW+jOkVar } & \text { OkW+j0kVar }\end{array}$

Table 15a - Black Start Test Results, 20kW + j20kVAr

Event Results

Mode $\quad$ Open SS and Gen-set A1 Start

Load

LOAD $35 \mathrm{~kW}+\mathrm{j} 20 \mathrm{kVar}$

Start End

A1

Unit

OkW

$-2.3 k W-j 2.75 k$ Var

A1

Injected P OkW

$32.5 \mathrm{~kW}+\mathrm{j} 20 \mathrm{kVar}$ 
A2

B1

$\mathrm{Fq}$

L3

L4

L5

L6

SS

Grid
Off

Off

$\mathrm{OHz}$

OkW

Off

Off

Off

OkW+j0kVar $\quad 0 k W+j 0 k V a r$

OkW+j0kVar OkW+j0kVar

Table 15b - Black Start Test Results, 35kW + j20kVAr

Event Results

Mode Open SS and Gen-set A1 Start

Load

A1

A2

B1

$\mathrm{Fq}$

L3

L4

L5

L6

SS

Grid

LOAD 55kW+j20kVar

Start End

Zone

OkW

$-2.35-j 2.75 k \operatorname{Var}$

Injected P

OkW

$50 \mathrm{~kW}+\mathrm{j} 20.5 \mathrm{kVar}$

Off

Off

Off Off

$\mathrm{OHz} \quad 59.9 \mathrm{~Hz}$

OkW $\quad 47 \mathrm{~kW}+\mathrm{j} 17.8 \mathrm{kVar}$

Off Off

Off Off

Off Off

OkW+j0kVar $\quad 0 k W+j 0 k V a r$

$0 k W+j 0 k V a r \quad 0 k W+j 0 k V a r$

Table 15c - Black Start Test Results, 55kW + j20kVAr

Event Results

Mode Open SS and Gen-set A1 Start 


$\begin{array}{llll} & & \text { Start } & \text { End } \\ \text { A1 } & \text { Zone } & \text { OkW } & -2.25 \mathrm{~kW}-\mathrm{j} 2.5 \mathrm{kVar} \\ \text { A1 } & \text { Injected P } & \text { OkW } & 52.5 \mathrm{~kW}+\mathrm{j} 29 \mathrm{kVar} \\ \text { A2 } & \text { Off } & \text { Off } \\ \text { B1 } & \text { Off } & \text { Off } \\ \text { Fq } & \text { OHz } & 59.75 \mathrm{~Hz} \\ \text { L3 } & \text { OkW } & 50 \mathrm{~kW}+\mathrm{j} 26.5 \mathrm{kVar} \\ \text { L4 } & \text { Off } & \text { Off } \\ \text { L5 } & \text { Off } & \text { Off } \\ \text { L6 } & \text { Off } & \text { Off } \\ \text { S5 } & \text { OkW+j0kVar } & \text { OkW+j0kVar } \\ \text { Grid } & \text { OkW+j0kVar } & \text { OkW+jOkVar }\end{array}$

Table 15d - Black Start Test Results, 60kW + j30kVAr

\section{Event Results}

Mode Open SS and Gen-set A1 Start

Load

$$
\text { LOAD } 70 \mathrm{~kW}+\mathrm{j} 30 \mathrm{kVar}
$$

Start End

A1

Zone

OkW

$-2.19 k W-j 2.35 k V a r$

A1

Injected P

OkW

$61 \mathrm{~kW}+\mathrm{j} 29 \mathrm{kVar}$

A2

Off

Off

B1

Off

Off

$\mathrm{Fq}$

$\mathrm{OHz}$

$59.8 \mathrm{~Hz}$

L3

OkW

$59 \mathrm{~kW}+\mathrm{j} 26.5 \mathrm{kVar}$

L4

Off

Off

L5

Off

Off

L6

Off

Off

SS

OkW+j0kVar $0 k W+j 0 k V a r$ 


\subsection{CONCLUSION}

The tests performed in section 8.0 "Reduced System Tests" were successfully performed which provided confidence that the Gen-set inverter controls were working properly and prepared for the power control test in section 9.0.

The tests proved to be more robust than previously predicted in the Black Start Tests in section 6.1.13 when the load was increased to a demand greater than the rating of the Gen-sets and all components remained online. 


\title{
CERTS MICROGRID LABORATORY TEST BED
}

\author{
Test Plan Section 9.0 \\ Power Flow Control Tests
}

Prepared For:

California Energy Commission

Public Interest Energy Research Program

Arnold Schwarzenegger Governor

$\frac{1}{x}$

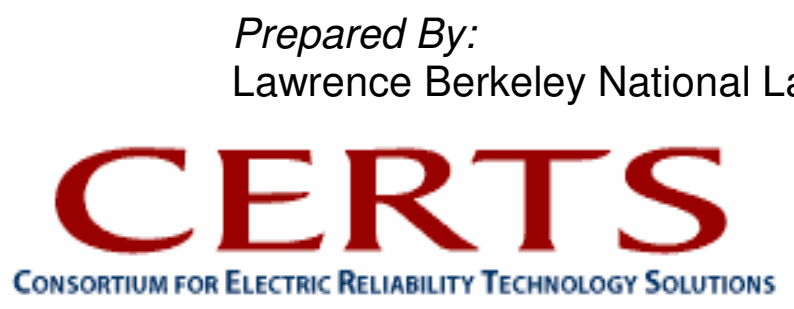



Prepared By:

Lawrence Berkeley National Laboratory

Joseph H. Eto, Principal Investigator

Berkeley, CA 94720

Ben Schenkman, Sandia National Laboratory

Harry Volkommer and Dave Klapp, American Electric Power

Commission Contract No. 500-03-024

Prepared For:

Public Interest Energy Research (PIER)

California Energy Commission

Bernard Treanton

Contract Manager

Mike Gravely

Program Area Lead

ENERGY SYSTEMS INTEGRATION

Mike Gravely

Office Manager

ENERGY SYSTEMS RESEARCH

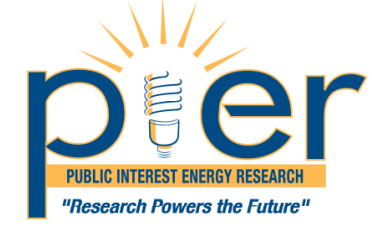

Martha Krebs, Ph.D.

PIER Director

Thom Kelly, Ph.D.

Deputy Director

ENERGY RESEARCH \& DEVELOPMENT DIVISION

Melissa Jones

Executive Director

\section{DISCLAIMER}

This report was prepared as the result of work sponsored by the California Energy Commission. It does not necessarily represent the views of the Energy Commission, its employees or the State of California. The Energy Commission, the State of California, its employees, contractors and subcontractors make no warrant, express or implied, and assume no legal liability for the information in this report; nor does any party represent that the uses of this information will not infringe upon privately owned rights. This report has not been approved or disapproved by the California Energy Commission nor has the California Energy Commission passed upon the accuracy or adequacy of the information in this report. 



\section{CERTS MICROGRID TEST REPORT}

\section{SECTION 9.0}

"Power Flow Control Tests" 


\section{$\underline{\text { Table of Contents }}$}

1.0

2.0

3.0

4.0

5.0

6.0

6.1

INTRODUCTION

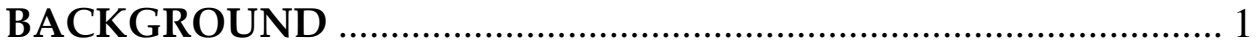

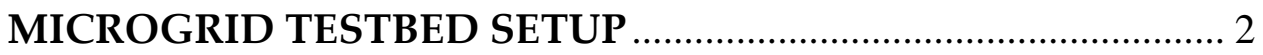

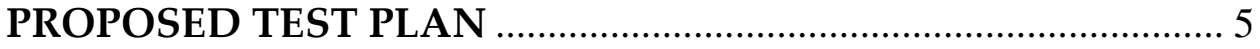

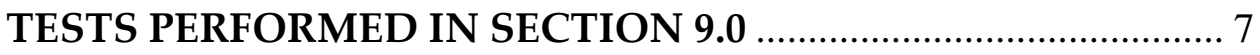

ANALYSES OF TEST RESULTS ………………...................... 8

SECTION 9 - POWER FLOW CONTROL TESTS ……………...... 8

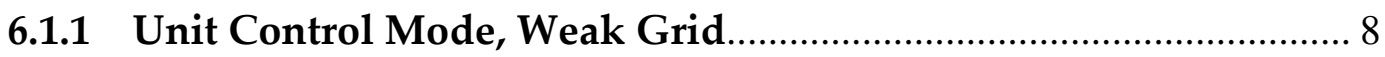

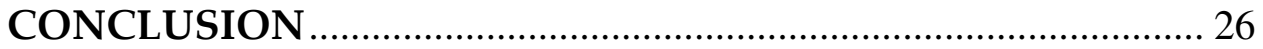




\section{$\underline{\text { List Of Figures }}$}

Figure 1 - CERTS Microgrid Aerial Photo................................................................... 3

Figure 2 - One Line Diagram of CERTS Microgrid Test Bed...................................... 3

Figure 3 - Simplified diagram of Test Bed showing Meter and Relay locations .... 4

Figure 4 - Diagram of DAS \& EMS Data networks..................................................... 5

Figure 29a - Meter 3 voltage before/after the static switch opened, islanding the microgrid from the utility grid.................................................................. 12

Figure $29 b$ - Meter 3 frequency before/after the static switch opened, islanding the microgrid from the utility grid................................................................. 13

Figure 29c - Meter 3 voltage before/after the static switch opened, islanding the microgrid from the utility grid................................................................. 16

Figure $29 \mathrm{~d}$ - Meter 3 frequency before/after the static switch opened, islanding the microgrid from the utility grid.

Figure 29e - Meter 3 voltage before/after the static switch opened, islanding the microgrid from the utility grid.

Figure 29f - Meter 3 frequency before/after the static switch opened, islanding the microgrid from the utility grid................................................................. 21

Figure $29 \mathrm{~g}$ - Meter 3 voltage before/after the static switch opened, islanding the microgrid from the utility grid

Figure $29 \mathrm{~h}$ - Meter 3 frequency before/after the static switch opened, islanding the microgrid from the utility grid. 


\section{List Of Tables}

Table 16a - Unit Control Mode, Weak Grid Settings for Load Banks and Gen-sets

Table 16b - Test 9.1.7 results with dark squares in the table meaning that measurements were not needed.

Table 16c - Test 9.1.8 results with dark squares in the table meaning that

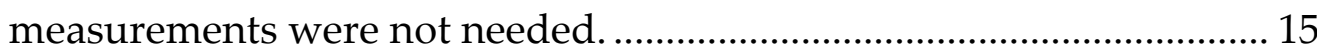

Table 16d - Test 9.1.9 results with dark squares in the table meaning that measurements were not needed.

Table 16e - Test 9.1.9 results with dark squares in the table meaning that measurements were not needed. 


\subsection{INTRODUCTION}

A series of tests were performed on the CERTS Microgrid by American Electric Power at the Walnut test site in Groveport, Ohio with support from Lawrence Berkeley National laboratory, Sandia National Laboratory, TECOGEN, The Switch (originally Youtility), Distributed-Energy (originally Northern Power) and University of Wisconsin. These tests were designed to demonstrate the CERTS Microgrid concepts of control and protection while connected to the utility electrical system and isolated (i.e., referred to as "islanded" from it. This paper describes the tests that were performed in Section 9.0 "Power Flow Control Tests" of the CERTS Micro-grid Test Plan.

\subsection{BACKGROUND}

The CERTS Microgrid Concept is an advanced approach for enabling integration of, in principle, an unlimited quantity of DER (e.g., distributed generation (DG), energy storage, etc.) into the electric utility grid. A key feature of a microgrid is its ability to separate and island itself from the utility system, during a utility grid disturbance. This is accomplished via intelligent power electronic interfaces and a single, high-speed, switch which is used for disconnection from the grid and synchronization to the grid. During a disturbance, the DER and corresponding loads can autonomously be separated from the utility's distribution system, isolating the microgrid's load from the disturbance (and thereby maintaining high level of service) without harming the integrity of the utility's electrical system. Thus, when the utility grid returns to normal, the microgrid automatically synchronizes and reconnects itself to the grid, in an equally seamless fashion. Intentional islanding of DER and loads has the potential to provide a higher level of reliability than that provided by the distribution system as a whole.

What is unique about the CERTS Microgrid is that it can provide this technically challenging functionality without extensive (i.e., expensive) custom engineering. In addition, the design of the CERTS Microgrid provides a high level of system reliability and great flexibility in the placement of DER within the microgrid. The CERTS Microgrid offers these functionalities at much lower costs than traditional approaches by incorporating peer-to-peer and plug-and-play concepts for each component within the microgrid.

The original concept was driven by two fundamental principles: 1.) A systems perspective was necessary for customers, utilities, and society to capture the full benefits of integrating DER into an energy system; and 2.) The business case for accelerating 
adoption of these advanced concepts will be driven, primarily, by lowering the up-front cost and enhancing the value offered by microgrids.

Each innovation was created specifically to lower the cost and improve the reliability of small-scale DG systems (i.e., installed systems with capacities ranging from less than $100 \mathrm{~kW}$ to $1000 \mathrm{~kW}$ ). The goal was to increase and accelerate realization of the many benefits offered by small-scale DG, such as their ability to supply waste heat at the point of need or to provide a higher level of reliability to some but not all loads within a facility. From an electric utility perspective, the CERTS Microgrid Concept is attractive because it recognizes that the nation's distribution system is extensive, aging, and will change over time which impacts power quality. The CERTS Microgrid Concept enables high penetration of DG systems without requiring re-design or re-engineering of the utility's distribution system.

Prospective applications of the CERTS Microgrid include industrial parks, commercial and institutional campuses, situations that require uninterrupted power supplies and high power quality, CHP systems, Greenfield communities, and remote applications. In short, wherever economic and DG location considerations indicate the need for multiple DG units within a (or among) site, the CERTS Microgrid offers the potential for a much more reliable, flexible, and lower cost solution compared to traditional engineering approaches for integrating DG.

\subsection{MICROGRID TESTBED SETUP}

The CERTS Microgrid Test Bed is operated at 480/277 volts (i.e., three-phase, four-wire) and consists of three TECOGEN Generators at 480 volts capable of producing $60 \mathrm{~kW}$ plus 60kVAr (Gen-set A1, Gen-set A2 and Gen-set B1) and four load banks (Load Bank 3, Load Bank 4, Load Bank 5 and Load Bank 6) capable of consuming 100kW plus 20kVAr each, as shown in Figure 2. Each of the generators are connected to a 112kVA isolation transformer and interfaced to the CERTS Microgrid through an inverter, developed by The Switch, where the algorithms for the CERTS Microgrid controls are embedded. . A semiconductor switch made by S\&C Electric Company, known as the static switch, connects the CERTS Microgrid to the utility grid. Load Banks 3 - 5 are the local loads in zones located beyond the static switch; and Load Bank 6 is a customer load in Zone 6 located on the utility side of the static switch. 


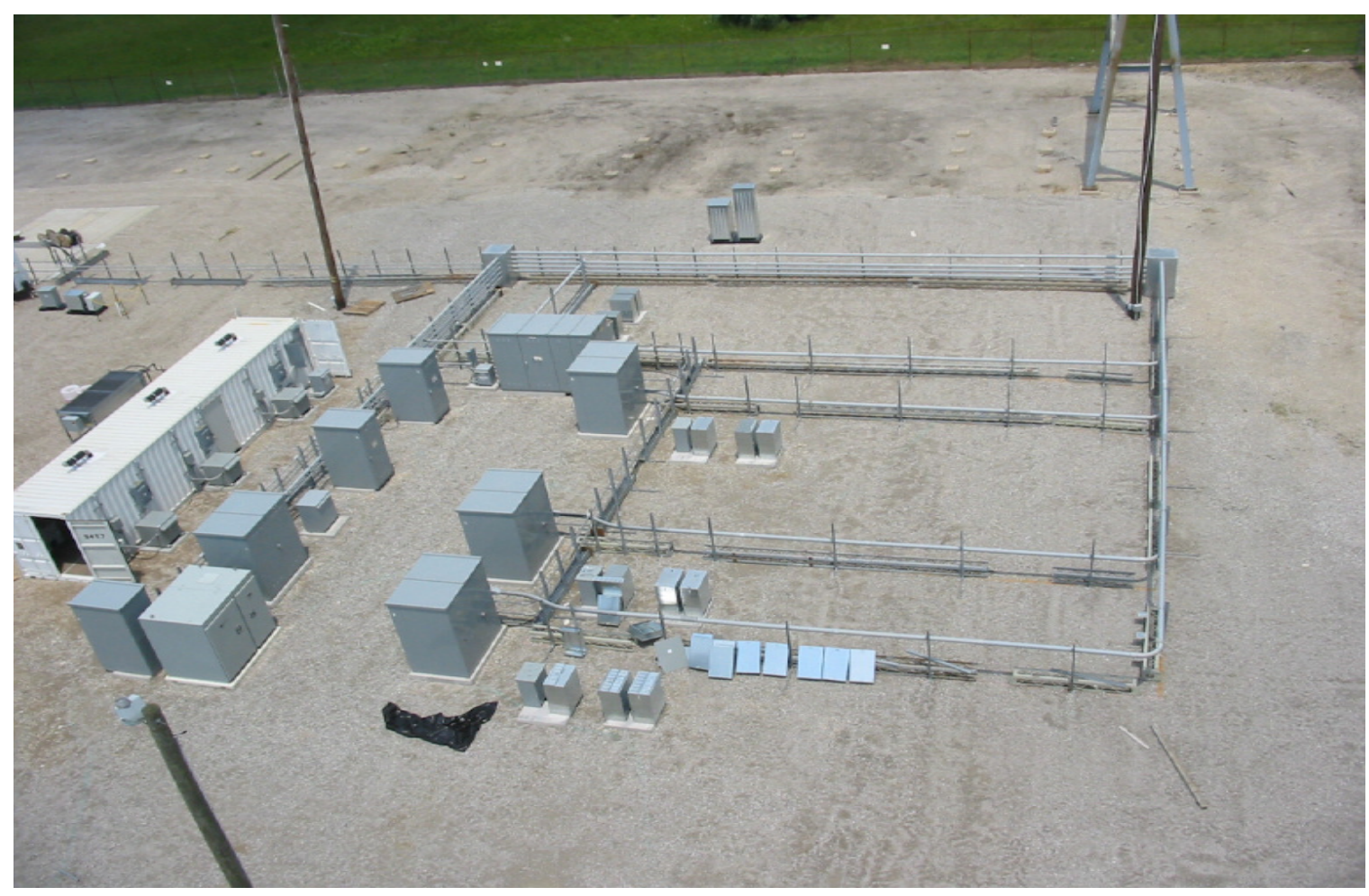

Figure 1 - CERTS Microgrid Aerial Photo

There are 6 zones in the Test Bed with Zones 2 - 6 contained within the CERTS Microgrid design and Zone 1 being the utility interface and referred to as the point-ofcommon coupling (PCC) to the grid. Each zone is protected by a Schweitzer SEL-351 relay. Faults of varying magnitude can be applied to each zone through an additional breaker which allows fault application and removal.

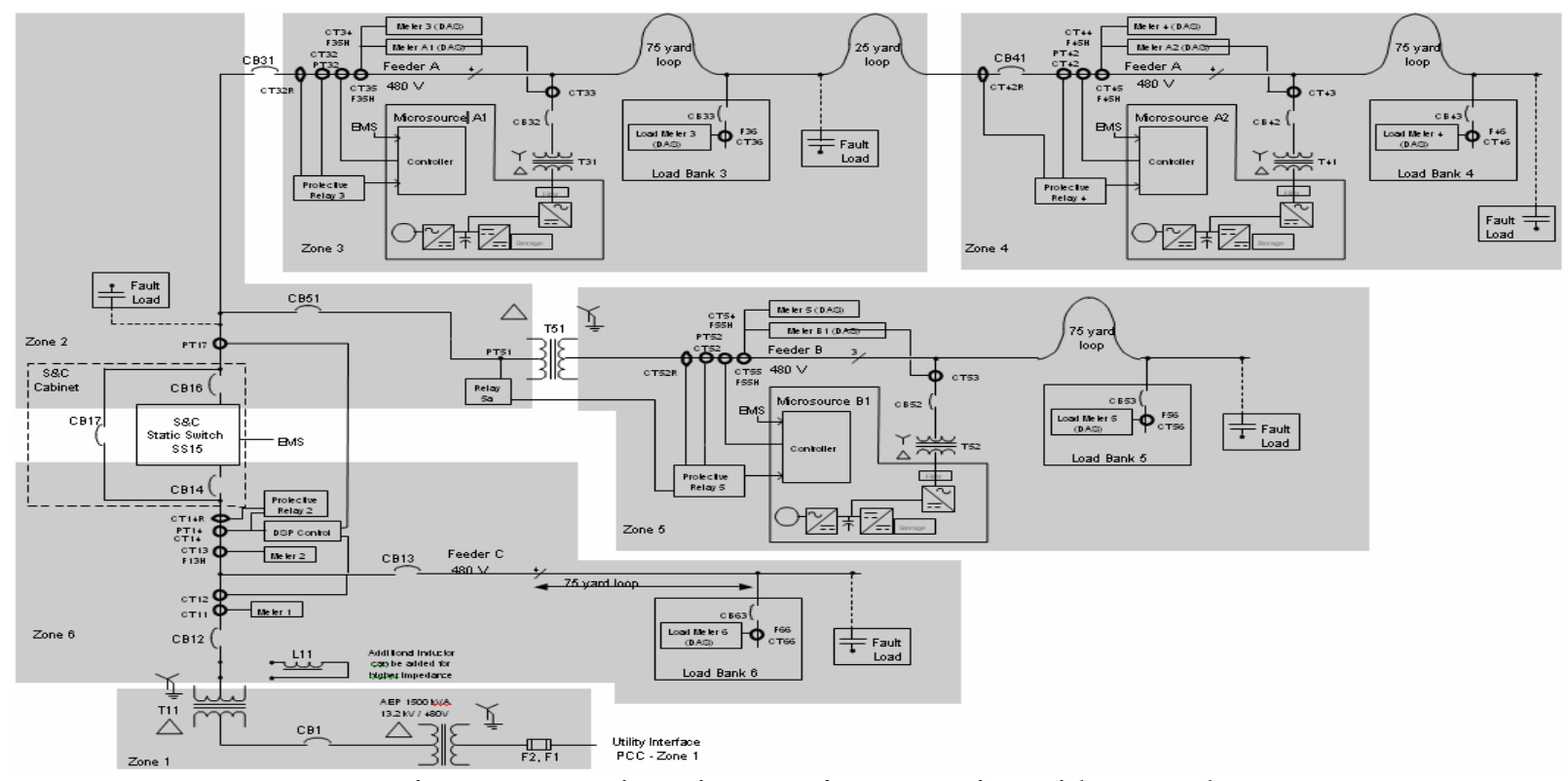

Figure 2 - One Line Diagram of CERTS Microgrid Test Bed 


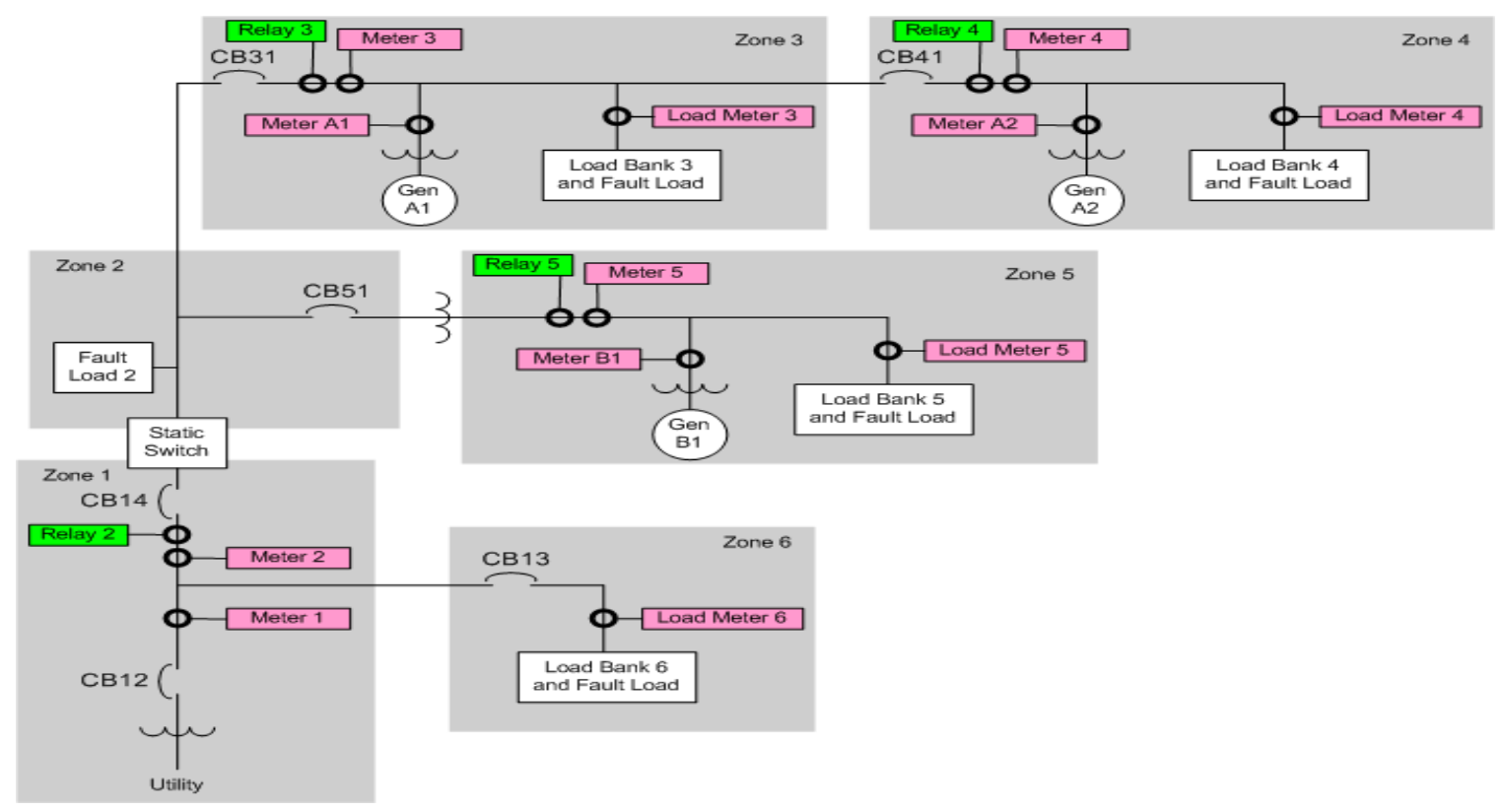

Figure 3 - Simplified diagram of Test Bed showing Meter and Relay locations

There are twelve PML ION 7650 meters placed through out the microgrid and shown in Figure 3, which monitor electrical system conditions, plus acquire phase current and voltage waveforms; and calculate RMS values of voltage, current, active power, reactive power, and frequency. 


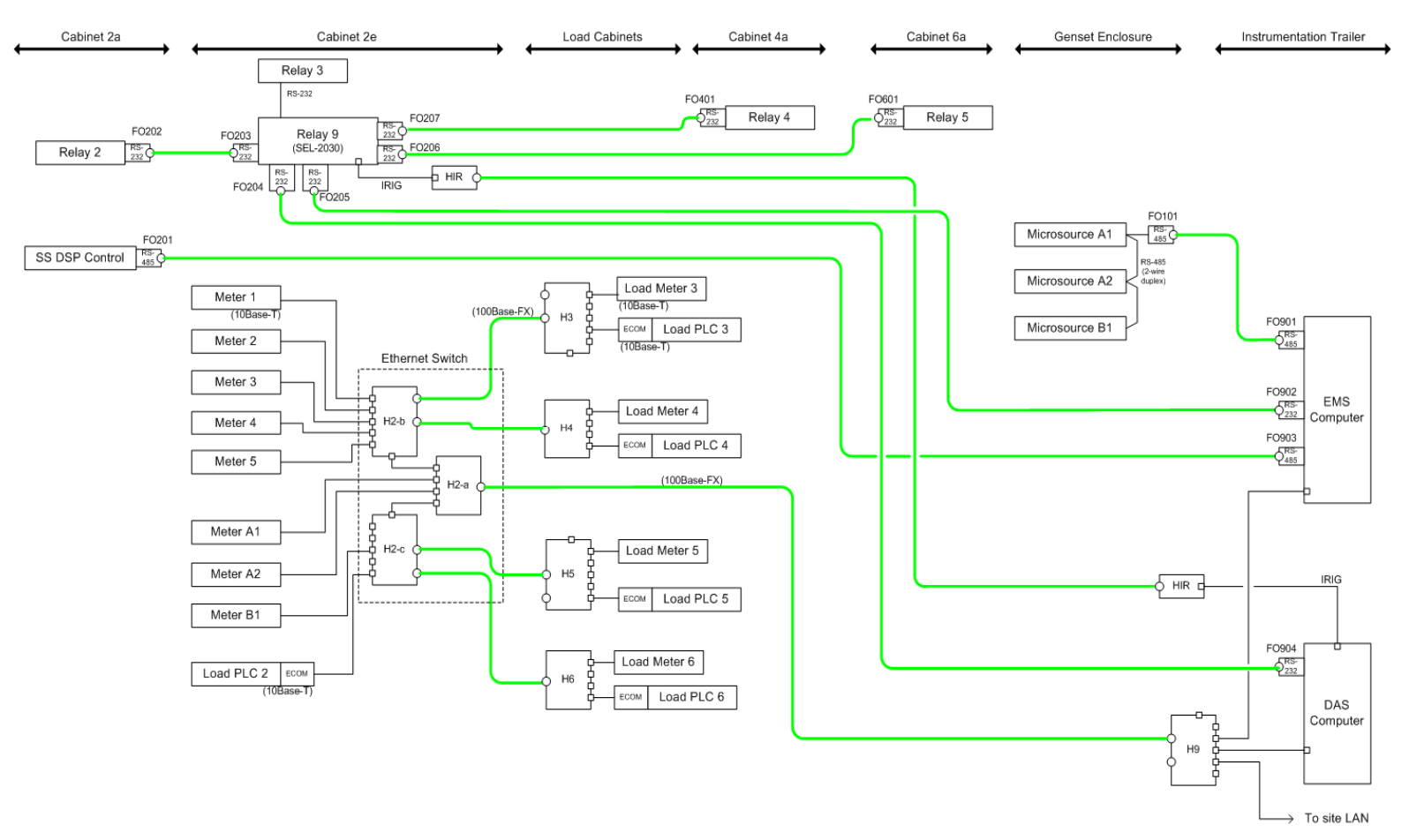

Figure 4 - Diagram of DAS \& EMS Data networks

An Ethernet network was provided as shown in Figure 4, for communications between all meters, load control PLCs, and the Data Acquisition System (DAS) computer, using fiber-optic links and switches. The DAS and Energy Management System (EMS) computers were also networked into the local Dolan Local Area Network (LAN) and to a secure Website with user ID and password protection. Additional serial links, using fiber optic converters, connect all relays, static switch Digital Signal Processor (DSP) controller, and TECOGEN Gen-set controls to the EMS computer.

\subsection{PROPOSED TEST PLAN}

The CERTS Microgrid Test Plan was developed by the CERTS Microgrid Team to demonstrate the unique concepts of control and protection of the CERTS Microgrid. This test plan was reviewed by a Technical Advisory Committee outside the CERTS Microgrid Team and then implemented by American Electric Power. CERTS Microgrid Test Plan consists of 12 sections with 5 of them detailing desired tests, starting at section 6.0, to demonstrate the controls and concepts of the CERTS Microgrid. The other 7 sections pertain to safety procedures, equipment calibration, and documentation. Each section of the test plan is described below.

- Section 1 - "Purpose, References, and Definitions" describes the purpose of the test plan, helpful references for further explanation of how the test bed was created, and definitions used through out the test plan. 
- Section 2 - "Responsibilities" informs personnel of their responsibilities while working on or near the CERTS Microgrid test site.

- Section 3 - “Training - Team Members" lists the mandatory training needed by personnel before they can work on or near the CERTS Microgrid test site.

- Section 4 - "Procedure - CERTS Microgrid Test Bed Lockout/Tagout" entails how to safely shut down the equipment and lockout/tagout the closest upstream disconnect to work on or near equipment.

- Section 5 - "Procedure - General" is the daily procedures performed at the CERTS Microgrid Test Site, prior to performing a test from Section 6 through Section 10.

- Section 6 - "Procedure - Microgrid Test Bed System Checkout" was designed to check control and operation of the static switch, basic power and voltage control of the Gen-sets, and a preliminary check of the protection scheme. The goal is to assure that the test bed is operating and ready to perform the tests described in the remaining sections of the test plan document.

- Section 7 - "Validate Protection Settings \& Initial Fault Testing" is designed to examine a preliminary set of fault (i.e. overload simulating a fault) condition tests to ensure protection and safety of the Micro-grid test Bed, while performing other planned tests. The goal is to test and adjust protection settings to achieve the most ideal conditions and protection design.

- Section 8 - "Procedure - Reduced System Tests" is a limited set of tests to build confidence that the Gen-set inverter controls are working correctly. This includes unit control, zone control, and mixed power controls, in conjunction with limit controls and synchronized closing of the static switch. These tests are based on the TECOGEN/THE SWITCH factory acceptance testing.

- Section 9 - "Procedure - Demonstration Tests of Control Power Flow" demonstrates the flexibility of the Micro-grid both grid connected and islanded for different loads, power flows and impact on the utility. 
- Section 10 - "Procedure - Test Difficult Loads" determines operation limits of the Micro-grid (i.e. power quality, protection and inverter limits) with low pf loads, motor loads, harmonic loads and unbalance loads.

- Section 11 - "Hazards \& Mitigation" informs the personnel of hazards that may exist while working on or near the CERTS Micro-grid test site and how to mitigate them.

- Section 12 - “Quality Assurance” ensures quality for the acquiring data results by providing a checklist reminder for personnel.

\subsection{TESTS PERFORMED IN SECTION 9.0}

Prior to each test day, the person in charge performed a job safety briefing (JSB) with barricades and test setup inspected for safety and compliance. A minimum of two people were on-site during each planned test.

Visual and audible alarms were used to warn persons that energized testing was being performed in the Microgrid Test Bed area. The visual alarm consisted of a portable red flashing light, located between the Control Trailer and Gen-set Enclosure. An audible alarm, consisting of a portable wireless motion detector, was located at the front gate of the Walnut Test Site with the fence gate "Closed", not locked, and audible alarm in the trailer operational during test(s).

Barricades were set up around the Micro-grid Test Bed area (i.e., saw-horse style barricades with a "Red" plastic chain surrounded the test area containing the Gen-set Enclosure, Micro-grid switching cabinets, plus load and fault bank cabinets).

Prior to performing tests, the Test Engineer or Technical Consultant verified that all personnel and visitors were properly protected and in assigned locations. Personnel were in or adjacent to the Control Trailer while tests were being performed. All nonessential personnel either left the main site or were sheltered in the Control Trailer.

For all tests the following waveforms were captured and recorded in the DAS for voltage $(\mathrm{V})$ and current $(\mathrm{I})$. From these waveforms real power $(\mathrm{kW})$, reactive power $(\mathrm{kVAr})$, and frequency (freq) were post calculated by the PQView software. Frequency measurements in this report should be used for steady state information and not used for transient analysis, due to the calculation and filtering methods employed. Below is a list of the meters capturing this data. 
- Meters 1, 2, 3, 4 \& 5

- Load Meters 3, 4, 5, \& 6

- Meters A1, A2 \& B1

- Meter 2 also measures the voltage across the static switch on phase A

Schweitzer event reports were also captured for each event, along with breaker and static switch status, such as Open or Close.

Section 9 tests were designed to demonstrate the flexibility of the CERTS Microgrid connected to the utility grid and islanded. Various loads are applied to the microgrid while in unit control and zone control mode along with different power settings of the Gen-sets.

Tests performed in this section included inductor L11 in the circuit, reflecting weak utility grid conditions. Load on Feeder A and Feeder B were less than 170kW and Feeder $\mathrm{C}$ was such that there was no power going into the utility grid. All the loads included an approximate 0.9 power factor (pf) when possible and the motor load was connected and included as part of Zone 3.

Weak grid conditions were tested in the beginning of the test plan to minimize any damage to equipment. Time constraints forced a decision by the Team to postpone strong grid testing to a later date with concentration on weak grid testing. Therefore, strong grid tests were postponed from Tests 9.1, 9.2, 9.3 and 9.4. Tests 9.1-9.3 were required to verify and document power flow and Microgrid frequency changes when transitioning from utility connected to an islanded mode of operation. In each test, 9.1 9.3, a series of tests were performed that vary in the amount of load that is applied to the microgrid in a weak grid scenario along with the power settings of each Gen-set. The difference between each test is the type of control mode that the Gen-sets are in. In Test 9.1, all the Gen-sets are set for unit control mode and then the next test, 9.2, all the Gensets are set for zone control mode. Test 9.3 mixes the unit and zone control modes of the Gen-sets during each test.

\subsection{ANALYSES OF TEST RESULTS}

\subsection{SECTION 9 - POWER FLOW CONTROL TESTS}

\subsubsection{Unit Control Mode, Weak Grid}

Performance Goal: 
Verify and document power flow and micro-grid frequency changes when transitioning from utility connected mode to an islanded mode of operation.

Four tests were completed in this section with each test event and settings listed in Table 16a. During each test Gen-sets A1, A2, and B1 were set for unit power control mode of operation. Each of the four tests varied the output power command set-point in each Gen-set, along with varying the load settings in load banks LB3, LB4, LB5 and LB6. During each test, inductor L11 was in series with the utility grid to simulate a weak grid condition at the PCC to the microgrid.

\begin{tabular}{|c|c|c|c|c|c|c|c|c|c|c|c|}
\hline $\begin{array}{c}\text { Test } \\
\text { Event }\end{array}$ & $\begin{array}{c}\text { Gen- } \\
\text { set } \\
\text { A1 }\end{array}$ & $\begin{array}{c}\text { Gen } \\
\text {-set } \\
\text { A2 }\end{array}$ & $\begin{array}{c}\text { Gen } \\
\text {-set } \\
\text { B1 }\end{array}$ & $\begin{array}{c}\text { Meter } \\
2\end{array}$ & $\begin{array}{c}\text { Meter } \\
3\end{array}$ & $\begin{array}{c}\text { Meter } \\
4\end{array}$ & $\begin{array}{c}\text { Meter } \\
5\end{array}$ & LB 3 & LB 4 & LB 5 & LB 6 \\
\hline 9.1 .7 & $\begin{array}{c}\text { Unit, } \\
30 \\
\text { kW }\end{array}$ & $\begin{array}{c}\text { Unit, } \\
30 \\
\mathrm{~kW}\end{array}$ & $\begin{array}{c}\text { Unit, } \\
30 \\
\text { kW }\end{array}$ & $\begin{array}{l}+30 \\
\mathrm{~kW}\end{array}$ & $\begin{array}{l}+20 \\
\mathrm{~kW}\end{array}$ & $\begin{array}{l}+10 \\
\mathrm{~kW}\end{array}$ & $\begin{array}{l}+10 \\
\mathrm{~kW}\end{array}$ & $\begin{array}{c}40 \mathrm{~kW} \\
20 \\
\mathrm{kVAr}\end{array}$ & $\begin{array}{c}40 \mathrm{~kW} \\
20 \\
\mathrm{kVAr}\end{array}$ & $\begin{array}{c}40 \mathrm{~kW} \\
20 \\
\mathrm{kVAr}\end{array}$ & $\begin{array}{c}20 \mathrm{~kW} \\
10 \\
\mathrm{kVAr}\end{array}$ \\
\hline 9.1 .8 & $\begin{array}{c}\text { Unit, } \\
40 \\
\text { kW }\end{array}$ & $\begin{array}{c}\text { Unit, } \\
30 \\
\mathrm{~kW}\end{array}$ & $\begin{array}{c}\text { Unit, } \\
25 \\
\text { kW }\end{array}$ & $\begin{array}{l}+15 \\
\mathrm{~kW}\end{array}$ & $\begin{array}{l}-10 \\
\mathrm{~kW}\end{array}$ & $\begin{array}{l}-15 \\
k W\end{array}$ & $\begin{array}{l}+25 \\
\mathrm{~kW}\end{array}$ & $\begin{array}{c}20 \mathrm{~kW} \\
10 \\
\mathrm{kVAr}\end{array}$ & $\begin{array}{c}40 \mathrm{~kW} \\
20 \\
\mathrm{kVAr}\end{array}$ & $\begin{array}{c}50 \mathrm{~kW} \\
20 \\
\mathrm{kVAr}\end{array}$ & $\begin{array}{c}20 \mathrm{~kW} \\
10 \\
\mathrm{kVAr}\end{array}$ \\
\hline 9.1 .9 & $\begin{array}{c}\text { Unit, } \\
40 \\
\text { kW }\end{array}$ & $\begin{array}{c}\text { Unit, } \\
30 \\
\text { kW }\end{array}$ & $\begin{array}{c}\text { Unit, } \\
30 \\
\text { kW }\end{array}$ & $\begin{array}{l}-10 \\
k W\end{array}$ & $\begin{array}{l}-30 \\
k W\end{array}$ & $\begin{array}{l}+10 \\
\mathrm{~kW}\end{array}$ & $\begin{array}{l}+20 \\
\mathrm{~kW}\end{array}$ & $\begin{array}{c}25 \mathrm{~kW} \\
10 \\
\mathrm{kVAr}\end{array}$ & $\begin{array}{c}15 \mathrm{~kW} \\
10 \\
\mathrm{kVAr}\end{array}$ & $\begin{array}{c}50 \mathrm{~kW} \\
20 \\
\mathrm{kVAr}\end{array}$ & $\begin{array}{c}30 \mathrm{~kW} \\
10 \\
\mathrm{kVAr}\end{array}$ \\
\hline 9.1 .10 & $\begin{array}{c}\text { Unit, } \\
30 \\
\mathrm{~kW}\end{array}$ & $\begin{array}{c}\text { Unit, } \\
20 \\
\text { kW }\end{array}$ & $\begin{array}{c}\text { Unit, } \\
45 \\
\text { kW }\end{array}$ & $\begin{array}{l}+20 \\
\mathrm{~kW}\end{array}$ & $\begin{array}{l}+40 \\
\mathrm{~kW}\end{array}$ & $\begin{array}{c}20 \\
\mathrm{~kW}\end{array}$ & $\begin{array}{l}-20 \\
k W\end{array}$ & $\begin{array}{c}50 \mathrm{~kW} \\
20 \\
\mathrm{kVAr}\end{array}$ & $\begin{array}{c}40 \mathrm{~kW} \\
20 \\
\mathrm{kVAr}\end{array}$ & $\begin{array}{c}25 \mathrm{~kW} \\
10 \\
\mathrm{kVAr}\end{array}$ & $\begin{array}{c}30 \mathrm{~kW} \\
10 \\
\mathrm{kVAr}\end{array}$ \\
\hline
\end{tabular}

Table 16a - Unit Control Mode, Weak Grid Settings for Load Banks and Gen-sets

The procedure for each test event began with setting the $\mathrm{kW}$ and $\mathrm{kVAr}$ load (i.e., balanced load) conditions, as indicated in Table 16a, using the EMS at each Load Bank to achieve an approximate 0.9 power factor. Before loads were brought on-line, Gen-sets A1, A2 and B1 were started to allow them to warm up. After the Gen-sets ran for a few minutes and steady-state conditions established, the loads were brought on-line, the static switch was allowed to close, and measurements were taken from Meters 2, 3, 4 and 
5. The measurements were then compared with the expected values in Table 16a. When the measured values coincided with the expected values from the table, then a manual open of the static switch was initiated to isolate/island the microgrid from the utility grid. Gen-sets were observed at this time to make sure they increased or decreased their output power as planned to satisfy the loads at load banks (i.e., LB3, LB4, LB5 and LB6) and that a smooth transition occurred. When all the measurements were verified and recorded in the DAS Database, the manual open signal to the static switch was released, allowing it to synchronize and close back into the utility grid. As soon as the static switch synchronized and re-closed, all data (i.e., from the manual open event and the reconnection event) was verified and recorded in the DAS Database. Loads and Unit Power Control set-points were then changed to the settings for the next test. Test results are explained in the following paragraphs.

For Test 9.1.7 the measured values, after the Gen-sets were warmed up and load banks brought on-line, were approximately $40 \mathrm{~kW}$ at Meter $1,22 \mathrm{~kW}$ at Meter 2, $12.5 \mathrm{~kW}$ at Meter $3,3.5 \mathrm{~kW}$ at Meter $4,7 \mathrm{~kW}$ at Meter 5, shown in Table 16b. From the microgrid, $26.5 \mathrm{~kW}$ was produced by Gen-set A1, $31.5 \mathrm{~kW}$ produced by Gen-set A2, and $28 \mathrm{~kW}$ produced by Gen-set B1. The load banks were slightly less than planned with $35.5 \mathrm{~kW}$ at LB3, $34.7 \mathrm{~kW}$ at LB4, $34.8 \mathrm{~kW}$ at LB5, and $18.32 \mathrm{~kW}$ at LB6. These measurements were relatively close to the expected values in Table 16a, but not exact due to temperature, phase voltages and electrical losses in conductors. In addition, the $40 \mathrm{~kW}$ setting for LB3, LB4 and LB5; and 20kW setting for LB6 were also below the selected set values. The deviation of selected values versus actual values was consistent for the other three tests in this section. At the time of these measurements, the voltage and frequency was $276 \mathrm{~V}$ and $59.99 \mathrm{~Hz}$ at the static switch (i.e., Meter 2) when connected to the utility grid; and $276 \mathrm{~V}$ and $59.99 \mathrm{~Hz}$ at Meter 3. The power factor at each load was approximately 0.87 which is a $3 \%$ difference from the desired 0.9 power factor.

\begin{tabular}{|c|c|c|c|c|c|c|c|c|c|c|c|c|}
\hline & GENSET A1 & GENSET A2 & GENSET B1 & METER 1 & METER 2 & METER 3 & METER 4 & METER 5 & LB 3 & LB 4 & LB 5 & LB 6 \\
\hline INITIAL SETUP & UNIT +30kW & UNIT +30kW & UNIT +30kW & & $+30 \mathrm{~kW}$ & $+20 \mathrm{~kW}$ & & $+10 \mathrm{~kW}$ & $40 \mathrm{~kW}$ & $40 \mathrm{~kW}$ & $40 \mathrm{~kW}$ & $20 \mathrm{~kW}$ \\
\hline \multicolumn{13}{|c|}{ GRID-TO-ISLAND } \\
\hline \multicolumn{13}{|l|}{ Start } \\
\hline Voltage (V) & & & & & 276 & 276 & & & & & & \\
\hline Current (A) & 32 & 40 & 39 & 80 & 56 & 41 & 15 & 11 & & & & \\
\hline Real Power (kW) & 26.5 & 31.5 & 28 & 40 & 22 & 12.5 & 3.5 & 7 & 35.15 & 34.7 & 34.8 & 18.32 \\
\hline
\end{tabular}




\begin{tabular}{|c|c|c|c|c|c|c|c|c|c|c|c|c|}
\hline Reactive Power (kVAr) & 0 & 8 & 14.5 & 51 & 41 & 31.5 & 11 & 5 & 19.7 & 19.7 & 19.7 & 10.675 \\
\hline Power Factor (pf) & 1.00 & 0.97 & 0.89 & & & & & & 0.87 & 0.87 & 0.87 & 0.86 \\
\hline Frequency $(\mathrm{Hz})$ & & & & & 59.99 & 59.99 & & & & & & \\
\hline \multicolumn{13}{|l|}{ End } \\
\hline Voltage (V) & & & & & 281 & 268 & & & & & & \\
\hline Current (A) & 42 & 54 & 52.5 & 26 & 0 & 5 & 6.5 & 9 & & & & \\
\hline Real Power (kW) & 32 & 37 & 33.6 & 19 & 0 & -2.5 & -4 & -0.25 & 33.3 & 32.85 & 33.3 & 19 \\
\hline Reactive Power (kVAr) & 13 & 21 & 25 & 12 & 0 & 3 & -3 & -6 & 18.7 & 18.7 & 18.9 & 11.25 \\
\hline Power Factor (pf) & 0.93 & 0.87 & 0.80 & & & & & & 0.87 & 0.87 & 0.87 & 0.86 \\
\hline Frequency $(\mathrm{Hz})$ & & & & & 59.988 & 59.944 & & & & & & \\
\hline \multicolumn{13}{|c|}{ ISLAND-TO-GRID } \\
\hline \multicolumn{13}{|l|}{ Start } \\
\hline Voltage (V) & & & & & 280 & 268 & & & & & & \\
\hline Current (A) & 42 & 52 & 52 & 26 & 0 & 5 & 6 & 8 & & & & \\
\hline Real Power (kW) & 32 & 36.5 & 33.5 & 19 & 0 & -2 & -4 & -0.25 & 33.35 & 32.7 & 33.1 & 18.75 \\
\hline Reactive Power (kVAr) & 13 & 22 & 25 & 12 & 0 & 4 & -3 & -6 & 18.7 & 18.75 & 18.95 & 11.24 \\
\hline Power Factor (pf) & 0.93 & 0.86 & 0.80 & & & & & & 0.87 & 0.87 & 0.87 & 0.86 \\
\hline Frequency $(\mathrm{Hz})$ & & & & & 60.009 & 59.942 & & & & & & \\
\hline \multicolumn{13}{|l|}{ End } \\
\hline Voltage (V) & & & & & 276 & 272 & & & & & & \\
\hline Current (I) & 30 & 30 & 35 & 85 & 60 & 45 & 16 & 13 & & & & \\
\hline Real Power (kW) & 23.5 & 28.5 & 25 & 47 & 30 & 17 & 6 & 9.5 & 35.1 & 34.45 & 34.55 & 17.9 \\
\hline Reactive Power (kVAr) & 0 & 9 & 15.5 & 50 & 39 & 31 & 10 & 4 & 19.7 & 19.75 & 19.75 & 10.7 \\
\hline Power Factor (pf) & 1.00 & 0.95 & 0.85 & & & & & & 0.87 & 0.87 & 0.87 & 0.86 \\
\hline Frequency $(\mathrm{Hz})$ & & & & & 60.01 & 60.01 & & & & & & \\
\hline
\end{tabular}

Table 16b - Test 9.1.7 results with dark squares in the table meaning that measurements were not needed. 
According to the measurements in Table $16 \mathrm{~b}$ when connected to the utility grid, each Gen-set in the microgrid was set at $30 \mathrm{~kW}$ which was not enough to satisfy the loads in LB3, LB4 and LB5 and needed approximately 22kW from the utility grid, as measured by Meter 2. Total power from the utility grid was measured at approximately $40 \mathrm{~kW}$ from Meter 1, because it was meeting the demands of not only the microgrid loads, but the load at LB6. Once all data was verified and recorded into the DAS Database, the static switch was directed by the EMS to manually open.

When the static switch opened, Meter 1 decreased to approximately $19 \mathrm{~kW}$ satisfying the load demand in LB6 which was approximately 19kW and not supplying any power beyond the static switch to the microgrid loads. $0 \mathrm{~kW}$ was recorded at Meter 2, showing that power was not flowing through the static switch. LB3, LB4 and LB5 loads reduced slightly to $33.3 \mathrm{~kW}, 32.85 \mathrm{~kW}$ and $33.3 \mathrm{~kW}$, respectively. This load reduction resulted from a voltage drop in the microgrid, shown in Figure 29a, from approximately $276 \mathrm{~V}$ (i.e., $276 \mathrm{~V}$ on A-phase and $273 \mathrm{~V}$ on B- \& C-phases) when connected to the utility grid to $268 \mathrm{~V}$ at Meter 3 (i.e., on A-, B-, and C-phases) when islanded.

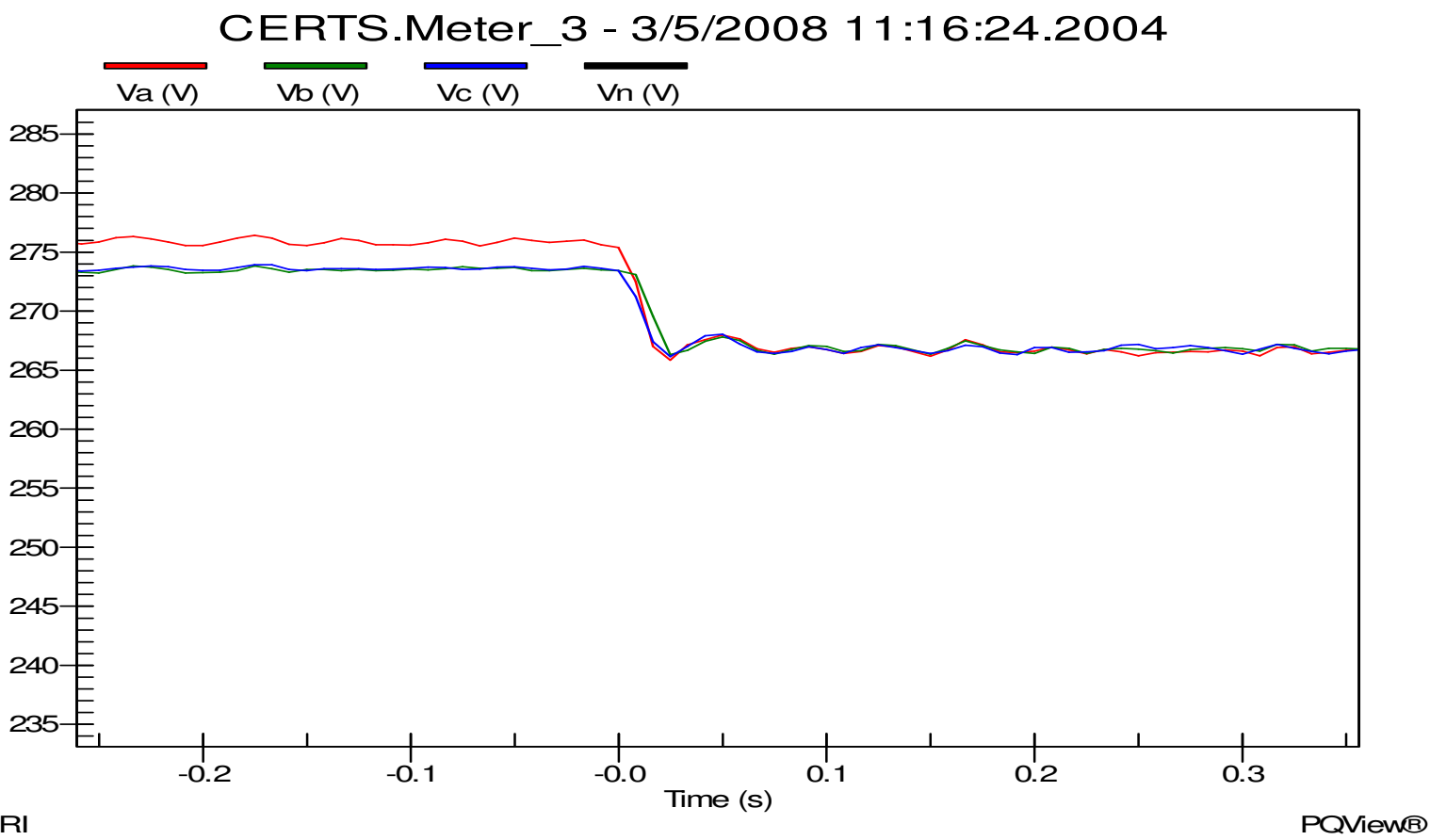

Figure 29a - Meter 3 voltage before/after the static switch opened, islanding the microgrid from the utility grid

Frequency change in the microgrid, shown in Figure 29b, dropped from $59.99 \mathrm{~Hz}$ when connected to the utility grid to approximately $59.94 \mathrm{~Hz}$ when islanded. This change in frequency was part of the CERTS algorithm which allowed the Gen-sets to increase their output power to satisfy the load demands. Gen-set A1, A2, and B1 increased their output power to approximately $32 \mathrm{~kW}, 37 \mathrm{~kW}$ and $33.6 \mathrm{~kW}$, respectively. Meters 3,4 and 
5 were negative values $(-2.5 \mathrm{~kW},-4 \mathrm{~kW}$ and $-0.25 \mathrm{~kW}$, respectively), indicating the Gensets were satisfying the loads in the microgrid and the power losses in the line. All data was verified and recorded into the DAS Database. The microgrid ran for a couple of minutes in this electrical state before the manual open signal was released and allowed the static switch to synchronize to the utility grid.

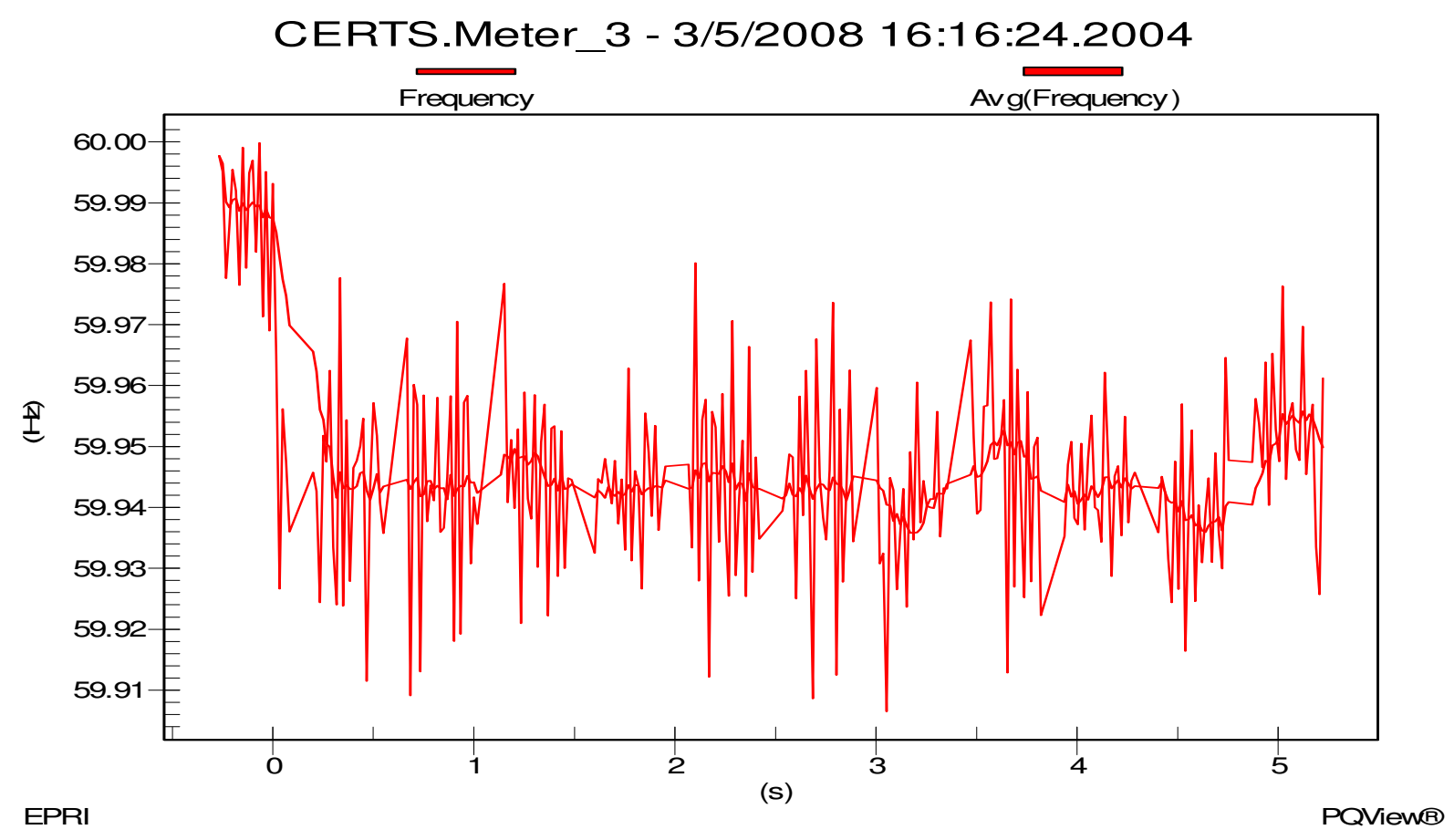

Figure 29b - Meter 3 frequency before/after the static switch opened, islanding the microgrid from the utility grid.

As soon as the static switch closed and steady-state conditions established, the measurements at the Gen-sets, Meters and Load Banks were compared to the initial conditions at the beginning of the test. The measured values when synchronized to the utility grid were very similar to the initial conditions. Voltage and frequency in the microgrid increased from $268 \mathrm{~V}$ to $276 \mathrm{~V}$ and $59.944 \mathrm{~Hz}$ to $60.01 \mathrm{~Hz}$. The power factor of approximately 0.87 remained the same throughout the test. After all the data was verified and recorded into the DAS Database the Gen-sets and Load Banks set-points were changed according to the next test (9.1.8) in Table 16a.

For Test 9.1.8 the measured values, after the Gen-sets were warmed up and load banks brought on-line, were approximately $35 \mathrm{~kW}$ at Meter 1, 17kW at Meter 2, $-10 \mathrm{~kW}$ at Meter $3,6 \mathrm{~kW}$ at Meter 4 , and $24 \mathrm{~kW}$ at Meter 5, shown in Table 16c. From the microgrid, $33.75 \mathrm{~kW}$ was produced by Gen-set A1, $29 \mathrm{~kW}$ produced by Gen-set A2, and $20.5 \mathrm{~kW}$ produced by Gen-set B1. The load banks were slightly less than planned with $18.05 \mathrm{~kW}$ at LB3, 34.6kW at LB4, $44.5 \mathrm{~kW}$ at LB5, and $18.1 \mathrm{~kW}$ at LB6. These measurements were 
relatively close to the expected values in Table 16a but not exact, due to temperature, phase voltages and electrical losses in the conductors. In addition, the desired $\mathrm{kW}$ settings for LB3, LB4, LB5 and LB6 were also below the set values. This deviation of set values verses actual values was consistent for the other tests in this section. At the time of these measurements, the voltage and frequency when connected to the utility grid was $277 \mathrm{~V}$ and $60.008 \mathrm{~Hz}$ at the static switch (i.e., Meter 2); and $277 \mathrm{~V}$ and $60.008 \mathrm{~Hz}$ at Meter 3. The power factor at LB3, LB4 and LB6 was approximately 0.87 and 0.91 at LB5 which is close to the desired 0.9 power factor.

\begin{tabular}{|c|c|c|c|c|c|c|c|c|c|c|c|c|}
\hline \multicolumn{13}{|l|}{9.1 .8} \\
\hline & GENSET A1 & GENSET A2 & GENSET B1 & METER 1 & METER 2 & METER 3 & METER 4 & METER 5 & LB 3 & LB 4 & LB 5 & LB 6 \\
\hline INITIAL SETUP & UNIT +40kW & UNIT +30kW & UNIT +25kW & & $+15 \mathrm{~kW}$ & $-10 \mathrm{~kW}$ & & $+25 \mathrm{~kW}$ & $20 \mathrm{~kW}$ & $40 \mathrm{~kW}$ & $50 \mathrm{~kW}$ & $20 \mathrm{~kW}$ \\
\hline \multicolumn{13}{|c|}{ GRID-TO-ISLAND } \\
\hline \multicolumn{13}{|l|}{ Start } \\
\hline Voltage (V) & & & & & 277 & 277 & & & & & & \\
\hline Current (A) & 41 & 37 & 30 & 71 & 50 & 35 & 18 & 30 & & & & \\
\hline Real Power (kW) & 33.75 & 29 & 20.5 & 35 & 17 & -10 & 6 & 24 & 18.05 & 34.6 & 44.5 & 18.1 \\
\hline Reactive Power (kVAr) & -3 & 6 & 15 & 47 & 37 & 28 & 0 & 4.8 & 10.65 & 19.95 & 19.8 & 10.8 \\
\hline Power Factor (pf) & 1.00 & 0.98 & 0.81 & & & & & & 0.86 & 0.87 & 0.91 & 0.86 \\
\hline Frequency $(\mathrm{Hz})$ & & & & & 60.008 & 60.008 & & & & & & \\
\hline \multicolumn{13}{|l|}{ End } \\
\hline Voltage (V) & & & & & 280 & 269 & & & & & & \\
\hline Current (A) & 47 & 46 & 44 & 26 & 0 & 26 & 3 & 24 & & & & \\
\hline Real Power (kW) & 37.5 & 33.25 & 25 & 19 & 0 & -21 & 0 & 18 & 17.15 & 33 & 42.8 & 18.85 \\
\hline Reactive Power (kVAr) & 9 & 18 & 24.5 & 12 & 0 & 2 & 1.5 & -5.5 & 10.1 & 19 & 19 & 11.3 \\
\hline Power Factor ( $p f)$ & 0.97 & 0.88 & 0.71 & & & & & & 0.86 & 0.87 & 0.91 & 0.86 \\
\hline Frequency $(\mathrm{Hz})$ & & & & & 60.008 & 59.974 & & & & & & \\
\hline \multicolumn{13}{|c|}{ ISLAND-TO-GRID } \\
\hline \multicolumn{13}{|l|}{ Start } \\
\hline Voltage (V) & & & & & 281 & 269 & & & & & & \\
\hline
\end{tabular}




\begin{tabular}{|c|c|c|c|c|c|c|c|c|c|c|c|c|}
\hline Current (A) & 47 & 46 & 44 & 26 & 0 & 26 & 3 & 24 & & & & \\
\hline Real Power (kW) & 38 & 33.5 & 25 & 19 & 0 & -21 & 0 & 18 & 17.25 & 33.1 & 42.9 & 18.9 \\
\hline Reactive Power (kVAr) & 9 & 18 & 24.5 & 12 & 0 & 2.5 & 1 & -6 & 10.1 & 19 & 19.05 & 11.35 \\
\hline Power Factor (pf) & 0.97 & 0.88 & 0.71 & & & & & & 0.86 & 0.87 & 0.91 & 0.86 \\
\hline Frequency $(\mathrm{Hz})$ & & & & & 59.985 & 59.975 & & & & & & \\
\hline \multicolumn{13}{|l|}{ End } \\
\hline Voltage (V) & & & & & 276 & 276 & & & & & & \\
\hline Current (I) & 42 & 39 & 32 & 70 & 50 & 38 & 19 & 28 & & & & \\
\hline Real Power (kW) & 35.5 & 31 & 22.5 & 30 & 13 & -12.5 & 4.5 & 22.5 & 18.2 & 34.9 & 44.7 & 18.1 \\
\hline Reactive Power (kVAr) & -4 & 5 & 14 & 50 & 39 & 29.5 & 15 & 5 & 10.7 & 20 & 19.8 & 10.8 \\
\hline Power Factor (pf) & 0.99 & 0.99 & 0.85 & & & & & & 0.86 & 0.87 & 0.91 & 0.86 \\
\hline Frequency $(\mathrm{Hz})$ & & & & & 59.995 & 59.995 & & & & & & \\
\hline
\end{tabular}

Table 16c - Test 9.1.8 results with dark squares in the table meaning that measurements were not needed.

According to the measurements in Figure 16c when connected to the utility grid, each Gen-set in the microgrid was set at $40 \mathrm{~kW}$ for Gen-set A1, 30kW for Gen-set A2 and $25 \mathrm{~kW}$ for Gen-set B1, which was not enough to satisfy the loads in LB4 and LB5, and needed approximately $17 \mathrm{~kW}$ from the utility grid, measured by Meter 2. Total power from the utility grid was measured at approximately $35 \mathrm{~kW}$ in Meter 1, because it was meeting the demands of not only the microgrid loads, but the load at LB6. In addition, Meter 3 recorded approximately $-10 \mathrm{~kW}$, meaning Gen-set A1 was supplying power for all of LB3, 6kW to LB4, measured by Meter 4, and approximately $10 \mathrm{~kW}$ to LB5. Once all data was verified and recorded into the DAS Database, the static switch was directed by the EMS to manually open.

When the static switch opened, Meter 1 decreased to approximately $19 \mathrm{~kW}$ satisfying the load demand in LB6 which was approximately $18.85 \mathrm{~kW}$ and not supplying any power beyond the static switch to the microgrid loads. $0 \mathrm{~kW}$ was measured at Meter 2, showing that power was not flowing through the static switch. LB3, LB4 and LB5 loads reduced slightly to $17.15 \mathrm{~kW}, 33 \mathrm{~kW}$ and $42.8 \mathrm{~kW}$, respectively. This load reduction resulted from a voltage drop in the microgrid, shown in Figure 29c, from approximately 277V (i.e., 277V on A-phase, $275 \mathrm{~V}$ on B- and C-phases) when connected to the utility grid to $269 \mathrm{~V}$ on all three phases when islanded. 


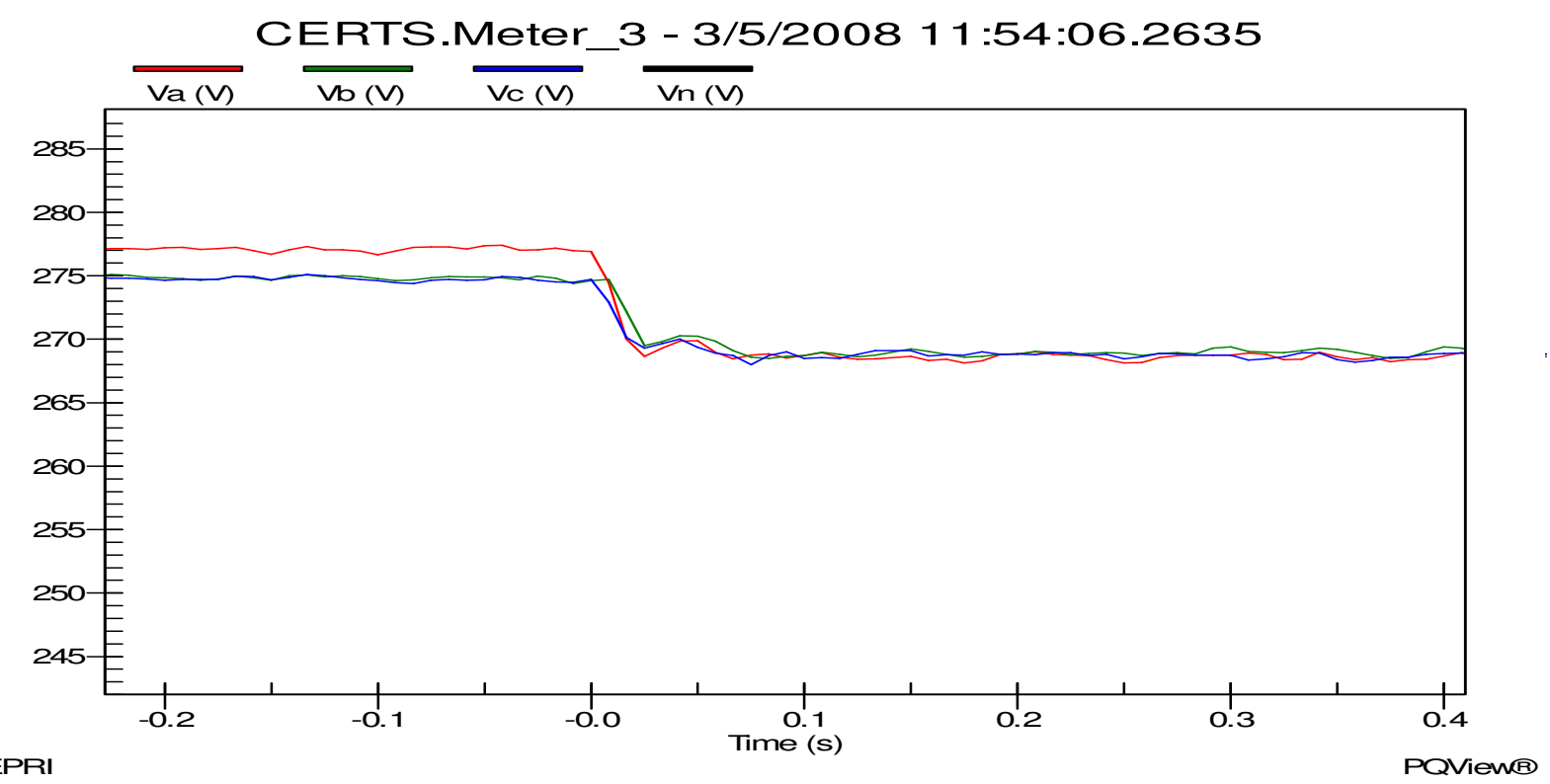

Figure 29c - Meter 3 voltage before/after the static switch opened, islanding the microgrid from the utility grid.

Frequency changed in the microgrid, shown in Figure 29d, from $60.008 \mathrm{~Hz}$ to $59.974 \mathrm{~Hz}$ when islanded. This change in frequency was part of the CERTS algorithm which allowed the Gen-sets to increase their output power to satisfy the load demands. Genset A1, A2 and B1 increased their output power to $37.5 \mathrm{~kW}, 33.25 \mathrm{~kW}$ and $25 \mathrm{~kW}$, respectively. Meter 3 indicated a negative value of $-21 \mathrm{~kW}$, meaning it supplied all of LB3 load and 18kW of LB5 at Meter 5. Meter 4 indicated 0kW showing that Gen-set A2 supplied all of LB4 load. All data was verified and recorded into the DAS Database. The microgrid ran for a couple of minutes in this electrical state before the manual open signal was released and allowed the static switch to synchronize to the utility grid. 
CERTS.Meter_3 - 3/5/2008 16:54:06.2635

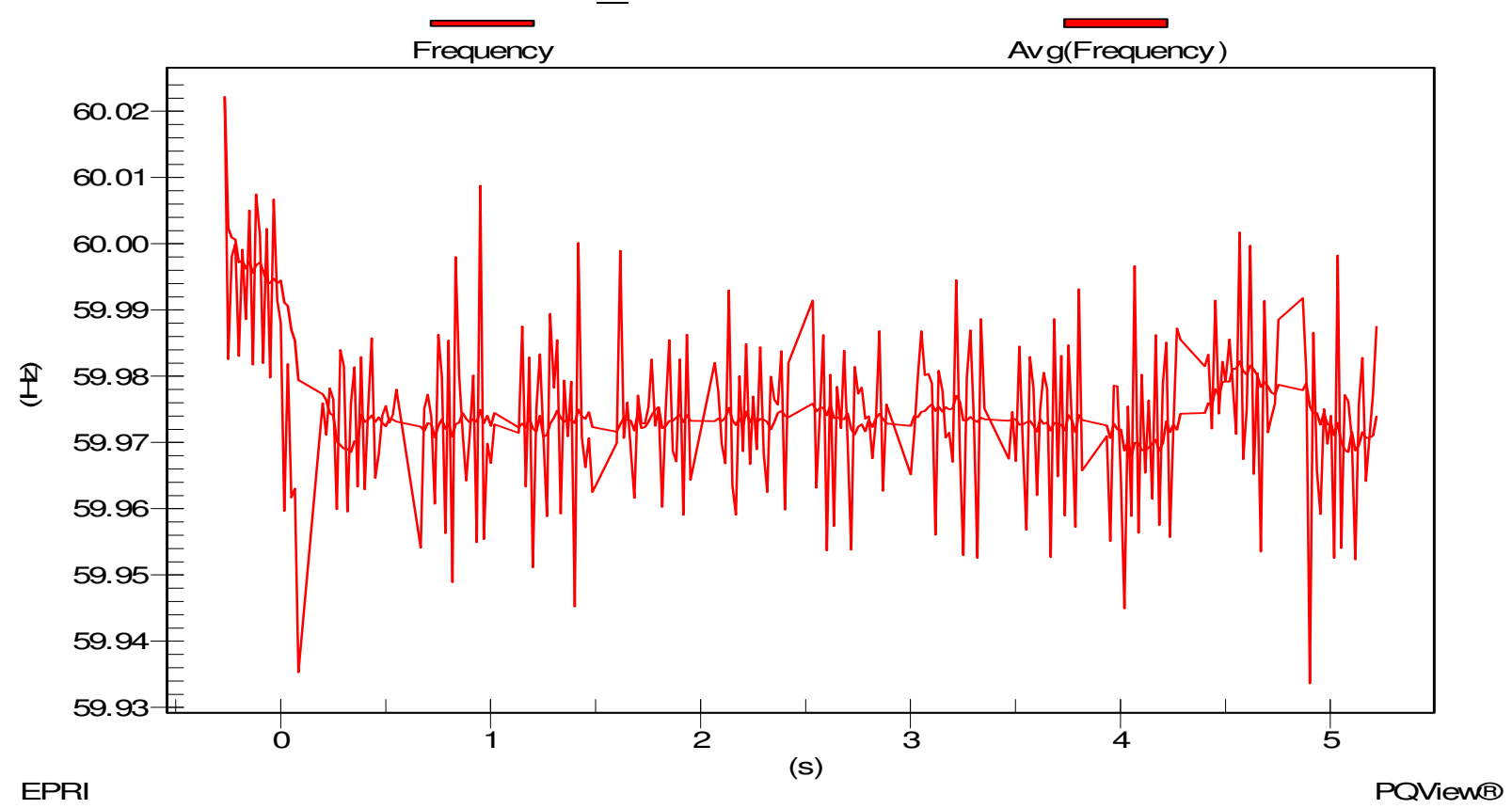

Figure 29d - Meter 3 frequency before/after the static switch opened, islanding the microgrid from the utility grid.

As soon as the static switch closed and steady-state conditions established, the measurements at the Gen-sets, Meters and Load Banks were compared to the initial conditions at the beginning of the test. The measured values were very similar to the initial test conditions which were approximately $30 \mathrm{~kW}$ at Meter 1, 13kW at Meter 2, $12.5 \mathrm{~kW}$ at Meter $3,4.5 \mathrm{~kW}$ at Meter 4 , and $22.5 \mathrm{~kW}$ at Meter 5 with $35.5 \mathrm{~kW}$ produced by Gen-set A1, 31kW produced by Gen-set A2, and $22.5 \mathrm{~kW}$ produced by Gen-set B1. Likewise, $18.2 \mathrm{~kW}$ was at LB3, $34.9 \mathrm{~kW}$ at LB4, $44.7 \mathrm{~kW}$ at LB5 and $18.1 \mathrm{~kW}$ at LB6. The microgrid voltage increased from $269 \mathrm{~V}$ to $276 \mathrm{~V}$ and frequency increased from $59.974 \mathrm{~Hz}$ to $59.995 \mathrm{~Hz}$. The power factor of approximately 0.87 remained the same throughout the test for LB3, LB4 and LB6 and 0.91 for LB5. After all data was verified and recorded into the DAS Database, Gen-sets A1, A2 and B1 were shut down manually and all load banks/alarms were reset and prepared for the next test (9.1.9) in Table 16a.

For Test 9.1.9 the measured values, after the Gen-sets were warmed up and load banks brought on-line, were approximately $15 \mathrm{~kW}$ at Meter $1,-13.5 \mathrm{~kW}$ at Meter 2, $-33 \mathrm{~kW}$ at Meter $3,-15 \mathrm{~kW}$ at Meter 4 , and $16.5 \mathrm{~kW}$ at Meter 5. From the microgrid $40.5 \mathrm{~kW}$ was produced by Gen-set A1, $32 \mathrm{~kW}$ produced by Gen-set A2, 28.5kW produced by Gen-set B1. The load banks were slightly less than planned with $23.25 \mathrm{~kW}$ at LB3, $16 \mathrm{~kW}$ at LB4, $45 \mathrm{~kW}$ at LB5, and $28.6 \mathrm{~kW}$ at LB6. These measurements were relatively close to the expected values in Table 16a but not exact, due to temperature, phase voltages and electrical losses in conductors. In addition, the desired $\mathrm{kW}$ settings for LB3, LB4, LB5 and LB6 were below the set values. This deviation of set values verses actual values was 
consistent for the other tests in this section. At the time of these measurements the voltage and frequency was $277 \mathrm{~V}$ and $59.99 \mathrm{~Hz}$ at the static switch (i.e., Meter 2) when connected to the utility grid; and $277 \mathrm{~V}$ and $59.99 \mathrm{~Hz}$ at Meter 3 . The power factor at LB4 was approximately $0.85,0.91$ at LB3 and LB5, and 0.94 at LB6 which was close to the desired 0.9 power factor.

\begin{tabular}{|c|c|c|c|c|c|c|c|c|c|c|c|c|}
\hline \multicolumn{13}{|l|}{9.1 .9} \\
\hline & GENSET A1 & GENSET A2 & GENSET B1 & METER 1 & METER 2 & METER 3 & METER 4 & METER 5 & LB 3 & LB 4 & LB 5 & LB 6 \\
\hline INITIAL SETUP & UNIT +40kW & UNIT +30kW & $\begin{array}{c}\text { UNIT } \\
+30 \mathrm{~kW}\end{array}$ & & $-10 \mathrm{~kW}$ & $-30 \mathrm{~kW}$ & & $+20 \mathrm{~kW}$ & $25 \mathrm{~kW}$ & $15 \mathrm{~kW}$ & $50 \mathrm{~kW}$ & $30 \mathrm{~kW}$ \\
\hline \multicolumn{13}{|c|}{ GRID-TO-ISLAND } \\
\hline \multicolumn{13}{|l|}{ Start } \\
\hline Voltage (V) & & & & & 277 & 277 & & & & & & \\
\hline Current (A) & 47 & 39 & 38 & 60 & 45 & 50 & 22 & 22 & & & & \\
\hline Real Power (kW) & 40.5 & 32 & 28.5 & 15 & -13.5 & -33 & -15 & 16.5 & 23.25 & 16 & 45 & 28.6 \\
\hline Reactive Power (kVAr) & -5 & 3 & 12.5 & 45 & 35 & 23 & 7 & 7 & 10.8 & 10 & 19.9 & 10.8 \\
\hline Power Factor (pf) & 0.99 & 1.00 & 0.92 & & & & & & 0.91 & 0.85 & 0.91 & 0.94 \\
\hline Frequency (Hz) & & & & & 59.99 & 59.99 & & & & & & \\
\hline \multicolumn{13}{|l|}{ End } \\
\hline Voltage (V) & & & & & 280 & 270 & & & & & & \\
\hline Current (A) & 44 & 37 & 39 & 39 & 30 & 0 & 15 & 26 & & & & \\
\hline Real Power (kW) & 35 & 26 & 23 & 30 & 0 & -23 & -11 & 21 & 22.25 & 15.3 & 43.4 & 29.65 \\
\hline Reactive Power (kVAr) & 6 & 15 & 22 & 12 & 0 & -1 & -5 & -3 & 10.3 & 9.55 & 19.2 & 11.2 \\
\hline Power Factor (pf) & 0.99 & 0.87 & 0.72 & & & & & & 0.91 & 0.85 & 0.91 & 0.94 \\
\hline Frequency $(\mathrm{Hz})$ & & & & & 59.986 & 60.03 & & & & & & \\
\hline \multicolumn{13}{|c|}{ ISLAND-TO-GRID } \\
\hline \multicolumn{13}{|l|}{ Start } \\
\hline Voltage (V) & & & & & 280 & 270 & & & & & & \\
\hline Current (A) & 44 & 37 & 39 & 39 & 0 & 30 & 15 & 26 & & & & \\
\hline Real Power (kW) & 35 & 26 & 23 & 30 & 0 & -23 & -10.5 & 21 & 22.3 & 15.35 & 43.4 & 29.6 \\
\hline
\end{tabular}




\begin{tabular}{|c|c|c|c|c|c|c|c|c|c|c|c|c|}
\hline Reactive Power (kVAr) & 6 & 15 & 22 & 12 & 0 & 0 & -5 & -3 & 10.3 & 9.55 & 19.2 & 11.25 \\
\hline Power Factor (pf) & 0.99 & 0.87 & 0.72 & & & & & & 0.91 & 0.85 & 0.91 & 0.93 \\
\hline Frequency $(\mathrm{Hz})$ & & & & & 59.997 & 60.06 & & & & & & \\
\hline \multicolumn{13}{|l|}{ End } \\
\hline Voltage (V) & & & & & 276 & 276 & & & & & & \\
\hline Current (I) & 50 & 40 & 38 & 60 & 47 & 50 & 21 & 22 & & & & \\
\hline Real Power (kW) & 40.5 & 32 & 29 & 13 & -15 & -34 & -16 & 16 & 23.25 & 16.05 & 45 & 28.55 \\
\hline Reactive Power (kVAr) & -5 & 3 & 12.5 & 46 & 45 & 23 & 7 & 7 & 10.8 & 10 & 19.95 & 10.8 \\
\hline Power Factor (pf) & 0.99 & 1.00 & 0.92 & & & & & & 0.91 & 0.85 & 0.91 & 0.94 \\
\hline Frequency $(\mathrm{Hz})$ & & & & & 59.98 & 59.98 & & & & & & \\
\hline
\end{tabular}

Table 16d - Test 9.1.9 results with dark squares in the table meaning that measurements were not needed. According to the measurements in Table $16 \mathrm{~d}$ when connected to the utility grid, each Gen-set in the microgrid was set at $40 \mathrm{~kW}$ for Gen-set A1, 30kW for Gen-set A2 and $30 \mathrm{~kW}$ for Gen-set B1 which was enough to satisfy the loads in LB3, LB4 and LB5 and provided $13.5 \mathrm{~kW}$ measured at Meter 2. The utility supplied approximately $15 \mathrm{~kW}$, measured in Meter 1, because it supplied the remaining portion of load at LB6. In addition, Meter 4 measured $-15 \mathrm{~kW}$ meaning that Gen-set A2 was supplying all the power to LB4, plus an additional 15kW. Meter 3 measured 33kW, meaning Gen-set A1 was supplying all the power to LB3 plus producing an additional $18 \mathrm{~kW}$. The excess of approximately 33kW, flowing out of Zone 3, was supplied to LB5 and LB6, measured by Meter 5 at $16.5 \mathrm{~kW}$ and Meter 1 at $15 \mathrm{~kW}$. Once all data was verified and recorded into the DAS Database, the static switch was manually opened by the EMS.

When the static switch opened, Meter 1 increased to approximately $30 \mathrm{~kW}$ satisfying the load demand in LB6, which was $29.65 \mathrm{~kW}$ and not supplying any power beyond the static switch to microgrid loads. 0kW was measured at Meter 2, showing that power was not flowing through the static switch. LB3, LB4 and LB5 loads reduced slightly to $22.25 \mathrm{~kW}, 15.3 \mathrm{~kW}$ and $43.4 \mathrm{~kW}$, respectively. This load reduction resulted from a voltage drop in the microgrid, shown in Figure 29e, from approximately $277 \mathrm{~V}$ (i.e., $277.5 \mathrm{~V}$ on Aphase and $275.5 \mathrm{~V}$ on B- and C-phases) when connected to the utility grid, to $270 \mathrm{~V}$ on all three phases when islanded. 


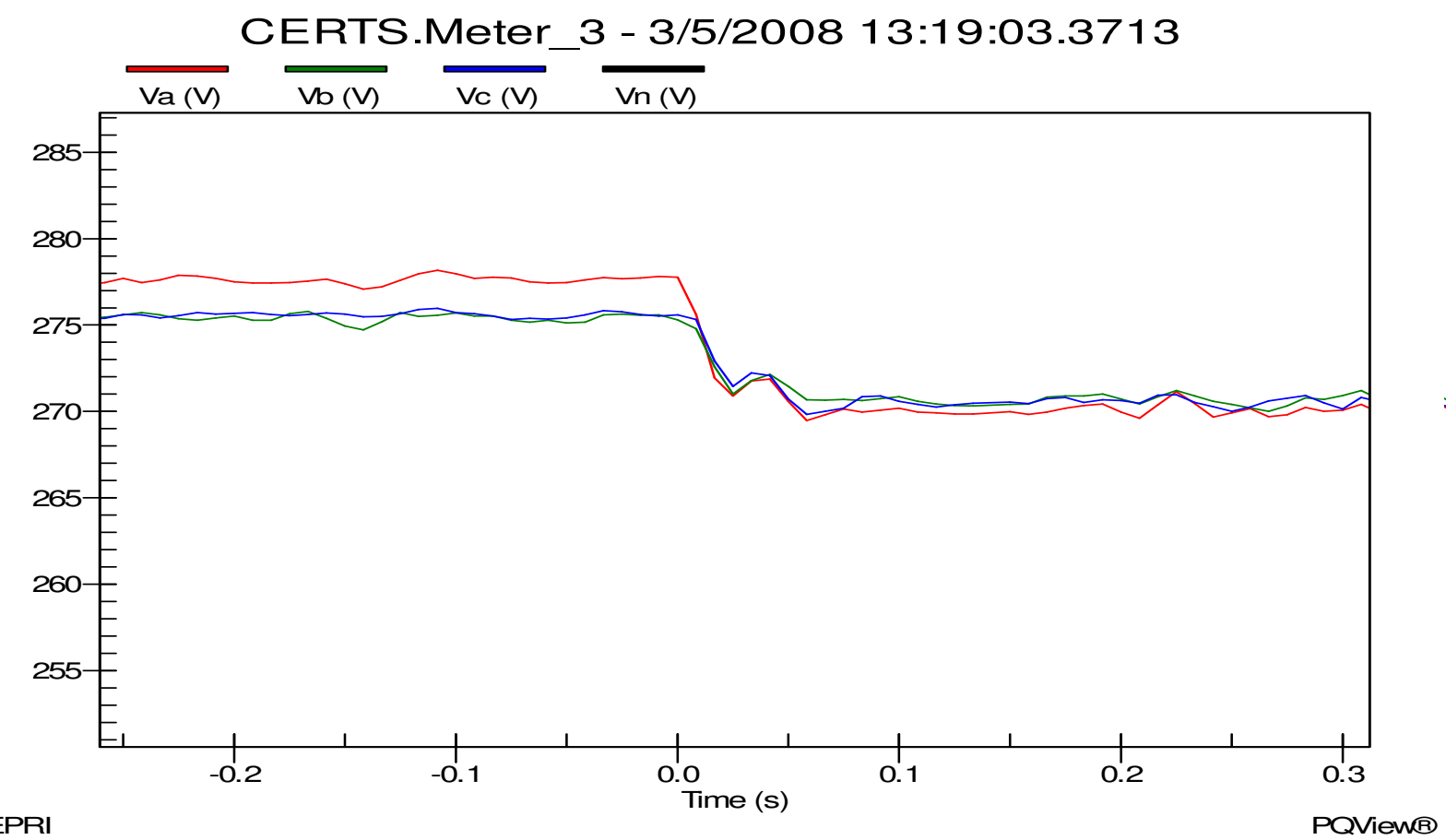

Figure 29e - Meter 3 voltage before/after the static switch opened, islanding the microgrid from the utility grid.

Frequency changed in the microgrid, shown in Figure 29f, and increased to approximately $60.03 \mathrm{~Hz}$ which was part of the CERTS algorithm which allowed the Gensets to decrease their output power to satisfy the load demands. Gen-set A1, A2 and B1 decreased their output power to approximately $35 \mathrm{~kW}, 26 \mathrm{~kW}$ and $23 \mathrm{~kW}$, respectively. Meter 4 measured approximately $-11 \mathrm{~kW}$ and Meter 3 measured $-23 \mathrm{~kW}$. Gen-sets A1 and A2 supplied all the power to LB3 and LB4 plus supplied approximately $21 \mathrm{~kW}$ of LB5 indicated on Meter5. All data was verified and recorded into the DAS Database. The microgrid ran for a couple of minutes in this electrical state before the manual open signal was released and allowed the static switch to synchronize to the utility grid. 


\section{CERTS.Meter_3 - 3/5/2008 18:19:03.3713}

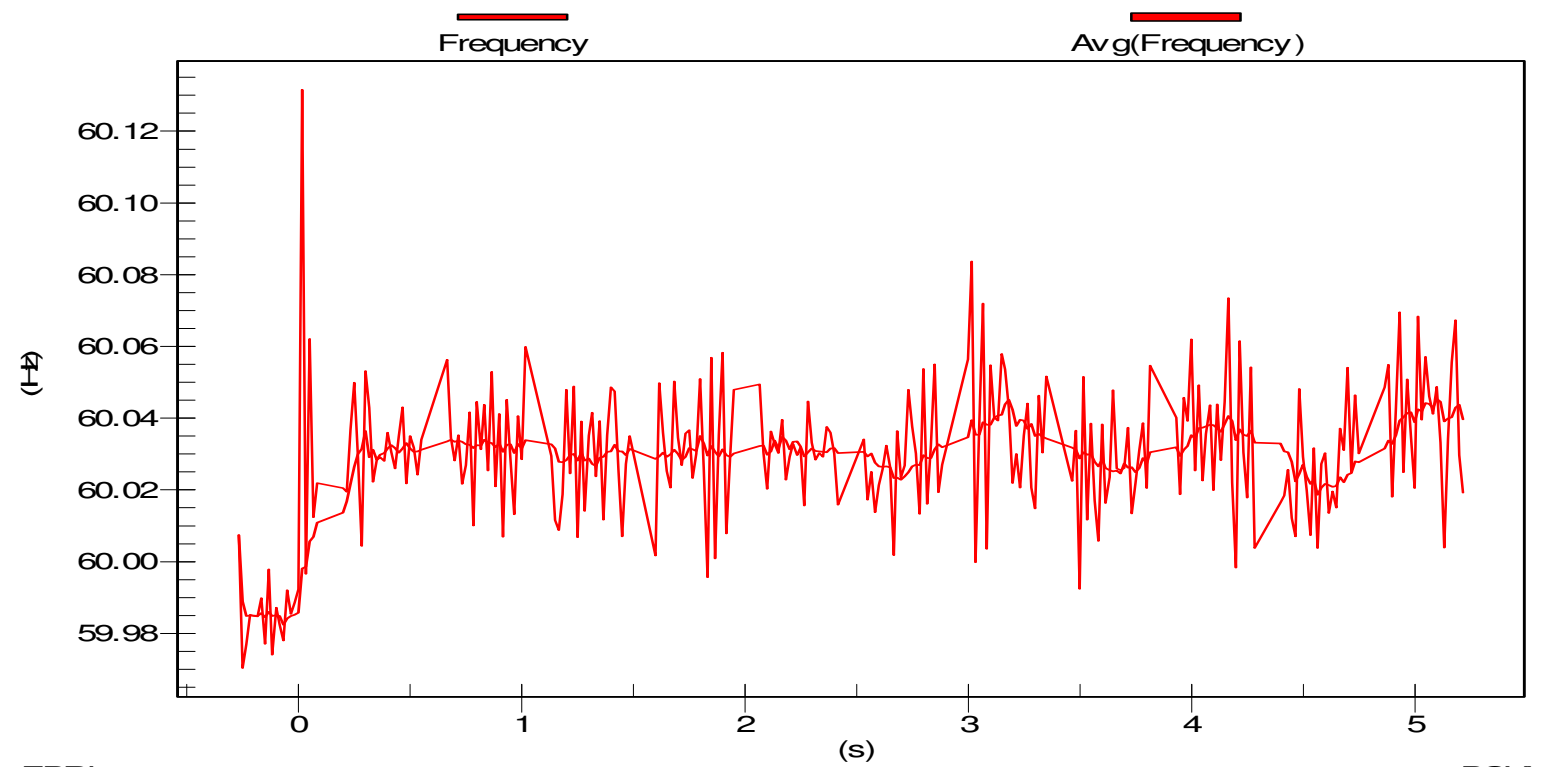

EPRI

$(\mathrm{s})$

PQVienA

Figure 29f - Meter 3 frequency before/after the static switch opened, islanding the microgrid from the utility grid.

As soon as the static switch closed and steady-state conditions established, the measurements at the Gen-sets, Meters and Load Banks were compared to the initial conditions at the beginning of the test. The measured values were very similar to the initial test conditions which were $13 \mathrm{~kW}$ at Meter 1, $-15 \mathrm{~kW}$ at Meter 2, $-34 \mathrm{~kW}$ at Meter 3, $16 \mathrm{~kW}$ at Meter 4 , and $16 \mathrm{~kW}$ at Meter 5 with $40.5 \mathrm{~kW}$ produced by Gen-set A1, 32kW produced by Gen-set A2, and $29 \mathrm{~kW}$ produced by Gen-set B1. Likewise, $23.25 \mathrm{~kW}$ was at LB3, $16.05 \mathrm{~kW}$ at LB4, $45 \mathrm{~kW}$ at LB5 and $28.55 \mathrm{~kW}$ at LB6. The microgrid voltage increased from $270 \mathrm{~V}$ to $276 \mathrm{~V}$ and frequency decreased from $60.03 \mathrm{~Hz}$ to $59.98 \mathrm{~Hz}$. The power factor of approximately 0.85 remained the same throughout the test for LB4, 0.91 for LB3 and LB5, and 0.94 for LB6. After all data was verified and recorded into the DAS Database, Gen-sets A1, A2 and B1 were shut down manually and all load banks/alarms were reset and prepared for the next test (9.1.10) in Table 16a.

For Test 9.1.10 the measured values, after the Gen-sets were warmed up and the load banks brought on-line, were approximately $36 \mathrm{~kW}$ at Meter 1, $7.5 \mathrm{~kW}$ at Meter 2, $26 \mathrm{~kW}$ at Meter $3,12 \mathrm{~kW}$ at Meter 4 , and $-21.5 \mathrm{~kW}$ at Meter 5 . From the microgrid $31.3 \mathrm{~kW}$ was produced by Gen-set A1, 22.6kW produced by Gen-set A2, and $44.5 \mathrm{~kW}$ produced by Gen-set B1. The load banks were slightly less than planned with $45.5 \mathrm{~kW}$ at LB3, $34.55 \mathrm{~kW}$ at LB4, $22.95 \mathrm{~kW}$ at LB5, and $28.35 \mathrm{~kW}$ at LB6. These measurements were relatively close to the expected values in Table 16a but not exact, due to temperature, phase voltages, and electrical losses in conductors. In addition, the desired $\mathrm{kW}$ setting for LB3, LB4, LB5 and LB6 were below the set values. This deviation of set values verses 
actual values was consistent with other tests in this section. At the time of these measurements the voltage and frequency was $276 \mathrm{~V}$ and $59.9785 \mathrm{~Hz}$ at the static switch (i.e., Meter 2) when connected to the utility grid; and $276 \mathrm{~V}$ and $59.977 \mathrm{~Hz}$ at Meter 3 . The power factor at LB4 was approximately $0.87,0.91$ at LB5, 0.92 at LB3, and 0.94 at LB6 which is close to the desired 0.9 power factor.

\begin{tabular}{|c|c|c|c|c|c|c|c|c|c|c|c|c|}
\hline \multicolumn{13}{|l|}{9.1 .10} \\
\hline & GENSET A1 & GENSET A2 & GENSET B1 & METER 1 & METER 2 & METER 3 & METER 4 & METER 5 & LB 3 & LB 4 & LB 5 & LB 6 \\
\hline INITIAL SETUP & UNIT +30kW & UNIT +20kW & UNIT +45kW & & $+20 \mathrm{~kW}$ & $+40 \mathrm{~kW}$ & & $-20 \mathrm{~kW}$ & $50 \mathrm{~kW}$ & $40 \mathrm{~kW}$ & $25 \mathrm{~kW}$ & $30 \mathrm{~kW}$ \\
\hline \multicolumn{13}{|c|}{ GRID-TO-ISLAND } \\
\hline \multicolumn{13}{|l|}{ Start } \\
\hline Voltage (V) & & & & & 276 & 276 & & & & & & \\
\hline Current (A) & 37 & 31 & 55 & 75 & 46 & 54 & 21.5 & 26.5 & & & & \\
\hline Real Power (kW) & 31.3 & 22.6 & 44.5 & 36 & 7.5 & 26 & 12 & -21.5 & 45.5 & 34.55 & 22.95 & 28.35 \\
\hline Reactive Power (kVAr) & -2 & 9 & 9.5 & 50 & 39 & 33 & 11 & 1 & 19.725 & 19.75 & 10.74 & 10.7 \\
\hline Power Factor (pf) & 1.00 & 0.93 & 0.98 & & & & & & 0.92 & 0.87 & 0.91 & 0.94 \\
\hline Frequency $(\mathrm{Hz})$ & & & & & 59.978 & 59.977 & & & & & & \\
\hline \multicolumn{13}{|l|}{ End } \\
\hline Voltage (V) & & & & & 279 & 268 & & & & & & \\
\hline Current (A) & 41 & 40 & 61 & 37 & 0 & 27 & 13 & 31 & & & & \\
\hline Real Power (kW) & 32 & 23.3 & 45.4 & 29.6 & 0 & 21 & 9.5 & -23 & 43.2 & 32.85 & 22 & 29.55 \\
\hline Reactive Power (kVAr) & 10.9 & 21.5 & 19.5 & 2 & 0 & 5 & -2.5 & -9 & 18.75 & 18.75 & 10.25 & 11.2 \\
\hline Power Factor (pf) & 0.95 & 0.73 & 0.92 & & & & & & 0.92 & 0.87 & 0.91 & 0.94 \\
\hline Frequency $(\mathrm{Hz})$ & & & & & 59.9775 & 59.97 & & & & & & \\
\hline \multicolumn{13}{|c|}{ ISLAND-TO-GRID } \\
\hline \multicolumn{13}{|l|}{ Start } \\
\hline Voltage $(V)$ & & & & & 281 & 270 & & & & & & \\
\hline Current (A) & 42 & 40 & 61 & 39 & 0 & 29 & 12 & 31 & & & & \\
\hline Real Power (kW) & 32.5 & 23.5 & 45.5 & 30 & 0 & 21 & 9 & -23.2 & 43.3 & 32.9 & 22.1 & 29.7 \\
\hline
\end{tabular}




\begin{tabular}{|c|c|c|c|c|c|c|c|c|c|c|c|c|}
\hline Reactive Power (kVAr) & 32.5 & 21 & 20 & 12 & 0 & 6 & -2.5 & -9.5 & 18.8 & 18.85 & 10.25 & 11.3 \\
\hline Power Factor (pf) & 0.71 & 0.75 & 0.92 & & & & & & 0.92 & 0.87 & 0.91 & 0.93 \\
\hline Frequency $(\mathrm{Hz})$ & & & & & 59.995 & 59.98 & & & & & & \\
\hline \multicolumn{13}{|l|}{ End } \\
\hline Voltage (V) & & & & & 275 & 277 & & & & & & \\
\hline Current (I) & 37 & 27 & 54 & 80 & 52 & 59 & 22.5 & 25 & & & & \\
\hline Real Power (kW) & 30 & 21.25 & 43 & 40 & 12 & 29.5 & 13.75 & -20 & 45.6 & 34.7 & 23.5 & 28.4 \\
\hline Reactive Power (kVAr) & 30 & 8 & 9 & 51 & 40 & 35 & 11 & 1.9 & 19.8 & 19.85 & 10.75 & 10.75 \\
\hline Power Factor (pf) & 0.71 & 0.94 & 0.98 & & & & & & 0.92 & 0.87 & 0.91 & 0.94 \\
\hline Frequency $(\mathrm{Hz})$ & & & & & 59.99 & 59.99 & & & & & & \\
\hline
\end{tabular}

Table 16e - Test 9.1.9 results with dark squares in the table meaning that measurements were not needed.

According to the measurements in Table 16e, Gen-sets in the microgrid were set at $30 \mathrm{~kW}$ for Gen-set A1, 20kW for Gen-set A2 and 45kW for Gen-set B1, which was not enough to satisfy the loads in LB3 and LB4 and needed approximately $7.5 \mathrm{~kW}$ from the utility grid, measured by Meter 2. The utility grid supplied a total power of approximately $36 \mathrm{~kW}$, measured in Meter 1, because it met the demands of not only the microgrid loads, but the load at LB61. Meter 5 measured approximately $-21.5 \mathrm{~kW}$, meaning Gen-set B1 supplied all the power to LB5 and exported some power to LB3 and LB4. The power exported from the utility grid and Gen-set B1 was approximately 26kW into LB3 and LB4, measured by Meter 3. Meter 4 measured 12kW meaning that LB3 needed an additional $14 \mathrm{~kW}$ on top of the power produced by Gen-set A1 and LB4 needed an additional $12 \mathrm{~kW}$ on top of the power produced by Gen-set A2. Once all data was verified and recorded into the DAS Database, the static switch was manually opened by the EMS. 


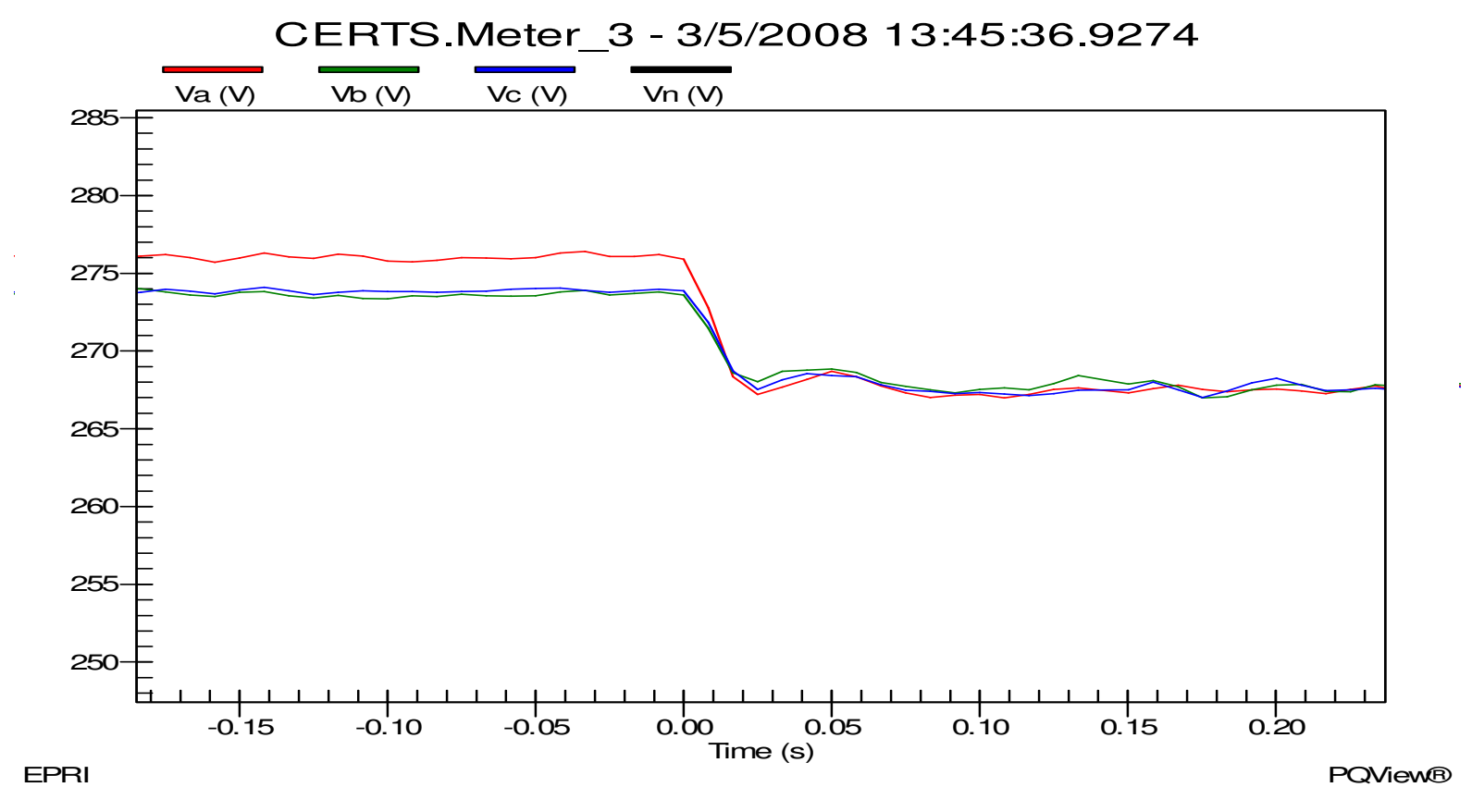

Figure $29 \mathrm{~g}$ - Meter 3 voltage before/after the static switch opened, islanding the microgrid from the utility grid.

When the static switch opened, Meter 1 decreased to approximately $29.6 \mathrm{~kW}$ satisfying the load demand in LB6, which was approximately $29.55 \mathrm{~kW}$ and not supplying any power beyond the static switch to microgrid loads. 0kW was measured at Meter 2, showing that power was not flowing through the static switch. LB3, LB4 and LB5 loads reduced slightly to $43.2 \mathrm{~kW}, 32.85 \mathrm{~kW}$ and $22 \mathrm{~kW}$, respectively. This load reduction resulted from a voltage drop in the microgrid, shown in Figure 29g, from approximately $276 \mathrm{~V}$ (i.e., $276 \mathrm{~V}$ on A-phase and $274 \mathrm{~V}$ on B- and C-phases), when connected to the grid, to $268 \mathrm{~V}$ on all three phases when islanded. 


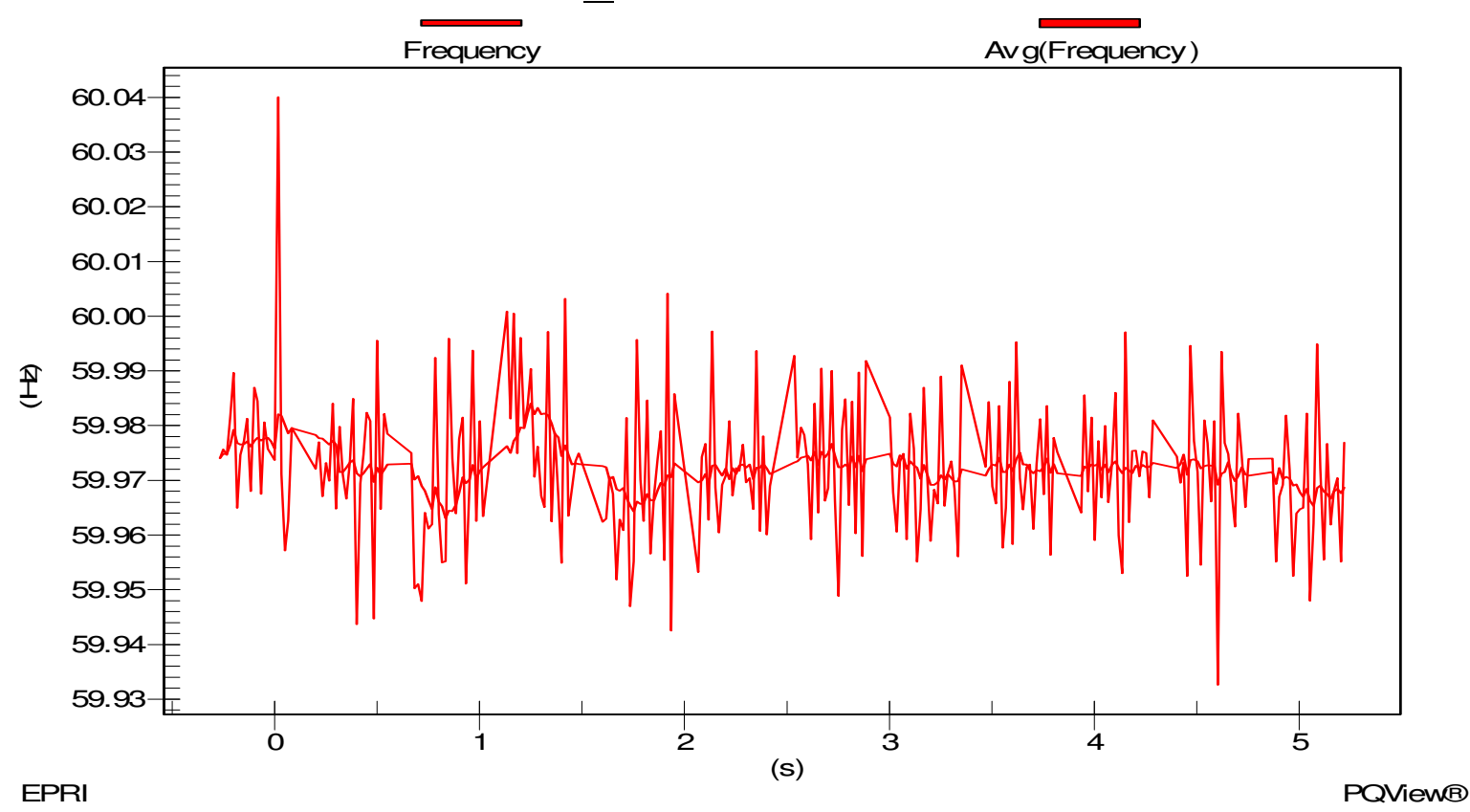

Figure $29 \mathrm{~h}$ - Meter 3 frequency before/after the static switch opened, islanding the microgrid from the utility grid.

Frequency in the microgrid changed slightly, as shown in Figure 29h to approximately $59.97 \mathrm{~Hz}$ which was part of the CERTS algorithm which allowed the Gen-sets to increase their output power to satisfy the load demands. Gen-set A1, A2 and B1 increased output power to approximately $32 \mathrm{~kW}, 23.3 \mathrm{~kW}$ and $45.5 \mathrm{~kW}$, respectively. Meter 5 measured approximately $-23 \mathrm{~kW}$ meaning that Gen-set B1 supplied all the power to LB5 and exported some of its power to LB3 and LB4. The power exported from Gen-set B1 was approximately $21 \mathrm{~kW}$ into LB3 and LB4, measured by Meter 3. Meter 4 measured approximately $9.5 \mathrm{~kW}$, meaning LB3 needed an additional $11.5 \mathrm{~kW}$ on top of the power produced by Gen-set A1 and LB4 needed and additional $9.5 \mathrm{~kW}$ on top of the power produced by Gen-set A2. All data was verified and recorded into the DAS Database. The microgrid ran for a couple of minutes in this electrical state before manual open signal was released and allowed the static switch to synchronize to the utility grid.

As soon as the static switch closed and steady-state conditions established, the measurement values in Table 16e were compared to the initial conditions at the beginning of the test. The measured values were very similar to initial test conditions which were approximately $40 \mathrm{~kW}$ at Meter 1, 12kW at Meter 2, 29.5kW at Meter 3, $13.75 \mathrm{~kW}$ at Meter 4 , and $-20 \mathrm{~kW}$ at Meter 5 with $30 \mathrm{~kW}$ produced by Gen-set A1, $21.25 \mathrm{~kW}$ produced by Gen-set A2, and $43 \mathrm{~kW}$ produced by Gen-set B1. Likewise, $45.6 \mathrm{~kW}$ was at LB3, $34.7 \mathrm{~kW}$ at LB4, $23.5 \mathrm{~kW}$ at LB5 and $28.4 \mathrm{~kW}$ at LB6. The microgrid voltage increased from $270 \mathrm{~V}$ to $277 \mathrm{~V}$ and frequency increased from $59.98 \mathrm{~Hz}$ to $59.99 \mathrm{~Hz}$. The power factor remained the same throughout the test with 0.87 for LB4, 0.91 for LB5, 0.92 for LB3 and 
0.94 for LB6. After all data was verified and recorded into the DAS Database the Gensets A1, A2 and B1 were shut down manually by the EMS, and all the loads/alarms were reset and prepared for the next planned tests.

\subsection{CONCLUSION}

The tests in section 9.0 "Demonstration Tests of Control Power Flow" were all successfully performed to as predicted. These successful tests provide confidence that the CERTS algorithms real power versus frequency and reactive power versus voltage work for the Gen-sets in unit and zone power control modes while utility connected and islanded. 


\title{
CERTS MICROGRID LABORATORY TEST BED
}

\author{
Test Plan Section 10.0 \\ Difficult Loads
}

Prepared For:

California Energy Commission

Public Interest Energy Research Program

Arnold Schwarzenegger Governor

$\frac{\sum}{x}$

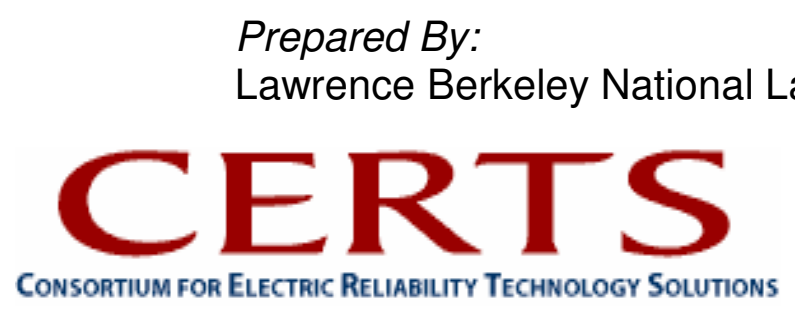



Prepared By:

Lawrence Berkeley National Laboratory

Joseph H. Eto, Principal Investigator

Berkeley, CA 94720

Ben Schenkman, Sandia National Laboratory

Harry Volkommer and Dave Klapp, American Electric Power

Commission Contract No. 500-03-024

Prepared For:

Public Interest Energy Research (PIER)

California Energy Commission

Bernard Treanton

Contract Manager

Mike Gravely

Program Area Lead

ENERGY SYSTEMS INTEGRATION

Mike Gravely

Office Manager

ENERGY SYSTEMS RESEARCH

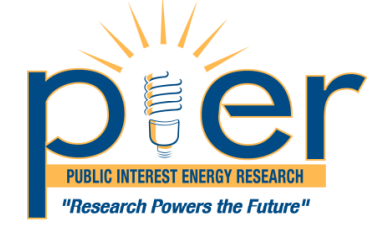

Martha Krebs, Ph.D.

PIER Director

Thom Kelly, Ph.D.

Deputy Director

ENERGY RESEARCH \& DEVELOPMENT DIVISION

Melissa Jones

Executive Director

\section{DISCLAIMER}

This report was prepared as the result of work sponsored by the California Energy Commission. It does not necessarily represent the views of the Energy Commission, its employees or the State of California. The Energy Commission, the State of California, its employees, contractors and subcontractors make no warrant, express or implied, and assume no legal liability for the information in this report; nor does any party represent that the uses of this information will not infringe upon privately owned rights. This report has not been approved or disapproved by the California Energy Commission nor has the California Energy Commission passed upon the accuracy or adequacy of the information in this report. 



\section{CERTS MICROGRID TEST REPORT}

\section{SECTION 10.0}

"Difficult Load Tests" 


\section{Table of Contents}

1.0

2.0

3.0

4.0

5.0

6.0

6.1

6.1.1

6.1.2

6.1.3

7.0
INTRODUCTION

BACKGROUND

MICROGRID TESTBED SETUP

PROPOSED TEST PLAN

TESTS PERFORMED IN SECTION 10.0

ANALYSES OF TEST RESULTS

SECTION 10 - DIFFICULT LOAD TESTS

Motor Start Tests, Weak Grid, Balanced Load with 0.9 Power

Factor

Motor Start Tests, Weak Grid, Unbalanced Load with 0.9 Power

Factor

Unbalanced Load Tests, Weak Grid, 0.9 Power Factor CONCLUSION 90

1

1 2 5 7 8 


\section{$\underline{\text { List Of Figures }}$}

Figure 1 - CERTS Microgrid Aerial Photo.................................................................... 3

Figure 2 - One Line Diagram of CERTS Microgrid Test Bed...................................... 4

Figure 3 - Simplified diagram of Test Bed showing Meter and Relay locations .... 4

Figure 4 - Diagram of DAS \& EMS Data networks................................................... 5

Figure 5a - Load Bank 3 Real Power Motor Start and Utility Connected for Test 10.2.12

Figure 5b - Load Bank 3 Reactive Power during Motor Start and Utility

Connected for Test 10.2.12

Figure $5 c$ - Static Switch Line-to-Ground Voltages during Motor Start and Utility

Connected for Test 10.2.12. 13

Figure 5d - Static Switch Frequency during Motor Start and Utility Connected

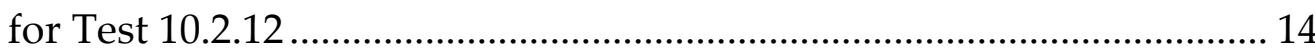

Figure 5e - Static Switch Real Power during Motor Start and Utility Connected

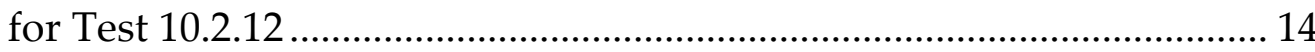

Figure 5f - Static Switch Reactive Power during Motor Start and Utility

Connected for Test 10.2.12 15

Figure 5g - Meter 3 Line-to-Ground Voltages during Utility Connected to Island mode for Test 10.2.12 ……………….......................................................... 16

Figure 5h - Meter 3 Frequency during Utility Connected to Island mode for Test 10.2.12. 17

Figure 5i - Load Bank 3 Real Power during Motor Start and Islanded for Test 10.2.12. 18

Figure 5j - Load Bank 3 Reactive Power during Motor Start and Islanded for Test 10.2.12. 18

Figure 5k - Meter 3 Line-to-Ground Voltage during Motor Start and Islanded for

Test 10.2.12 19

Figure 51 - Meter 3 Frequency during Motor Start and Islanded for Test 10.2.12 20

Figure 5m - Gen-set A1 Real Power during Motor Start and Islanded for Test 10.2.12 21

Figure 5n - Gen-Set A1 Reactive Power during Motor Start and Islanded for Test 10.2.12. 21

Figure 5o - Gen-set A2 Real Power during Motor Start and Islanded for Test 10.2.12.

Figure 5p - Gen-set A2 Reactive Power during Motor Start and Islanded for Test 10.2.12. 22

Figure 5q - Static Switch Real Power during Island to Utility Connected mode for Test 10.2.12 23 
Figure 5r - Static Switch Reactive Power during Island to Utility Connected mode for Test 10.2.12 .................................................................................... 24

Figure 5s - Meter 3 Line-to-Ground Voltages during Island to Utility Connected mode for Test 10.2.12 .................................................................................. 24

Figure 5t - Meter 3 Frequency during Island to Utility Connected mode for Test 10.2.12

Figure 6a - Load Bank 3 Real Power during Motor Start and Utility Connected for Test 10.2.14 26

Figure 6b - Load Bank 3 Reactive Power during Motor Start and Utility Connected for Test 10.2.14 ............................................................................. 26

Figure 6c - Static Switch Line-to-Ground Voltages during Motor Start and Utility Connected for Test 10.2.14 27

Figure 6d - Static Switch Frequency during Motor Start and Utility Connected for Test 10.2.14 28

Figure 6e - Static Switch Real Power during Motor Start and Utility Connected for Test 10.2.14 28

Figure 6f - Static Switch Reactive Power during Motor Start and Utility Connected for Test 10.2.14

Figure $6 \mathrm{~g}$ - Meter 3 Line-to-Ground Voltages during Utility Connected to Island

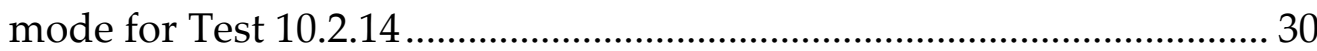

Figure $6 \mathrm{~h}$ - Meter 3 Frequency during Utility Connected to Island mode for Test 10.2.14 30

Figure 6i - Load Bank 3 Real Power during Motor Start and Islanded for Test 10.2.14. 31

Figure $6 \mathbf{j}$ - Load Bank 3 Reactive Power during Motor Start and Islanded for Test 10.2.14 32

Figure 6k - Meter 3 Line-to-Ground Voltages during Motor Start and Islanded for Test 10.2.14

Figure 61 - Meter 3 Frequency during Motor Start and Islanded for Test 10.2.14 33 Figure 6m - Gen-set A1 Real Power during Motor Start and Islanded for Test 10.2.14. 34

Figure 6n - Gen-set A1 Reactive Power during Motor Start and Islanded for Test 10.2.14. 34

Figure 6o - Static Switch Real Power during Island to Utility Connected mode for Test 10.2.14 35

Figure 6p - Static Switch Reactive Power during Island to Utility Connected mode for Test 10.2.14 ....................................................................................... 36

Figure 6q - Meter 3 Line-to-Ground Voltages during Island to Utility Connected

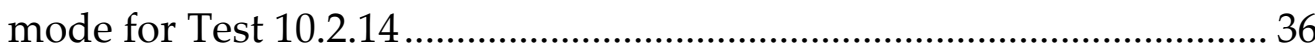


Figure 6r - Meter 3 Frequency during Island to Utility Connected mode for Test

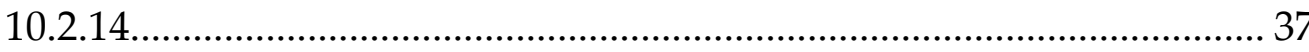

Figure 7a - Load Bank 3 Real Power during Motor Start Utility Connected for

Test 10.2.15 ................................................................................................. 38

Figure 7b - Load Bank 3 Reactive Power during Motor Start Utility Connected for Test 10.2.15 39

Figure 7c - Static Switch Line-to-Ground Voltages during Motor Start and Utility Connected for Test 10.2.15 40

Figure 7d - Static Switch Frequency during Motor Start and Utility Connected for Test 10.2.15 40

Figure 7e - Static Switch Real power during Motor Start and Utility Connected for Test 10.2.15

Figure 7f - Static Switch Reactive Power during Motor Start and Utility Connected for Test 10.2.15

Figure 7g - Meter 3 Line-to-Ground Voltages during Utility Connected to Island mode for Test 10.2.15

Figure 7h - Meter 3 Frequency during Utility Connected to Island mode for Test 10.2.15.

Figure 7i - Load Bank 3 Real Power during Motor Start and Islanded for Test 10.2.15 44

Figure 7j - Load Bank 3 Reactive Power during Motor Start and Islanded for Test 10.2.15. 44

Figure 7k - Meter 3 Line-to-Ground Voltages during Motor Start and Islanded for Test 10.2.15

Figure 7l - Meter 3 Frequency during Motor Start and Islanded for Test 10.2.15 46 Figure 7m - Gen-set A1 Real Power during Motor Start and Islanded for Test 10.2.15....... 47

Figure 7n - Gen-set A1 Reactive Power during Motor Start and Islanded for Test 10.2.15. 47

Figure 7o - Gen-set A2 Real Power during Motor Start and Islanded for Test 10.2.15. 48

Figure 7p - Gen-set A2 Reactive Power during Motor Start and Islanded for Test 10.2.15. 48

Figure 7q - Static Switch Real Power during Island to Utility Connected mode for Test 10.2.15 49

Figure $7 \mathrm{r}$ - Static Switch Reactive Power during Island to Utility Connected mode for Test 10.2.15 ..................................................................................... 50

Figure 7s - Meter 3 Line-to-Ground Voltages during Island to Utility Connected mode for Test 10.2.15 ............................................................................... 50 
Figure 7t - Meter 3 Frequency during Island to Utility Connected mode for Test 10.2.15

Figure 8a - Load Bank 3 Real Power during Motor Start and Utility Connected for

Test 10.2.17 52

Figure 8b - Load Bank 3 Reactive Power during Motor Start and Utility

Connected for Test 10.2.17 52

Figure $8 \mathrm{c}$ - Static Switch Line-to-Ground Voltages during Motor Start and Utility

Connected for Test 10.2.17 53

Figure 8d - Static Switch Frequency during Motor Start and Utility Connected for Test 10.2.17 54

Figure 8e - Static Switch Real Power during Motor Start and Utility Connected for Test 10.2.17 54

Figure 8f - Static Switch Reactive Power during Motor Start and Utility

Connected for Test 10.2.17 55

Figure 8g - Meter 3 Line-to-Ground Voltages during Utility Connected to Island mode for Test 10.2.17 56

Figure $8 \mathrm{~h}$ - Meter 3 Frequency during Utility Connected to Island mode for Test 10.2.17. 56

Figure 8i - Load Bank 3 Real Power during Motor Start and Islanded for Test 10.2.17. 57

Figure $8 \mathrm{j}$ - Load Bank 3 Reactive Power during Motor Start and Islanded for Test 10.2.17. 58

Figure 8k - Meter 3 Line-to-Ground Voltages during Motor Start and Islanded for

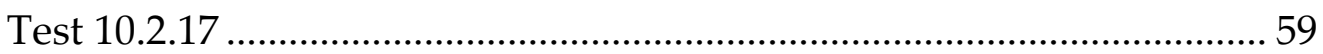

Figure 81 - Meter 3 Frequency during Motor Start and Islanded for Test 10.2.17 59 Figure 8m - Gen-set A1 Real Power during Motor Start and Islanded for Test 10.2.17. 60

Figure 8n - Gen-set A1 Reactive Power during Motor Start and Islanded for Test 10.2.17. 60

Figure 8o - Static Switch Real Power during Island to Utility Connected mode for Test 10.2.17 61

Figure $8 \mathrm{p}$ - Static Switch Reactive Power during Island to Utility Connected mode for Test 10.2.17. 62

Figure 8q - Meter 3 Line-to-Ground Voltages during Island to Utility Connected mode for Test 10.2.17 62

Figure 8r - Meter 3 Frequency during Island to Utility Connected mode for Test 10.2.17. 63

Figure 9a - Load Bank 3 Real Power during Motor Start and Utility Connected for Test 10.2.18 64 
Figure 9b - Load Bank 3 Reactive Power during Motor Start and Utility

Connected for Test 10.2.18.

Figure 9c - Static Switch Line-to-Ground Voltage during Motor Start and Utility

Connected for Test 10.2.18 66

Figure 9d - Static Switch Frequency during Motor Start and Utility Connected for

Test 10.2.18 66

Figure 9e - Static Switch Real Power during Motor Start and Utility Connected for Test 10.2.18

Figure 9f - Static Switch Reactive Power during Motor Start and Utility

Connected for Test 10.2.18

Figure 9g - Meter 3 Line-to-Ground Voltages during Utility Connected to Island

mode for Test 10.2.18. 69

Figure 9h - Meter 3 Frequency during Utility Connected to Island mode for Test 10.2.18. 69

Figure 9i - Load Bank 3 Real Power during Motor Start and Islanded for Test 10.2.18 70

Figure 9j - Load Bank 3 Reactive Power during Motor Start and Islanded for Test 10.2.18. 71

Figure 9k - Meter 3 Line-to-Ground Voltages during Motor Start and Islanded for Test 10.2.18 72

Figure 91 - Meter 3 Frequency during Motor Start and Islanded for Test 10.2.18 72 Figure 9m - Gen-set A1 Real Power during Motor Start and Islanded for Test 10.2.18 73

Figure 9n - Gen-set A1 Reactive Power during Motor Start and Islanded for Test 10.2.18. 73

Figure 9o - Gen-set A2 Real Power during Motor Start and Islanded for Test 10.2.18. 74

Figure 9p - Gen-set A2 Reactive Power during Motor Start and Islanded for Test 10.2.18...... 75

Figure $9 \mathrm{q}$ - Static Switch Real Power during Island to Utility Connected mode for Test 10.2.18 ................................................................................................... 76

Figure 9r - Static Switch Reactive Power during Island to Utility Connected mode for Test 10.2.18 76

Figure 9s - Meter 3 Line-to-Ground Voltages during Island to Utility Connected mode for Test 10.2.18 77

Figure $9 \mathrm{t}$ - Meter 3 Frequency during Island to Utility Connected mode for Test 10.2.18. 77

Figure 10a - Load Bank 3 Real Power during Motor Start and Utility Connected for Test 10.2.20...... 79 
Figure 10b - Load Bank 3 Reactive Power during Motor Start and Utility

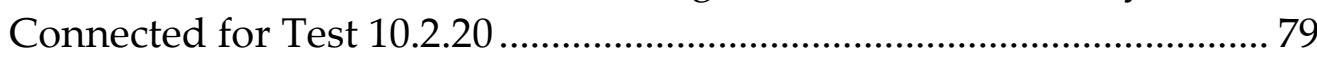

Figure 10c - Static Switch Line-to-Ground Voltage during Motor Start and Utility

Connected for Test 10.2.20 80

Figure 10d - Static Switch Frequency during Motor Start and Utility Connected

for Test 10.2.20

Figure 10e - Static Switch Real Power during Motor Start and Utility Connected

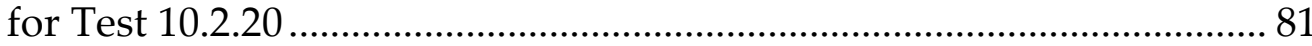

Figure 10f - Static Switch Reactive Power during Motor Start and Utility

Connected for Test 10.2.20

Figure 10g - Meter 3 Line-to-Ground Voltages during Utility Connected to Island

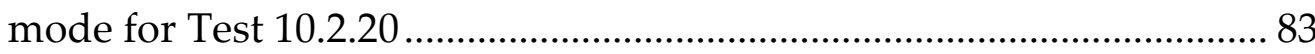

Figure 10h - Meter 3 Frequency during Utility Connected to Island mode for Test

10.2.20. 84

Figure 10i - Load Bank 3 Real Power during Motor Start and Islanded for Test

10.2.20. 85

Figure 10j - Load Bank 3 Reactive Power during Motor Start and Islanded for Test 10.2.20 85

Figure 10k - Meter 3 Line-to-Ground Voltages during Motor Start and Islanded for Test 10.2.20 86

Figure 101 - Meter 3 Frequency during Motor Start and Islanded for Test 10.2.20 86

Figure 10m - Gen-set A1 Real Power during Motor Start and Islanded for Test 10.2.20.

Figure 10n - Gen-set A1 Reactive Power during Motor Start and Islanded for Test 10.2.20. 87

Figure 10o - Static Switch Real Power during Island to Utility Connected mode for Test 10.2.20 88

Figure 10p - Static Switch Reactive Power during Island to Utility Connected

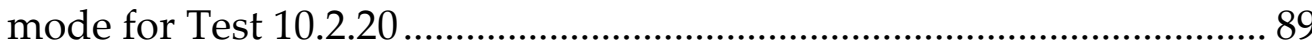

Figure 10q - Meter 3 Line-to-Ground Voltages during Island to Utility Connected mode for Test 10.2.20. 89

Figure 10r - Meter 3 Frequency during Island to Utility Connected mode for Test 10.2.20. 90

Figure 11a - Load Bank 3 Real Single-Phase Power during Motor Start and Utility Connected for Test 10.3.12. 93

Figure 11b - Load Bank 3 Reactive Single-Phase Power during Motor Start and Utility Connected for Test 10.3.12. 93 
Figure 11c - Static Switch Line-to-Ground Voltages during Motor Start and

Utility Connected for Test 10.3.12.....

Figure 11d - Static Switch Frequency during Motor Start and Utility Connected

for Test 10.3.12 ........................................................................................... 95

Figure 11e - Static Switch Real Single-Phase Power during Motor Start and Utility

Connected for Test 10.3.12 ..................................................................... 95

Figure 11f - Static Switch Reactive Single-Phase Power during Motor Start and

Utility Connected for Test 10.3.12.......................................................... 96

Figure 11g - Meter 3 Line-to-Ground Voltages during Utility Connected to Island

mode for Test 10.3.12 ................................................................................. 97

Figure $11 \mathrm{~h}$ - Meter 3 Frequency during Utility Connected to Island mode for Test

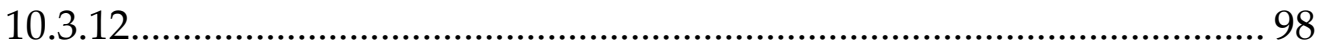

Figure 11i - Load Bank3 Real Single-Phase Power during Motor Start and

Islanded for Test 10.3.12 99

Figure 11j - Load Bank 3 Reactive Single-Phase Power during Motor Start and

Islanded for Test 10.3.12.

Figure 11k - Meter 3 Line-to-Ground Voltages during Motor Start and Islanded

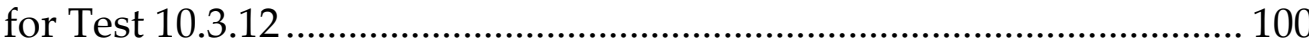

Figure 111 - Meter 3 Frequency during Motor Start and Islanded for Test 10.3.12 101

Figure 11m - Gen-set A1 Real Single-Phase Power during Motor Start and Islanded for Test 10.3.12 102

Figure 11n - Gen-set A1 Reactive Single-Phase Power during Motor Start and

Islanded for Test 10.3.12........................................................................ 102

Figure 11o - Gen-set A2 Real Single-Phase Power during Motor Start and

Islanded for Test 10.3.12. 103

Figure 11p - Gen-set A2 Reactive Single-Phase Power during Motor Start and Islanded for Test 10.3.12. 103

Figure 11q - Static Switch Real Single-Phase Power during Island to Utility Connected mode for Test 10.3.12 104

Figure 11r - Static Switch Reactive Single-Phase Power during Island to Utility Connected mode for Test 10.3.12 105

Figure 11s - Meter 3 Line-to-Ground Voltage during Island to Utility Connected

mode for Test 10.3.12. 105

Figure 11t - Meter 3 Frequency during Island to Utility Connected mode for Test 10.3.12 106

Figure 12a - Load Bank 3 Real Single-Phase Power during Motor Start and Utility Connected for Test 10.3.14. 108 
Figure 12b - Load Bank 3 Reactive Single-Phase Power during Motor Start and

Utility Connected for Test 10.3.14.............................................................. 108

Figure 12c - Static Switch Line-to-Ground Voltages during Motor Start and

Utility Connected for Test 10.3.14.

Figure 12d - Static Switch Frequency during Motor Start and Utility Connected

for Test 10.3.14 110

Figure 12e - Static Switch Real Single-Phase Power during Motor Start and Utility

Connected for Test 10.3.14 110

Figure 12f - Static Switch Reactive Single-Phase Power during Motor Start and

Utility Connected for Test 10.3.14 ............................................................ 111

Figure 12g - Meter 3 Line-to-Ground Voltages during Utility Connected to Island

mode for Test 10.3.14

Figure 12h - Meter 3 Frequency during Utility Connected to Island mode for Test

10.3.14.

Figure 12i - Load Bank 3 Real Single-Phase Power during Motor Start and

Islanded for Test 10.3.14.

Figure 12j - Load Bank 3 Reactive Single-Phase Power during Motor Start and

Islanded for Test 10.3.14.

Figure 12k - Meter 3 Line-to-Ground Voltages during Motor Start and Islanded

for Test 10.3.14

Figure 121 - Meter 3 Frequency during Motor Start and Islanded for Test 10.3.14

Figure 12m - Gen-set A1 Real Single-Phase Power during Motor Start and

Islanded for Test 10.3.14

Figure 12n - Gen-set A1 Reactive Single-Phase Power during Motor Start and

Islanded for Test 10.3.14

Figure 12o - Static Switch Real Single-Phase Power during Island to Utility

Connected mode for Test 10.3.14

Figure 12p - Static Switch Reactive Single-Phase Power during Island to Utility

Connected mode for Test 10.3.14

Figure 12q - Meter 3 Line-to-Ground Voltages during Island to Utility Connected for Test 10.3.14

Figure 12r - Meter 3 Frequency during Island to Utility Connected mode for Test 10.3.14.

Figure 13a - Load Bank 3 Real Single-Phase Power during Motor Start and Utility

Connected for Test 10.3.15.

Figure 13b - Load Bank 2 Reactive Single-Phase Power during Motor Start and

Utility Connected for Test 10.3.15. 
Figure 13c - Static Switch Line-to-Ground Voltage during Motor Start and Utility

Connected for Test 10.3.15.

Figure 13d - Static Switch Frequency during Motor Start and Utility Connected

for Test 10.3.15 124

Figure 13e - Static Switch Real Single-Phase Power during Motor Start and Utility

Connected for Test 10.3.15 124

Figure 13f - Static Switch Reactive Single-Phase Power during Motor Start and

Utility Connected for Test 10.3.15... 125

Figure 13g - Meter 3 Line-to-Ground Voltages during Utility Connected to Island mode for Test 10.3.15 126

Figure 13h - Meter 3 Frequency during Utility Connected to Island mode for Test 10.3.15 127

Figure 13i - Load Bank 3 Real Single-Phase Power during Motor Start and Islanded for Test 10.3.15. 128

Figure 13j - Load Bank 3 Reactive Single-Phase Power during Motor Start and Islanded for Test 10.3.15.

Figure 13k - Meter 3 Line-to-Ground Voltages during Motor Start and Islanded for Test 10.3.15

Figure 131 - Meter 3 Frequency during Motor Start and Islanded for Test 10.3.15 130

Figure 13m - Gen-set A1 Real Single-Phase Power during Motor Start and Islanded for Test 10.3.15. 131

Figure 13n - Gen-set A1 Reactive Single-Phase Reactive Power during Motor

Start and Islanded for Test 10.3.15. 131

Figure 13o - Gen-set A2 Real Single-Phase Power during Motor Start and Islanded for Test 10.3.15. 132

Figure 13p - Gen-set A2 Reactive Single-Phase Power during Motor Start and Islanded for Test 10.3.15.

Figure 13q - Static Switch Real Single-Phase Power during Island to Utility Connected mode for Test 10.3.15 133

Figure 13r - Static Switch Reactive Single-Phase Power during Island to Utility Connected mode for Test 10.3.15 134

Figure 13s - Meter 3 Line-to-Ground Voltages during Island to Utility Connected mode for Test 10.3.15. 134

Figure 13t - Meter 3 Frequency during Island to Utility Connected mode for Test 10.3.15. 135

Figure 14a - Load Bank 3 Real Single-Phase Power during Motor Start and Utility Connected for Test 10.3.17. 137 
Figure 14b - Load Bank 3 Reactive Single-Phase Power during Motor Start and

Utility Connected for Test 10.3.17............................................................. 137

Figure 14c - Static Switch Line-to-Ground Voltages during Motor Start and

Utility Connected for Test 10.3.17.

Figure 14d - Static Switch Frequency during Motor Start and Utility Connected

for Test 10.3.17

Figure 14e - Static Switch Real Single-Phase Power during Motor Start and Utility

Connected for Test 10.3.17

Figure 14f - Static Switch Reactive Single-Phase Power during Motor Start and

Utility Connected for Test 10.3.17............................................................ 140

Figure 14g - Meter 3 Line-to-Ground Voltages during Utility Connected to Island

mode for Test 10.3.17 ............................................................................... 141

Figure 14h - Meter 3 Frequency during Utility Connected to Island mode for Test 10.3.17.

Figure 14i - Load Bank3 Real Single-Phase Power during Motor Start and

Islanded for Test 10.3.17.

Figure 14j - Load Bank 3 Reactive Single-Phase Power during Motor Start and Islanded for Test 10.3.17.

Figure 14k - Meter 3 Line-to-Ground Voltages during Motor Start and Islanded for Test 10.3.17

Figure 141 - Meter 3 Frequency during Motor Start and Islanded for Test 10.3.17

Figure 14m - Gen-set A1 Real Single-Phase Power during Motor Start and

Islanded for Test 10.3.17

Figure 14n - Gen-set A1 Reactive Single-Phase Power during Motor Start and

Islanded for Test 10.3.17. 146

Figure 14o - Static Switch Real Single-Phase Power during Island to Utility

Connected mode for Test 10.3.17.

Figure 14p - Static Switch Reactive Single-Phase Power during Islanded to Utility

Connected mode for Test 10.3.17

Figure 14q - Meter 3 Line-to-Ground Voltages during Islanded to Utility

Connected mode for Test 10.3.17. 148

Figure 14r - Meter 3 Frequency during Islanded to Utility Connected mode for

Test 10.3.17 148

Figure 15a - Load Bank 3 Real Single-Phase Power during Motor Start and Utility

Connected for Test 10.3.18. 150

Figure 15b - Load Bank 3 Reactive Single-Phase Power during Motor Start and

Utility Connected for Test 10.3.18. 151 
Figure 15c - Static Switch Line-to-Ground Voltages during Motor Start and

Utility Connected for Test 10.3.18..... 152

Figure 15d - Static Switch Frequency during Motor Start and Utility Connected

for Test 10.3.18 152

Figure 15e - Static Switch Real Single-Phase Power during Motor Start and Utility

Connected for Test 10.3.18. 153

Figure $15 \mathrm{f}$ - Static Switch Reactive Single-Phase Power during Motor Start and

Utility Connected for Test 10.3.18. 153

Figure 15g - Meter 3 Line-to-Ground Voltages during Utility Connected to Island mode for Test 10.3.18 155

Figure 15h - Meter 3 Frequency during Utility Connected to Island mode for Test 10.3.18. 155

Figure 15i - Load Bank 3 Real Single-Phase Power during Motor Start and Islanded for Test 10.3.18. 156

Figure 15j - Load Bank 3 Reactive Single-Phase Power during Motor Start and Islanded for Test 10.3.18 157

Figure 15k - Meter 3 Line-to-Ground Voltages during Motor Start and Islanded for Test 10.3.18 158

Figure 151 - Meter 3 Frequency during Motor Start and Islanded for Test 10.3.18 158

Figure 15m - Gen-set A1 Real Single-Phase Power during Motor Start and Islanded for Test 10.3.18 159

Figure 15n - Gen-set A1 Reactive Single-Phase Power during Motor Start and

Islanded for Test 10.3.18. 160

Figure 15o - Gen-set A2 Real Single-Phase Power during Motor Start and

Islanded for Test 10.3.18. 160

Figure 15p - Gen-set A2 Reactive Single-Phase Power during Motor Start and Islanded for Test 10.3.18.

Figure 15q - Static Switch Real Single-Phase Power during Island to Utility Connected mode for Test 10.3.18 162

Figure 15r - Static Switch Reactive Single-Phase Power during Island to Utility Connected mode for Test 10.3.18 162

Figure 15s - Meter 3 Line-to-Ground Voltages during Island to Utility Connected mode for Test 10.3.18. 163

Figure 15t - Meter 3 Frequency during Island to Utility Connected mode for Test 10.3.18 163

Figure 16a - Load Bank 3 Real Single-Phase Power during Motor Start and Utility Connected for Test 10.3.20 165 
Figure 16b - Load Bank 3 Reactive Single-Phase Power during Motor Start and

Utility Connected for Test 10.3.20............................................................... 166

Figure 16c - Static Switch Line-to-Ground Voltages during Motor Start and

Utility Connected for Test 10.3.20.............................................................. 167

Figure 16d - Static Switch Frequency during Motor Start and Utility Connected

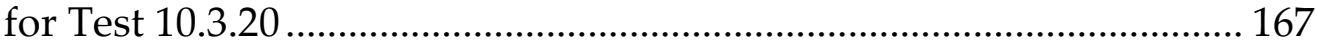

Figure 16e - Static Switch Real Single-Phase Power during Motor Start and Utility

Connected for Test 10.3.20 ....................................................................... 168

Figure 16f - Static Switch Reactive Single-Phase Power during Motor Start and

Utility Connected for Test 10.3.20.......................................................... 168

Figure 16g - Meter 3 Line-to-Ground Voltages during Utility Connected to Island

mode for Test 10.3.20 .............................................................................. 170

Figure 16h - Meter 3 Frequency during Utility Connected to Island mode for Test

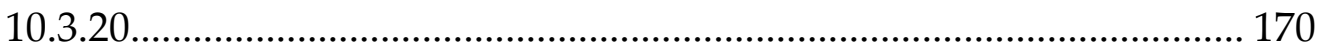

Figure 16i - Load Bank 3 Real Single-Phase Power during Motor Start and

Islanded for Test 10.3.20.

Figure 16j - Load Bank 3 Reactive Single-Phase Power during Motor Start and

Islanded for Test 10.3.20.......................................................................... 171

Figure 16k - Meter 3 Line-to-Ground Voltage during Motor Start and Islanded for Test 10.3.20

Figure 161 - Meter 3 Frequency during Motor Start and Islanded for Test 10.3.20

Figure 16m - Gen-set A1 Real Single-Phase Power during Motor Start and

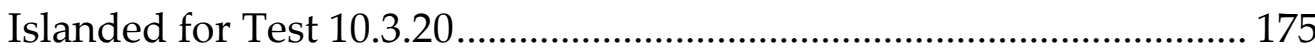

Figure 16n - Gen-set A1 Reactive Single-Phase Power during Motor Start and

Islanded for Test 10.3.20........................................................................ 175

Figure 16o - Static Switch Real Single-Phase Power during Island to Utility

Connected mode for Test 10.3.20 ............................................................ 176

Figure 16p - Static Switch Reactive Single-Phase Power during Island to Utility

Connected mode for Test 10.3.20 ........................................................... 177

Figure 16q - Meter 3 Line-to-Ground Voltages during Island to Utility Connected mode for Test 10.3.20 .................................................................................. 177

Figure 16r - Static Switch Frequency during Island to Utility Connected mode for

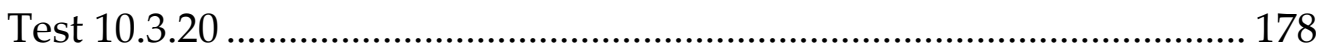

Figure 17a - Relay 2 (Static Switch) Tripping on a Reverse Power Condition.... 181

Figure $17 \mathrm{~b}$ - Meter 3 Line-to-Ground Voltages during Utility Connected to Island mode for Test 10.4.12 ............................................................................... 182

Figure 17c - Meter 3 Frequency before/after the static switch opened for Test

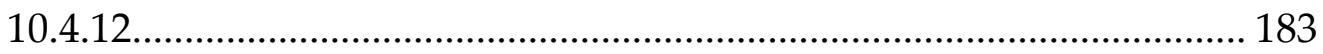


Figure 17d - Relay 2 (Static Switch) Reverse Power Condition Trip for Test 10.4.12 188

Figure 17e - Meter 3 Line-to-Ground Voltages during Islanded to Utility

Connected mode for Test 10.4.12. 190

Figure 17f - Meter 3 Frequency during Islanded to Utility Connected mode for

Test 10.4.12 190

Figure 17g - Static Switch Real Power during Islanded to Utility Connected mode for Test 10.4.12 191

Figure 17h - Meter 1 Real Power during Islanded to Utility Connected mode for

Test 10.4.12 192

Figure 18a - Relay 2 (Static Switch) Tripping on a Reverse Power Condition for

Test 10.4.14 194

Figure 18b - Meter 3 Line-to-Ground Voltages during Utility Connected to Island

mode for Test 10.4.14. 195

Figure 18c - Meter 3 Frequency Before/After the Static Switch Opened for Test

10.4.14. 196

Figure 18d - Relay 2 (Static Switch) Tripping on a Reverse Power Condition

during Island to Utility Connected mode for Test 10.4.14

Figure 18e - Meter 3 Line-to-Ground Voltages during Islanded to Utility

Connected mode for Test 10.4.14..... 201

Figure 18f - Meter 3 Frequency during Islanded to Utility Connected mode for

Test 10.4.14 201

Figure 18g - Static Switch Real Power during Islanded to Utility Connected mode for Test 10.4.14 202

Figure 18h - Meter 1 Real Power during Islanded to Utility Connected mode for

Test 10.4.14 202

Figure 19a - Meter 3 Line-to-Ground Voltages during Utility Connected to Island mode for Test 10.4.17 .................................................................................. 206

Figure 19b - Meter 3 Frequency Before/After the Static Switch Opened for Test 10.4.17. 207

Figure 19c - Relay 2 (Static Switch) Tripping on a Reverse Power Condition during Island to Utility Connected mode for Test 10.4.17

Figure 19d - Meter 3 Line-to-Ground Voltages during Islanded to Utility

Connected mode for Test 10.4.17.

Figure 19e - Meter 3 Frequency during Islanded to Utility Connected mode for

Test 10.4.17

Figure 19f - Static Switch Real Power during Islanded to Utility Connected mode for Test 10.4.17. 214 
Figure 19g - Meter 1 Real Power during Islanded to Utility Connected mode for

Test 10.4.17 ..................................................................................... 215 


\section{List of Tables}

Table 1 -Balance Load, Weak Grid, Before Motor Start settings of Gen-sets and

Load Banks

Table 2 -Unbalance Load, Weak Grid, Before Motor Start settings of Gen-sets and Load Banks 91

Table 3 - Measured Values after Start Up for Test 10.3.12 .................................... 92

Table 4 - Measured Values after Start Up for Test 10.3.14 ................................... 107

Table 5 - Measured Values after Start Up for Test 10.3.15 ................................. 121

Table 6 - Measured Values after Start Up for Test 10.3.17 ................................... 136

Table 7 - Measured Values after Start Up for Test 10.3.18 ................................... 149

Table 8 - Measured Values after Start Up for Test 10.3.20 .................................. 164

Table 9 -Unbalance Load Test, Weak Grid, settings of Gen-sets and Load Banks

Table 10 - Measured Values after Start Up for Test 10.4.12 and Load Banks 3 and 6 A-phase Reduced by $50 \%$

Table 11 - Measured Values after Static Switch Opened for Test 10.4.12 182

Table 12 - Measured Values with Static Switch Open and A-Phase Reduced in Load Bank 3 by 100\%and Load Bank 6 by 75\% for Test 10.4.12......... 184

Table 13 - Measured Values with Static Switch Open and A-Phase Reduced in Load Bank 4 by $75 \%$ for Test 10.4.12

Table 14 - Measured Values with Static Switch Open and A-Phase Reduced in Load Bank 6 by $100 \%$ for Test 10.4.12.

Table 15 - Measured Values with Static Switch Open and A-Phase Reduced in Load Bank 4 by $100 \%$ for Test 10.4.12. 187

Table 16 - Measured Values before/after Static Switch Synchronized and Closed back into the utility and after Static Switch Tripped on Reverse Power Condition for Test 10.4.12

Table 17 - Measured Values after Start Up for Test 10.4.14 and Load Banks 3 and 6 A-phase Reduced by $50 \%$

Table 18 - Measured Values after Static Switch Opened for Test 10.4.14..... 194

Table 19 - Measured Values after Static Switch Opened and A-phase Reduced in Load Bank 6 by $75 \%$ for Test 10.4.14 196

Table 20 - Measured Values after Static Switch Opened and A-phase Reduced in Load Bank 3 by $100 \%$ for Test 10.4.14.... 197

Table 21 - Measured Values after Static Switch Opened and A-phase Reduced in Load Bank 6 by $100 \%$ for Test 10.4.14 198 
Table 22 - Measured Values before/after Static Switch Synchronized and Closed back into the utility and after Static Switch Tripped on Reverse Power Condition for Test 10.4.14 200

Table 23 - Measured Values After Start Up for Test 10.4.17 and Load Bank 3 Aphase Reduced by 50\% ......................................................................... 204

Table 24 - Measured Values After Start Up for Test 10.4.17 and Load Bank 6 Aphase Reduced by 50\% ........................................................................ 205

Table 25 - Measured Values after Static Switch Opened for Test 10.4.17 ............ 205

Table 26 - Measured Values after Static Switch Opened and A-phase Reduced in Load Bank 6 by 75\% for Test 10.4.17 ..................................................... 208

Table 27 - Measured Values after Static Switch Opened and A-phase Reduced in Load Bank 3 by 75\% for Test 10.4.17 ...................................................... 208

Table 28 - Measured Values after Static Switch Opened and A-phase Reduced in Load Bank 6 by 100\% for Test 10.4.17 ................................................... 209

Table 29 - Measured Values after Static Switch Opened and A-phase Reduced in Load Bank 3 by $100 \%$ for Test 10.4.17 210

Table 30 - Measured Values before/after Static Switch Synchronized and Closed back into the utility and after Static Switch Tripped on Reverse Power Condition for Test 10.4.17 212 


\subsection{INTRODUCTION}

A series of tests were performed on the CERTS Microgrid by American Electric Power at the Walnut test site in Groveport, Ohio with support from Lawrence Berkeley National laboratory, Sandia National Laboratory, TECOGEN, The Switch (originally Youtility), Distributed-Energy (originally Northern Power) and University of Wisconsin. These tests were designed to demonstrate the CERTS Microgrid concepts of control and protection while connected to the utility electrical system and isolated (i.e., referred to as "islanded" from it. This paper describes the tests that were performed in Section 10.0 "Difficult Load Tests" of the CERTS Micro-grid Test Plan.

\subsection{BACKGROUND}

The CERTS Microgrid Concept is an advanced approach for enabling integration of, in principle, an unlimited quantity of DER (e.g., distributed generation (DG), energy storage, etc.) into the electric utility grid. A key feature of a microgrid is its ability to separate and island itself from the utility system, during a utility grid disturbance. This is accomplished via intelligent power electronic interfaces and a single, high-speed, switch which is used for disconnection from the grid and synchronization to the grid. During a disturbance, the DER and corresponding loads can autonomously be separated from the utility's distribution system, isolating the microgrid's load from the disturbance (and thereby maintaining high level of service) without harming the integrity of the utility's electrical system. Thus, when the utility grid returns to normal, the microgrid automatically synchronizes and reconnects itself to the grid, in an equally seamless fashion. Intentional islanding of DER and loads has the potential to provide a higher level of reliability than that provided by the distribution system as a whole.

What is unique about the CERTS Microgrid is that it can provide this technically challenging functionality without extensive (i.e., expensive) custom engineering. In addition, the design of the CERTS Microgrid provides a high level of system reliability and great flexibility in the placement of DER within the microgrid. The CERTS Microgrid offers these functionalities at much lower costs than traditional approaches by incorporating peer-to-peer and plug-and-play concepts for each component within the microgrid.

The original concept was driven by two fundamental principles: 1.) A systems perspective was necessary for customers, utilities, and society to capture the full benefits of integrating DER into an energy system; and 2.) The business case for accelerating adoption of these advanced concepts will be driven, primarily, by lowering the up-front cost and enhancing the value offered by microgrids. 
Each innovation was created specifically to lower the cost and improve the reliability of small-scale DG systems (i.e., installed systems with capacities ranging from less than $100 \mathrm{~kW}$ to $1000 \mathrm{~kW}$ ). The goal was to increase and accelerate realization of the many benefits offered by small-scale DG, such as their ability to supply waste heat at the point of need or to provide a higher level of reliability to some but not all loads within a facility. From an electric utility perspective, the CERTS Microgrid Concept is attractive because it recognizes that the nation's distribution system is extensive, aging, and will change over time which impacts power quality. The CERTS Microgrid Concept enables high penetration of DG systems without requiring re-design or re-engineering of the utility's distribution system.

Prospective applications of the CERTS Microgrid include industrial parks, commercial and institutional campuses, situations that require uninterrupted power supplies and high power quality, CHP systems, Greenfield communities, and remote applications. In short, wherever economic and DG location considerations indicate the need for multiple DG units within a (or among) site, the CERTS Microgrid offers the potential for a much more reliable, flexible, and lower cost solution compared to traditional engineering approaches for integrating DG.

\subsection{MICROGRID TESTBED SETUP}

The CERTS Microgrid Test Bed is operated at 480/277 volts (i.e., three-phase, four-wire) and consists of three TECOGEN Generators at 480 volts capable of producing $60 \mathrm{~kW}$ plus 60kVAr (Gen-set A1, Gen-set A2 and Gen-set B1) and four load banks (Load Bank 3, Load Bank 4, Load Bank 5 and Load Bank 6) capable of consuming 100kW plus 20kVAr each, as shown in Figure 2. Each of the generators are connected to a 112kVA isolation transformer and interfaced to the CERTS Microgrid through an inverter, developed by The Switch, where the algorithms for the CERTS Microgrid controls are embedded. . A semiconductor switch made by S\&C Electric Company, known as the static switch, connects the CERTS Microgrid to the utility grid. Load Banks $3-5$ are the local loads in zones located beyond the static switch; and Load Bank 6 is a customer load in Zone 6 located on the utility side of the static switch. 


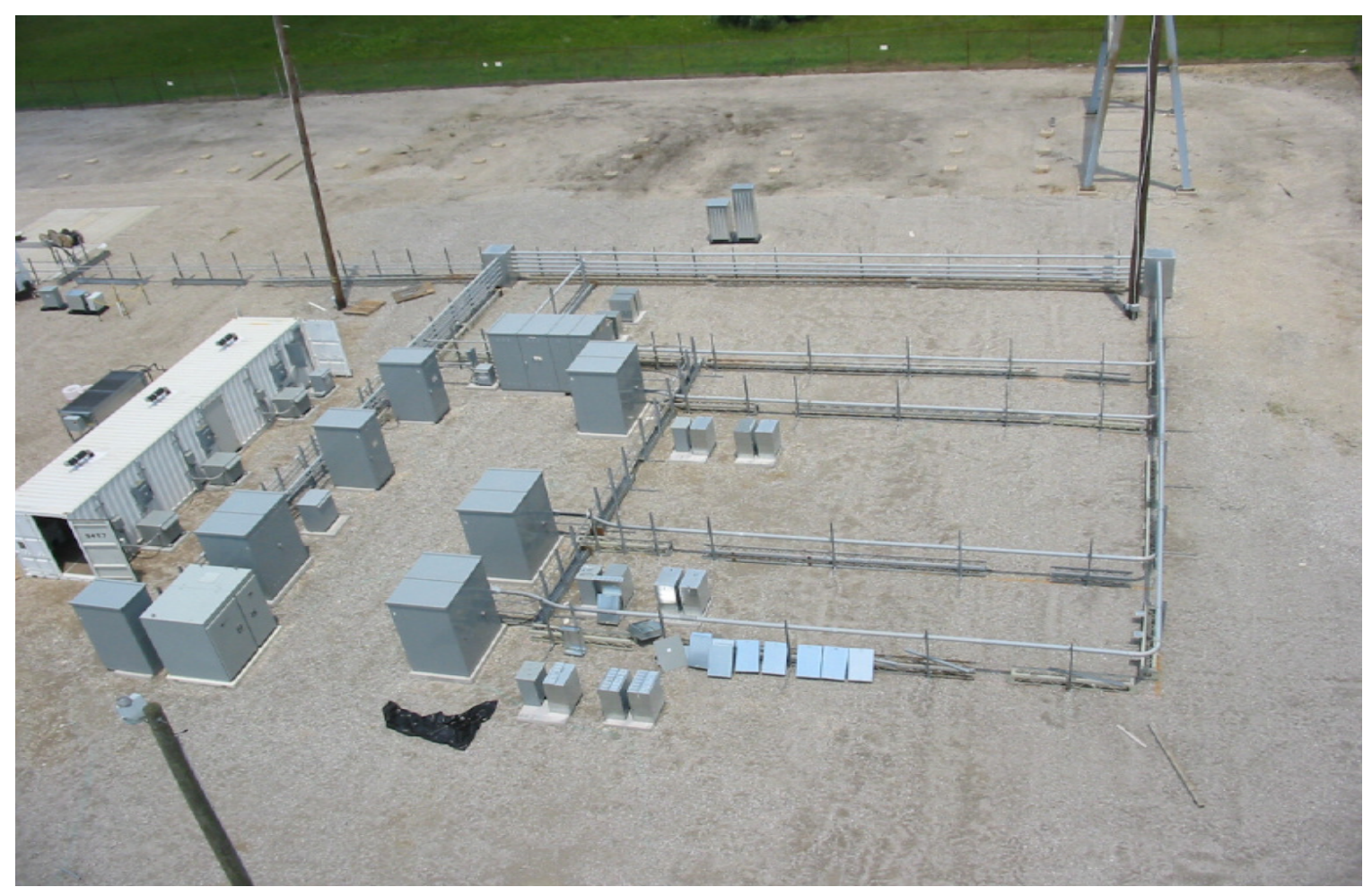

Figure 1 - CERTS Microgrid Aerial Photo

There are 6 zones in the Test Bed with Zones 2 - 6 contained within the CERTS Microgrid design and Zone 1 being the utility interface and referred to as the point-of-common coupling (PCC) to the grid. Each zone is protected by a Schweitzer SEL-351 relay. Faults of varying magnitude can be applied to each zone through an additional breaker which allows fault application and removal.

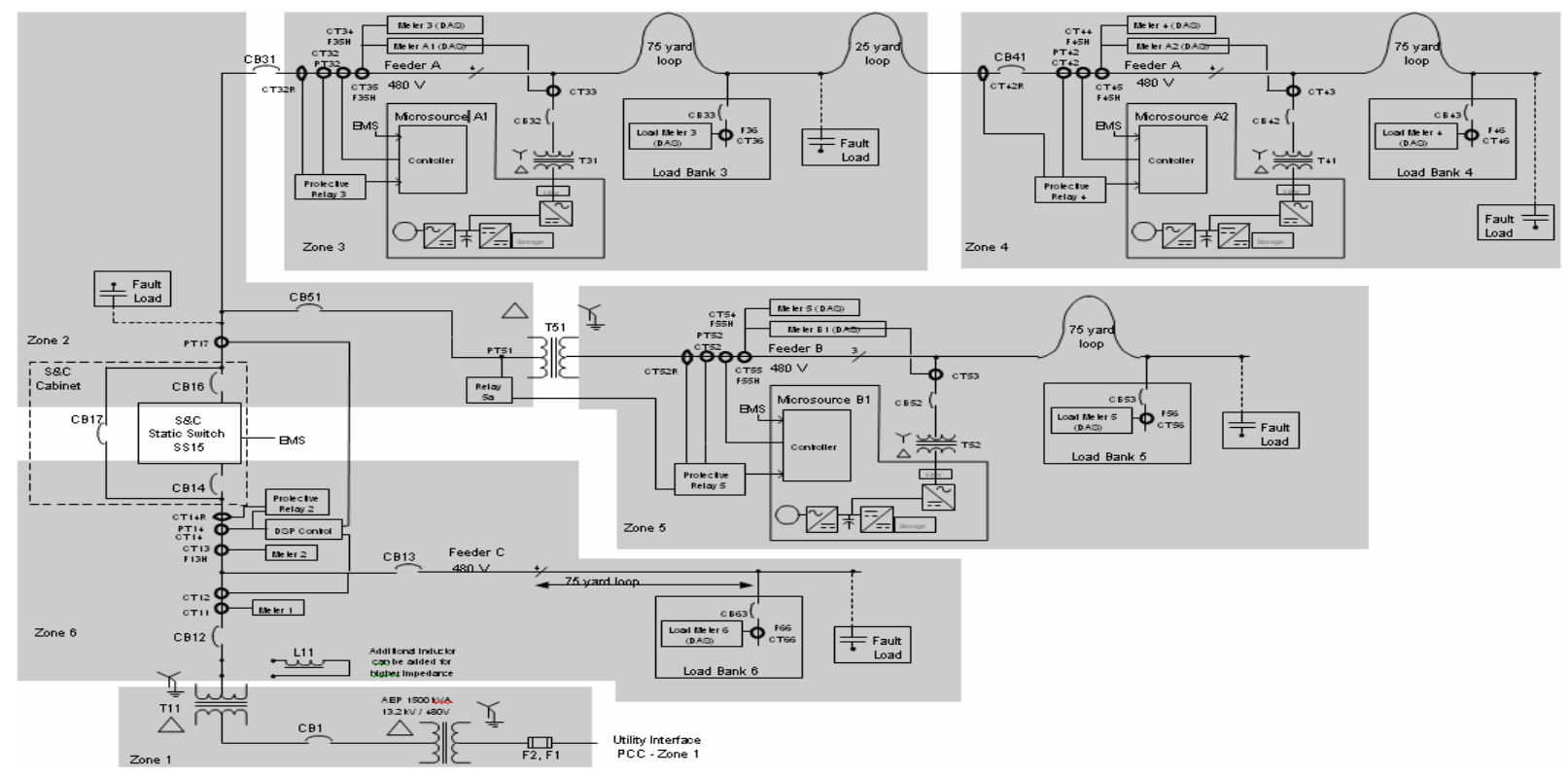




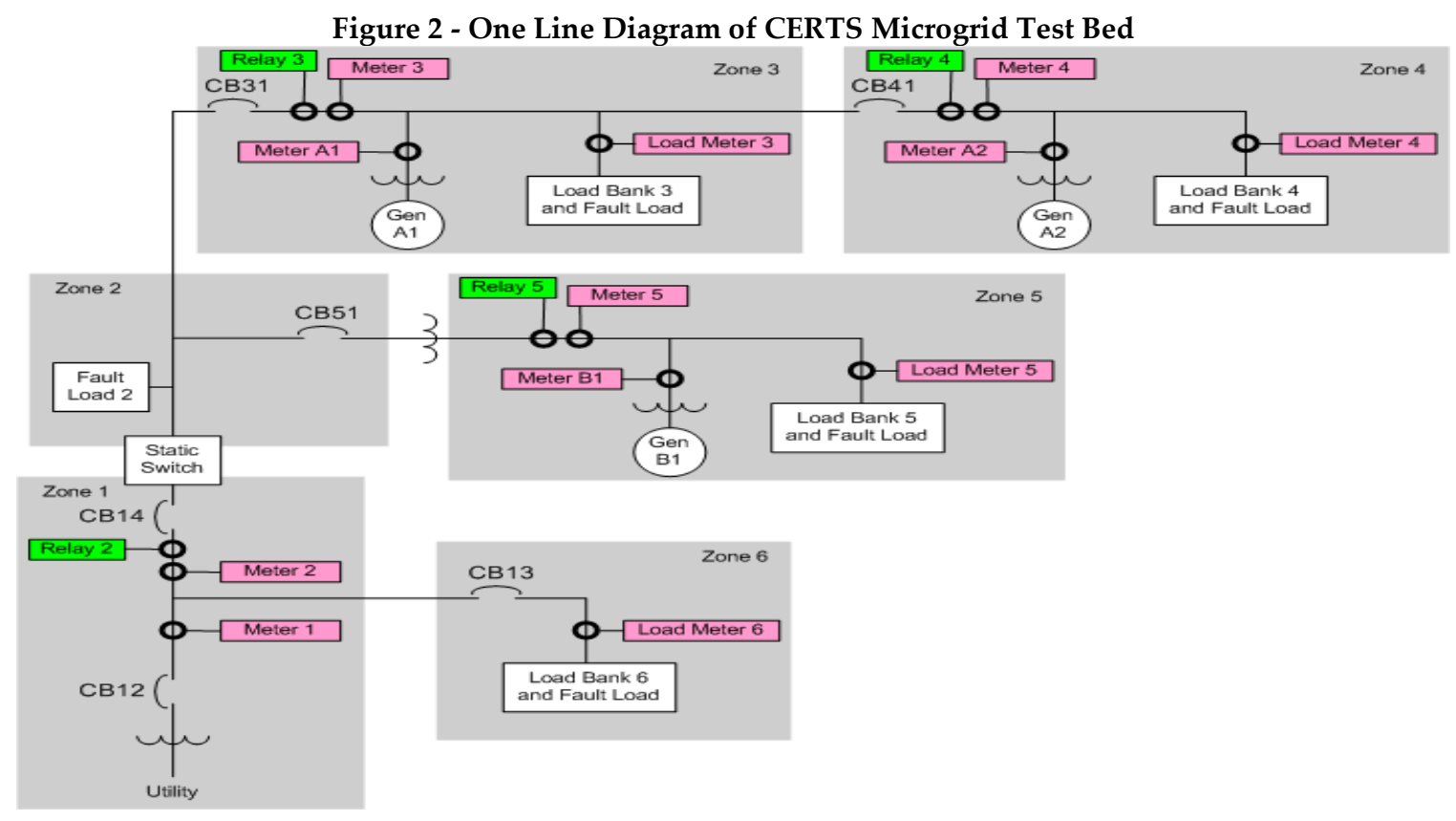

Figure 3 - Simplified diagram of Test Bed showing Meter and Relay locations

There are twelve PML ION 7650 meters placed through out the microgrid and shown in Figure 3, which monitor electrical system conditions, plus acquire phase current and voltage waveforms; and calculate RMS values of voltage, current, active power, reactive power, and frequency. 


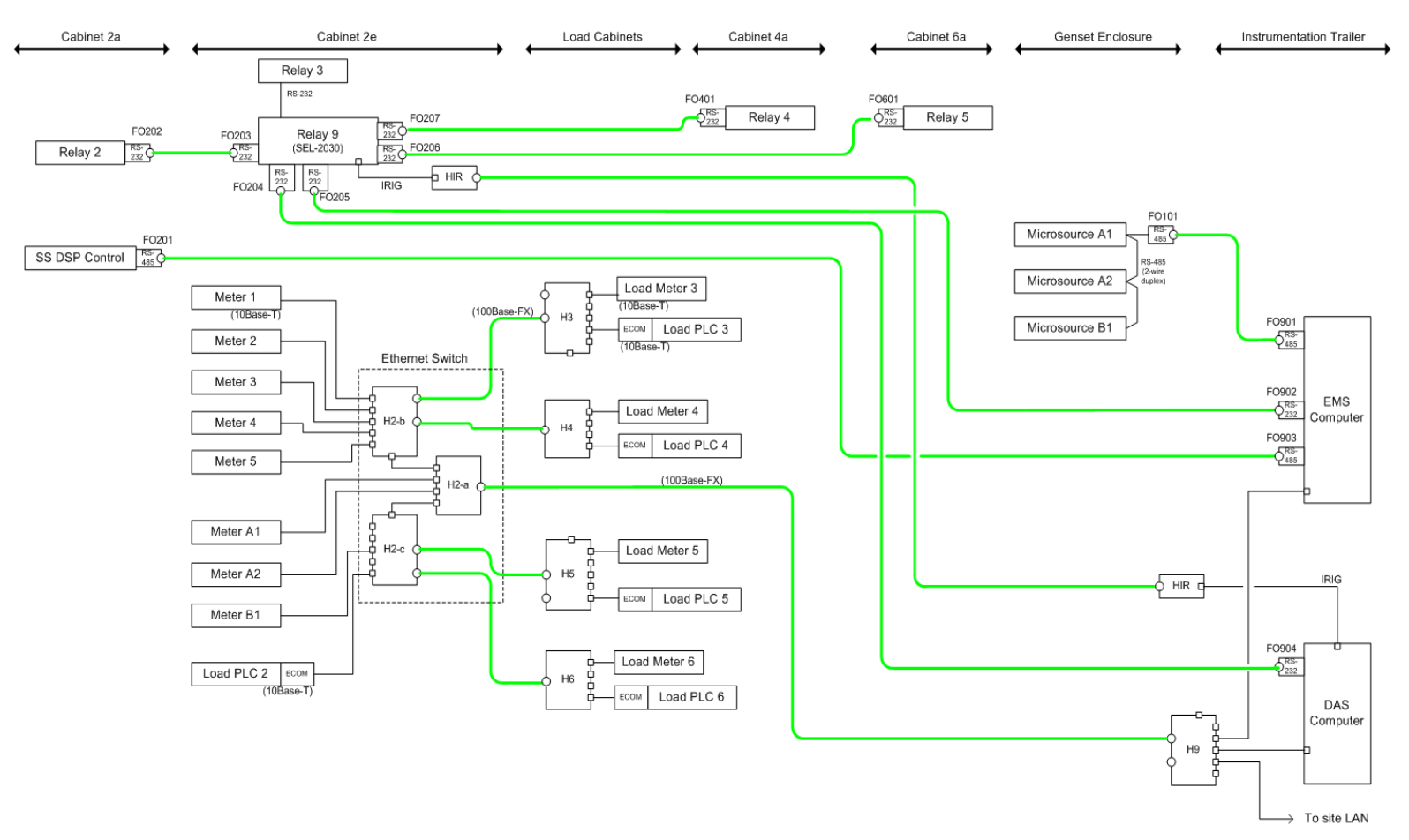

Figure 4 - Diagram of DAS \& EMS Data networks

An Ethernet network was provided as shown in Figure 4, for communications between all meters, load control PLCs, and the Data Acquisition System (DAS) computer, using fiber-optic links and switches. The DAS and Energy Management System (EMS) computers were also networked into the local Dolan Local Area Network (LAN) and to a secure Website with user ID and password protection. Additional serial links, using fiber optic converters, connect all relays, static switch Digital Signal Processor (DSP) controller, and TECOGEN Gen-set controls to the EMS computer.

\subsection{PROPOSED TEST PLAN}

The CERTS Microgrid Test Plan was developed by the CERTS Microgrid Team to demonstrate the unique concepts of control and protection of the CERTS Microgrid. This test plan was reviewed by a Technical Advisory Committee outside the CERTS Microgrid Team and then implemented by American Electric Power. CERTS Microgrid Test Plan consists of 12 sections with 5 of them detailing desired tests, starting at section 6.0, to demonstrate the controls and concepts of the CERTS Microgrid. The other 7 sections pertain to safety procedures, equipment calibration, and documentation. Each section of the test plan is described below.

- Section 1 - "Purpose, References, and Definitions" describes the purpose of the test plan, helpful references for further explanation of how the test bed was created, and definitions used through out the test plan. 
- Section 2 - "Responsibilities" informs personnel of their responsibilities while working on or near the CERTS Microgrid test site.

- Section 3 - “Training - Team Members" lists the mandatory training needed by personnel before they can work on or near the CERTS Microgrid test site.

- Section 4 - "Procedure - CERTS Microgrid Test Bed Lockout/Tagout" entails how to safely shut down the equipment and lockout/tagout the closest upstream disconnect to work on or near equipment.

- Section 5 - "Procedure - General" is the daily procedures performed at the CERTS Microgrid Test Site, prior to performing a test from Section 6 through Section 10.

- Section 6 - "Procedure - Microgrid Test Bed System Checkout" was designed to check control and operation of the static switch, basic power and voltage control of the Gen-sets, and a preliminary check of the protection scheme. The goal is to assure that the test bed is operating and ready to perform the tests described in the remaining sections of the test plan document.

- Section 7 - "Validate Protection Settings \& Initial Fault Testing" is designed to examine a preliminary set of fault (i.e. overload simulating a fault) condition tests to ensure protection and safety of the Micro-grid test Bed, while performing other planned tests. The goal is to test and adjust protection settings to achieve the most ideal conditions and protection design.

- Section 8 - "Procedure - Reduced System Tests" is a limited set of tests to build confidence that the Gen-set inverter controls are working correctly. This includes unit control, zone control, and mixed power controls, in conjunction with limit controls and synchronized closing of the static switch. These tests are based on the TECOGEN/THE SWITCH factory acceptance testing.

- Section 9 - "Procedure - Demonstration Tests of Control Power Flow" demonstrates the flexibility of the Micro-grid both grid connected and islanded for different loads, power flows and impact on the utility. 
- Section 10 - "Procedure - Test Difficult Loads" determines operation limits of the Micro-grid (i.e. power quality, protection and inverter limits) with low pf loads, motor loads, harmonic loads and unbalance loads.

- Section 11 - "Hazards \& Mitigation" informs the personnel of hazards that may exist while working on or near the CERTS Micro-grid test site and how to mitigate them.

- Section 12 - “Quality Assurance” ensures quality for the acquiring data results by providing a checklist reminder for personnel.

\subsection{TESTS PERFORMED IN SECTION 10.0}

Prior to each test day, the person in charge performed a job safety briefing (JSB) with barricades and test setup inspected for safety and compliance. A minimum of two people were on-site during each planned test.

Visual and audible alarms were used to warn persons that energized testing was being performed in the Microgrid Test Bed area. The visual alarm consisted of a portable red flashing light, located between the Control Trailer and Gen-set Enclosure. An audible alarm, consisting of a portable wireless motion detector, was located at the front gate of the Walnut Test Site with the fence gate "Closed", not locked, and audible alarm in the trailer operational during test(s).

Barricades were set up around the Micro-grid Test Bed area (i.e., saw-horse style barricades with a "Red" plastic chain surrounded the test area containing the Gen-set Enclosure, Micro-grid switching cabinets, plus load and fault bank cabinets).

Prior to performing tests, the Test Engineer or Technical Consultant verified that all personnel and visitors were properly protected and in assigned locations. Personnel were in or adjacent to the Control Trailer while tests were being performed. All nonessential personnel either left the main site or were sheltered in the Control Trailer.

For all tests the following waveforms were captured and recorded in the DAS for voltage $(\mathrm{V})$ and current $(\mathrm{I})$. From these waveforms real power $(\mathrm{kW})$, reactive power $(\mathrm{kVAr})$, and frequency (freq) were post calculated by the PQView software. Frequency measurements in this report should be used for steady state information and not used for transient analysis, due to the calculation and filtering methods employed. Below is a list of the meters capturing this data. 
- Meters 1, 2, 3, 4 \& 5

- Load Meters 3, 4, 5, \& 6

- Meters A1, A2 \& B1

- Meter 2 also measures the voltage across the static switch on phase A

Schweitzer event reports were also captured for each event, along with breaker and static switch status, such as Open or Close.

Section 10 tests were developed to determine operation limits of the Microgrid (i.e., power quality, protection and inverter limits) with low power factor (pf) loads, motor loads, harmonic load and unbalance loads.

For Section 10, weak grid conditions were planned in the beginning of the test plan to minimize any damage to equipment. However, time constraints forced a decision by the Team to postpone strong grid testing to a later date with concentration on weak grid testing. Motor start tests done in Test 10.2 had balanced loads at 0.9 in power factor in a weak grid scenario, while connected to the utility grid and islanded. These tests were to verify and document power flow, Microgrid frequency changes and protection design with different Gen-set settings during motor starts when utility connected and then repeat motor start test during an islanded mode of operation. Test 10.3 is similar to Test 10.2 , except that the loads are now unbalanced with a 0.9 power factor.

In Test 10.4 tests were created to verify and document power flow, Microgrid frequency changes and protection design with different Gen-set settings during changes of unbalanced load in Load Banks. Test 10.5 is similar to Test 10.4, except that the loads are balanced. Power factor tests with unbalanced loads were tested in Test 10.6 which was designed to verify and document power flow, Microgrid frequency changes and protection design with different Gen-set settings during changes of power factor of unbalanced load in Load Banks.

Tests $10.7-10.15$ are similar tests that verify and document power flow, Microgrid frequency changes and protection design with different Gen-set settings. Changes of harmonic loads in different Zones with a motor start and balanced load in Load Banks were planned.

\subsection{ANALYSES OF TEST RESULTS}

\subsection{SECTION 10 - DIFFICULT LOAD TESTS}

To maintain a common understanding of Gen-set control modes and power flows during tests described in the following sections, it is important to know the difference 
between unit power control mode and zone control mode and positive versus negative power flow.

Unit power control mode involves the amount of power (i.e., $\mathrm{kW}$ ) injected into the Zone from the Gen-set being controlled. Zone power control mode involves the amount of power (i.e., $\mathrm{kW}$ ) entering/exiting the Zone which controls the output of the Gen-set in that zone.

Positive power flow recorded by Meter 1, Meter 2, Meter 3, Meter 4 and Meter 5 are in the direction from the utility going downstream into the microgrid. Negative power flow through these meters means that the power is flowing from the Gen-sets out towards the utility. For example, if Meter 2 (i.e., static switch) recorded a power flow of $-20 \mathrm{~kW}+\mathrm{j} 35 \mathrm{kVAr}$ then real power is flowing from the Gen-sets through the static switch out into Zone 6 and reactive power is flowing in the opposite direction from Zone 6 into the critical loads of the microgrid (Zones 2, 3, 4 and 5). Positive power flow recorded by Meters A1, A2 and B1 are what the Gen-sets are producing and negative power flow is what the Gen-sets are absorbing. This is vice versa for Meters LB3, LB4, LB5 and LB6 meaning that a positive power flow is what the Load Bank is absorbing. Load Bank meters will never have a negative power flow.

\subsubsection{Motor Start Tests, Weak Grid, Balanced Load with 0.9 Power Factor Performance Goal:}

Verify and document power flow, Micro-grid frequency changes and protection design with different Gen-set settings during motor starts when utility connected and then repeat motor start test during an islanded mode of operation.

Six tests were completed in this section with each test event and settings listed in Table 1. During each test Gen-sets A1 and A2 were both set for either unit or zone power control mode of operation. Each of the six tests varied the output power command setpoint in each Gen-set, along with varying the load settings in load banks LB3, LB4 and LB6. During each test, inductor L11 was in series with the utility grid to simulate a weak grid condition at the PCC to the microgrid.

\begin{tabular}{|c|c|c|c|c|c|c|c|c|c|}
\hline $\begin{array}{c}\text { Test } \\
\text { Event }\end{array}$ & $\begin{array}{c}\text { Gen- } \\
\text { set A1 }\end{array}$ & $\begin{array}{c}\text { Gen - } \\
\text { set A2 }\end{array}$ & Meter 1 & Meter 2 & Meter 3 & Meter 4 & LB 3 & LB 4 & LB 6 \\
\hline 10.2 .12 & Unit & Unit & $+20 \mathrm{~kW}$ & $-20 \mathrm{~kW}$ & $-20 \mathrm{~kW}$ & $+10 \mathrm{~kW}$ & $10 \mathrm{~kW}$ & $40 \mathrm{~kW}$ & $40 \mathrm{~kW}$ \\
\hline
\end{tabular}




\begin{tabular}{|c|c|c|c|c|c|c|c|c|c|}
\hline & $+40 \mathrm{~kW}$ & $+30 \mathrm{~kW}$ & & & & & \\
\hline 10.2 .14 & $\begin{array}{c}\text { Unit } \\
+40 \mathrm{~kW}\end{array}$ & $0 \mathrm{~kW}$ & $+10 \mathrm{~kW}$ & $-30 \mathrm{~kW}$ & $-30 \mathrm{~kW}$ & $0 \mathrm{~kW}$ & $10 \mathrm{~kW}$ & $0 \mathrm{~kW}$ & $40 \mathrm{~kW}$ \\
\hline 10.2 .15 & $\begin{array}{c}\text { Unit } \\
+40 \mathrm{~kW}\end{array}$ & $\begin{array}{c}\text { Unit } \\
+30 \mathrm{~kW}\end{array}$ & $+70 \mathrm{~kW}$ & $+30 \mathrm{~kW}$ & $+30 \mathrm{~kW}$ & $+20 \mathrm{~kW}$ & $50 \mathrm{~kW}$ & $50 \mathrm{~kW}$ & $40 \mathrm{~kW}$ \\
\hline 10.2 .17 & $\begin{array}{c}\text { Unit } \\
+40 \mathrm{~kW}\end{array}$ & $0 \mathrm{~kW}$ & $+20 \mathrm{~kW}$ & $-20 \mathrm{~kW}$ & $-20 \mathrm{~kW}$ & $0 \mathrm{~kW}$ & $20 \mathrm{~kW}$ & $0 \mathrm{~kW}$ & $40 \mathrm{~kW}$ \\
\hline 10.2 .18 & $\begin{array}{c}\text { Zone } \\
10 \mathrm{~kW}\end{array}$ & $\begin{array}{c}\text { Zone } \\
+10 \mathrm{~kW}\end{array}$ & $+30 \mathrm{~kW}$ & $-10 \mathrm{~kW}$ & $-10 \mathrm{~kW}$ & $+10 \mathrm{~kW}$ & $20 \mathrm{~kW}$ & $40 \mathrm{~kW}$ & $40 \mathrm{~kW}$ \\
\hline 10.2 .20 & $\begin{array}{c}\text { Zone } \\
20 \mathrm{~kW}\end{array}$ & $0 \mathrm{~kW}$ & $+20 \mathrm{~kW}$ & $-20 \mathrm{~kW}$ & $-20 \mathrm{~kW}$ & $0 \mathrm{~kW}$ & $20 \mathrm{~kW}$ & $0 \mathrm{~kW}$ & $40 \mathrm{~kW}$ \\
\hline
\end{tabular}

Table 1 -Balance Load, Weak Grid, Before Motor Start settings of Gen-sets and Load Banks

The procedure for each test event began with setting the $\mathrm{kW}$ and $\mathrm{kVAr}$ load (i.e., balanced load) conditions, as indicated in Table 1. Before loads were brought on-line, Gen-sets A1 and A2 or just Gen-set A1 depending on the test setup, were started to allow them to warm up. After the Gen-sets ran for a few minutes and steady-state conditions established, the loads were brought on-line, the static switch was allowed to close, and measurements were taken from Meters 1, 2, 3 and 4 . The measurements were then compared with the expected values in Table 1 . When the measured values coincided with the expected values from the table, then a $10 \mathrm{Hp}$ motor was started in Zone 3 with the dynamometer set in $\mathrm{ft}-\mathrm{lbs}$ at $100 \%$ motor rating. When all measurements were verified and recorded in the DAS Database for before and after the motor start, the motor was turned off and the manual open signal to the static switch was initiated. This allowed the static switch to open and separate the microgrid from the utility grid. Gen-sets were observed at this time to make sure they increased or decreased their output power as planned to satisfy the loads at load banks (i.e., LB3, LB4 and LB6) and that a smooth transition occurred. When all the measurements were verified and recorded in the DAS Database, the $10 \mathrm{Hp}$ motor was started again with the dynamometer set in $\mathrm{ft}-\mathrm{lbs}$ for $100 \%$ motor rating. Once all measurements were verified and recorded in the DAS Database for before and after the motor start, the manual open signal to the static switch was released. Releasing the manual open signal allowed the static switch to synchronize and reclose. All data (i.e., from the manual open event and the reconnection event) was verified and recorded in the DAS Database. Loads, power control set-points and operation modes were then changed to the settings for the next test. Test results are explained in the following paragraphs. 
For Test 10.2.12 the measured values, after the Gen-sets were warmed up and load banks brought on-line, were approximately $12 \mathrm{~kW}+\mathrm{j} 24 \mathrm{kVAr}$ at Meter 1, $-24 \mathrm{~kW}+$ $\mathrm{j} 22 \mathrm{kVAr}$ at Meter 2, $-27 \mathrm{~kW}+\mathrm{j} 16 \mathrm{kVAr}$ at Meter 3 and $3.5 \mathrm{~kW}+\mathrm{j} 5 \mathrm{kVAr}$ at Meter 4 . From the microgrid, $41 \mathrm{~kW}-\mathrm{j} 12 \mathrm{kVAr}$ was produced by Gen-set A1 and $33 \mathrm{~kW}-\mathrm{j} 3 \mathrm{kVAr}$ was produced by Gen-set A2. The load banks were $11 \mathrm{~kW}+\mathrm{j} 0 \mathrm{kVAr}$ at LB3, 35.9kW + j0.645kVAr at LB4 and $35.9 \mathrm{~kW}+\mathrm{j} 0.6975 \mathrm{kVAr}$ at LB6. These measurements were relatively close to the expected values in Table 1 , but not exact due to temperature, phase voltages and electrical losses in conductors. In addition, the $40 \mathrm{~kW}$ settings for LB4 and LB6 were also below selected set values. The deviation of selected values versus actual values was consistent for the other five tests in this section. At the time of these measurements, the voltage and frequency was $281.5 \mathrm{~V}$ on A-phase, $279 \mathrm{~V}$ on Bphase and $279 \mathrm{~V}$ on $\mathrm{C}$-phase and $59.98 \mathrm{~Hz}$ at the static switch (i.e., Meter 2) when connected to the utility grid; and $282 \mathrm{~V}$ on A-phase, $280 \mathrm{~V}$ on B-phase and $280 \mathrm{~V}$ on Cphase and $59.98 \mathrm{~Hz}$ at Meter 3.

The Gen-sets in this test were set up to produce more power than Load Banks 3 and 4 needed which approximately $24 \mathrm{~kW}$ of excess power was exported through the static switch to Load Bank 6. Since Load Bank 6 was approximately $35.9 \mathrm{~kW}+\mathrm{j} 0.6975 \mathrm{kVAr}$, the utility had to supply approximately $12 \mathrm{~kW}$ to satisfy the load. Reactive power had to be imported in from the utility of approximately 22kVAr because Gen-sets A1 and A2 needed approximately 15kVAr between the two and the reactive power of Load Banks 3 and 4. Once all data was verified and recorded into the DAS Database, the $10 \mathrm{Hp}$ induction motor was started in Zone 3. 


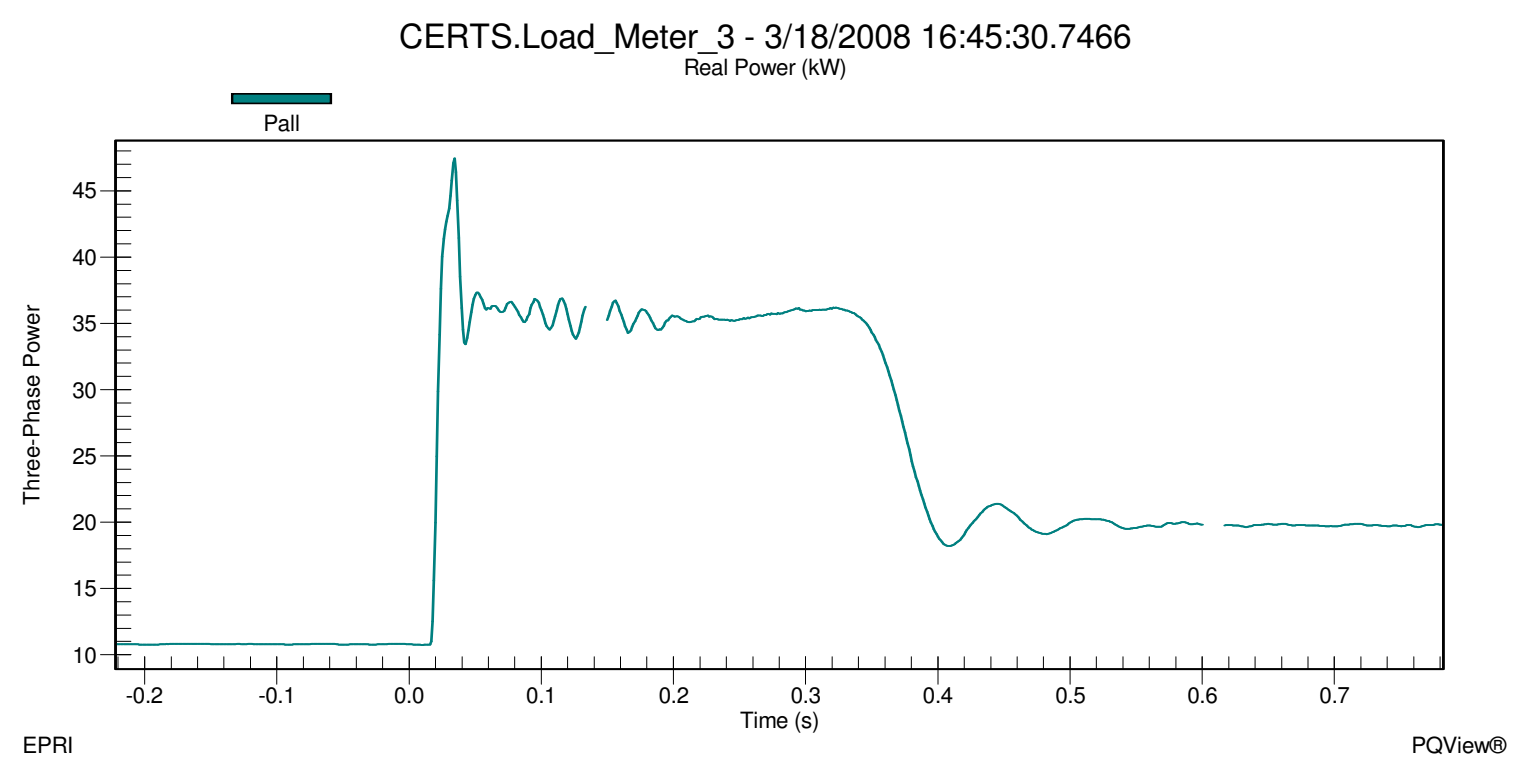

Figure 5a - Load Bank 3 Real Power Motor Start and Utility Connected for Test 10.2.12

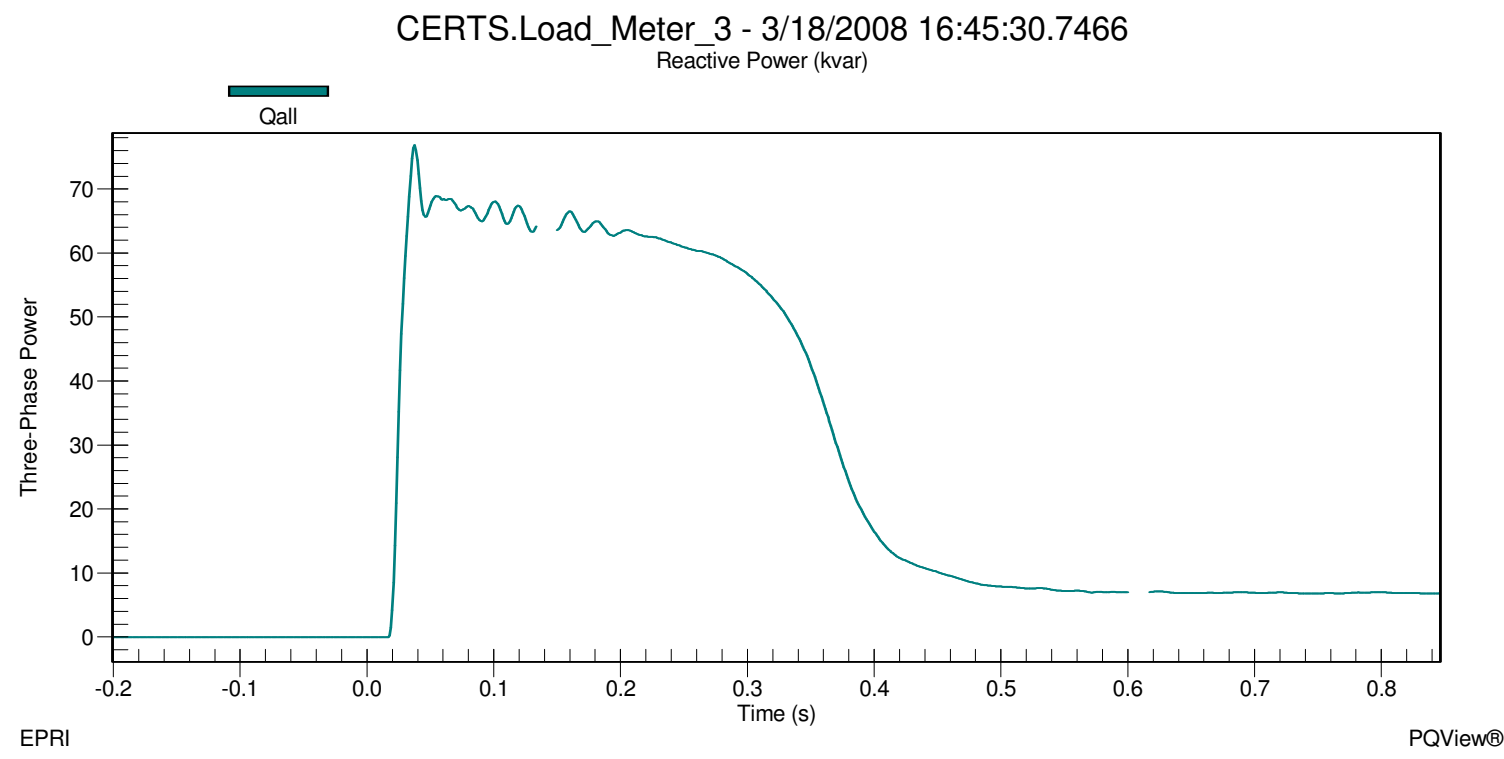

Figure 5b - Load Bank 3 Reactive Power during Motor Start and Utility Connected for Test 10.2.12

In Figures $5 \mathrm{a}$ and $5 \mathrm{~b}$ it can be seen that the load in Zone 3 was approximately $10.9 \mathrm{~kW}+$ j0kVAr before the start of the induction motor and increased to approximately $47.4 \mathrm{~kW}+$ j77kVAr during the inrush phase of the motor start. After about 1.5 cycles, the motor settled down to approximately $35.6 \mathrm{~kW}+\mathrm{j} 64 \mathrm{kVAr}$ during the warm up phase which 
lasted about 24 cycles ( 0.40 seconds). When the motor reached steady state, the load in Zone 3 was approximately $19.9 \mathrm{~kW}+\mathrm{j} 6.9 \mathrm{kVAr}$.

The voltage and frequency at the static switch before the motor start was approximately 281.2V on A-phase, $278.7 \mathrm{~V}$ on B-phase and $279 \mathrm{~V}$ on C-phase shown in Figure 5c and approximately $59.97 \mathrm{~Hz}$ shown in Figure $5 \mathrm{~d}$. When the motor started, the voltage at the static switch during the inrush decreased to approximately $274.3 \mathrm{~V}$ on A-phase, $271.4 \mathrm{~V}$ on B-phase and $272.4 \mathrm{~V}$ on C-phase for about 1.5 cycles. Frequency dropped during the inrush to approximately $59.90 \mathrm{~Hz}$ and quickly increased back to the starting frequency of approximately $59.97 \mathrm{~Hz}$. Voltage increased as the motor was warming up and eventually settled at a steady state voltage at approximately $280.6 \mathrm{~V}$ on A-phase, $278.4 \mathrm{~V}$ on B-phase and 278V on C-phase at an approximate frequency of $59.97 \mathrm{~Hz}$.

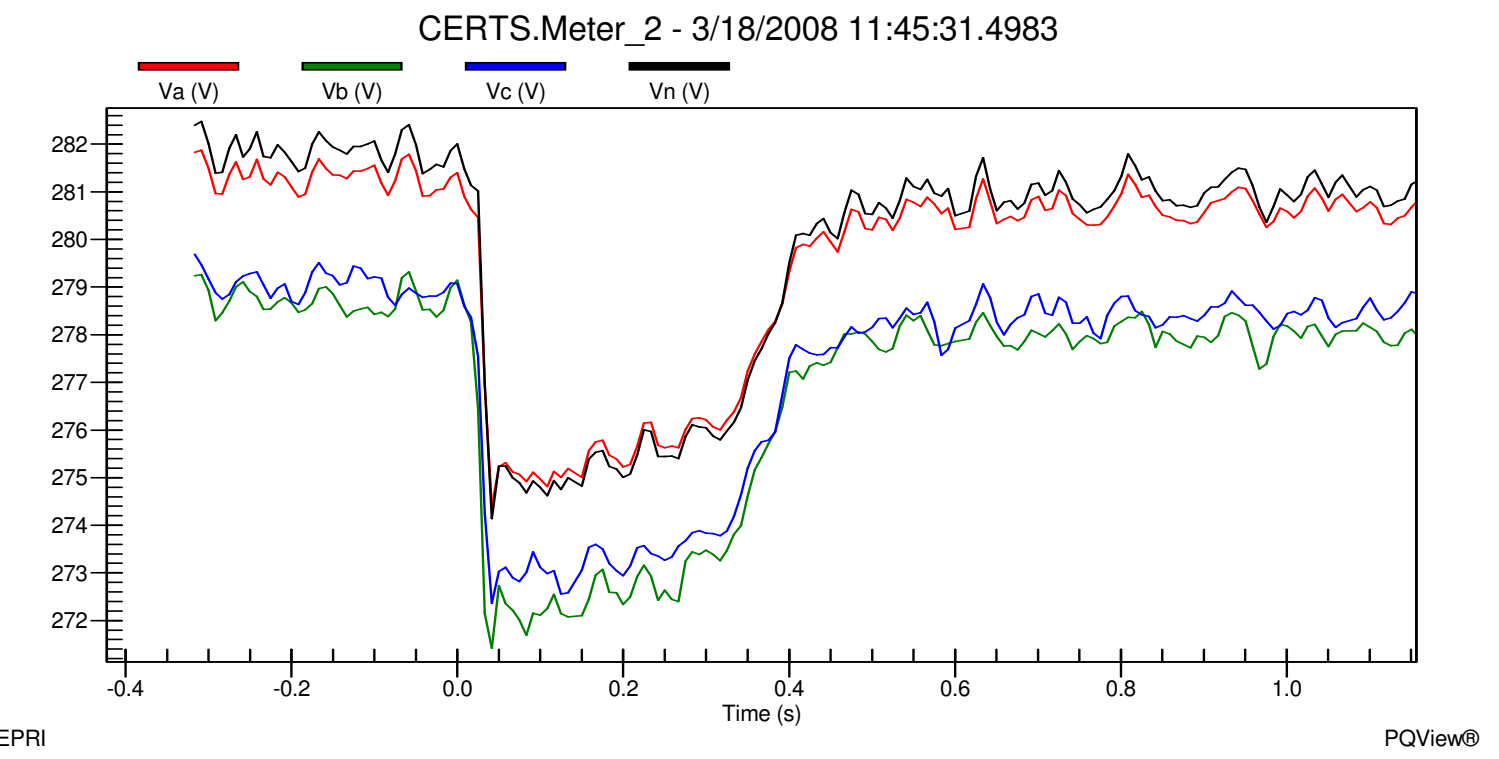

Figure 5c - Static Switch Line-to-Ground Voltages during Motor Start and Utility Connected for Test 10.2.12 


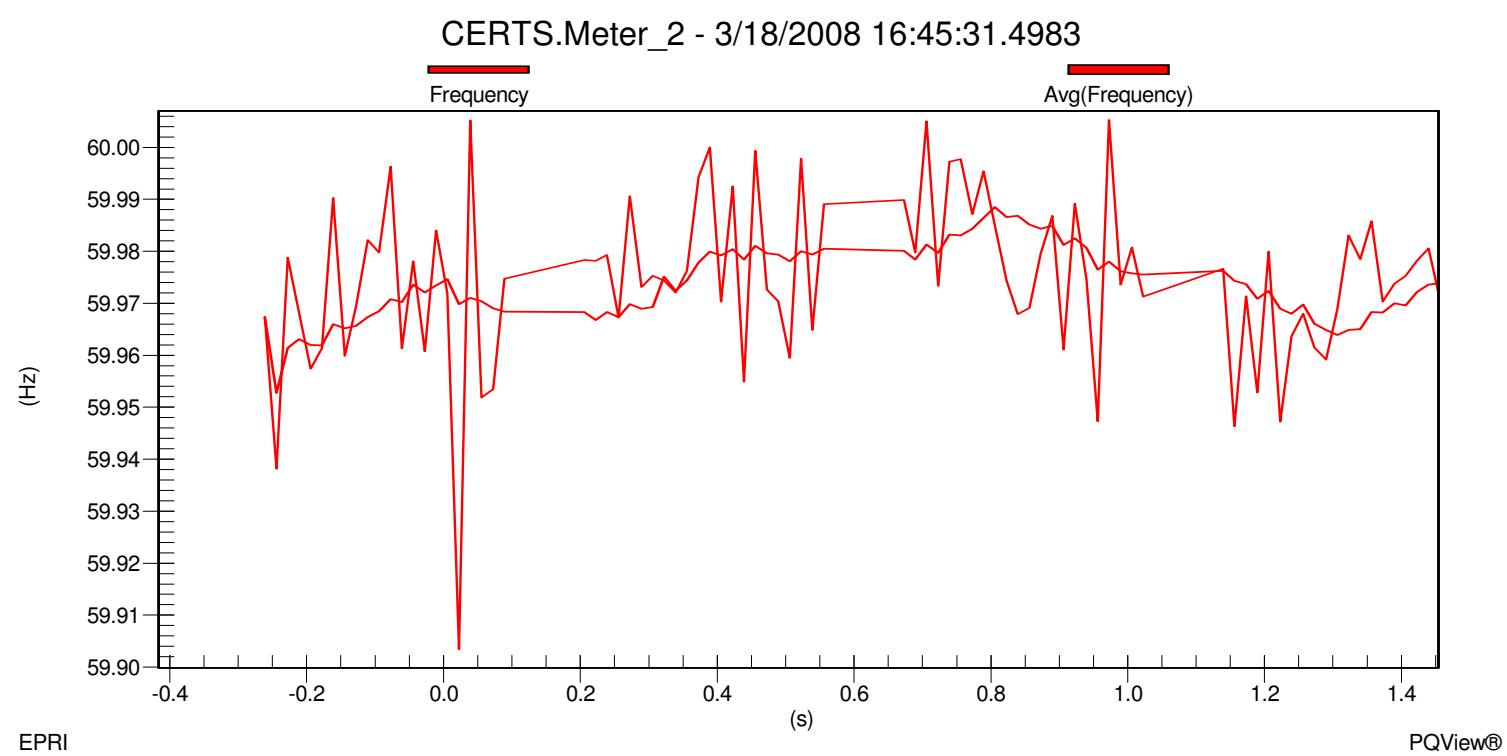

Figure 5d - Static Switch Frequency during Motor Start and Utility Connected for Test 10.2.12

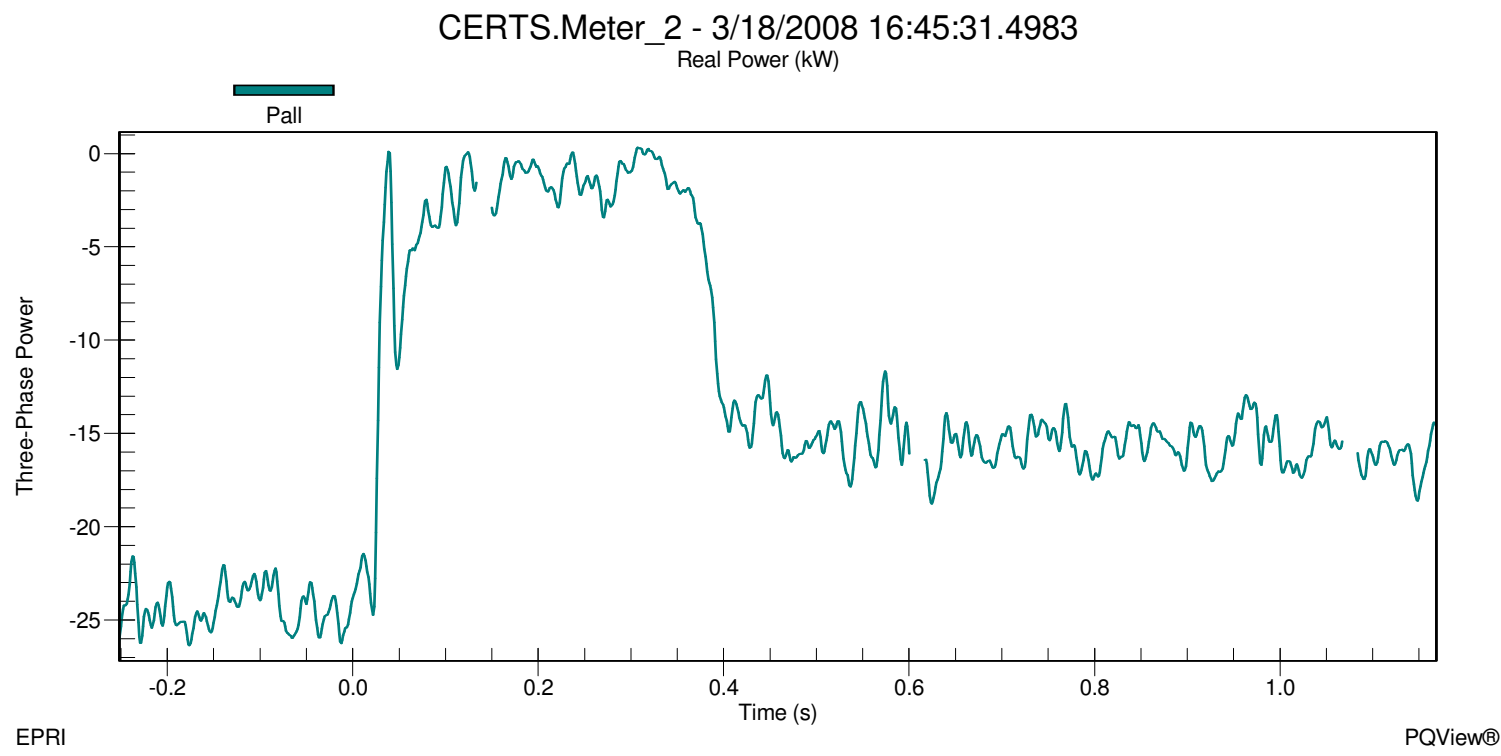

Figure 5e - Static Switch Real Power during Motor Start and Utility Connected for Test 10.2.12 


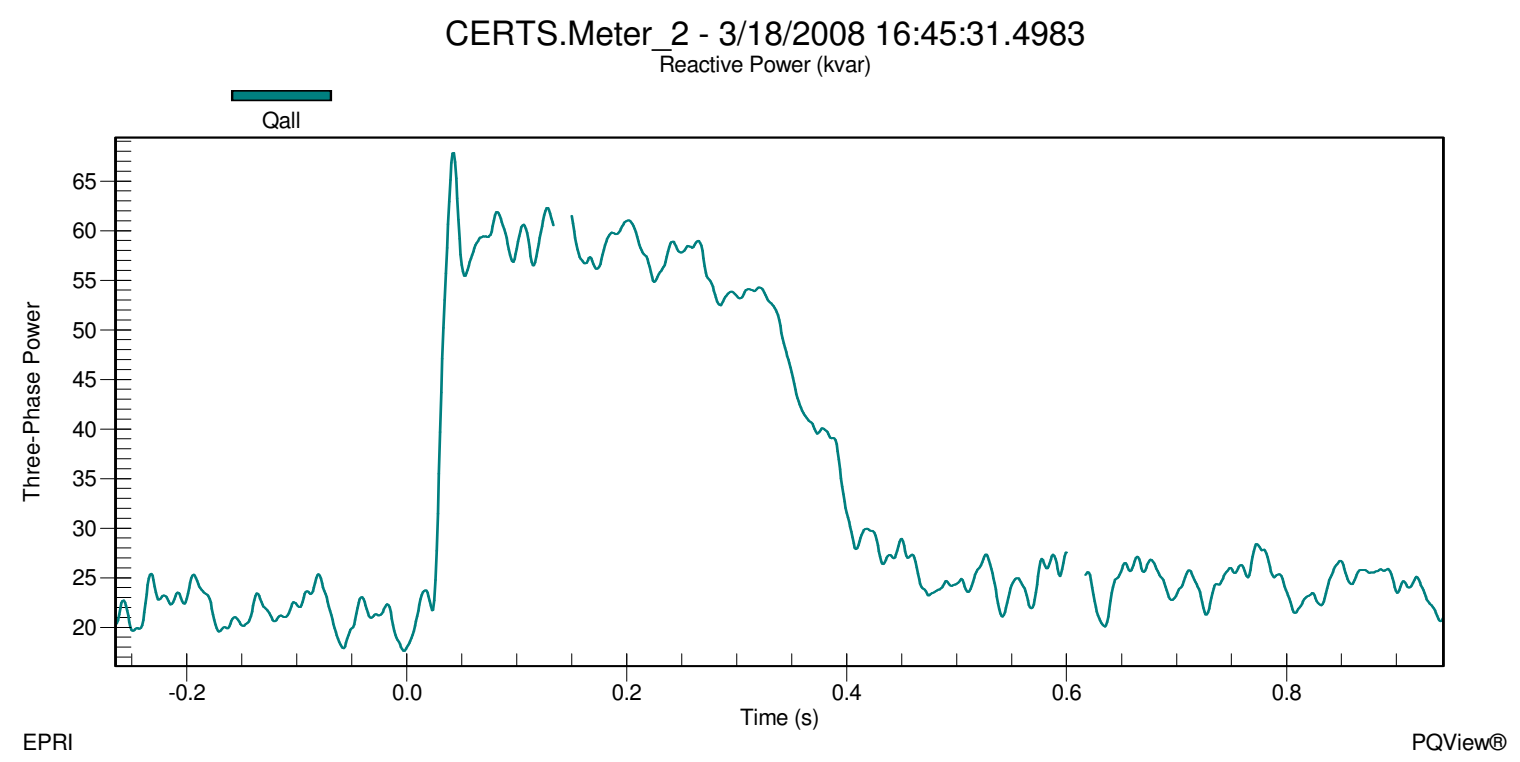

Figure 5f - Static Switch Reactive Power during Motor Start and Utility Connected for Test 10.2.12

Before the motor started Gen-sets A1 and A2 were producing approximately $41.1 \mathrm{~kW}-$ $\mathrm{j} 12 \mathrm{kVAr}$ and $32.6 \mathrm{~kW}-\mathrm{j} 3.8 \mathrm{kVAr}$, respectively. This was enough for the load demands of Zones 3 and 4 with real power being exported to Zone 6 of approximately $24 \mathrm{~kW}$ shown in Figure 5e. The grid was supporting the reactive power of the microgrid with approximately $22 \mathrm{kVAr}$ shown in Figure $5 \mathrm{f}$. When the motor started the inrush caused the utility to supply approximately $68 \mathrm{kVAr}$ and to pick up the load demand of Load Bank 6. The power that was being supplied by Gen-sets A1 and A2 to Load Bank 6 was now supplying the motor inrush which can be seen in Figure $5 \mathrm{e}$ as the real power through the static switch becomes $0 \mathrm{~kW}$. Gen-sets A1 and A2 increased their real and reactive power output levels to approximately $45.9 \mathrm{~kW}+\mathrm{j} 1.5 \mathrm{kVAr}$ and $40.4 \mathrm{~kW}+$ j15.9kVAr, respectively. Notice the signs of the VAr output changed from negative to positive for both Gen-sets in order to support the induction motor load in Zone 3.

When the motor reached steady state, the real and reactive power through the static switch was approximately $-15.8 \mathrm{~kW}+\mathrm{j} 24.2 \mathrm{kVAr}$ which meant that Gen-sets A1 and A2 were supplying the power for the induction motor and approximately $15.8 \mathrm{~kW}$ of Load Bank 6. Gen-sets A1 and A2 real power returned to the values before the motor started of approximately $41.1 \mathrm{~kW}$ and $32.6 \mathrm{~kW}$, respectively, and the reactive power increased to approximately $-10.5 \mathrm{kVAr}$ in Gen-set A1 and -1.9 in Gen-set A2. Once all data was 
verified and recorded into the DAS Database, the motor was shut down and the static switch was directed by the EMS to manually open.

As soon as the static switch opened, Meter 1 recorded real power increased to approximately $37 \mathrm{~kW}$ and reactive power decreased to $0 \mathrm{kVAr}$ satisfying the load demand in Load Bank 6 which was approximately 36.6kW + j0kVAr and not supplying any power beyond the static switch to Load Banks 3 and $4.0 \mathrm{~kW}+\mathrm{j} 0 \mathrm{kVAr}$ was recorded at the static switch, indicating that power was not flowing through the static switch. Load Banks 3 and 4 loads reduced slightly to $10.5 \mathrm{~kW}-\mathrm{j} 0.039 \mathrm{kVAr}$ and $34.5 \mathrm{~kW}+$ j0.685kVAr, respectively. This load reduction resulted from a voltage drop in the microgrid, shown in Figure 5g, from approximately 282V on A-phase, 280V on B-phase and $280 \mathrm{~V}$ on C-phase when connected to the utility grid to $275 \mathrm{~V}$ on A-phase, $275 \mathrm{~V}$ on Bphase and 276V on C-phase at Meter 3 when islanded.

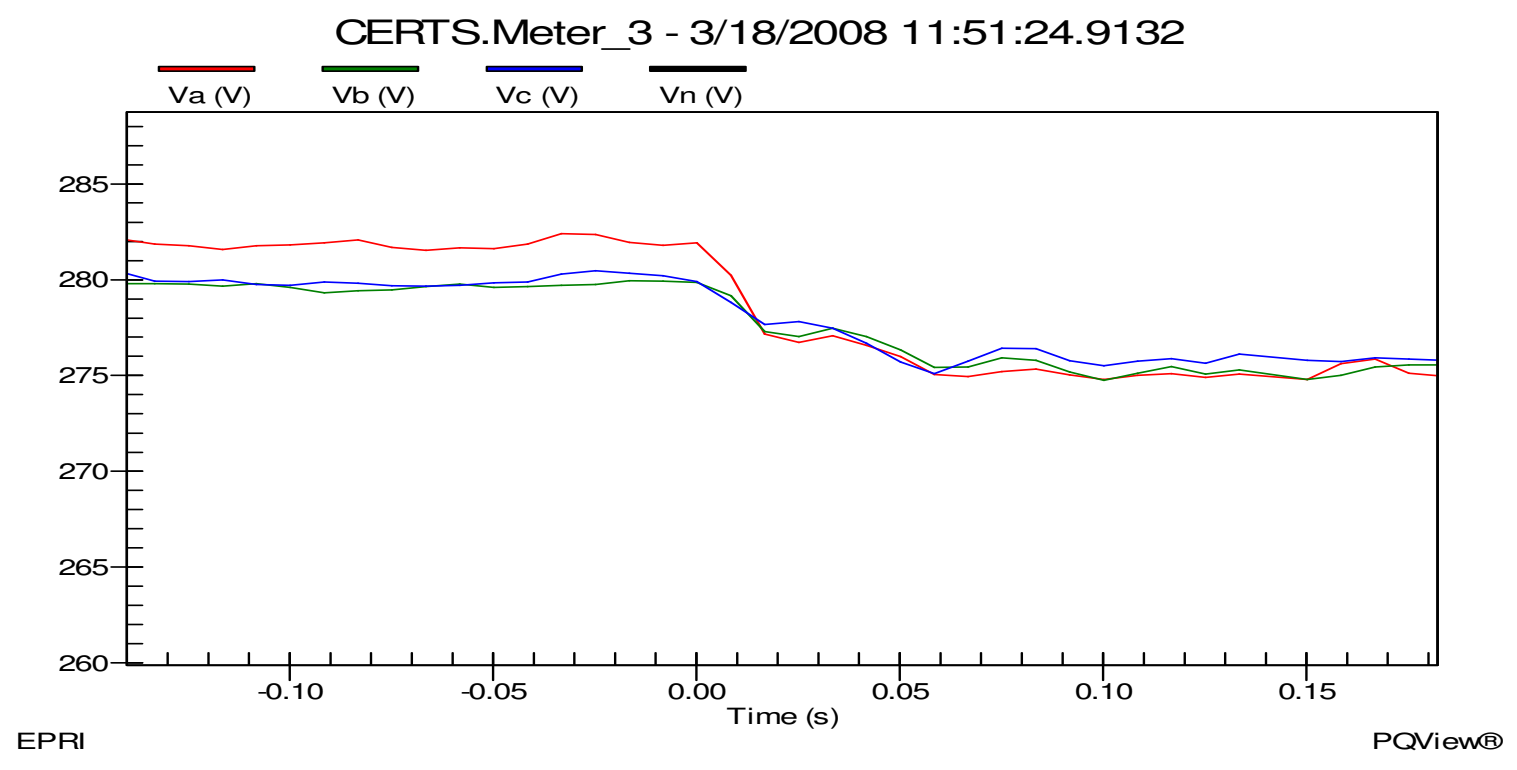

Figure 5g - Meter 3 Line-to-Ground Voltages during Utility Connected to Island mode for Test 10.2.12

Frequency change in the microgrid, shown in Figure 5h, increased from approximately $60.02 \mathrm{~Hz}$ when connected to the utility grid to approximately $60.08 \mathrm{~Hz}$ when islanded. This change in frequency was part of the CERTS algorithm which allowed the Gen-sets to decrease their output power to satisfy the load demands. Gen-set A1 and A2 decreased their output real power to approximately $28.5 \mathrm{~kW}$ and $19.5 \mathrm{~kW}$, respectively and increased their output reactive power to approximately $2.5 \mathrm{kVAr}$ and $2.5 \mathrm{kVAr}$, 
respectively. Meter 3 was approximately $-3.8 \mathrm{~kW}+\mathrm{j} 0.4 \mathrm{kVAr}$ indicating that the Gen-sets were satisfying the loads in the microgrid and the power losses in the electrical lines. All data was verified and recorded into the DAS Database. The microgrid ran for a couple of minutes in this electrical state before the $10 \mathrm{Hp}$ induction motor was started in Zone 3.

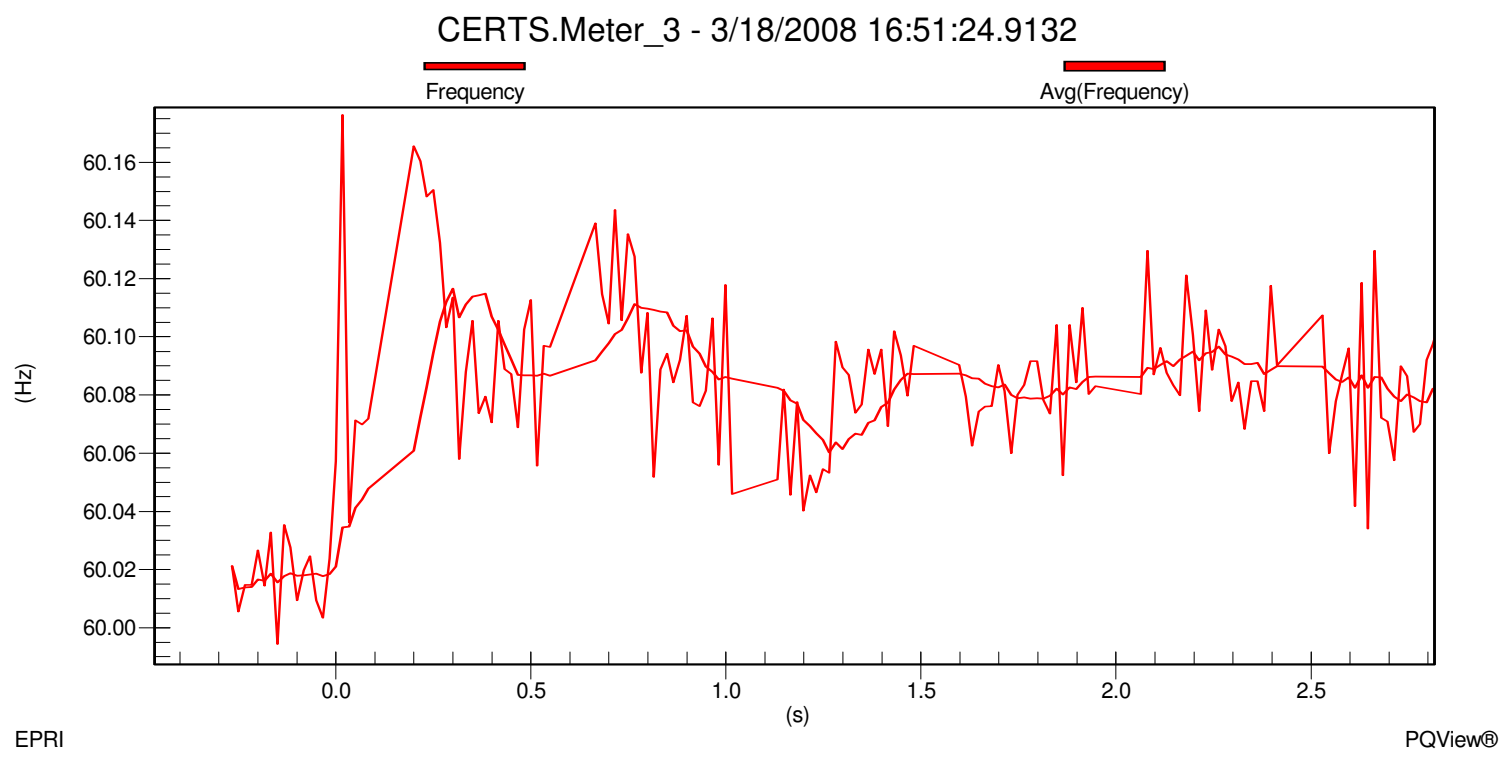

Figure 5h - Meter 3 Frequency during Utility Connected to Island mode for Test 10.2.12 


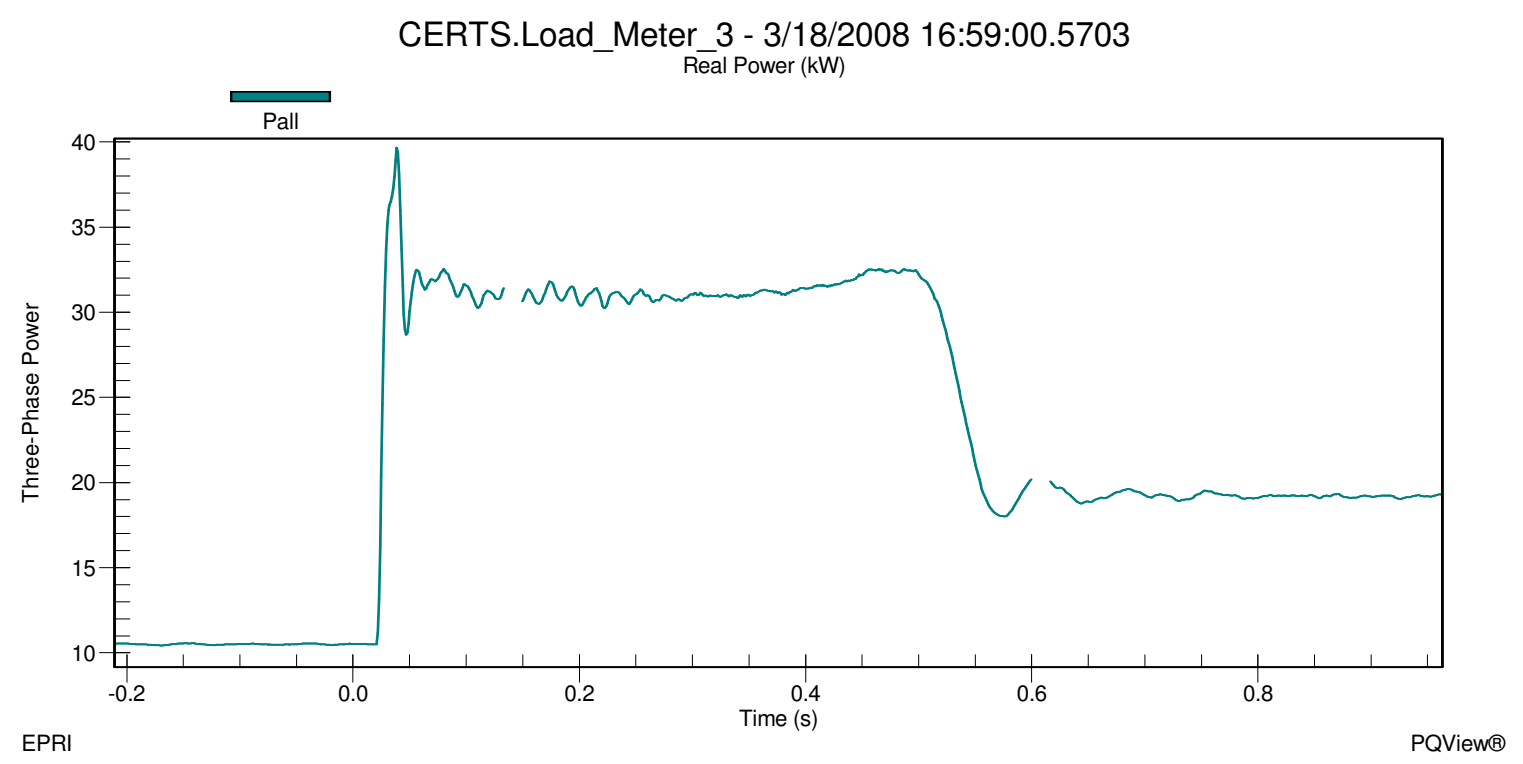

Figure 5i - Load Bank 3 Real Power during Motor Start and Islanded for Test 10.2.12

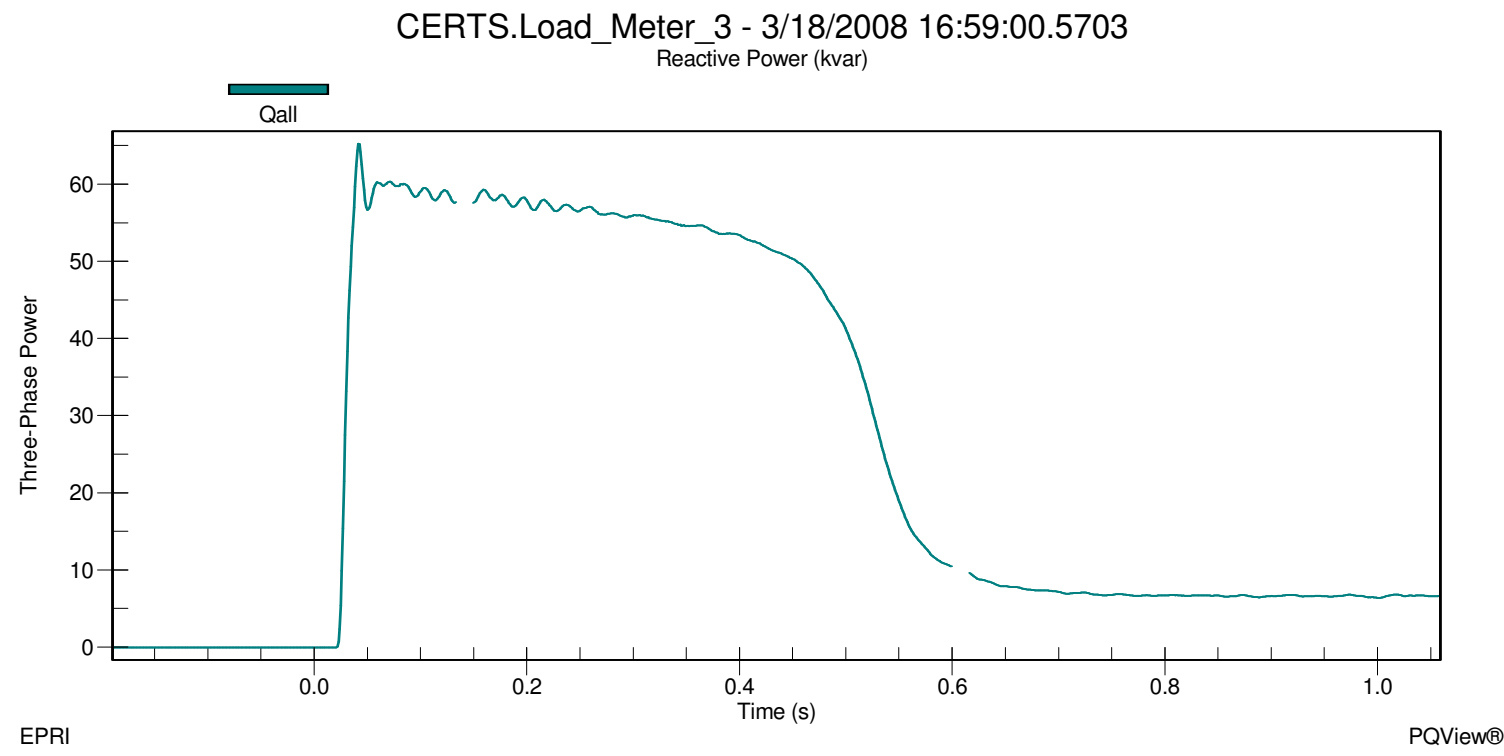

Figure 5j - Load Bank 3 Reactive Power during Motor Start and Islanded for Test 10.2.12

In Figures $5 \mathrm{i}$ and $5 \mathrm{j}$ it can be seen that the load in Zone 3 was approximately $10.5 \mathrm{~kW}+$ j0kVAr before the start of the induction motor and increased to approximately $39.7 \mathrm{~kW}+$ j65kVAr during the inrush phase of the motor start. After about 1.5 cycles, the motor settled down to approximately $31 \mathrm{~kW}+\mathrm{j} 56 \mathrm{kVAr}$ during the warm up phase which lasted 
about 39 cycles ( 0.65 Seconds). When the motor reached steady state, the load in Zone 3 was approximately $19.2 \mathrm{~kW}+\mathrm{j} 6 \mathrm{kVAr}$.

The voltage and frequency at Meter 3 before the motor start was approximately $275 \mathrm{~V}$ (i.e., $275 \mathrm{~V}$ on A-Phase, $275 \mathrm{~V}$ on B-Phase and $275 \mathrm{~V}$ on C-phase) shown in Figure 5k and approximately $60.09 \mathrm{~Hz}$ shown in Figure 5l. When the motor started, the voltage at Meter 3 during the inrush decreased to approximately 256V (i.e., 256V on A-Phase, 256V on B-Phase and $256 \mathrm{~V}$ on C-Phase) for about 1.5 cycles. Frequency dropped during the inrush to approximately $59.99 \mathrm{~Hz}$ and quickly increased to approximately $60.01 \mathrm{~Hz}$. Voltage increased as the motor was warming up and eventually settled at a steady state voltage at approximately $273 \mathrm{~V}$ (i.e., $273 \mathrm{~V}$ on A-Phase, $273 \mathrm{~V}$ on B-Phase and 273V on CPhase) at an approximate frequency of $60.05 \mathrm{~Hz}$.

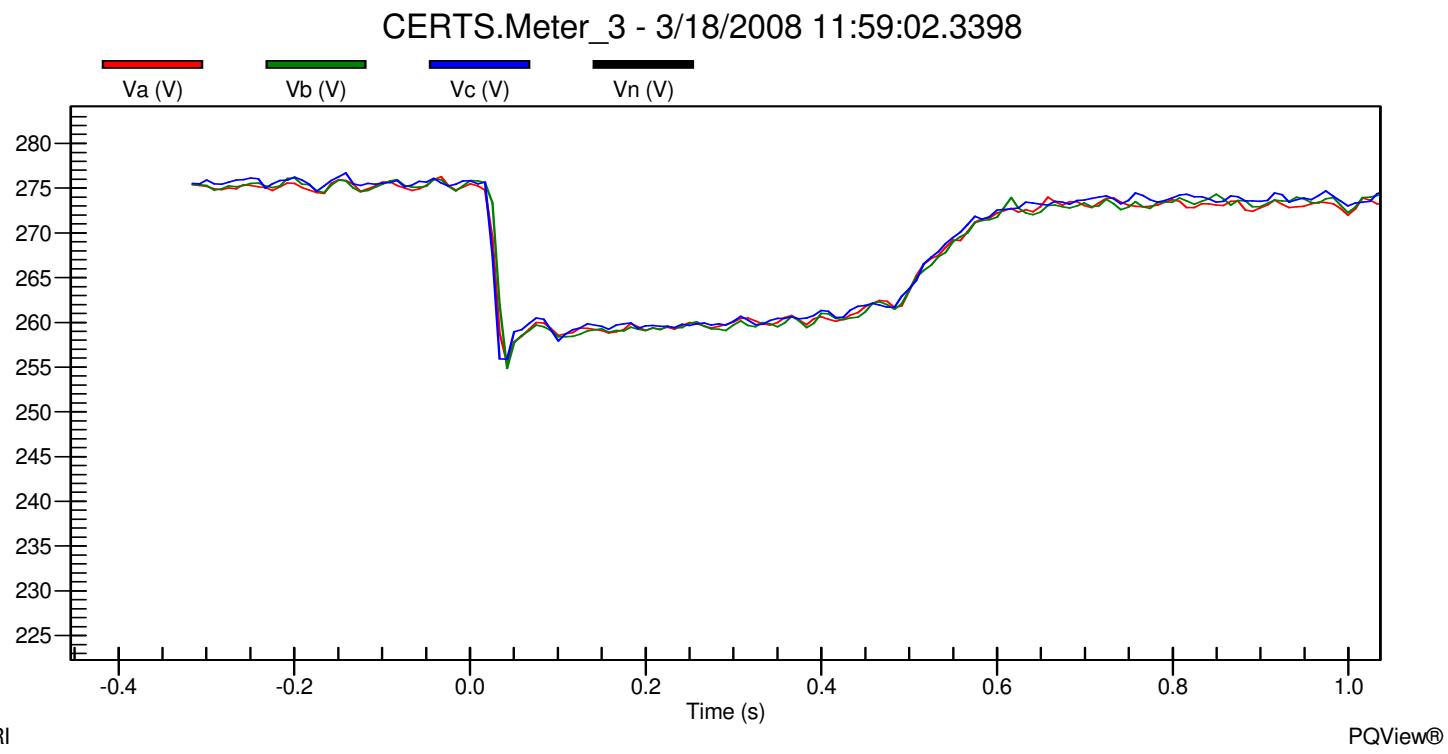

Figure 5k - Meter 3 Line-to-Ground Voltage during Motor Start and Islanded for Test 10.2.12 


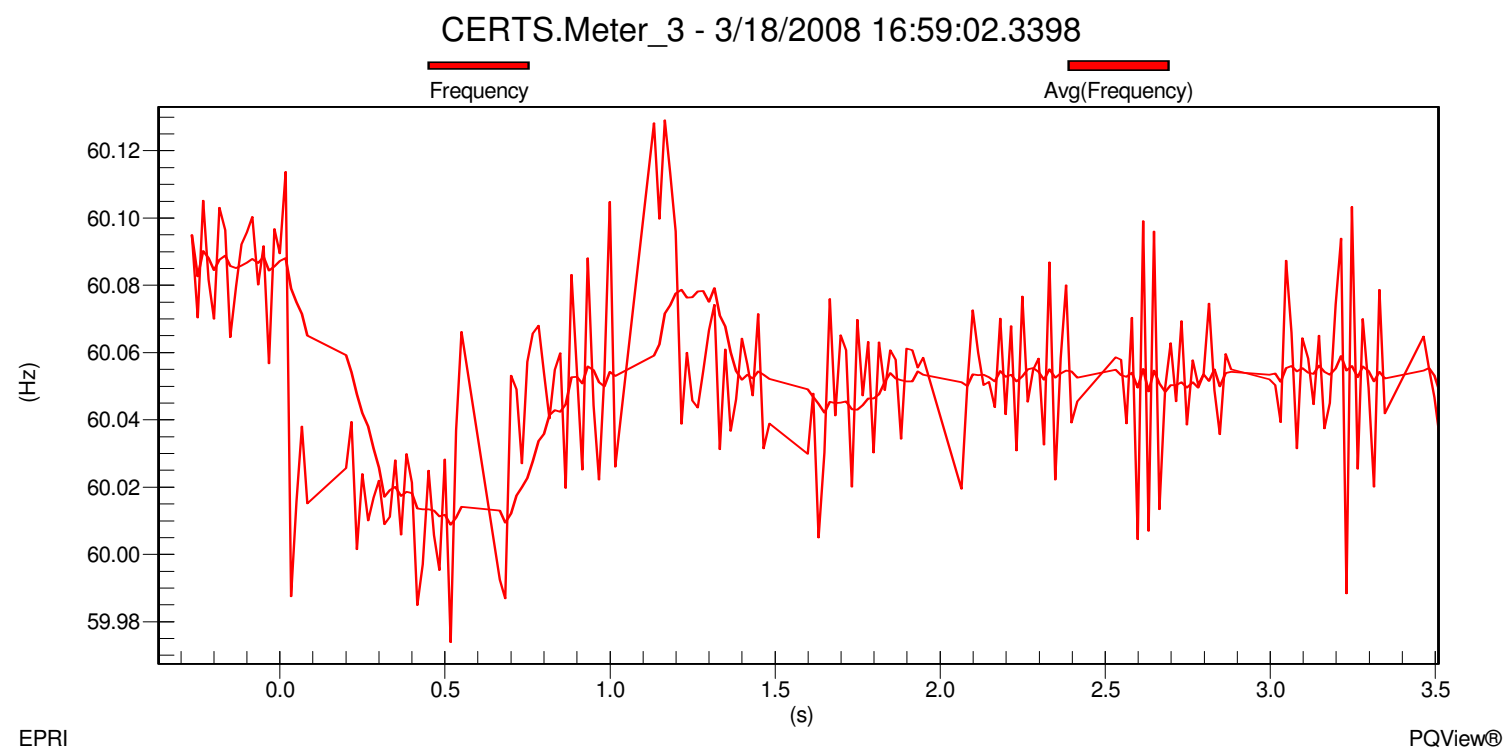

Figure 51 - Meter 3 Frequency during Motor Start and Islanded for Test 10.2.12

Before the motor started Gen-sets A1 and A2 were producing approximately $28.1 \mathrm{~kW}$ $\mathrm{j} 2.8 \mathrm{kVAr}$ and $19.4 \mathrm{~kW}+\mathrm{j} 7 \mathrm{kVAr}$, respectively shown in Figures $5 \mathrm{~m}-5 \mathrm{p}$. The power generated by both Gen-sets was satisfying the loads in Load Banks 3 and 4 and all the electrical losses in the microgrid system. When the motor started the inrush caused the Gen-sets to increase their output levels to $38.9 \mathrm{~kW}+\mathrm{j} 30.5 \mathrm{kVAr}$ for Gen-set A1 and $32.5 \mathrm{~kW}+\mathrm{j} 37.7 \mathrm{kVAr}$ for Gen-set A2. The Gen-sets decreased their output levels while the motor was warming up and eventually dropped to $32 \mathrm{~kW}+\mathrm{j} 0.2 \mathrm{kVAr}$ for Gen-set A1 and $23.6 \mathrm{~kW}+\mathrm{j} 10 \mathrm{kVAr}$ for Gen-set A2 when the motor reached steady state. Once all the data was verified and recorded into the DAS Database, the static switch was directed by the EMS to manually close. 


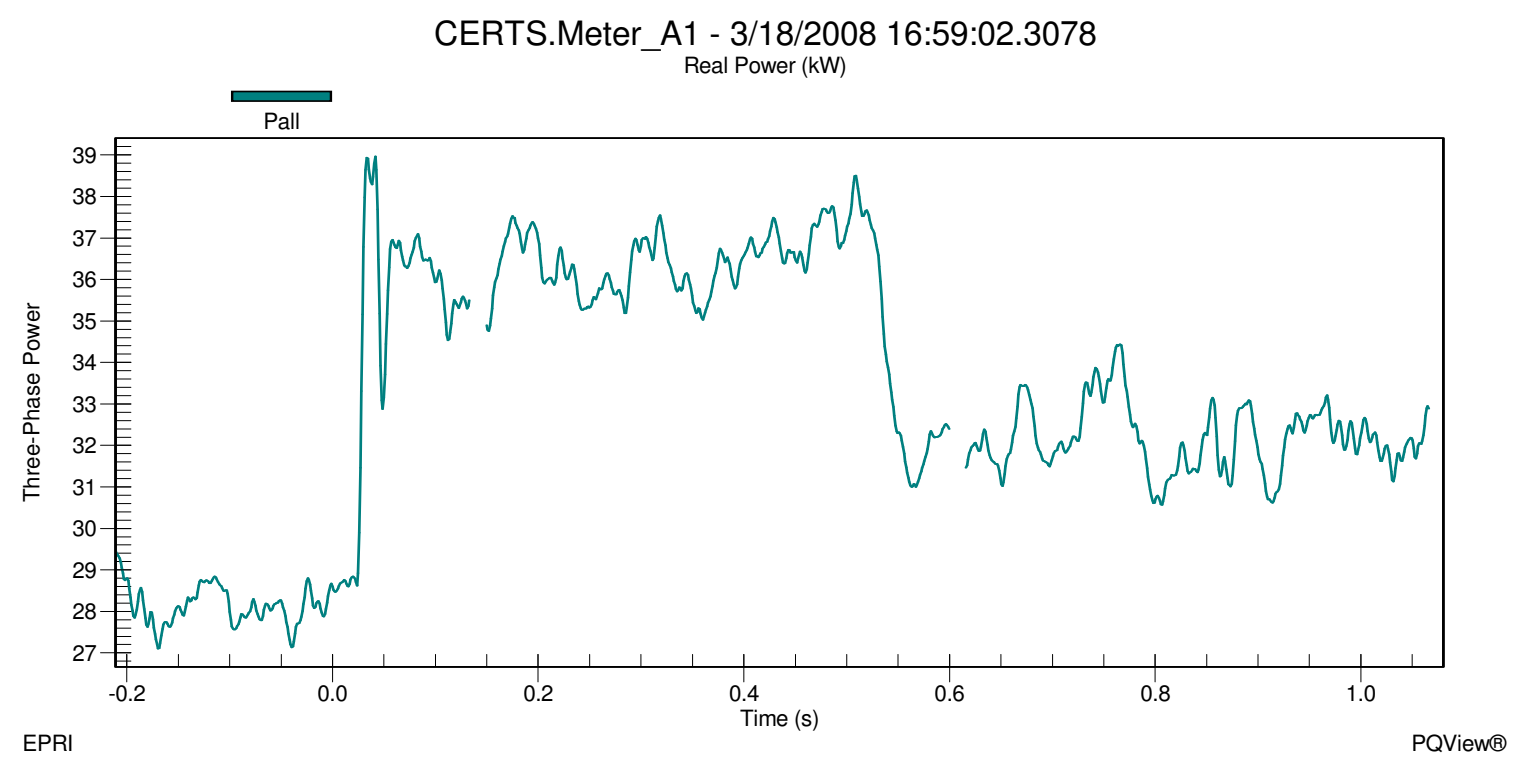

Figure 5m - Gen-set A1 Real Power during Motor Start and Islanded for Test 10.2.12

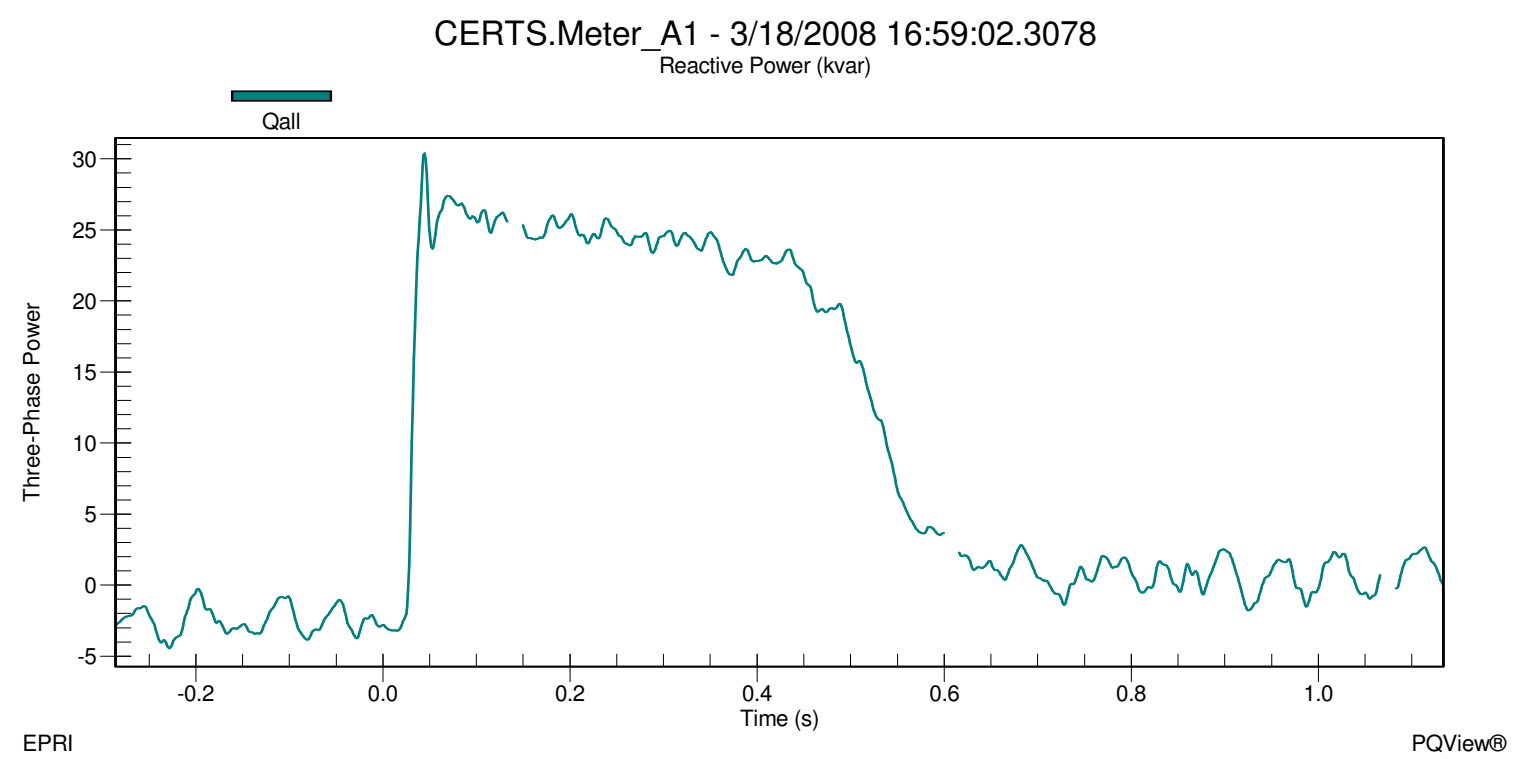

Figure 5n - Gen-Set A1 Reactive Power during Motor Start and Islanded for Test 10.2.12 


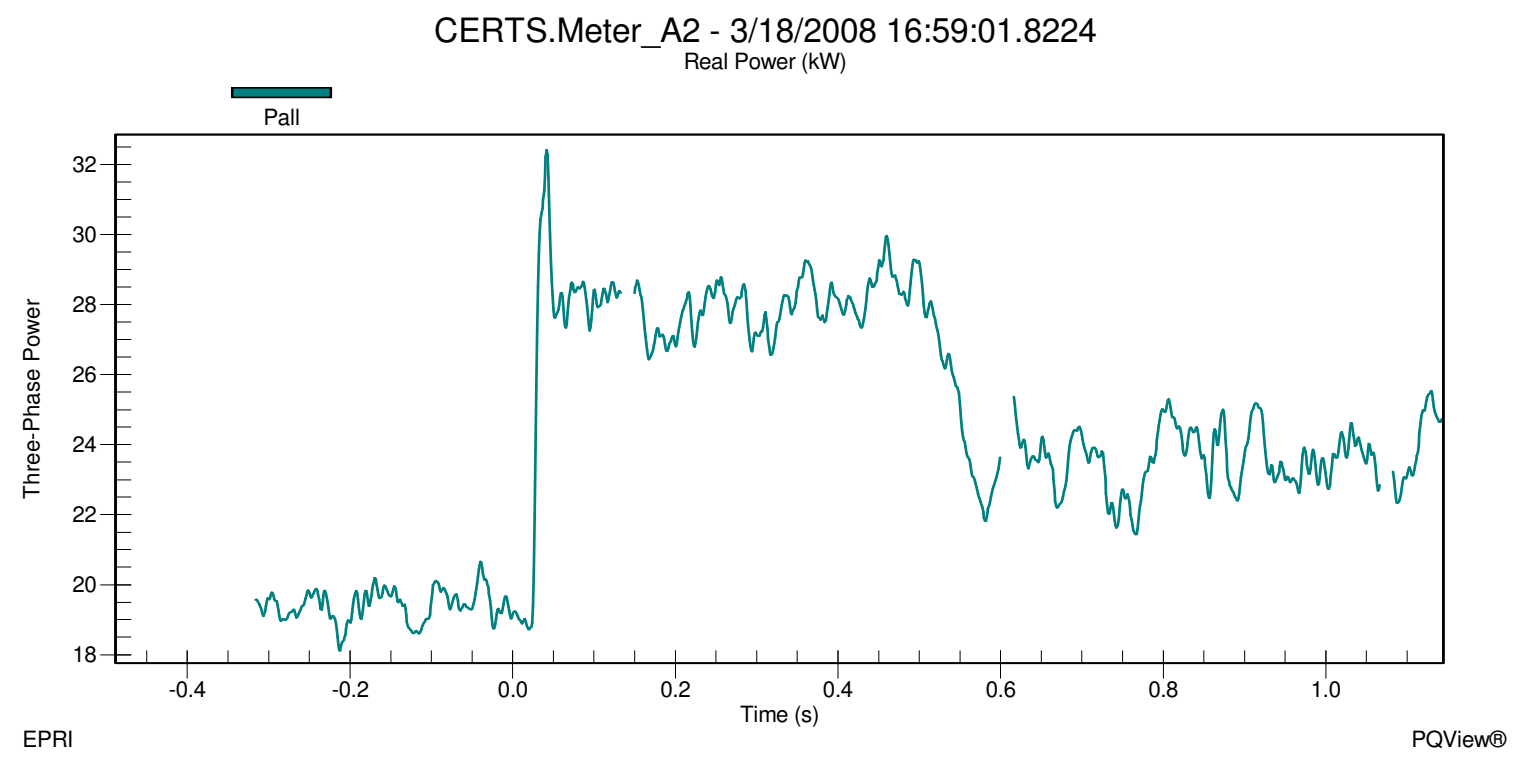

Figure 5o - Gen-set A2 Real Power during Motor Start and Islanded for Test 10.2.12

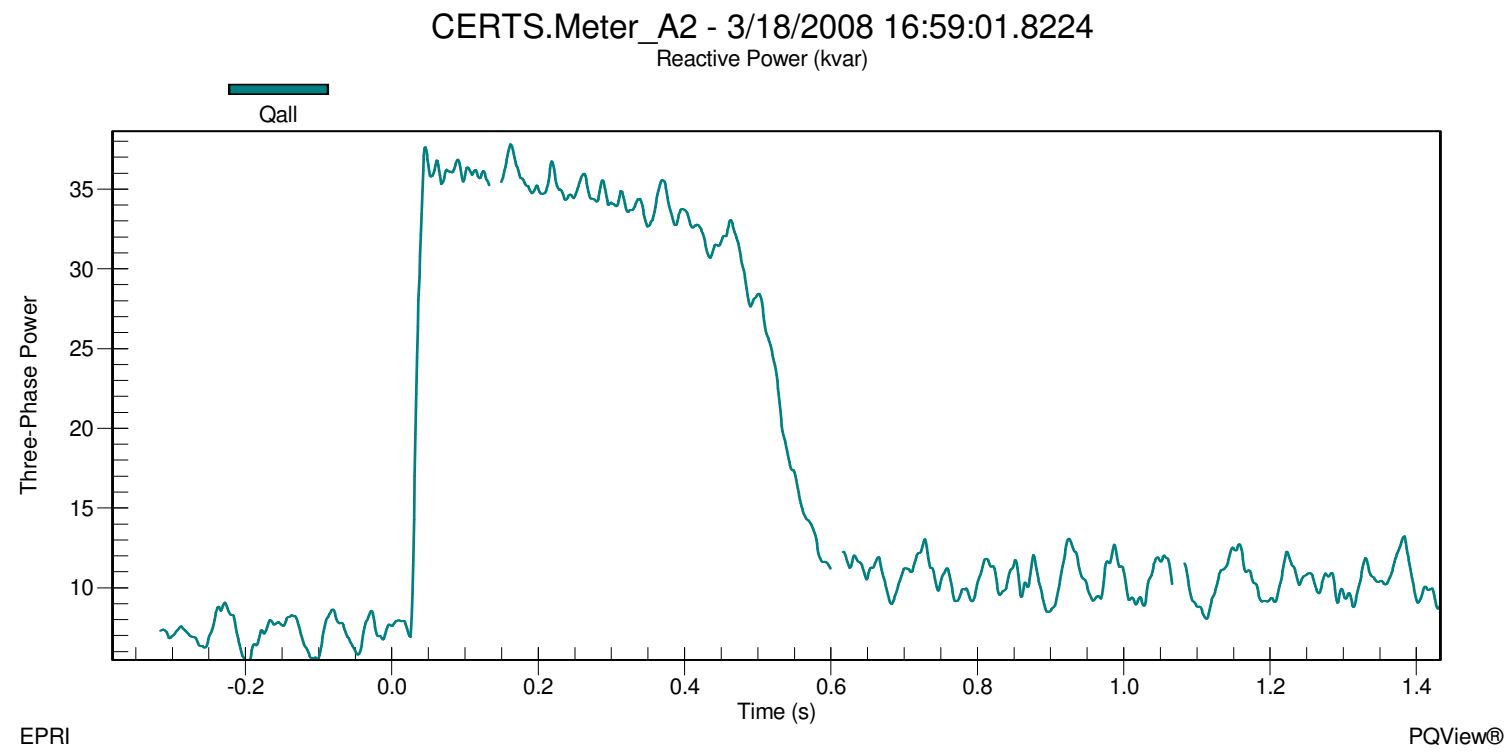

Figure 5p - Gen-set A2 Reactive Power during Motor Start and Islanded for Test 10.2.12

As soon as the static switch closed, Meter 1 recorded real power decreased from approximately $37 \mathrm{~kW}$ to $24 \mathrm{~kW}$ and reactive power increased from approximately $0 \mathrm{kVAr}$ to $28 \mathrm{kVAr}$ which means that the utility was satisfying a portion of the load demand in Load Bank 6 and all the reactive power in the microgrid. Figures $5 q$ and $5 \mathrm{r}$ show the static switch decreasing from approximately $0 \mathrm{~kW}$ to $-11.5 \mathrm{~kW}$ and increasing from approximately $0 \mathrm{kVAr}$ to $27 \mathrm{kVAr}$. At the beginning of the test, the initial power flow through the static switch was $-24 \mathrm{~kW}+\mathrm{j} 22 \mathrm{kVAr}$ which is not the same recorded at this 
point in the test because the $10 \mathrm{Hp}$ motor load is on in Zone 3. The Gen-sets have picked up the motor load and supporting Load Bank6 with approximately $11.5 \mathrm{~kW}$.

Load Banks 3 and 4 loads increased slightly to $35.7 \mathrm{~kW}+\mathrm{j} 0.71 \mathrm{kVAr}$ and $17.95 \mathrm{~kW}+$ j6.15kVAr, respectively. This slight load increase is a result from a voltage rise in the microgrid, shown in Figure 5s, from approximately $273.8 \mathrm{~V}$ (i.e., $273.8 \mathrm{~V}$ on A-phase, 273.8V on B-phase and 273.8V on C-phase) when islanded to $281.8 \mathrm{~V}$ on A-phase, $279.2 \mathrm{~V}$ on B-phase and 279.2V on C-phase at Meter 3 when connected to the utility grid.

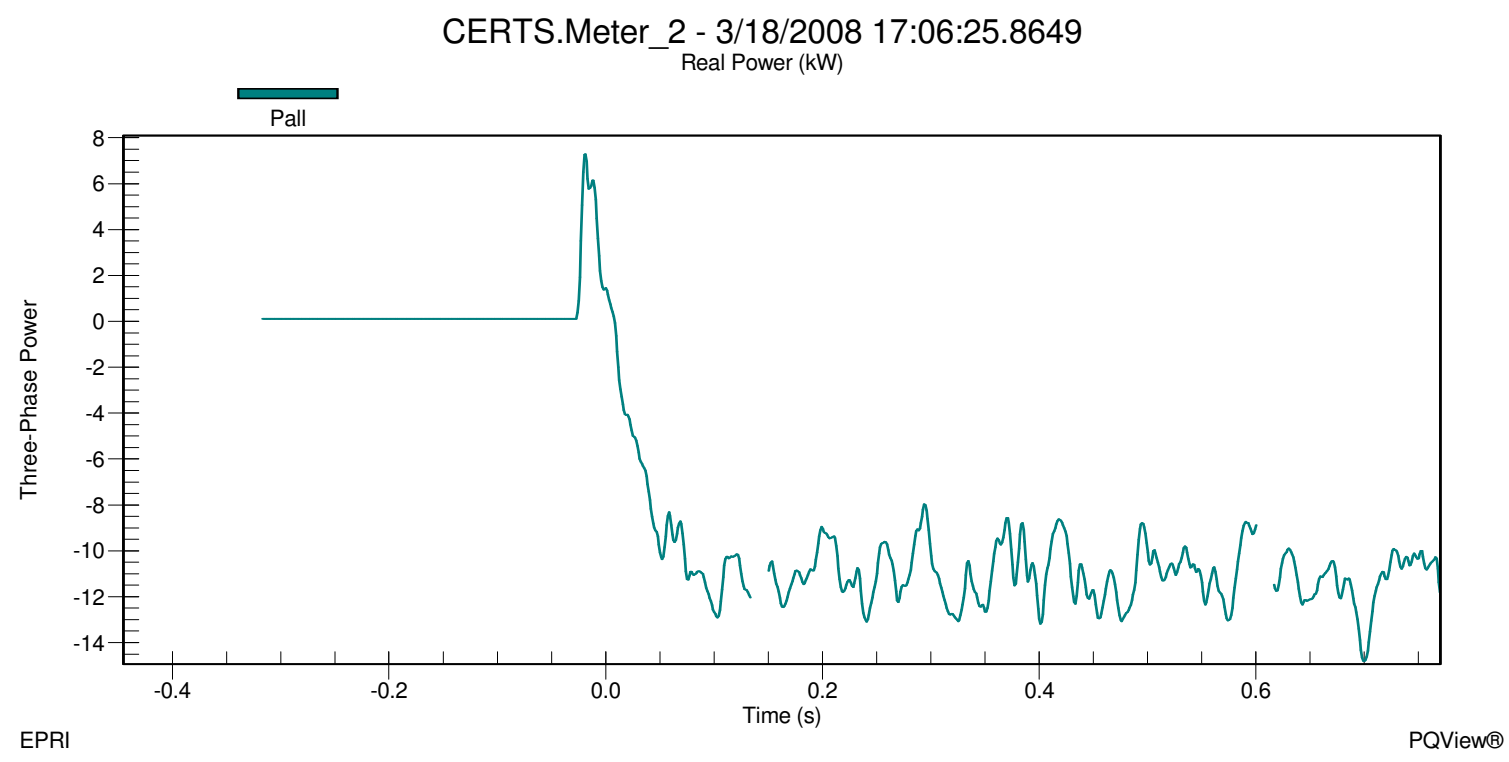

Figure 5q-Static Switch Real Power during Island to Utility Connected mode for Test 10.2.12 


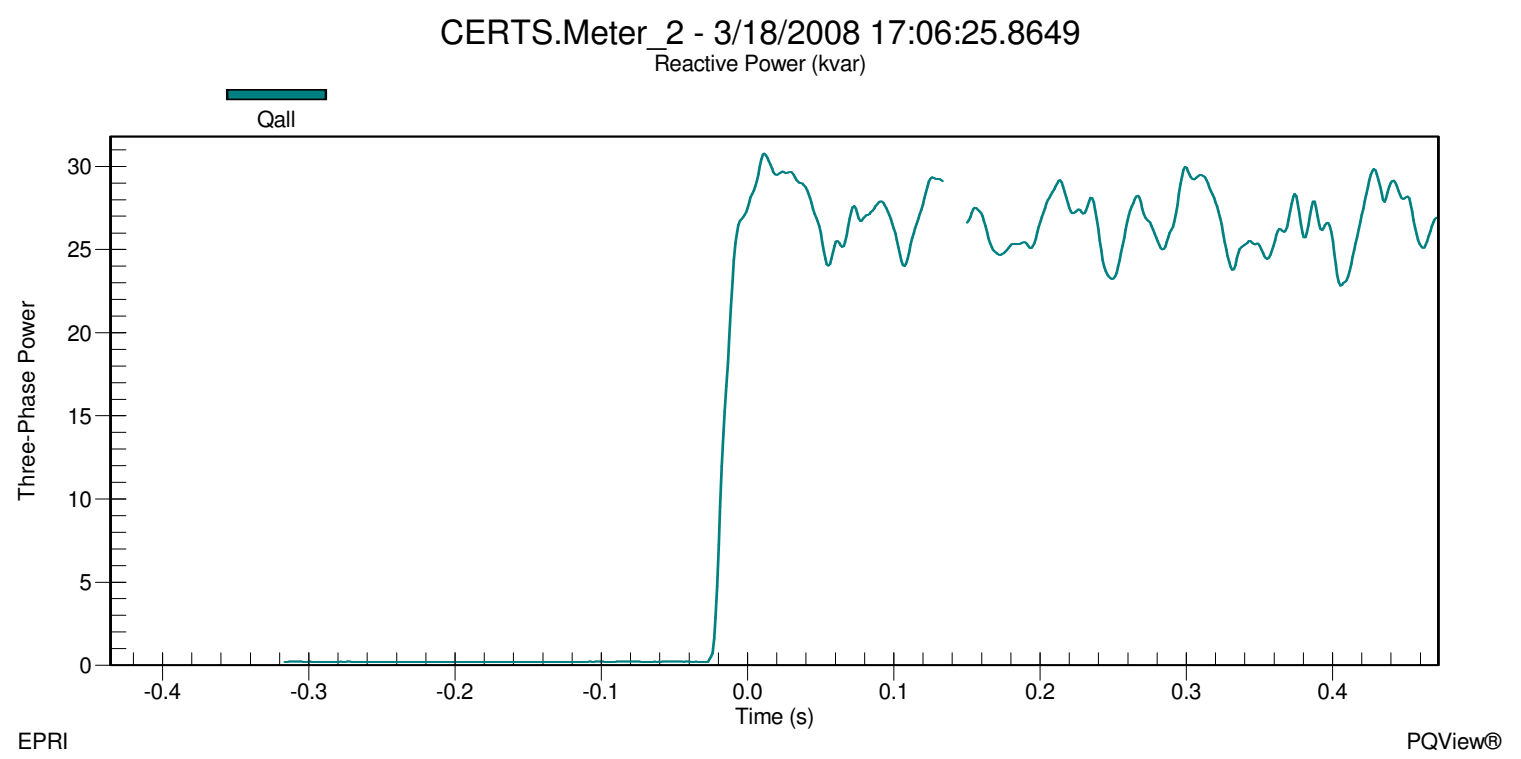

Figure 5r - Static Switch Reactive Power during Island to Utility Connected mode for Test 10.2.12

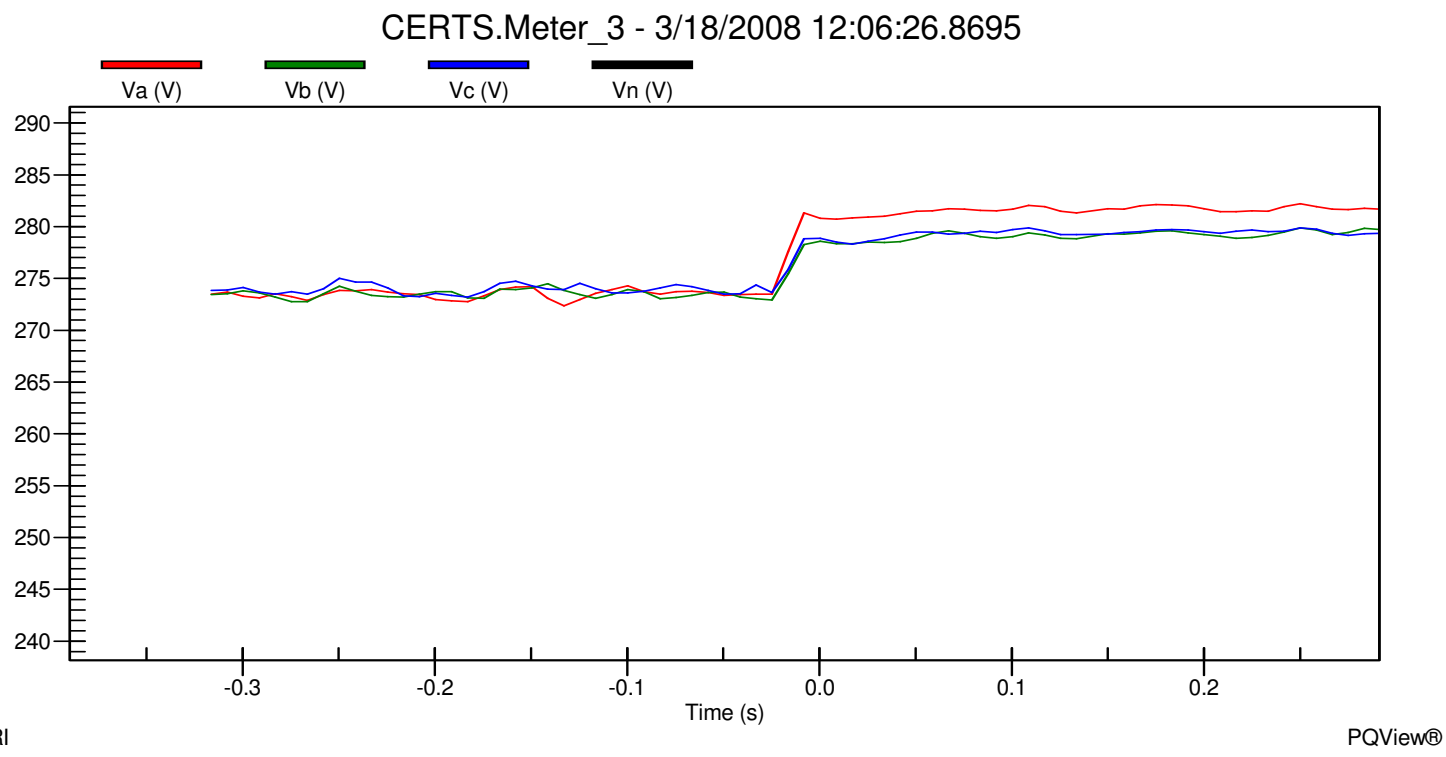

Figure 5s - Meter 3 Line-to-Ground Voltages during Island to Utility Connected mode for Test 10.2.12 


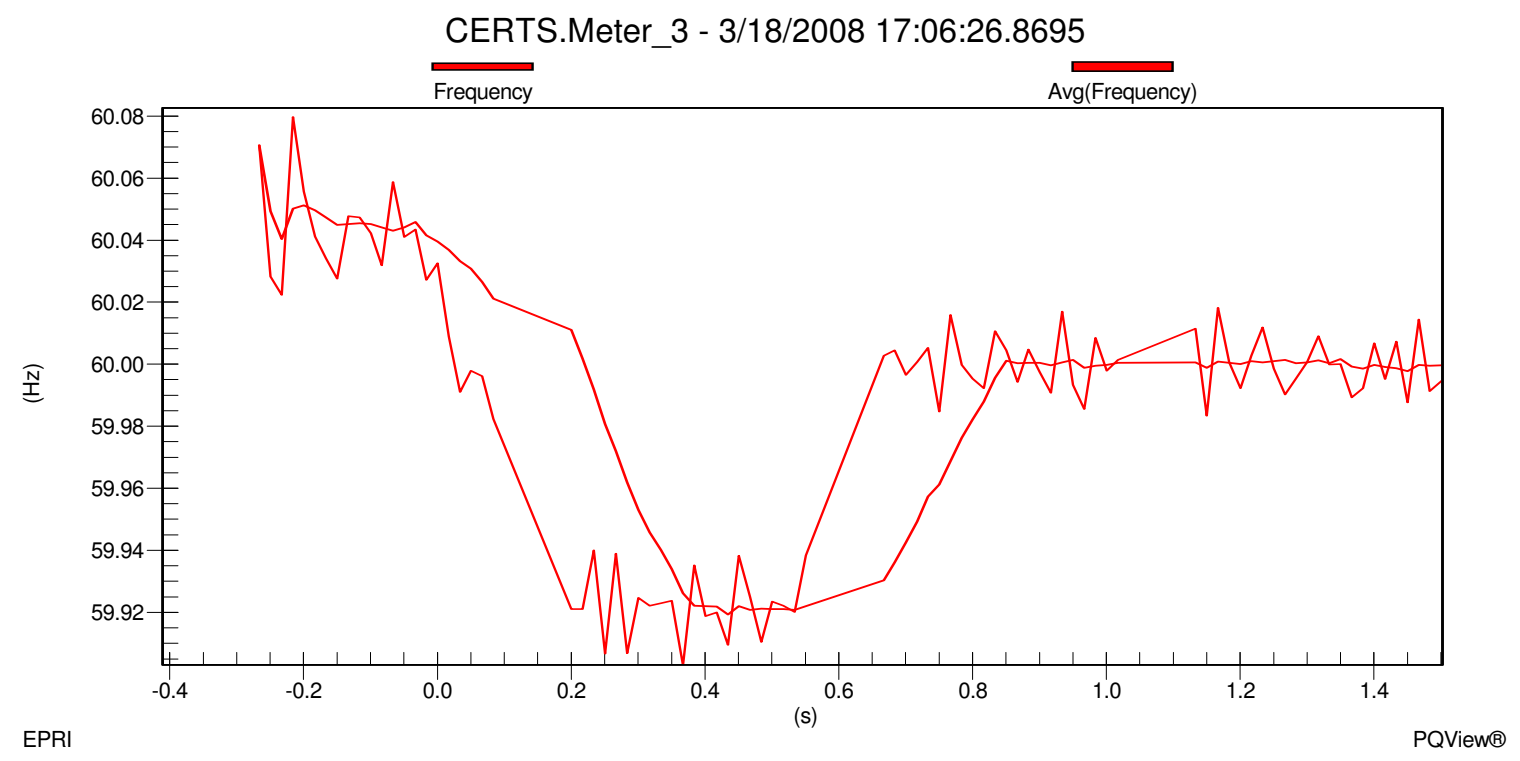

Figure 5t - Meter 3 Frequency during Island to Utility Connected mode for Test 10.2.12

Frequency change in the microgrid, shown in Figure 5t, decreased from approximately $60.05 \mathrm{~Hz}$ when islanded to approximately $60.00 \mathrm{~Hz}$ when connected to the utility grid. This change in frequency is due to the frequency no longer being established by the Gensets using the CERTS algorithm but by the utility. Gen-sets A1 and A2 are in unit power control mode therefore when the static switch closed back into the utility the Gen-sets produced real power based on their set-points initialized at the beginning of the test. The output power for both Gen-sets were relatively close to the values at the beginning of the test with Gen-set A1 producing approximately $38 \mathrm{~kW}-\mathrm{j} 12 \mathrm{kVAr}$ and Gen-set A2 producing approximately $30 \mathrm{~kW}-\mathrm{j} 3 \mathrm{kVAr}$. After all the data was verified and recorded into the DAS Database, the motor was turned off and the Gen-sets and Load Banks setpoints were changed according to the next test (10.2.14) in Table 1.

For Test 10.2.14 the measured values, after Gen-set A1 was warmed up and load banks brought on-line, were approximately $10 \mathrm{~kW}+\mathrm{j} 20 \mathrm{kVAR}$ at Meter $1,-26 \mathrm{~kW}+\mathrm{j} 20 \mathrm{kVAr}$ at Meter 2 and $-29 \mathrm{~kW}+\mathrm{j} 15 \mathrm{kVAR}$ at Meter 3. From the microgrid, $40 \mathrm{~kW}-\mathrm{j} 14 \mathrm{kVAr}$ was produced by Gen-set A1. The Load Banks were $11 \mathrm{~kW}+\mathrm{j} 0 \mathrm{kVAr}$ at LB3 and $36.1 \mathrm{~kW}+$ j0.745kVAr at LB6. At the time of these measurements, the voltage and frequency was 282.8 on A-phase, $279.6 \mathrm{~V}$ on B-phase and $279.8 \mathrm{~V}$ on C-phase and $59.986 \mathrm{~Hz}$ at the static switch (i.e., Meter 2) when connected to the utility grid; and $283 \mathrm{~V}$ on A-phase, $280 \mathrm{~V}$ on B-phase and $280 \mathrm{~V}$ on C-phase at Meter 3.

Gen-set A1 was setup in this test to produce more power than Load Bank 3 needed which approximately $26 \mathrm{~kW}$ of excess power was exported through the static switch to Load Bank 6. Since Load Bank 6 was approximately $36.1 \mathrm{~kW}+\mathrm{j} 0.0745 \mathrm{kVAr}$, the utility 
had to supply approximately $10 \mathrm{~kW}$ to satisfy the load. Reactive power had to be imported in from the utility of approximately 20kVAr because Gen-set A1 needed approximately $14 \mathrm{kVAr}$ and the reactive power absorbed in the electrical lines. Once all data was verified and recorded into the DAS Database, the $10 \mathrm{Hp}$ induction motor was started in Zone 3.

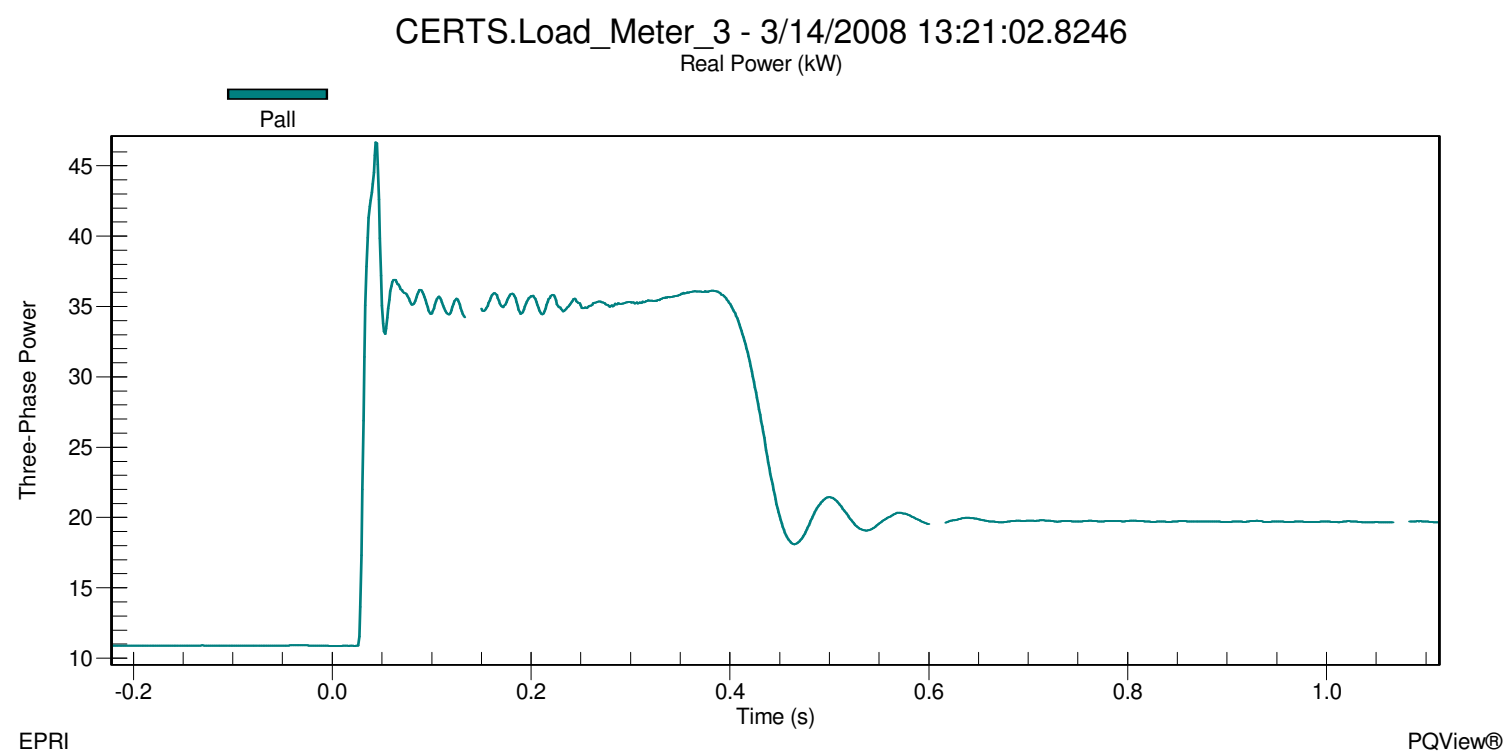

Figure 6a - Load Bank 3 Real Power during Motor Start and Utility Connected for Test 10.2.14

CERTS.Load_Meter_3 - 3/14/2008 13:21:02.8246

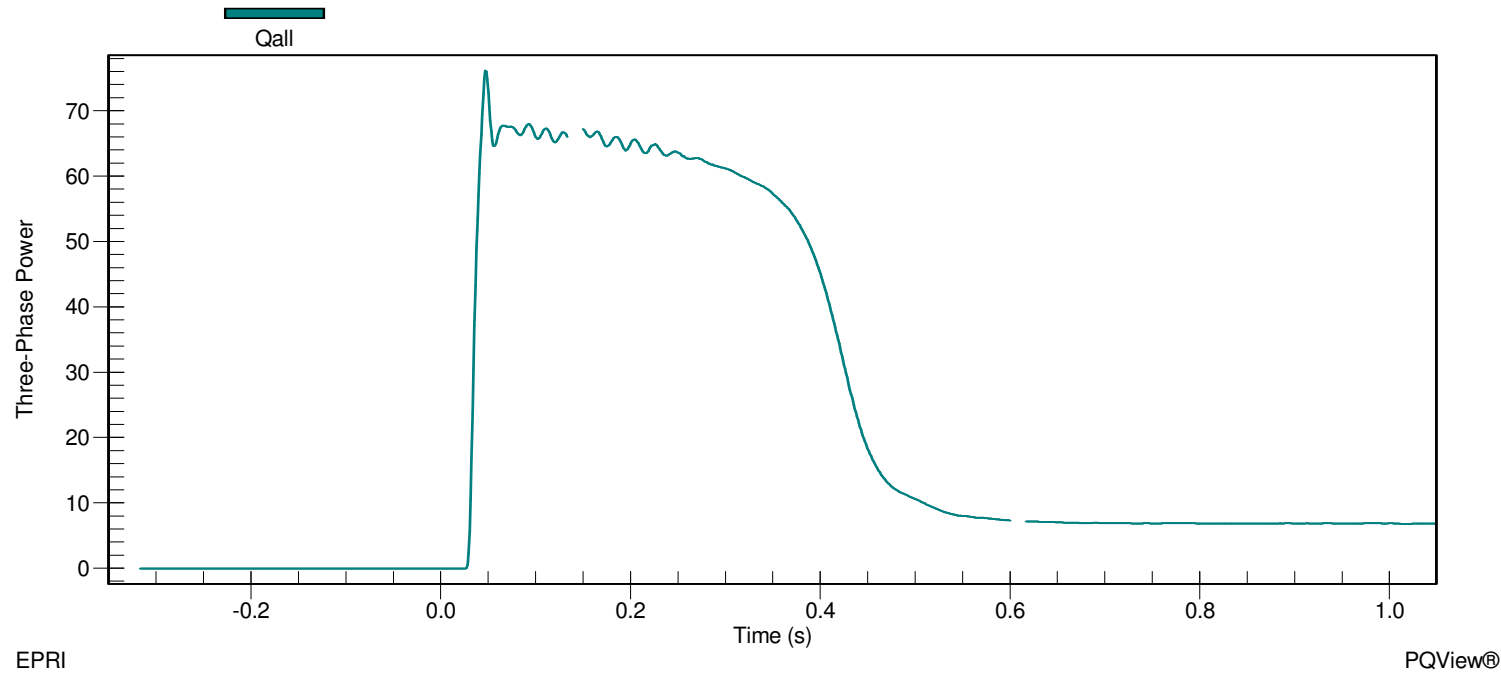

Figure 6b - Load Bank 3 Reactive Power during Motor Start and Utility Connected for Test 10.2.14 
In Figures $6 \mathrm{a}$ and $6 \mathrm{~b}$ it can be seen that the load in Zone 3 was approximately $11 \mathrm{~kW}+$ j0kVAr before the start of the induction motor and increased to approximately $46.8 \mathrm{~kW}+$ j76kVAr during the inrush phase of the motor start. After about 1.5 cycles, the motor settled down to approximately $35 \mathrm{~kW}+\mathrm{j} 63 \mathrm{kVAr}$ during the warm up phase which lasted about 33 cycles ( 0.55 Seconds). When the motor reached steady state, the load in Zone 3 was approximately $19.8 \mathrm{~kW}+\mathrm{j} 7 \mathrm{kVAr}$.

The voltage and frequency at the static switch before the motor start was approximately $282.8 \mathrm{~V}$ on A-phase, $279.6 \mathrm{~V}$ on B-phase and $279.8 \mathrm{~V}$ on C-phase shown in Figure $6 \mathrm{c}$ and approximately $59.99 \mathrm{~Hz}$ shown in Figure $6 \mathrm{~d}$. When the motor started, the voltage at the static switch during the inrush decreased to approximately $274.6 \mathrm{~V}$ on A-phase, $271.7 \mathrm{~V}$ on B-phase and $271 \mathrm{~V}$ on C-phase for about 1.5 cycles. Frequency dropped during the inrush to approximately $59.92 \mathrm{~Hz}$ and quickly increased back to the starting frequency of approximately $59.99 \mathrm{~Hz}$. Voltage increased as the motor was warming up and eventually settled at a steady state voltage at approximately $282 \mathrm{~V}$ on A-phase, $278.8 \mathrm{~V}$ on B-phase and $279 \mathrm{~V}$ on C-phase at an approximate frequency of $59.98 \mathrm{~Hz}$.

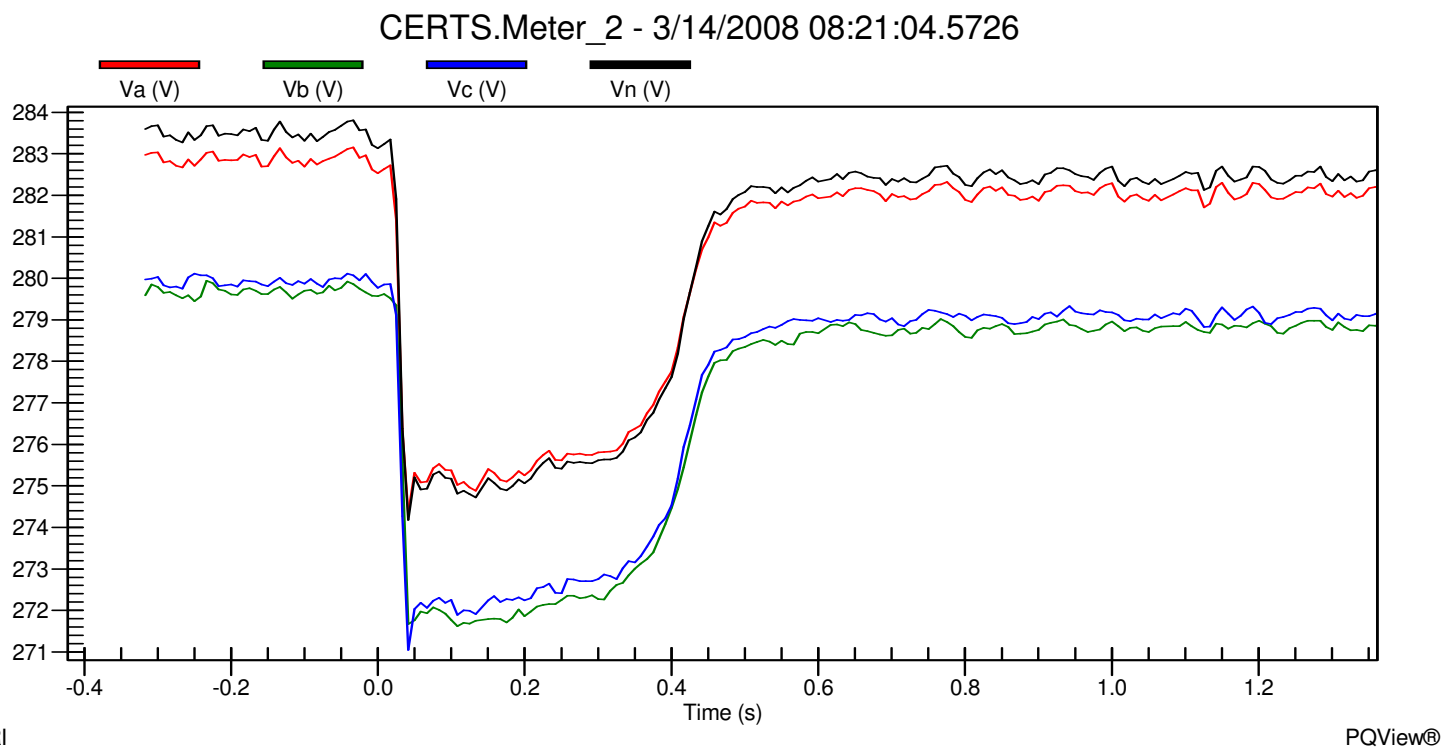

Figure 6c - Static Switch Line-to-Ground Voltages during Motor Start and Utility Connected for Test 10.2.14 


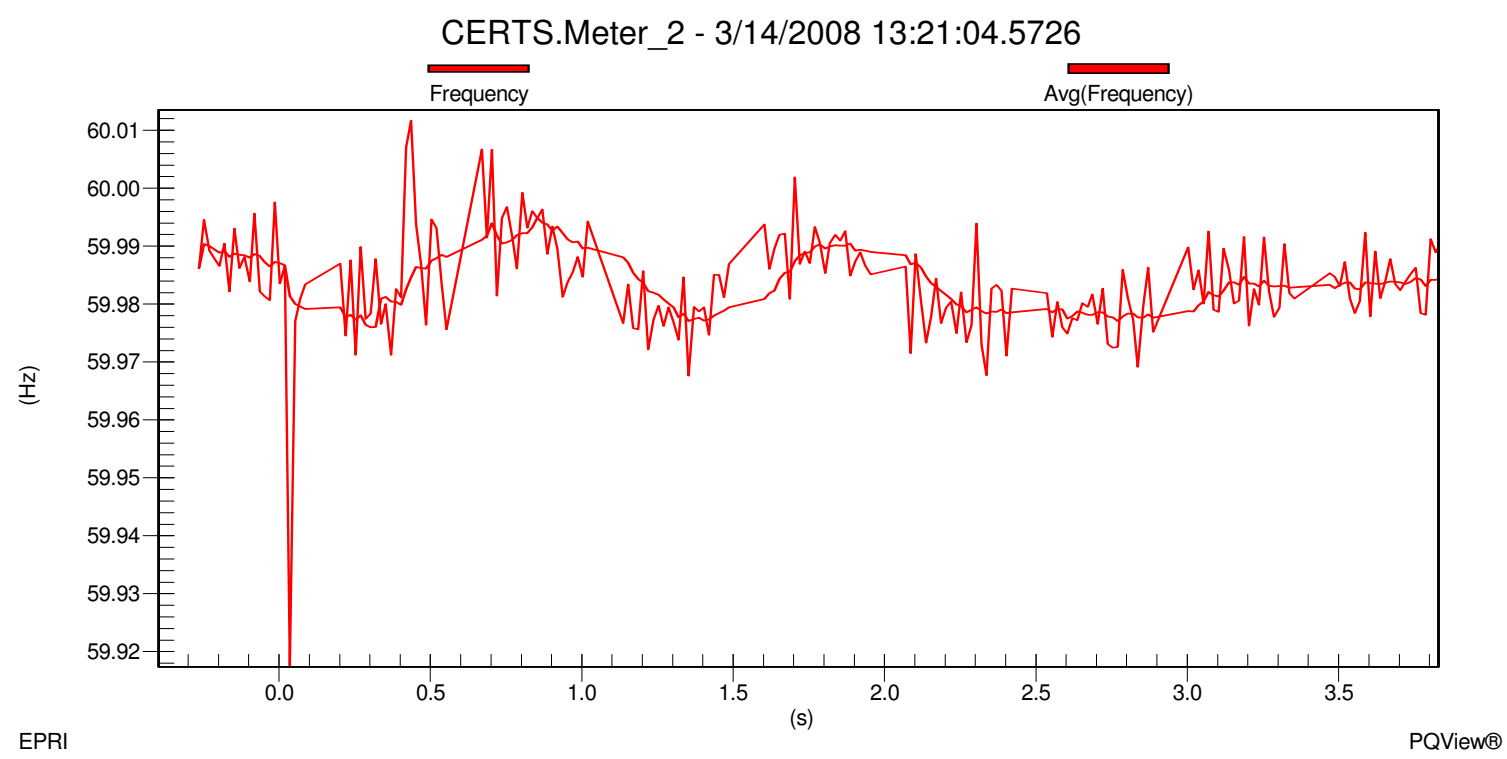

Figure 6d - Static Switch Frequency during Motor Start and Utility Connected for Test 10.2.14

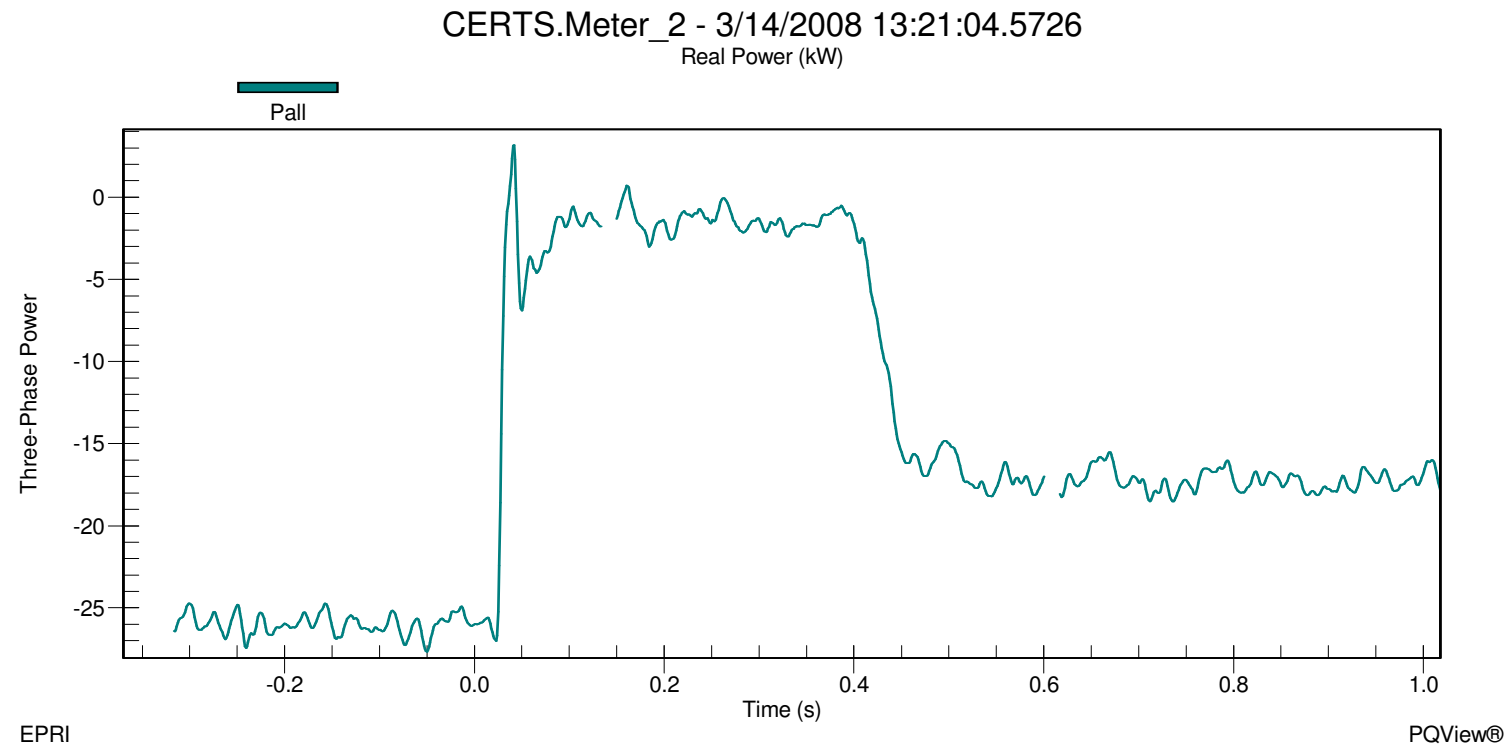

Figure 6e - Static Switch Real Power during Motor Start and Utility Connected for Test 10.2.14 


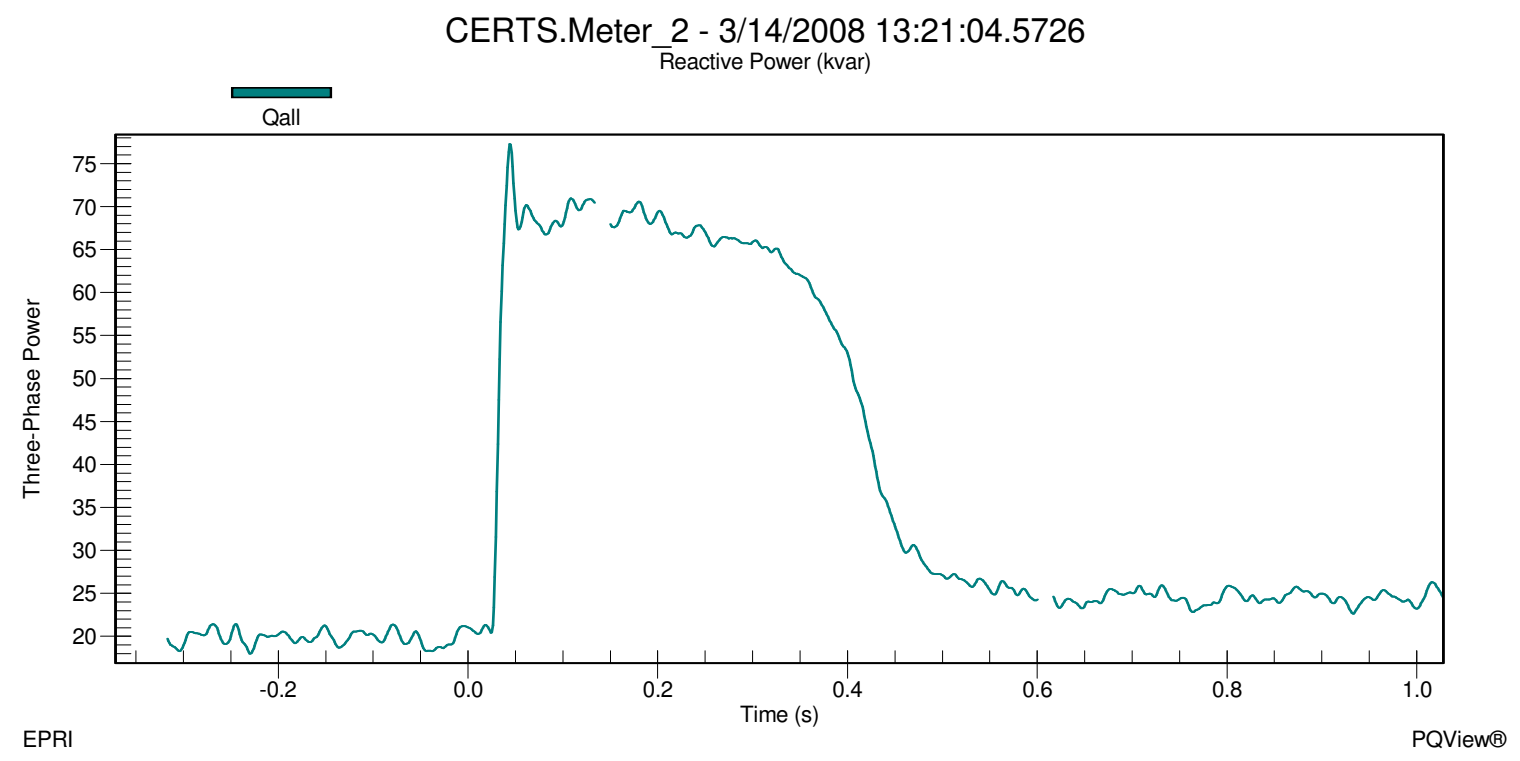

Figure 6f - Static Switch Reactive Power during Motor Start and Utility Connected for Test 10.2.14

Before the motor started Gen-set A1 was producing approximately 40kW - j14kVAr. This was enough for the load demands of Zone 3 with real power being exported to Zone 6 of approximately $26 \mathrm{~kW}$ shown in Figure 6e. The grid was supporting the reactive power of the microgrid with approximately 20kVAr shown in Figure 6f. When the motor started the inrush caused the utility to supply approximately $77.1 \mathrm{kVAr}$ and to pick up the load demand in Load Bank 6 along with approximately $3 \mathrm{~kW}$ of the load in Zone 3. The power being supplied by Gen-set A1 to Load Bank 6 was now supplying the motor inrush which can be seen in Figure 6e as the real power through the static switch becomes positive. Gen-set A1 increased its real and reactive power output level to approximately $46.9 \mathrm{~kW}+\mathrm{j} 5 \mathrm{kVAr}$. Notice the sign of the VAr output changed from negative to positive for Gen-set A1 in order to support the induction motor load in Zone 3.

When the motor reached steady state, the real and reactive power through the static switch was approximately $-17.2 \mathrm{~kW}+\mathrm{j} 24 \mathrm{kVAr}$ which meant that Gen-set A1 was supplying the power for the induction motor and approximately $17.2 \mathrm{~kW}$ of Load Bank 6 . Gen-set A1 real power returned to the value before the motor started of approximately $40 \mathrm{~kW}$ and the reactive power increased to approximately $-12 \mathrm{kVAr}$. Once all the data was verified and recorded into the DAS Database, the motor was shut down and the static switch was directed by the EMS to manually open.

As soon as the static switch opened, Meter 1 recorded real power increased to approximately $37 \mathrm{~kW}$ and reactive power decreased to $0 \mathrm{kVAr}$ satisfying the load demand in Load Bank 6 which was approximately 36.6kW + j0kVAr and not supplying 
any power beyond the static switch to Load Bank 3. $0 \mathrm{~kW}+\mathrm{j} 0 \mathrm{kVAr}$ was recorded at the static switch, indicating that power was not flowing through the static switch. Load Bank 3 loads was slightly reduced to $10.3 \mathrm{~kW}-\mathrm{j} 0.041 \mathrm{kVAr}$. This load reduction resulted from a voltage drop in the microgrid, shown in Figure $6 \mathrm{~g}$, from approximately $283 \mathrm{~V}$ on A-phase, $280 \mathrm{~V}$ on B-phase and $280 \mathrm{~V}$ on C-phase when connected to the utility grid to $273 \mathrm{~V}$ on A-phase, $271.5 \mathrm{~V}$ on B-phase and 273V on C-phase at Meter 3 when islanded.

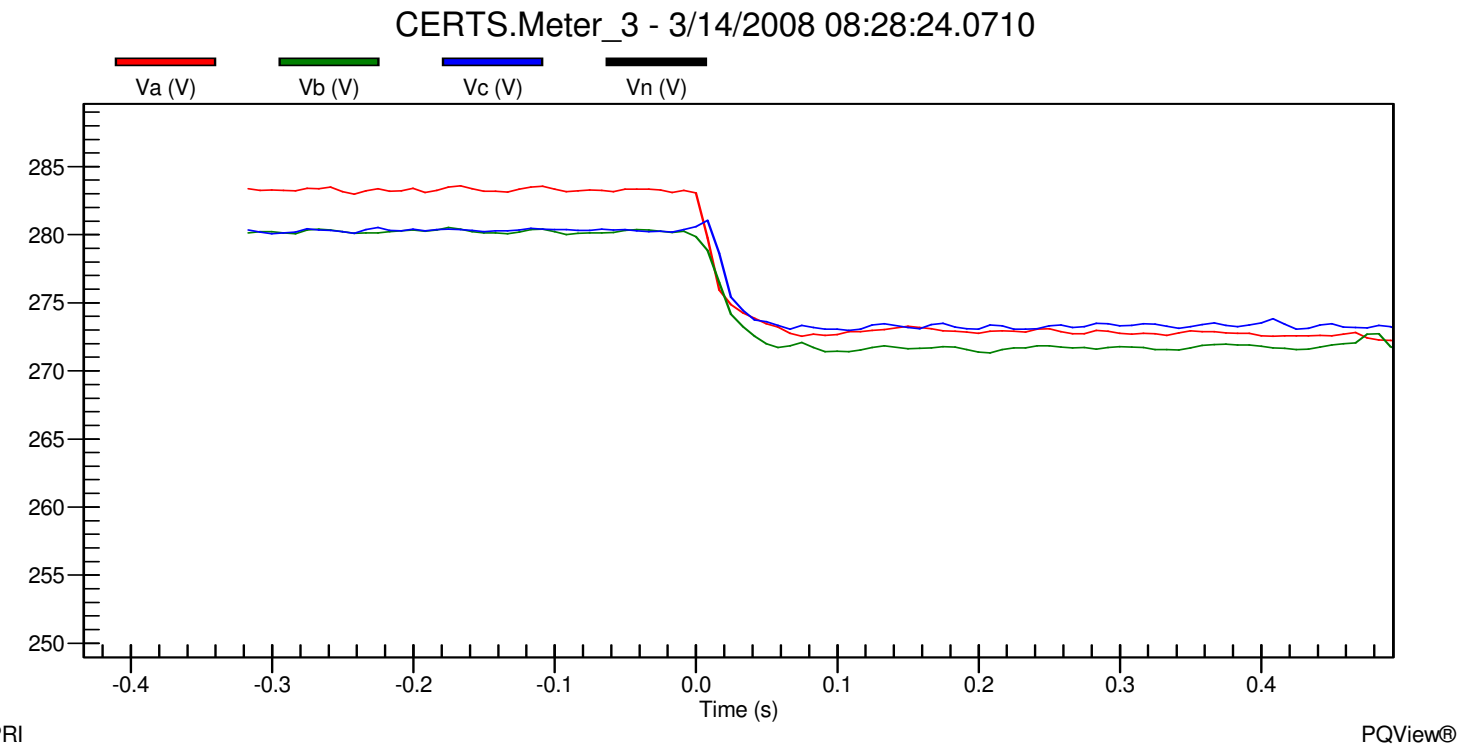

Figure $6 \mathrm{~g}$ - Meter 3 Line-to-Ground Voltages during Utility Connected to Island mode for Test 10.2.14

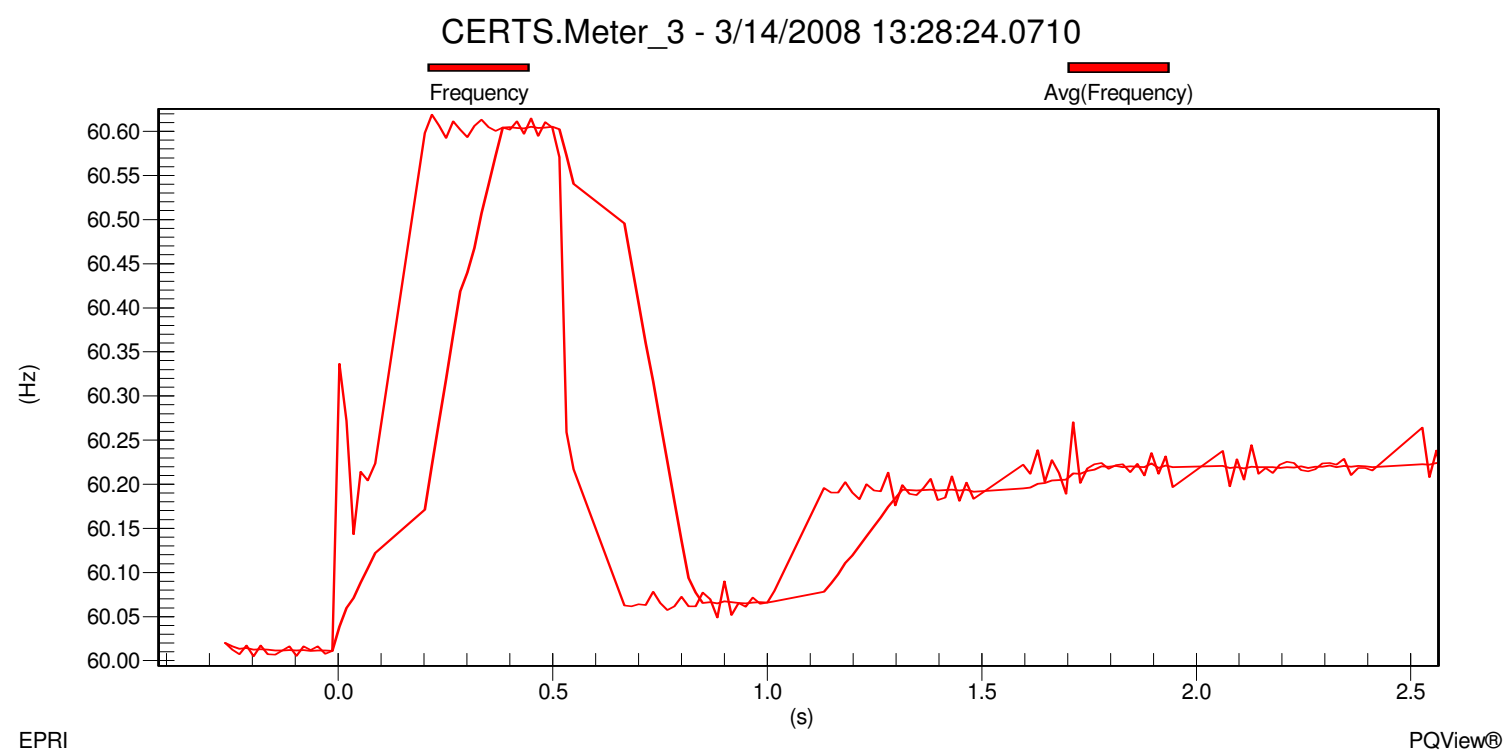

Figure $6 \mathrm{~h}$ - Meter 3 Frequency during Utility Connected to Island mode for Test 10.2.14 
Frequency change in the microgrid, shown in Figure 6h, increased from approximately $60.01 \mathrm{~Hz}$ when connected to the utility grid to approximately $60.22 \mathrm{~Hz}$ when islanded. This change in frequency was part of the CERTS algorithm which allowed Gen-set A1 to decrease its output power to satisfy the load demands. Gen-set A1 decreased its output real power to approximately $13 \mathrm{~kW}$ and increased its output reactive power to approximately $3.5 \mathrm{kVAr}$. Meter 3 was approximately $-3.5 \mathrm{~kW}-\mathrm{j} 3.9 \mathrm{kVAr}$ indicating that Gen-set A1 was satisfying the load in Load Bank 3 and the power losses in the electrical lines. All data was verified and recorded into the DAS Database. The microgrid ran for a couple of minutes in this electrical state before the $10 \mathrm{Hp}$ induction motor was started in Zone 3.

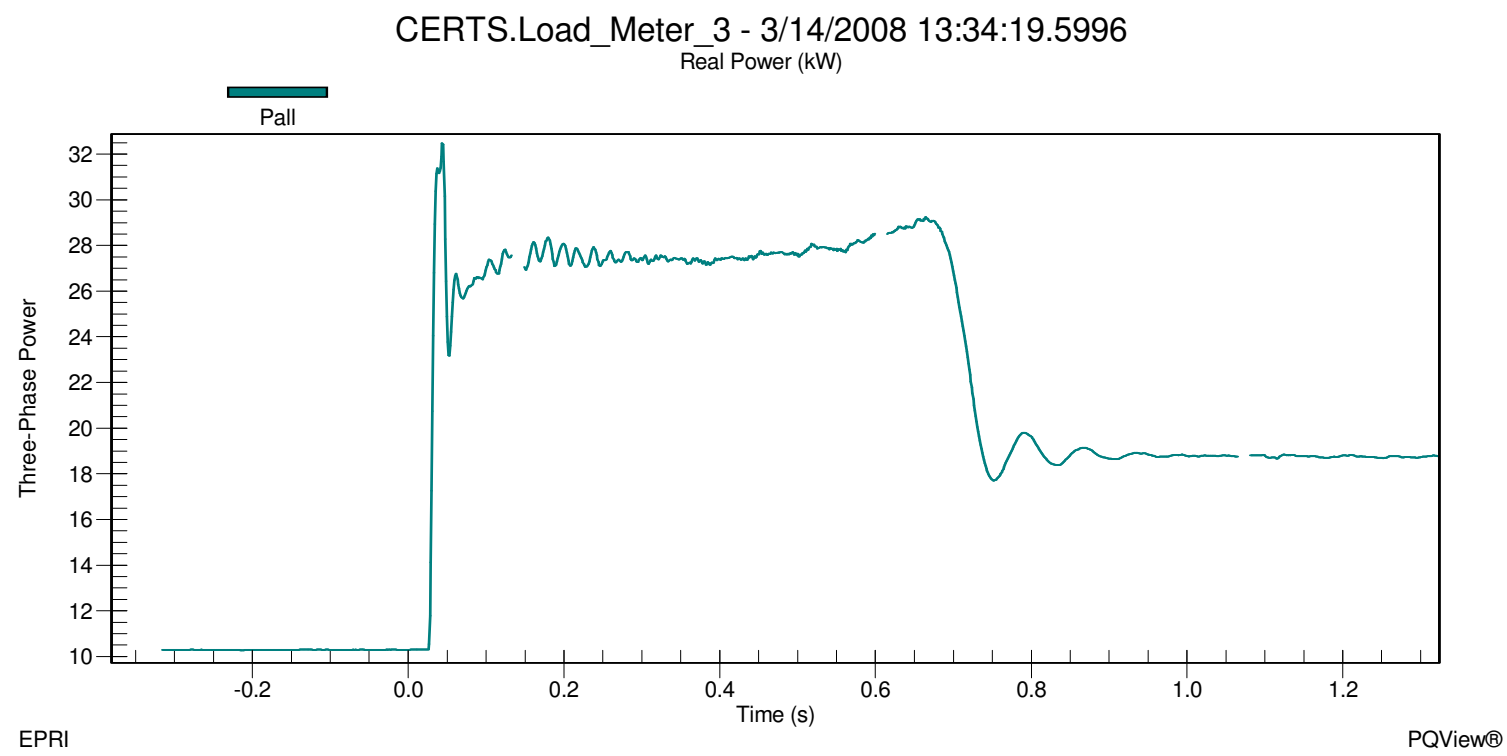

Figure 6i - Load Bank 3 Real Power during Motor Start and Islanded for Test 10.2.14 


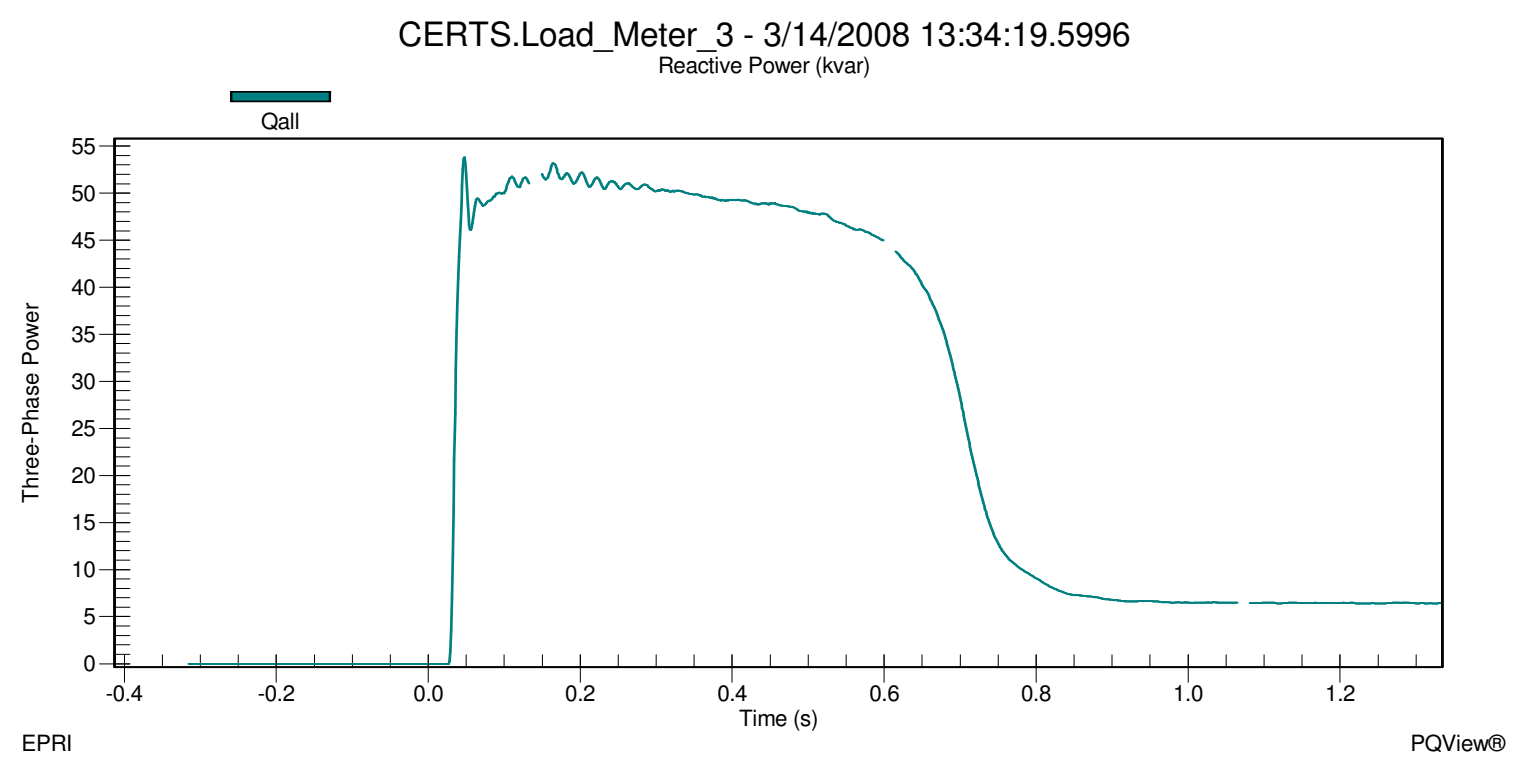

Figure 6j - Load Bank 3 Reactive Power during Motor Start and Islanded for Test 10.2.14

In Figures $6 \mathrm{i}$ and $6 \mathrm{j}$ it can be seen that the load in Zone 3 was approximately $10.4 \mathrm{~kW}+$ j0kVAr before the start of the induction motor and increased to approximately $32.5 \mathrm{~kW}+$ j54kVAr during the inrush phase of the motor start. After about 1.5 cycles, the motor settled down to approximately $28 \mathrm{~kW}+\mathrm{j} 50 \mathrm{kVAr}$ during the warm up phase which lasted about 51 cycles ( 0.85 Seconds). When the motor reached steady state, the load in Zone 3 was approximately $18.8 \mathrm{~kW}+\mathrm{j} 6.8 \mathrm{kVAr}$.

The voltage and frequency at Meter 3 before the motor start was approximately $272.5 \mathrm{~V}$ on A-phase, $272.5 \mathrm{~V}$ on B-phase and $272.5 \mathrm{~V}$ on C-phase shown in Figure $6 \mathrm{k}$ and approximately $60.22 \mathrm{~Hz}$ shown in Figure 61 . When the motor started, the voltage at Meter 3 during the inrush decreased to approximately $235 \mathrm{~V}$ on A-phase, 235V on Bphase and $235 \mathrm{~V}$ on C-phase for about 1.5 cycles. Frequency dropped during the inrush to approximately $59.88 \mathrm{~Hz}$ and quickly increased to approximately $60.15 \mathrm{~Hz}$. Voltage increased as the motor was warming up and eventually settled at a steady state voltage at approximately $268.8 \mathrm{~V}$ on A-phase, $268.8 \mathrm{~V}$ on B-phase and $268.8 \mathrm{~V}$ on C-phase at an approximate frequency of $60.15 \mathrm{~Hz}$. 


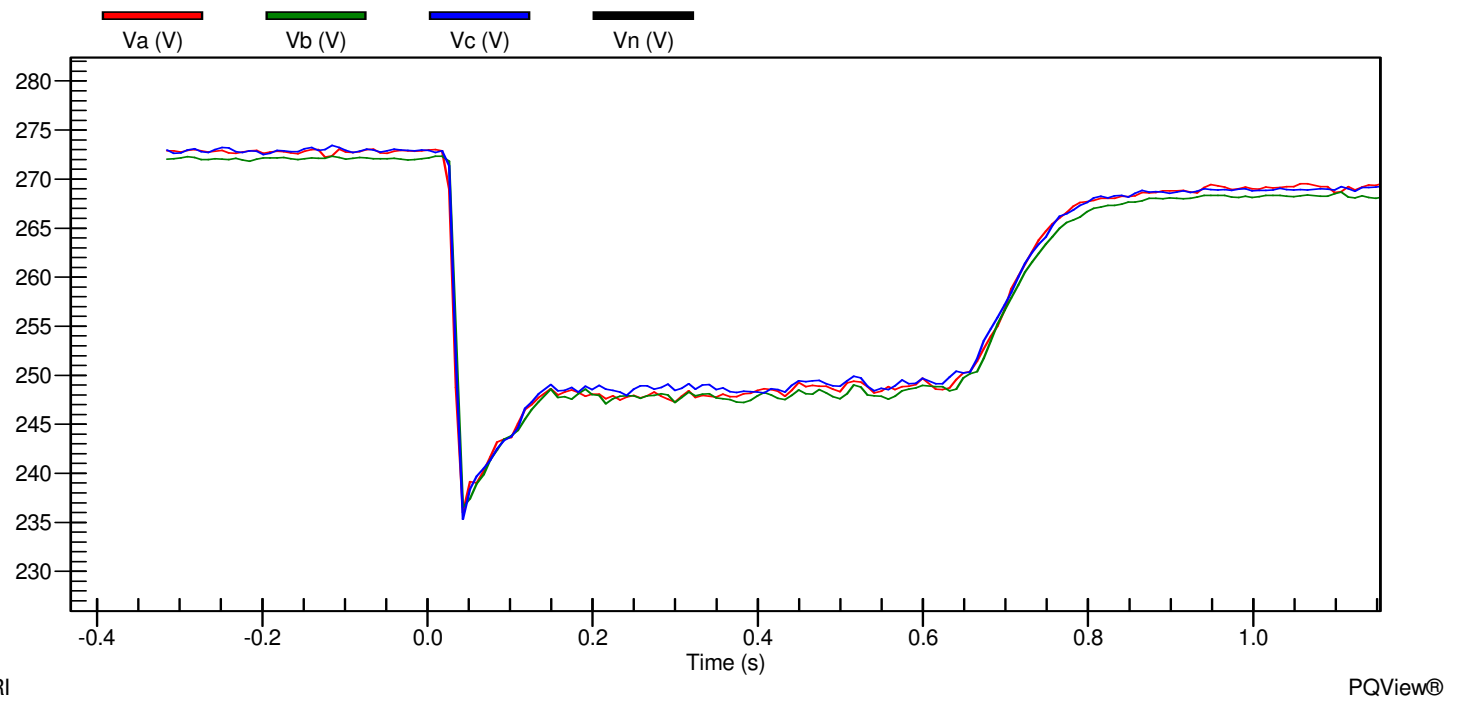

Figure 6k - Meter 3 Line-to-Ground Voltages during Motor Start and Islanded for Test 10.2.14

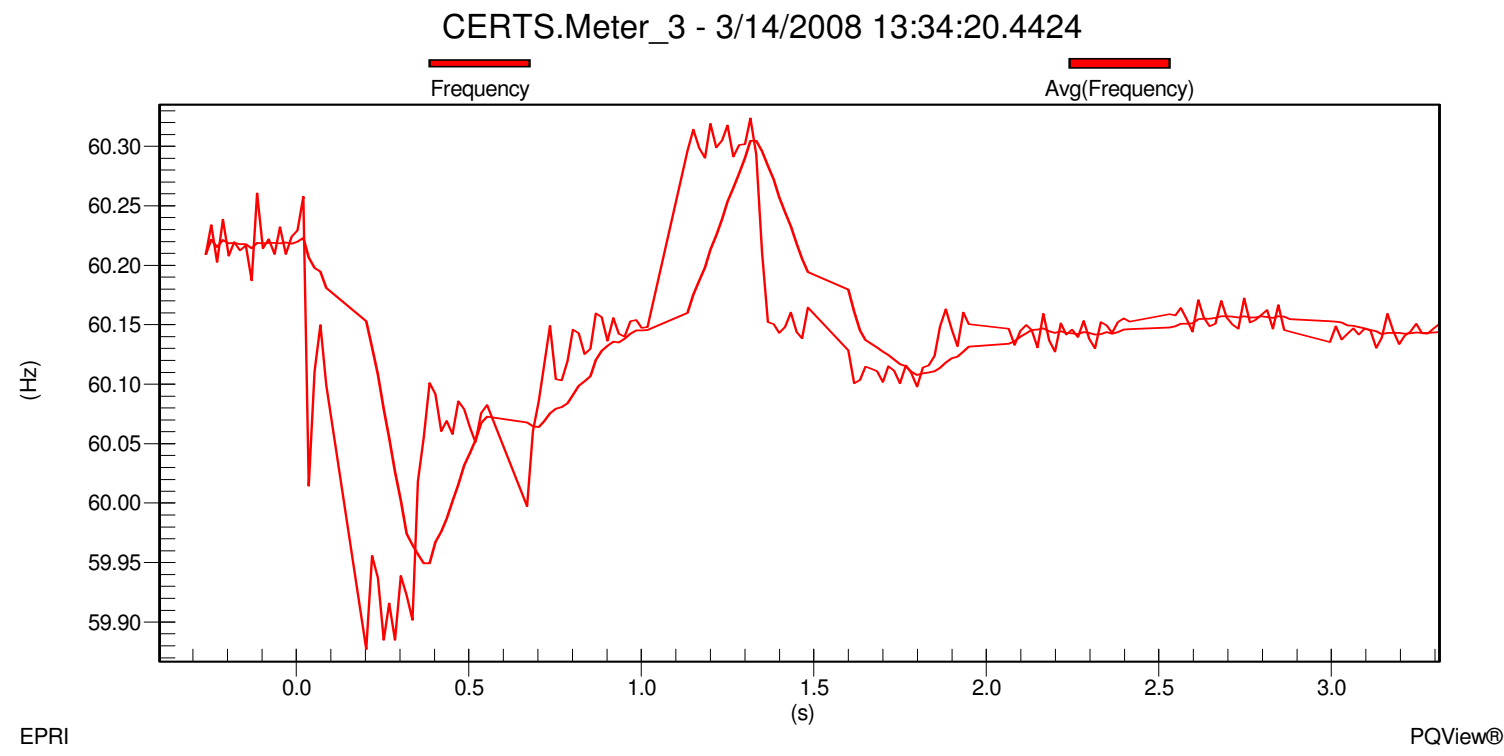

Figure 61 - Meter 3 Frequency during Motor Start and Islanded for Test 10.2.14

Before the motor started, Gen-set A1 was producing approximately $12.5 \mathrm{~kW}+\mathrm{j} 3.5 \mathrm{kVAr}$ shown in Figures $6 \mathrm{~m}$ and $6 \mathrm{n}$. The power generated by Gen-set A1 was satisfying the loads in Load Bank 3 and all the electrical losses in the microgrid system. When the motor started the inrush caused the Gen-set to increase its output level to $34.3 \mathrm{~kW}+$ j55kVAr. Gen-set A1 decreased its output level while the motor was warming up and eventually dropped to $21 \mathrm{~kW}+\mathrm{j} 9.8 \mathrm{kVAr}$ when the motor reached steady state. Once all the data was verified and recorded into the DAS Database, the static switch was directed by the EMS to manually close. 


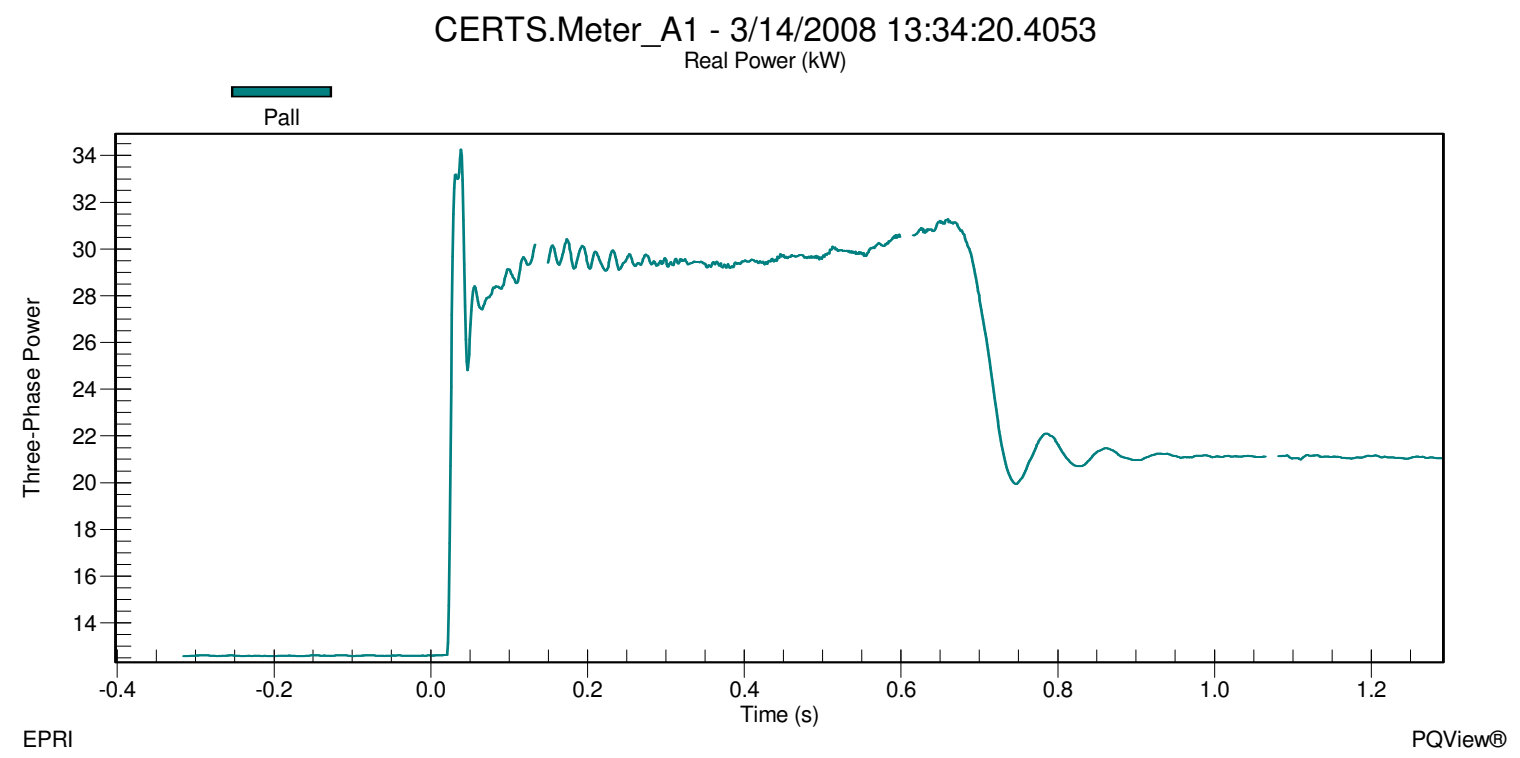

Figure 6m - Gen-set A1 Real Power during Motor Start and Islanded for Test 10.2.14

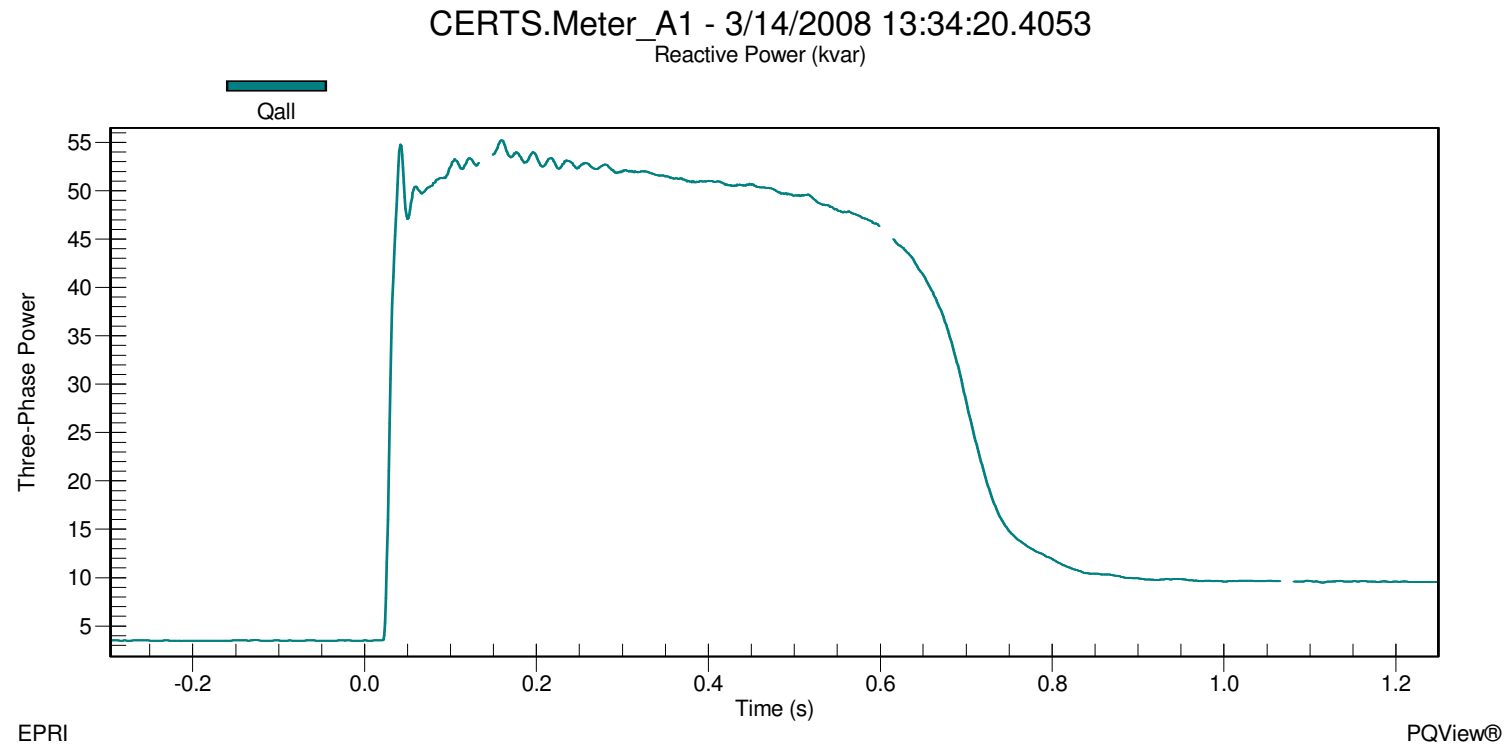

Figure 6n - Gen-set A1 Reactive Power during Motor Start and Islanded for Test 10.2.14

As soon as the static switch closed, Meter 1 recorded real power decreased from approximately $37 \mathrm{~kW}$ to $18 \mathrm{~kW}$ and reactive power increased from approximately $0 \mathrm{kVAr}$ to $25 \mathrm{kVAr}$ which means that the utility was satisfying a portion of the load demand in Load Bank 6 and all the reactive power in the microgrid. Figures 60 and $6 \mathrm{p}$ show the static switch decreasing from approximately $0 \mathrm{~kW}$ to $-17.7 \mathrm{~kW}$ and increasing from approximately $0 \mathrm{kVAr}$ to $24 \mathrm{kVAr}$. At the beginning of the test, the initial power flow through the static switch was $-26 \mathrm{~kW}+\mathrm{j} 20 \mathrm{kVAr}$ which was not the same recorded at this 
point in the test because the $10 \mathrm{Hp}$ motor load is on in Zone 3. Gen-set A1 has picked up the motor load and is supporting Load Bank 6 with approximately $17.7 \mathrm{~kW}$.

Load Bank 3 loads increased slightly to $17.65 \mathrm{~kW}+\mathrm{j} 6.1 \mathrm{kVAr}$. This slight increase is a result from a voltage rise in the microgrid, shown in Figure $6 \mathrm{q}$, from approximately $269 \mathrm{~V}$ on A-phase, $269 \mathrm{~V}$ on B-phase and $269 \mathrm{~V}$ on C-phase when islanded to $282 \mathrm{~V}$ on Aphase, $280 \mathrm{~V}$ on B-phase and $280 \mathrm{~V}$ on C-phase at Meter 3 when connected to the utility grid.

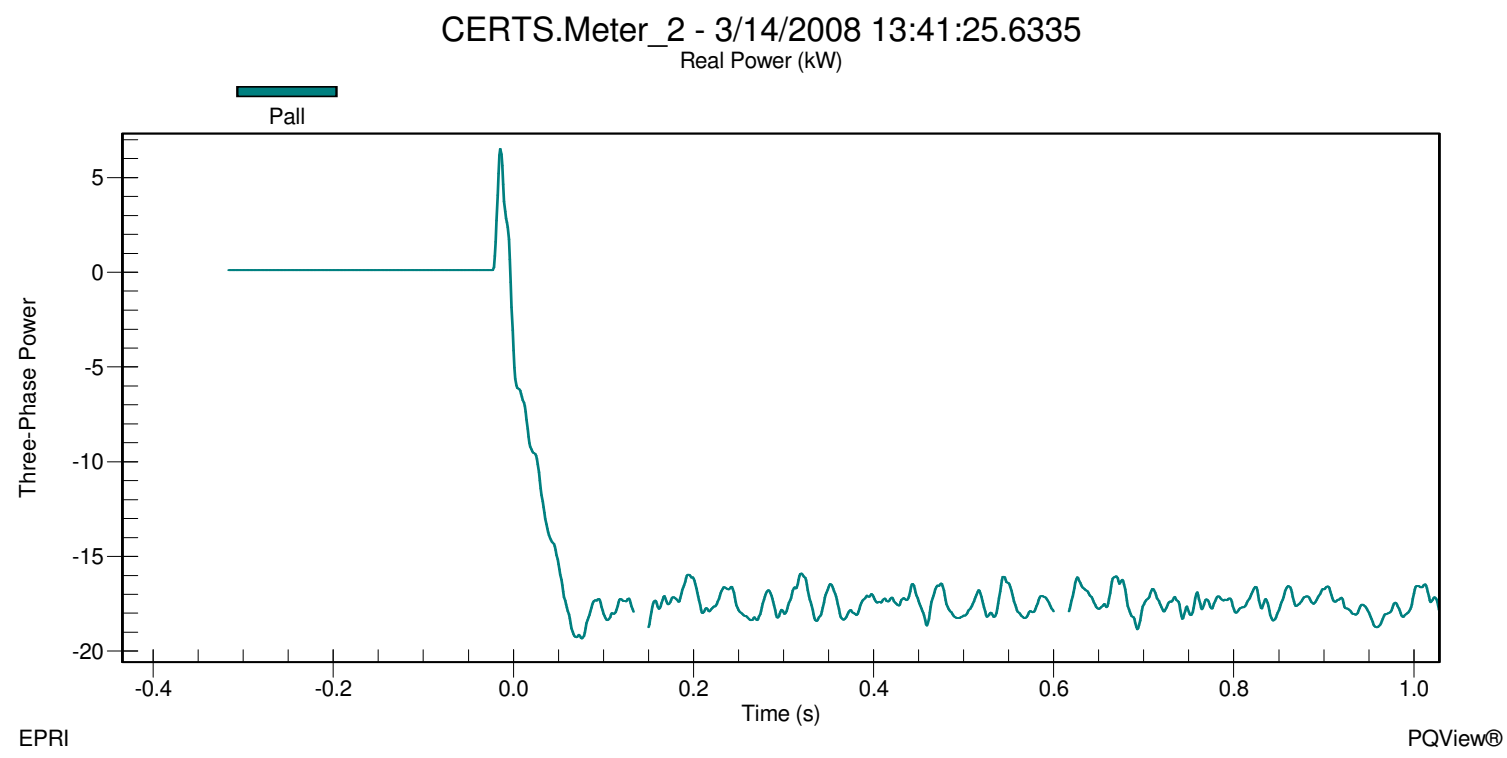

Figure 6o - Static Switch Real Power during Island to Utility Connected mode for Test 10.2.14 


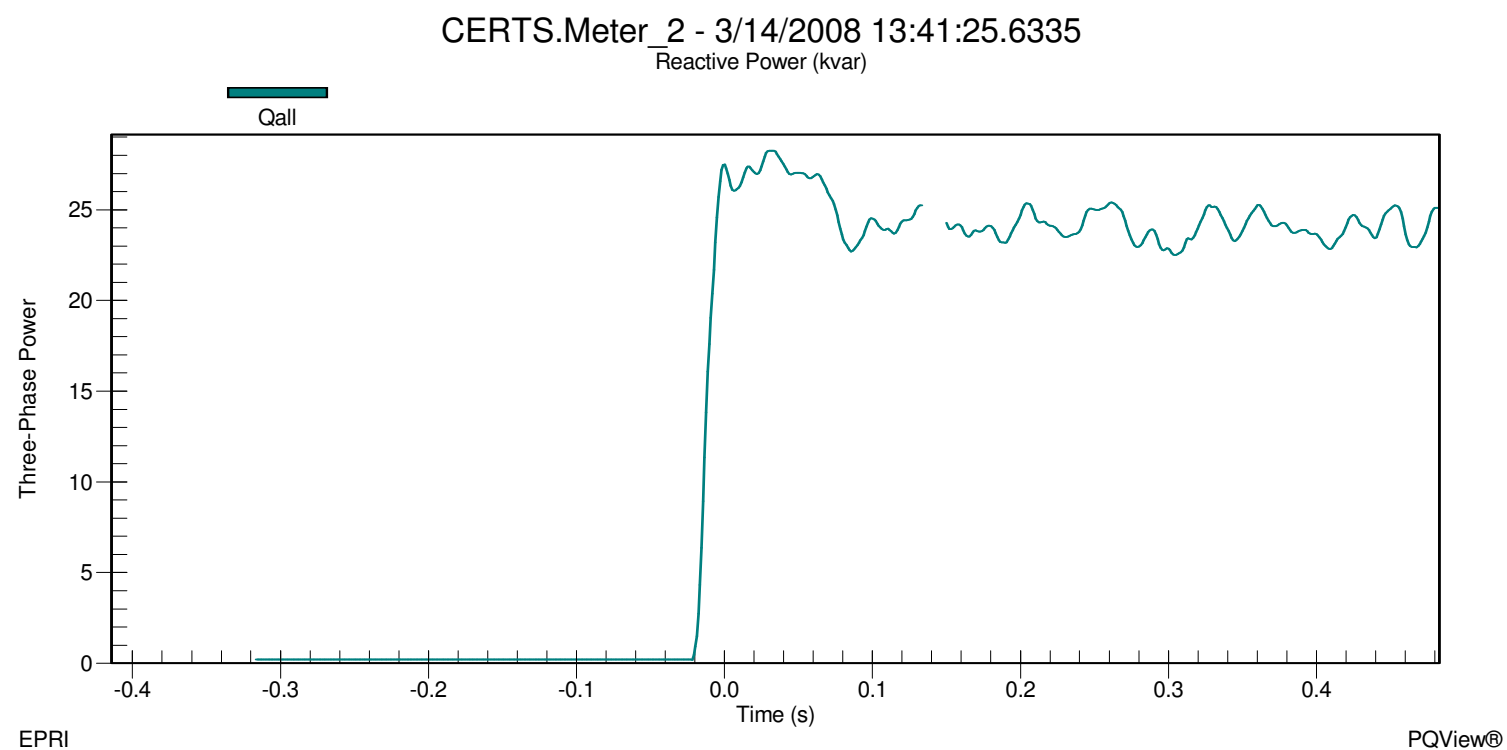

Figure 6p - Static Switch Reactive Power during Island to Utility Connected mode for Test 10.2.14

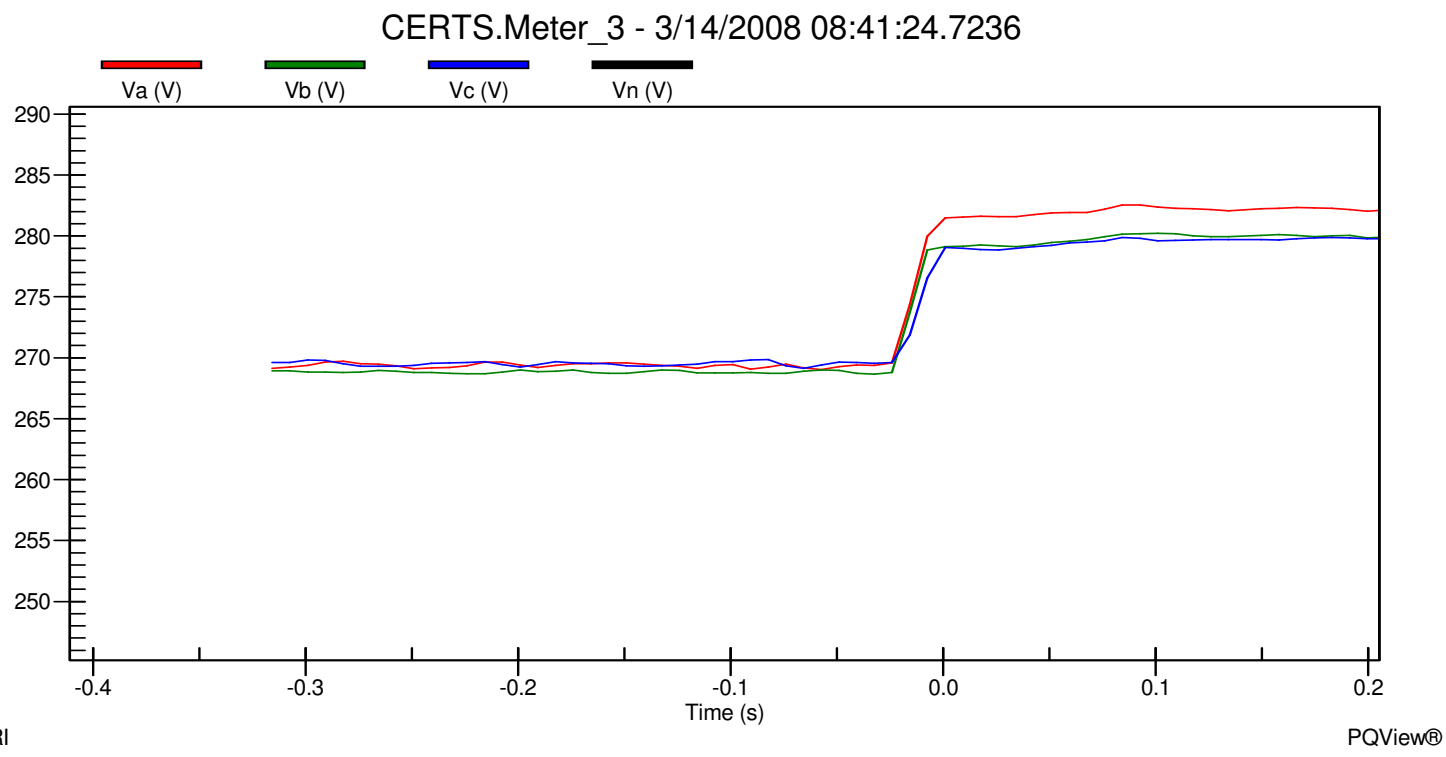

Figure 6q - Meter 3 Line-to-Ground Voltages during Island to Utility Connected mode for Test 10.2.14 


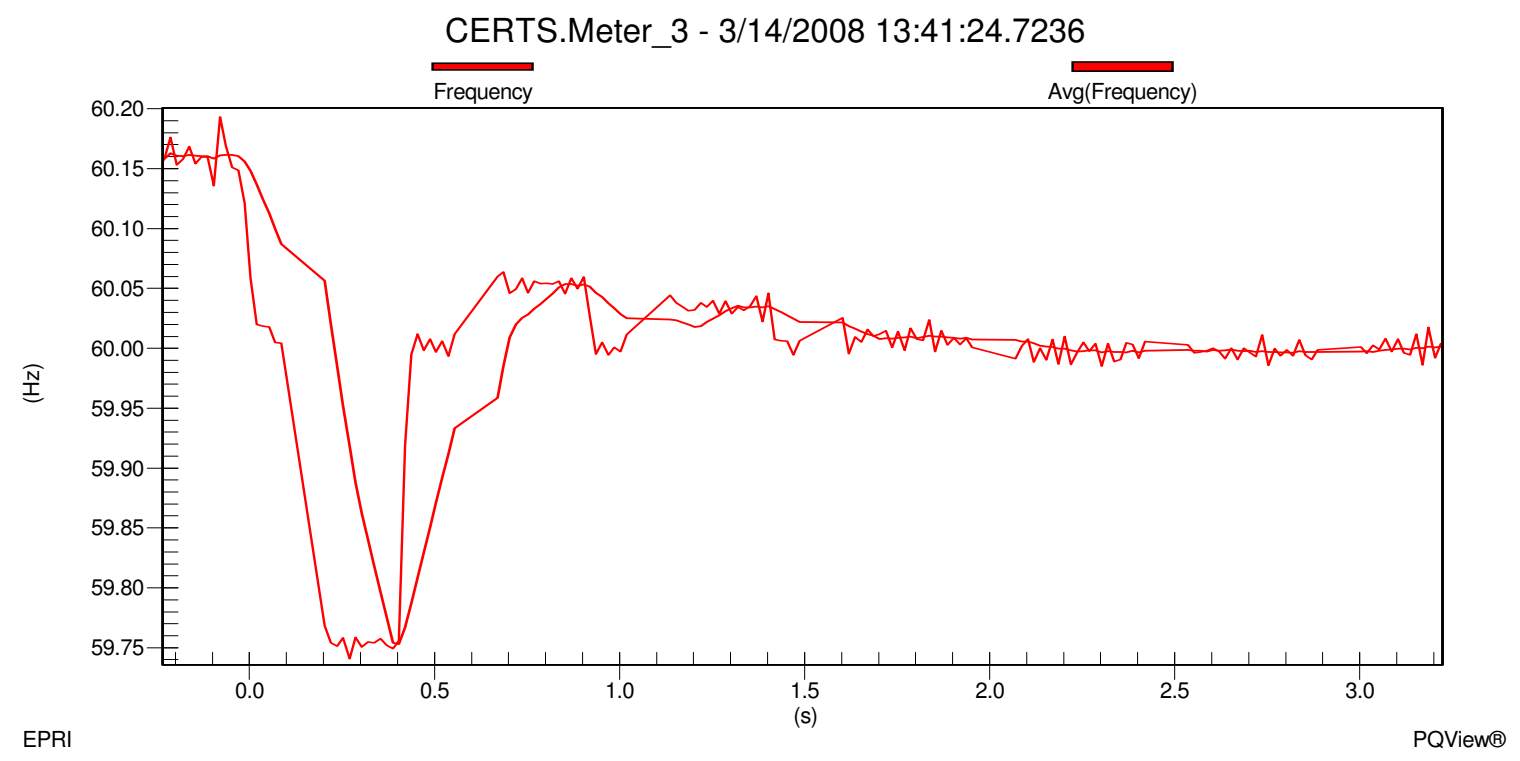

Figure 6r - Meter 3 Frequency during Island to Utility Connected mode for Test 10.2.14

Frequency change in the microgrid, shown in Figure 6r, decreased from approximately $60.16 \mathrm{~Hz}$ when islanded to approximately $60.00 \mathrm{~Hz}$ when connected to the utility grid. This change in frequency is due to the frequency no longer being established by Gen-set A1 using the CERTS algorithm but by the utility. Gen-set A1 is in unit power control mode therefore when the static switch closed back into the utility the Gen-set produced real power based on the set-point initialized at the beginning of the test. The output power for Gen-set A1 was relatively close to the values at the beginning of the test with Gen-set A1 producing approximately $38 \mathrm{~kW}-\mathrm{j} 12 \mathrm{kVAr}$. After all the data was verified and recorded into the DAS Database, the motor was turned off and the Gen-sets and Load Banks set-points were changed according to the next test (10.2.15) in Table 1.

For Test 10.2.15 the measured values, after the Gen-sets were warmed up and load bank brought on-line, were approximately $64 \mathrm{~kW}+17 \mathrm{kVAr}$ at Meter $1,28 \mathrm{~kW}+\mathrm{j} 16 \mathrm{kVAr}$ at Meter 2, 25kW $+\mathrm{j} 10 \mathrm{kVAr}$ at Meter 3 and $16 \mathrm{~kW}+\mathrm{j} 1 \mathrm{kVAr}$. From the microgrid, $38.4 \mathrm{~kW}-$ j10kVAr was produced by Gen-set A1 and 30kW + j0kVAr was produced by Gen-set A2. The load banks were $47 \mathrm{~kW}+\mathrm{j} 0 \mathrm{kVAr}$ at LB3, $46.9 \mathrm{~kW}+\mathrm{j} 0.715 \mathrm{kVAr}$ at LB4 and $35.8 \mathrm{~kW}+$ j0.766kVAr at LB6. These measurements were relatively close to the expected values in Table 1, but not exact due to temperature, phase voltages and electrical losses in conductors. In addition, the $50 \mathrm{~kW}$ settings for LB3 and LB4 and the $40 \mathrm{~kW}$ setting for LB6 were also below selected set values. At the time of these measurements, the voltage and frequency was $281.6 \mathrm{~V}$ on A-phase, $279.1 \mathrm{~V}$ on B-phase and $279.1 \mathrm{~V}$ on C-phase and $60.02 \mathrm{~Hz}$ at the static switch (i.e., Meter 2) when connected to the utility grid; and $281.6 \mathrm{~V}$ on A-phase, 279.1V on B-phase and 279.1V on C-phase and 60.02Hz at Meter 3. 
The Gen-sets in this test were set up to produce less power than the microgrid loads needed which approximately $28 \mathrm{~kW}$ of power was imported from the utility through the static switch to support Load Banks 3 and 4. Since Load Bank 6 was approximately $35.8 \mathrm{~kW}+\mathrm{j} 0.766 \mathrm{kVAr}$, the utility had to supply approximately $64 \mathrm{~kW}$ to satisfy the load demand of Load Bank 6 and the microgrid loads. Reactive power had to be imported in from the utility of approximately 16kVAr because Gen-set A1 and A2 needed approximately 10kVAr between the two and the reactive power of Load Banks 3 and 4 and electrical wires. Once all data was verified and recorded into the DAS Database, the $10 \mathrm{Hp}$ induction motor was started in Zone 3.

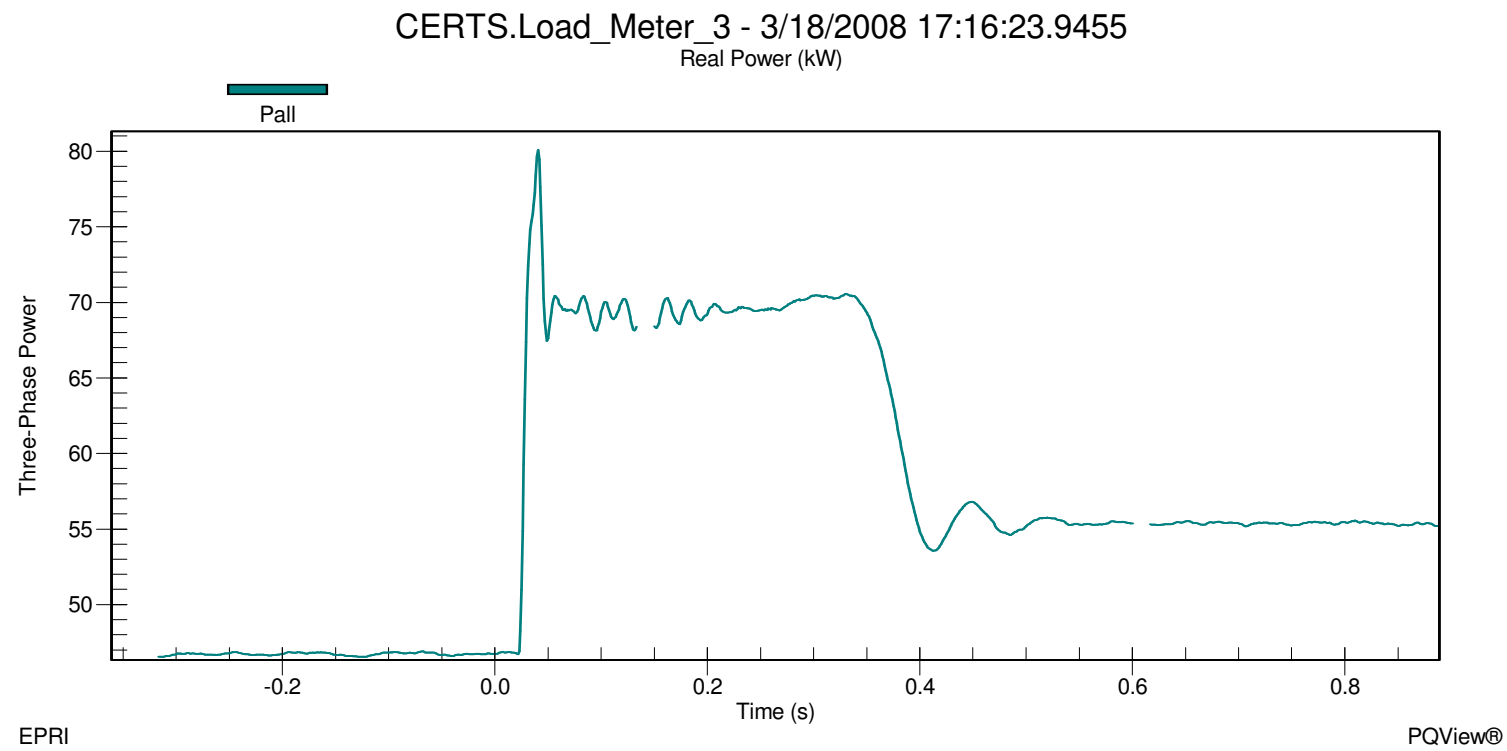

Figure 7a - Load Bank 3 Real Power during Motor Start Utility Connected for Test 10.2.15 


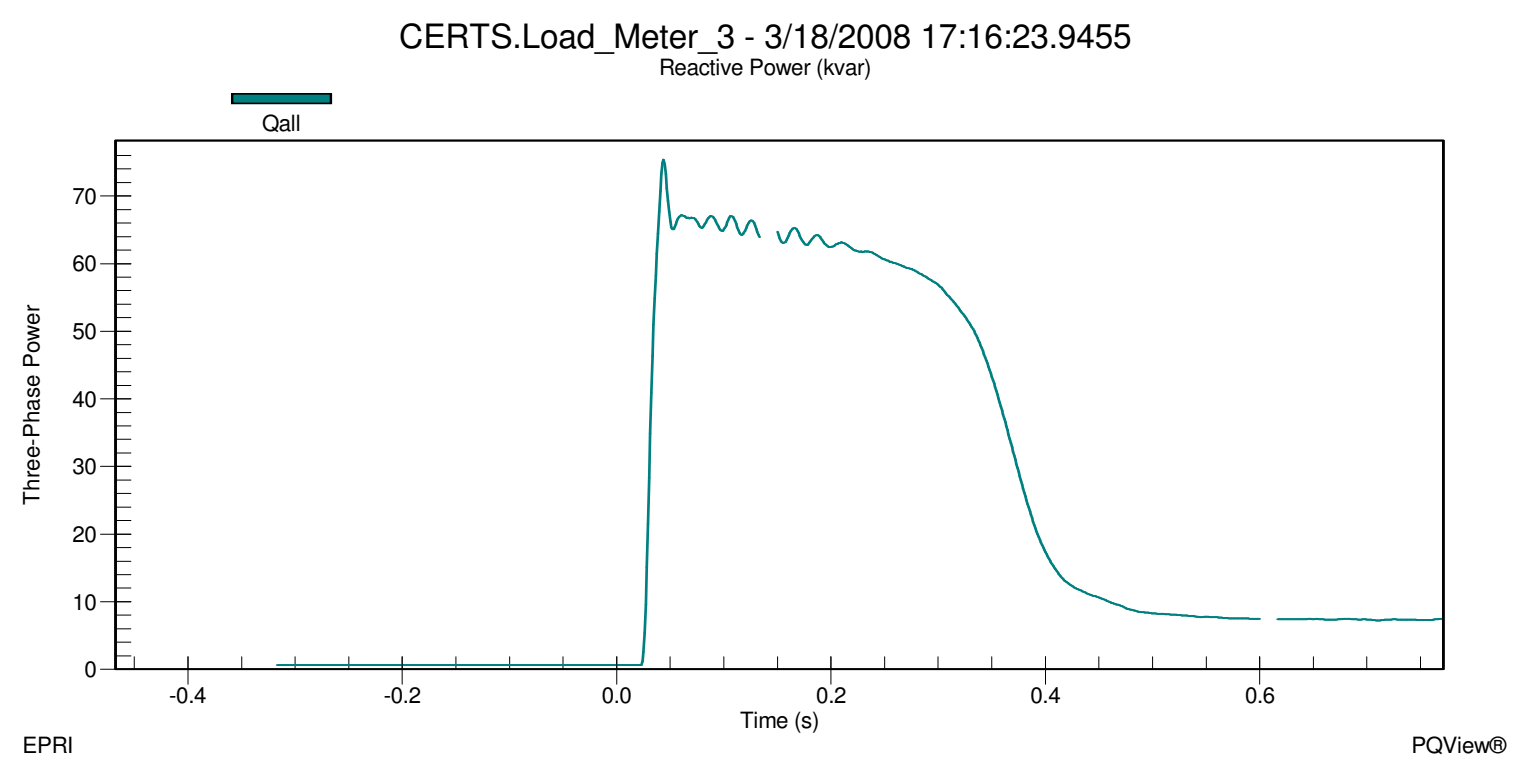

Figure 7b - Load Bank 3 Reactive Power during Motor Start Utility Connected for Test 10.2.15

In Figures $7 \mathrm{a}$ and $7 \mathrm{~b}$ it can be seen that the load in Zone 3 was approximately $46.9 \mathrm{~kW}+$ j0.715kVAr before the start of the induction motor and increased to approximately $80 \mathrm{~kW}$ $+\mathrm{j} 75 \mathrm{kVAr}$ during the inrush phase of the motor start. After about 1.5 cycles, the motor settled down to approximately $69.5 \mathrm{~kW}+\mathrm{j} 64 \mathrm{kVAr}$ during the warm up phase which lasted about 33 cycles ( 0.55 Seconds). When the motor reached steady state, the load in Zone 3 was approximately $55.5 \mathrm{~kW}+\mathrm{j} 7 \mathrm{kVAr}$.

The voltage and frequency at the static switch before the motor start was approximately 281.6V on A-phase, 279.1V on B-phase and 279.1V on C-phase shown in Figure 7c and approximately $60.02 \mathrm{~Hz}$ shown in Figure $7 \mathrm{~d}$. When the motor started, the voltage at the static switch during the inrush decreased to approximately $274.6 \mathrm{~V}$ on A-phase, $271.6 \mathrm{~V}$ on B-phase and $272.1 \mathrm{~V}$ on C-phase for about 1.5 cycles. Frequency dropped during the inrush to approximately $59.95 \mathrm{~Hz}$ and quickly increased back to approximately $60.00 \mathrm{~Hz}$. Voltage increased as the motor was warming up and eventually settled at a steady state voltage at approximately $280.8 \mathrm{~V}$ on A-phase, $278.2 \mathrm{~V}$ on B-phase and $278.4 \mathrm{~V}$ on C-phase at an approximate frequency of $60.00 \mathrm{~Hz}$. 


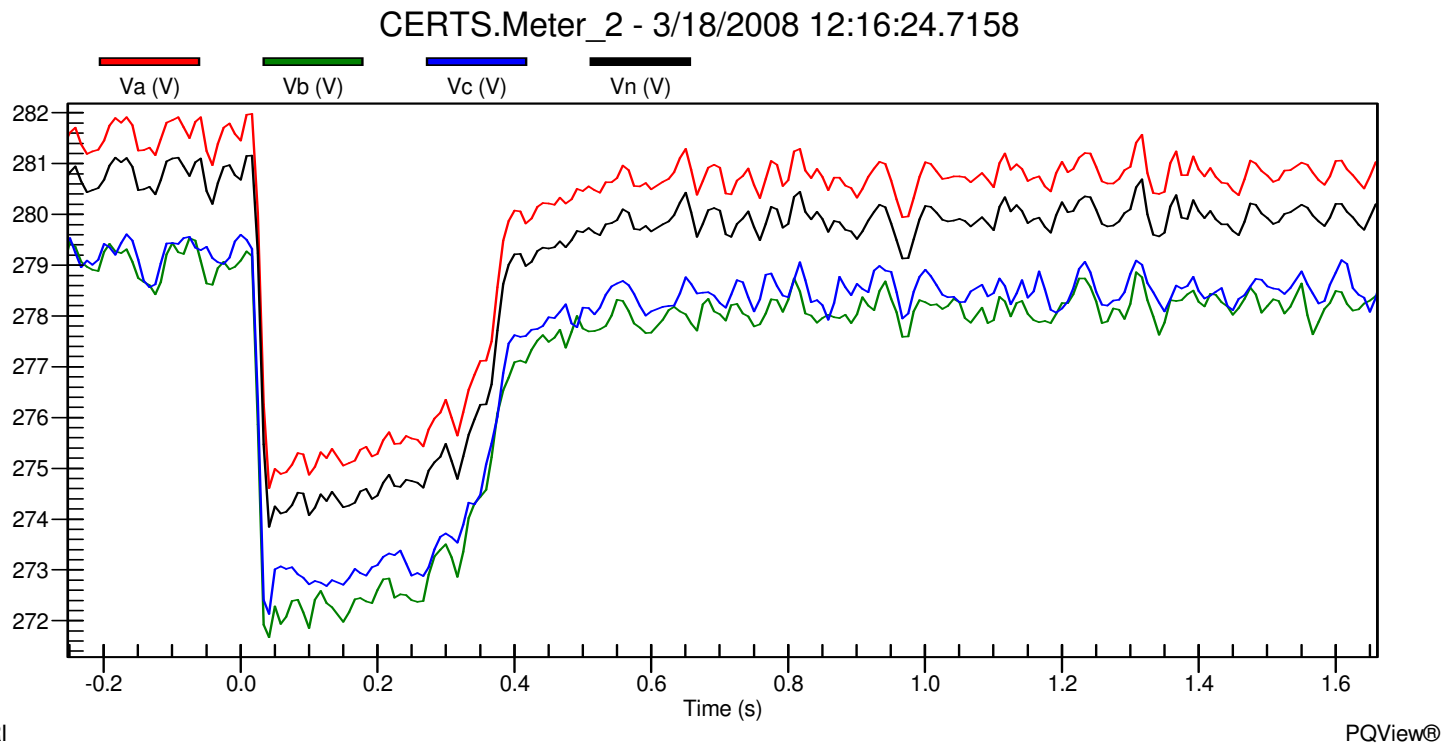

Figure 7c - Static Switch Line-to-Ground Voltages during Motor Start and Utility Connected for Test 10.2.15

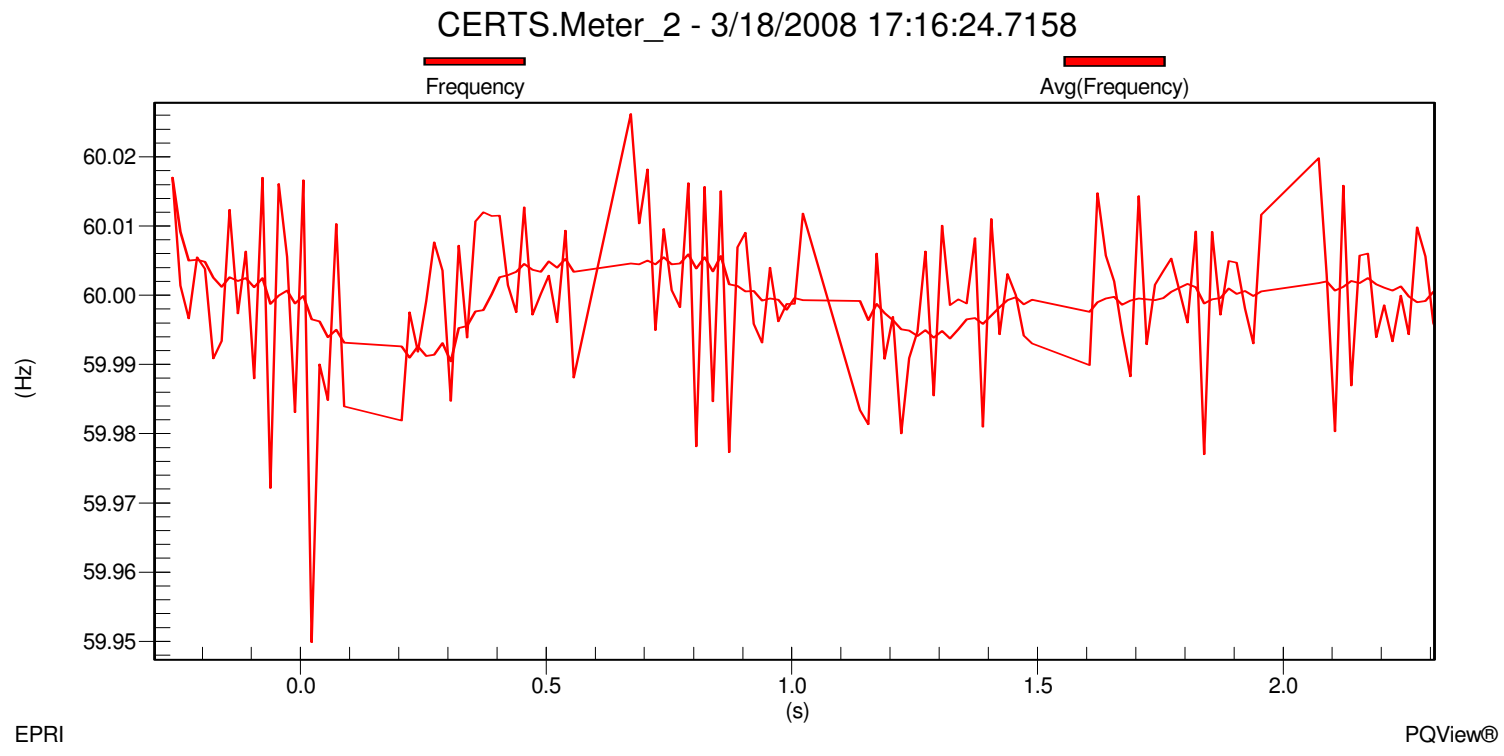

Figure 7d - Static Switch Frequency during Motor Start and Utility Connected for Test 10.2.15 


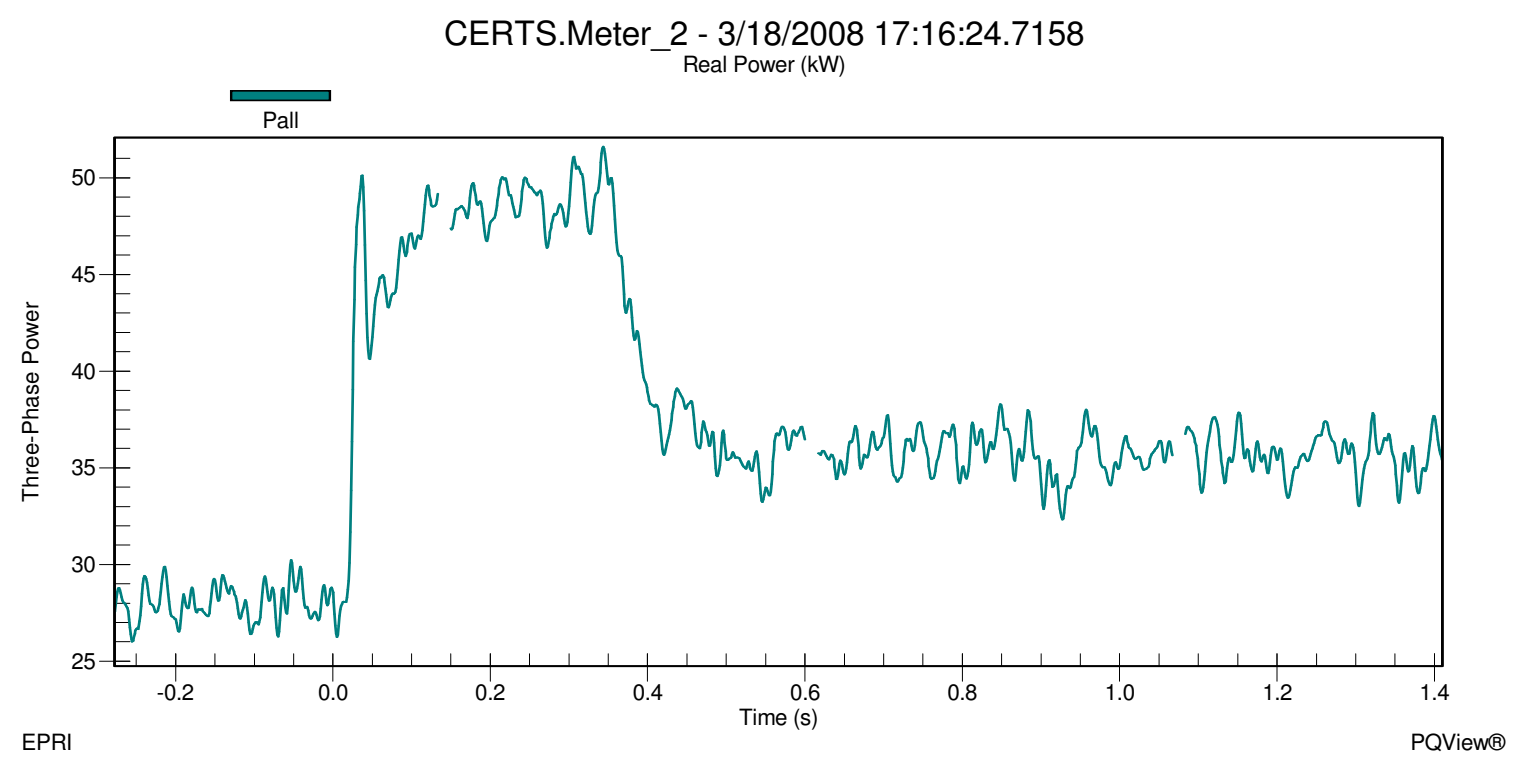

Figure 7e - Static Switch Real power during Motor Start and Utility Connected for Test 10.2.15

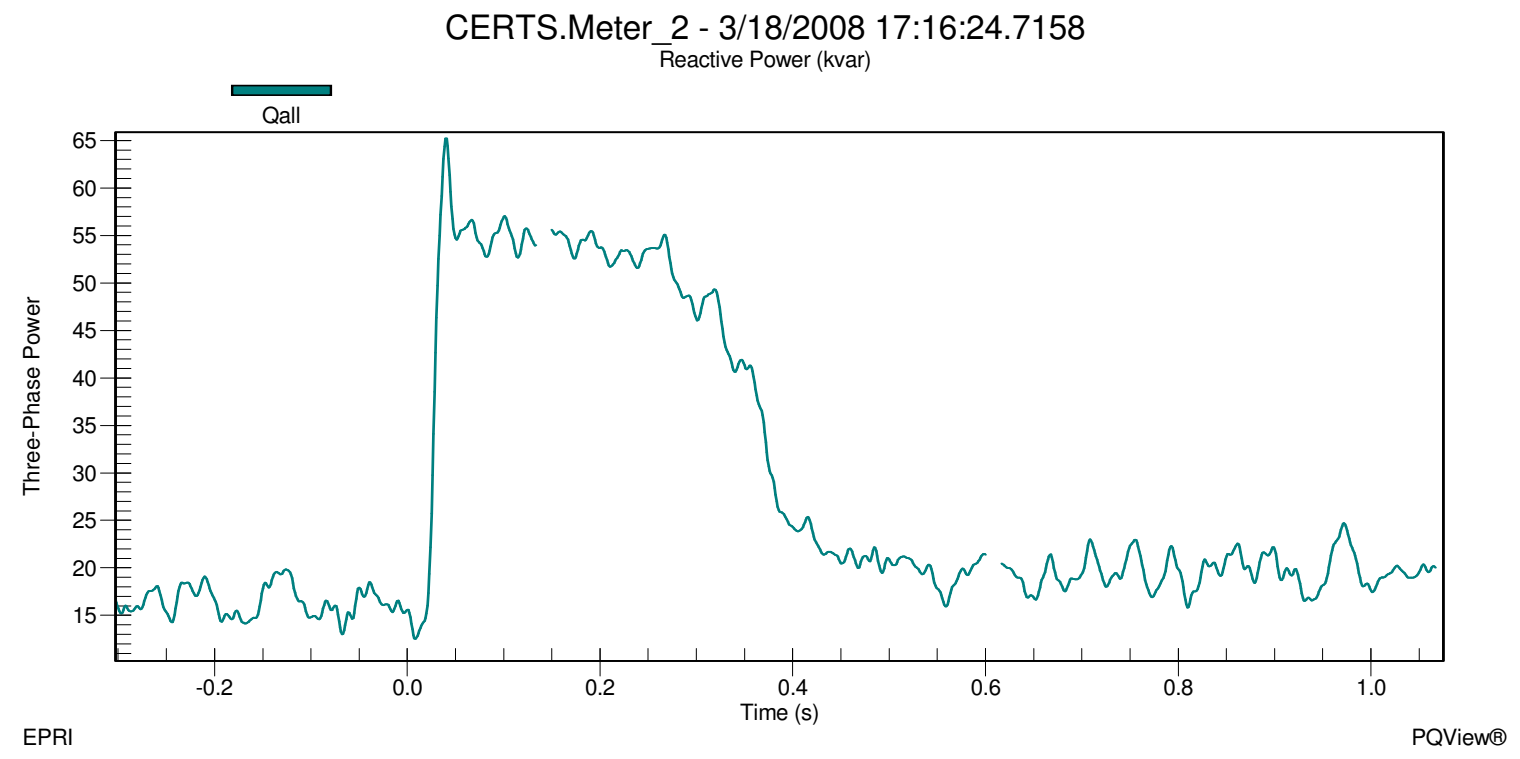

Figure 7f - Static Switch Reactive Power during Motor Start and Utility Connected for Test 10.2.15

Before the motor started Gen-sets A1 and A2 were producing approximately $38.4 \mathrm{~kW}-$ j10kVAr and 30kW + j0kVAr, respectively. The load demands of Load Banks 3 and 4 were greater than the power being produced by both Gen-sets therefore the utility had to supply approximately $28 \mathrm{~kW}$ shown in Figure 7e. The utility was supporting the reactive power of the microgrid with approximately 16kVAr shown in Figure 7f. When the motor started the inrush caused the utility to supply approximately $50 \mathrm{~kW}+\mathrm{j} 65 \mathrm{kVAr}$ to the microgrid loads, shown in Figures 7e and 7f, and Gen-sets A1 and A2 increased their output power levels to $41.8 \mathrm{~kW}+\mathrm{j} 5 \mathrm{kVAr}$ and $35.5 \mathrm{~kW}+\mathrm{j} 14.5 \mathrm{kVAr}$, respectively. 
Notice the sign of the VAr output changed from negative to positive for Gen-set A1 and $0 \mathrm{kVAr}$ to $14.5 \mathrm{kVAr}$ for Gen-set A2 in order to support the induction motor load in Zone 3.

When the motor reached steady state, the real and reactive power through the static switch was approximately $35.5 \mathrm{~kW}+\mathrm{j} 19 \mathrm{kVAr}$ which meant that the utility was supplying the power for the induction motor. Gen-sets A1 and A2 real power returned to the values before the motor started of approximately $38.4 \mathrm{~kW}$ and $30 \mathrm{~kW}$, respectively, and the reactive power slightly increased to approximately $-9 \mathrm{kVAr}$ in Gen-set A1 and 2kVAr in Gen-set A2. Once all the data was verified and recorded into the DAS Database, the motor was shut down and the static switch was directed by the EMS to manually open.

As soon as the static switch opened, Meter 1 recorded real and reactive power decreased to approximately $37 \mathrm{~kW}+\mathrm{j} 1.4 \mathrm{kVAr}$ satisfying the load demand in Load Bank 6 which was approximately $36.6 \mathrm{~kW}+\mathrm{j} 0.78 \mathrm{kVAr}$ and not supplying any power beyond the static switch to Load Banks 3 and 4 . 0kW + j0kVAr was recorded at the static switch, indicating that power was not flowing through the static switch. Load Banks 3 and 4 loads reduced slightly to $44.9 \mathrm{~kW}+\mathrm{j} 0.645 \mathrm{kVAr}$ and $44.4 \mathrm{~kW}+\mathrm{j} 0.688 \mathrm{kVAr}$, respectively. This load reduction resulted from a voltage drop in the microgrid, shown in Figure $7 \mathrm{~g}$, from approximately $280.8 \mathrm{~V}$ on A-phase, $278.2 \mathrm{~V}$ on B-phase and $278.2 \mathrm{~V}$ on C-phase when connected to the utility grid to $274.1 \mathrm{~V}$ on A-phase, $274.1 \mathrm{~V}$ on B-phase and $274.1 \mathrm{~V}$ on Cphase at Meter 3 when islanded.

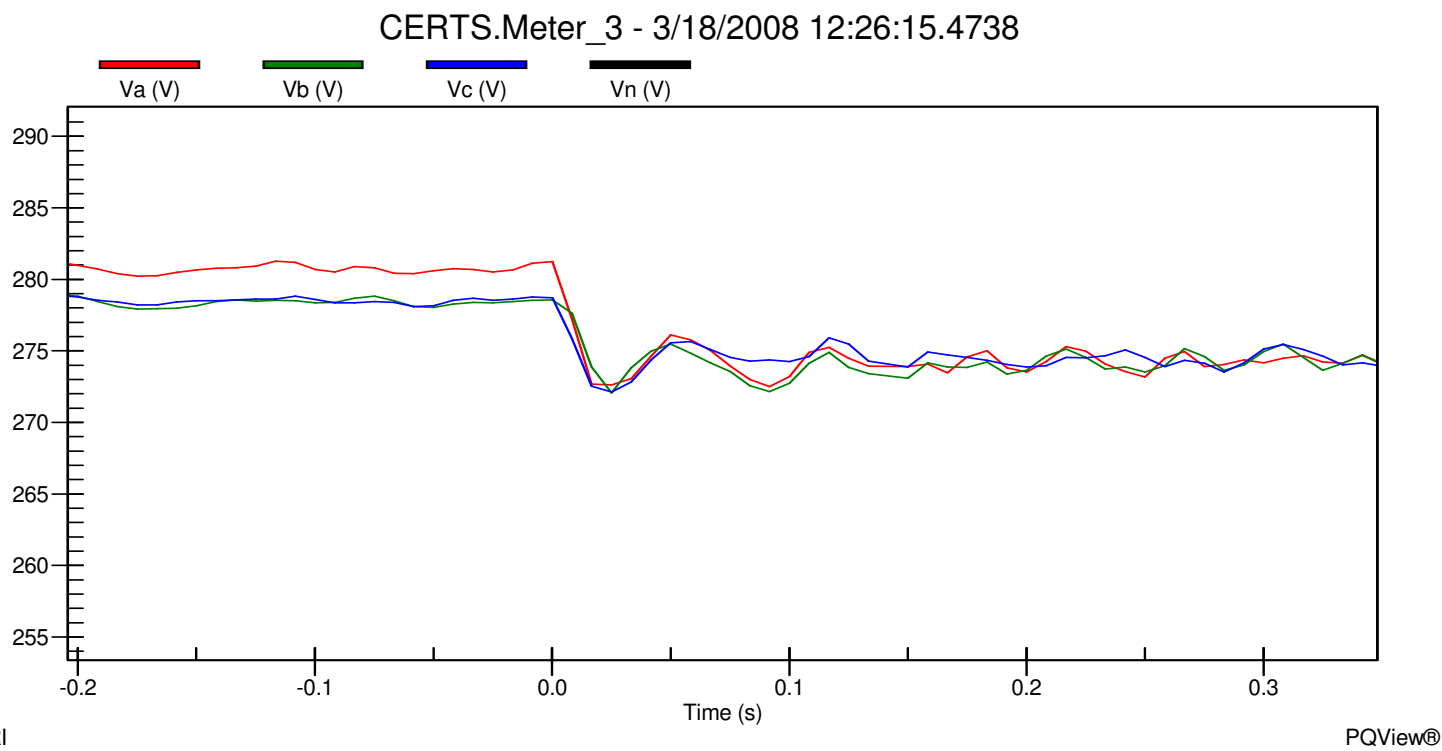

Figure 7g - Meter 3 Line-to-Ground Voltages during Utility Connected to Island mode for Test 10.2.15 


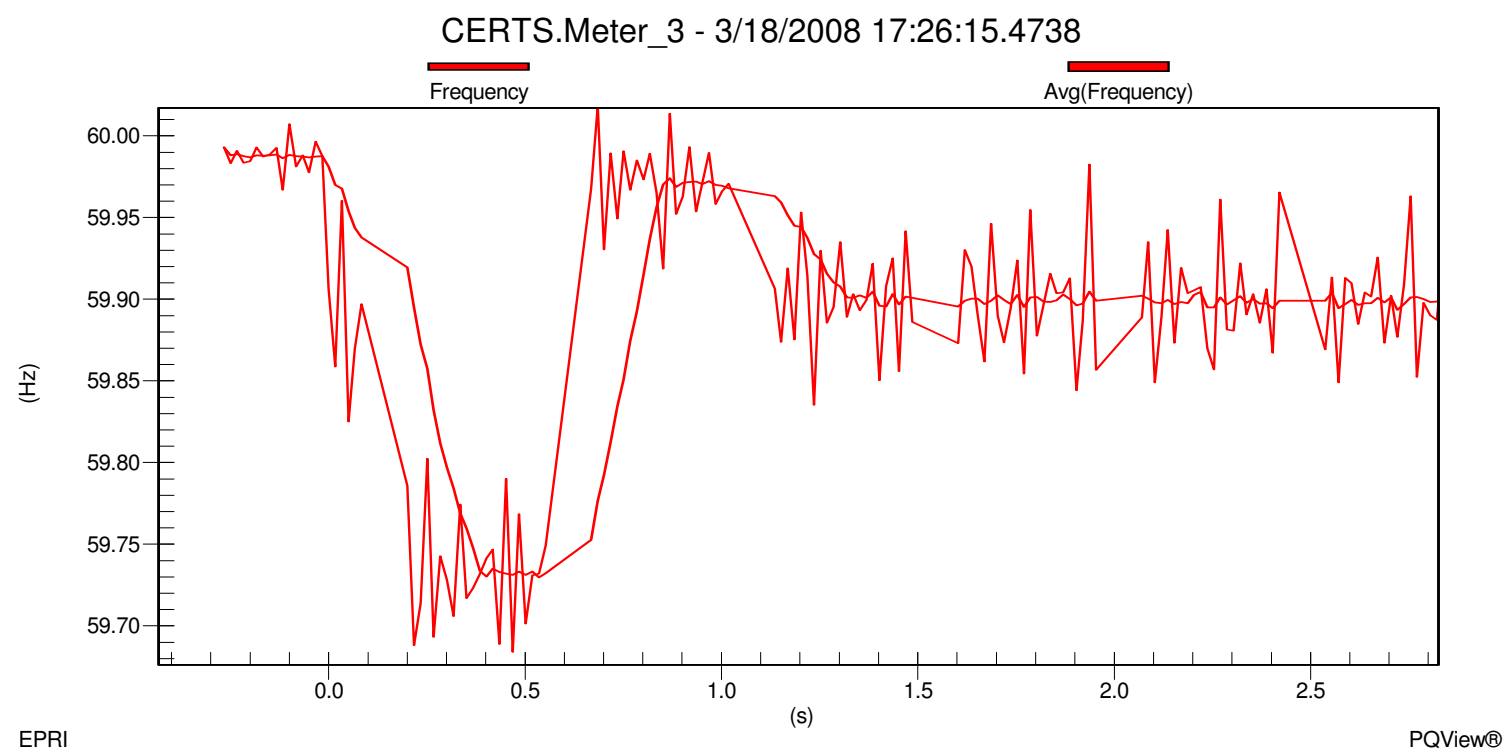

Figure 7h - Meter 3 Frequency during Utility Connected to Island mode for Test 10.2.15

Frequency change in the microgrid, shown in Figure 7h, decreased from approximately $59.99 \mathrm{~Hz}$ when connected to the utility grid to approximately $59.90 \mathrm{~Hz}$ when islanded. This change in frequency was part of the CERTS algorithm which allowed the Gen-sets to increase their output power to satisfy the load demands. Gen-set A1 and A2 increased their output real and reactive powers approximately $49.6 \mathrm{~kW}-\mathrm{j} 2 \mathrm{kVAr}$ and $41.9 \mathrm{~kW}+\mathrm{j} 7 \mathrm{kVAr}$, respectively. Meter 3 was approximately $-2 \mathrm{~kW}-\mathrm{j} 3 \mathrm{kVAr}$ indicating that the Gen-sets were satisfying the loads in the microgrid and the power losses in the electrical lines. All data was verified and recorded into the DAS Database. The microgrid ran for a couple of minutes in this electrical state before the $10 \mathrm{Hp}$ induction motor was started in Zone 3. 


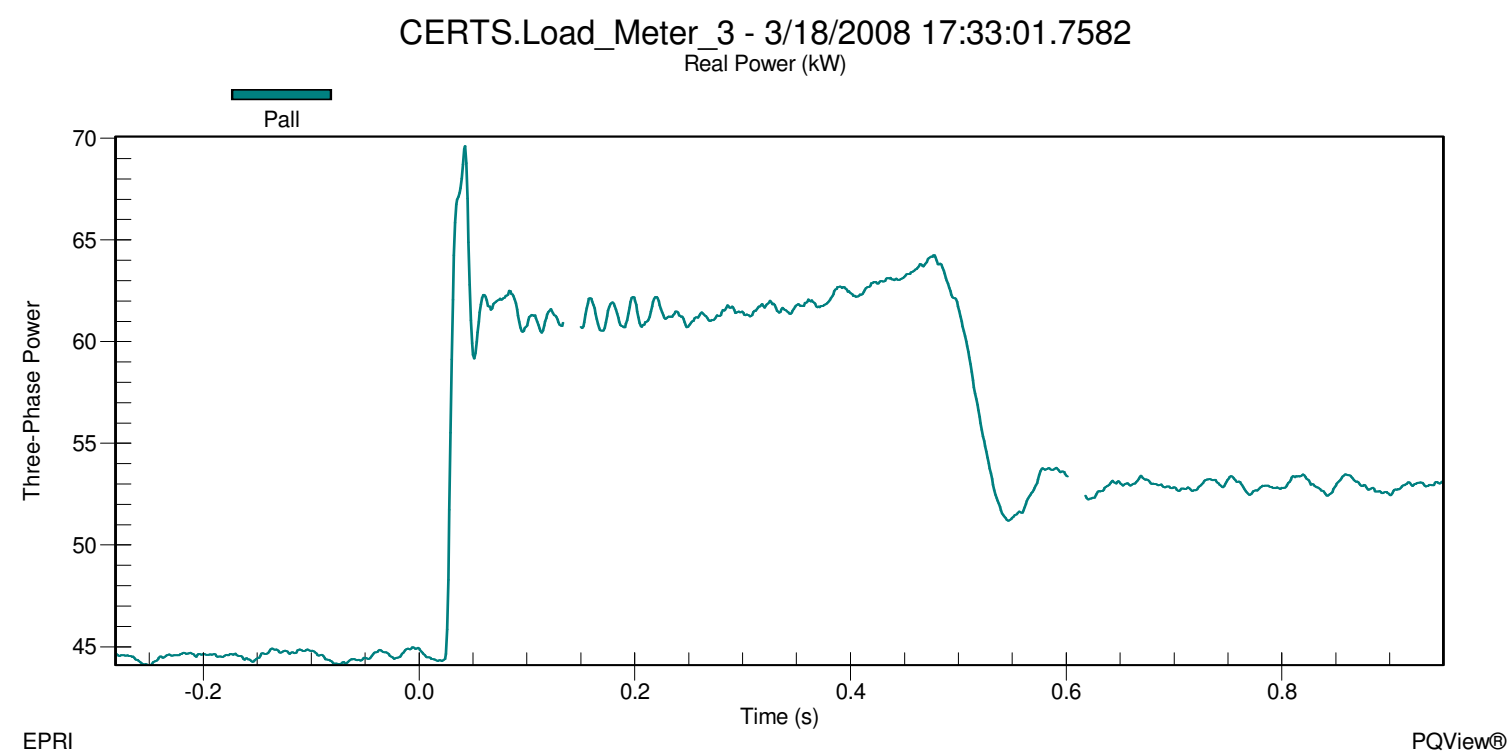

Figure 7i - Load Bank 3 Real Power during Motor Start and Islanded for Test 10.2.15

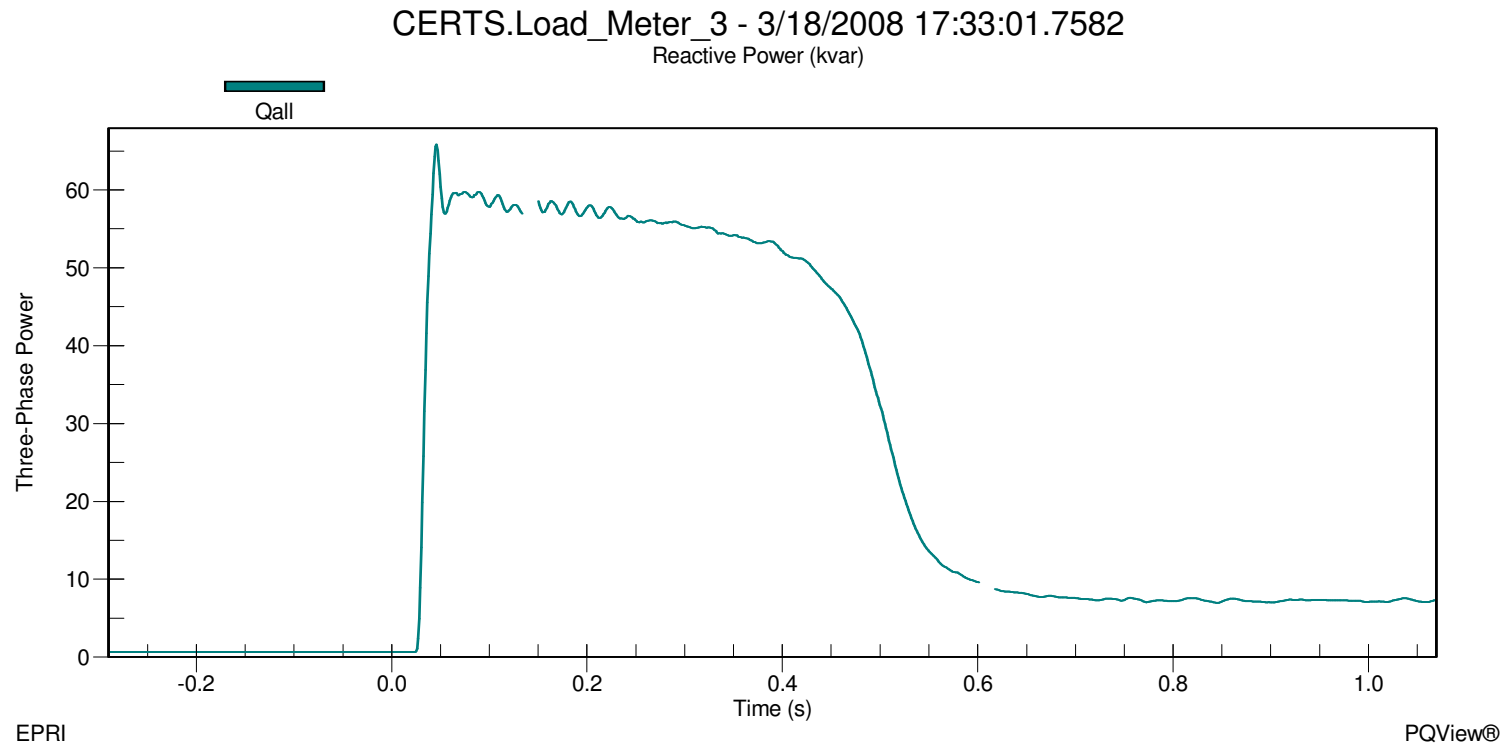

Figure 7j - Load Bank 3 Reactive Power during Motor Start and Islanded for Test 10.2.15

In Figures $7 \mathrm{i}$ and $7 \mathrm{j}$ it can be seen that the load in Zone 3 was approximately $44.5 \mathrm{~kW}+$ $j 1 \mathrm{kVAr}$ before the start of the induction motor and increased to approximately $69.6 \mathrm{~kW}+$ j66kVAr during the inrush phase of the motor start. After about 1.5 cycles, the motor settled down to approximately $61.8 \mathrm{~kW}+\mathrm{j} 56 \mathrm{kVAr}$ during the warm up phase which lasted about 39 cycles ( 0.65 Seconds). When the motor reached steady state, the load in Zone 3 was approximately $53 \mathrm{~kW}+\mathrm{j} 7.5 \mathrm{kVAr}$. 
The voltage and frequency at Meter 3 before the motor start was approximately $274 \mathrm{~V}$ on A-Phase, $274 \mathrm{~V}$ on B-Phase and $274 \mathrm{~V}$ on C-phase shown in Figure $7 \mathrm{k}$ and approximately $59.90 \mathrm{~Hz}$ shown in Figure 7l. When the motor started, the voltage at Meter 3 during the inrush decreased to approximately $254.8 \mathrm{~V}$ on A-Phase, $254.8 \mathrm{~V}$ on B-Phase and $254.8 \mathrm{~V}$ on C-Phase for about 1.5 cycles. Frequency dropped during the inrush to approximately $59.81 \mathrm{~Hz}$ and quickly increased to approximately $59.84 \mathrm{~Hz}$. Voltage increased as the motor was warming up and eventually settled at a steady state voltage at approximately $272 \mathrm{~V}$ on A-Phase, $272 \mathrm{~V}$ on B-Phase and $272 \mathrm{~V}$ on C-Phase at an approximate frequency of $59.87 \mathrm{~Hz}$.

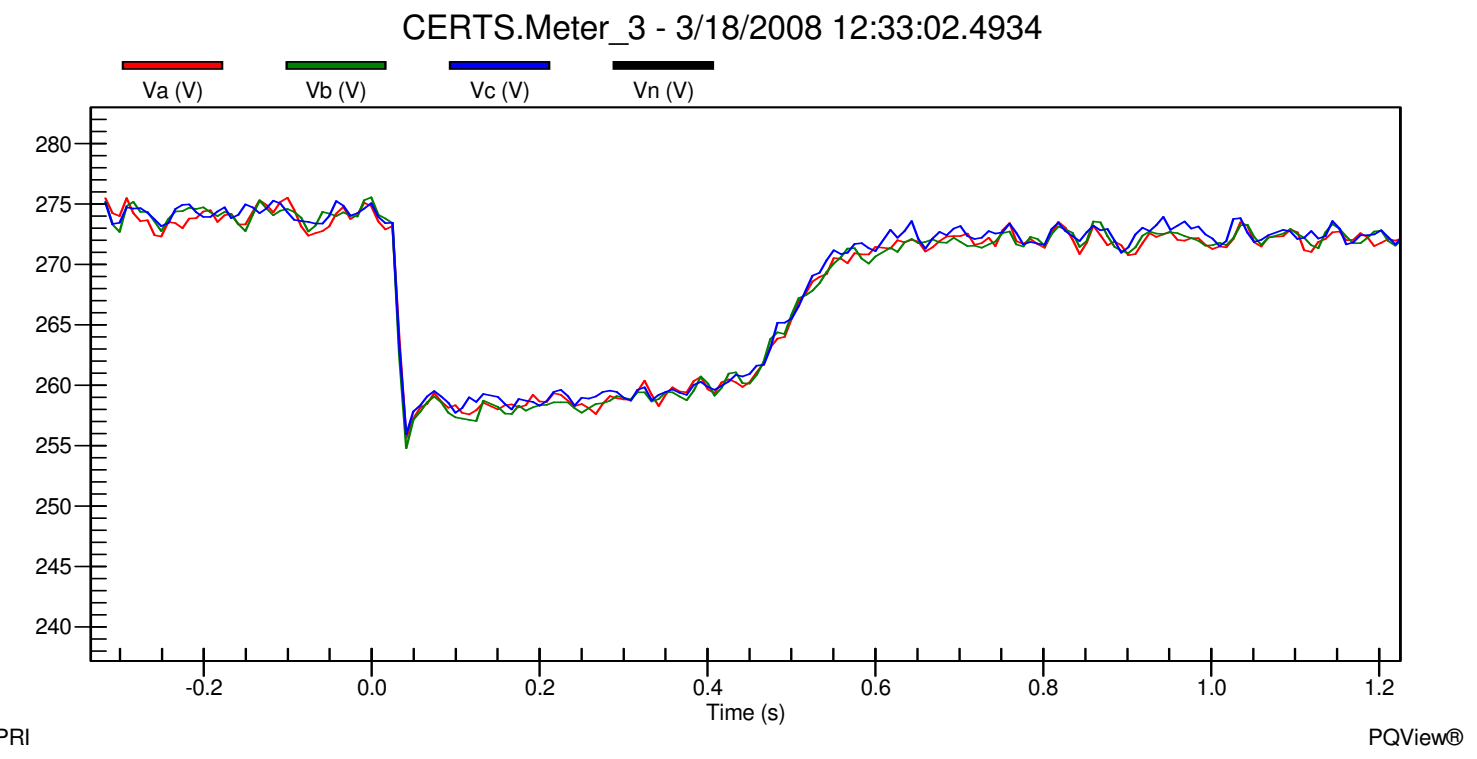

Figure 7k - Meter 3 Line-to-Ground Voltages during Motor Start and Islanded for Test 10.2.15 


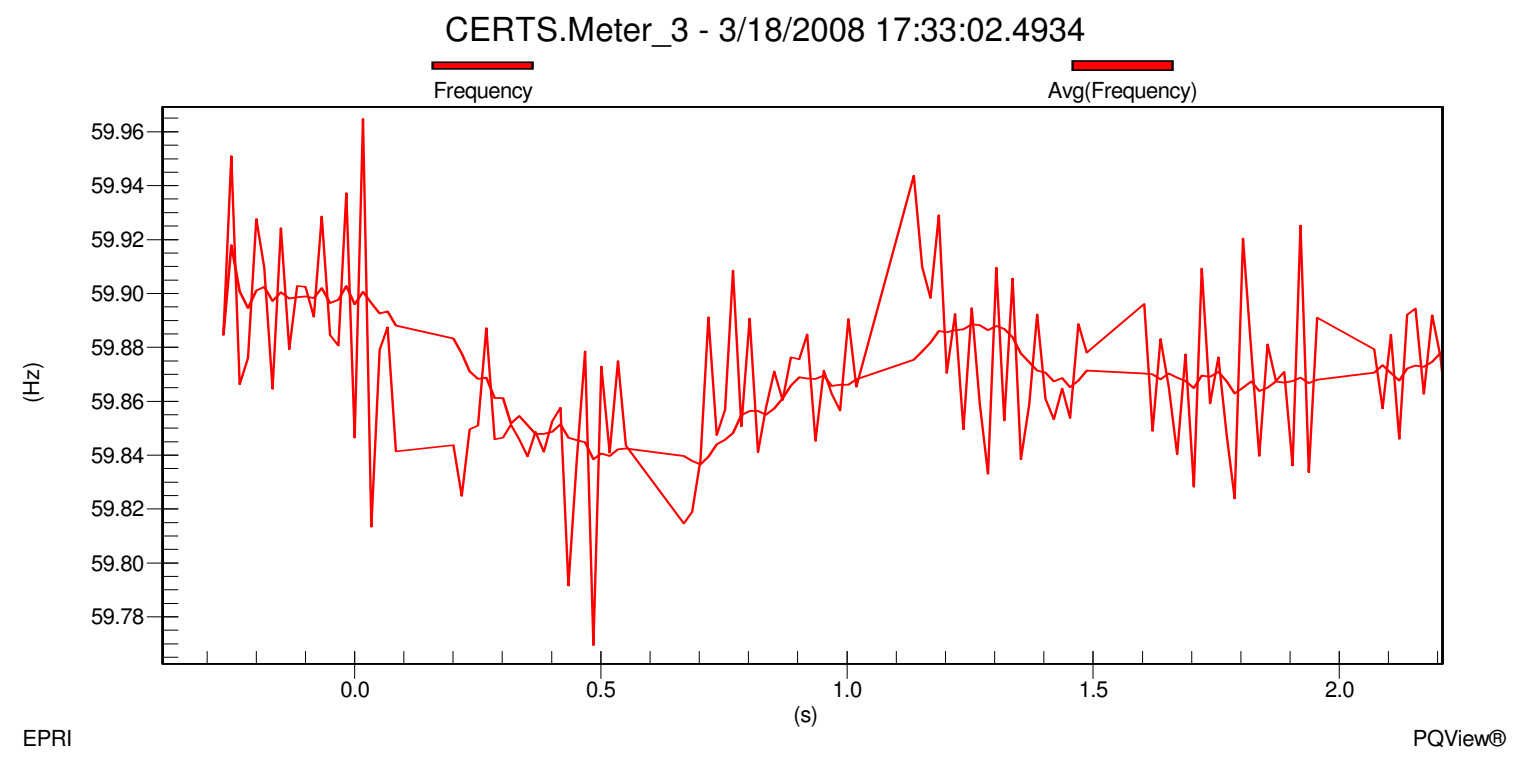

Figure 71 - Meter 3 Frequency during Motor Start and Islanded for Test 10.2.15

Before the motor started Gen-sets A1 and A2 were producing approximately $49.6 \mathrm{~kW}-$ $\mathrm{j} 2 \mathrm{kVAr}$ and $41.9 \mathrm{~kW}+\mathrm{j} 7 \mathrm{kVAr}$, respectively shown in Figures $7 \mathrm{~m}-7 \mathrm{p}$. The power generated by both Gen-sets was satisfying the loads in Load Banks 3 and 4 and all the electrical losses in the microgrid system. When the motor started the inrush caused the Gen-sets to increase their output levels to $59 \mathrm{~kW}+\mathrm{j} 30.7 \mathrm{kVAr}$ for Gen-set A1 and $52 \mathrm{~kW}+$ j38kVAr for Gen-set A2. The Gen-sets decreased their output levels while the motor was warming up and eventually dropped to $53.4 \mathrm{~kW}+\mathrm{j} 1 \mathrm{kVAr}$ for Gen-set A1 and $45.5 \mathrm{~kW}+$ j10.5 for Gen-set A2 when the motor reached steady state. Once all the data was verified and recorded into the DAS Database, the static switch was directed by the EMS to manually close. 


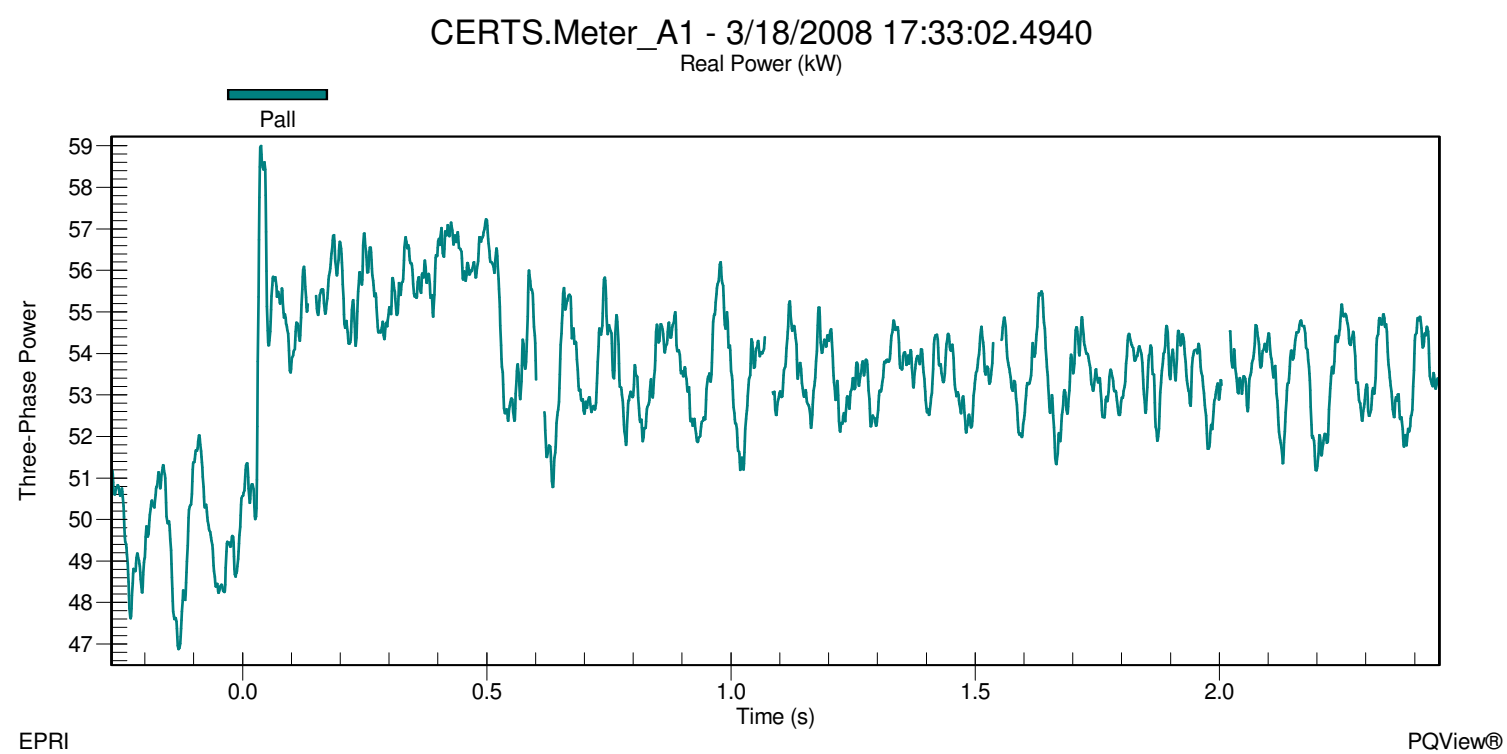

Figure 7m - Gen-set A1 Real Power during Motor Start and Islanded for Test 10.2.15

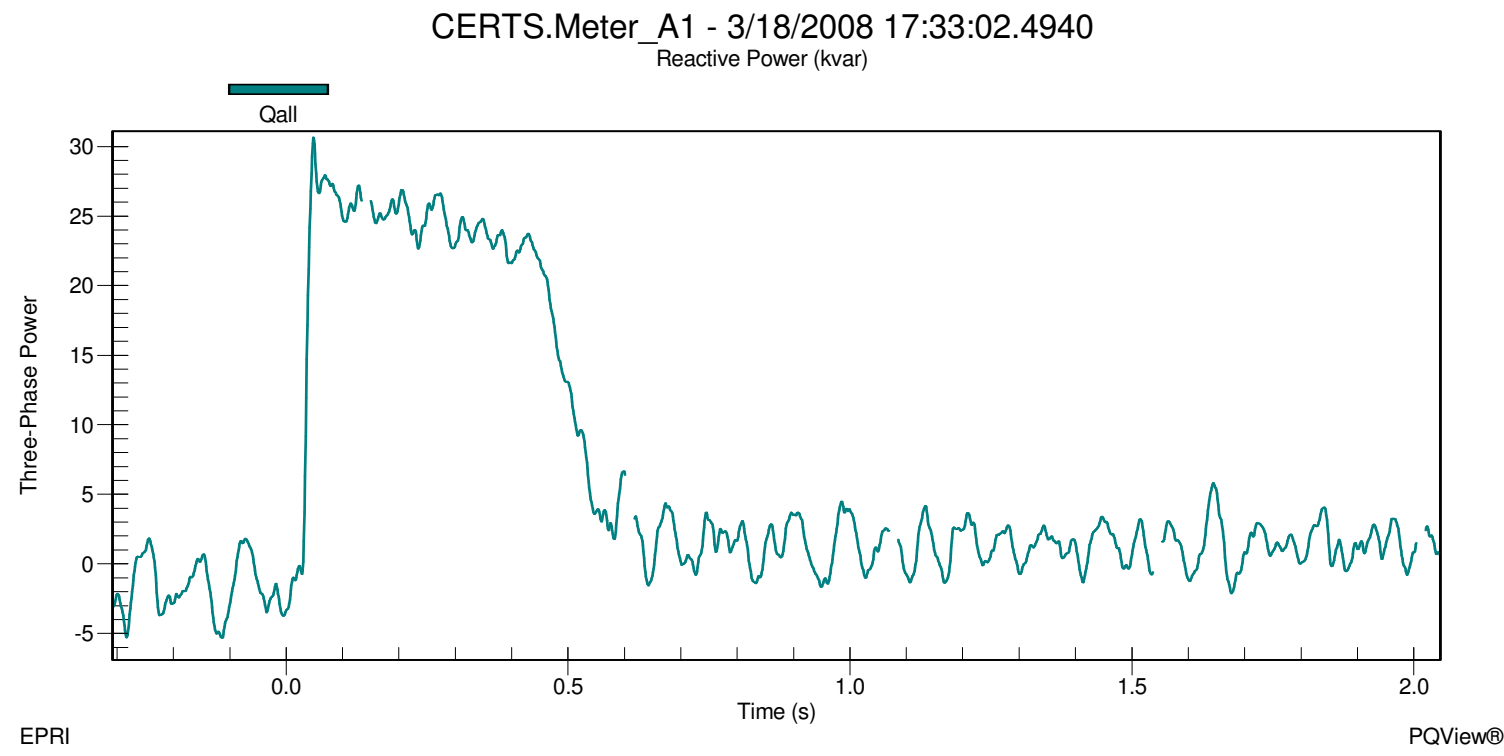

Figure 7n - Gen-set A1 Reactive Power during Motor Start and Islanded for Test 10.2.15 


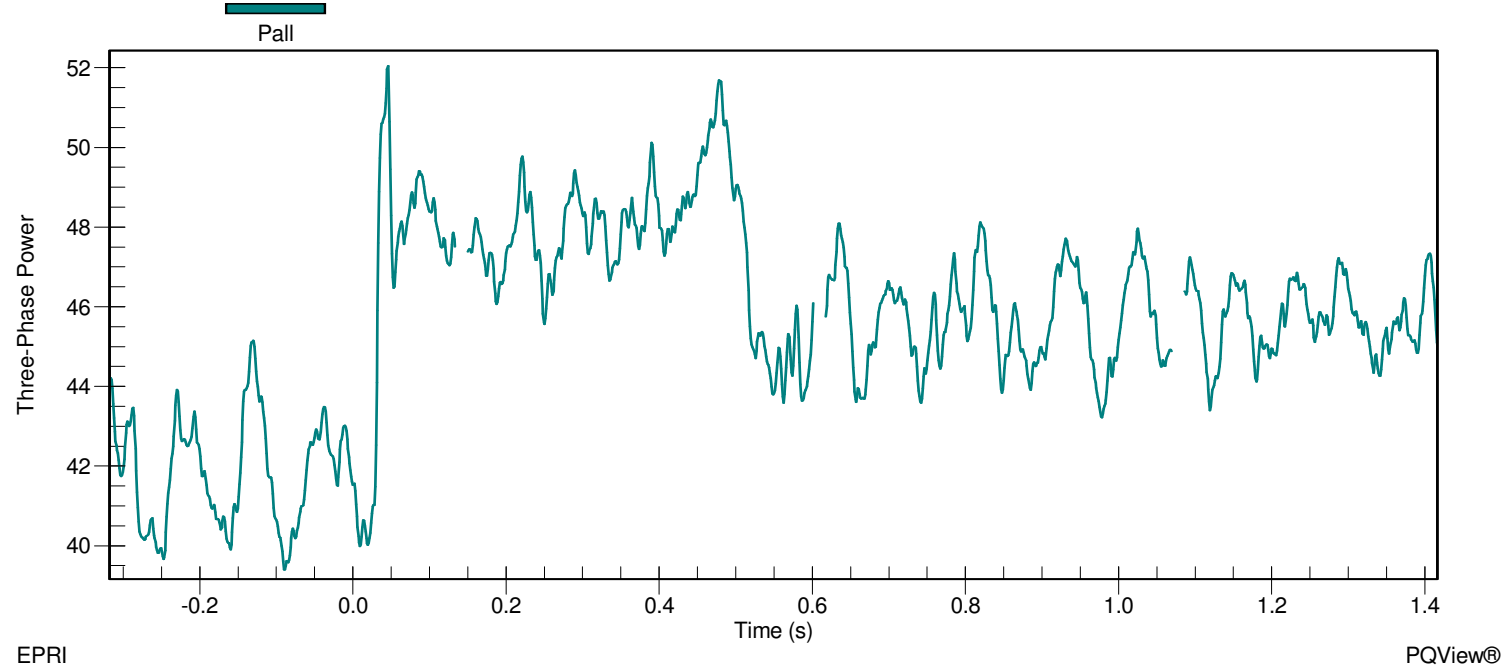

Figure 7o - Gen-set A2 Real Power during Motor Start and Islanded for Test 10.2.15

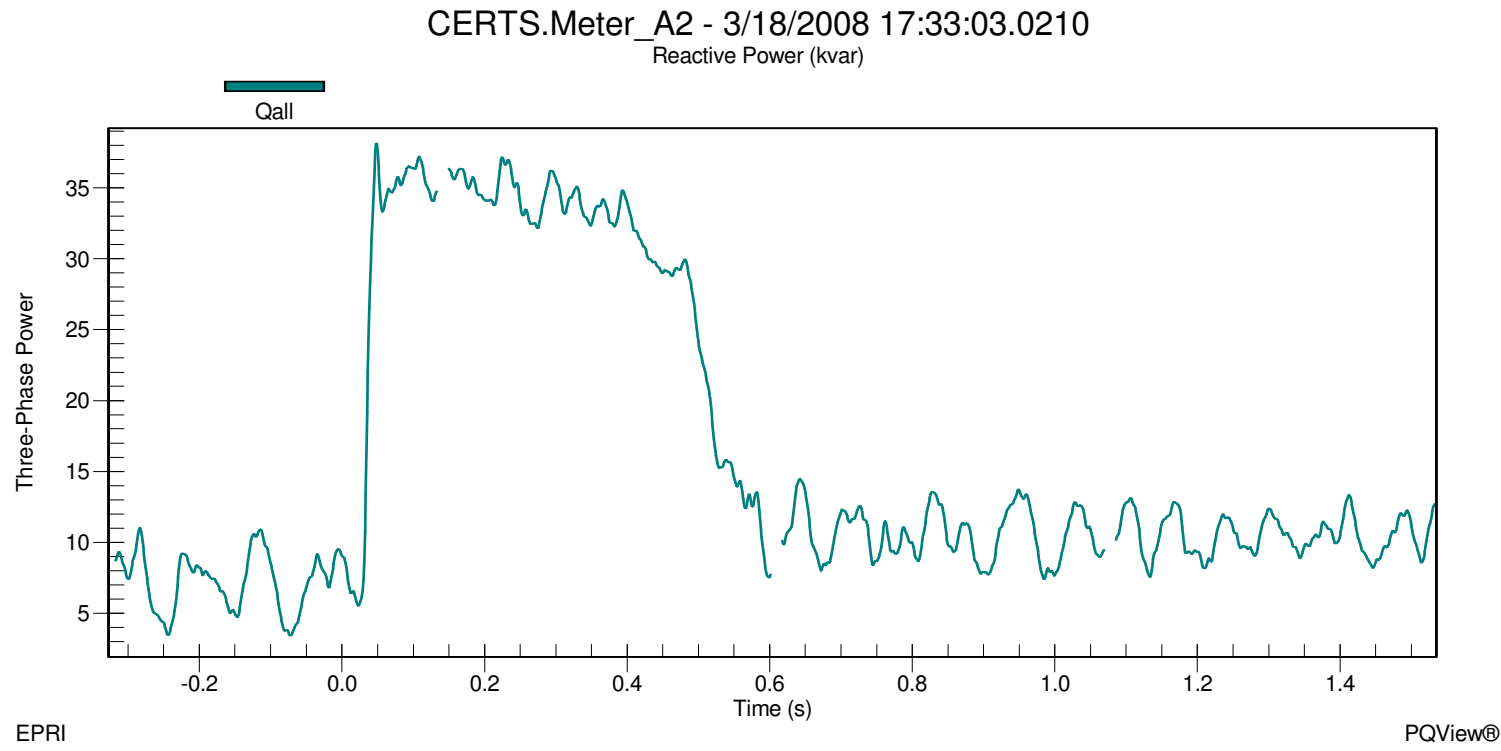

Figure 7p - Gen-set A2 Reactive Power during Motor Start and Islanded for Test 10.2.15

As soon as the static switch closed, Meter 1 recorded real and reactive powers increased from approximately $37 \mathrm{~kW}+\mathrm{j} 1.1 \mathrm{kVArto} 70 \mathrm{~kW}+\mathrm{j} 23 \mathrm{kAVr}$ which means that the utility was satisfying not only the load demand of Load Bank 6 but also all the reactive power and a portion of the real power for the microgrid loads. Figures $7 \mathrm{q}$ and $7 \mathrm{r}$ show the static switch increasing from approximately $0 \mathrm{~kW}+\mathrm{j} 0 \mathrm{kVAr}$ to $34.5 \mathrm{~kW}+\mathrm{j} 21 \mathrm{kVAr}$. At the beginning of the test, the initial power flow through the static switch was $28 \mathrm{~kW}+$ j16kVAr which is not the same recorded at this point in the test because the $10 \mathrm{Hp}$ motor load is on in Zone 3. The Gen-sets A1 and A2 output power levels reduced to 
approximately $37.2 \mathrm{~kW}-\mathrm{j} 9.5 \mathrm{kVAr}$ and $30 \mathrm{~kW}+\mathrm{j} 1.5 \mathrm{kVAr}$, respectively, which are relatively close to the initial values at the beginning of the test. Since the motor is on in Zone 3 and the Gen-sets returned to their initial values at the beginning of the test before the motor was turned on means that the induction motor is completely being powered by the utility.

Load Banks 3 and 4 loads increased slightly to $53 \mathrm{~kW}+\mathrm{j} 6.7 \mathrm{kVAr}$ and $45.7 \mathrm{~kW}+$ j0.707kVAr, respectively. This slight load increase is a result from a voltage rise in the microgrid, shown in Figure $7 \mathrm{~s}$, from approximately $272.5 \mathrm{~V}$ on A-phase, 272.5V on Bphase and $272.5 \mathrm{~V}$ on C-phase when islanded to $280 \mathrm{~V}$ on A-phase, $278 \mathrm{~V}$ on B-phase and $278 \mathrm{~V}$ on C-phase at Meter 3 when connected to the utility grid.

CERTS.Meter_2 - 3/18/2008 17:38:32.3902
Real Power (kW)

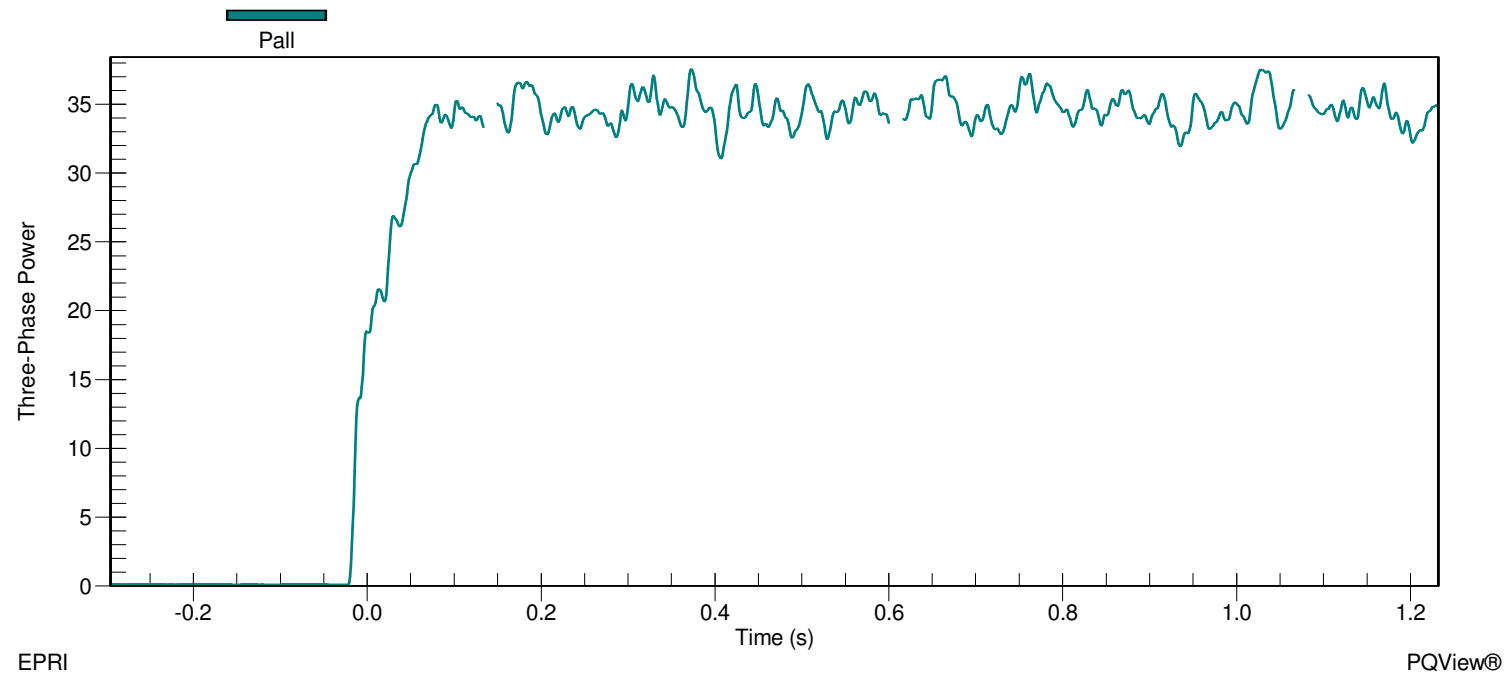

Figure 7q - Static Switch Real Power during Island to Utility Connected mode for Test 10.2.15 


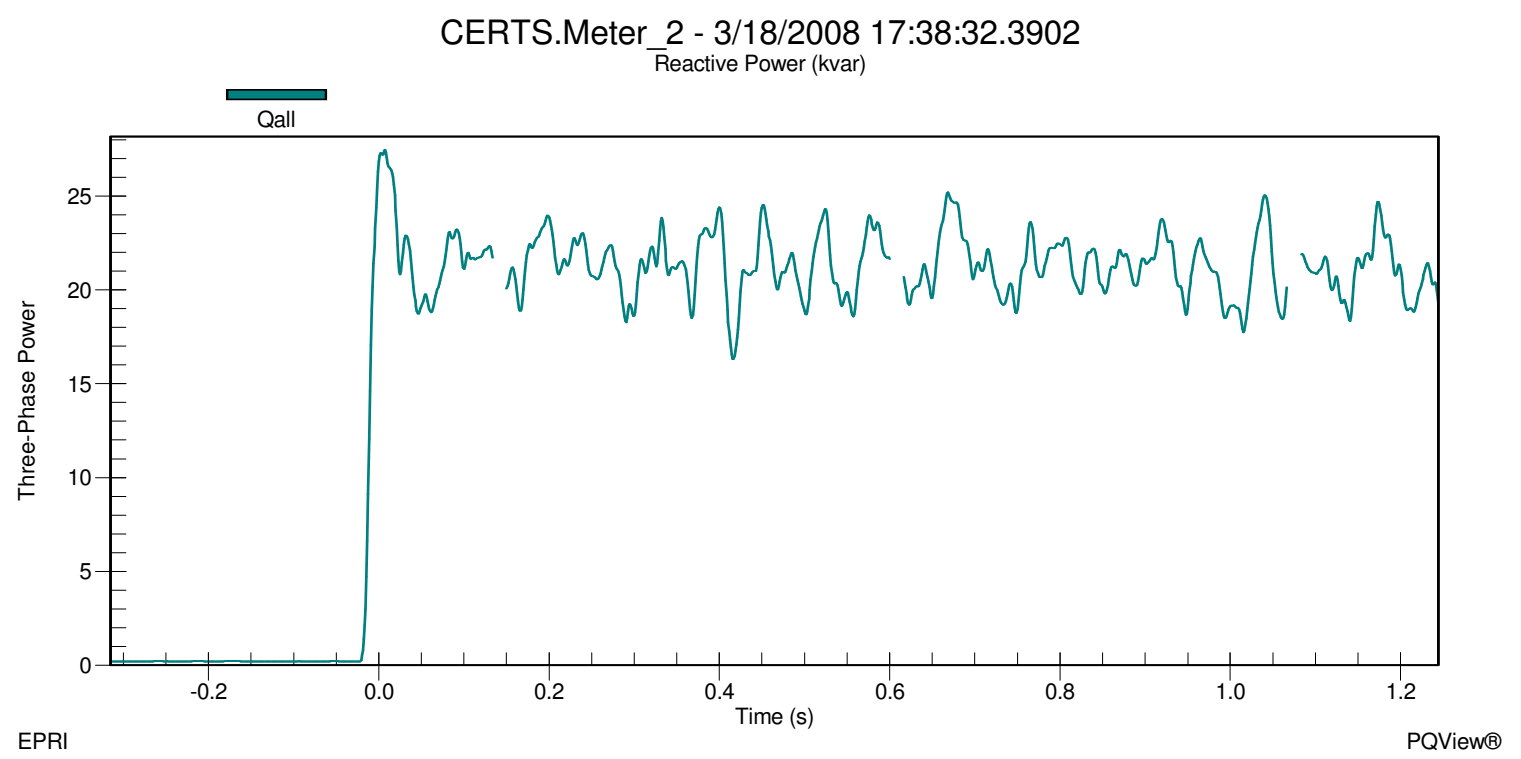

Figure 7r - Static Switch Reactive Power during Island to Utility Connected mode for Test 10.2.15

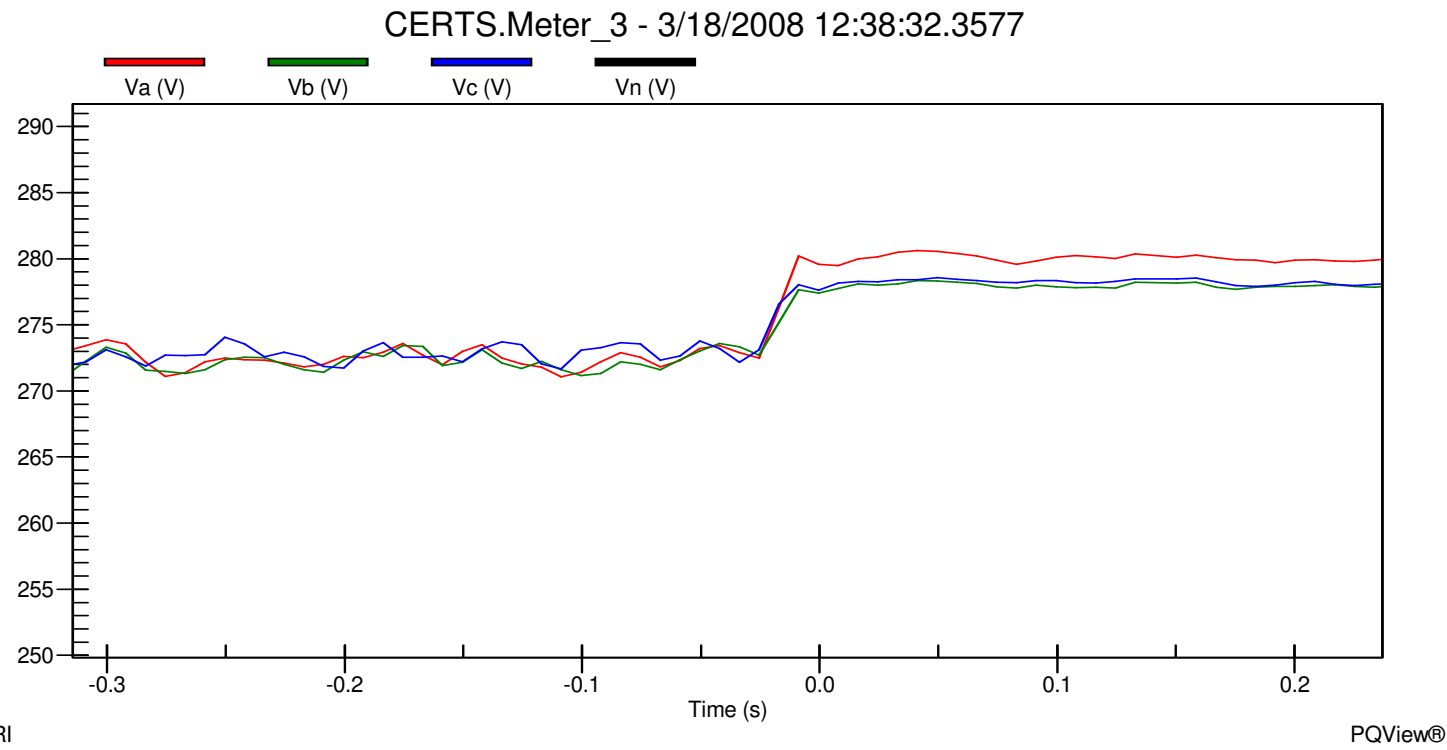

Figure 7s - Meter 3 Line-to-Ground Voltages during Island to Utility Connected mode for Test 10.2.15 


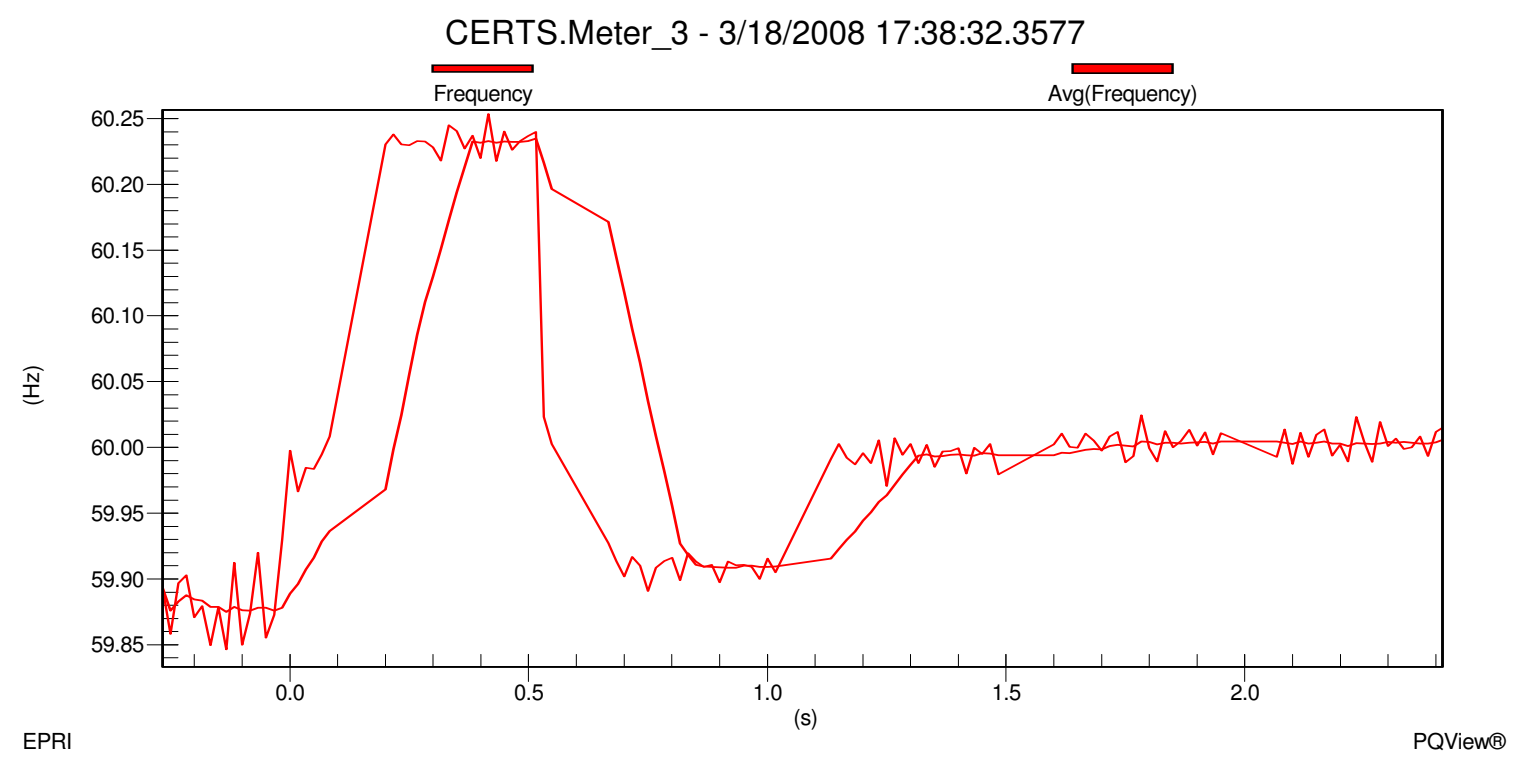

Figure $7 \mathrm{t}$ - Meter 3 Frequency during Island to Utility Connected mode for Test 10.2.15

Frequency change in the microgrid, shown in Figure $7 \mathrm{t}$, increased from approximately $59.88 \mathrm{~Hz}$ when islanded to approximately $60.00 \mathrm{~Hz}$ when connected to the utility grid. This change in frequency is due to the frequency no longer being established by the Gensets using the CERTS algorithm but by the utility. Gen-sets A1 and A2 are in unit power control mode therefore when the static switch closed back into the utility the Gen-sets produced real power based on their set-points initialized at the beginning of the test. After all the data was verified and recorded into the DAS Database, the motor was turned off and the Gen-sets and Load Banks set-points were changed according to the next test (10.2.17) in Table 1.

For Test 10.2.17 the measured values, after Gen-set A1 was warmed up and load banks brought on-line, were approximately $19 \mathrm{~kW}+\mathrm{j} 20 \mathrm{kVAr}$ at Meter $1,-17 \mathrm{~kW}+\mathrm{j} 19.8 \mathrm{kVAr}$ at Meter 2 and $-20 \mathrm{~kW}+\mathrm{j} 14 \mathrm{kVAr}$ at Meter 3. From the microgrid, $38.5 \mathrm{~kW}-\mathrm{j} 13 \mathrm{kVAr}$ was produced by Gen-set A1. The Load Banks were 18.2kW + j0kVAr at LB3 and 35.92kW + j0.78kVAr at LB6. At the time of these measurements, the voltage and frequency was $282.6 \mathrm{~V}$ on A-phase, $279.2 \mathrm{~V}$ on B-phase and $279.4 \mathrm{~V}$ on C-phase and $60.00 \mathrm{~Hz}$ at the static switch (i.e., Meter 2) when connected to the utility grid; and $282.6 \mathrm{~V}$ on A-phase, $280 \mathrm{~V}$ on B-phase and 280V on C-phase at Meter 3.

Gen-set A1 was setup in this test to produce more power than Load Bank 3 needed which approximately $17 \mathrm{~kW}$ of excess power was exported through the static switch to Load Bank 6. Since Load Bank 6 was approximately $35.92 \mathrm{~kW}+\mathrm{j} 0.78 \mathrm{kVAr}$, the utility had to supply approximately $19 \mathrm{~kW}$ to satisfy the load. Reactive power had to be imported in from the utility of approximately 19.8kVAr because Gen-set A1 needed 
approximately $13 \mathrm{kVAr}$ and the reactive power absorbed in the electrical lines. Once all data was verified and recorded into the DAS Database, the $10 \mathrm{Hp}$ induction motor was started in Zone 3.

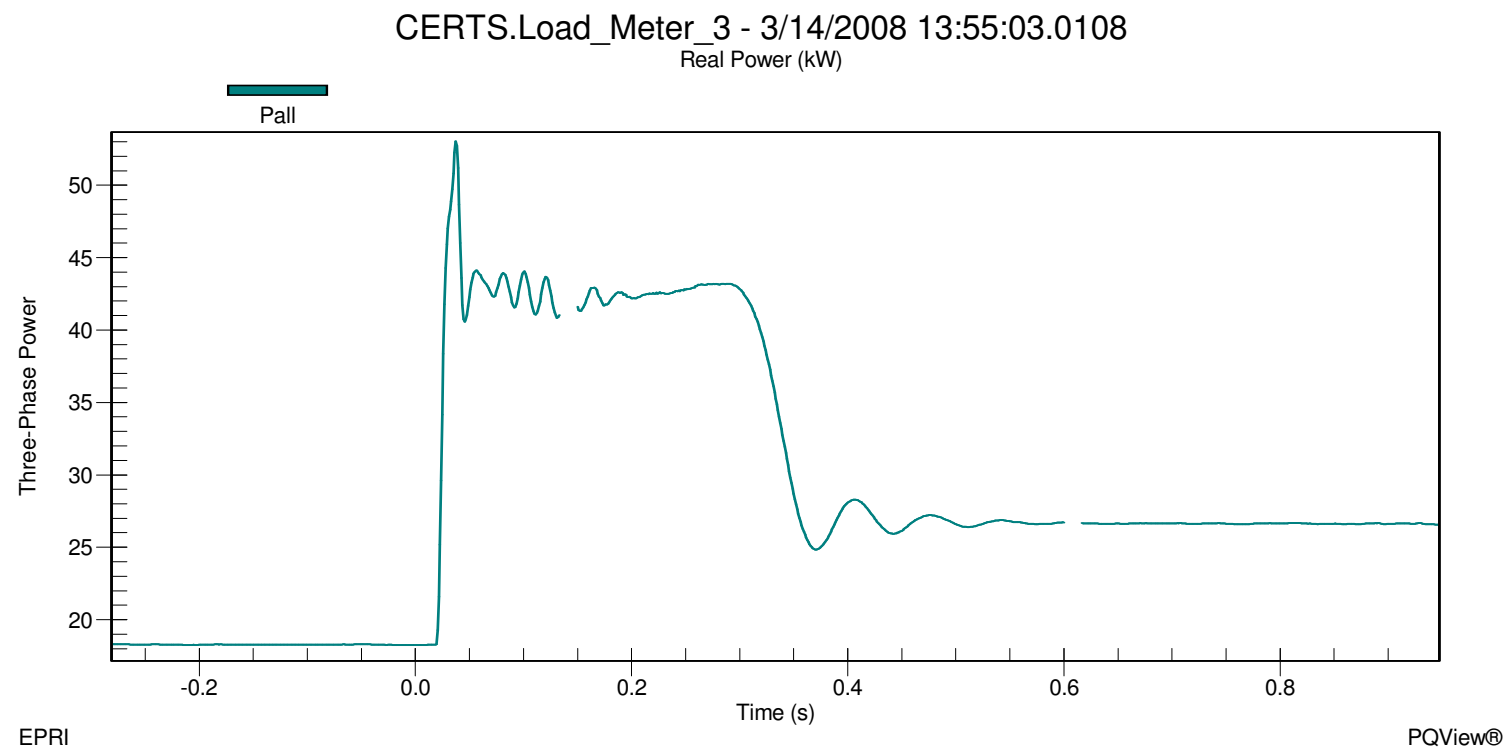

Figure 8a - Load Bank 3 Real Power during Motor Start and Utility Connected for Test 10.2.17

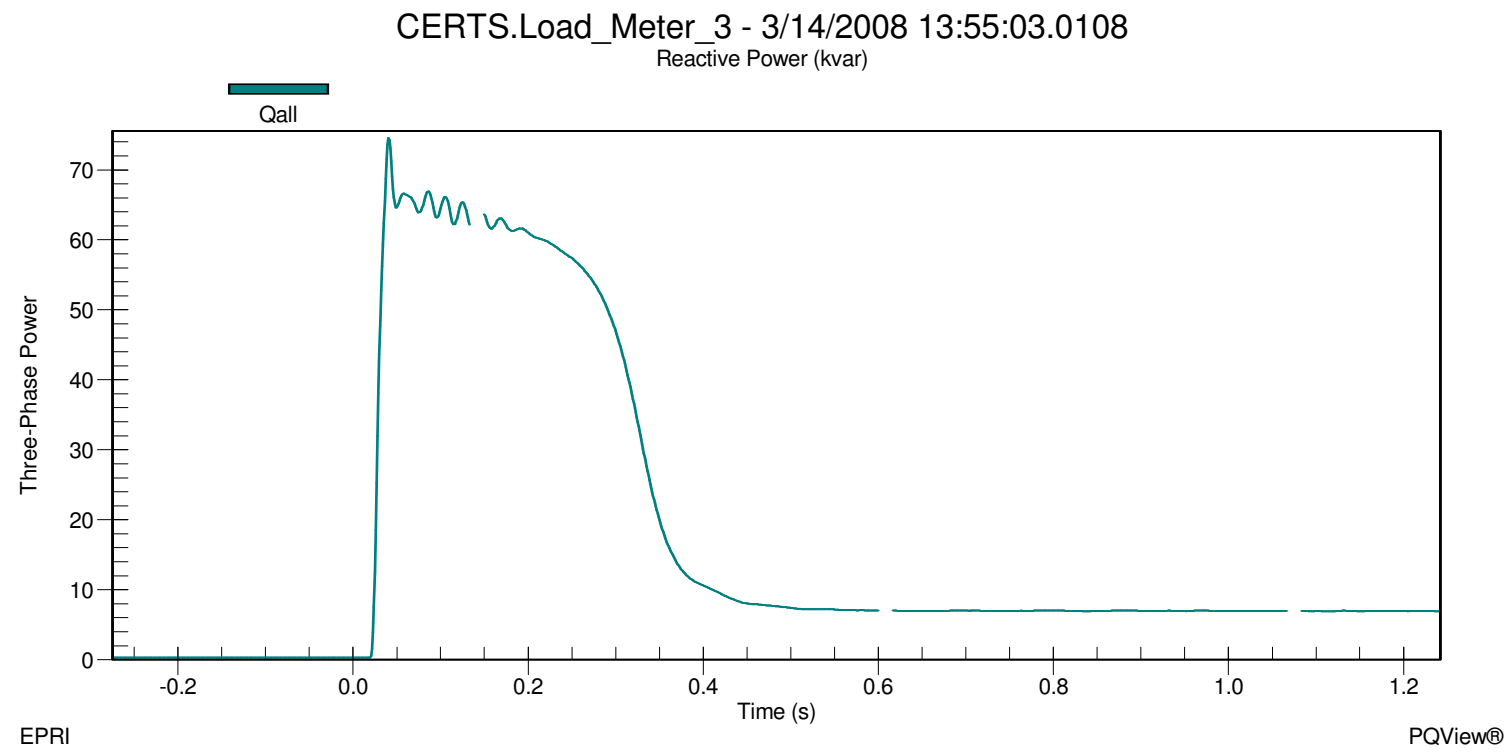

Figure 8b - Load Bank 3 Reactive Power during Motor Start and Utility Connected for Test 10.2.17

In Figures $8 \mathrm{a}$ and $8 \mathrm{~b}$ it can be seen that the load in Zone 3 was approximately $18.2 \mathrm{~kW}+$ j0kVAr before the start of the induction motor and increased to approximately $53 \mathrm{~kW}+$ j75kVAr during the inrush phase of the motor start. After about 1.5 cycles, the motor 
settled down to approximately $42.8 \mathrm{~kW}+\mathrm{j} 62 \mathrm{kVAr}$ during the warm up phase which lasted about 27 cycles ( 0.45 Seconds). When the motor reached steady state, the load in Zone 3 was approximately $26.7 \mathrm{~kW}+\mathrm{j} 7 \mathrm{kVAr}$.

The voltage and frequency at the static switch before the motor start was approximately 282.6V on A-phase, $279.2 \mathrm{~V}$ on B-phase and $279.4 \mathrm{~V}$ on C-phase shown in Figure 8c and approximately $60.00 \mathrm{~Hz}$ shown in Figure $8 \mathrm{~d}$. When the motor started, the voltage at the static switch during the inrush decreased to approximately $274.4 \mathrm{~V}$ on A-phase, $270.9 \mathrm{~V}$ on B-phase and $271.2 \mathrm{~V}$ on C-phase for about 1.5 cycles. Frequency dropped during the inrush to approximately $59.90 \mathrm{~Hz}$. Voltage increased as the motor was warming up and eventually settled at a steady state voltage at approximately $281.6 \mathrm{~V}$ on A-phase, $278.2 \mathrm{~V}$ on B-phase and $278.8 \mathrm{~V}$ on C-phase at an approximate frequency of $60.00 \mathrm{~Hz}$.

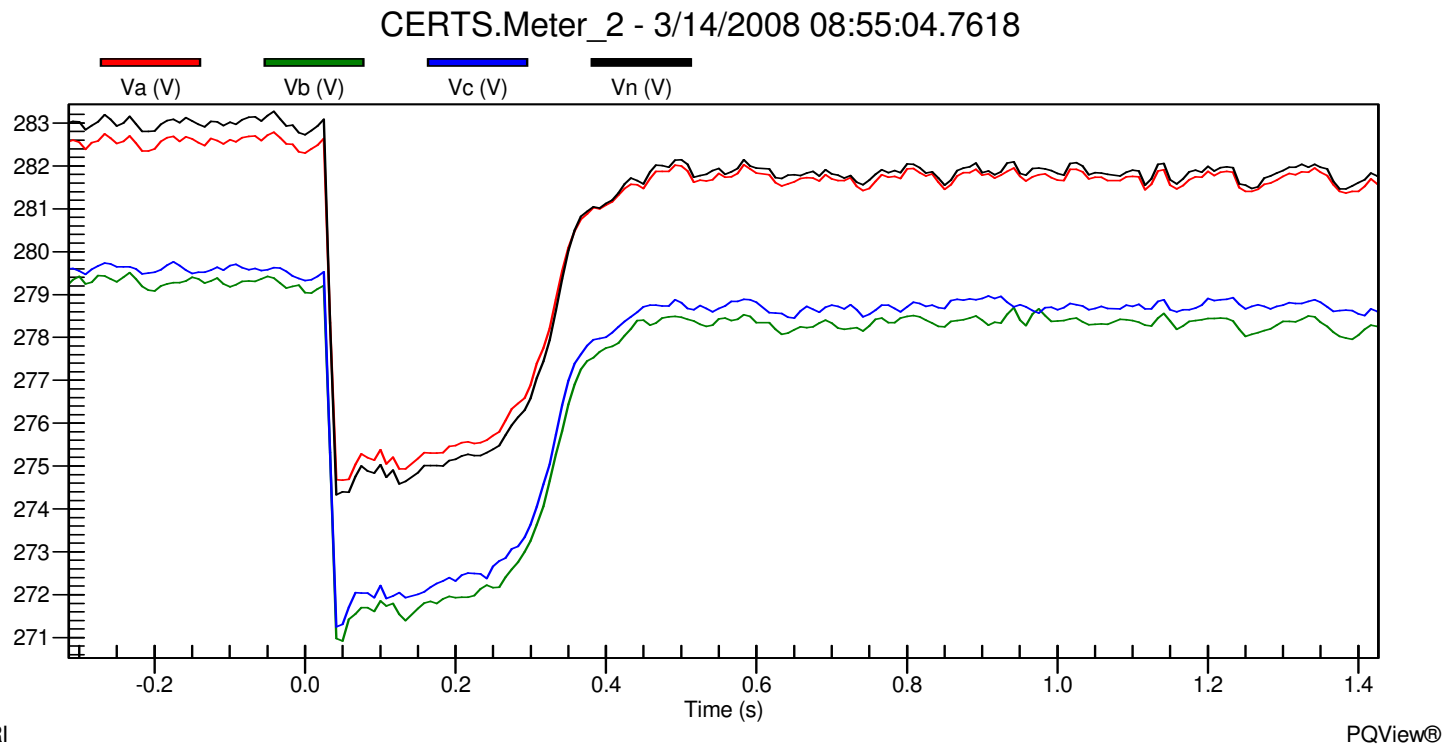

Figure 8c - Static Switch Line-to-Ground Voltages during Motor Start and Utility Connected for Test 10.2.17 


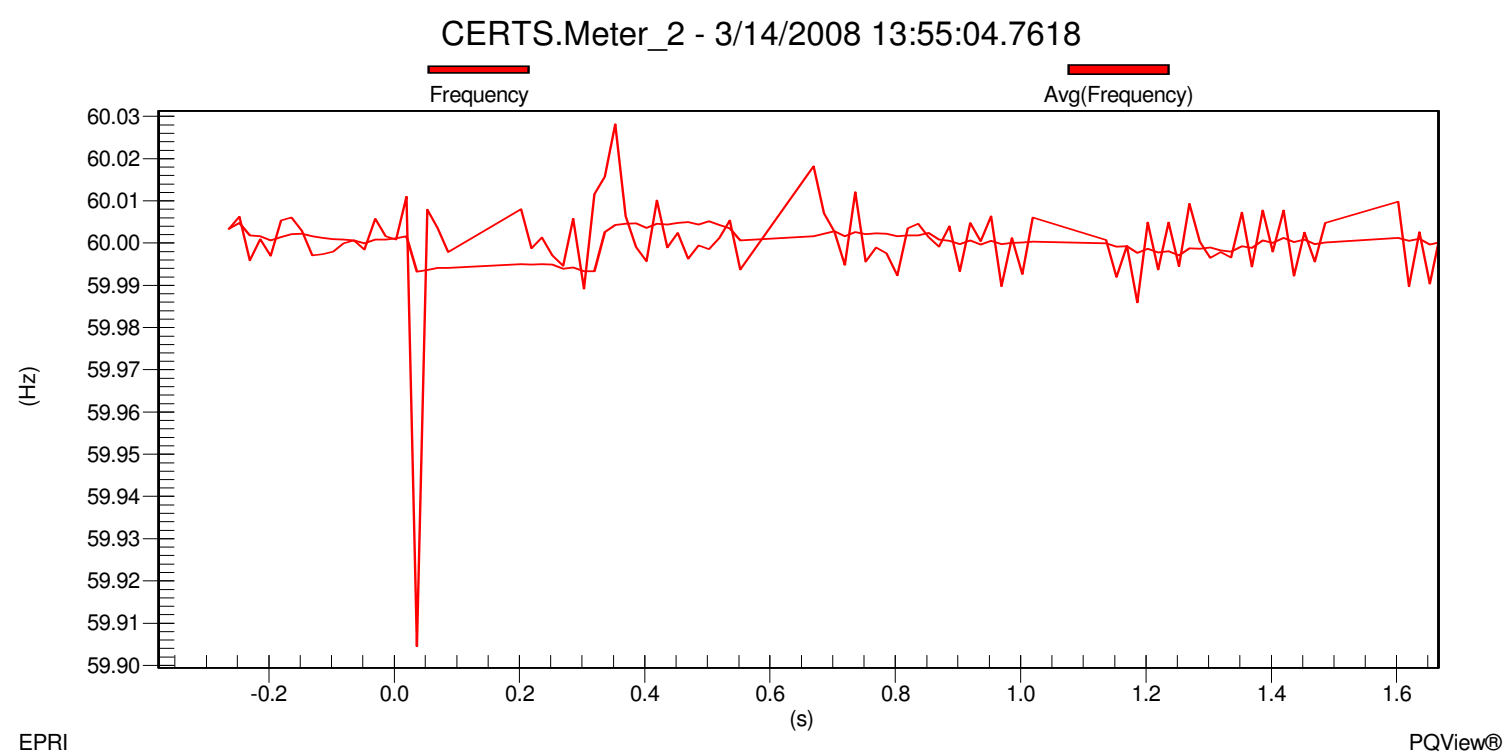

Figure 8d - Static Switch Frequency during Motor Start and Utility Connected for Test 10.2.17

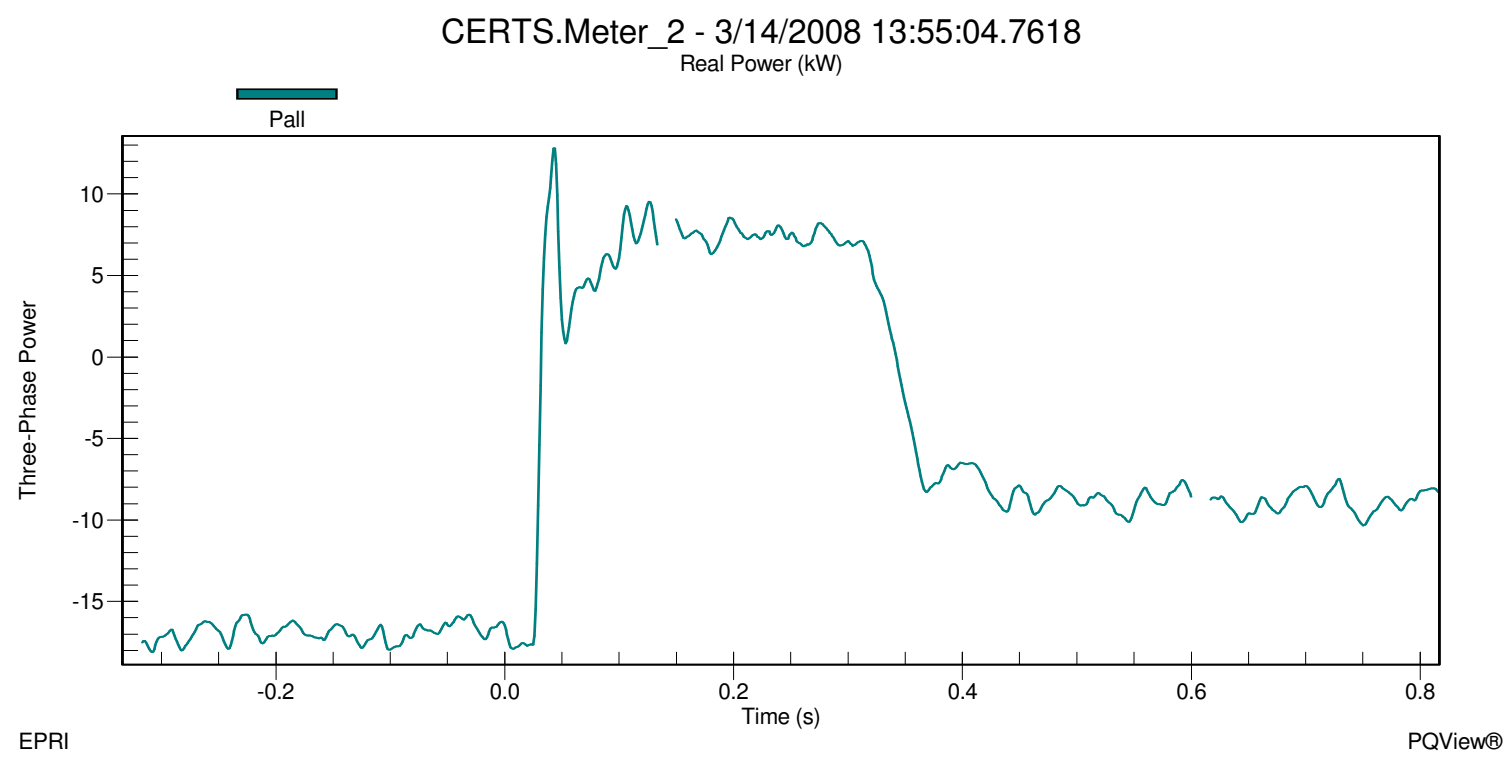

Figure 8e - Static Switch Real Power during Motor Start and Utility Connected for Test 10.2.17 


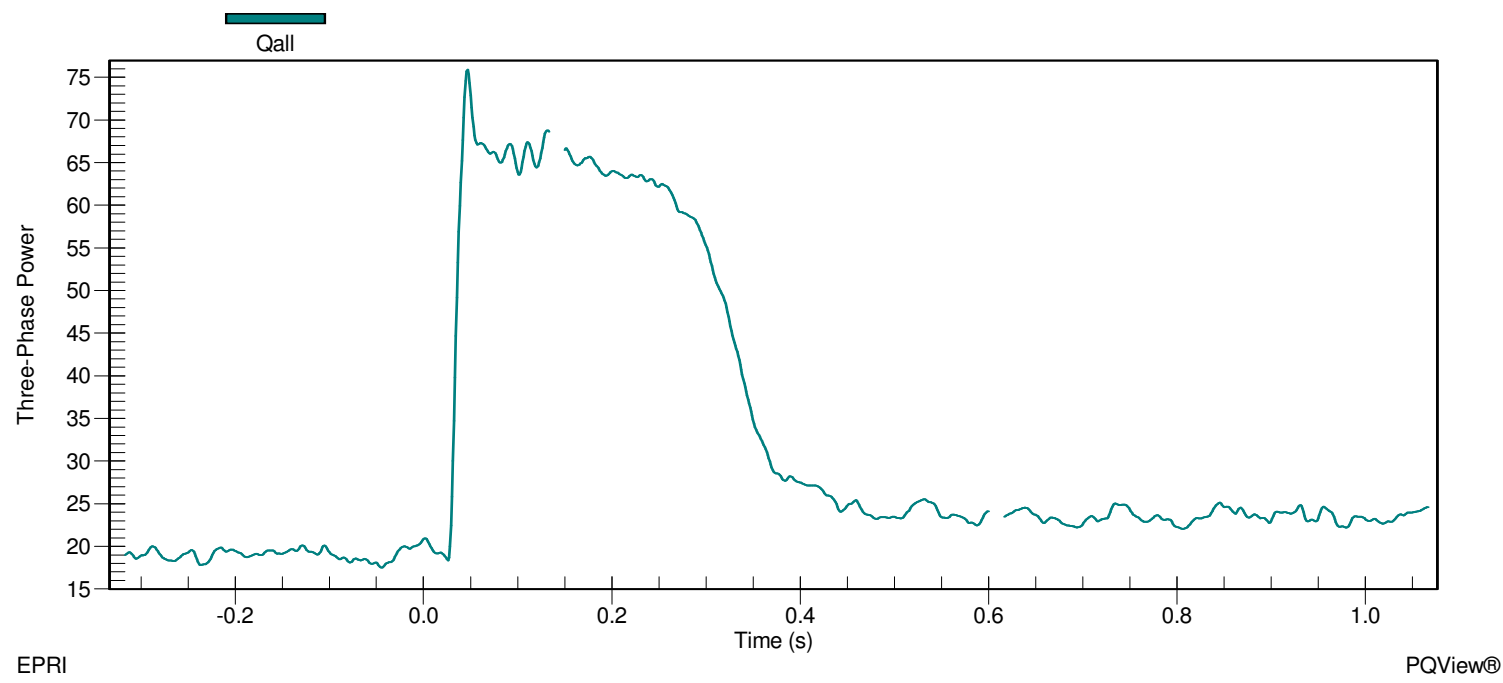

Figure 8f - Static Switch Reactive Power during Motor Start and Utility Connected for Test 10.2.17

Before the motor start Gen-set A1 was producing approximately $38.5 \mathrm{~kW}-\mathrm{j} 13 \mathrm{kVAr}$. This was enough for the load demands of Zone 3 with real power being exported to Zone 6 of approximately 17kW shown in Figure 8e. The grid was supporting the reactive power of the microgrid with approximately 19.8 shown in Figure $8 \mathrm{f}$. When the motor started the inrush caused the utility to supply approximately $76 \mathrm{kVAr}$ and to pick up the load demand in Load Bank 6 along with approximately $13 \mathrm{~kW}$ of the load in Zone 3. The power being supplied by Gen-set A1 to Load Bank 6 was now supplying the motor inrush which can be seen in Figure 8e as the real power through the static switch becomes positive. Gen-set A1 increased its real and reactive power output level to approximately $44 \mathrm{~kW}+\mathrm{j} 4.6 \mathrm{kVAr}$. Notice the sign of the VAr output changed from negative to positive for Gen-set A1 in order to support the induction motor load in Zone 3.

When the motor reached steady state, the real and reactive power through the static switch was approximately $-9 \mathrm{~kW}+\mathrm{j} 23 \mathrm{kVAr}$ which meant that Gen-set A1 was supplying the power for the induction motor and approximately $9 \mathrm{~kW}$ of Load Bank 6. Gen-set A1 real power returned to the value before the motor started of approximately $38.5 \mathrm{~kW}$ and the reactive power increased to approximately $-11.4 \mathrm{kVAr}$. Once all the data was verified and recorded into the DAS Database, the motor was shut down and the static switch was directed by the EMS to manually open.

As soon as the static switch opened, Meter 1 recorded real power increased to approximately $36.9 \mathrm{~kW}$ and reactive power decreased to $1 \mathrm{kVAr}$ satisfying the load demand in Load Bank6 which was approximately $36.82 \mathrm{~kW}+\mathrm{j} 0.787 \mathrm{kVAr}$ and not 
supplying any power beyond the static switch to Load Bank 3. 0kW + j0kVAr was recorded at the static switch, indicating that power was not flowing through the static switch. Load Bank 3 loads was slightly reduced to $23.3 \mathrm{~kW}+\mathrm{j} 5.9 \mathrm{kVAr}$. This load reduction resulted from a voltage drop in the microgrid, shown in Figure $8 \mathrm{~g}$, from approximately $282 \mathrm{~V}$ on A-phase, $279 \mathrm{~V}$ on B-phase and $279 \mathrm{~V}$ on C-phase when connected to the utility grid to $269 \mathrm{~V}$ on A-phase, $268 \mathrm{~V}$ on B-phase, and $269 \mathrm{~V}$ on C-phase at Meter 3 when islanded.

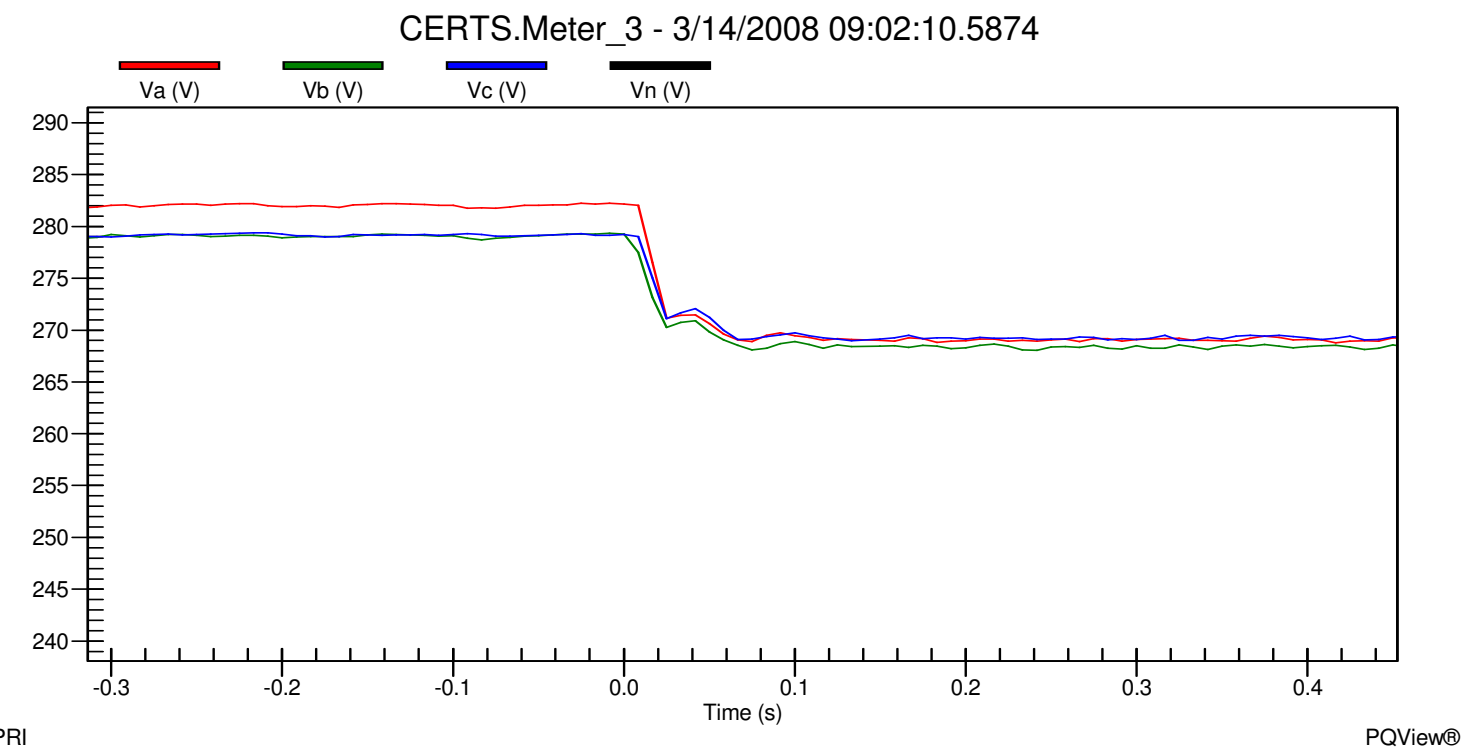

Figure 8g - Meter 3 Line-to-Ground Voltages during Utility Connected to Island mode for Test 10.2.17

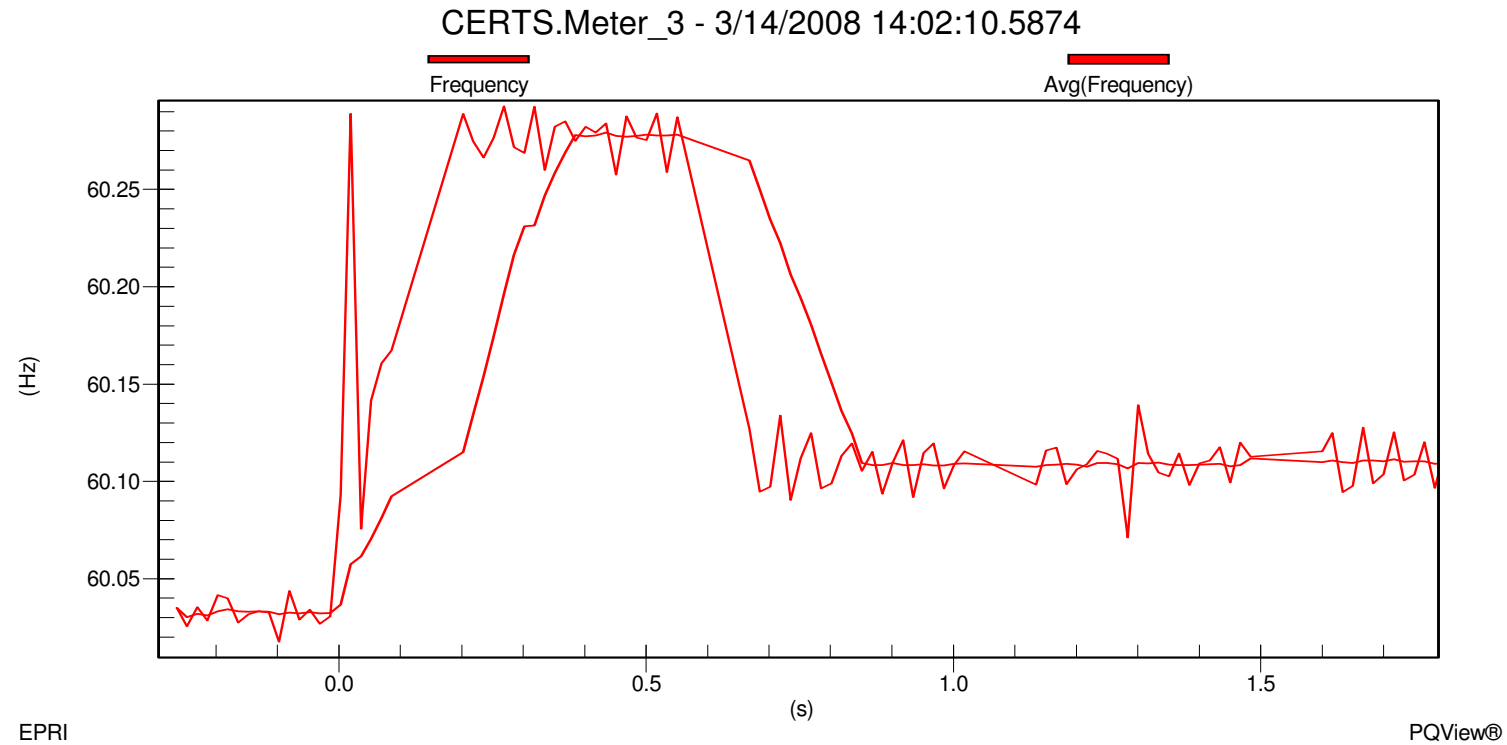

Figure 8h - Meter 3 Frequency during Utility Connected to Island mode for Test 10.2.17 
Frequency change in the microgrid, shown in Figure 8h, increased from approximately $60.03 \mathrm{~Hz}$ when connected to the utility grid to approximately $60.11 \mathrm{~Hz}$ when islanded. This change in frequency was part of the CERTS algorithm which allowed Gen-set A1 to decrease its output power to satisfy the load demands. Gen-set A1 decreased its output power to approximately $25.5 \mathrm{~kW}$ and increased its output reactive power to approximately 9kVAr. Meter 3 was approximately $-2.3 \mathrm{~kW}-\mathrm{j} 3.0 \mathrm{kVAr}$ indicating that Gen-set A1 was satisfying the load in Load Bank 3 and the power losses in the electrical lines. All data was verified and recorded into the DAS Database. The microgrid ran for a couple of minutes in this electrical state before the $10 \mathrm{Hp}$ induction motor was started in Zone 3.

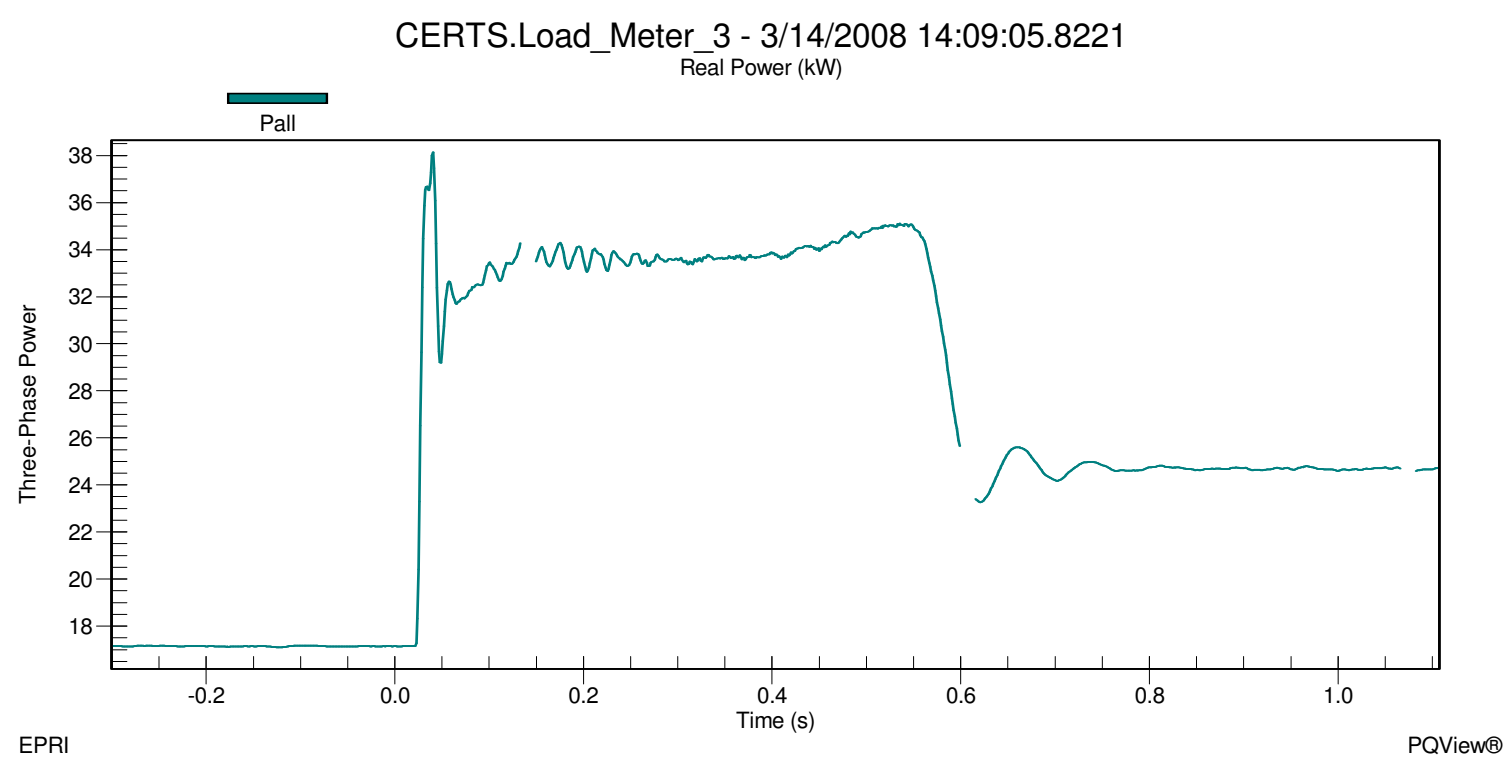

Figure 8i - Load Bank 3 Real Power during Motor Start and Islanded for Test 10.2.17 


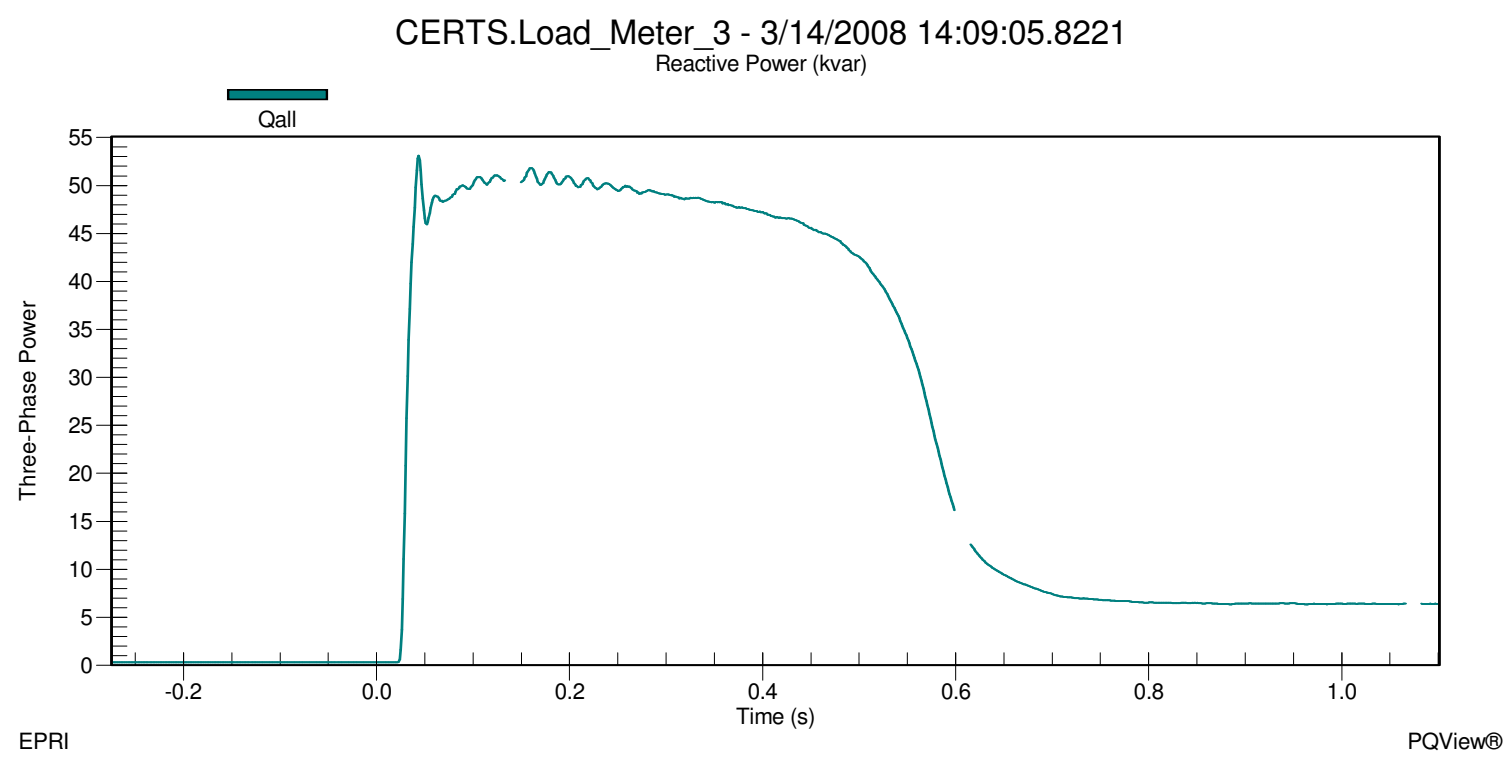

Figure 8j - Load Bank 3 Reactive Power during Motor Start and Islanded for Test 10.2.17

In Figures $8 \mathrm{i}$ and $8 \mathrm{j}$ it can be seen that the load in Zone 3 was approximately $16.4 \mathrm{~kW}+$ j0.2kVAr before the start of the induction motor and increased to approximately $38 \mathrm{~kW}+$ j53kVAr during the inrush phase of the motor start. After about 1.5 cycles, the motor settled down to approximately $33.5 \mathrm{~kW}+\mathrm{j} 49 \mathrm{kVAr}$ during the warm up phase which lasted about 43.8 cycles ( 0.73 Seconds). When the motor reached steady state the load in Zone 3 was approximately $24.6 \mathrm{~kW}+\mathrm{j} 6.5 \mathrm{kVAr}$.

The voltage and frequency at Meter 3 before the motor start was approximately $272 \mathrm{~V}$ on A-phase, 272V on B-phase and 272V on C-phase shown in Figure 8k and approximately $60.18 \mathrm{~Hz}$ shown in Figure 81. When the motor started, the voltage at Meter 3 during the inrush decreased to approximately $238.5 \mathrm{~V}$ on A-phase, $237.5 \mathrm{~V}$ on B-phase and $238.5 \mathrm{~V}$ on C-phase for about 1.5 cycles. Frequency dropped during the inrush to approximately $59.75 \mathrm{~Hz}$ and quickly increased to approximately $60.03 \mathrm{~Hz}$. Voltage increased as the motor was warming up and eventually settled at a steady state voltage at approximately $269 \mathrm{~V}$ on A-phase, $268 \mathrm{~V}$ on B-phase and $269 \mathrm{~V}$ on C-phase at an approximate frequency of $60.10 \mathrm{~Hz}$. 


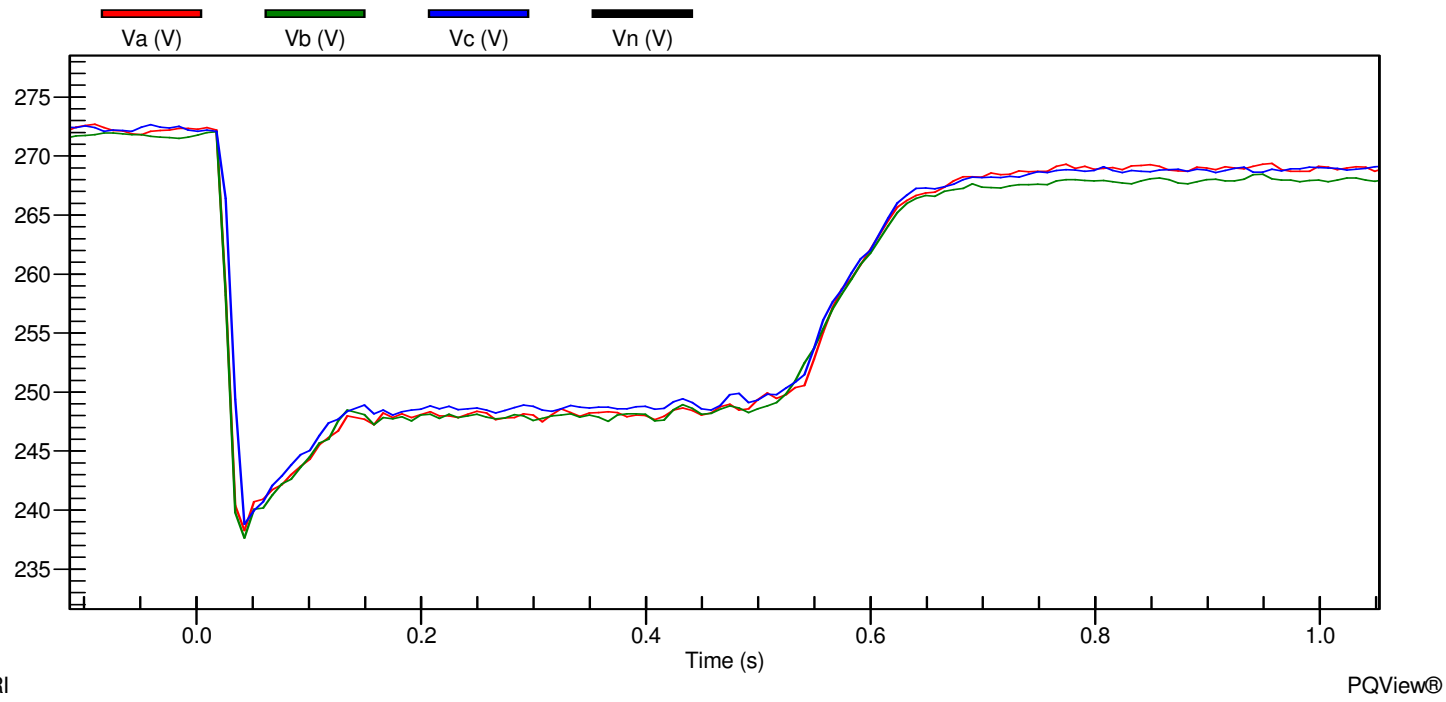

Figure 8k - Meter 3 Line-to-Ground Voltages during Motor Start and Islanded for Test 10.2.17

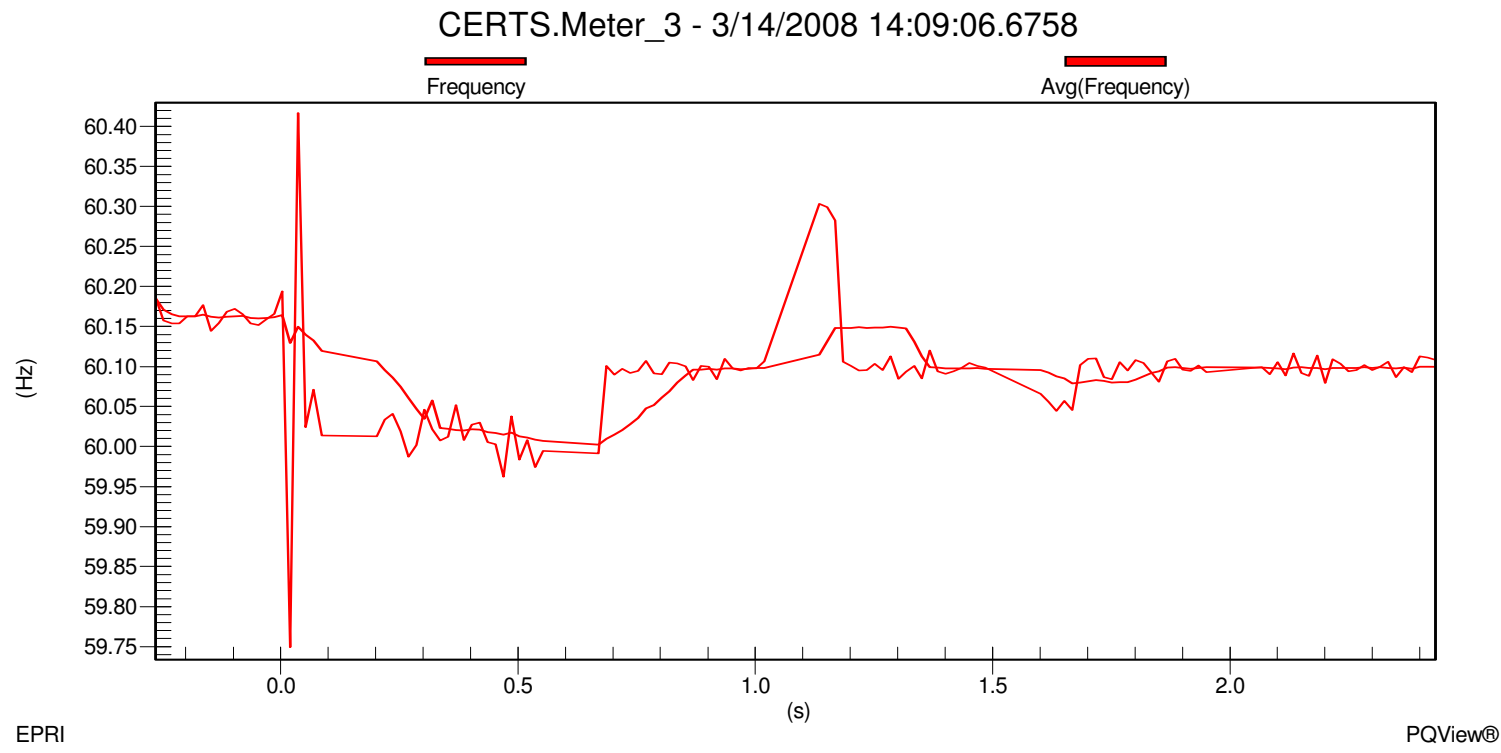

Figure 81 - Meter 3 Frequency during Motor Start and Islanded for Test 10.2.17

Before the motor started, Gen-set A1 was producing approximately $18.9 \mathrm{~kW}+\mathrm{j} 4 \mathrm{kvAr}$ shown in Figures $8 \mathrm{~m}$ and $8 \mathrm{n}$. The power generated by Gen-set A1 was satisfying the loads in Load Bank 3 and all the electrical losses in the microgrid system. When the motor started the inrush caused the Gen-set to increase its output level to $40 \mathrm{~kW}+$ j54kVAr. Gen-set A1 decreased its output level when the motor was warming up and eventually dropped to $27 \mathrm{~kW}+\mathrm{j} 9.8 \mathrm{kVAr}$ when the motor reached steady state. Once all the data was verified and recorded into the DAS Database, the static switch was directed by the EMS to manually close. 


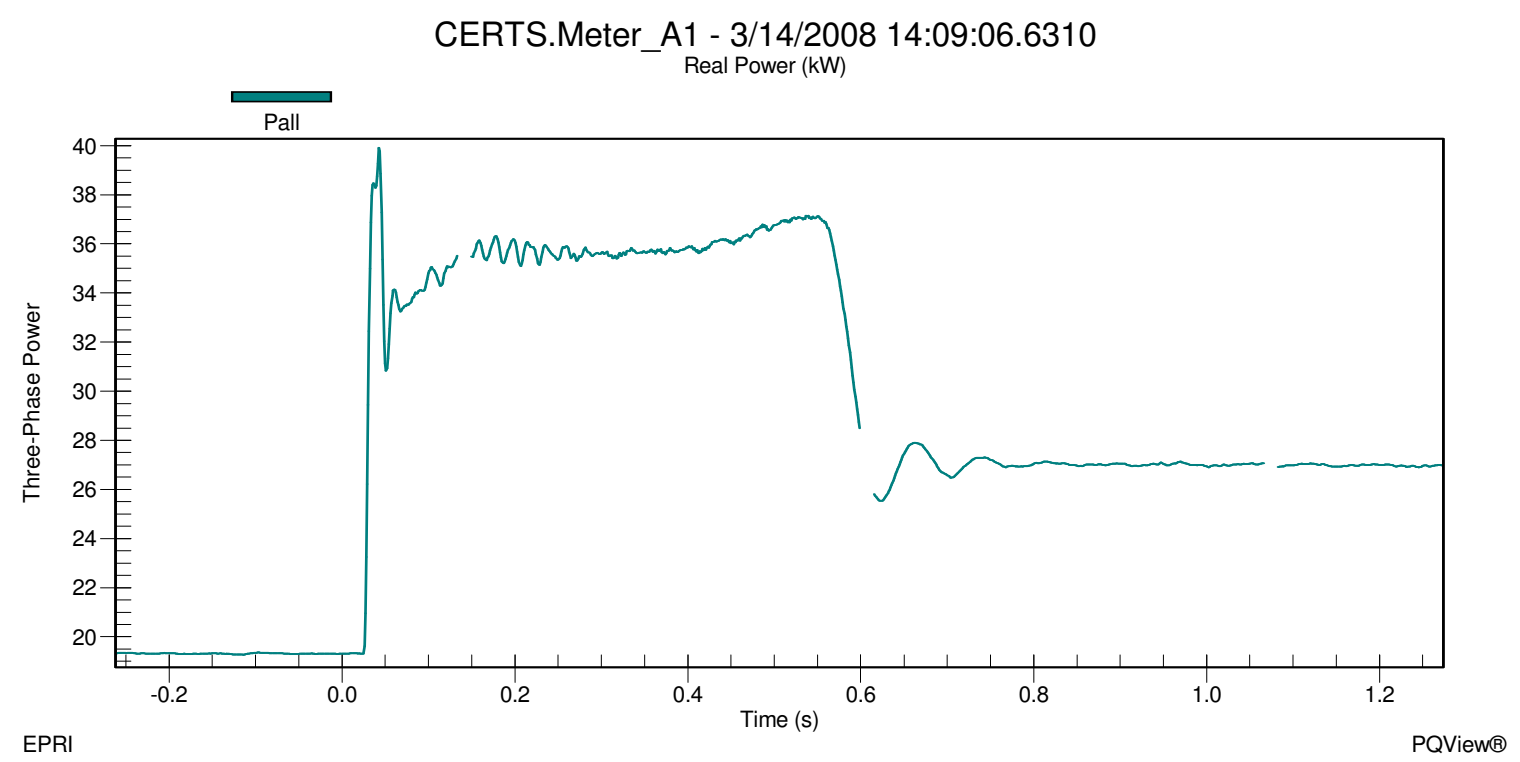

Figure 8m - Gen-set A1 Real Power during Motor Start and Islanded for Test 10.2.17

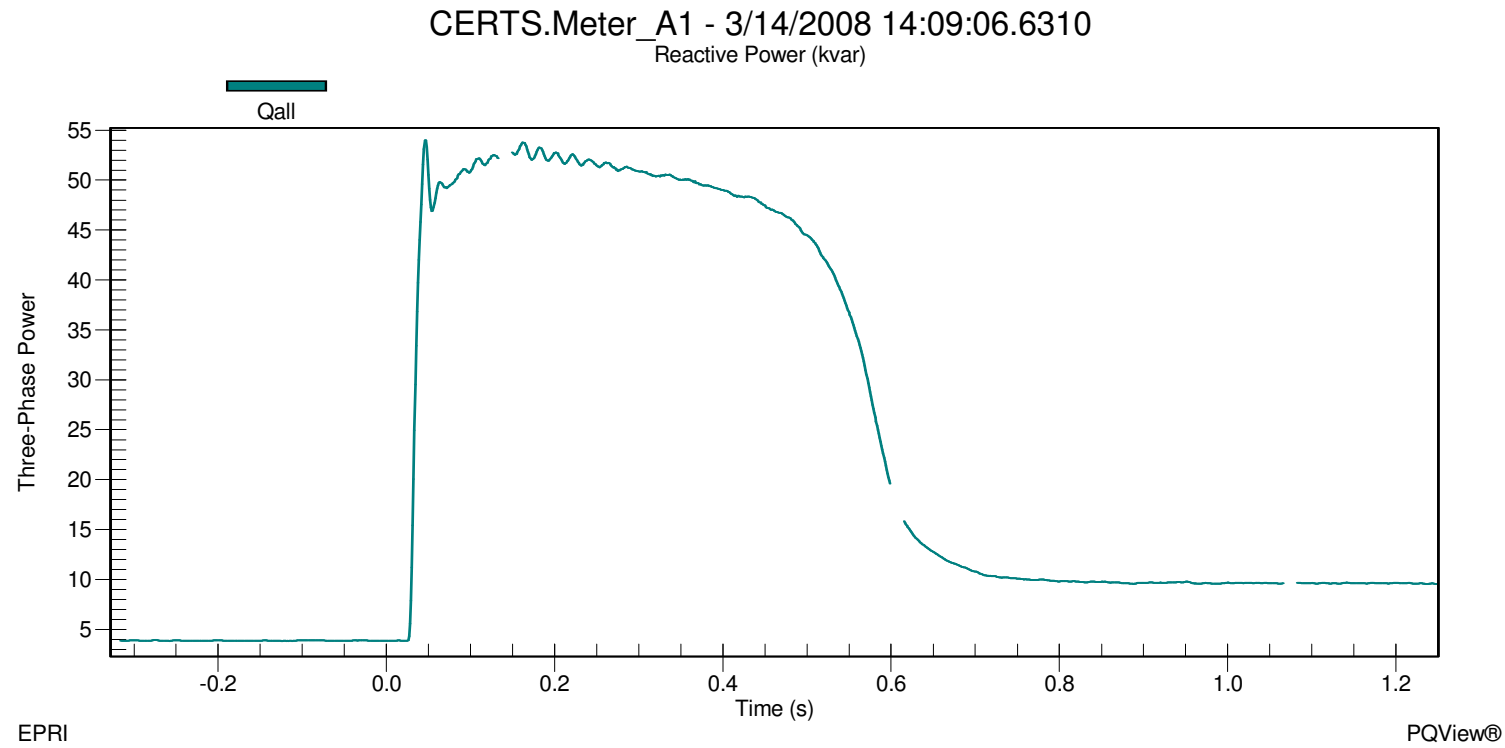

Figure 8n - Gen-set A1 Reactive Power during Motor Start and Islanded for Test 10.2.17

As soon as the static switch closed, Meter 1 recorded real power decreased from approximately $36.8 \mathrm{~kW}$ to $25 \mathrm{~kW}$ and reactive power increased from approximately $1 \mathrm{kVAr}$ to $23.7 \mathrm{kVAr}$ which means that the utility was satisfying a portion of the load demand in Load Bank 6 and all the reactive power in the microgrid. Figures 8o and 8p show the static switch real power decreasing from approximately $0 \mathrm{~kW}$ to $-11 \mathrm{~kW}$ and reactive power increasing from approximately $0 \mathrm{kVAr}$ to $22.2 \mathrm{kVAr}$. At the beginning of the test, the initial power flow through the static switch was $-17 \mathrm{~kW}+\mathrm{j} 19.8 \mathrm{kVAr}$ which is 
not the same recorded at this point in the test because the $10 \mathrm{Hp}$ motor load is on in Zone 3. Gen-set A1 has picked up the motor load and is supporting Load Bank6 with approximately $11 \mathrm{~kW}$.

Load Bank 3 loads increased slightly to $25 \mathrm{~kW}+\mathrm{j} 6.4 \mathrm{kVAr}$. This slight increase is a result from a voltage rise in the microgrid, shown in Figure 8q, from approximately $269 \mathrm{~V}$ on A-phase, $268 \mathrm{~V}$ on B-phase and $269 \mathrm{~V}$ on C-phase when islanded to $281.5 \mathrm{~V}$ on A-phase, $279 \mathrm{~V}$ on B-phase and $279 \mathrm{~V}$ on C-phase at Meter 3 when connected to the utility grid.

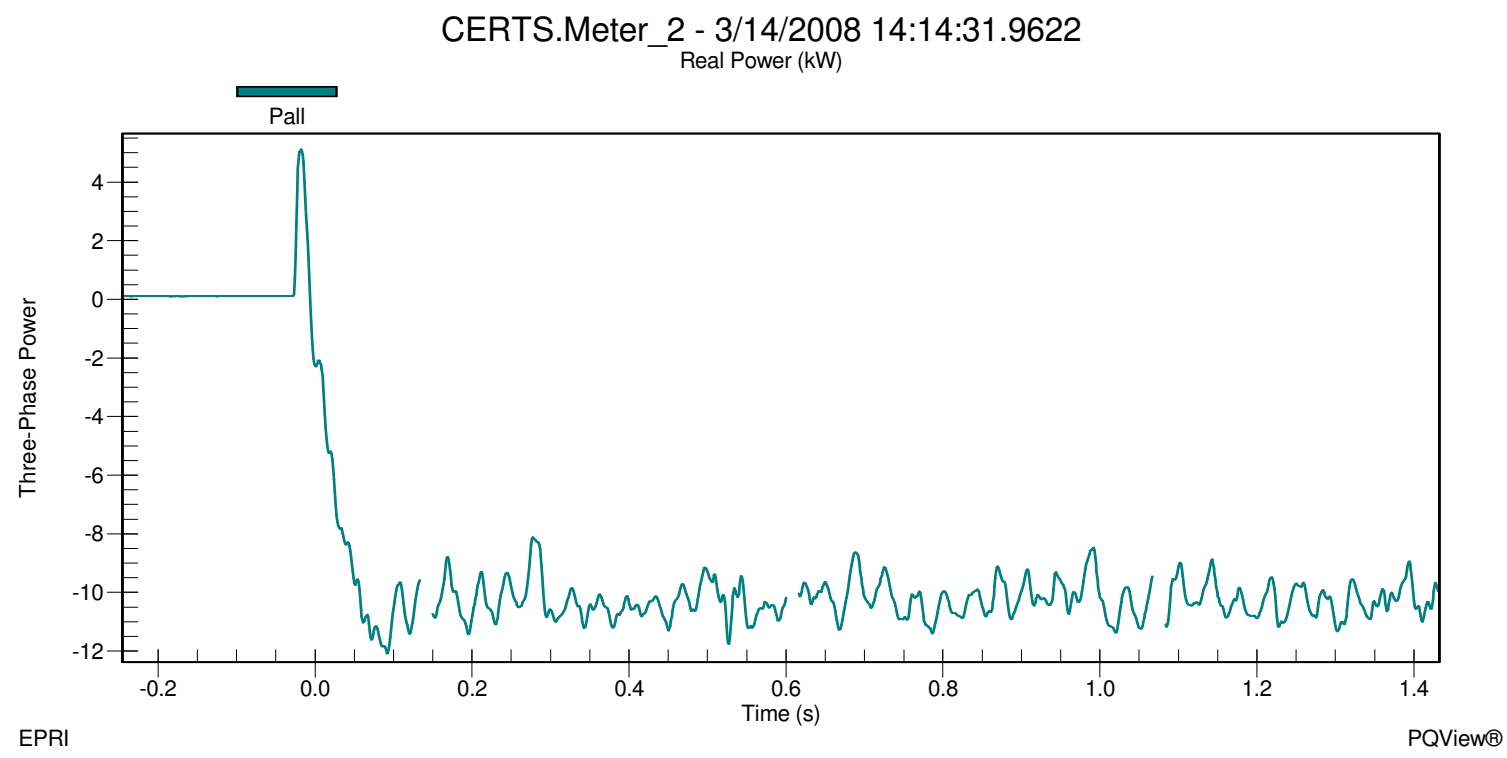

Figure 8o - Static Switch Real Power during Island to Utility Connected mode for Test 10.2.17 


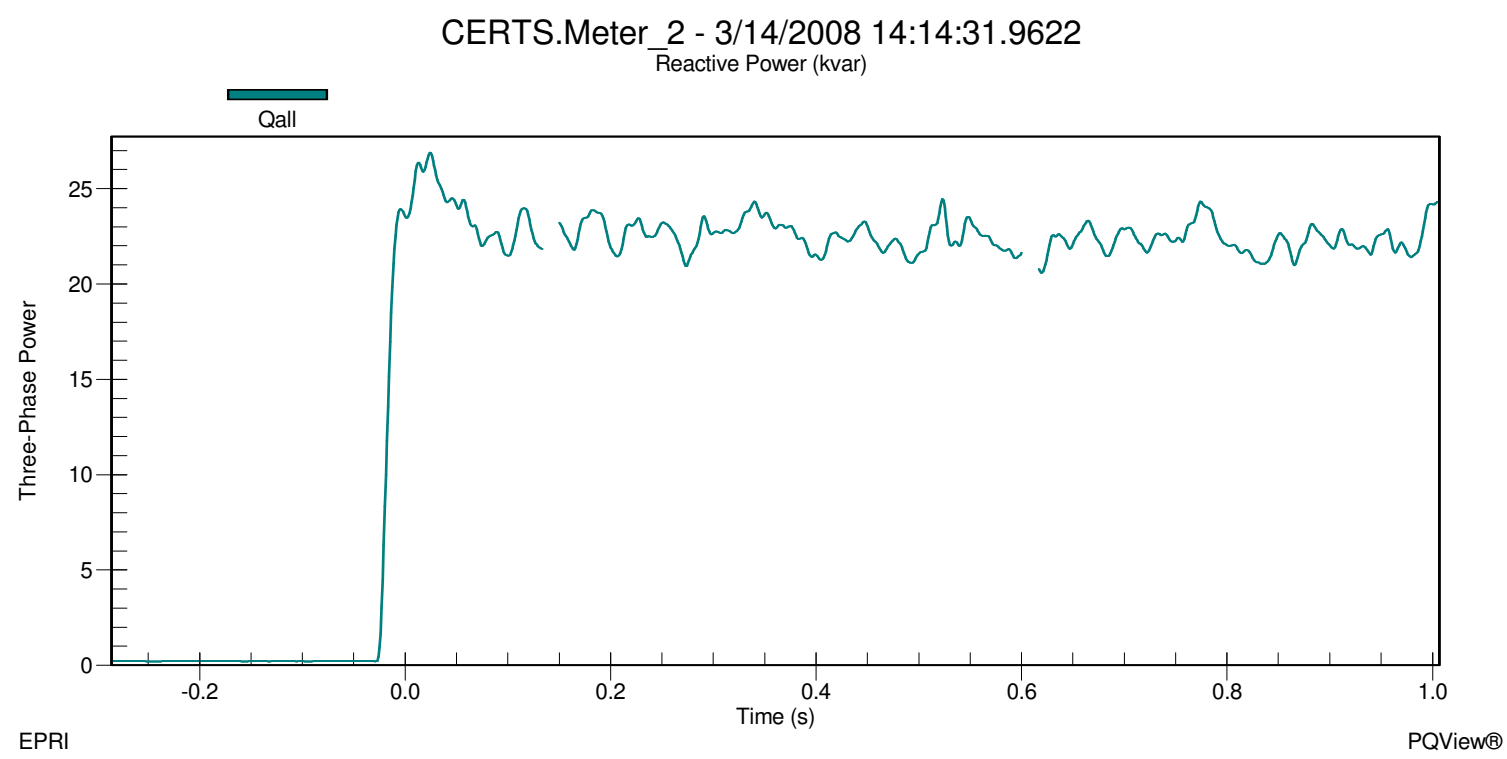

Figure 8p - Static Switch Reactive Power during Island to Utility Connected mode for Test 10.2.17

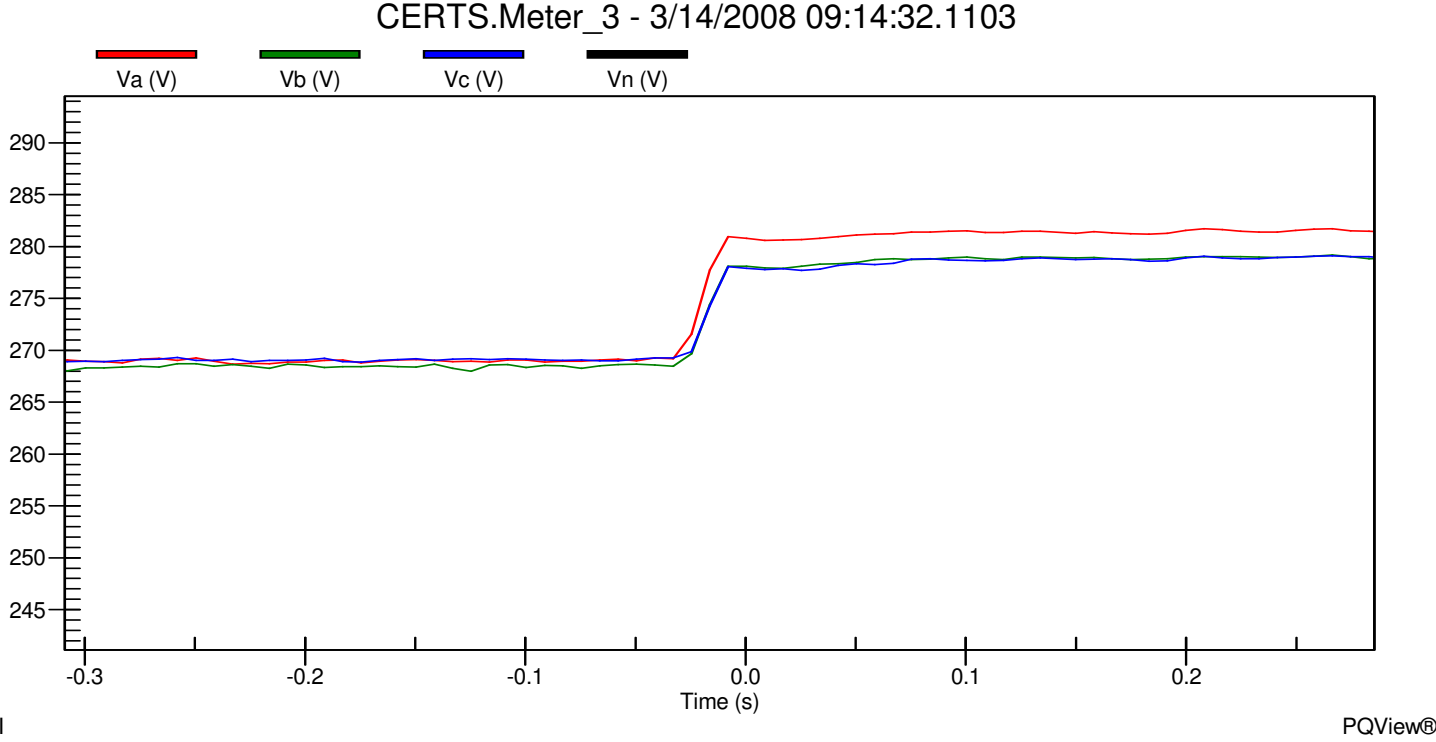

Figure 8q - Meter 3 Line-to-Ground Voltages during Island to Utility Connected mode for Test 10.2.17 


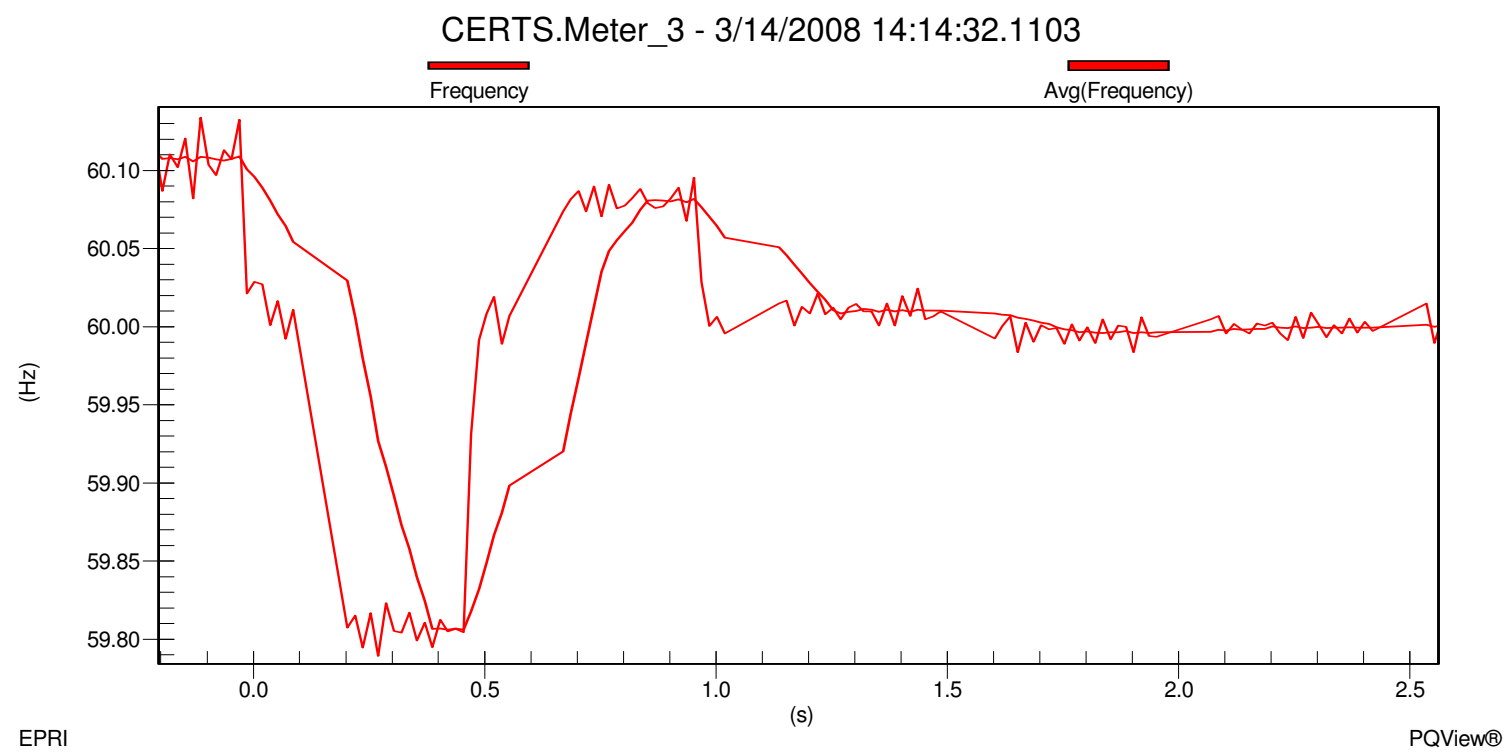

Figure 8r - Meter 3 Frequency during Island to Utility Connected mode for Test 10.2.17

Frequency change in the microgrid, shown in Figure 8r, decreased from approximately $60.11 \mathrm{~Hz}$ when islanded to approximately $60.00 \mathrm{~Hz}$ when connected to the utility grid. This change in frequency is due to the frequency no longer being established by Gen-set A1 using the CERTS algorithm but by the utility. Gen-set A1 is in unit power control mode therefore when the static switch closed back into the utility the Gen-set produced real power based on the set-point initialized at the beginning of the test. The output power for Gen-set A1 was relatively close to the value at the beginning of the test with Gen-set A1 producing approximately $38 \mathrm{~kW}-\mathrm{j} 11.5 \mathrm{kVAr}$. After all the data was verified and recorded into the DAS Database, the motor was turned off and the Gen-sets and Load Banks set-points were changed according to the next test (10.2.18) in Table 1.

For Test 10.2.18 the measured values, after the Gen-sets were warmed up and load banks brought on-line, were approximately $33 \mathrm{~kW}+\mathrm{j} 22 \mathrm{kVAr}$ at Meter $1,-2.5 \mathrm{~kW}+$ $\mathrm{j} 20 \mathrm{kVAr}$ at Meter 2, $-6 \mathrm{~kW}+\mathrm{j} 15 \mathrm{kVAr}$ at Meter 3 and $12.5 \mathrm{~kW}+\mathrm{j} 3 \mathrm{kVAr}$ at Meter 4 . From the microgrid, $36 \mathrm{~kW}-\mathrm{j} 12 \mathrm{kVAr}$ was produced by Gen-set A1 and $23.5 \mathrm{~kW}-\mathrm{j} 2 \mathrm{kVAr}$ was produced by Gen-set A2. The load banks were $18 \mathrm{~kW}+\mathrm{j} 0 \mathrm{kVAr}$ at LB3, 35.78kW + j0.714kVAr at LB4 and $35.9 \mathrm{~kW}+\mathrm{j} 0.77 \mathrm{kVAr}$ at LB6. These measurements were relatively close to the expected values in Table 1, but not exact due to temperature, phase voltages and electrical losses in conductors. In addition, the $40 \mathrm{~kW}$ settings for LB4 and LB6 and $20 \mathrm{~kW}$ setting for LB3 were also below selected set values. At the time of these measurements, the voltage and frequency was $281.8 \mathrm{~V}$ on A-phase, $279.7 \mathrm{~V}$ on B-phase and $279.7 \mathrm{~V}$ on $\mathrm{C}$-phase and $60.02 \mathrm{~Hz}$ at the static switch (i.e., Meter 2) when connected to the utility grid; and $281.9 \mathrm{~V}$ on A-phase, $279.7 \mathrm{~V}$ on B-phase and $279.2 \mathrm{~V}$ on C-phase and $60.00 \mathrm{~Hz}$ at Meter 3 . 
The Gen-sets in this test were set up to produce $-10 \mathrm{~kW}$ going through Zone 3 (i.e., Meter 3) which means they produced $10 \mathrm{~kW}$ more power than Load Banks 3 and 4 needed. Approximately $6 \mathrm{~kW}$ of excess power was exported out of Zone 3 and $2.5 \mathrm{~kW}$ of that power went through the static switch to Load Bank 6 and the remaining $3.5 \mathrm{~kW}$ was due to power losses in the conductors. Since Load Bank 6 was approximately $35.9 \mathrm{~kW}+$ j0.77kVAr, the utility had to supply approximately $33 \mathrm{~kW}$ to satisfy the load. Reactive power had to be imported in from the utility of approximately 22kVAr because Gen-sets A1 and A2 needed approximately 14lkVAr between the two and the reactive power of Load Bank 4. Once all the data was verified and recorded into the DAS Database, the $10 \mathrm{Hp}$ induction motor was started in Zone 3.

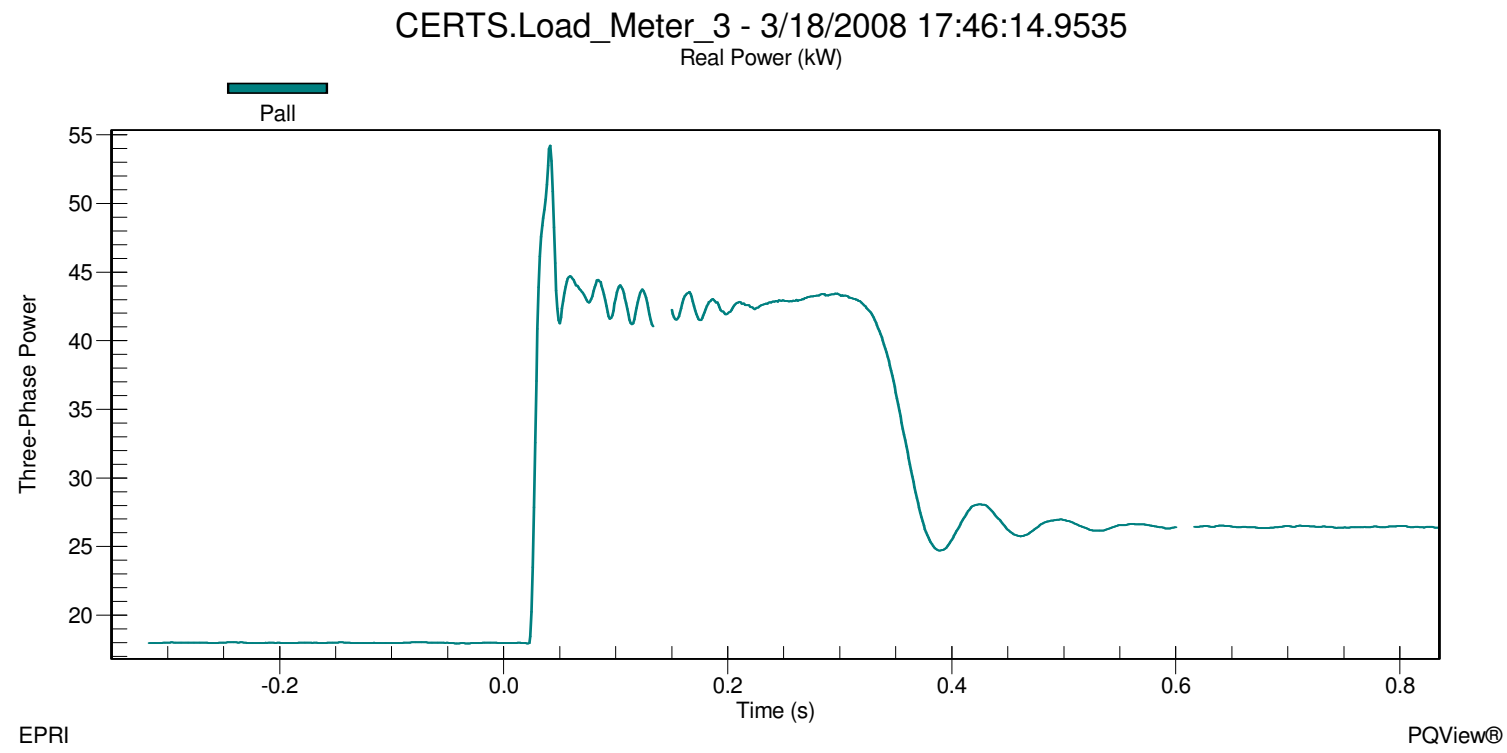

Figure 9a - Load Bank 3 Real Power during Motor Start and Utility Connected for Test 10.2.18 


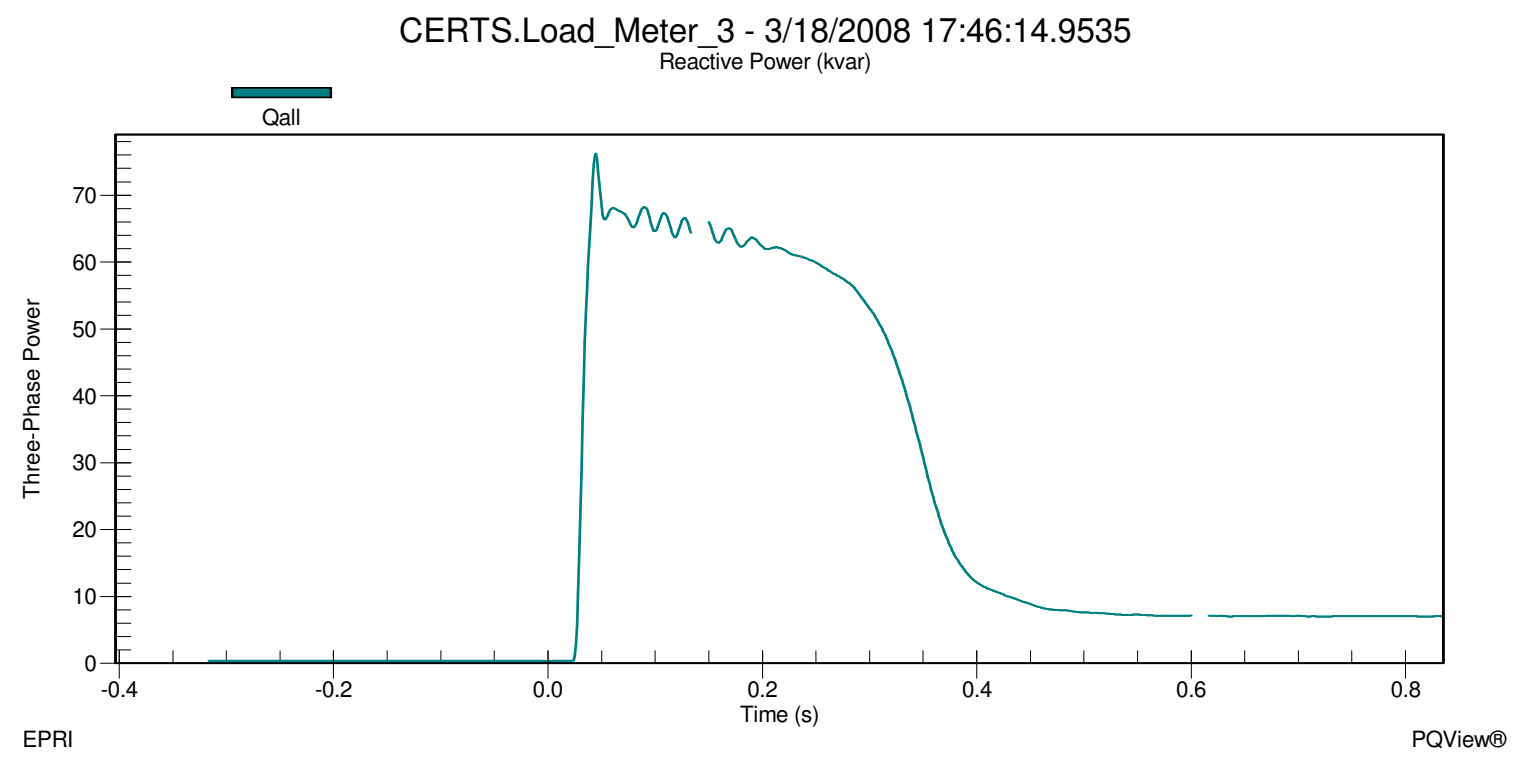

Figure 9b - Load Bank 3 Reactive Power during Motor Start and Utility Connected for Test 10.2.18

In Figures 9a and 9b it can be seen that the load in Zone 3 was approximately $18 \mathrm{~kW}+$ j0kVAr before the start of the induction motor and increased to approximately $54.2 \mathrm{~kW}+$ j76kVAr during the inrush phase of the motor start. After about 1.5 cycles, the motor settled down to approximately $42.8 \mathrm{~kW}+\mathrm{j} 63 \mathrm{kVAr}$ during the warm up phase which lasted about 28.2 cycles ( 0.47 Seconds). When the motor reached steady state, the load in Zone 3 was approximately $26.5 \mathrm{~kW}+\mathrm{j} 7 \mathrm{kVar}$.

The voltage and frequency at the static switch before the motor start was approximately 281.8V on A-phase, 279.7V on B-phase and 279.7V on C-phase shown in Figure 9c and approximately $60.02 \mathrm{~Hz}$ shown in Figure $9 \mathrm{~d}$. When the motor started, the voltage at the static switch during the inrush decreased to approximately $274.5 \mathrm{~V}$ on A-phase, $271.5 \mathrm{~V}$ on B-phase and $271.5 \mathrm{~V}$ on C-phase for about 1.5 cycles. Frequency dropped during the inrush to approximately $59.94 \mathrm{~Hz}$ and quickly increased to approximately $60.02 \mathrm{~Hz}$. Voltage increased as the motor was warming up and eventually settled at a steady state voltage at approximately $280.8 \mathrm{~V}$ on A-phase, $278.8 \mathrm{~V}$ on B-phase and $278.8 \mathrm{~V}$ on C-phase at an approximate frequency $60.02 \mathrm{~Hz}$. 


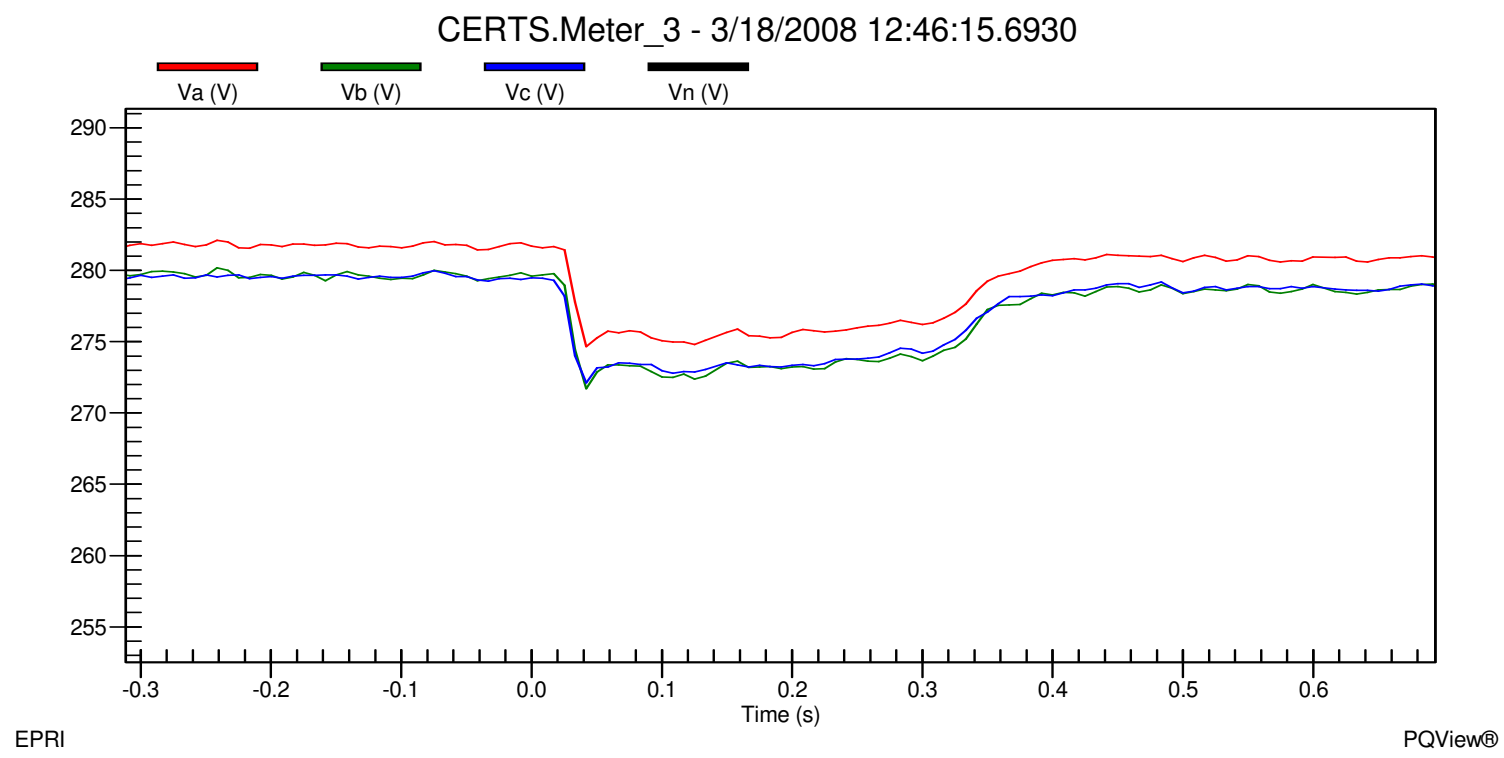

Figure 9c - Static Switch Line-to-Ground Voltage during Motor Start and Utility Connected for Test 10.2.18

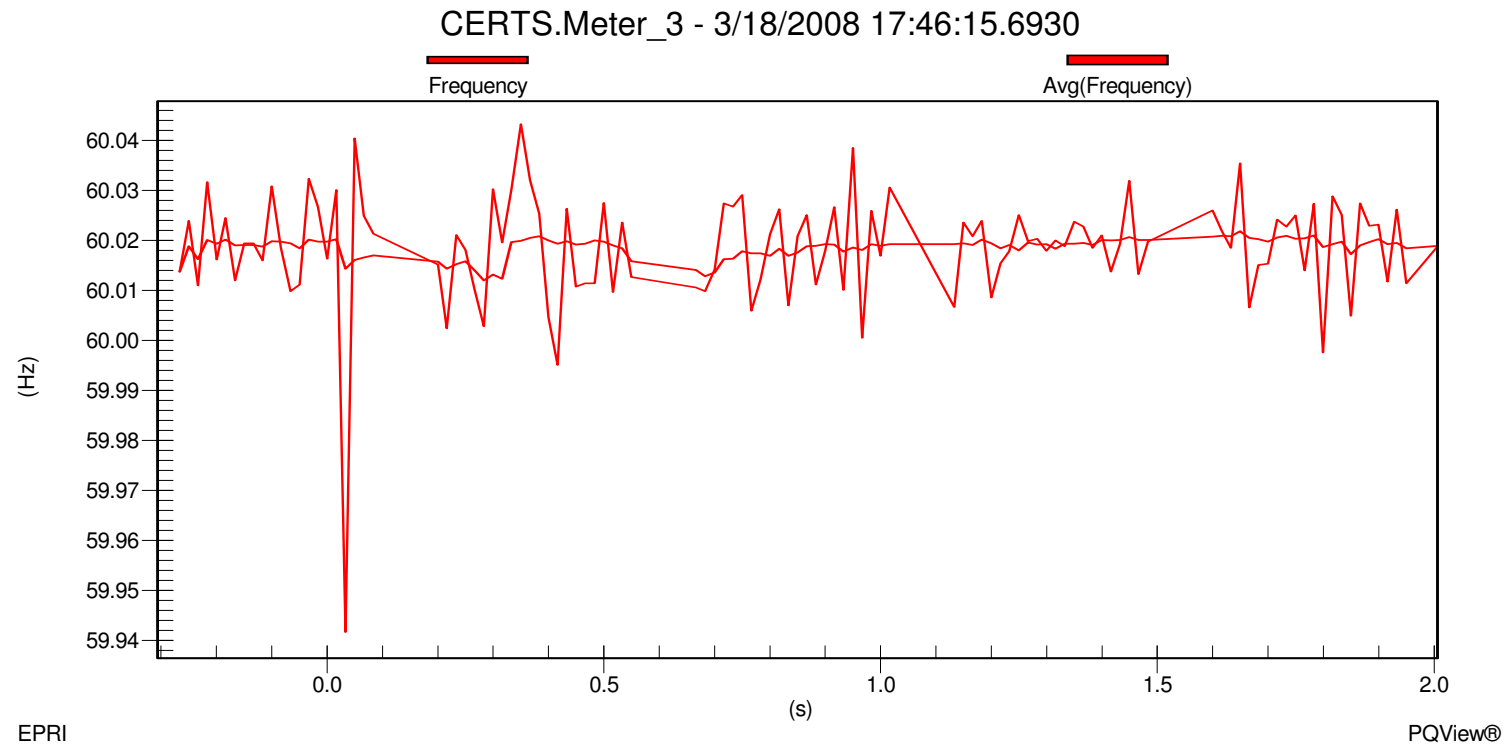

Figure 9d - Static Switch Frequency during Motor Start and Utility Connected for Test 10.2.18 


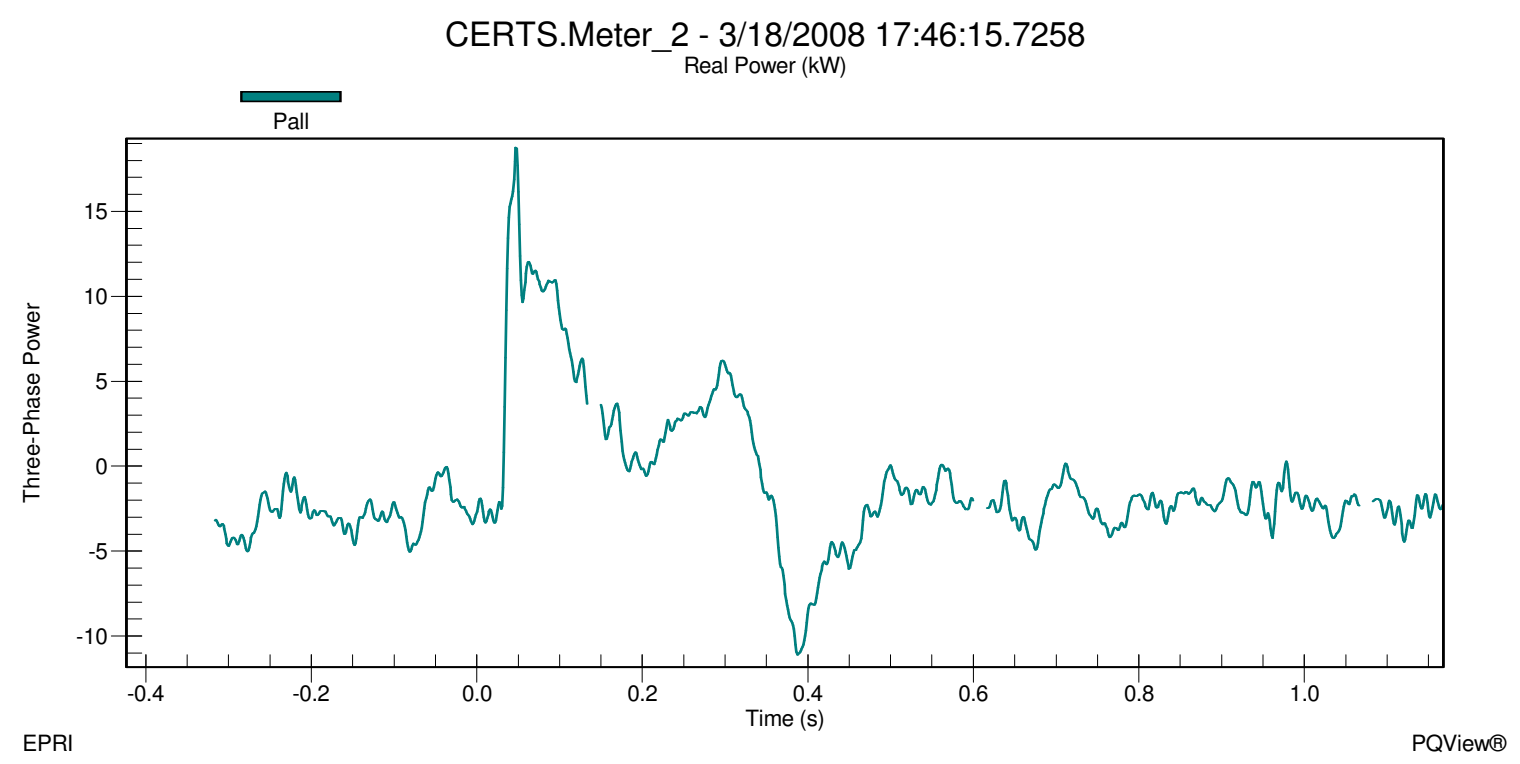

Figure 9e - Static Switch Real Power during Motor Start and Utility Connected for Test 10.2.18

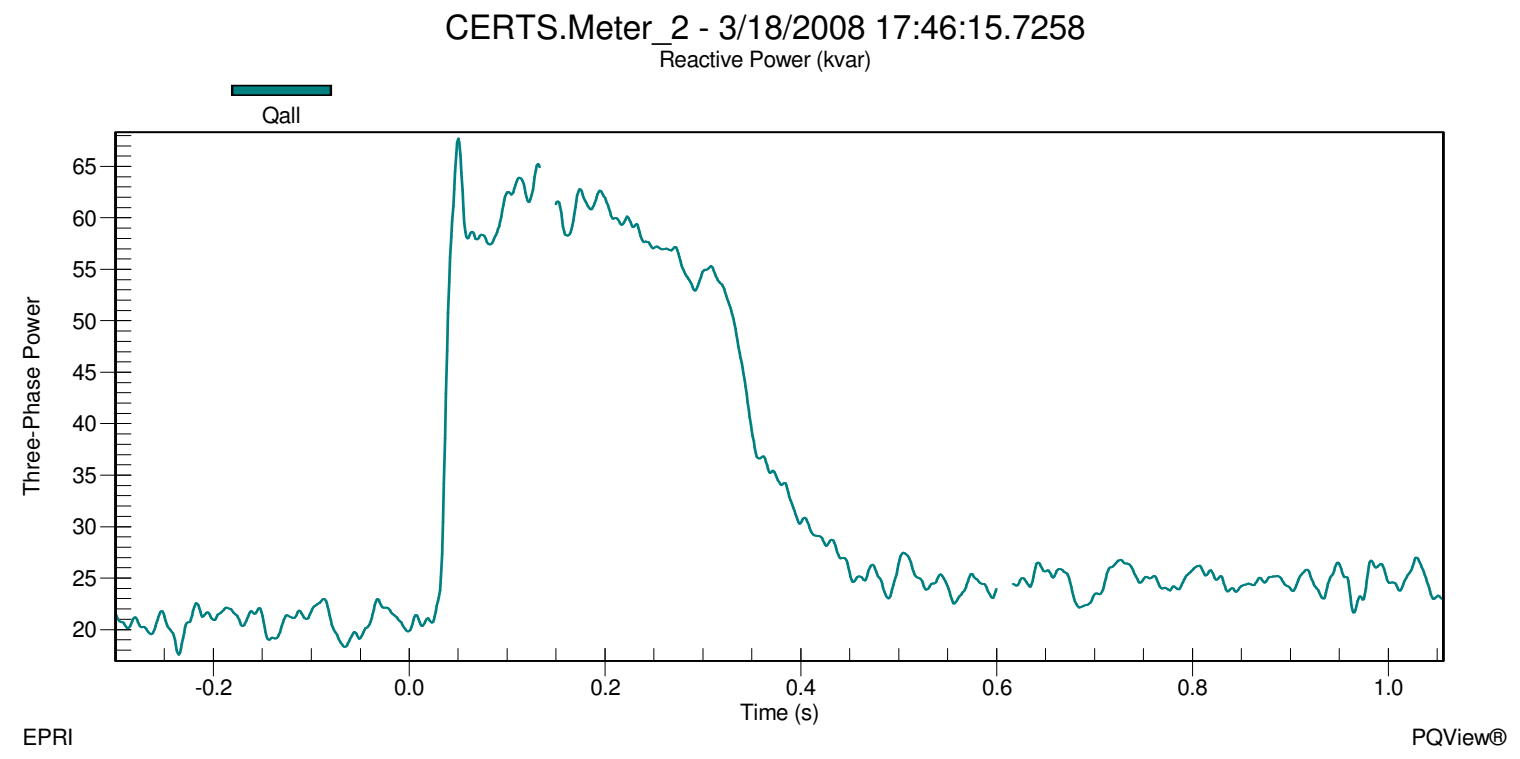

Figure 9f - Static Switch Reactive Power during Motor Start and Utility Connected for Test 10.2.18

Before the motor started Gen-sets A1 and A2 were producing approximately $36 \mathrm{~kW}-$ $\mathrm{j} 12 \mathrm{kVAr}$ and $23.5 \mathrm{~kW}-\mathrm{j} 2 \mathrm{kVAr}$, respectively. This was enough power for the load demands of Load Banks 3 and 4 with real power being exported to Zone 6 of approximately $2.5 \mathrm{~kW}$ shown in Figure 9e. The grid was supporting the reactive power of the microgrid with approximately 20kVAr shown in Figure 9f. When the motor started the inrush caused the utility to supply approximately $68 \mathrm{kVAr}$ and to pick up the load demand of Load Bank 6 and approximately $18.8 \mathrm{~kW}$ of the microgrid loads. The power that was being supplied by Gen-set A1 to Load Bank 6 was now supplying the 
motor inrush which can be seen in Figure 9e as the real power through the static switch becomes positive. Gen-sets A1 and A2 increased their real and reactive power output levels to approximately $59 \mathrm{~kW}-\mathrm{j} 0.6 \mathrm{kVAr}$ and $31.8 \mathrm{~kW}+\mathrm{j} 18 \mathrm{kVAr}$, respectively. Notice the signs of the VAr output changed from negative to positive for both Gen-sets in order to support the induction motor load in Zone 3.

When the motor reached steady state, the real and reactive power through the static switch was approximately $-2.5 \mathrm{~kW}+\mathrm{j} 24 \mathrm{kVAr}$ which meant that Gen-sets A1 and A2 were supplying the power for the induction motor and approximately $2.5 \mathrm{~kW}$ of Load Bank 6 . The real power in Gen-set A1 increased from its initial value at the beginning of the test to approximately $44 \mathrm{~kW}$ and the real power for Gen-set A2 returned to the value before the motor started which was approximately $23.5 \mathrm{~kW}$. Reactive power in Gen-set A1 and A2 increased from its initial value at the beginning of the test to approximately $-9 \mathrm{kVAr}$ and $-0.5 \mathrm{kVAr}$, respectively. Once all the data was verified and recorded into the DAS Database, the motor was shut down and the static switch was directed by the EMS to manually open.

As soon as the static switch opened, Meter 1 recorded real power increased to approximately $37 \mathrm{~kW}$ and reactive power decreased to $1.2 \mathrm{kVAr}$ satisfying the load demand in Load Bank 6 which was approximately $36.78 \mathrm{~kW}+\mathrm{j} 0.785 \mathrm{kVAr}$ and not supplying any power beyond the static switch to Load Banks 3 and 4 . $0 \mathrm{~kW}+\mathrm{j} 0 \mathrm{kVAr}$ was recorded at the static switch, indicating that power was not flowing through the static switch. Load Banks 3 and 4 loads reduced slightly to $17.4 \mathrm{~kW}+j 0.3285 \mathrm{kVAr}$ and $34.45 \mathrm{~kW}+\mathrm{j} 0.686 \mathrm{kVAr}$, respectively. This load reduction resulted from a voltage drop in the microgrid, shown in Figure $9 \mathrm{~g}$, from approximately $281.9 \mathrm{~V}$ on A-phase, $279.8 \mathrm{~V}$ on Bphase and $279.8 \mathrm{~V}$ on C-phase when connected to the utility grid to $275 \mathrm{~V}$ on A-phase, $275 \mathrm{~V}$ on B-phase and 275V on C-phase at Meter 3 when islanded. 


\section{CERTS.Meter_3 - 3/18/2008 12:53:05.9709}

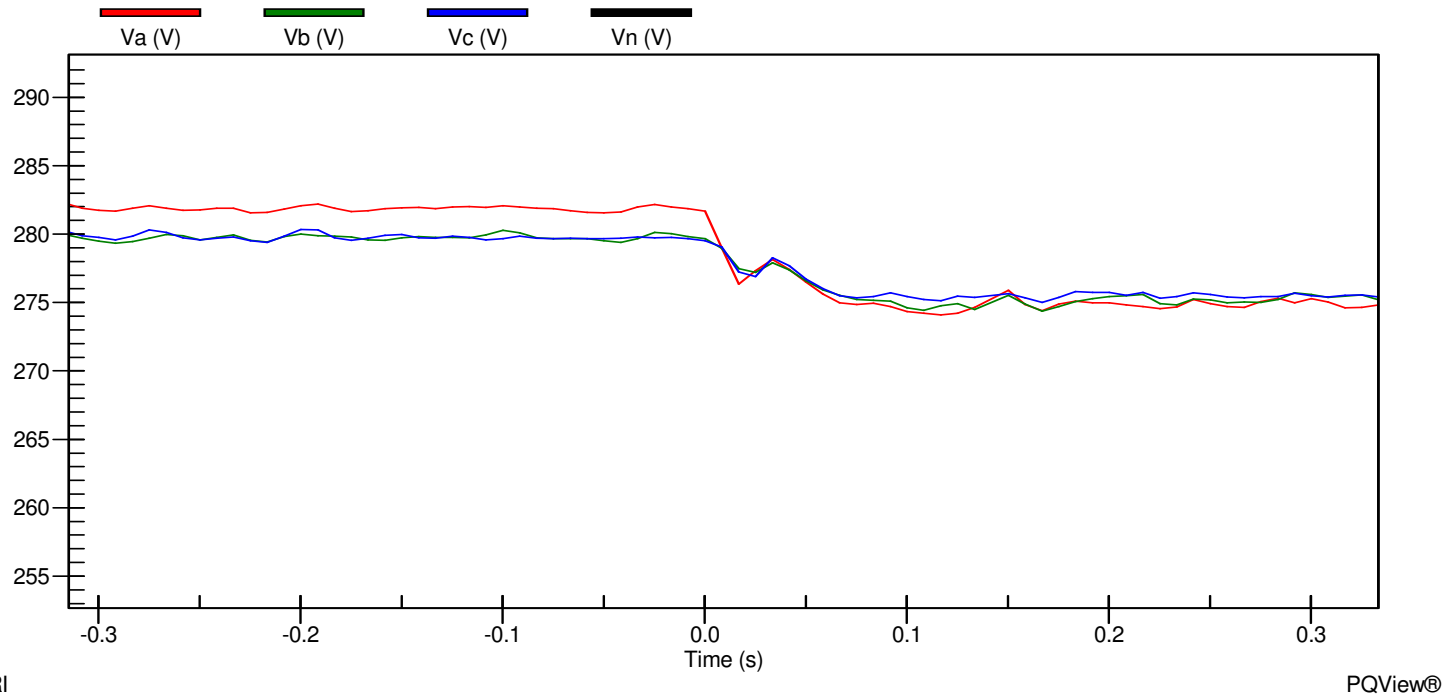

Figure 9g - Meter 3 Line-to-Ground Voltages during Utility Connected to Island mode for Test 10.2.18

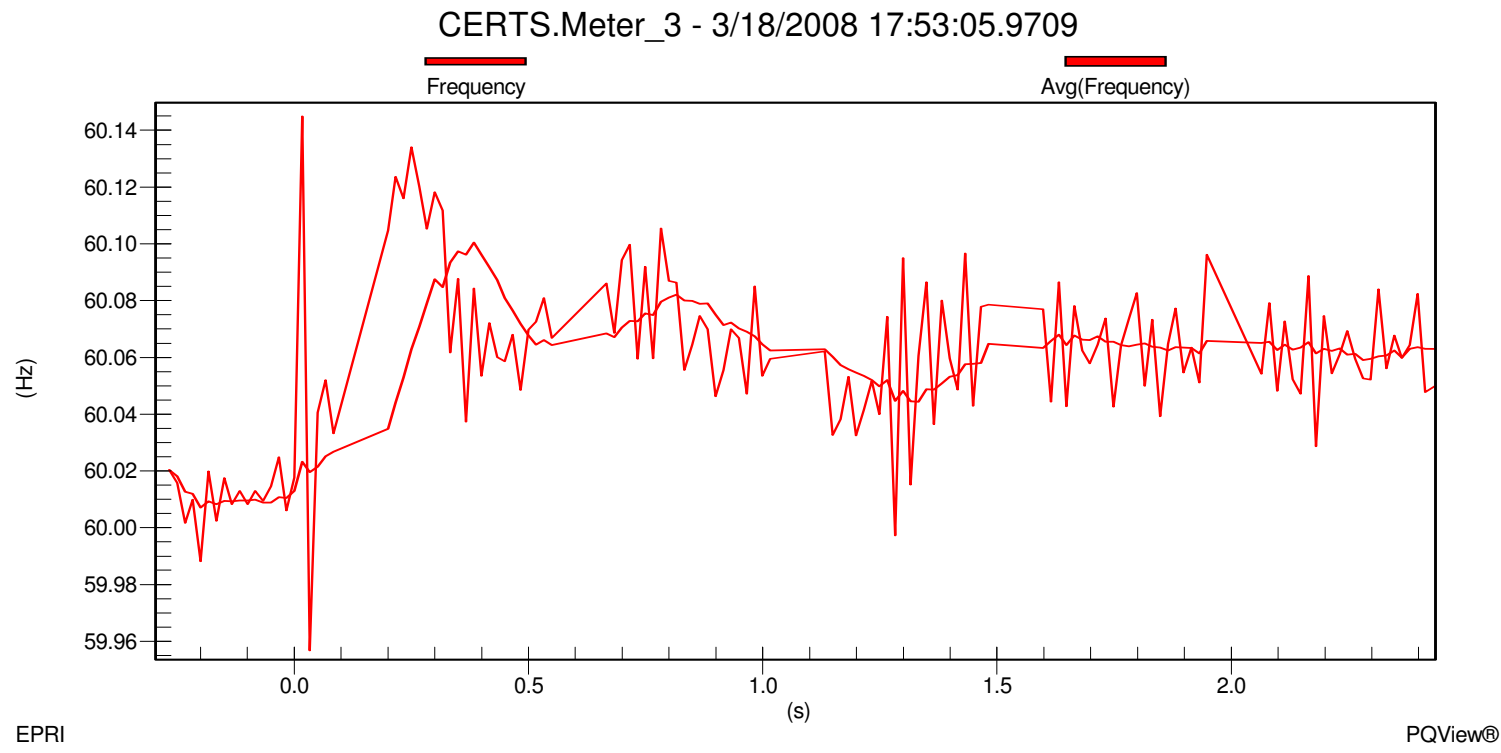

Figure 9h - Meter 3 Frequency during Utility Connected to Island mode for Test 10.2.18 
Frequency change in the microgrid, shown in Figure 9h, increased from approximately $60.01 \mathrm{~Hz}$ when connected to the utility grid to approximately $60.06 \mathrm{~Hz}$ when islanded. This change in frequency was part of the CERTS algorithm which allowed Gen-set A2 to decrease its output real power to approximately $17.5 \mathrm{~kW}$. Gen-set A1 remained relatively at the same level which was approximately $37 \mathrm{~kW}$. Reactive power in Gen-sets $\mathrm{A} 1$ and A2 increased to approximately $-3 \mathrm{kVAr}$ and $8 \mathrm{kVAr}$, respectively. Meter 3 was approximately $-2.03 \mathrm{~kW}-\mathrm{j} 2.05 \mathrm{kAr}$ indicating that the Gen-sets were satisfying the loads in the microgrid and the power losses in the electrical lines. All data was verified and recorded into the DAS Database. The microgrid ran for a couple of minutes in this electrical state before the $10 \mathrm{Hp}$ induction motor was started in Zone 3.

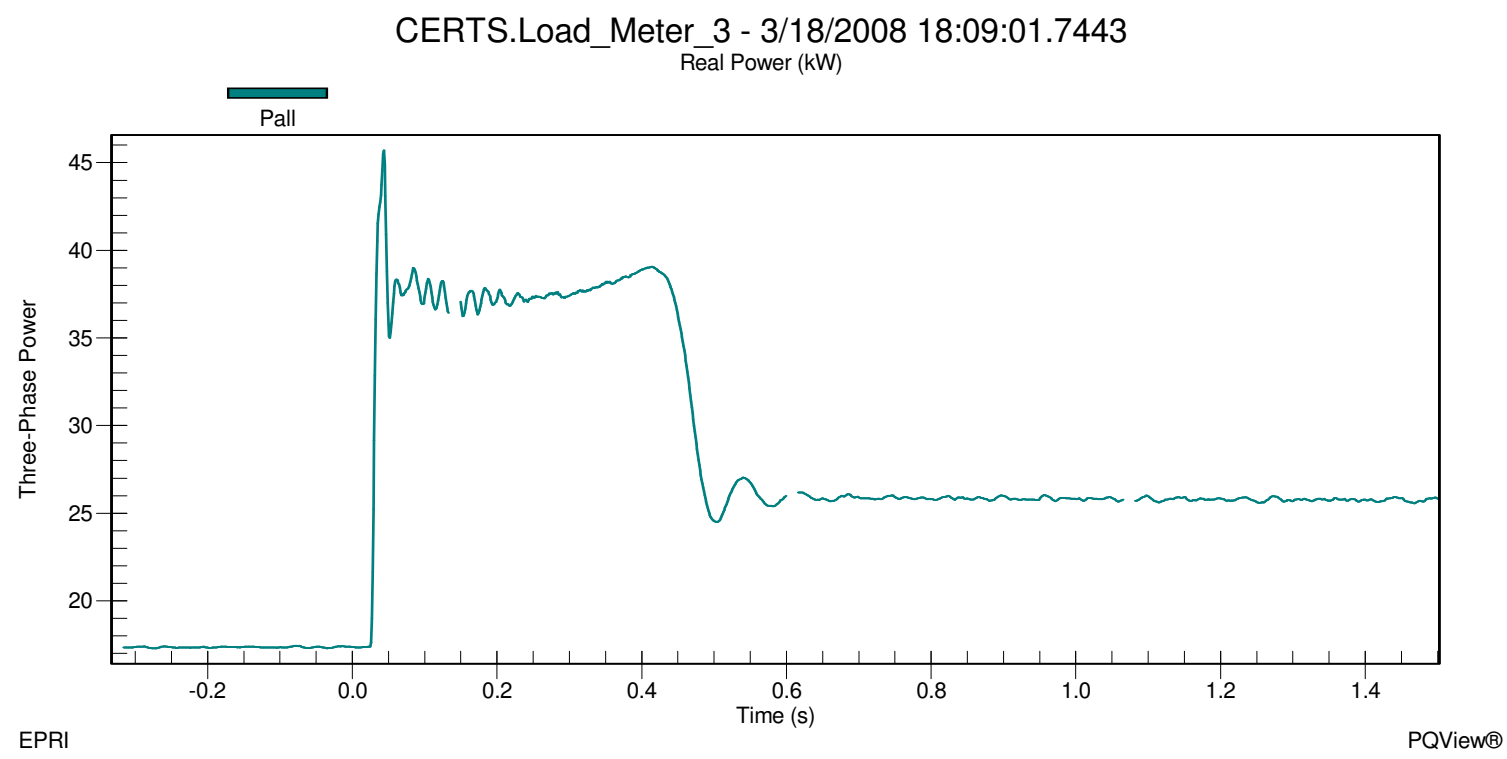

Figure 9i - Load Bank 3 Real Power during Motor Start and Islanded for Test 10.2.18 


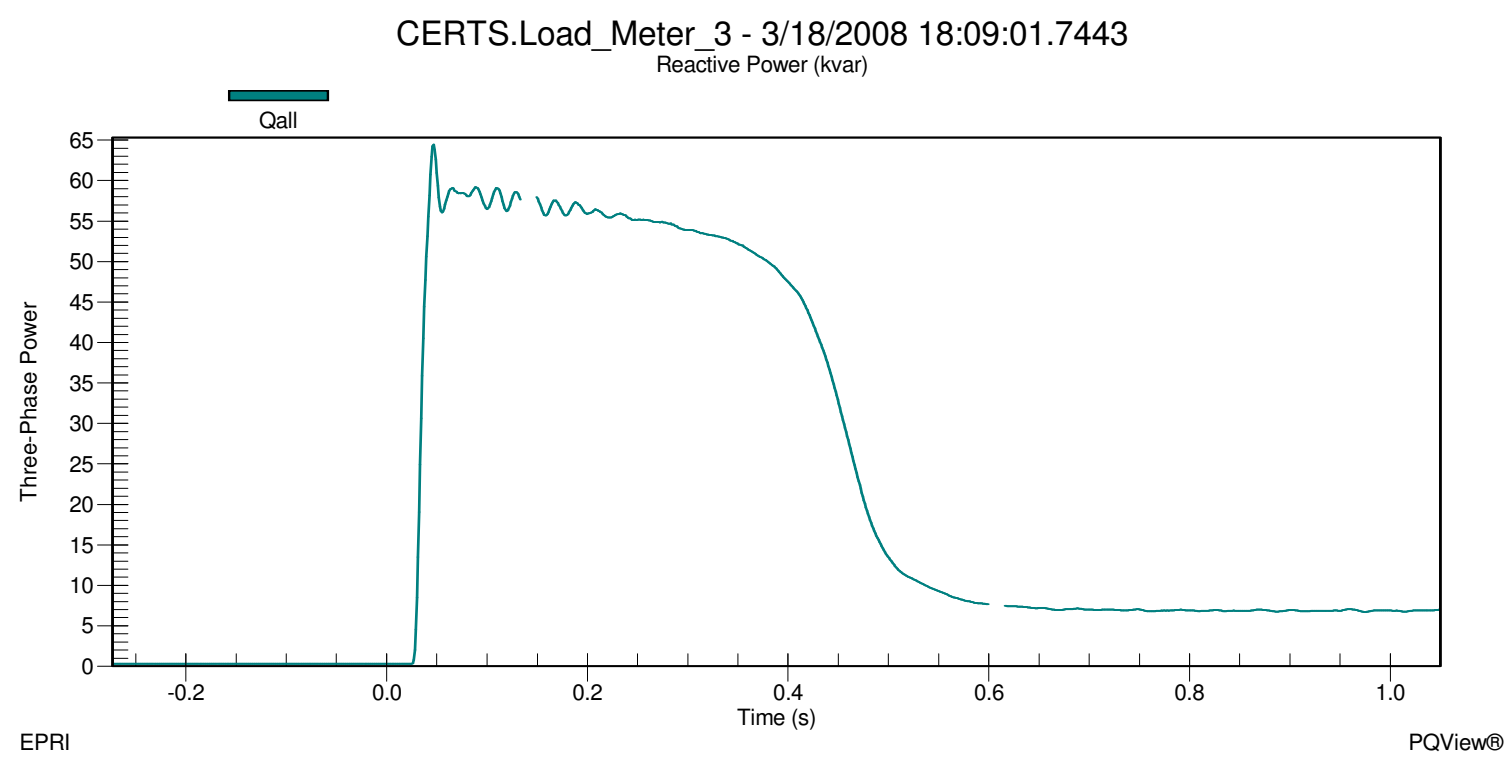

Figure 9j - Load Bank 3 Reactive Power during Motor Start and Islanded for Test 10.2.18

In Figures 9i and 9j it can be seen that the load in Zone 3 was approximately $17.5 \mathrm{~kW}+$ j0kVAr before the start of the induction motor and increased to approximately $46 \mathrm{~kW}+$ j65kVAr during the inrush phase of the motor start. After about 1.5 cycles, the motor settled down to approximately $37.8 \mathrm{~kW}+\mathrm{j} 55 \mathrm{kVAr}$ during the warm up phase which lasted about 37.8 cycles ( 0.63 Seconds). When the motor reached steady state, the load in Zone 3 was approximately $25.8 \mathrm{~kW}+\mathrm{j} 7 \mathrm{kVAr}$.

The voltage and frequency at Meter 3 before the motor start was approximately $275 \mathrm{~V}$ on A-phase, $275 \mathrm{~V}$ on B-phase and 275V on C-phase shown in Figure 9k and approximately $60.07 \mathrm{~Hz}$ shown in Figure 91. When the motor started, the voltage at Meter 3 during the inrush decreased to approximately $254.5 \mathrm{~V}$ on A-phase, $255.9 \mathrm{~V}$ on B-phase and $255.9 \mathrm{~V}$ on C-phase for about 1.5 cycles. Frequency dropped during the inrush to approximately $59.97 \mathrm{~Hz}$ and quickly increased to approximately $60.03 \mathrm{~Hz}$. Voltage increased as the motor was warming up and eventually settled at a steady state voltage at approximately $273 \mathrm{~V}$ on A-phase, $273 \mathrm{~V}$ on B-phase and $273 \mathrm{~V}$ on C-phase at an approximate frequency of $60.06 \mathrm{~Hz}$. 


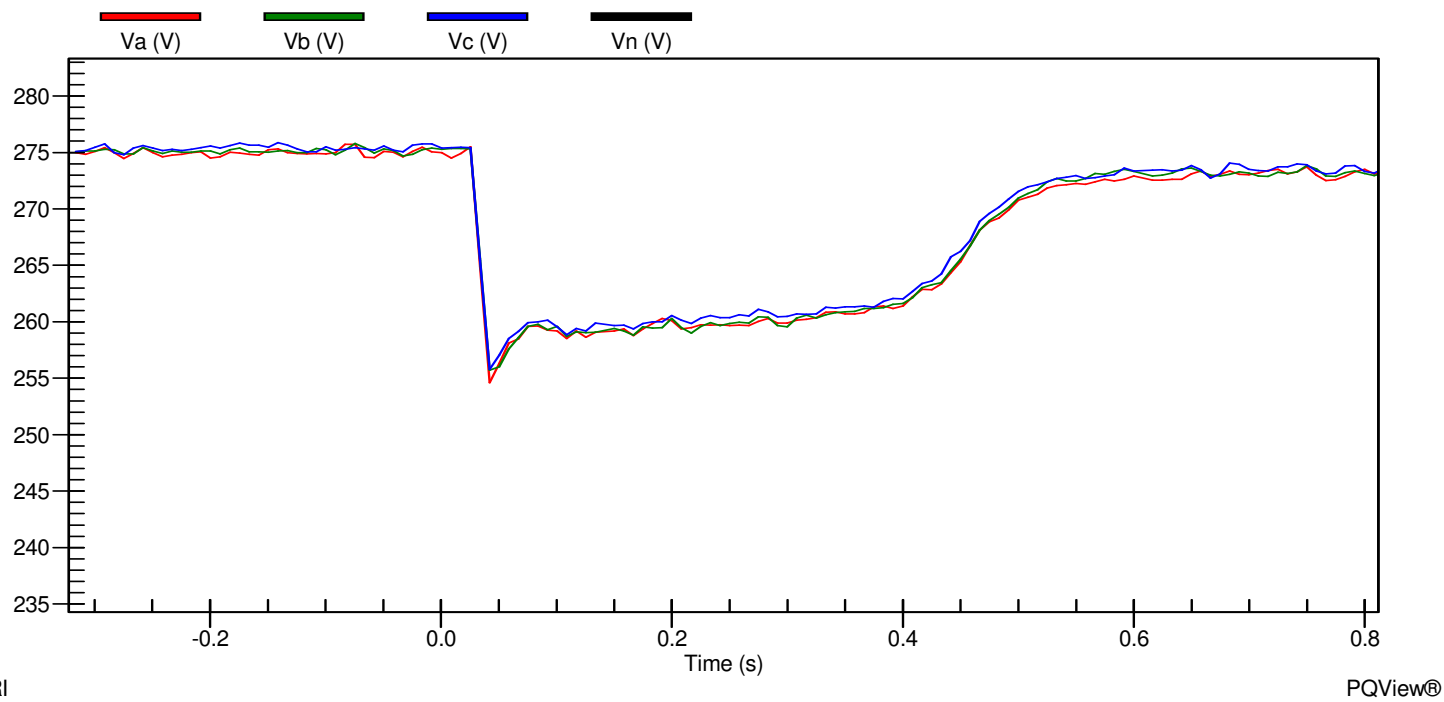

Figure 9k - Meter 3 Line-to-Ground Voltages during Motor Start and Islanded for Test 10.2.18

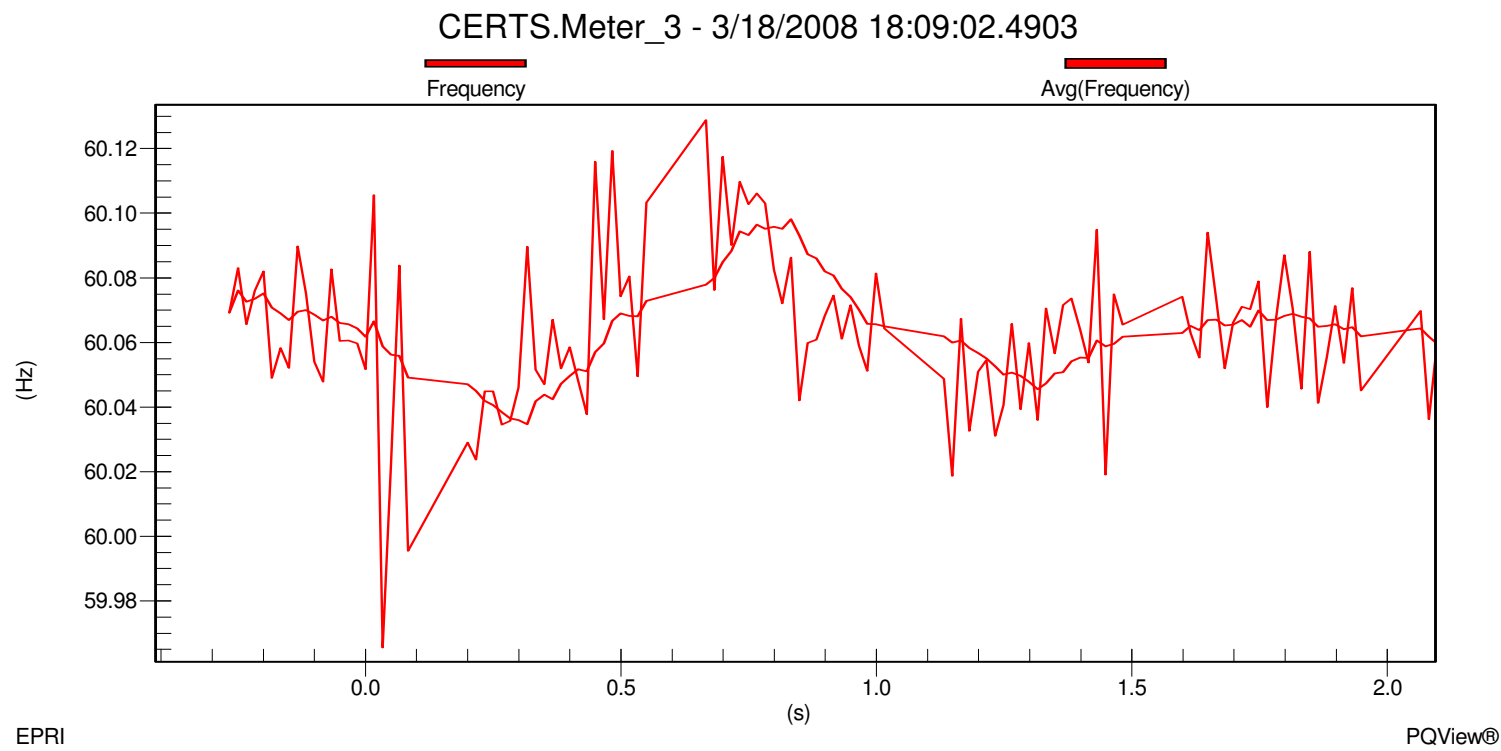

Figure 91 - Meter 3 Frequency during Motor Start and Islanded for Test 10.2.18

Before the motor started Gen-sets A1 and A2 were producing approximately $37 \mathrm{~kW}-$ $\mathrm{j} 3 \mathrm{kVAr}$ and $17.5 \mathrm{~kW}+\mathrm{j} 8 \mathrm{kVAr}$, respectively shown in Figures $9 \mathrm{~m}-9 \mathrm{p}$. The power generated by both Gen-sets was satisfying the loads in Load Banks 3 and 4 and all the electrical losses in the microgrid system. When the motor started the inrush caused the Gen-sets to increase their output power levels to $47.5 \mathrm{~kW}+\mathrm{j} 30 \mathrm{kVAr}$ for Gen-set A1 and $30 \mathrm{~kW}+\mathrm{j} 37 \mathrm{kVAr}$ for Gen-set A2. The increase in real and reactive power due to the motor start inrush was shared by both Gen-sets and Gen-set A2 picked up most of the real power. Gen-set A1 increased its output real power during the warm up phase to approximately $57 \mathrm{~kW}$ and decreased its reactive power. Gen-set A2 decreased real and reactive power output during the warm up phase. The Gen-sets eventually dropped 
their output levels to $45.4 \mathrm{~kW}-\mathrm{j} 0.2 \mathrm{kVAr}$ for Gen-set A1 and $16.5 \mathrm{~kW}+11.1 \mathrm{kVAr}$ for Genset A2 when the motor reached steady state. Once all the data was verified and recorded into the DAS Database, the static switch was directed by the EMS to manually close.

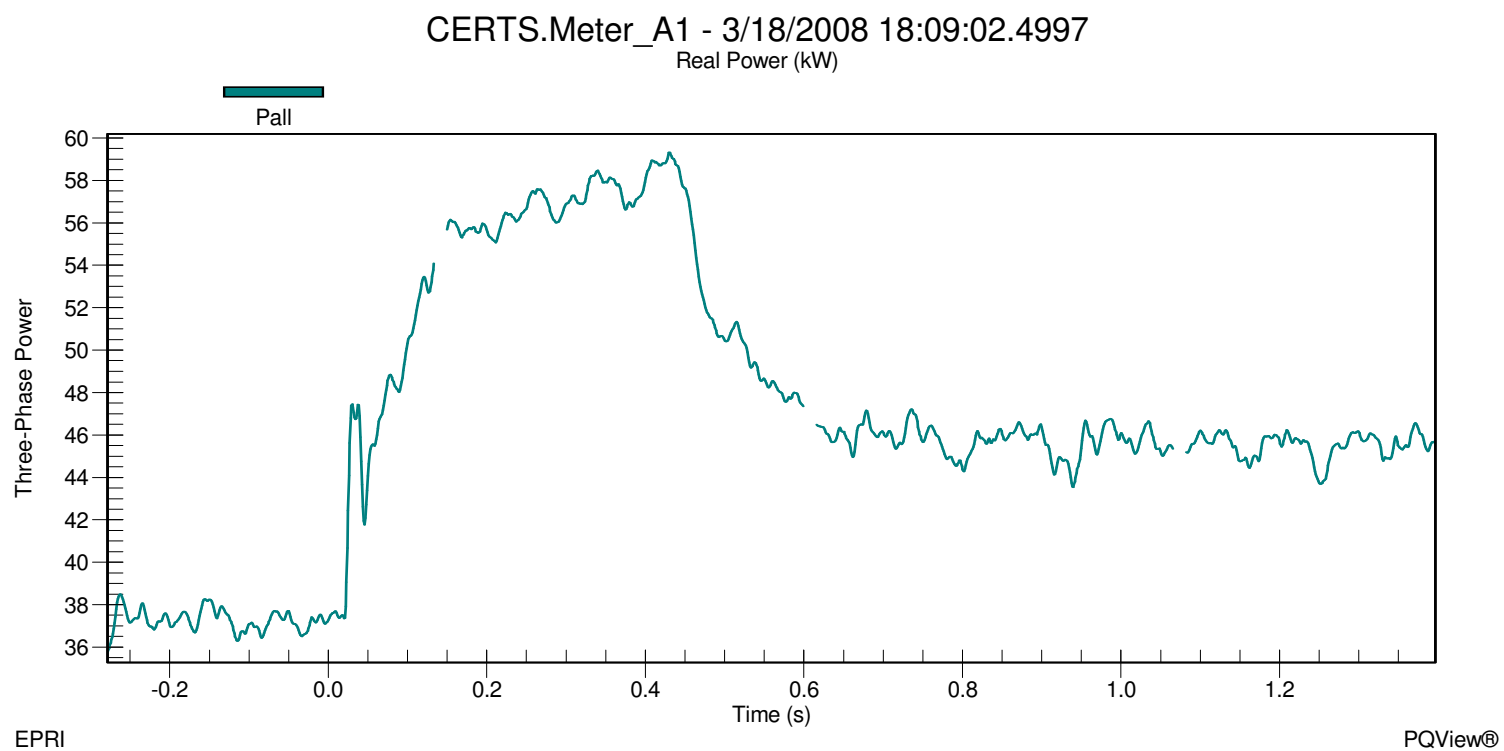

Figure 9m - Gen-set A1 Real Power during Motor Start and Islanded for Test 10.2.18

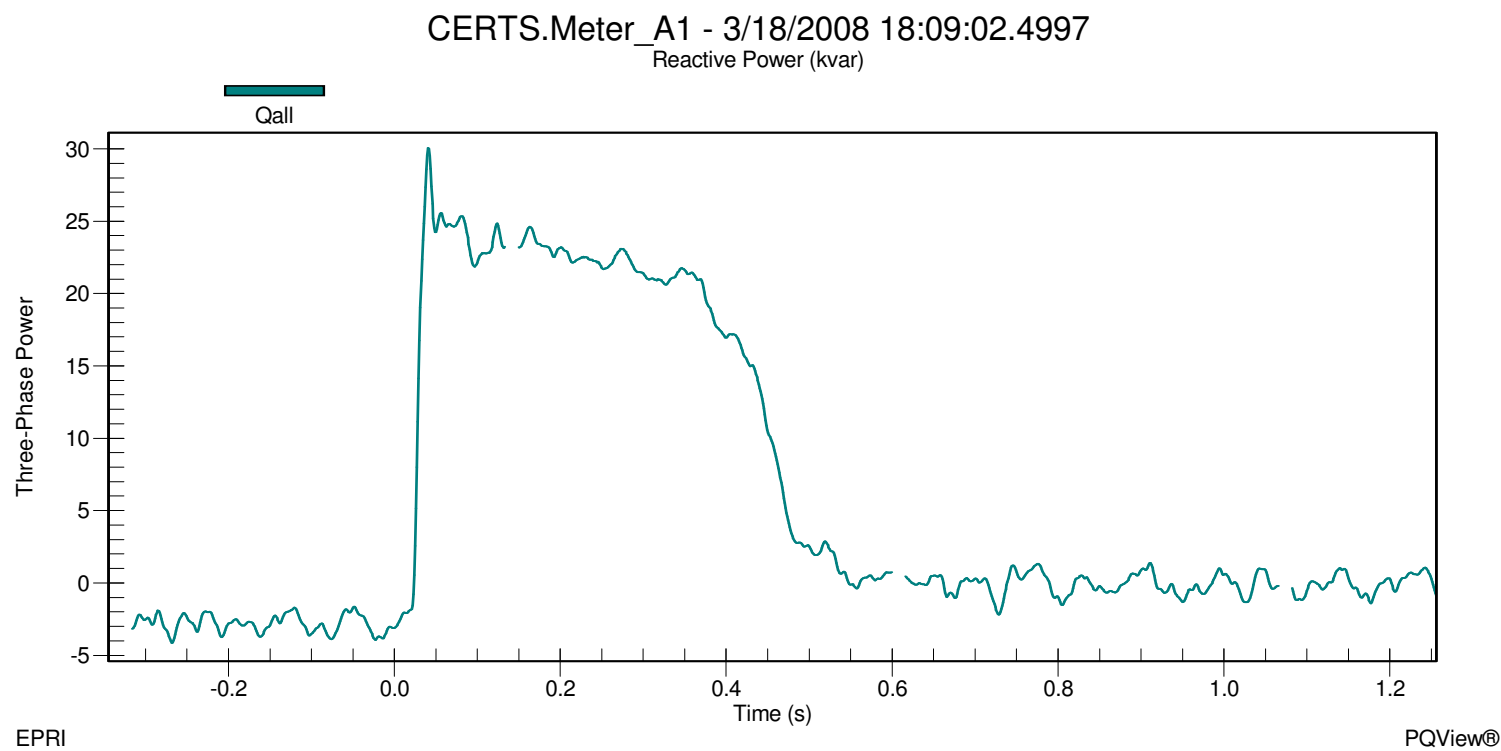

Figure 9n - Gen-set A1 Reactive Power during Motor Start and Islanded for Test 10.2.18 


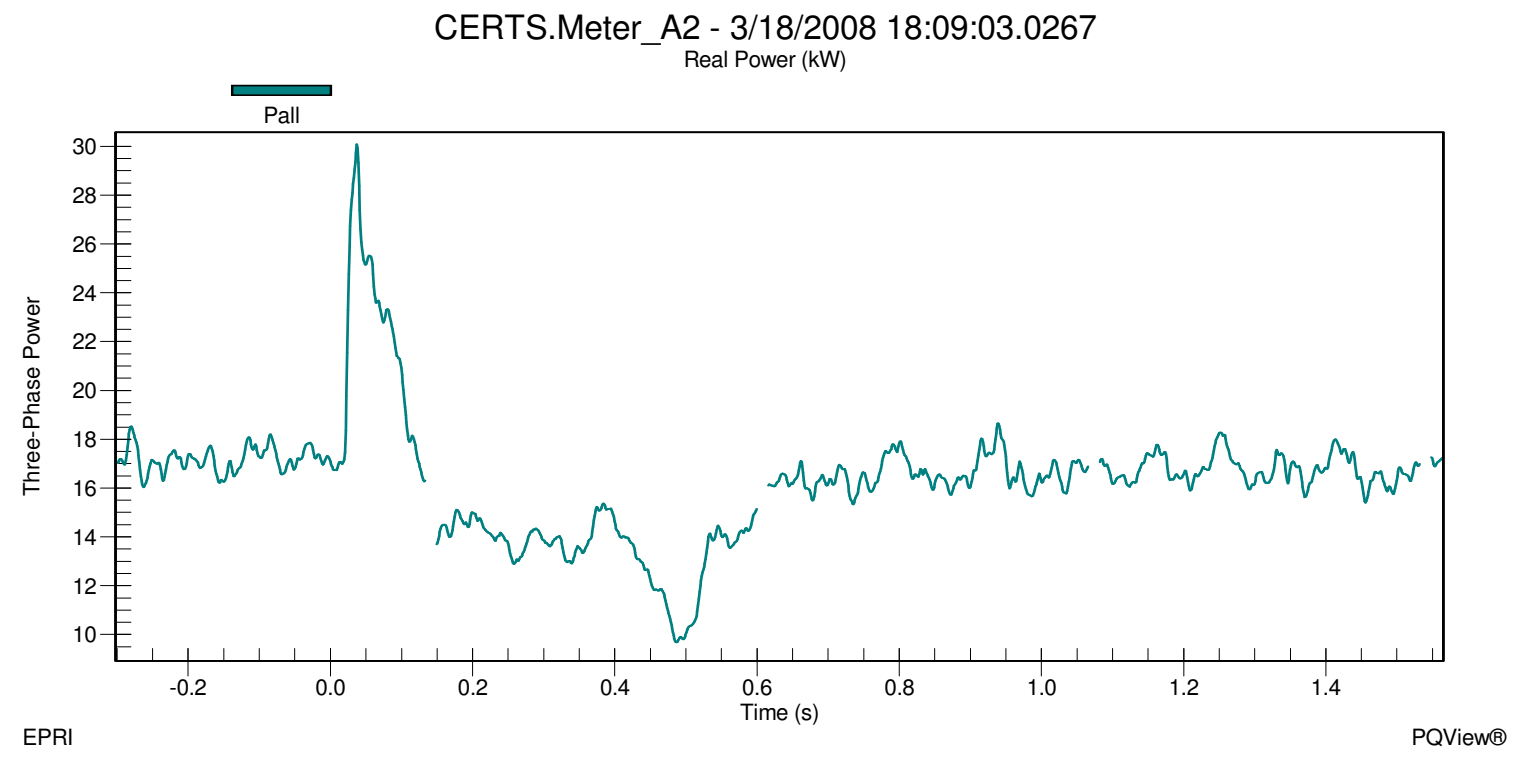

Figure 9o - Gen-set A2 Real Power during Motor Start and Islanded for Test 10.2.18 


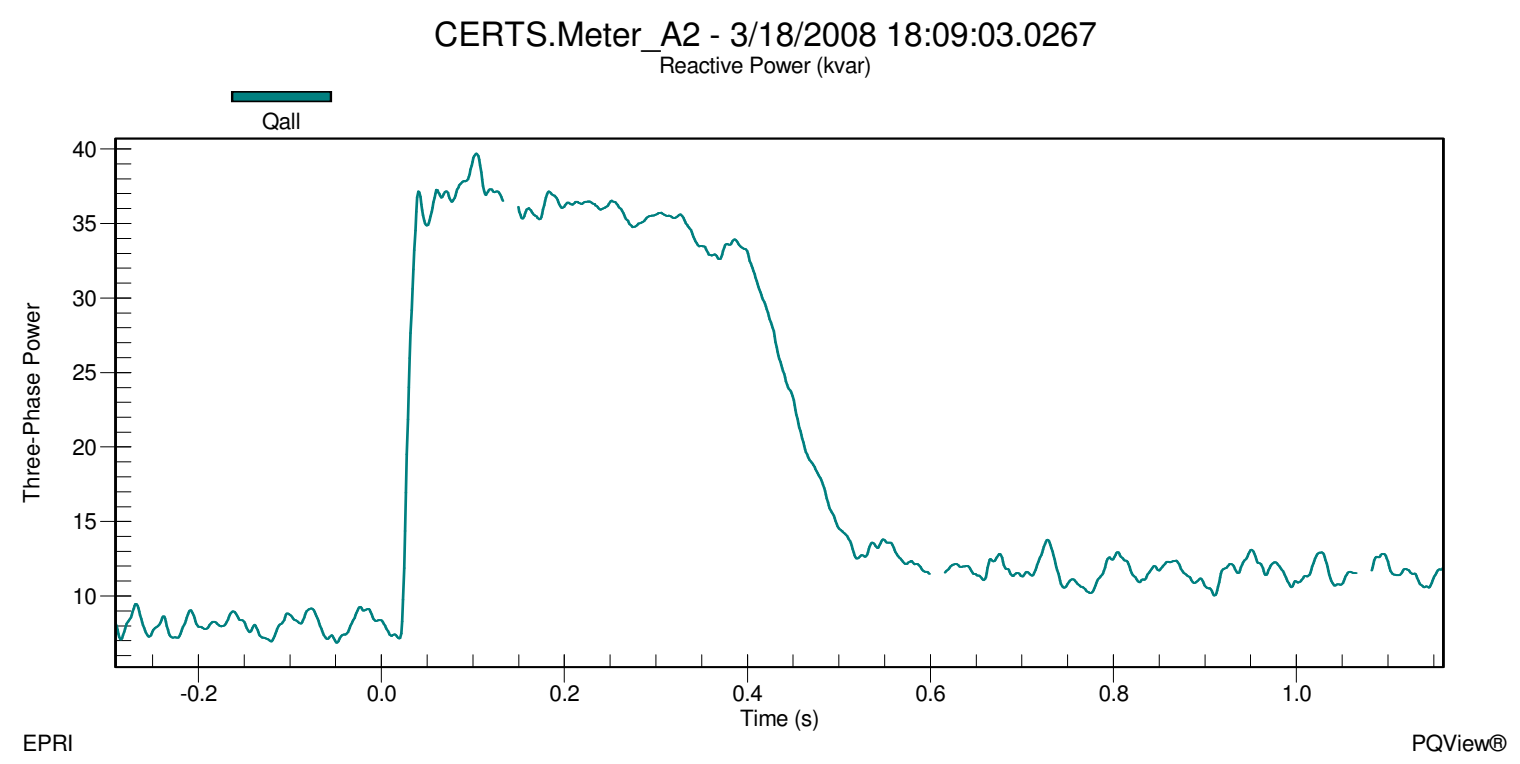

Figure 9p - Gen-set A2 Reactive Power during Motor Start and Islanded for Test 10.2.18

As soon as the static switch closed, Meter 1 recorded real power decreased from approximately $37 \mathrm{~kW}$ to $30 \mathrm{~kW}$ and reactive power increased from approximately $1.5 \mathrm{kVAr}$ to $29 \mathrm{kVAr}$ which means that the utility was satisfying a portion of the load demand in Load Bank 6 and all the reactive power in the microgrid. Figures $9 \mathrm{q}$ and $9 \mathrm{r}$ show the static switch real power decreasing from approximately $0 \mathrm{~kW}$ to $-5 \mathrm{~kW}$ and reactive power increasing from approximately $0 \mathrm{kVAr}$ to $27.2 \mathrm{kVAr}$. At the beginning of the test, the initial power flow through the static switch was $-2.5 \mathrm{~kW}+\mathrm{j} 20 \mathrm{kVAr}$ which is not the same recorded at this point in the test because the $10 \mathrm{Hp}$ motor load is on in Zone 3. The Gen-sets have picked up the motor load and supporting Load Bank 6 with approximately $5 \mathrm{~kW}$.

Load Banks 3 and 4 loads increased slightly to $25.1 \mathrm{~kW}+\mathrm{j} 6.49 \mathrm{kVAr}$ and $35.8 \mathrm{~kW}+$ j0.714kVAr, respectively. This slight load increase is a result from a voltage rise in the microgrid, shown in Figure 9s, from approximately $273.2 \mathrm{~V}$ on A-phase, $273.2 \mathrm{~V}$ on Bphase and $273.2 \mathrm{~V}$ on C-phase when islanded to $282 \mathrm{~V}$ on A-phase, $279.5 \mathrm{~V}$ on B-phase and $279.5 \mathrm{~V}$ on C-phase at Meter 3 when connected to the utility grid. 


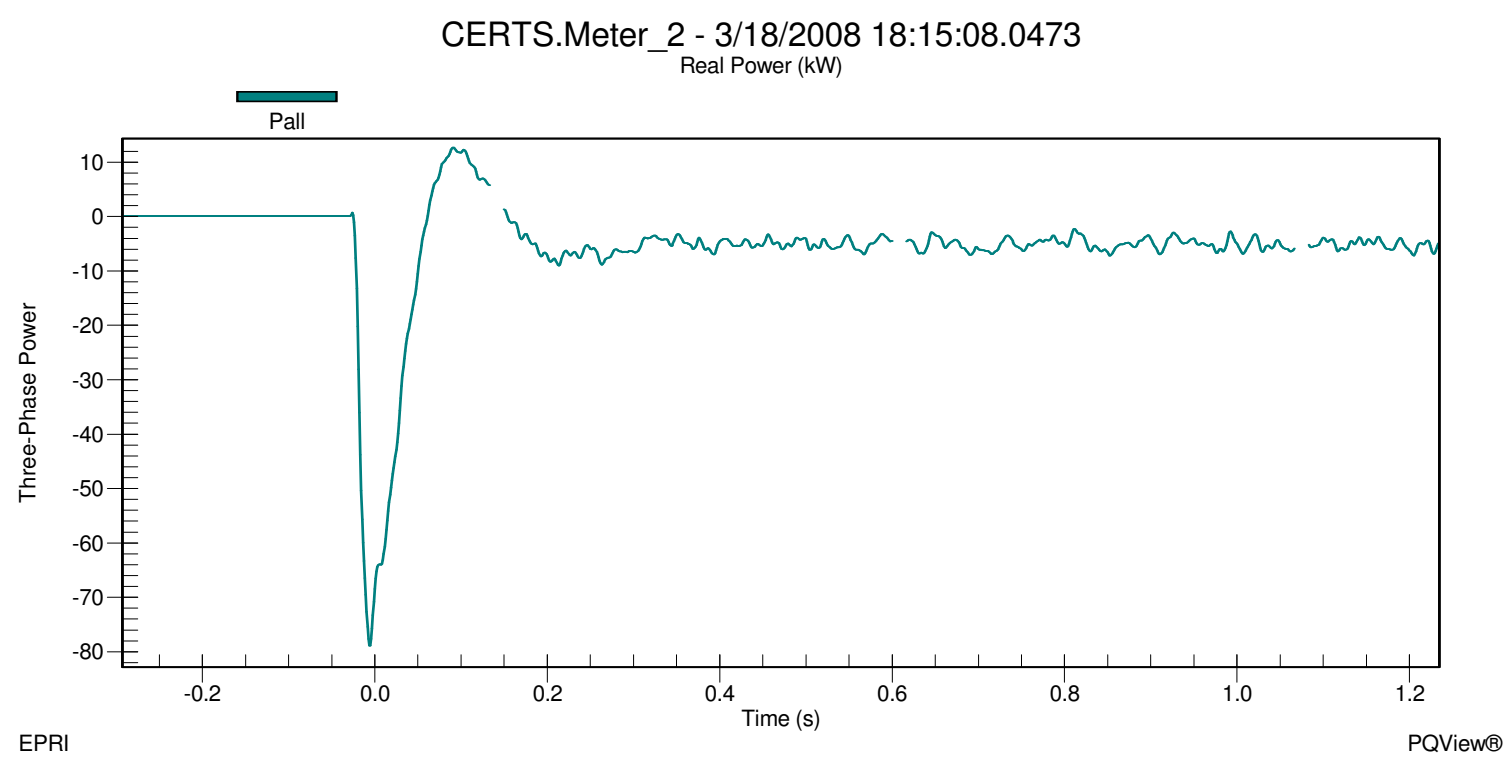

Figure 9q - Static Switch Real Power during Island to Utility Connected mode for Test 10.2.18

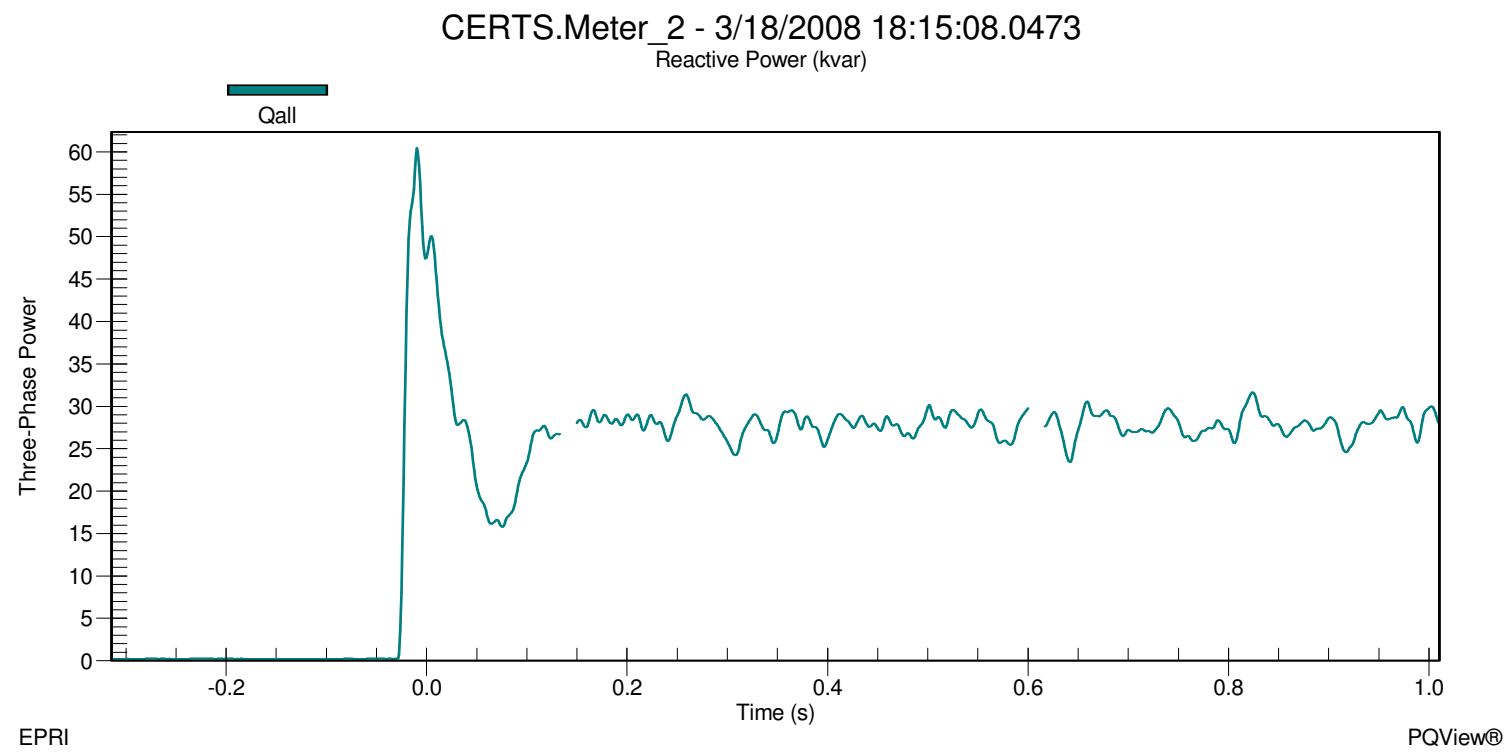

Figure 9r - Static Switch Reactive Power during Island to Utility Connected mode for Test 10.2.18 


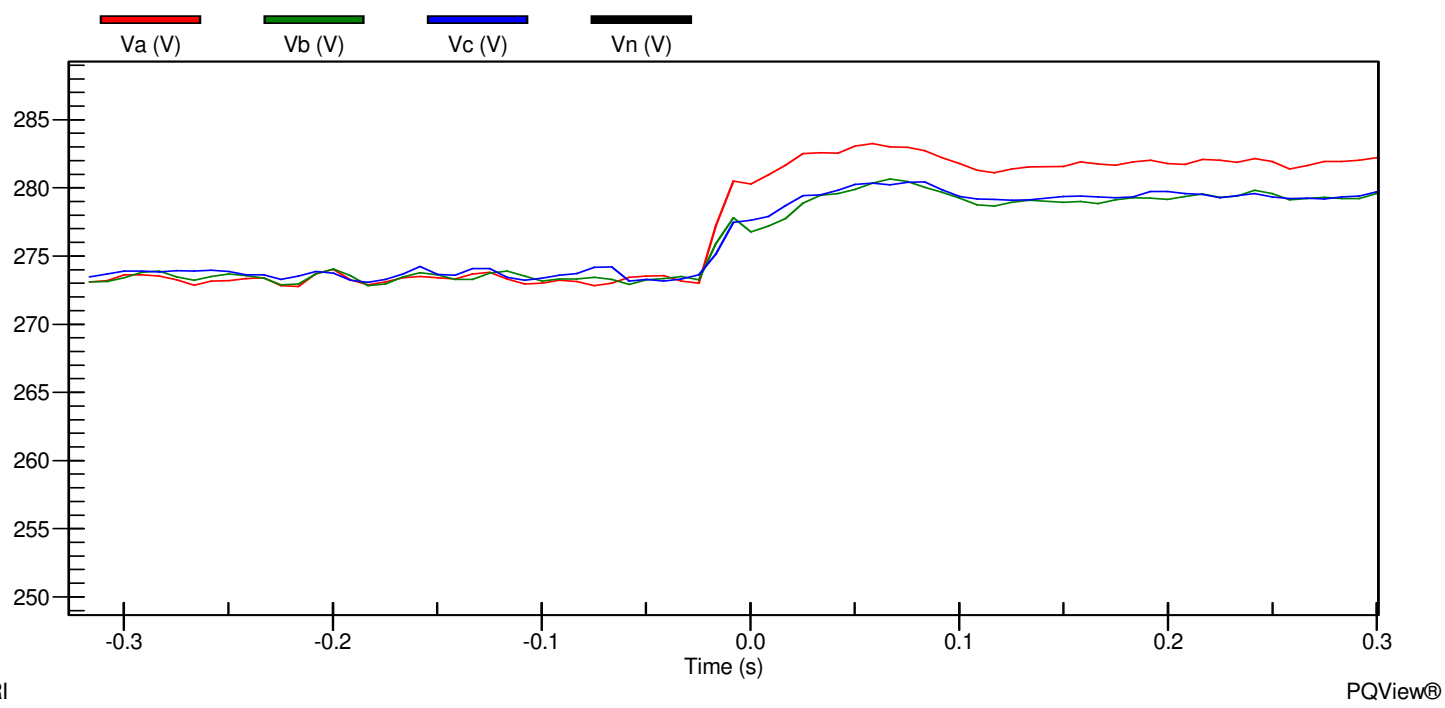

Figure 9s - Meter 3 Line-to-Ground Voltages during Island to Utility Connected mode for Test 10.2.18

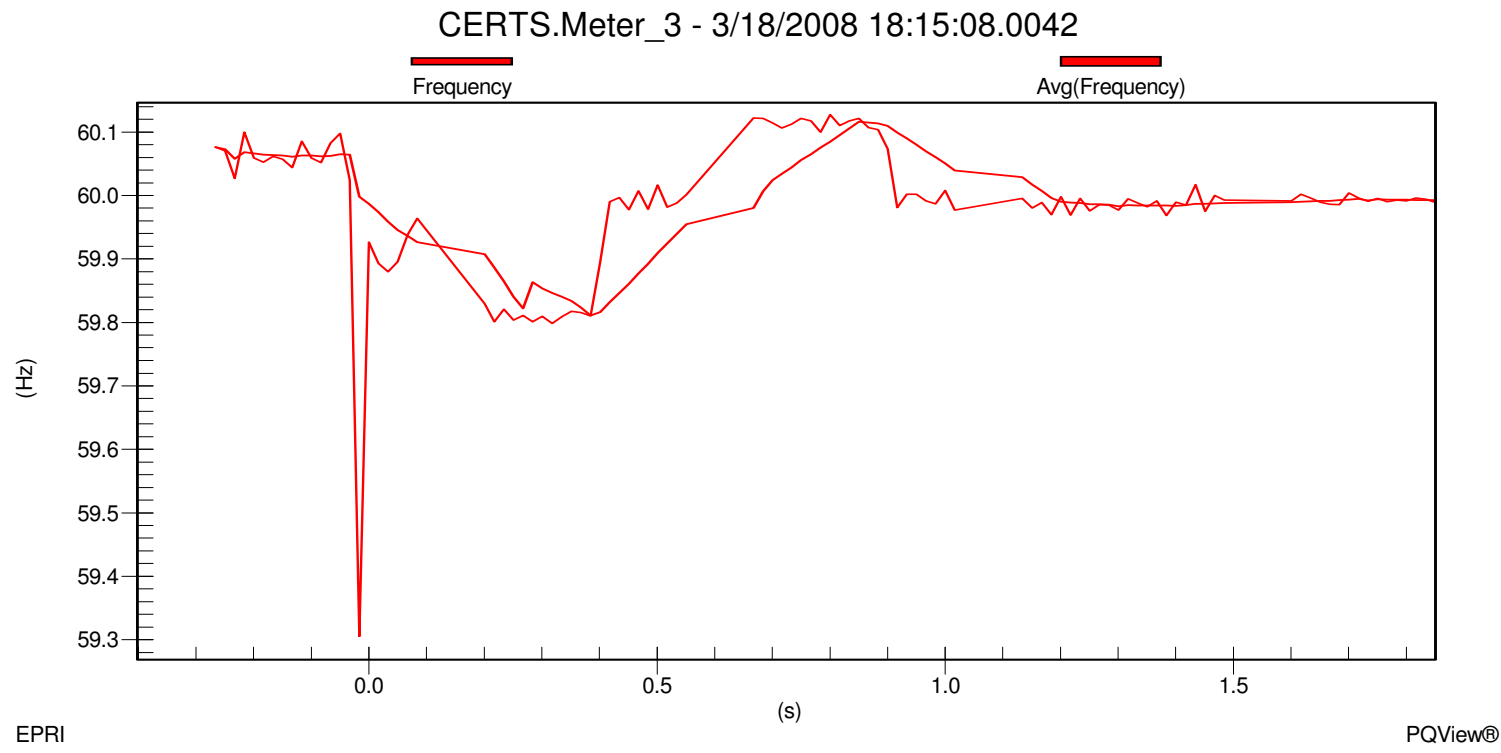

Figure 9t - Meter 3 Frequency during Island to Utility Connected mode for Test 10.2.18

Frequency change in the microgrid, shown in Figure 9t, decreased from approximately $60.06 \mathrm{~Hz}$ when islanded to approximately $59.99 \mathrm{~Hz}$ when connected to the utility grid. This change in frequency is due to the frequency no longer being established by the Gensets using the CERTS algorithm but by the utility. Gen-sets A1 and A2 are in zone power control mode therefore when the static switch closed back into the utility the Gen-sets produced real power based on set-points for the power flow through Zones 3 and 4 initialized at the beginning of the test. The real power in both Gen-sets increased from their initial values at the beginning of the test to approximately $42 \mathrm{~kW}$ for Gen-set 
A1 and 26kW for Gen-set A2. This increase is because the Gen-sets have picked up the real power of the induction motor in Zone 3. Reactive powers for both Gen-sets were relatively close to the values at the beginning of the test with approximately $-12.5 \mathrm{kVAr}$ for Gen-set A1 and -3kVAr for Gen-set A2. After all the data was verified and recorded into the DAS Database, the motor was turned off and the Gen-sets and Load Banks setpoints were changed according to the next test (10.2.20) in Table 1.

For Test 10.2.20 the measured values, after Gen-set A1 was warmed up and load banks brought on-line, were approximately $22 \mathrm{~kW}+\mathrm{j} 19 \mathrm{kVAr}$ at Meter $1,-15 \mathrm{~kW}+\mathrm{j} 18 \mathrm{kVAr}$ at Meter 2 and $-17 \mathrm{~kW}+\mathrm{j} 8 \mathrm{kVAr}$ at Meter 3. From the microgrid, $35.8 \mathrm{~kW}-\mathrm{j} 12 \mathrm{kVAr}$ was produced by Gen-set A1. The load banks were $18 \mathrm{~kW}+\mathrm{j} 0 \mathrm{kVAr}$ at LB3 and $35.8 \mathrm{~kW}+$ j0.775kVAr at LB6. These measurements were relatively close to the expected values in Table 1, but not exact due to temperature, phase voltages and electrical losses in conductors. In addition, the $40 \mathrm{~kW}$ setting for LB6 and $20 \mathrm{~kW}$ setting for LB3 were also below selected set values. At the time of these measurements, the voltage and frequency was $282.2 \mathrm{~V}$ on A-phase, $278.8 \mathrm{~V}$ on B-phase and $278.8 \mathrm{~V}$ on C-phase and $60.00 \mathrm{~Hz}$ at the static switch (i.e., Meter 2) when connected to the utility grid; and 282.5V on A-phase, $279.5 \mathrm{~V}$ on B-phase and $279.5 \mathrm{~V}$ on C-phase and $60.00 \mathrm{~Hz}$ at Meter 3.

Gen-set A1 in this test was set up to produce -20kW going through Zone 3 (i.e., Meter 3) which means it produced $20 \mathrm{~kW}$ more power than Load Bank 3 needed. Approximately $17 \mathrm{~kW}$ of excess power was exported out of Zone 3 and $15 \mathrm{~kW}$ of that power went through the static switch to Load Bank 6 and the remaining $2 \mathrm{~kW}$ was due to power losses in the conductors. Since Load Bank 6 was approximately $35.8 \mathrm{~kW}+\mathrm{j} 0.775 \mathrm{kVAr}$, the utility had to supply approximately $22 \mathrm{~kW}$ to satisfy the load. Reactive power had to be imported in from the utility of approximately 19kVAr because Gen-set A1 needed approximately $12 \mathrm{kVAr}$ and losses in the conductors. 


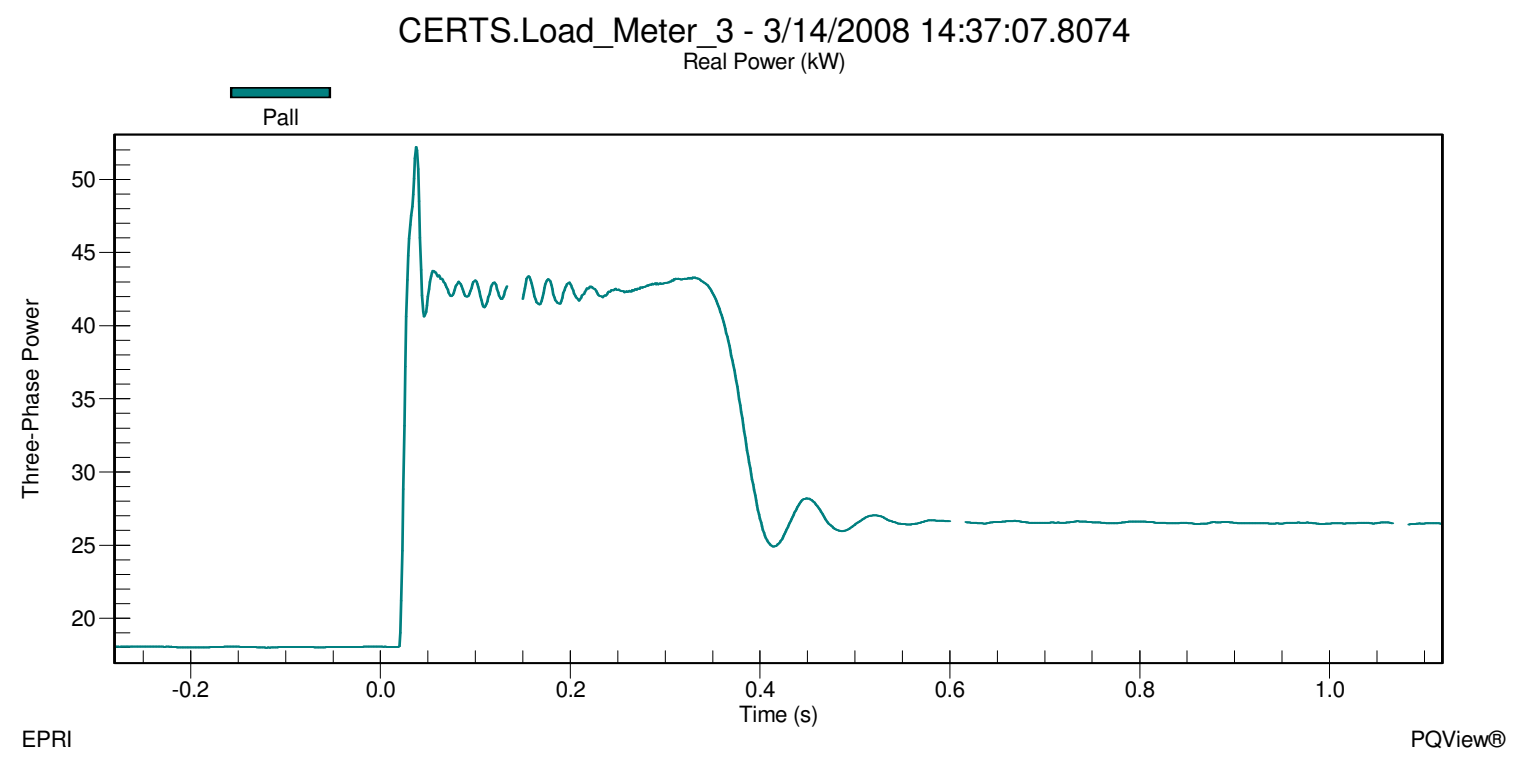

Figure 10a - Load Bank 3 Real Power during Motor Start and Utility Connected for Test 10.2.20

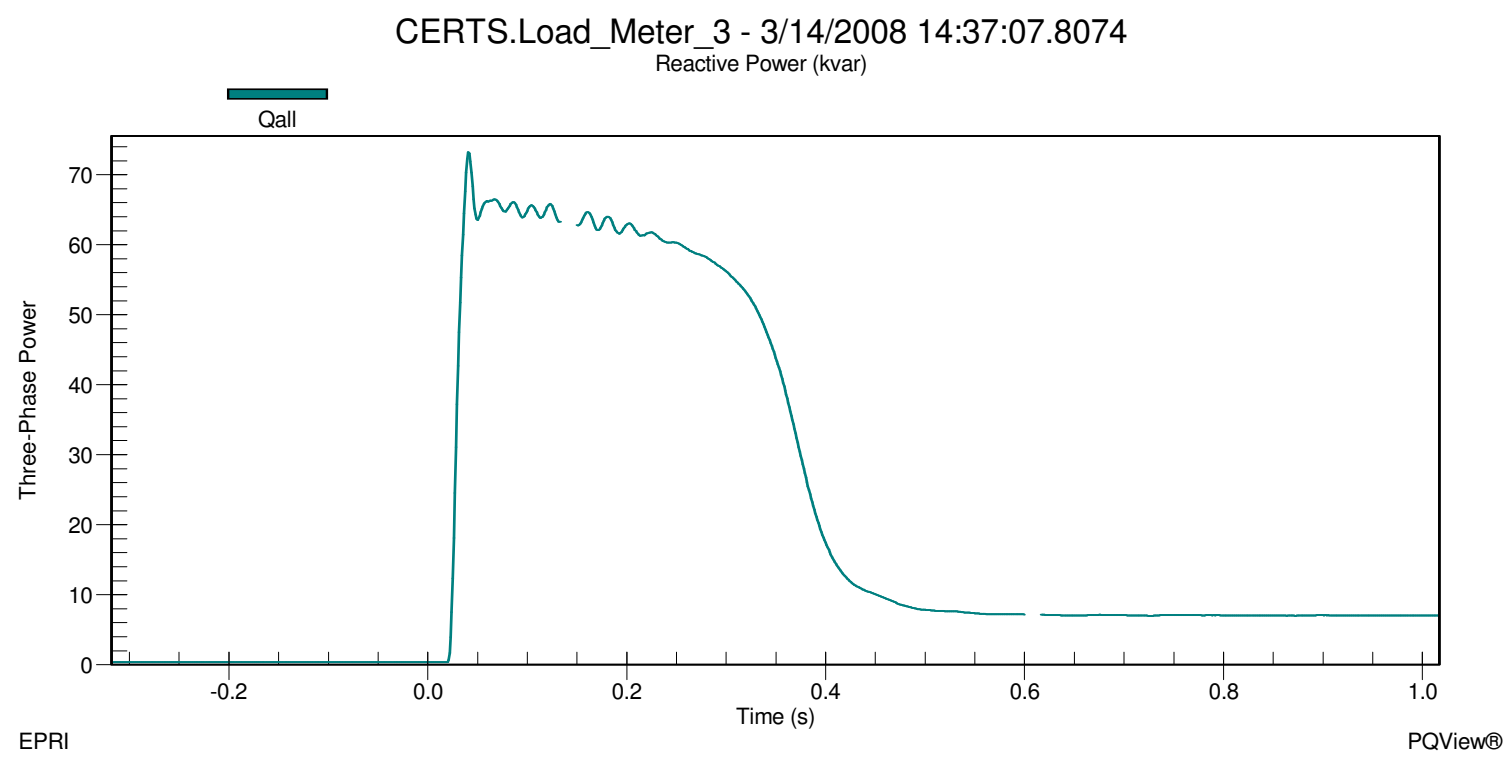

Figure 10b - Load Bank 3 Reactive Power during Motor Start and Utility Connected for Test 10.2.20 
In Figures 10a and 10b it can be seen that the load in Zone 3 was approximately $18 \mathrm{~kW}+$ j0kVAr before the start of the induction motor and increased to approximately $52.2 \mathrm{~kW}+$ j73.1kVAr during the inrush phase of the motor start. After about 1.5 cycles, the motor settled down to approximately $42.5 \mathrm{~kW}+\mathrm{j} 62 \mathrm{kVAr}$ during the warm up phase which lasted about 33 cycles ( 0.55 Seconds). When the motor reached steady state, the load in Zone 3 was approximately $26.5 \mathrm{~kW}+\mathrm{j} 7 \mathrm{kVAr}$.

The voltage and frequency at the static switch before the motor start was approximately 282.2V on A-phase, $278.8 \mathrm{~V}$ on B-phase and $278.8 \mathrm{~V}$ on C-phase shown in Figure 10c and approximately $60.00 \mathrm{~Hz}$ shown in Figure 10d. When the motor started, the voltage at the static switch during the inrush decreased to approximately $274.3 \mathrm{~V}$ on A-phase, $270.6 \mathrm{~V}$ on B-phase and $270.8 \mathrm{~V}$ on C-phase for about 1.5 cycles. Frequency dropped during the inrush to approximately $59.90 \mathrm{~Hz}$ and quickly increased to approximately $60.00 \mathrm{~Hz}$. Voltage increased as the motor was warming up and eventually settled at a steady state voltage at approximately $281.6 \mathrm{~V}$ on A-phase, $278.2 \mathrm{~V}$ on B-phase and $278.2 \mathrm{~V}$ on C-phase at an approximate frequency $60.00 \mathrm{~Hz}$.

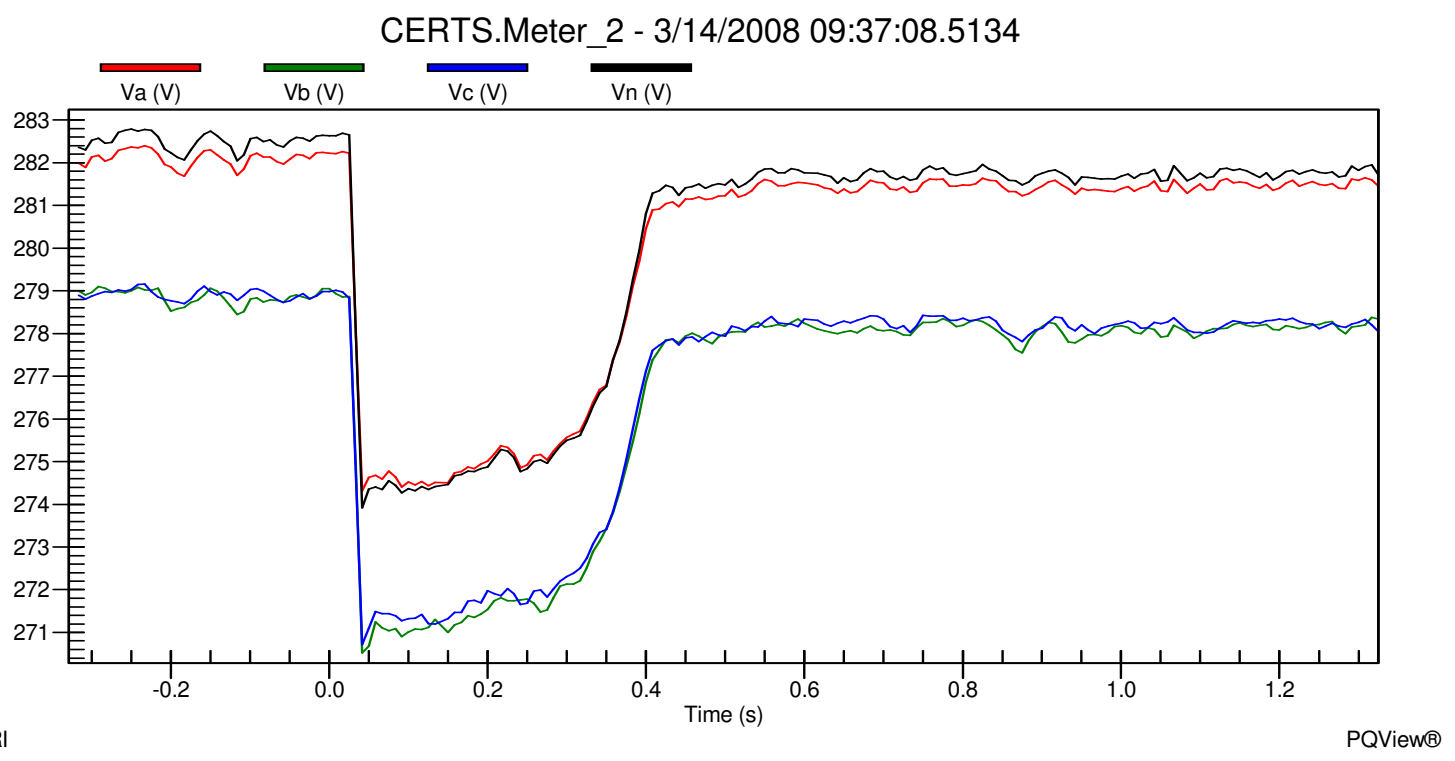

Figure 10c - Static Switch Line-to-Ground Voltage during Motor Start and Utility Connected for Test 10.2.20 


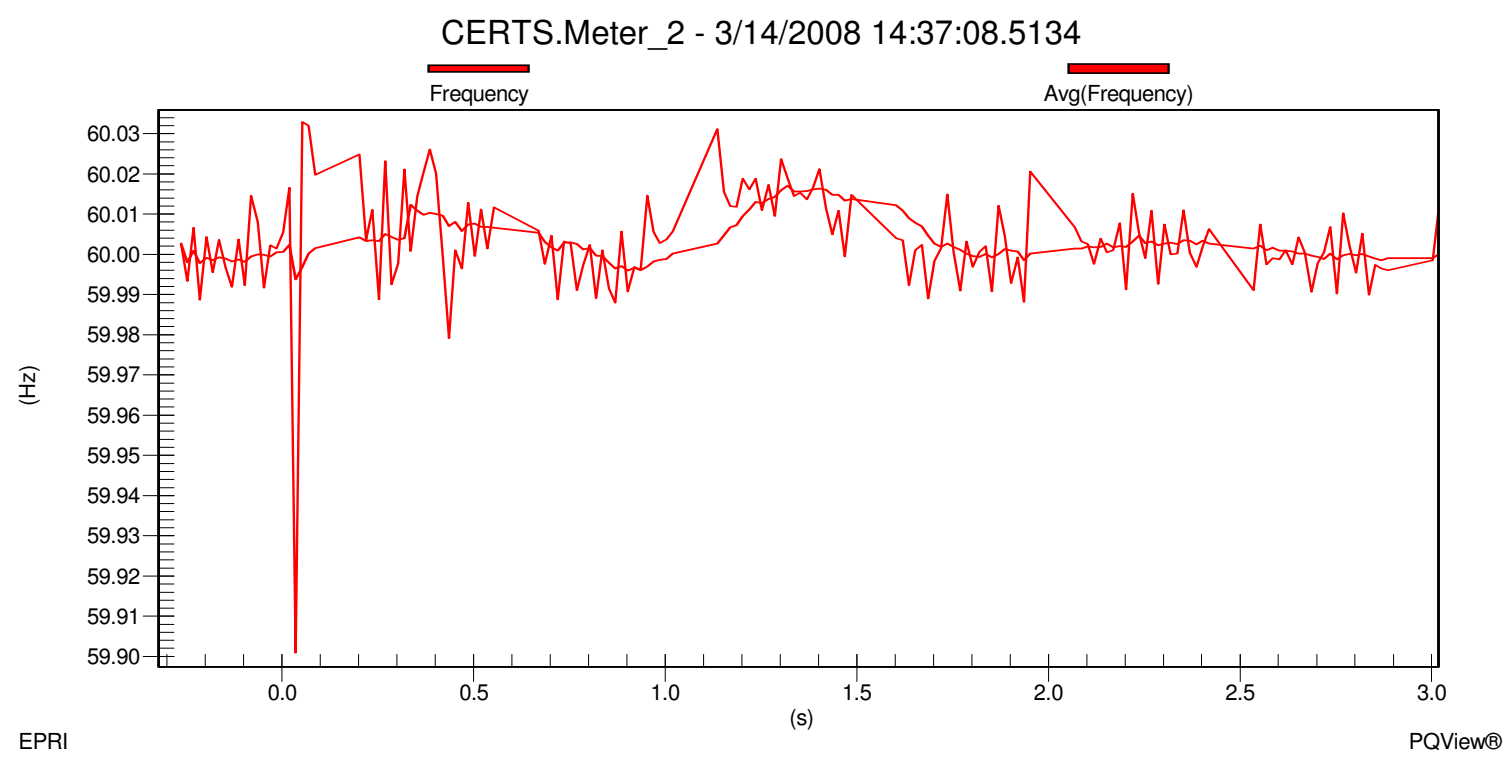

Figure 10d - Static Switch Frequency during Motor Start and Utility Connected for Test 10.2.20

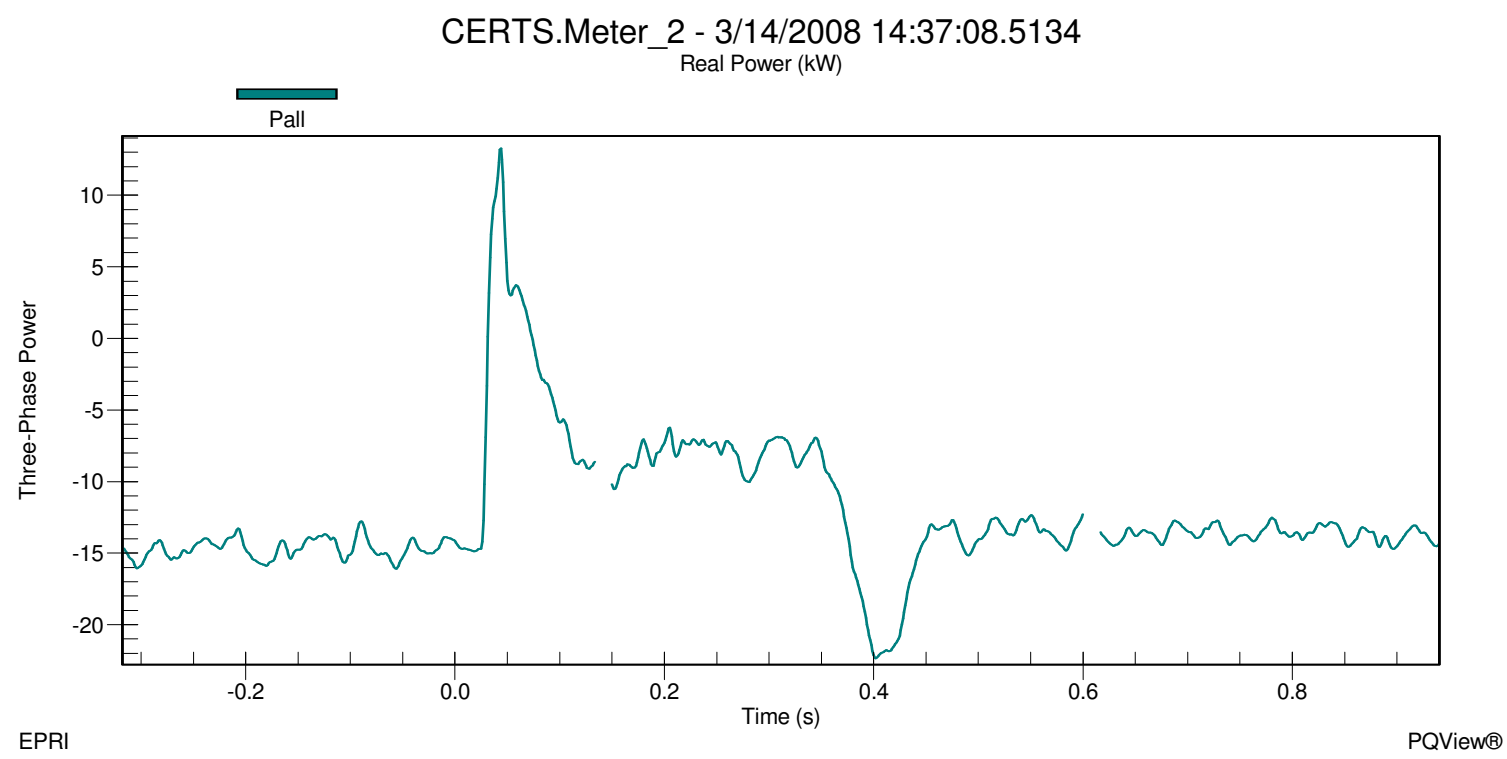

Figure 10e - Static Switch Real Power during Motor Start and Utility Connected for Test 10.2.20 


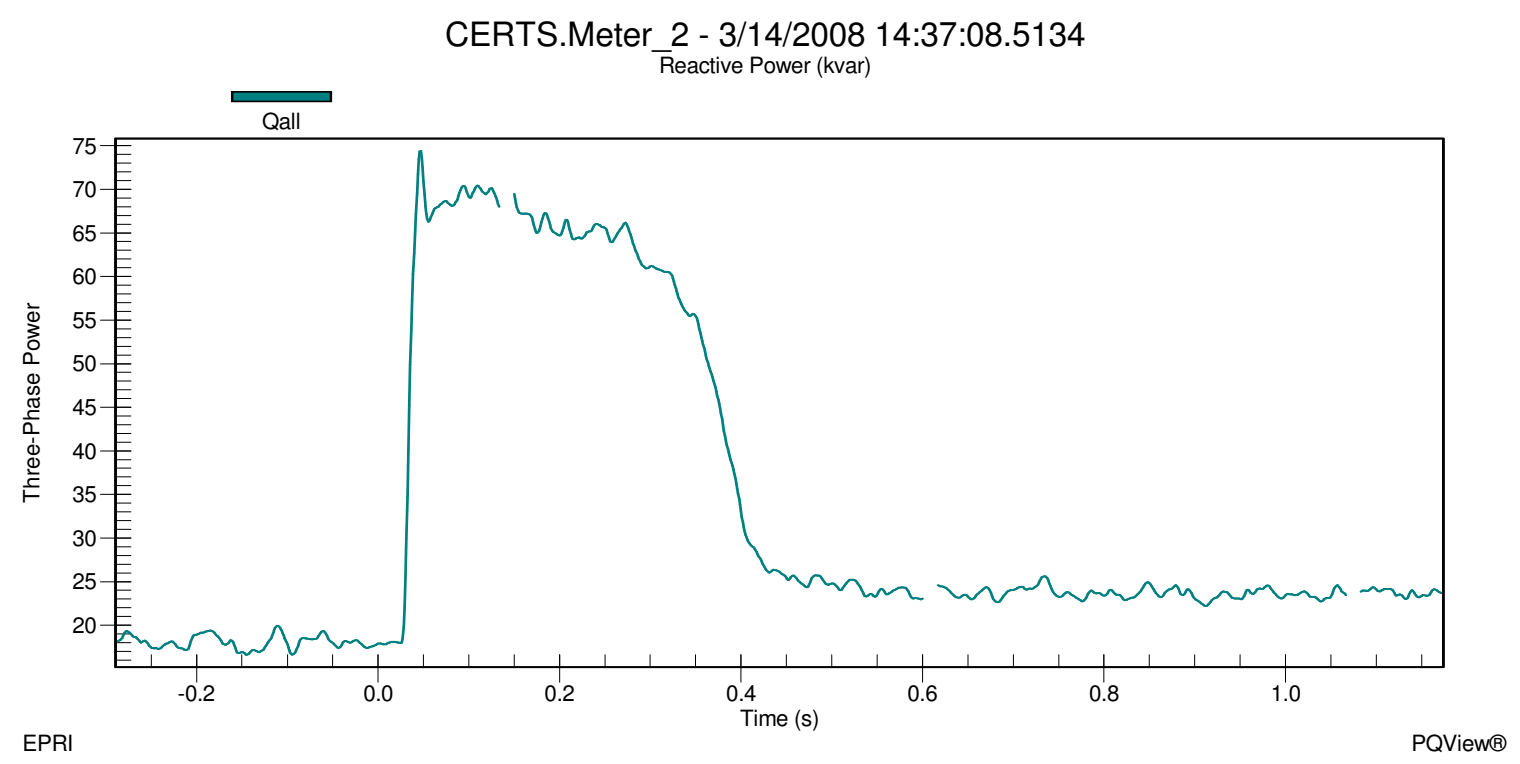

Figure 10f - Static Switch Reactive Power during Motor Start and Utility Connected for Test 10.2.20

Before the motor started Gen-set A1 was producing approximately $35.8 \mathrm{~kW}$ - j12kVAr. This was enough power for the load demands of Load Bank 3 with real power being exported to Zone 6 of approximately 15kW shown in Figure 10e. The grid was supporting the reactive power of the microgrid with approximately $19 \mathrm{kVAr}$ shown in Figure 10f. When the motor started the inrush caused the utility to supply approximately $74.5 \mathrm{kVAr}$ and to pick up the load demand of Load Bank 6 and approximately $13.3 \mathrm{~kW}$ of the load demand in Zone 3. The power that was being supplied by Gen-set A1 to Load Bank 6 was now supplying the motor inrush which can be seen in Figure 9e as the real power through the static switch becomes positive. Genset A1 increased its real and reactive power output levels to approximately $55.1 \mathrm{~kW}+$ j2.9kVAr. Notice the signs of the VAr output changed from negative to positive for Genset A1 in order to support the induction motor load in Zone 3.

When the motor reached steady state, the real and reactive power through the static switch was approximately $-14 \mathrm{~kW}+\mathrm{j} 23.5 \mathrm{kVAr}$ which meant that Gen-set A1 was supplying the power for the induction motor and approximately $14 \mathrm{~kW}$ of Load Bank 6 . The real power in Gen-set A1 increased from its initial value at the beginning of the test to approximately $43.2 \mathrm{~kW}$ and the reactive power went relatively back to the initial value before the motor started of approximately $-11 \mathrm{kVAr}$. Once all the data was verified and recorded into the DAS Database, the motor was shut down and the static switch was directed by the EMS to manually open.

As soon as the static switch opened, Meter 1 recorded real power increased to approximately $36.9 \mathrm{~kW}$ and reactive power decreased to $1 \mathrm{kVAr}$ satisfying the load 
demand in Load Bank 6 which was approximately $36.5 \mathrm{~kW}+$ j0.781kVAr and not supplying any power beyond the static switch to Load Bank 3. 0kW + j0kVAr was recorded at the static switch, indicating that power was not flowing through the static switch. Load Bank 3 loads reduced slightly to $17.05 \mathrm{~kW}+\mathrm{j} 0 \mathrm{kVAr}$. This load reduction resulted from a voltage drop in the microgrid, shown in Figure $10 \mathrm{~g}$, from approximately $282.5 \mathrm{~V}$ on A-phase, $279.5 \mathrm{~V}$ on B-phase and $279.5 \mathrm{~V}$ on C-phase when connected to the utility to $272 \mathrm{~V}$ on A-phase, $272 \mathrm{~V}$ on B-phase and $272 \mathrm{~V}$ on C-phase at Meter 3 when islanded.

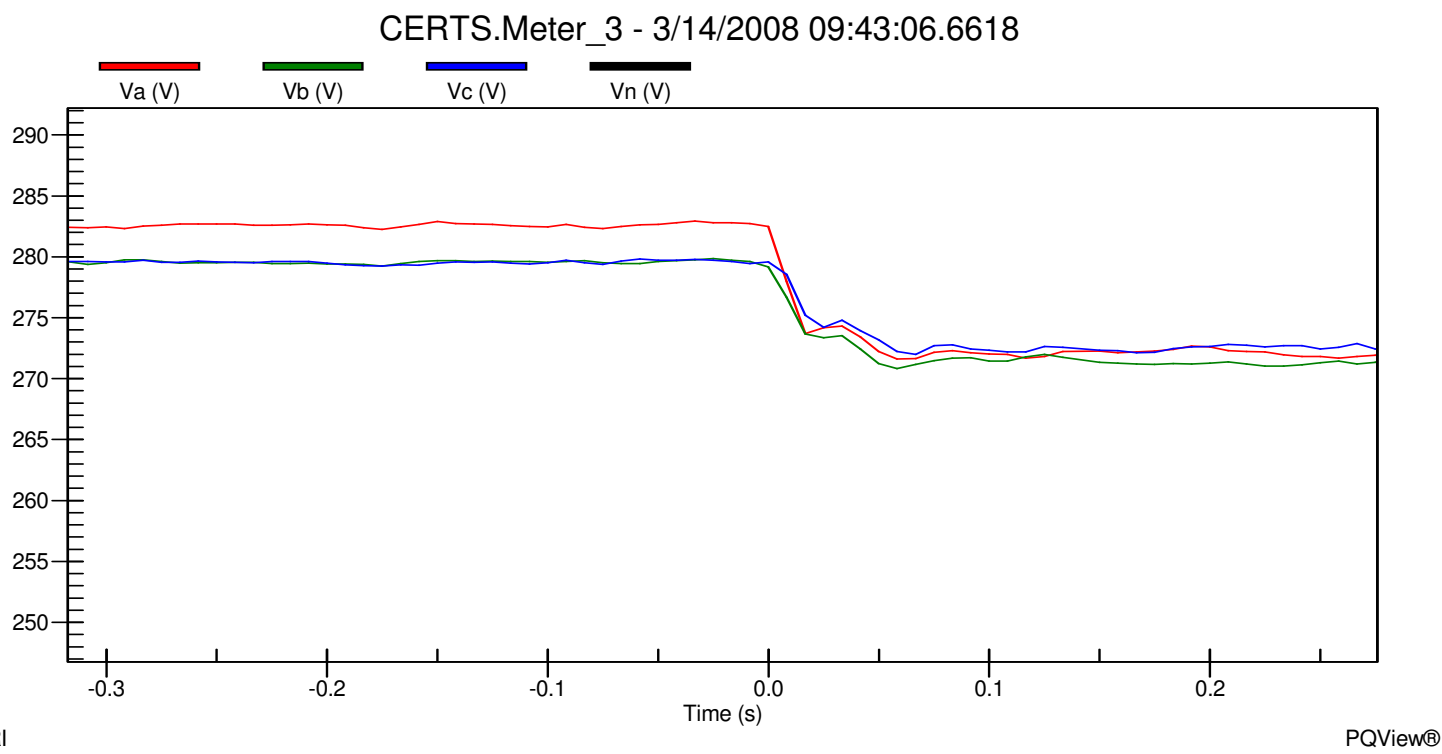

Figure 10g - Meter 3 Line-to-Ground Voltages during Utility Connected to Island mode for Test 10.2.20 


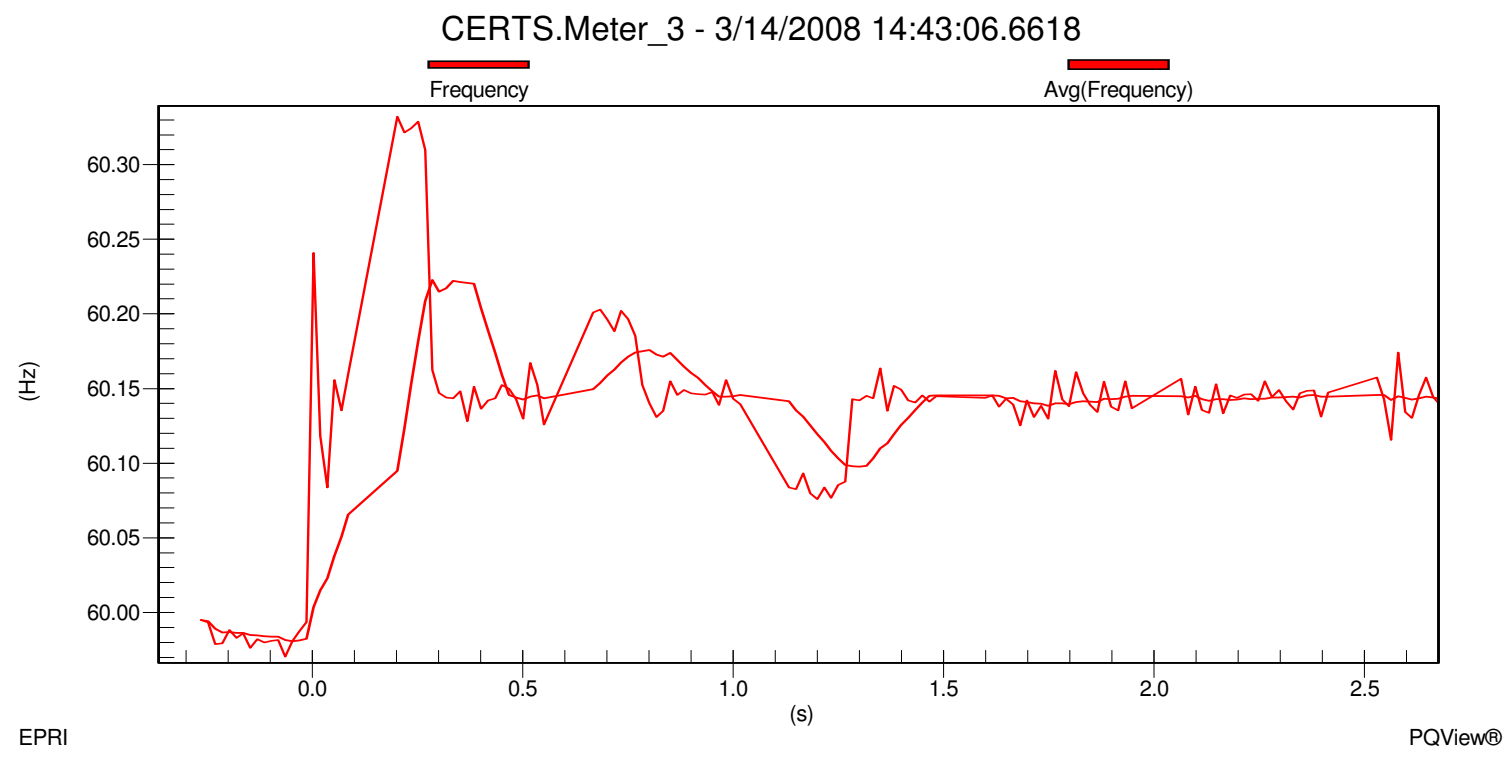

Figure 10h - Meter 3 Frequency during Utility Connected to Island mode for Test 10.2.20

Frequency change in the microgrid, shown in Figure 10h, increased from approximately $59.99 \mathrm{~Hz}$ when connected to the utility grid to approximately $60.15 \mathrm{~Hz}$ when islanded. This change in frequency was part of the CERTS algorithm which allowed Gen-set A1 to decrease its output real power to approximately $18.8 \mathrm{~kW}$. Reactive power in Gen-set A1 increased to approximately $4 \mathrm{kVAr}$. Meter 3 was approximately $-2.5 \mathrm{~kW}-3.2 \mathrm{kVAr}$ indicating that Gen-set A1 was satisfying the load in Load Bank 3 and the power losses in the electrical lines. All data was verified and recorded into the DAS Database. The microgrid ran for a couple of minutes in this electrical state before the $10 \mathrm{Hp}$ induction motor was started in Zone 3. 


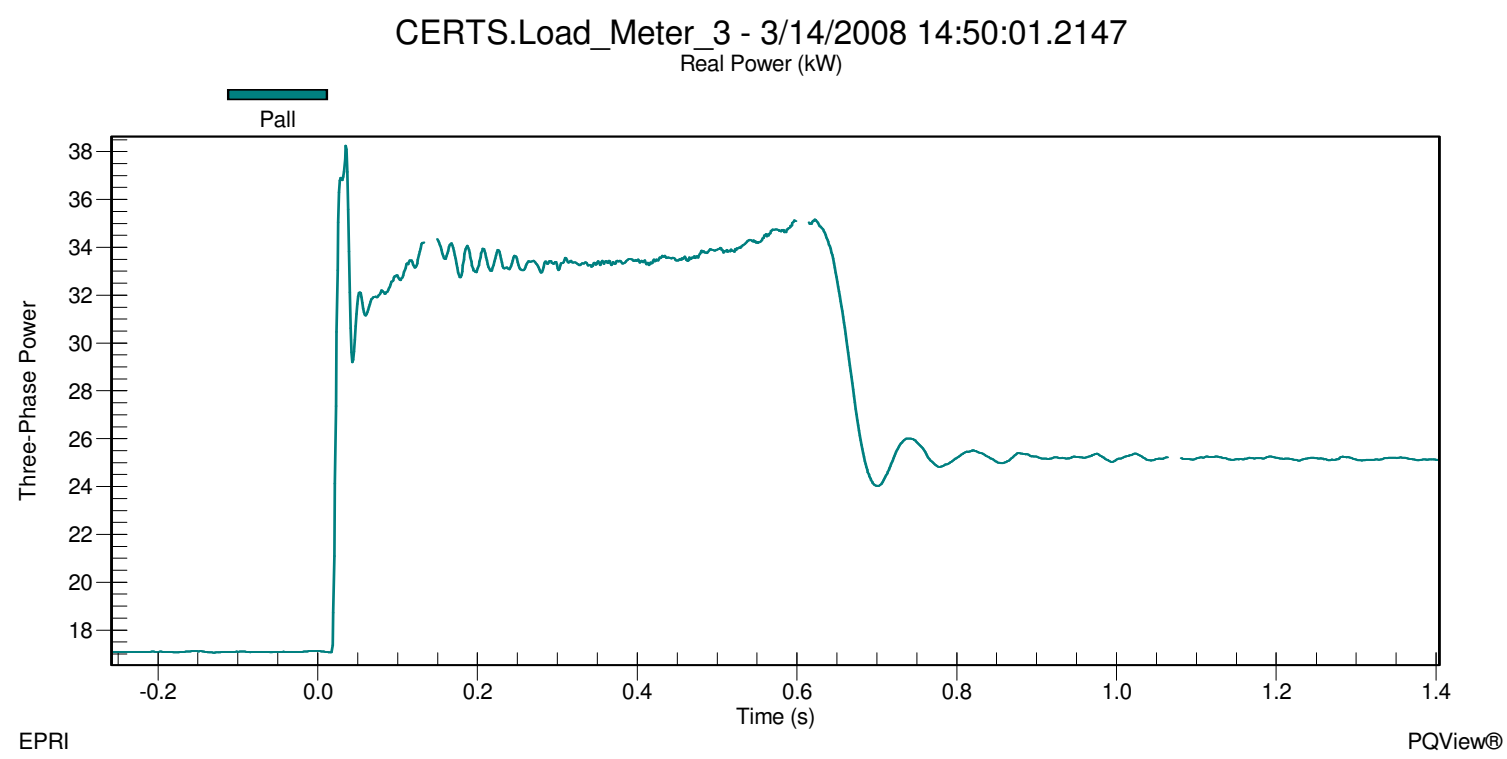

Figure 10i - Load Bank 3 Real Power during Motor Start and Islanded for Test 10.2.20

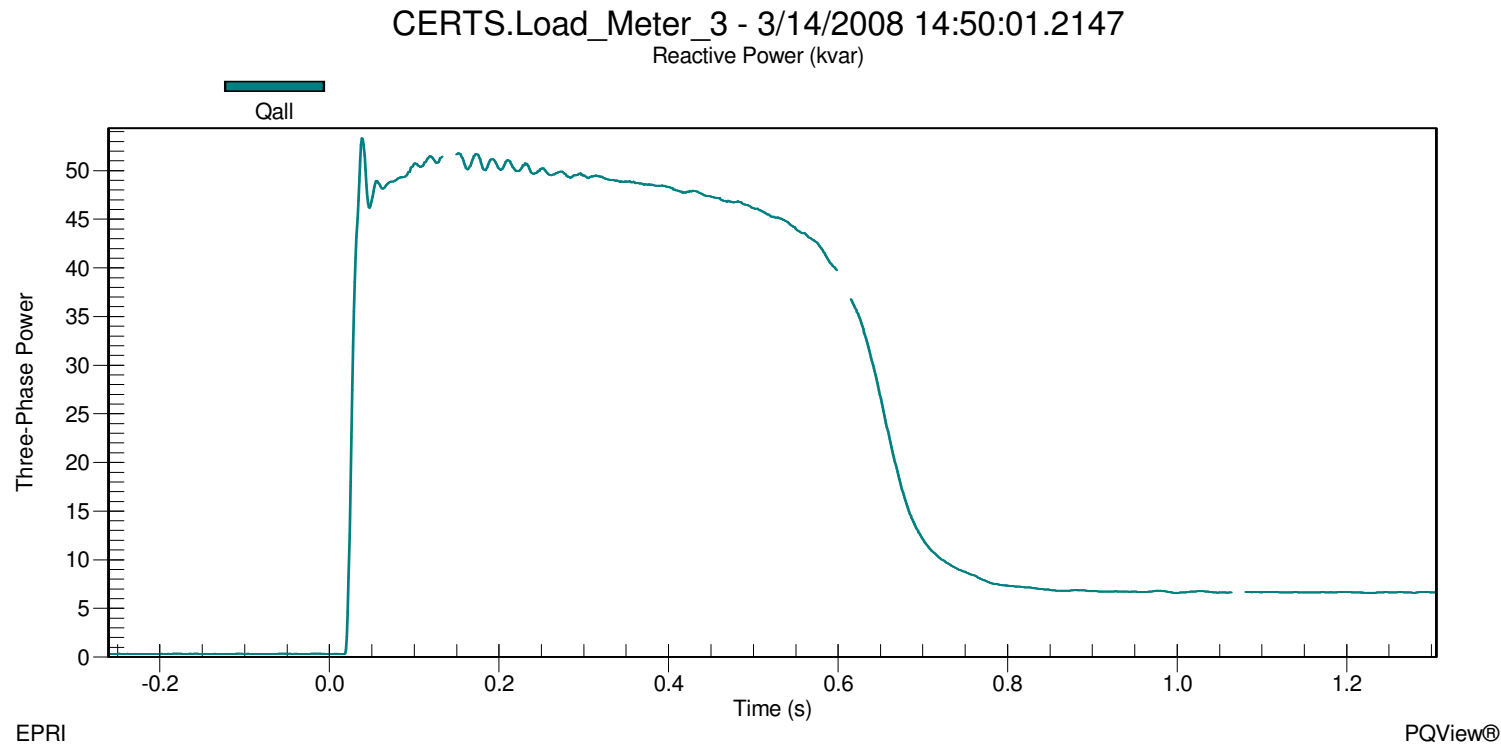

Figure 10j - Load Bank 3 Reactive Power during Motor Start and Islanded for Test 10.2.20

In Figures 10i and 10j it can be seen that the load in Zone 3 was approximately $16 \mathrm{~kW}+$ j0.3kVAr before the start of the induction motor and increased to approximately $38.3 \mathrm{~kW}$ + j53.2kVAr during the inrush phase of the motor start. After about 1.5 cycles, the motor settled down to approximately $34 \mathrm{~kW}+\mathrm{j} 48.3 \mathrm{kVAr}$ during the warm up phase which lasted about 49.2 cycles ( 0.82 Seconds). When the motor reached steady state, the load in Zone 3 was approximately $25.2 \mathrm{~kW}+\mathrm{j} 7 \mathrm{kVAr}$. 
The voltage and frequency at Meter 3 before the motor start was approximately $272 \mathrm{~V}$ on A-phase, 272V on B-phase and 272V on C-phase shown in Figure 10k and approximately $60.17 \mathrm{HZ}$ shown in Figure 101. When the motor started, the voltage at Meter 3 during the inrush decreased to approximately $235.5 \mathrm{~V}$ on A-phase, $235.5 \mathrm{~V}$ on B-phase and $235.5 \mathrm{~V}$ on C-phase for about 1.5 cycles. Frequency dropped during the inrush to approximately $59.99 \mathrm{~Hz}$. Voltage increased as the motor was warming up and eventually settled at a steady state voltage at approximately $269 \mathrm{~V}$ on A-phase, $269 \mathrm{~V}$ on B-phase and $269 \mathrm{~V}$ on C-phase at an approximate frequency of $60.15 \mathrm{~Hz}$.

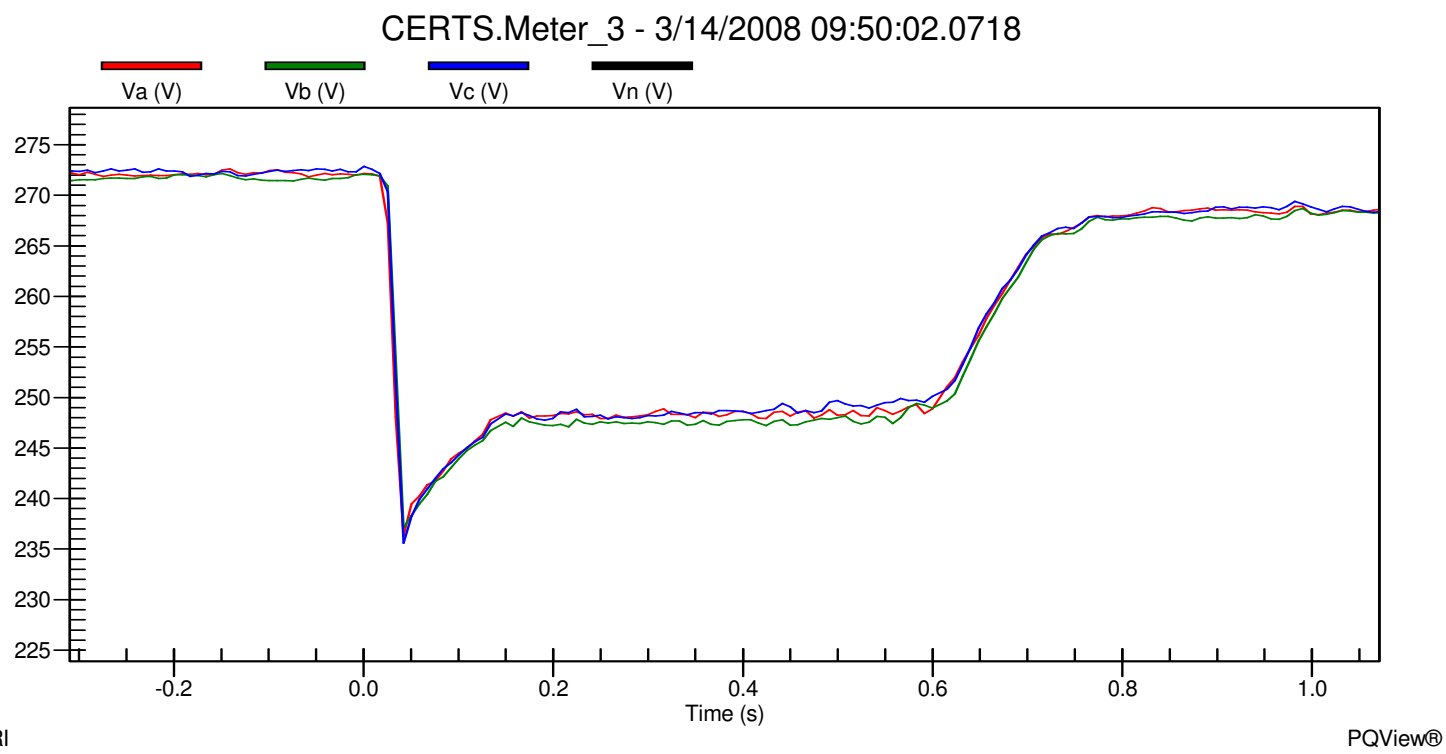

Figure 10k - Meter 3 Line-to-Ground Voltages during Motor Start and Islanded for Test 10.2.20

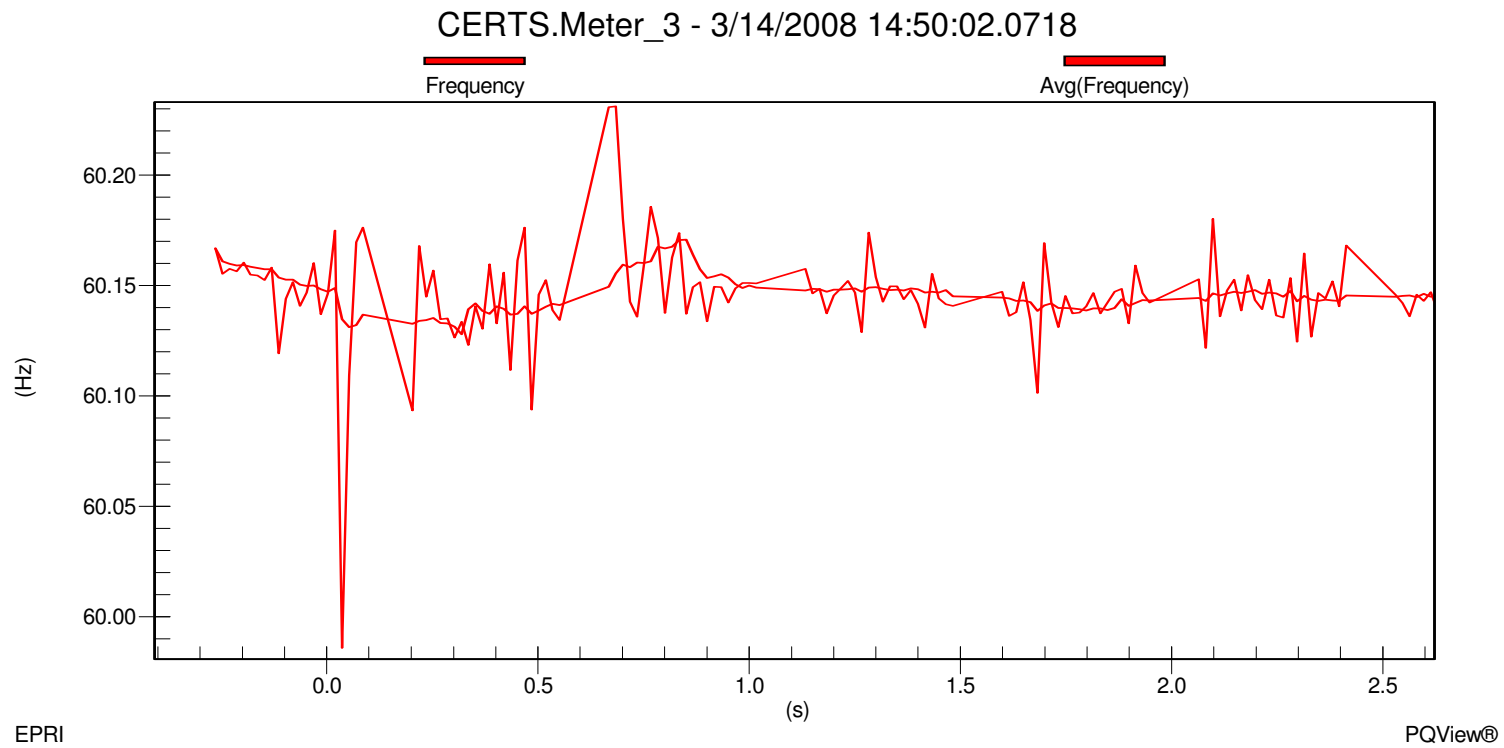

Figure 101 - Meter 3 Frequency during Motor Start and Islanded for Test 10.2.20 
Before the motor started, Gen-set A1 was producing approximately $18.8 \mathrm{~kW}+\mathrm{j} 4 \mathrm{kVAr}$ shown in Figures $10 \mathrm{~m}$ and $10 \mathrm{n}$. The power generated by Gen-set A1 was satisfying the loads in Load Bank 3 and all the electrical losses in the microgrid system. When the motor started the inrush caused Gen-set A1 to increase its output power level to $40 \mathrm{~kW}+$ j54.5kVAr. Gen-set A1 decreased its output level while the motor was warming up and eventually dropped to $27.5 \mathrm{~kW}+\mathrm{j} 10 \mathrm{kVAr}$ when the motor reached steady state. Once all the data was verified and recorded into the DAS Database, the static switch was directed by the EMS to manually close.

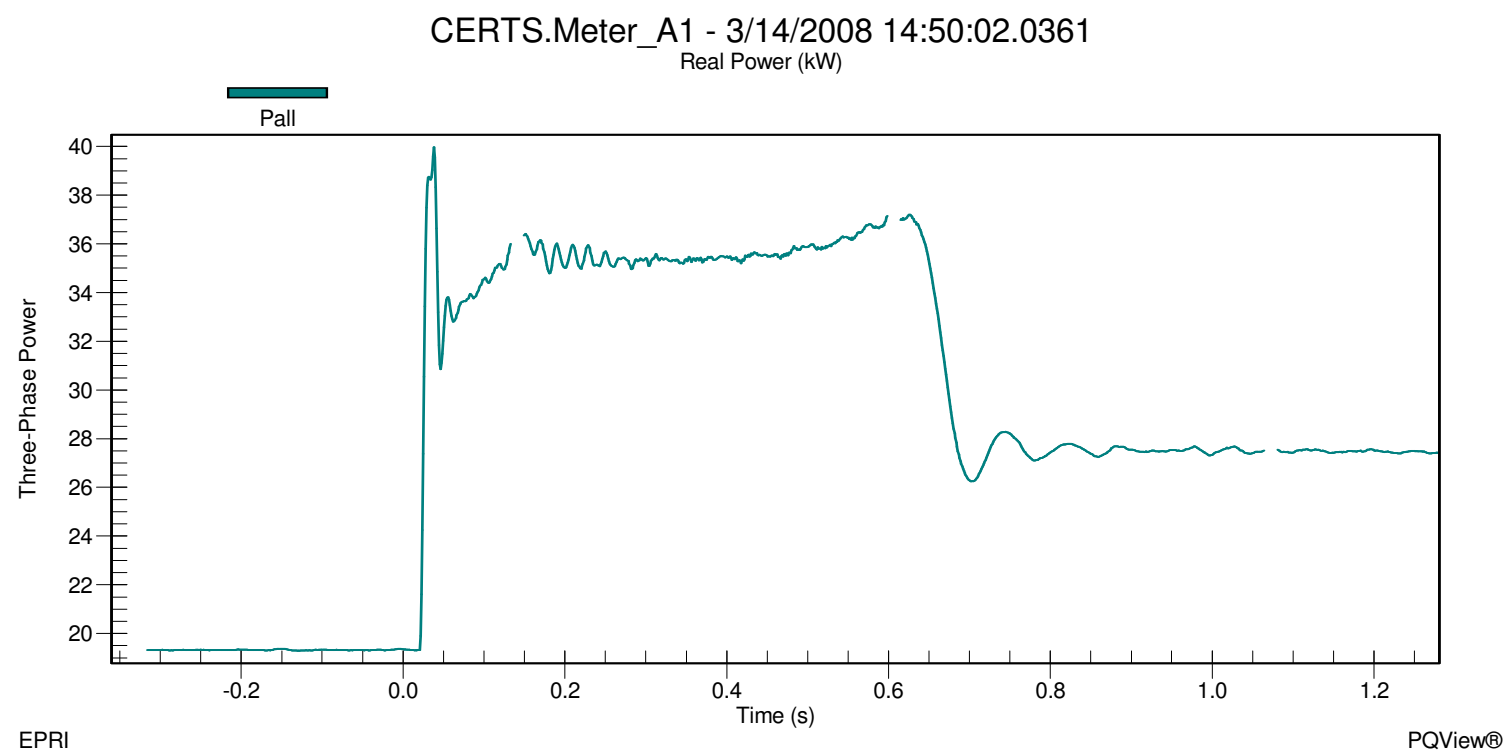

Figure 10m - Gen-set A1 Real Power during Motor Start and Islanded for Test 10.2.20

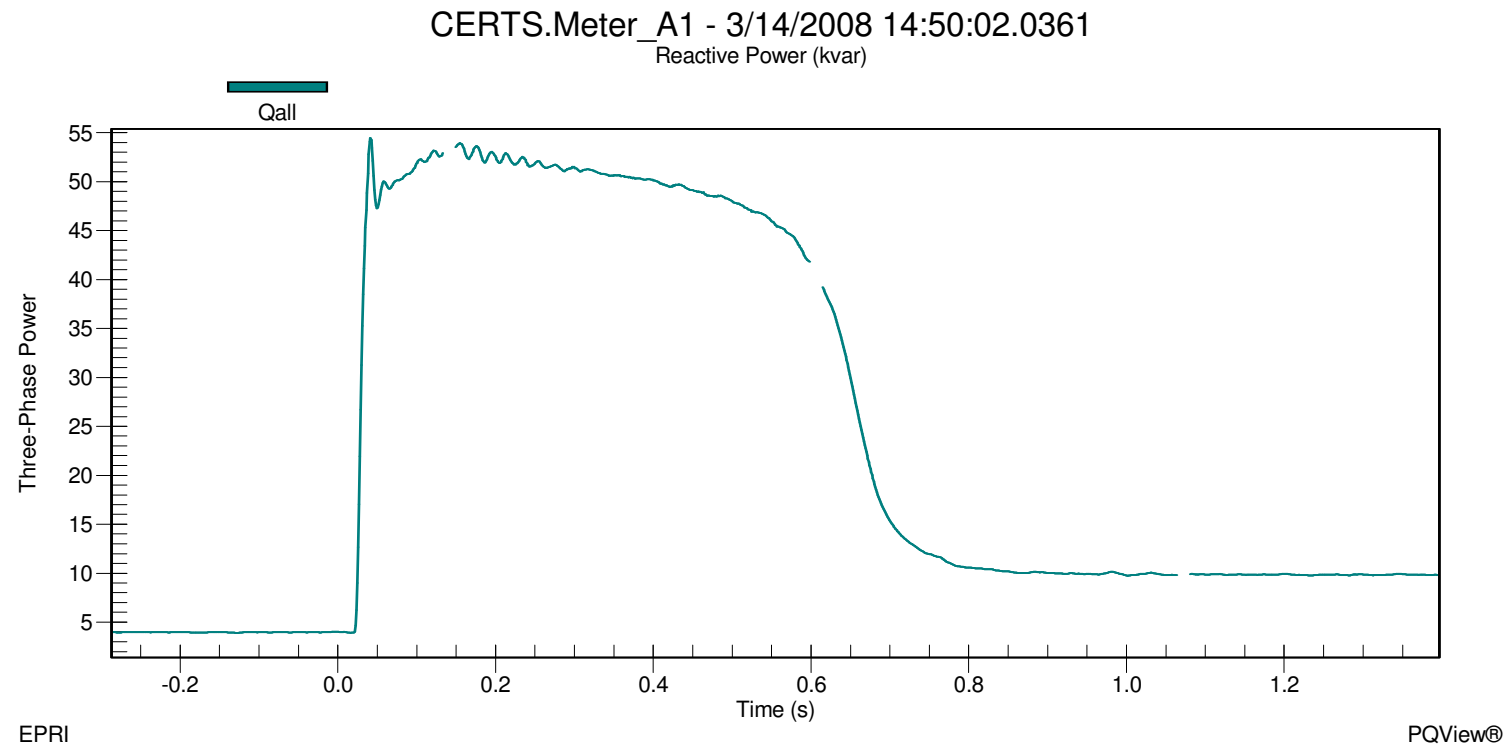

Figure 10n - Gen-set A1 Reactive Power during Motor Start and Islanded for Test 10.2.20 
As soon as the static switch closed, Meter 1 recorded real power decreased from approximately $36.8 \mathrm{~kW}$ to $26.7 \mathrm{~kW}$ and reactive power increased from approximately $1 \mathrm{kVAr}$ to $25 \mathrm{kVAr}$ which means that the utility was satisfying a portion of the load demand in Load Bank6 and all the reactive power in the microgrid. Figures 10o and 10p show the static switch real power decreasing from approximately $0 \mathrm{~kW}$ to $-10.6 \mathrm{~kW}$ and reactive power increasing from approximately $0 \mathrm{kVAr}$ to $24 \mathrm{kVAr}$. At the beginning of the test, the initial power flow through the static switch was $-15 \mathrm{~kW}+\mathrm{j} 18 \mathrm{kVAr}$ which is not the same recorded at this point in the test because the $10 \mathrm{Hp}$ motor load is on in Zone 3. Gen-set A1 has picked up the motor load and supporting Load Bank 6 with approximately $10.6 \mathrm{~kW}$.

Load Bank 3 increased slightly to $24.9 \mathrm{~kW}+\mathrm{j} 6.4 \mathrm{kAVr}$. This slight load increase is a result from a voltage rise in the microgrid, shown in Figure 10q, from approximately $269 \mathrm{~V}$ on A-phase, $269 \mathrm{~V}$ on B-phase and $269 \mathrm{~V}$ on C-phase when islanded to $282 \mathrm{~V}$ on A-phase, 279.3V on B-phase and 279.3V on C-phase at Meter 3 when connected to the utility grid.

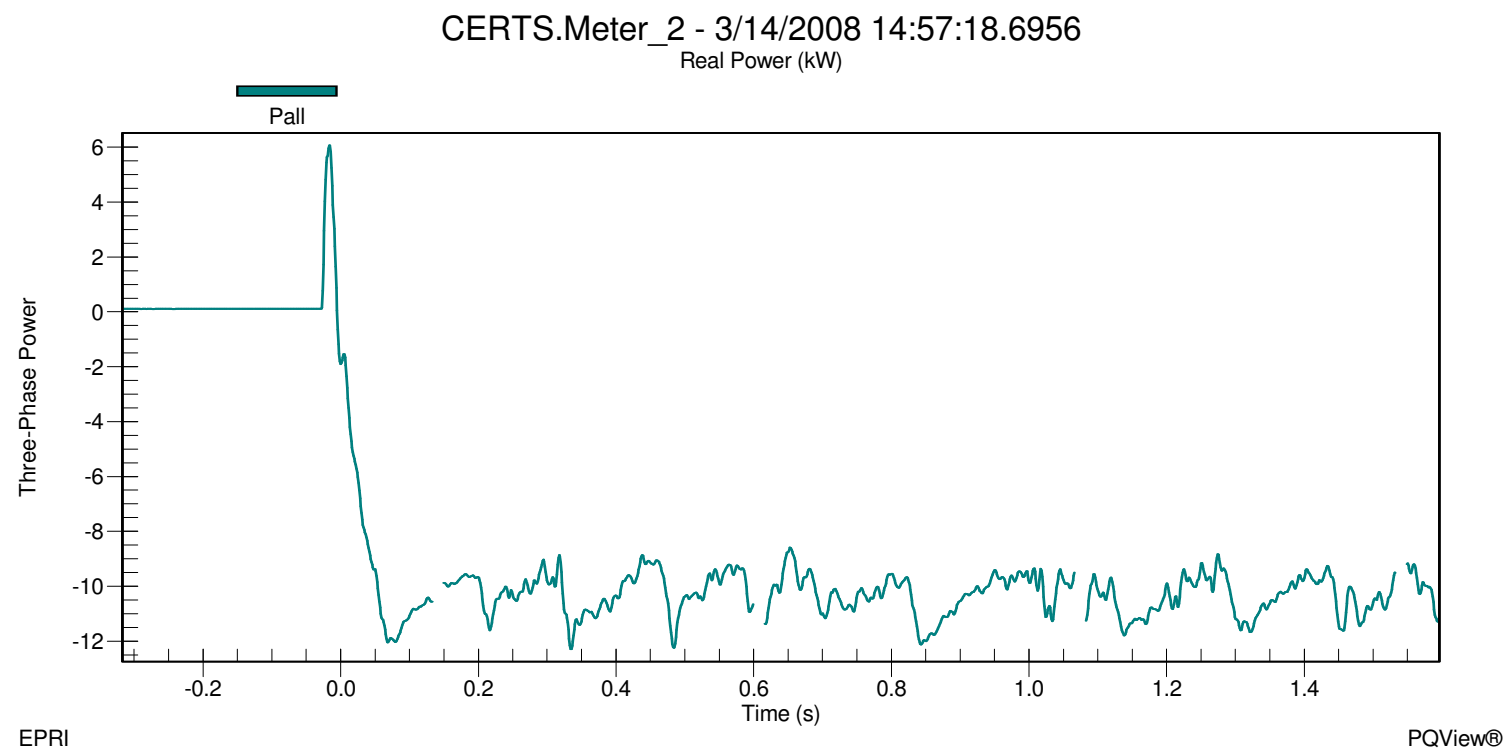

Figure 10o - Static Switch Real Power during Island to Utility Connected mode for Test 10.2.20 


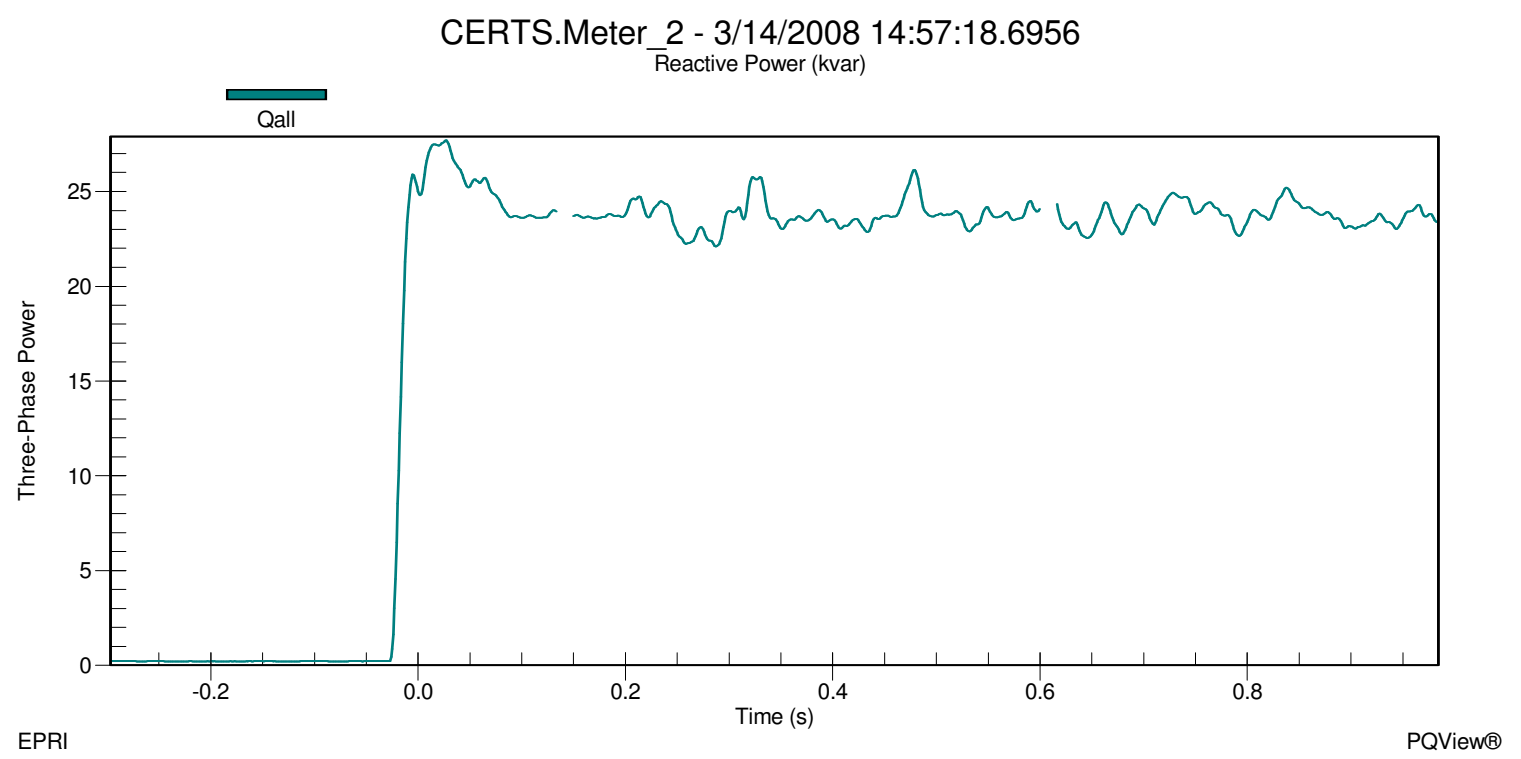

Figure 10p - Static Switch Reactive Power during Island to Utility Connected mode for Test 10.2.20

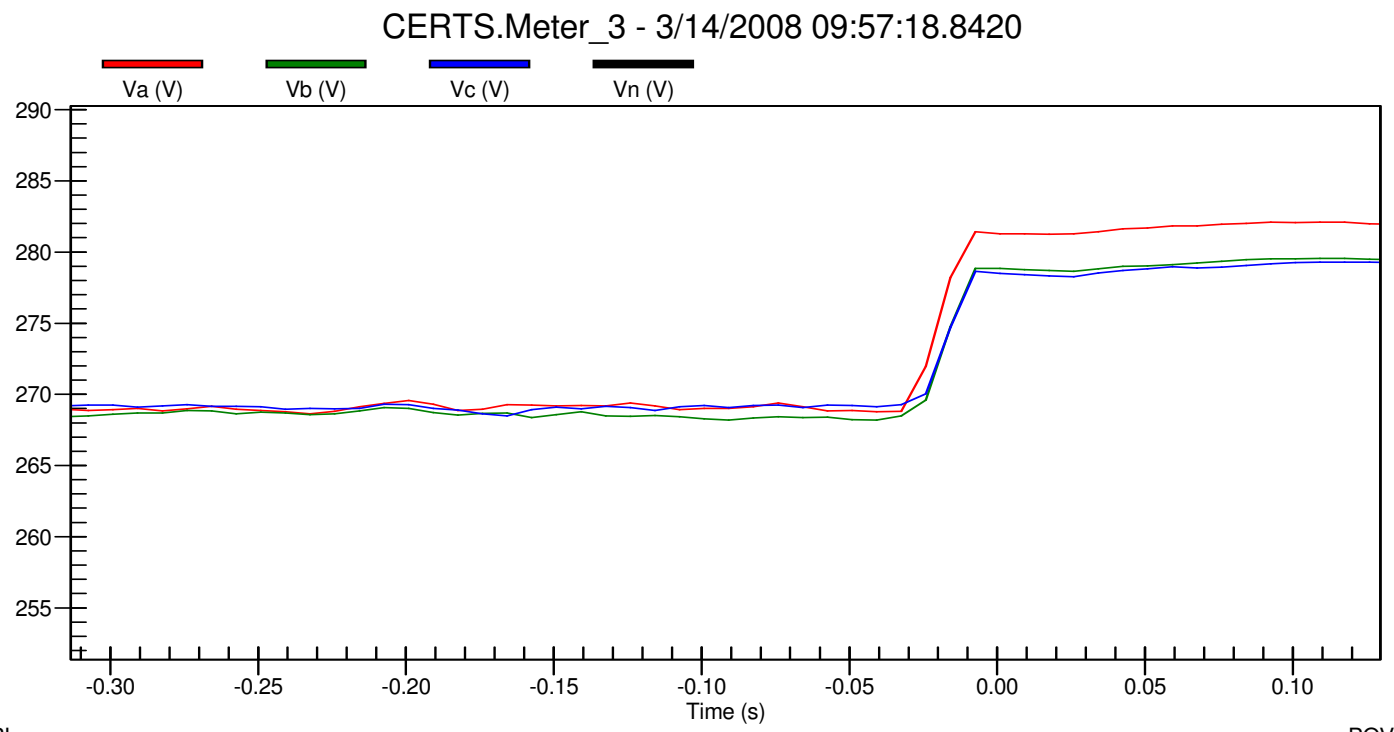

EPRI

$\mathrm{PQView}{ }^{\circledR}$

Figure 10q - Meter 3 Line-to-Ground Voltages during Island to Utility Connected mode for Test 10.2.20 


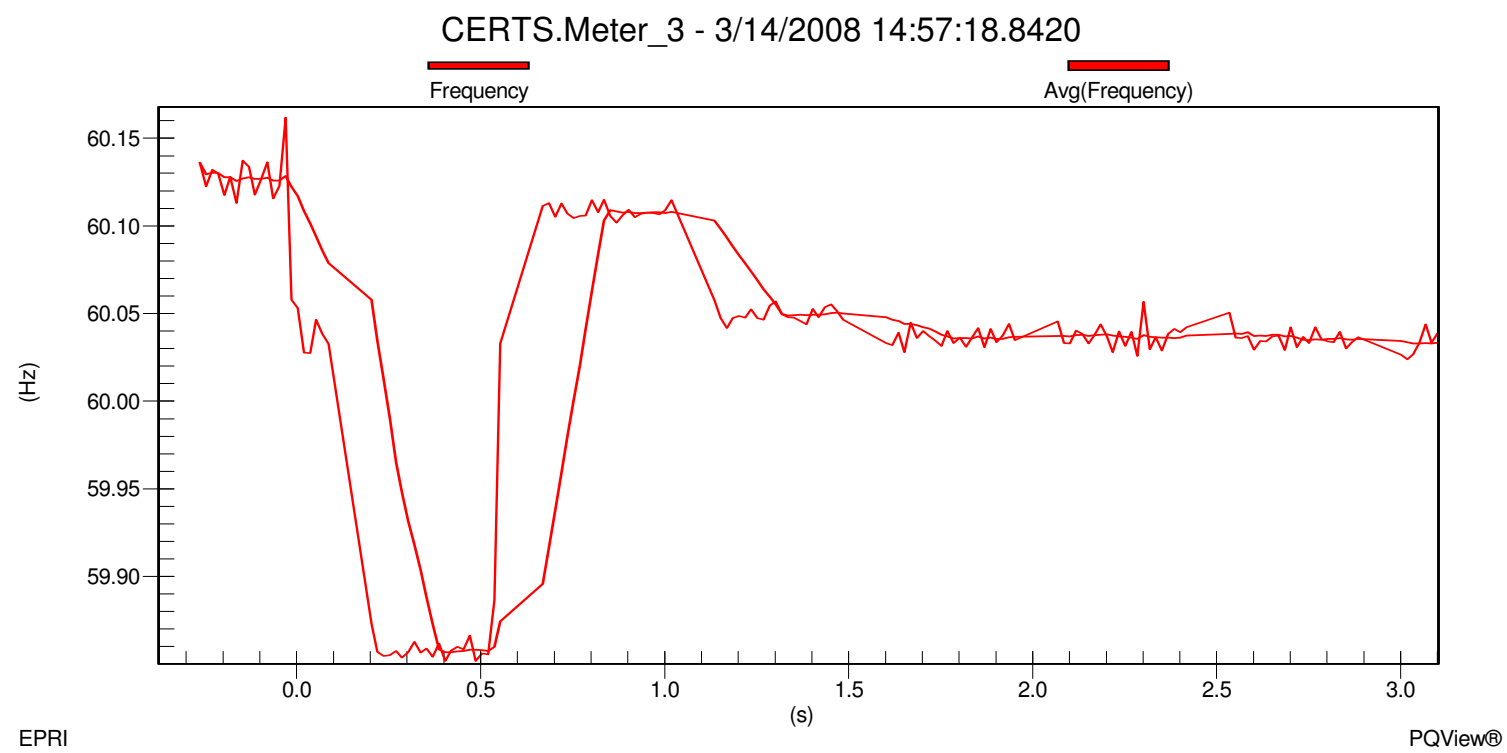

Figure 10r - Meter 3 Frequency during Island to Utility Connected mode for Test 10.2.20

Frequency change in the microgrid, shown in Figure 10r, decreased from approximately $60.13 \mathrm{~Hz}$ when islanded to approximately $60.03 \mathrm{~Hz}$ when connected to the utility grid. This change in frequency is due to the frequency no longer being established by the Genset using the CERTS algorithm but by the utility. Gen-set A1 is in zone power control mode therefore when the static switch closed back into the utility Gen-set A1 produced real power based on set-points for the power flow through Zone 3 initialized at the beginning of the test. The real power increased from its initial value at the beginning of the test to approximately $38.4 \mathrm{~kW}$ for Gen-set A1. This increase is because Gen-set A1 has picked up the real power of the induction motor in Zone 3. Reactive power for Genset A1 was relatively close to the value at the beginning of the test with approximately $11.2 \mathrm{kVAr}$. After all the data was verified and recorded into the DAS Database, the motor, Gen-set A1, and Load Banks 3 and 6 were turned off.

\subsubsection{Motor Start Tests, Weak Grid, Unbalanced Load with 0.9 Power Factor Performance Goal:}

Verify and document power flow, Micro-grid frequency changes and protection design with different Gen-set settings during motor starts when utility connected and then repeat motor start test during an islanded mode of operation.

Six tests were completed in this section with each test event and settings listed in Table 1. During each test Gen-sets A1 and A2 were both set for either unit or zone power 
control mode of operation. Each of the six tests varied the output power command setpoint in each Gen-set, along with varying the load settings in load banks LB3, LB4 and LB6. During each test, inductor L11 was in series with the utility grid to simulate a weak grid condition at the PCC to the microgrid.

\begin{tabular}{|c|c|c|c|c|c|c|c|c|c|}
\hline $\begin{array}{c}\text { Test } \\
\text { Event }\end{array}$ & $\begin{array}{c}\text { Gen- } \\
\text { set } A 1\end{array}$ & $\begin{array}{l}\text { Gen - } \\
\text { set A2 }\end{array}$ & Meter 1 & Meter 2 & Meter 3 & Meter 4 & LB 3 & LB 4 & LB 6 \\
\hline 10.3.12 & $\begin{array}{c}\text { Unit } \\
+40 \mathrm{~kW}\end{array}$ & $\begin{array}{c}\text { Unit } \\
+30 \mathrm{~kW}\end{array}$ & $+30 \mathrm{~kW}$ & $-10 \mathrm{~kW}$ & $-10 \mathrm{~kW}$ & $+10 \mathrm{~kW}$ & $20 \mathrm{~kW}$ & $\begin{array}{l}A=10 \mathrm{~kW} \\
B=15 \mathrm{~kW} \\
C=15 \mathrm{~kW}\end{array}$ & $\begin{array}{l}A=10 \mathrm{~kW} \\
B=20 \mathrm{~kW} \\
C=10 \mathrm{~kW}\end{array}$ \\
\hline 10.3.14 & $\begin{array}{c}\text { Unit } \\
+40 \mathrm{~kW}\end{array}$ & $0 \mathrm{~kW}$ & $+10 \mathrm{~kW}$ & $-30 \mathrm{~kW}$ & $-30 \mathrm{~kW}$ & $0 \mathrm{~kW}$ & $10 \mathrm{~kW}$ & $0 \mathrm{~kW}$ & $\begin{array}{l}A=10 \mathrm{~kW} \\
B=20 \mathrm{~kW} \\
C=10 \mathrm{~kW}\end{array}$ \\
\hline 10.3.15 & $\begin{array}{l}\text { Unit } \\
+40 \mathrm{~kW}\end{array}$ & $\begin{array}{c}\text { Unit } \\
+30 \mathrm{~kW}\end{array}$ & $+70 \mathrm{~kW}$ & $+30 \mathrm{~kW}$ & $+30 \mathrm{~kW}$ & $+20 \mathrm{~kW}$ & $50 \mathrm{~kW}$ & $\begin{array}{l}A=10 \mathrm{~kW} \\
B=20 \mathrm{~kW} \\
C=20 \mathrm{~kW}\end{array}$ & $\begin{array}{l}A=10 \mathrm{~kW} \\
B=20 \mathrm{~kW} \\
C=10 \mathrm{~kW}\end{array}$ \\
\hline 10.3.17 & $\begin{array}{c}\text { Unit } \\
+40 \mathrm{~kW}\end{array}$ & $0 \mathrm{~kW}$ & $+20 \mathrm{~kW}$ & $-20 \mathrm{~kW}$ & $-20 \mathrm{~kW}$ & $0 \mathrm{~kW}$ & $20 \mathrm{~kW}$ & $0 \mathrm{~kW}$ & $\begin{array}{l}A=10 \mathrm{~kW} \\
B=20 \mathrm{~kW} \\
C=10 \mathrm{~kW}\end{array}$ \\
\hline 10.3.18 & $\begin{array}{c}\text { Zone - } \\
10 \mathrm{~kW}\end{array}$ & $\begin{array}{l}\text { Zone } \\
+10 \mathrm{~kW}\end{array}$ & $+30 \mathrm{~kW}$ & $-10 \mathrm{~kW}$ & $-10 \mathrm{~kW}$ & $+10 \mathrm{~kW}$ & $20 \mathrm{~kW}$ & $\begin{array}{l}A=10 \mathrm{~kW} \\
B=15 \mathrm{~kW} \\
C=15 \mathrm{~kW}\end{array}$ & $\begin{array}{l}A=10 \mathrm{~kW} \\
B=20 \mathrm{~kW} \\
C=10 \mathrm{~kW}\end{array}$ \\
\hline 10.3.20 & $\begin{array}{c}\text { Zone - } \\
20 \mathrm{~kW}\end{array}$ & $0 \mathrm{~kW}$ & $+20 \mathrm{~kW}$ & $-20 \mathrm{~kW}$ & $-20 \mathrm{~kW}$ & $0 \mathrm{~kW}$ & $20 \mathrm{~kW}$ & $0 \mathrm{~kW}$ & $\begin{array}{l}A=10 \mathrm{~kW} \\
B=20 \mathrm{~kW} \\
C=10 \mathrm{~kW}\end{array}$ \\
\hline
\end{tabular}

Table 2 -Unbalance Load, Weak Grid, Before Motor Start settings of Gen-sets and Load Banks

The test procedures for these six tests were exactly the same as in the tests performed in Section 6.1.1 (Tests 10.2.12 - 10.2.20). 
For Test 10.3.12 the measured values, after the Gen-sets were warmed up and load banks brought on-line are provided in Table 3.

\begin{tabular}{|c|c|c|c|c|c|c|c|c|c|}
\hline Meter & $\begin{array}{l}\text { Gen- } \\
\text { set A1 }\end{array}$ & $\begin{array}{l}\text { Gen - } \\
\text { set A2 }\end{array}$ & Meter 1 & Meter 2 & Meter 3 & Meter 4 & LB 3 & LB 4 & LB 6 \\
\hline $\begin{array}{c}3 \text { Phase } \\
\text { kW }\end{array}$ & 37.5 & 28.5 & 30 & -7.8 & -10.5 & 8.5 & 18.2 & 36.7 & 36.9 \\
\hline \multirow{3}{*}{$\begin{array}{c}1 \text { Phase } \\
\text { kW }\end{array}$} & $A=11$ & $A=8$ & $A=8$ & $A=-2$ & $A=-3$ & $A=2$ & $A=6$ & $\mathrm{~A}=9.7$ & $\mathrm{~A}=9.7$ \\
\hline & $\mathrm{B}=15.5$ & $B=12$ & $B=11$ & $B=-7$ & $B=-8$ & $B=1.5$ & $B=6.2$ & $B=13.5$ & $B=17.6$ \\
\hline & $C=10.5$ & $C=8.5$ & $\mathrm{C}=11$ & $C=1.2$ & $C=0.05$ & $C=5$ & $C=6$ & $C=13.5$ & $C=9.6$ \\
\hline $\begin{array}{c}3 \text { Phase } \\
\text { kVAr }\end{array}$ & -12 & -4 & 24.5 & 22.4 & 17.9 & 5 & 0.3 & 0.615 & 0.638 \\
\hline \multirow{3}{*}{$\begin{array}{l}1 \text { Phase } \\
\text { kVAr }\end{array}$} & $\mathrm{A}=-4.5$ & $A=-2$ & $A=9$ & $A=8.7$ & $\mathrm{~A}=7.5$ & $\mathrm{~A}=2.4$ & $\mathrm{~A}=0.1$ & $\mathrm{~A}=0.13$ & $\mathrm{~A}=0.122$ \\
\hline & $B=-4$ & $B=-1$ & $B=8$ & $B=7$ & $B=5.2$ & $\mathrm{~B}=1.2$ & $\mathrm{~B}=0.1$ & $\mathrm{~B}=0.28$ & $B=0.394$ \\
\hline & $C=-3.5$ & $C=-1$ & $C=7.5$ & $C=6.7$ & $C=5.2$ & $C=1.4$ & $C=0.1$ & $C=0.205$ & $C=0.122$ \\
\hline
\end{tabular}

Table 3 - Measured Values after Start Up for Test 10.3.12

These measurements were relatively close to the expected values in Table 2, but not exact due to temperature, phase voltages and electrical losses in conductors. In addition, the $40 \mathrm{~kW}$ settings for LB3 and LB4 and the 20kW setting for LB3 were also below selected set values. At the time of the measurements, the voltage and frequency was 282.7V on A-phase, $279 \mathrm{~V}$ on B-phase and $279.6 \mathrm{~V}$ on C-phase and $60.02 \mathrm{~Hz}$ at the static switch (i.e., Meter 2) when connected to the utility grid; and $282.7 \mathrm{~V}$ on A-phase, $279.5 \mathrm{~V}$ on B-phase and $279.5 \mathrm{~V}$ on C-phase and $60.02 \mathrm{~Hz}$ at Meter 3.

The Gen-sets in this test were set up to produce more power than Load Banks 3 and 4 needed which approximately $7.8 \mathrm{~kW}$ of excess power was exported through the static with to Load Bank 6. Since Load Bank 6 was approximately $36.9 \mathrm{~kW}(A=9.7 \mathrm{~kW}$ $B=17.6 \mathrm{~kW} \mathrm{C}=9.6 \mathrm{~kW})+\mathrm{j} 0.638 \mathrm{kVAr}(\mathrm{A}=0.122 \mathrm{kVAr} B=0.394 \mathrm{kVAr} C=0.122 \mathrm{kVAr})$, the utility had to supply approximately $30 \mathrm{~kW}(\mathrm{~A}=8 \mathrm{~kW} B=11 \mathrm{~kW} C=11 \mathrm{~kW})$ to satisfy the load. Reactive power had to be imported in from the utility of approximately $24.5 \mathrm{kVAr}$ ( $\mathrm{A}=9 \mathrm{kVAr} \mathrm{B}=8 \mathrm{kVAr} \mathrm{C}=7.5 \mathrm{kVAr}$ ) because of Gen-set $\mathrm{A} 1$ and $\mathrm{A} 2$ needed approximately $16 \mathrm{kVAr}$ between both and the reactive power absorbed in the electrical lines. Once all the data was verified and recorded into the DAS Database, the $10 \mathrm{Hp}$ induction motor was started in Zone 3. 


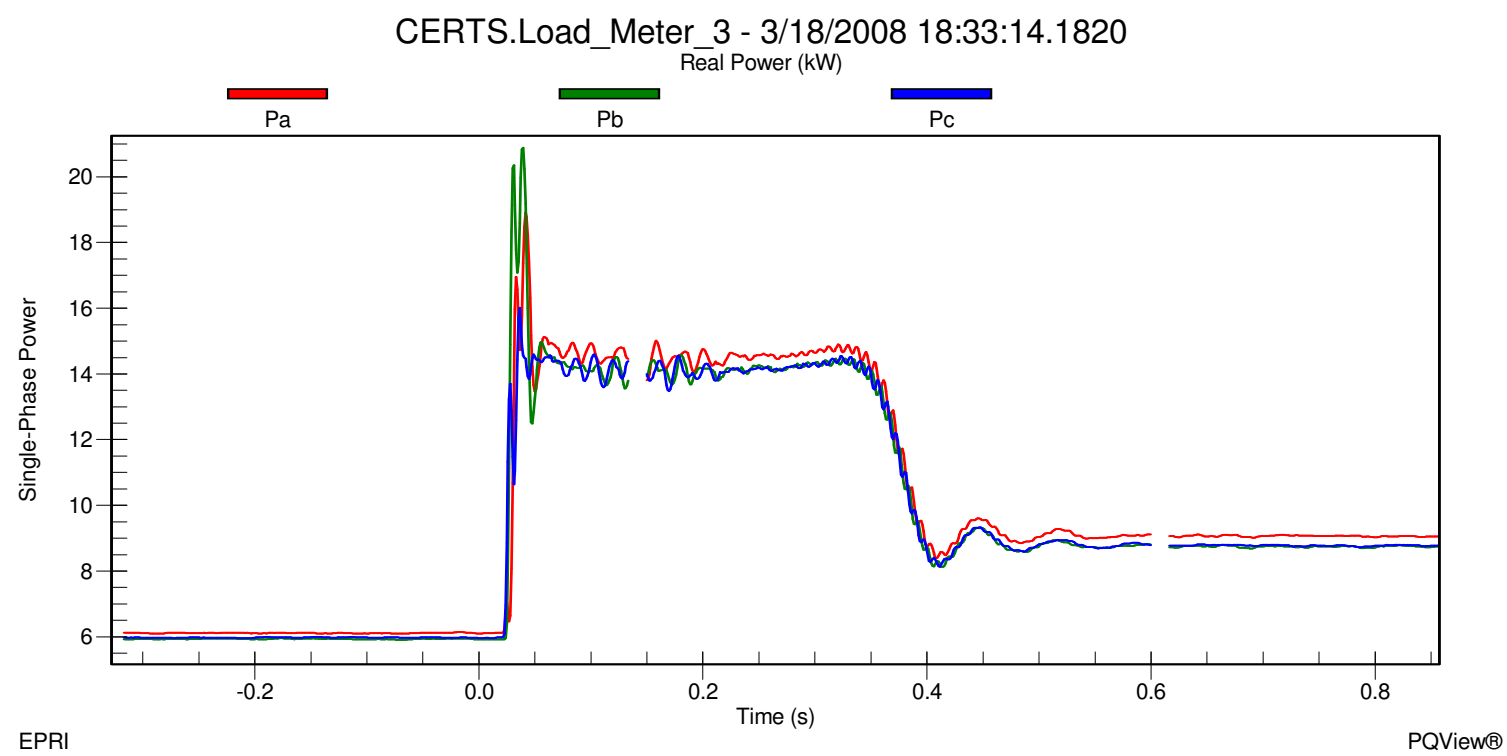

Figure 11a - Load Bank 3 Real Single-Phase Power during Motor Start and Utility Connected for Test 10.3.12

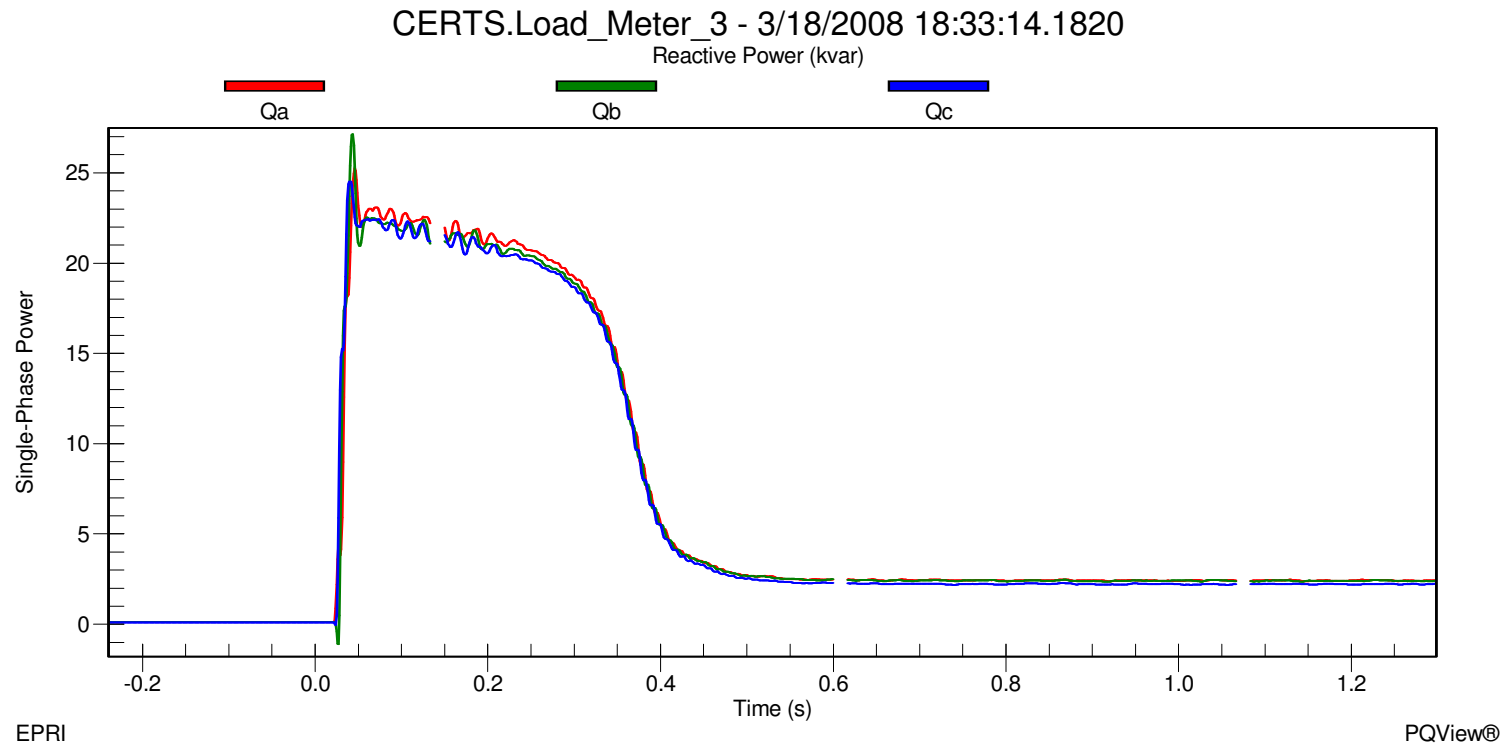

Figure 11b - Load Bank 3 Reactive Single-Phase Power during Motor Start and Utility Connected for Test 10.3.12

In Figures $11 \mathrm{a}$ and $11 \mathrm{~b}$ it can be seen that the load in Zone 3 was approximately $18.2 \mathrm{~kW}$ $(A=6 \mathrm{~kW} \mathrm{~B}=6.2 \mathrm{~kW} C=6 \mathrm{~kW})+j 0.3 \mathrm{kVAr}(\mathrm{A}=0.1 \mathrm{kVAr} B=0.1 \mathrm{kVAr} C=0.1 \mathrm{kVAr})$ before the start of the induction motor and increased to approximately $55.6 \mathrm{~kW}(\mathrm{~A}=19 \mathrm{~kW} \mathrm{~B}=20.9 \mathrm{~kW}$ $\mathrm{C}=15.7 \mathrm{~kW})+\mathrm{j} 76.1 \mathrm{kVAr}(\mathrm{A}=24.5 \mathrm{kVAr} B=27.1 \mathrm{kVAr} C=24.5 \mathrm{kVAr})$ during the inrush phase of the motor start. After about 1.5 cycles, the motor settled down to approximately 
$43.1 \mathrm{~kW} \quad(\mathrm{~A}=14.7 \mathrm{~kW} \quad \mathrm{~B}=14.2 \mathrm{~kW} \quad \mathrm{C}=14.2 \mathrm{~kW})+\mathrm{j} 63.5 \mathrm{kVAr} \quad(\mathrm{A}=21.5 \mathrm{kVAr} \quad \mathrm{B}=21 \mathrm{kVAr}$ $\mathrm{C}=21 \mathrm{kAVr}$ ) during the warm up phase which lasted about 33 cycles (0.55 Seconds). When the motor reached steady state, the load in Zone 3 was approximately $26.5 \mathrm{~kW}$ $(A=9.1 \mathrm{~kW} \mathrm{~B}=8.7 \mathrm{~kW} C=8.7 \mathrm{~kW})+\mathrm{j} 6.6 \mathrm{kVAr}(\mathrm{A}=2.2 \mathrm{kVAr} B=2.2 \mathrm{kVAr} \mathrm{C}=2.2 \mathrm{kVAr})$.

The voltage and frequency at the static switch before the motor start was approximately 282.7V on A-phase 279V, on B-phase and 279.6V on C-phase shown in Figure 11c and approximately $60.02 \mathrm{~Hz}$ shown in Figure 11d. When the motor started, the voltage at the static switch during the inrush decreased to approximately $276.3 \mathrm{~V}$ on A-phase, $272.4 \mathrm{~V}$ on B-phase and $273 \mathrm{~V}$ on C-phase for about 1.5 cycles. Frequency dropped during the inrush to approximately $59.96 \mathrm{~Hz}$ and quickly increased to approximately $60.00 \mathrm{~Hz}$. Voltage increased as the motor was warming up and eventually settled at a steady state voltage at approximately $282.1 \mathrm{~V}$ on A-phase, $278.2 \mathrm{~V}$ on B-phase and $278.9 \mathrm{~V}$ on C-phase at an approximate frequency of $60.01 \mathrm{~Hz}$.

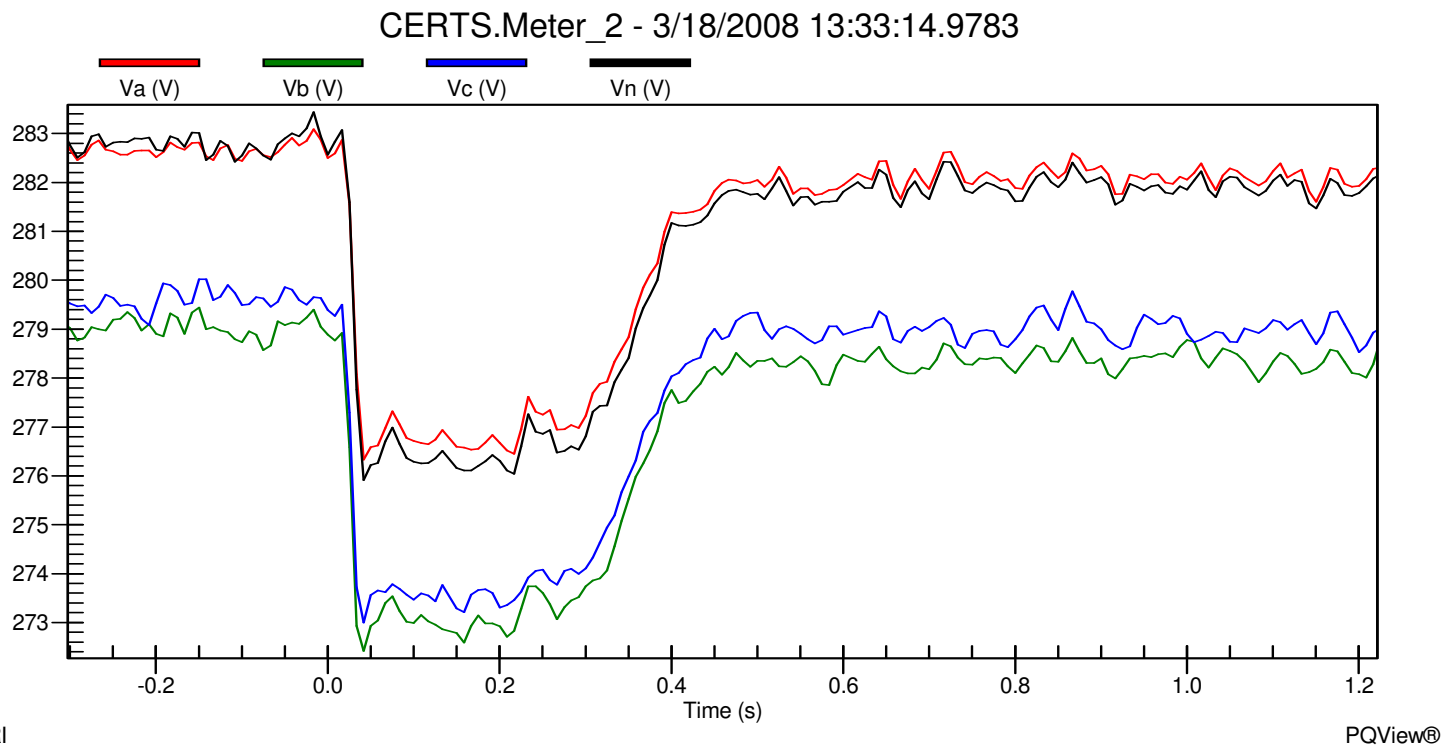

Figure 11c - Static Switch Line-to-Ground Voltages during Motor Start and Utility Connected for Test 10.3.12 


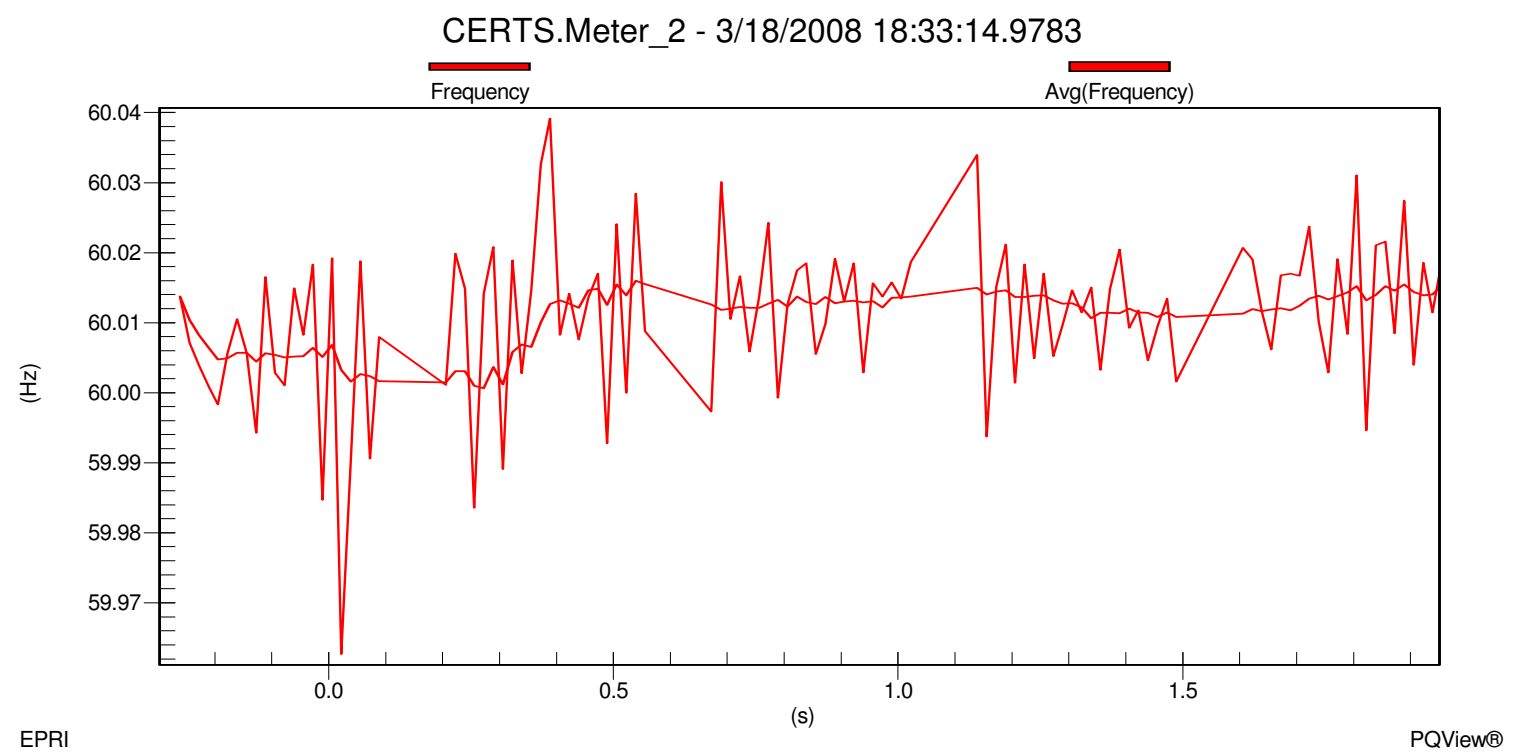

Figure 11d - Static Switch Frequency during Motor Start and Utility Connected for Test 10.3.12

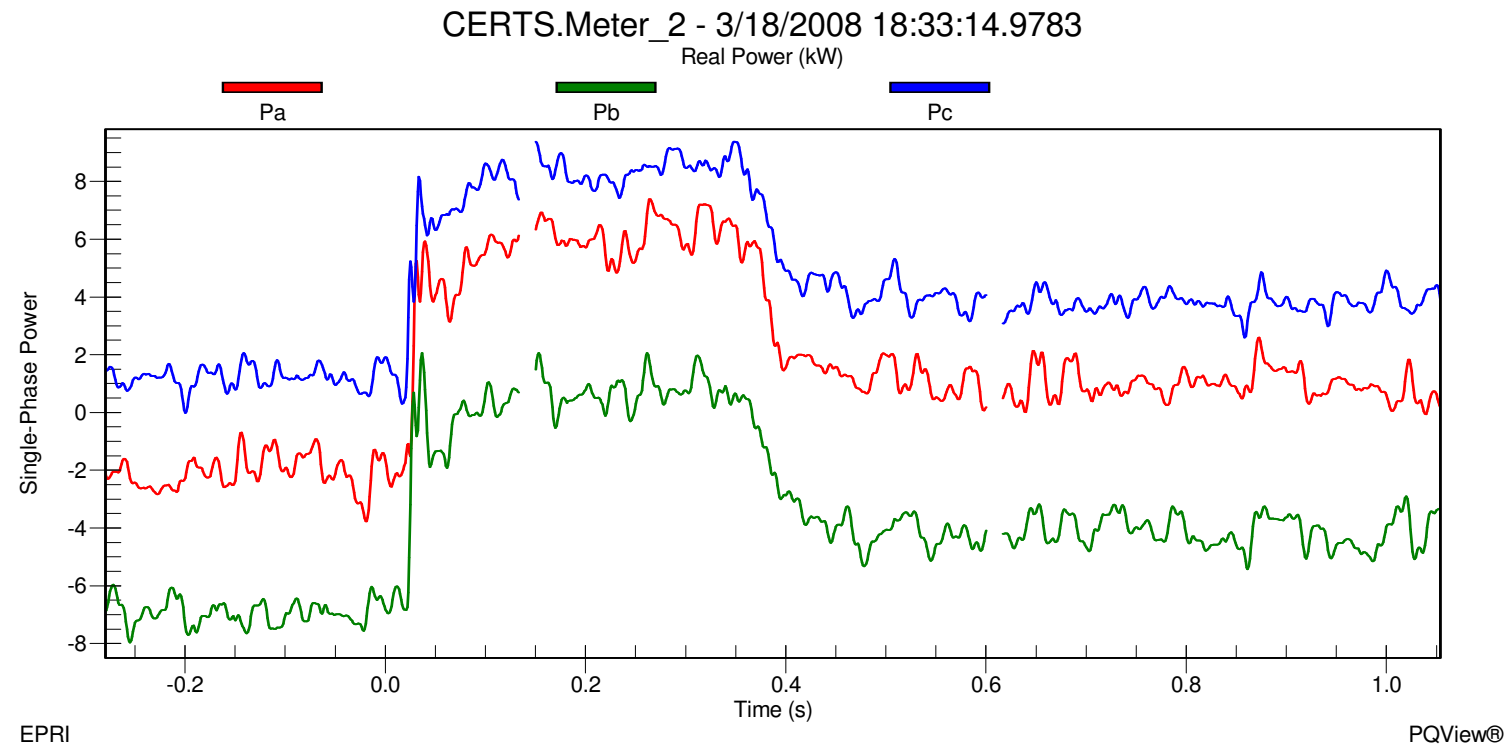

Figure 11e - Static Switch Real Single-Phase Power during Motor Start and Utility Connected for Test 10.3.12 


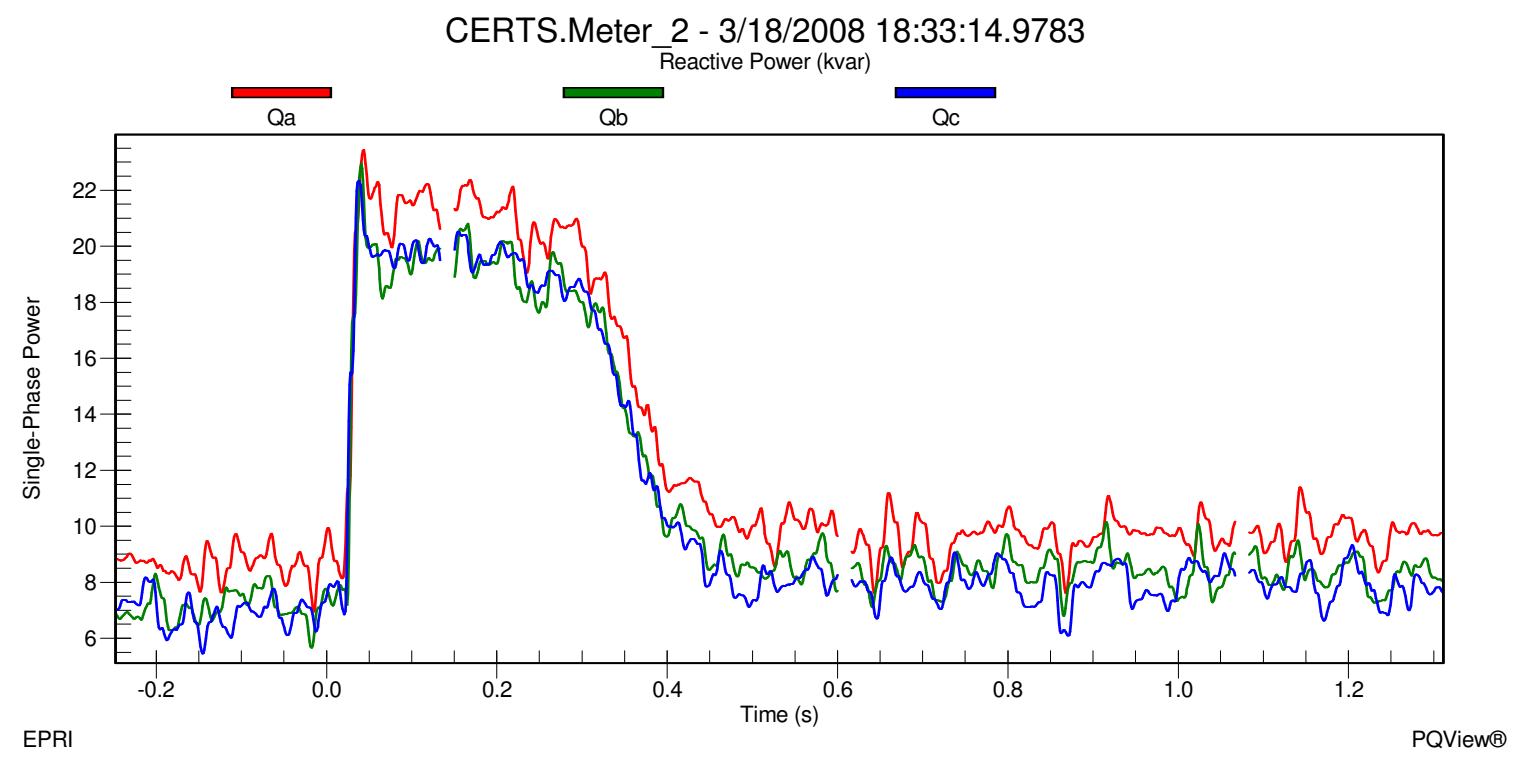

Figure 11f - Static Switch Reactive Single-Phase Power during Motor Start and Utility Connected for Test 10.3.12

Before the motor started Gen-sets $\mathrm{A} 1$ and $\mathrm{A} 2$ were producing approximately $37.5 \mathrm{~kW}$ $(A=11 \mathrm{~kW} B=15.5 \mathrm{~kW} \quad \mathrm{C}=10.5 \mathrm{~kW})-\mathrm{j} 12 \mathrm{kVAr}(\mathrm{A}=-4.5 \mathrm{kVAr} B=-4 \mathrm{kVAr} C=-3.5 \mathrm{kVAr})$ and $28.5 \mathrm{~kW} \quad(A=8 \mathrm{~kW} \quad B=12 \mathrm{~kW} \quad \mathrm{C}=8.5 \mathrm{~kW})-j 4 \mathrm{kVAr} \quad(A=-2 \mathrm{kVAr} \quad B=-1 \mathrm{kVAr} \quad \mathrm{C}=-1 \mathrm{kVAr})$, respectively. This was enough power for the load demands of Zones 3 and 4 with real power being exported to Zone 6 of approximately $7.8 \mathrm{~kW}$ shown in Figure 11e. The grid was supporting the reactive power of the microgrid with approximately $22.4 \mathrm{kVAr}$ $(\mathrm{A}=8.7 \mathrm{kVAr} \mathrm{B}=7 \mathrm{kVAr} \mathrm{C}=6.7 \mathrm{kVAr}$ ) shown in Figure 11f. When the motor started the inrush caused the utility to supply approximately $68.4 \mathrm{kVAr}(\mathrm{A}=23.5 \mathrm{kVAr} \mathrm{B}=22.6 \mathrm{kVAr}$ $\mathrm{C}=22.3 \mathrm{kVAr}$ ) and to pick up the load demands of Load Bank 6 along with approximately $15.3 \mathrm{~kW}(\mathrm{~A}=5.3 \mathrm{~kW} \mathrm{~B}=1.8 \mathrm{~kW} \mathrm{C}=8.2 \mathrm{~kW})$ of the microgrid critical loads. The power being supplied by Gen-sets A1 and A2 to Load Bank 6 was now supplying a portion of the motor inrush which can be seen in Figure 11e as the real power becomes positive. Gensets $\mathrm{A} 1$ and $\mathrm{A} 2$ increased their real and reactive power output levels to approximately $43 \mathrm{~kW}(\mathrm{~A}=13 \mathrm{~kW} B=18 \mathrm{~kW} C=12 \mathrm{~kW})+\mathrm{j} 0.3 \mathrm{kVAr}(\mathrm{A}=-1 \mathrm{kVAr} B=0.9 \mathrm{kVAr} C=0.4 \mathrm{kVAr})$ and $36.5 \mathrm{~kW} \quad(\mathrm{~A}=10.1 \mathrm{~kW} \quad \mathrm{~B}=15.8 \mathrm{~kW} \quad \mathrm{C}=10.6 \mathrm{~kW})+\mathrm{j} 12 \mathrm{kVAr} \quad(\mathrm{A}=3.7 \mathrm{kVAr} \quad \mathrm{B}=4.6 \mathrm{kVAr}$ $\mathrm{C}=3.7 \mathrm{kVAr}$ ), respectively. Notice the signs of the $\mathrm{VAr}$ output changed from negative to positive for both Gen-sets in order to support the induction motor load in Zone 3.

When the motor reached steady state, the real and reactive power through the static switch was approximately $0.4 \mathrm{~kW} \quad(\mathrm{~A}=0.9 \mathrm{~kW} \quad \mathrm{~B}=-4.3 \mathrm{~kW} \quad \mathrm{C}=3.8 \mathrm{~kW})+\mathrm{j} 25.8 \mathrm{kVAr}$ $(\mathrm{A}=9.7 \mathrm{kVAr} \mathrm{B}=8.4 \mathrm{kVAr} \mathrm{C}=7.7 \mathrm{kVAr})$ which meant that Gen-sets $\mathrm{A} 1$ and $\mathrm{A} 2$ were supplying enough power for the induction motor and Load Banks 3 and 4 but needed approximately $0.4 \mathrm{~kW}(\mathrm{~A}=0.9 \mathrm{~kW} \mathrm{~B}=4.3 \mathrm{~kW} \mathrm{C}=3.8 \mathrm{~kW})$ from the utility grid for the power losses in the conductors. Gen-sets A1 and A2 real power returned to the values before 
the motor started of approximately $37.5 \mathrm{~kW}(\mathrm{~A}=11 \mathrm{~kW} \mathrm{~B}=15.5 \mathrm{~kW} \mathrm{C}=10.5 \mathrm{~kW})$ and $28.5 \mathrm{~kW}$ $(\mathrm{A}=8 \mathrm{~kW} \quad \mathrm{~B}=12 \mathrm{~kW} \mathrm{C}=8.5 \mathrm{~kW})$, respectively, and the reactive power increased to approximately $-10.8 \mathrm{kVAr}(\mathrm{A}=4 \mathrm{kVAr} \mathrm{B}=-3.3 \mathrm{kVAr} \mathrm{C}=-3.5 \mathrm{kVAr})$ in Gen-set $\mathrm{A} 1$ and $1 \mathrm{kVAr}(\mathrm{A}=-1.4 \mathrm{kVAr} \mathrm{B}=0.2 \mathrm{kVAr} \mathrm{C}=0.2 \mathrm{kVAr})$ in Gen-set $\mathrm{A} 2$. Once all the data was verified and recorded into the DAS Database, the motor was shut down and the static switch was directed by the EMS to manually open.

As soon as the static switch opened, Meter 1 recorded real power increased to approximately $38.1 \mathrm{~kW}(\mathrm{~A}=10.1 \mathrm{~kW} \mathrm{~B}=18.1 \mathrm{~kW} \mathrm{C}=9.9 \mathrm{~kW})$ and reactive power decreased to $-0.5 \mathrm{kVAr}(\mathrm{A}=-0.3 \mathrm{kVAr} \mathrm{B}=0.1 \mathrm{kVAr} \mathrm{C}=-0.3 \mathrm{kVAr})$ satisfying the load demand in Load Bank 6 which was approximately $37.9 \mathrm{~kW}(\mathrm{~A}=10.1 \mathrm{~kW} \mathrm{~B}=9.8 \mathrm{~kW} \mathrm{C}=18 \mathrm{~kW})+\mathrm{j} 0.655 \mathrm{kVAr}$ $(\mathrm{A}=0.123 \mathrm{kVAr} \mathrm{B}=0.405 \mathrm{kVAr} \mathrm{C}=0.127 \mathrm{kVAr})$ and not supplying any power beyond the static switch to Load Banks 3 and 4 . $0 \mathrm{~kW}(\mathrm{~A}=0 \mathrm{~kW} B=0 \mathrm{~kW} \mathrm{C}=0 \mathrm{~kW})+\mathrm{j} 0 \mathrm{kVAr}(\mathrm{A}=0 \mathrm{kVAr}$ $\mathrm{B}=0 \mathrm{kVAr} \mathrm{C}=0 \mathrm{kVAr}$ ) was recorded at the static switch indicating that power was not flowing through the static switch. Load banks 3 and 4 loads reduced slightly to $17.35 \mathrm{~kW}$ $(\mathrm{A}=5.76 \mathrm{~kW} \quad \mathrm{~B}=5.84 \mathrm{~kW} \quad \mathrm{C}=5.75 \mathrm{~kW})+j 0.321 \mathrm{kVAr} \quad(\mathrm{A}=0.105 \mathrm{kVAr} \quad \mathrm{B}=0.123 \mathrm{kVAr}$ $C=0.093 \mathrm{kVAr})$ and $35.4 \mathrm{~kW}(\mathrm{~A}=9.3 \mathrm{~kW} B=13.1 \mathrm{~kW} C=13 \mathrm{~kW})+j 0.59 \mathrm{kVAr}(\mathrm{A}=0.115 \mathrm{kVAr}$ $\mathrm{B}=0.275 \mathrm{kVAr} \mathrm{C}=0.2 \mathrm{kVAr})$, respectively. This load reduction resulted from a voltage drop in the microgrid, shown in Figure 11g, from approximately $283 \mathrm{~V}$ on A-phase, $280 \mathrm{~V}$ on B-phase and $280 \mathrm{~V}$ on C-phase when connected to the utility grid to $275 \mathrm{~V}$ on A-phase, $276 \mathrm{~V}$ on B-phase and $274 \mathrm{~V}$ on C-phase at Meter 3 when islanded.

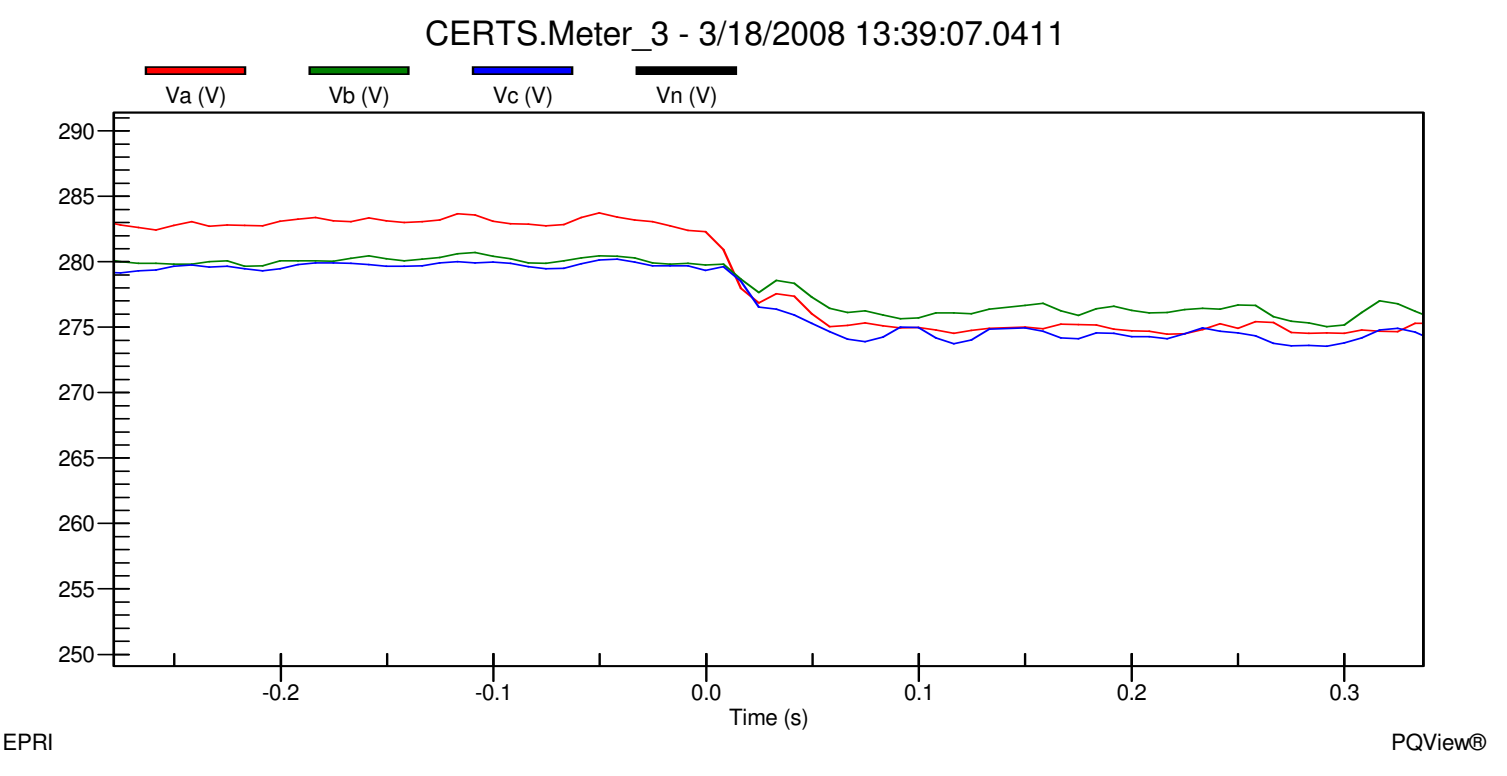

Figure 11g - Meter 3 Line-to-Ground Voltages during Utility Connected to Island mode for Test 10.3.12 


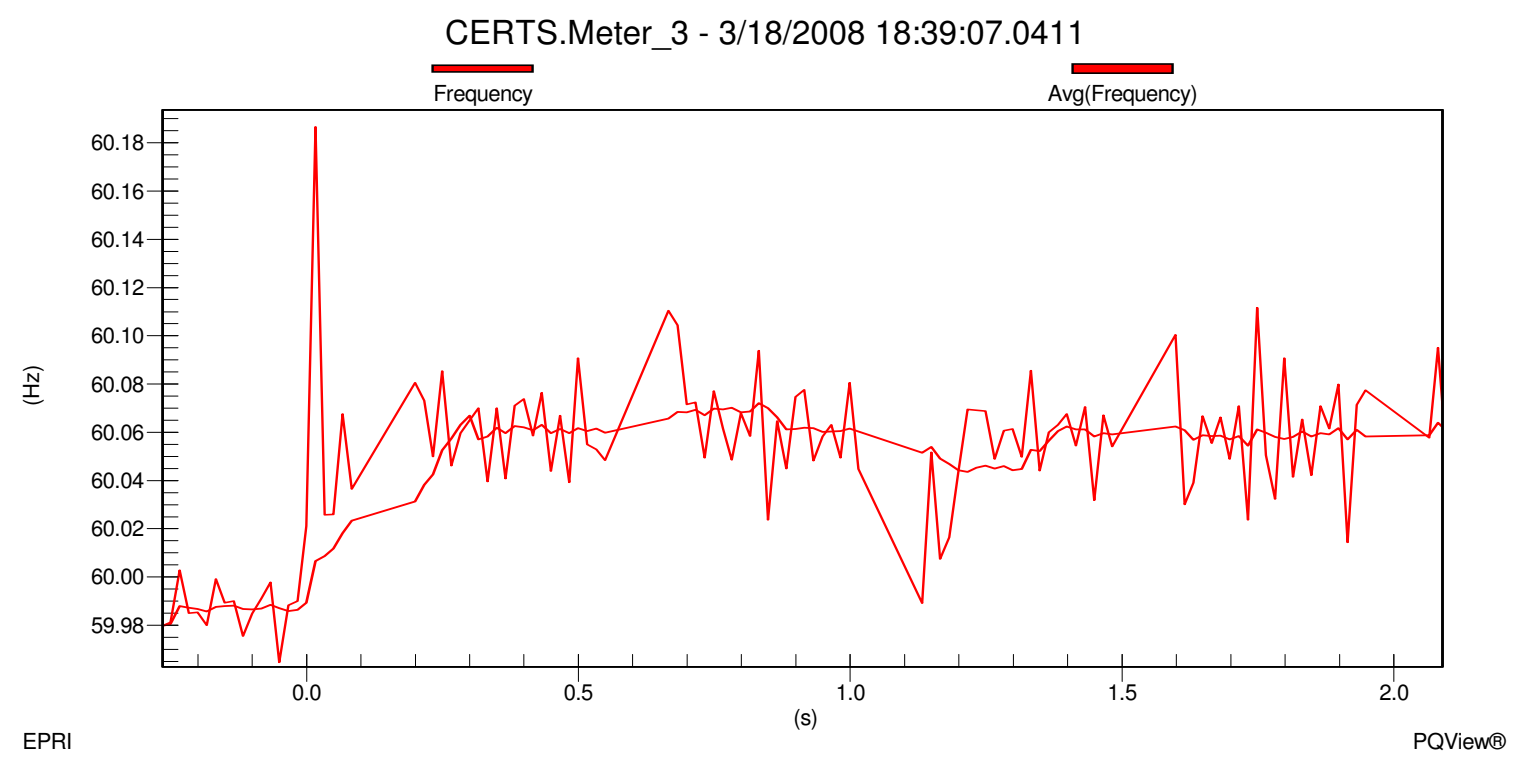

Figure 11h - Meter 3 Frequency during Utility Connected to Island mode for Test 10.3.12

Frequency change in the microgrid, shown in Figure 11h, increased from approximately $59.99 \mathrm{~Hz}$ when connected to the utility grid to approximately $60.06 \mathrm{~Hz}$ when islanded. This change in frequency was part of the CERTS algorithm which allowed the Gen-sets to decrease their output power to satisfy the load demands. Gen-set A1 and A2 decreased their output real power to approximately $31.4 \mathrm{~kW}(\mathrm{~A}=9 \mathrm{~kW} \quad \mathrm{~B}=11.4 \mathrm{~kW}$ $\mathrm{C}=11 \mathrm{~kW})$ and $22.7 \mathrm{~kW}(\mathrm{~A}=6.8 \mathrm{~kW} \mathrm{~B}=2 \mathrm{~kW} \mathrm{C}=8.5 \mathrm{~kW})$, respectively and increased their output reactive power to approximately $-2.2 \mathrm{kVAr}(\mathrm{A}=-0.8 \mathrm{kVAr} \mathrm{B}=-1 \mathrm{kVAr} \mathrm{C}=-0.4 \mathrm{kVAr})$ and $7.5 \mathrm{kVAr}(\mathrm{A}=2.5 \mathrm{kVAr} \mathrm{B}=2.5 \mathrm{kVAr} \mathrm{C}=2.5 \mathrm{kVAr})$, respectively. Meter 3 was approximately $-1.1 \mathrm{~kW}(\mathrm{~A}=-0.1 \mathrm{~kW} \quad \mathrm{~B}=-0.5 \mathrm{~kW} \quad \mathrm{C}=-0.5 \mathrm{~kW})-\mathrm{j} 4.2 \mathrm{kVAr} \quad(\mathrm{A}=-1.5 \mathrm{kVAr} \mathrm{B}=-$ $1.1 \mathrm{kVAr} \mathrm{C}=-1.6 \mathrm{kVAr}$ ) indicating that the Gen-sets were satisfying the loads in the microgrid and the power losses in the electrical lines. All the data was verified and recorded into the DAS Database. The microgrid ran for a couple of minutes in this electrical state before the $10 \mathrm{Hp}$ induction motor was started in Zone 3. 


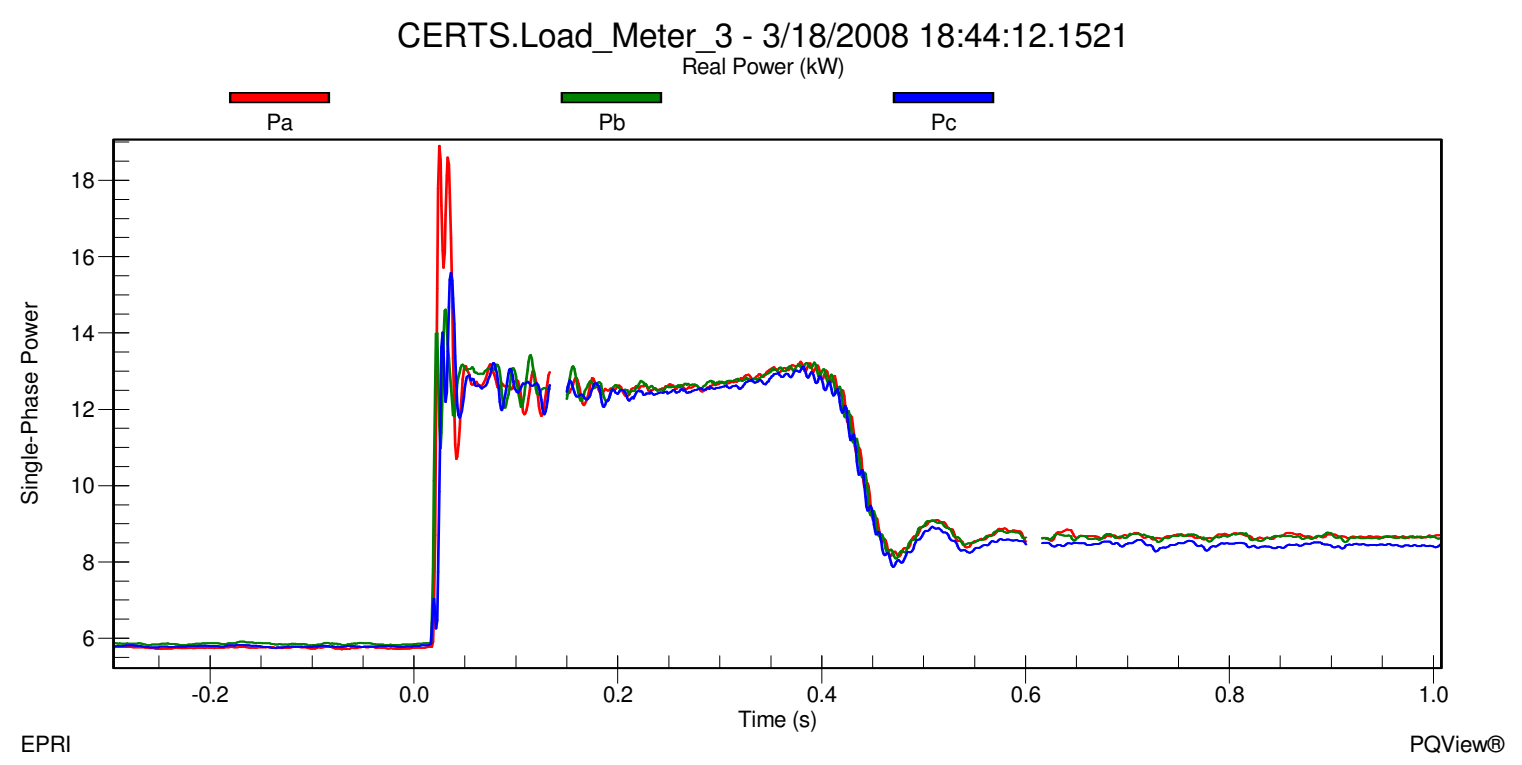

Figure 11i - Load Bank3 Real Single-Phase Power during Motor Start and Islanded for Test 10.3.12

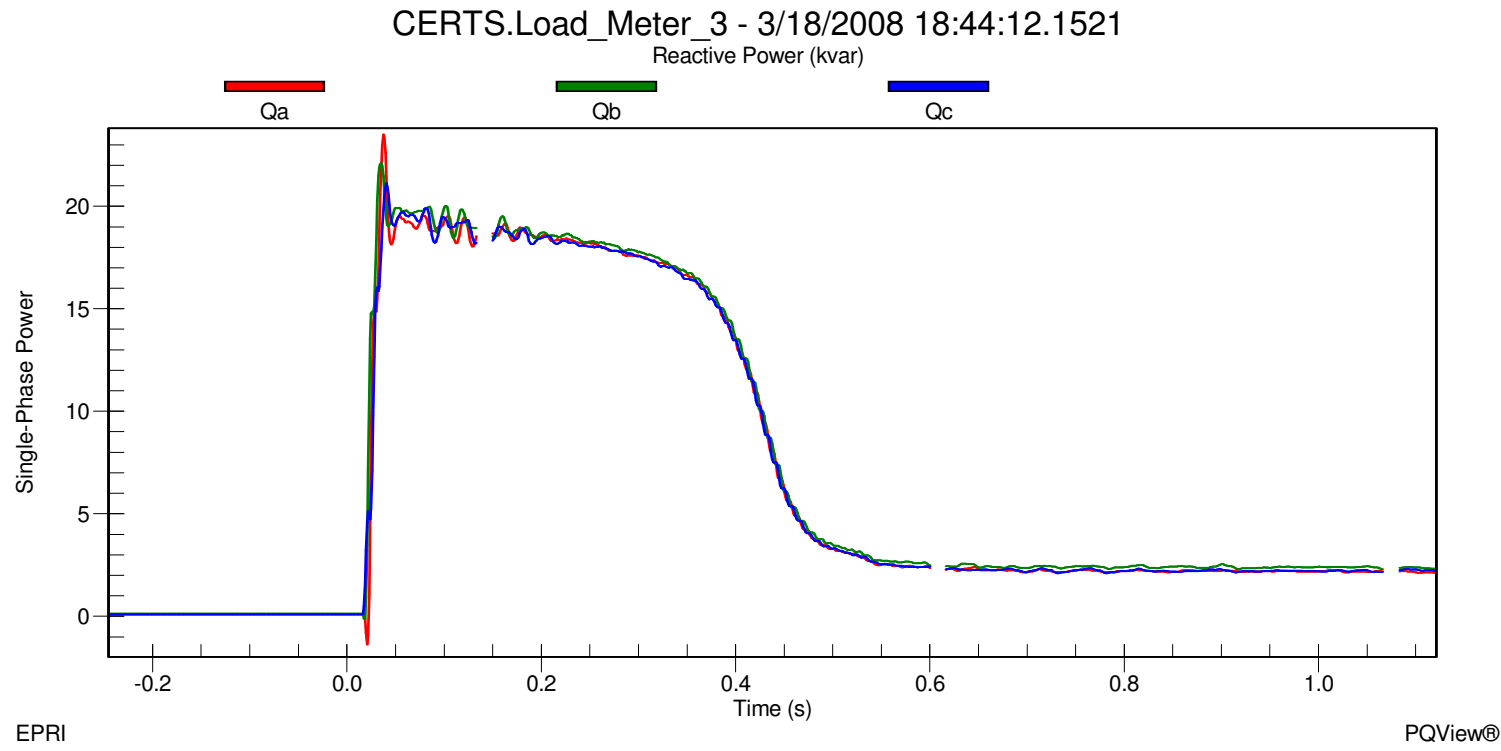

Figure 11j - Load Bank 3 Reactive Single-Phase Power during Motor Start and Islanded for Test 10.3.12

In Figures $11 \mathrm{i}$ and $11 \mathrm{j}$ it can be seen that the load in Zone 3 was approximately $17.4 \mathrm{~kW}$ $(\mathrm{A}=5.8 \mathrm{~kW} \mathrm{~B}=5.8 \mathrm{~kW} \mathrm{C}=5.8 \mathrm{~kW})+\mathrm{j} 0.15 \mathrm{kVAr}(\mathrm{A}=0.05 \mathrm{kVAr} \mathrm{B}=0.05 \mathrm{kVAr} \mathrm{C}=0.05 \mathrm{kVAr})$ before the start of the induction motor and increased to approximately $47 \mathrm{~kW}(\mathrm{~A}=19 \mathrm{~kW} B=14 \mathrm{~kW}$ $\mathrm{C}=14 \mathrm{~kW})+\mathrm{j} 66.8 \mathrm{kVAr}(\mathrm{A}=23.7 \mathrm{kVAr} \mathrm{B}=22 \mathrm{kVAr} \mathrm{C}=21.1 \mathrm{kVAr})$ during the inrush phase of the motor start. After about 1.5 cycles, the motor settled down to approximately $37.8 \mathrm{~kW}$ $(A=12.6 \mathrm{~kW} B=12.6 \mathrm{~kW} C=12.6 \mathrm{~kW})+j 56.1 \mathrm{kVAr}(\mathrm{A}=18.7 \mathrm{kVAr} B=18.7 \mathrm{kVAr} C=18.7 \mathrm{kVAr})$ during the warm up phase which lasted about 36 cycles (0.6 Seconds). When the motor 
reached steady state, the load in Zone 3 was approximately $25.8 \mathrm{~kW}(\mathrm{~A}=8.7 \mathrm{~kW} \mathrm{~B}=8.4 \mathrm{~kW}$ $\mathrm{C}=8.7 \mathrm{~kW})+\mathrm{j} 6.9 \mathrm{kVAr}(\mathrm{A}=2.3 \mathrm{kVAr} \mathrm{B}=2.3 \mathrm{kVAr} \mathrm{C}=2.3 \mathrm{kVAr})$.

The voltage and frequency at Meter 3 before the motor start was approximately $275 \mathrm{~V}$ on A-phase, $275.5 \mathrm{~V}$ on B-phase and $274 \mathrm{~V}$ on C-phase shown in Figure $11 \mathrm{k}$ and approximately $60.05 \mathrm{~Hz}$ shown in Figure 111. When the motor started, the voltage at Meter 3 during the inrush decreased to approximately 255V on A-phase, 254.3V on Bphase and $254.3 \mathrm{~V}$ on C-phase for about 1.5 cycles. Frequency dropped during the inrush to approximately $59.81 \mathrm{~Hz}$ and quickly increased to approximately $60.00 \mathrm{~Hz}$. Voltage increased as the motor was warming up and eventually settled at a steady state voltage at approximately $273.2 \mathrm{~V}$ on A-phase, $273.2 \mathrm{~V}$ on B-phase and $272.7 \mathrm{~V}$ on C-phase at an approximate frequency of $60.02 \mathrm{~Hz}$.

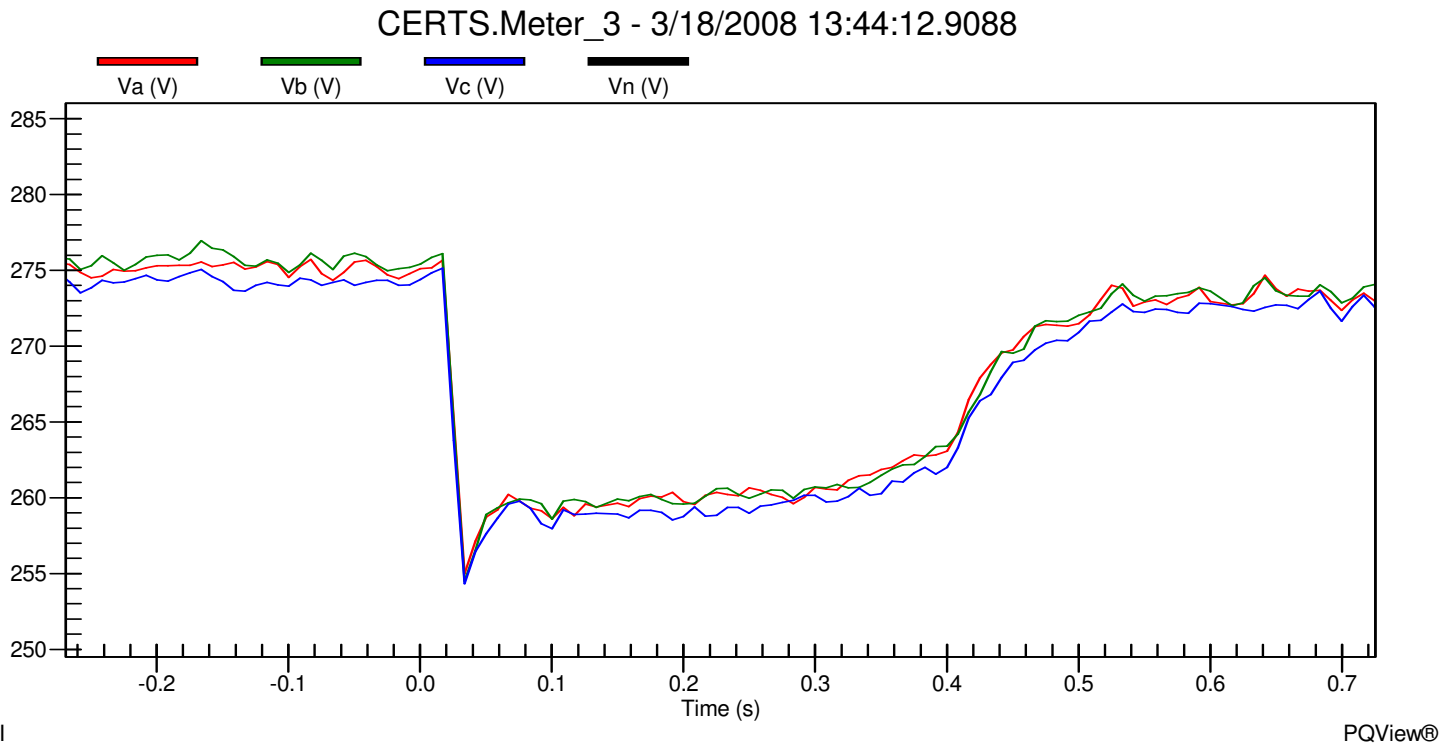

Figure 11k - Meter 3 Line-to-Ground Voltages during Motor Start and Islanded for Test 10.3.12 


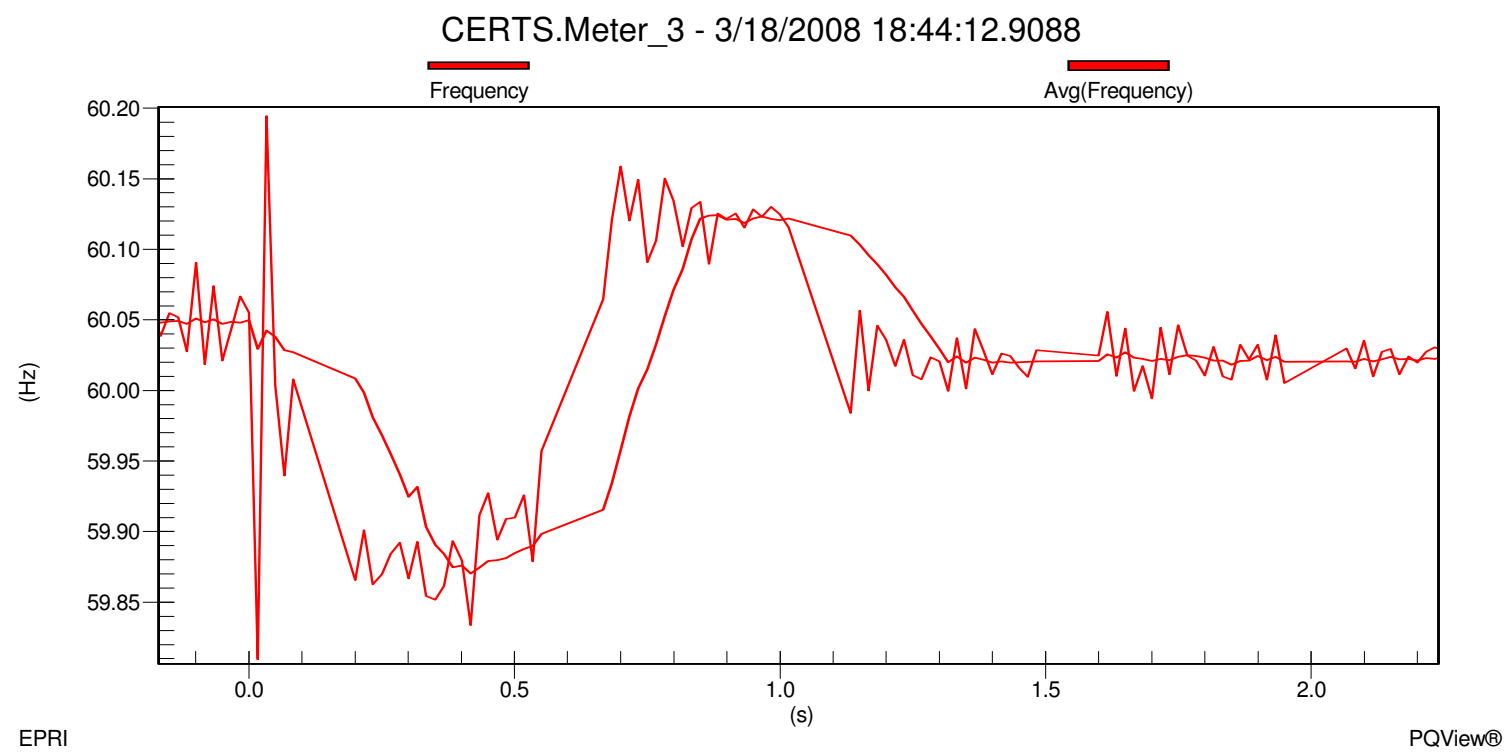

Figure 111 - Meter 3 Frequency during Motor Start and Islanded for Test 10.3.12

Before the motor started Gen-sets $\mathrm{A} 1$ and $\mathrm{A} 2$ were producing approximately $32 \mathrm{~kW}$ $(\mathrm{A}=9.5 \mathrm{~kW} \quad \mathrm{~B}=11.7 \mathrm{~kW} \quad \mathrm{C}=10.8 \mathrm{~kW})-\mathrm{j} 2 \mathrm{kVAr} \quad(\mathrm{A}=-1 \mathrm{kVAr} \quad \mathrm{B}=-0.8 \mathrm{kVAr} \quad \mathrm{C}=-0.2 \mathrm{kVAr})$ and $23.5 \mathrm{~kW}(\mathrm{~A}=6.5 \mathrm{~kW} B=8.5 \mathrm{~kW} C=8.5 \mathrm{~kW})+\mathrm{j} 7.1 \mathrm{kVAr}(\mathrm{A}=2.3 \mathrm{kVAr} B=2.5 \mathrm{kVAr} C=2.3 \mathrm{kVAr})$, respectively shown in Figures $11 \mathrm{~m}-11 \mathrm{p}$. The power generated by both Gen-sets was satisfying the loads in Load Banks 3 and 4 and all the electrical losses in the microgrid system. When the motor started the inrush caused the Gen-sets to increase their output levels to $46.1 \mathrm{~kW}(\mathrm{~A}=16.2 \mathrm{~kW} B=15.8 \mathrm{~kW} \mathrm{C}=14.1 \mathrm{~kW})+\mathrm{j} 27.6 \mathrm{kVAr}(\mathrm{A}=7.5 \mathrm{kVAr} B=11 \mathrm{kVAr}$ $\mathrm{C}=9.1 \mathrm{kVAr})$ for Gen-set $\mathrm{A} 1$ and $35.4 \mathrm{~kW}(\mathrm{~A}=10.7 \mathrm{~kW} \mathrm{~B}=11.5 \mathrm{~kW} \mathrm{C}=13.2 \mathrm{~kW})+\mathrm{j} 37.8 \mathrm{kVAr}$ $(\mathrm{A}=13.5 \mathrm{kVAr} B=11.3 \mathrm{kVAr} \mathrm{C}=13 \mathrm{kVAr})$ for Gen-set A2. The Gen-sets decreased their output levels while the motor was warming up and eventually dropped to $35.5 \mathrm{~kW}$ $(\mathrm{A}=11 \mathrm{~kW} B=12.6 \mathrm{~kW} C=11.9 \mathrm{~kW})+\mathrm{j} 1.3 \mathrm{kVAr}(\mathrm{A}=0 \mathrm{kVAr} B=0.5 \mathrm{kVAr} \mathrm{C}=0.8 \mathrm{kVAr})$ for Genset $\mathrm{A} 1$ and $27.5 \mathrm{~kW}(\mathrm{~A}=7.7 \mathrm{~kW} B=9.9 \mathrm{~kW} C=9.9 \mathrm{~kW})+\mathrm{j} 10.3 \mathrm{kVAr}(\mathrm{A}=3.4 \mathrm{kVAr} B=3.5 \mathrm{kVAr}$ $\mathrm{C}=3.4 \mathrm{kVAr}$ ) for Gen-set A2 when the motor reached steady state. Once all the data was verified and recorded into the DAS Database, the static switch was directed by the EMS to manually close. 


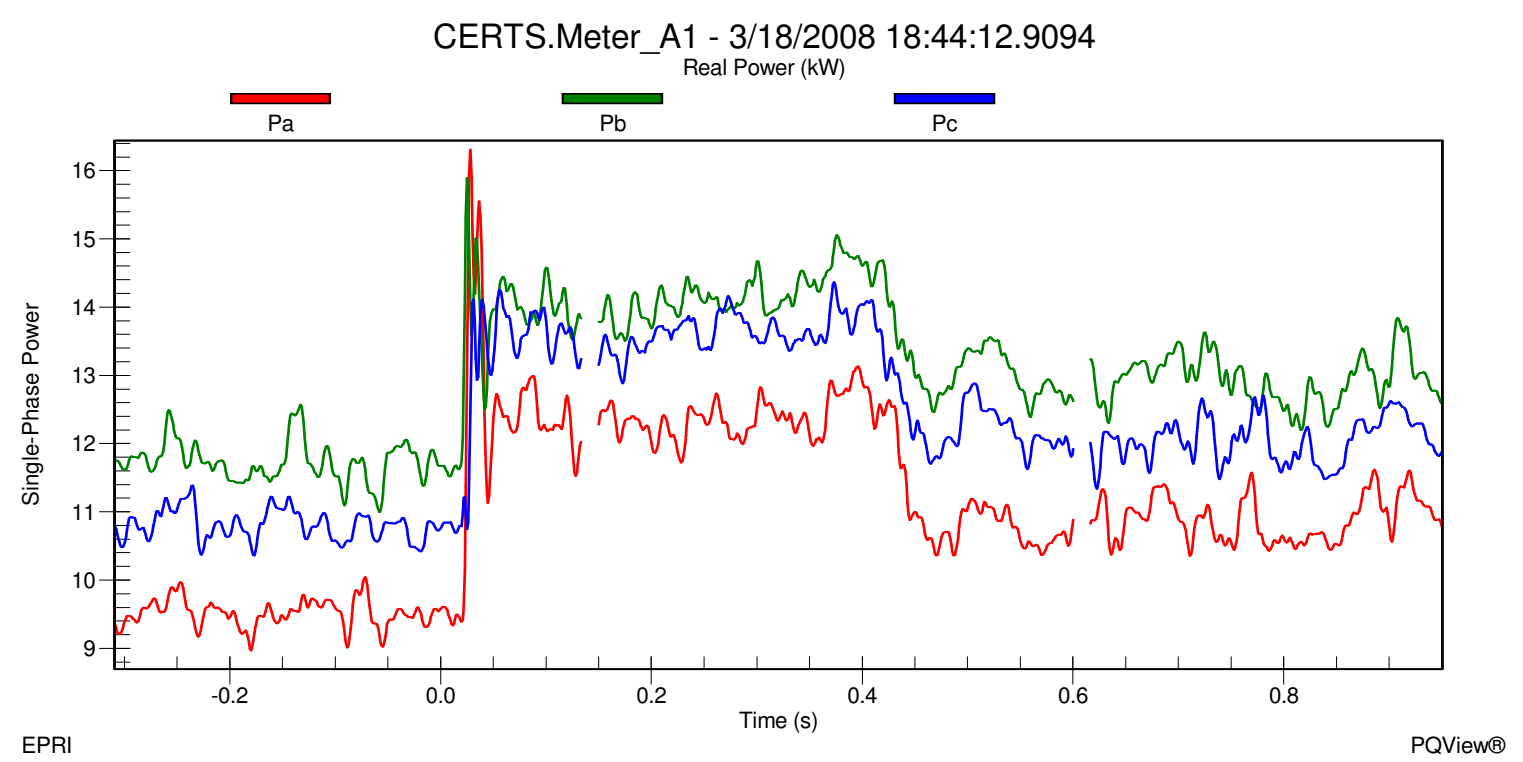

Figure 11m - Gen-set A1 Real Single-Phase Power during Motor Start and Islanded for Test 10.3.12

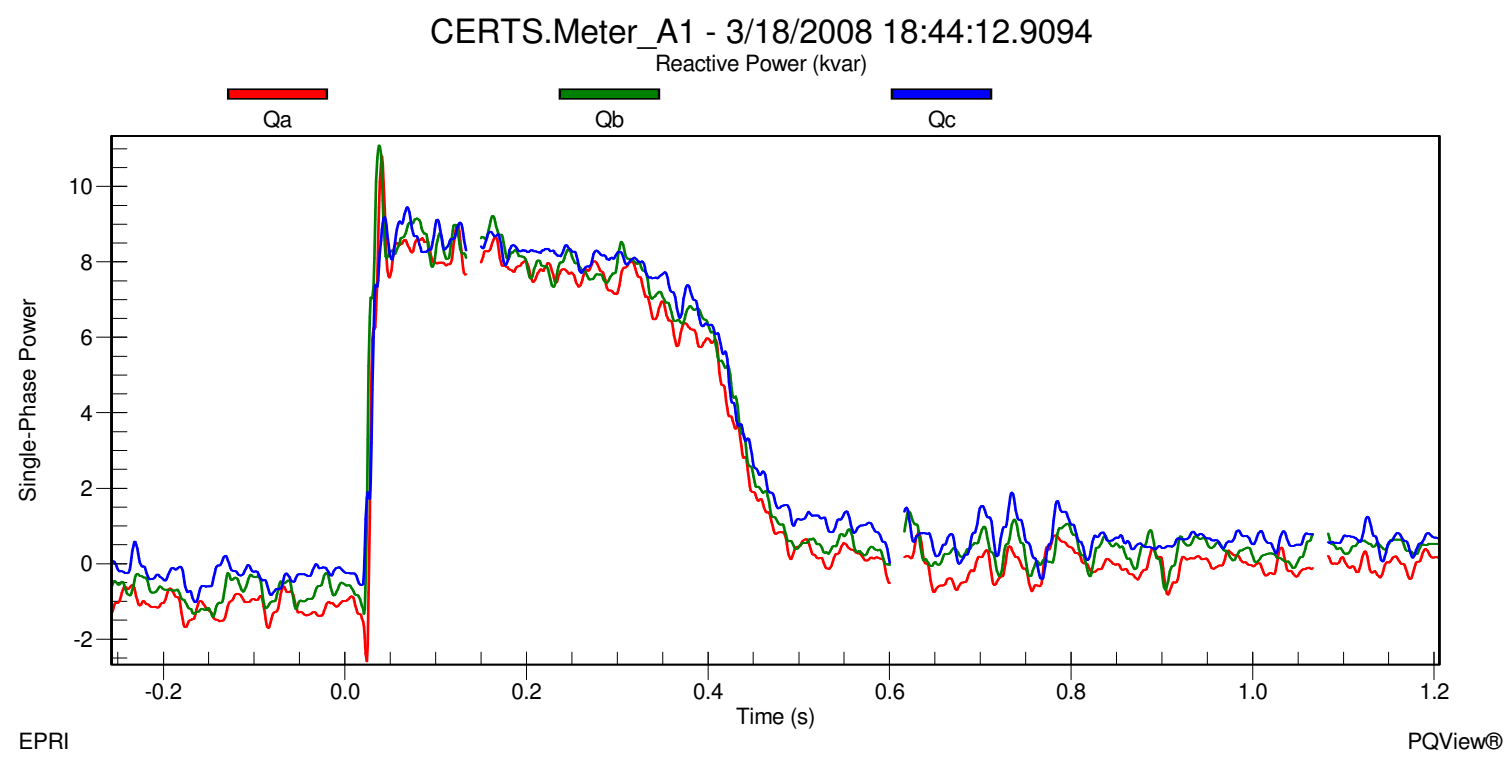

Figure 11n - Gen-set A1 Reactive Single-Phase Power during Motor Start and Islanded for Test 10.3.12 


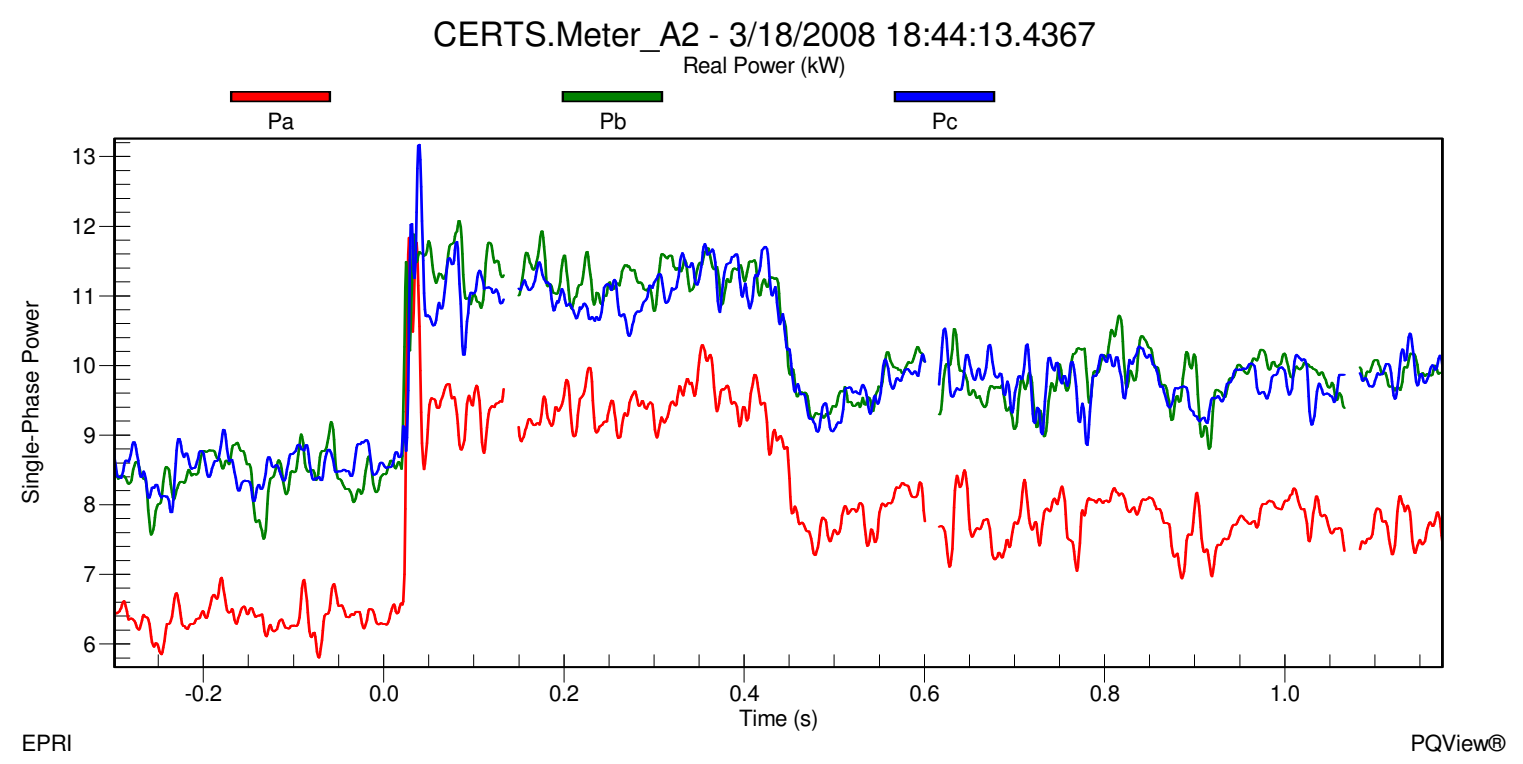

Figure 11o - Gen-set A2 Real Single-Phase Power during Motor Start and Islanded for Test 10.3.12

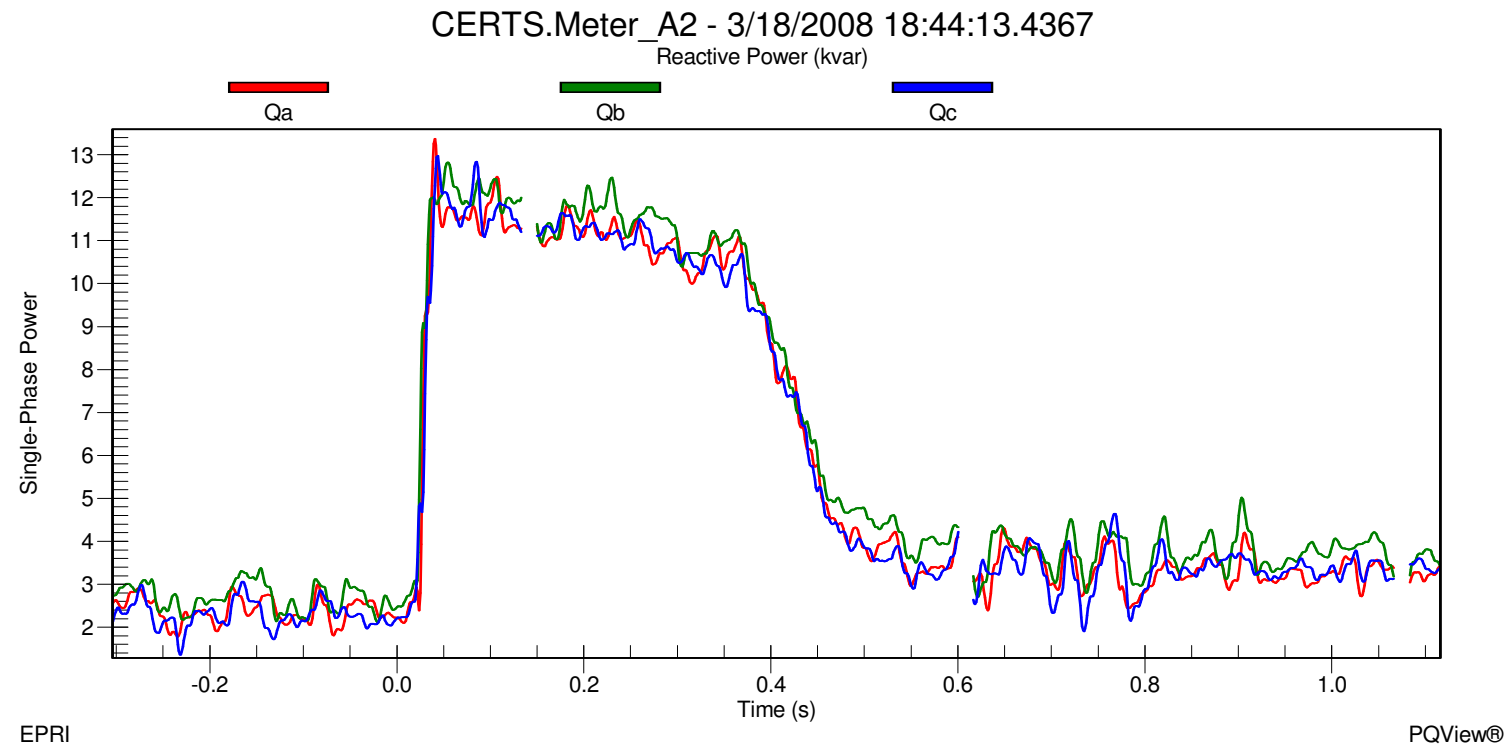

Figure 11p - Gen-set A2 Reactive Single-Phase Power during Motor Start and Islanded for Test 10.3.12

As soon as the static switch closed, Meter 1 recorded real power decreased from approximately $37.7 \mathrm{~kW}(\mathrm{~A}=10 \mathrm{~kW} \quad \mathrm{~B}=18 \mathrm{~kW} \quad \mathrm{C}=9.7 \mathrm{~kW})$ to $33.2 \mathrm{~kW} \quad(\mathrm{~A}=9.2 \mathrm{~kW} \quad \mathrm{~B}=12 \mathrm{~kW}$ $\mathrm{C}=12 \mathrm{~kW})$ and reactive power increased from approximately $0.9 \mathrm{kVAr}(\mathrm{A}=0.2 \mathrm{kVAr}$ $\mathrm{B}=0.5 \mathrm{kVAr} \mathrm{C}=0.2 \mathrm{kVAr})$ to $24 \mathrm{kVAr}(\mathrm{A}=8.7 \mathrm{kVAr} \mathrm{B}=8 \mathrm{kVAr} \mathrm{C}=7.3 \mathrm{kVAr})$ which means that the utility was satisfying a portion of the load demand in Load Bank 6 and all the reactive power in the microgrid. Figures $11 \mathrm{q}$ and $11 \mathrm{r}$ show the static switch decreasing in real power from approximately $0 \mathrm{~kW}(\mathrm{~A}=0 \mathrm{~kW} B=0 \mathrm{~kW} \mathrm{C}=0 \mathrm{~kW})$ to $-3.7 \mathrm{~kW}(\mathrm{~A}=-0.5 \mathrm{~kW}$ $\mathrm{B}=-5.6 \mathrm{~kW} \mathrm{C}=2.4 \mathrm{~kW})$ and increasing in reactive power from approximately $0 \mathrm{kVAr}$ 
( $\mathrm{A}=0 \mathrm{kVAr} \mathrm{B}=0 \mathrm{kVAr} \mathrm{C}=0 \mathrm{kVAr})$ to $23.3 \mathrm{kVAr}(\mathrm{A}=8.6 \mathrm{kVAr} \mathrm{B}=7.6 \mathrm{kVAr} \mathrm{C}=7.3 \mathrm{kVAr})$. At the beginning of the test, the initial power flow through the static switch was $-7.8 \mathrm{~kW}(\mathrm{~A}=-$ $2 \mathrm{~kW} \mathrm{~B}=-7 \mathrm{~kW} \mathrm{C}=1.2 \mathrm{~kW})+\mathrm{j} 22.4 \mathrm{kVAr}(\mathrm{A}=8.7 \mathrm{kVAr} \mathrm{B}=7 \mathrm{kVAr} \mathrm{C}=6.7 \mathrm{kVAr})$ which is not the same recorded at this point in the test because the $10 \mathrm{Hp}$ motor load is on in Zone 3 . The Gen-sets have picked up the motor load and supporting Load Bank 6 with approximately $3.7 \mathrm{~kW}$.

Load Banks 3 and 4 loads increased slightly to $24.8 \mathrm{~kW}(\mathrm{~A}=8.45 \mathrm{~kW} B=8.2 \mathrm{~kW} \mathrm{C}=8.15 \mathrm{~kW})+$ j6.34kVAr $\quad(A=2.17 \mathrm{kVAr} \quad \mathrm{B}=2.17 \mathrm{kVAr} \quad \mathrm{C}=2 \mathrm{kVAr})$ and $36.4 \mathrm{~kW} \quad(\mathrm{~A}=9.7 \mathrm{~kW} \quad \mathrm{~B}=13.4 \mathrm{~kW}$ $C=13.3 \mathrm{~kW})+j 0.61 \mathrm{kVAr}(\mathrm{A}=0.12 \mathrm{kVAr} B=0.28 \mathrm{kVAr} C=0.21 \mathrm{kVAr})$, respectively. This slight load increase is a result from a voltage rise in the microgrid, shown in Figure 11s, from approximately $273.6 \mathrm{~V}$ on A-phase, $273.6 \mathrm{~V}$ on B-phase and $272.5 \mathrm{~V}$ on C-phase when islanded to $281 \mathrm{~V}$ on A-phase, $278.1 \mathrm{~V}$ on B-phase and $277.9 \mathrm{~V}$ on C-phase at Meter 3 when connected to the utility grid.

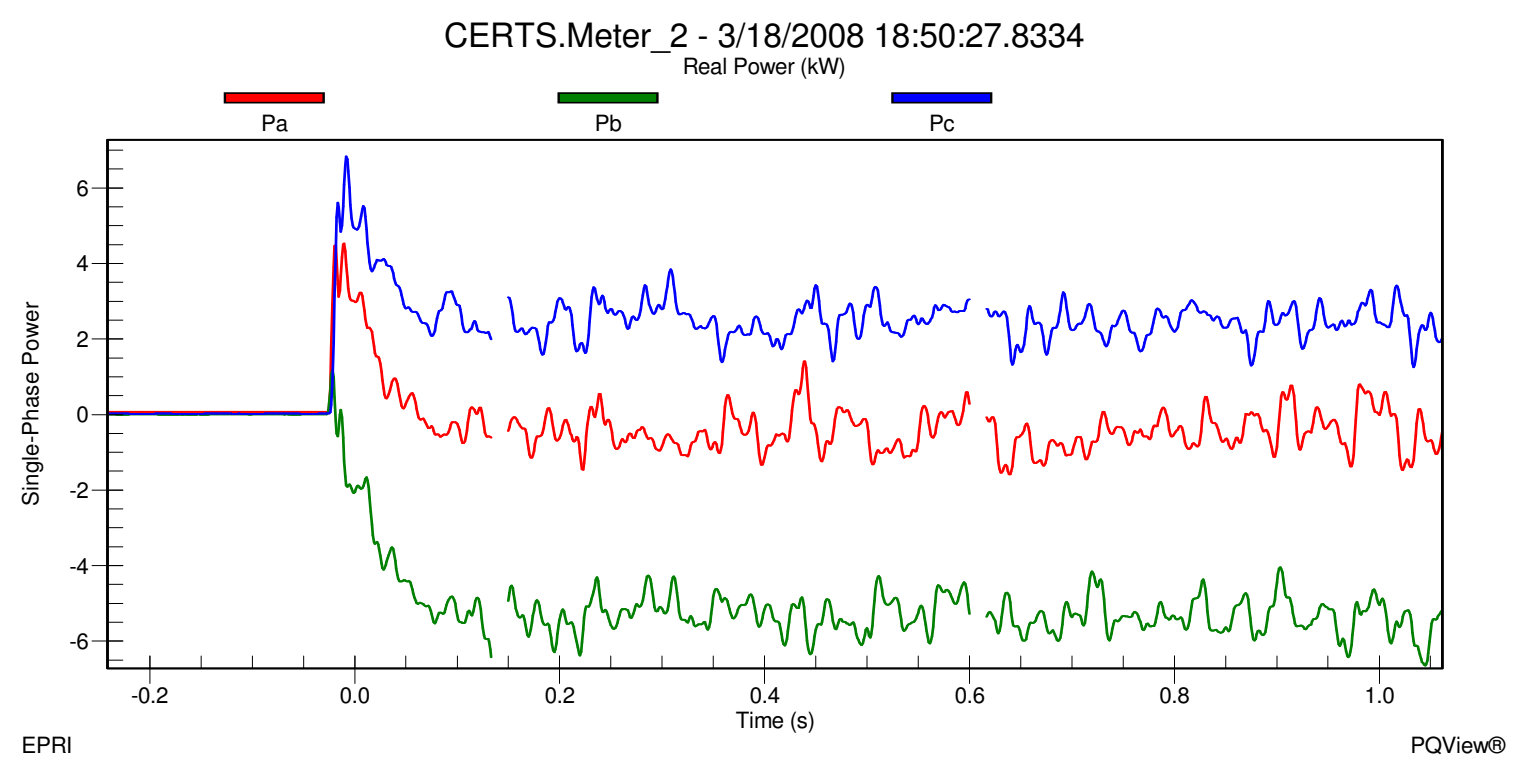

Figure 11q - Static Switch Real Single-Phase Power during Island to Utility Connected mode for Test 10.3.12 


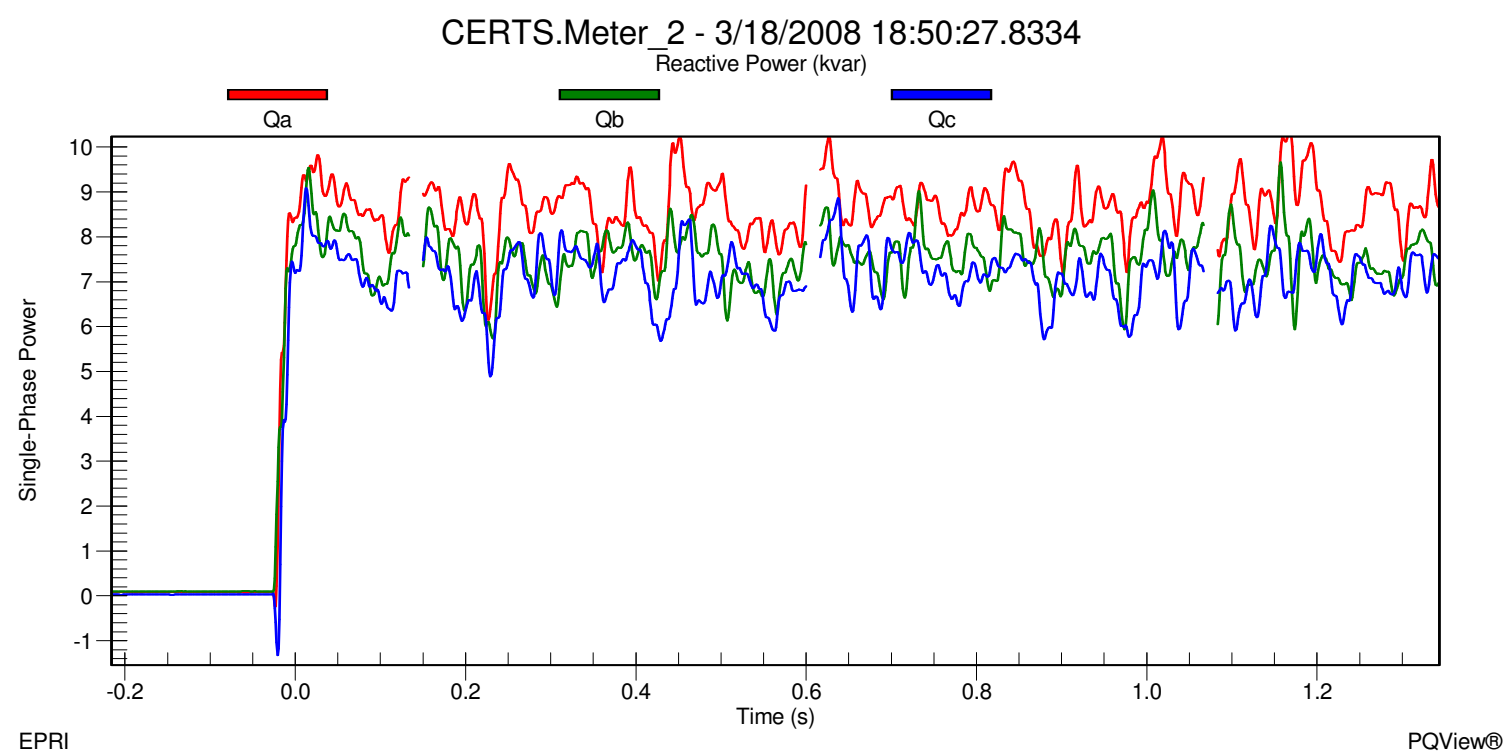

Figure 11r - Static Switch Reactive Single-Phase Power during Island to Utility Connected mode for Test 10.3.12

CERTS.Meter 3 - 3/18/2008 13:50:27.7899

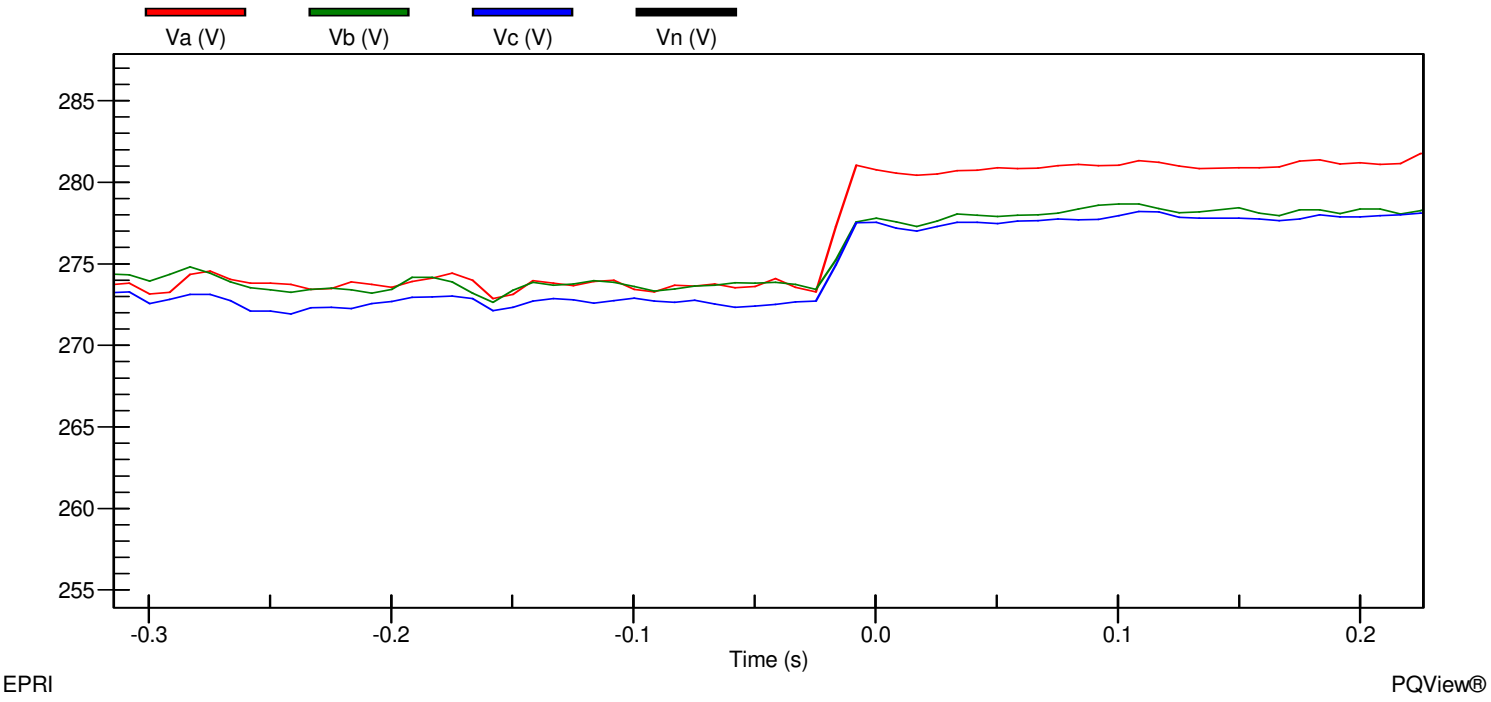

Figure 11s - Meter 3 Line-to-Ground Voltage during Island to Utility Connected mode for Test 10.3.12 


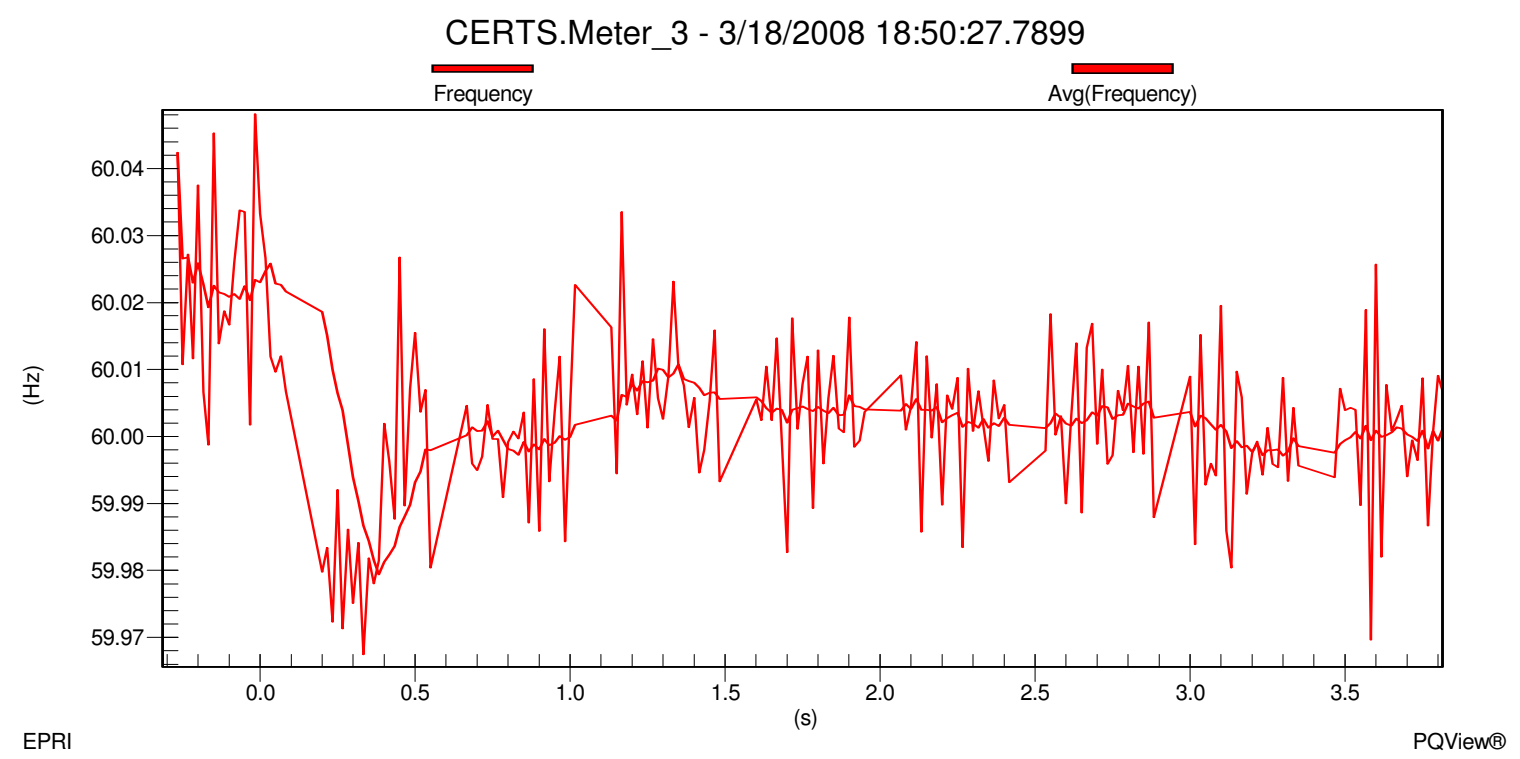

Figure 11t - Meter 3 Frequency during Island to Utility Connected mode for Test 10.3.12

Frequency change in the microgrid, shown in Figure 11t, decreased from approximately $60.02 \mathrm{~Hz}$ when islanded to approximately $60.00 \mathrm{~Hz}$ when connected to the utility grid. This change in frequency is due to the frequency no longer being established by the Gensets using the CERTS algorithm but by the utility. Gen-sets A1 and A2 are in unit power control mode therefore when the static switch closed back into the utility the Gen-sets produced real power based on their set-points initialized at the beginning of the test. The output power for both Gen-sets were relatively close to the values at the beginning of the test with Gen-set A1 producing approximately $38.5 \mathrm{~kW}(\mathrm{~A}=11.5 \mathrm{~kW} B=16 \mathrm{~kW}$ $\mathrm{C}=11 \mathrm{~kW})-\mathrm{j} 10 \mathrm{kVAr}(\mathrm{A}=-3.6 \mathrm{kVAr} \mathrm{B}=-3.5 \mathrm{kVAr} \mathrm{C}=-2.9 \mathrm{kVAr})$ and Gen-set $\mathrm{A} 2$ producing approximately $29.4 \mathrm{~kW}(\mathrm{~A}=8.4 \mathrm{~kW} B=12.2 \mathrm{~kW} C=8.8 \mathrm{~kW})-\mathrm{j} 1 \mathrm{kVAr}(\mathrm{A}=-1 \mathrm{kVAr} B=0 \mathrm{kVAr}$ $\mathrm{C}=0 \mathrm{kVAr})$. After all the data was verified and recorded into the DAS Database, the motor was turned off and the Gen-sets and Load Banks set-points were changed according to the next test (10.3.14) in Table 2.

For Test 10.3.14 the measured values, after Gen-set A1 was warmed up and load banks brought on-line are provided in Table 4.

\begin{tabular}{|c|c|c|c|c|c|c|c|c|c|}
\hline Meter & $\begin{array}{c}\text { Gen- } \\
\text { set A1 }\end{array}$ & $\begin{array}{c}\text { Gen - } \\
\text { set A2 }\end{array}$ & Meter 1 & Meter 2 & Meter 3 & Meter 4 & LB 3 & LB 4 & LB 6 \\
\hline $\begin{array}{c}\text { 3 Phase } \\
\text { kW }\end{array}$ & 37.3 & 0 & 14.1 & -23.4 & -26.5 & 0 & 10.95 & 0 & 37.1 \\
\hline 1 Phase & $\mathrm{A}=11.3$ & $\mathrm{~A}=0$ & $\mathrm{~A}=3.1$ & $\mathrm{~A}=-6.6$ & $\mathrm{~A}=-7.6$ & $\mathrm{~A}=0$ & $\mathrm{~A}=3.75$ & $\mathrm{~A}=0$ & $\mathrm{~A}=9.8$ \\
\hline
\end{tabular}




\begin{tabular}{|c|c|c|c|c|c|c|c|c|c|}
\hline $\mathbf{k W}$ & $\mathrm{B}=16.1$ & $\mathrm{~B}=0$ & $\mathrm{~B}=6.5$ & $\mathrm{~B}=-11.2$ & $\mathrm{~B}=-12.8$ & $\mathrm{~B}=0$ & $\mathrm{~B}=3.6$ & $\mathrm{~B}=0$ & $\mathrm{~B}=17.8$ \\
$\mathrm{C}=9.9$ & $\mathrm{C}=0$ & $\mathrm{C}=4.5$ & $\mathrm{C}=-5.6$ & $\mathrm{C}=-6.1$ & $\mathrm{C}=0$ & $\mathrm{C}=3.6$ & $\mathrm{C}=0$ & $\mathrm{C}=9.5$ \\
\hline $\begin{array}{c}\text { 3 Phase } \\
\mathbf{k V A r}\end{array}$ & -13.1 & 0 & 19.5 & 18.9 & 13.4 & 0 & 0 & 0 & 0.645 \\
\hline $\begin{array}{c}\text { 1 Phase } \\
\mathbf{k V A r}\end{array}$ & $\mathrm{B}=-4.6$ & $\mathrm{~B}=0$ & $\mathrm{~B}=7$ & $\mathrm{~B}=6.5$ & $\mathrm{~B}=4.6$ & $\mathrm{~B}=0$ & $\mathrm{~B}=0$ & $\mathrm{~B}=0$ & $\mathrm{~B}=0.39$ \\
& $\mathrm{C}=-3.8$ & $\mathrm{C}=0$ & $\mathrm{C}=6$ & $\mathrm{C}=5.9$ & $\mathrm{C}=3.9$ & $\mathrm{C}=0$ & $\mathrm{C}=0$ & $\mathrm{C}=0$ & $\mathrm{C}=0.13$ \\
\hline
\end{tabular}

Table 4 - Measured Values after Start Up for Test 10.3.14

These measurements were relatively close to the expected values in Table 2, but not exact due to temperature, phase voltages and electrical losses in conductors. In addition, the $40 \mathrm{~kW}$ setting for LB6 was below the selected set value. At the time of the measurements, the voltage and frequency was $283.1 \mathrm{~V}$ on A-phase, $278.9 \mathrm{~V}$ on B-phase and $279.2 \mathrm{~V}$ on C-phase and $60.00 \mathrm{~Hz}$ at the static switch (i.e., Meter 2) when connected to the utility grid; and $283.8 \mathrm{~V}$ on A-phase, $279.8 \mathrm{~V}$ on B-phase and $279.9 \mathrm{~V}$ on C-phase and $60.02 \mathrm{~Hz}$ at Meter 3.

Gen-set A1 was setup in this test to produce more power than Load Bank 3 needed which approximately $23.4 \mathrm{~kW}$ of excess power was exported through the static switch to Load Bank 6. Since Load Bank 6 was approximately $37.1 \mathrm{~kW}(\mathrm{~A}=9.8 \mathrm{~kW} \quad \mathrm{~B}=17.8 \mathrm{~kW}$ $\mathrm{C}=9.5 \mathrm{~kW})+\mathrm{j} 0.645 \mathrm{kVAr}(\mathrm{A}=0.125 \mathrm{kVAr} \mathrm{B}=0.39 \mathrm{kVAr} \mathrm{C}=0.13 \mathrm{kVAr})$, the utility had to supply approximately $14.1 \mathrm{~kW}$ to satisfy the load. Reactive power had to be imported in from the utility of approximately 18.9kVAr because Gen-sets A1 needed approximately j13.1kVAr $(\mathrm{A}=-4.7 \mathrm{kVAr} \mathrm{B}=-4.6 \mathrm{kVAr} \mathrm{C}=-3.8 \mathrm{kVAr})$ and the reactive power absorbed in the electrical lines. Once all data was verified and recorded into the DAS Database, the $10 \mathrm{Hp}$ induction motor was started in Zone 3. 


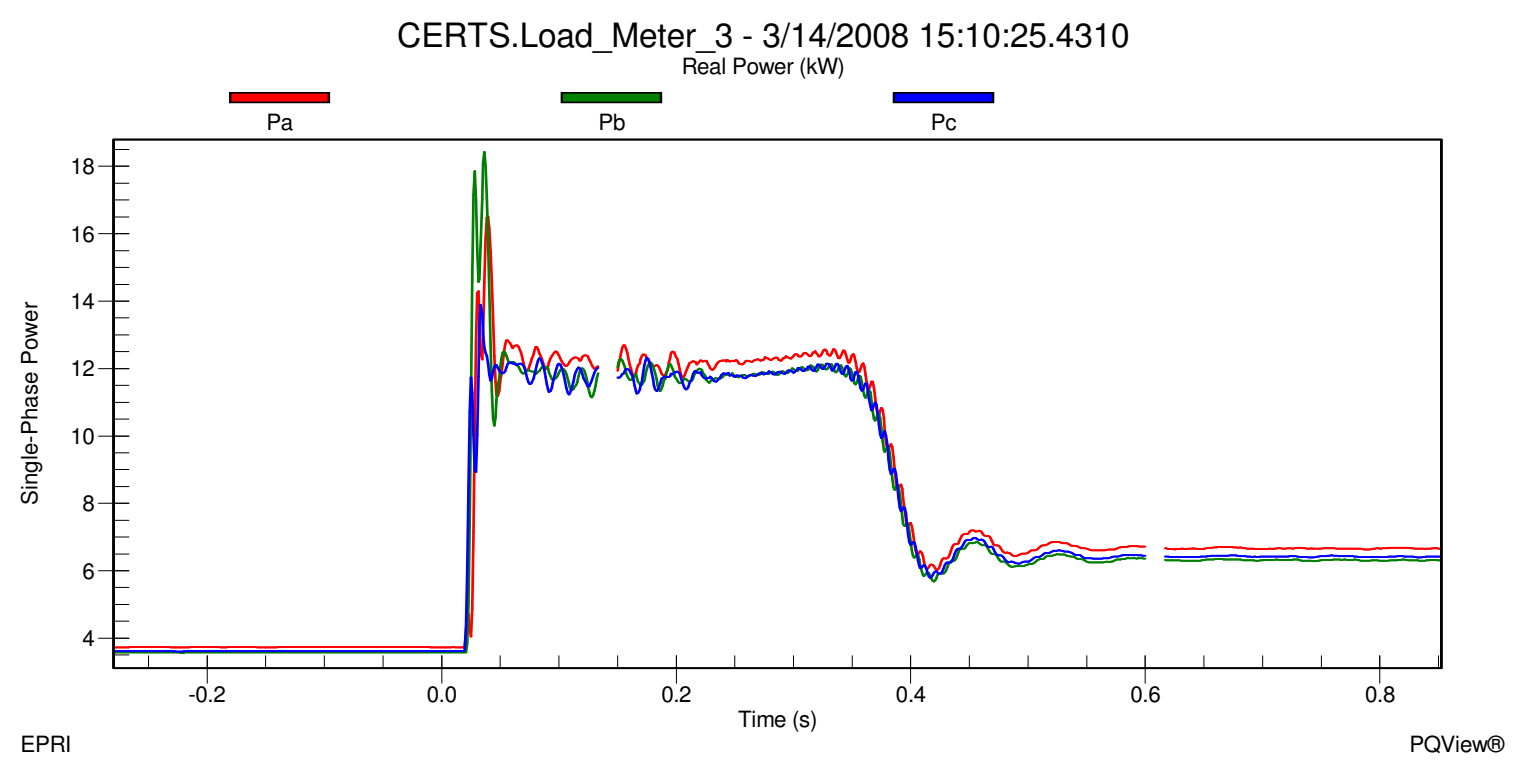

Figure 12a - Load Bank 3 Real Single-Phase Power during Motor Start and Utility Connected for Test 10.3.14

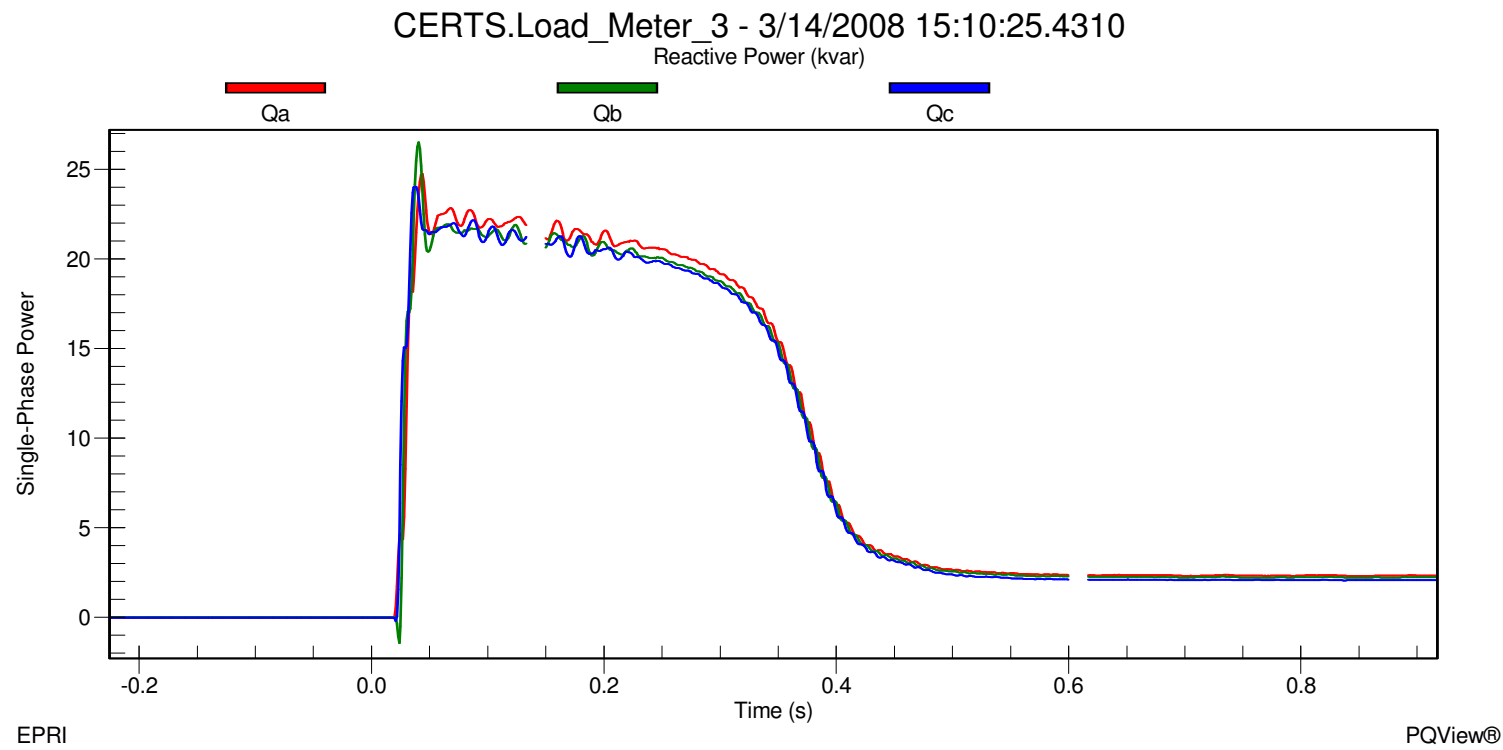

Figure 12b - Load Bank 3 Reactive Single-Phase Power during Motor Start and Utility Connected for Test 10.3.14

In Figures $12 \mathrm{a}$ and $12 \mathrm{~b}$ it can be seen that the load in Zone 3 was approximately $10.95 \mathrm{~kW}$ $(\mathrm{A}=3.75 \mathrm{~kW} \mathrm{~B}=3.6 \mathrm{~kW} \mathrm{C}=3.6 \mathrm{~kW})+\mathrm{j} 0 \mathrm{kVAr}(\mathrm{A}=0 \mathrm{kVAr} \mathrm{B}=0 \mathrm{kVAr} \mathrm{C}=0 \mathrm{kVAr})$ before the start of the induction motor and increased to approximately $48.45 \mathrm{~kW}(\mathrm{~A}=16.25 \mathrm{~kW} \mathrm{~B}=18.5 \mathrm{~kW}$ $\mathrm{C}=13.7 \mathrm{~kW})+\mathrm{j} 75.1 \mathrm{kVAr}(\mathrm{A}=24.6 \mathrm{kVAr} \mathrm{B}=26.5 \mathrm{kVAr} \mathrm{C}=24 \mathrm{kVAr})$ during the inrush phase of the motor start. After about 1.5 cycles, the motor settled down to approximately $35.8 \mathrm{~kW}$ $(A=12.2 \mathrm{~kW} \quad \mathrm{~B}=11.8 \mathrm{~kW} \quad \mathrm{C}=11.8 \mathrm{~kW})+\mathrm{j} 62 \mathrm{kVAr} \quad(\mathrm{A}=21 \mathrm{kVAr} \quad \mathrm{B}=20.5 \mathrm{kVAr} \quad \mathrm{C}=20.5 \mathrm{kVAr})$ during the warm up phase which lasted about 30 cycles (0.5 Seconds). When the motor 
reached steady state, the load in Zone 3 was approximately $19.4 \mathrm{~kW}(\mathrm{~A}=6.7 \mathrm{~kW} \mathrm{~B}=6.3 \mathrm{~kW}$ $\mathrm{C}=6.4 \mathrm{~kW})+\mathrm{j} 6.7 \mathrm{kVAr}(\mathrm{A}=2.4 \mathrm{kVAr} \mathrm{B}=2.2 \mathrm{kVAr} \mathrm{C}=2.1 \mathrm{kVAr})$.

The voltage and frequency at the static switch before the motor start was approximately 283.1V on A-phase, 278.9V on B-phase and 279.2V on C-phase shown in Figure 12c and approximately $60.00 \mathrm{~Hz}$ shown in Figure $12 \mathrm{~d}$. When the motor started, the voltage at the static switch during the inrush decreased to approximately $274.8 \mathrm{~V}$ on A-phase, $271.6 \mathrm{~V}$ on B-phase and $271.6 \mathrm{~V}$ on C-phase for about 1.5 cycles. Frequency dropped during the inrush to approximately $59.91 \mathrm{~Hz}$ and quickly increased to approximately $59.99 \mathrm{~Hz}$. Voltage increased as the motor was warming up and eventually settled at a steady state voltage at approximately $282.8 \mathrm{~V}$ on A-phase, $278 \mathrm{~V}$ on B-phase and $278.3 \mathrm{~V}$ on C-phase at an approximate frequency of $60.01 \mathrm{~Hz}$.

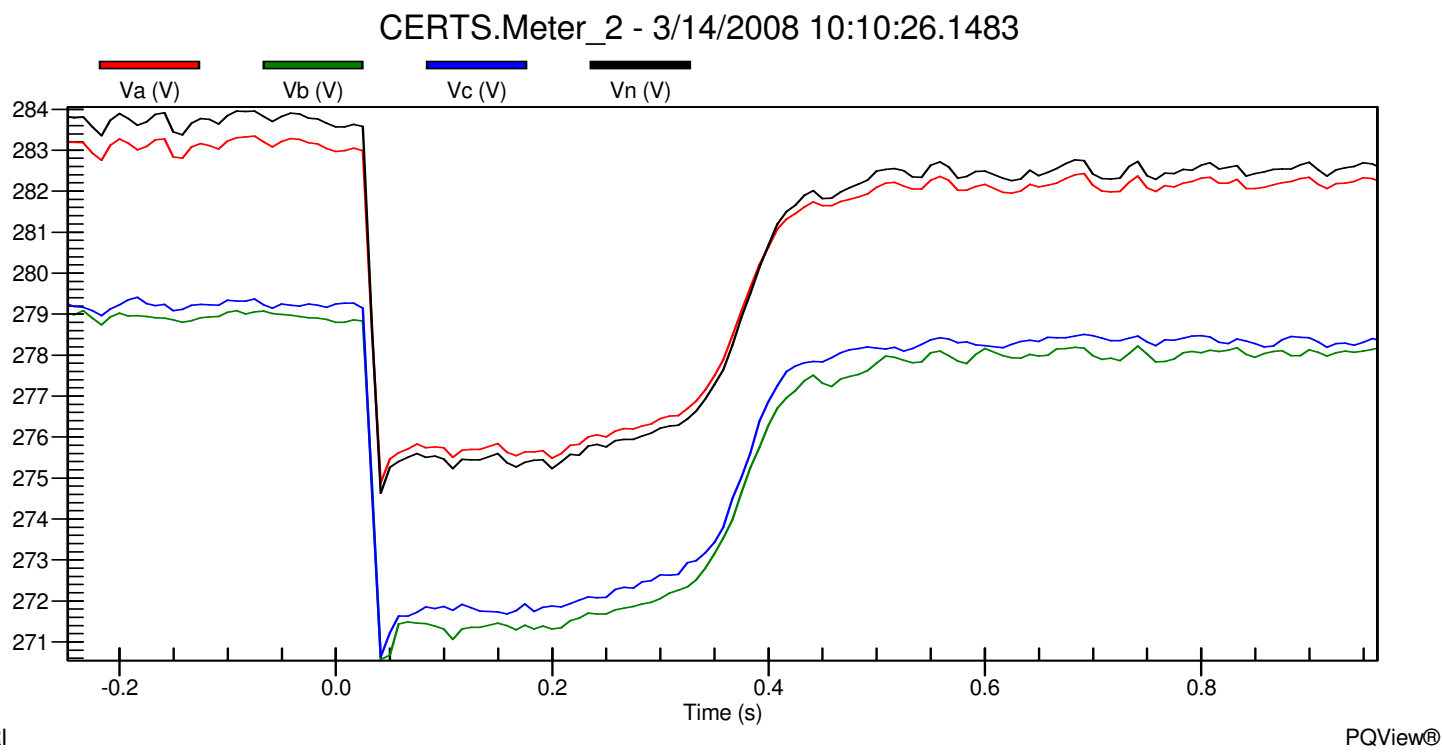

Figure 12c - Static Switch Line-to-Ground Voltages during Motor Start and Utility Connected for Test 10.3.14 


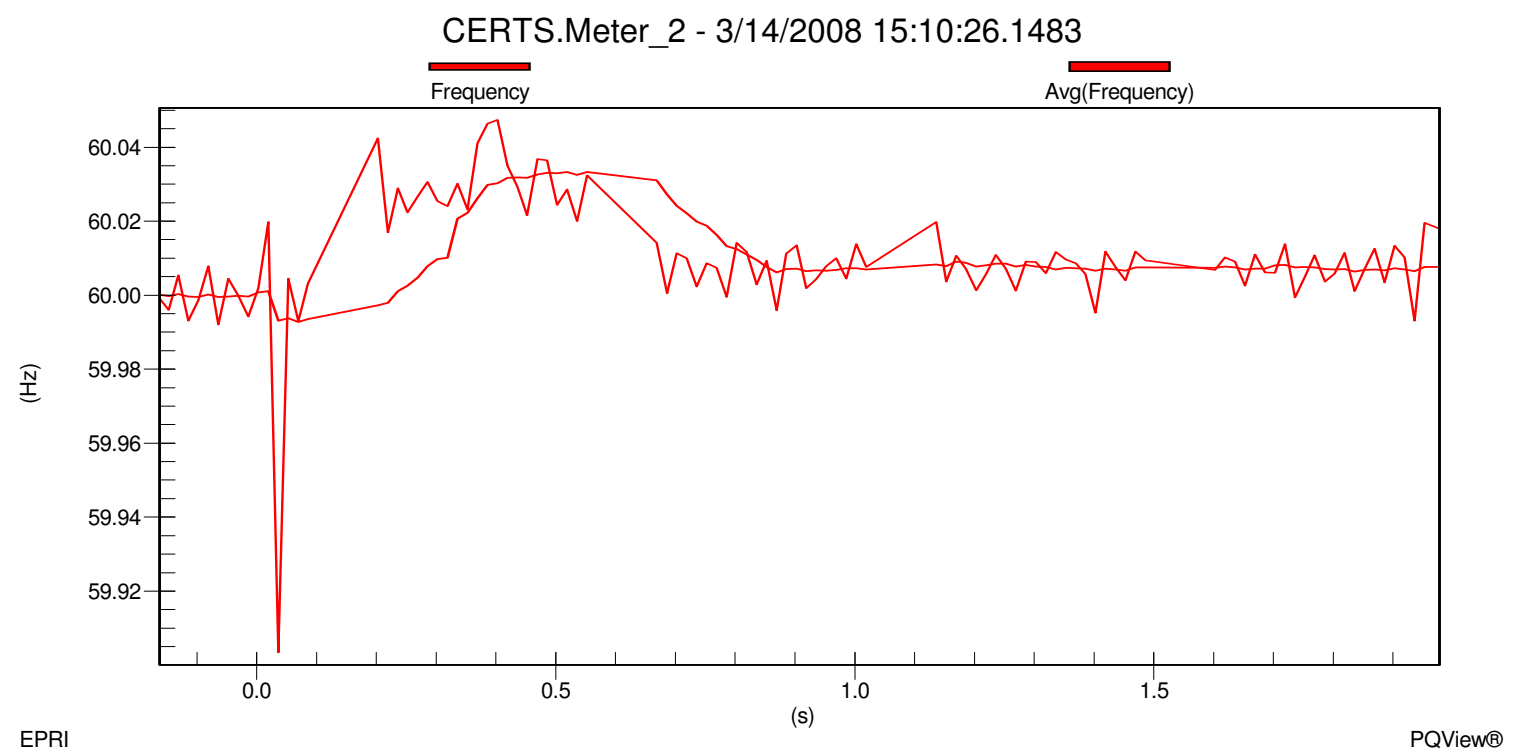

Figure 12d - Static Switch Frequency during Motor Start and Utility Connected for Test 10.3.14

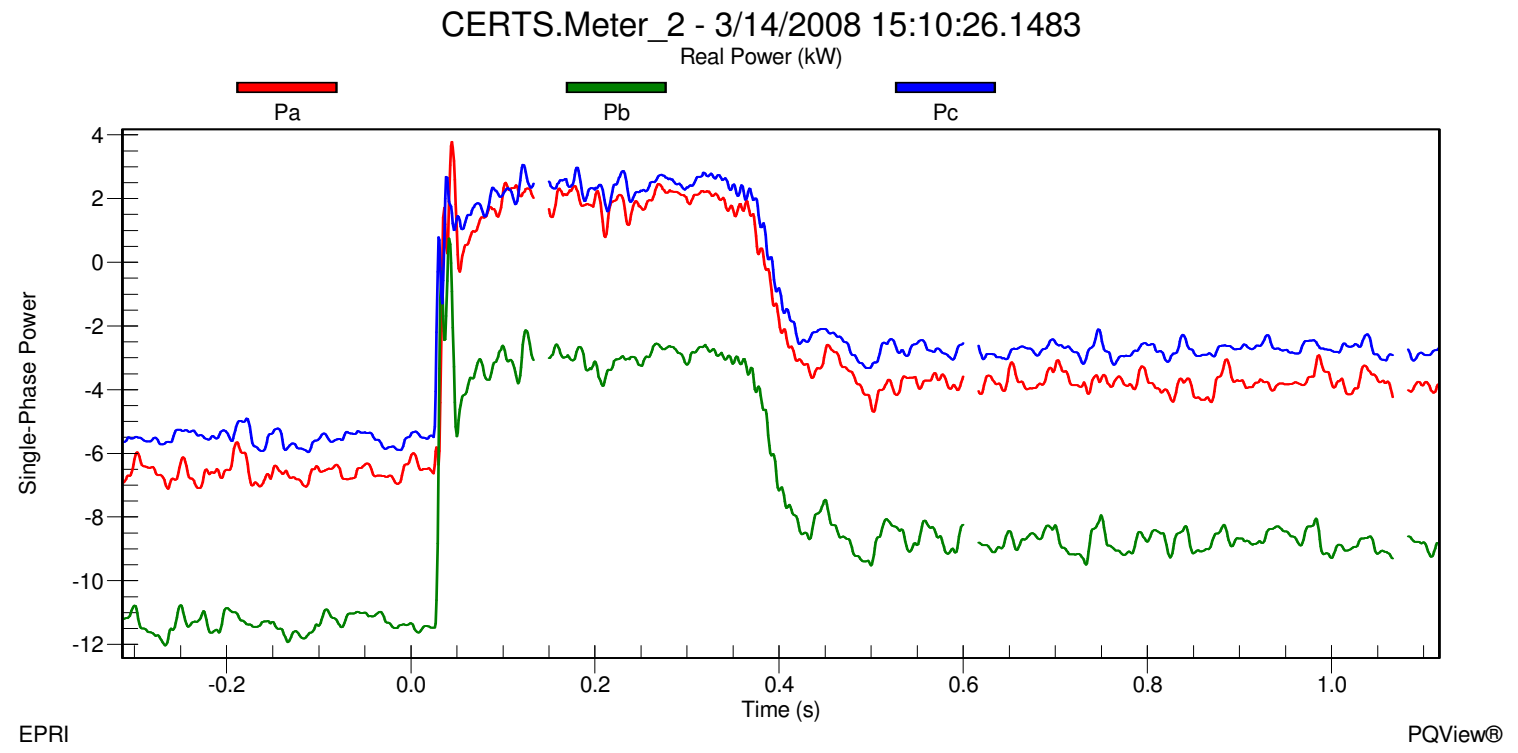

Figure 12e - Static Switch Real Single-Phase Power during Motor Start and Utility Connected for Test 10.3.14 


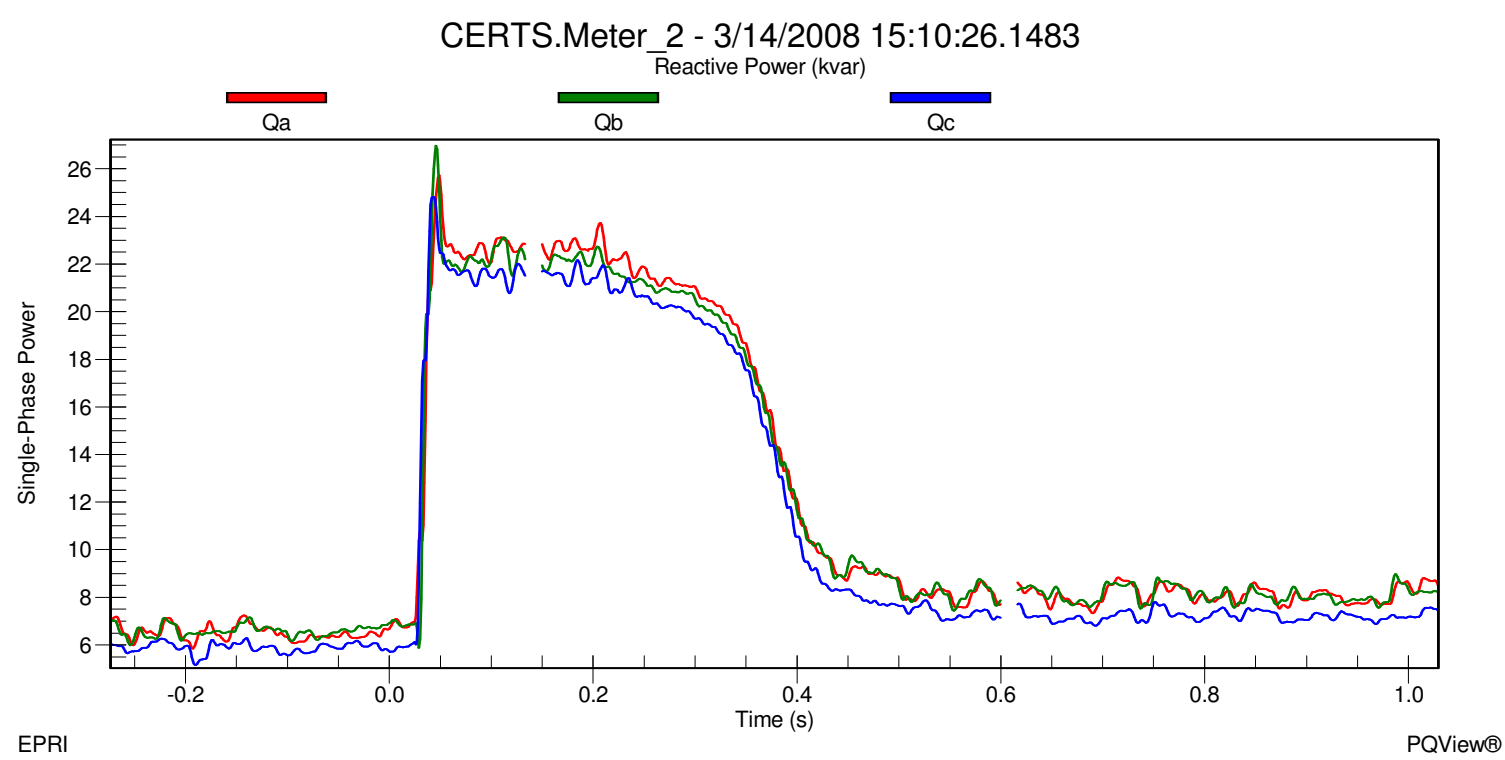

Figure 12f - Static Switch Reactive Single-Phase Power during Motor Start and Utility Connected for Test 10.3.14

Before the motor started Gen-set A1 was producing approximately $37.3 \mathrm{~kW}(\mathrm{~A}=11.3 \mathrm{~kW}$ $B=16.1 \mathrm{~kW} C=9.9 \mathrm{~kW})-\mathrm{j} 13.1 \mathrm{kVAr}(\mathrm{A}=-4.7 \mathrm{kVAr} B=-4.6 \mathrm{kVAr} C=-3.8 \mathrm{kVAr})$. This was enough for the load demands of Zone 3 with real power being exported to Zone 6 of approximately $23.4 \mathrm{~kW}(\mathrm{~A}=6.6 \mathrm{~kW} \mathrm{~B}=11.2 \mathrm{~kW} \mathrm{C}=5.6 \mathrm{~kW})$ shown in Figure 12e. The grid was supporting the reactive power of the microgrid with approximately $18.9 \mathrm{kVAr}$ $(\mathrm{A}=6.5 \mathrm{kVAr} \mathrm{B}=6.5 \mathrm{kVAr} \mathrm{C}=5.9 \mathrm{kVAr})$ shown in Figure $12 \mathrm{f}$. When the motor started the inrush caused the utility to supply approximately 77.3kVAr $(A=25.7 \mathrm{kVAr} B=26.8 \mathrm{kVAr}$ $\mathrm{C}=24.8 \mathrm{kVAr}$ ) and to pick up the load demand in Load Bank 6 along with approximately $6.35 \mathrm{~kW}(\mathrm{~A}=3.8 \mathrm{~kW} \mathrm{~B}=0.75 \mathrm{~kW} \mathrm{C}=1.8 \mathrm{~kW})$ of the load in Zone 3. The power being supplied by Gen-set A1 to Load Bank 6 was now supplying the motor inrush which can be seen in Figure $12 \mathrm{e}$ as the real power through the static switch becomes positive. Gen-set A1 increased its real and reactive power output level to approximately $44.5 \mathrm{~kW}(\mathrm{~A}=13.6 \mathrm{~kW}$ $\mathrm{B}=19.3 \mathrm{~kW} \mathrm{C}=11.6 \mathrm{~kW})+\mathrm{j} 5.7 \mathrm{kVAr}(\mathrm{A}=1.5 \mathrm{kVAr} \mathrm{B}=2.1 \mathrm{kVAr} \mathrm{C}=2.1 \mathrm{kVAr})$. Notice the sign of the VAr output changed from negative to positive for Gen-set A1 in order to support the induction motor load in Zone 3.

When the motor reached steady state, the real and reactive power through the static switch was approximately $-15.3 \mathrm{~kW}(\mathrm{~A}=-3.8 \mathrm{~kW} \mathrm{~B}=-8.75 \mathrm{~kW} \mathrm{C}=-2.75 \mathrm{~kW})+\mathrm{j} 23.45 \mathrm{kVAr}$ $(\mathrm{A}=8.1 \mathrm{kVAr} \mathrm{B}=8.1 \mathrm{kVAr} \mathrm{C}=7.25 \mathrm{kVAr})$ which meant that Gen-set $\mathrm{A} 1$ was supplying the power for the induction motor and approximately $15.3 \mathrm{~kW}(\mathrm{~A}=3.8 \mathrm{~kW} \quad \mathrm{~B}=8.75 \mathrm{~kW}$ $\mathrm{C}=2.75 \mathrm{~kW}$ ) of Load Bank 6 . Gen-set A1 real power returned relative close to the value before the motor started of approximately $37.6 \mathrm{~kW}(\mathrm{~A}=11.4 \mathrm{~kW} \mathrm{~B}=16.3 \mathrm{~kW} \mathrm{C}=9.9 \mathrm{~kW})$ and the reactive power increased to approximately $-j 11.6 \mathrm{kVAr}(\mathrm{A}=-4.25 \mathrm{kVAr} B=-4.1 \mathrm{kVAr} \mathrm{C}=$ 
3.25kVAr). Once all the data was verified and recorded into the DAS Database, the motor was shut down and the static switch was directed by the EMS to manually open.

As soon as the static switch opened, Meter 1 recorded real power increased to approximately $38.1 \mathrm{~kW}(\mathrm{~A}=10.1 \mathrm{~kW} \mathrm{~B}=18.1 \mathrm{~kW} \mathrm{C}=9.9 \mathrm{~kW})$ and reactive power decreased to j1.1kVAr $(A=0.25 \mathrm{kVAr} B=0.6 \mathrm{kVAr} C=0.25 \mathrm{kVAr})$ satisfying the load demand in Load

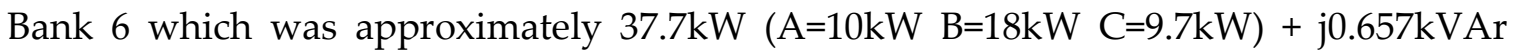
$(\mathrm{A}=0.129 \mathrm{kVAr} \mathrm{B}=0.399 \mathrm{kVAr} \mathrm{C}=0.129 \mathrm{kVAr})$ and not supplying any power beyond the

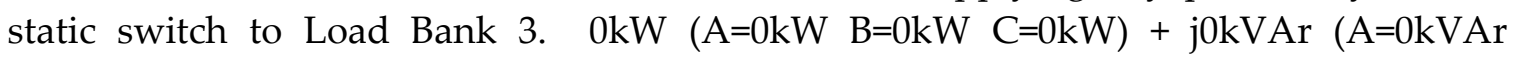
$\mathrm{B}=0 \mathrm{kVAr} \mathrm{C}=0 \mathrm{kVAr}$ ) was recorded at the static switch, indicating that power was not flowing through the static switch. Load Bank 3 loads was slightly reduced to $10.3 \mathrm{~kW}$ $(\mathrm{A}=3.44 \mathrm{~kW} \quad \mathrm{~B}=3.43 \mathrm{~kW} \quad \mathrm{C}=3.43 \mathrm{~kW})-\mathrm{j} 0.041 \mathrm{kVAr} \quad(\mathrm{A}=-0.016 \mathrm{kVAr} \quad \mathrm{B}=-0.002 \mathrm{kVAr} \quad \mathrm{C}=$ $0.023 \mathrm{kVAr}$ ). This load reduction resulted from a voltage drop in the microgrid, shown in Figure $12 \mathrm{~g}$, from approximately $284 \mathrm{~V}$ on A-phase, $279.5 \mathrm{~V}$ on B-phase and $279.5 \mathrm{~V}$ on C-phase when connected to the utility grid to $272.8 \mathrm{~V}$ on A-phase, $271.6 \mathrm{~V}$ on B-phase and 272.8V on C-phase at Meter 3 when islanded.

CERTS.Meter_3 - 3/14/2008 10:16:10.7131

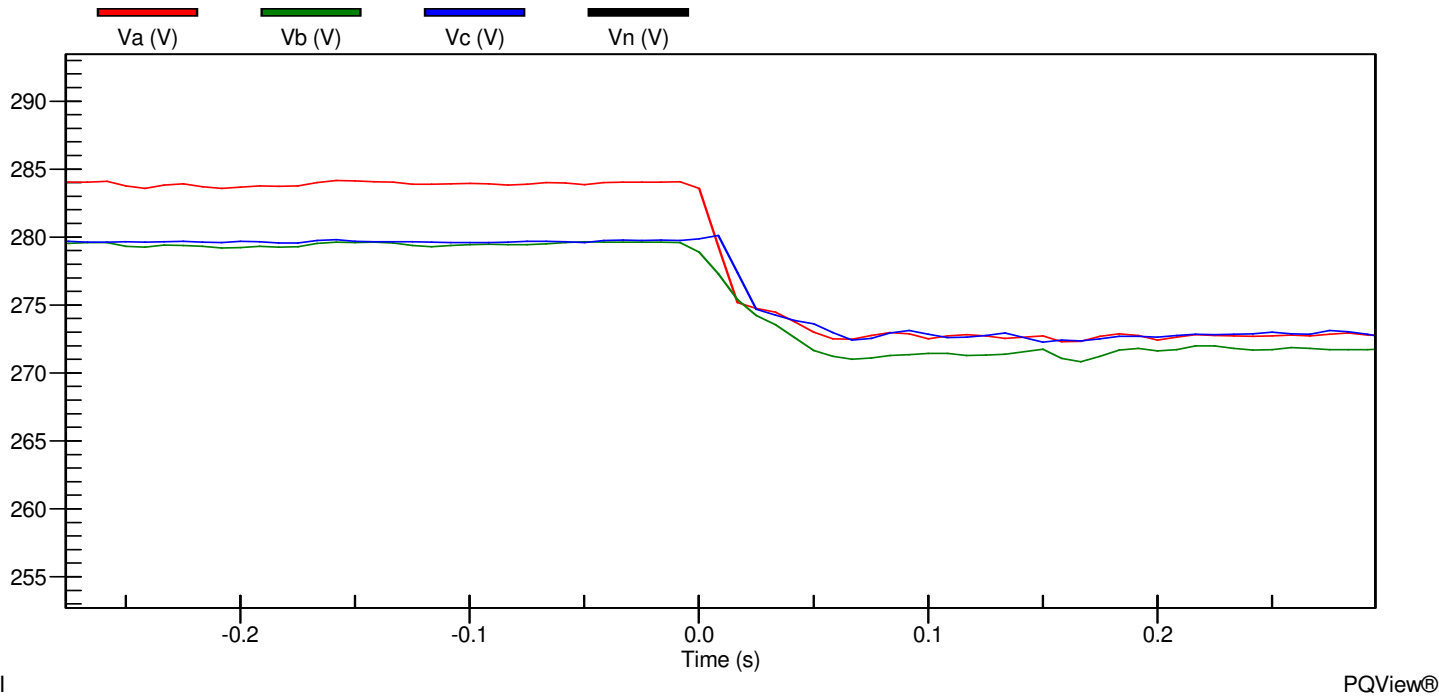

Figure 12g - Meter 3 Line-to-Ground Voltages during Utility Connected to Island mode for Test 10.3.14 


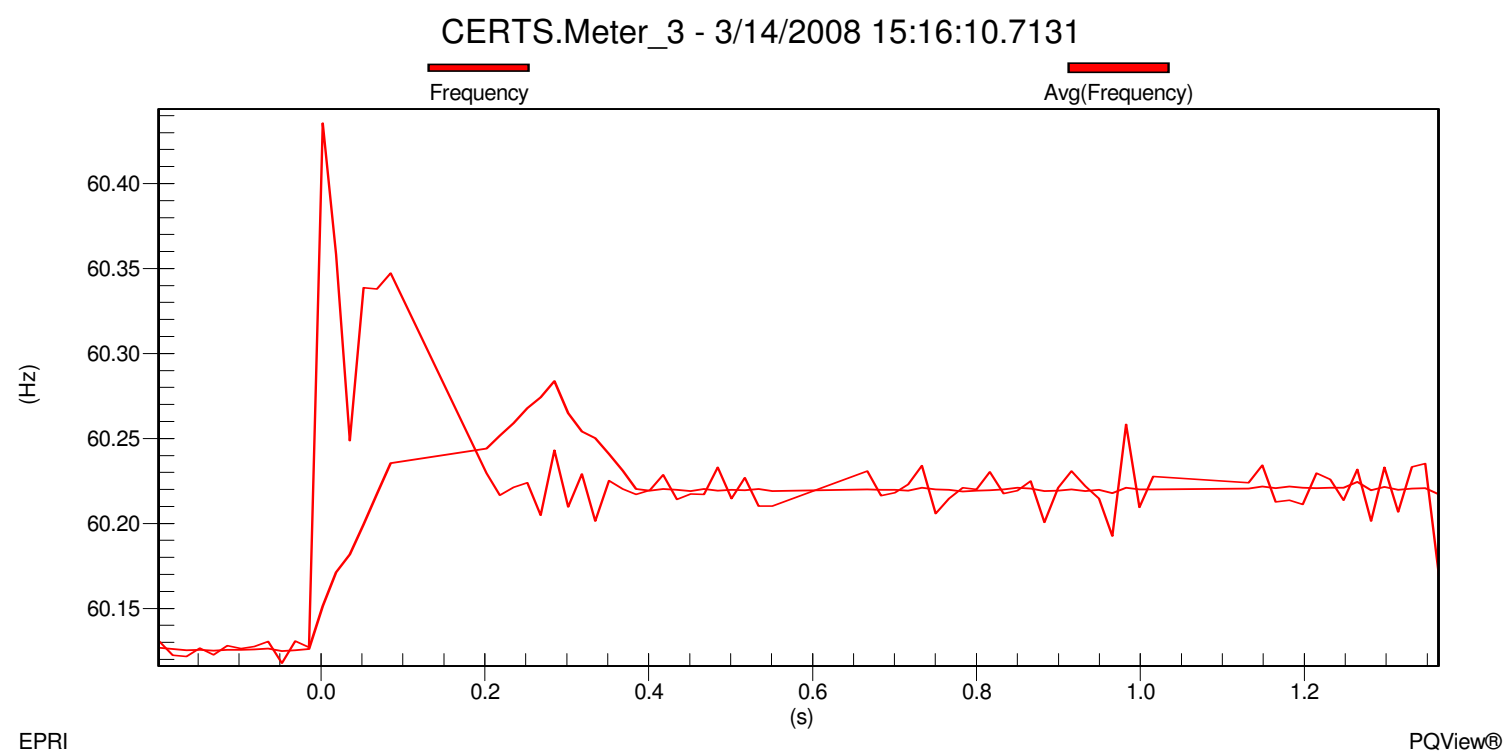

Figure 12h - Meter 3 Frequency during Utility Connected to Island mode for Test 10.3.14

Frequency change in the microgrid, shown in Figure 12h, increased from approximately $60.13 \mathrm{~Hz}$ when connected to the utility grid to approximately $60.22 \mathrm{~Hz}$ when islanded. This change in frequency was part of the CERTS algorithm which allowed Gen-set A1 to decrease its output power to satisfy the load demands. Gen-set A1 decreased its output real power to approximately $12.8 \mathrm{~kW}(\mathrm{~A}=4.2 \mathrm{~kW} \mathrm{~B}=4.6 \mathrm{~kW} \mathrm{C}=4 \mathrm{~kW})$ and increased its output reactive power to approximately $\mathrm{j} 3.6 \mathrm{kVAr}(\mathrm{A}=0.8 \mathrm{kVAr} B=1.4 \mathrm{kVAr} C=1.4 \mathrm{kVAr})$. Meter 3 was approximately $-2.2 \mathrm{~kW}(\mathrm{~A}=-0.7 \mathrm{~kW} \quad \mathrm{~B}=-1 \mathrm{~kW} \quad \mathrm{C}=-0.5 \mathrm{~kW})-\mathrm{j} 3.6 \mathrm{kVAr}(\mathrm{A}=-$ $0.8 \mathrm{kVAr} B=-1.4 \mathrm{kVAr} C=-1.4 \mathrm{kVAr}$ ) indicating that Gen-set $\mathrm{A} 1$ was satisfying the load in Load Bank 3 and the power losses in the electrical lines. All data was verified and recorded into the DAS Database. The microgrid ran for a couple of minutes in this electrical state before the $10 \mathrm{Hp}$ induction motor was started in Zone 3. 


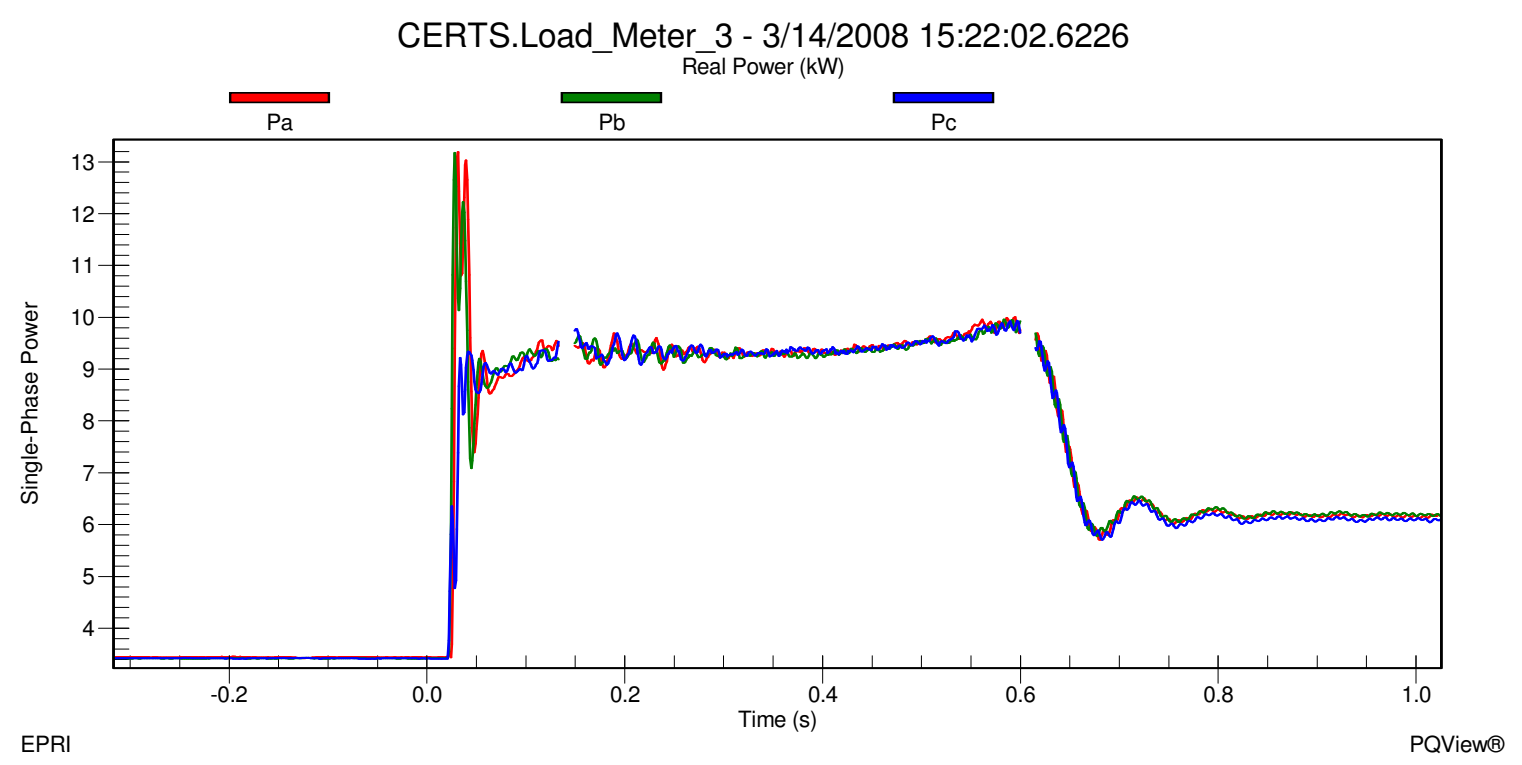

Figure 12i - Load Bank 3 Real Single-Phase Power during Motor Start and Islanded for Test 10.3.14

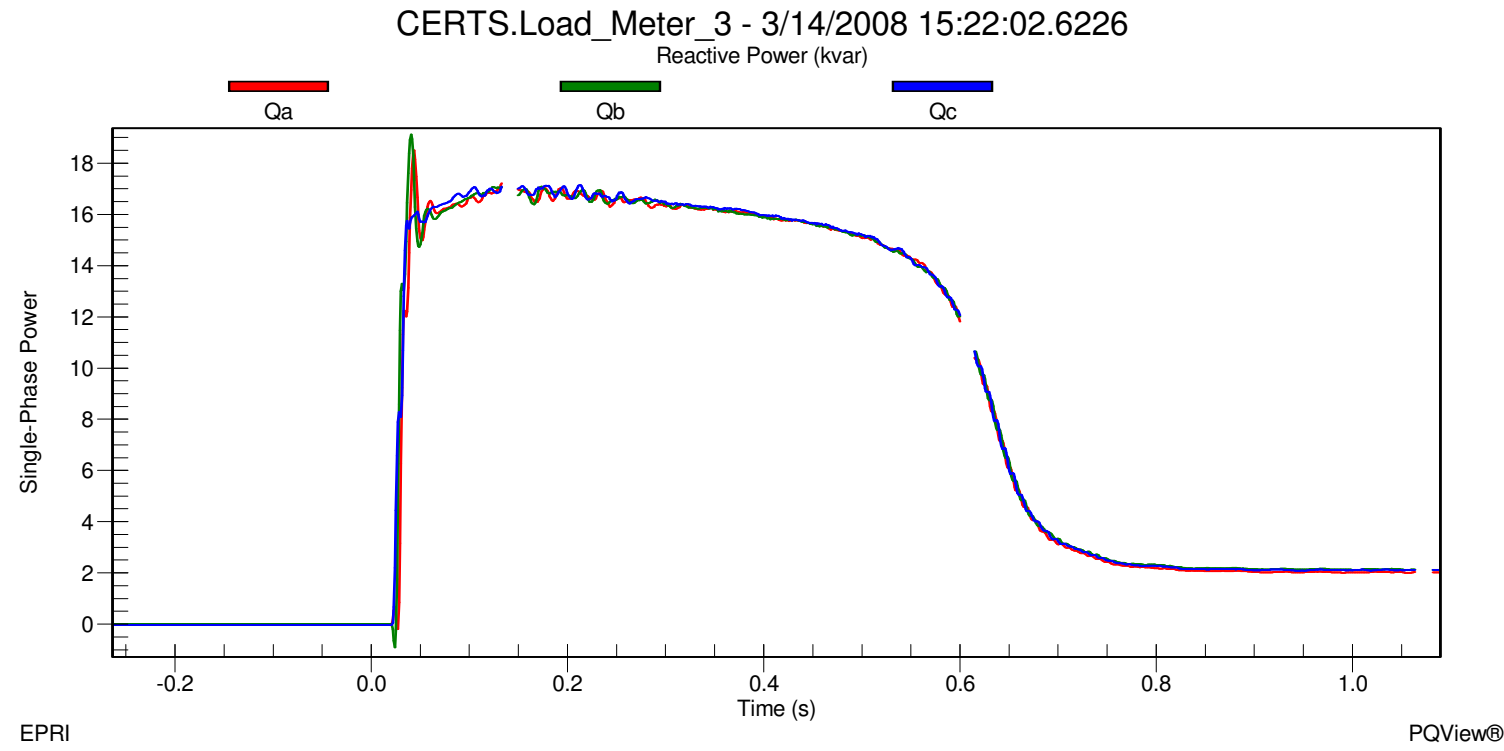

Figure 12j - Load Bank 3 Reactive Single-Phase Power during Motor Start and Islanded for Test 10.3.14

In Figures $12 \mathrm{i}$ and $12 \mathrm{j}$ it can be seen that the load in Zone 3 was approximately $10.2 \mathrm{~kW}$ $(\mathrm{A}=3.4 \mathrm{~kW} \mathrm{~B}=3.4 \mathrm{~kW} \mathrm{C}=3.4 \mathrm{~kW})+\mathrm{j} 0 \mathrm{kVAr}(\mathrm{A}=0 \mathrm{kVAr} \mathrm{B}=0 \mathrm{kVAr} \mathrm{C}=0 \mathrm{kVAr})$ before the start of the induction motor and increased to approximately $34.5 \mathrm{~kW}(\mathrm{~A}=13.2 \mathrm{~kW} \mathrm{~B}=12.1 \mathrm{~kW}$ $\mathrm{C}=9.2 \mathrm{~kW})+\mathrm{j} 53.3 \mathrm{kVAr}(\mathrm{A}=19.2 \mathrm{kVAr} B=18.1 \mathrm{kVAr} \mathrm{C}=16 \mathrm{kVAr})$ during the inrush phase of the motor start. After about 1.5 cycles, the motor settled down to approximately $28.5 \mathrm{~kW}$ $(\mathrm{A}=9.5 \mathrm{~kW} \quad \mathrm{~B}=9.5 \mathrm{~kW} \quad \mathrm{C}=9.5 \mathrm{~kW})+\mathrm{j} 48.3 \mathrm{kVAr} \quad(\mathrm{A}=16.1 \mathrm{kVAr} \quad \mathrm{B}=16.1 \mathrm{kVAr} \quad \mathrm{C}=16.1 \mathrm{kVAr})$ during the warm up phase which lasted about 48 cycles ( 0.8 Seconds). When the motor 
reached steady state, the load in Zone 3 was approximately $18.6 \mathrm{~kW}(\mathrm{~A}=6.2 \mathrm{~kW} \mathrm{~B}=6.2 \mathrm{~kW}$ $\mathrm{C}=6.2 \mathrm{~kW})+\mathrm{j} 6 \mathrm{kVAr}(\mathrm{A}=2 \mathrm{kVAr} \mathrm{B}=2 \mathrm{kVAr} \mathrm{C}=2 \mathrm{kVAr})$.

The voltage and frequency at Meter 3 before the motor start was approximately $272.8 \mathrm{~V}$ on A-phase, 272V on B-phase and $272.7 \mathrm{~V}$ on C-phase shown in Figure $12 \mathrm{k}$ and approximately $60.22 \mathrm{~Hz}$ shown in Figure 121 . When the motor started, the voltage at Meter 3 during the inrush decreased to approximately $238.1 \mathrm{~V}$ on A-phase, 237.6V on Bphase and $236 \mathrm{~V}$ on C-phase for about 1.5 cycles. Frequency dropped during the inrush to approximately $59.8 \mathrm{~Hz}$ and quickly increased to approximately $60.38 \mathrm{~Hz}$. Voltage increased as the motor was warming up and eventually settled at a steady state voltage at approximately $269 \mathrm{~V}$ on A-phase, $269 \mathrm{~V}$ on B-phase and $269.1 \mathrm{~V}$ on C-phase at an approximate frequency of $60.15 \mathrm{~Hz}$.

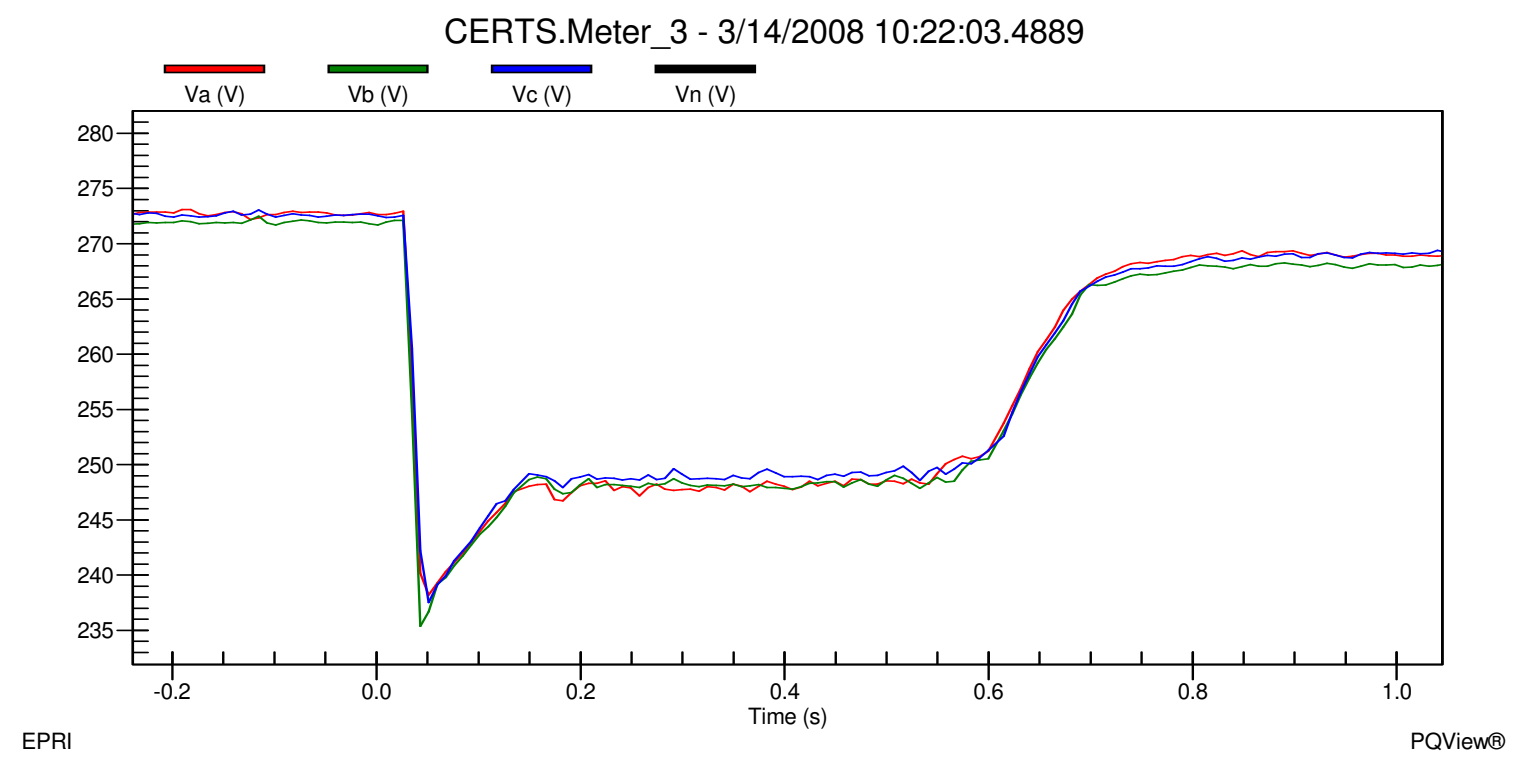

Figure 12k - Meter 3 Line-to-Ground Voltages during Motor Start and Islanded for Test 10.3.14 


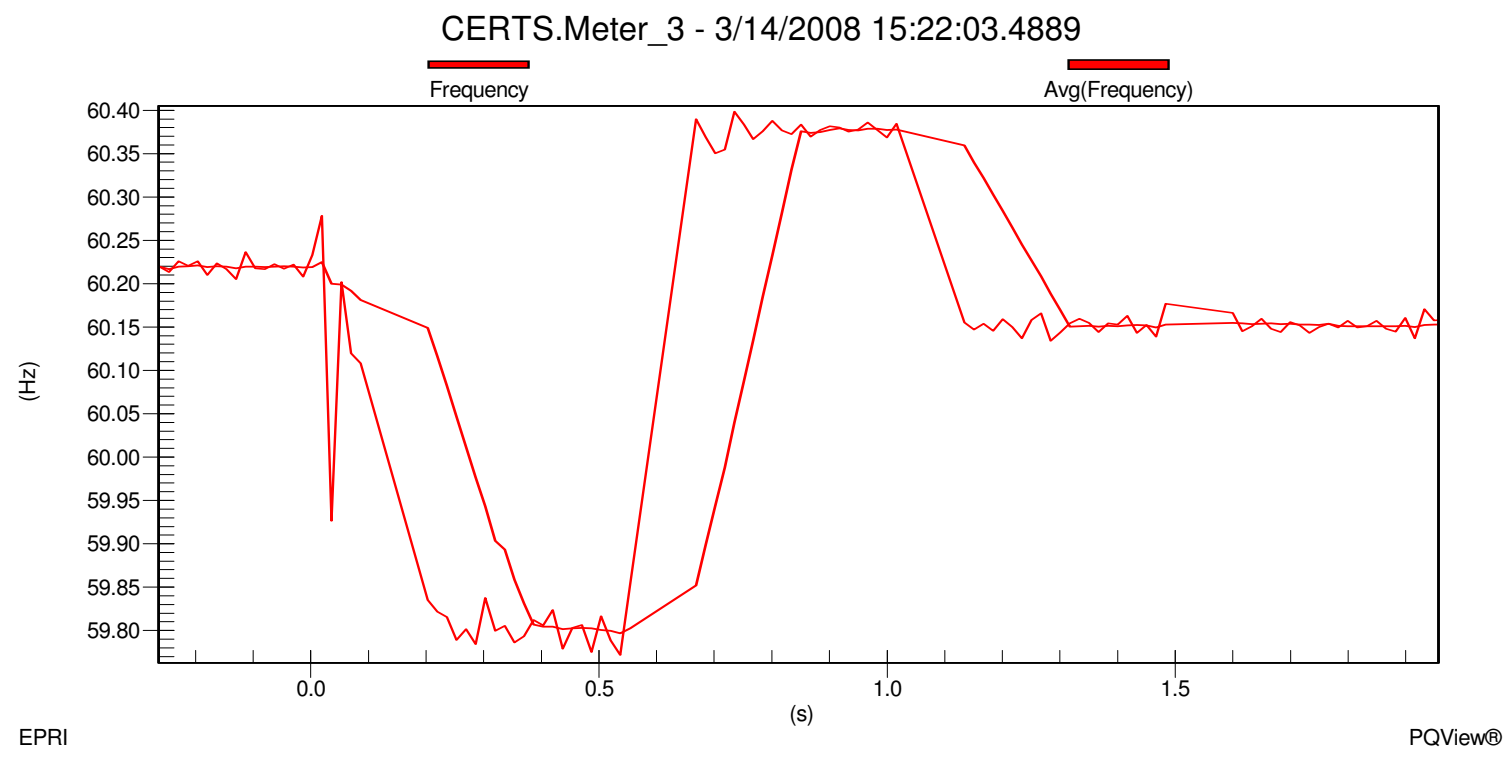

Figure 121 - Meter 3 Frequency during Motor Start and Islanded for Test 10.3.14

Before the motor started Gen-set A1 was producing approximately $12.6 \mathrm{~kW}(\mathrm{~A}=4.2 \mathrm{~kW}$ $\mathrm{B}=4.5 \mathrm{~kW} \mathrm{C}=3.9 \mathrm{~kW})+\mathrm{j} 3.6 \mathrm{kVAr}(\mathrm{A}=0.8 \mathrm{kVAr} B=1.4 \mathrm{kVAr} \mathrm{C}=1.4 \mathrm{kVAr})$ shown in Figure $12 \mathrm{~m}$ and $12 \mathrm{n}$. The power generated by Gen-set A1 was satisfying the loads in Load Bank 3 and all the electrical losses in the microgrid system. When the motor started the inrush caused the Gen-set to increase its output level to $36.5 \mathrm{~kW}(\mathrm{~A}=13.9 \mathrm{~kW} B=13.9 \mathrm{~kW}$ $\mathrm{C}=8.7 \mathrm{~kW})+\mathrm{j} 54.2 \mathrm{kVAr}(\mathrm{A}=18.2 \mathrm{kVAr} B=19.6 \mathrm{kVAr} \mathrm{C}=16.4 \mathrm{kVAr})$. Gen-set A1 decreased its output level while the motor was warming up and eventually dropped to $20.8 \mathrm{~kW}$ $(A=6.9 \mathrm{~kW} \mathrm{~B}=7.3 \mathrm{~kW} \mathrm{C}=6.6 \mathrm{~kW})+\mathrm{j} 9.5 \mathrm{kVAr}(\mathrm{A}=2.75 \mathrm{kVAr} B=3.4 \mathrm{kVAr} \mathrm{C}=3.4 \mathrm{kVAr})$ when the motor reached steady state. Once all the data was verified and recorded into the DAS Database, the static switch was directed by the EMS to manually close. 


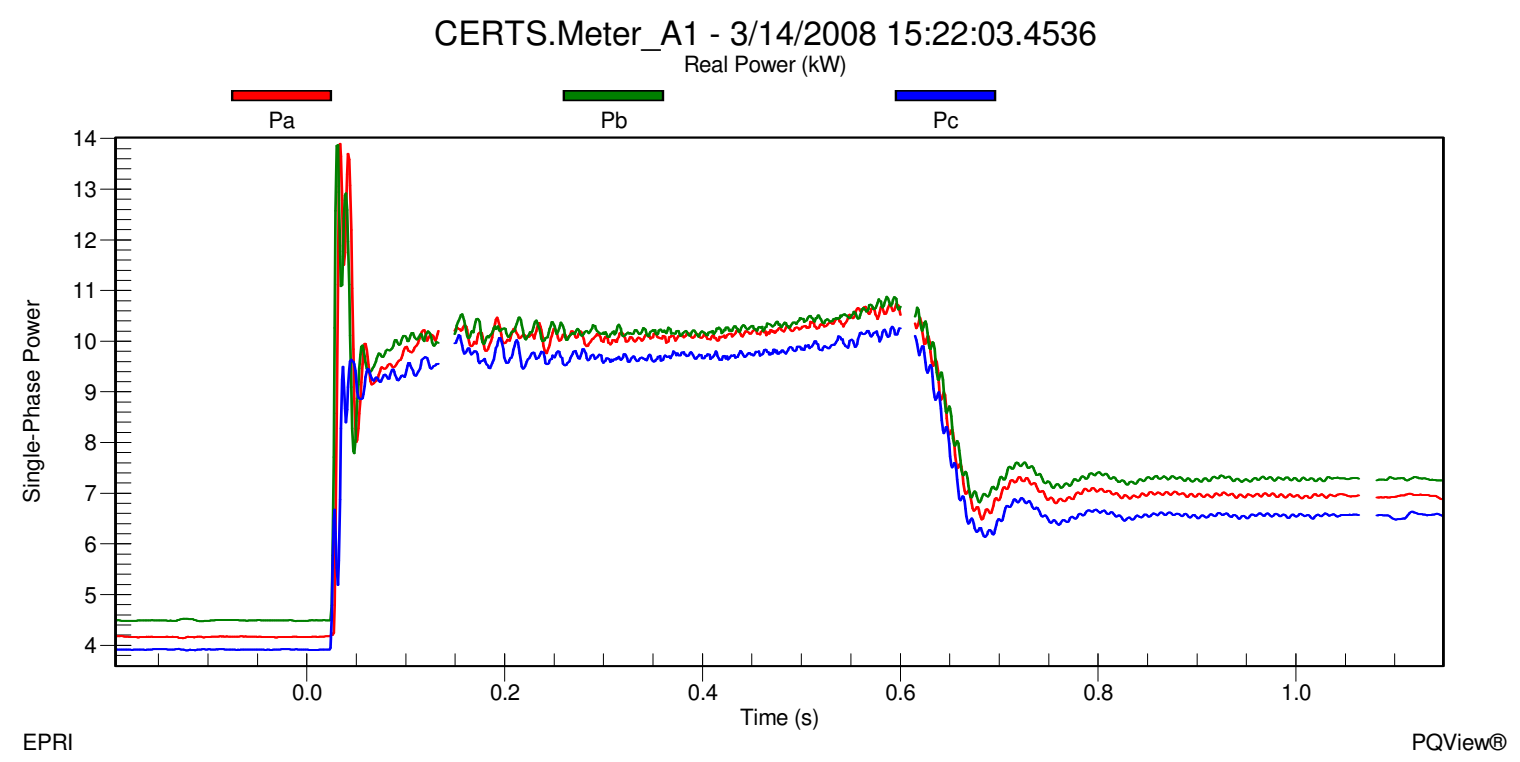

Figure 12m - Gen-set A1 Real Single-Phase Power during Motor Start and Islanded for Test 10.3.14

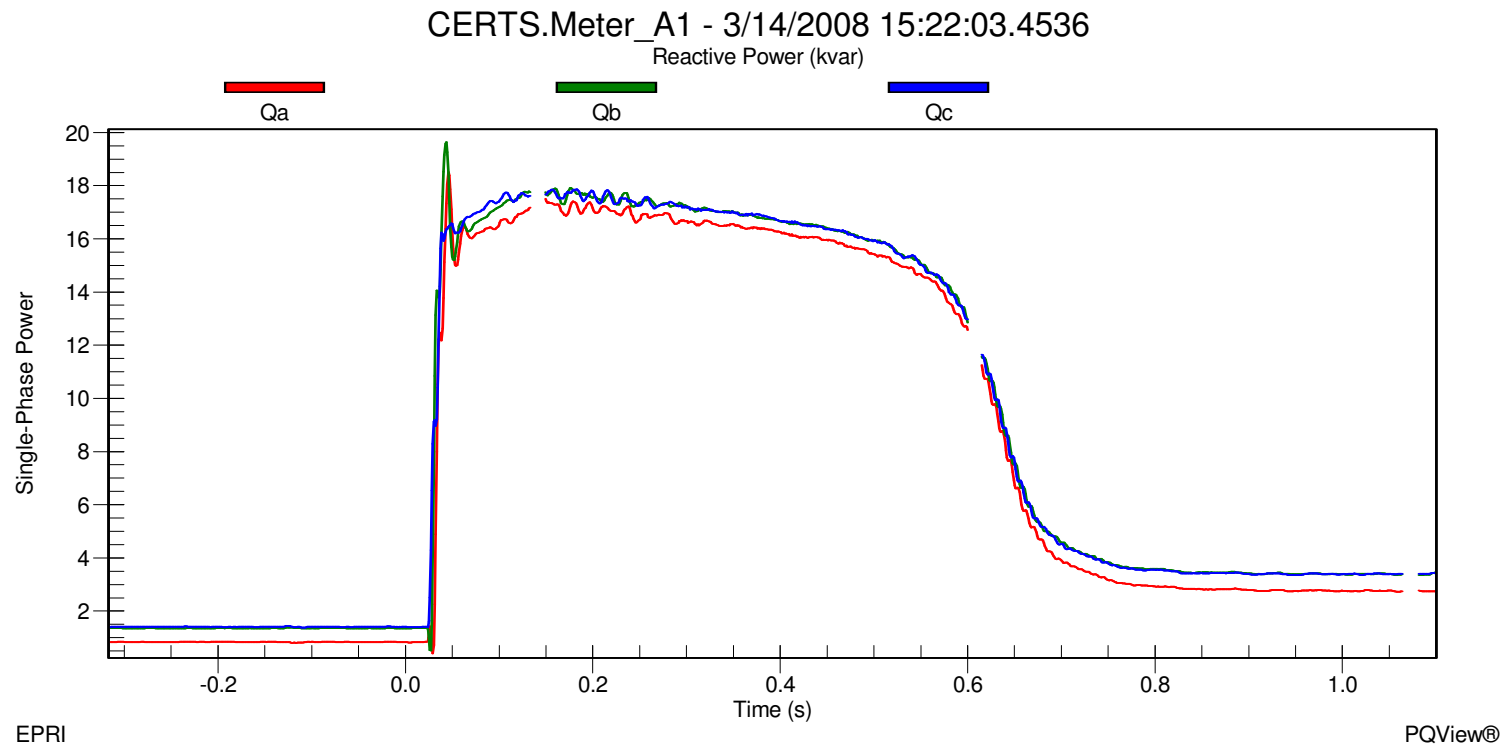

Figure 12n - Gen-set A1 Reactive Single-Phase Power during Motor Start and Islanded for Test 10.3.14

As soon as the static switch closed, Meter 1 recorded real power decreased from approximately $38.2 \mathrm{~kW}(\mathrm{~A}=10.2 \mathrm{~kW} \mathrm{~B}=18.1 \mathrm{~kW} \mathrm{C}=9.9 \mathrm{~kW})$ to $20.9 \mathrm{~kW}(\mathrm{~A}=5.7 \mathrm{~kW} \mathrm{~B}=8.6 \mathrm{~kW}$ $\mathrm{C}=6.6 \mathrm{~kW})$ and reactive power increased from approximately $\mathrm{j} 1 \mathrm{kVAr}(\mathrm{A}=0.2 \mathrm{kVAr}$ $\mathrm{B}=0.6 \mathrm{kVAr} \mathrm{C}=0.2 \mathrm{kVAr})$ to $\mathrm{j} 25 \mathrm{kVAr}(\mathrm{A}=8.5 \mathrm{kVAr} \mathrm{B}=8.7 \mathrm{kVAr} \mathrm{C}=7.8 \mathrm{kVAr})$ which means that the utility was satisfying a portion of the load demand in Load Bank 6 and all the reactive power in the microgrid. Figures $12 \mathrm{o}$ and $12 \mathrm{p}$ show the static switch decreasing from approximately $0 \mathrm{~kW}(\mathrm{~A}=0 \mathrm{~kW} B=0 \mathrm{~kW} \mathrm{C}=0 \mathrm{~kW})$ to $-16.45 \mathrm{~kW}(\mathrm{~A}=-4.25 \mathrm{~kW} \mathrm{~B}=-9.2 \mathrm{~kW}$ $\mathrm{C}=-3 \mathrm{~kW})$ and increasing from approximately j0kVAr $(\mathrm{A}=0 \mathrm{kVAr} \mathrm{B}=0 \mathrm{kVAr} \mathrm{C}=0 \mathrm{kVAr})$ to 
j24.1kVAr $(A=8.3 \mathrm{kVAr} B=8.1 \mathrm{kVAr} C=7.7 \mathrm{kVAr})$. At the beginning of the test, the initial power flow through the static switch was $-23.4 \mathrm{~kW}(\mathrm{~A}=-6.6 \mathrm{~kW} \mathrm{~B}=-11.2 \mathrm{~kW} \mathrm{C}=-5.6 \mathrm{~kW})+$ j18.9kVAr $(A=6.5 \mathrm{kVAr} B=6.5 \mathrm{kVAr} C=5.9 \mathrm{kVAr})$ which is not the same recorded at this point in the test because the $10 \mathrm{Hp}$ motor load is on in Zone 3. Gen-set A1 has picked up the motor load and is supporting Load Bank 6 with approximately $16.45 \mathrm{~kW}(\mathrm{~A}=4.25 \mathrm{~kW}$ $\mathrm{B}=9.2 \mathrm{~kW} \mathrm{C}=3 \mathrm{~kW})$.

Load Bank 3 loads increased slightly to $17.65 \mathrm{~kW}(\mathrm{~A}=6.06 \mathrm{~kW} \mathrm{~B}=5.74 \mathrm{~kW} \mathrm{C}=5.85 \mathrm{~kW})+$ $\mathrm{j} 6 \mathrm{kVAr}(\mathrm{A}=2.12 \mathrm{kVAr} B=2.01 \mathrm{kVAr} C=1.87 \mathrm{kVAr})$. This slight increase is a result from a voltage rise in the microgrid, shown in Figure 12q, from approximately 269.3V on Aphase, $269 \mathrm{~V}$ on B-phase and $269.1 \mathrm{~V}$ on C-phase when islanded to $283 \mathrm{~V}$ on A-phase, $279.4 \mathrm{~V}$ on B-phase and $279.4 \mathrm{~V}$ on C-phase at Meter 3 when connected to the utility grid.

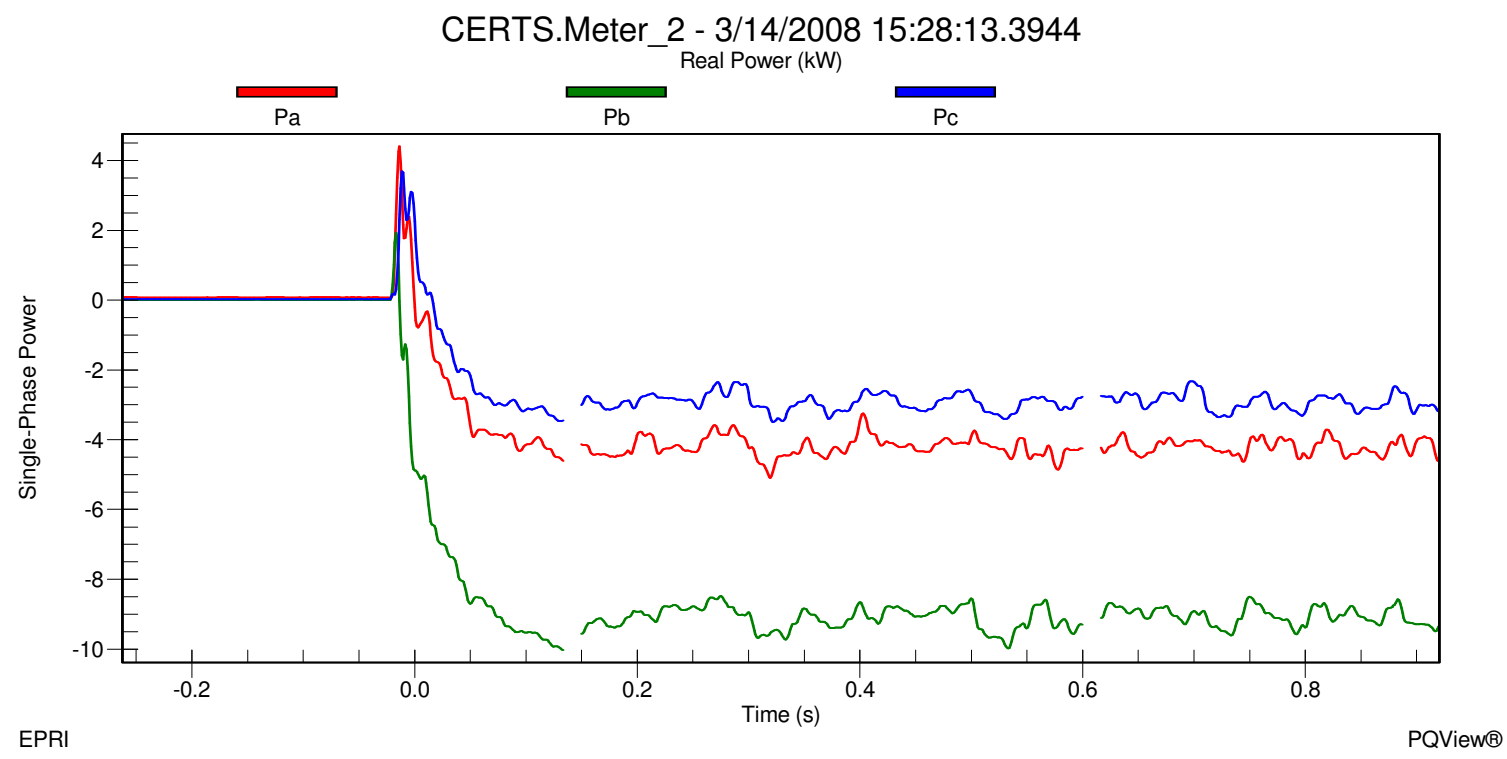

Figure 120 - Static Switch Real Single-Phase Power during Island to Utility Connected mode for Test 10.3.14 


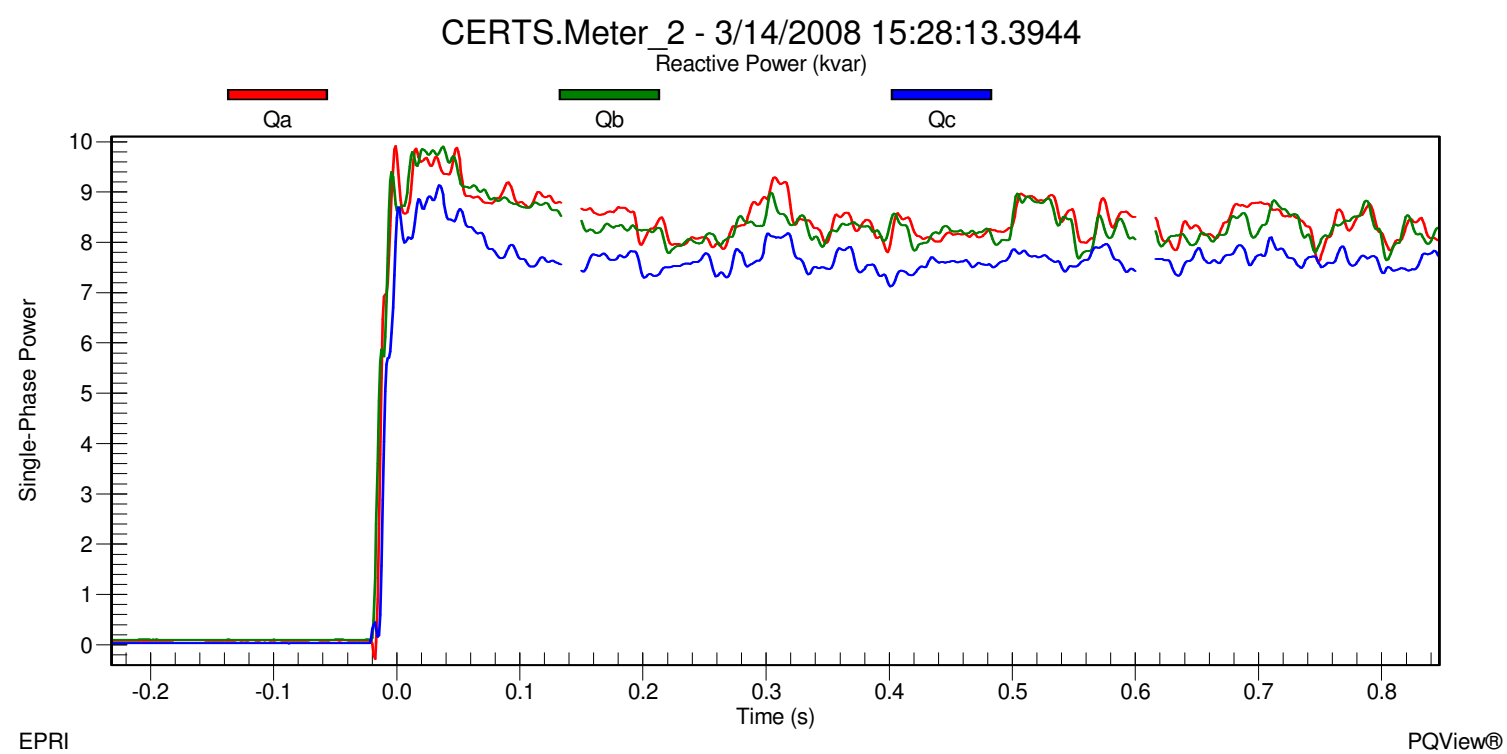

Figure 12p - Static Switch Reactive Single-Phase Power during Island to Utility Connected mode for Test 10.3.14

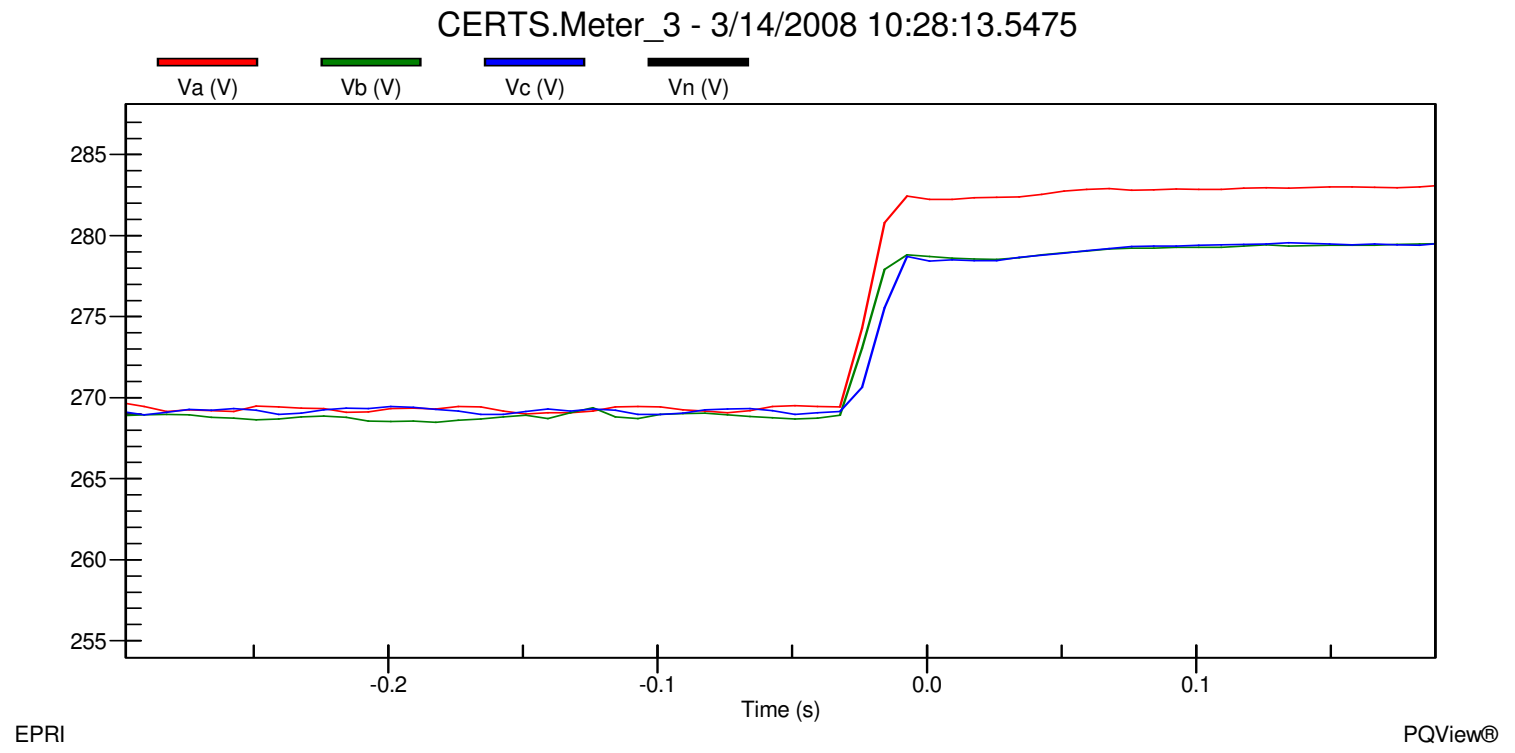

Figure 12q - Meter 3 Line-to-Ground Voltages during Island to Utility Connected for Test 10.3.14 


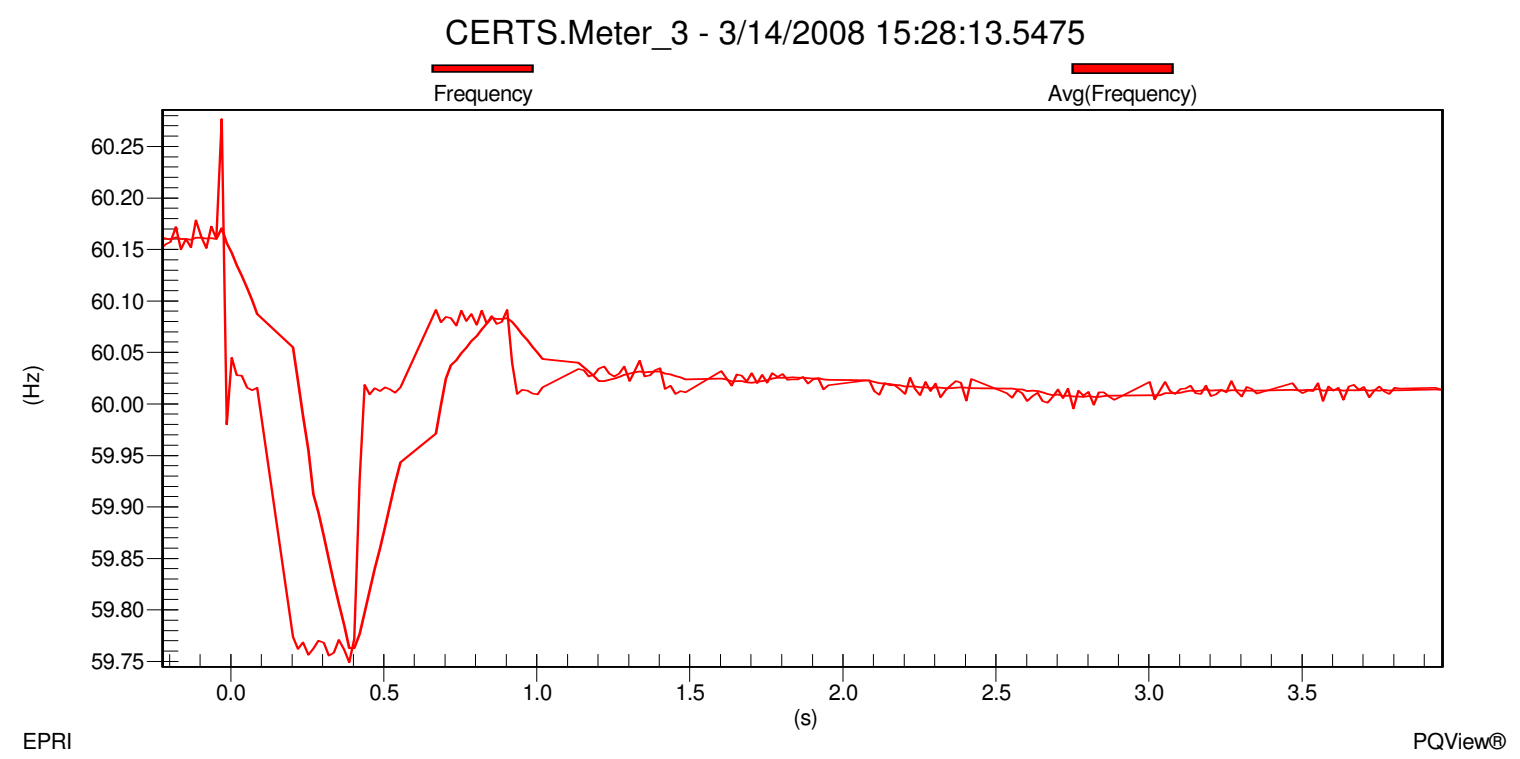

Figure 12r - Meter 3 Frequency during Island to Utility Connected mode for Test 10.3.14

Frequency change in the microgrid, shown in Figure 12r, decreased from approximately $60.16 \mathrm{~Hz}$ when islanded to approximately $60.01 \mathrm{~Hz}$ when connected to the utility grid. This change in frequency is due to the frequency no longer being established by Gen-set A1 using the CRETS algorithm but by the utility. Gen-set A1 is in unit power control mode therefore when the static switch closed back into the utility the Gen-set produced real power based on the set-point initialized at the beginning of the test. The output power for Gen-set A1 was relatively close to the value at the beginning of the test with Gen-set A1 producing approximately $36.6 \mathrm{~kW}(\mathrm{~A}=11.1 \mathrm{~kW} B=16 \mathrm{~kW} \mathrm{C}=9.5 \mathrm{~kW})-\mathrm{j} 12.7 \mathrm{kVAr}$ $(\mathrm{A}=-4.6 \mathrm{kVAr} \mathrm{B}=-4.4 \mathrm{kVAr} \mathrm{C}=-3.7 \mathrm{kVAr})$. After all the data was verified and recorded into the DAS Database, the motor was turned off and the Gen-sets and Load Banks set-points were changed according to the next test (10.3.15) in Table 2.

For Test 10.3.15 the measured values, after the Gen-sets were warmed up and load banks brought on-line are provided in Table 5.

\begin{tabular}{|c|c|c|c|c|c|c|c|c|c|}
\hline Meter & $\begin{array}{c}\text { Gen- } \\
\text { set A1 }\end{array}$ & $\begin{array}{c}\text { Gen - } \\
\text { set A2 }\end{array}$ & Meter 1 & Meter 2 & Meter 3 & Meter 4 & LB 3 & LB 4 & LB 6 \\
\hline $\begin{array}{c}\text { 3 Phase } \\
\text { kW }\end{array}$ & 38.3 & 29.8 & 62.8 & 25.95 & 23.2 & 14.9 & 46.2 & 44.65 & 36.8 \\
\hline $\begin{array}{c}\mathbf{1} \text { Phase } \\
\mathbf{k W}\end{array}$ & $\mathrm{A}=10.5$ & $\mathrm{~A}=7.1$ & $\mathrm{~A}=18.4$ & $\mathrm{~A}=8.75$ & $\mathrm{~A}=7.9$ & $\mathrm{~A}=2.6$ & $\mathrm{~A}=15.6$ & $\mathrm{~A}=9.75$ & $\mathrm{~A}=9.7$ \\
$\mathrm{~B}=16.6$ & $\mathrm{~B}=13.1$ & $\mathrm{~B}=22.2$ & $\mathrm{~B}=4.6$ & $\mathrm{~B}=3.2$ & $\mathrm{~B}=4.4$ & $\mathrm{~B}=15.3$ & $\mathrm{~B}=17.5$ & $\mathrm{~B}=17.6$ \\
\hline
\end{tabular}




\begin{tabular}{|c|c|c|c|c|c|c|c|c|c|}
\hline & $\mathrm{C}=11.2$ & $\mathrm{C}=9.6$ & $\mathrm{C}=22.2$ & $\mathrm{C}=12.6$ & $\mathrm{C}=12.1$ & $\mathrm{C}=7.9$ & $\mathrm{C}=15.3$ & $\mathrm{C}=17.4$ & $\mathrm{C}=9.5$ \\
\hline $\begin{array}{c}\text { 3 Phase } \\
\text { kVAr }\end{array}$ & -9 & 0.1 & 16.8 & 15.05 & 10.5 & 0.5 & 0.6 & 0.803 & 0.641 \\
\hline \multirow{2}{*}{ Phase } \\
kVAr & $\mathrm{A}=-3.4$ & $\mathrm{~A}=-0.9$ & $\mathrm{~A}=6.5$ & $\mathrm{~A}=5.8$ & $\mathrm{~A}=4.8$ & $\mathrm{~A}=1$ & $\mathrm{~A}=0.2$ & $\mathrm{~A}=0.119$ & $\mathrm{~A}=0.12$ \\
& $\mathrm{C}=-2.8$ & $\mathrm{~B}=0.1$ & $\mathrm{~B}=4.8$ & $\mathrm{~B}=4$ & $\mathrm{~B}=2.2$ & $\mathrm{~B}=-0.7$ & $\mathrm{~B}=0.2$ & $\mathrm{~B}=0.38$ & $\mathrm{~B}=0.4$ \\
& $\mathrm{C}=5.5$ & $\mathrm{C}=5.25$ & $\mathrm{C}=3.5$ & $\mathrm{C}=0.2$ & $\mathrm{C}=0.2$ & $\mathrm{C}=0.304$ & $\mathrm{C}=0.121$ \\
\hline
\end{tabular}

Table 5 - Measured Values after Start Up for Test 10.3.15

These measurements were relatively close to the expected values in Table 2, but not exact due to temperature, phase voltages and electrical losses in conductors. In addition, the 50kW settings for LB3 and LB4 and the 40kW setting for LB6 were also below selected set values. At the time of the measurements, the voltage and frequency was 281.7V on A-phase, $278.5 \mathrm{~V}$ on B-phase and $278 \mathrm{~V}$ on C-phase and $60.00 \mathrm{~Hz}$ at the static switch (i.e., Meter 2) when connected to the utility grid; and $281 \mathrm{~V}$ on A-phase, $278 \mathrm{~V}$ on B-phase and 277V on C-phase and $60.00 \mathrm{~Hz}$ at Meter 3.

The Gen-sets in this test were set up to produce less power than the microgrid loads needed which approximately $25.95 \mathrm{~kW}(\mathrm{~A}=8.75 \mathrm{~kW} \mathrm{~B}=4.6 \mathrm{~kW} \mathrm{C}=12.6 \mathrm{~kW})$ of power was imported from the utility through the static switch to support Load Banks 3 and 4 . Since Load Bank 6 was approximately 36.8kW $(A=9.7 \mathrm{~kW} \mathrm{~B}=17.6 \mathrm{~kW} \mathrm{C}=9.5 \mathrm{~kW})+\mathrm{j} 0.641 \mathrm{kVAr}$ $(\mathrm{A}=0.12 \mathrm{kVAr} \mathrm{B}=0.4 \mathrm{kVAr} \mathrm{C}=0.121 \mathrm{kVAr})$, the utility had to supply approximately $62.8 \mathrm{~kW}$ $(\mathrm{A}=18.4 \mathrm{~kW} \mathrm{~B}=22.2 \mathrm{~kW} \mathrm{C}=22.2 \mathrm{~kW})$ to satisfy the load demand of Load Bank 6 and the microgrid loads. Reactive power had to be imported in from the utility of approximately $16.8 \mathrm{kVAr}(\mathrm{A}=6.5 \mathrm{kVAr} \mathrm{B}=4.8 \mathrm{kVAr} \mathrm{C}=5.5 \mathrm{kVAr})$ because Gen-sets $\mathrm{A} 1$ and A2 needed approximately $8.9 \mathrm{kVAr}$ between the two and the reactive power of Load Banks 3 and 4 and electrical wires. Once all data was verified and recorded into the DAS Database, the 10Hp induction motor was started in Zone 3. 


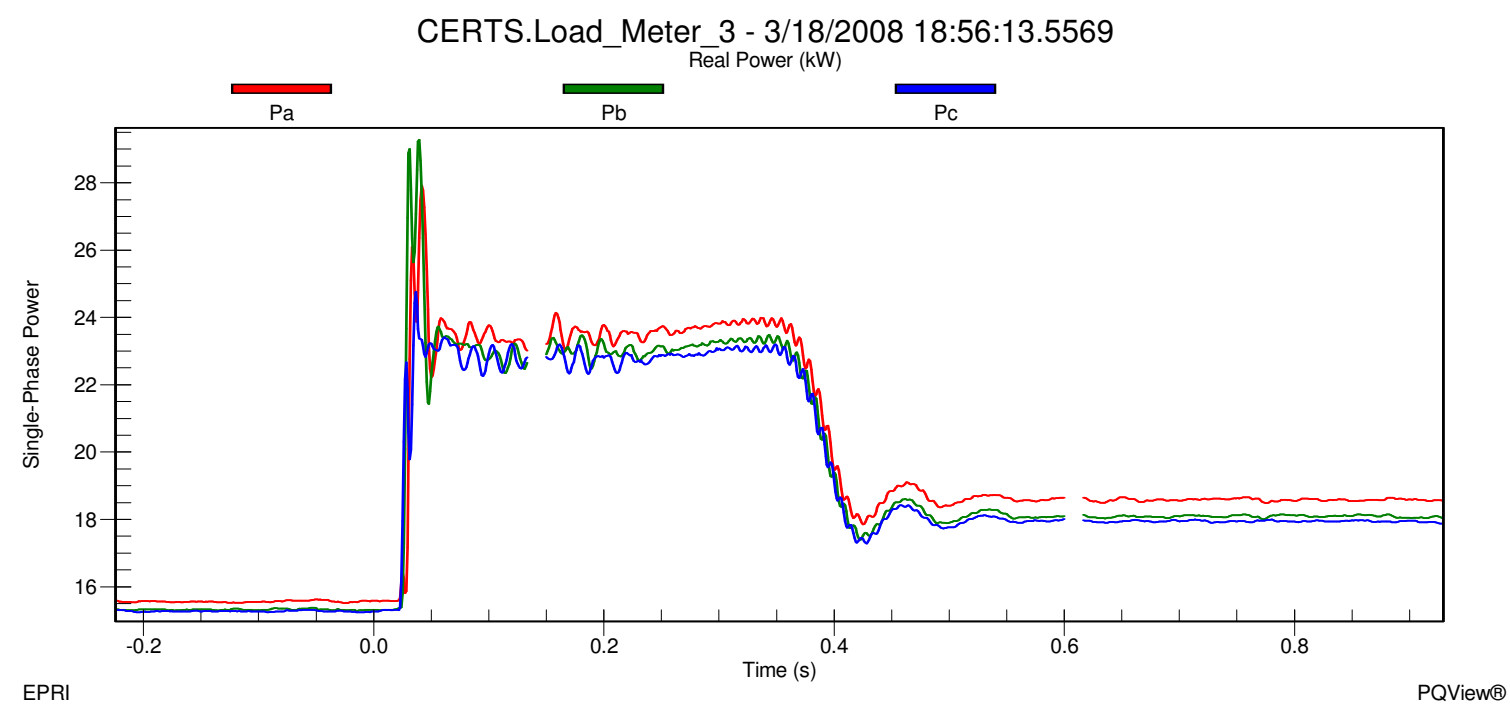

Figure 13a - Load Bank 3 Real Single-Phase Power during Motor Start and Utility Connected for Test 10.3.15

CERTS.Load_Meter_3 - 3/18/2008 18:56:13.5569

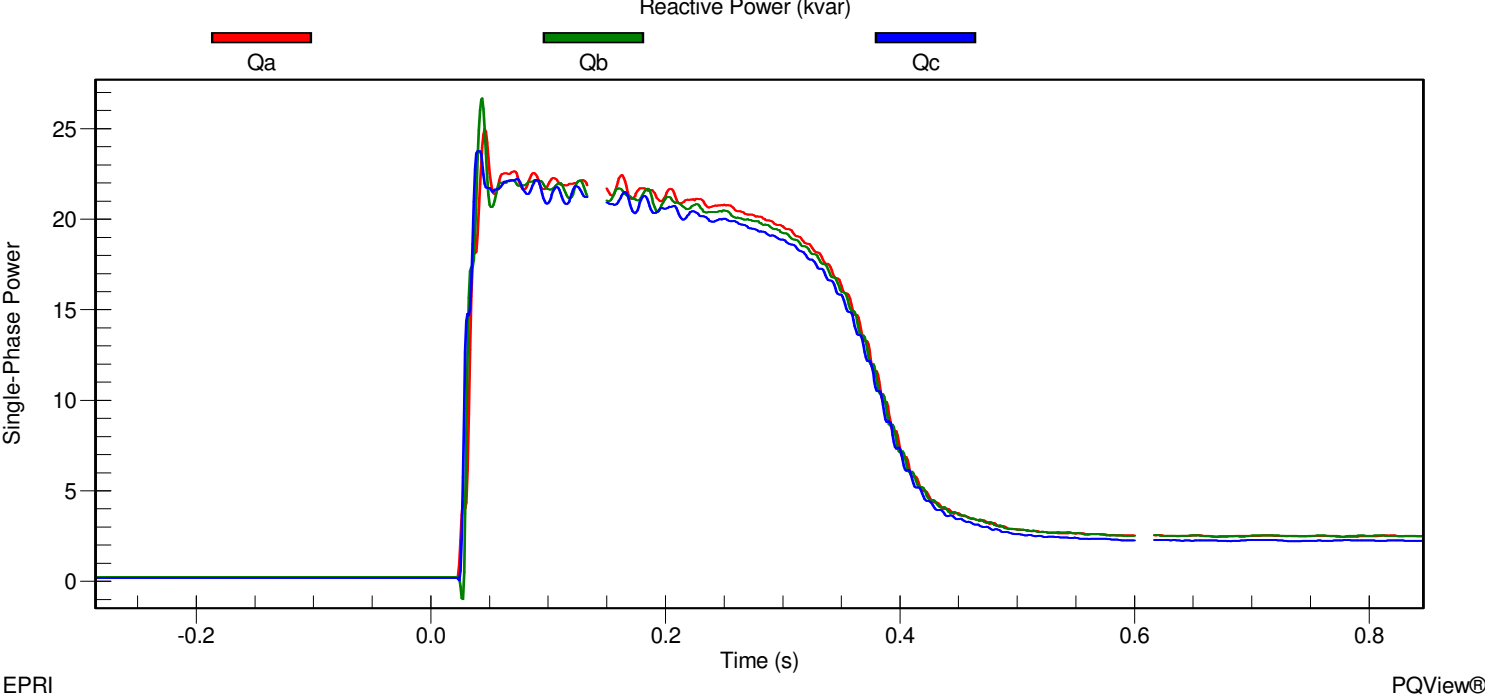

Figure 13b - Load Bank 2 Reactive Single-Phase Power during Motor Start and Utility Connected for Test 10.3.15

In Figures $13 \mathrm{a}$ and $13 \mathrm{~b}$ it can be seen that the load in Zone 3 was approximately $46.2 \mathrm{~kW}$ $(A=15.6 \mathrm{~kW} \mathrm{~B}=15.3 \mathrm{~kW} C=15.3 \mathrm{~kW})+\mathrm{j} 0.6 \mathrm{kVAr}(\mathrm{A}=0.2 \mathrm{kVAr} B=0.2 \mathrm{kVAr} C=0.2 \mathrm{kVAr})$ before the start of the induction motor and increased to approximately $79.45 \mathrm{~kW}(\mathrm{~A}=25.5 \mathrm{~kW}$ $\mathrm{B}=29.2 \mathrm{~kW} \mathrm{C}=24.75 \mathrm{~kW})+\mathrm{j} 75.6 \mathrm{kVAr}(\mathrm{A}=24.9 \mathrm{kVAr} \mathrm{B}=26.8 \mathrm{kVAr} \mathrm{C}=23.9 \mathrm{kVAr})$ during the inrush phase of the motor start. After about 1.5 cycles, the motor settled down to approximately $69.7 \mathrm{~kW}(\mathrm{~A}=23.6 \mathrm{~kW} \quad \mathrm{~B}=23.2 \mathrm{~kW} \quad \mathrm{C}=22.9 \mathrm{~kW})+\mathrm{j} 63.3 \mathrm{kVAr} \quad(\mathrm{A}=21.3 \mathrm{kVAr}$ $\mathrm{B}=21 \mathrm{kVAr} \mathrm{C}=21 \mathrm{kVAr})$ during the warm up phase which lasted about 33 cycles $(0.55$ Seconds). When the motor reached steady state, the load in Zone 3 was approximately 
$54.6 \mathrm{~kW} \quad(\mathrm{~A}=18.6 \mathrm{~kW} \quad \mathrm{~B}=18.1 \mathrm{~kW} \quad \mathrm{C}=17.9 \mathrm{~kW})+j 7.2 \mathrm{kVAr} \quad(\mathrm{A}=2.5 \mathrm{kVAr} \quad \mathrm{B}=2.5 \mathrm{kVAr}$ $\mathrm{C}=2.2 \mathrm{kVAr})$.

The voltage and frequency at the static switch before the motor start was approximately 281.7V on A-phase, 278.5V on B-phase and 278V on C-phase shown in Figure 13c and approximately $60.00 \mathrm{~Hz}$ shown in Figure 13d. When the motor started, the voltage at the static switch during the inrush decreased to approximately $274.9 \mathrm{~V}$ on A-phase, $271.8 \mathrm{~V}$ on B-phase and $271 \mathrm{~V}$ on C-phase for about 1.5 cycles. Frequency dropped during the inrush to approximately $59.96 \mathrm{~Hz}$ and quickly increased back to approximately $59.99 \mathrm{~Hz}$. Voltage increased as the motor was warming up and eventually settled at a steady state voltage at approximately $281.1 \mathrm{~V}$ on A-phase, $277.7 \mathrm{~V}$ on B-phase and $277.4 \mathrm{~V}$ on C-phase at an approximate frequency of $60.00 \mathrm{~Hz}$.

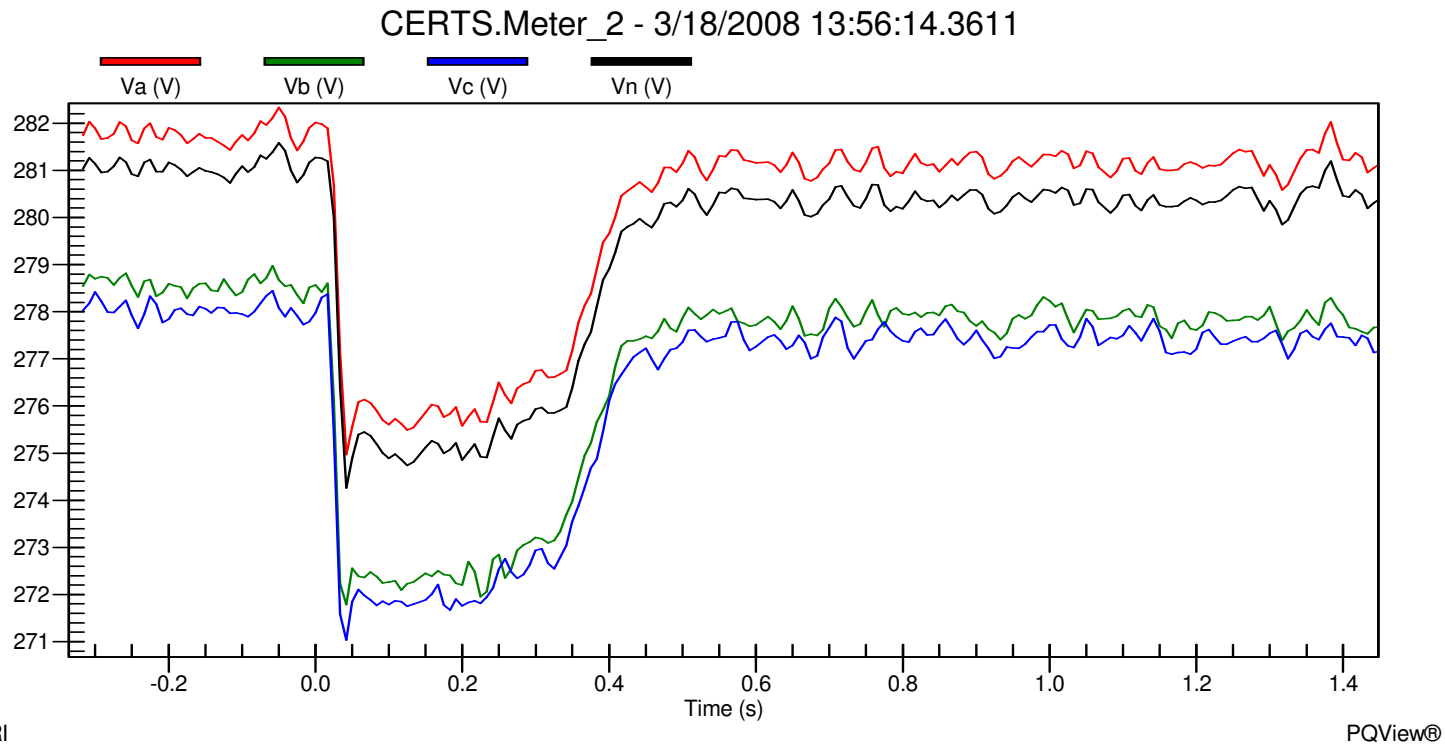

Figure 13c - Static Switch Line-to-Ground Voltage during Motor Start and Utility Connected for Test 10.3.15 


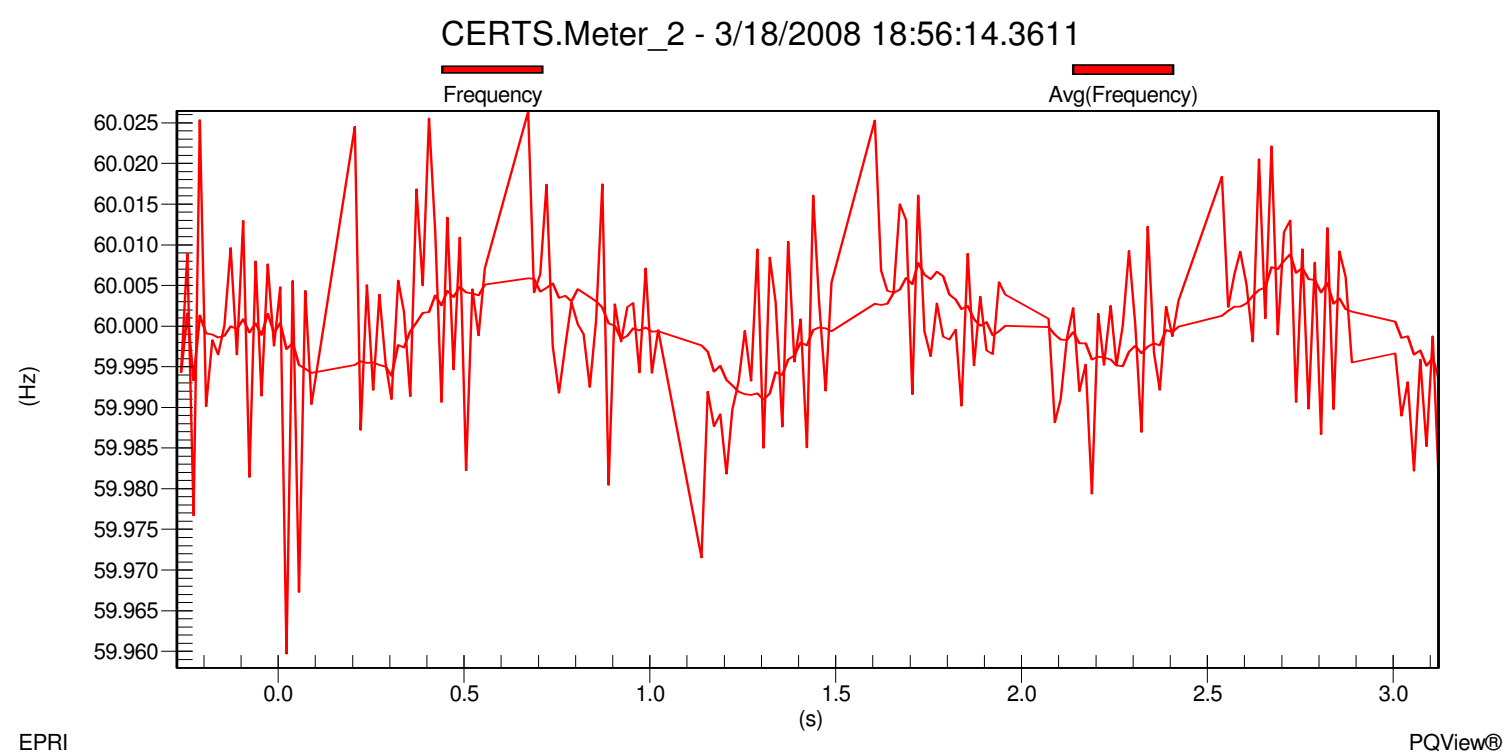

Figure 13d - Static Switch Frequency during Motor Start and Utility Connected for Test 10.3.15 CERTS.Meter_2 - 3/18/2008 18:56:14.3611

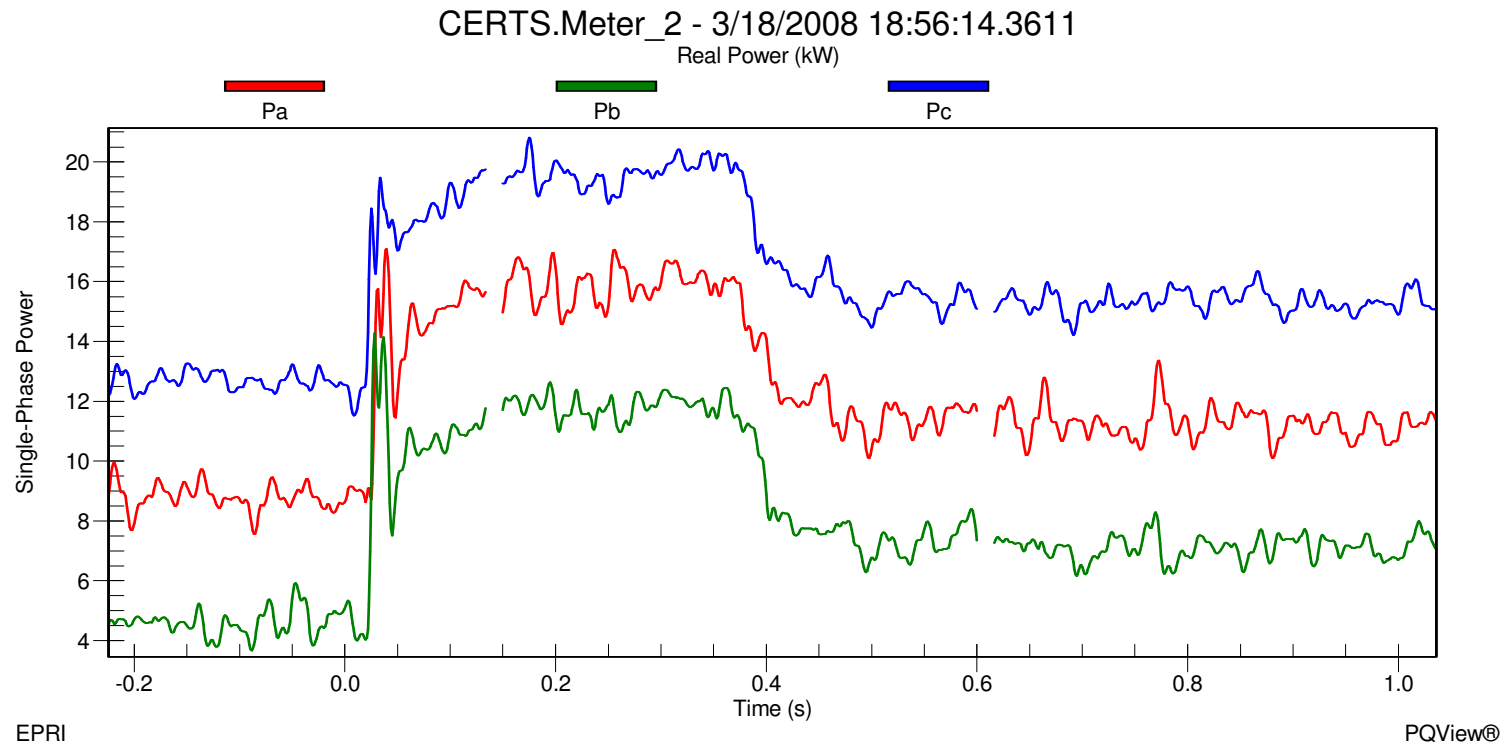

Figure 13e - Static Switch Real Single-Phase Power during Motor Start and Utility Connected for Test 10.3.15 


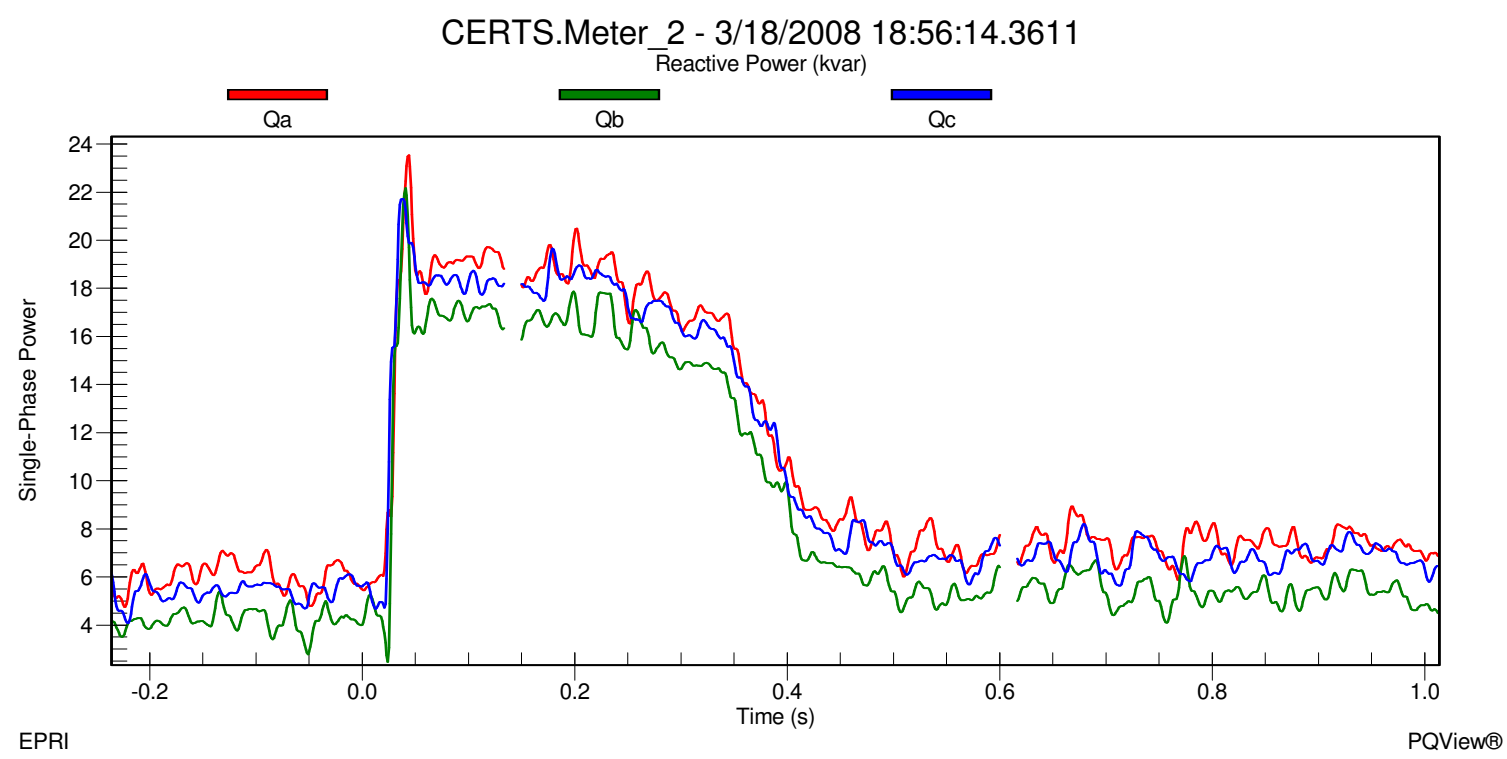

Figure 13f - Static Switch Reactive Single-Phase Power during Motor Start and Utility Connected for Test 10.3.15

Before the motor started Gen-sets $\mathrm{A} 1$ and $\mathrm{A} 2$ were producing approximately $38.3 \mathrm{~kW}$ $(\mathrm{A}=10.5 \mathrm{~kW} B=16.6 \mathrm{~kW} \mathrm{C}=11.2 \mathrm{~kW})-\mathrm{j} 9 \mathrm{kVAr}(\mathrm{A}=-3.4 \mathrm{kVAr} B=-2.8 \mathrm{kVAr} \mathrm{C}=-2.8 \mathrm{kVAr})$ and $29.8 \mathrm{~kW}(\mathrm{~A}=7.1 \mathrm{~kW} \mathrm{~B}=13.1 \mathrm{~kW} \mathrm{C}=9.6 \mathrm{~kW})+\mathrm{j} 0.1 \mathrm{kVAr}(\mathrm{A}=-0.9 \mathrm{kVAr} B=0.1 \mathrm{kVAr} C=0.9 \mathrm{kVAr})$, respectively. The load demands of Load Banks 3 and 4 were greater than the power being produced by both Gen-sets therefore the utility had to supply approximately 25.95 $(\mathrm{A}=8.75 \mathrm{~kW} B=4.6 \mathrm{~kW} \mathrm{C}=12.6 \mathrm{~kW})$ shown in Figure $13 \mathrm{e}$. The utility was supporting the reactive power of the microgrid with approximately $15.05 \mathrm{kVAr}(\mathrm{A}=5.8 \mathrm{kVAr} B=4 \mathrm{kVAr}$ $\mathrm{C}=5.25 \mathrm{kVAr}$ ) shown in Figure 13f. When the motor started the inrush caused the utility to supply approximately $50.25 \mathrm{~kW}(\mathrm{~A}=16.75 \mathrm{~kW} \quad \mathrm{~B}=14.2 \mathrm{~kW} \mathrm{C}=19.3 \mathrm{~kW})+\mathrm{j} 67.2 \mathrm{kVAr}$ $(\mathrm{A}=23.5 \mathrm{kVAr} \mathrm{B}=22.2 \mathrm{kVAr} \mathrm{C}=21.5 \mathrm{kVAr})$ to the microgrid loads, shown in Figure 13e and $13 \mathrm{f}$, and Gen-sets $\mathrm{A} 1$ and $\mathrm{A} 2$ increased their output power levels to $42.4 \mathrm{~kW}(\mathrm{~A}=12.6 \mathrm{~kW}$ $B=17.2 \mathrm{~kW} \quad C=12.6 \mathrm{~kW})+j 3.4 \mathrm{kVAr}(\mathrm{A}=1 \mathrm{kVAr} B=1.6 \mathrm{kVAr} \quad \mathrm{C}=0.8 \mathrm{kVAr})$ and $35.1 \mathrm{~kW}$ $(A=9.6 \mathrm{~kW} \quad B=14.4 \mathrm{~kW} \quad C=11.1 \mathrm{~kW})+j 16.9 \mathrm{kVAr}(A=4.9 \mathrm{kVAr} B=6.75 \mathrm{kVAr} \quad \mathrm{C}=5.25 \mathrm{kVAr})$, respectively. Notice the sign of the $\mathrm{VAr}$ output changed from negative to positive for Gen-set $\mathrm{A} 1$ and $0.1 \mathrm{kVAr}(\mathrm{A}=-0.9 \mathrm{kVAr} B=0.1 \mathrm{kVAr} C=0.9 \mathrm{kVAr})$ to $16.9 \mathrm{kVAr}(\mathrm{A}=4.9 \mathrm{kVAr}$ $\mathrm{B}=6.75 \mathrm{kVAr} \mathrm{C}=5.25 \mathrm{kVAr}$ ) in order to support the induction motor load in Zone 3.

When the motor reached steady state, the real and reactive power through the static switch was approximately $33.6 \mathrm{~kW}(\mathrm{~A}=11.25 \mathrm{~kW} B=7.1 \mathrm{~kW} C=15.25 \mathrm{~kW})+\mathrm{j} 18.85 \mathrm{kVAr}$ $(\mathrm{A}=7 \mathrm{kVAr} \mathrm{B}=5.25 \mathrm{kVAr} \mathrm{C}=6.6 \mathrm{kVAr})$ which meant the utility was supplying the power for the induction motor. Gen-set A1 and A2 real power returned relatively close to the values before the motor started of approximately $38.5 \mathrm{~kW} \quad(A=10.7 \mathrm{~kW} \quad B=16.6 \mathrm{~kW}$ $\mathrm{C}=11.2 \mathrm{~kW})$ and $29.95 \mathrm{~kW}(\mathrm{~A}=7.1 \mathrm{~kW} \mathrm{~B}=13.1 \mathrm{~kW} \mathrm{C}=9.75 \mathrm{~kW})$, respectively, and the reactive power slightly increased to approximately $-j 7.6 \mathrm{kVAr}(\mathrm{A}=-2.8 \mathrm{kVAr} B=-2.3 \mathrm{kVAr} \mathrm{C}=-$ 
2.5kVAr) in Gen-set $\mathrm{A} 1$ and j1.75kVAr $(\mathrm{A}=-0.25 \mathrm{kVAr} B=1.5 \mathrm{kVAr} \mathrm{C}=0.5 \mathrm{kVAr})$ in Gen-set A2. Once all the data was verified and recorded into the DAS Database, the motor was shut down and the static switch was directed by the EMS to manually open.

As soon as the static switch opened, Meter 1 recorded real and reactive power decreased to approximately $37.9 \mathrm{~kW}(\mathrm{~A}=10 \mathrm{~kW} \quad \mathrm{~B}=18.1 \mathrm{~kW} \quad \mathrm{C}=9.8 \mathrm{~kW})+\mathrm{j} 1.1 \mathrm{kVAr} \quad(\mathrm{A}=0.25 \mathrm{kVAr}$ $\mathrm{B}=0.6 \mathrm{kVAr} \mathrm{C}=0.25 \mathrm{kVAr})$ satisfying the load demand in Load Bank 6 which was approximately $37.65 \mathrm{~kW}(\mathrm{~A}=10 \mathrm{~kW} B=17.9 \mathrm{~kW} C=9.75 \mathrm{~kW})+j 0.651 \mathrm{kVAr}(\mathrm{A}=0.121 \mathrm{kVAr}$ $\mathrm{B}=0.409 \mathrm{kVAr} \mathrm{C}=0.121 \mathrm{kVAr})$ and not supplying any power beyond the static switch to Load Banks 3 and 4. $0 \mathrm{~kW}(\mathrm{~A}=0 \mathrm{~kW} B=0 \mathrm{~kW} C=0 \mathrm{~kW})+\mathrm{j} 0 \mathrm{kVAr}(\mathrm{A}=0 \mathrm{kVAr} B=0 \mathrm{kVAr}$ $\mathrm{C}=0 \mathrm{kVAr}$ ) was recorded at the static switch, indicating that power was not flowing through the static switch. Load Banks 3 and 4 loads reduced slightly to $51.35 \mathrm{~kW}$ $(\mathrm{A}=17.3 \mathrm{~kW} B=17.3 \mathrm{~kW} \mathrm{C}=16.75 \mathrm{~kW})+\mathrm{j} 6.55 \mathrm{kVAr}(\mathrm{A}=2.14 \mathrm{kVAr} \mathrm{B}=2.35 \mathrm{kVAr} \mathrm{C}=206 \mathrm{kVAr})$ and $42.5 \mathrm{~kW}(\mathrm{~A}=9.2 \mathrm{~kW} B=16.8 \mathrm{~kW} C=16.5 \mathrm{~kW})+\mathrm{j} 0.775 \mathrm{kVAr}(\mathrm{A}=0.11 \mathrm{kVAr} B=0.375 \mathrm{kVAr}$ $\mathrm{C}=0.29 \mathrm{kVAr})$, respectively. This load reduction resulted from a voltage drop in the microgrid, shown in Figure 13g, from approximately $280.5 \mathrm{~V}$ on A-phase, $277.4 \mathrm{~V}$ on Bphase and $276.9 \mathrm{~V}$ on C-phase when connected to the utility grid to $273.2 \mathrm{~V}$ on A-phase, $273.2 \mathrm{~V}$ on B-phase and $270.4 \mathrm{~V}$ on C-phase at Meter 3 when islanded.

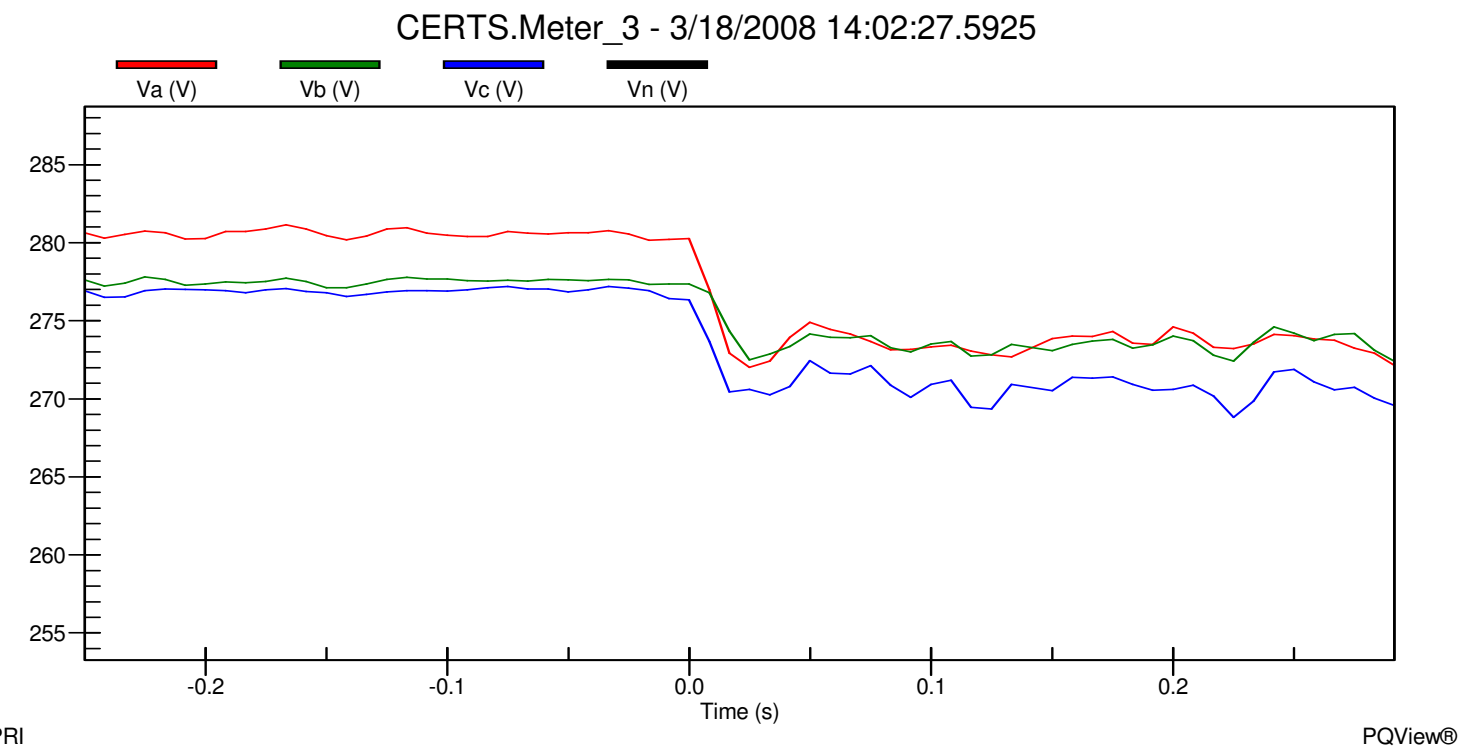

Figure 13g - Meter 3 Line-to-Ground Voltages during Utility Connected to Island mode for Test 10.3.15 


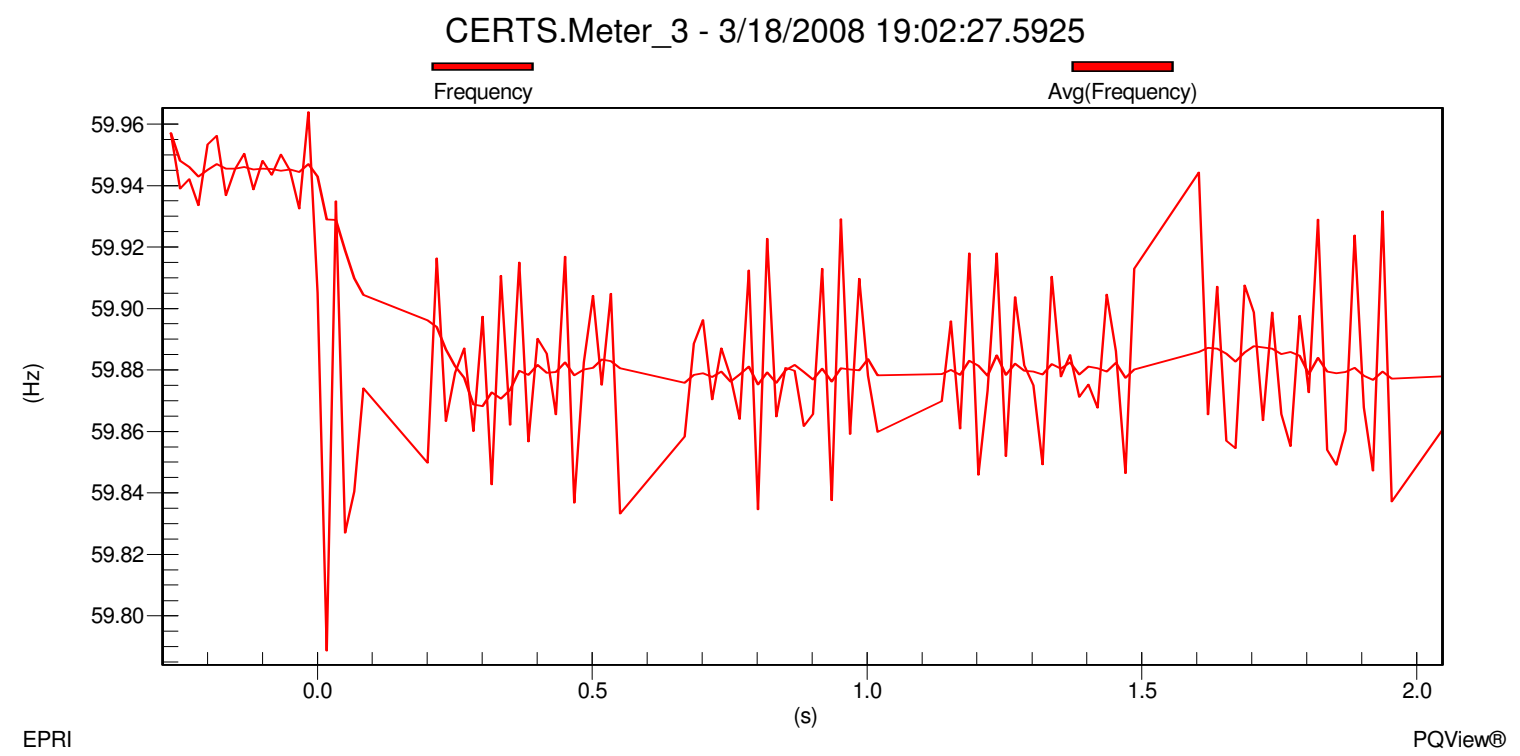

Figure 13h - Meter 3 Frequency during Utility Connected to Island mode for Test 10.3.15

Frequency change in the microgrid, shown in Figure 13h, decreased from approximately $59.95 \mathrm{~Hz}$ when connected to the utility grid to approximately $59.88 \mathrm{~Hz}$ when islanded. This change in frequency was part of the CERTS algorithm which allowed the Gen-sets to increase their output power to satisfy the load demands. Gen-set A1 and A2 increased their output real and reactive powers to approximately $52.1 \mathrm{~kW}(A=15.25 \mathrm{~kW}$ $B=19.1 \mathrm{~kW} C=17.75 \mathrm{~kW})+\mathrm{j} 1.2 \mathrm{kVAr}(\mathrm{A}=0.1 \mathrm{kVAr} B=0.2 \mathrm{kVAr} C=0.9 \mathrm{kVAr})$ and $44.2 \mathrm{~kW}$ $(\mathrm{A}=12 \mathrm{~kW} \quad \mathrm{~B}=16.2 \mathrm{~kW} \quad \mathrm{C}=16 \mathrm{~kW})+\mathrm{j} 10 \mathrm{kVAr} \quad(\mathrm{A}=3.1 \mathrm{kVAr} \quad \mathrm{B}=3.8 \mathrm{kVAr} \quad \mathrm{C}=3.1 \mathrm{kVAr})$, respectively. Meter 3 was approximately $-1.25 \mathrm{~kW}(\mathrm{~A}=-0.4 \mathrm{~kW} B=-0.6 \mathrm{~kW} \mathrm{C}=-0.25 \mathrm{~kW})+\mathrm{j}-$ $4 \mathrm{kVAr}(\mathrm{A}=-1 \mathrm{kVAr} \mathrm{B}=-1.5 \mathrm{kVAr} \mathrm{C}=-1.5 \mathrm{kVAr})$ indicating that the Gen-sets were satisfying the loads in the microgrid and the power losses in the electrical lines. All data was verified and recorded into the DAS Database. The microgrid ran for a couple of minutes in this electrical state before the $10 \mathrm{Hp}$ induction motor was started in Zone 3. 


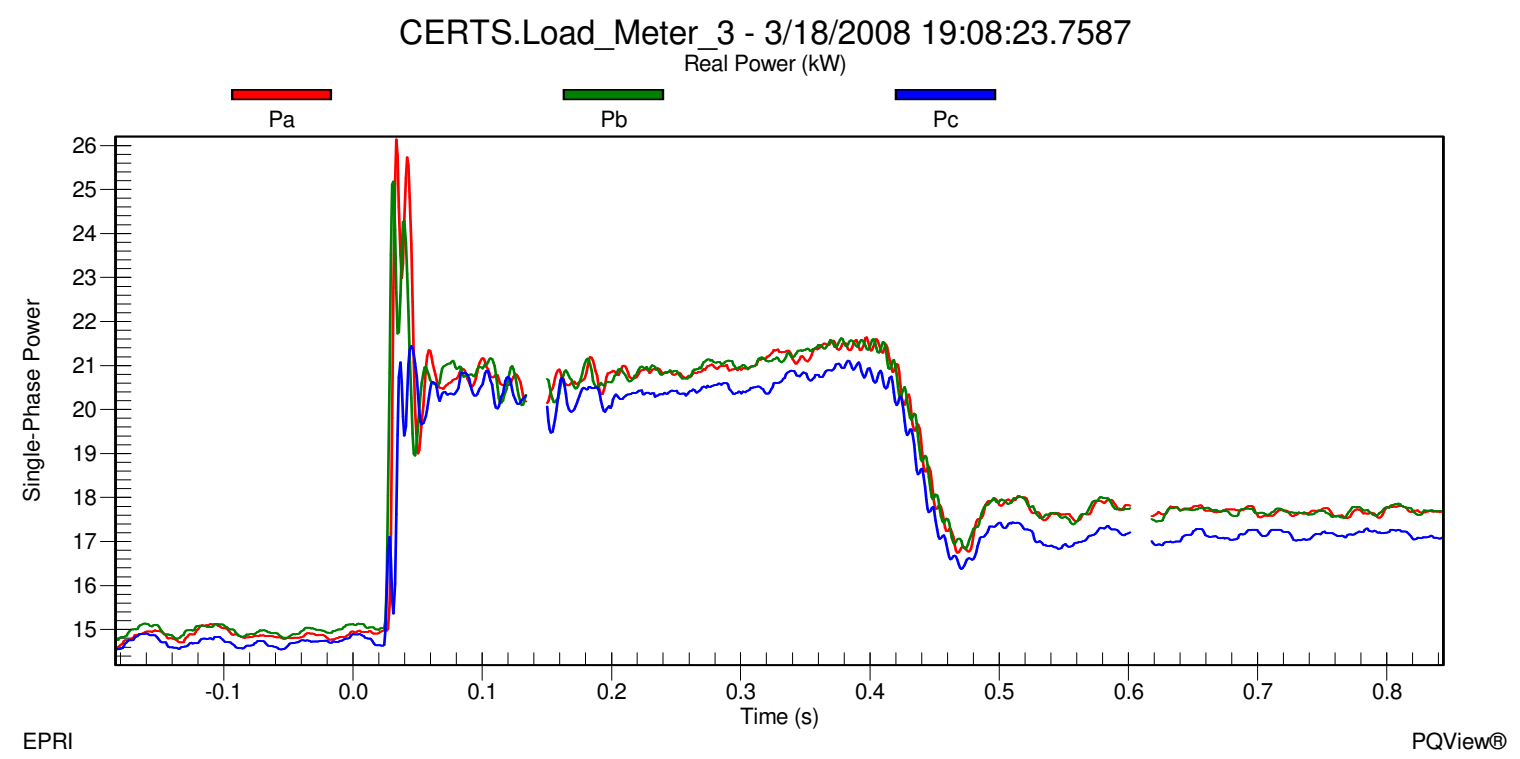

Figure 13i - Load Bank 3 Real Single-Phase Power during Motor Start and Islanded for Test 10.3.15

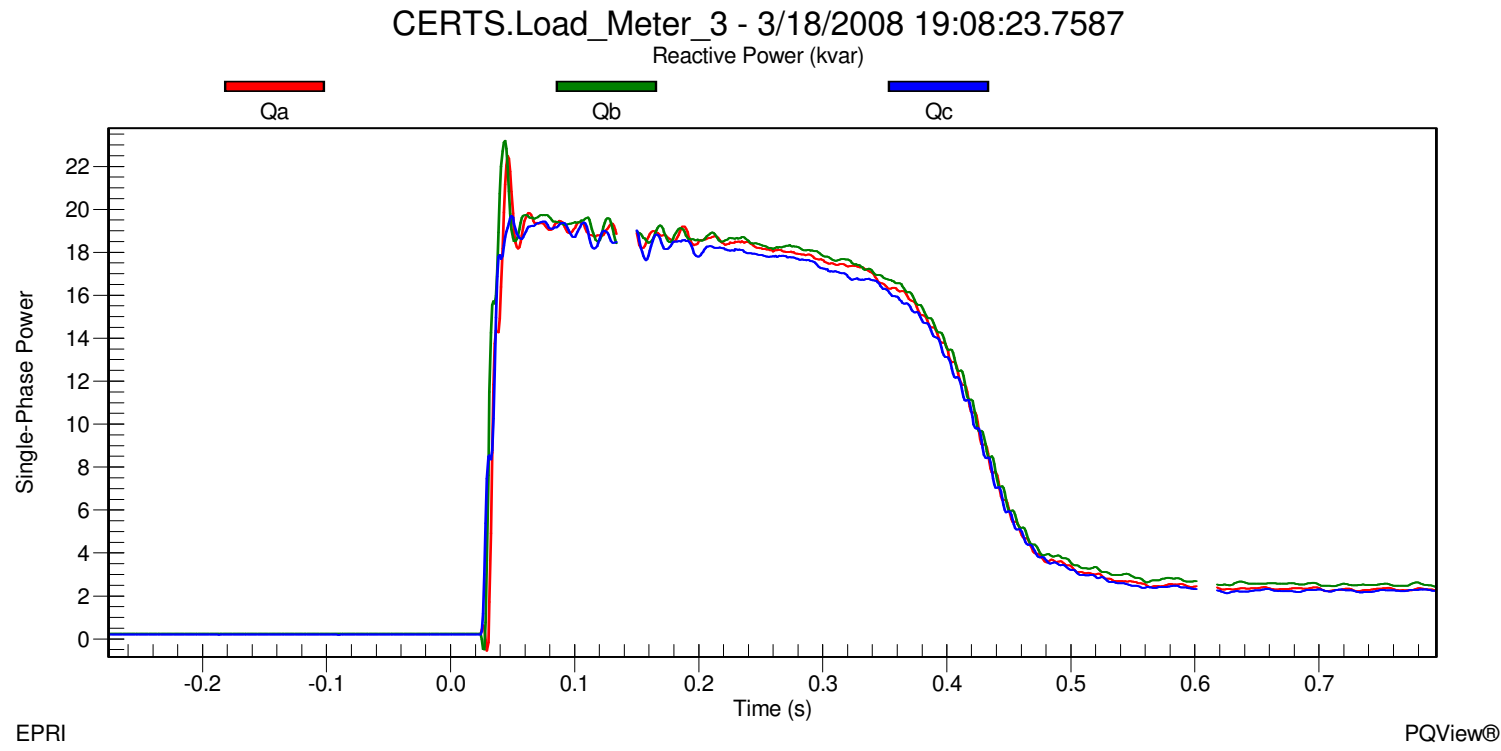

Figure 13j - Load Bank 3 Reactive Single-Phase Power during Motor Start and Islanded for Test 10.3.15

In Figures 13i and 13j it can be seen that the load in Zone 3 was approximately $44.5 \mathrm{~kW}$ $(\mathrm{A}=14.8 \mathrm{~kW} \quad \mathrm{~B}=15 \mathrm{~kW} \mathrm{C}=14.7 \mathrm{~kW})+\mathrm{j} 0.75 \mathrm{kVAr}(\mathrm{A}=0.25 \mathrm{kVAr} \quad \mathrm{B}=0.25 \mathrm{kVAr} \quad \mathrm{C}=0.25 \mathrm{kVAr})$ before the start of the induction motor and increased to approximately $71.4 \mathrm{~kW}$ $(\mathrm{A}=26.2 \mathrm{~kW} B=24.4 \mathrm{~kW} \mathrm{C}=20.8 \mathrm{~kW})+\mathrm{j} 64.75 \mathrm{kVAr}(\mathrm{A}=22.5 \mathrm{kVAr} \mathrm{B}=23.25 \mathrm{kVAr} \mathrm{C}=19 \mathrm{kVAr})$ during the inrush phase of the motor start. After about 1.2 cycles, the motor settled down to approximately $62.5 \mathrm{~kW}(\mathrm{~A}=21 \mathrm{~kW} \mathrm{~B}=21 \mathrm{~kW} \mathrm{C}=20.5 \mathrm{~kW})+\mathrm{j} 55.1 \mathrm{kVAr}(\mathrm{A}=18.5 \mathrm{kVAr}$ $\mathrm{B}=18.5 \mathrm{kVAr} \mathrm{C}=18.1 \mathrm{kVAr})$ during the warm up phase which lasted about 32 cycles $(0.53$ Seconds). When the motor reached steady state, the load in Zone 3 was approximately 
$52.6 \mathrm{~kW} \quad(\mathrm{~A}=17.7 \mathrm{~kW} \quad \mathrm{~B}=17.7 \mathrm{~kW} \quad \mathrm{C}=17.2 \mathrm{~kW})+j 7.1 \mathrm{kVAr} \quad(\mathrm{A}=2.3 \mathrm{kVAr} \quad \mathrm{B}=2.5 \mathrm{kVAr}$ $\mathrm{C}=2.3 \mathrm{kVAr})$.

The voltage and frequency at Meter 3 before the motor start was approximately $275 \mathrm{~V}$ on A-phase, $275 \mathrm{~V}$ on B-phase and $272.8 \mathrm{~V}$ on C-phase shown in Figure $13 \mathrm{~K}$ and approximately $59.91 \mathrm{~Hz}$ shown in Figure 131. When the motor started, the voltage at Meter 3 during the inrush decreased to approximately $255 \mathrm{~V}$ on A-phase, $255 \mathrm{~V}$ on Bphase and $253.2 \mathrm{~V}$ on $\mathrm{C}$-phase for about 1.2 cycles. Frequency dropped during the inrush to approximately $59.74 \mathrm{~Hz}$ and quickly increased to approximately $59.82 \mathrm{~Hz}$. Voltage increased as the motor was warming up and eventually settled at a steady state voltage at approximately $273 \mathrm{~V}$ on A-phase, $273 \mathrm{~V}$ on B-phase and $270.9 \mathrm{~V}$ on C-phase at an approximate frequency of $59.87 \mathrm{~Hz}$.

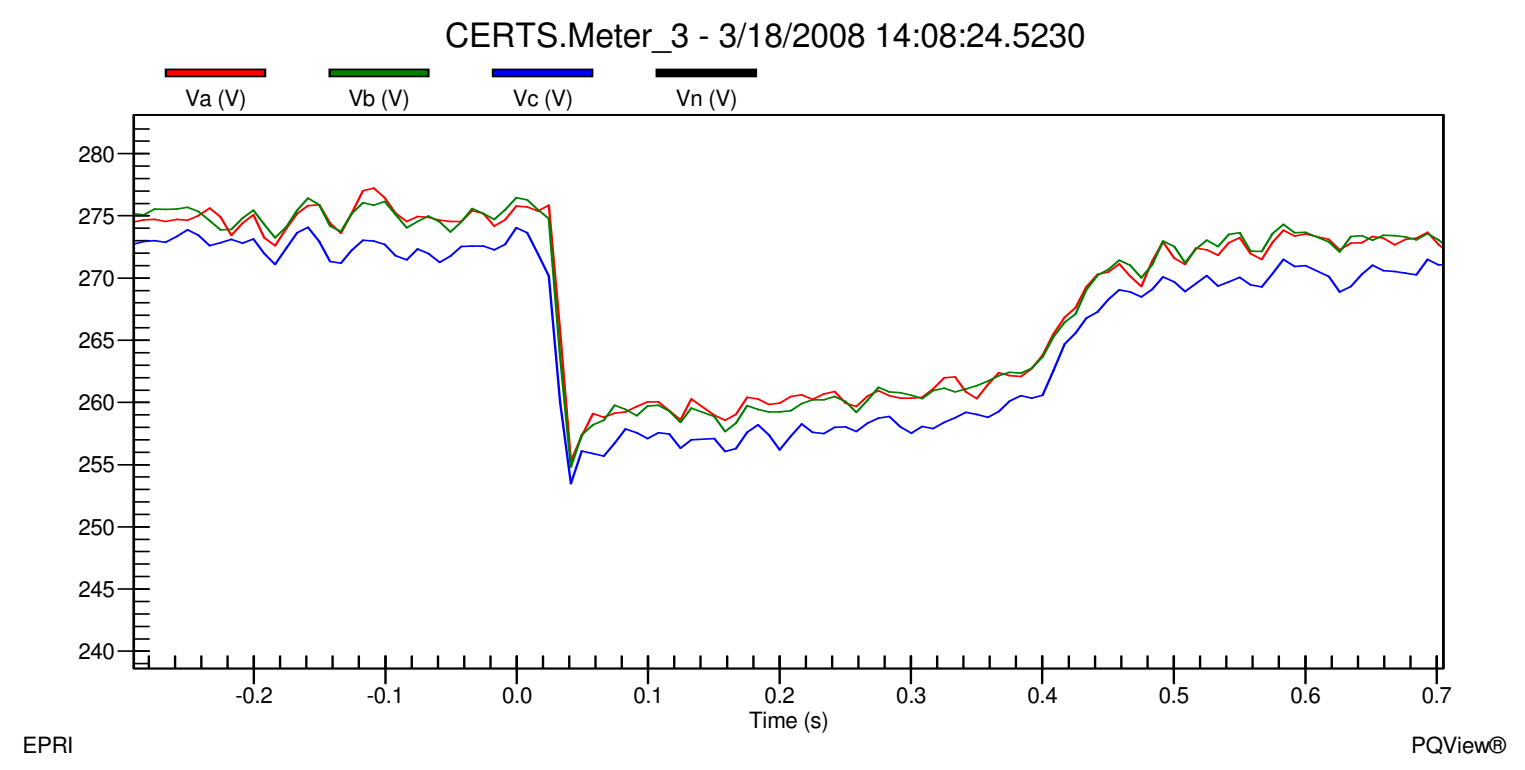

Figure 13k - Meter 3 Line-to-Ground Voltages during Motor Start and Islanded for Test 10.3.15 


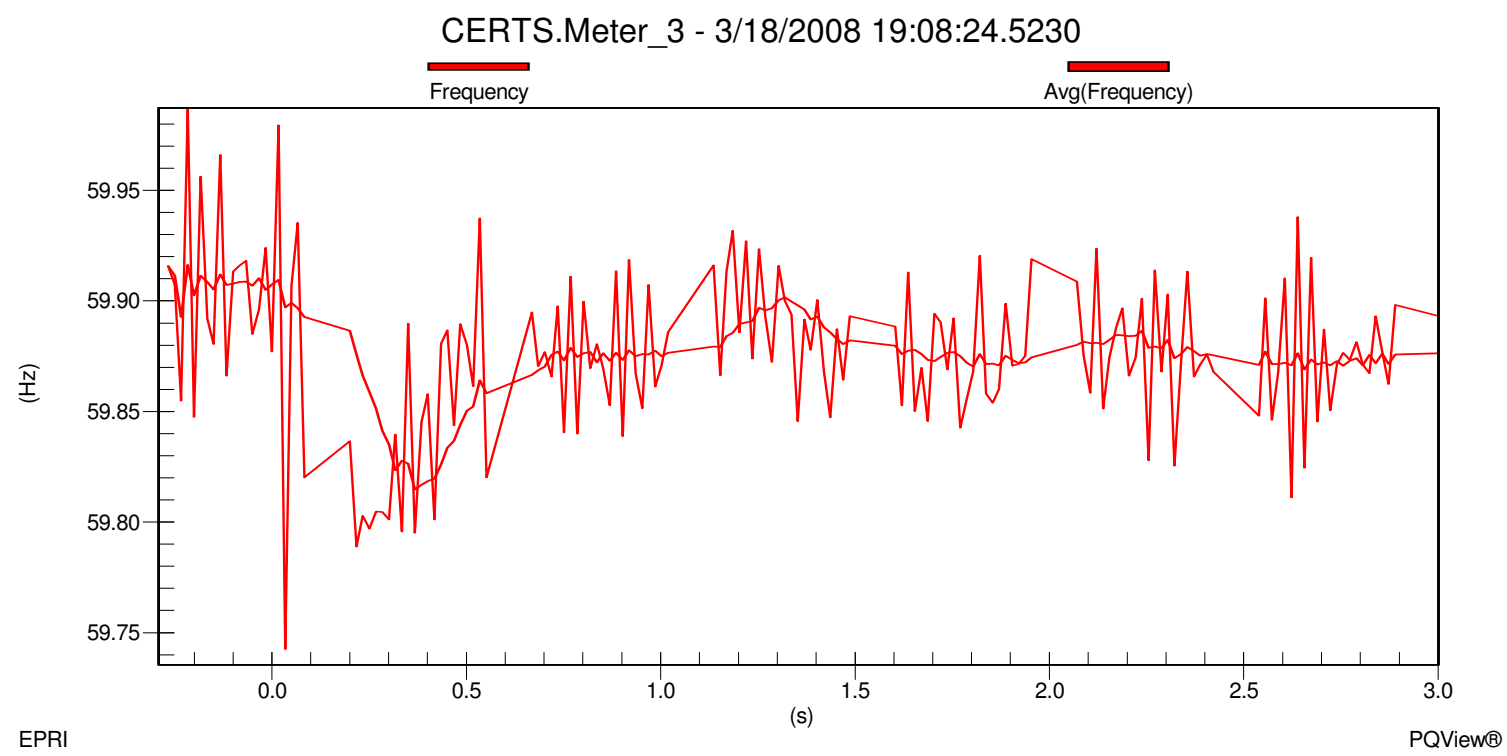

Figure 131 - Meter 3 Frequency during Motor Start and Islanded for Test 10.3.15

Before the motor started Gen-sets $\mathrm{A} 1$ and $\mathrm{A} 2$ were producing approximately $49.1 \mathrm{~kW}$ $(A=14.1 \mathrm{~kW} \mathrm{~B}=18.1 \mathrm{~kW} C=16.9 \mathrm{~kW})-\mathrm{j} 3.7 \mathrm{kVAr}(\mathrm{A}=-1.5 \mathrm{kVAr} B=-1.5 \mathrm{kVAr} C=-0.7 \mathrm{kVAr})$ and $41 \mathrm{~kW}(\mathrm{~A}=10.7 \mathrm{~kW} \mathrm{~B}=15.2 \mathrm{~kW} \mathrm{C}=15.1 \mathrm{~kW})+\mathrm{j} 7.2 \mathrm{kVAr}(\mathrm{A}=2.2 \mathrm{kVAr} B=2.6 \mathrm{kVAr} C=2.4 \mathrm{kVAr})$, respectively shown in Figures $13 \mathrm{~m}-13 \mathrm{p}$. The power generated by both Gen-sets was satisfying the loads in Load Banks 3 and 4 and all the electrical losses in the microgrid system. When the motor started the inrush caused the Gen-sets to increase their output levels to $58.3 \mathrm{~kW}(\mathrm{~A}=18.3 \mathrm{~kW} \mathrm{~B}=22.6 \mathrm{~kW} \mathrm{C}=17.4 \mathrm{~kW})+\mathrm{j} 28.5 \mathrm{kVAr}(\mathrm{A}=8.9 \mathrm{kVAr} \mathrm{B}=10.9 \mathrm{kVAr}$ $\mathrm{C}=8.7 \mathrm{kVAr})$ for Gen-set $\mathrm{A} 1$ and $50.4 \mathrm{~kW}(\mathrm{~A}=15.6 \mathrm{~kW} B=19 \mathrm{~kW} C=15.8 \mathrm{~kW})+\mathrm{j} 37 \mathrm{kVAr}$ $(\mathrm{A}=12.1 \mathrm{kVAr} B=13.6 \mathrm{kVAr} \mathrm{C}=11.3 \mathrm{kVAr})$ for Gen-set $\mathrm{A} 2$. The Gen-sets decreased their output levels while the motor was warming up and eventually dropped to $52.7 \mathrm{~kW}$ $(\mathrm{A}=15.4 \mathrm{~kW} \mathrm{~B}=19.4 \mathrm{~kW} \mathrm{C}=17.9 \mathrm{~kW})+\mathrm{j} 1.3 \mathrm{kVAr}(\mathrm{A}=0 \mathrm{kVAr} \mathrm{B}=0.5 \mathrm{kVAr} \mathrm{C}=0.8 \mathrm{kVAr})$ for Genset $\mathrm{A} 1$ and $44.8 \mathrm{~kW}(\mathrm{~A}=12.2 \mathrm{~kW} B=16.4 \mathrm{~kW} \mathrm{C}=16.2 \mathrm{~kW})+\mathrm{j} 10.1 \mathrm{kVAr}(\mathrm{A}=3.2 \mathrm{kVAr} B=4.1 \mathrm{kVAr}$ $\mathrm{C}=3.2 \mathrm{kVAr}$ ) for Gen-set $\mathrm{A} 2$ when the motor reached steady state. Once all the data was verified and recorded into the DAS Database, the static switch was directed by the EMS to manually close. 


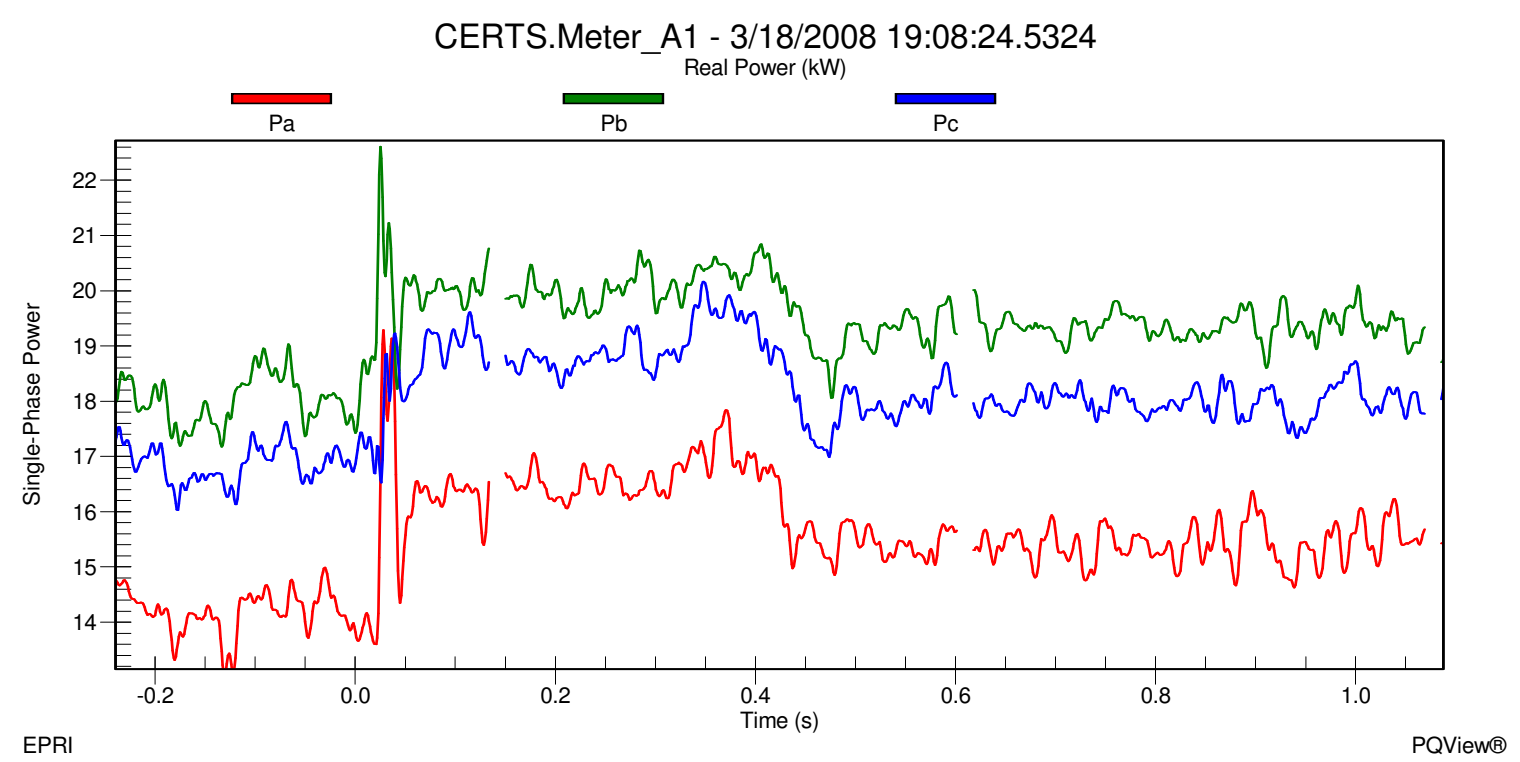

Figure 13m - Gen-set A1 Real Single-Phase Power during Motor Start and Islanded for Test 10.3.15

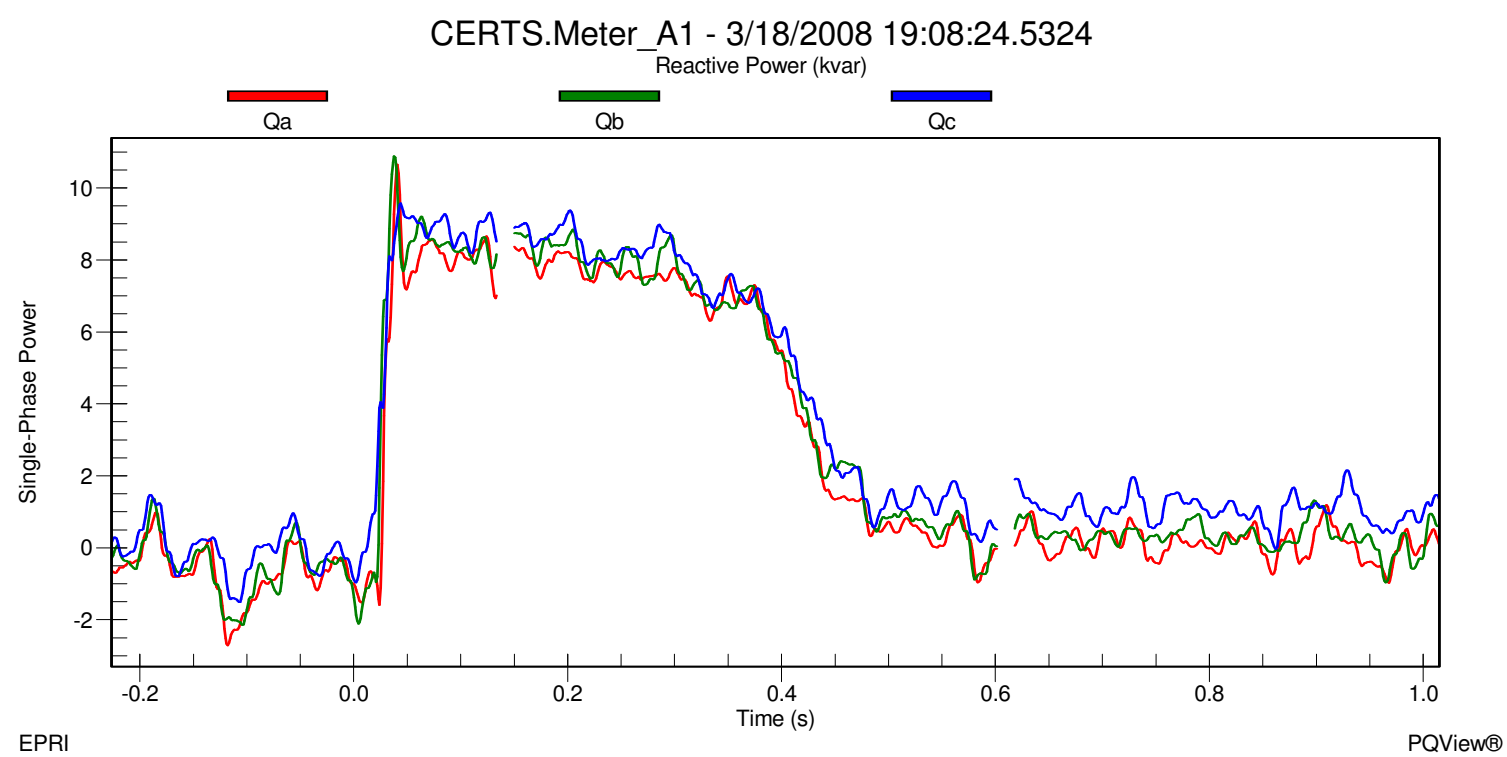

Figure 13n - Gen-set A1 Reactive Single-Phase Reactive Power during Motor Start and Islanded for Test 10.3.15 


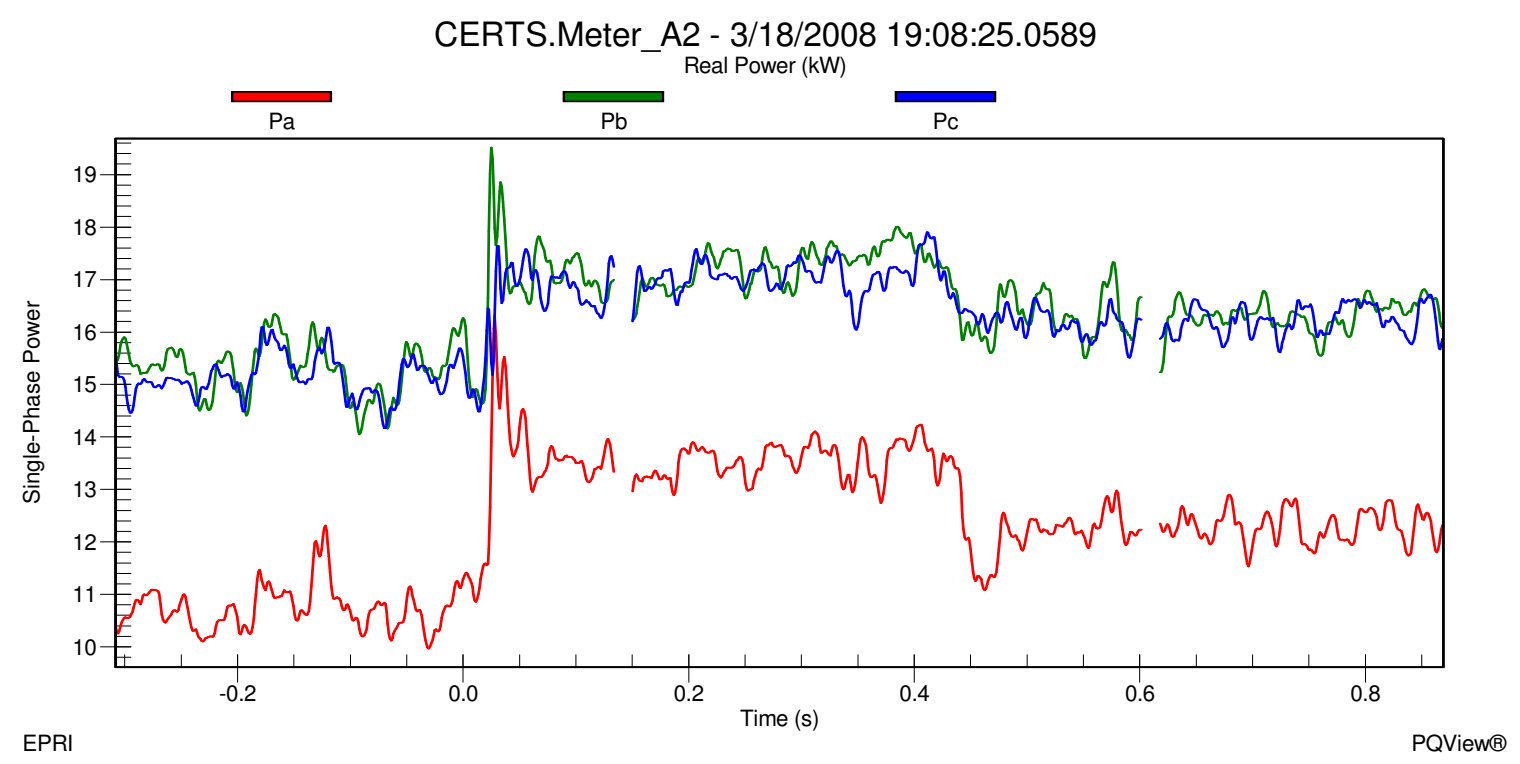

Figure 13o - Gen-set A2 Real Single-Phase Power during Motor Start and Islanded for Test 10.3.15

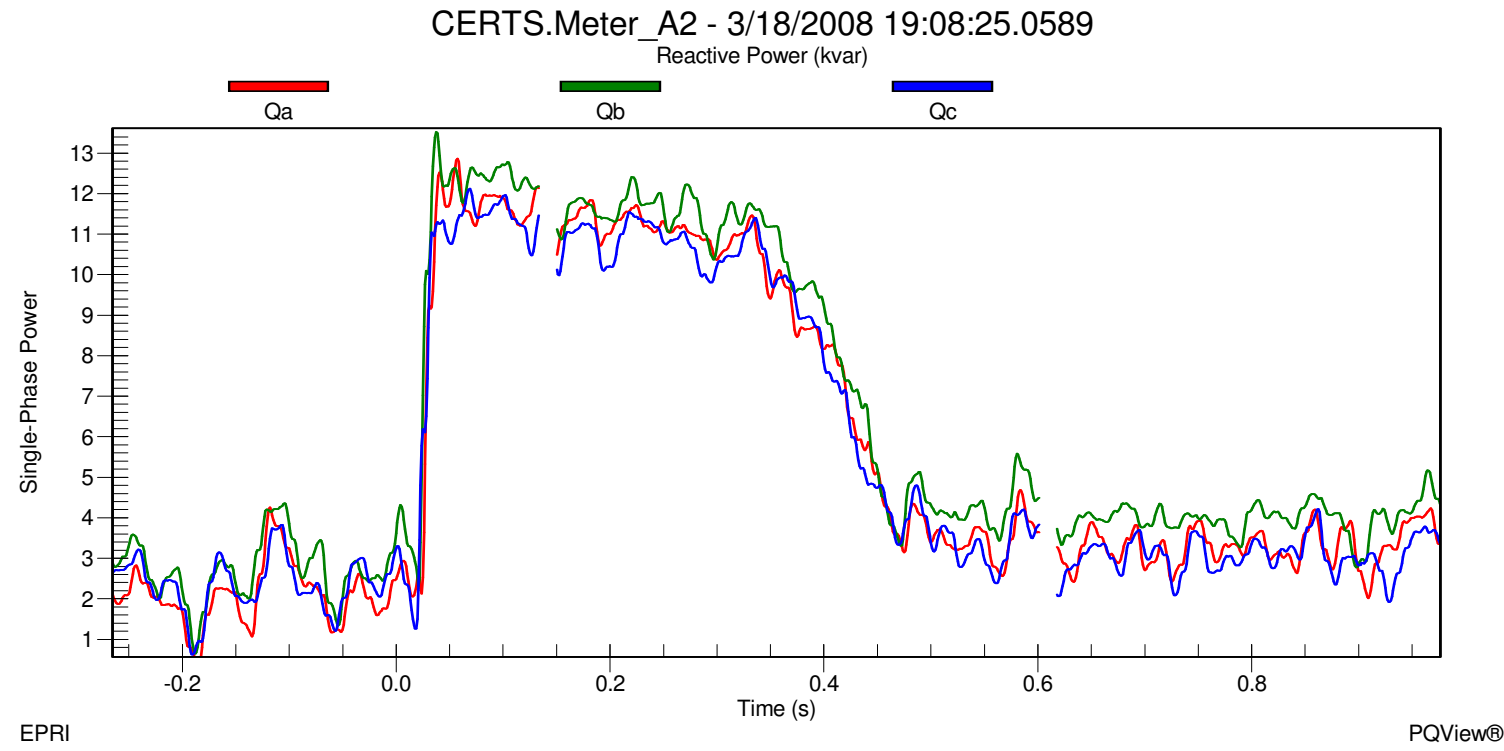

Figure 13p - Gen-set A2 Reactive Single-Phase Power during Motor Start and Islanded for Test 10.3.15

As soon as the static switch closed, Meter 1 recorded real and reactive powers increased from approximately $38 \mathrm{~kW}(\mathrm{~A}=10 \mathrm{~kW} B=18.1 \mathrm{~kW} C=9.9 \mathrm{~kW})+\mathrm{j} 1.1 \mathrm{kVAr}(\mathrm{A}=0.25 \mathrm{kVAr}$ $\mathrm{B}=0.6 \mathrm{kVAr} \quad \mathrm{C}=0.25 \mathrm{kVAr})$ to $65.5 \mathrm{~kW}(\mathrm{~A}=19.4 \mathrm{~kW} \quad \mathrm{~B}=23 \mathrm{~kW} \quad \mathrm{C}=23.1 \mathrm{~kW})+\mathrm{j} 22.65 \mathrm{kVAr}$ $(\mathrm{A}=8.2 \mathrm{kVAr} \mathrm{B}=6.75 \mathrm{kVAr} \mathrm{C}=7.7 \mathrm{kVAr})$ which means that the utility was satisfying not only the load demand of Load Bank 6 but also all the reactive power except $0.7 \mathrm{kVAr}$ from Gen-set A2 and a portion of the real power for the microgrid loads.

Figures $13 \mathrm{q}$ and $13 \mathrm{r}$ show the static switch increasing from approximately $0 \mathrm{~kW}(\mathrm{~A}=0 \mathrm{~kW}$ $\mathrm{B}=0 \mathrm{~kW} \mathrm{C}=0 \mathrm{~kW})+\mathrm{j} 0 \mathrm{kVAr}(\mathrm{A}=0 \mathrm{kVAr} \mathrm{B}=0 \mathrm{kVAr} \mathrm{C}=0 \mathrm{kVAr})$ to $28.6 \mathrm{~kW}(\mathrm{~A}=9.6 \mathrm{~kW} \mathrm{~B}=5.25 \mathrm{~kW}$ 
$\mathrm{C}=13.75 \mathrm{~kW})+\mathrm{j} 21.8 \mathrm{kVAr}(\mathrm{A}=8 \mathrm{kVAr} \mathrm{B}=6.4 \mathrm{kVAr} \mathrm{C}=7.4 \mathrm{kVAr})$. At the beginning of the test, the initial power flow through the static switch was $25.95 \mathrm{~kW}(\mathrm{~A}=8.75 \mathrm{~kW} B=4.6 \mathrm{~kW}$ $\mathrm{C}=12.6 \mathrm{~kW})+\mathrm{j} 15.05 \mathrm{kVAr}(\mathrm{A}=5.8 \mathrm{kVAr} \mathrm{B}=4 \mathrm{kVAr} \mathrm{C}=5.25 \mathrm{kVAr})$ which is not the same recorded at this point in the test because the $10 \mathrm{Hp}$ motor load is on in Zone 3. The Genssets $\mathrm{A} 1$ and $\mathrm{A} 2$ output power levels reduced to approximately $40 \mathrm{~kW}(\mathrm{~A}=11.3 \mathrm{~kW}$ $B=17.2 \mathrm{~kW} \quad C=11.5 \mathrm{~kW})-\mathrm{j} 9.3 \mathrm{kVAr}(\mathrm{A}=-3.4 \mathrm{kVAr} B=-3 \mathrm{kVAr} \quad \mathrm{C}=-2.9 \mathrm{kVAr})$ and $31.5 \mathrm{~kW}$ $(\mathrm{A}=7.8 \mathrm{~kW} \quad \mathrm{~B}=13.6 \mathrm{~kW} \quad \mathrm{C}=10.1 \mathrm{~kW})+\mathrm{j} 0.7 \mathrm{kVAr} \quad(\mathrm{A}=-0.5 \mathrm{kVAr} \quad \mathrm{B}=1.2 \mathrm{kVAr} \quad \mathrm{C}=0 \mathrm{kVAr})$, respectively. These power levels are relatively close to the initial values at the beginning of the test. Since the motor is on in Zone 3 and the Gen-sets returned to their initial values at the beginning of the test before the motor was turned on means that the induction motor is completely being powered by the utility.

Load Banks 3 and 4 loads increased slightly to $53.12 \mathrm{~kW}(\mathrm{~A}=18.08 \mathrm{~kW} \quad \mathrm{~B}=17.6 \mathrm{~kW}$ $\mathrm{C}=17.44 \mathrm{~kW})+\mathrm{j} 6.77 \mathrm{kVAr}(\mathrm{A}=2.34 \mathrm{kVAr} B=2.34 \mathrm{kVAr} \mathrm{C}=2.09 \mathrm{kVAr})$ and $44.3 \mathrm{~kW}(\mathrm{~A}=9.75 \mathrm{~kW}$ $\mathrm{B}=17.3 \mathrm{~kW} \quad \mathrm{C}=17.25 \mathrm{~kW})+\mathrm{j} 0.83 \mathrm{kVAr} \quad(\mathrm{A}=0.119 \mathrm{kVAr} \quad \mathrm{B}=0.391 \mathrm{kVAr} \quad \mathrm{C}=0.32 \mathrm{kVAr})$, respectively. This slight load increase is a result from a voltage rise in the microgrid, shown in Figure 13s, from approximately $273.1 \mathrm{~V}$ on A-phase, $273.1 \mathrm{~V}$ on B-phase and $270.9 \mathrm{~V}$ on C-phase when islanded to $280.7 \mathrm{~V}$ on A-phase, $278 \mathrm{~V}$ on B-phase and $277.3 \mathrm{~V}$ on C-phase at Meter 3 when connected to the utility grid.

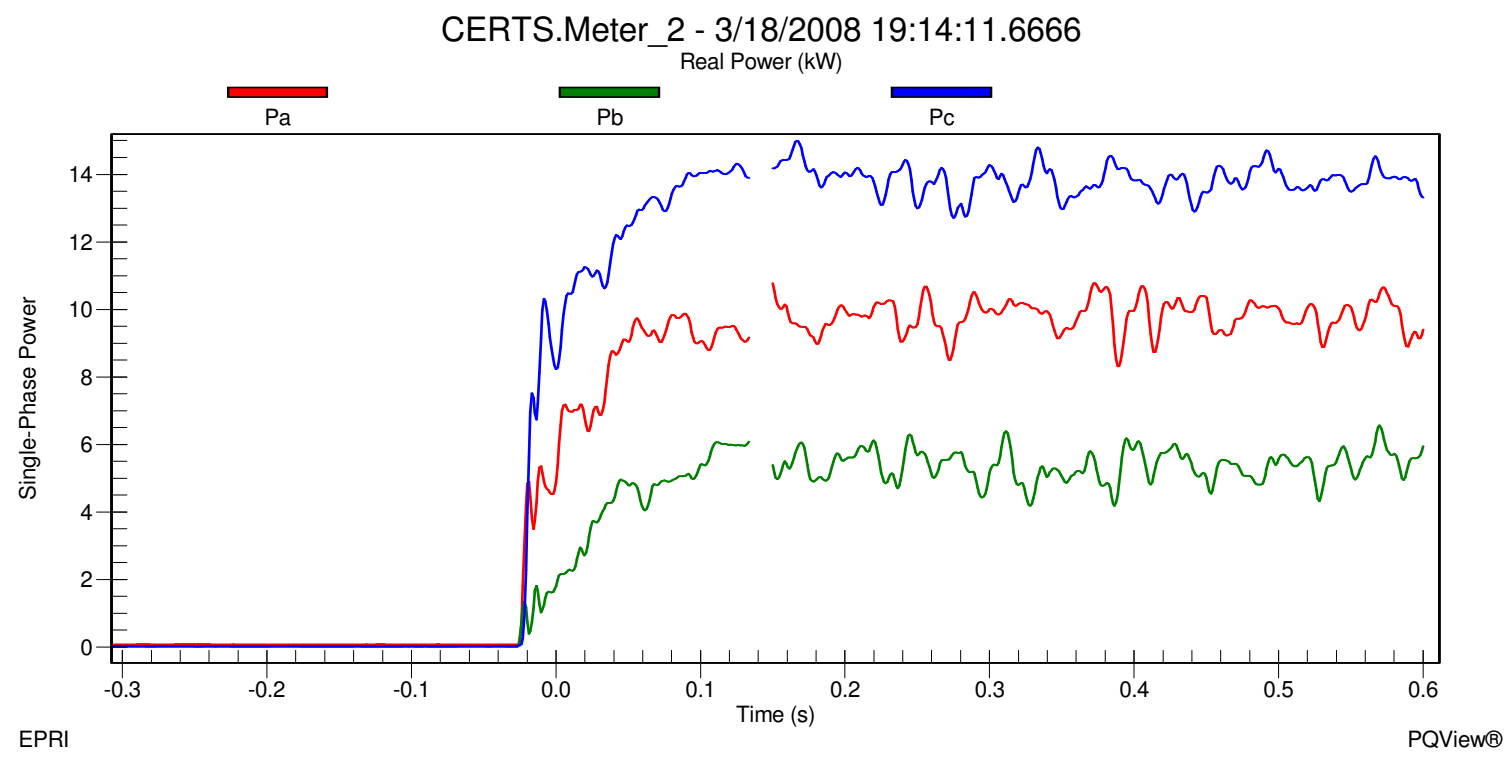

Figure 13q - Static Switch Real Single-Phase Power during Island to Utility Connected mode for Test 10.3.15 


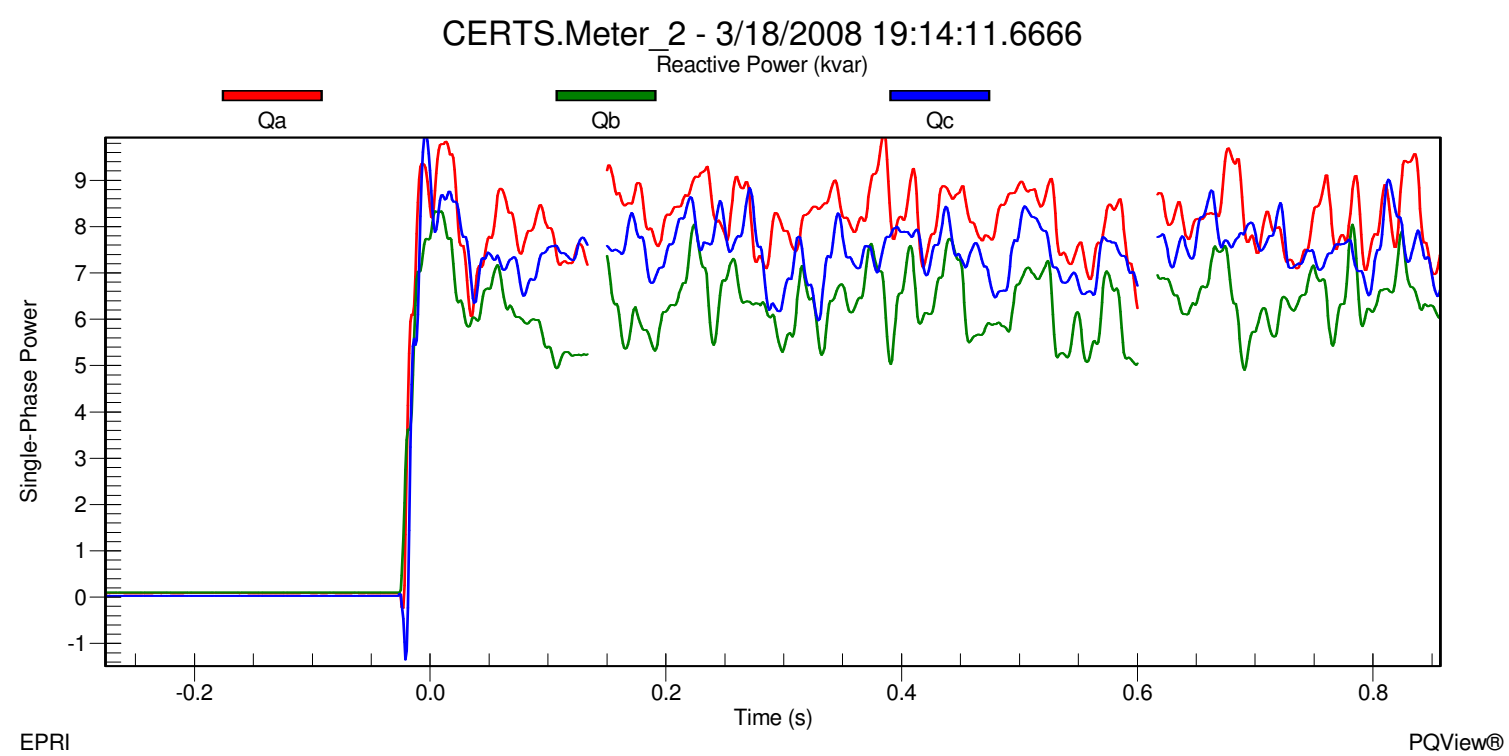

Figure 13r - Static Switch Reactive Single-Phase Power during Island to Utility Connected mode for Test 10.3.15

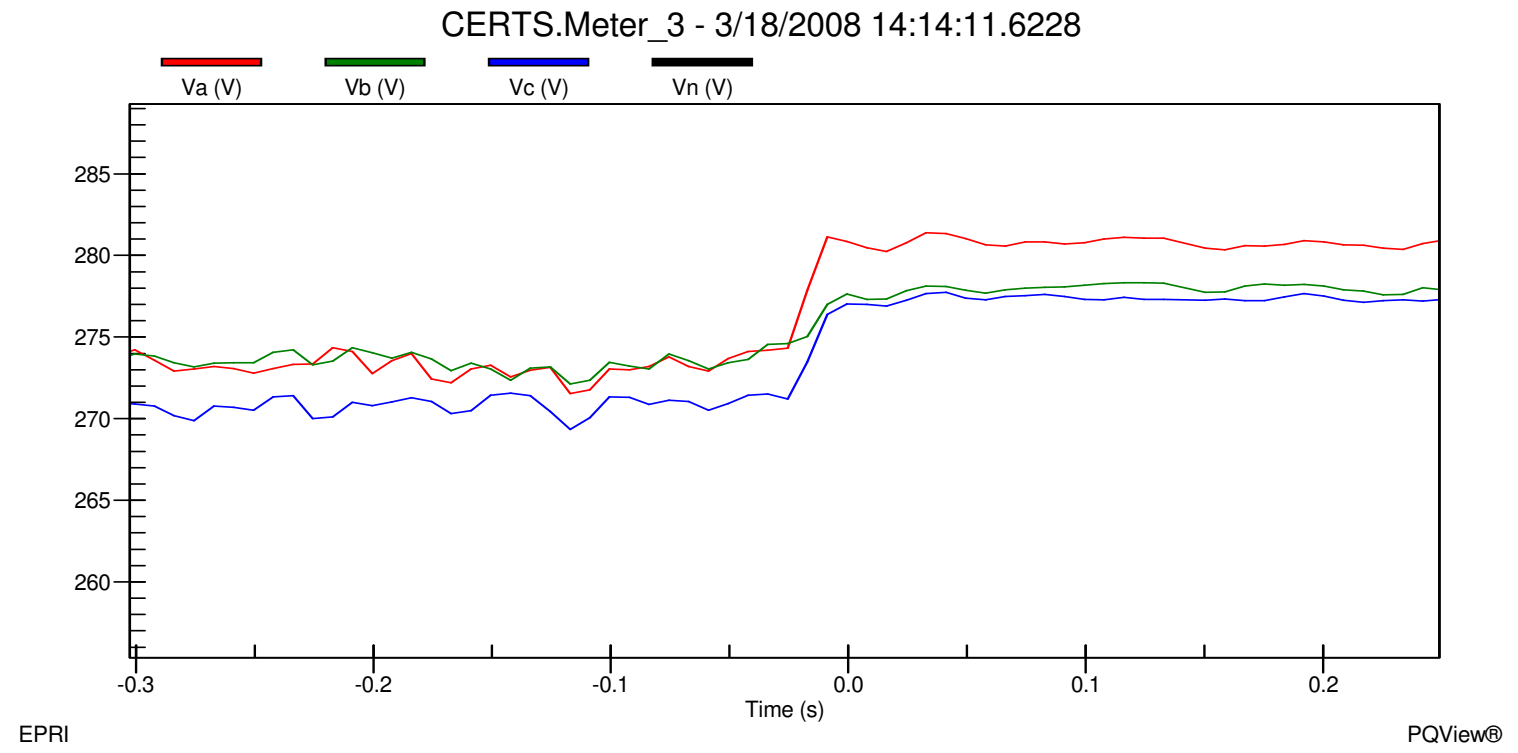

Figure 13s - Meter 3 Line-to-Ground Voltages during Island to Utility Connected mode for Test 10.3.15 


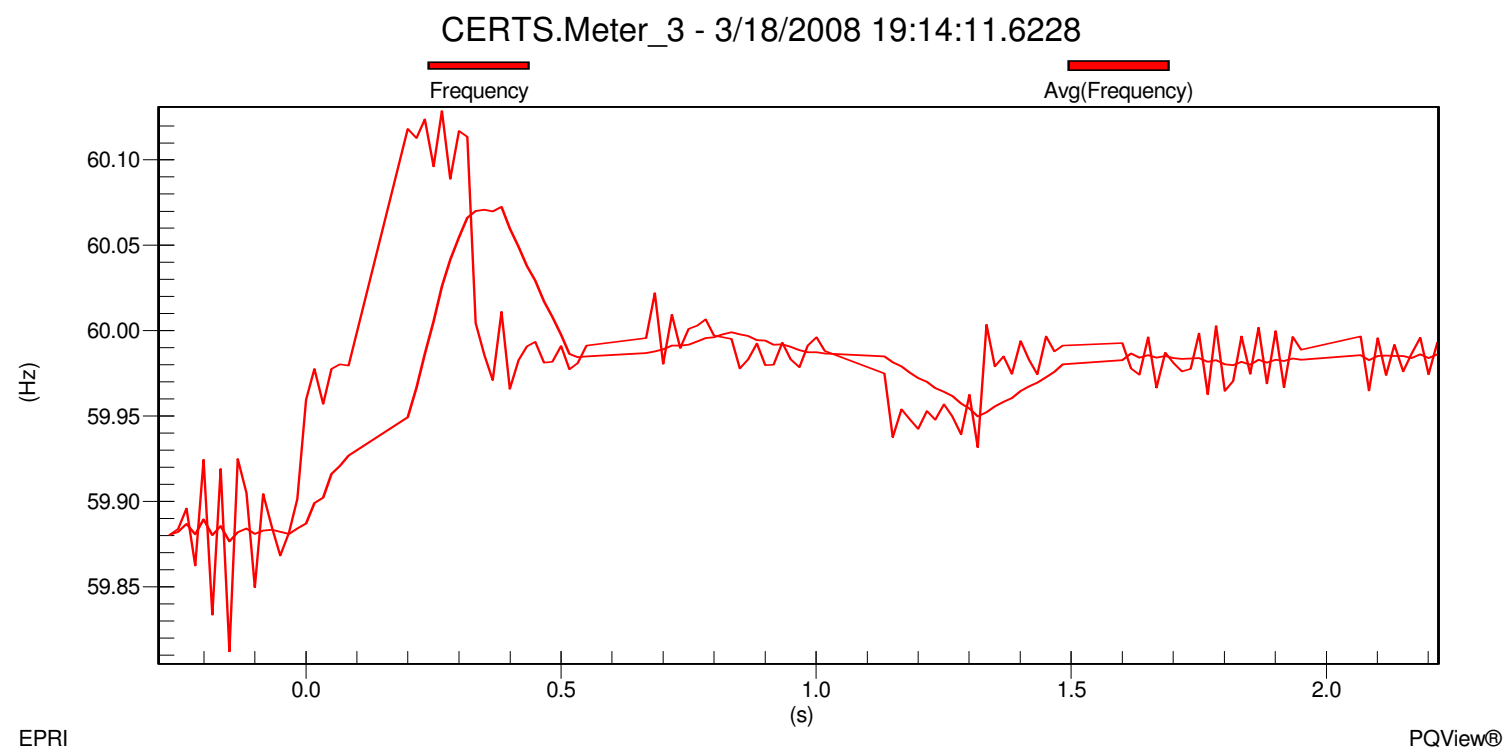

Figure 13t - Meter 3 Frequency during Island to Utility Connected mode for Test 10.3.15

Frequency change in the microgrid, shown in Figure 13t, increased from approximately $59.88 \mathrm{~Hz}$ when islanded to approximately $59.98 \mathrm{~Hz}$ when connected to the utility grid. This change in frequency is due to the frequency no longer being established by the Gensets using the CERTS algorithm but by the utility. Gen-sets A1 and A2 are in unit power control mode therefore when the static switch closed back into the utility the Gen-sets produced real power based on their set-points initialized at the beginning of the test. After all the data was verified and recorded into the DAS Database, the motor was turned off and the Gen-sets and Load Banks set-points were changed according to the next test (10.3.17) in Table 2.

For Test 10.3.17 the measured values, after Gen-set A1 was warmed up and load banks brought on-line are provided in Table 6.

\begin{tabular}{|c|c|c|c|c|c|c|c|c|c|}
\hline Meter & $\begin{array}{c}\text { Gen- } \\
\text { set A1 }\end{array}$ & $\begin{array}{c}\text { Gen - } \\
\text { set A2 }\end{array}$ & Meter 1 & Meter 2 & Meter 3 & Meter 4 & LB 3 & LB 4 & LB 6 \\
\hline $\begin{array}{c}\text { 3 Phase } \\
\mathbf{k W}\end{array}$ & 35.4 & 0 & 23.6 & -14.25 & -17.2 & 0 & 18.45 & 0 & 37.4 \\
\hline $\begin{array}{c}\mathbf{1} \text { Phase } \\
\mathbf{k W}\end{array}$ & $\mathrm{B}=15.6$ & $\mathrm{~B}=0$ & $\mathrm{~B}=9.8$ & $\mathrm{~B}=-8.25$ & $\mathrm{~B}=-9.6$ & $\mathrm{~B}=0$ & $\mathrm{~B}=6.1$ & $\mathrm{~B}=0$ & $\mathrm{~B}=17.8$ \\
& $\mathrm{C}=9.1$ & $\mathrm{C}=0$ & $\mathrm{C}=7.3$ & $\mathrm{C}=-2.5$ & $\mathrm{C}=-3.1$ & $\mathrm{C}=0$ & $\mathrm{C}=6.1$ & $\mathrm{C}=0$ & $\mathrm{C}=9.7$ \\
\hline
\end{tabular}




\begin{tabular}{|c|c|c|c|c|c|c|c|c|c|}
\hline $\begin{array}{c}3 \text { Phase } \\
\text { kVAr }\end{array}$ & -13.6 & 0 & 20.3 & 19.7 & 14.3 & 0 & 0.3 & 0 & 0.574 \\
\hline \multirow{3}{*}{$\begin{array}{c}1 \text { Phase } \\
\text { kVAr }\end{array}$} & $\mathrm{A}=-4.9$ & $A=0$ & $A=6.9$ & $A=6.8$ & $A=5.2$ & $A=0$ & $A=0.1$ & $A=0$ & $\mathrm{~A}=0.104$ \\
\hline & $\mathrm{B}=-4.8$ & $\mathrm{~B}=0$ & $B=7.1$ & $B=6.9$ & $B=5$ & $B=0$ & $\mathrm{~B}=0.1$ & $B=0$ & $B=0.359$ \\
\hline & $C=-3.9$ & $C=0$ & $C=6.3$ & $C=6$ & $C=4.1$ & $\mathrm{C}=0$ & $C=0.1$ & $\mathrm{C}=0$ & $C=0.111$ \\
\hline
\end{tabular}

Table 6 - Measured Values after Start Up for Test 10.3.17

These measurements were relatively close to the expected values in Table 2, but not exact due to temperature, phase voltages and electrical losses in conductors. In addition, the $40 \mathrm{~kW}$ settings for LB6 and the $20 \mathrm{~kW}$ setting for LB3 were also below selected set values. At the time of the measurements, the voltage and frequency was $283.6 \mathrm{~V}$ on Aphase, $279.3 \mathrm{~V}$ on B-phase and $279.9 \mathrm{~V}$ on C-phase and $60.02 \mathrm{~Hz}$ at the static switch (i.e., Meter 2) when connected to the utility grid; and $284 \mathrm{~V}$ on A-phase, $280 \mathrm{~V}$ on B-phase and $280.1 \mathrm{~V}$ on C-phase and $60.02 \mathrm{~Hz}$ at Meter 3.

Gen-set A1 was setup in this test to produce more power than Load Bank 3 needed which approximately $14.25 \mathrm{~kW}(\mathrm{~A}=3.5 \mathrm{~kW} \mathrm{~B}=8.25 \mathrm{~kW} \mathrm{C}=2.5 \mathrm{~kW})$ of excess power was exported through the static switch to load Bank 6. Since Load Bank 6 was approximately $37.4 \mathrm{~kW}(\mathrm{~A}=9.9 \mathrm{~kW} \quad \mathrm{~B}=17.8 \mathrm{~kW} \quad \mathrm{C}=9.7 \mathrm{~kW})+j 0.574 \mathrm{kVAr} \quad(\mathrm{A}=0.104 \mathrm{kVAr}$ $\mathrm{B}=0.359 \mathrm{kVAr} \mathrm{C}=0.111 \mathrm{kVAr})$, the utility had to supply approximately $23.6 \mathrm{~kW}(\mathrm{~A}=6.5 \mathrm{~kW}$ $\mathrm{B}=9.8 \mathrm{~kW} \mathrm{C}=7.3 \mathrm{~kW}$ ) to satisfy the load. Reactive power had to be imported in from the utility of approximately j19.7kVAr $(A=6.8 \mathrm{kVAr} B=6.9 \mathrm{kVAr} C=6 \mathrm{kVAr})$ because Gen-set A1 needed approximately j13.6kVAr $(A=4.9 \mathrm{kVAr} B=4.8 \mathrm{kVAr} C=3.9 \mathrm{kVAr})$ and the reactive power absorbed in the electrical lines. Once all data was verified and recorded into the DAS Database, the $10 \mathrm{Hp}$ induction motor was started in Zone 3. 


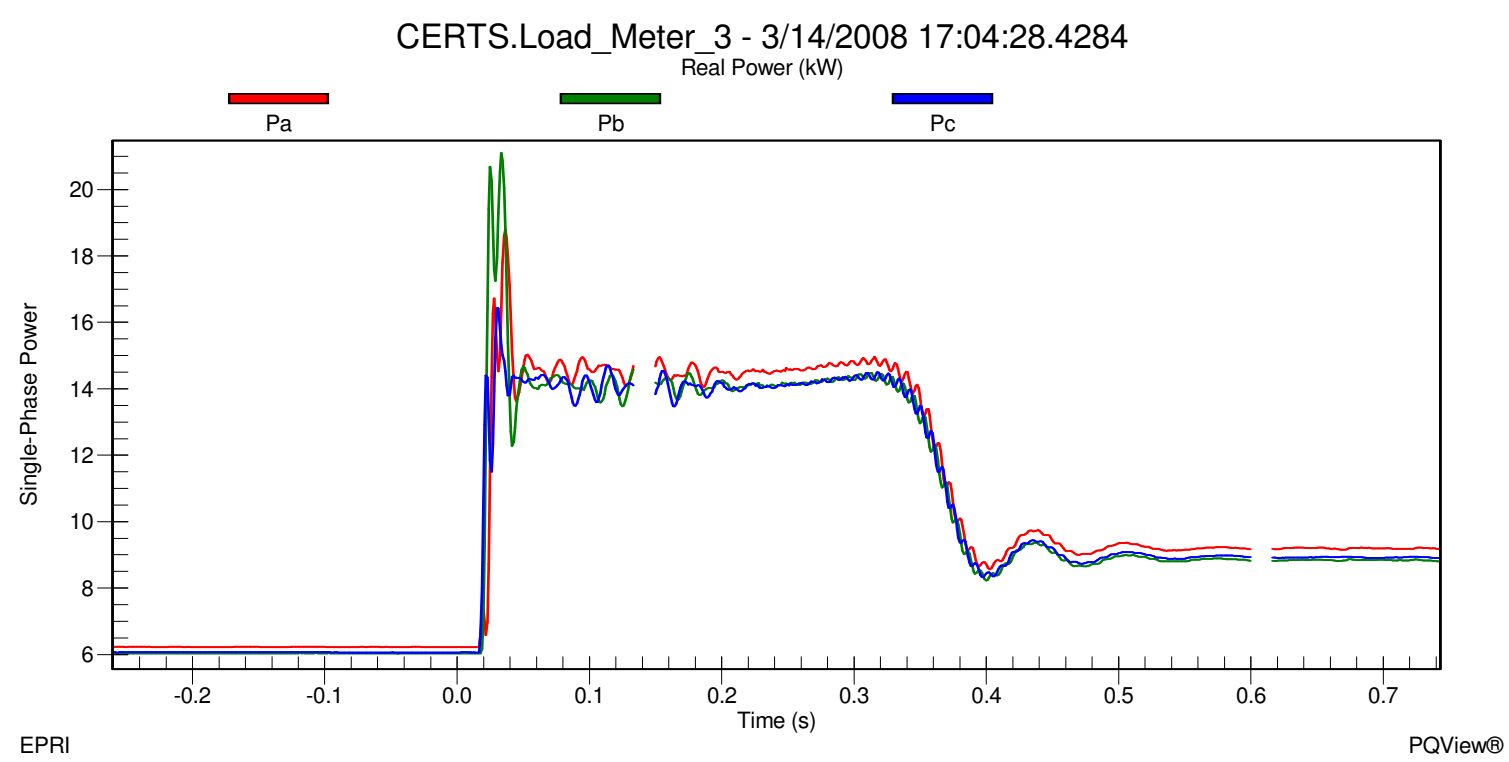

Figure 14a - Load Bank 3 Real Single-Phase Power during Motor Start and Utility Connected for Test 10.3.17

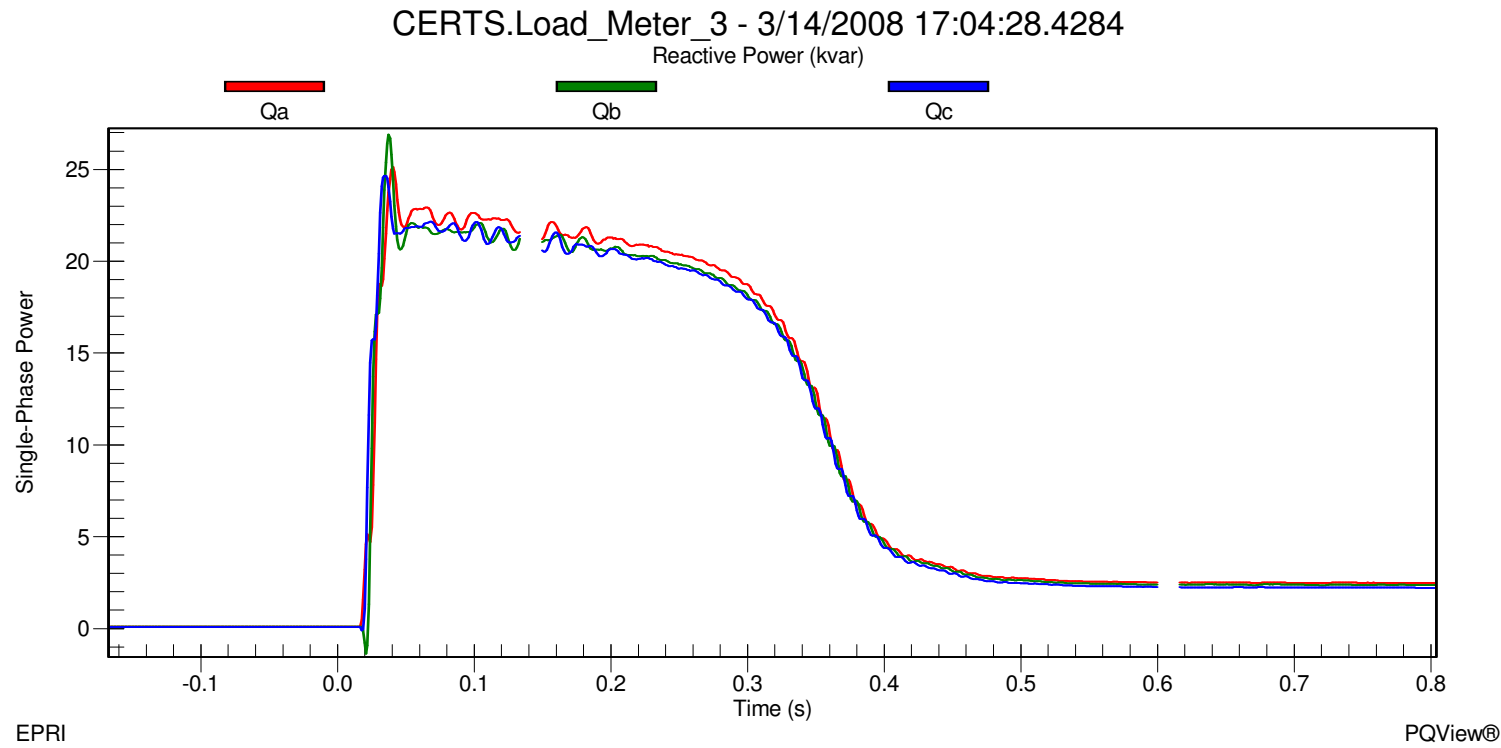

Figure 14b - Load Bank 3 Reactive Single-Phase Power during Motor Start and Utility Connected for Test 10.3.17

In Figures $14 \mathrm{a}$ and $14 \mathrm{~b}$ it can be seen that the load in Zone 3 was approximately $18.45 \mathrm{~kW}$ $(A=6.25 \mathrm{~kW} \mathrm{~B}=6.1 \mathrm{~kW} C=6.1 \mathrm{~kW})+\mathrm{j} 0.3 \mathrm{kVAr}(\mathrm{A}=0.1 \mathrm{kVAr} B=0.1 \mathrm{kVAr} C=0.1 \mathrm{kVAr})$ before the start of the induction motor and increased to approximately $54.7 \mathrm{~kW}(\mathrm{~A}=17.1 \mathrm{~kW}$ $\mathrm{B}=21.2 \mathrm{~kW} \mathrm{C}=16.4 \mathrm{~kW})+\mathrm{j} 75.8 \mathrm{kVAr}(\mathrm{A}=24.5 \mathrm{kVAr} \mathrm{B}=26.8 \mathrm{kVAr} \mathrm{C}=24.5 \mathrm{kVAr})$ during the inrush phase of the motor start. After about 1.5 cycles, the motor settled down to approximately $43.1 \mathrm{~kW}(\mathrm{~A}=14.6 \mathrm{~kW} B=14.25 \mathrm{~kW} \quad \mathrm{C}=14.25 \mathrm{~kW})+\mathrm{j} 63.5 \mathrm{kVAr}(\mathrm{A}=21.5 \mathrm{kVAr}$ $\mathrm{B}=21 \mathrm{kVAr} \mathrm{C}=21 \mathrm{kVAr})$ during the warm up phase which lasted about 30 cycles (0.5 
Seconds). When the motor reached steady state, the load in Zone 3 was approximately $26.95 \mathrm{~kW}(\mathrm{~A}=9.25 \mathrm{~kW} \mathrm{~B}=8.9 \mathrm{~kW} \mathrm{C}=8.8 \mathrm{~kW})+\mathrm{j} 7.1 \mathrm{kVAr}(\mathrm{A}=2.5 \mathrm{kVAr} \mathrm{B}=2.4 \mathrm{kVAr} \mathrm{C}=2.2 \mathrm{kVAr})$.

The voltage and frequency at the static switch before the motor start was approximately 283.6V on A-phase, 279.3V on B-phase and 279.9V on C-phase shown in Figure 14c and approximately $60.02 \mathrm{~Hz}$ shown in Figure $14 \mathrm{~d}$. When the motor started, the voltage at the static switch during the inrush decreased to approximately $275.6 \mathrm{~V}$ on A-phase, $271.2 \mathrm{~V}$ on B-phase and $271.4 \mathrm{~V}$ on C-phase for about 1.5 cycles. Frequency dropped during the inrush to approximately $59.92 \mathrm{~Hz}$ and quickly increased to $60.01 \mathrm{~Hz}$. Voltage increased as the motor was warming up and eventually settled at a steady state voltage at approximately $282.8 \mathrm{~V}$ on A-phase, $278.4 \mathrm{~V}$ on B-phase and $279.2 \mathrm{~V}$ on C-phase at an approximate frequency of $60.03 \mathrm{~Hz}$.

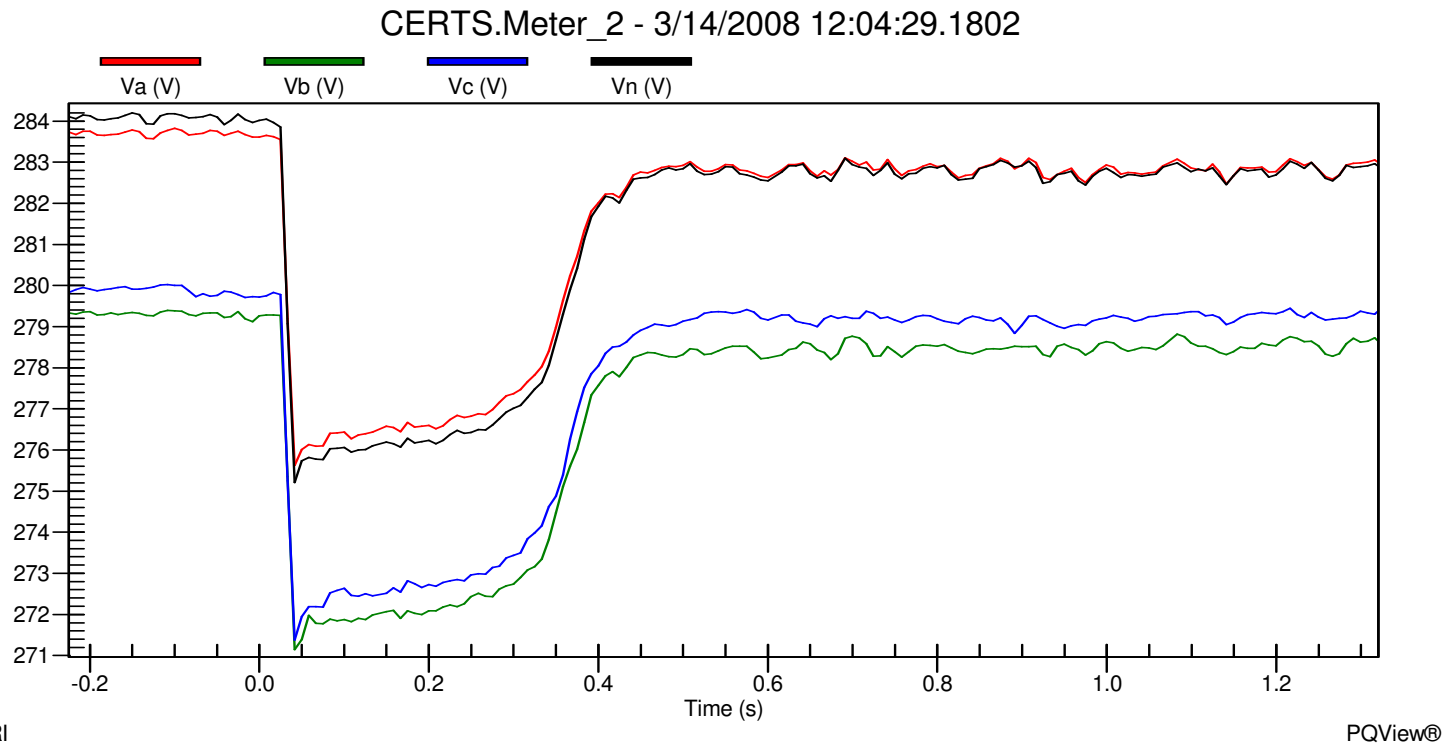

Figure 14c - Static Switch Line-to-Ground Voltages during Motor Start and Utility Connected for Test 10.3.17 


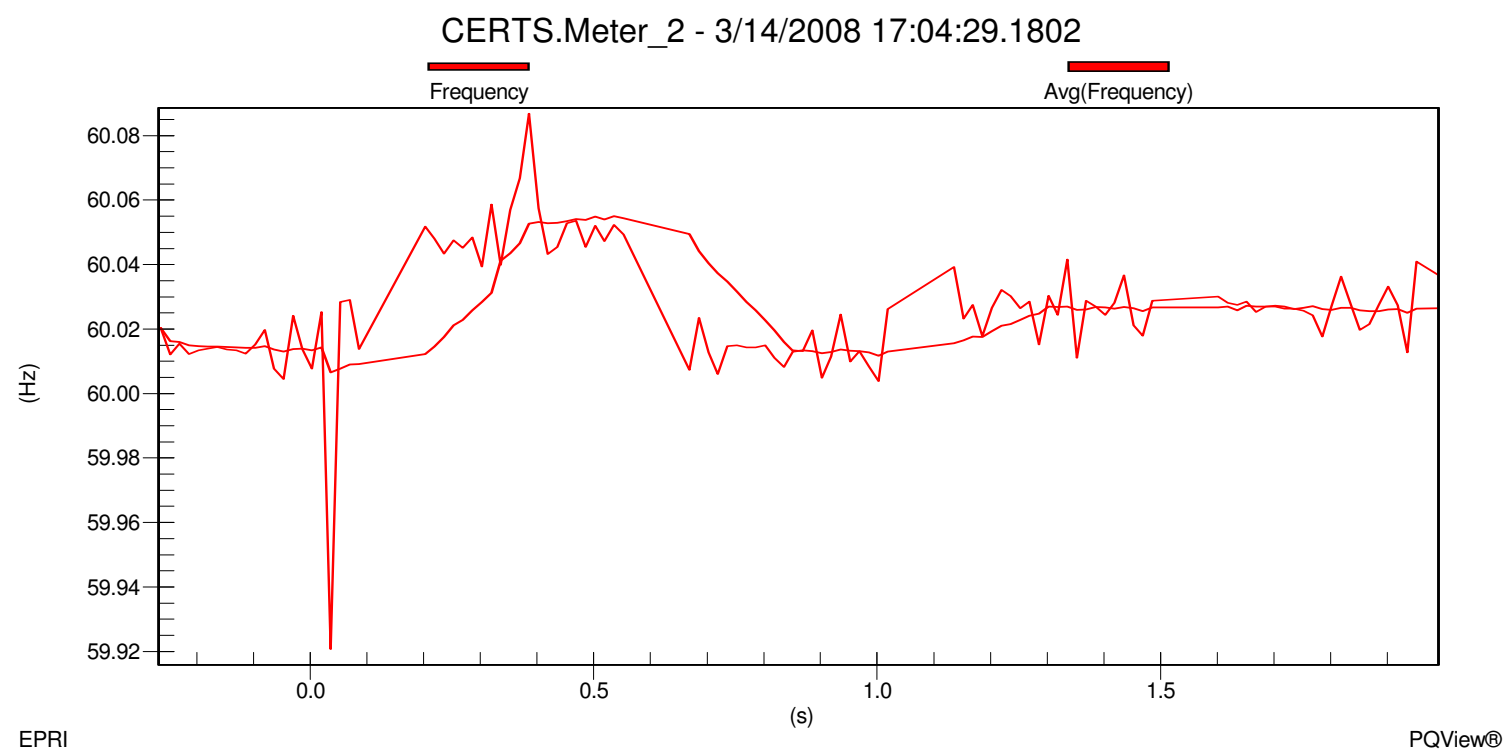

Figure 14d - Static Switch Frequency during Motor Start and Utility Connected for Test 10.3.17

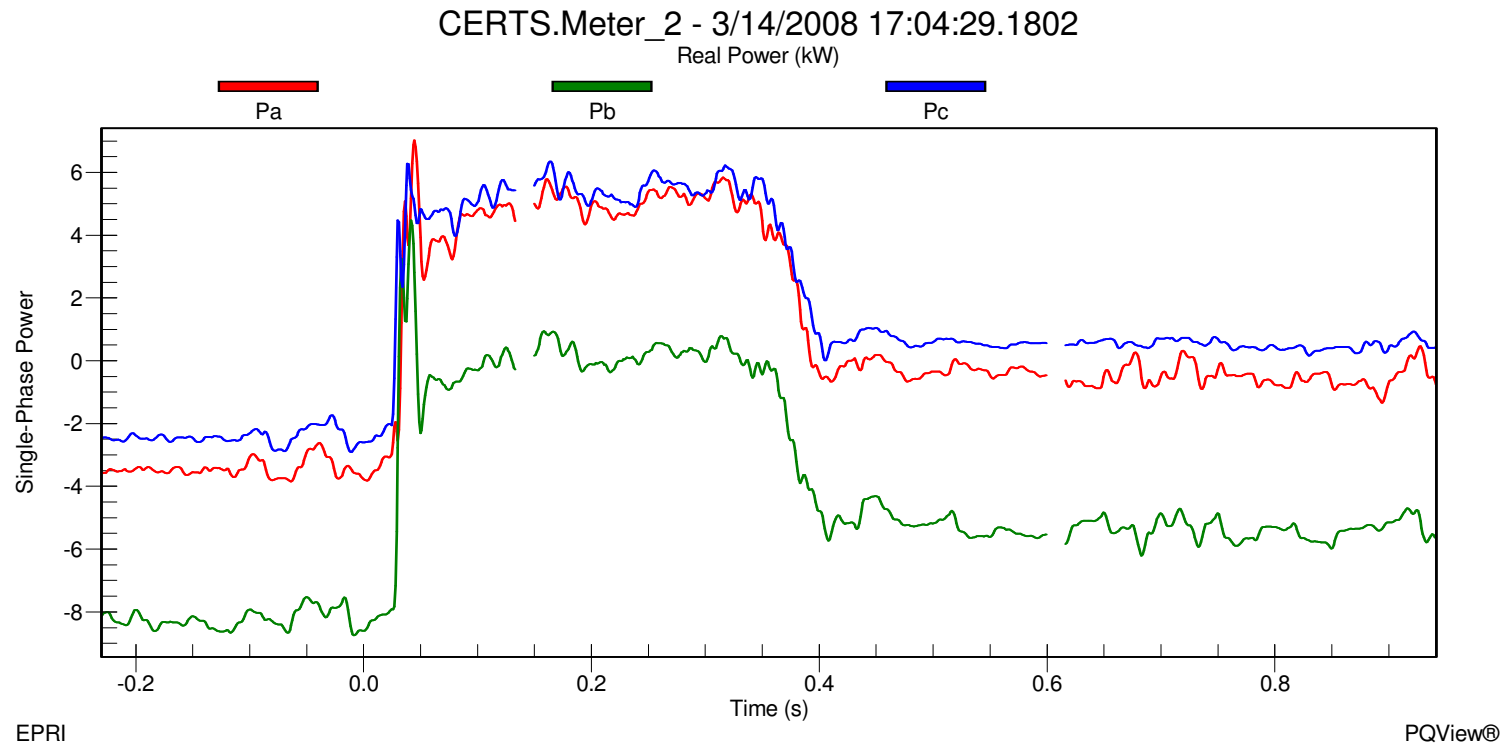

Figure 14e - Static Switch Real Single-Phase Power during Motor Start and Utility Connected for Test 10.3.17 


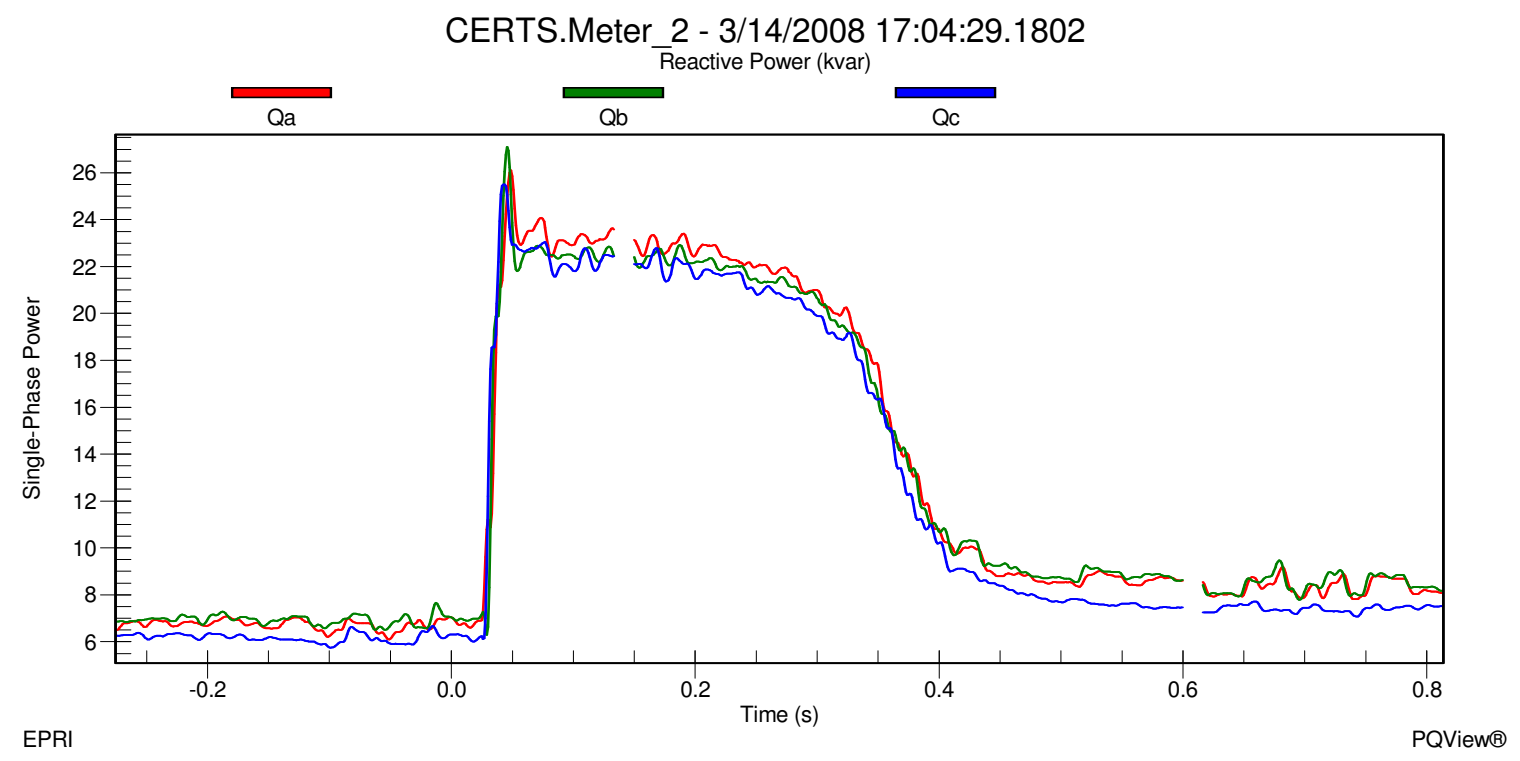

Figure 14f - Static Switch Reactive Single-Phase Power during Motor Start and Utility Connected for Test 10.3.17

Before the motor start Gen-set A1 was producing approximately $35.4 \mathrm{~kW}(\mathrm{~A}=10.7 \mathrm{~kW}$ $\mathrm{B}=15.6 \mathrm{~kW} \quad \mathrm{C}=9.1 \mathrm{~kW})-\mathrm{j} 13.6 \mathrm{kVAr}(\mathrm{A}=-4.9 \mathrm{kVAr} \mathrm{B}=-4.8 \mathrm{kVAr} \mathrm{C}=-3.9 \mathrm{kVAr})$. This was enough for the load demands of Zone 3 with real power being exported to Zone 6 of approximately $14.25 \mathrm{~kW}(\mathrm{~A}=3.5 \mathrm{~kW} B=8.25 \mathrm{~kW} \mathrm{C}=2.5 \mathrm{~kW})$ shown in Figure $14 \mathrm{e}$. The grid was supporting the reactive power of the microgrid with approximately $\mathrm{j} 19.7 \mathrm{kVAr}$ ( $A=6.8 \mathrm{kVAr} B=6.9 \mathrm{kVAr} \mathrm{C}=6 \mathrm{kVAr}$ ) shown in Figure 14f. When the motor started the inrush caused the utility to pick up the load demand in Load Bank 6 along with approximately $\quad 16.8 \mathrm{~kW} \quad(\mathrm{~A}=7.1 \mathrm{~kW} \quad \mathrm{~B}=4.5 \mathrm{~kW} \quad \mathrm{C}=5.2 \mathrm{~kW})+j 78 \mathrm{kVAr} \quad(\mathrm{A}=25.4 \mathrm{kVAr}$ $\mathrm{B}=27.2 \mathrm{kVAr} \mathrm{C}=25.4 \mathrm{kVAr}$ ) of the microgrid critical loads. The power being supplied by Gen-set A1 to Load Bank 6 was now supplying the motor inrush which can be seen in Figure $14 \mathrm{e}$ as the real power through the static switch becomes positive. Gen-set A1 increased its real and reactive power output levels to approximately $41.7 \mathrm{~kW}(\mathrm{~A}=17.9 \mathrm{~kW}$ $B=12.9 \mathrm{~kW} C=10.9 \mathrm{~kW})+\mathrm{j} 3.5 \mathrm{kVAr}(\mathrm{A}=0.75 \mathrm{kVAr} B=1 \mathrm{kVAr} C=1.75 \mathrm{kVAr})$. Notice the sign of the VAr output changed from negative to positive for Gen-set A1 in order to support the induction motor load in Zone 3.

When the motor reached steady state, the real and reactive power through the static switch was approximately $-7 \mathrm{~kW} \quad(\mathrm{~A}=-0.6 \mathrm{~kW} \quad \mathrm{~B}=-5.5 \mathrm{~kW} \quad \mathrm{C}=-0.9 \mathrm{~kW})+\mathrm{j} 24.2 \mathrm{kVAr}$ $(\mathrm{A}=8.4 \mathrm{kVAr} \mathrm{B}=8.4 \mathrm{kVAr} \mathrm{C}=7.4 \mathrm{kVAr})$ which meant that Gen-set $\mathrm{A} 1$ was supplying the power for the induction motor and approximately $7 \mathrm{~kW}(\mathrm{~A}=0.6 \mathrm{~kW} \mathrm{~B}=5.5 \mathrm{~kW} \mathrm{C}=0.9 \mathrm{~kW})$ of Load Bank 6. Gen-set A1 real power returned to the value before the motor started of approximately $35.1 \mathrm{~kW} \quad(\mathrm{~A}=10.7 \mathrm{~kW} \quad \mathrm{~B}=15.3 \mathrm{~kW} \quad \mathrm{C}=9.1 \mathrm{~kW})$ and the reactive power increased to approximately $-\mathrm{j} 11.9 \mathrm{kVAr}(\mathrm{A}=-4.4 \mathrm{kVAr} \mathrm{B}=-4.2 \mathrm{kVAr} \mathrm{C}=-3.3 \mathrm{kVAr})$. Once all 
the data was verified and recorded into the DAS Database, the motor was shut down and the static switch was directed by the EMS to manually open.

As soon as the static switch opened, Meter 1 recorded real power increased to approximately $38.4 \mathrm{~kW}(\mathrm{~A}=10.2 \mathrm{~kW} \mathrm{~B}=18.3 \mathrm{~kW} \mathrm{C}=9.9 \mathrm{~kW})$ and reactive power decreased to j1.1kVAr $(A=0.25 \mathrm{kVAr} B=0.6 \mathrm{kVAr} C=0.25 \mathrm{kVAr})$ satisfying the load demand in Load Bank 6 which was approximately $38.1 \mathrm{~kW}(\mathrm{~A}=10.1 \mathrm{~kW} \mathrm{~B}=18.2 \mathrm{~kW} \mathrm{C}=9.8 \mathrm{~kW})+\mathrm{j} 0.617 \mathrm{kVAr}$ $(\mathrm{A}=0.117 \mathrm{kVAr} \mathrm{B}=0.381 \mathrm{kVAr} \mathrm{C}=0.119 \mathrm{kVAr})$ and not supplying any power beyond the static switch to Load Bank 3. 0kW (A=0kW B=0kW C=0kW) + j0kVAr (A=0kVAr $\mathrm{B}=0 \mathrm{kVAr} \mathrm{C}=0 \mathrm{kVAr}$ ) was recorded at the static switch, indicating that power was not flowing through the static switch. Load Bank 3 loads was slightly reduced to $17.065 \mathrm{~kW}$ $(A=5.675 \mathrm{~kW} \quad \mathrm{~B}=5.71 \mathrm{~kW} \quad \mathrm{C}=5.68 \mathrm{~kW})+j 0.294 \mathrm{kVAr} \quad(\mathrm{A}=0.095 \mathrm{kVAr} \quad \mathrm{B}=0.112 \mathrm{kVAr}$ $\mathrm{C}=0.087 \mathrm{kVAr})$. This load reduction resulted from a voltage drop in the microgrid, shown in Figure 14g, from approximately $284.3 \mathrm{~V}$ on A-phase, $280.1 \mathrm{~V}$ on B-phase and $280.5 \mathrm{~V}$ on C-phase when connected to the utility grid to $272.2 \mathrm{~V}$ on A-phase, $271.9 \mathrm{~V}$ on Bphase and 271.7V on C-phase at Meter 3 when islanded.

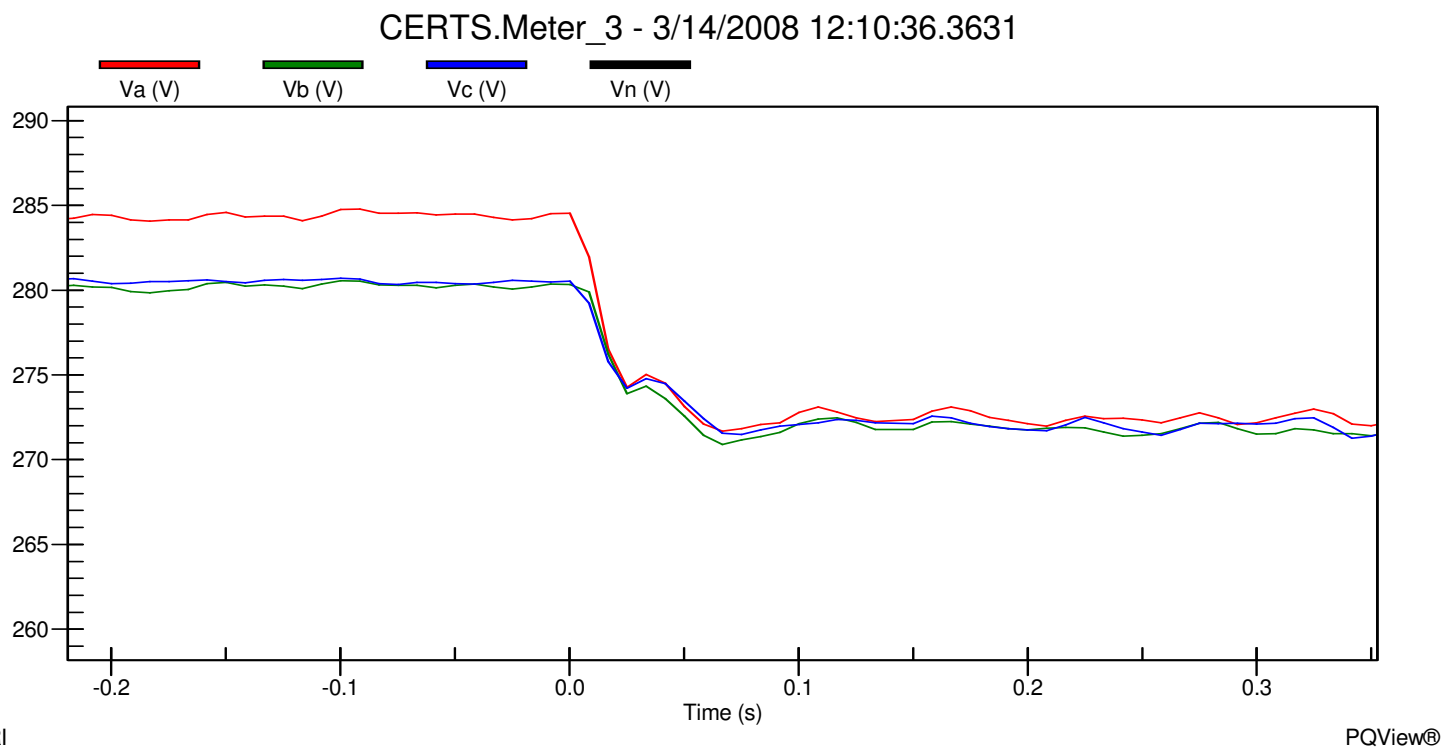

Figure 14g - Meter 3 Line-to-Ground Voltages during Utility Connected to Island mode for Test 10.3.17 


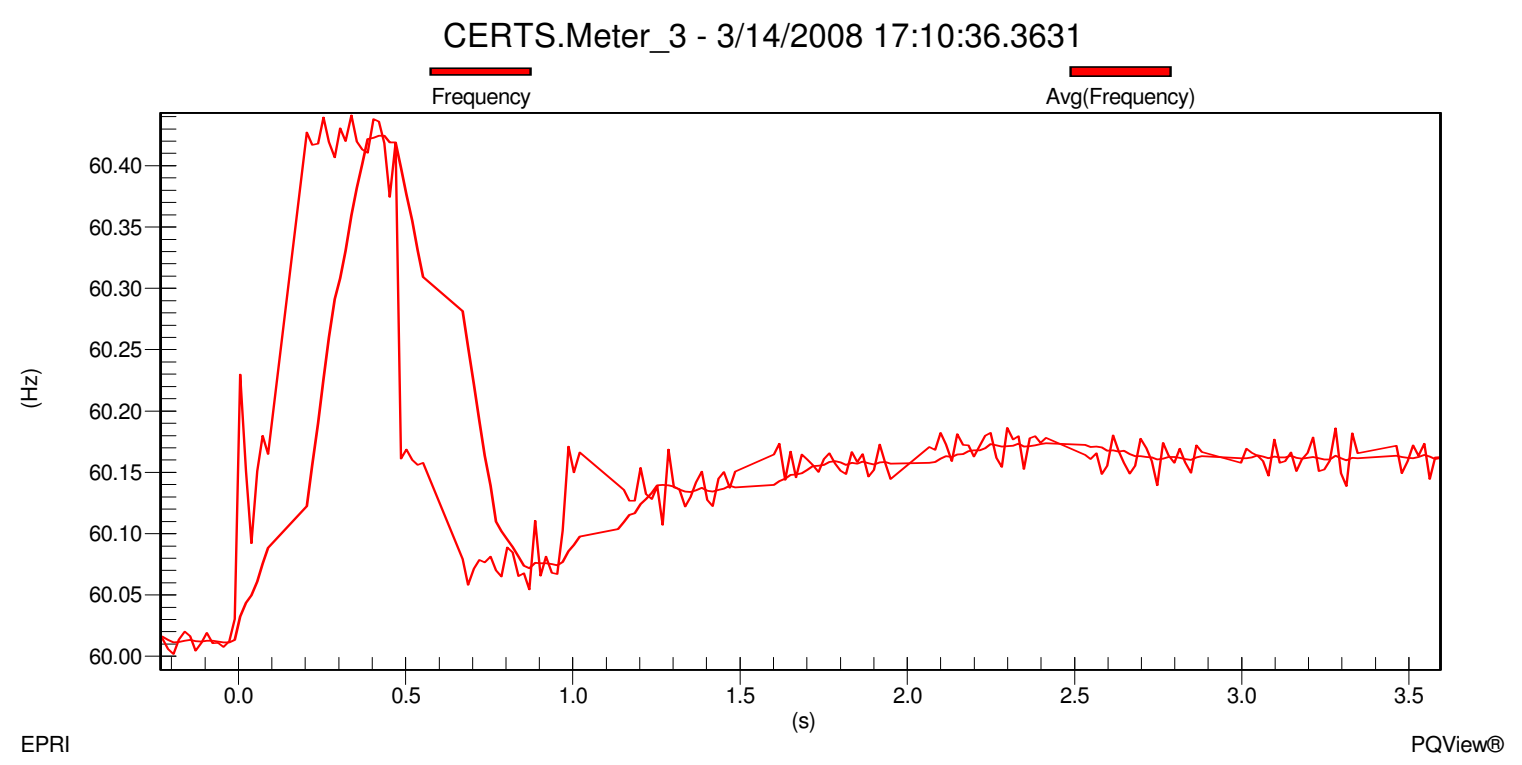

Figure 14h - Meter 3 Frequency during Utility Connected to Island mode for Test 10.3.17

Frequency change in the microgrid, shown in Figure 14h, increased from approximately $60.01 \mathrm{~Hz}$ when connected to the utility grid to approximately $60.16 \mathrm{~Hz}$ when islanded. This change in frequency was part of the CERTS algorithm which allowed Gen-set A1 to decrease its output power to satisfy the load demands. Gen-set A1 decreased its output power to approximately $19.4 \mathrm{~kW}(\mathrm{~A}=6.4 \mathrm{~kW} \mathrm{~B}=6.8 \mathrm{~kW} \mathrm{C}=6.2 \mathrm{~kW})$ and increased its output reactive power to approximately $\mathrm{j} 4 \mathrm{kVAr}(\mathrm{A}=1 \mathrm{kVAr} \mathrm{B}=1.5 \mathrm{kVAr} \mathrm{C}=1.5 \mathrm{kVAr})$. Meter 3 was approximately $-2.45 \mathrm{~kW}(\mathrm{~A}=-0.75 \mathrm{~kW} B=-1.2 \mathrm{~kW} C=-0.5 \mathrm{~kW})-\mathrm{j} 3.4 \mathrm{kVAr}(\mathrm{A}=-0.8 \mathrm{kVAr}$ $\mathrm{B}=-1.3 \mathrm{kVAr} \mathrm{C}=-1.3 \mathrm{kVAr}$ ) indicating that Gen-set A1 was satisfying the load in Load Bank 3 and the power losses in the electrical lines. All data was verified and recorded into the DAS Database. The microgrid ran for a couple of minutes in this electrical state before the $10 \mathrm{Hp}$ induction motor was started in Zone 3. 


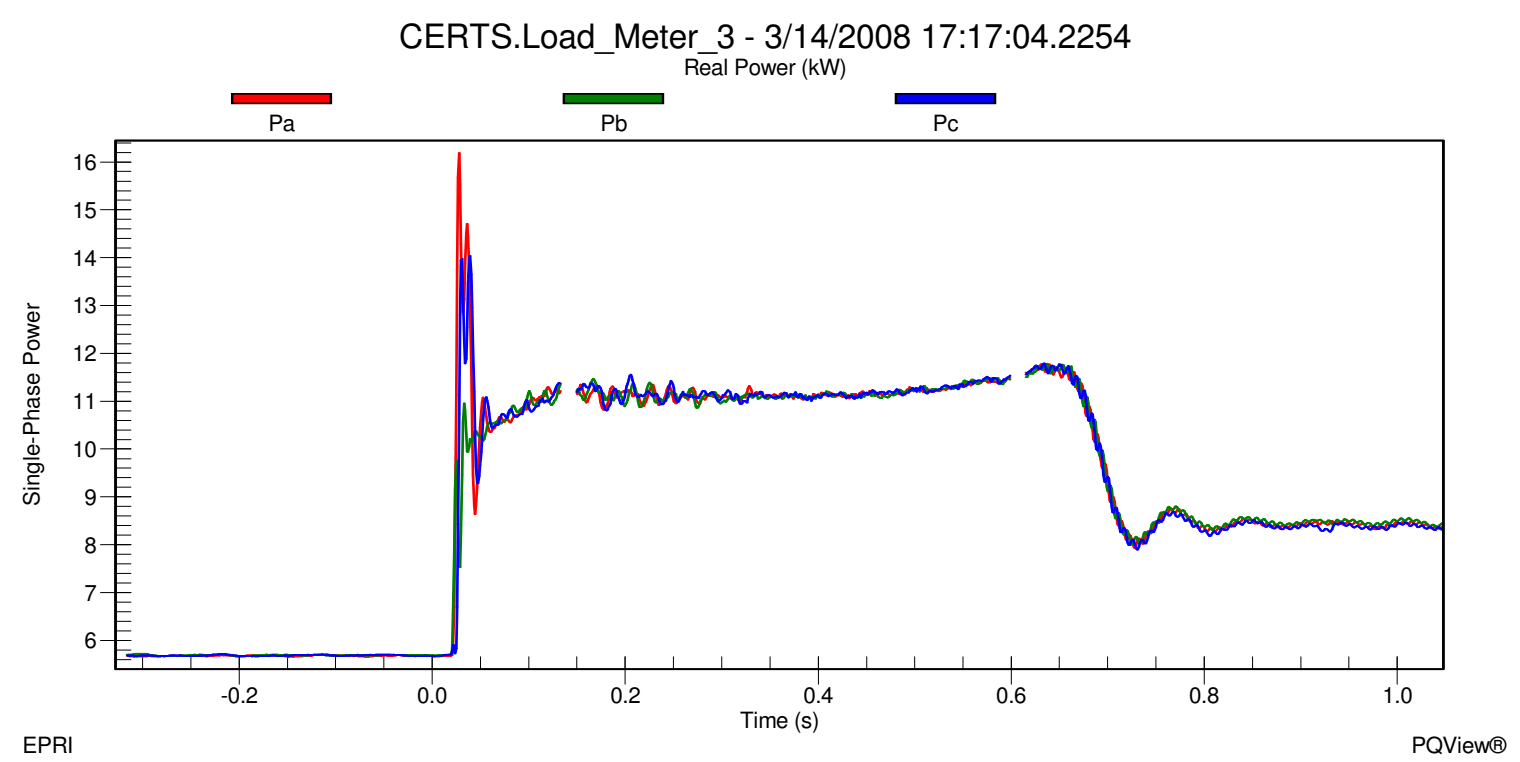

Figure 14i - Load Bank3 Real Single-Phase Power during Motor Start and Islanded for Test 10.3.17

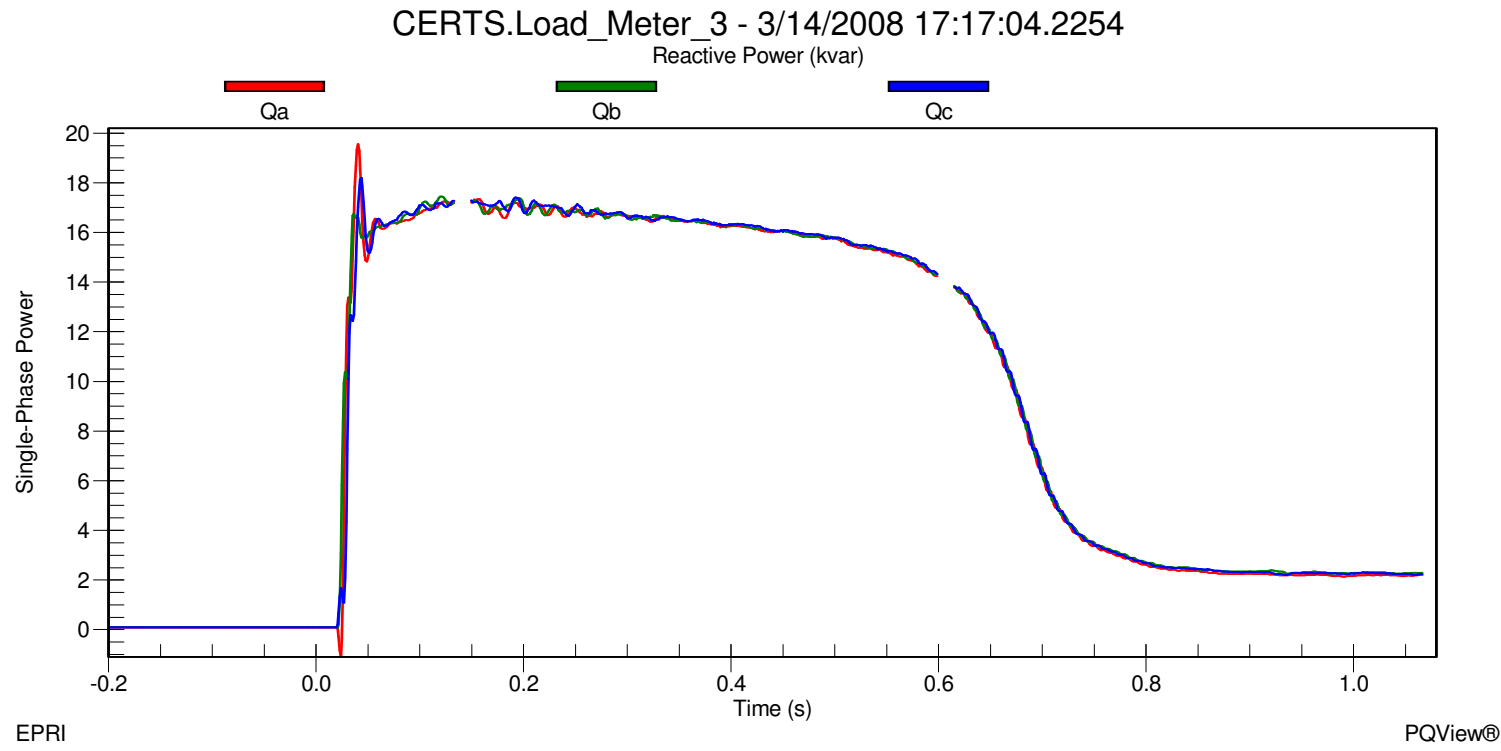

Figure 14j - Load Bank 3 Reactive Single-Phase Power during Motor Start and Islanded for Test 10.3.17

If Figures $14 \mathrm{i}$ and $14 \mathrm{j}$ it can be seen that the load in Zone 3 was approximately $17.1 \mathrm{~kW}$ $(\mathrm{A}=5.7 \mathrm{~kW} \mathrm{~B}=5.7 \mathrm{~kW} \mathrm{C}=5.7 \mathrm{~kW})+\mathrm{j} 0.6 \mathrm{kVAr}(\mathrm{A}=0.2 \mathrm{kVAr} \mathrm{B}=0.2 \mathrm{kVAr} \mathrm{C}=0.2 \mathrm{kVAr})$ before the start of the induction motor and increased to approximately $40.7 \mathrm{~kW}(\mathrm{~A}=16.2 \mathrm{~kW}$ $\mathrm{B}=10.5 \mathrm{~kW} \mathrm{C}=14 \mathrm{~kW})+\mathrm{j} 53.95 \mathrm{kVAr}(\mathrm{A}=19.6 \mathrm{kVAr} \mathrm{B}=16.6 \mathrm{kVAr} \mathrm{C}=17.75 \mathrm{kVAr})$ during the inrush phase of the motor start. After about 1.5 cycles, the motor settled down to approximately $33.9 \mathrm{~kW}(\mathrm{~A}=11.3 \mathrm{~kW} \quad \mathrm{~B}=11.3 \mathrm{~kW} \quad \mathrm{C}=11.3 \mathrm{~kW})+\mathrm{j} 49.5 \mathrm{kVAr} \quad(\mathrm{A}=16.5 \mathrm{kVAr}$ $\mathrm{B}=16.5 \mathrm{kVAr} \mathrm{C}=16.5 \mathrm{kVAr})$ during the warm up phase which lasted about 49.2 cycles $(0.82$ Seconds). When the motor reached steady state the load in Zone 3 was approximately 
$25.2 \mathrm{~kW} \quad(\mathrm{~A}=8.4 \mathrm{~kW} \quad \mathrm{~B}=8.4 \mathrm{~kW} \quad \mathrm{C}=8.4 \mathrm{~kW})+\mathrm{j} 6.75 \mathrm{kVAr} \quad(\mathrm{A}=2.25 \mathrm{kVAr} \quad \mathrm{B}=2.25 \mathrm{kVAr}$ $\mathrm{C}=2.25 \mathrm{kVAr})$.

The voltage and frequency at Meter 3 before the motor start was approximately $272 \mathrm{~V}$ on A-phase, $271.4 \mathrm{~V}$ on B-phase and $272 \mathrm{~V}$ on C-phase shown in Figure $14 \mathrm{k}$ and approximately $60.16 \mathrm{~Hz}$ shown in Figure 141 . When the motor started, the voltage at Meter 3 during the inrush decreased to approximately $235.7 \mathrm{~V}$ on A-phase, 235.7V on Bphase and $237.1 \mathrm{~V}$ on $\mathrm{C}$-phase for about 1.5 cycles. Frequency dropped during the inrush to approximately $59.76 \mathrm{~Hz}$ and quickly increased to approximately $60.02 \mathrm{~Hz}$. Voltage increased as the motor was warming up and eventually settled at a steady state voltage at approximately $269.2 \mathrm{~V}$ on A-phase, $268.5 \mathrm{~V}$ on B-phase and $268.9 \mathrm{~V}$ on C-phase at an approximate frequency of $60.10 \mathrm{~Hz}$.

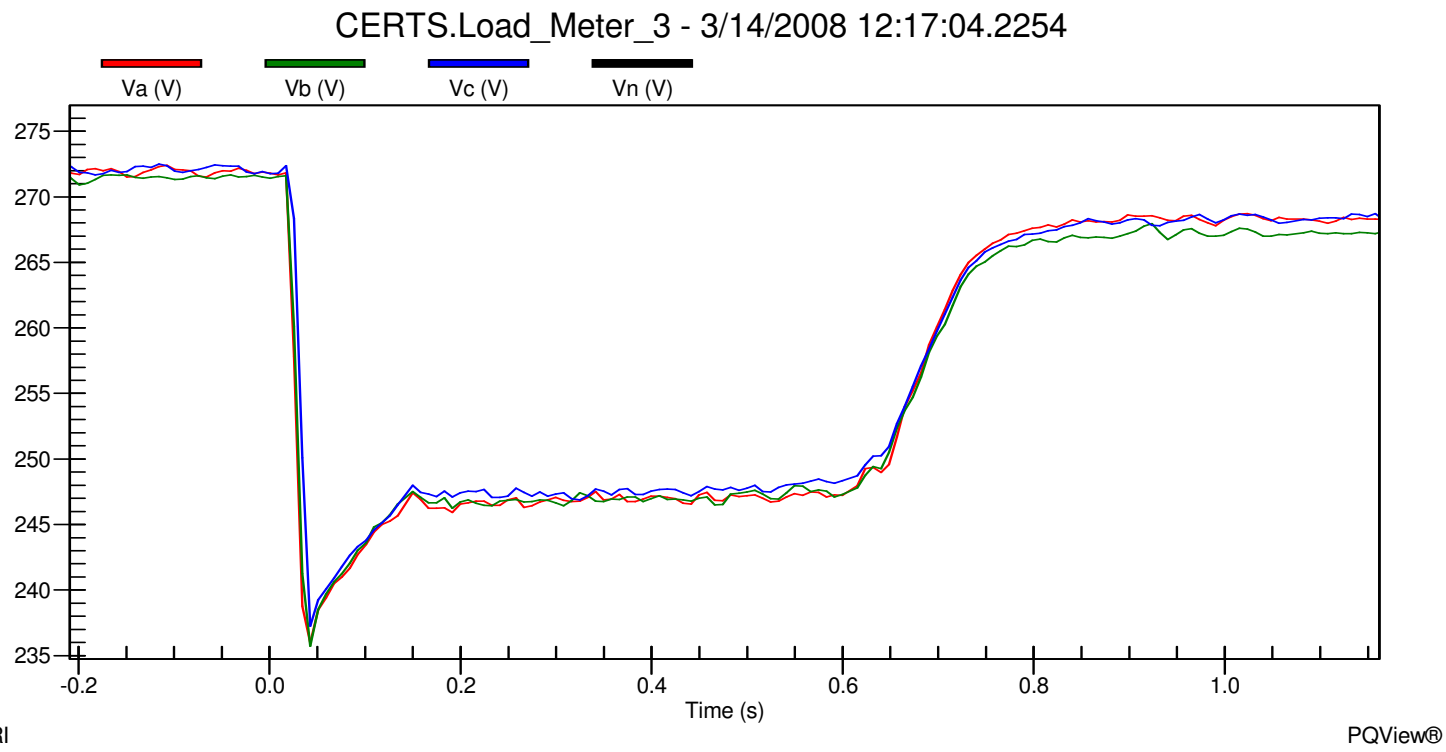

Figure 14k - Meter 3 Line-to-Ground Voltages during Motor Start and Islanded for Test 10.3.17 


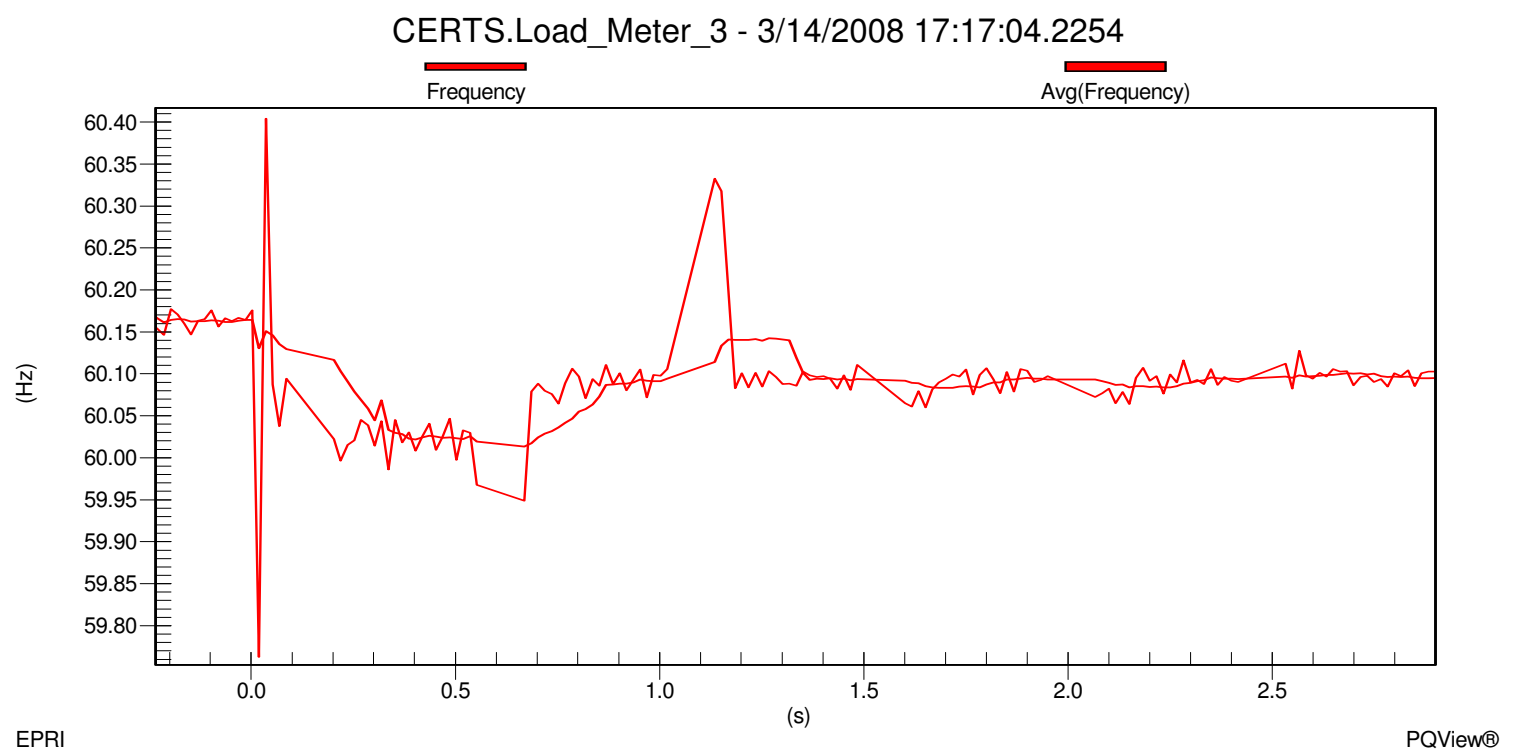

Figure 141 - Meter 3 Frequency during Motor Start and Islanded for Test 10.3.17

Before the motor started, Gen-set A1 was producing approximately $19.3 \mathrm{~kW}(\mathrm{~A}=6.4 \mathrm{~kW}$ $B=6.8 \mathrm{~kW} C=6.1 \mathrm{~kW})+\mathrm{j} 4 \mathrm{kVAr}(\mathrm{A}=1 \mathrm{kVAr} B=1.5 \mathrm{kVAr} C=1.5 \mathrm{kVAr})$ shown in Figures $14 \mathrm{~m}$ and $14 \mathrm{n}$. The power generated by Gen-set A1 was satisfying the loads in Load Bank 3 and all the electrical losses in the microgrid system. When the motor started the inrush caused the Gen-set to increase its output level to $41.6 \mathrm{~kW}(\mathrm{~A}=16.7 \mathrm{~kW} \quad \mathrm{~B}=10.6 \mathrm{~kW}$ $\mathrm{C}=14.3 \mathrm{~kW})+\mathrm{j} 55.05 \mathrm{kVAr}(\mathrm{A}=19.75 \mathrm{kVAr} \mathrm{B}=17.3 \mathrm{kVAr} \mathrm{C}=18 \mathrm{kVAr})$. Gen-set A1 decreased its output level when the motor was warming up and eventually dropped to $27.5 \mathrm{~kW}$ $(\mathrm{A}=9.2 \mathrm{~kW} B=9.5 \mathrm{~kW} \mathrm{C}=8.8 \mathrm{~kW})+\mathrm{j} 9.9 \mathrm{kVAr}(\mathrm{A}=2.9 \mathrm{kVAr} \mathrm{B}=3.5 \mathrm{kVAr} \mathrm{C}=3.5 \mathrm{kVAr})$ when the motor reached steady state. Once all the data was verified and recorded into the DAS Database, the static switch was directed by the EMS to manually close.

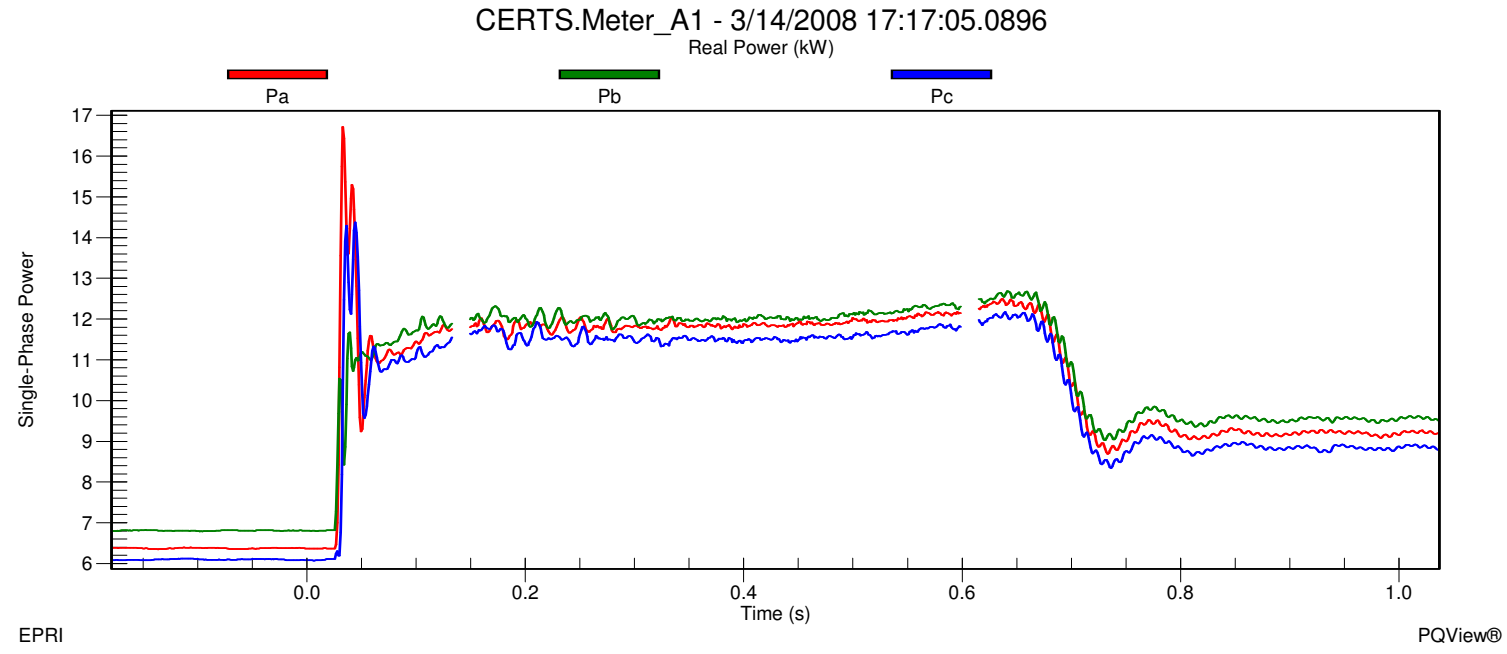

Figure 14m - Gen-set A1 Real Single-Phase Power during Motor Start and Islanded for Test 10.3.17 


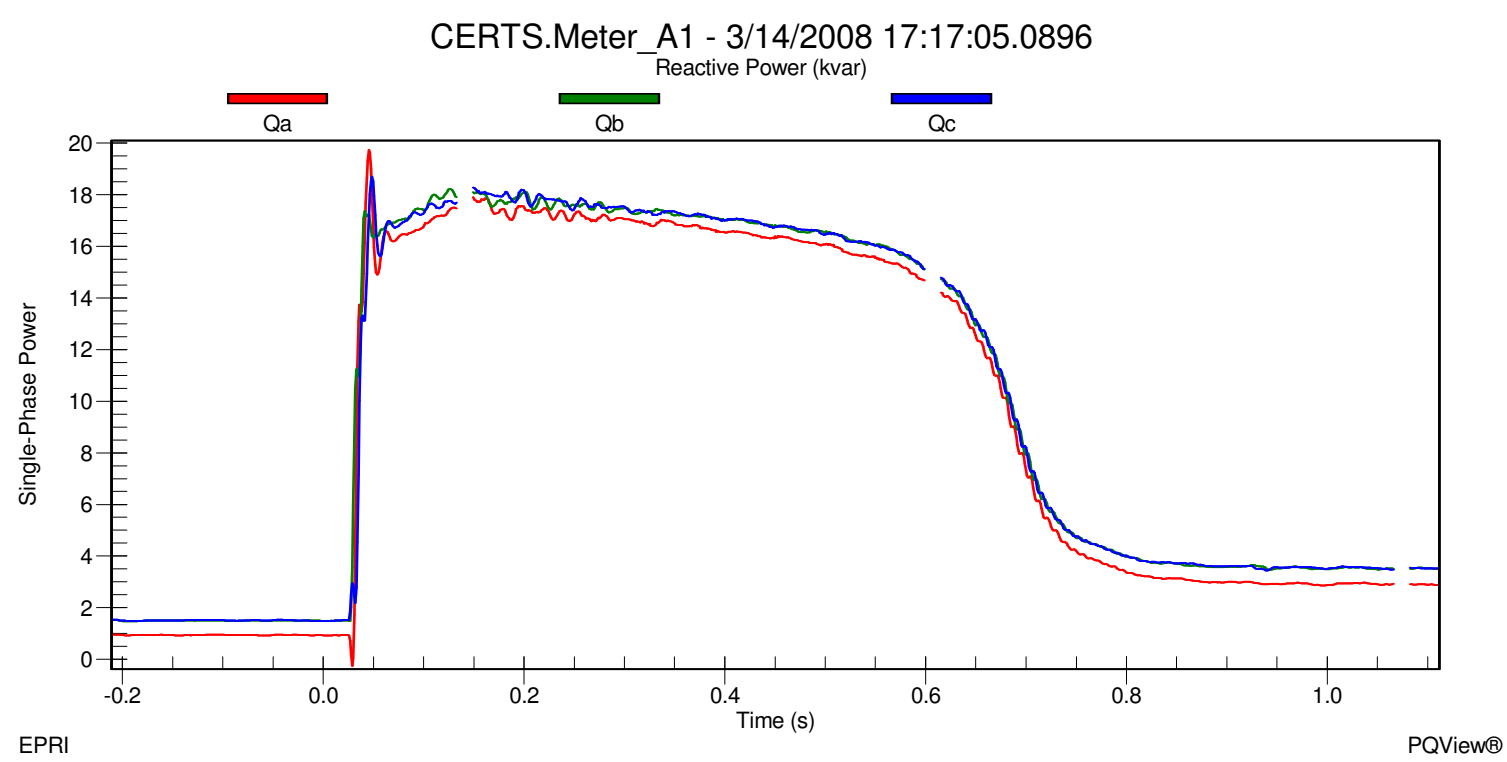

Figure 14n - Gen-set A1 Reactive Single-Phase Power during Motor Start and Islanded for Test 10.3.17

As soon as the static switch closed, Meter 1 recorded real power decreased from approximately $38.1 \mathrm{~kW} \quad(\mathrm{~A}=10.2 \mathrm{~kW} \quad \mathrm{~B}=18.1 \mathrm{~kW} \quad \mathrm{C}=9.8 \mathrm{~kW})$ to $28.65 \mathrm{~kW} \quad(\mathrm{~A}=8.4 \mathrm{~kW}$ $B=11.25 \mathrm{~kW} \quad C=9 \mathrm{~kW})$ and reactive power increased from approximately $j 1.1 \mathrm{kVAr}$ $(\mathrm{A}=0.25 \mathrm{kVAr} \quad \mathrm{B}=0.6 \mathrm{kVAr} \quad \mathrm{C}=0.25 \mathrm{kVAr}) \quad$ to $\mathrm{j} 24.3 \mathrm{kVAr} \quad(\mathrm{A}=8.25 \mathrm{kVAr} \quad \mathrm{B}=8.75 \mathrm{kVAr}$ $\mathrm{C}=7.3 \mathrm{kVAr}$ ) which means that the utility was satisfying a portion of the load demand in Load Bank 6 and all the reactive power in the microgrid. Figures 14o and 14p show the static switch real power decreasing from approximately $0 \mathrm{~kW}(\mathrm{~A}=0 \mathrm{~kW} \mathrm{~B}=0 \mathrm{~kW} \mathrm{C}=0 \mathrm{~kW})$ to $-8.1 \mathrm{~kW} \quad(\mathrm{~A}=-1.5 \mathrm{~kW} \quad \mathrm{~B}=-6.2 \mathrm{~kW} \quad \mathrm{C}=-0.4 \mathrm{~kW})$ and reactive power increasing from approximately j0kVAr $(A=0 \mathrm{kVAr} \quad \mathrm{B}=0 \mathrm{kVAr} \quad \mathrm{C}=0 \mathrm{kVAr})$ to $\mathrm{j} 23.4 \mathrm{kVAr} \quad(\mathrm{A}=8.1 \mathrm{kVAr}$ $\mathrm{B}=8.2 \mathrm{kVAr} \mathrm{C}=7.1 \mathrm{kVAr})$. At the beginning of the test, the initial power flow through the static switch was $-14.25 \mathrm{~kW}(\mathrm{~A}=-3.5 \mathrm{~kW} \mathrm{~B}=-8.25 \mathrm{~kW} C=-2.5 \mathrm{~kW})+j 19.7 \mathrm{kVAr}(\mathrm{A}=6.8 \mathrm{kVAr}$ $\mathrm{B}=6.9 \mathrm{kVAr} \mathrm{C}=6 \mathrm{kVAr}$ ) which is not the same recorded at this point in the test because the $10 \mathrm{Hp}$ motor load is on in Zone 3. Gen-set A1 has picked up the motor load and is supporting Load Bank 6 with approximately $8.1 \mathrm{~kW}(\mathrm{~A}=1.5 \mathrm{~kW} \mathrm{~B}=6.2 \mathrm{~kW} \mathrm{C}=0.4 \mathrm{~kW})$.

Load Bank 3 loads increased slightly to $25.1 \mathrm{~kW}(\mathrm{~A}=8.58 \mathrm{~kW} \mathrm{~B}=8.22 \mathrm{~kW} \mathrm{C}=8.3 \mathrm{~kW})+$ $6.485 \mathrm{kVAr}(\mathrm{A}=2.275 \mathrm{kVAr} \mathrm{B}=2.18 \mathrm{kVAr} \mathrm{C}=2.03 \mathrm{kVAr})$. This slight increase is a result from a voltage rise in the microgrid, shown in Figure 14q, from approximately $268.9 \mathrm{~V}$ on Aphase, $268.5 \mathrm{~V}$ on B-phase and $268.9 \mathrm{~V}$ on C-phase 3 when islanded to $282.5 \mathrm{~V}$ on A-phase, $279.1 \mathrm{~V}$ on B-phase and $278.8 \mathrm{~V}$ on C-phase at Meter 3 when connected to the utility grid. 


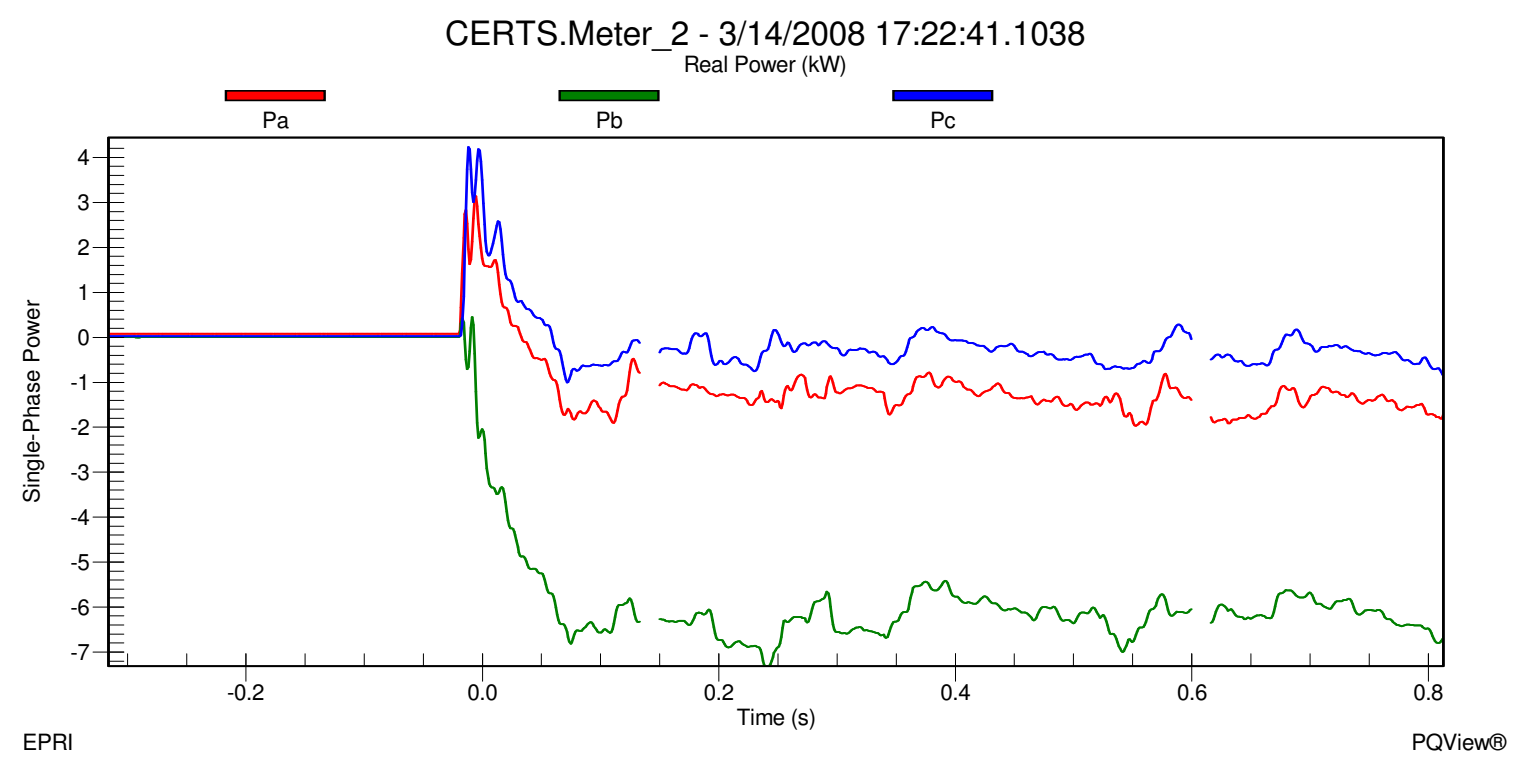

Figure 14o - Static Switch Real Single-Phase Power during Island to Utility Connected mode for Test 10.3.17

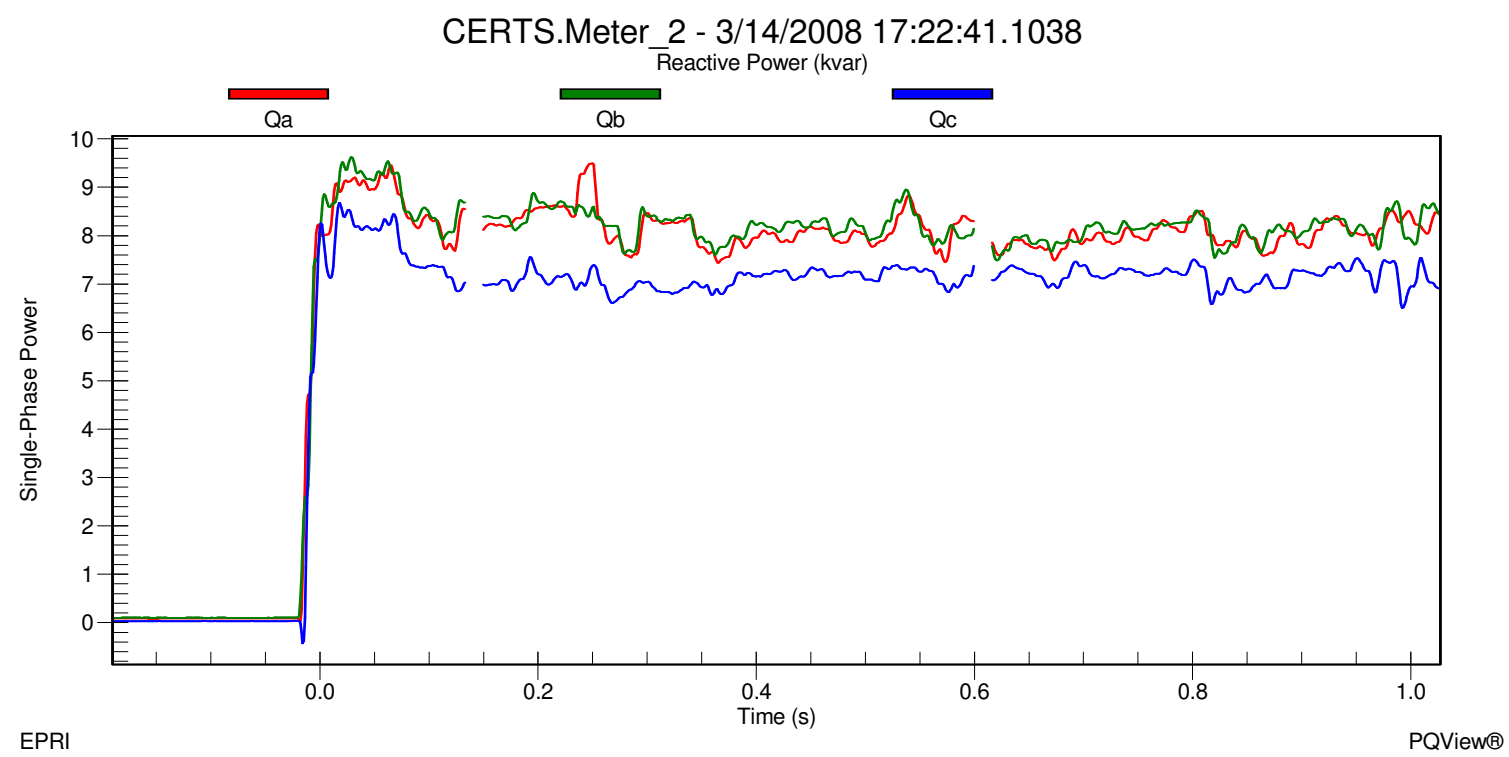

Figure 14p - Static Switch Reactive Single-Phase Power during Islanded to Utility Connected mode for Test 10.3.17 


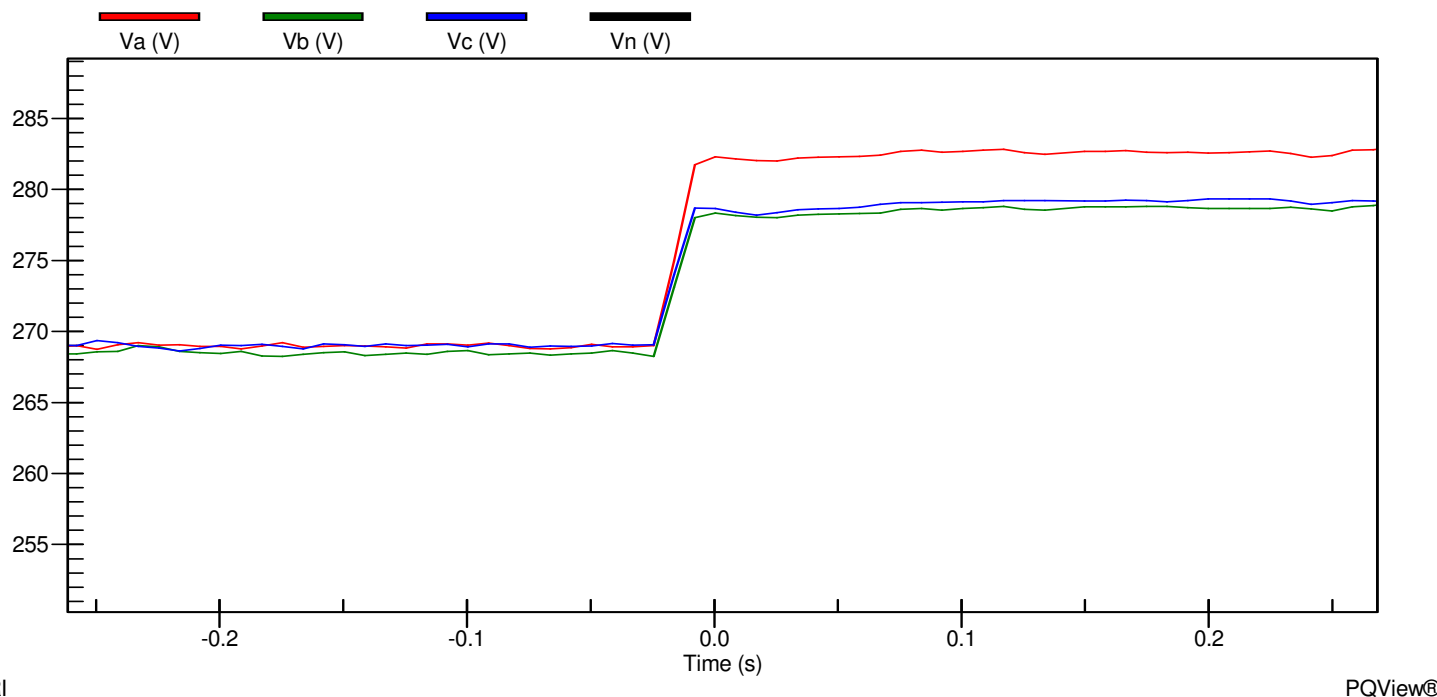

Figure 14q - Meter 3 Line-to-Ground Voltages during Islanded to Utility Connected mode for Test 10.3.17

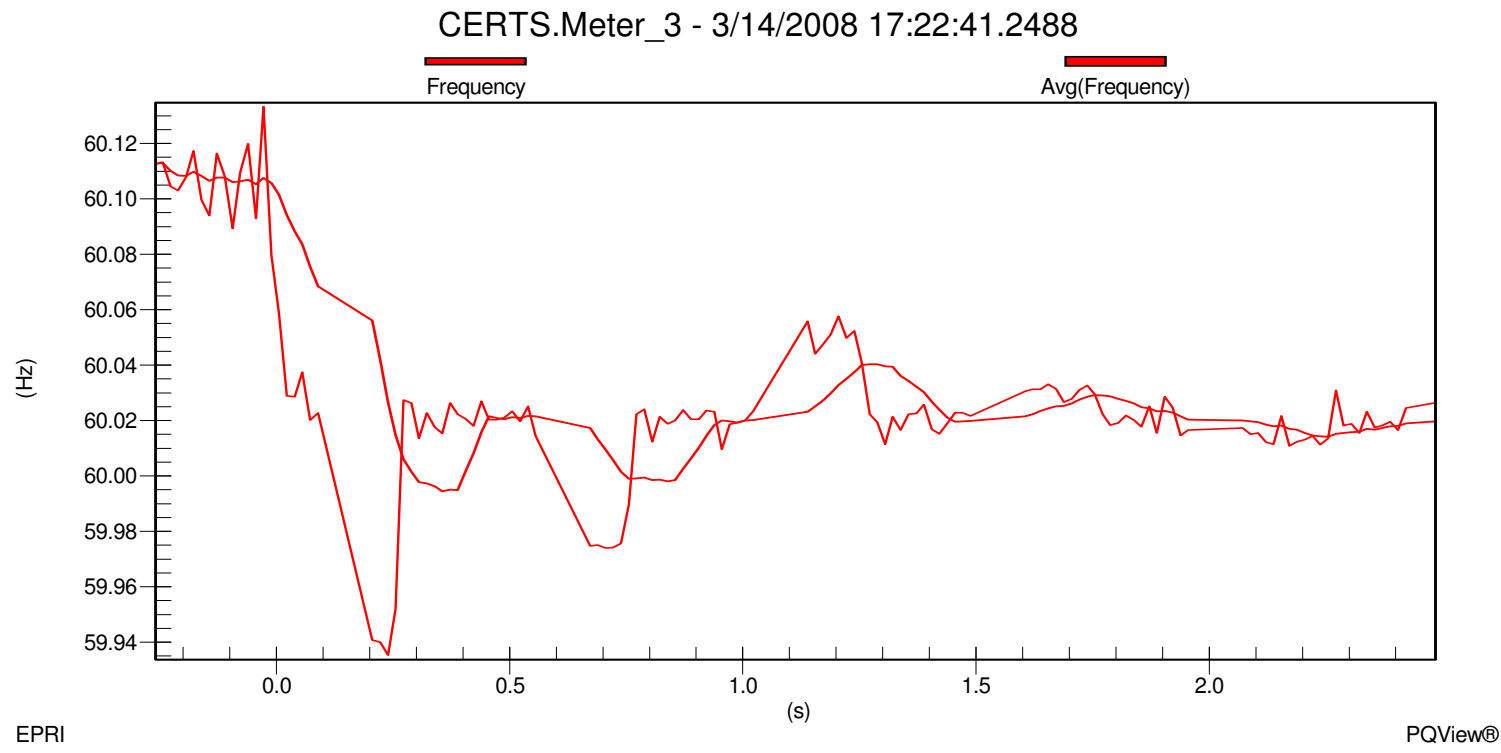

Figure 14r - Meter 3 Frequency during Islanded to Utility Connected mode for Test 10.3.17

Frequency change in the microgrid, shown in Figure 14r, decreased from approximately $60.11 \mathrm{~Hz}$ when islanded to approximately $60.02 \mathrm{~Hz}$ when connected to the utility grid. This change in frequency is due to the frequency no longer being established by Gen-set A1 using the CERTS algorithm by the utility. Gen-set A1 is in unit power control mode therefore when the static switch closed back into the utility the Gen-set produced real power based on the set-point initialized at the beginning of the test. The output power for Gen-set A1 was relatively close to the value at the beginning of the test with Gen-set A1 producing approximately $36 \mathrm{~kW}(\mathrm{~A}=10.9 \mathrm{~kW} B=15.7 \mathrm{~kW} \mathrm{C}=9.4 \mathrm{~kW})-\mathrm{j} 11.8 \mathrm{kVAr}(\mathrm{A}=-$ 
4.4kVAr $B=-4.2 \mathrm{kVAr} C=-3.2 \mathrm{kVAr})$. After all the data was verified and recorded into the DAS Database, the motor was turned off and the Gen-sets and Load Banks set-points were changed according to the next test (10.3.18) in Table 2.

For Test 10.3.18 the measured values, after Gen-set A1 was warmed up and load banks brought on-line are provided in Table 7.

\begin{tabular}{|c|c|c|c|c|c|c|c|c|c|}
\hline Meter & $\begin{array}{c}\text { Gen- } \\
\text { set A1 }\end{array}$ & $\begin{array}{c}\text { Gen - } \\
\text { set A2 }\end{array}$ & Meter 1 & Meter 2 & Meter 3 & Meter 4 & LB 3 & LB 4 & LB 6 \\
\hline $\begin{array}{c}\text { 3 Phase } \\
\text { kW }\end{array}$ & 36.2 & 27 & 31.3 & -6.05 & -8.5 & 9.3 & 17.9 & 36.6 & 36.95 \\
\hline $\begin{array}{c}\mathbf{1} \text { Phase } \\
\mathbf{k W}\end{array}$ & $\mathrm{B}=15.4$ & $\mathrm{~B}=11.6$ & $\mathrm{~B}=11.4$ & $\mathrm{~B}=-6.25$ & $\mathrm{~B}=-7.5$ & $\mathrm{~B}=1.8$ & $\mathrm{~B}=5.9$ & $\mathrm{~B}=13.45$ & $\mathrm{~B}=17.6$ \\
\hline $\begin{array}{c}\mathbf{3} \text { Phase } \\
\mathbf{k V A r}\end{array}$ & -11.5 & -2.5 & 22 & 20.1 & 15 & 3 & 0 & 0.615 & 0.641 \\
\hline $\begin{array}{c}\mathrm{C} \text { Phase } \\
\mathbf{k V A r}\end{array}$ & $\mathrm{B}=-3.9$ & $\mathrm{~B}=-0.5$ & $\mathrm{~B}=7.2$ & $\mathrm{~B}=6.9$ & $\mathrm{~B}=4.8$ & $\mathrm{~B}=0.7$ & $\mathrm{~B}=0$ & $\mathrm{~B}=0.28$ & $\mathrm{~B}=0.4$ \\
& $\mathrm{C}=-3.4$ & $\mathrm{C}=-0.5$ & $\mathrm{C}=6.5$ & $\mathrm{C}=6$ & $\mathrm{C}=4.2$ & $\mathrm{C}=0.7$ & $\mathrm{C}=0$ & $\mathrm{C}=0.215$ & $\mathrm{C}=0.121$ \\
\hline
\end{tabular}

Table 7 - Measured Values after Start Up for Test 10.3.18

These measurements were relatively close to the expected values in Table 2, but not exact due to temperature, phase voltages and electrical losses in conductors. In addition, the $40 \mathrm{~kW}$ settings for LB4 and LB6 and the $20 \mathrm{~kW}$ setting for LB3 were also below selected set values. At the time of the measurements, the voltage and frequency was 282.2V on A-phase, $278.6 \mathrm{~V}$ on B-phase and $279.2 \mathrm{~V}$ on C-phase and $60.01 \mathrm{~Hz}$ at the static switch (i.e., Meter 2) when connected to the utility grid; and $282 \mathrm{~V}$ on A-phase, $279.3 \mathrm{~V}$ on B-phase and $279 \mathrm{~V}$ on C-phase and $60.01 \mathrm{~Hz}$ at Meter 3.

The Gen-sets in this test were set up to produce $-10 \mathrm{~kW}$ through Zone 3 (i.e., Meter 3 ) which means they produced 10kW more power than Load Banks 3 and 4 needed. Approximately $8.5 \mathrm{~kW}(\mathrm{~A}=2 \mathrm{~kW} \mathrm{~B}=7.5 \mathrm{~kW} \mathrm{C}=-1 \mathrm{~kW})$ of excess power was exported out of Zone 3 and $6.05 \mathrm{~kW}(\mathrm{~A}=1.4 \mathrm{~kW} \mathrm{~B}=6.25 \mathrm{~kW} \mathrm{C}=-1.6 \mathrm{~kW})$ of that power went through the static switch to Load Bank 6 and the remaining $2.45 \mathrm{~kW}(\mathrm{~A}=0.6 \mathrm{~kW} \mathrm{~B}=1.25 \mathrm{~kW} \mathrm{C}=0.6 \mathrm{~kW})$ was loss to the electrical characteristics of the conductors. Since Load Bank 6 was 
approximately $36.95 \mathrm{~kW}(\mathrm{~A}=9.75 \mathrm{~kW} \quad \mathrm{~B}=17.6 \mathrm{~kW} \quad \mathrm{C}=9.6 \mathrm{~kW})+\mathrm{j} 0.641 \mathrm{kVAr}(\mathrm{A}=0.12 \mathrm{kVAr}$ $\mathrm{B}=0.4 \mathrm{kVAr} C=0.121 \mathrm{kVAr})$, the utility had to supply approximately $31.3 \mathrm{~kW}(\mathrm{~A}=8.5 \mathrm{~kW}$ $\mathrm{B}=11.4 \mathrm{~kW} \mathrm{C}=11.4 \mathrm{~kW}$ ) real power to satisfy the load. Reactive power had to be imported in from the utility of approximately j20.1kVAr $(A=7.5 \mathrm{kVAr} B=6.6 \mathrm{kVAr} C=6 \mathrm{kVAr})$ because Gen-sets $A 1$ and $A 2$ needed approximately $-j 14 k V A r(A=-5.7 k V A r ~ B=-4.4 k V A r$ $\mathrm{C}=-3.9 \mathrm{kVAr}$ ) between the two and the reactive power of Load Bank 4. Once all the data was verified and recorded into the DAS Database, the $10 \mathrm{Hp}$ induction motor was started in Zone 3.

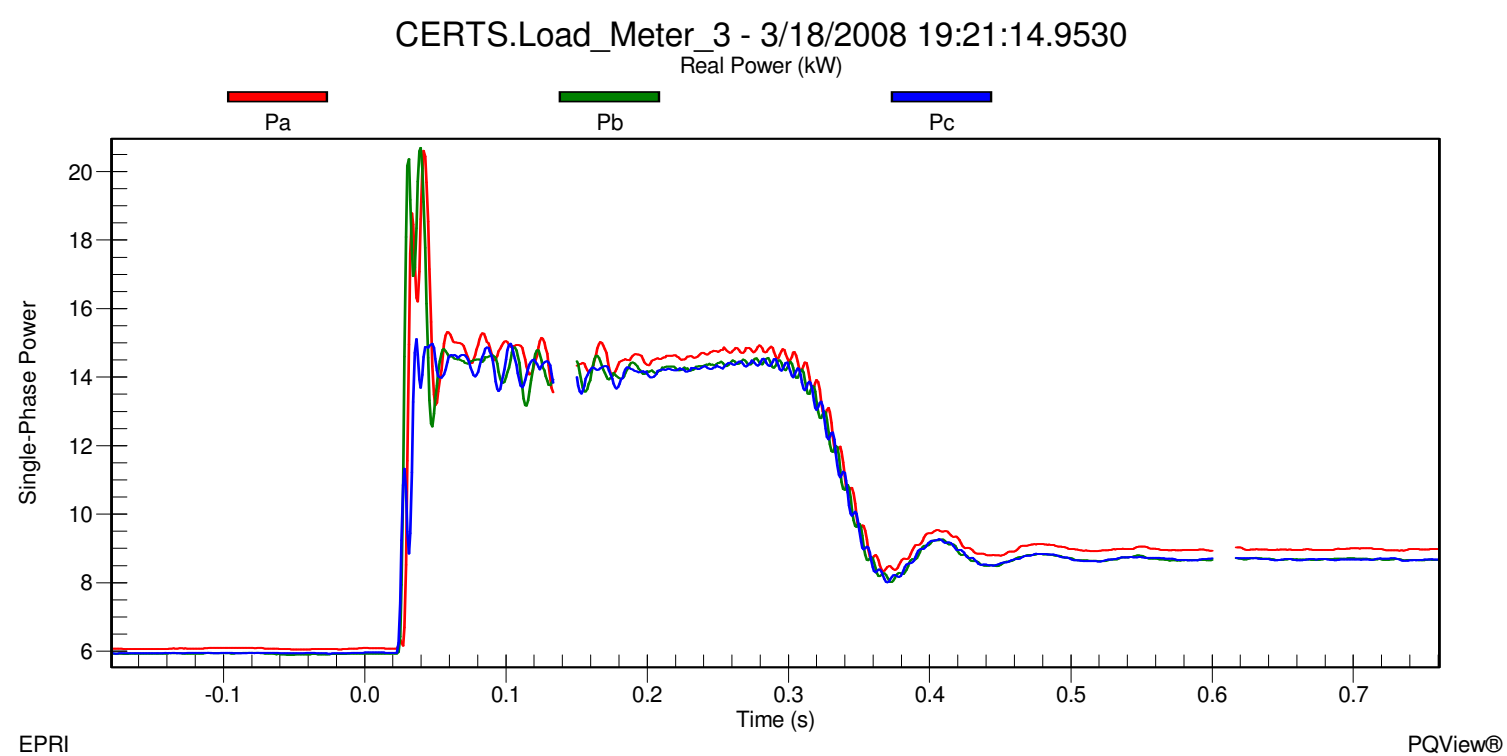

Figure 15a - Load Bank 3 Real Single-Phase Power during Motor Start and Utility Connected for Test 10.3.18 


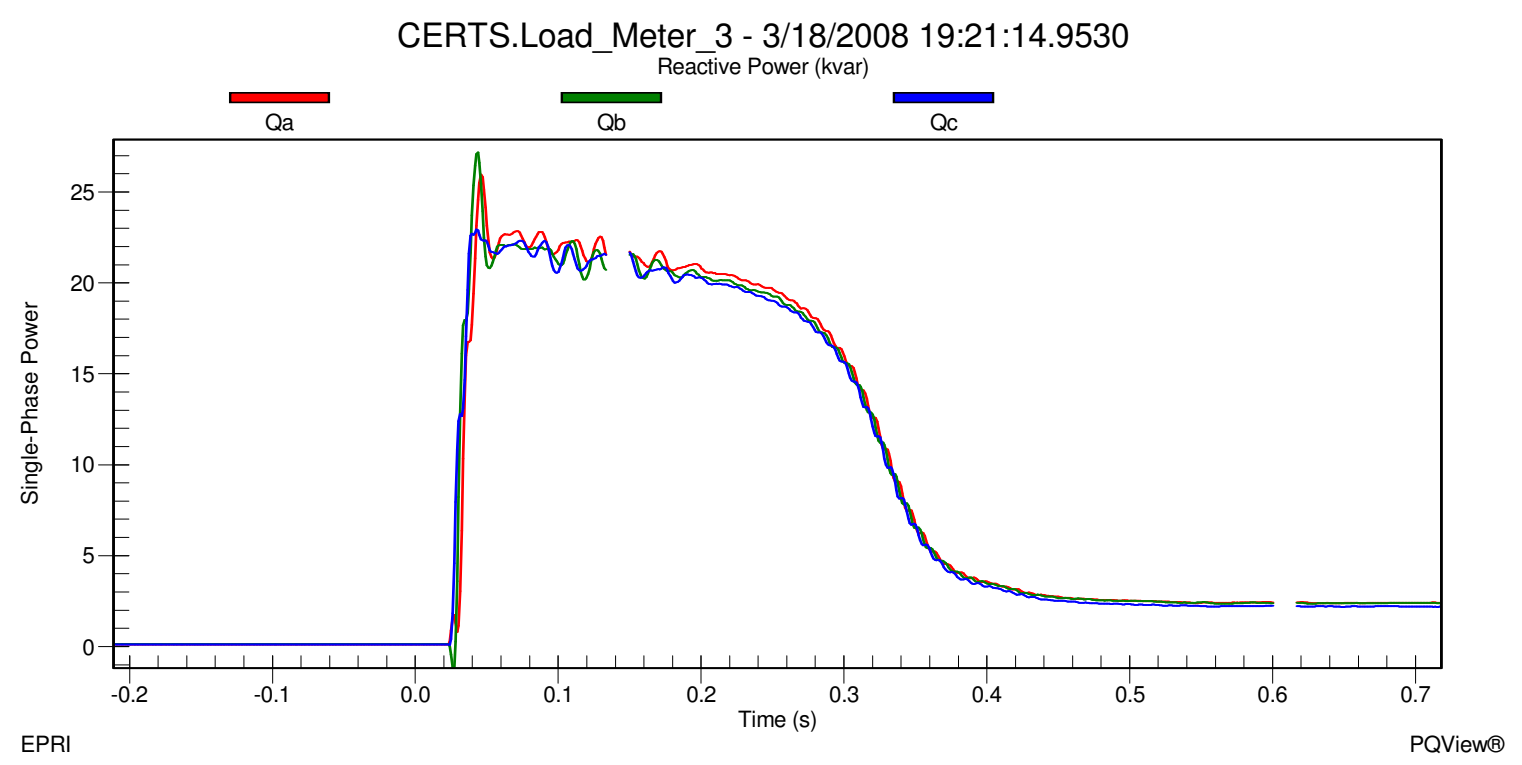

Figure 15b - Load Bank 3 Reactive Single-Phase Power during Motor Start and Utility Connected for Test 10.3.18

In Figures 15a and 15b it can be seen that the load in Zone 3 was approximately $17.9 \mathrm{~kW}$ $(\mathrm{A}=6.1 \mathrm{~kW} \mathrm{~B}=5.9 \mathrm{~kW} \mathrm{C}=5.9 \mathrm{~kW})+\mathrm{j} 0 \mathrm{kVAr}(\mathrm{A}=0 \mathrm{kVAr} \mathrm{B}=0 \mathrm{kVAr} \mathrm{C}=0 \mathrm{kVAr})$ before the start of the induction motor and increased to approximately $56.1 \mathrm{~kW}(\mathrm{~A}=20.6 \mathrm{~kW} \mathrm{~B}=21.4 \mathrm{~kW}$ $\mathrm{C}=14.1 \mathrm{~kW})+\mathrm{j} 74.7 \mathrm{kVAr}(\mathrm{A}=24.5 \mathrm{kVAr} \mathrm{B}=27.2 \mathrm{kVAr} \mathrm{C}=23 \mathrm{kVAr})$ during the inrush phase of the motor start. After about 1.32 cycles, the motor settled down to approximately $43.1 \mathrm{~kW} \quad(\mathrm{~A}=14.6 \mathrm{~kW} \quad \mathrm{~B}=14.25 \mathrm{~kW} \quad \mathrm{C}=14.25 \mathrm{~kW})+\mathrm{j} 61.2 \mathrm{kVAr} \quad(\mathrm{A}=20.8 \mathrm{kVAr} B=20.2 \mathrm{kVAr}$ $\mathrm{C}=20.2 \mathrm{kVAr}$ ) during the warm up phase which lasted about 30 cycles (0.5 Seconds). When the motor reached steady state, the load in Zone 3 was approximately $26.4 \mathrm{~kW}$ $(\mathrm{A}=9 \mathrm{~kW} \mathrm{~B}=8.7 \mathrm{~kW} \mathrm{C}=8.7 \mathrm{~kW})+\mathrm{j} 7 \mathrm{kVAr}(\mathrm{A}=2.4 \mathrm{kVAr} \mathrm{B}=2.4 \mathrm{kVAr} \mathrm{C}=2.2 \mathrm{kVAr})$.

The voltage and frequency at the static switch before the motor start was approximately 282.2V on A-phase, 278.6V on B-phase and 279.2V on C-phase shown in Figure 15c and approximately $60.01 \mathrm{~Hz}$ shown in Figure 15d. When the motor started, the voltage at the static switch during the inrush decreased to approximately $276 \mathrm{~V}$ on A-phase, $271.8 \mathrm{~V}$ on B-phase and $272.8 \mathrm{~V}$ on C-phase for about 1.32 cycles. Frequency dropped during the inrush to approximately $59.91 \mathrm{~Hz}$ and quickly increased to approximately $59.99 \mathrm{~Hz}$. Voltage increased as the motor was warming up and eventually settled at a steady state voltage at approximately $281.6 \mathrm{~V}$ on A-phase, $277.9 \mathrm{~V}$ on B-phase and $278.4 \mathrm{~V}$ on C-phase at an approximate frequency $60.00 \mathrm{~Hz}$. 
CERTS.Meter_2 - 3/18/2008 14:21:15.7568

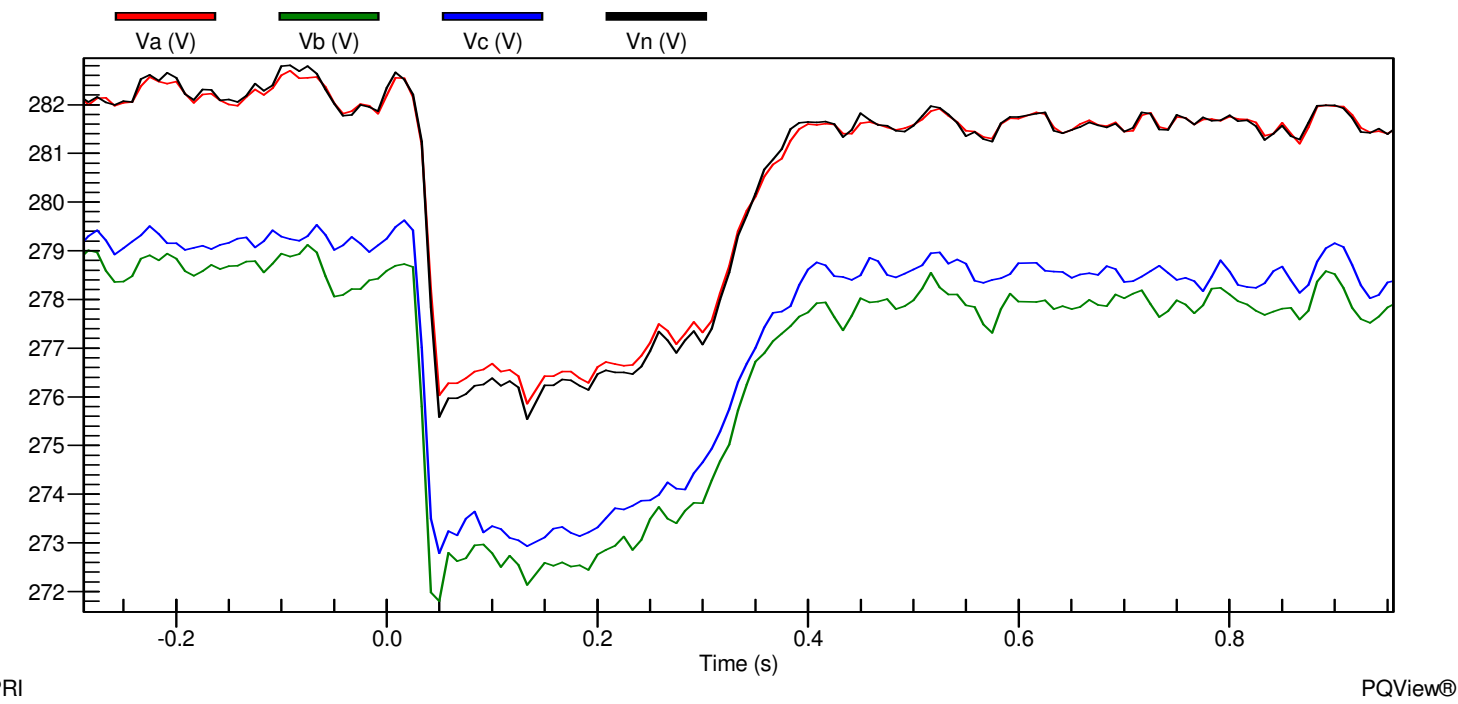

Figure 15c - Static Switch Line-to-Ground Voltages during Motor Start and Utility Connected for Test 10.3.18

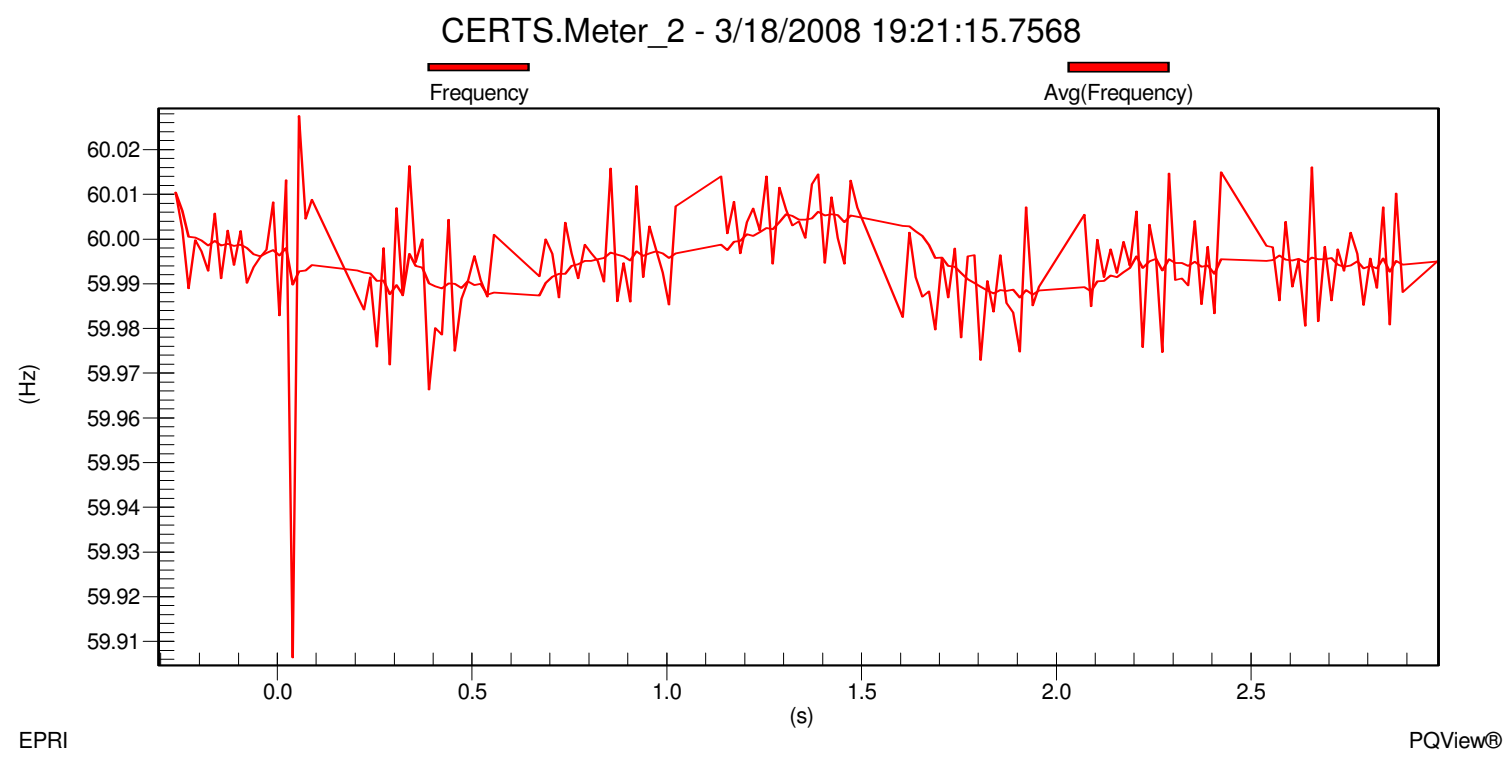

Figure 15d - Static Switch Frequency during Motor Start and Utility Connected for Test 10.3.18 


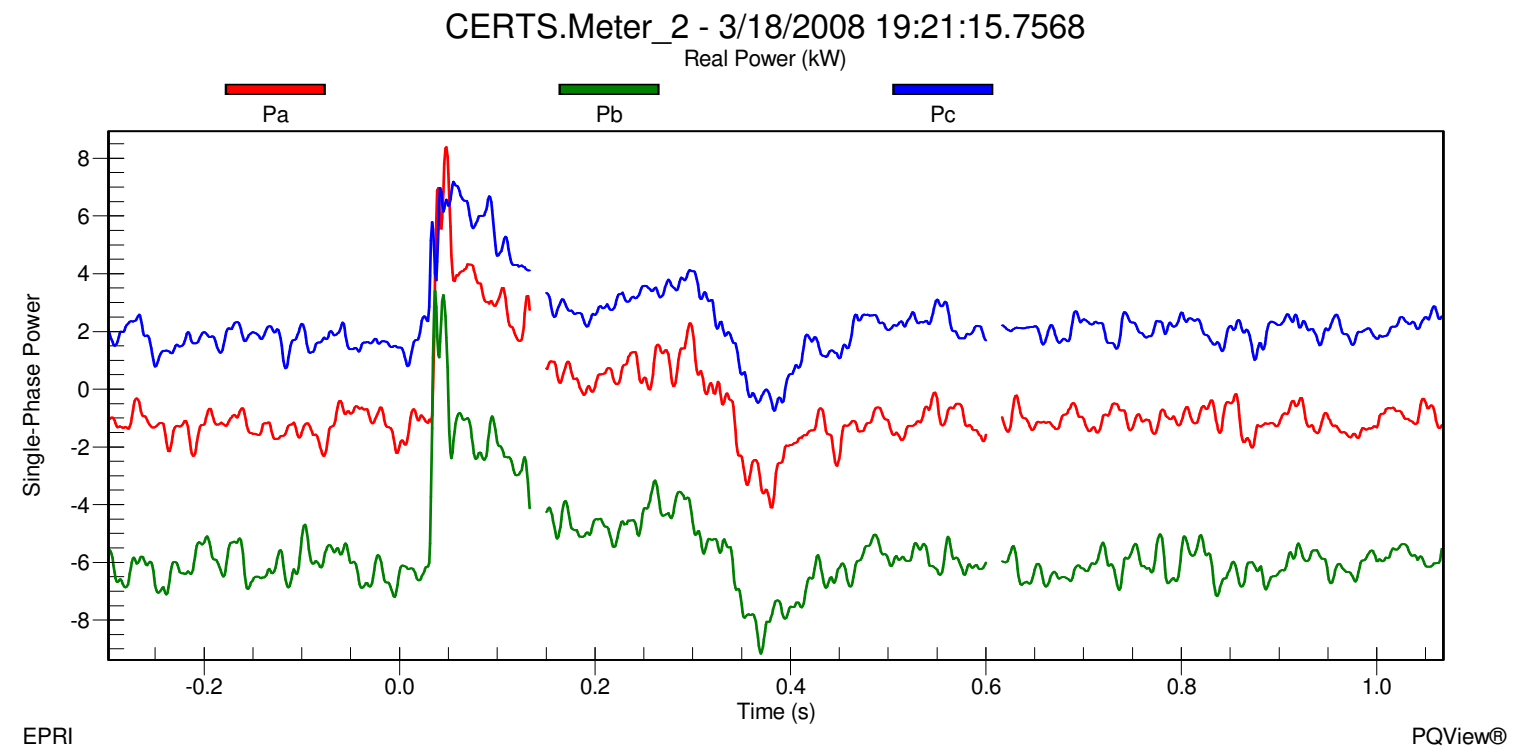

Figure 15e - Static Switch Real Single-Phase Power during Motor Start and Utility Connected for Test 10.3.18

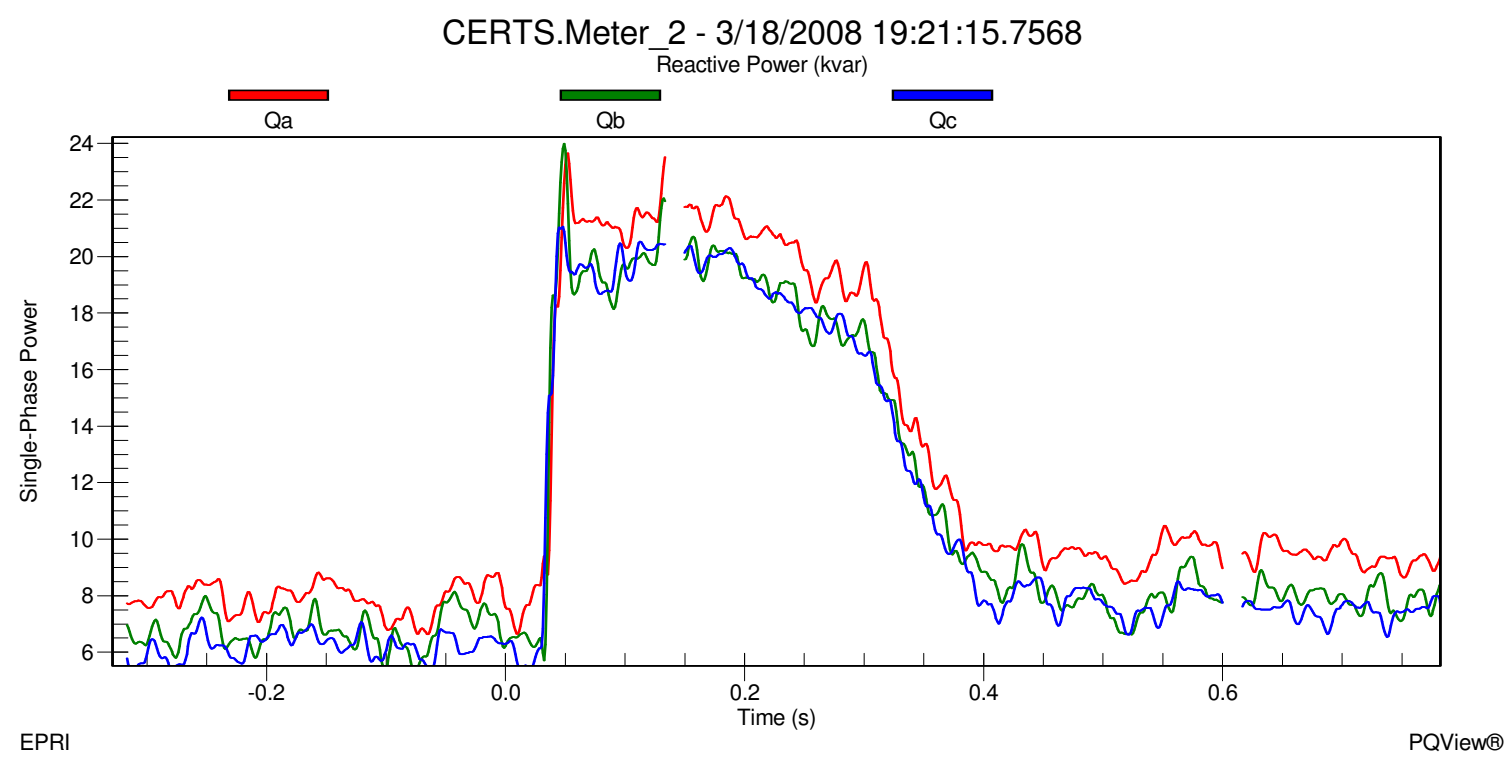

Figure 15f - Static Switch Reactive Single-Phase Power during Motor Start and Utility Connected for Test 10.3.18

Before the motor started Gen-sets A1 and A2 were producing approximately $36.2 \mathrm{~kW}$ $(A=10.6 \mathrm{~kW} \mathrm{~B}=15.4 \mathrm{~kW} C=10.2 \mathrm{~kW})-\mathrm{j} 11.5 \mathrm{kVAr}(\mathrm{A}=-4.2 \mathrm{kVAr} B=-3.9 \mathrm{kVAr} C=-3.4 \mathrm{kVAr})$ and $27 \mathrm{~kW}(\mathrm{~A}=7.4 \mathrm{~kW} B=11.6 \mathrm{~kW} \mathrm{C}=8 \mathrm{~kW})-\mathrm{j} 2.5 \mathrm{kVAr}(\mathrm{A}=-1.5 \mathrm{kVAr} B=-0.5 \mathrm{kVAr} \mathrm{C}=-0.5 \mathrm{kVAr})$, respectively. This was enough power for the load demands of Load Banks 3 and 4 with 
real power being exported to Zone 6 of approximately $6.05 \mathrm{~kW}(\mathrm{~A}=1.4 \mathrm{~kW} \mathrm{~B}=6.25 \mathrm{~kW} \mathrm{C}=-$ $1.6 \mathrm{~kW})$ shown in Figure 15e. The grid was supporting the reactive power of the microgrid with approximately j20.1kVAr $(A=7.5 \mathrm{kVAr} B=6.6 \mathrm{kVAr} C=6 \mathrm{kVAr})$ shown in Figure 15f. When the motor started the inrush caused the utility to pick up the load demand of Load Bank 6 and supply approximately $18.05 \mathrm{~kW}(\mathrm{~A}=8.3 \mathrm{~kW} B=3.25 \mathrm{~kW}$ $\mathrm{C}=6.5 \mathrm{~kW})+\mathrm{j} 68.7 \mathrm{kVAr}(\mathrm{A}=23.6 \mathrm{kVAr} \mathrm{B}=24 \mathrm{kVAr} \mathrm{C}=21.1 \mathrm{kVAr})$ to the microgrid critical load banks (i.e., Load Banks 3 and 4). The power that was being supplied by Gen-set A1 to Load Bank 6 was now supplying the motor inrush which can be seen in Figure 15e as the real power through the static switch becomes positive. Gen-sets A1 and A2 increased their real and reactive power output levels to approximately $59 \mathrm{~kW}(\mathrm{~A}=18.4 \mathrm{~kW}$ $B=22.9 \mathrm{~kW} C=17.7 \mathrm{~kW})+\mathrm{j} 0.5 \mathrm{kVAr}(\mathrm{A}=-0.55 \mathrm{kVAr} B=1.25 \mathrm{kVAr} C=-0.2 \mathrm{kVAr})$ and $33.4 \mathrm{~kW}$ $(A=8.9 \mathrm{~kW} \quad B=14.25 \mathrm{~kW} \quad \mathrm{C}=10.25 \mathrm{~kW})+j 17.7 \mathrm{kVAr} \quad(\mathrm{A}=4.7 \mathrm{kVAr} \quad \mathrm{B}=7 \mathrm{kVAr} \quad \mathrm{C}=6 \mathrm{kVAr})$, respectively. Notice the signs of the $\mathrm{VAr}$ output changed from negative to positive for both Gen-sets in order to support the induction motor load in Zone 3.

When the motor reached steady state, the real and reactive power through the static switch was approximately $-5.65 \mathrm{~kW}(\mathrm{~A}=-1.2 \mathrm{~kW} B=-6.25 \mathrm{~kW} \quad \mathrm{C}=1.8 \mathrm{~kW})+\mathrm{j} 24.35 \mathrm{kVAr}$ $(\mathrm{A}=9.3 \mathrm{kVAr} \mathrm{B}=7.8 \mathrm{kVAr} \mathrm{C}=7.25 \mathrm{kVAr})$ which meant that Gen-sets $\mathrm{A} 1$ and $\mathrm{A} 2$ were supplying the real power for the induction motor and approximately $5.65 \mathrm{~kW}(\mathrm{~A}=1.2 \mathrm{~kW}$ $\mathrm{B}=6.25 \mathrm{~kW} \mathrm{C}=-1.8 \mathrm{~kW}$ ) of Load Bank 6. The real power in Gen-set A1 increased from it initial value at the beginning of the test to approximately $43.2 \mathrm{~kW}(\mathrm{~A}=13.1 \mathrm{~kW} \mathrm{~B}=17.7 \mathrm{~kW}$ $\mathrm{C}=12.4 \mathrm{~kW}$ ) and the real power for Gen-set A2 returned relatively close to the value before the motor started which was approximately $27.2 \mathrm{~kW} \quad(A=7.5 \mathrm{~kW} \quad B=11.5 \mathrm{~kW}$ $\mathrm{C}=8.2 \mathrm{~kW}$ ). Reactive power in Gen-set $\mathrm{A} 1$ and $\mathrm{A} 2$ slightly increased from its initial value at the beginning of the test to approximately $-\mathrm{j} 11.05 \mathrm{kVAr}(\mathrm{A}=-4.2 \mathrm{kVAr} B=-3.75 \mathrm{kVAr} \mathrm{C}=$ $3.1 \mathrm{kVAr})$ and $-\mathrm{j} 1.2 \mathrm{kVAr}(\mathrm{A}=-1.2 \mathrm{kVAr} B=0 \mathrm{kVAr} C=0 \mathrm{kVAr})$, respectively. Once all the data was verified and recorded into the DAS Database, the motor was shut down and the static switch was directed by the EMS to manually open.

As soon as the static switch opened, Meter 1 recorded real power increased to approximately $38.1 \mathrm{~kW}(\mathrm{~A}=10.1 \mathrm{~kW} \mathrm{~B}=18.1 \mathrm{~kW} \mathrm{C}=9.9 \mathrm{~kW})$ and reactive power decreased to $\mathrm{j} 1 \mathrm{kVAr}(\mathrm{A}=0.2 \mathrm{kVAr} B=0.6 \mathrm{kVAr} C=0.2 \mathrm{kVAr})$ satisfying the load demand in Load bank6 which was approximately $37.8 \mathrm{~kW} \quad(\mathrm{~A}=10 \mathrm{~kW} \quad \mathrm{~B}=18 \mathrm{~kW} \quad \mathrm{C}=9.8 \mathrm{~kW})+\mathrm{j} 0.655 \mathrm{kVAr}$ $(\mathrm{A}=0.12 \mathrm{kVAr} \mathrm{B}=0.41 \mathrm{kVAr} \mathrm{C}=0.125 \mathrm{kVAr})$ and not supplying any power beyond the static switch to Load Banks 3 and 4. $0 \mathrm{~kW}(\mathrm{~A}=0 \mathrm{~kW} B=0 \mathrm{~kW} C=0 \mathrm{~kW})+\mathrm{j} 0 \mathrm{kVAr}(\mathrm{A}=0 \mathrm{kVAr}$ $\mathrm{B}=0 \mathrm{kVAr} \mathrm{C}=0 \mathrm{kVAr}$ ) was recorded at the static switch, indicating that power was not flowing through the static switch. Load Banks 3 and 4 loads reduced slightly to $17.39 \mathrm{~kW}$ $(\mathrm{A}=5.78 \mathrm{~kW} \quad \mathrm{~B}=5.84 \mathrm{~kW} \quad \mathrm{C}=5.77 \mathrm{~kW})+j 0.3284 \mathrm{kVAr} \quad(\mathrm{A}=0.107 \mathrm{kVAr} \quad \mathrm{B}=0.1225 \mathrm{kVAr}$ $\mathrm{C}=0.989 \mathrm{kVAr}) \quad$ and $\quad 35.35 \mathrm{~kW} \quad(\mathrm{~A}=9.3 \mathrm{~kW} \quad \mathrm{~B}=13.1 \mathrm{~kW} \quad \mathrm{C}=12.95 \mathrm{~kW})+\mathrm{j} 0.603 \mathrm{kVAr}$ $(\mathrm{A}=0.114 \mathrm{kVAr} \mathrm{B}=0.275 \mathrm{kVAr} \mathrm{C}=0.214 \mathrm{kVAr})$, respectively. This load reduction resulted from a voltage drop in the microgrid, shown in Figure 15g, from approximately $282.2 \mathrm{~V}$ 
on A-phase, $279.4 \mathrm{~V}$ on B-phase and $278.9 \mathrm{~V}$ on C-phase when connected to the utility grid to $275.1 \mathrm{~V}$ on A-phase, $275.8 \mathrm{~V}$ on B-phase and $274.7 \mathrm{~V}$ on C-phase at Meter 3 when islanded.

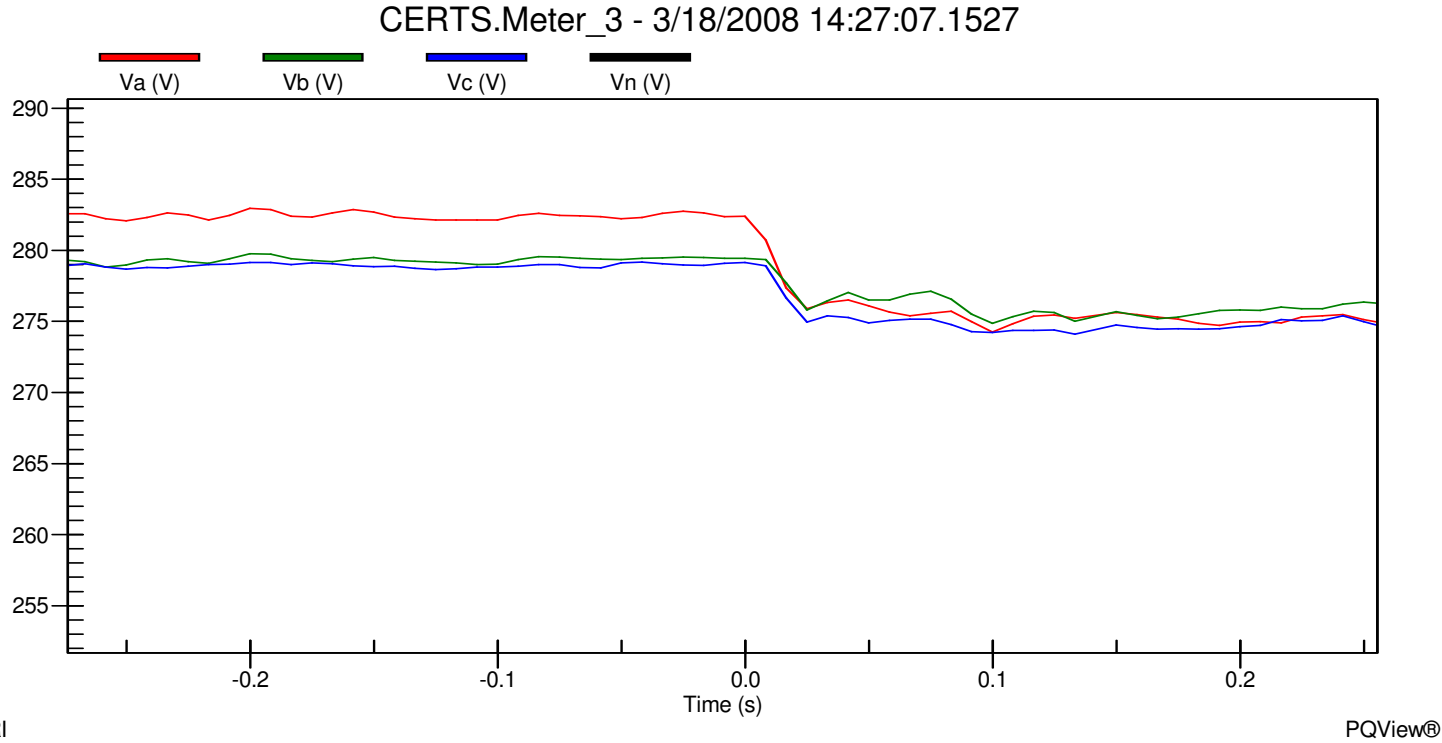

Figure 15g - Meter 3 Line-to-Ground Voltages during Utility Connected to Island mode for Test 10.3.18

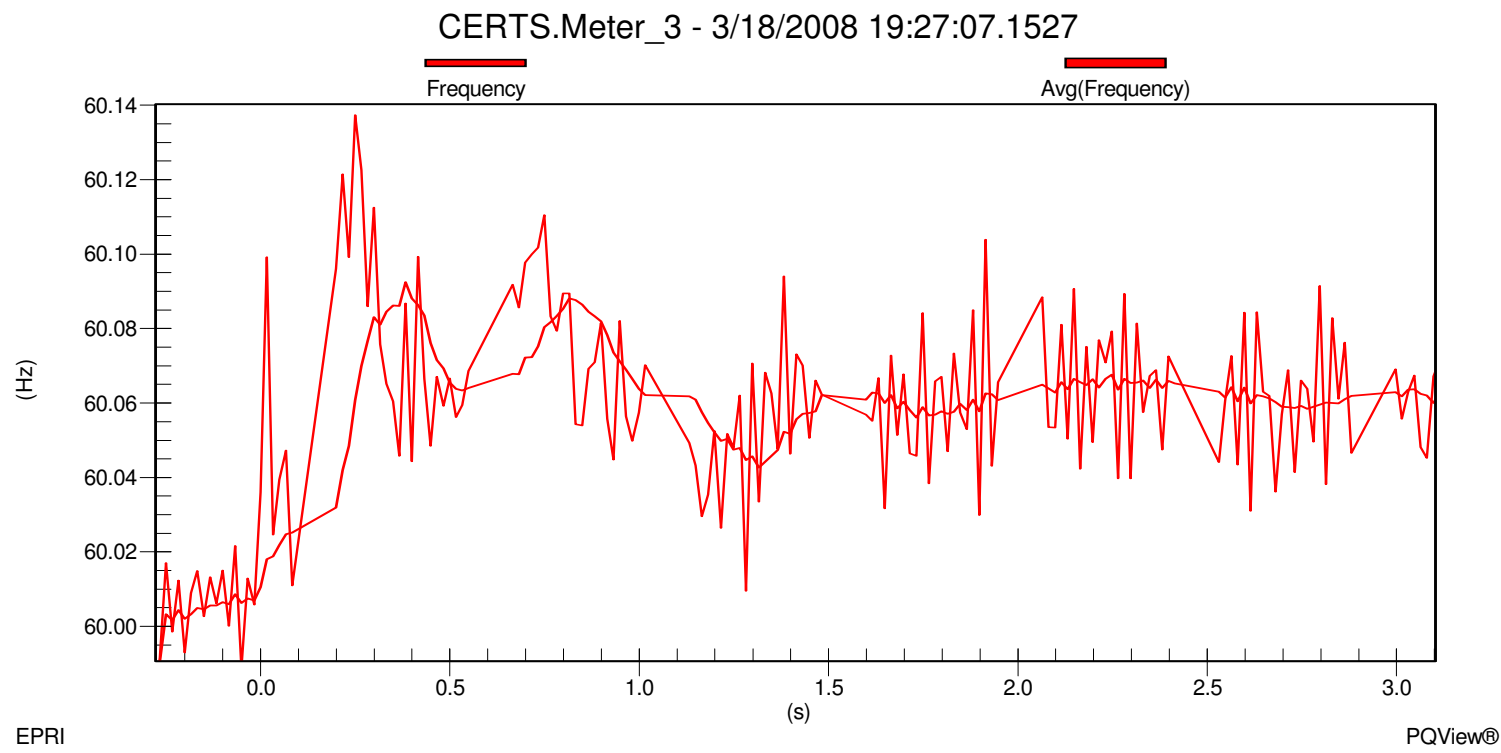

Figure 15h - Meter 3 Frequency during Utility Connected to Island mode for Test 10.3.18

Frequency change in the microgrid, shown in Figure 15h, increased from approximately $60.00 \mathrm{~Hz}$ when connected to the utility grid to approximately $60.06 \mathrm{~Hz}$ when islanded. This change in frequency was part of the CERTS algorithm which allowed Gen-set A2 to 
decrease its output real power to approximately $18.1 \mathrm{~kW} \quad(A=4.6 \mathrm{~kW} \quad B=6.75 \mathrm{~kW}$ $\mathrm{C}=6.75 \mathrm{~kW})$. Gen-set $\mathrm{A} 1$ remained relatively at the same level which was approximately $37.2 \mathrm{~kW}(\mathrm{~A}=11.3 \mathrm{~kW} \mathrm{~B}=13.4 \mathrm{~kW} \mathrm{C}=12.5 \mathrm{~kW})$. Reactive power in Gen-sets A1 and A2 increased to approximately $-\mathrm{j} 2.8 \mathrm{kVAr}(\mathrm{A}=-1.3 \mathrm{kVAr} B=-1 \mathrm{kVAr} \mathrm{C}=-0.5 \mathrm{kVAr})$ and $\mathrm{j} 8 \mathrm{kVAr}$ $(\mathrm{A}=2.5 \mathrm{kVAr} \mathrm{B}=3 \mathrm{kVAr} \mathrm{C}=2.5 \mathrm{kVAr})$, respectively. Meter 3 was approximately $-2.5 \mathrm{~kW}$ $(\mathrm{A}=-0.8 \mathrm{~kW} \quad \mathrm{~B}=-1.2 \mathrm{~kW} \quad \mathrm{C}=-0.5 \mathrm{~kW})-\mathrm{j} 4.2 \mathrm{kVAr} \quad(\mathrm{A}=-1 \mathrm{kVAr} \quad \mathrm{B}=-1.6 \mathrm{kVAr} \quad \mathrm{C}=-1.6 \mathrm{kVAr})$ indicating that the Gen-sets were satisfying the loads in the microgrid and the power losses in the electrical lines. All data was verified and recorded into the DAS Database. The microgrid ran fro a couple of minutes in this electrical state before the $10 \mathrm{Hp}$ induction motor was started in Zone 3.

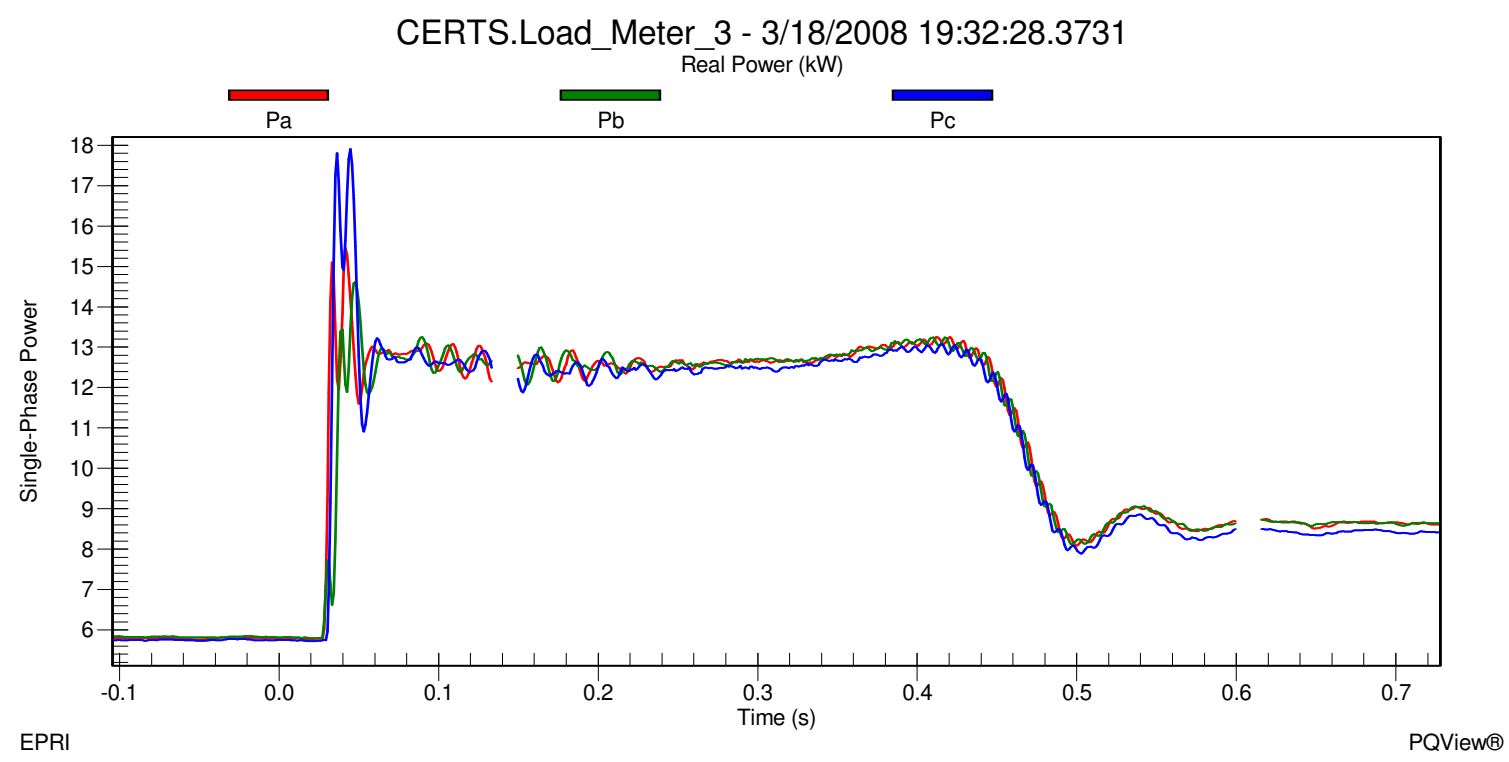

Figure 15i - Load Bank 3 Real Single-Phase Power during Motor Start and Islanded for Test 10.3.18 


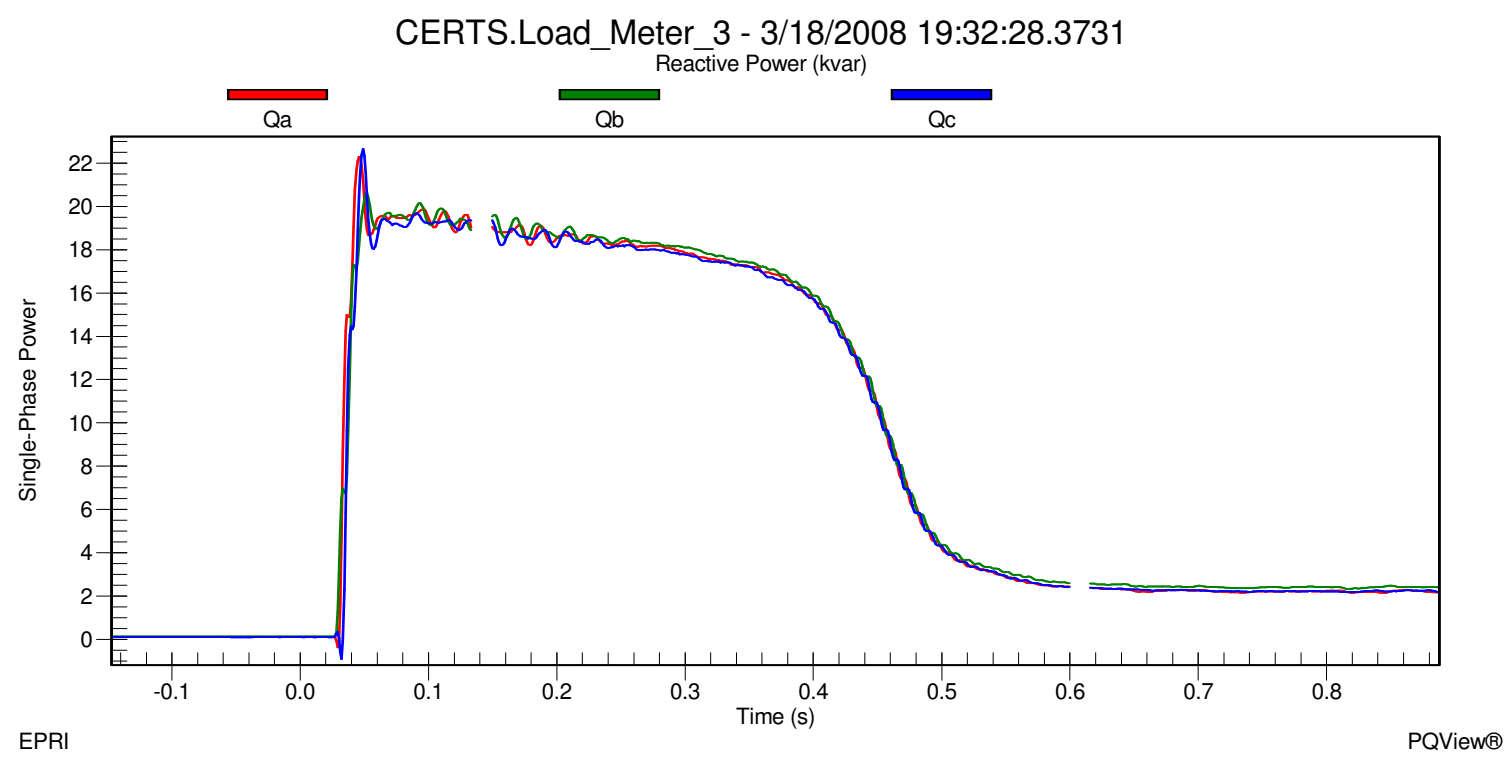

Figure 15j - Load Bank 3 Reactive Single-Phase Power during Motor Start and Islanded for Test 10.3.18

In Figures 15i and 15j it can be seen that the load in Zone 3 was approximately $17.3 \mathrm{~kW}$ $(\mathrm{A}=5.8 \mathrm{~kW} B=5.8 \mathrm{~kW} \mathrm{C}=5.7 \mathrm{~kW})+\mathrm{j} 0.3 \mathrm{kVAr}(\mathrm{A}=0.1 \mathrm{kVAr} B=0.1 \mathrm{kVAr} C=0.1 \mathrm{kVAr})$ before the start of the induction motor and increased to approximately $47 \mathrm{~kW}(\mathrm{~A}=14.8 \mathrm{~kW} \mathrm{~B}=14.3 \mathrm{~kW}$ $\mathrm{C}=17.9 \mathrm{~kW})+\mathrm{j} 64.95 \mathrm{kVAr}(\mathrm{A}=21.75 \mathrm{kVAr} \mathrm{B}=20.5 \mathrm{kVAr} \mathrm{C}=22.7 \mathrm{kVAr})$ during the inrush phase of the motor start. After about 1.5 cycles, the motor settled down to approximately $37.8 \mathrm{~kW}(\mathrm{~A}=12.6 \mathrm{~kW} \quad \mathrm{~B}=12.6 \mathrm{~kW} \quad \mathrm{C}=12.6 \mathrm{~kW})+\mathrm{j} 55.2 \mathrm{kVAr} \quad(\mathrm{A}=18.4 \mathrm{kVAr}$ $\mathrm{B}=18.4 \mathrm{kVAr} \mathrm{C}=18.4 \mathrm{kVAr})$ during the warm up phase which lasted about 36cycles $(0.6$ Seconds). When the motor reached steady state, the load in Zone 3 was approximately $25.6 \mathrm{~kW}(\mathrm{~A}=8.6 \mathrm{~kW} \mathrm{~B}=8.6 \mathrm{~kW} \mathrm{C}=8.4 \mathrm{~kW})+\mathrm{j} 6.9 \mathrm{kVAr}(\mathrm{A}=2.25 \mathrm{kVAr} B=2.4 \mathrm{kVAr} \mathrm{C}=2.25 \mathrm{kVAr})$.

The voltage and frequency at Meter 3 before the motor start was approximately $275.4 \mathrm{~V}$ on A-phase, $275.7 \mathrm{~V}$ on B-phase and $274 \mathrm{~V}$ on C-phase shown in Figure $15 \mathrm{k}$ and approximately $60.06 \mathrm{~Hz}$ shown in Figure 15l. When the motor started, the voltage at Meter 3 during the inrush decreased to approximately $256 \mathrm{~V}$ on A-phase, $255.5 \mathrm{~V}$ on Bphase and $255.3 \mathrm{~V}$ on $\mathrm{C}$-phase for about 1.5 cycles. Frequency dropped during the inrush to approximately $59.96 \mathrm{~Hz}$ and quickly increased to approximately $60.03 \mathrm{~Hz}$. Voltage increased as the motor was warming up and eventually settled at a steady state voltage at approximately $273.6 \mathrm{~V}$ on A-phase, $273.6 \mathrm{~V}$ on B-phase and $272.5 \mathrm{~V}$ on C-phase at an approximate frequency of $60.07 \mathrm{~Hz}$. 


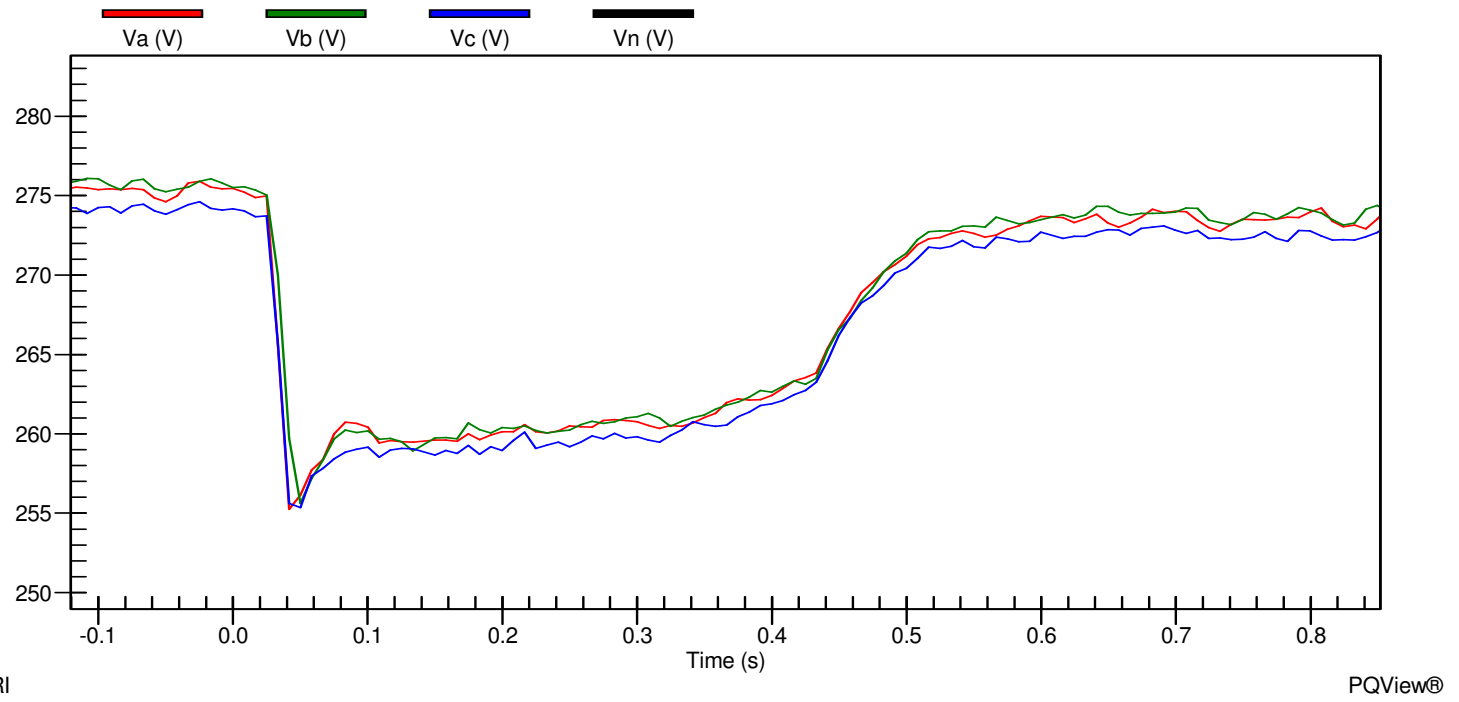

Figure 15k - Meter 3 Line-to-Ground Voltages during Motor Start and Islanded for Test 10.3.18

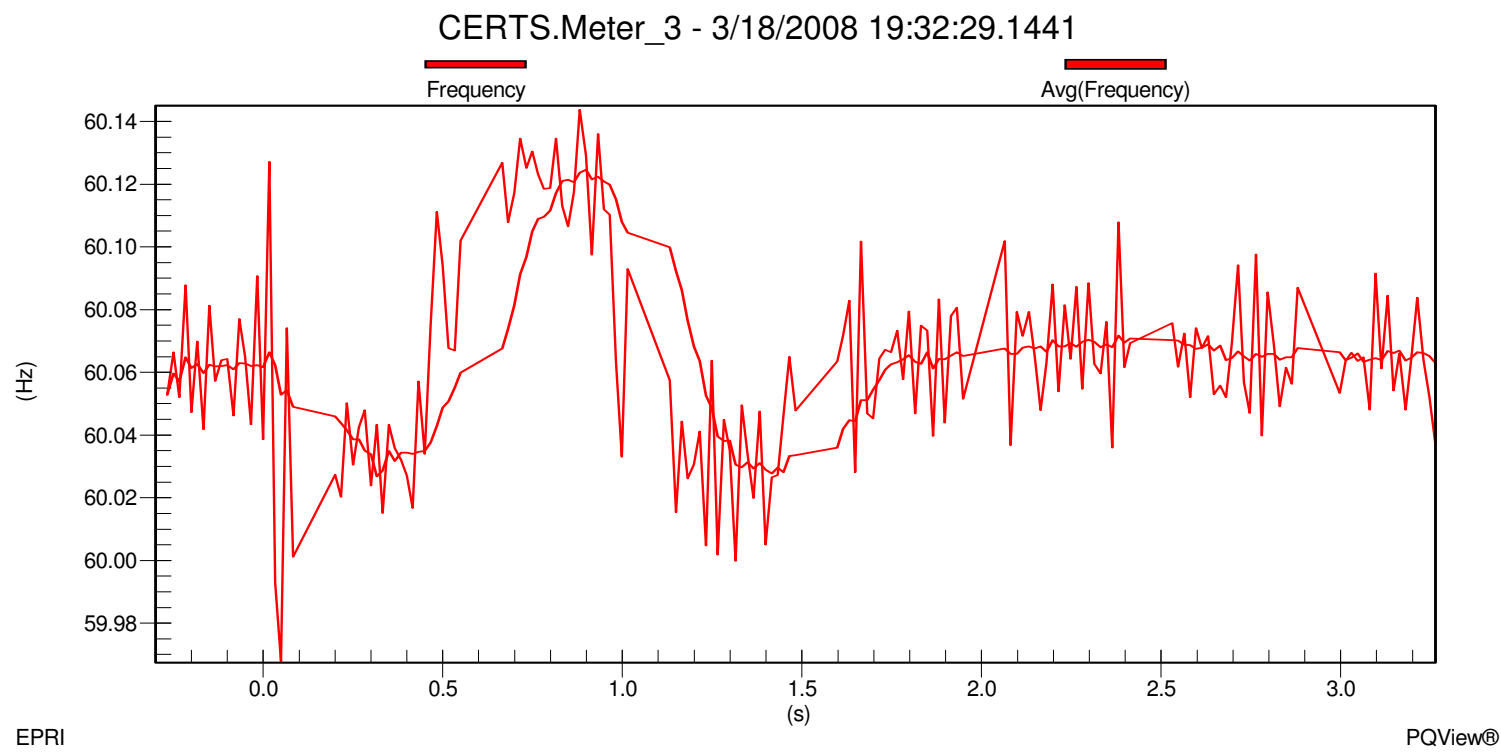

Figure 151 - Meter 3 Frequency during Motor Start and Islanded for Test 10.3.18

Before the motor started Gen-sets $\mathrm{A} 1$ and $\mathrm{A} 2$ were producing approximately $37.1 \mathrm{~kW}$ $(\mathrm{A}=11.2 \mathrm{~kW} B=13.5 \mathrm{~kW} C=12.4 \mathrm{~kW})-\mathrm{j} 2.9 \mathrm{kVAr}(\mathrm{A}=-1.4 \mathrm{kVAr} B=-1 \mathrm{kVAr} \mathrm{C}=-0.5 \mathrm{kVAr})$ and $18 \mathrm{~kW}(\mathrm{~A}=4.7 \mathrm{~kW} B=6.5 \mathrm{~kW} C=6.8 \mathrm{~kW})+\mathrm{j} 7.6 \mathrm{kVAr}(\mathrm{A}=2.4 \mathrm{kVAr} B=2.8 \mathrm{kVAr} \quad \mathrm{C}=2.4 \mathrm{kVAr})$, respectively shown in Figures $15 \mathrm{~m}-15 \mathrm{p}$. The power generated by both Gen-sets was satisfying the loads in Load Banks 3 and 4 and all the electrical losses in the microgrid system. When the motor started the inrush caused the Gen-sets to increase their output power levels to $47 \mathrm{~kW} \quad(\mathrm{~A}=13.4 \mathrm{~kW} \quad \mathrm{~B}=15.9 \mathrm{~kW} \quad \mathrm{C}=17.7 \mathrm{~kW})+\mathrm{j} 29 \mathrm{kVAr} \quad(\mathrm{A}=9.5 \mathrm{kVAr}$ $B=9.1 \mathrm{kVAr} C=10.4 \mathrm{kVAr})$ for Gen-set $\mathrm{A} 1$ and $32.1 \mathrm{~kW}(\mathrm{~A}=9.6 \mathrm{~kW} \mathrm{~B}=9.9 \mathrm{~kW} \mathrm{C}=12.6 \mathrm{~kW})+$ 
j38.7kVAr $(A=13.2 \mathrm{kVAr} B=12.2 \mathrm{kVAr} C=13.3 \mathrm{kVAr})$ for Gen-set $\mathrm{A} 2$. The increase in real and reactive power due to the motor start inrush was shared by Gen-sets A1 and A2. Gen-sets A1 increased its output real power during the warm up phase to approximately $57.4 \mathrm{~kW}(\mathrm{~A}=18.2 \mathrm{~kW} \mathrm{~B}=20.1 \mathrm{~kW} \mathrm{C}=19.1 \mathrm{~kW})$ and decreased its reactive power. Gen-set A2 decreased real and reactive power output during the warm up phase. The Gen-sets eventually dropped their output levels to $45.3 \mathrm{~kW}(\mathrm{~A}=14.1 \mathrm{~kW}$ $B=16.2 \mathrm{~kW} C=15 \mathrm{~kW})-\mathrm{j} 0.1 \mathrm{kVAr}(\mathrm{A}=-0.4 \mathrm{kVAr} B=0 \mathrm{kVAr} C=0.3 \mathrm{kVAr})$ for Gen-set $\mathrm{A} 1 \mathrm{and}$ $17.4 \mathrm{~kW}(\mathrm{~A}=4.4 \mathrm{~kW} B=6.4 \mathrm{~kW} \mathrm{C}=6.6 \mathrm{~kW})+\mathrm{j} 11.1 \mathrm{kVAr}(\mathrm{A}=3.6 \mathrm{kVAr} B=3.9 \mathrm{kVAr} C=3.6 \mathrm{kVAr})$ for Gen-set A2 when the motor reached steady state. Once all the data was verified and recorded into the DAS Database, the static switch was directed by the EMS to manually close.

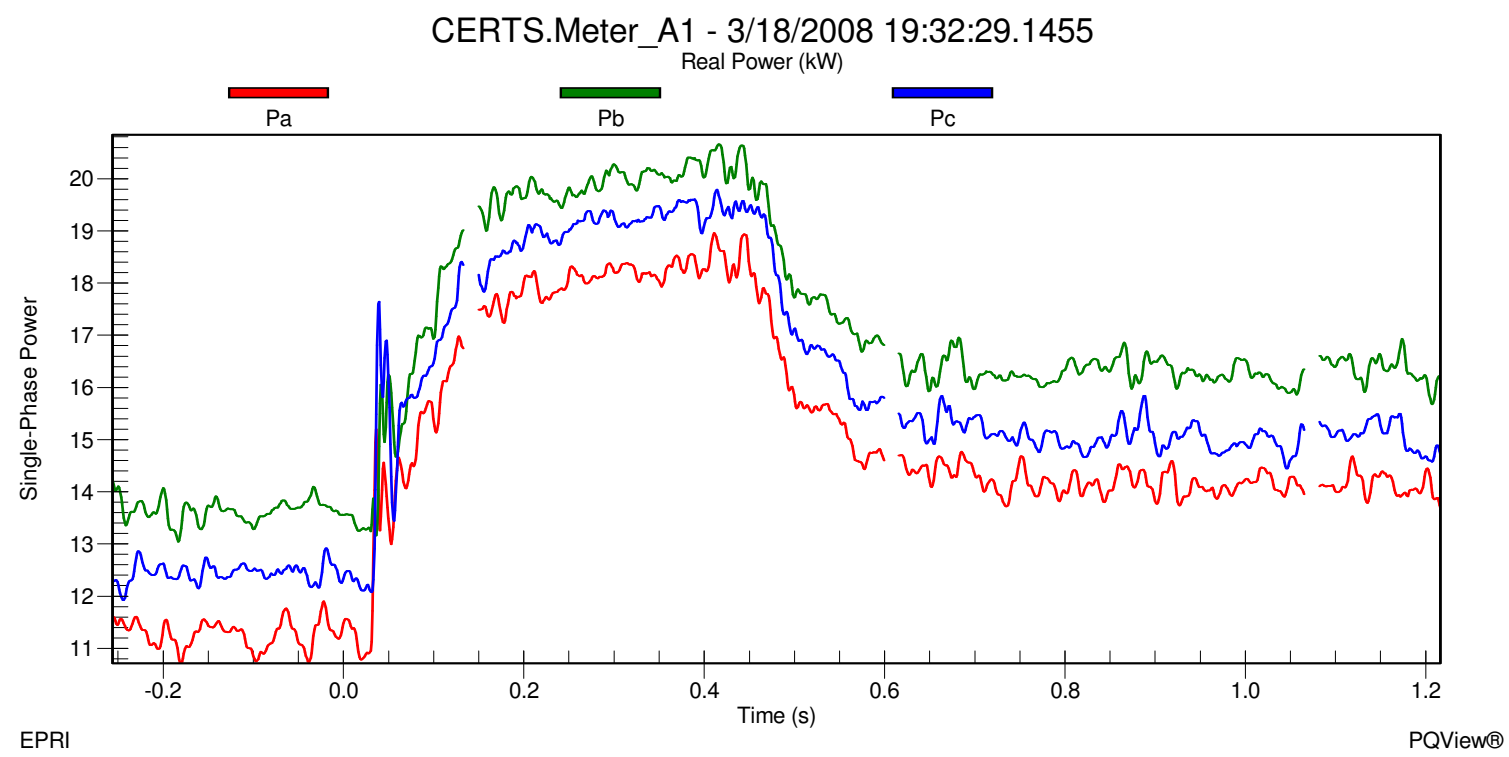

Figure 15m - Gen-set A1 Real Single-Phase Power during Motor Start and Islanded for Test 10.3.18 


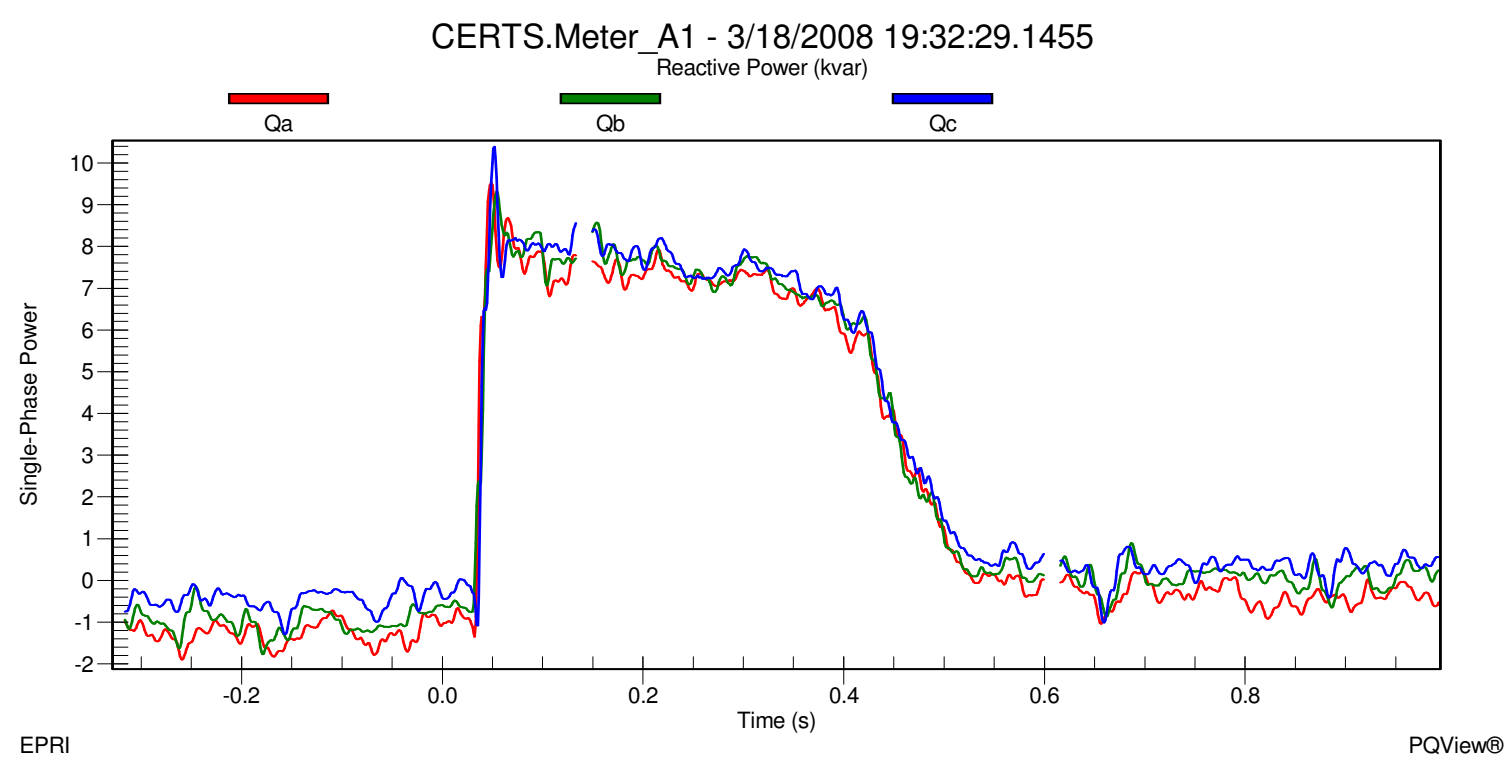

Figure 15n - Gen-set A1 Reactive Single-Phase Power during Motor Start and Islanded for Test 10.3.18

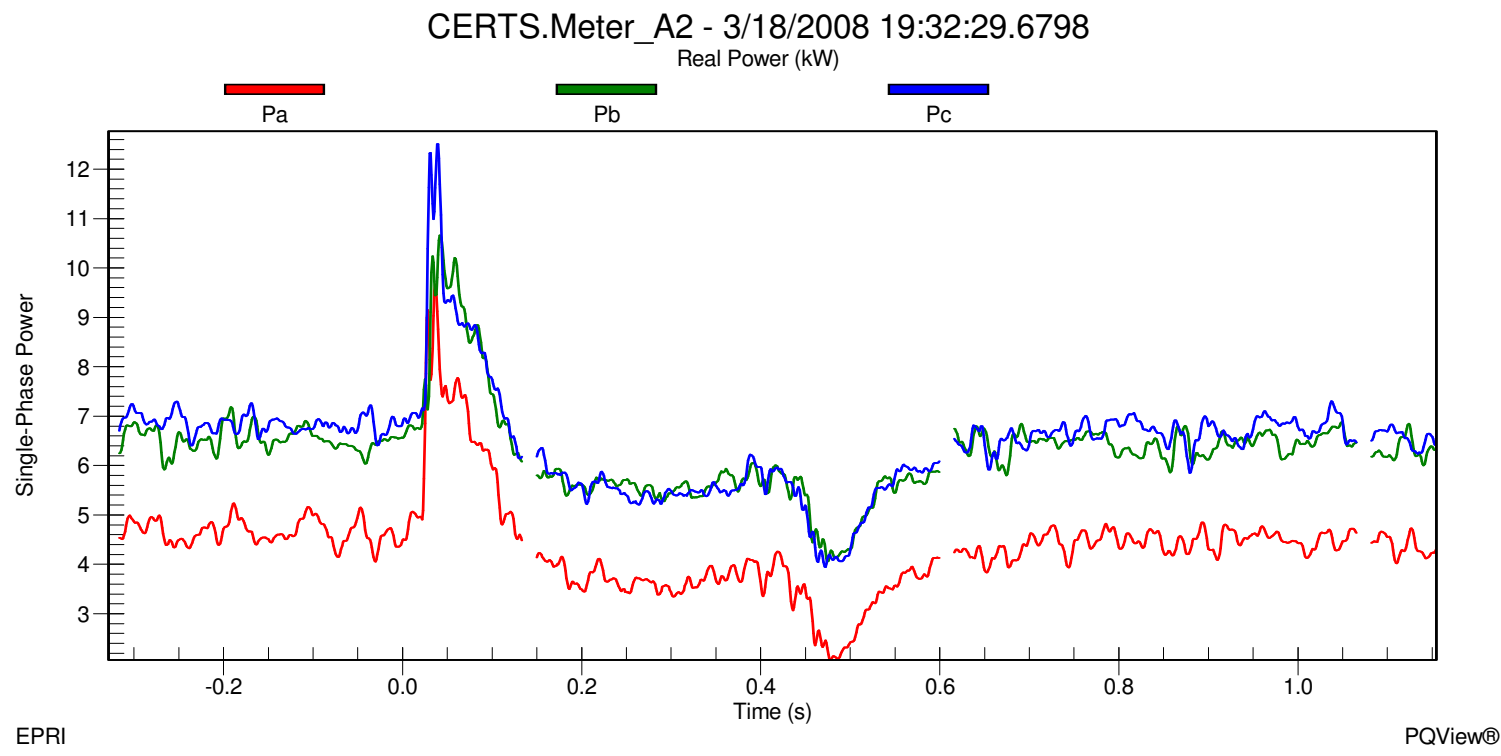

Figure 15o - Gen-set A2 Real Single-Phase Power during Motor Start and Islanded for Test 10.3.18 


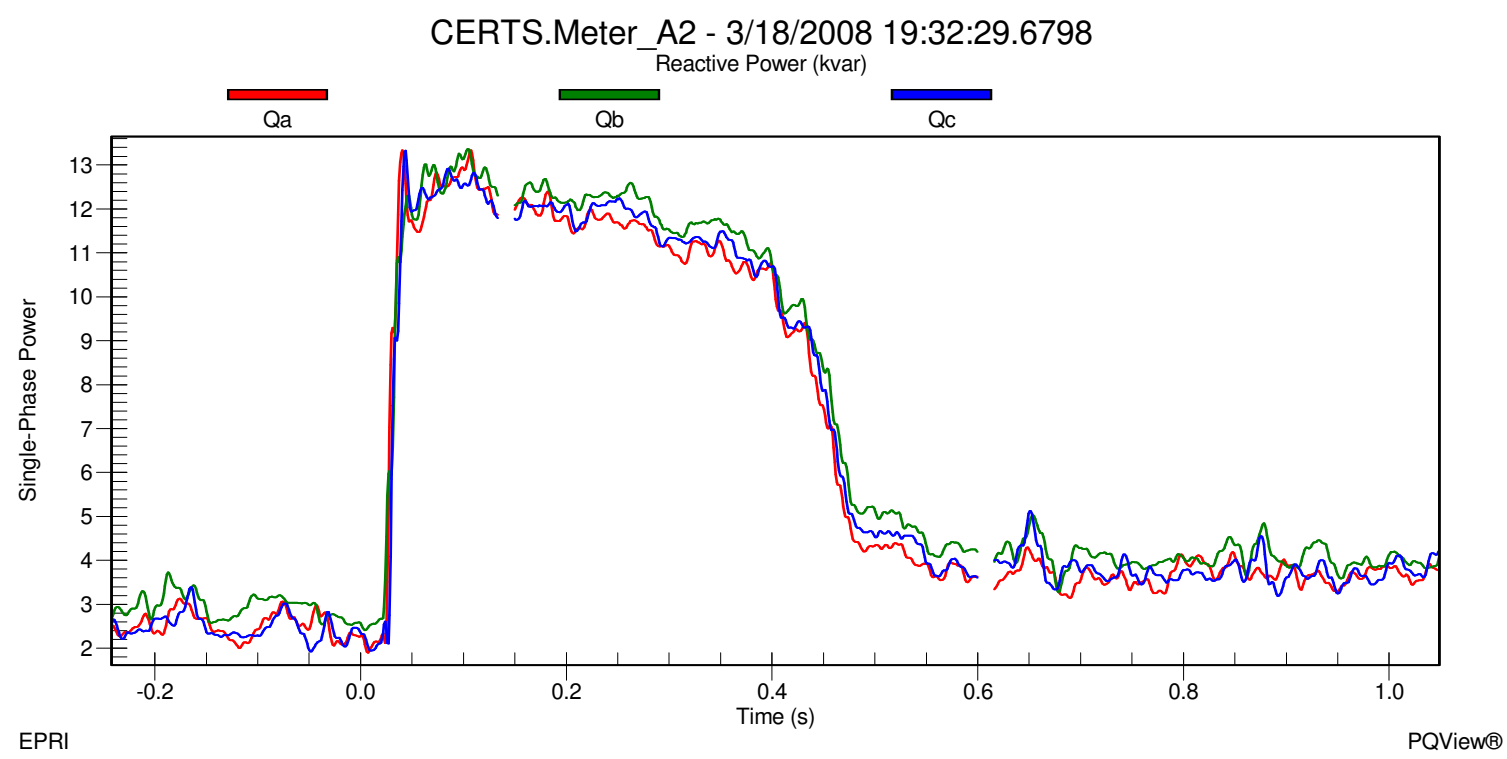

Figure 15p - Gen-set A2 Reactive Single-Phase Power during Motor Start and Islanded for Test 10.3.18

As soon as the static switch closed, Meter 1 recorded real power decreased from $38 \mathrm{~kW}$ $(\mathrm{A}=10.1 \mathrm{~kW} \mathrm{~B}=18.1 \mathrm{~kW} \mathrm{C}=9.8 \mathrm{~kW})$ to $32.5 \mathrm{~kW}(\mathrm{~A}=8.9 \mathrm{~kW} \mathrm{~B}=11.8 \mathrm{~kW} \mathrm{C}=11.8 \mathrm{~kW})$ and reactive power increased from approximately j1kVAr $(A=0.2 \mathrm{kVAr} B=0.6 \mathrm{kVAr} C=0.2 \mathrm{kVAr})$ to j27.4kVAr $(A=10 \mathrm{kVAr} B=9.2 \mathrm{kVAr} \quad \mathrm{C}=8.2 \mathrm{kVAr})$ which means that the utility was satisfying a portion of the load demand in Load Bank 6 and all the reactive power in the microgrid. Figures $15 \mathrm{q}$ and $15 \mathrm{r}$ show the static switch real power decreasing from approximately $0 \mathrm{~kW}(\mathrm{~A}=0 \mathrm{~kW} \mathrm{~B}=0 \mathrm{~kW} \mathrm{C}=0 \mathrm{~kW})$ to $-4.75 \mathrm{~kW}(\mathrm{~A}=-1 \mathrm{~kW} B=-6 \mathrm{~kW} C=2.25 \mathrm{~kW})$ and reactive power increasing from approximately j0kVAr $(A=0 \mathrm{kVAr} B=0 \mathrm{kVAr}$ $\mathrm{C}=0 \mathrm{kVAr})$ to $\mathrm{j} 26.1 \mathrm{kVAr}(\mathrm{A}=9.6 \mathrm{kVAr} \mathrm{B}=8.5 \mathrm{kVAr} \mathrm{C}=8 \mathrm{kVAr})$. At the beginning of the test, the initial power flow through the static switch was $-6.05 \mathrm{~kW}(\mathrm{~A}=-1.4 \mathrm{~kW} B=-6.25 \mathrm{~kW}$ $\mathrm{C}=1.6 \mathrm{~kW})+\mathrm{j} 20.1 \mathrm{kVAr}(\mathrm{A}=7.5 \mathrm{kVAr} \mathrm{B}=6.6 \mathrm{kVAr} \mathrm{C}=6 \mathrm{kVAr})$ which is not the same recorded at this point in the test because the $10 \mathrm{Hp}$ motor load is on in Zone 3. The Gen-sets have picked up the motor load and supporting Load Bank 6 with approximately $4.75 \mathrm{~kW}$ $(\mathrm{A}=1 \mathrm{~kW} \mathrm{~B}=6 \mathrm{~kW} \mathrm{C}=-2.25 \mathrm{~kW})$.

Load Banks 3 and 4 loads increased slightly to $24.9 \mathrm{~kW}(\mathrm{~A}=8.5 \mathrm{~kW} \mathrm{~B}=8.2 \mathrm{~kW} \mathrm{C}=8.2 \mathrm{~kW})+$ j6.43kVAr $(A=2.205 \mathrm{kVAr} B=2.205 \mathrm{kVAr} C=2.02 \mathrm{kVAr})$ and $36.62 \mathrm{~kW}(\mathrm{~A}=9.8 \mathrm{~kW} \mathrm{~B}=13.47 \mathrm{~kW}$ $\mathrm{C}=13.35 \mathrm{~kW})+\mathrm{j} 0.613 \mathrm{kVAr}(\mathrm{A}=0.12 \mathrm{kVAr} \mathrm{B}=0.28 \mathrm{kVAr} \mathrm{C}=0.213 \mathrm{kVAr})$, respectively. This slight load increase is a result from a voltage rise in the microgrid, shown in Figure 15s, from approximately $273.9 \mathrm{~V}$ on A-phase, $273.9 \mathrm{~V}$ on B-phase and $272.6 \mathrm{~V}$ on C-phase when islanded to $282.4 \mathrm{~V}$ on A-phase, $279 \mathrm{~V}$ on B-phase and $279 \mathrm{~V}$ on C-phase at Meter 3 when connected to the utility grid. 


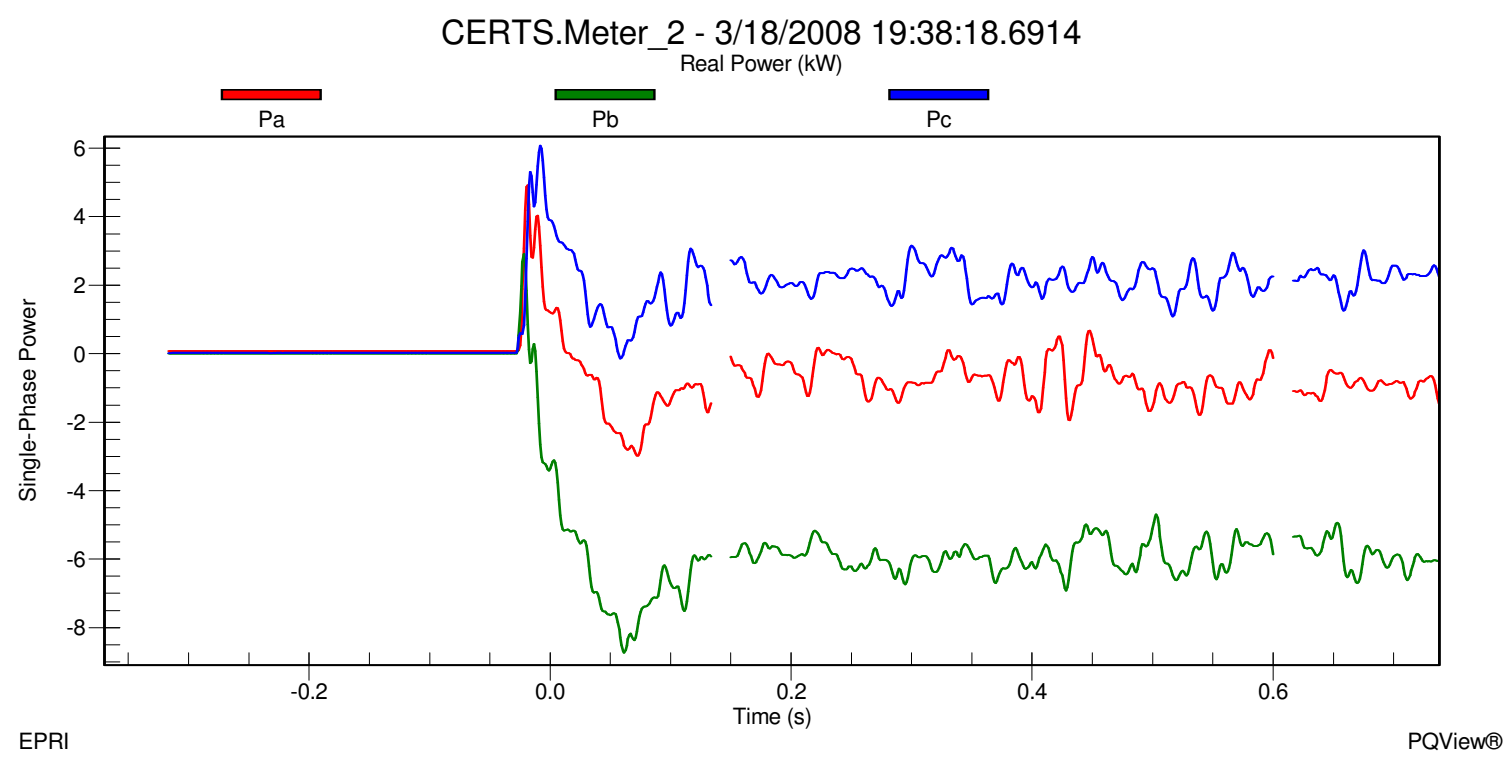

Figure 15q - Static Switch Real Single-Phase Power during Island to Utility Connected mode for Test 10.3.18

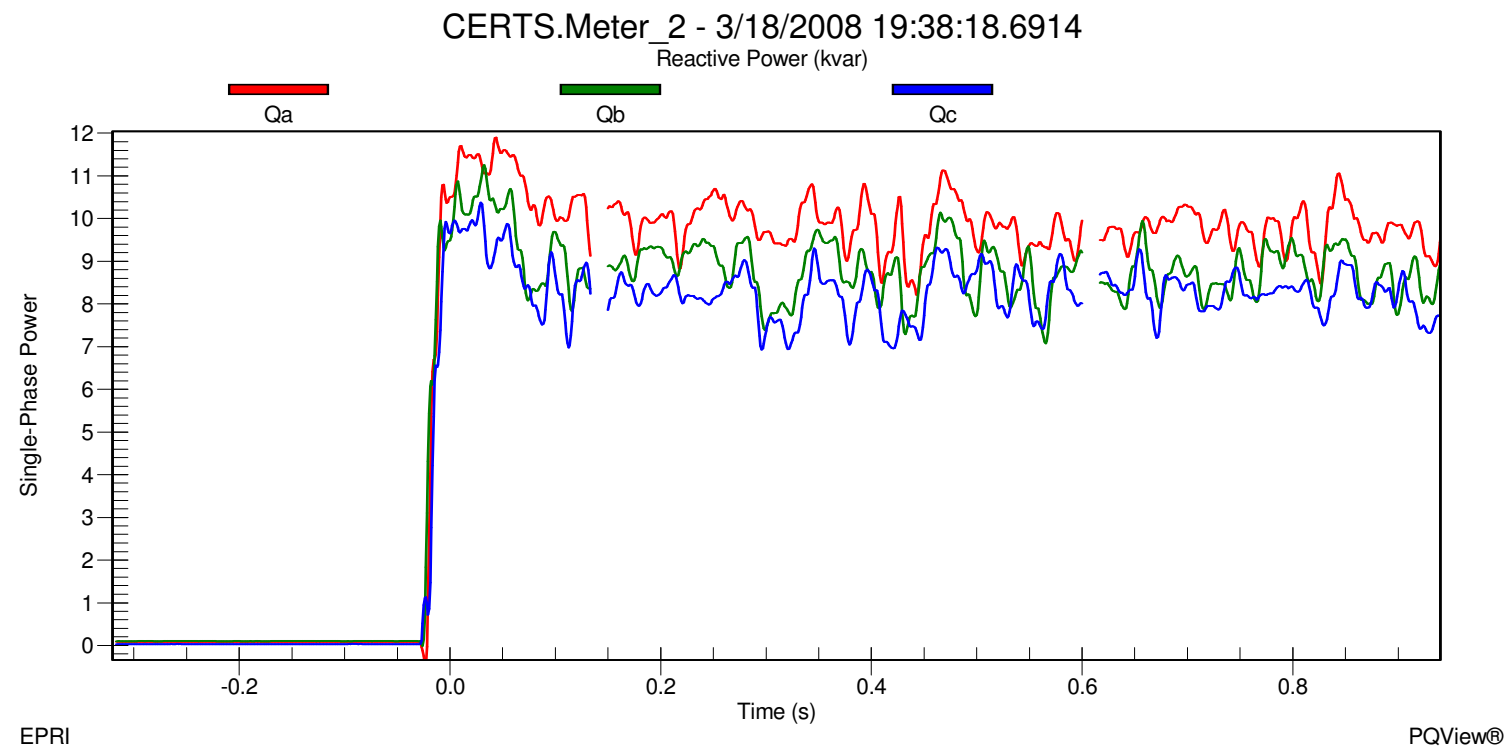

Figure 15r - Static Switch Reactive Single-Phase Power during Island to Utility Connected mode for Test 10.3.18 


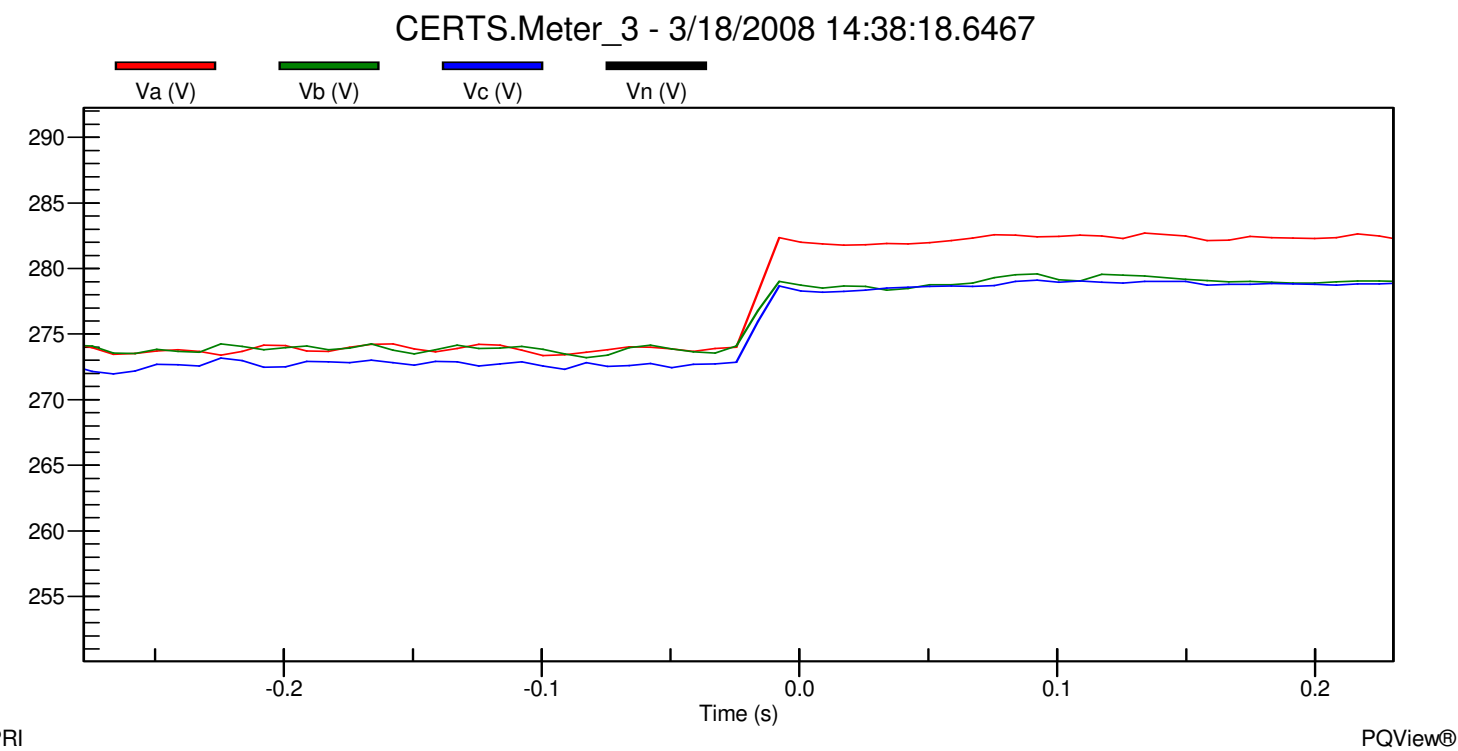

Figure 15s - Meter 3 Line-to-Ground Voltages during Island to Utility Connected mode for Test 10.3.18

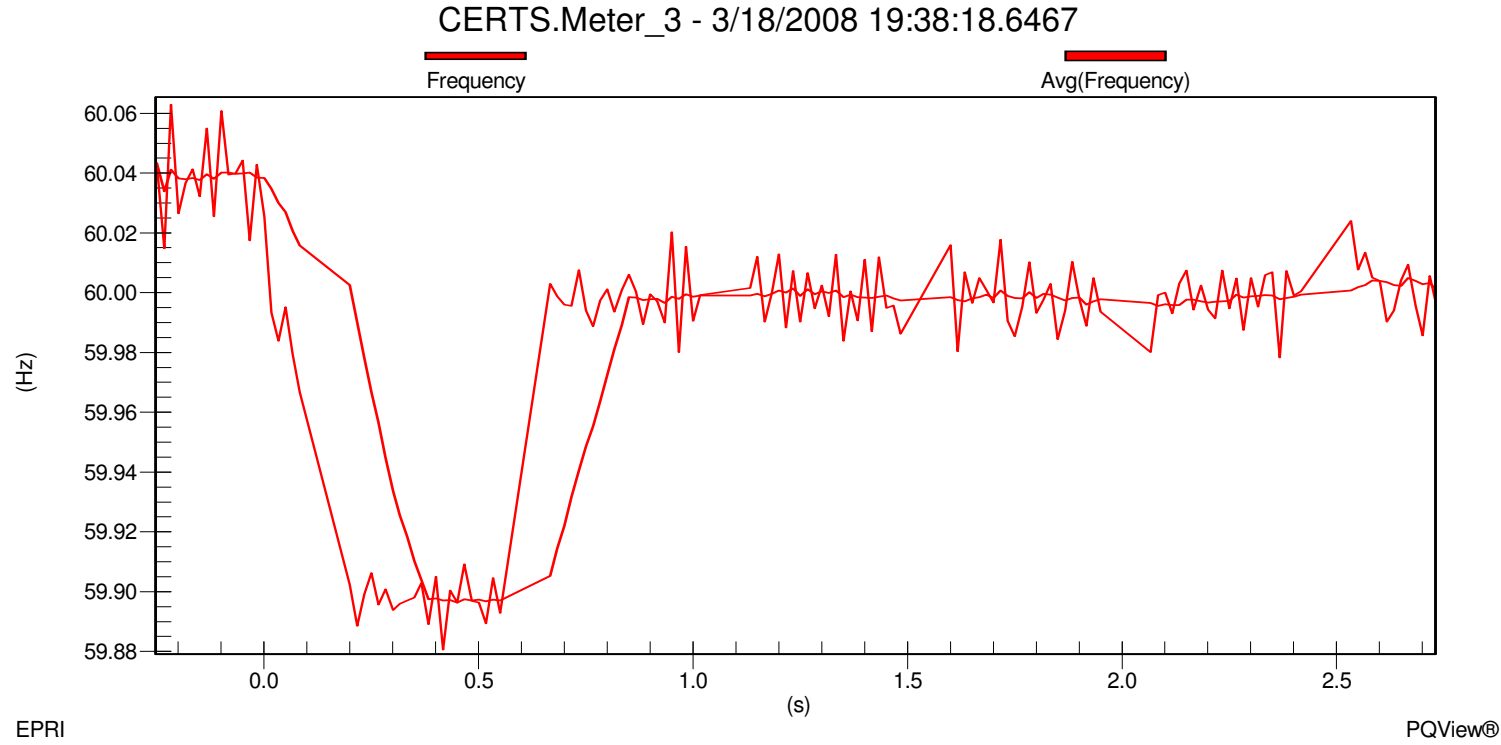

Figure 15t - Meter 3 Frequency during Island to Utility Connected mode for Test 10.3.18

Frequency change in microgrid, shown in Figure 15t, decreased from approximately $60.04 \mathrm{~Hz}$ when islanded to approximately $60.00 \mathrm{~Hz}$ when connected to the utility grid. This change in frequency is due to the frequency no longer being established by the Gensets using the CERTS algorithm but by the utility. Gen-sets A1 and A2 are in zone power control mode therefore when the static switch closed back into the utility the Gen-sets produced real power based on set-points for the power flow through Zones 3 and 4 initialized at the beginning of the test. The real power in Gen-set A1 increased 
from its initial value at the beginning of the test to approximately $42.25 \mathrm{~kW}(\mathrm{~A}=12.75 \mathrm{~kW}$ $\mathrm{B}=17.5 \mathrm{~kW} \mathrm{C}=12 \mathrm{~kW})$. Gen-set $\mathrm{A} 2$ real power was relatively close to its initial value at the beginning of the test which was approximately $26.4 \mathrm{~kW} \quad(\mathrm{~A}=7.25 \mathrm{~kW} \quad \mathrm{~B}=11.25 \mathrm{~kW}$ $\mathrm{C}=7.9 \mathrm{~kW})$. Reactive powers for both Gen-sets were relatively close to the values at the beginning of the test with approximately $-j 11.85 \mathrm{kVAr}(\mathrm{A}=-4.25 \mathrm{kVAr} B=-4 \mathrm{kVAr} \mathrm{C}=-$ 3.6kVAr) for Gen-set $\mathrm{A} 1$ and $-\mathrm{j} 2 \mathrm{kVAr}(\mathrm{A}=-1.6 \mathrm{kVAr} B=-0.2 \mathrm{kVAr} C=-0.2 \mathrm{kVAr})$ for Gen-set A2. After all the data was verified and recorded into the DAS Database, the motor was turned off and the Gen-sets and Load Banks set-points were changed according to the next test (10.3.20) in Table 2.

For Test 10.3.20 the measured values, after Gen-set A1 was warmed up and load banks brought on-line are provided in Table 7.

\begin{tabular}{|c|c|c|c|c|c|c|c|c|c|}
\hline Meter & $\begin{array}{l}\text { Gen- } \\
\text { set A1 }\end{array}$ & $\begin{array}{l}\text { Gen - } \\
\text { set A2 }\end{array}$ & Meter 1 & Meter 2 & Meter 3 & Meter 4 & LB 3 & LB 4 & LB 6 \\
\hline $\begin{array}{c}3 \text { Phase } \\
\text { kW }\end{array}$ & 33.1 & 0 & 25.5 & -12.4 & -14.9 & 0 & 17.9 & 18.2 & 37.25 \\
\hline \multirow{3}{*}{$\begin{array}{c}1 \text { Phase } \\
\text { kW }\end{array}$} & $A=10$ & $\mathrm{~A}=0$ & $A=7$ & $A=-3$ & $A=-3.9$ & $A=0$ & $A=6.1$ & $A=6.2$ & $\mathrm{~A}=9.9$ \\
\hline & $\mathrm{B}=14.7$ & $\mathrm{~B}=0$ & $B=10.5$ & $B=-7.5$ & $B=-8.8$ & $B=0$ & $B=5.9$ & $B=6$ & $B=17.75$ \\
\hline & $C=8.4$ & $C=0$ & $C=8$ & $C=-1.9$ & $C=-2.2$ & $\mathrm{C}=0$ & $C=5.9$ & $C=6$ & $C=9.6$ \\
\hline $\begin{array}{c}3 \text { Phase } \\
\text { kVAr }\end{array}$ & -13.8 & 0 & 21 & 20 & 14.45 & 0 & 0 & 0.3 & 0.656 \\
\hline \multirow{3}{*}{$\begin{array}{l}1 \text { Phase } \\
\text { kVAr }\end{array}$} & $A=-5$ & $A=0$ & $A=7$ & $A=6.75$ & $A=5.2$ & $\mathrm{~A}=0$ & $A=0$ & $\mathrm{~A}=0.1$ & $\mathrm{~A}=0.12$ \\
\hline & $B=-4.8$ & $B=0$ & $\mathrm{~B}=7.5$ & $B=7$ & $B=5$ & $\mathrm{~B}=0$ & $\mathrm{~B}=0$ & $B=0.1$ & $B=0.411$ \\
\hline & $C=-4$ & $C=0$ & $C=6.5$ & $C=6.25$ & $C=4.25$ & $\mathrm{C}=0$ & $\mathrm{C}=0$ & $C=0.1$ & $C=0.125$ \\
\hline
\end{tabular}

Table 8 - Measured Values after Start Up for Test 10.3.20

These measurements were relatively close to the expected values in Table 2, but not exact due to temperature, phase voltages and electrical losses in conductors. In addition, the $40 \mathrm{~kW}$ settings for LB6 and the $20 \mathrm{~kW}$ setting for LB3 were also below selected set values. At the time of the measurements, the voltage and frequency was $284 \mathrm{~V}$ on Aphase, $279.6 \mathrm{~V}$ on B-phase and $280 \mathrm{~V}$ on C-phase and $60.03 \mathrm{~Hz}$ at the static switch (i.e., Meter 2) when connected to the utility grid; and $284.3 \mathrm{~V}$ on A-phase, $280.1 \mathrm{~V}$ on B-phase and $280.1 \mathrm{~V}$ on C-phase and $60.03 \mathrm{~Hz}$ at Meter 3. 
Gen-set A1 in this test was set up to produce $-20 \mathrm{~kW}$ going through Zone 3 (i.e., Meter 3) which means it produced $20 \mathrm{~kW}$ more power than Load Bank 3 needed. Approximately $14.9 \mathrm{~kW}(\mathrm{~A}=3.9 \mathrm{~kW} \mathrm{~B}=8.8 \mathrm{~kW} \mathrm{C}=2.2 \mathrm{~kW})$ of excess power was exported out of Zone 3 and $12.4 \mathrm{~kW}(\mathrm{~A}=3 \mathrm{~kW} \mathrm{~B}=7.5 \mathrm{~kW} \mathrm{C}=1.9 \mathrm{~kW})$ of that power went through the static switch to Load Bank 6 and the remaining $2.5 \mathrm{~kW}(\mathrm{~A}=0.9 \mathrm{~kW} \mathrm{~B}=1.3 \mathrm{~kW} \mathrm{C}=0.3 \mathrm{~kW})$ was due to power losses in the conductors. Since Load Bank 6 was approximately $37.25 \mathrm{~kW}(\mathrm{~A}=9.9 \mathrm{~kW}$ $\mathrm{B}=17.75 \mathrm{~kW} \mathrm{C}=9.6 \mathrm{~kW})+\mathrm{j} 0.656 \mathrm{kVAr}(\mathrm{A}=0.12 \mathrm{kVAr} B=0.411 \mathrm{kVAr} C=0.125 \mathrm{kVAr})$, the utility had to supply approximately $25.5 \mathrm{~kW}(\mathrm{~A}=7 \mathrm{~kW} \mathrm{~B}=10.5 \mathrm{~kW} \mathrm{C}=8 \mathrm{~kW})$ to satisfy the load. Reactive power had to be imported in from the utility of approximately j20kVAr ( $\mathrm{A}=6.75 \mathrm{kVAr} \mathrm{B}=7 \mathrm{kVAr} \mathrm{C}=6.25 \mathrm{kVAr}$ ) because Gen-set A1 needed approximately j13.8kVAr $(A=5 \mathrm{kVAr} B=4.8 \mathrm{kVAr} C=4 \mathrm{kVAr})$ and losses in the conductors.

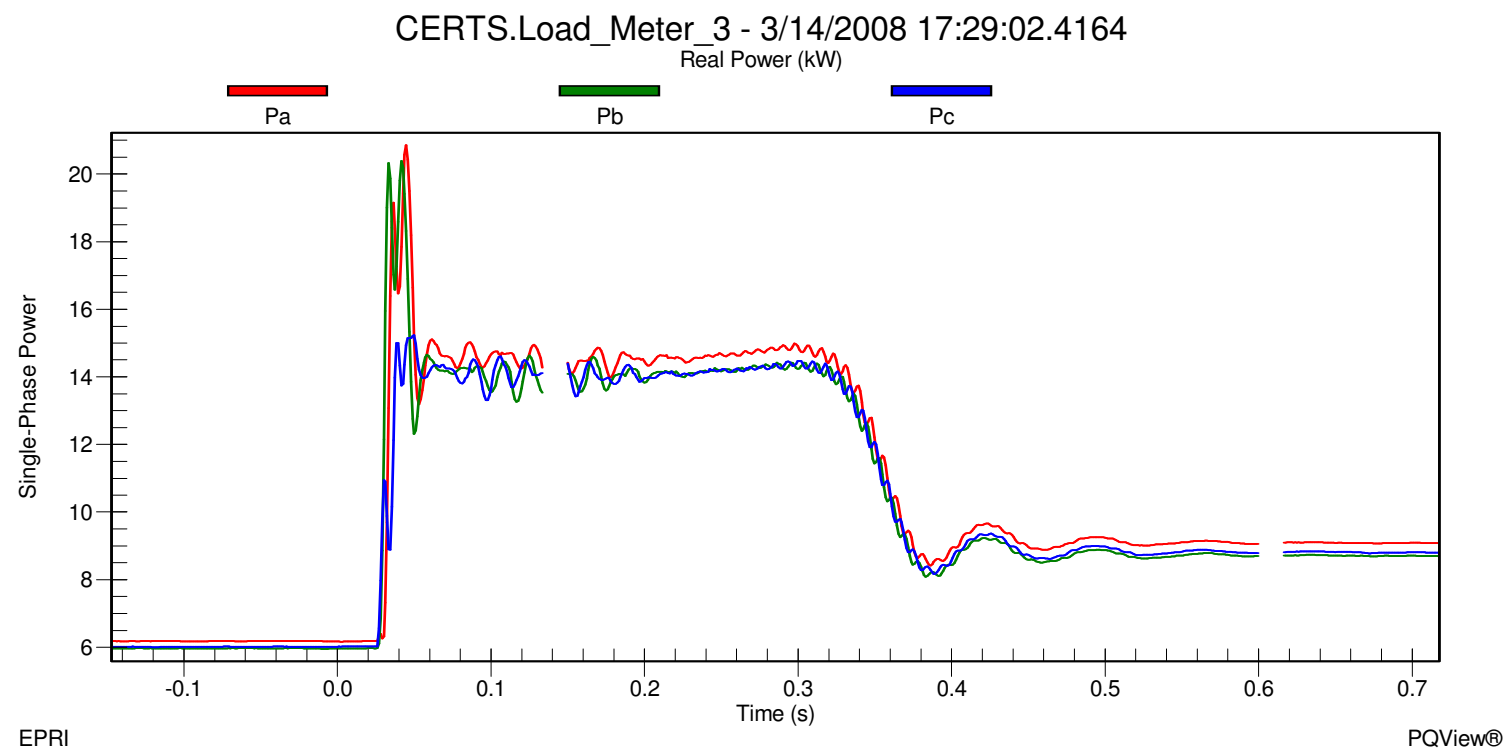

Figure 16a - Load Bank 3 Real Single-Phase Power during Motor Start and Utility Connected for Test 10.3.20 


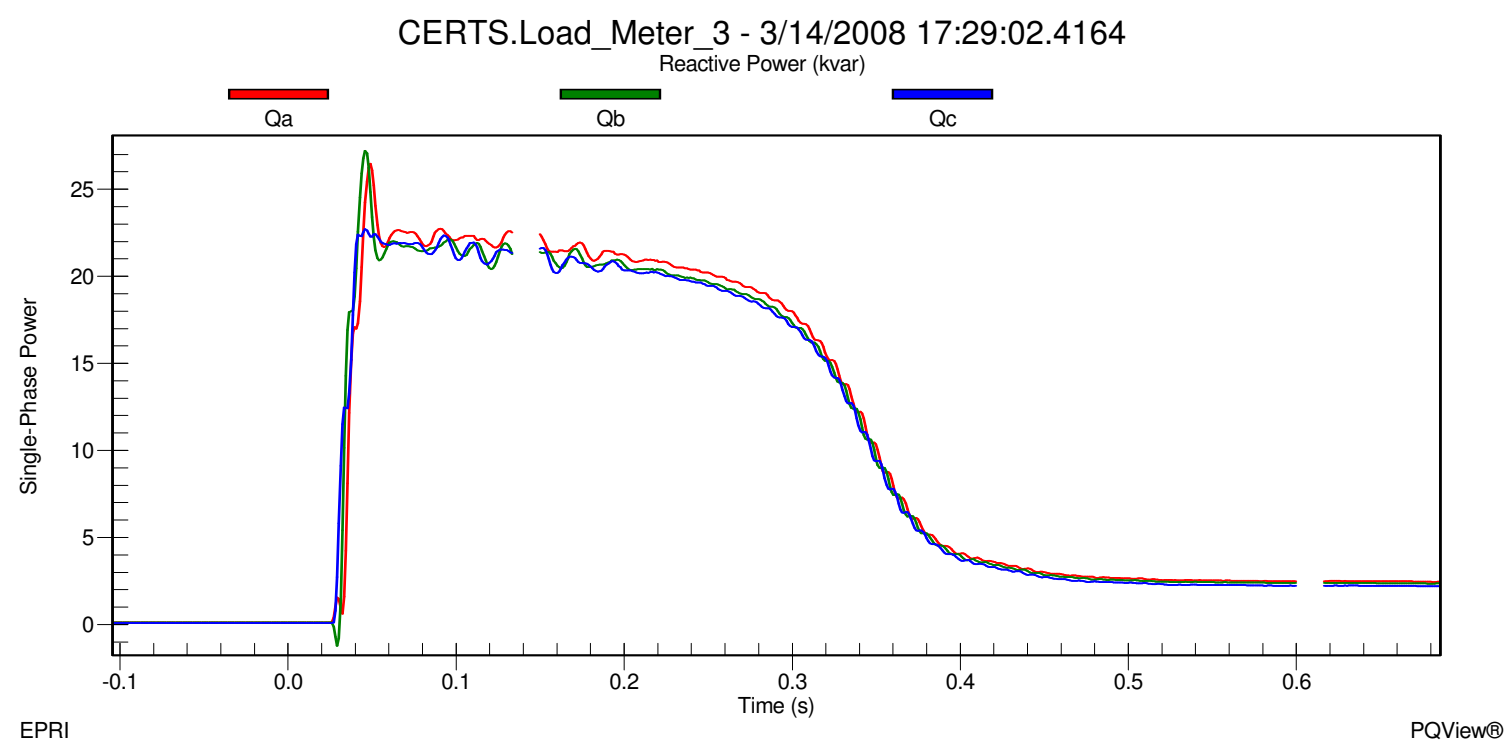

Figure 16b - Load Bank 3 Reactive Single-Phase Power during Motor Start and Utility Connected for Test 10.3.20

In Figures $16 \mathrm{a}$ and $16 \mathrm{~b}$ it can be seen that the load in Zone 3 was approximately $18.2 \mathrm{~kW}$ $(\mathrm{A}=6.2 \mathrm{~kW} \mathrm{~B}=6 \mathrm{~kW} \mathrm{C}=6 \mathrm{~kW})+\mathrm{j} 0.3 \mathrm{kVAr}(\mathrm{A}=0.1 \mathrm{kVAr} B=0.1 \mathrm{kVAr} C=0.1 \mathrm{kVAr})$ before the start of the induction motor and increased to approximately $55.8 \mathrm{~kW}(\mathrm{~A}=21 \mathrm{~kW} \mathrm{~B}=19.6 \mathrm{~kW}$ $\mathrm{C}=15.2 \mathrm{~kW})+\mathrm{j} 75.8 \mathrm{kVAr}(\mathrm{A}=25.9 \mathrm{kVAr} \mathrm{B}=27.2 \mathrm{kVAr} \mathrm{C}=22.7 \mathrm{kVAr})$ during the inrush phase of the motor start. After about 1.555 cycles, the motor settled down to approximately $42.9 \mathrm{~kW} \quad(\mathrm{~A}=14.5 \mathrm{~kW} \quad \mathrm{~B}=14.2 \mathrm{~kW} \quad \mathrm{C}=14.2 \mathrm{~kW})+\mathrm{j} 61.4 \mathrm{kVAr} \quad(\mathrm{A}=20.8 \mathrm{kVAr} \quad \mathrm{B}=20.3 \mathrm{kVAr}$ $\mathrm{C}=20.3 \mathrm{kVAr}$ ) during the warm up phase which lasted about 36.6 cycles ( 0.61 Seconds). When the motor reached steady state, the load in Zone 3 was approximately $27.15 \mathrm{~kW}$ $(A=9.6 \mathrm{~kW} B=8.75 \mathrm{~kW} \mathrm{C}=8.8 \mathrm{~kW})+\mathrm{j} 7 \mathrm{kVAr}(\mathrm{A}=2.5 \mathrm{kVAr} B=2.3 \mathrm{kVAr} C=2.2 \mathrm{kVAr})$.

The voltage and frequency at the static switch before the motor start was approximately $284 \mathrm{~V}$ on A-phase, $279.6 \mathrm{~V}$ on B-phase and $280 \mathrm{~V}$ on C-phase shown in Figure 16c and approximately $60.03 \mathrm{~Hz}$ shown in Figure 16d. When the motor started, the voltage at the static switch during the inrush decreased to approximately $275.8 \mathrm{~V}$ on A-phase, $270.9 \mathrm{~V}$ on B-phase and $272.25 \mathrm{~V}$ on C-phase for about 1.5 cycles. Frequency dropped during the inrush to approximately $59.93 \mathrm{~Hz}$ and quickly increased to approximately $60.03 \mathrm{~Hz}$. Voltage increased as the motor was warming up and eventually settled at a steady state voltage at approximately $283 \mathrm{~V}$ on A-phase, $278.8 \mathrm{~V}$ on B-phase and $279.25 \mathrm{~V}$ on C-phase at an approximate frequency $60.02 \mathrm{~Hz}$. 


\section{CERTS.Meter_2 - 3/14/2008 12:29:03.1856}

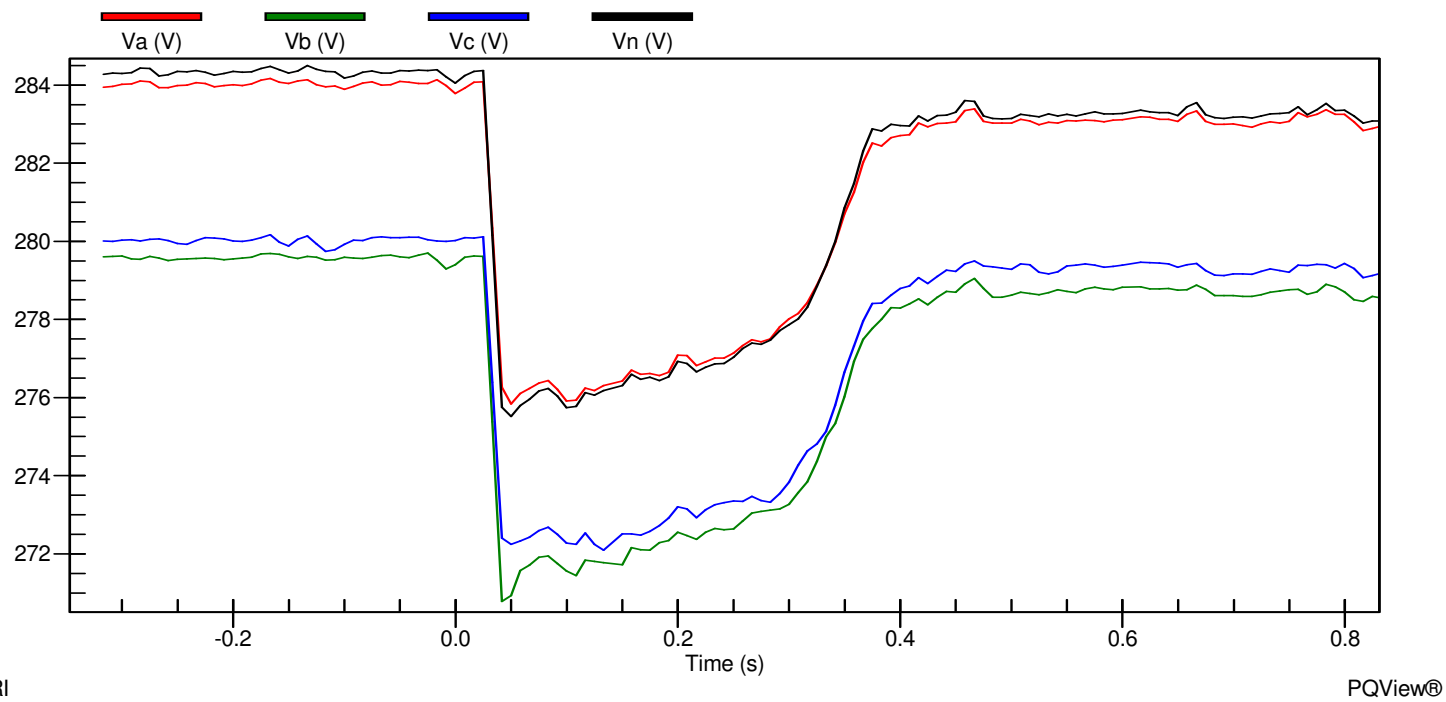

Figure 16c - Static Switch Line-to-Ground Voltages during Motor Start and Utility Connected for Test 10.3.20

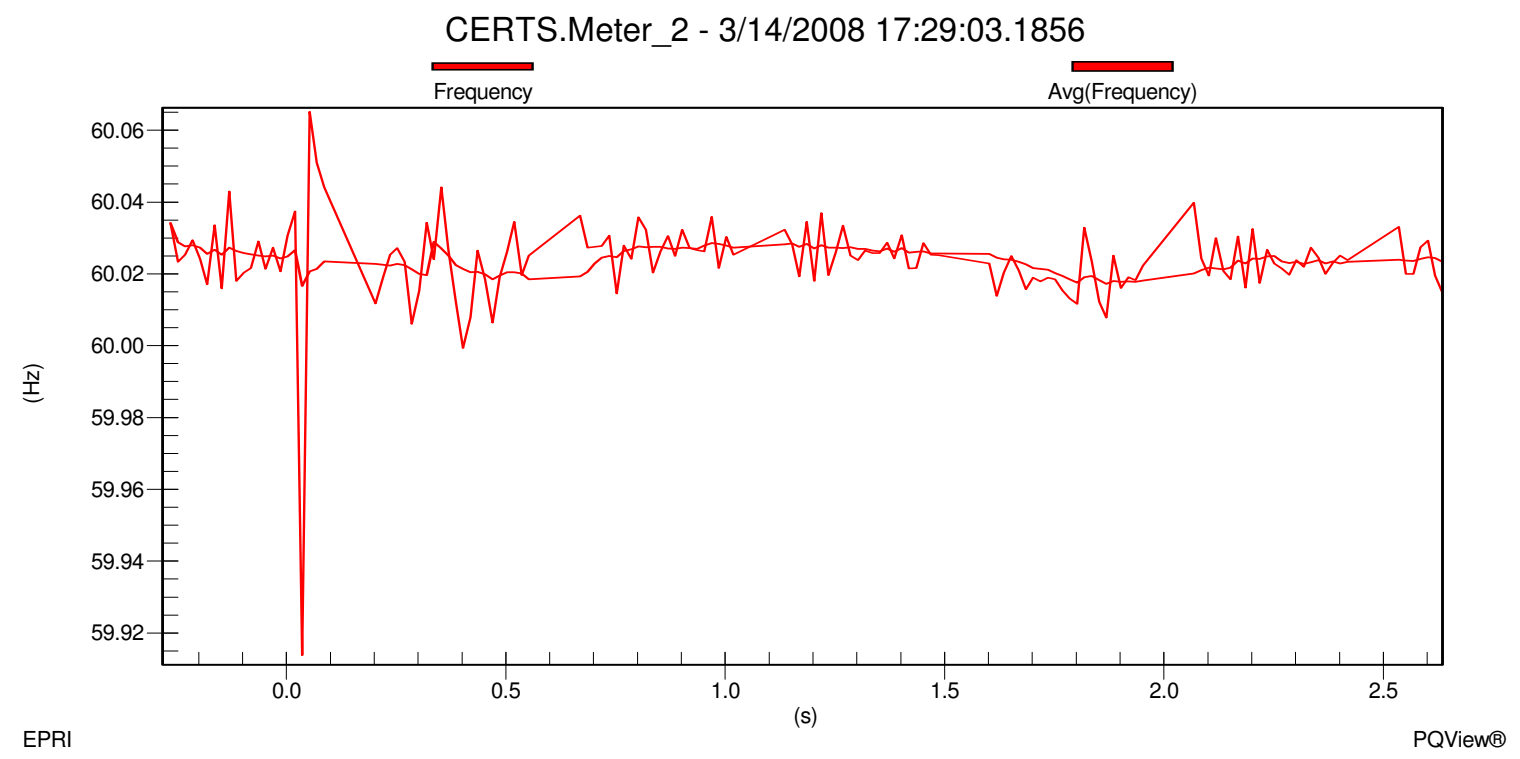

Figure 16d - Static Switch Frequency during Motor Start and Utility Connected for Test 10.3.20 


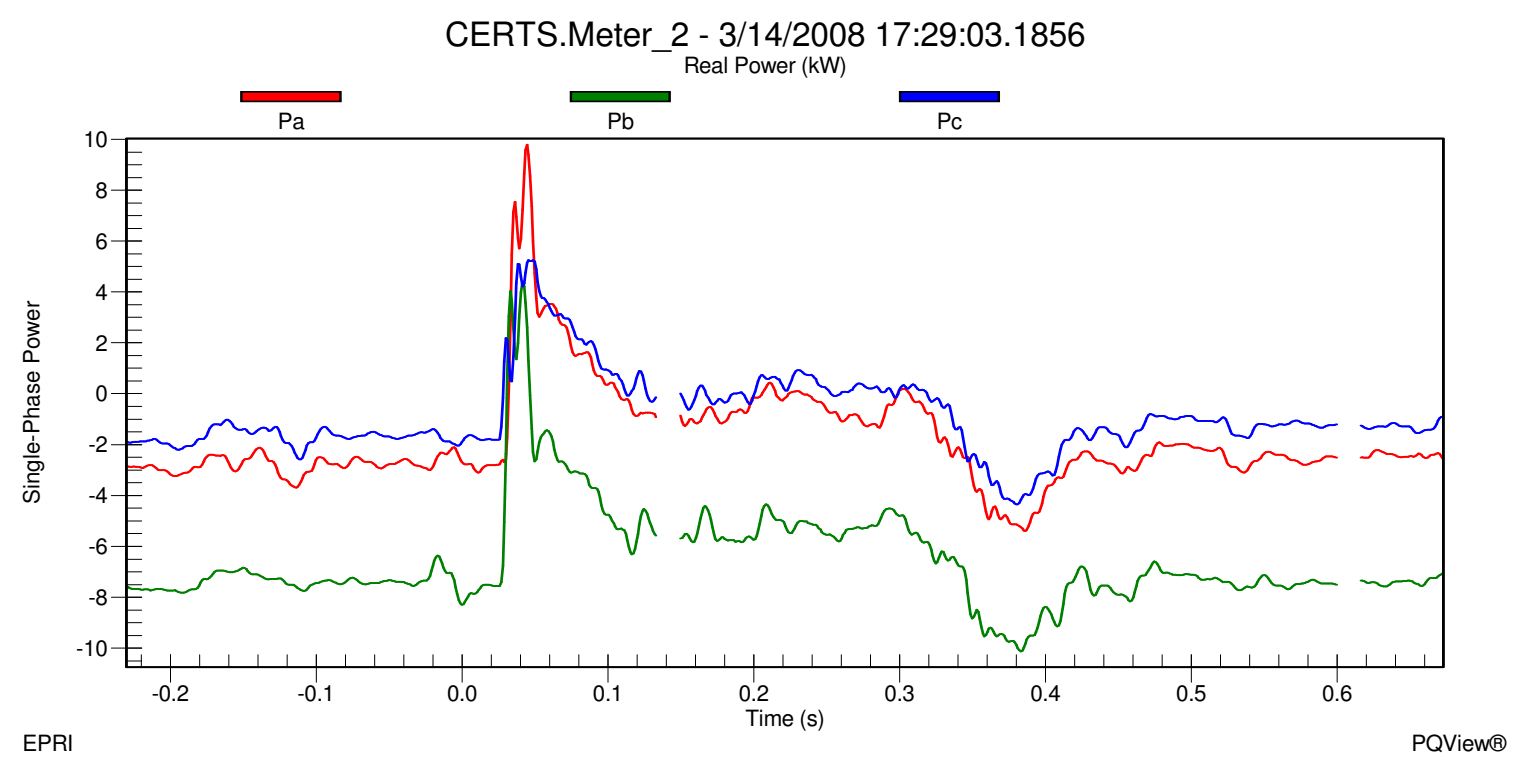

Figure 16e - Static Switch Real Single-Phase Power during Motor Start and Utility Connected for Test 10.3.20

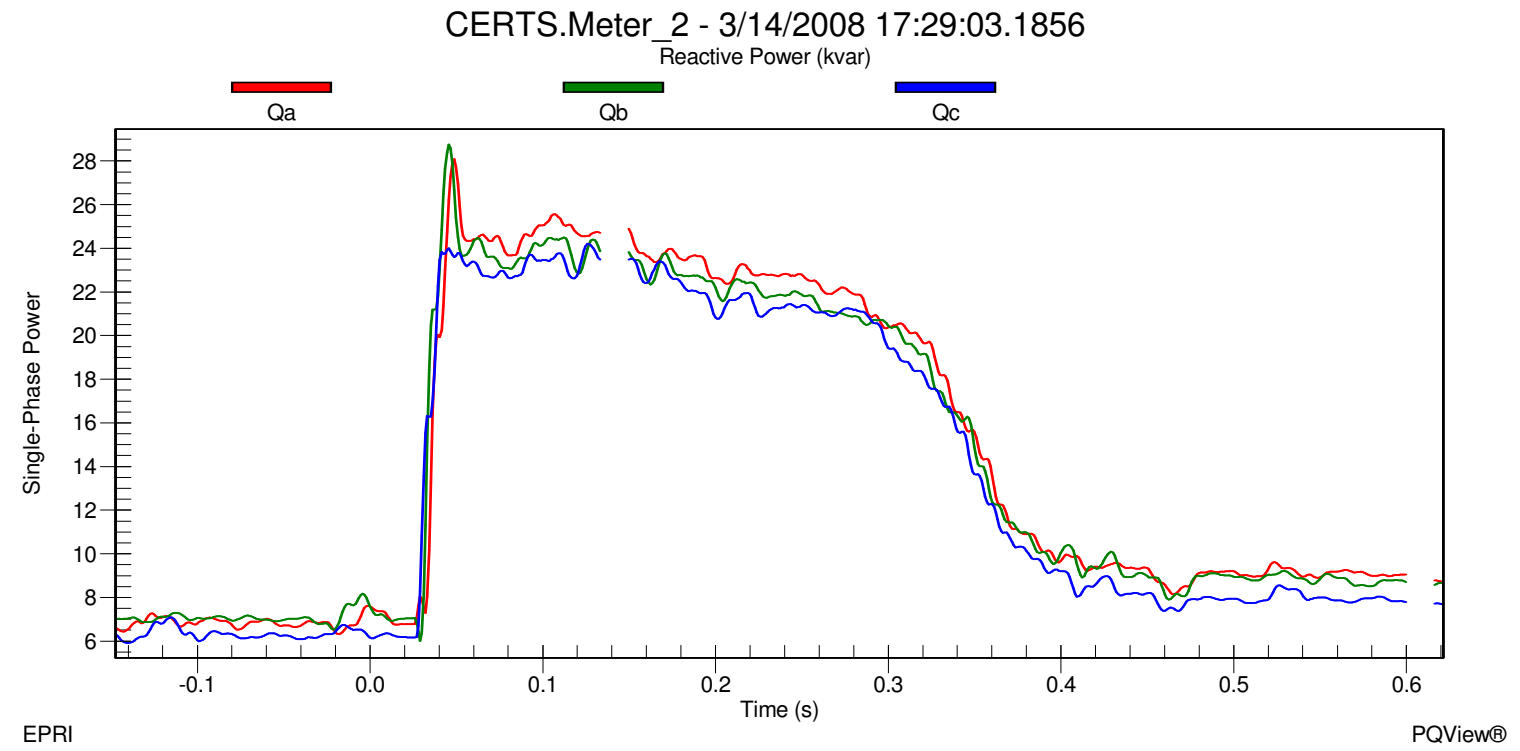

Figure 16f - Static Switch Reactive Single-Phase Power during Motor Start and Utility Connected for Test 10.3.20

Before the motor started Gen-set A1 was producing approximately $33.1 \mathrm{~kW}(\mathrm{~A}=10 \mathrm{~kW}$ $\mathrm{B}=14.7 \mathrm{~kW} \mathrm{C}=8.4 \mathrm{~kW})-\mathrm{j} 13.8 \mathrm{kVAr}(\mathrm{A}=5 \mathrm{kVAr} \mathrm{B}=4.8 \mathrm{kVAr} \mathrm{C}=-4 \mathrm{kVAr})$. This was enough power for the load demands of Load Bank 3 with real power being exported to Zone 6 of approximately $-12.4 \mathrm{~kW}(\mathrm{~A}=-3 \mathrm{~kW} \mathrm{~B}=-7.5 \mathrm{~kW} \mathrm{C}=-1.9 \mathrm{~kW})$ shown in Figure 16e. The grid was supporting the reactive power of the microgrid with approximately $\mathrm{j} 20 \mathrm{kVAr}$ ( $\mathrm{A}=6.75 \mathrm{kVAr} \mathrm{B}=7 \mathrm{kVAr} \mathrm{C}=6.25 \mathrm{kVAr}$ ) shown in Figure 16f. When the motor started the inrush caused the utility to supply approximately j81.5kVAr $(A=28.3 \mathrm{kVAr} B=29.2 \mathrm{kVAr}$ $\mathrm{C}=24 \mathrm{kVAr}$ ) and to pick up the load demand of Load Bank 6 and approximately $18.6 \mathrm{~kW}$ 
$(\mathrm{A}=9.9 \mathrm{~kW} \mathrm{~B}=3.4 \mathrm{~kW} \mathrm{C}=5.3 \mathrm{~kW})$ of the load demand in Zone 3 . The power that was being supplied by Gen-set A1 to Load Bank 6 was now supplying the motor inrush which can be seen in Figure 16e as the real power through the static switch becomes positive. Genset $\mathrm{A} 1$ increased its real and reactive power output levels to approximately $54.15 \mathrm{~kW}$ $(A=17.1 \mathrm{~kW} \quad B=21.8 \mathrm{~kW} \quad \mathrm{C}=15.25 \mathrm{~kW})+\mathrm{j} 1.8 \mathrm{kVAr} \quad(\mathrm{A}=-0.3 \mathrm{kVAr} B=0.3 \mathrm{kVAr} \quad \mathrm{C}=1.8 \mathrm{kVAr})$. Notice the signs of the VAr output changed from negative to positive for Gen-set A1 in order to support the induction motor load in Zone 3.

When the motor reached steady state, the real and reactive power through the static switch was approximately $-11.3 \mathrm{~kW}(\mathrm{~A}=-2.6 \mathrm{~kW} \quad \mathrm{~B}=-7.4 \mathrm{~kW} \quad \mathrm{C}=-1.3 \mathrm{~kW})+\mathrm{j} 25.8 \mathrm{kVAr}$ $(\mathrm{A}=9 \mathrm{kVAr} \mathrm{B}=8.8 \mathrm{kVAr} \mathrm{C}=8 \mathrm{kVAr})$ which meant that $\mathrm{Gen}$-set $\mathrm{A} 1$ was supplying the power for the induction motor and approximately $11.3 \mathrm{~kW}(\mathrm{~A}=2.6 \mathrm{~kW} \mathrm{~B}=7.4 \mathrm{~kW} \mathrm{C}=1.3 \mathrm{~kW})$ of Load Bank 6. The real power in Gen-set A1 increased from its initial value at the beginning of the test to approximately $40.5 \mathrm{~kW}(\mathrm{~A}=12.4 \mathrm{~kW} \mathrm{~B}=17.1 \mathrm{~kW} \mathrm{C}=11 \mathrm{~kW})$ and the reactive power went relatively back to the initial value before the motor started of approximately $-\mathrm{j} 12.5 \mathrm{kVAr}(\mathrm{A}=-4.6 \mathrm{kVAr} B=-4.4 \mathrm{kVAr} \mathrm{C}=-3.5 \mathrm{kVAr})$. Once all the data was verified and recorded into the DAS Database, the motor was shut down and the static switch was directed by the EMS to manually open.

As soon as the static switch opened, Meter 1 recorded real power increased to approximately $38.1 \mathrm{~kW}(\mathrm{~A}=10.1 \mathrm{~kW} \mathrm{~B}=18.2 \mathrm{~kW} \mathrm{C}=9.8 \mathrm{~kW})$ and reactive power decreased to $j 1 \mathrm{kVAr}(\mathrm{A}=0.2 \mathrm{kVAr} B=0.6 \mathrm{kVAr} C=0.2 \mathrm{kVAr})$ satisfying the load demand in Load Bank 6 which was approximately $37.85 \mathrm{~kW}(\mathrm{~A}=10.05 \mathrm{~kW} \quad \mathrm{~B}=18 \mathrm{~kW} \mathrm{C}=9.8 \mathrm{~kW})+\mathrm{j} 0.659 \mathrm{kVAr}$ $(\mathrm{A}=0.124 \mathrm{kVAr} \mathrm{B}=0.41 \mathrm{kVAr} \mathrm{C}=0.125 \mathrm{kVAr})$ and not supplying any power beyond the static switch to Load Bank 3. $0 \mathrm{~kW}(\mathrm{~A}=0 \mathrm{~kW} \mathrm{~B}=0 \mathrm{~kW} C=0 \mathrm{~kW})+\mathrm{j} 0 \mathrm{kVAr}(\mathrm{A}=0 \mathrm{kVAr}$ $\mathrm{B}=0 \mathrm{kVAr} \mathrm{C}=0 \mathrm{kVAr}$ ) was recorded at the static switch, indicating that power was not flowing through the static switch. Load Bank 3 loads reduced slightly to $16.97 \mathrm{~kW}$ $(\mathrm{A}=5.645 \mathrm{~kW} \quad \mathrm{~B}=5.66 \mathrm{~kW} \quad \mathrm{C}=5.665 \mathrm{~kW})+\mathrm{j} 0.3205 \mathrm{kVAr} \quad(\mathrm{A}=0.103 \mathrm{kVAr} \quad \mathrm{B}=0.12 \mathrm{kVAr}$ $\mathrm{C}=0.0975 \mathrm{kVAr}$ ). This load reduction resulted from a voltage drop in the microgrid, shown in Figure $16 \mathrm{~g}$, from approximately $284.1 \mathrm{~V}$ on A-phase, $279.9 \mathrm{~V}$ on B-phase and $280 \mathrm{~V}$ on C-phase when connected to the utility to $272.2 \mathrm{~V}$ on A-phase, $271.2 \mathrm{~V}$ on B-phase and $281.9 \mathrm{~V}$ on C-phase at Meter 3 when Islanded. 


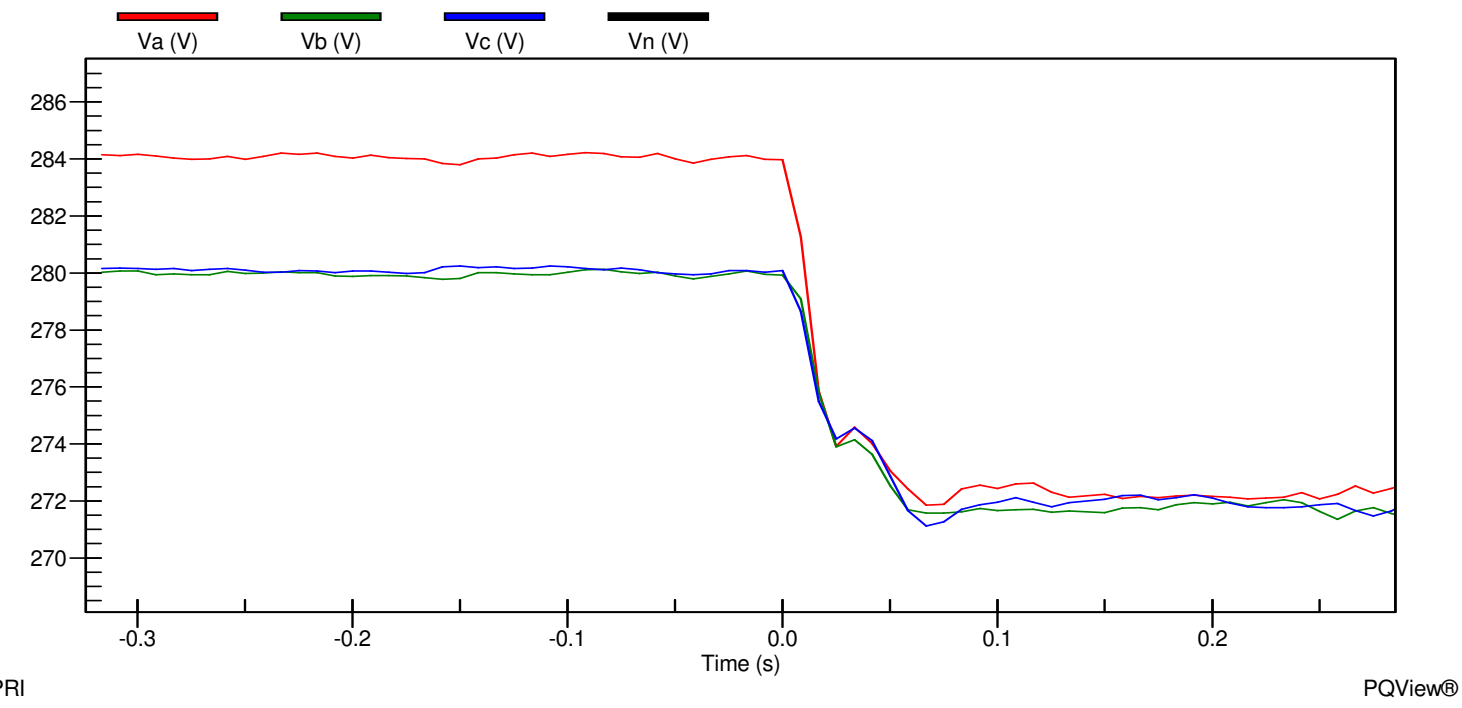

Figure 16g - Meter 3 Line-to-Ground Voltages during Utility Connected to Island mode for Test 10.3.20

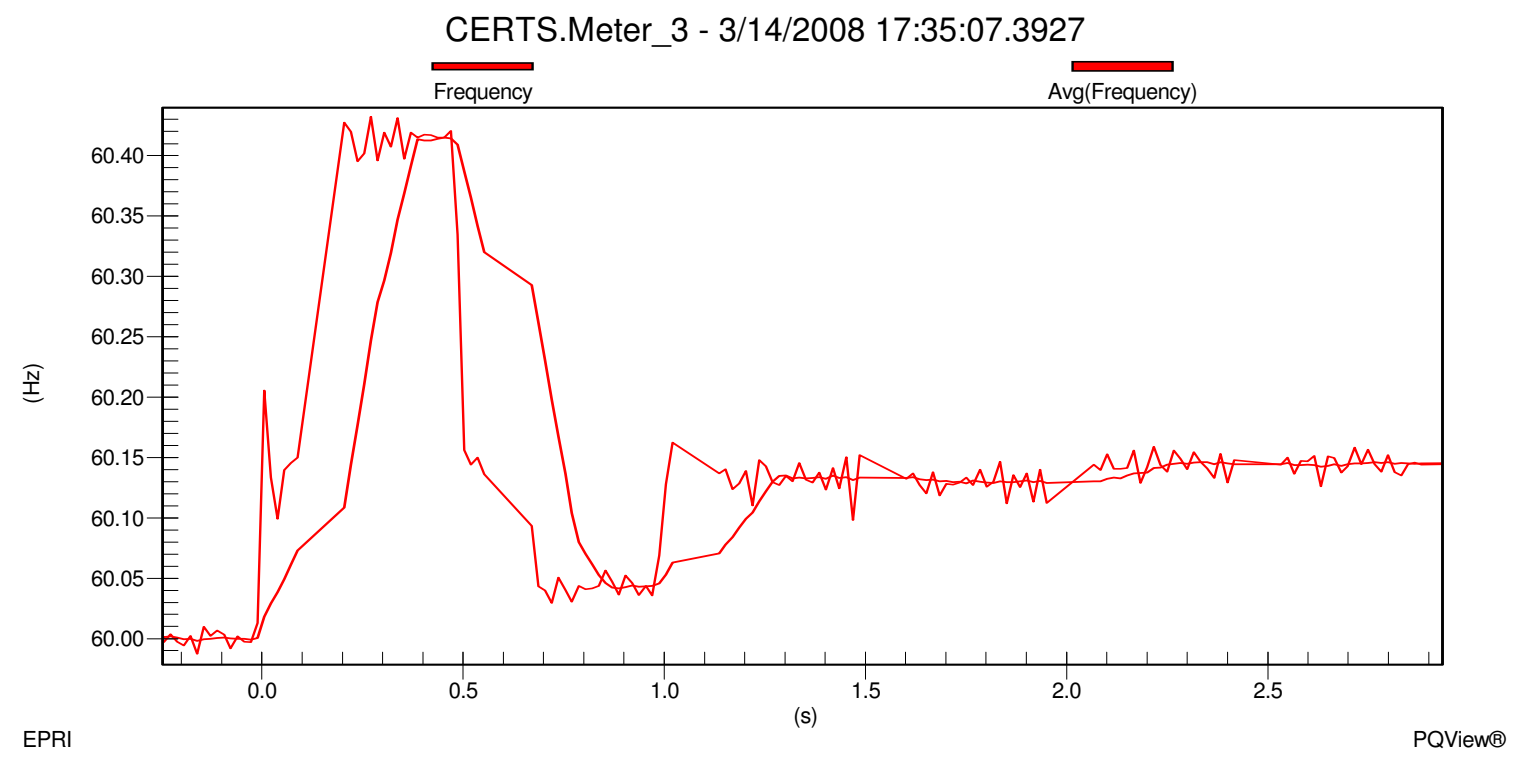

Figure 16h - Meter 3 Frequency during Utility Connected to Island mode for Test 10.3.20

Frequency change in the microgrid, shown in Figure 16h, increased from approximately $60.00 \mathrm{~Hz}$ when connected to the utility grid to approximately $60.14 \mathrm{~Hz}$ when islanded. 
This change in frequency was part of the CERTS algorithm which allowed Gen-set A1 to decrease its output real power to approximately $19.4 \mathrm{~kW}(\mathrm{~A}=6.4 \mathrm{~kW} \mathrm{~B}=6.8 \mathrm{~kW} \mathrm{C}=6.2 \mathrm{~kW})$. Reactive power in Gen-set A1 increased to approximately j4kVAr $(A=1 \mathrm{kVAr} B=1.5 \mathrm{kVAr}$ $\mathrm{C}=1.5 \mathrm{kVAr})$. Meter 3 was approximately $-2.4 \mathrm{~kW}(\mathrm{~A}=-0.8 \mathrm{~kW} B=-1.1 \mathrm{~kW} \quad \mathrm{C}=-0.5 \mathrm{~kW})-$ j3.69kVAr $(A=-0.85 \mathrm{kVAr} B=-1.4 \mathrm{kVAr} C=-1.44 \mathrm{kVAr})$ indicating that Gen-set A1 was satisfying the load in Load Bank 3 and the power losses in the electrical lines. All data was verified and recorded into the DAS Database. The microgrid ran for a couple of minutes in this electrical state before the $10 \mathrm{Hp}$ induction motor was started in Zone 3.

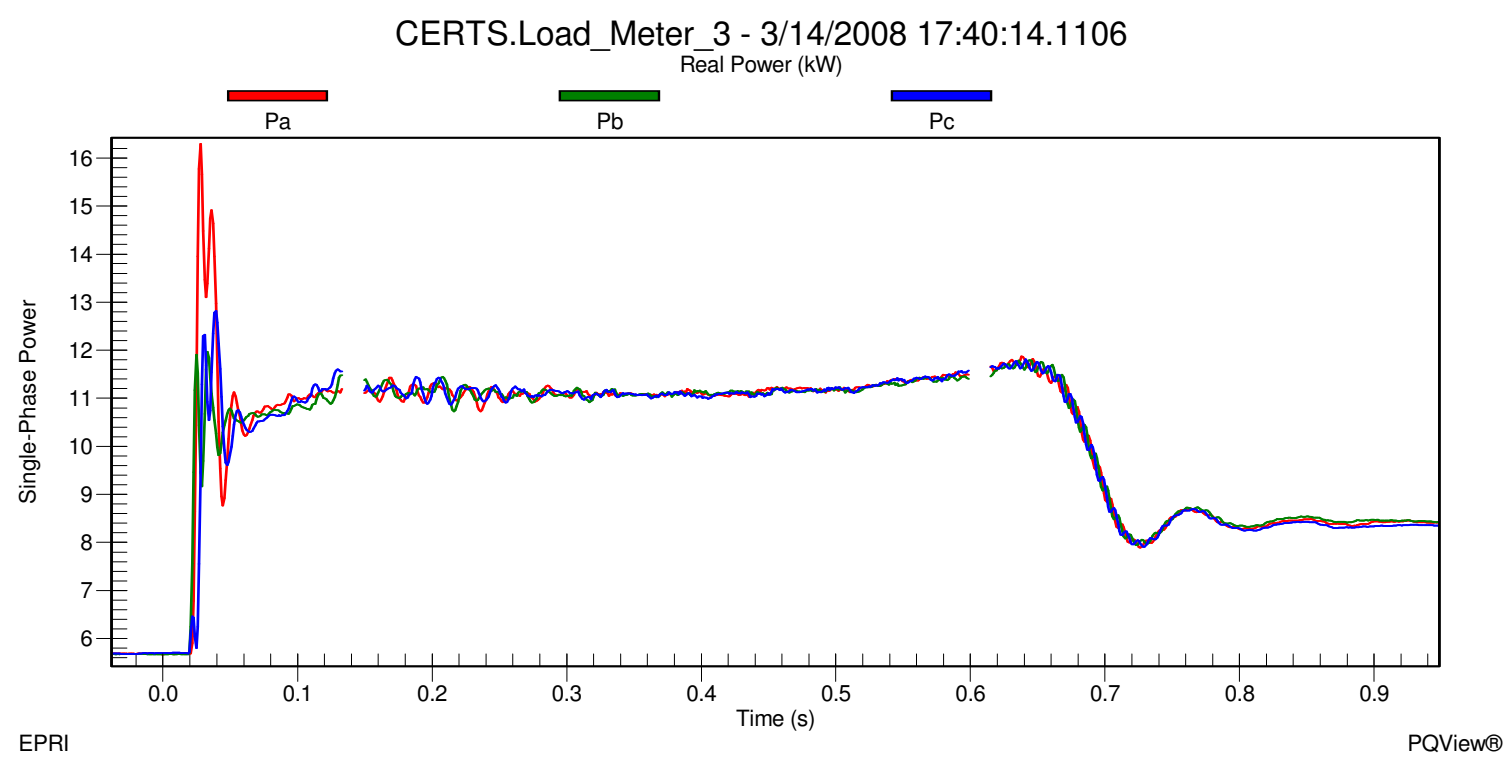

Figure 16i - Load Bank 3 Real Single-Phase Power during Motor Start and Islanded for Test 10.3.20

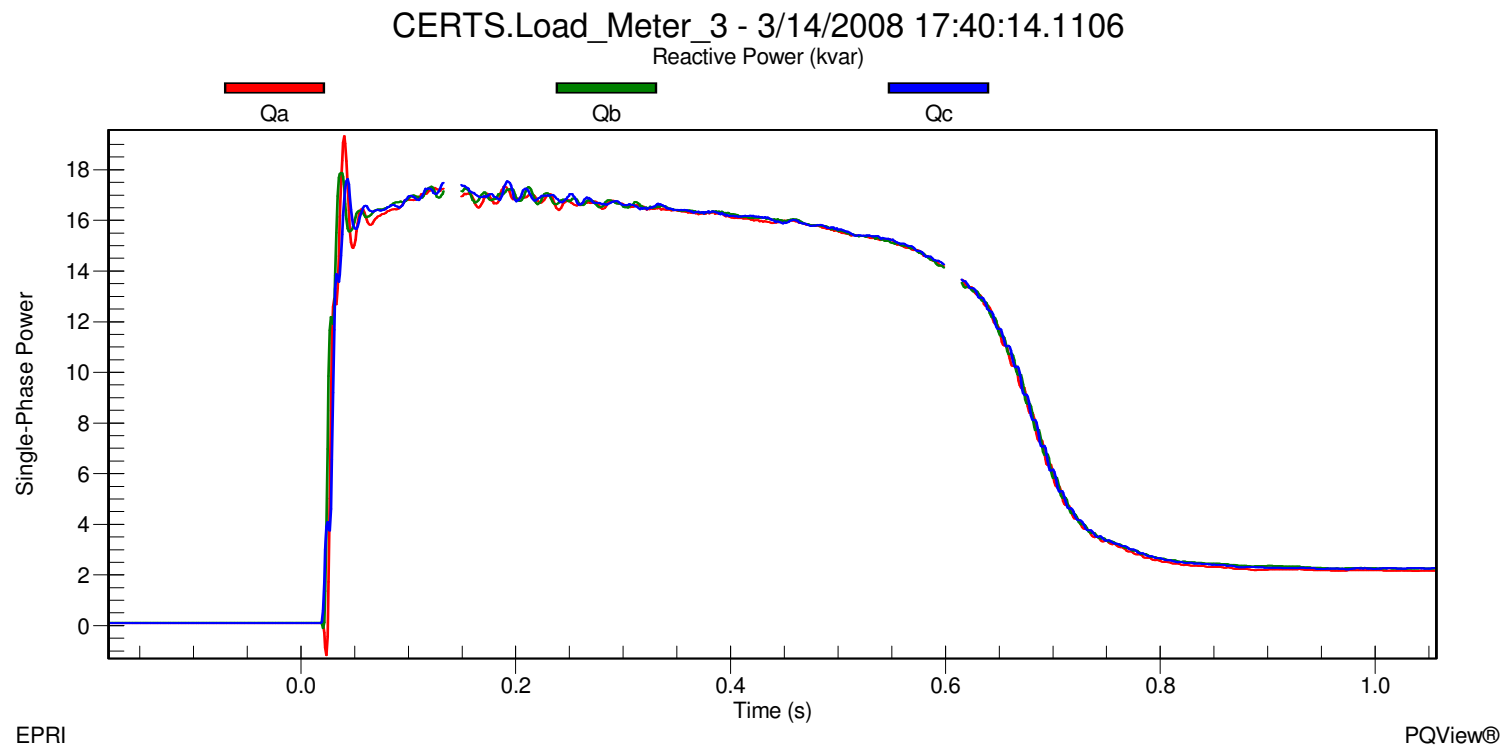

Figure 16j - Load Bank 3 Reactive Single-Phase Power during Motor Start and Islanded for Test 10.3.20 
In Figures 16i and 16j it can be seen that the load in Zone 3 was approximately $17.1 \mathrm{~kW}$ $(\mathrm{A}=5.7 \mathrm{~kW} \mathrm{~B}=5.7 \mathrm{~kW} \mathrm{C}=5.7 \mathrm{~kW})+\mathrm{j} 0.6 \mathrm{kVAr}(\mathrm{A}=0.2 \mathrm{kVAr} \mathrm{B}=0.2 \mathrm{kVAr} \mathrm{C}=0.2 \mathrm{kVAr})$ before the start of the induction motor and increased to approximately $38.6 \mathrm{~kW}(\mathrm{~A}=16.4 \mathrm{~kW}$ $B=10.2 \mathrm{~kW} C=12 \mathrm{~kW})+j 54.75 \mathrm{kVAr}(\mathrm{A}=19.4 \mathrm{kVAr} B=17.75 \mathrm{kVAr} C=17.6 \mathrm{kVAr})$ during the inrush phase of the motor start. After about 1.5 cycles, the motor settled down to approximately $33.6 \mathrm{~kW}(\mathrm{~A}=11.2 \mathrm{~kW} \quad \mathrm{~B}=11.2 \mathrm{~kW} \quad \mathrm{C}=11.2 \mathrm{~kW})+\mathrm{j} 48.6 \mathrm{kVAr} \quad(\mathrm{A}=16.2 \mathrm{kVAr}$ $\mathrm{B}=16.2 \mathrm{kVAr} \mathrm{C}=16.2 \mathrm{kVAr})$ during the warm up phase which lasted about 48 cycles $(0.8$ Seconds). When the motor reached steady state, the load in Zone 3 was approximately $25.2 \mathrm{~kW} \quad(\mathrm{~A}=8.4 \mathrm{~kW} \quad \mathrm{~B}=8.4 \mathrm{~kW} \quad \mathrm{C}=8.4 \mathrm{~kW})+\mathrm{j} 6.75 \mathrm{kVAr} \quad(\mathrm{A}=2.25 \mathrm{kVAr} \quad \mathrm{B}=2.25 \mathrm{kVAr}$ $\mathrm{C}=2.25 \mathrm{kVAr})$.

The voltage and frequency at Meter 3 before the motor start was approximately $272.1 \mathrm{~V}$ on A-phase, $271.8 \mathrm{~V}$ on B-phase and $272.1 \mathrm{~V}$ on C-phase shown in Figure 16k and approximately $60.13 \mathrm{~Hz}$ shown in Figure 161. When the motor started, the voltage at Meter 3 during the inrush decreased to approximately $236.4 \mathrm{~V}$ on A-phase, $235.8 \mathrm{~V}$ on Bphase and $237.6 \mathrm{~V}$ on $\mathrm{C}$-phase for about 1.5 cycles. Frequency dropped during the inrush to approximately $59.76 \mathrm{~Hz}$. Voltage increased as the motor was warming up and eventually settled at a steady state voltage at approximately $268.5 \mathrm{~V}$ on A-phase, $268 \mathrm{~V}$ on B-phase and 268.9V on C-phase at an approximate frequency of $60.15 \mathrm{~Hz}$. 


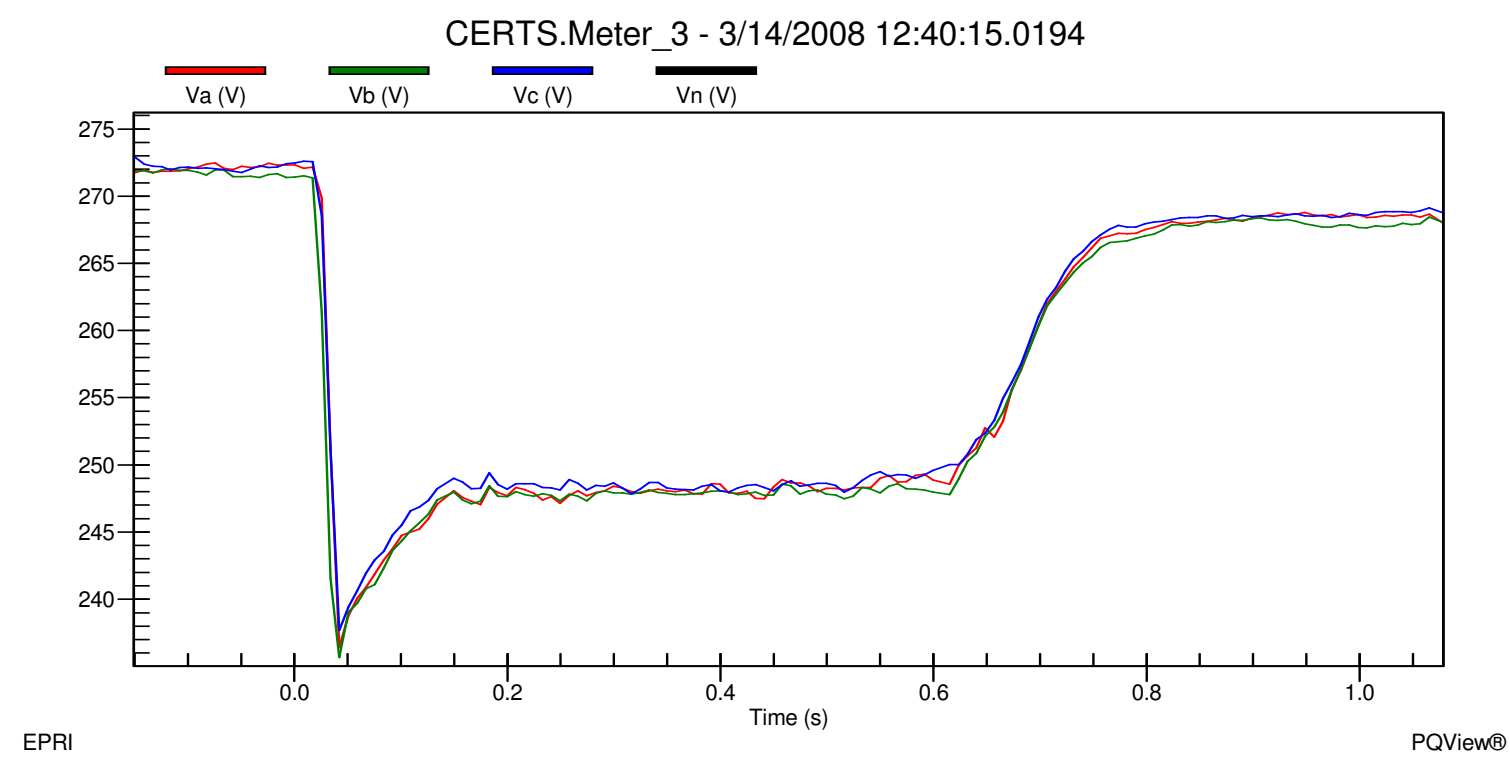

Figure 16k - Meter 3 Line-to-Ground Voltage during Motor Start and Islanded for Test 10.3.20 


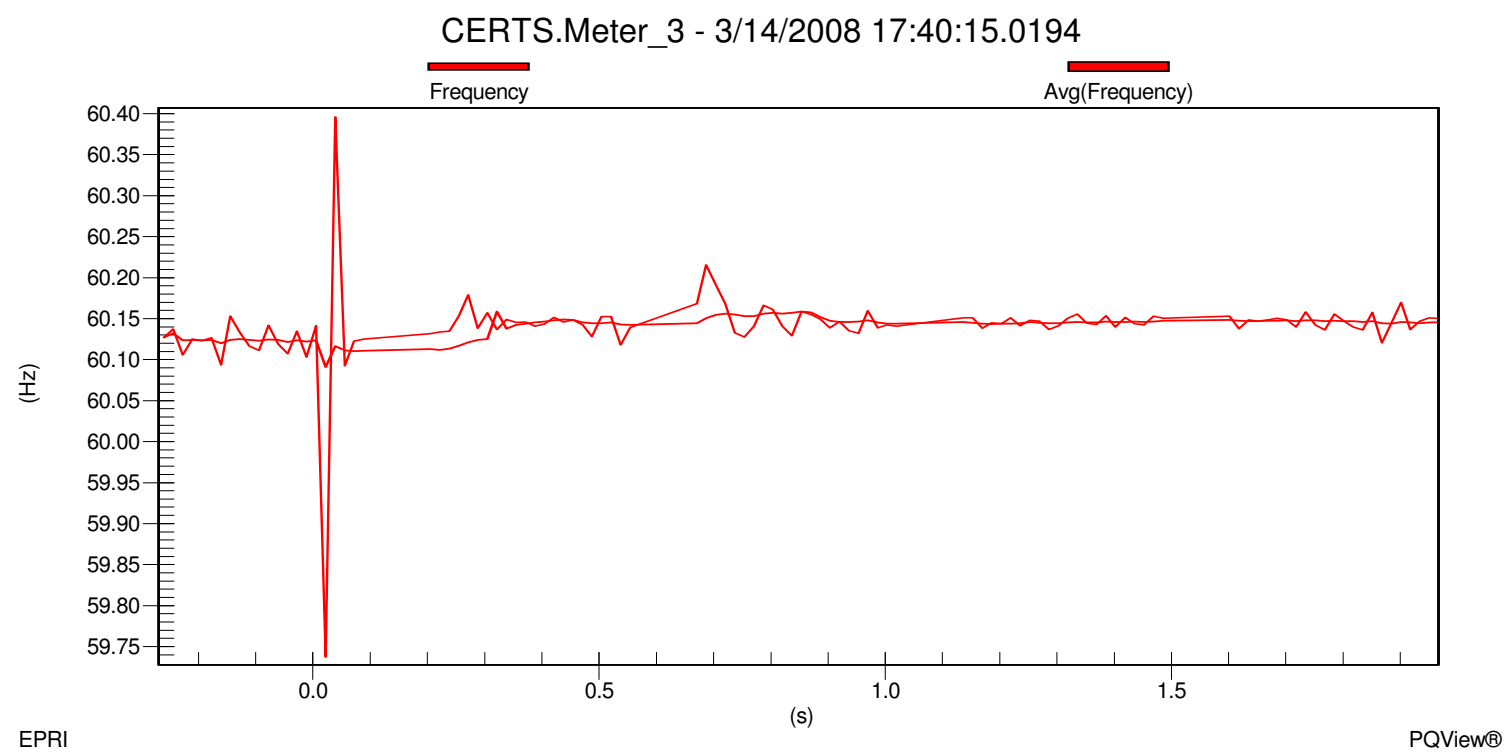

Figure 161 - Meter 3 Frequency during Motor Start and Islanded for Test 10.3.20

Before the motor started, Gen-set A1 was producing approximately $19.28 \mathrm{~kW}(\mathrm{~A}=6.4 \mathrm{~kW}$ $B=6.78 \mathrm{~kW} \mathrm{C}=6.1 \mathrm{~kW})+\mathrm{j} 4 \mathrm{kVAr}(\mathrm{A}=1 \mathrm{kVAr} B=1.5 \mathrm{kVAr} C=1.5 \mathrm{kVAr})$ shown in Figures $16 \mathrm{~m}$ and 16n. The power generated by Gen-set A1 was satisfying the loads in Load Bank 3 and all the electrical losses in the microgrid system. When the motor started the inrush caused Gen-set A1 to increase its output power level to $41.4 \mathrm{~kW}$ (A=16.8kW B=12.3kW $\mathrm{C}=12.3 \mathrm{~kW})+\mathrm{j} 55.6 \mathrm{kVAr}(\mathrm{A}=19.4 \mathrm{kVAr} \mathrm{B}=18.3 \mathrm{kVAr} \mathrm{C}=17.9 \mathrm{kVAr})$. Gen-set A1 decreased its output level while the motor was warming up and eventually dropped to $27.5 \mathrm{~kW}$ $(A=9.2 \mathrm{~kW} \mathrm{~B}=9.5 \mathrm{~kW} C=8.8 \mathrm{~kW})+j 9.9 \mathrm{kVAr}(\mathrm{A}=2.9 \mathrm{kVAr} B=3.5 \mathrm{kVAr} C=3.5 \mathrm{kVAr})$ when the motor reached steady state. Once all the data was verified and recorded into the DAS Database, the static switch was directed by the EMS to manually close. 


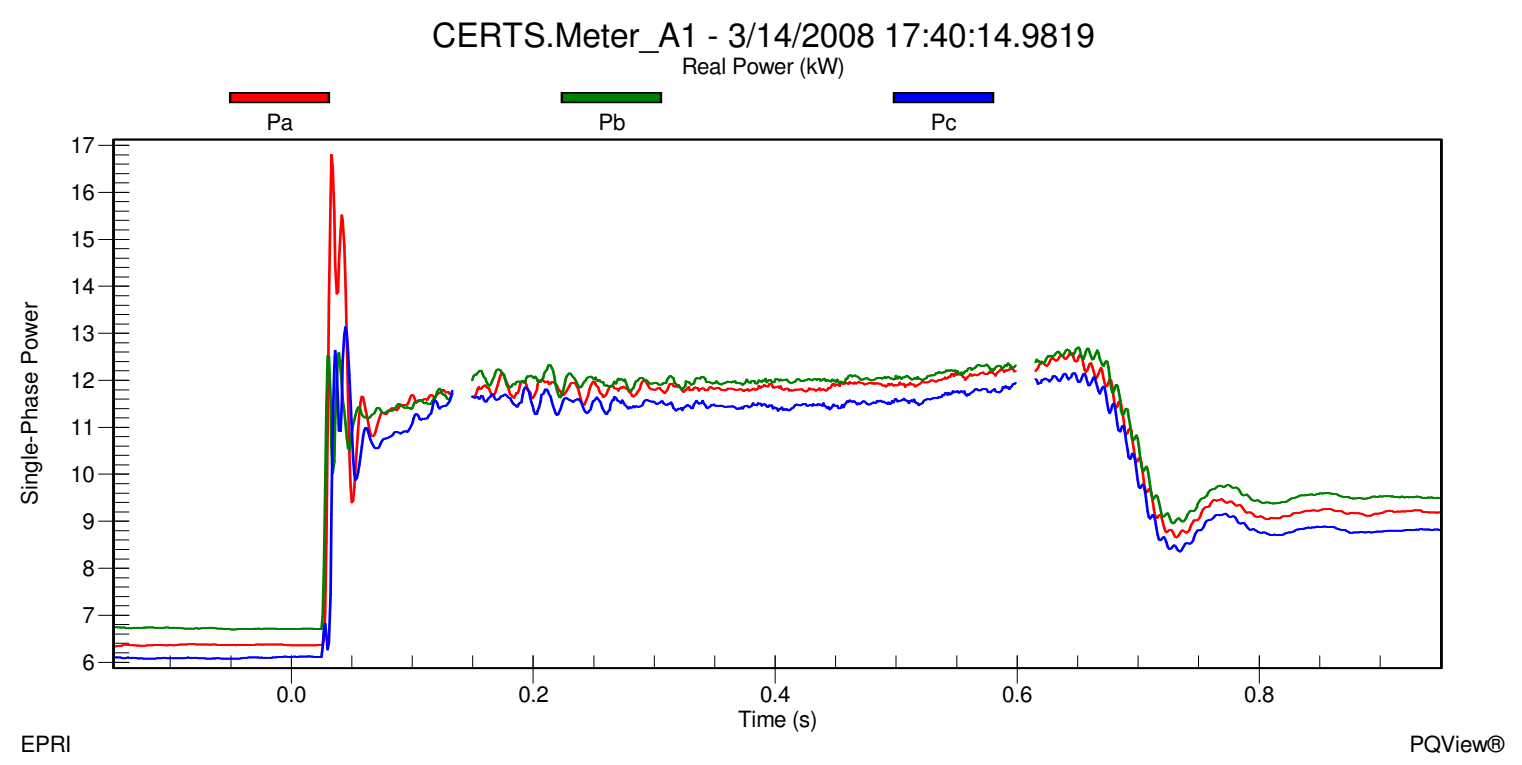

Figure 16m - Gen-set A1 Real Single-Phase Power during Motor Start and Islanded for Test 10.3.20

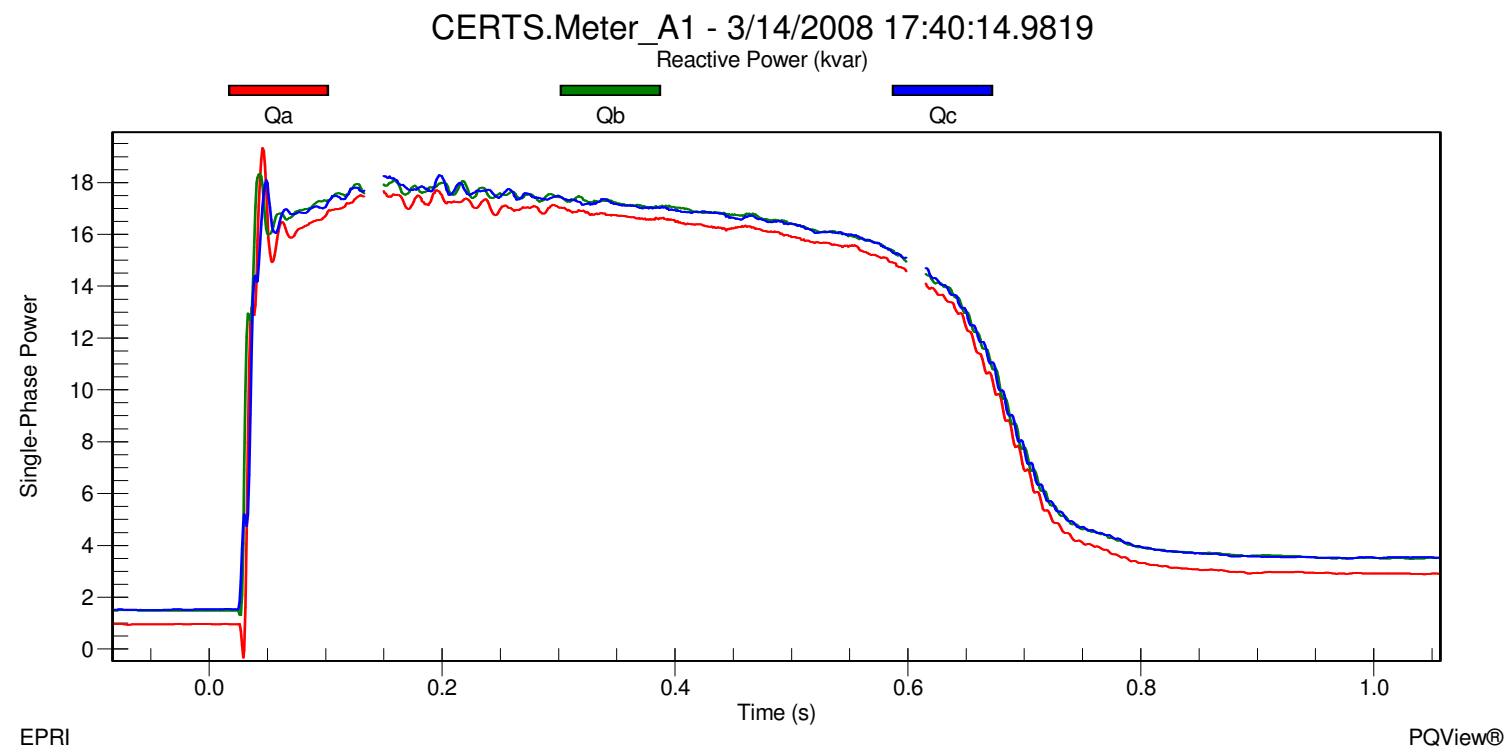

Figure 16n - Gen-set A1 Reactive Single-Phase Power during Motor Start and Islanded for Test 10.3.20

As soon as the static switch closed, Meter 1 recorded real power decreased from approximately $38.09 \mathrm{~kW} \quad(\mathrm{~A}=10.1 \mathrm{~kW} \quad \mathrm{~B}=18.17 \mathrm{~kW} \quad \mathrm{C}=9.82 \mathrm{~kW})$ to $24.1 \mathrm{~kW} \quad(\mathrm{~A}=6.7 \mathrm{~kW}$ $\mathrm{B}=9.7 \mathrm{~kW} \quad \mathrm{C}=7.7 \mathrm{~kW})$ and reactive power increased from approximately $\mathrm{j} 1 \mathrm{kVAr}$ $(\mathrm{A}=0.2 \mathrm{kVAr} \quad \mathrm{B}=0.6 \mathrm{kVAr} \quad \mathrm{C}=0.2 \mathrm{kVAr})$ to $\mathrm{j} 25.9 \mathrm{kVAr} \quad(\mathrm{A}=8.7 \mathrm{kVAr} \quad \mathrm{B}=9 \mathrm{kVAr} \quad \mathrm{C}=8.2 \mathrm{kVAr})$ which means that the utility was satisfying a portion of the load demand in Load Bank 6 and all the reactive power in the microgrid. Figures 160 and 16p show the static switch real power decreasing from approximately $0 \mathrm{~kW}(\mathrm{~A}=0 \mathrm{~kW} B=0 \mathrm{~kW} C=0 \mathrm{~kW})$ to $-13.5 \mathrm{~kW}$ $(A=-3.4 \mathrm{~kW} B=-8 \mathrm{~kW} C=-2.1 \mathrm{~kW})$ and reactive power increasing from $\mathrm{j} 0 \mathrm{kVAr}(\mathrm{A}=0 \mathrm{kVAr}$ 
$\mathrm{B}=0 \mathrm{kVAr} \mathrm{C}=0 \mathrm{kVAr})$ to $\mathrm{j} 24.8 \mathrm{kVAr}(\mathrm{A}=8.5 \mathrm{kVAr} \mathrm{B}=8.5 \mathrm{kVAr} \mathrm{C}=7.8 \mathrm{kVAr})$. At the beginning of the test, the initial power flow through the static switch was $-12.4 \mathrm{~kW}(\mathrm{~A}=-3 \mathrm{~kW} B=-$ $7.5 \mathrm{~kW} \mathrm{C}=-1.9 \mathrm{~kW})+\mathrm{j} 20 \mathrm{kVAr}(\mathrm{A}=6.75 \mathrm{kVAr} B=7 \mathrm{kVAr} \mathrm{C}=6.25 \mathrm{kVAr})$ which is not the same recorded at this point in the test because the $10 \mathrm{Hp}$ motor load is on in Zone 3. Gen-set A1 has picked up the motor load and supporting Load Bank 6 with approximately $13.5 \mathrm{~kW}(\mathrm{~A}=3.4 \mathrm{~kW} \mathrm{~B}=8 \mathrm{~kW} \mathrm{C}=2.1 \mathrm{~kW})$.

Load Bank 3 increased slightly to $25.25 \mathrm{~kW}(\mathrm{~A}=8.62 \mathrm{~kW} \mathrm{~B}=8.27 \mathrm{~kW} \mathrm{C}=8.36 \mathrm{~kW})+\mathrm{j} 6.53 \mathrm{kVAr}$ $(\mathrm{A}=2.27 \mathrm{kVAr} \mathrm{B}=2.2 \mathrm{kVAr} \mathrm{C}=2.06 \mathrm{kVAr})$. This slight load increase is a result from a voltage rise in the microgrid, shown in Figure 16q, from approximately $268.5 \mathrm{~V}$ on Aphase, $268.5 \mathrm{~V}$ on B-phase and $268.5 \mathrm{~V}$ on C-phase when islanded to $283 \mathrm{~V}$ on A-phase, $279 \mathrm{~V}$ on B-phase and $279.5 \mathrm{~V}$ on C-phase at Meter 3 when connected to the utility grid.

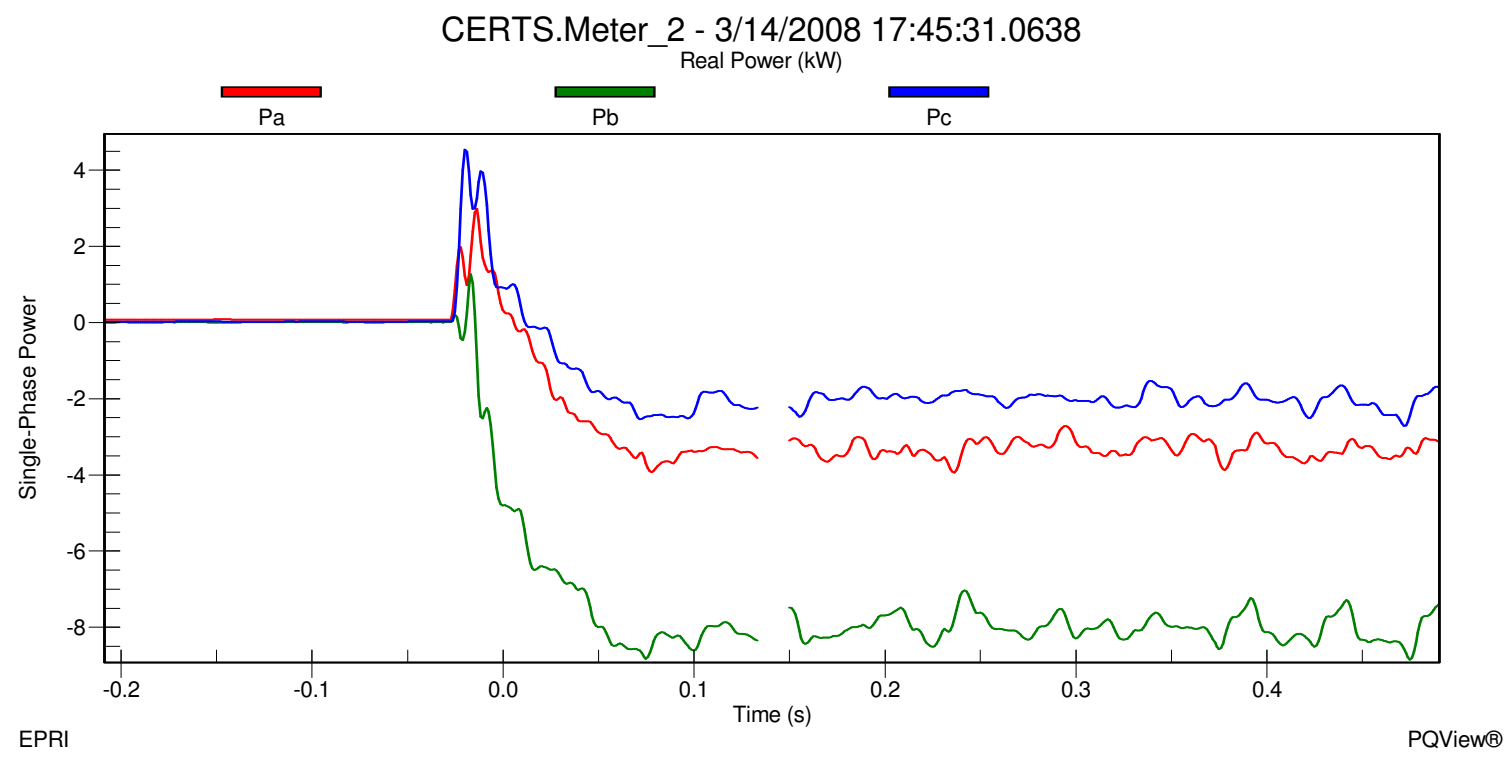

Figure 160 - Static Switch Real Single-Phase Power during Island to Utility Connected mode for Test 10.3.20 


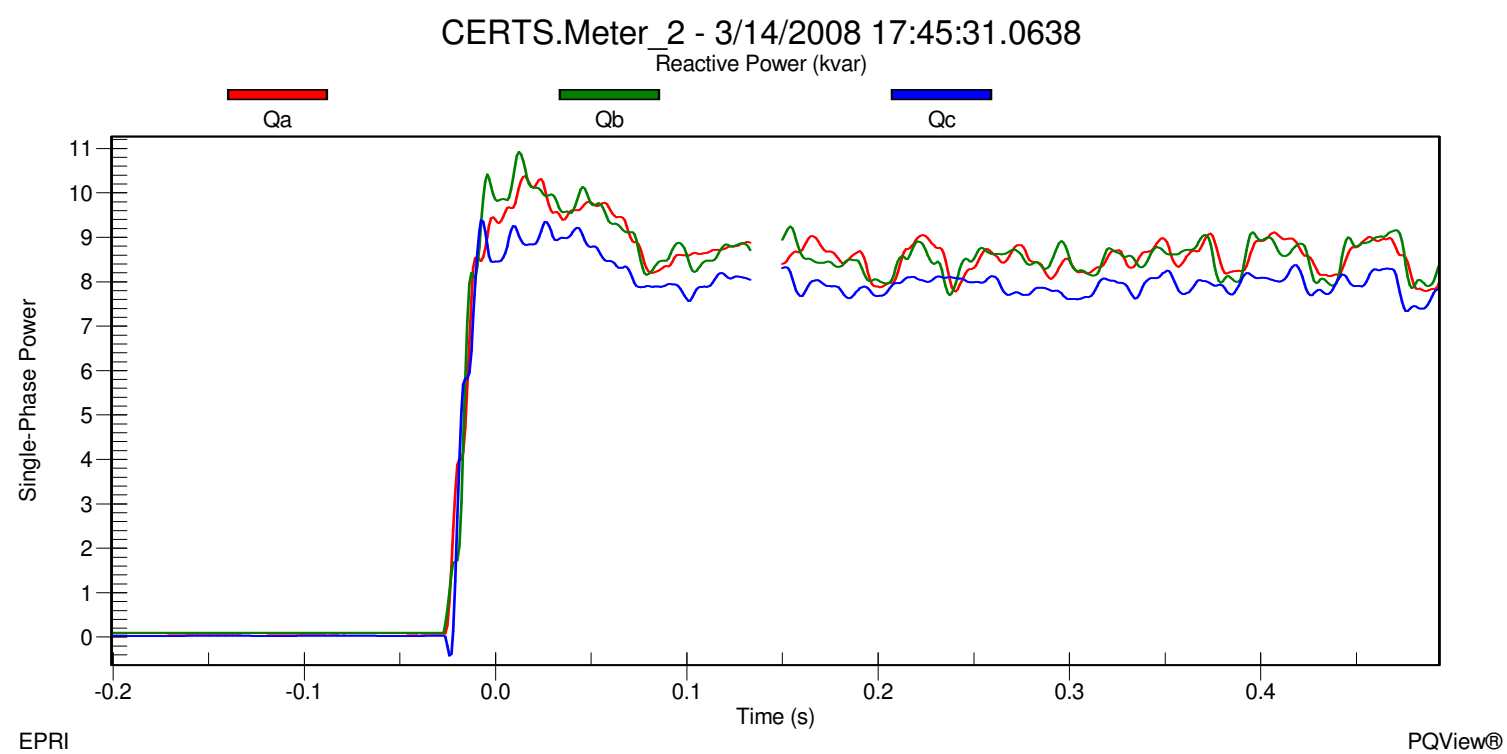

Figure 16p - Static Switch Reactive Single-Phase Power during Island to Utility Connected mode for Test 10.3.20

CERTS.Meter 3 - 3/14/2008 12:45:31.1988

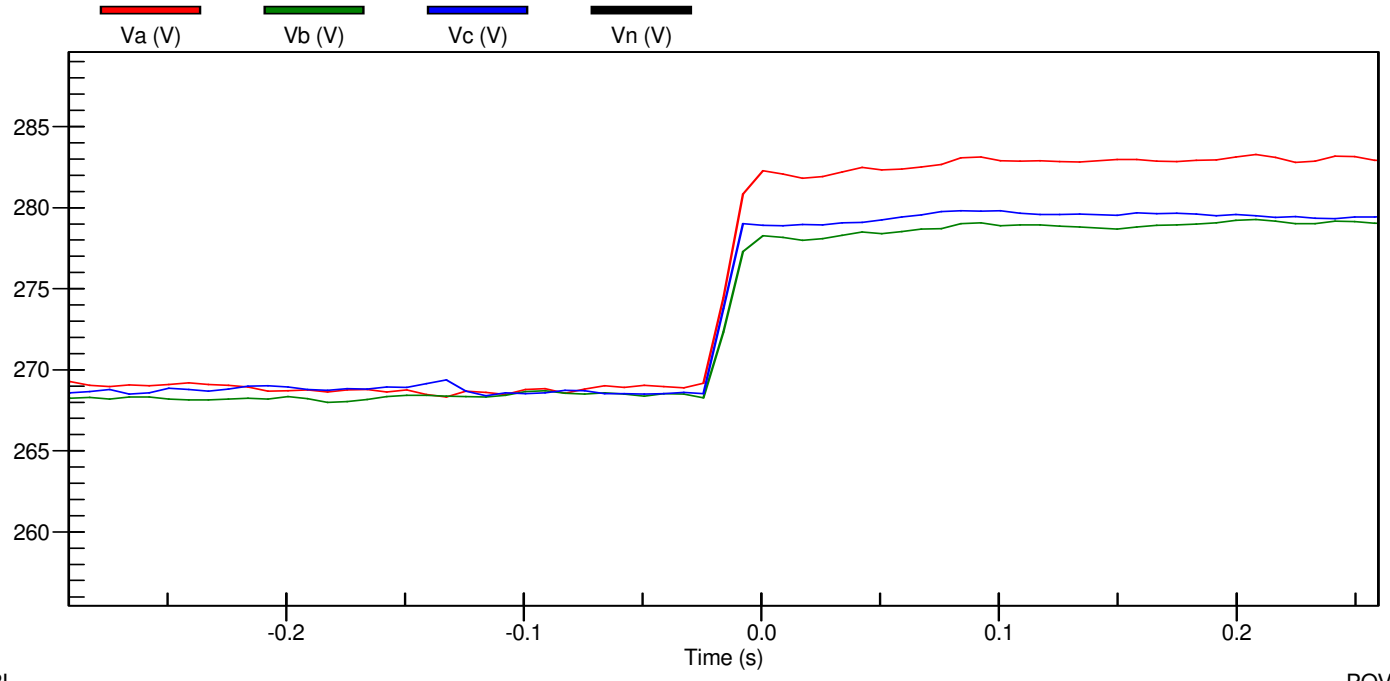

EPRI

PQView®

Figure 16q - Meter 3 Line-to-Ground Voltages during Island to Utility Connected mode for Test 10.3.20 


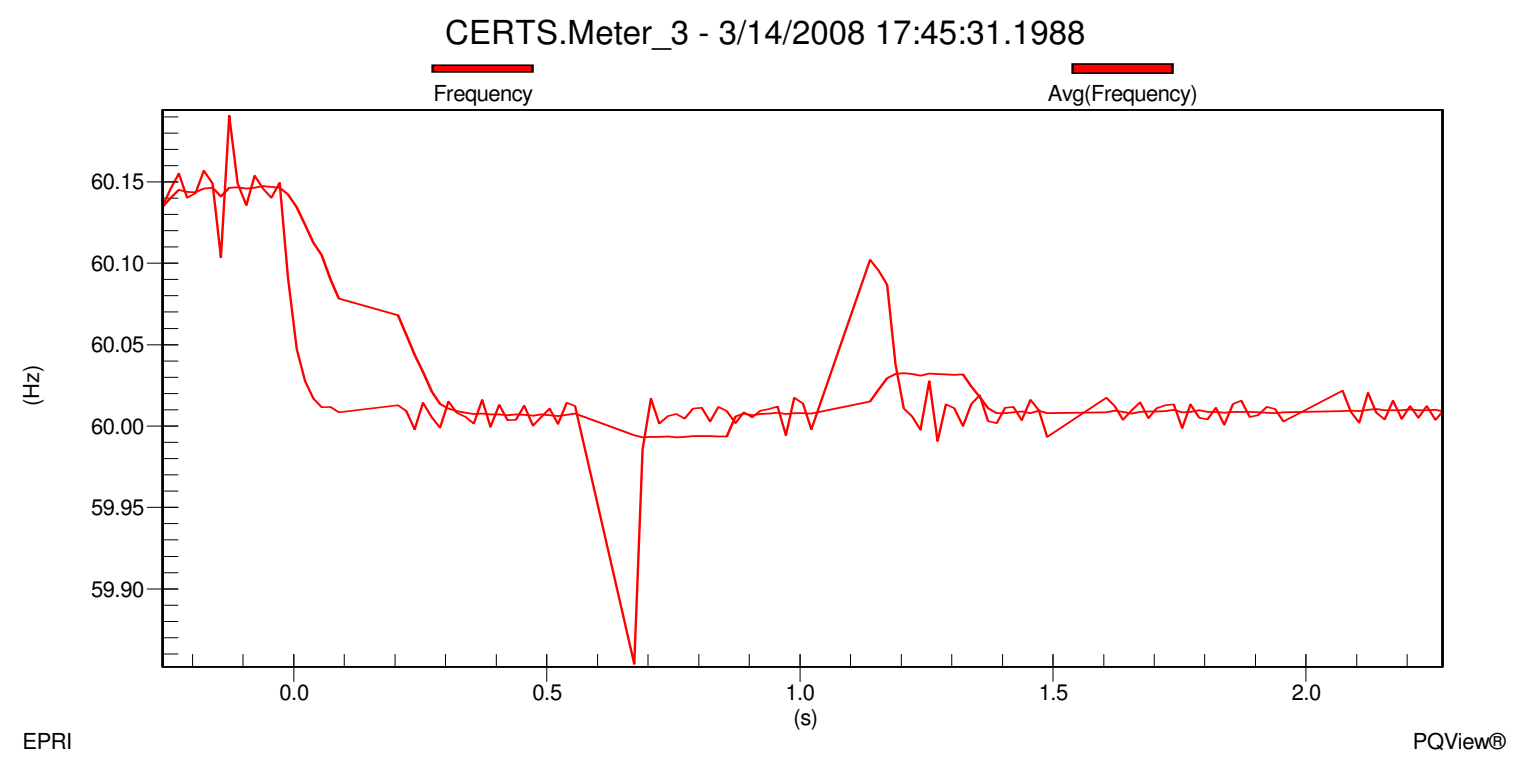

Figure 16r - Static Switch Frequency during Island to Utility Connected mode for Test 10.3.20

Frequency change in the microgrid, shown in Figure 16r, decreased from approximately $60.14 \mathrm{~Hz}$ when islanded to approximately $60.01 \mathrm{~Hz}$ when connected to the utility grid. This change in frequency is due to the frequency no longer being established by the Genset using the CERTS algorithm but by the utility. Gen-set A1 is in zone power control mode therefore when the static switch closed back into the utility Gen-set A1 produced real power based on set-points for the power flow through Zone 3 initialized at the beginning of the test. The real power increased from its initial value at the beginning of the test to approximately $41.3 \mathrm{~kW}(\mathrm{~A}=12.8 \mathrm{~kW} \mathrm{~B}=17.5 \mathrm{~kW} \mathrm{C}=11 \mathrm{~kW})$ for Gen-set $\mathrm{A} 1$. This increase is because Gen-set A1 has picked up the real power of the induction motor in Zone 3. Reactive power for Gen-set A1 was relatively close to the value at the beginning of the test with approximately $-\mathrm{j} 12.6 \mathrm{kVAr}(\mathrm{A}=-4.6 \mathrm{kVAr} \mathrm{B}=-4.2 \mathrm{kVAr} \mathrm{C}=-3.8 \mathrm{kVAr})$. After all the data was verified and recorded into the DAS Database, the motor, Gen-set A1, and Load Banks 3 and 6 were turned off.

\subsubsection{Unbalanced Load Tests, Weak Grid, 0.9 Power Factor} Performance Goal:

Verify and document power flow, Micro-grid frequency changes and protection design with different Gen-set settings during changes of unbalanced load in Load Banks. Continue tests with additional reduction of unbalanced load during an islanded mode of operation.

Three tests were completed in this section with each test event and settings listed in Table 9. During each test Gen-sets A1 and A2 were both set for either unit or zone 
power control mode of operation. Each of the three tests varied the output power command set-point in each Gen-set, along with varying the load settings in load banks LB3, LB4 and LB6. During each test, inductor L11 was in series with the utility grid to simulate a weak grid condition at the PCC to the microgrid.

\begin{tabular}{|c|c|c|c|c|c|c|c|c|c|}
\hline $\begin{array}{c}\text { Test } \\
\text { Event }\end{array}$ & $\begin{array}{c}\text { Gen- } \\
\text { set A1 }\end{array}$ & $\begin{array}{c}\text { Gen - } \\
\text { set A2 }\end{array}$ & Meter 1 & Meter 2 & Meter 3 & Meter 4 & LB 3 & LB 4 & LB 6 \\
\hline 10.4 .12 & $\begin{array}{c}\text { Unit } \\
+40 \mathrm{~kW}\end{array}$ & $\begin{array}{c}\text { Unit } \\
+30 \mathrm{~kW}\end{array}$ & $+20 \mathrm{~kW}$ & $-20 \mathrm{~kW}$ & $-20 \mathrm{~kW}$ & $+10 \mathrm{~kW}$ & $10 \mathrm{~kW}$ & $40 \mathrm{~kW}$ & $40 \mathrm{~kW}$ \\
\hline 10.4 .14 & $\begin{array}{c}\text { Unit } \\
+40 \mathrm{~kW}\end{array}$ & $0 \mathrm{~kW}$ & $+10 \mathrm{~kW}$ & $-30 \mathrm{~kW}$ & $-30 \mathrm{~kW}$ & $0 \mathrm{~kW}$ & $10 \mathrm{~kW}$ & $0 \mathrm{~kW}$ & $40 \mathrm{~kW}$ \\
\hline 10.3 .17 & $\begin{array}{c}\text { Zone } \\
20 \mathrm{~kW}\end{array}$ & $0 \mathrm{~kW}$ & $+20 \mathrm{~kW}$ & $-20 \mathrm{~kW}$ & $-20 \mathrm{~kW}$ & $0 \mathrm{~kW}$ & $20 \mathrm{~kW}$ & $0 \mathrm{~kW}$ & $40 \mathrm{~kW}$ \\
\hline
\end{tabular}

Table 9 -Unbalance Load Test, Weak Grid, settings of Gen-sets and Load Banks

The procedure for each test event began with setting the $\mathrm{kW}$ and $\mathrm{kVAr}$ (i.e., balanced load) conditions, as indicated in Table 9. Before loads were brought on-line, Gen-sets A1 and A2 or just Gen-set A1 depending on the test setup, were started to allow them to warm up. After the Gen-sets ran for a few minutes and steady-state conditions established, the loads were brought on-line, the static switch was allowed to close, and measurements were taken from Meters 1, 2, 3 and 4. The measurements were then compared with the expected values in Table 9. When the measured values coincided with the expected values from the table, then the A-phase load was reduced by $50 \%$ in Load Banks 3, 4 and 6. When all measurements were verified and recorded in the DAS Database for before and after the A-phase reduction, the manual open signal to the static switch was initiated. Gen-sets were observed at this time to make sure they increased or decreased their output power as planned to satisfy the loads at load banks (i.e., LB3, LB4 and LB6) and that a smooth transition occurred. Frequency on the microgrid is also observed for an increase or decrease depending on the Gen-set and load settings. When all measurement were verified and recorded in the DAS Database, the A-phase load was reduced by another 50\% (i.e., 75\% from balanced load value) in Load Banks 3, 4 and 6 . The measurement were verified and recorded into the DAS Database and then Aphase load was reduced by 100\% (i.e., zero load in A-phase) in Load Banks 3, 4 and 6. After all the measurements were verified and recorded into the DAS Database, the manual static switch "Open" condition was released and allowed to self synchronize and reclose into the utility with the unbalanced load. All data (i.e., from the manual open event and the reconnection event) was verified and recorded into the DAS Database. Loads, power control set-points and operation modes were then changed to the settings for the next test. Test results are explained in the following paragraphs. 
For Test 10.4.12 the measured values, after the Gen-sets were warmed up and load banks brought on-line with the A-phase in Load Banks 3 and 6 reduced by $50 \%$ are provided in Table 10.

\begin{tabular}{|c|c|c|c|c|c|c|c|c|c|}
\hline Meter & $\begin{array}{c}\text { Gen- } \\
\text { set A1 }\end{array}$ & $\begin{array}{c}\text { Gen }- \\
\text { set A2 }\end{array}$ & Meter 1 & Meter 2 & Meter 3 & Meter 4 & LB 3 & LB 4 & LB 6 \\
\hline $\begin{array}{c}\text { 3 Phase } \\
\text { kW }\end{array}$ & 40.35 & 31.8 & 4.8 & -25.2 & -28 & 2.7 & 9 & 35.945 & 29.9 \\
\hline $\begin{array}{c}\mathbf{1} \text { Phase } \\
\mathbf{k W}\end{array}$ & $\mathrm{B}=15.75$ & $\mathrm{~B}=12.1$ & $\mathrm{~B}=0.5$ & $\mathrm{~B}=-11.4$ & $\mathrm{~B}=-12.8$ & $\mathrm{~B}=-1$ & $\mathrm{~B}=3.61$ & $\mathrm{~B}=11.92$ & $\mathrm{~B}=11.9$ \\
\hline $\begin{array}{c}\mathbf{3} \text { Phase } \\
\mathbf{k V A r}\end{array}$ & -12.55 & -4 & 21.7 & 21.45 & 15.9 & 4.2 & -0.028 & 0.635 & 0.569 \\
\hline $\begin{array}{c}\mathbf{1} \text { Phase } \\
\mathbf{k V A r}\end{array}$ & $\mathrm{B}=-3.25$ & $\mathrm{~B}=-1$ & $\mathrm{~B}=6.2$ & $\mathrm{~B}=6$ & $\mathrm{~B}=4$ & $\mathrm{~B}=1.1$ & $\mathrm{~B}=-0.003$ & $\mathrm{~B}=0.236$ & $\mathrm{~B}=0.234$ \\
& $\mathrm{C}=-4$ & $\mathrm{C}=-1$ & $\mathrm{C}=6.9$ & $\mathrm{C}=6.7$ & $\mathrm{C}=4.7$ & $\mathrm{C}=1.1$ & $\mathrm{C}=-0.027$ & $\mathrm{C}=0.175$ & $\mathrm{C}=0.227$ \\
\hline
\end{tabular}

Table 10 - Measured Values after Start Up for Test 10.4.12 and Load Banks 3 and 6 A-phase Reduced by $50 \%$

These measurements were relatively close to the expected values, but not exact due to temperature, phase voltages and electrical losses in conductors. At the time of the measurements, the voltage and frequency was $281.6 \mathrm{~V}$ on A-phase, $279.1 \mathrm{~V}$ on B-phase and $278.6 \mathrm{~V}$ on C-phase and $59.98 \mathrm{~Hz}$ at the static switch (i.e., Meter 2) when connected to the utility grid; and $282 \mathrm{~V}$ on A-phase, $280 \mathrm{~V}$ on B-phase and $279.4 \mathrm{~V}$ on C-phase and $59.98 \mathrm{~Hz}$ at Meter 3.

The Gen-sets in this test were set up to produce more power than Load Banks 3 and 4 needed which approximately $25.2 \mathrm{~kW}(\mathrm{~A}=6.6 \mathrm{~kW} \mathrm{~B}=11.4 \mathrm{~kW} \mathrm{C}=7.2 \mathrm{~kW})$ of excess power was exported through the static switch to Load Bank 6. Since Load Bank 6 was approximately $29.9 \mathrm{~kW}(\mathrm{~A}=6.1 \mathrm{~kW} B=11.9 \mathrm{~kW} \mathrm{C}=11.9 \mathrm{~kW})+\mathrm{j} 0.569 \mathrm{kVAr}(\mathrm{A}=0.108 \mathrm{kVAr}$ $\mathrm{B}=0.234 \mathrm{kVAr} \mathrm{C}=0.227 \mathrm{kVAr})$, the utility had to supply approximately $4.8 \mathrm{~kW}(\mathrm{~A}=-0.4 \mathrm{~kW}$ $\mathrm{B}=0.5 \mathrm{~kW} \mathrm{C}=4.7 \mathrm{~kW})$ to satisfy the load in Load Bank 6. Reactive power had to be imported in from the utility of approximately $21.45 \mathrm{kVAr}(\mathrm{A}=8.75 \mathrm{kVAr} B=6 \mathrm{kVAr}$ $\mathrm{C}=6.7 \mathrm{kVAr}$ ) because of Gen-set $\mathrm{A} 1$ and $\mathrm{A} 2$ needed approximately j16.55kVAr 
$(\mathrm{A}=7.3 \mathrm{kVAr} \mathrm{B}=4.25 \mathrm{kVAr} \mathrm{C}=5 \mathrm{kVAr})$ between both and the reactive power absorbed in the electrical lines.

Very soon after the A-phase in Load Banks 3 and 6 were reduced by 50\%, the static switch tripped on a reverse power condition shown in Figure 17a isolating the CERTS microgrid from the utility grid. The static switch was reset and started with a manual open and all data was verified and recorded into the DAS Database. Table 11 shows the measured values after the static switch was open.

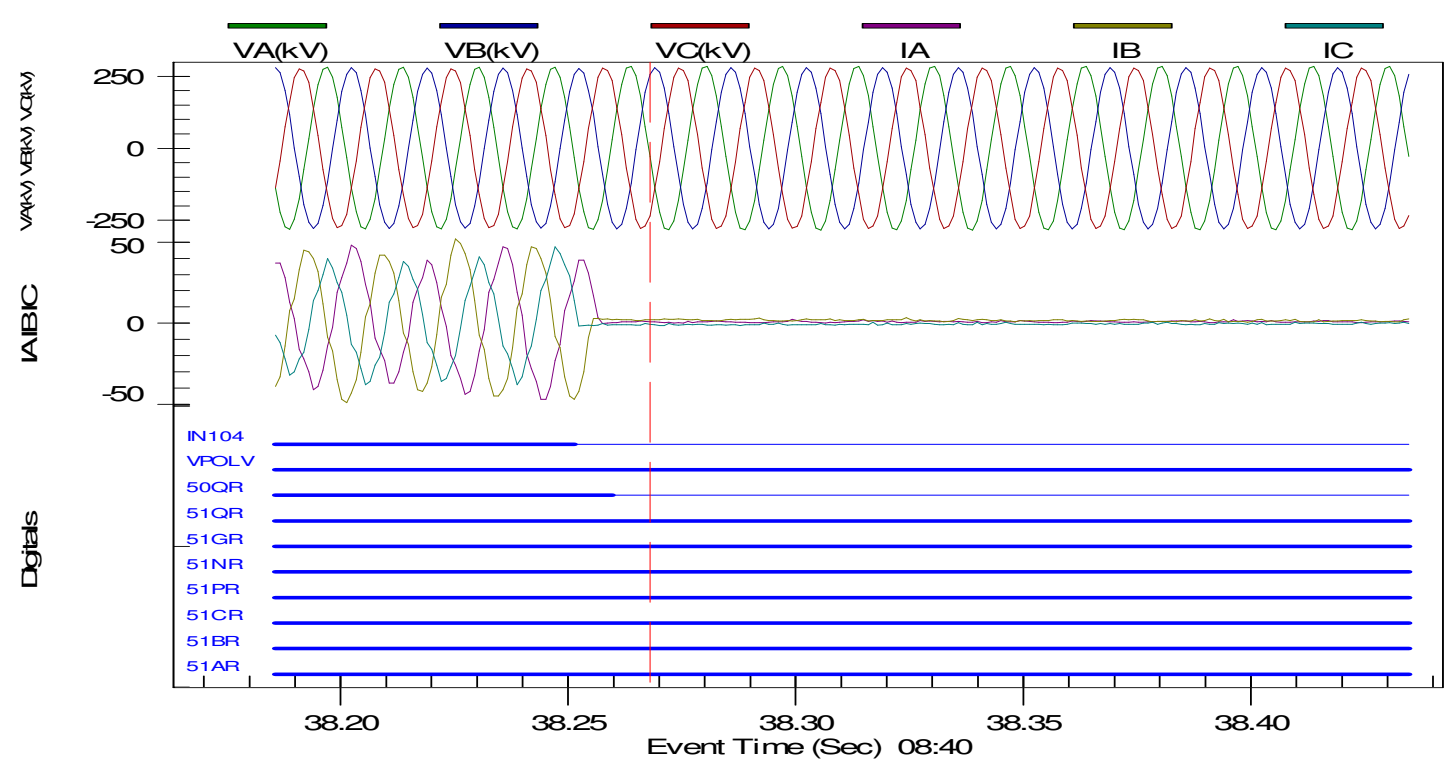

Figure 17a - Relay 2 (Static Switch) Tripping on a Reverse Power Condition

\begin{tabular}{|c|c|c|c|c|c|c|c|c|c|}
\hline Meter & $\begin{array}{c}\text { Gen- } \\
\text { set A1 }\end{array}$ & $\begin{array}{c}\text { Gen - } \\
\text { set A2 }\end{array}$ & Meter 1 & Meter 2 & Meter 3 & Meter 4 & LB 3 & LB 4 & LB 6 \\
\hline $\begin{array}{c}\text { 3 Phase } \\
\mathbf{~ k W}\end{array}$ & 27 & 18.6 & 30.7 & 0 & -2.4 & 15.8 & 8.7 & 35.645 & 30.55 \\
\hline $\begin{array}{c}\mathbf{1} \text { Phase } \\
\mathbf{k W}\end{array}$ & $\mathrm{B}=9.6$ & $\mathrm{~B}=6.5$ & $\mathrm{~B}=12.2$ & $\mathrm{~B}=0$ & $\mathrm{~B}=-1.2$ & $\mathrm{~B}=5$ & $\mathrm{~B}=3.51$ & $\mathrm{~B}=11.55$ & $\mathrm{~B}=12.1$ \\
\hline $\begin{array}{c}\mathbf{3} \text { Phase } \\
\mathbf{k V A r}\end{array}$ & -2.75 & 7.5 & 0.8 & 0 & -4 & -6.9 & -0.03 & 0.6205 & 0.575 \\
\hline $\begin{array}{c}\mathbf{1} \text { Phase } \\
\mathbf{k V A r}\end{array}$ & $\mathrm{A}=-1.25$ & $\mathrm{~A}=2.5$ & $\mathrm{~A}=0.2$ & $\mathrm{~A}=0$ & $\mathrm{~A}=-1$ & $\mathrm{~A}=-2.3$ & $\mathrm{~A}=0.001$ & $\mathrm{~A}=0.216$ & $\mathrm{~A}=0.108$ \\
\hline
\end{tabular}




\begin{tabular}{|c|c|c|c|c|c|c|c|c|c|}
\hline & $\mathrm{B}=-0.75$ & $\mathrm{~B}=2.5$ & $\mathrm{~B}=0.3$ & $\mathrm{~B}=0$ & $\mathrm{~B}=-1.5$ & $\mathrm{~B}=-2.3$ & $\mathrm{~B}=-0.004$ & $\mathrm{~B}=0.2325$ & $\mathrm{~B}=0.236$ \\
& $\mathrm{C}=-0.75$ & $\mathrm{C}=2.5$ & $\mathrm{C}=0.3$ & $\mathrm{C}=0$ & $\mathrm{C}=-1.5$ & $\mathrm{C}=-2.3$ & $\mathrm{C}=-0.027$ & $\mathrm{C}=0.172$ & $\mathrm{C}=0.231$ \\
\hline
\end{tabular}

Table 11 - Measured Values after Static Switch Opened for Test 10.4.12

In Table 11, $0 \mathrm{~kW}(\mathrm{~A}=0 \mathrm{~kW} \mathrm{~B}=0 \mathrm{~kW} \mathrm{C}=0 \mathrm{~kW})+\mathrm{j} 0 \mathrm{kVAr}(\mathrm{A}=0 \mathrm{kVAr} \mathrm{B}=0 \mathrm{kVAr} \mathrm{C}=0 \mathrm{kVAr})$ was recorded at Meter 2 indicating that power was not flowing through the static switch. Meter 1 real power increased from $4.8 \mathrm{~kW}(\mathrm{~A}=-0.4 \mathrm{~kW} \mathrm{~B}=0.5 \mathrm{~kW} \mathrm{C}=4.7 \mathrm{~kW})$ to $30.7 \mathrm{~kW}$ $(A=6.3 \mathrm{~kW} \quad B=12.2 \mathrm{~kW} \quad \mathrm{C}=12.2 \mathrm{~kW})$ and reactive power decreased from $\mathrm{j} 21.7 \mathrm{kVAr}$ $(\mathrm{A}=8.6 \mathrm{kVAr} \mathrm{B}=6.2 \mathrm{kVAr} \mathrm{C}=6.9 \mathrm{kVAr})$ to $\mathrm{j} 0.8 \mathrm{kVAr}(\mathrm{A}=0.2 \mathrm{kVAr} \mathrm{B}=0.3 \mathrm{kVAr} \mathrm{C}=0.3 \mathrm{kVAr})$. The change in power is because the microgrid is no longer supplying power for Load Bank 6 which was approximately $30.55 \mathrm{~kW} \quad(\mathrm{~A}=6.25 \mathrm{~kW} \quad \mathrm{~B}=12.1 \mathrm{~kW} \quad \mathrm{C}=12.2 \mathrm{~kW})+$ j0.575kVAr $(A=0.108 \mathrm{kVAr} B=0.236 \mathrm{kVAr} C=0.231 \mathrm{kVAr})$. Load Banks 3 and 4 reduced slightly to $8.7 \mathrm{~kW}(\mathrm{~A}=1.7 \mathrm{~kW} \quad \mathrm{~B}=3.51 \mathrm{~kW} \quad \mathrm{C}=3.41 \mathrm{~kW})-\mathrm{j} 0.03 \mathrm{kVAr} \quad(\mathrm{A}=0.001 \mathrm{kVAr} \mathrm{B}=-$ $0.004 \mathrm{kVAr} \quad \mathrm{C}=-0.027 \mathrm{kVAr})$ and $35.645 \mathrm{~kW} \quad(\mathrm{~A}=11.575 \mathrm{~kW} \quad \mathrm{~B}=11.55 \mathrm{~kW} \quad \mathrm{C}=11.52 \mathrm{~kW})+$ j0.6205kVAr $(A=0.216 \mathrm{kVAr} B=0.2325 \mathrm{kVAr} C=0.172 \mathrm{kVAr})$, respectively. This load reduction resulted from a voltage drop in the microgrid, shown in Figure $17 \mathrm{~b}$, from approximately $282 \mathrm{~V}$ on A-phase, $280 \mathrm{~V}$ on B-phase and $279.4 \mathrm{~V}$ on C-phase when connected to the utility grid to $275 \mathrm{~V}$ on A-phase, $276 \mathrm{~V}$ on B-phase and $275.2 \mathrm{~V}$ on Cphase when islanded at Meter 3.

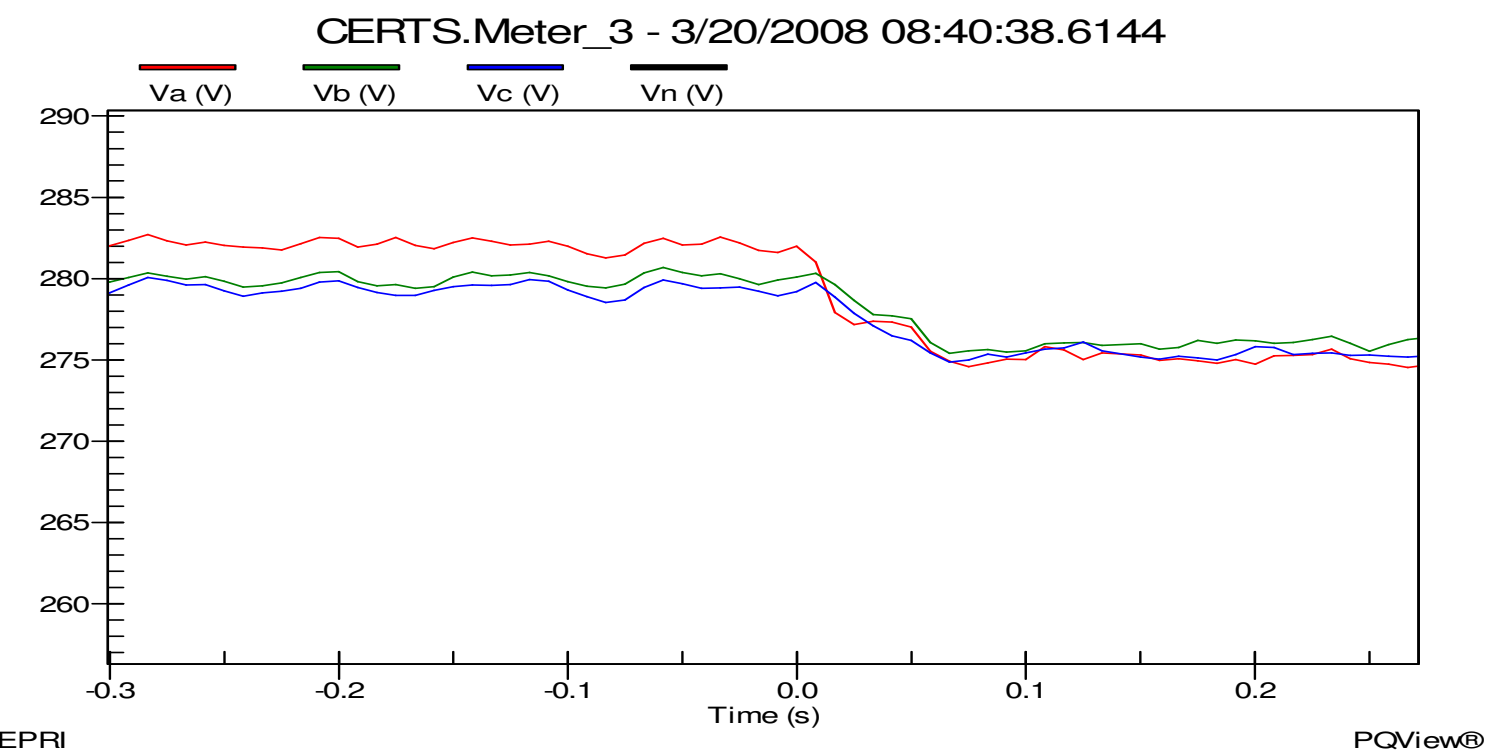

Figure 17b - Meter 3 Line-to-Ground Voltages during Utility Connected to Island mode for Test 10.4.12

Frequency change in the microgrid, shown in Figure 17c, increased from approximately $59.98 \mathrm{~Hz}$ when connected to the utility grid to approximately $60.09 \mathrm{~Hz}$ when islanded. This change in frequency was part of the CERTS algorithm which allowed the Gen-sets 
to decrease their output power to satisfy the critical load demands. Gen-sets A1 and A2 decreased their output power to approximately $27 \mathrm{~kW}(\mathrm{~A}=8.4 \mathrm{~kW} B=9.6 \mathrm{~kW} \mathrm{C}=9 \mathrm{~kW})-$ $\mathrm{j} 2.75 \mathrm{kVAr}(\mathrm{A}=-1.25 \mathrm{kVAr} B=-0.75 \mathrm{kVAr} C=-0.75 \mathrm{kVAr})$ and $18.6 \mathrm{~kW}(\mathrm{~A}=5.6 \mathrm{~kW} \quad \mathrm{~B}=6.5 \mathrm{~kW}$ $\mathrm{C}=6.5 \mathrm{~kW})+\mathrm{j} 7.5 \mathrm{kVAr}(\mathrm{A}=2.5 \mathrm{kVAr} \mathrm{B}=2.5 \mathrm{kVAr} \mathrm{C}=2.5 \mathrm{kVAr})$, respectively. Meters 3 and 4 were approximately $-2.4 \mathrm{~kW}(\mathrm{~A}=-0.8 \mathrm{~kW} B=-1.2 \mathrm{~kW} C=-1.4 \mathrm{~kW})-\mathrm{j} 4 \mathrm{kVAr}(\mathrm{A}=-1 \mathrm{kVAr} B=-$ 1.5kVAr $C=-1.5 \mathrm{kVAr})$ and $15.8 \mathrm{~kW}(\mathrm{~A}=5.8 \mathrm{~kW} \mathrm{~B}=5 \mathrm{~kW} C=5 \mathrm{~kW})-\mathrm{j} 6.9 \mathrm{kVAr}(\mathrm{A}=-2.3 \mathrm{kVAr}$ $\mathrm{B}=-2.3 \mathrm{kVAr} \mathrm{C}=-2.3 \mathrm{kVAr})$, respectively. Negative power recorded at Meter 3 indicates that all the critical loads in the microgrid were being satisfied by Gen-sets A1 and A2. Meter 4 indicates that Gen-set A2 was not supplying enough real power for Load Bank 4 load demand and needed approximately $15.8 \mathrm{~kW}(\mathrm{~A}=5.8 \mathrm{~kW} \mathrm{~B}=5 \mathrm{~kW} \mathrm{C}=5 \mathrm{~kW})$ from Genset A1. All the reactive power in the critical loads was supplied by Gen-set A2 of approximately j7.5kVAr $(\mathrm{A}=2.5 \mathrm{kVAr} B=2.5 \mathrm{kVAr} C=2.5 \mathrm{kVAr})$. All data was verified and recorded into the DAS Database. The microgrid ran for a couple of minutes in this electrical state before the A-phase was reduced in Load Bank 3 by $100 \%$ and A-phase in Load Bank 6 by an additional 50\% (i.e., 75\% from balanced load value). The measured values for the A-phase load reduction in Load Banks 3 and 6 are in Table 12.

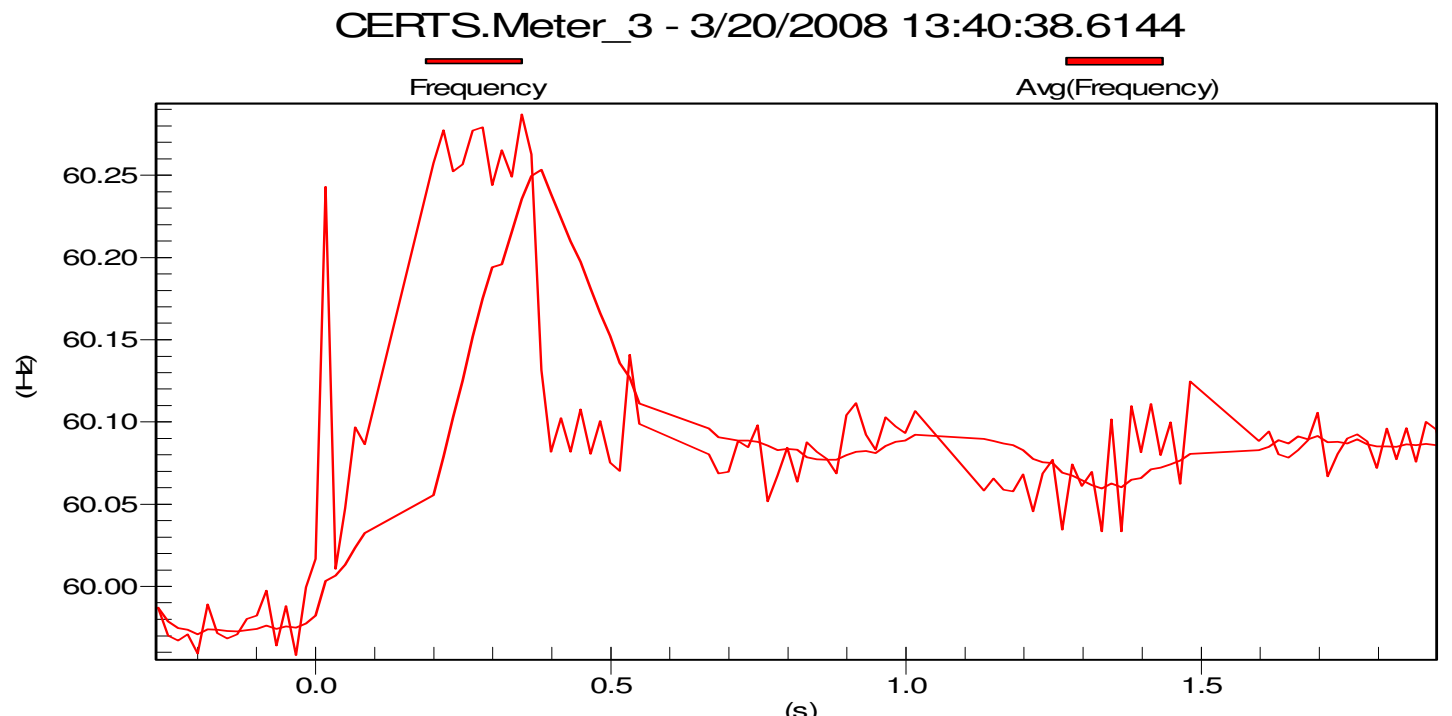

EPRI

(s)

PQVienß

Figure 17c - Meter 3 Frequency before/after the static switch opened for Test 10.4.12

\begin{tabular}{|c|c|c|c|c|c|c|c|c|c|}
\hline Meter & $\begin{array}{c}\text { Gen- } \\
\text { set A1 }\end{array}$ & $\begin{array}{c}\text { Gen - } \\
\text { set A2 }\end{array}$ & Meter 1 & Meter 2 & Meter 3 & Meter 4 & LB 3 & LB 4 & LB 6 \\
\hline $\begin{array}{c}\text { 3 Phase } \\
\text { kW }\end{array}$ & 26.45 & 17.6 & 28.25 & 0 & -2.475 & 16.75 & 6.99 & 39.2 & 28.1 \\
\hline $\begin{array}{c}\mathbf{1} \text { Phase } \\
\mathbf{k W}\end{array}$ & $\mathrm{A}=7.6$ & $\mathrm{~A}=4.8$ & $\mathrm{~A}=3.8$ & $\mathrm{~A}=0$ & $\mathrm{~A}=-0.82$ & $\mathrm{~A}=6.75$ & $\mathrm{~A}=0$ & $\mathrm{~A}=13.1$ & $\mathrm{~A}=3.8$ \\
\hline
\end{tabular}




\begin{tabular}{|c|c|c|c|c|c|c|c|c|c|}
\hline & $\mathrm{B}=9.75$ & $\mathrm{~B}=6.4$ & $\mathrm{~B}=12.2$ & $\mathrm{~B}=0$ & $\mathrm{~B}=-1.175$ & $\mathrm{~B}=5$ & $\mathrm{~B}=3.5$ & $\mathrm{~B}=13.1$ & $\mathrm{~B}=12.1$ \\
$\mathrm{C}=9.1$ & $\mathrm{C}=6.4$ & $\mathrm{C}=12.25$ & $\mathrm{C}=0$ & $\mathrm{C}=-0.48$ & $\mathrm{C}=5$ & $\mathrm{C}=3.49$ & $\mathrm{C}=13$ & $\mathrm{C}=12.2$ \\
\hline $\begin{array}{c}\text { 3 Phase } \\
\text { kVAr }\end{array}$ & -2.5 & 7.1 & 0.738 & 0 & -4.06 & -6.87 & -0.0305 & 0.68 & 0.506 \\
\hline $\begin{array}{c}\text { 1 Phase } \\
\text { kVAr }\end{array}$ & $\mathrm{B}=-0.7$ & $\mathrm{~B}=2.4$ & $\mathrm{~B}=0.345$ & $\mathrm{~B}=0$ & $\mathrm{~B}=-1.58$ & $\mathrm{~B}=-2.4$ & $\mathrm{~B}=-.0025$ & $\mathrm{~B}=0.25$ & $\mathrm{~B}=0.25$ \\
& $\mathrm{C}=-0.6$ & $\mathrm{C}=2.3$ & $\mathrm{C}=0.323$ & $\mathrm{C}=0$ & $\mathrm{C}=-1.58$ & $\mathrm{C}=-2.22$ & $\mathrm{C}=-0.026$ & $\mathrm{C}=0.19$ & $\mathrm{C}=0.246$ \\
\hline
\end{tabular}

Table 12 - Measured Values with Static Switch Open and A-Phase Reduced in Load Bank 3 by 100\%and Load Bank 6 by $75 \%$ for Test 10.4 .12

In Table 12, Meter 1 real power decreased from approximately $30.7 \mathrm{~kW}(\mathrm{~A}=6.3 \mathrm{~kW}$ $\mathrm{B}=12.2 \mathrm{~kW} \mathrm{C}=12.2 \mathrm{~kW})$ to $28.25 \mathrm{~kW}(\mathrm{~A}=3.8 \mathrm{~kW} \mathrm{~B}=12.2 \mathrm{~kW} \mathrm{C}=12.25 \mathrm{~kW})$ because of the Aphase reduction in Load Bank 6 which was approximately $28.1 \mathrm{~kW}(\mathrm{~A}=3.8 \mathrm{~kW} B=12.1 \mathrm{~kW}$ $\mathrm{C}=12.2 \mathrm{~kW})+\mathrm{j} 0.506 \mathrm{kVAr}(\mathrm{A}=0.01 \mathrm{kVAr} \mathrm{B}=0.25 \mathrm{kVAr} \mathrm{C}=0.246 \mathrm{kVAr})$. The reduction in Load Bank 3 from approximately 8.7kW $(A=1.7 \mathrm{~kW} \mathrm{~B}=3.51 \mathrm{~kW} \mathrm{C}=3.49 \mathrm{~kW})-\mathrm{j} 0.03 \mathrm{kVAr}$ $(\mathrm{A}=0.001 \mathrm{kVAr} \mathrm{B}=-0.004 \mathrm{kVAr} \mathrm{C}=-0.027 \mathrm{kVAr})$ to $6.99 \mathrm{~kW}(\mathrm{~A}=0 \mathrm{~kW} \mathrm{~B}=3.5 \mathrm{~kW} \mathrm{C}=3.49 \mathrm{~kW})-$ j0.0305kVAr $(A=-0.002 \mathrm{kVAr} B=-0.0025 \mathrm{kVAr} C=-0.026 \mathrm{kVAr})$ caused the frequency to increase within the microgrid from approximately $60.09 \mathrm{~Hz}$ to $60.13 \mathrm{~Hz}$. The increase in frequency is part of the CERTS algorithm which caused the Gen-sets to decrease their power level outputs due to the decrease in the A-phase load demand of Load Bank 3. Gen-set A1 decreased from approximately 27kW (A=8.4kW B=9.6kW C=9kW) j2.75kVAr $(A=-1.25 \mathrm{kVAr} B=-0.75 \mathrm{kVAr} C=-0.75 \mathrm{kVAr})$ to $26.45 \mathrm{~kW}(\mathrm{~A}=7.6 \mathrm{~kW} \quad \mathrm{~B}=9.75 \mathrm{~kW}$ $\mathrm{C}=9.1 \mathrm{~kW})-\mathrm{j} 2.5 \mathrm{kVAr}(\mathrm{A}=-1.2 \mathrm{kVAr} B=-0.7 \mathrm{kVAr} \mathrm{C}=-0.6 \mathrm{kVAr})$ and Gen-set $\mathrm{A} 2$ decreased from approximately $18.6 \mathrm{~kW}(\mathrm{~A}=5.6 \mathrm{~kW} B=6.5 \mathrm{~kW} C=6.5 \mathrm{~kW})+j 7.5 \mathrm{kVAr} \quad(\mathrm{A}=2.5 \mathrm{kVAr}$ $B=2.5 \mathrm{kVAr} C=2.5 \mathrm{kVAr})$ to $17.6 \mathrm{~kW}(\mathrm{~A}=4.8 \mathrm{~kW} \mathrm{~B}=6.4 \mathrm{~kW} C=6.4 \mathrm{~kW})+j 7.1 \mathrm{kVAr}(\mathrm{A}=2.4 \mathrm{kVAr}$ $B=2.4 \mathrm{kVAr} C=2.3 \mathrm{kVAr}$ ). Since both Gen-sets decreased their real power outputs, power being supplied by Gen-set A1 to Load Bank 4 load demand increased from approximately $15.8 \mathrm{~kW} \quad(\mathrm{~A}=5.8 \mathrm{~kW} \quad \mathrm{~B}=5 \mathrm{~kW} \quad \mathrm{C}=5 \mathrm{~kW})$ to $16.75 \mathrm{~kW} \quad(\mathrm{~A}=6.75 \mathrm{~kW} \quad \mathrm{~B}=5 \mathrm{~kW}$ $\mathrm{C}=5 \mathrm{~kW})$ measured by Meter 4 . The voltage within the microgrid during these measurements was approximately $275.7 \mathrm{~V}$ on A-phase, $276.2 \mathrm{~V}$ on B-phase and $274.9 \mathrm{~V}$ on C-phase.

All data was verified and recorded into the DAS Database. The microgrid ran for a couple of minutes in this electrical state before the A-phase was reduced in Load Bank 4 by $75 \%$. The measured values for the A-phase load reduction for Load Bank 4 are in Table 13. 


\begin{tabular}{|c|c|c|c|c|c|c|c|c|c|}
\hline Meter & $\begin{array}{c}\text { Gen- } \\
\text { set A1 }\end{array}$ & $\begin{array}{c}\text { Gen - } \\
\text { set A2 }\end{array}$ & Meter 1 & Meter 2 & Meter 3 & Meter 4 & LB 3 & LB 4 & LB 6 \\
\hline $\begin{array}{c}\text { 3 Phase } \\
\text { kW }\end{array}$ & 22.6 & 13.45 & 28.25 & 0 & -2.52 & 12.75 & 7 & 29.2 & 28.1 \\
\hline $\begin{array}{c}\mathbf{1} \text { Phase } \\
\mathbf{k W}\end{array}$ & $\mathrm{B}=9.7$ & $\mathrm{~B}=6.6$ & $\mathrm{~B}=12.2$ & $\mathrm{~B}=0$ & $\mathrm{~B}=-1.17$ & $\mathrm{~B}=4.8$ & $\mathrm{~B}=3.6$ & $\mathrm{~B}=13.1$ & $\mathrm{~B}=12.1$ \\
\hline $\begin{array}{c}\mathbf{3} \text { Phase } \\
\mathbf{k V A r}\end{array}$ & -3 & 7.4 & 0.738 & 0 & -3.92 & -7.08 & -0.0293 & 0.43 & 0.506 \\
\hline $\begin{array}{c}\mathbf{1} \text { Phase } \\
\mathbf{k V A r}\end{array}$ & $\mathrm{B}=-1.3$ & $\mathrm{~B}=3.1$ & $\mathrm{~B}=0.345$ & $\mathrm{~B}=0$ & $\mathrm{~B}=-1.62$ & $\mathrm{~B}=-2.82$ & $\mathrm{~B}=-.0019$ & $\mathrm{~B}=0.25$ & $\mathrm{~B}=0.25$ \\
& $\mathrm{C}=-0.3$ & $\mathrm{C}=2.05$ & $\mathrm{C}=0.323$ & $\mathrm{C}=0$ & $\mathrm{C}=-1.5$ & $\mathrm{C}=-1.96$ & $\mathrm{C}=-.0255$ & $\mathrm{C}=0.18$ & $\mathrm{C}=0.246$ \\
\hline
\end{tabular}

Table 13 - Measured Values with Static Switch Open and A-Phase Reduced in Load Bank 4 by $75 \%$ for Test 10.4.12

In Table 13, Meter 4 real power decreased from approximately $16.75 \mathrm{~kW}(\mathrm{~A}=6.75 \mathrm{~kW}$ $\mathrm{B}=5 \mathrm{~kW} \mathrm{C}=5 \mathrm{~kW})$ to $12.75 \mathrm{~kW}(\mathrm{~A}=3.25 \mathrm{~kW} \mathrm{~B}=4.8 \mathrm{~kW} \mathrm{C}=4.7 \mathrm{~kW})$ because of the A-phase reduction in Load Bank 4 which was approximately $29.2 \mathrm{~kW}(\mathrm{~A}=3.5 \mathrm{~kW} \quad B=13.1 \mathrm{~kW}$ $\mathrm{C}=12.6 \mathrm{~kW})+\mathrm{j} 0.43 \mathrm{kVAr}(\mathrm{A}=0 \mathrm{kVAr} \mathrm{B}=0.25 \mathrm{kVAr} \mathrm{C}=0.18 \mathrm{kVAr})$. The reduction in Load Bank 4 caused the frequency to increase within the microgrid from approximately $60.13 \mathrm{~Hz}$ to $60.14 \mathrm{~Hz}$. The increase in frequency is part of the CERTS algorithm which caused the Gen-sets to decrease their power level output due to the decrease in the Aphase load demand of Load Bank 4. Gen-set A1 decreased from approximately $26.45 \mathrm{~kW}$ $(A=7.6 \mathrm{~kW} \quad B=9.75 \mathrm{~kW} \quad \mathrm{C}=9.1 \mathrm{~kW})-\mathrm{j} 2.5 \mathrm{kVAr}(\mathrm{A}=-1.2 \mathrm{kVAr} B=-0.7 \mathrm{kVAr} \quad \mathrm{C}=-0.6 \mathrm{kVAr})$ to $22.6 \mathrm{~kW}(\mathrm{~A}=4.2 \mathrm{~kW} B=9.7 \mathrm{~kW} C=8.7 \mathrm{~kW})-\mathrm{j} 3 \mathrm{kVAr}(\mathrm{A}=-1.4 \mathrm{kVAr} B=-1.3 \mathrm{kVAr} C=-0.3 \mathrm{kVAr})$ and Gen-set A2 decreased from approximately $17.6 \mathrm{~kW}(\mathrm{~A}=4.8 \mathrm{~kW} \mathrm{~B}=6.4 \mathrm{~kW} \mathrm{C}=6.4 \mathrm{~kW})+$ j7.1kVAr $(\mathrm{A}=2.4 \mathrm{kVAr} B=2.4 \mathrm{kVAr} C=2.3 \mathrm{kVAr})$ to $13.45 \mathrm{~kW}(\mathrm{~A}=0.25 \mathrm{~kW} \mathrm{~B}=6.6 \mathrm{~kW} \mathrm{C}=6.6 \mathrm{~kW})$ $+j 7.4 \mathrm{kVAr}(\mathrm{A}=2.25 \mathrm{kVAr} B=3.1 \mathrm{kVAr} C=2.05 \mathrm{kVAr})$. The voltage within the microgrid during these measurements was approximately 276.7V on A-phase, 277.6V on B-phase and $272.8 \mathrm{~V}$ on C-phase. Load Bank 6 load demands was not changed at this time, therefore, all the measured values for Load Bank 6, Meter 1 and Meter 2 did not change from the previous load reduction in Load Banks 3 and 6.

All data was verified and recorded into the DAS Database. The microgrid ran for a couple of minutes in this electrical state before the A-phase was reduced in Load Bank 6 by $100 \%$. The measured values for the A-phase load reduction for Load Bank 6 are in Table 14 . 


\begin{tabular}{|c|c|c|c|c|c|c|c|c|c|}
\hline Meter & $\begin{array}{c}\text { Gen- } \\
\text { set A1 }\end{array}$ & $\begin{array}{c}\text { Gen - } \\
\text { set A2 }\end{array}$ & Meter 1 & Meter 2 & Meter 3 & Meter 4 & LB 3 & LB 4 & LB 6 \\
\hline $\begin{array}{c}\text { 3 Phase } \\
\text { kW }\end{array}$ & 22.45 & 13.5 & 24.31 & 0 & -2.515 & 12.65 & 7 & 26.35 & 24.1 \\
\hline $\begin{array}{c}\mathbf{1} \text { Phase } \\
\mathbf{k W}\end{array}$ & $\mathrm{B}=9.5$ & $\mathrm{~B}=6.5$ & $\mathrm{~B}=12.1$ & $\mathrm{~B}=0$ & $\mathrm{~B}=-1.18$ & $\mathrm{~B}=4.75$ & $\mathrm{~B}=3.6$ & $\mathrm{~B}=11.5$ & $\mathrm{~B}=12.1$ \\
\hline $\begin{array}{c}\mathbf{3} \text { Phase } \\
\mathbf{k V A r}\end{array}$ & -2.65 & 7 & 0.79 & 0 & -3.92 & -6.7 & -0.0273 & 0.45 & 0.509 \\
\hline $\begin{array}{c}\mathbf{1} \text { Phase } \\
\mathbf{k V A r}\end{array}$ & $\mathrm{B}=-1.05$ & $\mathrm{~B}=2.7$ & $\mathrm{~B}=0.35$ & $\mathrm{~B}=0$ & $\mathrm{~B}=-1.62$ & $\mathrm{~B}=-2.75$ & $\mathrm{~B}=-.0013$ & $\mathrm{~B}=0.26$ & $\mathrm{~B}=0.255$ \\
& $\mathrm{C}=-0.3$ & $\mathrm{C}=1.9$ & $\mathrm{C}=0.35$ & $\mathrm{C}=0$ & $\mathrm{C}=-1.5$ & $\mathrm{C}=-1.75$ & $\mathrm{C}=-.0245$ & $\mathrm{C}=0.19$ & $\mathrm{C}=0.254$ \\
\hline
\end{tabular}

Table 14 - Measured Values with Static Switch Open and A-Phase Reduced in Load Bank 6 by 100\% for Test 10.4.12

In Table 14, Meter 1 real power decreased from approximately $28.25 \mathrm{~kW}(\mathrm{~A}=3.8 \mathrm{~kW}$ $\mathrm{B}=12.2 \mathrm{~kW} \mathrm{C}=12.25 \mathrm{~kW})$ to $24.31 \mathrm{~kW}(\mathrm{~A}=0.1 \mathrm{~kW} \mathrm{~B}=12.1 \mathrm{~kW} \mathrm{C}=12.11 \mathrm{~kW})$ because of the Aphase reduction in Load Bank 6 which was approximately $24.1 \mathrm{~kW}(\mathrm{~A}=0 \mathrm{~kW} B=12 \mathrm{~kW}$ $\mathrm{C}=12.1 \mathrm{~kW})+\mathrm{j} 0.509 \mathrm{kVAr} \quad(\mathrm{A}=0 \mathrm{kVAr} \mathrm{B}=0.255 \mathrm{kVAr} \quad \mathrm{C}=0.254 \mathrm{kVAr})$. Voltages and frequencies remained the same in both the utility and microgrid when Load Bank 6 Aphase was reduced to $0 \mathrm{~kW}$ because nothing changed in the CERTS microgrid downstream of the static switch and the utility is a stiff system.

All data was verified and recorded into the DAS Database. The microgrid ran for a couple of minutes in this electrical state before the A-phase was reduced in Load Bank 4 by $100 \%$. The measured values for the A-phase load reduction for Load Bank 4 are in Table 15.

\begin{tabular}{|c|c|c|c|c|c|c|c|c|c|}
\hline Meter & $\begin{array}{c}\text { Gen- } \\
\text { set A1 }\end{array}$ & $\begin{array}{c}\text { Gen - } \\
\text { set A2 }\end{array}$ & Meter 1 & Meter 2 & Meter 3 & Meter 4 & LB 3 & LB 4 & LB 6 \\
\hline $\begin{array}{c}\text { 3 Phase } \\
\text { kW }\end{array}$ & 20.8 & 11.65 & 24.31 & 0 & -2.54 & 11 & 7.1 & 22.6 & 24.1 \\
\hline 1 Phase & $\mathrm{A}=2.6$ & $\mathrm{~A}=-1.75$ & $\mathrm{~A}=0.1$ & $\mathrm{~A}=0$ & $\mathrm{~A}=-0.91$ & $\mathrm{~A}=1.6$ & $\mathrm{~A}=0$ & $\mathrm{~A}=0$ & $\mathrm{~A}=0$ \\
\hline
\end{tabular}




\begin{tabular}{|c|c|c|c|c|c|c|c|c|c|}
\hline $\mathbf{k W}$ & $\mathrm{B}=9.5$ & $\mathrm{~B}=6.7$ & $\mathrm{~B}=12.1$ & $\mathrm{~B}=0$ & $\mathrm{~B}=-1.17$ & $\mathrm{~B}=4.8$ & $\mathrm{~B}=3.7$ & $\mathrm{~B}=11.5$ & $\mathrm{~B}=12$ \\
$\mathrm{C}=8.7$ & $\mathrm{C}=6.7$ & $\mathrm{C}=12.11$ & $\mathrm{C}=0$ & $\mathrm{C}=-0.46$ & $\mathrm{C}=4.6$ & $\mathrm{C}=3.4$ & $\mathrm{C}=11.1$ & $\mathrm{C}=12.1$ \\
\hline $\begin{array}{c}\text { 3 Phase } \\
\text { kVAr }\end{array}$ & -2.95 & 7.07 & 0.79 & 0 & -4.125 & -7.28 & -0.0303 & 0.43 & 0.509 \\
\hline $\begin{array}{c}\text { 1 Phase } \\
\text { kVAr }\end{array}$ & $\mathrm{B}=-1.45$ & $\mathrm{~B}=3$ & $\mathrm{~B}=0.35$ & $\mathrm{~B}=0$ & $\mathrm{~B}=-1.64$ & $\mathrm{~B}=-3.22$ & $\mathrm{~B}=-0.004$ & $\mathrm{~B}=0.25$ & $\mathrm{~B}=0.255$ \\
& $\mathrm{C}=-0.1$ & $\mathrm{C}=1.68$ & $\mathrm{C}=0.35$ & $\mathrm{C}=0$ & $\mathrm{C}=-1.51$ & $\mathrm{C}=-1.65$ & $\mathrm{C}=-0.025$ & $\mathrm{C}=0.18$ & $\mathrm{C}=0.254$ \\
\hline
\end{tabular}

Table 15 - Measured Values with Static Switch Open and A-Phase Reduced in Load Bank 4 by 100\% for Test 10.4.12

In Table 15, Meter 4 real power decreased from approximately $12.65 \mathrm{~kW}(\mathrm{~A}=3.3 \mathrm{~kW}$ $\mathrm{B}=4.75 \mathrm{~kW} \mathrm{C}=4.6 \mathrm{~kW})$ to $11 \mathrm{~kW}(\mathrm{~A}=1.6 \mathrm{~kW} \mathrm{~B}=4.8 \mathrm{~kW} \mathrm{C}=4.6 \mathrm{~kW})$ because of the A-phase reduction in Load Bank 4 which was approximately $22.6 \mathrm{~kW}(\mathrm{~A}=0 \mathrm{~kW} B=11.5 \mathrm{~kW}$ $\mathrm{C}=11.1 \mathrm{~kW})+\mathrm{j} 0.43 \mathrm{kVAr}(\mathrm{A}=0 \mathrm{kVAr} \mathrm{B}=0.25 \mathrm{kVAr} \mathrm{C}=0.18 \mathrm{kVAr})$. The reduction in Load Bank 4 caused the frequency to increase within the microgrid from approximately $60.14 \mathrm{~Hz}$ to $60.15 \mathrm{~Hz}$. The increase in frequency is part of the CERTS algorithm which caused the Gen-sets to decrease their power level output due to the decrease in the Aphase load demand of Load Bank 4. Gen-set A1 decreased from approximately $22.45 \mathrm{~kW}$ $(\mathrm{A}=4.25 \mathrm{~kW} B=9.5 \mathrm{~kW} C=8.7 \mathrm{~kW})-\mathrm{j} 2.65 \mathrm{kVAr}(\mathrm{A}=-1.3 \mathrm{kVAr} B=-1.05 \mathrm{kVAr} C=-0.3 \mathrm{kVAr})$ to $20.8 \mathrm{~kW} \quad(\mathrm{~A}=2.6 \mathrm{~kW} \quad \mathrm{~B}=9.5 \mathrm{~kW} \quad \mathrm{C}=8.7 \mathrm{~kW})-\mathrm{j} 2.95 \mathrm{kVAr} \quad(\mathrm{A}=-1.4 \mathrm{kVAr} \quad \mathrm{B}=-1.45 \mathrm{kVAr} \quad \mathrm{C}=-$ $0.1 \mathrm{kVAr})$ and Gen-set $\mathrm{A} 2$ decreased from approximately $13.5 \mathrm{~kW}(\mathrm{~A}=0.25 \mathrm{~kW} B=6.5 \mathrm{~kW}$ $\mathrm{C}=6.75 \mathrm{~kW})+\mathrm{j} 7 \mathrm{kVAr}(\mathrm{A}=2.4 \mathrm{kVAr} \mathrm{B}=2.7 \mathrm{kVAr} \mathrm{C}=1.9 \mathrm{kVAr})$ to $11.65 \mathrm{~kW} \quad(\mathrm{~A}=-1.75 \mathrm{~kW}$ $\mathrm{B}=6.7 \mathrm{~kW} \mathrm{C}=6.7 \mathrm{~kW})+j 7.07 \mathrm{kVAr}(\mathrm{A}=2.39 \mathrm{kVAr} \mathrm{B}=3 . \mathrm{kVAr} \mathrm{C}=1.68 \mathrm{kVAr})$. The voltage within the microgrid during these measurements was approximately $276.9 \mathrm{~V}$ on Aphase, $278.4 \mathrm{~V}$ on B-phase and $271.1 \mathrm{~V}$ on C-phase. Load Bank 6 load demands was not changed at this time, therefore, all the measured values for Load Bank 6, Meter 1 and Meter 2 did not change from the previous load reduction in Load Banks 3 and 6.

All data was verified and recorded into the DAS Database. The microgrid ran for a couple of minutes in this electrical state before the manual open signal was released and allowed the static switch to synchronize and close back into the utility grid. Shortly after the static switch closed back into the utility it tripped on a reverse power due to the Aphase on Load Banks 3, 4 and 6 equaling 0kW shown in Figure 17d. The measured values are provided in Table 16. 


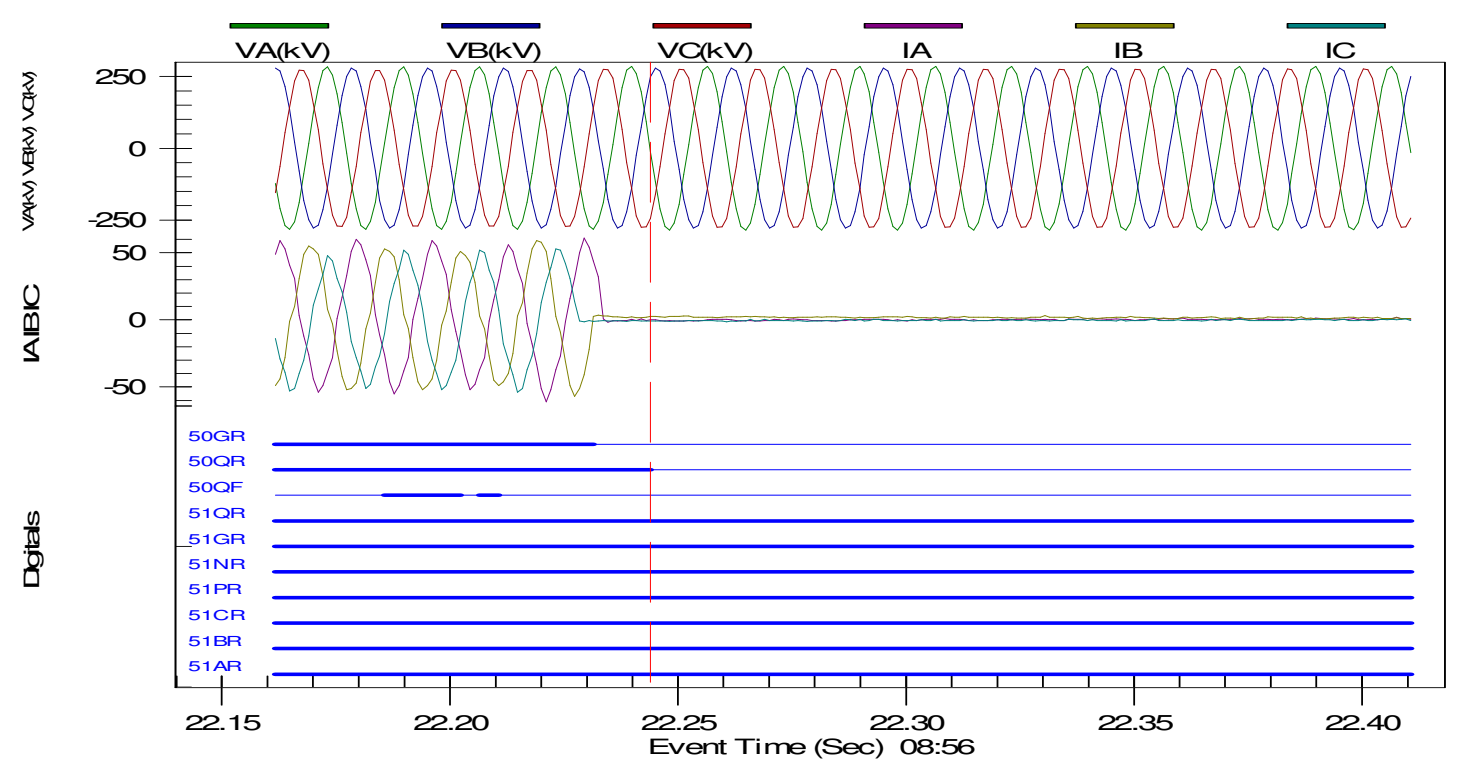

Figure 17d - Relay 2 (Static Switch) Reverse Power Condition Trip for Test 10.4.12

\begin{tabular}{|c|c|c|c|c|c|c|c|c|c|}
\hline Meter & $\begin{array}{c}\text { Gen- } \\
\text { set A1 }\end{array}$ & $\begin{array}{l}\text { Gen - } \\
\text { set A2 }\end{array}$ & Meter 1 & Meter 2 & Meter 3 & Meter 4 & LB 3 & LB 4 & LB 6 \\
\hline \multicolumn{10}{|c|}{ Before Static Switch Synchronization and Close } \\
\hline $\begin{array}{c}3 \text { Phase } \\
\text { kW }\end{array}$ & 20.6 & 11.5 & 24 & 0 & -2.5 & 11.1 & 7 & 22.7 & 24 \\
\hline \multirow{3}{*}{$\begin{array}{c}1 \text { Phase } \\
\text { kW }\end{array}$} & $A=2.6$ & $A=-1.6$ & $A=0$ & $\mathrm{~A}=0$ & $A=-1$ & $\mathrm{~A}=1.7$ & $\mathrm{~A}=0$ & $\mathrm{~A}=0$ & $\mathrm{~A}=0$ \\
\hline & $\mathrm{B}=9.5$ & $B=6.6$ & $\mathrm{~B}=12$ & $\mathrm{~B}=0$ & $B=-1.2$ & $\mathrm{~B}=4.9$ & $B=3.6$ & $B=11.6$ & $\mathrm{~B}=12$ \\
\hline & $C=8.5$ & $C=6.5$ & $\mathrm{C}=12$ & $\mathrm{C}=0$ & $C=-0.3$ & $\mathrm{C}=4.5$ & $C=3.4$ & $\mathrm{C}=11.1$ & $\mathrm{C}=12$ \\
\hline $\begin{array}{c}3 \text { Phase } \\
\text { kVAr }\end{array}$ & -2.8 & 7.15 & 0.9 & 0 & -4.25 & -7.2 & -0.028 & 0.454 & 0.519 \\
\hline \multirow{3}{*}{$\begin{array}{c}1 \text { Phase } \\
\text { kVAr }\end{array}$} & $\mathrm{A}=-1.3$ & $A=2.25$ & $\mathrm{~A}=0.1$ & $A=0$ & $A=-1$ & $\mathrm{~A}=-2.4$ & $\mathrm{~A}=-0.002$ & $\mathrm{~A}=0$ & $\mathrm{~A}=0$ \\
\hline & $B=-1.3$ & $B=3.2$ & $\mathrm{~B}=0.4$ & $\mathrm{~B}=0$ & $B=-1.75$ & $B=-3.2$ & $B=-0.001$ & $B=0.26$ & $B=0.26$ \\
\hline & $C=-0.2$ & $\mathrm{C}=1.7$ & $\mathrm{C}=0.4$ & $\mathrm{C}=0$ & $C=-1.5$ & $C=-1.6$ & $C=-0.025$ & $\mathrm{C}=0.194$ & $C=0.259$ \\
\hline \multicolumn{10}{|c|}{ Static Switch Synchronized and Closed } \\
\hline $\begin{array}{c}3 \text { Phase } \\
\text { kW }\end{array}$ & 38.9 & 30.3 & -12.5 & -36.7 & -39.5 & -7.3 & 7.35 & 23.55 & 23.5 \\
\hline \multirow{2}{*}{$\begin{array}{c}1 \text { Phase } \\
\text { kW }\end{array}$} & $\mathrm{A}=8.4$ & $\mathrm{~A}=4.7$ & $A=-12.5$ & $\mathrm{~A}=-12.4$ & $A=-13.3$ & $\mathrm{~A}=-4.8$ & $\mathrm{~A}=0$ & $\mathrm{~A}=0$ & $A=0$ \\
\hline & $\mathrm{B}=17$ & $\mathrm{~B}=14.1$ & $B=-5$ & $B=-14.8$ & $B=-16.1$ & $B=-2.5$ & $B=3.75$ & $\mathrm{~B}=11.8$ & $\mathrm{~B}=11.8$ \\
\hline
\end{tabular}




\begin{tabular}{|c|c|c|c|c|c|c|c|c|c|}
\hline & $C=13.5$ & $C=11.5$ & $C=5$ & $C=-9.5$ & $C=-10.1$ & $C=0$ & $C=3.6$ & $C=11.75$ & $C=11.7$ \\
\hline $\begin{array}{c}3 \text { Phase } \\
\text { kVAr }\end{array}$ & -13.3 & -5.15 & 23.7 & 23 & 17.6 & 4.2 & -0.0306 & 0.461 & 0.506 \\
\hline \multirow{3}{*}{$\begin{array}{c}1 \text { Phase } \\
\text { kVAr }\end{array}$} & $A=-5.1$ & $A=-2.5$ & $A=8.8$ & $A=8.7$ & $A=7.3$ & $\mathrm{~A}=2.4$ & $A=-0.001$ & $\mathrm{~A}=0$ & $A=0$ \\
\hline & $B=-3.1$ & $B=-2.9$ & $\mathrm{~B}=4.7$ & $\mathrm{~B}=4.5$ & $B=2.5$ & $B=-1$ & $B=-.0033$ & $\mathrm{~B}=0.26$ & $B=0.256$ \\
\hline & $C=-5.1$ & $C=0.25$ & $C=10.2$ & $C=9.8$ & $\mathrm{C}=7.8$ & $C=2.8$ & $C=-.0263$ & $C=0.2$ & $C=0.25$ \\
\hline \multicolumn{10}{|c|}{ Static Switch After Reverse Power Condition Trip } \\
\hline $\begin{array}{c}3 \text { Phase } \\
\text { kW }\end{array}$ & 20.6 & 11.5 & 24 & 0 & -2.5 & 11.2 & 7 & 22.6 & 24 \\
\hline \multirow{3}{*}{$\begin{array}{c}1 \text { Phase } \\
\text { kW }\end{array}$} & $A=2.5$ & $A=-1.6$ & $A=0$ & $A=0$ & $A=-1$ & $\mathrm{~A}=1.7$ & $A=0$ & $A=0$ & $A=0$ \\
\hline & $B=9.6$ & $\mathrm{~B}=6.6$ & $\mathrm{~B}=12$ & $B=0$ & $B=-1.2$ & $\mathrm{~B}=4.9$ & $B=3.6$ & $\mathrm{~B}=11.5$ & $B=12$ \\
\hline & $C=8.5$ & $C=6.5$ & $C=12$ & $\mathrm{C}=0$ & $C=-0.3$ & $C=4.6$ & $C=3.4$ & $\mathrm{C}=11.1$ & $\mathrm{C}=12$ \\
\hline $\begin{array}{c}3 \text { Phase } \\
\text { kVAr }\end{array}$ & -2.8 & 7.15 & 0.9 & 0 & -4.25 & -7.2 & -0.0303 & 0.45 & 0.519 \\
\hline \multirow{3}{*}{$\begin{array}{c}1 \text { Phase } \\
\text { kVAr }\end{array}$} & $A=-1.3$ & $A=2.25$ & $\mathrm{~A}=0.1$ & $A=0$ & $A=-1$ & $\mathrm{~A}=-2.4$ & $A=-.0019$ & $A=0$ & $A=0$ \\
\hline & $B=-1.3$ & $B=3.2$ & $\mathrm{~B}=0.4$ & $B=0$ & $B=-1.75$ & $B=-3.2$ & $\mathrm{~B}=-.0039$ & $\mathrm{~B}=0.26$ & $\mathrm{~B}=0.26$ \\
\hline & $C=-0.2$ & $\mathrm{C}=1.7$ & $C=0.4$ & $\mathrm{C}=0$ & $C=-1.5$ & $C=-1.6$ & $C=-.0245$ & $\mathrm{C}=0.19$ & $C=0.259$ \\
\hline
\end{tabular}

Table 16 - Measured Values before/after Static Switch Synchronized and Closed back into the utility and after Static Switch Tripped on Reverse Power Condition for Test 10.4.12

Voltage and frequency in the microgrid increased from approximately $276.5 \mathrm{~V}$ on Aphase, $278.4 \mathrm{~V}$ on B-phase and $271.5 \mathrm{~V}$ on C-phase and $60.15 \mathrm{~Hz}$ when islanded to $282.9 \mathrm{~V}$ on A-phase, $281 \mathrm{~V}$ on B-phase and $278.1 \mathrm{~V}$ on C-phase and $59.99 \mathrm{~Hz}$ when utility connected at Meter 3 shown in Figures 17e and 17f. This change in frequency is due to the frequency no longer being established by the Gen-set using the CERTS algorithm but by the utility. Gen-sets A1 and A2 are in unit power control mode therefore when the static switch closed back into the utility the Gen-sets produced real power based on the set-point initialized at the beginning of the test. The output power for both Gen-sets were relatively close to the value at the beginning of the test with Gen-set A1 producing approximately $38.9 \mathrm{~kW}(\mathrm{~A}=8.4 \mathrm{~kW} \quad \mathrm{~B}=17 \mathrm{~kW} \quad \mathrm{C}=13.5 \mathrm{~kW})-\mathrm{j} 13.3 \mathrm{kVAr} \quad(\mathrm{A}=-5.1 \mathrm{kVAr} \mathrm{B}=-$ $3.1 \mathrm{kVAr} C=-5.1 \mathrm{kVAr})$ and $\mathrm{Gen}-$ set $\mathrm{A} 2$ producing approximately $30.3 \mathrm{~kW}(\mathrm{~A}=4.7 \mathrm{~kW}$ $B=14.1 \mathrm{~kW} C=11.5 \mathrm{~kW})-j 5.15 \mathrm{kVAr}(\mathrm{A}=-2.5 \mathrm{kVAr} B=-2.9 \mathrm{kVAr} C=0.25 \mathrm{kVAr})$. 


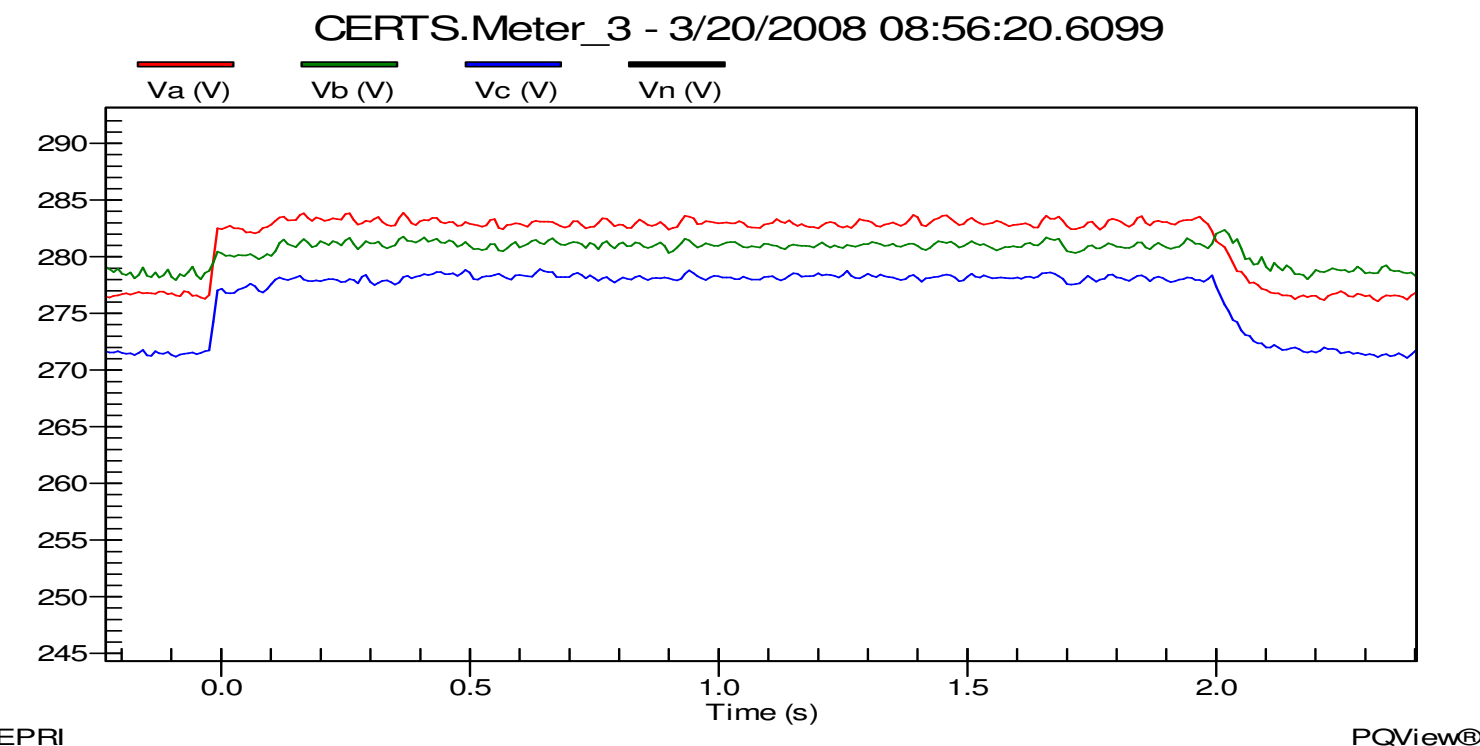

Figure 17e - Meter 3 Line-to-Ground Voltages during Islanded to Utility Connected mode for Test 10.4.12

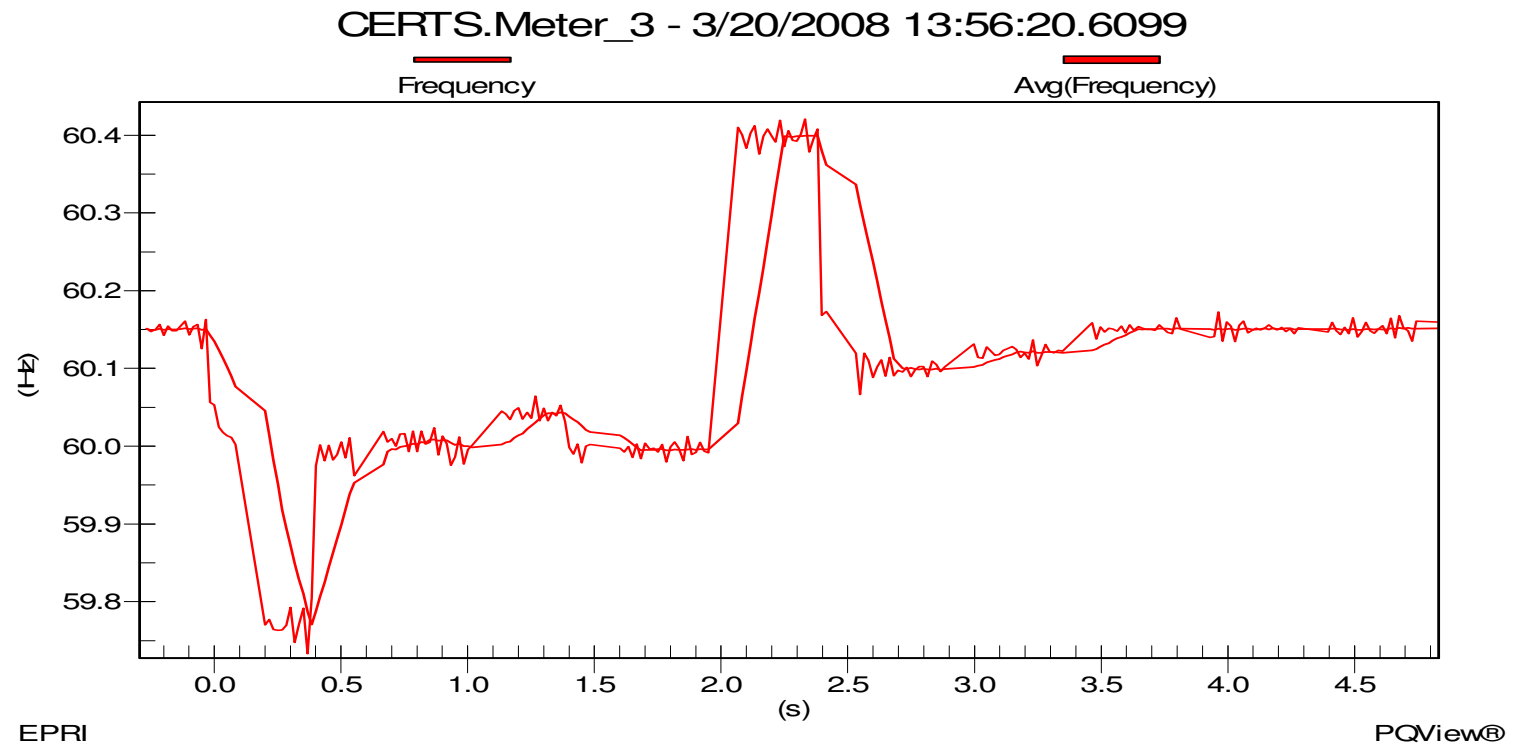

Figure 17f - Meter 3 Frequency during Islanded to Utility Connected mode for Test 10.4.12

Meter 2 recorded real power decreasing from approximately $0 \mathrm{~kW}(\mathrm{~A}=0 \mathrm{~kW} \quad \mathrm{~B}=0 \mathrm{~kW}$ $\mathrm{C}=0 \mathrm{~kW})$ to $-36.7 \mathrm{~kW}(\mathrm{~A}=-12.4 \mathrm{~kW} \mathrm{~B}=-14.8 \mathrm{~kW} \mathrm{C}=-9.5 \mathrm{~kW})$ and reactive power increasing 
from approximately j0kVAr $(\mathrm{A}=0 \mathrm{kVAr} B=0 \mathrm{kVAr} \mathrm{C}=0 \mathrm{kVAr})$ to $\mathrm{j} 23 \mathrm{kVAr} \quad(\mathrm{A}=8.7 \mathrm{kVAr}$ $\mathrm{B}=4.5 \mathrm{kVAr} \mathrm{C}=9.8 \mathrm{kVAr}$ ) after the static switch closed shown in Figure $17 \mathrm{~g}$. Gen-sets A1 and A2 were producing power for all the critical loads in the microgrid and approximately $36.7 \mathrm{~kW}(\mathrm{~A}=12.4 \mathrm{~kW} \mathrm{~B}=14.8 \mathrm{~kW} \mathrm{C}=9.5 \mathrm{~kW})$ of Load Bank 6 load demands. Load Bank 6 was approximately 23.5kW $(A=0 \mathrm{~kW} \mathrm{~B}=11.8 \mathrm{~kW} \mathrm{C}=11.7 \mathrm{~kW})+\mathrm{j} 0.506 \mathrm{kVAr}$ ( $A=0 \mathrm{kVAr} B=0.256 \mathrm{kVAr} C=0.25 \mathrm{kVAr}$ ) which is less than the power supplied by Gen-set $\mathrm{A} 1$ and $\mathrm{A} 2$ and Meter 1 recorded $-12.5 \mathrm{~kW}(\mathrm{~A}=-12.5 \mathrm{~kW} \mathrm{~B}=-5 \mathrm{~kW} \mathrm{C}=5 \mathrm{~kW})$ being exported to the utility. This caused a reverse power condition which opened the static switch in 2.0 seconds islanding the CERTS microgrid from the utility grid shown in Figure 17h.

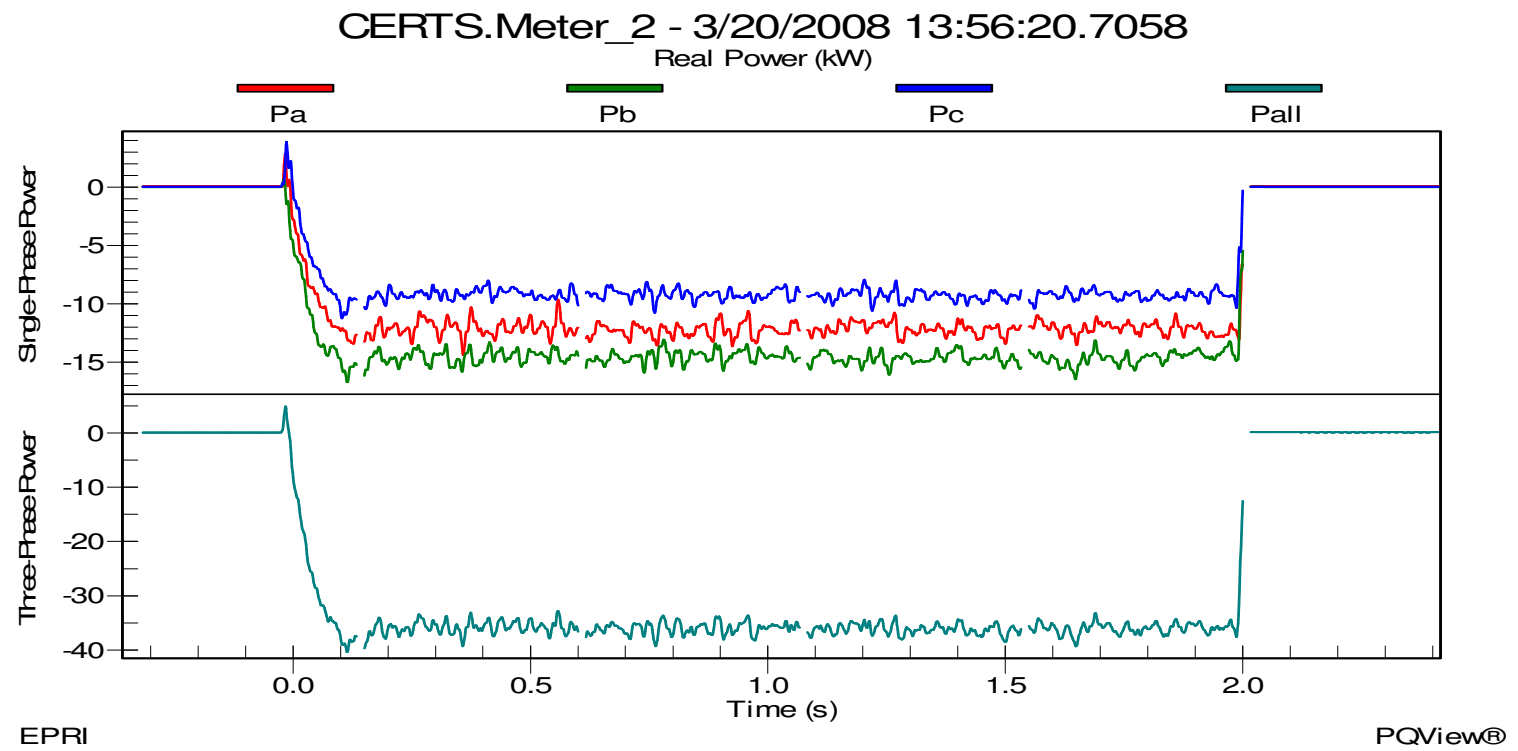

Figure 17g - Static Switch Real Power during Islanded to Utility Connected mode for Test 10.4.12 


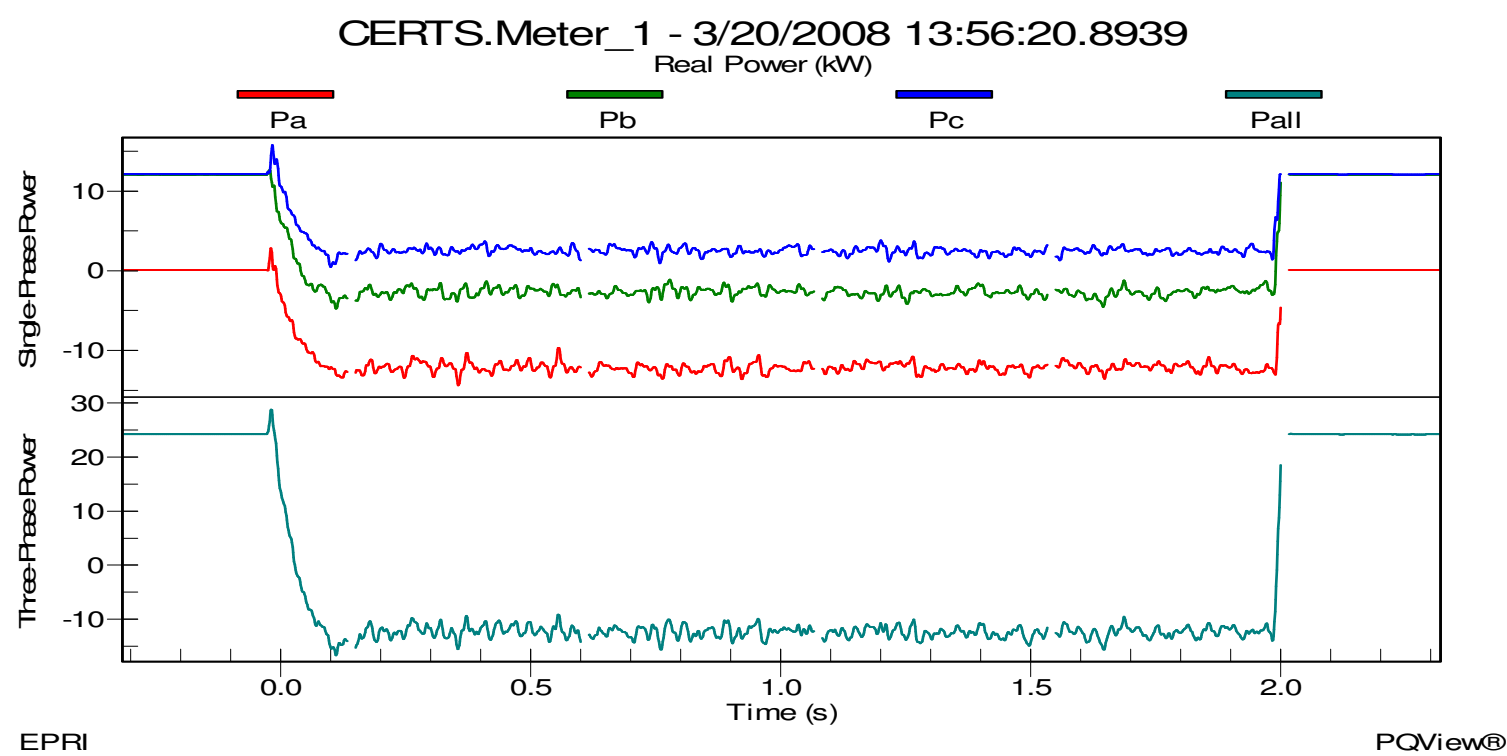

Figure 17h - Meter 1 Real Power during Islanded to Utility Connected mode for Test 10.4.12

In Table 16 after the static switch opened due to the reverse trip condition, $0 \mathrm{~kW}(\mathrm{~A}=0 \mathrm{~kW}$ $\mathrm{B}=0 \mathrm{~kW} \mathrm{C}=0 \mathrm{~kW})+\mathrm{j} 0 \mathrm{kVAr}(\mathrm{A}=0 \mathrm{kVAr} \mathrm{B}=0 \mathrm{kVAr} \mathrm{C}=0 \mathrm{kVAr})$ was recorded at Meter 2 indicating that power was not flowing through the static switch. Meter 1 real power increased from approximately $-12.5 \mathrm{~kW}(\mathrm{~A}=-12.5 \mathrm{~kW} \mathrm{~B}=-5 \mathrm{~kW} \mathrm{C}=5 \mathrm{~kW})$ to $24 \mathrm{~kW}(\mathrm{~A}=0 \mathrm{~kW}$ $\mathrm{B}=12 \mathrm{~kW} \quad \mathrm{C}=12 \mathrm{~kW})$ and reactive power decreased from approximately j23.7kVAr ( $\mathrm{A}=8.8 \mathrm{kVAr} \mathrm{B}=4.7 \mathrm{kVAr} \mathrm{C}=10.2 \mathrm{kVAr})$ to $\mathrm{j} 0.9 \mathrm{kVAr}$ ( $\mathrm{A}=0.1 \mathrm{kVAr} \mathrm{B}=0.4 \mathrm{kVAr} \mathrm{C}=0.4 \mathrm{kVAr})$. The change in power is because the microgrid is no longer supplying power for the load demands of Load Bank 6 which was approximately $24 \mathrm{~kW}(\mathrm{~A}=0 \mathrm{~kW} B=12 \mathrm{~kW} \mathrm{C}=12 \mathrm{~kW})+$ j0.519kVAr ( $\mathrm{A}=0 \mathrm{kVAr} B=0.26 \mathrm{kVAr} C=0.259 \mathrm{kVAr})$.

The power produced by the Gen-sets were very similar to the measured values before the static switch manual open was released which was approximately $20.6 \mathrm{~kW}(\mathrm{~A}=2.5 \mathrm{~kW}$ $\mathrm{B}=9.6 \mathrm{~kW} C=8.5 \mathrm{~kW})-\mathrm{j} 2.8 \mathrm{kVAr}(\mathrm{A}=-1.3 \mathrm{kVAr} \mathrm{B}=-1.3 \mathrm{kVAr} \mathrm{C}=-0.2 \mathrm{kVAr})$ for Gen-set $\mathrm{A} 1$ and $11.5 \mathrm{~kW}(\mathrm{~A}=-1.6 \mathrm{~kW} B=6.6 \mathrm{~kW} C=6.5 \mathrm{~kW})+j 7.15 \mathrm{kVAr}(\mathrm{A}=2.25 \mathrm{kVAr} B=3.2 \mathrm{kVAr} \mathrm{C}=1.7 \mathrm{kVAr})$ for Gen-set A2. Also, the voltage and frequency returned to measured values before the static switch manual open was released which was $276.5 \mathrm{~V}$ on A-phase, $278.8 \mathrm{~V}$ on Bphase and $271.4 \mathrm{~V}$ on C-phase and $60.15 \mathrm{~Hz}$. After all the data was verified and recorded into the DAS Database the Gen-sets and Load Banks set-points were changed according to the next test (10.4.14) in Table 9.

For Test 10.4.14 the measured values after Gen-set A1 was warmed up and load banks brought on-line with the A-phase in Load Banks 3 and 6 reduced by $50 \%$ are provided in Table 17. 


\begin{tabular}{|c|c|c|c|c|c|c|c|c|c|}
\hline Meter & $\begin{array}{c}\text { Gen- } \\
\text { set A1 }\end{array}$ & $\begin{array}{c}\text { Gen - } \\
\text { set A2 }\end{array}$ & Meter 1 & Meter 2 & Meter 3 & Meter 4 & LB 3 & LB 4 & LB 6 \\
\hline $\begin{array}{c}\text { 3 Phase } \\
\text { kW }\end{array}$ & 38.2 & 0 & 3.85 & -26 & -29.1 & 0 & 9.1 & 0 & 30.2 \\
\hline $\begin{array}{c}\mathbf{1} \text { Phase } \\
\mathbf{k W}\end{array}$ & $\mathrm{B}=15.8$ & $\mathrm{~B}=0$ & $\mathrm{~B}=1.25$ & $\mathrm{~B}=-10.7$ & $\mathrm{~B}=-12.1$ & $\mathrm{~B}=0$ & $\mathrm{~B}=3.68$ & $\mathrm{~B}=0$ & $\mathrm{~B}=12$ \\
\hline $\begin{array}{c}\mathbf{3} \text { Phase } \\
\mathbf{k V A r}\end{array}$ & -15.75 & 0 & 22.65 & 22.1 & 15.75 & 0 & -0.024 & 0 & 0.82 \\
\hline $\begin{array}{c}\mathbf{1} \text { Phase } \\
\mathbf{k V A r}\end{array}$ & $\mathrm{B}=-4.25$ & $\mathrm{~B}=0$ & $\mathrm{~B}=6.75$ & $\mathrm{~B}=6.5$ & $\mathrm{~B}=4.25$ & $\mathrm{~B}=0$ & $\mathrm{~B}=-0.003$ & $\mathrm{~B}=0$ & $\mathrm{~B}=0.35$ \\
& $\mathrm{C}=-5.1$ & $\mathrm{C}=0$ & $\mathrm{C}=7.6$ & $\mathrm{C}=7.4$ & $\mathrm{C}=5.1$ & $\mathrm{C}=0$ & $\mathrm{C}=-0.024$ & $\mathrm{C}=0$ & $\mathrm{C}=0.32$ \\
\hline
\end{tabular}

Table 17 - Measured Values after Start Up for Test 10.4.14 and Load Banks 3 and 6 A-phase Reduced by $50 \%$

These measurements were relatively close to the expected values, but not exact due to temperature, phase voltages and electrical losses in conductors. At the time of the measurements, the voltage and frequency was $284.2 \mathrm{~V}$ on A-phase, $281 \mathrm{~V}$ on B-phase and $280.2 \mathrm{~V}$ on C-phase and $60.00 \mathrm{~Hz}$ at the static switch (i.e., Meter 2) when connected to the utility grid; and $284.8 \mathrm{~V}$ on A-phase, $281.8 \mathrm{~V}$ on B-phase and $281 \mathrm{~V}$ on C-phase and $60.00 \mathrm{~Hz}$ at Meter 3.

Gen-set A1 in this test was set up to produce more power than Load Bank 3 needed which approximately $26 \mathrm{~kW}(\mathrm{~A}=7.5 \mathrm{~kW} \quad \mathrm{~B}=10.7 \mathrm{~kW} \quad \mathrm{C}=7.8 \mathrm{~kW})$ of excess power was exported through the static switch to Load Bank 6 . Since Load Bank 6 was approximately $30.2 \mathrm{~kW} \quad(\mathrm{~A}=6.2 \mathrm{~kW} \quad \mathrm{~B}=12 \mathrm{~kW} \quad \mathrm{C}=12 \mathrm{~kW})+\mathrm{j} 0.82 \mathrm{kVAr} \quad(\mathrm{A}=0.15 \mathrm{kVAr}$ $\mathrm{B}=0.35 \mathrm{kVAr} \mathrm{C}=0.32 \mathrm{kVAr})$, the utility had to supply approximately $3.85 \mathrm{~kW}(\mathrm{~A}=-1.5 \mathrm{~kW}$ $B=1.25 \mathrm{~kW} \mathrm{C}=4.1 \mathrm{~kW}$ ) to satisfy the load demands of Load Bank 6 . Reactive power had to be imported in from the utility of approximately $\mathrm{j} 22.1 \mathrm{kVAr}(\mathrm{A}=8.2 \mathrm{kVAr} B=6.5 \mathrm{kVAr}$ $\mathrm{C}=7.4 \mathrm{kVAr})$ because Gen-set $\mathrm{A} 1$ needed approximately j15.75kVAr $(\mathrm{A}=6.4 \mathrm{kVAr}$ $\mathrm{B}=4.25 \mathrm{kVAr} \mathrm{C}=5.1 \mathrm{kVAr}$ ) and the reactive power absorbed in the electrical lines.

Very soon after the A-phase in Load Banks 3 and 6 were reduced by $50 \%$, the static switch tripped on a reverse power condition shown in Figure 18a isolating the CERTS microgrid from the utility grid. The static switch was reset and started with a manual 
open and all data was verified and recorded into the DAS Database. Table 18 shows the measured values after the static switch was open.

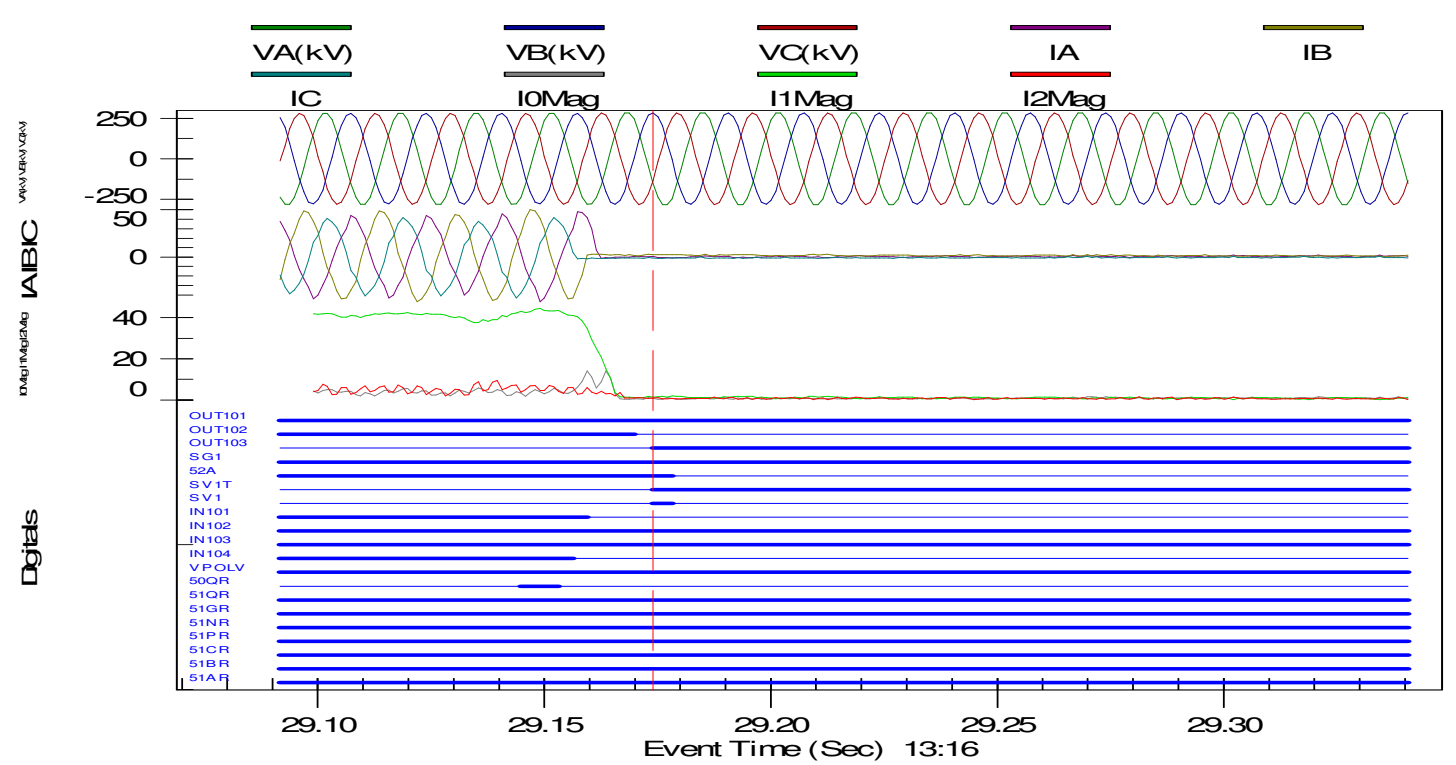

Figure 18a - Relay 2 (Static Switch) Tripping on a Reverse Power Condition for Test 10.4.14

\begin{tabular}{|c|c|c|c|c|c|c|c|c|c|}
\hline Meter & $\begin{array}{l}\text { Gen- } \\
\text { set A1 }\end{array}$ & $\begin{array}{l}\text { Gen - } \\
\text { set A2 }\end{array}$ & Meter 1 & Meter 2 & Meter 3 & Meter 4 & LB 3 & LB 4 & LB 6 \\
\hline $\begin{array}{c}3 \text { Phase } \\
\text { kW }\end{array}$ & 11 & 0 & 30.9 & 0 & -2.4 & 0 & 8.5 & 0 & 30.7 \\
\hline \multirow{3}{*}{$\begin{array}{c}1 \text { Phase } \\
\text { kW }\end{array}$} & $\mathrm{A}=2.5$ & $\mathrm{~A}=0$ & $A=6.5$ & $\mathrm{~A}=0$ & $A=-0.8$ & $A=0$ & $A=1.65$ & $A=0$ & $A=6.3$ \\
\hline & $B=4.6$ & $\mathrm{~B}=0$ & $B=12.2$ & $B=0$ & $B=-1.1$ & $B=0$ & $B=3.45$ & $\mathrm{~B}=0$ & $\mathrm{~B}=12.2$ \\
\hline & $C=3.9$ & $\mathrm{C}=0$ & $C=12.2$ & $\mathrm{C}=0$ & $C=-0.5$ & $\mathrm{C}=0$ & $C=3.4$ & $\mathrm{C}=0$ & $C=12.2$ \\
\hline $\begin{array}{c}3 \text { Phase } \\
\text { kVAr }\end{array}$ & 3.9 & 0 & 0.95 & 0 & -3.6 & 0 & -0.024 & 0 & 0.82 \\
\hline \multirow{3}{*}{$\begin{array}{l}1 \text { Phase } \\
\text { kVAr }\end{array}$} & $\mathrm{A}=0.9$ & $\mathrm{~A}=0$ & $\mathrm{~A}=0.25$ & $A=0$ & $\mathrm{~A}=-0.8$ & $\mathrm{~A}=0$ & $\mathrm{~A}=0.0015$ & $A=0$ & $\mathrm{~A}=0.15$ \\
\hline & $\mathrm{B}=1.5$ & $B=0$ & $\mathrm{~B}=0.4$ & $B=0$ & $B=-1.4$ & $\mathrm{~B}=0$ & $B=-0.003$ & $\mathrm{~B}=0$ & $\mathrm{~B}=0.35$ \\
\hline & $C=1.5$ & $C=0$ & $C=0.3$ & $C=0$ & $C=-1.4$ & $\mathrm{C}=0$ & $C=-.0225$ & $\mathrm{C}=0$ & $C=0.32$ \\
\hline
\end{tabular}

Table 18 - Measured Values after Static Switch Opened for Test 10.4.14

In Table 18, $0 \mathrm{~kW}(\mathrm{~A}=0 \mathrm{~kW} \mathrm{~B}=0 \mathrm{~kW} \mathrm{C}=0 \mathrm{~kW})+\mathrm{j} 0 \mathrm{kVAr}(\mathrm{A}=0 \mathrm{kVAr} \mathrm{B}=0 \mathrm{kVAr} \mathrm{C}=0 \mathrm{kVAr})$ was recorded at Meter 2 indicating that power was not flowing through the static switch. Meter 1 real power increased from $3.85 \mathrm{~kW}(\mathrm{~A}=-1.5 \mathrm{~kW} \mathrm{~B}=1.25 \mathrm{~kW} \mathrm{C}=4.1 \mathrm{~kW})$ to $30.9 \mathrm{~kW}$ 
$(\mathrm{A}=6.5 \mathrm{~kW} \quad \mathrm{~B}=12.2 \mathrm{~kW} \quad \mathrm{C}=12.2 \mathrm{~kW})$ and reactive power decreased from $\mathrm{j} 22.65 \mathrm{kVAr}$ $(\mathrm{A}=8.3 \mathrm{kVAr} \mathrm{B}=6.75 \mathrm{kVAr} \mathrm{C}=7.6 \mathrm{kVAr})$ to $j 0.95 \mathrm{kVAr}(\mathrm{A}=0.25 \mathrm{kVAr} \mathrm{B}=0.4 \mathrm{kVAr} \mathrm{C}=0.3 \mathrm{kVAr})$. The change in power is because the microgrid is no longer supplying power for Load Bank 6 which was approximately $30.7 \mathrm{~kW}(\mathrm{~A}=6.3 \mathrm{~kW} \mathrm{~B}=12.2 \mathrm{~kW} \mathrm{C}=12.2 \mathrm{~kW})+\mathrm{j} 0.82 \mathrm{kVAr}$ $(\mathrm{A}=0.15 \mathrm{kVAr} B=0.35 \mathrm{kVAr} \mathrm{C}=0.32 \mathrm{kVAr})$. Load Bank 3 reduced slightly to $8.5 \mathrm{~kW}$ $(\mathrm{A}=1.65 \mathrm{~kW} \quad \mathrm{~B}=3.45 \mathrm{~kW} \quad \mathrm{C}=3.4 \mathrm{~kW})-\mathrm{j} 0.024 \mathrm{kVAr} \quad(\mathrm{A}=0.0015 \mathrm{kVAr} \quad \mathrm{B}=0.003 \mathrm{kVAr} \quad \mathrm{C}=-$ $0.0225 \mathrm{kVAr}$ ). This load reduction resulted from a voltage drop in the microgrid, shown in Figure 18b, from approximately $284.8 \mathrm{~V}$ on A-phase, $281 \mathrm{~V}$ on B-phase and $280.2 \mathrm{~V}$ on C-phase when connected to the utility grid to $273 \mathrm{~V}$ on A-phase, $272.5 \mathrm{~V}$ on B-phase and $271.5 \mathrm{~V}$ on C-phase when islanded at Meter 3.

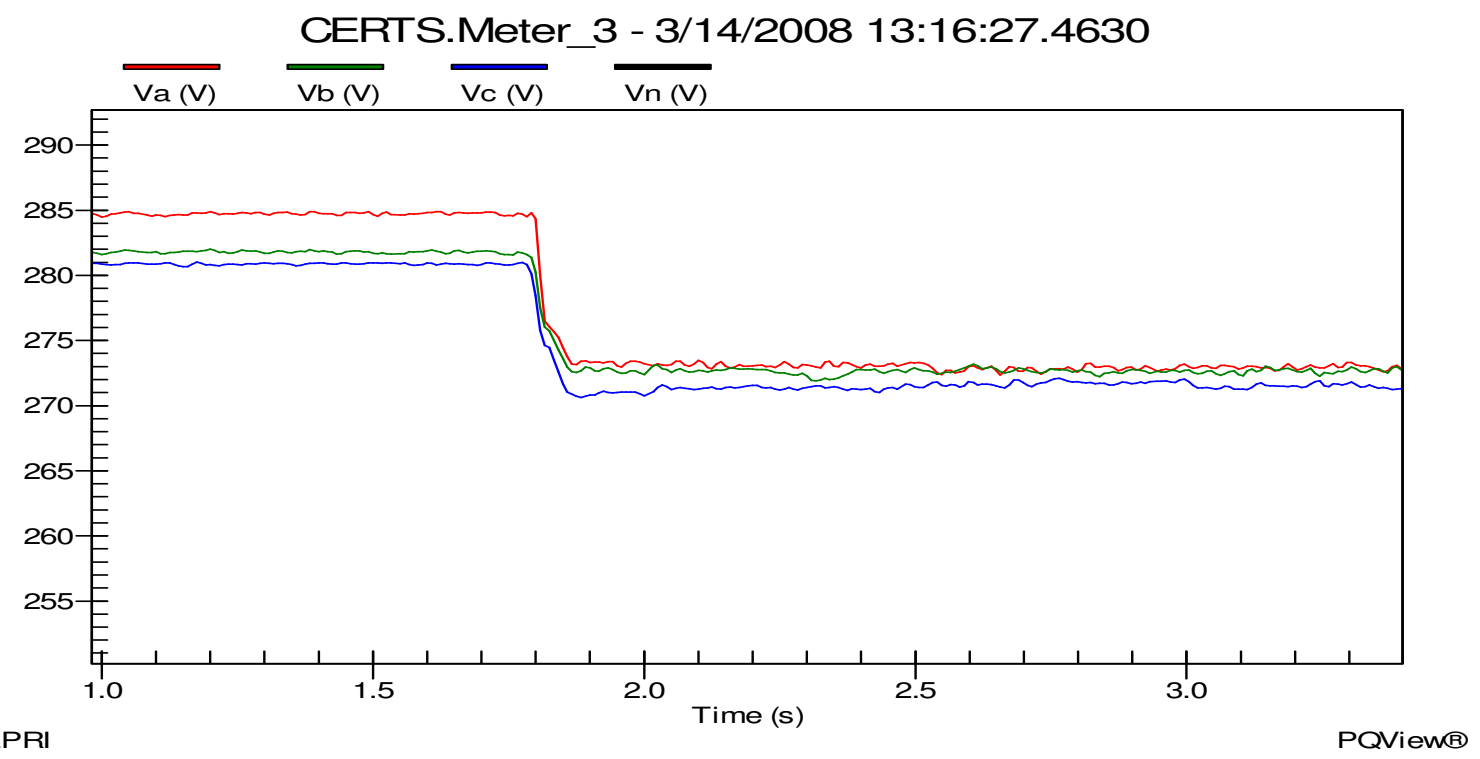

Figure 18b - Meter 3 Line-to-Ground Voltages during Utility Connected to Island mode for Test 10.4.14

Frequency change in the microgrid, shown in Figure 18c, increased from approximately $60.00 \mathrm{~Hz}$ when connected to the utility grid to approximately $60.24 \mathrm{~Hz}$ when islanded. This change in frequency was part of the CERTS algorithm which allowed Gen-set A1 to decrease its output power to satisfy the critical load demands. Gen-set A1 decreased its real output power to approximately $11 \mathrm{~kW}(\mathrm{~A}=2.5 \mathrm{~kW} \mathrm{~B}=4.6 \mathrm{~kW} \mathrm{C}=3.9 \mathrm{~kW})$ and increased its reactive output power to approximately $\mathrm{j} 3.9 \mathrm{kVAr} \quad(\mathrm{A}=0.9 \mathrm{kVAr} \quad \mathrm{B}=1.5 \mathrm{kVAr}$ $\mathrm{C}=1.5 \mathrm{kVAr}$ ) to satisfy the critical loads. Note the sign change from negative to positive for Gen-set A1 reactive power when the microgrid is isolated from the utility. This sign change is due to the utility no longer supplying the reactive power for the critical loads but by Gen-set A1. Meter 3 was approximately $-2.4 \mathrm{~kW}(\mathrm{~A}=-0.8 \mathrm{~kW} B=-1.1 \mathrm{~kW} \mathrm{C}=-0.5 \mathrm{~kW})$ - j3.6kVAr $(A=-0.8 \mathrm{kVAr} B=-1.4 \mathrm{kVAr} C=-1.4 \mathrm{kVAr})$. Negative power recorded at Meter 3 indicates that all the critical loads in the microgrid were being satisfied by Gen-set A1. All data was verified and recorded into the DAS Database. The microgrid ran for a couple of minutes in this electrical state before the A-phase was reduced in Load Bank 6 
by $50 \%$ (i.e., $75 \%$ from balanced load value). The measured values for the A-phase load reduction in Load Bank 6 are in Table 19.

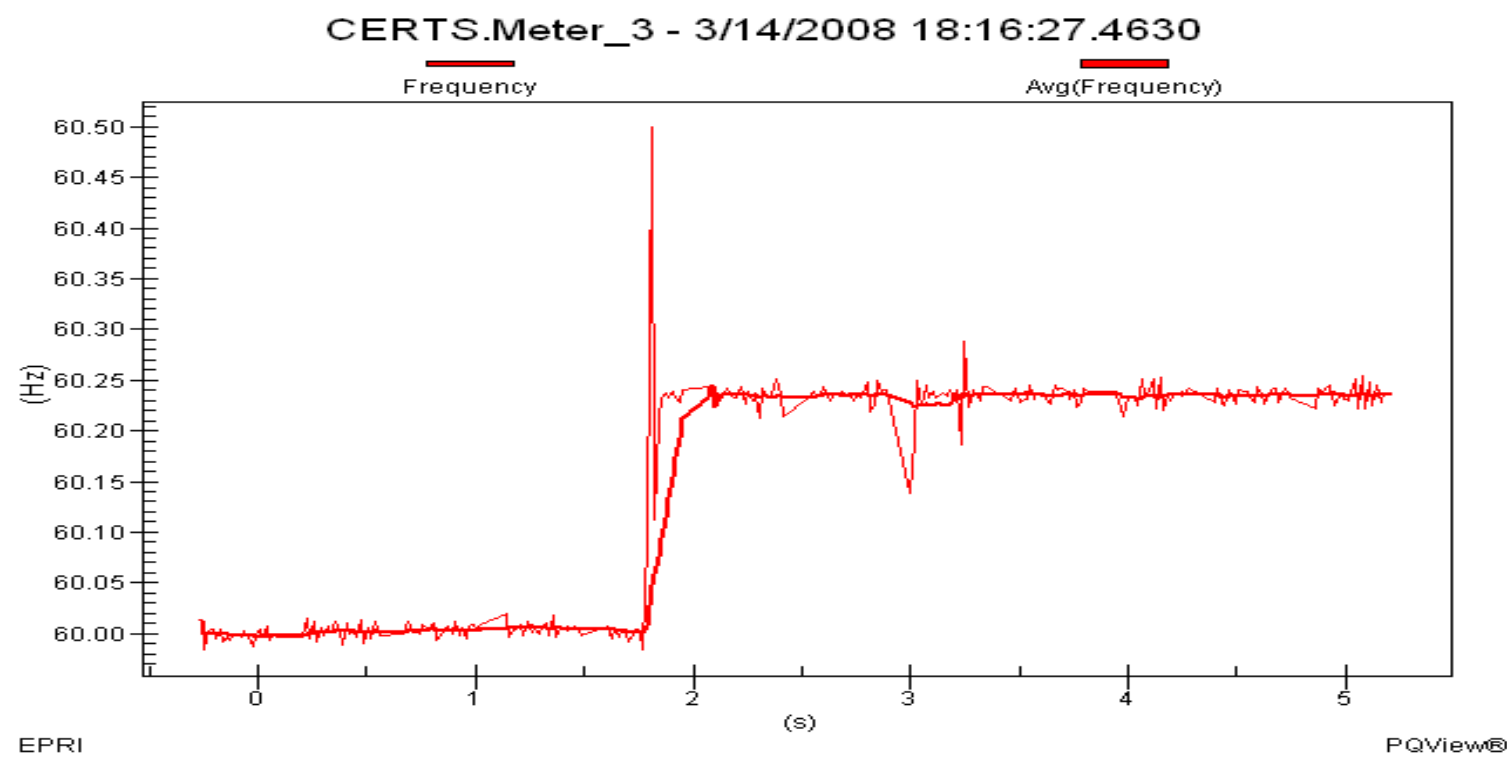

Figure 18c - Meter 3 Frequency Before/After the Static Switch Opened for Test 10.4.14

\begin{tabular}{|c|c|c|c|c|c|c|c|c|c|}
\hline Meter & $\begin{array}{l}\text { Gen- } \\
\text { set A1 }\end{array}$ & $\begin{array}{l}\text { Gen - } \\
\text { set A2 }\end{array}$ & Meter 1 & Meter 2 & Meter 3 & Meter 4 & LB 3 & LB 4 & LB 6 \\
\hline $\begin{array}{c}3 \text { Phase } \\
\text { kW }\end{array}$ & 10.9 & 0 & 28.18 & 0 & -2.405 & 0 & 8.465 & 0 & 28 \\
\hline \multirow{3}{*}{$\begin{array}{c}1 \text { Phase } \\
\text { kW }\end{array}$} & $\mathrm{A}=2.4$ & $\mathrm{~A}=0$ & $A=3.9$ & $\mathrm{~A}=0$ & $\mathrm{~A}=-0.8$ & $A=0$ & $A=1.65$ & $A=0$ & $\mathrm{~A}=3.8$ \\
\hline & $\mathrm{B}=4.7$ & $B=0$ & $B=12.08$ & $B=0$ & $B=-1.13$ & $B=0$ & $B=3.425$ & $\mathrm{~B}=0$ & $\mathrm{~B}=12$ \\
\hline & $C=3.8$ & $\mathrm{C}=0$ & $C=12.2$ & $\mathrm{C}=0$ & $C=-0.475$ & $\mathrm{C}=0$ & $C=3.39$ & $\mathrm{C}=0$ & $C=12.2$ \\
\hline $\begin{array}{c}3 \text { Phase } \\
\text { kVAr }\end{array}$ & 3.695 & 0 & 0.8 & 0 & -3.725 & 0 & -0.02 & 0 & 0.52 \\
\hline \multirow{3}{*}{$\begin{array}{l}1 \text { Phase } \\
\text { kVAr }\end{array}$} & $\mathrm{A}=0.88$ & $\mathrm{~A}=0$ & $\mathrm{~A}=0.08$ & $A=0$ & $\mathrm{~A}=-0.875$ & $\mathrm{~A}=0$ & $\mathrm{~A}=0.001$ & $A=0$ & $\mathrm{~A}=-0.01$ \\
\hline & $\mathrm{B}=1.425$ & $B=0$ & $B=0.37$ & $B=0$ & $B=-1.43$ & $\mathrm{~B}=0$ & $B=-0.001$ & $\mathrm{~B}=0$ & $B=0.269$ \\
\hline & $C=1.39$ & $C=0$ & $C=0.35$ & $C=0$ & $C=-1.42$ & $\mathrm{C}=0$ & $C=-0.02$ & $\mathrm{C}=0$ & $C=0.261$ \\
\hline
\end{tabular}

Table 19 - Measured Values after Static Switch Opened and A-phase Reduced in Load Bank 6 by 75\% for Test 10.4.14

In Table 19, Meter 1 real power decreased from approximately $30.9 \mathrm{~kW}(\mathrm{~A}=6.5 \mathrm{~kW}$ $\mathrm{B}=12.2 \mathrm{~kW} \mathrm{C}=12.2 \mathrm{~kW})$ to $28.18 \mathrm{~kW}(\mathrm{~A}=3.9 \mathrm{~kW} \mathrm{~B}=12.08 \mathrm{~kW} \mathrm{C}=12.2 \mathrm{~kW})$ because of the A- 
phase reduction in Load Bank 6 which was approximately $28 \mathrm{~kW}(\mathrm{~A}=3.8 \mathrm{~kW} B=12 \mathrm{~kW}$ $\mathrm{C}=12.2 \mathrm{~kW})+\mathrm{j} 0.52 \mathrm{kVAr}(\mathrm{A}=-0.01 \mathrm{kVAr} \mathrm{B}=0.269 \mathrm{kVAr} \mathrm{C}=0.261 \mathrm{kVAr})$. Voltages and frequencies remained the same in both the utility and microgrid when Load Bank 6 Aphase was reduced to $3.8 \mathrm{~kW}$ because nothing changed in the CERTS microgrid downstream of the static switch and the utility is a stiff system.

All data was verified and recorded into the DAS Database. The microgrid ran for a couple of minutes in this electrical state before the A-phase was reduced in Load Bank 3 by $100 \%$. The measured values for the A-phase reduction for Load Bank 3 are in Table 20.

\begin{tabular}{|c|c|c|c|c|c|c|c|c|c|}
\hline Meter & $\begin{array}{l}\text { Gen- } \\
\text { set A1 }\end{array}$ & $\begin{array}{l}\text { Gen - } \\
\text { set A2 }\end{array}$ & Meter 1 & Meter 2 & Meter 3 & Meter 4 & LB 3 & LB 4 & LB 6 \\
\hline $\begin{array}{c}3 \text { Phase } \\
\text { kW }\end{array}$ & 9.25 & 0 & 28.18 & 0 & -2.4 & 0 & 6.86 & 0 & 28 \\
\hline \multirow{3}{*}{$\begin{array}{c}1 \text { Phase } \\
\text { kW }\end{array}$} & $A=0.75$ & $A=0$ & $A=3.9$ & $A=0$ & $A=-0.82$ & $A=0$ & $\mathrm{~A}=0$ & $A=0$ & $\mathrm{~A}=3.8$ \\
\hline & $\mathrm{B}=4.7$ & $\mathrm{~B}=0$ & $B=12.08$ & $\mathrm{~B}=0$ & $B=-1.12$ & $B=0$ & $B=3.48$ & $\mathrm{~B}=0$ & $\mathrm{~B}=12$ \\
\hline & $C=3.8$ & $\mathrm{C}=0$ & $\mathrm{C}=12.2$ & $\mathrm{C}=0$ & $C=-0.46$ & $\mathrm{C}=0$ & $C=3.38$ & $\mathrm{C}=0$ & $C=12.2$ \\
\hline $\begin{array}{c}3 \text { Phase } \\
\text { kVAr }\end{array}$ & 3.63 & 0 & 0.8 & 0 & -3.7 & 0 & -0.02 & 0 & 0.52 \\
\hline \multirow{3}{*}{$\begin{array}{l}1 \text { Phase } \\
\text { kVAr }\end{array}$} & $A=0.87$ & $A=0$ & $\mathrm{~A}=0.08$ & $A=0$ & $\mathrm{~A}=-0.87$ & $A=0$ & $\mathrm{~A}=0.001$ & $A=0$ & $A=-0.01$ \\
\hline & $B=1.39$ & $\mathrm{~B}=0$ & $B=0.37$ & $\mathrm{~B}=0$ & $B=-1.45$ & $B=0$ & $B=-0.001$ & $\mathrm{~B}=0$ & $B=0.269$ \\
\hline & $C=1.37$ & $C=0$ & $C=0.35$ & $C=0$ & $C=-1.38$ & $\mathrm{C}=0$ & $C=-0.02$ & $\mathrm{C}=0$ & $C=0.261$ \\
\hline
\end{tabular}

Table 20 - Measured Values after Static Switch Opened and A-phase Reduced in Load Bank 3 by $100 \%$ for Test 10.4.14

In Table 20, Load Bank 3 was approximately $6.86 \mathrm{~kW}(\mathrm{~A}=0 \mathrm{~kW} \mathrm{~B}=3.48 \mathrm{~kW} \mathrm{C}=3.48 \mathrm{~kW})-$ j0.02kVAr $(A=0.001 \mathrm{kVAr} B=-0.001 \mathrm{kVAr} C=-0.02 \mathrm{kVAr})$ after the A-phase was reduced by $100 \%$. The reduction in Load Bank 3 caused the frequency to increase within the microgrid from approximately $60.24 \mathrm{~Hz}$ to $60.25 \mathrm{~Hz}$. The increase in frequency is part of the CERTS algorithm which caused Gen-set A1 to decrease its power level output due to the decrease in the A-phase load demand of Load Bank 3. Gen-set A1 decreased from approximately $10.9 \mathrm{~kW} \quad(\mathrm{~A}=2.4 \mathrm{~kW} \quad \mathrm{~B}=4.7 \mathrm{~kW} \quad \mathrm{C}=3.8 \mathrm{~kW})+j 3.695 \mathrm{kVAr} \quad(\mathrm{A}=0.88 \mathrm{kVAr}$ $\mathrm{B}=1.425 \mathrm{kVAr} \quad \mathrm{C}=1.39 \mathrm{kVAr})$ to $9.25 \mathrm{~kW} \quad(\mathrm{~A}=0.75 \mathrm{~kW} \quad \mathrm{~B}=4.7 \mathrm{~kW} \quad \mathrm{C}=3.8 \mathrm{~kW})+\mathrm{j} 3.63 \mathrm{kVAr}$ $(\mathrm{A}=0.87 \mathrm{kVAr} \mathrm{B}=1.39 \mathrm{kVAr} \mathrm{C}=1.37 \mathrm{kVAr})$. The voltage within the microgrid during these measurements was approximately $273.5 \mathrm{~V}$ on A-phase, $273.5 \mathrm{~V}$ on B-phase and $270.1 \mathrm{~V}$ on 
C-phase. Load Bank 6 load demands were not changed at this time, therefore, all the measured values for Load Bank 6, Meter 1 and Meter 2 did not change from the previous load reduction in Load Bank 6.

All data was verified and recorded into the DAS Database. The microgrid ran for a couple of minutes in this electrical state before the A-phase was reduced in Load Bank 6 by $100 \%$. The measured values for the A-phase load reduction for Load Bank 6 are in Table 21.

\begin{tabular}{|c|c|c|c|c|c|c|c|c|c|}
\hline Meter & $\begin{array}{l}\text { Gen- } \\
\text { set A1 }\end{array}$ & $\begin{array}{l}\text { Gen - } \\
\text { set A2 }\end{array}$ & Meter 1 & Meter 2 & Meter 3 & Meter 4 & LB 3 & LB 4 & LB 6 \\
\hline $\begin{array}{c}3 \text { Phase } \\
\text { kW }\end{array}$ & 9.1 & 0 & 24.33 & 0 & -2.4 & 0 & 6.87 & 0 & 24.2 \\
\hline \multirow{3}{*}{$\begin{array}{c}1 \text { Phase } \\
\text { kW }\end{array}$} & $A=0.6$ & $\mathrm{~A}=0$ & $\mathrm{~A}=0$ & $\mathrm{~A}=0$ & $A=-0.82$ & $\mathrm{~A}=0$ & $A=0$ & $\mathrm{~A}=0$ & $A=0$ \\
\hline & $\mathrm{B}=4.7$ & $\mathrm{~B}=0$ & $B=12.08$ & $\mathrm{~B}=0$ & $B=-1.12$ & $\mathrm{~B}=0$ & $B=3.49$ & $\mathrm{~B}=0$ & $\mathrm{~B}=12$ \\
\hline & $C=3.8$ & $C=0$ & $C=12.25$ & $C=0$ & $C=-0.46$ & $C=0$ & $C=3.38$ & $\mathrm{C}=0$ & $C=12.2$ \\
\hline $\begin{array}{c}3 \text { Phase } \\
\text { kVAr }\end{array}$ & 3.66 & 0 & 0.78 & 0 & -3.69 & 0 & -0.0255 & 0 & 0.54 \\
\hline \multirow{3}{*}{$\begin{array}{c}1 \text { Phase } \\
\text { kVAr }\end{array}$} & $\mathrm{A}=0.86$ & $A=0$ & $A=0.08$ & $A=0$ & $A=-0.87$ & $A=0$ & $A=-0.002$ & $A=0$ & $A=-0.01$ \\
\hline & $\mathrm{B}=1.44$ & $\mathrm{~B}=0$ & $B=0.35$ & $\mathrm{~B}=0$ & $\mathrm{~B}=-1.44$ & $\mathrm{~B}=0$ & $B=-0.003$ & $\mathrm{~B}=0$ & $B=0.26$ \\
\hline & $C=1.36$ & $C=0$ & $C=0.35$ & $C=0$ & $C=-1.38$ & $C=0$ & $C=-.0205$ & $C=0$ & $C=0.27$ \\
\hline
\end{tabular}

Table 21 - Measured Values after Static Switch Opened and A-phase Reduced in Load Bank 6 by $100 \%$ for Test 10.4.14

In Table 21, Meter 1 real power decreased from approximately $28.33 \mathrm{~kW}(\mathrm{~A}=4 \mathrm{~kW}$ $\mathrm{B}=12.08 \mathrm{~kW} \mathrm{C}=12.25 \mathrm{~kW})$ to $24.33 \mathrm{~kW}(\mathrm{~A}=0 \mathrm{~kW} \mathrm{~B}=12.08 \mathrm{~kW} \mathrm{C}=12.25 \mathrm{~kW})$ because of the Aphase reduction in Load Bank 6 which was approximately $24.2 \mathrm{~kW}(\mathrm{~A}=0 \mathrm{~kW} B=12 \mathrm{~kW}$ $\mathrm{C}=12.2 \mathrm{~kW})+\mathrm{j} 0.54 \mathrm{kVAr} \quad(\mathrm{A}=0.01 \mathrm{kVAr} \quad \mathrm{B}=0.26 \mathrm{kVAr} \quad \mathrm{C}=0.27 \mathrm{kVAr}) . \quad$ Voltages and frequencies remained the same in both the utility and microgrid when Load Bank 6 Aphase was reduced to $0 \mathrm{~kW}$ because nothing changed in the CERTS microgrid downstream of the static switch and the utility is a stiff system.

All data was verified and recorded into the DAS Database. The microgrid ran for a couple of minutes in this electrical state before the manual open signal was released and allowed the static switch to synchronize and close back into the utility grid. Shortly after 
the static switch closed back into the utility it tripped on a reverse power due to the Aphase on Load Banks 3 and 6 equaling 0kW shown in Figure 18d. The measured values are provided in Table 22.

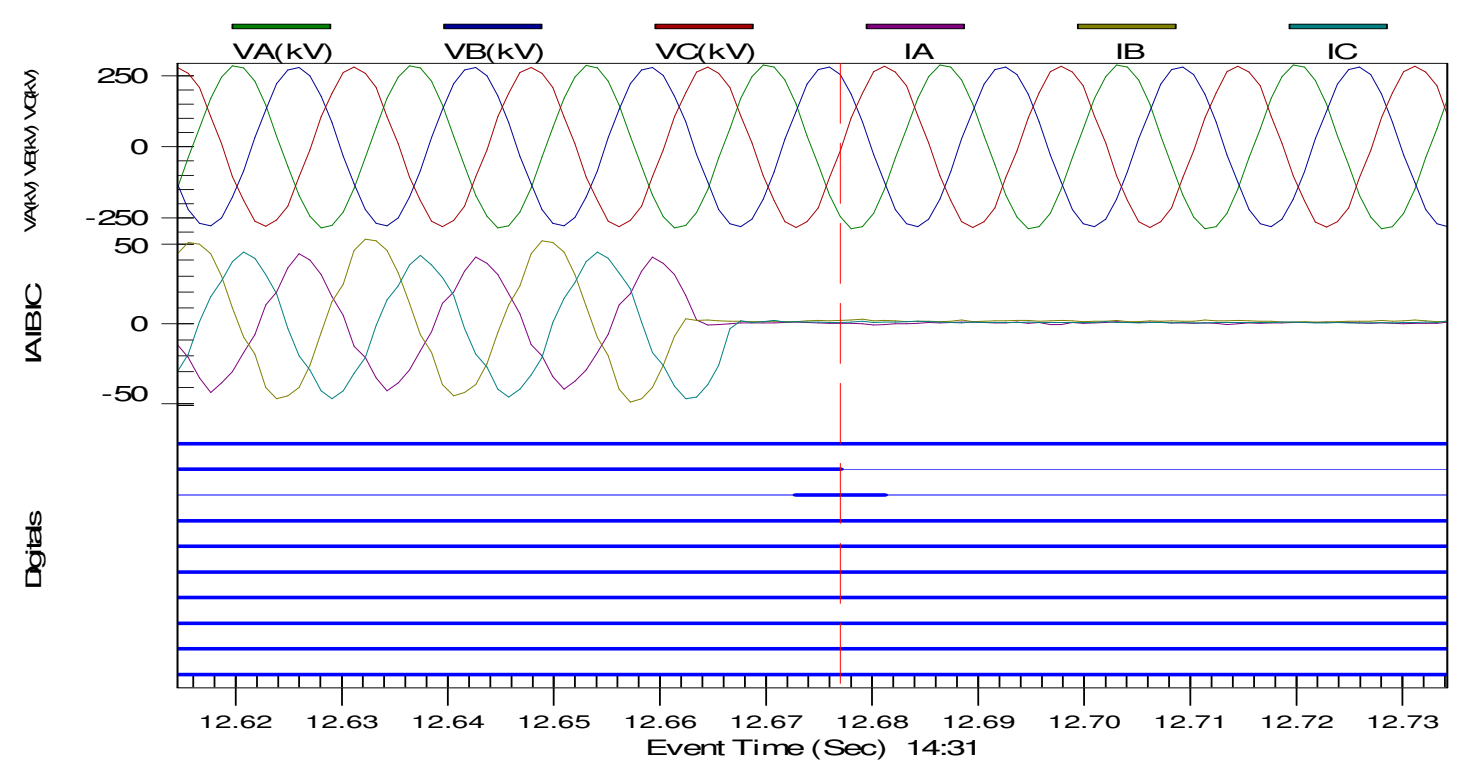

Figure 18d - Relay 2 (Static Switch) Tripping on a Reverse Power Condition during Island to Utility Connected mode for Test 10.4.14

\begin{tabular}{|c|c|c|c|c|c|c|c|c|c|}
\hline Meter & $\begin{array}{l}\text { Gen- } \\
\text { set A1 }\end{array}$ & $\begin{array}{l}\text { Gen - } \\
\text { set A2 }\end{array}$ & Meter 1 & Meter 2 & Meter 3 & Meter 4 & LB 3 & LB 4 & LB 6 \\
\hline \multicolumn{10}{|c|}{ Before Static Switch Synchronization and Close } \\
\hline $\begin{array}{c}3 \text { Phase } \\
\text { kW }\end{array}$ & 9.4 & 0 & 24.4 & 0 & -2.2 & 0 & 6.87 & 0 & 24.2 \\
\hline \multirow{3}{*}{$\begin{array}{c}1 \text { Phase } \\
\text { kW }\end{array}$} & $A=1$ & $\mathrm{~A}=0$ & $A=0$ & $\mathrm{~A}=0$ & $\mathrm{~A}=-0.8$ & $\mathrm{~A}=0$ & $A=0$ & $\mathrm{~A}=0$ & $\mathrm{~A}=0$ \\
\hline & $\mathrm{B}=4.5$ & $\mathrm{~B}=0$ & $B=12.2$ & $\mathrm{~B}=0$ & $B=-1$ & $\mathrm{~B}=0$ & $B=3.47$ & $\mathrm{~B}=0$ & $\mathrm{~B}=12$ \\
\hline & $C=3.9$ & $C=0$ & $C=12.2$ & $C=0$ & $C=-0.4$ & $C=0$ & $C=3.4$ & $\mathrm{C}=0$ & $C=12.2$ \\
\hline $\begin{array}{l}3 \text { Phase } \\
\text { kVAr }\end{array}$ & 3.7 & 0 & 1 & 0 & -3.5 & 0 & -0.027 & 0 & 0.533 \\
\hline \multirow{3}{*}{$\begin{array}{c}1 \text { Phase } \\
\text { kVAr }\end{array}$} & $\mathrm{A}=0.8$ & $\mathrm{~A}=0$ & $A=0.1$ & $\mathrm{~A}=0$ & $\mathrm{~A}=-0.8$ & $\mathrm{~A}=0$ & $\mathrm{~A}=-0.002$ & $\mathrm{~A}=0$ & $\mathrm{~A}=0.001$ \\
\hline & $B=1.5$ & $B=0$ & $\mathrm{~B}=0.5$ & $B=0$ & $B=-1.4$ & $\mathrm{~B}=0$ & $\mathrm{~B}=-0.003$ & $\mathrm{~B}=0$ & $B=0.269$ \\
\hline & $\mathrm{C}=1.4$ & $C=0$ & $C=0.4$ & $C=0$ & $C=-1.3$ & $C=0$ & $C=-0.022$ & $\mathrm{C}=0$ & $C=0.263$ \\
\hline
\end{tabular}




\begin{tabular}{|c|c|c|c|c|c|c|c|c|c|}
\hline $\begin{array}{c}3 \text { Phase } \\
\text { kW }\end{array}$ & 39.05 & 0 & -4.95 & -29 & -32 & 0 & 7.45 & 0 & 23.9 \\
\hline \multirow{3}{*}{$\begin{array}{c}1 \text { Phase } \\
\text { kW }\end{array}$} & $\mathrm{A}=8.8$ & $A=0$ & $A=-7.9$ & $A=-7.9$ & $A=-8.8$ & $\mathrm{~A}=0$ & $\mathrm{~A}=0$ & $A=0$ & $\mathrm{~A}=0$ \\
\hline & $B=17.25$ & $\mathrm{~B}=0$ & $B=-0.25$ & $B=-12.4$ & $B=-13.8$ & $\mathrm{~B}=0$ & $\mathrm{~B}=3.75$ & $\mathrm{~B}=0$ & $\mathrm{~B}=12$ \\
\hline & $\mathrm{C}=13$ & $\mathrm{C}=0$ & $C=3.2$ & $C=-8.7$ & $C=-9.4$ & $\mathrm{C}=0$ & $C=3.7$ & $\mathrm{C}=0$ & $C=11.9$ \\
\hline $\begin{array}{l}3 \text { Phase } \\
\text { kVAr }\end{array}$ & -16.15 & 0 & 22.95 & 22.5 & 16.35 & 0 & -0.027 & 0 & 0.53 \\
\hline \multirow{3}{*}{$\begin{array}{c}1 \text { Phase } \\
\text { kVAr }\end{array}$} & $A=-6.2$ & $\mathrm{~A}=0$ & $\mathrm{~A}=7.9$ & $A=8$ & $A=6.3$ & $\mathrm{~A}=0$ & $\mathrm{~A}=-0.002$ & $\mathrm{~A}=0$ & $\mathrm{~A}=0.001$ \\
\hline & $B=-3.75$ & $\mathrm{~B}=0$ & $B=6.25$ & $\mathrm{~B}=6$ & $\mathrm{~B}=3.75$ & $\mathrm{~B}=0$ & $B=-0.003$ & $\mathrm{~B}=0$ & $B=0.269$ \\
\hline & $C=-6.2$ & $\mathrm{C}=0$ & $\mathrm{C}=8.8$ & $\mathrm{C}=8.5$ & $C=6.3$ & $\mathrm{C}=0$ & $C=-0.022$ & $\mathrm{C}=0$ & $C=0.26$ \\
\hline \multicolumn{10}{|c|}{ Static Switch After Reverse Power Condition Trip } \\
\hline $\begin{array}{c}3 \text { Phase } \\
\text { kW }\end{array}$ & 9.4 & 0 & 24.4 & 0 & -2.2 & 0 & 6.87 & 0 & 24.2 \\
\hline \multirow{3}{*}{$\begin{array}{c}1 \text { Phase } \\
\text { kW }\end{array}$} & $A=1$ & $A=0$ & $A=0$ & $\mathrm{~A}=0$ & $A=-0.8$ & $A=0$ & $\mathrm{~A}=0$ & $A=0$ & $A=0$ \\
\hline & $\mathrm{B}=4.5$ & $\mathrm{~B}=0$ & $\mathrm{~B}=12.2$ & $\mathrm{~B}=0$ & $B=-1$ & $\mathrm{~B}=0$ & $\mathrm{~B}=3.47$ & $\mathrm{~B}=0$ & $\mathrm{~B}=12$ \\
\hline & $\mathrm{C}=3.9$ & $\mathrm{C}=0$ & $C=12.2$ & $\mathrm{C}=0$ & $C=-0.34$ & $\mathrm{C}=0$ & $C=3.4$ & $\mathrm{C}=0$ & $C=12.2$ \\
\hline $\begin{array}{l}3 \text { Phase } \\
\text { kVAr }\end{array}$ & 3.7 & 0 & 1 & 0 & -3.5 & 0 & -0.027 & 0 & 0.533 \\
\hline \multirow{3}{*}{$\begin{array}{l}1 \text { Phase } \\
\text { kVAr }\end{array}$} & $\mathrm{A}=0.8$ & $\mathrm{~A}=0$ & $\mathrm{~A}=0.1$ & $\mathrm{~A}=0$ & $\mathrm{~A}=-0.8$ & $\mathrm{~A}=0$ & $\mathrm{~A}=-0.002$ & $\mathrm{~A}=0$ & $\mathrm{~A}=0.001$ \\
\hline & $\mathrm{B}=1.5$ & $\mathrm{~B}=0$ & $B=0.5$ & $\mathrm{~B}=0$ & $B=-1.4$ & $\mathrm{~B}=0$ & $B=-0.003$ & $\mathrm{~B}=0$ & $B=0.269$ \\
\hline & $\mathrm{C}=1.4$ & $\mathrm{C}=0$ & $C=0.4$ & $\mathrm{C}=0$ & $C=-1.3$ & $\mathrm{C}=0$ & $C=-0.022$ & $\mathrm{C}=0$ & $C=0.263$ \\
\hline
\end{tabular}

Table 22 - Measured Values before/after Static Switch Synchronized and Closed back into the utility and after Static Switch Tripped on Reverse Power Condition for Test 10.4.14

Voltage and frequency in the microgrid increased from approximately $273.4 \mathrm{~V}$ on Aphase, $273.4 \mathrm{~V}$ on B-phase and $270.1 \mathrm{~V}$ on C-phase and $60.13 \mathrm{~Hz}$ when islanded to $285.25 \mathrm{~V}$ on A-phase, $282 \mathrm{~V}$ on B-phase and $280.4 \mathrm{~V}$ on C-phase and $60.00 \mathrm{~Hz}$ when utility connected at Meter 3 shown in Figures 18e and 18f. This change in frequency is due to the frequency no longer being established by Gen-set A1 using the CERTS algorithm but by the utility. Gen-set A1 is in unit power control mode therefore when the static switch closed back into the utility the Gen-set produced real power based on the set-point initialized at the beginning of the test. The output power for Gen-set A1 was relatively close to the value at the beginning of the test with Gen-set A1 producing approximately 
$39.05 \mathrm{~kW}(\mathrm{~A}=8.8 \mathrm{~kW} B=17.25 \mathrm{~kW} C=13 \mathrm{~kW})-j 16.15 \mathrm{kVAr}(A=-6.2 \mathrm{kVAr} B=-3.75 \mathrm{kVAr} C=$ $6.2 \mathrm{kVAr})$.

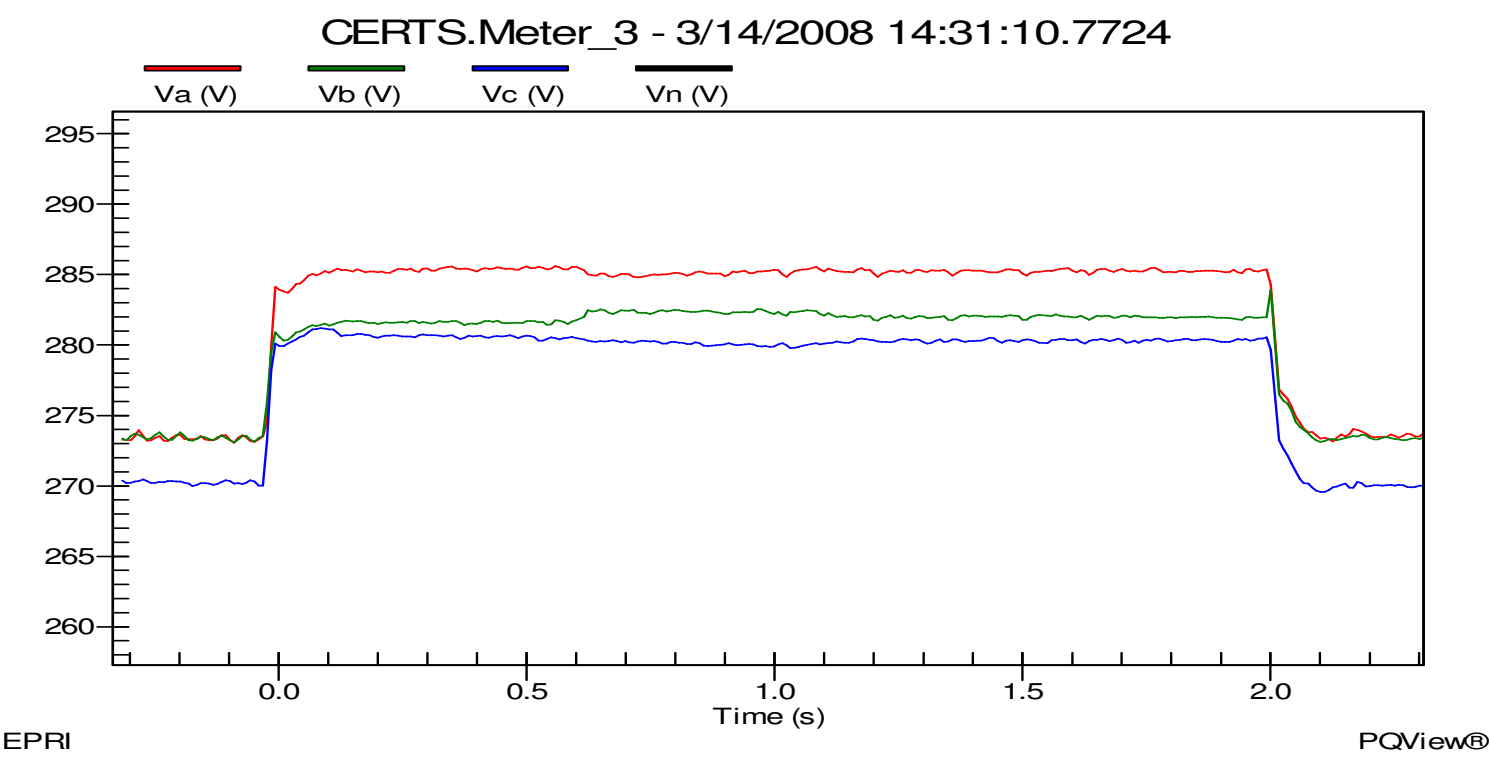

Figure 18e - Meter 3 Line-to-Ground Voltages during Islanded to Utility Connected mode for Test 10.4.14

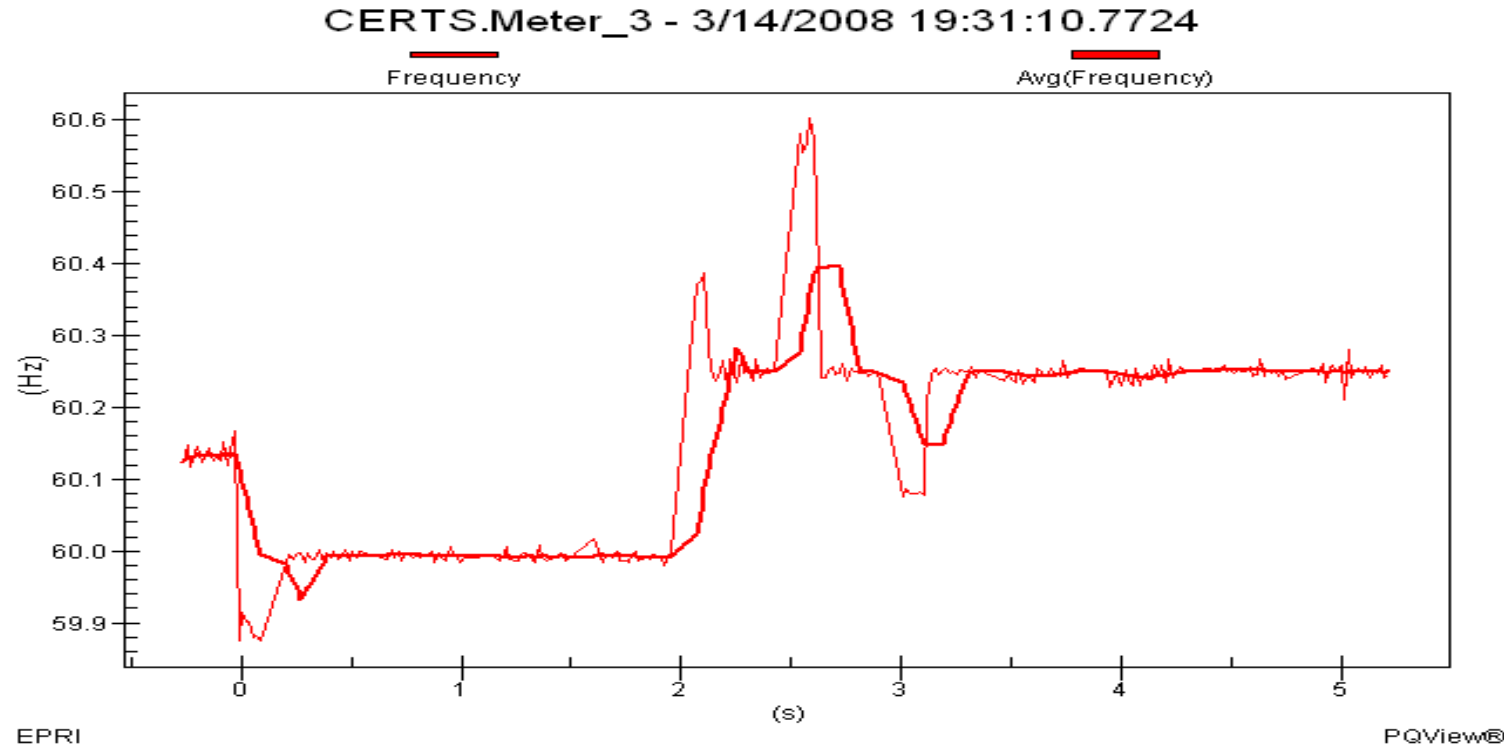

Figure 18f - Meter 3 Frequency during Islanded to Utility Connected mode for Test 10.4.14

Meter 2 recorded real power decreasing from approximately $0 \mathrm{~kW}(\mathrm{~A}=0 \mathrm{~kW} B=0 \mathrm{~kW}$ $\mathrm{C}=0 \mathrm{~kW})$ to $-29 \mathrm{~kW}(\mathrm{~A}=-7.9 \mathrm{~kW} \mathrm{~B}=-12.4 \mathrm{~kW} \mathrm{C}=-8.7 \mathrm{~kW})$ and reactive power increasing from approximately j0kVAr ( $\mathrm{A}=0 \mathrm{kVAr} B=0 \mathrm{kVAr} C=0 \mathrm{kVAr})$ to $\mathrm{j} 22.5 \mathrm{kVAr}(\mathrm{A}=8 \mathrm{kVAr} B=6 \mathrm{kVAr}$ $\mathrm{C}=8.5 \mathrm{kVAr}$ ) after the static switch closed shown in Figure 18g. Gen-set A1 was 
producing power for all the critical loads in the microgrid and approximately $29 \mathrm{~kW}$ $(\mathrm{A}=7.9 \mathrm{~kW} \mathrm{~B}=12.4 \mathrm{~kW} \mathrm{C}=8.7 \mathrm{~kW})$ of Load Bank 6 load demands. Load Bank 6 was approximately $23.9 \mathrm{~kW} \quad(\mathrm{~A}=0 \mathrm{~kW} \quad \mathrm{~B}=12 \mathrm{~kW} \quad \mathrm{C}=11.9 \mathrm{~kW})+j 0.53 \mathrm{kVAr} \quad(\mathrm{A}=0.001 \mathrm{kVAr}$ $\mathrm{B}=0.269 \mathrm{kVAr} \mathrm{C}=0.26 \mathrm{kVAr}$ ) which is less than the power supplied by Gen-set $\mathrm{A} 1$ and Meter 1 recorded $-4.95 \mathrm{~kW}(\mathrm{~A}=-7.9 \mathrm{~kW} \mathrm{~B}=-0.25 \mathrm{~kW} \mathrm{C}=3.2 \mathrm{~kW})$ being exported to the utility. This caused a reverse power condition which opened the static switch in 2.0 seconds islanding the CERTS microgrid from the utility grid shown in Figure 18h.

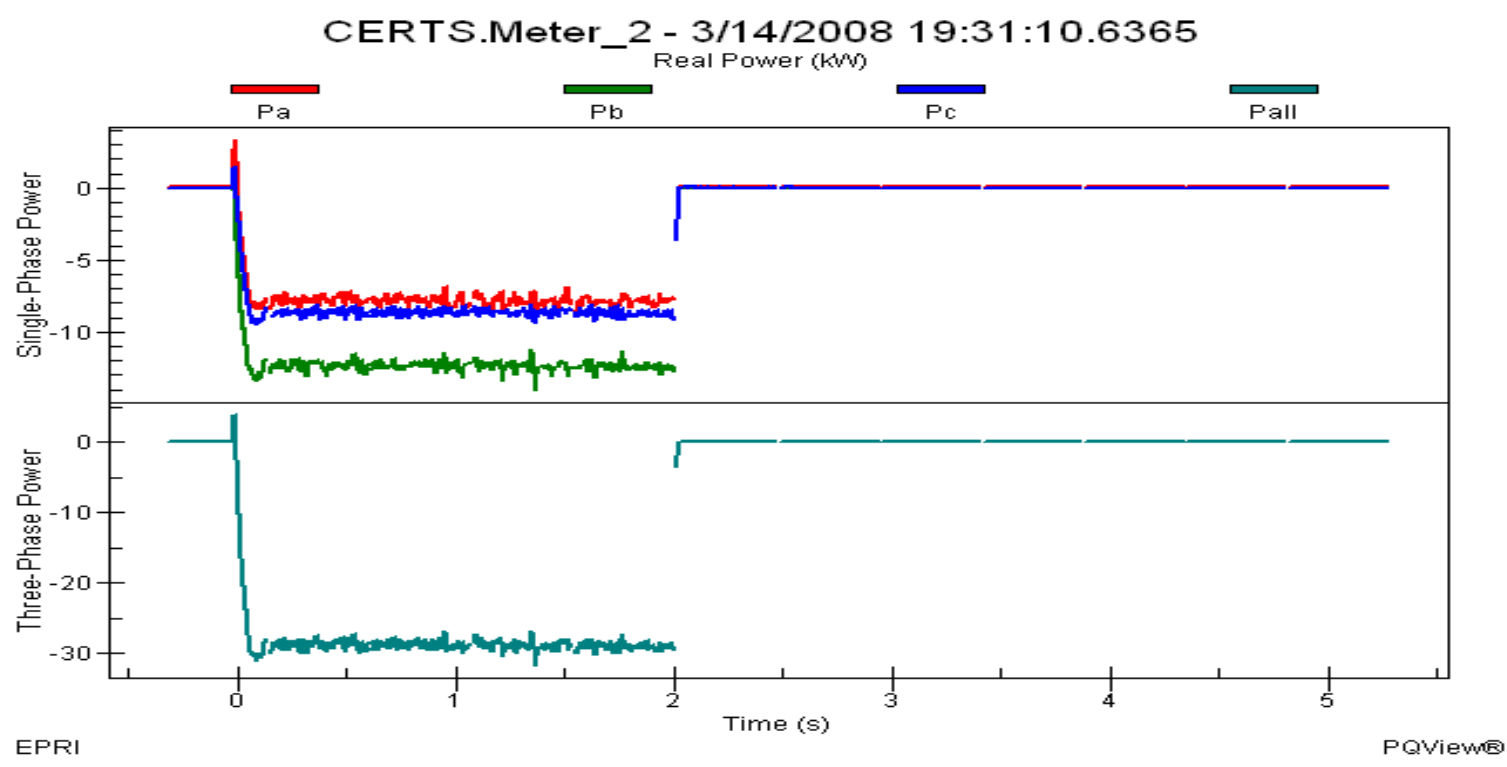

Figure 18g - Static Switch Real Power during Islanded to Utility Connected mode for Test 10.4.14

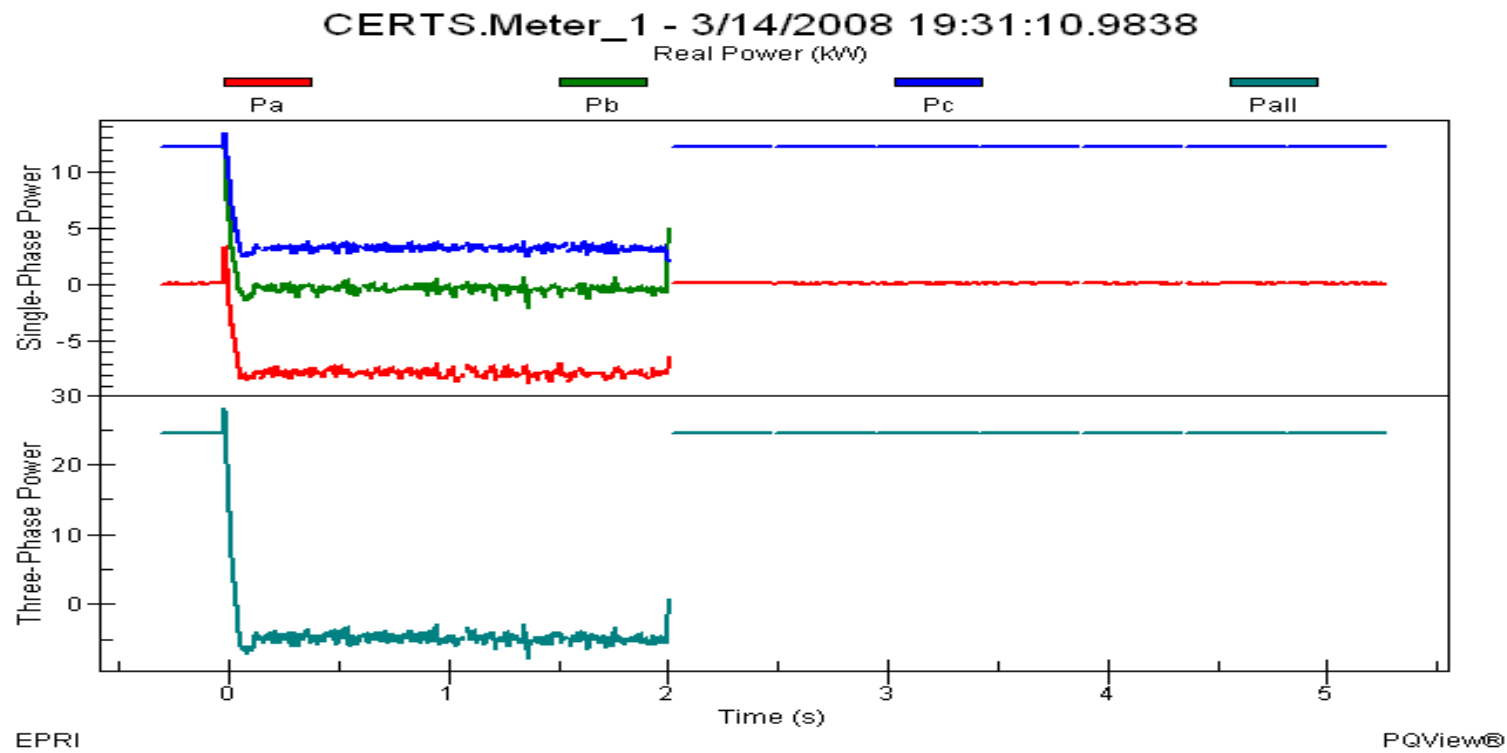

Figure 18h - Meter 1 Real Power during Islanded to Utility Connected mode for Test 10.4.14 
In Table 22 after the static switch opened due to the reverse trip condition, $0 \mathrm{~kW}(\mathrm{~A}=0 \mathrm{~kW}$ $\mathrm{B}=0 \mathrm{~kW} \mathrm{C}=0 \mathrm{~kW})+\mathrm{j} 0 \mathrm{kVAr}(\mathrm{A}=0 \mathrm{kVAr} \mathrm{B}=0 \mathrm{kVAr} \mathrm{C}=0 \mathrm{kVAr})$ was recorded at Meter 2 indicating that power was not flowing through the static switch. Meter 1 real power increased from approximately $-4.95 \mathrm{~kW}(\mathrm{~A}=-7.9 \mathrm{~kW} B=-0.25 \mathrm{~kW} \mathrm{C}=3.2 \mathrm{~kW})$ to $24.4 \mathrm{~kW}$ $(\mathrm{A}=0 \mathrm{~kW} \quad \mathrm{~B}=12.2 \mathrm{~kW} \mathrm{C}=12.2 \mathrm{~kW})$ and reactive power decreased from approximately j22.95kVAr $(A=7.9 \mathrm{kVAr} B=6.25 \mathrm{kVAr} C=8.8 \mathrm{kVAr})$ to j1kVAr $(A=0.1 \mathrm{kVAr} B=0.5 \mathrm{kVAr}$ $\mathrm{C}=0.4 \mathrm{kVAr})$. The change in power is because the microgrid is no longer supplying power for the load demands of Load Bank 6 which was approximately $24.2 \mathrm{~kW}$ (A=0kW $B=12 \mathrm{~kW} C=12.2 \mathrm{~kW})+j 0.533 \mathrm{kVAr}(\mathrm{A}=0.001 \mathrm{kVAr} B=0.269 \mathrm{kVAr} C=0.263 \mathrm{kVAr})$.

The power produced by the Gen-set was very similar to the measured value before the static switch manual open was released which was approximately $9.4 \mathrm{~kW}(\mathrm{~A}=1 \mathrm{~kW}$ $\mathrm{B}=4.5 \mathrm{~kW} \quad \mathrm{C}=3.9 \mathrm{~kW})+\mathrm{j} 3.7 \mathrm{kVAr}(\mathrm{A}=0.8 \mathrm{kVAr} \mathrm{B}=1.5 \mathrm{kVAr} \mathrm{C}=1.4 \mathrm{kVAr})$ for Gen-set $\mathrm{A} 1$. Also, the voltage and frequency returned to measured values before the static switch manual open was released which was $273.4 \mathrm{~V}$ on A-phase, $273.4 \mathrm{~V}$ on B-phase and 270V on C-phase and $60.25 \mathrm{~Hz}$. After all the data was verified and recorded into the DAS Database the Gen-sets and Load Banks set-points were changed according to the next test (10.4.17) in Table 9.

For Test 10.4.17 the measured values, after Gen-set A1 was warmed up and load banks brought on-line with the A-phase in Load Bank 3 reduced by $50 \%$ are provided in Table 23.

\begin{tabular}{|c|c|c|c|c|c|c|c|c|c|}
\hline Meter & $\begin{array}{c}\text { Gen- } \\
\text { set A1 }\end{array}$ & $\begin{array}{c}\text { Gen - } \\
\text { set A2 }\end{array}$ & Meter 1 & Meter 2 & Meter 3 & Meter 4 & LB 3 & LB 4 & LB 6 \\
\hline $\begin{array}{c}\text { 3 Phase } \\
\text { kW }\end{array}$ & 32 & 0 & 22.8 & -13.15 & -16.4 & 0 & 16.03 & 0 & 36.05 \\
\hline $\begin{array}{c}\text { 1 Phase } \\
\mathbf{k W}\end{array}$ & $\mathrm{B}=12.7$ & $\mathrm{~B}=0$ & $\mathrm{~B}=6.7$ & $\mathrm{~B}=-5.25$ & $\mathrm{~B}=-6.5$ & $\mathrm{~B}=0$ & $\mathrm{~B}=6.18$ & $\mathrm{~B}=0$ & $\mathrm{~B}=11.9$ \\
\hline $\begin{array}{c}\text { 3 Phase } \\
\mathbf{k V A r}\end{array}$ & -14.85 & 0 & 22.16 & 21 & 14.9 & 0 & 0.183 & 0 & 0.77 \\
\hline $\begin{array}{c}\text { 1 Phase } \\
\mathbf{k V A r}\end{array}$ & $\mathrm{B}=-4.25$ & $\mathrm{~B}=0$ & $\mathrm{~B}=6.6$ & $\mathrm{~B}=6.5$ & $\mathrm{~B}=4.4$ & $\mathrm{~B}=0$ & $\mathrm{~B}=0.111$ & $\mathrm{~B}=0$ & $\mathrm{~B}=0.27$ \\
& $\mathrm{C}=-6.2$ & $\mathrm{C}=0$ & $\mathrm{C}=7.4$ & $\mathrm{C}=6.75$ & $\mathrm{C}=4.4$ & $\mathrm{C}=0$ & $\mathrm{C}=0.086$ & $\mathrm{C}=0$ & $\mathrm{C}=0.26$ \\
\hline
\end{tabular}


Table 23 - Measured Values After Start Up for Test 10.4.17 and Load Bank 3 A-phase Reduced by 50\%

These measurements were relatively close to the expected values, but not exact due to temperature, phase voltages and electrical losses in conductors. At the time of the measurements, the voltage and frequency was $283.55 \mathrm{~V}$ on A-phase, $280.4 \mathrm{~V}$ on B-phase and $280.5 \mathrm{~V}$ on C-phase and $60.03 \mathrm{~Hz}$ at the static switch (i.e., Meter 2) when connected to the utility grid; and $284 \mathrm{~V}$ on A-phase, $281 \mathrm{~V}$ on B-phase and $281 \mathrm{~V}$ on C-phase and $60.03 \mathrm{~Hz}$ at Meter 3.

Gen-set A1 in this test was set up to produce $-20 \mathrm{~kW}$ going through Zone 3 (i.e., Meter 3) which means it will produce $20 \mathrm{~kW}$ more power than Load Bank 3 needed. Approximately $16.4 \mathrm{~kW}(\mathrm{~A}=6 \mathrm{~kW} \mathrm{~B}=6.5 \mathrm{~kW} \mathrm{C}=3.9 \mathrm{~kW})$ of excess power was exported out of Zone 3 and $13.15 \mathrm{~kW}(\mathrm{~A}=4.7 \mathrm{~kW} \mathrm{~B}=5.25 \mathrm{~kW} \mathrm{C}=3.2 \mathrm{~kW})$ of that power went through the static switch to Load Bank 6 and the remaining $3.25 \mathrm{~kW}(\mathrm{~A}=1.3 \mathrm{~kW} \mathrm{~B}=1.25 \mathrm{~kW} \mathrm{C}=0.7 \mathrm{~kW})$ was due to power losses in the conductors. Since Load Bank 6 was approximately $36.05 \mathrm{~kW} \quad(\mathrm{~A}=12.25 \mathrm{~kW} \quad \mathrm{~B}=11.9 \mathrm{~kW} \quad \mathrm{C}=11.9 \mathrm{~kW})+\mathrm{j} 0.77 \mathrm{kVAr} \quad(\mathrm{A}=0.24 \mathrm{kVAr} \quad \mathrm{B}=0.27 \mathrm{kVAr}$ $\mathrm{C}=0.26 \mathrm{kVAr})$, the utility had to supply approximately $22.8 \mathrm{~kW}(\mathrm{~A}=7.1 \mathrm{~kW} B=6.7 \mathrm{~kW}$ $\mathrm{C}=9 \mathrm{~kW})$ to satisfy the load. Reactive power had to be imported in from the utility of approximately j21kVAr $(A=7.75 \mathrm{kVAr} B=6.5 \mathrm{kVAr} C=6.75 \mathrm{kVAr})$ because Gen-set $\mathrm{A} 1$ needed approximately $\mathrm{j} 14.85 \mathrm{kVAr}(\mathrm{A}=4.4 \mathrm{kVAr} \mathrm{B}=4.25 \mathrm{kVAr} \mathrm{C}=6.2 \mathrm{kVAr})$ and losses in the conductors.

All Data was verified and recorded into the DAS Database. The microgrid ran for a couple of minutes in this electrical state before the A-phase was reduced in Load Bank 6 by $50 \%$. The measured values for the A-phase load reduction in Load Bank 6 are in Table 24.

\begin{tabular}{|c|c|c|c|c|c|c|c|c|c|}
\hline Meter & $\begin{array}{l}\text { Gen- } \\
\text { set A1 }\end{array}$ & $\begin{array}{l}\text { Gen - } \\
\text { set A2 }\end{array}$ & Meter 1 & Meter 2 & Meter 3 & Meter 4 & LB 3 & LB 4 & LB 6 \\
\hline $\begin{array}{c}3 \text { Phase } \\
\text { kW }\end{array}$ & 31.9 & 0 & 16.95 & -13.1 & -16.3 & 0 & 16.03 & 0 & 30 \\
\hline \multirow{3}{*}{$\begin{array}{c}1 \text { Phase } \\
\text { kW }\end{array}$} & $A=8$ & $\mathrm{~A}=0$ & $\mathrm{~A}=2.7$ & $\mathrm{~A}=-3.4$ & $\mathrm{~A}=-4.4$ & $\mathrm{~A}=0$ & $\mathrm{~A}=3.75$ & $\mathrm{~A}=0$ & $A=6.2$ \\
\hline & $\mathrm{B}=13.7$ & $B=0$ & $\mathrm{~B}=5.75$ & $B=-6.3$ & $B=-7.7$ & $\mathrm{~B}=0$ & $\mathrm{~B}=6.18$ & $B=0$ & $B=11.9$ \\
\hline & $C=10.2$ & $\mathrm{C}=0$ & $C=8.5$ & $C=-3.4$ & $C=-4.2$ & $C=0$ & $C=6.1$ & $C=0$ & $C=11.9$ \\
\hline $\begin{array}{c}3 \text { Phase } \\
\text { kVAr }\end{array}$ & -14.5 & 0 & 21.86 & 21.19 & 15 & 0 & 0.183 & 0 & 0.655 \\
\hline
\end{tabular}




\begin{tabular}{|c|c|c|c|c|c|c|c|c|c|}
\hline 1 Phase & $\mathrm{A}=-5.8$ & $\mathrm{~A}=0$ & $\mathrm{~A}=7.75$ & $\mathrm{~A}=7.6$ & $\mathrm{~A}=5.8$ & $\mathrm{~A}=0$ & $\mathrm{~A}=-0.014$ & $\mathrm{~A}=0$ & $\mathrm{~A}=0.125$ \\
kVAr & $\mathrm{B}=-3.8$ & $\mathrm{~B}=0$ & $\mathrm{~B}=7.61$ & $\mathrm{~B}=6.2$ & $\mathrm{~B}=4$ & $\mathrm{~B}=0$ & $\mathrm{~B}=0.111$ & $\mathrm{~B}=0$ & $\mathrm{~B}=0.27$ \\
& $\mathrm{C}=-4.9$ & $\mathrm{C}=0$ & $\mathrm{C}=6.5$ & $\mathrm{C}=7.39$ & $\mathrm{C}=5.2$ & $\mathrm{C}=0$ & $\mathrm{C}=0.086$ & $\mathrm{C}=0$ & $\mathrm{C}=0.26$ \\
\hline
\end{tabular}

Table 24 - Measured Values After Start Up for Test 10.4.17 and Load Bank 6 A-phase Reduced by 50\%

In Table 24, Meter 1 real power decreased from approximately $22.8 \mathrm{~kW}(\mathrm{~A}=7.1 \mathrm{~kW}$ $\mathrm{B}=6.7 \mathrm{~kW} \mathrm{C}=9 \mathrm{~kW})$ to $16.95 \mathrm{~kW}(\mathrm{~A}=2.7 \mathrm{~kW} \mathrm{~B}=5.75 \mathrm{~kW} \mathrm{C}=8.5 \mathrm{~kW})$ because of the A-phase reduction in Load Bank 6 which was approximately $30 \mathrm{~kW}(\mathrm{~A}=6.2 \mathrm{~kW} \quad \mathrm{~B}=11.9 \mathrm{~kW}$ $\mathrm{C}=11.9 \mathrm{~kW})+\mathrm{j} 0.655 \mathrm{kVAr}(\mathrm{A}=0.125 \mathrm{kVAr} B=0.27 \mathrm{kVAr} \mathrm{C}=0.26 \mathrm{kVAr})$. Voltage increased to approximately $283.9 \mathrm{~V}$ on A-phase and $280.6 \mathrm{~V}$ on B-phase at the static and $284.2 \mathrm{~V}$ on Aphase and 281.2V on B-phase at Meter 3. C-phase voltage decreased to approximately $280.1 \mathrm{~V}$ at the static switch and $280.6 \mathrm{~V}$ at Meter 3. Frequency decreased from approximately $60.03 \mathrm{~Hz}$ to $60.01 \mathrm{~Hz}$ which was caused by the frequency fluctuation in the utility grid. Gen-set A1 did not change its output power level since its primary purpose is to provide $20 \mathrm{~kW}$ through Zone 3 which is done by producing $20 \mathrm{~kW}$ more than the load demands of Load Bank 3 which remained constant during the A-phase reduction in Load Bank 6.

All data was verified and recorded into the DAS Database. The microgrid ran for a couple of minutes in this electrical state and then the static switch was directed by the EMS to manually open.

\begin{tabular}{|c|c|c|c|c|c|c|c|c|c|}
\hline Meter & $\begin{array}{c}\text { Gen- } \\
\text { set A1 }\end{array}$ & $\begin{array}{c}\text { Gen - } \\
\text { set A2 }\end{array}$ & Meter 1 & Meter 2 & Meter 3 & Meter 4 & LB 3 & LB 4 & LB 6 \\
\hline $\begin{array}{c}\text { 3 Phase } \\
\mathbf{k W}\end{array}$ & 17.25 & 0 & 30.95 & 0 & -2.35 & 0 & 14.86 & 0 & 30.9 \\
\hline $\begin{array}{c}\mathbf{1} \text { Phase } \\
\mathbf{k W}\end{array}$ & $\mathrm{B}=6.8$ & $\mathrm{~B}=0$ & $\mathrm{~B}=12.25$ & $\mathrm{~B}=0$ & $\mathrm{~B}=-1.2$ & $\mathrm{~B}=0$ & $\mathrm{~B}=5.75$ & $\mathrm{~B}=0$ & $\mathrm{~B}=12.25$ \\
\hline $\begin{array}{c}\mathbf{3} \text { Phase } \\
\mathbf{k V A r}\end{array}$ & 4 & 0 & 2.2 & 0 & -3.6 & 0 & 0.186 & 0 & 0.658 \\
\hline $\begin{array}{c}\text { 1 Phase } \\
\mathbf{k V A r}\end{array}$ & $\mathrm{B}=1.6$ & $\mathrm{~B}=0$ & $\mathrm{~B}=0.9$ & $\mathrm{~B}=0$ & $\mathrm{~B}=0-1.4$ & $\mathrm{~B}=0$ & $\mathrm{~B}=0.113$ & $\mathrm{~B}=0$ & $\mathrm{~B}=0.271$ \\
& $\mathrm{C}=1.5$ & $\mathrm{C}=0$ & $\mathrm{C}=0.8$ & $\mathrm{C}=0$ & $\mathrm{C}=-1.4$ & $\mathrm{C}=0$ & $\mathrm{C}=0.087$ & $\mathrm{C}=0$ & $\mathrm{C}=0.265$ \\
\hline
\end{tabular}

Table 25 - Measured Values after Static Switch Opened for Test 10.4.17 
In Table 25, 0kW $(A=0 \mathrm{~kW} B=0 \mathrm{~kW} \mathrm{C}=0 \mathrm{~kW})+\mathrm{j} 0 \mathrm{kVAr}(\mathrm{A}=0 \mathrm{kVAr} \mathrm{B}=0 \mathrm{kVAr} \mathrm{C}=0 \mathrm{kVAr})$ was recorded at Meter 2 indicating that power was not flowing through the static switch. Meter 1 real power increased from $16.6 \mathrm{~kW}(\mathrm{~A}=2.6 \mathrm{~kW} \mathrm{~B}=5.6 \mathrm{~kW} \mathrm{C}=8.4 \mathrm{~kW})$ to $30.95 \mathrm{~kW}$ $(A=6.4 \mathrm{~kW} \quad B=12.25 \mathrm{~kW} \quad \mathrm{C}=12.3 \mathrm{~kW})$ and reactive power decreased from $\mathrm{j} 22.55 \mathrm{kVAr}$ ( $A=7.9 \mathrm{kVAr} B=6.75 \mathrm{kVAr} C=7.9 \mathrm{kVAr})$ to $\mathrm{j} 2.2 \mathrm{kVAr}(\mathrm{A}=0.5 \mathrm{kVAr} B=0.9 \mathrm{kVAr} \mathrm{C}=0.8 \mathrm{kVAr})$. The change in power is because the microgrid is no longer supplying power for Load Bank 6 which was approximately $30.9 \mathrm{~kW} \quad(\mathrm{~A}=6.4 \mathrm{~kW} \quad \mathrm{~B}=12.25 \mathrm{~kW} \quad \mathrm{C}=12.25 \mathrm{~kW})+$ j0.658kVAr $(A=0.122 \mathrm{kVAr} B=0.271 \mathrm{kVAr} C=0.265 \mathrm{kVAr})$. Load Bank 3 reduced slightly to $14.86 \mathrm{~kW}(\mathrm{~A}=3.47 \mathrm{~kW} B=5.75 \mathrm{~kW} C=5.64 \mathrm{~kW})+\mathrm{j} 0.186 \mathrm{kVAr}(\mathrm{A}=-0.014 \mathrm{kVAr} B=0.113 \mathrm{kVAr}$ $\mathrm{C}=0.087 \mathrm{kVAr})$. This load reduction resulted from a voltage drop in the microgrid, shown in Figure 19a, from approximately $284.5 \mathrm{~V}$ on A-phase, $281.6 \mathrm{~V}$ on B-phase and $281 \mathrm{~V}$ on C-phase when connected to the utility grid to $273 \mathrm{~V}$ on A-phase, $272.4 \mathrm{~V}$ on Bphase and 270.5V on C-phase when islanded at Meter 3.

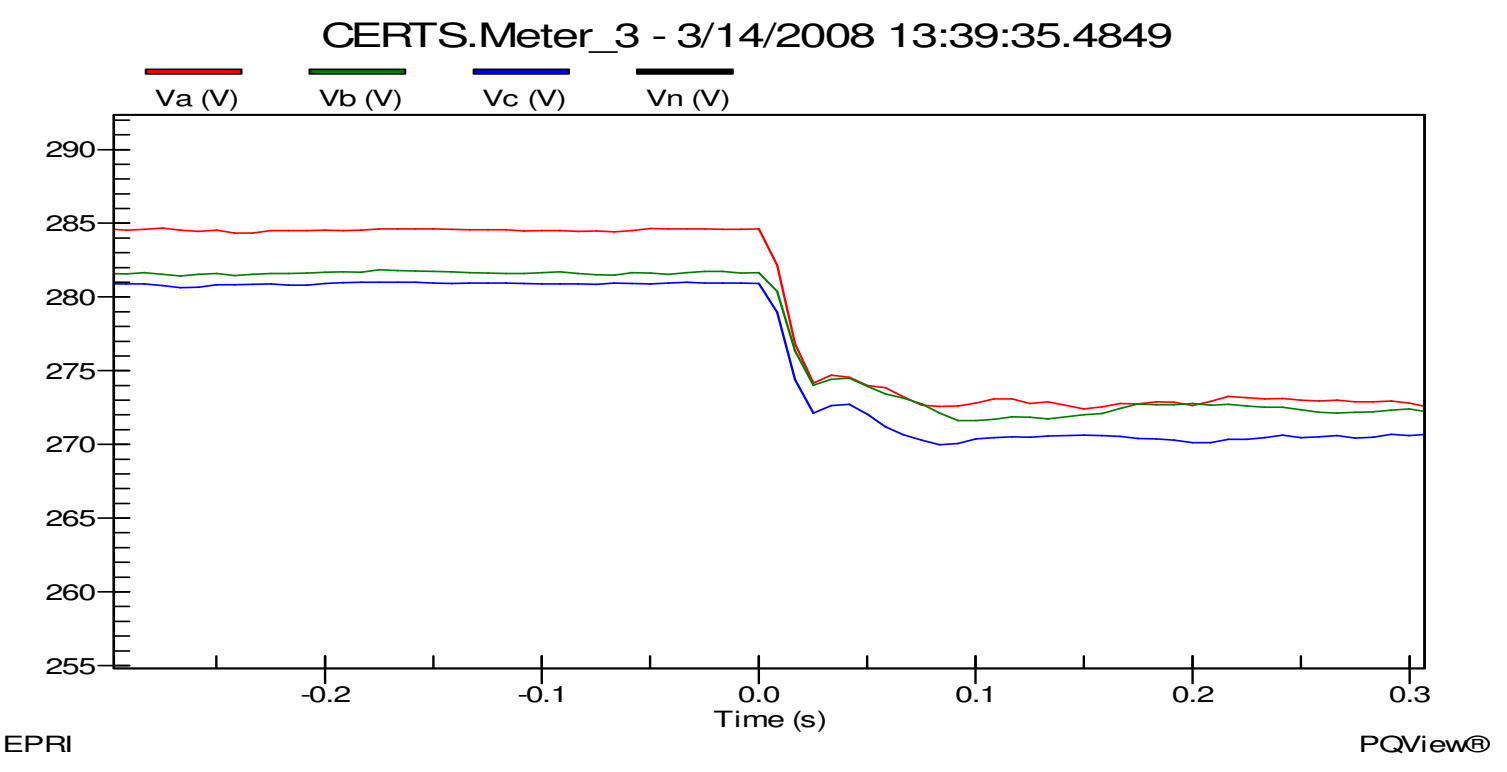

Figure 19a - Meter 3 Line-to-Ground Voltages during Utility Connected to Island mode for Test 10.4.17

Frequency change in the microgrid, shown in Figure 19b, increased from approximately $60.01 \mathrm{~Hz}$ when connected to the utility grid to approximately $60.14 \mathrm{~Hz}$ when islanded. This change in frequency was part of the CERTS algorithm which allowed Gen-set A1 to decrease its output power to satisfy the critical load demands. Gen-set A1 decreased its real output power to approximately $17.25 \mathrm{~kW}(\mathrm{~A}=4.25 \mathrm{~kW} B=6.8 \mathrm{~kW} \mathrm{C}=6.2 \mathrm{~kW})$ and increased its reactive output power to approximately j4kVAr $(A=0.9 \mathrm{kVAr} B=1.6 \mathrm{kVAr}$ $\mathrm{C}=1.5 \mathrm{kVAr}$ ) to satisfy the critical loads. Not the sign change from negative to positive for Gen-set A1 reactive power when the microgrid is isolated from the utility. This sign change is due to the utility no longer supplying the reactive power for the critical loads 
but by Gen-set A1. Meter 3 was approximately $-2.35 \mathrm{~kW}(\mathrm{~A}=-0.75 \mathrm{~kW} \mathrm{~B}=-1.2 \mathrm{~kW} \mathrm{C}=$ $0.4 \mathrm{~kW})-\mathrm{j} 3.6 \mathrm{kVAr}(\mathrm{A}=-0.8 \mathrm{kVAr} B=-1.4 \mathrm{kVAr} C=-1.4 \mathrm{kVAr})$. Negative power recorded at Meter 3 indicates that all the critical loads in the microgrid were being satisfied by Genset A1. All data was verified and recorded into the DAS Database. The microgrid ran for a couple of minutes in this electrical state before the A-phase was reduced in Load Bank 6 by $50 \%$ (i.e., 75\% from balanced load value). The measured values for the Aphase load reduction in Load Bank 6 are in Table 26.

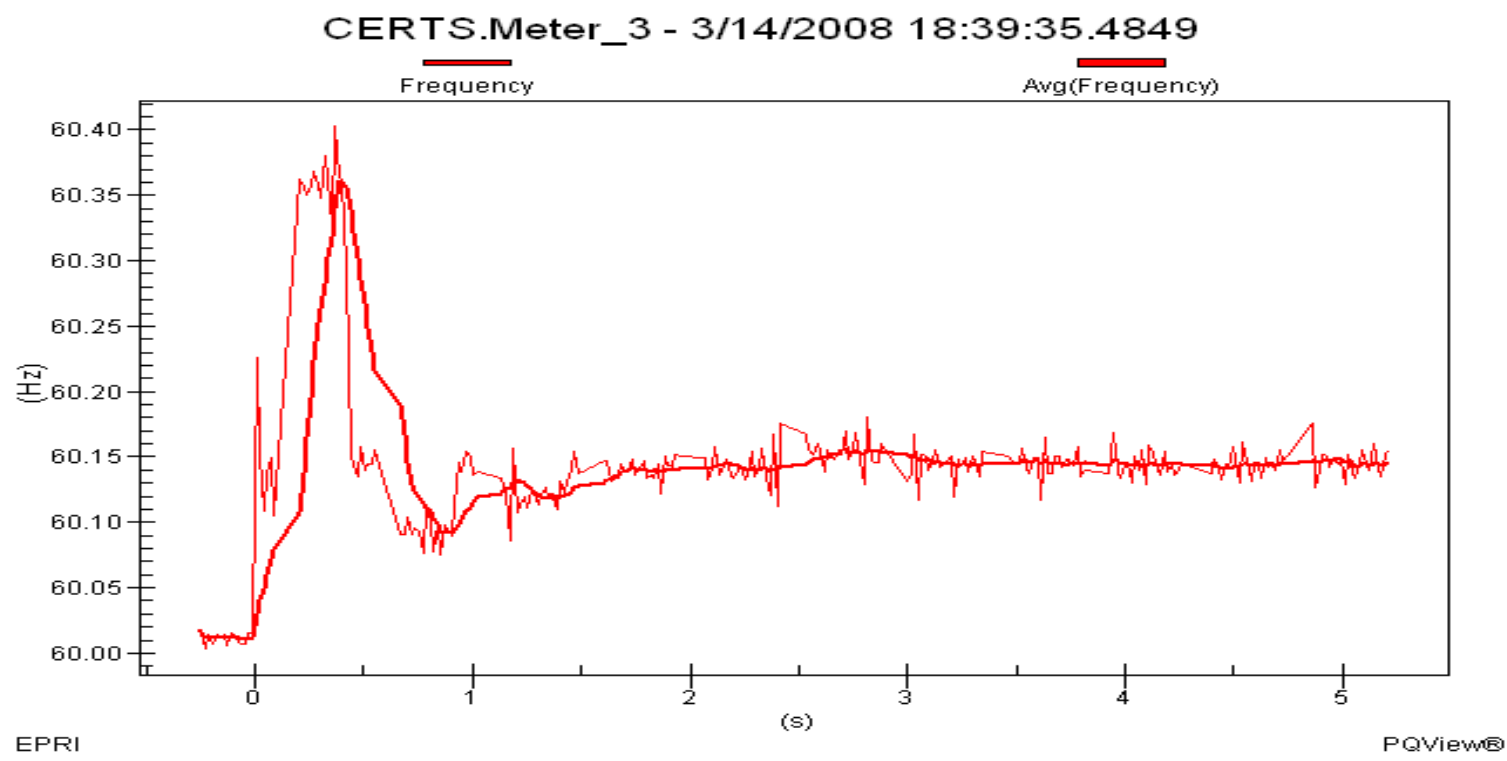

Figure 19b - Meter 3 Frequency Before/After the Static Switch Opened for Test 10.4.17

\begin{tabular}{|c|c|c|c|c|c|c|c|c|c|}
\hline Meter & $\begin{array}{c}\text { Gen- } \\
\text { set A1 }\end{array}$ & $\begin{array}{c}\text { Gen - } \\
\text { set A2 }\end{array}$ & Meter 1 & Meter 2 & Meter 3 & Meter 4 & LB 3 & LB 4 & LB 6 \\
\hline $\begin{array}{c}\text { 3 Phase } \\
\mathbf{k W}\end{array}$ & 17.25 & 0 & 28.1 & 0 & -2.395 & 0 & 14.75 & 0 & 27.8 \\
\hline $\begin{array}{c}\mathbf{1} \text { Phase } \\
\mathbf{k W}\end{array}$ & $\mathrm{B}=6.8$ & $\mathrm{~B}=0$ & $\mathrm{~B}=12.1$ & $\mathrm{~B}=0$ & $\mathrm{~B}=-1.125$ & $\mathrm{~B}=0$ & $\mathrm{~B}=5.75$ & $\mathrm{~B}=0$ & $\mathrm{~B}=12$ \\
\hline $\begin{array}{c}\text { 3 Phase } \\
\mathbf{k V A r}\end{array}$ & 3.935 & 0 & 0.788 & 0 & -3.73 & 0 & 0.215 & 0 & 0.519 \\
\hline $\begin{array}{c}\mathbf{1} \text { Phase } \\
\mathbf{k V A r}\end{array}$ & $\mathrm{B}=1.55$ & $\mathrm{~B}=0$ & $\mathrm{~B}=0.368$ & $\mathrm{~B}=0$ & $\mathrm{~B}=-1.44$ & $\mathrm{~B}=0$ & $\mathrm{~B}=0.125$ & $\mathrm{~B}=0$ & $\mathrm{~B}=0.268$ \\
& $\mathrm{C}=1.525$ & $\mathrm{C}=0$ & $\mathrm{C}=0.342$ & $\mathrm{C}=0$ & $\mathrm{C}=-1.42$ & $\mathrm{C}=0$ & $\mathrm{C}=0.1$ & $\mathrm{C}=0$ & $\mathrm{C}=0.261$ \\
\hline
\end{tabular}


Table 26 - Measured Values after Static Switch Opened and A-phase Reduced in Load Bank 6 by $75 \%$ for Test 10.4.17

In Table 26, Meter 1 real power decreased from approximately $30.95 \mathrm{~kW}(\mathrm{~A}=6.4 \mathrm{~kW}$ $B=12.25 \mathrm{~kW} C=12.3 \mathrm{~kW})$ to $28.1 \mathrm{~kW}(\mathrm{~A}=3.8 \mathrm{~kW} \mathrm{~B}=12.1 \mathrm{~kW} \mathrm{C}=12.2 \mathrm{~kW})$ because of the Aphase reduction in Load Bank 6 which was approximately $27.8 \mathrm{~kW}(\mathrm{~A}=3.8 \mathrm{~kW} B=12 \mathrm{~kW}$ $\mathrm{C}=12 \mathrm{~kW})+\mathrm{j} 0.519 \mathrm{kVAr}(\mathrm{A}=0.01 \mathrm{kVAr} \mathrm{B}=0.268 \mathrm{kVAr} \quad \mathrm{C}=0.261 \mathrm{kVAr}) . \quad$ Voltages and frequencies remained relatively the same in both the utility and microgrid when Load Bank 6 A-phase was reduced to $3.8 \mathrm{~kW}$ because nothing changed in the CERTS microgrid down stream of the static switch and the utility is a stiff system.

All data was verified and recorded into the DAS Database. The microgrid ran for a couple of minutes in this electrical state before the A-phase was reduced in Load Bank 3 by $50 \%$ (i.e., $75 \%$ from balanced load value). The measured values for the A-phase reduction for Load Bank 3 are in Table 27.

\begin{tabular}{|c|c|c|c|c|c|c|c|c|c|}
\hline Meter & $\begin{array}{c}\text { Gen- } \\
\text { set A1 }\end{array}$ & $\begin{array}{c}\text { Gen - } \\
\text { set A2 }\end{array}$ & Meter 1 & Meter 2 & Meter 3 & Meter 4 & LB 3 & LB 4 & LB 6 \\
\hline $\begin{array}{c}\text { 3 Phase } \\
\text { kW }\end{array}$ & 15.5 & 0 & 28.1 & 0 & -2.435 & 0 & 13.1 & 0 & 27.8 \\
\hline $\begin{array}{c}\mathbf{1} \text { Phase } \\
\mathbf{k W}\end{array}$ & $\mathrm{B}=6.8$ & $\mathrm{~B}=0$ & $\mathrm{~B}=12.1$ & $\mathrm{~B}=0$ & $\mathrm{~B}=-1.125$ & $\mathrm{~B}=0$ & $\mathrm{~B}=5.75$ & $\mathrm{~B}=0$ & $\mathrm{~B}=12$ \\
\hline $\begin{array}{c}\mathbf{3} \text { Phase } \\
\mathbf{k V A r}\end{array}$ & 3.93 & 0 & 0.788 & 0 & -3.695 & 0 & 0.235 & 0 & 0.519 \\
\hline $\begin{array}{c}\mathrm{C} \text { Phase } \\
\mathbf{k V A r}\end{array}$ & $\mathrm{B}=1.575$ & $\mathrm{~B}=0$ & $\mathrm{~B}=0.368$ & $\mathrm{~B}=0$ & $\mathrm{~B}=-1.45$ & $\mathrm{~B}=0$ & $\mathrm{~B}=0.125$ & $\mathrm{~B}=0$ & $\mathrm{~B}=0.268$ \\
& $\mathrm{C}=1.48$ & $\mathrm{C}=0$ & $\mathrm{C}=0.342$ & $\mathrm{C}=0$ & $\mathrm{C}=-1.375$ & $\mathrm{C}=0$ & $\mathrm{C}=0.1$ & $\mathrm{C}=0$ & $\mathrm{C}=0.261$ \\
\hline
\end{tabular}

Table 27 - Measured Values after Static Switch Opened and A-phase Reduced in Load Bank 3 by $75 \%$ for Test 10.4.17

In Table 27, Load Bank 3 was approximately $13.1 \mathrm{~kW}(\mathrm{~A}=1.75 \mathrm{~kW} \mathrm{~B}=5.75 \mathrm{~kW} \mathrm{C}=5.6 \mathrm{~kW})+$ j0.235kVAr $(A=0.01 \mathrm{kVAr} B=0.125 \mathrm{kVAr} C=0.1 \mathrm{kVAr})$ after the $\mathrm{A}-$ phase was reduced by $50 \%$ (i.e., $75 \%$ from balanced load value). Gen-set $\mathrm{A} 1$ decreased from approximately $17.25 \mathrm{~kW} \quad(\mathrm{~A}=4.25 \mathrm{~kW} \quad \mathrm{~B}=6.8 \mathrm{~kW} \quad \mathrm{C}=6.2 \mathrm{~kW})+j 3.935 \mathrm{kVAr} \quad(\mathrm{A}=0.86 \mathrm{kVAr} \quad \mathrm{B}=1.55 \mathrm{kVAr}$ $\mathrm{C}=1.525 \mathrm{kVAr})$ to $15.5 \mathrm{~kW}(\mathrm{~A}=2.6 \mathrm{~kW} \mathrm{~B}=6.8 \mathrm{~kW} \mathrm{C}=6.1 \mathrm{~kW})+j 3.93 \mathrm{kVAr}(\mathrm{A}=0.875 \mathrm{kVAr}$ 
$\mathrm{B}=1.575 \mathrm{kVAr} \mathrm{C}=1.48 \mathrm{kVAr}$ ) due to the reduction of $1.65 \mathrm{~kW}$ in the A-phase of Load Bank 3. The reduction in Load Bank 3 did not cause the frequency to increase which was approximately $60.14 \mathrm{~Hz}$. Voltage within the microgrid during these measurements was approximately $273 \mathrm{~V}$ on A-phase, $273 \mathrm{~V}$ on B-phase and $269.8 \mathrm{~V}$ on C-phase. Load Bank 6 load demands were not changed at this time, therefore, all the measured values for Load Bank 6, Meter 1 and Meter 2 did not change from the previous load reduction in Load Bank 6.

All data was verified and recorded into the DAS Database. The microgrid ran for a couple of minutes in this electrical state before the A-phase was reduced in Load Bank 6 by $100 \%$. The measured values for the A-phase load reduction for Load Bank 6 are in Table 28.

\begin{tabular}{|c|c|c|c|c|c|c|c|c|c|}
\hline Meter & $\begin{array}{l}\text { Gen- } \\
\text { set A1 }\end{array}$ & $\begin{array}{l}\text { Gen - } \\
\text { set A2 }\end{array}$ & Meter 1 & Meter 2 & Meter 3 & Meter 4 & LB 3 & LB 4 & LB 6 \\
\hline $\begin{array}{c}3 \text { Phase } \\
\text { kW }\end{array}$ & 15.4 & 0 & 24.3 & 0 & -2.435 & 0 & 13.05 & 0 & 24.3 \\
\hline \multirow{3}{*}{$\begin{array}{c}1 \text { Phase } \\
\text { kW }\end{array}$} & $\mathrm{A}=2.5$ & $\mathrm{~A}=0$ & $\mathrm{~A}=0$ & $\mathrm{~A}=0$ & $A=-0.84$ & $A=0$ & $\mathrm{~A}=1.7$ & $A=0$ & $\mathrm{~A}=0$ \\
\hline & $\mathrm{B}=6.8$ & $\mathrm{~B}=0$ & $B=12.1$ & $\mathrm{~B}=0$ & $\mathrm{~B}=-1.125$ & $\mathrm{~B}=0$ & $B=5.75$ & $\mathrm{~B}=0$ & $\mathrm{~B}=12.1$ \\
\hline & $C=6.1$ & $C=0$ & $\mathrm{C}=12.2$ & $\mathrm{C}=0$ & $C=-0.47$ & $\mathrm{C}=0$ & $C=5.6$ & $\mathrm{C}=0$ & $C=12.2$ \\
\hline $\begin{array}{c}3 \text { Phase } \\
\text { kVAr }\end{array}$ & 3.935 & 0 & 0.808 & 0 & -3.67 & 0 & 0.2 & 0 & 0.535 \\
\hline \multirow{3}{*}{$\begin{array}{l}1 \text { Phase } \\
\text { kVAr }\end{array}$} & $\mathrm{A}=0.88$ & $A=0$ & $\mathrm{~A}=0.09$ & $A=0$ & $A=-0.85$ & $A=0$ & $\mathrm{~A}=0.004$ & $A=0$ & $\mathrm{~A}=0.001$ \\
\hline & $\mathrm{B}=1.575$ & $B=0$ & $B=0.359$ & $B=0$ & $B=-1.37$ & $\mathrm{~B}=0$ & $B=0.102$ & $\mathrm{~B}=0$ & $B=0.269$ \\
\hline & $C=1.48$ & $C=0$ & $C=0.359$ & $C=0$ & $C=-1.45$ & $\mathrm{C}=0$ & $C=0.094$ & $\mathrm{C}=0$ & $C=0.265$ \\
\hline
\end{tabular}

Table 28 - Measured Values after Static Switch Opened and A-phase Reduced in Load Bank 6 by $100 \%$ for Test 10.4.17

In Table 28, Meter 1 real power decreased from approximately $28.1 \mathrm{~kW}(\mathrm{~A}=3.8 \mathrm{~kW}$ $\mathrm{B}=12.1 \mathrm{~kW} \mathrm{C}=12.2 \mathrm{~kW})$ to $24.3 \mathrm{~kW}(\mathrm{~A}=0 \mathrm{~kW} \mathrm{~B}=12.1 \mathrm{~kW} \mathrm{C}=12.2 \mathrm{~kW})$ because of the A-phase reduction in Load Bank 6 which was approximately $24.3 \mathrm{~kW}(\mathrm{~A}=0 \mathrm{~kW} \quad \mathrm{~B}=12.1 \mathrm{~kW}$ $\mathrm{C}=12.2 \mathrm{~kW})+\mathrm{j} 0.535 \mathrm{kVAr}(\mathrm{A}=0.001 \mathrm{kVAr} \mathrm{B}=0.269 \mathrm{kVAr} \quad \mathrm{C}=0.265 \mathrm{kVAr}) . \quad$ Voltages and frequencies remained the same in both the utility and microgrid when Load Bank 6 Aphase was reduced to $0 \mathrm{~kW}$ because nothing changed in the CERTS microgrid downstream of the static switch and the utility is a stiff system. 
All data was verified and recorded into the DAS Database. The microgrid ran for a couple of minutes in this electrical state before the A-phase was reduced in Load Bank 3 by $100 \%$. The measured values for the A-phase load reduction for Load Bank 6 are in Table 28.

\begin{tabular}{|c|c|c|c|c|c|c|c|c|c|}
\hline Meter & $\begin{array}{c}\text { Gen- } \\
\text { set A1 }\end{array}$ & $\begin{array}{c}\text { Gen - } \\
\text { set A2 }\end{array}$ & Meter 1 & Meter 2 & Meter 3 & Meter 4 & LB 3 & LB 4 & LB 6 \\
\hline $\begin{array}{c}\mathbf{3} \text { Phase } \\
\mathbf{k W}\end{array}$ & 13.7 & 0 & 24.3 & 0 & -2.41 & 0 & 11.4 & 0 & 24.3 \\
\hline $\begin{array}{c}\mathbf{1} \text { Phase } \\
\mathbf{k W}\end{array}$ & $\mathrm{B}=6.9$ & $\mathrm{~B}=0$ & $\mathrm{~B}=12.1$ & $\mathrm{~B}=0$ & $\mathrm{~B}=-1.12$ & $\mathrm{~B}=0$ & $\mathrm{~B}=5.8$ & $\mathrm{~B}=0$ & $\mathrm{~B}=12.1$ \\
\hline $\begin{array}{c}\mathbf{3} \text { Phase } \\
\mathbf{k V A r}\end{array}$ & 3.9 & 0 & 0.808 & 0 & -3.68 & 0 & 0.196 & 0 & 0.535 \\
\hline $\begin{array}{c}\mathbf{1} \text { Phase } \\
\mathbf{k V A r}\end{array}$ & $\mathrm{B}=1.59$ & $\mathrm{~B}=0$ & $\mathrm{~B}=0.359$ & $\mathrm{~B}=0$ & $\mathrm{~B}=0-1.47$ & $\mathrm{~B}=0$ & $\mathrm{~B}=0.102$ & $\mathrm{~B}=0$ & $\mathrm{~B}=0.269$ \\
& $\mathrm{C}=1.45$ & $\mathrm{C}=0$ & $\mathrm{C}=0.359$ & $\mathrm{C}=0$ & $\mathrm{C}=-1.34$ & $\mathrm{C}=0$ & $\mathrm{C}=0.094$ & $\mathrm{C}=0$ & $\mathrm{C}=0.265$ \\
\hline
\end{tabular}

Table 29 - Measured Values after Static Switch Opened and A-phase Reduced in Load Bank 3 by $100 \%$ for Test 10.4.17

In Table 29, Load Bank 3 was approximately $11.4 \mathrm{~kW}(\mathrm{~A}=0 \mathrm{~kW} \mathrm{~B}=5.8 \mathrm{~kW} \mathrm{C}=5.6 \mathrm{~kW})+$ j0.196kVAr $(A=0 \mathrm{kVAr} B=0.102 \mathrm{kVAr} C=0.094 \mathrm{kVAr})$ after the A-phase was reduced by $100 \%$. Gen-set $\mathrm{A} 1$ decreased from approximately $15.4 \mathrm{~kW}(\mathrm{~A}=2.5 \mathrm{~kW} \mathrm{~B}=6.8 \mathrm{~kW} \mathrm{C}=6.1 \mathrm{~kW})$ + j3.935kVAr $(A=0.88 \mathrm{kVAr} B=1.575 \mathrm{kVAr} C=1.548 \mathrm{kVAr})$ to $13.7 \mathrm{~kW}(\mathrm{~A}=0.8 \mathrm{~kW} \mathrm{~B}=6.9 \mathrm{~kW}$ $\mathrm{C}=6 \mathrm{~kW})+\mathrm{j} 3.9 \mathrm{kVAr}(\mathrm{A}=0.86 \mathrm{kVAr} B=1.59 \mathrm{kVAr} C=1.45 \mathrm{kVAr})$ due to the reduction of $1.7 \mathrm{~kW}$ in the A-phase of Load Bank 3. The reduction in Load Bank 3 did not cause the frequency to increase which was approximately $60.14 \mathrm{~Hz}$. Voltage within the microgrid during these measurements was approximately $273.3 \mathrm{~V}$ on A-phase, $274 \mathrm{~V}$ on B-phase and $268.8 \mathrm{~V}$ on C-phase. Load Bank 6 load demands were not changed at this time, therefore, all the measured values for Load Bank 6, Meter 1 and Meter 2 did not change from the previous load reduction in Load Bank 6.

All data was verified and recorded into the DAS Database. The microgrid ran for a couple of minutes in this electrical state before the manual open signal was released and allowed the static switch to synchronize and close back into the utility grid. Shortly after the static switch closed back into the utility it tripped on a reverse power due to the A- 
phase on Load Banks 3 and 6 equaling 0kW shown in Figure 19c. The measured values are provided in Table 30.

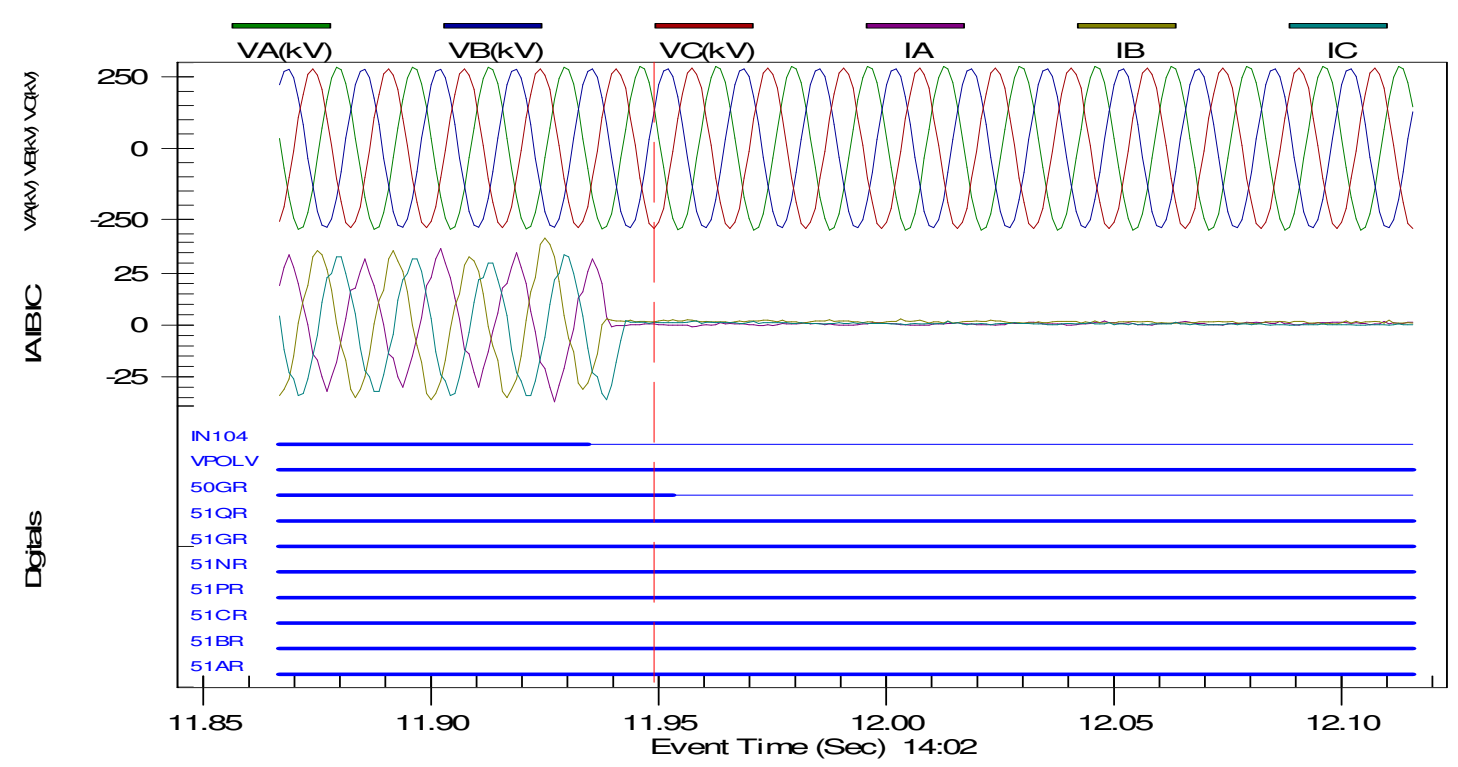

Figure 19c - Relay 2 (Static Switch) Tripping on a Reverse Power Condition during Island to Utility Connected mode for Test 10.4.17

\begin{tabular}{|c|c|c|c|c|c|c|c|c|c|}
\hline Meter & $\begin{array}{c}\text { Gen- } \\
\text { set A1 }\end{array}$ & $\begin{array}{l}\text { Gen - } \\
\text { set A2 }\end{array}$ & Meter 1 & Meter 2 & Meter 3 & Meter 4 & LB 3 & LB 4 & LB 6 \\
\hline \multicolumn{10}{|c|}{ Before Static Switch Synchronization and Close } \\
\hline $\begin{array}{c}3 \text { Phase } \\
\text { kW }\end{array}$ & 14 & 0 & 24.2 & 0 & -2.15 & 0 & 11.4 & 0 & 24.2 \\
\hline \multirow{3}{*}{$\begin{array}{c}1 \text { Phase } \\
\text { kW }\end{array}$} & $A=1$ & $\mathrm{~A}=0$ & $\mathrm{~A}=0$ & $\mathrm{~A}=0$ & $\mathrm{~A}=-0.75$ & $\mathrm{~A}=0$ & $\mathrm{~A}=0$ & $\mathrm{~A}=0$ & $\mathrm{~A}=0$ \\
\hline & $B=7$ & $B=0$ & $\mathrm{~B}=12.1$ & $\mathrm{~B}=0$ & $B=-1$ & $\mathrm{~B}=0$ & $B=5.8$ & $\mathrm{~B}=0$ & $\mathrm{~B}=12$ \\
\hline & $C=6$ & $\mathrm{C}=0$ & $C=12.1$ & $\mathrm{C}=0$ & $C=-0.4$ & $C=0$ & $C=5.6$ & $\mathrm{C}=0$ & $C=12.2$ \\
\hline $\begin{array}{c}3 \text { Phase } \\
\text { kVAr }\end{array}$ & 4 & 0 & 0.9 & 0 & -3.6 & 0 & 0.215 & 0 & 0.533 \\
\hline \multirow{3}{*}{$\begin{array}{c}1 \text { Phase } \\
\text { kVAr }\end{array}$} & $\mathrm{A}=0.9$ & $A=0$ & $\mathrm{~A}=0.1$ & $\mathrm{~A}=0$ & $\mathrm{~A}=-0.8$ & $A=0$ & $\mathrm{~A}=0$ & $A=0$ & $A=0$ \\
\hline & $B=1.6$ & $\mathrm{~B}=0$ & $\mathrm{~B}=0.4$ & $\mathrm{~B}=0$ & $B=-1.5$ & $B=0$ & $B=0.122$ & $\mathrm{~B}=0$ & $B=0.269$ \\
\hline & $C=1.5$ & $\mathrm{C}=0$ & $C=0.4$ & $\mathrm{C}=0$ & $C=-1.3$ & $\mathrm{C}=0$ & $\mathrm{C}=0.093$ & $\mathrm{C}=0$ & $C=0.264$ \\
\hline
\end{tabular}




\begin{tabular}{|c|c|c|c|c|c|c|c|c|c|}
\hline $\begin{array}{c}3 \text { Phase } \\
\text { kW }\end{array}$ & 32.25 & 0 & 6.7 & -17 & -20 & 0 & 12.1 & 0 & 23.9 \\
\hline \multirow{3}{*}{$\begin{array}{c}1 \text { Phase } \\
\text { kW }\end{array}$} & $\mathrm{A}=5.75$ & $A=0$ & $\mathrm{~A}=-4.8$ & $A=-4.7$ & $\mathrm{~A}=-5.75$ & $\mathrm{~A}=0$ & $\mathrm{~A}=0$ & $A=0$ & $A=0$ \\
\hline & $\mathrm{B}=15.5$ & $\mathrm{~B}=0$ & $\mathrm{~B}=4$ & $B=-8$ & $B=-9.25$ & $\mathrm{~B}=0$ & $\mathrm{~B}=6.1$ & $B=0$ & $B=12$ \\
\hline & $C=11$ & $\mathrm{C}=0$ & $C=7.5$ & $C=-4.3$ & $C=-5$ & $\mathrm{C}=0$ & $C=6$ & $\mathrm{C}=0$ & $C=11.9$ \\
\hline $\begin{array}{c}3 \text { Phase } \\
\text { kVAr }\end{array}$ & -13.65 & 0 & 20.55 & 19.95 & 14.1 & 0 & 0.227 & 0 & 0.526 \\
\hline \multirow{3}{*}{$\begin{array}{c}1 \text { Phase } \\
\text { kVAr }\end{array}$} & $A=-5$ & $\mathrm{~A}=0$ & $A=6.75$ & $A=6.75$ & $A=5.2$ & $\mathrm{~A}=0$ & $A=0$ & $A=0$ & $\mathrm{~A}=0$ \\
\hline & $B=-2.9$ & $\mathrm{~B}=0$ & $B=5.4$ & $\mathrm{~B}=5.2$ & $B=3.1$ & $\mathrm{~B}=0$ & $\mathrm{~B}=0.127$ & $B=0$ & $B=0.266$ \\
\hline & $C=-5.75$ & $\mathrm{C}=0$ & $C=8.4$ & $C=8$ & $C=5.8$ & $\mathrm{C}=0$ & $C=0.1$ & $C=0$ & $C=0.26$ \\
\hline \multicolumn{10}{|c|}{ Static Switch After Reverse Power Condition Trip } \\
\hline $\begin{array}{c}3 \text { Phase } \\
\text { kW }\end{array}$ & 14 & 0 & 24.2 & 0 & -2.15 & 0 & 11.4 & 0 & 24.2 \\
\hline \multirow{3}{*}{$\begin{array}{c}1 \text { Phase } \\
\text { kW }\end{array}$} & $A=1$ & $\mathrm{~A}=0$ & $\mathrm{~A}=0$ & $\mathrm{~A}=0$ & $A=-0.75$ & $\mathrm{~A}=0$ & $\mathrm{~A}=0$ & $\mathrm{~A}=0$ & $\mathrm{~A}=0$ \\
\hline & $B=7$ & $\mathrm{~B}=0$ & $B=12.1$ & $B=0$ & $B=-1$ & $\mathrm{~B}=0$ & $\mathrm{~B}=5.8$ & $B=0$ & $B=12$ \\
\hline & $C=6$ & $\mathrm{C}=0$ & $C=12.1$ & $C=0$ & $C=-0.4$ & $\mathrm{C}=0$ & $C=5.6$ & $C=0$ & $C=12.2$ \\
\hline $\begin{array}{c}3 \text { Phase } \\
\text { kVAr }\end{array}$ & 4 & 0 & 0.9 & 0 & -3.6 & 0 & 0.215 & 0 & 0.533 \\
\hline \multirow{3}{*}{$\begin{array}{c}1 \text { Phase } \\
\text { kVAr }\end{array}$} & $A=0.9$ & $\mathrm{~A}=0$ & $\mathrm{~A}=0.1$ & $A=0$ & $\mathrm{~A}=-0.8$ & $\mathrm{~A}=0$ & $\mathrm{~A}=0$ & $A=0$ & $\mathrm{~A}=0$ \\
\hline & $B=1.6$ & $\mathrm{~B}=0$ & $\mathrm{~B}=0.4$ & $\mathrm{~B}=0$ & $\mathrm{~B}=-1.5$ & $\mathrm{~B}=0$ & $\mathrm{~B}=0.122$ & $B=0$ & $B=0.269$ \\
\hline & $C=1.5$ & $\mathrm{C}=0$ & $C=0.4$ & $C=0$ & $C=-1.3$ & $\mathrm{C}=0$ & $C=0.093$ & $C=0$ & $C=0.264$ \\
\hline
\end{tabular}

Table 30 - Measured Values before/after Static Switch Synchronized and Closed back into the utility and after Static Switch Tripped on Reverse Power Condition for Test 10.4.17

Voltage and frequency in the microgrid increased from approximately $273.3 \mathrm{~V}$ on Aphase, $274 \mathrm{~V}$ on B-phase and $268.8 \mathrm{~V}$ on C-phase and $60.08 \mathrm{~Hz}$ when islanded to $284 \mathrm{~V}$ on A-phase, $281 \mathrm{~V}$ on B-phase and $278.9 \mathrm{~V}$ on C-phase and $59.98 \mathrm{~Hz}$ when utility connected at Meter 3 shown in Figures 19d and 19e. This change in frequency is due to the frequency 
no longer being established by Gen-set A1 using the CERTS algorithm but by the utility. Gen-set A1 is in zone power control mode therefore when the static switch closed back into the utility Gen-set A1 produced real power based on a set-point for the power flow through Zone 3 initialized at the beginning of the test. The output power for Gen-set A1 was relatively close to the value at the beginning of the test with Gen-set A1 producing approximately $32.25 \mathrm{~kW}(A=5.75 \mathrm{~kW} B=15.5 \mathrm{~kW} C=11 \mathrm{~kW})-j 13.65 \mathrm{kVAr}(A=-5 \mathrm{kVAr} B=-$ 2.9kVAr C=-5.75kVAr).

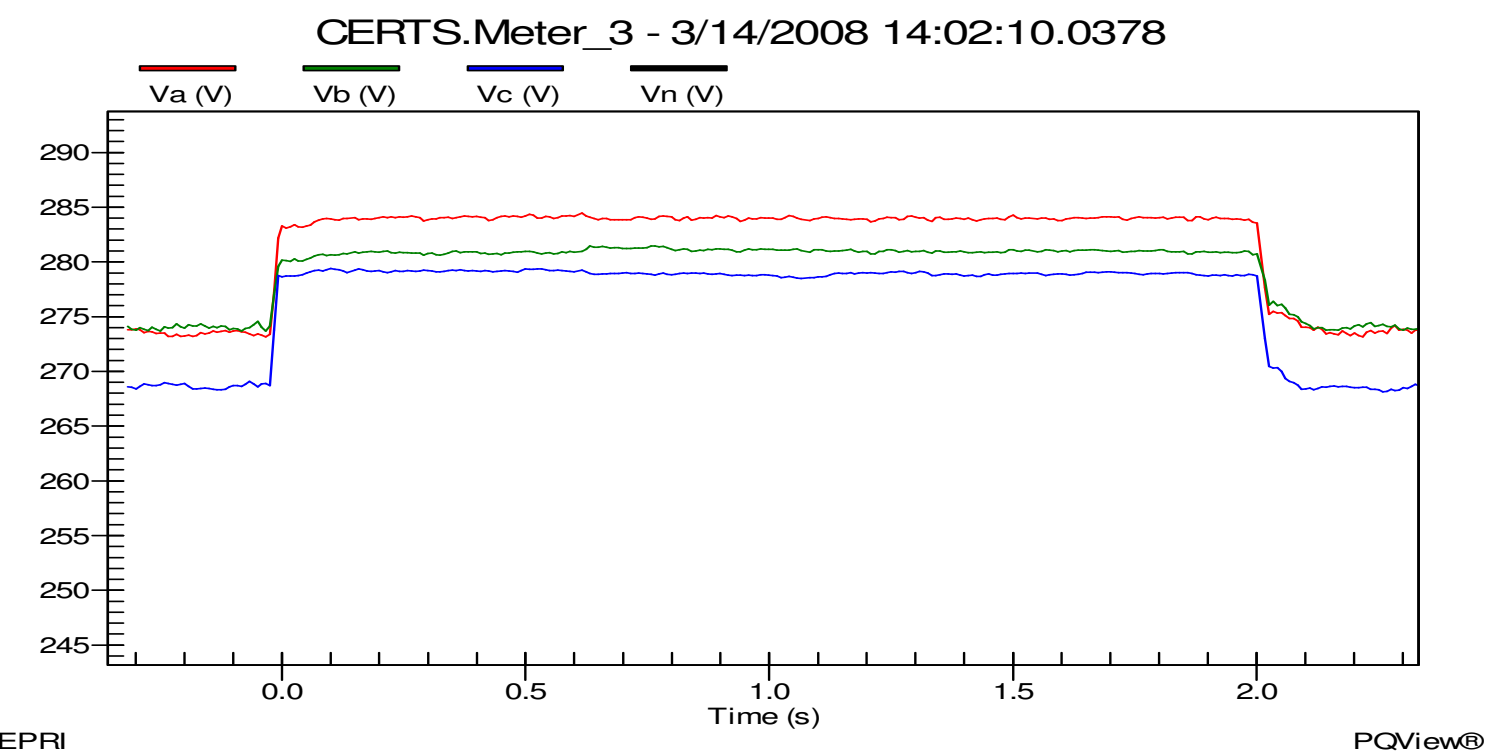

Figure 19d - Meter 3 Line-to-Ground Voltages during Islanded to Utility Connected mode for Test 10.4.17

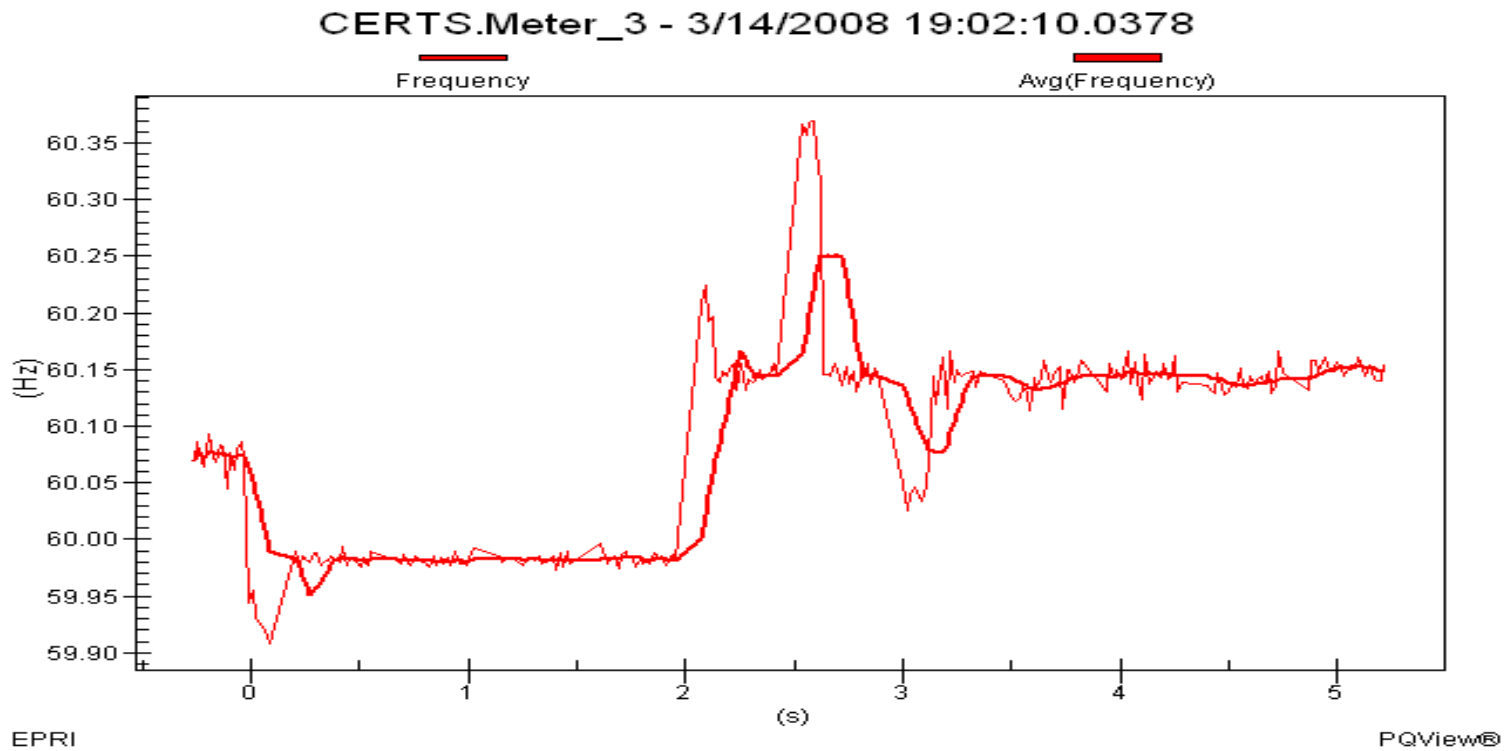

Figure 19e - Meter 3 Frequency during Islanded to Utility Connected mode for Test 10.4.17 
Meter 2 recorded real power decreasing from approximately $0 \mathrm{~kW}(\mathrm{~A}=0 \mathrm{~kW} B=0 \mathrm{~kW}$ $\mathrm{C}=0 \mathrm{~kW})$ to $-17 \mathrm{~kW}(\mathrm{~A}=-4.7 \mathrm{~kW} \mathrm{~B}=-8 \mathrm{~kW} \mathrm{C}=-4.3 \mathrm{~kW})$ and reactive power increasing from approximately j0kVAr $(A=0 \mathrm{kVAr} B=0 \mathrm{kVAr} \quad \mathrm{C}=0 \mathrm{kVAr})$ to j19.95kVAr $(\mathrm{A}=6.75 \mathrm{kVAr}$ $\mathrm{B}=5.2 \mathrm{kVAr} \mathrm{C}=8 \mathrm{kVAr}$ ) after the static switch closed shown in Figure 19f. Gen-set A1 was producing power for all the critical loads in the microgrid and approximately $17 \mathrm{~kW}$ $(\mathrm{A}=4.7 \mathrm{~kW} \mathrm{~B}=8 \mathrm{~kW} \mathrm{C}=4.3 \mathrm{~kW})$ of Load Bank 6 load demands. Load Bank 6 was approximately $23.9 \mathrm{~kW} \quad(\mathrm{~A}=0 \mathrm{~kW} \quad \mathrm{~B}=12 \mathrm{~kW} \quad \mathrm{C}=11.9 \mathrm{~kW})+\mathrm{j} 0.526 \mathrm{kVAr} \quad(\mathrm{A}=0 \mathrm{kVAr}$ $\mathrm{B}=0.266 \mathrm{kVAr} \mathrm{C}=0.26 \mathrm{kVAr}$ ) which is less than the power supplied by Gen-set A1 on the A-phase recorded by Meter 1 which was approximately $6.7 \mathrm{~kW}(\mathrm{~A}=-4.8 \mathrm{~kW} B=4 \mathrm{~kW}$ $\mathrm{C}=7.5 \mathrm{~kW})$. This caused a reverse power condition which opened the static switch in 2.0 seconds islanding the CERTS microgrid from the utility grid shown in Figure 19g.

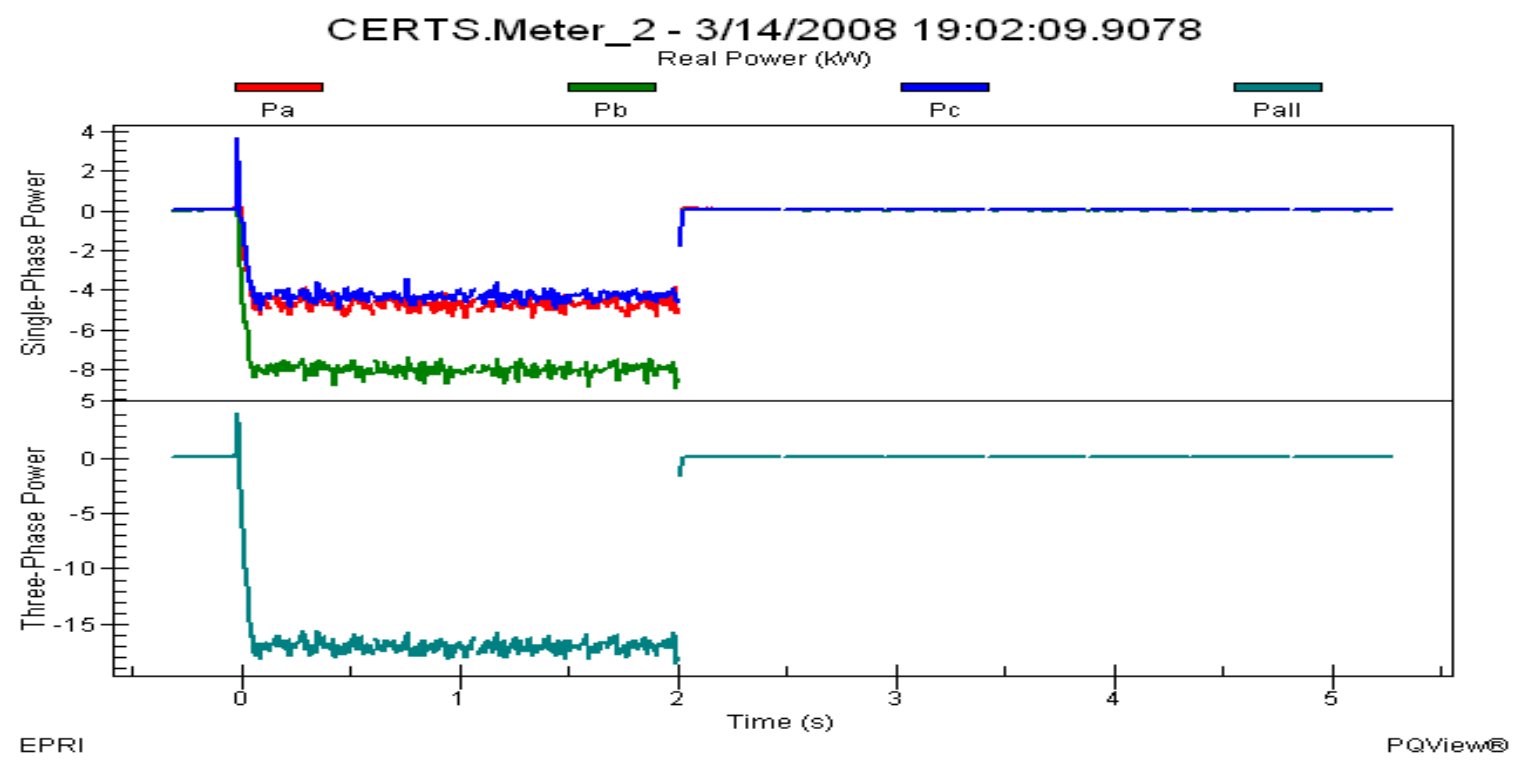

Figure 19f - Static Switch Real Power during Islanded to Utility Connected mode for Test 10.4.17 


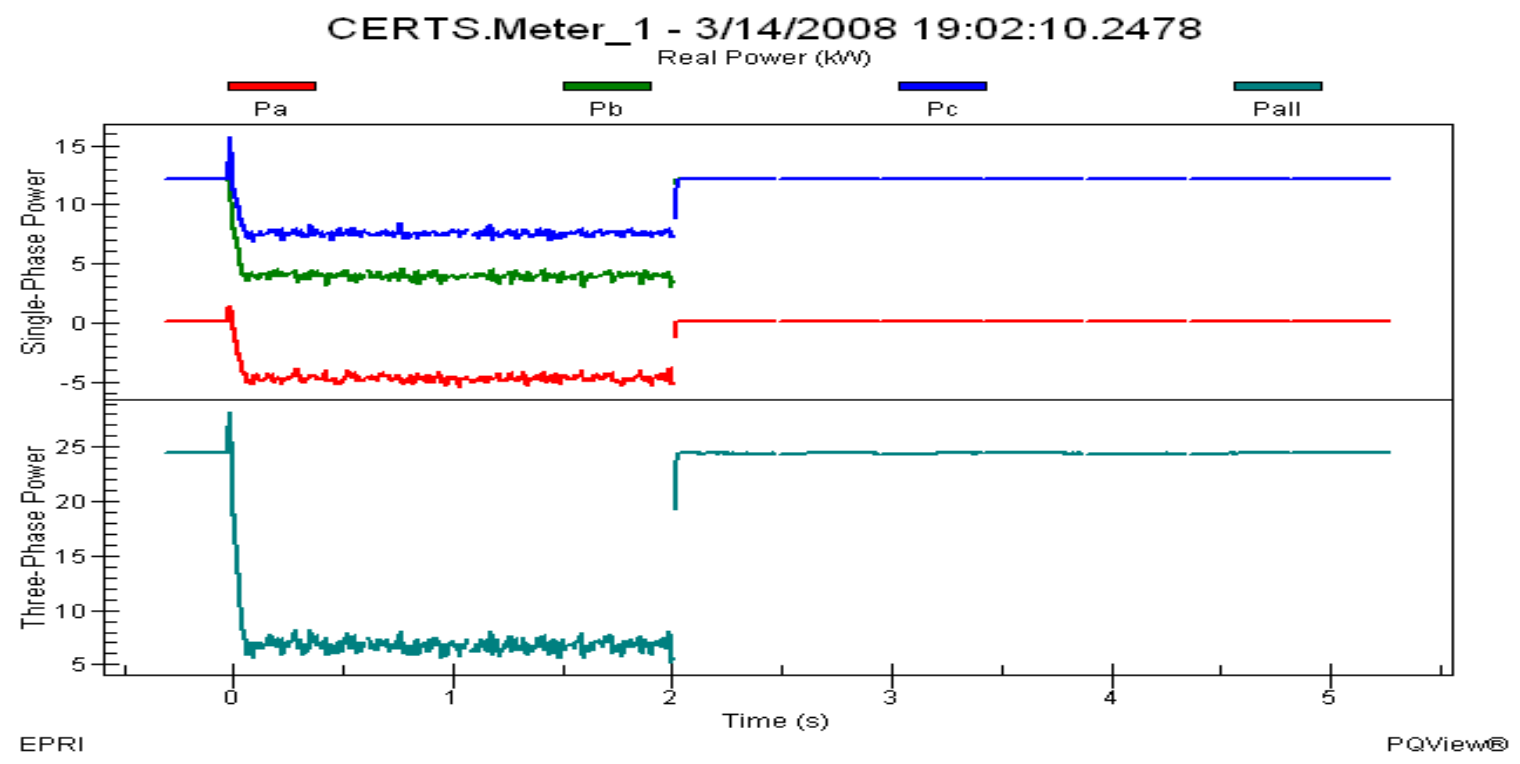

Figure 19g - Meter 1 Real Power during Islanded to Utility Connected mode for Test 10.4.17

In Table 30 after the static switch opened due to the reverse power trip condition, $0 \mathrm{~kW}$ $(\mathrm{A}=0 \mathrm{~kW} \mathrm{~B}=0 \mathrm{~kW} \mathrm{C}=0 \mathrm{~kW})+\mathrm{j} 0 \mathrm{kVAr}(\mathrm{A}=0 \mathrm{kVAr} \mathrm{B}=0 \mathrm{kVAr} \mathrm{C}=0 \mathrm{kVAr})$ was recorded at Meter 2 indicating that power was not flowing through the static switch. Meter 1 real power increased from approximately $6.7 \mathrm{~kW}(\mathrm{~A}=-4.8 \mathrm{~kW} \mathrm{~B}=4 \mathrm{~kW} \mathrm{C}=7.5 \mathrm{~kW})$ to $24.2 \mathrm{~kW}(\mathrm{~A}=0 \mathrm{~kW}$ $B=12.1 \mathrm{~kW} C=12.1 \mathrm{~kW})$ and reactive power decreased from approximately $\mathrm{j} 20.55 \mathrm{kVAr}$ $(\mathrm{A}=6.75 \mathrm{kVAr} B=5.4 \mathrm{kVAr} \mathrm{C}=8.4 \mathrm{kVAr})$ to $\mathrm{j} 0.9 \mathrm{kVAr}(\mathrm{A}=0.1 \mathrm{kVAr} \mathrm{B}=0.4 \mathrm{kVAr} \mathrm{C}=0.4 \mathrm{kVAr})$. The change in power is because the microgrid is no longer supplying the power for the load demands of Load Bank 6 which was approximately $24.2 \mathrm{~kW}(\mathrm{~A}=0 \mathrm{~kW} B=12 \mathrm{~kW}$ $\mathrm{C}=12.2 \mathrm{~kW})+\mathrm{j0} 0.533 \mathrm{kVAr}(\mathrm{A}=0 \mathrm{kVAr} \mathrm{B}=0.269 \mathrm{kVAr} \mathrm{C}=0.264 \mathrm{kVAr})$.

The power produced by the Gen-set was very similar to the measured value before the static switch manual open was released which was approximately $14 \mathrm{~kW}(\mathrm{~A}=1 \mathrm{~kW}$ $\mathrm{B}=7 \mathrm{~kW} \mathrm{C}=6 \mathrm{~kW})+\mathrm{j} 4 \mathrm{kVAr}(\mathrm{A}=0.9 \mathrm{kVAr} B=1.6 \mathrm{kVAr} C=1.5 \mathrm{kVAr})$ for Gen-set A1. Also, the voltage and frequency returned to measured values before the static switch manual open was released which was $273.5 \mathrm{~V}$ on A-phase, $274 \mathrm{~V}$ on B-phase and $268.7 \mathrm{~V}$ on Cphase and $60.15 \mathrm{~Hz}$. After all the data was verified and recorded into the DAS Database Gen-set and Load Banks 3 and 6 were turned off.

\subsection{CONCLUSION}

The tests in section 10.0 "Difficult Load Tests" were all successfully performed to as predicted. These tests have proven that the CERTS microgrid can handle a 10Hp 
induction motor turning on while utility connected or islanded with balanced or unbalanced loads. Also, the static switch will trip on reverse power if a phase is reduced to zero and that the microgrid is still functional and electrically stable while isolated from the utility grid as the critical load demand of a single phase is reduced to $0 \mathrm{~kW}$. 


\section{CERTS MICROGRID LABORATORY TEST BED}

Test Log

Prepared For:

California Energy Commission

Public Interest Energy Research Program

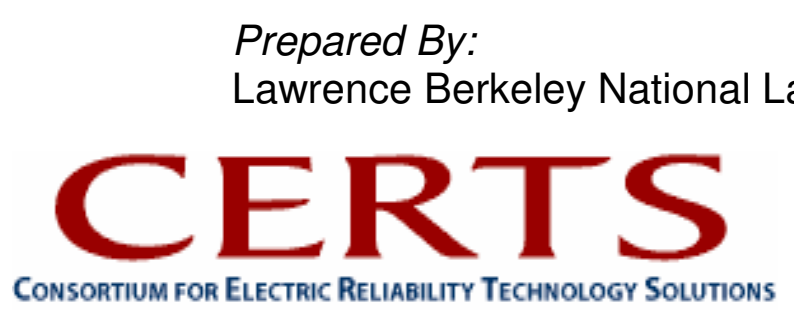

Arnold Schwarzenegger Governor

$z$
$\frac{x}{0}$
$\frac{1}{4}$
$\frac{\alpha}{\alpha}$ 

Prepared By:

Lawrence Berkeley National Laboratory

Joseph H. Eto, Principal Investigator

Berkeley, CA 94720

Ben Schenkman and John Stevens, Sandia National Laboratory

Harry Volkommer and Dave Klapp, American Electric Power

Commission Contract No. 500-03-024

Prepared For:

Public Interest Energy Research (PIER)

California Energy Commission

Bernard Treanton

Contract Manager

Mike Gravely

Program Area Lead

ENERGY SYSTEMS INTEGRATION

Mike Gravely

Office Manager

ENERGY SYSTEMS RESEARCH

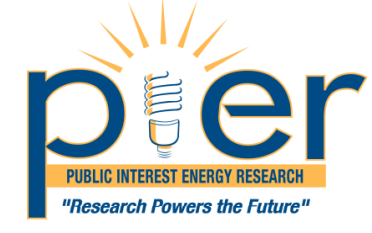

Martha Krebs, Ph.D.

PIER Director

Thom Kelly, Ph.D.

Deputy Director

ENERGY RESEARCH \& DEVELOPMENT DIVISION

Melissa Jones

Executive Director

\section{DISCLAIMER}

This report was prepared as the result of work sponsored by the California Energy Commission. It does not necessarily represent the views of the Energy Commission, its employees or the State of California. The Energy Commission, the State of California, its employees, contractors and subcontractors make no warrant, express or implied, and assume no legal liability for the information in this report; nor does any party represent that the uses of this information will not infringe upon privately owned rights. This report has not been approved or disapproved by the California Energy Commission nor has the California Energy Commission passed upon the accuracy or adequacy of the information in this report. 



\begin{tabular}{|c|c|c|}
\hline \multicolumn{3}{|c|}{$\begin{array}{l}\text { DTC Registered Procedure - CERTS Microgrid Test Plan Appendix C: } \\
\text { TEST LOG }\end{array}$} \\
\hline Dolan Technology Center & \multicolumn{2}{|c|}{ Document No: DTC212812.207.01A } \\
\hline Written by: AEP & Effective Date: 23 Feb 2007 & Target Group: Assigned \\
\hline Approved by: K. P. Loving & Procedure Review Date: 23 Feb. 2008 & \\
\hline
\end{tabular}

\begin{tabular}{|c|c|c|c|c|c|c|c|c|}
\hline $\begin{array}{l}\text { Start } \\
\text { Test } \\
\text { Sequence } \\
\text { No. }\end{array}$ & $\begin{array}{l}\text { Test } \\
\text { Date }\end{array}$ & $\begin{array}{l}\text { Start } \\
\text { Time }\end{array}$ & $\begin{array}{l}\text { Verify/ } \\
\text { Action }\end{array}$ & $\begin{array}{l}\text { Verify/ } \\
\text { Action }\end{array}$ & \begin{tabular}{|l|} 
Status \\
S or U
\end{tabular} & $\begin{array}{l}\text { End } \\
\text { Time }\end{array}$ & $\begin{array}{l}\text { Initial } \\
\mathrm{S}\end{array}$ & Test Event Comments: \\
\hline 6.1.1.1 & $3 / 9 / 07$ & & & & $\mathbf{S}$ & & GWP & LB3:40kW,LB6:40kW,59.86Hz Islanded \\
\hline 6.1.1.2 & 3/9/07 & $14: 23$ & 6.1.1.3 & $\begin{array}{l}\text { 6.1.1.4, } \\
\text { 6.1.1.5 }\end{array}$ & $\mathbf{S}$ & $14: 28$ & GWP & $\begin{array}{l}\text { 60.01Hz Connected, Meter } 152 \mathrm{~kW} \text {, Meter } 216 \mathrm{~kW} \text {, } \\
\text { Meter A1 20kW, }\end{array}$ \\
\hline 6.1.2.1 & $3 / 9 / 07$ & $14: 29$ & 6.1.2.2 & 6.1.2.3 & $\mathbf{S}$ & $14: 35$ & GWP & \\
\hline 6.1.2.4 & $3 / 9 / 07$ & $14: 36$ & 6.1.2.5 & 6.1.2.6 & $\mathbf{S}$ & $14: 37$ & GWP & $\begin{array}{l}\text { Event logger didn't capture } 1547 \text { Voltage test, SS } \\
\text { indicates that it did occur, Caused CB51 to Under } \\
\text { voltage Trip: Recommend Increasing Under voltage } \\
\text { delay to } 30 \text { cycles }\end{array}$ \\
\hline 6.1.2.7 & $3 / 9 / 07$ & $14: 48$ & 6.1.2.8 & $\begin{array}{l}\text { 6.1.2.9, } \\
\text { 6.1.2.10 }\end{array}$ & $\mathbf{S}$ & $14: 49$ & GWP & Waited 5mins. \\
\hline 6.1.3.1 & $3 / 9 / 07$ & 15:07 & 6.1.3.2, & 6.1.3.3 & $\mathbf{S}$ & $15: 08$ & GWP & Triggered at $3.33 \mathrm{~kW}$ \\
\hline 6.1.3.4 & $3 / 9 / 07$ & & 6.1.3.5 & 6.1.3.6 & $\mathbf{S}$ & & GWP & \\
\hline 6.1.3.7 & $3 / 9 / 07$ & 3:39 & 6.1.3.8 & 6.1.3.9 & $\mathbf{S}$ & $15: 40$ & GWP & \\
\hline 6.1.4.1 & 3/13/07 & 9:03 & 6.1.4.2 & $\begin{array}{l}\text { 6.1.4.3, } \\
\text { 6.1.4.4 }\end{array}$ & $\mathbf{S}$ & 9:11 & DAK & $\begin{array}{l}\text { LB3:40kW, LB4:40kW, Meter } 1 \text { Initial 53kW, Final } \\
\text { 18kW, No reverse trip occured }\end{array}$ \\
\hline
\end{tabular}

5 of 65

This document contains information confidential and proprietary to AEPSC. It shall not be reproduced in whole or in part or released to any third party without expressed written consent of AEPSC. 


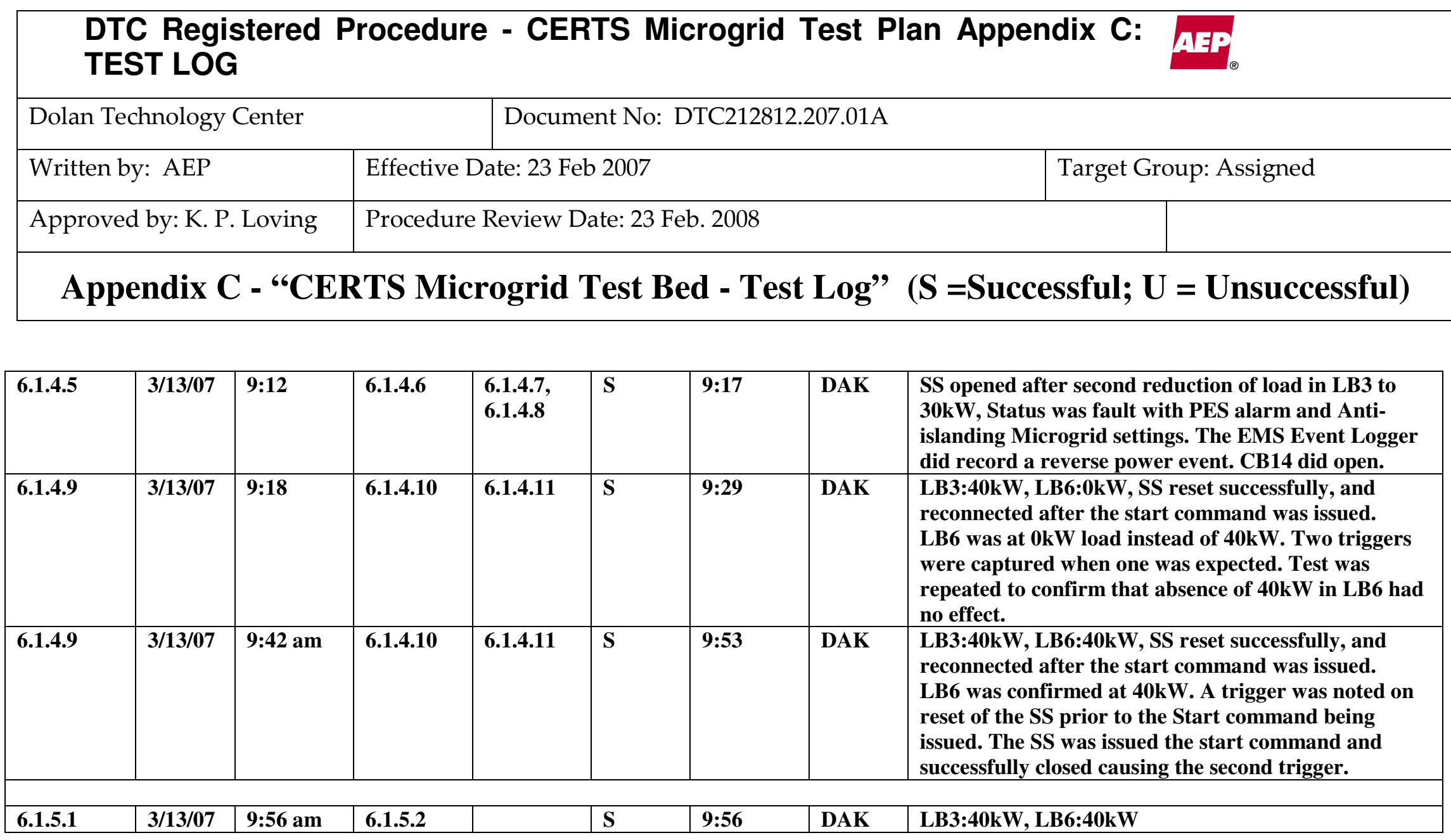

\begin{tabular}{|l|l|l|l|l|l|l|l|l|}
\hline $\begin{array}{l}\text { Start } \\
\text { Test } \\
\text { Sequence } \\
\text { No. }\end{array}$ & $\begin{array}{l}\text { Test } \\
\text { Date }\end{array}$ & $\begin{array}{l}\text { Start } \\
\text { Time }\end{array}$ & $\begin{array}{l}\text { Verify/ } \\
\text { Action }\end{array}$ & $\begin{array}{l}\text { Verify/ } \\
\text { Action }\end{array}$ & $\begin{array}{l}\text { Status } \\
\text { S or U }\end{array}$ & $\begin{array}{l}\text { End } \\
\text { Time }\end{array}$ & Initials & Test Event Comments: \\
\hline
\end{tabular}

This document contains information confidential and proprietary to AEPSC. It shall not be reproduced in whole or in part or released to any third party without expressed written consent of AEPSC. 


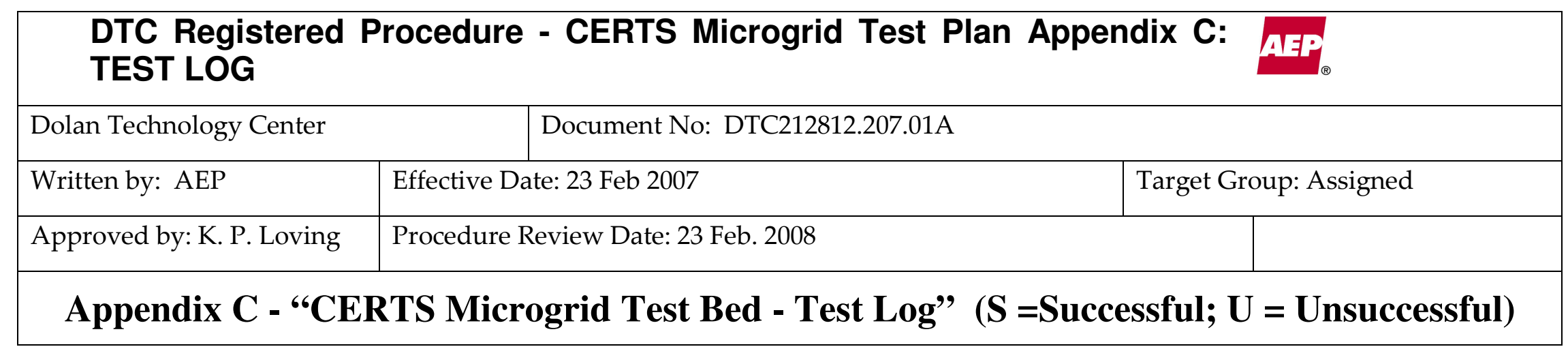

\begin{tabular}{|c|c|c|c|c|c|c|c|c|}
\hline 6.1.5.3 & 3/13/07 & 9:57 am & 6.1.5.4 & 6.1.5.5 & $\mathbf{S}$ & 10:02 am & DAK & $\begin{array}{l}\text { SS opened with a PES Alarm. Gen A1 shutdown } \\
\text { successfully. DG Side Dead Bus and DG PLL not } \\
\text { locked events were active. CB } 51 \text { opened when Gen } \\
\text { A1 shutdown. SS remained open with a dead bus. }\end{array}$ \\
\hline 6.1.5.6 & 3/13/07 & 10:04 am & 6.1.5.7 & & $\mathbf{S}$ & 10:15 am & DAK & $\begin{array}{l}\text { SS was reset to remove PES Alarm. Manual Open } \\
\text { removed and SS remained Open. Dead Bus Reclose } \\
\text { was Enabled, the SS closed and with all events } \\
\text { disappeared. }\end{array}$ \\
\hline 6.1.6.1 & $4 / 16 / 07$ & $1: 25 \mathrm{pm}$ & 6.1.6.2 & 6.1.6.3 & $\mathbf{S}$ & $2: 00 \mathrm{pm}$ & DAK & $\begin{array}{l}\text { Loads correct, Genset setup correctly, } 59.77 \mathrm{~Hz} \\
\text { prior to close of } \mathrm{SS}, 60.00 \mathrm{~Hz} \text { after. }\end{array}$ \\
\hline 6.1.6.4 & 4/16/07 & 2:02 pm & 6.1.6.5 & 6.1.6.6 & $\mathbf{S}$ & 2:08 pm & DAK & $\begin{array}{l}\text { Event logger didn't capture Power Quality, Relay2 } \\
\text { Trip or IEEE } 1547 \text { Voltage Trip, SS indicates that a } \\
\text { PQ event did occur. Data collection triggered. }\end{array}$ \\
\hline 6.1.6.7 & $4 / 16 / 07$ & 2:13 pm & 6.1.6.8 & $\begin{array}{l}\text { 6.1.6.9, } \\
\text { 6.1.6.10 }\end{array}$ & $\mathbf{S}$ & 2:18 pm & DAK & $\begin{array}{l}\text { IEEE } 1547 \text { Voltage Test shut off after CB1 was } \\
\text { reclosed, SS waited } 5 \text { mins to reconnect, Data } \\
\text { collection was triggered on the SS closure }\end{array}$ \\
\hline 7.1 .1 & $4 / 23 / 07$ & $12: 30 \mathrm{pm}$ & $\begin{array}{l}7.1 .2, \\
7.1 .3\end{array}$ & $\begin{array}{l}7.1 .4, \\
7.1 .5\end{array}$ & $\mathbf{S}$ & 2:00 pm & DAK & \\
\hline 7.1.6 & 4/25/07 & $8: 30$ am & $\begin{array}{l}\text { 7.1.7, } \\
\text { 7.1.8 }\end{array}$ & 7.1.9 & $\mathbf{S}$ & 10:00 am & DAK & $\begin{array}{l}\text { Found blown fuse on PT } 17 \text { Phase B secondary. } \\
\text { Corrected Meter CT ratios to correct values instead } \\
\text { of x10. Corrected Kep server on EMS to fix readout } \\
\text { of MG unit and feeder mode droop settings. Fault } \\
\text { key armed. }\end{array}$ \\
\hline
\end{tabular}

7 of 65

This document contains information confidential and proprietary to AEPSC. It shall not be reproduced in whole or in part or released to any third party without expressed written consent of AEPSC. 


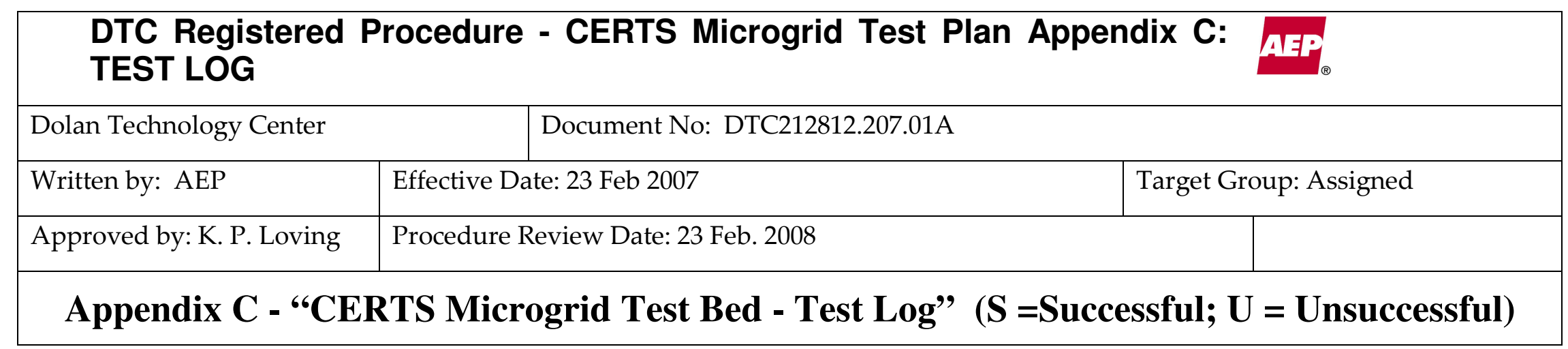

\begin{tabular}{|c|c|c|c|c|c|c|c|c|}
\hline 7.1.10 & $4 / 25 / 07$ & 10:01 am & 7.1.11 & $\begin{array}{l}\text { 7.1.12, } \\
\text { 7.1.13 }\end{array}$ & & $10: 20 \mathrm{am}$ & DAK & $\begin{array}{l}\text { Timer set to } 99 \text { secs. Once fault was initiated the } \\
\text { meter trigger remained on for the duration of the } \\
\text { fault timer. When the protection relays opened due } \\
\text { to the tests event their meter triggers were missed } \\
\text { because the fault timer remained high. Also the SS } \\
\text { and CB41 opened during the test. The SS was not } \\
\text { expected to open. }\end{array}$ \\
\hline 7.1.6R & $4 / 27 / 07$ & 9:37 am & $\begin{array}{l}\text { 7.1.7R, } \\
\text { 7.1.8R }\end{array}$ & 7.1.9R & $\mathbf{S}$ & 9:53 am & DAK & $\begin{array}{l}\text { Corrected PLC Trigger wiring and code to allow } \\
\text { proper protection triggers during faults. } \\
\text { LB4: } 60 \mathrm{~kW}, \text { SS closed, Load verified, Fault key } \\
\text { armed }\end{array}$ \\
\hline 7.1.10R & $4 / 27 / 07$ & 9:53 am & 7.1.11R & $\begin{array}{l}\text { 7.1.12R, } \\
\text { 7.1.13R }\end{array}$ & $\mathbf{S}$ & 10:10 am & DAK & $\begin{array}{l}\text { Timer set to } 99 \text { sec. Fault initiated CB41 opened, } \\
\text { Also SS and CB51 Opened }\end{array}$ \\
\hline 7.1.19 & $4 / 27 / 07$ & $10: 30 \mathrm{am}$ & 7.1.20 & 7.1.21 & $\mathbf{S}$ & 11:10 am & DAK & $\begin{array}{l}\text { LB4 : 60kW SS closed, Load verified. Removed } \\
\text { phases B and C from within fault load bank. } \\
\text { Fault key armed. }\end{array}$ \\
\hline 7.1 .22 & $4 / 27 / 07$ & $11: 10$ am & 7.1 .23 & $\begin{array}{l}7.1 .24 \\
7.1 .25\end{array}$ & $\mathbf{S}$ & $11: 15$ am & DAK & $\begin{array}{l}\text { Timer set to } 10 \text { sec. Fault initiated, SS opened, } \\
\text { CB41 opened, CB } 51 \text { opened. }\end{array}$ \\
\hline 7.1 .26 & $4 / 27 / 07$ & $12: 15 \mathrm{pm}$ & $\begin{array}{l}\text { 7.1.27, } \\
\text { 7.1.28 }\end{array}$ & $\begin{array}{l}7.1 .29 \\
7.1 .30\end{array}$ & $\mathbf{S}$ & $12: 40 \mathrm{pm}$ & DAK & Reconnected all 3 phases within fault load bank \\
\hline 7.2 .1 & $4 / 27 / 07$ & $12: 40 \mathrm{pm}$ & 7.2.2, & 7.2.4, & $\mathbf{S}$ & $12: 45 \mathrm{pm}$ & DAK & Moved fault load bank to Zone 3 . \\
\hline
\end{tabular}

This document contains information confidential and proprietary to AEPSC. It shall not be reproduced in whole or in part or released to any third party without expressed written consent of AEPSC. 


\begin{tabular}{|c|c|c|c|c|c|c|c|c|}
\hline \multicolumn{9}{|c|}{$\begin{array}{l}\text { DTC Registered } \\
\text { TEST LOG }\end{array}$} \\
\hline \multicolumn{4}{|c|}{ Dolan Technology Center } & \multicolumn{5}{|c|}{ Document No: DTC212812.207.01A } \\
\hline \multicolumn{3}{|c|}{ Written by: AEP } & \multicolumn{5}{|c|}{ Effective Date: 23 Feb 2007} & Target Group: Assigned \\
\hline \multicolumn{3}{|c|}{ Approved by: K. P. Loving } & \multicolumn{5}{|c|}{ Procedure Review Date: 23 Feb. 2008} & \\
\hline \multicolumn{9}{|c|}{ Appendix C - “CERTS Microgrid Test Bed - Test Log” (S =Successful; U = Unsuccessful) } \\
\hline & & & 7.2.3 & 7.2.5 & & & & Fault key armed. \\
\hline 7.2 .6 & $4 / 27 / 07$ & $12: 45 \mathrm{pm}$ & $\begin{array}{ll}\text { 7.2.7, } \\
\text { 7.2.8 }\end{array}$ & 7.2.9 & $\mathbf{S}$ & $12: 49 \mathrm{pm}$ & DAK & LB3: 60kW, LB4: 60kW, SS closed, Load verified \\
\hline 7.2.10 & $4 / 27 / 07$ & $12: 49 \mathrm{pm}$ & 7.2.11 & $\begin{array}{l}7.2 .12 \\
7.2 .13\end{array}$ & $\mathbf{S}$ & $12: 57 \mathrm{pm}$ & DAK & $\begin{array}{l}\text { Timer set to } 99 \text { sec. Fault initiated, SS opened, } \\
\text { CB31 did not open, CB51 opened. Relay } 2 \text { appears } \\
\text { to be causing the SS to open. The SS seems to open } \\
\text { when the fault breaker opens, there may be a large } \\
\text { transient associated with this which causes the SS to } \\
\text { open. }\end{array}$ \\
\hline 7.2.14 & $4 / 27 / 07$ & $12: 58 \mathrm{pm}$ & $\begin{array}{l}7.2 .15 \\
7.2 .16\end{array}$ & $\begin{array}{l}\text { 7.2.17, } \\
\text { 7.2.18 }\end{array}$ & $\mathbf{S}$ & $1: 10 \mathrm{pm}$ & DAK & Disconnected phases A and C from fault load bank. \\
\hline
\end{tabular}

\begin{tabular}{|c|c|c|c|c|c|c|c|c|}
\hline $\begin{array}{l}\text { Start Test } \\
\text { Sequence } \\
\text { No. }\end{array}$ & $\begin{array}{l}\text { Test } \\
\text { Date }\end{array}$ & $\begin{array}{l}\text { Start } \\
\text { Time }\end{array}$ & $\begin{array}{l}\text { Verify/ } \\
\text { Action }\end{array}$ & $\begin{array}{l}\text { Verify/ } \\
\text { Action }\end{array}$ & $\begin{array}{l}\text { Status } \\
\text { S or U }\end{array}$ & $\begin{array}{l}\text { End } \\
\text { Time }\end{array}$ & Initials & Test Event Comments: \\
\hline 7.2.19 & 4/27/07 & $1: 25 \mathrm{pm}$ & 7.2.20 & 7.2.21 & $\mathbf{S}$ & $1: 33 \mathrm{pm}$ & DAK & LB3: 60kW, LB4: 60kW, SS closed, Load verified \\
\hline 7.2 .22 & $4 / 27 / 07$ & $1: 33 \mathrm{pm}$ & 7.2 .23 & $\begin{array}{l}7.2 .24 \\
7.2 .25\end{array}$ & $\mathbf{S}$ & $1: 40 \mathrm{pm}$ & DAK & $\begin{array}{l}\text { Timer set to } 10 \text { sec. Fault initiated, SS opened, } \\
\text { CB31 did not open, CB51 opened, CB41 did not } \\
\text { open }\end{array}$ \\
\hline 7.2 .26 & $4 / 27 / 07$ & $1: 40 \mathrm{pm}$ & $\begin{array}{l}7.2 .27 \\
7.2 .28\end{array}$ & $\begin{array}{l}7.2 .29 \\
7.2 .30\end{array}$ & $\mathbf{S}$ & $1: 55 \mathrm{pm}$ & DAK & $\begin{array}{l}\text { Phases } A \text { and } C \text { reconnected within fault load bank } \\
\text { and fault load bank removed from Zone } 3\end{array}$ \\
\hline
\end{tabular}

This document contains information confidential and proprietary to AEPSC. It shall not be reproduced in whole or in part or released to any third party without expressed written consent of AEPSC. 


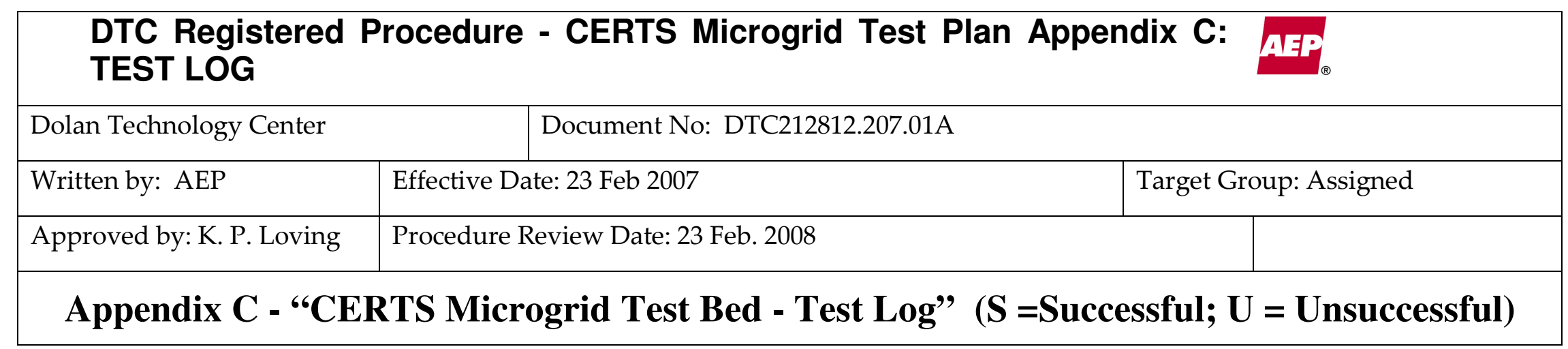

\begin{tabular}{|c|c|c|c|c|c|c|c|c|}
\hline 7.3.1 & 4/30/07 & $12: 30 \mathrm{pm}$ & $\begin{array}{l}\text { 7.3.2, } \\
\text { 7.3.3 }\end{array}$ & $\begin{array}{l}\text { 7.3.4, } \\
\text { 7.3.5 }\end{array}$ & $\mathbf{S}$ & $12: 35 \mathrm{pm}$ & DAK & $\begin{array}{l}\text { Fault load bank moved to zone } 5 \text {. All three phases } \\
\text { connected within fault load bank. }\end{array}$ \\
\hline$\overline{7.3 .6}$ & 4/30/07 & $12: 35 \mathrm{pm}$ & $\begin{array}{l}\text { 7.3.7, } \\
\text { 7.3.8 }\end{array}$ & 7.3.9 & $\mathbf{S}$ & $12: 39 \mathrm{pm}$ & DAK & LB5: 60kW, SS closed, Load verified. \\
\hline 7.3.10 & 4/30/07 & $12: 39 \mathrm{pm}$ & 7.3.11 & $\begin{array}{l}\text { 7.3.12, } \\
\text { 7.2.13 }\end{array}$ & $\mathbf{S}$ & $12: 53 \mathrm{pm}$ & DAK & $\begin{array}{l}\text { Timer set to } 99 \text { sec. Fault key armed. Fault } \\
\text { initiated. } \\
\text { SS opened, CB51 opened. CB41 and CB31 remained } \\
\text { closed. EMS event log shows Reverse power as } \\
\text { reason for SS opening. }\end{array}$ \\
\hline 7.3.14 & 4/30/07 & $12: 53 \mathrm{pm}$ & $\begin{array}{l}\text { 7.3.15, } \\
\text { 7.3.16 }\end{array}$ & $\begin{array}{l}\text { 7.3.17, } \\
\text { 7.3.18 }\end{array}$ & $\mathbf{S}$ & $1: 05$ pm & DAK & $\begin{array}{l}\text { Phases A and B were removed within the fault load } \\
\text { bank. }\end{array}$ \\
\hline 7.3.19 & 4/30/07 & $1: 20 \mathrm{pm}$ & 7.3.20 & 7.3.21 & $\mathbf{S}$ & $1: 25$ pm & DAK & LB5: 60kW, SS closed, Load verified. \\
\hline 7.3.22 & 4/30/07 & $1: 25 \mathrm{pm}$ & 7.3 .23 & $\begin{array}{l}\text { 7.3.24, } \\
\text { 7.3.25 }\end{array}$ & $\mathbf{S}$ & $1: 32 \mathrm{pm}$ & DAK & $\begin{array}{l}\text { Timer set to } 10 \text { sec. Fault key armed. Fault } \\
\text { initiated. } \\
\text { SS opened. CB51 opened. CB31 and CB41 remained } \\
\text { closed. EMS event logger shows SS opened due to } \\
\text { relay } 2 \text {. }\end{array}$ \\
\hline 7.3.26 & 4/30/07 & $1: 32$ pm & $\begin{array}{l}\text { 7.3.27, } \\
\text { 7.3.28 }\end{array}$ & $\begin{array}{l}\text { 7.3.29, } \\
\text { 7.3.30 }\end{array}$ & $\mathbf{S}$ & $1: 45 \mathrm{pm}$ & DAK & Fault load bank was removed from zone 5. \\
\hline 7.1.1R & 7/06/07 & 9:00 am & $\begin{array}{l}\text { 7.1.2R, } \\
\text { 7.1.3R }\end{array}$ & $\begin{array}{l}\text { 7.1.4R, } \\
\text { 7.1.5R }\end{array}$ & S & 9:07 am & DAK & \\
\hline 7.1.6R & $7 / 06 / 07$ & 9:07 am & $\begin{array}{l}\text { 7.1.7R, } \\
\text { 7.1.8R }\end{array}$ & 7.1.9R & S & 9:13 am & DAK & $\begin{array}{l}\text { Fault key armed. Dead Bus close successful. LB4: } \\
\text { 60kW. }\end{array}$ \\
\hline
\end{tabular}

10 of 65

This document contains information confidential and proprietary to AEPSC. It shall not be reproduced in whole or in part or released to any third party without expressed written consent of AEPSC. 


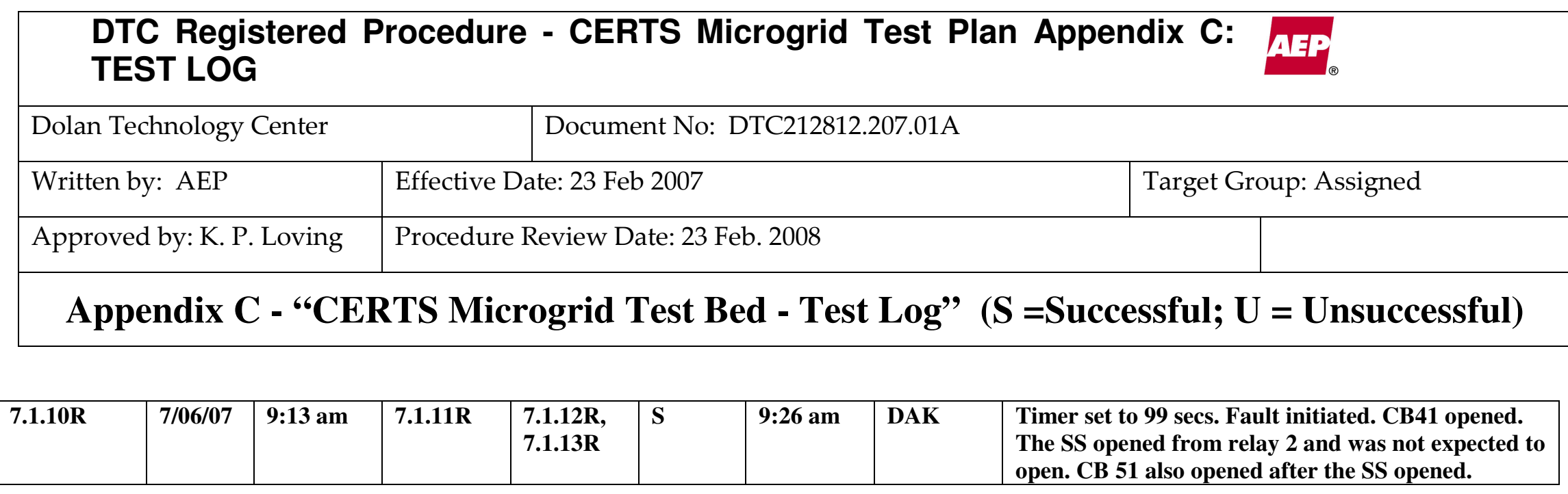

\begin{tabular}{|c|c|c|c|c|c|c|c|c|}
\hline $\begin{array}{l}\text { Start Test } \\
\text { Sequence } \\
\text { No. }\end{array}$ & $\begin{array}{l}\text { Test } \\
\text { Date }\end{array}$ & $\begin{array}{l}\text { Start } \\
\text { Time }\end{array}$ & $\begin{array}{l}\text { Verify/ } \\
\text { Action }\end{array}$ & $\begin{array}{l}\text { Verify/ } \\
\text { Action }\end{array}$ & $\begin{array}{l}\text { Status } \\
\text { S or U }\end{array}$ & $\begin{array}{l}\text { End } \\
\text { Time }\end{array}$ & Initials & Test Event Comments: \\
\hline & & & & & & & & $\begin{array}{l}\text { The ground over-current and neutral over-current } \\
\text { have all been delayed } 2 \text { cycles from original settings. } \\
\text { Note the negative sequence trip for relay } 2 \text { had } \\
\text { previously and continues to be delayed } 8 \text { cycles due } \\
\text { to problems from the inrush when dead bus } \\
\text { energizing the microgrid. }\end{array}$ \\
\hline 7.1.6R & 7/11/07 & 9:24 am & $\begin{array}{l}\text { 7.1.7R, } \\
\text { 7.1.8R }\end{array}$ & 7.1.9R & S & 9:27 am & DAK & $\begin{array}{l}\text { Fault key armed. Dead Bus close successful. LB4: } \\
60 \mathrm{~kW} \text {. }\end{array}$ \\
\hline 7.1.10R & 711/07 & 9:27 am & 7.1.11R & $\begin{array}{l}\text { 7.1.12R, } \\
\text { 7.1.13R }\end{array}$ & $\mathbf{S}$ & 9:35 am & DAK & $\begin{array}{l}\text { Timer set to } 99 \text { secs. Fault initiated. CB41 opened. } \\
\text { The SS opened from Reverse Power. CB } 51 \text { also } \\
\text { opened after the SS opened. The test procedure was } \\
\text { modified to add a small load in LB6 to prevent }\end{array}$ \\
\hline
\end{tabular}

11 of 65

This document contains information confidential and proprietary to AEPSC. It shall not be reproduced in whole or in part or released to any third party without expressed written consent of AEPSC. 


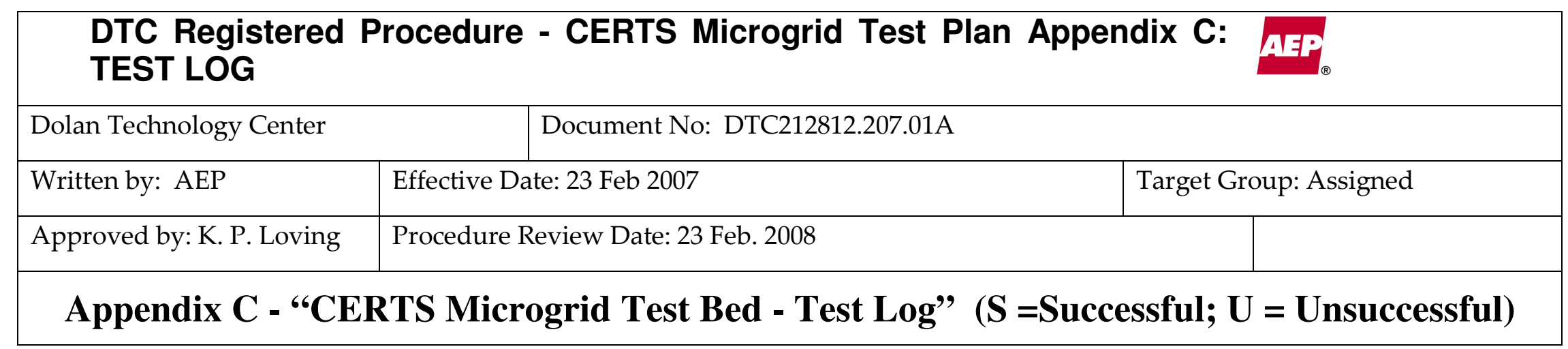

\begin{tabular}{|c|c|c|c|c|c|c|c|c|}
\hline & & & & & & & & $\begin{array}{l}\text { reverse power trips. Changed SS reverse power } \\
\text { setting to zero. }\end{array}$ \\
\hline 7.1.1R & 7/11/07 & 10:38: am & $\begin{array}{l}\text { 7.1.2R, } \\
\text { 7.1.3R }\end{array}$ & $\begin{array}{l}\text { 7.1.4R, } \\
\text { 7.1.5R }\end{array}$ & $\mathbf{S}$ & 10:40 am & DAK & \\
\hline 7.1.6R & 7/11/07 & 10:40 am & $\begin{array}{l}\text { 7.1.7R, } \\
\text { 7.1.8R }\end{array}$ & 7.1.9R & $\mathbf{S}$ & 10:42am & DAK & $\begin{array}{l}\text { Fault key armed. Dead Bus close successful. LB4: } \\
\text { 60kW. LB6:10kW. }\end{array}$ \\
\hline 7.1.10R & 711/07 & $10: 42$ am & 7.1.11R & $\begin{array}{l}\text { 7.1.12R, } \\
\text { 7.1.13R }\end{array}$ & $\mathbf{S}$ & 10:50 am & DAK & $\begin{array}{l}\text { Timer set to } 99 \text { secs. Fault initiated. CB41 opened. } \\
\text { The SS and CB } 51 \text { remained closed as expected. }\end{array}$ \\
\hline 7.1.19R & 711/07 & 11:47: am & 7.1.20R & 7.1.21R & $\mathbf{S}$ & 11:51 am & DAK & $\begin{array}{l}\text { LB4:60kW LB6:10kW SS closed, Load verified. } \\
\text { Removed phases B and C from within fault load } \\
\text { bank. } \\
\text { Fault key armed. }\end{array}$ \\
\hline 7.1.22R & 7/11/07 & 11:51 am & 7.1.23R & $\begin{array}{l}\text { 7.1.24R, } \\
\text { 7.1.25R }\end{array}$ & $\mathbf{S}$ & 12:00 pm & DAK & $\begin{array}{l}\text { Timer set to } 10 \text { sec. Fault initiated, SS opened from } \\
\text { a relay } 2 \text { trip, CB } 51 \text { opened after SS. CB41 } \\
\text { remained closed. }\end{array}$ \\
\hline 7.1.26R & 7/11/07 & 12:00 pm & $\begin{array}{l}\text { 7.1.27R, } \\
\text { 7.1.28R }\end{array}$ & $\begin{array}{l}\text { 7.1.29R, } \\
\text { 7.1.30R }\end{array}$ & $\mathbf{S}$ & 12:15 pm & DAK & Reconnected all 3 phases within fault load bank \\
\hline 7.2.1R & 7/11/07 & $12: 15 \mathrm{pm}$ & $\begin{array}{l}\text { 7.2.2R, } \\
\text { 7.2.3R }\end{array}$ & $\begin{array}{l}\text { 7.2.4R, } \\
\text { 7.2.5R }\end{array}$ & $\mathbf{S}$ & $12: 20 \mathrm{pm}$ & DAK & $\begin{array}{l}\text { Moved fault load bank to Zone } 3 \text {. } \\
\text { Fault key armed. }\end{array}$ \\
\hline 7.2.6R & 7/11/07 & $12: 20 \mathrm{pm}$ & $\begin{array}{l}\text { 7.2.7R, } \\
\text { 7.2.8R }\end{array}$ & 7.2.9R & $\mathbf{S}$ & $12: 24 \mathrm{pm}$ & DAK & $\begin{array}{l}\text { LB3: 60kW, LB4: 60kW, LB6:10kW SS closed, } \\
\text { Load verified }\end{array}$ \\
\hline 7.2.10R & 7/11/07 & $12: 24$ pm & 7.2.11R & 7.2.12R, & $\mathbf{S}$ & $12: 34 \mathrm{pm}$ & DAK & Timer set to 99 sec. Fault initiated, No protection \\
\hline
\end{tabular}
12 of 65

This document contains information confidential and proprietary to AEPSC. It shall not be reproduced in whole or in part or released to any third party without expressed written consent of AEPSC. 


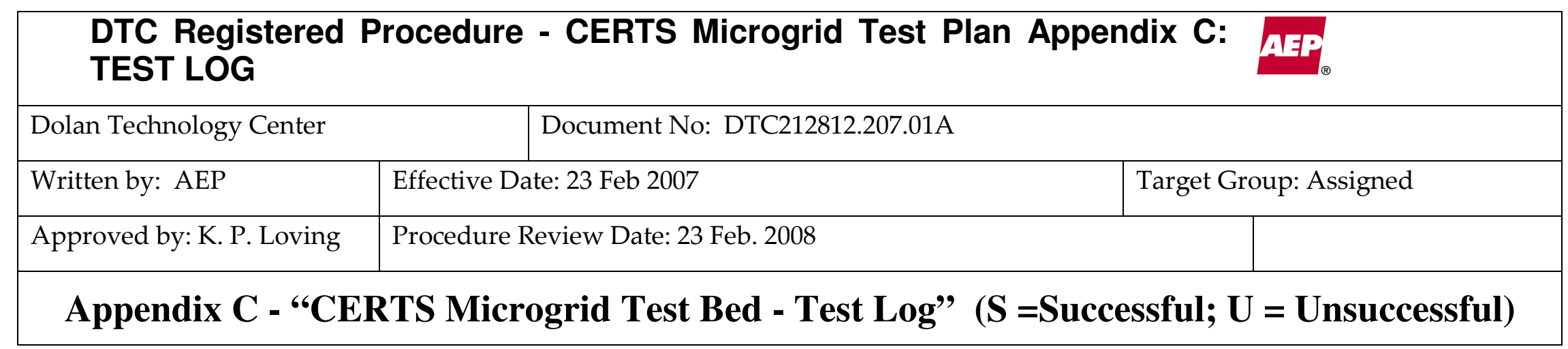

\begin{tabular}{|c|c|c|c|c|c|c|c|c|}
\hline & & & & 7.2.13R & & & & $\begin{array}{l}\text { operated. The overload is likely to small to trip the } \\
\text { protection. The trip point for breaker } 3 \text { is } 225 \mathrm{~A} \text { and } \\
\text { we were just below that at } 216 \mathrm{~A} \text {. }\end{array}$ \\
\hline 7.2.1R & 7/11/07 & $1: 10$ pm & $\begin{array}{l}\text { 7.2.2R, } \\
\text { 7.2.3R }\end{array}$ & $\begin{array}{l}\text { 7.2.4R, } \\
\text { 7.2.5R }\end{array}$ & $\mathbf{S}$ & $1: 12$ pm & DAK & $\begin{array}{l}\text { Moved fault load bank to Zone } 3 \text {. } \\
\text { Fault key armed. }\end{array}$ \\
\hline 7.2.6R & 7/11/07 & $1: 12 \mathrm{pm}$ & $\begin{array}{l}\text { 7.2.7R, } \\
\text { 7.2.8R }\end{array}$ & 7.2.9R & $\mathbf{S}$ & 1:15 pm & DAK & $\begin{array}{l}\text { LB3: 75kW, LB4: 75kW, LB6:10kW SS closed, } \\
\text { Load verified }\end{array}$ \\
\hline 7.2.10R & 7/11/07 & $1: 15$ pm & 7.2.11R & $\begin{array}{l}\text { 7.2.12R, } \\
\text { 7.2.13R }\end{array}$ & $\mathbf{S}$ & $1: 30 \mathrm{pm}$ & DAK & $\begin{array}{l}\text { Timer set to } 99 \text { sec. Fault initiated, CB } 31 \text { opened. } \\
\text { SS, CB41, and CB51 remained closed. }\end{array}$ \\
\hline 7.2.14R & 7/11/07 & $1: 30 \mathrm{pm}$ & $\begin{array}{l}\text { 7.2.15R, } \\
\text { 7.2.16R }\end{array}$ & $\begin{array}{l}\text { 7.2.17R, } \\
\text { 7.2.18R }\end{array}$ & $\mathbf{S}$ & $1: 45 \mathrm{pm}$ & DAK & Disconnected phases A and C from fault load bank. \\
\hline 7.2.19R & 7/11/07 & $1: 45 \mathrm{pm}$ & 7.2.20R & 7.2.21R & $\mathbf{S}$ & 2:01 pm & DAK & LB3: 60kW, LB4: 60kW, SS closed, Load verified \\
\hline 7.2.22R & 7/11/07 & 2:01 pm & 7.2.23R & $\begin{array}{l}\text { 7.2.24R, } \\
\text { 7.2.25R }\end{array}$ & $\mathbf{S}$ & 2:10 pm & DAK & $\begin{array}{l}\text { Timer set to } 10 \text { sec. Fault initiated, SS opened, all } \\
\text { other breakers remained closed. }\end{array}$ \\
\hline 7.2.26R & 711/07 & $2: 10 \mathrm{pm}$ & $\begin{array}{l}\text { 7.2.27R, } \\
\text { 7.2.28R }\end{array}$ & $\begin{array}{l}\text { 7.2.29R, } \\
\text { 7.2.30R }\end{array}$ & $\mathbf{S}$ & $2: 25$ pm & DAK & $\begin{array}{l}\text { Phases } A \text { and } C \text { reconnected within fault load bank } \\
\text { and fault load bank removed from Zone } 3\end{array}$ \\
\hline 7.3.1R & 7/18/07 & 10:34 am & $\begin{array}{l}\text { 7.3.2R, } \\
\text { 7.3.3R }\end{array}$ & $\begin{array}{l}\text { 7.3.4R, } \\
\text { 7.3.5R }\end{array}$ & $\mathbf{S}$ & 10:35 am & DAK & $\begin{array}{l}\text { Fault load bank moved to zone } 5 \text {. All three phases } \\
\text { connected within fault load bank. }\end{array}$ \\
\hline 7.3.6R & 7/18/07 & $10: 35$ am & $\begin{array}{l}\text { 7.3.7R, } \\
\text { 7.3.8R }\end{array}$ & 7.3.9R & $\mathbf{S}$ & $10: 37$ am & DAK & LB5: 60kW, SS closed, Load verified. \\
\hline 7.3.10R & 7/18/07 & 10:37 am & 7.3.11R & $\begin{array}{l}\text { 7.3.12R, } \\
\text { 7.2.13R }\end{array}$ & $\mathbf{S}$ & $10: 43$ am & DAK & $\begin{array}{l}\text { Timer set to } 99 \text { sec. Fault key armed. Fault } \\
\text { initiated. }\end{array}$ \\
\hline
\end{tabular}

13 of 65

This document contains information confidential and proprietary to AEPSC. It shall not be reproduced in whole or in part or released to any third party without expressed written consent of AEPSC. 


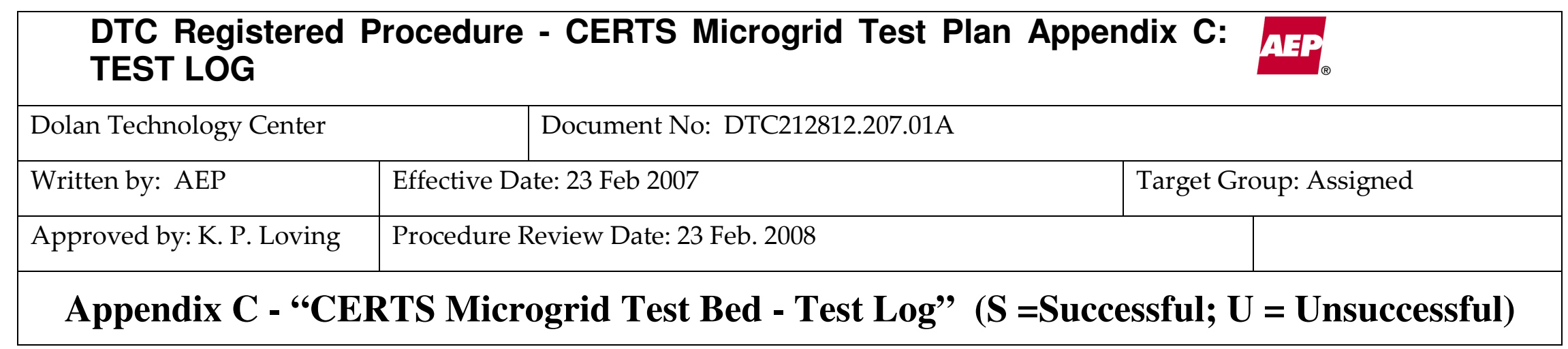

\begin{tabular}{|c|c|c|c|c|c|c|c|c|}
\hline & & & & & & & & $\begin{array}{l}\text { CB51 opened. The SS, CB41 and CB31 remained } \\
\text { closed. }\end{array}$ \\
\hline 7.3.14R & $7 / 18 / 07$ & $11: 48$ am & $\begin{array}{l}\text { 7.3.15R, } \\
\text { 7.3.16R }\end{array}$ & $\begin{array}{l}\text { 7.3.17R, } \\
\text { 7.3.18R }\end{array}$ & $\mathbf{S}$ & $11: 50$ am & DAK & $\begin{array}{l}\text { Phases A and B were removed within the fault load } \\
\text { bank. }\end{array}$ \\
\hline 7.3.19R & 7/18/07 & 11:50 am & 7.3.20R & 7.3.21R & $\mathbf{S}$ & $11: 52$ am & DAK & LB5: 60kW, SS closed, Load verified. \\
\hline 7.3.22R & 7/18/07 & $11: 52 \mathrm{am}$ & 7.3.23R & $\begin{array}{l}\text { 7.3.24R, } \\
\text { 7.3.25R }\end{array}$ & $\mathbf{S}$ & 12:02 pm & DAK & $\begin{array}{l}\text { Timer set to } 10 \text { sec. Fault key armed. Fault } \\
\text { initiated. } \\
\text { CB51 opened. The SS, CB31 and CB41 remained } \\
\text { closed. }\end{array}$ \\
\hline 7.3.26R & 7/18/07 & 12:02 pm & $\begin{array}{l}\text { 7.3.27R, } \\
\text { 7.3.28R }\end{array}$ & $\begin{array}{l}\text { 7.3.29R, } \\
\text { 7.3.30R }\end{array}$ & $\mathbf{S}$ & 12:10 pm & DAK & Fault load bank was removed from zone 5. \\
\hline 7.4.1 & 7/30/07 & 9:49 am & $\begin{array}{l}7.4 .2 \\
7.4 .3\end{array}$ & $\begin{array}{l}\text { 7.4.4 } \\
\text { 7.4.5 }\end{array}$ & $\mathbf{S}$ & 10:10 am & DAK & Fault load connected, armed, and verified. \\
\hline 7.4.6 & 7/30/07 & 10:10 am & $\begin{array}{l}\text { 7.4.7, } \\
\text { 7.4.8 }\end{array}$ & 7.4.9 & $\mathbf{S}$ & 10:18 am & DAK & $\begin{array}{l}\text { SS Open, Gensets setup and verified. Loads } \\
\text { connected and verified }\end{array}$ \\
\hline 7.4.10 & 7/30/07 & 10:18 am & 7.4.11 & 7.4.12 & $\mathbf{S}$ & 11:55 am & DAK & $\begin{array}{l}\text { Genset A1 connected at 10:25. Genset A2 connected } \\
\text { at 10:31, causing CB41 to open and shutdown A2. } \\
\text { Genset A1 remained online serving zones } 2,3 \text {, and } 5 \text {. } \\
\text { The primary side fuses of PT42 were found blown. } \\
\text { The primary and secondary fuses were swapped on } \\
\text { the PT and new fuses were installed. CB41 was } \\
\text { reclosed without triggering and the test sequence } \\
\text { was picked up where we left off. }\end{array}$ \\
\hline
\end{tabular}

14 of 65

This document contains information confidential and proprietary to AEPSC. It shall not be reproduced in whole or in part or released to any third party without expressed written consent of AEPSC. 


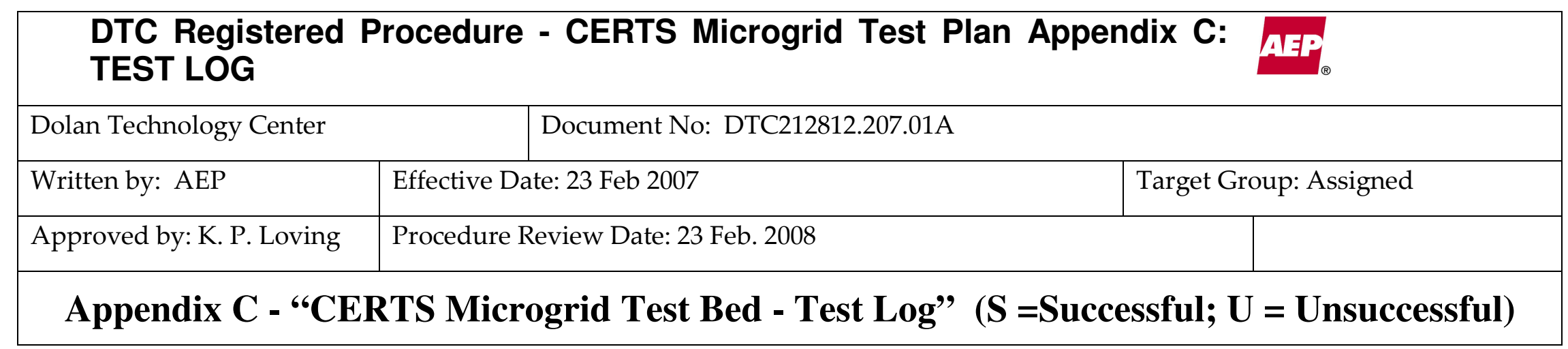

\begin{tabular}{|c|c|c|c|c|c|c|c|c|}
\hline & & & & & & & & $\begin{array}{l}\text { Genset A2 was restarted and successfully connected } \\
\text { at 11:42. SS was enabled and connected at 11:48 }\end{array}$ \\
\hline 7.4 .13 & $7 / 30 / 07$ & 11:55 am & $\begin{array}{l}\text { 7.4.14, } \\
\text { 7.4.15 }\end{array}$ & 7.4.16 & $\mathbf{S}$ & $1: 00 \mathrm{pm}$ & DAK & $\begin{array}{l}\text { Fault timer at } 99 \text { sec. LB4 was increased to } 65 \mathrm{~kW} \\
\text { due to load drift and generation offset. This was } \\
\text { done to insure enough load to trip CB41 under } \\
\text { overload. The fault was applied at } 11: 55 \text { am. Gen } \\
\text { A1 tripped off on a low oil pressure alarm which } \\
\text { appears as a false alarm. LB4 was reduced to } 0 \text { to } \\
\text { prevent CB41 from opening and causing another } \\
\text { trigger. Genset A1 was checked and restarted. Once } \\
\text { started Genset A1 seemed to operate well off its } \\
\text { RPM range. Tecogen was contacted. }\end{array}$ \\
\hline 7.4.1R & $7 / 31 / 07$ & $8: 45$ am & $\begin{array}{l}\text { 7.4.2R, } \\
\text { 7.4.3R }\end{array}$ & $\begin{array}{l}\text { 7.4.4R, } \\
\text { 7.4.5R }\end{array}$ & $\mathbf{S}$ & 9:01 am & DAK & Fault load connected, armed, and verified. \\
\hline 7.4.6R & $7 / 31 / 07$ & 9:01 am & $\begin{array}{l}\text { 7.4.7R, } \\
\text { 7.4.8R }\end{array}$ & 7.4.9R & $\mathbf{S}$ & 9:09 am & DAK & $\begin{array}{l}\text { SS Open, Gensets setup and verified. Genset A2 } \\
\text { changes its Power Flow mode to “0” constantly, } \\
\text { even though the Microgrid Control Status returns } \\
\text { unit control mode. Loads connected and verified }\end{array}$ \\
\hline 7.4.10R & $7 / 31 / 07$ & 9:09 am & 7.4.11R & 7.4.12R & $\mathbf{S}$ & 10:00 am & DAK & $\begin{array}{l}\text { Genset A1 connected at 9:15. Genset A2 connected } \\
\text { at 9:20, causing CB41 to open and shutdown A2. } \\
\text { Genset A1 remained online serving zones } 2,3 \text {, and } 5 \text {. } \\
\text { The secondary side phase A fuse of PT42 was found } \\
\text { blown. A new fuse was installed. CB41 was reclosed, } \\
\text { triggering a capture at 9:33 am and the test }\end{array}$ \\
\hline
\end{tabular}

15 of 65

This document contains information confidential and proprietary to AEPSC. It shall not be reproduced in whole or in part or released to any third party without expressed written consent of AEPSC. 


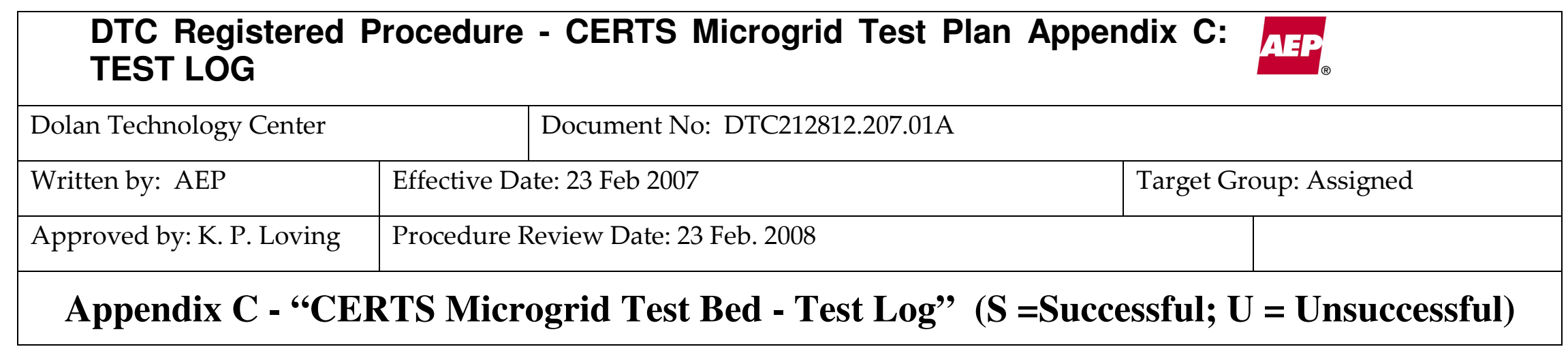

\begin{tabular}{|c|c|c|c|c|c|c|c|c|}
\hline & & & & & & & & $\begin{array}{l}\text { sequence was picked up where we left off. } \\
\text { Genset A2 was restarted and successfully connected } \\
\text { at 9:49. SS was enabled and connected at 9:55. }\end{array}$ \\
\hline 7.4.13R & 7/31/07 & 10:00 am & $\begin{array}{l}\text { 7.4.14R, } \\
\text { 7.4.15R }\end{array}$ & 7.4.16R & $\mathbf{S}$ & 10:03 am & DAK & $\begin{array}{l}\text { Fault timer at } 99 \text { sec. LB4 was increased to } 65 \mathrm{~kW} \\
\text { due to load drift and generation offset. This was } \\
\text { done to insure enough load to trip CB41 under } \\
\text { overload. The fault was applied at 10: } 01 \mathrm{am} \text {. CB41 } \\
\text { opened tripping off Genset A2. The SS opened } \\
\text { shortly afterwards on a reverse power trip. CB31 } \\
\text { and CB51 remained closed and Genset A1 remained } \\
\text { online serving the remaining load. }\end{array}$ \\
\hline 7.4.17 & 7/31/07 & 10:03 am & & & $\mathbf{S}$ & 10:08am & DAK & $\begin{array}{l}\text { Genset A1 was shutdown causing CB51 to open on } \\
\text { undervoltage trip at 10:03 }\end{array}$ \\
\hline 7.5.1 & 7/31/07 & 11:27 am & $\begin{array}{l}7.5 .2 \\
7.5 .3\end{array}$ & $\begin{array}{l}\text { 7.5.4, } \\
\text { 7.5.5, } \\
\text { 7.5.6 }\end{array}$ & $\mathbf{S}$ & $11: 28$ am & DAK & Fault load connected and verified. Fault key armed. \\
\hline 7.5.7 & 7/31/07 & 11:28 am & 7.5 .8 & 7.5.9 & $\mathbf{S}$ & 11:29 am & DAK & LB3: 40kW, LB6:40kW \\
\hline 7.5.10 & 7/31/07 & 11:29 am & 7.5.11 & 7.5.12 & $\mathbf{S}$ & 11:41 am & DAK & $\begin{array}{l}\text { Genset A1 connected at 11:32, SS connected at } \\
\text { 11:37. Meter } 1: 51 \mathrm{~kW} \text {, Meter2:15kW }\end{array}$ \\
\hline 7.5.13 & $7 / 31 / 07$ & 11:41 am & $\begin{array}{l}\text { 7.5.14, } \\
\text { 7.5.15 }\end{array}$ & 7.5.16 & $\mathbf{S}$ & 11:59 am & DAK & $\begin{array}{l}\text { Fault timer set to } 10 \text { sec. SS opened by relay } 2 \text { trip, } \\
\text { CB31 opened and Genset A1 shutdown. CB51 } \\
\text { opened on undervoltage. Ignore accidentally } \\
\text { created event at 11:44. Meter1:36kW, Meter2:0kW }\end{array}$ \\
\hline 7.5 .17 & 7/31/07 & 11:59 am & & & $\mathbf{S}$ & 12:02 pm & DAK & \\
\hline
\end{tabular}

16 of 65

This document contains information confidential and proprietary to AEPSC. It shall not be reproduced in whole or in part or released to any third party without expressed written consent of AEPSC. 


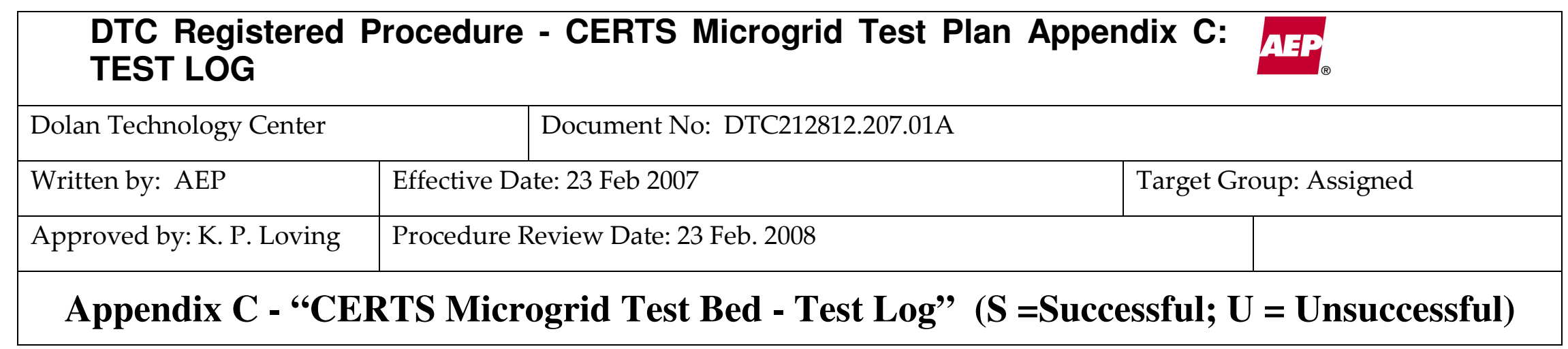

\begin{tabular}{|c|c|c|c|c|c|c|c|c|}
\hline 7.6.1 & 7/31/07 & 12:02 pm & 7.6 .2 & 76.3 & $\mathbf{S}$ & $12 \cdot 10 \mathrm{am}$ & DAK & Gensets $A 1$ and $A 2$ setun, Fault kev armed. \\
\hline 7.6 .4 & $7 / 31 / 07$ & $12: 10 \mathrm{pm}$ & 7.6 .5 & $\begin{array}{l}\text { 7.6.6, } \\
\text { 7.6.7 }\end{array}$ & $\mathbf{S}$ & $12: 23 \mathrm{pm}$ & DAK & $\begin{array}{l}\text { Triggers disabled, Genset A1 connected at 12:15, } \\
\text { Genset A2 connected at 12:19. LB3:40kW, } \\
\text { LB4:40kW, LB6:40kW. SS connected at 12:22. } \\
\text { Meter1:69kW, Meter2:35kW. Triggers armed }\end{array}$ \\
\hline 7.6.8 & 7/31/07 & $12: 23 \mathrm{pm}$ & $\begin{array}{l}\text { 7.6.9, } \\
7.6 .10\end{array}$ & 7.6.11 & $\mathbf{S}$ & $12: 30 \mathrm{pm}$ & DAK & $\begin{array}{l}\text { Fault timer set to } 10 \text { sec Fault initiated at 12:25. SS } \\
\text { opened, CB31 opened, CB41 Opened, and CB51 } \\
\text { opened. Gensets A1 and A2 shutdown. } \\
\text { Meter1:36kW, Meter2:0kW }\end{array}$ \\
\hline
\end{tabular}

\begin{tabular}{|c|c|c|c|c|c|c|c|c|}
\hline $\begin{array}{l}\text { Start Test } \\
\text { Sequence } \\
\text { No. }\end{array}$ & $\begin{array}{l}\text { Test } \\
\text { Date }\end{array}$ & $\begin{array}{l}\text { Start } \\
\text { Time }\end{array}$ & $\begin{array}{l}\text { Verify/ } \\
\text { Action }\end{array}$ & $\begin{array}{l}\text { Verify/ } \\
\text { Action }\end{array}$ & $\begin{array}{l}\text { Status } \\
\text { S or U }\end{array}$ & $\begin{array}{l}\text { End } \\
\text { Time }\end{array}$ & Initials & Test Event Comments: \\
\hline \multirow[t]{2}{*}{ 7.6.12 } & 7/31/07 & 12:30 & & & $\mathbf{S}$ & $12: 35 \mathrm{pm}$ & DAK & \\
\hline & & & & & & & & $\begin{array}{l}\text { Test } 7.7 \text { and } 7.8 \text { were skipped on } 7 / 31 / 07 \text { because } \\
\text { Genset } B 1 \text { was not operational and replacement } \\
\text { parts had not yet arrived. Replacement parts } \\
\text { installed } 8 / 7 / 07\end{array}$ \\
\hline 7.7.1 & 8/7/07 & 9:22 am & $\begin{array}{l}7.7 .2 \\
7.7 .3\end{array}$ & $\begin{array}{l}\text { 7.7.4, } \\
\text { 7.7.5, } \\
\text { 7.7.6 }\end{array}$ & $\mathbf{S}$ & 9:30 am & DAK & Fault load verified and connected, Fault key armed \\
\hline 7.7.7 & 8/7/07 & 9:30 am & 7.7.8 & 7.7 .9 & $\mathbf{S}$ & 9:37 am & DAK & LB3:40kW, LB6:40kW, Genset B1 setup \\
\hline
\end{tabular}

17 of 65

This document contains information confidential and proprietary to AEPSC. It shall not be reproduced in whole or in part or released to any third party without expressed written consent of AEPSC. 


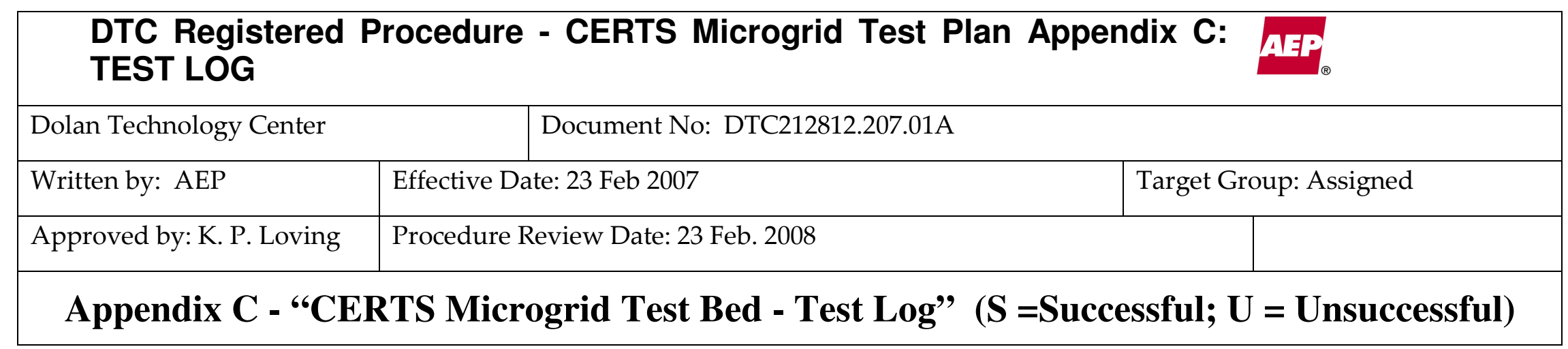

\begin{tabular}{|c|c|c|c|c|c|c|c|c|}
\hline 7.7.10 & 8/7/07 & 9:37 am & 7.7 .11 & 7.7 .12 & $\mathbf{S}$ & 9:56 am & DAK & $\begin{array}{l}\text { Genset connected at 9:55 with SS shortly after in } \\
\text { same trigger Meter1:52kW Meter2:16kW }\end{array}$ \\
\hline 7.8 .1 & 8/7/07 & 11:20 am & 7.8 .2 & $\mathbf{7 . 8 . 3}$ & $\mathbf{S}$ & 11:22 am & DAK & Breakers closed, Fault key armed. \\
\hline 7.8 .4 & $8 / 7 / 07$ & $11: 23 \mathrm{am}$ & 7.8 .5 & $\begin{array}{l}\text { 7.8.6, } \\
\text { 7.8.7 }\end{array}$ & $\mathbf{S}$ & $11: 42 \mathrm{am}$ & DAK & $\begin{array}{l}\text { Gensets started, LB3:40kW, LB4:40kW, LB6:40kW } \\
\text { SS connected, Meter1:61kW, Meter2:26kW. Note } \\
\text { while starting the gensets the SS was left in a } \\
\text { disconnect state with manual open asserted. After } \\
\text { starting the gensets the SS was found to be in the } \\
\text { OFF state. This happened a few times before it } \\
\text { would remain in the disconnect state reliable for a } \\
\text { period of time. There were no alarms present } \\
\text { during this process. }\end{array}$ \\
\hline 7.8 .8 & $8 / 7 / 07$ & 11:42 am & $\begin{array}{l}\text { 7.8.9, } \\
\text { 7.8.10 }\end{array}$ & 7.8 .11 & $\mathbf{S}$ & 11:47 am & DAK & $\begin{array}{l}\text { Fault timer at } 10 \text { sec. Fault triggered at 11:44. CB51 } \\
\text { opened and Genset B1 shutdown. SS and remaining } \\
\text { breakers remained closed. Meter1:83kW, } \\
\text { Meter2:49kW }\end{array}$ \\
\hline
\end{tabular}

18 of 65

This document contains information confidential and proprietary to AEPSC. It shall not be reproduced in whole or in part or released to any third party without expressed written consent of AEPSC. 


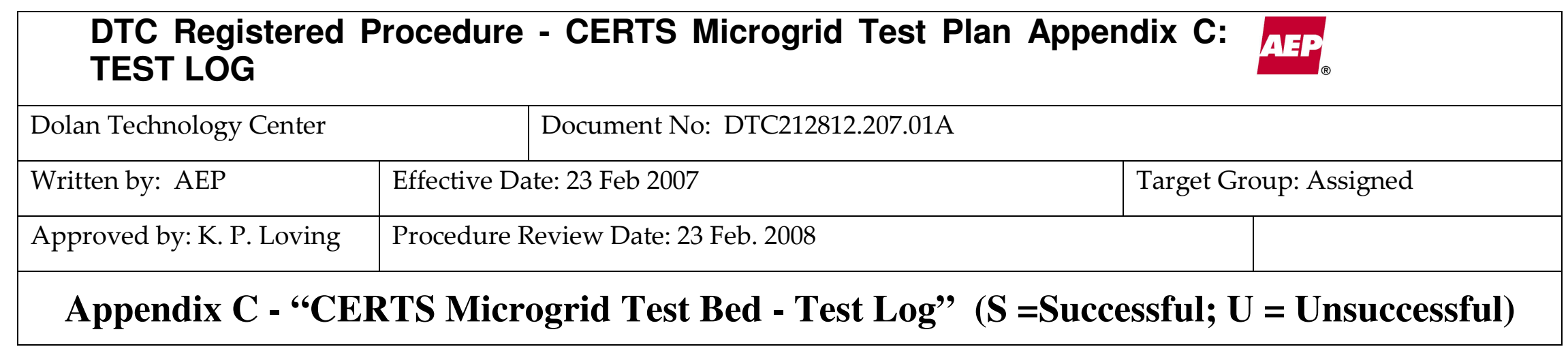

\begin{tabular}{|c|c|c|c|c|c|c|c|c|}
\hline & & & & & & & & $\begin{array}{l}\text { relay files it appears that the SS opened first on an } \\
\text { SCR alarm, then Genset A1 shutdown due to the } \\
\text { overload that remained. }\end{array}$ \\
\hline 7.9.1 & 7/31/07 & $12: 36 \mathrm{pm}$ & $\begin{array}{l}7.9 .2, \\
7.9 .3\end{array}$ & $\begin{array}{l}\text { 7.9.4, } \\
\text { 7.9.5, } \\
\mathbf{7 . 9 . 6}\end{array}$ & $\mathbf{S}$ & $12: 42 \mathrm{pm}$ & DAK & Fault load verified, key armed, All breakers closed. \\
\hline 7.9.7 & 7/31/07 & $12: 42 \mathrm{pm}$ & $\mathbf{7 . 9 . 8}$ & 7.9.9 & $\mathbf{S}$ & 12:49 pm & DAK & $\begin{array}{l}\text { Gensets setup, Triggers disabled, Genset A1 } \\
\text { connected at 12:46. Genset A2 connected at 12:48 }\end{array}$ \\
\hline
\end{tabular}

\begin{tabular}{|c|c|c|c|c|c|c|c|c|}
\hline $\begin{array}{l}\text { Start Test } \\
\text { Sequence } \\
\text { No. }\end{array}$ & $\begin{array}{l}\text { Test } \\
\text { Date }\end{array}$ & $\begin{array}{l}\text { Start } \\
\text { Time }\end{array}$ & $\begin{array}{l}\text { Verify/ } \\
\text { Action }\end{array}$ & $\begin{array}{l}\text { Verify/ } \\
\text { Action }\end{array}$ & \begin{tabular}{|l} 
Status \\
S or U
\end{tabular} & $\begin{array}{l}\text { End } \\
\text { Time }\end{array}$ & Initials & Test Event Comments: \\
\hline 7.9.10 & 7/31/07 & $12: 49 \mathrm{pm}$ & 7.9 .11 & 7.9 .12 & $\mathbf{S}$ & $12: 55 \mathrm{pm}$ & DAK & $\begin{array}{l}\text { LB3:40kW, LB4:40kW, LB6:40kW, SS connected } \\
\text { at 12:54. Meter1:71kW, Meter2:36kW }\end{array}$ \\
\hline 7.9.13 & 7/31/07 & $12: 55 \mathrm{pm}$ & 7.9.14 & $\begin{array}{l}\text { 7.9.15, } \\
\text { 7.9.16 }\end{array}$ & $\mathbf{S}$ & 1:05 pm & DAK & $\begin{array}{l}\text { Triggers enabled Fault timer set to } 10 \text { sec. Fault } \\
\text { initiated at 12:57. SS, CB12, and CB51 opened. } \\
\text { Genset A1 and A2 shutdown with Skipp } 1 \text { faults. } \\
\text { CB12 only opens under manual command or under } \\
\text { high instantaneous fault current from the utility. } \\
\text { This was not the expected outcome of this test. }\end{array}$ \\
\hline 7.9.1R & 7/31/07 & $1: 35$ pm & 7.9.2R, & 7.9.4R, & $\mathbf{S}$ & $1: 42 \mathrm{pm}$ & DAK & Fault load verified, key armed, All breakers closed. \\
\hline
\end{tabular}

19 of 65

This document contains information confidential and proprietary to AEPSC. It shall not be reproduced in whole or in part or released to any third party without expressed written consent of AEPSC. 


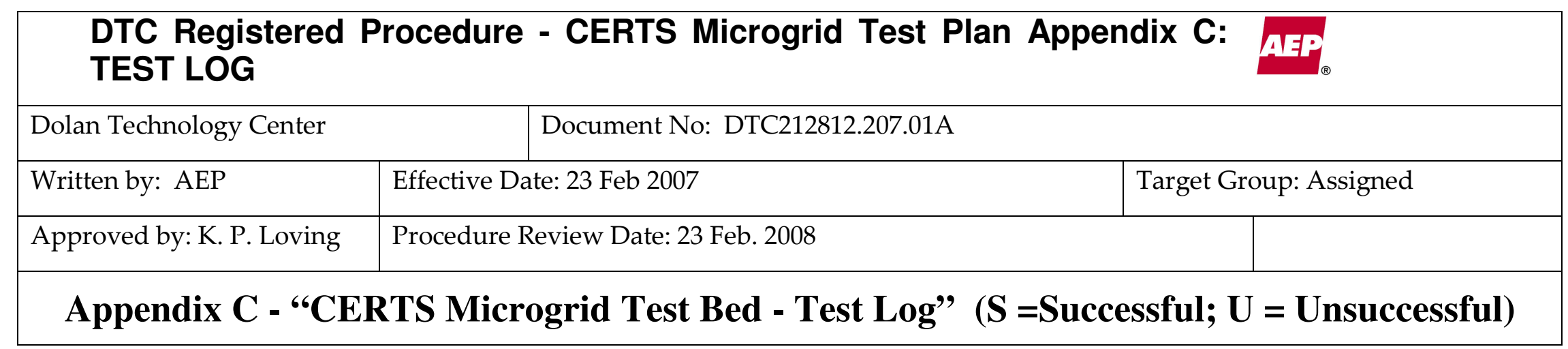

\begin{tabular}{|c|c|c|c|c|c|c|c|c|}
\hline & & & 7.9.3R & $\begin{array}{l}\text { 7.9.5R, } \\
\text { 7.9.6R }\end{array}$ & & & & \\
\hline 7.9.7R & 7/31/07 & $1: 42 \mathrm{pm}$ & 7.9.8R & 7.9.9R & $\mathbf{S}$ & $1: 47 \mathrm{pm}$ & DAK & $\begin{array}{l}\text { Gensets setup, Triggers disabled, Genset A1 } \\
\text { connected at 1:44. Genset A2 connected at 1:47 }\end{array}$ \\
\hline 7.9.10R & 7/31/07 & $1: 47 \mathrm{pm}$ & 7.9.11R & 7.9.12R & $\mathbf{S}$ & $1: 51 \mathrm{pm}$ & DAK & $\begin{array}{l}\text { LB3:40kW, LB4:40kW, LB6:40kW, SS connected } \\
\text { at 1:50. Meter1:69kW, Meter2:34kW }\end{array}$ \\
\hline 7.9.13R & 7/31/07 & $1: 51 \mathrm{pm}$ & 7.9.14R & $\begin{array}{l}\text { 7.9.15R, } \\
\text { 7.9.16R }\end{array}$ & $\mathbf{S}$ & 2:08 pm & DAK & $\begin{array}{l}\text { Triggers enabled. Fault timer set to } 10 \text { sec. Fault } \\
\text { initiated at 12:57. SS opened, CB41 opened. Genset } \\
\text { A2 shutdown. CB31 and CB51 remained closed, } \\
\text { Genset A1 remained online serving the remaining } \\
\text { load. The SS then reclosed. Also of note even those } \\
\text { the fault timer was set to } 10 \text { seconds the fault } \\
\text { remained on for approximately } 1.5 \text { mins. A check } \\
\text { needs to be made to determine of the fault timers } \\
\text { are working properly. }\end{array}$ \\
\hline 7.9 .17 & 7/31/07 & $2: 08 \mathrm{pm}$ & & & $\mathbf{S}$ & 2:12 pm & DAK & \\
\hline 7.10 .1 & 8/7/07 & $12: 10 \mathrm{pm}$ & $\begin{array}{l}7.10 .2 \\
7.10 .3\end{array}$ & $\begin{array}{l}\text { 7.10.4, } \\
\text { 7.10.5, } \\
\text { 7.10.6 }\end{array}$ & $\mathbf{S}$ & $12: 39 \mathrm{pm}$ & DAK & Fault load verified and connected, Fault key armed \\
\hline 7.10 .7 & $8 / 7 / 07$ & $12: 39 \mathrm{pm}$ & 7.10 .8 & 7.10 .9 & $\mathbf{S}$ & $12: 46 \mathrm{pm}$ & DAK & $\begin{array}{l}\text { SS continues to put itself in an OFF state without } \\
\text { being commaneded to do so. Gensets started at } \\
12: 45\end{array}$ \\
\hline 7.10 .10 & 8/7/07 & $12: 46 \mathrm{pm}$ & 7.10 .11 & 7.10 .12 & $\mathbf{S}$ & $12: 48 \mathrm{pm}$ & DAK & LB3:40kW, LB4:40kW, LB6:40kW SS closed at \\
\hline
\end{tabular}

20 of 65

This document contains information confidential and proprietary to AEPSC. It shall not be reproduced in whole or in part or released to any third party without expressed written consent of AEPSC. 


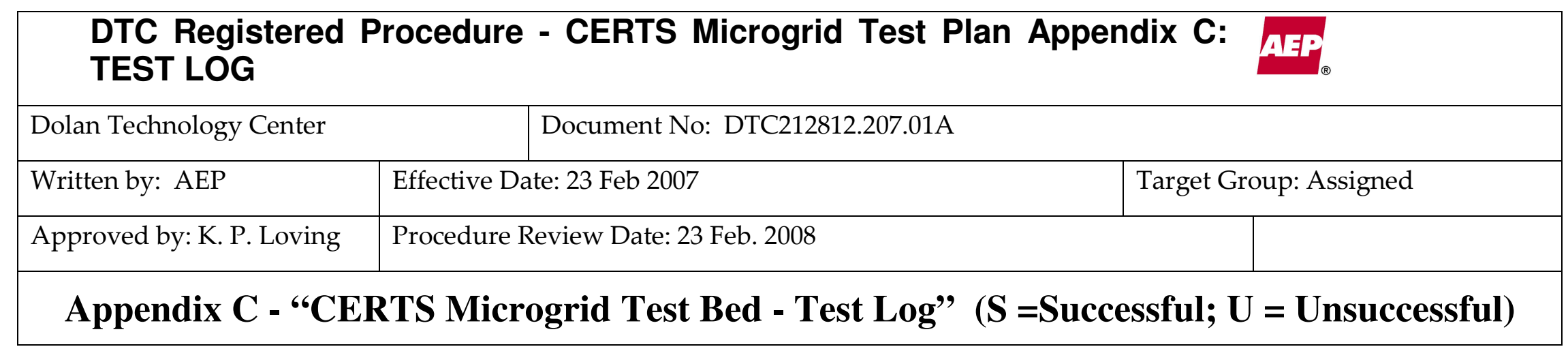

\begin{tabular}{|c|c|c|c|c|c|c|c|c|}
\hline & & & & & & & & 12:47. Meter1:71kW, Meter2:35kW \\
\hline 7.10 .13 & $8 / 7 / 07$ & 12:48 pm & $\begin{array}{l}7.10 .14, \\
7.10 .15\end{array}$ & 7.10 .16 & $\mathbf{S}$ & $12: 55 \mathrm{pm}$ & DAK & $\begin{array}{l}\text { Fault timer set to } 10 \text { sec. Fault initiated at 12:49. SS, } \\
\text { CB31, and CB51 opened, Gensets A1 and B1 } \\
\text { shutdown. }\end{array}$ \\
\hline 7.10 .17 & $8 / 7 / 07$ & $12: 55 \mathrm{pm}$ & & & $\mathbf{S}$ & 1:05 pm & DAK & \\
\hline 7.11.1 & $8 / 15 / 07$ & 11:32 am & $\begin{array}{l}7.11 .2 \\
7.11 .3\end{array}$ & $\begin{array}{l}\text { 7.11.4, } \\
\text { 7.11.5, } \\
\text { 7.11.6 }\end{array}$ & $\mathbf{S}$ & $12: 34 \mathrm{pm}$ & DAK & Fault load verified and connected, Fault key armed. \\
\hline 7.11.7 & $8 / \mathbf{1 5 / 0 7}$ & $12: 34 \mathrm{pm}$ & 7.11 .8 & 7.11 .9 & $\mathbf{S}$ & $12: 36 \mathrm{pm}$ & DAK & $\begin{array}{l}\text { Genset A1 was found non responsive to the EMS } \\
\text { system. An open fuse on the } 208 \mathrm{~V} \text { control power } \\
\text { feed was found and replaced. The control power } \\
\text { battery with the genset was found to be below } 6 \mathrm{~V} \\
\text { due to a prolonged power outage. After refusing } \\
\text { the battery was allowed to charge for } 2 \text { hours before } \\
\text { testing was attempted. LB3:40kW, LB6:40kW }\end{array}$ \\
\hline 7.11.10 & $8 / 15 / 07$ & $12: 36 \mathrm{pm}$ & 7.11 .11 & 7.11 .12 & & & DAK & $\begin{array}{l}\text { Genset faulted on a "DC Undervoltage fault". } \\
\text { Genset fault cleared and restarted. It was found } \\
\text { that due to the significant depletion of the control } \\
\text { battery the genset was not able to remain } \\
\text { operational. It was decided to allow the battery to } \\
\text { continue to charge overnight and to test at a later } \\
\text { date. }\end{array}$ \\
\hline 7.11.1R & $8 / 20 / 07$ & $12: 20 \mathrm{pm}$ & $\begin{array}{l}7.11 .2 \\
7.11 .3\end{array}$ & $\begin{array}{l}\text { 7.11.4, } \\
\text { 7.11.5, }\end{array}$ & $\mathbf{S}$ & $12: 26 \mathrm{pm}$ & DAK & $\begin{array}{l}\text { Genset } \mathrm{A1} \text { was test run successfully prior to } \\
\text { beginning this testing. }\end{array}$ \\
\hline
\end{tabular}

21 of 65

This document contains information confidential and proprietary to AEPSC. It shall not be reproduced in whole or in part or released to any third party without expressed written consent of AEPSC. 


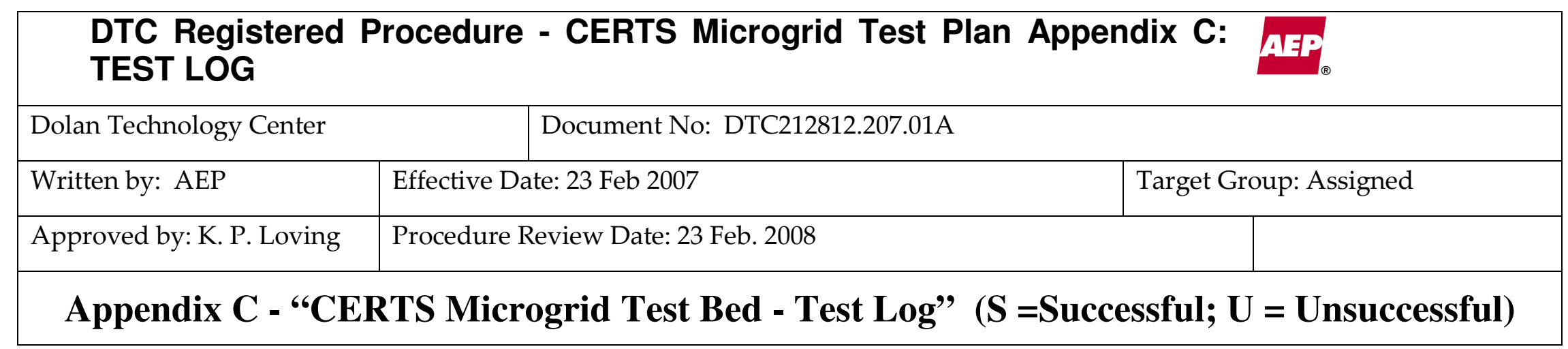

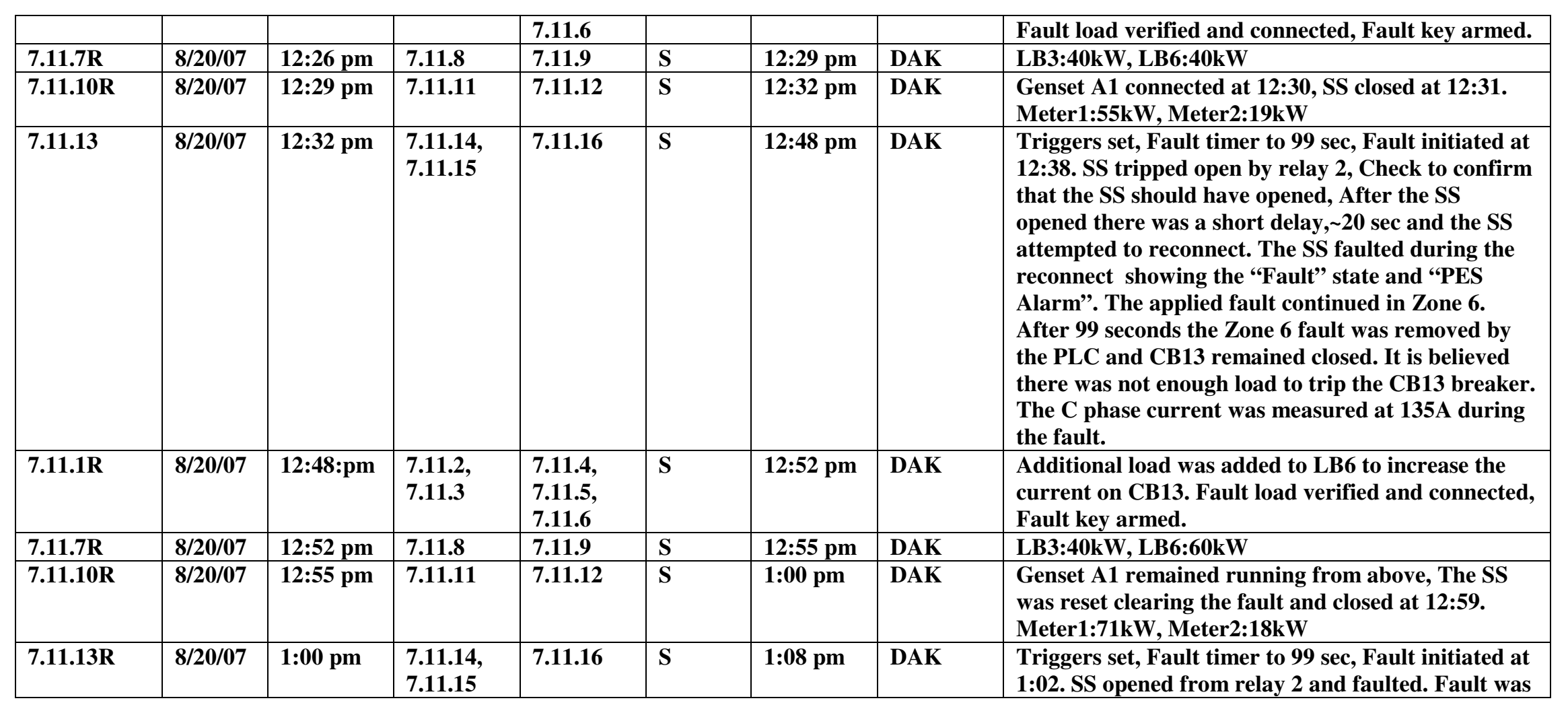

22 of 65

This document contains information confidential and proprietary to AEPSC. It shall not be reproduced in whole or in part or released to any third party without expressed written consent of AEPSC. 


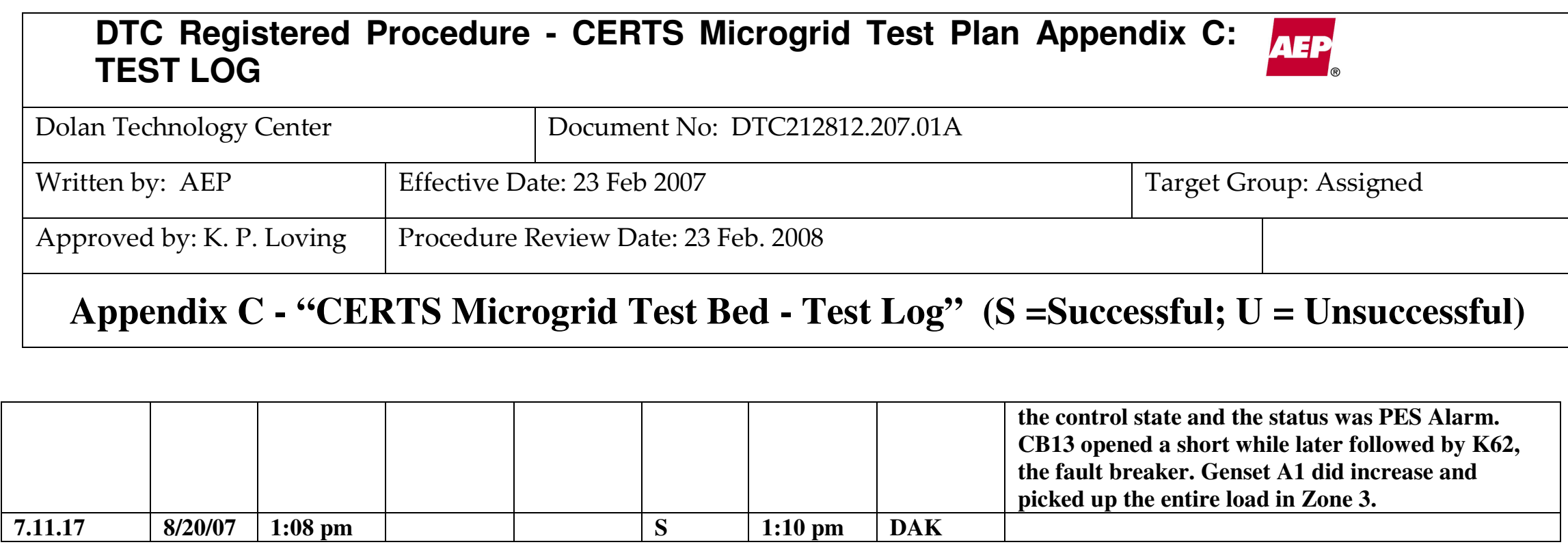

\begin{tabular}{|l|l|l|l|l|l|l|l|l|}
\hline $\begin{array}{l}\text { Start Test } \\
\text { Sequence } \\
\text { No. }\end{array}$ & $\begin{array}{l}\text { Test } \\
\text { Date }\end{array}$ & $\begin{array}{l}\text { Start } \\
\text { Time }\end{array}$ & $\begin{array}{l}\text { Verify/ } \\
\text { Action }\end{array}$ & $\begin{array}{l}\text { Verify/ } \\
\text { Action }\end{array}$ & $\begin{array}{l}\text { Status } \\
\text { S or U }\end{array}$ & $\begin{array}{l}\text { End } \\
\text { Time }\end{array}$ & Initials & Test Event Comments: \\
\hline 7.12 .1 & $\mathbf{8 / 2 0 / 0 7}$ & $\mathbf{1 : 1 0} \mathrm{pm}$ & $\begin{array}{l}\mathbf{7 . 1 2 . 2 ,} \\
\mathbf{7 . 1 2 . 3}\end{array}$ & $\mathbf{7 . 1 2 . 4}$ & $\mathrm{S}$ & $\mathbf{1 : 2 8} \mathrm{pm}$ & DAK & $\begin{array}{l}\text { Fault load verified and Fault key armed, Gensets } \\
\text { A1 and B1 started first prior to load to make sure } \\
\text { generation was greater than load. Genset A1 still } \\
\text { running from above, Genset B1 connected at 1:28. } \\
\text { LB3:40kW, LB5:40kW, LB6:60kW }\end{array}$ \\
\hline 7.12 .5 & $\mathbf{8 / 2 0 / 0 7}$ & $\mathbf{1 : 2 8} \mathrm{pm}$ & $\mathbf{7 . 1 2 . 6}$ & $\mathbf{7 . 1 2 . 7}$ & $\mathrm{S}$ & $\mathbf{1 : 4 5} \mathrm{pm}$ & DAK & $\begin{array}{l}\text { SS placed itself in the OFF state position. Reset and } \\
\text { Start commands resulted in TEST state followed by } \\
\text { OFF state. While troubleshooting the SS both } \\
\text { Genset A1 and B1 shut down. A1 showed a No Field } \\
\text { Signal, which in the past has meant overload, and } \\
\text { Genset B1 showed an External Trip. As the Meter } \\
\text { triggers were disabled only the relay files may be of }\end{array}$ \\
\hline
\end{tabular}

23 of 65

This document contains information confidential and proprietary to AEPSC. It shall not be reproduced in whole or in part or released to any third party without expressed written consent of AEPSC. 


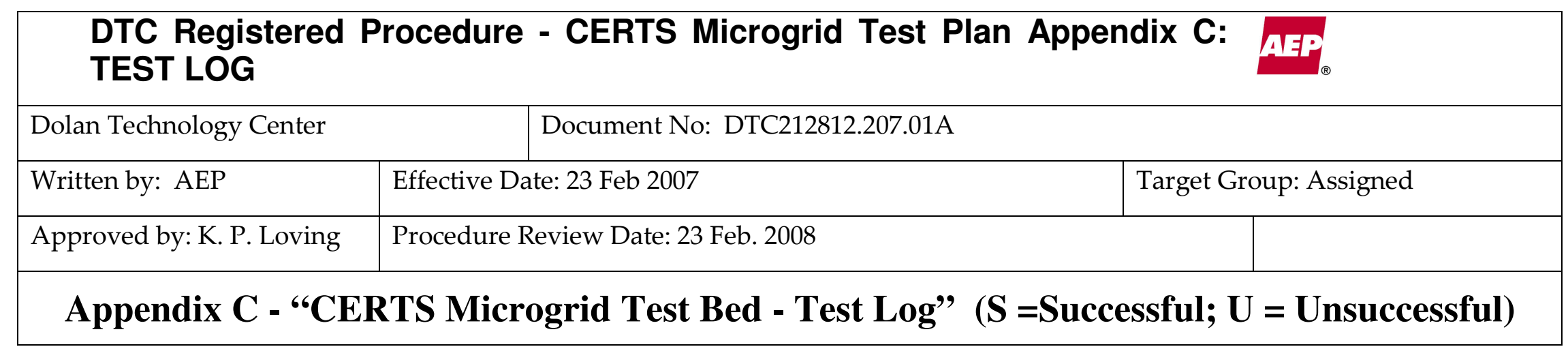

\begin{tabular}{|c|c|c|c|c|c|c|c|c|}
\hline & & & & & & & & use. \\
\hline 7.12.1R & $8 / 20 / 07$ & 1:45 pm & $\begin{array}{l}7.12 .2 \\
7.12 .3\end{array}$ & 7.12 .4 & $\mathbf{S}$ & 2:00 pm & DAK & $\begin{array}{l}\text { Fault load verified and Fault key armed, Gensets } \\
\text { A1 and B1 started first prior to load to make sure } \\
\text { generation was greater than load. Genset A1 still } \\
\text { running from above, Genset B1 connected at 1:28. } \\
\text { LB3:40kW, LB5:40kW, LB6:60kW }\end{array}$ \\
\hline \multirow[t]{2}{*}{$7.12 .5 \mathrm{R}$} & 8/20/07 & 2:00 pm & 7.12 .6 & 7.12 .7 & $\mathbf{S}$ & $2: 45 \mathrm{pm}$ & DAK & $\begin{array}{l}\text { SS placed itself in the OFF state position. Reset and } \\
\text { Start commands resulted in TEST state followed by } \\
\text { OFF state. While troubleshooting the SS both } \\
\text { Genset A1 and B1 shut down. A1 showed a No Field } \\
\text { Signal, which in the past has meant overload, and } \\
\text { Genset B1 showed an External Trip. As the Meter } \\
\text { triggers were disabled only the relay files may be of } \\
\text { use. We were not able to get the SS to function } \\
\text { properly and are waiting for further assistance } \\
\text { from the team. }\end{array}$ \\
\hline & & & & & & & & $\begin{array}{l}\text { The source of the SS problem was found to be an } \\
24 v \text { input module between the NPS DSP and the } \\
\text { SandC SS. The enable input component was } \\
\text { replaced within the SandC SS and the SS was } \\
\text { returned to normal operation. }\end{array}$ \\
\hline $7.12 .1 \mathrm{R} 2$ & 9/05/07 & $12: 15 \mathrm{pm}$ & $\begin{array}{l}7.12 .2 \\
7.12 .3\end{array}$ & 7.12 .4 & $\mathbf{S}$ & $1: 35 \mathrm{pm}$ & DAK & $\begin{array}{l}\text { Fault load verified and Fault key armed. The SS } \\
\text { was returned to normal service however Genset A1 } \\
\text { would not operate correctly. At first the generator } \\
\text { would attempt to start and then give a }\end{array}$ \\
\hline
\end{tabular}

24 of 65

This document contains information confidential and proprietary to AEPSC. It shall not be reproduced in whole or in part or released to any third party without expressed written consent of AEPSC. 


\begin{tabular}{|l|l|l|}
\hline \multicolumn{2}{|c|}{$\begin{array}{l}\text { DTC Registered Procedure - CERTS Microgrid Test Plan Appendix C: } \\
\text { TEST LOG }\end{array}$ Dolan Technology Center } & Document No: DTC212812.207.01A \\
\hline Written by: AEP & Effective Date: 23 Feb 2007 & \multicolumn{1}{|c|}{} \\
\hline Approved by: K. P. Loving & Procedure Review Date: 23 Feb. 2008 & \\
\hline \multicolumn{2}{|l|}{ Appendix C - “CERTS Microgrid Test Bed - Test Log” (S =Successful; U = Unsuccessful) } \\
\hline
\end{tabular}

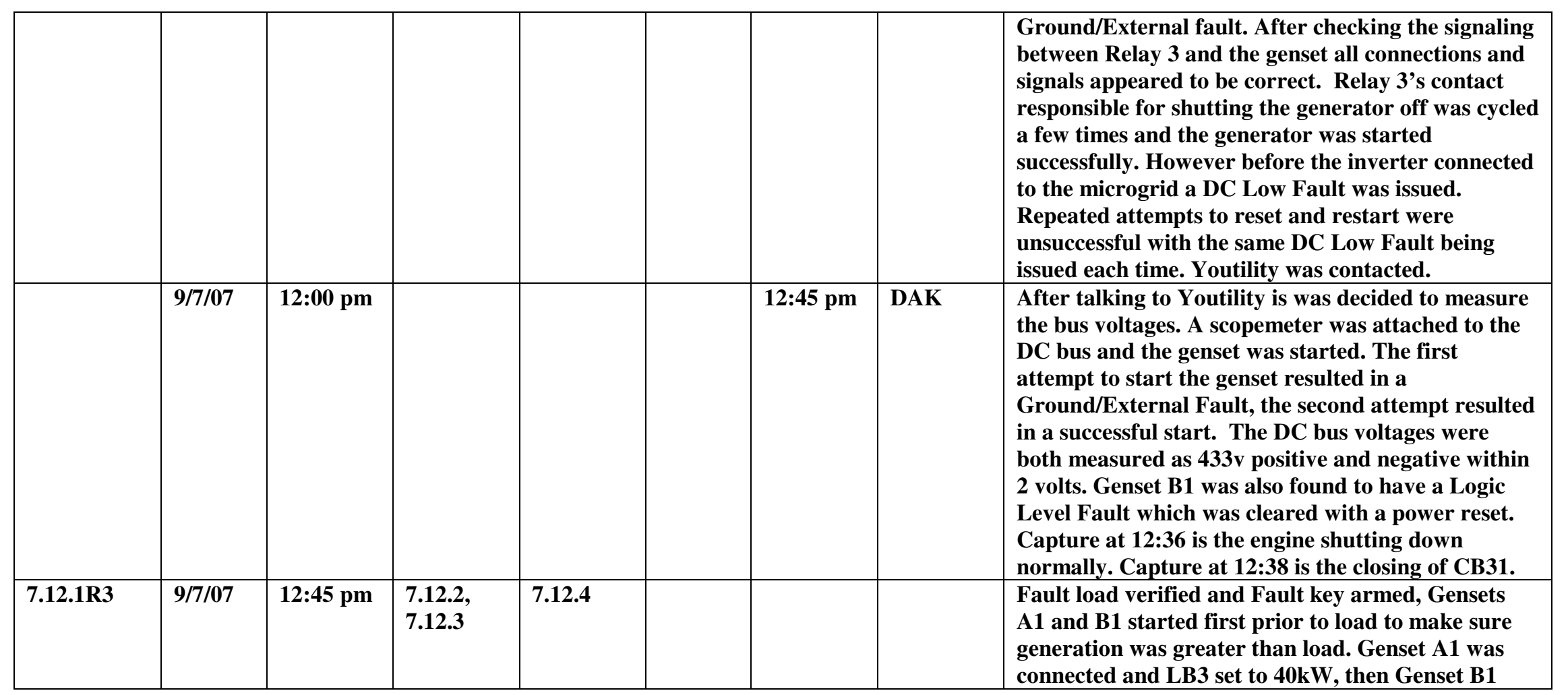

25 of 65

This document contains information confidential and proprietary to AEPSC. It shall not be reproduced in whole or in part or released to any third party without expressed written consent of AEPSC. 


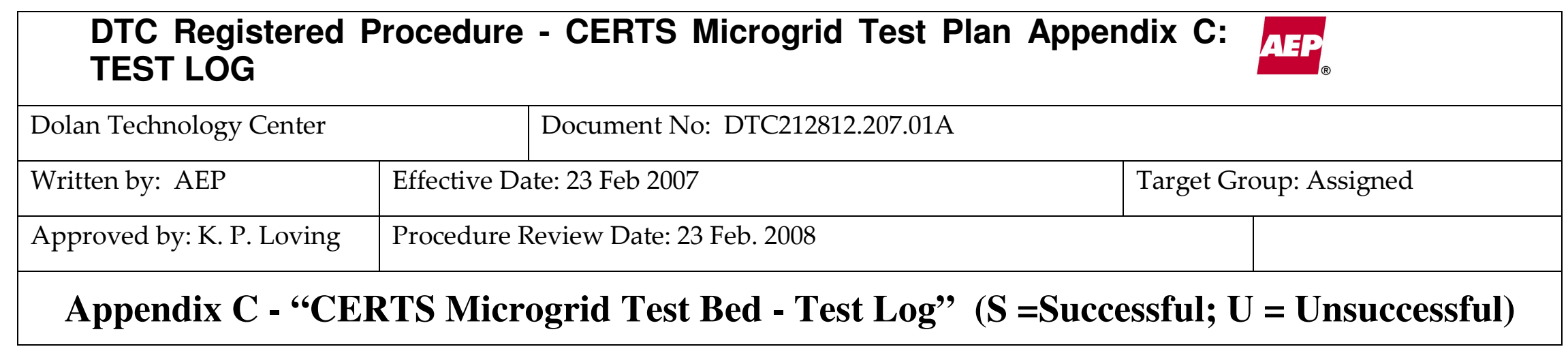

\begin{tabular}{|c|c|c|c|c|c|c|c|c|}
\hline & & & & & & & & $\begin{array}{l}\text { was connected. Next } 40 \mathrm{~kW} \text { of load was applied to } \\
\text { LB5 which caused the shutdown of both gensets. } \\
\text { Genset A1 had a CAN Bus Comms fault and Genset } \\
\text { B1 had a Low Oil Pressure alarm. The gensets were } \\
\text { restarted. Loads were removed and genset A1 was } \\
\text { restarted. Genset A1 then faulted on SA Operation } \\
\text { fault. Genset A1 was reset and restarted. Genset A1 } \\
\text { then faulted again on SA Operation fault. Genset } \\
\text { A1 was reset and restarted. }\end{array}$ \\
\hline 7.12.5R3 & 8/20/07 & $1: 28 \mathrm{pm}$ & 7.12.6 & 7.12.7 & & & & $\begin{array}{l}\text { SS placed itself in the OFF state position. Reset and } \\
\text { Start commands resulted in TEST state followed by } \\
\text { OFF state. While troubleshooting the SS both } \\
\text { Genset A1 and B1 shut down. A1 showed a No Field } \\
\text { Signal, which in the past has meant overload, and } \\
\text { Genset B1 showed an External Trip. As the Meter } \\
\text { triggers were disabled only the relay files may be of } \\
\text { use. }\end{array}$ \\
\hline $7.12 .1 \mathrm{R} 4$ & $10 / 3 / 07$ & $10: 26$ am & $\begin{array}{l}7.12 .2 \mathrm{R} 4 \\
7.12 .3 \mathrm{R} 4\end{array}$ & 7.12.4R4 & $\mathbf{S}$ & $10: 35$ am & DAK & $\begin{array}{l}\text { Fault load verified and Fault key armed, Gensets } \\
\text { A1 and B1 started first prior to load to make sure } \\
\text { generation was greater than load. LB3:40kW, } \\
\text { LB5:40kW, LB6:40kW }\end{array}$ \\
\hline 7.12.5R4 & 10/3/07 & $10: 35$ am & 7.12.6R4 & 7.12.7R4 & $\mathbf{S}$ & 10:42am & DAK & $\begin{array}{l}\text { SS connected at 10:36. Meter1:70kW, Meter2:34kW } \\
\text { Fault triggered at 10:39. The SS opened issuing a } \\
\text { PES alarm fault. The fault load cleared but CB13 } \\
\text { remained closed. The SS remained open and }\end{array}$ \\
\hline
\end{tabular}

26 of 65

This document contains information confidential and proprietary to AEPSC. It shall not be reproduced in whole or in part or released to any third party without expressed written consent of AEPSC. 


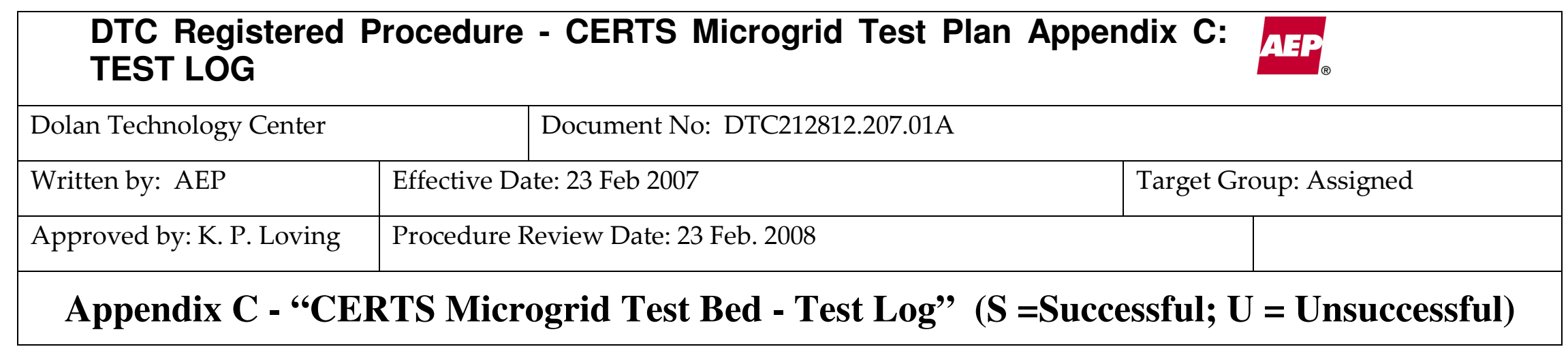

\begin{tabular}{|c|c|c|c|c|c|c|c|c|}
\hline & & & & & & & & $\begin{array}{l}\text { faulted, the gensets remained online, increased from } \\
20 \mathrm{~kW} \text { to } 36 \mathrm{~kW} \text { to serve LB3 and LB5 }\end{array}$ \\
\hline 7.12.1R5 & 10/3/07 & 10:42 am & $\begin{array}{l}\text { 7.12.2R5, } \\
\text { 7.12.3R5 }\end{array}$ & 7.12.4R5 & $\mathbf{S}$ & $10: 45$ am & DAK & $\begin{array}{l}\text { Fault load verified and Fault key armed, Gensets } \\
\text { A1 and B1 started first prior to load to make sure } \\
\text { generation was greater than load. LB3:40kW, } \\
\text { LB5:40kW, LB6:60kW }\end{array}$ \\
\hline 7.12.5R5 & 10/3/07 & 10:45 am & 7.12.6R5 & 7.12.7R5 & $\mathbf{S}$ & $10: 58 \mathrm{am}$ & DAK & $\begin{array}{l}\text { SS fault cleared and reconnected at 10:46. LB } 6 \\
\text { increased to } 60 \mathrm{~kW} \text { Meter1:88kW, Meter2:34kW } \\
\text { Fault triggered at 10:56. The SS opened issuing a } \\
\text { PES alarm fault. CB12 opened and the fault was } \\
\text { cleared. }\end{array}$ \\
\hline 7.12 .8 & 10/3/07 & 10:58 am & $\begin{array}{l}7.12 .9 \\
7.12 .10\end{array}$ & $\begin{array}{l}\text { 7.12.11, } \\
\text { 7.12.12 } \\
7.12 .13\end{array}$ & $\mathbf{S}$ & 11:01 am & DAK & $\begin{array}{l}\text { Prior to intentionally shutting down the gensets } \\
\text { both shutdown with a No field fault on A1 and a } \\
\text { Boost fault on B1. Waveforms were captured at } \\
\text { 10:59 }\end{array}$ \\
\hline 7.13.1 & 10/3/07 & $1: 26 \mathrm{pm}$ & $\begin{array}{l}\text { 7.13.2, } \\
\mathbf{7 . 1 3 . 3}\end{array}$ & $\begin{array}{l}\text { 7.13.4, } \\
\text { 7.13.5, } \\
\text { 7.13.6 }\end{array}$ & $\mathbf{S}$ & $1: 37 \mathrm{pm}$ & DAK & Fault load connected and verified. Fault key armed. \\
\hline 7.13.7 & 10/3/07 & $1: 37 \mathrm{pm}$ & 7.13.8 & $\begin{array}{l}\text { 7.13.9, } \\
\text { 7.13.10 }\end{array}$ & $\mathbf{S}$ & $1: 43 \mathrm{pm}$ & DAK & $\begin{array}{l}\text { Genset A1 and A2 setup and connected. LB3:40kW, } \\
\text { LB4:40kW, LB6:40kW }\end{array}$ \\
\hline 7.13.11 & 10/3/07 & $1: 43 \mathrm{pm}$ & 7.13.12 & & $\mathbf{S}$ & $1: 47 \mathrm{pm}$ & DAK & SS closed. Meter1:63kW, Meter2:27kW \\
\hline 7.13.13 & 10/3/07 & $1: 48 \mathrm{pm}$ & $\begin{array}{l}\text { 7.13.14, } \\
\text { 7.13.15 }\end{array}$ & 7.13.16 & $\mathbf{S}$ & $1: 55 \mathrm{pm}$ & DAK & $\begin{array}{l}\text { Fault initiated at 1:48. SS remained closed, CB41 } \\
\text { and CB31 opened shutting down both gensets. }\end{array}$ \\
\hline 7.13.17 & 10/3/07 & $1: 55 \mathrm{pm}$ & & & $\mathbf{S}$ & $1: 57 \mathrm{pm}$ & DAK & \\
\hline
\end{tabular}

27 of 65

This document contains information confidential and proprietary to AEPSC. It shall not be reproduced in whole or in part or released to any third party without expressed written consent of AEPSC. 


\begin{tabular}{|l|l|l|}
\hline \multicolumn{2}{|c|}{$\begin{array}{l}\text { DTC Registered Procedure - CERTS Microgrid Test Plan Appendix C: } \\
\text { TEST LOG }\end{array}$ Dolan Technology Center } & Document No: DTC212812.207.01A \\
\hline Written by: AEP & Effective Date: 23 Feb 2007 & \multicolumn{1}{|c|}{} \\
\hline Approved by: K. P. Loving & Procedure Review Date: 23 Feb. 2008 & \\
\hline \multicolumn{2}{|l|}{ Appendix C - “CERTS Microgrid Test Bed - Test Log” (S =Successful; U = Unsuccessful) } \\
\hline
\end{tabular}

\begin{tabular}{|c|c|c|c|c|c|c|c|c|}
\hline 7.13.1R1 & $10 / 3 / 07$ & $2: 00 \mathrm{pm}$ & $\begin{array}{l}\text { 7.13.2R1, } \\
\text { 7.13.3R1 }\end{array}$ & $\begin{array}{l}\text { 7.13.4, } \\
\text { 7.13.5, } \\
\text { 7.13.6 }\end{array}$ & $\mathbf{S}$ & $2: 06 \mathrm{pm}$ & DAK & Fault load connected and verified. Fault key armed. \\
\hline 7.13.7R1 & $10 / 3 / 07$ & 2:06 pm & 7.13.8R1 & $\begin{array}{l}\text { 7.13.9 R1, } \\
\text { 7.13.10 } \\
\text { R1 }\end{array}$ & $\mathbf{S}$ & 2:09 pm & DAK & $\begin{array}{l}\text { Genset A1 and A2 setup and connected. LB3:40kW, } \\
\text { LB4:40kW, LB5:20kW, LB6:40kW }\end{array}$ \\
\hline 7.13.11R1 & 10/3/07 & 2:09 pm & $\begin{array}{l}\text { 7.13.12 } \\
\text { R1 }\end{array}$ & & $\mathbf{S}$ & $2: 11 \mathrm{pm}$ & DAK & SS closed. Meter1:79kW, Meter2:43kW \\
\hline 7.13.13R1 & 10/3/07 & $1: 48 \mathrm{pm}$ & $\begin{array}{l}\text { 7.13.14 } \\
\text { R1, } \\
\text { 7.13.15 } \\
\text { R1 }\end{array}$ & $\begin{array}{l}\text { 7.13.16 } \\
\text { R1 }\end{array}$ & & & & $\begin{array}{l}\text { Fault initiated at 2:12. SS remained closed, CB41 } \\
\text { and CB31 opened shutting down both gensets. }\end{array}$ \\
\hline 7.13.17R1 & 10/3/07 & 2:11 pm & & & $\mathbf{S}$ & $2: 12 \mathrm{pm}$ & DAK & \\
\hline 7.14 .1 & 10/3/07 & $2: 25 \mathrm{pm}$ & $\begin{array}{l}\text { 7.14.2, } \\
\text { 7.14.3 }\end{array}$ & $\begin{array}{l}\text { 7.14.4, } \\
\text { 7.14.5, } \\
\text { 7.14.6 }\end{array}$ & $\mathbf{S}$ & $2: 28 \mathrm{pm}$ & DAK & Fault load connected and verified. Fault key armed. \\
\hline 7.14.7 & 10/3/07 & 2:28pm & 7.14 .8 & $\begin{array}{l}\text { 7.14.9, } \\
\text { 7.14.10 }\end{array}$ & $\mathbf{S}$ & 4:01 pm & DAK & $\begin{array}{l}\text { Gensets A1 and A2 connected at 4:00. LB3:40kW, } \\
\text { LB4:40kW, LB6:40kW }\end{array}$ \\
\hline 7.14.11 & 10/3/07 & 4:01 pm & 7.14.12 & & $\mathbf{S}$ & $4: 08 \mathrm{pm}$ & DAK & SS closed, Meter1:65kW, Meter2:30kW \\
\hline 7.14.13 & 10/3/07 & 4:08 pm & $\begin{array}{l}\text { 7.14.14, } \\
\text { 7.14.15 }\end{array}$ & 7.14.16 & $\mathbf{S}$ & $4: 15 \mathrm{pm}$ & DAK & $\begin{array}{l}\text { Fault initiated at 4:09. SS remained closed. CB31 } \\
\text { and CB41 opened. Both gensets shutdown. }\end{array}$ \\
\hline 7.14.17 & 10/3/07 & $4: 15 \mathrm{pm}$ & & & $\mathbf{S}$ & $4: 18 \mathrm{pm}$ & DAK & \\
\hline
\end{tabular}

This document contains information confidential and proprietary to AEPSC. It shall not be reproduced in whole or in part or released to any third party without expressed written consent of AEPSC. 


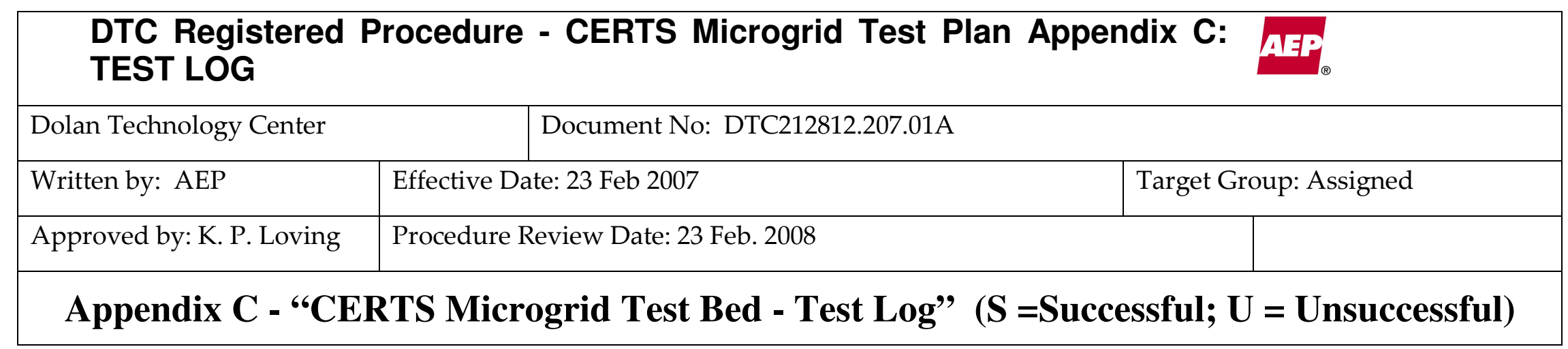

\begin{tabular}{|c|c|c|c|c|c|c|c|c|}
\hline $\begin{array}{l}\text { Start Test } \\
\text { Sequence } \\
\text { No. }\end{array}$ & $\begin{array}{l}\text { Test } \\
\text { Date }\end{array}$ & $\begin{array}{l}\text { Start } \\
\text { Time }\end{array}$ & $\begin{array}{l}\text { Verify/ } \\
\text { Action }\end{array}$ & $\begin{array}{l}\text { Verify/ } \\
\text { Action }\end{array}$ & $\begin{array}{l}\text { Status } \\
\text { S or U }\end{array}$ & $\begin{array}{l}\text { End } \\
\text { Time }\end{array}$ & Initials & Test Event Comments: \\
\hline 7.15.1 & 10/3/07 & $4: 18 \mathrm{pm}$ & $\begin{array}{l}7.15 .2, \\
7.15 .3\end{array}$ & $\begin{array}{l}\text { 7.15.4, } \\
\text { 7.15.5, } \\
\text { 7.15.6 }\end{array}$ & $\mathbf{S}$ & $4: 20 \mathrm{pm}$ & DAK & Fault load connected and verified. Fault key armed. \\
\hline 7.15 .7 & 10/3/07 & $4: 20 \mathrm{pm}$ & 7.15 .8 & $\begin{array}{l}7.15 .9, \\
7.15 .10\end{array}$ & $\mathbf{S}$ & $4: 25 \mathrm{pm}$ & DAK & $\begin{array}{l}\text { Gensets A1 and B1 connected at 4:24. LB3:40kW, } \\
\text { LB5:40kW, LB6:40kW }\end{array}$ \\
\hline 7.15.11 & 10/3/07 & $4: 25 \mathrm{pm}$ & 7.15.12 & & $\mathbf{S}$ & $4: 26 \mathrm{pm}$ & DAK & SS closed, Meter1:64kW, Meter2:28kW \\
\hline 7.15.13 & 10/3/07 & $4: 26 \mathrm{pm}$ & $\begin{array}{l}7.15 .14, \\
7.15 .15\end{array}$ & 7.15 .16 & $\mathbf{S}$ & $4: 28 \mathrm{pm}$ & DAK & $\begin{array}{l}\text { Fault initiated at 4:28. SS opened. Gensets picked } \\
\text { up load. Fault removed after } 10 \text { seconds. SS } \\
\text { reconnected. The setup returned to the pre-fault } \\
\text { conditions }\end{array}$ \\
\hline 7.15.1R1 & 10/3/07 & $4: 28 \mathrm{pm}$ & $\begin{array}{l}\text { 7.15.2R1, } \\
\text { 7.15.3R1 }\end{array}$ & $\begin{array}{l}\text { 7.15.4R1, } \\
\text { 7.15.5R1, } \\
\text { 7.15.6R1 }\end{array}$ & $\mathbf{S}$ & $4: 30 \mathrm{pm}$ & DAK & $\begin{array}{l}\text { Retested to confirm results. Fault load connected } \\
\text { and verified. Fault key armed. }\end{array}$ \\
\hline 7.15.7R1 & 10/3/07 & $4: 30 \mathrm{pm}$ & 7.15 .8 & $\begin{array}{l}\text { 7.15.9R1, } \\
\text { 7.15.10R1 }\end{array}$ & $\mathbf{S}$ & $4: 32 \mathrm{pm}$ & DAK & $\begin{array}{l}\text { Gensets A1 and B1 still connected. LB3:40kW, } \\
\text { LB5:40kW, LB6:40kW }\end{array}$ \\
\hline 7.15.11R1 & 10/3/07 & $4: 32 \mathrm{pm}$ & 7.15.12R1 & & $\mathbf{S}$ & $4: 34$ pm & DAK & SS still closed, Meter1:64kW, Meter2:28kW \\
\hline
\end{tabular}

29 of 65

This document contains information confidential and proprietary to AEPSC. It shall not be reproduced in whole or in part or released to any third party without expressed written consent of AEPSC. 


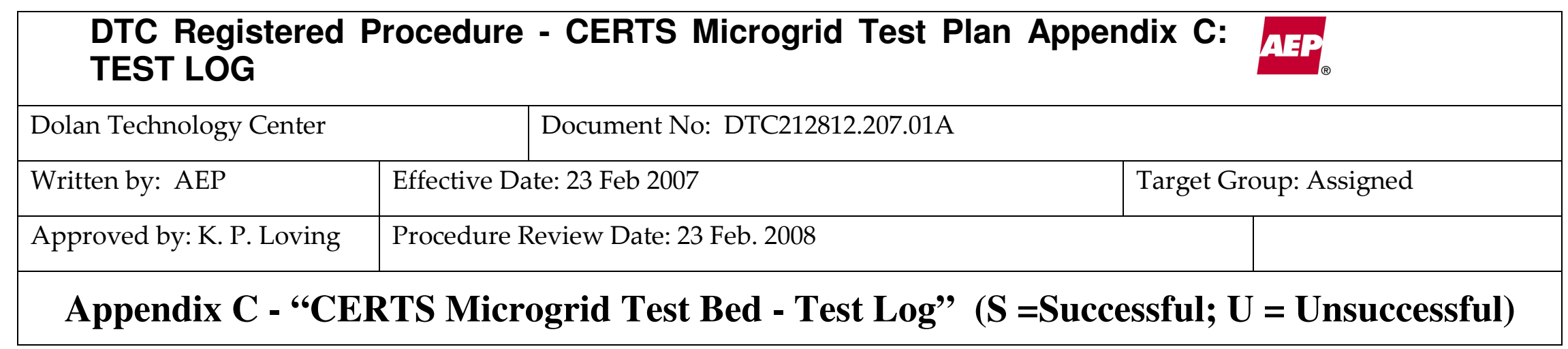

\begin{tabular}{|c|c|c|c|c|c|c|c|c|}
\hline 7.15.13 & 10/3/07 & $4: 33 p m$ & $\begin{array}{l}\text { 7.15.14R, } \\
\text { 7.15.15R1 }\end{array}$ & 7.15.16R1 & $\mathbf{S}$ & $4: 42 \mathrm{pm}$ & DAK & $\begin{array}{l}\text { Fault initiated at 4:41. SS opened. Gensets picked } \\
\text { up load. Fault removed after } 10 \text { seconds. SS } \\
\text { reconnected. }\end{array}$ \\
\hline 7.15.17 & 10/3/07 & $4: 42 \mathrm{pm}$ & & & $\mathbf{S}$ & $4: 45 \mathrm{pm}$ & DAK & \\
\hline 7.16 .1 & 10/3/07 & $4: 45 \mathrm{pm}$ & $\begin{array}{l}\text { 7.16.2, } \\
\text { 7.16.3 }\end{array}$ & $\begin{array}{l}\text { 7.16.4, } \\
\text { 7.16.5, } \\
\text { 7.16.6 }\end{array}$ & $\mathbf{S}$ & $4: 47 \mathrm{pm}$ & DAK & Fault load connected and verified. Fault key armed. \\
\hline 7.16.7 & 10/3/07 & $4: 47 \mathrm{pm}$ & 7.16 .8 & $\begin{array}{l}7.16 .9, \\
7.16 .10\end{array}$ & $\mathbf{S}$ & $4: 49 \mathrm{pm}$ & DAK & $\begin{array}{l}\text { Gensets A1 and B1 still connected. LB3:40kW, } \\
\text { LB5:40kW, LB6:40kW }\end{array}$ \\
\hline 7.16.11 & 10/3/07 & $4: 49 \mathrm{pm}$ & 7.16.12 & & $\mathbf{S}$ & 4:51 pm & DAK & SS still closed, Meter1:65kW, Meter2:28kW \\
\hline 7.16 .13 & 10/3/07 & $4: 51 \mathrm{pm}$ & $\begin{array}{l}7.16 .14, \\
7.16,15\end{array}$ & 7.16 .16 & & $4: 55 \mathrm{pm}$ & & $\begin{array}{l}\text { Fault initiated at 4:54. SS opened. CB51 opened and } \\
\text { shutdown genset B1. CB41, CB31 and SS remained } \\
\text { closed and genset A1 continued to serve load. }\end{array}$ \\
\hline 7.16.17 & 10/3/07 & $4: 55 \mathrm{pm}$ & & & $\mathbf{S}$ & $4: 56 \mathrm{pm}$ & DAK & \\
\hline 7.6.1R & 1/23/08 & 2:02 pm & 7.6.2R & 7.6.3R & $\mathbf{S}$ & $2: 10$ am & DAK & Gensets A1 and A2 setup. Fault key armed. \\
\hline 7.6.4R & 1/23/08 & $2: 10 \mathrm{pm}$ & 7.6.5R & $\begin{array}{l}\text { 7.6.6R, } \\
\text { 7.6.7R }\end{array}$ & $\mathbf{S}$ & 2:15 pm & DAK & $\begin{array}{l}\text { Triggers disabled, Genset A1 and A2 connected. } \\
\text { LB3:40kW, LB4:40kW, LB6:40kW. SS connected. } \\
\text { Meter1:66kW, Meter2:31kW. Triggers armed }\end{array}$ \\
\hline 7.6.8R & 1/23/08 & 2:15 pm & $\begin{array}{l}\text { 7.6.9R, } \\
\text { 7.6.10R }\end{array}$ & 7.6.11R & $\mathbf{S}$ & $2: 25 \mathrm{pm}$ & DAK & $\begin{array}{l}\text { Fault timer set to } 10 \text { sec Fault initiated at 2:16. SS } \\
\text { opened, CB31 opened, CB41 Opened, and CB51 } \\
\text { opened. Gensets A1 shutdown on External Trip and } \\
\text { A2 shutdown on Low Oil Pressure (Stall). } \\
\text { Meter1:37kW, Meter2:0kW }\end{array}$ \\
\hline
\end{tabular}

30 of 65

This document contains information confidential and proprietary to AEPSC. It shall not be reproduced in whole or in part or released to any third party without expressed written consent of AEPSC. 


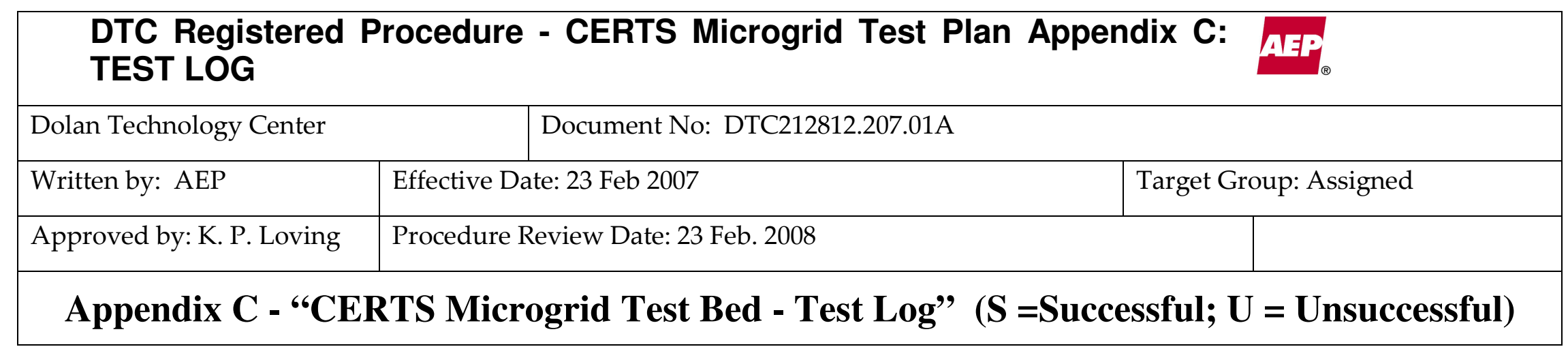

\begin{tabular}{|c|c|c|c|c|c|c|c|c|}
\hline 7.6.12 & 1/23/08 & $2: 25$ pm & & & $\mathbf{S}$ & 2:35 pm & DAK & \\
\hline 7.9.1R & $1 / 30 / 08$ & $2: 40 \mathrm{pm}$ & $\begin{array}{l}\text { 7.9.2R, } \\
\text { 7.9.3R }\end{array}$ & $\begin{array}{l}\text { 7.9.4R, } \\
\text { 7.9.5R, } \\
\text { 7.9.6R }\end{array}$ & $\mathbf{S}$ & $2: 45 \mathrm{pm}$ & DAK & Fault load verified, key armed, All breakers closed. \\
\hline 7.9.7R & 1/30/08 & $2: 45 \mathrm{pm}$ & 7.9.8R & 7.9.9R & $\mathbf{S}$ & $2: 50 \mathrm{pm}$ & DAK & $\begin{array}{l}\text { Gensets setup, Triggers disabled, Genset } \mathrm{A1} \text { and } \mathrm{A2} \\
\text { connected }\end{array}$ \\
\hline 7.9.10R & 1/30/08 & $2: 50 \mathrm{pm}$ & 7.9.11R & 7.9.12R & $\mathbf{S}$ & $3: 15$ pm & DAK & $\begin{array}{l}\text { LB3:40kW, LB4:40kW, LB6:40kW, SS connected } \\
\text { at 1:41. Meter1:73kW, Meter2:37kW }\end{array}$ \\
\hline \multirow[t]{2}{*}{ 7.9.13R } & 1/30/08 & $3: 15$ pm & 7.9.14R & $\begin{array}{l}\text { 7.9.15R, } \\
\text { 7.9.16R }\end{array}$ & $\mathbf{S}$ & 4:00 pm & DAK & $\begin{array}{l}\text { Triggers enabled. Fault timer set to } 10 \text { sec. Fault } \\
\text { initiated but breaker } \mathrm{K} 42 \text { failed to close in the fault } \\
\text { load. The breaker was manually exercised a few } \\
\text { times. During this Genset A2 suddenly shutdown } \\
\text { with a Skipp3 fault. The fault was cleared and the } \\
\text { genset restarted. The Genset shutdown again with a } \\
\text { Skipp3 fault and further investigation was } \\
\text { performed. The output filter cap was found to be } \\
\text { damaged and Youtility was contacted for } \\
\text { replacement. }\end{array}$ \\
\hline & & & & & & & & $\begin{array}{l}\text { The filter capacitors from A2 were replaced with } \\
\text { those from B1. The Genset was tested and returned } \\
\text { to service. Replacement capacitor filters were order } \\
\text { for B1. }\end{array}$ \\
\hline 7.9.1R & 1/31/08 & $1: 35$ pm & 7.9.2R, & 7.9.4R, & $\mathbf{S}$ & $1: 38 \mathrm{pm}$ & DAK & Fault load verified, key armed, All breakers closed. \\
\hline
\end{tabular}

31 of 65

This document contains information confidential and proprietary to AEPSC. It shall not be reproduced in whole or in part or released to any third party without expressed written consent of AEPSC. 


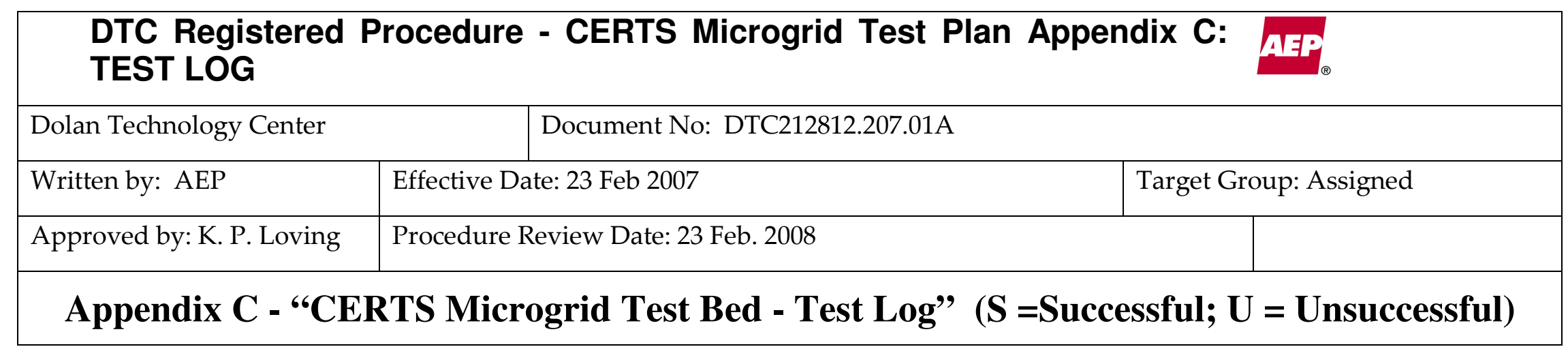

\begin{tabular}{|c|c|c|c|c|c|c|c|c|}
\hline & & & 7.9.3R & $\begin{array}{l}\text { 7.9.5R, } \\
\text { 7.9.6R }\end{array}$ & & & & \\
\hline 7.9.10R & $1 / 31 / 08$ & $1: 40 \mathrm{pm}$ & 7.9.11R & 7.9.12R & $\mathbf{S}$ & 1:42: pm & DAK & $\begin{array}{l}\text { LB3:40kW, LB4:40kW, LB6:40kW, SS connected } \\
\text { at 3:13. Meter1:71kW, Meter2:33kW }\end{array}$ \\
\hline 7.9.13R & 1/31/08 & $1: 42 \mathrm{pm}$ & 7.9.14R & $\begin{array}{l}\text { 7.9.15R, } \\
\text { 7.9.16R }\end{array}$ & $\mathbf{S}$ & $1: 46 \mathrm{pm}$ & DAK & $\begin{array}{l}\text { Triggers enabled. Fault timer set to } 10 \text { sec. Fault } \\
\text { initiated. SS opened. CB41 opened and Genset A2 } \\
\text { Shutdown. CB31 and } 51 \text { remained closed. Genset } \\
\text { A1 remained online serving the load in Zone } 3 \text {. The } \\
\text { SS reclosed shortly afterwards. }\end{array}$ \\
\hline 7.9.17R & $\mathbf{1 / 3 1 / 0 8}$ & $1: 46 \mathrm{pm}$ & & & $\mathbf{S}$ & 2:00 pm & DAK & \\
\hline 7.4.1R & 1/31/08 & 2:00 pm & $\begin{array}{l}\text { 7.4.2R, } \\
\text { 7.4.3R }\end{array}$ & $\begin{array}{l}\text { 7.4.4R, } \\
\text { 7.4.5R }\end{array}$ & $\mathbf{S}$ & 2:02 pm & DAK & Fault load connected, armed, and verified. \\
\hline 7.4.6R & $1 / 31 / 08$ & 2:02 pm & $\begin{array}{l}\text { 7.4.7R, } \\
\text { 7.4.8R }\end{array}$ & 7.4.9R & $\mathbf{S}$ & 2:07 pm & DAK & Genset $A 1$ and $A 2$ connected. SS closed. \\
\hline 7.4.10R & 1/31/08 & 2:07 pm & 7.4.11R & 7.4.12R & $\mathbf{S}$ & $2: 10 \mathrm{pm}$ & DAK & $\begin{array}{l}\text { LB3:10kW, LB4:65kW, LB6:40kW, Meter1:37kW, } \\
\text { Meter2:1kW }\end{array}$ \\
\hline
\end{tabular}

32 of 65

This document contains information confidential and proprietary to AEPSC. It shall not be reproduced in whole or in part or released to any third party without expressed written consent of AEPSC. 


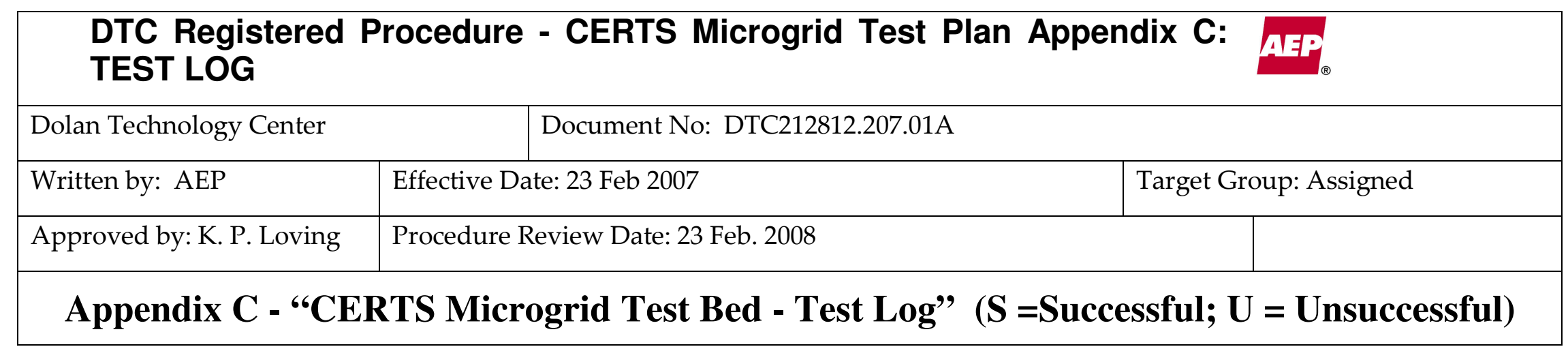

\begin{tabular}{|c|c|c|c|c|c|c|c|c|}
\hline & & & & & & & & $\begin{array}{l}\text { utility serving the load. The SS opened after about } \\
50 \text { seconds due to anti-islanding. Genset A1 } \\
\text { continued to serve the } 10 \mathrm{~kW} \text { load in Zone } 3\end{array}$ \\
\hline 7.4 .17 & 1/31/08 & $2: 15$ am & & & $\mathbf{S}$ & $2: 20 \mathrm{pm}$ & DAK & \\
\hline 7.5.1R & 1/31/08 & $2: 20 \mathrm{pm}$ & $\begin{array}{l}\text { 7.5.2R, } \\
\text { 7.5.3R }\end{array}$ & $\begin{array}{l}\text { 7.5.4R, } \\
\text { 7.5.5R, } \\
\text { 7.5.6R }\end{array}$ & $\mathbf{S}$ & $2: 36 \mathrm{pm}$ & DAK & Fault load connected and verified. Fault key armed. \\
\hline 7.5.7R & 1/31/08 & $2: 36 \mathrm{pm}$ & 7.5.8R & 7.5.9R & $\mathbf{S}$ & $2: 37 \mathrm{pm}$ & DAK & LB3: 40kW, LB6:40kW \\
\hline 7.5.10R & 1/31/08 & $2: 37$ pm & 7.5.11R & 7.5.12R & $\mathbf{S}$ & $2: 39$ pm & DAK & $\begin{array}{l}\text { Genset A1 connected, SS connected at 2:38pm. } \\
\text { Meter } 1: 56 \mathrm{~kW} \text {, Meter2:19kW }\end{array}$ \\
\hline 7.5.13R & $\mathbf{1 / 3 1 / 0 8}$ & $2: 39 \mathrm{pm}$ & $\begin{array}{l}\text { 7.5.14R, } \\
\text { 7.5.15R }\end{array}$ & 7.5.16R & $\mathbf{S}$ & $2: 55 \mathrm{pm}$ & DAK & $\begin{array}{l}\text { Fault timer set to } 10 \text { sec. Triggers armed. Fault } \\
\text { initiated at 2:50pm. The SS opened. CB31 opened } \\
\text { shutting down Genset A1. CB41 remained closed. } \\
\text { CB51 opened. Meter1:37kW, Meter2:0kW }\end{array}$ \\
\hline 7.5.17R & 1/31/08 & $2: 55$ pm & & & $\mathbf{S}$ & 3:00 pm & DAK & \\
\hline 7.6.1R & 1/31/08 & $3: 00 \mathrm{pm}$ & 7.6.2R & 7.6.3R & $\mathbf{S}$ & 3:03 pm & DAK & Gensets A1 and A2 setup. Fault key armed. \\
\hline 7.6.4R & 1/31/08 & 3:03 pm & 7.6.5R & $\begin{array}{l}\text { 7.6.6R, } \\
\text { 7.6.7R }\end{array}$ & $\mathbf{S}$ & 3:06 pm & DAK & $\begin{array}{l}\text { Genset A1 and A2 connected. LB3:40kW, } \\
\text { LB4:40kW, LB6:40kW. SS connected. } \\
\text { Meter1:70kW, Meter2:34kW. Triggers armed }\end{array}$ \\
\hline 7.6.8R & 1/31/08 & 3:06 pm & $\begin{array}{l}\text { 7.6.9R, } \\
\text { 7.6.10R }\end{array}$ & 7.6.11R & $\mathbf{S}$ & 3:09 pm & DAK & $\begin{array}{l}\text { Fault timer set to } 10 \text { sec Fault initiated at 3:07. SS } \\
\text { opened. CB41 opened and Genset A2 shutdown. CB } \\
31 \text { opened and Genset A1 shutdown. CB } 51 \text { opened. } \\
\text { Meter1:37kW, Meter2:0kW }\end{array}$ \\
\hline
\end{tabular}

33 of 65

This document contains information confidential and proprietary to AEPSC. It shall not be reproduced in whole or in part or released to any third party without expressed written consent of AEPSC. 


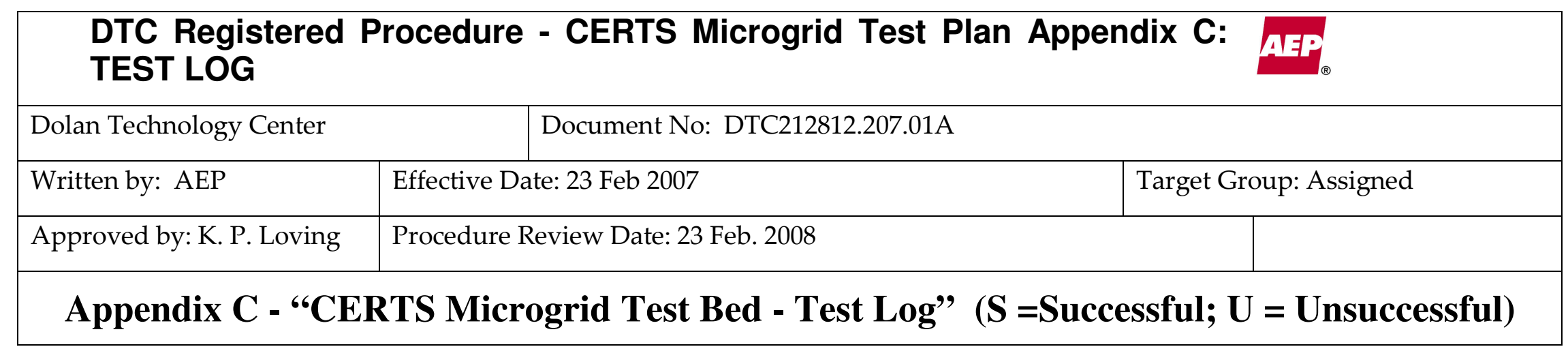

\begin{tabular}{|c|c|c|c|c|c|c|c|c|}
\hline 7.6.12R & 1/31/08 & 3:09 pm & & & $\mathbf{S}$ & $3: 15$ pm & DAK & \\
\hline 7.13.1R & $2 / 1 / 08$ & 2:00 pm & $\begin{array}{l}\text { 7.13.2R, } \\
\text { 7.13.3R }\end{array}$ & $\begin{array}{l}\text { 7.13.4, } \\
\text { 7.13.5, } \\
\text { 7.13.6 }\end{array}$ & $\mathbf{S}$ & $2: 10 \mathrm{pm}$ & DAK & Fault load connected and verified. Fault key armed. \\
\hline 7.13.7R & $2 / 1 / 08$ & $2: 10 \mathrm{pm}$ & 7.13.8R & $\begin{array}{l}\text { 7.13.9R, } \\
\text { 7.13.10R }\end{array}$ & $\mathbf{S}$ & $2: 14 \mathrm{pm}$ & DAK & $\begin{array}{l}\text { Genset A1 and A2 setup and connected. LB3:40kW, } \\
\text { LB4:40kW, LB6:40kW }\end{array}$ \\
\hline 7.13.11R & $2 / 1 / 08$ & $2: 14 \mathrm{pm}$ & 7.13.12 R & & $\mathbf{S}$ & $2: 15$ pm & DAK & SS closed at 2:15. Meter1:73kW, Meter2:36kW \\
\hline 7.13.13R & $2 / 1 / 08$ & $2: 15$ pm & $\begin{array}{l}\text { 7.13.14 R, } \\
\text { 7.13.15 R }\end{array}$ & 7.13.16R & $\mathbf{S}$ & 2:19 pm & DAK & $\begin{array}{l}\text { Fault timer set to } 10 \text { sec. Triggers armed. Fault } \\
\text { initiated at } 2: 17 \text {. SS opened. Genset } \mathrm{A} 1 \text { and } \mathrm{A2} \\
\text { picked up the load and remained online. The fault } \\
\text { load opened after } 10 \text { seconds and the SS } \\
\text { reconnected shortly thereafter. All breakers } \\
\text { remained closed, all generators remained running. }\end{array}$ \\
\hline 7.13.17R & $2 / 1 / 08$ & 2:19 pm & & & $\mathbf{S}$ & $2: 30 \mathrm{pm}$ & DAK & \\
\hline 7.14.1R & $2 / 1 / 08$ & $2: 30 \mathrm{pm}$ & $\begin{array}{l}\text { 7.14.2R, } \\
\text { 7.14.3R }\end{array}$ & $\begin{array}{l}\text { 7.14.4R, } \\
\text { 7.14.5R, } \\
\text { 7.14.6R }\end{array}$ & $\mathbf{S}$ & $2: 33$ pm & DAK & Fault load connected and verified. Fault key armed. \\
\hline 7.14.7R & $2 / 1 / 08$ & 2:33 pm & 7.14.8R & $\begin{array}{l}\text { 7.14.9R, } \\
\text { 7.14.10R }\end{array}$ & $\mathbf{S}$ & $2: 34 \mathrm{pm}$ & DAK & $\begin{array}{l}\text { Gensets A1 and A2 connected. LB3:40kW, } \\
\text { LB4:40kW, LB6:40kW }\end{array}$ \\
\hline 7.14.11R & $2 / 1 / 08$ & $2: 34 \mathrm{pm}$ & 7.14.12R & & $\mathbf{S}$ & $2: 35 \mathrm{pm}$ & DAK & SS closed, Meter1:71kW, Meter2:35kW \\
\hline 7.14.13R & $2 / 1 / 08$ & $2: 35 \mathrm{pm}$ & $\begin{array}{l}\text { 7.14.14R, } \\
\text { 7.14.15R }\end{array}$ & 7.14.16R & $\mathbf{S}$ & $2: 38 \mathrm{pm}$ & DAK & $\begin{array}{l}\text { Fault timer set } 10 \text { sec. Triggers enabled. Fault } \\
\text { initiated at 2:36. SS opened. CB41 opened and }\end{array}$ \\
\hline
\end{tabular}

34 of 65

This document contains information confidential and proprietary to AEPSC. It shall not be reproduced in whole or in part or released to any third party without expressed written consent of AEPSC. 


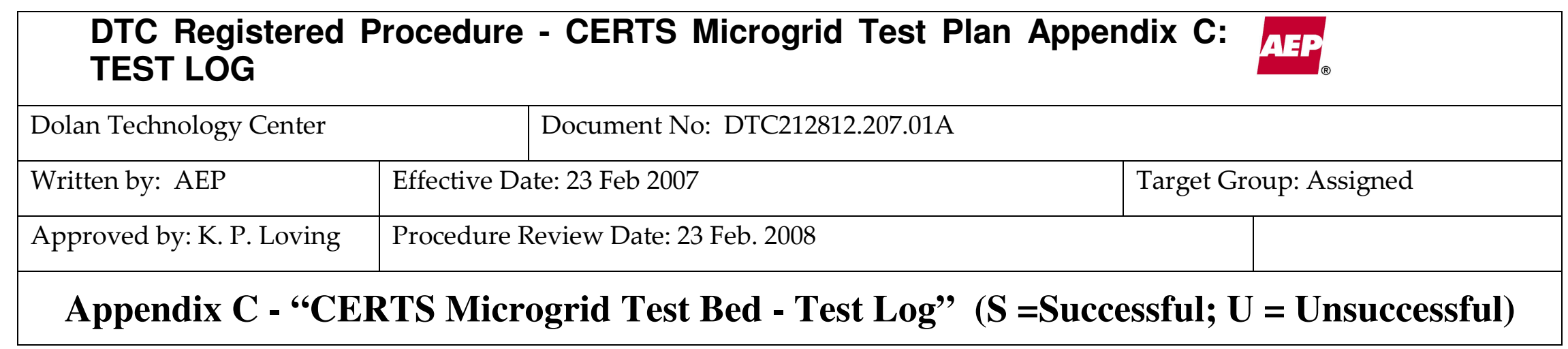

\begin{tabular}{|c|c|c|c|c|c|c|c|c|}
\hline & & & & & & & & $\begin{array}{l}\text { Genset A2 shutdown. CB31 and CB51 remained } \\
\text { close with Genset A1 serving the load. The SS } \\
\text { reconnect shortly thereafter. }\end{array}$ \\
\hline 7.14.17R & $2 / 1 / 08$ & $2: 38 \mathrm{pm}$ & & & $\mathbf{S}$ & & & \\
\hline 7.15.1R & $2 / 6 / 08$ & $12: 20 \mathrm{pm}$ & $\begin{array}{l}\text { 7.15.2R, } \\
\text { 7.15.3R }\end{array}$ & $\begin{array}{l}\text { 7.15.4R, } \\
\text { 7.15.5R, } \\
\text { 7.15.6R }\end{array}$ & $\mathbf{S}$ & $12: 25 \mathrm{pm}$ & DAK & Fault load connected and verified. Fault key armed \\
\hline 7.15.7R & $2 / 6 / 08$ & $12: 25 \mathrm{pm}$ & 7.15.8R & $\begin{array}{l}\text { 7.15.9R, } \\
\text { 7.15.10R }\end{array}$ & $\mathbf{S}$ & $12: 30 \mathrm{pm}$ & DAK & $\begin{array}{l}\text { Gensets A1 and B1 connected. LB3:40kW, } \\
\text { LB5:40kW, LB6:40kW }\end{array}$ \\
\hline 7.15.11R & $2 / 6 / 08$ & $12: 30 \mathrm{pm}$ & 7.15.12R & & $\mathbf{S}$ & $12: 35 \mathrm{pm}$ & DAK & SS closed at 12:35, Meter1:73kW, Meter2:37kW \\
\hline 7.15.13R & $2 / 6 / 08$ & $12: 35 \mathrm{pm}$ & $\begin{array}{l}\text { 7.15.14R, } \\
\text { 7.15.15R }\end{array}$ & 7.15.16R & $\mathbf{S}$ & $12: 43 \mathrm{pm}$ & DAK & $\begin{array}{l}\text { Triggers armed, Fault initiated at 12:38. The SS } \\
\text { remained closed. All Gensets and Breakers } \\
\text { remained online and closed. No operations, other } \\
\text { than the fault load application and removal, } \\
\text { occurred. }\end{array}$ \\
\hline 7.15.17R & $2 / 6 / 08$ & $12: 43 \mathrm{pm}$ & & & $\mathbf{S}$ & $12: 45 \mathrm{pm}$ & DAK & \\
\hline 7.16.1R & $2 / 6 / 08$ & $12: 45 \mathrm{pm}$ & $\begin{array}{l}\text { 7.16.2R, } \\
\text { 7.16.3R }\end{array}$ & $\begin{array}{l}\text { 7.16.4R, } \\
\text { 7.16.5R, } \\
\text { 7.16.6R }\end{array}$ & $\mathbf{S}$ & $12: 47 \mathrm{pm}$ & DAK & Fault load connected and verified. Fault key armed. \\
\hline $7.16 .7 \mathrm{R}$ & $2 / 6 / 08$ & $12: 47 \mathrm{pm}$ & $7.16 .8 \mathrm{R}$ & $\begin{array}{l}\text { 7.16.9R, } \\
\text { 7.16.10R }\end{array}$ & $\mathbf{S}$ & $12: 49 \mathrm{pm}$ & DAK & $\begin{array}{l}\text { Gensets A1 and B1 connected. LB3:40kW, } \\
\text { LB5:40kW, LB6:40kW }\end{array}$ \\
\hline 7.16.11R & $2 / 6 / 08$ & $12: 49 \mathrm{pm}$ & 7.16.12R & & $\mathbf{S}$ & $12: 50 \mathrm{pm}$ & DAK & SS closed, Meter1:70kW, Meter2:34kW \\
\hline 7.16.13R & 2/6/08 & $12: 50 \mathrm{pm}$ & 7.16.14R, & 7.16.16R & $\mathbf{S}$ & $12: 55 \mathrm{pm}$ & DAK & Triggers armed. Fault initiated at 12:53. SS opened. \\
\hline
\end{tabular}
35 of 65

This document contains information confidential and proprietary to AEPSC. It shall not be reproduced in whole or in part or released to any third party without expressed written consent of AEPSC. 


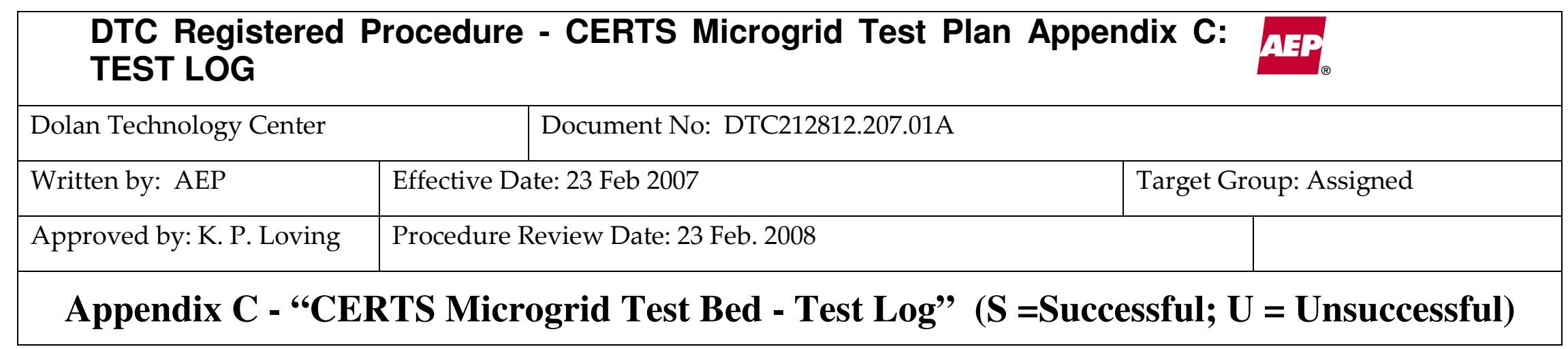

\begin{tabular}{|c|c|c|c|c|c|c|c|c|}
\hline & & & 7.16.15R & & & & & $\begin{array}{l}\text { CB51 opened and Genset B1 shutdown. Genset A1 } \\
\text { remained online carrying the load. CB } 31 \text { and } 41 \\
\text { remained closed. SS reconnected shortly thereafter. }\end{array}$ \\
\hline 7.16.17R & $2 / 6 / 08$ & $12: 55 \mathrm{pm}$ & & & $\mathbf{S}$ & $12: 58 \mathrm{pm}$ & DAK & \\
\hline 7.7.1R & $2 / 6 / 08$ & $1: 55$ pm & $\begin{array}{l}\text { 7.7.2R, } \\
\text { 7.7.3R }\end{array}$ & $\begin{array}{l}\text { 7.7.4R, } \\
\text { 7.7.5R, } \\
\text { 7.7.6R }\end{array}$ & $\mathbf{S}$ & 2:00 pm & DAK & Fault load verified and connected, Fault key armed \\
\hline 7.7.7R & $2 / 6 / 08$ & $2: 00 \mathrm{pm}$ & 7.7.8R & $7.7 .9 \mathrm{R}$ & $\mathbf{S}$ & $2: 01 \mathrm{pm}$ & DAK & LB3:40kW, LB6:40kW, Genset B1 connected \\
\hline 7.7.10R & $2 / 6 / 08$ & 2:01 pm & 7.7.11R & 7.7.12R & $\mathbf{S}$ & 2:02 pm & DAK & SS closed. Meter1:52kW Meter2:16kW \\
\hline 7.7.13R & $2 / 6 / 08$ & 2:02 pm & $\begin{array}{l}\text { 7.7.14R, } \\
\text { 7.7.15R }\end{array}$ & 7.7.16R & $\mathbf{S}$ & 2:06 pm & DAK & $\begin{array}{l}\text { Triggers armed. Fault initiated at 2:04. SS opened. } \\
\text { CB51 opened and genset B1 shutdown. CB31 and } \\
\text { CB41 remained closed. SS remained opened as a } \\
\text { Dead Bus was present. }\end{array}$ \\
\hline 7.7.17R & $2 / 6 / 08$ & 2:06 pm & & & $\mathbf{S}$ & 2:09 pm & DAK & \\
\hline $\mathbf{7 . 8 . 1}$ & $2 / 6 / 08$ & 2:09 pm & $\mathbf{7 . 8 . 2}$ & 7.8 .3 & $\mathbf{S}$ & $2: 11 \mathrm{pm}$ & DAK & Fault load verified and connected, Fault key armed \\
\hline 7.8 .4 & $2 / 6 / 08$ & 2:11 pm & 7.8 .5 & $\begin{array}{l}\text { 7.8.6, } \\
\text { 7.8.7 }\end{array}$ & $\mathbf{S}$ & $2: 15$ pm & DAK & $\begin{array}{l}\text { Genset A1 and B1 connected, LB3:40kW, } \\
\text { LB4:40kW, LB6:40kW. SS closed. Meter1:52kW } \\
\text { Meter2:16kW }\end{array}$ \\
\hline 7.8 .8 & $2 / 6 / 08$ & 2:15 pm & $\begin{array}{l}\text { 7.8.9, } \\
\text { 7.8.10 }\end{array}$ & 7.8.11 & $\mathbf{S}$ & 2:18 pm & DAK & $\begin{array}{l}\text { Triggers armed. Fault initiated at 2:17. CB51 } \\
\text { opened and Genset B1 shutdown. SS, CB31 and } \\
\text { CB41 remained closed and Genset A1 remained } \\
\text { connected. }\end{array}$ \\
\hline 7.8.12 & $2 / 6 / 08$ & 2:18 pm & & & $\mathbf{S}$ & $2: 25 \mathrm{pm}$ & DAK & \\
\hline
\end{tabular}

This document contains information confidential and proprietary to AEPSC. It shall not be reproduced in whole or in part or released to any third party without expressed written consent of AEPSC. 


\begin{tabular}{|c|c|c|c|c|c|c|c|c|}
\hline \multicolumn{9}{|c|}{$\begin{array}{l}\text { DTC Registered } \\
\text { TEST LOG }\end{array}$} \\
\hline \multicolumn{4}{|c|}{ Dolan Technology Center } & \multicolumn{5}{|c|}{ Document No: DTC212812.207.01A } \\
\hline \multicolumn{3}{|c|}{ Written by: AEP } & \multicolumn{5}{|c|}{ Effective Date: 23 Feb 2007} & Target Group: Assigned \\
\hline \multicolumn{3}{|c|}{ Approved by: K. P. Loving } & \multicolumn{5}{|c|}{ Procedure Review Date: 23 Feb. 2008} & \\
\hline \multicolumn{9}{|c|}{ Appendix C - "CERTS Microgrid Test Bed - Test Log” (S =Successful; U = Unsuccessful) } \\
\hline \multirow{2}{*}{$\overline{7.10 .1}$} & & & & & & & & \multirow[b]{2}{*}{ Fault load verified and connected, Fault key armed } \\
\hline & $2 / 6 / 08$ & $2: 25 \mathrm{pm}$ & $\begin{array}{l}7.10 .2 \\
7.10 .3\end{array}$ & $\begin{array}{l}7.10 .4, \\
7.10 .5, \\
7.10 .6\end{array}$ & $\mathbf{S}$ & $2: 28 p m$ & DAK & \\
\hline 7.10 .7 & $2 / 6 / 08$ & \begin{tabular}{|l|}
$2: 28 \mathrm{pm}$ \\
\end{tabular} & 7.10 .8 & 7.10 .9 & $\mathbf{S}$ & $2: 30 \mathrm{pm}$ & DAK & Gensets A1 and B1 connected \\
\hline 7.10 .10 & $2 / 6 / 08$ & $2: 30 \mathrm{pm}$ & 7.10 .11 & 7.10 .12 & $\mathbf{S}$ & $2: 33 \mathrm{pm}$ & DAK & $\begin{array}{l}\text { LB3:40kW, LB4:40kW, LB6:40kW. SS closed. } \\
\text { Meter1:70kW, Meter2:34kW }\end{array}$ \\
\hline 7.10 .13 & $2 / 6 / 08$ & $2: 33$ pm & $\begin{array}{l}7.10 .14 \\
7.10 .15\end{array}$ & 7.10 .16 & $\mathbf{S}$ & $2: 36 \mathrm{pm}$ & DAK & $\begin{array}{l}\text { Triggers armed, Fault initiated at 2:34. SS opened, } \\
\text { CB51 opened and Genset B1 shutdown, CB31 } \\
\text { opened and Genset A1 shutdown. CB41 remained } \\
\text { closed. }\end{array}$ \\
\hline 7.10 .17 & $2 / 6 / 08$ & 2:36 pm & & & $\mathbf{S}$ & $2: 40 \mathrm{pm}$ & DAK & \\
\hline
\end{tabular}

( $\mathrm{S}$ =Successful; $\mathrm{U}=$ Unsuccessful)

\begin{tabular}{|l|l|l|l|l|l|l|l|l|}
\hline $\begin{array}{l}\text { Start Test } \\
\text { Sequence } \\
\text { No. }\end{array}$ & $\begin{array}{l}\text { Test } \\
\text { Date }\end{array}$ & $\begin{array}{l}\text { Start } \\
\text { Time }\end{array}$ & $\begin{array}{l}\text { Verify/ } \\
\text { Action }\end{array}$ & $\begin{array}{l}\text { Verify/ } \\
\text { Action }\end{array}$ & $\begin{array}{l}\text { Status } \\
\text { S or U }\end{array}$ & $\begin{array}{l}\text { End } \\
\text { Time }\end{array}$ & Initials & Test Event Comments: \\
\hline 8.1 .1 & $2 / 19 / 08$ & $12: 30 ~ p m$ & 8.1 .2 & 8.1 .3 & S & $12: 33$ pm & DAK & $\begin{array}{l}\text { CB1, CB12, CB31, CB41, and CB51 Closed, All LBs } \\
\text { 0kW, SS Open }\end{array}$ \\
\hline 8.1 .4 & $2 / 19 / 08$ & $12: 33 ~ p m$ & 8.1 .5 & & S & $12: 40 ~ p m$ & DAK & Genset A1 connected, Triggers armed, LB3:20kW \\
\hline
\end{tabular}

This document contains information confidential and proprietary to AEPSC. It shall not be reproduced in whole or in part or released to any third party without expressed written consent of AEPSC. 


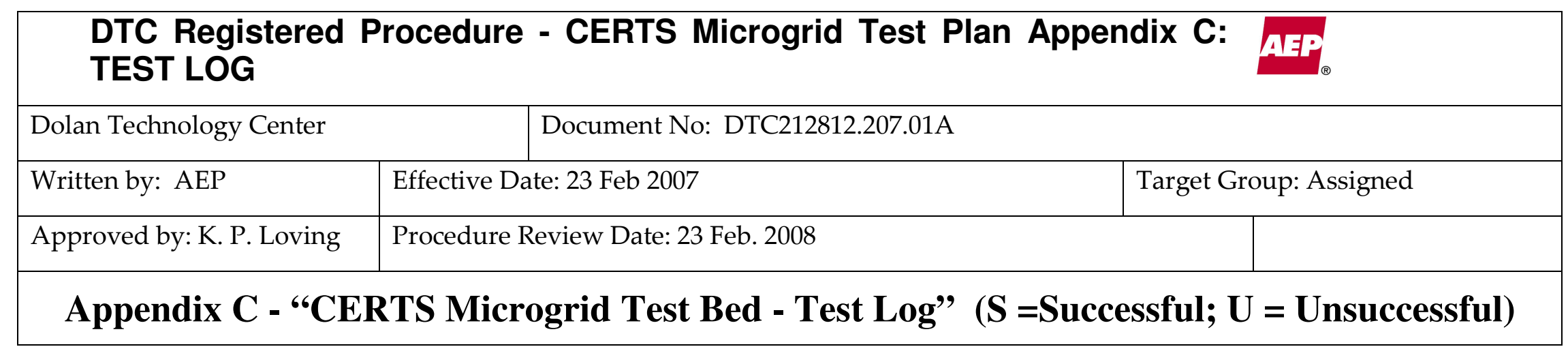

\begin{tabular}{|c|c|c|c|c|c|c|c|}
\hline & & & & & & & Load increased at 12:36 pm \\
\hline 8.1 .6 & $2 / 19 / 08$ & $12: 40 \mathrm{pm}$ & 8.1 .7 & $\mathbf{S}$ & $12: 46 \mathrm{pm}$ & DAK & $\begin{array}{l}\text { Triggers armed, LB3:40kW, Load increased at } \\
\text { 12:41 pm }\end{array}$ \\
\hline 8.1 .8 & $2 / 19 / 08$ & 12:46 pm & 8.1 .9 & $\mathbf{S}$ & 12:54 pm & DAK & $\begin{array}{l}\text { Triggers armed, LB3:60kW, Load increased at } \\
12: 48 \text { pm }\end{array}$ \\
\hline 8.1.10 & 2/19/08 & $12: 54 \mathrm{pm}$ & & $\mathbf{S}$ & $12: 59 \mathrm{pm}$ & DAK & LB3:0kW Voltage set to 291V (+5\%) \\
\hline 8.1.10(8.1.4) & $2 / 19 / 08$ & $12: 59 \mathrm{pm}$ & $\begin{array}{l}\text { 8.1.10(8.1 } \\
.5)\end{array}$ & $\mathbf{S}$ & 1:08 pm & DAK & $\begin{array}{l}\text { Triggers armed, LB3:20kW, Load increased at 1:04 } \\
\text { pm }\end{array}$ \\
\hline 8.1.10(8.1.6) & $2 / 19 / 08$ & 1:08 pm & $\begin{array}{l}\text { 8.1.10(8.1 } \\
.7)\end{array}$ & $\mathbf{S}$ & $1: 13 \mathrm{pm}$ & DAK & $\begin{array}{l}\text { Triggers armed, LB3:40kW, Load increased at 1:09 } \\
\text { pm }\end{array}$ \\
\hline 8.1.10(8.1.8) & $2 / 19 / 08$ & 1:13 pm & $\begin{array}{l}\text { 8.1.10(8.1 } \\
.9)\end{array}$ & $\mathbf{S}$ & $1: 20 \mathrm{pm}$ & DAK & $\begin{array}{l}\text { Triggers armed, LB3:60kW, Load increased at 1:14 } \\
\text { pm }\end{array}$ \\
\hline 8.1.11 & 2/19/08 & $1: 20 \mathrm{pm}$ & & $\mathbf{S}$ & 1:21 pm & DAK & LB3:0kW Voltage set to 263V (-5\%) \\
\hline 8.1.11(8.1.4) & $2 / 19 / 08$ & 1:21 pm & $\begin{array}{l}\text { 8.1.11(8.1 } \\
.5)\end{array}$ & $\mathbf{S}$ & $1: 27 \mathrm{pm}$ & DAK & $\begin{array}{l}\text { Triggers armed, LB3:20kW, Load increased at 1:23 } \\
\text { pm }\end{array}$ \\
\hline 8.1.11(8.1.6) & $2 / 19 / 08$ & $1: 27 \mathrm{pm}$ & $\begin{array}{l}\text { 8.1.11(8.1 } \\
.7)\end{array}$ & $\mathbf{S}$ & $1: 36 \mathrm{pm}$ & DAK & $\begin{array}{l}\text { Triggers armed, LB3:40kW, Load increased at 1:29 } \\
\text { pm }\end{array}$ \\
\hline 8.1.11(8.1.8) & $2 / 19 / 08$ & 1:36 pm & $\begin{array}{l}\text { 8.1.11(8.1 } \\
.9)\end{array}$ & $\mathbf{S}$ & $1: 50 \mathrm{pm}$ & DAK & $\begin{array}{l}\text { Triggers armed, LB3:60kW, Load increased at 1:45 } \\
\text { pm }\end{array}$ \\
\hline
\end{tabular}

\begin{tabular}{|l|l|l|l|l|l|l|l|l|}
\hline Start Test & Test & Start & Verify/ & Verify/ & Status & End & Initials & Test Event Comments: \\
\hline
\end{tabular}

This document contains information confidential and proprietary to AEPSC. It shall not be reproduced in whole or in part or released to any third party without expressed written consent of AEPSC. 


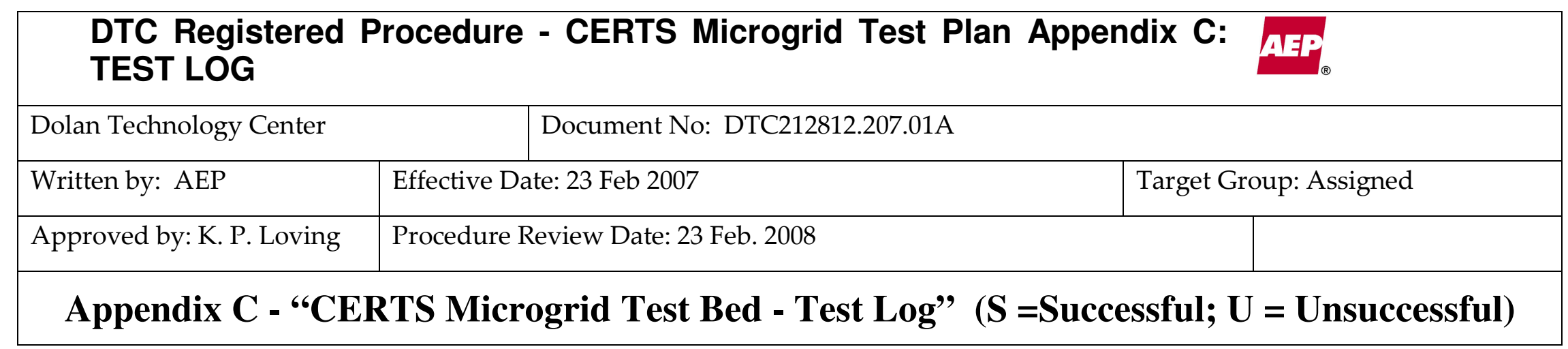

\begin{tabular}{|c|c|c|c|c|c|c|c|c|}
\hline $\begin{array}{l}\text { Sequence } \\
\text { No. }\end{array}$ & Date & Time & Action & Action & S or $\mathbf{U}$ & Time & & \\
\hline 8.1.12 & $2 / 19 / 08$ & $1: 50 \mathrm{pm}$ & & & $\mathbf{S}$ & 2:02 pm & DAK & LB3:0kW, Genset A1 shutdown \\
\hline 8.1.13(8.1.4) & $2 / 19 / 08$ & 2:02 pm & $\begin{array}{l}\text { 8.1.13(8.1 } \\
.5)\end{array}$ & & $\mathbf{S}$ & $2: 23 \mathrm{pm}$ & DAK & $\begin{array}{l}\text { Genset A2 connected, Triggers armed, LB4:20kW } \\
\text { Load increased at 2:19 pm }\end{array}$ \\
\hline 8.1.13(8.1.6) & $2 / 19 / 08$ & $2: 23 \mathrm{pm}$ & $\begin{array}{l}\text { 8.1.13(8.1 } \\
.7)\end{array}$ & & $\mathbf{S}$ & $2: 29 \mathrm{pm}$ & DAK & $\begin{array}{l}\text { Triggers armed, LB4:40kW, Load increased at 2:24 } \\
\text { pm }\end{array}$ \\
\hline 8.1.13(8.1.8) & $2 / 19 / 08$ & $2: 29 \mathrm{pm}$ & $\begin{array}{l}\text { 8.1.13(8.1 } \\
.9)\end{array}$ & & $\mathbf{S}$ & $2: 36 \mathrm{pm}$ & DAK & $\begin{array}{l}\text { Triggers armed, LB4:60kW, Load increased at 2:30 } \\
\text { pm }\end{array}$ \\
\hline $\begin{array}{l}\text { 8.1.13(8.1.1 } \\
\text { 0) }\end{array}$ & $2 / 19 / 08$ & $2: 36$ pm & & & $\mathbf{S}$ & 2:37 pm & DAK & LB4:0kW Voltage set to 291V (+5\%) \\
\hline $\begin{array}{l}\text { 8.1.13(8.1.1 } \\
\text { 0(8.1.4)) }\end{array}$ & $2 / 19 / 08$ & $2: 37 \mathrm{pm}$ & $\begin{array}{l}\text { 8.1.13(8.1 } \\
.10(8.1 .5))\end{array}$ & & $\mathbf{S}$ & 2:43 pm & DAK & $\begin{array}{l}\text { Triggers armed, LB4:20kW, Load increased at 2:38 } \\
\text { pm }\end{array}$ \\
\hline $\begin{array}{l}\text { 8.1.13(8.1.1 } \\
\text { 0(8.1.6)) }\end{array}$ & $2 / 19 / 08$ & $2: 43 \mathrm{pm}$ & $\begin{array}{l}\text { 8.1.13(8.1 } \\
.10(8.1 .7))\end{array}$ & & $\mathbf{S}$ & $2: 53 \mathrm{pm}$ & DAK & $\begin{array}{l}\text { Triggers armed, LB4:40kW, Load increased at 2:49 } \\
\text { pm }\end{array}$ \\
\hline $\begin{array}{l}\text { 8.1.13(8.1.1 } \\
\text { 0(8.1.8)) }\end{array}$ & $2 / 19 / 08$ & $2: 53 \mathrm{pm}$ & $\begin{array}{l}\text { 8.1.13(8.1 } \\
.10(8.1 .9))\end{array}$ & & $\mathbf{S}$ & $2: 59 \mathrm{pm}$ & DAK & $\begin{array}{l}\text { Triggers armed, LB4:60kW, Load increased at 2:54 } \\
\text { pm }\end{array}$ \\
\hline $\begin{array}{l}\text { 8.1.13(8.1.1 } \\
\text { 1) }\end{array}$ & $2 / 19 / 08$ & $2: 59 \mathrm{pm}$ & & & $\mathbf{S}$ & 3:00 pm & DAK & LB4:0kW Voltage set to 263V (-5\%) \\
\hline $\begin{array}{l}\text { 8.1.13(8.1.1 } \\
\text { 1(8.1.4)) }\end{array}$ & $2 / 19 / 08$ & 3:00 pm & $\begin{array}{l}\text { 8.1.13(8.1 } \\
.11(8.1 .5))\end{array}$ & & $\mathbf{S}$ & 3:04 pm & DAK & $\begin{array}{l}\text { Triggers armed, LB4:20kW, Load increased at 3:00 } \\
\text { pm }\end{array}$ \\
\hline $\begin{array}{l}\text { 8.1.13(8.1.1 } \\
\text { 1(8.1.6)) }\end{array}$ & $2 / 19 / 08$ & 3:04 pm & $\begin{array}{l}\text { 8.1.13(8.1 } \\
.11(8.1 .7))\end{array}$ & & $\mathbf{S}$ & $3: 12 \mathrm{pm}$ & DAK & $\begin{array}{l}\text { Triggers armed, LB4:40kW, Load increased at 3:07 } \\
\text { pm }\end{array}$ \\
\hline
\end{tabular}




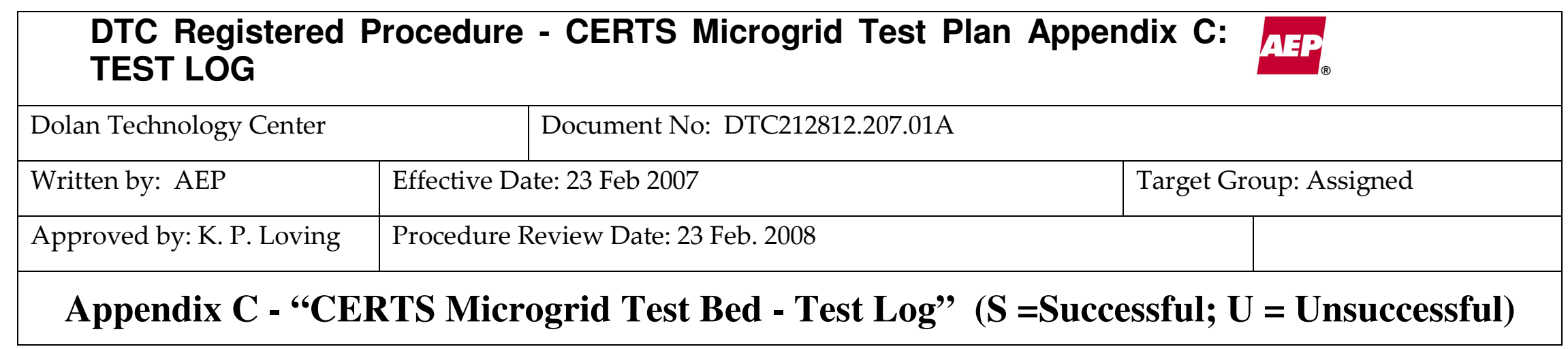

\begin{tabular}{|c|c|c|c|c|c|c|c|c|}
\hline $\begin{array}{l}\text { Start Test } \\
\text { Sequence } \\
\text { No. }\end{array}$ & $\begin{array}{l}\text { Test } \\
\text { Date }\end{array}$ & $\begin{array}{l}\text { Start } \\
\text { Time }\end{array}$ & $\begin{array}{l}\text { Verify/ } \\
\text { Action }\end{array}$ & $\begin{array}{l}\text { Verify/ } \\
\text { Action }\end{array}$ & $\begin{array}{l}\text { Status } \\
\text { S or U }\end{array}$ & $\begin{array}{l}\text { End } \\
\text { Time }\end{array}$ & Initials & Test Event Comments: \\
\hline $\begin{array}{l}\text { 8.1.13(8.1.1 } \\
\text { 1(8.1.8)) }\end{array}$ & $2 / 19 / 08$ & $3: 12$ pm & $\begin{array}{l}\text { 8.1.13(8.1 } \\
.11(8.1 .9))\end{array}$ & & $\mathbf{S}$ & 3:17 pm & DAK & $\begin{array}{l}\text { Triggers armed, LB4:60kW, Load increased at } \\
3: 12 \mathrm{pm}\end{array}$ \\
\hline 8.1.14 & $2 / 19 / 08$ & 3:17 pm & & & $\mathbf{S}$ & 3:25 pm & DAK & LB4:0kW, Genset A2 shutdown \\
\hline 8.1.15(8.1.4) & $2 / 21 / 08$ & 9:45 am & $\begin{array}{l}\text { 8.1.15(8.1 } \\
.5)\end{array}$ & & $\mathbf{S}$ & 9:57 am & DAK & $\begin{array}{l}\text { Genset B1 connected, Triggers armed, LB5:20kW } \\
\text { Load increased at 9:52 am }\end{array}$ \\
\hline 8.1.15(8.1.6) & $2 / 21 / 08$ & 9:57 am & $\begin{array}{l}\text { 8.1.15(8.1 } \\
.7)\end{array}$ & & $\mathbf{S}$ & 10:08 am & DAK & $\begin{array}{l}\text { Triggers armed, LB5:40kW, Load increased at } \\
\text { 10:03 am }\end{array}$ \\
\hline 8.1.15(8.1.8) & $2 / 21 / 08$ & 10:08 am & $\begin{array}{l}\text { 8.1.15(8.1 } \\
.9)\end{array}$ & & $\mathbf{S}$ & 10:14 am & DAK & $\begin{array}{l}\text { Triggers armed, LB5:60kW, Load increased at } \\
\text { 10:09 am }\end{array}$ \\
\hline $\begin{array}{l}\text { 8.1.15(8.1.1 } \\
\text { 0) }\end{array}$ & $2 / 21 / 08$ & 10:14 am & & & $\mathbf{S}$ & 10:15 am & DAK & LB5:0kW Voltage set to 291V (+5\%) \\
\hline
\end{tabular}

This document contains information confidential and proprietary to AEPSC. It shall not be reproduced in whole or in part or released to any third party without expressed written consent of AEPSC. 


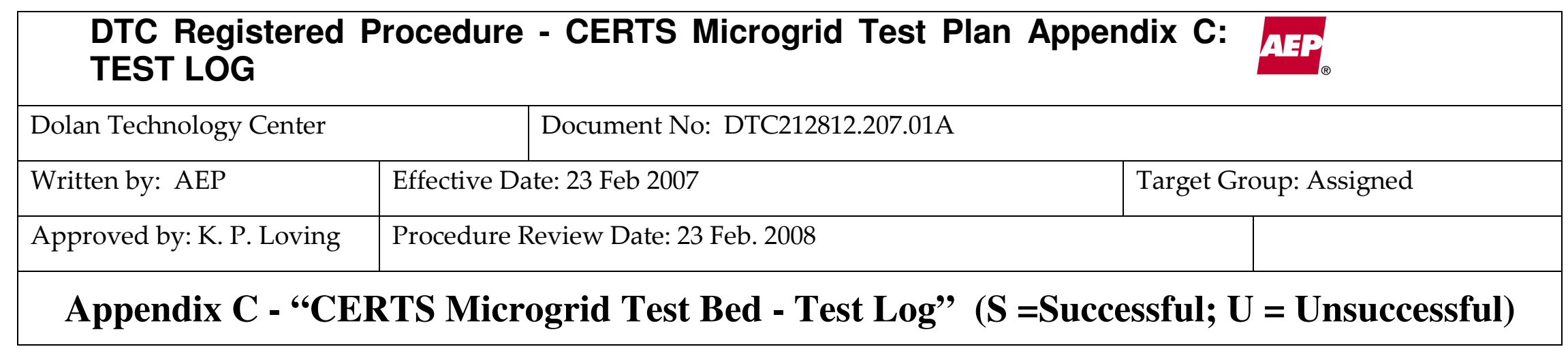

\begin{tabular}{|c|c|c|c|c|c|c|c|}
\hline $\begin{array}{l}\text { 8.1.15(8.1.1 } \\
\text { 0(8.1.4)) }\end{array}$ & $2 / 21 / 08$ & 10:15 am & $\begin{array}{l}\text { 8.1.15(8.1 } \\
.10(8.1 .5))\end{array}$ & $\mathbf{S}$ & $10: 22 \mathrm{am}$ & DAK & $\begin{array}{l}\text { Triggers armed, LB5:20kW, Load increased at } \\
\text { 10:17 am }\end{array}$ \\
\hline $\begin{array}{l}\text { 8.1.15(8.1.1 } \\
\text { 0(8.1.6)) }\end{array}$ & $2 / 21 / 08$ & $10: 22$ am & $\begin{array}{l}\text { 8.1.15(8.1 } \\
.10(8.1 .7))\end{array}$ & $\mathbf{S}$ & $10: 26$ am & DAK & $\begin{array}{l}\text { Triggers armed, LB5:40kW, Load increased at } \\
\text { 10:22 am }\end{array}$ \\
\hline $\begin{array}{l}\text { 8.1.15(8.1.1 } \\
0(8.1 .8))\end{array}$ & $2 / 21 / 08$ & $10: 26$ am & $\begin{array}{l}\text { 8.1.15(8.1 } \\
.10(8.1 .9))\end{array}$ & $\mathbf{S}$ & $10: 31$ am & DAK & $\begin{array}{l}\text { Triggers armed, LB5:60kW, Load increased at } \\
\text { 10:27 am }\end{array}$ \\
\hline $\begin{array}{l}\text { 8.1.15(8.1.1 } \\
\text { 1) }\end{array}$ & $2 / 21 / 08$ & 10:31 am & & $\mathbf{S}$ & 10:33 am & DAK & LB5:0kW Voltage set to 263V (-5\%) \\
\hline $\begin{array}{l}\text { 8.1.15(8.1.1 } \\
\text { 1(8.1.4)) }\end{array}$ & $2 / 21 / 08$ & $10: 33 \mathrm{am}$ & $\begin{array}{l}\text { 8.1.15(8.1 } \\
.11(8.1 .5))\end{array}$ & $\mathbf{S}$ & $10: 39$ am & DAK & $\begin{array}{l}\text { Triggers armed, LB5:20kW, Load increased at } \\
\text { 10:34 am }\end{array}$ \\
\hline
\end{tabular}

\begin{tabular}{|l|l|l|l|l|l|l|l|l|}
\hline $\begin{array}{l}\text { Start Test } \\
\text { Sequence } \\
\text { No. }\end{array}$ & $\begin{array}{l}\text { Test } \\
\text { Date }\end{array}$ & $\begin{array}{l}\text { Start } \\
\text { Time }\end{array}$ & $\begin{array}{l}\text { Verify/ } \\
\text { Action }\end{array}$ & $\begin{array}{l}\text { Verify/ } \\
\text { Action }\end{array}$ & $\begin{array}{l}\text { Status } \\
\text { S or U }\end{array}$ & $\begin{array}{l}\text { End } \\
\text { Time }\end{array}$ & Initials & Test Event Comments: \\
\hline $\begin{array}{l}\text { 8.1.15(8.1.1 } \\
\text { 1(8.1.6)) }\end{array}$ & $2 / 21 / 08$ & $\mathbf{1 0 : 3 9}$ am & $\begin{array}{l}\mathbf{8 . 1 . 1 5 ( 8 . 1} \\
\text {.11(8.1.7)) }\end{array}$ & & S & 10:44 am & DAK & $\begin{array}{l}\text { Triggers armed, LB5:40kW, Load increased at } \\
\text { 10:40 am }\end{array}$ \\
\hline
\end{tabular}




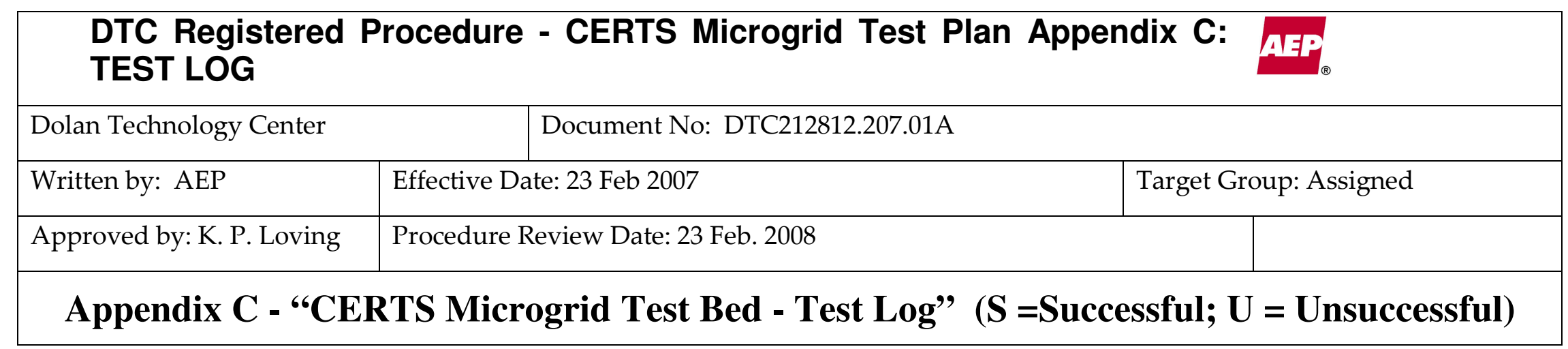

\begin{tabular}{|c|c|c|c|c|c|c|c|c|}
\hline $\begin{array}{l}\text { 8.1.15(8.1.1 } \\
1(8.1 .8))\end{array}$ & $2 / 21 / 08$ & 10:44 am & $\begin{array}{l}\text { 8.1.15(8.1 } \\
.11(8.1 .9))\end{array}$ & & $\mathbf{S}$ & $10: 49$ am & DAK & $\begin{array}{l}\text { Triggers armed, LB5:60kW, Load increased at } \\
\text { 10:45 am }\end{array}$ \\
\hline 8.1.16 & $2 / 21 / 08$ & 10:49 am & & & $\mathbf{S}$ & $10: 50$ am & DAK & LB5:0kW, Genset B1 shutdown \\
\hline 8.2.1 & $2 / 21 / 08$ & $10: 50$ am & 8.2 .2 & & $\mathbf{S}$ & $11: 10$ am & DAK & $\begin{array}{l}\text { Weak grid. SS, CB12, CB31, CB41, CB51 Closed. } \\
\text { Gensets in unit mode and setup. LB3:20kW, } \\
\text { LB4:20kW, LB6:30kW, LB6 increased to } 30 \mathrm{~kW} \text { to } \\
\text { prevent reverse power condition at SS. This was } \\
\text { necessary due to unbalance power at the PCC. } \\
\text { Meter 1:6kW }\end{array}$ \\
\hline 8.2.3 & 2/21/08 & 11:10 am & 8.2 .4 & 8.2 .5 & $\mathbf{S}$ & 11:16 am & DAK & $\begin{array}{l}\text { Triggers armed. SS manual open asserted at 11:11 } \\
\text { am. SS opened with a Fault Status and PES alarm. } \\
\text { Genset A1:0kW, Genset A2:37kW Meter1:0kW }\end{array}$ \\
\hline 8.2.6 & 2/21/08 & 11:16 am & 8.2.7 & 8.2.8 & $\mathbf{S}$ & $11: 28$ am & DAK & $\begin{array}{l}\text { SS was reset. Triggers armed. Manual open was } \\
\text { removed at 11:23 am. SS closed. Genset A1:5kW } \\
\text { Genset A2:55kW, Meter1:7kW. At 11:25 the SS } \\
\text { opened unexpectedly under reverse power. }\end{array}$ \\
\hline 8.2.9 & 2/21/08 & $11: 28$ am & & & $\mathbf{S}$ & $11: 40$ am & DAK & $\begin{array}{l}\text { Genset A1 was shutdown. The SS was allowed to } \\
\text { reconnect. A trigger was setup when Genset } \mathrm{A2} \text { was } \\
\text { shutdown to get a baseline of the unbalance due } \\
\text { solely to the utility. The event occurred at 11:36 am }\end{array}$ \\
\hline 8.3.1 & 2/21/08 & $11: 40$ am & 8.3.2 & & $\mathbf{S}$ & $11: 43 \mathrm{am}$ & DAK & $\begin{array}{l}\text { Weak grid. SS, CB12, CB31, CB41, CB51 Closed. } \\
\text { Gensets in unit mode and setup. LB3:60kW, }\end{array}$ \\
\hline
\end{tabular}

42 of 65

This document contains information confidential and proprietary to AEPSC. It shall not be reproduced in whole or in part or released to any third party without expressed written consent of AEPSC. 


\begin{tabular}{|c|c|c|c|c|c|c|c|c|}
\hline \multicolumn{9}{|c|}{$\begin{array}{l}\text { DTC Registered } \\
\text { TEST LOG }\end{array}$} \\
\hline \multicolumn{4}{|c|}{ Dolan Technology Center } & \multicolumn{5}{|c|}{ Document No: DTC212812.207.01A } \\
\hline \multicolumn{3}{|c|}{ Written by: AEP } & \multicolumn{5}{|c|}{ Effective Date: 23 Feb 2007} & Target Group: Assigned \\
\hline \multicolumn{3}{|c|}{ Approved by: K. P. Loving } & \multicolumn{5}{|c|}{ Procedure Review Date: 23 Feb. 2008} & \\
\hline \multicolumn{9}{|c|}{ Appendix C - "CERTS Microgrid Test Bed - Test Log” (S =Successful; U = Unsuccessful) } \\
\hline & & & & & & & & LB4:40kW. Meter 1:34kW \\
\hline 8.3 .3 & $2 / 21 / 08$ & 11:43 am & 8.3 .4 & 8.3 .5 & $\mathbf{S}$ & 11:49 am & DAK & $\begin{array}{l}\text { Triggers armed. SS manual open asserted at 11:45 } \\
\text { am. SS opened with a Fault Status and PES alarm. } \\
\text { Genset A1:29kW, Genset A2:60kW Meter1:0kW }\end{array}$ \\
\hline 8.3 .6 & $2 / 21 / 08$ & 11:49 am & 8.3 .7 & $\mathbf{8 . 3 . 8}$ & $\mathbf{S}$ & $12: 03 \mathrm{pm}$ & DAK & $\begin{array}{l}\text { SS was reset. Triggers armed. Manual open was } \\
\text { removed at } 11: 50 \text { am. SS closed. Genset A1:4kW } \\
\text { Genset A2:53kW, Meter1:35kW. Triggers disabled. } \\
\text { Genset A2 shutdown on an overfield fault at 11:54. }\end{array}$ \\
\hline 8.3.9 & $2 / 21 / 08$ & $12: 03 \mathrm{pm}$ & & & $\mathbf{S}$ & 12:05 pm & DAK & $\begin{array}{l}\text { Genset A1 was shutdown normally. All loads } \\
\text { removed. }\end{array}$ \\
\hline
\end{tabular}

( $\mathrm{S}$ =Successful; $\mathrm{U}=$ Unsuccessful)

\begin{tabular}{|l|l|l|l|l|l|l|l|l|}
\hline $\begin{array}{l}\text { Start Test } \\
\text { Sequence } \\
\text { No. }\end{array}$ & $\begin{array}{l}\text { Test } \\
\text { Date }\end{array}$ & $\begin{array}{l}\text { Start } \\
\text { Time }\end{array}$ & $\begin{array}{l}\text { Verify/ } \\
\text { Action }\end{array}$ & $\begin{array}{l}\text { Verify/ } \\
\text { Action }\end{array}$ & $\begin{array}{l}\text { Status } \\
\text { S or U }\end{array}$ & $\begin{array}{l}\text { End } \\
\text { Time }\end{array}$ & Initials & Test Event Comments: \\
\hline $\mathbf{8 . 4 . 1}$ & $\mathbf{2 / 2 6 / 0 8}$ & $\mathbf{1 0 : 2 6}$ am & $\mathbf{8 . 4 . 2}$ & & S & 10:46 am & DAK & $\begin{array}{l}\text { SS manual open asserted. CB12, CB31, CB41, CB51 } \\
\text { closed. Gensets setup and connected. LB3:60kW, } \\
\text { MeterA1:13kW, MeterA2:43kW }\end{array}$ \\
\hline $\mathbf{8 . 4 . 3}$ & $\mathbf{2 / 2 6 / 0 8}$ & $\mathbf{1 0 : 4 6}$ am & $\mathbf{8 . 4 . 4}$ & $\mathbf{8 . 4 . 5}$ & S & $\mathbf{1 0 : 5 1 \text { am }}$ & DAK & $\begin{array}{l}\text { Triggers armed. Load decreased at 10:48 am. } \\
\text { MeterA1:4kW, MeterA2:34kW }\end{array}$ \\
\hline $\mathbf{8 . 4 . 6}$ & $\mathbf{2 / 2 6 / 0 8}$ & $\mathbf{1 0 : 5 1}$ am & & & & & & LB3:0kW, Genset A1 and A2 shutdown. \\
\hline
\end{tabular}

43 of 65

This document contains information confidential and proprietary to AEPSC. It shall not be reproduced in whole or in part or released to any third party without expressed written consent of AEPSC. 


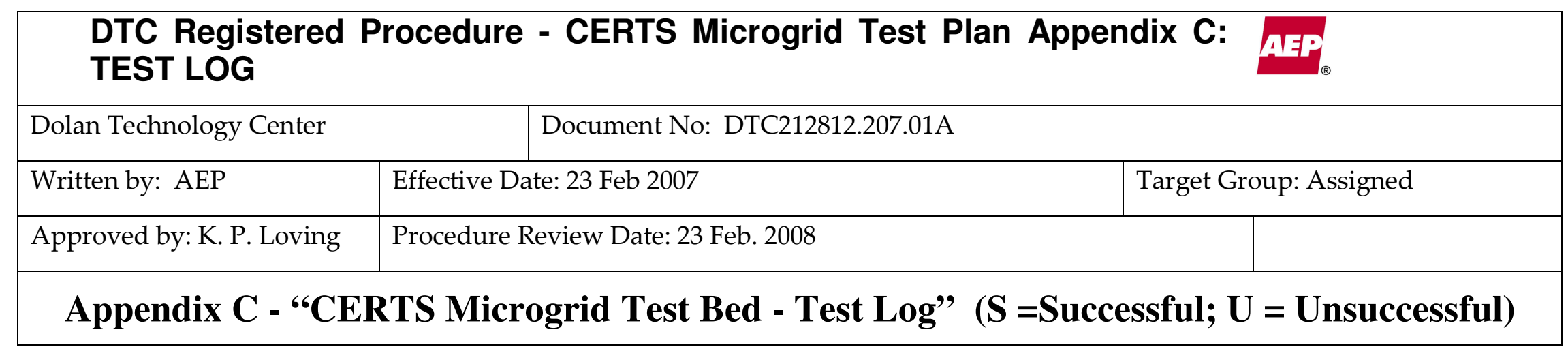

\begin{tabular}{|c|c|c|c|c|c|c|c|c|}
\hline 8.5 .1 & $2 / 26 / 08$ & 11:03 am & 8.5 .2 & & $\mathbf{S}$ & 11:05 am & $\overline{\text { DAK }}$ & $\begin{array}{l}\text { SS closed, All breakers closed, Gensets setup and } \\
\text { connected, LB3:35kW, LB4:35kW }\end{array}$ \\
\hline 8.5 .3 & $2 / 26 / 08$ & 11:05 am & $\begin{array}{l}8.5 .4 \\
\end{array}$ & 8.5 .5 & $\mathbf{S}$ & $11: 20 \mathrm{am}$ & DAK & $\begin{array}{l}\text { Meter3:57kW, MeterA1:5kW, MeterA2:6kW, } \\
\text { Triggers armed. LB3:85kW Load increased at } \\
\text { 11:15 am. Meter3:58kW, MeterA1:48kW, } \\
\text { MeterA2:6kW }\end{array}$ \\
\hline 8.5.6 & $2 / 26 / 08$ & $11: 20$ am & & & $\mathbf{S}$ & 11:22 am & & $\begin{array}{l}\text { A mistake with the dispatch was noticed. Genset } \mathrm{A1} \\
\text { was accidentally set for } 0 \mathrm{~kW} \text { in feeder mode. The } \\
\text { test was repeated. Gensets were not shutdown but } \\
\text { instead redispatched. }\end{array}$ \\
\hline 8.5.1R & $2 / 26 / 08$ & $11: 22$ am & 8.5.2R & & $\mathbf{S}$ & $11: 28$ am & DAK & $\begin{array}{l}\text { SS closed, All breakers closed, Gensets setup and } \\
\text { connected, LB3:35kW, LB4:35kW, Meter3:51kW, } \\
\text { MeterA1:10kW, MeterA2:6kW, }\end{array}$ \\
\hline 8.5.3R & $2 / 26 / 08$ & 11:28 am & 8.5.4R & 8.5.5R & $\mathbf{S}$ & 11:36 am & DAK & $\begin{array}{l}\text { Triggers armed. LB3:85kW Load increased at } \\
\text { 11:31 am. Meter3:52kW, MeterA1:52kW, } \\
\text { MeterA2:7kW }\end{array}$ \\
\hline $8.5 .6 \mathrm{R}$ & $2 / 26 / 08$ & $11: 36$ am & & & $\mathbf{S}$ & 11:37 am & DAK & \\
\hline 8.6 .1 & $2 / 26 / 08$ & $11: 37$ am & 8.6 .2 & & $\mathbf{S}$ & $11: 38$ am & DAK & $\begin{array}{l}\text { SS closed, All breakers closed, Gensets setup and } \\
\text { connected, LB3:75kW, LB4:35kW, Meter3:51kW, } \\
\text { MeterA1:44kW, MeterA2:7kW }\end{array}$ \\
\hline 8.6 .3 & $2 / 26 / 08$ & $11: 38$ am & 8.6 .4 & 8.6 .5 & $\mathbf{S}$ & $11: 45$ am & DAK & $\begin{array}{l}\text { Triggers armed. LB4:55kW Load increased at } \\
\text { 11:39 am. Meter3:58kW, MeterA1:58kW, }\end{array}$ \\
\hline
\end{tabular}

44 of 65

This document contains information confidential and proprietary to AEPSC. It shall not be reproduced in whole or in part or released to any third party without expressed written consent of AEPSC. 


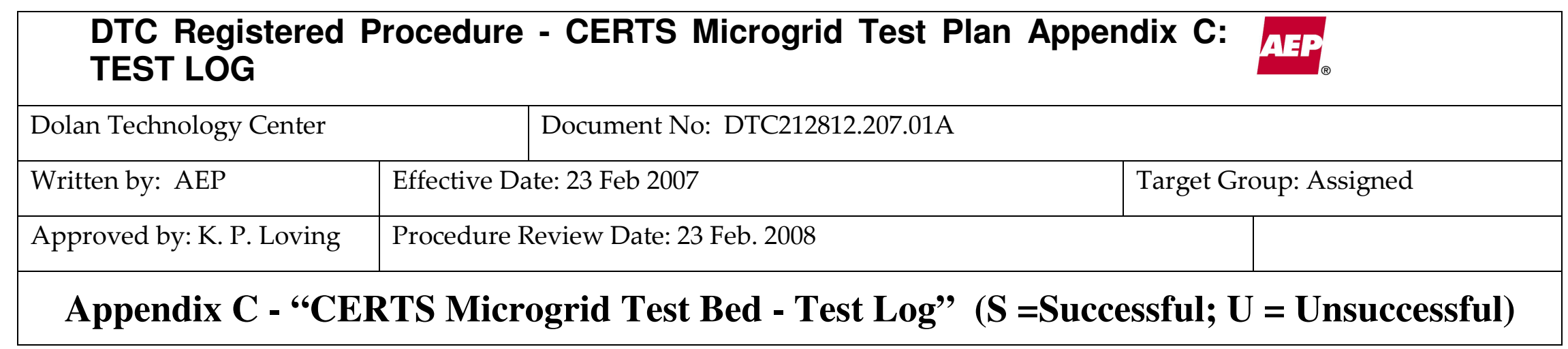

\begin{tabular}{|c|c|c|c|c|c|c|c|c|}
\hline & & & & & & & & MeterA2:5kW \\
\hline 8.6.6 & 2/26/08 & $11: 45 \mathrm{am}$ & & & $\mathbf{S}$ & $11: 46 \mathrm{am}$ & DAK & Gensets and loads redispatched instead of shutdown \\
\hline 8.7.1 & $2 / 26 / 08$ & 11:46 am & 8.7.2 & & $\mathbf{S}$ & $11: 49$ am & DAK & $\begin{array}{l}\text { SS closed, All breakers closed, Gensets setup and } \\
\text { connected, LB3:85kW, LB4:35kW, Meter3:54kW, } \\
\text { MeterA1:51kW, MeterA2:6kW }\end{array}$ \\
\hline 8.7 .3 & $2 / 26 / 08$ & 11:49 am & 8.7 .4 & 8.7 .5 & $\mathbf{S}$ & 12:02 pm & DAK & $\begin{array}{l}\text { Manually triggered at 11:52. Setpoint changed at } \\
\text { 11:53 am. Manually triggered at 11:57. } \\
\text { Meter3:103kW, MeterA1:0kW, MeterA2:6kW }\end{array}$ \\
\hline 8.7 .6 & $2 / 26 / 08$ & 12:02 pm & & & $\mathbf{S}$ & $12: 03$ pm & DAK & Gensets and loads redispatched instead of shutdown \\
\hline
\end{tabular}

\begin{tabular}{|l|l|l|l|l|l|l|l|l|}
\hline $\begin{array}{l}\text { Start Test } \\
\text { Sequence } \\
\text { No. }\end{array}$ & $\begin{array}{l}\text { Test } \\
\text { Date }\end{array}$ & $\begin{array}{l}\text { Start } \\
\text { Time }\end{array}$ & $\begin{array}{l}\text { Verify/ } \\
\text { Action }\end{array}$ & $\begin{array}{l}\text { Verify/ } \\
\text { Action }\end{array}$ & $\begin{array}{l}\text { Status } \\
\text { S or U }\end{array}$ & $\begin{array}{l}\text { End } \\
\text { Time }\end{array}$ & Initials & Test Event Comments: \\
\hline $\mathbf{8 . 8 . 1}$ & $\mathbf{2 / 2 6 / 0 8}$ & $12: 03 \mathrm{pm}$ & $\mathbf{8 . 8 . 2}$ & & $\mathrm{S}$ & $\mathbf{1 2 : 0 7} \mathrm{pm}$ & DAK & $\begin{array}{l}\text { SS closed, All breakers closed, Gensets setup and } \\
\text { connected, LB3:35kW, LB4:35kW, Meter3:47kW, } \\
\text { MeterA1:14kW, MeterA2:6kW }\end{array}$ \\
\hline $\mathbf{8 . 8 . 3}$ & $\mathbf{2 / 2 6 / 0 8}$ & $\mathbf{1 2 : 0 7} \mathrm{pm}$ & $\mathbf{8 . 8 . 4}$ & $\mathbf{8 . 8 . 5}$ & $\mathrm{S}$ & $\mathbf{1 2 : 1 5} \mathrm{pm}$ & DAK & $\begin{array}{l}\text { Triggers armed. Manual Open asserted at 12:08. } \\
\text { Meter3:-2kW, MeterA1:9kW, MeterA2:58kW }\end{array}$ \\
\hline
\end{tabular}

45 of 65

This document contains information confidential and proprietary to AEPSC. It shall not be reproduced in whole or in part or released to any third party without expressed written consent of AEPSC. 


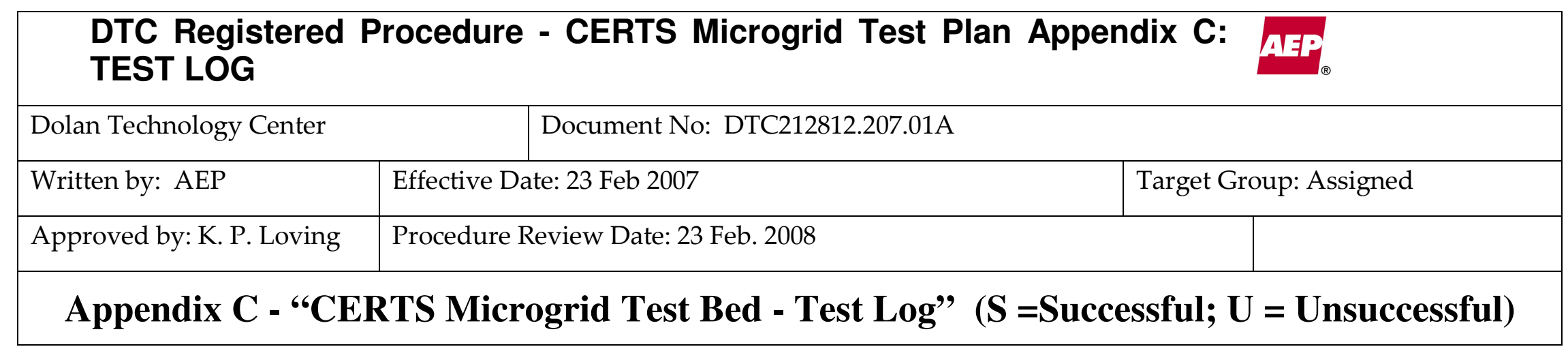

\begin{tabular}{|c|c|c|c|c|c|c|c|c|}
\hline & & & & & & & & $\begin{array}{l}\text { At approx 12:10 both gensets shutdown, Low Oil } \\
\text { Pressure on A1, and Underspeed on A2. Testing } \\
\text { repeated. }\end{array}$ \\
\hline 8.8 .6 & $2 / 26 / 08$ & $12: 15 \mathrm{pm}$ & $\begin{array}{l}8.8 .7 \\
\end{array}$ & 8.8 .8 & $\mathbf{S}$ & $12: 20 \mathrm{pm}$ & $\overline{\text { DAK }}$ & $\begin{array}{l}\text { Gensets shutdown unexpectedly, Loads were } \\
\text { removed. }\end{array}$ \\
\hline 8.8.1 & $2 / 26 / 08$ & 12:03 pm & 8.8 .2 & & $\mathbf{S}$ & 12:07 pm & DAK & $\begin{array}{l}\text { SS closed, All breakers closed, Gensets setup and } \\
\text { connected, LB3:35kW, LB4:35kW, Meter3:47kW, } \\
\text { MeterA1:14kW, MeterA2:6kW }\end{array}$ \\
\hline 8.8.3 & $2 / 26 / 08$ & 12:07 pm & 8.8.4 & 8.8.5 & $\mathbf{S}$ & 12:15 pm & DAK & $\begin{array}{l}\text { Triggers armed. Manual Open asserted at 12:08. } \\
\text { Meter3:-2kW, MeterA1:9kW, MeterA2:58kW } \\
\text { At approx 12:10 both gensets shutdown, Low Oil } \\
\text { Pressure on A1, and Underspeed on A2. Testing } \\
\text { repeated. }\end{array}$ \\
\hline 8.8.1R & $2 / 26 / 08$ & $1: 08 \mathrm{pm}$ & 8.8.2R & & $\mathbf{S}$ & 1:18 pm & DAK & $\begin{array}{l}\text { SS closed, All breakers closed, Gensets setup and } \\
\text { connected, LB3:35kW, LB4:35kW, Meter3:48kW, } \\
\text { MeterA1:14kW, MeterA2:56kW }\end{array}$ \\
\hline 8.8.3R & $2 / 26 / 08$ & $1: 18 \mathrm{pm}$ & 8.8.4R & 8.8.5R & $\mathbf{S}$ & $1: 25 \mathrm{pm}$ & DAK & $\begin{array}{l}\text { Triggers armed. Manual Open asserted at 1:21. } \\
\text { Meter3:-2kW, MeterA1:9kW, MeterA2:59kW, } \\
\text { Freq 59.57Hz }\end{array}$ \\
\hline 8.8.6R & $2 / 26 / 08$ & $1: 25 \mathrm{pm}$ & $8.8 .7 \mathrm{R}$ & 8.8.8R & $\mathbf{S}$ & $1: 32 \mathrm{pm}$ & DAK & $\begin{array}{l}\text { Manual Open removed at 1:27. SS Closed. } \\
\text { Meter3:49kW, MeterA1:14kW, MeterA2:4kW }\end{array}$ \\
\hline 8.8.9R & $2 / 26 / 08$ & $1: 32 \mathrm{pm}$ & & & $\mathbf{S}$ & $1: 33 \mathrm{pm}$ & DAK & $\begin{array}{l}\text { Gensets and loads redispatched instead of } \\
\text { shutdown. }\end{array}$ \\
\hline
\end{tabular}

46 of 65

This document contains information confidential and proprietary to AEPSC. It shall not be reproduced in whole or in part or released to any third party without expressed written consent of AEPSC. 


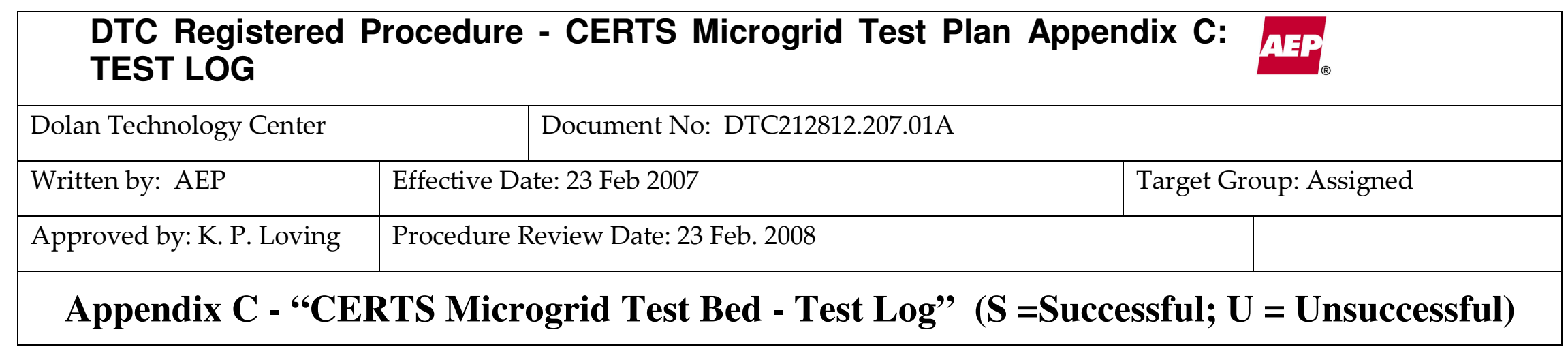

\begin{tabular}{|c|c|c|c|c|c|c|c|c|}
\hline 8.9.1 & $2 / 26 / 08$ & $1: 33$ pm & 8.9 .2 & & $\mathbf{S}$ & $1: 40 \mathrm{pm}$ & DAK & $\begin{array}{l}\text { SS closed, All breakers closed, Gensets setup and } \\
\text { connected, LB3:60kW, LB4:60kW, Meter3:55kW, } \\
\text { MeterA1:51kW, MeterA2:4kW }\end{array}$ \\
\hline 8.9 .3 & $2 / 26 / 08$ & $1: 40 \mathrm{pm}$ & 8.9 .4 & 8.9 .5 & $\mathbf{S}$ & $1: 52 \mathrm{pm}$ & DAK & $\begin{array}{l}\text { Triggers armed. Manual Open asserted at 1:42 pm. } \\
\text { SS opened. Genset A1 shutdown with a Overload } \\
\text { Alarm, Genset A2 shutdown with a Low Oil } \\
\text { Pressure alarm. Test repeated }\end{array}$ \\
\hline 8.9.1R & $2 / 26 / 08$ & 1:52 pm & 8.9.2R & & $\mathbf{S}$ & 2:02 pm & DAK & $\begin{array}{l}\text { SS closed, All breakers closed, Gensets setup and } \\
\text { connected, LB3:60kW, LB4:60kW, Meter3:51kW, } \\
\text { MeterA1:52kW, MeterA2:9kW }\end{array}$ \\
\hline 8.9.3R & $2 / 26 / 08$ & 2:02 pm & 8.9.4R & 8.9.5R & $\mathbf{S}$ & $208 \mathrm{pm}$ & DAK & $\begin{array}{l}\text { Triggers armed. Manual Open asserted at 2:03 pm. } \\
\text { SS opened. Meter3:-2kW, MeterA1:47kW, } \\
\text { MeterA2:60kW }\end{array}$ \\
\hline 8.9.6R & $2 / 26 / 08$ & 2:08 pm & 8.9.7R & 8.9.8R & $\mathbf{S}$ & 2:14 pm & DAK & $\begin{array}{l}\text { Manual Open removed at 2:09. SS Closed. } \\
\text { Meter3:56kW, MeterA1:48kW, MeterA2:3kW }\end{array}$ \\
\hline 8.9.9R & $2 / 26 / 08$ & 2:14 pm & & & $\mathbf{S}$ & 2:15 pm & DAK & $\begin{array}{l}\text { Genset A2 shutdown, Genset A1 and loads } \\
\text { redispatched. }\end{array}$ \\
\hline 8.10 .1 & $2 / 26 / 08$ & $2: 15$ pm & 8.10 .2 & & $\mathbf{S}$ & 2:27 pm & DAK & $\begin{array}{l}\text { SS closed, All breakers closed, Gensets setup and } \\
\text { connected, LB3:40kW, LB5:50kW, Meter3:36kW, } \\
\text { MeterA1:0kW, Meter5:10kW, MeterB1:37kW }\end{array}$ \\
\hline 8.10 .3 & $2 / 26 / 08$ & $2: 27 \mathrm{pm}$ & 8.10 .4 & 8.10 .5 & $\mathbf{S}$ & $2: 35 \mathrm{pm}$ & DAK & $\begin{array}{l}\text { Triggers armed. Manual Open asserted at 2:30 pm. } \\
\text { SS opened. Meter3:13kW, MeterA1:21kW, Meter5: } \\
\text {-15kW, MeterB1:61kW. }\end{array}$ \\
\hline
\end{tabular}

47 of 65

This document contains information confidential and proprietary to AEPSC. It shall not be reproduced in whole or in part or released to any third party without expressed written consent of AEPSC. 


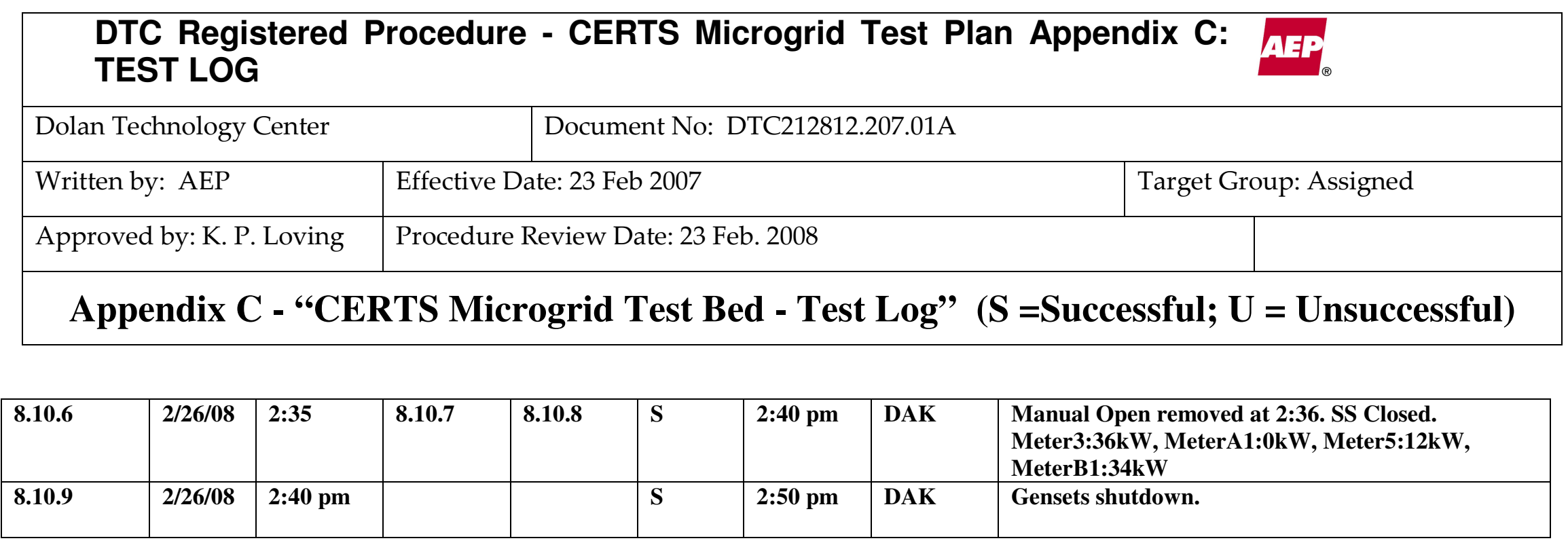

( $\mathrm{S}=$ Successful; $\mathbf{U}=$ Unsuccessful)

\begin{tabular}{|l|l|l|l|l|l|l|l|l|}
\hline $\begin{array}{l}\text { Start Test } \\
\text { Sequence } \\
\text { No. }\end{array}$ & $\begin{array}{l}\text { Test } \\
\text { Date }\end{array}$ & $\begin{array}{l}\text { Start } \\
\text { Time }\end{array}$ & $\begin{array}{l}\text { Verify/ } \\
\text { Action }\end{array}$ & $\begin{array}{l}\text { Verify/ } \\
\text { Action }\end{array}$ & $\begin{array}{l}\text { Status } \\
\text { S or U }\end{array}$ & $\begin{array}{l}\text { End } \\
\text { Time }\end{array}$ & Initials & Test Event Comments: \\
\hline $\mathbf{8 . 1 1 . 1}$ & $\mathbf{2 / 2 6 / 0 8}$ & $\mathbf{3 : 0 0} \mathrm{pm}$ & $\mathbf{8 . 1 1 . 2}$ & & $\mathrm{S}$ & $\mathbf{3 : 0 7} \mathrm{pm}$ & DAK & $\begin{array}{l}\text { SS closed, All breakers closed, Gensets setup and } \\
\text { connected, LB3:20kW, LB5:40kW, LB6:20kW. } \\
\text { Meter3:-25kW, MeterA1:43kW, Meter5:22kW, } \\
\text { MeterB1:14kW, Meter2:-1kW, Meter1:18kW }\end{array}$ \\
\hline $\mathbf{8 . 1 1 . 3}$ & $\mathbf{2 / 2 6 / 0 8}$ & $\mathbf{3 : 0 7} \mathrm{pm}$ & $\mathbf{8 . 1 1 . 4}$ & $\mathbf{8 . 1 1 . 5}$ & $\mathrm{S}$ & $\mathbf{3 : 1 5} \mathrm{pm}$ & DAK & $\begin{array}{l}\text { Triggers armed. Manual Open asserted at 3:11 pm. } \\
\text { SS opened with PES Alarm. Meter3:-25kW, } \\
\text { MeterA1:43kW, Meter5:23kW, MeterB1:12kW, } \\
\text { Meter2:0kW, Meter1:19kW }\end{array}$ \\
\hline $\mathbf{8 . 1 1 . 6}$ & $\mathbf{2 / 2 6 / 0 8}$ & $\mathbf{3 : 1 5} \mathrm{pm}$ & $\mathbf{8 . 1 1 . 7}$ & $\mathbf{8 . 1 1 . 8}$ & $\mathrm{S}$ & $\mathbf{3 : 2 1} \mathrm{pm}$ & DAK & $\begin{array}{l}\text { Manual Open removed at 3:16. SS Closed. Meter3:- } \\
\text { 26kW, MeterA1:45kW, Meter5:21kW, } \\
\text { MeterB1:15kW, Meter2:-2kW, Meter1:16kW }\end{array}$ \\
\hline $\mathbf{8 . 1 1 . 9}$ & $\mathbf{2 / 2 6 / 0 8}$ & $\mathbf{3 : 2 1} \mathrm{pm}$ & & & $\mathrm{S}$ & $\mathbf{3 : 2 2} \mathrm{pm}$ & DAK & Gensets shutdown and loads removed. \\
\hline
\end{tabular}

48 of 65

This document contains information confidential and proprietary to AEPSC. It shall not be reproduced in whole or in part or released to any third party without expressed written consent of AEPSC. 


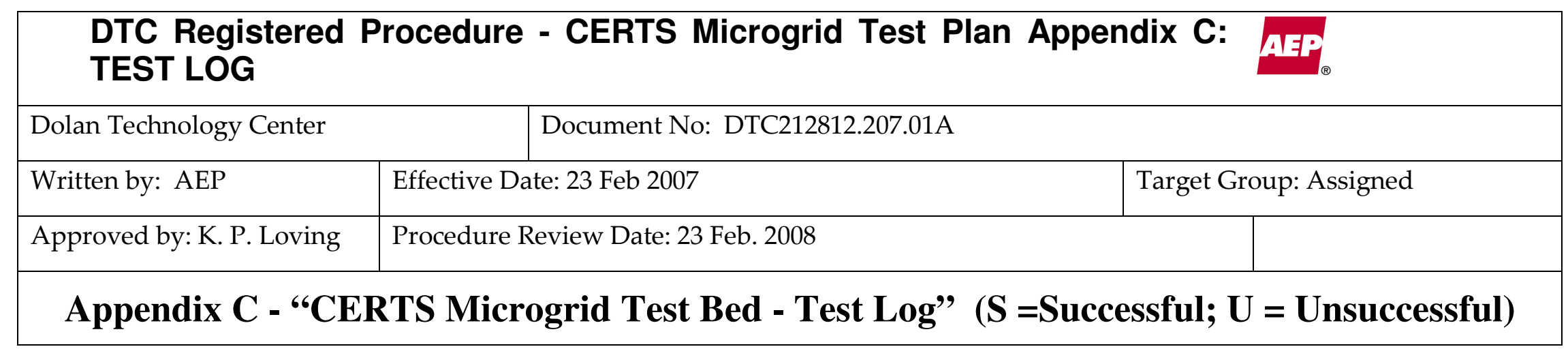

\begin{tabular}{|c|c|c|c|c|c|c|c|}
\hline 8.12 .1 & $2 / 27 / 08$ & 9:54 am & 8.12 .2 & $\mathbf{S}$ & $10: 31$ am & DAK & $\begin{array}{l}\text { CB12 open, Gensets setup to } 20 \mathrm{~kW} \text { in unit control } \\
\text { and connected. }\end{array}$ \\
\hline 8.12 .3 & $2 / 27 / 08$ & 10:31 am & & $\mathbf{S}$ & 10:34 am & DAK & $\begin{array}{l}\text { LB3:25kW, 10kVar, LB4:25kW, 10kVar, } \\
\text { LB5:25kW, 10kVar, LB6:5kW, 10kVar. Load was } \\
\text { added to LB6 to pull the isolated bus voltage down } \\
\text { to near zero volts, it was floating near } 80 \text { volts prior. } \\
\text { Manual open removed. }\end{array}$ \\
\hline 8.12 .4 & 2/27/08 & $10: 34$ am & 8.12 .5 & $\mathbf{S}$ & $10: 49$ am & & CB12 closed at 10:38:10. SS closed at 10:43:10 \\
\hline 8.12 .6 & $2 / 27 / 08$ & 10:49 am & & $\mathbf{S}$ & 11:12 am & & $\begin{array}{l}\text { DAS system did trigger on the closing of the SS. At } \\
\text { 11:01 the SS opened on reverse power and locked } \\
\text { out. The meter triggers were disabled and only the } \\
\text { relays captured this event. }\end{array}$ \\
\hline
\end{tabular}

\begin{tabular}{|l|l|l|l|l|l|l|l|l|}
\hline $\begin{array}{l}\text { Start Test } \\
\text { Sequence } \\
\text { No. }\end{array}$ & $\begin{array}{l}\text { Test } \\
\text { Date }\end{array}$ & $\begin{array}{l}\text { Start } \\
\text { Time }\end{array}$ & $\begin{array}{l}\text { Verify/ } \\
\text { Action }\end{array}$ & $\begin{array}{l}\text { Verify/ } \\
\text { Action }\end{array}$ & $\begin{array}{l}\text { Status } \\
\text { S or U }\end{array}$ & $\begin{array}{l}\text { End } \\
\text { Time }\end{array}$ & Initials & Test Event Comments: \\
\hline 8.13.1 & $2 / 27 / 08$ & $11: 12$ am & $\mathbf{8 . 1 3 . 2}$ & & S & $11: 16$ am & DAK & CB12 opened, Genset setup to 20kW unit control. \\
\hline 8.13 .3 .1 & $2 / 27 / 08$ & $11: 16$ am & $\mathbf{8 . 1 3 . 3 . 2}$ & & S & $11: 18$ am & DAK & Manual Open asserted. LB3:20kW ,20kVar \\
\hline
\end{tabular}

49 of 65

This document contains information confidential and proprietary to AEPSC. It shall not be reproduced in whole or in part or released to any third party without expressed written consent of AEPSC. 


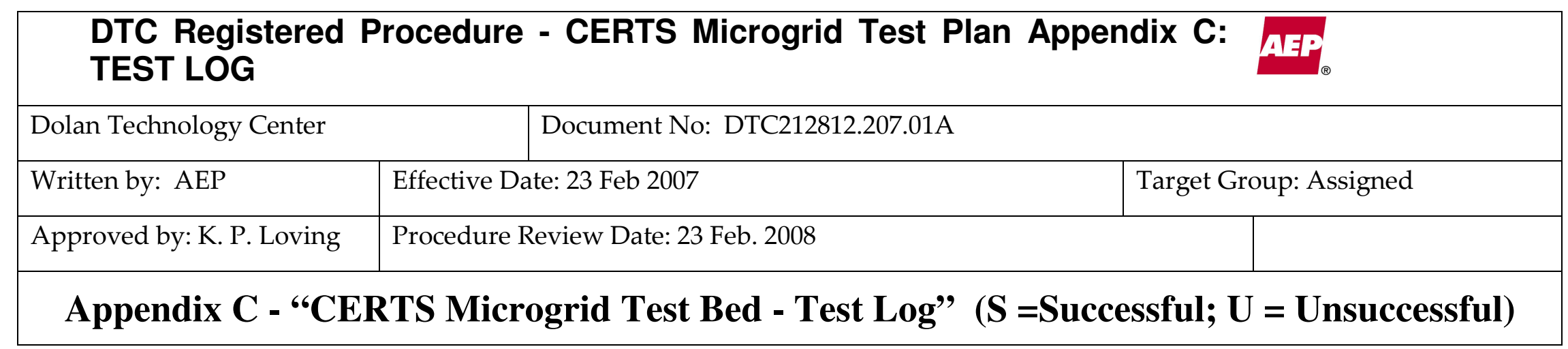

\begin{tabular}{|c|c|c|c|c|c|c|c|}
\hline 8.13.3.3 & $2 / 27 / 08$ & 11:18 am & 8.13.3.4 & $\mathbf{S}$ & 11:24 am & DAK & $\begin{array}{l}\text { Triggers armed. Genset A1 connected at 11:20 am } \\
\text { Meter3:-2kW, -3kVar, MeterA1:20kW 20kVar, } \\
\text { LoadMeter3:17kW 18kVar }\end{array}$ \\
\hline 8.13.3.5 & $2 / 27 / 08$ & $11: 24$ am & 8.13.3.6 & $\mathbf{S}$ & $11: 25$ am & DAK & Genset A1 shutdown. LB3:35kW, 20kVar \\
\hline 8.13.3.7 & $2 / 27 / 08$ & 11:25 am & 8.13.3.8 & $\mathbf{S}$ & 11:31 am & DAK & $\begin{array}{l}\text { Triggers armed. Genset A1 connected at 11:26 am } \\
\text { Meter3:-2kW, -3kVar, MeterA1:33kW, 21kVar } \\
\text { LoadMeter3:31kW, 18kVar }\end{array}$ \\
\hline 8.13.3.9 & $2 / 27 / 08$ & 11:31 am & 8.13.3.10 & $\mathbf{S}$ & $11: 45$ am & DAK & Genset A1 shutdown. LB3:55kW, 20kVar \\
\hline 8.13.3.11 & $2 / 27 / 08$ & $11: 45$ am & 8.13.3.12 & $\mathbf{S}$ & $11: 52$ am & DAK & $\begin{array}{l}\text { Triggers armed. Genset A1 connected at 11:47 am } \\
\text { Meter3:-2kW, -3kVar, MeterA1:51kW 21kVar, } \\
\text { LoadMeter3:47kW 18kVar }\end{array}$ \\
\hline 8.13.3.13 & $2 / 27 / 08$ & $11: 52$ am & 8.13.3.14 & $\mathbf{S}$ & $11: 54$ am & DAK & Genset A1 shutdown. LB3:60kW, 30kVar \\
\hline 8.13.3.15 & $2 / 27 / 08$ & 11:54 am & 8.13.3.16 & $\mathbf{S}$ & 12:00 pm & DAK & $\begin{array}{l}\text { Triggers armed. Genset A1 connected at 11:55 am } \\
\text { Meter3:-2kW, -2kVar, MeterA1:50kW 29kVar, } \\
\text { LoadMeter3:47kW 26kVar }\end{array}$ \\
\hline 8.13.3.13 B & $2 / 27 / 08$ & 12:00 am & $\begin{array}{l}\text { 8.13.3.14 } \\
\text { B }\end{array}$ & $\mathbf{S}$ & 12:02 pm & DAK & $\begin{array}{l}\text { Genset A1 shutdown. LB3:70kW, 30kVar. Load } \\
\text { was increased a final time as the actual load is } \\
\text { always less than that which is dispatched. }\end{array}$ \\
\hline 8.13.3.15B & $2 / 27 / 08$ & 12:02 pm & $\begin{array}{l}\text { 8.13.3.16 } \\
\text { B }\end{array}$ & $\mathbf{S}$ & 12:09 pm & DAK & $\begin{array}{l}\text { Triggers armed. Genset A1 connected at 12:04 pm } \\
\text { Meter3:-2kW, -2kVar, MeterA1:60kW 29kVar, } \\
\text { LoadMeter3:57kW 26kVar }\end{array}$ \\
\hline
\end{tabular}

50 of 65

This document contains information confidential and proprietary to AEPSC. It shall not be reproduced in whole or in part or released to any third party without expressed written consent of AEPSC. 


\begin{tabular}{|c|c|c|c|c|c|c|}
\hline \multicolumn{7}{|c|}{$\begin{array}{l}\text { DTC Registered Procedure - CERTS Microgrid Test Plan Appendix C: } \\
\text { TEST LOG }\end{array}$} \\
\hline \multicolumn{3}{|c|}{ Dolan Technology Center } & & \multicolumn{3}{|c|}{ Document No: DTC212812.207.01A } \\
\hline \multicolumn{3}{|c|}{ Written by: AEP } & \multicolumn{3}{|c|}{ Effective Date: 23 Feb 2007} & Target Group: Assigned \\
\hline \multicolumn{3}{|c|}{ Approved by: K. P. Loving } & \multicolumn{3}{|c|}{ Procedure Review Date: 23 Feb. 2008} & \\
\hline \multicolumn{7}{|c|}{ Appendix C - “CERTS Microgrid Test Bed - Test Log” (S =Successful; U = Unsuccessful) } \\
\hline 8.13.3.17 & $2 / 27 / 08$ & 12:09 pm & & $12: 20 \mathrm{pm}$ & DAK & Genset A1 shutdown and loads removed. \\
\hline
\end{tabular}

\begin{tabular}{|c|c|c|c|c|c|c|c|c|}
\hline $\begin{array}{l}\text { Start Test } \\
\text { Sequence } \\
\text { No. }\end{array}$ & $\begin{array}{l}\text { Test } \\
\text { Date }\end{array}$ & $\begin{array}{l}\text { Start } \\
\text { Time }\end{array}$ & $\begin{array}{l}\text { Verify/ } \\
\text { Action }\end{array}$ & $\begin{array}{l}\text { Verify/ } \\
\text { Action }\end{array}$ & $\begin{array}{l}\text { Status } \\
\text { S or U }\end{array}$ & $\begin{array}{l}\text { End } \\
\text { Time }\end{array}$ & Initials & Test Event Comments: \\
\hline 9.1.7(9.1.1) & $3 / 5 / 08$ & $10: 28$ am & $\begin{array}{l}\text { 9.1.7(9.1. } \\
\text { 2) }\end{array}$ & & $\mathbf{S}$ & $11: 12$ am & DAK & $\begin{array}{l}\text { Gensets setup an d connected. SS closed. LB3:40kW } \\
\text { 20kVar, LB4:40kW 20kVar, LB5:40kW 20kVar, } \\
\text { LB6:20kW 10kVar, Meter2:28kW, Meter3:17kW, } \\
\text { Meter4:6kW, Meter5:9kW }\end{array}$ \\
\hline 9.1.7(9.1.3) & $3 / 5 / 08$ & 11:12 am & $\begin{array}{l}\text { 9.1.7(9.1. } \\
4)\end{array}$ & & $\mathbf{S}$ & 11:37 am & DAK & $\begin{array}{l}\text { Triggers armed, Manual Open asserted at 11:16. SS } \\
\text { opened with PES Alarm. Meter2:0kW, Meter3:- } \\
\text { 2kW, Meter4:-4kW, Meter5:0kW Meters triggered } \\
\text { when SS was reset. }\end{array}$ \\
\hline 9.1.7(9.1.5) & $3 / 5 / 08$ & 11:37 am & $\begin{array}{l}\text { 9.1.7(9.1. } \\
6)\end{array}$ & & $\mathbf{S}$ & 11: 43 am & DAK & $\begin{array}{l}\text { Triggers armed, Manual Open removed at 11:38. } \\
\text { SS closed. Meter2:29kW, Meter3:17kW, } \\
\text { Meter4:6kW, Meter5:10kW }\end{array}$ \\
\hline 9.1.8(9.1.1) & $3 / 5 / 08$ & $11: 43$ am & $\begin{array}{l}\text { 9.1.8(9.1. } \\
2)\end{array}$ & & $\mathbf{S}$ & 11:51 am & DAK & $\begin{array}{l}\text { Gensets setup an d connected. SS closed. LB3:20kW } \\
\text { 10kVar, LB4:40kW 20kVar, LB5:50kW 20kVar, } \\
\text { LB6:20kW 10kVar, Meter2:21kW, Meter3:-8kW, }\end{array}$ \\
\hline
\end{tabular}

51 of 65

This document contains information confidential and proprietary to AEPSC. It shall not be reproduced in whole or in part or released to any third party without expressed written consent of AEPSC. 


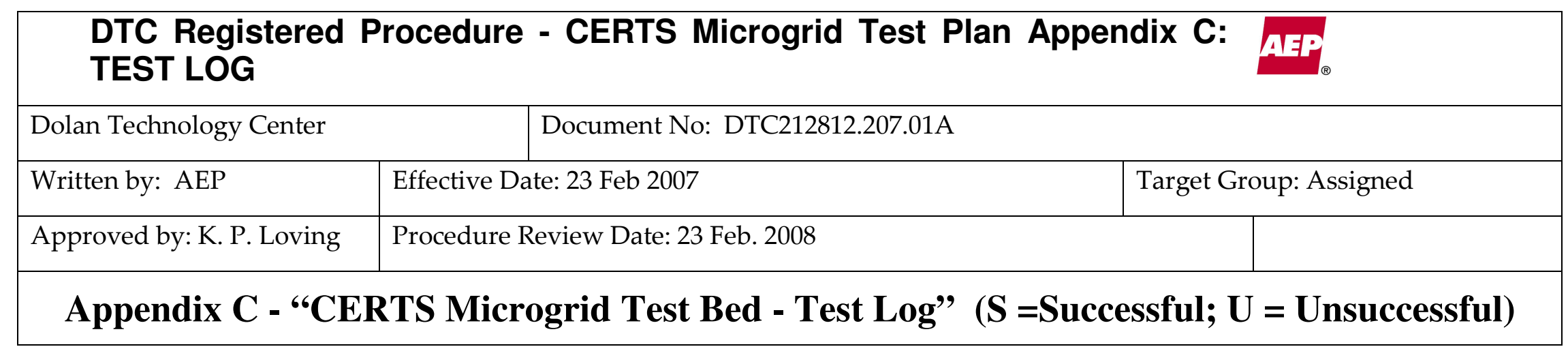

\begin{tabular}{|c|c|c|c|c|c|c|c|}
\hline & & & & & & & Meter4:7kW, Meter5:25kW \\
\hline 9.1.8(9.1.3) & $3 / \mathbf{5} / 08$ & 11:51 am & $\begin{array}{l}9.1 .8(9.1 \\
4)\end{array}$ & $\mathbf{S}$ & 12:01 pm & DAK & $\begin{array}{l}\text { Triggers armed, Manual Open asserted at 11:54. SS } \\
\text { opened with PES Alarm. Meter2:0kW, Meter3:- } \\
\text { 21kW, Meter4:0kW, Meter5:18kW }\end{array}$ \\
\hline 9.1.8(9.1.5) & $3 / 5 / 08$ & 12:01 pm & $\begin{array}{l}\text { 9.1.8(9.1. } \\
\text { 6) }\end{array}$ & $\mathbf{S}$ & $12: 15 \mathrm{pm}$ & DAK & $\begin{array}{l}\text { Triggers armed, Manual Open removed at 12:09. } \\
\text { SS closed. Meter2:14kW, Meter3:-12kW, } \\
\text { Meter4:5kW, Meter5:23kW }\end{array}$ \\
\hline $9.1 .9(9.1 .1)$ & $3 / 5 / 08$ & $1: 10 \mathrm{pm}$ & $\begin{array}{l}9.1 .9(9.1 . \\
2)\end{array}$ & $\mathbf{S}$ & $1: 17 \mathrm{pm}$ & DAK & $\begin{array}{l}\text { Gensets setup an d connected. SS closed. LB3:20kW } \\
\text { 10kVar, LB4:15kW 10kVar, LB5:50kW 20kVar, } \\
\text { LB6:30kW 10kVar, Meter2:-12kW, Meter3:-32kW, } \\
\text { Meter4:-15kW, Meter5:17kW }\end{array}$ \\
\hline 9.1.9(9.1.3) & $3 / 5 / 08$ & 1:17 pm & $\begin{array}{l}9.1 .9(9.1 . \\
4)\end{array}$ & $\mathbf{S}$ & $1: 24 \mathrm{pm}$ & DAK & $\begin{array}{l}\text { Triggers armed, Manual Open asserted at 1:19. SS } \\
\text { opened with PES Alarm. Meter2:0kW, Meter3:- } \\
\text { 23kW, Meter4:-11kW, Meter5:21kW }\end{array}$ \\
\hline 9.1.9(9.1.5) & $3 / 5 / 08$ & $1: 24 \mathrm{pm}$ & $\begin{array}{l}9.1 .9(9.1 \\
6)\end{array}$ & $\mathbf{S}$ & $1: 31 \mathrm{pm}$ & DAK & $\begin{array}{l}\text { Triggers armed, Manual Open removed at 1:26. SS } \\
\text { closed. Meter2:-17kW, Meter3:-35kW, Meter4:- } \\
\text { 17kW, Meter5:16kW }\end{array}$ \\
\hline 9.1.10(9.1.1) & $3 / 5 / 08$ & $1: 31 \mathrm{pm}$ & $\begin{array}{l}9.1 .10(9.1 \\
.2)\end{array}$ & $\mathbf{S}$ & $1: 43 \mathrm{pm}$ & \begin{tabular}{|l} 
DAK \\
\end{tabular} & $\begin{array}{l}\text { Gensets setup an d connected. SS closed. LB3:50kW } \\
\text { 20kVar, LB4:40kW 20kVar, LB5:25kW 10kVar, } \\
\text { LB6:30kW 10kVar, Meter2:19kW, Meter3:34kW, } \\
\text { Meter4:15kW, Meter5:-18kW }\end{array}$ \\
\hline $9.1 .10(9.1 .3)$ & $3 / 5 / 08$ & 1:43 pm & $\begin{array}{l}9.1 .10(9.1 \\
.4)\end{array}$ & $\mathbf{S}$ & $1: 54 \mathrm{pm}$ & DAK & $\begin{array}{l}\text { Triggers armed, Manual Open asserted at 1:45. SS } \\
\text { opened with PES Alarm. Meter2:0kW, }\end{array}$ \\
\hline
\end{tabular}

52 of 65

This document contains information confidential and proprietary to AEPSC. It shall not be reproduced in whole or in part or released to any third party without expressed written consent of AEPSC. 


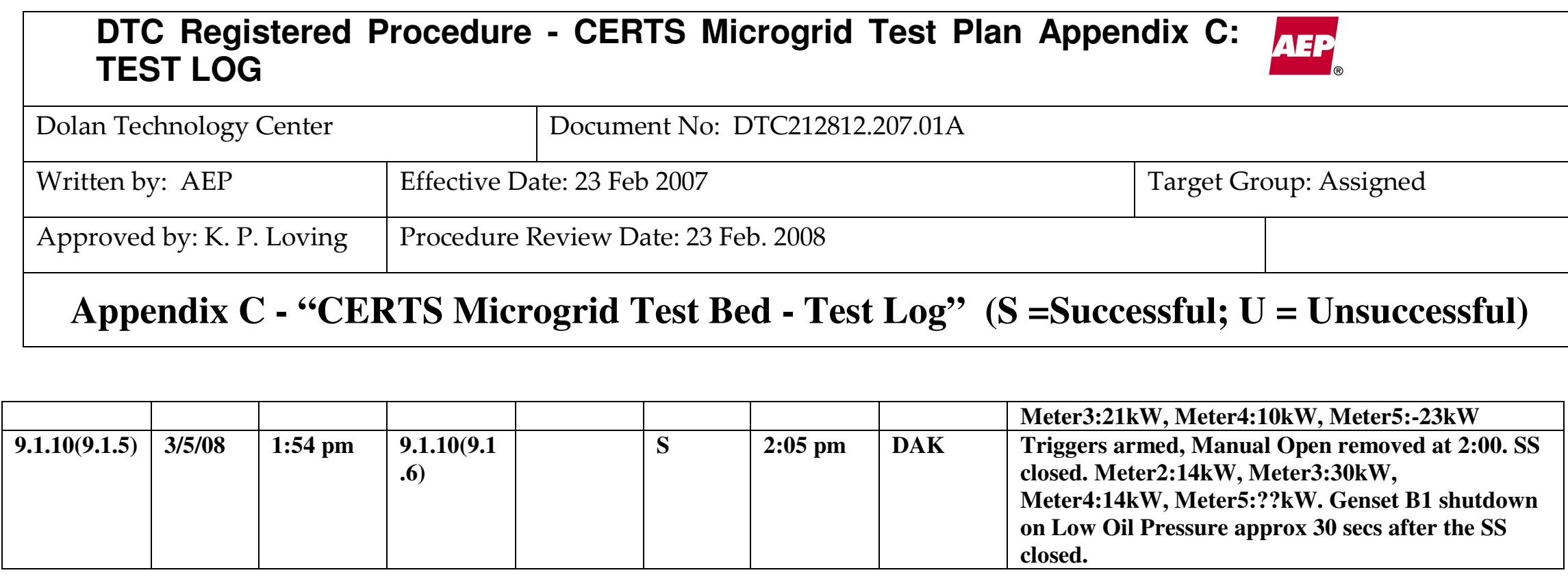




\begin{tabular}{|l|l|l|}
\hline \multicolumn{2}{|c|}{$\begin{array}{l}\text { DTC Registered Procedure - CERTS Microgrid Test Plan Appendix C: } \\
\text { TEST LOG }\end{array}$} \\
\hline Dolan Technology Center & Document No: DTC212812.207.01A \\
\hline Written by: AEP & Effective Date: 23 Feb 2007 & \multicolumn{1}{|c|}{} \\
\hline Approved by: K. P. Loving & Procedure Review Date: 23 Feb. 2008 & \\
\hline \multicolumn{2}{|l|}{ Appendix C - “CERTS Microgrid Test Bed - Test Log” (S =Successful; U = Unsuccessful) } \\
\hline
\end{tabular}

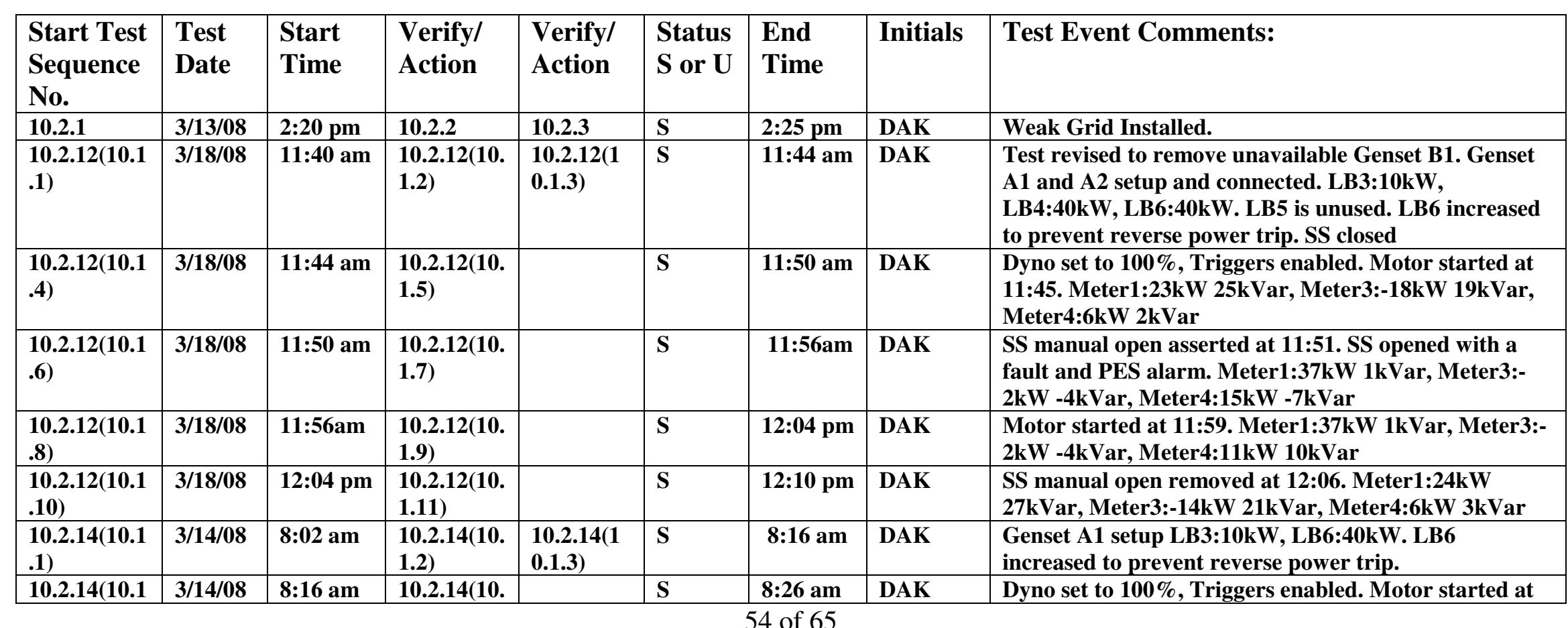

This document contains information confidential and proprietary to AEPSC. It shall not be reproduced in whole or in part or released to any third party without expressed written consent of AEPSC. 


\begin{tabular}{|c|c|c|}
\hline \multicolumn{3}{|c|}{$\begin{array}{l}\text { DTC Registered Procedure - CERTS Microgrid Test Plan Appendix C: } \\
\text { TEST LOG }\end{array}$} \\
\hline Dolan Technology Center & \multicolumn{2}{|c|}{ Document No: DTC212812.207.01A } \\
\hline Written by: AEP & Effective Date: 23 Feb 2007 & Target Group: Assigned \\
\hline Approved by: K. P. Loving & Procedure Review Date: 23 Feb. 2008 & \\
\hline \multicolumn{3}{|c|}{ Appendix C - “CERTS Microgrid Test Bed - Test Log” (S =Successful; U = Unsuccessful) } \\
\hline
\end{tabular}

\begin{tabular}{|c|c|c|c|c|c|c|c|c|}
\hline $\begin{array}{l}\text { Start Test } \\
\text { Sequence } \\
\text { No. }\end{array}$ & $\begin{array}{l}\text { Test } \\
\text { Date }\end{array}$ & $\begin{array}{l}\text { Start } \\
\text { Time }\end{array}$ & $\begin{array}{l}\text { Verify/ } \\
\text { Action }\end{array}$ & $\begin{array}{l}\text { Verify/ } \\
\text { Action }\end{array}$ & $\begin{array}{l}\text { Status } \\
\text { S or U }\end{array}$ & $\begin{array}{l}\text { End } \\
\text { Time }\end{array}$ & Initials & Test Event Comments: \\
\hline $\begin{array}{l}\text { 10.2.14(10.1 } \\
.8)\end{array}$ & $3 / 14 / 08$ & $8: 33$ am & $\begin{array}{l}\text { 10.2.14(1 } \\
\text { 0.1.9) }\end{array}$ & & $\mathbf{S}$ & 8:39 am & DAK & Motor started at 8:34. Meter3:-2kW -3kVar \\
\hline $\begin{array}{l}\text { 10.2.14(10.1 } \\
.10)\end{array}$ & $3 / 14 / 08$ & 8:39 am & $\begin{array}{l}\text { 10.2.14(1 } \\
\text { 0.1.11) }\end{array}$ & & $\mathbf{S}$ & $8: 46$ am & DAK & $\begin{array}{l}\text { SS manual open removed at 8:41. Meter3:-21kW } \\
\text { 19kVar }\end{array}$ \\
\hline $\begin{array}{l}\text { 10.2.15(10.1 } \\
.1)\end{array}$ & 3/18/08 & $12: 10 \mathrm{pm}$ & $\begin{array}{l}10.2 .15(1 \\
0.1 .2)\end{array}$ & $\begin{array}{l}\text { 10.2.15(1 } \\
0.1 .3)\end{array}$ & $\mathbf{S}$ & 12:15 am & DAK & $\begin{array}{l}\text { Test revised to remove unavailable Genset } B 1 \text { and test } \\
\text { the gensets closer to capacity. Genset } A 1 \text { and } A 2 \text { setup } \\
\text { and connected. } L B 3: 50 \mathrm{~kW}, \mathrm{LB} 4: 50 \mathrm{~kW}, \mathrm{LB6}: 40 \mathrm{~kW} \text {, } \\
\mathrm{LB5} \text { is unused. } \mathrm{LB} 6 \text { increased to prevent reverse } \\
\text { power trip. SS closed }\end{array}$ \\
\hline $\begin{array}{l}\text { 10.2.15(10.1 } \\
.4)\end{array}$ & $3 / 18 / 08$ & 12:15 am & $\begin{array}{l}\text { 10.2.15(1 } \\
0.1 .5)\end{array}$ & & $\mathbf{S}$ & $12: 21 \mathrm{pm}$ & DAK & $\begin{array}{l}\text { Dyno set to } 100 \% \text {, Triggers enabled. Motor started at } \\
\text { 12:16. Meter1:69kW 21kVar, Meter3:29kW 15kVar, } \\
\text { Meter4:14kW -1kVar }\end{array}$ \\
\hline $\begin{array}{l}\text { 10.2.15(10.1 } \\
.6)\end{array}$ & $3 / 18 / 08$ & $12: 21 \mathrm{pm}$ & $\begin{array}{l}\text { 10.2.15(1 } \\
0.1 .7)\end{array}$ & & $\mathbf{S}$ & $12: 32 \mathrm{pm}$ & DAK & $\begin{array}{l}\text { SS manual open asserted at 12:26. SS opened with a } \\
\text { fault and PES alarm. Meter1:37kW } 1 \mathrm{kVar} \text {, Meter3:- } \\
2 \mathrm{~kW}-4 \mathrm{kVar} \text {, Meter4:2kW -7kVar }\end{array}$ \\
\hline
\end{tabular}

55 of 65

This document contains information confidential and proprietary to AEPSC. It shall not be reproduced in whole or in part or released to any third party without expressed written consent of AEPSC. 


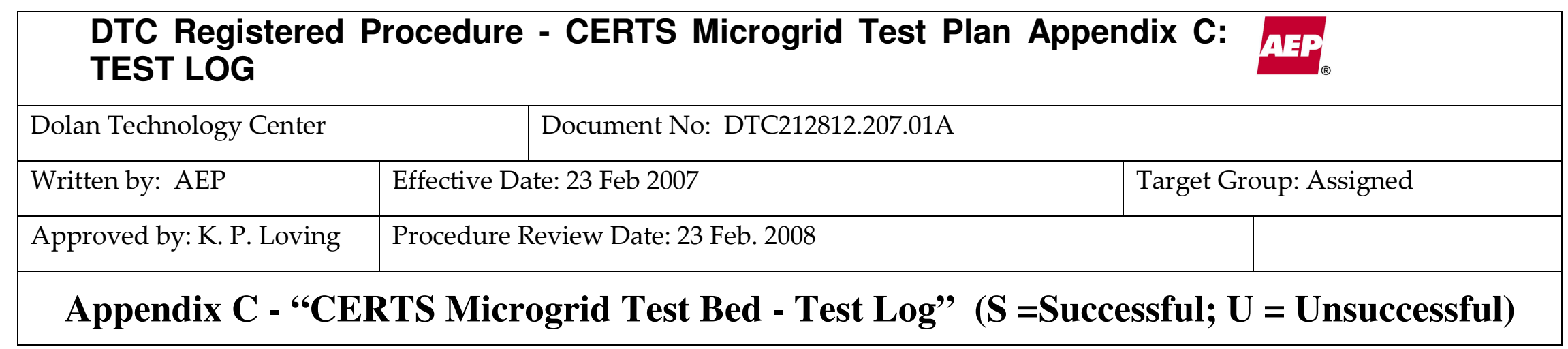

\begin{tabular}{|l|l|l|l|l|l|l|l|l|}
\hline $\begin{array}{l}\text { 10.2.15(10.1 } \\
\text {.8) }\end{array}$ & $3 / 18 / 08$ & $12: 32$ pm & $\begin{array}{l}\text { 10.2.15(1 } \\
\text { 0.1.9) }\end{array}$ & & S & 12:38 pm & DAK & $\begin{array}{l}\text { Motor started at 12:33. Meter1:37kW 1kVar, } \\
\text { Meter3:-2kW -4kVar, Meter4:-1kW 10kVar }\end{array}$ \\
\hline $\begin{array}{l}\text { 10.2.15(10.1 } \\
\text {.10) }\end{array}$ & $3 / 18 / 08$ & $12: 38 ~ p m$ & $\begin{array}{l}\text { 10.2.15(1 } \\
\text { 0.1.11) }\end{array}$ & & S & 12:43 pm & DAK & $\begin{array}{l}\text { SS manual open removed at 12:38. Meter1:70kW } \\
\text { 22kVar, Meter3:31kW 16kVar, Meter4:16kW 0kVar }\end{array}$ \\
\hline
\end{tabular}

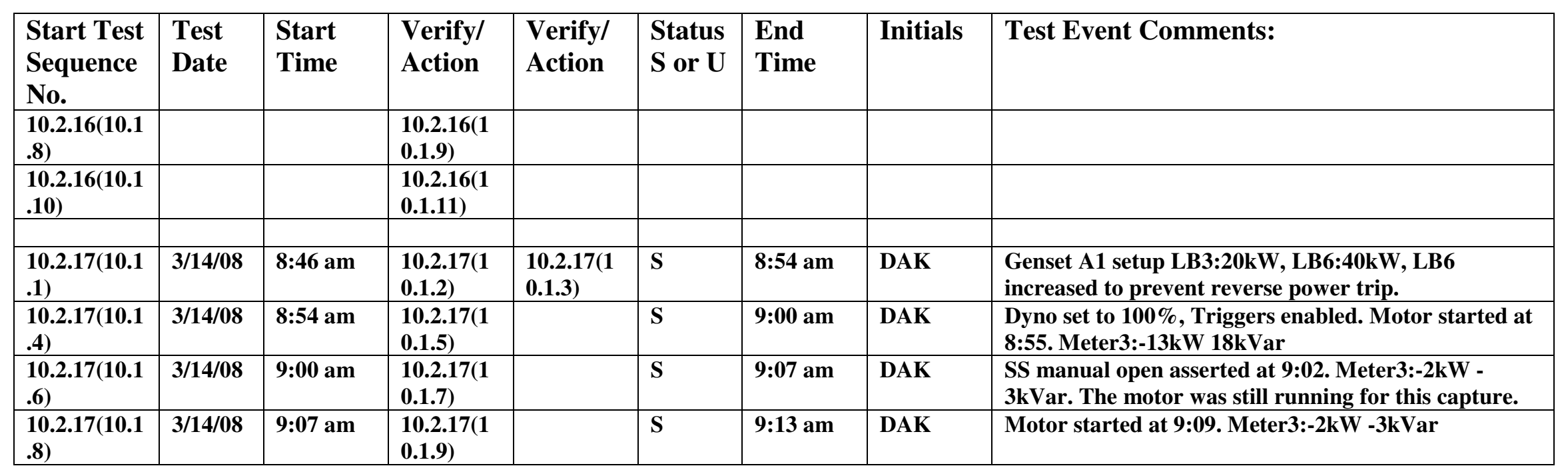

This document contains information confidential and proprietary to AEPSC. It shall not be reproduced in whole or in part or released to any third party without expressed written consent of AEPSC. 


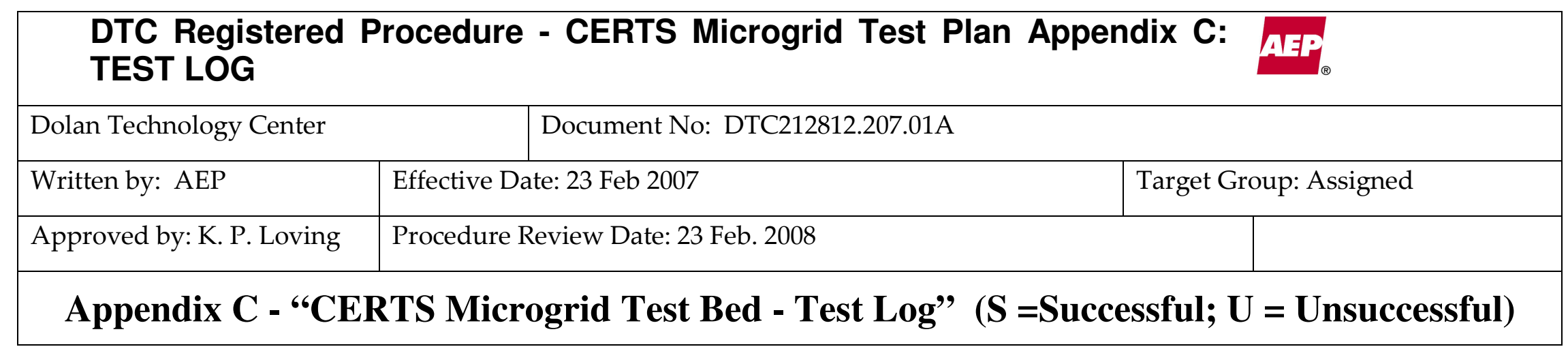

\begin{tabular}{|c|c|c|c|c|c|c|c|c|}
\hline $\begin{array}{l}\text { 10.2.17(10.1 } \\
.10)\end{array}$ & $3 / 14 / 08$ & 9:13 am & $\begin{array}{l}\text { 10.2.17(1 } \\
\text { 0.1.11) }\end{array}$ & & $\mathbf{S}$ & 9:20 am & DAK & $\begin{array}{l}\text { SS manual open removed at 9:14. Meter3:-11kW } \\
\text { 17kVar }\end{array}$ \\
\hline $\begin{array}{l}\text { 10.2.18(10.1 } \\
.1)\end{array}$ & $3 / 18 / 08$ & $12: 43 \mathrm{pm}$ & $\begin{array}{l}\text { 10.2.18(1 } \\
\text { 0.1.2) }\end{array}$ & \begin{tabular}{|l|}
$10.2 .18(1$ \\
$0.1 .3)$
\end{tabular} & $\mathbf{S}$ & $12: 46 \mathrm{pm}$ & DAK & $\begin{array}{l}\text { Test revised to remove unavailable Genset B1.Genset } \\
\text { A1 and A2 setup and connected. LB3:20kW, } \\
\text { LB4:40kW, LB6:40kW, LB5 is unused. LB6 increased } \\
\text { to prevent reverse power trip. SS closed }\end{array}$ \\
\hline $\begin{array}{l}\text { 10.2.18(10.1 } \\
\text {.4) }\end{array}$ & $3 / 18 / 08$ & $12: 46 \mathrm{pm}$ & $\begin{array}{l}\text { 10.2.18(1 } \\
\text { 0.1.5) }\end{array}$ & & S & $12: 51 \mathrm{pm}$ & DAK & $\begin{array}{l}\text { Dyno set to 100\%, Triggers enabled. Motor started at } \\
\text { 12:46. Meter1:33kW 25kVar, Meter3:-5kW 19kVar, } \\
\text { Meter4:13kW 1kVar }\end{array}$ \\
\hline $\begin{array}{l}\text { 10.2.18(10.1 } \\
.8)\end{array}$ & $3 / 18 / 08$ & $12: 58 \mathrm{pm}$ & $\begin{array}{l}\text { 10.2.18(1 } \\
\text { 0.1.9) }\end{array}$ & & S & 1:14 pm & DAK & $\begin{array}{l}\text { Motor started at 1:09. Meter1:37kW 1kVar, Meter3:- } \\
\text { 2kW -4kVar, Meter4:17kW 11kVar }\end{array}$ \\
\hline $\begin{array}{l}\text { 10.2.18(10.1 } \\
.10)\end{array}$ & $3 / 18 / 08$ & $1: 14 \mathrm{pm}$ & $\begin{array}{l}\text { 10.2.18(1 } \\
\text { 0.1.11) }\end{array}$ & & $\mathbf{S}$ & $1: 20 \mathrm{pm}$ & DAK & $\begin{array}{l}\text { SS manual open removed at 1:15. Meter1:32kW } \\
\text { 29kVar, Meter3:-8kW 21kVar, Meter4:10kW 3kVar }\end{array}$ \\
\hline
\end{tabular}

\begin{tabular}{|l|l|l|l|l|l|l|l|l|}
\hline $\begin{array}{l}\text { Start Test } \\
\text { Sequence } \\
\text { No. }\end{array}$ & $\begin{array}{l}\text { Test } \\
\text { Date }\end{array}$ & $\begin{array}{l}\text { Start } \\
\text { Time }\end{array}$ & $\begin{array}{l}\text { Verify/ } \\
\text { Action }\end{array}$ & $\begin{array}{l}\text { Verify/ } \\
\text { Action }\end{array}$ & $\begin{array}{l}\text { Status } \\
\text { S or U }\end{array}$ & $\begin{array}{l}\text { End } \\
\text { Time }\end{array}$ & Initials & Test Event Comments: \\
\hline
\end{tabular}

This document contains information confidential and proprietary to AEPSC. It shall not be reproduced in whole or in part or released to any third party without expressed written consent of AEPSC. 


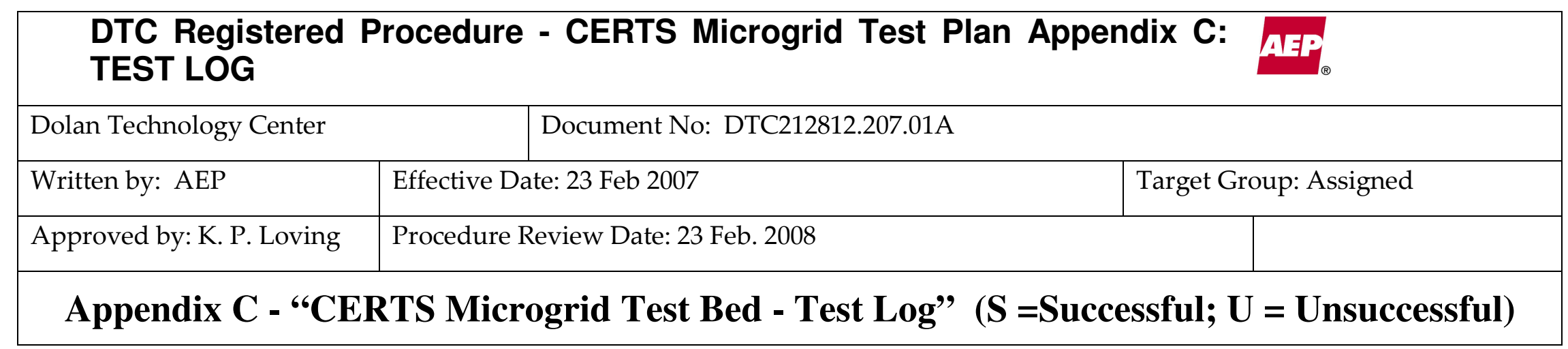

\begin{tabular}{|c|c|c|c|c|c|c|c|c|}
\hline $\begin{array}{l}\text { 10.2.19(10.1 } \\
.1)\end{array}$ & & & $\begin{array}{l}\text { 10.2.19(1 } \\
\text { 0.1.2) }\end{array}$ & $\begin{array}{l}\text { 10.2.19(1 } \\
0.1 .3)\end{array}$ & & & & \\
\hline $\begin{array}{l}10.2 .19(10.1 \\
.4)\end{array}$ & & & $\begin{array}{l}\text { 10.2.19(1 } \\
0.1 .5)\end{array}$ & & & & & \\
\hline $\begin{array}{l}10.2 .19(10.1 \\
.6)\end{array}$ & & & $\begin{array}{l}\text { 10.2.19(1 } \\
0.1 .7)\end{array}$ & & & & & \\
\hline $\begin{array}{l}\text { 10.2.19(10.1 } \\
.8)\end{array}$ & & & $\begin{array}{l}\text { 10.2.19(1 } \\
0.1 .9)\end{array}$ & & & & & \\
\hline $\begin{array}{l}10.2 .19(10.1 \\
.10)\end{array}$ & & & $\begin{array}{l}\text { 10.2.19(1 } \\
0.1 .11)\end{array}$ & & & & & \\
\hline $\begin{array}{l}10.2 .20(10.1 \\
.1)\end{array}$ & $3 / 14 / 08$ & $9: 20$ am & $\begin{array}{l}\text { 10.2.20(1 } \\
0.1 .2)\end{array}$ & $\begin{array}{l}\text { 10.2.20(1 } \\
0.1 .3)\end{array}$ & $\mathbf{S}$ & 9:36 am & DAK & $\begin{array}{l}\text { Genset A1 setup LB3:20kW, LB6:40kW. LB6 } \\
\text { increased to prevent reverse power trip. }\end{array}$ \\
\hline $\begin{array}{l}\text { 10.2.20(10.1 } \\
.4)\end{array}$ & $3 / 14 / 08$ & 9:36 am & $\begin{array}{l}\text { 10.2.20(1 } \\
0.1 .5)\end{array}$ & & $\mathbf{S}$ & 9:42 am & DAK & $\begin{array}{l}\text { Dyno set to } 100 \% \text {, Triggers enabled. Motor started at } \\
\text { 9:37. Meter3:-16kW 18kVar }\end{array}$ \\
\hline $\begin{array}{l}10.2 .20(10.1 \\
.6)\end{array}$ & $3 / 14 / 08$ & 9:42 am & $\begin{array}{l}\text { 10.2.20(1 } \\
0.1 .7)\end{array}$ & & $\mathbf{S}$ & 9:48 am & DAK & $\begin{array}{l}\text { SS manual open asserted at 9:43. Meter3:-2kW - } \\
\text { 4kVar. }\end{array}$ \\
\hline $\begin{array}{l}10.2 .20(10.1 \\
.8)\end{array}$ & $3 / 14 / 08$ & $9: 48$ am & $\begin{array}{l}\text { 10.2.20(1 } \\
0.1 .9)\end{array}$ & & $\mathbf{S}$ & 9:55 am & DAK & Motor started at 9:50. Meter3:-2kW -3kVar \\
\hline $\begin{array}{l}\text { 10.2.20(10.1 } \\
.10)\end{array}$ & $3 / 14 / 08$ & 9:55 am & $\begin{array}{l}\text { 10.2.20(1 } \\
0.1 .11)\end{array}$ & & $\mathbf{S}$ & 10:03 am & DAK & $\begin{array}{l}\text { SS manual open removed at 9:57. Meter3:-15kW } \\
\text { 18kVar }\end{array}$ \\
\hline
\end{tabular}




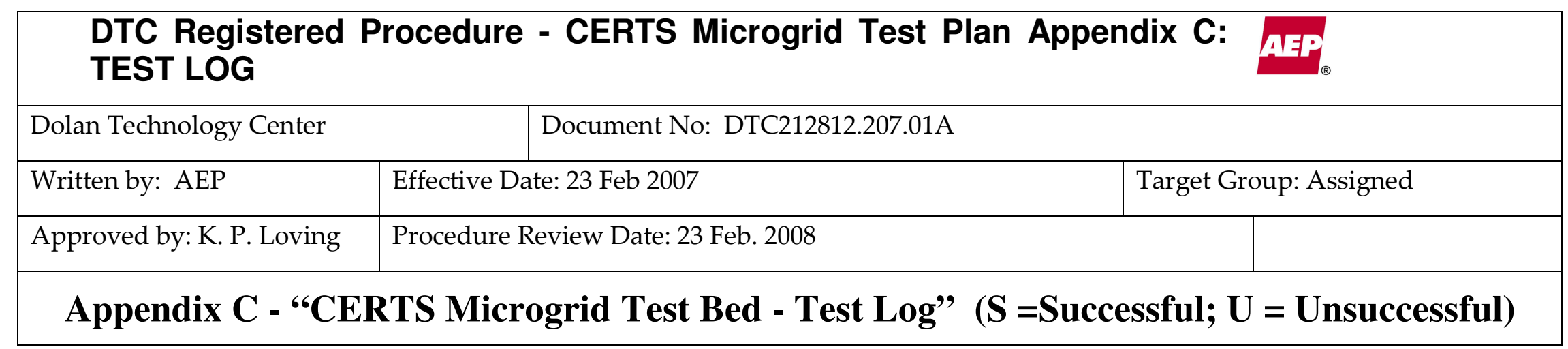

\begin{tabular}{|c|c|c|c|c|c|c|c|c|}
\hline $\begin{array}{l}\text { Start Test } \\
\text { Sequence } \\
\text { No. }\end{array}$ & $\begin{array}{l}\text { Test } \\
\text { Date }\end{array}$ & $\begin{array}{l}\text { Start } \\
\text { Time }\end{array}$ & $\begin{array}{l}\text { Verify/ } \\
\text { Action }\end{array}$ & $\begin{array}{l}\text { Verify/ } \\
\text { Action }\end{array}$ & \begin{tabular}{|l} 
Status \\
S or U
\end{tabular} & $\begin{array}{l}\text { End } \\
\text { Time }\end{array}$ & Initials & Test Event Comments: \\
\hline $\begin{array}{l}\text { 10.3.12(10.1 } \\
.6)\end{array}$ & $3 / 18 / 08$ & $1: 39 \mathrm{pm}$ & $\begin{array}{l}\text { 10.3.12(1 } \\
0.1 .7)\end{array}$ & & $\mathbf{S}$ & 1:44 pm & DAK & $\begin{array}{l}\text { SS manual open asserted at 1:39. SS opened. } \\
\text { Meter1:38kW 1kVar, Meter3:-2kW -4kVar, } \\
\text { Meter4:12kW -7kVar }\end{array}$ \\
\hline $\begin{array}{l}\text { 10.3.12(10.1 } \\
.8)\end{array}$ & $3 / 18 / 08$ & $1: 44 \mathrm{pm}$ & $\begin{array}{l}\text { 10.3.12(1 } \\
\text { 0.1.9) }\end{array}$ & & $\mathbf{S}$ & $1: 49 \mathrm{pm}$ & DAK & $\begin{array}{l}\text { Motor started at 1:44. Meter1:38kW 1kVar, Meter3:- } \\
\text { 1kW -4kVar, Meter4:9kW 10kVar }\end{array}$ \\
\hline $\begin{array}{l}\text { 10.3.12(10.1 } \\
.10)\end{array}$ & $3 / 18 / 08$ & $1: 49 \mathrm{pm}$ & $\begin{array}{l}\text { 10.3.12(1 } \\
\text { 0.1.11) }\end{array}$ & & $\mathbf{S}$ & $1: 55$ pm & DAK & $\begin{array}{l}\text { SS manual open removed at 1:50. Meter1:32kW } \\
\text { 24kVar, Meter3:-7kW 18kVar, Meter4:6kW 1kVar }\end{array}$ \\
\hline
\end{tabular}

\begin{tabular}{|l|l|l|l|l|l|l|l|l|}
\hline $\begin{array}{l}\text { Start Test } \\
\text { Sequence } \\
\text { No. }\end{array}$ & $\begin{array}{l}\text { Test } \\
\text { Date }\end{array}$ & $\begin{array}{l}\text { Start } \\
\text { Time }\end{array}$ & $\begin{array}{l}\text { Verify/ } \\
\text { Action }\end{array}$ & $\begin{array}{l}\text { Verify/ } \\
\text { Action }\end{array}$ & $\begin{array}{l}\text { Status } \\
\text { S or U }\end{array}$ & $\begin{array}{l}\text { End } \\
\text { Time }\end{array}$ & Initials & Test Event Comments: \\
\hline
\end{tabular}

This document contains information confidential and proprietary to AEPSC. It shall not be reproduced in whole or in part or released to any third party without expressed written consent of AEPSC. 


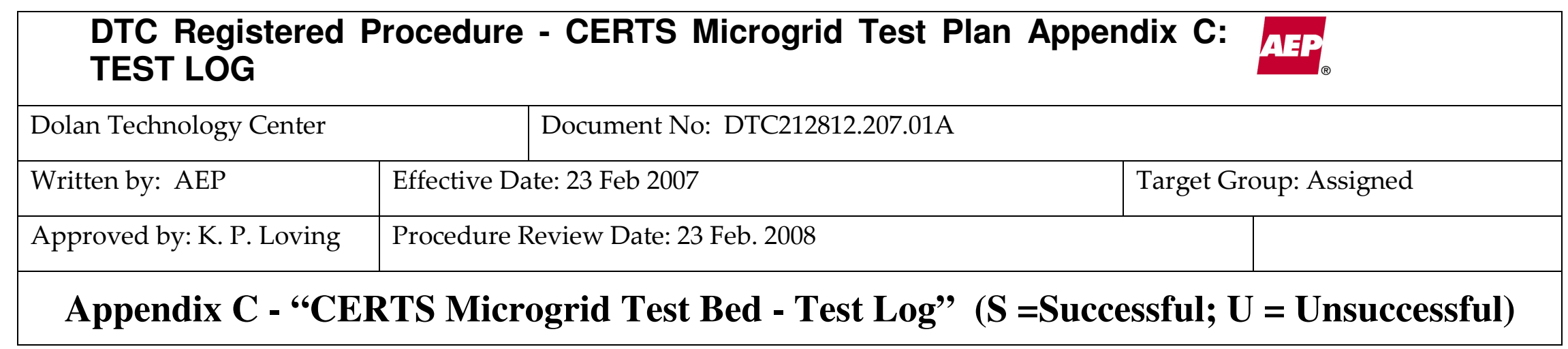

\begin{tabular}{|c|c|c|c|c|c|c|c|c|}
\hline $\begin{array}{l}10.3 .14(10.1 \\
.1)\end{array}$ & $3 / 14 / 08$ & 10:03 am & $\begin{array}{l}\text { 10.3.14(1 } \\
\text { 0.1.2) }\end{array}$ & $\begin{array}{l}\text { 10.3.14(1 } \\
0.1 .3)\end{array}$ & $\mathbf{S}$ & 10:09 am & DAK & $\begin{array}{l}\text { Genset A1 setup LB3:10kW, LB6:10AkW 20BkW } \\
\text { 10CkW. LB6 increased to prevent reverse power trip. }\end{array}$ \\
\hline $\begin{array}{l}\text { 10.3.14(10.1 } \\
.6)\end{array}$ & 3/14/08 & 10:15 am & $\begin{array}{l}\text { 10.3.14(1 } \\
0.1 .7)\end{array}$ & & $\mathbf{S}$ & $10: 21$ am & DAK & $\begin{array}{l}\text { SS manual open asserted at 10:16. Meter3:-2kW - } \\
\text { 4kVar. }\end{array}$ \\
\hline $\begin{array}{l}\text { 10.3.14(10.1 } \\
.8)\end{array}$ & $3 / 14 / 08$ & 10:21 am & $\begin{array}{l}\text { 10.3.14(1 } \\
\text { 0.1.9) }\end{array}$ & & $\mathbf{S}$ & 10:27 am & DAK & Motor started at 10:22. Meter3:-2kW -3kVar \\
\hline $\begin{array}{l}\text { 10.3.14(10.1 } \\
\text {.10) }\end{array}$ & $3 / 14 / 08$ & 10:27 am & $\begin{array}{l}\text { 10.3.14(1 } \\
0.1 .11)\end{array}$ & & $\mathbf{S}$ & 10:35 am & DAK & $\begin{array}{l}\text { SS manual open removed at 10:28. Meter3:-19kW } \\
\text { 19kVar }\end{array}$ \\
\hline $\begin{array}{l}\text { 10.3.15(10.1 } \\
.1)\end{array}$ & $3 / 18 / 08$ & $1: 55 \mathrm{pm}$ & $\begin{array}{l}\text { 10.3.15(1 } \\
0.1 .2)\end{array}$ & $\begin{array}{l}\text { 10.3.15(1 } \\
\text { 0.1.3) }\end{array}$ & $\mathbf{S}$ & $1: 56 \mathrm{pm}$ & DAK & $\begin{array}{l}\text { Test revised to remove unavailable Genset } B 1 \text { and test } \\
\text { the gensets closer to capacity. Genset } A 1 \text { and } A 2 \text { setup } \\
\text { and connected. LB3:50kW, LB4:10AkW 20BkW } \\
\text { 20CkW, LB6: 10AkW 20BkW 10CkW. LB5 is unused. } \\
\text { LB6 increased to prevent reverse power trip. SS } \\
\text { closed }\end{array}$ \\
\hline $\begin{array}{l}10.3 .15(10.1 \\
.4)\end{array}$ & $3 / 18 / 08$ & 1:56 pm & $\begin{array}{l}\text { 10.3.15(1 } \\
0.1 .5)\end{array}$ & & $\mathbf{S}$ & 2:01 pm & DAK & $\begin{array}{l}\text { Dyno set to } 100 \% \text {, Triggers enabled. Motor started at } \\
\text { 1:56. Meter1:69kW 20kVar, Meter3:29kW 14kVar, } \\
\text { Meter4:14kW -1kVar }\end{array}$ \\
\hline $\begin{array}{l}\text { 10.3.15(10.1 } \\
.8)\end{array}$ & $3 / 18 / 08$ & 2:07 pm & $\begin{array}{l}\text { 10.3.15(1 } \\
\text { 0.1.9) }\end{array}$ & & $\mathbf{S}$ & 2:13 pm & DAK & $\begin{array}{l}\text { Motor started at 2:08. Meter1:38kW 1kVar, Meter3:- } \\
\text { 2kW -4kVar, Meter4:-2kW -9kVar }\end{array}$ \\
\hline
\end{tabular}

60 of 65

This document contains information confidential and proprietary to AEPSC. It shall not be reproduced in whole or in part or released to any third party without expressed written consent of AEPSC. 


\begin{tabular}{|c|c|c|c|c|c|c|c|c|}
\hline \multicolumn{9}{|c|}{$\begin{array}{l}\text { DTC Registerec } \\
\text { TEST LOG }\end{array}$} \\
\hline \multicolumn{4}{|c|}{ Dolan Technology Center } & \multicolumn{5}{|c|}{ Document No: DTC212812.207.01A } \\
\hline \multicolumn{3}{|c|}{ Written by: AEP } & \multicolumn{5}{|c|}{ Effective Date: 23 Feb 2007} & Target Group: Assigned \\
\hline \multicolumn{3}{|c|}{ Approved by: K. P. Loving } & \multicolumn{5}{|c|}{ Procedure Review Date: 23 Feb. 2008} & \\
\hline \multicolumn{9}{|c|}{ Appendix C - "CERTS Microgrid Test Bed - Test Log" (S =Successful; U = Unsuccessful) } \\
\hline $\begin{array}{l}\text { 10.3.15(10.1 } \\
.10)\end{array}$ & 3/18/08 & $2: 13$ pm & $\begin{array}{l}\text { 10.3.15(1 } \\
\text { 0.1.11) }\end{array}$ & & $\mathbf{S}$ & $2: 19 \mathrm{pm}$ & DAK & \begin{tabular}{|l|} 
SS manual open removed at 2:14. Meter1:66kW \\
23kVar, Meter3:26kW 16kVar, Meter4:13kW 0kVar \\
\end{tabular} \\
\hline $\begin{array}{l}\text { Start Test } \\
\text { Sequence } \\
\text { No. }\end{array}$ & $\begin{array}{l}\text { Test } \\
\text { Date }\end{array}$ & $\begin{array}{l}\text { Start } \\
\text { Time }\end{array}$ & $\begin{array}{l}\text { Verify/ } \\
\text { Action }\end{array}$ & $\begin{array}{l}\text { Verify/ } \\
\text { Action }\end{array}$ & $\begin{array}{l}\text { Status } \\
\text { S or U }\end{array}$ & $\begin{array}{l}\text { End } \\
\text { Time }\end{array}$ & Initials & Test Event Comments: \\
\hline 10.3.17(10.1 & $3 / 14 / 08$ & 11:58 am & $\begin{array}{l}\text { 10.3.17(1 } \\
0.1 .2)\end{array}$ & $\begin{array}{l}\text { 10.3.17(1 } \\
\text { 0.1.3) }\end{array}$ & $\mathbf{S}$ & 12:02 pm & DAK & $\begin{array}{l}\text { Genset A1 setup LB3:20kW, LB6:10AkW 20BkW } \\
\text { 10CkW, LB6 increased to prevent reverse power trip. }\end{array}$ \\
\hline 10.3.17(10.1 & 3/14/08 & $12: 02$ pm & $\begin{array}{l}\text { 10.3.17(1 } \\
0.1 .5)\end{array}$ & & $\mathbf{S}$ & $12: 09$ pm & DAK & $\begin{array}{l}\text { Dyno set to 100\%, Triggers enabled. Motor started at } \\
\text { 12:04. Meter3:-9kW 18kVar }\end{array}$ \\
\hline $\begin{array}{ll}10.3 .17(10.1 \\
.6)\end{array}$ & 3/14/08 & 12:09 pm & $\begin{array}{l}\text { 10.3.17(1 } \\
0.1 .7)\end{array}$ & & $\mathbf{S}$ & $12: 16 \mathrm{pm}$ & DAK & $\begin{array}{l}\text { SS manual open asserted at 12:10. Meter3:-2kW - } \\
\text { 4kVar. }\end{array}$ \\
\hline $\begin{array}{l}\text { 10.3.17(10.1 } \\
.8)\end{array}$ & 3/14/08 & $12: 16$ pm & $\begin{array}{l}\text { 10.3.17(1 } \\
0.1 .9)\end{array}$ & & $\mathbf{S}$ & $12: 22 \mathrm{pm}$ & DAK & Motor started at 12:17. Meter3:-2kW -3kVar \\
\hline $\begin{array}{l}10.3 .17(10.1 \\
.10)\end{array}$ & 3/14/08 & $12: 22 \mathrm{pm}$ & $\begin{array}{l}\text { 10.3.17(1 } \\
\text { 0.1.11) }\end{array}$ & & $\mathbf{S}$ & $12: 28 \mathrm{pm}$ & DAK & $\begin{array}{l}\text { SS manual open removed at 12:22. Meter3:-12kW } \\
\text { 18kVar }\end{array}$ \\
\hline $\begin{array}{l}\text { 10.3.18(10.1 } \\
.1)\end{array}$ & 3/18/08 & $2: 19$ pm & $\begin{array}{l}\text { 10.3.18(1 } \\
0.1 .2)\end{array}$ & $\begin{array}{l}\text { 10.3.18(1 } \\
\text { 0.1.3) }\end{array}$ & $\mathbf{S}$ & $2: 20 \mathrm{pm}$ & DAK & $\begin{array}{l}\text { Test revised to remove unavailable Genset B1. Genset } \\
\text { A1 and A2 setup and connected. LB3:20kW, } \\
\text { LB4:10AkW 15BkW, 15CkW, LB6: 10AkW 20BkW, } \\
\text { 10CkW, LB5 is unused. LB6 increased to prevent } \\
\text { reverse power trip. SS closed }\end{array}$ \\
\hline
\end{tabular}

61 of 65

This document contains information confidential and proprietary to AEPSC. It shall not be reproduced in whole or in part or released to any third party without expressed written consent of AEPSC. 


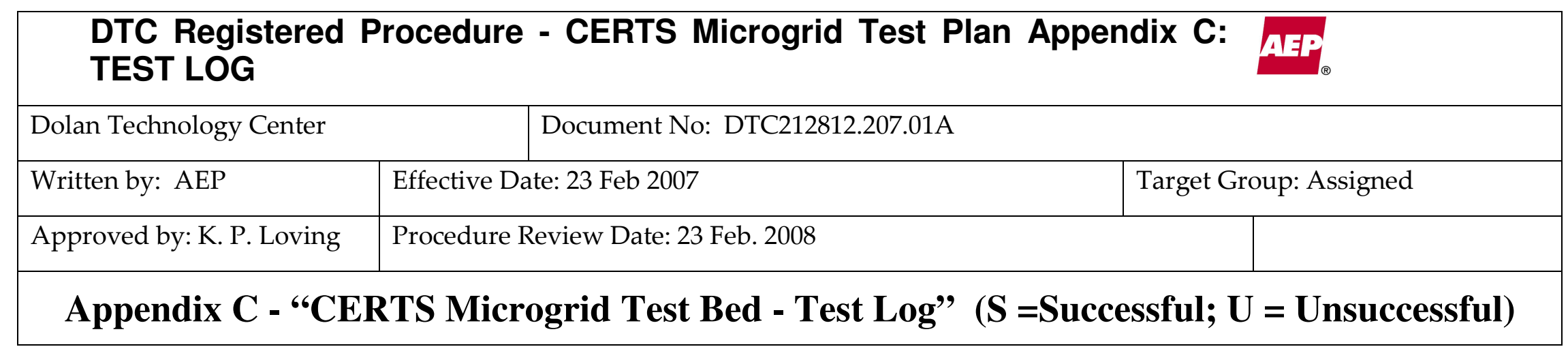

\begin{tabular}{|c|c|c|c|c|c|c|c|}
\hline $\begin{array}{l}\text { 10.3.18(10.1 } \\
\text {.4) }\end{array}$ & $3 / 18 / 08$ & 2:20 pm & $\begin{array}{l}\text { 10.3.18(1 } \\
\text { 0.1.5) }\end{array}$ & $\mathbf{S}$ & 2:26 pm & DAK & $\begin{array}{l}\text { Dyno set to 100\%, Triggers enabled. Motor started at } \\
\text { 2:21. Meter1:32kW 25kVar, Meter3:-8kW 19kVar, } \\
\text { Meter4:9kW 2kVar }\end{array}$ \\
\hline $\begin{array}{l}\text { 10.3.18(10.1 } \\
.6)\end{array}$ & $3 / 18 / 08$ & $2: 26 \mathrm{pm}$ & $\begin{array}{l}\text { 10.3.18(1 } \\
0.1 .7)\end{array}$ & $\mathbf{S}$ & 2:32 pm & DAK & $\begin{array}{l}\text { SS manual open asserted at 2:27. SS opened with a } \\
\text { fault and a PES alarm. Meter1:38kW 1kVar, } \\
\text { Meter3:-2kW -4kVar, Meter4:17kW -8kVar }\end{array}$ \\
\hline $\begin{array}{l}\text { 10.3.18(10.1 } \\
.8)\end{array}$ & $3 / 18 / 08$ & 2:32 pm & $\begin{array}{l}\text { 10.3.18(1 } \\
\text { 0.1.9) }\end{array}$ & $\mathbf{S}$ & $2: 37 \mathrm{pm}$ & DAK & $\begin{array}{l}\text { Motor started at 2:32. Meter1:38kW 1kVar, Meter3:- } \\
\text { 2kW -4kVar, Meter4:17kW 11kVar }\end{array}$ \\
\hline $\begin{array}{l}\text { 10.3.18(10.1 } \\
.10)\end{array}$ & $3 / 18 / 08$ & 2:37 pm & $\begin{array}{l}\text { 10.3.18(1 } \\
\text { 0.1.11) }\end{array}$ & $\mathbf{S}$ & 2: & DAK & $\begin{array}{l}\text { SS manual open removed at 2:38. Meter1:33kW } \\
\text { 27kVar, Meter3:-8kW 21kVar, Meter4:10kW 2kVar }\end{array}$ \\
\hline
\end{tabular}

\begin{tabular}{|c|c|c|c|c|c|c|c|c|}
\hline $\begin{array}{l}\text { Start Test } \\
\text { Sequence } \\
\text { No. }\end{array}$ & $\begin{array}{l}\text { Test } \\
\text { Date }\end{array}$ & $\begin{array}{l}\text { Start } \\
\text { Time }\end{array}$ & $\begin{array}{l}\text { Verify/ } \\
\text { Action }\end{array}$ & $\begin{array}{l}\text { Verify/ } \\
\text { Action }\end{array}$ & \begin{tabular}{|l} 
Status \\
S or U
\end{tabular} & $\begin{array}{l}\text { End } \\
\text { Time }\end{array}$ & Initials & Test Event Comments: \\
\hline $\begin{array}{l}10.3 .20(10.1 \\
.4)\end{array}$ & $3 / 14 / 08$ & $12: 29 \mathrm{pm}$ & $\begin{array}{l}\text { 10.3.20(1 } \\
\text { 0.1.5) }\end{array}$ & & $\mathbf{S}$ & $12: 34 \mathrm{pm}$ & DAK & $\begin{array}{l}\text { Dyno set to 100\%, Triggers enabled. Motor started at } \\
\text { 12:29. Meter3:-14kW, 19kVar }\end{array}$ \\
\hline $\begin{array}{l}\text { 10.3.20(10.1 } \\
.6)\end{array}$ & $3 / 14 / 08$ & $12: 34 \mathrm{pm}$ & $\begin{array}{l}\text { 10.3.20(1 } \\
\text { 0.1.7) }\end{array}$ & & $\mathbf{S}$ & $12: 40 \mathrm{pm}$ & DAK & $\begin{array}{l}\text { SS manual open asserted at 12:35. Meter3:-2kW, - } \\
\text { 4kVar. }\end{array}$ \\
\hline
\end{tabular}

62 of 65

This document contains information confidential and proprietary to AEPSC. It shall not be reproduced in whole or in part or released to any third party without expressed written consent of AEPSC. 


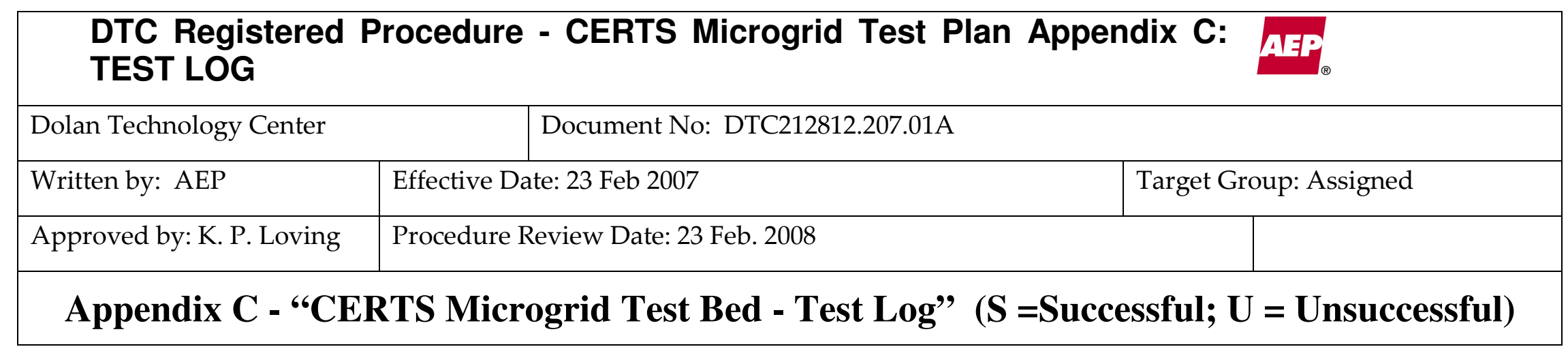

\begin{tabular}{|c|c|c|c|c|c|c|c|}
\hline $\begin{array}{l}\text { 10.3.20(10.1 } \\
.10)\end{array}$ & $3 / 14 / 08$ & $12: 45 \mathrm{pm}$ & $\begin{array}{l}\text { 10.3.20(1 } \\
\text { 0.1.11) }\end{array}$ & $\mathbf{S}$ & $12: 51 \mathrm{pm}$ & DAK & $\begin{array}{l}\text { SS manual open removed at 12:45. Meter3:-16kW, } \\
\text { 19kVar }\end{array}$ \\
\hline
\end{tabular}

\begin{tabular}{|c|c|c|c|c|c|c|c|c|}
\hline $\begin{array}{l}\text { Start Test } \\
\text { Sequence } \\
\text { No. }\end{array}$ & $\begin{array}{l}\text { Test } \\
\text { Date }\end{array}$ & $\begin{array}{l}\text { Start } \\
\text { Time }\end{array}$ & $\begin{array}{l}\text { Verify/ } \\
\text { Action }\end{array}$ & $\begin{array}{l}\text { Verify/ } \\
\text { Action }\end{array}$ & $\begin{array}{l}\text { Status } \\
\text { S or U }\end{array}$ & $\begin{array}{l}\text { End } \\
\text { Time }\end{array}$ & Initials & Test Event Comments: \\
\hline $\begin{array}{l}\text { 10.4.12(10.1 } \\
.1)\end{array}$ & $3 / 20 / 08$ & $8: 30$ am & $\begin{array}{l}10.4 .12(1 \\
0.1 .2)\end{array}$ & $\begin{array}{l}10.4 .12(1 \\
0.1 .3)\end{array}$ & $\mathbf{S}$ & $8: 35$ am & DAK & $\begin{array}{l}\text { Genset A1 setup. LB3:3.3AkW 3.3BkW 3.3CkW, } \\
\text { LB4:13.3AkW 13.3BkW 13.3CkW, LB6:13.3AkW } \\
\text { 13.3BkW 13.3CkW. LB6 increased to prevent reverse } \\
\text { power trip. }\end{array}$ \\
\hline $\begin{array}{l}\text { 10.4.12(10.4 } \\
.2)\end{array}$ & $3 / 20 / 08$ & $8: 35$ am & $\begin{array}{l}\text { 10.4.12(1 } \\
0.4 .3)\end{array}$ & & $\mathbf{S}$ & $8: 45$ am & DAK & $\begin{array}{l}\text { LB3:1.6AkW 3.3BkW 3.3CkW, LB6:6.66AkW } \\
\text { 13.3BkW 13.3CkW. Triggers enabled. SS opened } \\
\text { shortly afterward on reverse power. SS opened with a } \\
\text { fault and a PES alarm. LB4 was still at } 40 \mathrm{~kW} \text { load } \\
\text { prior to the SS opening. }\end{array}$ \\
\hline $\begin{array}{l}\text { 10.4.12(10.4 } \\
\text {.4) }\end{array}$ & $3 / 20 / 08$ & 8:45 am & $\begin{array}{l}\text { 10.4.12(1 } \\
0.4 .5)\end{array}$ & & $\mathbf{S}$ & $8: 46$ am & DAK & SS reset and started with manual open asserted \\
\hline $\begin{array}{l}\text { 10.4.12(10.4 } \\
.6)\end{array}$ & $3 / 20 / 08$ & $8: 46$ am & $\begin{array}{l}\text { 10.4.12(1 } \\
0.4 .7)\end{array}$ & & $\mathbf{S}$ & $8: 51$ am & DAK & $\begin{array}{l}\text { LB3:0AkW 3.3BkW 3.3CkW, LB6:3.3AkW 13.3BkW } \\
\text { 13.3CkW.Triggers enabled. LB4 reduced at 8:46. } \\
\text { LB4:3.33AkW, 13.3BkW, 13.3CkW }\end{array}$ \\
\hline $\begin{array}{l}\text { 10.4.12(10.4 } \\
.8)\end{array}$ & $3 / 20 / 08$ & $8: 51$ am & $\begin{array}{l}\text { 10.4.12(1 } \\
0.4 .9)\end{array}$ & & $\mathbf{S}$ & $8: 56$ am & DAK & $\begin{array}{l}\text { LB3:0AkW 3.3BkW 3.3CkW, LB6:3.3AkW 13.3BkW } \\
\text { 13.3CkW.Triggers enabled. LB4 reduced at 8:51. } \\
\text { LB4:3.33AkW, 13.3BkW, 13.3CkW }\end{array}$ \\
\hline
\end{tabular}

63 of 65

This document contains information confidential and proprietary to AEPSC. It shall not be reproduced in whole or in part or released to any third party without expressed written consent of AEPSC. 


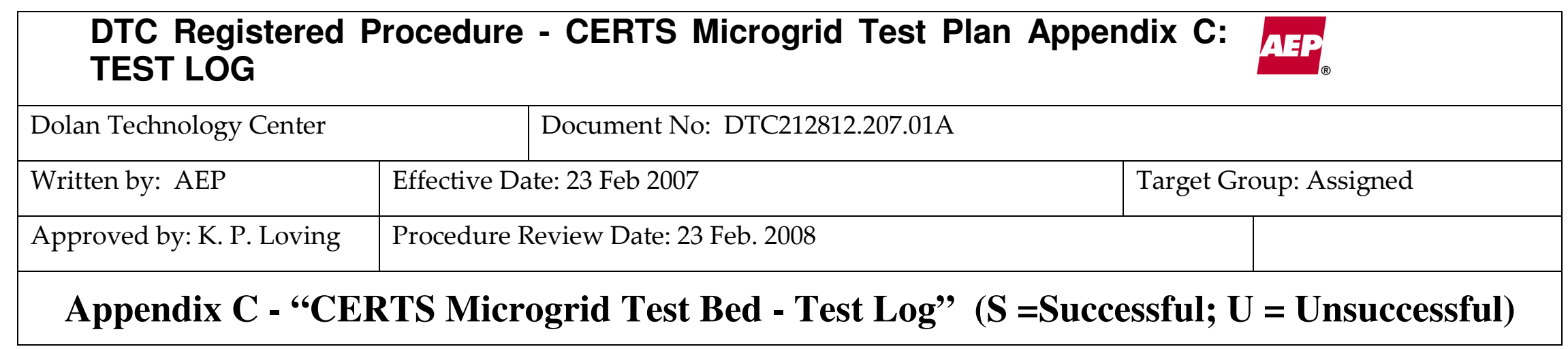

\begin{tabular}{|l|l|l|l|l|l|l|l|l|}
\hline $\begin{array}{l}\text { 10.4.12(10.4 } \\
\text {.10) }\end{array}$ & $3 / 20 / 08$ & $8: 56$ am & $\begin{array}{l}\text { 10.4.12(1 } \\
\text { 0.4.11) }\end{array}$ & & S & 9:01 am & DAK & $\begin{array}{l}\text { SS manual open removed at 8:56. SS closed. SS } \\
\text { opened shortly afterward on reverse power trip. SS } \\
\text { opened with a fault and a PES alarm. }\end{array}$ \\
\hline
\end{tabular}

\begin{tabular}{|c|c|c|c|c|c|c|c|c|}
\hline $\begin{array}{l}\text { Start Test } \\
\text { Sequence } \\
\text { No. }\end{array}$ & $\begin{array}{l}\text { Test } \\
\text { Date }\end{array}$ & $\begin{array}{l}\text { Start } \\
\text { Time }\end{array}$ & $\begin{array}{l}\text { Verify/ } \\
\text { Action }\end{array}$ & $\begin{array}{l}\text { Verify/ } \\
\text { Action }\end{array}$ & $\begin{array}{l}\text { Status } \\
\text { S or U }\end{array}$ & $\begin{array}{l}\text { End } \\
\text { Time }\end{array}$ & Initials & Test Event Comments: \\
\hline $\begin{array}{l}\text { 10.4.14(10.1 } \\
.1)\end{array}$ & 3/14/08 & $12: 51 \mathrm{pm}$ & $\begin{array}{l}\text { 10.4.14(1 } \\
\text { 0.1.2) }\end{array}$ & $\begin{array}{l}\text { 10.4.14(1 } \\
\text { 0.1.3) }\end{array}$ & $\mathbf{S}$ & 1:01 pm & DAK & $\begin{array}{l}\text { Genset A1 setup. LB3:3.3AkW, 3.3BkW, 3.3CkW, } \\
\text { LB6:13.3AkW, 13.3BkW, 13.3CkW. LB6 increased to } \\
\text { prevent reverse power trip. }\end{array}$ \\
\hline $\begin{array}{l}\text { 10.4.14(10.4 } \\
.2)\end{array}$ & 3/14/08 & 1:01 pm & $\begin{array}{l}\text { 10.4.14(1 } \\
0.4 .3)\end{array}$ & & $\mathbf{S}$ & $1: 13$ pm & DAK & $\begin{array}{l}\text { LB6:6.6AkW, 13.3BkW, } 13.3 \mathrm{CkW} \text {. Triggers enabled. } \\
\text { SS opened on reverse power at 1:08. Load step altered } \\
\text { to prevent reverse power trip. }\end{array}$ \\
\hline $\begin{array}{l}\text { 10.4.14(10.4 } \\
.2 \mathrm{R})\end{array}$ & 3/14/08 & $1: 13$ pm & $\begin{array}{l}\text { 10.4.14(1 } \\
\text { 0.4.3R) }\end{array}$ & & $\mathbf{S}$ & $1: 21 \mathrm{pm}$ & DAK & $\begin{array}{l}\text { LB3:1.6AkW, 3.3BkW, 3.3CkW. Triggers enabled. } \\
\text { LB6 reduced at 1:16. LB6:6.6AkW, 13.3BkW, } \\
\text { 13.3CkW. SS opened shortly afterward on reverse } \\
\text { power. SS opened with a fault and a PES alarm. }\end{array}$ \\
\hline $\begin{array}{l}\text { 10.4.14(10.4 } \\
\text {.4) }\end{array}$ & 3/14/08 & 2:07 pm & $\begin{array}{l}\text { 10.4.14(1 } \\
0.4 .5)\end{array}$ & & $\mathbf{S}$ & $2: 18 \mathrm{pm}$ & DAK & $\begin{array}{l}\text { Test skipped briefly and returned to at } 2: 10 \mathrm{pm} . \mathrm{SS} \\
\text { reset and started with manual open asserted }\end{array}$ \\
\hline $\begin{array}{l}\text { 10.4.14(10.4 } \\
.6)\end{array}$ & 3/14/08 & $2: 18 \mathrm{pm}$ & $\begin{array}{l}\text { 10.4.14(1 } \\
\text { 0.4.7) }\end{array}$ & & $\mathbf{S}$ & $2: 23 \mathrm{pm}$ & DAK & $\begin{array}{l}\text { LB6:3.3AkW, 13.3BkW, 13.3CkW. Triggers enabled. } \\
\text { LB3 reduced at 2:18. LB3:0AkW, 3.3BkW, 3.3CkW }\end{array}$ \\
\hline 10.4.14(10.4 & 3/14/08 & $2: 23 \mathrm{pm}$ & 10.4.14(1 & & $\mathbf{S}$ & $2: 29 \mathrm{pm}$ & DAK & LB3:0kW, 3.3BkW, 3.3CkW. Triggers enabled. LB6 \\
\hline
\end{tabular}

64 of 65

This document contains information confidential and proprietary to AEPSC. It shall not be reproduced in whole or in part or released to any third party without expressed written consent of AEPSC. 


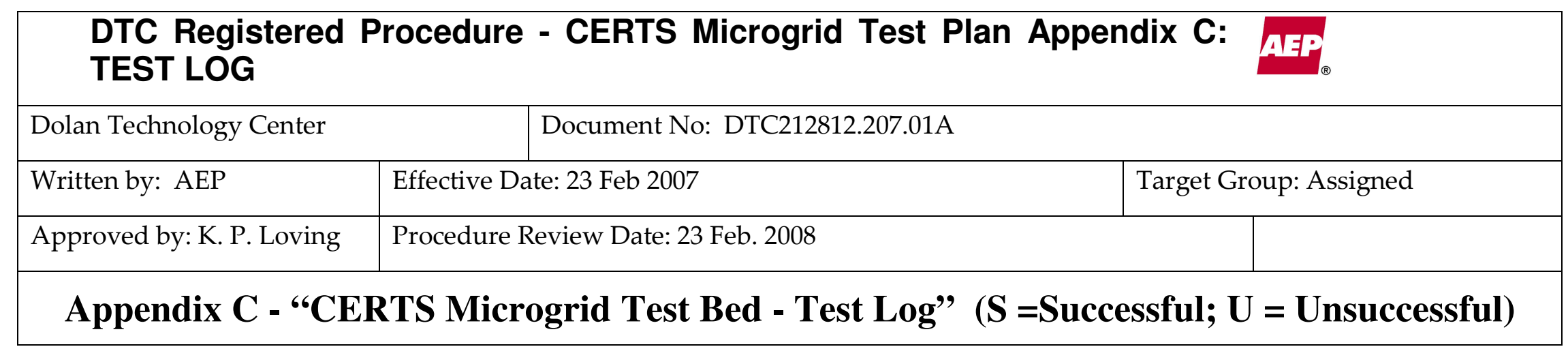

\begin{tabular}{|l|l|l|l|l|l|l|l|l|}
\hline .8$)$ & & & $0.4 .9)$ & & & & & reduced at 2:24. LB6:0AkW, 13.3BkW, 13.3CkW. \\
\hline $\begin{array}{l}\text { 10.4.14(10.4 } \\
.10)\end{array}$ & $3 / 14 / 08$ & $2: 29 \mathrm{pm}$ & $\begin{array}{l}\mathbf{1 0 . 4 . 1 4}(1 \\
\text { 0.4.11) }\end{array}$ & & $\mathrm{S}$ & $\mathbf{2 : 3 7} \mathrm{pm}$ & DAK & $\begin{array}{l}\text { SS manual open removed at 2:31. SS closed. SS } \\
\text { opened shortly afterward on reverse power trip. }\end{array}$ \\
\hline
\end{tabular}

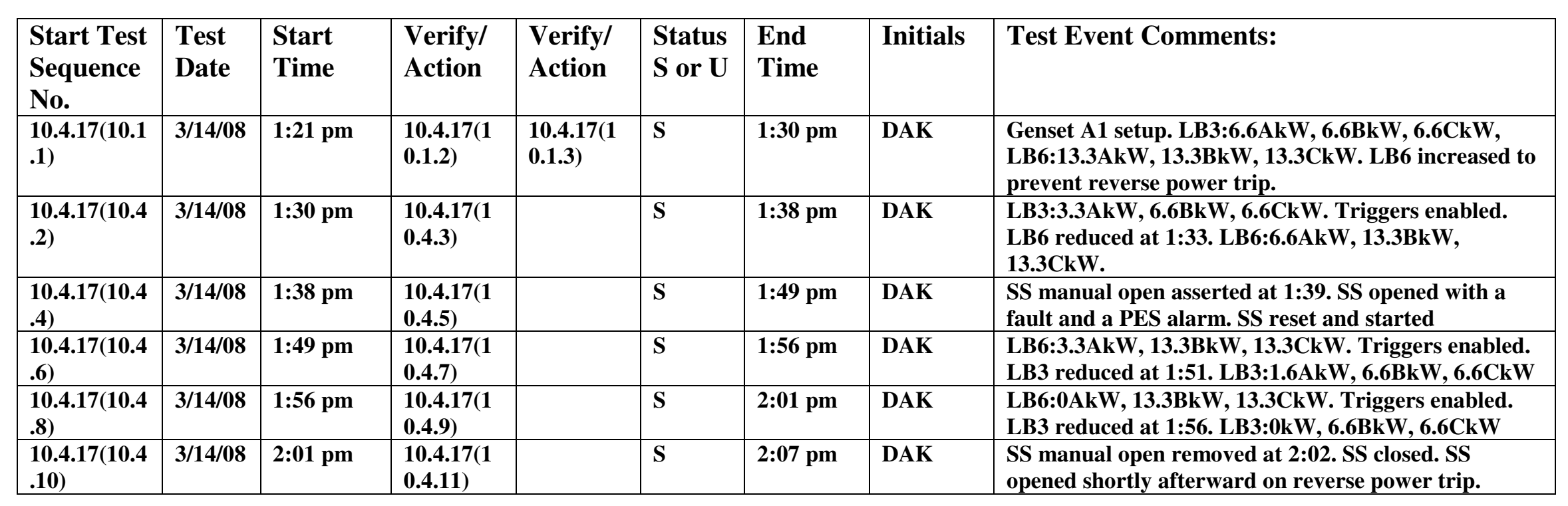




\section{CERTS MICROGRID LABORATORY TEST BED}

\section{Technical Advisory Committee Meeting Summary and Review Comments}

Prepared For:

California Energy Commission

Public Interest Energy Research Program

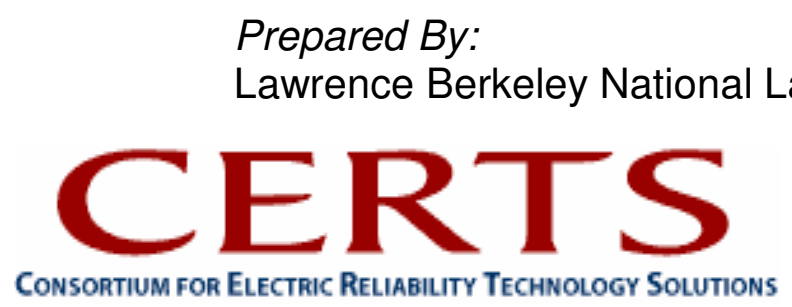

Arnold Schwarzenegger Governor 

Prepared By:

Lawrence Berkeley National Laboratory

Joseph H. Eto, Principal Investigator

Berkeley, CA 94720

Commission Contract No. 500-03-024

Prepared For:

Public Interest Energy Research (PIER) California Energy Commission

Bernard Treanton

Contract Manager

Mike Gravely

Program Area Lead

ENERGY SYSTEMS INTEGRATION

Mike Gravely

Office Manager

ENERGY SYSTEMS RESEARCH

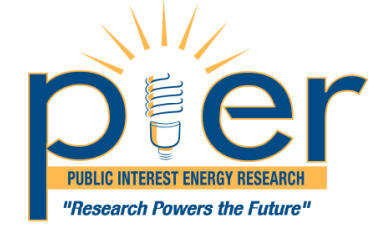

Martha Krebs, Ph.D.

PIER Director

Thom Kelly, Ph.D.

Deputy Director

ENERGY RESEARCH \& DEVELOPMENT DIVISION

Melissa Jones

Executive Director

\section{DISCLAIMER}

This report was prepared as the result of work sponsored by the California Energy Commission. It does not necessarily represent the views of the Energy Commission, its employees or the State of California. The Energy Commission, the State of California, its employees, contractors and subcontractors make no warrant, express or implied, and assume no legal liability for the information in this report; nor does any party represent that the uses of this information will not infringe upon privately owned rights. This report has not been approved or disapproved by the California Energy Commission nor has the California Energy Commission passed upon the accuracy or adequacy of the information in this report. 



\title{
Fifth Meeting of the \\ Technical Advisory Committee for the \\ California Energy Commission Public Interest Energy Research \\ CERTS Microgrid Test Bed Project
}

\author{
30 April 2008 \\ Lawrence Berkeley National Laboratory \\ Building 90 Room 4133 \\ 1 Cyclotron Road, Berkeley, CA
}

Meeting Summary

\section{Participants}

Technical Advisory Committee

Merrill Smith and Steve Waslo, U.S. Department of Energy

Ben Kroposki, National Renewable Energy Laboratory

Mohammed Vaziri, Pacific Gas and Electric Co.

Tom Dossey, Southern California Edison

Mark McGranaghan, EPRI Solutions

Guests Eric Wong, Cummins

Dave Potter, Chevron Energy Solutions

CEC Bernard Treanton, Steve Ghadiri

CERTS Joe Eto, LBNL

Ben Schenkman, Sandia National Laboratories

Robert Lasseter, University of Wisconsin/PSERC

Ed Linton, Northern Power

Bob Panora and Jean Roy, Tecogen

Dave Klapp and Ray Hayes, American Electric Power

Meeting Purpose: Introduce test bed results; initiate technical review process

\section{Summary of TAC, CEC, and guest's overall impressions/comments}

Concepts are elegant, yet simple. Simple translates to more affordable.

Demonstration that static switch can comply with 1547, with non-compliant generation behind it, is a major accomplishment. The team has confirmed that a static switch is very fast. It is recognized that ground fault testing on the utility side of the static switch is still missing from this aspect of 1547 compliance. 
Inverter controls are impressive.

Test plan was well laid-out. A wide range of concerns that were identified have now been successfully addressed. In particular, motor starting was a challenging test and the results are very encouraging. Should be a major contribution to the literature.

The test bed platform should be used for future research.

\section{Next Steps - CEC Final Report}

\section{Technical review comments due to J. Eto on May 23, 2008}

Be careful to ensure consistent presentation of transient information alongside of steady state information in graphics and tables reporting testing results

Explain clearly why inverters were observed to put out such apparently high fault currents and what options do or do not exist to limit them within the current inverter design.

Next Steps - DOE RDSI award (as part of already specified tasks to: reduce internal protection costs, optimize DC storage, examine AC storage, and incorporate non-inverter-based synchronous generation sources)

Determine whether and how to conduct ground fault testing on utility side of static switch.

Conduct harmonic testing.

Conduct longer term tests in an effort to capture microgrid response to power quality events.

\section{Next Steps - general}

Conduct outreach. Publish results in refereed archival literature (e.g., IEEE Transactions). Prepare functional specifications defining core elements and performance requirements of the CERTS Microgrid Concept. 
Support DOE and CEC in developing presentation materials they can use in describing work to lay- and non-technical audiences and to help defend research budgets.

Expand research focus to include role of energy manager. Consider, specifically, automatic adjustment of inverter power and voltage setpoints to regulate real and reactive power at the PCC

Examine flows onto the grid.

Explore physical and cyber security issues that might be addressed by microgrids. 
Additional comments from Southern California Edison, received May 16, 2008

Thank you for the opportunity to review the tests that have been performed on the CERTS Microgrid. As demonstrated in the documentation provided and especially through the detailed procedures of the test plan developed by AEP, this testing appears to have been comprehensive and adequate to demonstrate the operational capabilities of the systems and designs.

We note that for the most part the components of CERTS Microgrid performed as expected, and could transition from a grid-connected to an autonomous operating mode without adversely affecting the quality of the power supplied to the test loads. This is an important step and such operation will be a necessary feature of a commercial system. We would also like to highlight the very desirable concept demonstrated through your project and testing: i.e. the use of simple existing communication and control concepts where applicable, such as the use of local/device frequency droop controls. We applaud your technical progress that supports an outlook that a smart grid is not necessarily a complex grid dependent on extensive custom engineering and systems.

We look forward to the next steps in the development trail and will be pleased to continue to support the concept and project.

Please feel free to contact either of us if you have questions.

Tom Dossey, Charlie Vartanian

Southern California Edison

Distributed Energy Resources 
Additional comments from Pacific Gas and Electric, received May 24, 2008

Based on the notes I took during our April 30, 2008 advisory committee meeting, my comments are as follows:

1 - The results from the tests conducted so far seemed reasonable and in many cases better than anticipated.

2 - The static switch seemed to have successfully paralleled the systems without any troubles or need for a centralized communication system. This is certainly a positive result with good potential.

3 - For the phase and ground fault tests within the micro grid system, the results seemed correct and reasonable.

4 - For documentation purposes, the conditions (such open/closed switches, loading conditions, etc.) for each test case should be identified on the corresponding diagram to avoid any confusion.

5 - There was some confusion about the maximum output from the inverter based units. In some cases, up to 6.0 P.U. outputs was reported and discussed which was surprising to some of attendees. Careful measurements should be taken during faults to clarify this.

6 - Still to be verified is the following:

Tests need to be conducted to verify "Detection and timely clearing of Phase-Phase, 3 Phase, and especially Line-Ground faults on the Utility System, while the micro grid is in parallel operation with the utility." With a "Delta" connection on the utility side windings of the main interface transformer, conventional detection of ground faults on the utility system would require a grounding transformer. This has been pointed out all along from the beginning of the project. All Generating facilities are required by IEEE 1547 to detect and isolate their systems from an electrical fault on the utility system.

Regards, Moh 
Response to Pacific Gas and Electric, sent by project team on June 4, 2008

Thank you for attending the TAC meeting at LBNL and your comments below. Let me see if I can help clarify some of the questions that you mention below regarding the testing done so far.

4 - For documentation purposes, the conditions (such open/closed switches, loading conditions, etc.) for each test case should be identified on the corresponding diagram to avoid any confusion.

We completely agree with this. We will be working on the information presentation for the future phases of the project so that its both easier to understand as well as explain to others.

5 - There was some confusion about the maximum output from the inverter based units. In some cases, up to 6.0 P.U. outputs was reported and discussed which was surprising to some of attendees. Careful measurements should be taken during faults to clarify this.

We have discussed this further with Tecogen and Youtility. Their position is that this test result was expected. The inverters are $125 \mathrm{~kW}$ rated devices although we are artificially limiting them to half that. They are also currently setup to deliver $\sim 2.5 \mathrm{pu}$ fault current. This works out to $\sim 550 \mathrm{~A}$ peak, close to the $\sim 600 \mathrm{~A}$ number we were discussing. Tecogen is formalizing a more detailed response for the TAC group which will be included in the final report for this phase. In the next phase we are planning to adjust the inverters and retest to prove that the inverter fault current can be controlled to a desired level as needed by installed application. If you have suggestions as to what this fault contribution should be please let me know.

6 - Tests need to be conducted to verify "Detection and timely clearing of PhasePhase, 3 Phase, and especially Line-Ground faults on the Utility System, while the micro grid is in parallel operation with the utility." With a "Delta" connection on the utility side windings of the main interface transformer, conventional detection of ground faults on the utility system would require a grounding transformer. This has been pointed out all along from the beginning of the project. All Generating facilities are required by IEEE 1547 to detect and isolate their systems from an electrical fault on the utility system. 
The detection and times of the clearing for the different types of faults on the system are in the CERTS Microgrid Report Section 7.0 that I have attached to this email. We will have to figure out how to test a single line-to-ground fault on the utility side because I don't think AEP will just allow us to deliberately put a fault on their system.

The protection testing performed to date does show that the microgrid detects; phase to phase, 3 phase, and phase to ground faults within the microgrid. As you know in a wye-wye system these types of faults would appear very similar whether in the utility or within the microgrid. The biggest difference would be the measured magnitude of the fault which depends on a large number of variables. To date the faults we have tested have also been very low grade faults better described as overloads to the system. This was done to minimize the risk to the system while we worked out the protection scheme. At some point in the future we will be performing higher grade faults up to and including bolted faults. This has been the plan all along but we have just not reached it yet. Your point about the delta transformer is also well taken and we are looking into alternatives, a grounding transformer is one possibility. My hope is to replace the delta this with a wye-wye and monitor the microgrid operation during unscheduled utility faults. As Ben stated our company will not let us intentionally fault our own distribution system but there is no reason we can't try to capture a naturally occurring one.

Ben Schenkman

Dave Klapp 
Additional comments from National Renewable Energy Laboratory, received May 28, 2008

Sorry for the delay. I have been too busy getting ready for Kythnos. I am in Greece now, so my comments will be short.

1) Add labels to the components of the aerial photo of the CERTS microgrid. This would make the good photo used throughout the report more useful to people not familiar with the project.

2) Add conclusions section to Sections 6,7,8 and 9. In the conclusions section add a summary table that summarizes the tests and important results. This is in the text, but difficult to find as presently written.

3) Recommendations: additional test to determine fault current level and time from the DGs.

4) Overall this is very important work. I think it would be useful to include a report or link to a description of the droop control method that is being used by the DGs. Bob's presentation at the meeting discussed this a little but I don't see it included in the reports. This would make for a more complete package.

Ben Kroposki

NREL 


\section{CERTS MICROGRID LABORATORY TEST BED}

\section{Microgrid Fault Protection Based on Symmetrical and Differential Current Components}

Prepared For:

California Energy Commission

Public Interest Energy Research Program

Arnold Schwarzenegger Governor

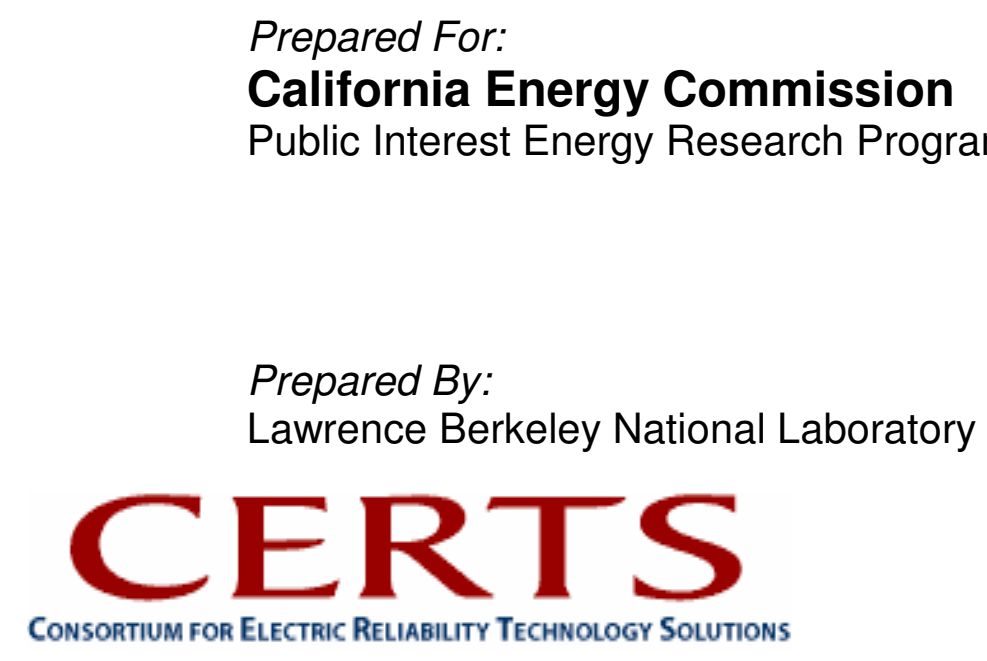



Prepared By:

Lawrence Berkeley National Laboratory

Joseph H. Eto, Principal Investigator

Berkeley, CA 94720

Hassan Nikkhajoei and Robert H. Lasseter, University of Wisconsin

Nancy J. Lewis, Lawrence Berkeley National Laboratory

Commission Contract No. 500-03-024

Prepared For:

Public Interest Energy Research (PIER)

California Energy Commission

Bernard Treanton

Contract Manager

Mike Gravely

Program Area Lead

ENERGY SYSTEMS INTEGRATION

Mike Gravely

Office Manager

ENERGY SYSTEMS RESEARCH

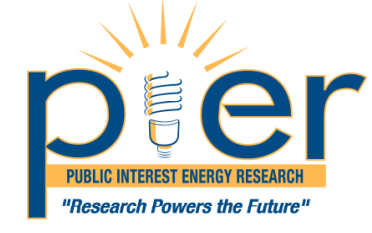

Martha Krebs, Ph.D.

PIER Director

Thom Kelly, Ph.D.

Deputy Director

ENERGY RESEARCH \& DEVELOPMENT DIVISION

Melissa Jones

Executive Director

\section{DISCLAIMER}

This report was prepared as the result of work sponsored by the California Energy Commission. It does not necessarily represent the views of the Energy Commission, its employees or the State of California. The Energy Commission, the State of California, its employees, contractors and subcontractors make no warrant, express or implied, and assume no legal liability for the information in this report; nor does any party represent that the uses of this information will not infringe upon privately owned rights. This report has not been approved or disapproved by the California Energy Commission nor has the California Energy Commission passed upon the accuracy or adequacy of the information in this report. 



\title{
Microgrid Fault Protection Based on Symmetrical and Differential Current Components
}

\author{
Prepared for \\ Public Interest Energy Research \\ California Energy Commission
}

Prepared by

Hassan Nikkhajoei and Robert H. Lasseter

Wisconsin Power Electronics Research Center

Department of Electrical and Computer Engineering

University of Wisconsin-Madison

December 2006

The work described in this report was coordinated by the Consortium for Electric Reliability Technology Solutions with funding support from the California Energy Commission, Public Interest Energy Research Program, under Contract No. 500-03-024. 


\section{Table of Contents}

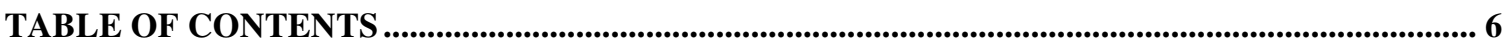

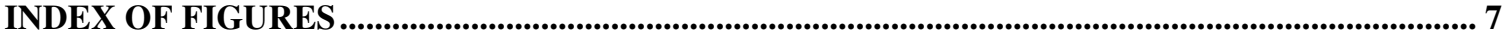

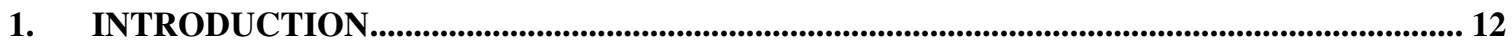

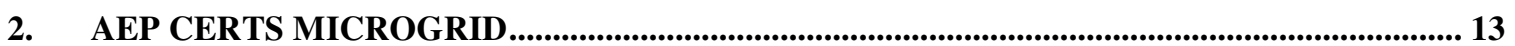

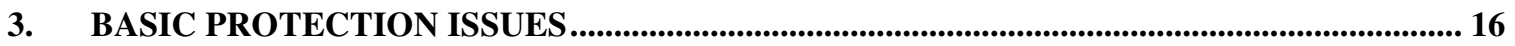

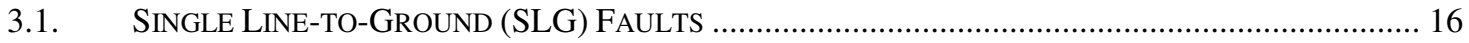

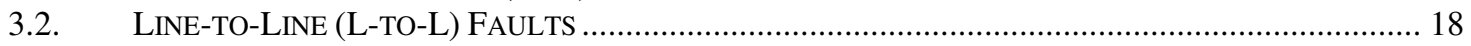

4. PROTECTION BASED ON SYMMETRICAL COMPONENTS ................................................. 18

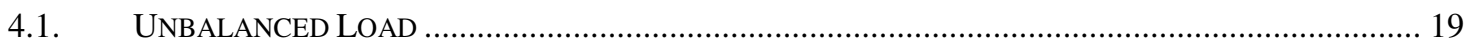

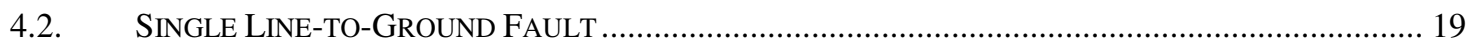

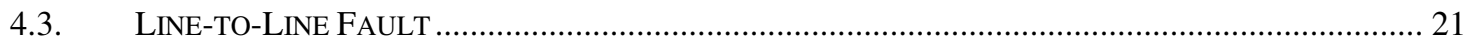

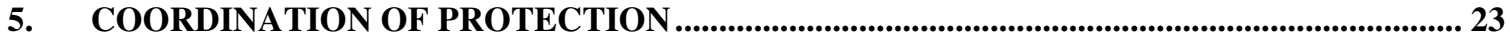

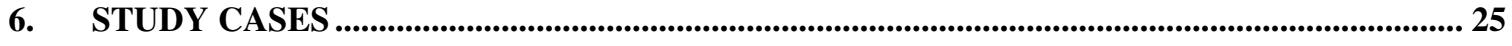

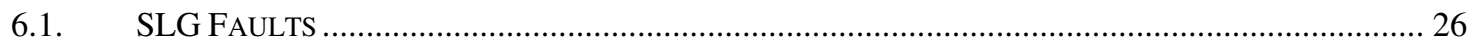

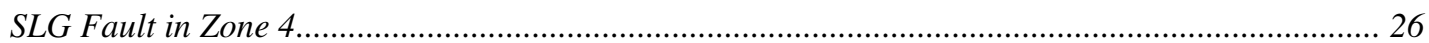

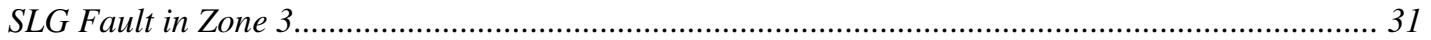

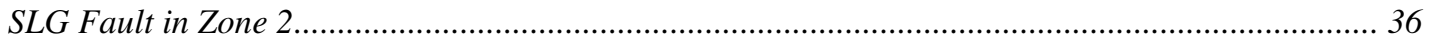

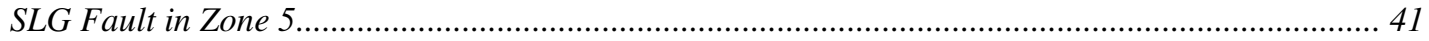

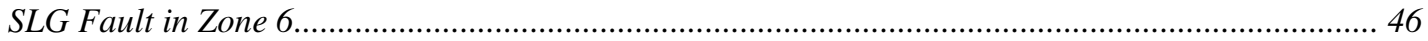

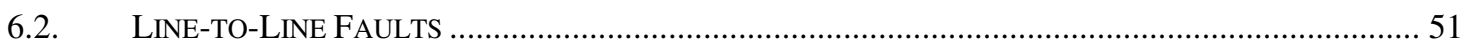

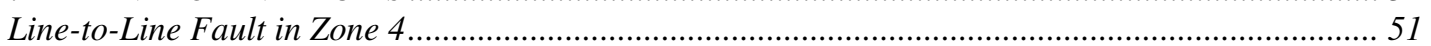

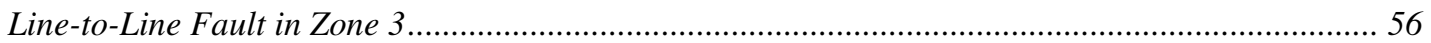

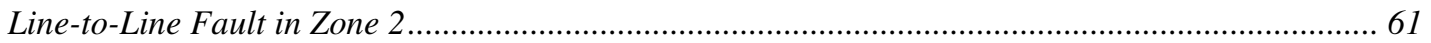

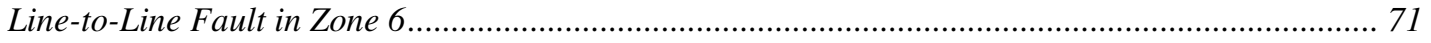




\section{Index of Figures}

Figure 1: Schematic representation of the AEP CERTS microgrid

Figure 2 : Response of the Relay 2 to a $28.5 \mathrm{~kW} \mathrm{SLG} \mathrm{fault} \mathrm{in} \mathrm{Zone} \mathrm{4:} \mathrm{(a,b)} \mathrm{three-phase}$ voltages and currents, (c,d) real and reactive power components, (e) frequency, and (f) differential and zero- and negative-sequence current components and output command of the relay

Figure 3: Response of the Relay 3 to a $28.5 \mathrm{~kW} \mathrm{SLG} \mathrm{fault} \mathrm{in} \mathrm{Zone} \mathrm{4:} \mathrm{(a,b)} \mathrm{three-phase}$ voltages and currents, (c,d) real and reactive power components, (e) frequency, and (f) differential and zero- and negative-sequence current components and output command of the relay

Figure 4: Response of the Relay 4 to a $28.5 \mathrm{~kW}$ SLG fault in Zone 4: (a,b) three-phase voltages and currents, $(\mathrm{c}, \mathrm{d})$ real and reactive power components, (e) frequency, and (f) differential and zero- and negative-sequence current components and output command of the relay.

Figure 5: Response of the Relay 5 to a $28.5 \mathrm{~kW}$ SLG fault in Zone 4: (a,b) three-phase voltages and currents, (c,d) real and reactive power components, (e) frequency, and (f) differential and zero- and negative-sequence current components, negativesequence voltage component at the transformer $T_{51}$ and output command of the relay

Figure 6: Response of the Relay 2 to a $28.5 \mathrm{~kW}$ SLG fault in Zone 3: $(\mathrm{a}, \mathrm{b})$ three-phase voltages and currents, (c,d) real and reactive power components, (e) frequency, and (f) differential and zero- and negative-sequence current components and output command of the relay.....

Figure 7: Response of the Relay 3 to a $28.5 \mathrm{~kW}$ SLG fault in Zone 3: $(\mathrm{a}, \mathrm{b})$ three-phase voltages and currents, (c,d) real and reactive power components, (e) frequency, and (f) differential and zero- and negative-sequence current components and output command of the relay.....

Figure 8: Response of the Relay 4 to a $28.5 \mathrm{~kW}$ SLG fault in Zone 3: $(\mathrm{a}, \mathrm{b})$ three-phase voltages and currents, (c,d) real and reactive power components, (e) frequency, and (f) differential and zero- and negative-sequence current components and output command of the relay.

Figure 9: Response of the Relay 5 to a $28.5 \mathrm{~kW}$ SLG fault in Zone 3: $(\mathrm{a}, \mathrm{b})$ three-phase voltages and currents, (c,d) real and reactive power components, (e) frequency, and (f) differential and zero- and negative-sequence current components, negativesequence voltage component at the transformer $T_{51}$ and output command of the relay 
Figure 10: Response of the Relay 2 to a $28.5 \mathrm{~kW}$ SLG fault in Zone 2: $(\mathrm{a}, \mathrm{b})$ three-phase voltages and currents, $(\mathrm{c}, \mathrm{d})$ real and reactive power components, (e) frequency, and (f) differential and zero- and negative-sequence current components and output command of the relay.

Figure 11: Response of the Relay 3 to a $28.5 \mathrm{~kW}$ SLG fault in Zone 2: (a,b) three-phase voltages and currents, (c,d) real and reactive power components, (e) frequency, and (f) differential and zero- and negative-sequence current components and output command of the relay.

Figure 12: Response of the Relay 4 to a $28.5 \mathrm{~kW} \mathrm{SLG} \mathrm{fault} \mathrm{in} \mathrm{Zone} \mathrm{2:} \mathrm{(a,b)} \mathrm{three-phase}$ voltages and currents, (c,d) real and reactive power components, (e) frequency, and (f) differential and zero- and negative-sequence current components and output command of the relay.....

Figure 13: Response of the Relay 5 to a $28.5 \mathrm{~kW}$ SLG fault in Zone 2: (a,b) three-phase voltages and currents, (c,d) real and reactive power components, (e) frequency, and (f) differential and zero- and negative-sequence current components, negativesequence voltage component at the transformer $T_{51}$ and output command of the relay

Figure 14: Response of the Relay 2 to a $28.5 \mathrm{~kW} \mathrm{SLG} \mathrm{fault} \mathrm{in} \mathrm{Zone} \mathrm{5:} \mathrm{(a,b)} \mathrm{three-phase}$ voltages and currents, (c,d) real and reactive power components, (e) frequency, and (f) differential and zero- and negative-sequence current components and output command of the relay.....

Figure 15: Response of the Relay 3 to a $28.5 \mathrm{~kW} \mathrm{SLG} \mathrm{fault} \mathrm{in} \mathrm{Zone} \mathrm{5:} \mathrm{(a,b)} \mathrm{three-phase}$ voltages and currents, $(\mathrm{c}, \mathrm{d})$ real and reactive power components, (e) frequency, and (f) differential and zero- and negative-sequence current components and output command of the relay.....

Figure 16: Response of the Relay 4 to a $28.5 \mathrm{~kW} \mathrm{SLG} \mathrm{fault} \mathrm{in} \mathrm{Zone} \mathrm{5:} \mathrm{(a,b)} \mathrm{three-phase}$ voltages and currents, (c,d) real and reactive power components, (e) frequency, and (f) differential and zero- and negative-sequence current components and output command of the relay.....

Figure 17: Response of the Relay 5 to a $28.5 \mathrm{~kW} \mathrm{SLG} \mathrm{fault} \mathrm{in} \mathrm{Zone} \mathrm{5:} \mathrm{(a,b)} \mathrm{three-phase}$ voltages and currents, (c,d) real and reactive power components, (e) frequency, and (f) differential and zero- and negative-sequence current components, negativesequence voltage component at the transformer $T_{51}$ and output command of the relay

Figure 18: Response of the Relay 2 to a $28.5 \mathrm{~kW}$ SLG fault in Zone 6: $(\mathrm{a}, \mathrm{b})$ three-phase voltages and currents, (c,d) real and reactive power components, (e) frequency, and 
(f) differential and zero- and negative-sequence current components and output command of the relay.

Figure 19: Response of the Relay 3 to a $28.5 \mathrm{~kW}$ SLG fault in Zone 6: (a,b) three-phase voltages and currents, (c,d) real and reactive power components, (e) frequency, and (f) differential and zero- and negative-sequence current components and output command of the relay..... 48

Figure 20: Response of the Relay 4 to a $28.5 \mathrm{~kW}$ SLG fault in Zone 6: (a,b) three-phase voltages and currents, $(c, d)$ real and reactive power components, (e) frequency, and (f) differential and zero- and negative-sequence current components and output command of the relay.....

Figure 21: Response of the Relay 5 to a $28.5 \mathrm{~kW}$ SLG fault in Zone 6: $(\mathrm{a}, \mathrm{b})$ three-phase voltages and currents, (c,d) real and reactive power components, (e) frequency, and (f) differential and zero- and negative-sequence current components, negativesequence voltage component at the transformer $T_{51}$ and output command of the relay

Figure 22: Response of the Relay 2 to a $85.5 \mathrm{~kW} \mathrm{LL}$ fault in Zone 4: (a,b) three-phase voltages and currents, (c,d) real and reactive power components, (e) frequency, and (f) differential and zero- and negative-sequence current components and output command of the relay

Figure 23: Response of the Relay 3 to a $85.5 \mathrm{~kW}$ LL fault in Zone 4: (a,b) three-phase voltages and currents, (c,d) real and reactive power components, (e) frequency, and (f) differential and zero- and negative-sequence current components and output command of the relay.....

Figure 24: Response of the Relay 4 to a $85.5 \mathrm{~kW}$ LL fault in Zone 4: (a,b) three-phase voltages and currents, $(\mathrm{c}, \mathrm{d})$ real and reactive power components, (e) frequency, and (f) differential and zero- and negative-sequence current components and output command of the relay

Figure 25: Response of the Relay 5 to a $85.5 \mathrm{~kW}$ LL fault in Zone 4: (a,b) three-phase voltages and currents, (c,d) real and reactive power components, (e) frequency, and (f) differential and zero- and negative-sequence current components, negativesequence voltage component at the transformer $T_{51}$ and output command of the relay

Figure 26: Response of the Relay 2 to a $85.5 \mathrm{~kW}$ LL fault in Zone 3: (a,b) three-phase voltages and currents, (c,d) real and reactive power components, (e) frequency, and (f) differential and zero- and negative-sequence current components and output command of the relay.

Figure 27: Response of the Relay 3 to a $85.5 \mathrm{~kW}$ LL fault in Zone 3: (a,b) three-phase voltages and currents, (c,d) real and reactive power components, (e) frequency, and 
(f) differential and zero- and negative-sequence current components and output command of the relay.

Figure 28: Response of the Relay 4 to a $85.5 \mathrm{~kW}$ LL fault in Zone 3: (a,b) three-phase voltages and currents, $(\mathrm{c}, \mathrm{d})$ real and reactive power components, (e) frequency, and (f) differential and zero- and negative-sequence current components and output command of the relay.....

Figure 29: Response of the Relay 5 to a $85.5 \mathrm{~kW}$ LL fault in Zone 3: (a,b) three-phase voltages and currents, $(\mathrm{c}, \mathrm{d})$ real and reactive power components, (e) frequency, and (f) differential and zero- and negative-sequence current components, negativesequence voltage component at the transformer $T_{51}$ and output command of the relay

Figure 30: Response of the Relay 2 to a $85.5 \mathrm{~kW}$ LL fault in Zone 2: (a,b) three-phase voltages and currents, (c,d) real and reactive power components, (e) frequency, and (f) differential and zero- and negative-sequence current components and output command of the relay.....

Figure 31: Response of the Relay 3 to a $85.5 \mathrm{~kW}$ LL fault in Zone 2: (a,b) three-phase voltages and currents, (c,d) real and reactive power components, (e) frequency, and (f) differential and zero- and negative-sequence current components and output command of the relay.

Figure 32: Response of the Relay 4 to a $85.5 \mathrm{~kW}$ LL fault in Zone 2: (a,b) three-phase voltages and currents, (c,d) real and reactive power components, (e) frequency, and (f) differential and zero- and negative-sequence current components and output command of the relay

Figure 33: Response of the Relay 5 to a $85.5 \mathrm{~kW}$ LL fault in Zone 2: (a,b) three-phase voltages and currents, (c,d) real and reactive power components, (e) frequency, and (f) differential and zero- and negative-sequence current components, negativesequence voltage component at the transformer $T_{51}$ and output command of the relay

Figure 34: Response of the Relay 2 to a $85.5 \mathrm{~kW}$ LL fault in Zone 5: (a,b) three-phase voltages and currents, (c,d) real and reactive power components, (e) frequency, and (f) differential and zero- and negative-sequence current components and output command of the relay

Figure 35: Response of the Relay 3 to a $85.5 \mathrm{~kW} \mathrm{LL}$ fault in Zone 5: (a,b) three-phase voltages and currents, $(\mathrm{c}, \mathrm{d})$ real and reactive power components, (e) frequency, and (f) differential and zero- and negative-sequence current components and output command of the relay.....

Figure 36: Response of the Relay 4 to a $85.5 \mathrm{~kW}$ LL fault in Zone 5: (a,b) three-phase voltages and currents, (c,d) real and reactive power components, (e) frequency, and 
(f) differential and zero- and negative-sequence current components and output command of the relay

Figure 37: Response of the Relay 5 to a $85.5 \mathrm{~kW} \mathrm{LL}$ fault in Zone 5: (a,b) three-phase voltages and currents, (c,d) real and reactive power components, (e) frequency, and (f) differential and zero- and negative-sequence current components, negativesequence voltage component at the transformer T51 and output command of the relay

Figure 38: Response of the Relay 2 to a $85.5 \mathrm{~kW}$ LL fault in Zone 6: (a,b) three-phase voltages and currents, $(\mathrm{c}, \mathrm{d})$ real and reactive power components, (e) frequency, and (f) differential and zero- and negative-sequence current components and output command of the relay.

Figure 39: Response of the Relay 3 to a $85.5 \mathrm{~kW}$ LL fault in Zone 6: (a,b) three-phase voltages and currents, (c,d) real and reactive power components, (e) frequency, and (f) differential and zero- and negative-sequence current components and output command of the relay.

Figure 40: Response of the Relay 4 to a $85.5 \mathrm{~kW}$ LL fault in Zone 6: (a,b) three-phase voltages and currents, (c,d) real and reactive power components, (e) frequency, and (f) differential and zero- and negative-sequence current components and output command of the relay.

Figure 41: Response of the Relay 5 to a $85.5 \mathrm{~kW}$ LL fault in Zone 6: (a,b) three-phase voltages and currents, (c,d) real and reactive power components, (e) frequency, and (f) differential and zero- and negative-sequence current components, negativesequence voltage component at the transformer T51 and output command of the relay 


\section{Introduction}

The objective of this study is to develop a systematic approach for protecting a microgrid, in particular the AEP microgrid, against Single Line-to-Ground (SLG) and Line-to-Line (LL) faults. A microgrid is a part of a large power system in which a number of sources, usually attached to a power electronic converter, and loads are clustered so that the microgrid can operate independent of the main power system [1], [2]. In general, a microgrid can operate in both the grid-connected mode and the islanded mode where the microgrid is disconnected from the main power system by a fast semiconductor switch called static switch, Fig. 1.

It is essential to protect a microgrid in both the grid-connected and the islanded modes of operation against for all SLG and LL faults. The philosophy of protection is to disconnect the static switch for all classes of faults. With the static switch open (islanded mode), output currents of the microgrid sources are limited by the corresponding power electronic converter to $2.0 \mathrm{pu}$. Therefore, a microgrid cannot be protected against faults based on traditional over-current protection since there is not adequate fault currents in the islanded mode of operation.

The main challenge for protecting a microgrid arises from the fact that power can flow in both directions in each feeder of a microgrid. Close to each local load, there may exist two or more sources that contribute to the load power, Fig. 1. The sources can be located in both sides of the load and therefore, power flows in opposite directions from the two sources towards the load. As a results, in a section of the feeder, e.g. Zone 3 of Fig. 1, that is between the source $A_{1}$ and the load, power flows in one direction and in the other section, i.e. Zone 4, power flows in an opposite direction. It is to be noted that, in conventional distribution systems, power always flows in one direction, from the distribution substation towards the loads.

It was initially thought that a microgrid can be protected, at least for SLG faults, based on differential components of phase currents. However, studies showed that each relay, which corresponds to a microgrid protection zone, e.g. the Relay 4 of Fig. 1, can only detect SLG faults that occur in the up-stream zone of the relay, i.e. Zone 4 of Fig. 1. The relays do not detect an SLG fault in a down-stream zone, e.g. Zone 3 for the Relay 4, since the fault current does not pass through Zone 4 and therefore, the differential current component detected by the Relay 4 is zero. Details of a study that demonstrates the inability of protecting a microgrid based on differential current components can be found in [3]. In addition to inability for protecting against down-stream SLG faults, the relays do not detect a differential current component for LL faults in any of the microgrid protection zones, Fig. 1.

An alternative solution is to protect a microgrid against SLG and LL faults based on symmetrical current components [4]. A broad range of studies have been conducted on the AEP microgrid and it was concluded that SLG faults in down-stream zones of relays and all LL faults can be detected based on symmetrical current components. The SLG 
and LL faults are detected from the zero-sequence component and the negative-sequence components of currents, respectively. However, zero- and negative-sequence components of current are also non-zero under normal operating conditions of a microgrid. This is due to the fact that a microgrid may, in general, include single-phase loads or three-phase unbalanced loads.

A study has been conducted for the AEP microgrid, in which the values of zero- and negative-sequence components of currents flowing through all relays of the microgrid were obtained for a maximum unbalanced load power of 20 percents. To ensure that the microgrid protection does not command for a trip under unbalanced load conditions, a threshold was assigned for each of the symmetrical current components. With the thresholds being set for all relays, each of them commands for breaker trip only when the corresponding zero-sequence or negative-sequence component exceeds a threshold. This guarantees that the protection system clears the SLG and LL faults in all protective zones while it does not command for an unwanted trip under unbalanced load conditions.

Based on the studies carried out for differential and symmetrical current components, a symmetric approach for protecting a microgrid against all SLG and LL faults has been developed. The developed microgrid protection approach employs the differential and zero-sequence current components as primary protection against SLG faults and the negative-sequence current component as a primary protection for LL faults.

If the microgrid primary protection fails, the time integral of current, i.e. $I^{2} t$, as a back-up protects the microgrid for all classes of fault [5]. A secondary back-up for the protection system is the conventional over-current protection. The over-current $\left(\mathrm{I}_{\max }\right)$ protection usually commands for a trip after the $\mathrm{I}^{2} \mathrm{t}$ protection, except for extremely high-current faults for which the over-current protection detects a fault fast. It is to be noted that, the $\mathrm{I}^{2} \mathrm{t}$ and over-current protections are also back-up for the static switch in case that it fails to trip based on differential and symmetrical current protections.

In the following sections, first, structure of the AEP microgrid and its protection zones are described. Then, the results obtained for the symmetrical current components related to unbalanced load conditions and SLG and LL faults in all protection zones are presented. Coordination of the protective relays of the AEP microgrid, based on the results obtained for symmetrical current components, are also discussed. The results of studies carried out on the AEP microgrid for SLG and LL faults in all protection zones of the microgrid are presented. Conclusions are stated in the last section of the report.

\section{AEP CERTS Microgrid}

Fig. 1 shows a single-line representation of the AEP CERTS microgrid. The microgrid includes three microsources, specified as $\mathrm{A}_{1}, \mathrm{~A}_{2}$ and $\mathrm{B}_{1}$, and three local loads, specified as $\mathrm{L}_{1}, \mathrm{~L}_{2}$ and $\mathrm{L}_{3}$. Each microsource can provide a maximum output power of $60 \mathrm{~kW}$ and each load can consume a power up to a maximum amount of $120 \mathrm{~kW}$, where the total load of microgrid should not exceed $180 \mathrm{~kW}$. The microgrid is connected through a fast 
semiconductor switch, that is called static switch, to the main grid. The combination of microgrid and the main grid has six protection zones that are specified as Zone 1 to Zone 6, Fig. 1.

CERTS Microgrid has two critical components, the static switch and the microsource. The static switch has the ability to autonomously island the microgrid from disturbances such as faults, IEEE 1547 events or power quality events. After islanding, the reconnection of the microgrid is achieved autonomously after the tripping event is no longer present. This synchronization is achieved by using the frequency difference between the islanded microgrid and the utility grid insuring a transient free operation without having to match frequency and phase angles at the connection point. Each microsource can seamlessly balance the power on the islanded microgrid using a power vs. frequency droop controller. This frequency droop also insures that the microgrid frequency is different from the grid frequency to facilitate reconnection to the utility.

To enhance reliability, a peer-to-peer and plug-and-play model is used for each component of the microgrid. The peer-to-peer concept insures that there are no components, such as a master controller or central storage unit that is critical for operation of the microgrid. This implies that the microgrid can continue operating with loss of any component or generator. With one additional source, $(\mathrm{N}+1)$, we can insure complete functionality with the loss of any source. Plug-and-play implies that a unit can be placed at any point on the electrical system without re-engineering the controls thereby reducing the chance for engineering errors. The plug-and-play model facilitates placing generators near the heat loads thereby allowing more effective use of waste heat without complex heat distribution systems such as steam and chilled water pipes.

Peer-to-peer and plug-and-play concepts also impact the protection design. The peer-topeer concept insures that there are no protection components, such as a master coordinator or communication system critical to the protection of the microgrid. Plugand-play implies that a unit can be placed at any point on the electrical system without reengineering the protection thereby reducing the chance for engineering errors. This implies that microgrid protection is part of each source. 


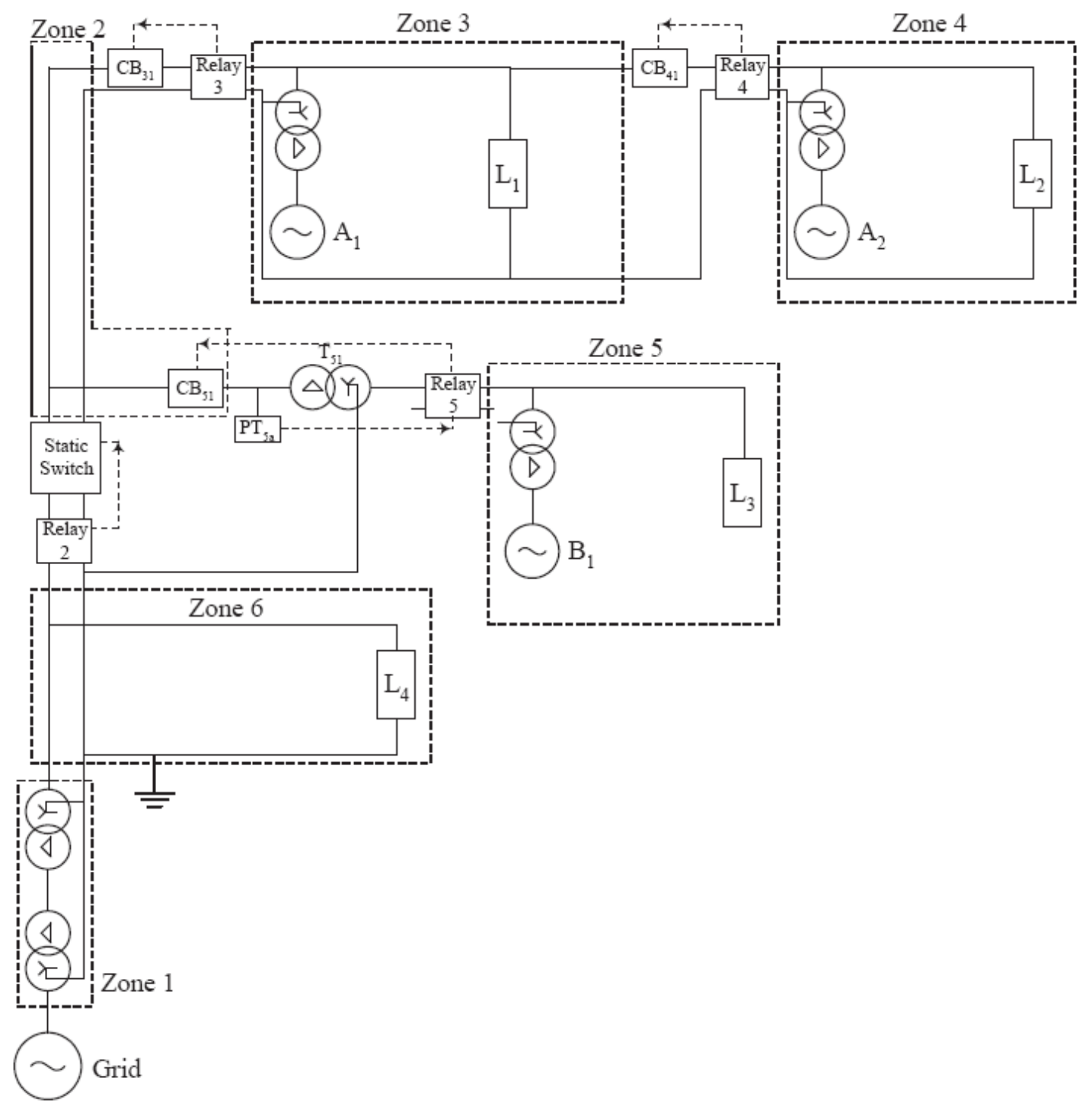

Figure 1: Schematic representation of the AEP CERTS microgrid 


\section{Basic Protection Issues}

In general, a microgrid can operate in both the grid-connected mode and the islanded mode where the microgrid is interfaced to the main power system by a fast semiconductor switch called static switch, (SS). It is essential to protect a microgrid in both the grid-connected and the islanded modes of operation against all types of faults. The major issue arises in island operation with inverter-based sources. Inverter fault currents are limited by the ratings of the silicon devices to around 2 p.u. rated current. Fault currents in islanded inverter based microgrids may not have adequate magnitudes to use traditional over-current protection techniques. This possibility requires an expanded protection strategy.

The philosophy for protection is to have the same protection strategies for both islanded and grid-connected operation. The static switch is designed to open for all faults. With the static switch open, faults within the microgrid need to be cleared with techniques that do not rely on high fault currents. Fig. 1 shows a simple microgrid. The SS (static switch) separates the utility from the microgrid. The islanded part contains two loads and the inverter based sources. The microgrid assumes that the sources have adequate ratings to meet the load demands while in island mode. This example shows two sources in series with impedances between the sources using a four-wire configuration with a common ground point and one source in parallel.

In this example, we assume four protection zones with circuit breakers between Zone 2 and Zone 3, Zone 3 and Zone 4 and between Zone 2 and Zone 5. The system could be designed without these circuit breakers but the protection zones remain the same. In either case, sources feeding the fault must shut down without communications. For example, for a fault in Zone 2 we would expect the SS in Zone 2 to open in $1 / 2$ to 2 cycles, the circuit breakers between Zones 2-3, 3-4 and 2-5 to open with the inverter-based sources in all Zones shutting down. If the fault was in Zone 4, ideally the SS would open and the circuit breaker between Zones 3 and 4 would open but the breaker between Zones 2 and 3 would not. The source in Zone 4 would also shut down. This would result in Zone 2, Zone 3 and Zone 5 operating as an island.

\subsection{Single Line-to-Ground (SLG) Faults}

The basic fault sensors for each zone are; a current sensors for each phase and a current sensor summing the three phases and the neutral in zones 2, 3 and 40. It was initially thought that a microgrid could be protected, at least for SLG faults, based on differential

component of current $I_{d},\left(I_{d}=\sum_{k=a, b, c, n}\left|I_{k}\right|\right)$ for any number of sources in series. For example,

in Fig.1, a SLG fault in Zone 4 would generate a non-zero differential current in zones 2, 3 and 4 . This would result in Relay-2 opening the SS in $1 / 2-2$ cycles followed by Relay-4 opening its circuit breaker and shutting down the source in Zone 4. Using a delay in Relay-3 would allow the section(s) of the microgrid up-stream of the fault to remain operating. This section could then reconnect to the utility autonomously since the faulted section is no longer connected. The problem with this concept is that any zones downstream of the fault will not see the effect of ground currents. The $I_{d}$, sum of the three phase currents and neutral current, will be zero. 
For example a SLG fault in Zone 3 will create differential current signals for the SS and Relay-3 to open and shut down the source in Zone 3, but the Zone 4 relay will not see the fault. The fault's ground current does not pass through Zone 4 and therefore, the differential current component detected by the Relay-4 is zero. This would leave Zones 3 and 4 energized as a faulted sub-island. For the case of a microgrid, which does not have breakers between zones, the SS would open and source in Zone 3 would be shut down but the source in Zone 4 would not shut down. It would continue to operate and feed the fault. If the fault were of high impedance, such back-up detection methods as over current and $\mathrm{I}^{2} \mathrm{t}$ would not trip the source in Zone 4. Under-voltage protection could possible provide some back up, but again this would have problems. This problem requires using zero-sequence current detection for SLG faults. In this case, this is defined as the vector sum of the three phase currents.

As an example, consider the system in Fig. 1 where the loads in all zones are $40 \mathrm{~kW}$ and the source in Zone 3 is dispatched at $40 \mathrm{~kW}$ and the Zones 4 and 5 are at $20 \mathrm{~kW}$. The nominal line-to-line voltage is 480 volts. This implies that the grid provides $40 \mathrm{~kW}$. When a high impedance SLG $(\sim 28 \mathrm{~kW})$ fault is applied to phase A, in Zone 3, Relay-2 will see the fault, $I_{d}>0$, and open the SS in 5-10 milliseconds. The resulting sequence voltages and currents seen by each relay are shown below;

FAULT CURRENTS SEEN BY THE RELAYS FOR A SLG FAULT IN ZONE 3 AND SS OPEN

\begin{tabular}{|c|c|c|c|}
\hline Signal & Relay 2 & Relay 3 & Relay 4 \\
\hline$\left|I_{a}\right|(A)$ & 0 & 3.5 & 59.6 \\
\hline$\left|I_{b}\right|(A)$ & 0 & 20.8 & 4.8 \\
\hline$\left|I_{c}\right|(A)$ & 0 & 21.4 & 9.2 \\
\hline$I_{d}(A)$ & 0 & 32.2 & 0 \\
\hline $3\left|I_{0}\right|(A)$ & 0 & 0 & 54.4 \\
\hline$\left|V_{+}\right|($volts $)$ & 391.6 & 392.1 & 391.2 \\
\hline$|V|($ volts $)$ & 0 & 4.8 & 5 \\
\hline
\end{tabular}

Relay-4 has a 3-cycle delay to insure that the SS will open first. Relay 3 has a 10-cycle delay to insure that Zone 4 will shut down first. From the table, we see that Relay-4's differential current is zero but the zero sequence current provides the trip signal. Note that the voltage unbalance is small, $(\sim 1 \%)$ due to the local voltage control at each source. The usual relationship between the differential currents and the zero sequence currents is a function of the placement of the cts and the neutral conductors connection to the inverters' transformers.

Coordination between unbalanced loads and zero sequence currents is important. Unbalanced loads cause a zero sequence trip. The differential current detection does not see unbalanced loads. 


\subsection{Line-to-Line (L-to-L) Faults}

Using $\mathrm{I}^{2} \mathrm{t}$ protection for L-to-L faults is effective for low impedance faults but in many cases the time to trip will be long causing unnecessary disturbances to the loads and utility. High impedance fault current magnitudes will not be adequate to provide a trip. The our approach is to use negative sequence current to detect line-to-line faults.

Using the negative-sequence current, all L-to-L faults can be detected except the fault in Zone 3. In the microgrid of Fig. 1, no load exists between the static switch and Relay 3. When the static switch trips in response to a L-to-L fault in Zone 3, no current flows through Relay 3 and therefore, the relay does not command the corresponding breaker to trip. As a result, the microsource $A_{1}$ is not shut down and continues to provide the fault current.

\section{Protection Based on Symmetrical Components}

An SLG or LL fault imbalances phase currents of a number of protection zones of a microgrid. For the analysis of unbalanced currents, the concept of symmetrical components is usually employed [4]. The symmetrical components analysis uses a transformation matrix which converts phase components of an unbalanced three-phase current to equivalent positive-, negative- and zero-sequence current components [4]. The positive-sequence component represents a three-phase current that rotates in the same direction as the unbalanced three-phase current, while the negative-sequence component represents a three-phase current that rotates in an opposite direction with respect to the unbalanced three-phase currents. The zero-sequence component represents a system of three currents that have the same phase-angle.

An SLG fault generates usually zero- and negative-sequence current components, and an LL fault generates only a negative-sequence current component. The zero- and negativesequence components of currents at the protective relays can be employed to detect SLG and LL faults in different protection zones of a microgrid. It is to be emphasized that zero- and negative-sequence current components are also generated and detected by relays under unbalanced load conditions. The microgrid concept is currently used for the distribution level of power systems which usually operates under unbalanced load conditions. Therefore, a protection approach based on symmetrical current components must distinguish between faulty and unbalanced operating conditions. In other words, the microgrid protection system must be coordinated such that it does not command for a trip under unbalanced load conditions while the protection system should detect SLG and LL faults in all zones of the microgrid.

For the AEP microgrid of Fig. 1, the zero- and negative-sequence components of currents at all relays, including Relay 2 that is attached to the static switch, are obtained for unbalanced load and SLG and LL fault conditions. The values of symmetrical current components are obtained based on digital-time domain simulations of the microgrid in the EMTP software environment. To comply with values measured by the real-world protective relays [6]-[8], magnitudes of all symmetrical components measured in the EMTP are multiplied by a factor of 3 . Thus, each relay detects three times of the 
magnitudes of symmetrical current components, i.e. $3\left|I_{+}\right|, 3\left|I_{-}\right|$and $3\left|I_{0}\right|$ which are referred to as magnitudes of positive, negative and zero components of currents herein after.

Magnitudes of the positive, negative and zero current components at all relays for a maximum load power unbalance of 20 percents and for SLG and LL faults in all protection zones have been obtained for the AEP microgrid and the results are presented in the following sections. For all the study cases, powers of the microsources $A_{1}, A_{2}$ and $B_{1}$ and powers of the loads $L_{1}, L_{2}$ and $L_{3}$ have been set at the following values: $\mathrm{P}_{\mathrm{A} 1}=40 \mathrm{~kW}, \mathrm{P}_{\mathrm{A} 2}=20 \mathrm{~kW}, \mathrm{P}_{\mathrm{B} 1}=0 \mathrm{~kW}, \mathrm{P}_{\mathrm{L} 1}=40 \mathrm{~kW}, \mathrm{P}_{\mathrm{L} 2}=60 \mathrm{~kW}$, and $\mathrm{P}_{\mathrm{L} 3}=40 \mathrm{~kW}$.

\subsection{Unbalanced Load}

In this case study, the phase resistances of load $\mathrm{L}_{3}$ are changed such that it provides a power unbalance of 20 percents, while its total power remains at $60 \mathrm{~kW}$. Table I gives the values of three-phase currents at the Relays 2, 3, 4 and 5 and magnitudes of the corresponding positive-, negative- and zero-sequence current components as well as the positive- and negative-sequence voltage components at all relays. It is seen from the results that the zero- and negative-sequence current components have maximum values of 11.9 and $26 \mathrm{~A}$, respectively, and they correspond to the currents flowing through the Relay 4. If one of the other loads, i.e. $\mathrm{L}_{1}$ or $\mathrm{L}_{2}$, are 20 percents unbalanced, then current of the closest relay to the unbalanced load will have maximum zero- and negativesequence components which do not exceed the maximum values obtained for the case of an unbalanced load $\mathrm{L}_{3}$. When using symmetrical current components for detecting faults, the threshold of all relays must be selected above the maximum zero- and negativesequence current magnitudes of 11.9 and $26 \mathrm{~A}$. This ensures that the protection system does not trip any of the breakers when the AEP microgrid operates under a maximum load power unbalance of 20 percents.

TABLE I: SYMMETRICAL SEQUENCE CURRENT COMPONENTS AT THE AEP MICROGRID RELAYS FOR A 20 PERCENTS UNBALANCED LOAD L3

\begin{tabular}{c|c|c|c|c|c|c|c|c}
\hline Device & $\left|I_{a}\right|(\mathrm{A})$ & $\left|I_{b}\right|(\mathrm{A})$ & $\left|I_{c}\right|(\mathrm{A})$ & $3\left|I_{+}\right|(\mathrm{A})$ & $3\left|I_{-}\right|(\mathrm{A})$ & $3\left|I_{0}\right|(\mathrm{A})$ & $\left|V_{+}\right|(\mathrm{A})$ & $\left|V_{-}\right|(\mathrm{A})$ \\
\hline \hline Relay 2 & 179.2 & 162.7 & 164.9 & 506.8 & 21.8 & 9.3 & 391.3 & 0.22 \\
\hline Relay 3 & 112.3 & 94.7 & 97.3 & 304.2 & 23.7 & 9.3 & 391.3 & 0.22 \\
\hline Relay 4 & 114 & 93.9 & 96.7 & 304.4 & 26 & 11.9 & 389.3 & 0.37 \\
\hline Relay 5 & 67.3 & 67.4 & 68.3 & 203 & 2 & 0 & 390.8 & 0.18 \\
\hline
\end{tabular}

\subsection{Single Line-to-Ground Fault}

This section presents the results obtained from the EMTP based simulation of the AEP microgrid of Fig. 1 for an SLG fault in one of the protection Zones 2, 3, 4 and 5. The AEP microgrid operates under steady-state conditions with the source and load parameters given in Section 3. An SLG fault with a power of $28.5 \mathrm{~kW}\left(R_{f}=2.695 \mathrm{Ohms}\right)$ occurs in one of the protection zones at $t=1 \mathrm{~s}$ when the microgrid operates under steadystate conditions. Magnitudes of the phase currents, the symmetrical current components $3\left|I_{+}\right|, 3\left|I_{-}\right|$and $3\left|I_{0}\right|$ and the symmetrical voltage components $\left|V_{+}\right|$and $\left|V_{-}\right|$at all relays are 
recorded when the microgrid operates under steady-state conditions subsequent to the SLG fault. Values of the parameters for the grid-connected (SS open) and the islanded (SS open) modes, when the static switch trips after the fault, are given in Tables II, III, IV and V for an SLG fault in Zones 4, 3, 2 and 5, respectively.

TABLE II: SYMMETRICAL SEQUENCE CURRENT COMPONENTS AT THE AEP MICROGRID RELAYS FOR AN SLG FAULT IN ZONE 4

\begin{tabular}{c|c|c|c|c|c|c|c|c}
\hline Device & $\left|I_{a}\right|(\mathrm{A})$ & $\left|I_{b}\right|(\mathrm{A})$ & $\left|I_{c}\right|(\mathrm{A})$ & $3\left|I_{+}\right|(\mathrm{A})$ & $3\left|I_{-}\right|(\mathrm{A})$ & $3\left|I_{0}\right|(\mathrm{A})$ & $\left|V_{+}\right|(\mathrm{A})$ & $\left|V_{-}\right|(\mathrm{A})$ \\
\hline SS Closed & \multicolumn{7}{|c}{} \\
\hline \hline Relay 2 & 211.6 & 117 & 116.9 & 445 & 102.8 & 88.1 & 391.3 & 1.05 \\
\hline Relay 3 & 147 & 47.6 & 50 & 242.7 & 112 & 88.1 & 391.3 & 1.05 \\
\hline Relay 4 & 158.8 & 42.4 & 42.7 & 242.8 & 123.3 & 111.4 & 389.6 & 1.76 \\
\hline Relay 5 & 66 & 66.3 & 70.7 & 202.9 & 9.2 & 0 & 390.9 & 0.88 \\
\hline \hline SS Open & \multicolumn{7}{|c|}{} \\
\hline \hline Relay 2 & 0 & 0 & 0 & 0 & 0 & 0 & 391.6 & 0 \\
\hline Relay 3 & 2.4 & 20.8 & 21.6 & 33 & 40 & 0 & 392.1 & 4.5 \\
\hline Relay 4 & 81.5 & 4 & 8.5 & 72 & 88.4 & 85.4 & 391.1 & 5 \\
\hline Relay 5 & 13 & 11.6 & 24.4 & 33.2 & 40 & 0 & 392 & 3.8 \\
\hline
\end{tabular}

TABLE III: SYMMETRICAL SEQUENCE CURRENT COMPONENTS AT THE AEP MICROGRID RELAYS FOR AN SLG FAULT IN ZONE 3

\begin{tabular}{c|c|c|c|c|c|c|c|c}
\hline Device & $\left|I_{a}\right|(\mathrm{A})$ & $\left|I_{b}\right|(\mathrm{A})$ & $\left|I_{c}\right|(\mathrm{A})$ & $3\left|I_{+}\right|(\mathrm{A})$ & $3\left|I_{-}\right|(\mathrm{A})$ & $3\left|I_{0}\right|(\mathrm{A})$ & $\left|V_{+}\right|(\mathrm{A})$ & $\left|V_{-}\right|(\mathrm{A})$ \\
\hline SS Closed & \multicolumn{7}{|c|}{} \\
\hline \hline Relay 2 & 215.6 & 116.6 & 116.2 & 448 & 106.5 & 93.5 & 391.3 & 1.1 \\
\hline Relay 3 & 151.2 & 47.2 & 49.2 & 245.6 & 116 & 93.5 & 391.3 & 1.09 \\
\hline Relay 4 & 22.6 & 41.8 & 41.5 & 104.8 & 17 & 26 & 389.8 & 1.6 \\
\hline Relay 5 & 66 & 66.2 & 70.8 & 202.9 & 9.5 & 0 & 390.9 & 0.9 \\
\hline \hline SS Open & \multicolumn{7}{|c|}{0} \\
\hline \hline Relay 2 & 0 & 0 & 0 & 0 & 0 & 0 & 391.6 & 0 \\
\hline Relay 3 & 3.5 & 20.8 & 21.4 & 31.2 & 41.5 & 0 & 392.1 & 4.8 \\
\hline Relay 4 & 59.6 & 4.8 & 9.2 & 72 & 53 & 54.4 & 391.2 & 5 \\
\hline Relay 5 & 13 & 11.9 & 24.2 & 31.3 & 41.5 & 0 & 392 & 4.8 \\
\hline
\end{tabular}

The results given in Tables II, III, IV and V indicate that magnitudes of zero-sequence current components of all relays that must trip for a close-by up-stream SLG fault are well above the maximum zero-sequence current component of $11.9 \mathrm{~A}$ that is related to the maximum load power unbalance of 20 percents. This means that a zero-sequence current threshold can be selected for all relays such that they can detect up-stream SLG faults in all microgrid protection zones. It is to be noted that the relays do not detect SLG faults that are located in their down-stream area after the static switch trips. However, the 
down-stream faults are detected based on the differential current components at the relays.

TABLE IV: SYMMETRICAL SEQUENCE CURRENT COMPONENTS AT THE AEP MICROGRID RELAYS FOR AN SLG FAULT IN ZONE 2

\begin{tabular}{|c|c|c|c|c|c|c|c|c|}
\hline Device & $\left|I_{a}\right|(\mathrm{A})$ & $\left|I_{b}\right|(\mathrm{A})$ & $\left|I_{c}\right|(\mathrm{A})$ & $3\left|I_{+}\right|(\mathrm{A})$ & $3\left|I_{-}\right|(\mathrm{A})$ & $3\left|I_{0}\right|(\mathrm{A})$ & $\left|V_{+}\right|(\mathrm{A})$ & $\left|V_{-}\right|(\mathrm{A})$ \\
\hline \multicolumn{9}{|c|}{ SS Closed } \\
\hline Relay 2 & 221.6 & 114.5 & 113.2 & 4449 & 112 & 105.8 & 391.3 & 1.1 \\
\hline Relay 3 & 16 & 45 & 45.8 & 105 & 23.3 & 39.6 & 391.1 & 1.1 \\
\hline Relay 4 & 26.7 & 39.3 & 37.3 & 103.2 & 11.3 & 12.2 & 390.2 & 1.1 \\
\hline Relay 5 & 65.8 & 66.3 & 71 & 203 & 10 & 0 & 390.8 & 1.0 \\
\hline \multicolumn{9}{|l|}{ SS Open } \\
\hline Relay 2 & 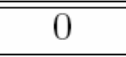 & "0 & 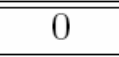 & 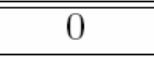 & 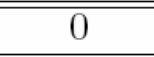 & 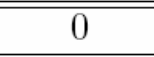 & 391.6 & 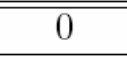 \\
\hline Relay 3 & 140.6 & 21.7 & 21.4 & 175.6 & 101.5 & 144.8 & 392 & 5 \\
\hline Relay 4 & 55 & 7.5 & 11.2 & 71.6 & 49.4 & 44.7 & 391.6 & 4.8 \\
\hline Relay 5 & 12.6 & 13.2 & 24.7 & 30.7 & 43.5 & 0 & 391.8 & 4.2 \\
\hline
\end{tabular}

TABLE V: SYMMETRICAL SEQUENCE CURRENT COMPONENTS AT THE AEP MICROGRID RELAYS FOR AN SLG FAULT IN ZONE 5

\begin{tabular}{c|c|c|c|c|c|c|c|c}
\hline Device & $\left|I_{a}\right|(\mathrm{A})$ & $\left|I_{b}\right|(\mathrm{A})$ & $\left|I_{c}\right|(\mathrm{A})$ & $3\left|I_{+}\right|(\mathrm{A})$ & $3\left|I_{-}\right|(\mathrm{A})$ & $3\left|I_{0}\right|(\mathrm{A})$ & $\left|V_{+}\right|(\mathrm{A})$ & $\left|V_{-}\right|(\mathrm{A})$ \\
\hline SS Closed & \multicolumn{7}{|c|}{} \\
\hline \hline Relay 2 & 161.5 & 118.4 & 168.6 & 444 & 90.4 & 0 & 391.3 & 0.92 \\
\hline Relay 3 & 34.5 & 40.8 & 30.3 & 104.8 & 18.9 & 0 & 391.3 & 0.92 \\
\hline Relay 4 & 33.7 & 37.4 & 32.3 & 103.2 & 9.2 & 0 & 390.2 & 0.87 \\
\hline Relay 5 & 198 & 74.6 & 72.2 & 343.2 & 109.3 & 141.7 & 390.4 & 3 \\
\hline \hline SS Open & \multicolumn{7}{|c|}{} \\
\hline \hline Relay 2 & 0 & 0 & 0 & 0 & 0 & 0 & 391.6 & 0 \\
\hline Relay 3 & 74.6 & 30.4 & 75.8 & 173 & 82 & 0 & 392 & 4 \\
\hline Relay 4 & 32.2 & 9.8 & 31.7 & 69.4 & 40 & 0 & 391.7 & 3.8 \\
\hline Relay 5 & 132 & 28.1 & 25.3 & 173.2 & 82.3 & 142 & 391.4 & 5.5 \\
\hline
\end{tabular}

\subsection{Line-to-Line Fault}

This section presents the results obtained from the EMTP based simulation of the AEP microgrid of Fig. 1 for an LL fault in one of the protection Zones 2, 3, 4 and 5. The AEP microgrid operates under steady-state conditions with the source and load parameters given in Section 3. An LL fault with a power of $85.5 \mathrm{~kW}\left(R_{f}=2.695 \mathrm{Ohms}\right)$ occurs in one of the protection zones at $t=1 \mathrm{~s}$ when the microgrid operates under steady-state conditions. Magnitudes of the phase currents, the symmetrical current components $3 \mid I_{+} I_{\text {, }}$ 3|I_. and $3\left|I_{0}\right|$ and the symmetrical voltage components $\left|V_{+}\right|$and $\left|V_{-}\right|$at all relays are recorded when the microgrid operates under steady-state conditions subsequent to the LL fault. Values of the parameters for the grid-connected (SS closed) and the islanded (SS open) modes, when the static switch trips after the fault, are given in Tables VI, VII, VIII 
and IX for an LL fault in Zones 4, 3, 2 and 5, respectively. The results indicate that magnitudes of negative-sequence current components of all relays that must trip for a close-by LL fault are well above the maximum negative-sequence current component of 26 A that is related to the maximum load power unbalance of 20 percents. In particular, magnitudes of the negative-sequence current components become much higher after the static switch trips. This means that a negative-sequence current threshold can be selected for all relays such that the AEP microgrid is protected against LL faults in all microgrid protection zones.

TABLE VI: SYMMETRICAL SEQUENCE CURRENT COMPONENTS AT THE AEP MICROGRID RELAYS FOR AN LL FAULT IN ZONE 4

\begin{tabular}{c|c|c|c|c|c|c|c|c}
\hline Device & $\left|I_{a}\right|(\mathrm{A})$ & $\left|I_{b}\right|(\mathrm{A})$ & $\left|I_{c}\right|(\mathrm{A})$ & $3\left|I_{+}\right|(\mathrm{A})$ & $3\left|I_{-}\right|(\mathrm{A})$ & $3\left|I_{0}\right|(\mathrm{A})$ & $\left|V_{+}\right|(\mathrm{A})$ & $\left|V_{-}\right|(\mathrm{A})$ \\
\hline SS Closed & \multicolumn{7}{|c|}{} \\
\hline \hline Relay 2 & 310 & 296 & 140 & 719 & 304 & 0 & 391 & 3.1 \\
\hline Relay 3 & 253 & 239 & 63.7 & 516 & 330 & 0 & 391 & 3.1 \\
\hline Relay 4 & 262 & 249 & 52.5 & 517 & 364 & 0 & 388.1 & 5.2 \\
\hline Relay 5 & 72 & 58.6 & 73.2 & 203 & 27 & 0 & 390.6 & 2.6 \\
\hline \hline SS Open & \multicolumn{7}{|l|}{} \\
\hline \hline Relay 2 & 0 & 0 & 0 & 0 & 0 & 0 & 391.6 & 0 \\
\hline Relay 3 & 75 & 72 & 6.7 & 135 & 118 & 0 & 391.4 & 14 \\
\hline Relay 4 & 157 & 152 & 6.9 & 275 & 260 & 0 & 389.5 & 15 \\
\hline Relay 5 & 45.2 & 85 & 40 & 136 & 118 & 0 & 391.8 & 11 \\
\hline
\end{tabular}

TABLE VII: SYMMETRICAL SEQUENCE CURRENT COMPONENTS AT THE AEP MICROGRID RELAYS FOR AN LL FAULT IN ZONE 3

\begin{tabular}{c|c|c|c|c|c|c|c|c}
\hline Device & $\left|I_{a}\right|(\mathrm{A})$ & $\left|I_{b}\right|(\mathrm{A})$ & $\left|I_{c}\right|(\mathrm{A})$ & $3\left|I_{+}\right|(\mathrm{A})$ & $3\left|I_{-}\right|(\mathrm{A})$ & $3\left|I_{0}\right|(\mathrm{A})$ & $\left|V_{+}\right|(\mathrm{A})$ & $\left|V_{-}\right|(\mathrm{A})$ \\
\hline SS Closed & \multicolumn{7}{|c|}{} \\
\hline \hline Relay 2 & 318 & 304 & 139 & 733 & 318 & 0 & 391 & 3.1 \\
\hline Relay 3 & 262 & 247 & 63 & 530 & 346 & 0 & 391 & 3.2 \\
\hline Relay 4 & 22.1 & 43.4 & 51.3 & 112 & 50.7 & 0 & 388.5 & 4.8 \\
\hline Relay 5 & 72.2 & 58.2 & 73.4 & 203 & 28.4 & 0 & 390.6 & 2.7 \\
\hline \hline SS Open & \multicolumn{7}{|l|}{} \\
\hline \hline Relay 2 & 0 & 0 & 0 & 0 & 0 & 0 & 391.6 & 0 \\
\hline Relay 3 & 77.6 & 75 & 6.4 & 140 & 124 & 0 & 391.3 & 14 \\
\hline Relay 4 & 85.6 & 90 & 5.6 & 147 & 157 & 0 & 390 & 15 \\
\hline Relay 5 & 46.6 & 88 & 42 & 140 & 124 & 0 & 391.7 & 12 \\
\hline
\end{tabular}


TABLE VIII: SYMMETRICAL SEQUENCE CURRENT COMPONENTS AT THE AEP MICROGRID RELAYS FOR AN LL FAULT IN ZONE 2

\begin{tabular}{c|c|c|c|c|c|c|c|c}
\hline Device & $\left|I_{a}\right|(\mathrm{A})$ & $\left|I_{b}\right|(\mathrm{A})$ & $\left|I_{c}\right|(\mathrm{A})$ & $3\left|I_{+}\right|(\mathrm{A})$ & $3\left|I_{-}\right|(\mathrm{A})$ & $3\left|I_{0}\right|(\mathrm{A})$ & $\left|V_{+}\right|(\mathrm{A})$ & $\left|V_{-}\right|(\mathrm{A})$ \\
\hline SS Closed & \multicolumn{7}{|c|}{} \\
\hline \hline Relay 2 & 324 & 309 & 135 & 737 & 335 & 0 & 390.9 & 3.4 \\
\hline Relay 3 & 21.6 & 41.6 & 58.2 & 109 & 70 & 0 & 390.9 & 3.4 \\
\hline Relay 4 & 27.8 & 34 & 45.8 & 105 & 34 & 0 & 389.8 & 3.2 \\
\hline Relay 5 & 72.3 & 57.8 & 74 & 203 & 30 & 0 & 390.5 & 2.8 \\
\hline \hline SS Open & \multicolumn{7}{|c|}{0} \\
\hline \hline Relay 2 & 0 & 0 & 0 & 0 & 0 & 0 & 391.6 & 0 \\
\hline Relay 3 & 170 & 172 & 4.5 & 290 & 302 & 0 & 390.8 & 15 \\
\hline Relay 4 & 83 & 85 & 2.3 & 145 & 147 & 0 & 390.8 & 14 \\
\hline Relay 5 & 47.2 & 90 & 44 & 142 & 130 & 0 & 391.4 & 12 \\
\hline
\end{tabular}

TABLE IX: SYMMETRICAL SEQUENCE CURRENT COMPONENTS AT THE AEP MICROGRID RELAYS FOR AN LL FAULT IN ZONE 5

\begin{tabular}{c|c|c|c|c|c|c|c|c}
\hline Device & $\left|I_{a}\right|(\mathrm{A})$ & $\left|I_{b}\right|(\mathrm{A})$ & $\left|I_{c}\right|(\mathrm{A})$ & $3\left|I_{+}\right|(\mathrm{A})$ & $3\left|I_{-}\right|(\mathrm{A})$ & $3\left|I_{0}\right|(\mathrm{A})$ & $\left|V_{+}\right|(\mathrm{A})$ & $\left|V_{-}\right|(\mathrm{A})$ \\
\hline SS Closed & \multicolumn{7}{|c|}{} \\
\hline \hline Relay 2 & 327 & 196 & 222 & 716 & 266 & 0 & 390.9 & 2.7 \\
\hline Relay 3 & 22 & 53 & 41.1 & 109 & 55.6 & 0 & 390.9 & 2.7 \\
\hline Relay 4 & 26.6 & 42.1 & 37.6 & 105 & 27 & 0 & 389.8 & 2.6 \\
\hline Relay 5 & 280 & 270 & 99 & 616 & 322 & 0 & 389 & 8.8 \\
\hline \hline SS Open & \multicolumn{7}{|l|}{} \\
\hline \hline Relay 2 & 0 & 0 & 0 & 0 & 0 & 0 & 391.6 & 0 \\
\hline Relay 3 & 173 & 85 & 89 & 276 & 242 & 0 & 391.2 & 12 \\
\hline Relay 4 & 85.2 & 43 & 43 & 138 & 117 & 0 & 391.2 & 11 \\
\hline Relay 5 & 151 & 148 & 11.5 & 276 & 242 & 0 & 390.1 & 16 \\
\hline
\end{tabular}

\section{Coordination of Protection}

This section presents a summary of the items that are considered when the protection system of the AEP microgrid is coordinated. Based on the results presented in Section 3.1 and Section 3.2, a threshold of $3 \mathrm{I}_{0} \mid=35 \mathrm{~A}$ is selected for all relays for detecting a downstream SLG fault based on a zero-sequence current component. The up-stream SLG faults that are close to a relay are detected based on a differential current component for which a threshold of $\left|I_{d}\right|=15$ A is chosen. Also, based on the result presented in Section 3.1 and Section 3.3, a threshold of 3|I $\mid=95 \mathrm{~A}$ is considered for all relays when detecting an LL fault based on a negative-sequence current component. The thresholds considered for the symmetrical and differential current components guarantee that the protection system detects all SLG faults with a power more than $28.5 \mathrm{~kW} /$ phase and all LL faults with a power greater than $85.5 \mathrm{~kW}$ that occur in any of the microgrid protection zones.

To prevent detecting instantaneous or short faults and transients, a delay of $50 \mathrm{~ms}$ (3 cycles) is set for all relays before detecting an SLG or LL fault. In other words, if a 
symmetrical component remains greater than the corresponding threshold longer than $50 \mathrm{~ms}$, then the relay will command the corresponding circuit breaker to trip. The delay of $50 \mathrm{~ms}$ is considered for all relays except the Relay 3, for the case of SLG faults only, for which a delay of $167 \mathrm{~ms}$ (10 cycles) is chosen. The longer delay considered for the Relay 3 ensures that the relay does not command for a trip for SLG faults in Zone 4, Fig. 1, as such faults are cleared by the Relay 4 in less than 10 cycles. It is to be noted that the delay considered for detecting an LL fault by the Relay 3 is the same as that of the other relays, i.e. $50 \mathrm{~ms}$. It should also be noted that no delay is considered for the SLG and LL faults that are detected by the Relay 2, that is attached to the static switch, since the switch is expected to clear a fault within a maximum period of $8 \mathrm{~ms}$ (half a cycle).

When a trip signal is sent by a relay to the corresponding breaker, a delay of $30 \mathrm{~ms}$ is considered before starting to open phase switches of the corresponding breaker. The reason for considering the delay of $30 \mathrm{~ms}$ is that magnitudes of the symmetrical current components of the relays have different rise times and thus, each of them exceeds the corresponding threshold at a different time than the others. If no delay is considered, one of the relays may not command for a necessary trip since the corresponding symmetrical component does not stay above the threshold more than $50 \mathrm{~ms}$ prior to the fault clearance by another breaker. This causes one of the microsources to continue its operation in a stand-alone mode after the fault clearance, which is not desirable. To ensure that all relays which are expected to command for a trip have sufficient time to detect a fault, the delay of $30 \mathrm{~ms}$ is considered before disconnecting a circuit breaker.

Once a symmetrical current component with a magnitude greater than the corresponding threshold lasts more than 3 cycles, the corresponding relay sends a trip command to the circuit breaker. The 3-cycle delay is considered for all relays, except the Relay 3 which has a 10-cycle delay for SLG faults, and the Relay 2 which has no delay for all types of fault. When the breaker receives a trip command, its controller considers a delay of $30 \mathrm{~ms}$ before starting the procedure of disconnecting the breaker phases. The $30 \mathrm{~ms}$ delay is considered for all the breakers except the static switch which has no delay. After a delay of $30 \mathrm{~ms}$, the three-phase circuit breaker who has received a trip command is opened, one phase at a time. To open a circuit breaker, currents of all three phases of the breaker are measured and when a phase current crosses zero, the corresponding phase switch is opened. The disconnection procedure is the same for all the breakers including the static switch. 
TABLE X: RELAY SETTINGS

\begin{tabular}{|c|c|c|c|c|c|}
\hline Protection & Relay & $\begin{array}{c}\text { Up-stream SLG } \\
\text { faults } \\
\end{array}$ & \multicolumn{2}{|c|}{$\begin{array}{c}\text { Down-stream SLG } \\
\text { faults }\end{array}$} & $\begin{array}{c}\text { Line-to-Line } \\
\text { faults }\end{array}$ \\
\hline \multirow[t]{4}{*}{ Primary } & 2 & $\begin{array}{c}I_{d}>15 A \\
\text { delay }=0 \mathrm{~ms}\end{array}$ & \multicolumn{2}{|c|}{$\begin{array}{c}3\left|I_{0}\right|>35 A \\
\text { delay }=0 \mathrm{~ms}\end{array}$} & $\begin{array}{l}3\left|I_{-}\right|>95 A \\
\text { Delay }=0 \mathrm{~ms}\end{array}$ \\
\hline & 3 & $\begin{array}{c}I_{d}>15 A \\
\text { delay }=167 \mathrm{~ms}\end{array}$ & \multicolumn{2}{|c|}{$\begin{array}{c}3\left|I_{0}\right|>35 A \\
\text { delay }=167 \mathrm{~ms}\end{array}$} & $\begin{array}{l}3\left|I_{-}\right|>95 A \\
\text { delay }=50 \mathrm{~ms}\end{array}$ \\
\hline & 4 & $\begin{array}{c}I_{d}>15 \mathrm{~A} \\
\text { delay }=50 \mathrm{~ms}\end{array}$ & \multicolumn{2}{|c|}{$\begin{array}{c}3\left|I_{0}\right|>35 A \\
\text { delay }=50 \mathrm{~ms}\end{array}$} & $\begin{array}{c}3\left|I_{-}\right|>95 A \\
\text { Delay }=50 \mathrm{~ms}\end{array}$ \\
\hline & 5 & $\begin{array}{c}I_{d}>15 A \\
\text { delay }=50 \mathrm{~ms}\end{array}$ & \multicolumn{2}{|c|}{$\begin{array}{c}3\left|I_{0}\right|>35 A \\
\text { delay=50 ms } \\
50 \% \text { volts dip }\end{array}$} & $\begin{array}{c}3\left|I_{-}\right|>95 A \\
\text { Delay }=50 \mathrm{~ms}\end{array}$ \\
\hline \multirow[t]{4}{*}{$1^{\text {st }}$ Back-up } & 2 & \multicolumn{3}{|c|}{$I^{2} t$ activation level } & $80 A$ \\
\hline & 3 & \multicolumn{3}{|c|}{$I^{2} t$ activation level } & $25 A$ \\
\hline & 4 & \multicolumn{3}{|c|}{$I^{2} t$ activation level } & $25 A$ \\
\hline & 5 & \multicolumn{4}{|c|}{$I^{2} t$ activation level } \\
\hline \multirow{4}{*}{$\begin{array}{l}2^{\text {nd }} \text { Back-up } \\
\text { and under } \\
\text { voltage } \\
\text { protection }\end{array}$} & 2 & \multicolumn{2}{|c|}{$\begin{array}{ll}\text { Peak current } & |I|>750 A\end{array}$} & \multicolumn{2}{|c|}{ Voltage power quality levels } \\
\hline & 3 & & & \multicolumn{2}{|c|}{$\begin{array}{l}50 \% \text { under voltage delay } 30 \\
\text { cycles }\end{array}$} \\
\hline & 4 & & & \multicolumn{2}{|c|}{$\begin{array}{l}50 \% \text { under voltage delay } 20 \\
\text { cycles }\end{array}$} \\
\hline & 5 & & & \multicolumn{2}{|c|}{$\begin{array}{l}50 \% \text { under voltage delay } 20 \\
\text { cycles }\end{array}$} \\
\hline
\end{tabular}

\section{Study Cases}

This section evaluates performance of the protection system of the AEP microgrid based on digital time-domain simulation studies in the EMTP software environment. Performance of the protection system is studied for SLG and LL faults in all the microgrid protection zones, i.e. Zones 2, 3, 4, 5 and 6 of Fig. 1. All SLG and LL faults have a resistance of $2.695 \mathrm{Ohms}$ which corresponds to a $28.5 \mathrm{~kW} /$ phase power for SLG faults and a $85.5 \mathrm{~kW}$ power for LL faults, respectively. The fault resistance is equal to the resistance used for experimenting faults at the real-world microgrid test site in Ohio.

For all study cases of this section, powers of the microsources $A_{1}, A_{2}$ and $B_{1}$ and powers of the loads $\mathrm{L}_{1}, \mathrm{~L}_{2}$ and $\mathrm{L}_{3}$ are set at the following values: $\mathrm{P}_{\mathrm{A} 1}=40 \mathrm{~kW}, \mathrm{P}_{\mathrm{A} 2}=20 \mathrm{~kW}$, $\mathrm{P}_{\mathrm{B} 1}=10 \mathrm{~kW}, \mathrm{P}_{\mathrm{L} 1}=40 \mathrm{~kW}, \mathrm{P}_{\mathrm{L} 2}=40 \mathrm{~kW}$, and $\mathrm{P}_{\mathrm{L} 3}=40 \mathrm{~kW}$. For each study case, the results are shown in four figures and each figure shows the operating parameters related to the relay that is attached to the static switch or one of the Relays 3, 4 and 5. Each figure shows the three-phase voltages and currents, the real and reactive components of power, and the frequency at one of the relays. Each figure also shows normalized magnitudes of the differential and zero- and negative-sequence current components 
measured at a relay as well as the relay output signal that can command a breaker to trip or to continue its operation. The figure that corresponds to the Relay 5 also shows normalized magnitude of the phase voltage that is measured at the delta side of the transformer $\mathrm{T}_{51}$, Fig. 1 . The phase voltage is measured for detecting SLG faults in Zone 2 for which the Relay 5 does not detect a differential or zero-sequence current component.

\subsection{SLG Faults}

\section{SLG Fault in Zone 4}

The AEP microgrid of Fig. 1 is operating at steady-state conditions with the source and load settings as explained in Section 5 and an SLG fault with a resistance of 2.695 Ohms is applied in Zone 4 at t=1.0 s. Figs. 3(a)-(e) to Figs. 6(a)-(e) show variations of the threephase voltages and currents, the real and reactive power components, and the frequencies measured at the Relays 2, 3, 4 and 5, respectively, in response to the SLG fault.

Fig. 2(f) shows that the Relay 2 commands the static switch to trip right after the fault since the relay detects a differential current component that exceeds 1.0 pu (15 A). Fig. 3(f) shows that the Relay 3 does not command for a trip since it has a built-in delay of 10 cycles and magnitude of the differential current component detected by the relay falls below 1.0 pu before 10 cycle when the breaker $\mathrm{CB}_{41}$ clears the fault. Fig. 4(f) shows that the Relay 4 sends a trip command to the circuit breaker $\mathrm{CB}_{41}$ since magnitudes of both the differential and zero-sequence current components at the relay are greater than $1.0 \mathrm{pu}$ for a period more than $50 \mathrm{~ms}$ ( 3 cycles). However, the relay trip command is due to the differential current component since it exceeds $1.0 \mathrm{pu}$ earlier than the zero-sequence current component. Fig. 5(f) shows that the Relay 5 does not command for a trip since none of its fault detection parameters, i.e. the differential and symmetrical current components, exceed the threshold of $1.0 \mathrm{pu}$.

For the case of SLG fault in Zone 4, the static switch islands the microgrid and the breaker $\mathrm{CB}_{41}$ disconnects Zone 4 of the microgrid and shuts down the microsource $\mathrm{A}_{2}$. The Zones 2, 3 and 5 of the microgrid and the microsources $A_{1}$ and $B_{1}$ continue the operation subsequent to the fault detection and its clearance. The results shown in Fig. 2 to Fig. 5 demonstrate that the developed protection system is able to protect the AEP microgrid against an SLG fault in Zone 4 with a power of $28.5 \mathrm{~kW} /$ phase by clearing the fault and isolating the microgrid from the main grid. Since the fault current increases as the fault power increases, it is concluded that the microgrid protection system is able to protect all SLG faults in Zone 4 with a power more than or equal to $28.5 \mathrm{~kW} /$ phase. 


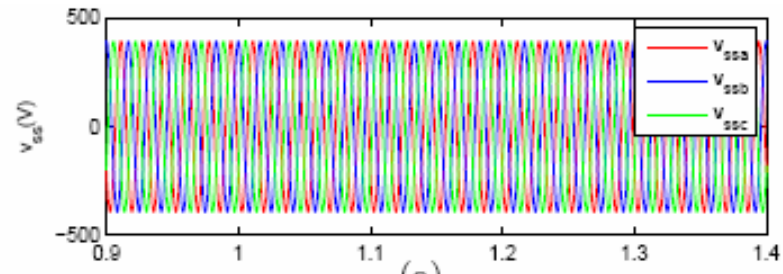

(a)

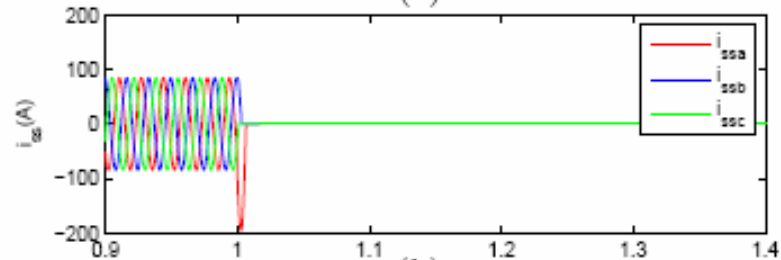

(b)

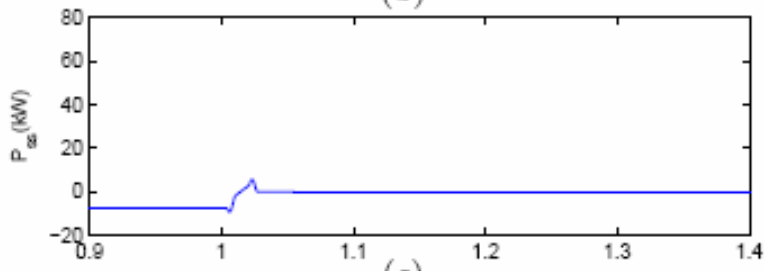

(c)

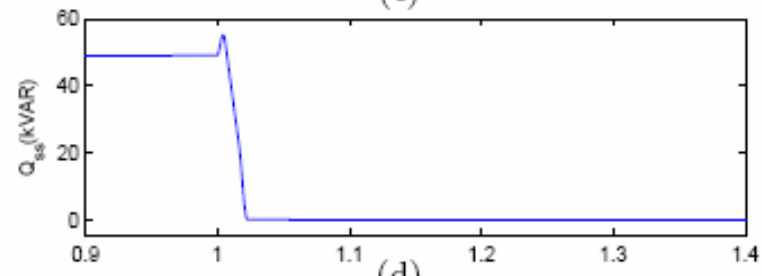

(d)

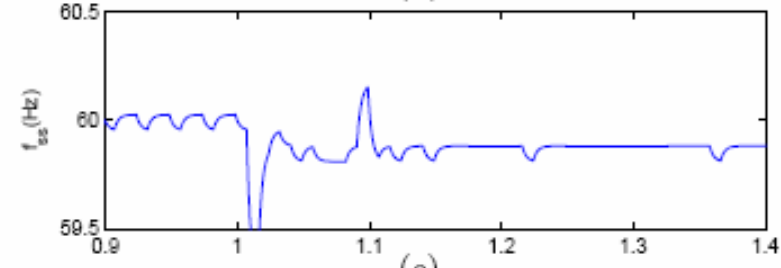

(e)

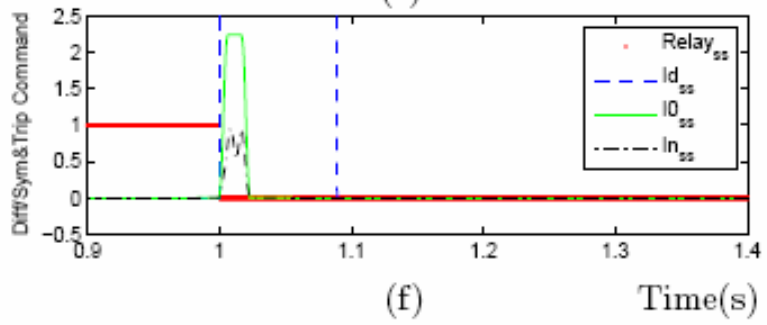

Figure 2 : Response of the Relay 2 to a $28.5 \mathrm{~kW}$ SLG fault in Zone 4: (a,b) threephase voltages and currents, $(\mathrm{c}, \mathrm{d})$ real and reactive power components, (e) frequency, and (f) differential and zero- and negative-sequence current components and output command of the relay 

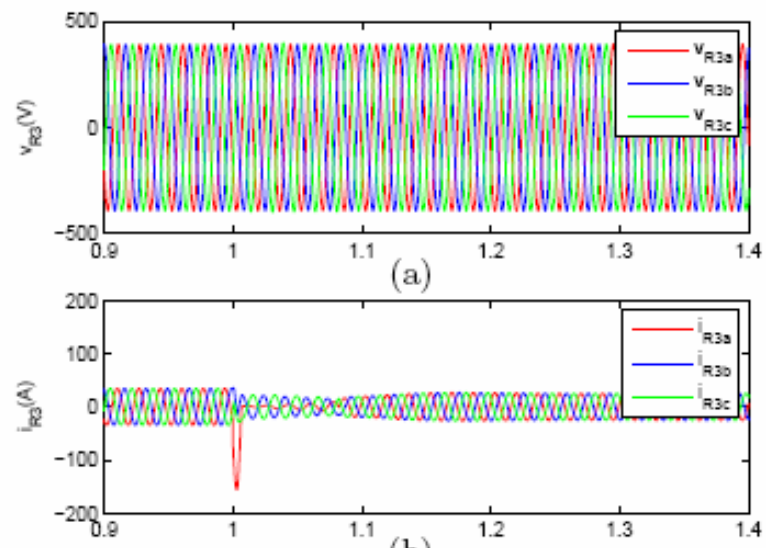

(b)

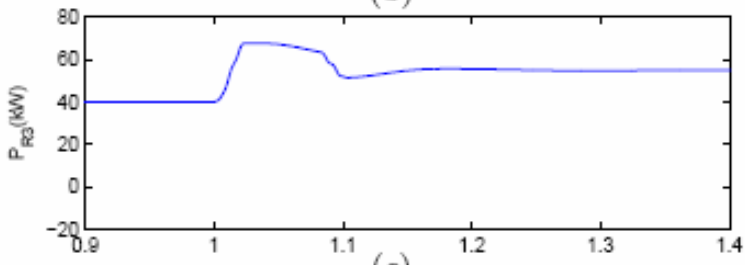

(c)

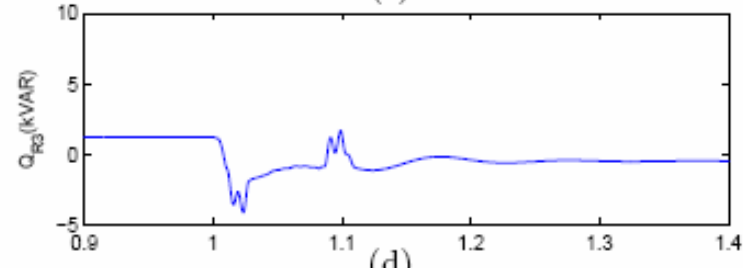

(d)

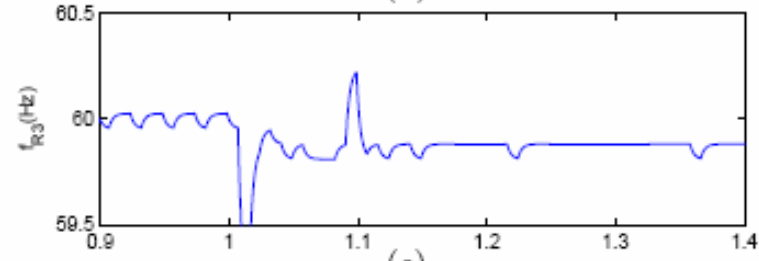

(e)

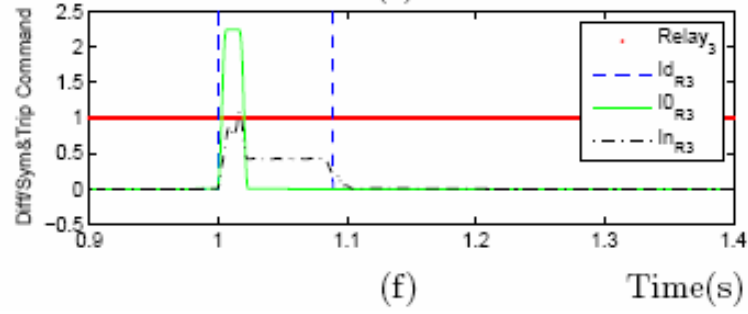

Figure 3: Response of the Relay 3 to a $28.5 \mathrm{~kW}$ SLG fault in Zone 4: (a,b) threephase voltages and currents, $(c, d)$ real and reactive power components, (e) frequency, and (f) differential and zero- and negative-sequence current components and output command of the relay 

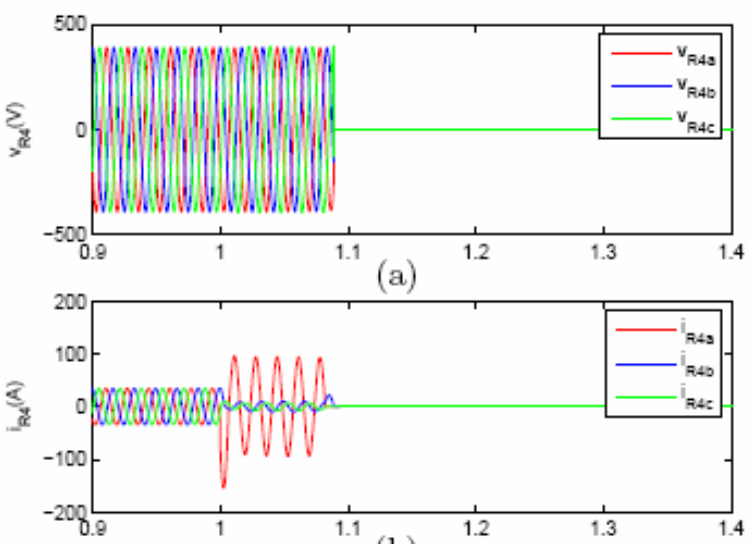

(b)

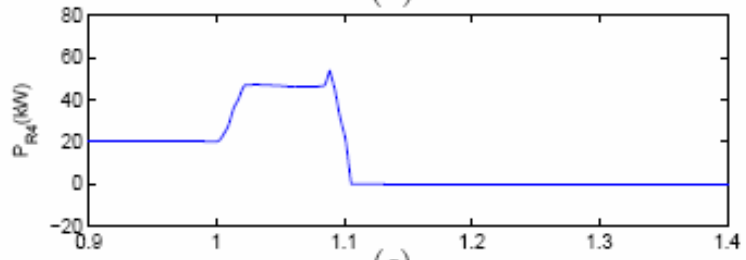

(c)
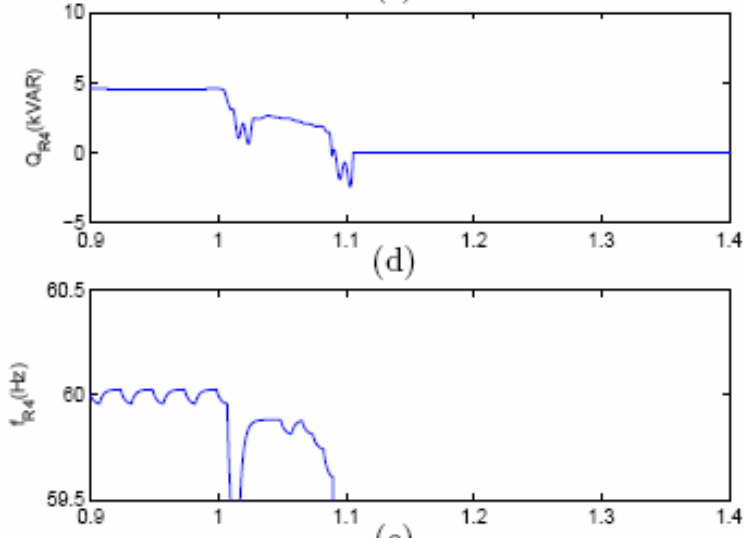

(e)

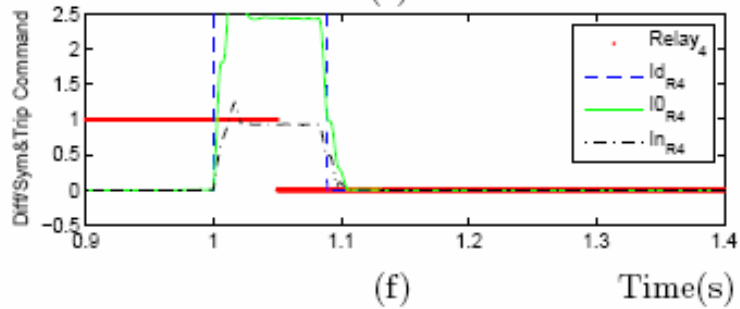

Figure 4: Response of the Relay 4 to a $28.5 \mathrm{~kW}$ SLG fault in Zone 4: (a,b) threephase voltages and currents, $(\mathrm{c}, \mathrm{d})$ real and reactive power components, (e) frequency, and (f) differential and zero- and negative-sequence current components and output command of the relay 


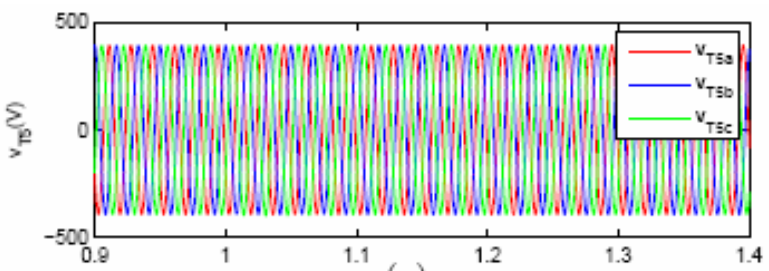

(a)

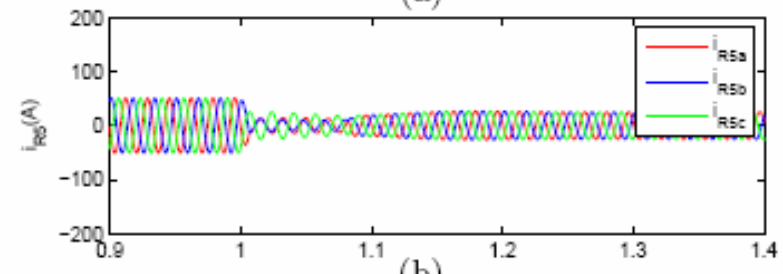

(b)

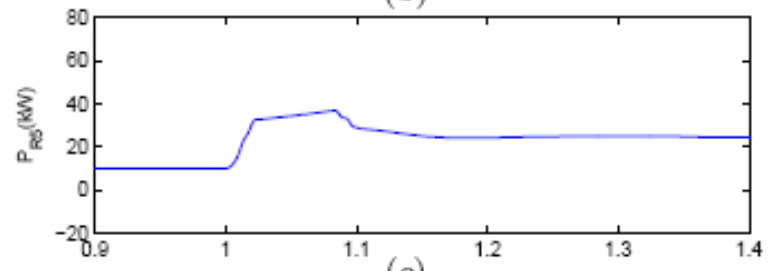

(c)
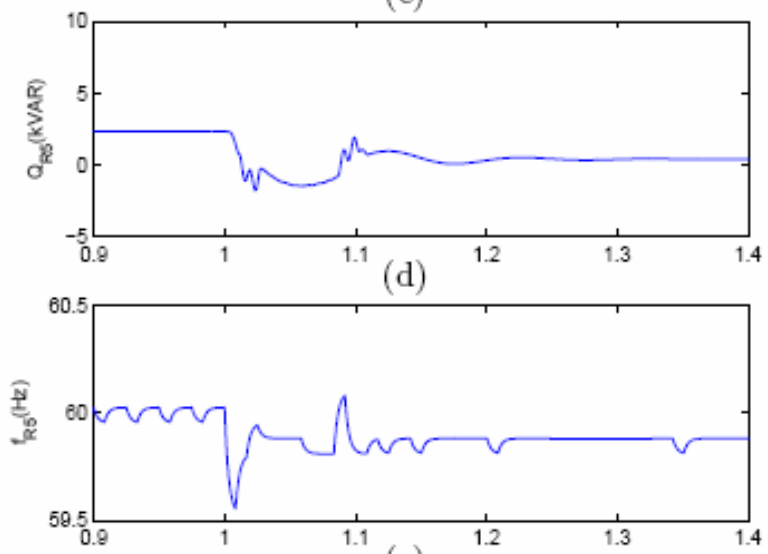

(e)

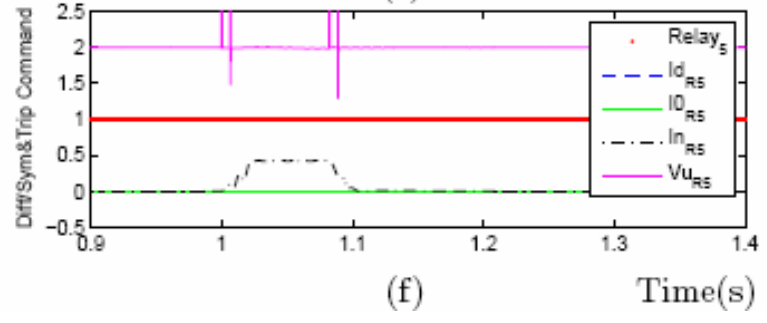

Figure 5: Response of the Relay 5 to a $28.5 \mathrm{~kW}$ SLG fault in Zone 4: (a,b) threephase voltages and currents, $(\mathrm{c}, \mathrm{d})$ real and reactive power components, (e) frequency, and (f) differential and zero- and negative-sequence current components, negative-sequence voltage component at the transformer $T_{51}$ and output command of the relay 


\section{SLG Fault in Zone 3}

The AEP microgrid of Fig. 1 is operating at steady-state conditions with the source and load settings as explained in Section 0.5 and an SLG fault with a resistance of 2.695 Ohms is applied in Zone 3 at $\mathrm{t}=1.0 \mathrm{~s}$. Figs. 7(a)-(e) to Figs. 10(a)-(e) show variations of the three-phase voltages and currents, the real and reactive power components, and the frequencies measured at the Relays 2, 3, 4 and 5, respectively, in response to the SLG fault.

Fig. 6(f) shows that the Relay 2 commands the static switch to trip right after the fault since the relay detects a differential current component that exceeds $1.0 \mathrm{pu}(15 \mathrm{~A})$. Fig. 7 (f) shows that the Relay 3 commands for a trip since even when the circuit breaker $\mathrm{CB}_{41}$ trips, the fault is not cleared in the protection Zone 3 and the relay still detects a differential current component. Fig. 8(f) shows that the Relay 4 sends a trip command to the circuit breaker $\mathrm{CB}_{41}$ since magnitude of the zero-sequence current component at the relay is greater than $1.0 \mathrm{pu}(35 \mathrm{~A})$ for a period more than $50 \mathrm{~ms}$ (3 cycles). Fig. 9(f) shows that the Relay 5 does not command for a trip since none of its fault detection parameters, i.e. the differential and symmetrical current components, exceeds the threshold of $1.0 \mathrm{pu}$.

For the case of SLG fault in Zone 3, the static switch islands the microgrid and the breakers $\mathrm{CB}_{31}$ and $\mathrm{CB}_{41}$ disconnect Zones 3 and 4 of the microgrid and shut down the microsources $A_{1}$ and $A_{2}$. The Zones 2 and 5 of the microgrid and the microsource $B_{1}$ continue the operation subsequent to the fault detection and its clearance. The results shown in Fig. 6 to Fig. 9 demonstrate that the developed protection system is able to protect the AEP microgrid against an SLG fault in Zone 3 with a power of $28.5 \mathrm{~kW} /$ phase by clearing the fault and isolating the microgrid from the main grid. Since the fault current increases as the fault power increases, it is concluded that the microgrid protection system is able to protect all SLG faults in Zone 3 with a power more than or equal to $28.5 \mathrm{~kW} /$ phase. 

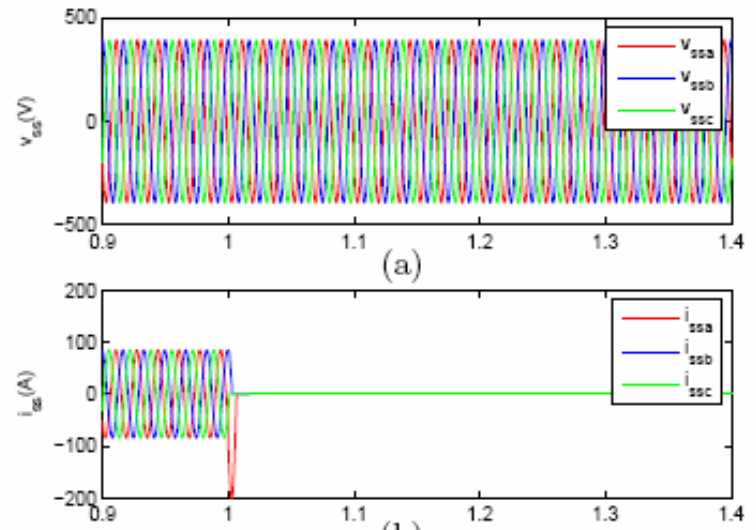

(b)

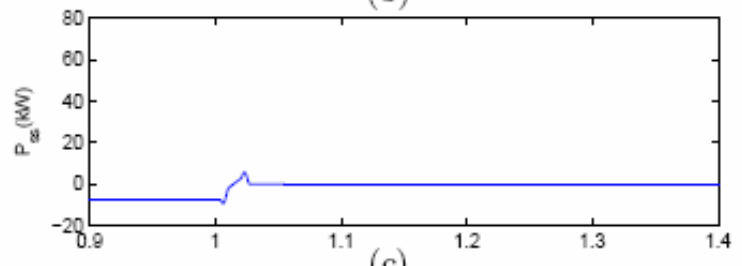

(c)
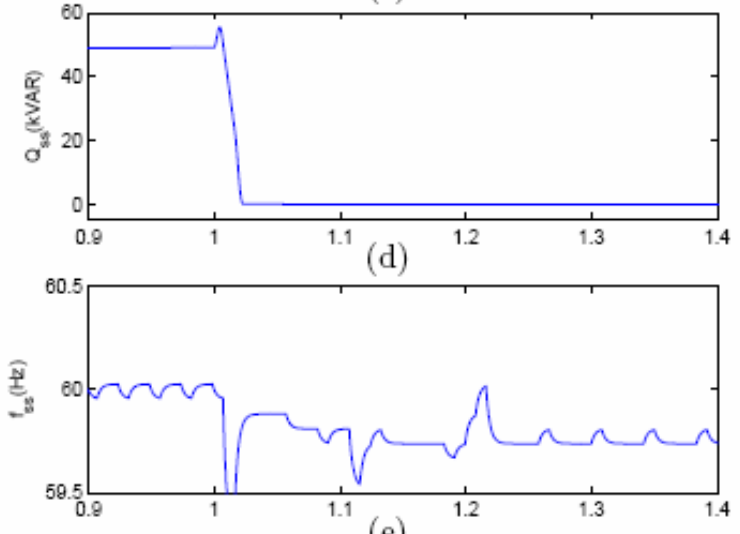

(e)

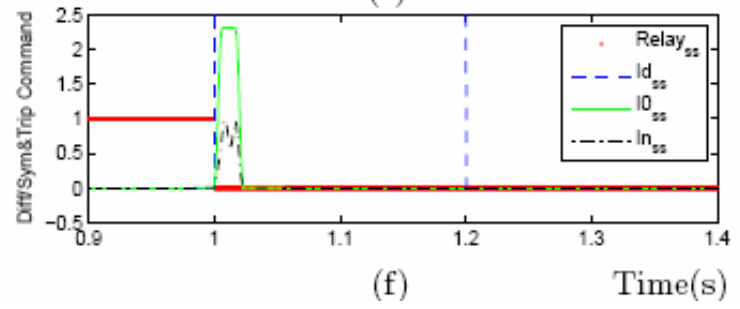

Figure 6: Response of the Relay 2 to a $28.5 \mathrm{~kW}$ SLG fault in Zone 3: (a,b) threephase voltages and currents, $(\mathrm{c}, \mathrm{d})$ real and reactive power components, (e) frequency, and (f) differential and zero- and negative-sequence current components and output command of the relay 


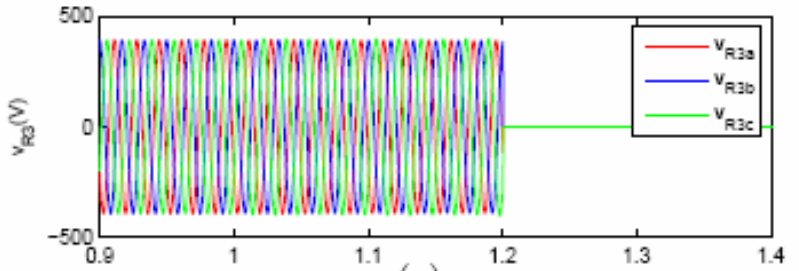

(a)

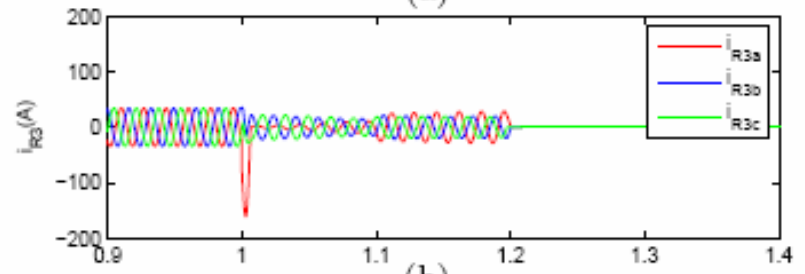

(b)

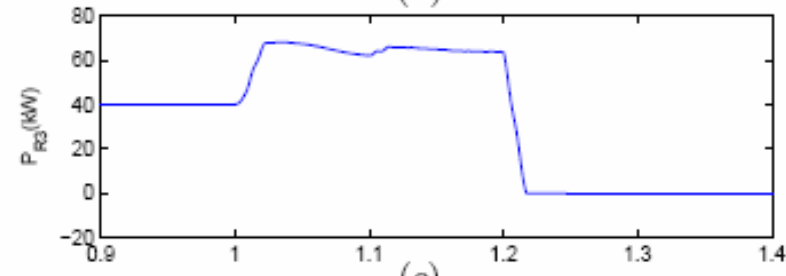

(c)
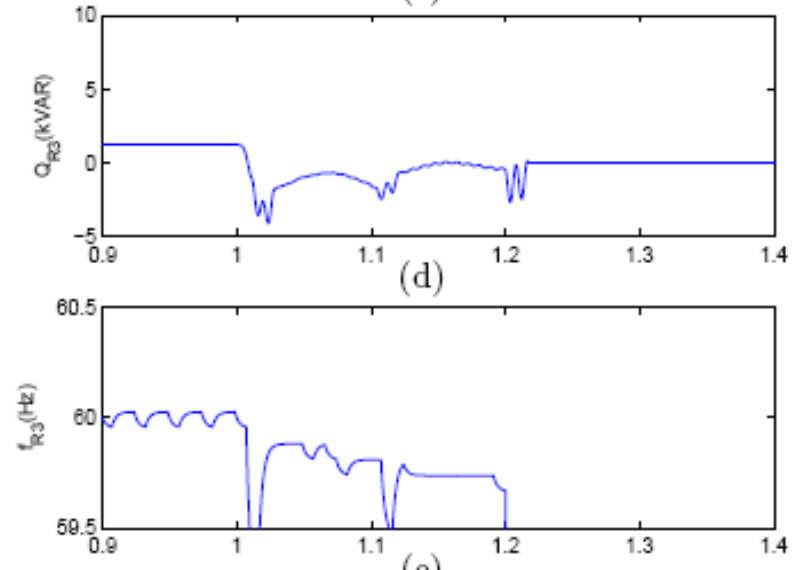

(e)

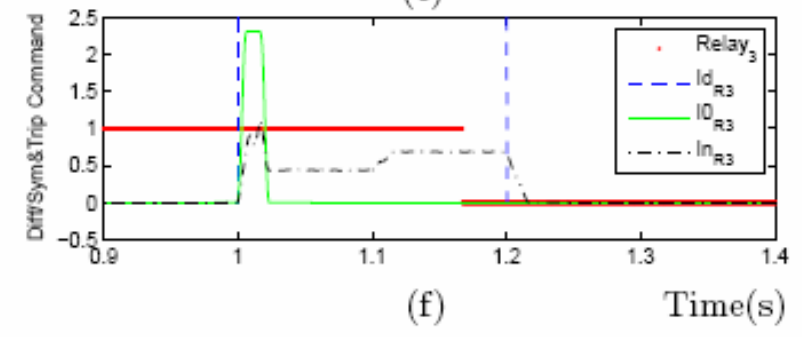

Figure 7: Response of the Relay 3 to a $28.5 \mathrm{~kW}$ SLG fault in Zone 3: (a,b) threephase voltages and currents, $(\mathrm{c}, \mathrm{d})$ real and reactive power components, (e) frequency, and (f) differential and zero- and negative-sequence current components and output command of the relay 

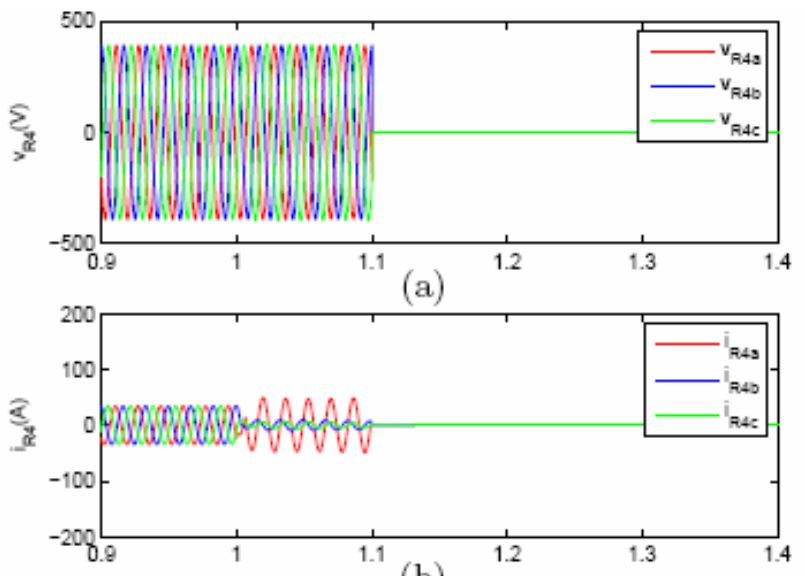

(b)
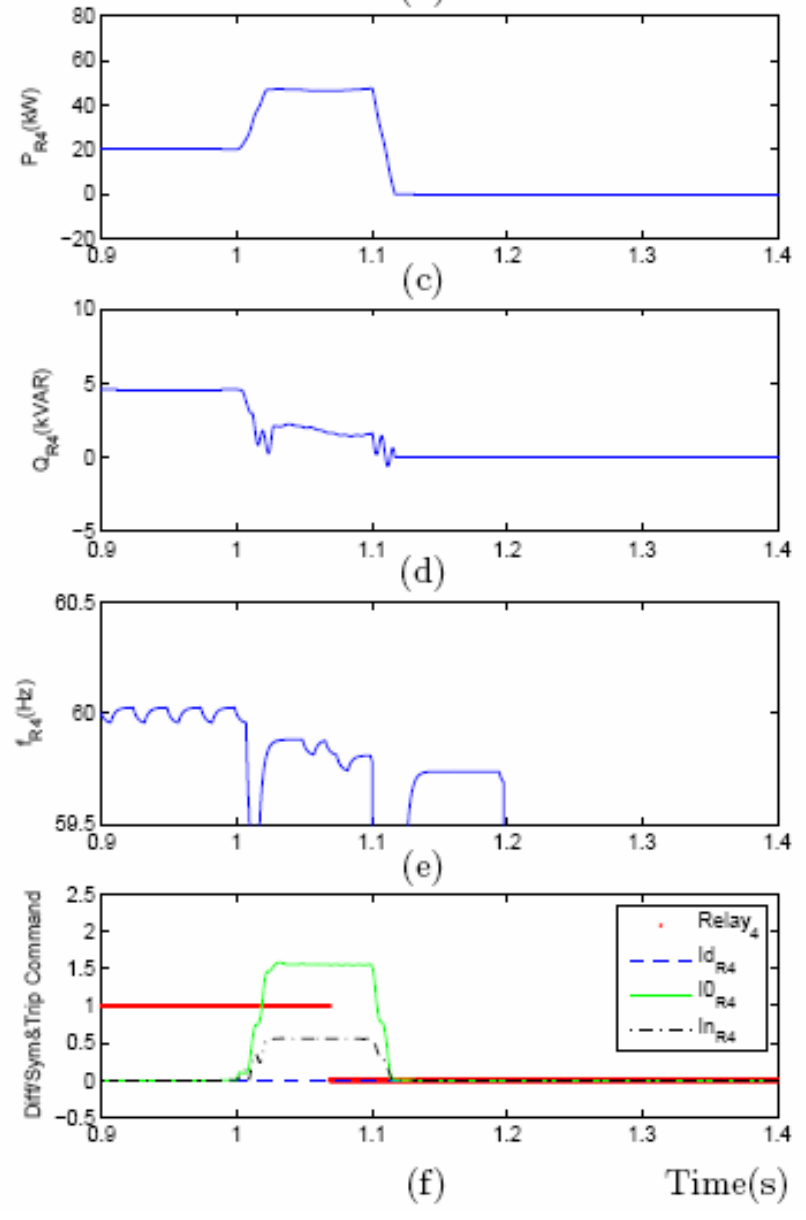

Figure 8: Response of the Relay 4 to a $28.5 \mathrm{~kW} \mathrm{SLG} \mathrm{fault} \mathrm{in} \mathrm{Zone} \mathrm{3:} \mathrm{(a,b)} \mathrm{three-}$ phase voltages and currents, $(\mathrm{c}, \mathrm{d})$ real and reactive power components, (e) frequency, and (f) differential and zero- and negative-sequence current components and output command of the relay 


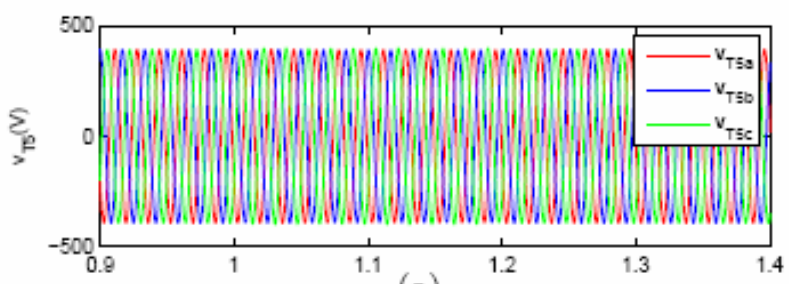

(a)

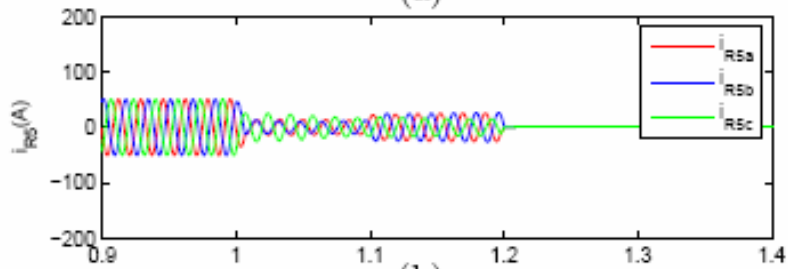

(b)

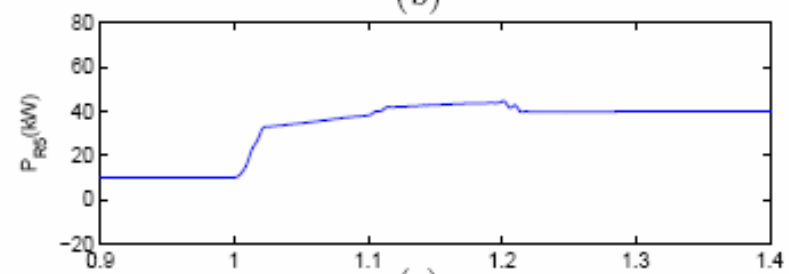

(c)
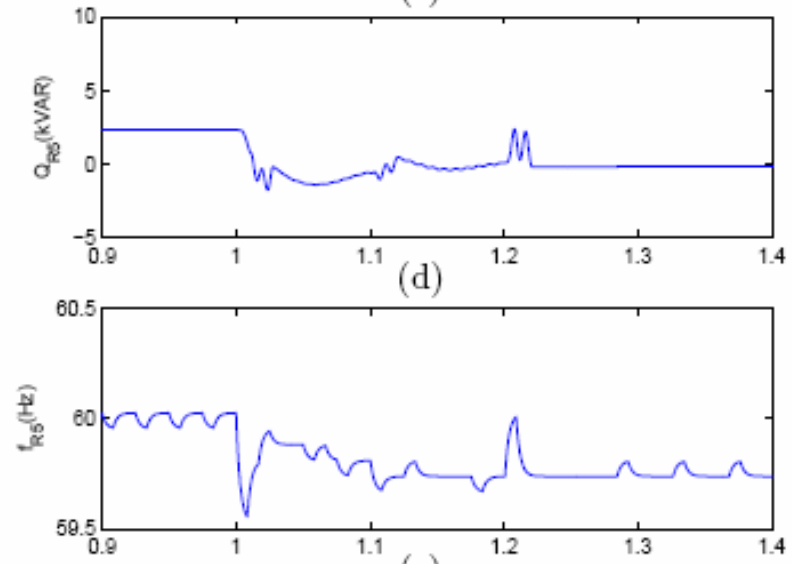

(e)

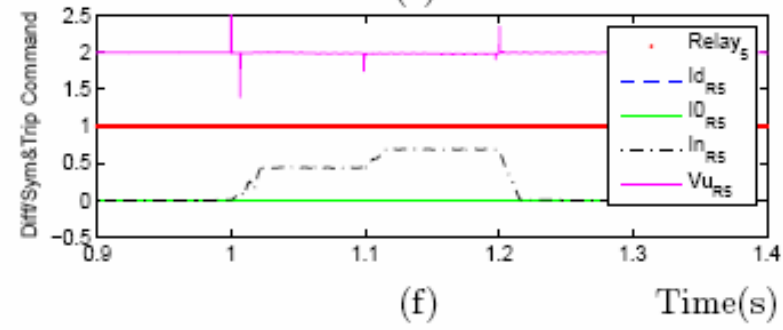

Figure 9: Response of the Relay 5 to a $28.5 \mathrm{~kW}$ SLG fault in Zone 3: (a,b) threephase voltages and currents, $(c, d)$ real and reactive power components, (e) frequency, and (f) differential and zero- and negative-sequence current components, negative-sequence voltage component at the transformer $T_{51}$ and output command of the relay 


\section{SLG Fault in Zone 2}

The AEP microgrid of Fig. 1 is operating at steady-state conditions with the source and load settings as explained in Section 5 and an SLG fault with a resistance of $2.695 \mathrm{Ohms}$ is applied in Zone 2 at $\mathrm{t}=1.0$ s. Figs. 11(a)-(e) to Figs. 14(a)-(e) show variations of the three-phase voltages and currents, the real and reactive power components, and the frequencies measured at the Relays 2, 3, 4 and 5, respectively, in response to the SLG fault.

Fig. 10(f) shows that the Relay 2 commands the static switch to trip right after the fault since the relay detects a differential current component that exceeds $1.0 \mathrm{pu}(15 \mathrm{~A})$. Fig. 11(f) shows that the Relay 3 sends a trip command to the circuit breaker $\mathrm{CB}_{31}$ since magnitude of the negative-sequence current component at the relay is greater than $1.0 \mathrm{pu}$ (95 A) for a period more than $50 \mathrm{~ms}$ (3 cycles). Fig. 12(f) shows that the Relay 4 sends a trip command to the circuit breaker $\mathrm{CB}_{41}$ since magnitude of the zero-sequence current component at the relay is greater than $1.0 \mathrm{pu}(35 \mathrm{~A})$ for a period more than $50 \mathrm{~ms}$. Fig. 13(f) shows that the Relay 5 sends a trip command to the circuit breaker CB51 since magnitude of the phase voltage at the delta-side of transformer $\mathrm{T}_{51}$ falls below $1.0 \mathrm{pu}$ $(196 \mathrm{~V})$ for a period more than 20 cycles.

For the case of SLG fault in Zone 2, the static switch islands the microgrid and the breakers $\mathrm{CB}_{31}, \mathrm{CB}_{41}$ and $\mathrm{CB}_{51}$ disconnect all three zones of the microgrid and shut down all the microsource located inside the microgrid. The results shown in Fig. 10 to Fig. 13 demonstrate that the developed protection system is able to protect the AEP microgrid against an SLG fault in Zone 2 with a power of $28.5 \mathrm{~kW} /$ phase by clearing the fault and isolating the microgrid from the main grid. Since the fault current increases as the fault power increases, it is concluded that the microgrid protection system is able to protect all SLG faults in Zone 2 with a power more than or equal to $28.5 \mathrm{~kW} / \mathrm{phase}$. 


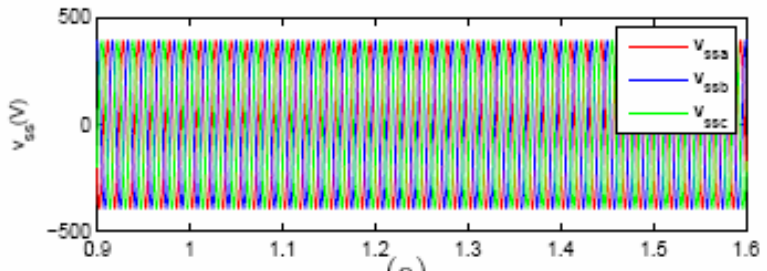

(a)

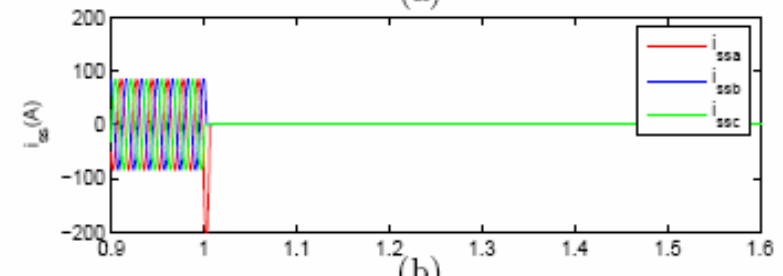

(b)

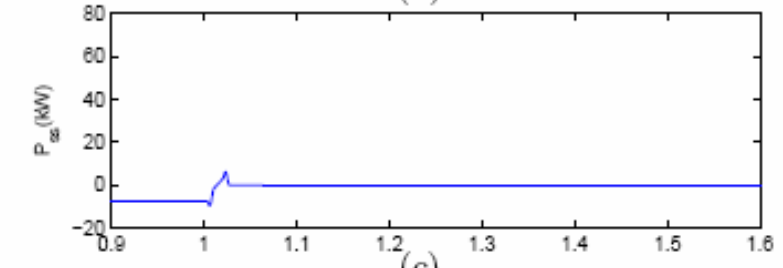

(c)
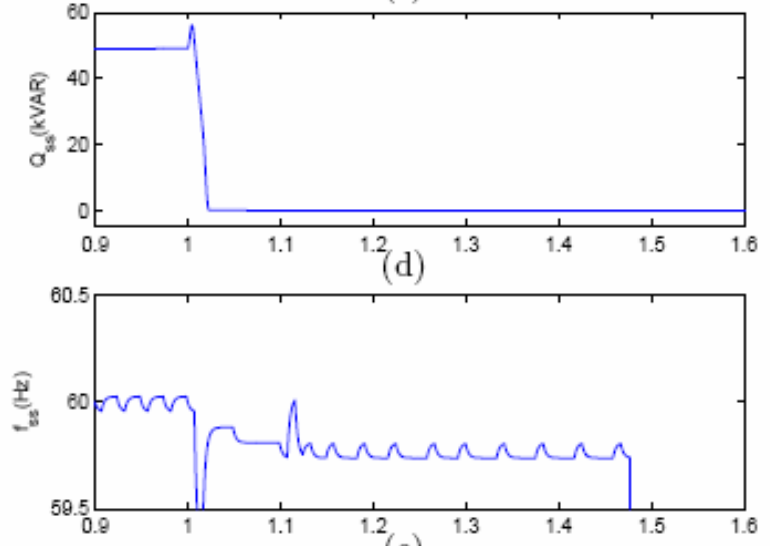

(e)

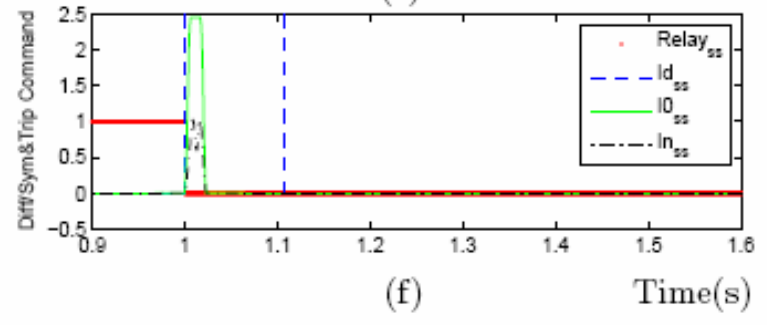

Figure 10: Response of the Relay 2 to a $28.5 \mathrm{~kW}$ SLG fault in Zone 2: (a,b) threephase voltages and currents, $(\mathrm{c}, \mathrm{d})$ real and reactive power components, (e) frequency, and (f) differential and zero- and negative-sequence current components and output command of the relay 

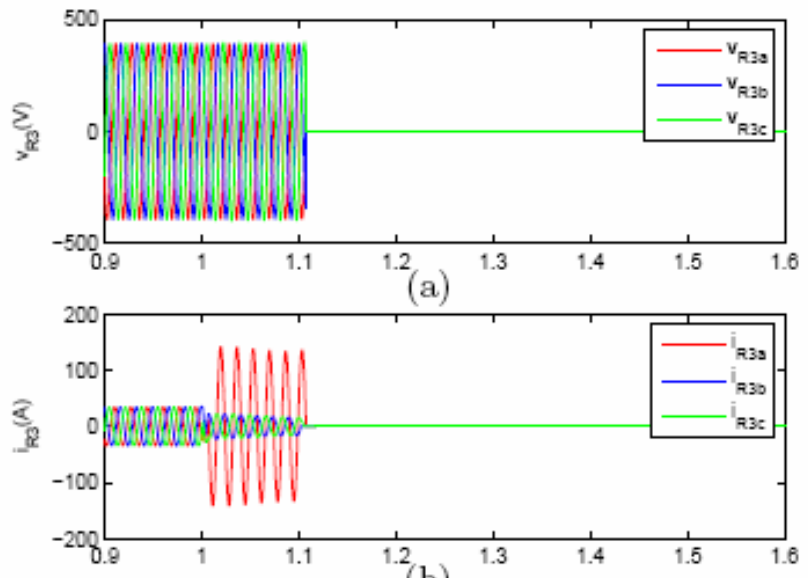

(b)

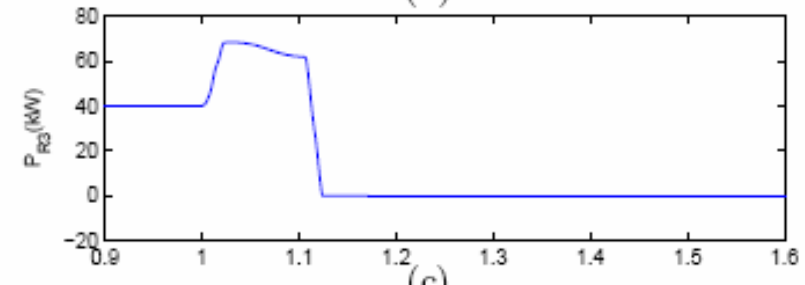

(c)
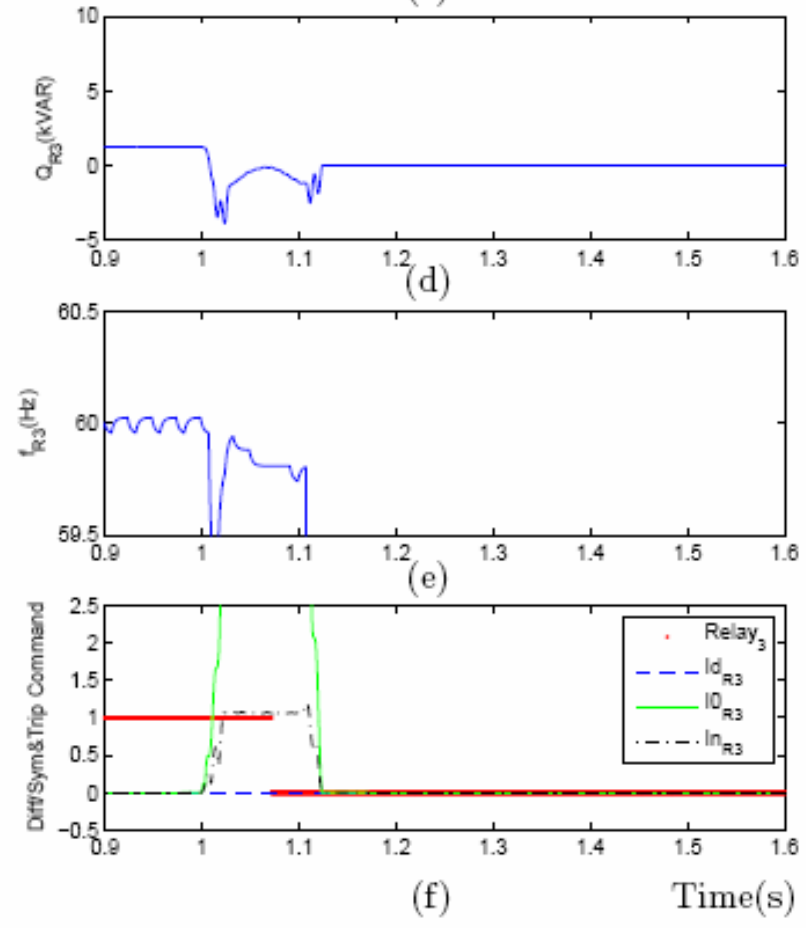

Figure 11: Response of the Relay 3 to a $28.5 \mathrm{~kW}$ SLG fault in Zone 2: (a,b) threephase voltages and currents, (c,d) real and reactive power components, (e) frequency, and (f) differential and zero- and negative-sequence current components and output command of the relay 

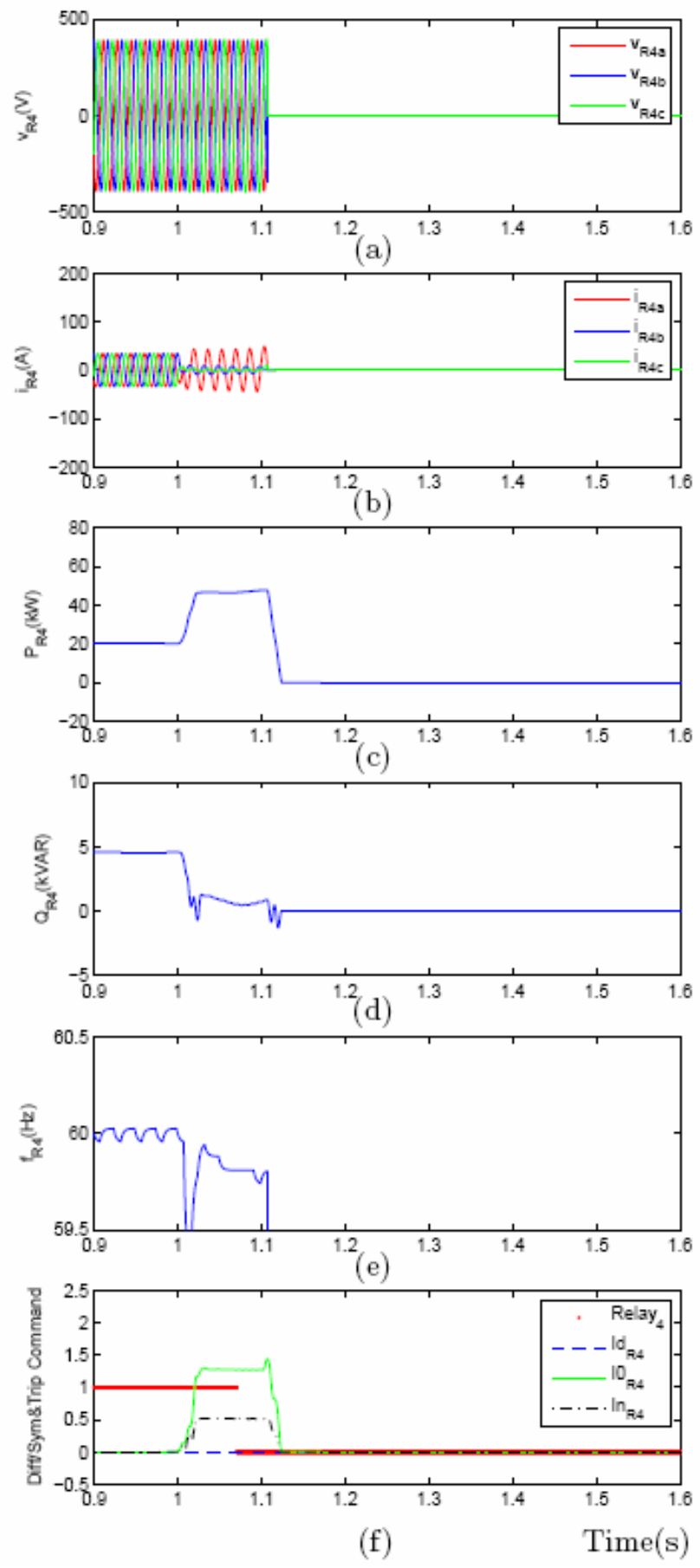

Figure 12: Response of the Relay 4 to a $28.5 \mathrm{~kW}$ SLG fault in Zone 2: (a,b) threephase voltages and currents, $(\mathrm{c}, \mathrm{d})$ real and reactive power components, (e) frequency, and (f) differential and zero- and negative-sequence current components and output command of the relay 


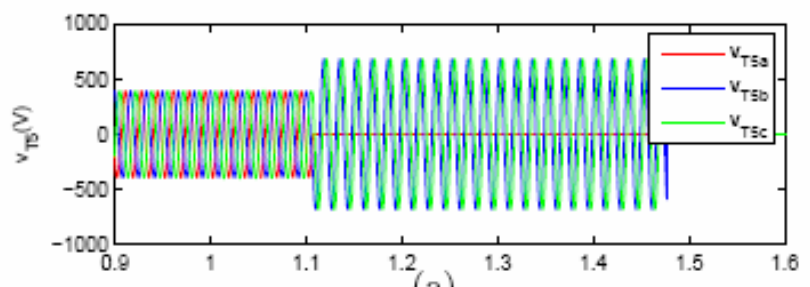

(a)
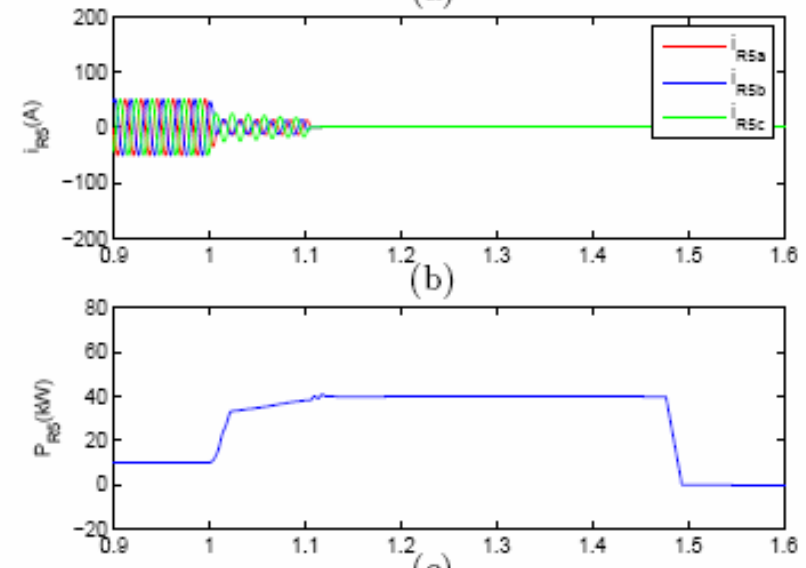

(c)
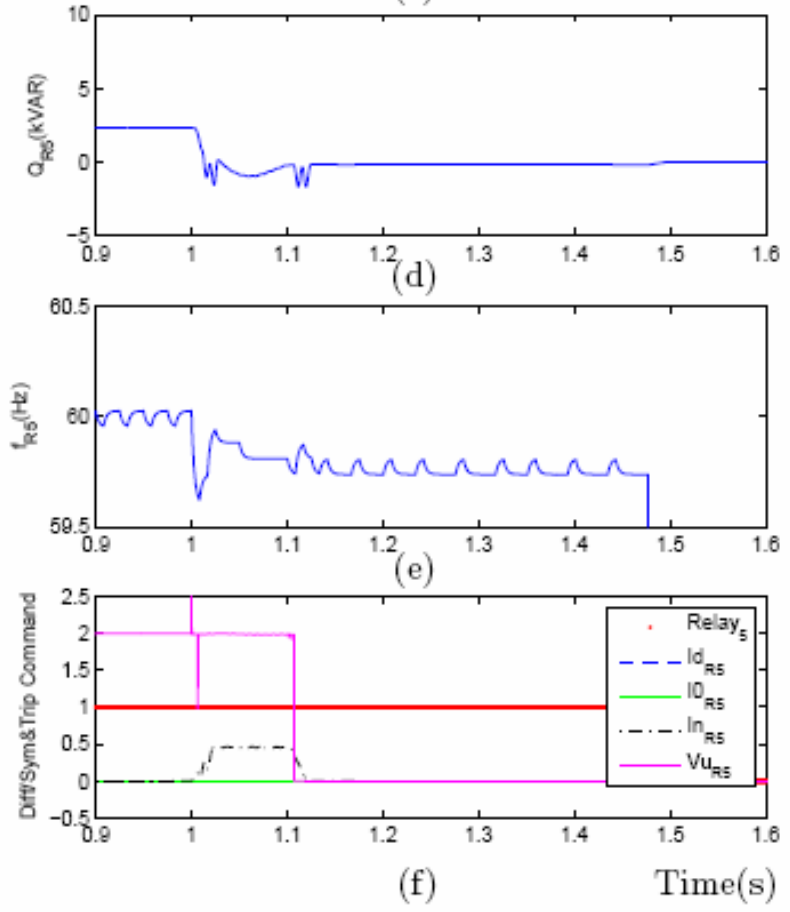

Figure 13: Response of the Relay 5 to a $28.5 \mathrm{~kW}$ SLG fault in Zone 2: (a,b) threephase voltages and currents, $(c, d)$ real and reactive power components, (e) frequency, and (f) differential and zero- and negative-sequence current components, negative-sequence voltage component at the transformer $T_{51}$ and output command of the relay 


\section{SLG Fault in Zone 5}

The AEP microgrid of Fig. 1 is operating at steady-state conditions with the source and load settings as explained in Section 5 and an SLG fault with a resistance of $2.695 \mathrm{Ohms}$ is applied in Zone 5 at $\mathrm{t}=1.0$ s. Figs. 15(a)-(e) to Figs. 18(a)-(e) show variations of the three- phase voltages and currents, the real and reactive power components, and the frequencies measured at the Relays 2, 3, 4 and 5, respectively, in response to the SLG fault.

Fig. 14(f) shows that the Relay 2 commands the static switch to trip when $\mathrm{CB}_{51}$ trips since the relay detects an instantaneous negative-sequence current component that exceeds $1.0 \mathrm{pu}$ (95 A). Fig. 15(f) shows that the Relay 3 does not command for a trip since none of its fault detection parameters, i.e. the differential and symmetrical current components, exceeds the threshold of 1.0 pu. Fig. 16(f) shows that the Relay 4 does not command for a trip since none of its fault detection parameters, i.e. the differential and symmetrical current components, exceeds the threshold of 1.0 pu. Fig. 17(f) shows that the Relay 5 commands for a trip to the circuit breaker $\mathrm{CB}_{51}$ since magnitude of the differential current component at the relay is above the threshold of $1.0 \mathrm{pu}(15 \mathrm{~A})$ for a period more than $50 \mathrm{~ms}$.

For the case of SLG fault in Zone 5, the static switch islands the microgrid and the breaker $\mathrm{CB}_{51}$ disconnects Zone 5 of the microgrid and shuts down the microsource $\mathrm{B}_{1}$. The Zones 2, 3 and 4 of the microgrid and the microsources $A_{1}$ and $A_{2}$ continue the operation subsequent to the fault detection and its clearance. The results shown in Fig. 14 to Fig.17 demonstrate that the developed protection system is able to protect the AEP microgrid against an SLG fault in Zone 5 with a power of $28.5 \mathrm{~kW} /$ phase by clearing the fault and isolating the microgrid from the main grid. Since the fault current increases as the fault power increases, it is concluded that the microgrid protection system is able to protect all SLG faults in Zone 5 with a power more than or equal to $28.5 \mathrm{~kW} /$ phase. 


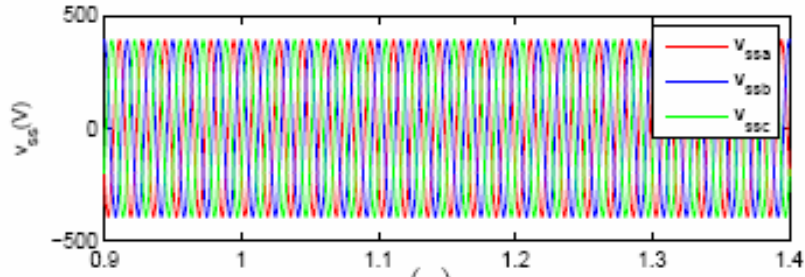

(a)

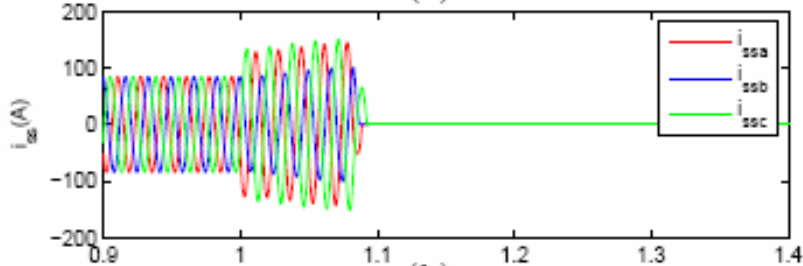

(b)

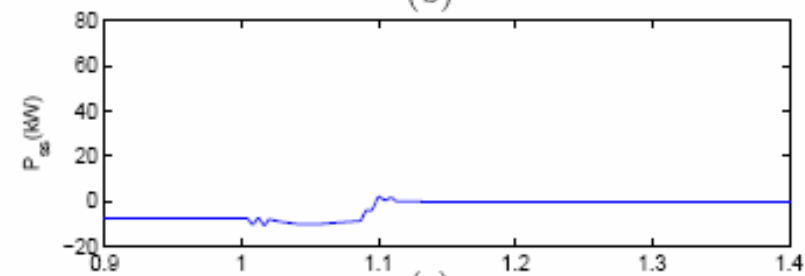

(c)

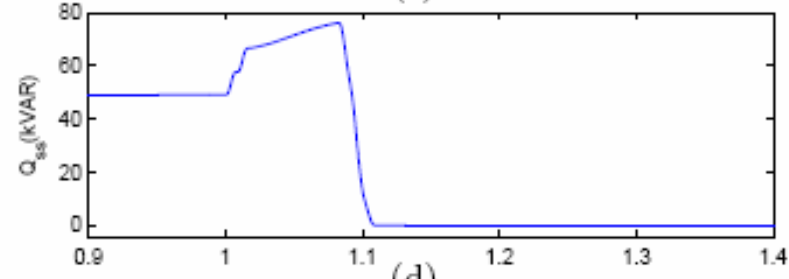

(d)

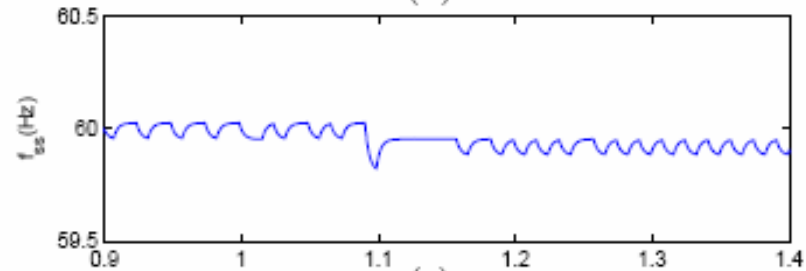

(e)

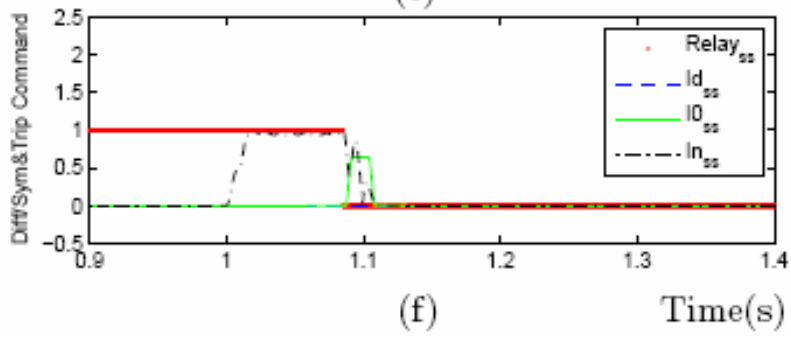

Figure 14: Response of the Relay 2 to a $28.5 \mathrm{~kW}$ SLG fault in Zone 5: (a,b) threephase voltages and currents, $(c, d)$ real and reactive power components, (e) frequency, and (f) differential and zero- and negative-sequence current components and output command of the relay 


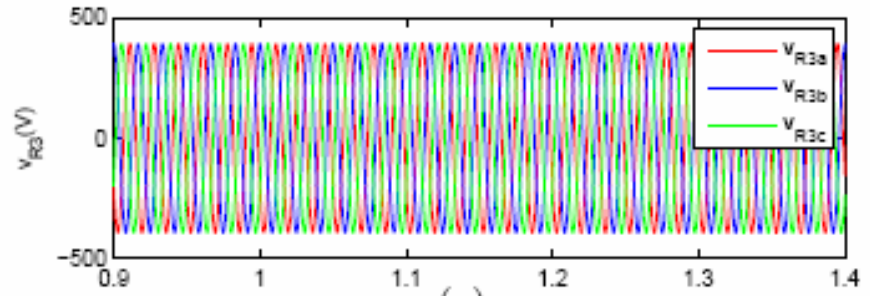

(a)

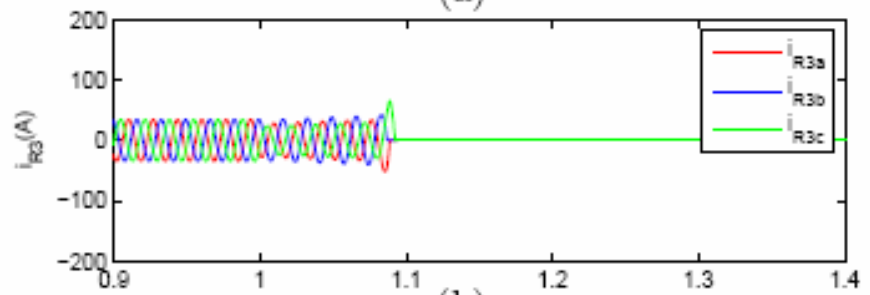

(b)

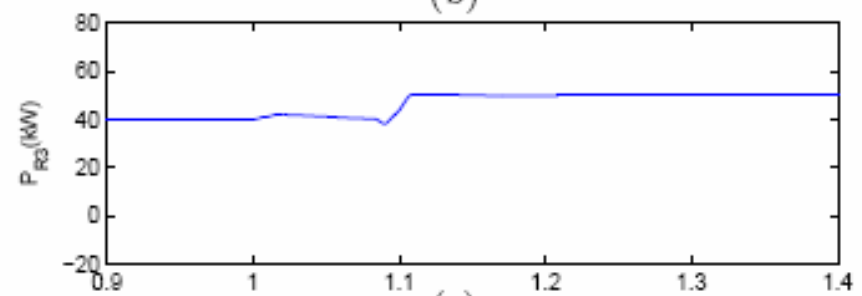

(c)

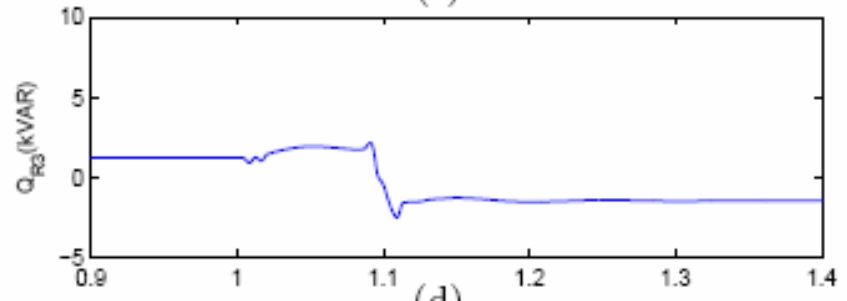

(d)

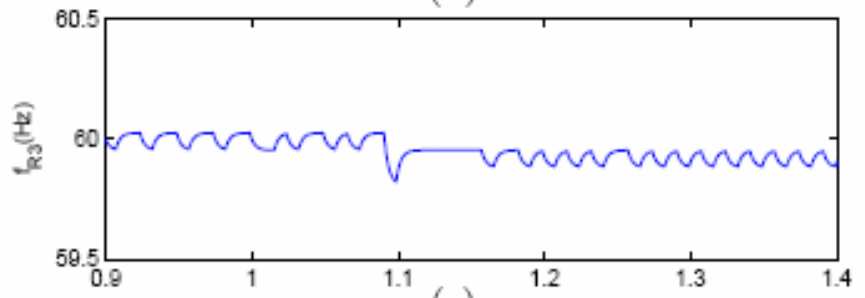

(e)

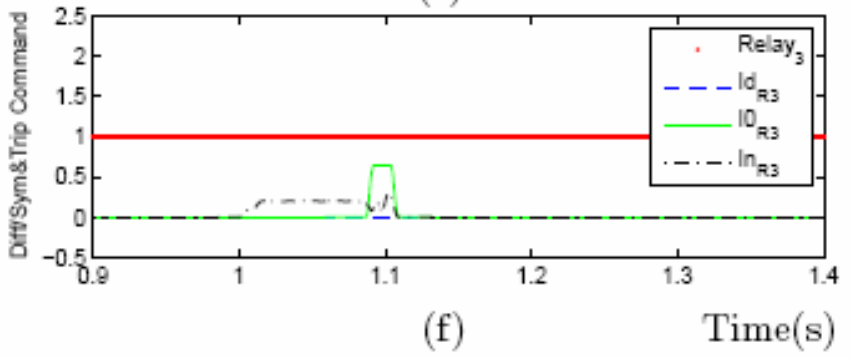

Figure 15: Response of the Relay 3 to a $28.5 \mathrm{~kW}$ SLG fault in Zone 5: (a,b) threephase voltages and currents, (c,d) real and reactive power components, (e) frequency, and (f) differential and zero- and negative-sequence current components and output command of the relay 

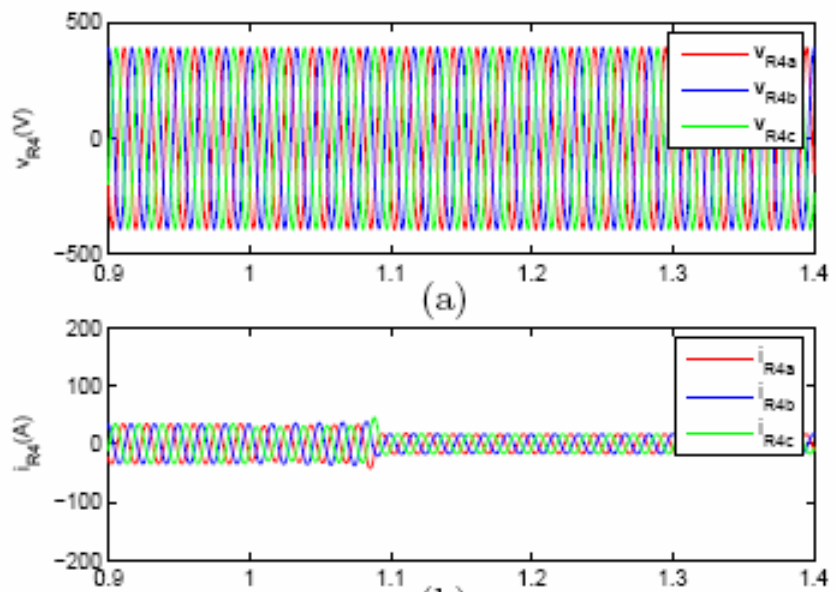

(b)

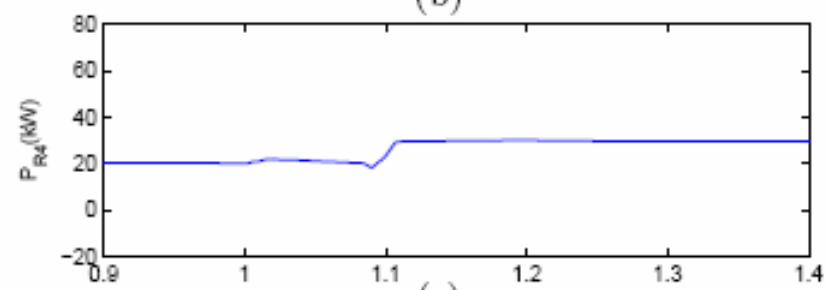

(c)
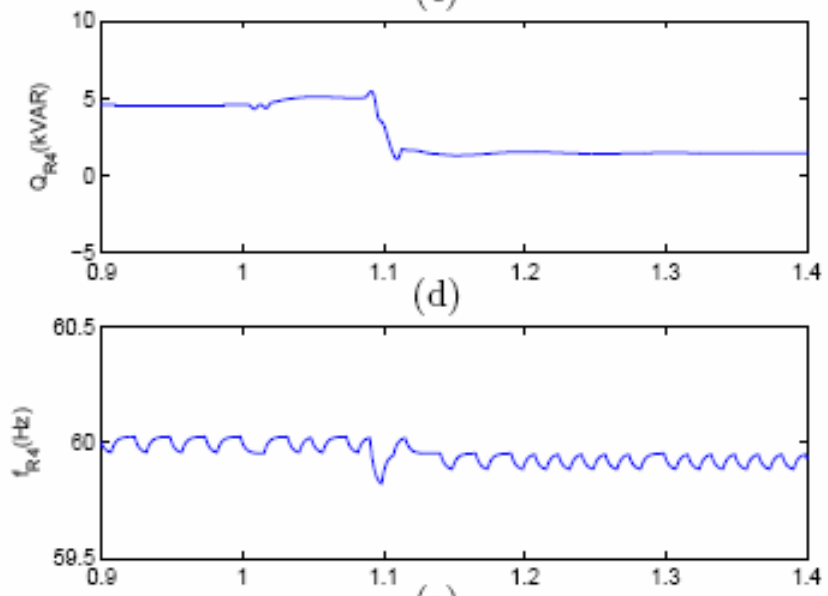

(e)

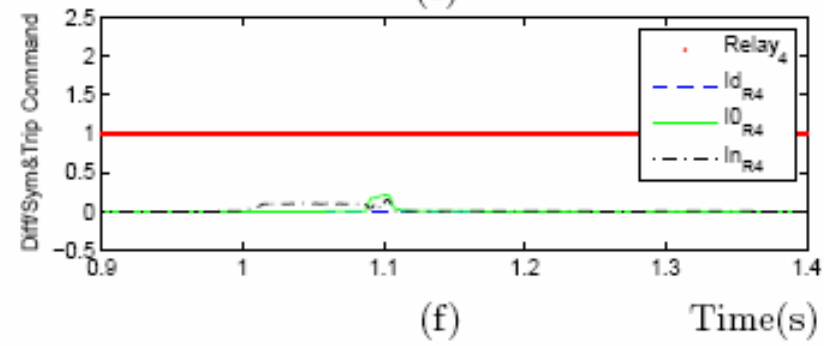

Figure 16: Response of the Relay 4 to a $28.5 \mathrm{~kW}$ SLG fault in Zone 5: (a,b) threephase voltages and currents, (c,d) real and reactive power components, (e) frequency, and (f) differential and zero- and negative-sequence current components and output command of the relay 


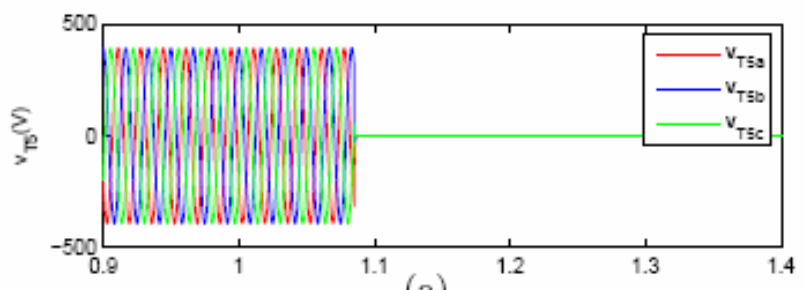

(a)

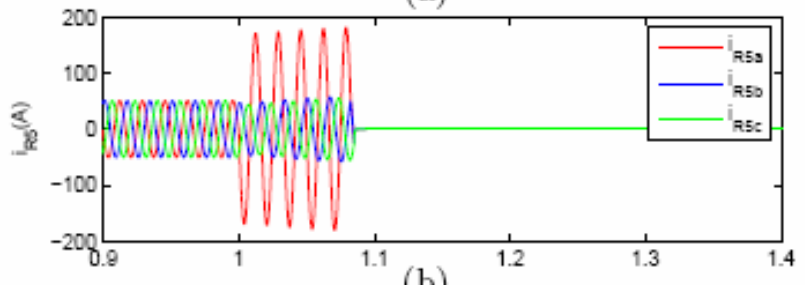

(b)

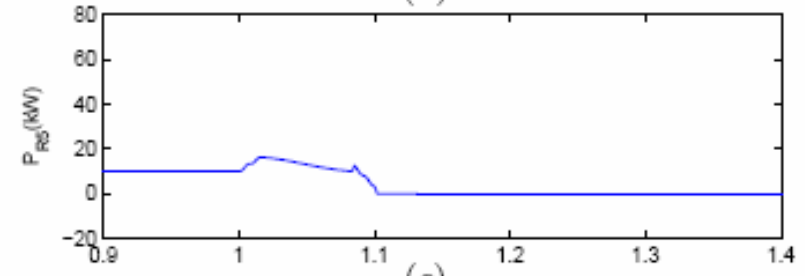

(c)

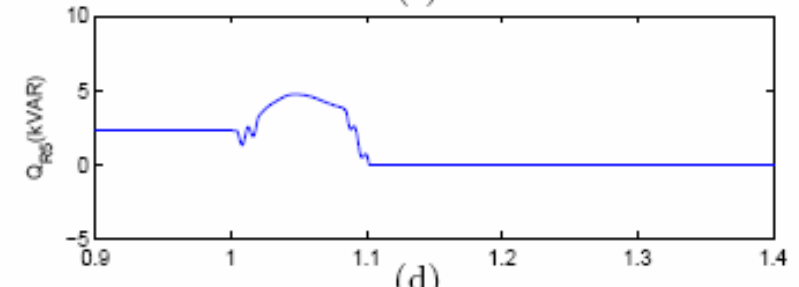

(d)

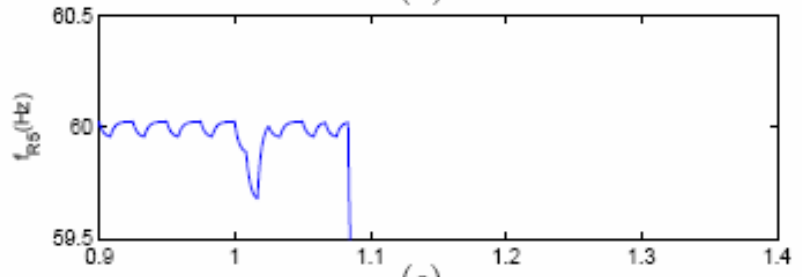

(e)

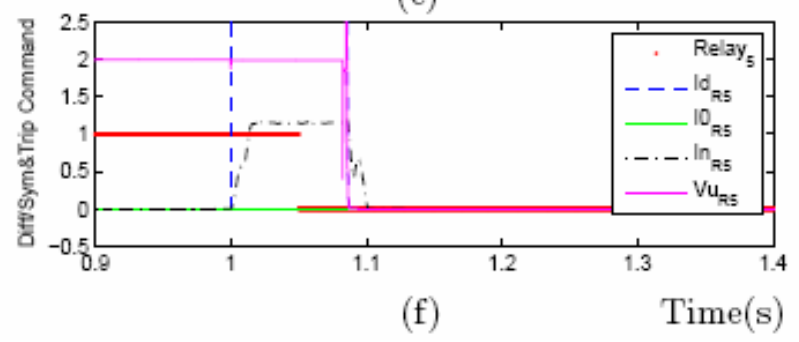

Figure 17: Response of the Relay 5 to a $28.5 \mathrm{~kW}$ SLG fault in Zone 5: (a,b) threephase voltages and currents, (c,d) real and reactive power components, (e) frequency, and (f) differential and zero- and negative-sequence current components, negative-sequence voltage component at the transformer $T_{51}$ and output command of the relay 


\section{SLG Fault in Zone 6}

The AEP microgrid of Fig. 1 is operating at steady-state conditions with the source and load settings as explained in Section 5 and an SLG fault with a resistance of $2.695 \mathrm{Ohms}$ is applied in Zone 6 at $t=1.0$ s. Figs. 19(a)-(e) to Figs. 22(a)-(e) show variations of the three- phase voltages and currents, the real and reactive power components, and the frequencies measured at the Relays 2, 3, 4 and 5, respectively, in response to the SLG fault.

Fig. 18(f) shows that the Relay 2 commands the static switch to trip since the relay detects an instantaneous negative-sequence current component that exceeds $1.0 \mathrm{pu}$ (95 A). Fig. 19(f) shows that the Relay 3 does not command for a trip since none of its fault detection parameters, i.e. the differential and symmetrical current components, exceeds the threshold of 1.0 pu. Fig. 20(f) shows that the Relay 4 does not command for a trip since none of its fault detection parameters, i.e. the differential and symmetrical current components, exceeds the threshold of $1.0 \mathrm{pu}$. Fig. 21(f) shows that the Relay 5 does not command for a trip since none of its fault detection parameters, i.e. the differential and symmetrical current components, exceeds the threshold of $1.0 \mathrm{pu}$.

For the case of SLG fault in Zone 6, the static switch islands the microgrid. The Zones 2, 3,4 and 5 of the microgrid and the microsources $A_{1}, A_{2}$ and $B_{1}$ continue the operation subsequent to the fault detection and its clearance. The results shown in Fig. 18 to Fig. 21 demonstrate that the developed protection system is able to protect the AEP microgrid against an SLG fault in Zone 6 with a power of $28.5 \mathrm{~kW} /$ phase by isolating the microgrid from the main grid. Since the fault current increases as the fault power increases, it is concluded that the microgrid protection system is able to protect all SLG faults in Zone 6 with a power more than or equal to $28.5 \mathrm{~kW} /$ phase. 


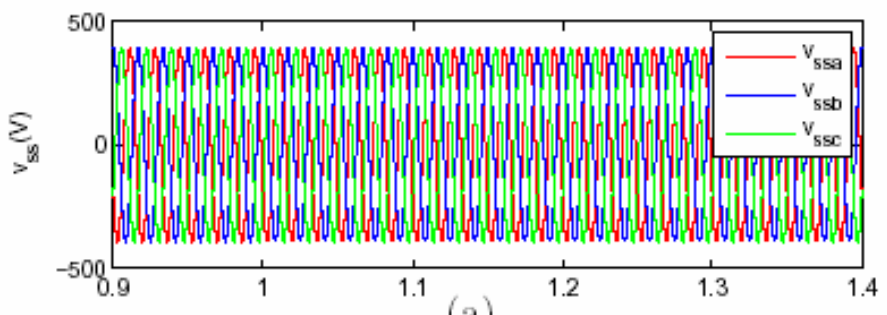

(a)

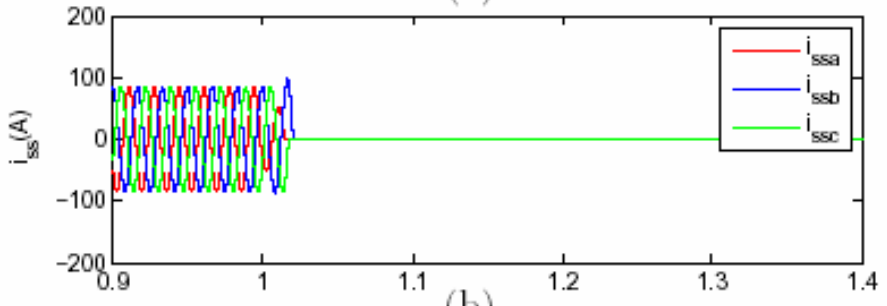

(b)
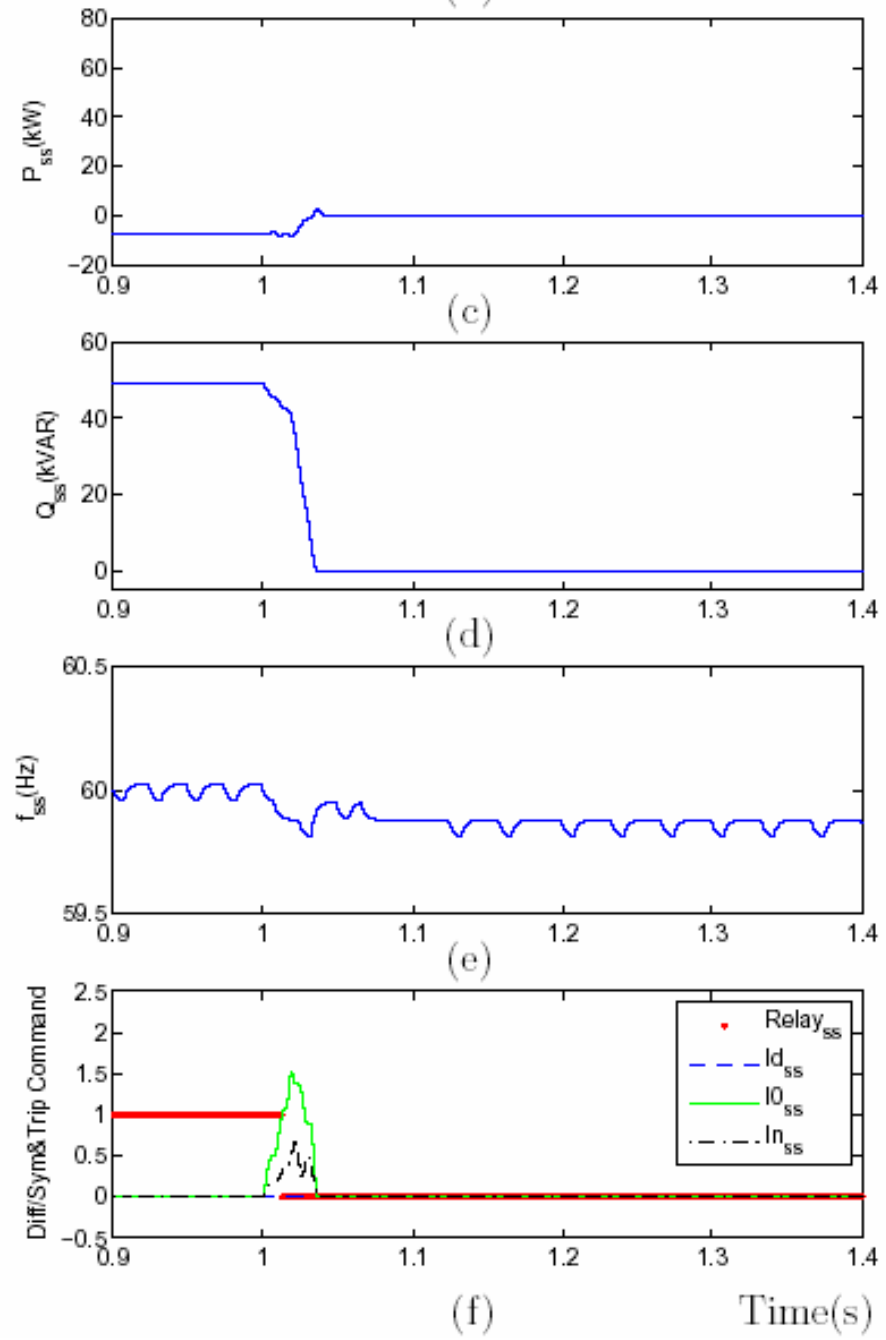

Figure 18: Response of the Relay 2 to a $28.5 \mathrm{~kW}$ SLG fault in Zone 6: (a,b) threephase voltages and currents, $(\mathrm{c}, \mathrm{d})$ real and reactive power components, (e) frequency, and (f) differential and zero- and negative-sequence current components and output command of the relay 


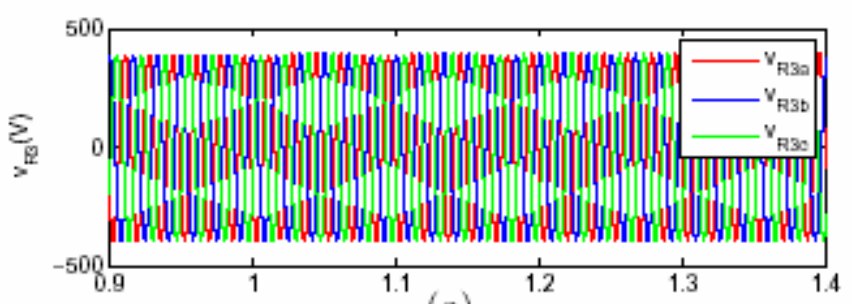

(a)

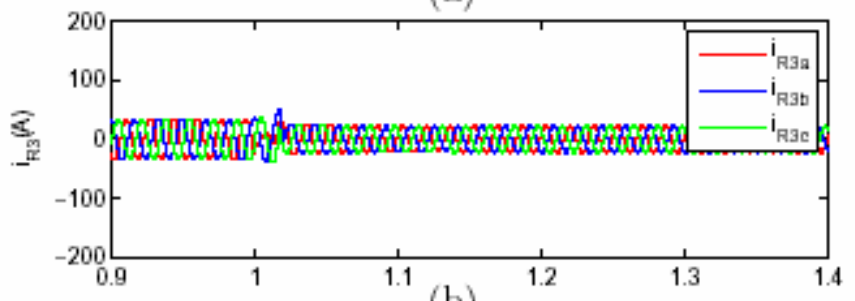

(b)
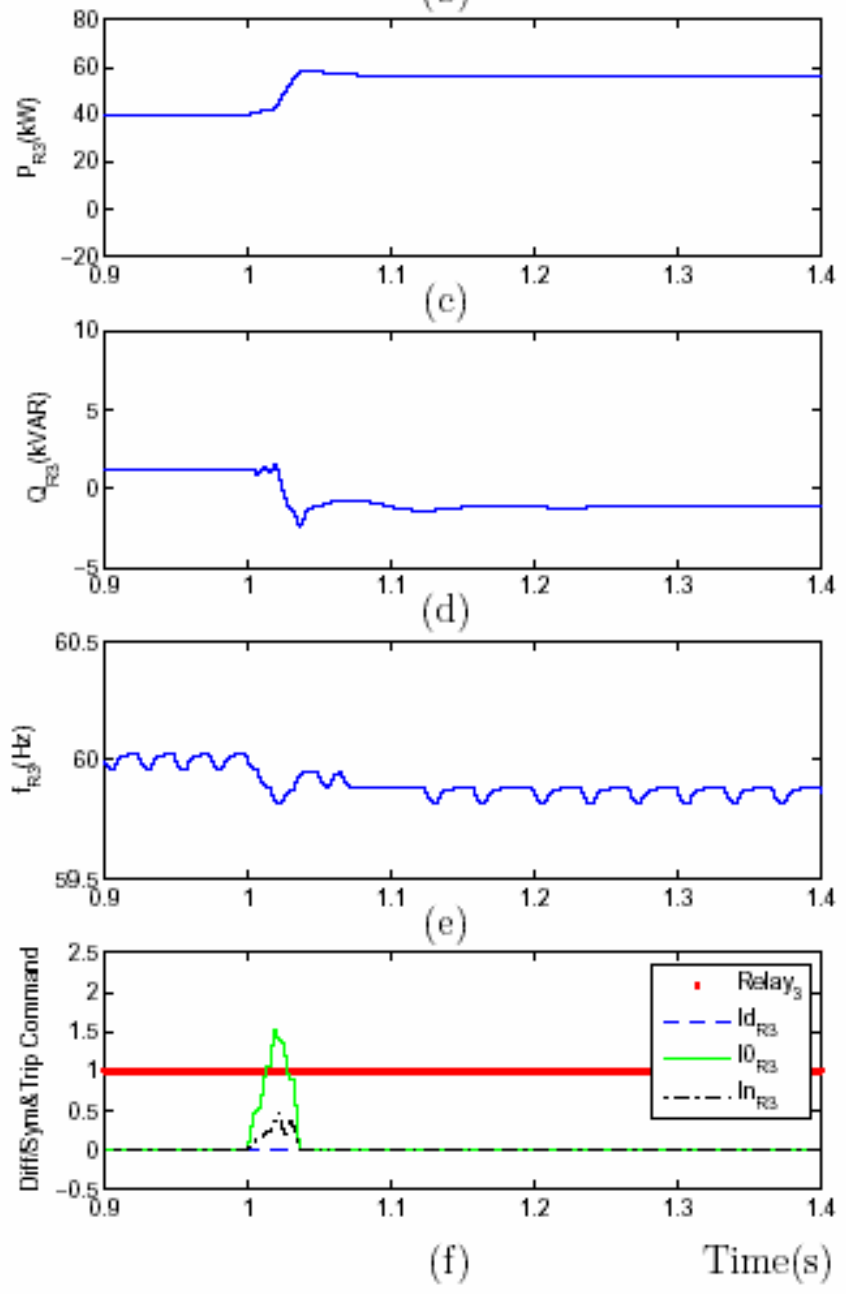

Figure 19: Response of the Relay 3 to a $28.5 \mathrm{~kW}$ SLG fault in Zone 6: (a,b) threephase voltages and currents, (c,d) real and reactive power components, (e) frequency, and (f) differential and zero- and negative-sequence current components and output command of the relay 


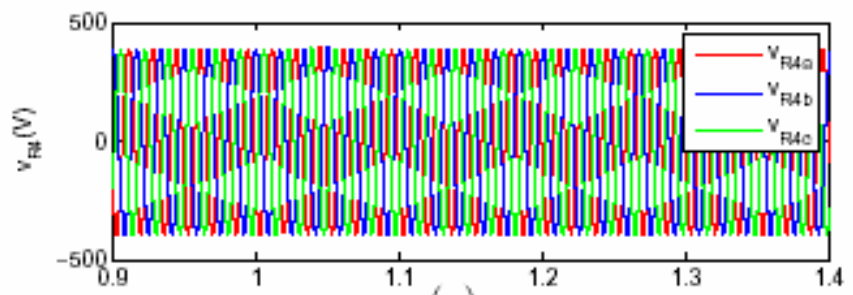

(a)
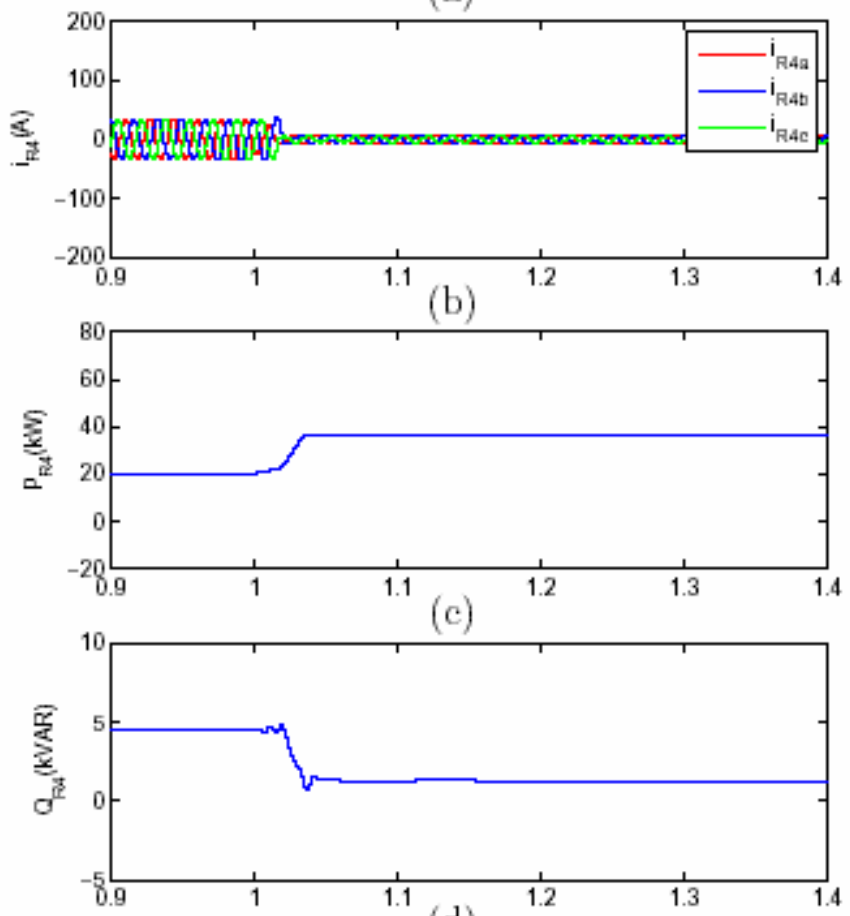

(d)
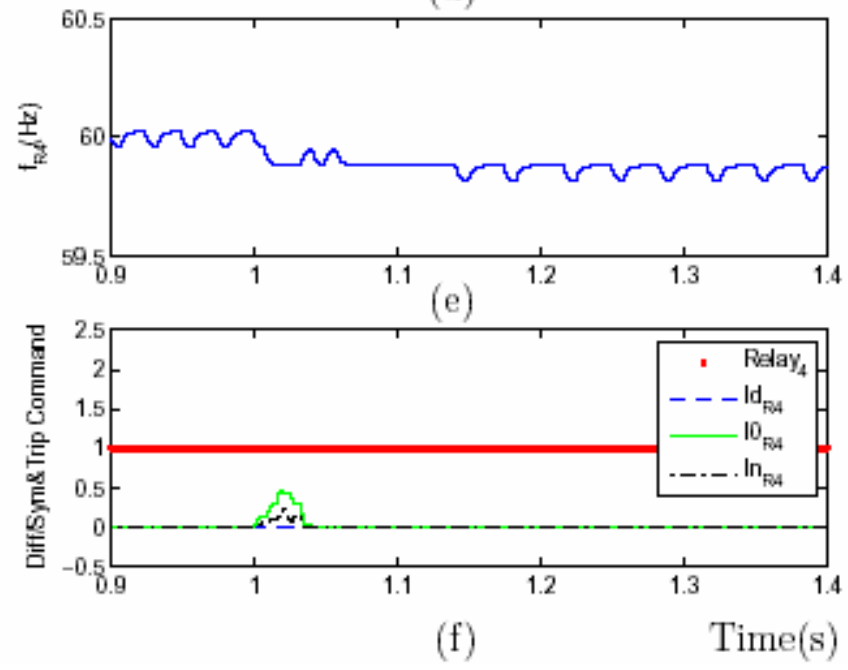

Figure 20: Response of the Relay 4 to a $28.5 \mathrm{~kW}$ SLG fault in Zone 6: (a,b) threephase voltages and currents, (c,d) real and reactive power components, (e) frequency, and (f) differential and zero- and negative-sequence current components and output command of the relay 

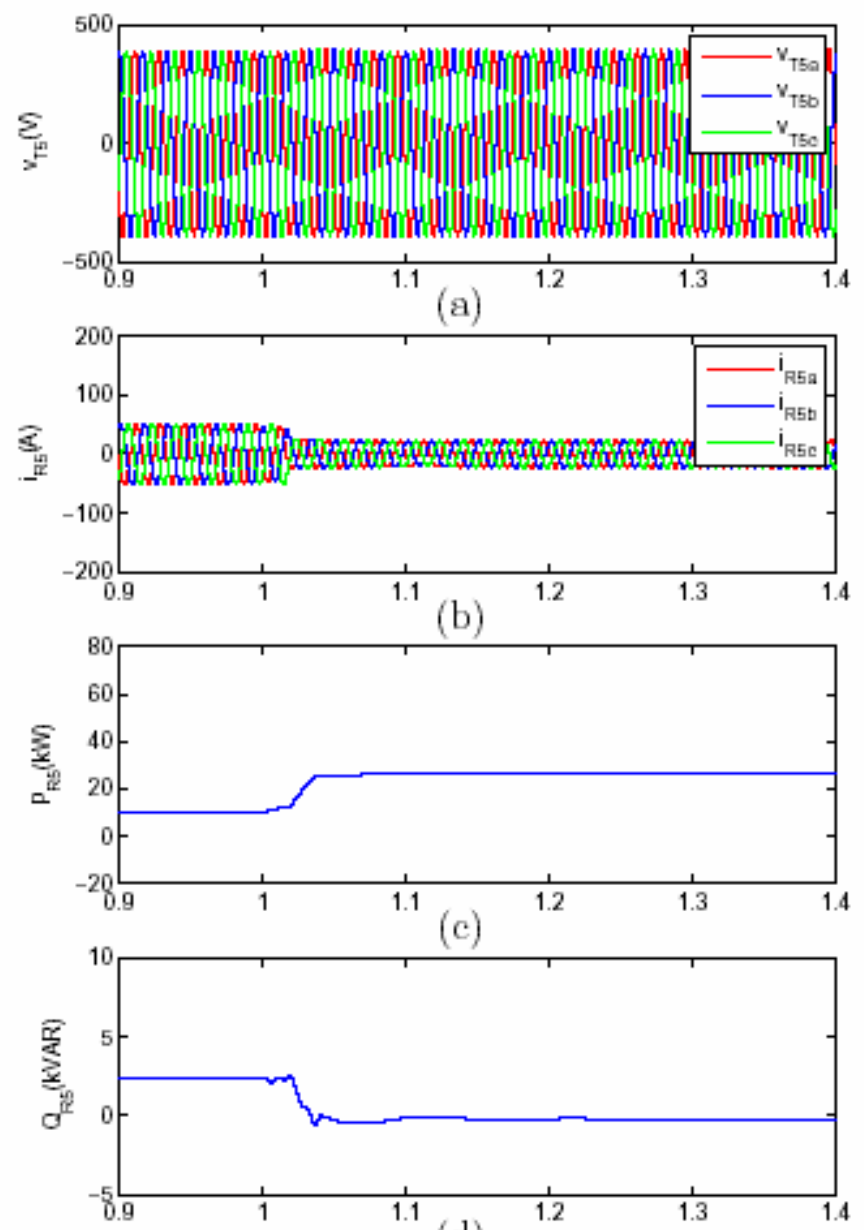

(d)
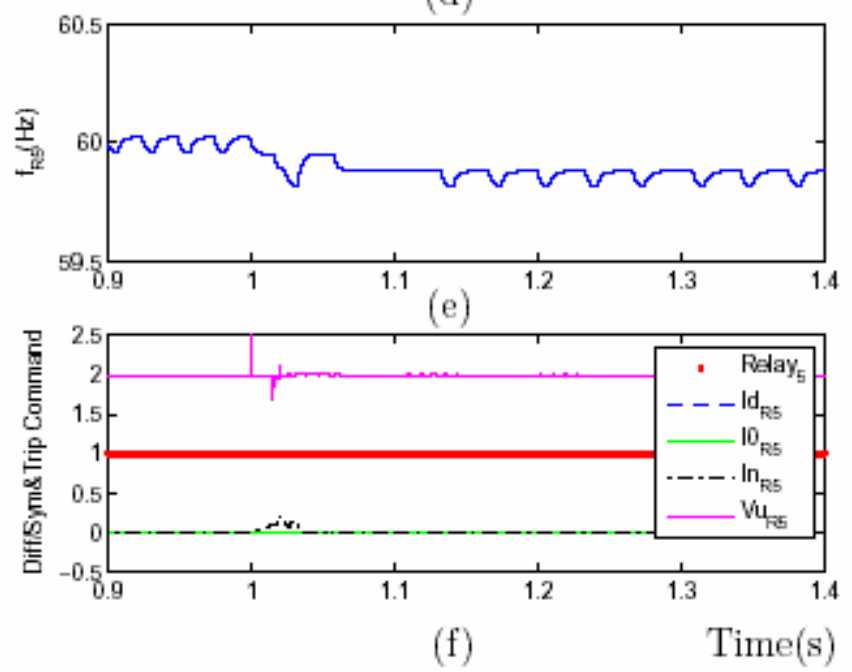

Figure 21: Response of the Relay 5 to a $28.5 \mathrm{~kW}$ SLG fault in Zone 6: (a,b) threephase voltages and currents, $(c, d)$ real and reactive power components, (e) frequency, and (f) differential and zero- and negative-sequence current components, negative-sequence voltage component at the transformer $T_{51}$ and output command of the relay 


\subsection{Line-to-Line Faults}

\section{Line-to-Line Fault in Zone 4}

The AEP microgrid of Fig. 1 is operating at steady-state conditions with the source and load settings as explained in Section 5 and an LL fault with a resistance of $2.695 \mathrm{Ohms}$ is applied in Zone 4 at $t=1.0$ s. Figs. 23(a)-(e) to Figs. 26(a)-(e) show variations of the threephase voltages and currents, the real and reactive power components, and the frequencies measured at the Relays 2, 3, 4 and 5, respectively, in response to the LL fault.

Fig. 22(f) shows that the Relay 2 commands the static switch to trip shortly after the fault when the relay detects a negative-sequence current component that exceeds $1.0 \mathrm{pu}$ (95 A). Fig. 23(f) shows that the Relay 3 commands $\mathrm{CB}_{31}$ to trip since magnitude of the negative-sequence current component at the relay is above $1.0 \mathrm{pu}$ for a period more than $50 \mathrm{~ms}$ (3 cycles). Fig. 24(f) shows that the Relay 4 sends a trip command to the circuit breaker $\mathrm{CB}_{41}$ since magnitude of the negative-sequence current component at the relay is greater than $1.0 \mathrm{pu}$ for a period more than $50 \mathrm{~ms}$. Fig. 25(f) shows that the Relay 5 commands $\mathrm{CB}_{51}$ to trip since magnitude of the negative-sequence current component at the relay is above $1.0 \mathrm{pu}$ for a period more than $50 \mathrm{~ms}$. Magnitudes of the differential and zero-sequence current components of all relays do not exceed the corresponding threshold of $15 \mathrm{~A}$ and $35 \mathrm{~A}$.

For the case of an LL fault in Zone 4, the static switch islands the microgrid and the breakers $\mathrm{CB}_{31}, \mathrm{CB}_{41}$ and $\mathrm{CB}_{51}$ disconnects all zones of the microgrid and shut down all the microsource located inside the microgrid. The results shown in Fig. 22 to Fig. 25 demonstrate that the developed protection system is able to protect the AEP microgrid against an LL fault in Zone 4 with a power of $85.5 \mathrm{~kW}$ by clearing the fault and disconnecting all protection zones of the microgrid. Since the fault current increases as the fault power increases, it is concluded that the microgrid protection system is able to protect all LL faults in Zone 4 with a power more than or equal to $85.5 \mathrm{~kW}$. 

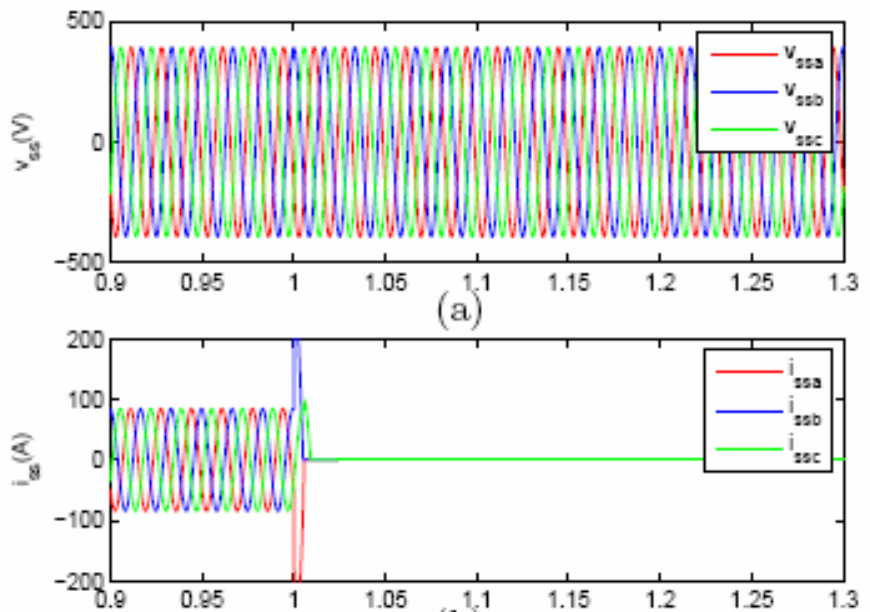

(b)

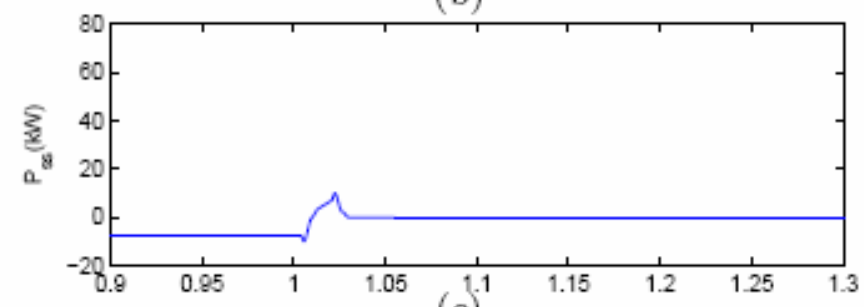

(c)
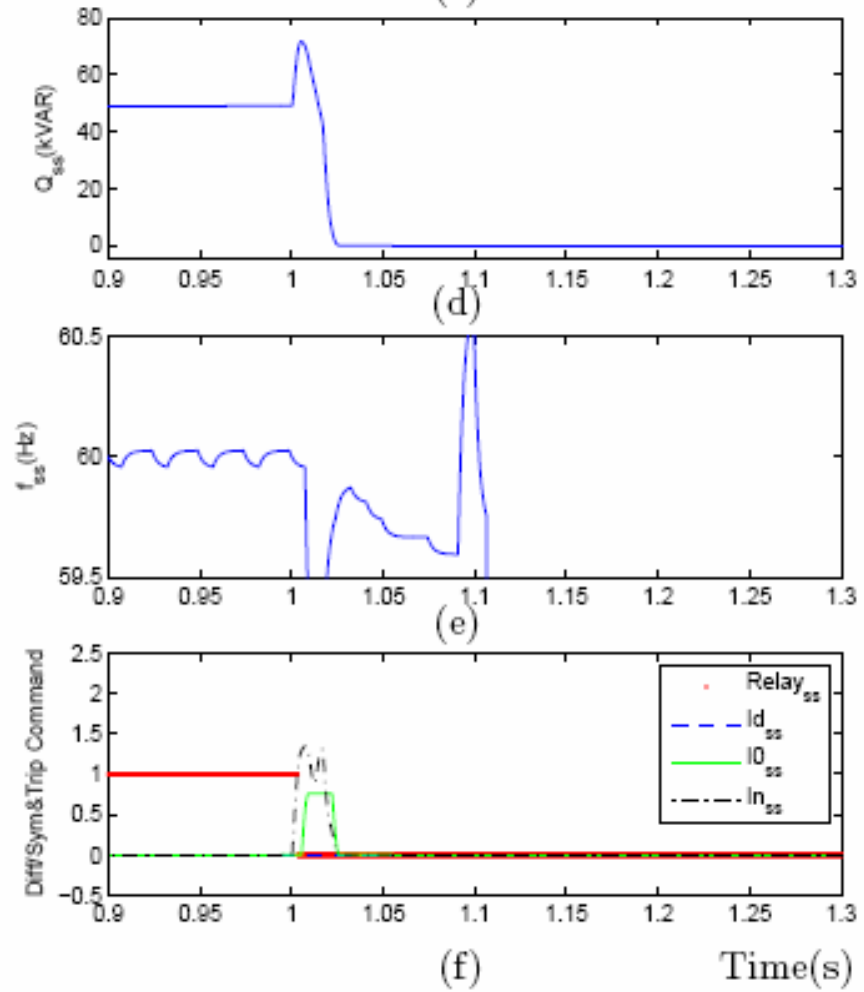

Figure 22: Response of the Relay 2 to a $85.5 \mathrm{~kW}$ LL fault in Zone 4: (a,b) threephase voltages and currents, (c,d) real and reactive power components, (e) frequency, and (f) differential and zero- and negative-sequence current components and output command of the relay 

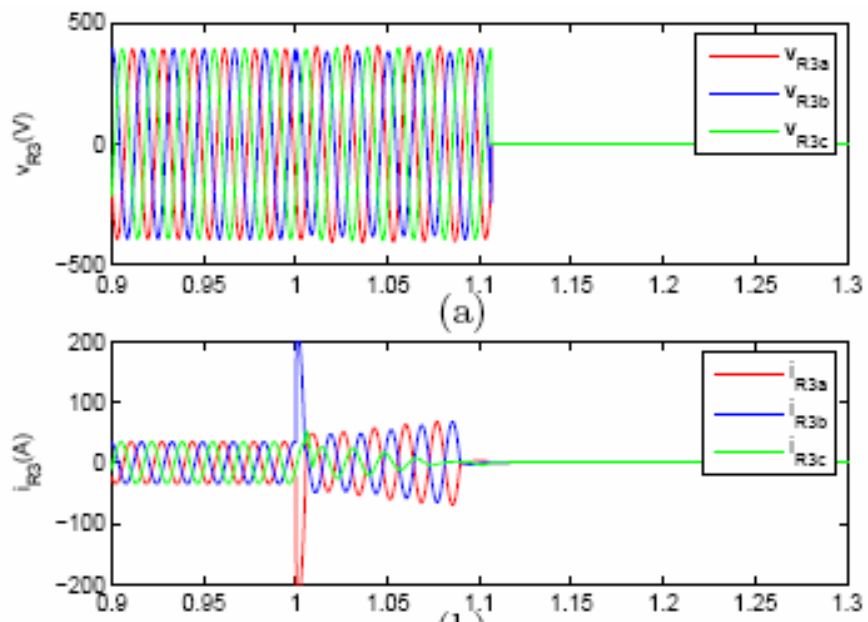

(b)
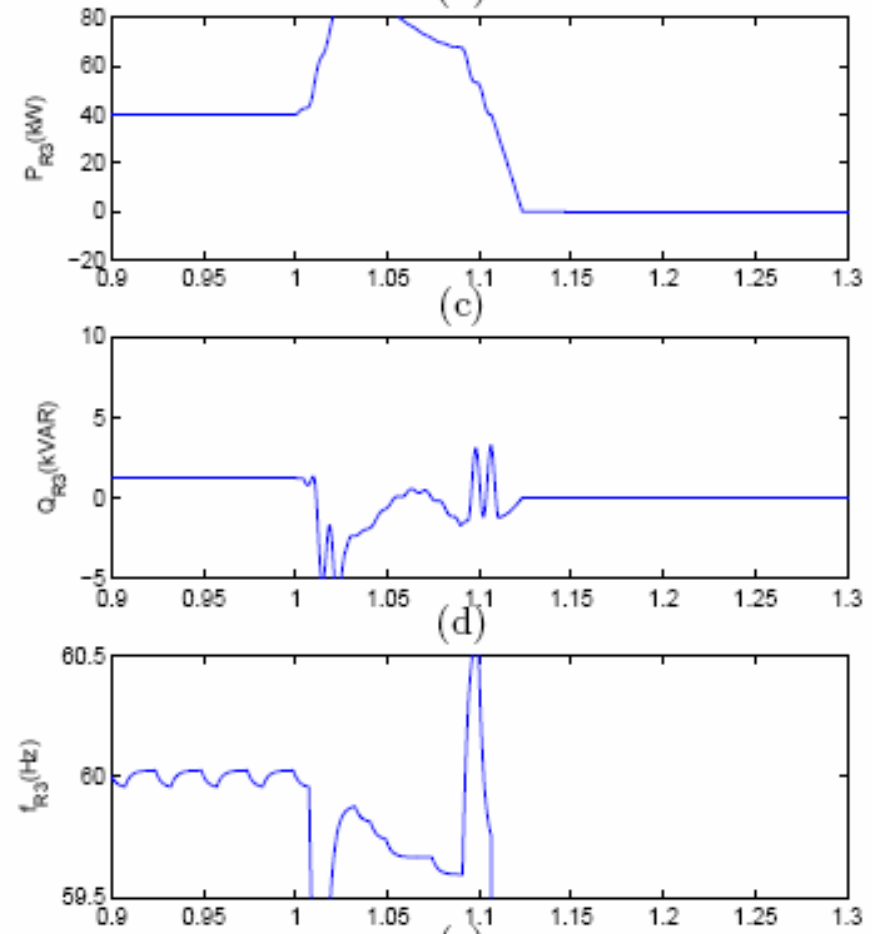

(e)

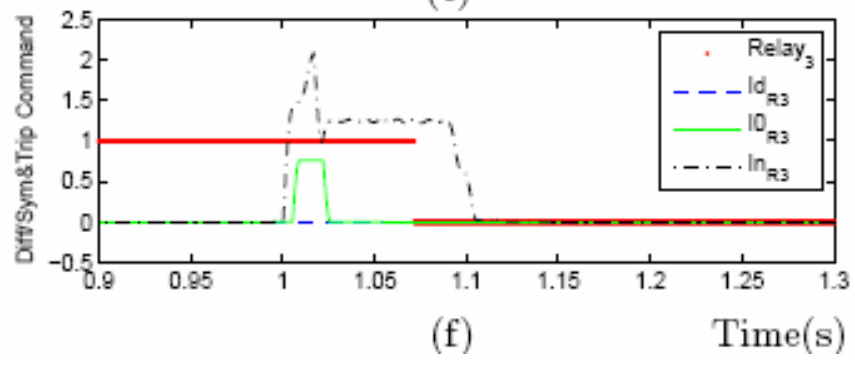

Figure 23: Response of the Relay 3 to a $85.5 \mathrm{~kW}$ LL fault in Zone 4: (a,b) threephase voltages and currents, $(\mathrm{c}, \mathrm{d})$ real and reactive power components, (e) frequency, and (f) differential and zero- and negative-sequence current components and output command of the relay 

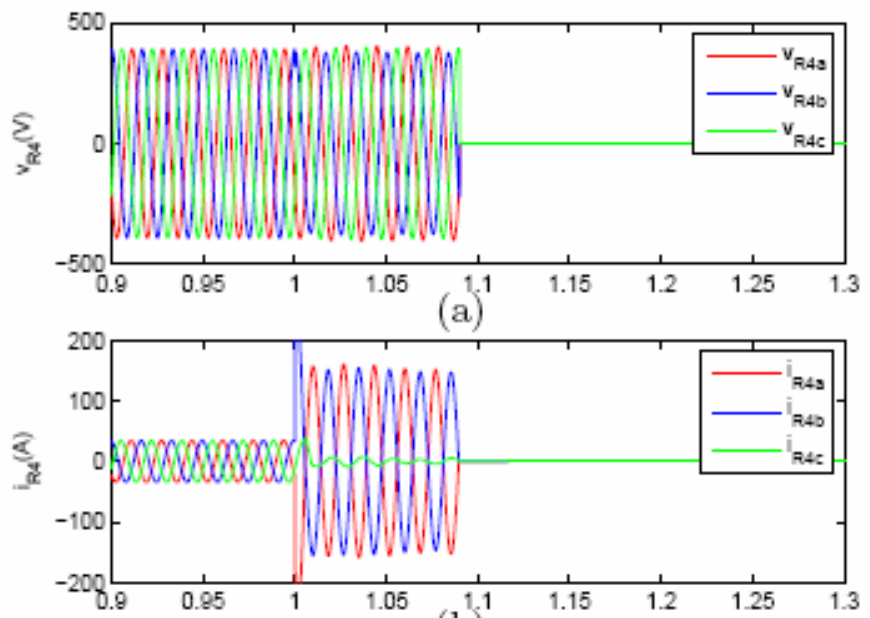

(b)

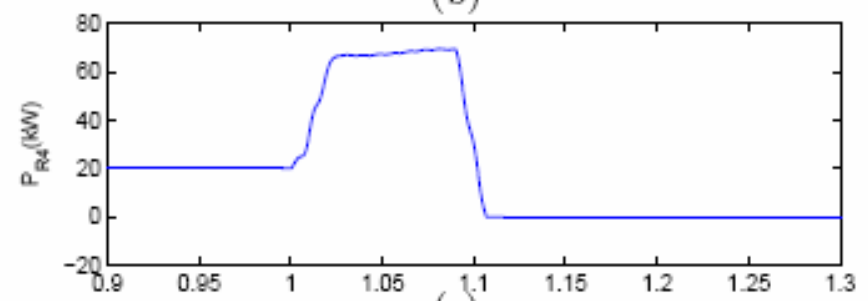

(c)
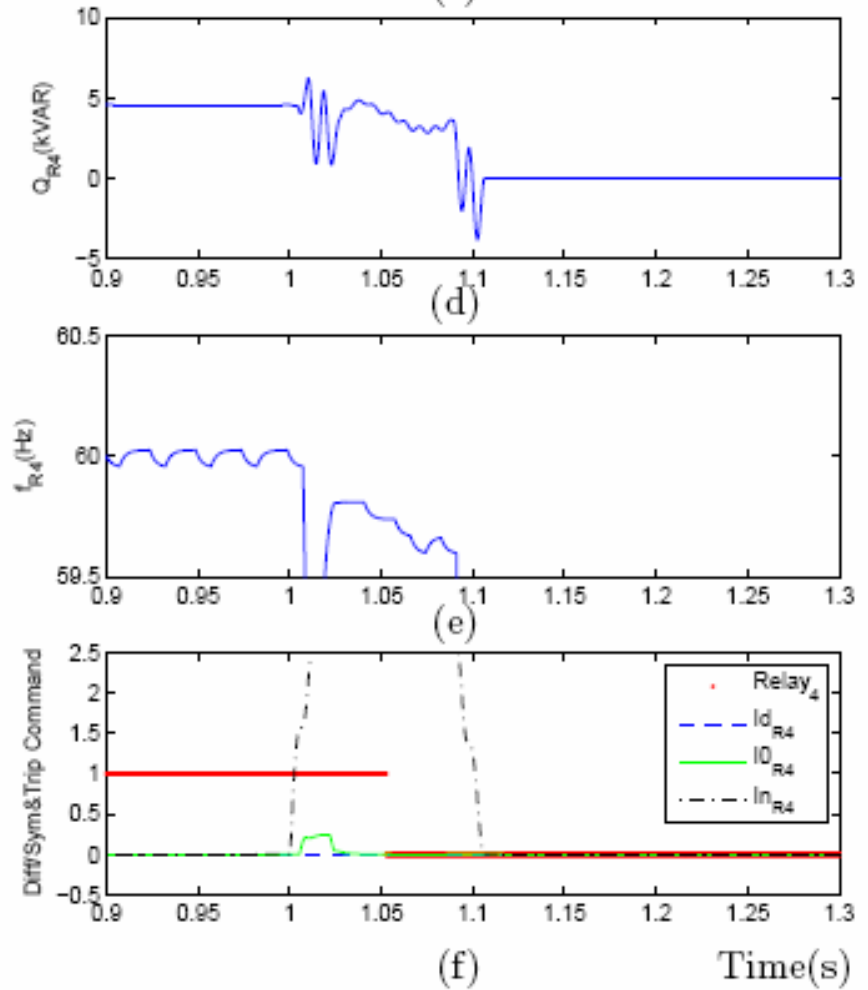

Figure 24: Response of the Relay 4 to a $85.5 \mathrm{~kW}$ LL fault in Zone 4: (a,b) threephase voltages and currents, (c,d) real and reactive power components, (e) frequency, and (f) differential and zero- and negative-sequence current components and output command of the relay 


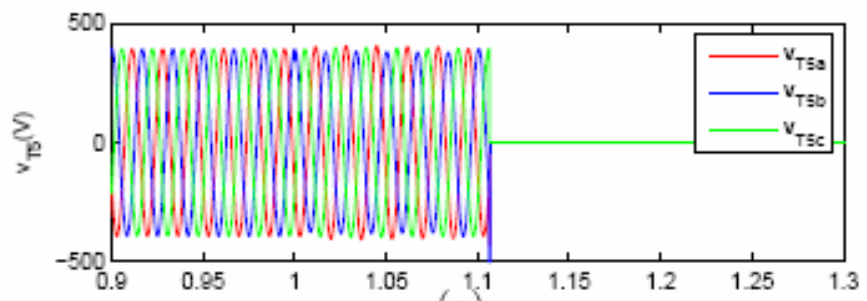

(a)

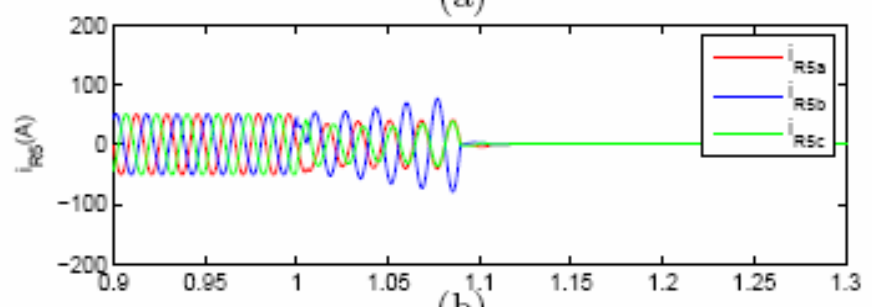

(b)

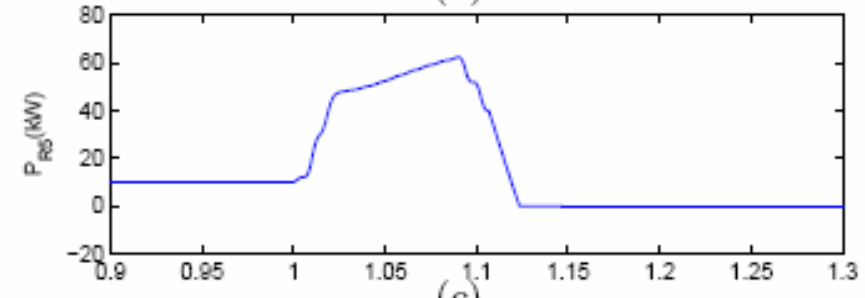

(c)
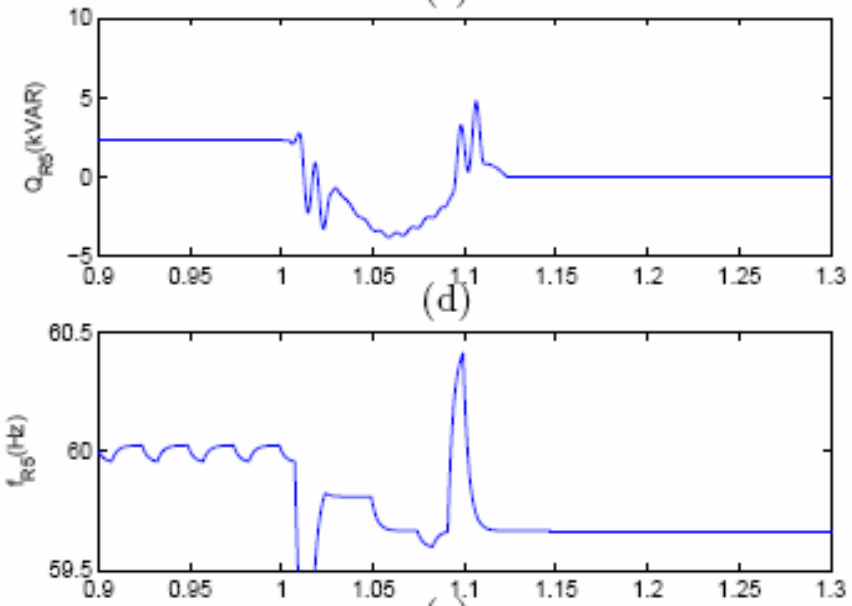

(e)

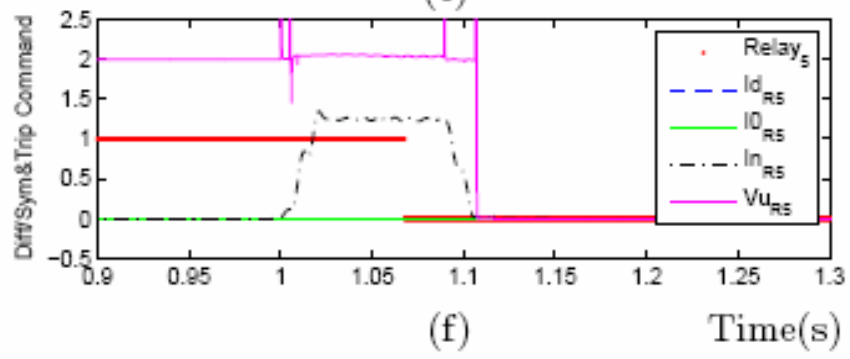

Figure 25: Response of the Relay 5 to a $85.5 \mathrm{~kW}$ LL fault in Zone 4: (a,b) threephase voltages and currents, (c,d) real and reactive power components, (e) frequency, and (f) differential and zero- and negative-sequence current components, negative-sequence voltage component at the transformer $T_{51}$ and output command of the relay 


\section{Line-to-Line Fault in Zone 3}

The AEP microgrid of Fig. 1 is operating at steady-state conditions with the source and load settings as explained in Section 5 and an LL fault with a resistance of $2.695 \mathrm{Ohms}$ is applied in Zone 3 at $t=1.0$ s. Figs. 27(a)-(e) to Figs. 20(a)-(e) show variations of the threephase voltages and currents, the real and reactive power components, and the frequencies measured at the Relays 2, 3, 4 and 5, respectively, in response to the LL fault.

Fig. 26(f) shows that the Relay 2 commands the static switch to trip shortly after the fault when the relay detects a negative-sequence current component that exceeds $1.0 \mathrm{pu}$ (95 A). Fig. 27(f) shows that the Relay 3 commands $\mathrm{CB}_{31}$ to trip since magnitude of the negative-sequence current component at the relay is above $1.0 \mathrm{pu}$ for a period more than $50 \mathrm{~ms}$ (3 cycles). Fig. 28(f) shows that the Relay 4 sends a trip command to the circuit breaker $\mathrm{CB}_{41}$ since magnitude of the negative-sequence current component at the relay is greater than $1.0 \mathrm{pu}$ for a period more than $50 \mathrm{~ms}$. Fig. 29(f) shows that the Relay 5 commands $\mathrm{CB}_{51}$ to trip since magnitude of the negative-sequence current component at the relay is above $1.0 \mathrm{pu}$ for a period more than $50 \mathrm{~ms}$. Magnitudes of the differential and zero-sequence current components of all relays do not exceed the corresponding thresholds of $15 \mathrm{~A}$ and $35 \mathrm{~A}$.

For the case of an LL fault in Zone 3, the static switch islands the microgrid and the breakers $\mathrm{CB}_{31}, \mathrm{CB}_{41}$ and $\mathrm{CB}_{51}$ disconnects all zones of the microgrid and shut down all the microsource located inside the microgrid. The results shown in Fig. 26 to Fig. 29 demonstrate that the developed protection system is able to protect the AEP microgrid against an LL fault in Zone 3 with a power of $85.5 \mathrm{~kW}$ by clearing the fault and disconnecting all protection zones of the microgrid. Since the fault current increases as the fault power increases, it is concluded that the microgrid protection system is able to protect all LL faults in Zone 3 with a power more than or equal to $85.5 \mathrm{~kW}$. 


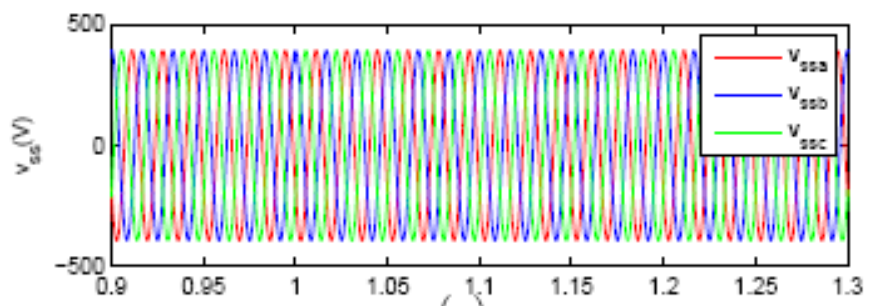

(a)
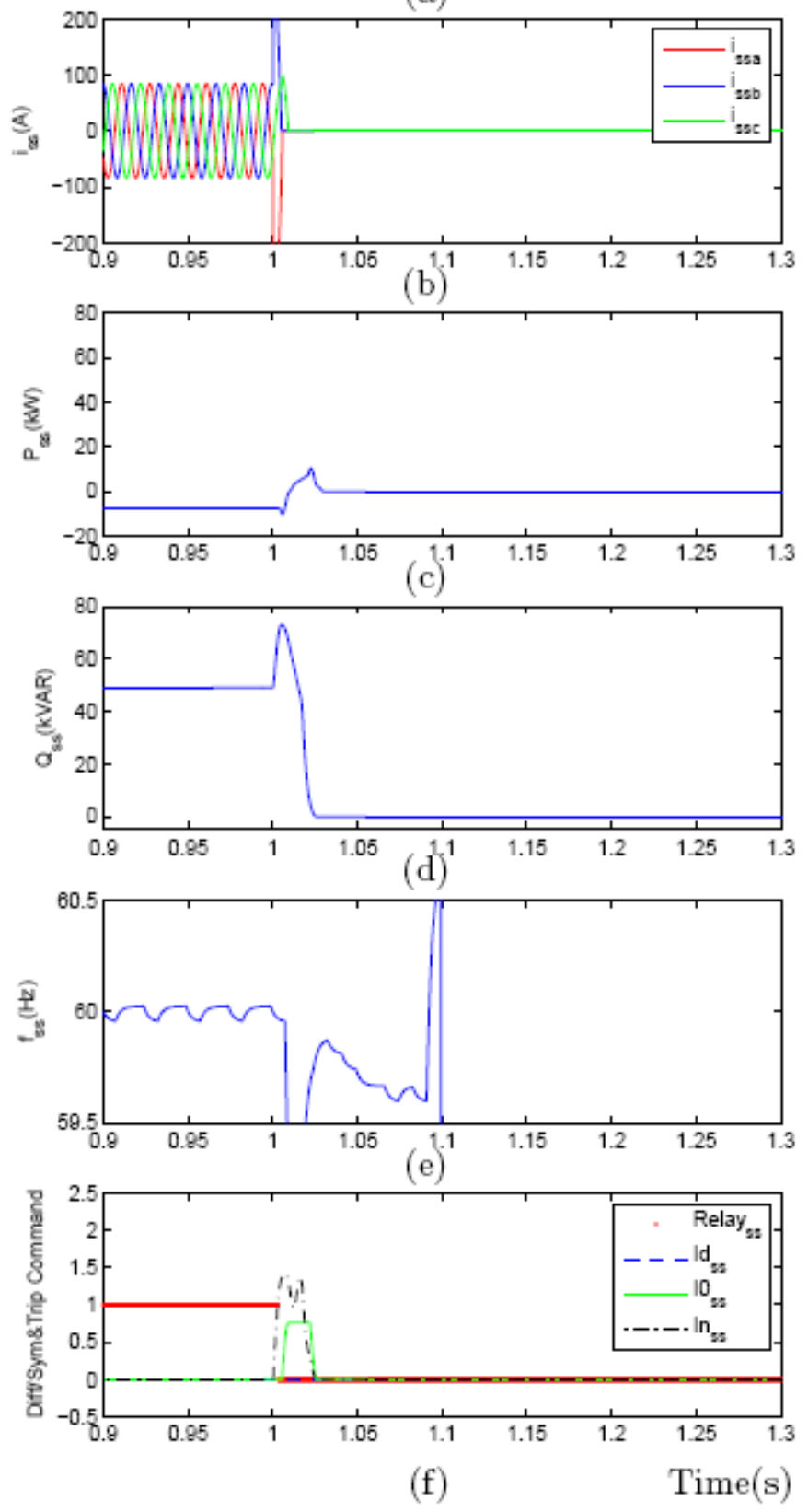

Figure 26: Response of the Relay 2 to a $85.5 \mathrm{~kW}$ LL fault in Zone 3: (a,b) threephase voltages and currents, $(\mathrm{c}, \mathrm{d})$ real and reactive power components, (e) frequency, and (f) differential and zero- and negative-sequence current components and output command of the relay 

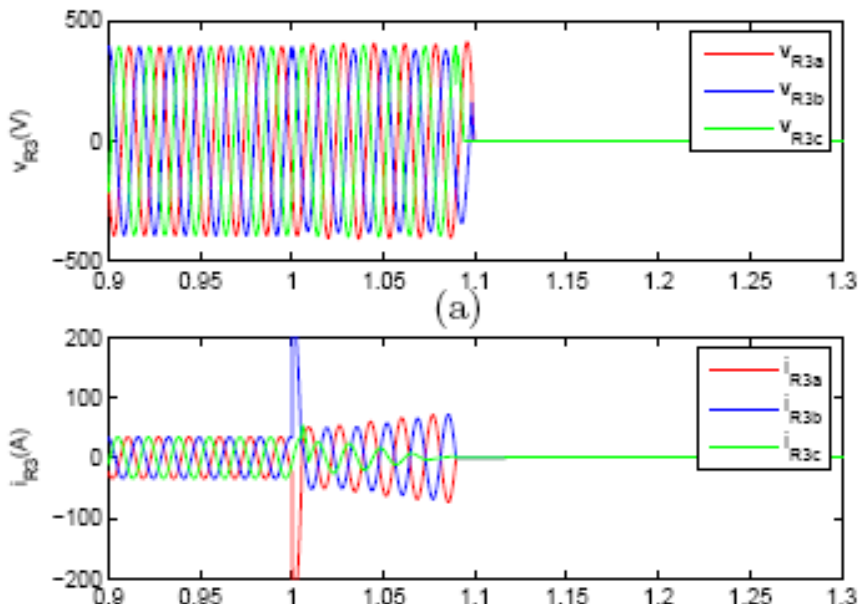

(b)

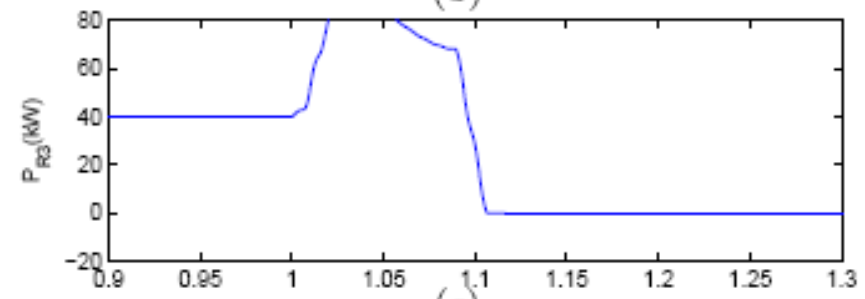

(c)
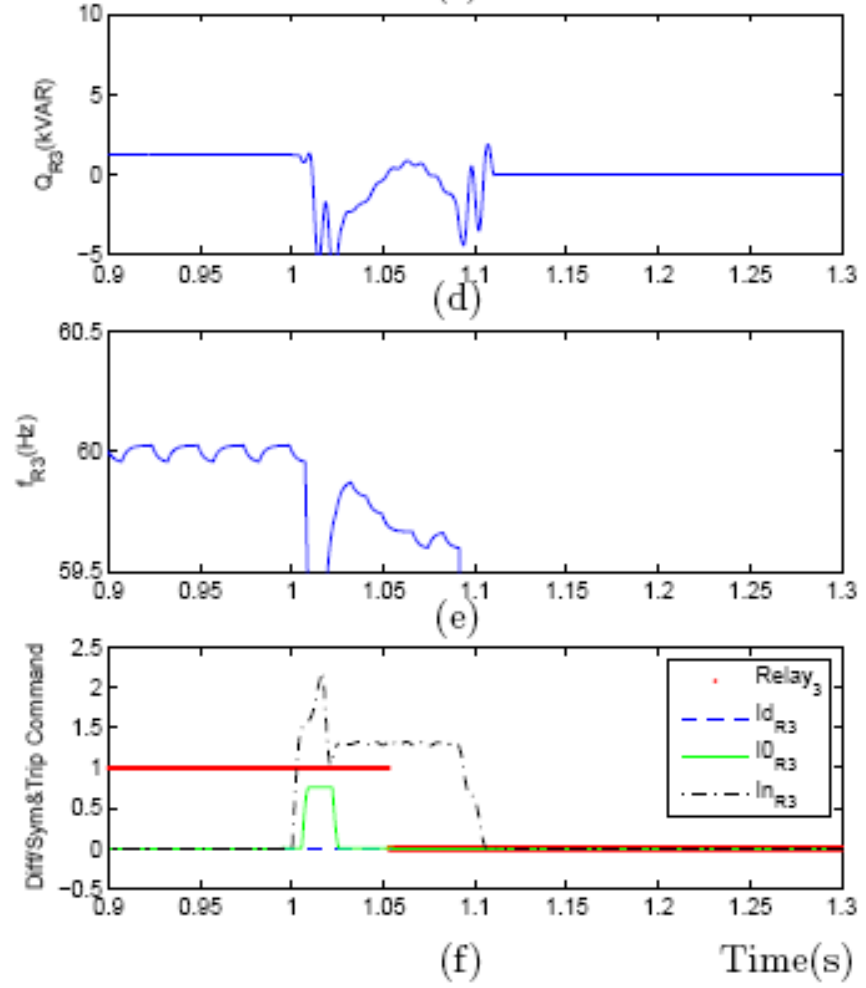

Figure 27: Response of the Relay 3 to a $85.5 \mathrm{~kW}$ LL fault in Zone 3: (a,b) threephase voltages and currents, $(c, d)$ real and reactive power components, (e) frequency, and (f) differential and zero- and negative-sequence current components and output command of the relay 


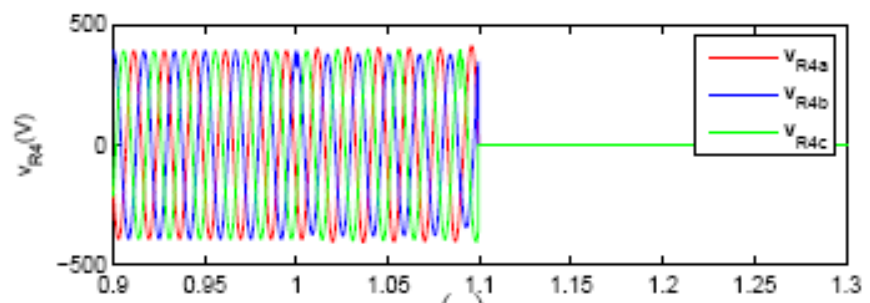

(a)

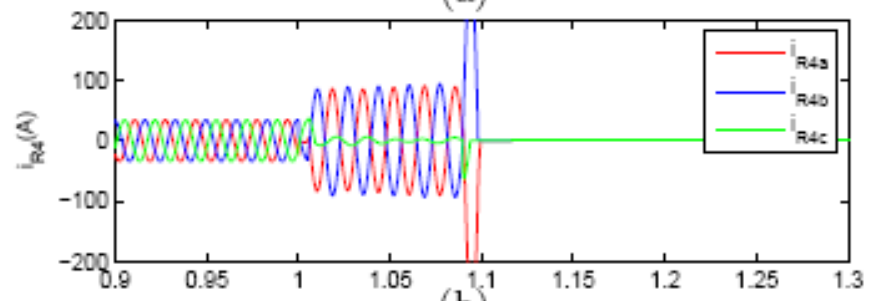

(b)
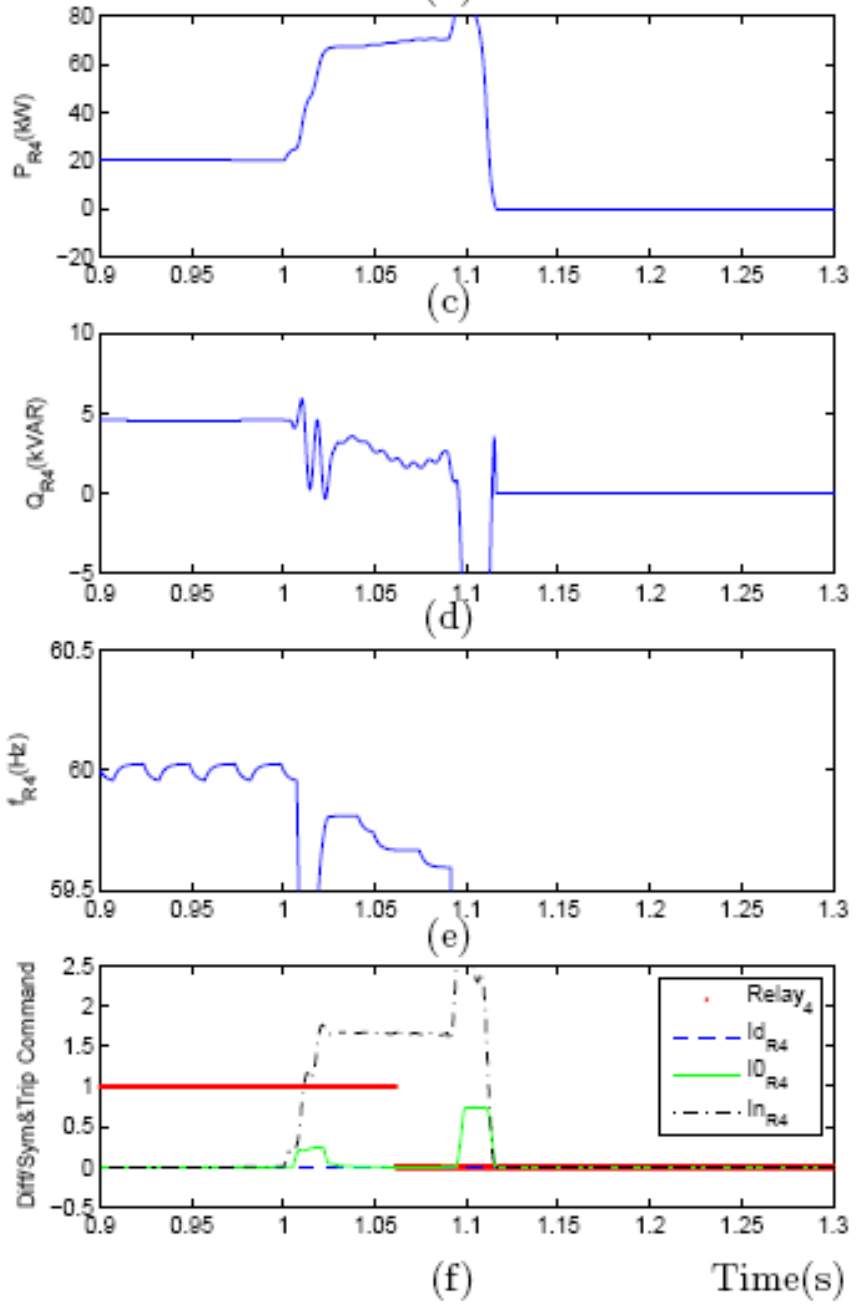

Figure 28: Response of the Relay 4 to a $85.5 \mathrm{~kW}$ LL fault in Zone 3: (a,b) threephase voltages and currents, (c,d) real and reactive power components, (e) frequency, and (f) differential and zero- and negative-sequence current components and output command of the relay 


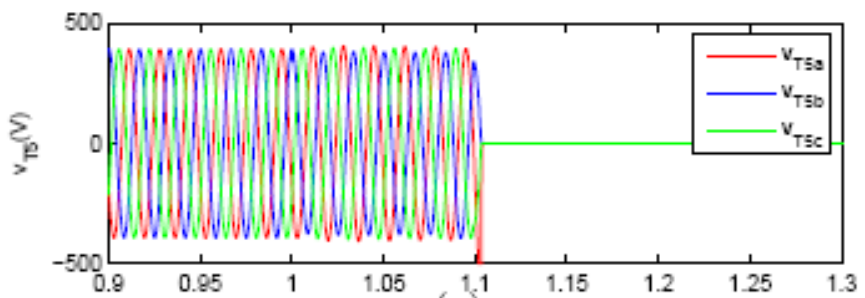

(a)

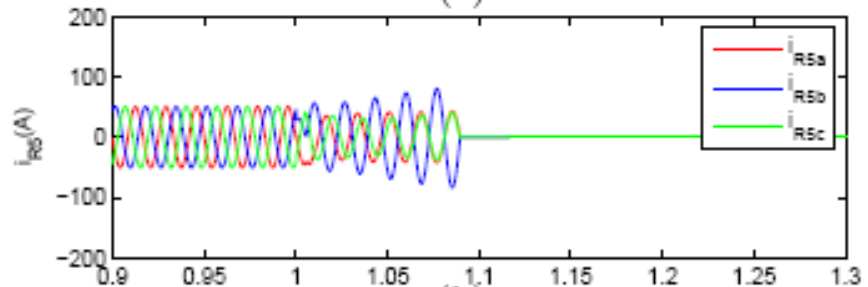

(b)

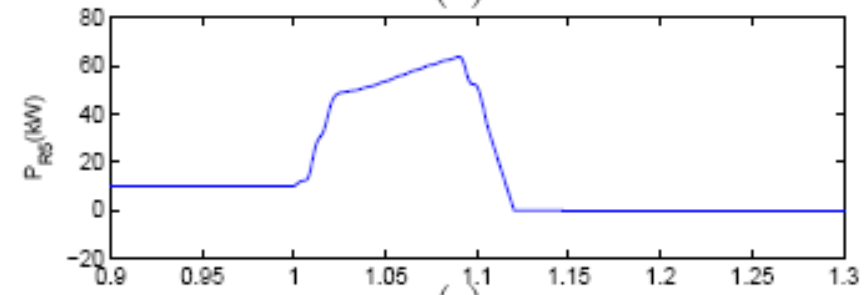

(c)
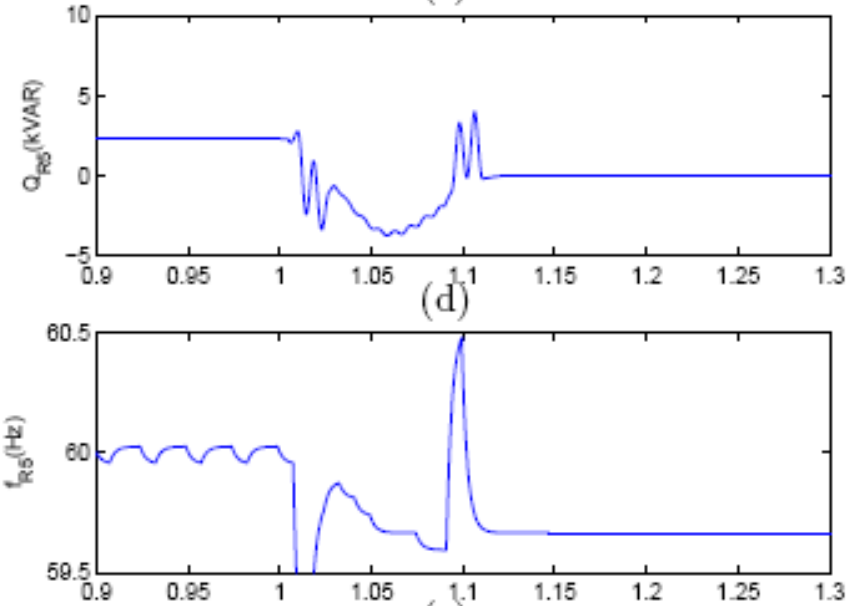

(e)

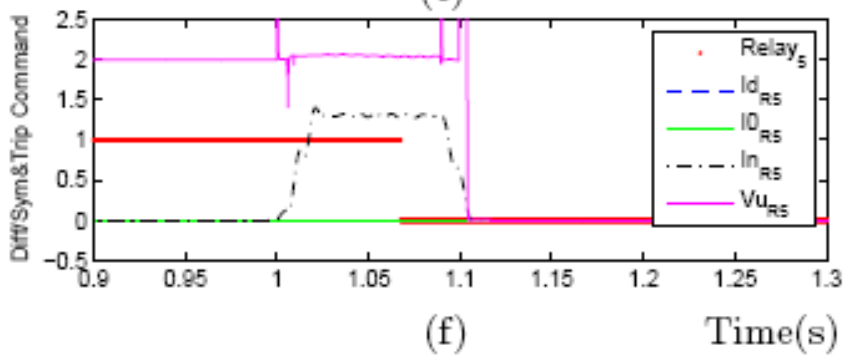

Figure 29: Response of the Relay 5 to a $85.5 \mathrm{~kW}$ LL fault in Zone 3: (a,b) threephase voltages and currents, (c,d) real and reactive power components, (e) frequency, and (f) differential and zero- and negative-sequence current components, negative-sequence voltage component at the transformer $T_{51}$ and output command of the relay 


\section{Line-to-Line Fault in Zone 2}

The AEP microgrid of Fig. 1 is operating at steady-state conditions with the source and load settings as explained in Section 5 and an LL fault with a resistance of $2.695 \mathrm{Ohms}$ is applied in Zone 2 at $\mathrm{t}=1.0 \mathrm{~s}$. Figs. 31(a)-(e) to Figs. 34(a)-(e) show variations of the threephase voltages and currents, the real and reactive power components, and the frequencies measured at the Relays 2, 3, 4 and 5, respectively, in response to the LL fault.

Fig. 30(f) shows that the Relay 2 commands the static switch to trip shortly after the fault when the relay detects a negative-sequence current component that exceeds $1.0 \mathrm{pu}$ (95 A). Fig. 31(f) shows that the Relay 3 commands $\mathrm{CB}_{31}$ to trip since magnitude of the negative-sequence current component at the relay is above $1.0 \mathrm{pu}$ for a period more than $50 \mathrm{~ms}$ (3 cycles). Fig. 32(f) shows that the Relay 4 sends a trip command to the circuit breaker $\mathrm{CB}_{41}$ since magnitude of the negative-sequence current component at the relay is greater than $1.0 \mathrm{pu}$ for a period more than $50 \mathrm{~ms}$. Fig. 33(f) shows that the Relay 5 commands $\mathrm{CB}_{51}$ to trip since magnitude of the negative-sequence current component at the relay is above $1.0 \mathrm{pu}$ for a period more than $50 \mathrm{~ms}$. Magnitudes of the differential and zero-sequence current components of all relays do not exceed the corresponding thresholds of $15 \mathrm{~A}$ and $35 \mathrm{~A}$.

For the case of an LL fault in Zone 2, the static switch islands the microgrid and the breakers $\mathrm{CB}_{31}, \mathrm{CB}_{41}$ and $\mathrm{CB}_{51}$ disconnects all zones of the microgrid and shut down all the microsource located inside the microgrid. The results shown in Fig. 30 to Fig. 33 demonstrate that the developed protection system is able to protect the AEP microgrid against an LL fault in Zone 2 with a power of $85.5 \mathrm{~kW}$ by clearing the fault and disconnecting all protection zones of the microgrid. Since the fault current increases as the fault power increases, it is concluded that the microgrid protection system is able to protect all LL faults in Zone 2 with a power more than or equal to $85.5 \mathrm{~kW}$. 

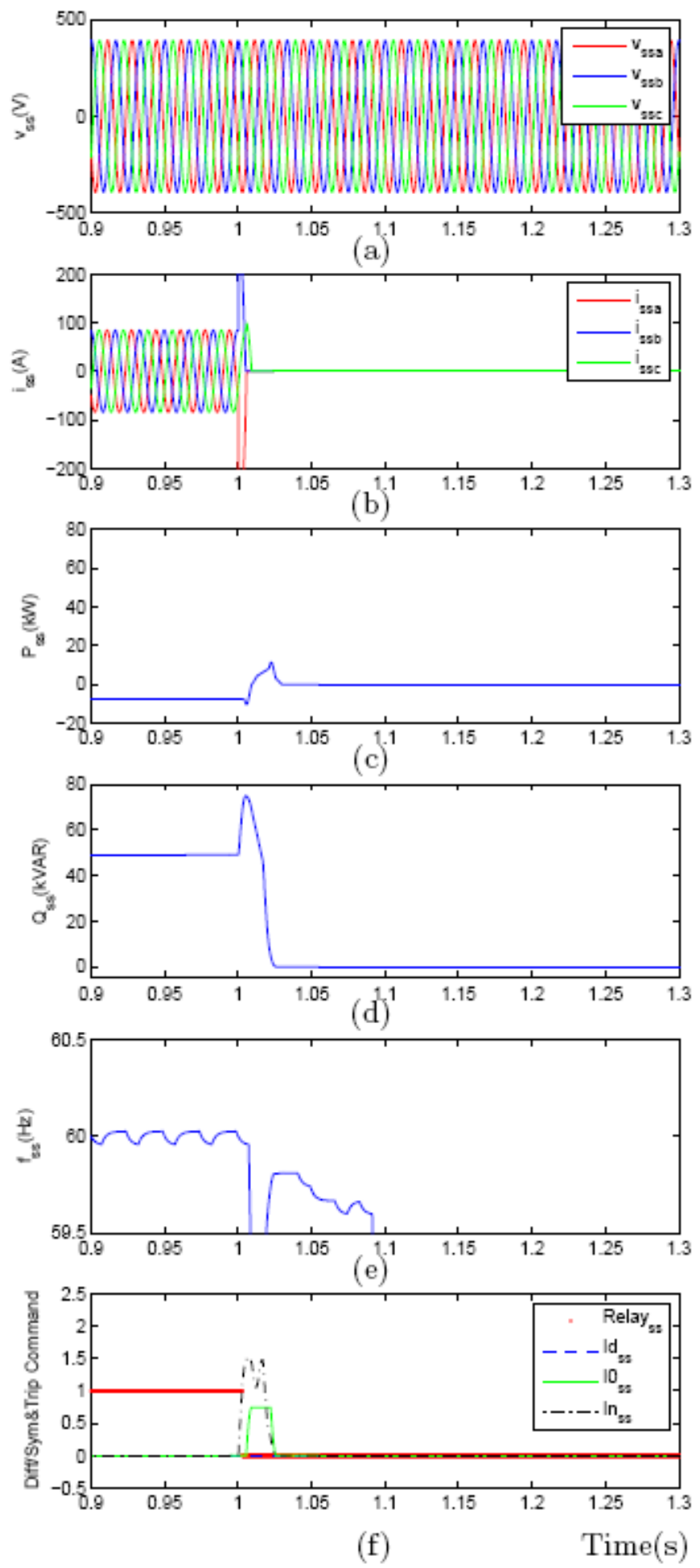

Figure 30: Response of the Relay 2 to a $85.5 \mathrm{~kW}$ LL fault in Zone 2: (a,b) threephase voltages and currents, $(\mathrm{c}, \mathrm{d})$ real and reactive power components, (e) frequency, and (f) differential and zero- and negative-sequence current components and output command of the relay 

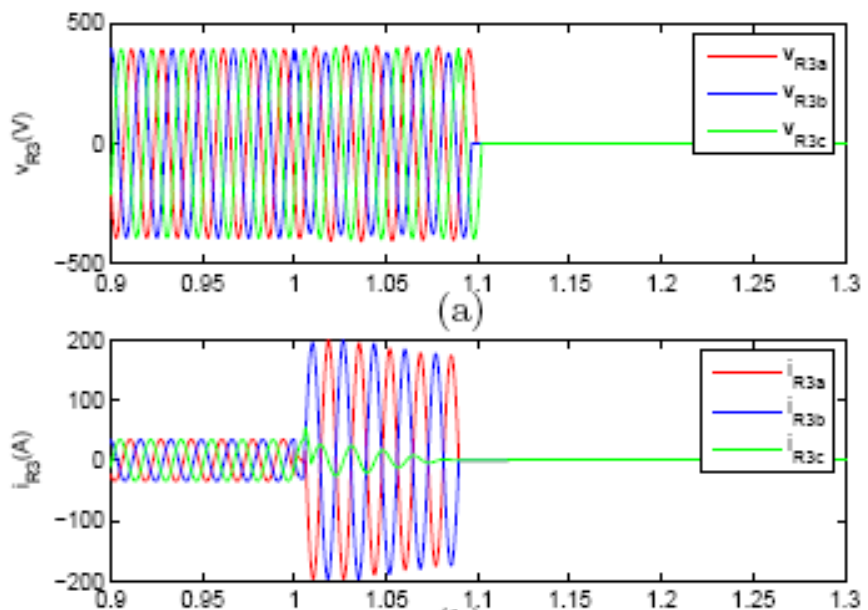

(b)
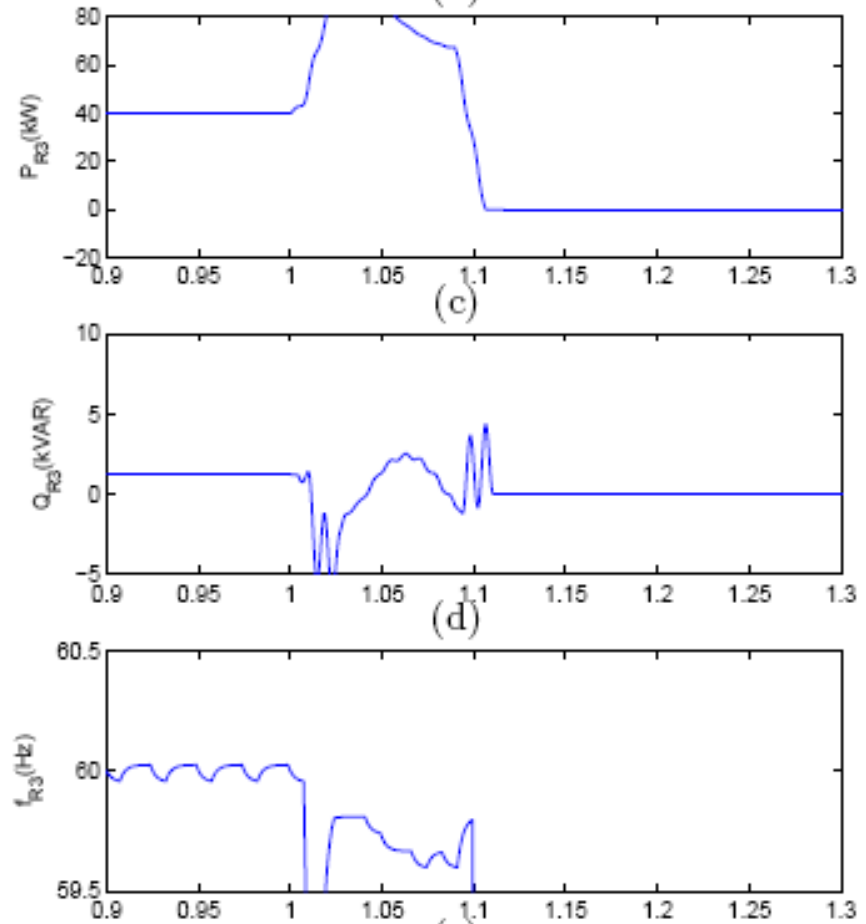

(e)

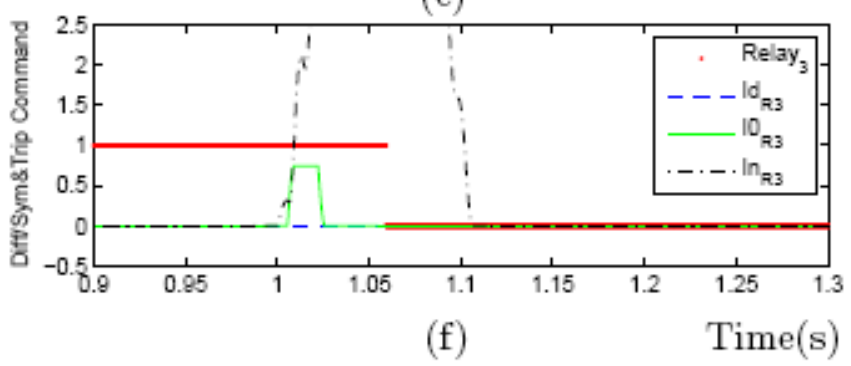

Figure 31: Response of the Relay 3 to a $85.5 \mathrm{~kW}$ LL fault in Zone 2: (a,b) threephase voltages and currents, (c,d) real and reactive power components, (e) frequency, and (f) differential and zero- and negative-sequence current components and output command of the relay 


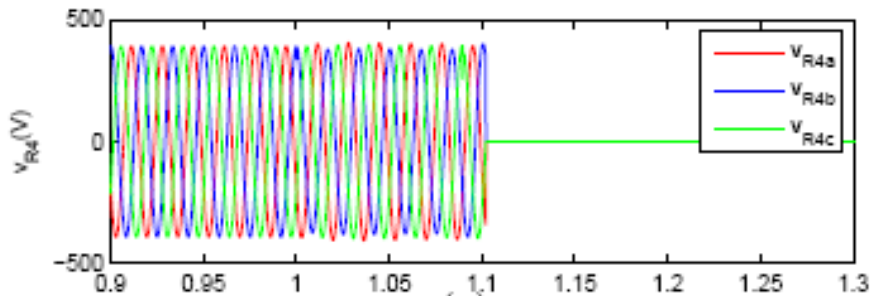

(a)

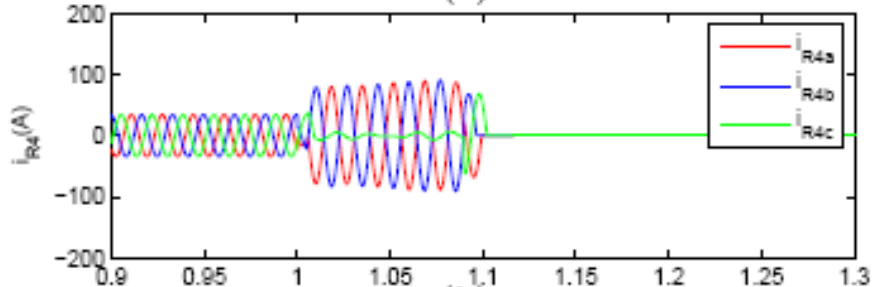

(b)
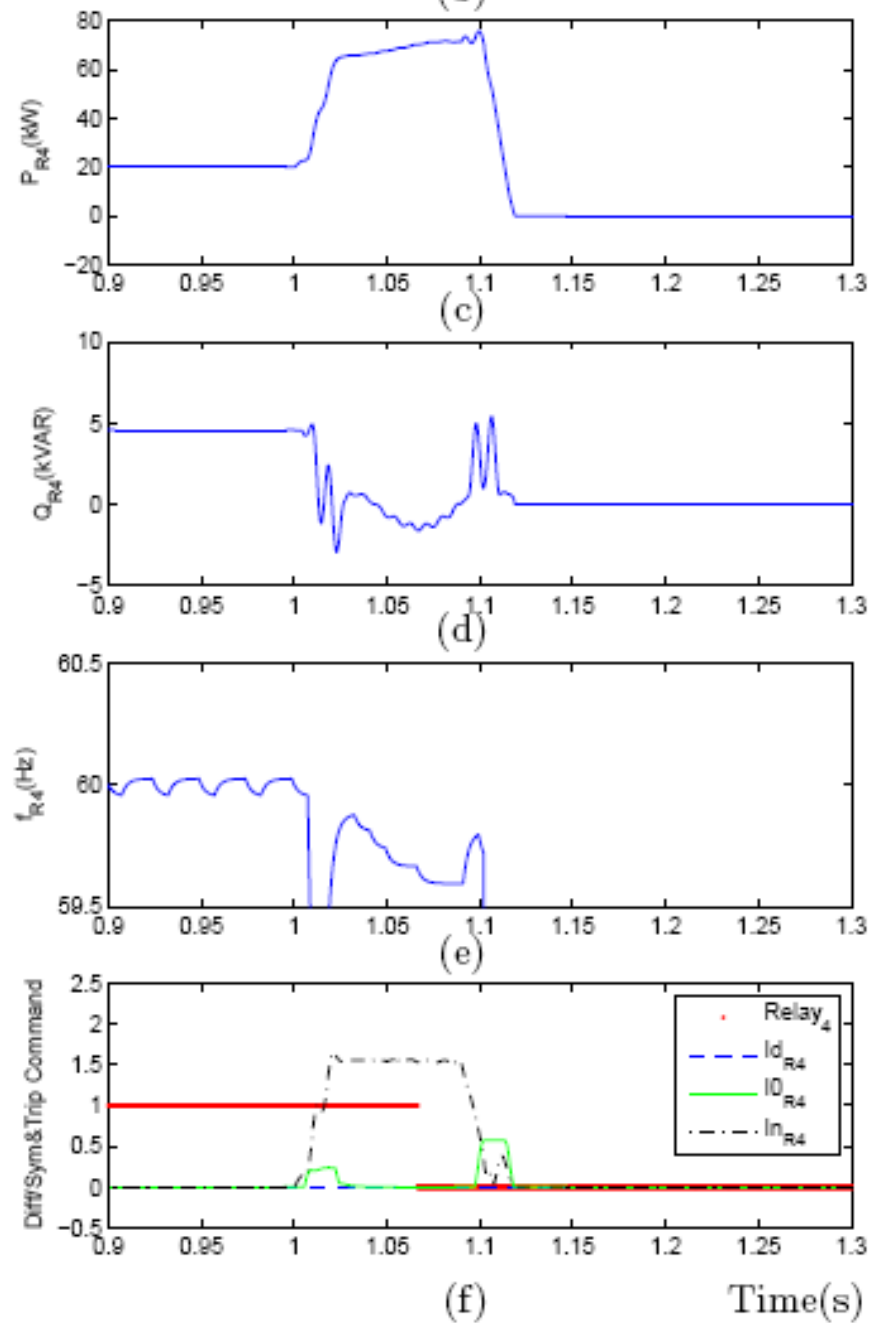

Figure 32: Response of the Relay 4 to a $85.5 \mathrm{~kW}$ LL fault in Zone 2: (a,b) threephase voltages and currents, (c,d) real and reactive power components, (e) frequency, and (f) differential and zero- and negative-sequence current components and output command of the relay 


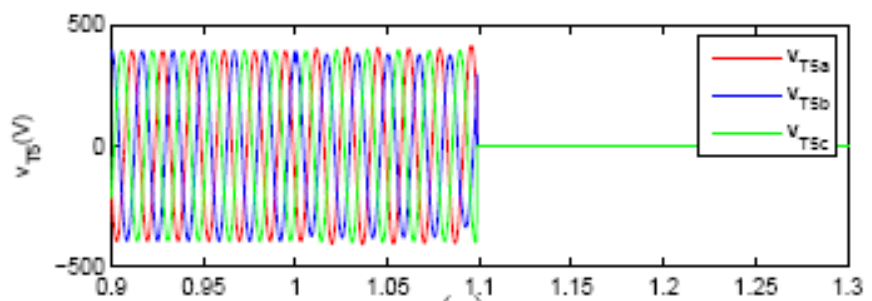

(a)

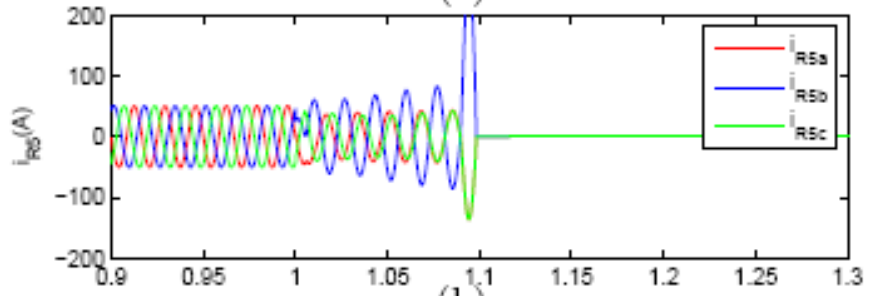

(b)

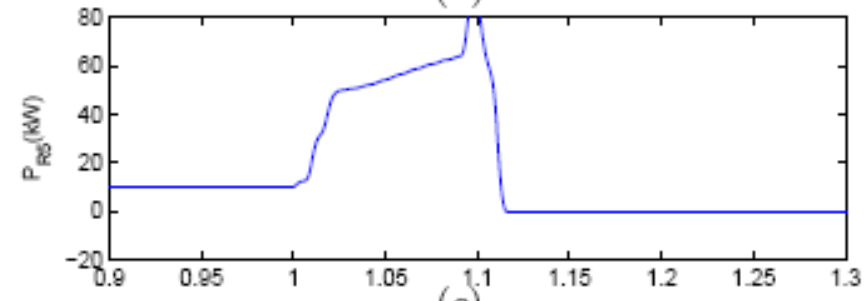

(c)
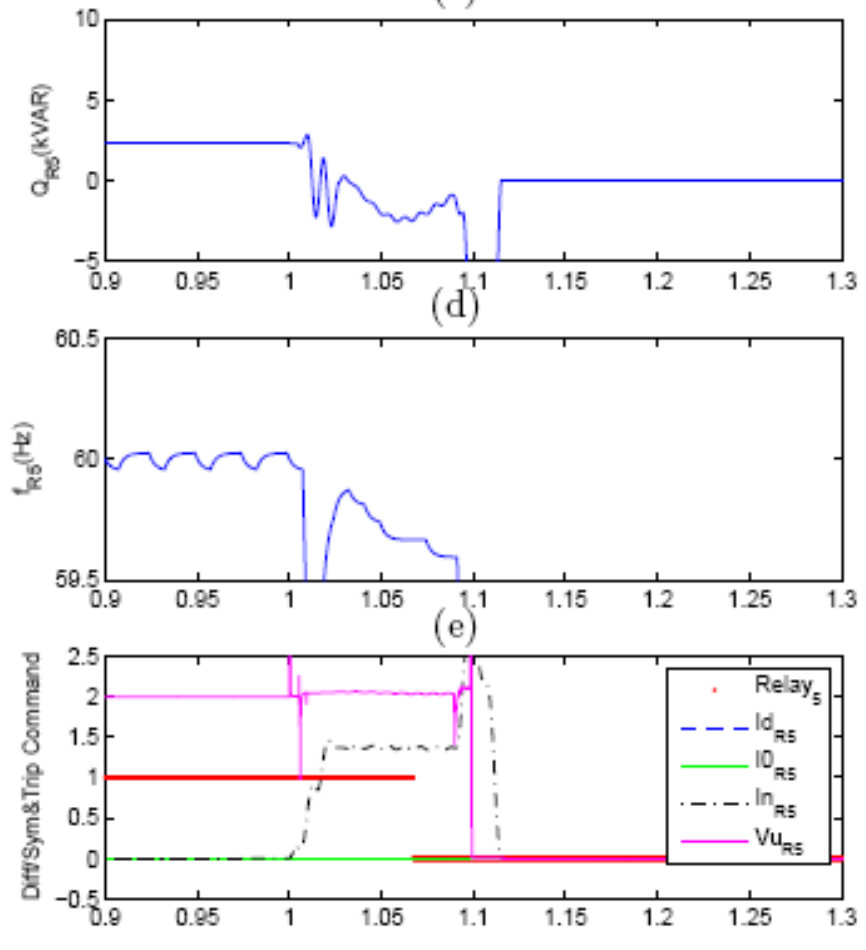

(f)

Time(s)

Figure 33: Response of the Relay 5 to a $85.5 \mathrm{~kW}$ LL fault in Zone 2: (a,b) threephase voltages and currents, (c,d) real and reactive power components, (e) frequency, and (f) differential and zero- and negative-sequence current components, negative-sequence voltage component at the transformer $T_{51}$ and output command of the relay 


\section{Line-to-Line Fault in Zone 5}

The AEP microgrid of Fig. 1 is operating at steady-state conditions with the source and load settings as explained in Section 5 and an LL fault with a resistance of $2.695 \mathrm{Ohms}$ is applied in Zone 5 at $t=1.0$ s. Figs. 35(a)-(e) to Figs. 38(a)-(e) show variations of the threephase voltages and currents, the real and reactive power components, and the frequencies measured at the Relays 2, 3, 4 and 5, respectively, in response to the LL fault.

Fig. 34(f) shows that the Relay 2 commands the static switch to trip shortly after the fault when the relay detects a negative-sequence current component that exceeds $1.0 \mathrm{pu}$ (95 A). Fig. 35(f) shows that the Relay 3 commands $\mathrm{CB}_{31}$ to trip since magnitude of the negative-sequence current component at the relay is above $1.0 \mathrm{pu}$ for a period more than $50 \mathrm{~ms}$ ( 3 cycles). Fig. 36(f) shows that the Relay 4 sends a trip command to the circuit breaker $\mathrm{CB}_{41}$ since magnitude of the negative-sequence current component at the relay is greater than $1.0 \mathrm{pu}$ for a period more than $50 \mathrm{~ms}$. Fig. 37(f) shows that the Relay 5 commands $\mathrm{CB}_{51}$ to trip since magnitude of the negative-sequence current component at the relay is above $1.0 \mathrm{pu}$ for a period more than $50 \mathrm{~ms}$. Magnitudes of the differential and zero-sequence current components of all relays do not exceed the corresponding thresholds of $15 \mathrm{~A}$ and $35 \mathrm{~A}$.

For the case of an LL fault in Zone 5, the static switch islands the microgrid and the breakers $\mathrm{CB}_{31}, \mathrm{CB}_{41}$ and $\mathrm{CB}_{51}$ disconnects all zones of the microgrid and shut down all the microsource located inside the microgrid. The results shown in Fig. 34 to Fig. 37 demonstrate that the developed protection system is able to protect the AEP microgrid against an LL fault in Zone 5 with a power of $85.5 \mathrm{~kW}$ by clearing the fault and disconnecting all protection zones of the microgrid. Since the fault current increases as the fault power increases, it is concluded that the microgrid protection system is able to protect all LL faults in Zone 5 with a power more than or equal to $85.5 \mathrm{~kW}$. 


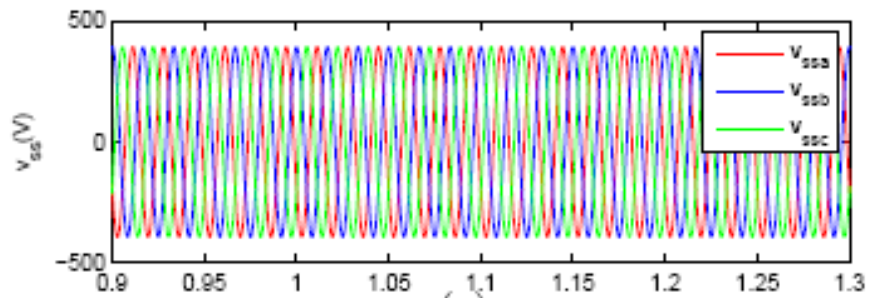

(a)

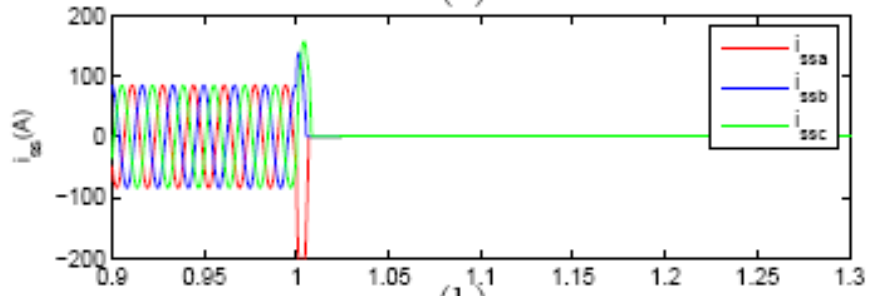

(b)
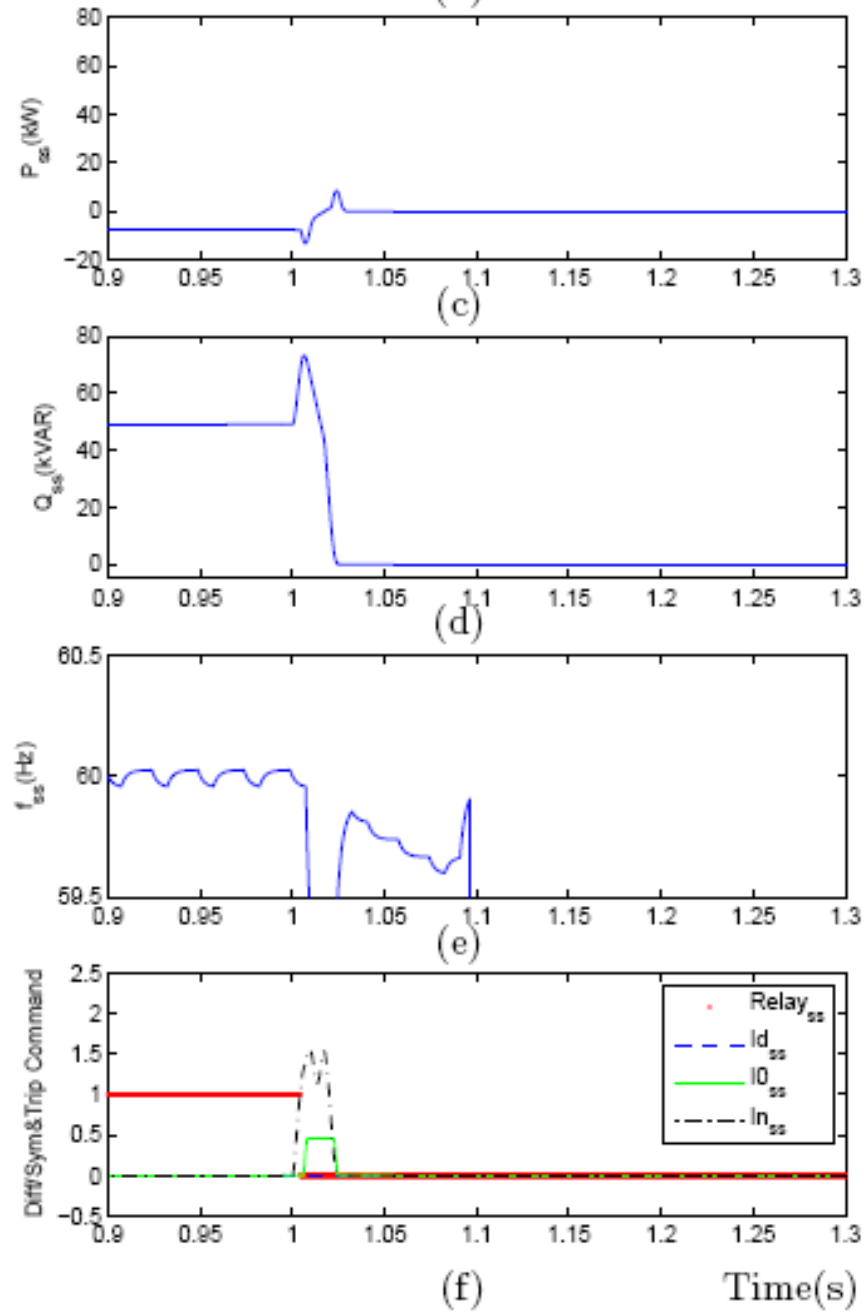

Figure 34: Response of the Relay 2 to a $85.5 \mathrm{~kW}$ LL fault in Zone 5: (a,b) threephase voltages and currents, $(\mathrm{c}, \mathrm{d})$ real and reactive power components, (e) frequency, and (f) differential and zero- and negative-sequence current components and output command of the relay 


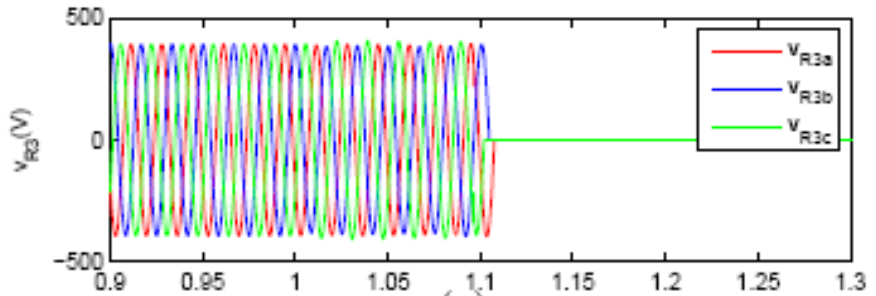

(a)

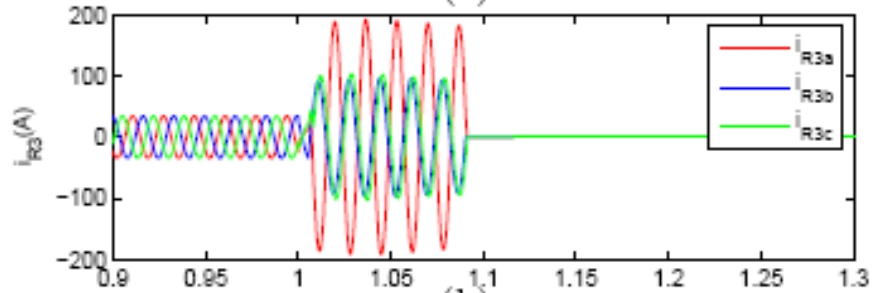

(b)

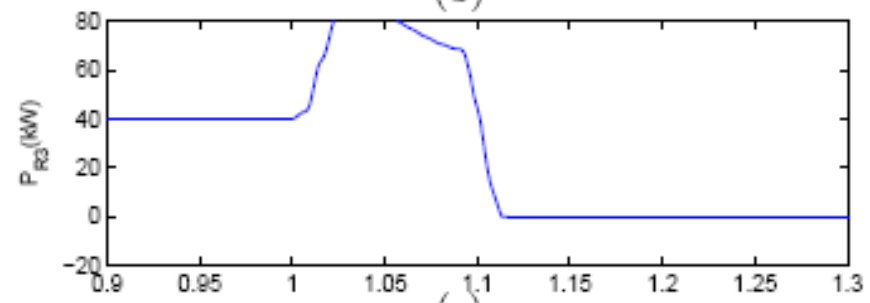

(c)
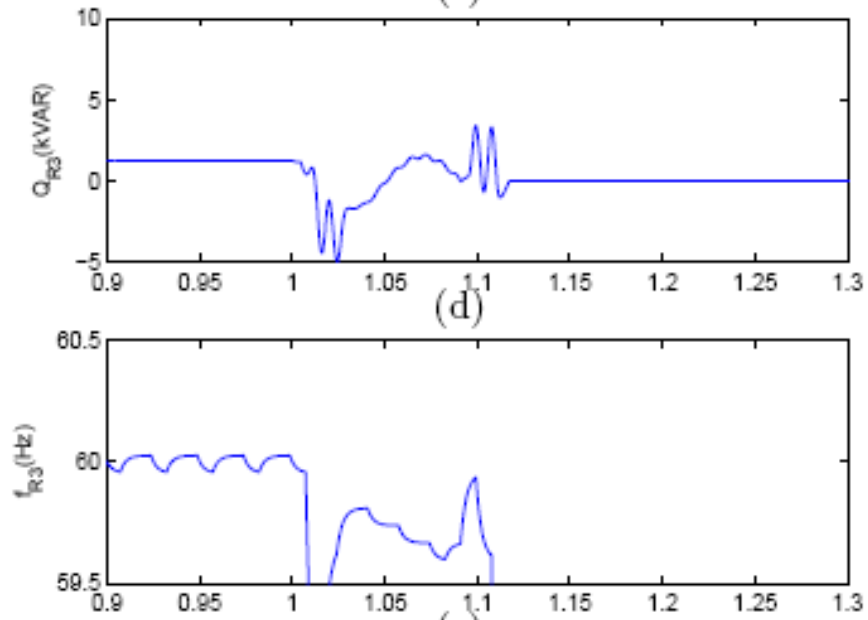

(e)

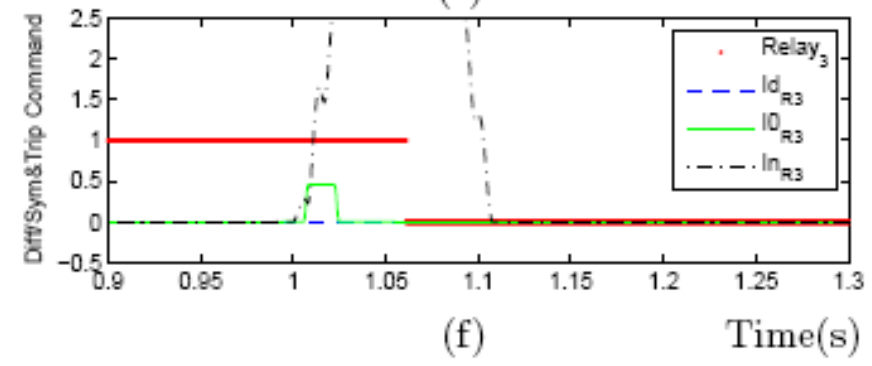

Figure 35: Response of the Relay 3 to a $85.5 \mathrm{~kW}$ LL fault in Zone 5: (a,b) threephase voltages and currents, (c,d) real and reactive power components, (e) frequency, and (f) differential and zero- and negative-sequence current components and output command of the relay 

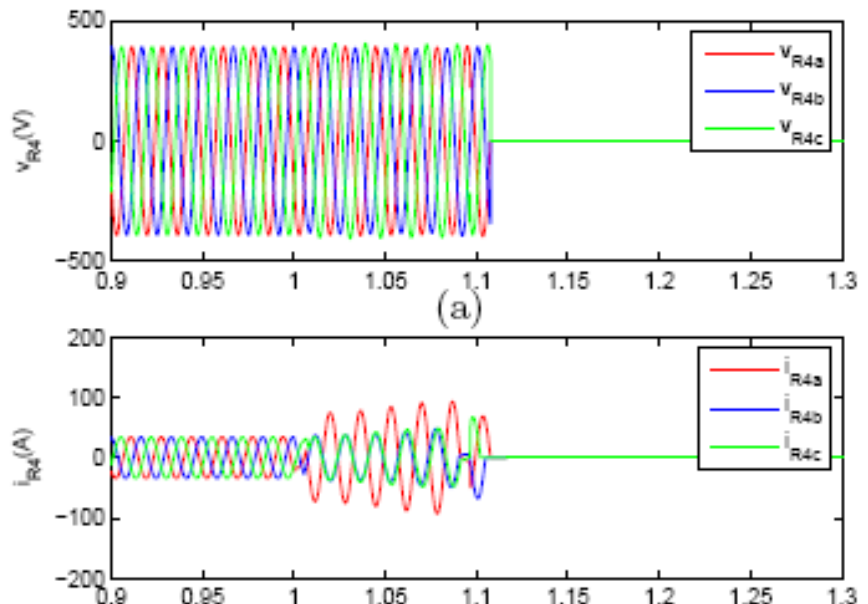

(b)

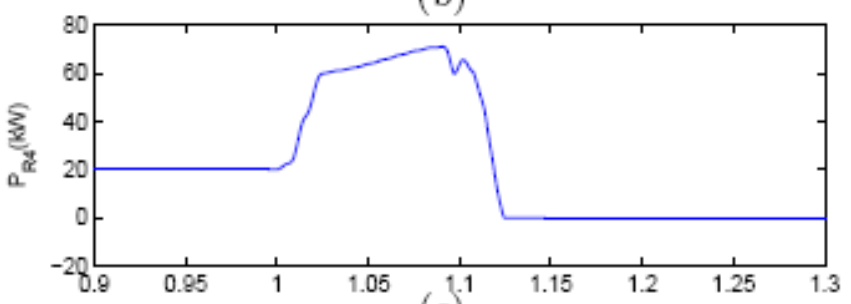

(c)
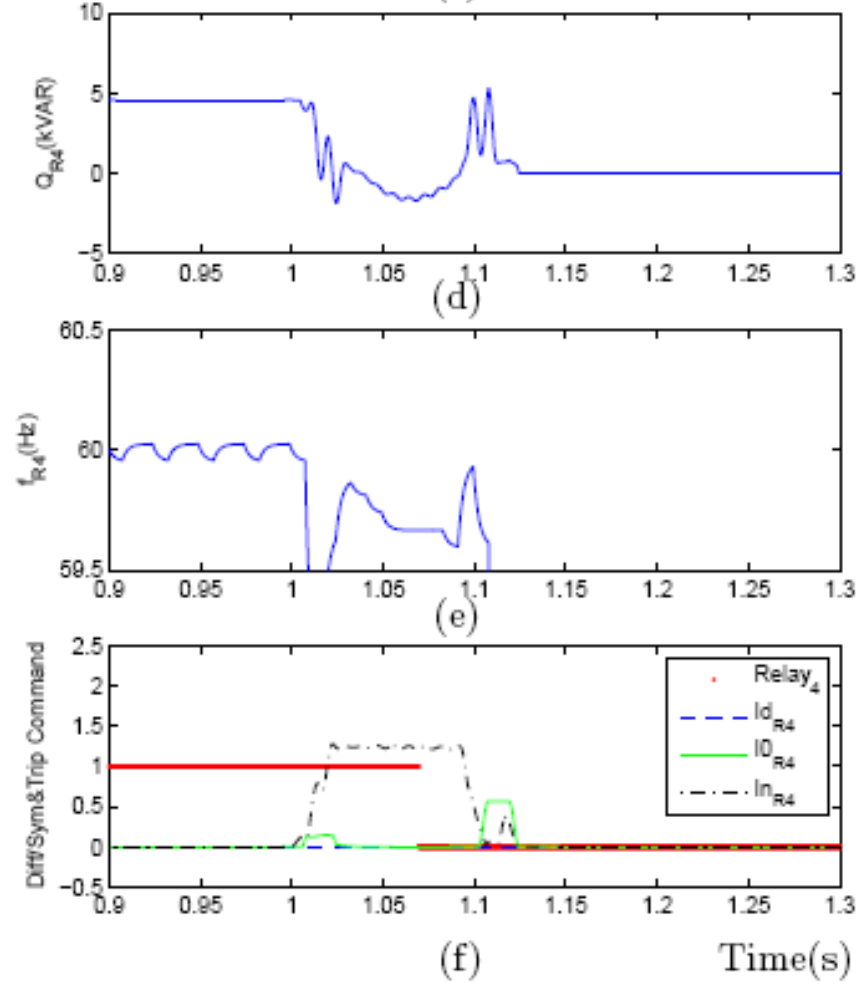

Figure 36: Response of the Relay 4 to a $85.5 \mathrm{~kW}$ LL fault in Zone 5: (a,b) threephase voltages and currents, $(\mathrm{c}, \mathrm{d})$ real and reactive power components, (e) frequency, and (f) differential and zero- and negative-sequence current components and output command of the relay 


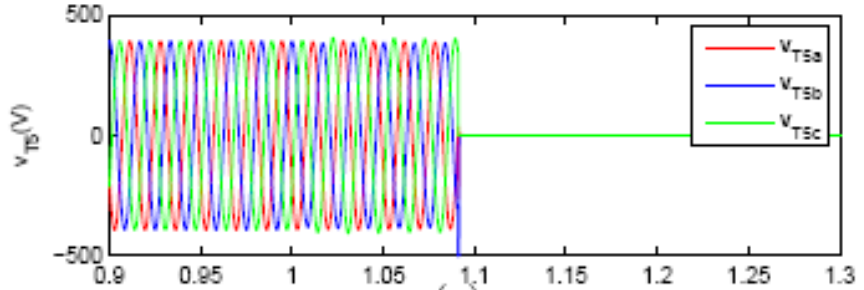

(a)

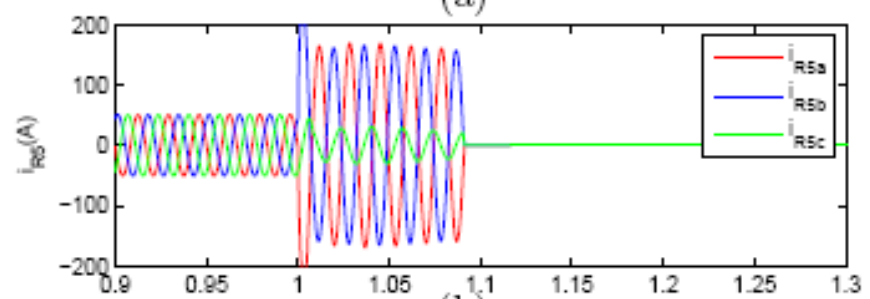

(b)
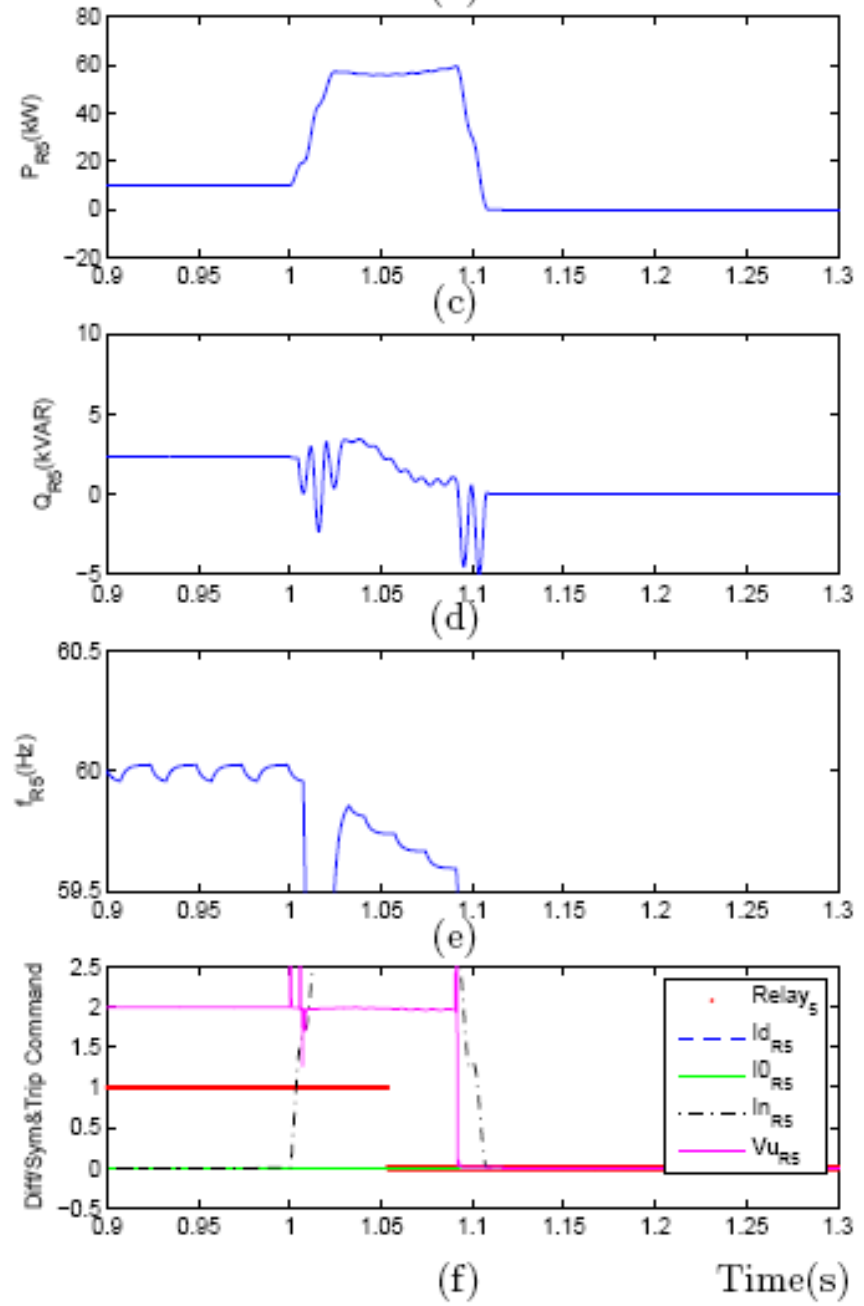

Figure 37: Response of the Relay 5 to a $85.5 \mathrm{~kW}$ LL fault in Zone 5: (a,b) threephase voltages and currents, (c,d) real and reactive power components, (e) frequency, and (f) differential and zero- and negative-sequence current components, negative-sequence voltage component at the transformer $T 51$ and output command of the relay 


\section{Line-to-Line Fault in Zone 6}

The AEP microgrid of Fig. 1 is operating at steady-state conditions with the source and load settings as explained in Section 5 and an LL fault with a resistance of $2.695 \mathrm{Ohms}$ is applied in Zone 6 at $t=1.0$ s. Figs. 39(a)-(e) to Figs. 42(a)-(e) show variations of the threephase voltages and currents, the real and reactive power components, and the frequencies measured at the Relays 2, 3, 4 and 5, respectively, in response to the LL fault.

Fig. 38(f) shows that the Relay 2 commands the static switch to trip shortly after the fault when the relay detects a negative-sequence current component that exceeds $1.0 \mathrm{pu}$ (95 A). Fig. 39(f) shows that the Relay 3 does not command for a trip since none of its fault detection parameters, i.e. the differential and symmetrical current components, exceeds the threshold of $1.0 \mathrm{pu}$. Fig. 40(f) shows that the Relay 4 does not command for a trip since none of its fault detection parameters, i.e. the differential and symmetrical current components, exceeds the threshold of $1.0 \mathrm{pu}$. Fig. 41(f) shows that the Relay 5 does not command for a trip since none of its fault detection parameters, i.e. the differential and symmetrical current components, exceeds the threshold of $1.0 \mathrm{pu}$.

For the case of an LL fault in Zone 6, the static switch islands the microgrid. The Zones 2, 3, 4 and 5 of the microgrid and the microsources $A_{1}, A_{2}$ and $B_{1}$ continue the operation subsequent to the fault detection and its clearance. The results shown in Fig. 38 to Fig. 41 demonstrate that the developed protection system is able to protect the AEP microgrid against an LL fault in Zone 6 with a power of $85.5 \mathrm{~kW}$ by disconnecting the static switch. Since the fault current increases as the fault power increases, it is concluded that the microgrid protection system is able to protect all LL faults in Zone 6 with a power more than or equal to $85.5 \mathrm{~kW}$. 

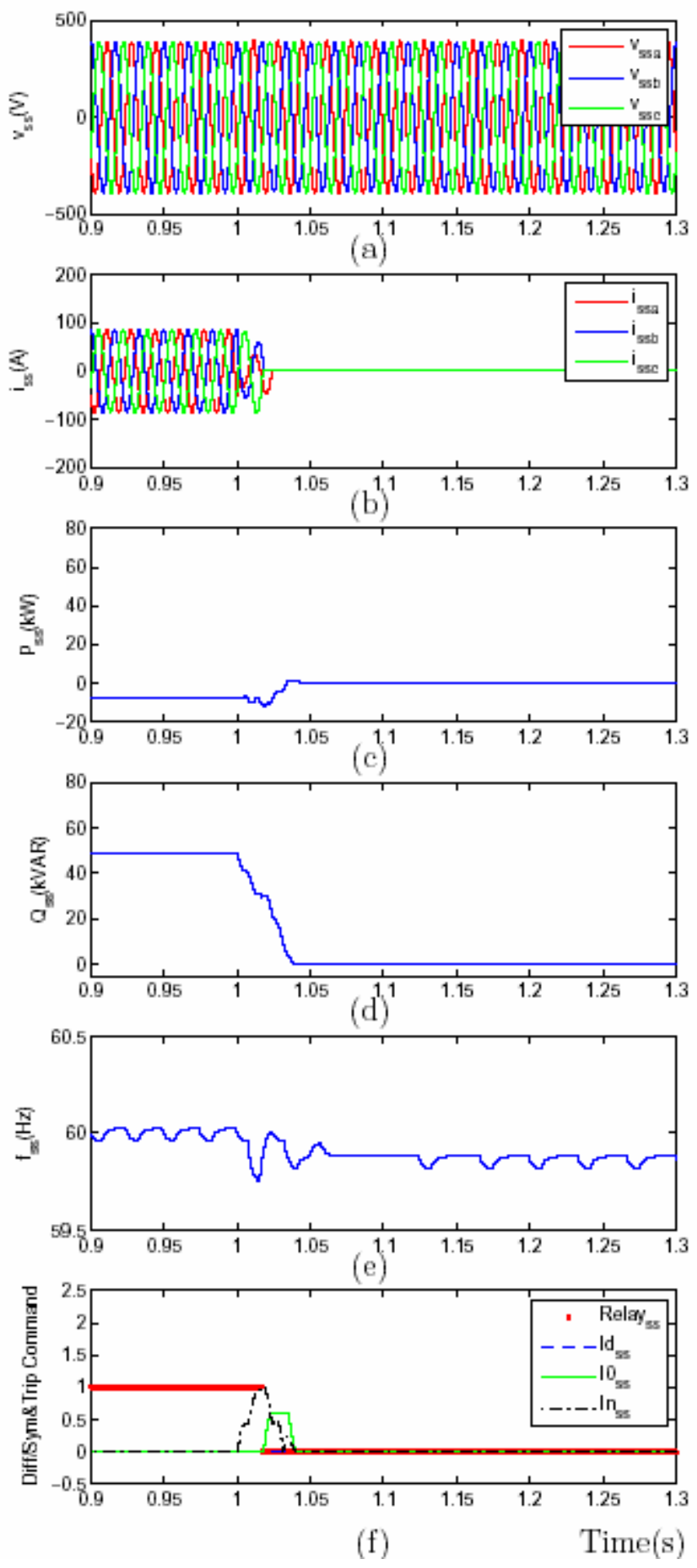

Figure 38: Response of the Relay 2 to a $85.5 \mathrm{~kW}$ LL fault in Zone 6: (a,b) threephase voltages and currents, (c,d) real and reactive power components, (e) frequency, and (f) differential and zero- and negative-sequence current components and output command of the relay 

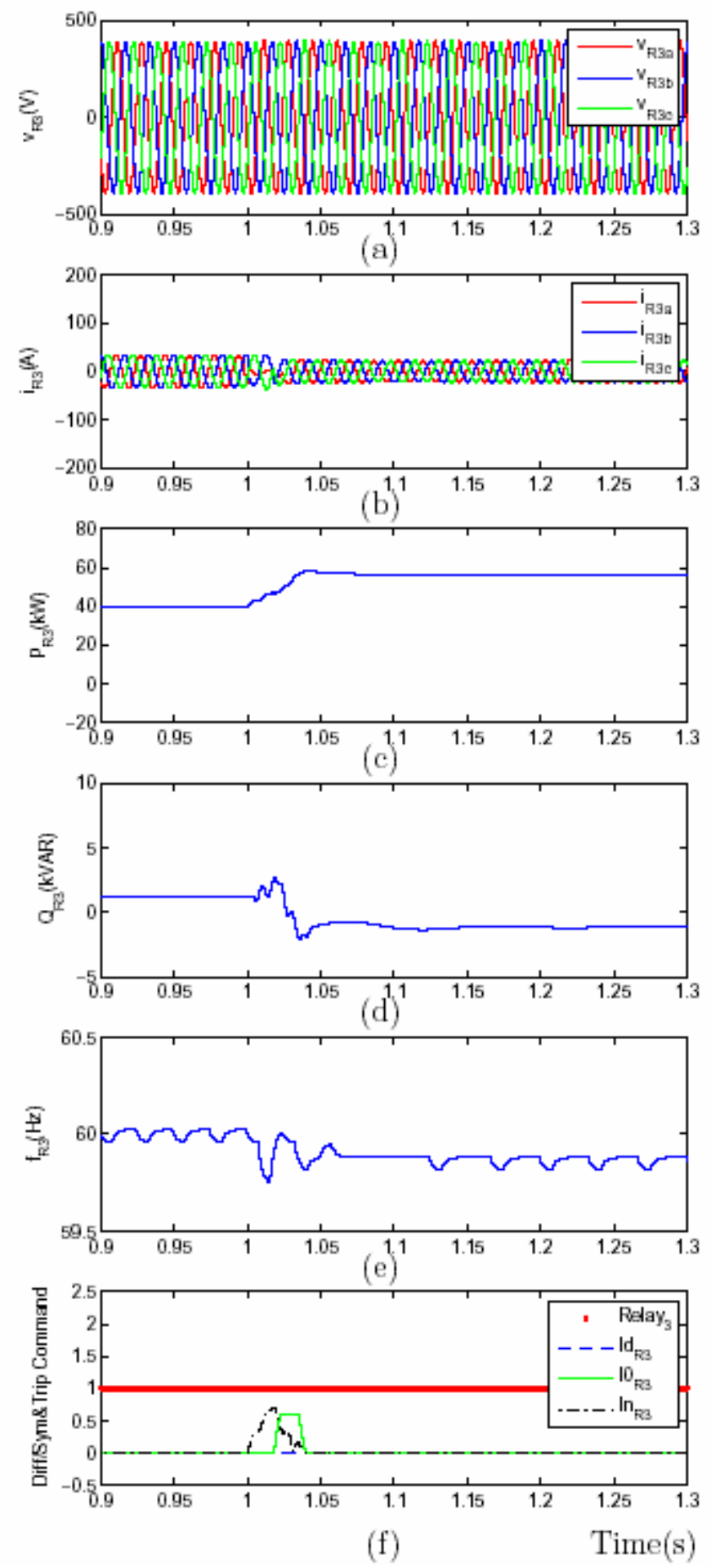

Figure 39: Response of the Relay 3 to a $85.5 \mathrm{~kW}$ LL fault in Zone 6: (a,b) threephase voltages and currents, $(\mathrm{c}, \mathrm{d})$ real and reactive power components, (e) frequency, and (f) differential and zero- and negative-sequence current components and output command of the relay 

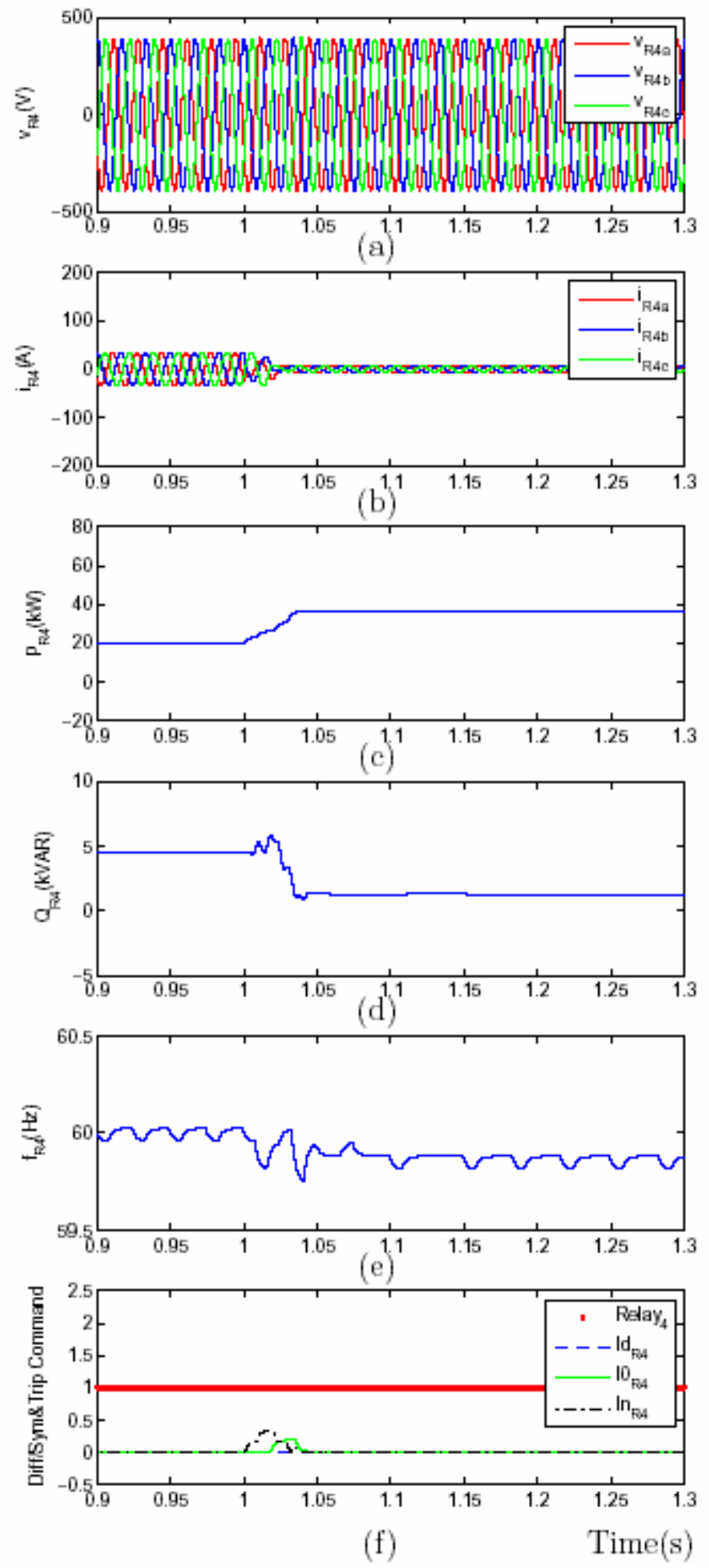

Figure 40: Response of the Relay 4 to a $85.5 \mathrm{~kW} \mathrm{LL}$ fault in Zone 6: (a,b) threephase voltages and currents, $(\mathrm{c}, \mathrm{d})$ real and reactive power components, (e) frequency, and (f) differential and zero- and negative-sequence current components and output command of the relay 

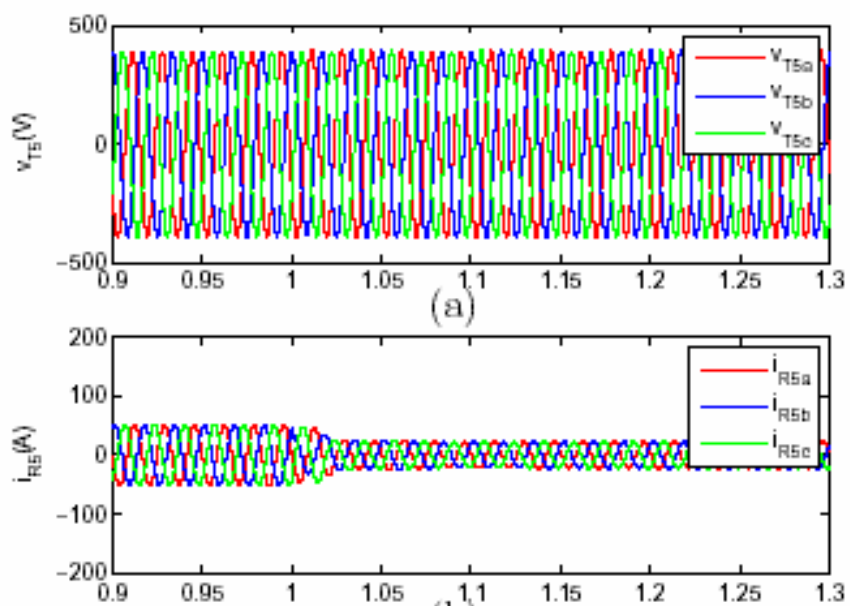

(b)
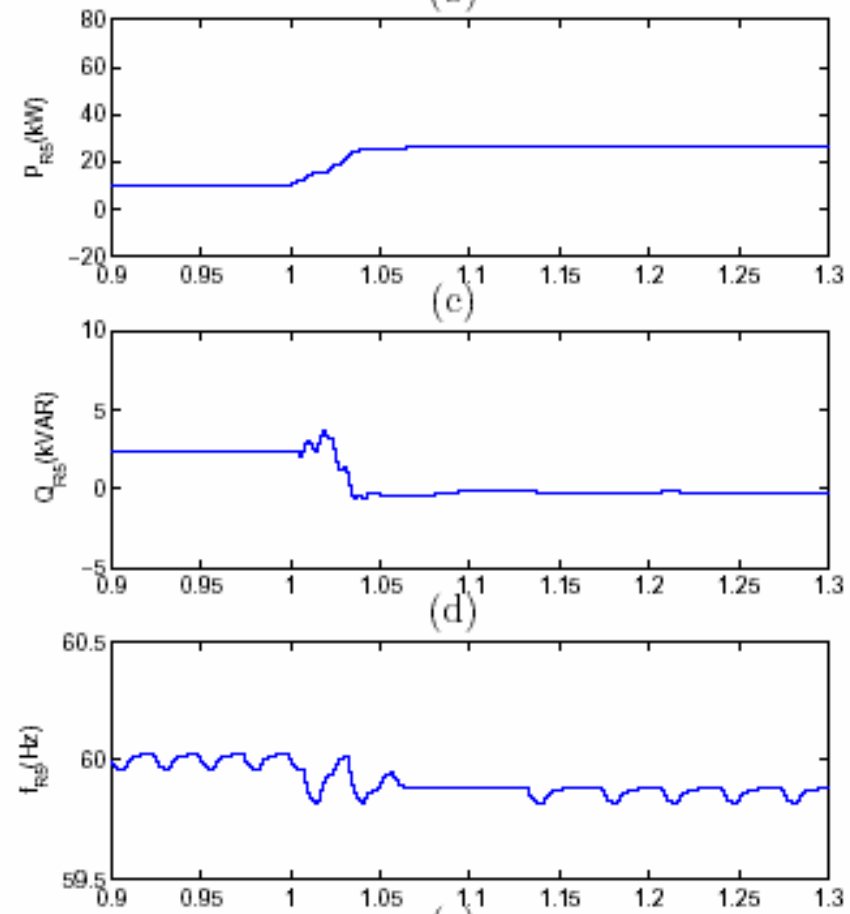

(e)

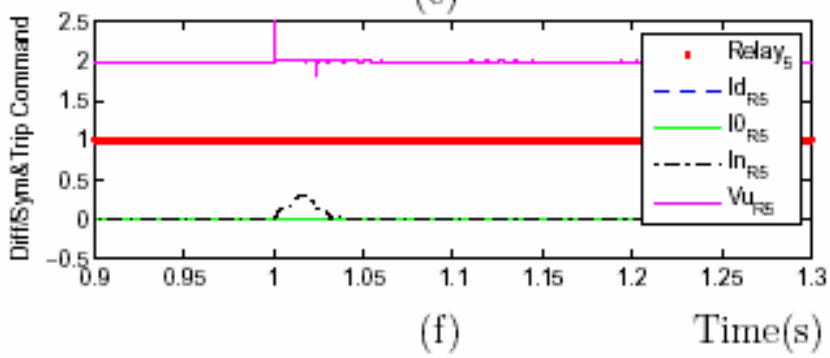

Figure 41: Response of the Relay 5 to a $85.5 \mathrm{~kW}$ LL fault in Zone 6: (a,b) threephase voltages and currents, $(c, d)$ real and reactive power components, (e) frequency, and (f) differential and zero- and negative-sequence current components, negative-sequence voltage component at the transformer $T 51$ and output command of the relay 


\section{Bibliography}

[1] P. Piagi, "Microgrid control," Ph.D. dissertation, University of Wisconsin-Madison, 2005

[2] R. H. Lasseter and P. Piagi, "Control and design of microgrid components," PSERC Publication 06-03, University of Wisconsin-Madison, January 2006

[3] H. Nikkhajoei, P. Piagi, and R. H. Lasseter, "EMTP Simulation of Single Line to Ground Fault in Zone 3 of AEP Microgrid System," Wisconsin Power Electronics Research Center, University of Wisconsin-Madison, August 2006

[4] A. R. Bergen, "Power Systems Analysis", Prentice-Hall Publications, 1986

[5] "CERTS Test Bed Protection Device Selection and Settings," Northern Power Systems Energy Technology Laboratory, 2005

[6] B. Fleming, "Creating a Phase Current Unbalance Alarm/Supervision Function in the SEL-351 Relay,” Schweitzer Engineering Laboratories, Inc., 1998

[7] F. Calero, "Rebirth of negative-Sequence Quantities in protective Relaying with microprocessor-Based relays," Schweitzer Engineering Laboratories, Inc., 2003

[8] A. F. Elneweihi, E. O. Scweitzer, and M. W. Feltis, "Negative-Sequence Overcurrent Element Application and Coordination in distribution Protection," IEEE Trans. On Power Delivery, Vol. 8, No. 3, pp. 915-924, July 1993 